PROJECT TITLE:

DOE AWARD \#:

REPORT:

REPORTING PERIOD:

ISSUED:

SUBMITTED TO:

PREPARED BY:

PIs:
Atmospheric Aerosol Source-Receptor Relationships: The Role of Coal-Fired Power Plants

DE-FC26-01NT41017

Final Technical Report

February 2001 - December 2005

August 2006

US Department of Energy

National Energy Technology Laboratory

P.O. Box 10940

626 Cochrans Mill Rd

Pittsburgh, PA 15236-0940

Attn: Bill Aljoe

Carnegie Mellon University

5000 Forbes Ave

Pittsburgh, PA 15213

Allen L. Robinson, Spyros N. Pandis, Cliff I. Davidson (412) 268-3657; (412) 268-3348 (fax)

e-mail: alr@andrew.cmu.edu 


\section{DISCLAIMER}

This report was prepared as an account of work sponsored by an agency of the United States Government. Neither the United States Government nor any agency thereof, nor any of their employees, makes any warranty, express or implied, or assumes any legal liability or responsibility for the accuracy, completeness, or usefulness of any information, apparatus, product, or process disclosed, or represents that its use would not infringe privately owned rights. Reference herein to any specific commercial product, process, or service by trade name, trademark, manufacturer, or otherwise does not necessarily constitute or imply its endorsement, recommendation, or favoring by the United States Government or any agency thereof. The views and opinions of authors expressed herein do not necessarily state or reflect those of the United States Government or any agency thereof. 


\section{ABSTRACT}

This document serves as the final report for the project "Atmospheric Aerosol Source-Receptor Relationships: The Role of Coal-Fired Power Plants" supported by the US Department of Energy. The project involved measurement of the ambient fine particle concentrations in the Pittsburgh metropolitan area, development of source profiles for important source classes in the Pittsburgh region, source apportionment using statistical and deterministic air quality models, and investigation of the response in ambient fine particle concentrations to changes in emissions. The project was led by Carnegie Mellon University in collaboration with universities, companies, national laboratories, and regional, state and local air quality agencies. This report describes the overall approach of the project and its major findings. 


\section{TABLE OF CONTENTS}

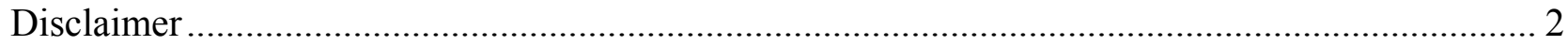

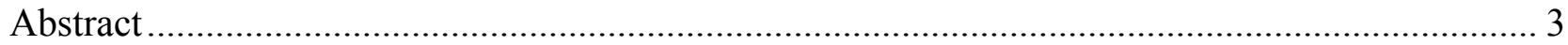

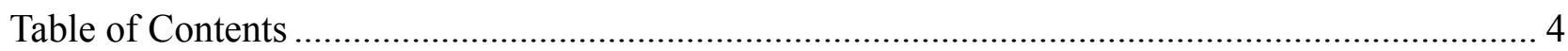

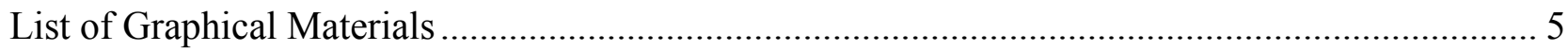

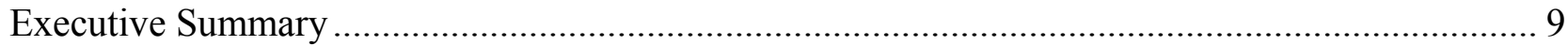

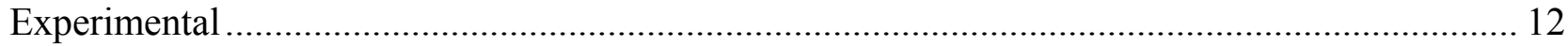

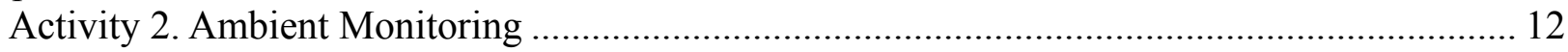

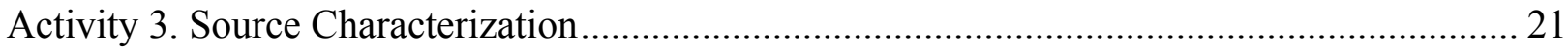

Characterization of coke production emissions ……………............................................. 22

Characterization of motor vehicle emissions.................................................................... 25

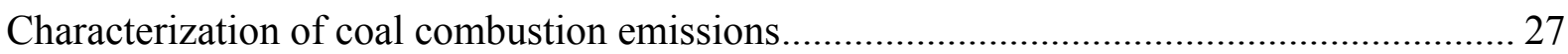

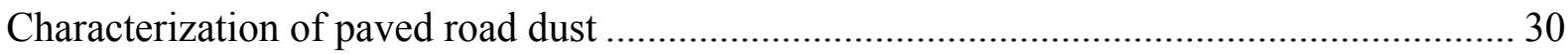

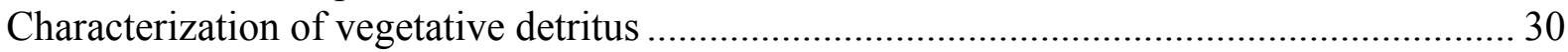

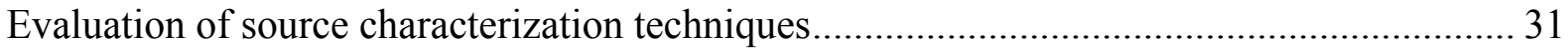

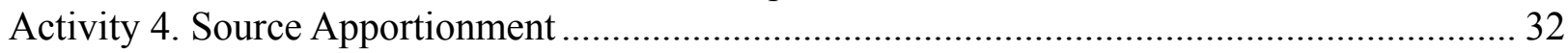

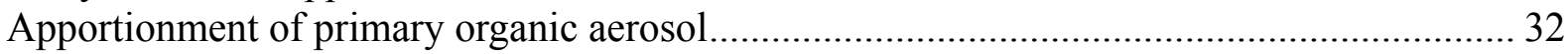

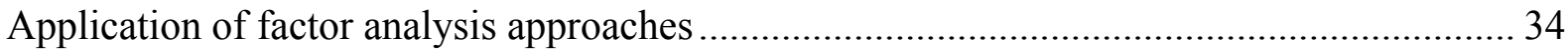

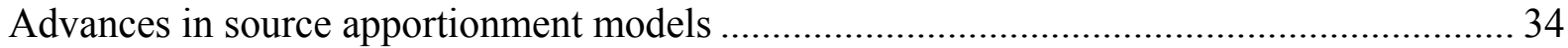

Activity 5. Three-Dimensional Deterministic Modeling ........................................................ 35

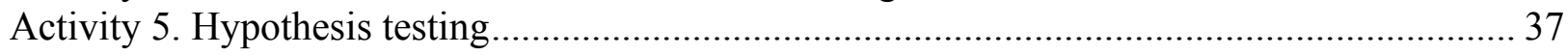

Ambient aerosol characterization ......................................................................................... 37

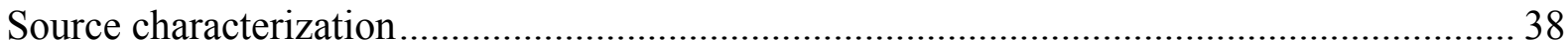

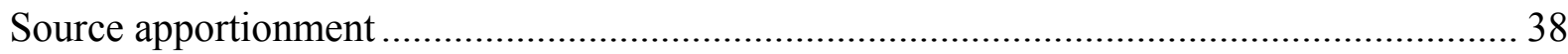

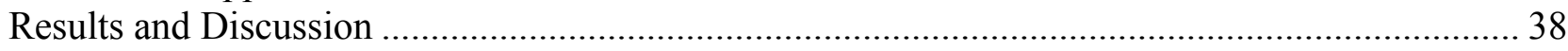

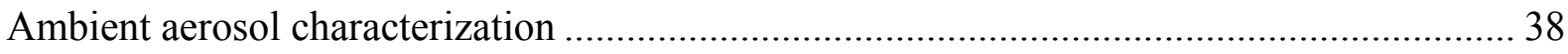

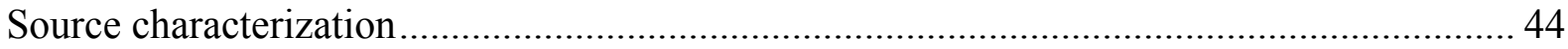

Source apportionment .................................................................................................. 47

Three-dimensional chemical transport modeling ……….................................................... 51

$\mathrm{PM}_{2.5}$ Response to Changes in the Emissions of its Precursors in the Eastern United States . 66

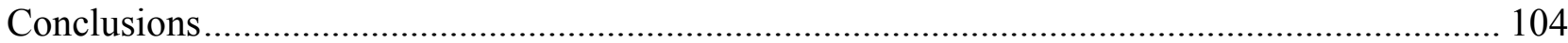

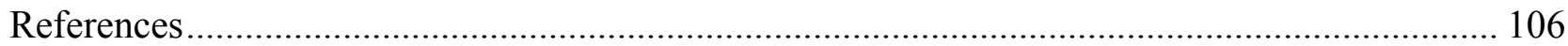

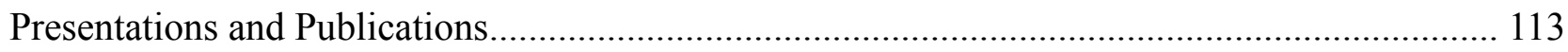

Publications:

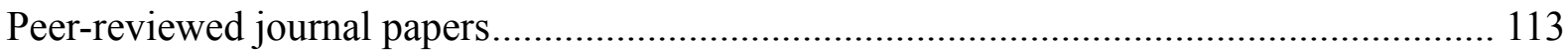

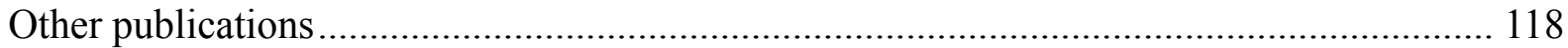

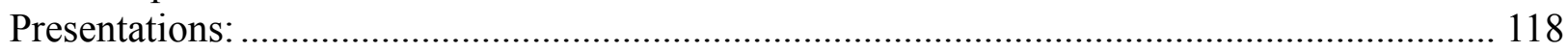

Appendix A Quality Assurance Final Report..............................................

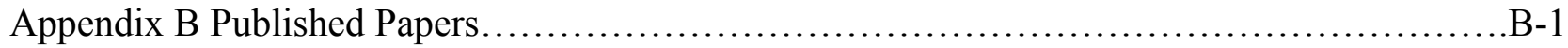




\section{LIST OF GRAPHICAL MATERIALS}

Figure 1. Map of Pittsburgh showing the approximate location of the central site (indicated by a star) and a photograph of the site from the northwest.

Figure 2. Map of the Pittsburgh region showing the approximate location of the central site, the Florence, Lawrenceville, Hazelwood, Greensburg, and Athens satellite sites, and the NETL, Holbrook, and Steubenville sites independently operated by other researchers during PAQS.

Figure 3. a) Top view of coking facility and sampling site; and b) wind rose for entire study period. The coking facility influences the sample concentrations for wind directions from $175^{\circ}$ to $300^{\circ}$. The coke batteries and other major operations are located between $240^{\circ}$ and $300^{\circ}$. 24

Figure 4. Side view of coking facility and sampling site. 24

Figure 5. Schematic of the Squirrel Hill Tunnel sampling location. The tunnel is $1.3 \mathrm{~km}$ long and has a $2.5 \%$ up-grade. Arrows in upper portion of schematic represent air flow in mechanical ventilation system. 26

Figure 6. Schematic of CMU Dynamic Dilution Sampler (Dilution Tunnel).............................. 28

Figure 7. The PMCAMx+ modeling domain for the eastern United States. The locations of the monitoring stations for the IMPROVE network are shown. 36

Figure 8. $\mathrm{PM}_{2.5}$ composition on a monthly average basis at the central site from July 2001 to June 2002. Also shown is the $\mathrm{PM}_{2.5}$ total mass measured using the FRM. Organic matter concentrations for July 2001 are based on 24 hour integrated denuder sampler measurements and the experimentally determined multiplier of 1.8. For other periods, organic matter concentrations are based on the difference between the OC collected on the upstream ('bare quartz') and downstream quartz filters of the CMU TQQQ sampler double-quartz filter pack. Sulfate, nitrate, and ammonium concentrations are composites of measurements made from several methods, including the CMU inorganic sampler, the PC-BOSS, the steam sampler and the Rupprecht and Patashnick 8400 instruments. Crustal component concentrations for August 2001 through June 2002 were estimated from daily July 2001 measurements collected at the PAQS satellite sites to be approximately $1 \mu \mathrm{g} \mathrm{m}^{-3}$....

Figure 9. Time series plots of $\mathrm{PM}_{2.5}$ concentrations during summer 2001 intensive sampling period.

Figure 10. Aerosol surface area and volume size distributions composition on a monthly average basis at the central site from June 2001 to May 2002. Also shown are the distributions based on a logarithmic scale.

Figure 11. SAS emission factors as a fraction of $\mathrm{PM}_{2.5}$ mass measured during pilot-scale and fullscale boiler testing.

Figure 12. Fuel-based emissions from low-load diesel (left column), medium-load diesel (middle column), and wood smoke (right column) experiments. Plots a1-a3 show $\mathrm{PM}_{2.5}$ mass emission factor; plots b1-b3 compare organic carbon emission factors measured with the Bare-Q and Q - QBT approaches; plots c1-c3 show particulate organic carbon (OC) and elemental carbon (EC) emission factors measured using the Q - QBT approach. Bare-Q is carbon measured with a quartz filter; Q - QBT is carbon measured with a backup-corrected quartz filter. Q QBT is particulate OC, while Bare-Q has substantial positive artifact, as discussed in the text. In plots $\mathrm{c} 1-\mathrm{c} 3$ symbols indicate total carbon emissions and shading indicates the contribution of $\mathrm{OC}$ and $\mathrm{EC}$ to the emissions. Plots c1-c3 also show the carbon fractions from the OC/EC 
analysis: $\mathrm{He} 1 \mathrm{OC}$ is the $\mathrm{OC}$ that evolves at $340^{\circ} \mathrm{C}$, and $\mathrm{He} 2-\mathrm{He} 4 \mathrm{OC}$ is the $\mathrm{OC}$ that evolves at temperatures greater than $340^{\circ} \mathrm{C}$ during the $\mathrm{OC} / \mathrm{EC}$ analysis. The diesel plots combine data from back-to-back experiments conducted on the same day. Lines are intended as a visual aid. Vertical bars in (a) are experimental uncertainties determined from intercomparison experiments (Lipsky and Robinson, 2005b).

Figure 13. Winter and summer averages of $\mathrm{CMB}$ predictions using molecular markers of primary sources of organic aerosol. The colored bars indicate the range of statistically strong CMB solutions using different combinations of source profiles.

Figure 14. Average predicted $\mathrm{PM}_{2.5}$ concentrations over the period of July 12-28 2001 for $\mathrm{PM}_{2.5}$ mass, sulphate, nitrate, ammonium, elemental carbon and organic mass $\left(\mu \mathrm{g} / \mathrm{m}^{3}\right) \ldots \ldots \ldots \ldots \ldots . . . . .52$

Figure 15. Average predicted $\mathrm{PM}_{2.5}$ concentrations over the period of October 01-31 2001 for $\mathrm{PM}_{2.5}$ mass, ammonium, nitrate, sulphate, elemental carbon and organic mass $\left(\mu \mathrm{g} / \mathrm{m}^{3}\right) \ldots \ldots . .53$

Figure 16. Average predicted $\mathrm{PM}_{2.5}$ concentrations over the period of January 01-31 2002 for $\mathrm{PM}_{2.5}$ mass, ammonium, nitrate, sulphate, elemental carbon and organic mass $\left(\mu \mathrm{g} / \mathrm{m}^{3}\right) \ldots \ldots . .54$

Figure 17. Average predicted $\mathrm{PM}_{2.5}$ concentrations over the period of April 03-30 2002 for $\mathrm{PM}_{2.5}$ mass, ammonium, nitrate, sulphate, elemental carbon and organic mass $\left(\mu \mathrm{g} / \mathrm{m}^{3}\right) \ldots \ldots \ldots \ldots \ldots .55$

Figure 18. Primary (POC) and Secondary (SOA) OC contribution to Organic Mass (OM) in all four seasons $\left(\mu \mathrm{g} / \mathrm{m}^{3}\right)$.

Figure 19. Comparison of predicted $\mathrm{PM}_{2.5}$ sulfate, $\mathrm{PM}_{2.5}$ nitrate, $\mathrm{PM}_{2.5}$ ammonium, organic mass, elemental carbon and total $\mathrm{PM}_{2.5}$ mass to data from the AIRS and IMPROVE Networks during July 2001. Also shown are the 1:1 and $\pm 30 \%$ lines.

Figure 20. Comparison of predicted $\mathrm{PM}_{2.5}$ sulfate, $\mathrm{PM}_{2.5}$ nitrate, $\mathrm{PM}_{2.5}$ ammonium, organic mass, elemental carbon and total $\mathrm{PM}_{2.5}$ mass to data from the AIRS and IMPROVE Networks during October 2001. Also shown are the 1:1 and $\pm 30 \%$ lines.

Figure 21. Comparison of predicted $\mathrm{PM}_{2.5}$ sulfate, $\mathrm{PM}_{2.5}$ nitrate, $\mathrm{PM}_{2.5}$ ammonium, organic mass, elemental carbon and total $\mathrm{PM}_{2.5}$ mass to data from the AIRS and IMPROVE Networks during January 2002. Also shown are the $1: 1$ and $\pm 30 \%$ lines.

Figure 22. Comparison of predicted $\mathrm{PM}_{2.5}$ sulfate, $\mathrm{PM}_{2.5}$ nitrate, $\mathrm{PM}_{2.5}$ ammonium, organic mass, elemental carbon and total $\mathrm{PM}_{2.5}$ mass to data from the AIRS and IMPROVE Networks during April 2002. Also shown are the 1:1 and $\pm 30 \%$ lines.

Figure 23. Predicted average base-case sulfate concentrations $\left(\mu \mathrm{g} \mathrm{m}^{-3}\right)$ for July $2001 \ldots \ldots \ldots \ldots \ldots . . . . .67$

Figure 24. Predicted change in sulfate concentration for a 50\% reduction in $\mathrm{SO}_{2}$ emissions for July 2001. A positive change corresponds to a concentration reduction.

Figure 25. Predicted fractional change in sulfate concentration for a $50 \%$ reduction in $\mathrm{SO}_{2}$ emissions for July 2001. A positive change corresponds to a concentration reduction........... 68

Figure 26. Predicted average base-case nitrate concentrations $\left(\mu \mathrm{g} \mathrm{m}^{-3}\right)$ for July $2001 \ldots \ldots \ldots \ldots \ldots . . . .69$

Figure 27. Predicted change $\left(\mu \mathrm{g} \mathrm{m}^{-3}\right)$ in nitrate concentration for a $50 \%$ reduction in $\mathrm{SO}_{2}$ emissions for July 2001. A positive change corresponds to a concentration reduction......

Figure 28. Predicted average base-case ammonium concentrations $\left(\mu \mathrm{g} \mathrm{m}^{-3}\right)$ for July 2001 ......... 70

Figure 29. Predicted change $\left(\mu \mathrm{g} \mathrm{m}^{-3}\right)$ in ammonium concentration for a $50 \%$ reduction in $\mathrm{SO}_{2}$ emissions for July 2001. A positive change corresponds to a concentration reduction.......... 71

Figure 30. Predicted average base-case $\mathrm{PM}_{2.5}$ concentrations $\left(\mu \mathrm{g} \mathrm{m}^{-3}\right)$ for July $2001 \ldots \ldots \ldots \ldots \ldots . . . . .72$

Figure 31. Predicted change $\left(\mu \mathrm{g} \mathrm{m}^{-3}\right)$ in $\mathrm{PM}_{2.5}$ concentration for a $50 \%$ change in $\mathrm{SO}_{2}$ emissions for July 2001. A positive change corresponds to a concentration reduction.

Figure 32. Predicted fractional change in $\mathrm{PM}_{2.5}$ concentration for a $50 \%$ change in $\mathrm{SO}_{2}$ emissions for July 2001. A positive change corresponds to a concentration reduction. 
Figure 33. Predicted average base-case sulfate concentrations $\left(\mu \mathrm{g} \mathrm{m}^{-3}\right)$ for January 2002 ........... 74

Figure 34. Predicted change in sulfate concentration $\left(\mu \mathrm{g} \mathrm{m} \mathrm{m}^{-3}\right.$ ) for a $50 \%$ reduction in $\mathrm{SO}_{2}$ emissions for January 2002. A positive change corresponds to a concentration reduction.... 74

Figure 35. Predicted fractional change in sulfate concentration for a $50 \%$ reduction in $\mathrm{SO}_{2}$ emissions for January 2002. A positive change corresponds to a concentration reduction.... 75

Figure 36. Predicted average base-case nitrate concentrations $\left(\mu \mathrm{g} \mathrm{m}^{-3}\right)$ for January 2002 .......... 76

Figure 37. Predicted change $(\mu \mathrm{g} \mathrm{m}-3)$ in nitrate concentration for a $50 \%$ reduction in $\mathrm{SO}_{2}$ emissions for January 2002. A positive change corresponds to a concentration reduction..... 76

Figure 38. Predicted average base-case ammonium concentrations $\left(\mu \mathrm{g} \mathrm{m}^{-3}\right)$ for January 2002 ... 77

Figure 39. Predicted change $\left(\mu \mathrm{g} \mathrm{m}^{-3}\right)$ in ammonium concentration for a $50 \%$ reduction in $\mathrm{SO}_{2}$ emissions for January 2002. A positive change corresponds to a concentration reduction..... 77

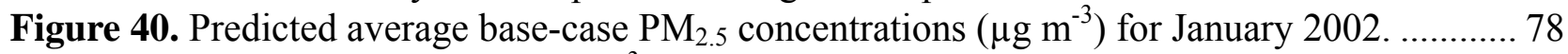

Figure 41. Predicted change $\left(\mu \mathrm{g} \mathrm{m}^{-3}\right)$ in $\mathrm{PM} 2.5$ concentration for a $50 \%$ reduction in $\mathrm{SO}_{2}$ emissions for January 2002. A positive change corresponds to a concentration reduction..... 79

Figure 42. Predicted fractional change in $\mathrm{PM}_{2.5}$ concentration for a $50 \%$ reduction in $\mathrm{SO}_{2}$ emissions for January 2002. A positive change corresponds to a concentration reduction..... 79

Figure 43. Predicted change $\left(\mu \mathrm{g} \mathrm{m} \mathrm{m}^{-3}\right)$ in nitrate concentration for a $50 \%$ reduction in $\mathrm{NO}_{\mathrm{x}}$ emissions for July 2001. A positive change corresponds to a concentration reduction.......... 81

Figure 44. Predicted fractional change in nitrate concentration for a $50 \%$ reduction in $\mathrm{NO}_{\mathrm{x}}$ emissions for July 2001. A positive change corresponds to a concentration reduction........... 81

Figure 45. Predicted change $\left(\mu \mathrm{g} \mathrm{m}^{-3}\right)$ in sulfate concentration for a $50 \%$ reduction in $\mathrm{NO}_{\mathrm{x}}$ emissions for July 2001. A positive change corresponds to a concentration reduction.......... 82

Figure 46. Predicted change $\left(\mu \mathrm{g} \mathrm{m}^{-3}\right)$ in ammonium concentration for a $50 \%$ reduction of $\mathrm{NO}_{\mathrm{x}}$ emissions for July 2001. A positive change corresponds to a concentration reduction........... 82

Figure 47. Predicted change $\left(\mu \mathrm{g} \mathrm{m}^{-3}\right)$ in $\mathrm{PM}_{2.5}$ concentration for a $50 \%$ reduction of $\mathrm{NO}_{\mathrm{x}}$ emissions for July 2001. A positive change corresponds to a concentration reduction.......... 83

Figure 48. Predicted fractional change $\left(\mu \mathrm{g} \mathrm{m}^{-3}\right)$ in PM2.5 concentration for a $50 \%$ reduction of $\mathrm{NO}_{\mathrm{x}}$ emissions for July 2001. A positive change corresponds to a concentration reduction... 83

Figure 49. Predicted change $\left(\mu \mathrm{g} \mathrm{m} \mathrm{m}^{-3}\right)$ in nitrate concentration for a $50 \%$ reduction of $\mathrm{NO}_{\mathrm{x}}$ emissions for January 2002. A positive change corresponds to a concentration reduction..... 84

Figure 50. Predicted change $\left(\mu \mathrm{g} \mathrm{m} \mathrm{m}^{-3}\right)$ in sulfate concentration for a $50 \%$ reduction of $\mathrm{NO}_{\mathrm{x}}$ emissions for January 2002. A positive change corresponds to a concentration reduction..... 85

Figure 51. Predicted change $\left(\mu \mathrm{g} \mathrm{m}^{-3}\right)$ in ammonium concentration for a $50 \%$ reduction of $\mathrm{NO}_{\mathrm{x}}$ emissions for January 2002. A positive change corresponds to a concentration reduction..... 85

Figure 52. Predicted change $\left(\mu \mathrm{g} \mathrm{m} \mathrm{m}^{-3}\right)$ in $\mathrm{PM}_{2.5}$ concentration for a $50 \%$ reduction of $\mathrm{NO}_{\mathrm{x}}$ emissions for January 2002. A positive change corresponds to a concentration reduction..... 86

Figure 53. Predicted change $\left(\mu \mathrm{g} \mathrm{m}^{-3}\right)$ in ammonium concentration for a $50 \%$ reduction of $\mathrm{NH}_{3}$ emissions for June 2001. A positive change corresponds to a concentration reduction......... 87

Figure 54. Predicted change $\left(\mu \mathrm{g} \mathrm{m}^{-3}\right)$ in nitrate concentration for a $50 \%$ reduction of $\mathrm{NH}_{3}$ emissions for June 2001. A positive change corresponds to a concentration reduction......... 88

Figure 55. Predicted change $\left(\mu \mathrm{g} \mathrm{m}{ }^{-3}\right)$ in $\mathrm{PM}_{2.5}$ concentration for a $50 \%$ reduction of $\mathrm{NH}_{3}$ emissions for June 2001. A positive change corresponds to a concentration reduction......... 88

Figure 56. Predicted fractional change $\left(\mu \mathrm{g} \mathrm{m}^{-3}\right)$ in $\mathrm{PM}_{2.5}$ concentration for a $50 \%$ reduction of $\mathrm{NH}_{3}$ emissions for June 2001. A positive change corresponds to a concentration reduction......... 89

Figure 57. Predicted change $\left(\mu \mathrm{g} \mathrm{m}^{-3}\right)$ in ammonium concentration for a $50 \%$ reduction of $\mathrm{NH}_{3}$ emissions for January 2002. A positive change corresponds to a concentration reduction..... 90 
Figure 58. Predicted change $\left(\mu \mathrm{g} \mathrm{m} \mathrm{m}^{-3}\right)$ in nitrate concentration for a $50 \%$ reduction of $\mathrm{NH}_{3}$ emissions for January 2002. A positive change corresponds to a concentration reduction..... 90

Figure 59. Predicted change $\left(\mu \mathrm{g} \mathrm{m}^{-3}\right)$ in sulfate concentration for a $50 \%$ reduction of $\mathrm{NH}_{3}$ emissions for January 2002. A positive change corresponds to a concentration reduction.... 91

Figure 60. Predicted change $\left(\mu \mathrm{g} \mathrm{m}^{-3}\right)$ in $\mathrm{PM}_{2.5}$ concentration for a $50 \%$ reduction of $\mathrm{NH}_{3}$ emissions for January 2002. A positive change corresponds to a concentration reduction..... 91

Figure 61. Predicted fractional change $\left(\mu \mathrm{g} \mathrm{m}^{-3}\right)$ in $\mathrm{PM}_{2.5}$ concentration for a $50 \%$ reduction of $\mathrm{NH}_{3}$ emissions for January 2002. A positive change corresponds to a concentration reduction..... 92

Figure 62. Predicted change $\left(\mu \mathrm{g} \mathrm{m}^{-3}\right)$ in sulfate concentration for a $50 \%$ reduction in $\mathrm{SO}_{2}$ and $\mathrm{NOx}$ emissions for July 2001. A positive change corresponds to a concentration reduction.......... 93

Figure 63. Predicted fractional change in sulfate concentration for a $50 \%$ reduction in $\mathrm{SO}_{2}$ and NOx emissions for July 2001. A positive change corresponds to a concentration reduction. . 94

Figure 64. Predicted change $\left(\mu \mathrm{g} \mathrm{m}^{-3}\right)$ in nitrate concentration for a $50 \%$ reduction in $\mathrm{SO}_{2}$ and $\mathrm{NOx}$ emissions for July 2001. A positive change corresponds to a concentration reduction.......... 95

Figure 65. Predicted fractional change in nitrate concentration for a $50 \%$ reduction in $\mathrm{SO}_{2}$ and NOx emissions for July 2001. A positive change corresponds to a concentration reduction. . 95

Figure 66. Predicted change $\left(\mu \mathrm{g} \mathrm{m}^{-3}\right)$ in ammonium concentration for a $50 \%$ reduction in $\mathrm{SO}_{2}$ and NOx emissions for July 2001. A positive change corresponds to a concentration reduction. . 96

Figure 67. Predicted change $\left(\mu \mathrm{g} \mathrm{m}^{-3}\right)$ in organic aerosol concentration for a $50 \%$ reduction in $\mathrm{SO}_{2}$ and NOx emissions for July 2001. A positive change corresponds to a concentration reduction.

Figure 68. Predicted change $\left(\mu \mathrm{g} \mathrm{m}^{-3}\right)$ in $\mathrm{PM}_{2.5}$ concentration for a $50 \%$ reduction in $\mathrm{SO}_{2}$ and $\mathrm{NOx}$ emissions for July 2001. A positive change corresponds to a concentration reduction.......... 98

Figure 69. Predicted fractional change in $\mathrm{PM}_{2.5}$ concentration for a $50 \%$ reduction in $\mathrm{SO}_{2}$ and NOx emissions for July 2001. A positive change corresponds to a concentration reduction. . 98

Figure 70. Predicted change $\left(\mu \mathrm{g} \mathrm{m}^{-3}\right)$ in sulfate concentration for a $50 \%$ reduction in $\mathrm{SO}_{2}$ and $\mathrm{NOx}$ emissions for January 2002. A positive change corresponds to a concentration reduction.... 99

Figure 71. Predicted fractional change in sulfate concentration for a $50 \%$ reduction in $\mathrm{SO}_{2}$ and NOx emissions for January 2002. A positive change corresponds to a concentration reduction.

Figure 72. Predicted change $\left(\mu \mathrm{g} \mathrm{m}^{-3}\right)$ in nitrate concentration for a $50 \%$ reduction in $\mathrm{SO}_{2}$ and $\mathrm{NOx}$ emissions for January 2002. A positive change corresponds to a concentration reduction... 100

Figure 73. Predicted change $\left(\mu \mathrm{g} \mathrm{m}^{-3}\right)$ in organic aerosol concentration for a $50 \%$ reduction in $\mathrm{SO}_{2}$ and NOx emissions for January 2002. A positive change corresponds to a concentration reduction.

Figure 74. Predicted change $\left(\mu \mathrm{g} \mathrm{m}^{-3}\right)$ in $\mathrm{PM}_{2.5}$ concentration for a $50 \%$ reduction in $\mathrm{SO}_{2}$ and $\mathrm{NOx}$ emissions for January 2002. A positive change corresponds to a concentration reduction... 102

Figure 75. Predicted fractional change in $\mathrm{PM}_{2.5}$ concentration for a $50 \%$ reduction in $\mathrm{SO}_{2}$ and NOx emissions for January 2002. A positive change corresponds to a concentration reduction. 


\section{EXECUTIVE SUMMARY}

With support from the US Department of Energy and the US Environmental Protection Agency, Carnegie Mellon University is conducting detailed studies of the ambient particulate matter in the Pittsburgh, PA metropolitan area - the Pittsburgh Air Quality Study (PAQS). The research was conducted by an integrated team of engineers and scientists from universities, companies, national laboratories, and regional, state and local air quality agencies. This document is the final technical report for the DOE-supported component of the project. Major activities of the project included:

- Measurements of ambient air quality at a central monitoring site located in an urban park in Pittsburgh, PA and five satellite sites located in Pittsburgh, Southwestern Pennsylvania, and Eastern Ohio. Baseline monitoring took place over a fourteen month period that began July 1, 2001 and provided data for a wide variety of PM physical and chemical characteristics, gaseous species, fogwater and biological aerosol composition, and meteorological parameters. Two intensive sampling periods from July 1 to August 3, 2001 (ESP 01) and January 1 to January 15, 2002 (ESP 02) involved an increase in temporal resolution of many of the baseline measurements as well as measurements of size resolved aerosol composition, speciated organic composition and particle morphology, as well as semi-continuous aerosol metal composition and gaseous volatile organic compound (VOC) species.

- Measurement of source profiles for important sources in the Pittsburgh region. Profiles for emissions from motor vehicles, coke production, coal combustion, paved road dust, and vegetative detritus were developed through a combination of source testing, fenceline measurements, and analysis of highly time resolved data collected at the central site.

- Source apportionment of the ambient aerosol using statistical and deterministic air quality models.

- Predictions of three-dimensional aerosol chemical transport model, PMCAMx+, were compared to highly time-resolved measurements collected in Pittsburgh and to spatially resolved measurements by the EPA STN and IMPROVE networks. The model was used to predict the response of ambient PM concentrations to changes in emissions.

Major findings of the project include:

- The annual average $\mathrm{PM}_{2.5}$ concentration (July 1, 2001 through June 30, 2002) was $15.8 \mu \mathrm{g} \mathrm{m}^{-3}$, indicating that the region will likely violate the annual average National Ambient Air Quality Standard for $\mathrm{PM}_{2.5}\left(15 \mu \mathrm{g} \mathrm{m}^{-3}\right)$. The daily average $\mathrm{PM}_{2.5}$ standard $\left(65 \mu \mathrm{g} \mathrm{m}^{-3}\right)$ was never exceeded during the approximately 400 days of measurements during PAQS. However, on 9 days the daily average $\mathrm{PM}_{2.5}$ concentration was greater than $50 \mu \mathrm{g} \mathrm{m} \mathrm{m}^{-3}$. All 9 days occurred during the summer months of 2001 and 2002. Roughly half of the episodes were due to high sulfate concentrations while the other half were mainly due to high organic matter concentrations.

- Seasonal variations in $\mathrm{PM}_{2.5}$ mass and composition in Western Pennsylvania are driven by differences in the seasonal variations of organic carbon and sulfate, with sulfate being 
dominant in the summer and organic matter and sulfate showing similar concentrations in the cooler months. In the cooler months, nitrate also is important and approximately equal to about half the sulfate concentration on average.

- During the study roughly $90 \%$ of the $\mathrm{PM}_{2.5}$ concentration came from areas outside Pittsburgh. There are important local sources of fine particulate metals, and roughly half of the particle number concentration was due to local transportation sources.

- Emissions from coal-fired power plants contribute significantly to $\mathrm{PM}_{2.5}$ concentrations in Pittsburgh. The vast majority of this contribution is to secondary sulfate which represents $38 \%$ of annual average $\mathrm{PM}_{2.5}$ mass.

- Direct emissions of $\mathrm{PM}_{2.5}$ mass from coal-fired plants is a minor source to fine particle mass, estimated to contribute on average $0.25 \mu \mathrm{g} \mathrm{m}-3$ to the PM2.5 mass in Pittsburgh (or 1.5\% of annual average $\mathrm{PM}_{2.5}$ ).

- No major enhancement of the organic aerosol concentration is observed during periods with elevated inorganic acidity. If we assume a causal relationship between inorganic acidity and $\mathrm{OC}$, reductions in OC for Western Pennsylvania that might result from reductions in inorganic acidity were estimated to be $2 \pm 4 \%$ based on regression analysis of the ambient data with an upper bound of $5 \pm 7 \%$ based on calculations from laboratory measurements.

- Dilution sampler measurements indicate that organic and elemental carbon contributes less than $5 \%$ of the $\mathrm{PM}_{2.5}$ mass directly emitted from coal-fired power plants. In addition, the majority of the OC emissions appear to be sampling artifacts.

- A three-dimensional aerosol chemical transport model $(\mathrm{PMCAMx}+)$ was used to investigate the sensitivity of $\mathrm{PM}_{2.5}$ mass to changes in $\mathrm{SO}_{2}, \mathrm{NO}_{\mathrm{x}}$ and $\mathrm{NH}_{3}$ emissions in both the summer and winter. The model was evaluated by comparing predictions to daily measurements made by EPA STN and IMPROVE monitoring networks during July 2001, October 2001, January 2002 and April 2002. The average fractional bias of the model predictions during each of these periods was less than $30 \%$ for most aerosol species.

- During the summer (July 2001), a 50\% reduction in $\mathrm{SO}_{2}$ emissions is predicted to lead to reductions of the average $\mathrm{PM}_{2.5}$ levels by 3-5 $\mu \mathrm{g} \mathrm{m}^{-3}$, which corresponds to $20-30 \%$ reduction in PM2.5 mass across much of the Eastern US. During the winter (January 2002), a 50\% reduction in $\mathrm{SO}_{2}$ emissions is predicted to reduce ambient $\mathrm{PM}_{2.5}$ mass by up to $1.5 \mu \mathrm{g} \mathrm{m}^{-3}$. The Southeastern US is predicted to benefit the most from wintertime controls on $\mathrm{SO}_{2}$ emissions while changes in $\mathrm{PM}_{2.5}$ mass in the Northeastern US are rather small (less than $0.5 \mu \mathrm{g} \mathrm{m}^{-3}$ for a $50 \%$ reduction in $\mathrm{SO}_{2}$ emissions) mainly because the sulfate reduction is balanced by an increase in nitrate. Therefore, $\mathrm{SO}_{2}$ emission controls are most effective in the summer.

- During the summer (July 2001), a 50\% reduction in $\mathrm{NO}_{\mathrm{x}}$ emissions is predicted to reduce $\mathrm{PM}_{2.5}$ levels by as much as $2 \mu \mathrm{g} \mathrm{m}^{-3}$ in the Midwest, Pennsylvania, and parts of the South. These changes correspond to reductions of $\mathrm{PM}_{2.5}$ of $5-15 \%$ in most of the Eastern US. During the wintertime (January 2002), PMCAMx predicts that a 50\% reduction of NOx emissions can lead to average reductions in particulate nitrate of 0.4 to $0.8 \mu \mathrm{g} \mathrm{m}^{-3}$. 
- During the summer (July 2001), a $50 \%$ reduction in $\mathrm{NH}_{3}$ emissions is predicted to reduce $\mathrm{PM}_{2.5}$ levels by up $1.5 \mu \mathrm{g} \mathrm{m}^{-3}$, which corresponds to 5 to $10 \%$ of the $\mathrm{PM}_{2.5}$ mass in the Eastern US. Similar levels of reduction are predicted for winter, indicating that changes in ammonia emissions represent an interesting emission control strategy.

- During the summer (July 2001), a simultaneous 50\% reduction in both $\mathrm{SO}_{2}$ and $\mathrm{NO}_{\mathrm{x}}$ emissions is predicted to lead to reductions of the average $\mathrm{PM}_{2.5}$ levels during July by $3-9 \mu \mathrm{g} \mathrm{m}^{-3}$, which corresponds to a $20 \%$ of the $\mathrm{PM}_{2.5}$ in the large urban areas and more than $40 \%$ in rural areas of both the Northeast and Southeast US. Substantial reductions in $\mathrm{PM}_{2.5}$ are also predicted for the winter for control scenarios which simultaneously reduce $\mathrm{SO}_{2}$ and $\mathrm{NO}_{\mathrm{x}}$ emissions.

- Regional scale formation of ultrafine particles (nucleation) takes place in Western Pennsylvania on $30 \%$ of the days of the year during all seasons, but it is most frequent in fall and spring and least frequent in winter. Regional nucleation is most common on sunny days with below average $\mathrm{PM}_{2.5}$ concentrations. The frequent nucleation events can be explained by a ternary sulfuric acid-ammonia-water nucleation model.

- As of April 2006, the findings of this study have been described in fifty papers either already published or submitted to peer-reviewed journals. The ambient data collected by the project are available in publicly accessible on-line relational databases. 


\section{EXPERIMENTAL}

\section{Activity 2. Ambient Monitoring}

The purpose of this activity is to create an extensive database of ambient PM measurements for source apportionment, examination of aerosol processes, evaluation of instrumentation, and air quality model development and evaluation. The central monitoring site was located on a grassy hill in Schenley Park, located roughly 6 kilometers east of downtown Pittsburgh. The site is more than several hundred meters from any major source of air pollution, and roughly one kilometer of parkland exists between the site and the city in the predominant upwind direction (south and west). The site was positioned approximately 50 meters past the end of a dead end street, and several hundred meters from the nearest heavily traveled street (Forbes Avenue). Sampling equipment was housed in a 33 square meter air pollution monitoring station, with some samplers and sampler inlets on the station rooftop. The location of the central site in relation to the downtown Pittsburgh area and a picture of the central site are shown in Figure 1.
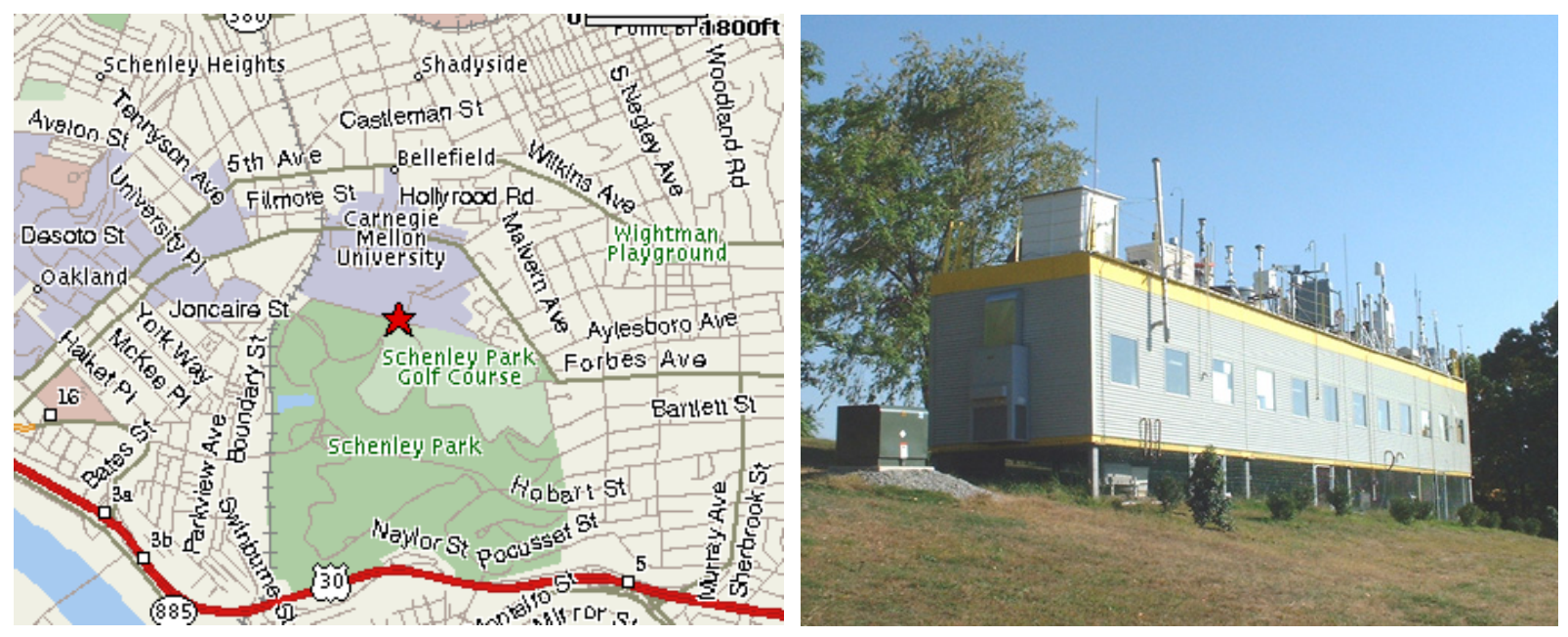

Figure 1. Map of Pittsburgh showing the approximate location of the central site (indicated by a star) and a photograph of the site from the northwest.

Figure 2 shows the location of satellite sites used during the site. The PAQS team operated satellite sites in five locations. Satellite sites located in Florence, PA and Greensburg, PA were maintained by the Pennsylvania Department of Environmental Protection (PA DEP). The Florence site was about 50 kilometers west of the main site in a rural area with no nearby sources. The Greensburg site was about 50 kilometers east of the main site in a suburban area, close to a heavily traveled road but otherwise in an area of only moderate traffic and few stationary sources. Satellite sites were also located in the Lawrenceville and Hazelwood neighborhoods of the City of Pittsburgh, and were maintained by the Allegheny County Health Department (ACHD). The final satellite site was located in Athens, Ohio, and operated by Ohio University. Additional air quality measurements were conducted concurrently by others at the NETL laboratories, approximately 15 kilometers south of downtown Pittsburgh, at Holbrook, PA and at Steubenville, OH. Table 1 lists 
the latitude, longitude, and elevation of these sites. In addition to the ground-based measurements, two aircraft provided air quality and meteorological data aloft during the intensive periods. R. B. Jacko and Associates, Inc. operated a Cessna 210 Turbo Centurion and the University of Maryland operated a Piper 23-250 Aztec-F.

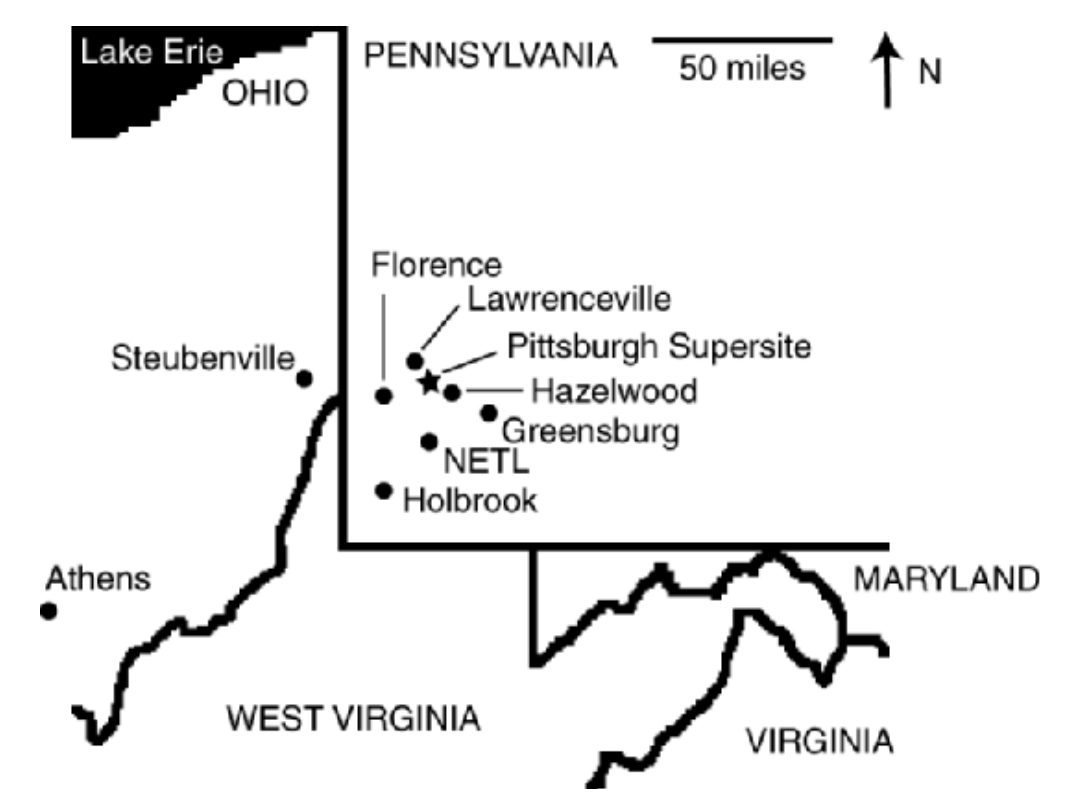

Figure 2. Map of the Pittsburgh region showing the approximate location of the central site, the Florence, Lawrenceville, Hazelwood, Greensburg, and Athens satellite sites, and the NETL, Holbrook, and Steubenville sites independently operated by other researchers during PAQS.

Baseline monitoring took place over a fourteen month period that began July 1, 2001 and provided data for a wide variety of PM physical and chemical characteristics, gaseous species, fogwater and biological aerosol composition, and meteorological parameters. Two intensive sampling periods from July 1 to August 3, 2001 (ESP 01) and January 1 to January 15, 2002 (ESP 02) involved an increase in temporal resolution of many of the baseline measurements as well as measurements of size resolved aerosol composition, speciated organic composition and particle morphology, as well as semi-continuous aerosol metal composition and gaseous volatile organic compound (VOC) species. The frequency, resolution, and dates over which each method was used at the central site are summarized in Table 1. Quality control procedures used to verify the measurements are presented in Appendix A.

Measurements of $\mathrm{PM}_{2.5}$ aerosol mass, inorganic ions, organic and elemental carbon, and metal concentrations were obtained at the satellite sites during both intensive periods. The sampling period of measurements at each of the satellite sites is summarized in Table 3. Two additional special experiments were performed at the Florence, PA satellite site during January and July of 2002. One experiment involved the collection of 24 hour integrated $\mathrm{PM}_{2.5}$ organic speciation samples and the second experiment involved 10 minute resolution measurement of the aerosol size distribution from 0.012 to $0.28 \mu \mathrm{m}$. Aircraft high time resolution measurements of meteorology, aerosol properties, and gas-phase species were obtained during select days of July and August 2001 and 2002. The Jacko aircraft conducted 4 flights in 2 days over Pittsburgh, PA (July 23, 2001 
and August 2, 2001). The Maryland aircraft conducted ten flights in July and August of 2001 and 2002 over the western PA region (six of the flights conducted measurements above Pittsburgh, PA).

The ambient data from this project have been submitted to the NARSTO database for permanent archive (http://cdiac.esd.ornl.gov/programs/NARSTO/access.htm). The data are also available in two publicly available online databases: the EPA-supported Supersites relational database (http://supersitesdata.umd.edu/) and the DOE-supported relational database (http://www.pmdata.org/).

Table 1. Locations of ambient measurements collected during PAQS.

\begin{tabular}{|c|c|c|c|}
\hline Site name & Group & $\begin{array}{l}\text { Latitude/Longitude } \\
\text { (decimal degrees) }\end{array}$ & Period \\
\hline Central site & CMU & $40.4395 /-79.9405$ & $6 / 30 / 01-7 / 31 / 02$ \\
\hline \multicolumn{4}{|l|}{ Satellite sites } \\
\hline Hazelwood, PA & CMU/ACHD & $40.4124 /-79.9424$ & $6 / 30-7 / 30 / 01,1 / 2-1 / 22 / 02$ \\
\hline Lawrenceville, PA & CMU/ACHD & $40.4432 /-79.9595$ & $6 / 30-7 / 30 / 01,1 / 2-1 / 22 / 02$ \\
\hline Florence, PA & CMU/DEP & $40.4454 /-80.4212$ & $\begin{array}{l}\text { 6/30-7/30/01, } 1 / 2-1 / 22 / 02 \\
1 / 3-1 / 22 / 02,7 / 14-8 / 3 / 02^{1} \\
2 / 24 / 02 \text { to } 3 / 28 / 02^{2}\end{array}$ \\
\hline Greensburg, PA & CMU/DEP & $40.3043 /-79.5059$ & $6 / 30-7 / 30 / 01,1 / 2-1 / 22 / 02$ \\
\hline Athens, $\mathrm{OH}$ & Ohio Univ. & $39.3283 /-82.9067$ & $6 / 30-8 / 8 / 01$ \\
\hline \multicolumn{4}{|l|}{ Aircraft } \\
\hline Cessna & Jacko & Various & $7 / 6-8 / 2 / 01$ \\
\hline Piper & UM & Various & $7 / 6-8 / 1 / 01,7 / 2-8 / 4 / 02$ \\
\hline \multicolumn{4}{|l|}{ Non-PAQS sites } \\
\hline NETL & NETL & 40.3065 / -79.9794 & $6 / 30-8 / 8 / 01$ \\
\hline Holbrook, PA & ATS & $39.8160 /-80.2850$ & $6 / 30-8 / 8 / 01$ \\
\hline Steubenville, $\mathrm{OH}$ & CONSOL & $40.3617 /-80.6147$ & $6 / 30-8 / 8 / 01$ \\
\hline
\end{tabular}

Groups - ACHD: Allegheny County Health Department; ATS: Advanced Technology Systems; DEP: Pennsylvania Department of Environmental Planning; NETL: National Energy and Technology Laboratory; UM: University of Maryland; Jacko: R. B. Jacko and Associates.

Central site measurements - See Table 2.

Satellite site measurements $-\mathrm{PM}_{2.5}$ mass, $\mathrm{PM}_{2.5}$ elements, and $\mathrm{PM}_{2.5}$ ammonium, nitrate, potassium, sodium, sulfate, elemental carbon, and organic carbon. ${ }^{1} \mathrm{~A} \mathrm{PM}_{2.5}$ quartz-PUFF organic sampler was used to measure 24 hour organic aerosol concentrations on 17 days within the stated range. ${ }^{2}$ Particle sizing instruments (TSI 3071 DMA and 3010 $\mathrm{CPC}$ ) were used to measure 10 minute aerosol size distribution semi-continuously from 0.012 to $0.280 \mu \mathrm{m}$. 
Table 2. Summary of PAQS ambient measurements.

\begin{tabular}{|c|c|c|c|c|c|}
\hline Observable & Method & Group & Resolution & Frequency & Period of Operation \\
\hline $\begin{array}{l}\text { Aerosol Mass } \\
\mathrm{PM}_{10} \text { mass } \\
\mathrm{PM}_{2.5} \text { mass } \\
\mathrm{PM}_{2.5} \text { mass } \\
\mathrm{PM}_{2.5} \text { mass } \\
\mathrm{PM}_{\mathrm{x}} \text { mass } \\
\mathrm{PM}_{\mathrm{x}} \text { mass }\end{array}$ & $\begin{array}{l}\text { Dichotomous sampler with gravimetry } \\
\text { Dichotomous sampler with gravimetry } \\
\text { Federal Reference Method sampler with gravimetry } \\
\text { R\&P 1400A TEOM monitor with SES } \\
\text { MOUDI sampler with gravimetry } \\
\text { MOUDI sampler with gravimetry }\end{array}$ & $\begin{array}{l}\text { CMU } \\
\text { CMU } \\
\text { CMU } \\
\text { CMU } \\
\text { CMU } \\
\text { CMU }\end{array}$ & $\begin{array}{l}24 \mathrm{hr} \\
24 \mathrm{hr} \\
24 \mathrm{hr} \\
10 \mathrm{~min} \\
24 \mathrm{hr} \\
8 \mathrm{hr}\end{array}$ & $\begin{array}{l}\text { Daily } \\
\text { Daily } \\
\text { Daily } \\
\text { Continuous } \\
\text { Daily } \\
3 \text { per day }\end{array}$ & $\begin{array}{l}7 / 1 / 01-7 / 1 / 02 \\
7 / 1 / 01-7 / 1 / 02 \\
7 / 1 / 01-7 / 1 / 02 \\
7 / 1 / 01-9 / 1 / 02 \\
7 / 1 / 01-7 / 1 / 02 * \\
7 / 22-25 / 01,7 / 31-8 / 3 / 01\end{array}$ \\
\hline $\begin{array}{l}\text { Aerosol Size Distribution } \\
\text { Aerosol number distribution } \\
\text { Aerosol surface area distribution } \\
\text { Aerosol surface area distribution } \\
\text { Aerosol volume distribution }\end{array}$ & $\begin{array}{l}\text { TSI ultra fine SMPS, SMPS and APS } \\
\text { TSI ultra fine SMPS, SMPS and APS } \\
\text { Epiphaniometer } \\
\text { TSI ultra fine SMPS, SMPS and APS }\end{array}$ & $\begin{array}{l}\text { CMU } \\
\text { CMU } \\
\text { PSI } \\
\text { CMU }\end{array}$ & $\begin{array}{l}10 \mathrm{~min} \\
10 \mathrm{~min} \\
30 \mathrm{~min} \\
10 \mathrm{~min}\end{array}$ & $\begin{array}{l}\text { Continuous } \\
\text { Continuous } \\
\text { Continuous } \\
\text { Continuous }\end{array}$ & $\begin{array}{l}7 / 1 / 01-7 / 1 / 02 \\
7 / 1 / 01-7 / 1 / 02 \\
6 / 11 / 01-9 / 18 / 01 \\
7 / 1 / 01-7 / 1 / 02\end{array}$ \\
\hline $\begin{array}{l}\text { Aerosol Chemical Composition } \\
\text { PM2.5 inorganic ions } \\
\text { PM2.5 inorganic ions } \\
\text { PMx inorganic ions } \\
\text { PMx inorganic ions } \\
\text { PM10 elements } \\
\text { PM2.5 elements } \\
\text { PMx elements } \\
\text { PMx elements } \\
\text { PM2.5 organic and elemental carbon } \\
\text { PM2.5 organic and elemental carbon } \\
\text { PM2.5 organic and elemental carbon } \\
\text { PM2.5 organic and elemental carbon } \\
\text { PM2.5 organic and elemental carbon } \\
\text { PMx organic and elemental carbon } \\
\text { PMx organic and elemental carbon } \\
\text { PM2.5 speciated organics } \\
\text { PM2.5 speciated organics } \\
\text { PM2.5 speciated organics } \\
\text { PM2.5 biological material }\end{array}$ & $\begin{array}{l}\text { CMU sampler with IC analysis } \\
\text { CMU sampler with IC analysis } \\
\text { MOUDI sampler with IC analysis } \\
\text { MOUDI sampler with IC analysis } \\
\text { Hi-Vol sampler with ICP-MS analysis } \\
\text { Hi-Vol sampler with ICP-MS analysis } \\
\text { MOUDI sampler with ICP-MS analysis } \\
\text { MOUDI sampler with ICP-MS analysis } \\
\text { CMU TQQQ sampler with TOT analysis } \\
\text { CMU TQQQ sampler with TOT analysis } \\
\text { CMU denuded organic sampler with TOT analysis } \\
\text { CMU denuded organic sampler with TOT analysis } \\
\text { MOUDI sampler with TOT analysis } \\
\text { MOUDI sampler with TOT analysis } \\
\text { BYU PC-BOSS } \\
\text { LPI sampler with FTIR analysis } \\
\text { CMU organic speciation sampler } \\
\text { CMU organic speciation sampler } \\
\text { Epi-fluorescent microscopy with assays }\end{array}$ & $\begin{array}{l}\text { CMU } \\
\text { CMU } \\
\text { CMU } \\
\text { CMU } \\
\text { CMU } \\
\text { CMU } \\
\text { CMU } \\
\text { CMU } \\
\text { CMU } \\
\text { CMU } \\
\text { CMU } \\
\text { CMU } \\
\text { CMU } \\
\text { CMU } \\
\text { BYU } \\
\text { CMU } \\
\text { FIU } \\
\text { FIU } \\
\text { UC }\end{array}$ & $\begin{array}{l}24 \mathrm{hr} \\
4-6 \mathrm{hr} \\
24 \mathrm{hr} \\
8 \mathrm{hr} \\
24 \mathrm{hr} \\
24 \mathrm{hr} \\
24 \mathrm{hr} \\
8 \mathrm{hr} \\
24 \mathrm{hr} \\
4-6 \mathrm{hr} \\
24 \mathrm{hr} \\
24 \mathrm{hr} \\
24 \mathrm{hr} \\
8 \mathrm{hr} \\
24 \mathrm{hr} \\
24 \mathrm{hr} \\
24 \mathrm{hr} \\
24 \mathrm{hr} \\
24 \mathrm{hr}\end{array}$ & $\begin{array}{l}\text { Daily } \\
5 \text { per day } \\
\text { Daily } \\
3 \text { per day } \\
\text { Daily } \\
\text { Daily } \\
\text { Daily } \\
3 \text { per day } \\
\text { Daily } \\
5 \text { per day } \\
\text { 6th day } \\
\text { Daily } \\
\text { Daily } \\
3 \text { per day } \\
\text { Daily } \\
\text { Daily } \\
6 \text { th Day } \\
\text { Daily } \\
\text { Daily }\end{array}$ & $\begin{array}{l}\text { 7/1/01-7/1/02* } \\
\text { ESP01 Intensive } \\
7 / 1 / 01-7 / 1 / 02 * \\
7 / 22-25 / 01,7 / 31-8 / 3 / 01 \\
7 / 12 / 01-8 / 02 / 02 \\
7 / 11 / 01-9 / 30 / 02 \\
\text { ESP01, ESP02 Intensives* } \\
\text { 7/22-25/01, 7/31-8/3/01 } \\
7 / 1 / 01-7 / 31 / 02 * \\
\text { ESP01 Intensive } \\
7 / 1 / 01-6 / 1 / 02 * \\
\text { ESP01, ESP02 Intensives } \\
\text { ESP01 Intensive * } \\
7 / 22-25 / 01,7 / 31-8 / 3 / 01 \\
\text { ESP01, ESP02 Intensives } \\
\text { ESP01, ESP02 Intensives } \\
\text { 7/1/01-7/1/02* } \\
\text { ESP01, ESP02 Intensives } \\
\text { 7/7/01-7/1/02 }\end{array}$ \\
\hline
\end{tabular}


Table 2. Summary of PAQS measurements, continued.

\begin{tabular}{|c|c|c|c|c|c|}
\hline Observable & Method & Group & Resolution & Frequency & Period of Operation \\
\hline $\begin{array}{l}\text { Aerosol Chemical Composition, } c \\
\mathrm{PM}_{2.5} \text { nitrate } \\
\mathrm{PM}_{2.5} \text { sulfate } \\
\mathrm{PM}_{2.5} \text { carbon } \\
\mathrm{PM}_{2.5} \text { carbon } \\
\mathrm{PM}_{2.5} \text { water soluble ions } \\
\mathrm{PM}_{2.5} \text { water soluble ammonium } \\
\mathrm{PM}_{1.3} \text { metals } \\
\mathrm{PM}_{1.0} \text { size resolved composition }\end{array}$ & $\begin{array}{l}\text { ntinued } \\
\text { R\&P } 8400 N \text { with flash volatilization } \\
\text { R\&P } 8400 \text { S with flash volatilization } \\
\text { ADI monitor with flash volatilization } \\
\text { Denuded Sunset Labs in situ TOT analyzer } \\
\text { Khylstov steam sampler with IC analysis } \\
\text { Ammonium online detector } \\
\text { SEAS with GFAA analysis } \\
\text { Aerodyne Mass Spectrometer (AMS) }\end{array}$ & $\begin{array}{l}\text { ADI } \\
\text { ADI } \\
\text { ADI } \\
\text { RU } \\
\text { CMU } \\
\text { CMU } \\
\text { UMD } \\
\text { UC, AERODYNE }\end{array}$ & $\begin{array}{l}10 \mathrm{~min} \\
10 \mathrm{~min} \\
30 \mathrm{~min} \\
2-4 \mathrm{hr} \\
1-2 \mathrm{hr} \\
10 \mathrm{~min} \\
30 \mathrm{~min} \\
5 \mathrm{~min}\end{array}$ & $\begin{array}{l}\text { Semi-continuous } \\
\text { Semi-continuous } \\
\text { Semi-continuous } \\
\text { Semi-continuous } \\
\text { Semi-continuous } \\
\text { Continuous } \\
\text { Semi-continuous } \\
\text { Semi-continuous }\end{array}$ & $\begin{array}{l}7 / 1 / 01-8 / 1 / 02 \\
7 / 1 / 01-9 / 1 / 02 \\
1 / 1 / 02-9 / 1 / 02 \\
7 / 1 / 01-9 / 1 / 02 \\
7 / 1 / 01-9 / 21 / 02 \\
7 / 1 / 01-9 / 21 / 02 \\
7 / 8-8 / 10 / 01,3 / 29-4 / 17 / 02 \\
9 / 6 / 02-9 / 21 / 02\end{array}$ \\
\hline $\begin{array}{l}\text { Aerosol Characteristics } \\
\text { Particle light scattering } \\
\text { Particle hygroscopicity } \\
\text { Cloud condensation }\end{array}$ & $\begin{array}{l}\text { Optec NGN-3 ambient nephelometer } \\
\text { CMU DAASS } \\
\text { DH Associates M1 CCN }\end{array}$ & $\begin{array}{l}\text { CMU } \\
\text { CMU } \\
\text { CMU }\end{array}$ & $\begin{array}{l}10 \mathrm{~min} \\
1 \mathrm{hr} \\
4 \mathrm{hr}\end{array}$ & $\begin{array}{l}\text { Continuous } \\
\text { Semi-continuous } \\
\text { Semi-Continuous }\end{array}$ & $\begin{array}{l}7 / 16 / 01-6 / 30 / 02 \\
7 / 1-8 / 31 / 01,1 / 1-7 / 1 / 02 \\
9 / 01\end{array}$ \\
\hline $\begin{array}{l}\text { Single Particle Characteristics } \\
\text { Single particle polar organics } \\
\text { Single particle ion composition } \\
\text { Particle morphology }\end{array}$ & $\begin{array}{l}\text { RSMS-III } \\
\text { RSMS-III } \\
\text { Nuclepore filter with SEM }\end{array}$ & $\begin{array}{l}\text { UCD, UD } \\
\text { UCD, UD } \\
\text { RJL }\end{array}$ & $\begin{array}{l}10 \mathrm{~min} \\
10 \mathrm{~min} \\
24 \mathrm{hr}\end{array}$ & $\begin{array}{l}\text { Semi-continuous } \\
\text { Semi-continuous } \\
\text { Daily }\end{array}$ & $\begin{array}{l}9 / 20 / 01-10 / 1 / 02 \\
9 / 20 / 01-10 / 1 / 02 \\
\text { ESP01, ESP02 Intensives }\end{array}$ \\
\hline $\begin{array}{l}\text { Gaseous Species } \\
\text { Light }\left(\mathrm{C}_{2}-\mathrm{C}_{12}\right) \text { hydrocarbons } \\
\text { Light }\left(\mathrm{C}_{2}-\mathrm{C}_{12}\right) \text { hydrocarbons } \\
\text { All hydrocarbons } \\
\mathrm{CO} \\
\mathrm{SO}_{2} \\
\mathrm{NO} \text { and } \mathrm{NO}_{2} \\
\mathrm{O}_{3} \\
\text { Water soluble inorganic gases } \\
\text { Inorganic gases } \\
\text { Inorganic gases } \\
\text { Total soluble peroxides } \\
\text { Hydrogen and organic peroxides }\end{array}$ & $\begin{array}{l}\text { Canister with GC-FID analysis } \\
\text { Canister with GC-FID analysis } \\
\text { UCB GC-FID/MSD } \\
\text { API } 300 \text { infrared absorption } \\
\text { API } 100 \text { A pulsed fluorescence } \\
\text { API 200A chemiluminescence } \\
\text { API 400A UV absorption } \\
\text { Khlystov steam sampler with IC analysis } \\
\text { CMU sampler with IC analysis } \\
\text { CMU sampler with IC analysis } \\
\text { CSU monitor } \\
\text { CSU monitor }\end{array}$ & $\begin{array}{l}\text { CMU } \\
\text { CMU } \\
\text { UCB } \\
\text { CMU } \\
\text { CMU } \\
\text { CMU } \\
\text { CMU } \\
\text { CMU } \\
\text { CMU } \\
\text { CMU } \\
\text { CSU } \\
\text { CSU }\end{array}$ & $\begin{array}{l}24 \mathrm{hr} \\
24 \mathrm{hr} \\
1 \mathrm{hr} \\
10 \mathrm{~min} \\
10 \mathrm{~min} \\
10 \mathrm{~min} \\
10 \mathrm{~min} \\
1-2 \mathrm{hr} \\
24 \mathrm{hr} \\
4-6 \mathrm{hr} \\
1 \mathrm{hr} \\
\quad 1 \mathrm{hr}\end{array}$ & $\begin{array}{l}3^{\text {rd }} \text { day } \\
\text { Daily } \\
\text { Semi-Continuous } \\
\text { Continuous } \\
\text { Continuous } \\
\text { Continuous } \\
\text { Continuous } \\
\text { Semi-Continuous } \\
\text { Daily } \\
5 \text { per day } \\
\text { Continuous } \\
\text { Continuous }\end{array}$ & $\begin{array}{l}\text { 9/1/01-7/31/02* } \\
\text { ESP02 Intensive } \\
1 / 9-2 / 12,7 / 10-8 / 10 / 02 \\
7 / 1 / 01-9 / 1 / 02 \\
7 / 1 / 01-9 / 1 / 02 \\
7 / 1 / 01-9 / 1 / 02 \\
7 / 1 / 01-9 / 1 / 02 \\
7 / 1 / 01-9 / 21 / 02 \\
7 / 1 / 01-7 / 1 / 02 * \\
\text { ESP01 Intensive } \\
7 / 1 / 01-8 / 18 / 02 * \\
\text { ESP01, ESP02 Intensives }\end{array}$ \\
\hline
\end{tabular}


Table 2. Summary of PAQS measurements, continued.

\begin{tabular}{|c|c|c|c|c|c|}
\hline Observable & Method & Group & Resolution & Frequency & Period of Operation \\
\hline $\begin{array}{l}\text { Fog } \\
\text { Fog composition }\end{array}$ & CASCC2 collector with IC, TOC, and $\mathrm{pH}$ analyses & $\mathrm{CSU}$ & Per event & $\begin{array}{ll}8 & \text { events } \\
\text { captured } & \\
\end{array}$ & $7 / 1 / 01-9 / 1 / 02$ \\
\hline $\begin{array}{l}\text { Meteorology } \\
\text { Wind speed/direction } \\
\text { Temperature } \\
\text { Relative humidity } \\
\text { UV solar radiation } \\
\text { Total solar radiation } \\
\text { Precipitation } \\
\text { Pressure }\end{array}$ & $\begin{array}{l}\text { Met One 014A wind vane and cup anemometer } \\
\text { Campbell HMP45C thermistor-based sensor } \\
\text { Campbell HMP45C capacitance thin film sensor } \\
\text { Kipp \& Zonen CUV3 broadband UV radiometer } \\
\text { Kipp \& Zonen CM3 pyranometer } \\
\text { Met One } 370 \text { rain gauge } \\
\text { Campbell CS105 Aneroid barometer }\end{array}$ & $\begin{array}{l}\text { CMU } \\
\text { CMU } \\
\text { CMU } \\
\text { CMU } \\
\text { CMU } \\
\text { CMU } \\
\text { CMU }\end{array}$ & $\begin{array}{l}10 \mathrm{~min} \\
10 \mathrm{~min} \\
10 \mathrm{~min} \\
10 \mathrm{~min} \\
10 \mathrm{~min} \\
10 \mathrm{~min} \\
10 \mathrm{~min}\end{array}$ & $\begin{array}{l}\text { Continuous } \\
\text { Continuous } \\
\text { Continuous } \\
\text { Continuous } \\
\text { Continuous } \\
\text { Continuous } \\
\text { Continuous }\end{array}$ & $\begin{array}{l}7 / 1 / 01-9 / 1 / 02 \\
7 / 1 / 01-9 / 1 / 02 \\
7 / 1 / 01-9 / 1 / 02 \\
7 / 1 / 01-9 / 1 / 02 \\
7 / 1 / 01-9 / 1 / 02 \\
7 / 1 / 01-9 / 1 / 02 \\
7 / 1 / 01-9 / 1 / 02\end{array}$ \\
\hline
\end{tabular}

Groups - ADI: Aerosol Dynamics, Inc.; BYU: Brigham Young University; CMU: Carnegie Mellon University; CSU: Colorado State University; FIU: Florida International University; PSI: Paul Scherrer Institute; RJL: R. J. Lee Instruments, RU: Rutgers University; UC: University of Colorado; UCD: University of California at Davis; UD: University of Delaware; UMD: University of Maryland; UW: University of Wisconsin.

Methods - APS: Aerodynamic Particle Sizer; CASCC2: Caltech Active Strand Cloudwater Collector; DAASS: Dry-Ambient Aerosol Size Spectrometer; FRM: Federal Reference Method; TEOM with SES: Tapered Element Oscillating Microbalance with a Sample Equilibration System; FTIR: Fourier Transform Infra Red spectrometry; GC-FID: Gas Chromatography with Flame Ionization Detection; IC: Ion Chromatography; ICP-MS: Inductively Coupled Plasma Mass Spectrometry; LPI: Low Pressure Impactor; R\&P: Rupprecht and Patashnick; RSMS: Rapid Single-particle Mass Spectrometer; SEAS: Semi-continuous Environmental Aerosol Sampler; SEM: Scanning Electron Microscopy; SMPS: Scanning Mobility Particle Sizer; TOC: Total Organic Carbon; TOT: Total Optical Transmittance.

Period of operation - ESP01 Intensive: July 1, 2001 - August 3, 2001; ESP02 Intensive: January 1, 2002 - January 15, 2002; * Except during periods when samples were collected at a higher time resolution. 
PM Mass Characterization. Integrated samples of $\mathrm{PM}_{2.5}$ mass were collected onto Teflon filters using a Partisol®-Federal Reference Method (FRM) Model 2000 $\mathrm{PM}_{2.5}$ Air Sampler (Rupprecht \& Patashnick, Albany, NY). Integrated samples of $\mathrm{PM}_{2.5}$ and $\mathrm{PM}_{10}$ mass were also collected on Teflon filters using a Dichotomous (Dichot) sampler (Thermo Andersen, Model 241). Both samplers were operated using standard procedures (USEPA, 1998). A Micro-Orifice Uniform Deposit Impactor (MOUDI) (MSP Corp., Model 110) was used to collect time-integrated samples of particles in several size ranges on Teflon substrates using standard procedures (MSP Corp., 1998). This permitted estimates of concentrations of particles with diameters less than $\mathrm{x} \mu \mathrm{m}\left(\mathrm{PM}_{\mathrm{x}}\right)$. The $\mathrm{PM}_{\mathrm{x}}$ estimates included mass concentrations of particles with diameters less than 2.5, 1.8, 1.0, $0.56,0.32,0.18,0.10$, and $0.056 \mu \mathrm{m}$. The FRM, Dichot, and MOUDI filters were analyzed for mass concentration by gravimetric analysis using standard protocols (Code of Federal Regulations, 2002) in a humidity-controlled equilibration chamber in the CMU Air Quality Laboratory.

Semi-continuous $\mathrm{PM}_{2.5}$ mass was measured using a Tapered Element Oscillating Microbalance (TEOM) monitor with a Sample Equilibration System (SES) (Rupprecht and Patashnick, Model 1400A). The Pallflex tapered element was maintained at a collection temperature of $30^{\circ} \mathrm{C}$.

PM Size Distributions. A suite of particle sizing instruments that included a nanoScanning Mobility Particle Sizer (SMPS) (TSI, Inc., Model 3936N25), standard SMPS (TSI, Inc., Model 3936L10), and Aerodynamic Particle Sizer (APS) (TSI, Inc., Model 3320) were used to measure particle number size distributions semi-continuously for particles from 0.003 to $10 \mu \mathrm{m}$ (Stanier, Khlystov et al. 2004). The separate overlapping distributions from these instruments were combined, after inversion, by using the nanoSMPS data up to $0.03 \mu \mathrm{m}$, the SMPS data from 0.03 to $0.6 \mu \mathrm{m}$, and the APS data beginning at $0.6 \mu \mathrm{m}$. Aerodynamic diameters measured by the APS were converted to mobility diameters prior to combining data using an effective density and a least-squares fitting algorithm (Khlystov, Stanier et al. 2004).

$P M$ Inorganic Ions. Integrated samples of $\mathrm{PM}_{10}$ and $\mathrm{PM}_{2.5}$ were collected onto filter packs of PTFE Teflon, nylon, and cellulose-fiber filters using a sampler that consisted of a combination of cyclones, denuders, and filter packs (Takahama, Wittig et al. 2004). Size resolved $\mathrm{PM}_{\mathrm{x}}$ inorganic ion samples were collected onto Teflon substrates using a MOUDI. The filter pack and MOUDI samples were analyzed for nitrate, sulfate, ammonium and chloride by Ion Chromatography (IC) using procedures similar to those described by Jaffrezo et al. (1998).

Semi-continuous measurements of total nitrate $\left(\mathrm{PM}_{2.5}\right.$ nitrate plus $\left.\mathrm{HNO}_{3}\right)$, total sulfate $\left(\mathrm{PM}_{2.5}\right.$ sulfate plus $\left.\mathrm{SO}_{2}\right)$, and total ammonium $\left(\mathrm{PM}_{2.5}\right.$ ammonium plus $\left.\mathrm{NH}_{3}\right)$ were made using a steam sampler (Khlystov, Wyers et al. 1995) with analysis by IC. Total ammonium was measured continuously on-line using the steam sampler and the detector of Slanina et al. (2001). 
Semi-continuous measurements of $\mathrm{PM}_{2.5}$ nitrate and sulfate were made using Rupprecht and Patashnick Integrated Collection and Vaporization Cell (ICVC) instruments (Rupprecht and Patashnick, Models 8400S and 8400N) and processed using the method described by Wittig et al. (2004).

PM Trace Metals. Integrated $\mathrm{PM}_{10}$ and $\mathrm{PM}_{2.5}$ samples were collected on cellulose filters using High-Volume (High Vol) samplers (Thermo Andersen) operated according to standard procedures (Thermo Andersen, 1999; USEPA, 1999). The filters were digested in a combination of nitric acid, hydrofluoric acid, and hydrogen peroxide in a sealed microwave vessel and analyzed according to standard procedures by Inductively Coupled Plasma Mass Spectrometry (ICP-MS) (USEPA, 1991; USEPA, 1994) to determine concentrations of Al, As, Ba, Ca, Cd, Cr, Cu, Fe, Ga, K, Mg, Mn, Mo, Na, Ni, Pb, Rb, Sb, Se, Sr, Ti, V and Zn.

Semi-continuous measurements of 11 metals (Al, As, Cd, Cr, Cu, Fe, Mn, Ni, Pb, Se and $\mathrm{Zn}$ ) were also made using the Semi-continuous Environmental Aerosol Sampler (SEAS) (Kidwell and Ondov 2001) with offline analysis using Graphite Furnace Atomic Absorption (GFAA).

PM Carbonaceous Material. Integrated samples of $\mathrm{PM}_{2.5}$ were collected on bare quartz filters and two backup quartz filters, one downstream of the bare quartz filter and the other in a parallel port behind a Teflon filter (Subramanian, Khlystov et al. 2004). Integrated samples of $\mathrm{PM}_{2.5}$ were also denuded and collected on quartz fiber filters with a carbon impregnated graphite backup filter (Subramanian, Khlystov et al. 2004). Size resolved $\mathrm{PM}_{\mathrm{x}}$ samples were collected on ungreased foil substrates using a MOUDI sampler. The quartz and foil filters were analyzed for organic carbon (OC) and elemental carbon (EC) using the Thermal Optical Transmittance (TOT) method and the NIOSH thermal evolution protocol (NIOSH 1999; Subramanian, Khlystov et al. 2004) in the CMU Air Quality Laboratory. Integrated samples of $\mathrm{PM}_{2.5} \mathrm{OC}$ and $\mathrm{EC}$ were also collected using the PC-BOSS diffusion denuder sampler (Ding, Pang et al. 2002) and analyzed at Brigham Young University.

Additional size-resolved samples were collected on zinc selenide disks using a Hering Low Pressure Impactor (LPI) sampler (Aerosol Dynamics) and analyzed for organic functional groups using Fourier Transform Infrared Spectroscopy (FTIR) at Rutgers University (Blando, Porcja et al. 2001).

Semi-continuous measurements of $\mathrm{PM}_{2.5} \mathrm{OC}$ and $\mathrm{EC}$ were conducted using the Sunset Labs in-situ carbon analyzer (Sunset Laboratory) with a denuder placed upstream of the instrument (Turpin, Cary et al. 1990). Semi-continuous measurements of $\mathrm{PM}_{2.5}$ total carbon were obtained using the Aerosol Dynamics ICVC total carbon instrument which is similar in design to the $\mathrm{R} \& \mathrm{P}$ instruments used to measure $\mathrm{PM}_{2.5}$ nitrate and sulfate semi-continuously (Stolzenburg and Hering 2000). The carbon instrument uses platinum flash strips and a $\mathrm{Li}-\mathrm{Cor} \mathrm{CO}_{2}$ detector downstream of a $650{ }^{\circ} \mathrm{C} \mathrm{MnO}_{2}$ oven that converts $\mathrm{CO}$ to $\mathrm{CO}_{2}$. 
PM Biological Material. Ambient bioaerosols were collected using a High Vol sampler during July 2001, and a low volume sampler for all other periods. The samples were analyzed at Colorado State University using direct epi-fluorescent microscopy and newer molecular biology methods to obtain microbioaerosol concentrations of bacteria, fungi, and their spores.

PM Hygroscopicity. The ability of ambient fine particles to absorb water and grow was measured using the Dry-Ambient Aerosol Size Spectrometer (DAASS) (Stanier, Khlystov et al. 2004). The DAASS measures the aerosol number distribution from 0.003 to $10 \mu \mathrm{m}$ at ambient $\mathrm{RH}$ and also after drying the air containing the aerosols to less than 30 percent RH. The measurements at low RH were obtained by sampling ambient air though a system of Nafion dryers prior to analysis by the system of particle sizing instruments. Aerosol water content is based on the difference between the dried and ambient RH volume distributions.

Cloud Condensation Nuclei $(\mathrm{CCN})$ concentration was measured using a $\mathrm{CCN}$ counter (DH Associates, Model M1). The CCN concentration is directly related to the ability of the particles to become cloud droplets.

Aerosol Light Scattering. Continuous $\mathrm{PM}_{2.5}$ scattering coefficients from $5^{\circ}$ to $175^{\circ}$ at a wavelength of 550 nanometers were measured at ambient conditions using an integrating nephelometer (Optec, Inc., NGN-3) with a $\mathrm{PM}_{2.5}$ cyclone at the inlet.

Aerosol Mass Spectroscopy. The Aerodyne Mass Spectrometer (AMS) was used to measure size resolved aerosol chemical composition semi-continuously (Jayne, Leard et al. 2000; Jimenez, Jayne et al. 2003). The instrument determined the size distributions from 0.050 to $1 \mu \mathrm{m}$ of sulfate, nitrate, ammonium, chloride, total organics, and water chemical components.

Single Particle Mass Spectroscopy. The RSMS-III was used to measure the size and composition of individual particles for nine size ranges from 0.04 to $2 \mu \mathrm{m}$ (Carson, Neubauer et al. 1995; Ge, Wexler et al. 1998). The method is able to identify metals and metal oxides, refractory crustal materials, sulfates and nitrates, aromatic and amine organic compounds, and elemental carbon.

Single Particle SEM Analyses. $\mathrm{PM}_{10}$ and $\mathrm{PM}_{2.5}$ aerosol samples were collected onto Nuclepore filters for offsite analysis by SEM at the laboratories of R.J. Lee, Inc. The SEM analyses focus on the elemental composition and morphology of individual larger particles, including the identification of bioaerosol, soot, and spherical aluminosilicate particles.

Gaseous Species. Integrated VOC samples were collected in 6 liter fused silica-lined canisters (Entech Instruments, Part 29-10621) using a Passive canister sampler inlet (Entech Instruments, Model CS1200) and analyzed in the CMU Air Quality Laboratory using standard GC-FID techniques (Lewis, Stevens et al. 1999). This approach permitted the quantification of over 70 compounds, including some of the standard set of Photochemical Assessment Monitoring Station (PAMS) species. 
Semi-continuous VOC measurements were made by the UC Berkeley group using a fully automated, in-situ, two-channel GC/MS/FID system. Two separate channels (FID and MSD) were configured with different preconditioning systems, preconcentration traps, and chromatography columns, enabling quantification of over 60 compounds, including C3-C6 alkanes, alkenes, alkynes, and aromatic, oxygenated and halogenated compounds.

Continuous measurements of $\mathrm{O}_{3}, \mathrm{NO}$ and $\mathrm{NO}_{2}, \mathrm{CO}$, and $\mathrm{SO}_{2}$ gas concentrations were made using high-sensitivity gas analyzers (Teledyne Advanced Pollution Instrumentation, respective Models 400A, 200A, 300 and 100A). Continuous measurements of organic peroxide, hydrogen peroxide, and total soluble peroxide were made using the CSU monitor based on the method of Lazrus et al. (1986).

Fog. Fog composition was measured using a compact version of the Caltech Active Strand Cloudwater Collector known as the CASCC2 (Demoz et al., 1996). Eight fog events long enough in duration to collect a liquid sample were observed during the fourteen month sampling period. Fog samples were analyzed onsite for $\mathrm{pH}$. Aliquots of the collected fog were prepared and analyzed offsite for major anions and cations using IC and total organic carbon (TOC) and dissolved organic carbon (DOC) using a TOC analyzer (Shimadzu, Model 5000-A).

Meteorology. Precipitation, temperature, relative humidity, wind speed and direction, and UV intensity were measured using standard methods.

\section{Activity 3. Source Characterization}

The purpose of this activity is to develop and evaluate state-of-the-art techniques for source characterization and to update emission data for important source categories around Pittsburgh. Updated source profiles were developed through a combination of source testing, fenceline measurements, and analysis of highly time resolved data collected at the central site. Source profiles were developed using the same analytical techniques as the ambient measurements to ensure that the data are compatible for source-apportionment calculations. Table 3 summarizes the source characterization activities. 
Table 3. Summary of source characterization activities

\begin{tabular}{lcc}
\hline Source & Method & Date \\
\hline Metallurgical coke & Fence-line measurements & $8 / 02-9 / 02$ \\
production & & \\
Motor vehicle emissions & Tunnel study & $10 / 02-12 / 02 \& 7 / 04$ \\
$\begin{array}{l}\text { Coal combustion } \\
\text { Paved road dust }\end{array}$ & Dilution sampling & $9 / 03 \& 11 / 03$ \\
& Collection and resuspension & Summer 03 \\
Vegetative detritus & in laboratory & Sollection and resuspension \\
& in laboratory & \\
\hline
\end{tabular}

\section{Characterization of coke production emissions}

A fenceline study was conducted to develop an integrated emission profile for a metallurgical coke production facility. A fenceline approach was employed given the significant fugitive emissions and large number of individual emission points in a coke facility. The coking facility is the largest point source of fine particle pollution in Allegheny County. It consists of 12 batteries with more than 850 coking ovens which annually convert more than 6 million tons of coal to more than 4 million tons of coke using slot oven by-product batteries. This facility, like essentially all modern coke plants, is based on the by-product recovery process, in which a portion of the volatiles driven off during the coking process are collected and burned on site for energy or converted into chemicals. At this plant, the byproduct recovery operations include a tar refinery that converts crude coke oven tars into liquid pitch and other liquid products. The plant uses carbonate scrubbing to remove hydrogen sulfide from the coke oven gas, and an on-site Claus plant converts the hydrogen sulfide to molten sulfur. Finally the ammonia is separated from the coke oven gas and processed into anhydrous ammonia. The balance of the coke oven gas is used for energy, either on site in boilers at or at other nearby facilities. The plant operates essentially at full capacity throughout the year. $\mathrm{NO}_{\mathrm{x}}$ emission rates from boilers burning coke oven gas suggest normal plant operations throughout the measurement period (USEPA 2003).

There are numerous points in the coking process at which air pollutants are emitted to the atmosphere. Significant emissions are associated with the coke ovens. The coke ovens are sealed during most of the coking process, but leaks are not uncommon. This facility is required to visually track and report door, lid, and off-take leaks. In 2002, the average percentage of the doors with leaks was $1.3 \%$ and $1.4 \%$ during the study period. The percentage of off-take leaks was $0.6 \%$ for 2002 and $0.7 \%$ for the study period. Reported percentage of lids leaking is low, $0.03 \%$. This information is used to estimate annual emissions from the facility. During pushing, both doors are removed from the coke oven and emissions are difficult to control. This facility has emission control devices installed on the coke side of the ovens. Traveling canopy hood in conjunction with a bag house are used to control emissions during pushing on eleven of the twelve batteries. The twelfth battery uses a coke-side shed and bag house to help control pushing emissions. Sheds are viewed as a much more effective control technology than 
traveling hoods. Though coke production is a batch process, the large number of ovens $(>850)$ dictate that all the steps in the coking process (such as pushing of an oven) are happening every few minutes.

The sampling site was located at the edge of a plateau across a river from the coke plant (Figures 3 and 4). The facility is not in a valley, but rather at the edge of a sharp change in elevation. The plant is upwind of the sampling site for wind directions between $175^{\circ}$ and $300^{\circ}$ magnetic (Figure 3). The transit time of emission between the plant and the sampling site is on the order of 5 minutes under typical sampling conditions. The sampling site is surrounded by scrub brush and is approximately $1 / 2 \mathrm{~km}$ from the public access road.

Table 4 lists the pollutants measured at the fenceline site in addition to instrumentation, measurement frequency and period of operation. The instrumentation and analytical procedures used for the coke facility fenceline study were identical to those used for the Pittsburgh Supersite to ensure compatibility of the results for source apportionment analysis of ambient data.

Background concentrations for the fenceline study were measured at the central Pittsburgh Supersite and several other sites in Allegheny County that are not strongly impacted by local sources. Although these sites are often more than $10 \mathrm{~km}$ away from the fenceline site, they provide suitable background data because of the strong regional character of fine particle concentrations in the Pittsburgh region. Tang et al. (2004) found little variation in daily samples collected across the Pittsburgh region further supporting the use of regional concentrations as an estimate of background levels. 


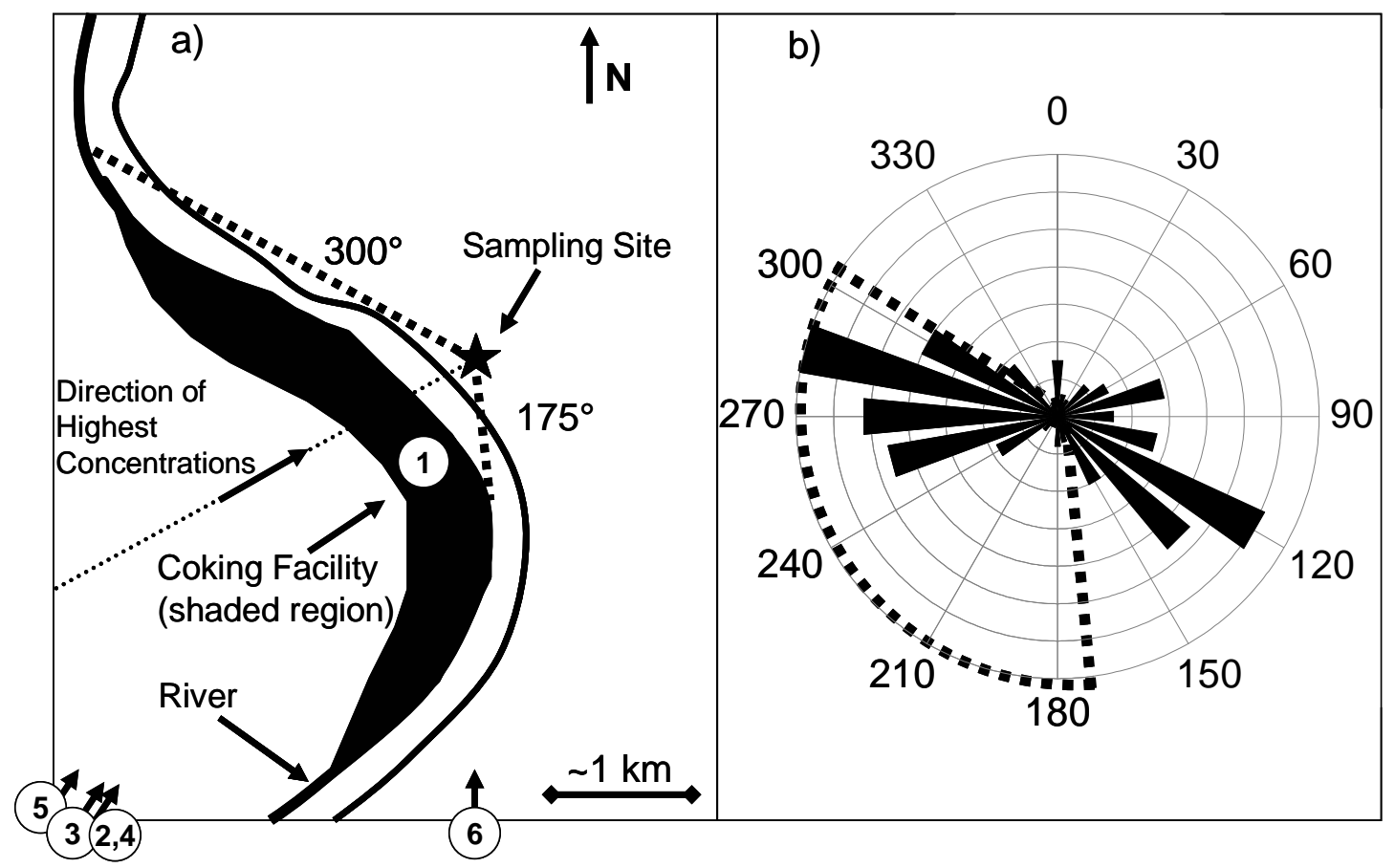

Figure 3. a) Top view of coking facility and sampling site; and b) wind rose for entire study period. The coking facility influences the sample concentrations for wind directions from $175^{\circ}$ to $300^{\circ}$. The coke batteries and other major operations are located between $240^{\circ}$ and $300^{\circ}$.

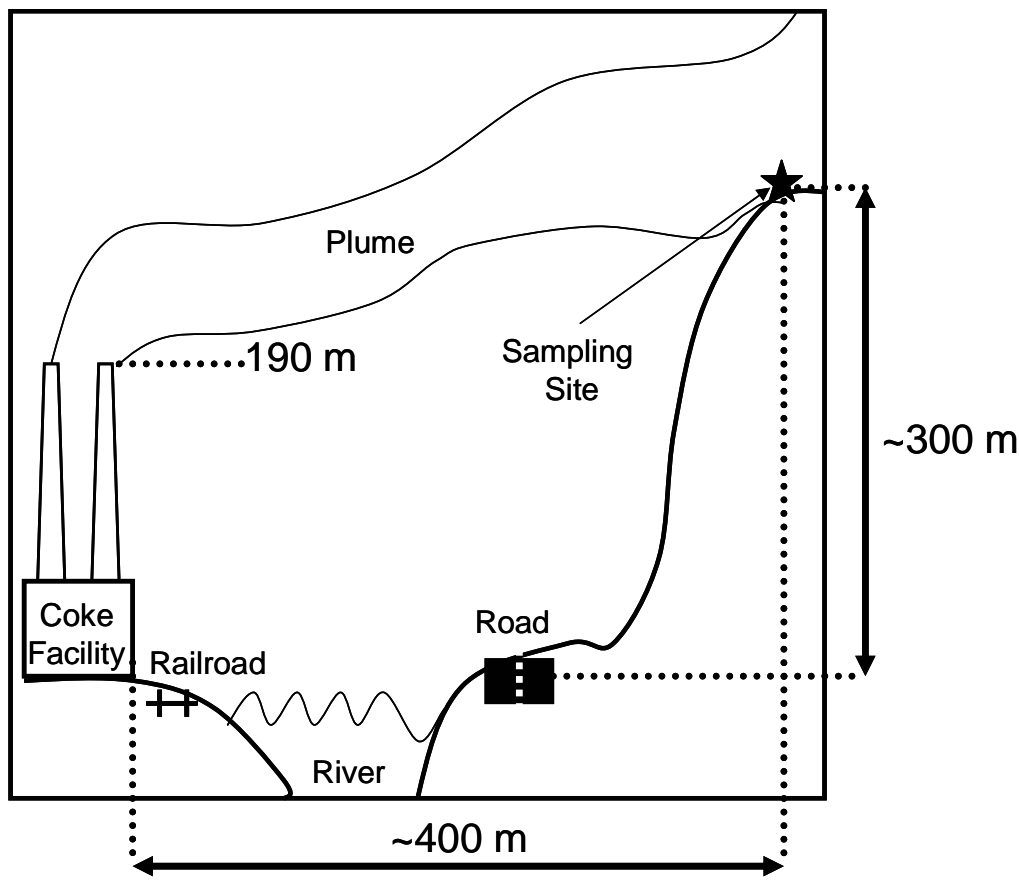

Figure 4. Side view of coking facility and sampling site. 
Table 4: Monitoring Instrumentation used at coke facility fenceline sampling site

\begin{tabular}{|c|c|c|c|}
\hline Instrument & Parameter & Frequency & Period of Operation \\
\hline \multicolumn{4}{|l|}{ Semi-continuous measurements: } \\
\hline API Model 100A SO $\mathrm{S}_{2}$ Analyzer & $\mathrm{SO}_{2}$ & $5 \mathrm{~min}$ & $8 / 15 / 02-9 / 4 / 02$ \\
\hline API Model 200A NO $\mathrm{A}_{\mathrm{x}}$ Analyzer & $\mathrm{NO}_{\mathrm{x}}, \mathrm{NO}$ & $5 \mathrm{~min}$ & $8 / 15 / 02-9 / 4 / 02$ \\
\hline R\&P Model 1400a TEOM® & $\mathrm{PM}_{2.5}$ Mass & $10 \mathrm{~min}$ & $8 / 15 / 02-9 / 5 / 02$ \\
\hline R\&P Model 1400a TEOM® & $\mathrm{PM}_{10}$ Mass & $1 \mathrm{hr}$ & 8/15/02-9/5/02 \\
\hline SMPS, APS & $\begin{array}{c}\text { Number size distribution from } \\
0.013 \text { to } 20 \mu \mathrm{m}\end{array}$ & $7 \mathrm{~min}$ & $\begin{array}{c}8 / 15 / 02-8 / 24 / 02 \\
8 / 26 / 04-9 / 5 / 02\end{array}$ \\
\hline $\begin{array}{l}\text { Semi-continuous Element in } \\
\text { Aerosol Sampler (SEAS) }\end{array}$ & $\begin{array}{c}\mathrm{Al}, \mathrm{As}, \mathrm{Cd}, \mathrm{Cr}, \mathrm{Cu}, \mathrm{Ni} \\
\mathrm{Mn}, \mathrm{Fe}, \mathrm{Pb}, \mathrm{Se}, \mathrm{Zn}\end{array}$ & $30 \mathrm{~min}$ & $8 / 20 / 02-8 / 22 / 02$ \\
\hline $\begin{array}{l}\text { Sunset Laboratory Carbon } \\
\text { Analyzer (Model 2) }\end{array}$ & $\mathrm{OC}, \mathrm{EC}$ & $65 / 90 \mathrm{~min}$ & $8 / 17 / 02-8 / 26 / 02$ \\
\hline Meteorological Tower & $\begin{array}{l}\text { Wind speed and direction } \\
\text { at } 10 \text { and } 33 \mathrm{~m}\end{array}$ & $15 \mathrm{~min}$ & $8 / 15 / 02-9 / 5 / 02$ \\
\hline \multicolumn{4}{|l|}{ Integrated measurements: } \\
\hline $\begin{array}{l}\text { Thermo Anderson High Volume } \\
\mathrm{PM}_{2.5} \text { Sampler }\end{array}$ & $\begin{array}{c}\mathrm{Al}, \mathrm{As}, \mathrm{Ba}, \mathrm{Ca}, \mathrm{Cd}, \mathrm{Cr}, \mathrm{Cu}, \mathrm{Fe} \\
\mathrm{Ga}, \mathrm{K}, \mathrm{Mg}, \mathrm{Mn}, \mathrm{Mo}, \mathrm{Na}, \mathrm{Ni} \\
\mathrm{Pb}, \mathrm{Rb}, \mathrm{Sb}, \mathrm{Se}, \mathrm{Sr}, \mathrm{Ti}, \mathrm{V}, \mathrm{Zn}\end{array}$ & $\begin{array}{c}\text { 7, 6-hr } \\
\text { samples }\end{array}$ & $8 / 22 / 02-9 / 5 / 02$ \\
\hline $\begin{array}{l}\text { TE } 1000 \mathrm{PM}_{2.5} \text { quartz/PUF } \\
\text { Sampler }\end{array}$ & 113 Species & $\begin{array}{l}\text { 5, 6-hr } \\
\text { samples }\end{array}$ & $8 / 21 / 02-9 / 4 / 02$ \\
\hline $\begin{array}{l}\text { CMU Inorganic Sampler, a } \\
\text { denuder/filter-based speciation }\end{array}$ & $\begin{array}{c}\mathrm{Na}^{+}, \mathrm{NH}_{4}^{+}, \mathrm{K}^{+}, \mathrm{Mg}^{2+}, \mathrm{Ca}^{2+}, \mathrm{Cl}^{-} \\
, \mathrm{SO}_{4}^{2-}, \mathrm{NO}_{3}^{-}, \mathrm{C}_{2} \mathrm{H}_{2} \mathrm{O}_{4}, \mathrm{NH}_{3}, \\
\mathrm{HCl}, \mathrm{HNO}_{3}\end{array}$ & $\begin{array}{l}13,6-\mathrm{hr} \\
\text { samples }\end{array}$ & $8 / 21 / 02-9 / 4 / 02$ \\
\hline
\end{tabular}

\section{Characterization of motor vehicle emissions}

A tunnel study was performed to characterize in-use motor vehicle emissions in the Pittsburgh Region. The experiments were conducted in the two-lane west-bound (inbound) bore of the Squirrel Hill Tunnel on Interstate 376 in Pittsburgh, Pennsylvania. The tunnel is $1.3 \mathrm{~km}$ long and has a $2.5 \%$ up-grade in the westerly direction. The tunnel is ventilated mechanically through ducts situated in the tunnel ceiling and by the effects of traffic motion. The sampling location was roughly 50 meters from the tunnel exit; at this position the mechanical ventilation system was under positive pressure and thus pushing fresh air into the tunnel. The sample inlets were inserted through an otherwise sealed ventilation slit in the tunnel ceiling; several slits up- and down-stream of the sample location were also blocked. A schematic of the tunnel is shown in Figure 1.

The majority of the measurements were made during a two-week period in November of 2002. A more limited study was also performed in the summer of 2004 to examine seasonal dependence of organic aerosol emissions.

Traffic count and speed data were gathered via a RTMS (Remote Traffic Microwave Sensor) operated for the Pennsylvania Department of Transportation (PennDOT). Fleet composition was determined from PennDOT video recorded at the entrance and exit of the tunnel's west-bound tube. Vehicles were manually classified as light-duty (LD) and heavy-duty (HD) based on visual inspection of video. Heavy-duty vehicles include 
tractor-trucks, large single-unit trucks and buses. City buses did not regularly pass through the tunnel. While this approach separates vehicles by size and not fuel type, the assumption that most large vehicles are diesel-powered and smaller ones gasolinepowered is reasonable. Less than $5 \%$ of heavy-duty vehicles with more than three axles in the U.S. fleet are gasoline-powered (VIUS 2002). The portion of light-duty vehicles powered by diesel fuel is similarly small; around $1 \%$ of vehicles in US households were diesel powered (EIA 1994) and at most 15\% of light-duty trucks are diesel powered (VIUS 2002). Therefore, we assume that all of the heavy-duty vehicles are diesels (HDDV) and light-duty vehicles (LDV) are gasoline powered.

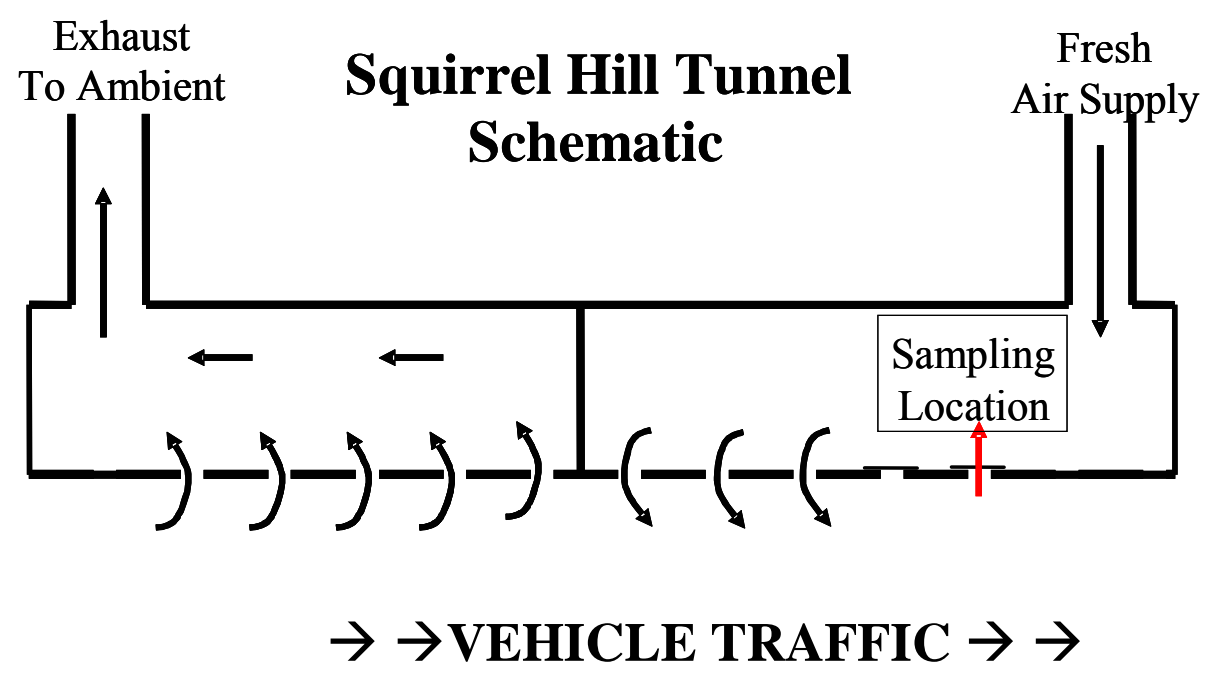

Figure 5. Schematic of the Squirrel Hill Tunnel sampling location. The tunnel is $1.3 \mathrm{~km}$ long and has a $2.5 \%$ up-grade. Arrows in upper portion of schematic represent air flow in mechanical ventilation system.

A suite of continuous instruments were operated during the November 2002 period to measure $\mathrm{CO}, \mathrm{CO}_{2}, \mathrm{NO}, \mathrm{NO}_{2}$ and $\mathrm{PM}_{2.5}$ mass. Measurement and instrument details are shown in Table 5. The instrumentation and analytical procedures used for the tunnel study were identical to those used for the Pittsburgh Supersite to ensure compatibility of the results for source apportionment analysis of ambient data. 
Table 5: Instrumentation used in Tunnel Study

\begin{tabular}{lccc}
\hline Observable & Method & Instrument & $\begin{array}{c}\text { Time } \\
\text { Resolution }\end{array}$ \\
\hline $\mathrm{CO}_{2}$ & Non-dispersive infrared & Li-Cor LI-820 & $1 \mathrm{~min}$. \\
$\mathrm{NOx} / \mathrm{NO}$ & Chemiluminensce & API 200A & $1 \mathrm{~min}$. \\
$\mathrm{PM}_{2.5}$ & TEOM with SES & R\&P 1400a & $5 \mathrm{~min}$. \\
$\mathrm{CO}$ & Chemiluminensce & API 300A & $1 \mathrm{~min}$. \\
Organic and Elemental & Quartz / Quartz-Behind Teflon & Sunset Laboratory TOT & $2 / 4 / 6.5$ hours \\
Carbon & & & $15 \mathrm{~min}$ \\
Aerosol Size Distribution & DAASS & TSI SMPS / nano- & \\
& & SMPS / APS & $2 / 4 / 6.5$ hours \\
Size resolved OC/EC/mass & MOUDI with foil filters & Sunset Laboratory & $2 / 4 / 6.5$ hours \\
Inorganic ions & Ion Chromatography & Dionex DX-600/120 & $2 / 8 / 12$ hours \\
Metals & ICP-MS & Agilent 4500 ICP-MS & $6.5 / 4 / 6.5$ hours \\
Size resolved metals & MOUDI with Teflon filters & Agilent 4500 ICP-MS & $2 / 4 / 6$ \\
\hline
\end{tabular}

\section{Characterization of coal combustion emissions}

Measurements were made on two commercial coal boilers to develop new emission profiles and to investigate changes in speciation of mercury emissions in the power plant plumes. The first boiler (Boiler \#1) was a $817 \mathrm{MW}$ unit firing a blend of $60 \%$ Powder River Basin sub-bituminous coal and 40\% mid-sulfur eastern bituminous coal. The boiler is wall-fired, with low-NOx burners and has an electrostatic precipitator with SO3 and NH4 conditioning. A selective catalytic reduction (SCR) unit is also used for NOx control. The second boiler (Boiler \#2) was a $500 \mathrm{MW}$ unit firing a blend of $87 \%$ Powder River Basin sub-bituminous coal and 13\% mid-sulfur eastern bituminous coal. The boiler is wall fired, with low-NOx burners and has an electrostatic precipitator for particle control. Experiments were performed while both units were operating at high load. One experiment was performed on Boiler \#2 at medium load.

Measurements were made on both boilers with a dynamic dilution sampler (Figure 2). The system is designed to simulate atmospheric dilution. Details on the design and operation of the system can be found in Lipsky et al. (2005). The sampler is constructed out of stainless steel; for the mercury testing all of the wetted components in contact with sample were coated with a $1200 \AA$ thick layer of Silcosteel, applied by Restek Performance Coatings. In addition to the main dilution tunnel, a residence time chamber (RTC, not shown in Figure 2) was brought inline during some testing periods. With the RTC inline, the overall aging time of the diluted exhaust can be varied between 2 seconds and 5 minutes. 


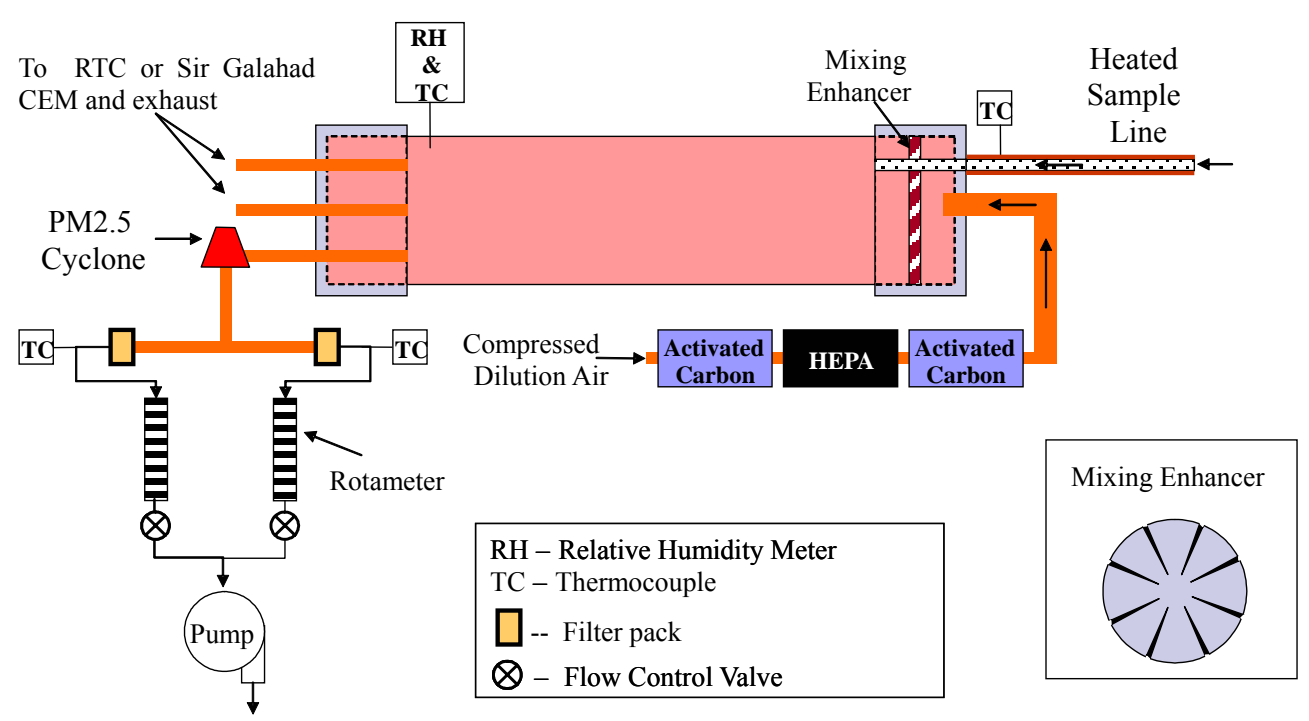

Figure 6. Schematic of CMU Dynamic Dilution Sampler (Dilution Tunnel)

The experiments performed on Boiler \#1 focused on measuring mercury emissions with more limited particle characterization using the instrumentation listed in Table 6. Mercury sampling and speciation were via Mercury Continuous Emission Monitors (CEMs). A PS Analytical Sir Galahad CEM measured gas-phase total and elemental mercury concentrations inside the dilution sampler. These concentrations ranged from 0 to $250 \mathrm{ng} / \mathrm{m}^{3}$. As operated, the Sir Galahad collected sample for 5 minutes before analysis; analysis required 3 to 4 minutes and alternated between elemental and total mercury analyses. A Tekran Mercury Vapor Analyzer was used to sample the un-diluted flue gas. The Tekran instrument measured concentrations in the range of 0 to $10 \mu \mathrm{g} / \mathrm{m} 3$ and alternately measured elemental mercury for 10 minutes and total mercury for 45 minutes with data points recorded every 2 minutes. The mercury instruments were operated by the University of North Dakota Energy and Environmental Research Center.

The experiments performed on Boiler \#2 focused on measuring fine particle emissions using the instrumentation listed in Table 7. The instrumentation and analytical procedures used for the tunnel study were identical to those used for the Pittsburgh Supersite to ensure compatibility of the results for source apportionment analysis of ambient data. 
Table 6: Instrumentation used for measurements of Boiler \#1

\begin{tabular}{|c|c|c|c|}
\hline Observable & Method & Instrument & $\begin{array}{c}\text { Time resolution / } \\
\text { Number of samples }\end{array}$ \\
\hline \multicolumn{4}{|l|}{ Flue gas measurements } \\
\hline $\mathrm{Hg}^{0}$, Total $\mathrm{Hg}$ & & $\begin{array}{c}\text { Tekran Mercury Vapor } \\
\text { Analyzer }\end{array}$ & $2 \min$ \\
\hline $\mathrm{CO}, \mathrm{CO} 2, \mathrm{HC}$ & Non-dispersive infrared & Nova 4765 & $2 \mathrm{~min}$ \\
\hline $\mathrm{O} 2, \mathrm{NOx} / \mathrm{NO}$ & Electrochemical & Nova 4765 & $2 \min$ \\
\hline \multicolumn{4}{|l|}{ Diluted measurements } \\
\hline Diluted $\mathrm{CO}_{2}$ & Non-dispersive infrared & Li-Cor LI-820 & $2 \min$ \\
\hline $\mathrm{Hg}^{0}$, Total Hg & & $\begin{array}{l}\text { PS Analytical Sir } \\
\text { Galahad CEM }\end{array}$ & $5 \mathrm{~min}$ \\
\hline $\mathrm{PM}_{2.5}$ filter samples & $\begin{array}{l}\text { Quartz, Teflon, } \\
\text { polycarbonate filters }\end{array}$ & $\begin{array}{l}\text { URG cyclones and } \\
\text { filter packs }\end{array}$ & 2 sets of samples \\
\hline $\begin{array}{l}\text { Organic and Elemental } \\
\text { Carbon }\end{array}$ & $\begin{array}{c}\text { Quartz / Quartz-Behind } \\
\text { Teflon }\end{array}$ & $\begin{array}{c}\text { Sunset Laboratory } \\
\text { TOT }\end{array}$ & 1 sample \\
\hline $\mathrm{PM}_{25}$ Mass & Gravimetric analysis & Mettler microbalance & 1 sample \\
\hline $\begin{array}{l}\text { Organic and elemental } \\
\text { carbon }\end{array}$ & Thermal-optical analysis & Sunset Laboratory & 1 sample \\
\hline Inorganic ions & Ion Chromatography & Dionex DX-600/120 & 1 sample \\
\hline Metals & ICP-MS & Agilent 4500 ICP-MS & 1 sample \\
\hline Spherical Alumina & SEM & RJ Lee PSEM & 2 samples \\
\hline Silicate & & & \\
\hline
\end{tabular}

Table 7: Instrumentation used for measurements of Boiler \#2

\begin{tabular}{|c|c|c|c|}
\hline Observable & Method & Instrument & $\begin{array}{l}\text { Time resolution / } \\
\text { Number of samples }\end{array}$ \\
\hline \multicolumn{4}{|l|}{ Flue gas measurements } \\
\hline $\mathrm{CO}, \mathrm{CO} 2, \mathrm{HC}$ & Non-dispersive infrared & Nova 4765 & $2 \mathrm{~min}$ \\
\hline $\mathrm{O} 2, \mathrm{NOx} / \mathrm{NO}$ & Electrochemical & Nova 4765 & $2 \min$ \\
\hline \multicolumn{4}{|l|}{ Diluted measurements } \\
\hline Diluted $\mathrm{CO}_{2}$ & Non-dispersive infrared & Li-Cor LI-820 & $2 \min$ \\
\hline Aerosol Size Distribution & & TSI SMPS & $5 \mathrm{~min}$ \\
\hline $\mathrm{PM}_{2.5}$ filter samples & $\begin{array}{l}\text { Quartz, Teflon, } \\
\text { polycarbonate filters }\end{array}$ & $\begin{array}{l}\text { URG cyclones and } \\
\text { filter packs }\end{array}$ & 7 sets of samples \\
\hline $\begin{array}{l}\text { Organic and Elemental } \\
\text { Carbon }\end{array}$ & $\begin{array}{c}\text { Quartz / Quartz-Behind } \\
\text { Teflon }\end{array}$ & $\begin{array}{c}\text { Sunset Laboratory } \\
\text { TOT }\end{array}$ & 7 samples \\
\hline $\mathrm{PM}_{2.5}$ Mass & Gravimetric analysis & Mettler microbalance & 7 samples \\
\hline $\begin{array}{l}\text { Organic and elemental } \\
\text { carbon }\end{array}$ & Thermal-optical analysis & Sunset Laboratory & 7 samples \\
\hline Inorganic ions & Ion Chromatography & Dionex DX-600/120 & 7 samples \\
\hline Metals & ICP-MS & Agilent 4500 ICP-MS & 7 samples \\
\hline $\begin{array}{l}\text { Spherical Alumina } \\
\text { Silicate }\end{array}$ & SEM & RJ Lee PSEM & 5 samples \\
\hline
\end{tabular}




\section{Characterization of paved road dust}

To develop a chemical fingerprint for road dust in the Pittsburgh region, paved road dust samples were collected from 5 rural and 6 urban/suburban sites in the summer of 2003 using a vacuum sampler constructed out of Teflon and stainless steel. Samples were dried in an oven at $100{ }^{\circ} \mathrm{C}$ and then passed through a $37 \mu \mathrm{m}$ sieve to remove large particles. The samples were combined to create a composite urban and rural dust sample.

The sieved road dust was then re-suspended in a glass vessel using HEPA and activated carbon cleaned air. The filter samples were analyzed for: $\mathrm{PM}_{2.5}$ mass via gravimetric analysis, OC \& EC: Thermal-Optical with Sunset Instrument and NIOSH protocol, elemental composition (acid digestion followed by ICP-MS), and organic speciation followed by GC-MS. Multiple samples were collected and analyzed for each composite dust sample to verify measurement repeatability.

\section{Characterization of vegetative detritus}

Due to wind-induced mechanical shear and the rubbing motion of leaves against each other, an unspecified amount of fine particulate epicuticular wax protrusions and leaf deposits are released to the atmosphere that have been identified in urban and rural ambient $\mathrm{PM}_{2.5}$ samples. As part of PAQS, we have developed a source profile for organic constituents associated with fine particulate leaf surface abrasion products.

Green leaves from 11 common tree species that are characteristic for the Pittsburgh area were harvested during September of 2003 and composited according to the tree distribution for that area. The distribution of leaves is given in Table 8. This tree distribution is generally representative of forests that are described as a "Mixed Oak Forest" or "Appalachian Oak Forest". The leaves were collected from Schenley Park, a park with 500 acres that is situated in Pittsburgh. The park is influenced by human activities, including picnic areas equipped with barbeque places.

To simulate a windblown generation process that dislodges waxy leaf surface protrusions, the leaf composite was placed in a clean Teflon bag and mechanically agitated while passing purified air through the bag, similar to the procedure used for the Los Angeles leaf samples. The fine waxy protrusions $(\mathrm{dp} \leq 2.5 \mu \mathrm{m})$ shed from the leaf surfaces were extracted and analyzed using gas chromatography/mass spectrometry. Individual organic compounds that were identified included: n-Alkanes, iso- and anteisoalkanes, n-alkanoic acids, n-alkenoic acids, alkanals, n-alkanones, steroids, pentacyclic triterpenoids, phenolic type compounds, and others. Trace amounts of PAH were identified and quantified as well. 
Table 8. Distribution of leaves in composite sample.

\begin{tabular}{llc}
\hline \multicolumn{1}{c}{ Pittsburgh Vegetation Sample Composite } & $\begin{array}{c}\text { Fraction of Mass in } \\
\text { Composite (\%) }\end{array}$ \\
\cline { 1 - 2 } Ailanthus & Simaroubaceae & 7.3 \\
White Ash & Fraxinus Americana & 6.3 \\
Ohio Buckeye & Aesculus Glabra & 1.0 \\
Wild Red Cherry & Prunus Pensylvanica & 2.2 \\
Black Oak & Quercus Velutina & 13.4 \\
American Elm & Ulmus Americana & 5.0 \\
Scarlet Hawthorne & Crataegus laevigata & 4.0 \\
Norway Maple & Acer Platanoides & 51.4 \\
Common Locust & Gleditsia triacanthos & 5.4 \\
Pin Oak & Quercus Palustris & 1.5 \\
Slippery Elm & Ulmus Rubra & 2.5 \\
\hline
\end{tabular}

\section{Evaluation of source characterization techniques}

A new, more portable dilution sampler has been designed, evaluated and used to characterize emissions from a wood stove, diesel engine, and coal boiler. The primary design objective for the new dilution sampler was to create a system that reproduces the results of the widely cited Caltech design but that is simpler and more portable. A schematic of the sampler is shown in Figure 6. Similar to other dilution samplers, exhaust is sampled isokinetically into a heated-inlet line that is maintained at a temperature slightly $\left(\sim 10^{\circ} \mathrm{C}\right)$ above the exhaust temperature to minimize thermophoretic losses. A stack-sampling cyclone can be installed on the inlet line to prevent large particles from entering the system. The sampled exhaust is then rapidly mixed by turbulence with filtered (HEPA and activated carbon) dilution air inside of a 0.9-m-long, 0.15 -m-diameter stainless dilution tunnel. The total flow rate through the dilution sampler is $174 \mathrm{lpm}$, and the dilution ratio is varied by changing the relative amount of exhaust and dilution air flow. Filter trains and other aerosol characterization instrumentation are connected to ports at the end of the dilution tunnel. The sampler is constructed out of stainless steel to minimize contamination.

To promote mixing, the dilution air is passed through a fan shaped mixing enhancer immediately upstream of where the dilution air and exhaust sample gas streams meet. The mixing enhancer breaks up the dilution air flow causing rapid and thorough mixing within the dilution tunnel. Mixing experiments were conducted using $\mathrm{CO}_{2}$ as a tracer to verify that the exhaust and dilution air are completely mixed at the end of the smaller tunnel across the entire operational range. The mixing rate in the smaller dilution tunnel was not characterized, only verified that the conditions were well mixed at the sampling ports. With the addition of a mixing enhancer, we expect more rapid mixing in the new dilution tunnel compared to the Caltech design.

Two changes have been made in the new design compared to the original Caltech design to reduce particle losses. First we measure the $\mathrm{CO}_{2}$ mixing ratios of the undiluted and diluted exhaust to determine dilution ratio. This eliminates the need for a venturi 
flow meter on the inlet line, which has been shown to be a significant cause of particle losses (Hildemann, Cass et al. 1989). Second, we changed the geometry at which the exhaust sample and dilution air are introduced inside the dilution tunnel. As shown in Figure 1, the exhaust sample passes through the end of the dilution tunnel flowing in parallel with the dilution air. In the Caltech design, the exhaust sample is introduced through the side of the tunnel, perpendicular to the dilution air flow. Visual inspection of the inside of the large tunnel indicates that losses occur on the opposite side of the dilution tunnel from the exhaust port and just downstream of the initial mixing point. The new flow orientation appears to reduce these losses.

A major design change was to eliminate the residence time tank. In the Caltech design and its variants, diluted exhaust flows through a large residence time tank before filter sampling or aerosol characterization. Eliminating the residence time tank significantly reduces the size and complexity of the new dilution sampler. The purpose of the residence time tank is to provide adequate time after dilution for microphysical processes such as condensation. As part of the evaluation of the new design, experiments were performed to assess the effects of additional residence time on the measured fine particle concentrations.

Inter-comparison experiments were performed using a diesel engine and wood stove to evaluate the comparability of the new design with a sampler based on the Caltech design. These experiments involved simultaneous operation of multiple dilution samplers from the same source, either a small single cylinder diesel engine or a wood stove. Filter based measurements included $\mathrm{PM}_{2.5}$ mass, organic carbon and elemental carbon emissions. Particle size distributions in the range from 10 to $480 \mathrm{~nm}$ were measured using a scanning mobility particle sizer.

Experiments were also conducted to examine the effects of dilution on fine particle mass emissions from a diesel engine and wood stove. Filter measurements were made simultaneously using three dilution sampling systems operating at dilution ratios ranging from 20:1 to 510:1. Denuders and backup filters were used to quantify organic sampling artifacts.

\section{Activity 4. Source Apportionment}

The purpose of this activity is to quantify the contribution of different sources to the fine $\mathrm{PM}_{2.5}$ levels in Pittsburgh. A variety of different existing computational tools as well as new approaches were developed for this project.

\section{Apportionment of primary organic aerosol}

The Chemical Mass Balance model was used to estimate the contribution of gasoline and diesel vehicles, wood combustion, meat cooking, and other primary sources to ambient organic carbon. The model was applied to the large data set of organic aerosol composition collected by the ambient monitoring portion of the project. CMB analysis is 
performed using data for individual days; seasonal and annual averages are then calculated from the $\mathrm{CMB}$ results for the individual days.

The selection of source profiles and species included in CMB are important considerations. Key factors are the inclusion of all dominant sources of each species in the model, and photo-chemical stability of the species in the atmosphere. Significant previous work has been performed to determine which molecular markers are appropriate for CMB analysis. Therefore, the compounds and source classes included in the model are largely the same as those used in previous applications of CMB with molecular markers (Schauer, Rogge et al. 1996; Schauer and Cass 2000; Schauer, Fraser et al. 2002; Zheng, Cass et al. 2002; Fraser, Yue et al. 2003).

Source profiles for the CMB analysis are primarily taken from the literature. The CMB calculations included source profiles for nine different source classes: gasoline vehicles, diesel vehicles, road dust, coke production, vegetative detritus, cigarette smoke, meat cooking, and biomass combustion. The road dust, coke production, and vegetative detritus source profiles have been developed specifically for the Pittsburgh region (Subramanian, Donahue et al. 2005). The cigarette profile is from Rogge et al. (1994). Extensive analysis was done to examine the sensitivity of the model predictions to the meat-cooking source profiles, gasoline and diesel vehicle source profiles, and wood combustion source profiles. These are thought to be the dominant source classes for primary organic aerosol in Pittsburgh.

Source profiles were selected based on comparisons with the ambient concentrations of different molecular markers using scatter and ratio-ratio plots. These plots allow a visual comparison of source profiles and ambient concentrations. Ratio-ratio plots are constructed using three species; one compound is selected as a reference to normalize the concentrations of the other two compounds (target species). The best reference compounds are relatively abundant, stable, and specific to the sources plotted. Source profiles appear as points on ratio-ratio plots and linear mixing lines can be drawn to visualize the effects of mixing of emissions from different sources. Although the three species in a ratio-ratio plot represent only a small fraction of the compounds included in $\mathrm{CMB}$, molecular markers are often highly source specific. Therefore, through the judicious groupings of compounds, ratio-ratio plots can often provide significant insight into the suitability of source profiles. More details on the construction, interpretation, and mathematics of ratio-ratio plots are provided in Robinson et al. $(2005 ; 2005)$.

Twenty-two species are included in the CMB model: n-heptacosane, n-nonacosane, n-hentriacontane and n-tritriacontane; iso-hentriacontane, anteiso-dotriacontane; 9hexadecenoic (palmitoleic) acid, cholesterol; syringaldehyde, sum of resin acids, acetosyringone, levoglucosan; 17a(H),21b(H)-29-norhopane, 17a(H),21b(H)-hopane, 22R+S-17a(H),21b(H)-30-homohopane, $\quad 22 \mathrm{R}+\mathrm{S}, 17 \mathrm{a}(\mathrm{H}), 21 \mathrm{~b}(\mathrm{H})-30$-bishomohopane; benzo[e]pyrene, indeno[1,2,3-cd]pyrene, benzo[g,h,i]perylene, coronene; iron, and elemental carbon (EC). Unless specifically noted, all of these species are included in every simulation. In certain simulations we also include n-tetracosane and n-hexacosane in the CMB model. Uncertainties for individual compounds are based on relative and 
absolute uncertainties determined from repeated analysis of parallel samples. Relative uncertainties range from $\pm 10 \%$ to $\pm 30 \%$ and absolute uncertainties are based on multiples of the minimum detection limits. OC is not included in the model as molecular markers for secondary organic aerosol and potentially other primary sources are not known. In order to determine the contribution of each source profile to ambient $\mathrm{OC}$, calculations are performed using source profiles which have normalized by the OC emissions.

\section{Application of factor analysis approaches}

Both the Unmix (Henry, 1997; Henry, 2001) and the PMF (Paatero and Tapper, 1993, 1994; Paatero, 1997) models were used to analyze the ambient data collected during PAQS. In addition, Conditional Probability Function and Potential Source Contribution Function analyses were performed to examine the spatial distribution of the factors determined by PMF analysis.

Both PMF and Unmix were applied to daily 24-hour averaged filter-based data for sulfate, nitrate, trace elements, organic and elemental carbon. This analysis was performed for data collected both at the PAQS central site and the NETL site. The predictions of the two approaches have been compared. To further identify and apportion the sources of $\mathrm{PM}_{2.5}$, specific OC compounds that are known tracers of some sources were added to the PMF analysis. Conditional probability function (CPF) and potential source contribution function analyses (PSCF) were performed for each source to ascertain the likely directions in which the sources were located.

The particulate size distribution data were analyzed as a bilinear receptor model solved by PMF. Each sample contained 165 size bins from 0.003 to $2.5 \mu \mathrm{m}$. Particle growth periods in nucleation events were identified and the data in these intervals were excluded from this study so that the size distribution profiles associated with the factors could be regarded as sufficiently constant to satisfy the assumptions of the receptor model. The values for each set of five consecutive size bins were averaged to produce 33 new size intervals. The factors from PMF could be assigned to particle sources by examination of the number size distributions associated with the factors, the time frequency properties of the contribution of each source (Fourier analysis of source contribution values), and the correlations of the contribution values with simultaneous gas phase measurements $\left(\mathrm{O}_{3}, \mathrm{NO}, \mathrm{NO}_{2}, \mathrm{SO}_{2}, \mathrm{CO}\right)$ and particle composition data (sulfate, nitrate, OC/EC). Seasonal trends and weekday/weekend effects were investigated. Conditional probability function (CPF) analyses were performed for each source to ascertain the likely directions in which the sources were located.

\section{Advances in source apportionment models}

The state-of-the-art monitoring technologies employed during PAQS permitted the measurement of a variety of chemical species in airborne particulate matter with time resolution as high as $10 \mathrm{~min}$ to $1 \mathrm{hr}$. There are still species that are measured with longer integration periods such as several hours to a day. These data from different measurement methods produce a data set of mixed time resolution. Traditional 
eigenvalue-based factor analysis methods are unable to analyze this kind of data set since these data cannot form a simple matrix. Averaging the high time resolution data or interpolating the low time resolution data to produce data on the same time schedule is not acceptable. The former method loses valuable temporal information and the latter produces unreliable high resolution series because of the invalid assumption of temporal smoothness. Zhou et al. (2004b) present a solution to the problem of multiple sampling time intervals. Each data value is used in its original time schedule without averaging or interpolation and the source contributions are averaged to the corresponding sampling interval. For data with the highest time resolution, the contributions are not actually averaged. The contribution series are smoothed by regularization auxiliary equations especially for sources containing very little high resolution species. This new model was developed using the PAQS ambient data.

A multivariate pseudo-deterministic receptor model (PDRM), combining mass balance and Gaussian plume dispersion equations, was applied to exploit highly-timeresolved ambient measurements to determine emission rates of $\mathrm{SO}_{2}$ and elemental constituents of particles and to predict their contributions to ambient levels from known stationary sources influencing air quality at the Carnegie Mellon University Supersite in Pittsburgh, PA. The PDRM exploits knowledge of the number and locations of major stationary sources, source and transport wind directions, stack gas emission parameters, and meteorological plume dispersion parameters during sample collections to constrain solutions for individual sources. The model was applied to ambient $\mathrm{SO}_{2}$ and particle measurements, the latter, made every 30-min for 11 elements ( $\mathrm{Al}, \mathrm{As}, \mathrm{Cd}, \mathrm{Cr}, \mathrm{Cu}, \mathrm{Fe}, \mathrm{Mn}$, $\mathrm{Ni}, \mathrm{Pb}, \mathrm{Se}$, and $\mathrm{Zn}$ ) during a 12.5-hr period on April $1^{\text {st }}$, when winds blew from direction of $290-330^{\circ}$ in which four small-scale coal-fired plants are situated.

\section{Activity 5. Three-Dimensional Deterministic Modeling}

The purpose of this activity is to evaluate the performance of the three-dimensional chemical transport model $(\mathrm{PMCAMx}+)$ with air quality data collected by this and other projects. After this evaluation, the model has been used to evaluate the response of ambient PM concentrations to changes in emissions.

PMCAMx + is a three-dimensional chemical transport model which uses the framework of CAMx (Environ, 2003) to simulate horizontal and vertical advection, horizontal and vertical dispersion, wet and dry deposition, and gas-phase chemistry. Three aerosol modules have been implemented in PMCAMx + to describe inorganic and organic aerosol dynamics and aerosol-cloud interactions using an operator-splitting approach (Gaydos, Pinder et al. 2005). The order of the processes is: emission, horizontal advection, vertical advection, vertical dispersion, horizontal dispersion, wet deposition, gas-phase chemistry, aerosol processes (nucleation, coagulation, inorganic aerosol condensation/evaporation), secondary organic aerosol formation, and aqueous phase-chemistry. This approach is further described in Gaydos et al. (2005). PMCAMx+ tracks thirteen different aerosol species: sulfate, nitrate, ammonium, aerosol water content, four secondary organic aerosol species, sodium, chloride, primary organic 
aerosol, primary elemental carbon, and all primary inert material. Each aerosol species has ten size sections, ranging from $40 \mathrm{~nm}$ to $40 \mu \mathrm{m}$.

For this research, PMCAMx+ was applied to a modeling domain that covers a 3492 x $3240 \mathrm{~km}$ region in the eastern United States with $36 \times 36 \mathrm{~km}$ grid resolution with 14 different levels up to $6 \mathrm{~km}$ (Gaydos, Pinder et al. 2005). The modeling domain is shown in Figure 7. Initial and boundary conditions for different aerosol species are listed in Table 9. Inputs to the model include horizontal wind components, temperature, pressure, water vapor, vertical diffusivity, clouds, and rainfall, all created using the meteorological model MM5 (Grell et al., 1995).

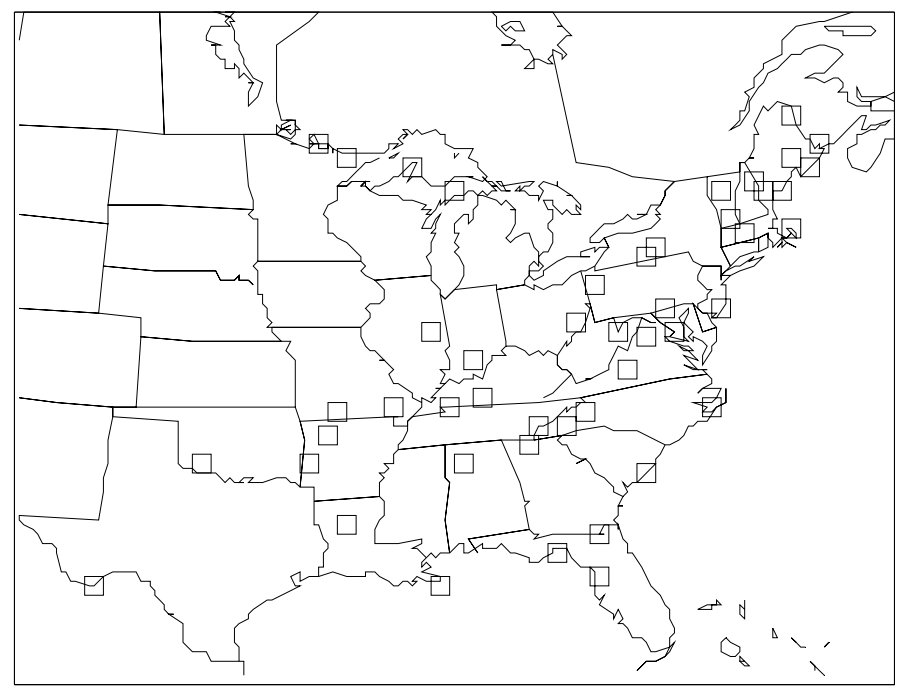

Figure 7. The PMCAMx+ modeling domain for the eastern United States. The locations of the monitoring stations for the IMPROVE network are shown.

The LADCO BaseE inventory generated using EMS-2003 (LADCO, 2003), is used to generate emission inventories. Emissions are derived primarily from the U.S. EPA's National Emission Inventory (NEI) 1999 Version 2.0 (U.S. EPA, 2002a), with the following changes: on-road transportation sources are from the U.S. EPA's MOBILE6 (U.S. 2002b); non-road sources are from U.S. EPA's NONROAD (U.S. EPA 2002c). The temporal profiles for electric power utility point sources are from an analysis of Continuous Emission Monitors (Janssen, 2003). Ammonia emissions are from the CMU Ammonia Emission Inventory (Goebes, Strader et al. 2003; Pinder, Strader et al. 2004). Biogenic emissions are from BIOME3 (Wilkinson and Janssen, 2001). A different emission inventory is used for weekdays, Saturdays, and Sundays.

The model has been applied to July 2001, October 2001, January 2002, and April 2002. During each of these periods, the model predictions have been evaluated against hourly measurements (for both particulate matter and gases) taken during PAQS. To 
cover a larger spatial scale, the model results are also compared to daily average measurements from the U.S. EPA's AIRS monitoring network (USEPA, 2002) and the IMPROVE network (IMPROVE, 1995). The primary organic carbon and elemental carbon inventories used by $\mathrm{PMCAMx}+$ have been evaluated by comparing source resolved predictions to results from Chemical Mass Balance Modeling using molecular markers (Lane, Pinder et al. 2005).

After this evaluation, PMCAMX+ was used to investigate changes in $\mathrm{PM}_{2.5}$ levels in the Eastern US to $50 \%$ reductions in $\mathrm{NOx}, \mathrm{SO}_{2}, \mathrm{NH}_{3}$, and VOC emissions. These simulations were performed for two periods: July 12-28, 2001 and January 1-31, 2002. These time periods are representative of typical summer and winter conditions.

Table 9. Boundary conditions for aerosol species.

\begin{tabular}{ccccc}
\hline & \multicolumn{4}{c}{ Concentration $\left(\mu \mathrm{g} / \mathrm{m}^{3}\right)$} \\
\cline { 2 - 5 } & July 2001 & October 2001 & January 2002 & April 2002 \\
\hline Organic aerosol & 0.8 & 0.8 & 0.5 & 0.5 \\
Elemental carbon & 0.1 & 0.1 & 0.1 & 0.1 \\
Sulfate & 0.9 & 0.9 & 0.7 & 0.7 \\
Nitrate & 0.1 & 0.1 & 0.3 & 0.3 \\
Ammonium & 0.37 & 0.37 & 0.35 & 0.35 \\
\hline
\end{tabular}

\section{Activity 5. Hypothesis testing}

The Pittsburgh Air Quality Study (PAQS) was designed to test a wide range of complementary hypotheses. The findings of the program provided strong support for some of these hypotheses and showed that some of them were false. A brief summary of the hypotheses relevant to the DOE portion of the project are presented below:

\section{Ambient aerosol characterization}

Hypothesis 1.1 The measured aerosol mass can be fully explained if one accounts for the water retained by organics and inorganics, the full organic aerosol contribution, and the full crustal contribution.

Hypothesis 1.2 The regional contributions to the $\mathrm{PM}_{2.5}$ levels in the Pittsburgh region for some compounds exceed the local contribution, whereas for others the local exceeds the regional. 


\section{Source characterization}

Hypothesis 2.1 The high time resolution, semi-continuous measurements will permit resolution of plumes from individual stationary sources impacting the site, and resolution of local and regional sources.

Hypothesis 2.2 Single particle instruments can directly determine the local air quality contributions from a broad range of sources.

Hypothesis 2.3 Dilution sampling can be used to provide better source fingerprints of combustion systems than traditional methods.

\section{Source apportionment}

Hypothesis 3.1 Aerosol nucleation (biogenic precursors or $\mathrm{SO}_{2}$ ) can be a major source of aerosol number in both urban and rural areas in the study region.

Hypothesis 3.2 Biogenic primary and secondary aerosols are a major component of the organic aerosol in the Pittsburgh region.

Hypothesis 3.3 The secondary aerosol contribution to OC exceeds $50 \%$ during the peak PM days, but is around $20 \%$ on a yearly average basis (based on similar contributions estimated for the Western US).

Hypothesis 3.4 The response of $\mathrm{PM}_{2.5}$ to changes in sulfate is highly non-linear during the winter and linear during the summer and is controlled by the ammonia availability.

\section{RESULTS AND DISCUSSION}

The key findings of the program are presented below. They are organized by activity. The findings are related to the corresponding hypotheses shown in brackets [ ]. Additional information about these findings can be found in the cited publications, which are included in Appendix B of this report.

\section{Ambient aerosol characterization}

- The annual average $\mathrm{PM}_{2.5}$ concentration (July 1, 2001 through June 30, 2002) was $15.8 \mu \mathrm{g} \mathrm{m}^{-3}$, suggesting that the region will violate the annual average $\mathrm{PM}_{2.5}$ standard.

- The daily average $\mathrm{PM}_{2.5}$ standard $\left(65 \mu \mathrm{g} \mathrm{m}^{-3}\right)$ was never exceeded during the approximately 400 days of measurements during PAQS. However, on 9 days the daily average $\mathrm{PM}_{2.5}$ concentration was greater than $50 \mu \mathrm{g} \mathrm{m} \mathrm{m}^{-3}$. All 9 days occurred during the summer months of 2001 and 2002. Roughly half of the episodes were due to high sulfate concentrations while the other half were mainly due to high organic matter concentrations (Wittig, Anderson et al. 2004). 
- The average FRM-measured $\mathrm{PM}_{2.5}$ concentration in Pittsburgh is on average $10 \%$ greater than the sum of the mass of the aerosol chemical components (Figure 8). The most significant discrepancies were observed during the summer. This discrepancy is mainly due to water retention on the FRM filters and can be corrected with the use of thermodynamic models. Accounting for the effects of water and volatilization losses closes the mass balance between the FRM and the sum of the $\mathrm{PM}_{2.5}$ chemical components (Rees, Robinson et al. 2004). [HYPOTHESIS 1.1]

- Seasonal variations in $\mathrm{PM}_{2.5}$ mass and composition in Western Pennsylvania are driven by differences in the seasonal variations of organic carbon and sulfate, with sulfate being dominant in the summer and organic matter and sulfate showing similar concentrations in the cooler months. In the cooler months, nitrate also is important and approximately equal to about half the sulfate concentration on average (Rees, Robinson et al. 2004; Wittig, Anderson et al. 2004). These trends are illustrated in Figure 8.

- The concentrations of $\mathrm{PM}_{2.5}$, sulfate, and $\mathrm{OC}$ were surprisingly uniform in Western Pennsylvania (three sites inside Pittsburgh, one upwind and one downwind of the city) during both the summer and winter (Tang, Raymond et al. 2004). The large regional contribution is supported by the weak daily patterns of the $\mathrm{PM}_{2.5}$, sulfate, and organic matter concentrations (Wittig, Anderson et al. 2004) and the average age (several days) of the particles estimated from natural radionuclide measurements (Gaffney, Marley et al. 2004). Time series of $\mathrm{PM}_{2.5}$ mass and composition at different satellite sites are shown in Figure 9. [HYPOTHESIS 1.2]

- $\mathrm{PM}_{2.5}$ represented on average two-thirds of the $\mathrm{PM}_{10}$ in Western Pennsylvania (Wittig, Anderson et al. 2004). The aerosol mass distribution was bimodal with most of the aerosol mass in the fine mode (Figure 10). The two modes were centered approximately at 0.3 and $3 \mu \mathrm{m}$. 


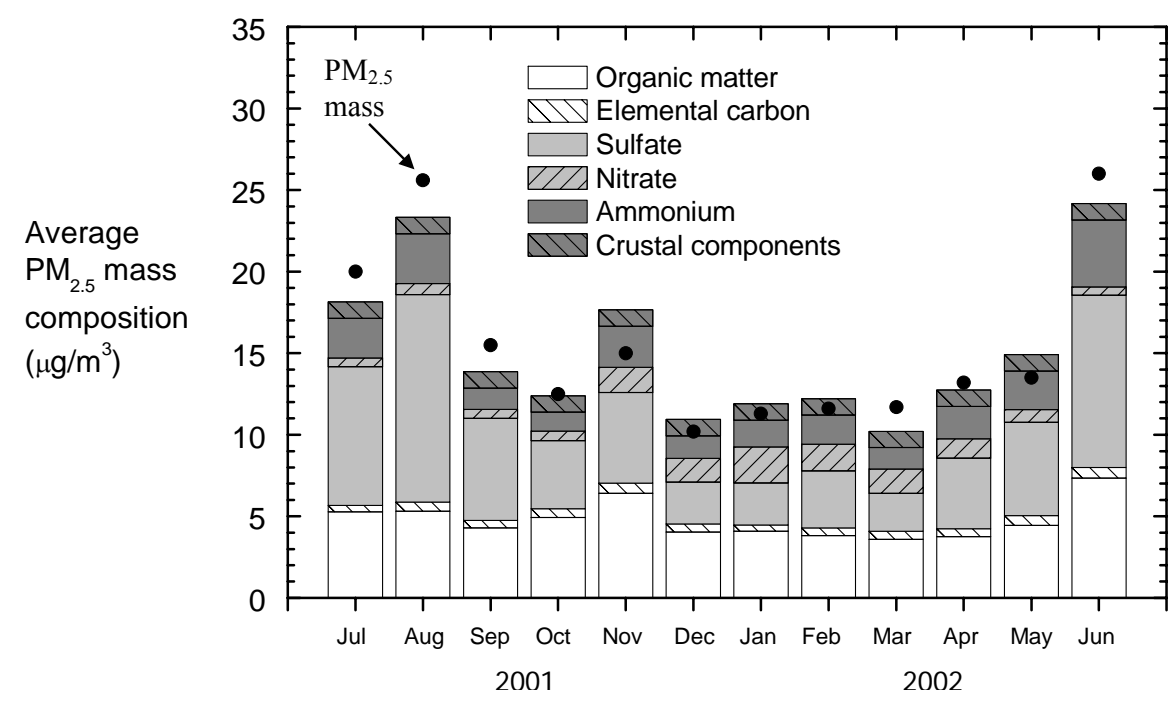

Figure 8. $\mathrm{PM}_{2.5}$ composition on a monthly average basis at the central site from July 2001 to June 2002. Also shown is the $\mathrm{PM}_{2.5}$ total mass measured using the FRM. Organic matter concentrations for July 2001 are based on 24 hour integrated denuder sampler measurements and the experimentally determined multiplier of 1.8. For other periods, organic matter concentrations are based on the difference between the OC collected on the upstream ('bare quartz') and downstream quartz filters of the CMU TQQQ sampler double-quartz filter pack. Sulfate, nitrate, and ammonium concentrations are composites of measurements made from several methods, including the CMU inorganic sampler, the PC-BOSS, the steam sampler and the Rupprecht and Patashnick 8400 instruments. Crustal component concentrations for August 2001 through June 2002 were estimated from daily July 2001 measurements collected at the PAQS satellite sites to be approximately $1 \mu \mathrm{g} \mathrm{m}^{-3}$.

- The correlation between $\mathrm{PM}_{2.5}$ and $\mathrm{PM}_{1}$ in Pittsburgh was very high $\left(\mathrm{R}^{2}=0.98\right)$. The correlation between $\mathrm{PM}_{2.5}$ and $\mathrm{PM}_{\mathrm{x}}$ decreased with decreasing size cut $x$ but $\mathrm{R}^{2}>0.75$ for $\mathrm{x}>0.56 \mu \mathrm{m}$. The correlations between $\mathrm{PM}_{0.1}, \mathrm{PM}_{0.2}$, and $\mathrm{PM}_{0.3}$ with $\mathrm{PM}_{2.5}$ were weak (Cabada, Rees et al. 2004).

- Ultrafine particles (below $100 \mathrm{~nm}$ ) account on average for less than 5\% of the $\mathrm{PM}_{2.5}$. During the summer the ultrafine mass is $50 \%$ carbonaceous material and $50 \%$ inorganic (mainly sulfate and ammonium); during the winter these percentages are $70 \%$ and $30 \%$ respectively (Cabada, Rees et al. 2004).

- PM mass distributions for all seasons are dominated by the accumulation mode with its sub-modes at $0.2 \mu \mathrm{m}$ (condensation mode) and $0.7 \mu \mathrm{m}$ (droplet mode). The droplet mode dominates in the summer (strong photochemical production of sulfate and cloud processing) while the condensation mode becomes as important in the winter and fall (significant influence of primary emissions) (Cabada, Rees et al. 2004; Zhang, Stanier et al. 2004). 


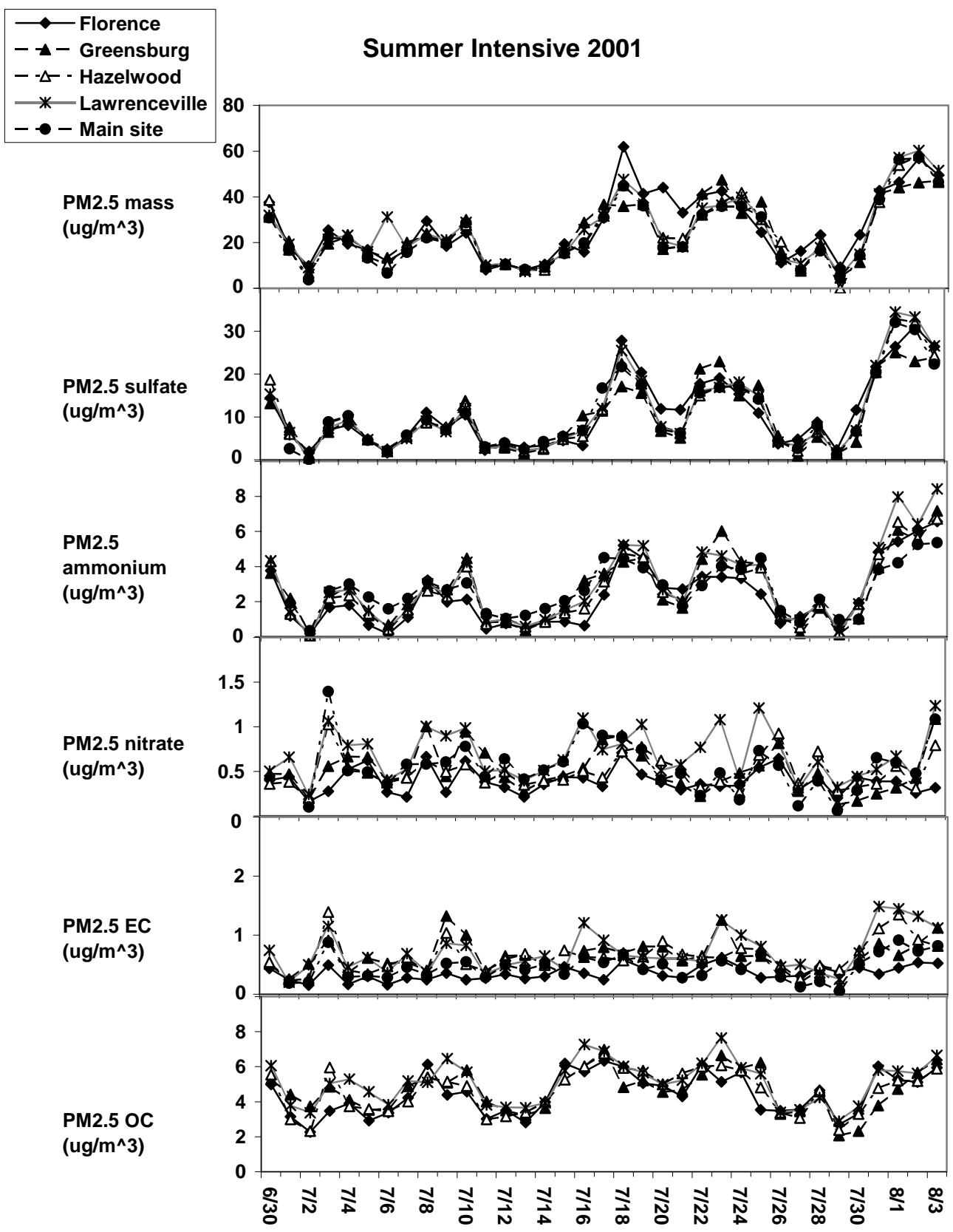

Figure 9. Time series plots of $\mathrm{PM}_{2.5}$ concentrations during summer 2001 intensive sampling period. 

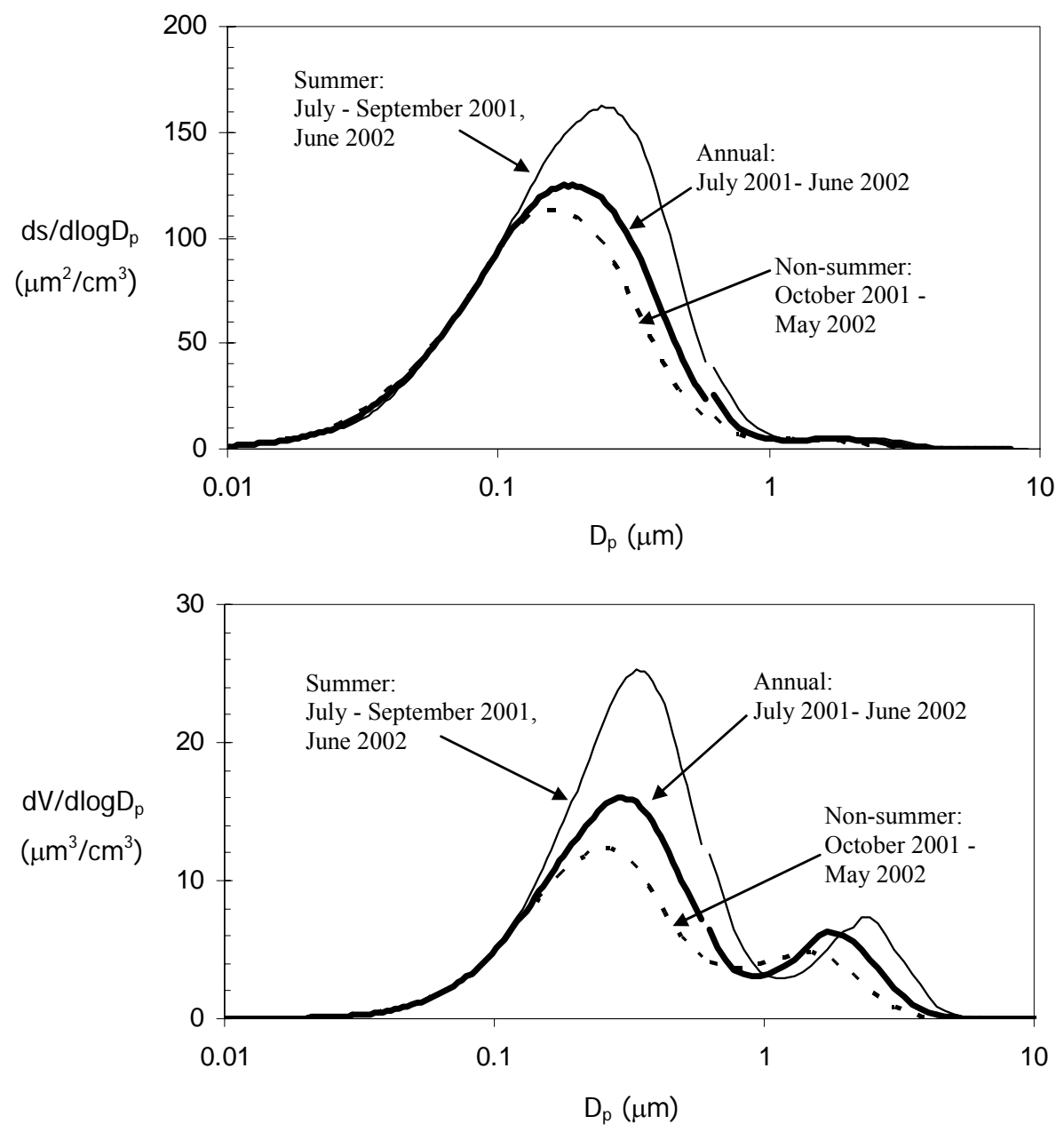

Figure 10. Aerosol surface area and volume size distributions composition on a monthly average basis at the central site from June 2001 to May 2002. Also shown are the distributions based on a logarithmic scale.

- The organic PM (both OC and EC) has a practically unimodal mass distribution during the whole year consistent with its regional aged character (Cabada, Rees et al. 2004).

- Almost the entire nitrate in the winter exists in submicrometer particles with a size distribution similar to that of sulfate and ammonium suggesting that it is mostly in the form of ammonium nitrate (Cabada, Rees et al. 2004).

- In the summer, most of the total nitrate in Pittsburgh is in the gas phase during the day and in the particulate phase during the night with maximum concentrations in the early morning before sunrise. Almost the entire available total nitrate is in the particulate phase in the winter (Wittig, Anderson et al. 2004). 
- Sulfate concentrations show little consistent diurnal variation throughout the year, except in the summer when sulfate peaks slightly during the afternoon due to photochemical production (Wittig, Anderson et al. 2004).

- The daily average sulfate concentration is well correlated with the hourly maximum sulfate concentration $\left(\mathrm{R}^{2}=0.89\right)$ (Wittig, Anderson et al. 2004).

- The average Pittsburgh number concentration is $22,000 \mathrm{~cm}^{-3}$ with an average mode size of $40 \mathrm{~nm}$. Rural number concentrations in Western Pennsylvania are a factor of 2-3 lower than the urban values. The highest number concentrations were observed during relatively clean days because of frequent nucleation events in the area. (Stanier, Khlystov et al. 2004).

- Regional scale formation of ultrafine particles (nucleation) takes place in Western Pennsylvania on $30 \%$ of the days of the year during all seasons, but it is most frequent in fall and spring and least frequent in winter. Regional nucleation is most common on sunny days with below average $\mathrm{PM}_{2.5}$ concentrations. Twenty-four-hour average number concentrations were approximately $40 \%$ higher on days with nucleation compared to those without. (Stanier, Khlystov et al. 2004). [HYPOTHESIS 3.1]

- High PM concentrations in Western Pennsylvania are associated with a transition from a high pressure to a low pressure regime in advance of an approaching frontal system indicating long-range transport of pollutants (Modey, Eatough et al. 2004).

- The nitrate and sulfate concentrations measured by the R\&P nitrate (model $8400 \mathrm{~N}$ ) and sulfate (model $8400 \mathrm{~S}$ ), after standard instrument calibration, had reasonably high correlations $\left(\mathrm{R}^{2}\right.$ about 0.85 ) with the filter-based methods. However, the sulfate and nitrate concentrations measured by the continuous instruments were low by $17 \%$ and $29 \%$ respectively. A final calibration step using filter-based measurements was recommended for these instruments (Wittig, Takahama et al. 2004).

- An automatic method for the semi-continuous measurement of the aerosol water content, the Dry Ambient Aerosol Size Spectrometer (DAASS) was developed and used continuously for a year (Stanier, Khlystov et al. 2004).

- The organic aerosol in Western Pennsylvania is aged and as a result the negative artifact in quartz filters was found to be small (typically less than $10 \%$ of the OC). The use of a backup quartz filter behind the front quartz filter provided a better estimate of daily average OC positive artifact than the use of a quartz backup filter behind a Teflon filter (Subramanian, Khlystov et al. 2004).

- A simple algorithm was developed to combine aerosol size distribution data measured with commercially available scanning mobility particle sizers and an aerodynamic particle sizer to produce the complete aerosol size distribution from 3 nanometers to 10 micrometers (Khlystov, Stanier et al. 2004). 
- The Versatile Aerosol Concentration Enrichment System (VACES) was tested during measurements of ambient aerosol in Pittsburgh. It was found that the shape of the sulfate distribution was preserved during passage through the concentrator with a mass enhancement factor of 10-20. The size distributions of organics, ammonium and nitrate were preserved in relatively clean days while artifacts smaller than $10 \%$ of the concentrated mass were introduced for dirty days (Khlystov, Stanier et al. 2005; Zhao, Bein et al. 2005).

- The density of aerosol in the Pittsburgh area was found to be $1.5 \pm 0.5 \mathrm{~g} \mathrm{~cm}^{-3}$ (Khlystov, Stanier et al. 2004).

- Agreement within experimental error was obtained between the measured scattering coefficient and the calculations using the high temporal-resolution $\mathrm{PM}_{2.5}$ composition measurements and aerosol water content. Approaches relying on estimation of the aerosol water content tended to underpredict the scattering coefficient by around $20 \%$ (Cabada, Khlystov et al. 2004).

- Aerosol sulfate and the associated water contribute around $70 \%$ to the scattering coefficient in the area during the summer with organics being responsible for most the remaining scattering. During the winter, sulfate accounts for approximately $40 \%$, nitrate roughly $25 \%$, and organics for $35 \%$ of the aerosol scattering (Cabada, Khlystov et al. 2004).

- During the summer months the ambient aerosol practically always contained water even when the relative humidity was as low as $30 \%$. In contrast, during the winter the aerosol was dry below $60 \% \mathrm{RH}$. The spring months were characterized by a transitional behavior between these two states. The observed seasonal behavior can be explained by the aerosol acidity. The summer aerosol was acidic and retained water at low RH. The winter aerosol was neutral and became wet when the relative humidity reached the deliquescence point of ammonium nitrate (Khlystov et al. 2005a).

\section{Source characterization}

- Spherical aluminum silicate (SAS) particles are thought to be a unique tracer for emissions from coal combustion. Based on tests performed at pilot and full-scale using 5 different types of coal (Figure 11), the average emission factor for SAS was $3.7 \times 10^{5}$ (number of SAS particles/ $\mu$ g primary $\mathrm{PM}_{2.5}$ emissions). There was reasonable agreement between pilot- and full-scale data, and some rank dependence of the SAS emission factor with lower rank coals having a higher SAS emission factor.

- Dilution sampler measurements indicate that coal fired boilers directly emit little carbonaceous aerosol. For the two full-scale boilers we tested, EC contributed on average less than $1 \%$ of the emitted PM2.5 mass. OC measured using a quartz filter 
contributed on average less than $2.5 \%$ of the emitted PM2.5 mass. In addition, backup filter measurements indicate that the majority of this OC is likely sampling artifact.

- Dilution sampler measurements indicate some conversion of oxidized mercury into its elemental state in the plume of power plants. Quantification of this effect is complicated by the wall losses inside the sampler (Robinson et al. 2005).

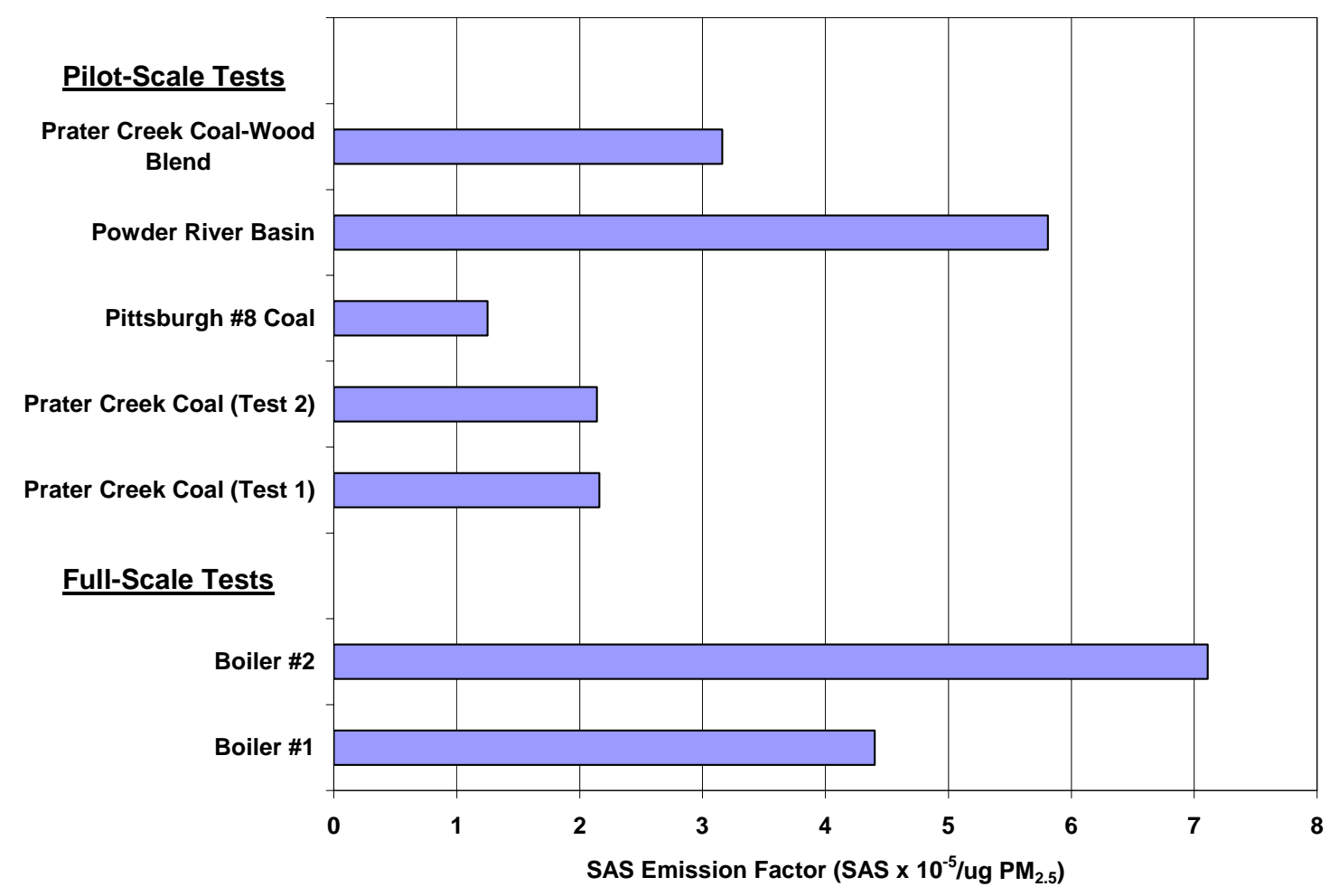

Figure 11. SAS emission factors as a fraction of $\mathrm{PM}_{2.5}$ mass measured during pilot-scale and full-scale boiler testing.

- The fine particle emissions profile of a large coke production facility is dominated by organic $\left(40 \% \pm 9 \%\right.$ of $\mathrm{PM}_{2.5}$ mass emissions) and elemental carbon $(25 \% \pm 5 \%$ of $\mathrm{PM}_{2.5}$ mass emissions). Significant contributions of inorganic ions and select trace metals were also observed. The particle emissions are dominated by the fine fraction, with $\mathrm{PM}_{2.5}$ estimated to contribute $84 \% \pm 14 \%$ of the $\mathrm{PM}_{10}$ mass. The profile can be used for source-receptor analysis of similar facilities (Weitkamp, Lipsky et al. 2005).

- On a fuel basis, emissions of $\mathrm{NO}_{\mathrm{x}}, \mathrm{PM}_{2.5}$, $\mathrm{EC}$ and $\mathrm{OC}$ from heavy-duty diesel vehicles inside the Squirrel Hill tunnel are significantly larger than those for light-duty vehicles. Emissions of particulate metals associated with brake wear $\mathrm{Cu}, \mathrm{Sb}, \mathrm{Ba}$ and potentially $\mathrm{Ga}$ ) were significantly higher during the rush-hour period when there was more brake usage (Grieshop, Lipsky et al. 2005). 
- There is a strong correlation between organic composition of motor vehicle exhaust measured inside the tunnel and the distribution of important motor vehicle markers (hopanes, steranes, PAHs) in the ambient air samples.

- A seasonal shift in the average $\mathrm{OC} / \mathrm{EC}$ ratio for the rush-hour period inside the Squirrel Hill Tunnel was observed; fall and summer OC/EC ratios are $0.83 \pm 0.15$ and $0.26 \pm 0.06$, respectively. Potential causes for this shift are seasonal changes in fuel formulation and an increased partitioning of semivolatile organic compounds into the gas phase during the summer months (Grieshop, Lipsky et al. 2005).

- Significant differences were observed in the $\mathrm{PM}_{2.5}$ fraction of urban and rural paved road dust in the Pittsburgh region. Urban road dust is enriched in organic compounds found associated with motor vehicles (hopanes, steranes, PAHs, benzothiazole and alkylcyclohexanes) and metals associated with anthropogenic sources $(\mathrm{Zn}, \mathrm{Cu}, \mathrm{Ni}$, and Mo). Rural road dust is enriched in sterols associated with biological sources (cholesterol and $\beta$-sitosterol).

- Waxy n-alkanes concentration levels for the Pittsburgh leaf abrasion sample are somewhat lower than found for Los Angeles (Rogge, Hildemann et al. 1993), possibly a result of the different plant distribution as well as differences in climate between the two locations.

- For diesel engines operating at low load and wood combustion, the $\mathrm{PM}_{2.5}$ mass emission rate decreases significantly when the aerosol is diluted because of changes in the partitioning of semivolatile organics (Figure 12). For example, the $\mathrm{PM}_{2.5}$ mass emission rate from a diesel engine operating at low load decreases by $50 \%$ when the dilution ratio increases from 20:1 to 350:1 (Lipsky and Robinson 2005).

- A new portable dilution sampler has been designed and tested for measurements of semivolatile aerosol emissions (Lipsky and Robinson 2005) [HYPOTHESIS 2.3]. 

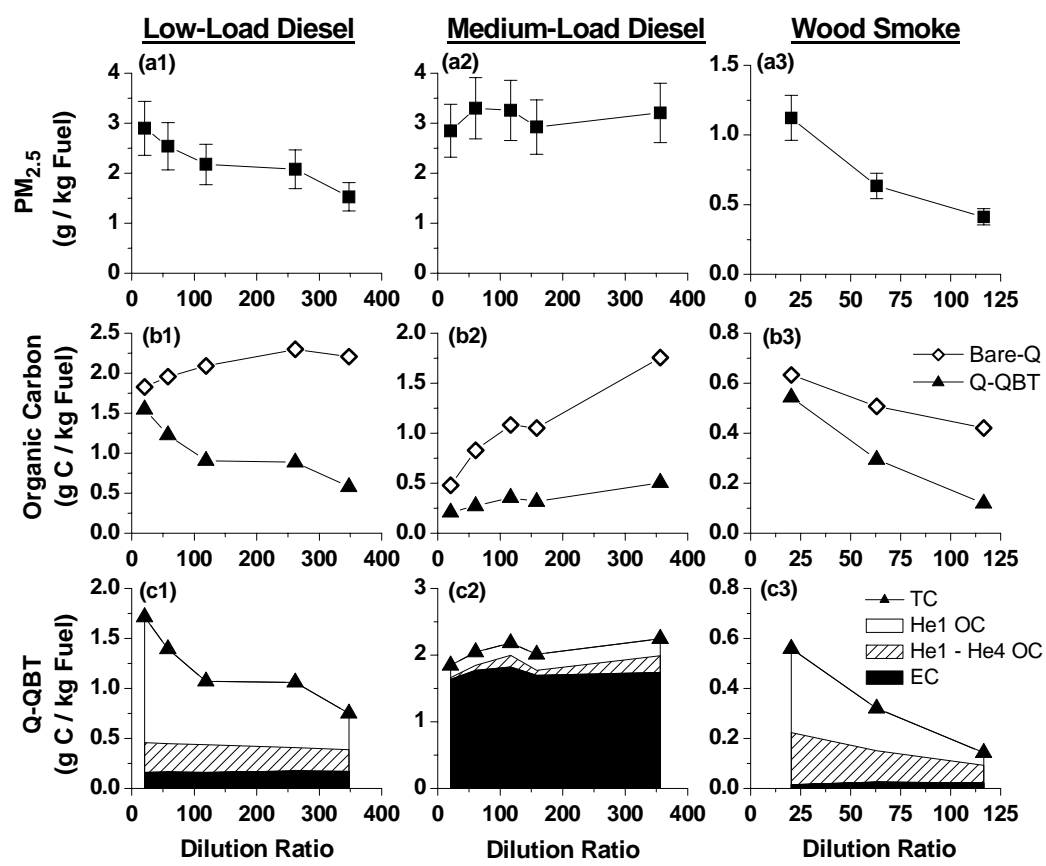

Figure 12. Fuel-based emissions from low-load diesel (left column), medium-load diesel (middle column), and wood smoke (right column) experiments. Plots a1-a3 show $\mathrm{PM}_{2.5}$ mass emission factor; plots b1-b3 compare organic carbon emission factors measured with the Bare-Q and Q - QBT approaches; plots c1-c3 show particulate organic carbon (OC) and elemental carbon (EC) emission factors measured using the Q - QBT approach. Bare-Q is carbon measured with a quartz filter; Q - QBT is carbon measured with a backup-corrected quartz filter. Q - QBT is particulate OC, while Bare-Q has substantial positive artifact, as discussed in the text. In plots $\mathrm{c} 1-\mathrm{c} 3$ symbols indicate total carbon emissions and shading indicates the contribution of $\mathrm{OC}$ and $\mathrm{EC}$ to the emissions. Plots c1-c3 also show the carbon fractions from the OC/EC analysis: He1 OC is the OC that evolves at $340^{\circ} \mathrm{C}$, and $\mathrm{He} 2-\mathrm{He} 4 \mathrm{OC}$ is the $\mathrm{OC}$ that evolves at temperatures greater than $340^{\circ} \mathrm{C}$ during the $\mathrm{OC} / \mathrm{EC}$ analysis. The diesel plots combine data from back-to-back experiments conducted on the same day. Lines are intended as a visual aid. Vertical bars in (a) are experimental uncertainties determined from intercomparison experiments (Lipsky and Robinson, 2005b).

\section{Source apportionment}

- During the study roughly $90 \%$ of the $\mathrm{PM}_{2.5}$ concentration came from areas outside Pittsburgh (Figure 9). Local mobile sources contributed only $7 \%$ to the average $\mathrm{PM}_{2.5}$ concentration (Zhou, Kim et al. 2004; Zhou, Kim et al. 2005). [HYPOTHESIS 1.2] 
- There are important local sources of fine particulate metals, particularly $\mathrm{Pb}, \mathrm{Mo}$ and $\mathrm{Cr}$ as well as a fraction of the $\mathrm{Fe}, \mathrm{Mn}$, and $\mathrm{Zn}$ (Bein, Zhao et al. 2005; Pekney, Davidson et al. 2005). These metals are associated with metal processing industries. In addition, roughly half of the particle number concentration was due to local transportation sources (Stanier, Khlystov et al. 2004; Zhou, Kim et al. 2004; Zhou, Kim et al. 2005). [HYPOTHESIS 1.2]

- Emissions from coal-fired power plants contribute significantly to $\mathrm{PM}_{2.5}$ concentrations in Pittsburgh. The vast majority of this contribution is to secondary sulfate which represents $38 \%$ of annual average $\mathrm{PM}_{2.5}$ mass (Rees et al. 2004). Ambient SAS concentrations indicate that primary emissions from coal-fired power are estimated to contribute on average $0.25 \mu \mathrm{g} \mathrm{m}^{-3}$ to fine particle mass (or $1.5 \%$ of annual average $\mathrm{PM}_{2.5}$ ). Factor-analysis based approaches applied to aerosol composition data yielded similarly low estimates for the contribution of primary coal combustion (Pekney, Davidson et al. 2005).

- The SOA contribution to the total organic PM concentration in Pittsburgh varies from around $10 \%$ during the winter months to around $50 \%$ of the total OC concentration in the summer months (Cabada, Pandis et al. 2002; Cabada, Pandis et al. 2004; Millet, Donahue et al. 2005). The SOA was estimated to be $35 \pm 15 \%$ of the OC during July 2001 (Millet, Donahue et al. 2005; Polidori, Turpin et al. 2005). On a daily basis the SOA contribution is quite variable from almost zero during the low OC days to more than 50\% during the high $\mathrm{PM}_{2.5}$ episodes (Cabada, Pandis et al. 2004). [HYPOTHESIS 3.2]

- The annual average SOA contribution to the organic PM was estimated to be around $30 \%$ (Polidori, Turpin et al. 2005). [HYPOTHESIS 3.2]

- No major enhancement of the organic concentration is observed during periods when the aerosol is acidic, which suggests that acid-catalyzed SOA formation was not an important process during this study (Zhang, Canagaratna et al. 2005).

- The entire record of semi-continuous measurements of organic carbon (OC), elemental carbon (EC), and inorganic species were carefully examined for evidence of coupling in the variations of inorganic acidity and OC. We were not able to detect significant enhancements of SOA production due to inorganic acidity most of the time, but the signal might have been lost in the noise. If we assume a causal relationship between inorganic acidity and OC, reductions in OC for Western Pennsylvania that might result from reductions in inorganic acidity were estimated to be $2 \pm 4 \%$ by a regression technique, and an upper bound for this geographic area was estimated to be $5 \pm 7 \%$ based on calculations from laboratory measurements (Takahama et al. 2006).

- Two systematic approaches (extensions of the EC tracer method) for the estimation of the SOA contribution to the OC levels were proposed (Cabada, Pandis et al. 2004; Millet, Donahue et al. 2005). The first method requires semi-continuous 
measurements of $\mathrm{OC}$ and $\mathrm{EC}$ and is applicable to areas where most of the $\mathrm{OC}$ is not emitted by local sources. The use of daily average OC and EC measurements results in an under-prediction of the SOA concentration. The second approach requires also continuous VOC measurements.

- Chemical Mass Balance modeling using molecular markers predicts that anthropogenic primary sources contribute on average more than two-thirds of the ambient OC in the winter and between one-third and two-thirds of the ambient OC in the summer (Figure 13). Variability in source profiles is a major source of uncertainty in the predictions. Meat cooking and gasoline vehicles are predicted to be the most important source classes.
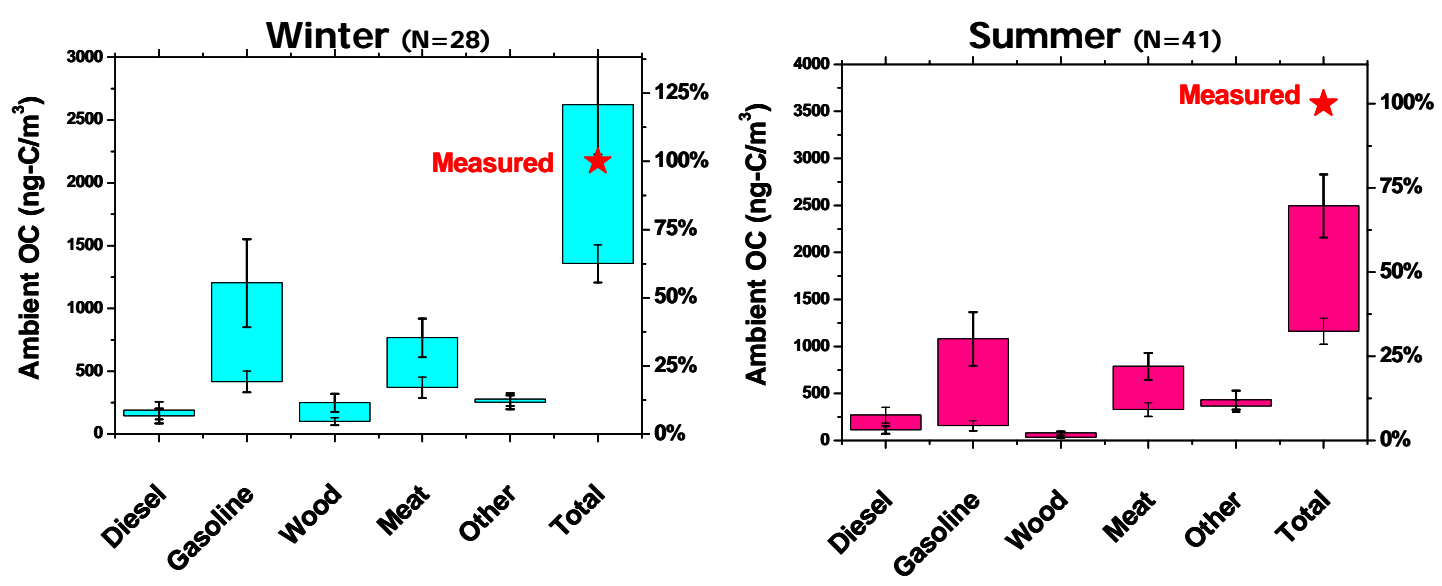

Figure 13. Winter and summer averages of CMB predictions using molecular markers of primary sources of organic aerosol. The colored bars indicate the range of statistically strong CMB solutions using different combinations of source profiles.

- Gasoline and diesel motor vehicles are estimated to contribute between $14 \%$ and $20 \%$ of the ambient $\mathrm{OC}$ in the winter. There is only modest day-to-day variability in the contribution of motor vehicles to ambient OC. However, the gasoline-diesel split is not well constrained given the variability in the source profiles. Estimates of the contribution of motor vehicle emissions in the summer are uncertain given the seasonal shift in the ambient data relative to the source profiles (Subramanian, Robinson et al. 2005).

- Biomass burning (including residential wood burning) is a relatively minor source in Pittsburgh, contributing roughly $14 \%$ of total OC in the winter and $7 \%$ of total OC in the summer, at the upper limit. There is large day-to-day variability in the contribution of biomass smoke to ambient PM, even in the winter.

- Emissions from meat cooking are estimated to be an important source of primary organic aerosol, contributing around $15 \%$ of the annual average OC. 
- Coke production contributes more than $80 \%$ of the ambient concentrations of high molecular weight PAH in Pittsburgh. The predicted contribution of coke production emissions to ambient OC is generally small (median contribution of $15 \mathrm{ng} \mathrm{C} \mathrm{m}^{-3}$ ), but large spikes, up to $710 \mathrm{ng} \mathrm{C} \mathrm{m}^{-3}$, are predicted on certain days (Robinson, Subramanian et al. 2005).

- Use of continuous size distribution, particle composition (sulfate, nitrate, and metals), and gas-phase concentrations in source-receptor analysis increased dramatically our ability to resolve source contributions to ambient $\mathrm{PM}_{2.5}$ concentrations. Eleven sources were identified for two summer episodes: remote traffic, local traffic, diesel traffic, secondary sulfate, secondary nitrate 1 and 2, a lead source, coal-fired power plants, steel mills, coke plants, and nucleation (Zhou, Hopke et al. 2005). [HYPOTHESIS 2.1]

- A positive matrix factorization (PMF) technique was developed for the analysis of the semi-continuous size distributions measured by scanning mobility particle spectrometers (SMPS) and aerodynamic particle sizers (APS) (Zhou, Kim et al. 2004). The sources were identified by using the number and volume distributions associated with these factors, the time frequency properties of the contribution of each source and the correlations of the contribution values with the gas-phase and $\mathrm{PM}_{2.5}$ composition data. [HYPOTHESIS 2.1]

- Use of continuous size distribution measurements and positive matrix factorization can provide valuable information about the contributions of local traffic and nucleation to the aerosol number and mass concentrations. However, additional information is needed to separate the rest of the sources (Zhou, Kim et al. 2005). [HYPOTHESIS 2.1]

- Semi-continuous measurements of PM metal concentrations together with continuous $\mathrm{PM}_{2.5}$ and major gas-phase pollutant concentrations can be used together with multivariate pseudo-deterministic receptor model (PDRM) can be used to predict the contributions to ambient levels of individual coal-fired boilers and other points sources (Park, Pancras et al. 2005). The method also allows the calculation of the emission rates of each source. [HYPOTHESIS 2.1]

- A new source-receptor analysis method (Advanced Factor Analysis) has been developed for datasets that have multiple resolution aerosol composition data (Zhou, Hopke et al. 2005). [HYPOTHESIS 2.1]

- Results from Unmix and PMF analysis of aerosol bulk composition data (OC, EC, ions, trace metals) showed reasonable agreement between the two approaches, both in composition of factors and factor contribution trends (Pekney, Davidson et al. 2005).

- Particles rich in $\mathrm{K}^{+}, \mathrm{Na}^{+}, \mathrm{Fe}^{+}$, and $\mathrm{Pb}^{+}$and to a lesser extent $\mathrm{Ga}^{+}$and $\mathrm{Zn}^{+}$were highly correlated with specific wind directions, facilitating the isolation of specific sources (Bein, Zhao et al. 2005). [HYPOTHESIS 2.2] 


\section{Three-dimensional chemical transport modeling}

Maps of the PMCAMx predictions of the average $\mathrm{PM}_{2.5}$ mass, sulfate, nitrate, ammonium, elemental carbon, and organic mass for July 2001, October 2001, January 2002, and April 2002 are shown in Figures 14, 15, 16, and 17 respectively. Large spatial and seasonal variations are predicted in the concentrations of $\mathrm{PM}_{2.5}$ mass and individual aerosol species. During July 2001 the peak $\mathrm{PM}_{2.5}$ mass concentrations are in the Midwest associated with high sulfate and ammonium concentrations. For example, in Illinois, Indiana and Ohio the predicted sulfate and ammonium levels are up to 12 and 4 $\mu \mathrm{g} / \mathrm{m}^{3}$, respectively. During the winter the highest $\mathrm{PM}_{2.5}$ mass concentrations are in the northeast associated with elevated organic aerosols.

PMCAMx predicts strong seasonal patterns in the $\mathrm{PM}_{2.5}$ composition. For example, during the summer nitrate concentrations are generally low (less than $1 \mu \mathrm{g} / \mathrm{m}^{3}$ in most areas) with the highest values in Indiana, eastern Ohio, and the Philadelphia area (up to $1.5 \mu \mathrm{g} / \mathrm{m}^{3}$ ). Peak nitrate concentrations are predicted in the winter with levels up to up to $3 \mu \mathrm{g} / \mathrm{m}^{3}$ in the northeast US. High nitrate concentrations are also predicted in the Midwest in the spring. Sulfate concentrations exhibit the opposite pattern with elevated concentrations (up to $12 \mu \mathrm{g} \mathrm{m}^{-3}$ ) in the summer and much lower levels in other seasons. This reflects the large seasonal changes in photochemical oxidation of $\mathrm{SO}_{2}$ emissions from power plants. Ammonium concentrations exhibit less of seasonal pattern than either nitrate or sulfate.

Figure 18 presents maps of monthly average concentrations total organic matter, primary organic aerosol, and secondary organic aerosol. In all four seasons the most of the organic mass is predicted to be primary, especially in urban areas. The highest monthly average organic mass concentrations are predicted in the winter along the heavily urbanized northeastern corridor.

$\mathrm{PM}_{2.5}$ mass is equal to the sum of the individual species (sulphate, nitrate, ammonium, organic matter, elemental carbon, and crustal material). During July 2001, sulfate is predicted to account for $40 \%$ of total $\mathrm{PM}_{2.5}$, followed by $\mathrm{OM}(22 \%)$, ammonium (13\%), EC (4\%), and finally nitrate (3\%). During October 2001, the total $\mathrm{PM}_{2.5}$ mass in the entire domain is comprised by sulfate (26\%), organic mass $(26 \%)$, ammonium (11\%), nitrate (6\%), and elemental carbon (5\%). During January 2002, the total PM mass consists of $25 \%$ organics, $19 \%$ sulphate, $12 \%$ nitrate, $10 \%$ ammonium and finally 5\% elemental carbon. During April 2002, sulphate accounts for $29 \%$ of total $\mathrm{PM}_{2.5}$ mass, followed by organic mass (22\%), ammonium (13\%), nitrate (11\%) and elemental carbon $(4 \%)$. 


\section{Summer}
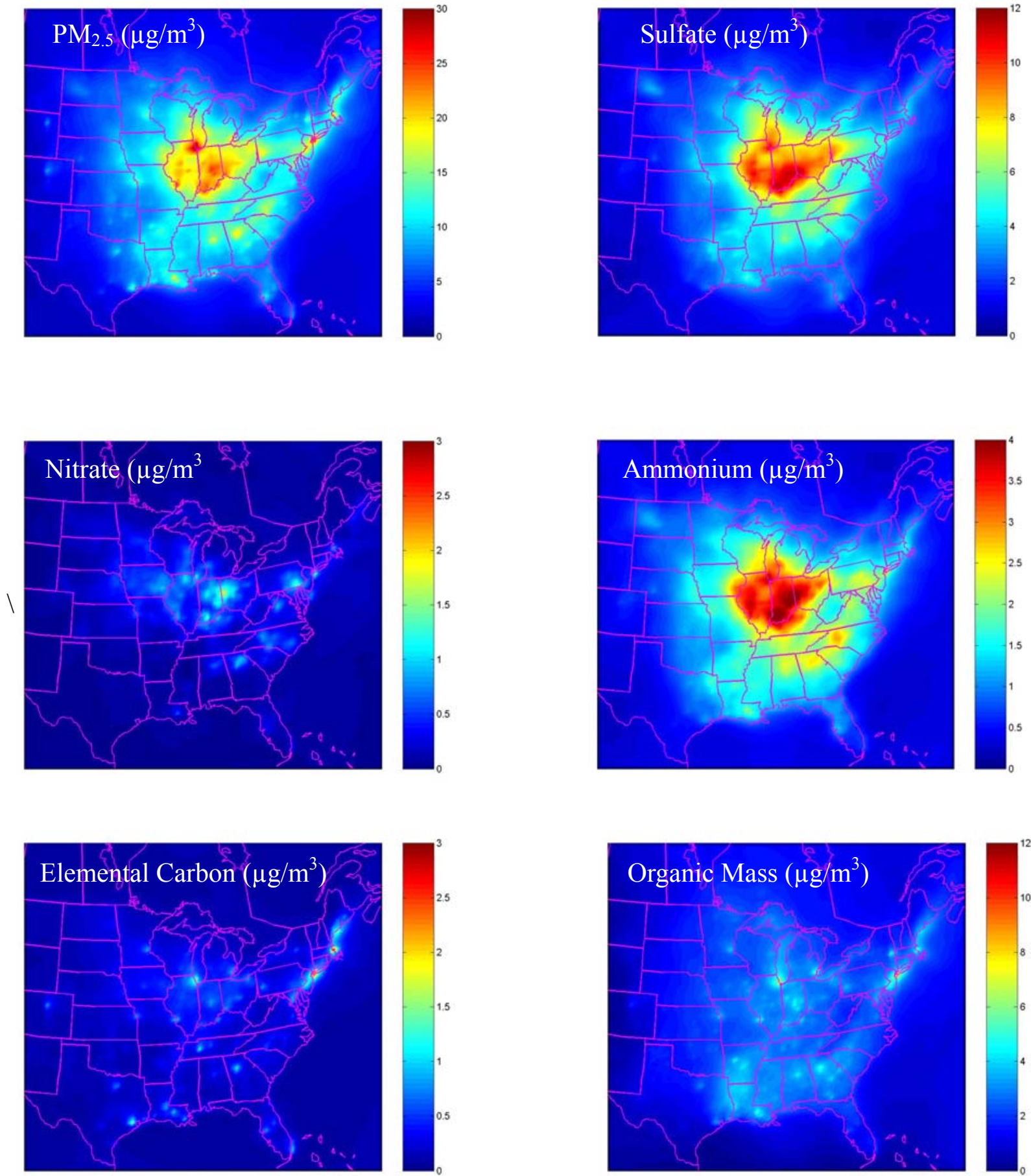

Figure 14. Average predicted $\mathrm{PM}_{2.5}$ concentrations over the period of July 12-28 2001 for $\mathrm{PM}_{2.5}$ mass, sulphate, nitrate, ammonium, elemental carbon and organic mass $\left(\mu \mathrm{g} / \mathrm{m}^{3}\right)$. 


\section{Fall}
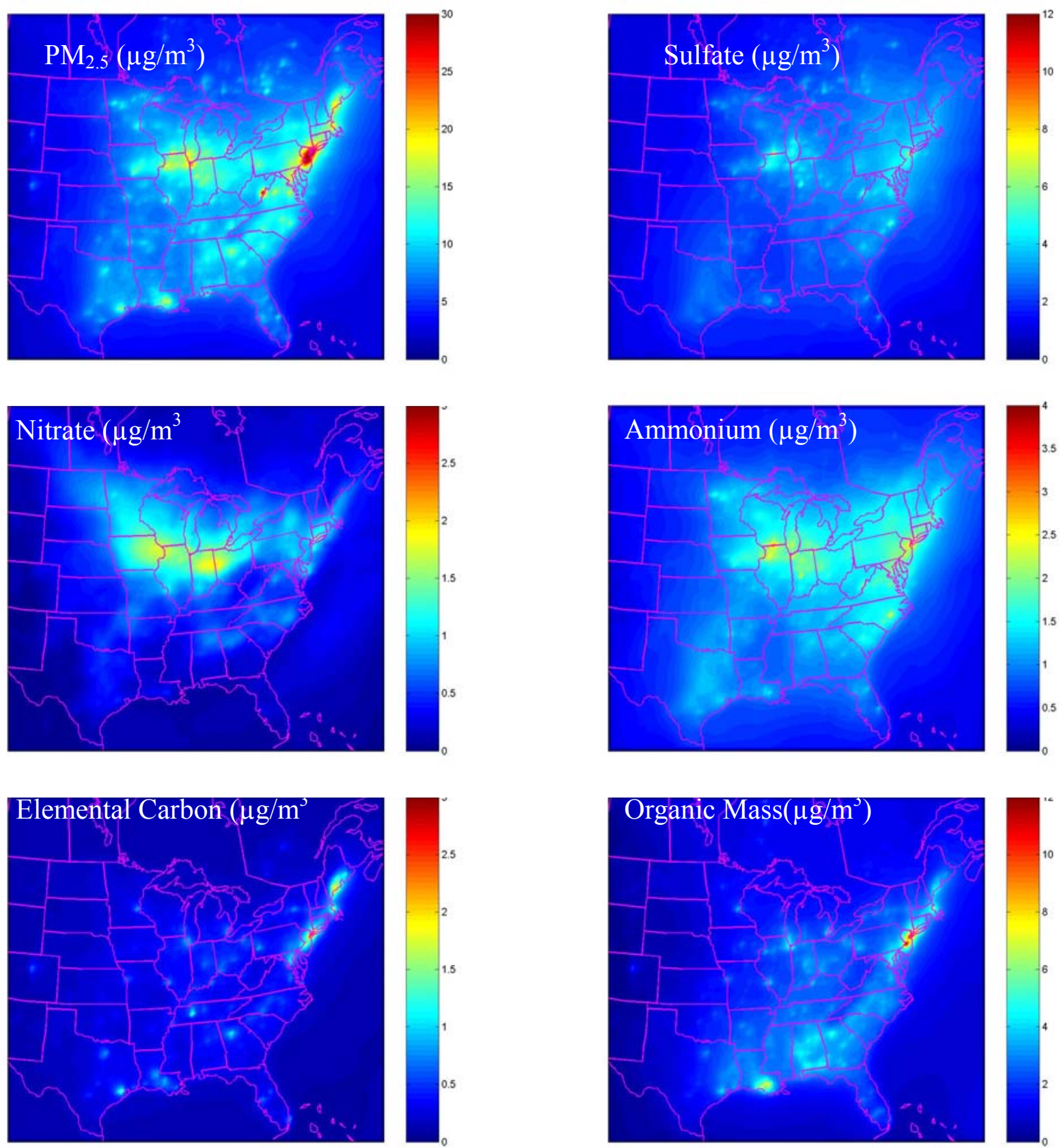

Figure 15. Average predicted $\mathrm{PM}_{2.5}$ concentrations over the period of October 01-31 2001 for $\mathrm{PM}_{2.5}$ mass, ammonium, nitrate, sulphate, elemental carbon and organic mass $\left(\mu \mathrm{g} / \mathrm{m}^{3}\right)$. 


\section{Winter}
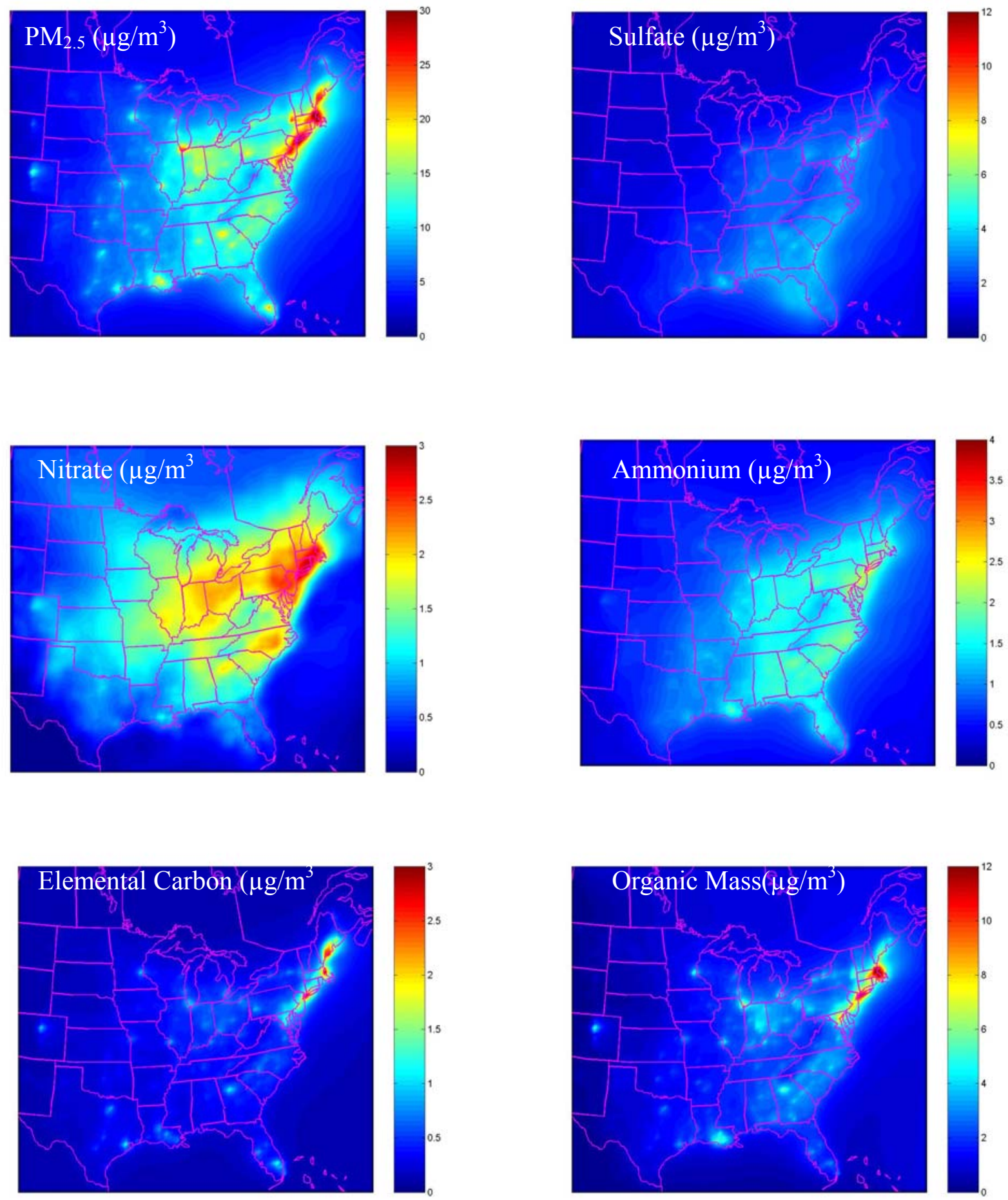

Figure 16. Average predicted $\mathrm{PM}_{2.5}$ concentrations over the period of January 01-31 2002 for $\mathrm{PM}_{2.5}$ mass, ammonium, nitrate, sulphate, elemental carbon and organic mass $\left(\mu \mathrm{g} / \mathrm{m}^{3}\right)$. 


\section{Spring}
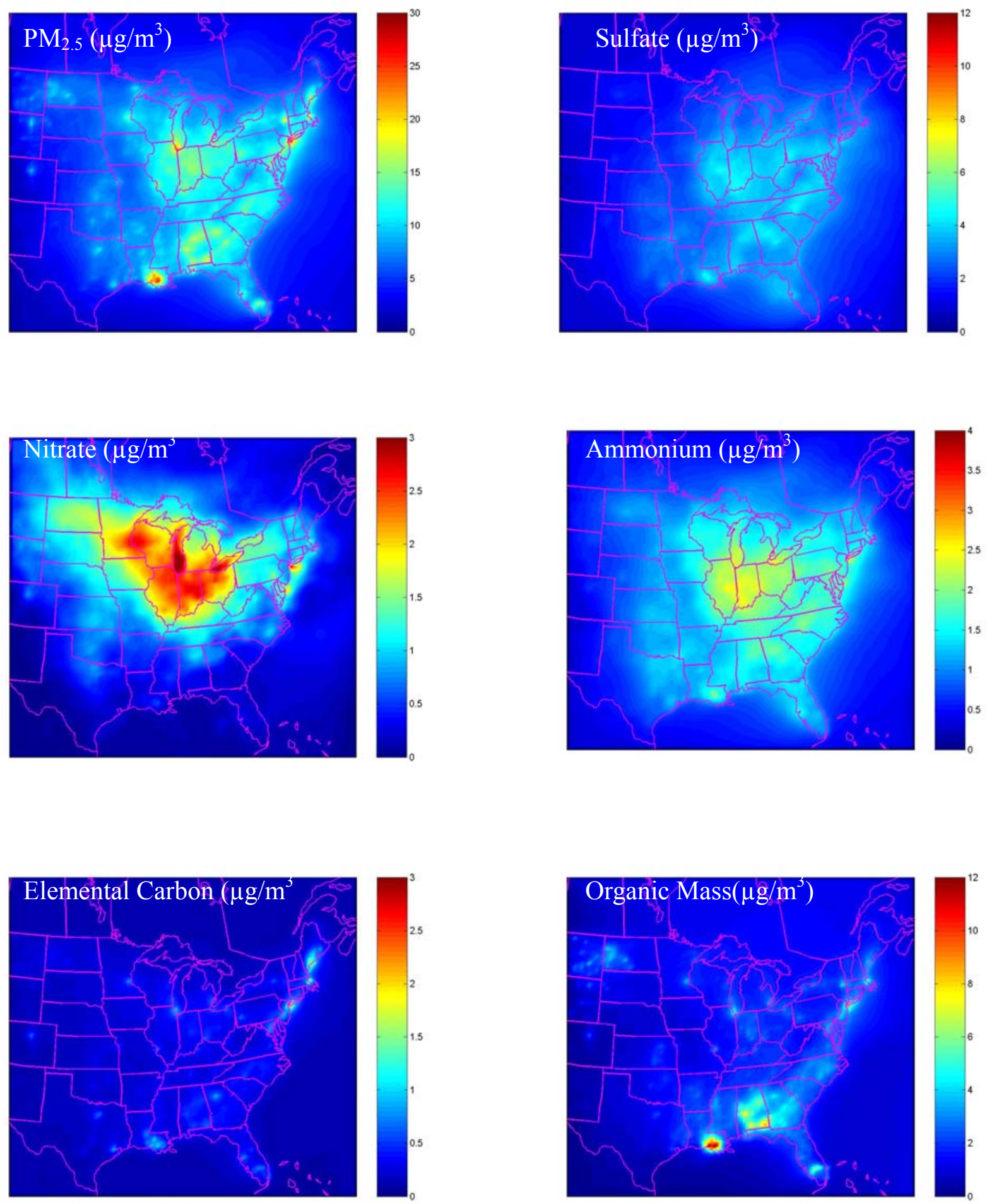

Figure 17. Average predicted $\mathrm{PM}_{2.5}$ concentrations over the period of April 03-30 2002 for $\mathrm{PM}_{2.5}$ mass, ammonium, nitrate, sulphate, elemental carbon and organic mass $\left(\mu \mathrm{g} / \mathrm{m}^{3}\right)$. 

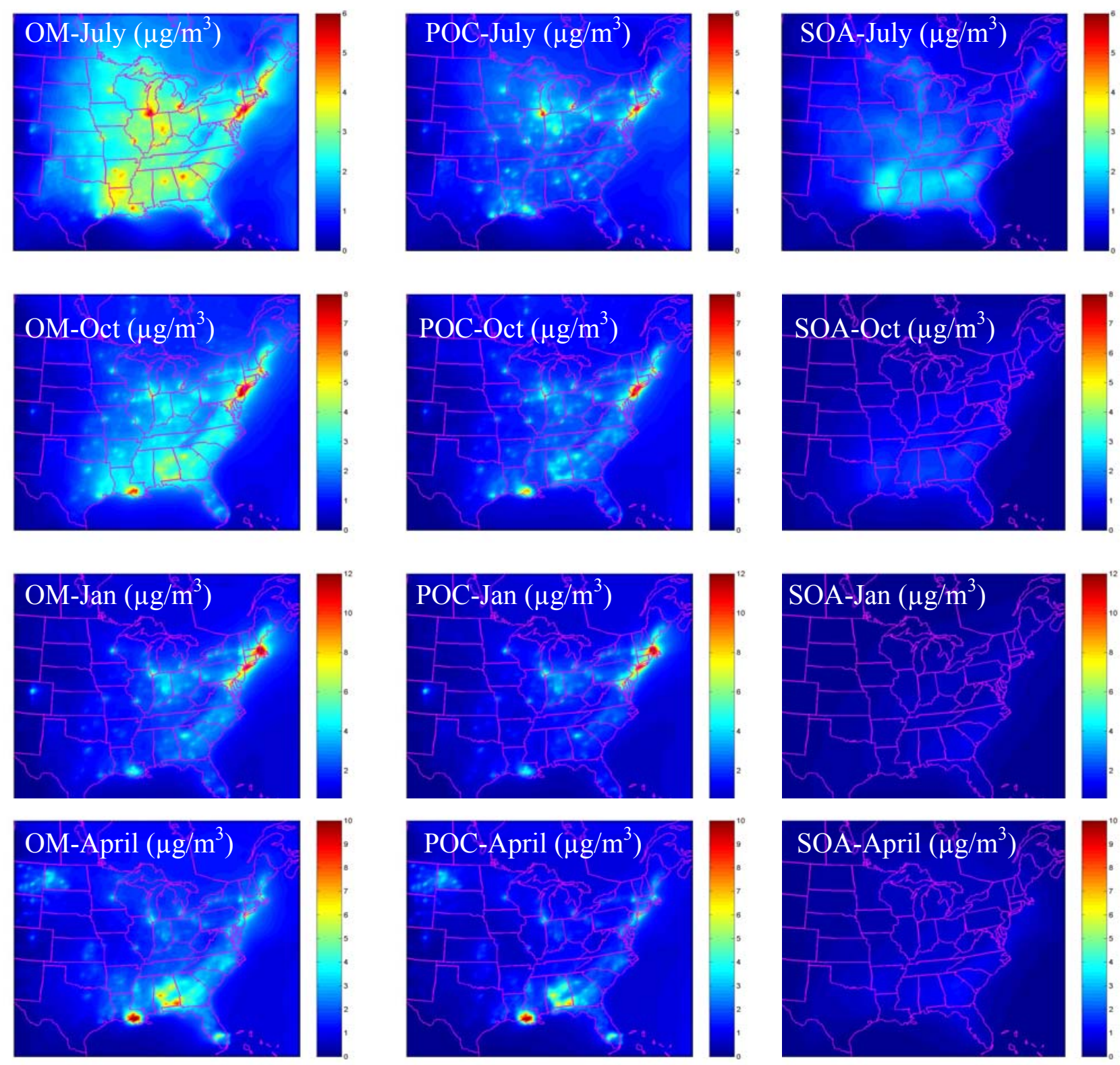

Figure 18. Primary (POC) and Secondary (SOA) OC contribution to Organic Mass (OM) in all four seasons $\left(\mu \mathrm{g} / \mathrm{m}^{3}\right)$. 
The performance of PMCAMx was evaluated by comparing model predictions to daily average measurements from the U.S. EPA's STN network and the IMPROVE network. Plots of the predicted versus measured concentrations are shown in Figures 5-8 for July 2001, October 2001, January 2002 and April 2002, respectively. These plots indicate relatively little bias in the model predictions for most species, but a reasonable amount of scatter.

To quantitatively assess the model performance we Icalculated the mean error (ERROR), mean bias (BIAS), fractional error (FERROR), and fractional bias (FBIAS) to assess the model performance:

$$
\begin{gathered}
\text { ERROR }=\frac{1}{N} \sum_{i=1}^{N}\left|P_{i}-O_{i}\right| \\
\text { BIAS }=\frac{1}{N} \sum_{i=1}^{N}\left(P_{i}-O_{i}\right) \\
\text { FERROR }=\frac{2}{N} \sum_{i=1}^{N} \frac{\left|P_{i}-O_{i}\right|}{P_{i}+O_{i}} \\
\text { FBIAS }=\frac{2}{N} \sum_{i=1}^{N}\left(\frac{P_{i}-O_{i}}{P_{i}+O_{i}}\right)
\end{gathered}
$$

where $P_{i}$ is the predicted value of the pollutant in a specific location, $\mathrm{O}_{\mathrm{i}}$ is the observed value of the pollutant in the same location, and $\mathrm{N}$ is the total number of the predictions used for the comparison. These parameters were evaluated separately for each month long simulations.

The bias and error between the model predictions and measured concentrations are summarized in Tables 10-13. The fractional bias for most species in most months is less than 0.3 indicating relatively little systematic bias in the model predictions. However, there is reasonable amount of scatter with most species having fractional errors values for many species being around 0.5 . This indicates that the model has difficulty reproducing the observed variation in the daily concentrations across the range of sites.

Sulfate: The best agreement between the model predictions and observations of sulfate are in January 2002 and April 2002. The fractional error for January and April are 0.44 and 0.39 , respectively. During July 2001, there is little systematic bias in the model predictions, but more scatter than the winter months. During October, PMCAMx 
overpredicts sulfate across the majority of the modeling domain. We attribute this to the high concentrations of ammonia that the model predicts that affect the cloud $\mathrm{pH}$ and accelerate the oxidation of $\mathrm{SO}_{2}$ to $\mathrm{H}_{2} \mathrm{SO}_{4}$.

Nitrate: During the summer $81 \%$ of the model nitrate predictions during summer diverge less than $\pm 30 \%$ or $\pm 0.5 \mu \mathrm{g} / \mathrm{m}^{3}$ from the measurements. In most of the domain the model slightly underpredicts the concentration of nitrate. This underprediction mainly occurs when the values of nitrate measurements are low levels, less than $1 \mu \mathrm{g} / \mathrm{m}^{3}$. Considering that the experimental measurements are uncertain by a $\pm 0.5 \mu \mathrm{g} / \mathrm{m}^{3}$, this discrepancy could be partially due to the measurements. However, there are few areas such as Baltimore, MD where the model significantly underpredicts the concentration of nitrate.

Model predictions of nitrate are sensitive to the ammonia concentration. Ammonia reacts preferentially with $\mathrm{H}_{2} \mathrm{SO}_{4}$ and, therefore only reacts with $\mathrm{HNO}_{3}$ to form particulate nitrate if sufficient $\mathrm{NH}_{3}$ is available. Thus, large underpredictions in nitrate concentrations are likely caused by underpredictions of ammonia emissions. In addition, there are some areas where sulfate is overpredicted by the model and consequently moves all the available $\mathrm{NH}_{3}$ to particulate phase which causes underprediction of nitrate.

Ammonium: The IMPROVE network does not measure ammonium, so the model performance can only be evaluated at the STN sites. Reasonable agreement between the predicted and measured ammonium concentrations is observed in all months but October 2001. During October the model overpredicts the concentrations of ammonium in most areas giving a fractional error of 0.91 and a mean bias $0.76 \mu \mathrm{g} / \mathrm{m}^{3}$. These problems are probably related to the ammonia emission inventory. The inventory predicts high emissions of ammonia in the fall associated with fertilizer application on farms. However, the agricultural activities on that season are uncertain and the ammonium emissions in such areas are probably overestimated. As a consequence, the emission inventory of ammonium over agricultural areas in the fall should be re-examined.

Organic PM: The model predictions for the organic mass are generally in agreement with the measurements in most areas and seasons. For example, in October 2001 PMCAMx predicts an average $3.1 \mu \mathrm{g} / \mathrm{m}^{3}$ for the organic mass concentration while the average of the measurements is $2.9 \mu \mathrm{g} / \mathrm{m}^{3}$. During July 2001 the model underpredicts the concentration of the organic mass, especially in comparison with most of the STN monitoring stations. However, a complication is the fact that the STN carbon data are not corrected for handling blanks which have been shown to create a significant bias in the data relative to the IMPROVE network. The overall bias is $-0.20 \mu \mathrm{g} / \mathrm{m}^{3}$, while the average concentration from the measurements is $2.92 \mu \mathrm{g} / \mathrm{m}^{3}$. During the winter, the model overpredicts the organic mass especially in comparison with some STN monitors in the east coast. The fractional bias during January is 0.31 while the fractional error is 0.58. During the spring, the model predicts high levels of organic mass in south Louisiana, which are not in agreement with the measurements from this area (STN stations). The evaluation of the model in April gives a fractional bias and error of 0.13 and 0.52 respectively. 
Elemental Carbon: During July 2001, the mean bias in the EC concentrations is 0.24 $\mu \mathrm{g} / \mathrm{m}^{3}$ when compared to the STN measurements and is unbiased $\left(-0.03 \mu \mathrm{g} / \mathrm{m}^{3}\right)$ when compared to the IMPROVE network. IMPROVE EC levels are much lower than STN because the IMPROVE sites are located in rural areas. Therefore, one explanation of the bias between the model predictions and the STN EC data is that the EC emissions in cities are overestimated. A complication is the well known inconsistencies in EC measured made using different analytical techniques. The EC analysis method by the IMPROVE network often measures more EC than that used by the STN network. The overall mean bias for the four months is $0.08 \mu \mathrm{g} / \mathrm{m}^{3}$ and $0.16 \mu \mathrm{g} / \mathrm{m}^{3}$, for the IMPROVE and the STN data respectively, while the overall average measurement is $0.31 \mu \mathrm{g} / \mathrm{m}^{3}$ and $0.59 \mu \mathrm{g} / \mathrm{m}^{3}$ respectively. During October 2001 and January 2002 the fractional error is 0.50 and 0.46 respectively. In spring (April 2002), the model has the better agreement with the measurements giving a fractional bias of 0.16 .

$\mathrm{PM}_{2.5}$ Concentration: As with most of the individual species, the model predictions generally agree with the measurements during the four seasons of the year giving an average of $12.2 \mu \mathrm{g} / \mathrm{m}^{3}$ across all sites while the average of the measured concentrations is $10.9 \mu \mathrm{g} / \mathrm{m}^{3}$. During July 2001 , the model underpredicts the $\mathrm{PM}_{2.5}$ mass concentration with a fractional bias of -0.17 . Part of this underprediction may be due to the measurements of water by the FRM PM 2.5 method (Rees et al. 2004). In certain periods regional biases in model predictions are observed. For example, in October 2001, the model overpredicts PM2.5 mass along the urbanized northeast corridor but underpredicts $\mathrm{PM}_{2.5}$ mass in the Midwest. The fractional error during the autumn is 0.50 . The fractional bias during this month is 0.35 while the fractional error is 0.49 . During April 2002, the model has the best agreement between the prediction and the observation in most of the PM species. That leads to a significant well prediction for the overall $\mathrm{PM}_{2.5}$ mass too. The fractional bias is 0.15 while the fractional error is 0.41 . 


\section{Summer}
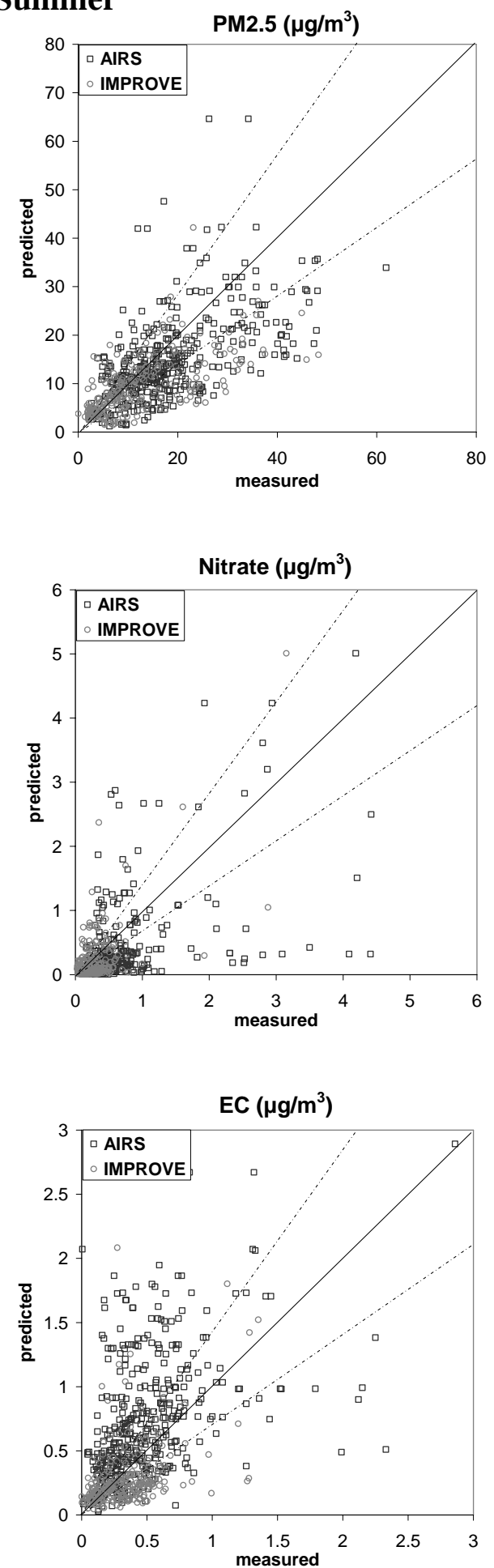
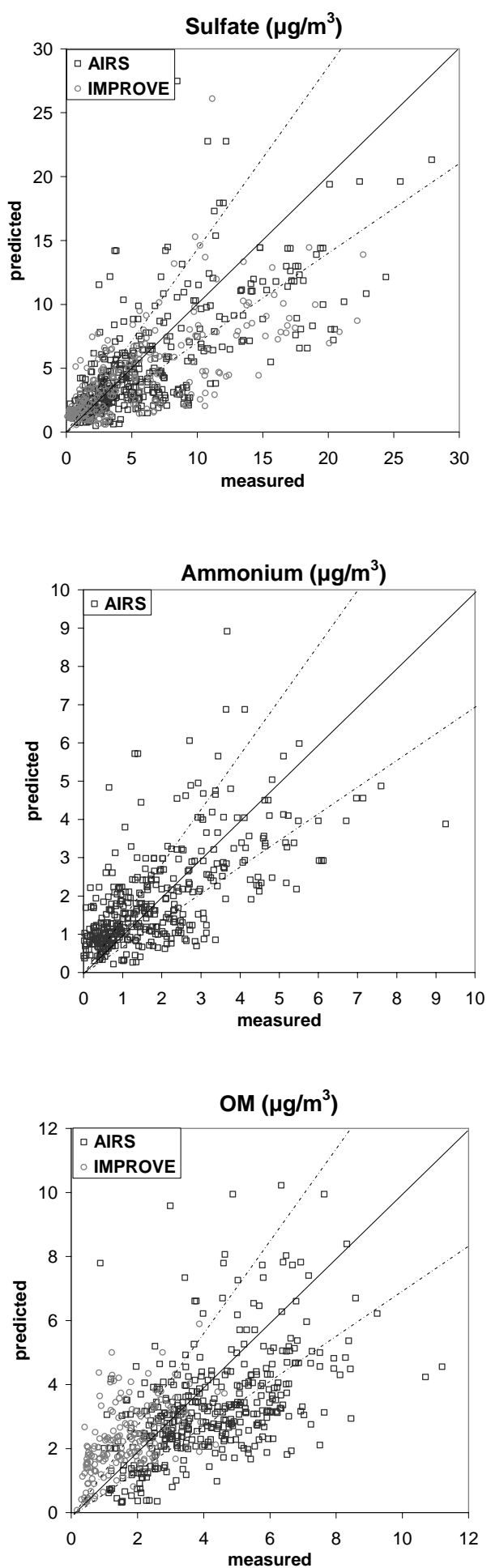

Figure 19. Comparison of predicted $\mathrm{PM}_{2.5}$ sulfate, $\mathrm{PM}_{2.5}$ nitrate, $\mathrm{PM}_{2.5}$ ammonium, organic mass, elemental carbon and total $\mathrm{PM}_{2.5}$ mass to data from the AIRS and IMPROVE Networks during July 2001. Also shown are the $1: 1$ and $\pm 30 \%$ lines. 


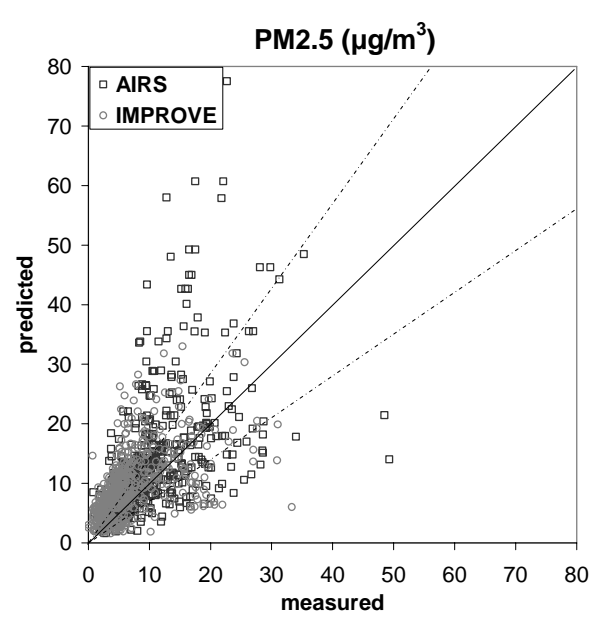

FALL
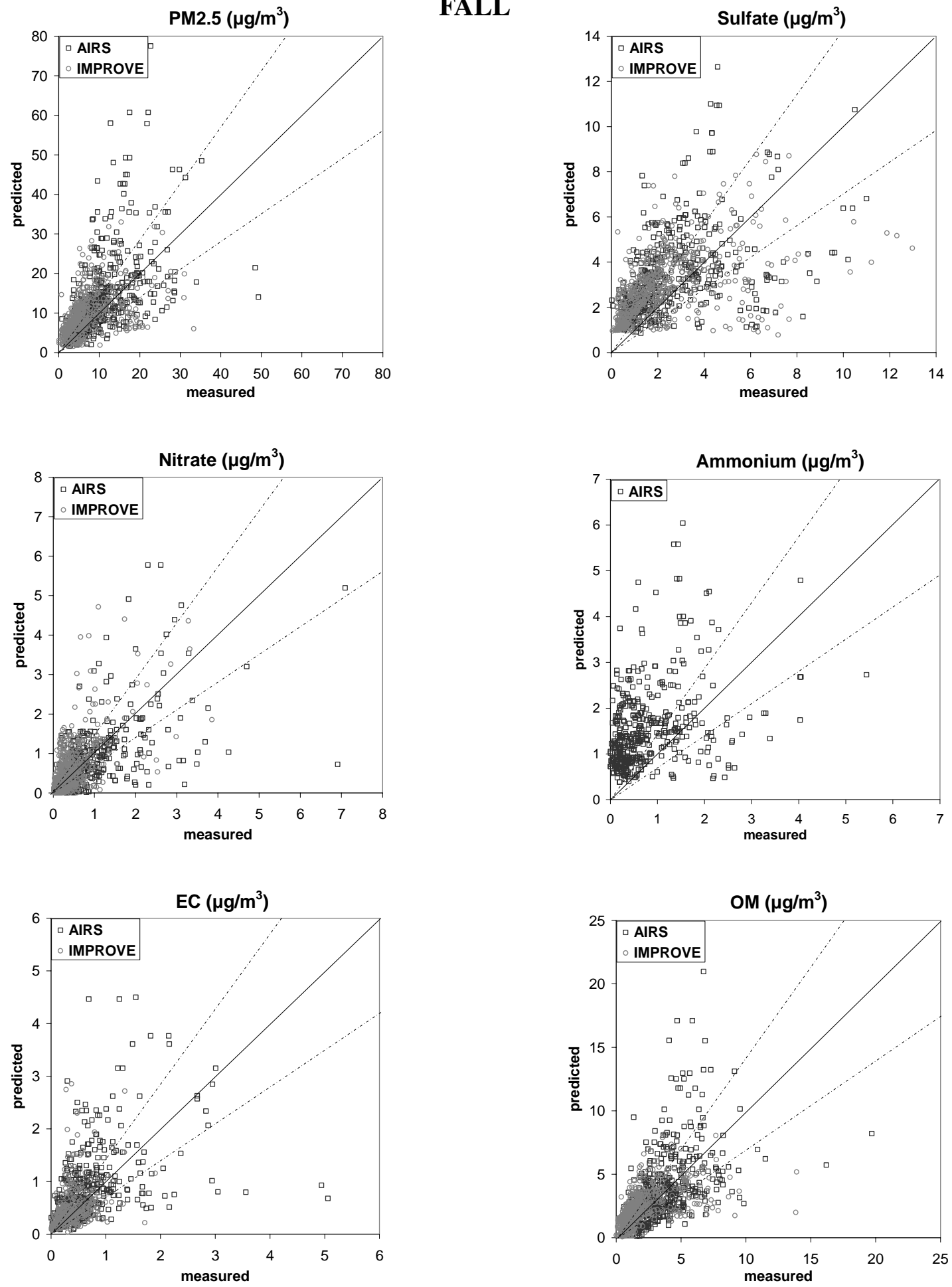

Figure 20. Comparison of predicted $\mathrm{PM}_{2.5}$ sulfate, $\mathrm{PM}_{2.5}$ nitrate, $\mathrm{PM}_{2.5}$ ammonium, organic mass, elemental carbon and total $\mathrm{PM}_{2.5}$ mass to data from the AIRS and IMPROVE Networks during October 2001. Also shown are the 1:1 and $\pm 30 \%$ lines. 


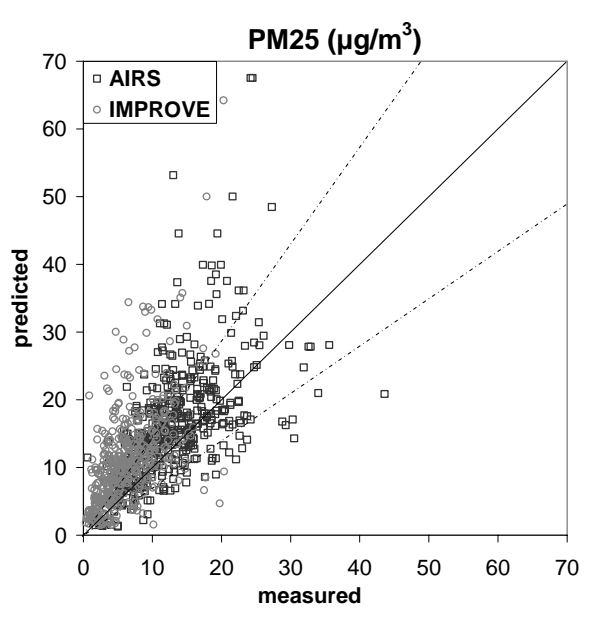

WINTER
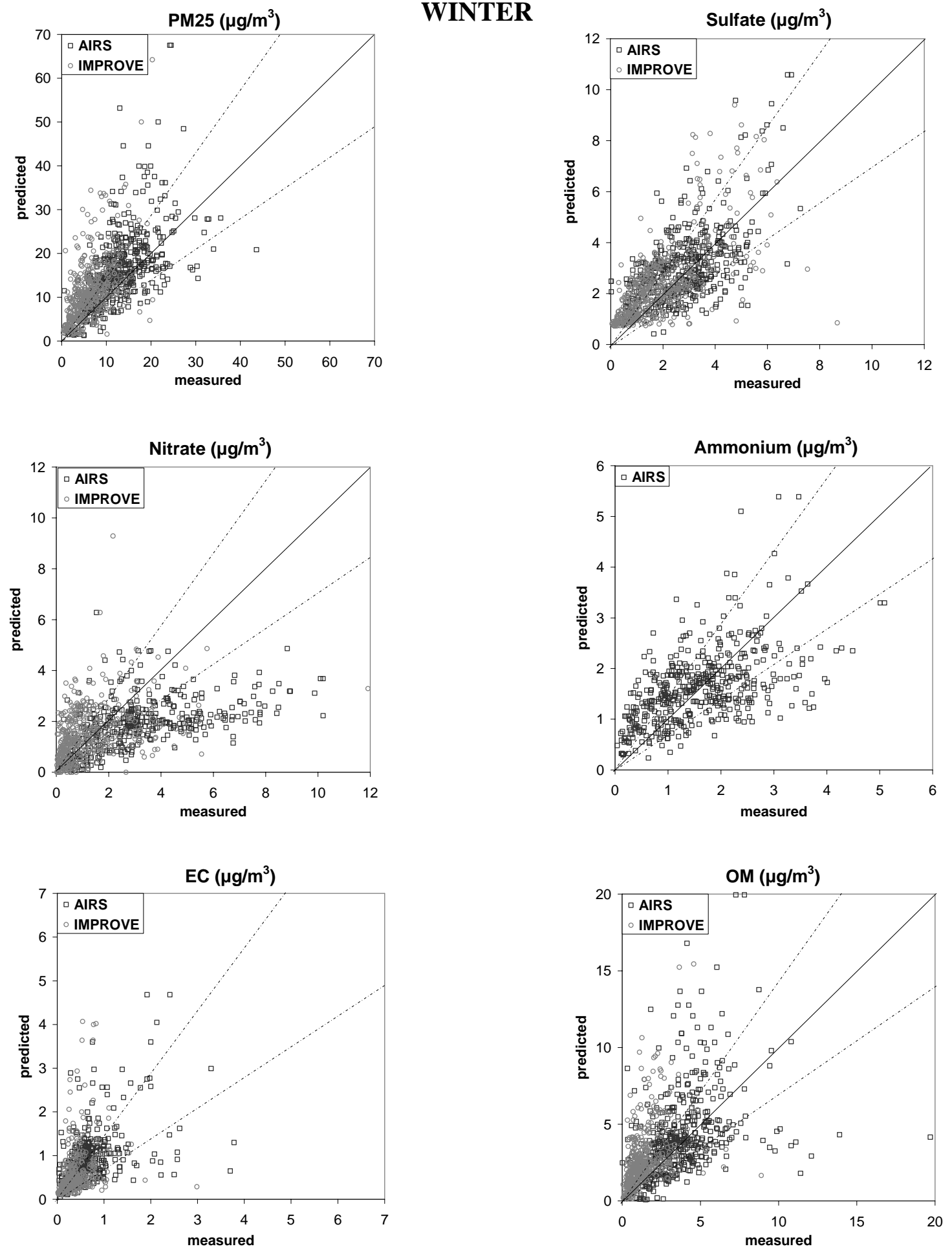

Figure 21. Comparison of predicted $\mathrm{PM}_{2.5}$ sulfate, $\mathrm{PM}_{2.5}$ nitrate, $\mathrm{PM}_{2.5}$ ammonium, organic mass, elemental carbon and total $\mathrm{PM}_{2.5}$ mass to data from the AIRS and IMPROVE Networks during January 2002. Also shown are the 1:1 and $\pm 30 \%$ lines. 


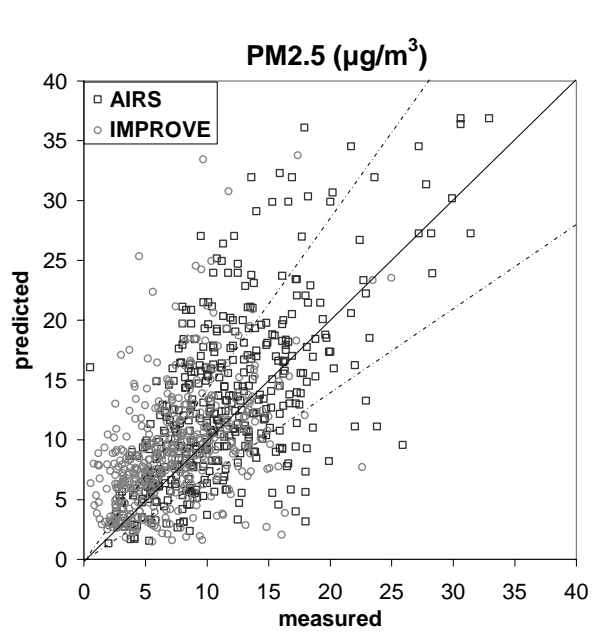

\section{SPRING}
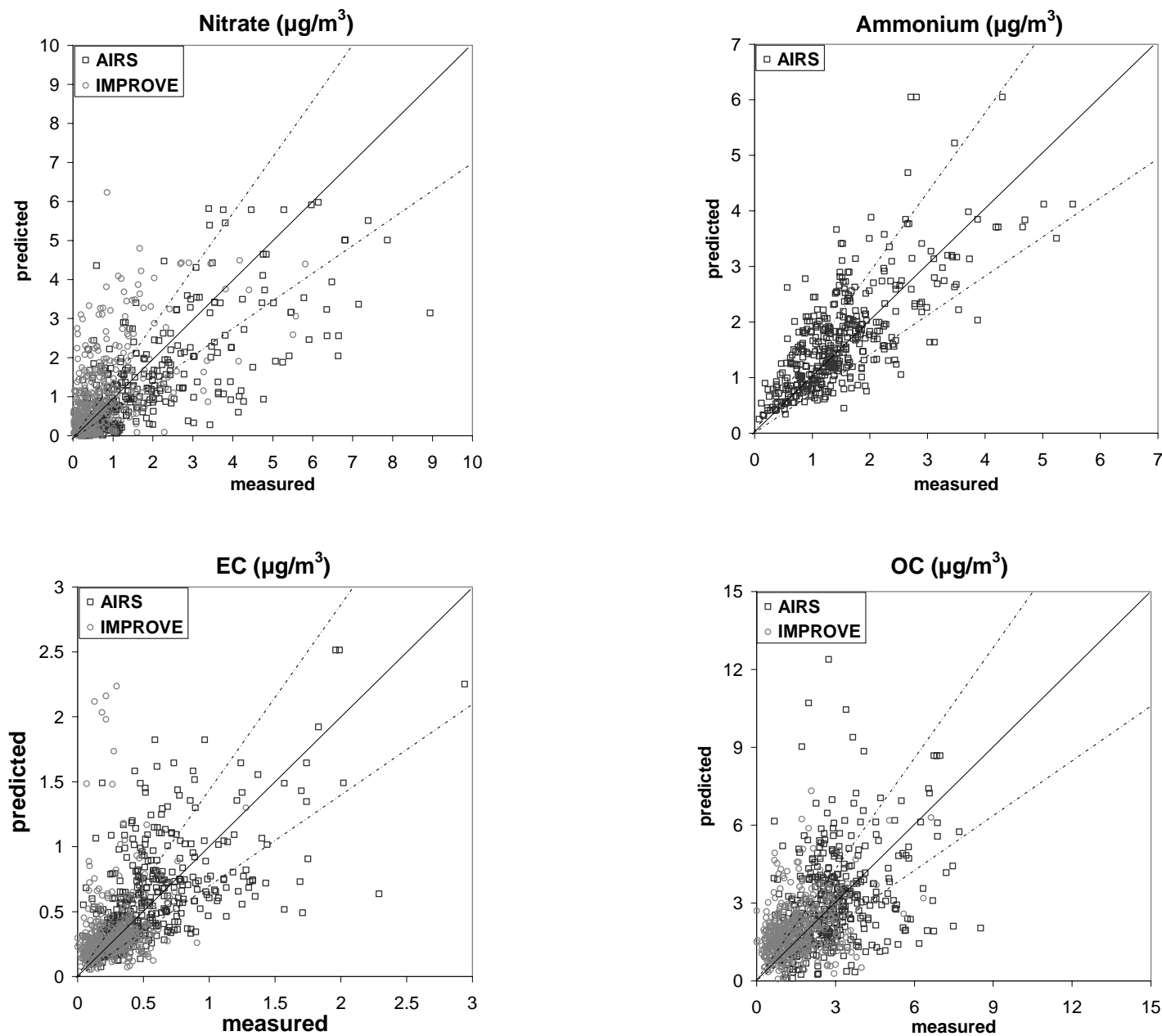

Figure 22. Comparison of predicted $\mathrm{PM}_{2.5}$ sulfate, $\mathrm{PM}_{2.5}$ nitrate, $\mathrm{PM}_{2.5}$ ammonium, organic mass, elemental carbon and total $\mathrm{PM}_{2.5}$ mass to data from the AIRS and IMPROVE Networks during April 2002. Also shown are the 1:1 and $\pm 30 \%$ lines. 
Table 10. Comparison of PMCAMx predictions with daily average measurements during the period of July 2001 .

\begin{tabular}{|c|c|c|c|c|c|c|c|}
\hline Element & $\begin{array}{c}\text { Predicted } \\
\text { Average } \\
\left(\mu \mathrm{g} / \mathrm{m}^{3}\right)\end{array}$ & $\begin{array}{c}\text { Measured } \\
\text { Average } \\
\left(\mu \mathrm{g} / \mathrm{m}^{3}\right)\end{array}$ & $\begin{array}{c}\text { Bias } \\
\left(\mu \mathrm{g} / \mathrm{m}^{3}\right)\end{array}$ & $\begin{array}{c}\text { Error } \\
\left(\mu \mathrm{g} / \mathrm{m}^{3}\right)\end{array}$ & $\begin{array}{c}\text { Fractional } \\
\text { Bias }\end{array}$ & $\begin{array}{c}\text { Fractional } \\
\text { Error }\end{array}$ & $\begin{array}{c}\text { Number of } \\
\text { comparisons } \\
(\mathrm{N})\end{array}$ \\
\hline Sulfate & 4.77 & 5.73 & -0.96 & 2.57 & -0.03 & 0.54 & 660 \\
\hline Nitrate & 0.34 & 0.51 & -0.17 & 0.36 & -0.62 & 0.92 & 530 \\
\hline Ammonium & 1.88 & 1.84 & 0.04 & 0.86 & 0.15 & 0.53 & 405 \\
\hline $\begin{array}{c}\text { Organic } \\
\text { Mass }\end{array}$ & 2.73 & 2.92 & -0.20 & 1.19 & 0.02 & 0.46 & 670 \\
\hline $\begin{array}{c}\text { Elemental } \\
\text { Carbon }\end{array}$ & 0.51 & 0.41 & 0.11 & 0.27 & 0.14 & 0.57 & 670 \\
\hline PM $_{2.5}$ mass & 12.21 & 15.39 & -3.18 & 6.22 & -0.17 & 0.46 & 661 \\
\hline
\end{tabular}

Table 11. Comparison of PMCAMx predictions with daily average measurements during October 2001.

\begin{tabular}{|c|c|c|c|c|c|c|c|}
\hline Element & $\begin{array}{c}\text { Predicted } \\
\text { Average } \\
\left(\mu \mathrm{g} / \mathrm{m}^{3}\right)\end{array}$ & $\begin{array}{c}\text { Measured } \\
\text { Average } \\
\left(\mu \mathrm{g} / \mathrm{m}^{3}\right)\end{array}$ & $\begin{array}{c}\text { Bias } \\
\left(\mu \mathrm{g} / \mathrm{m}^{3}\right)\end{array}$ & $\begin{array}{c}\text { Error } \\
\left(\mu \mathrm{g} / \mathrm{m}^{3}\right)\end{array}$ & $\begin{array}{c}\text { Fractional } \\
\text { Bias }\end{array}$ & $\begin{array}{c}\text { Fractional } \\
\text { Error }\end{array}$ & $\begin{array}{c}\text { Number of } \\
\text { comparisons } \\
(\mathrm{N})\end{array}$ \\
\hline Sulfate & 3.08 & 2.37 & -0.71 & 1.54 & 0.41 & 0.62 & 1015 \\
\hline Nitrate & 0.73 & 0.70 & 0.03 & 0.43 & -0.06 & 0.69 & 921 \\
\hline Ammonium & 1.54 & 0.78 & 0.76 & 0.98 & 0.78 & 0.91 & 443 \\
\hline $\begin{array}{c}\text { Organic } \\
\text { Mass }\end{array}$ & 3.07 & 2.90 & 0.17 & 1.42 & 0.06 & 0.50 & 1025 \\
\hline $\begin{array}{c}\text { Elemental } \\
\text { Carbon }\end{array}$ & 0.60 & 0.48 & 0.11 & 0.29 & 0.21 & 0.48 & 1025 \\
\hline PM $_{2.5}$ mass & 11.73 & 8.90 & 2.83 & 5.15 & 0.29 & 0.50 & 1021 \\
\hline
\end{tabular}


Table 12. Comparison of PMCAMx predictions with daily average measurements during January 2002.

\begin{tabular}{|c|c|c|c|c|c|c|c|}
\hline Element & $\begin{array}{c}\text { Predicted } \\
\text { Average } \\
\left(\mu \mathrm{g} / \mathrm{m}^{3}\right)\end{array}$ & $\begin{array}{c}\text { Measured } \\
\text { Average } \\
\left(\mu \mathrm{g} / \mathrm{m}^{3}\right)\end{array}$ & $\begin{array}{c}\text { Bias } \\
\left(\mu \mathrm{g} / \mathrm{m}^{3}\right)\end{array}$ & $\begin{array}{c}\text { Error } \\
\left(\mu \mathrm{g} / \mathrm{m}^{3}\right)\end{array}$ & $\begin{array}{c}\text { Fractional } \\
\text { Bias }\end{array}$ & $\begin{array}{c}\text { Fractional } \\
\text { Error }\end{array}$ & $\begin{array}{c}\text { Number of } \\
\text { comparisons } \\
(\mathrm{N})\end{array}$ \\
\hline Sulfate & 2.70 & 2.36 & 0.34 & 0.96 & 0.20 & 0.44 & 925 \\
\hline Nitrate & 1.66 & 2.20 & -0.54 & 1.26 & -0.09 & 0.69 & 853 \\
\hline Ammonium & 1.62 & 1.59 & 0.03 & 0.63 & 0.12 & 0.44 & 457 \\
\hline $\begin{array}{c}\text { Organic } \\
\text { Mass }\end{array}$ & 3.47 & 2.55 & 0.92 & 1.73 & 0.31 & 0.58 & 920 \\
\hline $\begin{array}{c}\text { Elemental } \\
\text { Carbon }\end{array}$ & 0.69 & 0.50 & 0.20 & 0.34 & 0.30 & 0.50 & 920 \\
\hline $\mathrm{PM}_{2.5}$ mass & 13.89 & 9.80 & 4.08 & 5.54 & 0.35 & 0.49 & 920 \\
\hline
\end{tabular}

Table 13. Comparison of PMCAMx predictions with daily average measurements during April 2002.

\begin{tabular}{|c|c|c|c|c|c|c|c|}
\hline Element & $\begin{array}{c}\text { Predicted } \\
\text { Average } \\
\left(\mu \mathrm{g} / \mathrm{m}^{3}\right)\end{array}$ & $\begin{array}{c}\text { Measured } \\
\text { Average } \\
\left(\mu \mathrm{g} / \mathrm{m}^{3}\right)\end{array}$ & $\begin{array}{c}\text { Bias } \\
\left(\mu \mathrm{g} / \mathrm{m}^{3}\right)\end{array}$ & $\begin{array}{c}\text { Error } \\
\left(\mu \mathrm{g} / \mathrm{m}^{3}\right)\end{array}$ & $\begin{array}{c}\text { Fractional } \\
\text { Bias }\end{array}$ & $\begin{array}{c}\text { Fractional } \\
\text { Error }\end{array}$ & $\begin{array}{c}\text { Number of } \\
\text { comparisons } \\
(\mathrm{N})\end{array}$ \\
\hline Sulfate & 3.16 & 3.17 & -0.01 & 1.14 & 0.02 & 0.39 & 881 \\
\hline Nitrate & 1.13 & 1.25 & -0.12 & 0.72 & -0.18 & 0.76 & 809 \\
\hline Ammonium & 1.69 & 1.50 & 0.19 & 0.51 & 0.14 & 0.34 & 418 \\
\hline $\begin{array}{c}\text { Organic } \\
\text { Mass }\end{array}$ & 2.49 & 2.22 & 0.27 & 1.15 & 0.13 & 0.52 & 882 \\
\hline $\begin{array}{c}\text { Elemental } \\
\text { Carbon }\end{array}$ & 0.47 & 0.41 & 0.06 & 0.21 & 0.16 & 0.46 & 882 \\
\hline $\mathrm{PM}_{2.5}$ mass & 11.08 & 9.41 & 1.67 & 3.96 & 0.15 & 0.41 & 888 \\
\hline
\end{tabular}




\section{$P M_{2.5}$ Response to Changes in the Emissions of its Precursors in the Eastern United}

\section{States}

Both observation based models and PMCAMx were used to investigate the sensitivity of fine particle concentrations to changes in emissions. First we summarize the major findings from the research based on observational models. Details on these findings are in the attached papers. We then describe in more detail the results from the PMCAMx simulations.

- An observation-based model, the thermodynamic model with removal (TMR) was developed to estimate responses of $\mathrm{PM}_{2.5}$ concentrations to changes in precursor concentrations. Use of the model requires semi-continuous measurements of sulfate, total nitrate, total ammonia, PM2.5 nitrate, and PM2.5 ammonium (Vayenas et al. 2005). [HYPOTHESIS 3.4]

- The ammonium nitrate concentrations in Western Pennsylvania are quite sensitive to the ammonia levels and reductions in $\mathrm{PM}_{2.5}$ concentrations may be assisted by reductions in ammonia emissions during both the summer and winter (Takahama et al. 2004). [HYPOTHESIS 3.4]

- The frequent nucleation events can be explained by a ternary sulfuric acidammonia-water nucleation model. The results are consistent with the composition of the fresh ultrafine particles of Zhang et al. (2004). Reductions of ammonia emissions are predicted to decrease the frequency of nucleation events during both summer and winter, with a more dramatic effect during the summer. Reductions of sulfur dioxide and the resulting sulfate by up to $40 \%$ are expected to increase the frequency of nucleation during the summer and decrease it during the winter (Gaydos et al. 2005b). [HYPOTHESIS 3.1]

- During the winter sulfate reductions will lead to increases in the nitrate concentrations in Pittsburgh. It is predicted that a 50\% sulfate reduction will lead to a $10 \%$ decrease of inorganic $\mathrm{PM}_{2.5}$ mass concentrations. For a $50 \%$ reduction in ammonia availability, inorganic $\mathrm{PM}_{2.5}$ was reduced by approximately $30 \%$, while for a $50 \%$ reduction in total nitric acid a $15-20 \%$ reduction in inorganic PM2.5 is predicted (Vayenas et al. 2005). [HYPOTHESIS 3.4]

- The available aerosol thermodynamic models can reproduce the observed nitrate partitioning within experimental error (Takahama et al. 2004)

The PMCAMx model was used to predict the changes in the concentrations of PM2.5 and its components after changes of the major precursors $\left(\mathrm{SO}_{2}, \mathrm{NH}_{3}, \mathrm{NO}_{\mathrm{x}}, \mathrm{VOCs}\right) \mathrm{A}$ spatially and temporally uniform emission change of $50 \%$ was assumed so these simulations should be viewed more as sensitivity tests exploring the sensitivity of the system to certain changes and not as analysis of detailed policy options. 
The concentration changes were estimated as:

Absolute Concentration Change $=[$ Basecase Concentration $]-[$ Concentration after Change $]$

Fractional Concentration Change $=\frac{[\text { Basecase Concentration }]-[\text { Concentration after Change }]}{[\text { Basecase Concentration }]}$

therefore positive responses correspond to concentration reductions and negative responses correspond to increases in concentration (even if the precursor emission was reduced).

\section{Changes in $\mathrm{SO}_{2}$ Emissions}

In these simulations a uniform $50 \%$ reduction in $\mathrm{SO}_{2}$ emissions was assumed for the whole modeling domain. Coal fired power plants are dominant source of $\mathrm{SO}_{2}$ emissions in Eastern US.

\subsection{Summertime (July 2001)}

The predicted average sulfate concentration for July 2001 is shown in Figure 23. The highest average concentrations are predicted in the Midwest US with values up to $12 \mu \mathrm{g}$ $\mathrm{m}^{-3}$. PMCAMx predicts that summertime reductions in emissions of $\mathrm{SO}_{2}$ will be quite efficient in reducing the sulfate concentrations, especially in the areas characterized by the highest concentrations (Figure 24).

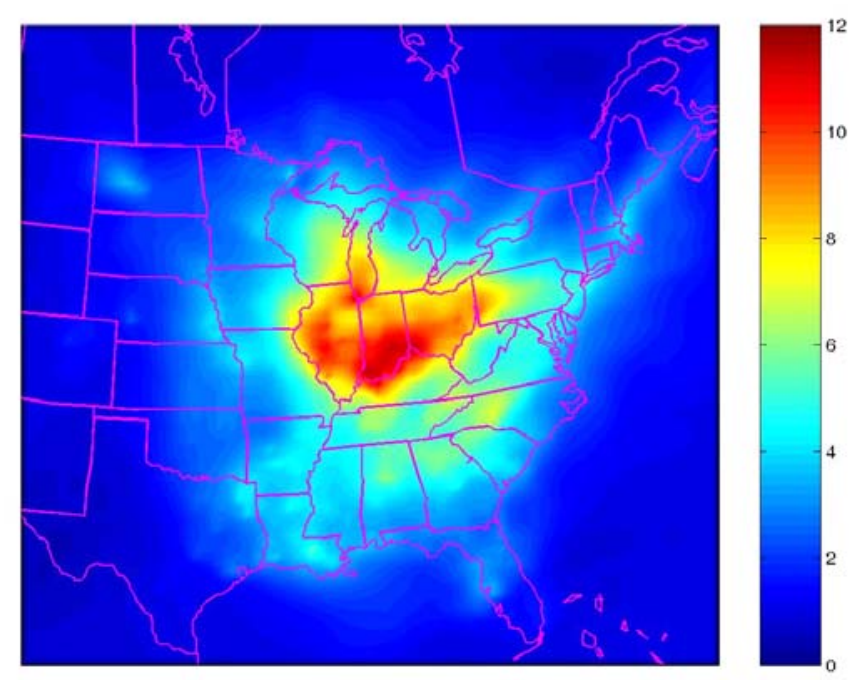

Figure 23. Predicted average base-case sulfate concentrations $\left(\mu \mathrm{g} \mathrm{m}^{-3}\right)$ for July 2001. 

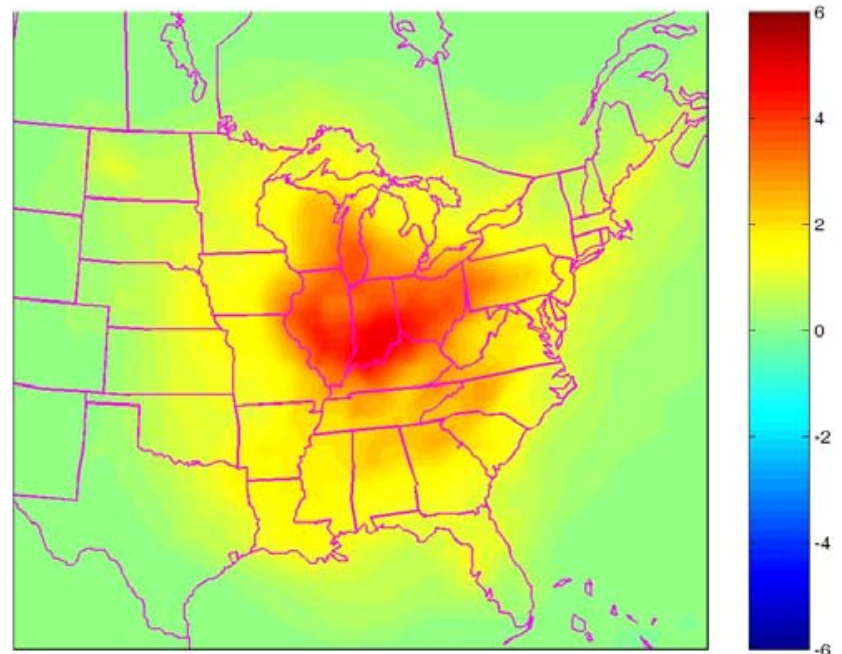

Figure 24. Predicted change in sulfate concentration for a $50 \%$ reduction in $\mathrm{SO}_{2}$ emissions for July 2001. A positive change corresponds to a concentration reduction.
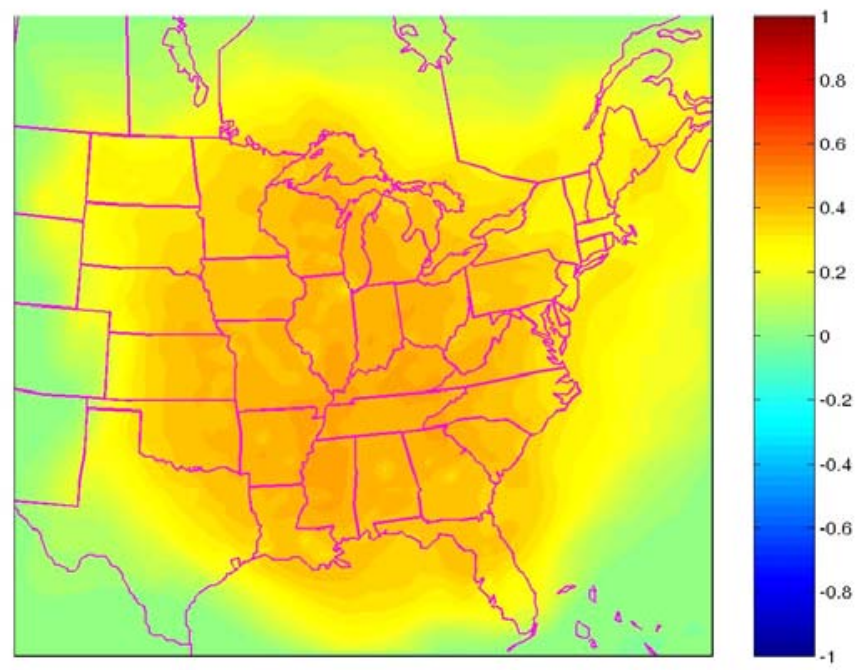

Figure 25. Predicted fractional change in sulfate concentration for a $50 \%$ reduction in $\mathrm{SO}_{2}$ emissions for July 2001. A positive change corresponds to a concentration reduction.

The fractional changes in the sulfate concentrations (Figure 25) are close to proportional (a 50\% reduction) for all areas that have an average sulfate concentration above $1 \mu \mathrm{g} \mathrm{m}^{-3}$. Smaller fractional changes smaller are observed near the boundaries of the modeling domain and are due to the long range transport of sulfate from areas outside the Eastern US. 
The changes in sulfate concentrations result in changes in the partitioning of nitrate between the gas and particulate phases. The base-case nitrate concentrations during the summer are relatively low (less than $1 \mu \mathrm{g} \mathrm{m}^{-3}$ ) in most of the modeling domain (Figure 26). However, there are areas with average nitrate concentrations above $1 \mu \mathrm{g} \mathrm{m} \mathrm{m}^{-3}$ in locations in the Midwest, North Carolina, eastern Pennsylvania, etc. The higher nitrate concentrations are predicted during the nighttime in agreement with the observations.

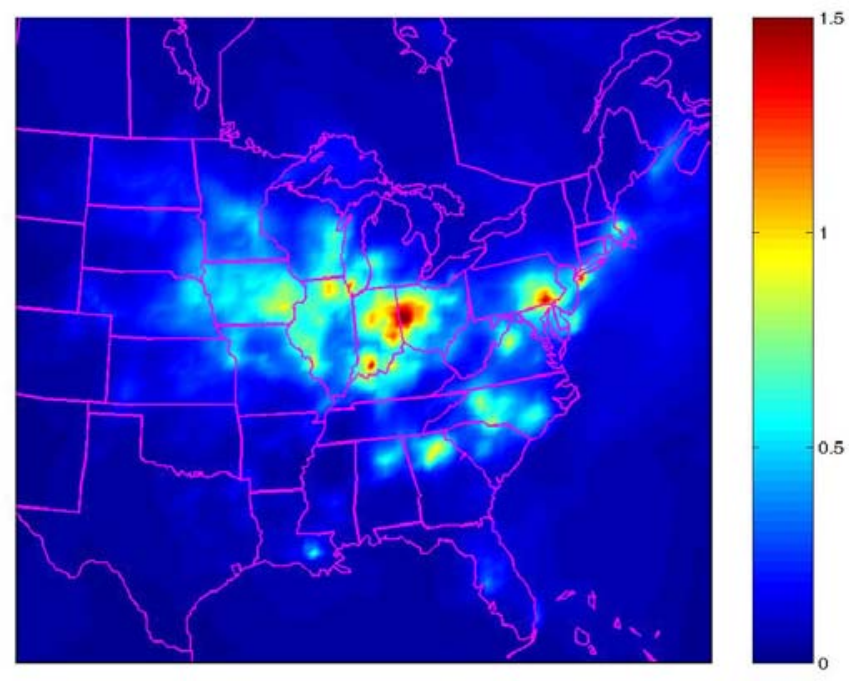

Figure 26. Predicted average base-case nitrate concentrations $\left(\mu \mathrm{g} \mathrm{m}^{-3}\right)$ for July 2001.

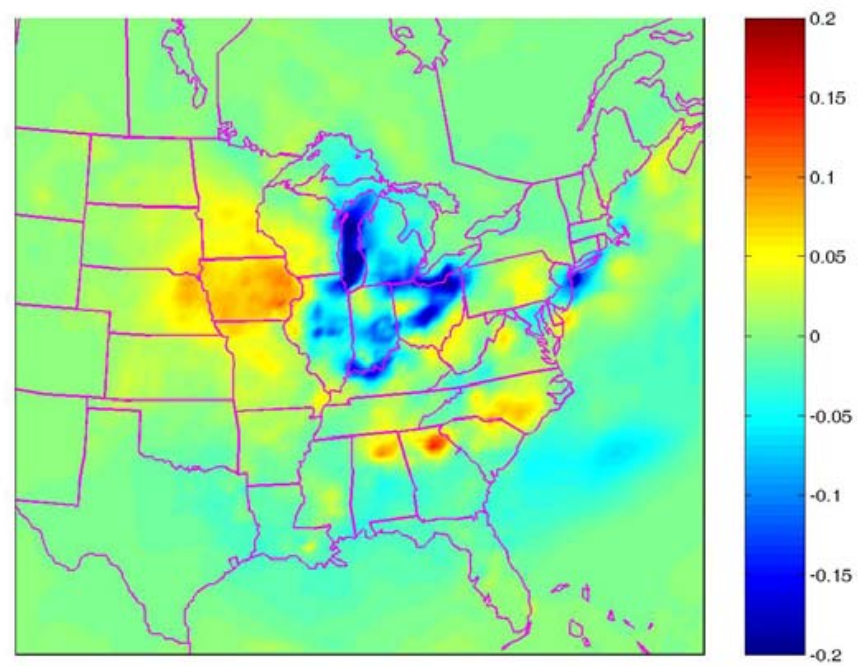

Figure 27. Predicted change $\left(\mu \mathrm{g} \mathrm{m}^{-3}\right)$ in nitrate concentration for a $50 \%$ reduction in $\mathrm{SO}_{2}$ emissions for July 2001. A positive change corresponds to a concentration reduction.

A decrease of the sulfate concentrations may lead to increases in the nitrate levels. The ammonia that used to be in the form of ammonium sulfate salts is released and may react with the available nitric acid vapor to form ammonium nitrate. At the same time 
reductions in sulfate ions in aqueous particles reduce the ionic strength of the solution and can lead to decreases of the nitrate levels.

Figure 27 shows that PMCAMx predicts that a 50\% reduction in $\mathrm{SO} 2$ emissions will cause only modest change in the average nitrate concentrations during the summertime (between +0.2 and $-0.2 \mu \mathrm{g} \mathrm{m}^{-3}$ ). The major increases in nitrate concentration are predicted to take place in the Midwest especially around Chicago but also in the eastern seaboard in the areas between Baltimore and Boston. In these areas, there are significant concentrations of all the major inorganic aerosol precursors (sulfur dioxide, nitric acid, and ammonia). On the other hand, reductions of the average nitrate are predicted for parts of the South such as Atlanta and also parts of the Midwest (Iowa, Nebraska, Minnesota, Wisconsin, etc.). These areas are characterized by relatively low levels of NOx and high ammonia therefore most of the available nitric acid is already in the particulate phase. Reductions of sulfate drive some of this aerosol nitrate to the gas phase by increasing its activity coefficient in the aqueous solution that has now fewer ions.

The highest ammonium concentrations are predicted to be in the Midwest with levels up to $4 \mu \mathrm{g} \mathrm{m}^{-3}$ (Figure 28). The reduction in sulfate is predicted to lead to a significant reduction in the ammonium which is transfer to the gas phase as ammonia (Figure 29).

The largest ammonium reductions (up to $1.5 \mu \mathrm{g} \mathrm{m}^{-3}$ ) are predicted to take place in the Midwest (Figure 29) that is in the area where the base-case concentrations are the highest. These ammonium reductions are an additional benefit of the $\mathrm{SO}_{2}$ emission reductions in the effort to reduce $\mathrm{PM}_{2.5}$ levels.

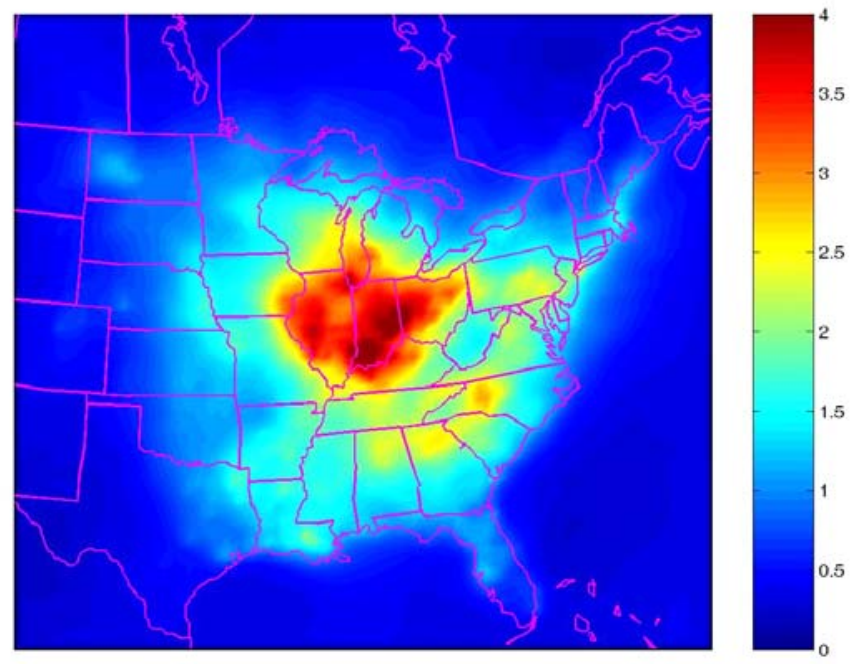

Figure 28. Predicted average base-case ammonium concentrations $\left(\mu \mathrm{g} \mathrm{m}^{-3}\right)$ for July 2001. 

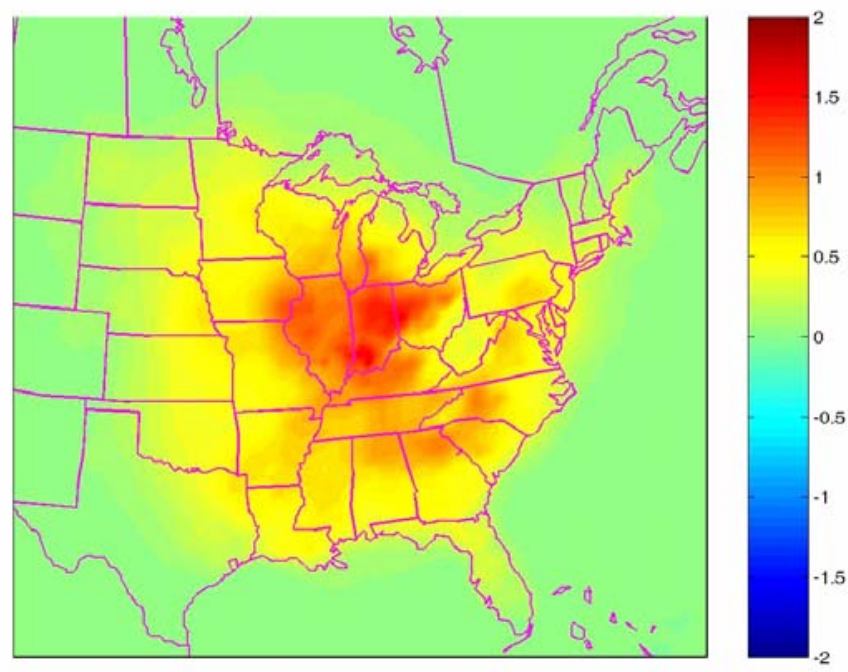

Figure 29. Predicted change $\left(\mu \mathrm{g} \mathrm{m}^{-3}\right)$ in ammonium concentration for a $50 \%$ reduction in $\mathrm{SO}_{2}$ emissions for July 2001. A positive change corresponds to a concentration reduction.

The concentrations of the other major $\mathrm{PM}_{2.5}$ components (primary and secondary organic aerosol, elemental carbon, dust) are predicted to remain the same for all practical purposes (less than $1 \%$ change) as $\mathrm{SO}_{2}$ emissions are reduced.

The net predicted change in the $\mathrm{PM}_{2.5}$ concentrations is the sum of the changes in sulfate, nitrate, and ammonium and is clearly dominated by the reductions in sulfate. The highest PM2.5 concentrations are predicted to be in a large area including Illinois, Indiana, Ohio, and Western Pennsylvania while high concentrations also exist in major metropolitan areas such as New York, Boston, and Atlanta (Figure 30). 


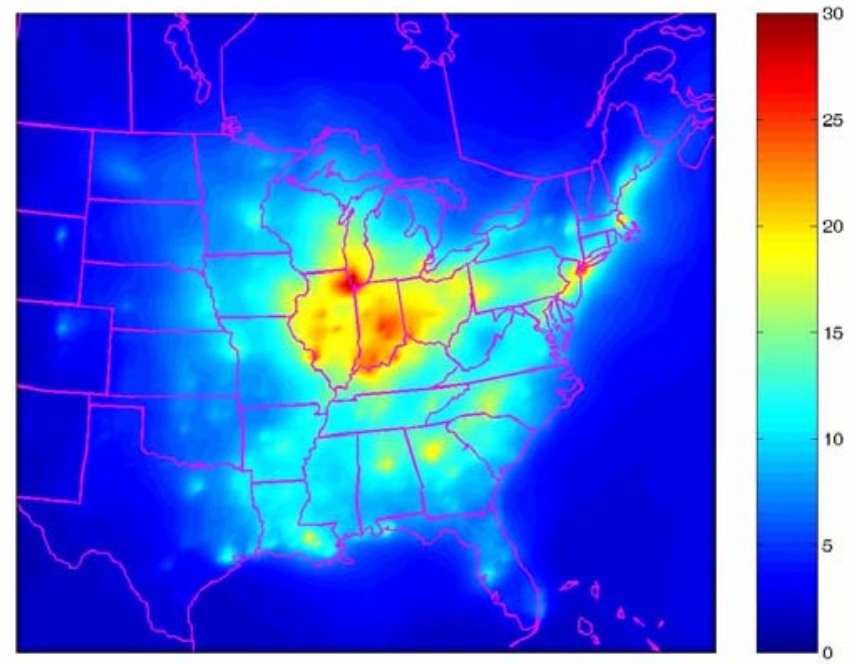

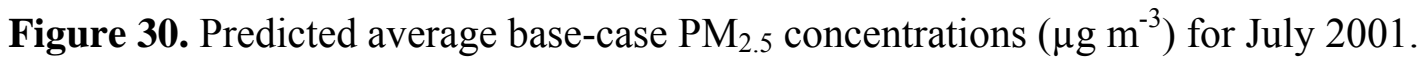
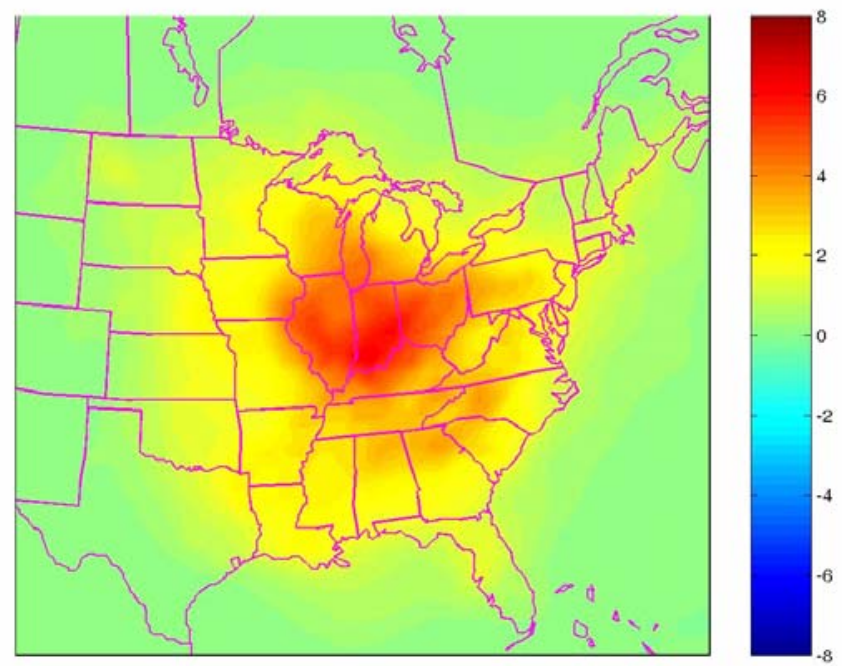

Figure 31. Predicted change $\left(\mu \mathrm{g} \mathrm{m}^{-3}\right)$ in $\mathrm{PM}_{2.5}$ concentration for a $50 \%$ change in $\mathrm{SO}_{2}$ emissions for July 2001. A positive change corresponds to a concentration reduction.

The $50 \%$ reduction in $\mathrm{SO}_{2}$ emissions is predicted to lead to reductions of the average $\mathrm{PM}_{2.5}$ levels during July by $3-5 \mu \mathrm{g} \mathrm{m}^{-3}$ (Figure 31 ). The highest reductions are once more in the Midwest in the areas where sulfate and PM2.5 levels are the highest.

The fractional change in $\mathrm{PM}_{2.5}$ concentrations is depicted in Figure 32. For most of the Eastern US the predicted reductions in average $\mathrm{PM}_{2.5}$ during July are in the $20-30 \%$ 
range. These reductions suggest strongly that reductions of $\mathrm{SO}_{2}$ emissions from coal-fired power plants during the summer will lead to significant reductions of the average fine particulate matter concentrations during the summer in the Eastern US.
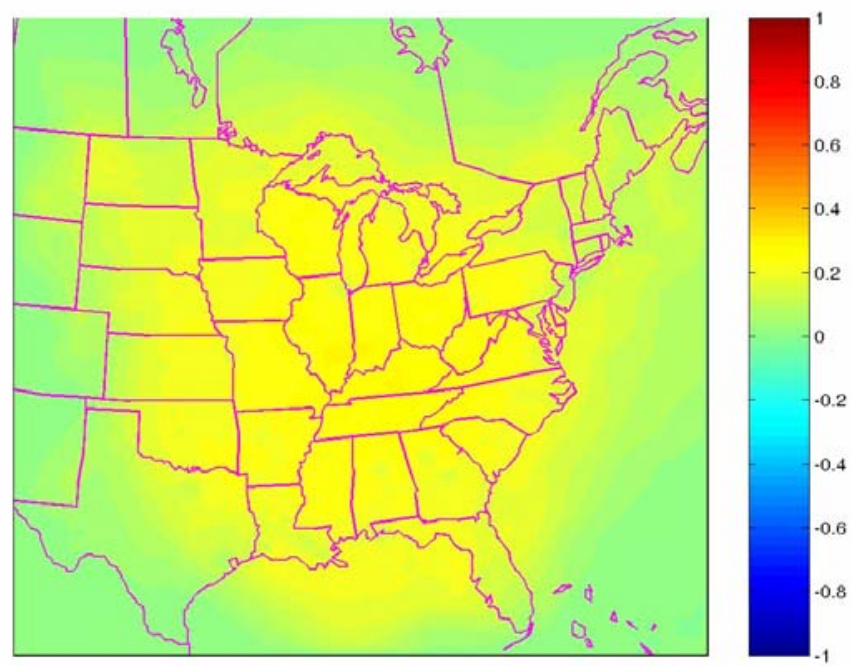

Figure 32. Predicted fractional change in $\mathrm{PM}_{2.5}$ concentration for a $50 \%$ change in $\mathrm{SO}_{2}$ emissions for July 2001. A positive change corresponds to a concentration reduction.

\subsection{Wintertime (January 2002)}

The sulfate concentrations during winter (Figure 33) are significantly lower compared to the summertime levels (Figure 23). Also the highest concentrations are now predicted to be in the Southeastern part of the country.

The assumed $50 \%$ spatially and temporally uniform reduction in $\mathrm{SO}_{2}$ emissions once more results in reductions of the predicted sulfate concentrations (Figure 34) with values up to $1.5 \mu \mathrm{g} \mathrm{m}^{-3}$ over Florida. 


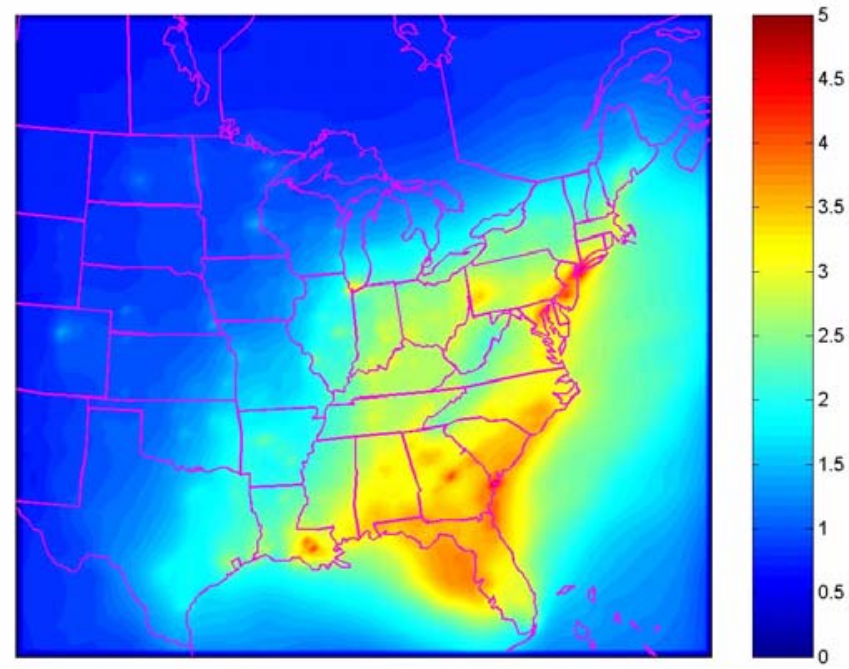

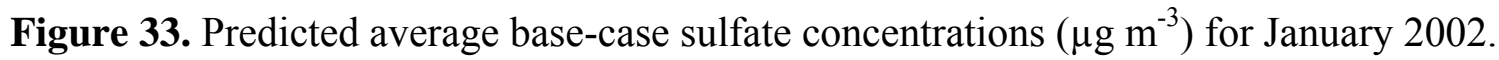
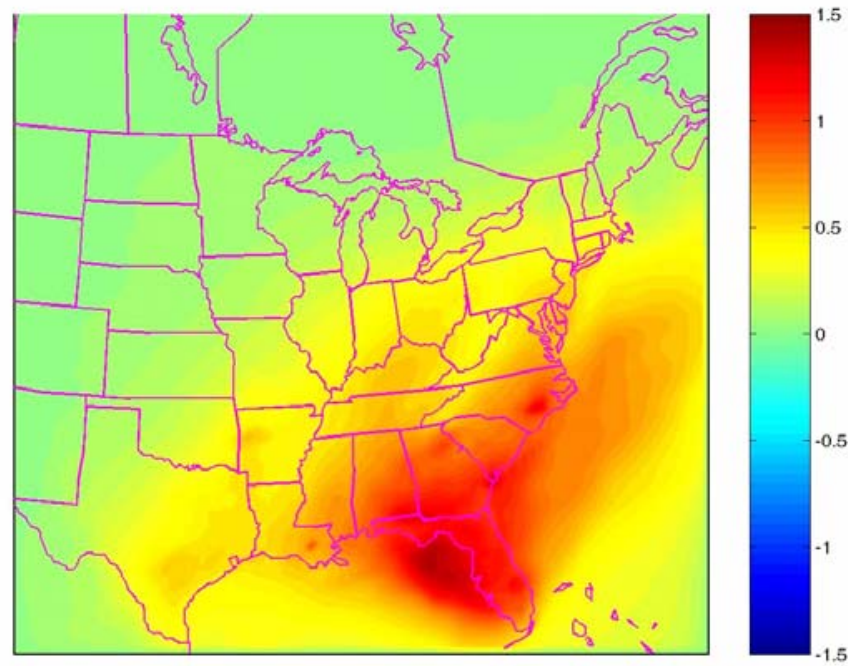

Figure 34. Predicted change in sulfate concentration $\left(\mu \mathrm{g} \mathrm{m}^{-3}\right)$ for a $50 \%$ reduction in $\mathrm{SO}_{2}$ emissions for January 2002. A positive change corresponds to a concentration reduction.

This predicted sulfate reduction is not proportional to the $\mathrm{SO}_{2}$ emission reduction. For most of the Eastern US the reduction is only 20-30\% (Figure 35) and only in the southeastern US it approaches $40 \%$. This lack of proportionality is due to the nonlinear cloud chemistry (it is limited by the hydrogen peroxide availability) and also to the fact that a more significant fraction of the sulfate is transported to the Eastern US from other areas. 


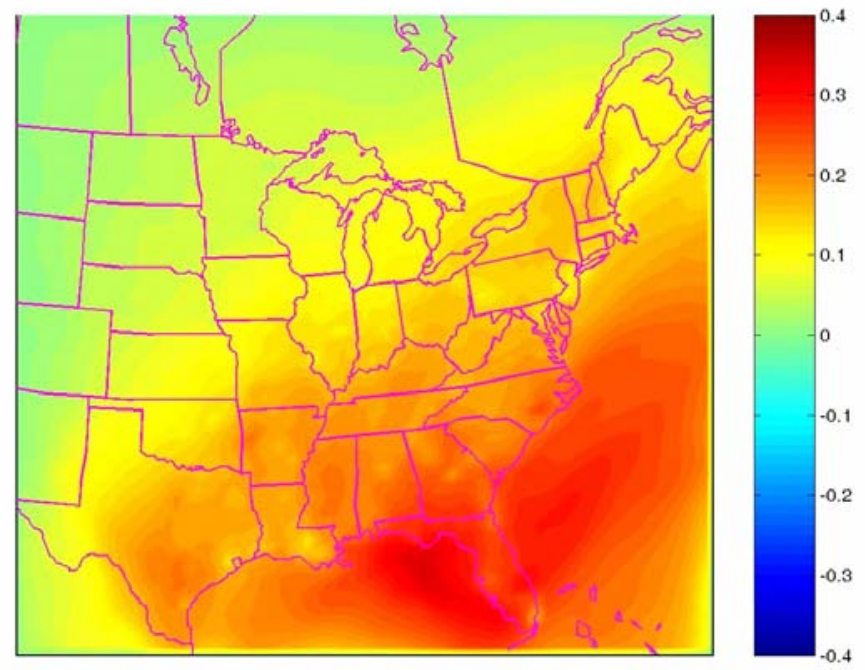

Figure 35. Predicted fractional change in sulfate concentration for a $50 \%$ reduction in $\mathrm{SO}_{2}$ emissions for January 2002. A positive change corresponds to a concentration reduction.

The fine particulate matter nitrate concentrations in the winter (Figure 36) are significantly higher than in the summer (Figure 26) with values up to $2.5 \mu \mathrm{g} \mathrm{m}^{-3}$. The average concentrations exceed $1.5 \mu \mathrm{g} \mathrm{m}^{-3}$ in most of the Northeastern US and are also quite high in North Carolina, and in the Atlanta area. These higher concentrations are mainly due to the change in partitioning of the total nitrate. The lower wintertime temperatures favor the existence of this total nitrate in the particulate phase.

The assumed $50 \%$ reduction in $\mathrm{SO}_{2}$ emissions and the corresponding sulfate reduction are accompanied mainly by increases of the aerosol nitrate concentrations (Figure 37). These increases are predicted to take place mainly in the Northeastern US and to be as much as $0.3 \mu \mathrm{g} \mathrm{m}^{-3}$. The nitrate concentration is predicted to increase over the entire Eastern continental US while it is predicted to decrease only over the Atlantic ocean and over the Gulf of Mexico. The predicted increases are due to the substitution of the sulfate by nitrate in the particulate phase and are consistent with the predictions of simpler thermodynamic models. 


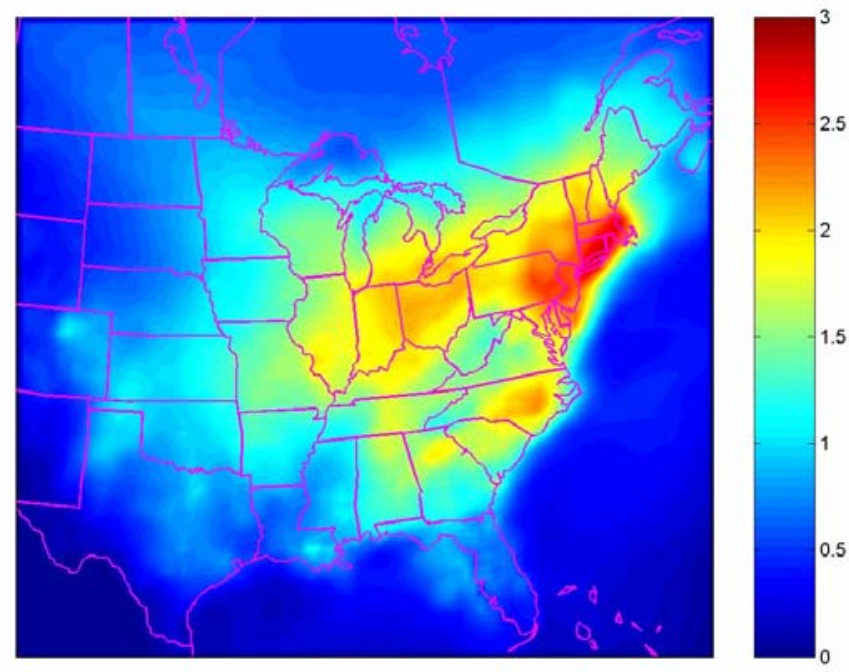

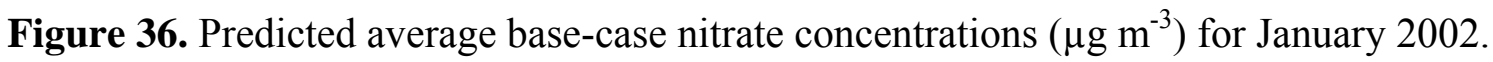

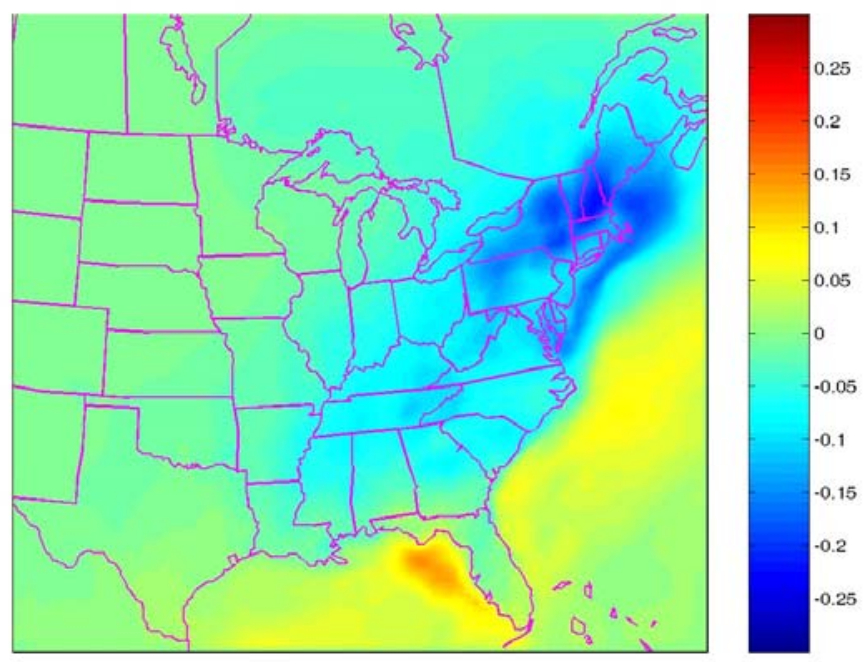

Figure 37. Predicted change $\left(\mu \mathrm{g} \mathrm{m}^{-3}\right)$ in nitrate concentration for a $50 \%$ reduction in $\mathrm{SO}_{2}$ emissions for January 2002. A positive change corresponds to a concentration reduction.

The base-case wintertime ammonium concentrations are shown in Figure 38. The predicted concentrations are higher in the Eastern Coast starting from South Carolina and extending all the way to Massachusetts. These areas are characterized by high concentrations of either sulfate or nitrate and also availability of ammonia to form ammonium sulfate or ammonium nitrate. 


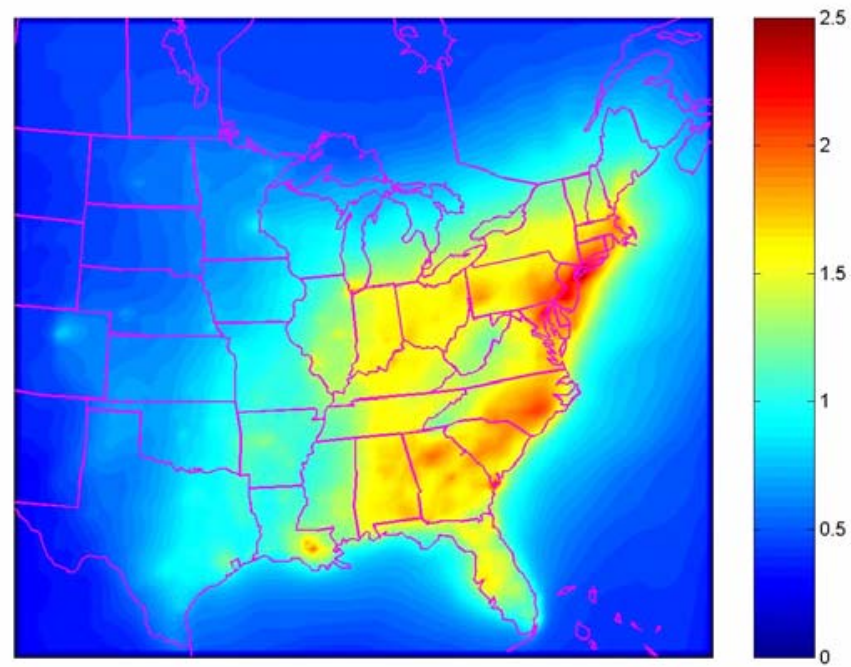

Figure 38. Predicted average base-case ammonium concentrations $\left(\mu \mathrm{g} \mathrm{m} \mathrm{m}^{-3}\right)$ for January 2002.

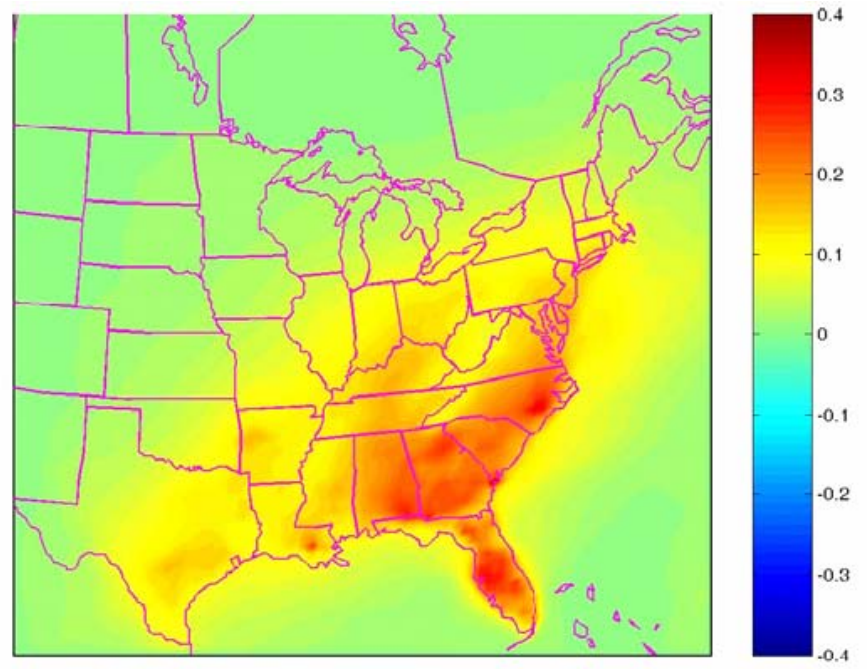

Figure 39. Predicted change $\left(\mu \mathrm{g} \mathrm{m}^{-3}\right)$ in ammonium concentration for a $50 \%$ reduction in $\mathrm{SO}_{2}$ emissions for January 2002. A positive change corresponds to a concentration reduction.

The $50 \%$ reduction in $\mathrm{SO}_{2}$ emissions is predicted to cause a significant reduction in the ammonium levels in the Southeastern US with values as much as $0.3 \mu \mathrm{g} \mathrm{m}^{-3}$ (Figure 39). The predicted ammonium concentration change in the Northeast, Midwest and Central US is rather modest at around $0.1 \mu \mathrm{g} \mathrm{m}-3$ or less partially because the decreased ammonium sulfate is replaced by ammonium nitrate. 
The rest of the major aerosol components (organics, dust) are predicted once more to remain the same in this $50 \% \mathrm{SO}_{2}$ emission reduction scenario.

The predicted average $\mathrm{PM}_{2.5}$ concentrations by PMCAMx are shown in Figure 40 . The high primary organic concentrations lead to a high concentration area (values above $20 \mu \mathrm{g} \mathrm{m}^{-3}$ ) extending from Baltimore to Maine. High concentrations are also predicted for the large urban areas such as Chicago and Atlanta.

Once more the overall effects of the $50 \% \mathrm{SO}_{2}$ emission change can be viewed for all practical purposes as the sum of the corresponding changes in the sulfate, nitrate, and ammonium concentrations. Figure 41 indicates that the $\mathrm{PM}_{2.5}$ concentration is predicted to decrease everywhere in the domain by values up to $1.5 \mu \mathrm{g} \mathrm{m}^{-3}$. The Southeast US is predicted to benefit the most from the reductions. In the Northeast there is a net reduction of $\mathrm{PM}_{2.5}$ but it is rather small (less than $0.5 \mu \mathrm{g} \mathrm{m}^{-3}$ ) mainly because the sulfate reduction is balanced by an increase in nitrate.

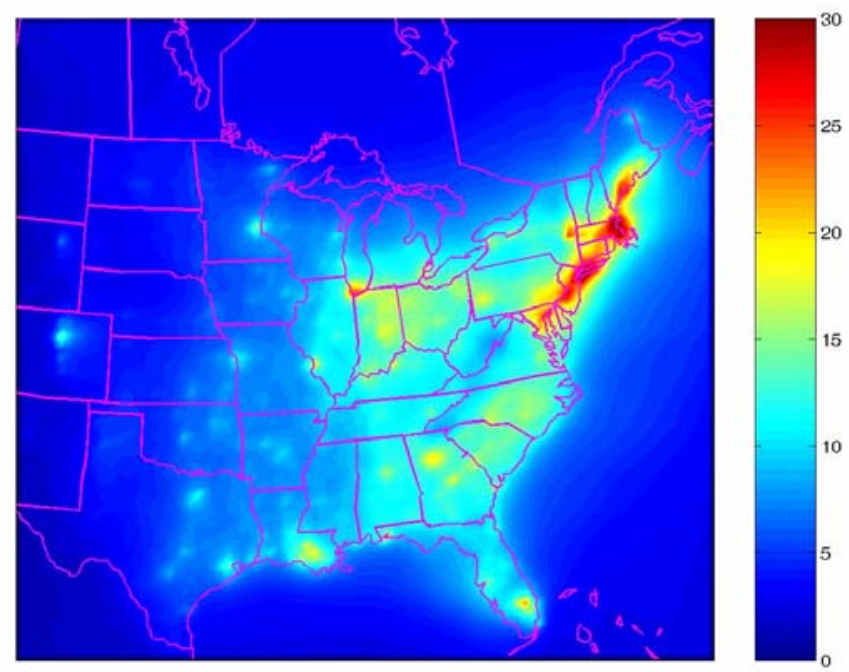

Figure 40. Predicted average base-case $\mathrm{PM}_{2.5}$ concentrations $\left(\mu \mathrm{g} \mathrm{m}^{-3}\right)$ for January 2002. 

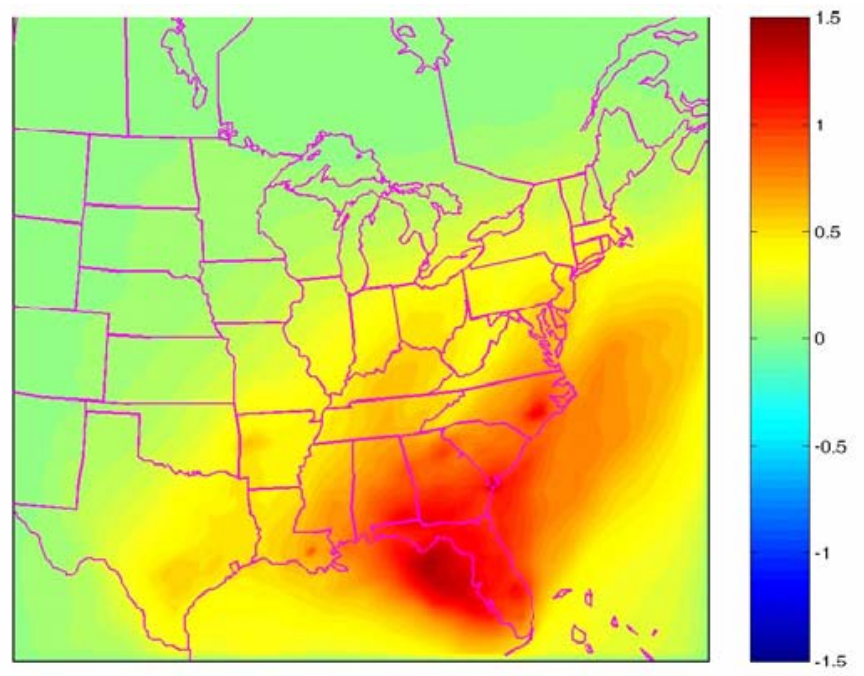

Figure 41. Predicted change $\left(\mu \mathrm{g} \mathrm{m}^{-3}\right)$ in $\mathrm{PM}_{2.5}$ concentration for a $50 \%$ reduction in $\mathrm{SO}_{2}$ emissions for January 2002. A positive change corresponds to a concentration reduction.
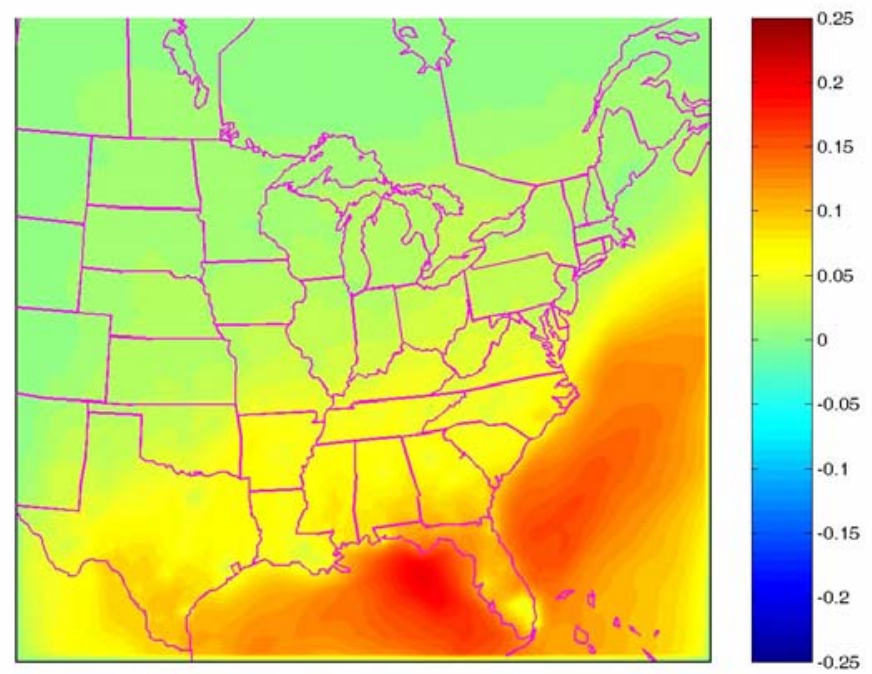

Figure 42. Predicted fractional change in $\mathrm{PM}_{2.5}$ concentration for a $50 \%$ reduction in $\mathrm{SO}_{2}$ emissions for January 2002. A positive change corresponds to a concentration reduction.

The net predicted fractional change in the $\mathrm{PM}_{2.5}$ concentrations after a $50 \%$ reduction in $\mathrm{SO}_{2}$ emissions is quite variable (Figure 42). The maximum effect is predicted over Florida with reductions around $15 \%$, is around $5-10 \%$ in the rest of the South US and is less than $5 \%$ in the North. These results suggest that a $\mathrm{SO}_{2}$ emissions reduction strategy would help reduce wintertime $\mathrm{PM}_{2.5}$ but the changes would be modest in the South and rather small in the North. 


\section{Changes in $\mathrm{NO}_{\mathrm{x}}$ Emissions}

In these simulations a uniform $50 \%$ reduction in $\mathrm{NO}_{\mathrm{x}}$ emissions was assumed for the whole modeling domain. Coal fired power plants together with transportation sources are the most significant contributors to $\mathrm{NO}_{\mathrm{x}}$ emissions in the Eastern US.

\subsection{Summertime (July 2001)}

A $50 \%$ reduction in $\mathrm{NO}_{\mathrm{x}}$ emissions is predicted to be quite effective in reducing the $\mathrm{PM}_{2.5}$ nitrate concentrations during the summer. The predicted changes in nitrate concentrations for this scenario are shown in Figure 43. The reductions have the same spatial distribution as the average nitrate concentrations (Figure 26).

The fractional reduction of nitrate (Figure 44) is around 60\% for most of the Eastern US. Therefore the reduction is more than proportional to the emission change. This strong response of the aerosol nitrate to $\mathrm{NO}_{\mathrm{x}}$ emissions is caused by the fact the concentrations of the hydroxyl radical are also reduced.

The decrease in the hydroxyl concentrations in most areas leads to a reduction of the sulfuric acid production in the gas phase from the reaction of $\mathrm{SO}_{2}$ with $\mathrm{OH}$. This decreased sulfuric acid production reduces sulfate levels for the $50 \%$ reduction in $\mathrm{NO}_{\mathrm{x}}$ emission scenario (Figure 45). The reductions have the same spatial distribution as the average sulfate concentration (Figure 23) and are up to $1.5 \mu \mathrm{g} \mathrm{m} \mathrm{m}^{-3}$. These changes correspond to a $10-20 \%$ reduction in the sulfate levels with the lowest fractional reductions close to the urban areas and the highest fractions reductions in the rural areas.

The reductions in sulfate and nitrate levels associated with the decrease in $\mathrm{NO}_{\mathrm{x}}$ emissions also cause a reduction in the corresponding ammonium concentrations as shown Figure 46. The decrease in ammonium is up to $0.5 \mu \mathrm{g} \mathrm{m}^{-3}$.

The modest reductions in the sulfate, nitrate, and ammonium levels lead to reductions of the PM2.5 levels of up to $2 \mu \mathrm{g} \mathrm{m}^{-3}$ in the Midwest, Pennsylvania, and parts of the South (Figure 47). These changes correspond to reductions of $\mathrm{PM}_{2.5}$ of 5-15\% in most of the Eastern US (Figure 48). These results indicate that reductions in $\mathrm{NO}_{\mathrm{x}}$ emissions can also reduce the $\mathrm{PM}_{2.5}$ levels in most areas but the reductions for the same control level will be smaller than the effect of $\mathrm{SO}_{2}$ emission reductions (Figure 31 ). 


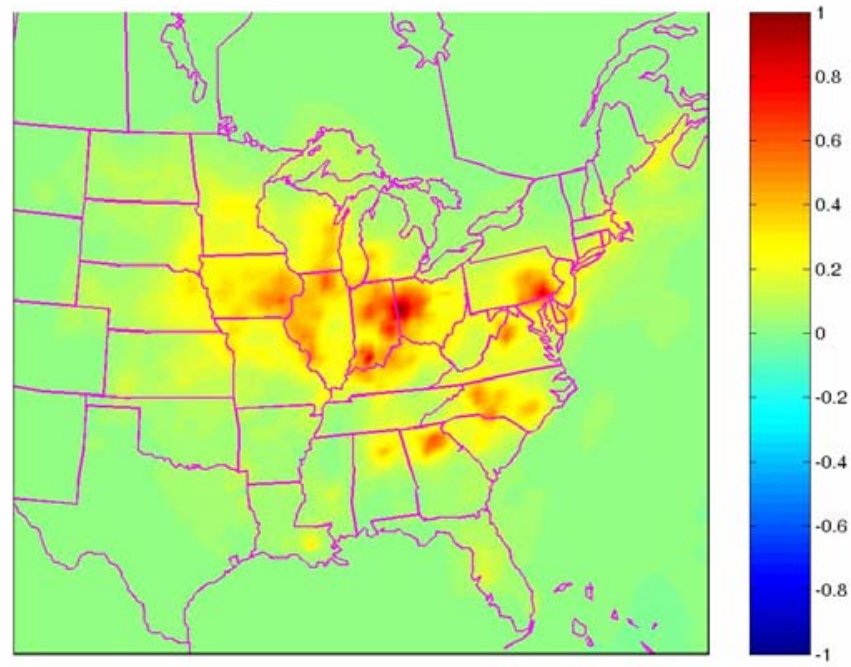

Figure 43. Predicted change $\left(\mu \mathrm{g} \mathrm{m}^{-3}\right)$ in nitrate concentration for a $50 \%$ reduction in $\mathrm{NO}_{\mathrm{x}}$ emissions for July 2001. A positive change corresponds to a concentration reduction.
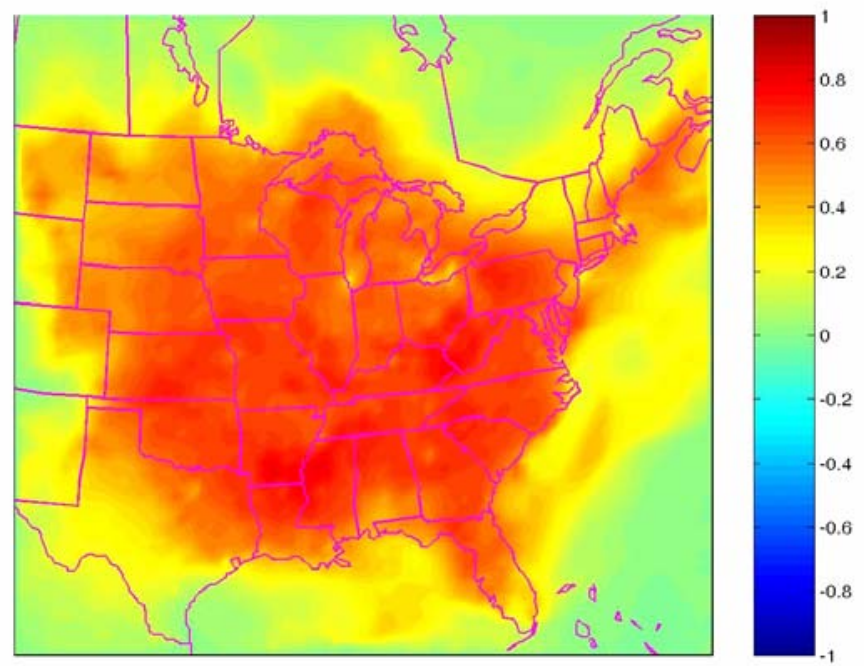

Figure 44. Predicted fractional change in nitrate concentration for a $50 \%$ reduction in $\mathrm{NO}_{\mathrm{x}}$ emissions for July 2001. A positive change corresponds to a concentration reduction. 


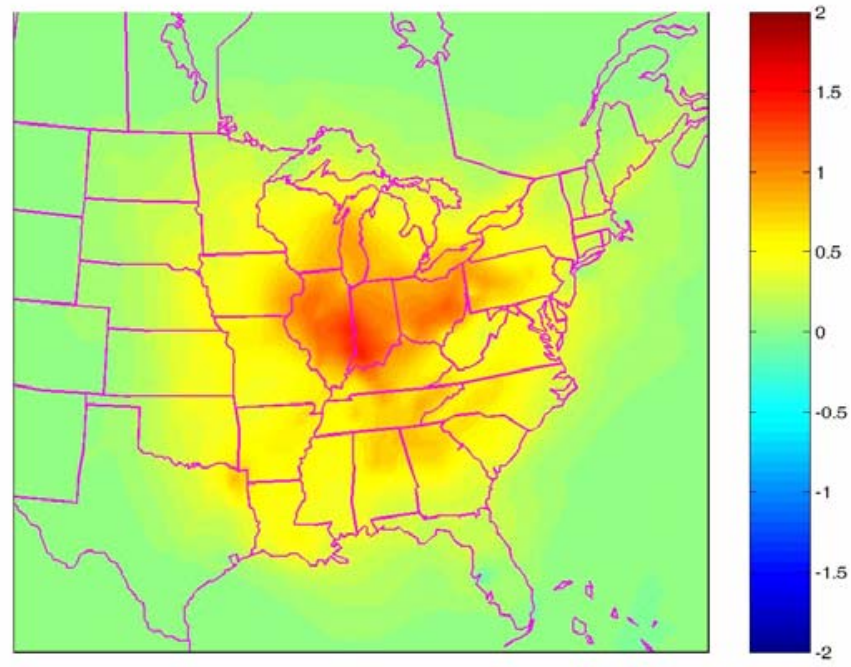

Figure 45. Predicted change $\left(\mu \mathrm{g} \mathrm{m}^{-3}\right)$ in sulfate concentration for a $50 \%$ reduction in $\mathrm{NO}_{\mathrm{x}}$ emissions for July 2001. A positive change corresponds to a concentration reduction.
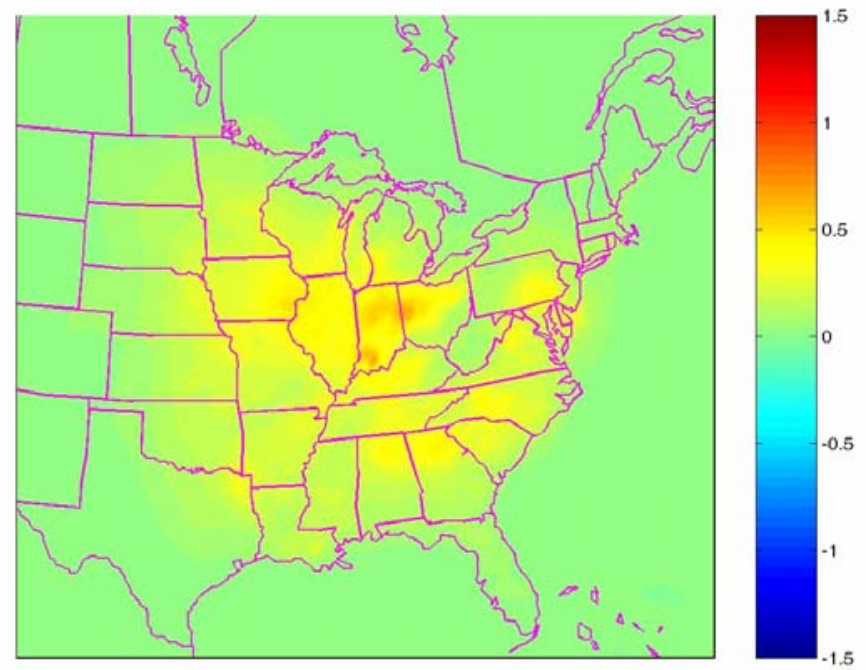

Figure 46. Predicted change $\left(\mu \mathrm{g} \mathrm{m}^{-3}\right)$ in ammonium concentration for a $50 \%$ reduction of $\mathrm{NO}_{\mathrm{x}}$ emissions for July 2001. A positive change corresponds to a concentration reduction. 


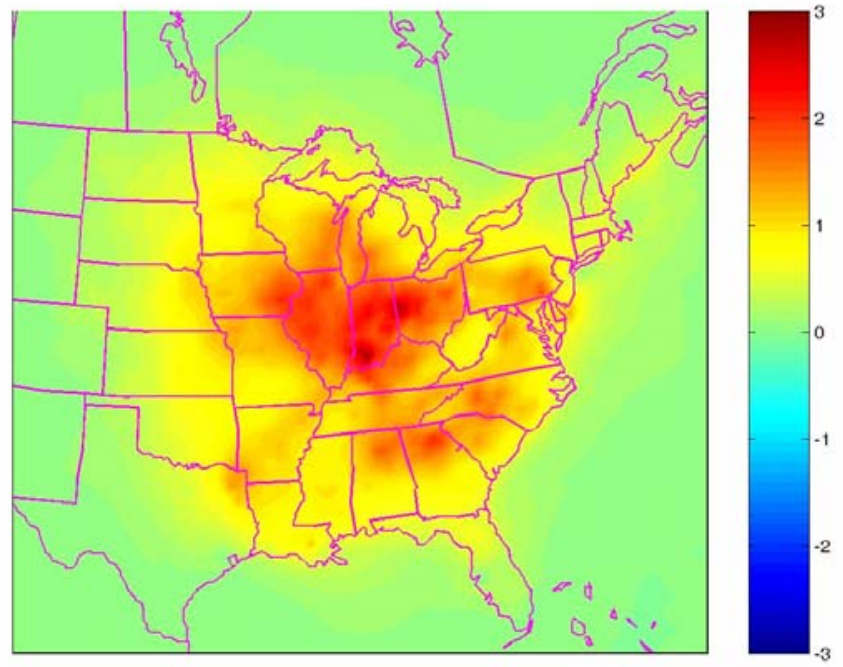

Figure 47. Predicted change $\left(\mu \mathrm{g} \mathrm{m}^{-3}\right)$ in $\mathrm{PM}_{2.5}$ concentration for a $50 \%$ reduction of $\mathrm{NO}_{\mathrm{x}}$ emissions for July 2001. A positive change corresponds to a concentration reduction.

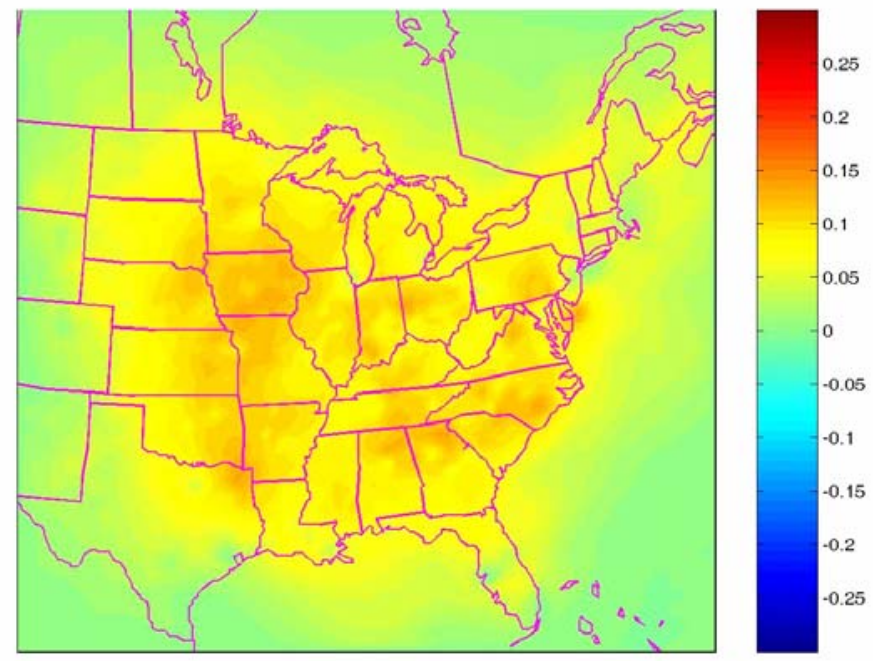

Figure 48. Predicted fractional change $\left(\mu \mathrm{g} \mathrm{m} \mathrm{m}^{-3}\right)$ in PM2.5 concentration for a $50 \%$ reduction of $\mathrm{NO}_{\mathrm{x}}$ emissions for July 2001. A positive change corresponds to a concentration reduction.

\subsection{Winter (January 2002)}

Reductions of $\mathrm{NO}_{\mathrm{x}}$ emissions is a potential strategy for reduction of the high wintertime nitrate concentrations (Figure 36) especially in the Northeast. PMCAMx predicts that indeed a $50 \%$ reduction of $\mathrm{NO}_{\mathrm{x}}$ emissions can lead to significant reductions of the nitrate concentrations (Figure 49). These reductions cover most of the Eastern part of the country and range from 0.4 to $0.8 \mu \mathrm{g} \mathrm{m}^{-3}$ on average. 
The corresponding reductions in nitrate are around $50 \%$ in the South and $20 \%$ in the North. The smaller change in the Northern part of the domain is due to an increase of the hydroxyl radical concentrations that offsets part of the $\mathrm{NO}_{\mathrm{x}}$ reduction. This increase in the hydroxyl radical levels accelerates the formation rate of sulfuric acid from the gas phase reaction of sulfur dioxide and $\mathrm{OH}$ leading to a rather unexpected increase in the sulfate concentrations in the Eastern part of the modeling domain (Figure 50). This unexpected increase is predicted to be up to $0.5 \mu \mathrm{g} \mathrm{m}^{-3}$ corresponding to $5-15 \%$ of the sulfate levels.
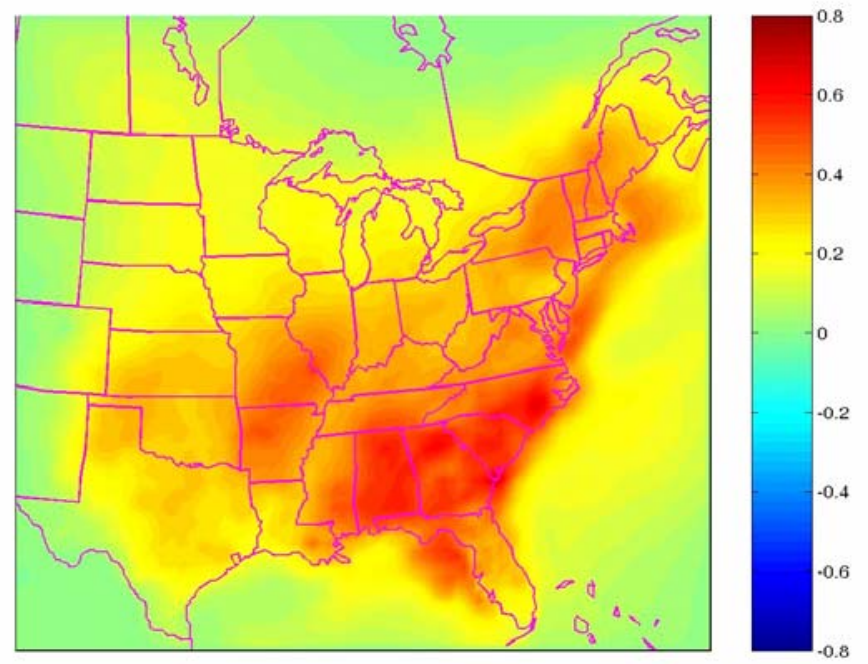

Figure 49. Predicted change $\left(\mu \mathrm{g} \mathrm{m}^{-3}\right)$ in nitrate concentration for a $50 \%$ reduction of $\mathrm{NO}_{\mathrm{x}}$ emissions for January 2002. A positive change corresponds to a concentration reduction. 


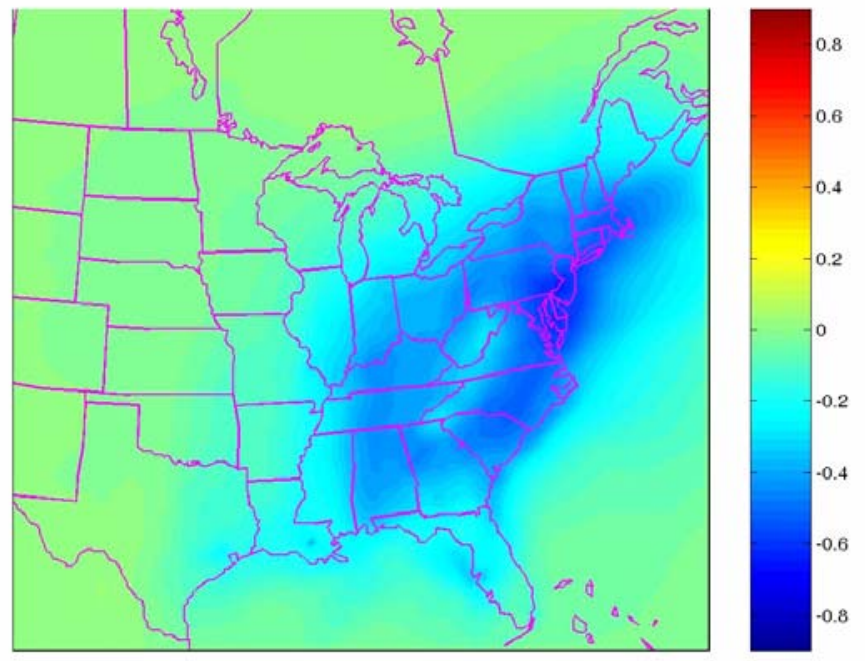

Figure 50. Predicted change $\left(\mu \mathrm{g} \mathrm{m}^{-3}\right)$ in sulfate concentration for a $50 \%$ reduction of $\mathrm{NO}_{\mathrm{x}}$ emissions for January 2002. A positive change corresponds to a concentration reduction.

The decreasing nitrate concentrations in most of the domain together with the increasing sulfate levels in the eastern part lead to a rather complex change in the corresponding ammonium levels (Figure 51). A 50\% $\mathrm{NO}_{\mathrm{x}}$ reduction in the winter is predicted to cause ammonium levels in the east especially in the Baltimore to Boston corridor by as much as $0.15 \mu \mathrm{g} \mathrm{m}^{-3}$. In the western part of the domain and parts of the South the reductions of ammonium up to $0.1 \mu \mathrm{g} \mathrm{m}^{-3}$ are predicted.
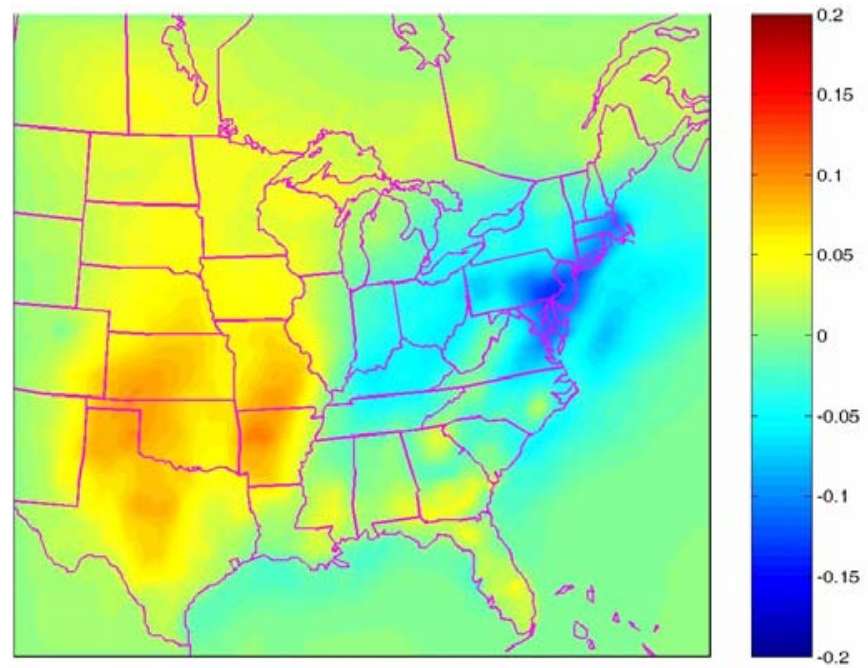

Figure 51. Predicted change $\left(\mu \mathrm{g} \mathrm{m}^{-3}\right)$ in ammonium concentration for a $50 \%$ reduction of $\mathrm{NO}_{\mathrm{x}}$ emissions for January 2002. A positive change corresponds to a concentration reduction. 
The overall effect of the $\mathrm{NO}_{\mathrm{x}}$ emission reductions is a rather unexpected increase in the $\mathrm{PM}_{2.5}$ levels in the Northeast of around $0.5 \mu \mathrm{g} \mathrm{m}^{-3}$ (Figure 52) with reductions in the western part of the modeling domain of up to $0.5 \mu \mathrm{g} \mathrm{m}^{-3}$. These results suggest that $\mathrm{NO}_{\mathrm{x}}$ emission reductions in the winter may not be an efficient way to reduce $\mathrm{PM}_{2.5}$ levels in the Northeast due to the predicted increases in sulfate.

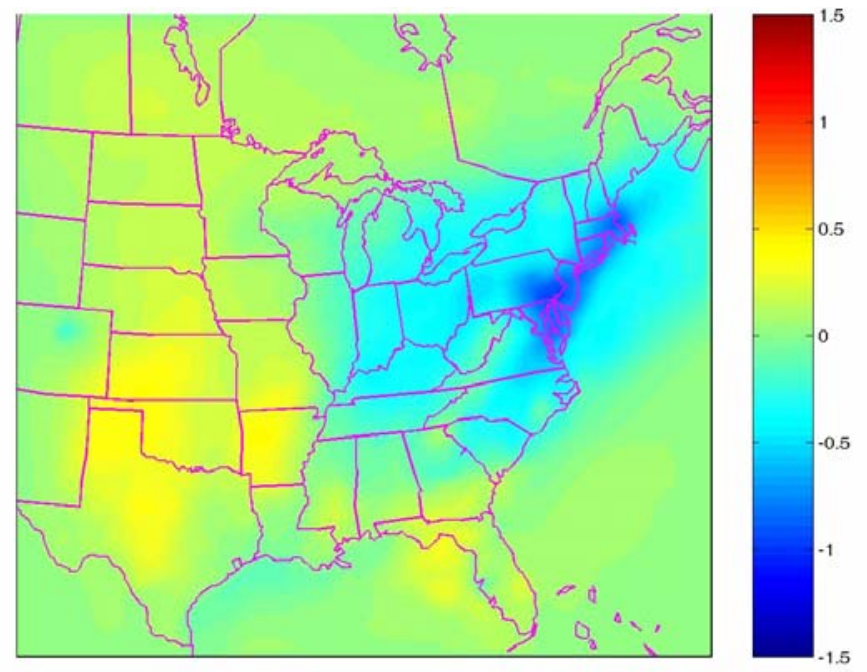

Figure 52. Predicted change $\left(\mu \mathrm{g} \mathrm{m}^{-3}\right)$ in $\mathrm{PM}_{2.5}$ concentration for a $50 \%$ reduction of $\mathrm{NO}_{\mathrm{x}}$ emissions for January 2002. A positive change corresponds to a concentration reduction.

\section{Changes in $\mathrm{NH}_{3}$ Emissions}

Coal-fired power plants are currently a small source of ammonia. However, ammonia controls in animal feeding operations and agriculture presents a potentially cost-effective way to reduce the $\mathrm{PM}_{2.5}$ levels. This could be especially important during the winter, when as we have seen neither $\mathrm{SO}_{2}$ nor $\mathrm{NO}_{\mathrm{x}}$ emission reductions are especially effective in reducing the inorganic $\mathrm{PM}_{2.5}$ concentrations. For these scenarios we assumed a uniform $50 \%$ reduction in ammonia emissions.

The primary role of ammonia in the atmosphere is to neutralize the acids and especially sulfuric acid. As soon as sulfuric acid is formed it reacts with ammonia to form sulfate salts (ammonium sulfate, letovicite, ammonium bisulfate). Ammonia is a volatile gas and stays in the gas phase if left alone (when there are not sufficient concentrations of acid vapors to react and form the corresponding salts).

When $\mathrm{SO}_{2}$ emissions are reduced, sulfate is reduced and the corresponding ammonia returns to the gas phase (it becomes "free ammonia"). A fraction of this ammonia may then react with the available nitric acid and return to the particulate phase as ammonium nitrate. However, due to aerosol thermodynamics, the ammonia reacting with nitric acid is usually less than the ammonia liberated from the sulfate reduction and $\mathrm{SO}_{2}$ emission 
reductions result in ammonium reductions. Gas-phase ammonia concentrations do increase in this case. The increase is usually less than the ammonium decrease because of the ammonium nitrate increase and the increase in gas-phase ammonia deposition.

\subsection{Summer (July 2001)}

Reductions of ammonia emissions lead, as expected, to significant reductions of the aerosol ammonium levels especially in the Midwest (Figure 53). The predicted reductions are up to $1.5 \mu \mathrm{g} \mathrm{m}^{-3}$ and represent $0-50 \%$ changes of the ammonium concentration levels.

The changes in ammonia levels have a relatively small effect on the predicted sulfate concentrations. Sulfate is predicted to decrease a little (less than $0.1 \mu \mathrm{g} \mathrm{m}^{-3}$ ) in certain area. These very small changes are mainly due to changes in the cloud $\mathrm{pH}$ and correspond to reductions in sulfate of only $1-2 \%$. The effect of ammonia in nitrate levels is more significant, especially in the areas characterized by high nitrate levels (Figure 54). Nitrate reductions of up to $0.6 \mu \mathrm{g} \mathrm{m}^{-3}$ are predicted for Illinois, Indiana, Atlanta, Baltimore, Philadelphia, and New York.

The reductions in the ammonium and nitrate concentrations lead to an overall reduction of up to $1.5 \mu \mathrm{g} \mathrm{m}^{-3}$ in the $\mathrm{PM}_{2.5}$ concentration (Figure 55). The resulting percentage reductions vary from 5 to $10 \%$ in the Eastern US (Figure 56).

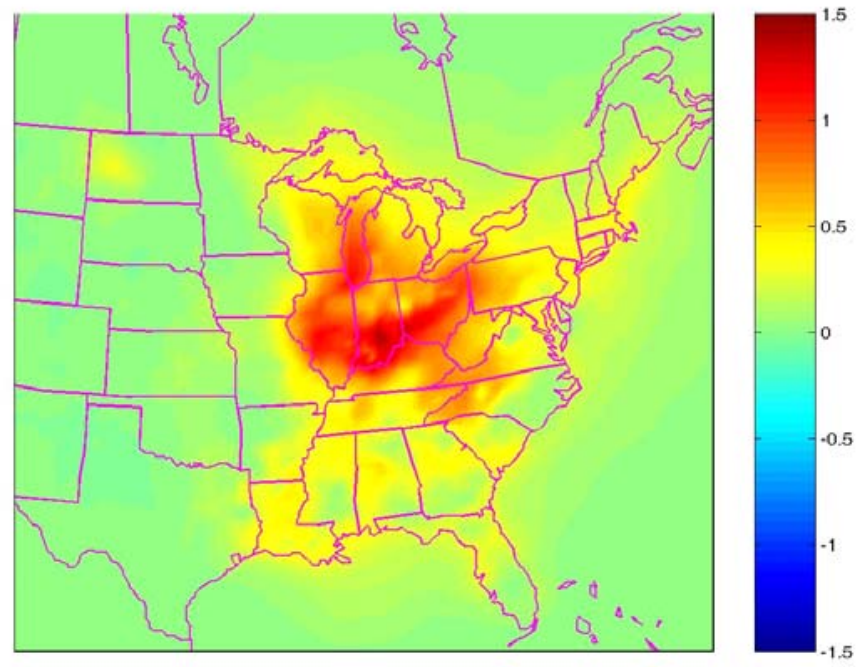

Figure 53. Predicted change $\left(\mu \mathrm{g} \mathrm{m}^{-3}\right)$ in ammonium concentration for a $50 \%$ reduction of $\mathrm{NH}_{3}$ emissions for June 2001. A positive change corresponds to a concentration reduction. 


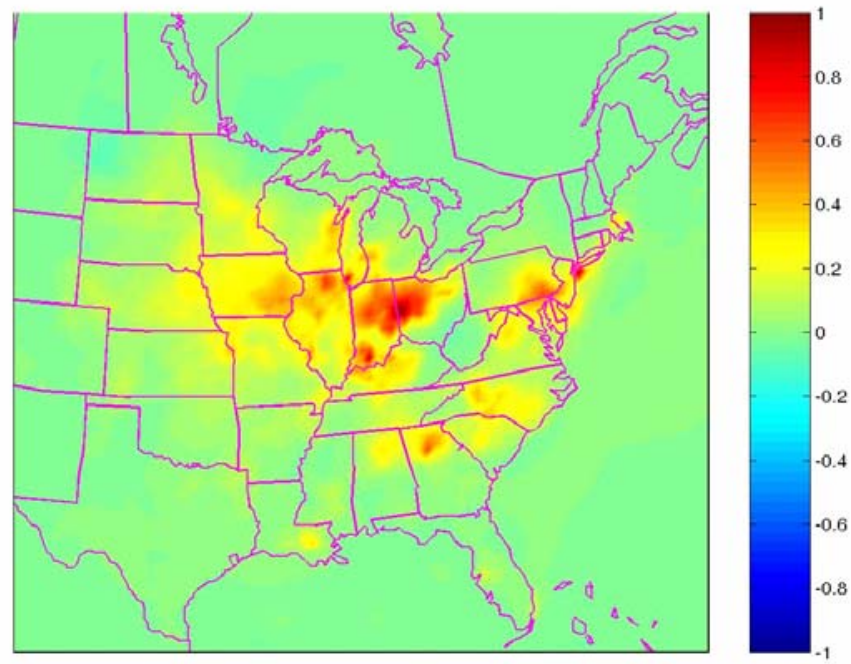

Figure 54. Predicted change $\left(\mu \mathrm{g} \mathrm{m}^{-3}\right)$ in nitrate concentration for a $50 \%$ reduction of $\mathrm{NH}_{3}$ emissions for June 2001. A positive change corresponds to a concentration reduction.

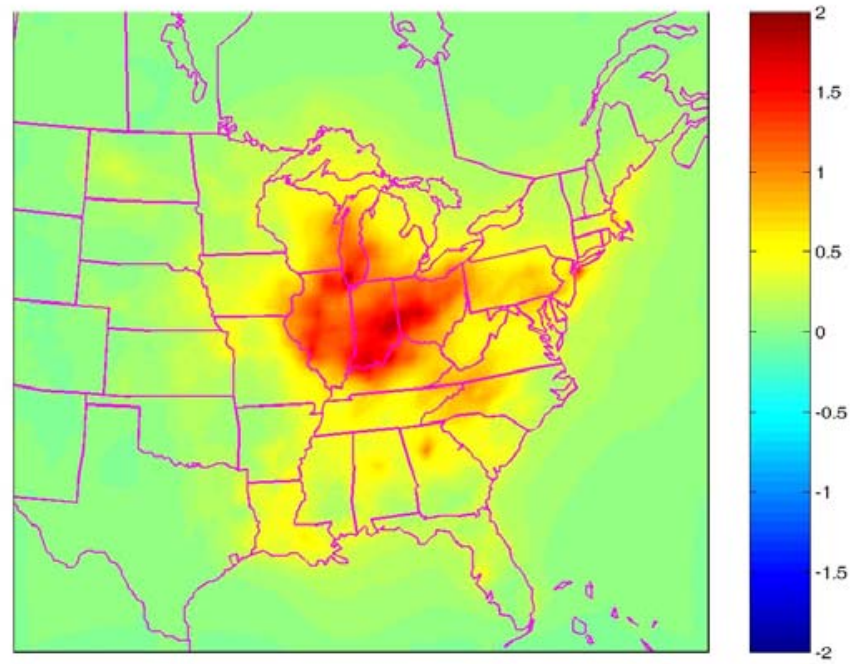

Figure 55. Predicted change $\left(\mu \mathrm{g} \mathrm{m}^{-3}\right)$ in $\mathrm{PM}_{2.5}$ concentration for a 50\% reduction of $\mathrm{NH}_{3}$ emissions for June 2001. A positive change corresponds to a concentration reduction. 


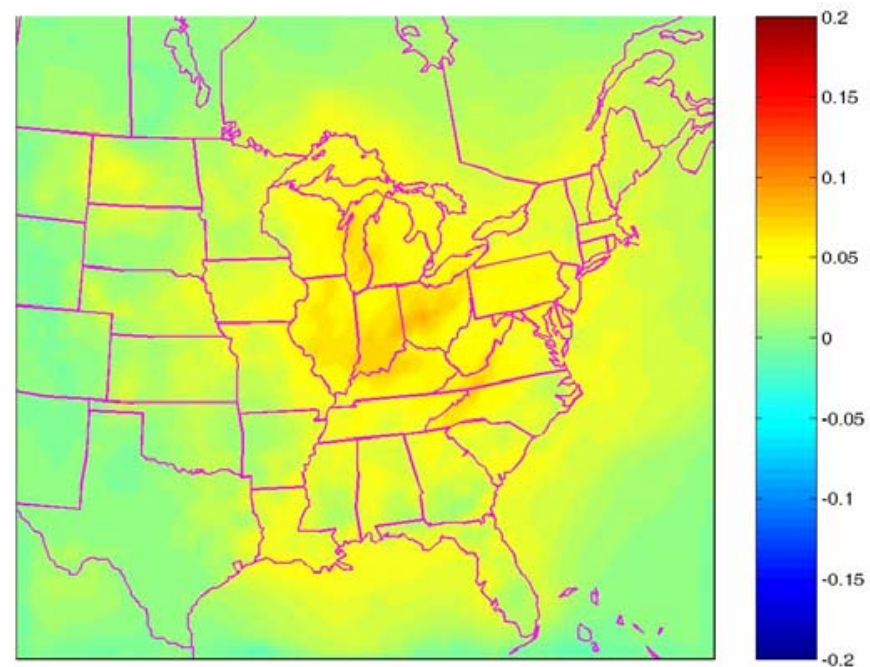

Figure 56. Predicted fractional change $\left(\mu \mathrm{g} \mathrm{m}^{-3}\right)$ in $\mathrm{PM}_{2.5}$ concentration for a $50 \%$ reduction of $\mathrm{NH}_{3}$ emissions for June 2001. A positive change corresponds to a concentration reduction.

\subsection{Winter (January 2002)}

The predicted wintertime ammonium concentrations are reduced significantly when the ammonia emissions are reduced by $50 \%$. The highest reductions take place in the eastern part of the country and are up to $0.6 \mu \mathrm{g} \mathrm{m}^{-3}$ (Figure 57). The corresponding changes are $20-40 \%$ of the base-case ammonium levels.

The reduction in ammonia availability leads to a significant reduction in the nitrate levels in most of the domain (Figure 58). The highest reductions are predicted for the northeast part of the country and exceed $1 \mu \mathrm{g} \mathrm{m}^{-3}$.

The ammonia emissions reductions are predicted to cause small reductions of the sulfate levels in parts of the domain (Figure 59). These small changes of up to $0.3 \mu \mathrm{g} \mathrm{m}^{-3}$ are due to changes in the cloud $\mathrm{pH}$ and corresponding reductions in the in-cloud sulfate production rate.

The overall predicted effect of the $50 \%$ reduction in ammonia emissions on $\mathrm{PM}_{2.5}$ is shown in Figure 60. The reductions vary from 0.5 to $2 \mu \mathrm{g} \mathrm{m}^{-3}$ with the highest reductions in the northeast. Reduction of the ammonia emissions appears to be the most efficient strategy for reducing the inorganic fine particulate mater during the winter in the eastern US.

The $50 \%$ reduction in ammonia emissions can lead to reductions in $\mathrm{PM}_{2.5}$ concentrations varying from 5 to $15 \%$ depending on the area (Figure 61). These results suggest strongly that changes in ammonia emissions represent an interesting emission control strategy especially in the winter but also during the summer. 

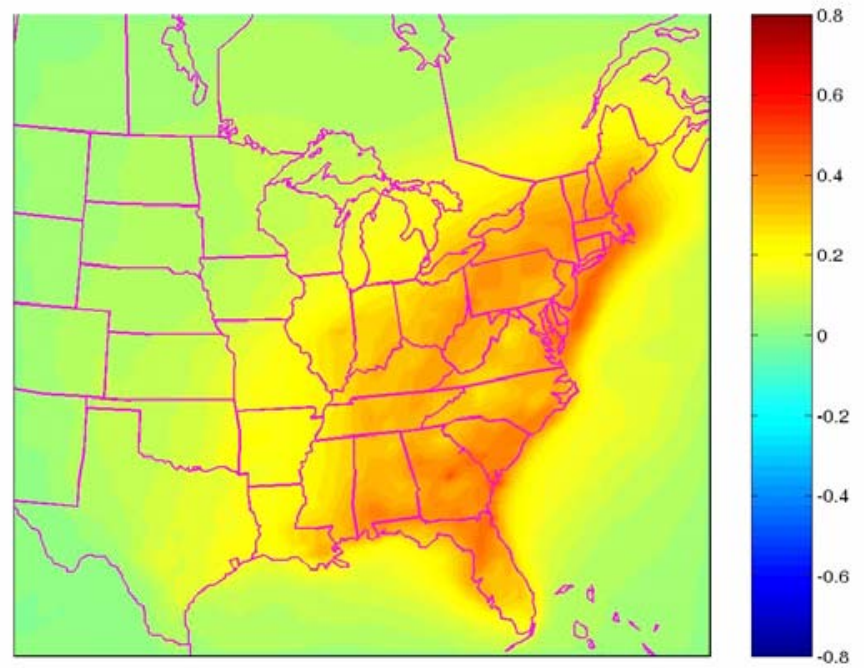

Figure 57. Predicted change $\left(\mu \mathrm{g} \mathrm{m}^{-3}\right)$ in ammonium concentration for a $50 \%$ reduction of $\mathrm{NH}_{3}$ emissions for January 2002. A positive change corresponds to a concentration reduction.
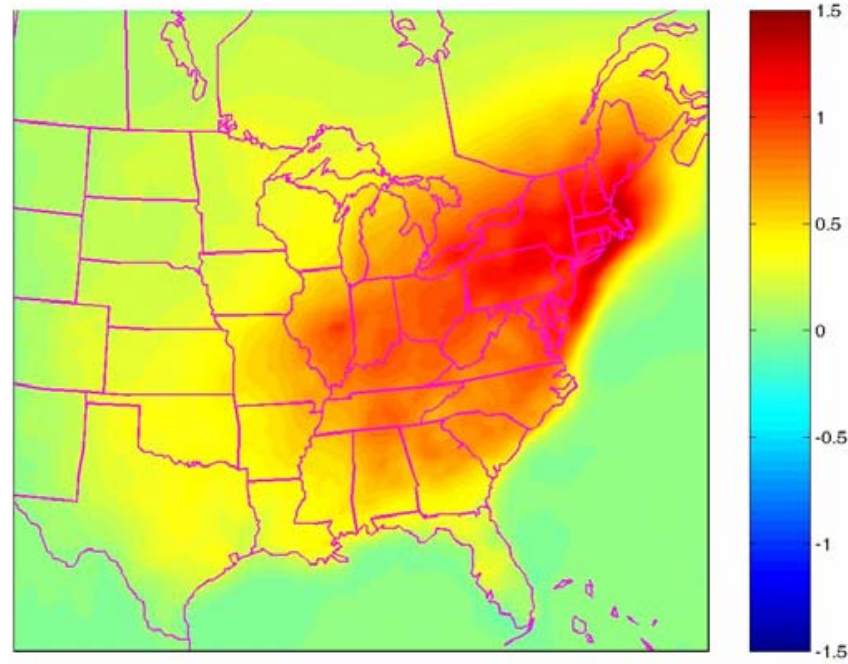

Figure 58. Predicted change $\left(\mu \mathrm{g} \mathrm{m}^{-3}\right)$ in nitrate concentration for a $50 \%$ reduction of $\mathrm{NH}_{3}$ emissions for January 2002. A positive change corresponds to a concentration reduction. 


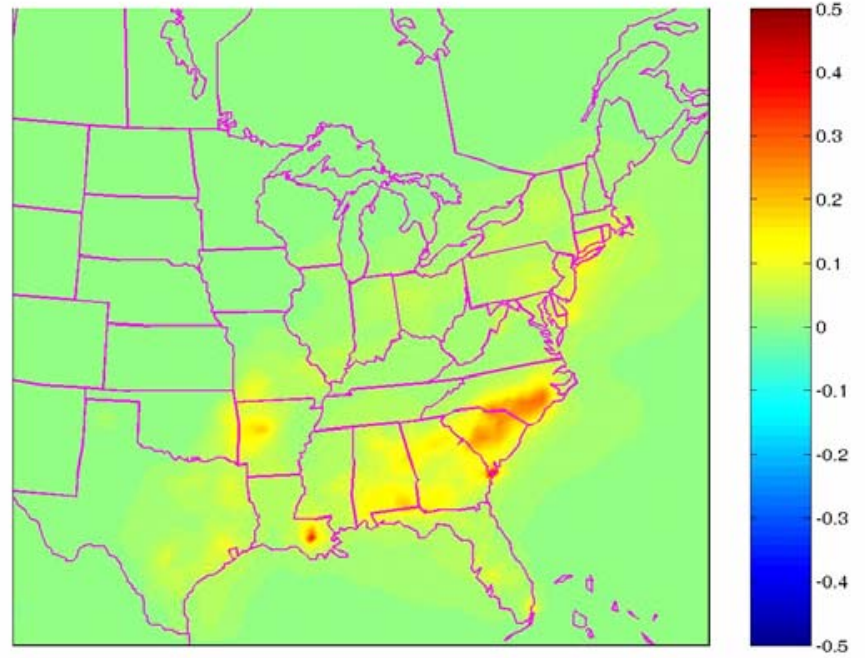

Figure 59. Predicted change $\left(\mu \mathrm{g} \mathrm{m}^{-3}\right)$ in sulfate concentration for a $50 \%$ reduction of $\mathrm{NH}_{3}$ emissions for January 2002. A positive change corresponds to a concentration reduction.
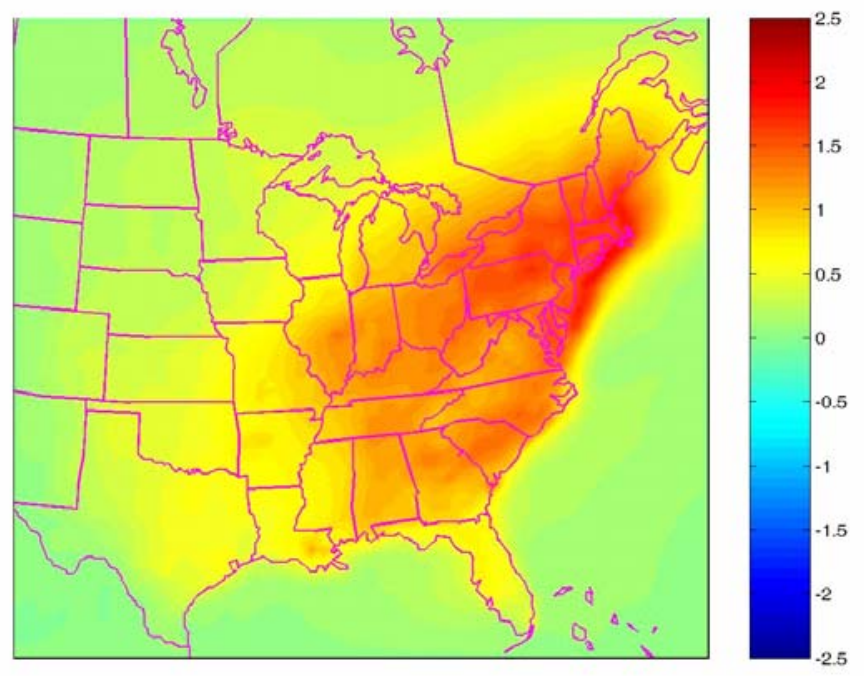

Figure 60. Predicted change $\left(\mu \mathrm{g} \mathrm{m}^{-3}\right)$ in $\mathrm{PM}_{2.5}$ concentration for a $50 \%$ reduction of $\mathrm{NH}_{3}$ emissions for January 2002. A positive change corresponds to a concentration reduction. 


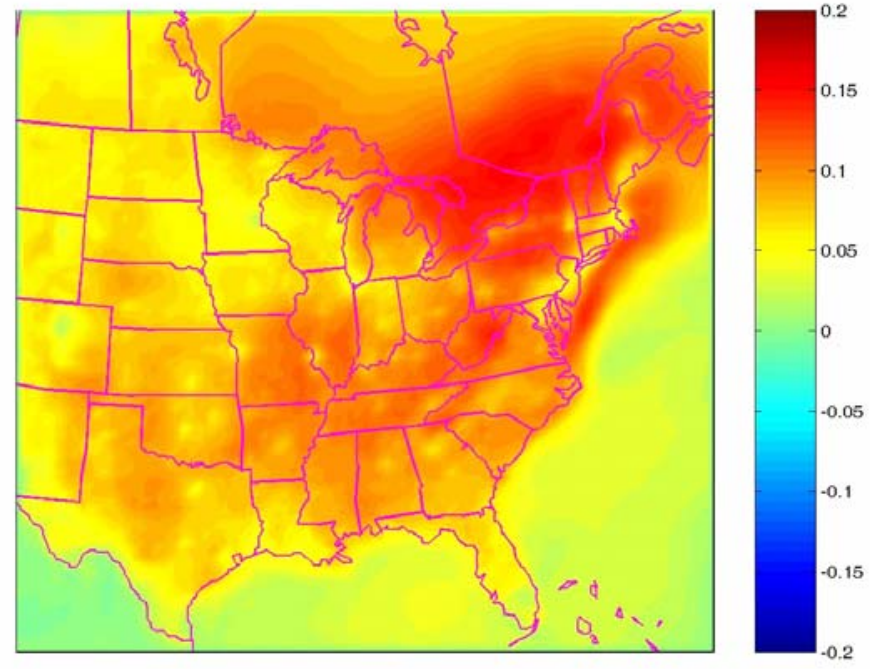

Figure 61. Predicted fractional change $\left(\mu \mathrm{g} \mathrm{m}^{-3}\right)$ in $\mathrm{PM}_{2.5}$ concentration for a $50 \%$ reduction of $\mathrm{NH}_{3}$ emissions for January 2002. A positive change corresponds to a concentration reduction. 


\section{COUPLED SO${ }_{2}$-NOx Emission Reductions}

Many potential emission control scenarios involving coal-fired plants involve simultaneous reduction of both $\mathrm{NO}_{\mathrm{x}}$ and $\mathrm{SO}_{2}$ emissions. To investigate potential synergies in such a scenario, PMCAMx was used to simulate a case in which both $\mathrm{SO}_{2}$ and $\mathrm{NO}_{\mathrm{x}}$ emissions were reduced uniformly by $50 \%$.

\section{Summertime (July 2001)}

The $\mathrm{SO}_{2}$ reductions by themselves are quite efficient in reducing sulfate concentrations during the summer (Figures 24 and 25). Coupling these reductions with $\mathrm{NO}_{\mathrm{x}}$ reductions decreases the sulfate a little more especially in the areas with the highest sulfate levels (Figure 62). The largest reduction, around $6 \mu \mathrm{g} \mathrm{m}^{-3}$, is in south Indiana. The net result is that in most of the modelling domain the summertime sulfate concentration reduction is more than proportional to the $\mathrm{SO}_{2}$ emission reductions. The net fractional reduction in sulfate is close to $60 \%$ throughout much of the modelling domain (Figure 63).
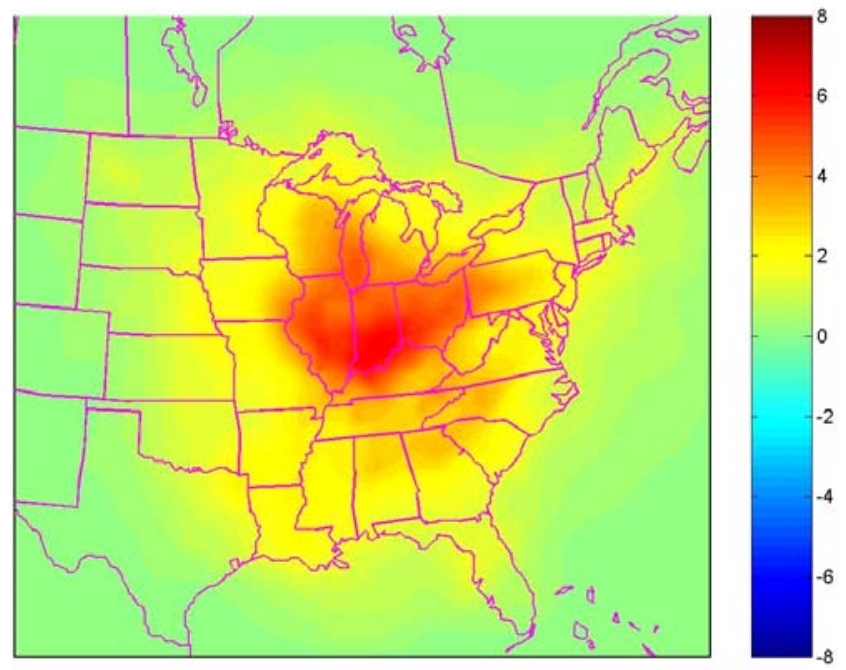

Figure 62. Predicted change $\left(\mu \mathrm{g} \mathrm{m}^{-3}\right)$ in sulfate concentration for a $50 \%$ reduction in $\mathrm{SO}_{2}$ and NOx emissions for July 2001. A positive change corresponds to a concentration reduction. 


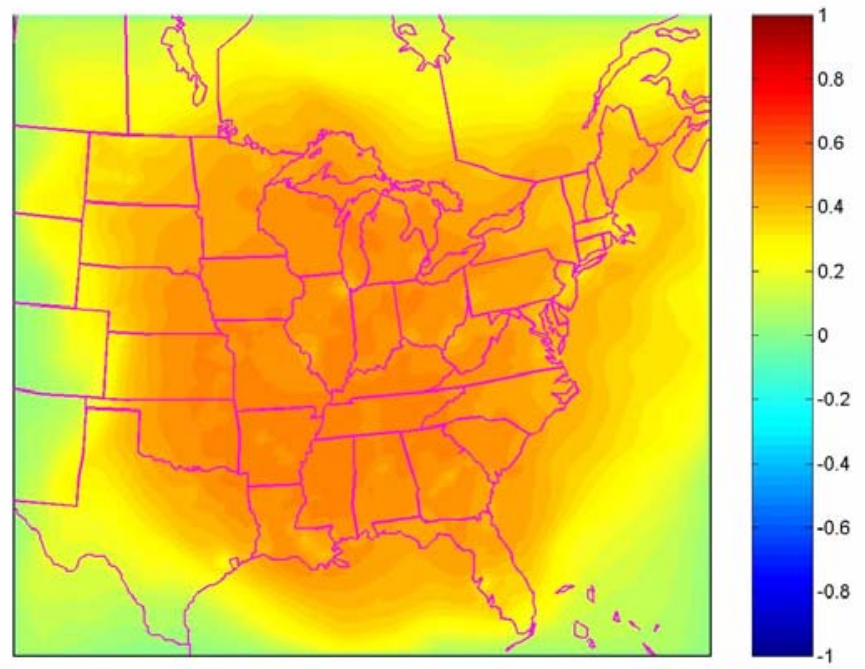

Figure 63. Predicted fractional change in sulfate concentration for a $50 \%$ reduction in $\mathrm{SO}_{2}$ and NOx emissions for July 2001. A positive change corresponds to a concentration reduction.

The decrease in just $\mathrm{SO}_{2}$ emissions results in small increases of nitrate during the summer (Figure 27). However, coupling the $\mathrm{SO}_{2}$ reductions with $\mathrm{NO}_{\mathrm{x}}$ emission reductions is predicted to lead in significant reduction of aerosol nitrate throughout the domain (Figure 64). In the Midwest, where nitrate concentration is the highest $(1.5 \mu \mathrm{g}$ $\left.\mathrm{m}^{-3}\right)$, nitrate concentration decreases by $1 \mu \mathrm{g} \mathrm{m}^{-3}$.

The nitrate reduction is around 70\% over most of the Eastern US (Figure 65) and the predicted concentrations of ammonium nitrate are close to zero almost everywhere. The $50 \%$ reduction of $\mathrm{NO}_{\mathrm{x}}$ not only prevents the increase of nitrate associated with a decrease in $\mathrm{SO}_{2}$ emissions, but also results in a significant reduction of the currently existing summertime nitrate. 


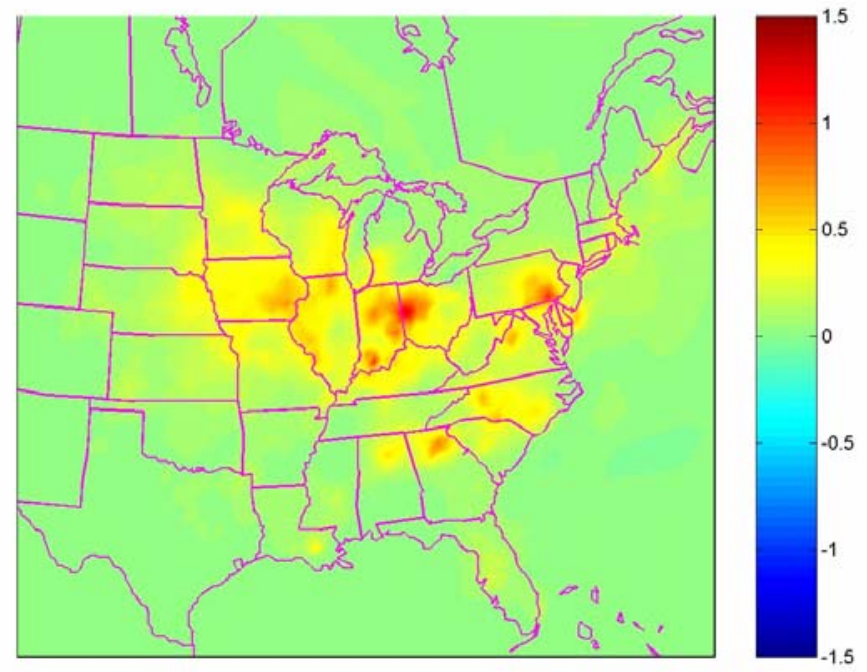

Figure 64. Predicted change $\left(\mu \mathrm{g} \mathrm{m}^{-3}\right)$ in nitrate concentration for a $50 \%$ reduction in $\mathrm{SO}_{2}$ and NOx emissions for July 2001. A positive change corresponds to a concentration reduction.

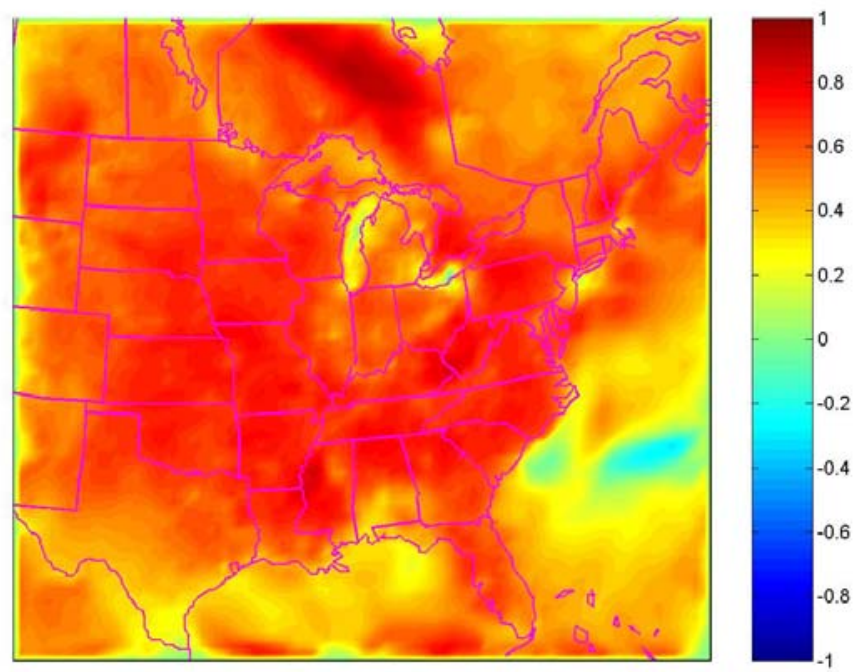

Figure 65. Predicted fractional change in nitrate concentration for a 50\% reduction in $\mathrm{SO}_{2}$ and NOx emissions for July 2001. A positive change corresponds to a concentration reduction. 
The significant reductions of both sulfate and nitrate are accompanied by a significant decrease of the aerosol ammonium (Figure 66), which is mostly transferred to the gas phase as ammonia vapour but is only partially deposited to the ground. The decrease is as large as $2 \mu \mathrm{g} \mathrm{m}^{-3}$ and its spatial pattern is similar to that of sulfate. Therefore the largest decreases take place at the areas of the highest concentrations. These reductions vary from 30 to $60 \%$ of the base case ammonium levels.

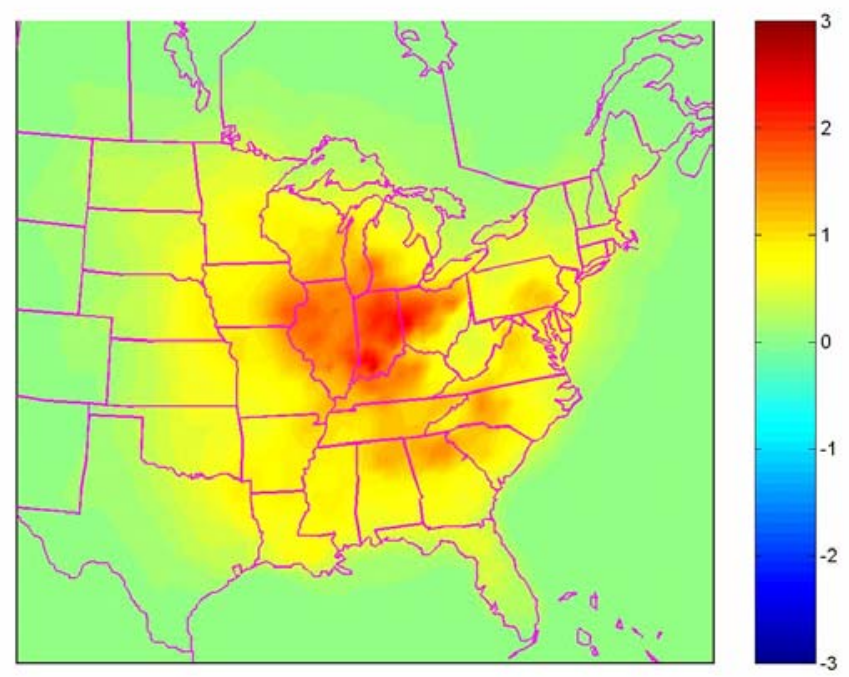

Figure 66. Predicted change $\left(\mu \mathrm{g} \mathrm{m}^{-3}\right)$ in ammonium concentration for a $50 \%$ reduction in $\mathrm{SO}_{2}$ and NOx emissions for July 2001. A positive change corresponds to a concentration reduction.

The changes in $\mathrm{SO}_{2}$ emissions have little effect on organic aerosol levels, but the reduction in $\mathrm{NO}_{\mathrm{x}}$ changes the gas-phase chemistry and results in rather complicated changes of secondary organic aerosol concentrations (Figure 67). Secondary organic aerosol concentrations are predicted to decrease in most of the domain especially in the South by as much as $0.3 \mu \mathrm{g} \mathrm{m}^{-3}$ (Louisiana, Arkansas). However increases of organic aerosol concentrations up to $0.5 \mu \mathrm{g} \mathrm{m}^{-3}$ are predicted for the major urban areas of the Northeast and Midwest (New York, Boston, Philadelphia, Chicago, Detroit). 

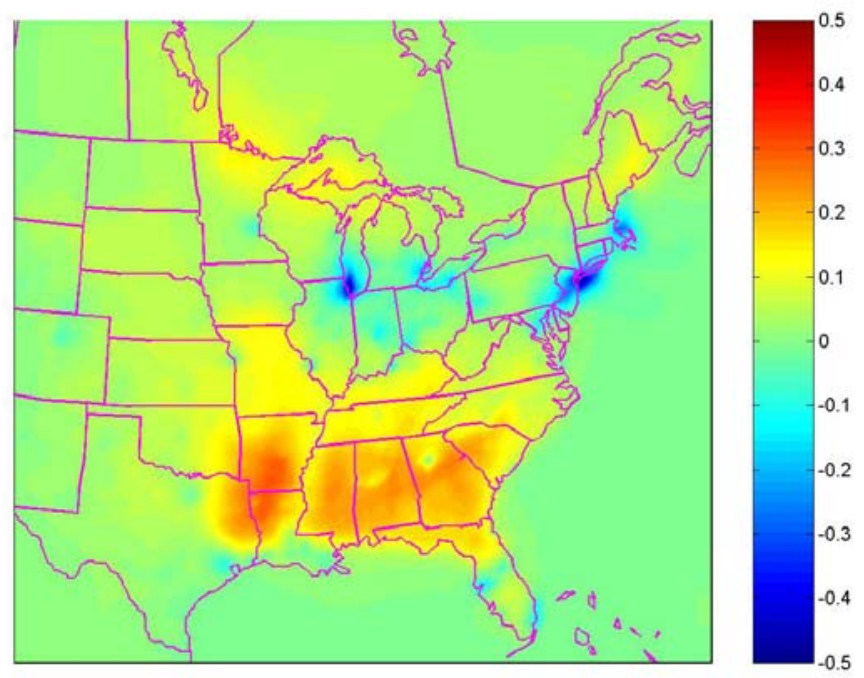

Figure 67. Predicted change $\left(\mu \mathrm{g} \mathrm{m}^{-3}\right)$ in organic aerosol concentration for a $50 \%$ reduction in $\mathrm{SO}_{2}$ and NOx emissions for July 2001. A positive change corresponds to a concentration reduction.

The photochemistry in these urban areas is limited by volatile organic vapors (VOCs) and the reduction in NOx is predicted to lead to an increase in ozone and secondary organic aerosol. As a fraction of the existing organic aerosol these changes are modest, corresponding to approximately $10 \%$ decreases in the South and $10 \%$ increases in the North.

The $50 \%$ reduction in $\mathrm{SO}_{2}$ and $\mathrm{NOx}$ emissions is predicted to lead to reductions of the average $\mathrm{PM}_{2.5}$ levels during July by 3-9 $\mu \mathrm{g} \mathrm{m}^{-3}$ (Figure 68) throughout the Eastern US. Even if there are small local increases in secondary organic aerosol, the net effect is a reduction of the $\mathrm{PM}_{2.5}$ levels in all areas. The predicted reductions vary from $20 \%$ of the $\mathrm{PM}_{2.5}$ in the large urban areas to more than $40 \%$ in rural areas of both the Northeast and Southeast US (Figure 69). 

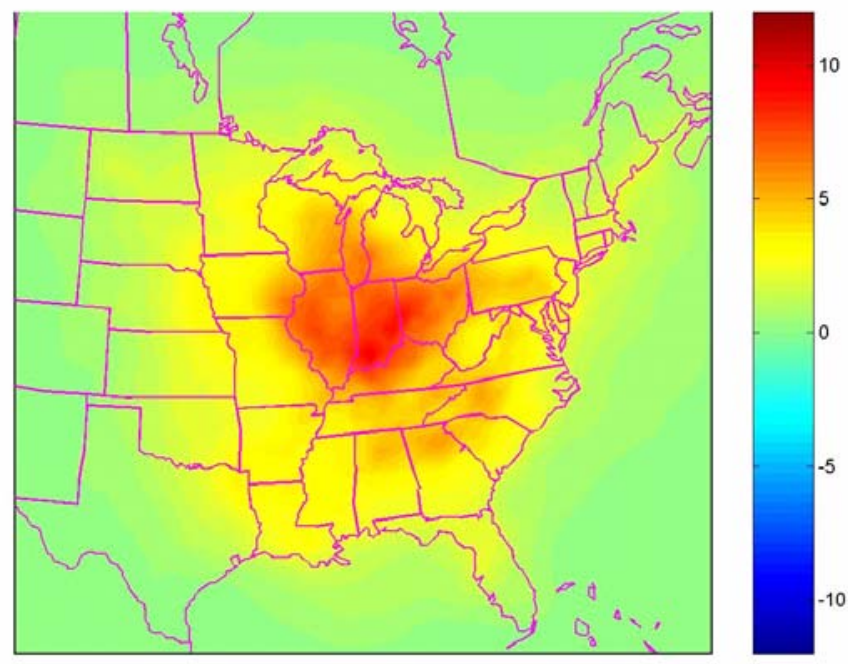

Figure 68. Predicted change $\left(\mu \mathrm{g} \mathrm{m}^{-3}\right)$ in $\mathrm{PM}_{2.5}$ concentration for a $50 \%$ reduction in $\mathrm{SO}_{2}$ and NOx emissions for July 2001. A positive change corresponds to a concentration reduction.

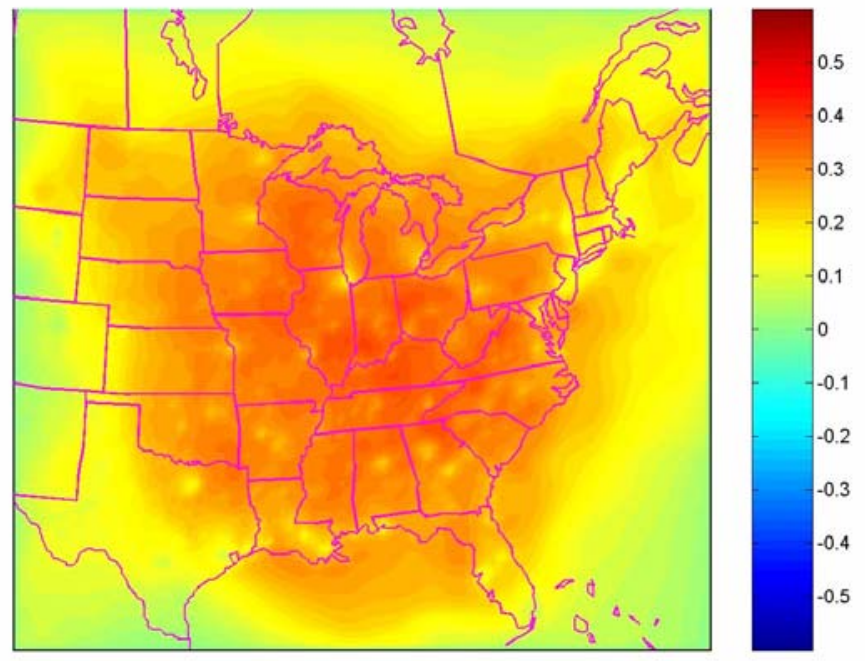

Figure 69. Predicted fractional change in $\mathrm{PM}_{2.5}$ concentration for a $50 \%$ reduction in $\mathrm{SO}_{2}$ and NOx emissions for July 2001. A positive change corresponds to a concentration reduction. 
Wintertime (January 2002)

Reductions of just $\mathrm{SO}_{2}$ during the winter are predicted to result in nitrate increases (Figure 37), while reductions of just $\mathrm{NO}_{\mathrm{x}}$ are predicted to cause an increase of the sulfate concentration (Figure 50). Simultaneous reduction of emissions of both pollutants by $50 \%$ is predicted to decrease the sulfate concentrations throughout the Eastern US (Figure 70). The reductions are small in the Northeast US $\left(0-0.5 \mu \mathrm{g} \mathrm{m}^{-3}\right)$ and larger in the Southeast $\left(0.5-1.5 \mu \mathrm{g} \mathrm{m}^{-3}\right)$. The predicted reductions correspond to around $5 \%$ of the basecase sulfate in the Northeast, around $15 \%$ in the Southeast, and up to $25 \%$ over Florida (Figure 71).

Reducing both the $\mathrm{SO}_{2}$ and $\mathrm{NO}_{\mathrm{x}}$ emissions also prevents the increases of nitrate (Figure 72) seen in the $\mathrm{SO}_{2}$ emissions reduction scenario (Figure 37). The decreases in nitrate concentrations in the coupled emissions reduction case are up to $1 \mu \mathrm{g} \mathrm{m}^{-3}$ with the largest reductions in the Southeast US.

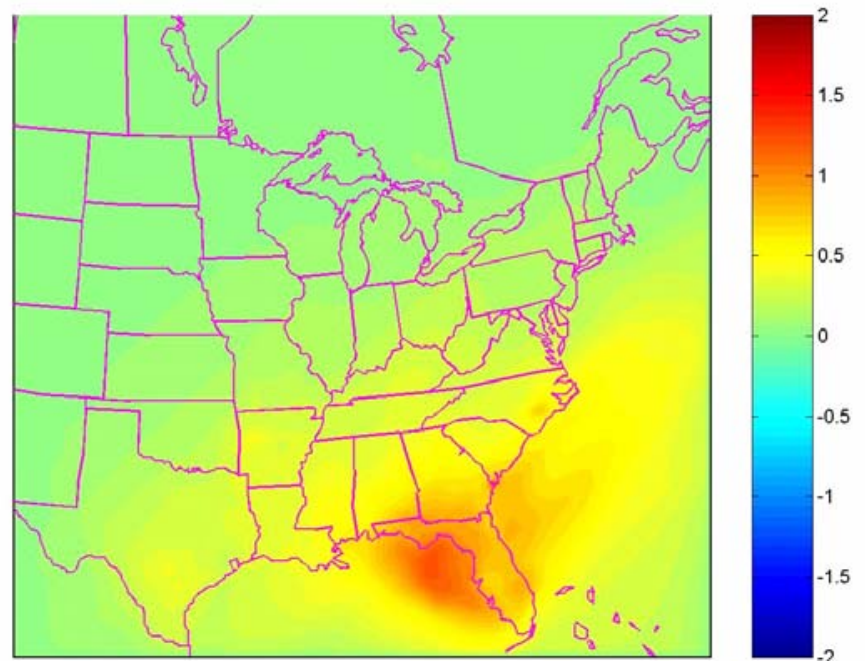

Figure 70. Predicted change $\left(\mu \mathrm{g} \mathrm{m}^{-3}\right)$ in sulfate concentration for a $50 \%$ reduction in $\mathrm{SO}_{2}$ and NOx emissions for January 2002. A positive change corresponds to a concentration reduction. 


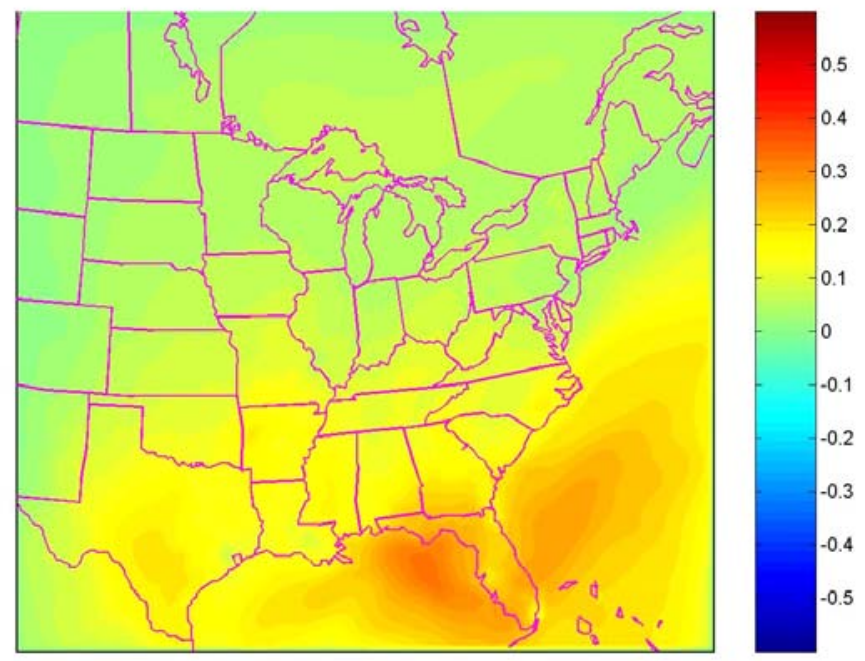

Figure 71. Predicted fractional change in sulfate concentration for a $50 \%$ reduction in $\mathrm{SO}_{2}$ and $\mathrm{NOx}$ emissions for January 2002. A positive change corresponds to a concentration reduction.

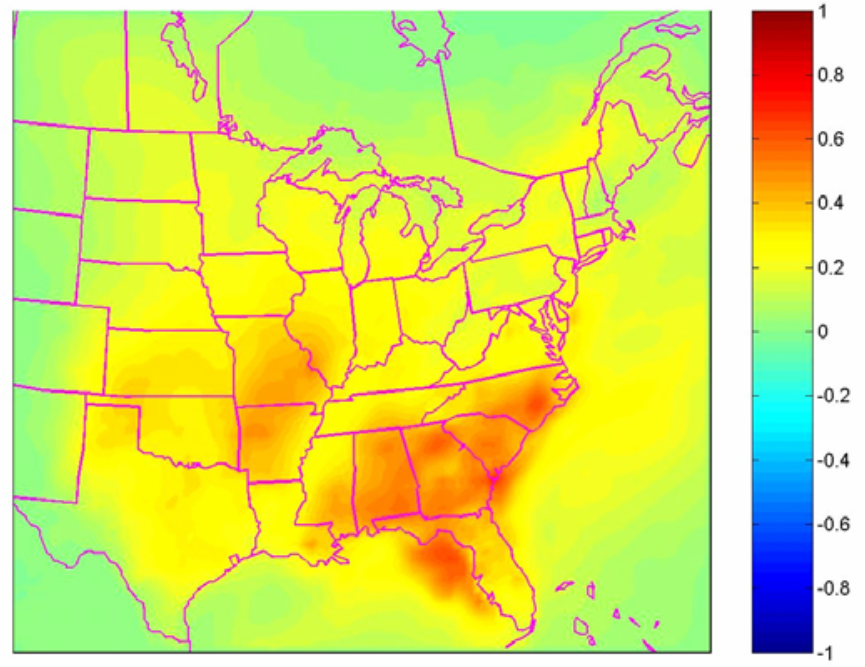

Figure 72. Predicted change $\left(\mu \mathrm{g} \mathrm{m}^{-3}\right)$ in nitrate concentration for a $50 \%$ reduction in $\mathrm{SO}_{2}$ and NOx emissions for January 2002. A positive change corresponds to a concentration reduction. 
The predicted changes correspond to nitrate reductions ranging from $10 \%$ in the Northeast up to $60 \%$ in Southeast. It is notable that in this case even if the highest nitrate levels are in the northeast, the largest reductions are achieved in the south. The significant reductions in both sulfate and nitrate levels result in corresponding reductions of ammonium especially in the Southeast (up to $30 \%$ ).

The only drawback of the coupled $\mathrm{SO}_{2}$ and NOx emissions reduction strategy appears to be the predicted increase in secondary organic aerosol in the Eastern US (Figure 73). This increase varies from around 0.1 up to $0.3 \mu \mathrm{g} \mathrm{m}^{-3}$, with the highest values in the coastal area extending from Southern Carolina to Maine.

The net change in $\mathrm{PM}_{2.5}$ from the coupled $\mathrm{SO}_{2}$ and $\mathrm{NO}_{\mathrm{x}}$ emissions reduction scenario is depicted in Figure 74. Significant concentrations reductions $\left(1.5-2.5 \mu \mathrm{g} \mathrm{m}^{-3}\right)$ are predicted for the Southeast corner of the US, moderate reductions of $0.5-1 \mu \mathrm{g} \mathrm{m}^{-3}$ are predicted for the South, and small reductions (up to $0.5 \mu \mathrm{g} \mathrm{m}{ }^{-3}$ ) for the North.

There are no areas where the $\mathrm{PM}_{2.5}$ is predicted to increase (as was the case in the $\mathrm{NO}_{\mathrm{x}}$ only emissions control strategy - Figure 52). The reductions vary from vary small in the Northeast to up to $25 \%$ of the $\mathrm{PM}_{2.5}$ in parts of the South US (Figure 75).

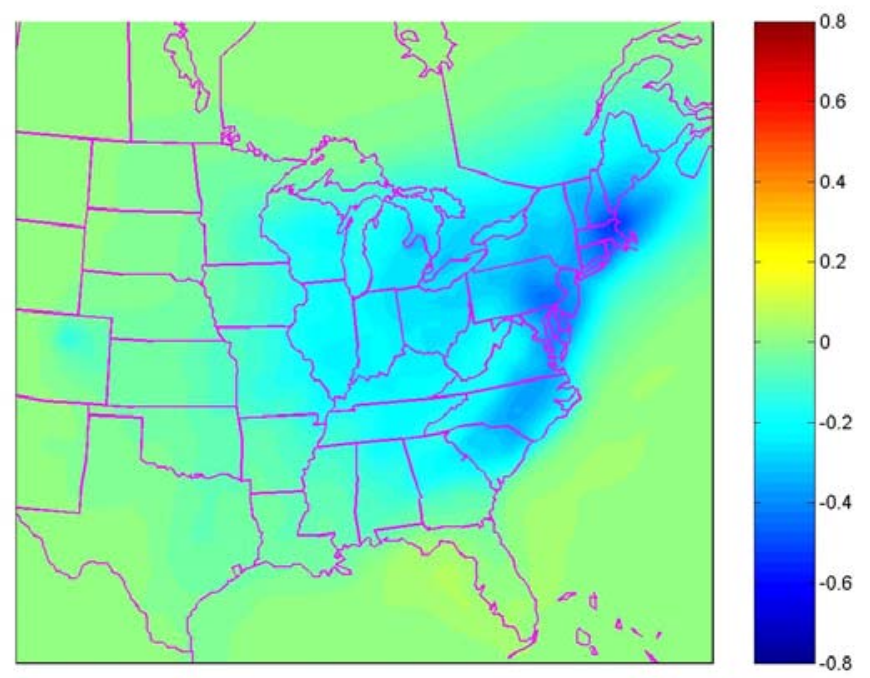

Figure 73. Predicted change $\left(\mu \mathrm{g} \mathrm{m}^{-3}\right)$ in organic aerosol concentration for a $50 \%$ reduction in $\mathrm{SO}_{2}$ and $\mathrm{NOx}$ emissions for January 2002. A positive change corresponds to a concentration reduction. 


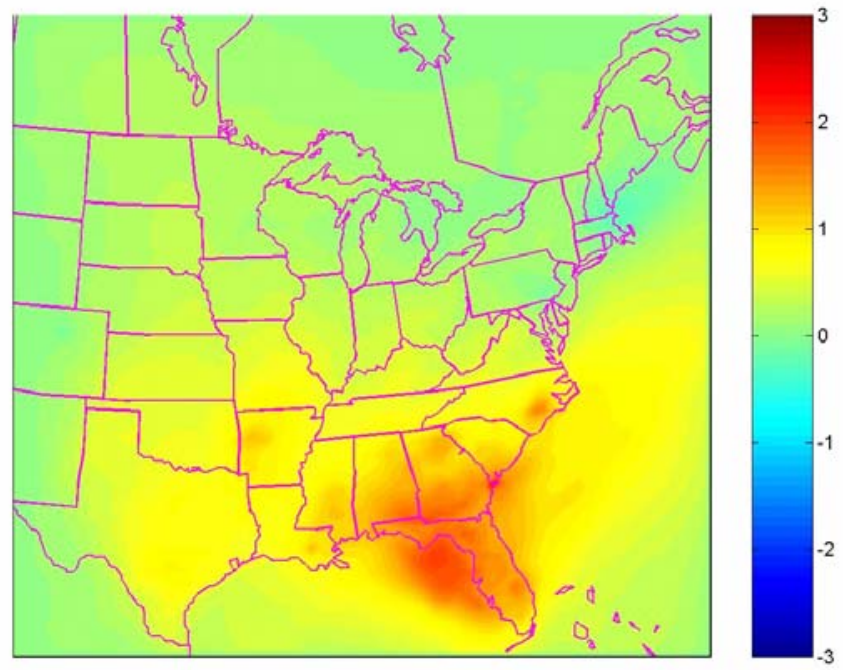

Figure 74. Predicted change $\left(\mu \mathrm{g} \mathrm{m}^{-3}\right)$ in $\mathrm{PM}_{2.5}$ concentration for a $50 \%$ reduction in $\mathrm{SO}_{2}$ and NOx emissions for January 2002. A positive change corresponds to a concentration reduction.

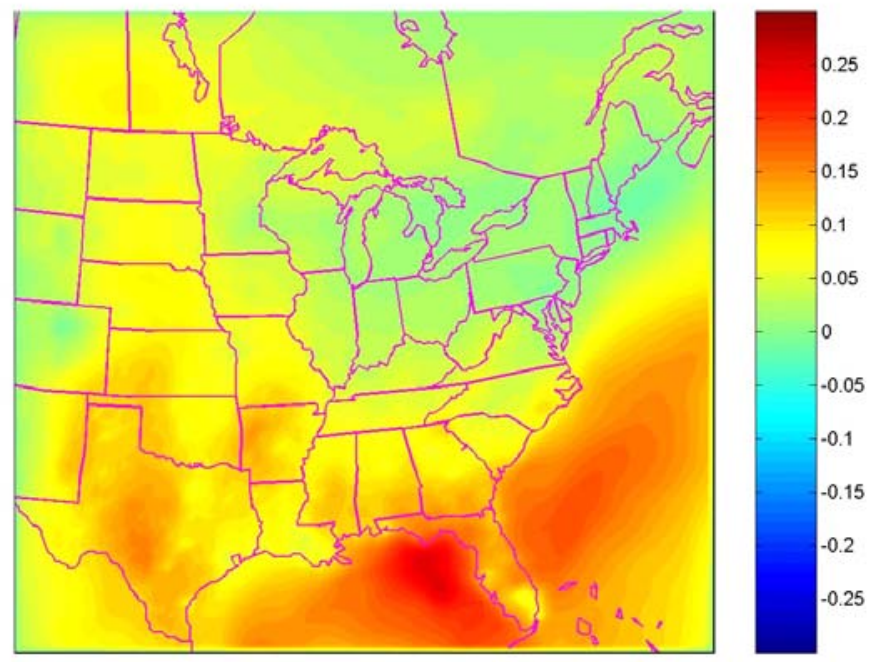

Figure 75. Predicted fractional change in $\mathrm{PM}_{2.5}$ concentration for a $50 \%$ reduction in $\mathrm{SO}_{2}$ and NOx emissions for January 2002. A positive change corresponds to a concentration reduction. 


\section{Predicted $\mathbf{P M}_{2.5}$ Reductions in Pittsburgh}

The maps presented in this section provide insight to both the magnitude and the spatial distribution of the response of $\mathrm{PM}_{2.5}$ with the different emission control scenarios. The responses of the $\mathrm{PM}_{2.5}$ to emission control strategies are different for different areas of the country. As an example, we show below the PMCAMx predictions for Pittsburgh for both summer (Tables 9 and 10) and winter (Tables 11 and 12).

Table 9. Predicted Reductions $\left(\mu \mathrm{g} \mathrm{m}^{-3}\right)$ in $\mathrm{PM}_{2.5}$ Concentrations for Pittsburgh (July 2001)

\begin{tabular}{|c|c|c|c|c|c|}
\hline & \multirow[b]{2}{*}{ Base Case } & \multicolumn{4}{|c|}{ Reduction in Emissions } \\
\hline & & $50 \% \mathrm{SO}_{2}$ & $50 \% \mathrm{NO}_{\mathrm{x}}$ & $\begin{array}{l}\mathbf{5 0 \%} \mathrm{SO}_{2} \\
\mathbf{5 0 \%} \mathbf{N O}_{\mathbf{x}}\end{array}$ & $50 \% \mathrm{NH}_{3}$ \\
\hline Sulfate & 9.58 & 3.85 & 0.73 & 4 & -0.008 \\
\hline Nitrate & 0.33 & 0.003 & 0.17 & 0.2 & 0.09 \\
\hline Ammonium & 2.65 & 0.63 & 0.12 & 0.7 & 0.91 \\
\hline Organic Mass & 3.67 & 0.005 & 0 & -0.1 & -0.002 \\
\hline Total $\mathbf{P M}_{2.5}$ & 20.92 & 4.48 & 1.02 & 4.8 & 0.99 \\
\hline
\end{tabular}

Table 10. Predicted Percentage Reductions in $\mathrm{PM}_{2.5}$ Concentrations for Pittsburgh (July 2001)

\begin{tabular}{|c|c|c|c|c|}
\hline \multirow{2}{*}{ Component } & \multicolumn{4}{|c|}{ Reduction in Emissions } \\
\cline { 2 - 5 } & $\mathbf{5 0 \%} \mathbf{~ S O}_{\mathbf{2}}$ & $\mathbf{5 0 \%}_{\mathbf{~ N O}}$ & $\begin{array}{c}\mathbf{5 0 \%} \mathbf{S O}_{\mathbf{2}} \\
\mathbf{5 0 \%} \mathbf{~ N O}_{\mathbf{x}}\end{array}$ & $\mathbf{5 0 \%} \mathbf{~ N H}_{\mathbf{3}}$ \\
\hline Sulfate & $40.2 \%$ & $8.4 \%$ & $41.8 \%$ & $-0.1 \%$ \\
\hline Nitrate & $0.1 \%$ & $57.4 \%$ & $59.2 \%$ & $26.9 \%$ \\
\hline Ammonium & $23.9 \%$ & $5.1 \%$ & $26.4 \%$ & $34.2 \%$ \\
\hline Organic Mass & $0.1 \%$ & $0 \%$ & $-2.8 \%$ & $-0.1 \%$ \\
\hline Total $\mathbf{P M}_{2.5}$ & $21.4 \%$ & $5.3 \%$ & $23 \%$ & $4.7 \%$ \\
\hline
\end{tabular}

For July the $50 \% \mathrm{SO}_{2}$ emission reductions are by far the most efficient for Pittsburgh, resulting in a decrease of the average $\mathrm{PM}_{2.5}$ concentration by $21.4 \%$ (or $4.48 \mu \mathrm{g} \mathrm{m}^{-3}$ ). Most of this reduction is due to the reduction of the ammonium sulfate concentration. The $50 \%$ reductions in $\mathrm{NO}_{\mathrm{x}}$ and $\mathrm{NH}_{3}$ reduce the $\mathrm{PM}_{2.5}$ by approximately the same amount, $1 \mu \mathrm{g} \mathrm{m}^{-3}$ or $5 \%$. The $\mathrm{NO}_{\mathrm{x}}$ reductions result in decreases of both ammonium sulfate and ammonium nitrate. On the other hand the $\mathrm{NH}_{3}$ reductions mainly reduce the aerosol ammonium resulting in more acidic particles during the summer in this area. Reducing both the $\mathrm{SO}_{2}$ and the $\mathrm{NO}_{\mathrm{x}}$ by $50 \%$ results in a $23 \%$ reduction of the $\mathrm{PM}_{2.5}$ but the reductions are not additive.

The response of the aerosol is quite different in the winter (Tables 3 and 4). In this case the $\mathrm{SO}_{2}$ emission reduction reduces the $\mathrm{PM}_{2.5}$ by only $0.56 \mu \mathrm{g} \mathrm{m}^{-3}$ (around $3 \%$ ), the 
NOx emission reduction increases the $\mathrm{PM}_{2.5}$ by $0.5 \mu \mathrm{g} \mathrm{m} \mathrm{m}^{-3}$ (around $3 \%$ ), and the combined $\mathrm{NOx} / \mathrm{SO}_{2}$ reduces $\mathrm{PM}_{2.5}$ by $0.3 \mu \mathrm{g} \mathrm{m} \mathrm{m}^{-3}$ (around $2 \%$ ). It is interesting that for this area the most efficient strategy examined is the ammonia emission reduction leading to a $1.5 \mu \mathrm{g} \mathrm{m}^{-3}(8 \%)$ reduction in $\mathrm{PM}_{2.5}$. Control of organic particulate matter is obviously the most efficient strategy for winter in this area.

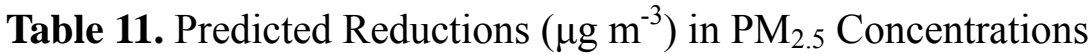
for Pittsburgh (January 2002)

\begin{tabular}{|c|c|c|c|c|c|}
\hline & \multicolumn{5}{|c|}{ Reduction in Emissions } \\
\hline & Base Case & $\mathbf{5 0 \%} \mathbf{S O}_{\mathbf{2}}$ & $\mathbf{5 0 \%} \mathbf{N O}_{\mathbf{x}}$ & $\begin{array}{c}\mathbf{5 0 \%} \mathbf{S O}_{\mathbf{2}} \\
\mathbf{5 0 \%} \mathbf{~ N O}_{\mathbf{x}}\end{array}$ & $\mathbf{5 0 \% ~}_{\mathbf{5 0}}$ \\
\hline Sulfate & 3.61 & 0.45 & -0.45 & 0.21 & 0.03 \\
\hline Nitrate & 2.03 & -0.09 & 0.32 & 0.19 & 1.06 \\
\hline Ammonium & 1.94 & 0.14 & -0.08 & 0.13 & 0.37 \\
\hline Organic Mass & 4.3 & 0.05 & -0.29 & -0.26 & 0.05 \\
\hline Total PM & 18.97 & 0.56 & -0.5 & 0.27 & 1.51 \\
\hline
\end{tabular}

Table 12. Predicted Percentage Reductions in $\mathrm{PM}_{2.5}$ Concentrations for Pittsburgh (January 2002)

\begin{tabular}{|c|c|c|c|c|}
\hline \multirow[b]{2}{*}{ Component } & \multicolumn{4}{|c|}{ Reduction in Emissions } \\
\hline & $50 \% \mathrm{SO}_{2}$ & $\begin{array}{l}50 \% \\
\text { NO }_{x}\end{array}$ & $\begin{array}{l}50 \% \mathrm{SO}_{2} \\
50 \% \mathrm{NO}_{\mathrm{x}}\end{array}$ & $50 \% \mathrm{NH}_{3}$ \\
\hline Sulfate & $12.5 \%$ & $-12.9 \%$ & $5.7 \%$ & $0.8 \%$ \\
\hline Nitrate & $-4.2 \%$ & $16 \%$ & $9.4 \%$ & $52 \%$ \\
\hline Ammonium & $7.4 \%$ & $-4.1 \%$ & $6.8 \%$ & $19.2 \%$ \\
\hline Organic Mass & $1.1 \%$ & $-7 \%$ & $-5.9 \%$ & $1.2 \%$ \\
\hline Total PM 2.5 & $3 \%$ & $-2.7 \%$ & $1.5 \%$ & $8 \%$ \\
\hline
\end{tabular}

\section{CONCLUSIONS}

The Pittsburgh Air Quality Study is a comprehensive multidisciplinary set of projects in the Pittsburgh region, led by Carnegie Mellon University with contributions by academics, consultants, national laboratories, and state and local air pollution agencies. Measurements of ambient air quality were made at a central monitoring site located in an urban park in Pittsburgh, PA and five satellite sites located in Pittsburgh, Southwestern Pennsylvania, and Eastern Ohio. Baseline monitoring took place over a fourteen-month period that began July 1, 2001 and provided data for a wide variety of PM physical and chemical characteristics, gaseous species, fogwater and biological aerosol composition, and meteorological parameters. Two intensive sampling periods were conducted from July 1 to August 3, 2001 and January 1 to January 15, 2002. The ambient data collected by the project are available through online relational databases. 
Updated source profiles were developed through a combination of source testing, fenceline measurements, and analysis of highly time resolved data collected at the central site. The profiles were developed using state-of-the-art sampling and analytical techniques. Profiles were developed for emissions from motor vehicles, coke production, coal combustion, paved road dust, and vegetative detritus.

A major focus of the project was investigating the contribution of different sources to ambient fine particle concentrations in the Pittsburgh region. This was done using a variety of statistical and deterministic air quality models. Part of this effort involved evaluating a three-dimensional aerosol chemical transport model, PMCAMx + . Predictions of this model were compared to highly time-resolved measurements collected in Pittsburgh and to spatially resolved measurements by the EPA STN and IMPROVE networks. After this evaluation, the model was used to investigate the response of ambient PM concentrations to changes in emissions of major aerosol precursors.

The study average $\mathrm{PM} 2.5$ mass concentration was $15.8 \mu \mathrm{g} \mathrm{m}^{-3}$. Nine days of relatively high $\mathrm{PM}_{2.5}$ mass concentration were experienced at PAQS, several of which formed multi-day episodes, and all of which occurred during the warmer summer months. Seasonal variations in $\mathrm{PM}_{2.5}$ mass and composition were the result of differences in monthly average organic carbon and sulfate components.

During the study roughly $90 \%$ of the $\mathrm{PM}_{2.5}$ concentration came from areas outside Pittsburgh. There are important local sources of fine particulate metals, and roughly half of the particle number concentration was due to local transportation sources. Emissions from coal-fired power plants contribute significantly to $\mathrm{PM}_{2.5}$ concentrations in Pittsburgh. The vast majority of this contribution is to secondary sulfate which represents $38 \%$ of annual average $\mathrm{PM}_{2.5}$ mass. Primary emissions from coal fired power are estimated to be a minor source to fine particle mass, contributing on average $0.25 \mu^{-3}$ to fine particle mass (or $1.5 \%$ of annual average $\mathrm{PM}_{2.5}$ ). Primary emissions from anthropogenic sources contributed on average more than two-thirds of the ambient OC in the winter and between one-third and two-thirds of the ambient OC. The secondary organic aerosol contribution to the total organic PM concentration in Pittsburgh varies from around $10 \%$ during the winter months to around $50 \%$ of the total OC concentration in the summer months. On a daily basis the SOA contribution is quite variable from almost zero during the low $\mathrm{OC}$ days to more than $50 \%$ during the high $\mathrm{PM}_{2.5}$ episodes.

Reasonable agreement is observed between predictions of a three-dimensional aerosol chemical transport model (PMCAMx + ) and observations. This model was used to investigate the response of ambient PM concentrations to changes in emissions. The reduction of $\mathrm{SO}_{2}$ emissions during the summer is predicted to decrease significantly sulfate and ammonium concentrations in the Eastern US with only small increases of nitrate concentrations in urban areas. In comparison, summertime reductions in $\mathrm{NO}_{\mathrm{x}}$ emissions result in a modest decrease in nitrate, sulfate, and ammonium in the Eastern US. Reductions of $\mathrm{NH}_{3}$ emissions in the winter are predicted to be quite effective in reducing the ammonium nitrate concentrations. 


\section{REFERENCES}

Bein, K. J., Zhao, Y. J., Wexler, A. S. and Johnston, M. V. (2005). "Speciation of sizeresolved individual ultrafine particles in Pittsburgh, Pennsylvania." Journal of Geophysical Research-Atmospheres 110(D7).

Blando, J. D., Porcja, R. J. and Turpin, B. J. (2001). "Issues in the quantitation of functional groups by FTIR spectroscopic analysis of impactor-collected aerosol samples." Aerosol Science and Technology 35(5): 899-908.

Cabada, J. C., Khlystov, A., Wittig, A. E., Pilinis, C. and Pandis, S. N. (2004a). "Light scattering by fine particles during the Pittsburgh Air Quality Study: measurements and modeling." Journal of Geophysical Research-Atmospheres 109(D16).

Cabada, J. C., Pandis, S. N. and Robinson, A. L. (2002). "Sources of atmospheric carbonaceous particulate matter in Pittsburgh, Pennsylvania." Journal of the Air \& Waste Management Association 52(6): 732-741.

Cabada, J. C., Pandis, S. N., Subramanian, R., Robinson, A. L., Polidori, A. and Turpin, B. (2004b). "Estimating the secondary organic aerosol contribution to PM2.5 using the EC tracer method." Aerosol Science and Technology 38: 140-155.

Cabada, J. C., Rees, S. L., Takahama, S., Khlystov, A., Pandis, S. N., Davidson, C. I. and Robinson, A. L. (2004c). "Mass size distributions and size resolved chemical composition of fine particulate matter at the Pittsburgh supersite." Atmospheric Environment 38: 3127-3141.

Carson, P. G., Neubauer, K. R., Johnston, M. V. and Wexler, A. S. (1995). "Online Chemical-Analysis of Aerosols By Rapid Single-Particle Mass-Spectrometry." Journal of Aerosol Science 26(4): 535-545.

Code of Federal Regulations Title 40 Subchapter C Part 50, 2002.

Ding, Y. M., Pang, Y. B. and Eatough, D. J. (2002). "High-volume diffusion denuder sampler for the routine monitoring of fine particulate matter: I. Design and optimization of the PC-BOSS." Aerosol Science and Technology 36(4): 369-382.

EIA (1994). Residential Transportation Energy Consumption Survey, U.S. Energy Information Administration.

Environ, 2003. User's Guide to the Comprehensive Air Quality Model With Extensions (CAMx). Version 4.02. Report prepared by ENVIRON International Corporation, Novato, CA.

Fraser, M. P., Yue, Z. W. and Buzcu, B. (2003). "Source apportionment of fine particulate matter in Houston, TX, using organic molecular markers." Atmospheric Environment 37(15): 2117-2123.

Gaffney, J. S., Marley, N. A. and Cunningham, M. M. (2004). "Natural radionuclides in fine aerosols in the Pittsburgh area." Atmospheric Environment 38(20): 31913200 . 
Gaydos, T. M., Pinder, R. W., Koo, B., Fahey, K. M. and Pandis, S. N. (2005a). "Development and Application of a Three-dimensional Aerosol Chemical Transport Model, PMCAMx+." Atmospheric Environment submitted.

Gaydos, T. M., Stanier, C. O. and Pandis, S. N. (2005b). "Modeling of in situ ultrafine atmospheric particle formation in the eastern United States." Journal of Geophysical Research-Atmospheres 110(D7).

Ge, Z. Z., Wexler, A. S. and Johnston, M. V. (1998). "Laser desorption/ionization of single ultrafine multicomponent aerosols." Environmental Science \& Technology 32(20): 3218-3223.

Goebes, M. D., Strader, R. and Davidson, C. (2003). "An ammonia emission inventory for fertilizer application in the United States." Atmospheric Environment 37(18): 2539-2550.

Grell, G.A.., J. Dudhia, and D.R. Stauffer, 1995. A Description of the Fifth-Generation Penn State/NCAR Mesoscale Model (MM5). NCAR/TN-398+STR. http://www.mmm.ucar.edu/mm5/documents/mm5-desc-doc.html.

Grieshop, A. P., Lipsky, E. M., Pekney, N. J., Takahama, S. and Robinson, A. L. (2005). "Diesel and Gasoline Vehicle Gaseous and Fine Particle Emission Factors Measured in a Highway Tunnel in Pittsburgh, PA." Atmospheric Environment Submitted.

Hildemann, L. M., Cass, G. R. and Markowski, G. R. (1989). "A Dilution Stack Sampler for Collection of Organic Aerosol Emissions - Design, Characterization and FieldTests." Aerosol Science and Technology 10(1): 193-204.

IMPROVE, 1995. IMPROVE Data Guide. University of California Davis, August, 1995.

http://vista.cira.colostate.edu/improve/Publications/OtherDocs/IMPROVEDataGu ide/IMPROVEDataGuide.htm.

Jaffrezo, J. L., Calas, T. and Bouchet, M. (1998). "Carboxylic acids measurements with ionic chromatography." Atmospheric Environment 32(14-15): 2705-2708.

Janssen, M., 2003. CEM Data Analysis and Use. Presented at the RPO National Technical Workgroup Meeting. St. Louis, MO. November 4-6, 2003.

Jayne, J. T., Leard, D. C., Zhang, X. F., Davidovits, P., Smith, K. A., Kolb, C. E. and Worsnop, D. R. (2000). "Development of an aerosol mass spectrometer for size and composition analysis of submicron particles." Aerosol Science and Technology 33(1-2): 49-70.

Jimenez, J. L., Jayne, J. T., Shi, Q., Kolb, C. E., Worsnop, D. R., Yourshaw, I., Seinfeld, J. H., Flagan, R. C., Zhang, X. F., Smith, K. A., Morris, J. W. and Davidovits, P. (2003). "Ambient aerosol sampling using the Aerodyne Aerosol Mass Spectrometer." Journal of Geophysical Research-Atmospheres 108(D7).

Khlystov, A., Stanier, C. and Pandis, S. N. (2004). "An algorithm for combining electrical mobility and aerodynamic size distributions data when measuring ambient aerosol." Aerosol Science and Technology 38: 229-238. 
Khlystov, A., Stanier, C. O., Takahama, S. and Pandis, S. N. (2005). "Water content of ambient aerosol during the Pittsburgh air quality study." Journal of Geophysical Research-Atmospheres 110(D7).

Khlystov, A., Wyers, G. P. and Slanina, J. (1995). "The Steam-Jet Aerosol Collector." Atmospheric Environment 29(17): 2229-2234.

Kidwell, C. B. and Ondov, J. M. (2001). "Development and evaluation of a prototype system for collecting sub-hourly ambient aerosol for chemical analysis." Aerosol Science and Technology 35(1): 596-601.

LADCO, 2003. Base E Modeling Inventory. Midwest Regional Planning Organization, $\begin{array}{llll}\text { Des Plaines, } & \text { IL, September }\end{array}$ http://www.ladco.org/tech/emis/BaseE/baseEreport.pdf

Lane, T. E., Pinder, R. W., Shrivastava, M., Robinson, A. L. and Pandis, S. N. (2005). "Source contributions to primary organic aerosol; Comparison of the results of a source-resolved model and the Chemical Mass Balance approach." Atmospheric Environment submitted.

Lazrus, A. L., Kok, G. L., Lind, J. A., Gitlin, S. N., Heikes, B. G. and Shetter, R. E. (1986). "Automated Fluorometric Method for Hydrogen-Peroxide in Air." Analytical Chemistry 58(3): 594-597.

Lewis, C. W., Stevens, R. K., Rasmussen, R. A., Cardelino, C. A. and Pierce, T. E. (1999). "Biogenic fraction of ambient VOC: Comparison of radiocarbon, chromatographic, and emissions inventory estimates for Atlanta, Georgia." Journal of the Air \& Waste Management Association 49(3): 299-307.

Lipsky, E. M. and Robinson, A. L. (2005a). "Design and evaluation of a portable dilution sampling system and the effects of residence time on mass emission rates." Aerosol Science and Technology 39(6): 542-553.

Lipsky, E. M. and Robinson, A. L. (2005b). "Effects of Dilution on Fine Particle Mass and Partitioning of Semivolatile Organics in Diesel Exhaust and Wood Smoke." Environmental Science \& Technology in press.

Millet, D. B., Donahue, N. M., Pandis, S. N., Polidori, A., Stanier, C. O., Turpin, B. J. and Goldstein, A. H. (2005). "Atmospheric volatile organic compound measurements during the Pittsburgh Air Quality Study: Results, interpretation, and quantification of primary and secondary contributions." Journal of Geophysical ResearchAtmospheres 110(D7).

Modey, W. K., Eatough, D. J., Anderson, R. R., Martello, D. V., Takahama, S., Lucas, L. J. and Davidson, C. I. (2004). "Ambient fine particulate concentrations and chemical composition at two sampling sites in metropolitan Pittsburgh: a 2001 intensive summer study." Atmospheric Environment 38(20): 3165-3178.

MSP Corp., Inc., 1998. Operator's Manual for the Micro-Orifice Uniform Deposit Impactor. Shoreview, MN. 
NIOSH (1999). Method 5040 Issue 3 (Interim): Elemental carbon (diesel exhaust). NIOSH Manual of Analytical Methods. Cincinnati, OH, National Institute of Occupational Safety and Health.

Park, S. S., Pancras, J. P., Ondov, J. M. and Robinson, A. L. (2005). "Application of the pseudo-deterministic receptor model to resolve power plant influences on air quality in Pittsburgh." Aerosol Science \& Technology submitted.

Pekney, N. J., Davidson, C. I., Robinson, A. L., Zhou, L., Hopke, P. K. and Eatough, D. (2005a). "Identification of Major Sources of PM2.5 in Pittsburgh using PMF and UNMIX." Aerosol Science \& Technology in press.

Pekney, N. J., Davidson, C. I., Zhou, L. and Hopke, P. K. (2005b). "Application of PSCF and CPF to PMF-modeled sources of PM2.5 in Pittsburgh." Aerosol Science \& Technology submitted.

Pinder, R. W., Strader, R., Davidson, C. I. and Adams, P. J. (2004). "A temporally and spatially resolved ammonia emission inventory for dairy cows in the United States." Atmospheric Environment 38(23): 3747-3756.

Polidori, A., Turpin, B. J., Lim, H. J., Cabada, J. C., Subramanian, R., Robinson, A. L. and Pandis, S. N. (2005). "Secondary organic aerosol formation in the Pittsburgh area using semi-continuous OC and EC measurements." Aerosol Science \& Technology submitted.

Rees, S. L., Robinson, A. L., Khlystov, A., Stanier, C. O. and Pandis, S. N. (2004). "Mass Balance Closure and the Federal Reference Method for PM2.5 in Pittsburgh, Pennsylvania." Atmospheric Environment 38(20): 3305-3318.

Robinson, A. L., Donahue, N. M. and Rogge, W. F. (2005a). "Photochemical oxidation and changes in molecular composition of organic aerosol in the regional context." Journal of Geophysical Research-Atmospheres in press.

Robinson, A. L., Subramanian, R., Donahue, N. M., Bernardo-Bricker, A. and Rogge, W. F. (2005b). "Methodology for selecting source profiles for chemical mass balance modeling using organic molecular markers." Environmental Science \& Technology submitted.

Rogge, W. F., Hildemann, L. M., Mazurek, M. A. and Cass, G. R. (1994). "Sources of Fine Organic Aerosol .6. Cigarette-Smoke in the Urban Atmosphere." Environmental Science \& Technology 28(7): 1375-1388.

Rogge, W. F., Hildemann, L. M., Mazurek, M. A., Cass, G. R. and Simoneit, B. R. T. (1993). "Sources of Fine Organic Aerosol .4. Particulate Abrasion Products From Leaf Surfaces of Urban Plants." Environmental Science \& Technology 27(13): 2700-2711.

Schauer, J. J. and Cass, G. R. (2000). "Source apportionment of wintertime gas-phase and particle-phase air pollutants using organic compounds as tracers." Environmental Science \& Technology 34(9): 1821-1832.

Schauer, J. J., Fraser, M. P., Cass, G. R. and Simoneit, B. R. T. (2002). "Source reconciliation of atmospheric gas-phase and particle-phase pollutants during a 
severe photochemical smog episode." Environmental Science \& Technology 36(17): 3806-3814.

Schauer, J. J., Rogge, W. F., Hildemann, L. M., Mazurek, M. A. and Cass, G. R. (1996). "Source apportionment of airborne particulate matter using organic compounds as tracers." Atmospheric Environment 30(22): 3837-3855.

Slanina, J., ten Brink, H. M., Otjes, R. P., Even, A., Jongejan, P., Khlystov, A., WaijersIjpelaan, A. and $\mathrm{Hu}, \mathrm{M}$. (2001). "The continuous analysis of nitrate and ammonium in aerosols by the steam jet aerosol collector (SJAC): extension and validation of the methodology." Atmospheric Environment 35(13): 2319-2330.

Stanier, C. O., Khlystov, A. Y. and Pandis, S. N. (2004b). "Ambient aerosol size distributions and number concentrations measured during the Pittsburgh Air Quality Study (PAQS)." Atmospheric Environment 38(20): 3275-3284.

Stanier, C. O., Khlystov, A. Y. and Pandis, S. N. (2004c). "Nucleation events during the Pittsburgh air quality study: Description and relation to key meteorological, gas phase, and aerosol parameters." Aerosol Science and Technology 38: 253-264.

Stanier, C. O., Khlystov, A. Y., Chan, W. R., Mandiro, M. and Pandis, S. N. (2004a). "A method for the in situ measurement of fine aerosol water content of ambient aerosols: The dry-ambient aerosol size spectrometer (DAASS)." Aerosol Science and Technology 38: 215-228.

Stolzenburg, M. R. and Hering, S. V. (2000). "Method for the automated measurement of fine particle nitrate in the atmosphere." Environmental Science \& Technology 34(5): 907-914.

Subramanian, R., Donahue, N. M., Bernardo-Bricker, A., Rogge, W. F. and Robinson, A. L. (2005a). "Source Apportionment of Primary Organic Aerosol in Pittsburgh, PA using organic molecular markers." Environmental Science \& Technology in preparation.

Subramanian, R., Khlystov, A. Y., Cabada, J. C. and Robinson, A. L. (2004). "Positive and Negative Artifacts in Particulate Organic Carbon Measurements with Denuded and Undenuded Sampler Configurations." Aerosol Science \& Technology 38(S1): 27-48.

Subramanian, R., Robinson, A. L., Donahue, N. M., Bernardo-Bricker, A. and Rogge, W. F. (2005b). "Contribution of motor vehicle emissions to the ambient organic carbon in Pittsburgh, PA: Effects of varying source profiles and seasonal trends in ambient marker concentrations." Atmospheric Environment submitted.

Takahama, S., Wittig, A. E., Vayenas, D. V., Davidson, C. I. and Pandis, S. N. (2004). "Modeling the diurnal variation of nitrate during the Pittsburgh Air Quality Study." Journal of Geophysical Research-Atmospheres 109(D16).

Takahama, S., Davidson, C. I. and Pandis, S. N. (2006). "Semi-Continuous Measurements of Organic Carbon and Acidity during the Pittsburgh Air Quality Study: Implications for Acid-Catalyzed Organic Aerosol Formation." Environmental Science \& Technology 40(7), 2191-2198. 
Tang, W. T. R., Beth Wittig, Cliff Davidson, Spyros Pandis, Allen RObinson, Kevin Crist (2004). "Spatial Variations of PM2.5 During thE Pittsburgh Air Quality Study." Aerosol Science and Technology submitted.

Tang, W., Raymond, T., Wittig, B., Davidson, C. I., Pandis, S. N., Robinson, A. L. and Crist, K. (2004). "Spatial Variations of PM2.5 during the Pittsburgh Air Quality Study." Aerosol Science \& Technology 38(S2): 80-90.

Thermo Andersen, 1999. Operator's Manual for PM10 High Volume Sampler. Smyrna, GA.

Turpin, B. J., Cary, R. A. and Huntzicker, J. J. (1990). "An In Situ, Time-Resolved Analyzer For Aerosol Organic and Elemental Carbon." Aerosol Science and Technology 12(1): 161-171.

USEPA, (1991). EPA Method 200.8 (rev 4.4): Determination of trace elements in waters and wastes by Inductively Coupled Plasma - Mass Spectrometry. Research Triangle Park, NC.

USEPA, (1994). EPA Method 6020: Inductively Coupled Plasma Mass Spectrometry. Research Triangle Park, NC.

USEPA, (1998). EPA Quality assurance guidance 2.12: Monitoring PM2.5 in ambient air using designated references or Class I equivalence methods. Research Triangle Park, NC.

USEPA, (1999). EPA Method 625 (rev R-96/01a): Compendium of methods for the determination of inorganic compounds in ambient air. Research Triangle Park, $\mathrm{NC}$.

USEPA. (2002). User Guide: Air Quality System. Report prepared by the U.S. EPA. April. http://www.epa.gov/ttn/airs/aqs/softw/AQSUserGuide_v1.pdf.

USEPA, 2002a. 1999 National Emission Inventory Documentation and Data, Office of Air Quality Planning and Standards, U.S. Environmental Protection Agency Research Triangle Park, NC. http://www.epa.gov/ttn/chief/net/1999inventory.html

USEPA, 2002b. NONROAD Model. U.S. Environmental Protection Agency, Office of Transportation and Air Quality: Ann Arbor, MI, 2002. http://www.epa.gov/oms/nonrdmdl.htm

USEPA, 2002c. User's Guide to MOBILE6.1 and MOBILE6.2: Mobile Source Emission Factor Model, Office of Transportation and Air Quality, Ann Arbor, MI, October 2002; Report No. EPA420-R-02-028. http://www.epa.gov/otaq/m6.htm

USEPA (2003). Acid Rain/OTC Program Hourly Emissions Data, USEPA.

Vayenas, D. V., Takahama, S., Davidson, C. I. and Pandis, S. N. (2005). "Simulation of the thermodynamics and removal processes in the sulfate-ammonia-nitric acid system during winter: Implications for PM2.5 control strategies." Journal of Geophysical Research-Atmospheres 110(D7).

VIUS (2002). Vehicle Inventory and Use Survey, U.S. Census Bureau. 
Weitkamp, E. A., Lipsky, E. M., Pancras, P., Ondov, J., Polidori, A., Turpin, B. J. and Robinson, A. L. (2005). "Fine Particle Emission Profile for a Large Coke Production Facility based on Highly Time Resolved Fenceline Measurements." Atmospheric Environment 39: 6719-6733.

Wilkinson, J. and Janssen, M., 2001. BIOME3. Proceedings from the 10th International Emission Inventory Conference. Denver, CO, April 30, 2001.

Wittig, A. E., Anderson, N., Khlystov, A. Y., Pandis, S. N., Davidson, C. I. and Robinson, A. L. (2004a). "Pittsburgh Air Quality Study Overview." Atmospheric Environment 38(20): 3107-3125.

Wittig, A. E., Takahama, S., Khlystov, A. Y., Pandis, S. N., Hering, S., Kirby, B. and Davidson, C. (2004b). "Semi-continuous PM2.5 inorganic composition measurements during the Pittsburgh air quality study." Atmospheric Environment 38(20): 3201-3213.

Zhang, Q., Canagaratna, M. R., Jayne, J. T., Worsnop, D. R. and Jimenez, J. L. (2005). "Time- and size-resolved chemical composition of submicron particles in Pittsburgh: Implications for aerosol sources and processes." Journal of Geophysical Research-Atmospheres 110(D7).

Zhang, Q., Stanier, C. O., Canagaratna, M. R., Jayne, J. T., Worsnop, D. R., Pandis, S. N. and Jimenez, J. L. (2004). "Insights into the chemistry of new particle formation and growth events in Pittsburgh based on aerosol mass spectrometry." Environmental Science \& Technology 38(18): 4797-4809.

Zhao, Y., Bein, K. J., Wexler, A. S., Misra, C., Fine, P. M. and Sioutas, C. (2005). "Field evaluation of the versatile aerosol concentration enrichment system (VACES) particle concentrator coupled to the rapid single-particle mass spectrometer (RSMS-3)." Journal of Geophysical Research-Atmospheres 110(D7).

Zheng, M., Cass, G. R., Schauer, J. J. and Edgerton, E. S. (2002). "Source apportionment of PM2.5 in the southeastern United States using solvent-extractable organic compounds as tracers." Environmental Science \& Technology 36(11): 2361-2371.

Zhou, L. M., Hopke, P. K., Stanier, C. O., Pandis, S. N., Ondov, J. M. and Pancras, J. P. (2005a). "Investigation of the relationship between chemical composition and size distribution of airborne particles by partial least squares and positive matrix factorization." Journal of Geophysical Research-Atmospheres 110(D7).

Zhou, L. M., Kim, E., Hopke, P. K., Stanier, C. and Pandis, S. N. (2005b). "Mining airborne particulate size distribution data by positive matrix factorization." Journal of Geophysical Research-Atmospheres 110(D7).

Zhou, L., Kim, E., Hopke, P. K., Stanier, C. O. and Pandis, S. N. (2004). "Advanced Factor Analysis on Pittsburgh Particle Size Distribution Data." Aerosol Science and Technology 38(S1): 118-132. 


\section{PRESENTATIONS AND PUBLICATIONS}

\section{Publications:}

\section{Peer-reviewed journal papers}

1. Bein K. J., Y. Zhao, A. S. Wexler and M.V. Johnston (2005) Speciation of sizeresolved individual ultrafine particles in Pittsburgh, Pennsylvania, J. Geophys. Res., 110, D07S05, doi:10.1029/2004JD004708.

2. Bein K. J., Y. Zhao, N. J. Pekney, C. I. Davidson, M.V. Johnston and A. S. Wexler (2006) Identification of Sources of Atmospheric PM at the Pittsburgh Supersite. Part II: Quantitative Comparisons of Single Particle, Particle Number, and Particle Mass Measurements, Atmospheric Environment, in press.

3. Bein K. J., Y. Zhao, N. J. Pekney, C. I. Davidson, M.V. Johnston and A. S. Wexler (2006) Identification of Sources of Atmospheric PM at the Pittsburgh Supersite. Part III: Source Characterization Atmospheric Environment, Atmospheric Environment, submitted.

4. Cabada J. C., A. Khlystov, A.E. Wittig, C. Pilinis, and S. N. Pandis (2004c) Light scattering by fine particles during the Pittsburgh Air Quality Study: Measurements and modeling, Journal of Geophysical Research, 109, D16S03, doi:10.1029/ 2003JD004155.

5. Cabada J. C., S. N. Pandis, and A. L. Robinson (2002) Sources of atmospheric particulate matter in Pittsburgh, Pennsylvania, JAWMA, 52, 732-741.

6. Cabada J. C., S. N. Pandis, R. Subramanian, A.L. Robinson, A. Polidori, and B. Turpin (2004a) Estimating the secondary organic aerosol contribution to $\mathrm{PM}_{2.5}$ using the EC tracer method, Aerosol Science and Technology, 38(S1), 140-155.

7. Cabada J. C., S. Rees, S. Takahama, A. Khlystov, S.N. Pandis, C.I. Davidson, and A.L. Robinson (2004b) Mass size distributions and size resolved chemical composition of fine particulate matter at the Pittsburgh Supersite, Atmospheric Environment, 38, 3127-3141.

8. Gaffney J. S., N. A. Marley, and M. M. Cunningham (2004) Natural radionuclides in fine aerosols in the Pittsburgh area, Atmospheric Environment, 38, 3191-3200.

9. Gaydos T. M., C.O. Stanier, and S. N. Pandis (2005a) Modeling of in situ ultrafine atmospheric particle formation in the eastern United States, J. Geophys. Res., 110, D07S12, doi:10.1029/2004JD004683. 
10. Gaydos T. M., R. W. Pinder, B. Koo, K. M. Fahey, and S. N. Pandis Development and Application of a Three-dimensional Aerosol Chemical Transport Model, PMCAMx+, Atmospheric Environment, submitted.

11. Grieshop A. P., E. M. Lipsky, N. J. Pekney, S. Takahama, and A. L. Robinson (2006) Fine Particle Emission Factors from Vehicles in a Highway Tunnel: Effects of Fleet Composition and Season, Atmospheric Environment, in press.

12. Hybl J., G. Lithgow, and S. G. Buckley (2003) Laser-Induced Breakdown Spectroscopy detection of biological material, Applied Spectroscopy, 57, 1207-1215

13. Kane D. B., J. J. Wang, K. Frost, M.V. Johnston (2002) Detection of negative ions from individual ultrafine particles, Analytical Chemistry, 74, 2092-2096.

14. Khlystov A., C. O. Stanier, and S.N. Pandis (2004) An algorithm for combining electrical mobility and aerodynamic size distributions data when measuring ambient aerosol, Aerosol Science and Technology, 38(S1), 229-238.

15. Khlystov A., C. O. Stanier, S. Takahama, and S. N. Pandis (2005a) Water content of ambient aerosol during the Pittsburgh Air Quality Study, J. Geophys. Res., 110, D07S10, doi:10.1029/2004JD004651.

16. Khlystov A., Q. Zhang, J. L. Jimenez, C. Stanier, S. N. Pandis, M. R. Canagaratna, P. Fine, C. Misra, and C. Sioutas (2005b) In-situ concentration of semivolatile aerosol using water-condensation technology, J. Aerosol Sci., 36(7), 866-880.

17. Lane T. E., R. W. Pinder, M. Shrivastava, A. L. Robinson, and S. N. Pandis (2006) Source contributions to primary organic aerosol: Comparison of the results of a source-resolved model and the Chemical Mass Balance approach, Atmospheric Environment, submitted.

18. Lipsky E. M. and A. L. Robinson (2006) Effects of dilution of fine particle mass and partitioning of semivolatile organics in diesel exhaust and wood smoke, Environ. Sci. Tech., 40(1), 155-162.

19. Lipsky E. M. and A. L. Robinson (2005) Design and evaluation of a portable dilution sampling system for measuring fine particle emissions from combustion systems, Aerosol Science \& Technology, 39(6), 542-553.

20. Lithgow G. A., A. L. Robinson, and S.G. Buckley (2004) Ambient measurements of metal-containing $\mathrm{PM}_{2.5}$ in an urban environment using laser-induced breakdown spectroscopy, Atmospheric Environment, 38, 3319-3328.

21. Millet D. B., N. M. Donahue, S. N. Pandis, A. Polidori, C. O. Stanier, B. J. Turpin, and A. H. Goldstein (2005) Partitioning VOCs and organic aerosols into primary and secondary sources: Results from the Pittsburgh Air Quality Study, J. Geophys. Res., 110, D07S07, doi:10.1029/2004JD004601. 
22. Modey W. K., D. J. Eatough, R. R. Anderson, D. V. Martello, S. Takahama, L. L. Lucas, and C. I. Davidson (2004) Ambient fine particulate concentrations and chemical composition at two sampling sites in metropolitan Pittsburgh: a 2001 intensive summer study, Atmospheric Environment, 38, 3165-3178.

23. Park S. S., J. P. Pancras, J. M. Ondov, and A.L. Robinson (2006) Application of the pseudo-deterministic receptor model to resolve power plant influences on air quality in Pittsburgh, Aerosol Science \& Technology, 40(10); 883-897.

24. Pekney N. J. and C. I. Davidson (2005) Determination of trace elements in ambient aerosol samples, Analytica Chimica Acta, 540(2), 269-277.

25. Pekney N. J., C. I. Davidson, A. L. Robinson, L. Zhou, P. Hopke, and D. Eatough (2006) Identification of major sources of $\mathrm{PM}_{2.5}$ in Pittsburgh using PMF and Unmix, Aerosol Science \& Technology, 40(10); 910 - 924.

26. Pekney N. J., C. I. Davidson, L. Zhou, P. K. Hopke Application of PSCF and CPF to PMF-modeled sources of $\mathrm{PM}_{2.5}$ in Pittsburgh, Aerosol Science \& Technology, submitted.

27. Pekney N. J., K. J. Bein, C.I. Davidson, A.S. Wexler and M.V. Johnston (2006) Identification of sources of atmospheric PM at the Pittsburgh Supersite Part I: Single Particle Analysis and Filter-based Positive Matrix Factorization, Atmospheric Environment, in press.

28. Polidori A., B. J. Turpin, H. J. Lim, J. C. Cabada, R. Subramanian, A. L. Robinson, and S. N. Pandis (2006) Local and Regional Secondary Organic Aerosol: Insights from a Year of Semi-continuous Carbon Measurements at Pittsburgh, Aerosol Science \& Technology, 40(10); 861-872.

29. Rees S. L., A. L. Robinson, A. Khlystov, C.O. Stanier, and S. N. Pandis (2004) Mass balance closure and the Federal Reference Method for $\mathrm{PM}_{2.5}$ in Pittsburgh, Pennsylvania, Atmospheric Environment, 38, 3305-3318.

30. Robinson A. L., N. M. Donahue, and W. F. Rogge (2006) Photochemical oxidation and changes in molecular composition of organic aerosol in the regional context, Journal of Geophysical Research, 111, D03302, doi:10.1029/2005JD006265.

31. Robinson A. L., Subramanian R., N. M. Donahue, A. Bernardo-Bricker, W. F. Rogge (2006) Methodology for selecting source profiles for chemical mass balance modeling using organic molecular markers, Environmental Science \& Technology, submitted.

32. Robinson A. L., R Subramanian, N.M. Donahue, A. Bernardo-Bricker, and W.F. Rogge, "Source apportionment of molecular markers and organic aerosol - 2. Biomass smoke," Environmental Science \& Technology, submitted. 
33. Robinson A. L., R Subramanian, N.M. Donahue, A. Bernardo-Bricker, and W.F. Rogge, "Source apportionment of molecular markers and organic aerosol - 3. Food cooking emissions," Environmental Science \& Technology, submitted.

34. Stanier C. O., A. Khlystov, W.R. Chan, M. Mandiro, and S. N. Pandis (2004b) A method for the in situ measurement of fine aerosol water content of ambient aerosols: The Dry-Ambient Aerosol Size Spectrometer (DAASS), Aerosol Science and Technology, 38(S1), 215-228.

35. Stanier C. O., A.Y. Khlystov, and S. N. Pandis (2004c) Ambient aerosol size distributions and number concentrations measured during the Pittsburgh Air Quality Study (PAQS), Atmospheric Environment, 38, 3275-3284.

36. Stanier C. O., A.Y. Khlystov, and S.N. Pandis (2004a) "Nucleation events during the Pittsburgh Air Quality Study: Description and relation to key meteorological, gas phase, and aerosol parameters, Aerosol Science and Technology, 38(S1), 253-264.

37. Subramanian R., A. Y. Khlystov, and A. L. Robinson (2006) Effect of Peak Inertmode Temperature on Elemental Carbon Measured Using Thermal-optical Analysis, Aerosol Science and Technology, 40(10); 763-780.

38. Subramanian R., A.Y. Khlystov, J. C. Cabada, and A.L. Robinson (2004) Positive and negative artifacts in particulate organic carbon measurements with denuded and undenuded sampler configurations, Aerosol Science and Technology, 38(S1), 27-48.

39. Subramanian R., N. M. Donahue, A. Bernardo-Bricker, W. F. Rogge and A. L. Robinson, (2006) Contribution of motor vehicle emissions to the ambient organic carbon in Pittsburgh, PA: Effects of varying source profiles and seasonal trends in ambient marker concentrations, Atmospheric Environment, in press.

40. Takahama S., A.E. Wittig, D.V. Vayenas, C.I. Davidson, and S.N. Pandis (2004) Modeling the diurnal variation of nitrate during the Pittsburgh Air Quality Study, Journal of Geophysical Research, 109, D16S06, doi:10.1029/2003JD004149.

41. Takahama, S., C.I. Davidson and S.N. Pandis, (2006). "Semi-Continuous Measurements of Organic Carbon and Acidity during the Pittsburgh Air Quality Study: Implications for Acid-Catalyzed Organic Aerosol Formation." Environmental Science \& Technology, 40(7), 2191-2198..

42. Tang W., T. Raymond, B. Wittig, C. Davidson, S. N. Pandis, A. Robinson, and K. Crist, (2004) Spatial variations of $\mathrm{PM}_{2.5}$ during the Pittsburgh Air Quality Study, Aerosol Science and Technology, 38(S2), 80-90.

43. Vayenas D., S. Takahama, C. Davidson, and S. N. Pandis (2005) Simulation of the thermodynamics and removal processes in the sulfate-ammonia-nitric acid system: Implications for $\mathrm{PM}_{2.5}$ control strategies, J. Geophys. Res., 110, D07S14, doi:10.1029/ 2005JD005983. 
44. Weitkamp E. A., E.M. Lipsky, P. Pancreas, J. Ondov, A. Polidori, B.J. Turpin, A.L. Robinson (2005) Fine particle emission profile for a large coke production facility based on highly time resolved fenceline measurements, Atmospheric Environment, 39, 6719-6733.

45. Wittig A. E., N. Anderson, A.Y. Khlystov, S. N. Pandis, C. Davidson, and A.L. Robinson (2004) Pittsburgh Air Quality Study overview, Atmospheric Environment, 38, 3107-3125.

46. Wittig A. E., S. Takahama, A.Y. Khlystov, S. N. Pandis, S. Hering, B. Kirby, and C. Davidson (2004) Semi-continuous $\mathrm{PM}_{2.5}$ inorganic composition measurements during the Pittsburgh Air Quality Study, Atmospheric Environment, 38, 3201-3213.

47. Zhang Q., C. O. Stanier, M. R. Canagaratna, J. T Jayne, D. R. Worsnop, S. N. Pandis, and J. L. Jimenez (2004) Insights into the chemistry of new particle formation and growth events in Pittsburgh based on aerosol mass spectrometry, Environ. Sci. Tech., 38, 4797-4809.

48. Zhang Q., M. R. Canagaratna, J. T. Jayne, D. R. Worsnop, and J. L. Jimenez (2005) Time and size-resolved chemical composition of submicron particles in Pittsburgh: Implications for aerosol sources and processes, J. Geophys. Res., 110, D07S09, doi:10.1029/2004JD004649.

49. Zhao Y., K. J. Bein, A. S. Wexler, C. Misra, P. M. Fine, and C. Sioutas (2005) Field evaluation of the VACES particle concentrator coupled to the RSMS-3 single particle mass spectrometer, J. Geophys. Res., 110, D07S02, doi:10.1029/2004JD004644.

50. Zhou L. M., P. K. Hopke, P. Paatero, J. M. Ondov, J. P. Pancras, N. J. Pekney, and C.I. Davidson (2004c) Advanced factor analysis for multiple time resolution aerosol composition data, Atmospheric Environment, 38, 4909-4920.

51. Zhou L., E. Kim, P. K. Hopke, C. Stanier, and S. N. Pandis (2005b) Mining airborne particulate size distribution data by positive matrix factorization, J. Geophys. Res., 110, D07S19, doi:10.1029/2004JD004707.

52. Zhou L., E. Kim, P.K. Hopke, C.O. Stanier, and S. Pandis (2004a) The advanced factor analysis on Pittsburgh particle size-distribution data, Aerosol Science and Technology, 38(S1), 118-132.

53. Zhou L., P. K. Hopke, C. O. Stanier, S. N. Pandis, J. M. Ondov, and J. P. Pancras (2005a) Investigation of the relationship between chemical composition and size distribution of airborne particles by Partial Least Square (PLS) and Positive Matrix Factorization (PMF), J. Geophys. Res., 110, D07S18, doi:10.1029/2004JD005050.

54. Zhou L., W. Liu, and P. K. Hopke (2004b) Comparison of two trajectory based models for locating particle sources for two rural New York sites, Atmospheric Environment, 38, 1955-1963. 


\section{Other publications}

1. Stanier C. O., A. Khlystov, and S. N. Pandis (2002) Chemical processes and longrange transport of aerosols: Insights from the Pittsburgh Air Quality Study, in Long Range Transport of Air Pollution, Kluwer.

2. Pandis S. N. (2003) Estimates of diesel and other emissions: Overview of the Supersite program, in Improving Estimates of Diesel and Other Emissions for Epidemiological Studies, HEI Communication 10, Health Effects Institute, Boston, MA.

3. Pandis S. N., P. A. Solomon, and R. Scheffe (2005) Preface to special section to Particulate Matter Supersites, J. Geophys. Res., 110, D07S01, doi:10.1029/ 2005JD005983.

4. Robinson A. L., A. P. Grieshop, D. Laudal, M. McCoy (2005) Investigation of Mercury Transformations in Coal Power Plant Plumes Using a Dilution Sampler, Proceedings of Air Quality V an International Conference on Mercury, Trace Elements, SO3, and Particulate Matter, University of North Dakota, Energy and Environmental Research Center, Grand Forks, ND.

5.

\section{Presentations:}

1. "Investigation of nucleation bursts in the Pittsburgh air quality study", 6th International Aerosol Conference, Taipei, Taiwan, September 2002 (C. O. Stanier, A. Y. Khlystov, and S. N. Pandis).

2. "Monitoring of water content of ambient aerosol during the Pittsburgh Air Quality Study" 6th International Aerosol Conference, Taipei, Taiwan, September 2002 (A. Y. Khlystov, C. O. Stanier, D. Vayenas, and S. N. Pandis).

3. Performance of the Aerodynamic Particle Sizer 3320 during the Pittsburgh Air Quality Study (PAQS)" 6th International Aerosol Conference, Taipei, Taiwan, September 2002 (A. Khlystov, C. Stanier, and S. N. Pandis).

4. "Sulfate-ammonia-nitric acid interactions in an urban area" 6th International Aerosol Conference, Taipei, Taiwan, September 2002 (S. Takahama, A. Khlystov, B. Wittig, S. V. Hering, C. Davidson, A. Robinson, and S. N. Pandis).

5. "Sampling artifacts during measurement of ambient carbonaceous aerosol" 6th International Aerosol Conference, Taipei, Taiwan, September 2002 (R. Subramanian, A. Y. Khlystov, J. C. Cabada, S. N. Pandis, and A. L. Robinson). 
6. "Formation and properties of regional aerosol: Some insights from the Pittsburgh Air Quality Study", NASA-GSFC, Greenbelt MD, May 2002,(C. Stanier, A. Khlystov, S. Rees, J. Cabada, A. Robinson, C. Davidson, and S. N. Pandis)

7. "Seasonal composition of PM2.5 and performance of the Federal Reference Method in Pittsburgh", PM2.5 and Electric Power Generation, Pittsburgh, April 2002 (S. L. Rees, S. Takahama, A. L. Robinson, A. Khlystov, and S. N. Pandis).

8. "Continuous measurements of ammonia, sulfate, and nitrate in Pittsburgh: Implications for PM2.5 control strategies", PM2.5 and Electric Power Generation, Pittsburgh, April 2002 (B. Wittig, A. Khlystov, S. Takahama, C. Davidson, A. Robinson, S. Hering, and S. N. Pandis).

9. "The contribution of long-range transport and secondary organic aerosol to PM2.5 in Pittsburgh", PM2.5 and Electric Power Generation, Pittsburgh, April 2002 (J. C. Cabada, R. Subramanian, S. N. Pandis, A. L. Robinson, W. Tang, N. J. Anderson, T. Raymond, and C. I. Davidson).

10. "The Dry-Ambient Size Spectrometer: A new technique for the automatic on-line measurement of the atmospheric aerosol water size distribution", Annual Meeting of American Geophysical Union, San Francisco, December 2001 (A. Khlystov, C. O. Stanier, S. N. Pandis).

11. "The July 2001 intensive of the Pittsburgh Air Quality Study", Annual Meeting of AAAR, Portland, Oregon, October 2001 (C. I. Davidson, A. L. Robinson, and A. Khlystov, S. N. Pandis).

12. "Sources of atmospheric carbonaceous particulate matter in Pittsburgh", Annual Meeting of AAAR, Portland, Oregon, October 2001 (J. Cabada, S. N. Pandis and A. L. Robinson).

13. "Automated measurements of dry and wet ambient aerosol distributions", Annual Meeting of AAAR, Portland, Oregon, October 2001 (A. Y. Klhystov, W. R. Chan, C. O. Stanier, M. Mandiro, and S. N. Pandis)

14. "Continuous measurements of ammonia and ammonium in ambient air", Annual Meeting of AAAR, Portland, Oregon, October 2001 (A. Khlystov, J. Sauser, R. Otjes, and S. N. Pandis).

15. The contribution of secondary organic aerosol to PM2.5 concentrations in Pittsburgh, AGU Fall Meeting 2002, San Francisco CA Dec. 2002 (J. C. Cabada, S. N. Pandis, A. L. Robinson, R. Subramanian, A. Polidori, and B. Turpin).

16. Preliminary results from the Pittsburgh Air Quality Study, AGU Fall Meeting 2002, San Francisco CA Dec. 2002 (S. N. Pandis, C. I. Davidson, A. L. Robinson, and A. Y. Khlystov) 
17. Monitoring of water content of ambient aerosol during the Pittsburgh Air Quality Study, AGU Fall Meeting 2002, San Francisco CA Dec. 2002 (A. Y. Khlystov, C. O. Stanier, D. Vayenas, and S. N. Pandis)

18. Investigation of nucleation bursts during the Pittsburgh Air Quality Study, AGU Fall Meeting 2002, San Francisco CA Dec. 2002 (C. O. Stanier, A. Y. Klhystov, B. Wittig, S. N. Pandis, Y. Zhou, K. Bein, A. S. Wexler, C. Misra, and C. Sioutas)

19. Atmospheric particulate matter: Physics, chemistry, and Chemical Transport Models, PM AAAR 2003, Pittsburgh PA March 2003 (B. Koo, K. Fahey, T. Gaydos, and S. N. Pandis)

20. Secondary organic aerosol contribution to carbonaceous PM2.5 concentrations in Pittsburgh, PM AAAR 2003, Pittsburgh PA March 2003 (J. C. Cabada, S. N. Pandis, B. Wittig, A. Robinson, R. Subramanian, A. Polidori, and B. J. Turpin)

21. Using ultrafine concentrators to increase the hit rates of single particle mass spectrometers, PM AAAR 2003, Pittsburgh PA March 2003 (Y. Zhao, K. J. Bein, A. S. Wexler, C. Misra, P. M. Fine, and C. Sioutas)

22. $\mathrm{PM}_{2.5}$ Federal Reference Method performance relative to mass balance closure, PM AAAR 2003, Pittsburgh PA March 2003 (S. L. Rees, A. L. Robinson, A. Khlystov, C. O. Stanier, and S. N. Pandis)

23. Examining the assumptions behind elemental carbon measurements using the thermal-optical transmittance technique, PM AAAR 2003, Pittsburgh PA March 2003 (R. Subramanian, A. Y. Khlystov, and A. L. Robinson)

24. Spatial variations of PM2.5 during intensive sampling of PAQS, PM AAAR 2003, Pittsburgh PA March 2003 (W. Tang, C. I. Davidson, T. R. Raymond, S. N. Pandis, B. Wittig, A. Khlystov, and A. L. Robinson)

25. sampling adjacent to a large coke production facility in Pittsburgh, PM AAAR 2003, Pittsburgh PA March 2003 (E. A. Weitkamp, E. Lipsky, A. Robinson, N. Anderson, H. Leifeste, R. Subramanian, J. Cabada, A. Khlystov, C. Stanier, L. Lucas, S. Takahama, B. Wittig, C. Davidson, S. Pandis, A. Polidori, H. J. Lim, B. Turpin, P. Pancras, and J. Ondov)

26. In-use vehicle emissions source characterization study: Squirrel Hill tunnel Pittsburgh, PM AAAR 2003, Pittsburgh PA March 2003 (E. M. Lipsky, A. Robinson, N. Anderson, H. Leifeste, R. Subramanian, J. Cabada, S. Rees, A. Khlystov, C. Stanier, L. Lucas, S. Takahama, B. Wittig, C. Davidson, S. N. Pandis, A. Polidori, H. J. Lim, and B. Turpin)

27. Water content of ambient aerosol during PAQS, PM AAAR 2003, Pittsburgh PA March 2003 (A. Khlystov, C. Stanier, and S. N. Pandis) 
28. Diurnal and seasonal trends in outdoor particle size distributions measured at urban and rural locations during PAQS (C. Stanier, A. Khlystov, and S. N. Pandis)

29. Mass and chemically resolved size compositions of fine particulate matter at the Pittsburgh Supersite, PM AAAR 2003, Pittsburgh PA March 2003, (J. C. Cabada, S. N. Pandis, S. Rees, S. Takahama, A. Khlystov, A. L. Robinson, and C. I. Davidson)

30. Simulation of the atmospheric aerosol size/composition distribution in a threedimensional chemical transport model, PM AAAR 2003, Pittsburgh PA March 2003 (T. M. Gaydos, K. M. Fahey, B. Koo, and S. N. Pandis)

31. Application of PMCAMx to the South Coast Air Basin and the Eastern United States, PM AAAR 2003, Pittsburgh PA March 2003 (B. Koo, K. M. Fahey, T. M. Gaydos, and S. N. Pandis)

32. Principal component analysis of trace elements in $\mathrm{PM}_{2.5}$ in Pittsburgh, PM AAAR 2003, Pittsburgh PA March 2003 (N. J. Anderson, C. I. Davidson, S. N. Pandis, A. Robinson, and A. Khlystov)

33. Source apportionment using particle size distribution data from PAQS, PM AAAR 2003, Pittsburgh PA March 2003 (L. Zhou, E. Kim, P. K. Hopke, C. Stanier, and S. N. Pandis)

34. Highly time-resolved measurements of elemental composition at the Baltimore, St. Louis, and Pittsburgh Supersites using the UM High Frequency Aerosol Slurry Sampler: Unprecedented resolution of the sources of primary atmospheric aerosol PM AAAR 2003, Pittsburgh PA March 2003 (J. M. Ondov, J. Pancras, S. Gazula, M. Yu, J. Turner, A. Robinson, S. N. Pandis, N. D. Poor, and R. K. Stevens)

35. "Size Resolved Chemical Classification of Dual Polarity Single-Ultrafine-Particle Mass Spectrometry Data Collected During Pittsburgh Supersite Experiment”, K.J. Bein, Y. Zhao, A.S. Wexler and M.V. Johnston, American Association for Aerosol Research Annual Conference, Anaheim, CA, October 2003.

36. "Dynamic Data Classification Using a Component-Weighted Similarity Algorithm", *K.J. Bein, Y. Zhao, A.S. Wexler and M.V. Johnston, American Association for Aerosol Research Annual Conference, Anaheim, CA, October 2003.

37. "Diurnal Variations of Ultra-fine Particles in Pittsburgh Measured by a Rapid Single Particle Mass Spectrometer", *Y. Zhao, K.J. Bein, A.S. Wexler, and M.V. Johnston, American Association for Aerosol Research Annual Conference, Anaheim, CA, October 2003. 
38. "Rapid Single Particle Mass Spectrometry and EPA Supersite Experiments", *K.J. Bein, Y. Zhao, A.S. Wexler and M.V. Johnston, NYU Institute of Environmental Medicine, Tuxedo, NY, December 2002.

39. "Speciation of Size-Resolved Individual Ultrafine Particles in Pittsburgh, Pennsylvania", *K.J. Bein, Y. Zhao, A.S. Wexler, D.A. Lake, D.B. Kane, M.P. Tolocka, K.P. Rhoads, M.V. Johnston and D.J. Phares, Association for Aerosol Research Annual Conference, Charlotte, NC, October 2002.

40. L. Zhou, E. Kim, and P.K. Hopke Analysis of the Particle Size Distribution Data Collected During Pittsburgh Air Quality Study, Presented to 21st Annual Conference of the American Association for Aerosol Research, Charlotte, NC, October 7-11, 2002.

41. L. Zhou, E. Kim, P.K. Hopke, C. Stanier, and S. Pandis Source Apportionment Using Particle Size Distribution Data from the Pittsburgh Air Quality Study (PAQS), presented to 2003 AAAR PM Meeting on Particulate Matter: Atmospheric Sciences, Exposure and the Fourth Colloquium on PM and Human Health, Pittsburgh, PA, March 30 to April 4, 2003.

42. L. Zhou, P.K. Hopke, and W. Liu, Comparison of three back trajectory based models--PSCF, SQTBA, residence-time weighted concentration model for identifying particle sources for Potsdam and Stockton, New York, presented to 21st Annual Conference of the American Association for Aerosol Research, Anaheim, CA, October 20-24, 2003.

43. L. Zhou, P.K. Hopke, and P. Paatero, Advanced Factor Analysis for Aerosol Composition Data with Various Temporal Resolutions, presented to 21st Annual Conference of the American Association for Aerosol Research, Anaheim, CA, October 20-24, 2003.

44. S.G. Buckley, F. Ferioli, G.A. Lithgow, "Combustion System Analysis Using Laser-Induced Breakdown Spectroscopy," Paper 403, 22nd International Congress on Applications of Lasers and Electro-Optics, Jacksonville, FL, October 13-16, 2003.

45. G.A. Lithgow, A.L. Robinson, and S.G. Buckley, "Ambient Measurements of Inorganic Species in an Urban Environment Using LIBS," Second International Conference on Laser-Induced Plasma Spectroscopy, Orlando, FL, September 24 27, 2002.

46. G.A. Lithgow, A.L. Robinson, S.G. Buckley, "Ambient Particle Measurements in an Urban Environment Using Laser-Induced Breakdown Spectroscopy (LIBS)," Paper 11E4, American Association of Aerosol Research Annual Conference, Anaheim, CA, October 20-24, 2003. 
47. Ondov, J. M. (2001). Sources of Metals Influencing Air Quality at the Pittsburgh Supersite: Data collected with the UM High-Frequency Aerosol Slurry Sampler.

48. Ondov, J. M. (2002). Unprecedented Source Apportionment with the University of Maryland Semi-Continuous Elements in Aerosol System Presented at the Regional AQ Modeling \& Data Analysis Meeting, sponsored by MARAMA, OTC, NESCAUM and MANE-VU, January 23-24, 2002, Baltimore, MD.

49. Ondov, J. M., (2003) Short-Term Concentrations of Metals SEAS via SEAS and Selected Variables. Presented at the Mid-Atlantic Region Air Monitoring Association Conference in Baltimore, Jan 21-22, at the Harbor Court Hotel.

50. Ondov, J. M., Pancras, J. P., Gazula, S., Moore, J. A., Park, S. S., Chang, Y. C., Squib, K., Powel, J., Mitkus, R., Turner, J., Yu, M.N.S., Robinson, A., Pandis, S., Davidson, C. (2003). Identification of Sources From High-Frequency Elements Measurements at 4 Supersites. Semi-annual EPA Supersites meeting in Atlanta, Jan 22-23.

51. Ondov, J. M. (2003). High-frequency metals measurements at the Pittsburgh Supersite and Coke Plant site with the University of Maryland SEAS. Seminar presented at the Pittsburgh Supersite Project workshop, March 5, Carnegie Mellon University.

52. Ondov, J. M. (2003) Highlights of SEAS Metals Data for the Pittsburgh Supersite. Presented at the Pittsburgh Air Quality Study Meeting, Pittsburgh, PA, April 4,5, 2003.

53. Ondov, J. M. (2003) New Paradigm for Air Pollution Control: Pseudo Deterministic Receptor Modeling of Highly Time Resolved Ambient Aerosol Composition Data Derived from the UMCP SEAS. Seminar presented to the Source Apportionment Group, US EPA, Research Triangle Park, November 12.

54. Ondov, J. M., Emission Rates of Pollutants from Stationary Sources Using Highly Time Resolved Ambient Measuremetns and a New Pseduo Deterministic Hybrid Receptor Model. Presented at the EPA Center for Hazardous Substances in Urban Environments, Research Program Internal Workshop, January 5, 2004.

55. Ondov, J. M., New Pseudo Deterministic Model for Individual Source Apportionment Using Highly Time Resolved Data. Presented at the MARAMA MANE-VU Science Meeting, Baltimore, MD, January 27-29, 2004.

56. Park, S. S., Pancras, J. P., Gazula, S., Ondov, J. M. (2002) Sources of Elemental Aerosol Constituents in Pittsburgh Using Positive Matrix Factorization of Highly Time-resolved Data. Presented at the American Association of Aerosol Research meeting, 21st Annual AAAR Conference October 7-11, Charlotte. 
57. Ondov, J. M., Pancras, J. P., Gazula, S., Yu, M. N. S., Turner, J., Robinson, A., Pandis, S., Stevens, R. K., Poor (2003). Highly Time-Resolved Measurements of Elemental Composition at the Baltimore, St. Louis, Pittsburgh, and Tampa Supersites Using the UM High-Frequency Aerosol Slurry Sampler: Unprecedented Resolution of the Sources of Primary Atmospheric Aerosol. To be presented at the Association of Aerosol Research meeting, Particulate Matter: Atmospheric Sciences, Exposure, and the Fourth Colloquium on PM and Human Health, March, Pittsburgh.

58. Ondov, J. M., Pancras, J. P., Park, S. S., Poor, N., Turner, J. R., Yu, M., Lipsky, E., Weitkamp, E., Robinson, A. (2003) PM emission rates from highly time-resolved ambient concentration measurements. Presented at the October meeting of the American Society for Aerosol Research, San Diego.

59. Ondov, J. M., Poor, N. (2003) Emission Inventory Development through highlytime-resolved ambient sampling. NARSTO Workshop on Innovative Methods for Emission-Inventory Development and Evaluation, University of Texas, Austin; October 14-17, 2003.

60. G.A. Lithgow and S.G. Buckley, "Detection and Classification of Biological Aerosols Using Laser-Induced Breakdown Spectroscopy," Paper 10B4, American Association of Aerosol Research Annual Conference, Anaheim, CA, October 2024, 2003.

61. S.G. Buckley "Laser-Induced Breakdown Spectroscopy for Detection of Biological Aerosols - Potential and Perspective," PITTCON 2005, Orlando FL, Feb. 27-Mar. 4, 2005. (invited)

62. S.G. Buckley "LIBS as a Combustion and Aerosol Diagnostic," PacificChem 2005, Honolulu, HI, Dec 15-20, 2005. (invited)

63. Cabada, J.C., Pandis, S.N., Robinson, A.L., Davidson, C.I., Polidori, A., Turpin, B.J., and Subramanian, R., "The contribution of Secondary Organic Aerosol to $\mathrm{PM}_{2.5}$ in Pittsburgh," Presented at the American Association for Aerosol Research Annual Conference, Charlotte, NC, October 2002.

64. Offenberg, J.H., Polidori, A., Porcja, R., Turpin, B.J., "Functional group composition by size and polarity in Pittsburgh, PA and insights into aerosol processing," Presented at the American Association for Aerosol Research Annual Conference, Charlotte, NC, October 2002.

65. Polidori, A., Turpin, B.J., Lim, H.J., Robinson, A., Subramanian, R., Cabada, J.C., "Semi-continuous organic particulate matter measurements during Pittsburgh Air Quality Study (PAQS)," Poster presentation at the American Association for Aerosol Research Annual Conference, Charlotte, NC, October 2002. 
66. Subramanian, R., Robinson, A.L., Cabada, J.C., Pandis, S.N., Wittig, B., Polidori, A., Turpin, B.J., Hering, S.V., Modey, W.K., Eatough, D.J., "Intercomparision of ambient carbonaceous aerosol samplers used during the Pittsburgh Air Quality Study," Poster presentation at the American Association for Aerosol Research Annual Conference, Charlotte, NC, October 2002.

67. Cabada, J.C., Pandis, S.N., Robinson, A.L., Davidson, C.I., Polidori, A., Turpin, B.J., and Subramanian, R., "The contribution of Secondary Organic Aerosol to $\mathrm{PM}_{2.5}$ in Pittsburgh," Platform Presentation at the Annual Meeting of the American Institute of Chemical Engineers, Indianapolis, IN, November 2002.

68. Millet, D.B., Donahue, N.M., Polidori, A., Stanier, C.O., Turpin, B.J., Goldstein, A.H., "VOC-Aerosol Relationships at the Pittsburgh Air Quality Study", Presented at the Berkeley Atmospheric Science Center symposium, Berkeley, CA, September 2003.

69. Polidori, A., Turpin, B., Lim, H.J., Cabada, J.C., Subramanian, R., Robinson, A., "Secondary Organic Aerosol Formation During the Pittsburgh Air Quality Study (PAQS", Presented at the Environmental Sciences Graduate Student Association (ESGSA), New Brunswick, NJ, June 2004.

70. Polidori, A., Turpin, B.J., Lim, H.J., Totten L., Davidson, C, "Characterization of the Organic Fraction of Atmospheric Aerosols" Platform Presentation at the American Association for Aerosol Research Annual Conference, Atlanta, GA, October 2004.

71. Robinson, A.L., Subramanian, R., Gaydos, T., Pandis, S.N., Bernardo-Bricker, A., Rogge, W.F., Polidori, A., Turpin, B.J., Clarke, L., Hernandez, M., "Synthesis of Source Apportionment Estimates of Organic Aerosol in the Pittsburgh Region" Platform Presentation at the American Association for Aerosol Research Annual Conference, Atlanta, GA, October 2004.

72. Polidori, A., Turpin, B., Lim, H.J., Subramanian, R., Robinson, A., Pandis, S., Cabada, J.C., " "Local and Regional Secondary Organic Aerosol Formation: Insights from a Year at Pittsburgh and Comparisons with Los Angeles and Atlanta," Platform Presentation at the American Association for Aerosol Research "Supersite" Conference, Atlanta, GA, February 2005.

73. Weitkamp, E., Lipsky, E., Robinson, A., Polidori, A., Turpin, B., Pancras, P., Ondov, J., Bernado-Bricker, A., Vasquez, O., Rogge, W., "Fine Particle Emission Profile for a Large Coke Production," Poster presentation at the American Association for Aerosol Research "Supersite" Conference, Atlanta, GA, February 2005.

74. "Source Sampling and Characterization Using a Single Particle Mass Spectrometer during the Pittsburgh Supersite Experiment", K.J. Bein, Y. Zhao, A.S. Wexler, E. Lipsky, A.L. Robinson and M.V. Johnston, American Association 
for Aerosol Research International Specialty Conference, Atlanta, GA, February 7-11, 2005.

75. "Size-Resolved Chemical Classification of Single Particle Mass Spectrometry Data Collected during the Pittsburgh Supersite Experiment: Source Attribution", K.J. Bein, Y. Zhao, A.S. Wexler and M.V. Johnston, American Association for Aerosol Research International Specialty Conference, Atlanta, GA, February 711, 2005.

76. "Laboratory Experiments Examining Ultrafine Particle Production by Rebreathing of Road Dust through a Diesel Engine", K.J. Bein, Y. Zhao, A.S. Wexler, E. Lipsky and A.L. Robinson, American Association for Aerosol Research International Specialty Conference, Atlanta, GA, February 7-11, 2005.

77. "Detection of a Contaminating Plume during a Roadway Tunnel Source Sampling Experiment Using a Single Particle Mass Spectrometer", K.J. Bein, Y. Zhao, A.S. Wexler, E. Lipsky, A.L. Robinson and M.V. Johnston, American Association for Aerosol Research International Specialty Conference, Atlanta, GA, February 711, 2005.

78. "Detection and Characterization of a Smoke Plume from Canadian Forest Fires during the Pittsburgh Supersite Experiment", K.J. Bein, Y. Zhao, A.S. Wexler, N.J. Pekney, C.I. Davidson and M.V. Johnston, American Association for Aerosol Research International Specialty Conference, Atlanta, GA, February 7-11, 2005.

79. "Field Evaluation of the VACES Particle Concentrator Coupled to the RSMS-3 Single Particle Mass Spectrometer", Y. Zhao, K.J. Bein, A.S. Wexler, C. Misra, P.M. Fine and C. Sioutas, American Association for Aerosol Research International Specialty Conference, Atlanta, GA, February 7-11, 2005.

80. "Identification of Sources of Atmospheric PM at the Pittsburgh Supersite: RSMSIII and Filter-based Positive Matrix Factorization", N.J. Pekney, K.J. Bein, C.I. Davidson, A.S. Wexler and M.V. Johnston, American Association for Aerosol Research International Specialty Conference, Atlanta, GA, February 7-11, 2005.

81. "Laboratory Experiments Examining Ultrafine Particle Production by Rebreathing of Road Dust through a Diesel Engine", K.J. Bein, Y. Zhao, A.S. Wexler, E. Lipsky and A.L. Robinson, American Association for Aerosol Research Annual Conference, Atlanta, GA, October 4-8, 2004.

82. "Measurements of Nitrate Particles in Pittsburgh, PA, Using Rapid Single Particle Mass Spectrometry", Y. Zhao, K.J. Bein, A.S. Wexler, M.P. Tolocka and M.V. Johnston, American Association for Aerosol Research Annual Conference, Atlanta, GA, October 4-8, 2004.

83. "Size Resolved Chemical Classification of Dual Polarity Single-Ultrafine-Particle Mass Spectrometry Data Collected During the Pittsburgh Supersite Experiment", 
K.J. Bein, Y. Zhao, A.S. Wexler and M.V. Johnston, American Association for Aerosol Research Annual Conference, Anaheim, CA, October 20-24, 2003.

84. "Dynamic Data Classification Using a Component-Weighted Similarity Algorithm", K.J. Bein, Y. Zhao, A.S. Wexler and M.V. Johnston, American Association for Aerosol Research Annual Conference, Anaheim, CA, October 2024, 2003.

85. "Diurnal Variations of Ultra-fine Particles in Pittsburgh Measured by a Rapid Single Particle Mass Spectrometer", Y. Zhao, K.J. Bein, A.S. Wexler, and M.V. Johnston, American Association for Aerosol Research Annual Conference, Anaheim, CA, October 20-24, 2003.

86. "Experimental Validation of a High Transmission Rate Inlet for Ultra-fine Single Particle Mass Spectrometers", Y. Zhao, K.J. Bein, P. Middha and A.S. Wexler, American Association for Aerosol Research Annual Conference, Anaheim, CA, October 20-24, 2003.

87. "Synthesis of Research on In-situ Particle Nucleation in Western Pennsylvania: Which Hypotheses for Nuclei Formation and Growth are Consistent with Field Observations, Mass Spectrometry, and Modeling?.”, C. Stanier, T. Gaydos, A.Y. Khlystov, Q. Zhang, M. Caragaratna, J. Jayne, D. Worsnop, J. Jimenez, D. Millet, A. Goldstein, K.J. Bein, Y. Zhao, A.S. Wexler and S.N. Pandis, American Association for Aerosol Research Annual Conference, Anaheim, CA, October 2024, 2003.

88. "Speciation of Size-Resolved Individual Ultrafine Particles in Pittsburgh, Pennsylvania", K.J. Bein, Y. Zhao, A.S. Wexler, D.A. Lake, D.B. Kane, M.P. Tolocka, K.P. Rhoads, M.V. Johnston and D.J. Phares, Association for Aerosol Research Annual Conference, Charlotte, NC, October 7-11, 2002.

89. "Using Ultrafine Concentrators to Increase the Hit Rate of Single Particle Mass Spectrometers", Y. Zhao, K.J. Bein, A.S. Wexler, C. Misra, P.M. Fine and C. Sioutas, Association for Aerosol Research Annual Conference, Charlotte, NC, October 7-11, 2002.

90. "Investigation of Urban Aerosols at the Baltimore Particulate Matter Supersite by Single Particle Mass Spectrometry", D.A. Lake, M.P. Tolocka, M.V. Johnston, K.J. Bein, Y. Zhao, A.S. Wexler, K.P. Rhoads, and D.J. Phares, Association for Aerosol Research Annual Conference, Charlotte, NC, October 7-11, 2002.

91. "Investigation of Nucleation Bursts during the Pittsburgh Air Quality Study, C. Stanier, A.Y. Khlystov, B. Wittig, S.N. Pandis, K.J. Bein, Y. Zhao, A.S. Wexler, C. Misra and C. Sioutas, Association for Aerosol Research Annual Conference, Charlotte, NC, October 7-11, 2002. 
92. G. Casuccio, T. Lersch, R.J. Lee, A. Robinson, E. Weitkamp, and D. Martello, "Estimating of the Impact of Primary Coal Fired Boiler Emissions to PM2.5 And PM10 Using Computer Controlled Scanning Electron Microscopy Data," American Association of Aerosol Research (AAAR) Specialty Conference: Particulate Matter, Supersites Program \& Related Studies, Atlanta, GA, February 7-11, 2005.

93. K.J. Bein, Y. Zhao, A.S. Wexler, E. Lipsky, A.L. Robinson, and M.V. Johnston, "Source Sampling and Characterization Using a Single Particle Mass Spectrometer During the Pittsburgh Supersite Experiment," American Association of Aerosol Research (AAAR) Specialty Conference: Particulate Matter, Supersites Program \& Related Studies, Atlanta, GA, February 7-11, 2005.

94. M. Hernandez, L. Clarke, L. Angenent, and A. Robinson, "Primary Biopolymer Associations with Fine Particulate Matter," American Association of Aerosol Research (AAAR) Specialty Conference: Particulate Matter, Supersites Program \& Related Studies, Atlanta, GA, February 7-11, 2005.

95. S.S. Park, J.P. Pancras, J.M. Ondov, A. Robinson, and C. Davidson, “Application of the UM Multivariate Pseudo-Deterministic Receptor Model to Resolve Power Plant Influences on Air Quality at the CMU Supersite," American Association of Aerosol Research (AAAR) Specialty Conference: Particulate Matter, Supersites Program \& Related Studies, Atlanta, GA, February 7-11, 2005.

96. R. Subramanian, A. Robinson, and A. Khlystov, "OC/EC Analysis with ThermalOptical Methods: Effects of Temperature Protocol and Non-Carbonaceous Compounds," American Association of Aerosol Research (AAAR) Specialty Conference: Particulate Matter, Supersites Program \& Related Studies, Atlanta, GA, February 7-11, 2005.

97. A. Wittig, A. Robinson, and S. Pandis, "Continuous PM2.5 Mass Measurements at the Pittsburgh Air Quality Study Supersite," American Association of Aerosol Research (AAAR) Specialty Conference: Particulate Matter, Supersites Program $\&$ Related Studies, Atlanta, GA, February 7-11, 2005. (poster)

98. W. Tang, T. Raymond, B. Wittig, C. Davidson, S. Pandis, A. Robinson, and K. Crist, "Spatial Variations of PM2.5 in the Pittsburgh Region," American Association of Aerosol Research (AAAR) Specialty Conference: Particulate Matter, Supersites Program \& Related Studies, Atlanta, GA, February 7-11, 2005.

99. A. Polidori, B. Turpin, H.J. Lim, R. Subramanian, A. Robinson, S. Pandis, and J.C. Cabada, "Local and Regional Secondary Organic Aerosol Formation: Insights from a Year at Pittsburgh and Comparison with Los Angeles and Atlanta," American Association of Aerosol Research (AAAR) Specialty Conference: Particulate Matter, Supersites Program \& Related Studies, Atlanta, GA, February 7-11, 2005. 
100. T. Lane, R. Pinder, M. Shrivastava, S.N. Pandis, and A.L. Robinson, "Evaluation of the Primary Organic Carbon Emission Inventory for the Eastern United States," American Association of Aerosol Research (AAAR) Specialty Conference: Particulate Matter, Supersites Program \& Related Studies, Atlanta, GA, February 7-11, 2005.

101. O. Sevimoglu, W.F. Rogge, A. Bernardo, A. Robinson and R. Subramanian, "Fine Particulate Abrasion Products from Leaf Surfaces of Urban Plants: Comparison Between Los Angeles and Pittsburgh," American Association of Aerosol Research (AAAR) Specialty Conference: Particulate Matter, Supersites Program \& Related Studies, Atlanta, GA, February 7-11, 2005. (poster)

102. A. Bernardo-Bricker, W.F. Rogge, O. Sevimoglu, A. Robinson, E. Lipsky, and A. Grieshop, "Source Profiles for Organic PM2.5 for Diesel Trucks and Gasoline Vehicles Determined for the Squirrel Hill Tunnel in Pittsburgh, PA," American Association of Aerosol Research (AAAR) Specialty Conference: Particulate Matter, Supersites Program \& Related Studies, Atlanta, GA, February 7-11, 2005. (poster)

103. K.J. Bein, Y. Zhao, A.S. Wexler, E. Lipsky, and A.L. Robinson, "Laboratory Experiments Examining Ultrafine Particle Production by Re-Breathing of Road Dust Through a Diesel Engine," American Association of Aerosol Research (AAAR) Specialty Conference: Particulate Matter, Supersites Program \& Related Studies, Atlanta, GA, February 7-11, 2005. (poster)

104. K.J. Bein, Y. Zhao, A.S. Wexler, E. Lipsky, A.L. Robinson, and M.V. Johnston, "Detection of a Contaminating Plume During a Roadway Tunnel Source Sampling Experiment Using a Single Particle Mass Spectrometer," American Association of Aerosol Research (AAAR) Specialty Conference: Particulate Matter, Supersites Program \& Related Studies, Atlanta, GA, February 7-11, 2005. (poster)

105. A.L. Robinson, S.L. Rees, A. Khlystov, C.O. Stanier, and S.N. Pandis, "Mass Balance Closure and the Federal Reference Method for PM2.5 in Pittsburgh, Pennsylvania," American Association of Aerosol Research (AAAR) Specialty Conference: Particulate Matter, Supersites Program \& Related Studies, Atlanta, GA, February 7-11, 2005.

106. R. Subramanian, A. Robinson, A. Bernardo-Bricker, and W.F. Rogge, "Organic Carbon Mass Balance and Source Apportionment of Primary Organic Carbon in the Pittsburgh Region Using Molecular Markers," American Association of Aerosol Research (AAAR) Specialty Conference: Particulate Matter, Supersites Program \& Related Studies, Atlanta, GA, February 7-11, 2005.

107. A.L. Robinson, E.M. Lipsky, N. Pekney, W.F. Rogge, A. Bernardo-Bricker, and O. Sevimoglu, "Fine Particle Emission Profile for Road Dust in Pittsburgh, Pennsylvania," American Association of Aerosol Research (AAAR) Specialty 
Conference: Particulate Matter, Supersites Program \& Related Studies, Atlanta, GA, February 7-11, 2005. (poster)

108. E. Weitkamp, E. Lipsky, A. Robinson, A. Polidori, B. Turpin, P. Pancras, J. Ondov, A. Bernardo-Bricker, O.R. Vasquez, and W.F. Rogge, "Fine Particle Emission Profile for a Large Coke Production, American Association of Aerosol Research (AAAR) Specialty Conference: Particulate Matter, Supersites Program \& Related Studies, Atlanta, GA, February 7-11, 2005. (poster)

109. A. Grieshop, E. Lipsky, and A. Robinson, "Fuel-Based Particulate Matter and Gaseous Emission Factors Determined from Vehicles in Pittsburgh, PA's Squirrel Hill Tunnel," American Association of Aerosol Research (AAAR) Specialty Conference: Particulate Matter, Supersites Program \& Related Studies, Atlanta, GA, February 7-11, 2005. (poster)

110. A.L. Robinson, N.M. Donahue, K. Huff Hartz, A. Sage and E. Weitkamp, "Field and Laboratory Experiments Examining the Stability of Organic Molecular Markers Used for Source Apportionment," American Association of Aerosol Research (AAAR) Specialty Conference: Particulate Matter, Supersites Program \& Related Studies, Atlanta, GA, February 7-11, 2005.

111. A.L. Robinson, N.M. Donahue, A.M. Sage, K.E. Huff Hartz, and E.A. Weitkamp, "Field and Laboratory Experiments Examining the Stability of Organic Molecular Markers Used for Source Apportionment," 2004 AGU Fall Meeting, San Francisco, CA, December 13-17, 2004.

112. A.L. Robinson, R. Subramanian, A. Bernardo-Bricker, and W.F. Rogge, "Source Apportionment of Primary Organic Carbon in the Pittsburgh Region using Molecular Markers and Different Receptor Models," American Association of Aerosol Research 23rd Annual Conference (AAAR 2004), Atlanta, GA, October 4-8, 2004.

113. K.E. Huff Hartz, E.A. Weitkamp, A.M. Sage, A.A. Presto, A.L. Robinson, and N.M. Donahue, "Kinetics of Atmospheric Processing of Organic Particulate Matter: A Relative Rates Approach," American Association of Aerosol Research 23rd Annual Conference (AAAR 2004), Atlanta, GA, October 4-8, 2004. (poster)

114. E.A. Weitkamp, K.E. Huff Hartz, A.M. Sage, A.L. Robinson, N.M. Donahue, W.F. Rogge, and A. Bernardo-Bricker, "Evaluation of the Oxidation Kinetics of Molecular Markers Used for Source Apportionment of Primary Organic Aerosol," American Association of Aerosol Research 23rd Annual Conference (AAAR 2004), Atlanta, GA, October 4-8, 2004. (poster)

115. A.L. Robinson, R. Subramanian, T. Gaydos, S.N. Pandis, A. Bernardo-Bricker, W.F. Rogge, A. Polidori, B.J. Turpin; L. Clarke, and M. Hernandez, "Synthesis of Source Apportionment Estimates of Organic Aerosol in the Pittsburgh Region," 
American Association of Aerosol Research 23rd Annual Conference (AAAR 2004), Atlanta, GA, October 4-8, 2004.

116. E. M. Lipsky and A.L. Robinson, "Effects of Dilution Ratio and Residence Time on the Partitioning of Semivolatile Organic Carbon in Emissions from a Wood Stove And Diesel Engine," American Association of Aerosol Research 23rd Annual Conference (AAAR 2004), Atlanta, GA, October 4-8, 2004.

117. K.J. Bein,Y. Zhao, A.S. Wexler, E.M. Lipsky, and A.L. Robinson, "Laboratory Experiments Examining Ultrafine Particle Production by Rebreathing of Road Dust Through a Diesel Engine," American Association of Aerosol Research 23rd Annual Conference (AAAR 2004), Atlanta, GA, October 4-8, 2004. (poster)

118. A.M. Sage, K.E. Huff Hartz, E.A. Weitkamp, A.L. Robinson, and N.M. Donahue "Laboratory Measurement of Heterogeneous Oxidation Kinetics of Organic Aerosols," American Association of Aerosol Research 23rd Annual Conference (AAAR 2004), Atlanta, GA, October 4-8, 2004. (poster)

119. A.P. Grieshop, E.M. Lipsky, and A.L. Robinson, "Fuel-Based Particulate Matter and Gaseous Emission Factors determined from Vehicles in Pittsburgh, PA's Squirrel Hill Tunnel," American Association of Aerosol Research 23rd Annual Conference (AAAR 2004), Atlanta, GA, October 4-8, 2004. (poster)

120. N.M. Donahue and A.L. Robinson, "Theoretical, In Situ, and Laboratory Constraints on Organic Aerosol Oxidation," American Association of Aerosol Research 23rd Annual Conference (AAAR 2004), Atlanta, GA, October 4-8, 2004.

121. A.L. Robinson, “Are Organic Molecular Markers Used for Source Apportionment Stable?," presented atTelluride Workshop on Atmospheric Aerosol Aging, Telluride, CO, August 9-13, 2004. (invited)

122. A.L. Robinson, "Comparison of Emission- and Receptor-based Model Predictions of Primary Organic Aerosol in Pittsburgh, PA," presented at LADCO Academic Community Meeting, Chicago, IL, June 11, 2004. (invited)

123. A.L. Robinson, "Sources of Organic Particulate Matter in Pittsburgh, PA," presented at Mid-Atlantic Regional Air Management Association Science Meeting (MANE-VU/MARAMA 2004), Baltimore, MD, January 27-29, 2004 (invited).

124. Investigation of the Relationship Between Chemical Composition and Size Distribution of Airborne Particles by Partial Least Square (PLS) and Positive Matrix Factorization (PMF), L. Zhou, P.K. Hopke, C.O. Stanier, S.N. Pandis, J.M. Ondov, J. P. Pancras, presented to the 2004 Annual Meeting of the American Association for Aerosol Research, Atlanta, GA, October 4-8, 2004. 
125. Source Apportionment of Baltimore Aerosol from Combined Size Distribution and Chemical Composition Data, D. Ogulei, P.K. Hopke, L. Zhou, J. P. Pancras, N. Nair, J.M. Ondov, presented to the Particulate Matter Supersites Program \& Related Studies Conference, Atlanta, GA, February 7-11, 2005.

126. Identifying Sources of $\mathrm{PM}_{2.5}$ in Pittsburgh Using PMF and PSCF, N. Pekney, C. Davidson, L. Zhou and P.K Hopke, presented to the Particulate Matter Supersites Program \& Related Studies Conference, Atlanta, GA, February 7-11, 2005. 


\section{Appendix A Quality Assurance Final Report}

This report summarizes the quality of the measurement data sets and provides a context for interpretation of measurements collected during the Pittsburgh Air Quality Study (PAQS) Supersite. Data quality is evaluated using particular data quality indicators (DQIs), selected by the PAQS Quality Assurance Manager and Principal Investigators, and the findings of the technical system and performance audits conducted during the field campaign. Additional information about PAQS can be found in the Quality Assurance Program Plan (Khlystov et al., 2001), a publication which provides an overview of the PAQS measurements and preliminary scientific findings (Wittig et al., 2003a), and various publications focusing on measurements collected at the PAQS Supersite (Cabada et al., 2003a; 2003b; 2003c; Khlystov et al., 2001; 2003; Rees et al., 2003; Stanier et al., 2003a; 2003b; Subramanian et al., 2003; Takahama et al., 2003; Wittig et al., 2003b).

\section{Technical system and performance audits}

Data quality was assured by performing two types of audits of all instruments and systems used during PAQS. A single technical system audit of all sample custody forms, logs and standard operating procedures was performed at the beginning of the study. The intent of this audit was to refine the forms and procedures to be used for the duration of the study. Two performance audits were also performed during the field campaign to evaluate the performance of the field instruments by external personnel (who were not normally responsible for the instruments) using external standards (which were not normally used to evaluate the instrument performance). Audit findings were immediately communicated to and discussed with the investigators. In few cases, the performance audits helped to diagnose instrument issues before the measurements were compromised. In even fewer cases, measurements were invalidated as a result of performance audit findings. In a majority of cases, the audits confirmed the stable performance of the instruments.

\section{Data Quality Indicators}

The Data Quality Indicators (DQI) used to evaluate the PAQS data set include precision, accuracy, minimum detection limits (MDLs) and completeness. When appropriate, measurement comparability was also evaluated. Measurement representativeness was evaluated for the site as a whole.

A list of all the measurements collected during the PAQS field campaign and the actual DQI values for a majority of the indicators are presented first in Table 1. Data quality objectives (DQOs), determined when possible for each instrument and system prior to use during PAQS, are also listed in Table 1. A brief description of each indicator and the method of calculating the indicator at PAQS is presented next. The actual methodology for determining each indicator is stated in the individual SOPs and RPs. In a few instrances typically associated with newly developed instruments, the DQI was greater than the DQO. In these cases, the possible explanation for the discrepancy between the DQIs and DQOs is presented as well. 
Table 1. Observable Resolution, Frequency, Period of Operation, Data Quality Objectives (DQO) and Indicators (DQI) at the PAQS Supersite.

\begin{tabular}{|c|c|c|c|c|c|c|c|c|c|}
\hline Observable & Method $^{1}$ & ڤ̊ & 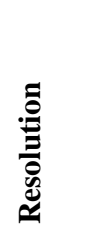 & 总 & مَّ & 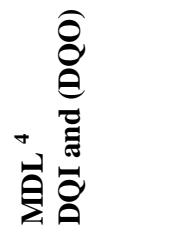 & 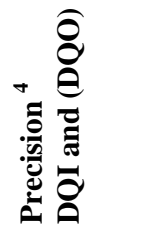 & 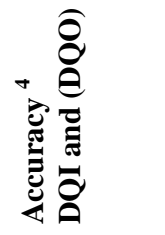 & 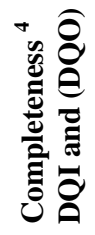 \\
\hline \multicolumn{10}{|l|}{ Aerosol mass } \\
\hline $\mathrm{PM}_{10}$ mass & Dichot sampler/Gravimetry & $\mathrm{CMU}$ & $24 \mathrm{hr}$ & Daily & $7 / 1 / 01-7 / 1 / 02$ & $\begin{array}{l}1.2 \mu \mathrm{g} / \mathrm{m}^{3} \\
\left(2 \mu \mathrm{g} / \mathrm{m}^{3}\right)\end{array}$ & $\begin{array}{l}1.43 \% \\
(10 \%)\end{array}$ & $\begin{array}{l}0.6 \% \\
(10 \%)\end{array}$ & $\begin{array}{l}86.6 \% \\
(70 \%)\end{array}$ \\
\hline $\mathrm{PM}_{2.5}$ mass & FRM sampler/Gravimetry & $\mathrm{CMU}$ & $24 \mathrm{hr}$ & Daily & $7 / 1 / 01-7 / 1 / 02$ & $\begin{array}{l}0.9 \mu \mathrm{g} / \mathrm{m}^{3} \\
\left(2 \mu \mathrm{g} / \mathrm{m}^{3}\right)\end{array}$ & $\begin{array}{l}1.43 \% \\
(10 \%)\end{array}$ & $\begin{array}{l}0.6 \% \\
(10 \%)\end{array}$ & $\begin{array}{l}92.5 \% \\
(70 \%)\end{array}$ \\
\hline $\mathrm{PM}_{2.5}$ mass & Dichot sampler/Gravimetry & $\mathrm{CMU}$ & $24 \mathrm{hr}$ & Daily & $7 / 1 / 01-7 / 1 / 02$ & $\begin{array}{l}1.2 \mu \mathrm{g} / \mathrm{m}^{3} \\
\left(2 \mu \mathrm{g} / \mathrm{m}^{3}\right)\end{array}$ & $\begin{array}{l}1.43 \% \\
(10 \%)\end{array}$ & $\begin{array}{l}0.6 \% \\
(10 \%)\end{array}$ & $\begin{array}{l}86.6 \% \\
(70 \%)\end{array}$ \\
\hline $\mathrm{PM}_{2.5}$ mass & R\&P 1400a TEOM with SES & $\mathrm{CMU}$ & $10 \mathrm{~min}$ & Continuous & $7 / 1 / 01-7 / 1 / 02$ & $\begin{array}{l}0.65 \mu \mathrm{g} / \mathrm{m}^{3} \\
\left(1 \mu \mathrm{g} / \mathrm{m}^{3}\right)\end{array}$ & $\begin{array}{l}2.2 \% \\
(10 \%)\end{array}$ & $\begin{array}{l}1.2 \% \\
(10 \%)\end{array}$ & $\begin{array}{l}94 \% \\
(70 \%)\end{array}$ \\
\hline $\mathrm{PM}_{\mathrm{x}}$ mass & MOUDI sampler/Gravimetry & $\mathrm{CMU}$ & $24 \mathrm{hr}$ & Daily & $7 / 1 / 01-7 / 1 / 02 *$ & $\begin{array}{l}1.7 \mu \mathrm{g} / \mathrm{m}^{3} \\
\left(2 \mu \mathrm{g} / \mathrm{m}^{3} \mathrm{PS}\right)\end{array}$ & $\begin{array}{l}1.43 \% \\
(10 \% \mathrm{PS})\end{array}$ & $\begin{array}{l}0.6 \% \\
(10 \% \mathrm{PS})\end{array}$ & $\begin{array}{l}84 \% \\
(70 \%)\end{array}$ \\
\hline $\mathrm{PM}_{\mathrm{x}}$ mass & MOUDI sampler/Gravimetry & $\mathrm{CMU}$ & $8 \mathrm{hr}$ & 3 per day & $7 / 22-25 / 01,7 / 31-8 / 3 / 01$ & $\begin{array}{l}1.5 \mu \mathrm{g} / \mathrm{m}^{3} \\
\left(2 \mu \mathrm{g} / \mathrm{m}^{3} \mathrm{PS}\right)\end{array}$ & $\begin{array}{l}1.43 \% \\
(10 \% \text { PS })\end{array}$ & $\begin{array}{l}0.6 \% \\
(10 \% \mathrm{PS})\end{array}$ & $\begin{array}{l}100 \% \\
(70 \%)\end{array}$ \\
\hline \multicolumn{10}{|l|}{ Aerosol size distribution } \\
\hline $\begin{array}{l}\text { Number, surface area, and } \\
\text { volume distribution }\end{array}$ & TSI SMPS & $\mathrm{CMU}$ & $10 \mathrm{~min}$ & Continuous & $7 / 1 / 01-7 / 1 / 02$ & N/A & $\begin{array}{l}30 \% \\
(30 \%)\end{array}$ & $\begin{array}{l}20 \% \text { size } \\
30 \% \text { count } \\
\text { (N/A) }\end{array}$ & $\begin{array}{l}70 \% \\
(70 \%)\end{array}$ \\
\hline $\begin{array}{l}\text { Number, surface area, and } \\
\text { volume distribution }\end{array}$ & TSI APS & $\mathrm{CMU}$ & $10 \mathrm{~min}$ & Continuous & $7 / 1 / 01-7 / 1 / 02$ & $\mathrm{~N} / \mathrm{A}$ & $\begin{array}{l}30 \% \\
(30 \%)\end{array}$ & $\begin{array}{l}10 \% \text { size } \\
30 \% \text { count }\end{array}$ & $\begin{array}{l}28 \% \\
(70 \%)\end{array}$ \\
\hline Surface area distribution & Epiphaniometer & PSI & $30 \mathrm{~min}$ & Semi-continuous & $6 / 11 / 01-9 / 18 / 01$ & N/A & $\begin{array}{l}- \\
(30 \%)\end{array}$ & N/A & - \\
\hline \multicolumn{10}{|l|}{ Aerosol Characteristics } \\
\hline Light scattering & Optec NGN-3 nephelometer & $\mathrm{CMU}$ & $10 \mathrm{~min}$ & Continuous & $7 / 16 / 01-6 / 30 / 02$ & N/A & $\begin{array}{l}- \\
(\mathrm{T} / \mathrm{D})\end{array}$ & $\begin{array}{l}- \\
(\mathrm{T} / \mathrm{D})\end{array}$ & $\begin{array}{l}- \\
(\mathrm{T} / \mathrm{D})\end{array}$ \\
\hline Hygroscopicity & CMU DAASS & $\mathrm{CMU}$ & $1 \mathrm{hr}$ & Semi-continuous & $7 / 1-8 / 31 / 01,1 / 1-7 / 1 / 02$ & $\mathrm{~N} / \mathrm{A}$ & $\begin{array}{l}30 \% \\
(\mathrm{~T} / \mathrm{D})\end{array}$ & $\begin{array}{l}20 \% \text { size } \\
30 \% \text { count } \\
\text { (T/D) }\end{array}$ & $\begin{array}{l}70 \% \\
(\mathrm{~T} / \mathrm{D})\end{array}$ \\
\hline Cloud condensation behavior & DH Associates M1 CCN & $\mathrm{CMU}$ & $4 \mathrm{hr}$ & Semi-continuous & $9 / 01$ & N/A & $\begin{array}{l}- \\
(\mathrm{T} / \mathrm{D})\end{array}$ & (T/D) & $\begin{array}{l}- \\
(\mathrm{T} / \mathrm{D})\end{array}$ \\
\hline
\end{tabular}




\begin{tabular}{|c|c|c|c|c|c|c|c|c|c|}
\hline Observable & Method $^{1}$ & 管 & 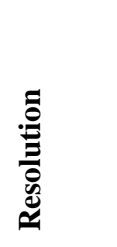 & 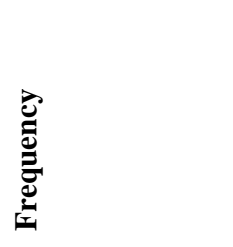 & 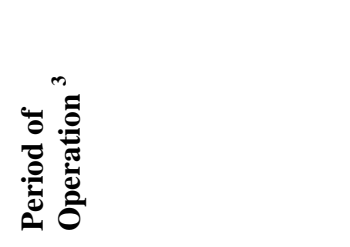 & 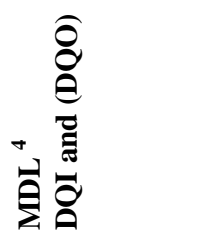 & 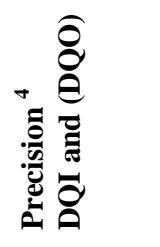 & 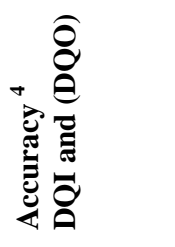 & 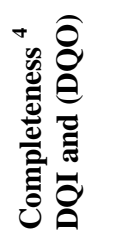 \\
\hline \multicolumn{10}{|l|}{ Aerosol chemical composition } \\
\hline $\mathrm{PM}_{10}$ inorganic ions & CMU Speciation sampler/ IC & CMU & $24 \mathrm{hr}$ & Daily & 7/1/01-7/21/02 & $\begin{array}{l}- \\
\left(0.1 \mu \mathrm{g} / \mathrm{m}^{3}\right)\end{array}$ & $-\bar{l}$ & $\begin{array}{l}- \\
(15 \%)\end{array}$ & $\begin{array}{l}- \\
(70 \%)\end{array}$ \\
\hline $\mathrm{PM}_{2.5}$ inorganic ions & CMU Speciation sampler/ IC & CMU & $24 \mathrm{hr}$ & Daily & $\begin{array}{l}7 / 1 / 01-7 / 21 / 02 * \text {-analysis } \\
\text { complete only to } 12 / 31 / 01\end{array}$ & $\begin{array}{l}0.01-0.5 \mu \mathrm{g} / \mathrm{m}^{3} \\
\left(0.1 \mu \mathrm{g} / \mathrm{m}^{3}\right)\end{array}$ & $\begin{array}{l}6.2-59.4 \% \\
(20 \%)\end{array}$ & $\begin{array}{l}-5.6 \text { to }+.1 \% \\
(15 \%)\end{array}$ & $\begin{array}{l}53-77 \% \\
(70 \%)\end{array}$ \\
\hline $\mathrm{PM}_{2.5}$ inorganic ions & CMU Speciation sampler/ IC & CMU & $4-6 \mathrm{hr}$ & 5 per day & ESP01 Intensive & $\begin{array}{l}0.03-0.62 \mu \mathrm{g} / \mathrm{m}^{3} \\
\left(0.1 \mu \mathrm{g} / \mathrm{m}^{3}\right)\end{array}$ & $\begin{array}{l}6.2-59.4 \% \\
(20 \%)\end{array}$ & $\begin{array}{l}-5.6 \text { to }+.1 \% \\
(20 \%)\end{array}$ & $\begin{array}{l}53-77 \% \\
(70 \%)\end{array}$ \\
\hline $\mathrm{PM}_{\mathrm{x}}$ inorganic ions & MOUDI sampler/IC & CMU & $24 \mathrm{hr}$ & Daily & $7 / 1 / 01-7 / 1 / 02 *$ & $\begin{array}{l}0.01-0.67 \mu \mathrm{g} / \mathrm{m}^{3} \\
\left(0.1 \mu \mathrm{g} / \mathrm{m}^{3} \mathrm{PS}\right)\end{array}$ & $\begin{array}{l}5 \% \\
(20 \% \mathrm{PS})\end{array}$ & (20\% PS) & $\begin{array}{l}78 \% \\
(70 \%)\end{array}$ \\
\hline $\mathrm{PM}_{2.5}$ nitrate & R\&P 8400N (ICVC) & ADI & $10 \mathrm{~min}$ & Semi-continuous & 7/1/01-8/1/02 & $\begin{array}{l}0.16 \mu \mathrm{g} / \mathrm{m}^{3} \\
\left(1.0 \mu \mathrm{g} / \mathrm{m}^{3}\right)\end{array}$ & $\begin{array}{l}19.5 \% \\
(10 \%)\end{array}$ & $\begin{array}{l}3.1 \% \\
(25 \%)\end{array}$ & $\begin{array}{l}89 \% \\
(85 \%)\end{array}$ \\
\hline $\mathrm{PM}_{2.5}$ sulfate & R\&P 8400S (ICVC) & ADI & $10 \mathrm{~min}$ & Semi-continuous & 7/1/01-9/1/02 & $\begin{array}{l}0.96 \mu \mathrm{g} / \mathrm{m}^{3} \\
\left(1.0 \mu \mathrm{g} / \mathrm{m}^{3}\right)\end{array}$ & $\begin{array}{l}20.9 \% \\
(10 \%)\end{array}$ & $\begin{array}{l}-8.1 \% \\
(25 \%)\end{array}$ & $\begin{array}{l}83 \% \\
(85 \%)\end{array}$ \\
\hline $\mathrm{PM}_{2.5}$ water soluble anions & CMU Steam sampler/IC & $\mathrm{CMU}$ & $1-2 \mathrm{hrs}$ & Semi-continuous & 7/1/01-9/21/02 & $\begin{array}{l}0.02-0.18 \mu \mathrm{g} / \mathrm{m}^{3} \\
\left(0.2 \mu \mathrm{g} / \mathrm{m}^{3}\right)\end{array}$ & $\begin{array}{l}15 \% \\
(10 \%)\end{array}$ & $\begin{array}{l}-8 \% \\
(20 \%)\end{array}$ & $\begin{array}{l}87 \% \\
(70 \%)\end{array}$ \\
\hline $\mathrm{PM}_{2.5}$ water soluble cations & CMU Steam sampler/IC & $\mathrm{CMU}$ & $1-2 \mathrm{hrs}$ & Semi-continuous & 7/1/01-9/21/02 & $\begin{array}{l}0.17-0.19 \mu \mathrm{g} / \mathrm{m}^{3} \\
\left(0.2 \mu \mathrm{g} / \mathrm{m}^{3}\right)\end{array}$ & $\begin{array}{l}15 \% \\
(10 \%)\end{array}$ & $\begin{array}{l}-17 \% \\
(20 \%)\end{array}$ & $\begin{array}{l}86 \% \\
(70 \%)\end{array}$ \\
\hline $\mathrm{PM}_{2.5}$ water soluble $\mathrm{NH}_{4}^{+}$ & CMU Steam sampler/OAD & $\mathrm{CMU}$ & $10 \mathrm{~min}$ & Continuous & 7/1/01-9/21/02 & $(\mathrm{T} / \mathrm{D})$ & $(\mathrm{T} / \mathrm{D})$ & (T/D) & $(\mathrm{T} / \mathrm{D})$ \\
\hline $\mathrm{PM}_{10}$ elements & Hi-Vol sampler/ICPMS & $\mathrm{CMU}$ & $24 \mathrm{hr}$ & Daily & $7 / 12 / 01-8 / 02 / 02$ & $\left(0.1 \mu \mathrm{g} / \mathrm{m}^{3}\right)$ & $\begin{array}{l}- \\
(20 \%)\end{array}$ & $\begin{array}{l}- \\
(20 \%)\end{array}$ & $\begin{array}{l}- \\
(70 \%)\end{array}$ \\
\hline $\mathrm{PM}_{2.5}$ elements & Hi-Vol sampler/ICPMS & $\mathrm{CMU}$ & $24 \mathrm{hr}$ & Daily & 7/11/01-9/30/02 & $\left(0.1 \mu \mathrm{g} / \mathrm{m}^{3}\right)$ & $-\overline{(20 \%)}$ & $-\bar{l}$ & $-70 \%) 10$ \\
\hline $\mathrm{PM}_{1.3}$ elements & UMD SEAS/GFAA & UMD & $30 \mathrm{~min}$ & Semi-continuous & $7 / 8-8 / 10 / 01,3 / 29-4 / 17 / 02$ & $\begin{array}{l}0.12-7.2 \mathrm{ppb} \\
(\mathrm{T} / \mathrm{D})\end{array}$ & $\begin{array}{l}5-10 \% \\
(T / D)\end{array}$ & $\begin{array}{l}10-15 \% \\
(\mathrm{~T} / \mathrm{D})\end{array}$ & $\begin{array}{l}0 \% \\
(\mathrm{~T} / \mathrm{D})\end{array}$ \\
\hline $\mathrm{PM}_{\mathrm{x}}$ elements & MOUDI sampler/ICPMS & $\mathrm{CMU}$ & $24 \mathrm{hr}$ & Daily & ESP01, 02 Intensives * & $\left(0.1 \mu \mathrm{g} / \mathrm{m}^{3} \mathrm{PS}\right)$ & $\overline{(20 \% \mathrm{PS})}$ & $\overline{-}(20 \% \mathrm{PS})$ & $-\bar{c}$ \\
\hline
\end{tabular}




\begin{tabular}{|c|c|c|c|c|c|c|c|c|c|}
\hline Observable & Method $^{1}$ & 官 & 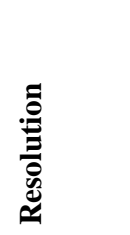 & 导 & סُ & 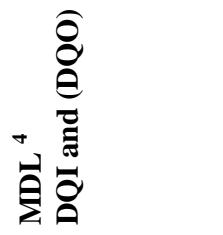 & 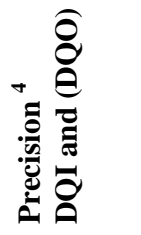 & 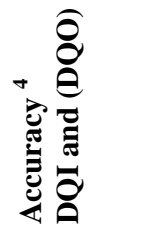 & 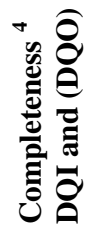 \\
\hline Aerosol chemical composition & & & & & & & & & \\
\hline $\mathrm{PM}_{2.5}$ organic/elemental carbon & CMU TQQQ sampler/TOT & $\mathrm{CMU}$ & $24 \mathrm{hr}$ & Daily & $7 / 1 / 01-7 / 31 / 02 *$ & $\begin{array}{l}0.17-0.53 \mu \mathrm{gC} / \mathrm{m}^{3} \\
\left(0.5 \mu \mathrm{gC} / \mathrm{m}^{3}\right)\end{array}$ & $\begin{array}{l}8 \% \\
(30 \%)\end{array}$ & $\begin{array}{l}2.3-5.7 \% \\
(30 \%)\end{array}$ & $\begin{array}{l}99 \% \\
(70 \%)\end{array}$ \\
\hline $\mathrm{PM}_{2.5}$ organic/elemental carbon & CMU TQQQ sampler/TOT & $\mathrm{CMU}$ & $4-6 \mathrm{hr}$ & 5 per day & ESP01 Intensive & $\begin{array}{l}0.17-0.53 \mu \mathrm{gC} / \mathrm{m}^{3} \\
\left(0.5 \mu \mathrm{gC} / \mathrm{m}^{3}\right)\end{array}$ & $\begin{array}{l}8 \% \\
(30 \%)\end{array}$ & $\begin{array}{l}2.3-5.7 \% \\
(30 \%)\end{array}$ & $\begin{array}{l}99 \% \\
(70 \%)\end{array}$ \\
\hline $\mathrm{PM}_{2.5}$ organic/elemental carbon & CMU Denuder sampler/TOT & $\mathrm{CMU}$ & $24 \mathrm{hr}$ & $6^{\text {th }}$ day & $7 / 1 / 01-6 / 1 / 02 *$ & $\begin{array}{l}0.3 \mu \mathrm{gC} / \mathrm{m}^{3} \\
\left(0.5 \mu \mathrm{gC} / \mathrm{m}^{3}\right)\end{array}$ & $(30 \%)$ & $\begin{array}{l}10 \% \\
(30 \%)\end{array}$ & $\begin{array}{l}75 \% \\
(70 \%)\end{array}$ \\
\hline $\mathrm{PM}_{2.5}$ organic/elemental carbon & CMU Denuder sampler/TOT & CMU & $24 \mathrm{hr}$ & Daily & ESP01, 02 Intensives & $\begin{array}{l}0.3 \mu \mathrm{gC} / \mathrm{m}^{3} \\
\left(0.5 \mu \mathrm{gC} / \mathrm{m}^{3}\right)\end{array}$ & $\begin{array}{l}2 \% \\
(30 \%)\end{array}$ & $\begin{array}{l}10 \% \\
(30 \%)\end{array}$ & $\begin{array}{l}95 \% \\
(70 \%)\end{array}$ \\
\hline $\mathrm{PM}_{2.5}$ organic/elemental carbon & BYU PC-BOSS & BYU & $24 \mathrm{hr}$ & Daily & $7 / 9 / 01-7 / 31 / 01$ & $\left(0.1 \mu \mathrm{gC} / \mathrm{m}^{3}\right)$ & - & - & $\begin{array}{l}- \\
(70 \%)\end{array}$ \\
\hline $\mathrm{PM}_{2.5}$ organic/elemental carbon & SLCarbon (TOT) analyzer & RU & $2-4 \mathrm{hr}$ & Semi-continuous & $7 / 1 / 01-9 / 1 / 02$ & $\left(0.3 \mu \mathrm{gC} / \mathrm{m}^{3}\right)$ & $(10 \%)$ & $(10 \%)$ & $\begin{array}{l}- \\
(70 \%)\end{array}$ \\
\hline $\mathrm{PM}_{\mathrm{x}}$ organic/elemental carbon & MOUDI/TOT & CMU & $24 \mathrm{hr}$ & Daily & ESP01, 02 Intensives * & $\begin{array}{l}.12-.21 \mu \mathrm{gC} / \mathrm{m}^{3} \\
\left(0.5 \mu \mathrm{gC} / \mathrm{m}^{3} \mathrm{PS}\right)\end{array}$ & $\begin{array}{l}8 \% \\
(30 \% \text { PS })\end{array}$ & $\begin{array}{l}2.3-5.7 \% \\
(30 \% \text { PS) }\end{array}$ & $\begin{array}{l}66 \% \\
(70 \%)\end{array}$ \\
\hline $\mathrm{PM}_{\mathrm{x}}$ organic/elemental carbon & MOUDI/TOT & $\mathrm{CMU}$ & $8 \mathrm{hr}$ & 3 per day & $7 / 22-25 / 01,7 / 31-8 / 3 / 01$ & ${ }^{-}\left(0.5 \mu \mathrm{gC} / \mathrm{m}^{3} \mathrm{PS}\right)$ & $\overline{-}(30 \% \mathrm{PS})$ & ${ }^{-}(30 \% \mathrm{PS})$ & $\begin{array}{l}- \\
(70 \%)\end{array}$ \\
\hline $\mathrm{PM}_{2.5}$ speciated organics & Organic sampler/GC-MS & FIU & $24 \mathrm{hr}$ & $6^{\text {th }}$ day & $7 / 1 / 01-7 / 1 / 02 *$ & $\begin{array}{l}- \\
(\mathrm{T} / \mathrm{D})\end{array}$ & $\begin{array}{l}- \\
(\mathrm{T} / \mathrm{D})\end{array}$ & $\begin{array}{l}- \\
(T / D)\end{array}$ & $\begin{array}{l}- \\
(\mathrm{T} / \mathrm{D})\end{array}$ \\
\hline $\mathrm{PM}_{2.5}$ speciated organics & Organic sampler/GC-MS & FIU & $24 \mathrm{hr}$ & Daily & ESP01, 02 Intensives & $(\mathrm{T} / \mathrm{D})$ & $\begin{array}{l}- \\
(\mathrm{T} / \mathrm{D})\end{array}$ & $\begin{array}{l}- \\
(\mathrm{T} / \mathrm{D})\end{array}$ & $\begin{array}{l}- \\
(\mathrm{T} / \mathrm{D})\end{array}$ \\
\hline $\mathrm{PM}_{\mathrm{x}}$ speciated organics & LPI/FTIR & RU & $24 \mathrm{hr}$ & Daily & ESP01, 02 Intensives & $\begin{array}{l}- \\
(\mathrm{T} / \mathrm{D})\end{array}$ & $\begin{array}{l}- \\
(\mathrm{T} / \mathrm{D})\end{array}$ & $\begin{array}{l}- \\
(\mathrm{T} / \mathrm{D})\end{array}$ & $\begin{array}{l}- \\
(\mathrm{T} / \mathrm{D})\end{array}$ \\
\hline $\mathrm{PM}_{2.5}$ biological material & $\begin{array}{l}\text { Epi-fluorescent microscopy } \\
\text { with assays }\end{array}$ & UColo & $24 \mathrm{hr}$ & Daily & 7/7/01-7/1/02 & $\overline{-}(\mathrm{T} / \mathrm{D})$ & $\begin{array}{l}- \\
(\mathrm{T} / \mathrm{D})\end{array}$ & $\begin{array}{l}- \\
(\mathrm{T} / \mathrm{D})\end{array}$ & $\begin{array}{l}- \\
(\mathrm{T} / \mathrm{D})\end{array}$ \\
\hline $\mathrm{PM}_{2.5}$ fog composition & Collector/IC/TOC/pH & $\mathrm{CSU}$ & Per event & 8 events & $7 / 1 / 01-9 / 1 / 02$ & $\begin{array}{l}1.3-27.9 \mu \mathrm{M} \\
(\mathrm{T} / \mathrm{D})\end{array}$ & $\begin{array}{l}0.5-6.7 \% \\
(\mathrm{~T} / \mathrm{D})\end{array}$ & $\begin{array}{l}1-19.6 \% \\
(T / D)\end{array}$ & $\begin{array}{l}100 \% \\
(\mathrm{~T} / \mathrm{D})\end{array}$ \\
\hline $\begin{array}{l}\mathrm{PM}_{1.0} \text { size resolved } \\
\text { composition }\end{array}$ & $\begin{array}{l}\text { Aerodyne Mass } \\
\text { Spectrometer (AMS) }\end{array}$ & $\begin{array}{l}\text { UCB, } \\
\text { Aerodyne }\end{array}$ & $5 \min$ & Semi-continuous & $9 / 6 / 02-9 / 21 / 02$ & $(\mathrm{~T} / \mathrm{D})$ & $(\mathrm{T} / \mathrm{D})$ & $(\mathrm{T} / \mathrm{D})$ & $(\mathrm{T} / \mathrm{D})$ \\
\hline \multicolumn{2}{|c|}{ Single Particle Chemical Composition } & & & & & & & & \\
\hline Polar organics & RSMS-III & UCD,UD & $10 \mathrm{~min}$ & Semi-continuous & $9 / 20 / 01-10 / 1 / 02$ & $\begin{array}{l}- \\
(\mathrm{T} / \mathrm{D})\end{array}$ & $\begin{array}{l}- \\
(\mathrm{T} / \mathrm{D})\end{array}$ & $\begin{array}{l}- \\
(\mathrm{T} / \mathrm{D})\end{array}$ & $\begin{array}{l}- \\
(\mathrm{T} / \mathrm{D})\end{array}$ \\
\hline Ion composition & RSMS-III & UCD,UD & $10 \mathrm{~min}$ & Semi-continuous & $9 / 20 / 01-10 / 1 / 02$ & $\begin{array}{l}- \\
(\mathrm{T} / \mathrm{D})\end{array}$ & $\begin{array}{l}- \\
(\mathrm{T} / \mathrm{D})\end{array}$ & $\begin{array}{l}- \\
(\mathrm{T} / \mathrm{D})\end{array}$ & $\begin{array}{l}- \\
(\mathrm{T} / \mathrm{D})\end{array}$ \\
\hline Particle morphology & Nuclepore filter/SEM & RJL & $24 \mathrm{hr}$ & Daily & ESP01, 02 Intensives & $\begin{array}{l}- \\
(\mathrm{T} / \mathrm{D})\end{array}$ & $\begin{array}{l}- \\
(\mathrm{T} / \mathrm{D})\end{array}$ & $\begin{array}{l}- \\
(\mathrm{T} / \mathrm{D})\end{array}$ & $\begin{array}{l}- \\
(\mathrm{T} / \mathrm{D})\end{array}$ \\
\hline
\end{tabular}




\begin{tabular}{|c|c|c|c|c|c|c|c|c|c|}
\hline Observable & Method $^{1}$ & 言 & 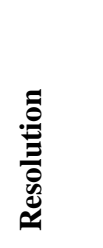 & 总 & 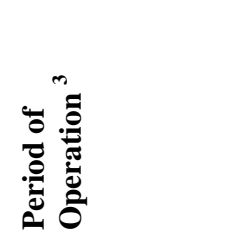 & 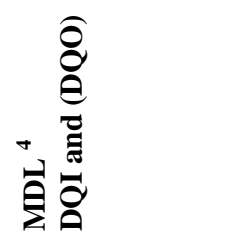 & 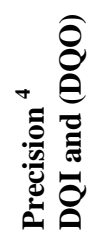 & 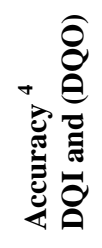 & 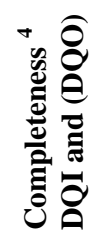 \\
\hline \multicolumn{10}{|l|}{ Gaseous Species } \\
\hline Light $\left(\mathrm{C}_{2}-\mathrm{C}_{12}\right)$ hydrocarbons & Canister/GC-FID & $\mathrm{CMU}$ & $24 \mathrm{hr}$ & $3^{\text {rd }}$ day & $9 / 1 / 01-7 / 31 / 02 *$ & $\begin{array}{l}0.0-0.25 \mu \mathrm{g} / \mathrm{m}^{3} \\
\text { (T/D) }\end{array}$ & $\begin{array}{l}33 \% \\
(\mathrm{~T} / \mathrm{D})\end{array}$ & $\begin{array}{l}20 \% \\
(\mathrm{~T} / \mathrm{D})\end{array}$ & $\begin{array}{l}65 \% \\
(\mathrm{~T} / \mathrm{D})\end{array}$ \\
\hline Light $\left(\mathrm{C}_{2}-\mathrm{C}_{12}\right)$ hydrocarbons & Canister/GC-FID & $\mathrm{CMU}$ & $24 \mathrm{hr}$ & Daily & ESP02 Intensive & & $(\mathrm{T} / \mathrm{D})$ & $(\mathrm{T} / \mathrm{D})$ & $\overline{(T / D)}$ \\
\hline Total peroxides & CSU Monitor & $\mathrm{CSU}$ & $1 \mathrm{hr}$ & Continuous & 7/1/01-9/1/02 & $\begin{array}{l}0.09 \mathrm{ppbv} \\
(0.2 \mathrm{ppbv})\end{array}$ & $\begin{array}{l}2.8 \% \\
(20 \%\end{array}$ & $\begin{array}{l}-2.2 \% \\
(20 \%)\end{array}$ & $\begin{array}{l}94 \% \\
(70 \%)\end{array}$ \\
\hline $\mathrm{O}_{3}$ & API $400 \mathrm{~A}$ & $\mathrm{CMU}$ & $10 \mathrm{~min}$ & Continuous & 7/1/01-9/1/02 & $\begin{array}{l}0.14 \mathrm{ppbv} \\
(0.6 \mathrm{ppbv})\end{array}$ & $\begin{array}{l}1.7 \% \\
(10 \%)\end{array}$ & $\begin{array}{l}1.1 \% \\
(10 \%)\end{array}$ & $\begin{array}{l}95 \% \\
(80 \%)\end{array}$ \\
\hline $\mathrm{NO}$ and $\mathrm{NO}_{\mathrm{x}}$ & API $200 \mathrm{~A}$ & $\mathrm{CMU}$ & $10 \mathrm{~min}$ & Continuous & 7/1/01-9/1/02 & $\begin{array}{l}0.39 \mathrm{ppbv} \\
(0.4 \mathrm{ppbv})\end{array}$ & $\begin{array}{l}2.3 \% \\
(10 \%)\end{array}$ & $\begin{array}{l}1.2 \% \\
(10 \%)\end{array}$ & $\begin{array}{l}90 \% \\
(80 \%)\end{array}$ \\
\hline $\mathrm{SO}_{2}$ & API 100A & $\mathrm{CMU}$ & $10 \mathrm{~min}$ & Continuous & 7/1/01-9/1/02 & $\begin{array}{l}0.34 \mathrm{ppbv} \\
(0.4 \mathrm{ppbv})\end{array}$ & $\begin{array}{l}6.9 \% \\
(10 \%)\end{array}$ & $\begin{array}{l}-0.8 \% \\
(10 \%)\end{array}$ & $\begin{array}{l}94 \% \\
(80 \%)\end{array}$ \\
\hline $\mathrm{CO}$ & API 300 & $\mathrm{CMU}$ & $10 \mathrm{~min}$ & Continuous & $7 / 1 / 01-9 / 1 / 02$ & $\begin{array}{l}0.1 \mathrm{ppmv} \\
(0.4 \mathrm{ppmv})\end{array}$ & $\begin{array}{l}1.1 \% \\
(10 \%) \\
\end{array}$ & $\begin{array}{l}1.1 \% \\
(10 \%) \\
\end{array}$ & $\begin{array}{l}94 \% \\
(80 \%) \\
\end{array}$ \\
\hline Inorganic gases & CMU Speciation sampler/ IC & $\mathrm{CMU}$ & $24 \mathrm{hr}$ & Daily & $7 / 1 / 01-7 / 1 / 02 *$ & $-\left(05 \mu \mathrm{g} / \mathrm{m}^{3}\right)$ & - & - & - \\
\hline Inorganic gases & CMU Speciation sampler/ IC & $\mathrm{CMU}$ & $4-6 \mathrm{hr}$ & 5 per day & ESP01 Intensive & - & & & - \\
\hline Water soluble inorganic gases & CMU Steam sampler/ IC & $\mathrm{CMU}$ & $1-2 \mathrm{hr}$ & Semi-continuous & $7 / 1 / 01-9 / 21 / 02$ & $\begin{array}{l}\left(0.5 \mu \mathrm{g} / \mathrm{m}^{3}\right) \\
0.02-0.19 \mu \mathrm{g} / \mathrm{m}^{3} \\
\left(0.2 \mu \mathrm{g} / \mathrm{m}^{3}\right)\end{array}$ & $\begin{array}{l}(30 \%) \\
15 \% \\
(10 \%)\end{array}$ & $\begin{array}{l}(30 \%) \\
86 \% \\
(20 \%)\end{array}$ & $\begin{array}{l}(70 \%) \\
- \\
(70 \%)\end{array}$ \\
\hline
\end{tabular}




\begin{tabular}{|c|c|c|c|c|c|c|c|c|c|}
\hline Observable & Method $^{1}$ & 言 & 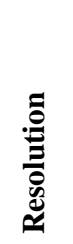 & 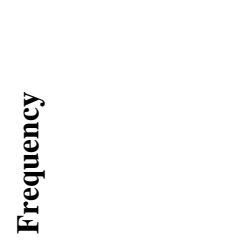 & 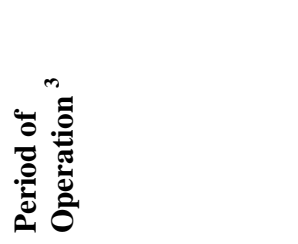 & 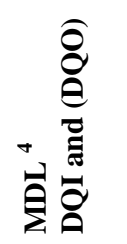 & 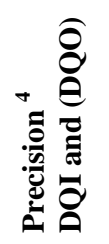 & 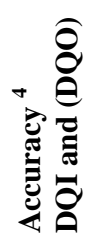 & 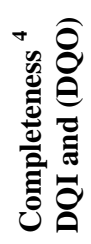 \\
\hline Hydrocarbons & UC Online GC-FID/MS & $\mathrm{UCB}$ & $1 \mathrm{hr}$ & Semi-continuous & $1 / 9-2 / 12,7 / 10-8 / 10 / 02$ & & & & \\
\hline $\begin{array}{l}\text { 1-butene } \\
\text { 1-methylcyclopentene } \\
\text { 1-pentene } \\
\text { 2-methyl-1-butene } \\
\text { 2-methylpropene }\end{array}$ & & & & & & $\begin{array}{l}1.0 \mathrm{ppt} \\
0.7 \mathrm{ppt} \\
0.8 \mathrm{pt} \\
0.8 \mathrm{ppt} \\
1.0 \mathrm{ppt}\end{array}$ & $\begin{array}{l}2 \% \\
2 \% \\
2 \% \\
2 \% \\
2 \%\end{array}$ & $\begin{array}{l}7 \% \\
7 \% \\
7 \% \\
7 \% \\
7 \%\end{array}$ & $\begin{array}{l}96.5 \% \\
99.2 \% \\
96.5 \% \\
96.5 \% \\
96.5 \%\end{array}$ \\
\hline $\begin{array}{l}\text { 3-methyl-1-butene } \\
\text { 3-methylfuran } \\
\text { acetone } \\
\text { alpha pinene } \\
\text { benzene }\end{array}$ & & & & & & $\begin{array}{l}0.8 \mathrm{ppt} \\
2.2 \mathrm{ppt} \\
47.3 \mathrm{ppt} \\
1.1 \mathrm{ppt} \\
25.6 \mathrm{ppt}\end{array}$ & $\begin{array}{l}2 \% \\
6 \% \\
4 \% \\
13 \% \\
7 \%\end{array}$ & $\begin{array}{l}7 \% \\
8 \% \\
7 \% \\
14 \% \\
9 \%\end{array}$ & $\begin{array}{l}96.5 \% \\
99.2 \% \\
96.1 \% \\
96.0 \% \\
96.3 \%\end{array}$ \\
\hline $\begin{array}{l}\text { butanol } \\
\text { butane } \\
\text { c-2-butene } \\
\text { perchloroethylene } \\
\text { c-2-pentene }\end{array}$ & & & & & & $\begin{array}{l}27.6 \mathrm{ppt} \\
1.0 \mathrm{pt} \\
1.0 \mathrm{ppt} \\
0.6 \mathrm{ppt} \\
0.8 \mathrm{ppt}\end{array}$ & $\begin{array}{l}6 \% \\
2 \% \\
2 \% \\
6 \% \\
2 \%\end{array}$ & $\begin{array}{l}8 \% \\
7 \% \\
7 \% \\
9 \% \\
7 \%\end{array}$ & $\begin{array}{l}99.1 \% \\
96.5 \% \\
96.5 \% \\
96.3 \% \\
99.2 \%\end{array}$ \\
\hline $\begin{array}{l}\text { dichloromethane } \\
\text { acetaldehyde } \\
\text { acetonitrile } \\
\text { chloroform } \\
\text { cyclopentane }\end{array}$ & & & & & & $\begin{array}{l}33.9 \mathrm{ppt} \\
82.2 \mathrm{ppt} \\
38.1 \mathrm{ppt} \\
1.0 \mathrm{ppt} \\
0.8 \mathrm{ppt}\end{array}$ & $\begin{array}{l}12 \% \\
9 \% \\
13 \% \\
4 \% \\
2 \%\end{array}$ & $\begin{array}{l}14 \% \\
11 \% \\
14 \% \\
7 \% \\
7 \%\end{array}$ & $\begin{array}{l}99.2 \% \\
94.0 \% \\
96.7 \% \\
96.2 \% \\
96.5 \%\end{array}$ \\
\hline $\begin{array}{l}\text { cyclopentene } \\
\text { dimethylsulfide } \\
\text { ethylbenzene } \\
\text { ethanol } \\
\text { hexane }\end{array}$ & & & & & & $\begin{array}{l}0.8 \mathrm{ppt} \\
3.2 \mathrm{pt} \\
1.6 \mathrm{ppt} \\
15.7 \mathrm{ppt} \\
0.7 \mathrm{ppt}\end{array}$ & $\begin{array}{l}2 \% \\
6 \% \\
6 \% \\
15 \% \\
2 \%\end{array}$ & $\begin{array}{l}7 \% \\
8 \% \\
9 \% \\
17 \% \\
7 \%\end{array}$ & $\begin{array}{l}99.2 \% \\
99.2 \% \\
96.3 \% \\
90.8 \% \\
96.5 \%\end{array}$ \\
\hline $\begin{array}{l}\text { isopropanol } \\
\text { isobutane } \\
\text { isopentane } \\
\text { isoprene } \\
\text { methacrolein }\end{array}$ & & & & & & $\begin{array}{l}22.8 \mathrm{ppt} \\
1.0 \mathrm{ppt} \\
0.8 \mathrm{pt} \\
0.8 \mathrm{ppt} \\
11.3 \mathrm{ppt}\end{array}$ & $\begin{array}{l}13 \% \\
2 \% \\
2 \% \\
2 \% \\
6 \%\end{array}$ & $\begin{array}{l}15 \% \\
7 \% \\
7 \% \\
7 \% \\
8 \%\end{array}$ & $\begin{array}{l}91.1 \% \\
96.5 \% \\
96.5 \% \\
99.2 \% \\
99.2 \%\end{array}$ \\
\hline
\end{tabular}




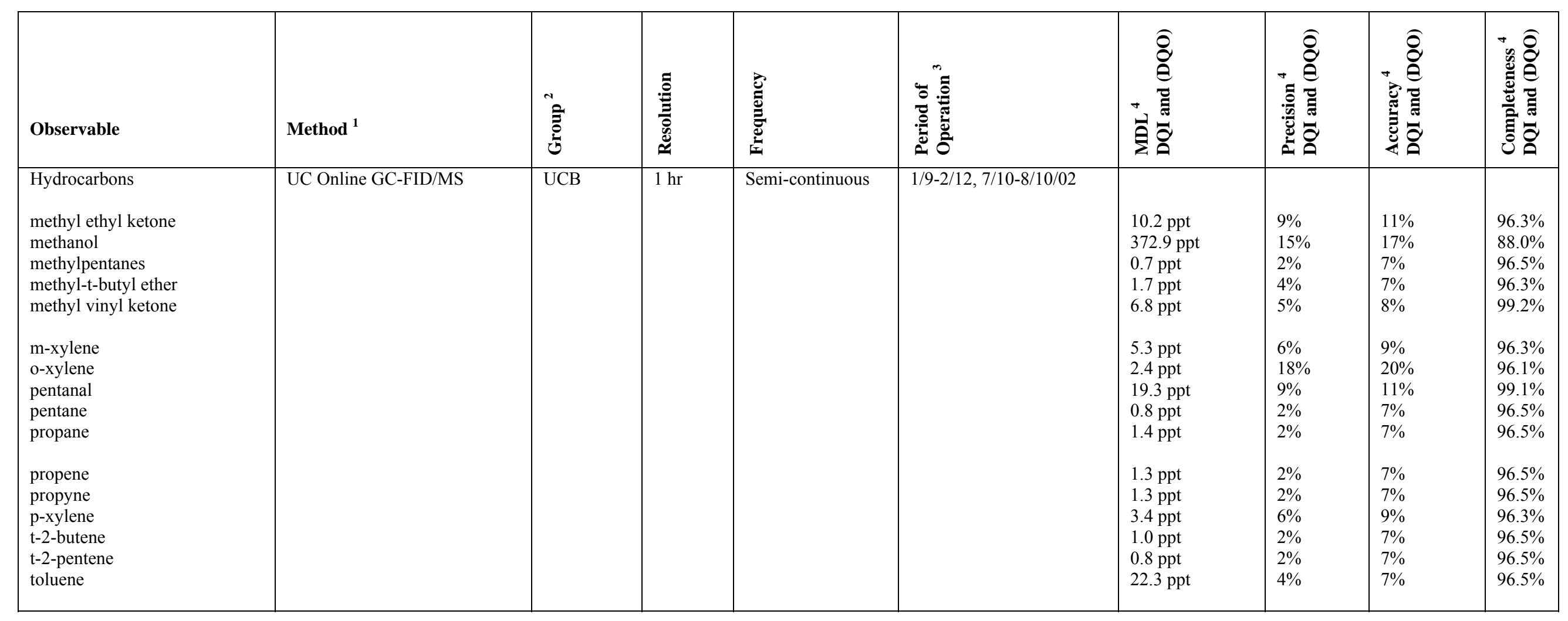




\begin{tabular}{|c|c|c|c|c|c|c|c|c|c|}
\hline Observable & Method $^{1}$ & 官 & 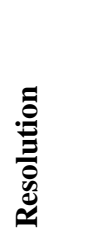 & 总 & 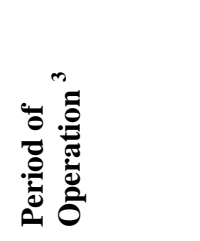 & 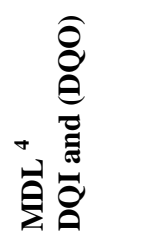 & 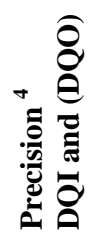 & 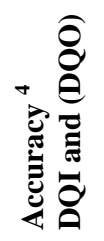 & 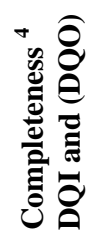 \\
\hline \multicolumn{10}{|l|}{ Meteorology } \\
\hline Wind speed & MetOne 014A & $\mathrm{CMU}$ & $10 \mathrm{~min}$ & Continuous & $7 / 1 / 01-9 / 1 / 02$ & $\begin{array}{l}0.5 \mathrm{~m} / \mathrm{s} \\
(\mathrm{N} / \mathrm{A})\end{array}$ & - & $\begin{array}{l}- \\
(10 \%)\end{array}$ & $\begin{array}{l}99 \% \\
(80 \%)\end{array}$ \\
\hline Wind direction & MetOne $014 \mathrm{~A}$ & $\mathrm{CMU}$ & $10 \mathrm{~min}$ & Continuous & $7 / 1 / 01-9 / 1 / 02$ & $\mathrm{~N} / \mathrm{A}$ & $(10 \%)$ & - & $\begin{array}{l}99 \% \\
(80 \%)\end{array}$ \\
\hline Temperature & Campbell HMP45C & CMU & $10 \mathrm{~min}$ & Continuous & 7/1/01-9/1/02 & N/A & $\begin{array}{l}- \\
(10 \%)\end{array}$ & $\begin{array}{l}- \\
(10 \%)\end{array}$ & $\begin{array}{l}99 \% \\
(80 \%)\end{array}$ \\
\hline Relative Humidity & Campbell HMP45C & $\mathrm{CMU}$ & $10 \mathrm{~min}$ & Continuous & $7 / 1 / 01-9 / 1 / 02$ & N/A & - & $\begin{array}{l}- \\
(10 \%)\end{array}$ & $\begin{array}{l}99 \% \\
(80 \%)\end{array}$ \\
\hline Pressure & Campbell CS105 & $\mathrm{CMU}$ & $10 \mathrm{~min}$ & Continuous & $7 / 1 / 01-9 / 1 / 02$ & N/A & 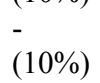 & $\begin{array}{l}- \\
(10 \%)\end{array}$ & $\begin{array}{l}99 \% \\
(80 \%)\end{array}$ \\
\hline Precipitation & MetOne 370 & $\mathrm{CMU}$ & $10 \mathrm{~min}$ & Continuous & 7/1/01-9/1/02 & $\begin{array}{l}0.254 \mathrm{~mm} \\
(\mathrm{~N} / \mathrm{A})\end{array}$ & $\begin{array}{l}- \\
(10 \%)\end{array}$ & $\begin{array}{l}- \\
(10 \%)\end{array}$ & $\begin{array}{l}99 \% \\
(80 \%)\end{array}$ \\
\hline UV Radiation & Kipp\&Zonen CUV3 & $\mathrm{CMU}$ & $10 \mathrm{~min}$ & Continuous & 7/1/01-9/1/02 & N/A & - & $(10 \%)$ & $\begin{array}{l}99 \% \\
(80 \%)\end{array}$ \\
\hline Solar Radiation & Kipp\&Zonen CM3 & $\mathrm{CMU}$ & $10 \mathrm{~min}$ & Continuous & $7 / 1 / 01-9 / 1 / 02$ & N/A & $(10 \%)$ & $\begin{array}{l}- \\
(10 \%)\end{array}$ & $\begin{array}{l}99 \% \\
(80 \%)\end{array}$ \\
\hline
\end{tabular}

1 Methods - ADI: Aerosol Dynamics Inc.; APS: Aerodynamic Particle Sizer; BYU: Brigham Young University; CMU: Carnegie Mellon University; CSU: Colorado State University; FRM: Federal Reference Method; FTIR: Fourier Transform Infrared Spectrometry; Grav: Gravimetry; IC: Ion Chromatography; ICPMS: Inductively Coupled Plasma Mass Spectrometry; ICVC: Integrated Collection and Vaporization Cell; LPI: Low Pressure Impactor; GC-FID: Gas Chromatography with Flame Ionization Spectroscopy; GC-MS: Gas Chromatography with Mass Spectroscopy; GFAA: Graphite Furnace with Atomic Absorption; OAD: Online Ammonium Detector; R\&P: Rupprecht and Patashnick, Co.; RSMS: Rapid Single particle Mass Spectrometer; SEAS: Semi-continuous Environmental Aerosol Sampler; SEM: Scanning Electron Microscopy; SL: Sunset Labs; SMPS: Scanning Mobility Particle Sizer; TEOM with SES: Tapered Element Oscillating Microbalance with a Sample Equilibration System; TOC: Total Organic Carbon; TOT: Total Optical Transmittance; UCB: University of California at Berkeley; UCD: University of California at Davis.

2 Groups - ADI: Aerosol Dynamics, Inc.; BYU: Brigham Young University; CMU: Carnegie Mellon University; CSU: Colorado State University; FIU: Florida International University; PSI: Paul Scherrer Institute; RJL: R. J. Lee Instruments, RU: Rutgers University; UC: University of California at Berkeley; UCB: University of Colorado at Boulder; UCD: University of California at Davis; UD: University of Delaware; UMD: University of Maryland.

3 Period of operation - ESP01 Intensive: July 1, 2001 - August 3, 2001; ESP02 Intensive: January 1, 2002 - January 15, 2002; *: Except during periods when samples were collected at a higher time resolution as noted in the entry below.

4 Data quality indicators and (Data quality objectives); N/A: Not applicable; T/D: To be determined; Values for MOUDIs and LPIs are per substrate (stage or filter). 


\subsection{Minimum detection limit (MDL)}

Analytical procedures and sampling equipment impose specific constraints on the determination of detection limits. MDL is defined as a statistically determined value above which the reported concentration can be differentiated from a zero concentration, and was calculated for a majority of measurements using Equation 1.

\section{Equation $1 \quad \mathrm{MDL}=\mathrm{t}_{(\mathrm{n}-1,0.99)} \bullet \mathrm{s}$}

where $\mathrm{s}$ is the standard deviation of the replicate zero analyses, and $\mathrm{t}$ is the student's t-test value for a standard deviation estimate with n-1 degrees of freedom at a $99 \%$ confidence level. Measurement results below MDLs of the instrument were reported as measured and to the level of precision of the instrument, but flagged accordingly.

For continuous gas monitors, the MDL accounts for all sampling and analytical procedures and therefore represents a detection limit that can be applied to ambient concentrations. For gas monitors, MDLs were based on the response of the instruments to purified air. MDLs for filter-based or canister-based instruments were determined from field and laboratory blank tests. At PAQS, approximately $10 \%$ of all substrates (filters or canisters) handled were field or laboratory blanks. The field blank was a substrate that underwent all the preparation, transportation, storage, and analysis activities as and with the sample substrate. A laboratory blank was a substrate that underwent the preparation and analysis activities as and with the sample substrate. However, because the analytical standards used to evaluate field blank and laboratory blank substrates for filter-based or canisterbased measurements are prepared and used in the laboratory, the MDL is not an ambient MDL but instead an instrument MDL.

\subsection{Precision}

Precision is a measure of the repeatability of results or of the agreement among individual measurements of the same parameter under the same prescribed conditions. The number of replicate analyses needed to properly assess the precision of each instrument was independently determined by each PAQS investigator.

Precision of analytical instruments was evaluated by repeated analysis of independent traceable standards that were separate from the standards used for instrument calibration. Precision of continuous gas monitors was evaluated using purified air. Precision of semi-continuous aerosol instruments was evaluated, when possible, by using artificially generated analytes. When possible, precision of filter-based methods was assessed by running collocated samplers. For each series of replicate analyses, the precision was calculated using Equation 2, where s is the standard deviation between the replicate analyses and $\{\mathrm{x}\}$ is the mean of the replicate analyses.

Equation 2 Precision $(\%)=100[2 \mathrm{~s}] /\{\mathrm{x}\}$

\subsection{Accuracy}

Accuracy (bias) is the closeness of a measurement to a reference value, and reflects the systematic distortion of a measurement process. To the extent possible, accuracy was determined from replicate analyses of authentic, traceable standards that were not used in the calibration of the instrument. For each instrument tested, multiple challenge data points were collected. The accuracy of the instrument was determined by:

Equation $3 \quad$ Accuracy $(\%)=(100 *[S-\{x\}]) / S$ 
where $\mathrm{S}$ is the standard value of the authentic traceable standard and $\{\mathrm{x}\}$ is the mean of the instrument responses to the replicate analysis.

\subsection{Completeness}

Completeness of a measurement data set indicates the percentage of the scheduled sample collections or measurements that resulted in ambient observations that were valid and met the data quality objectives established in the QAPP. Completeness was calculated using Equation 4, where N represents the number of measurements.

Equation. 1 Completeness $(\%)=(\mathrm{N}$ valid measurements/total $\mathrm{N}$ measurements $) \bullet 100$

\subsection{Comparability}

Comparability refers to how confidently one data set can be compared with another. Ideally, two instruments that measure the same observable should be statistically comparable. The existence of several overlapping techniques will allow the intercomparison of existing measurement approaches and also the evaluation of new and emerging approaches. Table 2 presents a list of observables for which multiple measurement methods were used.

Table 2. Comparison of methods

\begin{tabular}{|c|c|c|}
\hline Observable & Methods that will be compared ${ }^{\text {a }}$ & Methods that will not be compared ${ }^{\text {a }}$ \\
\hline $\mathrm{PM}_{10}$ Mass & Dichot/Gravimetry v. MOUDI/Gravimetry & - \\
\hline $\mathrm{PM}_{2.5}$ Mass & $\begin{array}{l}\text { FRM/Gravimetry v. Dichot/Gravimetry, } \\
\text { MOUDI/Gravimetry, and TEOM with SES }\end{array}$ & - \\
\hline $\begin{array}{l}\mathrm{PM}_{2.5} \text { plus gas } \\
\text { Ammonium }\end{array}$ & Speciation sampler/IC v. Steam sampler/IC & RSMS-III $^{1,2}$ \\
\hline $\mathrm{PM}_{2.5}$ Nitrate & Speciation sampler/IC v. ICVC ${ }^{1}$, and PC BOSS ${ }^{2}$ & RSMS-III ${ }^{1,2}$ \\
\hline $\mathrm{PM}_{2.5}$ Sulfate & $\begin{array}{l}\text { Speciation sampler/IC v. ICVC }{ }^{1}, \mathrm{PC} \mathrm{BOSS}^{2} \text {, and } \\
\text { Steam sampler/IC }\end{array}$ & RSMS-III $^{1,2}$ \\
\hline $\mathrm{PM}_{2.5}$ Carbon & $\begin{array}{l}\text { TQQQ sampler/TOT v. Denuder sampler/TOT and } \\
\text { TOT carbon analyzer }{ }^{1}\end{array}$ & $\mathrm{ICVC}^{1,4}, \mathrm{RSMS}^{\mathrm{IIII}}{ }^{1,2}$ \\
\hline $\begin{array}{l}\mathrm{PM}_{2.5} \\
\text { Elements }\end{array}$ & - & $\begin{array}{l}\text { Speciation Sampler/ICPMS } 6{ }^{6} \text { v. LIBS }^{1,2,4} \text {, } \\
\text { RSMS-III }^{1,2}, \text { SEAS/GFAA }\end{array}$ \\
\hline $\begin{array}{l}\mathrm{PM}_{2.5} \text { Polar } \\
\text { Organics }\end{array}$ & - & $\begin{array}{l}\text { Detailed Speciation/GC-FID v. LPI/FTIR }{ }^{2} \text {, } \\
\text { RSMS-III }^{1,2}\end{array}$ \\
\hline Particle sizing & MOUDI/Gravimetry v. APS $^{3}$ and SMPS $^{3}$ & RSMS-III $^{1,2}$, Epiphaniometer ${ }^{4}$ \\
\hline VOCs & Canister/GC-FID v. On-line GC-FID/MS & - \\
\hline \multicolumn{3}{|c|}{$\begin{array}{l}\text { 1: State-of-the-art measurement method } \\
\text { 2: Measurement method that is not quantitative } \\
\text { 3: Measurements only collected during intensive study periods (July } 2001 \text { and possibly January 2002) } \\
\text { 4: Limited availability of measurements due to excessive instrument malfunction } \\
\text { 5: At overlapping region only } \\
\text { 6: Measurement analysis not completed at the time the QAFR was written. }\end{array}$} \\
\hline
\end{tabular}

In this report, comparisons between measurement methods were performed only for data that met the precision, accuracy and completeness data quality objectives. These select comparisons are presented in the figures that follow, as are the major axis regression statistics (assuming a linear relationship) used to gage comparability. Comparability was not determined for state-of-the-art measurement methods that were not quantitative; when only one method was used to measure a particular observable; and if there was limited overlap of the particular observable due to excessive malfunction of an instrument. More detailed comparisons and instrument evaluations have already been performed by several PAQS investigators (Cabada et al., 2003a; 2003c; Rees et al., 2003; Stanier et al., 2003a; Subramanian et al., 2003; Wittig et al., 2003b). 
Figure 1. Comparison of aerosol mass measurements (Cabada et al., 2003c): a) 24-hour Dichot $\mathrm{PM}_{10}$ mass v. 24-hour MOUDI PM PM $_{10}$ mass, b) 24-hour FRM PM 2.5 mass v. 24-hour average of 5-min TEOM $\mathrm{PM}_{2.5}$ mass, c) 24-hour FRM PM $\mathrm{PM}_{2.5}$ mass v. 24-hour Dichot $\mathrm{PM}_{2.5}$ mass, and d) 24-hour FRM PM 2.5 mass v. 24-hour MOUDI PM 2.5 mass. Also shown are the 1:1 lines (dashed lines).
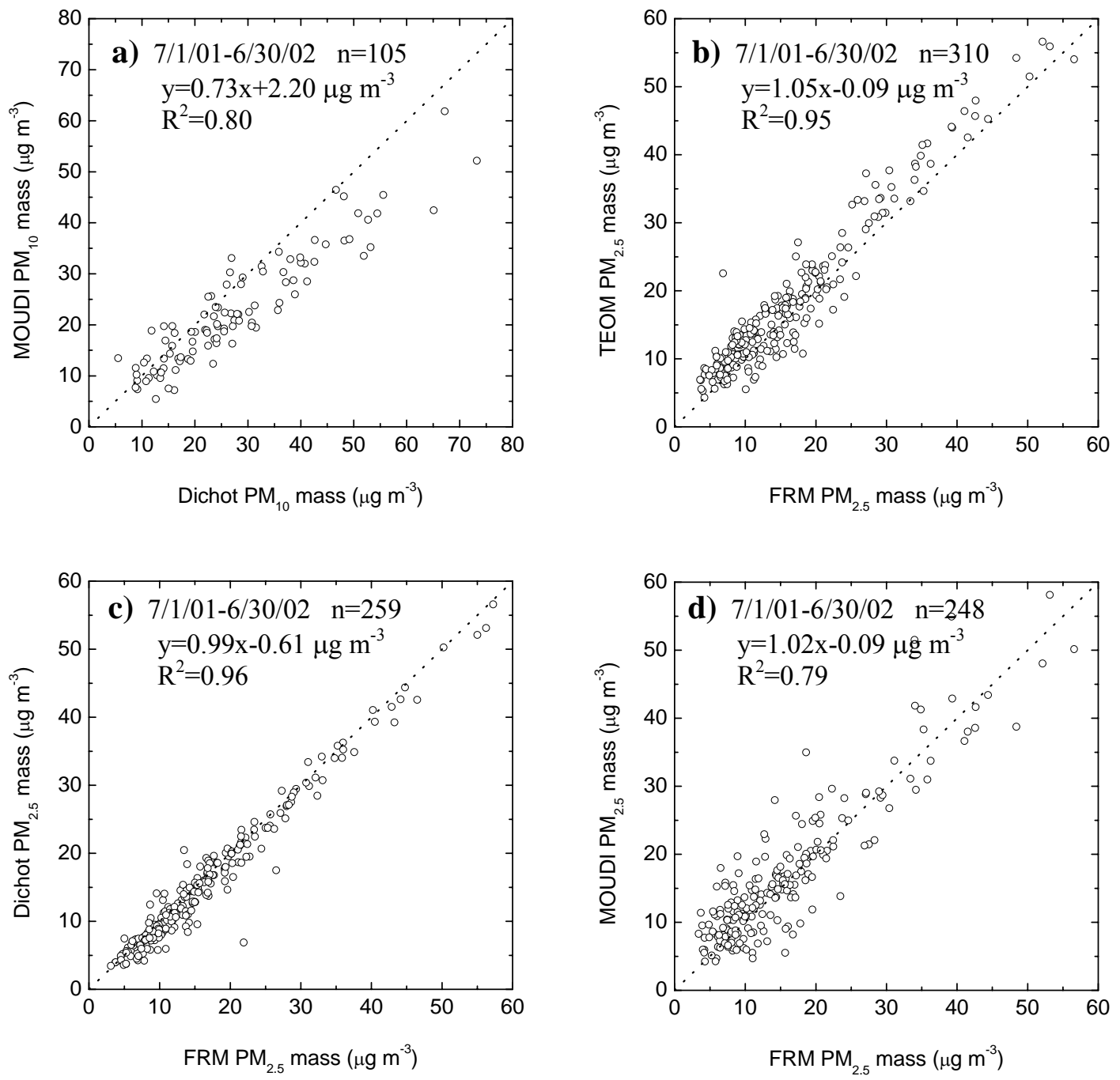
Figure 2. Comparison of $\mathrm{PM}_{2.5}$ plus gas ammonium measurements: a) 24-hour average of July 2001 4hour and 6-hour speciation sampler and 24-hour speciation sampler for remaining months v. 24-hour average of 1-hour or 2-hour steam sampler. Also shown is the 1:1 line (dashed line).

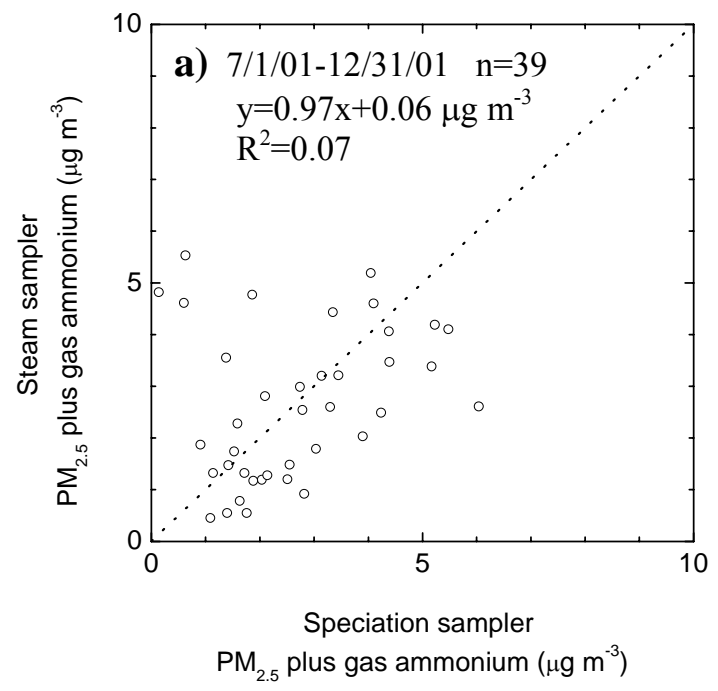

Figure 3. Comparison of $\mathrm{PM}_{2.5}$ nitrate measurements (Wittig et al., 2003a): a) 24-hour average of July 2001 4-hour and 6-hour speciation sampler and 24-hour speciation sampler for remaining months $\mathrm{V}$. 24-hour average of 1-hour R\&P 8400N and b) 24-hour average of July 2001 4-hour and 6-hour speciation sampler v. 24-hour PCBOSS. Also shown are the 1:1 lines (dashed lines).
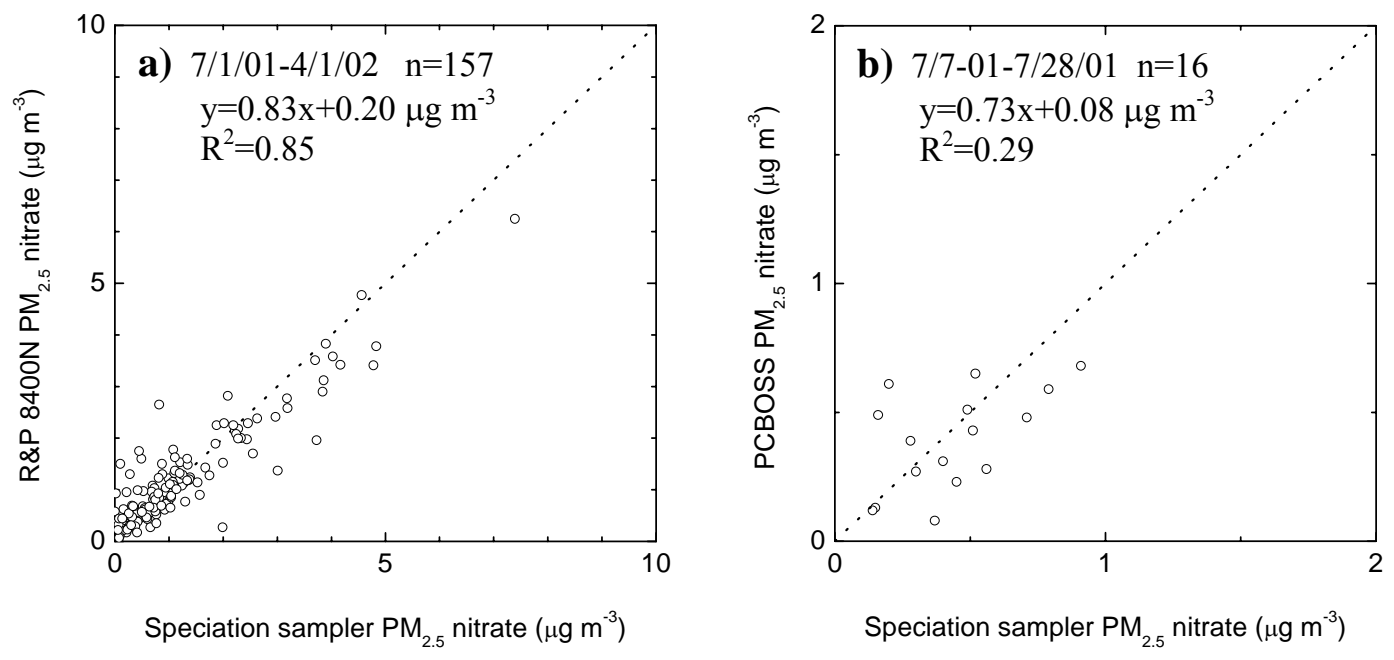
Figure 4. Comparison of $\mathrm{PM}_{2.5}$ sulfate measurements (Wittig et al., 2003a): a) 24-hour average of July 2001 4-hour and 6-hour speciation sampler and 24-hour speciation sampler for remaining months $\mathrm{v}$. 24-hour average of 1-hour or 2-hour steam sampler/IC, b) 24-hour average of July 2001 4-hour and 6hour speciation sampler and 24-hour speciation sampler for remaining months v. 24-hour average of 1-hour R\&P 8400N, and c) 24-hour average of July 2001 4-hour and 6-hour speciation sampler v. 24hour PCBOSS. Also shown are the 1:1 lines (dashed lines).
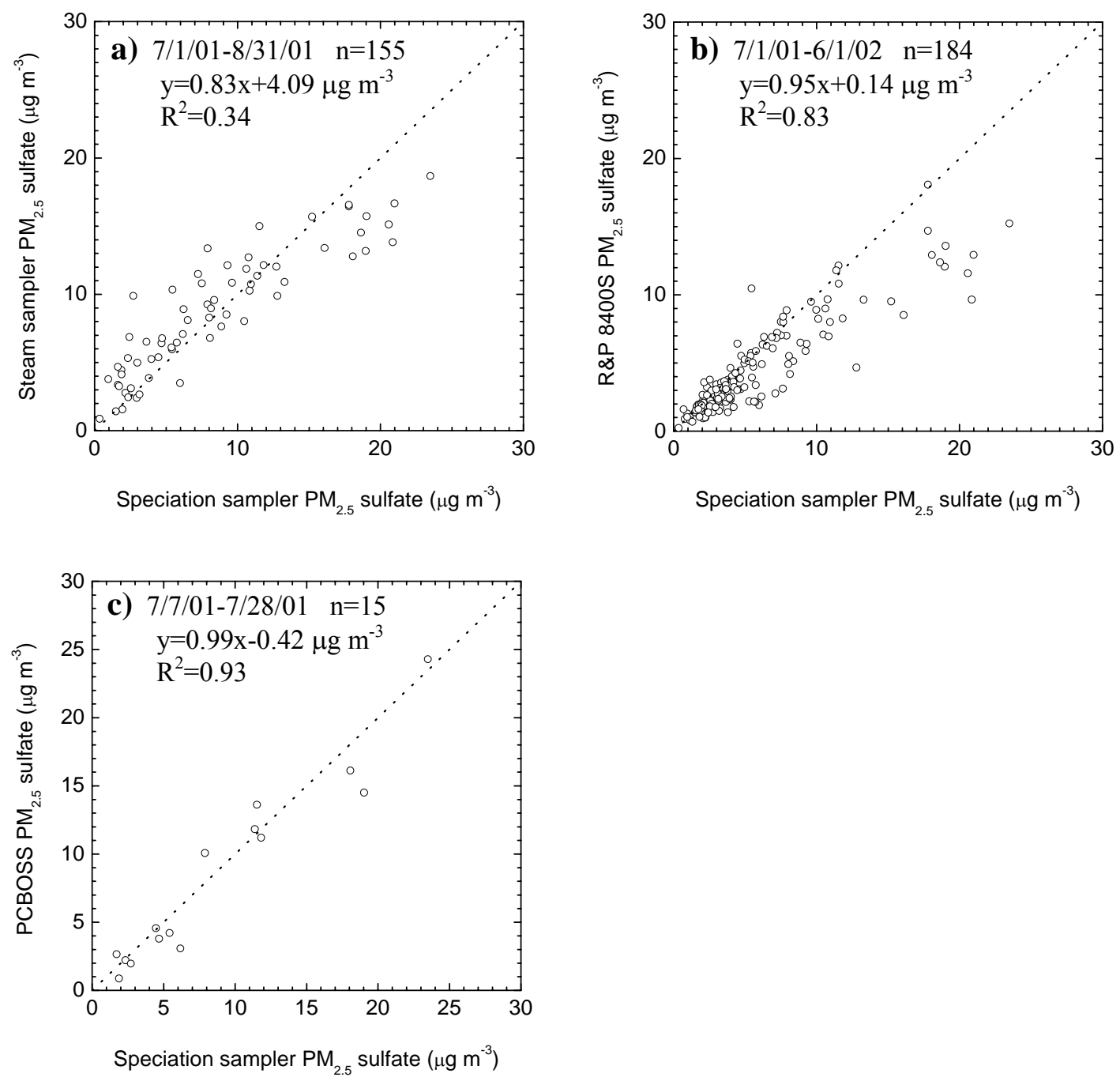
Figure 5. Comparison of $\mathrm{PM}_{2.5}$ organic carbon measurements: a) 24-hour TQQQ sampler v. 1-in-6 day 24-hour Denuder sampler, b) 24-hour TQQQ sampler v. 24-hour average of 2-hour to 4-hour TOT carbon analyzer. Also shown are the 1:1 lines (dashed lines).
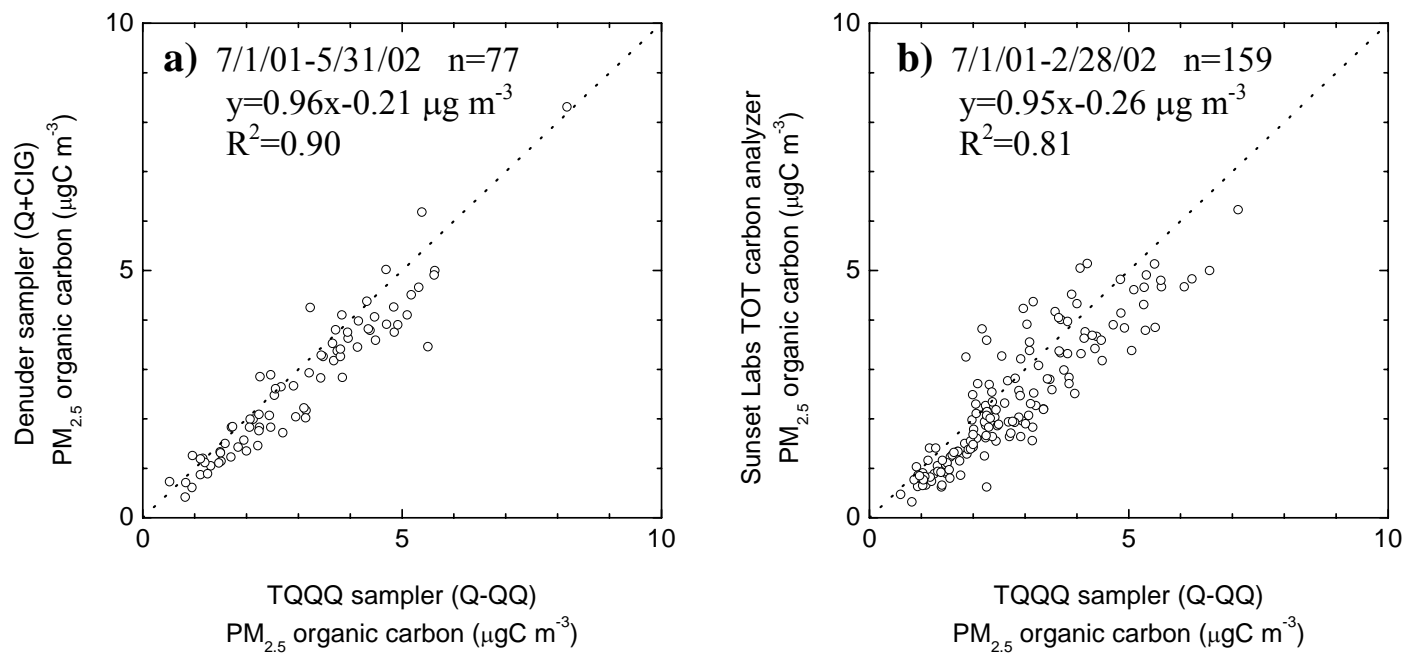

Figure 6. Comparison of $\mathrm{PM}_{2.5}$ elemental carbon measurements: a) 24-hour TQQQ sampler v. 24-hour Denuder sampler, b) 24-hour TQQQ sampler v. 24-hour average of 2-hour to 4-hour TOT carbon analyzer. Also shown are the 1:1 lines (dashed lines).
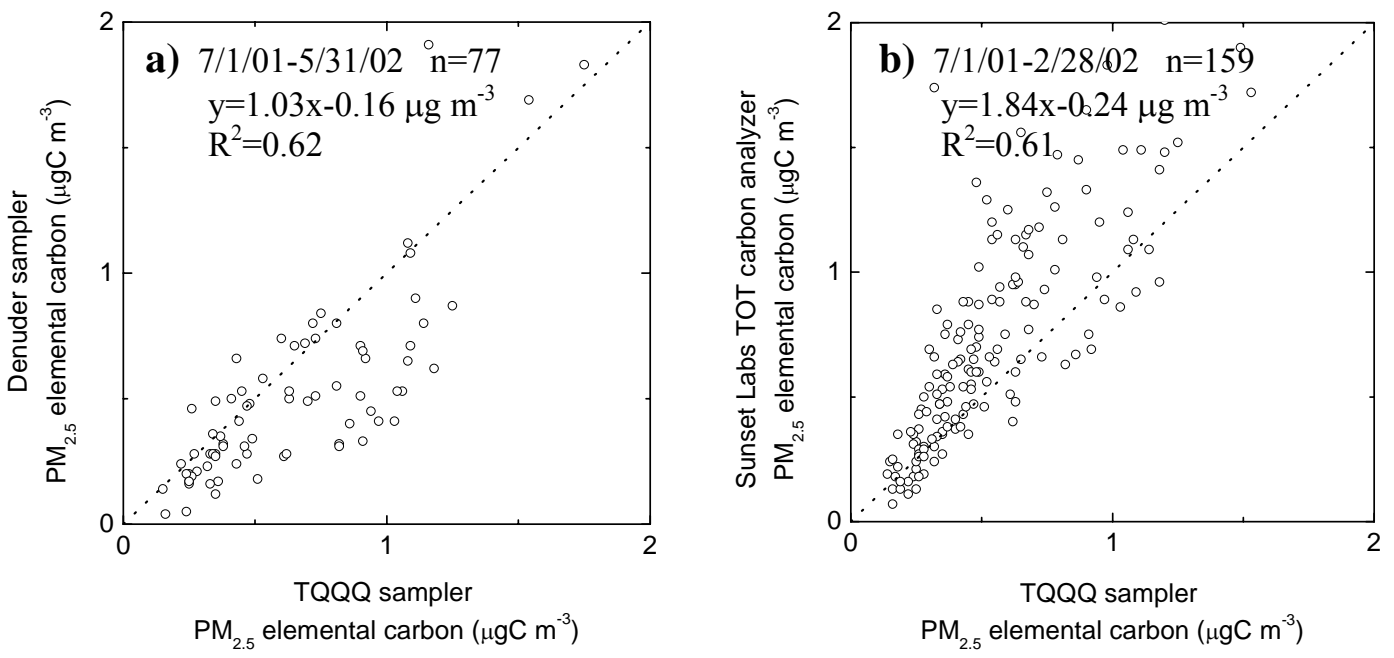
Figure 7. Comparison of the geometric mean of the aerosol diameter: 24-hour MOUDI sampler v. 24hour average of 5-minute SMPS measurements. Also shown is the 1:1 line (dashed line).

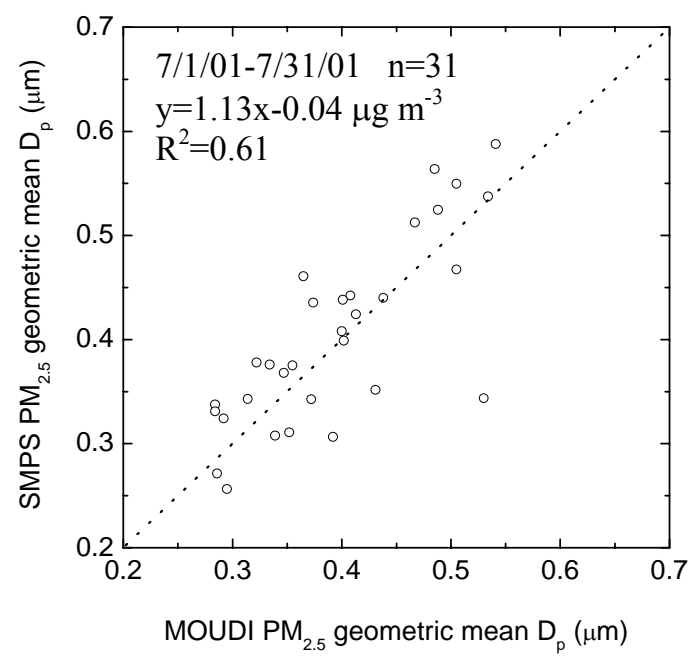

Figure 8. Comparison of gas-phase benzene measurements: 24-hour Canister sample with GC-FID analysis v. 24-hour average of 1-hour Online GC-MS measurements. Also shown is the 1:1 line (dashed line).

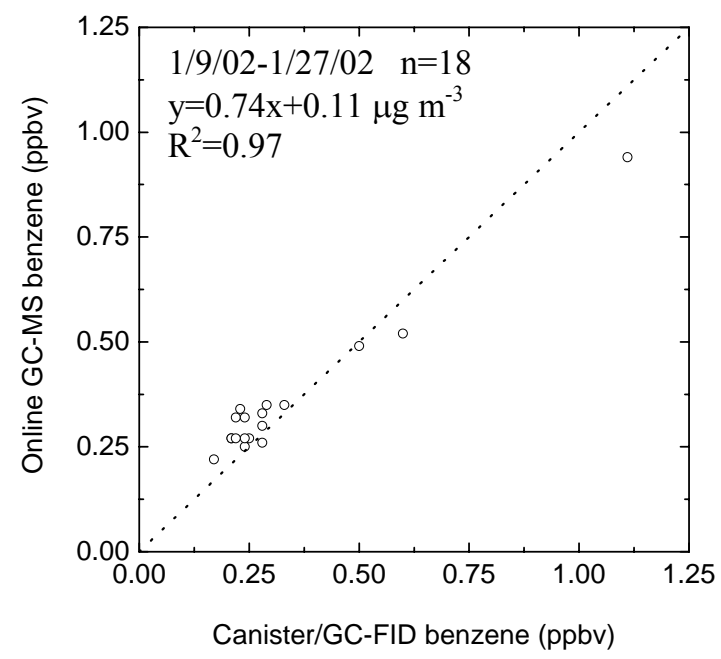

Overall, the select methods compare reasonably. In a few instances, best estimate data sets were produced to address the QA concerns of these methods ( $\mathrm{PM}_{2.5}$ sulfate, nitrate, and ammonium).

\subsection{Representativeness}

Representativeness expresses how closely a sample reflects the characteristics of the surrounding environment and can be quantified in terms of a spatial scale for monitoring. The main monitoring site is located in Schenley Park in the Oakland district of Pittsburgh. The site is on top of a grassy hill adjacent to the CMU campus, several hundred meters from the nearest heavily traveled street (Forbes Avenue), and fifty meters past the end of a dead end street on campus. There are no major sources within several hundred meters of the site. Schenley Park extends more than a kilometer 
to the south and west, the predominant upwind directions. The exposure of the surrounding environs represents both an 'urban' and 'neighborhood' scale for particle monitoring.

\subsection{Data quality objectives were not met during PAQs}

In a few instances, the data quality objectives were not met at PAQS. Typically, these instances were associated with newly developed instrumentation that did not perform as well as expected, and are summarized in Table 3. When possible, best estimate data sets were produced to address the QA concerns of these measurements ( $\mathrm{PM}_{2.5}$ sulfate, nitrate, and ammonium).

Table 3. Observables for which the DQI did not meet the DQO at PAQS.

\begin{tabular}{|c|c|c|}
\hline Observable & Method & Issue \\
\hline $\begin{array}{l}\text { Number, surface } \\
\text { area, and volume } \\
\text { distribution }\end{array}$ & TSI APS & $\begin{array}{l}\text { Data completeness - The instrument broke during fall } \\
\text { of } 2001 \text { and was not repaired and returned by the } \\
\text { manufacturer until March of } 2002 \text {. }\end{array}$ \\
\hline $\begin{array}{l}\mathrm{PM}_{10}, \mathrm{PM}_{2.5} \text {, and } \\
\mathrm{PM}_{\mathrm{x}} \text { mass }\end{array}$ & $\begin{array}{l}\text { FRM, Dichot, } \\
\text { and MOUDI } \\
\text { samplers/ } \\
\text { Gravimetry }\end{array}$ & $\begin{array}{l}\text { MDL - Table } 1 \text { shows the MDL DQO as } 2 \mu \mathrm{g} / \mathrm{m}^{3} \text { for } \\
\text { FRM, Dichot and MOUDI samplers. The DQOs for } \\
\text { these instruments were erroneously reported as } 0.2 \\
\mu \mathrm{g} / \mathrm{m}^{3} \text { in the QAPP. }\end{array}$ \\
\hline $\begin{array}{l}\mathrm{PM}_{2.5} \text { nitrate and } \\
\text { sulfate }\end{array}$ & $\begin{array}{l}\text { R\&P } 8400 \mathrm{~N} / \mathrm{S} \\
(\mathrm{ICVC})\end{array}$ & $\begin{array}{l}\text { Precision - The DQOs were overly optimistic, given } \\
\text { the fact that these instruments were newly } \\
\text { commercialized. In actuality, the instruments were less } \\
\text { precise than expected. However, a rigorous quality } \\
\text { control plan allowed these issues to be tracked over the } \\
\text { course of the study (Wittig et al., 2003b). }\end{array}$ \\
\hline $\mathrm{PM}_{2.5}$ sulfate & $\begin{array}{l}\text { R\&P 8400S } \\
(\mathrm{ICVC})\end{array}$ & $\begin{array}{l}\text { Data completeness - The instrument experienced more } \\
\text { frequent malfunctions than expected (typically strip } \\
\text { breakage) as well as a fatal error a month before the } \\
\text { end of the study. }\end{array}$ \\
\hline $\begin{array}{l}\mathrm{PM}_{10} \text { and } \mathrm{PM}_{2.5} \\
\text { elements }\end{array}$ & $\begin{array}{l}\text { Hi-Vol } \\
\text { sampler/ICPMS }\end{array}$ & $\begin{array}{l}\text { MDL, precision, accuracy, data completeness - } \\
\text { measurements were not finalized by the time this report } \\
\text { was compiled. }\end{array}$ \\
\hline $\begin{array}{l}\mathrm{PM}_{2.5} \text { water soluble } \\
\mathrm{NH}_{4}{ }^{+}\end{array}$ & $\begin{array}{l}\text { CMU Steam } \\
\text { sampler/OAD }\end{array}$ & $\begin{array}{l}\text { MDL, precision, accuracy, data completeness - } \\
\text { measurements were not finalized by the time this report } \\
\text { was compiled. }\end{array}$ \\
\hline $\begin{array}{l}\mathrm{PM}_{10} \text { and } \mathrm{PM}_{2.5} \\
\text { inorganic ions }\end{array}$ & $\begin{array}{l}\text { CMU Speciation } \\
\text { sampler/IC }\end{array}$ & $\begin{array}{l}\text { MDL, precision, accuracy, data completeness - } \mathrm{PM}_{10} \\
\text { measurements were not finalized by the time this report } \\
\text { was compiled. } \\
\text { Precision - } \mathrm{NH}_{3} \text { gas was present in extremely low } \\
\text { concentrations so the DQO was not achieved. } \\
\text { Completeness - } \mathrm{PM}_{2.5} \text { sampler malfunction as well as } \\
\text { loss or destruction of samples prior to analysis were } \\
\text { responsible for recovery levels below DQOs for all } \\
\text { species, except } \mathrm{SO}_{4} \text { which met the DQO. } \\
\text { The data have not yet been finalized, and thus DQIs for } \\
\mathrm{PM}_{10} \text { are not included in Table } 1 \text {. }\end{array}$ \\
\hline
\end{tabular}




\begin{tabular}{|l|l|l|}
\hline Observable & Method & Issue \\
\hline $\begin{array}{l}\text { Size segregated } \\
\text { chemistry: } \\
\text { inorganic ions, } \\
\text { EC/OC }\end{array}$ & MOUDI/IC,TOT & $\begin{array}{l}\text { MDL - A high limit of detection was experienced for } \\
\text { calcium; all other inorganic ions achieved the DQO. } \\
\text { Accuracy - No accuracy was determined for the } \\
\text { inorganic analyses. } \\
\text { Completeness - Instrument problems for the EC/OC } \\
\text { analyses resulted in 66\% completeness, just below the } \\
70 \% \text { target DQO. }\end{array}$ \\
\hline PM2.5 total carbon & $\begin{array}{l}\text { ADI Carbon } \\
\text { analyzer (ICVC) }\end{array}$ & $\begin{array}{l}\text { MDL, precision, accuracy, data completeness - Data } \\
\text { will not be submitted due to instrument difficulties. }\end{array}$ \\
\hline $\begin{array}{l}\text { Light hydrocarbons } \\
\text { (C1-C12) }\end{array}$ & Canister/GC-FID & $\begin{array}{l}\text { MDL, precision, accuracy, data completeness - } \\
\text { Measurements for ESP02 Intensive (sampling once } \\
\text { daily) not yet finalized by the time this report was } \\
\text { compiled. }\end{array}$ \\
\hline Meteorology & All methods & $\begin{array}{l}\text { MDL, precision, accuracy - The standard operating } \\
\text { procedures (and manufacturer recommended } \\
\text { procedures) did not allow these DQIs to be calculated. }\end{array}$ \\
\hline
\end{tabular}

\section{Conclusions}

- A majority of the data quality indicators showed the PAQS central site instruments performed as expected or better.

- In a few instances, PAQS central site instruments could not be evaluated because the data were not finalized by the time this report was produced $\left(\mathrm{PM}_{2.5}\right.$ water soluble $\mathrm{NH}_{4}{ }^{+}$using the CMU Steam sampler/IC and $\mathrm{PM}_{10}$ and $\mathrm{PM}_{2.5}$ elements using the Hi-Vol sampler/ICPMS).

- Most data quality objectives that were not met were associated with newly developed instrumentation that did not perform as well as expected. When possible, best estimate data sets were produced to address the QA concerns of these measurements $\left(\mathrm{PM}_{2.5}\right.$ sulfate, nitrate, and ammonium).

\section{References}

Cabada, J. C., Khlystov, A., Wittig, B., Pandis, S. N., 2003a. Fine particle light scattering reconstruction and measurements at PAQS. Submitted to Atmospheric Environment.

Cabada, J. C., Pandis, S. N., Subramanian, R., Robinson, A. L., Polidori, A., Turpin, B., $2003 b$. Estimating the secondary organic aerosol contribution to $\mathrm{PM}_{2.5}$ using the $\mathrm{EC}$ tracer method. Submitted to Atmospheric Environment.

Cabada, J. C., Takahama, S., Khlystov, A., Pandis, S. N., Rees, S., Davidson, C. I., Robinson, A. L., 2003c. Mass size distributions and size resolved chemical composition of fine particulate matter at the Pittsburgh Supersite. Submitted to Atmospheric Environment.

Khlystov, A., Stanier, C., Pandis, S. N., 2003. An algorithm for combining electrical mobility and aerodynamic size distributions data when measuring ambient aerosol. Submitted to Aerosol Science and Technology.

Khlystov, A., Wittig, B., Davidson, C., 2001. Quality Assurance Project Plan for the Pittsburgh Air Quality Study. Report prepared for the U. S. E. P. A., Research Triangle Park, NC.

Rees, S. L., Robinson, A. L., Khylstov, A., Stanier, C. O., Subramanian, R., Cabada, J. C., Wittig, B., Takahama, S., Davidson, C., Pandis, S., 2003. Mass balance closure and the Federal Reference Method for $\mathrm{PM}_{2.5}$ in Pittsburgh, PA. Submitted to Atmospheric Environment. 
Stanier, C. O., Khlystov, A., Chan, R., Mandiro, M., Pandis, S. N., 2003a. A method for the in-situ measurement of fine aerosol water content of ambient aerosols: The Dry-Ambient Aerosol Size Spectrometer (DAASS). Accepted by Aerosol Science and Technology.

Stanier, C. O., Khlystov, A., Pandis, S. N., 2003b. Nucleation events during the Pittsburgh Air Quality Study: Description and relation to key meteorological, gas-phase, and aerosol parameters. Accepted by Aerosol Science and Technology.

Subramanian, R., Khlystov, A., Y., Cabada, J. C., Robinson, A. L., 2003. Positive and negative artifacts in particulate organic carbon measurements with denuded and undenuded sampler configurations. Submitted to Aerosol Science and Technology.

Takahama, S., Khlystov, A., Wittig, B., Davidson, C., Pandis, S. N., 2003. Partitioning of nitrate during the Pittsburgh Air Quality Study and implications of time-averaging. Submitted to Atmospheric Environment.

Wittig, B., Anderson, N., Khlystov, A. Y., Pandis, S. N., Davidson, C., Robinson, A. L., $2003 a$. Pittsburgh Air Quality Study overview and preliminary scientific findings. Submitted to Atmospheric Environment.

Wittig, B., Takahama, S., Khlystov, A., Pandis, S. N., Hering, S., Kirby, B., Davidson, C., 2003 b. Semi-continuous $\mathrm{PM}_{2.5}$ inorganic composition measurements during the Pittsburgh Air Quality Study. Submitted to Atmospheric Environment. 
Appendix B

\section{Published Papers}

B - 1 


\title{
Investigation of the relationship between chemical composition and size distribution of airborne particles by partial least squares and positive matrix factorization
}

\author{
Liming Zhou ${ }^{1}$ and Philip K. Hopke \\ Center for Air Resources Engineering and Science and Department of Chemical Engineering, Clarkson University, Potsdam, \\ New York, USA \\ Charles O. Stanier ${ }^{2}$ and Spyros N. Pandis \\ Department of Chemical Engineering, Carnegie Mellon University, Pittsburgh, Pennsylvania, USA
}

John M. Ondov and J. Patrick Pancras

Department of Chemistry and Biochemistry, University of Maryland, College Park, Maryland, USA

Received 20 May 2004; revised 17 July 2004; accepted 11 October 2004; published 9 March 2005.

[1] Two multivariate data analysis methods, partial least square (PLS) and positive matrix factorization (PMF), were used to analyze aerosol size distribution data and composition data. The relationships between the size distribution data and composition data were investigated by PLS. Three latent variables summarized chemical composition data and most variations in size distribution data especially for large particles and proved the existence of the linearity between the two data sets. The three latent variables were associated with traffic and local combustion sources, secondary aerosol, and coal-fired power plants. The size distribution, particle composition, and gas composition data were combined and analyzed by PMF. Source information was obtained for each source using size distribution and chemical composition simultaneously. Eleven sources were identified: secondary nitrate 1 and 2, remote traffic, secondary sulfate, lead, diesel traffic, coal-fired power plant, steel mill, nucleation, local traffic, and coke plant.

Citation: Zhou, L., P. K. Hopke, C. O. Stanier, S. N. Pandis, J. M. Ondov, and J. P. Pancras (2005), Investigation of the relationship between chemical composition and size distribution of airborne particles by partial least squares and positive matrix factorization, J. Geophys. Res., 110, D07S18, doi:10.1029/2004JD005050.

\section{Introduction}

[2] Multivariate receptor models are widely used in source apportionment of airborne particles [Henry, 1997, 2002; Hopke, 2003]. The measured chemical composition data from the samples collected at the receptor site form a matrix and this matrix can then be analyzed by UNMIX [Henry, 2003], PMF [Paatero, 1997] or other techniques to obtain two matrices representing source contribution and source profile, respectively. Recently, efforts have been made to use the methods to analyze size distribution data to identify sources [Ruuskanen et al., 2001; Wahlin et al., 2001; Kim et al., 2004; Zhou et al., 2004a, 2005].

[3] Even over a short distance (or transit time), there can be substantial changes in the size distributions of the particles emitted [Zhu et al., 2002a, 2002b, 2004]. However, for the same location/transit time, the size distribution is

\footnotetext{
${ }^{1}$ Now at Providence Engineering and Environmental Group LLC, Baton Rouge, Louisiana, USA.

${ }^{2}$ Now at Department of Chemical and Biochemical Engineering, University of Iowa, Iowa City, Iowa, USA.

Copyright 2005 by the American Geophysical Union. 0148-0227/05/2004JD005050\$09.00
}

very similar. If the size distribution coming from a source does not vary much with time, then the number concentration series measured at the receptor site have a linear relationship with the number contribution from all sources and also with their mass contributions. A previous application of multivariate receptor model with size distribution data [Zhou et al., 2004a] has indicated that the number contribution of a source can be converted to its volume (mass) contribution by multiplying a constant determined by its size distribution profile.

[4] If there are linear relationships between the number concentrations and mass concentrations, it will be useful to combine the size distribution data and chemical composition data into a combined multivariate analysis. The source characteristics in both size distributions and chemical compositions may be obtained simultaneously and a better understanding of the source-receptor relationship will be provided.

[5] In this study, a small data set that includes both size distribution and composition data from the Pittsburgh Air Quality Study (PAQS) was analyzed by partial least squares (PLS) and positive matrix factorization (PMF). PLS is used to investigate the interrelationships between the number concentrations of all size intervals and the mass concen- 
Table 1. Missing Value Number (MN) of All Sizes and Species ${ }^{\mathrm{a}}$

\begin{tabular}{|c|c|c|c|c|c|c|c|c|c|}
\hline $\mathrm{MN}$ & Species & $\mathrm{MN}$ & Species & $\mathrm{MN}$ & Size, $\mu \mathrm{m}$ & $\mathrm{MN}$ & Size, $\mu \mathrm{m}$ & $\mathrm{MN}$ & Size, $\mu \mathrm{m}$ \\
\hline 9 & $\mathrm{Se}$ & 9 & sulfate & 24 & 0.168 & 29 & 0.0233 & 18 & 0.0032 \\
\hline 9 & $\mathrm{Zn}$ & 0 & nitrate & 24 & 0.202 & 29 & 0.0279 & 18 & 0.0039 \\
\hline 4 & $\mathrm{O}_{3}$ & 9 & $\mathrm{Al}$ & 24 & 0.242 & 29 & 0.0334 & 18 & 0.0046 \\
\hline 2 & $\mathrm{NO}$ & 61 & As & 24 & 0.289 & 29 & 0.0399 & 18 & 0.0055 \\
\hline 2 & $\mathrm{NO}_{\mathrm{x}}$ & 9 & $\mathrm{Cd}$ & 24 & 0.3461 & 27 & 0.0478 & 18 & 0.0066 \\
\hline 52 & $\mathrm{SO}_{2}$ & 9 & $\mathrm{Cr}$ & 24 & 0.414 & 26 & 0.0573 & 19 & 0.0079 \\
\hline 1 & $\mathrm{CO}$ & 9 & $\mathrm{Cu}$ & 24 & 0.496 & 24 & 0.068 & 20 & 0.0095 \\
\hline & & 9 & $\mathrm{Mn}$ & 8 & 0.898 & 32 & 0.098 & 25 & 0.0136 \\
\hline & & 9 & $\mathrm{Ni}$ & 8 & 1.286 & 28 & 0.118 & 25 & 0.0163 \\
\hline & & 9 & $\mathrm{~Pb}$ & 8 & 1.843 & 25 & 0.141 & 29 & 0.0194 \\
\hline
\end{tabular}

${ }^{\mathrm{a}}$ The total sample number is 240 .

trations of all chemical species. Only if the PLS analysis can find linear relationships between the two data sets, can the two types of data can reasonably be combined and analyzed with a two-way receptor model. The results of the PMF analysis will be compared with the results in the work of Zhou et al. [2004b].

\section{Data}

[6] All the data used in this study were measured at Pittsburgh Supersite (latitude 40.4395, longitude -79.9405) on 16, 17, 18, 23 and 24 July 2001. The Pittsburgh Supersite was located in a park, around 6 miles to the east of the city center. The interstate highway, I376, extending from west to east, is around 1 to $2 \mathrm{~km}$ to the south of the site. There are secondary streets and minor roads rather close $(<1 \mathrm{~km})$ to the site. These days were chosen during the July 2001 intensive since there were complete and simultaneous measurements of both particle size distributions and chemical compositions only on these days. In particular, there was measurement of elemental species with high temporal resolution. Although these days may not represent the full month, the results will give us insights into the relationship between the size distributions and compositions of the aerosol in Pittsburgh area, and will also be useful in more completely understanding the prior size distribution analyses [Zhou et al., 2004a, 2005]. The size distribution data were obtained from two scanning mobility particle spectrometers (SMPS) and an aerodynamic particle sampler (APS) with 15 min resolution. Above $583 \mathrm{~nm}$, the data used in this study represent electrical mobility diameter inferred from their aerodynamic mobility and estimated density [Khlystov et al., 2004]. The samples were collected at $25 \%$ relative humidity and "dry" particle distributions were obtained [Stanier et al., 2004].

[7] The original size distribution data include 165 logarithmically even-spaced intervals from $0.003 \mu \mathrm{m}$ to $2.5 \mu \mathrm{m}$. Every five consecutive size bins were combined into one and 33 new size intervals were produced [Zhou et al., 2004a]. The 15 min number concentrations were averaged to $30 \mathrm{~min}$ and 240 samples were produced. The detailed description of the measurement of size distributions at Pittsburgh Supersite can be found elsewhere [Stanier et al., 2004]. On 24 July there was a regional nucleation event with particle growth phenomenon and the number concentration data of that day were processed by the method introduced by Zhou et al. [2005].

[8] The composition data of $\mathrm{PM}_{2.5}$, including both particle phase and gas phase, are the same as was used before in a multi time factor analysis [Zhou et al., 2004b] except that all species with sampling period longer than $30 \mathrm{~min}$, such as organic carbon/elemental carbon (OC/EC), were excluded in this study and all the concentrations used in this work are $30 \mathrm{~min}$ average. The missing values were replaced by the regressed values obtained in our previous studies [Zhou et al., 2004b, 2005]. The aerosol composition data set includes sulfate and nitrate data obtained by continuous instruments of Aerosol Dynamics Inc. (ADI) [Stolzenburg and Hering, 2000] and metal species measured by the Semicontinuous Elements in Aerosol System (SEAS) [Kidwell and Ondov, 2001]. The complete description of all the measurement techniques can be found in the work of Wittig et al. [2003, 2004]. Table 1 summarizes the sizes and species that have been used as well as the number of missing values.

\section{PLS}

\subsection{Method}

[9] PLS is a basic tool of chemometrics for analyzing data with strongly collinear, noisy, and numerous $\mathrm{X}$ variables and simultaneously multiple response variables [Wold et al., 2001]. For this analysis, we use $X$ to stand for composition data and $Y$ for size distribution data, where $X \in R^{m \times n}$ and $Y \in R^{m \times p}$ with $m$ being the number of samples, $n$ is the number of chemical species and $p$ is the number of size intervals. The data in $X$ and $Y$ have been standardized from their original values so that each column vector in both matrices has a mean of 0 and a variance of 1 . The model equations are as following:

$$
\begin{aligned}
& X=T P^{\prime}+E \\
& Y=U C^{\prime}+D \\
& Y=T C^{\prime}+H,
\end{aligned}
$$

where $T$ and $U$ are score matrices, $P$ and $C$ are loading matrices, and $E, D$ and $H$ are residual matrices. In equations (1) and (2), $T$ and $U$ summarize the data in the $X$ and $Y$ matrices, respectively. Each of the column vectors in $T, t_{i}$, is called a latent variable (LV) that can be thought to be caused by a source or a source group, and so does the column vector in $U, u_{i}$. If $T$ and $U$ are very close, then $T$ can also be used to explain $Y$ and even to predict $Y$ as indicated by equation (3). The above model is solved by the nonlinear iterative partial least squares (NIPALS) 
Table 2. Correlations of Each Pair of Latent Variables $u$ and $v$

\begin{tabular}{cc} 
Correlation & LV \\
\hline Coefficient & 1 \\
0.80 & 2 \\
0.85 & 3 \\
0.71 & 4 \\
0.60 & 5 \\
0.51 & 6 \\
0.51 & 7 \\
0.37 & 8 \\
0.38 & 9 \\
0.42 & 10 \\
0.27 & 11 \\
0.36 & 12 \\
0.34 &
\end{tabular}

algorithm described by Wold et al. [2001]. Introductions to PLS are given in the work of Manne [1987] and Jong [1993]. An intuitive understanding of PLS but not a strict mathematical description can be provided as follows. The latent variables $t$ and $u$ in PLS analysis try to reproduce the variance in $X$ and $Y$, respectively, and at the same time, each pair of $t$ and $u$ try to maximize their similarity (covariance) to each other. The similarities between $t$ and $u$ indicate similar latent structures in $X$ and $Y$. For this specific study, similar $t$ and $u$ suggest they are both controlled by the same source or source group.

\subsection{Results and Discussion}

[10] Table 2 shows the correlation coefficients for each pair of latent variables (LV), $t_{i}$ and $u_{i}$. After the first three latent variables, the correlations become poor, suggesting that there is no further relationships between the residual matrices. We define the two variables $R x$ and $R y$ :

$$
\begin{aligned}
& R x=1-\operatorname{var}(E) / \operatorname{var}(X) \\
& R y=1-\operatorname{var}(H) / \operatorname{var}(Y),
\end{aligned}
$$

where var means variance. These two variables describe how much of the variance has been explained by the latent variables. As indicated in Table 3, when using three LVs, most of the variance in the $X$ matrix has been explained, but much less of the variance in the $Y$ matrix has been explained. This situation may suggest that the linear relationships amongst the chemical species are better than those amongst the sizes so that the size distribution data have larger residuals. Another reason is that number concentrations of all sizes were used in $Y$ matrix, but not all chemical species were included in $X$ matrix. Since some important species were not included in $X$ matrix, such as OC/EC, some number concentrations in $Y$ matrix will not be explained without those chemical species that characterize them. This is also one reason that PLS can identify less sources than PMF. Most of the variance of the small particles in the $Y$ matrix is not explained since the small particles have little mass contribution. Thus the chemical species reflect the mass concentrations rather than the number concentrations. If they have no unique marker species, they only produce small variations in $X$ matrix and hence are not summarized by the latent variables. This phenomenon also supports our prior results showing very weak correlations between number concentration and mass concentration for sources dominated by smallest sizes and largest number concentration [Zhou et al., 2004a, 2005].

[11] Figure 1 shows the loadings of the first LV (the first column vectors of $P$ and $C$ ). When the correlation coefficient between the LV score and the concentration series of a size interval or a species is over 0.7 , a black bar is used to denote that value. The first LV (LV1) explains most of the variations of nitrate, $\mathrm{NO}, \mathrm{NO}_{\mathrm{x}}$, and $\mathrm{CO}$ as well as several metal elements indicating emissions from traffic and other point combustion sources like the coke plant in the south. In our previous study [Zhou et al., 2004b], As, Cd and Mn are associated with point industrial sources such as metal working, and $\mathrm{Zn}$ was thought to be from traffic in our previous multi time analysis [Zhou et al., 2004b]. The size range of the first LV is wide, from $10 \mathrm{~nm}$ up to $200 \mathrm{~nm}$, and this size range is also found to be related to traffic and other point sources by the analyses with only size distribution data [Zhou et al., 2004a, 2005]. The high loadings between 1 to $2 \mu \mathrm{m}$ are consistent with the volume size distribution profiles of the traffic and combustion sources [Zhou et al., 2004a].

[12] The second LV (LV2) is mostly associated with sulfate and the size range 0.3 to $0.8 \mu \mathrm{m}$ as indicated in Figure 2. These are particles from distant sources, converted from the precursor $\mathrm{SO}_{2}$ via photochemical reactions during the transport [Zhou et al., 2004a, 2005].

[13] The third LV (LV3) also explains some sulfate but more $\mathrm{SO}_{2}$, as shown in Figure 3 and Table 4. The coal-fired power plants within $100 \mathrm{~km}$ from the receptor site are the probable sources. Because of the short distance, most of the $\mathrm{SO}_{2}$ cannot be converted during the transport. The trimodal distribution implied by the size loadings are most likely caused by the conversions when the plume traveled from the source to the receptor. The newly formed particles are small while the aged ones are large. Since the growth of the particles is susceptible to meteorological and other condi-

\begin{tabular}{|c|c|c|c|c|c|c|c|c|c|}
\hline$R x$ & Species & $R x$ & Species & Ry & Size, $\mu \mathrm{m}$ & Ry & Size, $\mu \mathrm{m}$ & Ry & Size, $\mu \mathrm{m}$ \\
\hline 0.45 & $\mathrm{Se}$ & 0.84 & sulfate & 0.62 & 0.168 & 0.26 & 0.0233 & 0.14 & 0.0032 \\
\hline 0.70 & $\mathrm{Zn}$ & 0.81 & nitrate & 0.63 & 0.202 & 0.28 & 0.0279 & 0.23 & 0.0039 \\
\hline 0.71 & $\mathrm{O}_{3}$ & 0.13 & $\mathrm{Al}$ & 0.67 & 0.242 & 0.24 & 0.0334 & 0.20 & 0.0046 \\
\hline 0.76 & $\mathrm{NO}$ & 0.59 & As & 0.75 & 0.289 & 0.18 & 0.0399 & 0.14 & 0.0055 \\
\hline 0.89 & $\mathrm{NO}_{\mathrm{x}}$ & 0.71 & $\mathrm{Cd}$ & 0.69 & 0.3461 & 0.21 & 0.0478 & 0.04 & 0.0066 \\
\hline 0.55 & $\mathrm{SO}_{2}$ & 0.44 & $\mathrm{Cr}$ & 0.63 & 0.414 & 0.22 & 0.0573 & 0.07 & 0.0079 \\
\hline \multirow[t]{5}{*}{0.71} & $\mathrm{CO}$ & 0.63 & $\mathrm{Cu}$ & 0.61 & 0.496 & 0.40 & 0.068 & 0.14 & 0.0095 \\
\hline & & 0.34 & $\mathrm{Fe}$ & 0.58 & 0.626 & 0.30 & 0.082 & 0.21 & 0.0113 \\
\hline & & 0.74 & $\mathrm{Mn}$ & 0.49 & 0.898 & 0.46 & 0.098 & 0.31 & 0.0136 \\
\hline & & 0.15 & $\mathrm{Ni}$ & 0.64 & 1.286 & 0.39 & 0.118 & 0.29 & 0.0163 \\
\hline & & 0.46 & $\mathrm{~Pb}$ & 0.77 & 1.843 & 0.58 & 0.141 & 0.31 & 0.0194 \\
\hline
\end{tabular}

Table 3. $R x$ and $R y$ of All Sizes and Species 


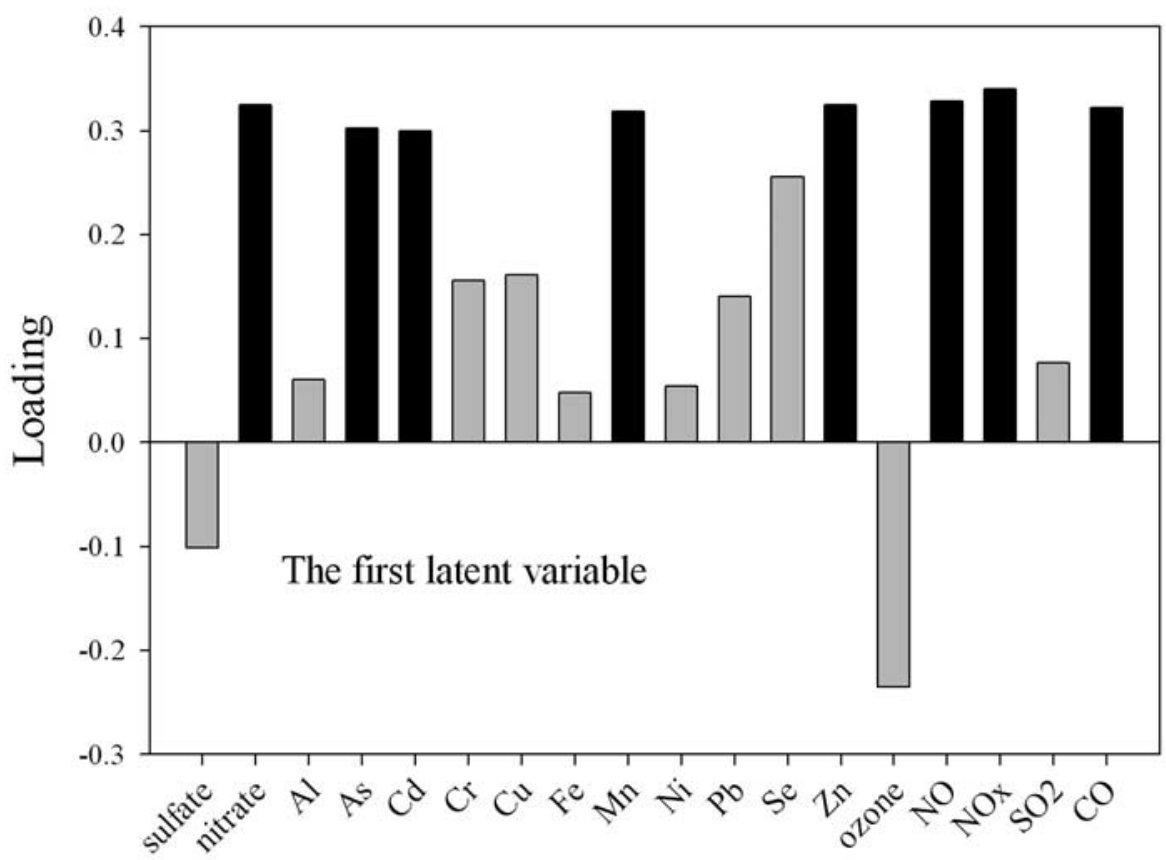

Species

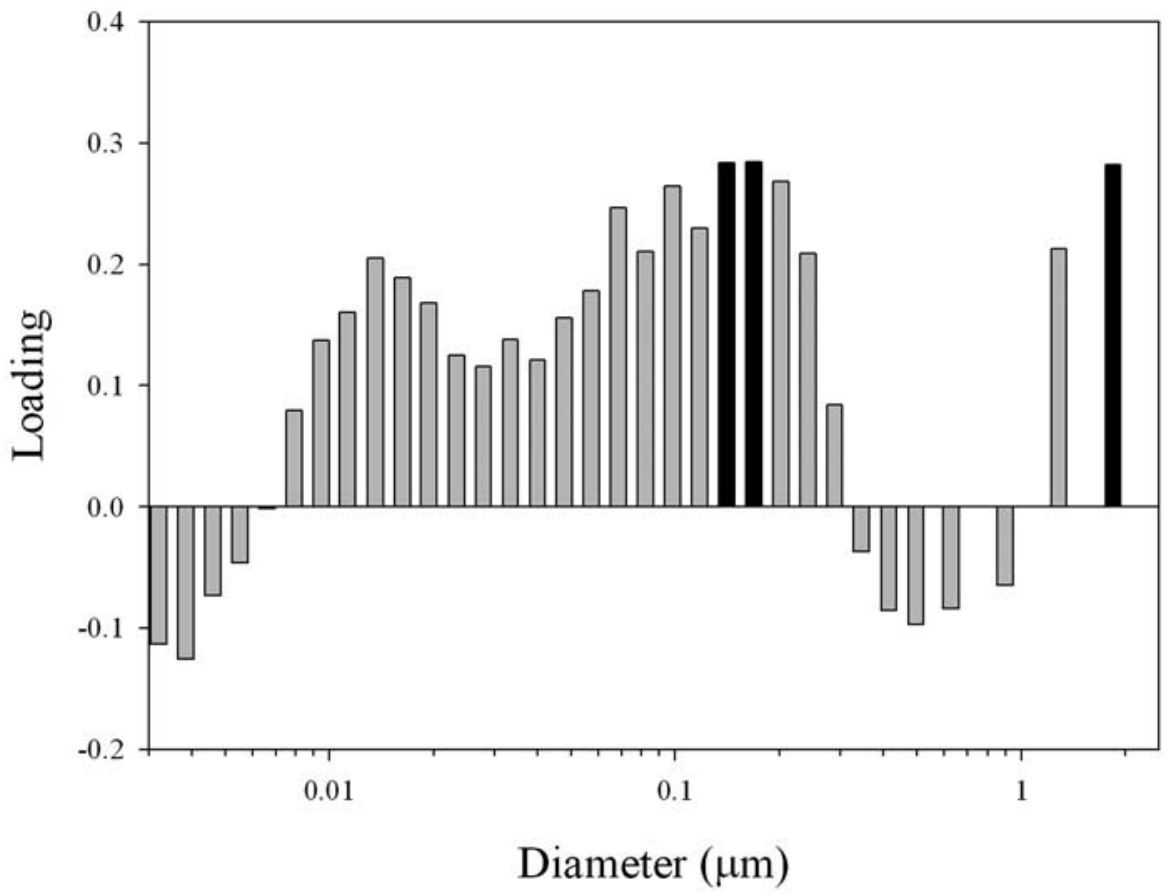

Figure 1. Loadings of the first LV for (top) chemical species and (bottom) sizes.

tions, the linearity between the number concentration and mass concentration is worse than for the first two LVs.

4. PMF

4.1. Method

[14] A two way receptor model was solved by PMF, an explicit least squares regression tool developed by Paatero [1997]. The model equation is:

$$
X=G F+E,
$$

or in the elemental form,

$$
x_{i j}=\sum_{k=1}^{p} g_{i k} f_{k j}+e_{i j},
$$




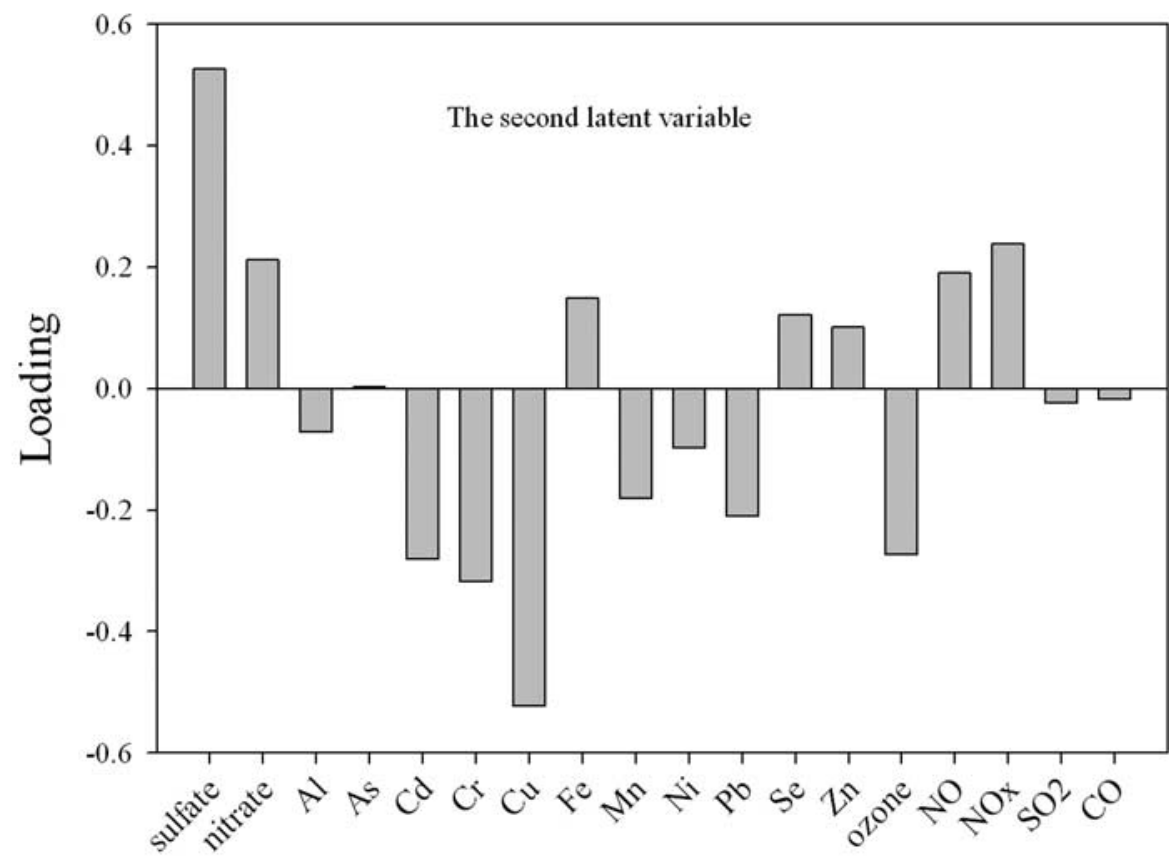

Species

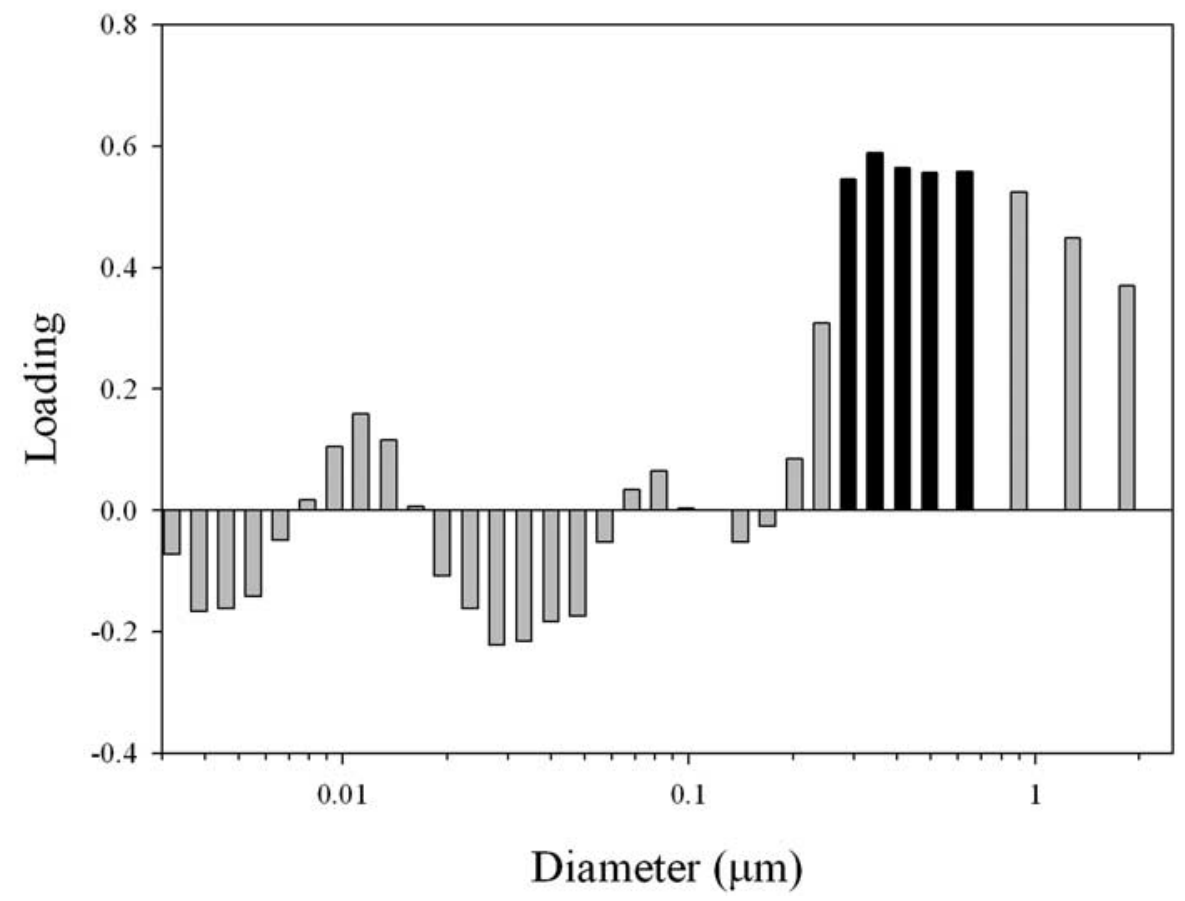

Figure 2. Loadings of the second LV for (top) chemical species and (bottom) sizes.

where $X$ is the matrix of observed data, the element $x_{i j}$ is the concentration value of the $i$ th sample at the $j$ th size interval or species. $G$ and $F$ are respectively the source contributions and source profiles to be estimated. $E$ is a matrix of residuals.
[15] The residual sum of squares $(Q)$ is defined by equation (8) and minimized by finding the optimal $F$ and $G$.

$$
Q=\left\|\frac{(X-G F)}{S}\right\|_{F, G}^{2}=\sum_{i} \sum_{j}\left(\frac{e_{i j}}{s_{i j}}\right)^{2} .
$$




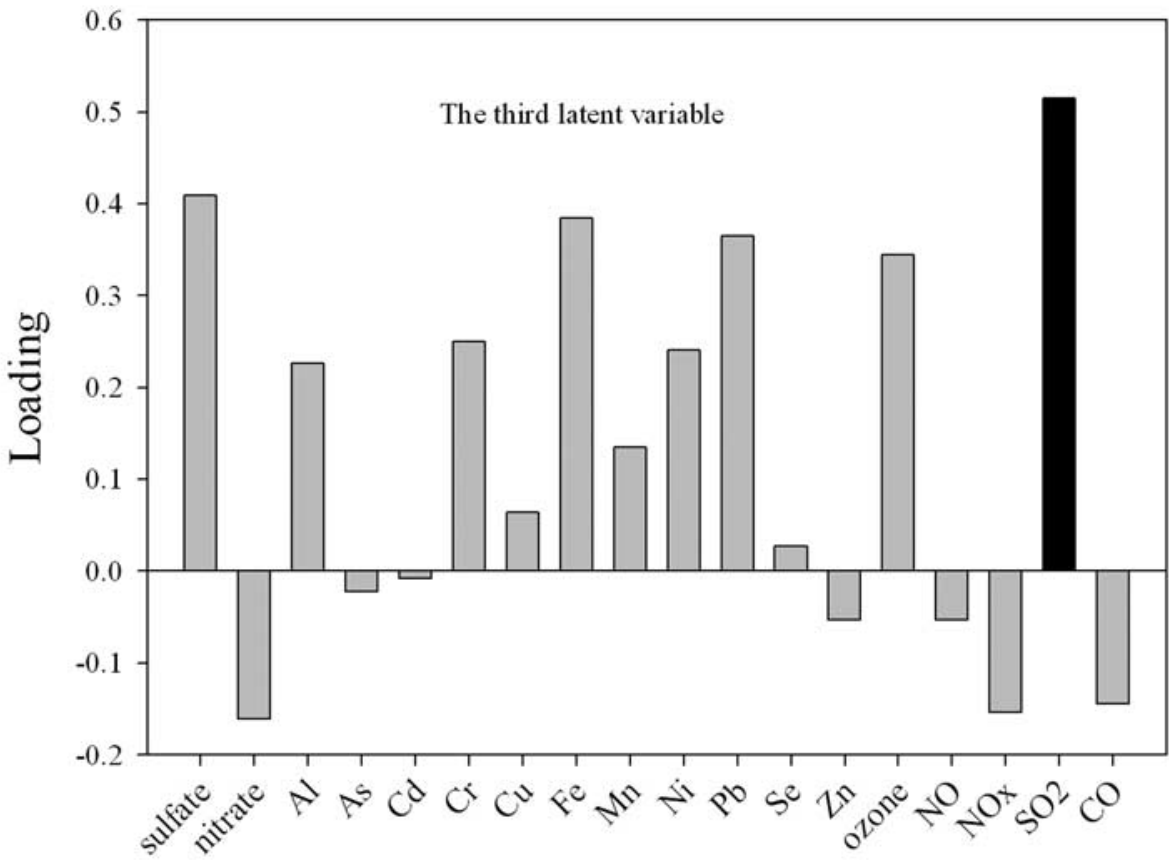

Species

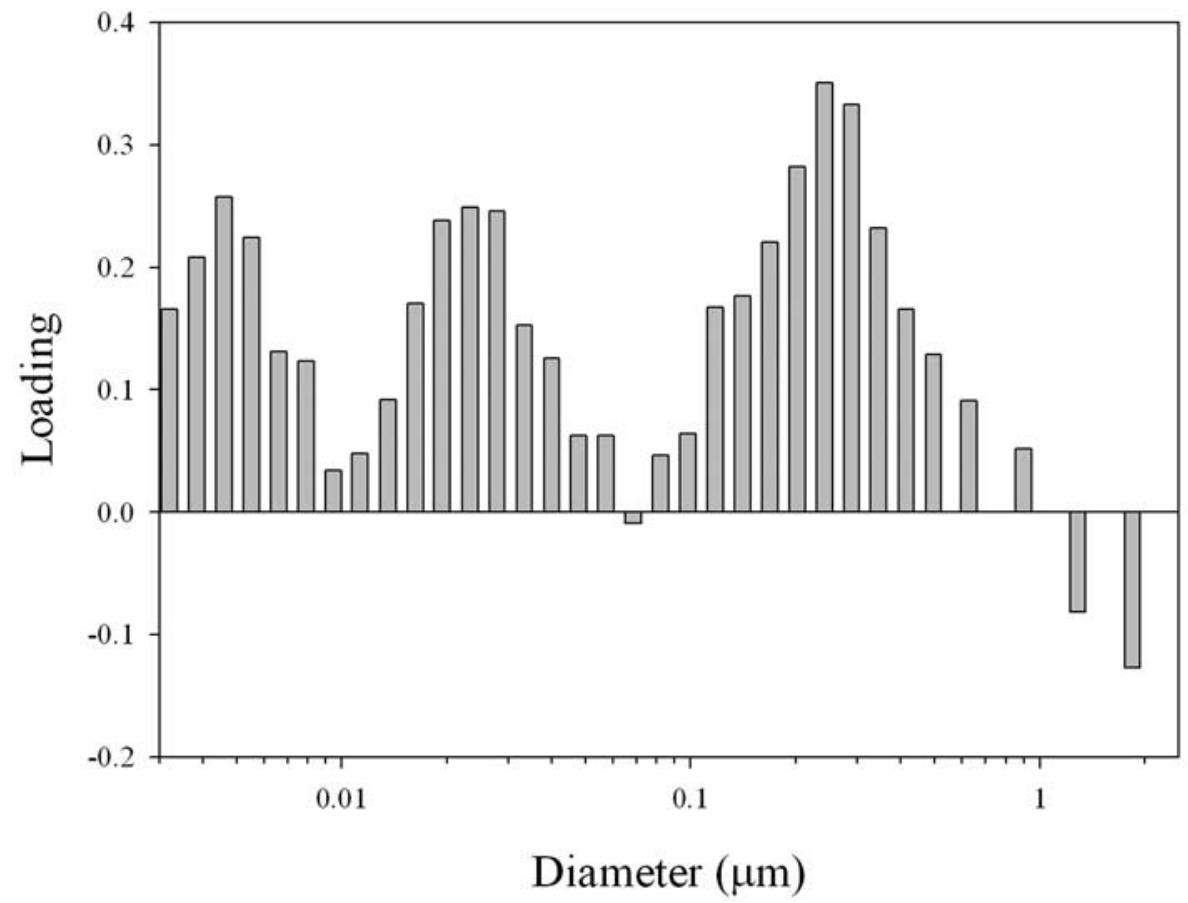

Figure 3. Loadings of the third LV for (top) chemical species and (bottom) sizes.

The uncertainties $s$ were computed based on the measurement errors by equation (9):

$$
s_{i j}=\sigma_{i j}+C_{3} \max \left(\left|x_{i j}\right|,\left|y_{i j}\right|\right),
$$

where $y_{i j}$ is the calculated value for $x_{i j}, \sigma_{i j}$ is the measurement error, and $C_{3}$ is a dimensionless constant value, 0.08 in this study. The estimation of the measurement errors of size distribution data were based on the combination of size bins and the detailed procedure was provided in the work of Zhou et al. [2004a]. $C_{3}$ is used as the estimation of the relative uncertainties of large values (see P. Paatero, User's Guide for positive matrix factorization programs PMF2 and PMF3, Part 2: Reference, 
Table 4. The Correlations of the Latent Variables by PLS With All Chemical Species

\begin{tabular}{lrrr}
\hline & LV1 & LV2 & LV3 \\
\hline Sulfate & -0.26 & 0.68 & 0.57 \\
$\mathrm{Nitrate}$ & 0.83 & 0.27 & -0.22 \\
$\mathrm{Al}$ & 0.15 & -0.09 & 0.31 \\
$\mathrm{As}$ & 0.77 & 0.00 & -0.03 \\
$\mathrm{Cd}$ & 0.76 & -0.36 & -0.01 \\
$\mathrm{Cr}$ & 0.40 & -0.41 & 0.35 \\
$\mathrm{Cu}$ & 0.41 & -0.67 & 0.09 \\
$\mathrm{Fe}$ & 0.12 & 0.19 & 0.53 \\
$\mathrm{Mn}$ & 0.81 & -0.23 & 0.19 \\
$\mathrm{Ni}$ & 0.14 & -0.13 & 0.33 \\
$\mathrm{~Pb}$ & 0.36 & -0.27 & 0.51 \\
$\mathrm{Se}$ & 0.65 & 0.16 & 0.04 \\
$\mathrm{Zn}$ & 0.83 & 0.13 & -0.07 \\
$\mathrm{Ozone}$ & -0.60 & -0.35 & 0.48 \\
$\mathrm{NO}$ & 0.83 & 0.25 & -0.07 \\
$\mathrm{NO}_{\mathrm{x}}$ & 0.86 & 0.31 & -0.21 \\
$\mathrm{SO}_{2}$ & 0.19 & -0.03 & 0.71 \\
$\mathrm{CO}$ & 0.82 & -0.02 & -0.20 \\
\hline
\end{tabular}

available by FTP at ftp://ftp.clarkson.edu/pub/hopkepk/ pmf/). FPEAK is a parameter in PMF for controlling rotations [Paatero et al., 2002]. When the FPEAK value is positive, the following additional term is included in the object function $Q$ :

$$
Q^{P}=\beta^{2}\left(\sum_{k=1}^{p} \sum_{j=1}^{n} f_{k j}\right)^{2},
$$

where $\beta^{2}$ corresponds to the FPEAK value. The term defined above attempts to pull the sum of all the elements of $F$ toward zero and makes the program do elementary transformations for $F$ and $G$ by subtracting the $F$ vectors from each other and adding corresponding $G$ vectors to obtain a more physically realistic solution. The FPEAK value was chosen as 0.1 since there was no clearly defined edges in $G$ space [Paatero et al., 2005].
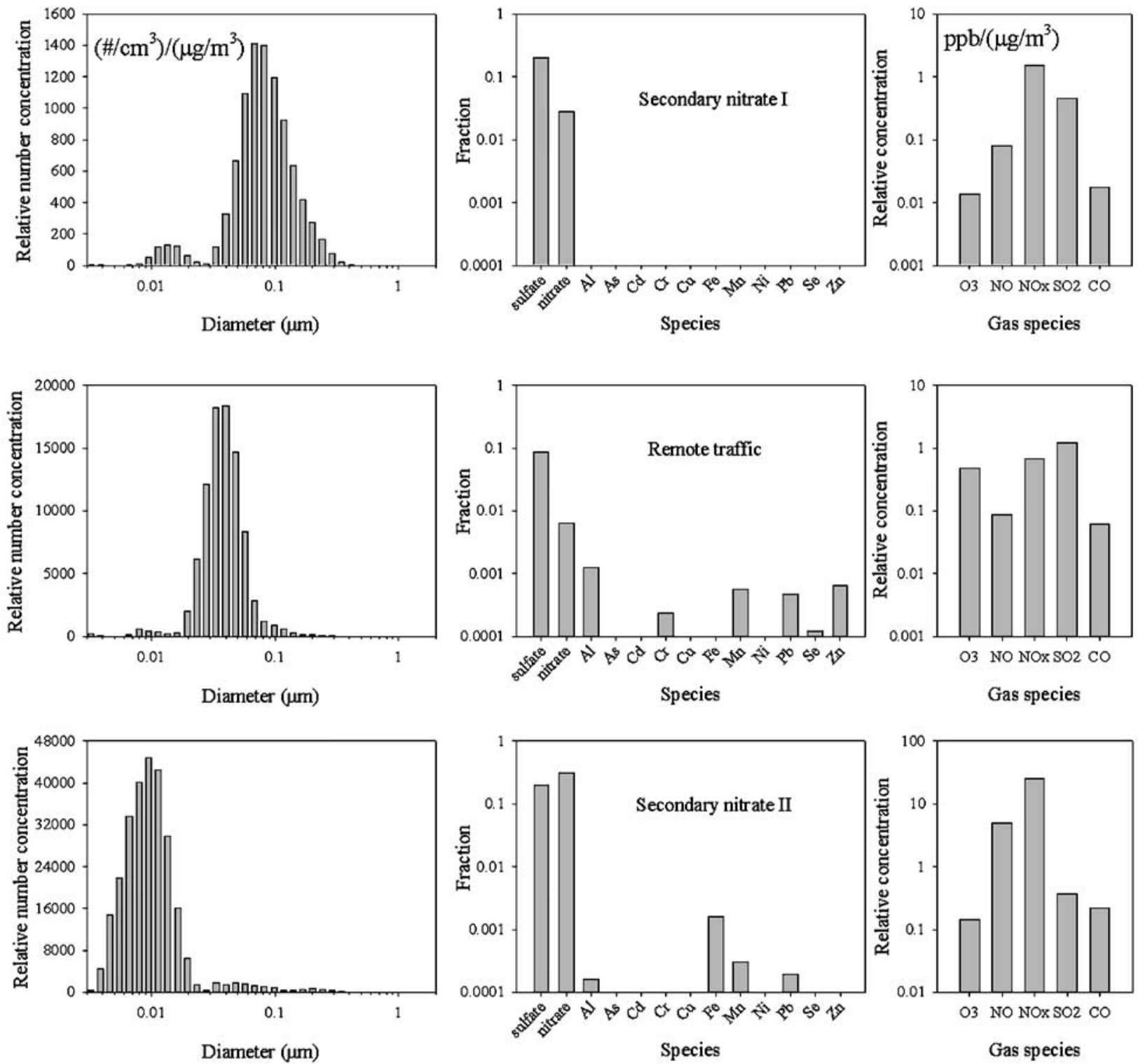

Figure 4. Source profiles from PMF analysis (secondary nitrate 1, remote traffic, and secondary nitrate 2). 

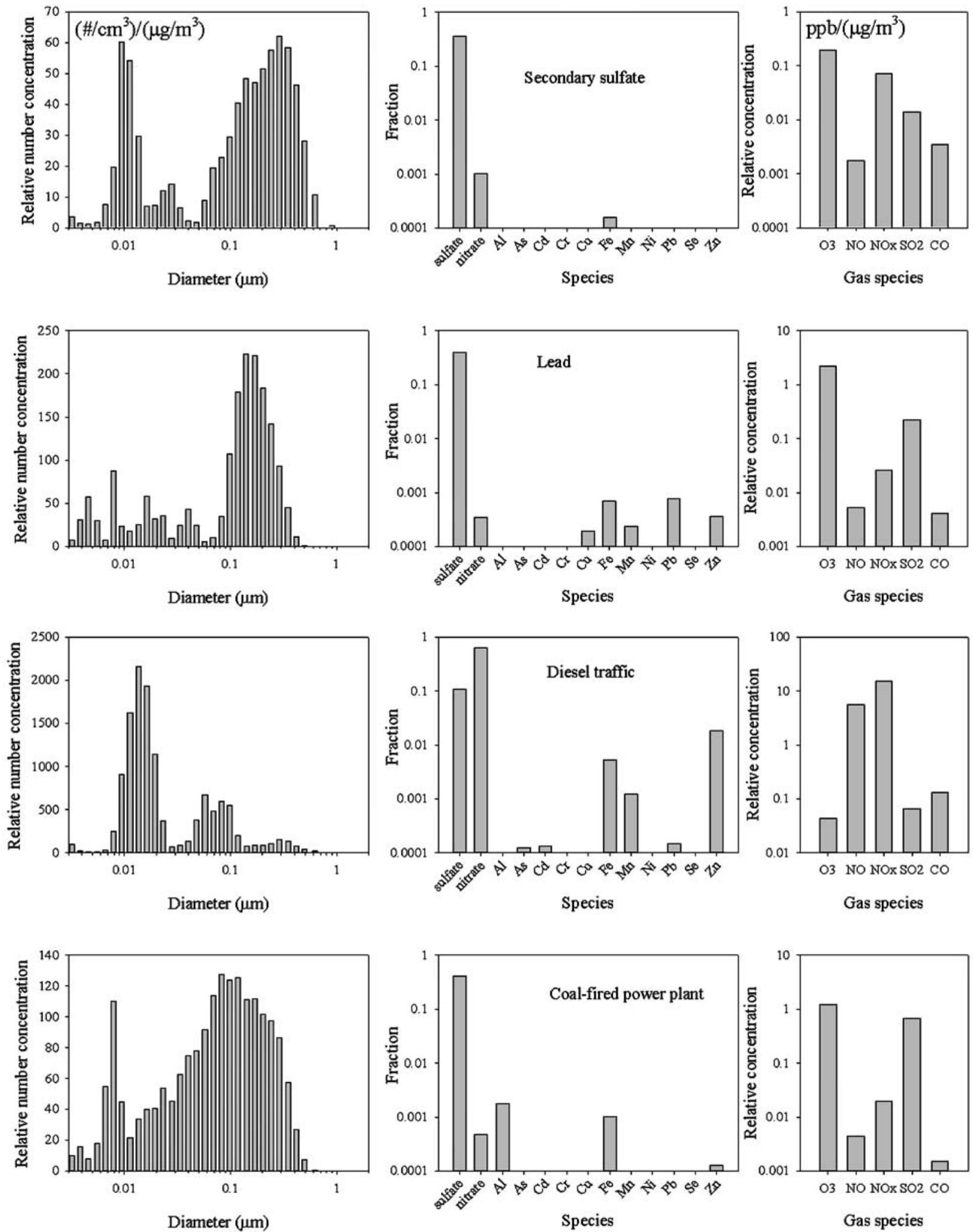

Figure 5. Source profiles from PMF analysis (secondary sulfate, lead, diesel traffic, and local coal-fired plant). 

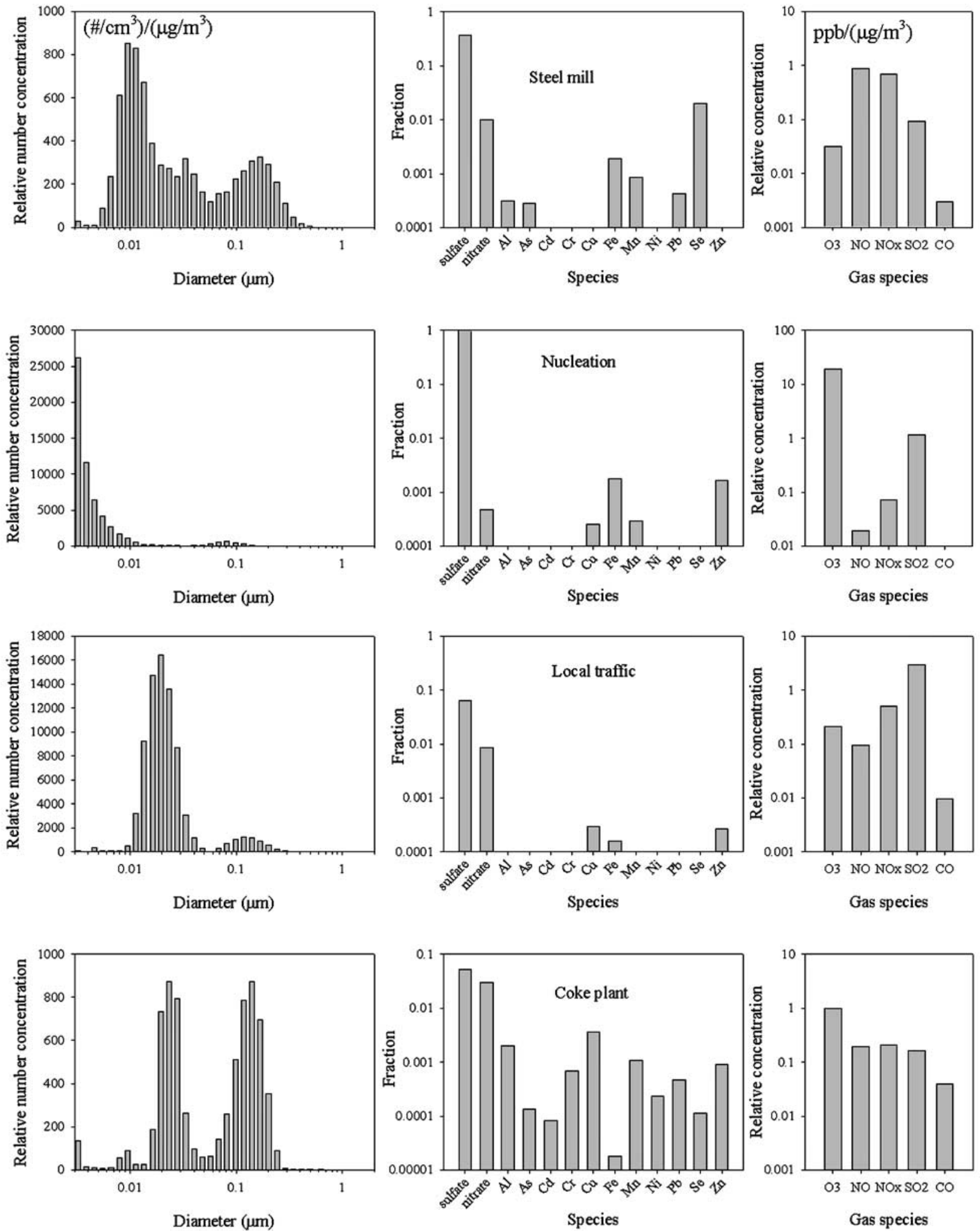

Figure 6. Source profiles from PMF analysis (steel mill, nucleation, local traffic, and coke plant). 


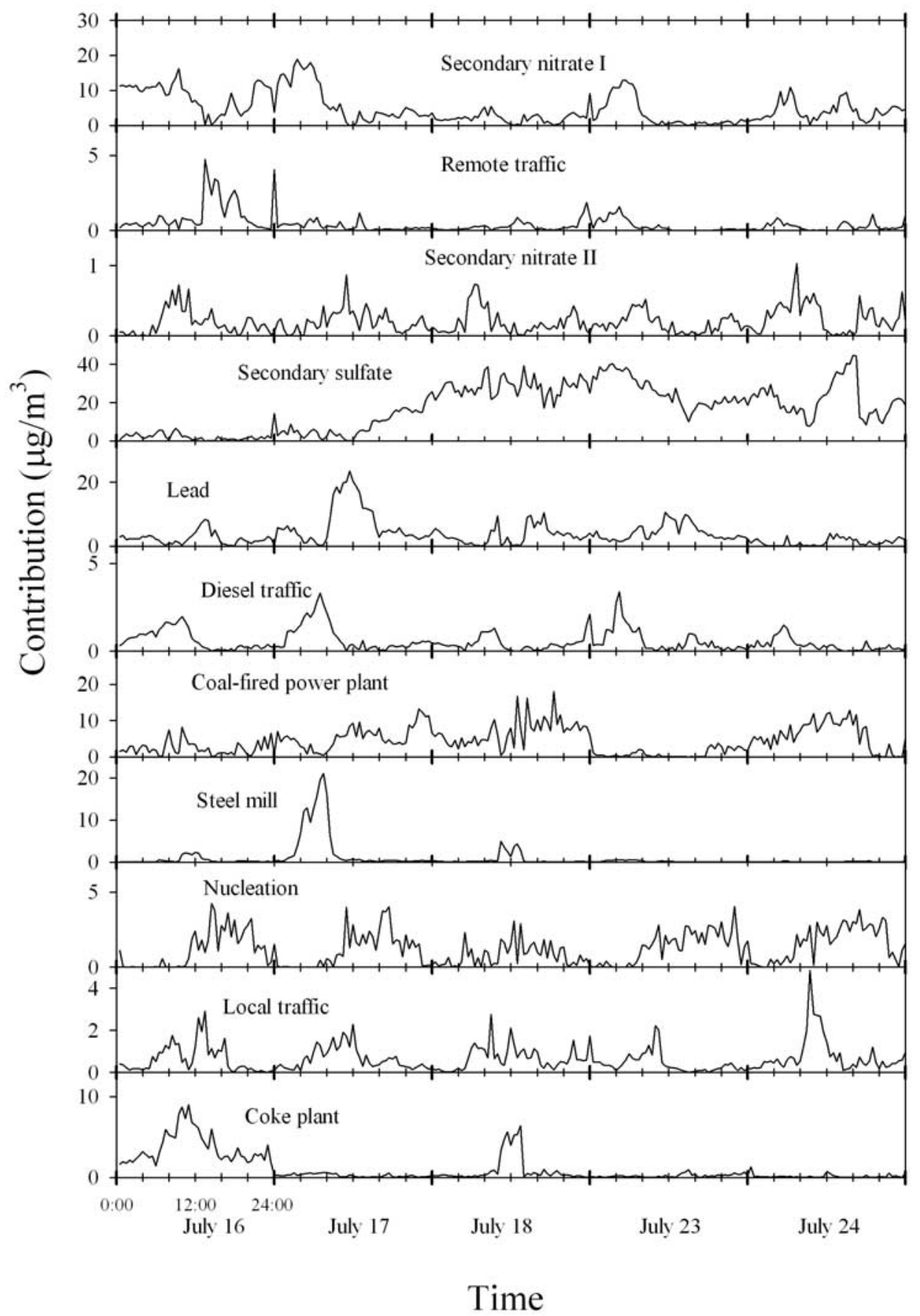

Figure 7. Source contributions from PMF analysis.

[16] The mass apportionment conditions [Hopke et al., 1980] are satisfied by re-scaling the source contribution series and source profiles as shown in equation (11).

$$
x_{i j}=\sum_{p=1}^{P} f_{i p} \cdot \frac{w_{p}}{w_{p}} \cdot g_{p j}
$$

The scaling constants in the above equation, $w_{p}$, were determined by regressing $\mathrm{PM}_{2.5}$ mass concentrations $\left(v_{j}\right)$ against the estimated source contributions as indicated in equation (12).

$$
v_{j}=\sum_{p=1}^{P} w_{p} \cdot g_{p j}
$$

[17] The source profiles include three parts, number concentrations for all size intervals, mass fractions for all species and volume concentration for all gases. In Figures 4-6, for each source profile, the unit of the 


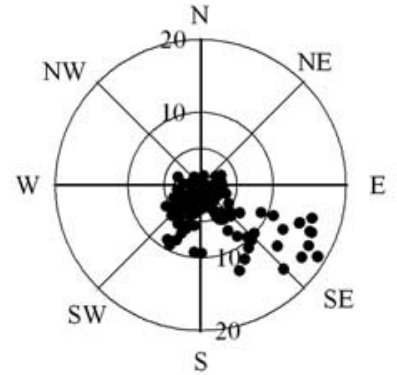

Secondary nitrate I

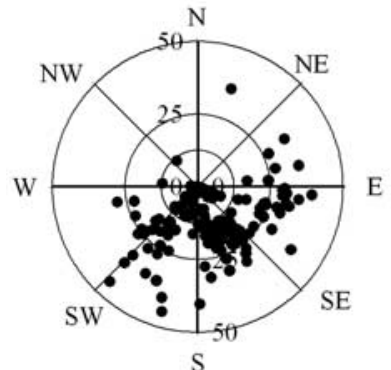

Secondary sulfate

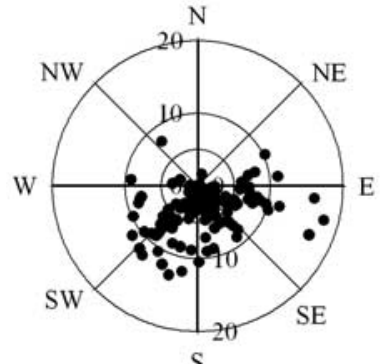

Coal-fired power plant

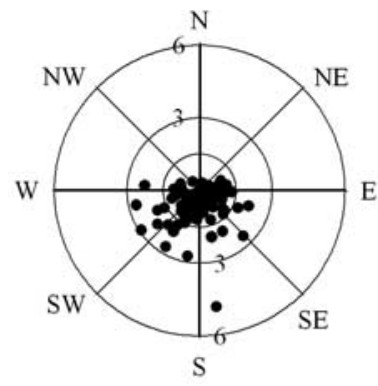

Local traffic

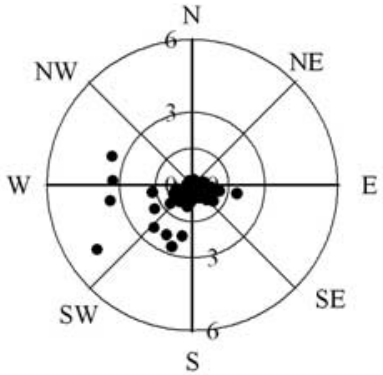

Remote traffic

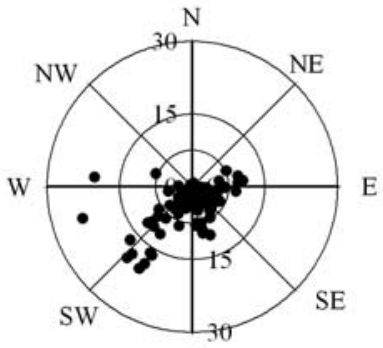

$\mathrm{S}$

Lead

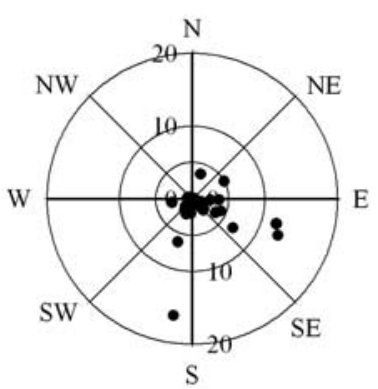

Steel mill

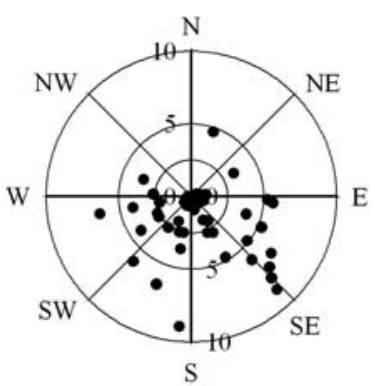

Coke plant

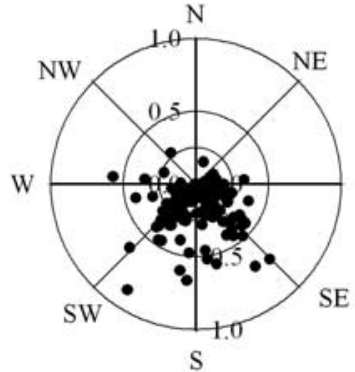

Secondary nitrate II

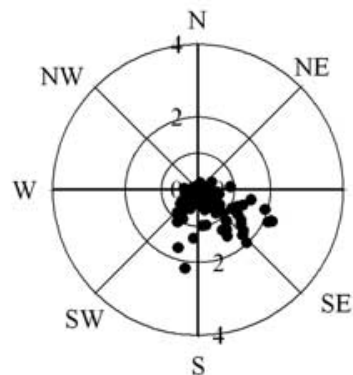

Diesel traffic

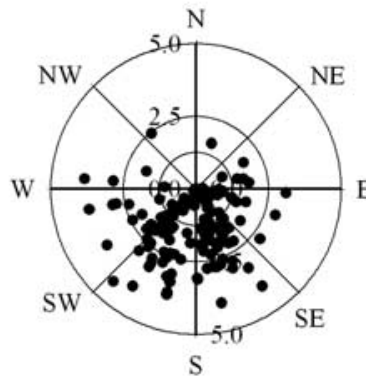

Nucleation

Figure 8. The relationships of source contributions with wind directions.

vertical axis in each of the three rows, from left to right, is (number $\left./ \mathrm{cm}^{3}\right) /\left(\mu \mathrm{g} / \mathrm{m}^{3}\right), 1$ and $\mathrm{ppb} /\left(\mu \mathrm{g} / \mathrm{m}^{3}\right.$ ) (for $\mathrm{CO}$, the unit is $\left.\mathrm{ppm} /\left(\mu \mathrm{g} / \mathrm{m}^{3}\right)\right)$, respectively.

\subsection{Results and Discussion}

[18] Eleven factors were found to provide the best solution. The sources are identified as secondary nitrate 1 and 2 , remote traffic, secondary sulfate, lead, diesel traffic, coalfired power plant, steel mill, nucleation, local traffic, and coke plant. When using an additional factor, the nucleation factor is separated into two factors and thus, additional factors were not warranted. With fewer factors, there were apparently mixed sources or poorly fit variables. The results will be discussed and compared with our previous PMF 

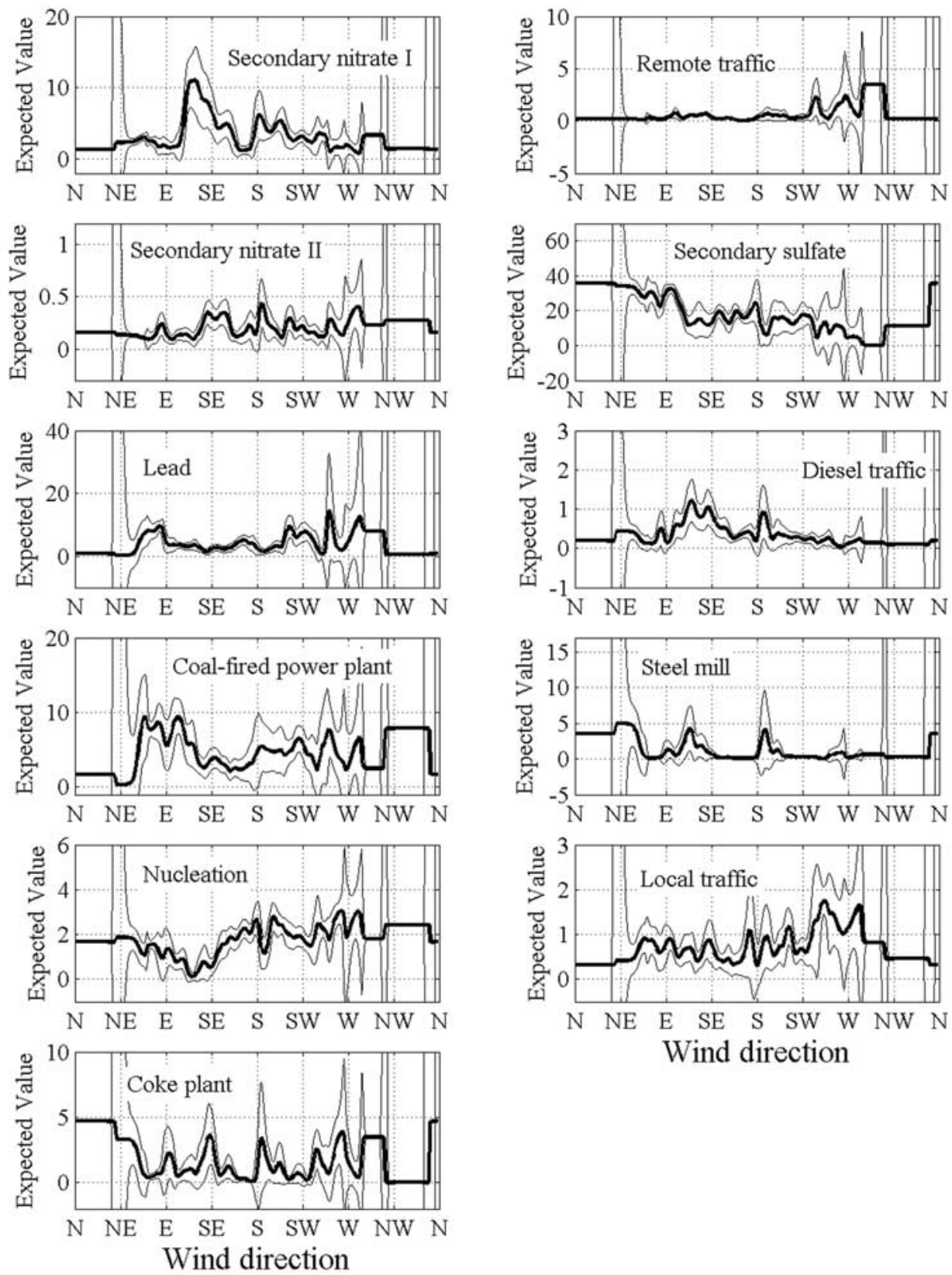

Figure 9. Nonparametric regression (NPR) analysis results for each source. (The unit of the expected value is $\mu \mathrm{g} / \mathrm{m}^{3}$.)

analyses of the size distribution data [Zhou et al., 2004a, 2005] and multi time analyses of composition data [Zhou et al., 2004b].

[19] The major mode of secondary nitrate 1 is at $0.08 \mu \mathrm{m}$ as indicated in Figure 4. Figure 7 shows that the source contribution is high in the early morning when the low temperature favors the formation of nitrate. These particles are associated with aged $\mathrm{NO}_{\mathrm{x}}$ emissions and grow into large sizes during the transport. In Figure 8, where the scatter plot of source contribution and corresponding wind directions are presented, the highest contribution is from southeast. Nonparametric regression (NPR) was also used to investigate the wind direction effects. In this method, averaged and smoothed source contributions are plotted against wind direction, confidential intervals are also given. High values indicate more transport from that direction. The detailed description of this method can be found elsewhere [Henry et al., 2002; Zhou et al., 2004b; Kim and Hopke, 2004] and will not be repeated here. The NPR results in Figure 9 also indicates that southeast is the major source direction of secondary nitrate 1 , where two thin lines give the $95 \%$ confidential intervals.

[20] The size range of remote traffic is similar to our previous results of the size distribution data analysis [Zhou et al., 2005], where the particles in this size range were found to be from traffic emissions several miles away or some other unknown point source emissions. The source contribution only has high peaks on the afternoon of 16 July 
Table 5. Correlations of the Source Contributions by PMF With All Chemical Species

\begin{tabular}{|c|c|c|c|c|c|c|c|c|c|}
\hline & Sulfate & Nitrate & $\mathrm{Al}$ & As & $\mathrm{Cd}$ & $\mathrm{Cr}$ & $\mathrm{Cu}$ & $\mathrm{Fe}$ & $\mathrm{Mn}$ \\
\hline Secondary nitrate 1 & -0.26 & 0.61 & -0.02 & 0.42 & 0.41 & 0.12 & 0.24 & -0.12 & 0.42 \\
\hline Remote traffic & -0.24 & 0.09 & 0.04 & 0.08 & 0.19 & 0.33 & 0.24 & -0.04 & 0.27 \\
\hline Secondary nitrate 2 & -0.10 & 0.26 & 0.06 & -0.09 & 0.17 & -0.01 & 0.07 & -0.08 & 0.05 \\
\hline Secondary sulfate & 0.77 & -0.11 & -0.11 & -0.34 & -0.42 & -0.23 & -0.47 & 0.20 & -0.50 \\
\hline Lead & 0.13 & -0.21 & 0.04 & 0.15 & 0.16 & -0.17 & 0.02 & -0.06 & 0.11 \\
\hline Diesel traffic & -0.10 & 0.79 & 0.02 & 0.62 & 0.54 & 0.09 & 0.13 & 0.03 & 0.65 \\
\hline Local coal-fired plant & 0.45 & -0.17 & 0.60 & -0.21 & -0.20 & -0.05 & -0.03 & 0.17 & -0.15 \\
\hline Nucleation & 0.09 & -0.55 & -0.08 & -0.34 & -0.31 & 0.01 & -0.01 & 0.06 & -0.14 \\
\hline Local traffic & -0.01 & 0.13 & 0.07 & -0.03 & 0.12 & 0.16 & 0.09 & 0.13 & 0.13 \\
\hline \multirow[t]{2}{*}{ Coke plant } & -0.40 & 0.24 & 0.24 & 0.21 & 0.64 & 0.88 & 0.70 & 0.28 & 0.48 \\
\hline & $\mathrm{Ni}$ & $\mathrm{Pb}$ & $\mathrm{Se}$ & $\mathrm{Zn}$ & $\mathrm{O}_{3}$ & $\mathrm{NO}$ & $\mathrm{NO}_{\mathrm{x}}$ & $\mathrm{SO}_{2}$ & $\mathrm{CO}$ \\
\hline Secondary nitrate 1 & -0.11 & -0.03 & 0.34 & 0.51 & -0.51 & 0.57 & 0.74 & 0.17 & 0.61 \\
\hline Remote traffic & 0.03 & 0.10 & 0.01 & 0.14 & -0.02 & 0.09 & 0.11 & 0.14 & 0.32 \\
\hline Secondary nitrate 2 & -0.24 & -0.01 & -0.01 & 0.11 & -0.26 & 0.30 & 0.33 & 0.17 & 0.18 \\
\hline Diesel traffic & 0.00 & 0.13 & 0.59 & 0.94 & -0.65 & 0.75 & 0.84 & -0.01 & 0.68 \\
\hline Local coal-fired plant & -0.08 & -0.02 & -0.14 & -0.18 & 0.17 & -0.21 & -0.23 & 0.40 & -0.19 \\
\hline Steel mill & 0.15 & 0.30 & 0.98 & 0.57 & -0.27 & 0.58 & 0.50 & 0.07 & 0.37 \\
\hline Nucleation & 0.03 & 0.07 & -0.19 & -0.35 & 0.70 & -0.34 & -0.51 & 0.18 & -0.43 \\
\hline Local traffic & -0.01 & 0.20 & 0.09 & 0.08 & 0.05 & 0.18 & 0.13 & 0.40 & 0.14 \\
\hline Coke plant & 0.43 & 0.20 & 0.02 & 0.21 & -0.08 & 0.24 & 0.19 & 0.12 & 0.44 \\
\hline
\end{tabular}

when there is much transport. The present species only explain a small fraction of the total particle mass contribution and some other species like OC/EC seem to be the major components. Figure 8 shows the source emission is from west, the direction of Pittsburgh city center, but the confidence interval at that direction in Figure 9 is large and this can be attributed to the short duration and small number of samples for the high source contribution episode. However, the major reason to think it as remote traffic is from the time series in the work of Zhou et al. [2005]. On the basis of the information from this study, we cannot exclude the possibility that it is from a point source.

[21] As shown in Figure 4, most particles of secondary nitrate 2 are at around $10 \mathrm{~nm}$. This source has very high relative concentrations of $\mathrm{NO}$ and $\mathrm{NO}_{\mathrm{x}}$ and also a very high fraction of nitrate. This source is associated with fresh $\mathrm{NO}_{\mathrm{x}}$ emissions and the particles are formed in the vicinity of the receptor site and thus they have not much time to grow into large ones. The source contribution is high around the morning rush hour, implying the relationship with $\mathrm{NO}_{\mathrm{x}}$ emissions from local traffic. Figures 8 and 9 indicate no clear dominating directions for this source.

[22] The secondary sulfate factor has a strong correlation with sulfate as shown in Table 5. It is composed of the largest particles and also has the largest mass contribution. This source corresponds to LV2 in the PLS analysis. The particles are formed during transport from distant sources. Compared with the other sources, this source has the lowest relative concentration of $\mathrm{SO}_{2}$ around $0.01 \mathrm{ppb} /\left(\mu \mathrm{g} / \mathrm{m}^{3}\right)$ as shown in Figure 5, while the relative concentration is 0.1 to $1 \mathrm{ppb} /\left(\mu \mathrm{g} / \mathrm{m}^{3}\right)$ for the other sources. This situation suggests that most of the $\mathrm{SO}_{2}$ is from local sources. It is shown in Figures 8 and 9 that this source seems to be from many directions.

[23] In our previous work [Zhou et al., 2004b], lead was found to be from an unidentified source. Table 5 indicates a strong correlation between the source contribution and lead concentration series. The lead source here may be a local point source. Both Figures 8 and 9 indicate that the source is from southwest, where a metal working plant is located.

[24] Diesel traffic is similar to the traffic source identified before [Zhou et al., 2004b] and both of them are strongly correlated with zinc. This source has the highest relative concentration for $\mathrm{NO}$ and $\mathrm{NO}_{\mathrm{x}}$ as shown in Figure 5, and also has the highest correlation with $\mathrm{NO}$ and $\mathrm{NO}_{\mathrm{x}}$ as shown in Table 5. In Figure 7, it can be found that the source contribution is only high in the early mornings. This phenomenon can be explained by the fact that the heavyduty truck drivers avoid driving in the morning rush hours. The southeast and south directions indicated in Figures 8 and 9 are the directions of highway I376.

[25] The mode of coal-fired power plant is around $0.1 \mu \mathrm{m}$. This source is corresponding to LV3 in the PLS analysis. Its particle composition is close to secondary sulfate and it also explains some sulfate. However, its gas composition and size distribution profile is different from the secondary sulfate factor and that enables the separation into two factors. The dominating direction is between southwest and south may also include the direction between east and southeast. Since there are more than one coal-fired power plants near Pittsburgh area especially in the south and southwest directions [Zhou et al., 2004a], this source may not be a single point source.

[26] Nucleation features the smallest particles. The small mode at $0.1 \mu \mathrm{m}$ explains most of the related mass concentration and this mode is probably caused by simultaneous condensation with nucleation. The major chemical component is sulfate and this is consistent with other theoretical and experimental results on nucleation studies. The concentration of ozone for nucleation is the highest of all sources. Obviously, ozone is not a primary emission. Thus this profile suggests a strong relationship between ozone as a measure of photochemical activity and nucleation events. The elevated source contribution and ozone concentration can both be attributed to the increased photochemical reaction activities around noon as shown 
in Figure 7. The nucleation source does not have clear dominating directions.

[27] The size range of local traffic, indicated in Figure 6, is similar to our previous results [Zhou et al., 2004b], where we found the particles in this range showed strong diurnal patterns, including the contribution peak around morning rush hours and significant weekday/weekend difference. Figure 7 indicates that the temporal variations of the source contribution show peaks at morning rush hours. The correlations of the source contribution with the gases $\left(\mathrm{NO}, \mathrm{NO}_{\mathrm{x}}\right.$ and $\mathrm{CO}$ ) are weak as indicated in Table 5 since most of these gases are emitted from other sources such as diesel traffic.

[28] A coke plant and a steel mill are two sources that were also found by analyses of composition data. They have two number modes at $10-20 \mathrm{~nm}$ to and $0.1-0.2 \mu \mathrm{m}$. In the analyses of size distribution data [Zhou et al., 2004a, 2005], the particles with the size range around $0.1 \mu \mathrm{m}$ were thought to be from the local combustion sources. This conclusion is consistent with the presence of the number modes at the large size while the number modes at $10-20 \mathrm{~nm}$ were not found to be related to point sources by the analysis with only size distribution data.

[29] The two point sources, coke plant and steel mill, as well as secondary nitrate 1 and the diesel traffic are included in LV1. Their size ranges are similar and are thus summarized in one latent variable in the PLS analysis. These sources explain most variations of $\mathrm{NO}, \mathrm{NO}_{\mathrm{x}}$ and $\mathrm{CO}$.

\section{Conclusion}

[30] Partial least squares and positive matrix factorization have been used to analyze aerosol sized distribution data and composition data together. PLS analyses found there are linear relationships between the number concentrations of large sized particles and the mass concentrations of most of the chemical species. Since the linear relationship between the two data sets was proved by PLS, PMF can be used for source apportionment and it can even identify the sources with small chemical mass concentrations but high number concentrations caused by small particle sizes, such as nucleation and local traffic.

[31] The two methods have revealed source information including both size distribution and chemical composition at the same time. These results are helpful for understanding the results by the analysis of size distribution data.

[32] Acknowledgments. This research was conducted as part of the Pittsburgh Air Quality Study which was supported by U.S. Environmental Protection Agency under contract R82806101 and the U.S. Department of Energy National Energy Technology Laboratory under contract DE-FC2601NT41017. This paper has not been subject to EPA's required peer and policy review, and therefore does not necessarily reflect the views of the agency. No official endorsement should be inferred.

\section{References}

Henry, R. C. (1997), History and fundamentals of multivariate air quality receptor models, Chemom. Intell. Lab. Syst., 37, 525-530.

Henry, R. C. (2002), Multivariate receptor models - Current practice and future trends, Chemom. Intell. Lab. Syst., 60, 43-48.

Henry, R. C. (2003), Multivariate receptor modeling by N-dimensional edge detection, Chemom. Intell. Lab. Syst., 65, 179-189.

Henry, R. C., Y.-S. Changa, and C. H. Spiegelman (2002), Locating nearby sources of air pollution by nonparametric regression of atmospheric concentrations on wind direction, Atmos. Environ., 36, 2237-2244.

Hopke, P. K. (2003), Recent developments in receptor modeling, J. Chemom., 17, 255-265.
Hopke, P. K., R. E. Lamb, and D. F. C. Natusch (1980), Multielemental characterization of urban roadway dust, Environ. Sci. Technol., 14, 164172.

Jong, S. (1993), SIMPLS: An alternative approach to partial least squares regression, Chemom. Intell. Lab. Syst., 18, 251-263.

Khlystov, A., C. Stanier, and S. N. Pandis (2004), An algorithm for combining electrical mobility and aerodynamic size distributions when measuring ambient aerosol, Aerosol. Sci. Technol., 38, 229-238.

Kidwell, C. B., and J. M. Ondov (2001), Elemental analysis of sub-hourly ambient aerosol collections, Aerosol Sci. Technol., 35, 596-601.

Kim, E., and P. K. Hopke (2004), Comparison between conditional probability function and nonparametric regression for fine particle source directions, Atmos. Environ., 38, 4667-4673.

Kim, E., P. K. Hopke, T. V. Larson, and D. S. Covert (2004), Analysis of ambient particle size distributions using UNMIX and positive matrix factorization, Environ. Sci. Technol., 38, 202-209.

Manne, R. (1987), Analysis of two partial-least-squares algorithms for multivariate calibration, Chemom. Intell. Lab. Syst., 2, 187-197.

Paatero, P. (1997), Least squares formulation of robust non-negative factor analysis, Chemom. Intell. Lab. Syst., 37, 23-35.

Paatero, P., P. K. Hopke, X. H. Song, and Z. Ramadan (2002), Understanding and controlling rotations in factor analytic models, Chemom. Intell. Lab. Syst., 60, 253-264.

Paatero, P., P. K. Hopke, B. A. Begum, and S. K. Biswas (2005), A graphical diagnostic method for assessing the rotation in factor analytical models of atmospheric pollution, Atmos. Environ., 39, 193-201.

Ruuskanen, J., et al. (2001), Concentrations of ultrafine, fine and PM2. 5 particles in three European cities, Atmos. Environ., 35, 3729-3738. Stanier, C., A. Khlystov, W. R. Chan, M. Mandiro, and S. N. Pandis (2004), A method for the in-situ measurement of fine aerosol water content of ambient aerosol: The Dry-Ambient Aerosol Spectrometer (DAASS), Aerosol Sci. Technol., 38(S1), 215-228.

Stolzenburg, M. R., and S. V. Hering (2000), Method for the automated measurement of fine particle nitrate in the atmosphere, Environ. Sci. Technol., 34, 907-914.

Wahlin, P., F. Palmgren, R. V. Dingenen, and F. Raes (2001), Experimental studies of ultrafine particles in streets and the relationship to traffic, Atmos. Environ., 35(S1), S63-S69.

Wittig, B., N. Anderson, A. Y. Khlystov, S. N. Pandis, C. Davidson, and A. L. Robinson (2003), Pittsburgh Air Quality Study overview and preliminary scientific findings, Atmos. Environ., 38, 3107-3125.

Wittig, A. E., S. Takahama, A. Y. Khlystov, S. N. Pandis, S. Heringe, B. Kirbye, and C. Davidson (2004), Semi-continuous PM2.5 inorganic composition measurements during the Pittsburgh Air Quality Study, Atmos. Environ., 38, 3201-3213.

Wold, S., M. Sjöström, and L. Eriksson (2001), PLS-regression: A basic tool of chemometrics, Chemom. Intell. Lab. Syst., 58, 109-130.

Zhou, L., E. Kim, P. K. Hopke, C. Stanier, and S. N. Pandis (2004a), Advanced factor analysis on Pittsburgh particle size distribution data, Aerosol Sci. Technol., 38, 118-132.

Zhou, L., P. K. Hopke, P. Paatero, J. M. Ondov, P. J. Pancras, N. J. Pekney, and C. I. Davidson (2004b), Advanced factor analysis for multiple time resolution aerosol composition data, Atmos. Environ., 38, 4909-4920.

Zhou, L., E. Kim, P. K. Hopke, C. Stanier, and S. N. Pandis (2005), Mining airborne particulate size distribution data by positive matrix factorization, J. Geophys. Res., doi:10.1029/2004JD004707, in press.

Zhu, Y., W. C. Hinds, S. Kim, S. Shen, and C. Sioutas (2002a), Study of ultrafine particles near a major highway with heavy-duty diesel traffic, Atmos. Environ., 36, 4323-4335.

Zhu, Y., W. C. Hinds, S. Kim, and C. Sioutas (2002b), Concentration and size distribution of ultrafine particles near a major highway, J. Air Waste Manage. Assoc., 52, 1032-1042.

Zhu, Y., W. C. Hinds, S. Kim, and C. Sioutas (2004), Seasonal trends of concentration and size distribution of ultrafine particles near major highways in Los Angeles, Aerosol Sci. Technol., 38(A1), 5-13.

P. K. Hopke, Center for Air Resources Engineering and Science, Department of Chemical Engineering, Clarkson University, PO Box 5708, Potsdam, NY 13699-5708, USA. (hopkepk@clarkson.edu)

J. M. Ondov and J. P. Pancras, Department of Chemistry and Biochemistry, University of Maryland, College Park, MD 20742, USA.

S. N. Pandis, Department of Chemical Engineering, Carnegie Mellon University, Pittsburgh, PA 15213, USA.

C. O. Stanier, Department of Chemical and Biochemical Engineering, University of Iowa, 4122 Seamans Center, Iowa City, IA 52242, USA. (cstanier@engineering.uiowa.edu)

L. Zhou, Providence Engineering and Environmental Group LLC, 6160 Perkins Road, Suite 100, Baton Rouge, LA 70808, USA. (limingzhou@) providencebr.com) 


\title{
Source Apportionment
}

\section{Advanced Factor Analysis on Pittsburgh Particle Size-Distribution Data}

\author{
Liming Zhou, ${ }^{1}$ Eugene Kim, ${ }^{1}$ Philip K. Hopke, ${ }^{1}$ Charles O. Stanier, ${ }^{2}$ \\ and Spyros Pandis ${ }^{2}$ \\ ${ }^{1}$ Department of Chemical Engineering, Clarkson University, Potsdam, New York \\ ${ }^{2}$ Department of Chemical Engineering, Carnegie Mellon University, Pittsburgh, Pennsylvania
}

\begin{abstract}
Positive matrix factorization (PMF) method was applied to particle size-distribution data acquired during the Pittsburgh Air Quality Study (PAQS) from July 2001 to August 2001. After removing those days with nucleation events, a total of 1632 samples, each with 165 evenly-sized intervals from 0.003 to $2.5 \mu \mathrm{m}$, were obtained from scanning mobility particle spectrometer (SMPS) and aerodynamic particle sampler (APS). The temporal resolution was $15 \mathrm{~min}$. The values for each set of five consecutive-size bins were averaged to produce 33 new size channels. The size distributions of particle number as well as volume were analyzed with a bilinear model. Three kinds of information were used to identify the sources: the number and volume size distributions associated with the factors, the time frequency properties of the contribution of each source (Fourier analysis of source contribution values) and the correlations of the contribution values with the gas-phase data and some composition data. Through these analyses, the sources were assigned as sparse nucleation, local traffic, stationary combustion, grown particles and remote traffic, and secondary aerosol in a sequence of decreasing number concentration contributions. Conditional probability function (CPF) analysis was performed for each source so as to ascertain the likely directions in which the sources were located.
\end{abstract}

Received 23 October 2002; accepted 31 March 2003.

This research was conducted as part of the Pittsburgh Air Quality Study which was supported by US Environmental Protection Agency under contract R82806101 and the US Department of Energy National Energy Technology Laboratory under contract DE-FC26-01NT41017. This paper has not been subject to EPA's required peer and policy review, and therefore does not necessarily reflect the views of the Agency. No official endorsement should be inferred.

Address correspondence to Philip K. Hopke, Department of Chemical Engineering, Clarkson University, P. O. Box 5708, Potsdam, NY 13699-5708. E-mail: hopkepk@ clarkson.edu

\section{INTRODUCTION}

Recently, substantial attention has been paid to the airborne particulate matter (PM), which is believed to be associated with increased morbidity and mortality, especially to high-risk groups (van Bree and Cassee 2000). Particles of different size have different deposition pattern in the airways. Ultrafine particles $(<0.1 \mu \mathrm{m})$ have a higher deposition fraction than fine particles $(0.1-2.5 \mu \mathrm{m})$. In general, pulmonary deposition increases with decreasing particle size (Venkataraman and Kao 1999) and number concentrations of ultrafine particles were also shown to be more closely associated with variations in lung function (Peters et al. 1997).

To implement effective strategies to control the emission of PM, a comprehensive data set is needed. The Pittsburgh Air Quality Study (PAQS) is a multidisciplinary set of projects in the Pittsburgh region that addresses issues including understanding of the PM health effects, establishing the PM source-receptor relationships, and finding the limitations of existing instrumentation for PM measurements (Wittig et al. 2004).

Previously, principal component analysis (PCA) has been widely used for the source apportionment and recently has been applied to size-distribution data (Ruuskanen et al. 2001; Wahlin et al. 2001; Kim et al. 2004). An alternative to PCA, positive matrix factorization (PMF) is a powerful factor analysis method and has been successfully used to solve the receptor model for the source apportionment of the aerosol particles (Xie et al. 1999; Lee et al. 1999; Ramadan et al. 2000; Chueinta et al. 2000; Polissar et al. 2001; Song et al. 2001). In this study, particle size-distribution data from PAQS will be analyzed by PMF method in terms of both the number and volume distributions.

The continuous measurement of aerosol size distributions has low labor costs and can provide very large data sets with a time resolution of minutes for a moderate investment. The number of sites around the world measuring aerosol size distributions 
has been increasing. The present article explores the possibility of using this information to gain insights about the sources contributing to the ambient PM levels. While it is possible to increase the power of the proposed method by combining the aerosol size distributions with additional measurements of the aerosol composition (metals, speciated organics, single-particle composition), here we focus on the use of the size distributions only. In future work the results of the present study will be compared with the more comprehensive analysis for a stricter test of the source apportionment power of the proposed method.

The aerosol may experience some changes in the size distribution during transport from the sources to the receptor site. The size distribution will change as particles coagulate and dry deposit. However, after some initial changes in the vicinity of the sources the most mobile particle sizes will be depleted, and the particle number concentrations will be sufficiently small so that further deposition and coagulation will be slow processes. Thus, it is reasonable to expect that the particle size distributions will become relatively stable at some appropriate distance from the emission sources. In this study, the events in which there is active growth of the particle size distribution have been explicitly eliminated from the analysis. Gas-phase data and some particlemass data, such as $\mathrm{PM}_{2.5}$, sulfate, and Organic carbon/elemented carbon (OC/EC), were also used to assist the identification of the potential sources.

\section{DESCRIPTION OF THE DATA SET}

The size-distribution measurements were conducted from 30 June to 4 August 2001 at the Pittsburgh Supersite (Latitude 40.4395 , Longitude -79.9405$)$. The location of the receptor site is shown in Figure 1 and is far from any major sources. Ambient air passed through Nafion dryers where the relative humidity (RH) was controlled to around 15\%, and thus the data obtained through these measurements were "dry" data in comparison to the data measured at the ambient RH. The frequency was four 15 min samples during each hour. The results obtained from three instruments were used to produce a single size distribution.

Measurements were made with a scanning mobility particle spectrometer (nano-SMPS; TSI 3083 DMA and TSI 3025 CPC) sampling from 3-83 nm, an SMPS (TSI 3081 DMA and TSI 3010 CPC) sampling from $13-583 \mathrm{~nm}$, and an aerodynamic particle size spectrometer (APS, TSI 3320) sampling from 0.562.5 microns. The SMPS instruments measured electrical mobility diameters. The APS reported aerodynamic diameters with the time of flight calibrated to a density of 1.0. The APS and long differential mobility analyzer (LDMA) data were merged by solving for an effective density for the APS particles, which gave the smallest error (in a least squares sense) in the APS-LDMA overlapping region $(560-583 \mathrm{~nm})$. Thus, above $583 \mathrm{~nm}$ the data represent electrical mobility diameter inferred from aerodynamic mobility and estimated density (Khlystov et al. 2004).

The meteorological (wind direction and wind speed), gas phase, and particle mass (including $\mathrm{PM}_{2.5}$, sulfate, and OC/EC) data were measured at the same time and location as the particle

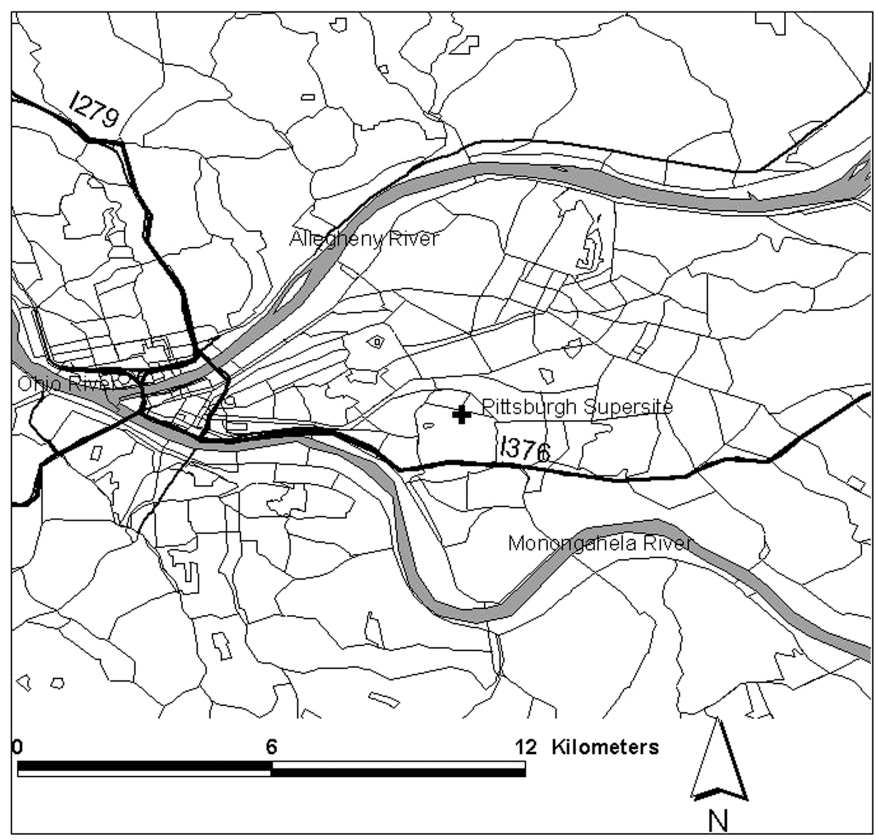

(a)

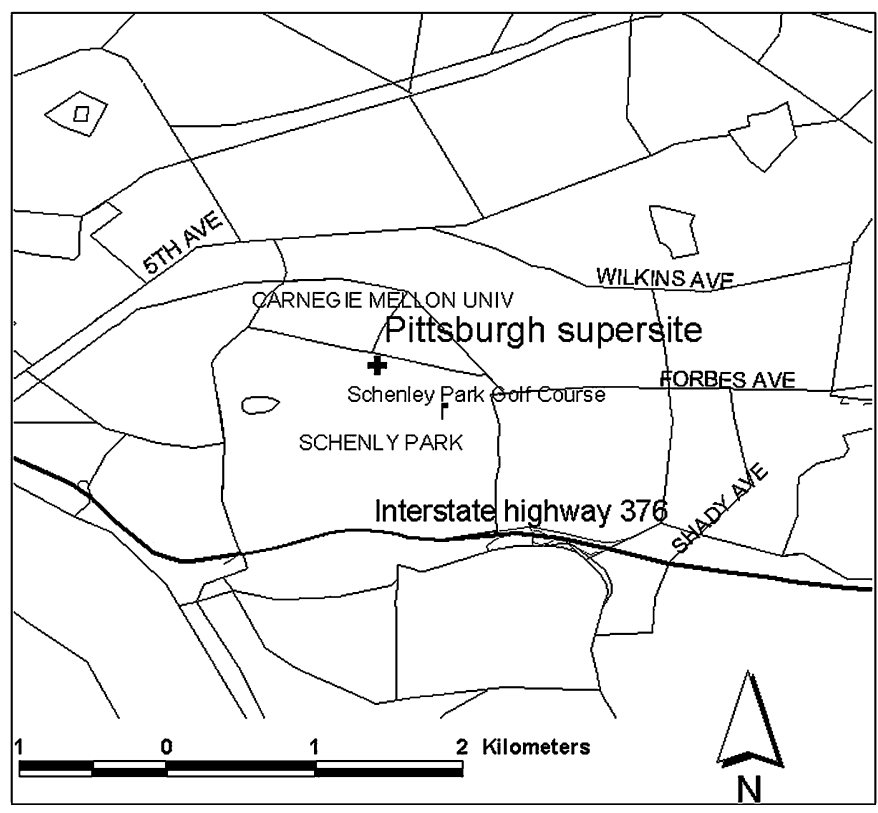

(b)

Figure 1. The map of the Pittsburgh region around the supersite.

number concentration measurements. The temporal resolution for meteorological data was $15 \mathrm{~min}$, and for gas phase and particle mass data was $10 \mathrm{~min}$. The sampling period for OC/EC was 4-5 h.

It appears that most of the sampling days have some degree of homogeneous nucleation occurring. In a typical nucleation event, the particle size initially grows rapidly $(4-5 \mathrm{~nm} / \mathrm{h})$ and then the growth slows as it reaches a size of $30-120 \mathrm{~nm}$. The 
Table 1

Sampling periods without nucleation events

\begin{tabular}{rll}
\hline No. & Date & Day \\
\hline 1 & Jul. 3 & Tue. \\
2 & Jul. 4 & Wed. \\
3 & Jul. 7 & Sat. \\
4 & Jul. 8 & Sun. \\
5 & Jul. 10 & Tue. \\
6 & Jul. 16 & Mon. \\
7 & Jul. 18 & Wed. \\
8 & Jul. 19 & Thu. \\
9 & Jul. 20 & Fri. \\
10 & Jul. 21 & Sat. \\
11 & Jul. 23 & Mon. \\
12 & Jul. 25 & Wed. \\
13 & Jul. 28 & Sat. \\
14 & Jul. 29 & Sun. \\
15 & Jul. 31 & Tue. \\
16 & Aug. 1 & Wed. \\
17 & Aug. 2 & Thu. \\
\hline
\end{tabular}

basic assumption of the receptor model is that the ambient data is the sum of constant profiles (size distributions from the contributing sources). Thus, the days with intense nucleation events (usually having particle growth) were excluded in this study. The definition of an intense nucleation is by investigating the temporal variation rate of the total particle number concentration between $3 \mathrm{~nm}$ and $10 \mathrm{~nm}$, denoted as $d N_{10} / d t$. If one day has a $d N_{10} / d t$ value over $4,000 /\left(\mathrm{cm}^{3} \mathrm{~h}\right)$, then it is thought that an intense nucleation events happens (Stanier et al. 2004) and that day is excluded from this study. Most of these days excluded have particle growth after the new particle formation. The remaining 17 days are indicated in Table 1, and they may still contain some weak and shortlived nucleation without observable particle growth.

The wind was generally from two directions during July, the northwest and southeast $\left(0^{\circ}\right.$ or $360^{\circ}$ for north, $90^{\circ}$ for east, etc. $)$. After those days with nucleation events were removed the wind from the northwest also disappeared, as shown by the wind profile subfigure in Figure 8. The reason may be that the transport from the Great Lakes area (northwest direction) brings clean and cooler air masses that provide good conditions for nucleation.

The missing values in the 165 size bins are replaced by the mean value of the samples in the same size interval. Since the maximum number of missing values for each size bin is 117 , about $7 \%$ of the total sample number, there is no necessity to omit any size bins. The volume concentration is then calculated by the number concentration.

\section{ESTIMATION OF MEASUREMENT ERRORS}

Since no measurement errors were provided with the original experimental data, the following method is used to estimate the

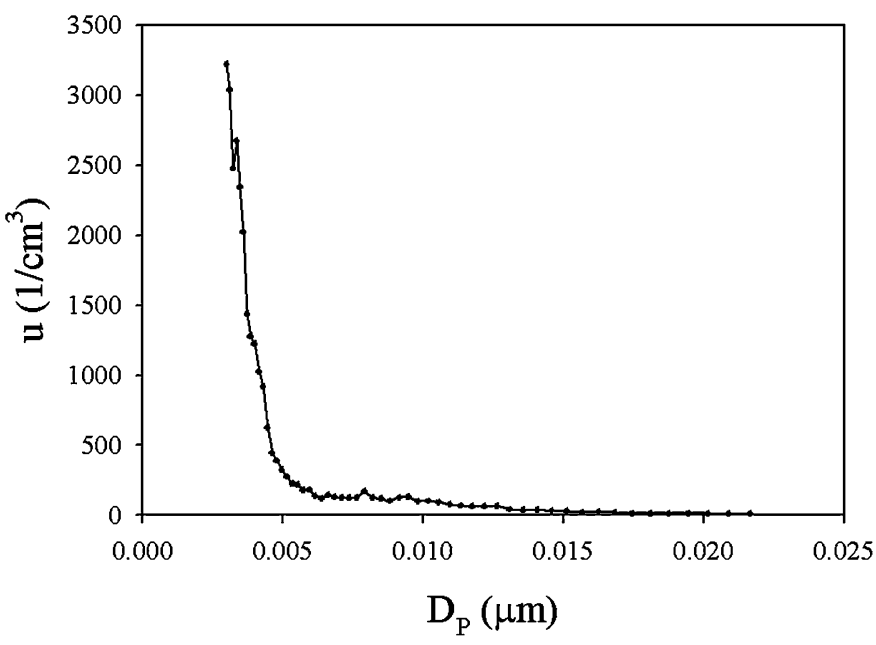

Figure 2. Estimated instrumental errors of small particles.

measurement errors to provide the needed inputs to the PMF program.

For the size range of 3-22 nm measured by the nano-SMPS, the smaller of the minimum nonzero value and the minimum difference of the concentration values within each size bin, $u_{h}$, was taken as the instrumental error for the $h$ th size interval. For larger particles, the instrumental error cannot be estimated through this method. Figure 2 illustrates these values, and it can be seen that the numbers of particles smaller than $5 \mathrm{~nm}$ have large $u$ values while they also have large particle numbers. Reischl (1991) discussed the instrumental error of the differential mobility analyzer (DMA) method and reported mean concentration determination errors for his DMA-Faraday cup electrometer (FCE) system. The condensation particle counter (CPC) has a counting efficiency that drops quickly toward zero for smaller particles and consequently has a higher instrumental error than the FCE, but the performance of CPC is much better for large particles, especially around $100 \mathrm{~nm}$.

Comparing the estimated instrumental error in this study and the instrumental errors reported by Reischl (1991), it can be found that in this study the errors for $3 \mathrm{~nm}$ particles are higher, but they are lower for the larger particle sizes.

Besides the instrumental error, there are other sources of measurement errors. A discussion about the measurement errors can be found in Wong (1997). Based on statistics, the true value of a quantity is given by the average of a large number of measurements. "If the uncertainties are associated with the measuring process are random, the values obtained will most likely be scattered around the true value with some definite distribution" (Wong 1997).

For the measurement of the $i$ th sample at the $j$ th size bin, the concentration increased from 0 with a step length $u_{j}$, and each increase is independent. Let $x_{i h}=N u_{h}$. Thus the probability distribution follows Poisson distribution with a mean of $N$ and a variance $N$. The best estimation of the measurement error is $\sqrt{N} u_{h}$ as indicated in Equation (1). To add 1 in the parentheses 


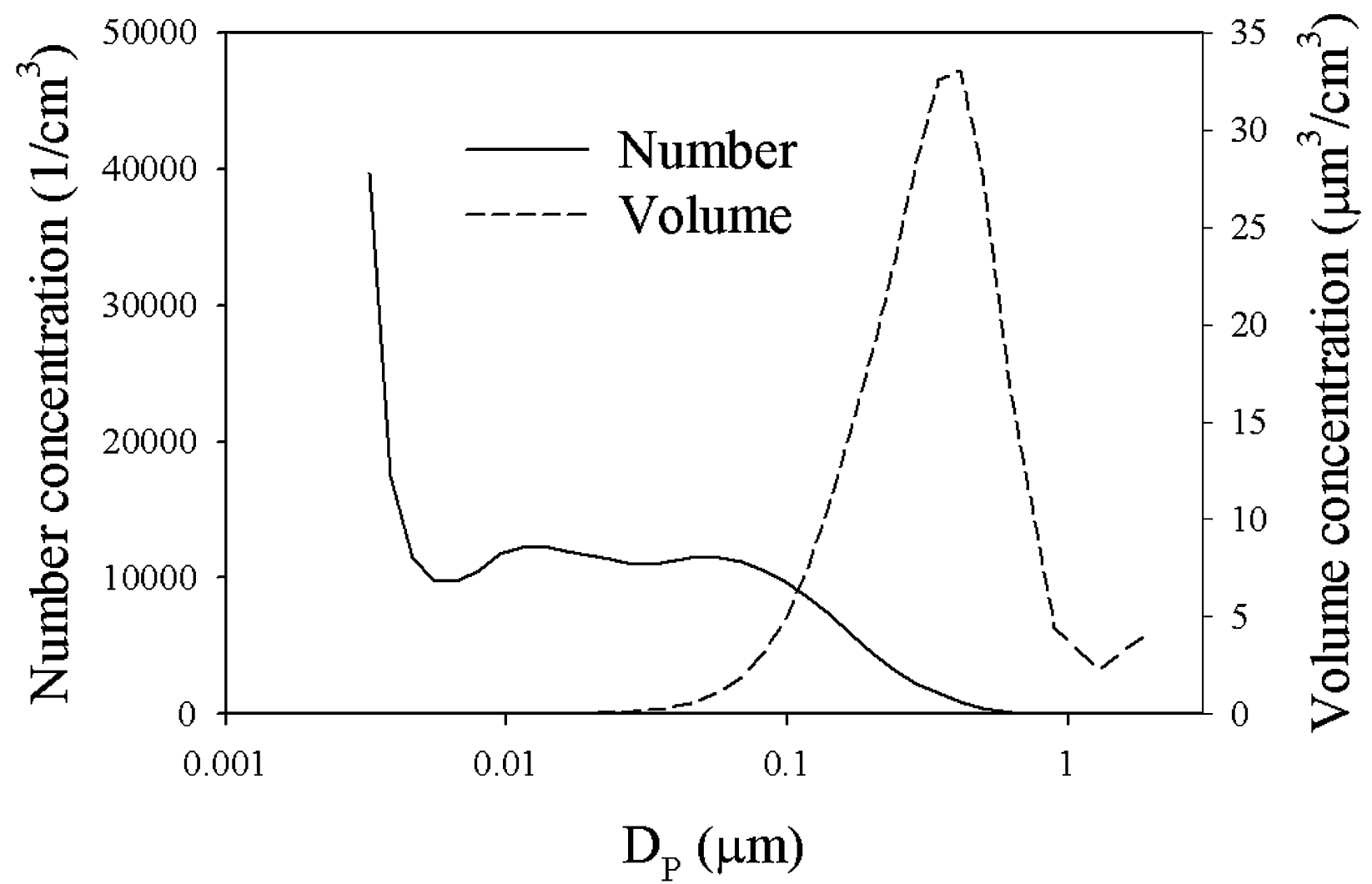

Figure 3. Average number and volume size distribution of all the samples.

is to avoid a zero uncertainty for a zero concentration value.

$$
\sigma_{i h}^{\prime}=\left(\sqrt{\frac{x_{i h}}{u_{h}}}+1\right) u_{h}, \quad h=1,2, \ldots, 55 .
$$

Every consecutive five size intervals were combined into a single new interval to make the size distribution smoother and minimize the error caused by the discontinuity between instruments, and thus 33 new size channels are created. Since in the original dataset the size bins were evenly spaced (in the sense of logarithm), the obtained 33 size channels are also evenly spaced. The diameter of each new size channel is the diameter from the middle one of the original five size bins. The measurement error for $x_{i j}$ after the combination is determined by the following equations:

$$
\begin{aligned}
D_{P} \leq 0.022 \mu \mathrm{m}, \sigma_{i j}= & \sum_{h=5 j-4}^{5 j} \sigma_{i h}^{\prime} \cdot \frac{1}{5}, j=1,2, \ldots, 11, \\
D_{P}>0.022 \mu \mathrm{m}, \sigma_{i j}= & {\left[\max \left(x_{i, 5 j-4}, \ldots, x_{i, 5 j}\right)\right.} \\
& \left.-\min \left(x_{i, 5 j-4}, \ldots, x_{i, 5 j}\right)\right] \\
& \times 0.5, \quad j=12,13, \ldots, 33 .
\end{aligned}
$$

For the 12 to 33 new size channels, the variation of the concentration is smooth. Since the variations between neighboring size bins are small, the concentrations of the five consecutive bins can be regarded as five measurements of the same true value. According to the central limit theorem, a large number of measurements conform a normal distribution whose mean is the true concentration value no matter what distribution each measurement follows. The five measurement are five samples of this normal distribution. The average of the five concentrations is then the best estimation of the mean. In Equation (3), the width of this distribution is approximated by the difference of the maximum and minimum concentration values, and the half width is referred to as the standard deviation of the normal distribution, which is taken as the measurement error. If any of the five original concentration values (165 size bins) is a missing value, the corresponding measurement error is assigned as 4 times the new-formed concentration value (33 size intervals).

Figure 3 gives the mean number and volume size distributions averaged over all of the samples used in these analyses. Table 2 contains detailed information about the maximum, minimum, and mean values for each combined size channel.

\section{POSITIVE MATRIX FACTORIZATION (PMF)}

The basic receptor model is expressed as

$$
X=G F+E,
$$


Table 2

Minimum, maximum, and mean value of the 33 size channels

\begin{tabular}{|c|c|c|c|c|c|c|}
\hline \multirow[b]{2}{*}{$D_{P}(\mu \mathrm{m})$} & \multicolumn{3}{|c|}{$\begin{array}{c}\text { Volume concentration } \\
d N_{V} / d \log D_{P}\left(\mu \mathrm{m}^{3} / \mathrm{cm}^{3}\right)\end{array}$} & \multicolumn{3}{|c|}{$\begin{array}{l}\text { Number concentration } \\
d N / d \log D_{P}\left(\mathrm{~cm}^{-3}\right)\end{array}$} \\
\hline & Minimum & Maximum & Mean & Minimum & Maximum & Mean \\
\hline 0.003 & 0 & 0.0144 & 0.0006 & 0 & $8.21 \mathrm{e}+05$ & $3.97 \mathrm{e}+04$ \\
\hline 0.004 & 0 & 0.0122 & 0.0006 & 0 & $4.22 \mathrm{e}+05$ & $1.75 e+04$ \\
\hline 0.005 & 0 & 0.0066 & 0.0006 & 0 & $1.34 \mathrm{e}+05$ & $1.15 \mathrm{e}+04$ \\
\hline 0.006 & 0 & 0.0096 & 0.0008 & 0 & $1.06 \mathrm{e}+05$ & $9.74 e+03$ \\
\hline 0.007 & 0 & 0.0216 & 0.0014 & 0 & $1.39 \mathrm{e}+05$ & $9.72 \mathrm{e}+03$ \\
\hline 0.008 & 0 & 0.031 & 0.0028 & 0 & $1.19 \mathrm{e}+05$ & $1.06 \mathrm{e}+04$ \\
\hline 0.009 & 0.0002 & 0.0638 & 0.0052 & 214 & $1.37 \mathrm{e}+05$ & $1.17 e+04$ \\
\hline 0.011 & 0.0006 & 0.07 & 0.0094 & 776 & $9.43 e+04$ & $1.22 \mathrm{e}+04$ \\
\hline 0.014 & 0.0014 & 0.101 & 0.0162 & 998 & $7.72 \mathrm{e}+04$ & $1.23 \mathrm{e}+04$ \\
\hline 0.016 & 0.0024 & 0.182 & 0.027 & 1043 & $8.02 \mathrm{e}+04$ & $1.19 \mathrm{e}+04$ \\
\hline 0.019 & 0.0022 & 0.327 & 0.0452 & 563 & $8.53 e+04$ & $1.16 \mathrm{e}+04$ \\
\hline 0.023 & 0.006 & 1.28 & 0.0758 & 880 & $1.87 \mathrm{e}+05$ & $1.13 \mathrm{e}+04$ \\
\hline 0.028 & 0.011 & 1.04 & 0.126 & 979 & $9.01 \mathrm{e}+04$ & $1.10 \mathrm{e}+04$ \\
\hline 0.033 & 0.0168 & 1.88 & 0.217 & 857 & $9.50 \mathrm{e}+04$ & $1.10 \mathrm{e}+04$ \\
\hline 0.040 & 0.0316 & 3.61 & 0.382 & 930 & $1.07 \mathrm{e}+05$ & $1.13 e+04$ \\
\hline 0.048 & 0.0724 & 5.75 & 0.670 & 1242 & $1.01 \mathrm{e}+05$ & $1.16 \mathrm{e}+04$ \\
\hline 0.057 & 0.179 & 5.93 & 1.14 & 1786 & $6.13 e+04$ & $1.15 \mathrm{e}+04$ \\
\hline 0.069 & 0.365 & 9.10 & 1.90 & 2120 & $5.34 \mathrm{e}+04$ & $1.12 \mathrm{e}+04$ \\
\hline 0.082 & 0.457 & 16.1 & 3.07 & 1600 & $5.50 \mathrm{e}+04$ & $1.05 \mathrm{e}+04$ \\
\hline 0.098 & 0.530 & 27.0 & 4.86 & 1070 & $5.41 \mathrm{e}+04$ & $9.73 e+03$ \\
\hline 0.118 & 0.713 & 37.4 & 7.39 & 830 & $4.40 \mathrm{e}+04$ & $8.63 e+03$ \\
\hline 0.141 & 1.33 & 42.23 & 10.8 & 893 & $2.93 e+04$ & $7.34 \mathrm{e}+03$ \\
\hline 0.169 & 2.42 & 51.2 & 14.6 & 958 & $2.05 e+04$ & $5.84 \mathrm{e}+03$ \\
\hline 0.202 & 2.37 & 50.8 & 18.7 & 564 & $1.21 \mathrm{e}+04$ & $4.36 e+03$ \\
\hline 0.241 & 1.79 & 70.2 & 23.3 & 252 & $9.51 \mathrm{e}+03$ & $3.17 e+03$ \\
\hline 0.289 & 1.13 & 87.3 & 28.4 & 91.7 & $6.92 \mathrm{e}+03$ & $2.25 \mathrm{e}+03$ \\
\hline 0.346 & 0.695 & 111.7 & 32.6 & 33.1 & $5.14 \mathrm{e}+03$ & $1.51 \mathrm{e}+03$ \\
\hline 0.414 & 0.450 & 134 & 33.1 & 12.3 & $3.62 \mathrm{e}+03$ & $9.00 \mathrm{e}+02$ \\
\hline 0.496 & 0.208 & 164 & 27.7 & 3.27 & $2.64 \mathrm{e}+03$ & $4.45 e+02$ \\
\hline 0.626 & 0.222 & 84.7 & 16.4 & 2.01 & $8.04 \mathrm{e}+02$ & $1.46 \mathrm{e}+02$ \\
\hline 0.898 & 0.116 & 28.0 & 4.42 & 0.328 & $8.80 \mathrm{e}+01$ & $1.35 \mathrm{e}+01$ \\
\hline 1.286 & 0.137 & 11.98 & 2.28 & 0.122 & $1.10 \mathrm{e}+01$ & $2.15 \mathrm{e}+00$ \\
\hline 1.843 & 0.280 & 19.0 & 3.98 & 0.082 & $5.87 e+00$ & $1.18 \mathrm{e}+00$ \\
\hline
\end{tabular}

or in the element form

$$
x_{i j}=\sum_{k=1}^{p} g_{i k} f_{k j}+e_{i j} \text {, }
$$

where $X$ is the matrix of observed data and the element $x_{i j}$ is the concentration value of the $i$ th sample at the $j$ th size bin. $G$ and $F$ are, respectively, the source contributions and size distribution profiles of the sources that are unknown and to be estimated from the analysis. To be specific, $g_{i k}$ is the concentration of particles from the $k$ th source associated with the $i$ th sample and $f_{k j}$ is the size distribution associated with $k$ th source. $E$ is a matrix of residuals. The model is solved by a least-square method using the program PMF2 (two-way PMF) (Paatero 1997). The mathematical expressions are

$$
\min _{G, F} Q
$$

and

$$
Q=\left\|\frac{(X-G F)}{s}\right\|_{F, G}^{2}=\sum_{i} \sum_{j}\left(\frac{e_{i j}}{s_{i j}}\right)^{2},
$$

where $s_{i j}$ is the uncertainty of each $x_{i j}$ value and the reciprocal of $s_{i j}$ serves as the weight. 
The details of the algorithm of PMF2 can be found elsewhere (Paatero 1997). The uncertainties inputted into PMF2 were computed based on the measurement errors with the expression

$$
s_{i j}=\sigma_{i j}+C_{3} \max \left(\left|x_{i j}\right|,\left|y_{i j}\right|\right),
$$

where $y_{i j}$ is the calculated value for $x_{i j}, \sigma_{i j}$ is the measurement error estimated in the previous section, and $\mathrm{C}_{3}$ is a dimensionless constant value between $0.1-0.2$. The additional uncertainty beyond the measurement errors estimated above is included to take into account in part the variability of the source profiles. The size distribution of particles emitted from a source cannot be expected to be perfectly constant, and some additional variation in the fit needs to be allowed to accommodate this variability. PMF2 was run separately for number and volume size distributions. The results of PMF2 analysis should be rescaled to satisfy the mass apportionment conditions (Hopke et al. 1980). For the sake of completeness, a brief introduction is given, as shown in the following equation:

$$
x_{i j}=\sum_{k=1}^{p} f_{i k} \cdot \frac{w_{k}}{w_{k}} \cdot g_{k j} .
$$

The scaling constant in the above equation, $w_{k}$, is determined by regressing the total number or volume contribution against the estimated source contributions:

$$
v_{j}=\sum_{k=1}^{p} w_{k} \cdot g_{k j} .
$$

The $G$ values discussed in this article are all rescaled for a number apportionment by this method.

\section{CONDITIONAL PROBABILITY FUNCTION (CPF)}

A conditional probability function (Ashbaugh et al. 1985; Kim et al. 2004) was calculated with the source contributions obtained by PMF2 and wind direction values as the following equation:

$$
\mathrm{CPF}=\frac{m_{\Delta \theta}}{n_{\Delta \theta}},
$$

where $m_{\Delta \theta}$ is the number of occurrences in the direction sector that exceed the threshold, upper $25 \%$ of the fractional contribution from each source; and $n_{\Delta \theta}$ is the total number of wind occurrences in the same direction sector. The fractional contribution is used instead of the volume or number contribution to avoid the influence of atmospheric dilution. Each direction sector is set 10 degrees, and thus there are 36 direction sectors. Those winds below $1.0 \mathrm{~m} / \mathrm{s}$ are excluded from this study. The sources are thought to be located in the direction sectors with high $\mathrm{CPF}$ values. It has to be pointed out that the $\mathrm{CPF}$ is not so dependable in finding the directions of far sources since the air mass may travel through a circuitous pathway.
The wind directions are concentrated within a few adjacent sectors; other direction sectors have a very low frequency of occurrence, and some have zero occurance. Those high CPF values with low values of $n_{\Delta \theta}$ are not reliable because of the uncertainties between the calculated contributions and the real contribution of each source. A threshold criteria, $n_{c}$, is needed. When $n_{c}>n_{\Delta \theta}$, the directional sector is neglected and CPF value is set to zero. In this study, $n_{c}$ is arbitrarily chosen to be 10 . The proper choice of the $n_{c}$ value remains an open question.

\section{RESULTS AND DISCUSSION}

Different numbers of factors and Fpeak values have been explored to obtain the most meaningful results. Fpeak is a parameter used in PMF2 to control the rotation. When the Fpeak is set to a positive value, the program adds one $G$ vector to another and subtracts the corresponding $F$ vectors from each other to obtain a more physically realistic solution. The details of the mathematical process can be found in Paatero et al. (2002). Five factors were selected, and the Fpeak value was set to 1.2 for number size-distribution and 0.2 for volume size-distribution analyses.

If the uncertainties are well estimated, the expected $Q$ value is approximately equal to the element number of $X$ matrix, which is 50,000 in this study. Since larger uncertainties were constructed (C3 is chosen as 0.2 ), the final $Q$ value is 25,013 , much smaller than the expected $Q$ value. The $Q$ value decreases when the factor number increases. When the factor number increases from 5 to 6 , the $Q$ value is not improved as much as it is when the factor number increases from 4 to 5 . Therefore, the final factor number was chosen as 5 .

Only the variation and time series analysis of the contribution values for the number concentration are presented here since the instruments directly measured the number concentration. The number and volume contributions of each factor were found to have quite similar temporal variations. For a given source, the size distribution profile for number concentration can be converted to obtain the profile for volume concentration, as indicated in Equation (12). In this equation, $N$ stands for number, $V$ stands for volume, $d_{j}$ is the diameter for the $j$ th size channel and $f_{j}$ is the fraction for the $j$ th channel. Multiplying the number contribution by the constant $C$ provides the corresponding volume contribution and vice versa. Since the source profiles and contributions for the number and volume distributions of each source are obtained through PMF with different rotations, the conversion is an approximate one. The match of the $G$ values by number and volume contribution is also based on the correlation coefficient of the $G$ values with gas-phase data and frequency properties:

$$
f_{j}^{V}=\frac{f_{j}^{N} d_{j}^{3} \cdot \pi / 6}{\sum_{j=1}^{n} f_{j}^{N} d_{j}^{3} \cdot \pi / 6}=\frac{f_{j}^{N} d_{j}^{3}}{\sum_{j=1}^{n} f_{j}^{N} d_{j}^{3}}=\frac{f_{j}^{N} d_{j}^{3}}{C} .
$$




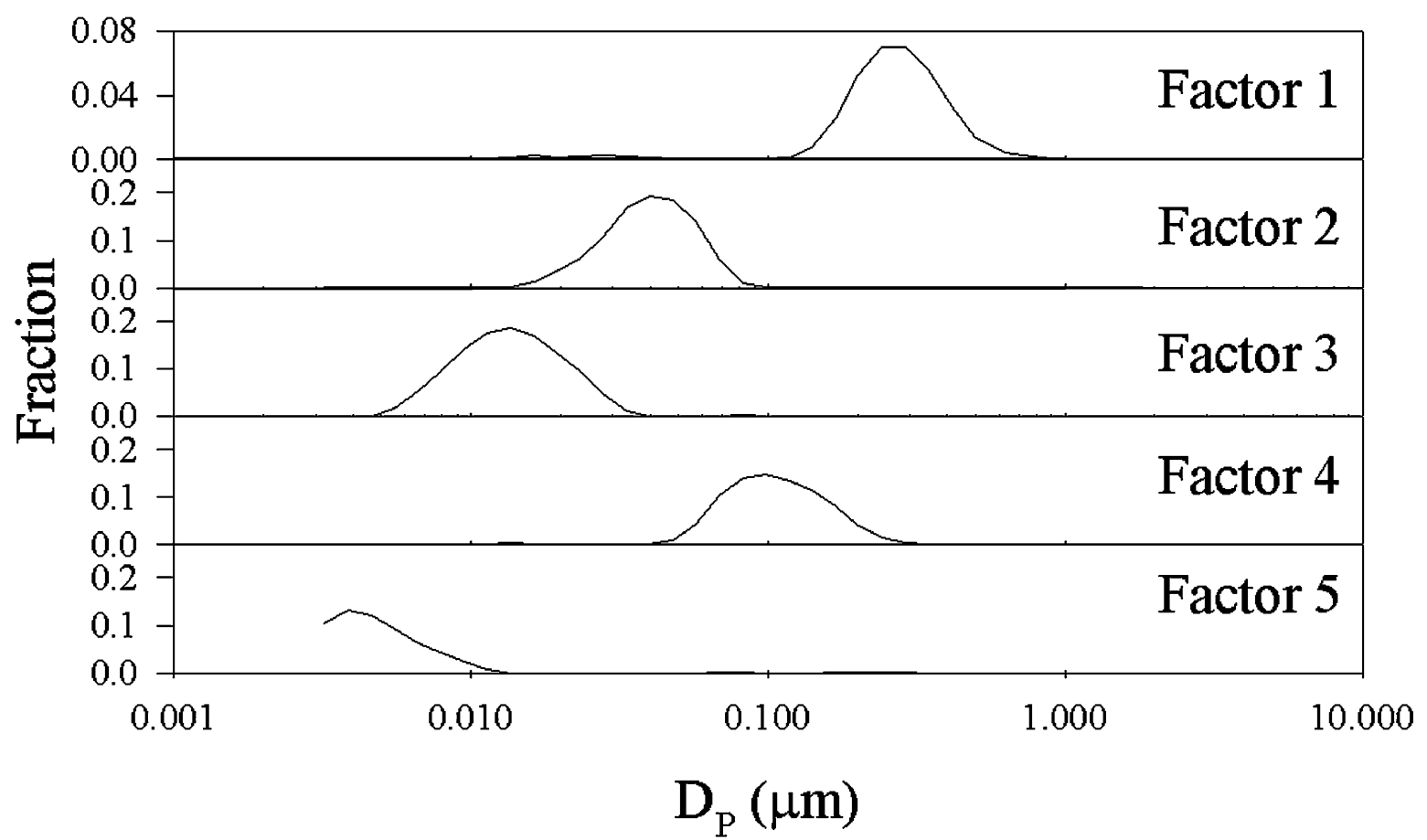

Figure 4. Normalized feature profile for number size distribution.

The sources were identified based on the profiles for both the number and volume size distributions of the factors, the time frequency properties of the contribution of each factor (Fourier analysis of $G$ values), and the correlations of the $G$ values with the gas phase and some composition data. The Fourier analysis identifies the strong frequencies in the data associated with periodic behavior, such as reoccurrences of rush hour traffic each day.

The number and volume size-distribution profiles of the five factors are shown in Figures 4 and 5, respectively. However,

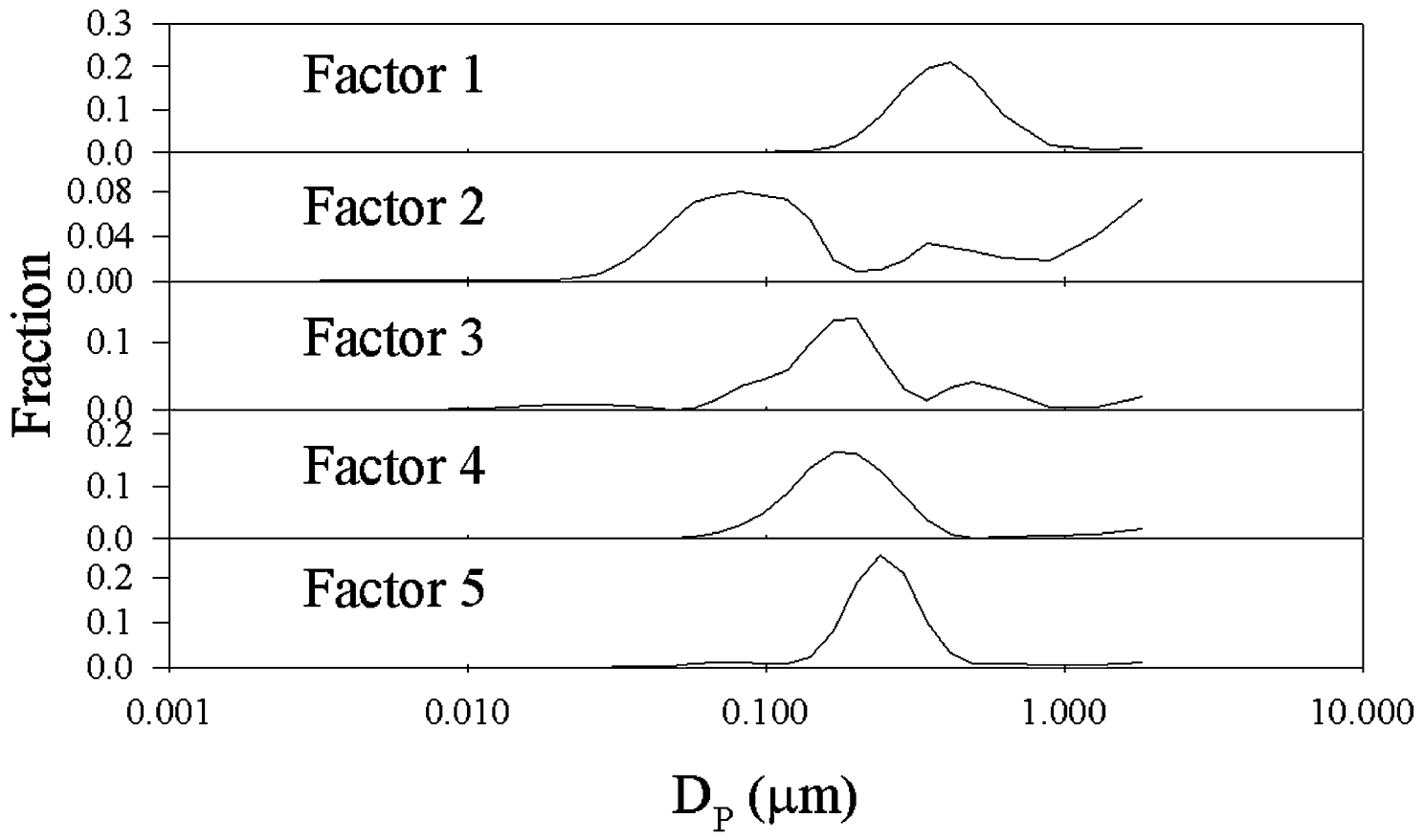

Figure 5. Normalized feature profile for volume size distribution. 


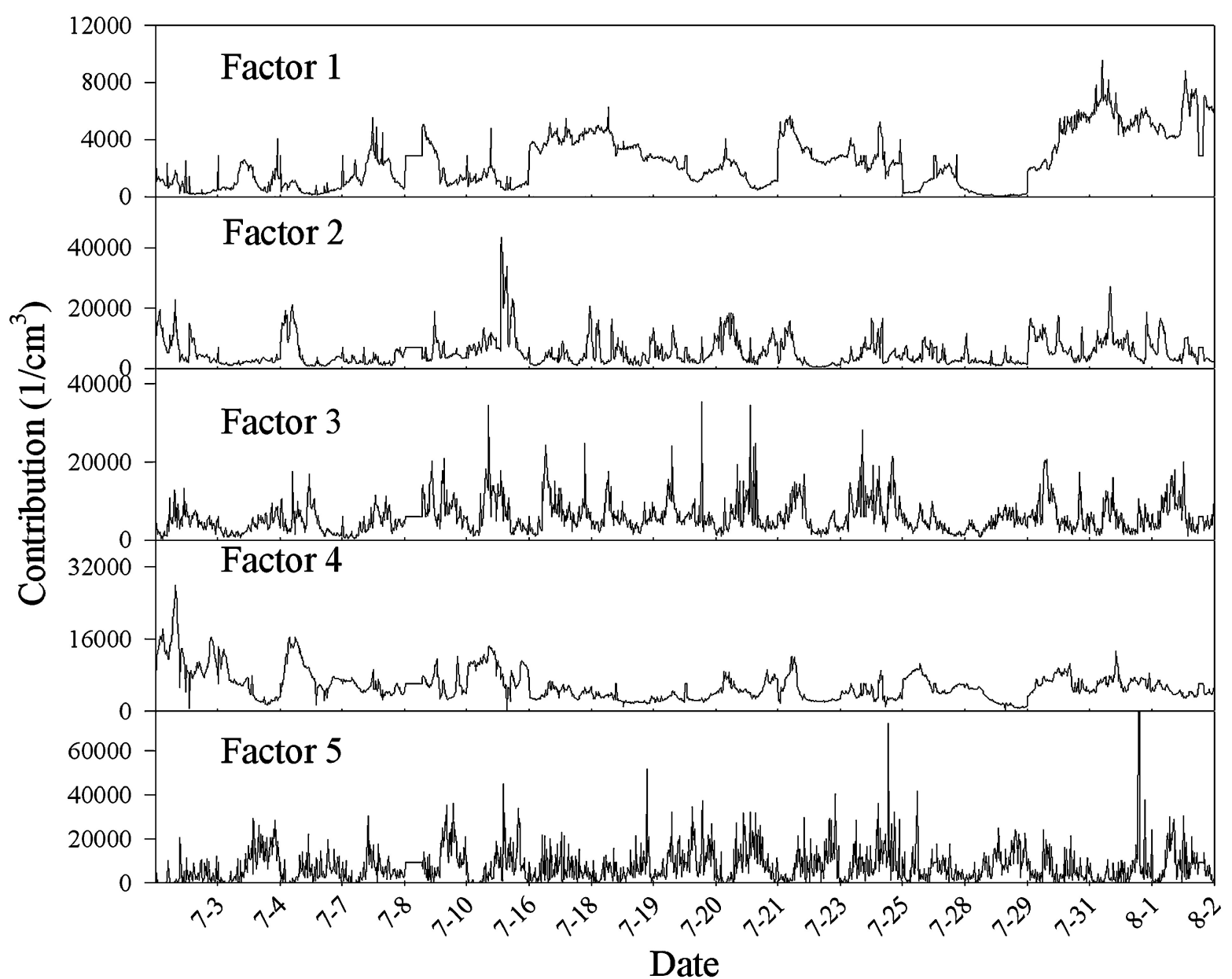

Figure 6. Number contribution for each factor.

size-distribution profiles alone are not sufficient since more than one source may have similar size-distribution profiles. The number of contributions for each factor are presented in Figure 6. The high temporal resolution of the experimental data make it possible to thoroughly investigate the frequency behavior of the contributions. It should be noted that those frequency peaks obtained by Fourier analysis do not absolutely reflect the actual temporal variations except those which are integral multiple of $1 / 24 \mathrm{~h}^{-1}$ because of the discontinuity of the dates resulting from the elimination of the nucleation events. However, the results of the Fourier transformation can still be used to identify daily patterns in the factors and to investigate the frequency properties qualitatively. The results of the Fourier transformation of the $G$ values (number) are shown in Figure 7. The results of the correlations of the $G$ values (number) with the gas-phase data and CPF function are presented in Table 3 and Figure 8, respectively. In Figure 8, the first subfigure indicates the number of occurrences in each wind direction sector is presented. Figure 9 shows about the $\mathrm{CPF}$ of gases for the same days as the particle. Because of their different temporal resolutions, the

Table 3

Correlations of $G$ (number) factors with gas-phase data and particle-mass data

\begin{tabular}{lcclllllll}
\hline & $\mathrm{O}_{3}$ & $\mathrm{NO}$ & $\mathrm{NO}_{\mathrm{x}}$ & $\mathrm{SO}_{2}$ & $\mathrm{CO}$ & $\mathrm{PM}_{2.5}$ & Sulfate & $\mathrm{OC}$ & $\mathrm{EC}$ \\
\hline Factor 1 & 0.07 & 0 & 0.146 & 0.332 & 0.148 & 0.907 & 0.858 & 0.527 & 0.463 \\
Factor 2 & -0.24 & 0.394 & 0.52 & 0.21 & 0.495 & 0.17 & 0.06 & 0.373 & 0.643 \\
Factor 3 & -0.19 & 0.198 & 0.31 & 0.01 & 0.145 & 0.113 & 0.04 & 0.09 & 0.394 \\
Factor 4 & -0.31 & 0.658 & 0.666 & 0.367 & 0.474 & 0.09 & -0.1 & 0.289 & 0.492 \\
Factor 5 & 0.221 & -0.19 & -0.22 & -0.12 & -0.2 & -0.16 & -0.14 & -0.43 & -0.21 \\
\hline
\end{tabular}




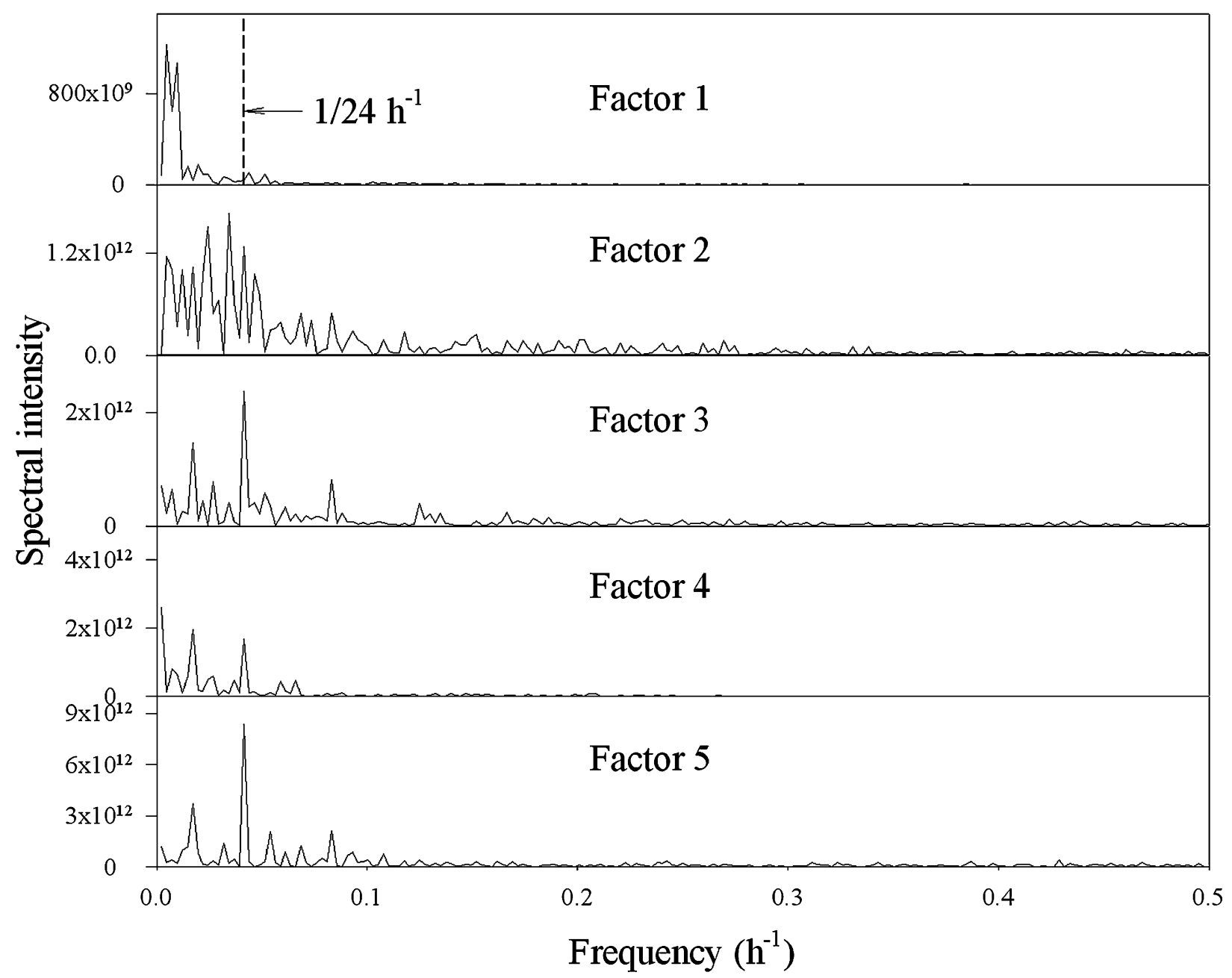

Figure 7. Fourier transformation of number contributions.

gas-phase data set and wind data were averaged to $30 \mathrm{~min}$, which caused some minor differences in the wind profiles of the two figures.

Factor 1 has a peak around $0.3 \mu \mathrm{m}$ and high correlations with $\mathrm{PM}_{2.5}$ and sulfate. The Fourier transformation shows that this factor has no obvious frequency peak. The total contribution to the receptor site does not change periodically with time. This information indicates a secondary aerosol. This factor is from distant sources; the particles were produced far from the receptor site and have accumulated secondary aerosol components (sulfate and organics) growing from their original size. The CPF function shows that these particles are from south of the site. Comparisons of the $\mathrm{PM}_{2.5}$ concentrations measured in Pittsburgh with the measurements in satellite sites around the city suggest that during July 2001 more than $80 \%$ of the Pittsburgh $\mathrm{PM}_{2.5}$ was transported into the city (Wittig et al. 2004). The present analysis also indicates that this factor is responsible for most of the aerosol volume as indicated in Table 4.
Factor 2 has a number mode at $0.04 \mu \mathrm{m}$, a volume mode around $0.09 \mu \mathrm{m}$, and is correlated with $\mathrm{NO}, \mathrm{NO}_{\mathrm{x}}$, and $\mathrm{CO}$. The correlation with EC is also strong, suggesting emissions from diesel trucks on the highway. The fact that the heavy-duty diesels also emit particles having this size range (Shi 2000) is consistent with the correlation with EC. There is a peak at $1 / 24 \mathrm{~h}^{-1}$, indicating a daily pattern. The $\mathrm{CPF}$ of the $\mathrm{NO}, \mathrm{NO}_{\mathrm{x}}$, and $\mathrm{CO}$ indicate that they are from the south, as shown in Figure 8, probably

Table 4

Average volume and number contribution of the sources

\begin{tabular}{lcc}
\hline Factor & Volume $\left(\mu \mathrm{m}^{3} / \mathrm{cm}^{3}\right)$ & Number $\left(\# / \mathrm{cm}^{3}\right)$ \\
\hline 1 & 14.09 & 2445 \\
2 & 0.93 & 5411 \\
3 & 0.36 & 5882 \\
4 & 3.26 & 5741 \\
5 & 0.24 & 7659 \\
\hline
\end{tabular}



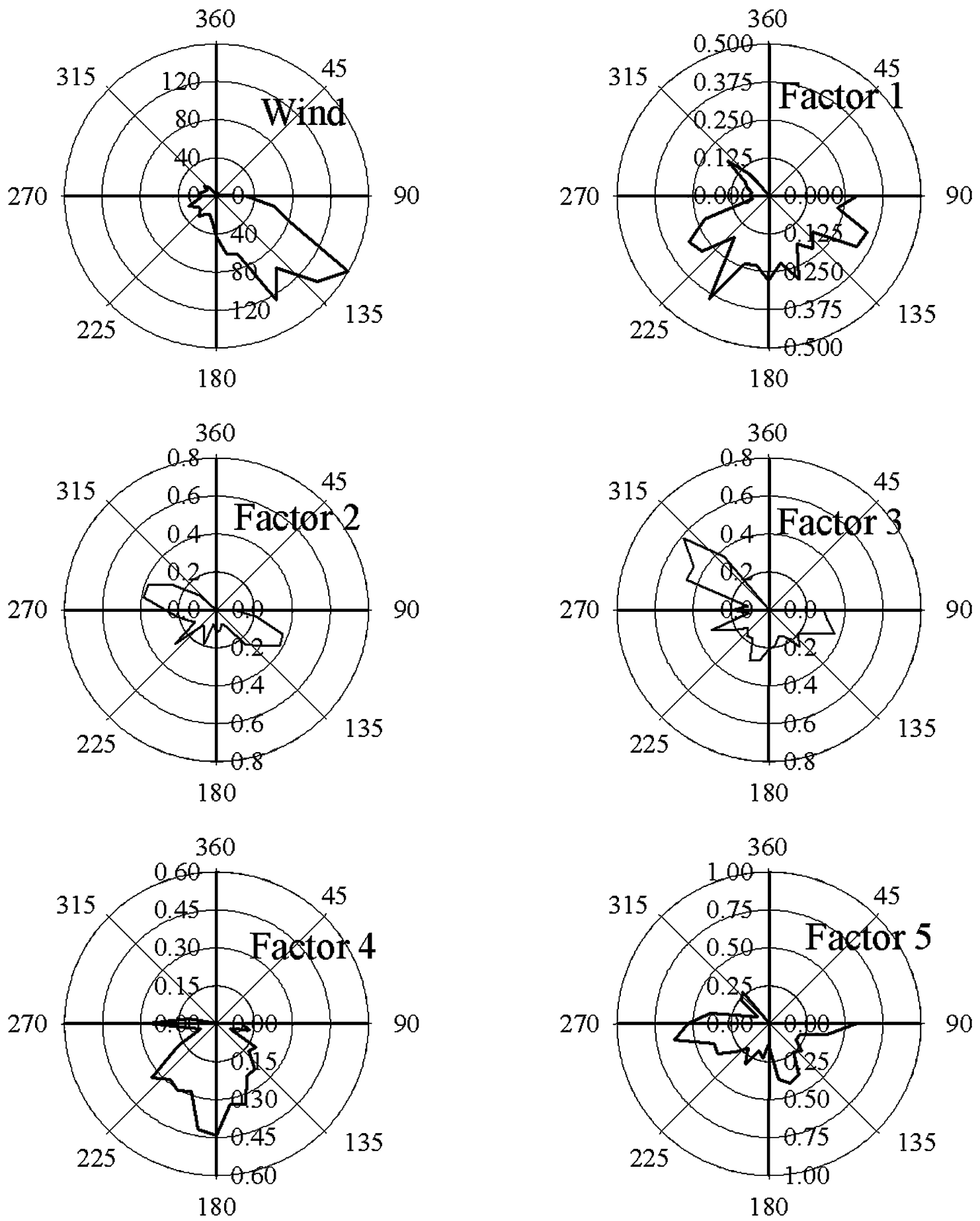

Figure 8. The conditional probability function for the number contributions.

from highway 376,1 mile south to the site, extending from west to east. This factor may contain particles from highway 376 . The distance from the source may explain the lack of a clear "transportation" diurnal profile. Meteorological variables such as mixing height and wind speed influence the profile more than the actual traffic pattern.

The number and volume modes are 0.015 and $0.2 \mu \mathrm{m}$ respectively, for Factor 3. Factor 3 is only weakly correlated with NO and $\mathrm{NO}_{\mathrm{x}}$, but it has an obvious frequency peak at $1 / 24 \mathrm{~h}^{-1}$. The two rush-hour peaks can be identified in Figure 10. Therefore, Factor 3 is assigned as a traffic aerosol. Because the particles are small (15 nm number size mode), this factor may be from the roads in the immediate vicinity of the site (Forbes Avenue, Schenley Drive, etc.), as shown in Figure 1. There is almost no diesel traffic in these places, which could explain the lack of correlation with $\mathrm{EC}$ and the weak correlation with $\mathrm{NO}_{\mathrm{x}}$. The 

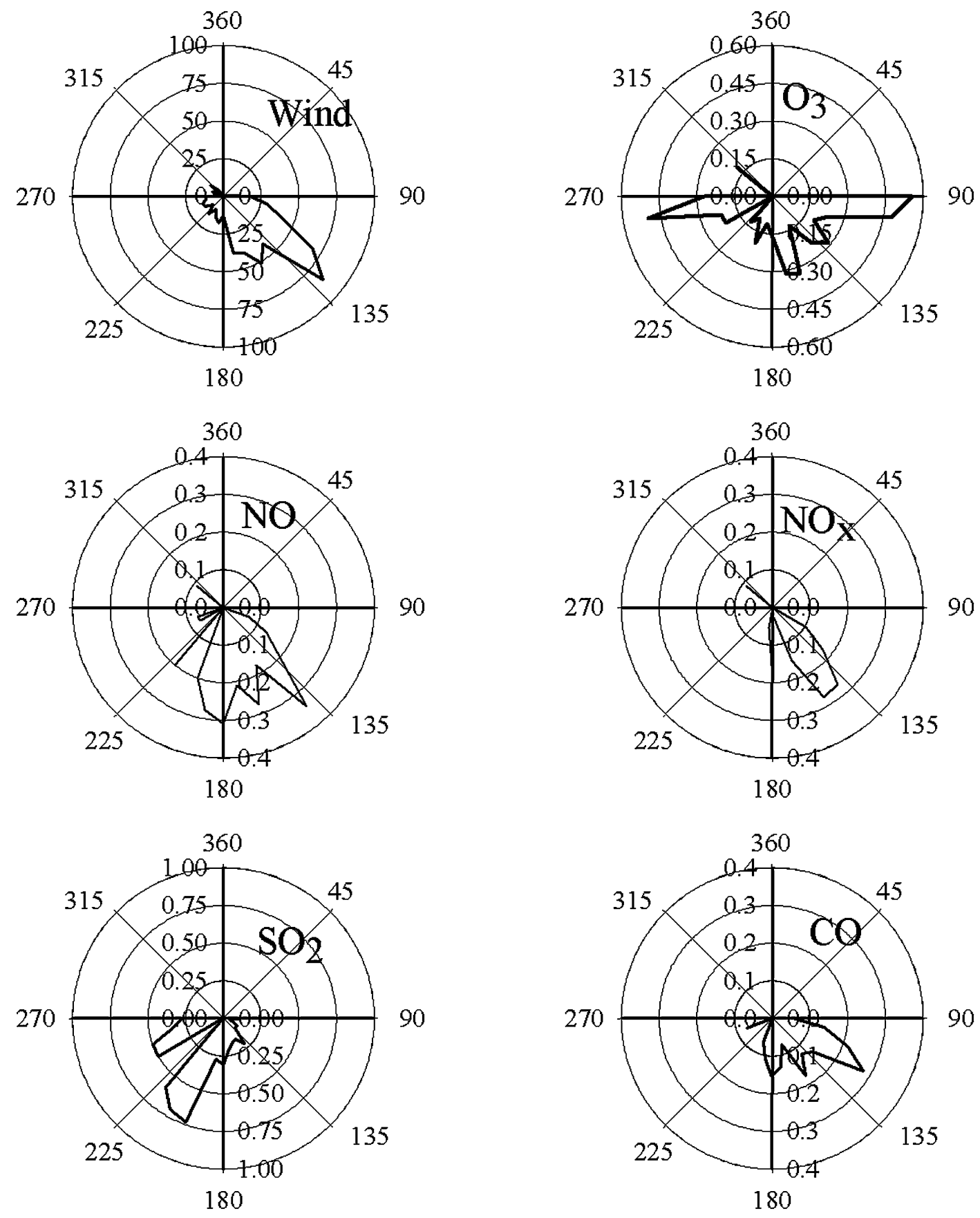

Figure 9. The conditional probability function for gases.

CPF has peaks at east and northwest, the directions of Forbes Avenue and other roads in the vicinity. The good diurnal traffic profile, as indicated in Figure 10, also suggests good proximity to the source. Figure 11 indicates the temporal variations of the gases ( $\mathrm{NO}, \mathrm{NO}_{\mathrm{x}}$, and $\mathrm{CO}$ ). The gases sometimes have high values at night, which could be caused by the inversion layer. These phenomena also occurred for factor 2 . The particles captured by the inversion layer were probably particles from the local traffic, which also weakens the "transportation" profile of factor 2. The traffic volume is lower on these smaller roads than on the highway, leading to a small contribution to the primary pollutants, which is also consistent with the small volume contribution of Factor 3. The low overall concentration contribution may explain the weak correlation of factor 3 with $\mathrm{NO}, \mathrm{NO}_{\mathrm{x}}, \mathrm{CO}$, and EC in another aspect. Conversely, factor 2 is named grown particles and remote traffic and factor 3 is titled local traffic.

Factor 4 has high correlations with $\mathrm{NO}, \mathrm{NO}_{\mathrm{x}}$, and $\mathrm{CO}$ but has no peak at $1 / 24 \mathrm{~h}^{-1}$. This factor may be related to combustion 


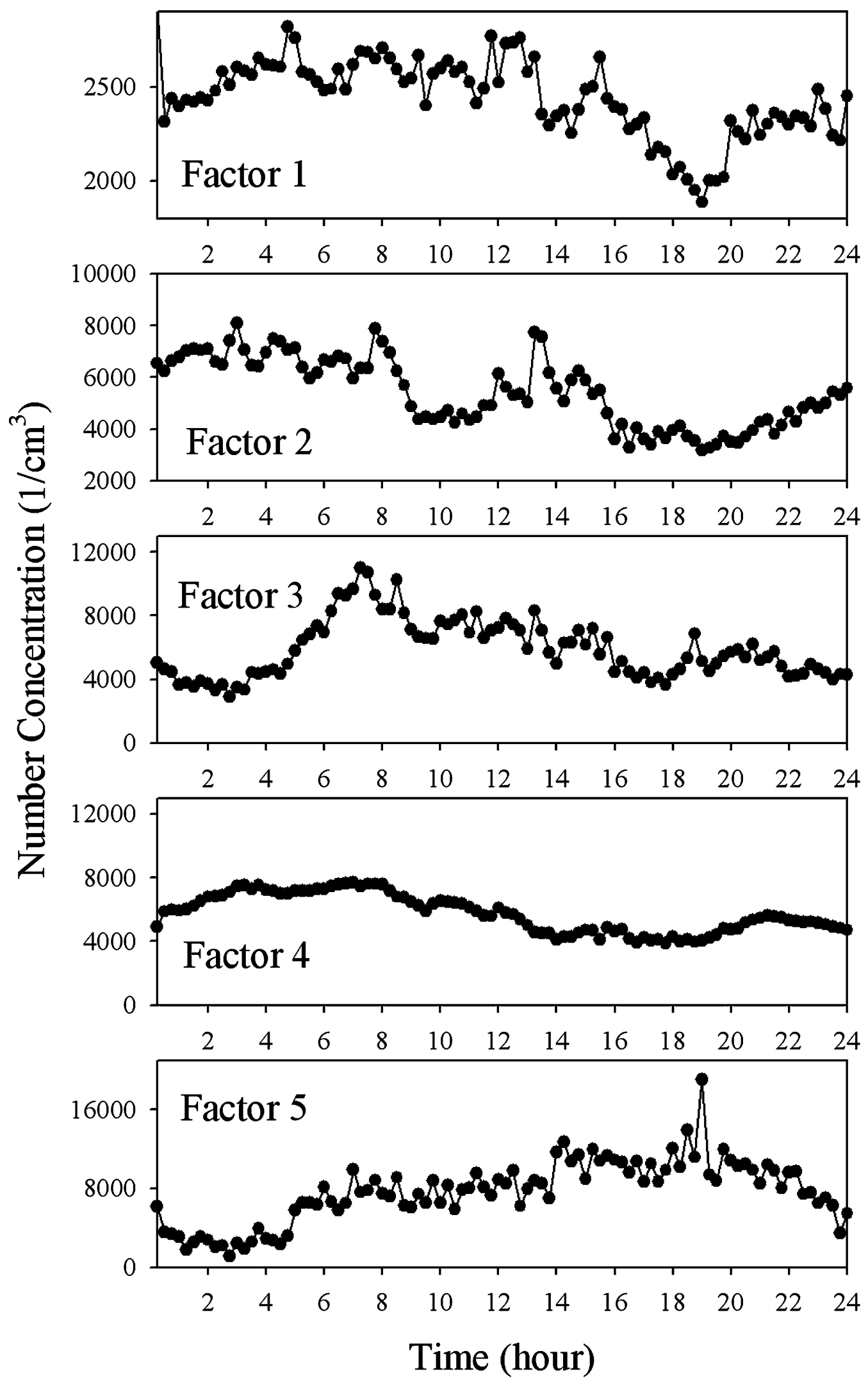

Figure 10. The average diurnal variation of the factors. 


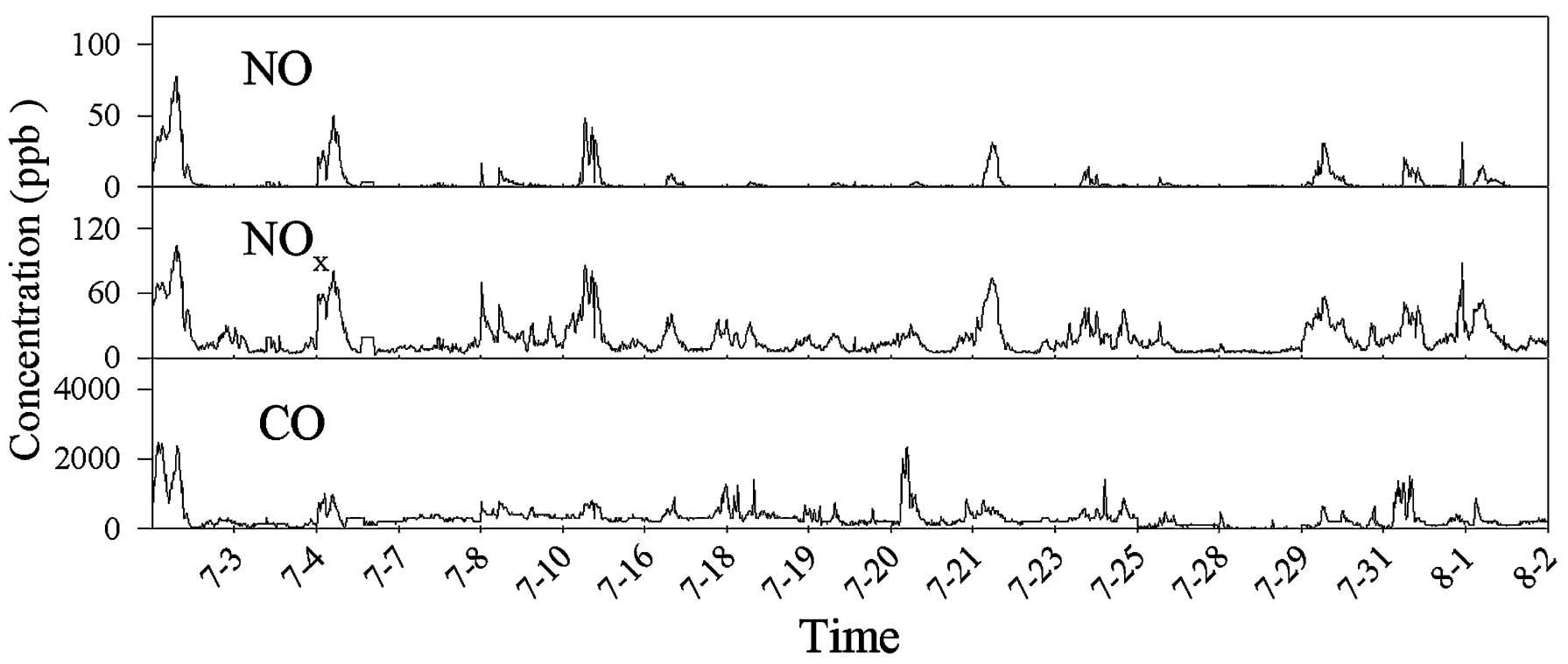

Figure 11. Temporal variations of $\mathrm{NO}, \mathrm{NO}_{\mathrm{x}}$, and $\mathrm{CO}$.

sources such as a power station or biomass fires (Morawska et al. 1998), but it is unlikely to be motor vehicle emissions. The mode around $0.1 \mu \mathrm{m}$ for the number distribution was suggested as representative of vegetation-burning-influenced aerosols by Morawska et al. (1999). The location of the volume mode is also in accordance with the reported measurement of wood burning (Morawska et al. 1999; Le Canut et al. 1996).

Wehner et al. (1999) reported that the number size distribution of a coal-fired heating power plant has a mode between 45 and $100 \mathrm{~nm}$. The mass mode of coal combustion is at $10 \mu \mathrm{m}$ (Lind et al. 1994), which is beyond the size range of this study. Factor 4 may be from local combustion sources and possibly vegetation burning as well. The CPF shows the sources of factor 4 are located to the south. The correlation of factor 4 with $\mathrm{SO}_{2}$ indicates coal combustion sources. Factor 4 has no correlation with sulfate, which means $\mathrm{SO}_{2}$ has not had enough time to oxidize to sulfate. Figure 12 is a map of the major emission sources in the eastern US. There are two major power plants to the south of Pittsburgh, close to the border with West Virginia, at a distance of less than $100 \mathrm{~km}$. A calculation is provided in the appendix to estimate the conversion rate of $\mathrm{SO}_{2}$. The result shows that within this range most $\mathrm{SO}_{2}$ is not reacted.

Factor 5 has a number mode at $3 \mathrm{~nm}$ and a volume mode at $0.25 \mu \mathrm{m}$. Factor 5 has a peak at $1 / 24 \mathrm{~h}^{-1}$ but no correlation with $\mathrm{NO}, \mathrm{NO}_{\mathrm{x}}$, or $\mathrm{CO}$. The contribution reaches a peak at midto late afternoon. The small particles, several nanometers large, with high number concentration, might be caused by nucleation events that were often observed to have peak activities in the afternoon. Photochemical reaction intensity increases in the afternoon and oxidants are produced, which can explain the positive correlation of factor 5 with ozone. These oxidants oxidize $\mathrm{SO}_{2}$ to form sulfuric acid which then nucleates, probably with water and possibly with ammonia. The volume mode at $0.25 \mu \mathrm{m}$, corresponding to the small "bumps" in the number distribution at the same size range, cannot be from nucleation. This volume mode can only be created locally together with the new particles by condensation of sulfuric acid and organic compounds. The anticorrelation of factor 5 with OC could be the result of the fact that most OC is primary. Factor 5 is called "sparse nucleation" since the nucleation occurs over limited time intervals with insufficient material present to permit growth in size up to the accumulation mode range (the excluded nucleation events). Factor 5 may have a composition similar to factor 1, and they are both secondary aerosol. The difference is that factor 5 is new-formed particles that have a short life time, while factor 1 are aged particles and more stable in the air.

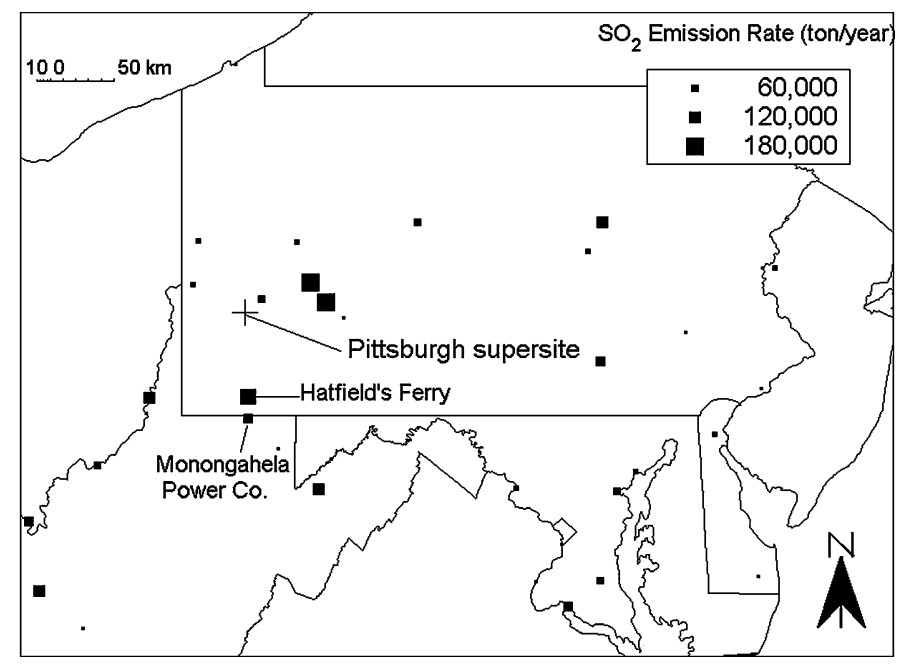

Figure 12. Map of region around the Pittsburgh area showing locations of power plants. 
Figure 5 shows that factors 1 and 4 have small peaks at high frequencies (over $0.1 \mathrm{~h}^{-1}$ ). This result may suggest that factors 1 and 4 form a kind of background that is stable and has no regular periodicity.

Table 4 summarizes the average contribution of those factors to the volume concentration and number concentration. The number concentration contributions are at the same order of magnitude, but the volume contributions are quite different. The volume contribution is mainly composed of secondary aerosol and stationary combustion.

\section{CONCLUSIONS}

Positive matrix factorization was used to explore sizedistribution data from Pittsburgh for source apportionment. From the investigation of the number and volume modes of the size distributions of the factors, the frequency properties, the correlations of the $G$ factors with gas phase data, and the CPF functions, five sources are identified: sparse nucleation, local traffic, stationary combustion, grown particles and remote traffic, and secondary aerosol. Although these factors are thus titled, they may still be contaminated by other unknown sources, especially the two traffic factors. Future analysis of the composition size data, such as micro-orifice uniform deposit impactor (MOUDI) and single-particle spectrum data, might provide additional, more specific source information.

\section{NOTATION}

$C_{3} \quad$ Coefficient used for a heuristic uncertainty in PMF2.

$D \quad$ Particle diameter, $\mu \mathrm{m}$

$E, e \quad$ Regression residual

$F, f \quad$ Size distribution profile associated with each source

$G, g$ Source contribution, $1 / \mathrm{cm}^{3}$ or $\mu \mathrm{m}^{3} / \mathrm{cm}^{3}$

$m$ The number of occurrences in the direction sector that exceed the threshold

$n \quad$ The total number of wind occurrences

$N \quad$ Number concentration, $1 / \mathrm{cm}^{3}$

$Q \quad$ Sum of the residual squares

$s \quad$ Uncertainty for each datum point used in PMF2

$t \quad$ Time

$u \quad$ Instrumental error for small particles, $1 / \mathrm{cm}^{3}$

$V \quad$ Volume concentration, $\mu \mathrm{m}^{3} / \mathrm{cm}^{3}$

$v \quad$ Total mass, number or volume concentration for each sample

$w \quad$ A constant for mass, number, or volume apportionment

$X, x \quad$ Data to be regressed

$y \quad$ Regressed concentration value used in PMF2

\section{Greek Letters}

\section{$\theta \quad$ Wind direction}

$\sigma \quad$ Measurement error for the combined size bins, $1 / \mathrm{cm}^{3}$

$\sigma^{\prime} \quad$ Measurement error for the original-size bins, $1 / \mathrm{cm}^{3}$

\section{REFERENCES}

Ashbaugh, L. L., Malm, W. C., and Sadeh, W. Z. (1985). A Residence Time Probability Analysis of Sulfur Concentrations at Ground Canyon National Park, Atmos. Environ. 19(8):1263-1270.

Chueinta, W., Hopke, P. K., and Paatero, P. (2000). Investigation of Sources of Atmospheric Aerosol at Urban and Suburban Residential Area in Thailand by Positive Matrix Factorization, Atmos. Environ. 34:33193329.

Hopke, P. K., Lamb, R. E., and Natusch, D. F. C. (1980). Multielemental Characterization of Urban Roadway Dust. Environ. Sci. Technol. 14:164172.

Khlystov, A., Stanier, C., and Pandis, S. N. (2004). An Algorithm for Combining Electrical Mobility and Aerodynamic Size Distributions When Measuring Ambient Aerosol, Aerosol Sci. Technol. 38:229-238.

Kim, E., Hopke, P. K., and Larson, T. V. (2004). Analysis of Ambient Particle Size Distributions Using Unmix and Positive Matrix Factorization, Environ. Sci. Technol. 38:202-209.

Le Canut, P., Andreae, M. O., Harris, G. W., Wienhold, F. G., and Zenker, T. (1996). Airborne Studies of Emissions From Savanna Fires in Southern Africa: Aerosol Emissions Measured with a Laser Optical Particle Counter, J. Geophys. Res. 101(D19):23615-23630.

Lee, E., Chun, C. K., and Paatero, P. (1999). Application of Positive Matrix Factorization in Source Apportionment of Particulate Pollutants, Atmos. Environ. 33:3201-3212.

Lind, T. M., Kauppinen, E. I., Jokiniemi, J. K., Lillieblad, L., and Klippel, N. (1994). Coal Combustion Aerosol Particle Size Distribution Determination Using Low-Pressure Impactor and CCSEM Methods, J. Aerosol Sci. 25(Suppl. 1):S327-S328.

Morawska, L., Thomas, S., Jamriska, M., and Johnson, G. (1999). The Modality of Particle Size Distributions of Environmental Aerosols, Atmos. Environ. 33:4401-4411.

Morawaska, L., Thomas, S., Bofinger, N., Wainwright, D., and Neale, D. (1998). Comprehensive Characterization of Aerosols in a Subtropical Urban Atmosphere: Particle Size Distribution and Correlation with Gaseous Pollutants, Atmos. Environ. 32:2467-2478.

Paatero, P. (1997). Least Squares Formulation of Robust, Non-Negative Factor Analysis, Chemometrics Intelligent Lab. Syst. 37:23-55.

Paatero, P., Hopke, P. K., Song, X. H., and Ramadan, Z. (2002) Understanding and Controlling Rotations in Factor Analytic Models, Chemometrics Intelligent Lab. Syst. 60:253-264.

Peters, A., Wichmann, H. E., Tuch, T., Heinrich, J., and Heyder, J. (1997). Respiratory Effects Are Associated with the Number of Ultrafine Particles, Am. J. Resp. Crit. Care Med. 155:1376-1383.

Polissar, A. V., Hopke, P. K., and Poirot, R. L. (2001). Atmospheric Aerosol over Vermont: Chemical Composition and Sources, Environ. Sci. Technol. 35:4604-4621.

Ramadan, Z., Song, X. H., and Hopke, P. K. (2000). Identification of Sources of Phoenix Aerosol by Positive Matrix Factorization, J. Air Waste Manag. Assoc. 50:1308-1320.

Reischl, G. P. (1991). Measurement of Ambient Aerosols by the Differential Mobility Analyzer Method: Concepts and Realization Criteria for the Size Range Between 2 and $500 \mathrm{~nm}$, Aerosol Sci. Technol. 14:5-24.

Ruuskanen, J., Tuch, T., Brink, H. T., Peters, A., Khlystov, A., Mirme, A., Kos, G. P. A., Brunekreef, B., Wichmann, H. E., Buzorius, G., Vallius, M., Kreyling, W. G., and Pekkanen. J. (2001). Concentrations of Ultrafine, Fine and PM2.5 Particles in Three European Cities, Atmos. Environ. 35:37293738.

Seinfeld, J. H., and Pandis, S. N. (1998). Atmospheric Chemistry and Physics. John Wiley \& Sons, New York, p. 365.

Shi, J. P., David, M., and Harrison, R. M. (2000). Characterization of Particles from a Current Technology Heavy-Duty Diesel Engine, Environ. Sci. Technol. 34:748-755.

Song, X. H., Polissar, A. V., and Hopke, P. K. (2001). Source of Fine Particle Composition in the Northeastern U.S., Atmos. Environ. 35:52775286. 
Stanier, C., Khlystov, A., and Pandis, S. N. (2004). Nucleation Events During the Pittsburgh Air Quality Study: Description and Relation to Key Meteorological, Gas Phase, and Aerosol Parameters, Aerosol Sci. Technol. 38:253-264.

van Bree, L., and Cassee, F. R. (2000). A Critical Review of Potentially Causative PM Properties and Mechanisms Associated with Health Effects, National Institute of Public Health and the Environment (RIVM) Research Report, report no. 650010015, Bilthoven, the Netherlands.

Venkataraman, C., and Kao, A. S. (1999). Comparison of Particles Lung Doses from the Fine and Coarse Fraction of Urban PM-10 Aerosols, Inhalation Toxic. 11:151-169.

Wahlin, P., Palmgren, F., Dingenen, R. V., and Raes, F. (2001). Experimental Studies of Ultrafine Particles in Streets and the Relationship to Traffic, Atmos. Environ. 35(S1):S63-S69.

Wehner, B., Bond, T. C., Birmili, W., Heintzenberg, J., Wiedensohler, A., and Charlson, R. J. (1999). Climate-Relevant Particulate Emission Characteristics of a Coal Fired Heating Plant, Environ. Sci. Technol. 33:3881-3886.

Wittig, B., Anderson, N., Khlystov, A. Y., Davidson, C., Robinson, A., and Pandis, S. N. (2004). The Pittsburgh Air Quality Study (PAQS), Atmos. Environ. in press.

Wong, S. S. M. (1997). Computational Methods in Physics and Engineering, 2nd ed. World Scientific Publishing Co. Pte. Ltd., Singapore, p. 237.

Xie, Y. L., Hopke, P. K., Paatero, P., Barrie, L. A., and Li, S. M. (1999). Identification of Source Nature and Seasonal Variations of Arctic Aerosol by Positive Matrix Factorization, J. Atmos. Sci. 56:249-260.

\section{APPENDIX: AN ESTIMATION OF $\mathrm{SO}_{2}$ CONVERSION RATE}

The distance of the coal power plant and other combustion sources emitting $\mathrm{SO}_{2}$ is $10-100 \mathrm{~km}$. Suppose the wind speed is $10 \mathrm{~km} / \mathrm{h}$. The traveling time of the pollutant is $1-10 \mathrm{~h}$.
The following equation is used to estimate the conversion rate:

$$
\frac{d C}{C}=-R d t,
$$

where $\mathrm{C}$ is the concentration of $\mathrm{SO}_{2}$ and $\mathrm{R}$ is the reaction rate with a unit of $\% \mathrm{~h}^{-1}$. The conversion rate of $\mathrm{SO}_{2} X$ is

$$
X=1-\frac{C}{C_{0}}=1-\exp (-R t) .
$$

A typical gas-phase $\mathrm{SO}_{2}$ oxidation rate by $\mathrm{OH}$ is on the order of $1 \% \mathrm{~h}^{-1}$ (Seinfeld and Pandis 1998). By substituting $\mathrm{R}=1$ into Equation (A2), the conversion rate is $0.006 \sim 1 \%$.

The oxidation of $\mathrm{SO}_{2}$ is dominated by dissolved hydrogen peroxide for $\mathrm{pH}<5$. Assume that the frequency of cloud occurrence is 0.4 , the areal coverage by cloud is 0.2 , and the average liquid water content of cloud is $0.2 \mathrm{~g} / \mathrm{m}^{3}$. In Seinfeld and Pandis (1998) the oxidation rate is estimated at around 500\%/h when the liquid water content is $1 \mathrm{~g} / \mathrm{m}^{3}$ and hydrogen peroxide has a concentration of $1 \mathrm{ppb}$. Thus the reaction rate of $\mathrm{SO}_{2}$ under the former assumptions should be $0.4 \times 0.2 \times 0.2 \times$ $500 \% / \mathrm{h}=8 \% / \mathrm{h}$.

In this situation, the conversion rate of $\mathrm{SO}_{2}$ is $7.7-55 \%$. In fact, the production of sulfuric acid decreases the $\mathrm{pH}$ value, which causes less dissolved $\mathrm{SO}_{2}$, so the conversion rate should be lower. 


\title{
Advanced factor analysis for multiple time resolution aerosol composition data
}

\author{
Liming Zhou ${ }^{\mathrm{a}}$, Philip K. Hopke ${ }^{\mathrm{a}, *}$, Pentti Paatero ${ }^{\mathrm{b}}$, John M. Ondov ${ }^{\mathrm{c}}$, \\ J. Patrick Pancras ${ }^{c}$, Natalie J. Pekney ${ }^{\mathrm{c}}$, Cliff I. Davidson ${ }^{\mathrm{d}}$ \\ ${ }^{a}$ Center for Air Resources Engineering and Science, and Department of Chemical Engineering, Clarkson University, \\ PO. Box 5708, Postdam, NY 13699-5708, USA \\ ${ }^{\mathrm{b}}$ Department of Physical Sciences, University of Helsinki, Finland \\ ${ }^{\mathrm{c}}$ Department of Chemistry and Biochemistry, University of Maryland at College Park, USA \\ ${ }^{\mathrm{d}}$ Department of Civil and Environmental Engineering, Carnegie Mellon University, USA
}

Received 3 February 2004; accepted 10 May 2004

\begin{abstract}
New monitoring technologies have now permitted the measurement of a variety of chemical species in airborne particulate matter with time resolution as high as $10 \mathrm{~min}$ to $1 \mathrm{~h}$. There are still species that are measured with longer integration periods such as several hours to a day. These data from different measurement methods produce a data set of mixed time resolution. Traditional eigenvalue-based methods used in solving multivariate receptor models are unable to analyze this kind of data set since these data cannot form a simple matrix. Averaging the high time resolution data or interpolating the low time resolution data to produce data on the same time schedule is not acceptable. The former method loses valuable temporal information and the latter produces unreliable high resolution series because of the invalid assumption of temporal smoothness. In the present work, a solution to the problem of multiple sampling time intervals has been developed and tested. Each data value is used in its original time schedule without averaging or interpolation and the source contributions are averaged to the corresponding sampling interval. For data with the highest time resolution, the contributions are not actually averaged. The contribution series are smoothed by regularization auxiliary equations especially for sources containing very little high resolution species. This new model will be explored using data from the Pittsburgh supersite.
\end{abstract}

(C) 2004 Elsevier Ltd. All rights reserved.

Keywords: Multiple time resolution; Aerosol; Source apportionment; Factor analysis; Multilinear Engine (ME); Non-parametric regression (NPR)

\section{Introduction}

Multivariate statistical receptor models have been extensively used for source apportionment of airborne particles (Henry, 1997, 2002; Hopke, 2003). For the conventional receptor model problem, aerosol compositional data containing a number of species are obtained

\footnotetext{
*Corresponding author. Fax: 315-268-4410.

E-mail address: hopkepk@clarkson.edu (P.K. Hopke).
}

for each sample. The concentrations of all species for all of the samples form a matrix. This matrix can be decomposed into two matrices representing source contributions and source profiles.

Recent developments of measurement methods for airborne particle species have provided higher time resolution. Automatic sampling analysis systems are commercially available for organic and elemental carbon, sulfate and nitrate with typical time resolution of 1 hour as summarized by Wilson et al. (2002). Several 
investigators have developed steam injection/impaction samplers that permit the collection of sufficient sample size to allow analyses for multiple species with time resolution as low as $30 \mathrm{~min}$ (Khlystov et al., 1995; Slanina et al., 2001; Kidwell and Ondov, 2001; Orsini et al., 2003). Recent work on Synchrotron X-ray fluorescence has also suggested that short time $(<1 \mathrm{~h})$ resolution data can be readily obtained (Bench et al., 2002). These data can be combined with longer term integrated samples analyzed by conventional techniques like XRF or PIXE to produce a data set of mixed time resolution.

Traditional multivariate techniques (PCA, PMF2) cannot utilize the full information content of data sets measured with multiple sample time schedules. It would

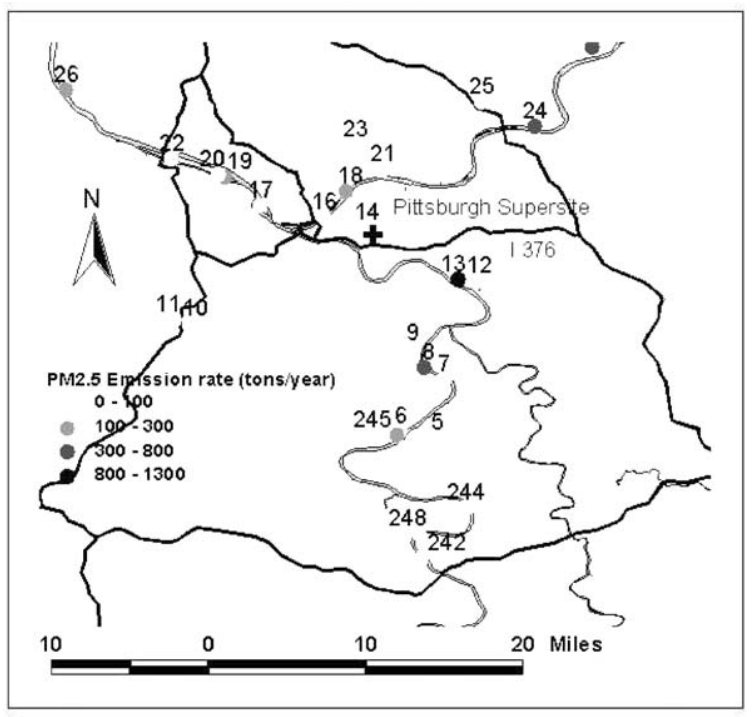

Fig. 1. Map of Pittsburgh supersite and major local PM2.5 sources (some sources are described in Table 6). be necessary to somehow transform the data to a single time schedule. Either the high-resolution data need to be averaged over the averaging intervals of the longest time-interval data, typically $24 \mathrm{~h}$, or the low-resolution data would have to be interpolated to the short time periods of the fastest measurements. Averaging would lose all of the valuable high time-resolution information. Alternatively, interpolation must be based on uncertain assumptions of the nature of temporal variation of the source emissions. Interpolation would typically ignore diurnal patterns and attribute similar amounts of concentration to all $24 \mathrm{~h}$ of the sampling period. Thus, it would be impossible to correctly deduce the proper connections between the 24-h interpolated aerosol data and high-resolution (e.g., hourly) concentration data displaying the true short term patterns.

In this work, aerosol compositional data from Pittsburgh air quality study (PAQS) have been used to test a new model in which each data value is preferentially used in its original time schedule. Neither averaging nor interpolation of original data is needed. Averaging of high resolution contribution series is only used for low resolution data.

\section{Data description}

The data are from the PAQS, 16, 17, 18, 23 and 24 July, 2001. The Pittsburgh supersite was located in a park close to the city center (Latitude 40.4395, Longitude -79.9405). The location of the site and major $\mathrm{PM}_{2.5}$ sources near the site are indicated in Fig. 1. Table 1 summarizes the data set that has been used in this study. The species and time resolution for each method are listed in Table 1. The total data set includes sulfate and nitrate data obtained by continuous instruments of Aerosol Dynamics Inc. ([ADI], Stolzenburg

Table 1

Summary of the data set used in this study

\begin{tabular}{|c|c|c|c|c|c|c|c|}
\hline & $\begin{array}{l}\text { ADI Sulfate, } \\
\text { nitrate }\end{array}$ & SEAS & $\begin{array}{l}\text { "Best } \\
\text { estimation" } \\
\text { of sulfate and } \\
\text { nitrate }\end{array}$ & Steam IC & $\mathrm{OC} / \mathrm{EC}$ & ICP-MS & $\begin{array}{l}\text { Sampling } \\
\text { date }\end{array}$ \\
\hline $\begin{array}{l}\text { Species } \\
\text { measured }\end{array}$ & $\begin{array}{l}\text { Sulfate, } \\
\text { nitrate }\end{array}$ & $\begin{array}{l}\mathrm{Al}, \mathrm{As}, \mathrm{Cd}, \\
\mathrm{Cr}, \mathrm{Cu}, \mathrm{Fe}, \\
\mathrm{Mn}, \mathrm{Ni}, \mathrm{Pb}, \\
\mathrm{Se}, \mathrm{Zn}\end{array}$ & $\begin{array}{l}\text { Sulfate, } \\
\text { nitrate }\end{array}$ & $\begin{array}{l}\text { Nitrite, } \\
\text { chloride, } \\
\text { ammonium } \\
\text { ion, } \\
\text { potassium }\end{array}$ & $\mathrm{OC} / \mathrm{EC}$ & $\begin{array}{l}\mathrm{As}, \mathrm{Cd}, \mathrm{Cu}, \\
\mathrm{Fe}, \mathrm{Mn}, \mathrm{Mo}, \\
\mathrm{Pb}, \mathrm{Sb} \mathrm{Se}, \mathrm{Ti}, \\
\text { V, Zn }\end{array}$ & - \\
\hline \multirow{5}{*}{$\begin{array}{l}\text { Time } \\
\text { resolution }\end{array}$} & $10 \mathrm{~min}$ & $0.5 \mathrm{~h}$ & $1 \mathrm{~h}$ & $1 \mathrm{~h}$ & $3,4,5 \mathrm{~h}$ & $1 \mathrm{~d}$ & 16 July \\
\hline & $10 \mathrm{~min}$ & $0.5 \mathrm{~h}$ & $1 \mathrm{~h}$ & $1 \mathrm{~h}$ & $3,4,5 \mathrm{~h}$ & $1 \mathrm{~d}$ & 17 July \\
\hline & $10 \mathrm{~min}$ & $0.5 \mathrm{~h}$ & $1 \mathrm{~h}$ & $1 \mathrm{~h}$ & $3,4,5 \mathrm{~h}$ & $1 \mathrm{~d}$ & 18 July \\
\hline & $10 \mathrm{~min}$ & $0.5 \mathrm{~h}$ & $1 \mathrm{~h}$ & $1 \mathrm{~h}$ & $2 \mathrm{~h}$ & $4,6 \mathrm{~h}$ & 23 July \\
\hline & $10 \mathrm{~min}$ & $0.5 \mathrm{~h}$ & $1 \mathrm{~h}$ & $1 \mathrm{~h}$ & $2 \mathrm{~h}$ & $4,6 \mathrm{~h}$ & 24 July \\
\hline
\end{tabular}


and Hering, 2000), with 10 min resolution; SEAS (Semicontinuous Elements in Aerosol System) data with $30 \mathrm{~min}$ resolution (Kidwell and Ondov, 2001); the $1 \mathrm{~h}$ sulfate and nitrate data regressed from several measurement methods, called "best estimation" (Wittig et al., 2004a, b); steam IC (Ionic Chromatography) data with $1 \mathrm{~h}$ resolution (Weber et al., 2001; Khlystov et al., 1995; Slanina et al., 2001); OC/EC data with varied sampling periods 2-4h (Turpin and Huntzicker, 1995); data from digestion and analysis of integrated filter by ICP-MS (Inductively Coupled Plasma Mass Spectrometry) with $24 \mathrm{~h}$ resolution or $4-6 \mathrm{~h}$ resolution on intensive measurement days. $\mathrm{PM}_{2.5}$ mass concentrations were measured every $10 \mathrm{~min}$ by TEOM (Tapered Element Oscillating Microbalance $\mathrm{PM}_{2.5}$ Sampler) running at $30^{\circ} \mathrm{C}$ with sample equilibration system. Gases, including $\mathrm{O}_{3}, \mathrm{NO}$, $\mathrm{NO}_{x}, \mathrm{CO}$ and $\mathrm{SO}_{2}$, as well as meteorological data were also obtained at the same time and location. Gases were measured every $10 \mathrm{~min}$ and meteorological conditions were measured every $15 \mathrm{~min}$. Details of the measurement methods and instruments can be found elsewhere (Stanier et al., 2004; Wittig et al., 2004a, b). The total sample number and the number of missing samples of all methods for every species are indicated in Table 2.

Table 2

Sum of squares of scaled residuals of Equation $1\left(Q_{\text {main }}\right)$ and adjustment factors

\begin{tabular}{|c|c|c|c|c|}
\hline Species & $\begin{array}{l}\text { Total } \\
\text { number of } \\
\text { samples }\end{array}$ & $\begin{array}{l}\text { Number } \\
\text { of missing } \\
\text { samples }\end{array}$ & $Q_{\text {main }}$ & $\begin{array}{l}\text { Adjustment } \\
\text { factors }\end{array}$ \\
\hline Sulfate & 840 & 31 & 175 & 0.78 \\
\hline Nitrate & 840 & 10 & 59 & 0.89 \\
\hline $\mathrm{Al}$ & 240 & 9 & 1007 & - \\
\hline As & 253 & 63 & 213 & 1.04 \\
\hline $\mathrm{Cd}$ & 253 & 17 & 209 & 1.29 \\
\hline $\mathrm{Cr}$ & 240 & 9 & 764 & - \\
\hline $\mathrm{Cu}$ & 253 & 19 & 463 & 2.01 \\
\hline $\mathrm{Fe}$ & 253 & 11 & 673 & 0.126 \\
\hline $\mathrm{Mn}$ & 253 & 11 & 581 & 0.70 \\
\hline $\mathrm{Ni}$ & 240 & 9 & 63 & - \\
\hline $\mathrm{Pb}$ & 253 & 11 & 1526 & 0.64 \\
\hline $\mathrm{Se}$ & 253 & 11 & 63 & 0.97 \\
\hline $\mathrm{Zn}$ & 253 & 13 & 95 & 1.04 \\
\hline Nitrite & 120 & 33 & 372 & - \\
\hline Chloride & 120 & 33 & 1135 & - \\
\hline Ammonium ion & 120 & 33 & 263 & - \\
\hline Sodium & 120 & 33 & 130 & - \\
\hline Potassium & 133 & 33 & 5 & - \\
\hline $\mathrm{OC}$ & 42 & 5 & 168 & - \\
\hline $\mathrm{EC}$ & 42 & 5 & 18 & - \\
\hline $\mathrm{Ti}$ & 13 & 1 & 8 & - \\
\hline V & 13 & 0 & 5 & - \\
\hline Mo & 13 & 3 & 34 & - \\
\hline $\mathrm{Sb}$ & 13 & 1 & 10 & - \\
\hline
\end{tabular}

\section{Model description}

The basic idea is to utilize the measured concentration data in its original time schedule. For each concentration value, there are contributions from several sources, and source contributions have to be averaged so that the concentration value and the source contribution are in the same sampling period. The main equation of the model is as below:

$x_{s j}=1 /\left(t_{s 2}-t_{s 1}+1\right) \sum_{p=1}^{P}\left(f_{j p} \sum_{i=t_{s 1}}^{t_{s 2}} g_{i p} \eta_{j m}\right)+e_{s j}$,

where $s$ is the sample number, $j$ stands for the species, $t_{s 2}$ is the end time and $t_{s 1}$ is the start time (described by the number of time units). The shortest sampling interval, $10 \mathrm{~min}$ for this data set, is chosen as the time unit. In Eq. (1), $x_{s j}$ is the concentration of $j$ th species in $s$ th sample, $f_{j p}$ is the mass fraction of species $j$ in particles from source $p, g_{i p}$ is the $p$ th source mass contribution during the time units for the sth sample and $e_{s j}$ is the residual. The source contributions are averaged over the sampling time of $x_{s j}$. If all species of all samples are measured within same durations, $t_{s 2}=t_{s 1}$, then Eq. (1) becomes the conventional two way receptor model.

Replicated species were measured by more than one method with different time resolutions. In Eq. (1), different values of the subscript $m$ correspond to different measurement methods (for ADI data $m=1$, for SEAS data $m=2$ and for steam IC $m=3$ ). For each sample $s$, the $m$ value corresponds to the method used for measuring the sample, $m=m(s)$. For a replicated species $j$, adjustment factors, $\eta_{j m}$ in Eq. (1), are used with the assumption that the concentration values measured by different methods are proportional. An adjustment factor close to 1 suggests a good agreement between the different measurements. Usually, the methods with longest periods provide better accuracy or are defined as reference methods. Thus, their adjustment factors are set to unity by default. For non-replicated species, no adjustment factors are needed, so that their $\eta_{j m}$ are set to unity by default.

If a source contains no species measured with high temporal resolution, then there is no way to obtain a reliable high time resolution contribution series. To solve this problem, a regularization equation is used to smooth the time series of source contributions, as indicated by Eq. (2), where $g_{i p}$ is the source contribution from the $p$ th source during the $i$ th time unit.

$g_{i+1, p}-g_{i, p}=0+\varepsilon_{i}$.

The total residual sum of squares is composed of residuals from both Eqs. (1) and (2). When a source includes few high time resolution species, there is limited influence from Eq. (1). Eq. (2) will eliminate the unreliable high time resolution details of the contribution 
series in order to minimize the residuals. When a source contains some high resolution species, reducing the residual in Eq. (2) leads to an increase in the residuals of Eq. (1) and the high temporal variations tend to be conserved. The balance between the two residuals can be controlled by multiplying the residual in Eq. (2) by a small coefficient, such as $\sim 0.1$. This balancing was implemented by weighting the residuals with their uncertainties.

The model was solved using the multilinear engine (Paatero, 1999). A script file was written to specify the model equations. The final weighted sum square of residuals $(Q)$ is optimized by a conjugate gradient method (Paatero, 1999). The uncertainties assigned for Eq. (1) were computed with the expression:

$\sigma_{s j}=C_{1}+C_{3} \max \left(\left|x_{s j}\right|,\left|y_{s j}\right|\right)$,

where $y_{s j}$ is the calculated value for $x_{s j}, C_{1}$ is the measurement error or an estimated error if no measurement error was available, and $C_{3}$ is a constant, 0.05 in this study. For Eq. (2), the uncertainties were assigned a constant value of 3 . Missing values were imputed, see below. For steam IC data, unrealistic and too small uncertainties were reported so the largest reported uncertainty from three consecutive values was chosen as the measurement uncertainty. The structure of the data set is displayed in Fig. 2, where the concentrations of the chemical species measured by the same method form a data sub block. One concentration value is represented by a dot in Fig. 2 and the dots of one species look like a line. The beginning and ending time units of these five subblocks are all 0 and 720 . The beginning and ending time unit of each sampling period were also included. Table 3 illustrates a part of the data table that has been inputted into ME.
This data set contains a significant number of missing values. Both the concentrations and measurement errors were imputed for all missing values. In the early phases of the analysis, median values of variables were imputed for concentrations and "large" values for measurement errors. This kind of imputation is practically equivalent to omitting the values in question from the analysis. However, in several factors, large artifact peaks appeared on occasions where a key variable was missing. Apparently the multiple-time model is more sensitive to missing data than the original PMF model. For this reason, an "intelligent" imputation of missing values was performed as follows. Linear interpolation was used in order to compute the values to be imputed for missing concentrations $x_{s j}$. For data errors, the values $k x_{s j}$, were imputed, where the coefficient $k$ was between 0.75 and 5 . The smallest value of $k$ was used for a variable with little overall variation, viz. ammonium ion. For other variables, $k$ was chosen large enough so that any likely variation during the imputation period would be contained within the error limits. Both the overall variation of the variable, and the variation immediately before and after the imputation period were considered. For most cases, $k$ was chosen to be approximately 3. This procedure decreased the spurious peaks to insignificant magnitude.

The contribution series and source profiles were rescaled to satisfy the mass apportionment conditions (Hopke et al., 1980) as shown in the following equation:

$x_{i j}=\sum_{p=1}^{P} f_{i p} \cdot \frac{w_{p}}{w_{p}} \cdot g_{p j}$.

The scaling constants in the above equation, $w_{k}$, were determined by regressing $\mathrm{PM}_{2.5}$ mass concentrations against the estimated source contributions. The final source contribution series are obtained after the

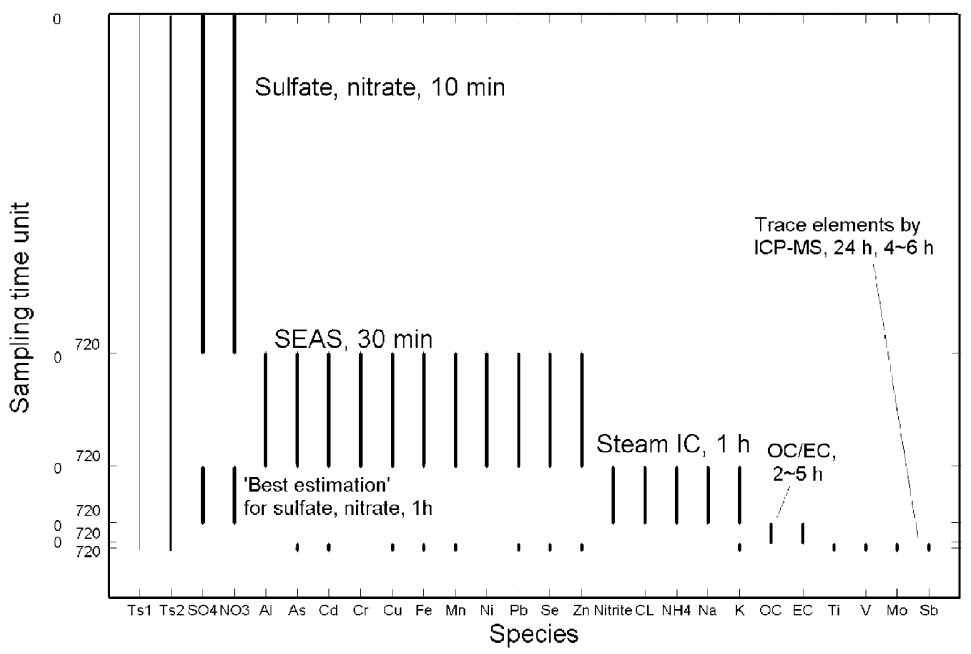

Fig. 2. Structure of the input data set. 
Table 3

Part of the data table as the input into ME

\begin{tabular}{|c|c|c|c|c|c|c|c|c|c|c|}
\hline $\begin{array}{l}\text { Start } \\
\text { time } T_{\mathrm{s} 1} \\
\text { yyyy } / \mathrm{mm} / \mathrm{dd} \\
\text { hh:mm }\end{array}$ & $\begin{array}{l}\text { End } \\
\text { time } T_{\mathrm{s} 2} \\
\text { yyyy } / \mathrm{mm} / \mathrm{dd} \\
\text { hh:mm }\end{array}$ & $\begin{array}{l}\text { Start of } \\
\text { sampling } \\
\text { Unit } \\
(10 \mathrm{~min})\end{array}$ & $\begin{array}{l}\text { Sampling } \\
\text { duration } \\
\text { Unit } \\
(10 \mathrm{~min})\end{array}$ & $\begin{array}{l}\text { End of } \\
\text { sampling } \\
\text { Unit } \\
(10 \mathrm{~min})\end{array}$ & $\begin{array}{l}\text { Sulfate } \\
\mu \mathrm{g} \mathrm{m}^{-3}\end{array}$ & $\begin{array}{l}\text { Sulfate_std } \\
\mu \mathrm{g} \mathrm{m}^{-3}\end{array}$ & $\begin{array}{l}\mathrm{Al} \\
\mu \mathrm{g} \mathrm{m}^{-3}\end{array}$ & $\begin{array}{l}\text { Al-std } \\
\mu \mathrm{g} \mathrm{m}^{-3}\end{array}$ & $\begin{array}{l}\mathrm{OC} \\
\mu \mathrm{g} \mathrm{m}^{-3}\end{array}$ & $\begin{array}{l}\text { OC-std } \\
\mu \mathrm{g} \mathrm{m}^{-3}\end{array}$ \\
\hline $7 / 16 / 010: 00$ & 7/16/01 0:10 & 1 & 1 & 1 & 5.16 & & & & & \\
\hline $7 / 17 / 010: 10$ & $7 / 17 / 01 \quad 0: 20$ & 2 & 1 & 2 & 5.31 & & & & & \\
\hline $7 / 18 / 010: 20$ & $7 / 18 / 010: 30$ & 3 & 1 & 3 & 5.80 & & & & & \\
\hline $7 / 19 / 01 \quad 0: 30$ & $7 / 19 / 01 \quad 0: 40$ & 4 & 1 & 4 & 5.73 & & & & & \\
\hline$\ldots$ & $\ldots$ & $\ldots$ & $\ldots$ & $\ldots$ & $\ldots$ & $\ldots$ & & & & \\
\hline $7 / 16 / 010: 00$ & $7 / 16 / 010: 30$ & 1 & 3 & 3 & & & 0.00788 & 0.00189 & & \\
\hline $7 / 16 / 01 \quad 0: 30$ & $7 / 16 / 01 \quad 1: 00$ & 4 & 3 & 6 & & & 0.00836 & 0.00038 & & \\
\hline $7 / 16 / 01 \quad 1: 00$ & $7 / 16 / 01 \quad 1: 30$ & 7 & 3 & 9 & & & 0.00681 & 0.00114 & & \\
\hline$\ldots$ & $\ldots$ & $\ldots$ & $\ldots$ & $\ldots$ & & & $\ldots$ & $\ldots$ & & \\
\hline 7/16/01 0:00 & $7 / 16 / 01$ 5:00 & 1 & 30 & 30 & & & & & 4.37 & \\
\hline $7 / 16 / 01$ 5:00 & $7 / 16 / 01 \quad 14: 00$ & 31 & 54 & 84 & & & & & 4.00 & 8.00 \\
\hline$\cdots$ & $\ldots$ & $\ldots$ & $\ldots$ & $\ldots$ & $\ldots$ & $\ldots$ & $\ldots$ & $\ldots$ & $\ldots$ & $\ldots$ \\
\hline
\end{tabular}

re-scaling as indicated in Eq. (5). When computing the regression, both the $\mathrm{PM}_{2.5}$ mass concentration and source contributions were averaged to two hour intervals values in order to reduce the possibly unreliable high time resolution details.

$v_{j}=\sum_{p=1}^{P} w_{p} \cdot g_{p j}$.

\section{Locating the sources with wind direction data}

The conditional probability function (CPF) has been applied for finding the directions of sources (Ashbaugh et al., 1985; Kim et al., 2003a). However, for this small data set, the CPF results proved misleading for the following two reasons: (1) there are many directions with very few (or no) wind occurrences as indicated in Fig. 3, (2) the distribution of concentrations is far from normal, with a small number of very large values. Instead of using CPF, the direct relationship of the source contributions and wind directions was graphically investigated (Chueinta et al., 2000) and nonparametric regression (NPR) analyses were also performed to locate the sources. All data with wind speed below $1.0 \mathrm{~m} \mathrm{~s}^{-1}$ were excluded in the application of both methods. The meteorological data (wind direction, wind speed, etc.) were obtained every $15 \mathrm{~min}$, so in the two analyses, both source contributions and meteorological data were averaged to $30 \mathrm{~min}$ resolution.

\section{NPR}

In NPR, the relationship of the contribution and wind direction is determined by NPR and confidence intervals

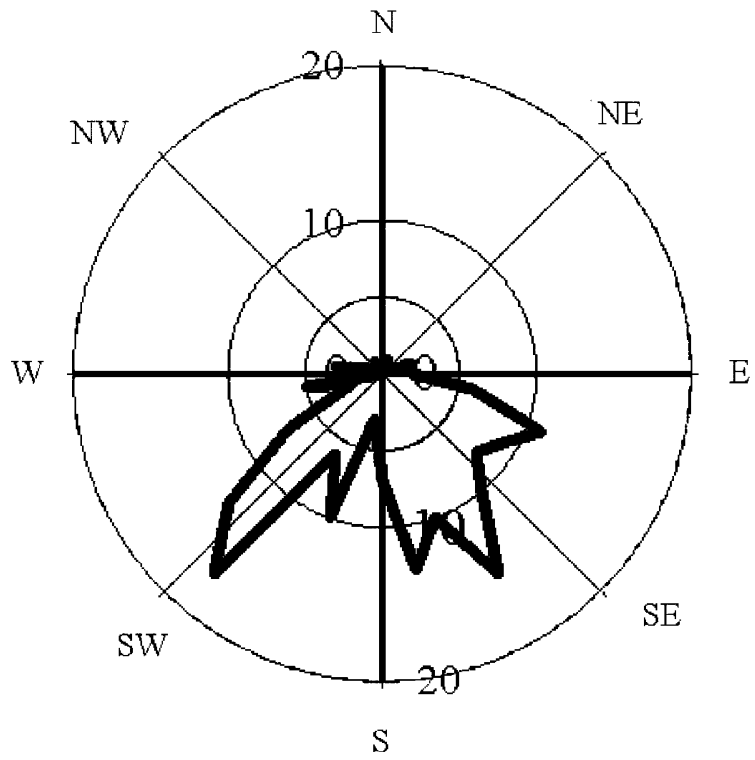

Fig. 3. Wind profile during 16, 17, 18, 23, and 24 July 2001.

are also given (Henry et al., 2002; Henry, 2002). The expected concentration $C$ at $\theta$ is computed by Eq. (6),

$\bar{C}(\theta, \Delta \theta)=\frac{\sum_{i=1}^{n} K((\theta-W) / \Delta \theta) C_{i}}{\sum_{i=1}^{n} K\left(\left(\theta-W_{i}\right) / \Delta \theta\right)}$,

where $K$ is a Gaussian kernel function, $W_{i}$ and $C_{i}$ are the wind direction and concentration of the $i$ th sample, and $\Delta \theta$ is the smoothing parameter, the only adjustable parameter in NPR. A cross validation method (Henry et al., 2002; Henry, 2002) was used to find the best $\Delta \theta$. However, the cross validation gave too small $\Delta \theta$ values like $2^{\circ}$, which may be caused by the small size of this data set, and too many meaningless small peaks were 
produced. In this study, $\Delta \theta=5^{\circ}$ was used. The $95 \%$ confidence intervals were also computed for the expected concentration by the method described by Henry et al. (2002) and Henry (2002).

\section{Results and discussion}

Six factors were found to be the best solution. When using five factors, zinc, selenium and lead had large residuals, and traffic and secondary nitrate were combined into one factor. Table 2 indicates the sum of squares of scaled residuals for the six factor solution to Eq. (1) for each species. For seven factors, the mass apportionment conditions cannot be satisfied and there is always a negative source contribution, suggesting that too many factors have been used, and the additional factor only improved the fitting of lead. There is a possibility that lead is contributed by an additional source, but the current data set is insufficient to test this hypothesis. In Fig. (4), the residuals scaled by the real measurement
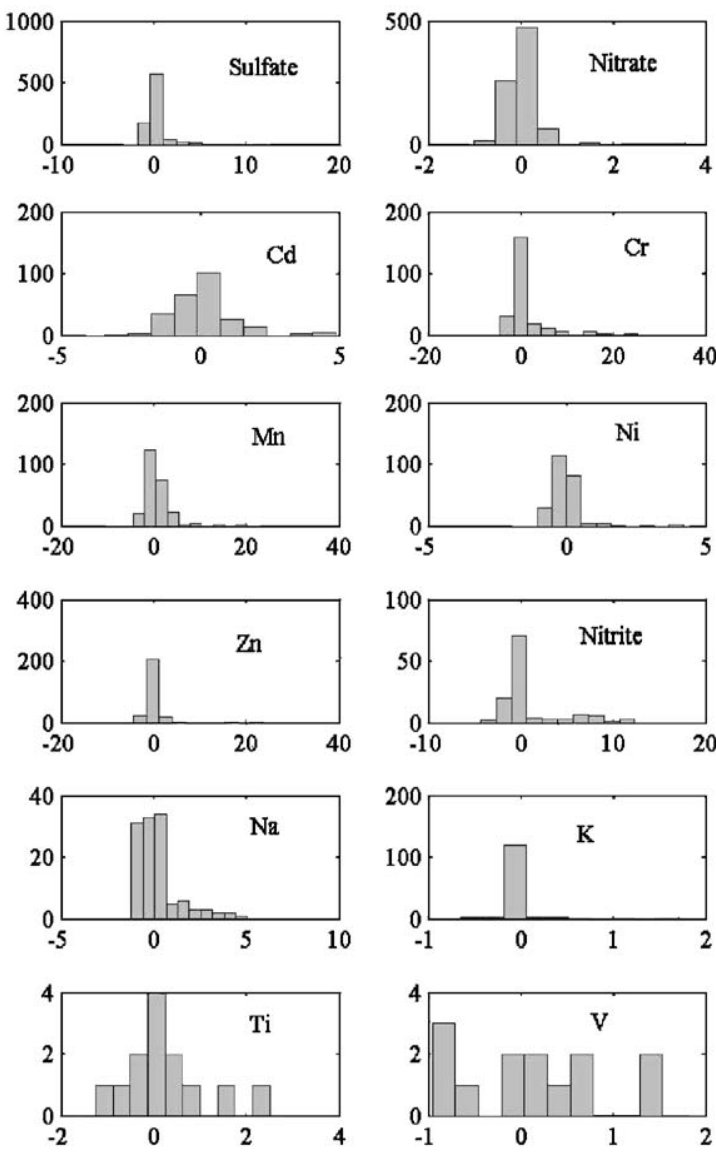

uncertainties $\left(C_{1}\right.$ in Eq. (3)) are presented. The existence of large positive residuals suggests that some species such as aluminum and lead were not well fit. There seem to be a few outliers in the residuals of copper, but they do not have much influence on the results since the program was running at robust mode (Paatero, 1999).

Factors 1-6 are assigned as: traffic, crustal, steel mill, secondary nitrate, secondary sulfate, and coke plant. The adjustment factors for the replicated species of the six factor solution are also presented in Table 2. Most of the adjustment factors are close to 1 except for copper and iron. For the SEAS data, only the particles smaller than $1.2 \mu \mathrm{m}$ were measured. Since the mass size distribution of the source emission is unlikely to experience significant changes during the five days, the variations measured by SEAS are likely to be consistent with other measurement methods. Since most particles have little mass in their size distribution between 1.2 and $2.5 \mu \mathrm{m}$, the measurements by SEAS are likely to be consistent with the other method and the adjustment factors are close to 1 .
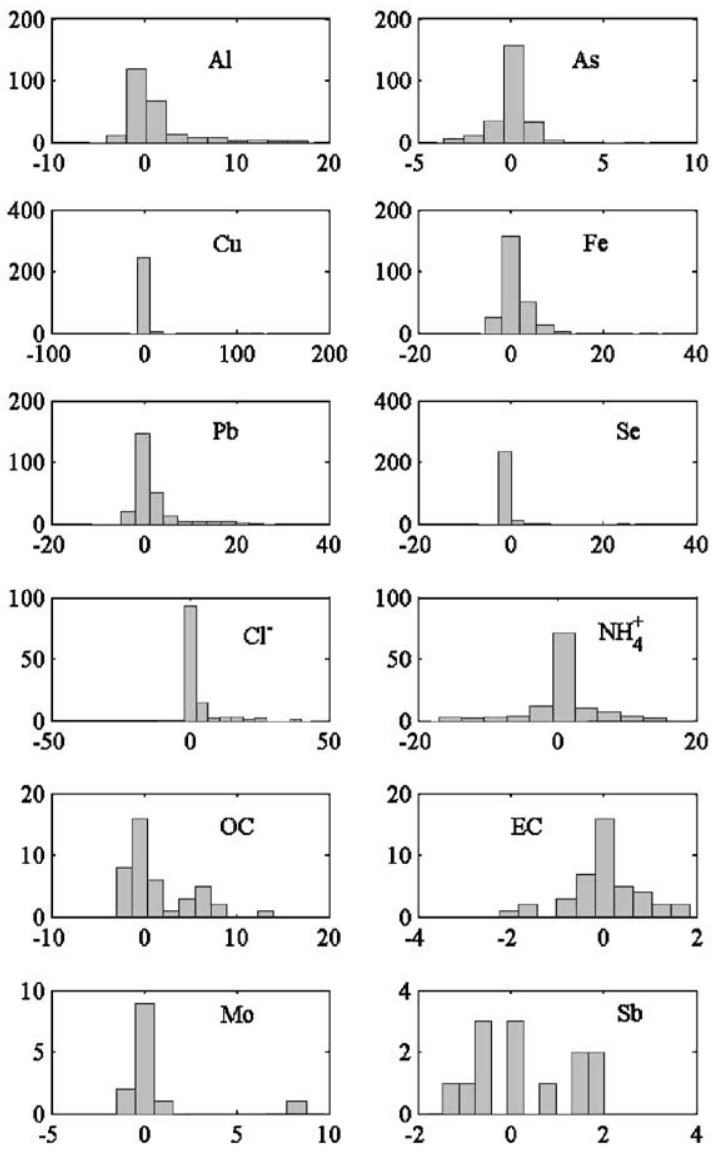

\section{Residuals rescaled by real measurement uncertainties}

Fig. 4. Histograms of scaled residuals. 
Fig. 5 presents the resolved source profiles. The black bars represent a correlation coefficient over 0.7 between the source contribution and the concentration series of that species. The correlation coefficients of source contributions and all species are given in Table 4. Zinc and EC are the two species explained mostly by Factor 1 . $\mathrm{Zn}$ was reported to be the most characteristic element from burnt and unburnt lubricant oil (Ristovski et al., 1999). Another possible origin of zinc is brake linings and tires (Sternbeck et al., 2002). EC is usually emitted by diesel engines. The source contribution series in Fig. 6 does not show clear traffic patterns. The transport from the highway to the site is influenced by wind and the transport brings high contributions occasionally, leading to the peaks in Fig. 6. Fig. 7 indicates that the traffic emissions were from the southeast and south, the direction of Interstate Highway 376. Although the total traffic is heavy on Forbes and Fifth Avenues through the middle of Oakland by the Cathedral of Learning, $1 \mathrm{~km}$ to the west of the site, there are not much transport to the site since the wind seldom blows from that direction. In Fig. 8, very large confidence intervals, indicated by the thin lines, are caused by no concentration values near that direction; when there is only one concentration value, the confidence interval is 0 . No source information can be drawn from these two situations. Considering the confidence interval in Fig. 8, it is not reliable to say

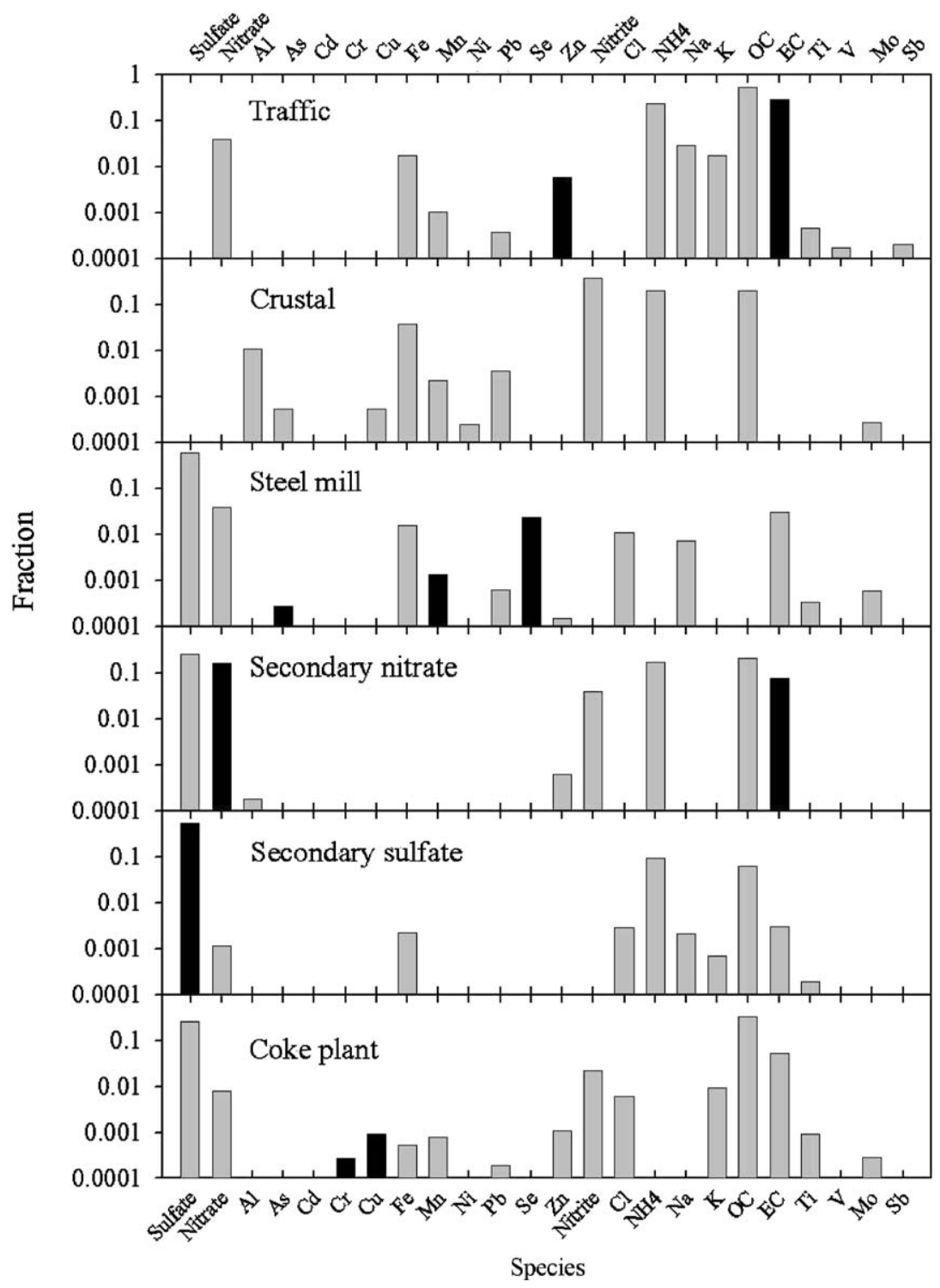

Fig. 5. The source profiles of all six factors. 
Table 4

Correlations of source contributions with all chemical species

\begin{tabular}{|c|c|c|c|c|c|c|c|c|}
\hline & Sulfate & Nitrate & $\mathrm{Al}$ & As & $\mathrm{Cd}$ & $\mathrm{Cr}$ & $\mathrm{Cu}$ & $\mathrm{Fe}$ \\
\hline Traffic & 0.12 & 0.37 & -0.02 & 0.45 & 0.26 & -0.01 & -0.11 & 0.55 \\
\hline Crustal & -0.23 & 0.15 & 0.50 & 0.38 & 0.51 & 0.51 & 0.53 & 0.06 \\
\hline Steel mill & -0.07 & 0.44 & 0.00 & 0.77 & 0.25 & 0.12 & -0.01 & 0.55 \\
\hline Secondary nitrate & -0.19 & 0.98 & 0.07 & 0.52 & 0.51 & 0.31 & 0.18 & 0.14 \\
\hline Secondary sulfate & 0.93 & -0.43 & -0.01 & -0.39 & -0.52 & -0.64 & -0.45 & 0.20 \\
\hline \multirow[t]{2}{*}{ Coke plant } & -0.54 & 0.27 & 0.22 & 0.22 & 0.71 & 0.93 & 0.81 & -0.16 \\
\hline & $\mathrm{Mn}$ & $\mathrm{Ni}$ & $\mathrm{Pb}$ & $\mathrm{Se}$ & $\mathrm{Zn}$ & Nitrite & $\mathrm{Cl}$ & $\mathrm{NH}_{4}^{+}$ \\
\hline Traffic & 0.59 & 0.09 & 0.28 & 0.60 & 0.86 & -0.03 & 0.02 & 0.21 \\
\hline Crustal & 0.41 & 0.23 & 0.47 & 0.06 & 0.16 & 0.69 & -0.29 & -0.13 \\
\hline Steel mill & 0.72 & 0.08 & 0.29 & 0.99 & 0.60 & 0.17 & -0.12 & -0.14 \\
\hline Secondary nitrate & 0.35 & 0.10 & 0.01 & 0.31 & 0.57 & 0.59 & -0.28 & 0.20 \\
\hline Secondary sulfate & -0.50 & -0.14 & -0.06 & -0.32 & -0.36 & -0.56 & 0.40 & 0.58 \\
\hline \multirow[t]{2}{*}{ Coke plant } & 0.51 & 0.25 & 0.22 & 0.02 & 0.29 & 0.45 & -0.15 & -0.37 \\
\hline & $\mathrm{Na}$ & $\mathrm{K}$ & $\mathrm{OC}$ & $\mathrm{EC}$ & $\mathrm{Ti}$ & $\mathrm{V}$ & Mo & $\mathrm{Sb}$ \\
\hline Traffic & 0.29 & 0.16 & 0.52 & 0.80 & -0.02 & 0.30 & -0.25 & 0.34 \\
\hline Crustal & -0.20 & -0.15 & 0.02 & 0.06 & -0.30 & -0.47 & 0.69 & 0.28 \\
\hline Steel mill & 0.05 & 0.02 & 0.27 & 0.63 & -0.17 & -0.25 & 0.58 & 0.26 \\
\hline Secondary nitrate & 0.01 & 0.02 & 0.55 & 0.74 & -0.38 & -0.16 & 0.14 & 0.75 \\
\hline Secondary sulfate & 0.14 & 0.03 & 0.01 & -0.25 & 0.18 & 0.45 & -0.60 & -0.49 \\
\hline Coke plant & -0.15 & -0.04 & -0.13 & 0.00 & -0.07 & -0.16 & 0.57 & 0.43 \\
\hline
\end{tabular}

the source is located to the south. Table 5 indicates a correlation of the source contributions with $\mathrm{NO}$ and $\mathrm{NO}_{x}$.

The crustal source is represented by $\mathrm{Al}$ and $\mathrm{Fe}$. Potassium was found by other researchers to be a major component of soil or road dust (Song et al., 2001). In this study, because of the poor quality of the steam-IC potassium data, these $1 \mathrm{~h}$ data were assigned very low weights and are not important in the fitting process. Although Fig. 5 indicates nearly no potassium component, the 13 ICP-MS potassium concentration values are insufficient to make this conclusion robust. The contribution from crustal materials is much smaller than other sources as shown in Fig. 6. Both Figs. 7 and 8 indicate that there seem to be no clearly dominating directions for this source. The source contribution drops to nearly zero on 23 and 24 July. The meteorological conditions of 16-18 July and 23-24 July have no significant differences. This situation is inconsistent with a local source, and the crustal is more likely to be from distant sources.

The steel mill source contribution has variations that are similar to selenium and has strong correlations with arsenic and manganese as well, as indicated by Fig. 5 and Table 4. There is a contribution peak in the early morning of July 17 and nearly no contribution at all other times, as shown by Fig. 6. This situation suggests a local transient source whose emission is transported to the site from a specific wind direction. Selenium is usually a marker element of coal power plant emissions (Morawska and Zhang, 2002). In Fig. 7 , four high-concentration samples point toward source 13 in Table 6, a steel mill. Fig. 8 also indicates that the source is located from east to southeast, the direction of the steel mill.

Secondary nitrate is dominated by nitrate and the correlation between the two series is very strong. The source contribution reaches its peak in the early morning when the low temperature favors the formation of ammonium nitrate and a diurnal pattern of nitrate is thus formed. The precursor gas of nitrate, $\mathrm{NO}_{x}$, is probably from traffic and other stationary combustion emissions because of the strong correlations with $\mathrm{NO}_{x}$ and $\mathrm{CO}$ indicated in Table 5. These two sources may also be major sources of $\mathrm{EC}$, and this may explain the strong correlation between the source contribution and EC. EC may serve as the nuclei on which $\mathrm{NH}_{4} \mathrm{NO}_{3}$ nucleates or condenses (Seinfeld and Pandis, 1998). The strong anti-correlation with ozone is the result of ozone having high concentrations during the daytime when there is increased photochemical activities. Thus, the two series have reversed variations. Southeast is the major direction for secondary nitrate in Fig. 7 as well as Fig. 8. The NPR analysis also found a small concentration peak to the south. Note, however, that the majority of samples with high nitrate concentration are rejected in the directional analysis because the wind speeds are below the acceptable lower limit. The directional pattern seen for nitrate may be influenced by the diurnal pattern of ammonium nitrate formation and dissociation. The variable equilibrium behavior may make the directional pattern less reliable. 


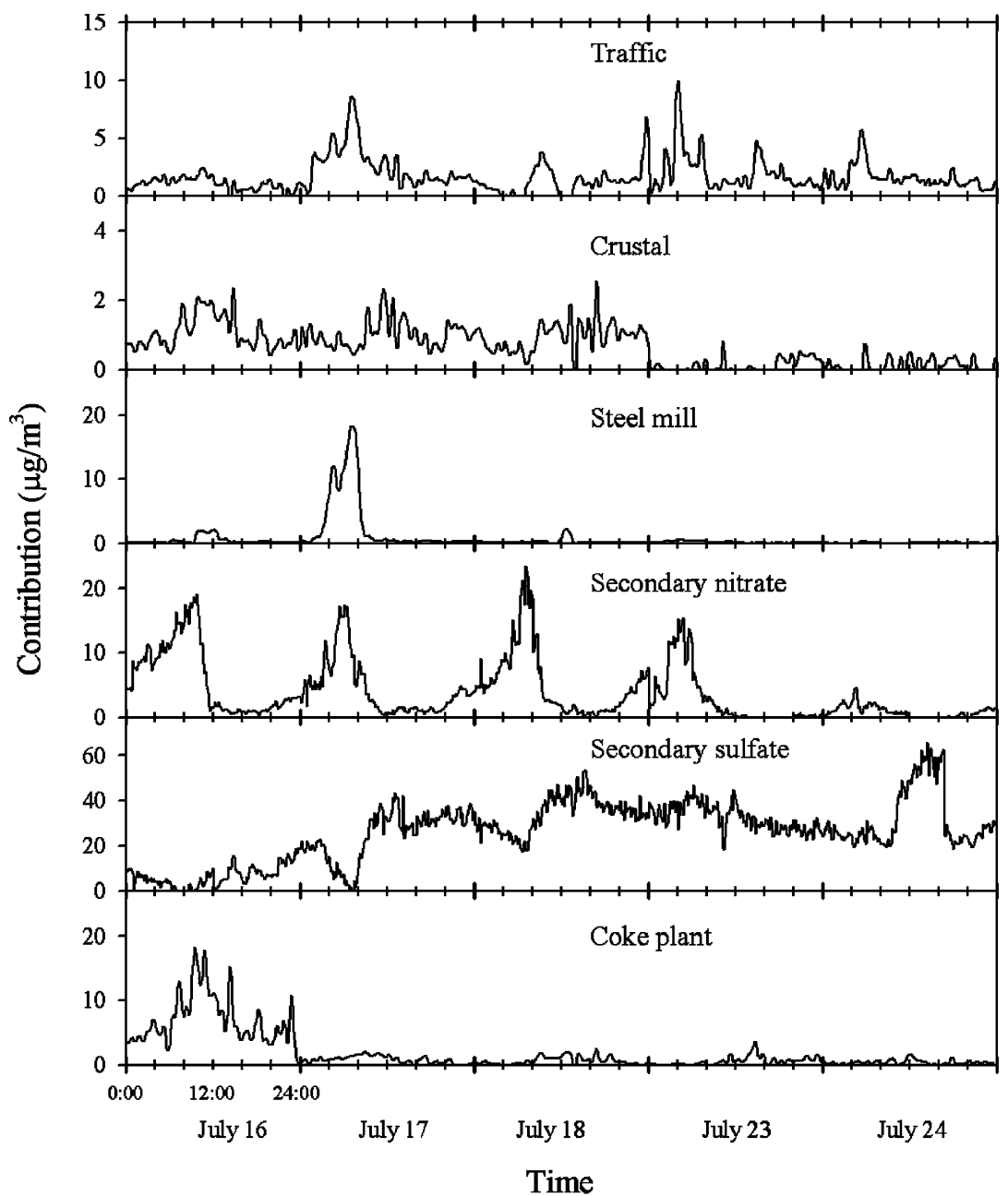

Fig. 6. The source contribution series of all six factors.

Secondary sulfate is dominated by sulfate, and its mass contribution dominates $\mathrm{PM}_{2.5}$ mass as indicated in Table 5. Since this source is dominated by sulfate, measured with $10 \mathrm{~min}$ resolution, the source contribution series provides the most details and is not as smooth as other source contributions. These particles are formed from the oxidation of $\mathrm{SO}_{2}$ transported from distant coal power plants (Zhou et al., 2004). OC and $\mathrm{EC}$ are the largest fractions of the PM mass except for sulfate and ammonium. Large OC/EC ratios indicate secondary $\mathrm{OC}$ that has condensed onto the sulfate particles during the transport. The source profile of secondary sulfate is close to the results from other eastern US sites (Song et al., 2001; Lee et al., 2002; Kim et al., 2003a, b; Kim and Hopke, 2004; Kim et al., 2004). Figs. 7 and 8 show that the secondary sulfate is transported from many directions and there is no clear dominating direction.
The coke plant explains most of the variation of cadmium, chromium and copper as shown in Table 4. In Fig. 6, it is found that most of its contribution is on 16 July. Both Figs. 7 and 8 indicate the emissions are from south where there are several sources located (Sources 5-9, and 245 in Table 6). Among the sources in those directions, the coke plant (source 8) has the highest emission rate for $\mathrm{PM}_{2.5}$ according to the emission inventory (EPA, 2003). The presence of sulfate and nitrate in the source profile suggests a combustion source and the high OC content is consistent with the emissions from a coke plant. The operations of the coke plant may be transient. When there are simultaneous emissions and appropriate wind directions, the source has the highest contributions. There are also some minor sources located close to the coke plant and may have some similar variations with the coke plant. These minor sources give relatively low $\mathrm{PM}_{2.5}$ mass contributions 


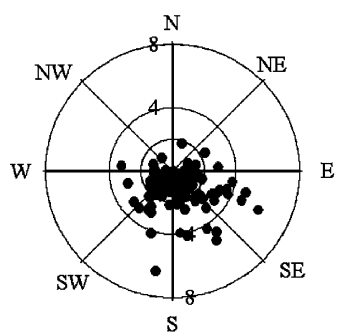

Traffic

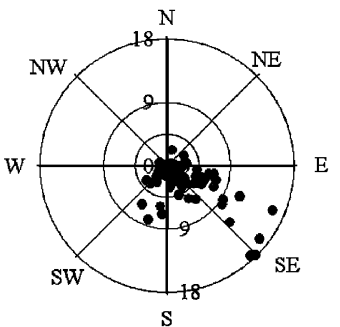

Secondary nitrate

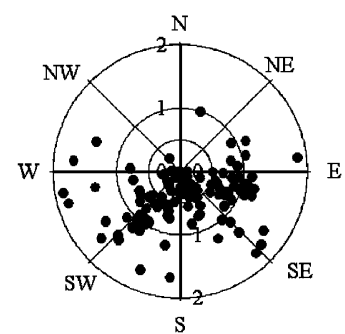

Crustal

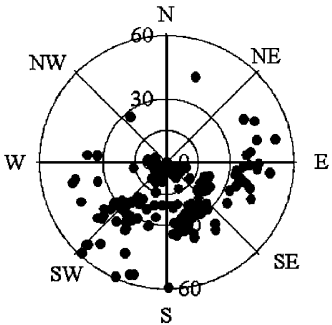

Secondary sulfate

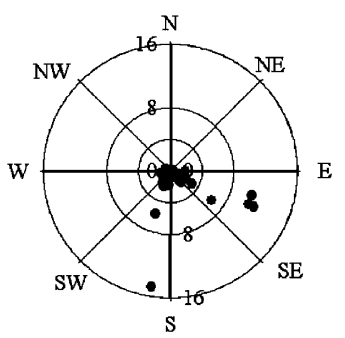

Steel mill

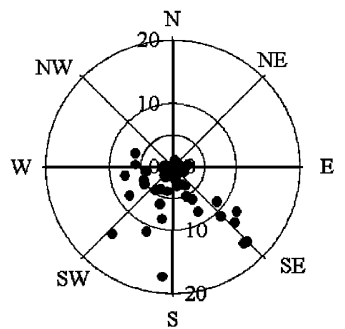

Coke plant

Fig. 7. The relationship of source contributions with wind direction.
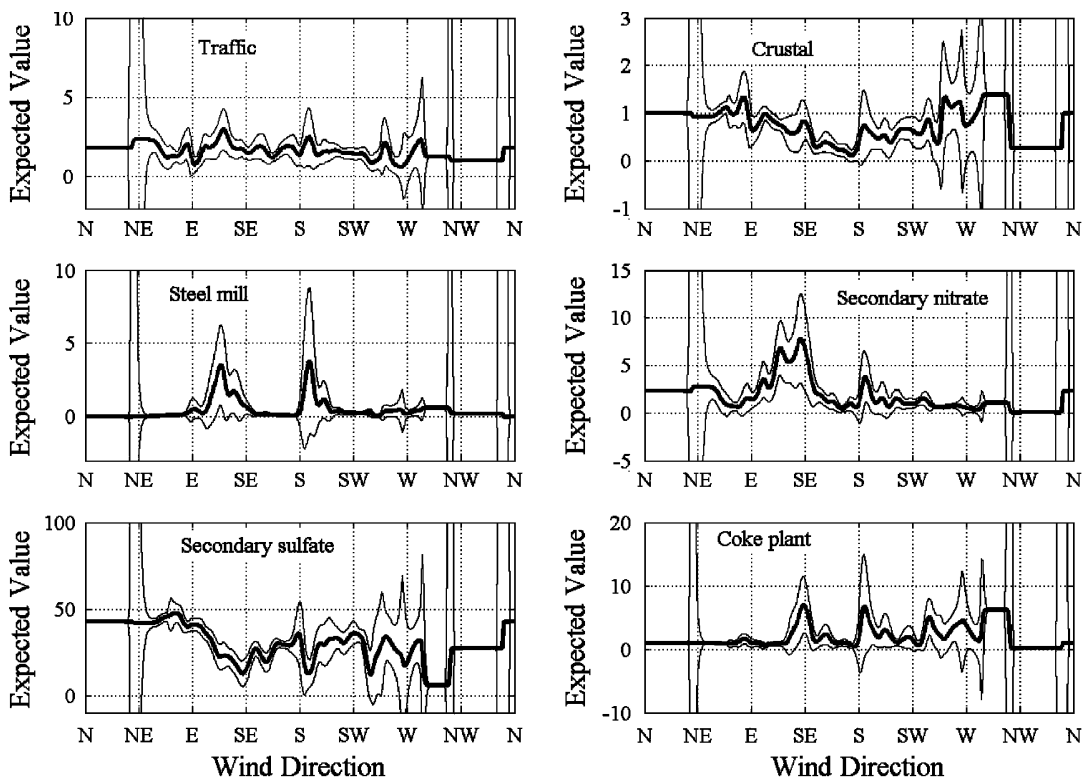

Fig. 8. NPR analysis results for each source. (The units of the expected value is $\mu \mathrm{g} \mathrm{m}^{-3}$ ).

and do not influence the mass apportionment much, but they may have high emissions of some trace elements and may influence the source profile of the coke plant. Because of the small size of the data set, these minor sources cannot be separated from the coke plant.
The mean $\mathrm{PM}_{2.5}$ mass concentration is $35.0 \mu \mathrm{g} \mathrm{m}^{-3}$. The mean contribution (in $\mu \mathrm{g} \mathrm{m}^{-3}$ ) from the six sources are: traffic (1.65), crustal (0.57), steel mill (0.75), secondary nitrate (3.93), secondary sulfate (26.23), and coke plant (1.91). It is found that the secondary sulfate dominates $\mathrm{PM}_{2.5}$ mass concentration during these five days. 
Table 5

Correlations of source contributions with gases and $\mathrm{PM}_{2.5}$

\begin{tabular}{lrrrrrr}
\hline & $\mathrm{O}_{3}$ & $\mathrm{NO}$ & $\mathrm{NO}_{x}$ & $\mathrm{SO}_{2}$ & $\mathrm{CO}$ & $\mathrm{PM}_{2.5}$ \\
\hline Traffic & -0.33 & 0.48 & 0.53 & 0.04 & 0.32 & 0.24 \\
Crustal & -0.08 & 0.04 & 0.04 & 0.30 & 0.32 & -0.10 \\
Steel mill & -0.26 & 0.58 & 0.52 & 0.07 & 0.37 & 0.05 \\
Secondary nitrate & -0.74 & 0.65 & 0.76 & 0.03 & 0.71 & 0.10 \\
Secondary sulfate & 0.38 & -0.34 & -0.40 & 0.17 & -0.52 & 0.71 \\
Coke plant & -0.05 & 0.27 & 0.24 & 0.14 & 0.48 & -0.35 \\
\hline
\end{tabular}

Table 6

Emission rate of some major $\mathrm{PM}_{2.5}$ sources near Pittsburgh supersite $\left(\right.$ ton $\mathrm{yr}^{-1}$ )

\begin{tabular}{|c|c|c|c|c|c|c|}
\hline $\begin{array}{l}\text { Map } \\
\text { no. }\end{array}$ & Category & $\mathrm{CO}$ & $\mathrm{NO}_{x}$ & VOC & $\mathrm{SO}_{2}$ & $\mathrm{PM}_{2.5}$ \\
\hline 5 & Sanitation & 21 & 34 & 7.3 & 2.9 & 11.2 \\
\hline 6 & Metal processing & 7.0 & 380 & 17.2 & 230 & 61 \\
\hline 7 & $\begin{array}{l}\text { Metal product } \\
\text { manufacture }\end{array}$ & 13.8 & 61 & 8.1 & 5.3 & 29 \\
\hline 8 & Coke plant & 3200 & 8700 & 370 & 1100 & 550 \\
\hline 9 & Steel mill & 220 & 640 & 97 & 70 & 100 \\
\hline 13 & Steel mill & 1600 & 310 & 28 & 746 & 990 \\
\hline 245 & Coal power plant & 720 & 7000 & 13.8 & 4930 & 287 \\
\hline
\end{tabular}

\section{Conclusion}

A new factor analysis method utilizing aerosol compositional data with multiple temporal resolution for source apportionment has been successfully developed in this study. The high resolution data are not averaged and all data are used in their original time schedule so that the information in the high resolution details of source contributions are extracted without loss. For sources with little component measured with high time resolution, their contribution series are smoothed to avoid unrealistic details. A small but complex aerosol composition data set from the Pittsburgh supersite has been analyzed with this model. Six sources have been identified: traffic, crustal, steel mill, secondary nitrate, secondary sulfate, and coke plant. There seem to be sources not fully separated and identified owing to the limitations of the information provided by this small data set. With larger data sets, this model is expected to give better performances in resolving source information for airborne particles.

\section{Acknowledgements}

This research was conducted as part of the PAQS, which was supported by US Environmental Protection Agency under contract R82806101 and the US
Department of Energy National Energy Technology Laboratory under contract DE-FC26-01NT41017. This paper has not been subject to EPA's required peer and policy review, and therefore does not necessarily reflect the views of the Agency. No official endorsement should be inferred.

\section{References}

Ashbaugh, L.L., Malm, W.C., Sadeh, W.Z., 1985. A residence time probability analysis of sulfur concentrations at Grand Canyon National Park. Atmospheric Environment 19, 1263-1270.

Bench, G., Grant, P.G., Ueda, D., Cliff, S.S., Perry, K.D., Cahill, T.A., 2002. The use of STIM and PESA to measure profiles of aerosol mass and hydrogen content, respectively, across Mylar rotating drum impactor samples. Aerosol Science and Technology 36, 642-651.

Chueinta, W., Hopke, P.K., Paatero, P., 2000. Investigation of sources of atmospheric aerosol at urban and suburban residential areas in Thailand by positive matrix factorization. Atmospheric Environment 34, 3319-3329.

EPA, 2003. http://www.epa.gov/air/data.

Henry, R.C., 1997. History and fundamentals of multivariate air quality receptor models. Chemometrics and Intelligent Laboratory Systems 37, 525-530.

Henry, R.C., 2002. Multivariate receptor models-current practice and future trends. Chemometrics and Intelligent Laboratory Systems 60, 43-48.

Henry, R.C., Changa, Y-S., Spiegelman, C.H., 2002. Locating nearby sources of air pollution by nonparametric regression of atmospheric concentrations on wind direction. Atmospheric Environment 36, 2237-2244.

Hopke, P.K., 2003. Recent developments in receptor modeling. Journal of Chemometrics 17, 255-265.

Hopke, P.K., Lamb, R.E., Natusch, D.F.C., 1980. Multielemental characterization of urban roadway dust. Environmental Science and Technology 14, 164-172.

Khlystov, A., Wyers, G.P., Slanina, J., 1995. The steam-jet aerosol collector. Atmospheric Environment 29, 2229-2234.

Kidwell, C.B., Ondov, J.M., 2001. Elemental analysis of subhourly ambient aerosol collections. Aerosol Science and Technology 35, 596-601.

Kim, E., Hopke, P.K., Edgerton, E., 2003a. Source identification of Atlanta aerosol by Positive Matrix Factorization. Journal of the Air Waste Management Association 53, 731-739.

Kim, E., Hopke, P.K., Paatero, P., Edgerton, E., 2003 b. Incorporation of Parametric Factors into Multilinear Receptor Model Studies of Atlanta Aerosol. Atmospheric Environment 37, 5009-5021.

Kim, E., Hopke, P.K., 2004. Source apportionment of fine Particles at Washington, DC utilizing temperature resolved carbon fractions. Journal of the Air and Waste Management Assocication 54, 773-785.

Kim, E., Hopke, P.K., Edgerton, E., 2004. Improving source identification of Atlanta aerosol using temperature resolved carbon fractions in Positive Matrix Factorization. Atmospheric Environment 38, 3349-3362. 
Lee, J.H., Yoshida, Y., Turpin, B.J., Hopke, P.K., Poirot, R.L., 2002. Identification of sources contributing to the MidAtlantic Regional Aerosol. Journal of Air and Waste Management Association 52, 1186-1205.

Morawska, L., Zhang, J., 2002. Combustion sources of particles. 1. Health relevance and source signatures. Chemosphere 49, 1045-1058.

Orsini, D.A., Ma, Y., Sullivan, A., Sierau, B., Baumann, K., Weber, R.J., 2003. Refinements to the particle-into-liquid sampler (PILS) for ground and airborne measurements of water soluble aerosol composition. Atmospheric Environment 37, 1243-1259.

Paatero, P., 1999. The multilinear engine - a table-driven least squares program for solving multilinear problems, including the $n$-way parallel factor analysis model. Journal of Computational and Graphical Statistics 8, 854-888.

Ristovski, Z., Agranovski, V., Bostrom, T., Thomas, S., Hitchins, J., Morawska, L., 1999. Elemental composition of combustion emissions from spark ignition vehicles. Journal of Aerosol Science 30 (Suppl. 1), S845-S846.

Seinfeld, J.H., Pandis, S.N., 1998. Atmospheric Chemistry and Physics, p. 708.

Slanina, J., ten Brink, H.M., Otjes, R.P., Even, A., Jongejuan, P., Khlystov, A., Waijers-Ijpelaan, A., Hu, M., Lu, Y., 2001. The continuous analysis of nitrate and ammonium in aerosols by the steam jet aerosol collector (SJAC): extension and validation of the methodology. Atmospheric Environment 35, 2319-2330.

Song, X-H., Polissar, V., Hopke, P.K., 2001. Sources of the particle composition in the northeastern US. Atmospheric Environment 35, 5277-5286.

Stanier, C., Khlystov, A., Pandis, S.N., 2004. Nucleation events during the Pittsburgh Air Quality Study: Description and relation to key meteorological, gas phase, and aerosol parameters. Aerosol Science and Technology 38 (S1), 253-264.

Sternbeck, J., Sjödin, Å., Andréasson, K., 2002. Metal emissions from road traffic and the influence of resuspension: results from two tunnel studies. Atmospheric Environment $36,4735-4744$.

Stolzenburg, M.R., Hering, S.V., 2000. Method for the automated measurement of fine particle nitrate in the atmosphere. Environmental Science and Technology 34, 907-914.

Turpin, B.J., Huntzicker, J.J., 1995. Identification of secondary organic aerosol episodes and quantitation of primary and secondary organic aerosol concentrations during SCAQS. Atmospheric Environment 29, 3527-3544.

Weber, R.J, Orsini, D., Daun, Y., Lee, Y.-N., Klotz, P.J., Brechtel, F., 2001. A particle-into-liquid collector for rapid measurement of aerosol bulk chemical composition. Aerosol Science and Technology 35, 718-727.

Wilson, W.E., Chow, J.C., Claiborn, C., Wei, F., Engelbrecht, J., Watson, J.G., 2002. Monitoring of particulate matter outdoors. Chemosphere 49, 1009-1043.

Wittig, B., Anderson, N., Khlystov, A.Y., Pandis, S.N., Davidson, C., Robinson, A.L., 2004a. Pittsburgh Air Quality Study overview. Atmospheric Environment 38, 3107-3125.

Wittig, B., Takahama, S., Khlystov, A., Pandis, S.N., Hering, S., Kirby, B., Davidson, C., 2004b. Semi-continuous PM2.5 inorganic composition measurements during the Pittsburgh Air Quality Study. Atmospheric Environment 38, 3201-3213.

Zhou, L., Kim, E., Hopke, P.K., Stanier, C., Pandis, S.N., 2004. Advanced factor analysis on Pittsburgh particle size distribution data. Aerosol Science and Technology 38 (S1), $118-132$. 


\section{AE International - North America}

Atmospheric Environment 38 (2004) 1955-1963
ATMOSPHERIC ENVIRONMENT

$\overline{\text { www.elsevier.com/locate/atmosenv }}$

\title{
Comparison of two trajectory based models for locating particle sources for two rural New York sites
}

\author{
Liming Zhou ${ }^{\mathrm{a}, \mathrm{b}}$, Philip K. Hopke ${ }^{\mathrm{a}, \mathrm{b}, *}$, Wei Liu ${ }^{\mathrm{c}}$ \\ ${ }^{a}$ Center for Air Resources Engineering and Science, Clarkson University, USA \\ ${ }^{\mathrm{b}}$ Department of Chemical Engineering, Clarkson University, Box 5708, POTSDAM, NY, 13699-5708, USA \\ ${ }^{\mathrm{c}}$ Department of Chemistry, Clarkson University, USA
}

Received 9 September 2003; accepted 27 December 2003

\begin{abstract}
Two back trajectory-based statistical models, simplified quantitative transport bias analysis and residence-time weighted concentrations (RTWC) have been compared for their capabilities of identifying likely locations of source emissions contributing to observed particle concentrations at Potsdam and Stockton, New York. Quantitative transport bias analysis (QTBA) attempts to take into account the distribution of concentrations around the directions of the back trajectories. In full QTBA approach, deposition processes (wet and dry) are also considered. Simplified QTBA omits the consideration of deposition. It is best used with multiple site data. Similarly the RTWC approach uses concentrations measured at different sites along with the back trajectories to distribute the concentration contributions across the spatial domain of the trajectories. In this study, these models are used in combination with the source contribution values obtained by the previous positive matrix factorization analysis of particle composition data from Potsdam and Stockton. The six common sources for the two sites, sulfate, soil, zinc smelter, nitrate, wood smoke and copper smelter were analyzed. The results of the two methods are consistent and locate large and clearly defined sources well. RTWC approach can find more minor sources but may also give unrealistic estimations of the source locations. (C) 2004 Elsevier Ltd. All rights reserved.
\end{abstract}

Keywords: Ambient aerosol; Receptor model; Back trajectory; Simplified quantitative transport bias analysis; Residence-time weighted concentrations

\section{Introduction}

Trajectory statistics methods have been designed in the past to derive information about the source locations and preferred transport directions of airborne particles or gas molecules (Ashbaugh et al., 1985; Keeler and Samson, 1989; Stohl, 1996; Poirot and Wishinaski, 1986). These methods use measured particulate or gaseous concentrations or the source contributions from multivariate receptor models, combined with air parcel back trajectories computed from the receptor site. Stohl

*Corresponding author. Department of Chemical Engineering, Clarkson University, PO. Box 5708, Potsdam, NY 136995708, USA. Fax: + 1-315-268-4410.

E-mail address: hopkepk@clarkson.edu (P.K. Hopke).
(1998) reviewed the computation and application of back trajectories and trajectory statistics.

One trajectory-based approach, potential source contribution function (PSCF) is a simple method that links residence time in upwind areas with high concentrations through a conditional probability field (Ashbaugh et al. 1985). This method has been extensively used in the past (Zeng and Hopke, 1989; Cheng et al., 1993; Stohl and Kromp-Kolb, 1994; Polissar et al., 1999; Xie et al., 1999; Lin et al., 2001). Vasconcelos et al. (1996) reported that the PSCF method gave good angular resolution but poor radial resolution. However, recent results (Begum et al., 2003) indicate that it is possible to obtain good radial and angular resolutions by PSCF. Cheng and Lin (2001) have found that using a larger data set and high-percentile criterion value 
provides a better estimation of source areas. Similar results have been observed by Poirot et al. (2001). Seibert et al. (1994) developed a method called "concentration weighted trajectory" (CWT). The CWT method assigns the concentration values at the receptor site to corresponding backward trajectories. The mean or logarithmic mean concentration is computed and used as the weight for the residence time of each grid cell. This method was refined by Stohl et al. (1996) into residence-time weighted concentrations (RTWC) method by redistributing the CWT field. Lupu and Maenhaut (2002) made an intercomparison of these two methods, PSCF and CWT, and found the two methods agreed well and correctly identified known emission sources. $\mathrm{PSCF} / \mathrm{CWT} / \mathrm{RTWC}$ comparisons were made by $\mathrm{Hsu}$ et al. (2003). They found PSCF and CWT were useful for distinguishing large and moderate sources and concluded that the three methods gave relative consistent results but each approach had its own advantage and to use them together provided better information on source areas.

There are various errors during the calculation of the trajectories and the uncertainties increase with the increasing trajectory length. Position errors of $20 \%$ of the traversed distance is considered typical (Stohl, 1998). The uncertainties of trajectories were considered in quantitative transport bias analysis (QTBA) by Keeler and Samson (1989). The transport potential of each grid cell for a given endpoint of a back trajectory is assumed to conform to a two-dimensional normal distribution. When applied to multi-site data, QTBA fields were overlaid to locate the sources (Keeler and Samson, 1989). Simplified QTBA (SQTBA) method employs the same basic framework but the effects of chemical reactions and depositions are neglected from the calculation of the probability function.

Stohl (1998) discussed the major assumptions of RTWC, the linear relationship between the emission rate and the measured concentration. For the methods directly using the concentration measured at the receptor site, including RTWC, CWT and SQTBA, the linearity assumption is needed.

The particle composition data from Potsdam and Stockton, New York have been analyzed by positive matrix factorization (PMF) and PSCF (Liu et al., 2003). In this study, the source contributions of the six common sources, sulfate, soil, zinc smelter, nitrate, wood smoke and copper smelter, combined with the back trajectories from the two sites, will be analyzed by RTWC and SQTBA. Our objective is to make an intercomparison of the capabilities of these methods on locating the sources.

\section{Data}

The particle samples were collected using a PM2.5 speciation sampler from May through August in 2000 and 2001 at Potsdam (Lat: $44.75^{\circ}$, Lon: $-75^{\circ}$ ) and Stockton (Lat: $42.27^{\circ}$, Lon: $-79.375^{\circ}$ ), New York. The locations of the two sites are indicated in Fig. 1. The sampling duration time of each sample is $23 \mathrm{~h}$. Particulate mass, black carbon, cations, anions, trace elements and poly-aromatic hydrocarbons (PAHs) were measured. PMF has been applied to analyze these data and six common sources have been found for these two sites: sulfate, soil, zinc smelter, nitrate, wood smoke and copper smelter (Liu et al., 2003). PSCF results are also presented in the same report (Liu et al., 2003). Table 1 summarizes the mean contribution of each sources at the respective site.

The 5-day back trajectories with hourly endpoints were computed by the Hybrid Single-Particle

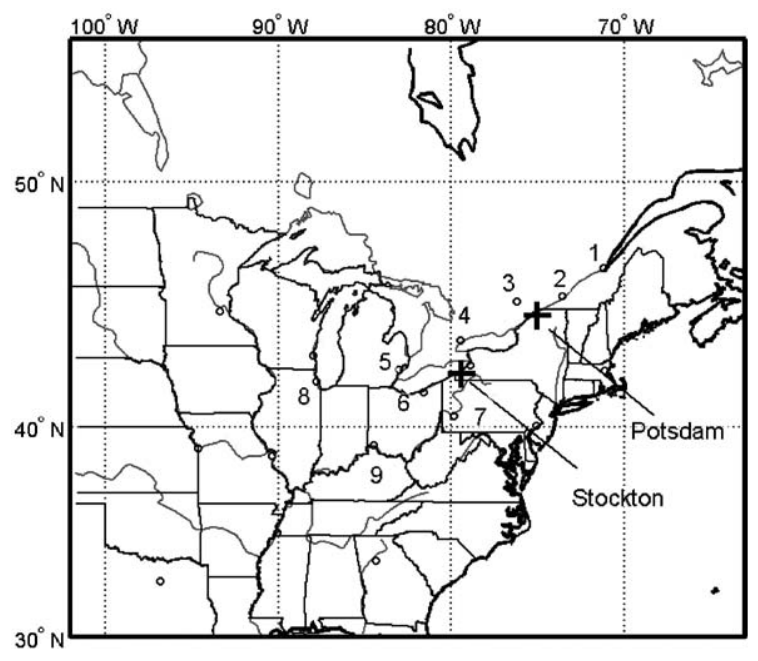

1 Quebec city, 2 Montreal, 3 Ottawa, 4 Toronto, 5 Detroit, 6 Cleveland, 7 Pittsburgh, 8 Chicago, 9 Cincinnati

Fig. 1. The position of the two receptor sites and major cities at possible source areas.

Table 1

Mean contribution of each source at the two receptor sites

\begin{tabular}{lccllll}
\hline Average concentration on $\left(\mu \mathrm{g} \mathrm{m}^{-3}\right)$ & Sulfate & Soil & Zinc smelter & Nitrate & Wood smoke & Copper smelter \\
\hline Potsdam & 6.03 & 1.58 & 0.213 & 0.988 & 0.953 & 0.218 \\
Stockton & 10.375 & 4.30 & 0.69 & 0.41 & 0.302 & 2.156 \\
\hline
\end{tabular}


Lagrangian Integrated Trajectory Model (Draxler and Hess, 1998; HYSPLIT4, 2002). The trajectories arrived at the receptor site every $6 \mathrm{~h}$ and the daily contribution of each source is assigned to the 4 trajectories belonging to that day. The computed trajectories were within the boundary layer. When the trajectory height exceeds the height of the top of the boundary layer, the trajectory was terminated (Draxler and Hess, 2002).

\section{Trajectory method}

\section{1. $S Q T B A$}

The probability of a tracer arriving at a point $(x, y)$ at time $t$ is given as

$A(x, t)=\int_{t-\tau}^{t} \int_{-\infty}^{\infty} \int_{-\infty}^{\infty} Q\left(x, y, t \mid x^{\prime}, y^{\prime}, t^{\prime}\right) \mathrm{d} x^{\prime} \mathrm{d} y^{\prime} \mathrm{d} t^{\prime}$,

where $Q\left(x, y, t \mid x^{\prime}, y^{\prime}, t^{\prime}\right)$ is the transition probability density function of an air parcel located at $\left(x^{\prime}, y^{\prime}\right)$ and time $t^{\prime}$ arriving at the receptor site $(x, y)$ at time $t$.

The transition probability $Q$ is assumed to be approximately normally distributed about the trajectory with a standard deviation that increases linearly with time upwind

$$
\begin{aligned}
Q\left(x, y, t \mid x^{\prime}, y^{\prime}, t^{\prime}\right)= & \frac{1}{2 \pi \sigma_{x} \sigma_{y}} \exp \left[-\frac{1}{2}\left(\left(\frac{X-x^{\prime}\left(t^{\prime}\right)}{\sigma_{x}}\right)^{2}\right.\right. \\
& \left.\left.+\left(\frac{Y-y^{\prime}\left(t^{\prime}\right)}{\sigma_{y}}\right)^{2}\right)\right]
\end{aligned}
$$

where $(X, Y)$ is the coordinate of the grid center and $x^{\prime}\left(t^{\prime}\right)$ and $y^{\prime}\left(t^{\prime}\right)$ are the coordinates of the center line of the trajectory. The $\sigma_{x}$ and $\sigma_{y}$ are approximated by

$\sigma_{x}\left(t^{\prime}\right)=\sigma_{y}\left(t^{\prime}\right)=a t^{\prime}$

with a dispersion speed, $a$, equal to $5.4 \mathrm{~km} \mathrm{~h}^{-1}$ (Samson, 1980). The potential mass-transfer field for a given trajectory, $\bar{T}_{k}\left(x, y, t \mid x^{\prime}, y^{\prime}, t^{\prime}\right)$, was integrated over the upwind period, $\tau$, of each trajectory to produce a twodimensional probability of natural transport field:

$\bar{T}_{k}\left(x, y \mid x^{\prime}, y^{\prime}\right)=\frac{\int_{t-\tau}^{t} Q\left(x, y, t \mid x^{\prime}, y^{\prime}, t^{\prime}\right) \mathrm{d} t^{\prime}}{\int_{t-\tau}^{t} \mathrm{~d} t^{\prime}}$.

The resulting natural transport potential field, $\bar{T}_{k}\left(x, y, t \mid x^{\prime}, y^{\prime}, t^{\prime}\right)$, for trajectory $k$, was weighted by the corresponding concentration, $\chi_{k}(x, y)$, yielding a concentration-weighted mass transfer potential field:

$\tilde{T}\left(x, y \mid x^{\prime}, y^{\prime}\right)=\sum_{k=1}^{K} T_{k}\left(x, y \mid x^{\prime}, y^{\prime}\right) \chi_{k}(x, y)$.

This definition of the weighted potential field is different from the definition given in Keeler (1987) since it is not divided by the sum of concentrations. Thus, the weighted potential field in Eq. (5) has the dimension of concentrations.

In PSCF, when cells are crossed by small number of trajectories, false source areas may be found if some of the trajectories also pass real source areas. This problem was solved by Cheng et al. (1993) by down-weighting the PSCF values. This "tailing effect" (Cheng and Lin, 2001) problem also exists for SQTBA and RTWC. In RTWC method, the concentrations of the cells that have a number of trajectory points less than an arbitrary threshold were discarded and a nine-point filter was used to smooth the concentration field (Stohl, 1996). To solve this problem in this study, the SQTBA field was downweighted empirically by the following method.

A coefficient $c_{r}$ is defined as

$c_{r}=\frac{10 K}{2 \pi\left(a t_{0}\right)^{2}}$,

where $K$ is total number of trajectories and $t_{0}$ is the length of the longest trajectory. The final SQTBA field is obtained by dividing the concentration-weighted field by unweighted field:

$$
\begin{aligned}
\operatorname{SQTBA}\left(x, y \mid x^{\prime}, y^{\prime}\right) \\
=\frac{\tilde{T}\left(x, y \mid x^{\prime}, y^{\prime}\right)}{\sum_{k=1}^{K} \bar{T}_{k}\left(x, y \mid x^{\prime}, y^{\prime}\right)} \\
\quad \times\left(1-\exp \left(-\frac{\sum_{k=1}^{K} \bar{T}_{k}\left(x, y \mid x^{\prime}, y^{\prime}\right)}{c_{r}}\right)\right) .
\end{aligned}
$$

The weighted field has the dimensions of concentrations and the unweighted natural field is dimensionless so the final SQTBA field has the dimensions of concentrations.

As indicated in Eq. (3), the transition probability is determined by the distance of each grid cell to the back trajectory endpoint, the width of each grid cell is $1^{\circ}$ of longitude and $0.74^{\circ}$ of latitude so that the grid cells have relatively uniform sizes.

\section{2. $R T W C$}

The rational of the redistribution in RTWC approach is no major pollutant sources are located along a "clean" trajectory (with very low concentration at the receptor site). The "polluted" trajectory (with high concentration at the receptor site) must have taken up the emission along its path where no "clean" trajectories pass. The complete detailed description of this method can be found in Stohl (1996).

For trajectory $k, c_{k}$ is the concentration measured (or computed source contribution for this study) upon the arrival of trajectory $k$. Let $C_{i k}, i=1, N_{k}$, be the mean concentrations of the grid cells which are hit by segments $i=1, N_{k}$ of trajectory $k((m, n)$ is the grid cell hit by trajectory segment $i)$. The redistribution for 

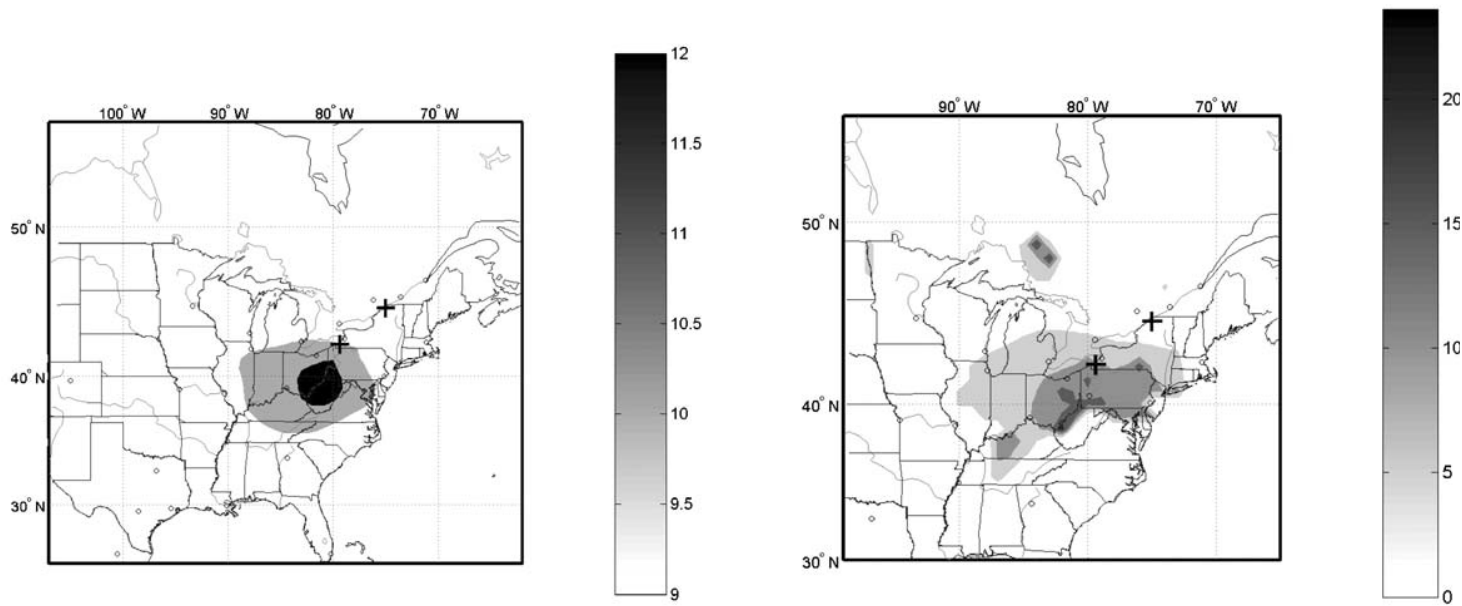

Fig. 2. Left: SQTBA field for sulfate. Right: RTWC field for sulfate.

trajectory $k$ is:

$c_{i k}=c_{k} \frac{C_{i k} N_{k}}{\sum_{j=1}^{N_{k}} C_{j k}}, \quad i=1, N_{k}$.

Eq. (8) is applied to all individual trajectories. After the redistribution, a new concentration field $\bar{C}_{m n}$ is computed:

$\log \bar{C}_{m n}=\frac{1}{\sum_{k=1}^{M} \sum_{i=1}^{N_{k l}} \tau_{m n i l}} \sum_{k=1}^{M} \sum_{i=1}^{N_{k}} \log \left(c_{i k}\right) \tau_{m n i l}$,

where $\tau_{\text {mnil }}$ is the residence time of segment $i$ of trajectory $k$ in grid cell $(m, n)$. The new field is redistributed again and these procedures are repeated until the average difference between the concentration fields of two successive iterations is below $0.5 \%$. The cells with less than a criterion number of trajectories passing are neglected. The grid cells have same sizes as those in SQTBA method, $0.74^{\circ}$ of latitude and $1^{\circ}$ of longitude.

\section{Results and discussion}

The results by SQTBA and RTWC for the six sources are presented in Figs. 2-7. From Eq. (7), it can be seen that the SQTBA field has the dimensions of concentrations. Unlike Stohl's (1996) original method, the RTWC field in this study uses concentrations instead of the logarithmic values of concentrations. The units of the scales in Figs. 2-8 are $\mu \mathrm{g} \mathrm{m}^{-3}$.

There are two major differences between the two methods. SQTBA assumes a normal distribution of the transport probability along the trajectories and RTWC features a redistribution for the concentration field. These differences may lead to different structures in their respective fields. All of the results by the two methods were evaluated by comparing their predictions with the known locations of the major sources. For RTWC method, the RTWC field was found to be influenced by the criterion number of trajectories and different criterion numbers have been tested. The best results were obtained when the criterion number were 50 , but for sulfate, this number is 30 .

\subsection{Sulfate}

As shown in Fig. 2, the result from SQTBA indicates the mid-west region is the potential source area, Ohio and western Pennsylvania. The RTWC field shows that Ohio and Pennsylvania as source areas and the highest concentrations appear in the Ohio River Valley, where many large coal power plants are located (Polissar et al., 2001). The hot spot at the border of New York state and Pennsylvania is probably from a coal power plant there (EPA, 2003). This point source was not found by SQTBA or PSCF. The annual $\mathrm{SO}_{2}$ emission rate of that power plant, $\sim 10,000 \mathrm{t}$, is small compared with the large coal power plants in the Ohio River Valley $\left(\sim 100,000 \mathrm{t} \mathrm{y}^{-1}\right)$. Either RTWC overestimates this point source or its real contribution is high since it is closer to the receptor sites. The two hot spots in RTWC field in Canada were found to be unrealistic. As shown in Table 1, the mean contribution of sulfate to Potsdam site is higher than that to Stockton site, suggesting the closer site may have more influence from the remote sources.

\subsection{Soil}

Fig. 3 presents the SQTBA and RTWC field for soil. The high concentration in the southern US shown in the SQTBA field, is similar to what has been found by Poirot et al. (2001) with residence-time analysis (RTA) at Underhill, VT. The high concentrations of RTWC field in the vicinity of Stockton site may be caused by 

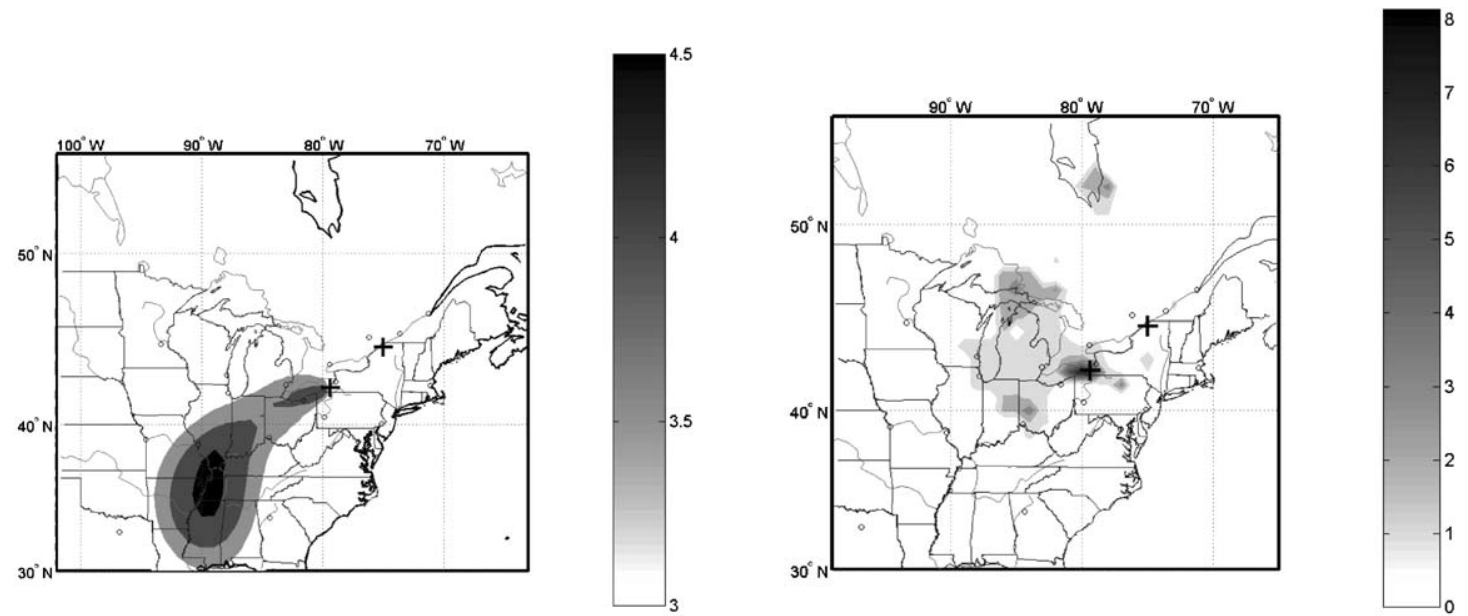

Fig. 3. Left: SQTBA field for soil. Right: RTWC field for soil.
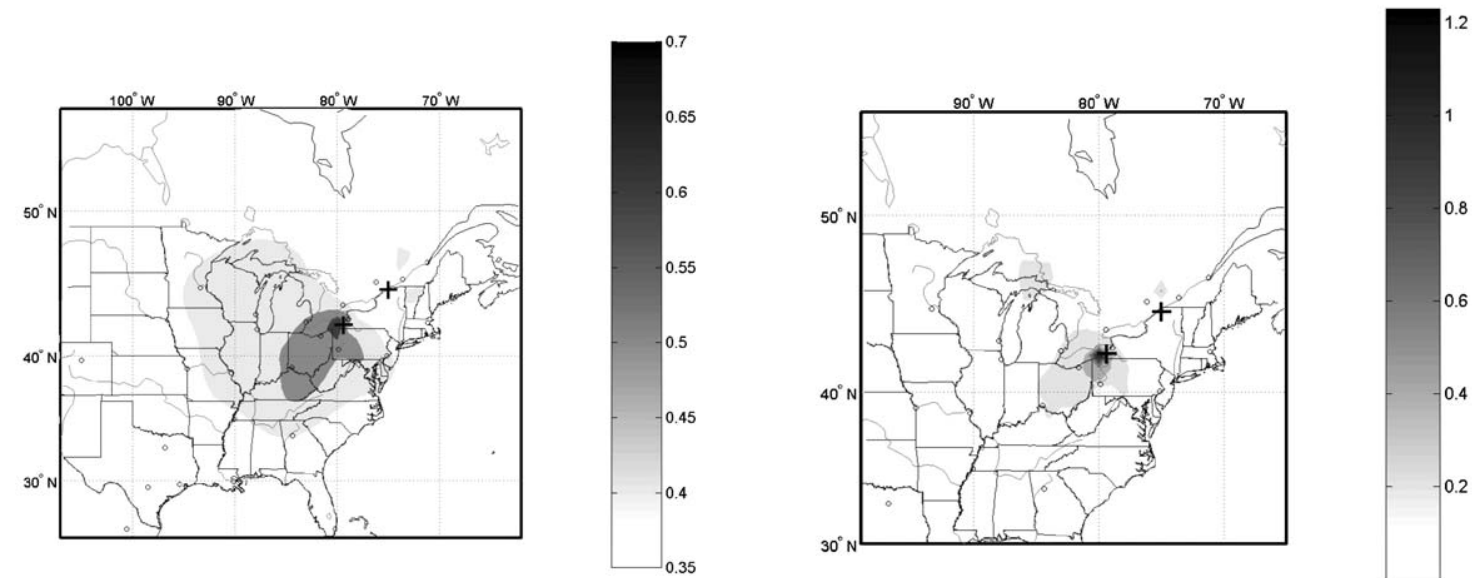

Fig. 4. Left: SQTBA field for zinc smelter. Right: RTWC field for zinc smelter.

local farming since the Stockton site was in a corn field. The much higher mean concentration at Stockton than Potsdam site also supports that soil is a local source for Stockton. RTWC field also shows wide source area including western and upper Michigan, western Ohio and northern Indiana. The farming area in these places could put soil in the air through cultivation activities. The source area predicted by RTWC in Canada may be from mining activities there.

The results by SQTBA, RTWC and PSCF are quite different, indicating fine soil emissions from a wide variety of emissions locations associated with dry and high wind speed conditions (Poirot et al., 2001).

\subsection{Zinc smelter}

The results of the two methods for zinc smelter are quite close as indicated by Fig. 4. The highest concentration of SQTBA field appears in Erie County, PA and a small part of Lake Erie near Erie County. RTWC only shows a hot spot at Erie County. There is no known zinc smelters in this location. The emission may also be from incinerators (Alexander et al., 2001; Chiaradia and Cupelin, 2000). The hot spot by RTWC may be from an incinerator there. SQTBA and RTWC both indicate source areas in Ohio, western Pennsylvania and the Detroit-Toronto area. Upper Michigan seems to be another source area by RTWC. The weak and isolated small source area, located north of the Potsdam site, may include the manufacture and primary metal industry facilities at Brownsburg-Chatham QC, and L'Orignal, ON. They should contribute primarily to Potsdam site because of the distance but their contributions are not large, which is in accordance with the fact that Stockton has much higher contribution from this source than Potsdam as indicated in Table 1. 


\subsection{Nitrate}

SQTBA and RTWC fields for nitrate are shown in Fig. 5. The contribution of nitrate to Potsdam is much higher than to Stockton, which implies that the major source is closer to Potsdam. The weather of Potsdam is cooler than Stockton. Particularly, in 2000, the average temperature at Potsdam was much lower. Ammonium nitrate tends to condense at low temperatures and in the presence of ammonia. Northern New York is primarily a dairy farming area that may contribute to Potsdam having higher nitrate concentrations. Although they both indicate local sources to Potsdam, the results from the two method do not agree well. SQTBA indicates the source area of nitrate is around North Bay-Ottawa area and Montreal area. Nitrate is formed from the oxidation of the precursor gas, $\mathrm{NO}_{x}$, from these areas. The major source area with the highest concentration by RTWC is located inside New York State. However, there are no major $\mathrm{NO}_{x}$ emission facilities in this area. The other two source areas with lower concentrations revealed by SQTBA are at the border of Ohio and West Virginia where the coal power plants produce the major $\mathrm{NO}_{x}$ emissions, and Maryland where $\mathrm{NO}_{x}$ emission is probably from traffic. These locations are not seen in RTWC field.

Wotawa and Kröger (1999) used simulated data from a Lagrangian box model to test the effect of RTWC in predicting nitric oxides source areas and found that temporal variations of emission and deposition decreased the accuracy of the predictions. The formation of nitrate is influenced by the concentrations of sulfuric acid and ammonia (Seinfeld and Pandis, 1998), and complex variations of nitrate concentration may be created by variations in the concentrations of other constituents as well as the rapid dry deposition of gaseous nitric acid. These factors may be the cause for the RTWC map making very little sense and it is recommended that this method should not be used for nitrate.

\subsection{Wood smoke}

Fig. 6 shows the wood smoke result. The two methods agree well. The highest concentration area is at the border of Canada and US, very close to Potsdam site. This source may be from home heating in June 2000. Other source areas predicted by RTWC including the Noranda area and the upper Michigan area are likely to have black carbon emissions from forest fire.

\subsection{Copper smelter}

Fig. 7 presents the results for the copper smelter at each site. The mean contribution at Stockton is much higher than at Potsdam as shown in Table 1, suggesting the source is close to Stockton. The RTWC field has the highest concentration very close to Stockton site, suggesting local source emissions such as the metal industries in Dunkirk NY (National Library of Medicine, 2003).

The SQTBA and RTWC results are quite different from the previous PSCF results (Liu et al., 2003): large sources in the area from Sudbury to Noranda was found for the Stockton site. When only using data from the Stockton site, SQTBA gives results close to PSCF as indicated in Fig. 8 which is more likely related to the large nickel smelter at Noranda, Quebec. The source profiles of copper smelters of the two sites have some differences (Liu et al., 2003). For example, the copper smelter source profile of the Potsdam site contained large fraction of black carbon but the source profile of the Stockton site contained little black carbon. A separate factor was separated at Potsdam and was
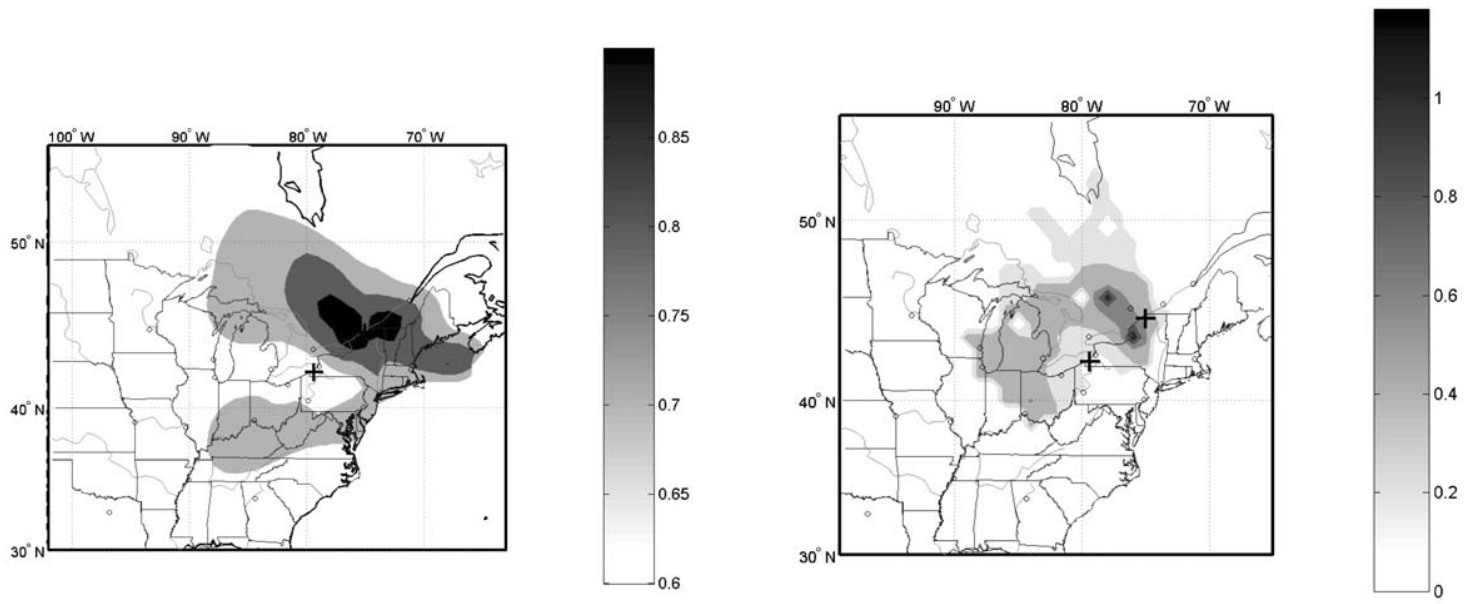

Fig. 5. Left: SQTBA field for nitrate. Right: RTWC field for nitrate. 

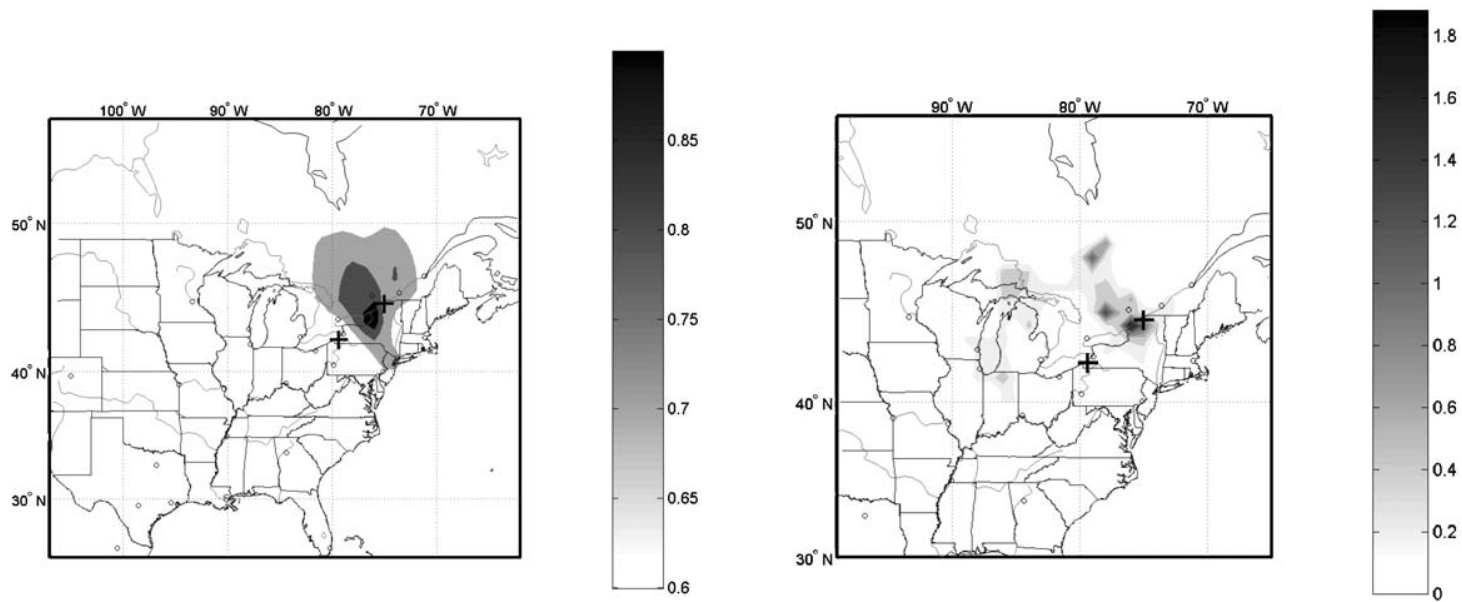

Fig. 6. Left: SQTBA field for wood smoke. Right: RTWC field for wood smoke.
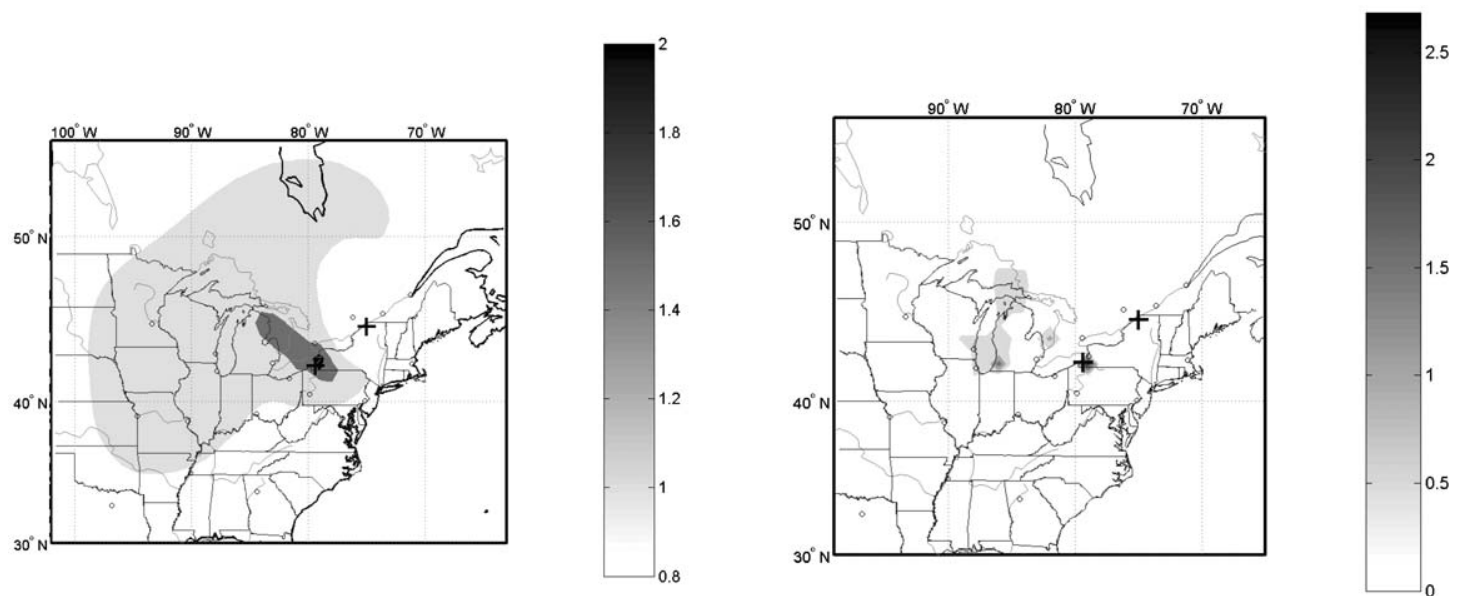

Fig. 7. Left: SQTBA field for copper smelter. Right: RTWC field for copper smelter.
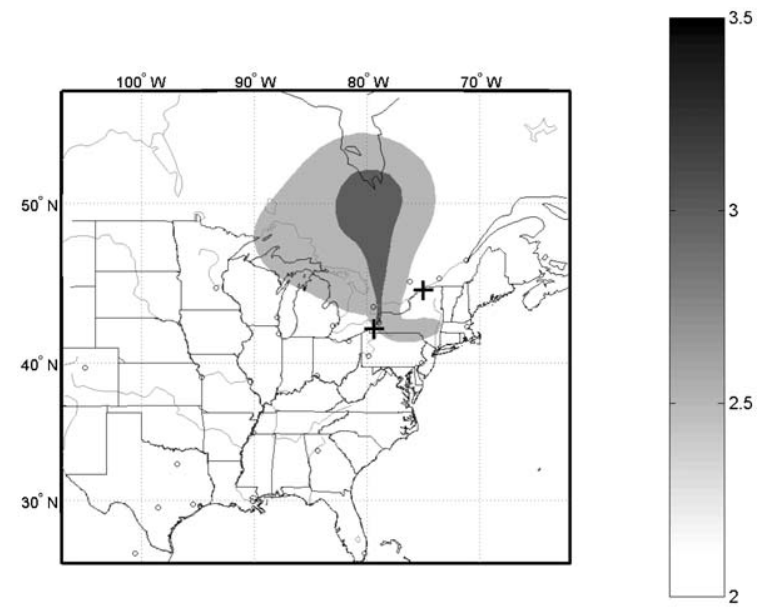

Fig. 8. SQTBA field for copper smelter for Stockton site. assigned to the nickel smelter based on the PSCF results. The results of this analysis do not agree well with the Stockton PSCF map for the "copper smelter" since those results did not clearly point to the Noranda location.

\section{Summary}

The source contributions from the factor analysis of particle composition data from Stockton and Potsdam sites were used in combination with back trajectories from the two sites to find the likely source locations. The two methods give consistent results in estimating the locations for large and clearly defined sources but less consistent for smaller and multiple sources. SQTBA can give reliable results with lower spatial resolution except 
that the "tailing effect" may lead to false source areas. By combining data from the two sites, local source areas can even be found by SQTBA. The redistribution of RTWC can give better spatial resolution and even emission hot spots as well as minor source areas but it seems to miss lots of source areas seen in other methods. The RTWC field is sensitive to the influence by many factors such as deposition, reaction or variations in emission rate and the analysis occasionally produces unrealistic structures or false source areas. One needs to be cautious when applying RTWC method, especially when there are significant variations in the source emissions. Besides checking the results by RTWC with the emission inventory, other methods without redistribution, such as PSCF, QTBA, or the CWT method, may also be used to ensure the reliability of the RTWC results.

\section{Acknowledgements}

This work was supported by the New York State Energy Research and Development Authority under contract 6083 and the US Department of Energy Technology Laboratory under contract DE-FC2601NT41017. We would like to thank Dr. Jeff Brook of Environment Canada for helping us get started with the SQTBA method.

\section{References}

Ashbaugh, L.L., Malm, W.C., Sadeh, W.Z., 1985. A residence time probability analysis of sulfur concentrations at Grand Canyon national park. Atmospheric Environment 19, 12631270.

Begum, A.B., Kim, E., Jeong, C.-H., Lee, D.-W., Hopke, P.K., 2003. Identification of source regions for atmospheric particles measured in Philadelphia, Pennsylvania. Atmospheric Environment, submitted for publication.

Cheng, M.-D., Lin, C.-J., 2001. Receptor modeling for smoke of 1998 biomass burning in Central America. Journal of Geophysical Research 106, 22871-22886.

Cheng, M.-D., Hopke, P.K., Zeng, Y., 1993. A receptororiented methodology for determining source regions of particulate sulfate at Doreset, Ontario. Journal of Geophysical Research 98, 16839-16849.

Chiaradia, M., Cupelin, F., 2000. Behavior of airborne lead and temporal variations of its sources effects in Geneva (Switzerland): comparison of anthropogenic versus natural processes. Atmospheric Environment 34, 959-971.

Draxler, R., Hess, G.D., 1998. An overview of the HYSPIT_4 modeling system for trajectories, dispersion and deposition. Australian Meteorological Magazine 47, 295-308.

Draxler, R., Hess., G.D., 2002. Description of the HYSPLIT 4 modeling system, NOAA Technical Memorandum ERL ARL-224.

EPA, 2003. http://www.epa.gov/triexplorer/facility.htm.
Hsu, Y-K., Holsen, T.M., Hopke, P.K., 2003. Comparison of hybrid receptor models to locate PCB sources in Chicago. Atmospheric Environment 37, 545-562.

HYSPLIT4, 2002. Hybrid single-particle Lagarangian integrated trajectory model. NOAA Air Resources Laboratory, Silver Spring, MD, Web address: http://www.arl.noaa.gov/ ready/hysplit4.html.

Keeler, G.J., 1987. A hybrid approach for source apportionment of atmospheric pollutants in the northeastern United States. Ph.D. Dissertation, University of Michigan, An Arbor MI.

Keeler, G.J., Samson, J., 1989. Spatial representativeness of trace element ratios. Environmental Science and Technology $23,1358-1364$.

Lin, C.-J., Cheng, M.-D., Schroeder, W.H., 2001. Transport patterns and potential sources of total gaseous mercury measured in Canadian high Arctic in 1995. Atmospheric Environment 35, 1141-1154.

Liu, W., Hopke, P.K., Han, Y.-J., Yi, S.M., Holsen, T.M., Cybart, S., Kozlowski, K., Milligan, M., 2003. Application of receptor modeling to atmospheric constituents at Potsdam and Stockton, NY. Atmospheric Environment 37, 4997-5007.

Lupu, A., Maenhaut, W., 2002. Application and composition of two statistical trajectory techniques for identification of source regions of atmospheric aerosol species. Atmospheric Environment 36, 5607-5618.

National Library of Medicine, Special Information Services, 2003. http://toxnet.nlm.nih.gov/cgi-bin/sis/.

Polissar, A.V., Hopke, P.K., Paatero, P., Kaufmann, Y.J., Hall, D.K., Bodhaine, B.A., Dutton, E.G., Harris, J.M., 1999. The aerosol at Barrow, Alaska: long-term trends and source locations. Atmospheric Environment 33, 2441-2458.

Polissar, A.V., Hopke, P.K., Poirot, R.L., 2001. Atmospheric aerosol over Vermont: chemical composition and sources. Environmental Science and Technology 35, 4604-4621.

Poirot, R.L., Wishinaski, P.R., 1986. Visibility, sulfate, and air mass history associated with the summertime aerosol in North Vermont. Atmospheric Environment 20, 1457-1469.

Poirot, R.L., Wishinski, P.R., Hopke, P.K., Polissar, A.V., 2001. Coparative application of multiple receptor methods to identify aerosol sources in northern Vermont. Environmental Science and Technology 35, 4622-4636.

Samson, P.J., 1980. Trajectory analysis of summer time sulfate concentrations in the northeastern United States. Journal of Applied Meteorology 19 (12), 1382-1394.

Seibert, P., Kromp-Kolb, H., Baltensperger, U., Jost, D.T., Schwikowski, M., Kasper, A., Puxbaum, H., 1994. Trajectory analysis of aerosol measurements at high alpine sites. In: Borrell, P.M., Borrell, P., Cvitaš, T. (Eds.), Transport and Transformation of Pollutants in the Troposphere. Academic Publishing, Den Haag, pp. 689-693.

Seinfeld, J.H., Pandis, S.N., 1998. Atmospheric Chemistry and Physics. Wiley, New York.

Stohl, A., 1996. Trajectory statistics - a new method to establish source-receptor relationships of air pollutants and its application to the transport of particulate sulfate in Europe. Atmospheric Environment 32, 947-966.

Stohl, A., 1998. Computation, accuracy and applications of trajectories - a review and bibliography. Atmospheric Environment 32 (6), 947-966. 
Stohl, A., Kromp-Kolb, H., 1994. Origin of ozone in Vienna and surroundings, Australia. Atmospheric Environment 28, 1255-1266.

Vasconcelos, L.A.P., Kahl, J.D.W., Liu, D., Macias, E.S., White, W.H., 1996. Spatial resolution of a transport inversion technique. Journal of Geophysical Research 101, 19337-19342.

Wotawa, G., Kröger, H., 1999. Testing the ability of trajectory statistics to reproduce emission inventories of air pollutants in cases of negligible measurement and transport errors. Atmospheric Environment 33, 3037-3043.

Xie, Y.-Y., Hopke, P.K., Paatero, P., Barrie, L.A., Li, S.-M., 1999. Locations and preferred pathways of possible sources of Arctic aerosol. Atmospheric Environment 34, 1367-1377.

Zeng, Y., Hopke, P.K., 1989. A study of the sources of acid precipitation in Ontario, Canada. Atmospheric Environment 23, 1499-1509. 


\title{
Field evaluation of the versatile aerosol concentration enrichment system (VACES) particle concentrator coupled to the rapid single-particle mass spectrometer (RSMS-3)
}

\author{
Y. Zhao, ${ }^{1}$ K. J. Bein, ${ }^{2}$ A. S. Wexler, ${ }^{1,2,3}$ C. Misra, ${ }^{4}$ P. M. Fine, ${ }^{4}$ and C. Sioutas ${ }^{4}$ \\ Received 13 February 2004; revised 28 July 2004; accepted 3 August 2004; published 17 February 2005.
}

[1] A field evaluation of versatile aerosol concentration enrichment system (VACES) coupled to a rapid single-particle mass spectrometer (RSMS-3) was conducted as part of the U.S. Environmental Protection Agency Supersite program in Pittsburgh during March 2002. RSMS-3 hit rate increases were measured, and possible particle composition changes introduced by the VACES were examined in the single-particle mass spectra. The hit rates increased by 5-20 times at particle sizes ranging from 40 to $640 \mathrm{~nm}$. VACES only enhances the hit rate by about a factor of 2 for large particle sizes because the RSMS-3 flow rates for these particles did not match the optimum operating condition of VACES. During the 3 days of measurements most of the particles were a mixture of carbonaceous material and ammonium nitrate with a variation across the spectrum from particles that were mostly carbonaceous to particles that were mostly ammonium nitrate. Both ambient and concentrated carbonaceous and ammonium nitrate composition distributions were indistinguishable with RSMS-3, suggesting that VACES introduces an insignificant artifact for those particles.

Citation: Zhao, Y., K. J. Bein, A. S. Wexler, C. Misra, P. M. Fine, and C. Sioutas (2005), Field evaluation of the versatile aerosol concentration enrichment system (VACES) particle concentrator coupled to the rapid single-particle mass spectrometer (RSMS-3), J. Geophys. Res., 110, D07S02, doi:10.1029/2004JD004644.

\section{Introduction}

[2] Atmospheric ultrafine particles are either formed by gas-to-particle conversion processes, in which hot and supersaturated vapors undergo condensation upon being cooled to ambient temperatures, or directly emitted as products of incomplete combustion processes [FinlaysonPitts and Pitts, 1986]. Although the mass fraction of the ultrafine mode is negligible, this size range contains the highest number of ambient particles as well as the highest total surface area. Because of their increased number and surface area, ultrafine particles are particularly important in atmospheric chemistry and environmental health.

[3] Recently, increasing toxicological and epidemiological evidence supports the link between respiratory health effects and exposures to ultrafine particles. Recent epidemiological studies [Heyder et al., 1996; Peters et al., 1997] demonstrate a stronger association between health effects and exposures to ultrafine particles compared to accumulation mode or coarse particles. Toxicological stud-

\footnotetext{
${ }^{1}$ Department of Mechanical and Aeronautical Engineering, University of California, Davis, California, USA.

${ }^{2}$ Department of Land, Air, and Water Resources, University of California, Davis, California, USA.

${ }^{3}$ Department of Civil and Environmental Engineering, University of California, Davis, California, USA.

${ }^{4}$ Department of Civil and Environmental Engineering, University of Southern California, Los Angeles, California, USA.
}

Copyright 2005 by the American Geophysical Union. 0148-0227/05/2004JD004644\$09.00 ies by Donaldson et al. [1998] indicate that ultrafine particles exerted a stronger physiological effect than the same mass of coarse or fine particles. A recent study by $\mathrm{Li}$ et al. [2003] indicates that ultrafine particulate matter (PM) is most potent in inducing cellular heme oxygenase-1 (HO-1) expression and depleting intracellular glutathione, both sensitive markers for oxidative stress, compared to concurrently collected accumulation and coarse mode PM. The same study showed that ultrafine particles, and to a lesser extent fine particles, localize in mitochondria where they induce major structural damage.

[4] A rapid single-particle mass spectrometer (RSMS) was developed at the University of California, Davis, and the University of Delaware [Phares et al., 2002] for measuring the size and chemical composition of individual atmospheric fine and ultrafine single particles. The second generation of the single-particle mass spectrometer, RSMS-2, was deployed at the U.S. Environmental Protection Agency (EPA) Supersite in Atlanta in August 1999 [Rhoads et al., 2003] and in Houston from 23 August to 18 September 2000 [Phares et al., 2003]. Over 15,000 individual particles were recorded covering $14-1300 \mathrm{~nm}$ size range and composing of 70 compound classes in the Atlanta Supersite experiment. In Houston, transient plumes of ultrafine particles that were present at the site for short duration were detected because of the instrument's fine temporal resolution and its ability to run continuously for a period of time. The RSMS-2 was modified to its third generation, RSMS-3, in 2001. In comparison with RSMS-2, there are two major improvements in RSMS-3: (1) both 
positive and negative ions can be detected and (2) two of the four digitizer channels are used to record each ion polarity signal to increase the spectral dynamic range (one channel is set at low sensitivity for strong signals and the other at high sensitivity for weak signals) and then signals in the two channels are combined by a computer program. One RSMS3 was installed at the U.S. EPA Supersite in Pittsburgh. A quarter of million single-particle mass spectra were analyzed over a 1 year period from September 2001 to October 2002. Results indicate that a rich array of multicomponent ultrafine particles were present [Bein et al., 2005]. Another RSMS-3 was installed at the U.S. EPA Supersite in Baltimore for semicontinuous operation over 9 months from 2001 to 2002 [Lake et al., 2003; Tolocka et al., 2005], where the characteristics of specific chemical components, such as metals [Tolocka et al., 2004a], sulfate [Lake et al., 2004], and nitrate [Tolocka et al., 2004b], and association among multiple components in the same particle were examined. A disadvantage of the RSMS-3 single-particle instrument is its insufficient hit rate for all but polluted urban conditions. Laboratory tests showed that the detection efficiency of RSMS was about one in a million and varied with particle size, shape, and composition [Kane and Johnston, 2000; Phares et al., 2002]. In order to increase the RSMS hit rate for cleaner conditions and therefore to broaden its applicability, several methods are under consideration. The goal is to either increase the sampling efficiency without changing its sizing ability or concentrate particles before they enter the instrument. Theoretical work shows that the hit rate may be increased by more than 10 times using a new inlet system with capped cone structure [Middha and Wexler, 2003]. Another way to increase the hit rate is to introduce a particle concentrator to the sampling inlet of the RSMS-3 mass spectrometer, which is the topic of this presentation.

[5] A particle concentrator (versatile aerosol concentration enrichment system, VACES) has been developed at the University of Southern California and deployed in many field experiments [Sioutas et al., 1999; Kim et al., 2001a, $2001 \mathrm{~b}$ ]. In its optimum configuration, VACES concentrates fine particles, including the ultrafine mode, by a factor up to 30 , depending on the ratio of total-to-minor flow rates of the virtual impactor [Sioutas et al., 1999; Kim et al., 2001a, $2001 \mathrm{~b}$. Evaluation of the VACES was previously performed in both laboratory and field and the results are described in a great detail by Kim et al. [2000, 2001a, 2001b] and Geller et al. [2002]. The ability of the VACES to concentrate particles has been laboratory tested using different type of particles, including polystyrene latex (PSL), silica beads, ammonia sulfate, and ammonia nitrate, in the size range from 50 to $1900 \mathrm{~nm}$ and at three minor flow rates of 7, 10, and 20 liters per minute (lpm) with the major intake flow rate of $220 \mathrm{lpm}$. TSI Condensation Particle Counter (CPC) was used to measure the number concentration of the original aerosols at upstream and concentrated aerosols at the downstream of the VACES. TSI Scanning Mobil Particle Sizer (SMPS) was used to measure the size distribution of those aerosols. The resulting enrichment factors (ratio of downstream aerosol number concentration to upstream) were very close to the ideal values (ratio of total-to-minor flow rate) and the aerosol size distribution was fairly well preserved during the concentra- tion enrichment process. Hygroscopic aerosols, such as ammonium sulfate and ammonia nitrate were concentrated as efficiently as hydrophobic PSL particles [Kim et al., 2001a]. Field evaluations of the VACES were conducted outdoors in Southern California [Geller et al., 2002; Kim et al., 2001b], where measurements of concentrationenriched aerosols were compared to direct ambient measurements made with micro-orifice uniform deposit impactor (MOUDI). Downstream and upstream measurements showed very good agreement (correlation coefficient $r^{2}=$ 0.80 for coarse particles, 0.66 for $\mathrm{PM}_{2.5}$ nitrate, 0.84 for $\mathrm{PM}_{2.5}$ sulfate, and 0.94 for ultrafine elemental carbon). Averaged concentration enrichment of those aerosols was very close to the ideal values. These experimental results indicated that the concentrator does not distort the size distribution of the original ultrafine aerosols on the basis of bulk measurements of particle chemical composition. Comparisons between the VACES and a reference monitor for ammonium nitrate, the Harvard/EPA Annular Denuder System, HEADS [Koutrakis et al., 1988], shows excellent agreement between the nitrate concentrations between HEADS and VACES [Kim et al., 2000].

[6] A field evaluation of the VACES concentrator coupled to the RSMS-3 ultrafine single-particle mass spectrometer was conducted at the Pittsburgh EPA Supersite in March 2002 to determine the hit rate increase and elucidate possible particle composition changes introduced by the concentrator, on the basis of single-particle mass spectra.

\section{Methods}

\subsection{Instrumental Setup}

[7] Figure 1 shows a schematic diagram of rapid singleparticle mass spectrometer, RSMS-3. The principle of RSMS-3 is nearly the same as RSMS-2, which has been described in detail previously [Phares et al., 2002], so only a brief description is given here. RSMS-3 consists of a Nafion dryer (PD-750-12SS, Perma Pure Inc., Toms River, New Jersey), a rotary valve orifice bank, an inlet system, two liners jointed with a source region, two Microchannel Plate (MCP) detectors (25 mm BiPolar Time-of-Flight, Burle Opto-Electronics Inc., Sturbridge, Massachusetts), and an UV ArF Excimer laser (EX10, GAM Laser Inc., Orlando, Florida). Sample air with particles passes through a dryer and a rotary valve orifice bank and then arrives at the inlet system composed of an aerodynamic lens with four vacuum stages. A $3 \mathrm{lpm}$ carrying dry air passes through the dryer and removes primarily water vapor from the sample. The orifice bank controls inlet pressure. The inlet system creates a particle beam with a narrow particle size range and skims off most of the gas. The optimum particle size that is focused depends on the upstream pressure, which is controlled by the rotary valve orifice bank. A $193 \mathrm{~nm}$, pulsed UV laser beam from an ArF Excimer laser is aligned coaxially with the particle beam by a $45^{\circ}$ folding-aligning mirror and focused at the source region by a lens. The laser emits laser pulses at 50 or $100 \mathrm{~Hz}$ and the laser energy is between 5 and $8 \mathrm{~mJ}$. When the laser beam hits a particle in the source region, the particle is ablated and ionized. Positive ions are accelerated by an electric field and fly inside the liner to the MCP detector on the positive side of the instrument. Negative ions fly in the opposite direction 


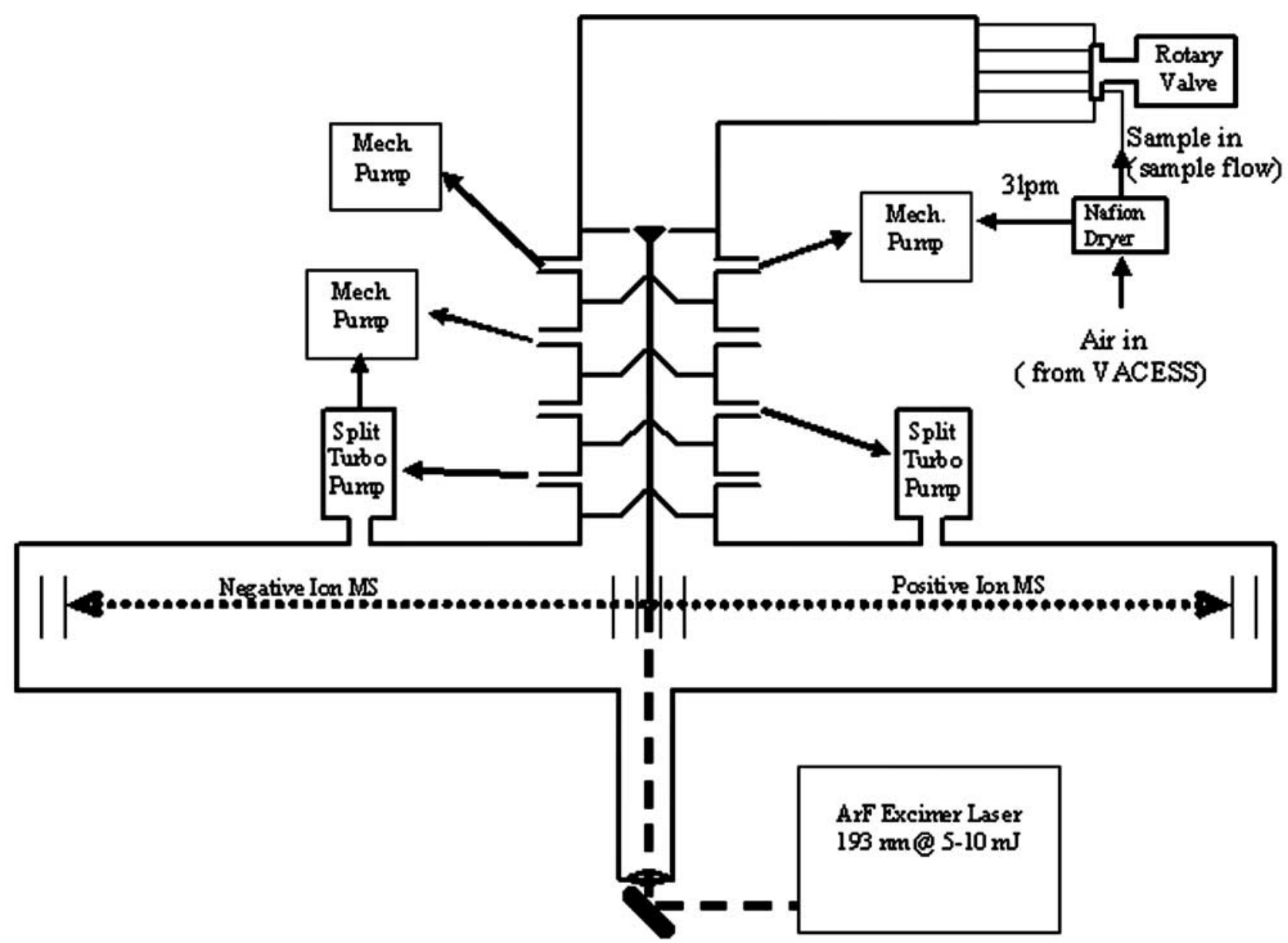

Figure 1. Schematic diagram of rapid single-particle mass spectrometer (RSMS-3).

and are detected by another MCP on the negative side. Signals from the MCP detectors are digitized by a fourchannel A/D converter (two A/D channels for each MCP signal to increase dynamic range) and recorded by a computer. There are nine orifices in the orifice bank, so RSMS-3 can measure nine particle sizes. Table 1 lists the inlet pressure, flow rates, and particle sizes (Stokes number $=1.14$ ) at each orifice. The $3 \mathrm{lpm}$ air drawn from the Nafion dryer was not included in Table 1, but shown in Figure 1.

[8] Figure 2 is a schematic diagram of VACES fine plus ultrafine particle concentrator. The VACES consists of a sample line, a saturation-condensation system, a virtual impactor, and a diffusion dryer (Model 3062, TSI Inc. Shoreview, Minnesota). Sample air is drawn through the sample line into a $35^{\circ} \mathrm{C}$ saturation chamber above a warm DI water bath where particles and air are humidified. This warm saturated aerosol is then introduced into a section cooled by $10^{\circ} \mathrm{C}$, thereby supersaturating the air and causing rapid condensation and particle growth. A virtual impactor concentrates the particles in its minor flow, which is then dehydrated so the particles return to their original sizes by means of a series of diffusion dryers. Particle enrichment by the VACES concentrator depends on the ratio of the virtual impactor's total-to-minor flow rates [Sioutas et al., 1999; Kim et al., 2001a]. The principle of a virtual impactor is similar to that of an inertial impactor [e.g., Willeke and Baron, 1993]: both methods use particulate inertia to separate particles from gases. A jet of particle-laden air is injected at a collection medium, which causes an abrupt deflection of the air streamlines. Particles larger than a certain size (the so-called cut point of the impactor) cross the air streamlines and, in the case of an inertial impactor, are collected on the medium, while particles smaller than a certain size follow the deflected streamlines. The main difference between an inertial and a virtual impactor is that in the latter, particles are injected into a collection probe rather than onto a collection medium. To separate larger particles continuously from the collection

Table 1. RSMS-3 Sizing Ability and Sample Flow Rates

\begin{tabular}{ccccc}
\hline Orifice & $\begin{array}{c}\text { Orifice ID, } \\
\text { inches/mm }\end{array}$ & $\begin{array}{c}\text { Inlet } \\
\text { Pressure, } \\
\text { torr }\end{array}$ & $\begin{array}{c}\text { Sample } \\
\text { Flow Rate, } \\
\text { lpm }\end{array}$ & $\begin{array}{c}\text { Sizing, } \\
\text { nm }\end{array}$ \\
\hline 1 & $0.063 / 1.600$ & 151.7 & 15.4 & 1100 \\
2 & $0.035 / 0.889$ & 60.5 & 4.96 & 640 \\
3 & $0.024 / 0.610$ & 30.2 & 2.82 & 354 \\
4 & $0.017 / 0.432$ & 15.2 & 1.44 & 184 \\
5 & $0.015 / 0.381$ & 9.6 & 1.09 & 117 \\
6 & $0.013 / 0.330$ & 9.0 & 0.74 & 109 \\
7 & $0.011 / 0.280$ & 6.0 & 0.55 & 73 \\
8 & $0.008 / 0.203$ & 3.2 & 0.23 & 40 \\
9 & $0.006 / 0.152$ & 1.5 & 0.10 & 18 \\
\hline
\end{tabular}

${ }^{\mathrm{a}} \mathrm{Here}, \mathrm{lpm}$, liters per minute. 


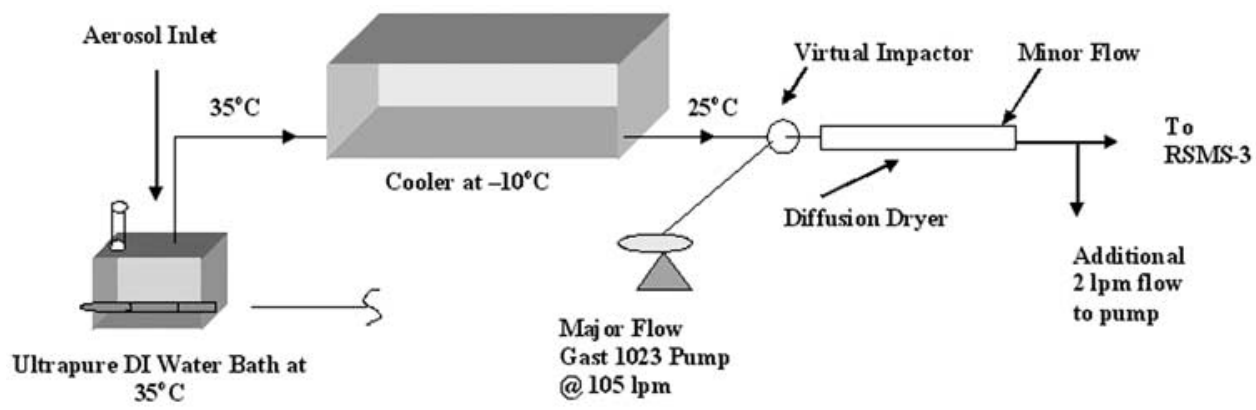

Figure 2. Schematic diagram of VACES fine plus ultrafine particle concentrator.

probe, a fraction of the total flow, referred to as the minor flow (typically $10-20 \%$ of the total flow), is allowed to pass through the probe, leaving particles larger than the cut point contained in a small fraction of the initial gases. The cut point of the virtual impactor used in VACES was $2.5 \mu \mathrm{m}$.

[9] In this experiment, the minor flow of the VACES concentrator was coupled to the RSMS-3 instrument; that is, the minor flow of the concentrator is directly connected to the sample port of the RSMS-3 mass spectrometer. The two instruments were operated at their original configurations, as described in the previous paragraphs in this section, without any modifications.

\subsection{Data Analysis}

[10] After firing each laser pulse, 5000 data points of the digitizer signals were acquired and examined by a computer. A single-particle mass spectrum was recorded when the height of any peak in the selected spectral region was greater than the predefined threshold value. Afterward, the data were transmitted from Pittsburgh Supersite to the UC Davis campus for storage and postprocessing. The singleparticle mass spectra were first mass calibrated (converted from time to mass coordinates) covering the spectral range from $\mathrm{m} / \mathrm{z}=-256$ to $\mathrm{m} / \mathrm{z}=+256$. Spectra with a broad peak centering at $\mathrm{m} / \mathrm{z}=149$ were caused by instrument emission and therefore were considered as background. After quality control to remove the background spectra, the calibrated spectra were integrated and normalized at integer $\mathrm{m} / \mathrm{z}$ values and finally classified using the Adaptive Resonance Theory-2a (Art-2a) algorithm, which was first introduced by Hopke and Song [1997] for mass spectra analysis.

[11] The Art-2a algorithm uses the vector dot product as its similarity metric to classify the particles and is controlled by two parameters. The vigilance factor sets the similarity condition and the learning rate determines the rate at which the parameters adjust. The algorithm first selects each normalized spectrum in a random order and compares it to an existing set of weight vectors. If a winning weight vector is found to have the largest degree of similarity with the selected spectrum and its dot product with the corresponding particle vector is greater than the predefined vigilance factor, the selected spectrum is considered to belong to the class that the winning weight vector represents and then it is incorporated into the winning weight vector. In this case, the weight vector components are shifted toward the added spectrum's by the learning rate. If no weight vector satisfies the vigilance criterion with the selected spectrum, the particle vector becomes a new weight vector and is then added to the set of existing weight vectors. The first selected spectrum must be a new weight vector because there are no existing weight vectors to compare at that time. Once all spectra were selected, the whole procedure was repeated with a set of weight vectors produced in the previous iteration. Phares et al. [2001] validated the application of Art-2a algorithm in the analysis of single-particle spectra generated in laboratory with aerosols composing of single and mixed know chemical components. It was shown that a higher vigilance factor tended to overclassify (more classes than the real number) while a lower vigilance factor tended underclassify (less classes than the real) these laboratory-generated mass spectra. A vigilance factor of 0.6 was recommended to produce a class number that was very close to the real number [Phares et al., 2001]; on this basis, the vigilance factor was predefined as 0.6 in this study. The final weight vectors are presented with equalweighted averages of all spectra belonging to that class, in order to calculate the standard deviations in the peak heights for each class. Therefore no learning rate was used in this data analysis.

[12] Although RSMS-3 is a bipolar mass spectrometer measuring both positive and negative ions simultaneously, only positive spectra were analyzed with Art-2a algorithm and presented in this study. Previous research shows that most of negative spectra were contributed by sulfate, which is very difficult to ablate and detect in the fine and ultrafine particles sampled here [Kane and Johnston, 2001; Lake et al., 2004]. Because of the large uncertainty in the detection of negative ion spectra, they were excluded in this study.

[13] The RSMS-3 hit rates were defined as the nonbackground particle hits divided by the corresponding measurement interval. Enhancements of the hit rate by VACESS concentrator were determined by ratios of the hit rate of concentrated air to that of ambient air. Ideal enrichments of particle concentration by VACES were predicted by the ratio of impactor's total-to-minor flow rate. The detection efficiency of RSMS-3 relative to the real aerosol concentration in the atmosphere will not be discussed in this work. Previously studies showed that the efficiency of RSMS instrument was about $10^{-6}$ and varied with particle size, shape, and chemical composition [Kane and Johnston, 2000; Phares et al., 2002].

\section{Results and Discussion}

[14] Experiments were performed during two sampling periods in spring of 2002. The first, 5 March, provided 


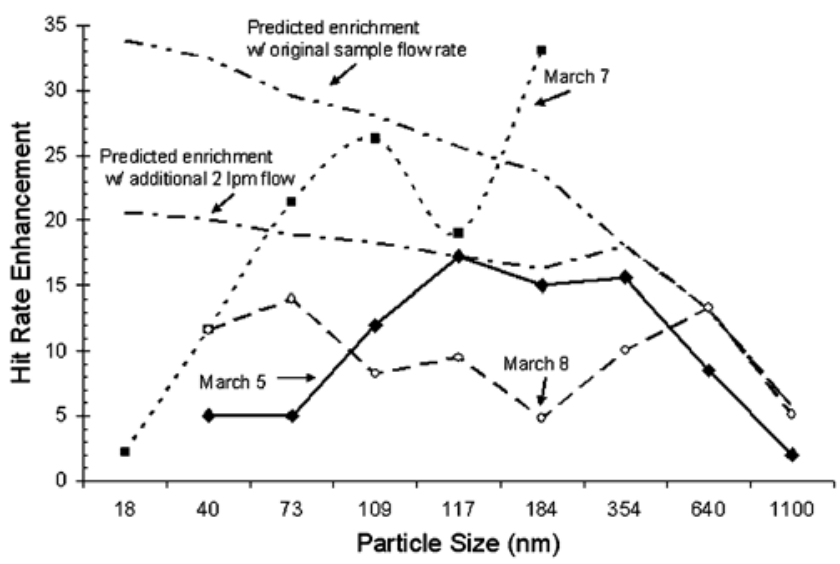

Figure 3. Enhancement of RSMS-3 hit rate by VACES particle concentrator. Predicted particle concentration enrichments were calculated using the ratio of VACES total-to-minor flow rate, where the $3 \mathrm{lpm}$ flow rate passing through the RSMS-3 Nafion dryer was taken into account.

preliminary data and understanding of the use of the instruments together. The second, 7-8 March, was a more thorough test of the coupled system.

\subsection{Data of 5 March 2002}

[15] RSMS-3 was scheduled to measure one sample of unconcentrated ambient air followed by a concentrated sample at each particle size. The measurement time for each orifice (each particle size bin) was terminated by a maximum of 30 particle hits or 5 minutes whichever came first. Each measurement was repeated 2-3 times. The VACES concentrator was operated with a fixed major flow of $105 \mathrm{lpm}$. In total, 342 mass spectra from ambient air sample and 462 from sample with concentrator were recorded on 5 March 2002 after removal of the background spectra.

[16] Figure 3 shows the enhancement of RSMS-3 hit rates by VACES concentrator at different particle sizes observed in this study. Enrichments of particle concentration by VACES were also predicted on the basis of the ratios of total-to-minor flow rates of the impactor. Since the minor flow of the VACES concentrator was directly coupled to the RSMS-3 sample port, the minor flow of VACES was equal to the RSMS-3 sample flow listed in Table 1 plus the $3 \mathrm{lpm}$ flow of the Nafion dryer which is not listed in Table 1. Diamonds connected with a solid line represent the $5 \mathrm{March}$ results. The hit rate enhancement of RSMS-3 varies with particle size for a number of reasons. Since the VACES minor flow rate changed when RSMS-3 was sampling different particle sizes (see Table 1), while its major flow rate was fixed, the ratio of total-to-minor flow rate, and therefore the VACES concentration enrichment, changed with particle sizes, which is consistent with the predicted enrichment of particle concentration by VACES at large particle sizes and can readily be seen in Figure 3. With the VACES concentrator, particle hit rates of the RSMS-3 were increased by $10-17$ times for the particles with sizes ranging from 109 to $354 \mathrm{~nm}$. From size $640 \mathrm{~nm}$ to $1100 \mathrm{~nm}$, the hit rate enhancements became smaller as the
RSMS-3 sampling flow rate, and therefore the minor flow rate of the concentrator, increased. For the particles of $1100 \mathrm{~nm}$, the enhancement of hit rates was only 2 . The predicted enrichment was 5 at this point. Table 1 indicates that the flow rate at this particle size was greater than $15 \mathrm{lpm}$, which plus the $3 \mathrm{lpm}$ of dryer flow was too high for the VACES concentrator to operate in its optimum range [Sioutas et al., 1999].

[17] At the small particle side, the hit rate enhancements also decreased as the flow rate decreased which is contrary to the predicted particle concentration enrichment that increased. At these small particle sizes, the flow rates (about $3 \mathrm{lpm}$ ) into RSMS-3 were lower than the design conditions for the concentrator, so particle losses to the walls of the diffusion dryer would be substantial. On the other hand, the enrichment of particle concentration by VACES is affected to a large extent by the actual minor flow ratio and deviates from its ideal value as this ratio becomes smaller (i.e., less than about 5\%). This is because as this minor flow decreases, particle losses mostly on the collection nozzle of the virtual impactor increase, thereby decreasing the overall enrichment [Marple and Chien, 1980; Sioutas et al., 1994]. This is the case also with the virtual impactors used in the VACES to concentrate the grown ultrafine particles [Sioutas et al., 1999]. Despite these losses, the hit rate enhancements at 40 and $73 \mathrm{~nm}$ were about 5 . Accuracy of the real flow rate measurement is another factor in difference between the predicted VACES concentration enrichment and RSMS-3 hit rate enhancement. The ablation ability of RSMS-3 for different particle sizes [Kane and Johnston, 2000] would be another cause for the difference. It should be emphasized that this experiment was conducted with the RSMS-3 and VACES in their original configurations and the main premise of this research was to find out problems in coupling the two instruments together and ways to fix them, but no efforts were made to achieve the maximum enhancement of RSMS-3 hit rates.

[18] All single-particle mass spectra obtained on 5, 7, and 8 March were divided into two groups, with and without the concentrator, to calculate the hit rate enhancements and

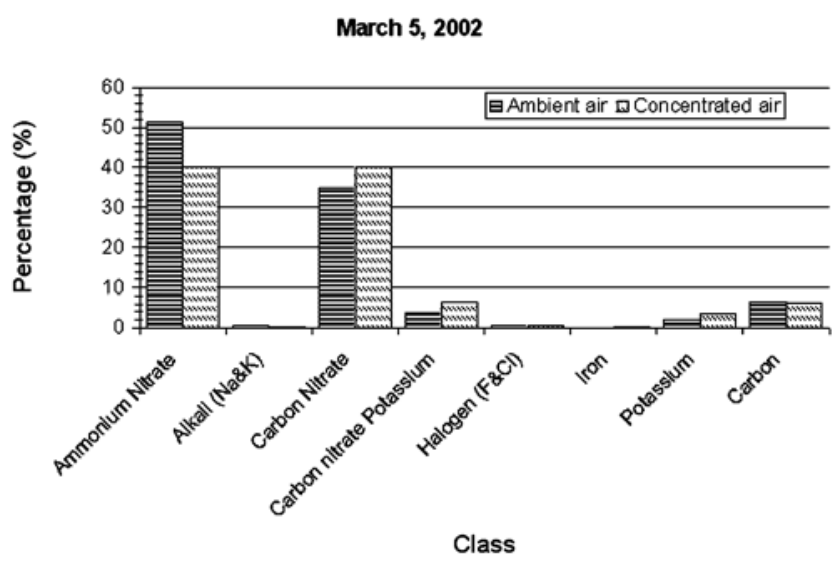

Figure 4. Comparison of chemical classes between ambient air and concentrated air measured on 5 March 2002. 
Class: Ammonium Nitrate

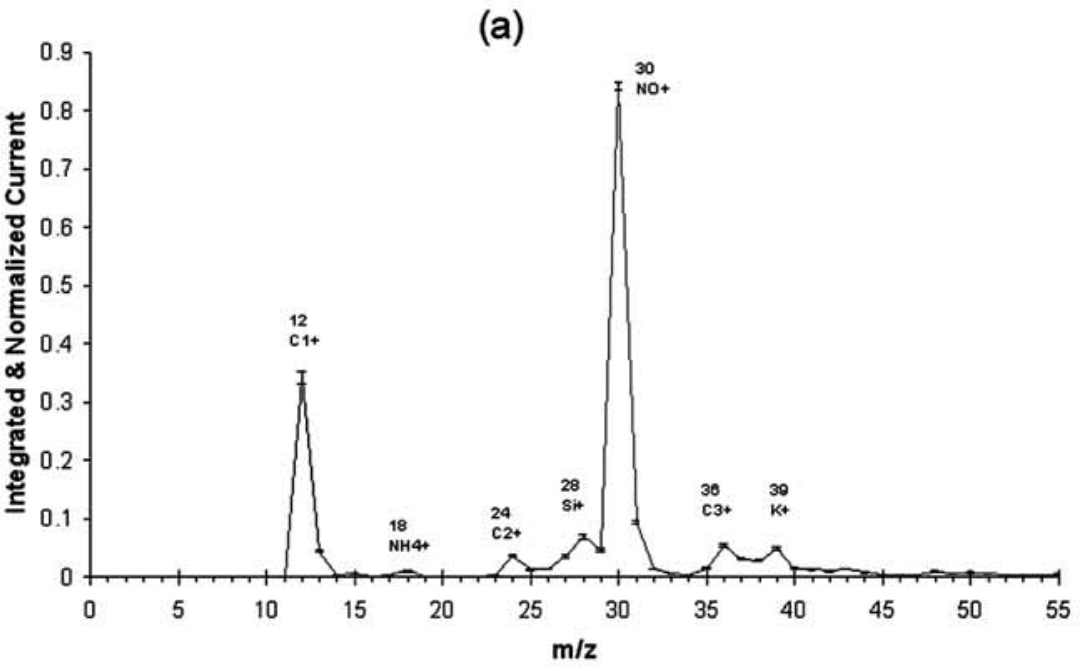

Class: Carbon Nitrate
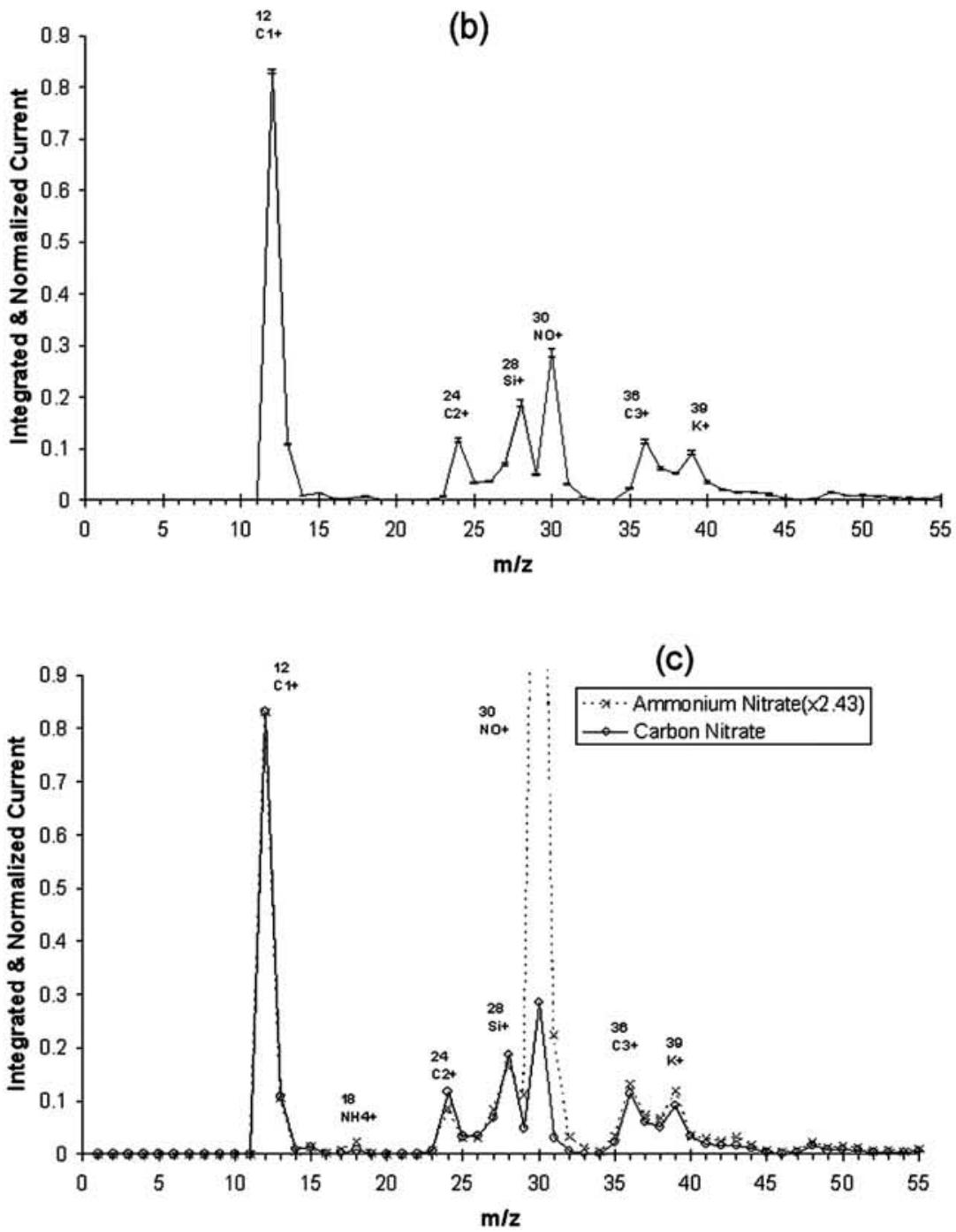

Figure 5. Average spectra of (a) ammonium nitrate and (b) carbon nitrate and (c) comparison between the two average spectra. The ammonium nitrate spectrum has been scaled up by 2.43 in Figure $5 \mathrm{c}$ to show the similarity between the two spectra. 


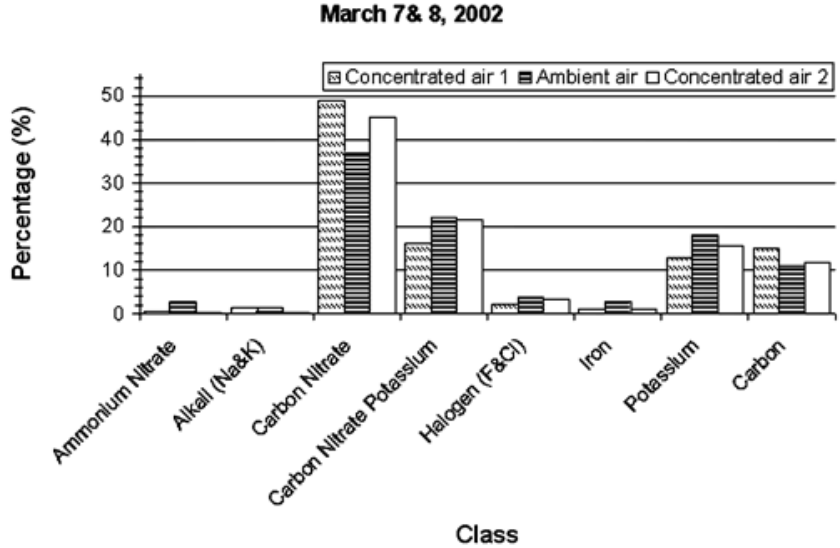

Figure 6. Same as Figure 4 but for 7-8 March 2002. The concentrated air was sampled immediately before (concentrated air 1) and after (concentrated air 2) the ambient air.

classified using Art-2a algorithm. All spectra were partitioned into 8 composition classes. Each of the eight classes represented more than 10 particles and those classes containing less 10 particles were considered minor and not presented. Figure 4 shows the fractions of total hits for each class observed on 5 March. The two major classes were ammonium nitrate (nitrate peak is domain in the spectra) and carbon nitrate (carbon peaks are domain in the spectra with a small nitrate peak), whose average spectra are shown in Figures $5 \mathrm{a}$ and $5 \mathrm{~b}$. Without the concentrator, about $51 \%$ of the particles were in the ammonium nitrate class and about $35 \%$ particles were in the carbon nitrate class, while their values were about $40 \%$ and $40 \%$, respectively, when the concentrator was used. Figure $5 \mathrm{c}$ compares the average spectrum of the ammonium nitrate class with the one in the carbon nitrate class. The two spectra are very similar except for the $\mathrm{NO}^{+}$peak at $\mathrm{m} / \mathrm{z}=30$ where the peak in the carbon nitrate class was much lower than in the ammonium nitrate class. Thus it appears that the concentrator might introduce a compositional change in a fraction of the particles, primarily shifting nitrate from particles in the ammonium nitrate class to those in the carbon nitrate class. This shift could be also due to a change in atmospheric composition during the experiment, so experiments on 7 and 8 March were designed to address this potential ambiguity. Including the effects of changes in chemical position of ambient air, the statistical uncertainty in RSMS-3 measurements, and the coupling with particle concentrator, in total about $8 \%$ particles were shifted from the ammonium nitrate class to the carbon nitrate class during the experiments on 5 March. More discussion regarding to this shift will be made in the following sections.

\subsection{Data of 7 and 8 March 2002}

[19] The RSMS-3 operating schedule was adjusted to measure one sample with concentrated air, one without, and again one with, at each particle size to separate changes in particle composition due to ambient conditions from those due to the concentrator. In order to obtain a more statistically significant sample, the measurement time was terminated at 30 particle hits at each particle size without setting a time limit. On 8 March, an additional 2 lpm was drawn from the minor flow port through diffusion dryer of the concentrator when RSMS-3 was sampling particles at sizes from 40 to $184 \mathrm{~nm}$, in order to keep the changes in the ratio of total-to-minor flow rates small and minimize small particle losses. In total, 227 particles were collected without the concentrator and 701 with after removal of the background spectra.

[20] The hit rate enhancements are shown in Figure 3 for 7 March (closed squares) and 8 March (open circles). Decrease of the hit rate enhancement for small particles can still be seen on 7 March, but this dependence on RSMS3 flow rate was partially corrected by pulling out the additional $2 \mathrm{lpm}$. On 7 March the hit rate enhancement of RSMS-3 at $184 \mathrm{~nm}$ was even higher than the predicted VACES enrichment of particle concentration, which is likely not true and might be caused by the removal of the background spectra. The causes for day-to-day hit rate variation, evident in Figure 3, may be due to variation in ambient conditions, RSMS-3 operating conditions such as laser intensity and laser beam alignment, or imprecise removal of background spectra.

[21] Class comparisons between samples collected with and without the concentrator on 7 and 8 March are shown in Figure 6. The four major classes were carbon nitrate (as shown in Figure 5b), carbon nitrate potassium, potassium, and carbon. Particles in the ammonium nitrate class were not observed on these days. The experiments of 7 and 8 March were designed to sample concentrated particles immediately before and after ambient particles to identify whether or not a shift in particle composition occurred during the sampling period. There is a $4 \%$ difference in the particle fractions between the two concentrated samples that could be due to either change in ambient air or instrument drift. A $10 \%$ difference is also seen in the carbon nitrate class between the ambient air and the average of the two concentrated samples indicating a possible class shift from other nitrate containing classes to carbon nitrate.

\subsection{Causes of the Class Shift}

[22] Figures 4 and 6 indicate that about $8-10 \%$ particles shifted from the ammonium nitrate class (or other nitrate containing classes) to the carbon nitrate class during the experiments on 5, 7, and 8 March when the RSMS-3 was coupled with the VACES particle concentrator. As discussed, changes in the ambient air, operational conditions of the RSMS-3, and artifacts caused by the VACES particle concentrator are the possible sources for the observed differences. This section will address more possible causes for the class shift.

[23] Figures $7 \mathrm{a}-7 \mathrm{~d}$ compare the chemical classes between concentrated particles and ambient particles at different particle sizes measured on 5, 7, and 8 March 2002. Although the RSMS-3 can measure nine sizes of particles as given in Table 1, only two size ranges, a small size (40$117 \mathrm{~nm})$ representing ultrafine particles and a large size $(184-1100 \mathrm{~nm})$ for the fine particles minus ultrafines, are grouped and shown in these figures in order to present statistically significant results. On 5 March the class shift from ammonium nitrate to carbon nitrate of ultrafine particles (Figure 7a) is the same pattern as the average of all sizes (Figure 4) but the shift of fine particles (Figure 7b) is 
(a) March 5, 2002

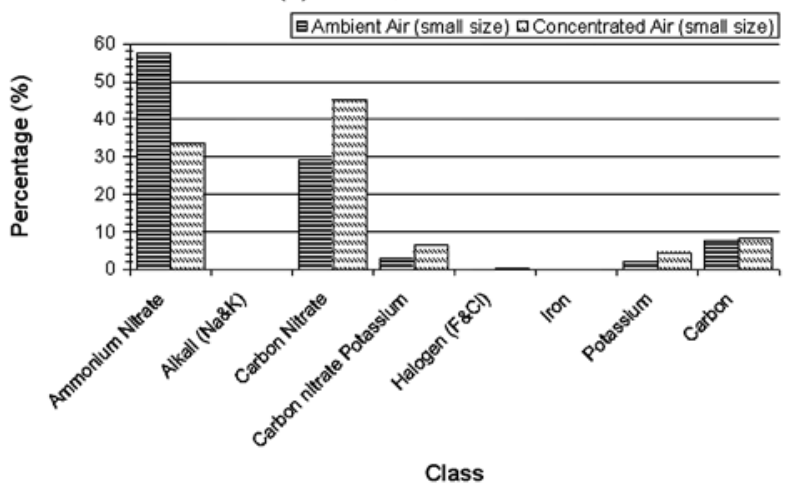

(c) March 7\& 8, 2002

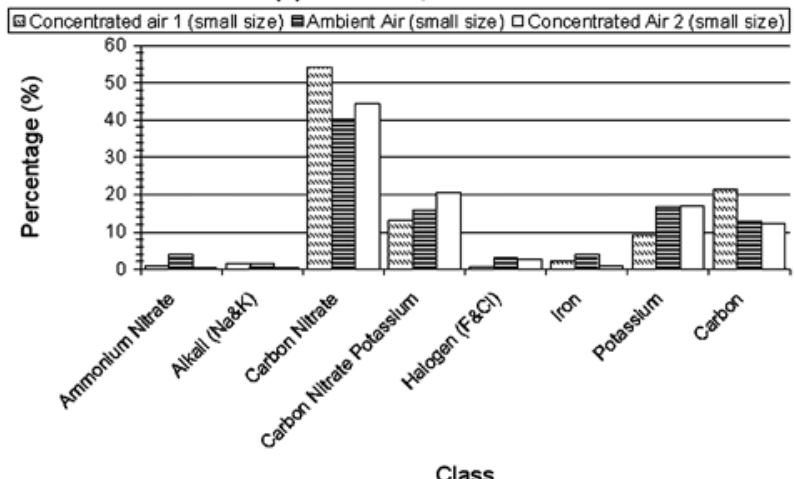

(b) March 5, 2002

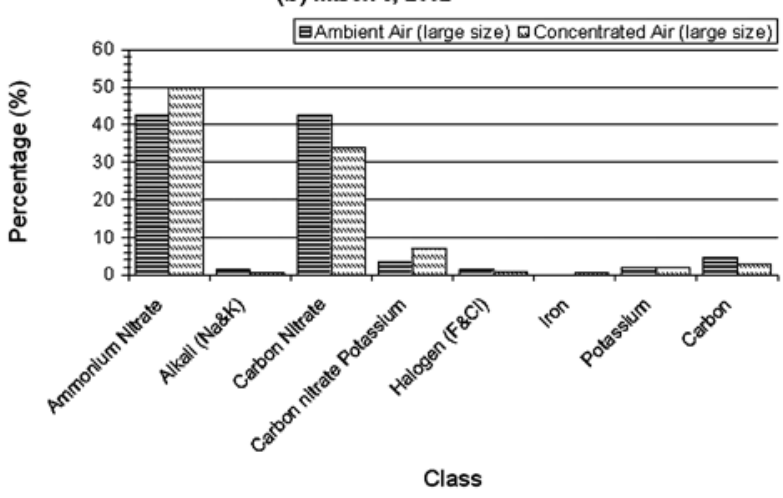

(d) March 7\& 8, 2002

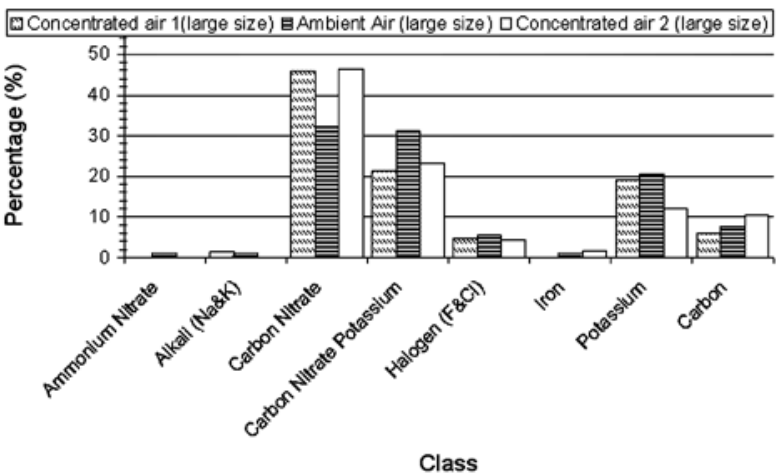

Figure 7. Same as Figure 4 but (a) for small size (ultrafine) particles of 5 March 2002 between 40 and $117 \mathrm{~nm}$, (b) for large size (fine) particles of 5 March 2002 between 184 and $1100 \mathrm{~nm}$, (c) for small size (ultrafine) particles of 7-8 March between 40 and $117 \mathrm{~nm}$, and (d) for large size (fine) particles of 7-8 March between 184 and $1100 \mathrm{~nm}$.

opposite. By comparison, on 7 and 8 March, the shifts at both fine and ultrafine particles (Figures $7 \mathrm{c}$ and $7 \mathrm{~d}$ ) are the same direction as that of the average. Observation by RSMS-3 on these days did not indicate any dependence of the class shift on particle sizes because the shift directions were random. From this point of view, it is thus unlikely that the VACES particle concentrator introduced the shift of particles from one class to another. If the concentrator did introduce such a shift, the patterns of the class shift should not change day-by-day.

[24] Figure 5c shows that the average spectra for the ammonium nitrate and carbon nitrate classes are identical when scaled to the $\mathrm{C}_{1}^{+}$peak heights, except for the height of the $\mathrm{NO}^{+}$peaks. Therefore it appears that the two classes have similar underlying carbonaceous cores with varying amounts of ammonium nitrate condensed, presumably due to varying particle age. Figure 8 shows the frequency distribution of particles from both classes as a function of the logarithm of the $\mathrm{NO}^{+} / \mathrm{C}_{1}^{+}$peak ratio. Areas under each curve in Figure 8 are normalized to 1 . It is seen that the frequency of ammonium nitrate class has a maximum at $\mathrm{NO}^{+} / \mathrm{C}_{1}^{+}>1$, while the maximum of carbon nitrate class is located at $\mathrm{NO}^{+} / \mathrm{C}_{1}^{+}<1$. There is a valley between the two maxima near $\mathrm{NO}^{+} / \mathrm{C}_{1}^{+}=1$. The Art-2a algorithm breaks the two classes near this valley. Since the valley is not very pronounced and in fact the distribution between the ammonium nitrate and carbon

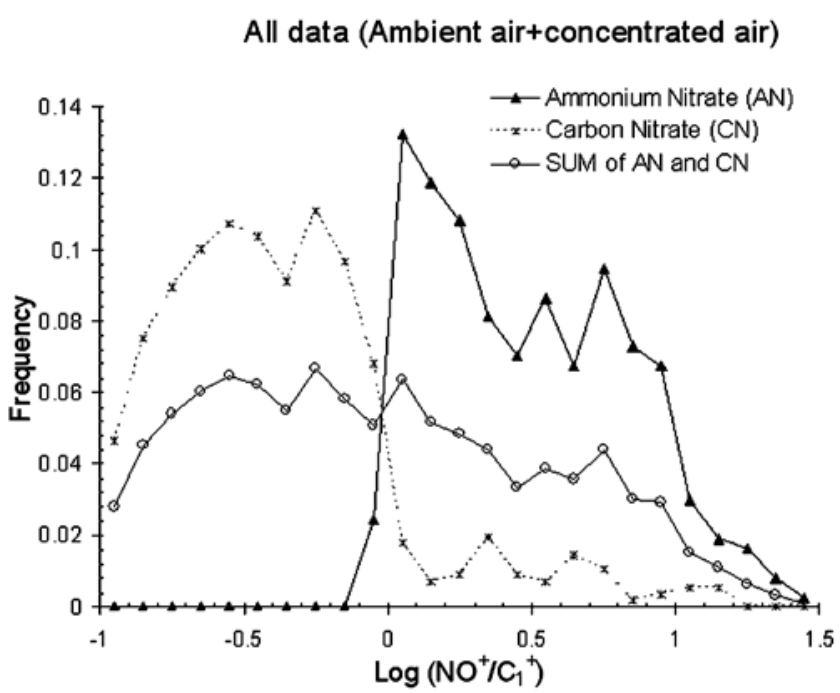

Figure 8. Frequency of the ratios of $\mathrm{NO}^{+}$peak to $\mathrm{C}_{1}^{+}$ peak of ammonium nitrate class, carbon nitrate class, and sum of the two classes. Areas under each curve are normalized to 1 . 
(a) Sum of Ammonium nitrate and Carbon nitrate, March 5

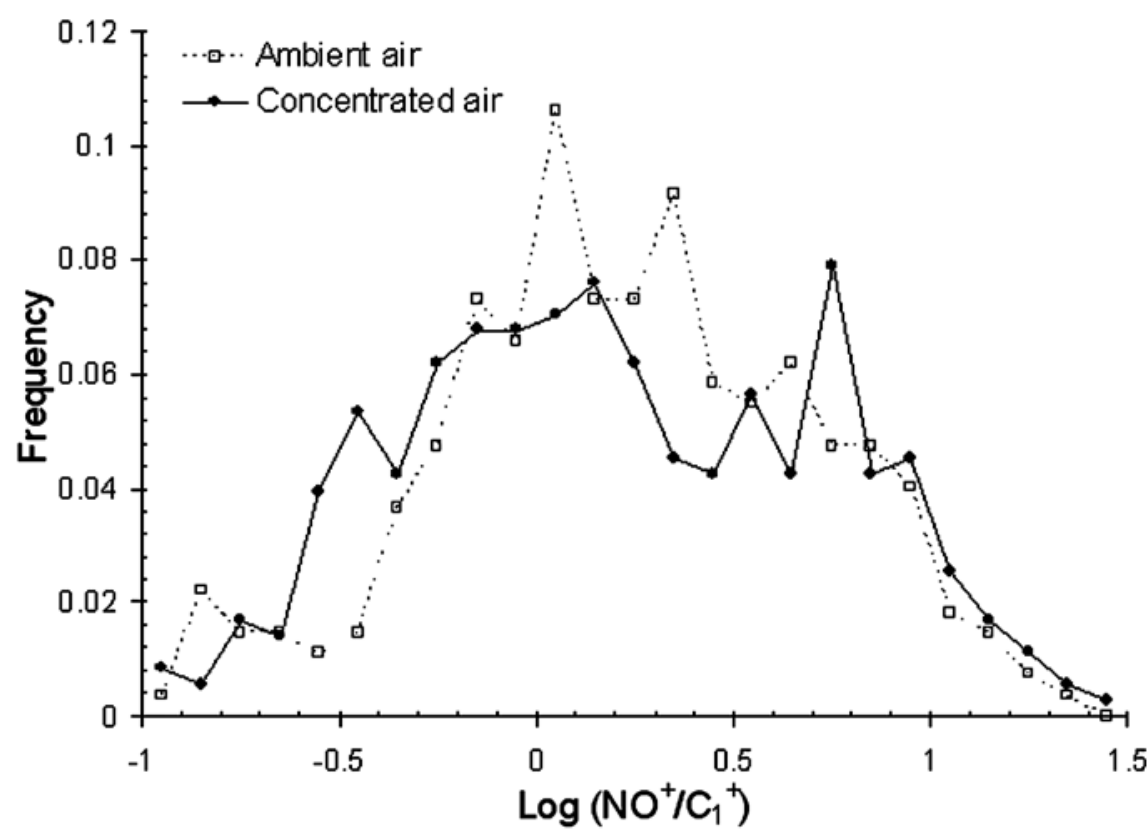

(b) Sum of Ammonium nitrate and Carbon nitrate, March 7\&8

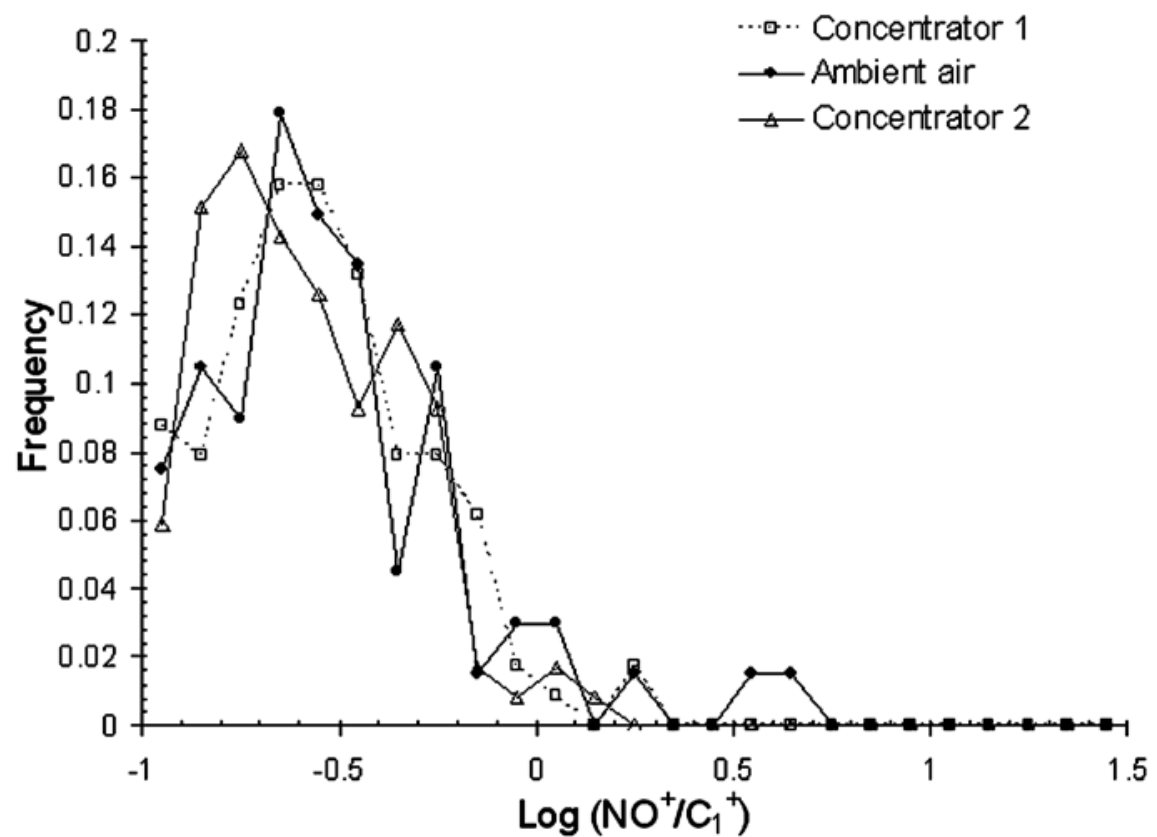

Figure 9. Comparisons of the frequency between ambient air and concentrated air measured on (a) 5 March 2002 and (b) 7-8 March 2002. Each curve is the sum of the ammonium nitrate class and the carbon nitrate class. Areas under each curve are normalized to 1 .

nitrate classes is more of a continuous distribution, subtle differences between ammonium nitrate class and carbon nitrate class near $\mathrm{NO}^{+} / \mathrm{C}_{1}^{+}=1$ can move particle spectra from one class to the other. The atmospheric conditions, instrument operating conditions, and the Art-2a initial conditions could cause this movement. It can also be seen in Figure 8 that some of the high $\mathrm{NO}^{+} / \mathrm{C}_{1}^{+}$particles were classified as carbon nitrate, which may be due to 
other peak information, since when the Art-2a algorithm compares a selected spectrum with the existing set of weight vector, all peaks in the spectrum make contribution to the vector dot product. Therefore, when the contribution of other peaks to the dot product becomes more significant, the Art-2a may shift the class from one to another.

[25] Comparisons of these frequency distributions for ambient air and concentrated air are shown in Figures 9a and $9 \mathrm{~b}$ for 5 March and 7 and 8 March, respectively. On 7 and 8 March the frequency distribution was skewed toward the $\mathrm{NO}^{+} / \mathrm{C}_{1}^{+}<1$ (see Figure 9b) indicating that on that day carbon nitrate particles (carbonaceous particles with a small amount of nitrate condensed on them) were observed much more frequently than ammonium nitrate particles (carbonaceous particles with a lot of nitrate condensed on them). Figure 6 shows the same results. Since both ammonium nitrate and carbon nitrate particle classes were observed in more equal proportions on 5 March (see Figure 4), the frequency distribution appears on both sides of $\mathrm{NO}^{+} / \mathrm{C}_{1}^{+}=$ 1 as shown in Figure 9a. There appear to be no significant difference in the frequency distributions between ambient and concentrated samples on both days and that the class shift from ammonium to carbon nitrate was not due to the particle concentrator.

\section{Summary and Conclusions}

[26] Our field evaluation of coupling VACES and RSMS3 resulted in the following conclusions:

[27] 1. By coupling with the VACES concentrator, hit rates of the RSMS-3 single-particle mass spectrometer increased by $5-20$ times except when RSMS-3 sampled the smallest and largest particle sizes where its flow rate was off the optimum configuration of the VACES concentrator.

[28] 2. Small differences in chemical composition were observed between samples with and without the VACES particle concentrator. The shift of $8-10 \%$ particles from one class to another could be caused by the changes in the composition of ambient air, or due to statistical variation in RSMS-3 measurements, or spectrum classification. There was no evidence showing that the VACES particle concentrator introduced the particle shift.

[29] 3. The minor flow rates of the VACES concentrator must be in its optimum range to effectively couple it to RSMS-3. Outside this range, the minor flow is either too high, resulting in a low concentration enhancement, or too low, causing ultrafine particle loss. Therefore it is necessary to control the minor flow rate of VACES in the coupling system when RSMS-3 samples different sizes of particles.

[30] 4. Since Art-2a judges class membership on the basis of a single vigilance factor, it broke a carbonaceous particle distribution with a wide range of ammonium nitrate content into two composition classes. Better algorithms should be developed that can identify distributions of composition within a class.

[31] Acknowledgments. This work was supported by EPA and DOE funding for the Pittsburgh Supersite (Y.Z., K.J.B., and A.S.W.) and the Southern California Particle Center and Supersite (SCPCS) funded by the U.S. EPA (STAR award R82735201) (C.M., P.M.F., and C.S.). This manuscript has not been subjected to the EPA peer and policy review and therefore does not necessarily reflect the views of the Agencies. No official endorsement should be inferred.

\section{References}

Bein, K. J., Y. Zhao, A. S. Wexler, and M. V. Johnston (2005), Speciation of size-resolved individual ultrafine particles in Pittsburgh, Pennsylvania, J. Geophys. Res., 110, D07S05, doi:10.1029/ 2004JD004708

Donaldson, K., X. Y. Li, and W. MacNee (1998), Ultrafine (nanometer) particle mediated lung injury, J. Aerosol Sci., 29, 553-560.

Finlayson-Pitts, B. J., and J. N. Pitts (1986), Atmospheric Chemistry: Fundamentals and Experimental Techniques, John Wiley, Hoboken, N. J.

Geller, M. D., S. Kim, C. Misra, C. Sioutas, B. A. Olson, and V. A. Marple (2002), A methodology for measuring size-dependent chemical composition of ultrafine particles, Aerosol Sci. Technol., 36, 748762 .

Heyder, J., P. Brand, J. Heinrich, A. Peters, G. Scheuh, T. Tuch, and E. Wichmann (1996), Size distribution of ambient particles and its relevance to human health, paper presented at 2nd Colloquium on Particulate Air Pollution and Health, U.S. Environ. Prot. Agency, Park City, Utah, 1-3 May.

Hopke, P. K., and X. Song (1997), Classification of single particles by neural networks based on the computer-controlled scanning electron microscopy data, Anal. Chim. Acta, 348, 375-388.

Kane, D. B., and M. V. Johnston (2000), Size and composition biases on the detection of individual ultrafine particles by aerosol mass spectrometry, Environ. Sci. Technol., 34, 4887-4893.

Kane, D. B., and M. V. Johnston (2001), Enhancing the detection of sulfate particles for laser ablation aerosol mass spectrometry, Anal. Chem., 73, $5356-5369$.

Kim, S., M. C. Chang, D. Kim, and C. Siouats (2000), A new generation of portable coarse, fine, and ultrafine particle concentrators for use in inhalation toxicology, Inhalation Toxicol., 12, 121-137.

Kim, S., P. Jaques, M. C. Chang, J. R. Froines, and C. Sioutas (2001a), A versatile aerosol concentrator for simultaneous in vivo and in vitro evaluation of toxic effects of coarse, fine and ultrafine particles: Part I: Laboratory evaluation, J. Aerosol Sci., 32, 1281-1297.

Kim, S., P. Jaques, M. C. Chang, C. Xiong, S. K. Friedlander, and C. Sioutas (2001b), A versatile aerosol concentrator for simultaneous in vivo and in vitro evaluation of toxic effects of coarse, fine and ultrafine particles: Part II: Field evaluation, J. Aerosol Sci., $32,1299-1314$

Koutrakis, P., J. M. Wolfson, J. L. Slater, M. Brauer, J. D. Spengler, R. K. Stevens, and C. L. Stone (1988), Evaluation of an annular denuder filter pack system to collect acidic aerosol and gases, Environ. Sci. Technol., $22,1463-1468$

Lake, D. A., M. P. Tolocka, M. V. Johnston, and A. S. Wexler (2003), Mass spectrometry of individual particles between 50 and $750 \mathrm{~nm}$ in diameter at the Baltimore Supersite, Environ. Sci. Technol., 37, $3268-3274$

Lake, D. A., M. P. Tolocka, M. V. Johnston, and A. S. Wexler (2004), The character of single particle sulfate in Baltimore, Atmos. Environ., 38, $5311-5320$.

Li, N., C. Sioutas, A. Cho, D. Schmitz, C. Misra, M. Wang, T. Oberley, J. R. Froines, and A. Nel (2003), Ultrafine particulate pollutants induce oxidative stress and mitochondrial damage, Environ. Health Perspect., 111, $455-460$.

Marple, V. A., and C. M. Chien (1980), Virtual impactors: A theoretical study, Environ. Sci. Technol., 14, 976-985.

Middha, P., and A. S. Wexler (2003), Particle focusing characteristics of sonic jets, Aerosol Sci. Technol., 37, 907-915.

Peters, A., D. W. Dockery, J. Heinrich, and H. E. Wichmann (1997), Shortterm effects of particulate air pollution on respiratory morbidity in asthmatic children, Eur. Respir. J., 10, 872-879.

Phares, D. J., K. P. Rhoads, A. S. Wexler, D. B. Kane, and M. V. Johnston (2001), Application of the Art-2a algorithm to laser ablation aerosol mass spectrometry of particle standards, Anal. Chem., 73, $2338-2344$.

Phares, D. J., K. P. Rhoads, and A. S. Wexler (2002), Performance of a single ultrafine particle mass spectrometer, Aerosol Sci. Technol., 36, $583-592$.

Phares, D. J., K. P. Rhoads, M. V. Johnston, and A. S. Wexler (2003), Sizeresolved ultrafine particle composition analysis: 2 . Houston, J. Geophys. Res, 108(D7), 8420, doi:10.1029/2001JD001212.

Rhoads, K. P., D. J. Phares, A. S. Wexler, and M. V. Johnston (2003), Sizeresolved ultrafine particle composition analysis: 1. Atlanta, J. Geophys. Res., 108(D7), 8418, doi:10.1029/2001JD001211. 
Sioutas, C., P. Koutrakis, and B. A. Olson (1994), Development of a low cutpoint virtual impactor, Aerosol Sci. Technol., 21, 223-235.

Sioutas, C., S. Kim, and M. C. Chang (1999), Development and evaluation of a prototype ultrafine particle concentrator, J. Aerosol Sci., 30, $1001-$ 1017.

Tolocka, M. P., D. A. Lake, M. V. Johnston, and A. S. Wexler (2004a), Number concentrations of fine and ultrafine particles containing metals, Atmos. Environ., 38, 3263-3273.

Tolocka, M. P., D. A. Lake, M. V. Johnston, and A. S. Wexler (2004b), Ultrafine nitrate particle events in Baltimore observed by real-time single particle mass spectrometry, Atmos. Environ., 38, 3215-3223.

Tolocka, M. P., D. A. Lake, M. V. Johnston, and A. S. Wexler (2005) Size-resolved fine and ultrafine particle composition in Baltimore, Maryland, J. Geophys. Res., 110, D07S04, doi:10.1029/2004JD004573.
Willeke, K., and P. A. Baron (1993), Aerosol Measurement: Principles, Techniques, and Applications, Van Nostrand Reinhold, Hoboken, N. J.

K. J. Bein, Department of Land, Air and Water Resources, University of California, Davis, One Shields Avenue, Davis, CA 95616, USA. (kjbein@ucdavis.edu)

P. M. Fine, C. Misra, and C. Sioutas, Department of Civil and Environmental Engineering, University of Southern California, $3620 \mathrm{~S}$. Vermont Avenue, Los Angeles, CA 90089, USA.

A. S. Wexler and Y. Zhao, Department of Mechanical and Aeronautical Engineering, University of California, Davis, One Shields Avenue, Davis, CA 95616, USA. (aswexler@ucdavis.edu; yjzhao@ucdavis.edu) 


\title{
Time- and size-resolved chemical composition of submicron particles in Pittsburgh: Implications for aerosol sources and processes
}

\author{
Qi Zhang \\ Cooperative Institute for Research in Environmental Sciences, University of Colorado, Boulder, Colorado, USA
}

Manjula R. Canagaratna, John T. Jayne, and Douglas R. Worsnop

Aerodyne Research Inc., Billerica, Massachusetts, USA

Jose-Luis Jimenez

Cooperative Institute for Research in Environmental Sciences, University of Colorado, Boulder, Colorado, USA

Department of Chemistry and Biochemistry, University of Colorado, Boulder, Colorado, USA

Received 15 February 2004; revised 4 August 2004; accepted 11 August 2004; published 25 January 2005.

[1] An Aerodyne aerosol mass spectrometer (AMS) was deployed at the Pittsburgh Environmental Protection Agency Supersite from 7 to 22 September 2002 as part of the Pittsburgh Air Quality Study (PAQS). The main objectives of this deployment were to characterize the concentrations, size distributions, and temporal variations of nonrefractory (NR) chemical species in submicron particles (approximately $\mathrm{PM}_{1}$ ) and to further develop and evaluate the AMS. Reasonably good agreement was observed on particle concentrations, composition, and size distributions between the AMS data and measurements from collocated instruments (given the difference between the $\mathrm{PM}_{1}$ and $\mathrm{PM}_{2.5}$ size cuts), including TEOM, semicontinuous sulfate, 2-hour- and 24-hour-averaged organic carbon, SMPS, 4-hour-averaged ammonium, and micro-orifice uniform deposit impactor. Total NR-PM 1 mass concentration in Pittsburgh accumulates over periods of several days punctuated with rapid cleaning due to rain or air mass changes. Sulfate and organics are the major NR-PM 1 components while the concentrations of nitrate and chloride are generally low. Significant amounts of ammonium, which most of the time are consistent with sulfate present as ammonium sulfate, are also present in particles.

However, there are periods when the aerosols are relatively acidic and more than $50 \%$ of sulfate is estimated to be in the form of ammonium bisulfate. No major enhancement of the organic concentration is observed during these acidic periods, which suggests that acid-catalyzed SOA formation was not an important process during this study. Size distributions of particulate sulfate, ammonium, organics, and nitrate vary on timescales of hours to days, showing unimodal, bimodal and even trimodal characteristics. The accumulation mode (peaking around $350-600 \mathrm{~nm}$ in vacuum aerodynamic diameter for the mass distributions) and the ultrafine mode $(<100 \mathrm{~nm})$ are observed most frequently. The accumulation mode is dominated by sulfate that appears to be internally mixed with oxidized organics, while combustion-emitted organics are often the main component of the ultrafine particles (except during nucleation events). The ultrafine-mode organic aerosols are mainly associated with combustion sources (likely traffic).

Citation: Zhang, Q., M. R. Canagaratna, J. T. Jayne, D. R. Worsnop, and J.-L. Jimenez (2005), Time- and size-resolved chemical composition of submicron particles in Pittsburgh: Implications for aerosol sources and processes, J. Geophys. Res., 110, D07S09, doi:10.1029/2004JD004649.

\section{Introduction}

[2] Atmospheric aerosols have important adverse impacts on human health [Dockery et al., 1993; Pope et al., 2002], visibility [Watson, 2002], and ecological integrity [Bytnerowicz and Fenn, 1996; Paerl et al., 1997; Schindler,

Copyright 2005 by the American Geophysical Union. 0148-0227/05/2004JD004649\$09.00
1988]. Aerosols also play important roles in atmospheric processes that are intrinsically linked to climate change [Intergovernmental Panel on Climate Change (IPCC), 2001] and stratospheric ozone depletion [Solomon, 1999]. While a thorough understanding of particle concentration, size distribution, chemical composition, state of mixing, and morphology is essential to address the radiative, ecological and human health effects of atmospheric particles, our knowledge of these parameters is usually limited by the 
coarse time and size resolution of most existing instrumentation for aerosol chemical measurements [McMurry, 2000].

[3] Several instruments that are capable of performing real-time and continuous (or semicontinuous) size-resolved measurement of ambient aerosol composition have been developed in the last decade [Jayne et al., 2000; Murphy et al., 1998; Suess and Prather, 1999]. Compared to the traditional multistage impactor technologies, online techniques are usually faster, less labor intensive, and less vulnerable to artifacts introduced during sample collection and processing. While most recently developed sizeresolved composition instruments measure the qualitative composition of single particles [Middlebrook et al., 2003], the Aerodyne aerosol mass spectrometer (AMS) can determine the sizes and chemical composition of the ensemble of submicron particles in situ, with time resolution of minutes for typical ambient measurements [Jayne et al., 2000; Jimenez et al., 2003c]. The AMS has been successfully employed in more than 30 field campaigns and laboratory studies to characterize the properties, identify the possible sources and elucidate the dynamics of ambient and laboratory aerosols [e.g., Alfarra et al., 2004; Allan et al., 2003a; Bahreini et al., 2003; Canagaratna et al., 2004; Drewnick et al., 2004b; Jimenez et al., 2003b, 2003c; Morris et al., 2002; Zhang et al., 2004].

[4] We deployed an AMS in Pittsburgh, Pennsylvania, during September 2002, as part of the Pittsburgh Air Quality Study (PAQS). PAQS was sponsored by the U.S. Environmental Protection Agency to address the relationship between particulate matter (PM) and health effects, establish the PM source-receptor relationships, and develop and evaluate the next generation of PM monitoring techniques [Wittig et al., 2004]. The focus of this AMS deployment was to characterize the chemistry and dynamics of ambient aerosols with high time and size resolution and therefore offer insights into the sources and processes of particles in the Pittsburgh region. In this paper, we mainly report the size-resolved chemical composition, concentrations, and temporal variations of the nonrefractory particle components (i.e., sulfate, organics, ammonium and nitrate). Quality control measures, such as corrections for nonideality of the AMS measurements and comparisons between the AMS results and various collocated measurements, are also reported. The chemistry and growth mechanisms of ultrafine particles during nucleation events in Pittsburgh [Zhang et al., 2004] and a more detailed organic analysis (Q. Zhang et al., Deconvolution and quantification of primary and oxygenated organic aerosols based on aerosol mass spectrometry: Part 1. Development and validation of the method, submitted to Environmental Science and Technology, 2004) (hereinafter referred to as Zhang et al., submitted manuscript, 2004) are discussed in separate papers.

\section{Experimental Methods}

\subsection{Sampling Site and Time}

[5] The AMS was operated at the PAQS central site $\left(40^{\circ} 27^{\prime} \mathrm{N}, 79^{\circ} 57^{\prime} \mathrm{W}\right)$ continuously, except for occasional maintenance and calibration, from 7 to 22 September 2002. This site was located $\sim 6 \mathrm{~km}$ east of downtown Pittsburgh, on a hill of Schenley Park next to Carnegie Mellon University [Wittig et al., 2004]. Baseline monitoring for PM, gas-phase and meteorological variables lasted for $\sim 15$ months at this site from July 2001 to October 2002 [Wittig et al., 2004]. An overview of PAQS and the Supersite operation was given by Wittig et al. [2004]. Other details can be found in the PAQS Quality Assurance Project Plan prepared by Khlystov et al. [2001].

[6] All dates and times are reported in Eastern Standard Time (EST). The local time during this study was Eastern Daylight Saving Time (EDT), which is 1 hour ahead of EST.

\subsection{AMS Instrument and Its Operation}

\subsubsection{Description of the AMS}

[7] The AMS has been described in detail in other publications [Alfarra et al., 2004; Allan et al., 2003b; Jayne et al., 2000; Jimenez et al., 2003c]; thus only a brief overview is given here. The AMS consists of three major parts: a particle beam generation inlet system, an aerodynamic sizing chamber, and a particle composition detection section. The inlet system contains a $100 \mu \mathrm{m}$ critical orifice that sets the airflow into the AMS at a nominal rate of $\sim 1.4 \mathrm{~cm}^{3} \mathrm{~s}^{-1}$ and an aerodynamic lens system that focuses particles into a narrow beam of about $100 \mu \mathrm{m}$ in diameter [Heberlein et al., 2001].

[8] Particles acquire size-dependent velocities upon supersonic expansion into the high-vacuum sizing chamber, where their vacuum aerodynamic diameters $\left(D_{v a}\right)$ are determined on the basis of calibration using particles of known sizes, densities, and shapes, such as polystyrene latex (PSL) spheres. Details on $D_{v a}$ (effectively the aerodynamic diameter measured in the free-molecular regime) and its relationships with mobility diameter $\left(D_{m}\right)$ and the traditional aerodynamic diameter (measured in the continuum regime) are given by Canagaratna et al. [2004], Jimenez et al. [2003a, 2003b], and DeCarlo et al. [2004].

[9] In the composition detection section, particles are flash vaporized upon impact with a heated surface $\left(\sim 600^{\circ} \mathrm{C}\right.$ during this study) under high vacuum $\left(\sim 10^{-8}\right.$ torr). The resulting vapor molecules are ionized by electron impact and the positive ions formed are analyzed by a quadrupole mass spectrometer (Balzers QMA410, Balzers, Liechtenstein). Because of the use of thermal vaporization, only nonrefractory (NR) particle components, such as $\mathrm{SO}_{4}^{2-}, \mathrm{NO}_{3}^{-}$, $\mathrm{NH}_{4}^{+}$and organics, are determined. "Nonrefractory" is defined operationally as those species that evaporate on timescales of a few seconds (i.e., one period of the blocked/open alternation of the particle beam chopper during the MS mode operation) or less under the AMS conditions. Minerals and elemental carbon are undetectable because of their very low vapor pressure at $\sim 600^{\circ} \mathrm{C}$. In addition, since the AMS used in this study has an approximate $1 \mu \mathrm{m}$ transmission size cut, the results of its measurements are referred to as NR-PM ${ }_{1}$.

[10] The AMS alternates between the particle time of flight (P-TOF) mode and the mass spectrum (MS) mode during operation [Jimenez et al., 2003c]. A set of preselected $\mathrm{m} / \mathrm{z}$ is scanned as a function of particle's time of flight in the P-TOF mode, from which the ensemble size distributions of aerosol species are derived. Mass loadings are calculated from the ensemble background-subtracted mass spectra $(\mathrm{m} / \mathrm{z} 1-300)$ acquired during the MS mode operation using a software package developed by Allan et al. 


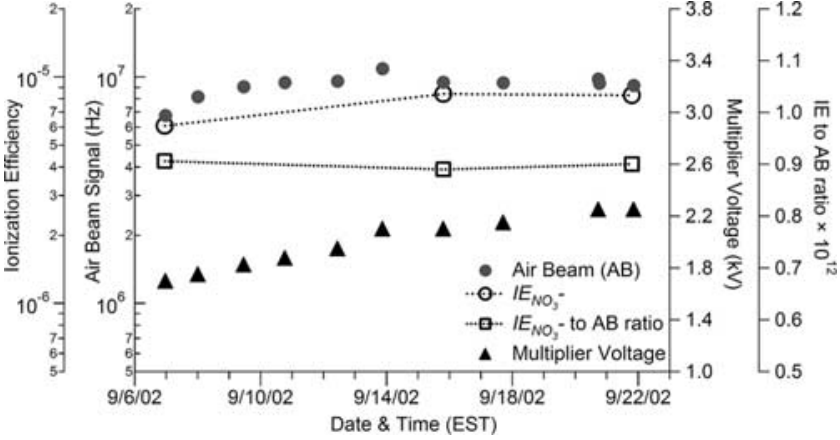

Figure 1. Ionization and ion transmission efficiency for nitrate $\left(I E_{\mathrm{NO}_{\overline{3}}}\right)$ determined during IE calibrations, multiplier voltage and air beam signal $\left(\mathrm{N}_{2}^{+}\right)$measured immediately after each EM calibration, and the ratio of $I E_{\mathrm{NO}_{3}}$ to air beam signal.

[2004b]. Interferences for the mass concentration and size distribution data are checked according to procedures described by Jimenez et al. [2003c] using a data diagnostics module (AMS Diagnostics 1.1.7a) developed by A. Delia (CU).

[11] Note that the AMS used here is not a single particle technique because the quadrupole MS measures only one $\mathrm{m} / \mathrm{z}$ at any one time, instead of the complete mass spectrum of individual particles. Reported AMS mass spectra and size distributions are the averages over specified intervals (5-10 min for this study; see section 2.2.2).

\subsubsection{Operation of the AMS}

[12] The AMS, along with a number of other PAQS sampling instruments, was housed in a $33 \mathrm{~m}^{2}$ trailer during this study [Wittig et al., 2004]. Ambient air was drawn into the AMS through a thermally insulated copper tube $(\sim 5 \mathrm{~m}$ long and $0.95 \mathrm{~cm}$ ID) from $\sim 2 \mathrm{~m}$ above the trailer rooftop. Coarse particles were removed using a cyclone with a $2.5 \mu \mathrm{m}$ cutoff (model URG-2000-30EN, URG, Chapel Hill, North Carolina). The total flow through the copper tube was $10 \mathrm{~L} \mathrm{~min}^{-1}$, out of which $\sim 0.1 \mathrm{~L} \mathrm{~min}^{-1}$ was isokinetically sampled from the center of the tube by the AMS and the rest was exhausted by the sampling pump. The residence time of sample air in the tube was $\sim 7 \mathrm{~s}$.

[13] The AMS alternated between the P-TOF and MS modes every $\sim 25 \mathrm{~s}$. During the P-TOF mode operation signals of twelve $\mathrm{m} / \mathrm{z}$ that are representative for $\mathrm{NO}_{3}^{-}(\mathrm{m} / \mathrm{z}$ 30 and 46), $\mathrm{SO}_{4}^{2-}$ ( $m / z 48$ and 64), organics $(m / z 43,44,55$, and 57), $\mathrm{NH}_{4}^{+}(\mathrm{m} / \mathrm{z} 15$ and 16$), \mathrm{H}_{2} \mathrm{O}(\mathrm{m} / \mathrm{z} 18)$, and $\mathrm{N}_{2}(\mathrm{~m} / \mathrm{z}$ 28) were recorded as a function of particle's time of flight. Four additional (mostly) organic fragments $(\mathrm{m} / \mathrm{z} 27,67,91$, and 95) were scanned after 8:55 pm on 13 September. Reported mass concentrations and size distributions are the averages over periods of $10 \mathrm{~min}$ before 9:50 am on 12 September and 5 min afterward.

\subsubsection{Instrument Calibrations}

[14] The AMS was calibrated several times for electron multiplier (EM) gain, ionization and ion transmission and detection efficiency (IE), and particle sizing during this study (Figure 1). The electron multiplier was calibrated every $1-2$ days to compensate for the normal reduction in sensitivity over time and to restore optimum ion signals. $I E$, which is defined as the ratio of ions detected by the multiplier to the number of the parent molecules vaporized in the AMS, was calibrated with monodisperse pure ammonium nitrate particles generated from an aqueous solution by a Collison atomizer (TSI model 3076, St. Paul, Minnesota) and a differential mobility analyzer custom built by Aerodyne Research (using the TSI DMA, model 3081). Monodisperse polystyrene latex spheres (PSL; Duke Scientific, Palo Alto, California) with nominal sizes of 50, 81, $155,350,600$, and $700 \mathrm{~nm}$ and density of $1.054 \mathrm{~g} \mathrm{~cm}^{-3}$ were used to calibrate the AMS sizing. Detailed quantification protocols are described in previous publications [Alfarra et al., 2004; Allan et al., 2003b; Jayne et al., 2000; Jimenez et al., 2003c].

\subsection{Quality Assurance and Quality Control (QA/QC)}

\subsubsection{AMS Detection Efficiency}

[15] In order to minimize uncertainties in the reported mass concentrations it is desirable that the fluctuations of the detection efficiency of the AMS are closely monitored and properly corrected throughout the whole campaign. The parameters that capture the AMS detection efficiency are $I E_{\mathrm{NO}_{3}^{-}}$, which is the ionization efficiency of a reference compound $\left(\mathrm{NO}_{3}^{-}\right)$, and the air beam signal $(A B)$, which is the ion rate $(\mathrm{Hz})$ detected for the direct beam of a major air component, e.g., $\mathrm{N}_{2}^{+}$[Allan et al., 2003b; Jimenez et al., $2003 \mathrm{c}]$. While $A B$ can be monitored continuously during instrument operation, the determination of $\mathrm{IE}_{\mathrm{NO}_{3}}$ requires interruption of sampling to perform a calibration experiment (typically 1-2 hours). Given this restriction and the expectation (based on previous experience) that variations in $I E$ can be corrected by changes in air beam signal (i.e., the ratio of IE to air beam signal was usually relatively stable) three $I E_{\mathrm{NO}_{3}}$ calibrations were conducted during this study (Figure 1 ). The measured $I E_{\mathrm{NO}_{3}}$ values increase by $\sim 50 \%$ between the first two calibrations but are constant between the second and the third, suggesting an improvement in the general detection efficiency of the AMS during the first few days of operation. This initial increase in detection efficiency has very often been observed after initial pump-down of the AMS and is possibly due to an enhancement in the detection efficiency (ratio of output signal pulses to ions impacting the first dynode surface) of the electron multiplier detector after its surfaces degas. Because the ratios of $I E_{N_{3}}$ to $A B$ remain remarkably constant during this study (r.s.d. $<1 \%$ ) the continuous $A B$ signal can be used to correct for the variations in the AMS detection efficiencies to a very good approximation. 2.3.2. AMS Collection Efficiency (CE) and Relative Ionization Efficiency (RIE)

[16] CE is introduced to correct for incomplete detection of NR-PM 1 by the AMS [Alfarra et al., 2004], e.g., due to the fact that a fraction of irregularly shaped particles may not reach the AMS vaporizer [Jayne et al., 2000; Tobias et al., 2000] or due to particle bounce from less-volatile particles (T. Onasch, Aerodyne Research, personal communication, 2004). Note that CE accounts only for the fraction of $\mathrm{PM}_{1}$ that may not be detected by the AMS, and is not intended to account for the difference in size cutoffs between the AMS (approximately $\mathrm{PM}_{1}$ ) and $\mathrm{PM}_{2.5}$ instruments. The AMS reports $\mathrm{PM}_{1}$ concentrations and we have made no attempt to predict $\mathrm{PM}_{2.5}$ concentrations on the basis of our $\mathrm{PM}_{1}$ measurement. Although strictly speaking 
Table 1. Relative Ionization Efficiencies With Respect to Measured $I E_{\mathrm{NO}_{3}}$ and Collection Efficiencies for Individual Species $^{\mathrm{a}}$

\begin{tabular}{lcc}
\hline Chemical Species & RIE & CE \\
\hline Sulfate & 1.15 & 0.5 \\
Ammonium & $3.8^{\mathrm{b}}$ & 0.5 \\
Organics & 1.4 & $0.7^{\mathrm{C}}$ \\
Nitrate & $1.1^{\mathrm{d}}$ & 0.5 \\
\hline
\end{tabular}

${ }^{\mathrm{a}} \mathrm{RIE}$, relative ionization efficiency; $\mathrm{CE}$, collection efficiency.

${ }^{\mathrm{b}}$ This value was measured during the IE calibrations in this study.

${ }^{\mathrm{c}}$ This value is the weighted average $\mathrm{CE}$ of primary and secondary organic aerosols (see section 2.3.2).

${ }^{\mathrm{d}} \mathrm{RIE}_{\mathrm{NO}_{3}^{-}}=$true IE of nitrate $/ I E_{\mathrm{NO}_{3}}$, where the true IE of nitrate was calculated from all fragments produced by $\mathrm{NO}_{3}^{-}$while the $I E_{\mathrm{NO}_{3}}$ was determined only on the basis of two major $\mathrm{NO}_{3}^{-}$fragments: $\mathrm{m} / z 30$ and 46 .

CE should be a function of particle size, composition, and shape, at present it is defined as the correction factor for the bulk mass concentrations, i.e., the fraction of the particle mass that is measured by the AMS, relative to what would have been measured if all particles were spherical and particle bounce was negligible. In addition, while the component of the AMS collection efficiency due to losses of nonspherical particles can now be measured in real time using a particle beam width probe (BWP) system (J. A. Huffman et al., manuscript in preparation, 2004) this device was not available at the time of this study. We therefore chose $\mathrm{CE}$ values on the basis of the general characteristics of the Pittsburgh aerosols and previous experience from multiple field campaigns and laboratory experiments.

[17] A CE value of 0.5 is assigned to sulfate (Table 1), on the basis of observations from several laboratory and field tests for sulfate aerosols [Alfarra et al., 2004; Allan et al., 2004a, 2003b; Drewnick et al., 2004a]. The same CE value (i.e., 0.5) is applied to nitrate and ammonium, because they appear to be internally mixed with sulfate in particles for most of the time in Pittsburgh.

[18] The CE value for total NR-PM $\mathrm{P}_{1}$ organics is estimated on the basis of their size distributions, which often show two modes: a larger accumulation mode that appears to be internally mixed with $\mathrm{SO}_{4}^{2-}, \mathrm{NO}_{3}^{-}$and $\mathrm{NH}_{4}^{+}$, and a smaller mode that seems to be mainly emitted from combustion sources (see section 3.2.2). A CE value of 0.5 is thus applied to the accumulation mode organics (due to the likely internal mixing with $\mathrm{SO}_{4}^{2-}$ ) while $\mathrm{CE}$ for the smaller mode is assumed to be 1.0 because a recent laboratory study has shown close to $100 \%$ AMS transmission and detection for sooty combustion particles [Slowik et al., 2004]. By studying the size distributions of total organics, as well as individual organic $\mathrm{m} / \mathrm{z}$, averaged over the whole sampling period we found that these two modes can be best separated at $D_{v a}=160 \mathrm{~nm}$ and that the mass ratio of the smaller $\left(D_{v a}<\right.$ $160 \mathrm{~nm})$ to the larger mode $\left(D_{v a}>160 \mathrm{~nm}\right)$ is roughly $2 / 3$. The CE value of the bulk organics is therefore set at 0.7 (Table 1).

[19] Relative ionization efficiency (RIE) is the ratio of the electron impact ionization efficiency of a given species to $I E_{\mathrm{NO}_{\overline{3}}}$ on a per unit mass basis. RIE values of individual species (Table 1) are determined following the approach described by Jimenez et al. [2003c] and Alfarra et al. [2004].

[20] Although CE and RIE for organic aerosols in this study are likely to be size dependent, at this point we have chosen to apply constant values (i.e., $\mathrm{CE}=0.7$ and $\mathrm{RIE}=$ 1.4) throughout the whole size range and time period of this study. Going beyond this simple approach requires a technique that can separate the contributions of the various organic aerosol types, and thus allow the application of separate values of $\mathrm{CE}$ and RIE to each type. One such technique has recently been developed by our group and is presented in a separate publication (Zhang et al., submitted manuscript, 2004). The direct quantification of the component of CE due to particle shape using the AMS BWP described above (J. A. Huffman et al., manuscript in preparation, 2004), and the use of particle humidification and/or the redesign of the AMS vaporizer to reduce particle bounce will further reduce the uncertainties in mass quantification for future studies.

[21] An important consequence of applying constant CE and RIE is that the proportion of the small mode and accumulation mode in the reported organic size distribution is likely distorted. First, the CE of the accumulation mode organic aerosols is likely smaller than 0.7 while that of the ultrafine mode is likely larger. Also because the average RIE of combustion-emitted organic species is larger than that of oxygenated organics [Jimenez et al., 2003c], RIE of accumulation mode organics is expected to be smaller than 1.4 while that of the ultrafine mode is larger. Thus the small mode, mainly primary organics are likely overestimated, while the accumulation mode, mostly oxygenated organics are likely underestimated.

\subsubsection{Detection Limits of the AMS Measurements}

[22] The detection limits (DL) of the AMS measurements of mass concentration are evaluated on the basis of the mass spectra of particle free ambient air (filtered with a HEPA filter). The species DLs are defined as 3 times the standard deviation of the corresponding species signals in the filtered air. The DLs of $\mathrm{SO}_{4}^{2-}, \mathrm{NH}_{4}^{+}, \mathrm{NO}_{3}^{-}$, and organics during this study are estimated to be $0.05,0.11,0.01$, and $0.15 \mu \mathrm{g} \mathrm{m}^{-3}$, respectively, for a $10 \mathrm{~min}$ averaging time (Table 2). Note that these values are $2-3$ times higher (i.e., worse) than the $3 \sigma$ instrumental detection limits defined by Allan et al. [2004a, 2004b, 2003b], but 1-2 orders of magnitude lower (i.e., better) than those of the ACE-Asia campaign [Bahreini et al., 2003]. The major reason for the $3 \sigma$ instrumental DLs estimated by Allan et al. [2004a, 2003b] to be smaller is that they only take into account uncertainties due to electronic noise and the ion counting statistics of the background [Allan et al., 2003a, 2003b]. The substantially lower sensitivity of the AMS during the ACE-Asia study was attributed to a relatively high level of background signals in the AMS due to a less optimized vacuum system and inability to

Table 2. Summary of AMS Mass Concentration Data

\begin{tabular}{lcccccc}
\hline & \multicolumn{5}{c}{ Mass Concentration, $\mu \mathrm{g} \mathrm{m}^{-3}$} \\
\cline { 2 - 7 } & Sulfate & Ammonium & Nitrate & Organics & Chloride & Total \\
\hline Average & 6.95 & 2.45 & 0.87 & 4.48 & 0.06 & 14.81 \\
$1 \sigma$ & 4.95 & 1.56 & 0.94 & 2.75 & 0.16 & 9.01 \\
Median & 5.99 & 2.31 & 0.56 & 4.24 & 0.01 & 13.75 \\
Minimum & 0.17 & 0.07 & 0.01 & 0.17 & -0.01 & 0.56 \\
Maximum & 23.92 & 10.49 & 13.48 & 51.17 & 2.45 & 70.59 \\
DL $^{\mathrm{a}}$ & 0.05 & 0.11 & 0.01 & 0.15 & 0.01 & 0.19 \\
\hline
\end{tabular}

${ }^{\mathrm{a}}$ Detection limit at an averaging time of $10 \mathrm{~min}$ (see section 2.3.3 for details). 

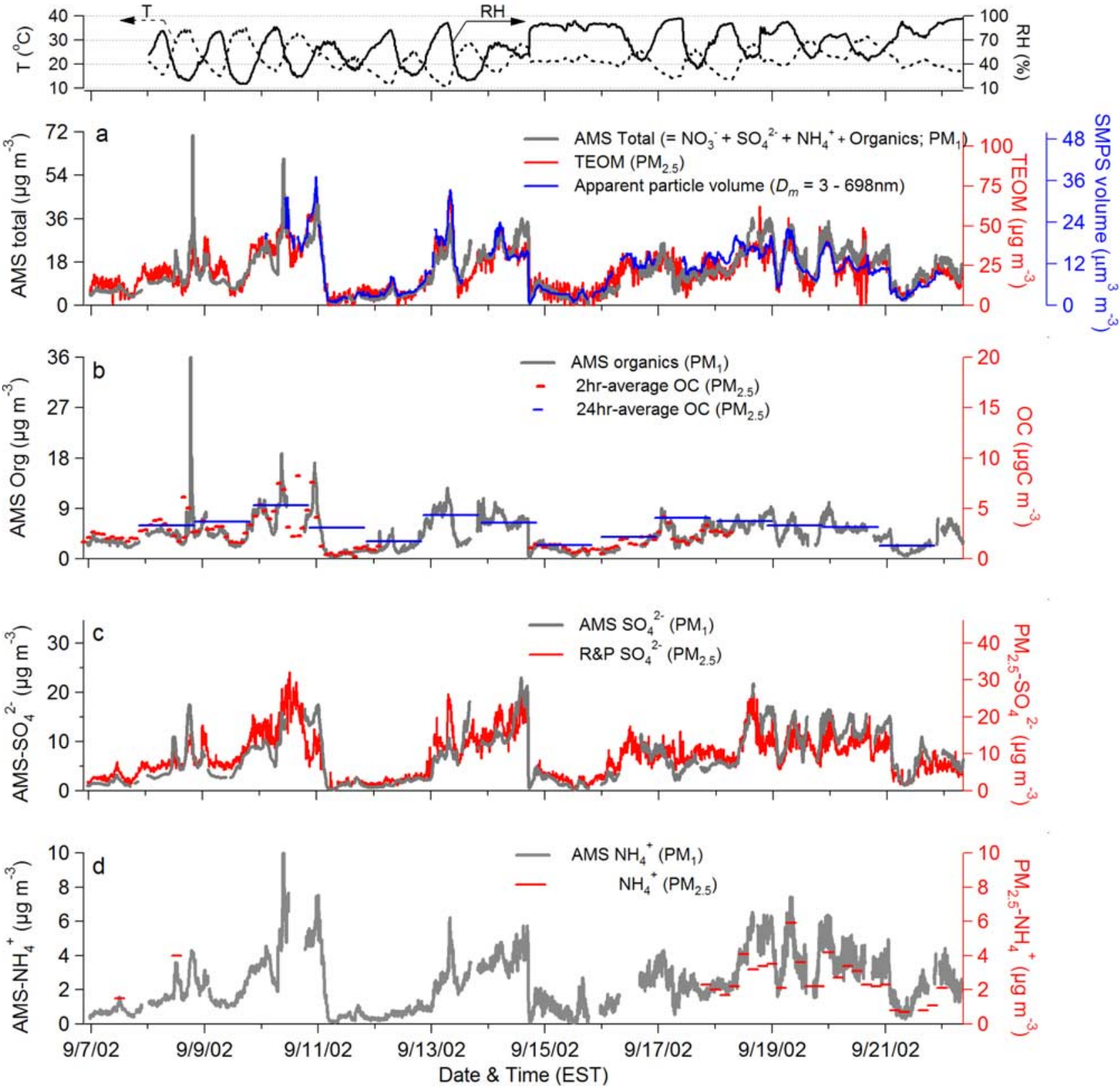

Figure 2. Comparisons of the AMS-measured mass concentrations to data reported by collocated instruments: (a) total $\left(=\mathrm{SO}_{4}^{2-}+\mathrm{NO}_{3}^{-}+\mathrm{NH}_{4}^{+}+\mathrm{Cl}^{-}+\right.$organics) versus $\mathrm{PM}_{2.5}$ mass and apparent particle volume $\left(D_{m}=3-698 \mathrm{~nm}\right)$; (b) organics versus 2-hour- and 24-hour-averaged $\mathrm{PM}_{2.5} \mathrm{OC}$; (c) sulfate versus $\mathrm{PM}_{2.5}$ sulfate; (d) ammonium versus 4-hour-averaged $\mathrm{PM}_{2.5}$ ammonium. See Table 2 for correlation coefficients. Plotted on the top of this figure are the time series of air temperature and relative humidity.

operate the vacuum system pumps continuously due to aircraft restrictions [Bahreini et al., 2003].

\section{Results and Discussions}

\subsection{Intercomparisons With Collocated Instruments}

[23] Figure 2 shows the time series of the concentrations of particle mass (approximately NR-PM ${ }_{1}$ ), organics, sulfate, and ammonium reported by the AMS, together with comparable traces from collocated instruments, including $\mathrm{PM}_{2.5}$ mass measured by a tapered element oscillating microbal- ance (TEOM, Rupprecht \& Patashnick Co., Inc, model $1400 \mathrm{~A}$, Albany, New York) that sampled at $30^{\circ} \mathrm{C}$ and was equipped with a Nafion diffusion dryer sample equilibration system (SES), along with a $\mathrm{PM}_{2.5}$ inlet, apparent particle volume (calculated assuming spherical particles) measured by a scanning mobility particle sizer system (models 3936N25 and 3936L10, TSI, Inc.), 2-hour $\mathrm{PM}_{2.5}$ OC measured in situ using a Sunset Laboratories thermal optical transmittance carbon analyzer [Turpin et al., 1990] and 24-hour OC from manual undenuded filter samples (sampling details given by Subramanian et al. [2004]), 
$\mathrm{PM}_{2.5}$ sulfate measured with a semicontinuous sulfate analyzer (Rupprecht and Patashnick, model 8400S) similar to the nitrate instrument described by Stolzenburg and Hering [2003] and Wittig et al. [2004], and semicontinuous $\mathrm{PM}_{2.5} \mathrm{NH}_{4}^{+}$measured using a steam sampler [Khlystov et al., 1995]. Note that $\mathrm{PM}_{2.5}$ is defined on the basis of aerodynamic diameter measured at atmospheric pressure, which to first approximation equals the ratio of $D_{v a}$ to the square root of particle density for spherical particles. Overall the agreement of these comparisons is fairly good given the differences in size cutoff (Figure 2) and the correlation coefficients $\left(\mathrm{r}^{2}\right)$ are in the range of $0.64-0.88$ (Figure 3 ).

[24] On the basis of the linear regression slopes, on average, the AMS reports $\sim 66 \%$ of the total $\mathrm{PM}_{2.5}$ mass observed by the TEOM and $\sim 75 \%$ of the $\mathrm{PM}_{2.5}$ sulfate from the semicontinuous $\mathrm{SO}_{4}^{2-}$ analyzer (Figure 3 ). These differences likely reflect the fact that the AMS measures $\mathrm{PM}_{1}$ rather than $\mathrm{PM}_{2.5}$ and that it has no response to refractory species, such as crustal, soot and metal components. Drewnick et al. [2004b] observed a similar regression slope (0.64) between the AMS and $\mathrm{PM}_{2.5}$ TEOM data at Queens, New York, during the summer of 2001.

[25] Compared to those reported during two other field studies, the correlation coefficients $\left(\mathrm{r}^{2}\right)$ of particle mass and sulfate data measured by the AMS compared to those by TEOM and sulfate analyzer observed during this study (0.71 and 0.68 , respectively) lie somewhat in the middle. During the PMTACS-NY summer 2001 campaign in Queens/New York, Drewnick et al. [2004a] reported very high $r^{2}(\sim 0.9)$ between AMS-SO ${ }_{4}^{2-}$ and three semicontinuous $\mathrm{PM}_{2.5}$ sulfate instruments, including particle into liquid sampler (PILS), R\&P sulfate monitor (R\&P 8400S), and a custom built continuous sulfate monitor. Allan et al. [2004a], however, observed a lower $\mathrm{r}^{2}(\sim 0.56)$ between $\mathrm{SO}_{4}^{2}$ data from the AMS and those from the PILS during the ITCT 2K2 study at Trinidad Head, California. Similarly, the correlation between AMS-measured $\mathrm{PM}_{1}$ mass concentrations and TEOM $\mathrm{PM}_{2.5}$ mass was also significantly better during the PMTACS-NY study $\left(\mathrm{r}^{2}=0.91\right)$ [Drewnick et al., $2004 \mathrm{~b}]$ than during this study $\left(\mathrm{r}^{2}=0.71\right)$. It is not yet clear the exact reason for these differences, but more variable amounts of material between $\mathrm{PM}_{1}$ and $\mathrm{PM}_{2.5}$ in Pittsburgh as compared to New York might have played a role.

[26] Good correlation is observed between the AMS organic mass concentrations and 2-hour-averaged organic carbon concentrations measured by the Sunset Labs in situ carbon analyzer $\left(\mathrm{r}^{2}=0.88\right.$; Figure 3$)$. This observation implies that the nominal particulate organic mass to carbon ratios (i.e., OM:OC ratios) are mostly in the range 1.2 to 2.2 , with a regression slope of $\sim 1.7$ (larger if differences in size cutoff, i.e., $\mathrm{PM}_{1}$ versus $\mathrm{PM}_{2.5}$, are accounted for). This value is similar to the $\mathrm{OM}: \mathrm{OC}$ ratio $(1.6 \pm 0.2)$ recently estimated for urban organic aerosols [Turpin and Lim, 2001]. Although the actual values are expected to change with time because of their dependence on organic aerosol types (the values are likely smaller in the morning when significant amounts of fresh combustion organic aerosols (OM:OC ratio $\sim 1.2$ ) are emitted and larger (e.g., OM:OC ratio $\sim 2.0$ ) in the afternoon when organics are more oxidized and secondary in nature [Russell, 2003; Turpin and Lim, 2001]), we did not observe significant diurnal variations in OM:OC ratios. One possible explanation for this is that ambient $\mathrm{PM}_{1}$ organics in Pittsburgh is often dominated by regional aged particles [Anderson et al., 2004; Tang et al., 2004], and thus show less pronounced difference in OM:OC ratios between morning and afternoon. Cabada et al. [2002], for instance, estimated that up to 50\% of the organic aerosol mass in Pittsburgh is of secondary origin during the summer. Our AMS mass spectra also suggest that there was a relatively high concentration of oxygenated organic aerosols during most periods in this study (Zhang et al., submitted manuscript, 2004).

[27] Present analytical uncertainties of the AMS might have also contributed to the lack of significant differences in the ratios of the AMS organic mass to the organic carbon content, between periods that appeared to be dominated by different types of organic aerosols. The AMS organic data may suffer from compensating systematic biases due to the application of constant relative ionization efficiency (RIE) and collection efficiency (CE; see section 2.3.2) for organic signals. According to Jimenez et al. [2003c] the expected RIE of oxygenated organics is somewhat lower than that of the hydrocarbons. However, we have used the average of these two types of organic aerosols (i.e., RIE = 1.4; Table 1), which probably has led to an overestimation of the mass concentrations of primary organic aerosols and an underestimation of those of the secondary. Similarly, using a constant $\mathrm{CE}$, which can be different for different organic aerosol types, might have introduced analytical errors as well. A procedure of separating the contributions of primary and oxidized organics aerosols (using both the size distributions and the mass spectra from the AMS) is currently under development and will be presented in a future publication (Zhang et al., submitted manuscript, 2004). This technique will permit the application of different RIE and $\mathrm{CE}$ factors to primary and oxidized aerosols, which may increase the variability of the OM:OC ratios.

[28] Given that the AMS and the SMPS during this study appear to measure a relatively similar particle population (detailed in paragraphs below and in Figure 4), the regression slope of the AMS mass concentrations and the SMPS volume concentration provides an estimation for the density of bulk particles in Pittsburgh, i.e., $\sim 1.4 \mathrm{~g} \mathrm{~cm}^{-3}$. However, as shown in Figure 3, the correlations between these two measurements appear to separate into two groups. The likely reasons for the separation are the effects of particle shape (see Figure $4 \mathrm{a}$ and associated discussions) and density. Further work in our group is in progress to address this apparent discrepancy by quantitative simultaneous modeling of the AMS and SMPS data [DeCarlo et al., 2004; Slowik et al., 2004].

[29] We estimated that the typical dry density of the nonrefractory $\mathrm{PM}_{1}$ in Pittsburgh is roughly $1.55 \mathrm{~g} \mathrm{~cm}^{-3}$ on the basis of the average particle composition of $\sim 65 \%$ inorganics (i.e., $\mathrm{SO}_{4}^{2-}, \mathrm{NH}_{4}^{+}$, and $\mathrm{NO}_{3}^{-}$) and $\sim 30 \%$ organics (see section 3.2), plus $5 \%$ of black carbon, and the assumption that the densities of $\left(\mathrm{NH}_{4}\right)_{2} \mathrm{SO}_{4}, \mathrm{NH}_{4} \mathrm{NO}_{3}$, bulk organics, and black carbon are $\sim 1.78,1.72, \sim 1.2 \mathrm{~g} \mathrm{~cm}^{-3}$ [Turpin and Lim, 2001], and $1.77 \mathrm{~g} \mathrm{~cm}^{-3}$ [Park et al., 2004] respectively. Black carbon was estimated from AMS $m / z$ 57 during the periods in which it was not available since these measurements were strongly correlated $\left(\mathrm{r}^{2}=0.78\right.$; slope $=0.11 \mu \mathrm{g} \mathrm{m}^{-3} / \mu \mathrm{gC} \mathrm{m}{ }^{-3}$ ) (Zhang et al., submitted manuscript, 2004). Note that the estimated densities 

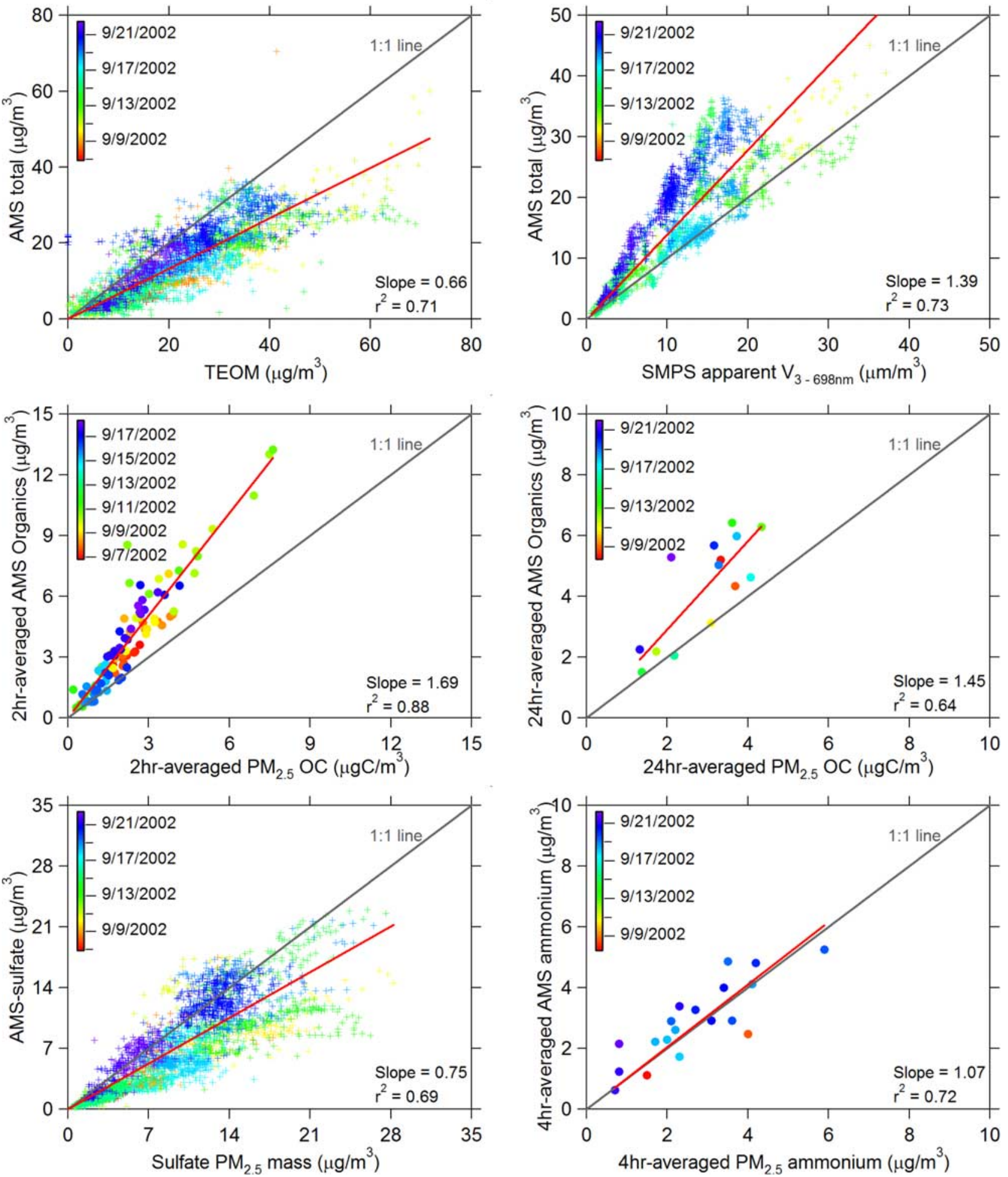

Figure 3. Correlation plots of AMS-measured mass concentrations versus data reported by collocated instruments. Data points are colored by time. Red lines are linear fits to the data.

reported in this study may contain systematic errors because refractory species other than black carbon, such as metals and crustal components, are not detectable by the AMS. However, refractory species other than black carbon on average only contribute $<10 \%$ of the total mass of $\mathrm{PM}_{1}$ in Pittsburgh [Cabada et al., 2004]. Thus the error in the estimated density due to the omission of metals and crustal components is expected to be small. Wittig et al. [2004] reported a similar value, $1.5 \mathrm{~g} \mathrm{~cm}^{-3}$, for the bulk density of the ambient particles in Pittsburgh, on the basis of TEOM and SMPS measurements.

[30] Figure 4 compares the AMS to the SMPS on the average size distributions of particle mass during 3 representative periods. The AMS data are the sum of $\mathrm{SO}_{4}^{2-}, \mathrm{NH}_{4}^{+}$, 
a. 9/12/2002 2:00 AM - 4:00 AM

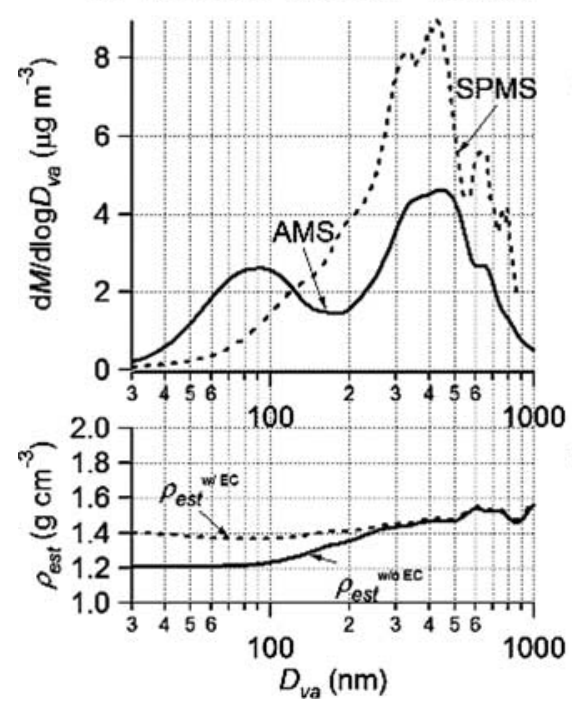

b. 9/12/2002 3:00 PM-5:00 PM
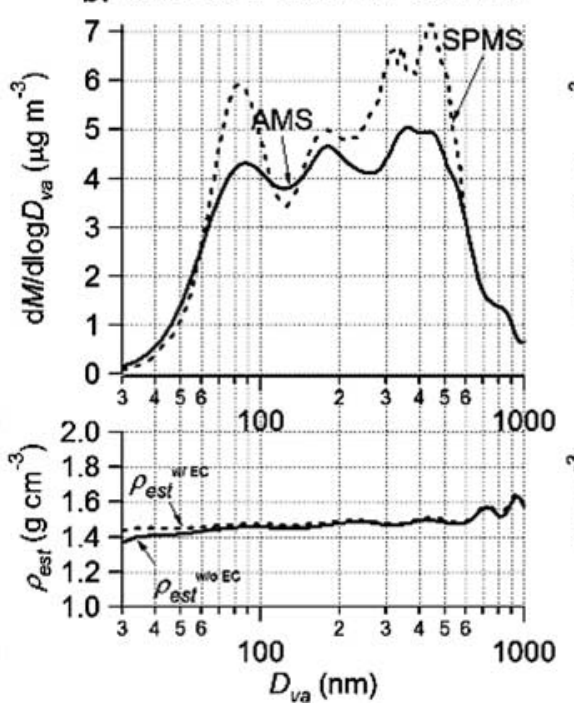

c. 9/14/2002 6:00 AM-8:00 AM
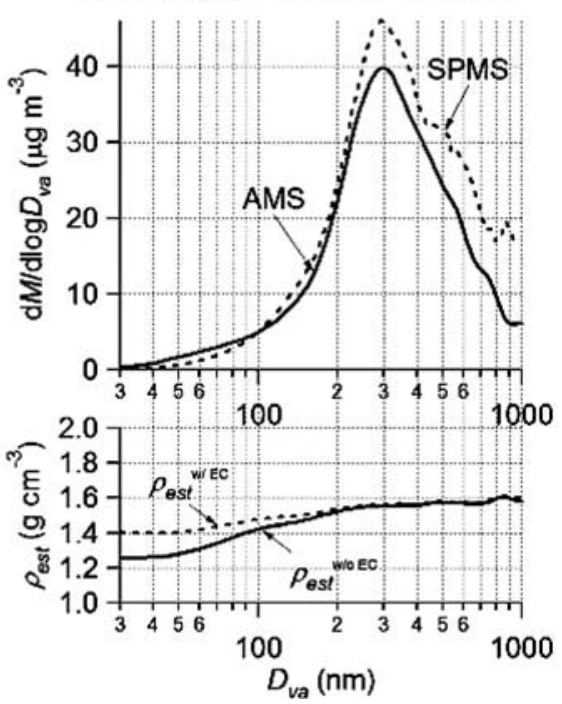

Figure 4. $(\mathrm{a}-\mathrm{c})$ Comparisons of 2-hour-averaged particle mass distributions measured by the AMS to those calculated from the SMPS number distribution data assuming spherical particles and $D_{v a} / D_{m}=$ $\rho_{e s t}^{w / E C} . \rho_{e s t}^{w / E C}$ (dashed lines) is the size-resolved particle density estimated on the basis of the mass distributions of particle species and assuming an average density of $1.77 \mathrm{~g} \mathrm{~cm}^{-3}$ for inorganics $\left(\mathrm{SO}_{4}^{2-}+\mathrm{NH}_{4}^{+}+\mathrm{NO}_{3}^{-}\right), 1.2 \mathrm{~g} \mathrm{~cm}^{-3}$ for organics, and $1.77 \mathrm{~g} \mathrm{~cm}^{-3}$ for black carbon. Since the black carbon size distribution was estimated (see text), $\rho_{e s t}^{w / o E C}$ (solid lines), which is the particle density estimated only using the AMS inorganic and organic data (i.e., not including the contributions of black carbon), is shown as a comparison.

$\mathrm{NO}_{3}^{-}$, and organics. The distributions of SMPS mass concentrations $(M)$ were calculated from the number distribution data [Jimenez et al., 2003a, 2003b]:

$$
\begin{gathered}
M=\pi / 6 \times D_{m}^{3} \times \rho_{e s t} \\
D_{v a} \approx D_{m} \times \rho_{e s t}
\end{gathered}
$$

where $D_{m}$ is the mobility diameter reported by the SMPS and $\rho_{\text {est }}$ is the estimated density of nonrefractory particles weighted by AMS-measured particle composition assuming an average density of $1.77 \mathrm{~g} \mathrm{~cm}^{-3}$ for inorganic species and $\sim 1.2 \mathrm{~g} \mathrm{~cm}^{-3}$ for organics [Turpin and Lim, 2001].

[31] In order to examine the influence of black carbon on estimated particle density, we have estimated the size distributions of elemental carbon (EC) during this study. Our approach is based on the findings that an AMS mass spectral signature, $\mathrm{m} / \mathrm{z} 57$, is a good tracer for primary combustion related organic aerosols [Alfarra et al., 2004; Zhang et al., submitted manuscript, 2004]. Good correlation has been observed between the mass concentrations of $\mathrm{m} / \mathrm{z}$ 57 and those of EC $\left(\mathrm{r}^{2}=0.78\right.$; slope $=0.11 \mu \mathrm{g} \mathrm{m} \mathrm{m}^{-3} / \mu \mathrm{gC}$ $\mathrm{m}^{-3}$ ) during this study (Zhang et al., submitted manuscript, 2004). While some dependence of the density, and thus OC/ EC ratio, of diesel soot particles versus mobility diameter has been shown [Park et al., 2004], this dependence is significantly reduced when considered versus $D_{v a}$ [Slowik et al., 2004]. Thus we estimated the size distribution of elemental carbon (in $D_{v a}$ space) to be the same as that of $\mathrm{m} / \mathrm{z}$ 57. We subsequently calculated the estimated density of particles including the EC contribution assuming an EC density of $1.77 \mathrm{~g} \mathrm{~cm}^{-3}$ [Park et al., 2004] and along with information about the inorganic and organic components. The effect of EC in the estimated density is very small for the accumulation mode, but is more important for smaller particles and for periods where combustion emission concentrations are larger (e.g., Figure 4a).

[32] The average mass distributions obtained by the AMS and the SMPS agree quite well for 2 periods (Figures $4 \mathrm{~b}$ and 4c), but at 2:00-4:00 am, 12 September, the AMS reported significantly larger mass of small particles $\left(D_{v a}<\sim 120 \mathrm{~nm}\right)$ and significantly lower mass of accumulation mode particles $\left(D_{v a} \sim 200-700 \mathrm{~nm}\right)$ than the SMPS did (Figure 4a). Such discrepancy has been frequently observed in urban areas when comparing the AMS and SMPS data, and is attributed to the presence of a significant amount of small irregularly shaped particles (e.g., soot from traffic emissions). This is because the AMS and the SMPS respond differently to particle shape: as a particle becomes less spherical, the AMS-measured $D_{v a}$ decreases while the SMPS-measured $D_{m}$ increases [Jimenez et al., 2003a, 2003b; DeCarlo et al., 2004]. This effect also leads to an overestimation of the accumulation mode by the SMPS, due to irregular particles that appear larger than they really are (and thus have much more apparent than real volume). Indeed, we found that the ultrafine particles during 2-4 am on 12 September were almost pure organic (average estimated density including EC contributions, i.e., $\rho_{e s t}^{w / E C}$, $\sim 1.4 \mathrm{~g} \mathrm{~cm}^{-3}$ ) while those from the other two periods contained significantly more inorganics (resulting in higher $\rho_{\text {est }}^{w / o E C}$; Figure $4 \mathrm{a}$ ).

[33] In general, these two instruments agree well on the size distributions of the accumulation mode particles for periods $\mathrm{b}$ and $\mathrm{c}$, although the AMS reports slightly lower 

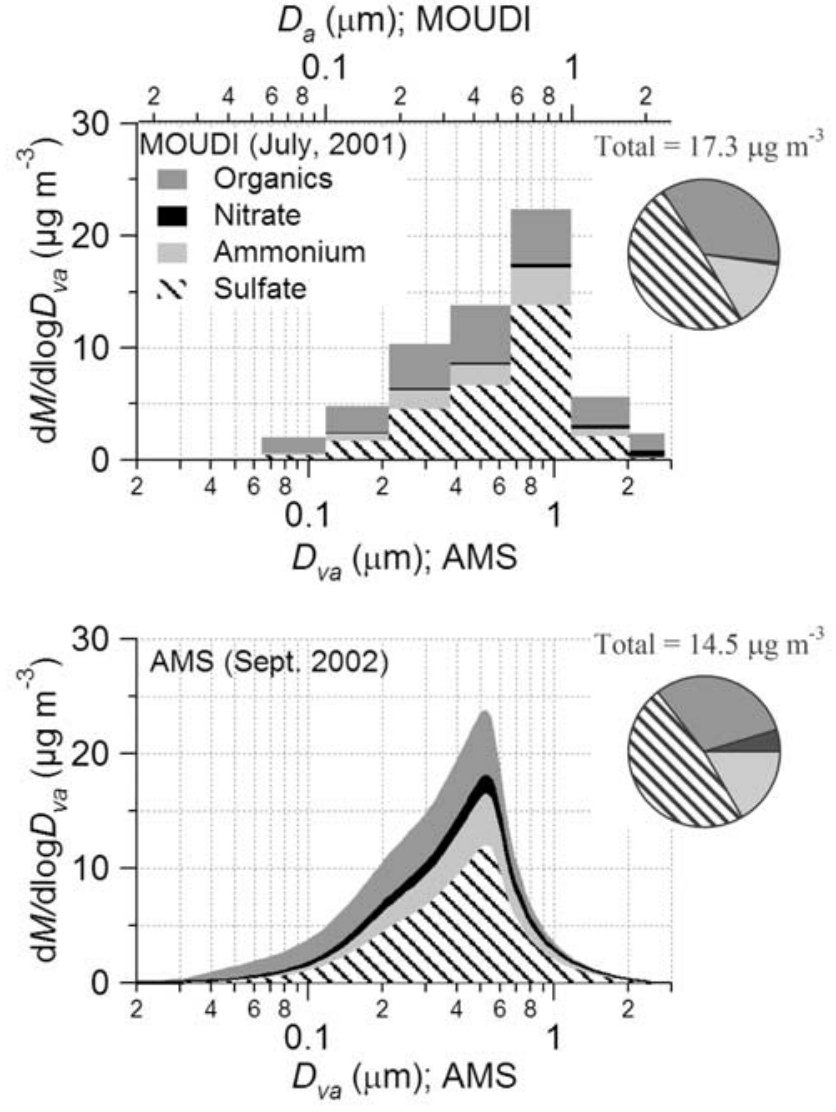

Figure 5. Comparisons of size-resolved chemical compositions measured by the AMS during September 2002 to those of a MOUDI during July 2001. Note that " $D_{a}$ " is the aerodynamic diameter measured by MOUDI. Given that diameter measurement in MOUDI is operated under higher pressure than that within the AMS to first approximation $D_{v a} / D_{a} \approx \rho_{e s t}^{1 / 2}$.

mass concentrations than the SMPS does at these sizes. Besides the possible influence from particle shape, another reason for such discrepancy is that the AMS does not measure refractory components, such as the mineral and crustal materials. It is interesting to note the comparatively good agreement between the SMPS and AMS on the distributions of the ultrafine particles during 3-5 pm, 12 September (Figure 4b). The reason is that a major fraction of these particles were the product of extensive condensational growth of the new particles that formed in a regional intense nucleation event that happened in the morning of 12 September [Zhang et al., 2004]. These particles appeared to be relatively spherical and as such the AMS and the SMPS detect them similarly.

[34] We also compared the average size-resolved chemical composition data determined by the AMS in this study (September 2002) to those obtained with a micro-orifice uniform deposit impactor (MOUDI) for July 2001 (Figure 5). The results from the two instruments are similar at $D_{v a}$ below $\sim 0.8 \mu \mathrm{m}$, despite the different sampling time periods. This is not surprising since the general characteristics of the particles during these two long averaging periods are expected to be similar given the major regional contribu- tions to fine particle concentrations in Pittsburgh, the likely similar local emission mix, and the similar season of sampling (July versus September) [Anderson et al., 2004; Tang et al., 2004; Wittig et al., 2004]. In addition, in order to better compare the AMS and MOUDI mass distributions, we plotted the MOUDI data onto $D_{v a}$ space using the following simplified equation [Canagaratna et al., 2004; Jimenez et al., 2003a, 2003b]:

$$
D_{v a} \approx D_{a} \times \rho_{e s t}^{1 / 2}
$$

where $D_{a}$ is the aerodynamic diameter measured by MOUDI and $\rho_{e s t}$ is the estimated density of particles (Figure 5). In this way, the average mass distribution of Pittsburgh particles in July 2001 appears to peak at $D_{v a}$ of $\sim 0.8-1 \mu \mathrm{m}$. The differences between the AMS and MOUDI at $D_{v a}>600 \mathrm{~nm}$ might be due to less than unit transmission of the AMS for particles larger than $600 \mathrm{~nm}$ (i.e., the approximately $\mathrm{PM}_{1}$ cut of the AMS versus the $\mathrm{PM}_{2.5}$ cut of the MOUDI). This comparison is consistent with the differences between the concentrations of particle mass and sulfate measured by the AMS and TEOM and the sulfate analyzer (Figures 2 and 3 ) are partly due to the difference size cuts of the instruments (i.e., $\mathrm{PM}_{1}$ versus $\mathrm{PM}_{2.5}$ ), although it is not a proof due to the different sampling periods. Similar findings have been reported by Alfarra et al. [2004].

[35] Note that a mass closure test, i.e., a thorough evaluation of the differences between the mass concentrations measured by the AMS and those of the TEOM would be very valuable. However, it is not possible to perform such a test for this data set since MOUDI data, as well as data for some refractory components such as minerals and metals, are not available for this period.

\subsection{Particle Characteristics and Dynamics}

\subsubsection{Mass Concentrations}

[36] The temporal variations of the mass concentrations of NR-PM $\mathrm{SO}_{4}^{2-}, \mathrm{NH}_{4}^{+}, \mathrm{NO}_{3}^{-}$, organics, $\mathrm{Cl}^{-}$, and total $\left(=\mathrm{SO}_{4}^{2-}+\mathrm{NH}_{4}^{+}+\mathrm{NO}_{3}^{-}+\right.$organics $\left.+\mathrm{Cl}^{-}\right)$are shown in Figure 6 . Sulfate (for $\sim 70 \%$ of the time during this study), and less frequently organics (for $\sim 30 \%$ ), dominates the particle composition. The mass concentrations of ammonium are relatively high as well, and are well correlated with those of sulfate $\left(r^{2}=0.83\right.$; Figure $S 1$ in the auxiliary material $\left.^{1}\right)$. These two species together account for more than half of the total NR-PM $M_{1}$ mass for over $75 \%$ of the sampling time of this study.

[37] The correlation between the mass concentrations of organics and sulfate is relatively weak $\left(\mathrm{r}^{2}=0.41\right.$; Figure $\mathrm{S} 1$ in the auxiliary material). Our interpretation for this observation is that local traffic emissions contribute significantly to the organic particle concentrations while the ambient concentrations of particulate sulfate are likely mainly driven by regional accumulation rather than local emissions. However, these two species appear to be internally mixed in the accumulation mode, as suggested by the good correlation between the mass concentrations of organics and sulfate in

${ }^{1}$ Auxiliary material is available at $\mathrm{ftp} / / \mathrm{ftp}$.agu.org/apend/jd/ 2004JD004649. 


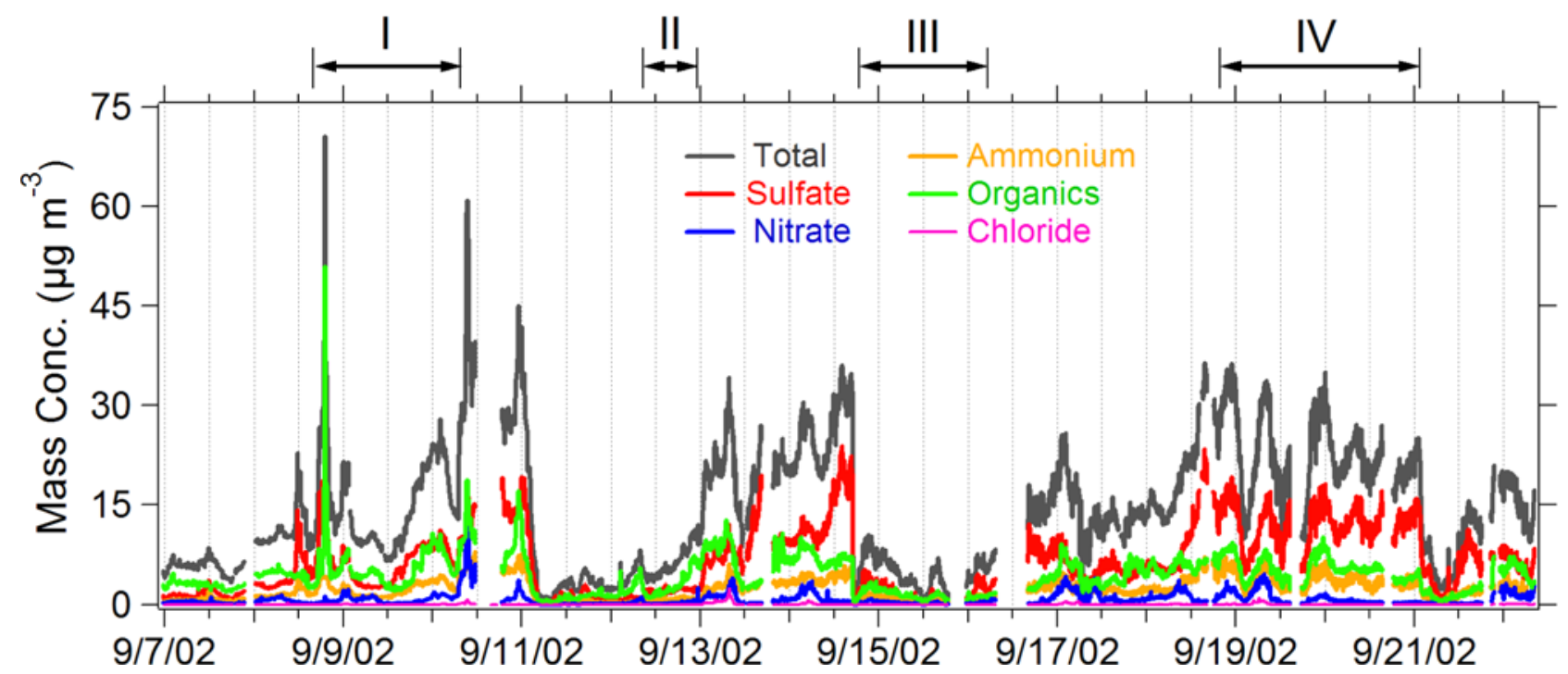

Figure 6. Times series of the mass concentrations of $\mathrm{SO}_{4}^{2-}, \mathrm{NO}_{3}^{-}, \mathrm{NH}_{4}^{+}, \mathrm{Cl}^{-}$, organics, and total $\left(=\mathrm{SO}_{4}^{2-}+\mathrm{NO}_{3}^{-}+\mathrm{NH}_{4}^{+}+\mathrm{Cl}^{-}+\right.$organics $)$. Periods I, II, III, and IV are marked as references for Figure 7.

the 250-1000 $\mathrm{nm}$ size range (see section 3.3.2.). Indeed, internally mixed organics and sulfate in Pittsburgh fine particles have been observed by Bein et al. [2005] using a rapid single-particle mass spectrometer (RSMS). In addition, similar findings have been reported in studies at other locations using single-particle MS techniques, such as particle analysis by laser mass spectrometry (PALMS) [Lee et al., 2003, and references therein].

[38] Nitrate and chloride are generally low and both show very weak correlations with sulfate $\left(\mathrm{r}^{2}<0.1\right)$. The mass concentrations of $\mathrm{NO}_{3}^{-}$are likely controlled by partitioning or formation of nitric acid. Since $\mathrm{NaCl}$ is not measured by the AMS at the vaporizer temperature used in this study the detected chloride must originate either from more volatile inorganic chlorides (such as $\mathrm{NH}_{4} \mathrm{Cl}$ ) or from organic chlorine-containing species. We observed some correlation $\left(\mathrm{r}^{2} \sim\right.$ 0.30 , Figure $\mathrm{S} 1$ in the auxiliary material) between $\mathrm{NO}_{3}^{-}$and $\mathrm{Cl}^{-}$, probably due to the fact that the formation of $\mathrm{NH}_{4} \mathrm{NO}_{3}$ and $\mathrm{NH}_{4} \mathrm{Cl}$ is favored by the same conditions: higher $\mathrm{NH}_{3}$ in the gas-phase and higher $\mathrm{RH}$.

[39] Multiday episodes of fine particle pollution, with intermittent cleaner periods, are observed (Figure 6). The cleaner periods often start with heavy rainfall (e.g., $\sim 5 \mathrm{pm}$ on 14 September and $\sim 6$ am on 21 September) and/or the arrival of clean air from Canada (e.g., 1-4 am on 11 September), which quickly reduce the mass concentrations of NR-PM 1 by one to two orders of magnitude. Afterward, the particle mass loading gradually increases but remains low for 1-3 days. Most significant and lasting increases of aerosol mass concentrations are associated with rapid increases of sulfate. Several short-lived spikes of organic aerosols are detected, likely due to the passage of relatively fresh local plumes by the sampling site. The mass spectra and size distributions of these organic aerosols suggest that they originated from combustion sources.

\subsubsection{Size Distributions}

[40] Figure 7 shows the average size distributions and chemical compositions of NR-PM 1 during 4 periods (marked as I, II, III, and IV on Figure 6) of this study. These periods were selected to represent various aerosol "climatologies" observed in this study: Period I is relatively polluted with approximately equal amounts of sulfate and organic aerosols; II corresponds to the growth stage of a strong regional nucleation event [see Zhang et al., 2004]; III is a relatively clean period after a rain shower; and IV is a sulfate-dominated regional pollution episode.

[41] The mass distributions are significantly different during these 4 periods, showing unimodal, bimodal and even trimodal characteristics. An accumulation mode peaking between $350-600 \mathrm{~nm}\left(D_{v a}\right)$ is constantly observed. This mode appears to be an internal mixture of ammonium sulfate with variable amounts of organics and a minor fraction of nitrate. In general, as the aerosol mass concentration increases and for more aged particles (e.g., in period IV), the peak diameter of the accumulation mode increases, the distribution appears to be narrower (e.g., the geometric standard deviations $\sigma_{g}$ of total mass distributions were $\sim 2.0$ and 1.6 for periods III and IV, respectively), and the sulfate content increases (Figure 7). However, the size distributions reported here are "as measured" and could be skewed by the limited aerodynamic lens transmissions for particles larger than $600 \mathrm{~nm}$ and smaller than $60 \mathrm{~nm}$ in $D_{v a}$ [Jayne et al., 2000]. Details in evolution of the mass distributions of particle species during this entire study are presented in Figures S4 and S5 in the auxiliary material.

[42] In addition to the accumulation mode, two smaller modes, one below $100 \mathrm{~nm}$ and one at $\sim 200 \mathrm{~nm}$, are also frequently observed (e.g., Figure 7, periods I and II). Given the essentially same size distributions of $\mathrm{NH}_{4}^{+}$and $\mathrm{SO}_{4}^{2-}$ throughout the entire campaign, and the good correlations between their mass concentrations (see section 3.2.1), these two species appear to be internally mixed, likely in the forms of $\left(\mathrm{NH}_{4}\right)_{2} \mathrm{SO}_{4}$ and $\mathrm{NH}_{4} \mathrm{HSO}_{4}$, in all modes. The organics, however, very often show an ultrafine mode $\left(D_{v a}<100 \mathrm{~nm}\right)$ that seems to be externally mixed with the other species. We have evidence that fuel combustion, 

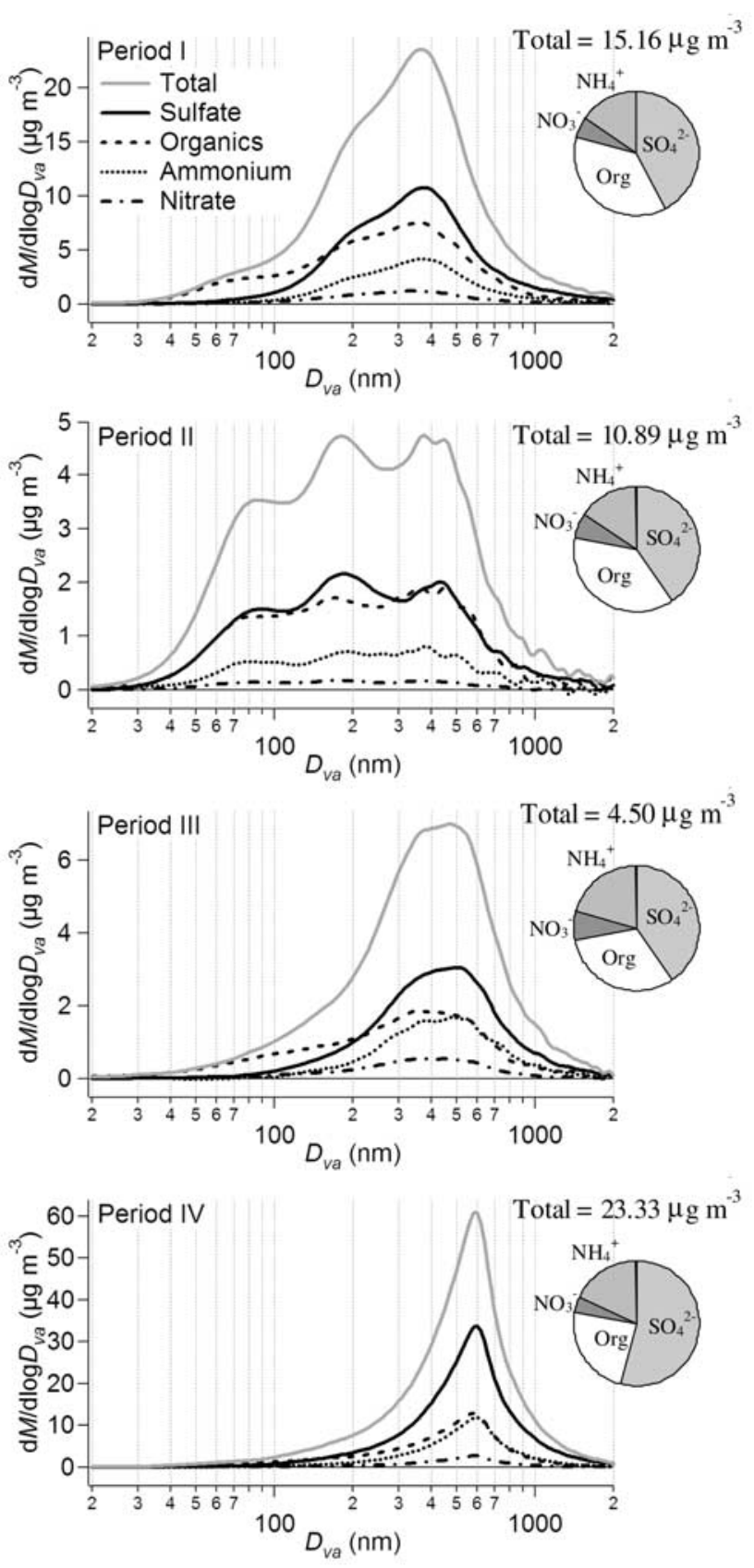

Figure 7. Average size distributions and chemical compositions of particle species during four time periods with similar characteristics (i.e., periods I, II, III, and IV as marked in Figure 6) of this study.

i.e., traffic emissions (see section 3.3.2) is a major source of these small mode organic aerosols. Similar findings have been reported for AMS measurements in other urban areas [e.g., Alfarra et al., 2004; Boudries et al., 2004; Drewnick et al., 2004c]. Canagaratna et al. [2004] also reported a peak $D_{v a}$ of $\sim 90 \mathrm{~nm}$ on the mass-weighted size distributions of the fresh diesel exhaust particles.

[43] Ultrafine particles behave rather differently during period II, the particle growth stage of a strong regional nucleation event that started at $\sim 8: 00 \mathrm{am}$. These particles consist of comparable amounts of sulfate and organics, which appear to be an internal mixture, together with $\mathrm{NH}_{4}^{+}$and $\mathrm{NO}_{3}^{-}$(Figure 7, period II). In fact, given the similarities in their size distributions, $\mathrm{SO}_{4}^{2-}, \mathrm{NH}_{4}^{+}, \mathrm{NO}_{3}^{-}$, and organics appear to be internally mixed over the entire size range during period II. Such homogeneity in aerosol composition was developed through extensive condensation of gas phase species including $\mathrm{H}_{2} \mathrm{SO}_{4}, \mathrm{NH}_{3}, \mathrm{HNO}_{3}$, and photochemically processed organic species, a phenomenon that is commonly observed during the growth stage of nucleation events in Pittsburgh. The reader is referred to Zhang et al. [2004] for a detailed description on the evolution of particle compositions and size distributions during this nucleation event.

[44] The average size distribution of particle mass concentration of the entire sampling period demonstrates a broad peak at $\sim 550 \mathrm{~nm}\left(D_{v a}\right)$, a slight shoulder at around $200 \mathrm{~nm}$, and a tail that extends below $100 \mathrm{~nm}$ (Figure 8a). The composition of the particles changes as a function of size in that larger particles tend to contain more $\mathrm{SO}_{4}^{2-}$ and $\mathrm{NH}_{4}^{+}$and less organics (Figure 8b). As a result, the estimated particle density is strongly size dependent as well, with higher values for the larger particles (Figure 8c). Note that mineral species have not been taken into account since they are not detectable by the AMS and their sizeresolved measurements were not available during this study. As described above, this omission should only cause a small error in the estimated density.

[45] Sulfate is the major component of the accumulation mode while organics dominate the mass of particles smaller than $200 \mathrm{~nm}\left(D_{v a}\right)$. Despite the high frequency of nucleation events in Pittsburgh [Stanier et al., 2004] and the fact that the ultrafine particles produced during the nucleation events are mainly composed of $\mathrm{SO}_{4}^{2-}$ and $\mathrm{NH}_{4}^{+}$[Zhang et al., 2004], organics overall account for up to $80-90 \%$ of the ultrafine mass $\left(D_{v a}<100 \mathrm{~nm}\right)$ (Figure 8b). This observation is consistent with combustion emissions being the major source of ultrafine mass in Pittsburgh, since the nonrefractory fraction of ultrafine particles from combustion processes are usually almost completely organic [Canagaratna et al., 2004]. A detailed analysis on the possible sources of organic species in Pittsburgh will be presented in a future publication (Zhang et al., submitted manuscript, 2004).

[46] In addition to the accumulation mode and the ultrafine mode particles, there also appears to be an intermediate mode $\left(D_{v a} \approx 100-250 \mathrm{~nm}\right)$ that is composed of comparable amounts of $\mathrm{SO}_{4}^{2-}$ and organics. The size ranges of these particles correspond to the so-called condensation mode [Pandis et al., 1995] and are likely the condensational growth products of the ultrafine particles from primary emissions or formed during the nucleation events. Conceptually these particles may be "on their way" to the accumulation mode, but not yet had enough time, or had not been through cloud or fog processes [Pandis et al., 1995], to grow to that size yet. These AMS-measured mass distributions are consistent with measurements by MOUDI at the same site during the summer of 2001 [Cabada et al., 2004].

\subsubsection{Diurnal Variations of Mass Concentrations} and Size Distributions

[47] A summary of the diurnal cycles of $\mathrm{SO}_{4}^{2-}, \mathrm{NH}_{4}^{+}$, $\mathrm{NO}_{3}^{-}$, and organics is shown in Figure 9. Note that the dips at the eighteenth hour on the plots are biased by a very large 

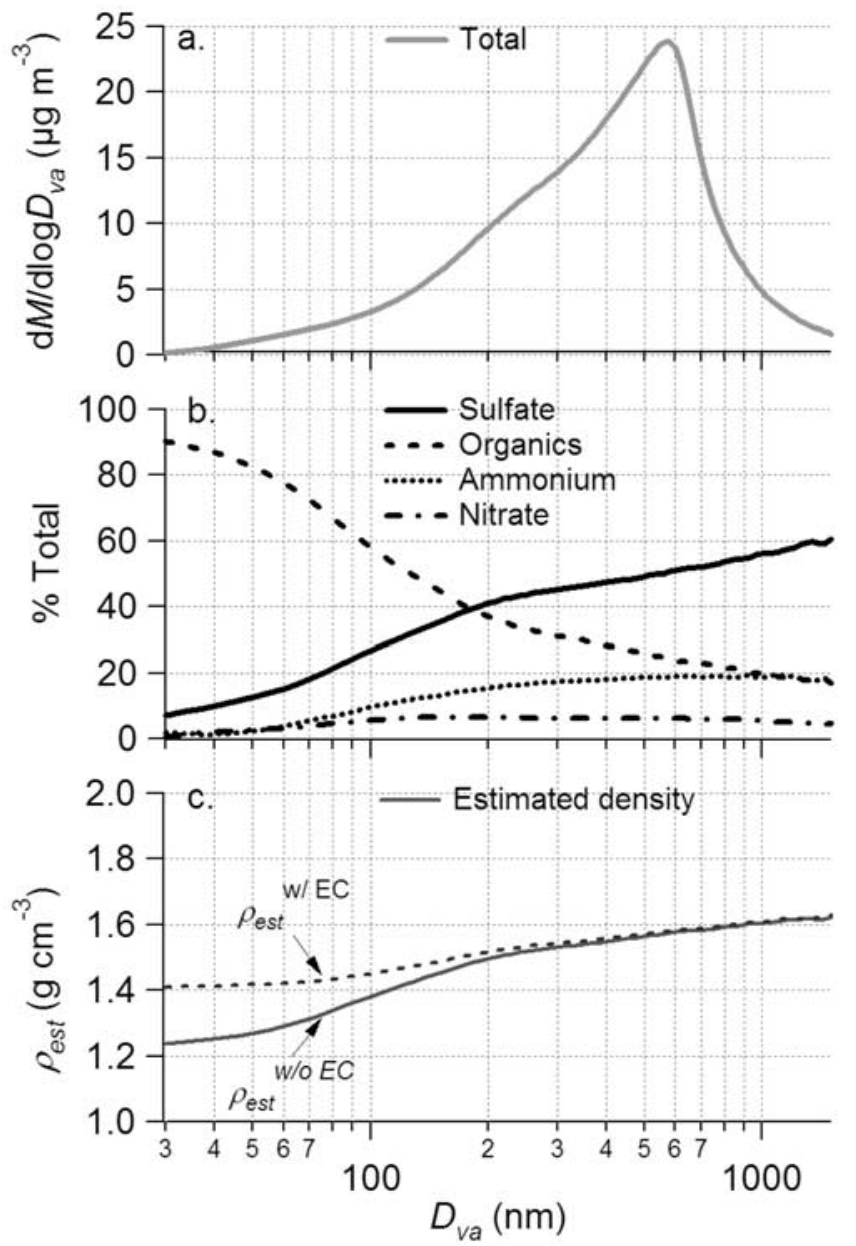

Figure 8. Averaged size distributions of (a) total nonrefractory mass, (b) particle chemical composition, and (c) estimated density for nonrefractory particle components (solid line) and estimated particle density including the contribution of elemental carbon (EC) (dashed line) averaged over the entire study (7-22 September 2002).

and sharp drop in particle concentration between 17:00 and 18:00 on 15 September, due to heavy rainfall (see Figure 6). The mass concentration of sulfate does not show a pronounced diurnal cycle, but nonetheless demonstrates a slight increase during the day (Figure 9a). Such increase appears to be driven by enhanced photochemical production of gaseous $\mathrm{H}_{2} \mathrm{SO}_{4}$. However, the overall lack of a clear diurnal cycle of sulfate suggests that multiday accumulation of sulfate on a regional scale dominates over its same-day photochemical production. The average concentrations of ammonium also slightly increased during the afternoon (Figure $9 \mathrm{~b}$ ), probably a result of neutralization of the enhanced $\mathrm{H}_{2} \mathrm{SO}_{4}$ uptake.

[48] The mass concentration of nitrate shows a pronounced diurnal profile that peaks in the early morning, drops after $10 \mathrm{am}$, and stays at low levels between 12:00 noon and 7:00 pm (Figure 9c). The observed cycle is likely partially driven by the gas-to-particle partitioning of ammonium nitrate precursors (gaseous $\mathrm{HNO}_{3}$ and $\mathrm{NH}_{3}$ ), which is favored by the lower temperature and higher relative humidity during the night and the early morning [Seinfeld and Pandis, 1998]. In addition, the higher level of nighttime gaseous $\mathrm{N}_{2} \mathrm{O}_{5}$ and $\mathrm{NO}_{3}$, which are quickly photolyzed during the day, might have acted as a significant source of $\mathrm{NO}_{3}^{-}$concentrations at night [Brown et al., 2003a, 2003b; Pandis et al., 1995] while enhanced higher particle acidity in the afternoon, as suggested by a relatively steeper increase of $\mathrm{SO}_{4}^{2-}$ than $\mathrm{NH}_{4}^{+}$between 1:00 pm and 4:00 pm (Figures $9 \mathrm{a}$ and $9 \mathrm{~b}$ ), might have played a role in reducing the concentration of $\mathrm{NO}_{3}^{-}$in the particles during that period.

[49] The average mass concentrations of organics are somewhat higher at night (Figure 9d), which might be attributed to the lower mixing layer depth that traps the city emissions. In addition, a small peak of organic mass is observed during the morning rush hour between 7:009:00, reflecting the fact that traffic emissions are usually an important source of organic aerosols in urban areas. Drewnick et al. [2004b] reported similar diurnal behaviors of $\mathrm{SO}_{4}^{2-}, \mathrm{NO}_{3}^{-}$, and organics at Queens, New York, during July 2001.

[50] Figures $9 \mathrm{e}-9 \mathrm{~h}$ are the average size distributions of the species during two time periods of the day, 6-9 am EST (i.e., 7-10 am EDT; the morning rush hours) and 1-4 pm (i.e., the period of most intense photochemistry and accumulation of photochemically produced aerosols). The average size distributions of $\mathrm{SO}_{4}^{2-}, \mathrm{NH}_{4}^{+}$, and $\mathrm{NO}_{3}^{-}$are similar to each other in the morning (6-9 am) as well as in the afternoon (1-4 pm). A much broader distribution of the organics, however, is observed during the morning rush hour, indicating the presence of large amounts of trafficrelated small mode organic aerosols.

[51] The average size distribution of organics is much narrower during $1-4 \mathrm{pm}$ and becomes very much alike to those of the inorganic species (Figure 9h). It appears that the organic aerosols from morning traffic emissions have been diluted by the increase of the mixing layer height and evolved by coagulation and/or condensation of gas phase species.

\subsubsection{Particle Acidity}

[52] We evaluated the acidity of the particles by comparing measured $\mathrm{NH}_{4}^{+}$mass concentration to the amount needed to fully neutralize the measured $\mathrm{SO}_{4}^{2-}, \mathrm{NO}_{3}^{-}$and $\mathrm{Cl}^{-}$. Particles are considered "more acidic" if the measured $\mathrm{NH}_{4}^{+}$concentration is significantly (by $25 \%$ or more) lower than the predicted values, and as bulk neutralized if the two values are similar. A measured-to-predicted $\mathrm{NH}_{4}^{+}$ratio of 0.75 suggests that roughly $50 \%$ of the $\mathrm{SO}_{4}^{2-}$ molecules in the particles are in the form of bisulfate $\left(\mathrm{HSO}_{4}^{-}\right)$. This approach should be relatively accurate because Pittsburgh particles generally contain very low amounts of the metal ions, such as $\mathrm{Na}^{+}, \mathrm{Ca}^{2+}$ and $\mathrm{K}^{+}$[Rees et al., 2004; Wittig et al., 2004].

[53] On the basis of this definition, aerosols appear to be bulk neutralized to slightly acidic for the majority of the time, but there are also episodes when they are "more acidic" (Figure 10). We estimate that for roughly 20\% of the time during this study the measured-to-predicted $\mathrm{NH}_{4}^{+}$ ratios are less than 0.75 , i.e., the mole ratio of $\mathrm{NH}_{4} \mathrm{HSO}_{4}$ to $\left(\mathrm{NH}_{4}\right)_{2} \mathrm{SO}_{4}$ is above 1 (Figure 10b). Because the acidic particles are often found during the high-mass-loading periods and only show a weak correlation with UV $\times$ $\mathrm{SO}_{2}$, which is a proxy for gas-phase $\mathrm{H}_{2} \mathrm{SO}_{4}$ production 

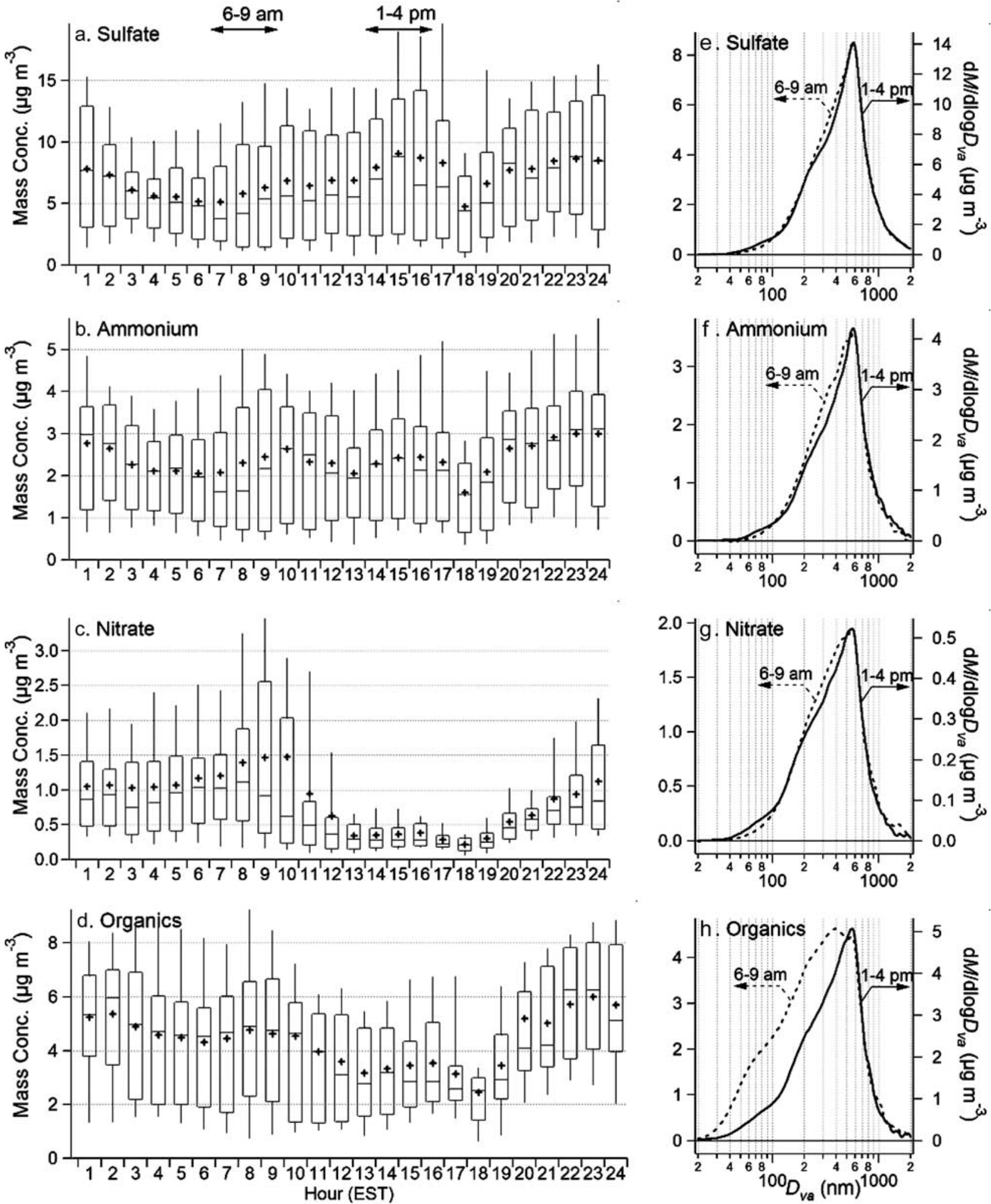

Figure 9. Average diurnal cycles of the mass concentrations of (a) $\mathrm{SO}_{4}^{2-}$, (b) $\mathrm{NH}_{4}^{+}$, (c) $\mathrm{NO}_{3}^{-}$and (d) organics and $(\mathrm{e}-\mathrm{h})$ the average size distributions of particle species during 6-9 am and 1-4 pm (722 September 2002). The box plots are read as follows: the upper and lower boundaries of the box indicate the 75th and the 25th percentiles, the line within the box marks the median, and the whiskers above and below the box indicate the 90th and 10th percentiles. Cross symbols represent the means. The $\mathrm{x}$-axis labels of the diurnal plots correspond to the hour of the day, e.g., "1" means from 00:00-01:00 am. 

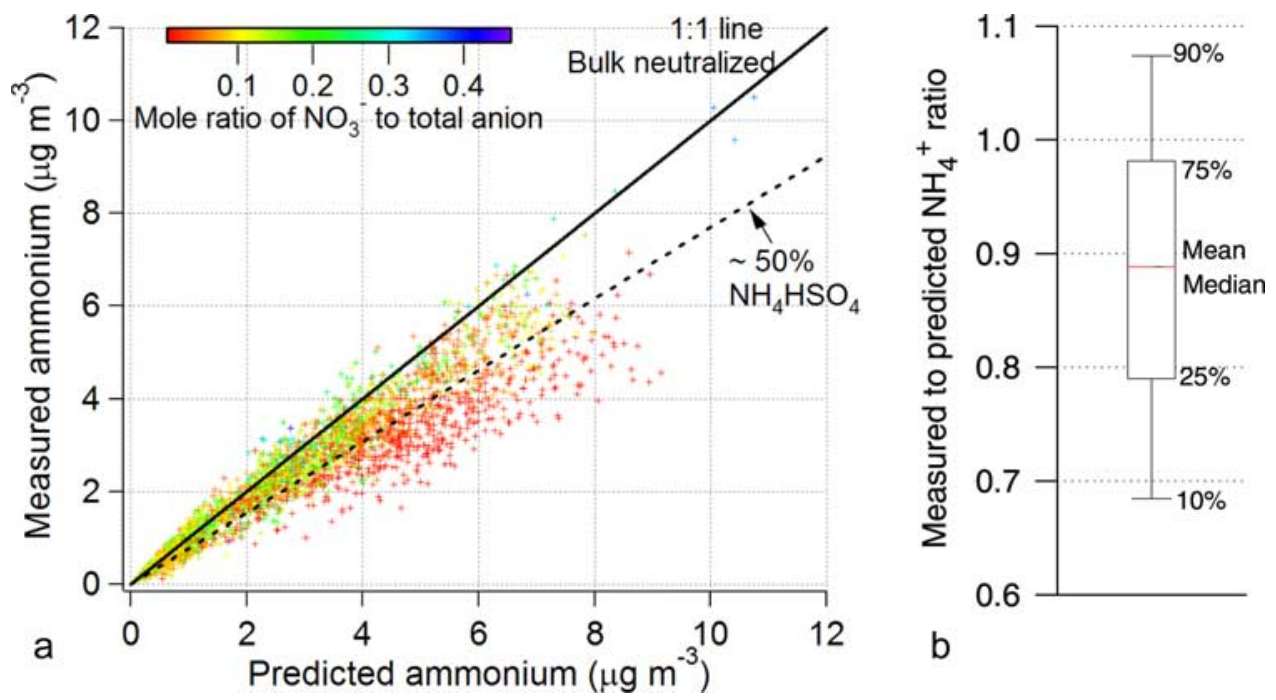

Figure 10. (a) Mass concentrations of measured $\mathrm{NH}_{4}^{+}$versus predicted $\mathrm{NH}_{4}^{+}$and (b) box plot of the ratio of measured to predicted $\mathrm{NH}_{4}^{+}$during the entire study (7-22 September $\left.2002 \mathrm{EST}\right)$. Note that predicted $\mathrm{NH}_{4}^{+}$concentrations were calculated from measured $\mathrm{SO}_{4}^{2-}, \mathrm{NO}_{3}^{-}$, and $\mathrm{Cl}^{-}$assuming full neutralization of these anions by $\mathrm{NH}_{4}^{+}$.

rate, we speculate that they are particles transported from large plumes relatively depleted of $\mathrm{NH}_{3}$.

[54] Summarized in Figure 11 are the average compositions of neutralized (i.e., measured-to-predicted $\mathrm{NH}_{4}^{+}$ ratio $\approx 1.0$ ) and "more acidic" (i.e., measured-to-predicted $\mathrm{NH}_{4}^{+}$ratio $\left.<0.75\right)$ particles. Compared to those considered as neutralized, the "more acidic" particles on average contain $\sim 30 \%$ more mass, which is almost exclusively due to the increase of $\mathrm{SO}_{4}^{2-}$. These two types of particles, however, contain comparable amounts of organics and $\mathrm{NH}_{4}^{+}$. Within the limitations of this analysis, we observe negligible enhancement on the organic concentration that could be attributed to acid-catalyzed secondary organic aerosol formation [Jang et al., 2002]. In addition, the concentrations of $\mathrm{NO}_{3}^{-}$and $\mathrm{Cl}^{-}$in the "more acidic" particles are roughly half of those in the neutralized particles, probably because of displacement of $\mathrm{HCl}$ and $\mathrm{HNO}_{3}$ by $\mathrm{H}_{2} \mathrm{SO}_{4}$ in the competition for $\mathrm{NH}_{3}$ [West et al., 1999].

\subsection{Primary and Secondary Aerosol Sources and Processes}

\subsubsection{Gas to Particle Partitioning of Nitrate}

[55] In order to investigate the diurnal cycles of the particulate nitrate mass, we plotted in Figure 12 the average diurnal patterns of $\mathrm{NO}_{3}^{-}$together with $\mathrm{UV} \times \mathrm{NO}_{2}$, which is a proxy for daytime $\mathrm{HNO}_{3}$ production rate, and the equilibrium formation constant of $\mathrm{NH}_{4} \mathrm{NO}_{3}$ from gas phase $\mathrm{HNO}_{3}$ and $\mathrm{NH}_{3}$ (i.e., $K_{p}, \mathrm{ppb}^{-2}$ ). The $K_{p}$ defined here is the inverse of the product of the partial pressures of $\mathrm{NH}_{3}$ and $\mathrm{HNO}_{3}$, and thus it is calculated using the inverse form of equation 9.91 of Seinfeld and Pandis [1998]:

$$
\ln K_{p}=1 /(84.6-24220 / T-6.1 \ln (T / 298))
$$

where $T$ is the ambient temperature in Kelvin. Although this equation only applies to dry particles, the relative trend of partitioning versus temperature is exactly the same when particles contain liquid water (equation 9.92 of Seinfeld and Pandis [1998]).

[56] The diurnal profile of the $\mathrm{CO}$ concentrations is also included as a first-order indicator for daily changes in the boundary layer height. Overall, the diurnal patterns of $\mathrm{NO}_{3}^{-}$, $K_{p}$ and $\mathrm{CO}$ positively correlate, but all anticorrelate with that of $\mathrm{UV} \times \mathrm{NO}_{2}$ (Figure 12). Although the morning peaks of $\mathrm{NO}_{3}^{-}, \mathrm{CO}$, and $K_{p}$ are shifted by about $1-2$ hours, these comparisons nonetheless suggest that variations in the

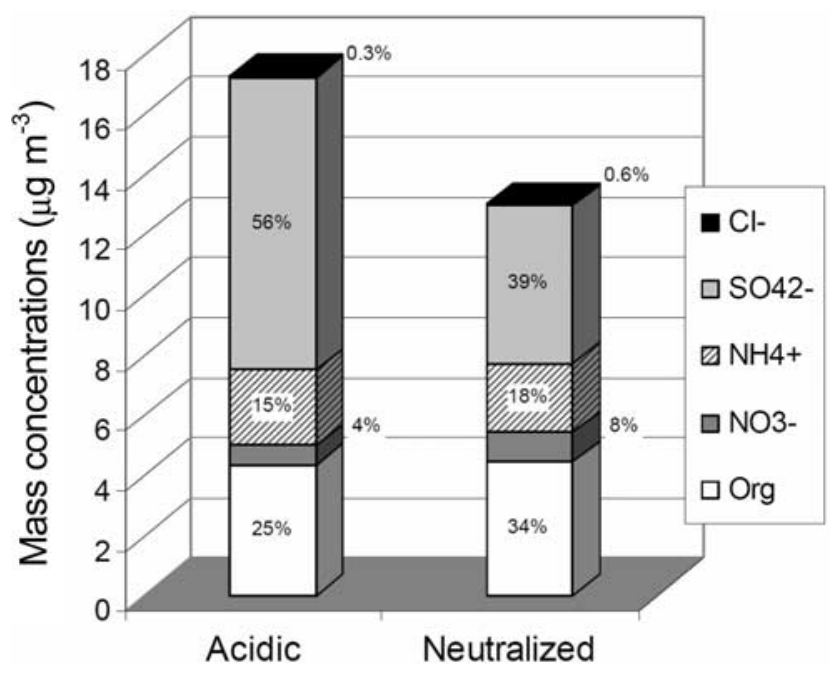

Figure 11. Average compositions of neutralized and "more acidic" NR-PM 1 particles during 7-22 September 2002. The neutralized particles are defined as those with measured-to-predicted ratio of $\mathrm{NH}_{4}{ }^{+}$between 0.93 (the mean value) and 1.15 (90th percentile), and the "more acidic" particles are those with a ratio of less than 0.75 , i.e., those that contain approximately equal moles of $\mathrm{NH}_{4} \mathrm{HSO}_{4}$ and $\left(\mathrm{NH}_{4}\right)_{2} \mathrm{SO}_{4}$. 


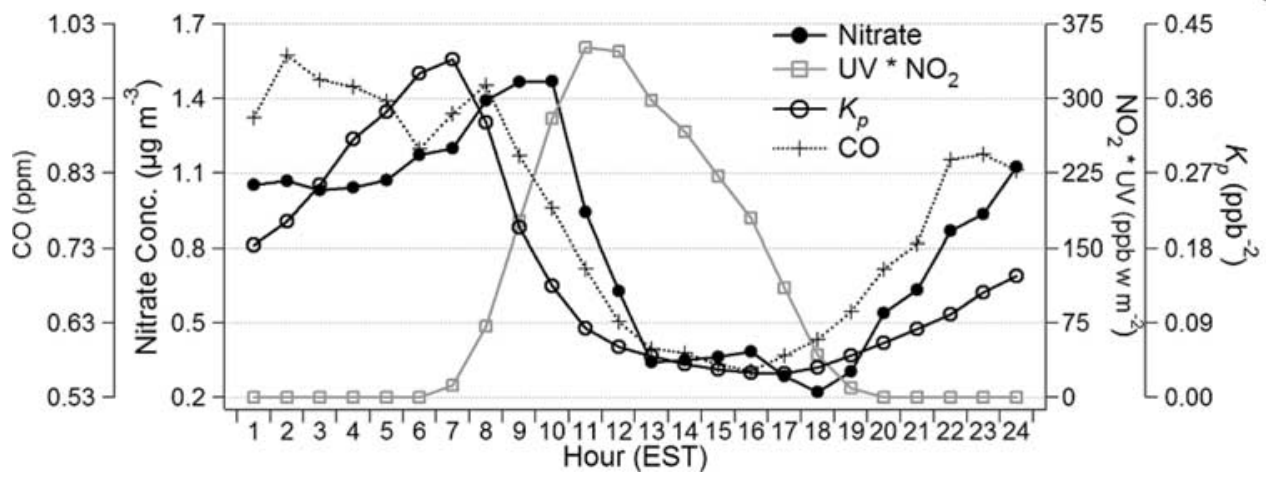

Figure 12. Average diurnal variations of 1-hour-averaged $\mathrm{NO}_{3}^{-}$concentrations, $\mathrm{CO}, \mathrm{UV} \times \mathrm{NO}_{2}$, and the equilibrium constant for $\mathrm{NH}_{4} \mathrm{NO}_{3}$ formation $\left(K_{s p}\right)$ during 7-22 September 2002.

mixing layer depth and gas to particle partitioning of $\mathrm{HNO}_{3}$ appear to have played important roles in driving the diurnal cycles of the particulate $\mathrm{NO}_{3}^{-}$concentrations. On the other hand, the fact that the $\mathrm{NO}_{3}^{-}$concentrations remain high for $\sim 2$ hours after $\sim 7: 00$ am, when in general $K_{p}$ starts to decline and boundary layer height begins to increase, suggests that either photochemical production of $\mathrm{HNO}_{3}$ or transport of nitrate particles are responsible for maintaining the $\mathrm{NO}_{3}^{-}$level during this period of time. Despite the relatively higher photochemical production of $\mathrm{HNO}_{3}$ in the afternoon, this mechanism does not result in increased $\mathrm{NO}_{3}^{-}$concentrations during that time period.

\subsubsection{Combustion Sources and Photochemical Formation of Organic Aerosols}

[57] In addition to the average size distributions (see section 3.2.3), the average mass spectra of organic aerosols are also considerably different between the morning rush hours (i.e., 6-9 am EST or 7-10 am local time) and the afternoon (1-4 pm; Figure 13). The mass spectrum of the morning organic aerosols shows prominent peaks at $\mathrm{m} / \mathrm{z} 55$ $\left(\mathrm{C}_{4} \mathrm{H}_{7}^{+}\right.$and $\left.\mathrm{C}_{3} \mathrm{H}_{3} \mathrm{O}^{+}\right)$and $57\left(\mathrm{C}_{4} \mathrm{H}_{9}^{+}\right)$, which are generally associated with primary (combustion-emitted) organics from combustion sources [Alfarra et al., 2004; Canagaratna et al., 2004], while that of the afternoon organic aerosols is dominated by $m / z 44\left(\mathrm{CO}_{2}^{+}\right)$, which is a pronounced peak in the AMS mass spectra of oxygenated organics [Alfarra et al., 2004; Drewnick et al., 2004b]. Note that $\mathrm{m} / \mathrm{z} 43$ $\left(\mathrm{C}_{3} \mathrm{H}_{7}^{+}\right.$and $\left.\mathrm{C}_{2} \mathrm{H}_{3} \mathrm{O}^{+}\right)$is a major peak in both mass spectra, because it is produced in roughly equal fractions from primary and secondary/oxidized organic aerosols and therefore is more of a surrogate for the bulk organics. In addition, mass fragments that are representative for combustion source organic species, such as $m / z 69,71,85,91$ and 95 [McLafferty and Turecek, 1993], are all comparatively higher in the mass spectrum of the morning organics (Figure 13). Overall these mass spectra are consistent with morning organic aerosols having a higher contribution of traffic-related combustion source emissions while the afternoon aerosols appear to be more oxidized.

[58] A study with two AMSs at 3 sites in the Vancouver area in Canada reported much more marked differences between the mass spectra of urban and rural organic aerosols [Alfarra et al., 2004] than between the morning and afternoon spectra in this study. The reason for the relatively smaller difference in the mass spectra in this study is that primary aerosols always contribute to the observed concentrations in Pittsburgh, while their contribution is very small for the rural aerosol in Vancouver. In addition, relatively high background of oxidized organic aerosols has been observed in both studies most of the time.

[59] Since aged organic species more likely reside in the accumulation mode, we compared the diurnal profiles of small mode $\left(D_{v a}=30-100 \mathrm{~nm}\right)$ and accumulation mode $\left(D_{v a}=250-1000 \mathrm{~nm}\right)$ organics (Figure 14). The mass concentrations of the ultrafine mode are elevated in the morning and evening rush hours and are much lower in the afternoon when photochemistry is usually intense. The accumulation mode on the other hand, did not show much of a diurnal variation. The average mass loading of the accumulation mode organic aerosols increased slightly between $1 \mathrm{pm}$ and $4 \mathrm{pm}$, suggesting the formation of secondary organic aerosols. We observed a comparatively good correlation between the accumulation mode organics and sulfate $\left(r^{2}=0.73\right.$; Figure 15$)$, which is an indication that these two species are likely internally mixed in the accumulation mode particles. The slope of the regression
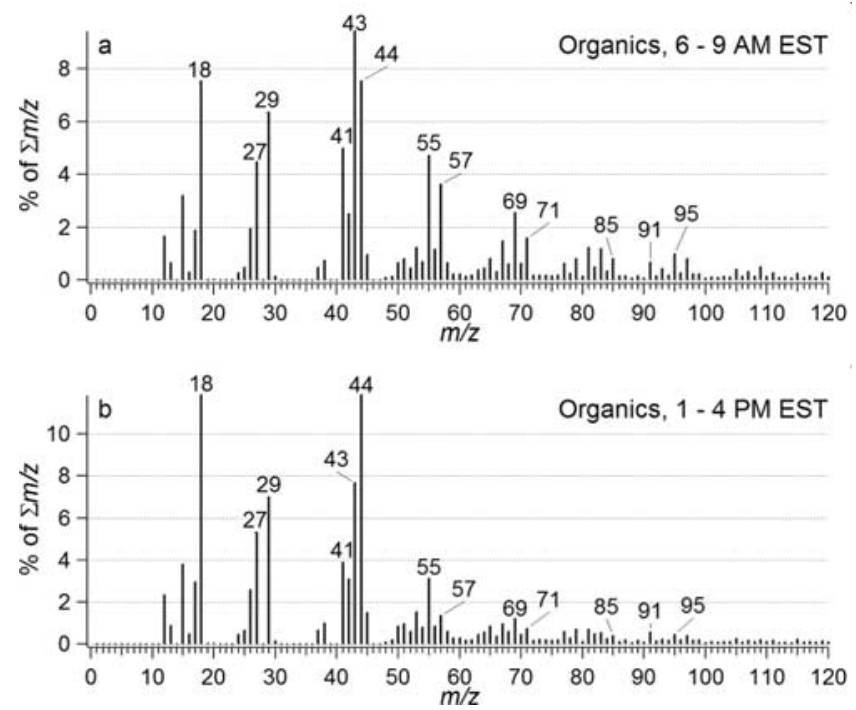

Figure 13. Average AMS mass spectra of organic particles during (a) 6-9 am and (b) 1-4 pm (7-22 September 2002 EST). Note that local time during this study was EDT, which is 1 hour ahead of EST. 

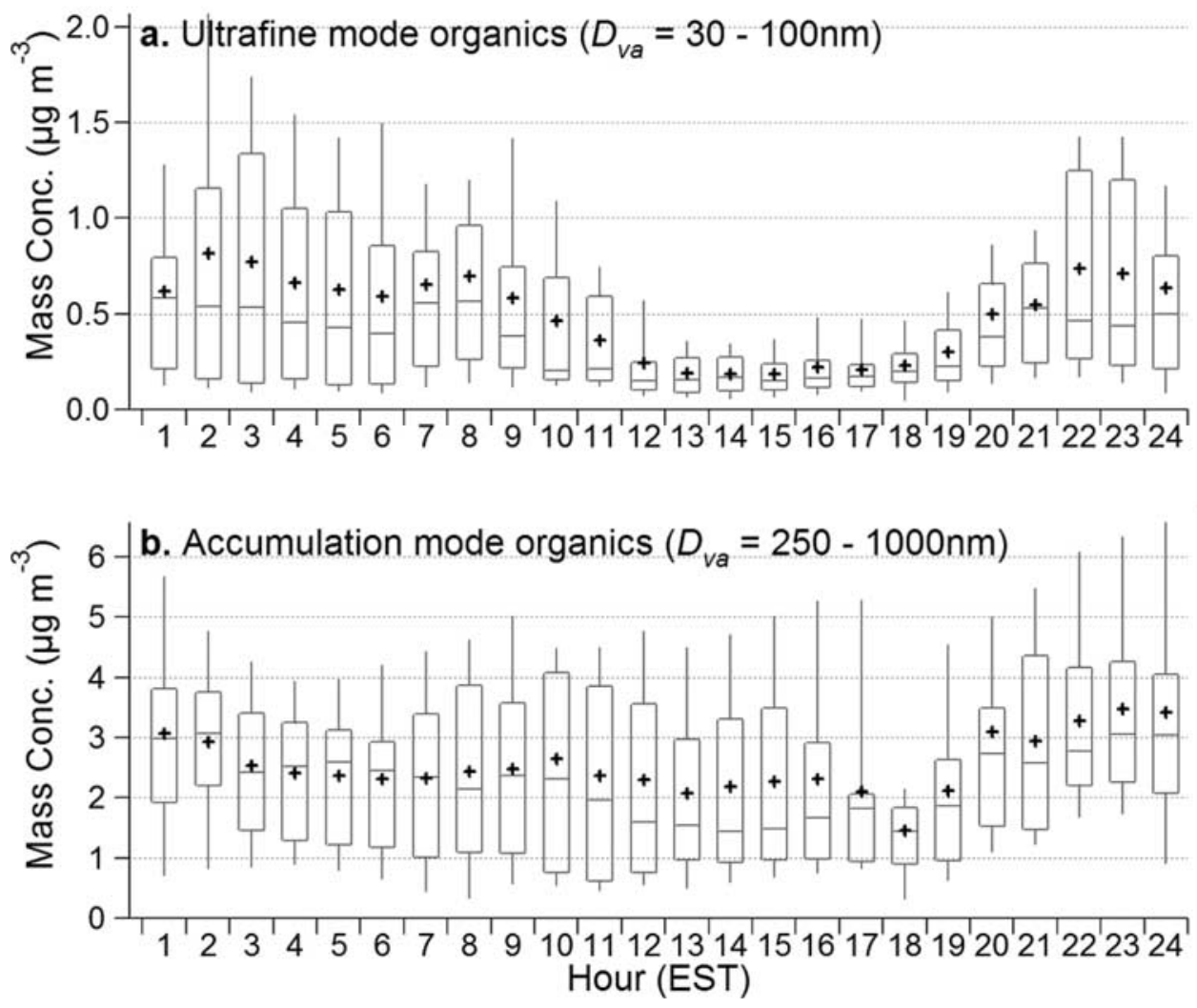

Figure 14. Average diurnal variations of (a) the ultrafine and (b) the accumulation mode organic particles during 7-22 September 2002 EST.

line likely represents the ratio of the source strengths of both aerosol types in the region.

\section{Conclusions}

[60] Nonrefractory submicron particles $\left(\mathrm{NR}-\mathrm{PM}_{1}\right)$ were measured in Pittsburgh from 7 to 22 September 2002 using an Aerodyne AMS. Reasonably good agreement was observed between the AMS and collocated instruments on total and species mass concentrations, and on size distributions, with smaller AMS concentrations likely due to the difference between $\mathrm{PM}_{1}$ and $\mathrm{PM}_{2.5}$. The comparisons of the size distributions obtained by the AMS and the SPMS, and the AMS composition data suggest that the average density of particles in Pittsburgh during this period was $\sim 1.5 \mathrm{~g} \mathrm{~cm}^{-3}$.

[61] Submicron particles in Pittsburgh during this time period were generally characterized by high contents of $\mathrm{SO}_{4}^{2-}$ and organics, and low levels of $\mathrm{NO}_{3}^{-}$and $\mathrm{Cl}^{-}$. The mass balance between $\mathrm{NH}_{4}^{+}$and the anions suggest that the majority of the particles are neutralized to slightly acidic. However, during relatively polluted periods significantly acidic particles (i.e., those estimated to contain approximately equal or larger amounts of $\mathrm{NH}_{4} \mathrm{HSO}_{4}$ than $\left.\left(\mathrm{NH}_{4}\right)_{2} \mathrm{SO}_{4}\right)$ are observed. Compared to neutralized particles, acidic particles generally contain significantly more sulfate, comparable amounts of organics and $\mathrm{NH}_{4}^{+}$, and less $\mathrm{NO}_{3}^{-}$and $\mathrm{Cl}^{-}$. We see no evidence of enhanced secondary organic aerosol formation due to acid-catalyzed organic chemistry.
[62] We observed a quickly changing aerosol population due to the combined influences from transport, local emissions, gas-to-particle conversion, and photochemistry. Through this entire study, the mass concentration of NR$\mathrm{PM}_{1}$ varies from below $1 \mu \mathrm{g} \mathrm{m}^{-3}$ to as high as $\sim 70 \mu \mathrm{g}$ $\mathrm{m}^{-3}$, with an average value of $\sim 15 \mu \mathrm{g} \mathrm{m}^{-3}$ and a

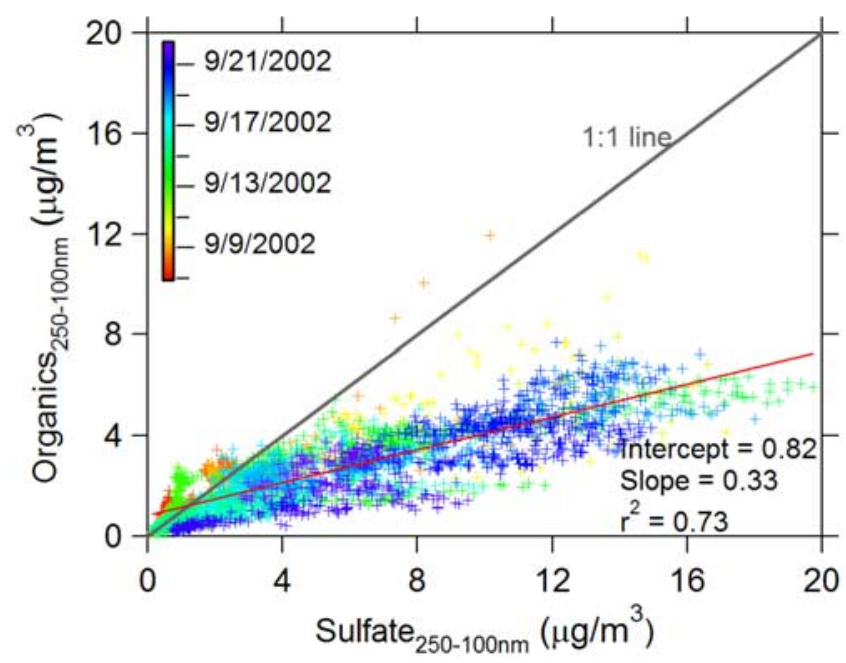

Figure 15. Correlation between organics and sulfate in the $D_{v a}=250-1500 \mathrm{~nm}$ particles. Data points are colored by the time. The red line is a linear fit to the data. 
standard deviation of $9 \mu \mathrm{g} \mathrm{m}^{-3}$. The mass concentrations and size distributions of particulate species vary on timescales between less than an hour to $\sim 2$ days. On the basis of the size distributions and diurnal profiles of the particle species, in addition to the mass spectra of the organic aerosols, we found that the NR-PM $\mathrm{P}_{1}$ particles in Pittsburgh are composed of 3 major modes, an ultrafine mode that is mainly composed of traffic organics, a relatively aged mode that likely grew by gaseous condensation, and an accumulation mode that is dominated by sulfate and ammonium.

\section{Appendix A: Removal of Gaseous Interference From the Size Distribution of Ammonium}

[63] The $\mathrm{NH}_{4}^{+}$size distribution was derived from the signal distribution of $\mathrm{m} / z 16\left(\mathrm{NH}_{2}^{+}\right)$in the P-TOF range corresponding to the sizes of the particles transmitted into the AMS. However, the $\mathrm{O}^{+}$(also $\mathrm{m} / z$ 16) signal from the ionization of gas phase $\mathrm{O}_{2}$ tails into the particle region, interfering with the measurement of $\mathrm{NH}_{4}^{+}$in small particles for $D_{v a}<100 \mathrm{~nm}$ (Figure A1). The tail of the $\mathrm{O}_{2}$ signal, which is attributed to a spread in the velocities of $\mathrm{O}_{2}$ molecules after the focusing lens, can be modeled using an exponential fit that has the same functional form as the one-dimensional Boltzmann velocity distribution function [Atkins, 1998; Morris, 2002],

$$
s=a \times \exp \left(-\frac{(v-b)^{2}}{c}\right)
$$

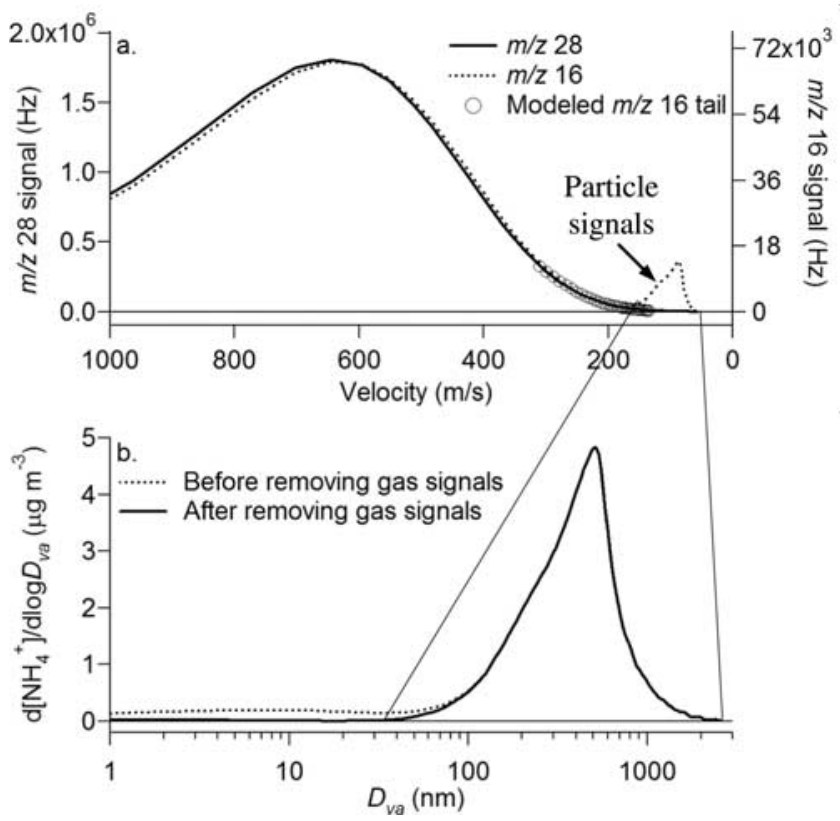

Figure A1. (a) Distributions of $m / z 16\left(\mathrm{NH}_{2}^{+}\right.$and $\left.\mathrm{O}^{+}\right)$and $28\left(\mathrm{~N}_{2}^{+}\right)$signals as a function of measured particle velocity; (b) mass distributions of ammonium as a function of $D_{v a}$ before and after the removal of gas phase $\mathrm{O}^{+}$signals.
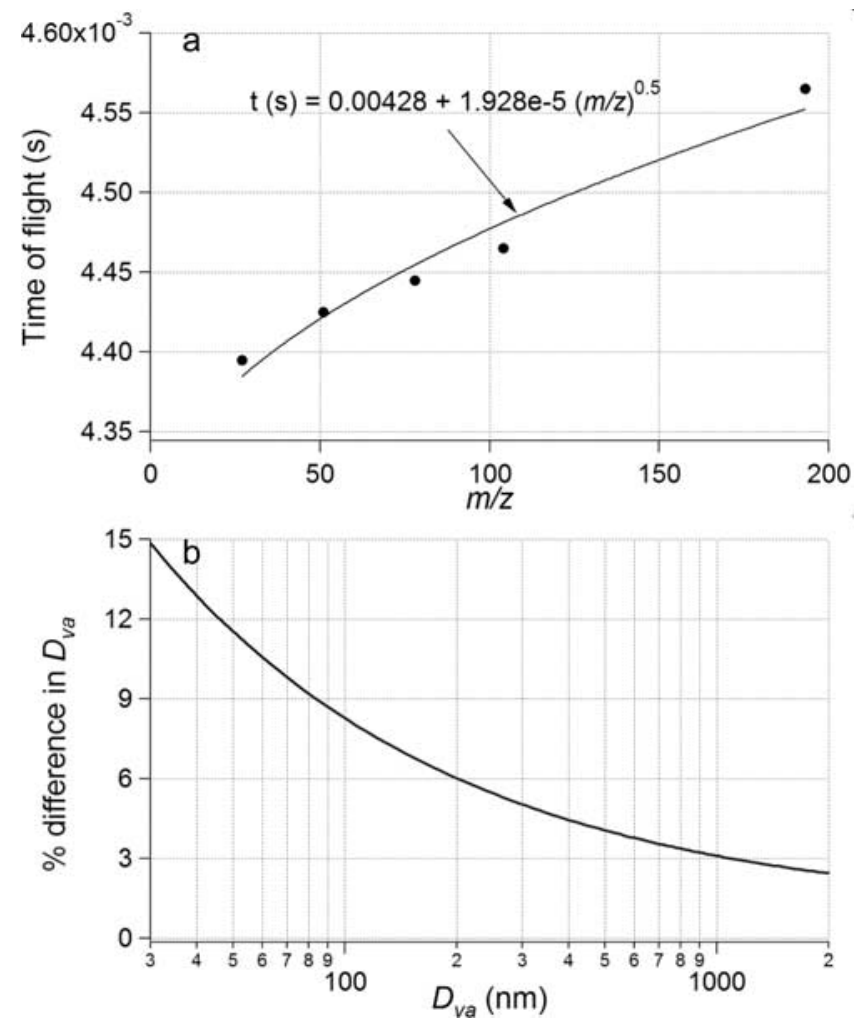

Figure B1. (a) P-TOF of ions $(m / z=27,51,78,104$ and 193; quadrupole extraction voltage $=180 \mathrm{~V}$ ) originated from monodisperse $500 \mathrm{~nm}$ PSL spheres; (b) differences in $D_{v a}$ due to $60 \mu$ s difference in P-TOF plotted as a function of particle size.

where $s$ is the ion rate $(\mathrm{Hz})$ detected for the signal, $a, b$, and $c$ are fit parameters, and $v$ is the velocity of the species measured in the P-TOF sizing chamber.

[64] Because the same function should apply to all gaseous species, $\mathrm{N}_{2}$ and $\mathrm{O}_{2}$ alike, we estimated the $a$, $b$, and $c$ values by fitting the signal distribution of $\mathrm{m} / \mathrm{z} 28$ $\left(\mathrm{N}_{2}^{+}\right)$to equation (A1), for P-TOFs corresponding to $1-$ $200 \mathrm{~nm}$ in $D_{v a}$ (Figure A1a). $\mathrm{m} / z 28$ was chosen because among all $\mathrm{m} / \mathrm{z}$ it has the highest signal-to-noise ratio and is the least affected by particle signals. Using the fitted $a, b$, and $c$ values, the gas phase signals of $\mathrm{m} / \mathrm{z} 16$ were then calculated and subtracted from the $1-200 \mathrm{~nm}$ region to give "filtered" $\mathrm{NH}_{4}^{+}$distributions (Figure A1a). All reported size distributions of $\mathrm{NH}_{4}^{+}$were corrected with this method. It needs to be noted that this correction is negligible for particles larger than $D_{v a}$ of $\sim 70 \mathrm{~nm}$ (Figure A1b), but is critical for a quantitative examination of the chemistry of new particles during the nucleation events [Zhang et al., 2004].

[65] The same technique is generally applicable to removal of gas signals from the size distributions of particle species, such as $m / z 28\left(\mathrm{CO}^{+}\right)$and $\mathrm{m} / \mathrm{z} 44$ $\left(\mathrm{CO}_{2}^{+}\right)$for organics and $18\left(\mathrm{H}_{2} \mathrm{O}^{+}\right)$for water. At ambient conditions, except for $\mathrm{m} / \mathrm{z} 28$, whose size distribution in the particle range is hugely influenced by gaseous $\mathrm{N}_{2}$, other $\mathrm{m} / \mathrm{z}$ generally chosen in P-TOF mode contain negligible amounts of gas signals compared to the particle signals because the aerodynamic lens and skimmers of 
the AMS reduce the concentration of gas phase species by a factor of $10^{7}$ relative to aerosol species [Allan et al., 2004b].

\section{Appendix B: Adjustment of the $\mathrm{NH}_{4}^{+}$Size Distributions for Faster Ion Flight in the Quadrupole}

[66] We observed a small (approximately $60 \mu \mathrm{s}$ ) shift in time of flight between the distributions of ammonium and sulfate throughout this campaign. The shift arises partly because the $\mathrm{NH}_{2}^{+}$ion $(\mathrm{m} / \mathrm{z} 16)$ travels slightly faster within the quadrupole than the $\mathrm{SO}^{+}$and $\mathrm{SO}_{2}^{+}$ions $(\mathrm{m} / \mathrm{z} 48$ and 64) do. This is expected since all singly charged ions have the same kinetic energy of about $14 \mathrm{eV}$ in the quadrupole (as used in AMS instruments), and thus the dwelling times of ions in the quadrupole are proportional to the square root of the mass to charge ratio of the ions. To adjust for this massdependent ion transition time in the quadrupole we measured the apparent P-TOF for various PSL ions originated from the same monodisperse particles. This experiment shows that the average difference between the P-TOFs of $\mathrm{SO}_{4}^{2-}$ ions and that of $\mathrm{NH}_{4}^{+}$was indeed $\sim 60 \mu$ s (Figure B1). Because no obvious shifts in P-TOF were observed between internally mixed $\mathrm{SO}_{4}^{2-}$ and $\mathrm{NO}_{3}^{-}$or organics we adjusted the size distributions of $\mathrm{NH}_{4}^{+}$by increasing its time of flight by $60 \mu \mathrm{s}$.

[67] As shown in Figure B1b, the relationship between $D_{v a}$ and P-TOF is nonlinear: a $60 \mu$ s difference in P-TOF translates into $\sim 10-15 \%$ difference in $D_{v a}$ in particles smaller than $70 \mathrm{~nm}$ but only $4-5 \%$ for the accumulation mode particles. As such, this is most important for the small size $\mathrm{NH}_{4}^{+}$, which is only significant during the new particle formation and growth events [Zhang et al., 2004].

[68] Acknowledgments. The authors thank Spyros Pandis (CMU) for organizing and directing the PAQS campaign and inviting us to participate in the study, Beth Wittig (CMU) for TEOM, continuous sulfate, gas phase and meteorological data, Charles Stanier (CMU) for particle number distribution data, R. Subramanian (CMU), Allen Robinson (CMU), Andrea Polidori (Rutgers University), and Barbara Turpin (Rutgers University) for OC data, Juan Cabada (CMU) for MOUDI data, Andrey Khlystov (CMU) for ammonium data and logistical assistance during this study, and James Allan (UMIST), Frank Drewnick (Max Planck Institute for Chemistry), and Alice Delia (CU) for AMS data analysis software. We also thank an anonymous reviewer for useful comments that helped us improve the manuscript. This research was conducted as part of the Pittsburgh Air Quality Study and was supported by the University of Colorado through J. L. Jimenez's Startup Fund.

\section{References}

Alfarra, M. R., et al. (2004), Characterization of urban and regional organic aerosols in the lower Fraser Valley using two Aerodyne aerosol mass spectrometers, Atmos. Environ., 38, 5745-5758.

Allan, J. D., et al. (2003a), Quantitative sampling using an Aerodyne aerosol mass spectrometer: 2. Measurements of fine particulate chemical composition in two UK cities, J. Geophys. Res., 108(D3), 4091, doi:10.1029/2002JD002359.

Allan, J. D., J. L. Jimenez, P. I. Williams, M. R. Alfarra, K. N. Bower, J. T. Jayne, H. Coe, and D. R. Worsnop (2003b), Quantitative sampling using an Aerodyne aerosol mass spectrometer: 1. Techniques of data interpretation and error analysis, J. Geophys. Res., 108(D3), 4090, doi:10.1029/ 2002JD002358

Allan, J. D., et al. (2004a), Submicron aerosol composition at Trinidad Head, California, during ITCT 2K2: Its relationship with gas phase volatile organic carbon and assessment of instrument performance, J. Geophys. Res., 109(D23), D23S24, doi:10.1029/2003JD004208.
Allan, J. D., et al. (2004b), A generalised method for the extraction of chemically resolved mass spectra from Aerodyne aerosol mass spectrometer data, J. Aerosol Sci., 35(7), 909-922 doi:10.1016/j.jaerosci. 2004.02.007.

Anderson, R. R., D. V. Martello, C. M. White, K. C. Crist, K. John, W. K. Modey, and D. J. Eatough (2004), The regional nature of $\mathrm{PM}_{2.5}$ episodes in the upper Ohio river valley, Air Waste Manage. Assoc., 54, 971-984.

Atkins, P. W. (1998), Physical Chemistry, Oxford Univ. Press, New York.

Bahreini, R., J. L. Jimenez, J. Wang, R. C. Flagan, J. H. Seinfeld, J. T. Jayne, and D. R. Worsnop (2003), Aircraft-based aerosol size and composition measurements during ACE-Asia using an Aerodyne aerosol mass spectrometer, J. Geophys. Res., 108(D23), 8645, doi:10.1029/ 2002JD003226

Bein, K. J., Y. Zhao, A. S. Wexler, and M. V. Johnston (2005), Speciation of size-resolved individual ultrafine particles in Pittsburgh, Pennsylvania, J. Geophys. Res., 110, D07S05, doi:10.1029/2004JD004708, in press.

Boudries, H., et al. (2004), Chemical and physical processes controlling the distribution of aerosols in the lower Fraser Valley, Canada, during the PACIFIC 2001 field campaign, Atmos. Environ., 38, 5759-5774.

Brown, S. S., H. Stark, and A. R. Ravishankara (2003a), Applicability of the steady state approximation to the interpretation of atmospheric observations of $\mathrm{NO}_{3}$ and $\mathrm{N}_{2} \mathrm{O}_{5}, J$. Geophys. Res., 108(D17), 4539, doi:10.1029/2003JD003407.

Brown, S. S., H. Stark, T. B. Ryerson, E. J. Williams, D. K. Nicks Jr., M. Trainer, F. C. Fehsenfeld, and A. R. Ravishankara (2003b), Nitrogen oxides in the nocturnal boundary layer: Simultaneous in situ measurements of $\mathrm{NO}_{3}, \mathrm{~N}_{2} \mathrm{O}_{5}, \mathrm{NO}_{2}, \mathrm{NO}$, and $\mathrm{O}_{3}, J$. Geophys. Res., 108(D9), 4299, doi:10.1029/2002JD002917.

Bytnerowicz, A., and M. E. Fenn (1996), Nitrogen deposition in California forests: A review, Environ. Pollut., 92(2), 127-146.

Cabada, J. C., S. N. Pandis, and A. L. Robinson (2002), Sources of atmospheric carbonaceous particulate matter in Pittsburgh, Pennsylvania, J. Air Waste Manage. Assoc., 52(6), 732-741.

Cabada, J. C., S. Rees, S. Takahama, A. Khlystov, S. N. Pandis, C. I. Davidson, and A. L. Robinson (2004), Mass size distributions and size resolved chemical composition of fine particulate matter at the Pittsburgh Supersite, Atmos. Environ., 38, 3127-3141.

Canagaratna, M. R., et al. (2004), Chase studies of particulate emissions from in-use New York city vehicles, Aerosol Sci. Technol., 38, 555-573, doi:10.1080/02786820490465504

DeCarlo, P., J. G. Slowik, D. R. Worsnop, P. Davidovits, and J. L. Jimenez (2004), Particle morphology and density characterization by combined mobility and aerodynamic diameter measurements. Part 1: Theory, Aerosol Sci. Technol., 38, 1185-1205, doi:10.1080/02786829093907.

Dockery, D. W., C. A. Pope, X. P. Xu, J. D. Spengler, J. H. Ware, M. E. Fay, B. G. Ferris, and F. E. Speizer (1993), An association between air pollution and mortality in 6 United States cities, N. Engl. J. Med., 329(24), 1753-1759.

Drewnick, F., J. J. Schwab, O. Hogrefe, S. Peters, L. Husain, D. Diamond, R. Weber, and K. L. Demerjian (2004a), Intercomparison and evaluation of four semi-continuous $\mathrm{PM}_{2.5}$ sulfate instruments, Atmos. Environ., 37(24), 3335-3350.

Drewnick, F., J. J. Schwab, J. T. Jayne, M. Canagaratna, D. R. Worsnop, and K. L. Demerjian (2004b), Measurement of ambient aerosol composition during the PMTACS-NY 2001 using an aerosol mass spectrometer. Part I: Mass concentrations, Aerosol Sci. Technol., 38, suppl. 1, 92-103.

Drewnick, F., J. J. Schwab, J. T. Jayne, M. Canagaratna, D. R. Worsnop, and K. L. Demerjian (2004c), Measurement of ambient aerosol composition during the PMTACS-NY 2001 using an aerosol mass spectrometer. Part II: Chemically speciated mass distributions, Aerosol Sci. Technol., 38, suppl. 1, 104-117.

Heberlein, J., et al. (2001), Thermal plasma deposition of nanophase hard coatings, Surface Coatings Technol., 142, 265-271.

Intergovernmental Panel on Climate Change (IPCC) (2001), Climate Change 2001: The Scientific Basis, Contribution of Working Group I to the Third Assessment Report of the Intergovernmental Panel on Climate Change, edited by J. T. Houghton et al., Cambridge Univ. Press, New York.

Jang, M. S., N. M. Czoschke, S. Lee, and R. M. Kamens (2002), Heterogeneous atmospheric aerosol production by acid-catalyzed particle-phase reactions, Science, 298(5594), 814-817.

Jayne, J. T., D. C. Leard, X. Zhang, P. Davidovits, K. A. Smith, C. E. Kolb, and D. R. Worsnop (2000), Development of an aerosol mass spectrometer for size and composition analysis of submicron particles, Aerosol Sci. Technol., 33, 49-70.

Jimenez, J. L., R. Bahreini, D. R. Cocker, H. Zhuang, V. Varutbangkul, R. C. Flagan, J. H. Seinfeld, C. O'Dowd, and T. Hoffmann (2003a), Correction to "New particle formation from photooxidation of diiodomethane $\left(\mathrm{CH}_{2} \mathrm{I}_{2}\right)$, ,J. Geophys. Res., 108(D23), 4733, doi:10.1029/ 2003JD004249. 
Jimenez, J. L., R. Bahreini, D. R. Cocker, H. Zhuang, V. Varutbangkul, R. C. Flagan, J. H. Seinfeld, C. O’Dowd, and T. Hoffmann (2003b), New particle formation from photooxidation of diiodomethane $\left(\mathrm{CH}_{2} \mathrm{I}_{2}\right)$, J. Geophys. Res., 108(D10), 4318, doi:10.1029/2002JD002452.

Jimenez, J. L., et al. (2003c), Ambient aerosol sampling with an aerosol mass spectrometer, J. Geophys. Res., 108(D7), 8425, doi:10.1029/ 2001JD001213.

Khlystov, A., G. P. Wyers, and J. Slanina (1995), The steam-jet aerosol collector, Atmos. Environ., 29(17), 2229-2234.

Khlystov, A. Y., B. Wittig, and C. Davidson (2001), Quality Assurance Project Plan for the Pittsburgh Air Quality Study, U.S. Environ. Prot. Agency, Research Triangle Park, N. C.

Lee, S., D. M. Murphy, D. S. Thomson, and A. M. Middlebrook (2003), Nitrate and oxidized organic ions in single particle mass spectra during the 1999 Atlanta Supersite Project, J. Geophys. Res., 108(D7), 8417, doi:10.1029/2001JD001455.

McLafferty, F. W., and F. Turecek (1993), Interpretation of Mass Spectra, Univ. Sci. Books, Mill Valley, Calif.

McMurry, P. H. (2000), A review of atmospheric aerosol measurements, Atmos. Environ., 34(12-14), 1959-1999.

Middlebrook, A., et al. (2003), An intercomparison of particle mass spectrometers during the 1999 Atlanta Supersite Project, J. Geophys. Res. 108(D7), 8424, doi:10.1029/2001JD000660.

Morris, J. W. (2002), Chemical kinetics and microphysics of atmospheric aerosols, Ph.D. thesis, Boston Coll., Boston, Mass.

Morris, J. W., P. Davidovits, J. T. Jayne, J. L. Jimenez, Q. Shi, C. E. Kolb, D. R. Worsnop, W. S. Barney, and G. Cass (2002), Kinetics of submicron oleic acid aerosols with ozone: A novel aerosol mass spectrometric technique, Geophys. Res. Lett., 29(9), 1357, doi:10.1029/2002GL014692.

Murphy, D. M., D. S. Thomson, and T. M. J. Mahoney (1998), In situ measurements of organics, meteoritic material, mercury, and other elements in aerosols at 5 to 19 kilometers, Science, 282(5394), 1664-1669.

Paerl, H. W., C. Aguilar, and M. L. Fogel (1997), Atmospheric nitrogen deposition in estuarine and coastal waters: Biogeochemical and water quality impacts, in Atmospheric Deposition of Contaminants to the Great Lakes and Coastal Waters, edited by J. E. Baker, pp. 415-429, SETAC Press, Pensacola, Fla.

Pandis, S. N., A. S. Wexler, and J. H. Seinfeld (1995), Dynamics of tropospheric aerosols, J. Physical Chemistry, 99(24), 9646-9659.

Park, K., D. B. Kittelson, M. R. Zachariah, and P. H. McMurry (2004), Measurement of inherent material density of nanoparticle agglomerates, J. Nanoparticle Res., 6(2), 267-272.

Pope, C. A., R. T. Burnett, M. J. Thun, E. E. Calle, D. Krewski, K. Ito, and G. D. Thurston (2002), Lung cancer, cardiopulmonary mortality, and long-term exposure to fine particulate air pollution, J. Am. Med. Assoc., 287(9), 1132-1141

Rees, S. L., A. L. Robinson, A. Khlystov, C. O. Stanier, and S. N. Pandis (2004), Mass balance closure and the Federal Reference Method for $\mathrm{PM}_{2.5}$ in Pittsburgh, Pennsylvania, Atmos. Environ., 28(20), 3305-3318.

Russell, L. M. (2003), Aerosol organic-mass-to-organic-carbon ratio measurements, Environ. Sci. Technol., 37(13), 2982-2987.

Schindler, D. W. (1988), Effects of acid-rain on fresh-water ecosystems, Science, 239(4836), 149-157.

Seinfeld, J. H., and S. N. Pandis (1998), Atmospheric Chemistry and Physics: From Air Pollution to Climate Change, 1326 pp., John Wiley, Hoboken, N. J.
Slowik, J. G., K. Stainken, P. Davidovits, L. R. Williams, J. T. Jayne, C. E. Kolb, D. R. Worsnop, Y. Rudich, P. DeCarlo, and J. L. Jimenez (2004), Particle morphology and density characterization by combined mobility and aerodynamic diameter measurements. Part 2: Application to combustion generated soot particles as a function of fuel equivalence ratio, Aerosol Sci. Technol., 38, 1206-1222, doi:10.1080/027868290903916.

Solomon, S. (1999), Stratospheric ozone depletion: A review of concepts and history, Rev. Geophys., 37(3), 275-316.

Stanier, C., A. Khlystov, and S. Pandis (2004), Nucleation events during the Pittsburgh Air Quality Study: Description and relation to key meteorological, gas phase, and aerosol parameters, Aerosol Sci. Technol., 38, suppl. 1, 253-264.

Stolzenburg, M., and S. V. Hering (2003), Method for the automated measurement of fine particle nitrate in the atmosphere, Environ. Sci. Technol., 34, 907-914.

Subramanian, R., A. Y. Khlystov, J. C. Cabada, and A. L. Robinson (2004), Positive and negative artifacts in particulate organic carbon measurements with denuded and undenuded sampler configurations, Aerosol Sci. Technol., 38, suppl. 1, 27-48.

Suess, D. T., and K. A. Prather (1999), Mass spectrometry of aerosols, Chem. Rev., 99(10), 3007-3035.

Tang, W., T. Raymond, B. Wittig, C. Davidson, S. Pandis, A. Robinson, and K. Crist (2004), Spatial variations of $\mathrm{PM}_{2.5}$ during the Pittsburgh Air Quality Study, Aerosol Sci. Technol., 38, suppl. 2, 80-90.

Tobias, H. J., P. M. Kooiman, K. S. Docherty, and P. J. Ziemann (2000), Real-time chemical analysis of organic aerosols using a thermal desorption particle beam mass spectrometer, Aerosol Sci. Technol., 33(1-2), 170-190

Turpin, B. J., and H. J. Lim (2001), Species contributions to $\mathrm{PM}_{2.5}$ mass concentrations: Revisiting common assumptions for estimating organic mass, Aerosol Sci. Technol., 35(1), 602-610.

Turpin, B. J., R. A. Cary, and J. J. Huntzicker (1990), An in-situ timeresolved analyzer for aerosol organic and elemental carbon, Aerosol Sci. Technol., 12, 161-171.

Watson, J. G. (2002), Visibility: Science and regulation, J. Air Waste Manage. Assoc., 52(6), 628-713.

West, J. J., A. S. Ansari, and S. N. Pandis (1999), Marginal PM 2.5 : Nonlinear aerosol mass response to sulfate reductions in the eastern United States, J. Air Waste Manage. Assoc., 49(12), 1415-1424.

Wittig, A. E., N. Anderson, A. Y. Khlystov, S. N. Pandis, C. Davidson, and A. L. Robinson (2004), Pittsburgh Air Quality Study overview and initial scientific findings, Atmos. Environ., 38, 3107-3125.

Zhang, Q., C. O. Stanier, M. R. Canagaratna, J. T. Jayne, D. R. Worsnop, S. N. Pandis, and J. L. Jimenez (2004), Insights into the chemistry of new particle formation and growth events in Pittsburgh based on aerosol mass spectrometry, Environ. Sci. Technol., 38(18), 4797-4809, doi:10.1021/ es $035417 \mathrm{u}$.

M. R. Canagaratna, J. T. Jayne, and D. R. Worsnop, Aerodyne Research Inc., Billerica, MA 01821-3976, USA.

J.-L. Jimenez and Q. Zhang, Cooperative Institute for Research in Environmental Sciences (CIRES), University of Colorado, Boulder, 216 UCB, Boulder, CO 80309-0216, USA. (jose.jimenez@colorado.edu; zhangq@cires.colorado.edu) 


\section{Insights into the Chemistry of New Particle Formation and Growth Events in Pittsburgh Based on Aerosol Mass Spectrometry}

\author{
Q I ZHANG, ${ }^{\dagger}$ CHARLES O. STANIER, \\ MANJULA R. CANAGARATNA, § \\ JOHN T. JAYNE, § \\ DOUGLAS R. WORSNOP, \\ SPYROS N. PANDIS, $¥$ AND \\ JOSE L. JIMENEZ*,t,\| \\ Cooperative Institute for Research in Environmental Sciences \\ (CIRES) and Department of Chemistry and Biochemistry, \\ University of Colorado, Boulder, Colorado 80309-0216, \\ Department of Chemical Engineering, Carnegie Mellon \\ University, Pittsburgh, Pennsylvania 15213-3890, and \\ Aerodyne Research Inc., Billerica, Massachusetts 01821-3976
}

New particle formation and growth events have been observed in several urban areas and are of concern due to their potential negative effects on human health. The main purpose of this study was to investigate the chemistry of ultrafine particles during the grow th phase of the frequently observed nucleation events in Pittsburgh ( 100 events per year) and therefore infer the mechanisms of new particle growth in the urban troposphere. An Aerodyne aerosol mass spectrometer (AMS) and two SMPS systems were deployed at the U.S. EPA Pittsburgh Supersite during September 2002. Significant nuc leation events were observed in 3 out of the 16 days of this deployment, including one of the 10 strongest nucleation events observed in Pittsburgh over a period of 15 months. These events appear to be representative of the climatology of new particle formation and growth in the Pittsburgh region. Distinctive growth of sulfate, ammonium, organics, and nitrate in the ultrafine mode (33-60 nm in a vacuum aerodynamic diameter or $\sim 18-$ $33 \mathrm{~nm}$ in physical diameter) was observed during each of these three events, with sulfate always being the first (and the fastest) species to increase. Ultrafine ammonium usually increased 10-40 min later than sulfate, causing the ultrafine mode particles to be more acidic during the initial stages of the nucleation events. Significant increase of ultrafine organics often happened after 11:00 a.m., when photochemistry is more intense. This observation coupled with a parallel increase of ultrafine $\mathrm{m} / \mathrm{z} 44$, a mass fragment generally representative of oxygenated organic compounds, indicates that secondary organic species contribute significantly to the growth of particles at a relatively later time of the event. Among all these four species, nitrate was always a minor component of the ultrafine

* Corresponding author telephone: (303)492-3557; fax: (303)4921149; e-mail: jose.jimenez@colorado.edu.

† CIRES, University of Colorado.

‡ Carnegie Mellon University.

$\S$ Aerodyne Research Inc.

"Department of Chemistry and Biochemistry, University of Colorado. particles and contributed the least to the new particle growth.

\section{Introduction}

New particle formation and growth events have been observed in many locations including forested $(2,3)$, coastal (4-6), rural/ remote (7-11), arctic $(12,13)$, and urban $(1,7$, 14-19) areas. These events are one of the major sources of ultrafine particles in both clean and polluted atmospheres and an important mechanism for sustaining the ambient aerosol population. Given the increased toxicity of ultrafine particles (20) and the role of ultrafine particles in particlerelated premature deaths and morbidity (21-24), the abundance of these particles after nucleation is considered as a potential human health hazard. In addition, the growth of nuclei from a detectable size of a few nanometers into particles that are optically active and efficient cloud condensation nuclei has important implications for visibility and climate $(4,25,26)$.

New particle formation events have been observed in Pittsburgh, PA-a polluted urban area in the Northeastern United States-during all seasons, most frequently in spring and fall (1). While these events are often associated with elevated $\mathrm{SO}_{2}$ and UV radiation, and thus presumably with high rates of $\mathrm{H}_{2} \mathrm{SO}_{4}(\mathrm{~g})$ production, classical binary $\mathrm{H}_{2} \mathrm{SO}_{4}-$ $\mathrm{H}_{2} \mathrm{O}$ nucleation theory considerably underestimates the frequency and intensity of the events (1). For this reason, additional species such as ammonia and/or organics have been suggested to participate in nucleation and nuclei growth (1). To some extent, this hypothesis is supported by recent model and field studies, which suggest that ternary nucleation $\left(\mathrm{H}_{2} \mathrm{SO}_{4}+\mathrm{NH}_{3}+\mathrm{H}_{2} \mathrm{O}\right)$ may be an efficient and prevalent new particle formation mechanism in the troposphere $(8,9,25$, $27,28)$ and that the growth of fresh nuclei $(1-3 \mathrm{~nm}$ in diameter) often requires the involvement of species other than $\mathrm{H}_{2} \mathrm{SO}_{4}$ and $\mathrm{H}_{2} \mathrm{O}(25,29,30)$. Nevertheless, to identify the compounds that are responsible for new particle formation and growth, it is necessary to study the chemistry and dynamics of ultrafine particles in the atmosphere with direct measurements.

The evolution of the particle number concentration and size distribution during nucleation has been characterized in a number of field studies, often with techniques such as scanning mobility particle sizers (SMPS; also known as differential mobility particle sizers or DMPS) $(1,31,32)$ and ultrafine condensation particle counters (UCPC) $(4,11,31$, 33). However, none of these techniques determine particle composition. Recently a thermal desorption chemical ionization mass spectrometer (TDCIMS) that is able to quantify the composition of nucleation mode particles has been described $(34,35)$. This instrument has been employed to study atmospheric nucleation in Atlanta, GA, where it was found that newly formed aerosol in the 8-15 nm diameter range were composed of sulfate that is neutralized to varying degrees by ammonium (36).

Besides TDCIMS, the aerosol mass spectrometer (AMS) developed by Aerodyne Research Inc. $(37,38)$ is also able to determine the composition of ultrafine particles. Despite its comparatively larger minimum-size cutoff ( 30-35 nm in a vacuum aerodynamic diameter or $\sim 20 \mathrm{~nm}$ in physical diameter, see section 2.3.1), the AMS is a powerful tool for studying new particle growth events in the atmosphere because of it fast time response, high sensitivity, and simultaneous measurement of the particle size distribution 
TABLE 1. Summary of Conditions during Nucleation Events and in Base Case Situations during September $7-22,2002^{\text {a }}$

\begin{tabular}{|c|c|c|c|c|c|c|c|c|c|c|c|c|c|c|c|c|}
\hline $\begin{array}{c}\text { nucleation } \\
\text { starting }^{\text {time }}{ }^{b}\end{array}$ & $\begin{array}{l}\text { event } \\
\text { intensity }\end{array}$ & $\begin{array}{c}N_{\text {tot }^{c}} \\
\left(\mathrm{~cm}^{-3}\right)\end{array}$ & $\begin{array}{c}\mathrm{dN} N_{\text {tot }} / \mathrm{dt}^{d} \\
\left(\mathrm{~cm}^{-3} \mathrm{~h}^{-1}\right)\end{array}$ & $\begin{array}{c}N_{10^{c}} \\
\left(\mathrm{~cm}^{-3}\right)\end{array}$ & $\begin{array}{c}\mathrm{d} N_{10} / \mathrm{d} t^{d} \\
\left(\mathrm{~cm}^{-3} \mathrm{~h}^{-1}\right)\end{array}$ & $\begin{array}{c}N_{30-78^{c}} \\
\left(\mathrm{~cm}^{3} \mathrm{~s}^{-1}\right)\end{array}$ & $\begin{array}{c}M^{\mathrm{e}} \\
\left(\mu \mathrm{g} \mathrm{m}^{-3}\right)\end{array}$ & $\begin{array}{c}M_{33-60^{f}} \\
\left(\mu g \mathrm{~m}^{-3}\right)\end{array}$ & $\begin{array}{l}\mathrm{RH} \\
(\%)\end{array}$ & $\begin{array}{c}T \\
\left({ }^{\top} \mathrm{C}\right)\end{array}$ & $\begin{array}{c}P \\
\text { (Torr) }\end{array}$ & $\begin{array}{l}\mathrm{SO}_{2} \\
(\mathrm{ppb})\end{array}$ & $\begin{array}{c}\mathrm{NO} \\
(\mathrm{ppb})\end{array}$ & $\underset{(\mathrm{ppm})}{\mathrm{CO}}$ & $\underset{\left(W m^{-2}\right)}{U V}$ & $\underset{\left(\mathrm{cm}^{-2}\right)}{\mathrm{CS}}$ \\
\hline \multicolumn{17}{|c|}{ Nucleation Periods } \\
\hline $8 / 02,10: 30$ & Strong & $1.2 \mathrm{E}+05^{9}$ & $2.4 \mathrm{E}+05$ & $2.8 \mathrm{E}+04$ & $1.2 \mathrm{E}+05$ & & & & 35 & 28 & 740 & 62 & 3.4 & 0.60 & 28.0 & \\
\hline & Moderate & $4.1 \mathrm{E}+049$ & $1.2 \mathrm{E}+04$ & $1.6 \mathrm{E}+04$ & & & 11 & & 36 & 27 & & 6.1 & 11.5 & & & \\
\hline $9 / 12 / 02,8: 15$ & Strong & $1.0 \mathrm{E}+05$ & $2.1 \mathrm{E}+05$ & $6.9 \mathrm{E}+04$ & $1.9 \mathrm{E}+05$ & $5.5 \mathrm{E}$ & 6. & 0.3 & 53 & 17 & 737 & 33 & 11 & 0.51 & 17.0 & $7.3 \mathrm{E}-$ \\
\hline \multicolumn{17}{|c|}{ Statistics for Nucleation Periods } \\
\hline average & & $8.9 E+04$ & $1.5 \mathrm{E}+05$ & $3.8 \mathrm{E}+04$ & & & 12.6 & 0. & 41 & 24 & 738 & 33 & 8.8 & 0.63 & 22.8 & 7.0 \\
\hline $1 \sigma$ & & $4.3 \mathrm{E}$ & $1.2 \mathrm{E}+$ & $2.8 \mathrm{E}$ & & & 7.1. & & 10 & 6 & 2 & 28 & 4.7 & 0. & 5 . & \\
\hline medi & & & & & & & & & 36 & 27 & 738 & & & & & \\
\hline nin & & $4.1 \mathrm{E}$ & $1.2 \mathrm{E}+$ & $1.6 \mathrm{E}+$ & & & 6. & 0. & 35 & 17 & 737 & 6.1 & 3.4 & 0. & 17 & 3.8 \\
\hline $\max$ & & $1.2 \mathrm{E}+05$ & $2.4 \mathrm{E}+05$ & $6.9 \mathrm{E}+04$ & $1.9 \mathrm{E}+05$ & $2.3 \mathrm{E}+04$ & 20.2 & 0.70 & 53 & 28 & 740 & 62 & 12 & 0.76 & 28.0 & $1.0 \mathrm{E}-0$ \\
\hline \multicolumn{17}{|c|}{ Statistics for Base Case Situationa } \\
\hline avera & & $2.5 \mathrm{E}$ & & & & & & & 69 & 22 & 735 & & & 0. & & \\
\hline & & $1.1 \mathrm{E}+$ & & $9.4 \mathrm{E}+03$ & & & 10 & & 16 & 4 & 2 & 5 & 1 & 0. & 8. & 5.9 \\
\hline ledian & & $2.3 \mathrm{E}+$ & & $6.9 \mathrm{E}+$ & & & & & 68 & 22 & 735 & & & 0. & & $7.9 \mathrm{E}-$ \\
\hline hin & & $6.9 E+03$ & & & & & 0.7 & & 29 & 15 & 730 & 0. & & 0. & & $1.2 \mathrm{E}-03$ \\
\hline $\max$ & & $9.1 \mathrm{E}+04$ & & $7.4 \mathrm{E}+04$ & & $1.8 \mathrm{E}+04$ & 60.9 & 1.26 & 96 & 31 & 738 & 36 & 133 & 1.66 & 30.1 & $3.1 \mathrm{E}-02$ \\
\hline
\end{tabular}

a The reported variables are the average of the 2-h period from the beginning of a given nucleation event (see footnote $b$ ). The base case situations are the average between 8:15 a.m. and 13:00 p.m. during nonnucleation days. All times are in Eatern Standard Time. ${ }^{b}$ Defined as the time when $N_{10}$ shows detectable increase. ${ }^{c}$ Particle number counts measured by the SMPS with mobility diameters indicated in the subscript. Specifically, $N_{\text {tot: }}$ total number of particles in 3-680 nm unless flagged (see footnote g); $N_{10}: D_{\mathrm{m}}=3-10 \mathrm{~nm} ; N_{30-78}: D_{\mathrm{m}}=30-78 \mathrm{~nm}$. ${ }^{d}$ Average increase rates (calculated by a linear fit) of particle number or total mass during the time period from the starting of the nucleation to when $N_{10}$ peaks (i.e., 10:45-11:30 a.m. on 9/8/2002; 9:45-11:15 a.m. on 9/9/2002; and 8:15-9:00 a.m. on 9/12/2002). ${ }^{\text {e }}$ Total fine particulate mass measured by the AMS; $[\mathrm{M}]=\left[\mathrm{SO}_{4}{ }^{2-}\right]+\left[\mathrm{NO}_{3}{ }^{-}\right]+\left[\mathrm{NH}_{4}{ }^{+}\right]+$[organics]. ${ }^{f}$ Total mass of ultrafine particles (i.e., $D_{\mathrm{va}}=33-60 \mathrm{~nm}$ ). ${ }^{g}$ No particle number data available above $78 \mathrm{~nm}$; therefore, $N_{\text {tot }}$ is the sum of particle number in $3-78 \mathrm{~nm}$.

for 10-20 ion mass to chargeratios (i.e., m/ z values). Indeed, the AMS has been successfully applied in a recent environmental ("smog") chamber study on new particle formation and growth $(6,39)$.

In September 2002, an Aerodyne AMS, together with two SMPS systems, was operated at the Pittsburgh U.S. EPA Supersite as part of the Pittsburgh Air Quality Study (PAQS) (40). One of the main purposes of this deployment was to characterize the chemistry of particles during the growth phase of the frequently observed nucleation events in Pittsburgh. We report here (i) the temporal variations of particlesize, number, and composition during the nucleation events; (ii) the dynamics of chemical species in the ultrafine particles; and (iii) the possible mechanisms responsible for the growth of ultrafine particles.

\section{Experimental Methods}

2.1. Sampling Timeand Location. All measurements in this study were conducted on the main PAQS samplingsite $\left(40^{\circ} 27^{\prime}\right.$ $\mathrm{N}, 79^{\circ} 57^{\prime} \mathrm{W}$ ) at Schenley Park right next to the campus of Carnegie Mellon University, which is about $6 \mathrm{~km}$ east of downtown Pittsburgh (40). The AMS operated continuously for 16 days from September 7 to September 22, 2002. The Supersite, including the SMPS systems and gas-phase and meteorology measurements, was operated far longer, almost continuously from J uly 2001 to October 2002 (40). The study described here only encompasses the AMSoperation period. All reported dates and times are Eastern Standard Time(EST).

\subsection{Instrumentation and Measurements.}

2.2.1. Measurements of Particle Composition. Mass concentrations and size distributions of submicron nonrefractory sulfate, nitrate, ammonium, and organics were measured with the AMS. Detailed descriptions of the Aerodyne AMS can be found elsewhere (37, 38, 41). Specific information on the AMS operation and data analysis during this study is presented in another paper (42), and only the main points aregiven here. Two new techniques were applied to improve the determination of the $\mathrm{NH}_{4}{ }^{+}$size distribution for ultrafine particles. These involved (a) removing the interference of gaseous $\mathrm{O}^{+}(\mathrm{m} / \mathrm{z} 16)$ signal on the size distributions of the $\mathrm{NH}_{2}{ }^{+}$fragment of ammonium below 100 $\mathrm{nm}(42)$ and (b) correcting the $\mathrm{NH}_{4}{ }^{+}$sizedistributions for the faster ion flight from ionizer to detector by $\sim 60 \mu \mathrm{s}$ as compared to sulfate (42). Details of the new techniques and of the standard technique for determining $\mathrm{NH}_{4}{ }^{+}$mass concentrations from AMS data are presented in two other publications $(42,43)$.

The averaging time for the AMS measurements was 10 min initially and 5 min after 9:40 a.m., September 12, 2002. TheAMShas nearly $100 \%$ transmission efficiency for particles in the size range of $60-600 \mathrm{~nm}$ vacuum aerodynamic diameters ( $D_{v a}$ ) and partial transmission up to $1500 \mathrm{~nm}$ (37). On the basis of our size calibrations, we estimated that the lower transmission limit of the AMS was $\sim 33 \mathrm{~nm}\left(D_{\mathrm{va}}\right)$ with an uncertainty of up to $7 \mathrm{~nm}$ (42). No attempt was made to correct the measured size distributions for partial transmission of larger and smaller particles. While this may lead to an underestimation of the growth rate of ultrafine species, it does not affect our ability of identifying the species that are responsible for the growth.

2.2.2. Measurements of Particle Number and Size Distributions. Two scanning mobility particlesizers(SMPS), a TSI 3936N 25 nano-SMPS (3-83 nm) and a TSI 3936L10 SMPS (10-680 nm), were operated to measure particle number concentration as a function of mobility diameter $\left(D_{m}\right)(1)$. The SMPS systems sampled alternatively between dry $(<30 \%)$ and humid (at ambient relatively humidity) modes but only the dry-mode data are reported here. Each SMPS size distribution used here is the average of two upscans ( 5 min each) onto a 15-min time interval. A single size distribution from 3 to $680 \mathrm{~nm} D_{m}$ was formed by merging data from these two SMPS systems and using data from the nano-SMPS up to $30 \mathrm{~nm}$. No multipliers are used to enforce continuity of the size distribution.

2.2.3. Measurement of Gaseous Pollutants and Meteorology. Data on concentrations of major gaseous pollutants $\left(\mathrm{SO}_{2}, \mathrm{O}_{3}, \mathrm{CO}, \mathrm{NO}\right.$, and $\left.\mathrm{NO}_{x}\right)$, UV radiation, and other meteorological parameters were collected at a timeresolution of 10 min during this study. Detailed information on these measurements is available elsewhere (40). 

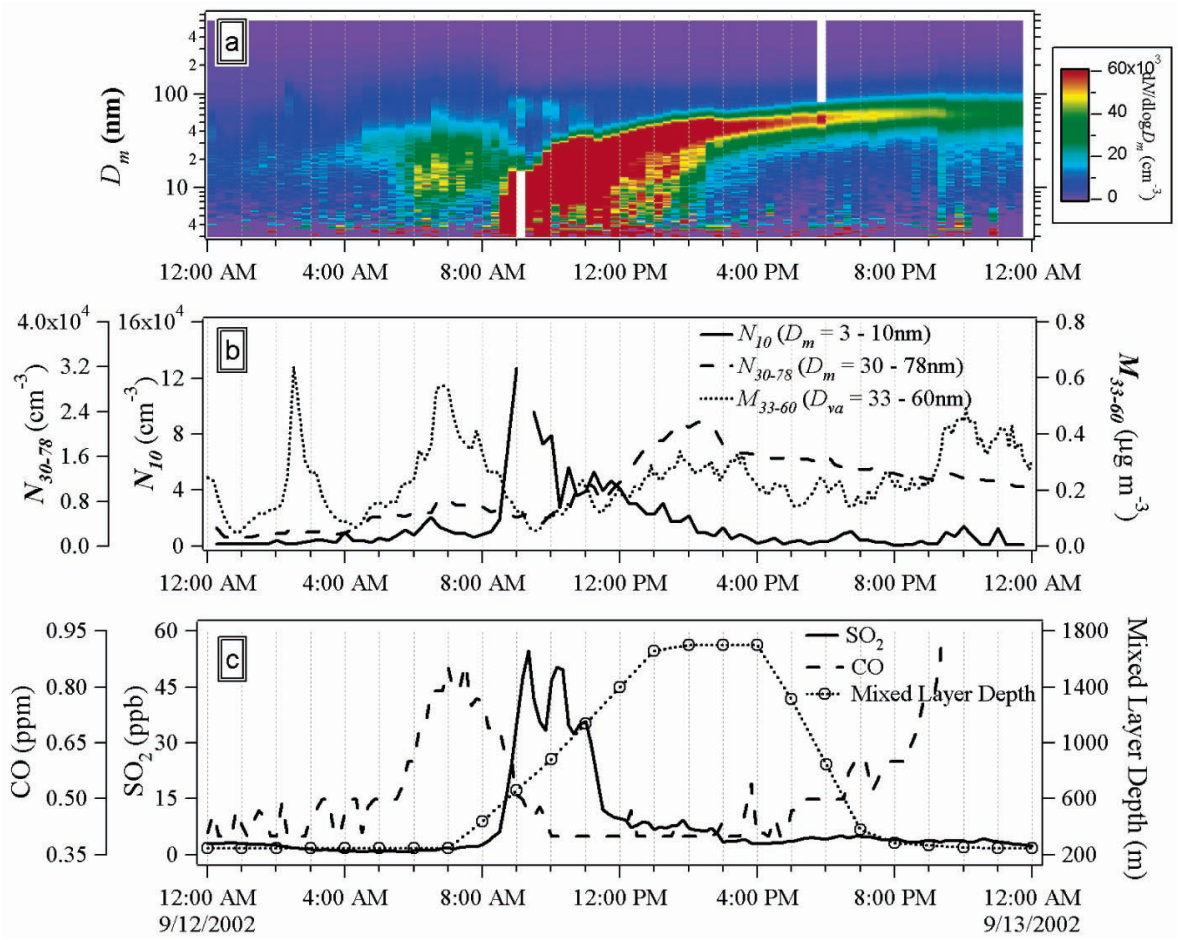

FIGURE 1. Change of (a) particle size distribution; (b) number concentration and total mass loading; and (c) $\mathrm{SO}_{2}, \mathrm{CO}_{\text {, and mixing layer }}$ depth as a function of time on September 12, 2002, a day with a well-defined and intense nucleation event. Mixing layer depth was calculated with the HYSPLIT4 trajectory model using EDAS wind field data.

\subsection{Data Analysis.}

2.3.1. Terminology. Mobility diameter $\left(D_{m}\right)$ and vacuum aerodynamic diameter $\left(D_{v a}\right)$ are used in parallel throughout the paper with $D_{m}$ denoting SMPS-measured sizes and $D_{v a}$ AMS sizes. Note that $D_{\text {va }}$ (the aerodynamic diameter of particles measured in thefree-molecular regime) differsfrom the classical aerodynamic diameter $\left(D_{\text {ca; }}\right.$ or aerodynamic diameter of particles in the continuum regime) in that (i) $D_{\text {va }}$ is proportional to particle material density instead of the squareroot of the density and (ii) $D_{\text {va }}$ and $D_{\text {ca }}$ relate differently to the dynamic shape factor $(\chi)(39,44)$. $\mathrm{D}_{\mathrm{m}}$ is also dependent on $\chi$ but not on particle material density. As a result, the ratio of $D_{v a}$ to $D_{m}$ is a function of size, composition, shape, and relative humidity for ambient particles. However, the $D_{v a}$ to $D_{m}$ ratio simplifies to particle density for spherical particles, for which $\chi=1$. The average density for the bulk Pittsburgh particles (usually dominated by theaccumulation mode) was estimated to be roughly 1.5 during this study, based on the average particlecomposition ( $\sim 70 \%$ ammonium, sulfate, and nitrate and $\sim 30 \%$ organics) in Pittsburgh (42).

Because the new particles formed during the nucleation events in Pittsburgh appear to be mainly composed of sulfuric acid neutralized to a varying degree by ammonium and likely containing some water (due to the high hygroscopicity of $\mathrm{H}_{2} \mathrm{SO}_{4}$ ) by the time they are first detected by the AMS, we assume that those particles are spheres with a density of $\sim 1.8 \mathrm{~g} / \mathrm{cm}^{3}$. In this way, weestimate that thesmallest particles (i.e., $\mathrm{D}_{\text {va }}=33 \mathrm{~nm}$ ) detectable by the AMS in these events have a physical diameter of about $18 \mathrm{~nm}$ and contain $\sim 200$ times more mass than a $3-\mathrm{nm}$ nucleus. Because of this limitation, we can directly address the question of which species contribute to the growth of new particles but not of which species formed the initial $1-3 \mathrm{~nm}$ nuclei. From this point of view, the AMS is complementary to the TDCIMS instrument for the study of new particleformation and growth $(34,35)$, since the later instrument can analyze smaller particles but of only one size and composition at a time.

In this paper, the modes of the observed particle size distribution are named according to the following conven- tion: "nucleation mode" $\left(D_{m}=3-10 \mathrm{~nm}\right)$, "ultrafine" or "Aitken mode" $\left(\mathrm{D}_{\mathrm{m}}=10-100 \mathrm{~nm}\right)$, and "accumulation mode" $\left(D_{m}=100-1000 \mathrm{~nm}\right)$ (33). Number concentrations of particles in a given size range (integrated from $\mathrm{dN} / \mathrm{dlog} \mathrm{D}_{m}$ ) were denoted as $N$ with their $D_{m}$ range as subscripts. Specifically, $\mathrm{N}_{10}$ stands for the number concentration of nucleation mode particles from $3 \mathrm{~nm}$ (the lower detection limit of the SMPS system) to $10 \mathrm{~nm} ; \mathrm{N}_{30-78}$ stands for the "growth mode" from 30 to $78 \mathrm{~nm}$; and $\mathrm{N}_{\text {tot }}$ stands for the total concentration of 3-680 $\mathrm{nm}$. These size ranges areslightly different from those listed above to maximize comparability of SMPS and AMS data and also due to operational considerations for the SMPS.

By the same convention, $\mathrm{SO}_{4}{ }^{2-}{ }_{33-60}, \mathrm{NH}_{4}{ }^{+} 33-60, \mathrm{NO}_{3}{ }^{-}{ }_{33-60}$, and $\mathrm{Org}_{33-60}$ stand for the mass concentrations of $\mathrm{SO}_{4}{ }^{2-}, \mathrm{NH}_{4}{ }^{+}$, $\mathrm{NO}_{3}{ }^{-}$, and organics, respectively, in particles with $\mathrm{D}_{\mathrm{va}}$ of 33 $\mathrm{nm}$ (thelower detection limit of the AMS) to $60 \mathrm{~nm}$. Thetotal mass of particles (referred to as $M$ ) is defined as the sum of these four species (e.g., $\mathrm{M}_{33-60}=\mathrm{SO}_{4}{ }^{2-}{ }_{33-60}+\mathrm{NH}_{4}{ }^{+} 33-60+$ $\mathrm{NO}_{3}{ }_{33-60}+\mathrm{Org}_{33-60}$. In addition, $\mathrm{m} / \mathrm{z} 43_{33-60}, \mathrm{~m} / \mathrm{z} 44_{33-60}$, $\mathrm{m} / \mathrm{z} 55_{33-60}$, and $\mathrm{m} / \mathrm{z} 57_{33-60}$ stand for the nitrate-equivalent mass concentrations of the organic fragments $\mathrm{m} / \mathrm{z} 43,44$, 55 , and 57, respectively, in 33-60 $\mathrm{nm}\left(\mathrm{D}_{\mathrm{va}}\right)$ particles. The nitrate-equivalent mass concentration of a given organic fragment equals the mass concentration of nitratethat would producean ion signal of the same intensity (by summing the $\mathrm{m} / \mathrm{z} 30$ and 46 signals from nitrate) (38). The nitrateequivalent mass concentration can be converted into the actual mass concentration by taking into account the ionization efficiency of the species relative to that of nitrate, its fragmentation pattern, and the collection efficiency of the particles $(38,42)$. Detailed information on AMS quantification is described in a separate paper (42).

2.3.2. Characterizing Nucleation Events. Nucleation events were identified based on the evolution of the size distributions and particle number concentrations. Primary emissions of nucleation mode particles (e.g., from traffic) were screened out if the increase of $\mathrm{N}_{10}$ was comparatively low and correlated with increases of CO and NO (1), and the sizedistributions were broader than thosetypically observed 

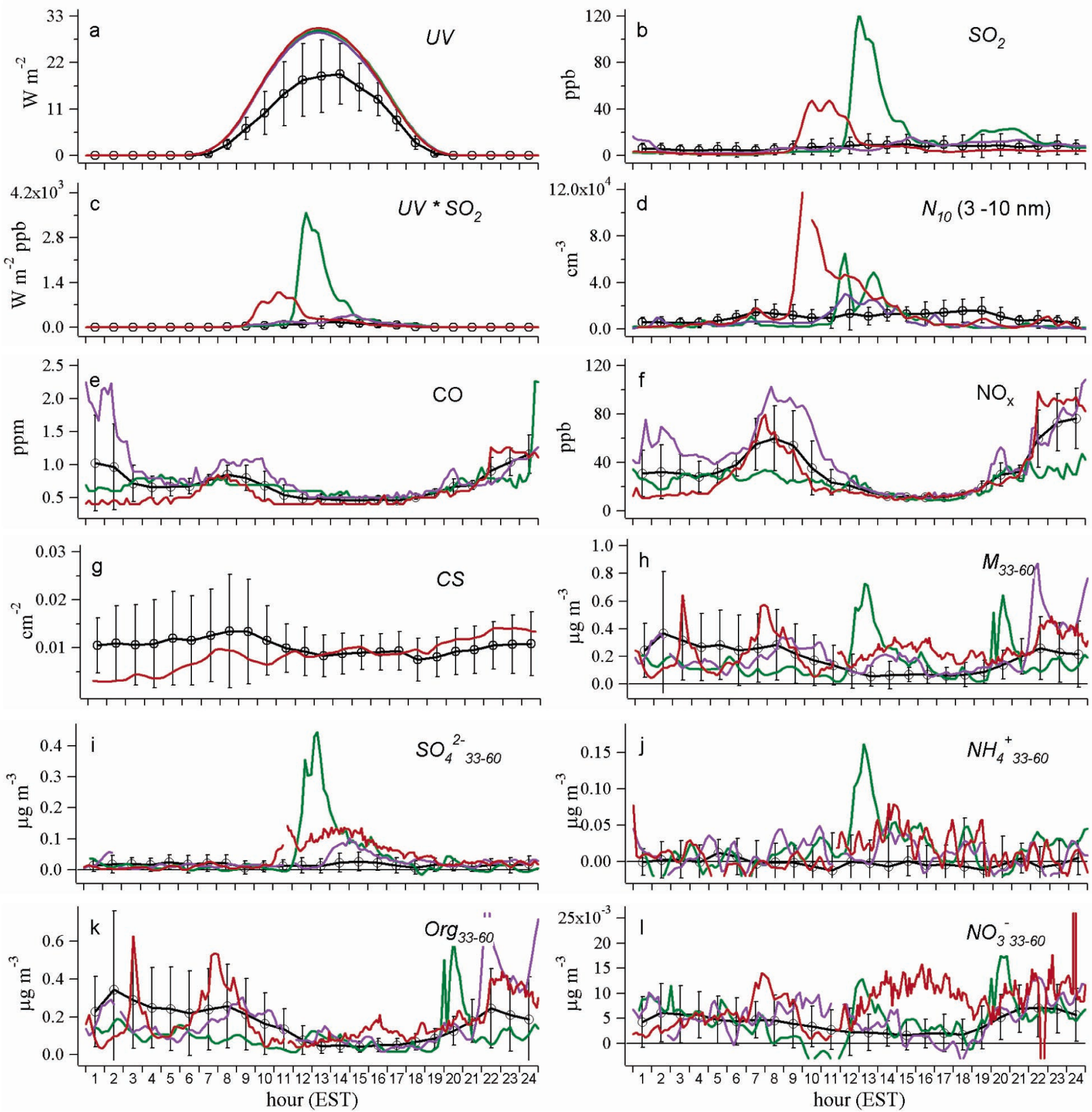

FIGURE 2. Average diurnal patterns of various parameters on days $w$ ith and without nucleation events. Symbols represent average values; errors bars are one standard deviation.

during nucleation events. Onceidentified, nucleation events were further categorized into "weak" $\left(\mathrm{dN}_{10} / \mathrm{dt}<4000 \mathrm{~cm}^{-3}\right.$ $\left.\mathrm{h}^{-1}\right)$, "moderate" $\left(15000>\mathrm{dN}_{10} / \mathrm{dt}>4000 \mathrm{~cm}^{-3} \mathrm{~s}^{-1}\right)$, or "strong" ( $\left.\mathrm{dN}_{10} / \mathrm{dt}>15000 \mathrm{~cm}^{-3} \mathrm{~h}^{-1}\right)$ following the classification of Stanier et al. (1).

2.3.3. Condensational Sink and Coagulation Time. The aerosol condensational sink (CS, in units of $\mathrm{cm}^{-2}$ ) is a measure of the available surface area for condensation. A larger CS is less favorable for nucleation. For this study CS was integrated from $D_{m}$ of $3-680 \mathrm{~nm}$, the size range of the SMPS measurement, using the formulation of Pirjola et al. (45). Because of this incomplete integration and because our $D_{m}$ values were for dry particles (see section 2.2.2), we likely underestimate the actual CS, especially for aged air masses that contain more larger particles and for periods that were relatively humid.
As an attempt to qualitatively evaluate the growth history of the ultrafine particles (i.e., $D_{\text {va }}>33 \mathrm{~nm}$; the AMS measurablesizerange), wecompared the characteristic time of coagulation to that of condensation for the nucleation mode particles. The characteristic time of coagulation was computed based on the theory of Brownian coagulation of polydisperse aerosols (46) using the Aerosol Calculator Program developed by Baron and Willeke (47).

2.3.4. Calculation of Mixed Layer Depth. Hourly values of mixed layer depths reported in this study were calculated using the Hybrid Single Particle Lagrangian Integrated Trajectory (HYSPLIT4) model and the EDAS meteorological datasetavailableat http:// www.arl.noaa.gov/ ready/hysplit4. html. Note that the mixing layer depth calculated by this method can have significant uncertainties and is treated qualitatively in this study. 

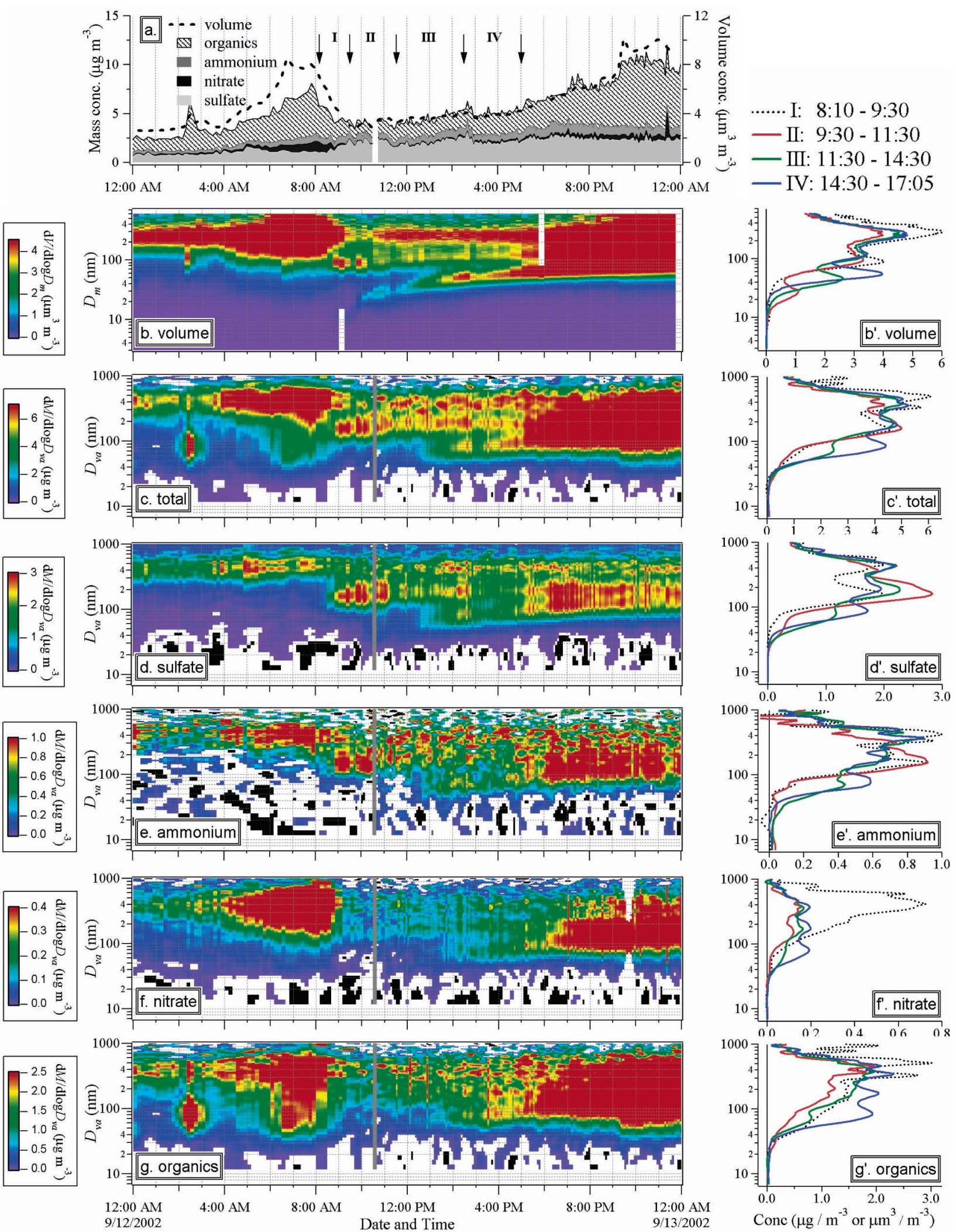

FIGURE 3. Time series of the mass concentrations of particle species and apparent volume (a) and evolutions of the size distributions for apparent volume (b), total mass concentration (c), and mass concentrations of chemical species (d-g) on September 12, 2002. The four stages (I-IV) of the nucleation and grow th are marked on plot a. To the right of the corresponding image plot are the average size distributions of given parameter during these four stages $\left(b^{\prime}-g^{\prime}\right)$. Particle apparent volume w as calculated using SM PS number distribution data assuming spherical particles. Missing data (w hite areas in plota and gray areas in plots $b-g$ ) are due to either occasional instrumental malfunction or maintenance/calibration. White areas in plots $b-g$ are due to the omission of data points that are below the detection limit $(1 \sigma)$ of the AMS. 

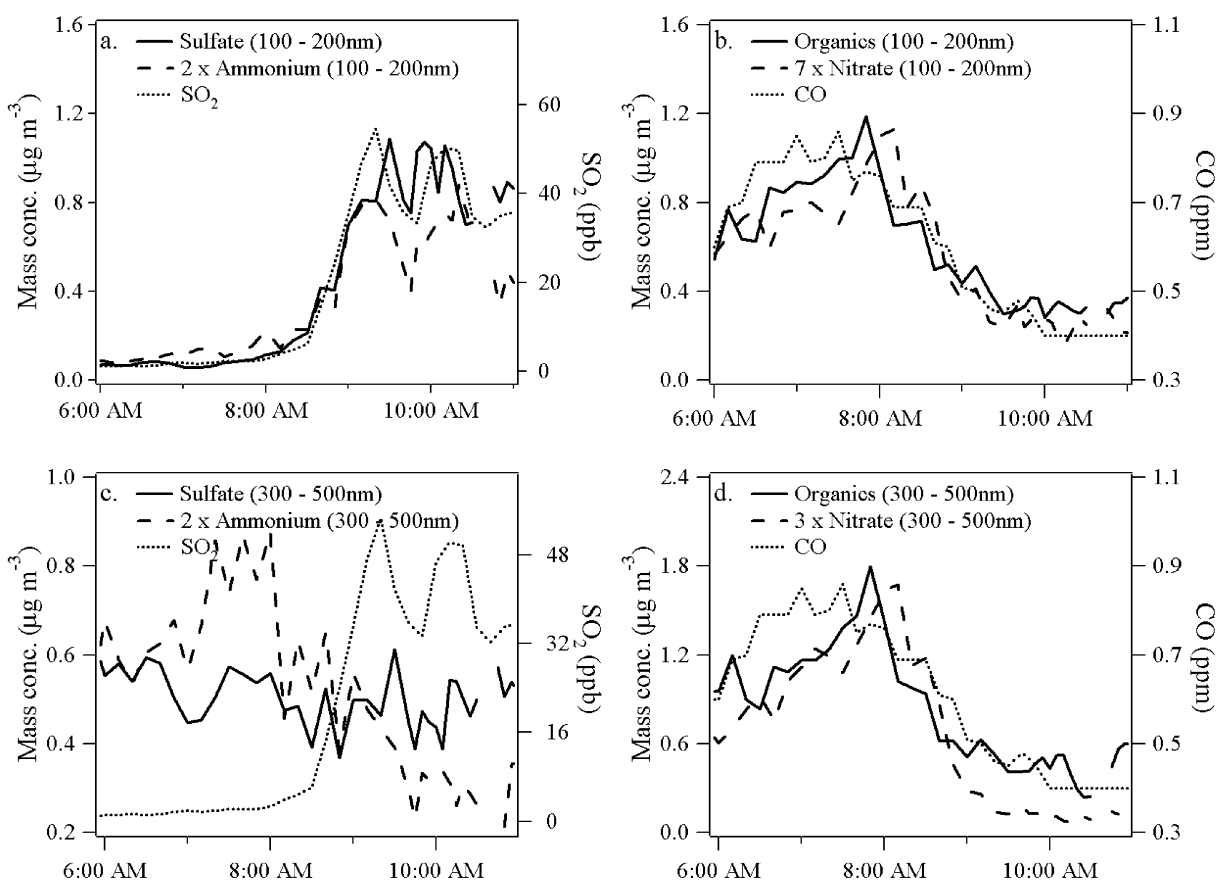

FIGURE 4. Variations in the mass concentrations of $\mathrm{SO}_{4}{ }^{2-}, \mathrm{NH}_{4}{ }^{+}, \mathrm{NO}_{3}{ }^{-}$, and organics in the accumulation mode $\left(D_{\mathrm{va}}=300-500 \mathrm{~nm}\right)$ and the intermediate mode $\left(D_{\mathrm{va}}=100-200 \mathrm{~nm}\right)$ together with those of $\mathrm{CO}$ and $\mathrm{SO}_{2}$ during the initial stage of nucleation on September 12, 2002. Note that ammonium and nitrate were scaled in the graph to match sulfate and organics, respectively; see the legends in the plots for scaling factors.

\section{Results and Discussion}

\subsection{Characteristics of the Nucleation Events.}

3.1.1. General Characteristics. Three significant nucleation and particle growth events (on September 8, 9, and 12, respectively) and one weak event (on September 7) were observed during September 7-22, 2002. Although another event may have occurred on September 13 (based on the increase of the number and mass concentrations of ultrafine particles larger than $15 \mathrm{~nm}$ ), we were not able to quantify it due to lack of information on particles below $15 \mathrm{~nm}\left(D_{m}\right)$. All events started in the morning between $~ 8: 00$ and 11:00 a.m. (Table 1). The average conditions during the initial $2-\mathrm{h}$ periods of the nucleation events on September 8, 9, and 12 along with those averaged from 8:00 to 13:00 EST on nonnucleation days are summarized in Table 1 . The September 7 event was not included due to large relative interference from traffic emissions. The nucleation periods are characterized by a substantial increase in the nucleation mode particles ( $D_{m}=3-10 \mathrm{~nm}$ ) (e.g., the average values of $\mathrm{N}_{10}$ ranged from 16000 up to $69000 \mathrm{~cm}^{-3}$ ) as compared to an average value of $\sim 10000 \pm 9400(1 \sigma)$ during nonnucleation periods (Table 1 ). $\mathrm{N}_{\text {tot }}$ and $\mathrm{N}_{30-78}$ as well as ultrafine mass (i.e., $M_{33-60}$ ) were also significantly higher, but the averagetotal massloading ( $M$; $D_{v a}=33-1000 \mathrm{~nm}$ ) wasslightly lower (Table 1), indicating a smaller average aerosol size during the nucleation periods.

Theevents on September 8 and 12 are classified as strong, and that on September 9 was classified as moderate based on their rates of increase in $\mathrm{N}_{10}$ (see section 2.3.2). Figure 1 shows the evolution of particle number and size distribution on September 12, one of the 10 strongest nucleation events observed in Pittsburgh over a period of 15 months (July 1 , 2001-September 30, 2002) (1). Sharp increase of nucleation mode particles started at 8:10 a.m. (e.g., N10 increased by roughly a factor of 10 within $\sim 1 \mathrm{~h}$ ) followed by a gradual growth of the new particles (Figure 1a). Significant increase of Aitken mode particles (e.g., $\mathrm{N}_{30-78}$ and $\mathrm{M}_{33-60}$ ) started roughly $1 \mathrm{~h}$ after that of $\mathrm{N}_{10}$ and lasted for $\sim 5 \mathrm{~h}$ (Figure $1 \mathrm{~b}$ ).
Meanwhile, synchronous increases of $\mathrm{SO}_{2}$, decrease of $\mathrm{CO}$, and rise of the calculated (HYSPLIT4) boundary layer height were observed at the start of this event (Figure 1c), indicating that these changes arelikely a result of breakup of the morning inversion that mixes down $\mathrm{SO}_{2}$-enriched but $\mathrm{CO}$-depleted air from aloft and dilutes the accumulated city emissions. According to Stanier et al. (1), this phenomenon is fairly common in Pittsburgh during the spring and fall. The welldefined nucleation and particle growth characteristics also suggest that the event on September 12 is occurring on a regional scale (1).

The major traits of the development of particle number concentrations and size distributions during the other two events are generally similar to this one (Table 1) and are consistent with observations from a 15-month study of Pittsburgh nucleation (1). Therefore, although we only characterized three events, they appear to be representative of the climatology and the strength of nucleation events in the Pittsburgh area.

3.1.2. Diurnal Patterns. Figure 2 summarizes the $1-h$ average diurnal profiles of 12 parameters on days in which nucleation was not observed, together with the individual time series for the three nucleation days. In general, the diurnal profiles of nonnucleation daysarecomparatively flat while those of nucleation days show much more variation. In addition to higher UV radiation and elevated $\mathrm{SO}_{2}$ (Figure $2 \mathrm{a}, \mathrm{b})$, we observed good correlation between $\mathrm{N}_{10}$ and the product of UV and $\mathrm{SO}_{2}$ (a proxy for the $\mathrm{H}_{2} \mathrm{SO}_{4}$ production rate) during nucleation events (Figure $2 \mathrm{c}, \mathrm{d}$; Table 1 ). This observation, as well as the fact that $\mathrm{N}_{10}$ and $\mathrm{UV} * \mathrm{SO}_{2}$ rise simultaneously, suggests an essential role played by $\mathrm{H}_{2} \mathrm{SO}_{4}$ in the nucleation and early growth. Particle condensational sink before the September 12 nucleation event (those on September 8 and 9 were not calculated due to lack of SM PS data for $D_{m}>78 \mathrm{~nm}$ ) was comparatively smaller than that during the same periods on nonnucleation days (Figure 2g). This observation is consistent with the finding of Stanier et al. (1) that low available aerosol surface favors the formation of new particles, probably through the accumulation of 

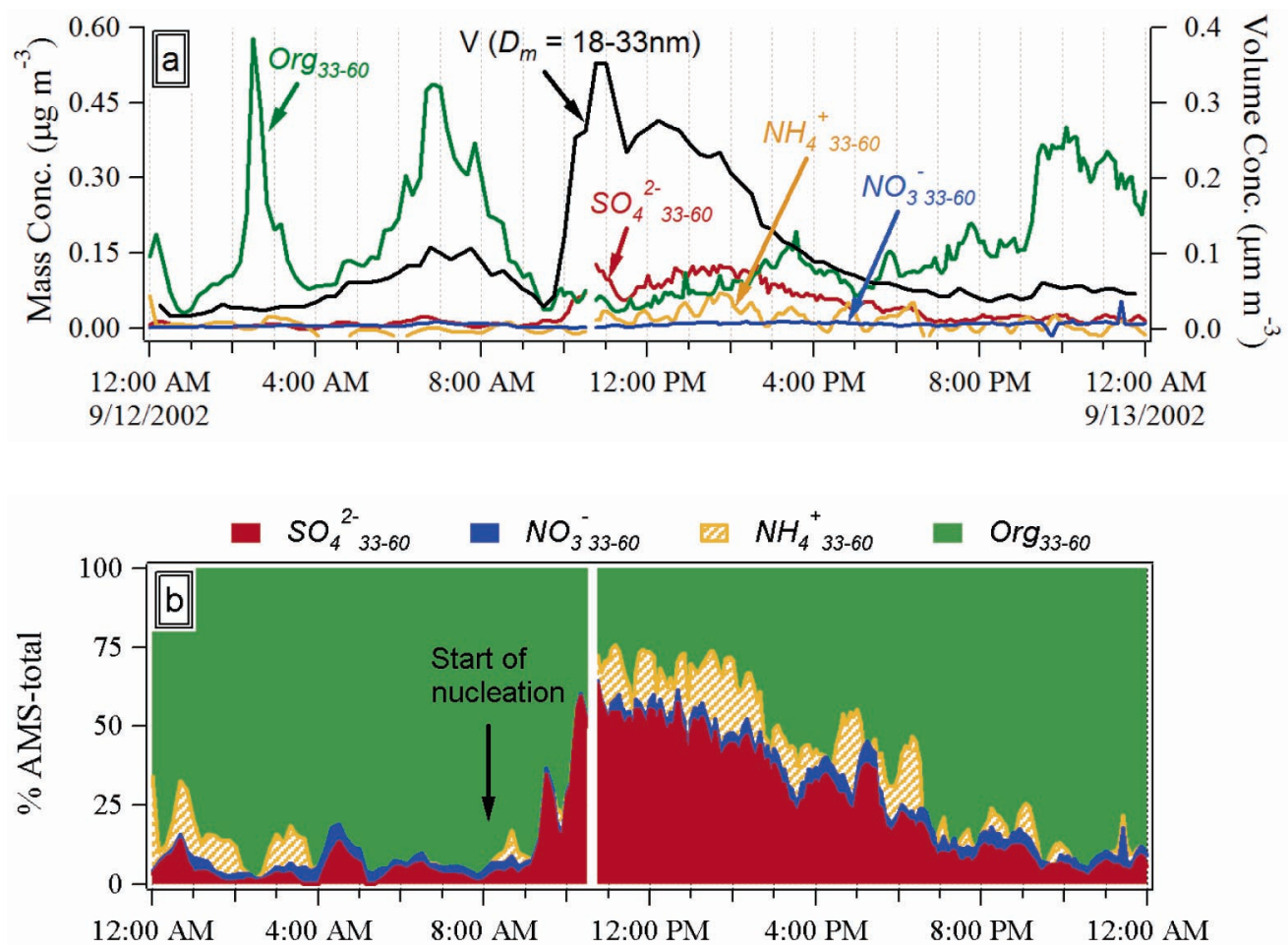

FIGURE 5. (a) Concentrations of $\mathrm{SO}_{4}{ }^{2-}, \mathrm{NH}_{4}{ }^{+}, \mathrm{NO}_{3}{ }^{-}$, organics, and particle volume in the 33-60 nm ( $D_{\text {va }}$ ) particles on September $12,2002$. Particle volume was calculated using SMPS data in the $D_{\mathrm{m}}$ range of $18-33 \mathrm{~nm}$ by assuming spherical particles with density of $1.8 \mathrm{~g} \mathrm{~cm}$. (b) Percent fraction of each species vs total (= sulfate + nitrate + ammonium + organics) in the $33-60 \mathrm{~nm}$ ( $D_{\text {va }}$ ) particles. Missing data are due to either occasional instrumental malfunction or maintenance/calibration.

condensable species in the gas phase that can be used to nucleate and grow new particles.

The mass concentrations of sulfate, ammonium, nitrate, and organics in the $33-60 \mathrm{~nm}\left(\mathrm{D}_{\mathrm{va}}\right)$ particles all increased considerably during nucleation days, but between 20 min to $1.5 \mathrm{~h}$ later than the rise of $\mathrm{N}_{10}$ (Figure $2 \mathrm{~h}-\mathrm{I}$ ). The lag corresponds to the time needed for the nucleation mode particles to grow into the size range detectable by the AMS. The average diurnal profile of $\operatorname{Org}_{33-60}$ during nonnucleation days resembles those of $\mathrm{NO}_{x}$ and $\mathrm{CO}$ (Figure $2 \mathrm{e}, \mathrm{f}, \mathrm{k}$ ), indicating a major combustion (probably traffic) source for the small mode organics $(38,42,48)$.

3.2. Evolution of Particle Chemistry during the Nucleation and Growth Event on September 12, 2002. We focus on the September 12 event because of its well-defined nucleation and growth characteristics (Figure 1) and the fact that it is one of the 10 strongest events observed in Pittsburgh in 15 months.

3.2.1. Chemistry and Dynamics of Size-Resolved Aerosol Species. Figure 3 provides an overview of the aerosol characteristics and their development on September 12, 2002. We have divided the nucleation event into four consecutive periods (I-IV) based on the dynamics of $\mathrm{N}_{10}$ and $\mathrm{N}_{30-78}$. Stage I (8:10-9:30) corresponds to the initial nucleation period from the beginning of the rise of $\mathrm{N}_{10}$ to its peak; stages II (9:30-11:30) and III (11:30-14:30) represent the growth period during which $\mathrm{N}_{10}$ was decreasing while $\mathrm{N}_{30-78}$ gradually grew to its maximum; and stage IV (14:30-17:05) represents a later period of the event when both $\mathrm{N}_{10}$ and $\mathrm{N}_{30-78}$ were decreasing. Average size distributions during thesefour stages areplotted to theright of thecorresponding image plot(Figure $\left.3 b^{\prime}-g^{\prime}\right)$.

As shown in Figure 3a, theaerosol mass loading increased in the early morning (likely due to rush hour traffic) and gradually decreased during stage $I$. This decrease coincides in time with a decrease in ambient $\mathrm{CO}$ concentration, an increase in $\mathrm{SO}_{2}$, and the (modeled) rise of the mixing layer height (Figure 1c) and therefore is likely a result of dilution of the accumulated city emissions by an $\mathrm{SO}_{2}$-enriched air mass from aloft. Particle mass concentration increased gradually after stage I but remained comparatively low for the rest of the day (Figure 3a).

The size distributions of particle apparent volume and non-refractory mass also changed considerably during this event (Figure $3 \mathrm{~b}-\mathrm{g}$ ). The distribution of aerosol apparent volume (calculated from the corresponding SMPS number data assuming spherical particles) was dominated by a single accumulation mode centered at $\sim 290 \mathrm{~nm}\left(D_{m}\right)$ before nucleation and developed into a trimodal distribution soon after, with the simultaneous appearance of an intermediate mode (centered at $\sim 90 \mathrm{~nm} D_{m}$ ) and the nucleation mode (Figure $\left.3 b, b^{\prime}\right)$. Both modes grew larger in size and in volume concentration during the rest of the event. The intermediate mode, for example, grew by $\sim 50 \%$ from a $D_{m}$ of 88 to $122 \mathrm{~nm}$ from stage I to stage IV, while the nucleation mode grew by a factor of 5 from 11 to $55 \mathrm{~nm}$. The accumulation mode, in contrast, showed a slight increase in concentration and almost no change in size.

The size distribution of the AMS-measured aerosol mass (= sulfate + ammonium + nitrate + organics) resembles that of SMPS-measured volume (Figure 3b,c). However, because the sizing limit of the AMS $\left(D_{v a}=33 \mathrm{~nm}\right)$ is larger than that of the SMPS ( $\left.D_{m}=3 \mathrm{~nm}\right)$, the smallest mode on the mass distributions appeared at a relatively later time.

The evolution of the size distributions of sulfate was very similar to those of mass and volume, exceptfor the dynamics of the intermediate mode that emerged shortly after the nucleation (Figure $3 b-d, b^{\prime}-d^{\prime}$ ). The intermediate mode on particle volume distribution $\left(D_{m}=60-120 \mathrm{~nm}\right)$ declined first and then grew higher (Figure 3b'), while that of sulfate $\left(D_{v a}=\sim 100-200 \mathrm{~nm}\right)$ did the opposite-increased significantly from stage I to stage II and gradually decreased afterward (Figure 3d'). The size distributions of ammonium, although noisier due to comparatively higher background 


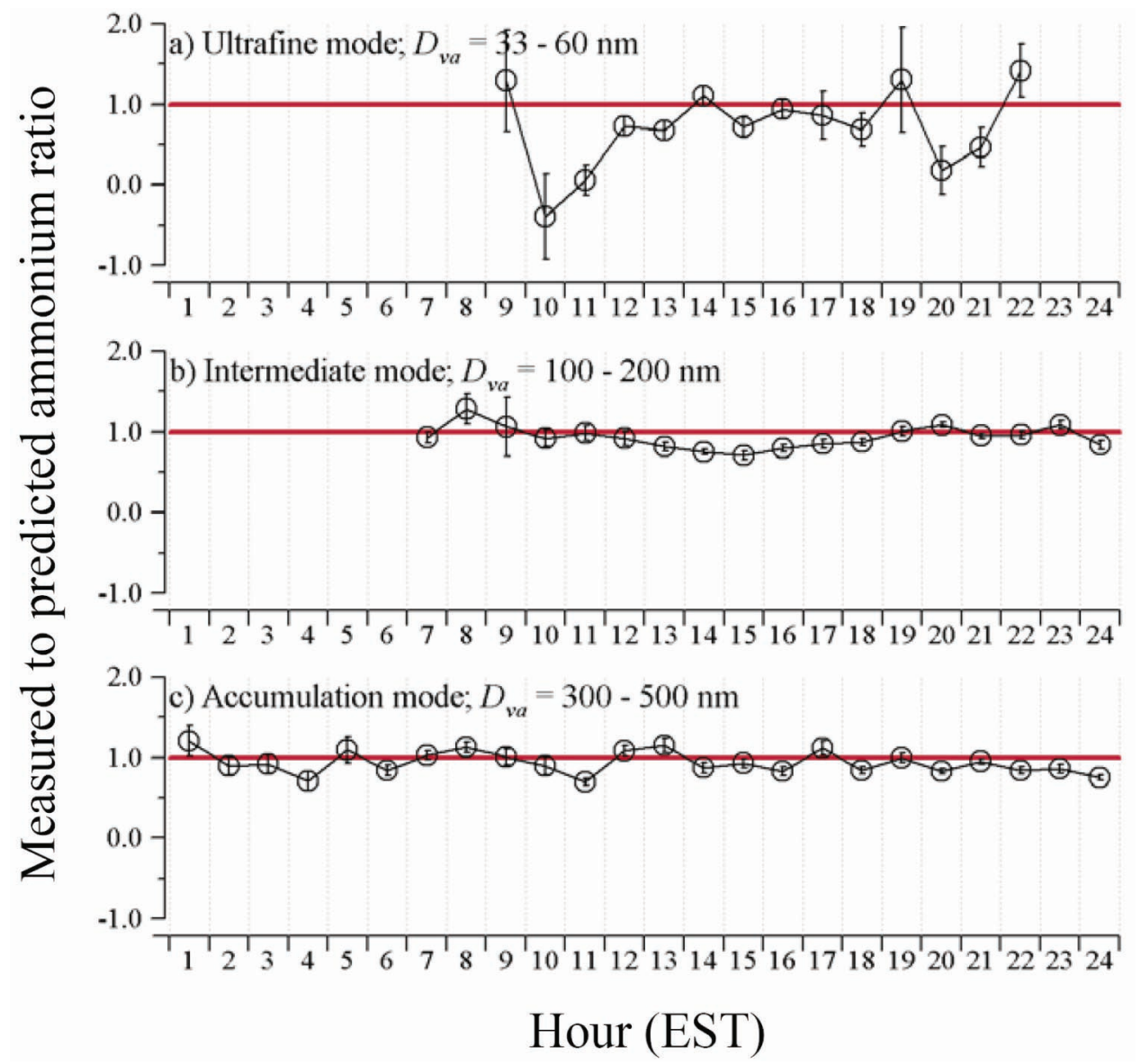

FIGURE 6. Hourly average of the mole ratio of measured $\mathrm{NH}_{4}{ }^{+}$vs predicted $\mathrm{NH}_{4}{ }^{+}$in three size bins of particles on September 12, 2002. Error bars are standard errors of the mean (calculated from propagation of the corresponding errors for $\mathrm{NH}_{4}{ }_{33}-60, \mathrm{SO}_{4}{ }^{2-}{ }_{33-60}, a^{2} \mathrm{~N} \mathrm{O}_{3}-{ }_{33-60}$ measurements and instrument noise). Note that only $\mathrm{SO}_{4}{ }^{2-}, \mathrm{NH}_{4}{ }^{+}$, and $\mathrm{NO}_{3}{ }^{-}$data that are 3 times above the AMS instrument detection limits were used to calculate the acidity values and that missing data are those below the detection limits. The predicted $\mathrm{NH}_{4}{ }^{+} \mathrm{W}$ as calculated from measured $\mathrm{SO}_{4}{ }^{2-}$ and $\mathrm{NO}_{3}{ }^{-}$assuming full neutralization of these ions by $\mathrm{NH}_{4}{ }^{+}$. A ratio of one suggests that the aerosols are neutralized.

signal for this species in the AMS (42), look similar to those of sulfate on almost every aspect, even in terms of the declining of its intermediate mode from stage II to stage IV (Figure $3 d, e, d^{\prime}, e^{\prime}$ ). The appearance and the increase of the intermediate mode sulfate and ammonium paralleled those of $\mathrm{SO}_{2}$ (Figure $4 a$ ), suggesting that both of them likely came from the same stable layer that capped the boundary layer before the breakup of the inversion.

The size distributions and evolution patterns of organics and nitrate are quite different from those of sulfate and ammonium, especially in the early morning before the start of the nucleation. First of all, in addition to the accumulation mode centered at $D_{\text {va }} \sim 400 \mathrm{~nm}$, organics and nitrate had an additional Aitken mode centered at $\sim 100 \mathrm{~nm}$ (Figure $3 \mathrm{f}, \mathrm{g}$ ). The organic component of these smaller mode particles, which contained much less ammonium and sulfate, appeared to come from traffic emissions based on their time variation and their strong correlation with $\mathrm{CO}$ and $\mathrm{NO}_{x}$ (42). This mode would likely contain internally mixed soot as well; however, the AMSis not capable of detecting this material at the aerosol vaporizer temperature employed for this study $\left(\sim 600^{\circ} \mathrm{C}\right)$. Increase of particulate nitrate in the early morning may be attributed to the favorable thermodynamic conditions for conversion of gaseous $\mathrm{HNO}_{3}$ and $\mathrm{NH}_{3}$ into $\mathrm{NH}_{4} \mathrm{NO}_{3}$ aerosol (low temperature and high $\mathrm{RH}$ ) or to the formation of nitrate via the $\mathrm{NO}_{3}$ radical and $\mathrm{N}_{2} \mathrm{O}_{5}$, a mechanism that is most active at night. Similar phenomenology for nitrate has been observed at other locations in the Eastern United States (38, 49).
During theinitial stage of the nucleation (e.g., from 8:009:30 a.m.), the mass concentrations of nitrate and organics in both the accumulation mode $\left(D_{v a}=300-500 \mathrm{~nm}\right)$ and the intermediate mode $\left(D_{\mathrm{va}}=100-200 \mathrm{~nm}\right.$ ) were gradually decreasing, together with a similar magnitude of decrease of $\mathrm{CO}$ (Figure 4b,d). Meanwhile, theintermediate modesulfate and ammonium were increasing, together with a similar magnitudeof increase of $\mathrm{SO}_{2}$ (Figure 4a). Becausethe increase of $\mathrm{SO}_{2}$ was likely due to mixing of $\mathrm{SO}_{2}$-enriched air mass from aloft while the decrease of $\mathrm{CO}$ was due to dilution of city emissions, this intermediate mode appears to be an externally mixed combination of a major portion of $\left(\mathrm{NH}_{4}\right)_{2} \mathrm{SO}_{4}$ particles (i.e., neutralized) that came from thesameair mass as $\mathrm{SO}_{2}$ and a minor portion of preexisting urban particles that were mainly composed of organics and nitrate.

Because the size distributions of all four species were similar at $D_{v a}$ above $300 \mathrm{~nm}$ before the nucleation, the accumulation mode was likely aged regional aerosols consistent with an internal mixture of sulfate, ammonium, organics, and a comparatively small amount of nitrate. During theinitial stage of the nucleation, all the accumulation mode species, except for sulfate, decreased in parallel with $\mathrm{CO}$ (Figure 4c,d) due to dilution from the air mixing down from aloft. The reason for sulfate decreasing less (Figure 4c) was probably its higher concentration in the air mass aloft and/ or the larger condensation of $\mathrm{H}_{2} \mathrm{SO}_{4}$.

Despite the initial differences, during stages III and IV both nitrate and organics gradually developed into a trimodal distribution that matched those of sulfate and ammonium 

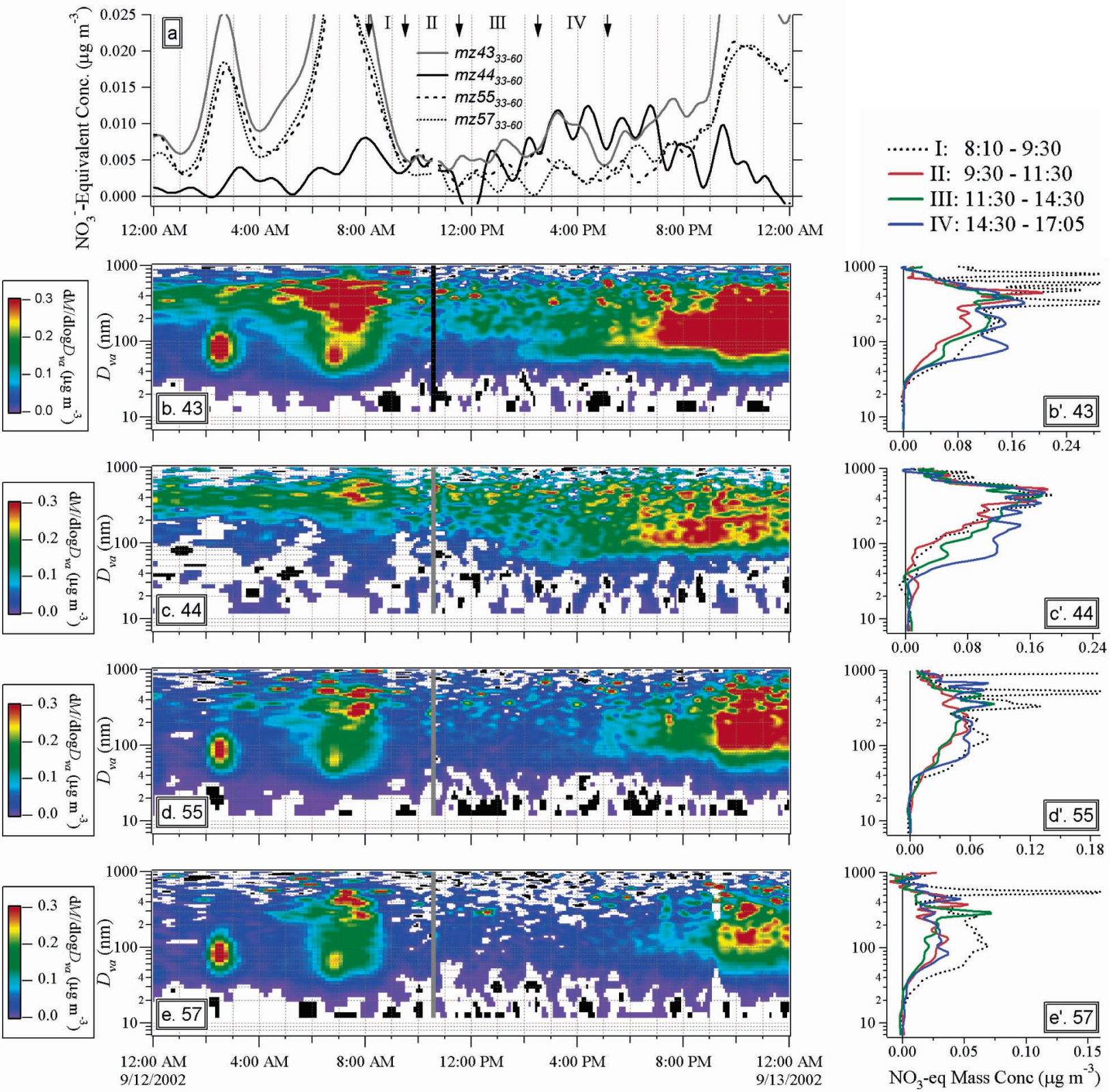

FIGURE 7. (a) Time series of the nitrate-equivalent mass concentrations of organic fragments $(m / z 43,44,55$, and 57$)$ in $33-60 \mathrm{~nm}\left(D_{\mathrm{va}}\right)$ particles on September 12, 2002 with the four stages of the nucleation event marked. (b-e) Evolution of the size distribution and mass concentration of these four fragments during September 12, 2002. $\left(b^{\prime}-e^{\prime}\right)$. The average size distribution of a given individual component during the four stages of the nucleation event. Gray areas on plots $b-e$ are due to either oc casional instrumental malfunction or maintenance/ calibration; w hite areas are due to the omission of data points that are below the detection limit $(1 \sigma)$ of the AM S. Note that $\mathrm{NO}_{3}{ }^{-}$equivalent mass concentration equals the mass concentration of nitrate that would produce a signal of same intensity, summing all of nitrate's $\mathbf{m} / \mathbf{z}$ (see section 2.3.1).

(Figure $3 c-g, c^{\prime}-g^{\prime}$ ), indicating extensive condensation of gaseous precursors of nitrate and organics onto preexisting particles. This process gradually diminished the heterogeneity of the particles so that they became more internally mixed.

3.2.2. Composition and Growth of Ultrafine Particles $\left(D_{\mathrm{va}}=33-60 \mathrm{~nm}\right)$. The growth of nucleation mode particles into theAitken mode was one of the most prominent features of thenucleation event (Figures la and 3). Thesmallest mode, which was originally the nucleation mode, grew from an average $D_{m}$ of $11 \mathrm{~nm}$ in stage I of the nucleation event gradually into 29,46 , and $55 \mathrm{~nm}$ subsequently across the other three stages (Figure 3b'). Due to a larger size cutoff of the AMS, this mode appeared at a later time on the mass distributions, but the growth of each species was evident. The growth appeared to proceed at different rates and probably through different mechanisms (Figure $3 d-f, d^{\prime}-$ $\left.f^{\prime}\right)$.

Variations of the mass concentration of $\mathrm{SO}_{4}{ }^{2-}, \mathrm{NH}_{4}{ }^{+}, \mathrm{NO}_{3}{ }^{-}$, and organics in the $D_{\text {va }}$ range 33 (the lower bound of the AMS detection) to $60 \mathrm{~nm}$ were thus carefully analyzed to gain insights into which species contributed to the growth, during which stage, and by what extent. Note that this size range is equivalent to $D_{m}$ of $18-33 \mathrm{~nm}$ assuming a density of $1.8 \mathrm{~g} / \mathrm{cm}^{3}$ (which would be appropriate for sulfuric acid or ammonium sulfate particles) and spherical particles. It took $1 \mathrm{~h}$ for the $3-10 \mathrm{~nm}\left(\mathrm{D}_{\mathrm{m}}\right)$ mode particles to grow into this size range during this event.

As shown in Figure $5 \mathrm{a}$, the concentrations of ultrafine sulfate (i.e., $\mathrm{SO}_{4}{ }^{2-}{ }_{33-60}$ ) were very low before the nucleation and started to increase at $\sim 9: 00$ a.m. A rapid increase of 
TABLE 2. Average Growth Rates of Particle Number $\left(\mathrm{cm}^{-3} \mathrm{~h}^{-1}\right)$ and Composition $\left(\mathrm{ng} \mathrm{m}^{-3} \mathrm{~h}^{-1}\right)$ in Ultrafine Particles during Nucleation ${ }^{\mathrm{a}}$

\begin{tabular}{|c|c|c|c|c|c|c|c|}
\hline start of grow th & peak of grow th & $\mathrm{d} N_{30-78} / \mathrm{d} t$ & $\mathrm{~d} M_{33-60} / \mathrm{d} t$ & $\mathrm{dSO}_{4}{ }^{2-}{ }_{33-60} / \mathrm{dt}$ & $\mathrm{dNH}_{4}{ }^{+}{ }_{33-60} / \mathrm{d} t$ & $\mathrm{dNO}_{3}{ }^{-}{ }_{33-60} / \mathrm{d} t$ & $\mathrm{dO} O \mathrm{rg}_{33-60} / \mathrm{d} t^{b}$ \\
\hline 9/8/02, 11:00 & 9/8/02 12:10 & 4.7E+04 & 621 & 353 & 138 & 15 & 116 \\
\hline $9 / 9 / 02,12: 00$ & 9/9/02 14:00 & $1.2 \bar{E}+04$ & 60 & 53 & 24 & 1.9 & 130 \\
\hline 9/12/02, 9:30 & 9/12/02 11:00 & $5.9 \mathrm{E}+03$ & 122 & 91 & 23 & 0.9 & 7 \\
\hline 9/12/02, 11:30 & 9/12/02 14:00 & 5.7E+03 & 70 & 24 & 24 & 3.5 & 19 \\
\hline average & & $1.8 \mathrm{E}+04$ & 218 & 130 & 52 & 5 & 68 \\
\hline $1 \sigma$ & & $2.0 \mathrm{E}+04$ & 270 & 151 & 57 & 6.6 & 64 \\
\hline median & & $8.9 E+03$ & 96 & 72 & 24 & 2.7 & 67 \\
\hline $\min$ & & $5.7 \bar{E}+03$ & 60 & 24 & 23 & 0.9 & 7 \\
\hline $\max$ & & 4.7E+04 & 621 & 353 & 138 & 15 & 130 \\
\hline
\end{tabular}

${ }^{a}$ Growth rates were determined by a linear fit to the data in the time periods listed in the first two columns unless flagged. ${ }^{b}$ Calculated for the period from when $\operatorname{Org}_{33-60}$ started to rise to when it peaked (i.e., 12:00-12:40 p.m.).

$\mathrm{SO}_{4}{ }^{2-}{ }_{33-60}$ was observed at $\sim 9: 50$ a.m., followed by the rise of $\mathrm{NH}_{4}^{+} 33-60$. Very high levels of ultrafine organics were present prior to the nucleation event, probably dueto traffic emissions(Figure 5a). As a result, Org $_{33-60}$ was more sensitive to changes of the boundary layer height and thus decreased dramatically during the initial stage of the nucleation (Figures $1 c$ and $5 a)$.

Org $_{33-60}$ went through a temporary rise from $\sim 9: 30$ to 9:50a.m. but declined again afterward (Figure 5a). Because this rise was coincident with a small increase in $\mathrm{CO}$ and decreases in $\mathrm{SO}_{2}$ and $\mathrm{SO}_{4}{ }^{2-}{ }_{33-60}$, it was probably a result of elevated detection of city emissions during this short period. While it is also possible that this rise of small mode organics was the result of organics being involved in the initial growth, the comparatively high background level of traffic ultrafine organics during this event and the relatively noisy signals of $\mathrm{m} / \mathrm{z} 44$, a mass spectral marker of oxygenated organic species (50), limited our ability to ascertain this point conclusively for this particular event.

A discernible increase of $\mathrm{NH}_{4}^{+}{ }_{33-60}$ was observed after 10:00a.m., about $45 \mathrm{~min}$ after the rapid increase of $\mathrm{SO}_{4}{ }^{2-}{ }_{33-60}$ (Figure 5a). This delay suggests that the growth of the new particles during this stage was dominated by condensation of $\mathrm{H}_{2} \mathrm{SO}_{4}$ without enough $\mathrm{NH}_{3}$ to neutralize the growing particles. The later uptake of $\mathrm{NH}_{3}$ by the particles occurred as it became available (e.g., from traffic emissions) (51). However, this observed lag does not ruleout theinvolvement of $\mathrm{NH}_{3}$ in the nucleation mechanism since the amount of $\mathrm{NH}_{3}$ needed for nucleating a particle is at least 3 orders of magnitude smaller than that needed for neutralizing $\mathrm{H}_{2} \mathrm{SO}_{4}$ in the ultrafineparticles measured by the AMS. It is estimated that given thehigh concentrations of $\mathrm{SO}_{2}$, and therefore $\mathrm{H}_{2} \mathrm{SO}_{4}$ production in sunny days, $\mathrm{NH}_{3}-\mathrm{H}_{2} \mathrm{SO}_{4}-\mathrm{H}_{2} \mathrm{O}$ ternary nucleation is favored in Pittsburgh for as low as 10 ppt $\mathrm{NH}_{3}$ (52).

Org $_{33-60}$ resumed a second rise at $\sim 11: 15$ a.m., when $\mathrm{NO}_{3}{ }^{-} 33-60$ also began to increase (Figure 5a). The increases seem to be a result of condensation of photochemical products onto ultrafine particles. Because of its very low concentration compared to the other threespecies (e.g., 1\% of the $\mathrm{M}_{33-60}$ ) and the comparatively later rise of $\mathrm{NO}_{3}{ }^{-}{ }_{33-60}$, nitrate seemed to have played a minor role in growing the new particles.

As mentioned earlier in thissection, thesmallest particles measured by the AMS during nucleation events were not freshly formed but rather grew from the nucleation mode that was formed $\sim 1-2 \mathrm{~h}$ before. We thus calculated the characteristic times of coagulation and condensation to compare the relative importance of these two mechanisms for the growth of the nuclei to the minimum size detectable by the AMS. According to our calculations, the growth of nucleation mode particles due to gaseous condensation was approximately an order of magnitude faster than that due to coagulation. The later would have required more than $10 \mathrm{~h}$ to grow the nucleation mode particles to the smallest size detectable by the AMS. Therefore, we are unable to derive the composition of original nuclei from these AMS measurements but rather identify the species that contributed to the condensational growth.

The mass fractions of each individual species in the 33$60 \mathrm{~nm}$ mode as a function of the total $\left(\mathrm{M}_{33-60}=\mathrm{SO}_{4}{ }^{2-}{ }_{33-60}\right.$ $+\mathrm{NO}_{3}{ }_{33-60}+\mathrm{NH}_{4}{ }_{33-60}+\mathrm{Org}_{33-60}$ ) shows that before nucleation $\operatorname{Org}_{33-60}$ was the dominant species, accounting for $\sim 90 \%$ of $\mathrm{M}_{33-60}$ (Figure $5 \mathrm{~b}$ ). $\mathrm{SO}_{4}{ }^{2-}{ }_{33-60}$ quickly overtook Org $_{33-60}$ after the nucleation started and became the major species (>50\% in mass) in the ultrafine mode between 10:30 a.m. and 2:00 p.m. (Figure 5b). $\mathrm{NH}_{4}{ }^{+} 33-60$ also rose substantially during the same period. Afterward, the concentrations of sulfate and ammonium in the $33-60 \mathrm{~nm}$ mode gradually declined back to their pre-nucleation levels while Org $_{33-60}$ kept rising and regained dominance (Figure $5 b$ ). There is evidence (seesection 3.2.4) that theultrafine organic aerosols present at high concentrations right before and after the nucleation was mainly generated from combustion processes (most likely traffic).

3.2.3. Particle Acidities. Particle acidity was examined based on the ratio of measured ammonium concentrations versus the amounts needed to fully neutral ize the measured sulfate and nitrate. A value of one suggests that sulfate and nitrate might be fully neutral ized by ammonium in the form of $\left(\mathrm{NH}_{4}\right)_{2} \mathrm{SO}_{4}$ and $\mathrm{NH}_{4} \mathrm{NO}_{3}$. A value close to zero suggests that the particles are predominately $\mathrm{H}_{2} \mathrm{SO}_{4}$. On the basis of this definition, ultrafine particles $\left(D_{\mathrm{va}}=33-60 \mathrm{~nm}\right)$ appeared to be acidic during the initial stage of the nucleation and gradually becameneutralized (Figure 6a). Very acidic particles seemed to exist between 9:00 and 12:00, consistent with the observation of an earlier and faster increase of $\mathrm{SO}_{4}{ }^{2-}{ }_{33-60}$ as compared to $\mathrm{NH}_{4}{ }_{33-60}$ (Figures 5a and 6a). It is interesting that although the start of the increase of ultrafine ammonium occurred $\sim 45 \mathrm{~min}$ later than that of sulfate, it took more than $2 \mathrm{~h}$ for the ultrafine particles to be fully neutralized. This observation reinforces the conclusion that $\mathrm{NH}_{3}$ was present at very low concentrations during the initial stages of the new particle formation event. In contrast, the two larger modes, the intermediate mode $\left(D_{\mathrm{va}}=100-200 \mathrm{~nm}\right)$ and the accumulation mode ( $\left.D_{v a}=300-500 \mathrm{~nm}\right)$, appeared to be nearly neutralized throughout the whole event (Figure $6 b, c)$, probably because they were aged.

3.2.4. Possible Role of Organic Vapors in Growth of Ultrafine Particles ( $\left(D_{\mathrm{va}}=33-60 \mathrm{~nm}\right)$. Size distributions of four organic fragments (i.e., $\mathrm{m} / \mathrm{z} 43,44,55$, and 57) were measured. Usually, $\mathrm{m} / \mathrm{z} 44$ (most likely the $\mathrm{CO}_{2}{ }^{+}$ion fragment) is a good tracer for photochemically formed secondary organic aerosol $(48,50)$, while $\mathrm{m} / \mathrm{z} 57\left(\mathrm{C}_{4} \mathrm{H}_{9}{ }^{+}\right)$is generally associated with primary organics from combustion sources $(38,42,44,50) . \mathrm{m} / \mathrm{z} 43$ and 55 can be produced by both primary combustion aerosols $\left(\mathrm{C}_{3} \mathrm{H}_{7}+\right.$ and $\mathrm{C}_{4} \mathrm{H}_{7}{ }^{+}$, respectively) 

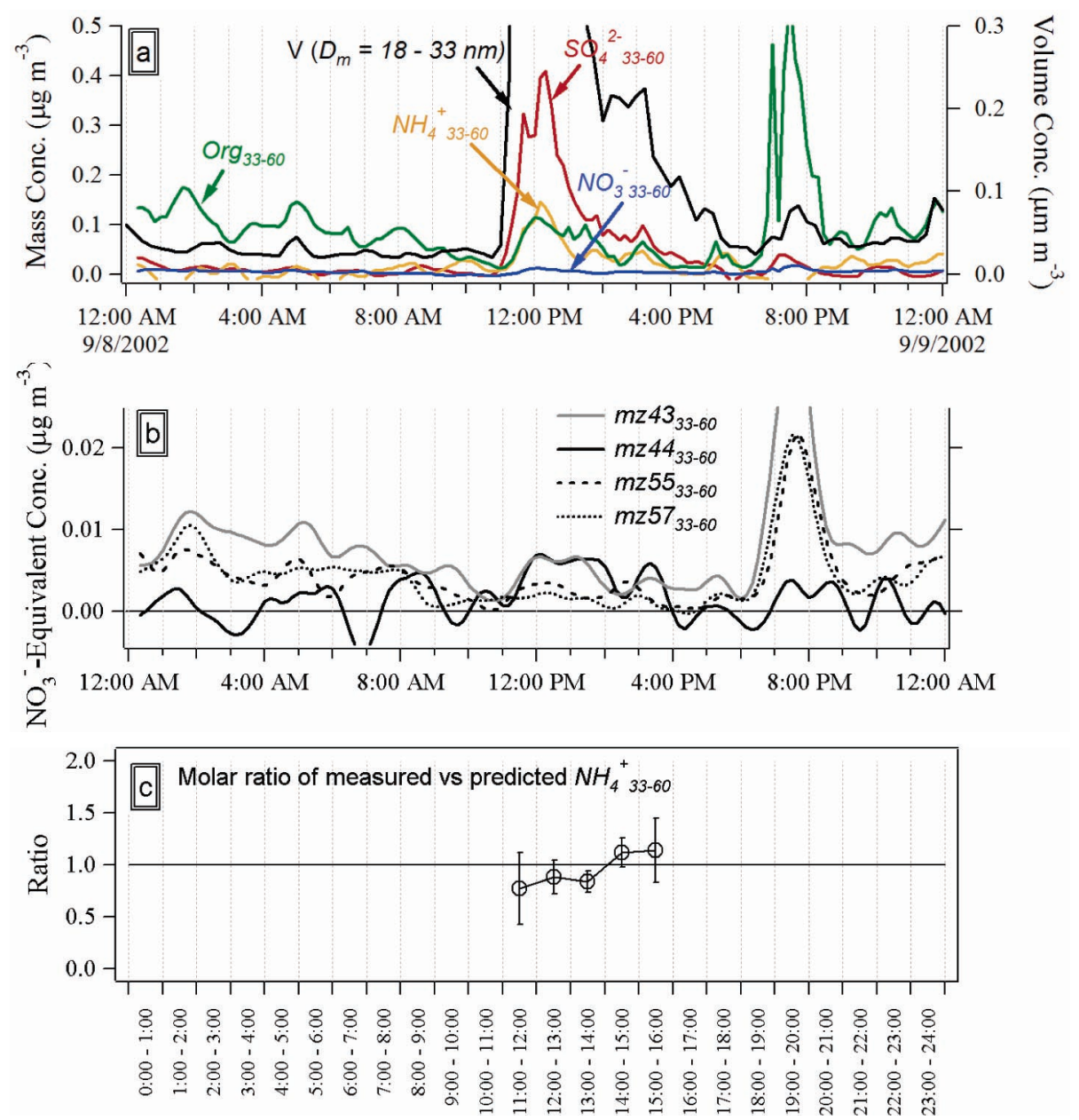

FIGURE 8. Variations of (a) concentrations of $\mathrm{SO}_{4}{ }^{2-}, \mathrm{NH}_{4}{ }^{+}, \mathrm{NO}_{3}{ }^{-}$, organics, and apparent particle volume: (b) $\mathrm{NO}_{3}{ }^{-}$equivalent mass

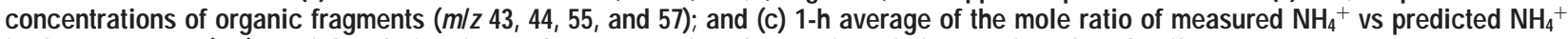
in the 33-60 nm $\left(D_{v a}\right)$ particles during September 8, 2002. See the caption of Figure 5 for other details.

and secondary photochemical aerosols $\left(\mathrm{C}_{2} \mathrm{H}_{3} \mathrm{O}^{+}\right.$and $\mathrm{C}_{3} \mathrm{H}_{3} \mathrm{O}^{+}$, respectively). $\mathrm{m} / \mathrm{z} 43$ seems to be produced in roughly equal fractions from primary and secondary organic aerosols; therefore, its time trend and size distributions most closely resemble those of the total organics. Indeed, we found that the mass ratio of $\mathrm{m} / \mathrm{z} 43$ to total organics was fairly constant throughout the whole campaign (42). $\mathrm{m} / \mathrm{z} 55$, on the other hand, seems to be produced more intensely from primary than from secondary organics (48). The time variations of these four fragments, in which $\mathrm{m} / \mathrm{z} 55$ and 57 peaked in the morning during rush hours while $\mathrm{m} / \mathrm{z} 44$ built up during the day when photochemistry is more intense, corroborate these qualitative associations (Figure 7a). A more in-depth discussion of theAM Sorganic fragmentsis given in separate papers $(42,50)$.

A distinctiveincrease of $\mathrm{m} / \mathrm{z} 44$ in the $33-60 \mathrm{~nm}$ particles was observed at $\sim 12: 00$ p.m., likely due to increased photochemical production of secondary organic aerosol (Figure 7a). Thetime series of $\mathrm{m} / \mathrm{z} 43_{33-60}$ tracked that of $\mathrm{m} / \mathrm{z}$ 4433-60 between 12:00 and 5:00 p.m. but instead tracked those of $\mathrm{m} / \mathrm{z} 5_{33}-60$ and $\mathrm{m} / \mathrm{z}$ 57 $_{33-60}$ nicely before and after the nucleation event (Figure 7a). Because $\mathrm{m} / \mathrm{z} 43$ often correlated well with bulk particulate organics, not only in mass loading but also in size distribution (42), the synchronous increase of $\mathrm{m} / \mathrm{z} 43$ and 44 in the ultrafine mode between 12:00 and 4:00 p.m. suggests that photochemically formed secondary organics contributed significantly to the growth of ultrafine particles during this period of time.

As shown in Figure 7, before the nucleation event $\mathrm{m} / \mathrm{z} 55$ and 57 were mainly in the ultrafine mode while $\mathrm{m} / \mathrm{z} 44$ was in the accumulation mode. These observations support our hypothesis that the Aitken mode organics prior to the nucleation were predominantly primary aerosols emitted from combustion processes. During stages I and II (8:1012:05), the mass concentrations of ultrafine $\mathrm{m} / \mathrm{z} 55$ and 57 were generally decreasing (Figure $7 d^{\prime}, e^{\prime}$ ), because of atmospheric dilution and reduced traffic emissions compared to morning rush hours. The concentrations of ultrafine $\mathrm{m} / \mathrm{z} 43$ and 44, however, increased after stage I (Figure $\left.7 b^{\prime}, c^{\prime}\right)$, probably due to photochemical production of their parent organic compounds. The development of the size distributions of these four organic fragments during the last two stages (III and IV) were fairly similar-all grew into three modes that match those of the sulfate (Figures $7 b^{\prime}-e^{\prime}$ and $\left.3 d^{\prime}\right)$, indicating either an extensive condensation of organic vapors during the later stages of the nucleation event or coagulation of the primary particles with the nucleation mode.

3.3. Evolution of Particle Chemistry during the Other Two Observed Nucleation Events. As mentioned in section 3.1, the general characteristics and meteorology of the other two nucleation days (September 8 and 9) resembled those of September 12. In addition, we found that the major traits of the growth dynamics of sulfate, ammonium, organics, and nitrate in the ultrafine mode $\left(D_{\text {va }}=33-60 \mathrm{~nm}\right)$ were similar amongthese three events as well. First of all, all of theultrafine speciesincreased significantly, although the absolute growth rates varied substantially from one event to another (Table 2). Such differences reflect variations in the intensity of the individual event (e.g., the increase rate of the number 
concentration of the nucleation mode particles; Table 1). Second, sulfate and ammonium appeared to be the major contributors to the growth of ultrafine particles during all three events (Table 2), and interestingly, a rapid increase of $\mathrm{SO}_{4}{ }^{2-}{ }_{33-60}$ al ways happened before that of $\mathrm{NH}_{4}{ }_{33-60}$, by $10-$ $40 \mathrm{~min}$. As a result, ultrafine particles were more acidic during the initial stages of the nucleation events and became more and moreneutralized later on (Figures $6 \mathrm{a}$ and $8 \mathrm{c}$ ). In addition, $\mathrm{NO}_{3}{ }^{-}$typically contributed very little $(<3 \%)$ to the growth of ultrafine particles (Table 2).

The average rates and duration of the growth of ultrafine sulfate and ammonium varied considerably among these three nucleation events (Table 2). In comparison to the September 12 event (Figure 8), the September 8 nucleation event was characterized with a weaker burst of $\mathrm{N}_{10}$ but a much faster, and relatively short-lived, growth of $\mathrm{SO}_{4}{ }^{2-}{ }_{33-60}$ and $\mathrm{NH}_{4}{ }^{+}{ }_{33-60}$ (Tables 1 and 2; Figure 8). The exceptionally high ambient $\mathrm{SO}_{2}$ concentration during the September 8 event (maximum $=156.4 \mathrm{ppb}$, vs maximum of $54.7 \mathrm{ppb}$ on September 12) and comparatively fewer new particles might be responsible for the fast growth. It also appears that the spatial extent of the September 8 event was smaller than that of the September 12 and that more intense nucleation on September 8 took place upwind of our sampling site. Distinctive growth of the Org $_{33-60}$ was observed during all three nucleation events (Table 2). Although the onset of the particle bursts varied by more than $2 \mathrm{~h}$ from one day to another (e.g., from 8:15 a.m. on September 12 to 10:30 a.m. on September 8; Table 1) significant increase in the mass of ultrafine organics $\left(D_{\mathrm{va}}=33-60 \mathrm{~nm}\right)$ all started between 11 : 00 a.m. and 12:00 p.m. (Table 2). In addition, the observed growth of ultrafine organics appeared to be mainlyattributed to the increases of $\mathrm{m} / \mathrm{z} 44_{33-60}$ and $\mathrm{m} / \mathrm{z} 43_{33-60}$ (e.g., Figures $7 \mathrm{a}$ and $8 \mathrm{~b}$ ), suggesting that secondary organic species played an important role in the growth of the new particles.

\section{Acknowledgments}

Wethank Dr. Beth Wittig (CMU) for providing meteorological and gas phase data, Dr. Andrey Khlystov (CMU) for logistical assistance, Frank Drewnick (MPI) and James Allan (UMIST) for AMSdata analysis software, and Dr. Owen Cooper (NOAA) for help with the HYSPLIT program. This research was conducted as part of the Pittsburgh Air Quality Study and was supported by the University of Colorado through startup funds for J.L.J., and by the U.S. Environmental Protection Agency under Contract R82806101.

\section{Literature Cited}

(1) Stanier, C.; Khlystov, A.; Pandis, S. Aerosol Sci. Technol. 2004, $38,253-264$.

(2) Kulmala, M.; Hameri, K.; Aalto, P. P.; Makela, J. M.; Pirjola, L.; Nilsson, E. D.; Buzorius, G.; Rannik, U.; Dal Maso, M.; Seidl, W.; Hoffman, T.; Janson, R.; Hansson, H. C.; Viisanen, Y.; Laaksonen, A.; O'Dowd, C. D. Tellus Ser. B 2001, 53, 324-343.

(3) Kulmala, M.; Toivonen, A.; Makela, J. M.; Laaksonen, A. Tellus Ser. B 1998, 50, 449-462.

(4) O'Dowd, C. D.; Geever, M.; Hill, M. K. Geophys. Res. Lett. 1998, $25,1661-1664$

(5) O'Dowd, C. D.; Hameri, K.; Makela, J. M.; Pirjola, L.; Kulmala, M.; Jennings, S. G.; Berresheim, H.; Hansson, H. C.; de Leeuw, G.; Kunz, G. J.; Allen, A. G.; Hewitt, C. N.; Jackson, A.; Viisanen, Y.; Hoffmann, T. J. Geophys. Res.-Atmos. 2002, 107, 8108.

(6) O'Dowd, C. D.; Jimenez, J. L.; Bahreini, R.; Flagan, R. C.; Seinfeld, J. H.; Hameri, K.; Pirjola, L.; Kulmala, M.; Jennings, S. G.; Hoffmann, T. Nature 2002, 417, 632-636.

(7) Wiedensohler, A.; Wehner, B.; Birmili, W. J. Aerosol Med. 2002, $15,237-243$.

(8) Eisele, F. L.; McMurry, P. H. Philos. Trans. R. Soc. London, Ser. B 1997, 352, 191-200.

(9) Weber, R. J.; McMurry, P. H.; Mauldin, R. L.;Tanner, D. J.; Eisele, F. L.; Clarke, A. D.; Kapustin, V. N. Geophys. Res. Lett. 1999, 26, 307-310.
(10) Weber, R. J.; McMurry, P. H.; Eisele, F. L.; Tanner, D. J. J. Atmos. Sci 1995, 52, 2242-2257.

(11) Weber, R. J.; Marti, J. J.; McMurry, P. H.; Eisele, F. L.; Tanner, D. J.; Jefferson, A. J. Geophys. Res.-Atmos. 1997, 102, 43754385.

(12) Pirjola, L.; Laaksonen, A.; Aalo, P.; Kulmala, M. J. Geophys. Res.Atmos. 1998, 103, 8309-8321.

(13) Weber, R. J.; Orsini, D.; Wang, B.; Scheuer, E.; Talbot, R. W.; Dibb, J. E.; Seid, G. K.; DeBell, L.; Mauldin, R. L.; Kosciuch, E.; Cantrell, C.; Eisele, F. J. Geophys. Res.-Atmos. 2003, 108, 8357

(14) Woo, K. S.; Chen, D. R.; Pui, D. Y. H.; McMurry, P. H. Aerosol Sci. Technol. 2001, 34, 75-87.

(15) Hameri, K.; Kulmala, M.; Aalto, P.; Leszczynski, K.; Visuri, R.; Hamekoski, K. Atmos. Res. 1996, 41, 281-298.

(16) Harrison, R. M.; Grenfell,J. L.; Savage, N.; Allen, A.; Chemitshaw, K. C.; Penkett, S.; Hewitt, C. N.; Davison, B. J. Geophys. Res.Atmos. 2000, 105, 17819-17832.

(17) Shi, J. P.; Evans, D. E.; Khan, A. A.; Harrison, R. M. Atmos. Environ. 2001, 35, 1193-1202.

(18) Alam, A.; Shi, J. P.; Harrison, R. M.J. Geophys. Res.-Atmos. 2003 108, 4093.

(19) Dunn, M. J.; Jiménez, J.-L.; Baumgardner, D.; Castro, T.; McMurry, P. H.; Smith, J. N. Geophys. Res. Lett. 2004, 31, L10102, doi: $10110.11029 / 12004 G L 019483$.

(20) Obersdorster, G.In UItrafineParticlesin theAtmosphere: Brown, L. M., Collings, N., Harrison, R. M., Maynard, A. D., Maynard, R. L., Eds.; Imperial College Press: London, 2003.

(21) Donaldson, K.; Brown, D.; Clouter, A.; Duffin, R.; MacNee, W. Renwick, L.; Tran, L.; Stone, V. J. Aerosol Med. 2002, 15, 213220.

(22) Donaldson, K.; Li, X. Y.; Macnee, W. J. Aerosol Sci. 1998, 29, 553-560.

(23) Brown, L. M.; Collings, N.; Harrison, R. M.; Maynard, A. D.; Maynard, R. L. Philos. Trans. R. Soc. London Ser. A 2000, 358, $2563-2565$.

(24) Li, N.; Sioutas, C.; Cho, A.; Schmitz, D.; Misra, C.; Sempf, J.; Wang, M. Y.; Oberley, T.; Froines, J.; Nel, A. Environ. Health Perspect 2003, 111, 455-460.

(25) Kulmala, M.; Pirjola, U.; Makela, J. M. Nature 2000, 404, 66-69.

(26) Adams, P. J.; Seinfeld, J. H. Geophys. Res. Lett. 2003, 30.

(27) Kulmala, M.; Korhonen, P.; Napari, I.; Karlsson, A.; Berresheim, H.; O'Dowd, C. D. J. Geophys. Res.-Atmos. 2002, 107, 8111.

(28) Lazaridis, M. Atmos. Environ. 2001, 35, 599-607.

(29) Zhang, K. M.; Wexler, A. S. J. Geophys. Res.-Atmos. 2002, 107, 4577.

(30) Kerminen, V. M. J. Aerosol Sci 1999, 30, 1069-1078.

(31) Kulmala, M.; Dal Maso, M.; Makela, J. M.; Pirjola, L.; Vakeva, M.; Aalto, P.; Miikkulainen, P.; Hameri, K.; O'Dowd, C. D. Tellus Ser. B 2001, 53, 479-490.

(32) Birmili, W.; Wiedensohler, A.; Heintzenberg, J.; Lehmann, K. J. Geophys. Res.-Atmos. 2001, 106, 32005-32018.

(33) McMurry, P. H.; Woo, K. S.; Weber, R.; Chen, D. R.; Pui, D. Y. H. Philos. Trans. R. Soc. London Ser. A 2000, 358, 2625-2642.

(34) Smith, J. N.; Moore, K. F.; McM urry, P. H.; Eisele, F. L. Aerosol Sci. Technol. 2004, 38, 100-110.

(35) Voisin, D.; Smith, J. N.; Sakurai, H.; McMurry, P. H.; Eisele, F. L. Aerosol Sci. Technol. 2003, 37, 471-475.

(36) Smith, J. N.; Moore, K. F.; Eisele, F. L.; Ghimire, A. K.; Sakurai, H.; McMurry, P. H. In Proceedings of the ACS Nanotechnology and the Environment Symposium (submitted for publication).

(37) Jayne, J. T.; Leard, D. C.; Zhang, X.; Davidovits, P.; Smith, K. A.; Kolb, C. E.; Worsnop, D. R. Aerosol Sci. Technol. 2000, 33, 4970.

(38) Jimenez, J. L.; Jayne, J. T.; Shi, Q.; Kolb, C. E.; Worsnop, D. R.; Yourshaw, I.; Seinfeld, J. H.; Flagan, R. C.; Zhang, X.; Smith, K. A.; Morris, J. W.; Davidovits, P. J. Geophys. Res.-Atmos. 2003, 108, 8425, doi: 8410:1029/2001JD001213.

(39) Jimenez, J. L.; Bahreini, R.; Cocker, D. R.; Zhuang, H.; Varutbangkul, V.; Flagan, R. C.; Seinfeld, J. H.; O'Dowd, C.; Hoffmann, T. J. Geophys. Res.-Atmos. 2003, 108, 4318, doi: 4310.1029/ 2002JD002452.

(40) Wittig, A. E.; Anderson, N.; Khlystov, A. Y.; Pandis, S. N.; Davidson C.; Robinson, A. L. Atmos. Environ. 2004, 38, 3107-3125.

(41) Allan, J. D.; Jimenez, J. L.; Williams, P. I.; Alfarra, M. R.; Bower, K. N.; Jayne, J. T.; Coe, H.; Worsnop, D. R. J. Geophys. Res.Atmos. 2003, 108, 4090, doi: 4010.1029/2002JD002358.

(42) Zhang, Q.; Canagaratna, M. C.; Jayne, J. T.; Worsnop, D. R.; Jimenez, J. J. Geophys. Res.-Atmos. (in press).

(43) Allan, J. D.; Coe, H.; Bower, K. N.; Alfarra, M. R.; Delia, A. E.; Jimenez, J. L.; Middlebrook, A. M.; Drewnick, F.; Onasch, T. B.; 
Canagaratna, M. R.; Jayne, J. T.; Worsnop, D. R. J. Aerosol Sci. 2004, 35, 909-922, doi: 910.1016/j.jaerosci.2004.1002.1007.

(44) Canagaratna, M. R.; Jayne, J. T.; Ghertner, D. A.; Herndon, S.; Shi, Q.; Jimenez, J. L.; Silva, P.J.; Williams, P.; Lanni, T.; Drewnick, F.; Demerjian, K. L.; Kolb, C. E.; Worsnop, D. R. Aerosol Sci. Technol. 2004, 38, 555-573, 510.1080/02786820490465504.

(45) Pirjola, L.; Kulmala, M.; Wilck, M.; Bischoff, A.; Stratmann, F.; Otto, E. J. Aerosol Sci. 1999, 30, 1079-1094.

(46) Park, S. H.; Lee, K. W.; Otto, E.; Fissan, H. J. Aerosol Sci. 1999, 30, 3-16.

(47) Baron, P. A.; Willeke, K. Aerosol Measurement: Principles, Techniques, and Applications; Wiley-Interscience: New York, 2001.

(48) Alfarra, M. R.; Coe, H.; Allan, J. D.; Bower, K. N.; Boudries, H.; Canagaratna, M. R.; Jimenez, J. L.; Jayne, J. T.; Garforth, A.; Li, S.-M.; Worsnop, D. R. Atmos. Environ. (in press).
(49) Drewnick, F.; Schwab, J. J.; Jayne, J. T.; Canagaratna, M.; Worsnop, D. R.; Demerjian, K. L. Aerosol Sci. Technol. 2004, 38, 92-103.

(50) Zhang, Q.; Alfarra, M. R.; Worsnop, D. R.; Allan, J. D.; Coe, H.; Canagaratna, M. R.; Jimenez, J. Environ. Sci. Technol. (manuscript in progress).

(51) Fraser, M. P.; Cass, G. R. Environ. Sci. Technol. 1998, 32, 10531057.

(52) Napari, I.; Noppel, M.; Vehkamaki, H.; Kulmala, M. J. Geophys. Res.-Atmos. 2002, 107, 4381.

Received for review December 17, 2003. Revised manuscript received June 22, 2004. Accepted June 24, 2004.

ES035417U 


\title{
Semi-continuous $\mathrm{PM}_{2.5}$ inorganic composition measurements during the Pittsburgh Air Quality Study
}

\author{
Ann E. Wittig ${ }^{\mathrm{a}, *}$, Satoshi Takahama ${ }^{\mathrm{b}}$, Andrei Y. Khlystov ${ }^{\mathrm{c}}$, Spyros N. Pandis ${ }^{\mathrm{b}, \mathrm{d}}$, \\ Susanne Hering ${ }^{\mathrm{e}}$, Brent Kirby ${ }^{\mathrm{e}}$, Cliff Davidson ${ }^{\mathrm{d}, \mathrm{f}}$ \\ ${ }^{a}$ Department of Civil Engineering, City College of New York, New York, NY 10031, USA \\ ${ }^{\mathrm{b}}$ Department of Chemical Engineering, Carnegie Mellon University, Pittsburgh, PA 15213, USA \\ ${ }^{\mathrm{c}}$ Department of Civil and Environmental Engineering, Duke University, Durham, NC 27708, USA \\ ${ }^{\mathrm{d}}$ Department of Engineering and Public Policy, Carnegie Mellon University, Pittsburgh, PA 15213, USA \\ ${ }^{\mathrm{e}}$ Aerosol Dynamics Inc., 2329 4th Street, Berkeley, CA 94710, USA \\ ${ }^{\mathrm{f}}$ Department of Civil and Environmental Engineering, Carnegie Mellon University, Pittsburgh, PA 15213, USA
}

\begin{abstract}
A method for semi-continuous (10 min time resolution) $\mathrm{PM}_{2.5}$ nitrate and sulfate measurements, based on the humidified impaction with flash volatilization design of Stolzenburg and Hering (Environ. Sci. Technol. 34 (2000) 907), was evaluated during the Pittsburgh Air Quality Study (PAQS) from July 2001 to August 2002. The semi-continuous measurements were corrected for several operating parameters. The overall corrections were less than $10 \%$ on average, but could be quite large for individual 10 min measurements. These corrections resulted in an improvement in the agreement of the measurements with the filter-based measurements, with a major axis regression relationship of $y=0.83 x+0.20 \mu \mathrm{g} \mathrm{m}^{-3}$ and $R^{2}$ of 0.84 for nitrate and $y=0.71 x+0.42 \mu \mathrm{g} \mathrm{m}^{-3}$ and $R^{2}$ of 0.83 for sulfate. The corrected semi-continuous measurements were calibrated over the entire year using collocated denuder/filterpack-based measurements. These calibrated semi-continuous measurements are used in conjunction with temporally resolved gas-phase measurements of total (gas- and aerosol-phase) nitrate and meteorological measurements to investigate short-term phenomena at the Pittsburgh Supersite. The gas-to-particle partitioning of nitrate varied daily and seasonally, with a majority of the nitrate in the particle phase at night and during the winter months.
\end{abstract}

(C) 2004 Elsevier Ltd. All rights reserved.

Keywords: Atmospheric aerosols; $\mathrm{PM}_{2.5}$ nitrate; $\mathrm{PM}_{2.5}$ sulfate; Continuous monitors

\section{Introduction}

Filter-based methods have traditionally been used to characterize water-soluble inorganic ions in $\mathrm{PM}_{2.5}$ and $\mathrm{PM}_{10}$. These filter methods typically involve the offline analysis of particle samples collected onto substrates by inertial impaction or filtration; The particles are then extracted from the substrate in water and the extract is analyzed by ion chromatography (IC) for the major inorganic PM components (Chow, 1995). Filter measurements are often performed daily, or at intervals of a

\footnotetext{
*Corresponding author.

E-mail address: wittig@ce.ccny.cuny.edu (A.E. Wittig).
}

few hours during intensive field studies. Inherent drawbacks to using filter-based methods for the measurement of inorganic aerosols include losses of volatile or reactive components from the substrate (Hering et al., 1988; Koutrakis et al., 1992; Suh et al., 1994; Hering and Cass, 1999; Babich et al., 2000; Pang et al., 2001), detection limit problems for high time temporal resolution measurements, and high labor costs.

In light of the above limitations of conventional filterbased methods, several alternate methods for temporally resolved inorganics measurements have been developed. Some methods bypass the filters and filter sampler used in the conventional method by collecting the particles directly into a liquid medium (Buhr et al., 1995; 
Khlystov et al., 1995; Liu and Dasgupta, 1996; Karlsson et al., 1997; Ito et al., 1998; Weber et al., 2001). These methods avoid the collection and extraction steps and their associated problems, although they typically employ a similar analytical technique, IC. Flame photometry has been used to perform in situ quantification of sulfate concentrations by Mueller and Collins (1980), D’Ottavio et al. (1981), Allen et al. (1984), and Lippmann et al. (2000). Another approach uses an aerodynamic particle time-of-flight to select the particle size range, followed by thermal vaporization and analysis using molecular mass spectrometry (Kolb et al., 2000). Other instruments perform single-particle composition analysis using mass spectrometry (McKeown et al., 1991; Carson et al., 1995; Marijinissen et al., 1988; Liu et al., 1999; Middlebrook et al., 2001; Prather et al., 1994). Single particle mass spectrometry measurements are generally not quantitative, although they provide valuable single-particle composition information.

We report here on an alternate method for temporally resolved inorganic aerosol measurement, that uses bulk collection followed by in situ analysis using a standard gas analyzer (Hering and Stolzenburg, 1998). Recently, Rupprecht and Patashnick (R\&P) commercialized this instrument design for semi-continuous $\mathrm{PM}_{2.5}$ nitrate (R\&P model $8400 \mathrm{~N}$ ) and $\mathrm{PM}_{2.5}$ sulfate (R\&P model 8400S) (Meyer et al., 2000). These commercialized versions of the Hering and Stolzenburg (1998) instrument are the subject of this work.

While the above semi-continuous instruments offer one to two orders of magnitude of improvement in timeresolution over the conventional filter-based techniques, their precision and accuracy relative to conventional methods has not been widely investigated over long time periods. Watson et al. (1998) used these instruments to collect high time-resolution $\mathrm{PM}_{2.5}$ nitrate and sulfate measurements across California during the California Regional PM Air Quality Study (CRPAQS). Watson et al. (2000) collected high time-resolution $\mathrm{PM}_{2.5}$ nitrate and sulfate measurements at the Fresno Supersite. Watson and Chow (2002) used these measurements to investigate a wintertime $\mathrm{PM}_{2.5}$ episode at the site. Hering et al. (2001) investigated differences in shortterm variability in ground and aloft nitrate measurements collected at the rural Angiola CRPAQS site. Hering et al. (2003) used high time-resolution $\mathrm{PM}_{2.5}$ sulfate measurements that were collected at Big Bend National Park to estimate light extinction coefficients. Several intercomparisons of the prototype instruments relative to standard methods have demonstrated the accuracy of the prototype systems (Hering and Stolzenburg, 1998; Stolzenburg and Hering; 2000; Liu et al., 2000). However, issues raised during the current intercomparison suggest that the commercialized versions of these instruments need to be investigated as well, and under various conditions.
In this work, the performance of the semi-continuous instruments is evaluated relative to $24 \mathrm{~h}$ integrated measurements collected using a conventional filter-based sampler and analyzed using IC. Comparisons were also performed relative to measurements collected using another temporally resolved semi-continuous method, the Khlystov et al. (1995) steam sampler, and analyzed using IC. The R\&P semi-continuous nitrate and sulfate instruments operated from July 2001 to August 2002, the steam sampler operated from July 2001 to March 2002, and the filter sampler operated from July 2001 to March 2002. The objectives of this paper are (1) to evaluate the accuracy of the semi-continuous measurements relative to the standard filter-based measurements, (2) to evaluate the correction and calibration techniques used to improve their accuracy, and (3) to discuss temporally resolved variations in nitrate and sulfate measured at the PAQS Supersite.

\section{Ambient inorganic PM and gas measurements}

The central site was located in Schenley Park, a 456 acre wooded park in the greater Pittsburgh area, approximately $6 \mathrm{~km}$ from the downtown area and $500 \mathrm{~m}$ from the nearest heavily traveled street.

\subsection{Semi-continuous inorganic $P M$ and gas measurements}

Semi-continuous nitrate and sulfate aerosol concentrations were measured on a $10 \mathrm{~min}$ basis using Rupprecht and Patashnick (R\&P) instrument models $8400 \mathrm{~N}$ and $8400 \mathrm{~S}$, respectively. The R\&P instrument model $8400 \mathrm{~N}$ follows a two-step process to measure $\mathrm{PM}_{2.5}$ nitrate (Stolzenburg and Hering, 2000). In the first step, the ambient $\mathbf{P M}_{2.5}$ is sampled through a sharpcut cyclone, denuder, and humidifier and impacted for $8 \mathrm{~min}$ on a Nichrome ${ }^{\mathbb{R}}$ flash strip that is mounted in an integrated collection and vaporization (ICV) cell. The sharp-cut cyclone, denuder, and humidifier remove larger particles and vapors and increase the collection efficiency of the remaining particles; particle collection is most efficient for particles above $0.1 \mu \mathrm{m}$. In the second step, the collected ambient $\mathrm{PM}_{2.5}$ is analyzed using a standard high-sensitivity $\mathrm{NO}_{x}$ gas analyzer. A flow path to the gas analyzer is established using a carrier gas of purified nitrogen, which also serves to purge the ICV cell of ambient gases. The baseline response of the gas analyzer is recorded. Then the collected particles are thermally vaporized in place, catalytically reducing the nitrate to $\mathrm{NO}_{x}$. The evolved gas is drawn into the carrier gas stream and delivered to the gas analyzer for analysis. The R\&P instrument model $8400 \mathrm{~S}$ is similar in design and operation, except that platinum flash strips are used to collect the $\mathrm{PM}_{2.5}$, purified air is used as the carrier 
gas, and a high-sensitivity $\mathrm{SO}_{2}$ gas analyzer is used to measure the evolved gas. For both instruments, the instrument output is an ambient concentration calculated as the difference between the integrated areas under the evolved gas response curve and the baseline, adjusted for theoretical conversion efficiency, sample flow rate and gas analyzer flow rate.

During routine operation, the instrument offset, actual conversion efficiency, gas analyzer efficiency, sample flow rate, and reaction cell vacuum were measured to assess the performance of the semicontinuous instruments. The instrument offset was measured on a biweekly basis during PAQS by placing a HEPA filter on the inlet of the instrument, and collecting and averaging three consecutive $10 \mathrm{~min}$ measurements. The instrument offset for the whole study was $0.18 \pm 0.15 \mu \mathrm{g} \mathrm{m}^{-3}$ (average \pm one standard deviation) for the nitrate instrument and $0.30 \pm 0.16 \mu \mathrm{g} \mathrm{m}^{-3}$ for the sulfate instrument. An evaluation of the daily variability over a 2 week period indicated that the instrument offset varied as much daily as it did biweekly.

The conversion efficiency was also quantified on a biweekly basis by performing aqueous standard calibrations. Conversion efficiency is a measure of the ability of the instrument to convert a microliter aliquot of a standard solution of ammonium nitrate to $\mathrm{NO}_{x}$ or ammonium sulfate to $\mathrm{SO}_{2}$. The aliquot of the standard solution is applied directly to the flash strip using a syringe. Three duplicate measurements were made at five points over the working range of the instruments (0-28 $\mathrm{g} \mathrm{m} \mathrm{m}^{-3}$ for nitrate, and $0-29.4 \mu \mathrm{g} \mathrm{m}^{-3}$ for sulfate). The study average conversion efficiency was $0.85 \pm 0.08$ for the nitrate and $0.65 \pm 0.07$ for the sulfate instrument; typical correlation coefficients of the relationships were greater than 0.99 . An evaluation of the daily variability of the conversion efficiency over a 2 week period indicated that like the instrument offset, the conversion efficiency varied as much daily as it did biweekly.

Gas analyzer efficiency was measured daily (nitrate) and every 4 days (sulfate) at PAQS. The gas analyzer efficiency is a measure of the accuracy of the gas analyzer and is determined by sequentially routing two calibration gases through the ICV cell to the gas analyzer. Purified nitrogen (nitrate) or air (sulfate) is sent to the gas analyzer first. A calibration gas mixture of $5080 \mathrm{ppbv} \mathrm{NO}$ in nitrogen (nitrate) or $790 \mathrm{ppbv} \mathrm{SO}_{2}$ in air (sulfate) is sent to the gas analyzer second. The gas analyzer efficiency was calculated as the ratio of the instrument response to calibration gas divided by the actual concentration of the calibration gas. The study average gas analyzer efficiency was stable at $0.99 \pm 0.04$ for the nitrate instrument and $0.99 \pm 0.06$ for the sulfate instrument.

The actual sample flow rate was measured on a monthly basis to quantify drift in the flow calibration using a certified flow standard. The study average ratio of the actual sample flow rate to the instrument indicated sample flow rate was $0.91 \pm 0.15$ for the nitrate instrument and $1.21 \pm 0.21$ for the sulfate instrument.

The nitrate gas analyzer reaction cell vacuum was recorded on a $10 \mathrm{~min}$ time basis to monitor deviations from the vacuum setpoint as a result of variable or declining pump performance. Vacuum deviations affect the accuracy of the semi-continuous measurements as well as the accuracy of the instrument offset, conversion efficiency and gas analyzer efficiency measurements. Kirby and Hering (2001) showed that $\mathrm{PM}_{2.5}$ nitrate measurements made during the CRPAQS field campaign decreased by $13 \%$ for every inch of $\mathrm{Hg}$ that the vacuum in the gas analyzer reaction cell increased from its setpoint.

Total ( $\mathrm{PM}_{2.5}$ and gas) nitrate measurements were also made on a $1-2 \mathrm{~h}$ basis using the steam sampler developed by Khlystov et al. (1995) with analysis by IC. The steam sampler inlet included a Teflon-coated $\mathrm{PM}_{2.5}$ cyclone and a Teflon-coated $20 \mathrm{~cm}$ length 1 -in ID aluminum pipe maintained at ambient conditions. Losses of total nitrate in the inlet were evaluated and found to be negligible. The steam sampler draws 16.7 LPM of ambient air through the inlet and mixes it with $2 \mathrm{~g} \mathrm{~min}^{-1}$ of steam, supersaturating the sample air with water vapor. The supersaturation causes a rapid growth of water droplets on the particles. At the same time, condensing water dissolves water soluble gases present in the sample air. The droplets and the condensed water, containing dissolved aerosol species and gases, are collected using two cyclones in series and automatically delivered into vials for offline analysis of major inorganic ions by IC.

\subsection{Filter-based inorganic measurements}

Measurements of total $\left(\mathrm{PM}_{2.5}\right.$ and gas) and $\mathrm{PM}_{2.5}$ nitrate, and $\mathrm{PM}_{2.5}$ sulfate were also made on a 4,6 , or $24 \mathrm{~h}$ time basis using a denuder- filter-based collection system and IC analysis (Chow, 1995). The filter-based sampler had two parallel lines, one with a $\mathrm{PM}_{2.5}$ cyclone and filter pack and one with a denuder upstream of the $\mathrm{PM}_{2.5}$ cyclone and filter pack. The filter packs contained a PTFE Teflon filter (Whatman Cat. No. 7592-104), a nylon filter (Whatman Cat. No. 7410-004), and a backup Cellulose-fiber filter (Whatman Cat. No. 1441047) in series. Ambient air was drawn through both lines at a flow rate of 16.7 LPM. $\mathrm{PM}_{2.5}$ sulfate and nitrate measurements were determined from the analysis of the Teflon filter and the Teflon and nylon filters, respectively, while inorganic gas-phase measurements were determined by difference of the same filters on the two parallel lines. Measurements of gas-phase and aerosol nitrate using this configuration have minimal artifacts 
and will therefore provide a basis for comparison for the semi-continuous method evaluation.

\section{Corrections to semi-continuous ambient PM measurements}

The raw semi-continuous measurements were corrected for sampling blanks (instrument offset) and analytical system calibrations (conversion efficiency). Corrections were also applied to the $10 \mathrm{~min}$ ambient nitrate and sulfate measurements to account for a software error, and correct for gas analyzer efficiency, vacuum drift, and sample flow rate drift.

\subsection{Raw semi-continuous measurements}

The output of the semi-continuous instruments is an ambient nitrate or sulfate measurement that is calculated as

$C_{i}^{*}=1000 \frac{\left(\int C_{\mathrm{s}, i} \mathrm{~d} t-\int C_{\mathrm{b}, i} \mathrm{~d} t\right)}{t_{\mathrm{s}, i} e f_{\mathrm{c}, i}} \frac{\mathrm{MW}_{i}}{V} \frac{Q_{\mathrm{c}, i}}{Q_{\mathrm{s}, i}}$,

where $i$ is nitrate or sulfate, $C_{i}^{*}$ the raw semi-continuous measurement corrected for the manufacturer software error in $\mu \mathrm{g} \mathrm{m}^{-3}, C_{\mathrm{s}, i}$ is the gas-phase concentration of $\mathrm{NO}_{x}$ or $\mathrm{SO}_{2}$ measured by the gas analyzer after the sample has been flash volatilized in ppb, $C_{\mathrm{b}, i}$ is the background gas-phase concentration of $\mathrm{NO}_{x}$ or $\mathrm{SO}_{2}$ measured by the gas analyzer in ppb, $t$ is the duration of the flash response measured by the gas analyzer in seconds, $\mathrm{MW}_{i}$ is molecular weight of nitrate $\left(62.01 \mathrm{~g} \mathrm{~mol}^{-1}\right)$ or sulfate $\left(96.06 \mathrm{~g} \mathrm{~mol}^{-1}\right), V$ is the molar volume of the carrier gas $\left(0.0224 \mathrm{~m}^{3} \mathrm{~mol}^{-1}\right), Q_{\mathrm{c}, i}$ is the carrier gas flow rate in $1 \mathrm{~min}^{-1}, Q_{\mathrm{s}, i}$ is the instrument indicated sample flow rate that is impinged on the flash strip in $1 \mathrm{~min}^{-1}, t_{\mathrm{s}, i}$ is the total sample duration in seconds, and $\mathrm{ef}_{\mathrm{c}, i}$ is the theoretical conversion efficiency (from zero to one) of the instrument to reduce aerosol nitrates to $\mathrm{NO}_{x}$ or sulfates to $\mathrm{SO}_{2}$; Values of 0.82 and 0.69 were determined from factory calibrations of the nitrate and sulfate instruments, respectively, and were programmed into the instrument software by the manufacturer.

\subsection{Software error correction}

A correction was applied to the raw ambient measurements to account for an error in $8400 \mathrm{~S}$ and $8400 \mathrm{~N}$ software versions prior to 0.703 . Version 0.702 was used during the study. The software error correction was $3 \%$ for nitrate and $11 \%$ for sulfate.

\subsection{Instrument offset correction}

Biweekly instrument offset measurements were used to assign a single instrument offset value to all times between the offset measurements. The resultant offset values were averaged over a rolling 30 day

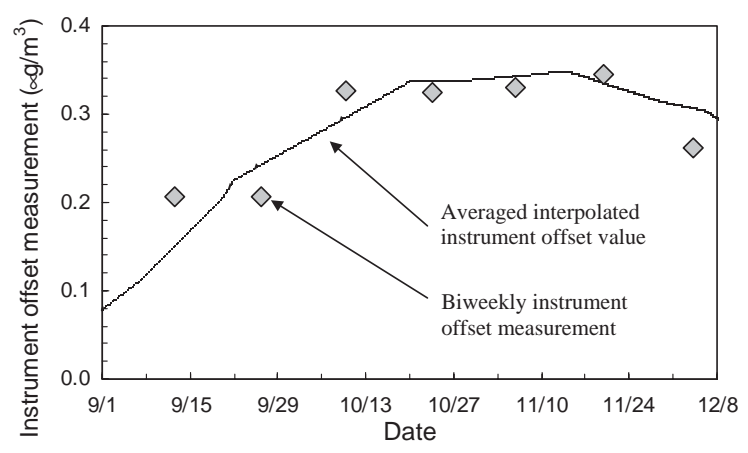

Fig. 1. Sulfate biweekly instrument offset measurements for the fall of 2001 and averaged instrument offset values used to adjust the ambient measurements, which were continuous and smoothed relative to the biweekly offset measurements.

period by

$C_{\mathrm{o}, i}(\tau)=\frac{\sum_{\tau-15 \text { days }}^{\tau+15 \text { days }} C_{\mathrm{o}, i \text {,assigned }}(\tau)}{N}$,

where $C_{\mathrm{o}, i}$ is the averaged instrument offset measurement at date $\tau, i$ is nitrate or sulfate, $C_{\mathrm{o}, i \text {,assigned }}$ is the instrument offset for nitrate or sulfate measured prior to date $\tau, \tau$ is the Julian date, and $N$ is the number of offset values averaged together over the rolling 30 day period centered at date $\tau$. This averaging procedure was used to incorporate daily variability into the biweekly instrument offset measurements, as shown in Fig. 1. Averaged $10 \mathrm{~min}$ instrument offset measurements were subtracted from the software corrected ambient measurements. The instrument offset correction ranged from $1 \%$ to $92 \%$ (18\% on average) for nitrate and $0.1 \%$ to $53 \%$ ( $9 \%$ on average) for sulfate.

\subsection{Conversion efficiency correction}

The raw semi-continuous measurements are calculated by the instrument software using the factory measured ('theoretical') conversion efficiencies. Deviations from the theoretical conversion efficiency were accounted for during data correction. The software corrected ambient measurements were multiplied by the theoretical conversion efficiency and divided by the actual measured conversion efficiency. Biweekly conversion efficiency measurements were used to assign a single conversion efficiency value to all times between the efficiency measurements. The resultant efficiency values were averaged over a rolling 30 day period. The conversion efficiency correction ranged from $-38 \%$ to $16 \%(-4 \%$ on average) for nitrate and $-32 \%$ to $36 \%$ $(-7 \%$ on average) for sulfate.

\subsection{Gas analyzer efficiency correction}

The gas analyzer efficiency is assumed to be unity in the raw semi-continuous measurement calculation. 
Deviations in the gas analyzer efficiency from unity were corrected for by dividing the nitrate and sulfate measurements by the actual gas analyzer efficiency. To account for slowly drifting efficiencies, gas analyzer efficiency measurements made daily or every 4 days were used to assign a single gas analyzer efficiency value to all times between the efficiency measurements. The resultant efficiency values were averaged over a rolling 2 day period (nitrate) or 8 day period (sulfate). The gas analyzer efficiency correction ranged from $-19 \%$ to $4 \%$ $(-1 \%$ on average) for nitrate and $-4 \%$ to $18 \%(1 \%$ on average) for sulfate.

\subsection{Sample flow drift correction}

Semi-continuous measurements were also corrected for discrepancies between the measured sample flow rate and the indicated (by the instrument) sample flow rate. The sample flow rate correction ranged from $-12 \%$ to $18 \%$ ( $-3 \%$ on average) for nitrate and $-90 \%$ to $98 \%$ $(-6 \%$ on average) for sulfate.

\subsection{Gas analyzer vacuum drift correction}

Differences between the $\mathrm{NO}_{x}$ gas analyzer reaction cell vacuum and the vacuum setpoint of $5.0 \mathrm{in} \mathrm{Hg}$ affected the semi-continuous nitrate measurements, and the nitrate instrument offset, conversion efficiency and gas analyzer efficiency measurements. These discrepancies were corrected by multiplying the software corrected ambient measurement, conversion efficiency or gas analyzer efficiency measurements by the linear relationship proposed by Kirby and Hering (2001). The reaction cell vacuum correction ranged from $-24 \%$ to $5 \%$ $(-1 \%$ on average) for the semi-continuous nitrate measurements.

\subsection{Corrected semi-continuous measurements}

The final corrected semi-continuous sulfate measurement was calculated from the raw semi-continuous measurement as

$C_{\mathrm{S}}=\left(C_{\mathrm{S}}^{*}-C_{\mathrm{o}, \mathrm{S}}\right) \frac{\mathrm{ef}_{\mathrm{c}, \mathrm{S}}}{\mathrm{ef}_{\mathrm{c}, \mathrm{S}}^{\prime}} \frac{1}{\mathrm{ef}_{\mathrm{ga}, \mathrm{S}}} \frac{Q_{\mathrm{s}, \mathrm{S}}}{Q_{\mathrm{s}, \mathrm{S}}^{\prime}}$,

where $C_{\mathrm{S}}$ is the corrected semi-continuous sulfate measurement in $\mu \mathrm{g} \mathrm{m}^{-3}, C_{\mathrm{S}}^{*}$ is the raw semi-continuous measurement corrected for the manufacturer software error in $\mu \mathrm{g} \mathrm{m}^{-3}, C_{\mathrm{o}, \mathrm{S}}$ is the averaged instrument offset measurement in $\mu \mathrm{g} \mathrm{m}^{-3}$, ef $\mathrm{c}, \mathrm{S}$ is the theoretical conversion efficiency, $\mathrm{ef}_{\mathrm{c}, \mathrm{S}}^{\prime}$ is the actual conversion efficiency, averaged, and corrected for gas analyzer efficiency, ef $\mathrm{ga}_{\mathrm{ga}} \mathrm{S}$ is the averaged actual gas analyzer efficiency, $Q_{\mathrm{s}, \mathrm{S}}$ is the instrument indicated sample flow rate in LPM, and $Q_{\mathrm{s}, \mathrm{S}}^{\prime}$ is the actual sample flow rate in LPM. The overall correction to the semi-continuous sulfate measurements was $-1 \%$ on average but ranged from $-90 \%$ to $100 \%$ for individual points.

The final corrected semi-continuous nitrate measurement was calculated from the raw semi-continuous measurement as

$$
\begin{aligned}
C_{\mathrm{N}}= & \left(C_{\mathrm{N}}^{*}-C_{\mathrm{o}, \mathrm{N}}\right) \frac{\mathrm{ef}_{\mathrm{c}, \mathrm{N}}}{\mathrm{ef}_{\mathrm{c}, \mathrm{N}}^{\prime}} \frac{1}{\mathrm{ef}_{\mathrm{ga}, \mathrm{N}}} \frac{Q_{\mathrm{s}, \mathrm{N}}}{Q_{\mathrm{s}, \mathrm{N}}^{\prime}} \\
& \times\left[1+0.13\left(V_{N}-5.0\right)\right],
\end{aligned}
$$

where $C_{\mathrm{N}}$ is the corrected semi-continuous nitrate measurement in $\mu \mathrm{g} \mathrm{m}^{-3}, C_{\mathrm{N}}^{*}$ is the raw semi-continuous measurement corrected for the manufacturer software error in $\mu \mathrm{g} \mathrm{m}^{-3}, C_{\mathrm{O}, \mathrm{N}}$ is the averaged instrument offset measurement in $\mu \mathrm{g} \mathrm{m}^{-3}$, ef $_{\mathrm{c}, \mathrm{N}}$ is the theoretical conversion efficiency, $\mathrm{ef}_{\mathrm{c}, \mathrm{N}}^{\prime}$ is the actual conversion efficiency, averaged, and corrected for gas analyzer efficiency and reaction cell vacuum drift, ef $f_{\mathrm{ga}, \mathrm{N}}$ is the actual gas analyzer efficiency, averaged, and corrected for reaction cell vacuum drift, $Q_{\mathrm{s}, \mathrm{N}}$ is the instrument indicated sample flow rate in LPM, $Q_{\mathrm{s}, \mathrm{N}}^{\prime}$ is the actual sample flow rate in LPM, and $V_{\mathrm{N}}$ is the nitrate instrument gas analyzer reaction cell vacuum in inch $\mathrm{Hg}$. The overall correction to the semi-continuous nitrate measurements was $8 \%$ on average but ranged from $-62 \%$ to $93 \%$ for individual points.

\section{Field comparison with filter-based measurements}

The $10 \mathrm{~min}$ measurements were averaged on a $24 \mathrm{~h}$ basis for comparison against the daily filter-based measurements. These $24 \mathrm{~h}$ averages were considered valid when more than $75 \%$ of the possible $10 \mathrm{~min}$ measurements were available. Data recovery for the $24 \mathrm{~h}$ average semi-continuous sulfate measurements was greater than $90 \%$ for all of the study months except November 2001, and for the nitrate was greater than $80 \%$ for all months except August 2002. Data loss was associated with vacuum pump failures or excessive flash strip breakages within a single month.

With only the software error correction and the typical ambient PM corrections, the semi-continuous measurements were found to be nonlinear (sulfate) or moderately correlated (nitrate) underpredictions of the true inorganic concentrations measured using the filter-based method, as shown in Fig. 2. With all of the corrections, the semi-continuous $\mathrm{PM}_{2.5}$ nitrate and sulfate measurements were found to correlate linearly and reasonably with the filter-based measurements, as shown in Fig. 3. However, the relationship of $y=0.83 x+0.20 \mathrm{\mu g} \mathrm{m}^{-3}$ for nitrate and $y=0.71 x+$ $0.42 \mu \mathrm{g} \mathrm{m}^{-3}$ for sulfate indicated that there was still a systematic bias in the measurements. The non-unity slope in these relationships could be due to incomplete collection of particles after humidification on the flash 

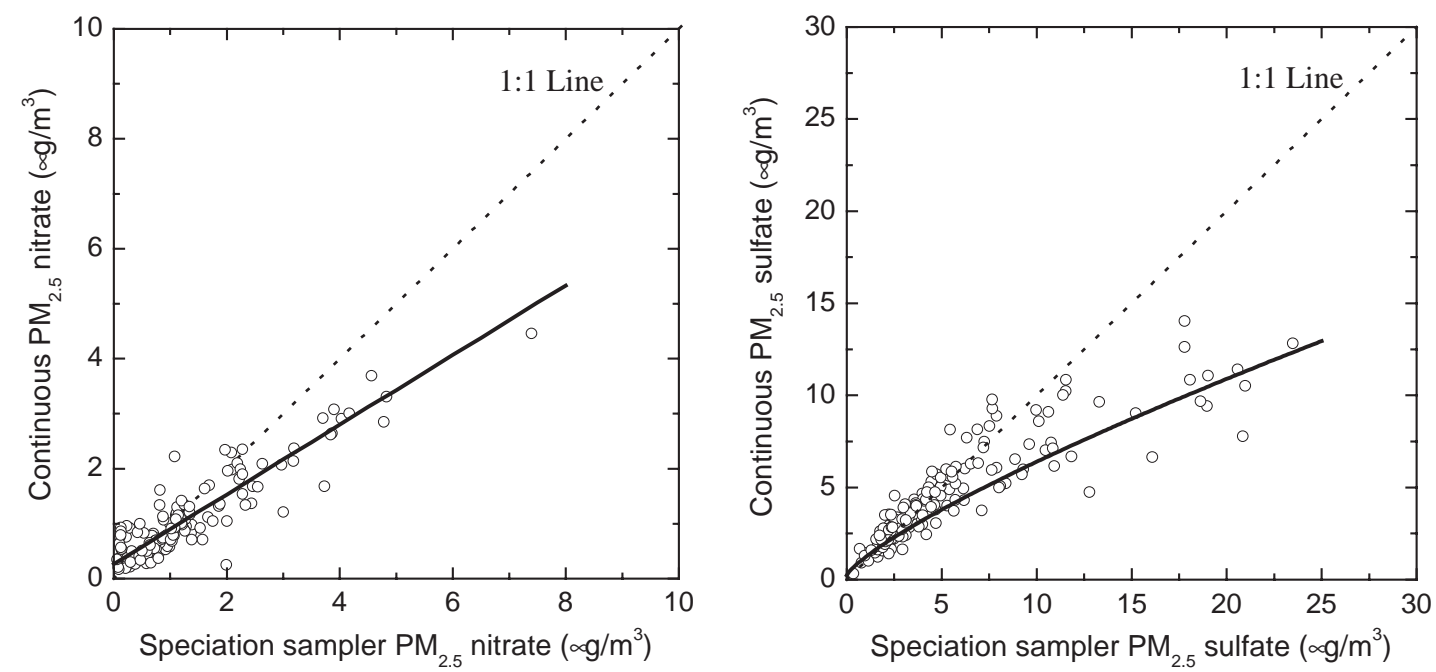

Fig. 2. Comparison of the daily averages of the instrument reported (raw measurements) semi-continuous nitrate and sulfate with the corresponding values of the filter-based speciation sampler from July 2001 to March 2002. The linear correlation (black line), $y=0.63 x+0.27 \mu \mathrm{g} \mathrm{m}^{-3}$ with $R^{2}=0.82$, was obtained for nitrate. The nonlinear correlation (black line), $y=x^{0.79}+0.23 \mu \mathrm{g} \mathrm{m}{ }^{-3}$ with $R^{2}=0.86$, was obtained for sulfate. Also shown are the $1: 1$ lines (dashed lines).
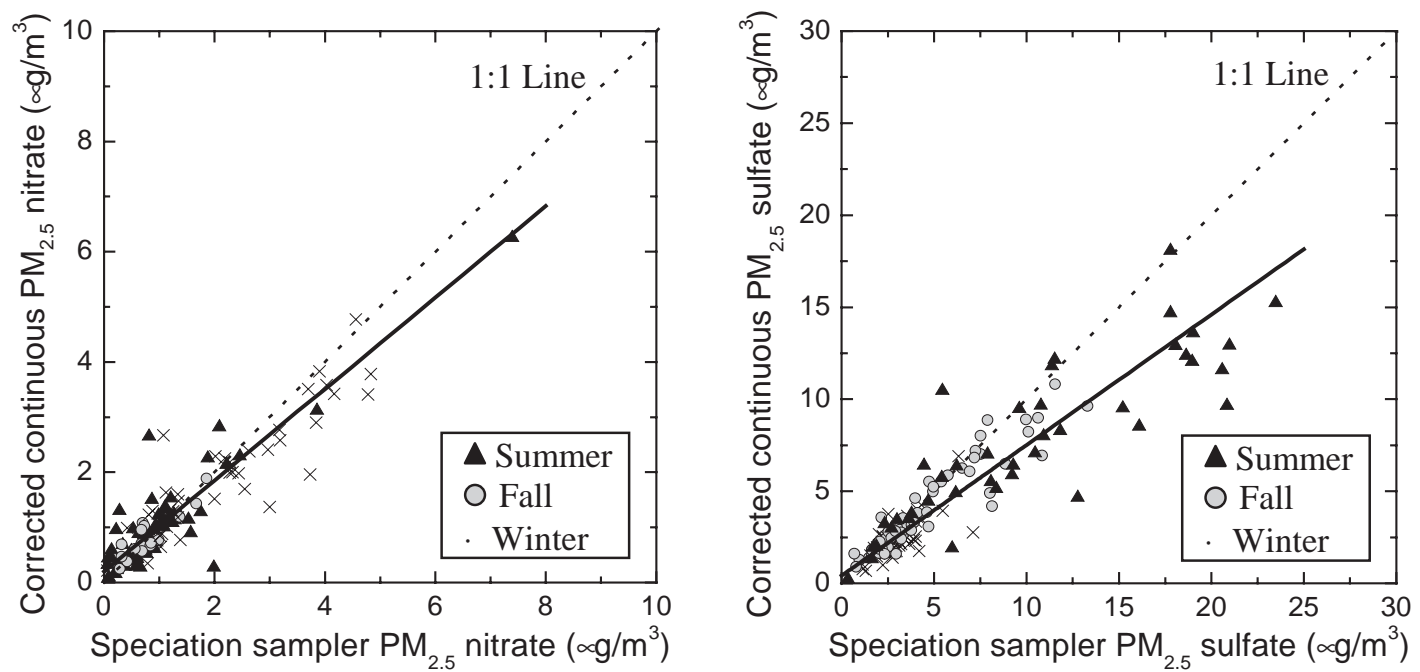

Fig. 3. Comparison of the daily averages of the corrected semi-continuous nitrate and sulfate with the corresponding values of the filter-based speciation sampler from July 2001 to March 2002. The linear correlation (black line), $y=0.83 x+0.20 \mu \mathrm{g} \mathrm{m}{ }^{-3}$ with $R^{2}=0.84$, was obtained for nitrate, and the linear correlation (black line), $y=0.71 x+0.42 \mu \mathrm{g} \mathrm{m}^{-3}$ with $R^{2}=0.83$, was obtained for sulfate. Also shown are the 1:1 lines (dashed lines).

strip or incomplete conversion of the various chemical forms of nitrate and sulfate to $\mathrm{NO}_{x}$ and $\mathrm{SO}_{2}$. The nonzero offset indicates a positive measurement artifact possibly due to organonitrates and organosulfates.

The apparent bias in the semi-continuous measurements can be reduced by calibrating the measurements against collocated filter-based measurements. The validity of this approach is based on two key assumptions: (1) individual points used to compute an average semicontinuous measurement have randomly distributed error, and (2) filter-based measurements should serve as the standard for evaluating the performance of the semi-continuous measurement methods. The first assumption is reasonable given that temporally resolved measurements of semi-continuous nitrate were consistent with independent temporally resolved measurements 

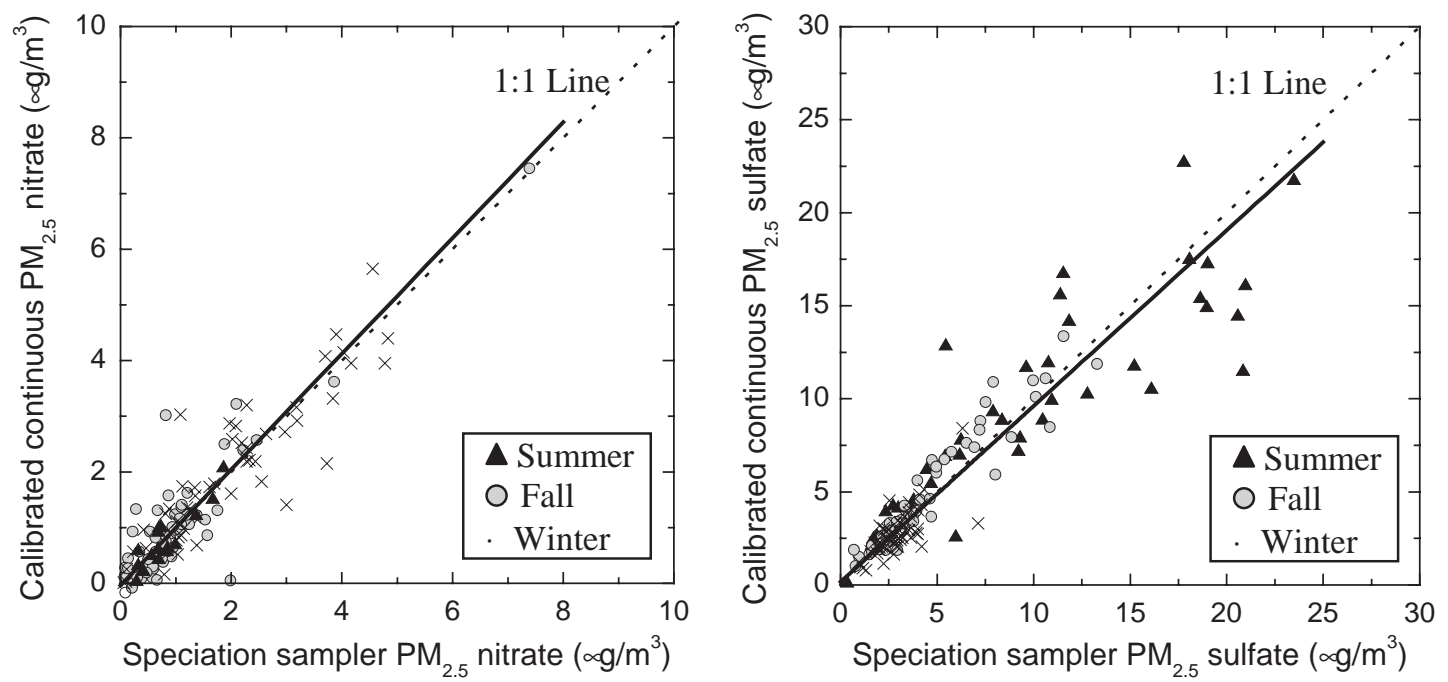

Fig. 4. Final comparison of the daily averages of the calibrated semi-continuous nitrate and sulfate with the corresponding values of the filter-based speciation sampler from July 2001 to March 2002. The linear correlation (black line), $y=1.04 x-0.04 \mu \mathrm{g} \mathrm{m}{ }^{-3}$ with $R^{2}=0.83$, was obtained for nitrate, and the linear correlation (black line), $y=0.94 x+0.17 \mu \mathrm{g} \mathrm{m}^{-3}$ with $R^{2}=0.84$, was obtained for sulfate. Also shown are the 1:1 lines (dashed lines).

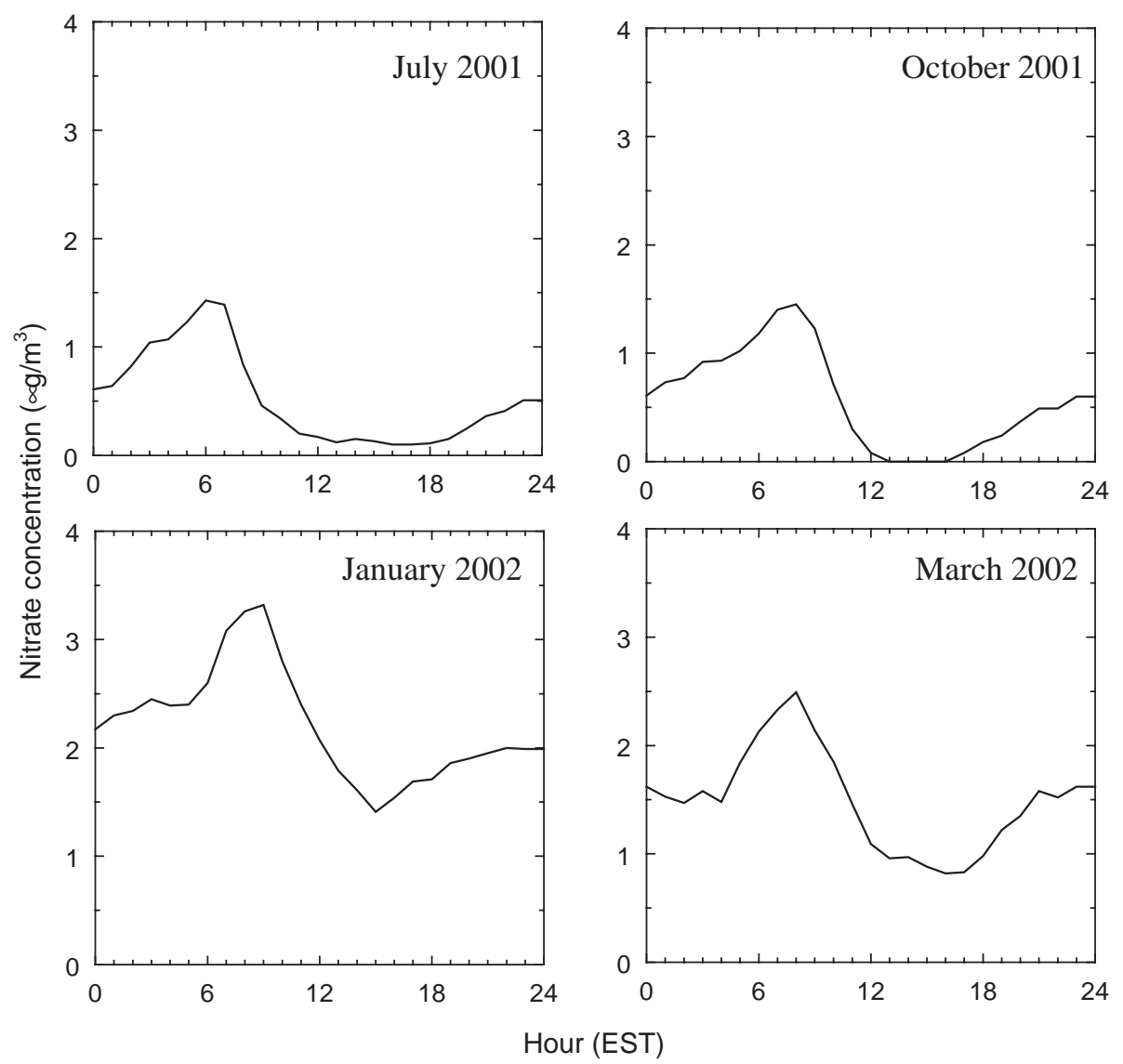

Fig. 5. Average diurnal $\mathrm{PM}_{2.5}$ nitrate monthly profiles for July 2001, October 2001, January 2002, and March 2002. 
of UV radiation and total nitrate. The second assumption is justified by the design of the filter-based sampler used in this study that minimized volatilization losses of nitrate from the filters.

Major axis regression was performed to account for measurement error in both measurements. In general, regressions were performed on $24 \mathrm{~h}$ filter-based measurements and $24 \mathrm{~h}$ averages of the semi-continuous measurements. In July 2001 and January 2002, 4 and $6 \mathrm{~h}$ filter-based measurements were available. During these intensive periods, regressions were performed on the $4-6 \mathrm{~h}$ filter-based measurements and identical averages of the semi-continuous measurements basis. A robust Huber's method (Meier and Zund, 2000) was used to identify gross outliers prior to regression when more than two measurements were available. High timeresolution concentrations were obtained by calibrating the $1 \mathrm{~h}$ semi-continuous concentrations using the corrected $24 \mathrm{~h}$ averages. The final relationship of $y=1.04 x-0.04 \mu \mathrm{g} \mathrm{m}^{-3}$ for nitrate and $y=0.94 x+$ $0.17 \mu \mathrm{g} \mathrm{m}^{-3}$ for sulfate indicated that the use of this calibration procedure resulted in a more accurate

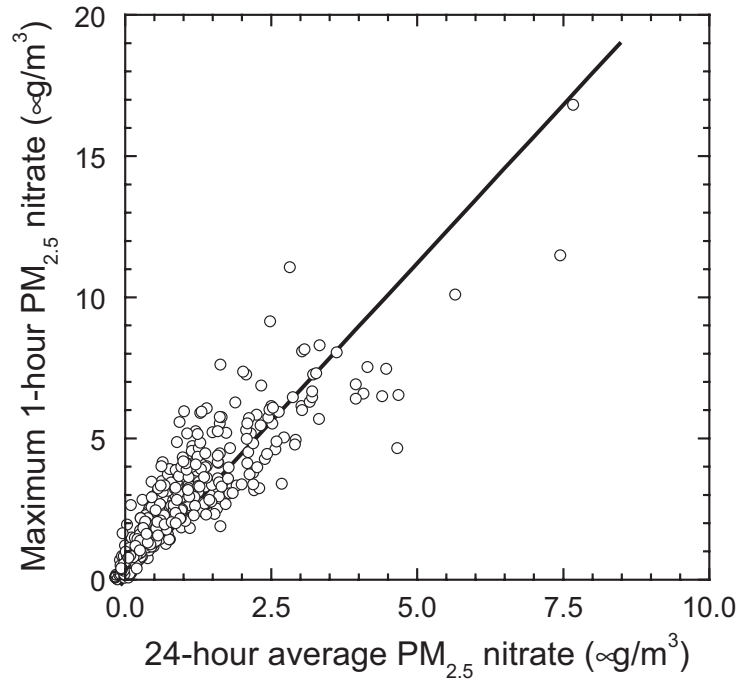

Fig. 6. The linear correlation of the 24-h average nitrate and the maximum daily 1 -h average nitrate for all points from July 2001 to August 2002: $y=2.24 x \mu \mathrm{g} \mathrm{m}^{-3}$ with $R^{2}=0.77$.
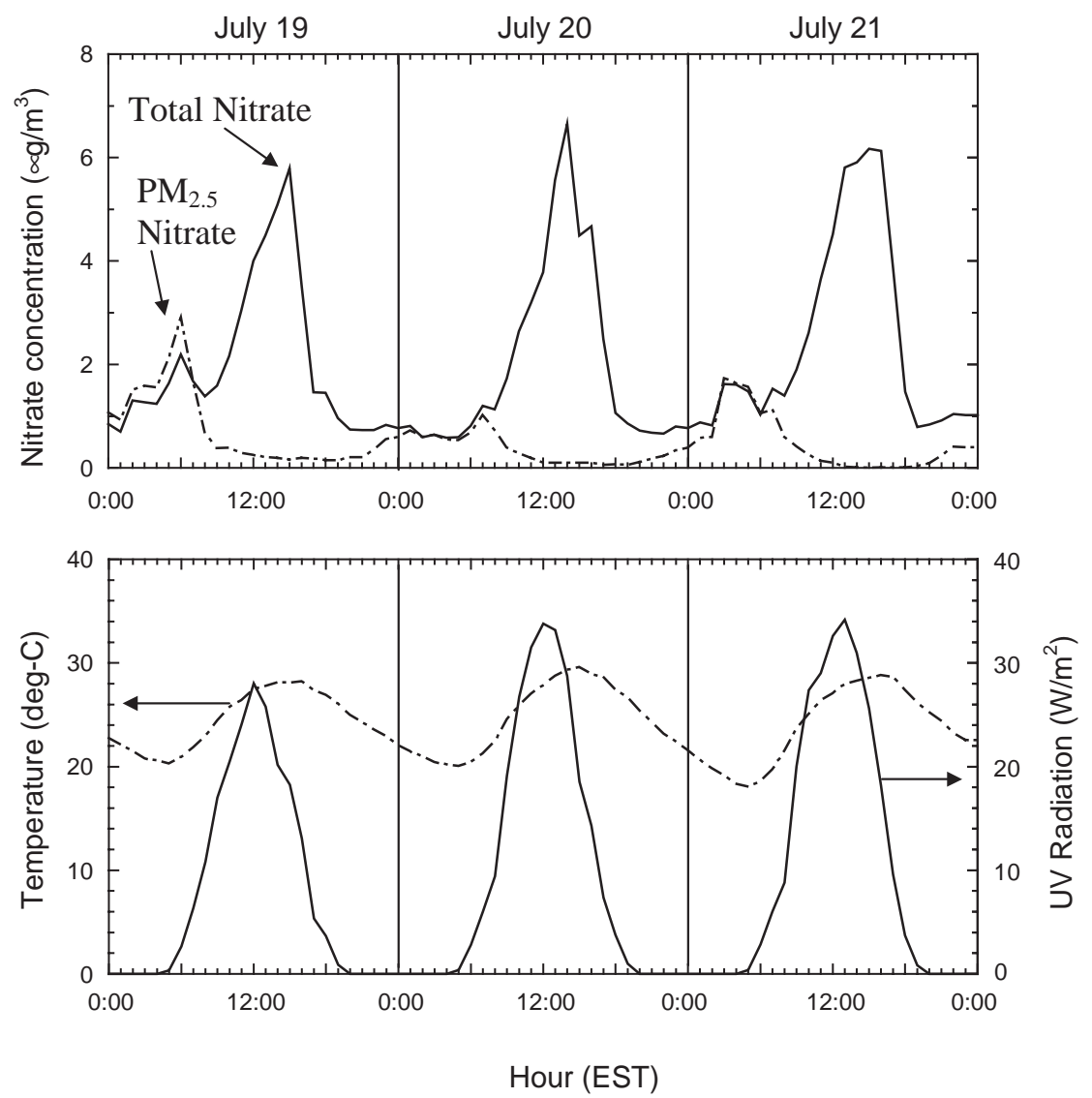

Fig. 7. Diurnal gas-to-particle partitioning of nitrate at PAQS for three consecutive days in July 2001. Also shown are hourly averaged temperature and ultraviolet (UV) radiation measurements made on the same days. 


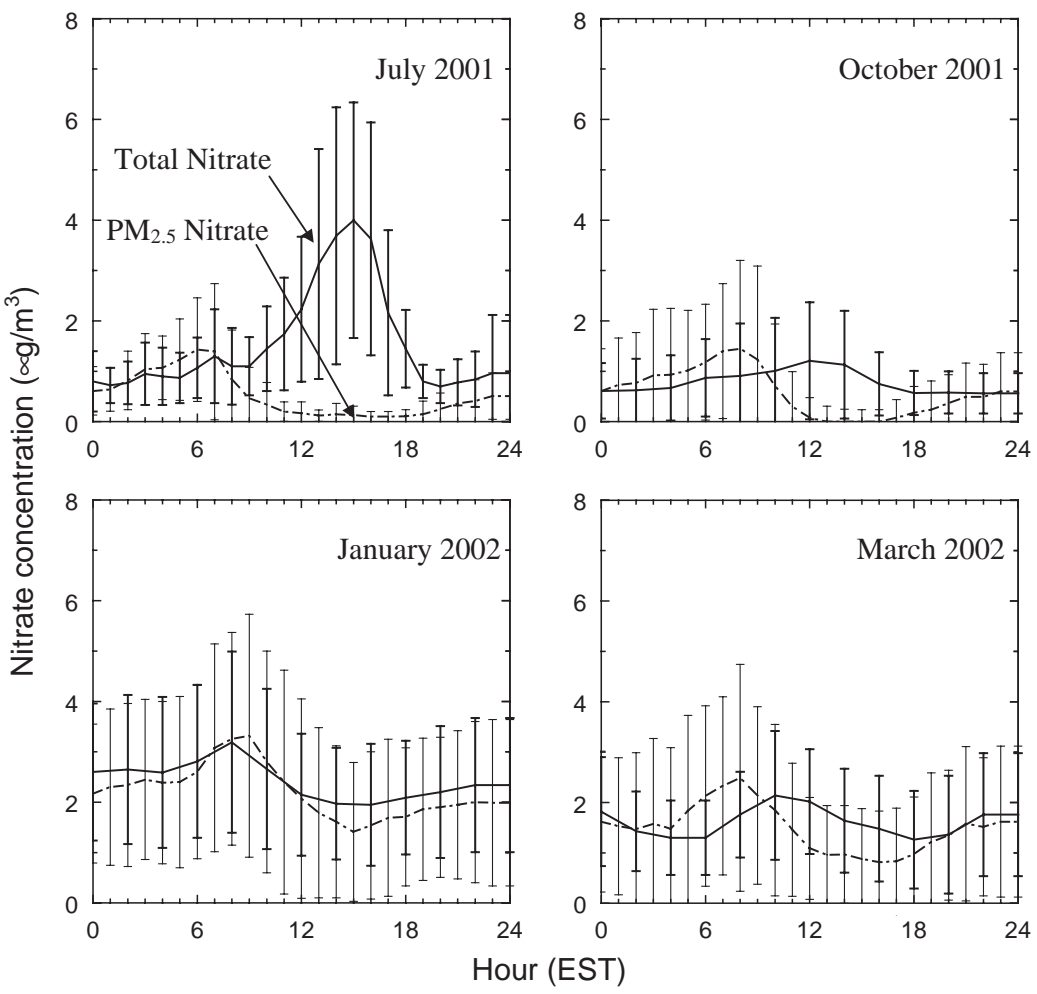

Fig. 8. Average diurnal gas-to-particle partitioning of nitrate for July 2001, October 2001, January 2002, and March 2002. Also shown is the standard deviation of the hourly measurements used to compute both monthly average diurnal profiles. $\mathrm{PM}_{2.5}$ nitrate diurnal profiles were computed from the final corrected nitrate semi-continuous measurements. Total $\left(\mathrm{PM}_{2.5}+\mathrm{Gas}_{)}\right.$nitrate diurnal profiles were computed from the steam sampler 1- to 2-h measurements.

temporally resolved data set. Fig. 4 shows these final results.

\section{Temporally resolved nitrate observations}

Average diurnal $\mathrm{PM}_{2.5}$ nitrate profiles were computed for each month in the study from the $1 \mathrm{~h}$ averages of the final semi-continuous concentrations; profiles for selected months are shown in Fig. 5. The profiles exhibit a strong diurnal pattern, with the maximum nitrate observed in the early morning a little before sunrise and minimum nitrate observed a couple hours before sunset. The time of the maximum and minimum nitrate shifted on a seasonal basis, consistent with changes in the ultraviolet radiation and temperature. The magnitudes of the maximum and minimum nitrate also changed on a seasonal basis and were greatest during the winter months. The minimum $\mathrm{PM}_{2.5}$ nitrate concentration during the winter months was non-zero and correlated with lower ambient temperatures measured at PAQS. Despite shifts in the position and magnitude of the maximum and minimum nitrate concentrations, the same general profile shape is observed regardless of

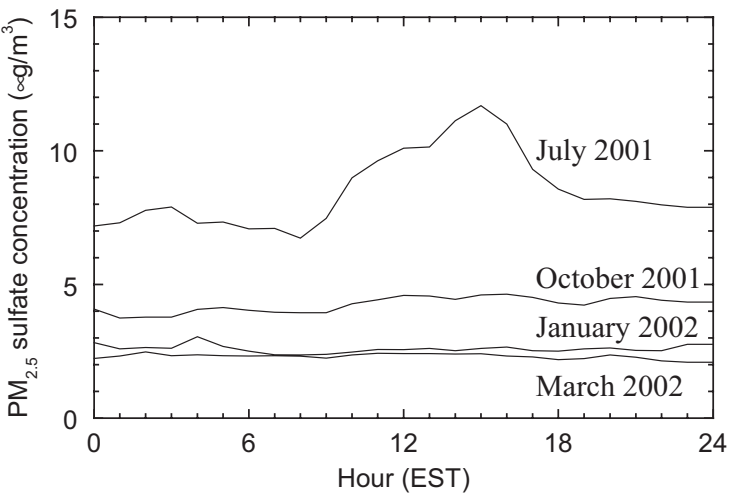

Fig. 9. Average diurnal $\mathrm{PM}_{2.5}$ sulfate profiles for July 2001, October 2001, January 2002, and March 2002.

season. This consistency is also present on a daily basis. The linear correlation of the $24 \mathrm{~h}$ average nitrate and the maximum daily $1 \mathrm{~h}$ average nitrate over the study period is shown in Fig. 6. Days that did not follow this consistent pattern are also indicated in Fig. 6 and were typically associated with a dramatic change in the air mass. 

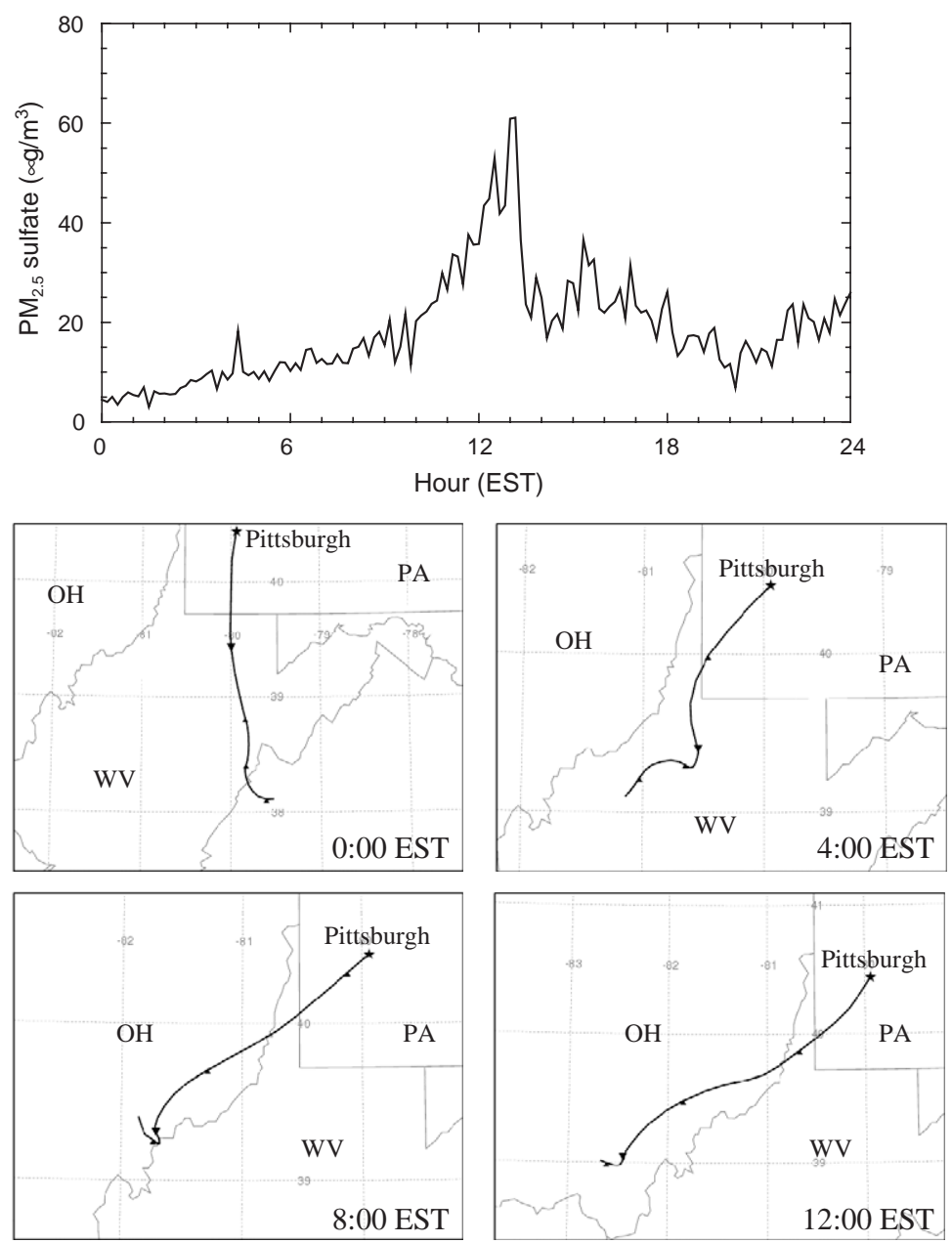

Fig. 10. 1-h average sulfate concentrations are shown for July 26, 2002. Also shown are 24-h back trajectories modeled using HYSPLIT to end at the PAQS Supersite at 0:00, 4:00, 8:00, 12:00, 14:00, 18:00, and 20:00. Triangles indicate 6-h of transport time.

The daily gas-to-particle partitioning of nitrate followed a consistent diurnal pattern as well, as shown in Fig. 7 for three consecutive days in July 2001. During the summer nights most of the nitrate was in the particle phase, while during the daytime practically all the nitrate existed in the gas phase as nitric acid vapor. Specific features of the diurnal gas-to-particle partitioning of nitrate varied seasonally, as shown in Fig. 8. During the winter almost all the nitrate was in the particle phase throughout the day. The fall and spring showed intermediate behavior, with almost all of the nitrate in the particle phase during the night and some nitrate in the gas phase during the day. At night, the nitrate is mostly in the particle phase because of the lower temperature and higher relative humidity (RH). As the sun rises and the atmosphere warms, nitrate is transferred to the gas phase. As the atmosphere continues to warm and the RH continues to decrease, the nitrate partitions almost completely to the gas phase. As the sun sets, some nitric acid vapor is partitioned back into the particle phase and some nitric acid vapor is lost by dry deposition and transport away from the city of Pittsburgh. These diurnal variations were not clear from integrated filter based measurements (even on a $6 \mathrm{~h}$ basis).

\section{Temporally resolved sulfate observations}

Average diurnal $\mathrm{PM}_{2.5}$ sulfate profiles were computed for each month in the study from the $1 \mathrm{~h}$ averages of the final sulfate measurements; profiles for selected months are shown in Fig. 9. During the summer months, the profiles exhibit a characteristic diurnal pattern, with maximum sulfate observed a couple hours before sunset. In fall, winter, and spring, the profiles on average do not exhibit a diurnal pattern. Fig. 10 shows sulfate concentrations and $24 \mathrm{~h}$ back-trajectories to the PAQS 


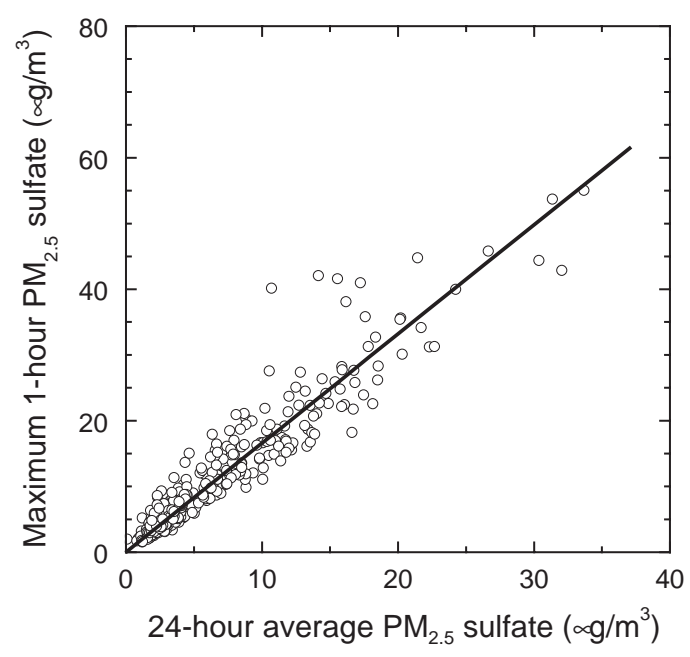

Fig. 11. The linear correlation of the 24 -h average sulfate and the maximum daily 1-h average sulfate for all points from July 2001 to August 2002: $y=1.66 x \mu \mathrm{g} \mathrm{m}^{-3}$ with $R^{2}=0.89$.

Supersite on July 26, 2002. Over $12 \mathrm{~h}$ the sulfate concentration increases from $4 \mu \mathrm{g} \mathrm{m}^{-3}$ to more than $60 \mu \mathrm{g} \mathrm{m}^{-3}$ and then drops to less than $20 \mu \mathrm{g} \mathrm{m}^{-3}$. The initial increase in concentration occurred as the wind direction shifted from southerly to southwesterly and the wind speeds increased. The decrease in concentration occurred after a front passed and the wind speed dramatically decreased at noon. For the remainder of the day, the sulfate concentrations are quite variable and decrease as the result of a short rain event at 16:00.

The average $\mathrm{PM}_{2.5}$ sulfate concentrations were highest during the summer months at PAQS. The $24 \mathrm{~h}$ average sulfate concentrations are well correlated with the maximum daily $1 \mathrm{~h}$ average sulfate over the study period (Fig. 11). Days that did not follow this consistent pattern are also indicated in this figure and were typically associated with rain events or a change in the air mass that resulted in a reduction of sulfate over a short period of time.

\section{Conclusions}

With only the software error correction and the typical ambient PM corrections (instrument offset and actual conversion efficiency), the semi-continuous measurements were nonlinear (sulfate) or underpredictions (nitrate) of the true inorganic concentrations measured using the filter-based method. To improve the accuracy of the continuous measurements, the instrument reported semi-continuous measurements were also corrected for gas analyzer efficiency, drift in reaction cell pressure (nitrate only), and drift in sample flow rate. The overall corrections were small on average (less than 10\% for most corrections), but were quite large for individual 10 minute measurements (varied from $-89 \%$ to $100 \%$ ). The corrected semi-continuous $\mathrm{PM}_{2.5}$ nitrate and sulfate measurements correlated linearly and reasonably with the filter-based measurements, although a strong systematic bias was still apparent in the measurements. This bias could be due to incomplete collection of particles, lower than expected conversion of particles to the corresponding gases, and the presence of a positive measurement artifact. The bias was minimized by calibrating the semi-continuous concentrations using collocated filter-based measurements. Once corrected and calibrated, the final semi-continuous inorganic measurements allowed two orders of magnitude increase in time resolution.

The final semi-continuous measurements were used to characterize short-term variations in $\mathrm{PM}_{2.5}$ inorganic measurements in the Pittsburgh region. When used in conjunction with temporally resolved steam sampler and meteorological measurements, several short-term phenomena were revealed that were not clear from the integrated filter-based measurements. Nitrate followed a consistent diurnal pattern throughout the study period, with maximum nitrate observed in the early morning and minimum nitrate observed in the late afternoon. The time of the maximum and minimum nitrate shifted with seasonal changes in ambient temperature and UV radiation. The maximum nitrate concentration was observed during the winter months when the minimum nitrate concentration was non-zero.

A majority of the nitrate was partitioned into the particle phase at night and into the gas phase during the day. Features of the diurnal patterns and the degree of gas-to-particle partitioning of the nitrate shifted seasonally with temperature, relative humidity, and ultraviolet radiation. During the summer, a majority of the nitrate partitioned into the gas phase during the day, while during winter, little if any nitrate partitioned into the gas phase. Fall and spring showed intermediate degrees of partitioning.

Sulfate concentrations varied diurnally only during the summer, consistent with the gas-phase photochemical production during the day. During the summer, maximum sulfate was observed a couple hours before sunset. During the fall, winter, and spring, sulfate concentrations were relatively stable over the course of a day.

\section{Acknowledgements}

This research was conducted as part of the Pittsburgh Air Quality Study, which was supported by US Environmental Protection Agency under contract R82806101 and the US Department of Energy National Energy Technology Laboratory under contract DE- 
FC26-01NT41017. This paper has not been subject to EPA's peer and policy review, and therefore does not necessarily reflect the views of the Agency. No official endorsement should be inferred.

\section{References}

Allen, G.A., Turner, W.A., Wolfson, J.M., Spengler, J.D., 1984. Description of a continuous sulfuric acid/sulfate monitor. In: Proceedings of the National Symposium on Recent Advances in Pollutant Monitoring of Ambient Air and Stationary Sources. US EPA, Research Triangle Park, North Carolina, p. 140.

Babich, P., Davey, M., Allen, G., Koutrakis, P., 2000. Method comparisons for particulate nitrate, elemental carbon, and $\mathrm{PM}_{2.5}$ mass in seven US cities. Journal of the Air and Waste Management Association 50, 1095-1105.

Buhr, S.M., Buhr, M.P., Fehsenfeld, F.C., Holloway, J.S., Karst, U., Norton, R.B., Parrish, D.D., Sievers, R.E., 1995. Development of a semi-continuous method for the measurement of nitric acid vapor and particulate nitrate and sulfate. Atmospheric Environment 29, 2609-2624.

Carson, P.G., Neubauer, K.R., Johnston, M.V., Wexler, A.S., 1995. On-line chemical analysis of aerosols by rapid singleparticle mass spectrometry. Journal of Aerosol Science 26, 535-545.

Chow, J.C., 1995. Measurement methods to determine compliance with ambient air quality standards for suspended particles. Journal of the Air and Waste Management Association 45, 320-382.

D’Ottavio, T., Garger, R., Tanner, R.L., Newman, L., 1981. Determination of ambient aerosol sulfur using a continuous flame photometric detection system. II. The measurement of low-level sulfur concentrations under varying atmospheric conditions. Atmospheric Environment 15, 197-203.

Hering, S., Cass, G., 1999. The magnitude of bias in the measurement of $\mathrm{PM}_{2.5}$ arising from volatilization of particulate nitrate from Teflon filters. Journal of the Air and Waste Management Association 49, 725-733.

Hering, S.V., Stolzenburg, M.R., 1998. Automated, high time resolution measurement of fine particle nitrate. Journal of Aerosol Science 29, S1189-S1190.

Hering, S.V., Lawson, D.R., Allegrini, I., Febo, A., Perrino, C., Possanzini, M., Sickles II, J.E., Anlauf, K.G., Wiebe, A., et al., 1988. The nitric acid shootout: field comparison of measurement methods. Atmospheric Environment 22, 1519-1539.

Hering, S.V., Kirby, B.W., Wittig, B., Magliano, K., 2001. Wintertime spatial and temporal distribution of fine particle nitrate in the San Joaquin Valley of California, USA. Journal of Aerosol Science 32, S631-S632.

Hering, S.V., Stolzenburg, M.R., Hand, J.L., Kreidenweis, S.M., Lee, T., Collett Jr., J.L., Dietrich, D., 2003. Hourly concentrations and light scattering efficiencies for fine particle sulfate at Big Bend National Park. Atmospheric Environment 37, 1175-1183.

Ito, L., Chasteen, C.C., Chung, H., Prouthoor, S.K., Genfa, Z., Dasgupta, P.K., 1998. A continuous monitoring system for strong acidity in aerosols. Analytical Chemistry 70, 2839-2847.

Karlsson, A., Irgum, K., Hansson, H., 1997. Single-stage flowing liquid film impactor for continuous on-line particle analysis. Journal of Aerosol Science 28, 1539-1551.

Khlystov, A., Wyers, G.P., Slanina, J., 1995. The steam-jet aerosol collector. Atmospheric Environment 29, 2229-2234.

Kirby, B.W., Hering, S.V., 2001. CRPAQS Nitrate measurement data validation summary. Report prepared by Aerosol Dynamics Incorporated for Sonoma Technology Incorporated.

Kolb, C.E., Worsnop, D.R., Jayne, J.T., Shi, Q., Jimenez, J.L., Davidovits, P., Morris, J., Yourshaw, I., Zhang, X., 2000. An aerosol mass spectrometer for real-time measurement of size resolved atmospheric particle composition and laboratory heterogeneous chemistry processes. In: Book of Abstracts of the 219th American Chemical Society National Meeting, March 26-30, San Francisco, CA, American Chemical Society, Washington, DC, pp. 368-369.

Koutrakis, P., Thompson, K.M., Wolfson, J.M., Spengler, J.D., Keeler, G.J., Slater, J.L., 1992. Determination of aerosol strong acidity losses due to interaction of collected particles: results from laboratory and field studies. Atmospheric Environment 26A, 987-995.

Lippmann, M., Xiong, J.Q., Li, W., 2000. Development of a continuous monitoring system for $\mathrm{PM}_{10}$ and components of $\mathrm{PM}_{2.5}$. Applied Occupational and Environmental Hygiene $15,57-67$.

Liu, S., Dasgupta, P.K., 1996. Automated system for chemical analysis of airborne particles based on corona-free electrostatic collection. Analytical Chemistry 68, 3638-3644.

Liu, D.-Y., Hering, S.V., Prather, K.A., 1999. Real-time monitoring of particulate nitrate in the troposphere. In: Book of Abstracts of the 217th American Chemical Society National Meeting, Anaheim, CA, 21-25 March. American Chemical Society, Washington, DC, p. 143923.

Liu, D.-Y., Prather, K.A., Hering, S.V., 2000. Variations in the size and chemical composition of nitrate-containing particles in Riverside, CA. Aerosol Science and Technology 33, 71-86.

Marijinissen, J.C.M., Scarlett, B., Verheijen, P.J.T., 1988. Proposed on-line aerosol analysis combining size determination, laser-induced fragmentation and time-of-flight mass spectroscopy. Journal of Aerosol Science 19, 1307.

McKeown, P.J., Johnston, M.V., Murphy, D.D., 1991. On-line single particle aerosol analysis by laser desorption mass spectroscopy. Analytical Chemistry 63, 2069.

Meier, P.C., Zund, R.E., 2000. Statistical Methods in Analytical Chemistry, 2nd edition. Wiley, New York.

Meyer, M.B., Patashnick, H., Rupprecht, E., 2000. New network-ready continuous monitors for the determination of ambient fine particle mass and chemical components. In: Proceedings of the Air and Waste Management Association's 93rd Annual Conference and Exhibition, Salt Lake City, UT, June 18-22. Air and Waste Management Association, Pittsburgh, PA, pp. 2977-2986.

Middlebrook, A.M., Lee, S-H., Murphy, D.M., Thomson, D.S., 2001. Measurements of single particles from Atlanta using Particle Analysis by Laser Mass Spectrometry (PALMS). In: Abstracts of Papers from the 222nd American Chemical Society National Meeting, Chicago, IL, August 26-30. American Chemical Society, Washington, DC, p. 641464. 
Mueller, P.K., Collins, J.F., 1980. Development of a particulate sulfate analyzer. Energy Research Abstracts 5, 143.

Pang, Y., Ren, Y., Obeidi, F., Hastings, R., Eatough, D.J., Wilson, W.E., 2001. Semi-volatile species in $\mathrm{PM}_{2.5}$ : comparison of integrated and continuous samplers for $\mathrm{PM}_{2.5}$ research or monitoring. Journal of the Air and Waste Management Association 51, 25-36.

Prather, K.A., Nordmeyer, T., Salt, K., 1994. Real-time characterization of individual aerosol particles using time-of-flight mass spectroscopy. Analytical Chemistry 66, 1403.

Stolzenburg, M.R., Hering, S.V., 2000. A new method for the automated measurement of atmospheric fine particle nitrate. Environmental Science and Technology 34, 907-914.

Suh, H.H., Allen, G.A., Aurian-Blajeni, B., Koutrakis, P., 1994. Field method comparison for the characterization of acid aerosols and gases. Atmospheric Environment 28, 2981-2989.
Watson, J.G., Chow, J.C., 2002. A wintertime $\mathrm{PM}_{2.5}$ episode at the Fresno, CA, Supersite. Atmospheric Environment 36, 465-475.

Watson, J.G., Chow, J.C., Bowen, J.L., Lowenthal, D.H., Hering, S., Ouchida, P., Oslund, W., 2000. Air quality measurements from the Fresno Supersite. Journal of the Air and Waste Management Association 50, 1321-1334.

Watson, J.G., DuBois, D.W., DeMandel, R., Kaduwela, A., Magliano, K., McDade, C., Mueller, P.K., Ranzieri, A., Roth, P.M., Tanrikulu, S., 1998. Aerometric monitoring program plan for the California Regional $\mathrm{PM}_{2.5} / \mathrm{PM}_{10}$ Air Quality Study. DRI Document No. 9801.1D5 prepared by Desert Research Institute for the California Regional $\mathrm{PM}_{2.5} / \mathrm{PM}_{10}$ Air Quality Study Technical Committee.

Weber, R.J., Orsini, D., Daun, Y., Lee, Y.-N., Klotz, P.J., Brechtel, F., 2001. A particle-into-liquid collector for rapid measurement of aerosol bulk chemical composition. Aerosol Science and Technology 35, 718-727. 


\title{
Pittsburgh air quality study overview
}

\author{
Ann E. Wittig ${ }^{\mathrm{a}, *}$, Natalie Anderson ${ }^{\mathrm{b}}$, Andrei Y. Khlystov ${ }^{\mathrm{c}}$, Spyros N. Pandis ${ }^{\mathrm{d}, \mathrm{e}}$, \\ Cliff Davidson $^{\mathrm{b}, \mathrm{e}}$, Allen L. Robinson ${ }^{\mathrm{e}, \mathrm{f}}$ \\ ${ }^{a}$ Department of Civil Engineering, City College of New York, New York, NY 10031, USA \\ ${ }^{\mathrm{b}}$ Department of Civil and Environmental Engineering, Carnegie Mellon University, Pittsburgh, PA 15213, USA \\ ${ }^{\mathrm{c}}$ Department of Civil and Environmental Engineering, Duke University, Durham, NC 27708, USA \\ ${ }^{\mathrm{d}}$ Department of Engineering and Public Policy, Carnegie Mellon University, Pittsburgh, PA 15213, USA \\ ${ }^{\mathrm{e}}$ Department of Chemical Engineering, Carnegie Mellon University, Pittsburgh, PA 15213, USA \\ ${ }^{\mathrm{f}}$ Department of Mechanical Engineering, Carnegie Mellon University, Pittsburgh, PA 15213, USA
}

\begin{abstract}
Ambient sampling for the Pittsburgh Air Quality Study (PAQS) was conducted from July 2001 to September 2002. The study was designed (1) to characterize particulate matter (PM) by examination of size, surface area, and volume distribution, chemical composition as a function of size and on a single particle basis, morphology, and temporal and spatial variability in the Pittsburgh region; (2) to quantify the impact of the various sources (transportation, power plants, biogenic sources, etc.) on the aerosol concentrations in the area; and (3) to develop and evaluate the next generation of atmospheric aerosol monitoring and modeling techniques. The PAQS objectives, study design, site descriptions and routine and intensive measurements are presented. Special study days are highlighted, including those associated with elevated concentrations of daily average $\mathrm{PM}_{2.5}$ mass. Monthly average and diurnal patterns in aerosol number concentration, and aerosol nitrate, sulfate, elemental carbon, and organic carbon concentrations, light scattering as well as gas-phase ozone, nitrogen oxides, and carbon monoxide are discussed with emphasis on the processes affecting them. Preliminary findings reveal day-to-day variability in aerosol mass and composition, but consistencies in seasonal average diurnal profiles and concentrations. For example, the seasonal average variations in the diurnal $\mathrm{PM}_{2.5}$ mass were predominately driven by the sulfate component.
\end{abstract}

(C) 2004 Elsevier Ltd. All rights reserved.

Keywords: Atmospheric aerosol; Supersite; PAQS

\section{Introduction}

Airborne particulate matter (PM) continues to pose serious health risks for susceptible members of the population and for sensitive ecosystems. The design of cost effective PM control strategies is limited by the lack of understanding of the relationship between PM and health effects. This lack of understanding is exacerbated by a paucity of physiological data, the difficulty of establishing the PM source-receptor relationships, and finally the limitations of existing instrumentation for PM measurements. The Pittsburgh Air Quality Study (PAQS) is a comprehensive multidisciplinary set of

\footnotetext{
*Corresponding author.
}

projects in the Pittsburgh region, designed to address the above issues. PAQS was led by Carnegie Mellon University (CMU) and included contributions by investigators from fifteen universities, a national laboratory, three private companies, and state and local air pollution agencies, as noted in Table 1. PAQS was supported by the Environmental Protection Agency (EPA) Supersites Program and the Department of Energy National Energy Technology Laboratory (NETL).

In this paper, we present the overall study design and features of the PAQS dataset. We begin by summarizing the PAQS program objectives and hypotheses, and describing the study area and measurement sites. We then describe the measurement methods and the 
Table 1

PAQS contributors

\begin{tabular}{ll}
\hline Contribution & Affiliation \\
\hline Overall project management, Aerosol physical and chemical characterization & Carnegie Mellon Univ.
\end{tabular}

Field manager

Quality assurance manager

Aircraft measurements

Satellite site measurements

Satellite site measurements

Satellite site measurements

Satellite site measurements

Additional instrumentation

Single particle morphology

Single particle mass spectroscopy

Semi-continuous size resolved aerosol composition

Semi-continuous metal measurements

Semi-continuous nitrate and sulfate measurements

Semi-continuous OC/EC measurements, FTIR spectroscopy of aerosols

Biaerosol composition

Integrated organic aerosol measurements

Integrated organic aerosol speciation

PM surface area concentration measurements

Semi-continuous VOC measurements

Continuous peroxide measurements, cloud and fog composition

Source-receptor relationships

Meteorology

Univ. of Maryland
ACHD
NETL
Ohio Univ.
PA DEP
CONSOL

RJ Lee Group, Ltd.

Univ. of California at Davis, Univ. of Delaware

Univ. of Colorado, AERODYNE

Univ. of Maryland

Aerosol Dynamics, Inc.

Rutgers Univ.

Univ. of Colorado

Brigham Young Univ.

Florida Intern. Univ.

Paul Scherrer Institute

Univ. of California at Berkeley

Colorado State Univ.

Clarkson Univ.

Univ. of Wisconsin
Contributor

Cliff Davidson, Spyros Pandis, Allen Robinson Andrey Khlystov Ann (Beth) Wittig

Bruce Doddridge

Darrell Stern

Richard Anderson

Kevin Crist

Various individuals

Steve Winter

Gary Casuccio

Anthony Wexler,

Murray Johnson

Jose Jimenez,

Doug Worsnop

John Ondov

Susanne Hering

Barbara Turpin

Mark Hernandez

Delbert Eatough

Wolfgang Rogge

Urs Baltensperger

Allen Goldstein

Jeff Collett

Phil Hopke

Jonathan Kahl availability of the measurements for both routine monitoring and intensive study periods. Next, we highlight the general features of the measurements collected at the central site, by examining monthly average and diurnal meteorological parameters and ambient concentrations of gases, aerosols, and aerosol species.

\section{Program objectives}

PAQS was designed to achieve several objectives: to determine the physical and chemical characteristics of PM in the Pittsburgh region; to develop and evaluate the next generation of atmospheric aerosol monitoring techniques; to update emission profiles for important regional sources; to quantify the impact of the various sources on the local PM concentrations; and to predict changes in the PM characteristics due to proposed changes in emissions. The last objective was based on concurrent modeling studies and was designed to support the development of regulations. These objectives were addressed through four components of the research: (1) ambient monitoring at a central site and a set of satellite sites in the region; (2) an instrument development and evaluation study; (3) a data analysis and synthesis component; and (4) a comprehensive modeling component. 


\section{Hypotheses and their testing}

Approximately 20 hypotheses were proposed to meet the goals of PAQS. These hypotheses address ambient aerosol measurement issues, questions regarding atmospheric processes and aerosol properties, and the need for refinement of source-receptor relationships. The key hypotheses are summarized in Table 2. A complete list of hypotheses and a description of the hypotheses testing can be found in the PAQS Quality Assurance Project Plan (Khlystov et al., 2001).

Table 2

Primary hypotheses to be investigated during PAQS

\section{Ambient aerosol characterization}

1.1: The measured aerosol mass can be fully explained if the water retained by organics and inorganics, the full organic aerosol contribution, and the full crustal contribution is accounted for.

1.2: The ambient aerosol surface area can be calculated with an error of less than $20 \%$ by using aerodynamic size measurements and by assuming that all particles are spherical.

\section{Measurement methods}

2.1: Single particle mass spectrometers can be used to obtain the full number and mass composition distributions of ambient aerosols.

2.2: Semi-continuous nitrate, sulfate, carbon and elements measurement techniques can quantify concentrations under conditions prevalent at the site.

\section{PAQS supersite description}

There are roughly two million people living in the Pittsburgh Metropolitan Statistical Area, and elderly people represent a significant fraction of the population. The area is located between the utilities and agricultural sources of the Midwest and the large urban centers of the East (Fig. 1). The central site was located on a grassy hill in Schenley Park, approximately $6 \mathrm{~km}$ east of downtown Pittsburgh. It was separated from the city in the predominant upwind direction (south and west) by roughly $1 \mathrm{~km}$ of parkland. It was at least several hundred meters from any other major

\section{Source-receptor relationships}

4.1: A complementary suite of instruments and techniques can be used to directly determine the local air quality contributions from a broad range of primary and secondary sources.

4.2: An increase in temporal resolution of elemental constituents of atmospheric aerosol coupled with sulfate and carbon analyses of comparable frequencies will permit unprecedented resolution of sources by receptor modeling techniques.

4.3: Specific aerosol signatures are associated with transport from specific source regions and along different altitudes.

\section{Aerosol properties}

5.1: Visibility in the area can be predicted from $\mathrm{RH}$ and size/ composition information obtained from aerosol sizing instruments and size bulk chemistry measurements.

5.2: Most particles in the area are liquid throughout the day in both winter and summer.

5.3: Aerosol in the area consists of two groups of particles based on the hygroscopic properties: those consisting mainly of sulfates that grow rapidly with relative humidity and those consisting of mainly carbonaceous material that grow slowly.

\section{Atmospheric processes}

3.1: Aerosol nucleation can be a major source of aerosol number in both urban and rural areas in the study region. 3.2: Biogenic primary and secondary aerosols are a major component of the organic aerosol in the Pittsburgh region. 3.3: Fogs and low clouds are responsible for extreme acid sulfate conditions in the Pittsburgh region.

3.4: The response of $\mathrm{PM}_{2.5}$ to changes in sulfate is highly nonlinear during the winter, is linear during the summer, and is controlled by the ammonia availability.

3.5: The secondary aerosol contribution to organic carbon (OC) exceeds $50 \%$ during the peak PM concentration days, but is approximately $20 \%$ on a yearly average basis.

3.6: The regional contributions to the $\mathrm{PM}_{2.5}$ levels in the Pittsburgh region for some compounds exceed the local contribution, whereas for others the local contributions exceed the regional contributions 
source of air pollution: the site was positioned approximately $50 \mathrm{~m}$ past the end of a dead end street, and several hundred meters from the nearest heavily traveled street (Forbes Avenue). The position of the central site in relation to the downtown Pittsburgh area is shown in Fig. 2. Sampling equipment was housed in an air pollution monitoring station, with samplers and instrument inlets on the station rooftop (Fig. 2).

Five additional sites were used for satellite measurements (Fig. 1). The Florence site was about $50 \mathrm{~km}$ west of the main site in a rural area with no nearby sources. The Greensburg site was about $50 \mathrm{~km}$ east of the main site in a suburban area, close to a heavily traveled road but otherwise in an area of only moderate traffic and few

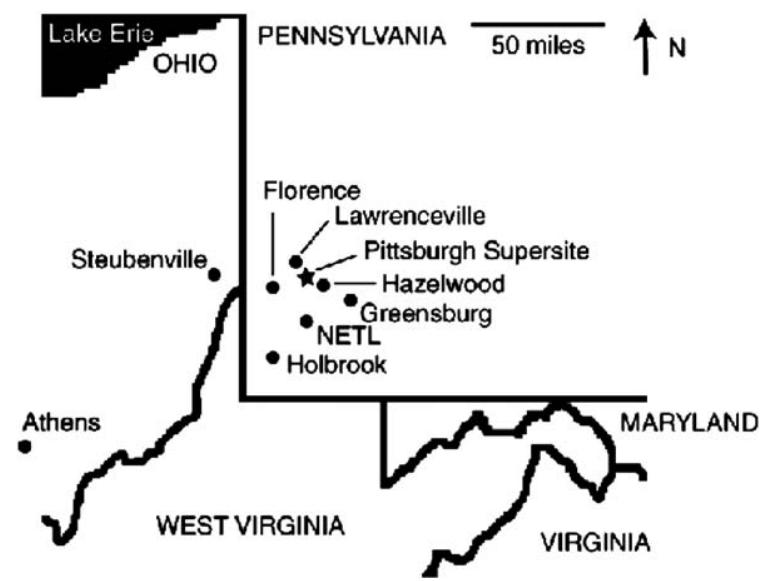

Fig. 1. Map of the PAQS region showing the location of the central site, the Florence, Lawrenceville, Hazelwood, Greensburg, and Athens satellite sites, and the NETL, Holbrook, and Steubenville sites independently operated by other researchers during PAQS. stationary sources. Satellite sites were also located in the Lawrenceville and Hazelwood neighborhoods of the City of Pittsburgh. The final satellite site was located in Athens, $\mathrm{OH}$. Additional air quality measurements were conducted concurrently by others at the NETL laboratories, approximately $15 \mathrm{~km}$ south of downtown Pittsburgh, at Holbrook, PA and at Steubenville, $\mathrm{OH}$ (Fig. 1). Table 3 lists the latitude, longitude, and period of operation of these sites. In addition to the groundbased measurements, two aircraft provided air quality and meteorological data aloft during the intensive periods. R. B. Jacko and Associates, Inc. operated a Cessna 210 Turbo Centurion and the University of Maryland operated a Piper 23-250 Aztec-F (Table 4).

\section{PAQS measurements}

Baseline monitoring took place over a 14-month period that began 1 July, 2001, and provided data of a wide variety of PM physical and chemical characteristics, including biological aerosol composition; gaseous species and fogwater composition; and meteorological parameters. Intensive monitoring was performed during two periods, from 1 July to 3 August, 2001 (ESP01) and 1 January to 15 January, 2002 (ESP02). During these periods, the temporal resolution of these baseline measurements was increased and the baseline measurements were supplemented with measurements of particle morphology, size resolved aerosol composition, organic aerosol speciation, semi-continuous aerosol metal composition and gaseous volatile organic compound (VOC) speciation. The frequency, resolution, and dates over which each method was used at the central site are summarized in Table 5. Quality control procedures used
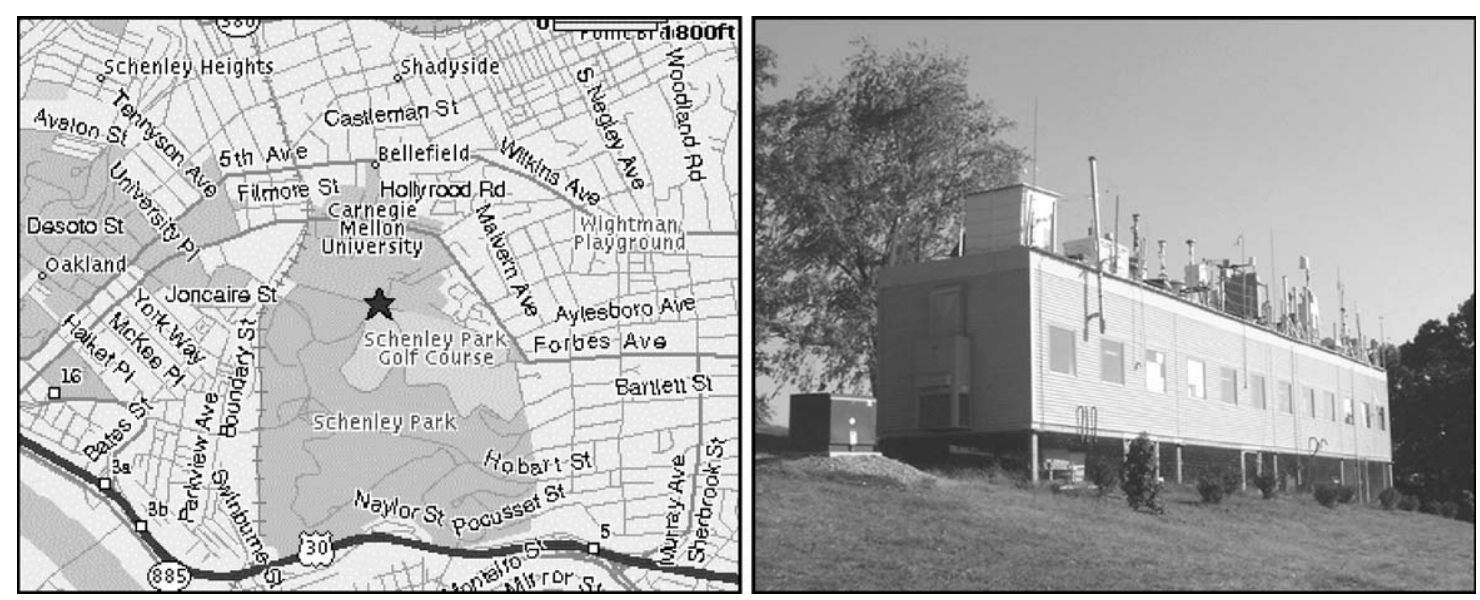

Fig. 2. Map of the downtown Pittsburgh area showing the approximate location of the central site (indicated by a star) and a photograph of the site from the northwest. 
Table 3

Locations of measurements collected during PAQS

\begin{tabular}{llll}
\hline Site name & Group $^{\text {a }}$ & Latitude/Longitude (decimal degrees) & Period \\
\hline Central site $^{\mathrm{b}}$ & CMU & $40.4395 /-79.9405$ & $6 / 30 / 01-7 / 31 / 02$ \\
Satellite sites & & & \\
Hazelwood, PA & & $40.4124 /-79.9424$ & $6 / 30-7 / 30 / 01,1 / 2-1 / 22 / 02$ \\
Lawrenceville, PA & CMU/ACHD & $40.4432 /-79.9595$ & $6 / 30-7 / 30 / 01,1 / 2-1 / 22 / 02$ \\
Florence, PA & CMU/ACHD & $40.4454 /-80.4212$ & $6 / 30-7 / 30 / 01,1 / 2-1 / 22 / 02$ \\
& & & $1 / 3-1 / 22 / 02,7 / 14-8 / 3 / 02^{\mathrm{d}}$ \\
Greensburg, PA & CMU/DEP & $40.3043 /-79.5059$ & $2 / 24 / 02$ to $3 / 28 / 02^{\mathrm{e}}$ \\
Athens, OH & Ohio Univ. & $39.3283 /-82.9067$ & $6 / 30-7 / 30 / 01,1 / 2-1 / 22 / 02$ \\
& & & $6 / 30-8 / 8 / 01$ \\
Aircraft & & & $7 / 6-8 / 2 / 01$ \\
Cessna & & Various & $7 / 6-8 / 1 / 01,7 / 2-8 / 4 / 02$ \\
Piper & Jacko & Various & $6 / 30-8 / 8 / 01$ \\
Non-PAQS sites & UM & & $6 / 30-8 / 8 / 01$ \\
NETL & & $40.3065 /-79.9794$ & $6 / 30-8 / 8 / 01$ \\
Holbrook, PA & NETL & $39.8160 /-80.2850$ & \\
Steubenville, OH & ATS & $40.3617 /-80.6147$ & \\
\hline
\end{tabular}

${ }^{a}$ Groups-ACHD: Allegheny County Health Department; ATS: Advanced Technology Systems; DEP: Pennsylvania Department of Environmental Planning; NETL: National Energy and Technology Laboratory; UM: University of Maryland; Jacko: R.B. Jacko and Associates.

${ }^{\mathrm{b}}$ Central site measurements - see Table 5 .

${ }^{\mathrm{c}}$ Satellite site measurements- $\mathrm{PM}_{2.5}$ mass, $\mathrm{PM}_{2.5}$ elements, and $\mathrm{PM}_{2.5}$ ammonium, nitrate, potassium, sodium, sulfate, elemental carbon, and organic carbon.

${ }^{\mathrm{d}}$ The CMU denuded organic sampler was used to measure $24 \mathrm{~h}$ organic carbon and elemental carbon concentrations on 17 days within the stated range.

${ }^{\mathrm{e}}$ Particle sizing instruments (TSI 3071 DMA and $3010 \mathrm{CPC}$ ) were used to measure $10 \mathrm{~min}$ aerosol size distribution semi-continuously from 0.012 to $0.280 \mu \mathrm{m}$.

${ }^{\mathrm{f}}$ Aircraft measurements-see Table 4.

Table 4

Summary of the aircraft flights completed during PAQS

\begin{tabular}{llll}
\hline Date of flight & Area of operation & Group & Flight details \\
\hline $7 / 6 / 2001 \mathrm{a}$ & Pittsburgh, PA & UM $^{\mathrm{a}}$ & Cadiz, OH to Sebring, OH; Sebring, OH to Cadiz, OH. \\
$7 / 6 / 2001 \mathrm{~b}$ & Western PA, IN & Jacko $^{\mathrm{b}}$ & Allegheny, PA to Pittsburgh, PA to Lafayette, IN \\
$7 / 22 / 2001$ & Pittsburgh, PA & SM & Steubenville, OH to Tarentum, PA to Monongahela, PA \\
$7 / 23 / 2001 \mathrm{a}$ & Pittsburgh, PA & UM & Steubenville, OH to Tarentum, PA to Monongahela, PA \\
$7 / 23 / 2001 \mathrm{~b}$ & Pittsburgh, PA & UM & Steubenville, OH to Tarentum, PA to Monongahela, PA \\
$7 / 23 / 2001 \mathrm{c}$ & Pittsburgh, PA & Jacko & Lafayette, IN to Pittsburgh, PA to Lafayette, IN \\
$8 / 1 / 2001 \mathrm{a}$ & Pittsburgh, PA & UM & Steubenville, OH \\
$8 / 1 / 2001 \mathrm{~b}$ & Pittsburgh, PA & UM & Monongahela, PA to Tarentum, PA \\
$8 / 2 / 2001 \mathrm{a}$ & IN, Western PA & Jacko & Lafayette, IN to Pittsburgh, PA \\
$8 / 2 / 2001 \mathrm{~b}$ & OH, Western PA & Jacko & Pittsburgh, PA to Columbus, OH \\
$7 / 2 / 2002 \mathrm{a}$ & MD, Western PA & UM & Fort Meade, MD to Bedford, PA \\
$7 / 2 / 2002 \mathrm{~b}$ & Western PA & UM & Clarion, PA to Corry, PA \\
$8 / 4 / 2002 \mathrm{a}$ & Western PA & UM & Fort Meade, MD to Indiana, PA \\
8/4/2002b & Western PA, Central NY & UM & Wellsboro, PA to Cortland, NY \\
\hline
\end{tabular}

${ }^{\mathrm{a}} \mathrm{UM}$ (University of Maryland) measurements-Ozone (4 s resolution); NO (10 s resolution); $\mathrm{NO}_{2}$ (undetermined resolution); $\mathrm{CO}$ and $\mathrm{SO}_{2}$ (1 min resolution); aerosol absorption and scattering (1 min resolution); temperature, relative humidity, pressure, latitude, longitude, elevation (10 s resolution).

${ }^{\mathrm{b}}$ Jacko (R. B. Jacko and Associates, Inc.) measurements-Ozone (4 s resolution); $\mathrm{NO}_{x}$ ( $10 \mathrm{~s}$ resolution); VOC and Carbonyls (15 min or longer samples); aerosol scattering (1 min resolution); $\mathrm{PM}_{2.5}$ and $\mathrm{SO}_{2}, \mathrm{NH}_{3}$, and $\mathrm{HNO}_{3}$ (2 $\mathrm{h}$ or longer samples); temperature, relative humidity, pressure, ultraviolet radiation, latitude, longitude, elevation (10 s resolution). 
Table 5

Summary of PAQS measurements

\begin{tabular}{|c|c|c|c|c|c|}
\hline Observable & Method $^{\mathrm{a}}$ & Group $^{b}$ & Resolution & Frequency & Period of operation $^{c}$ \\
\hline \multicolumn{6}{|l|}{ Aerosol mass } \\
\hline $\mathrm{PM}_{10}$ mass & $\begin{array}{l}\text { Dichotomous sampler with } \\
\text { gravimetry }\end{array}$ & $\mathrm{CMU}$ & $24 \mathrm{~h}$ & Daily & $7 / 1 / 01-7 / 1 / 02$ \\
\hline $\mathrm{PM}_{2.5}$ mass & $\begin{array}{l}\text { Dichotomous sampler with } \\
\text { gravimetry }\end{array}$ & $\mathrm{CMU}$ & $24 \mathrm{~h}$ & Daily & $7 / 1 / 01-7 / 1 / 02$ \\
\hline $\mathrm{PM}_{2.5}$ mass & $\begin{array}{l}\text { Federal Reference Method } \\
\text { sampler with gravimetry }\end{array}$ & CMU & $24 \mathrm{~h}$ & Daily & $7 / 1 / 01-7 / 1 / 02$ \\
\hline $\mathrm{PM}_{2.5}$ mass & $\begin{array}{l}\text { R\&P 1400A TEOM monitor } \\
\text { with SES }\end{array}$ & $\mathrm{CMU}$ & $10 \mathrm{~min}$ & Continuous & $7 / 1 / 01-9 / 1 / 02$ \\
\hline $\mathrm{PM}_{x}$ mass & $\begin{array}{l}\text { MOUDI sampler with } \\
\text { gravimetry }\end{array}$ & $\mathrm{CMU}$ & $24 \mathrm{~h}$ & Daily & $7 / 1 / 01-7 / 1 / 02^{\mathrm{d}}$ \\
\hline $\mathrm{PM}_{x}$ mass & $\begin{array}{l}\text { MOUDI sampler with } \\
\text { gravimetry }\end{array}$ & $\mathrm{CMU}$ & $8 \mathrm{~h}$ & 3 per day & $7 / 22-25 / 01,7 / 31-8 / 3 / 01$ \\
\hline \multicolumn{6}{|l|}{$\begin{array}{l}\text { Aerosol size } \\
\text { distribution }\end{array}$} \\
\hline $\begin{array}{l}\text { Aerosol number } \\
\text { distribution }\end{array}$ & $\begin{array}{l}\text { TSI ultra fine SMPS, SMPS } \\
\text { and APS }\end{array}$ & CMU & $10 \mathrm{~min}$ & Continuous & $7 / 1 / 01-7 / 1 / 02$ \\
\hline $\begin{array}{l}\text { Aerosol surface area } \\
\text { distribution }\end{array}$ & $\begin{array}{l}\text { TSI ultra fine SMPS, SMPS } \\
\text { and APS }\end{array}$ & $\mathrm{CMU}$ & $10 \mathrm{~min}$ & Continuous & $7 / 1 / 01-7 / 1 / 02$ \\
\hline $\begin{array}{l}\text { Aerosol surface area } \\
\text { distribution }\end{array}$ & Epiphaniometer & PSI & $30 \mathrm{~min}$ & Continuous & $6 / 11 / 01-9 / 18 / 01$ \\
\hline $\begin{array}{l}\text { Aerosol volume } \\
\text { distribution }\end{array}$ & $\begin{array}{l}\text { TSI ultra fine SMPS, SMPS } \\
\text { and APS }\end{array}$ & CMU & $10 \mathrm{~min}$ & Continuous & $7 / 1 / 01-7 / 1 / 02$ \\
\hline \multicolumn{6}{|l|}{$\begin{array}{l}\text { Aerosol chemical } \\
\text { composition }\end{array}$} \\
\hline $\mathrm{PM}_{2.5}$ inorganic ions & CMU sampler with IC analysis & $\mathrm{CMU}$ & $24 \mathrm{~h}$ & Daily & $7 / 1 / 01-7 / 1 / 02^{\mathrm{d}}$ \\
\hline $\mathrm{PM}_{2.5}$ inorganic ions & CMU sampler with IC analysis & CMU & $4-6 h$ & 5 per day & ESP01 Intensive \\
\hline $\mathrm{PM}_{x}$ inorganic ions & $\begin{array}{l}\text { MOUDI sampler with IC } \\
\text { analysis }\end{array}$ & $\mathrm{CMU}$ & $24 \mathrm{~h}$ & Daily & $7 / 1 / 01-7 / 1 / 02^{\mathrm{d}}$ \\
\hline $\mathrm{PM}_{x}$ inorganic ions & $\begin{array}{l}\text { MOUDI sampler with IC } \\
\text { analysis }\end{array}$ & CMU & $8 \mathrm{~h}$ & 3 per day & $7 / 22-25 / 01,7 / 31-8 / 3 / 01$ \\
\hline $\mathrm{PM}_{10}$ elements & $\begin{array}{l}\text { Hi-Vol sampler with ICP-MS } \\
\text { analysis }\end{array}$ & CMU & $24 \mathrm{~h}$ & Daily & $7 / 12 / 01-8 / 02 / 02$ \\
\hline $\mathrm{PM}_{2.5}$ elements & $\begin{array}{l}\text { Hi-Vol sampler with ICP-MS } \\
\text { analysis }\end{array}$ & $\mathrm{CMU}$ & $24 \mathrm{~h}$ & Daily & $7 / 11 / 01-9 / 30 / 02$ \\
\hline $\mathrm{PM}_{x}$ elements & $\begin{array}{l}\text { MOUDI sampler with ICP-MS } \\
\text { analysis }\end{array}$ & $\mathrm{CMU}$ & $24 \mathrm{~h}$ & Daily & $\begin{array}{l}\text { ESP01, ESP02 } \\
\text { Intensives }^{\mathrm{d}}\end{array}$ \\
\hline $\mathrm{PM}_{x}$ elements & $\begin{array}{l}\text { MOUDI sampler with ICP-MS } \\
\text { analysis }\end{array}$ & CMU & $8 \mathrm{~h}$ & 3 per day & $7 / 22-25 / 01,7 / 31-8 / 3 / 01$ \\
\hline $\begin{array}{l}\mathrm{PM}_{2.5} \text { organic and } \\
\text { elemental carbon }\end{array}$ & $\begin{array}{l}\text { CMU TQQQ sampler with } \\
\text { TOT analysis }\end{array}$ & $\mathrm{CMU}$ & $24 \mathrm{~h}$ & Daily & $7 / 1 / 01-7 / 31 / 02^{\mathrm{d}}$ \\
\hline $\begin{array}{l}\mathrm{PM}_{2.5} \text { organic and } \\
\text { elemental carbon }\end{array}$ & $\begin{array}{l}\text { CMU TQQQ sampler with } \\
\text { TOT analysis }\end{array}$ & $\mathrm{CMU}$ & $4-6 h$ & 5 per day & ESP01 Intensive \\
\hline $\begin{array}{l}\mathrm{PM}_{2.5} \text { organic and } \\
\text { elemental carbon }\end{array}$ & $\begin{array}{l}\text { CMU denuded organic sampler } \\
\text { with TOT analysis }\end{array}$ & CMU & $24 \mathrm{~h}$ & 6th day & $7 / 1 / 01-6 / 1 / 02^{\mathrm{d}}$ \\
\hline $\begin{array}{l}\mathrm{PM}_{2.5} \text { organic and } \\
\text { elemental carbon }\end{array}$ & $\begin{array}{l}\text { CMU denuded organic sampler } \\
\text { with TOT analysis }\end{array}$ & $\mathrm{CMU}$ & $24 \mathrm{~h}$ & Daily & ESP01, ESP02 Intensives \\
\hline $\begin{array}{l}\mathrm{PM}_{2.5} \text { organic and } \\
\text { elemental carbon }\end{array}$ & $\begin{array}{l}\text { MOUDI sampler with TOT } \\
\text { analysis }\end{array}$ & $\mathrm{CMU}$ & $24 \mathrm{~h}$ & Daily & ESP01 Intensive $^{\mathrm{d}}$ \\
\hline $\begin{array}{l}\mathrm{PM}_{x} \text { organic and } \\
\text { elemental carbon }\end{array}$ & $\begin{array}{l}\text { MOUDI sampler with TOT } \\
\text { analysis }\end{array}$ & $\mathrm{CMU}$ & $8 \mathrm{~h}$ & 3 per day & $7 / 22-25 / 01,7 / 31-8 / 3 / 01$ \\
\hline $\begin{array}{l}\mathrm{PM}_{x} \text { organic and } \\
\text { elemental carbon }\end{array}$ & BYU PC-BOSS & BYU & $24 \mathrm{~h}$ & Daily & $7 / 9 / 01-7 / 31 / 01$ \\
\hline $\mathrm{PM}_{2.5}$ speciated & LPI sampler with FTIR & CMU & $24 \mathrm{~h}$ & Daily & ESP01, ESP02 Intensives \\
\hline
\end{tabular}


Table 5 (continued)

\begin{tabular}{|c|c|c|c|c|c|}
\hline Observable & Method $^{\mathrm{a}}$ & Group $^{\mathrm{b}}$ & Resolution & Frequency & Period of operation $^{c}$ \\
\hline $\begin{array}{l}\mathrm{PM}_{2.5} \text { speciated } \\
\text { organics }\end{array}$ & $\begin{array}{l}\text { CMU organic speciation } \\
\text { sampler }\end{array}$ & FIU & $24 \mathrm{~h}$ & 6th Day & $7 / 1 / 01-7 / 1 / 02^{\mathrm{d}}$ \\
\hline $\begin{array}{l}\mathrm{PM}_{2.5} \text { speciated } \\
\text { organics }\end{array}$ & $\begin{array}{l}\text { CMU organic speciation } \\
\text { sampler }\end{array}$ & FIU & $24 \mathrm{~h}$ & Daily & ESP01, ESP02 Intensives \\
\hline $\begin{array}{l}\mathrm{PM}_{2.5} \text { biological } \\
\text { material }\end{array}$ & $\begin{array}{l}\text { Epi-fluorescent microscopy } \\
\text { with assays }\end{array}$ & $\mathrm{UC}$ & $24 \mathrm{~h}$ & Daily & $7 / 7 / 01-7 / 1 / 02$ \\
\hline $\mathrm{PM}_{2.5}$ nitrate & $\begin{array}{l}\mathrm{R} \& \mathrm{P} 8400 \mathrm{~N} \text { with flash } \\
\text { volatilization }\end{array}$ & ADI & $10 \mathrm{~min}$ & $\begin{array}{l}\text { Semi- } \\
\text { continuous }\end{array}$ & $7 / 1 / 01-8 / 1 / 02$ \\
\hline $\mathrm{PM}_{2.5}$ sulfate & $\begin{array}{l}\text { R\&P } 8400 \text { S with flash } \\
\text { volatilization }\end{array}$ & ADI & $10 \mathrm{~min}$ & $\begin{array}{l}\text { Semi- } \\
\text { continuous }\end{array}$ & $7 / 1 / 01-9 / 1 / 02$ \\
\hline $\mathrm{PM}_{2.5}$ carbon & $\begin{array}{l}\text { ADI monitor with flash } \\
\text { volatilization }\end{array}$ & ADI & $30 \mathrm{~min}$ & $\begin{array}{l}\text { Semi- } \\
\text { continuous }\end{array}$ & $1 / 1 / 02-9 / 1 / 02$ \\
\hline $\mathrm{PM}_{2.5}$ carbon & $\begin{array}{l}\text { Denuded Sunset Labs in situ } \\
\text { TOT analyzer }\end{array}$ & RU & $2-4 h$ & $\begin{array}{l}\text { Semi- } \\
\text { continuous }\end{array}$ & $7 / 1 / 01-9 / 1 / 02$ \\
\hline $\begin{array}{l}\mathrm{PM}_{2.5} \text { water soluble } \\
\text { ions }\end{array}$ & $\begin{array}{l}\text { Khylstov steam sampler with } \\
\text { IC analysis }\end{array}$ & CMU & $1-2 \mathrm{~h}$ & $\begin{array}{l}\text { Semi- } \\
\text { continuous }\end{array}$ & $7 / 1 / 01-9 / 21 / 02$ \\
\hline $\begin{array}{l}\mathrm{PM}_{2.5} \text { water soluble } \\
\text { ammonium }\end{array}$ & Ammonium online detector & CMU & $10 \mathrm{~min}$ & Continuous & $7 / 1 / 01-9 / 21 / 02$ \\
\hline $\mathrm{PM}_{1.3}$ metals & SEAS with GFAA analysis & UMD & $30 \mathrm{~min}$ & $\begin{array}{l}\text { Semi- } \\
\text { continuous }\end{array}$ & $\begin{array}{l}7 / 8 /-8 / 10 / 01,3 / 29-4 / 17 / \\
02\end{array}$ \\
\hline $\begin{array}{l}\mathrm{PM}_{1.0} \text { size resolved } \\
\text { composition }\end{array}$ & $\begin{array}{l}\text { Aerodyne Mass Spectrometer } \\
\text { (AMS) }\end{array}$ & $\mathrm{UC}$, & & & AERODYNE \\
\hline $5 \mathrm{~min}$ & Semi-continuous & $\begin{array}{l}9 / 6 / 02-9 / 21 / \\
02\end{array}$ & & & \\
\hline \multicolumn{6}{|l|}{ Aerosol characteristics } \\
\hline $\begin{array}{l}\text { Particle light } \\
\text { scattering }\end{array}$ & $\begin{array}{l}\text { Optec NGN-3 ambient } \\
\text { nephelometer }\end{array}$ & CMU & $10 \mathrm{~min}$ & Continuous & $7 / 16 / 01-6 / 30 / 02$ \\
\hline $\begin{array}{l}\text { Particle } \\
\text { hygroscopicity }\end{array}$ & CMU DAASS & CMU & $1 \mathrm{~h}$ & $\begin{array}{l}\text { Semi- } \\
\text { continuous }\end{array}$ & $7 / 1-8 / 31 / 01,1 / 1-7 / 1 / 02$ \\
\hline Cloud condensation & DH Associates M1 CCN & CMU & $4 \mathrm{~h}$ & $\begin{array}{l}\text { Semi- } \\
\text { continuous }\end{array}$ & $9 / 01$ \\
\hline \multicolumn{6}{|l|}{$\begin{array}{l}\text { Single particle } \\
\text { characteristics }\end{array}$} \\
\hline $\begin{array}{l}\text { Single particle polar } \\
\text { organics }\end{array}$ & RSMS-III & $\mathrm{UCD}, \mathrm{UD}$ & $10 \mathrm{~min}$ & $\begin{array}{l}\text { Semi- } \\
\text { continuous }\end{array}$ & $9 / 20 / 01-10 / 1 / 02$ \\
\hline $\begin{array}{l}\text { Single particle ion } \\
\text { composition }\end{array}$ & RSMS-III & $\mathrm{UCD}, \mathrm{UD}$ & $10 \mathrm{~min}$ & $\begin{array}{l}\text { Semi- } \\
\text { continuous }\end{array}$ & $9 / 20 / 01-10 / 1 / 02$ \\
\hline Particle morphology & Nuclepore filter with SEM & RJL & $24 \mathrm{~h}$ & Daily & ESP01, ESP02 Intensives \\
\hline \multicolumn{6}{|l|}{ Gaseous species } \\
\hline $\begin{array}{l}\text { Light }\left(\mathrm{C}_{2}-\mathrm{C}_{12}\right) \\
\text { hydrocarbons }\end{array}$ & Canister with GC-FID analysis & CMU & $24 \mathrm{~h}$ & 3rd day & $9 / 1 / 01-7 / 31 / 02^{\mathrm{d}}$ \\
\hline $\begin{array}{l}\text { Light }\left(\mathrm{C}_{2}-\mathrm{C}_{12}\right) \\
\text { hydrocarbons }\end{array}$ & Canister with GC-FID analysis & CMU & $24 \mathrm{~h}$ & Daily & ESP02 Intensive \\
\hline All hydrocarbons & UCB GC-FID/MSD & $\mathrm{UCB}$ & $1 \mathrm{~h}$ & $\begin{array}{l}\text { Semi- } \\
\text { continuous }\end{array}$ & $1 / 9-2 / 12,7 / 10-8 / 10 / 02$ \\
\hline $\mathrm{CO}$ & API 300 infrared absorption & CMU & $10 \mathrm{~min}$ & Continuous & $7 / 1 / 01-9 / 1 / 02$ \\
\hline $\mathrm{SO}_{2}$ & API 100A pulsed fluorescence & CMU & $10 \mathrm{~min}$ & Continuous & $7 / 1 / 01-9 / 1 / 02$ \\
\hline $\mathrm{NO}$ and $\mathrm{NO}_{2}$ & API 200A chemiluminescence & $\mathrm{CMU}$ & $10 \mathrm{~min}$ & Continuous & $7 / 1 / 01-9 / 1 / 02$ \\
\hline $\mathrm{O}_{3}$ & API 400A UV absorption & CMU & $10 \mathrm{~min}$ & Continuous & $7 / 1 / 01-9 / 1 / 02$ \\
\hline $\begin{array}{l}\text { Water soluble } \\
\text { inorganic gases }\end{array}$ & $\begin{array}{l}\text { Khlystov steam sampler with } \\
\text { IC analysis }\end{array}$ & $\mathrm{CMU}$ & $1-2 \mathrm{~h}$ & $\begin{array}{l}\text { Semi- } \\
\text { continuous }\end{array}$ & $7 / 1 / 01-9 / 21 / 02$ \\
\hline Inorganic gases & CMU sampler with IC analysis & $\mathrm{CMU}$ & $24 \mathrm{~h}$ & Daily & $7 / 1 / 01-7 / 1 / 02^{\mathrm{d}}$ \\
\hline Inorganic gases & CMU sampler with IC analysis & $\mathrm{CMU}$ & $4-6 h$ & 5 per day & ESP01 Intensive \\
\hline $\begin{array}{l}\text { Total soluble } \\
\text { peroxides }\end{array}$ & CSU monitor & $\mathrm{CSU}$ & $1 \mathrm{~h}$ & Continuous & $7 / 1 / 01-8 / 18 / 02^{\mathrm{d}}$ \\
\hline
\end{tabular}


Table 5 (continued)

\begin{tabular}{llllll}
\hline Observable & Method $^{\mathrm{a}}$ & Group $^{\mathrm{b}}$ & Resolution & Frequency $^{\text {Period of operation }^{\mathrm{c}}}$ \\
\hline $\begin{array}{l}\text { Hydrogen and } \\
\text { organic peroxides }\end{array}$ & CSU monitor & CSU & $1 \mathrm{~h}$ & Continuous & ESP01, ESP02 Intensives \\
$\begin{array}{l}\text { Fog } \\
\text { Fog composition }\end{array}$ & $\begin{array}{l}\text { CASCC2 collector with IC, } \\
\text { TOC } \text { and pH analyses }\end{array}$ & CSU & Per event & $\begin{array}{l}8 \text { events } \\
\text { captured }\end{array}$ & $7 / 1 / 01-9 / 1 / 02$
\end{tabular}

\begin{tabular}{|c|c|c|c|c|c|}
\hline $\begin{array}{l}\text { Meteorology } \\
\text { Wind speed/direction }\end{array}$ & $\begin{array}{l}\text { Met One } 014 \mathrm{~A} \text { wind vane and } \\
\text { cup anemometer }\end{array}$ & $\mathrm{CMU}$ & $10 \mathrm{~min}$ & Continuous & $7 / 1 / 01-9 / 1 / 02$ \\
\hline Temperature & $\begin{array}{l}\text { Campbell HMP45C thermistor- } \\
\text { based sensor }\end{array}$ & $\mathrm{CMU}$ & $10 \mathrm{~min}$ & Continuous & $7 / 1 / 01-9 / 1 / 02$ \\
\hline Relative humidity & $\begin{array}{l}\text { Campbell HMP45C } \\
\text { capacitance thin film sensor }\end{array}$ & $\mathrm{CMU}$ & $10 \mathrm{~min}$ & Continuous & $7 / 1 / 01-9 / 1 / 02$ \\
\hline UV solar radiation & $\begin{array}{l}\text { Kipp \& Zonen CUV3 } \\
\text { broadband UV radiometer }\end{array}$ & $\mathrm{CMU}$ & $10 \mathrm{~min}$ & Continuous & $7 / 1 / 01-9 / 1 / 02$ \\
\hline Total solar radiation & $\begin{array}{l}\text { Kipp \& Zonen CM3 } \\
\text { pyranometer }\end{array}$ & $\mathrm{CMU}$ & $10 \mathrm{~min}$ & Continuous & $7 / 1 / 01-9 / 1 / 02$ \\
\hline Precipitation & Met One 370 rain gauge & CMU & $10 \mathrm{~min}$ & Continuous & $7 / 1 / 01-9 / 1 / 02$ \\
\hline Pressure & $\begin{array}{l}\text { Campbell CS105 Aneroid } \\
\text { barometer }\end{array}$ & CMU & $10 \mathrm{~min}$ & Continuous & $7 / 1 / 01-9 / 1 / 02$ \\
\hline \multicolumn{6}{|c|}{ 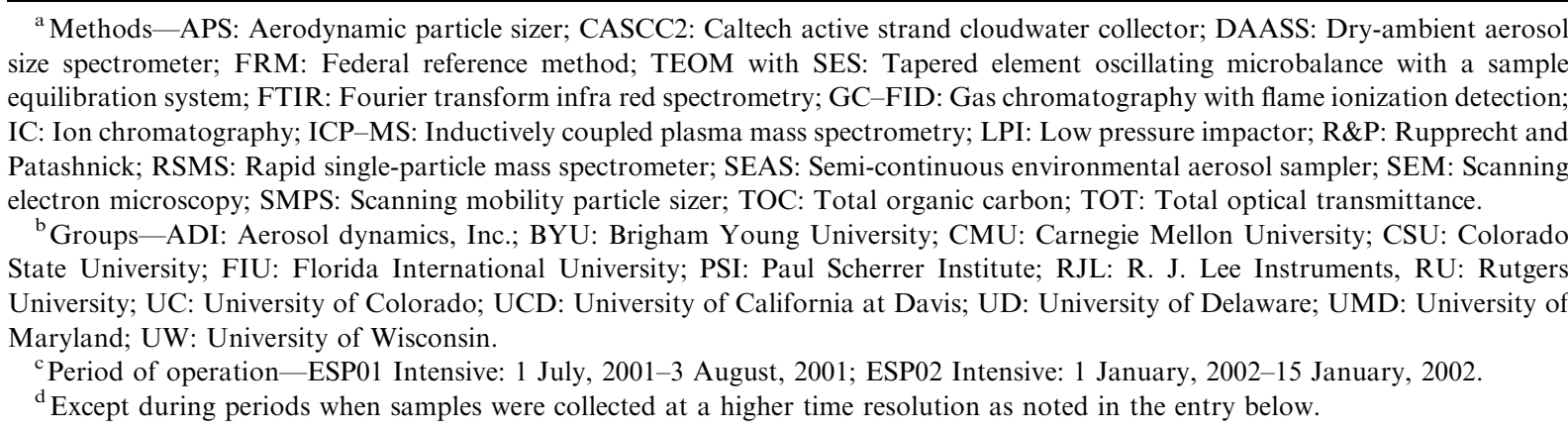 } \\
\hline
\end{tabular}

to verify the measurements are presented in detail by Khlystov et al. (2001).

During the intensive periods, $\mathrm{PM}_{2.5}$ aerosol mass, inorganic ions, organic and elemental carbon, and metal concentrations were measured at the satellite sites (Table 3). During two special experiments at the Florence PA satellite site in January and July of 2002, the $24 \mathrm{~h}$ integrated $\mathrm{PM}_{2.5}$ organic speciation and $10 \mathrm{~min}$ aerosol size distribution between 0.012 and $0.28 \mu \mathrm{m}$ was also measured. Aircraft high time resolution measurements of meteorology, aerosol properties, and gas-phase species were obtained during select days of July and August 2001 and 2002 (Table 4). The Jacko aircraft conducted 4 flights in 2 days over Pittsburgh, PA (July 23, 2001 and August 2, 2001). The Maryland aircraft conducted 10 flights in July and August of 2001 and 2002 over the western PA region.

PM mass characterization. Integrated samples of $\mathrm{PM}_{2.5}$ mass were collected onto Teflon filters using a Partisol $^{\mathrm{R}}$-Federal Reference Method (FRM) Model
$2000 \mathrm{PM}_{2.5}$ Air Sampler (Rupprecht \& Patashnick, Albany, NY). Integrated samples of $\mathrm{PM}_{2.5}$ and $\mathrm{PM}_{10}$ mass were also collected on Teflon filters using a Dichotomous (Dichot) sampler (Thermo Andersen, Model 241). Both samplers were operated using standard procedures (U.S.E.P.A., 1998). A MicroOrifice Uniform Deposit Impactor (MOUDI) (MSP Corp., Model 110) was used to collect size-resolved samples of particles on Teflon substrates using standard procedures (MSP Corp., 1998). This permitted estimates of concentrations of particles, $\mathrm{PM}_{x}$, with diameters less than $2.5,1.8,1.0,0.56,0.32,0.18,0.10$, and $0.056 \mu \mathrm{m}$. The FRM, Dichot, and MOUDI filters were analyzed for mass concentration by gravimetric analysis using standard protocols (Code of Federal Regulations, 2002) in a humidity-controlled equilibration chamber in the CMU Air Quality Laboratory.

Semi-continuous $\mathrm{PM}_{2.5}$ mass was measured using a Tapered Element Oscillating Microbalance (TEOM) monitor (Rupprecht and Patashnick, Model 1400A). 
The Sample Equilibration System (SES) permitted particles to be collected onto the Pallflex tapered element at temperature of $30^{\circ} \mathrm{C}$.

$P M$ size distributions. A suite of particle sizing instruments was used to semi-continuously measure the number size distributions for particles from 0.003 to $10 \mu \mathrm{m}$ (Stanier et al., 2004a). The suite included a nanoScanning Mobility Particle Sizer (SMPS) (TSI, Inc., Model 3936N25), a standard SMPS (TSI, Inc., Model 3936L10), and an Aerodynamic Particle Sizer (APS) (TSI, Inc., Model 3320). The 3 separate distributions were combined, after inversion, by using the nanoSMPS data up to $0.03 \mu \mathrm{m}$, the SMPS data from 0.03 to $0.6 \mu \mathrm{m}$, and the APS data beginning at $0.6 \mu \mathrm{m}$. Aerodynamic diameters measured by the APS were converted to mobility diameters prior to combining data using an effective density and a least-squares fitting algorithm (Khlystov et al., 2003). The average effective density used for the period from July to August of 2001 was $1.52 \mathrm{~g} \mathrm{~cm}^{-3}$, with 5 th and 95th percentile values of 0.94 and $1.94 \mathrm{~g} \mathrm{~cm}^{-3}$. Effective density was used over a density derived from speciated aerosol measurements for the following three reasons: $\mathrm{PM}_{2.5}$ speciation measurements were not always available; the density derived from speciated data requires an estimate of water content, which was not always available; and the effective density takes into account the unknown shape factor.

$P M$ inorganic ions. Integrated samples of $\mathrm{PM}_{10}$ and $\mathrm{PM}_{2.5}$ were drawn through cyclones and denuders and collected onto filter packs of PTFE Teflon, nylon, and cellulose-fiber filters (Takahama et al., 2004). Size resolved $\mathrm{PM}_{x}$ inorganic ion samples were collected onto Teflon substrates using a MOUDI. The filter pack and MOUDI samples were analyzed for nitrate, sulfate, ammonium and chloride by Ion Chromatography (IC) using procedures similar to those described by Jaffrezo et al. (1998).

Semi-continuous measurements of total nitrate $\left(\mathrm{PM}_{2.5}\right.$ nitrate plus $\left.\mathrm{HNO}_{3}\right)$, total sulfate $\left(\mathrm{PM}_{2.5}\right.$ sulfate plus $\left.\mathrm{SO}_{2}\right)$, and total ammonium $\left(\mathrm{PM}_{2.5}\right.$ ammonium plus $\mathrm{NH}_{3}$ ) were made using a steam sampler (Khlystov et al., 1995) with analysis by IC. Total ammonium was measured continuously on-line using the steam sampler and the detector of Slanina et al. (2001). Comparisons of steam sampler measurements against denuder-filter pack measurements did not reveal an ammonium artifact, consistent with the findings of Slanina et al. (2001), Erisman et al. (2001), and Zhang et al. (2002).

Semi-continuous measurements of $\mathrm{PM}_{2.5}$ nitrate and sulfate were made using Integrated Collection and Vaporization Cell (ICVC) instruments (Rupprecht and Patashnick, Models 8400 S and $8400 \mathrm{~N}$ ) and processed using the method described by Wittig et al. (2003).

PM Trace Metals. Integrated $\mathrm{PM}_{10}$ and $\mathrm{PM}_{2.5}$ samples were collected on cellulose filters using Thermo
Andersen High-Volume (High Vol) samplers operated using standard procedures (Thermo Andersen, 1999; U.S.E.P.A., 1999). The filters were digested in a combination of nitric acid, hydrofluoric acid, and hydrogen peroxide in a sealed microwave vessel and analyzed according to standard procedures by Inductively Coupled Plasma Mass Spectrometry (ICP-MS) (U.S.E.P.A., 1991; U.S.E.P.A., 1994) to determine concentrations of $\mathrm{Al}$, As, $\mathrm{Ba}, \mathrm{Ca}, \mathrm{Cd}, \mathrm{Cr}, \mathrm{Cu}, \mathrm{Fe}, \mathrm{Ga}, \mathrm{K}, \mathrm{Mg}, \mathrm{Mn}, \mathrm{Mo}, \mathrm{Na}, \mathrm{Ni}, \mathrm{Pb}$, $\mathrm{Rb}, \mathrm{Sb}, \mathrm{Se}, \mathrm{Sr}, \mathrm{Ti}, \mathrm{V}$ and $\mathrm{Zn}$.

Semi-continuous measurements of 11 metals (Al, As, $\mathrm{Cd}, \mathrm{Cr}, \mathrm{Cu}, \mathrm{Fe}, \mathrm{Mn}, \mathrm{Ni}, \mathrm{Pb}, \mathrm{Se}$ and $\mathrm{Zn}$ ) were also made using the Semi-continuous Environmental Aerosol Sampler (SEAS) (Kidwell and Ondov, 2001) with offline analysis using Graphite Furnace Atomic Absorption (GFAA).

$P M$ carbonaceous material. Integrated samples of $\mathrm{PM}_{2.5}$ were collected on bare quartz filters and two backup quartz filters, one downstream of the bare quartz filter and the other in a parallel port behind a Teflon filter (Subramanian et al., 2003). Integrated samples of $\mathrm{PM}_{2.5}$ were also denuded and collected on quartz fiber filters with a carbon impregnated graphite backup filter (Subramanian et al., 2003). Size resolved $\mathrm{PM}_{x}$ samples were collected on ungreased foil substrates using a MOUDI sampler. The quartz and foil filters were analyzed for organic carbon (OC) and elemental carbon (EC) using the Thermal Optical Transmittance (TOT) method and the NIOSH thermal evolution protocol (NIOSH, 1996; Cabada et al., 2004a) in the CMU Air Quality Laboratory. Integrated samples of $\mathrm{PM}_{2.5}$ OC and EC were also collected using the PCBOSS diffusion denuder sampler (Eatough et al., 2001) and analyzed at Brigham Young University.

Additional size-resolved samples were collected on zinc selenide disks using a Hering Low Pressure Impactor (LPI) sampler (Aerosol Dynamics Inc.) and analyzed for organic functional groups using Fourier Transform Infrared Spectroscopy (FTIR) at Rutgers University (Blando et al., 2001).

Semi-continuous measurements of $\mathrm{PM}_{2.5} \mathrm{OC}$ and EC were conducted using the Sunset Laboratory in situ carbon analyzer with a denuder placed upstream of the instrument (Turpin et al., 1990). Semi-continuous measurements of $\mathrm{PM}_{2.5}$ total carbon were obtained using an the Aerosol Dynamics ICVC total carbon instrument which is similar in design to the R\&P instruments used to measure $\mathrm{PM}_{2.5}$ nitrate and sulfate semi-continuously (Stolzenburg and Hering, 2000).

PM biological material. Ambient bioaerosols were collected using a High Vol sampler for July 2001, and using a low volume sampler for all other periods. The samples were analyzed at Colorado State University using direct epi-fluorescent microscopy and newer molecular biology methods to obtain microbioaerosol concentrations of bacteria, fungi, and their spores. 
PM hygroscopicity. The ability of ambient fine particles to absorb water and grow was measured using the DryAmbient Aerosol Size Spectrometer (DAASS) (Stanier et al., 2004a). The water content of aerosols from 0.003 to $10 \mu \mathrm{m}$ was computed as the difference in particle volume distributions at ambient $\mathrm{RH}$ and at 'dried' conditions. Dried conditions $(\mathrm{RH}<30 \%)$ were achieved by sampling ambient air though a system of Nafion dryers prior to analysis by the suite of particle sizing instruments. Sizedependent losses across the DAASS inlet were 17\% at $2.5 \mu \mathrm{m}$ and practically zero at $1 \mu \mathrm{m}$, determined as the difference in total particle counts across the dried and ambient RH inlets when a dry monodisperse ammonium sulfate was injected into both inlets. Particle losses below $20 \mathrm{~nm}$ were estimated using the empirical particle loss correlation of Willeke and Baron (1993).

Cloud Condensation Nuclei (CCN) concentration was measured using CCN counter (DH Associates, Model M1). The CCN concentration is directly related to the ability of the particles to become cloud droplets.

Aerosol light scattering. Continuous $\mathrm{PM}_{2.5}$ scattering coefficients from $5^{\circ}$ to $175^{\circ}$ at a wavelength of $550 \mathrm{~nm}$ were measured at ambient conditions using an integrating nephelometer (Optec, Inc., NGN-3) with a $\mathrm{PM}_{2.5}$ cyclone at the inlet. Temperature was measured at the inlet to the nephelometer and inside the nephelometer at the chamber outlet. Comparisons between these two measurements were used to correct for the decrease in relative humidity of the sampled air due to heating inside the chamber.

Aerosol mass spectroscopy. The Aerodyne Mass Spectrometer (AMS) was used to measure size resolved aerosol chemical composition semi-continuously (Jayne et al., 2000; Jimenez et al., 2003). The instrument determined the size distributions from 0.050 to $1 \mu \mathrm{m}$ of sulfate, nitrate, ammonium, chloride, total organics, and water chemical components.

Single particle mass spectroscopy. The Rapid Single Particle Mass Spectrometer (RSMS-III) measured the size and composition of individual particles for nine particle size ranges between 0.04 and $2 \mu \mathrm{m}$ (Carson et al.,
1997; Ge et al., 1998). This method was used to detect metals and metal oxides, refractory crustal materials, sulfates and nitrates, aromatic and amine organic compounds, and elemental carbon in single particles.

Single particle SEM analyses. $\mathrm{PM}_{10}$ and $\mathrm{PM}_{2.5}$ aerosol samples were collected onto Nuclepore filters for offsite analysis by Scanning Electron Microscopy (SEM) at the laboratories of R.J. Lee, Inc. The SEM analyses provided elemental composition and morphology of individual particles, and were used to identify bioaerosols, soot, and spherical aluminosilicate particles.

Gaseous species. Integrated VOC samples were collected in 61 fused silica-lined canisters (Entech Instruments, Part 29-10621) using a passive inlet (Entech Instruments, Model CS1200) and analyzed in the CMU Air Quality Laboratory using standard GC-FID techniques (Lewis et al., 1999). This approach permitted the quantification of over 70 compounds, including some of the standard set of Photochemical Assessment Monitoring Station (PAMS) species.

Semi-continuous VOC measurements were made by the UC Berkeley group using an in-situ two-channel GC/MS/FID system. The channels were configured with different preconditioning systems, preconcentration traps, and chromatography columns, enabling quantification of over 60 compounds, including C3-C6 alkanes, alkenes, alkynes, and aromatic, oxygenated and halogenated compounds.

Continuous measurements of $\mathrm{O}_{3}, \mathrm{NO}$ and $\mathrm{NO}_{2}, \mathrm{CO}$, and $\mathrm{SO}_{2}$ gas concentrations were made using highsensitivity gas analyzers (Advanced Pollution Instrumentation, respective Models 400A, 200A, 300 and 100A). Continuous measurements of organic peroxide, hydrogen peroxide, and total soluble peroxide were made using the CSU monitor based on the method of Lazrus et al. (1986).

Fog. Fog composition was measured using a compact version of the Caltech Active Strand Cloudwater Collector known as the CASCC2 (Demoz et al., 1996). Eight major fog events were captured during the 14month baseline study period and are listed in Table 6 .

Table 6

Days during which fog was collected at the central site

\begin{tabular}{|c|c|c|c|c|}
\hline Start date (EST) & Start hour (EST) & End date (EST) & End hour (EST) & $\begin{array}{l}\text { Liquid collected during } \\
\text { event }(\mathrm{ml})\end{array}$ \\
\hline $2001 / 08 / 10$ & $00: 15$ & $2001 / 08 / 10$ & $06: 50$ & 21.5 \\
\hline $2001 / 08 / 26$ & $18: 20$ & $2001 / 08 / 27$ & 06:10 & 88.2 \\
\hline $2001 / 11 / 02$ & $16: 00$ & $2001 / 11 / 02$ & $21: 20$ & 27.8 \\
\hline $2001 / 11 / 17$ & $03: 15$ & $2001 / 11 / 17$ & $07: 45$ & 36.8 \\
\hline $2001 / 11 / 17$ & 19:05 & $2001 / 11 / 18$ & $08: 10$ & 444.5 \\
\hline $2001 / 11 / 19$ & $18: 30$ & $2001 / 11 / 19$ & $19: 35$ & 24.0 \\
\hline $2002 / 05 / 07$ & $09: 50$ & $2002 / 05 / 08$ & $23: 45$ & 19.4 \\
\hline $2002 / 05 / 11$ & $22: 45$ & $2002 / 05 / 13$ & $11: 05$ & 18.6 \\
\hline
\end{tabular}


These fog samples were analyzed onsite for $\mathrm{pH}$. Aliquots of the collected fog were also prepared and analyzed offsite for major anions and cations using IC, and total organic carbon (TOC) and dissolved organic carbon (DOC) using a TOC analyzer (Shimadzu, Model 5000-A).

Meteorology. Precipitation, temperature, relative humidity, wind speed and direction, and UV intensity were measured using standard methods. Backward air mass trajectories were calculated using the methods of Harris and Kahl (1994).

\section{PAQS Source sampling experiments}

Many primary sources of atmospheric aerosols for the Pittsburgh region are inadequately characterized. Several of these sources have been selected for more complete characterization: vehicle exhaust (truck and diesel), coal-fired boilers, coke production, dust (soil and road), vegetative detritus, and fireplaces (waste wood). Field studies that include stack testing using the CMU dilution sampler (Lipsky et al., 2002), fence line monitoring with high time resolution instruments, and

24-hour
$\mathrm{PM}_{2.5}$ mass
$\left(\mu \mathrm{g} / \mathrm{m}^{3}\right)$

24-hour $\mathrm{PM}_{2.5}$ sulfate $\left(\mu \mathrm{g} / \mathrm{m}^{3}\right)$

24-hour $\mathrm{PM}_{2.5}$ nitrate $\left(\mu \mathrm{g} / \mathrm{m}^{3}\right)$

24-hour
$\mathrm{PM}_{2.5}$
organic
carbon
$\left(\mu \mathrm{g}-\mathrm{C} / \mathrm{m}^{3}\right)$

24-hour

$\mathrm{PM}_{2.5}$

elemental

carbon

$\left(\mu \mathrm{g}-\mathrm{C} / \mathrm{m}^{3}\right)$
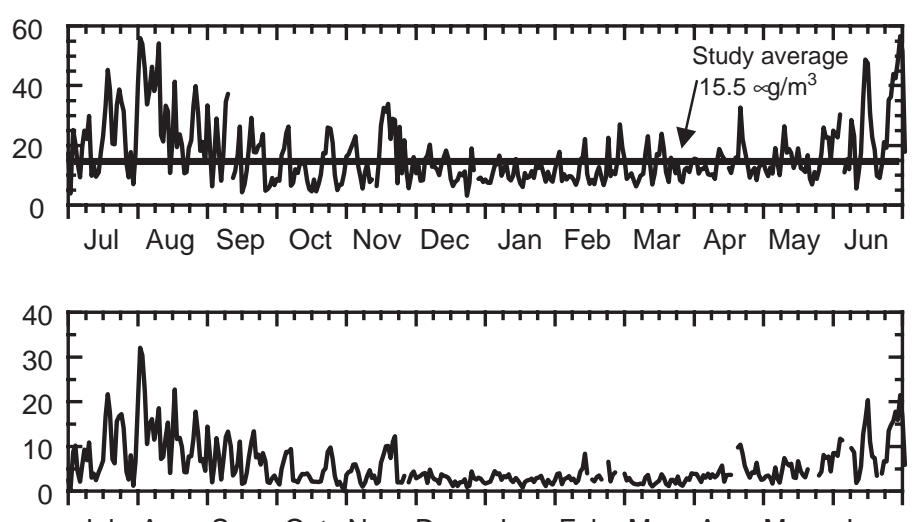

Jul Aug Sep Oct Nov Dec Jan Feb Mar Apr May Jun

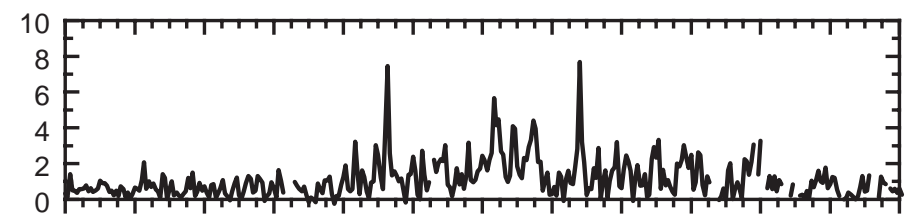

Jul Aug Sep Oct Nov Dec Jan Feb Mar Apr May Jun
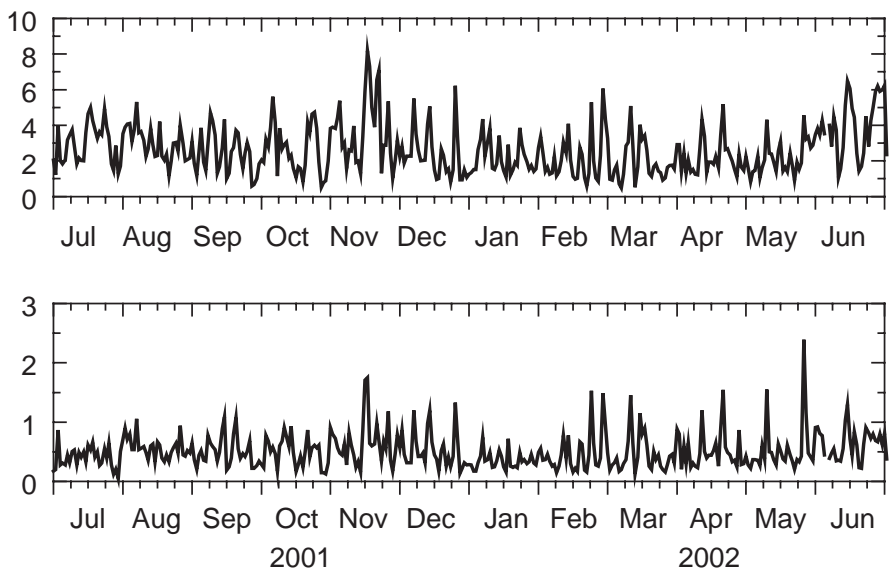

Fig. 3. Daily $24 \mathrm{~h}$ average mass, sulfate, nitrate, organic carbon, and elemental carbon concentrations at the central site from July 2001 to June 2002. Mass concentrations are measured using a TEOM. Organic carbon concentrations are based on the difference between the OC collected on the upstream ('bare quartz') and downstream quartz filters of the CMU TQQQ sampler double-quartz filter pack. Elemental carbon concentrations are based on the TQQQ upstream quartz filter alone. Nitrate and sulfate concentrations are composited from measurements made using the CMU inorganic sampler, the PC-BOSS, the steam sampler, and the Rupprecht and Patashnick 8400 instruments. 
sampling in a highway tunnel are being performed to develop updated emission profiles for these source categories. The source characterization uses the same approaches and instrumentation as the central site ambient monitoring, including detailed organic speciation and single-particle mass spectrometry. These source profiles will be compared to ambient measurements by compiling source activity data and by using a variety of source-receptor techniques.

\section{Daily aerosol measurements collected at the central site}

The study average $\mathrm{PM}_{2.5}$ mass concentration was $15.5 \mu \mathrm{g} \mathrm{m}^{-3}$. Average $24 \mathrm{~h}$ concentrations of mass, sulfate, nitrate, organic carbon, and elemental carbon are presented in Fig. 3. On average, all species exhibited day to day variations in concentration. For example, in July 2001, the maximum and minimum $24 \mathrm{~h}$ average concentrations were 60.9 and $3.7 \mu \mathrm{g} \mathrm{m}^{-3}\left(\mathrm{PM}_{2.5}\right.$ mass), 33.7 and $0.1 \mu \mathrm{g} \mathrm{m}^{-3}\left(\mathrm{PM}_{2.5}\right.$ sulfate), 2.5 and $0.0 \mu \mathrm{g} \mathrm{m}^{-3}$ $\left(\mathrm{PM}_{2.5}\right.$ nitrate), 11.4 and $1.2 \mu \mathrm{g} \mathrm{m}^{-3}\left(\mathrm{PM}_{2.5}\right.$ organic carbon), and 1.7 and $0.1 \mu \mathrm{g} \mathrm{m}^{-3} \quad\left(\mathrm{PM}_{2.5}\right.$ elemental carbon).

The $24 \mathrm{~h} \mathrm{PM}_{2.5}$ Federal Standard of $65 \mu \mathrm{g} \mathrm{m}{ }^{-3}$ was never exceeded during the study at the PAQS central site. However, on the nine days shown in Table 7, the $24 \mathrm{~h}$ average $\mathrm{PM}_{2.5}$ mass concentration was greater than $50 \mathrm{\mu g} \mathrm{m}^{-3}$. Six of these nine days were consecutive 2-day episodes. All 9 days occurred during the summer months of 2001 and 2002, when the $24 \mathrm{~h}$ average ambient temperature was higher than average. On three of the days, the episodes were associated with higher than average sulfate and lower than average organic carbon concentrations ( 1 and 2 August, 2001, and 18 July, 2002). On four other days, the episodes were associated with lower than average sulfate and higher than average organic carbon concentrations (26 June, 1 and 2 July, and 8 July, 2002).

In the remaining sections, seasonality in meteorology, gas-phase and aerosol-phase measurements is assessed using monthly average measurements. This approach allowed seasonal generalizations to be made that were not biased by inconsistencies observed during anomalous months, such as July 2001 and November 2001.

\section{Seasonal summary of measurements collected at the central site}

Meteorology. The seasonal variations in meteorological variables are shown in Fig. 4. Monthly average ambient relative humidity $(\mathrm{RH})$ did not exhibit a strong seasonal dependence and was approximately 60-65\%. On average, the predominant wind direction was
Table 7

Days on which the $24 \mathrm{~h}$ average $\mathrm{PM}_{2.5}$ mass was greater than $50 \mu \mathrm{g} \mathrm{m}^{-3}$

\begin{tabular}{lll}
\hline Date & $\begin{array}{l}\text { Maximum 1 h mass } \\
\text { concentration }\left(\mu \mathrm{g} / \mathrm{m}^{3}\right)\end{array}$ & $\begin{array}{l}\text { Average 24h mass } \\
\text { concentration }\left(\mu \mathrm{g} / \mathrm{m}^{3}\right)\end{array}$ \\
\hline $8 / 1 / 01$ & 63.8 & 55.9 \\
$8 / 2 / 01$ & 75.0 & 54.0 \\
$8 / 9 / 01$ & 72.6 & 54.2 \\
$6 / 25 / 02$ & 75.4 & 56.6 \\
$6 / 26 / 02$ & 74.2 & 51.5 \\
$7 / 1 / 02$ & 76.7 & 60.9 \\
$7 / 2 / 02$ & 79.5 & 56.1 \\
$7 / 8 / 02$ & 69.3 & 51.1 \\
$7 / 18 / 02$ & 70.3 & 53.3 \\
\hline
\end{tabular}

southeasterly or northwesterly in July 2001 and southwesterly in January 2002. The maximum wind speeds were higher, on average, in January 2002 than in July 2001.

$\mathrm{PM}_{10}$ and $\mathrm{PM}_{2.5}$ mass concentrations. Monthly average $\mathrm{PM}_{10}$ and $\mathrm{PM}_{2.5}$ mass concentrations are presented in Fig. 5. On average, approximately $65 \%$ of the $\mathrm{PM}_{10}$ mass measured at PAQS was in the fine $(<2.5 \mu \mathrm{m})$ particle range. The monthly average $\mathrm{PM}_{2.5}$ mass concentrations were robust, as four independent methods of measurement (MOUDI, FRM, Dichot, and TEOM with SES) were in close agreement $\left(R^{2}>0.95\right)$ throughout the study period (Wittig and Davidson, 2003; Cabada et al., 2004b). Fig. 6 illustrates this correlation between the FRM, Dichot, and TEOM with SES but points out that the TEOM slightly overestimated the $\mathrm{PM}_{2.5}$ mass.

$\mathrm{PM}_{2.5}$ composition. Seasonal differences in the average $\mathrm{PM}_{2.5}$ mass composition are presented in Fig. 7. Organic matter concentrations for July 2001 are based on $24 \mathrm{~h}$ integrated denuder sampler measurements and a multiplier of 1.8. For the rest of the study, organic matter concentrations are based on the difference between the OC collected on the upstream ('bare quartz') and downstream quartz filters of the CMU TQQQ sampler double-quartz filter pack. Sulfate, nitrate, and ammonium concentrations are composites of measurements made from several methods, including the CMU inorganic sampler, the PC-BOSS, the steam sampler and the Rupprecht and Patashnick 8400 instruments. $\mathrm{PM}_{2.5}$ crustal material concentrations were estimated from daily $24 \mathrm{~h} \mathrm{PM}_{2.5}$ metal species measurements at the PAQS satellite sites in July 2001 using the sum of oxides algorithm (Malm et al., 1994a, b). The $\mathrm{PM}_{2.5}$ metal species concentrations did not vary substantially across the satellite sites and the estimate of $\mathrm{PM}_{2.5}$ crustal material concentration of $\sim 1 \mu \mathrm{g} \mathrm{m}^{-3}$ compared well with estimates from similar studies (Tolocka et al., 2001). Because the Pittsburgh aerosol is of a regional 

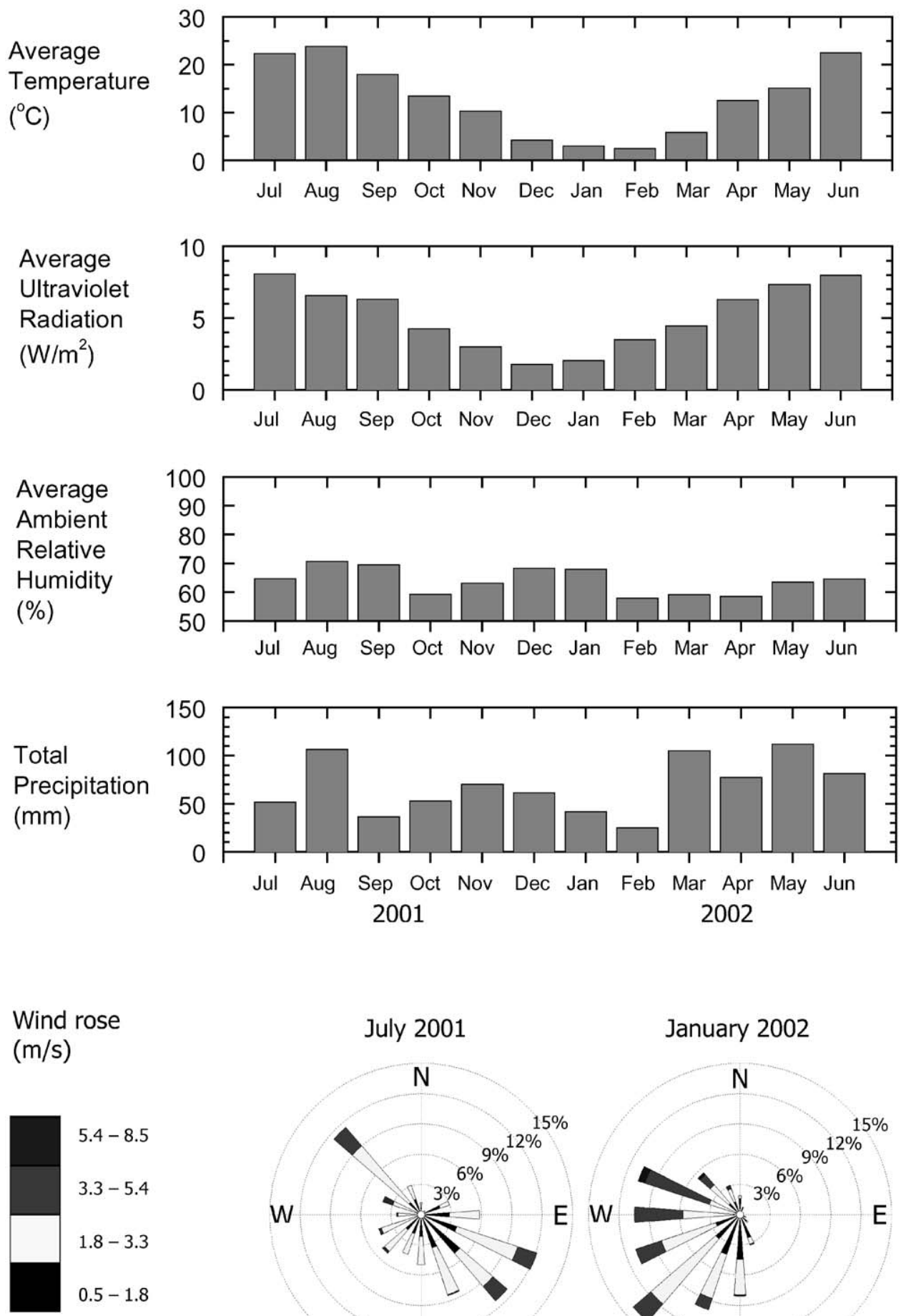

July 2001

$\mathrm{N}$

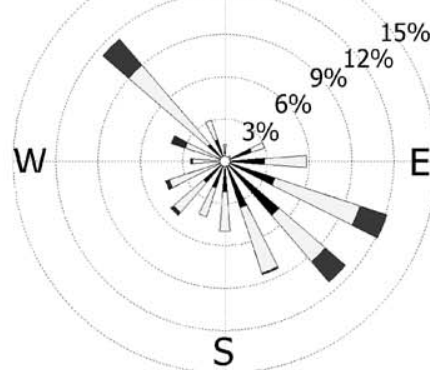

January 2002

N

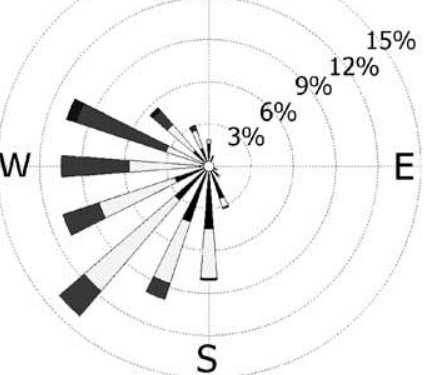

Fig. 4. Monthly average ambient temperature, ultraviolet radiation, and relative humidity, and monthly total precipitation measured at the central site from July 2001 to June 2002. Also shown is the relative occurrence of $1 \mathrm{~h}$ wind speeds for July 2001 and January 2002. 


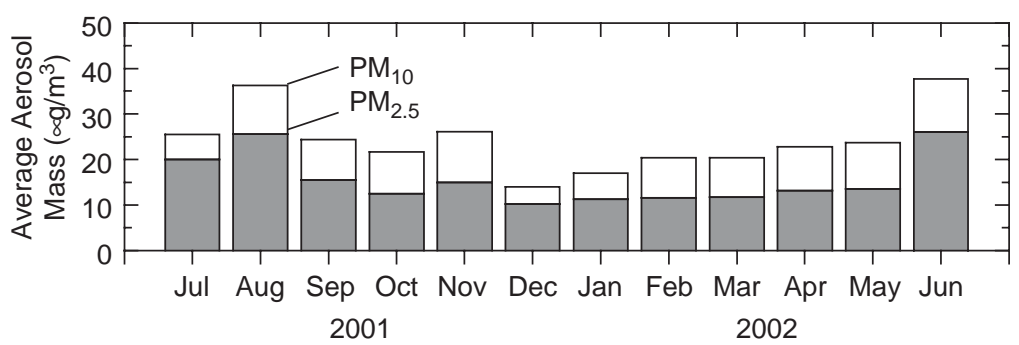

Fig. 5. Monthly average $\mathrm{PM}_{10}$ mass and $\mathbf{P M}_{2.5}$ mass measured at the central site from July 2001 to June 2002 . PM 10 mass was measured on a $24 \mathrm{~h}$ basis using the Dichot sampler, while $\mathrm{PM}_{2.5}$ mass was measured on a $24 \mathrm{~h}$ basis using the FRM sampler.

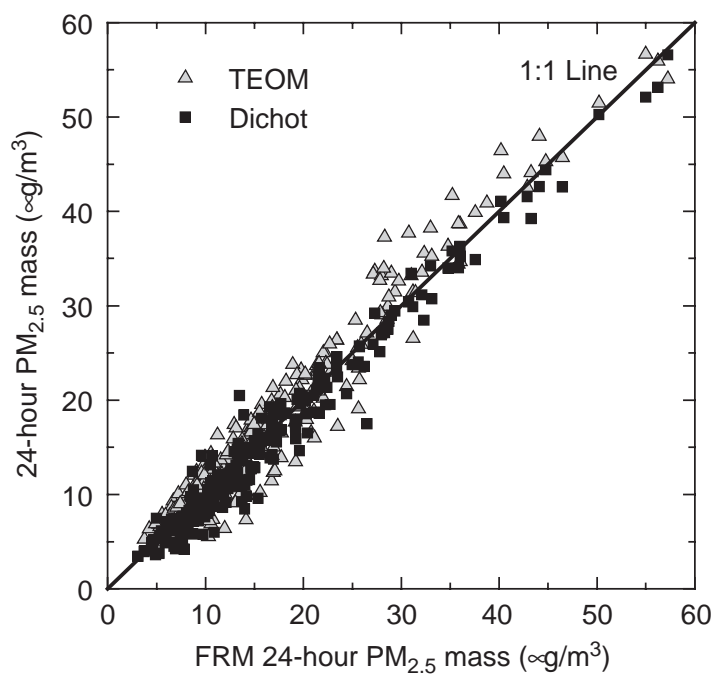

Fig. 6. Comparison of $24 \mathrm{~h}$ integrated $\mathrm{FRM} \mathrm{PM}_{2.5}$ mass measurements and other $\mathrm{PM}_{2.5}$ mass measurements collected at the central site from July 1, 2001 to June 30, 2002. Integrated Dichot measurements were collected on a daily basis, while TEOM measurements were collected continuously and averaged on a $24 \mathrm{~h}$ basis.

nature, this estimate was also used for the months of August 2001 through June 2002. Overall, the monthly average elemental and organic carbon concentrations were variable over the year, while the nitrate concentrations were highest during the winter months, and the mass and sulfate concentrations were highest during the summer months.

Although not noted in Fig. 7, seasonal variations in ultra fine aerosol composition (less than $0.1 \mu \mathrm{m}$ ), which comprise approximately $5 \%$ of the total $\mathrm{PM}_{2.5}$ mass, were also observed at the PAQS site. During the summer intensive, Cabada et al. (2004c) found that on average, $55 \%$ of the ultra fine aerosol mass was inorganic (mainly sulfate and ammonium) and $45 \%$ of the mass was carbonaceous. During the winter intensive, only $33 \%$ of

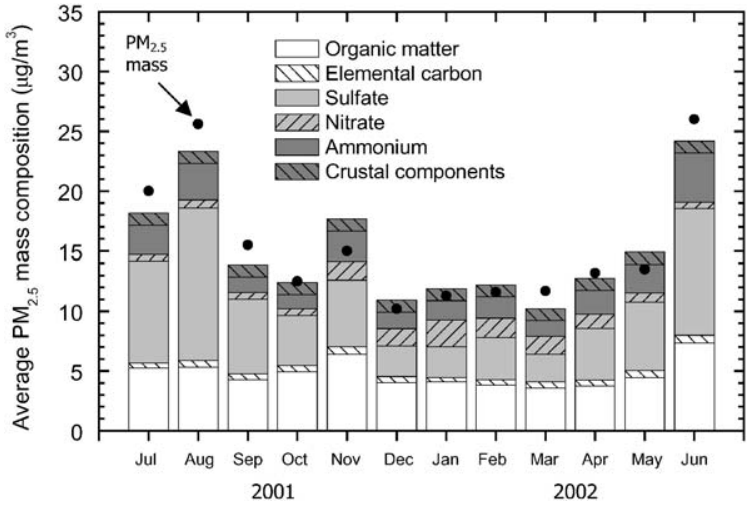

Fig. 7. $\mathrm{PM}_{2.5}$ composition on a monthly average basis at the central site from July 2001 to June 2002. Also shown is the $\mathrm{PM}_{2.5}$ total mass measured using the FRM. Organic matter concentrations for July 2001 are based on $24 \mathrm{~h}$ integrated denuder sampler measurements and the experimentally determined multiplier of 1.8. For other periods, organic matter concentrations are based on the difference between the OC collected on the upstream ('bare quartz') and downstream quartz filters of the CMU TQQQ sampler double-quartz filter pack. Sulfate, nitrate, and ammonium concentrations are composites of measurements made from several methods, including the CMU inorganic sampler, the PC-BOSS, the steam sampler and the Rupprecht and Patashnick 8400 instruments. Crustal component concentrations for August 2001 through June 2002 were estimated from daily July 2001 measurements collected at the PAQS satellite sites to be approximately $1 \mathrm{\mu g} \mathrm{m}^{-3}$.

the ultra fine aerosol mass was inorganic while $65 \%$ was carbonaceous in nature.

$P M$ size distributions. Average surface area and volume size distributions are presented in Fig. 8 for selected months. The aerosol surface area was centered at approximately $0.2 \mu \mathrm{m}$ in aerodynamic diameter. The aerosol volume distribution was bimodal, with a majority of the aerosol volume in the fine mode. The two modes were centered at approximately 0.3 and $3 \mu \mathrm{m}$. 

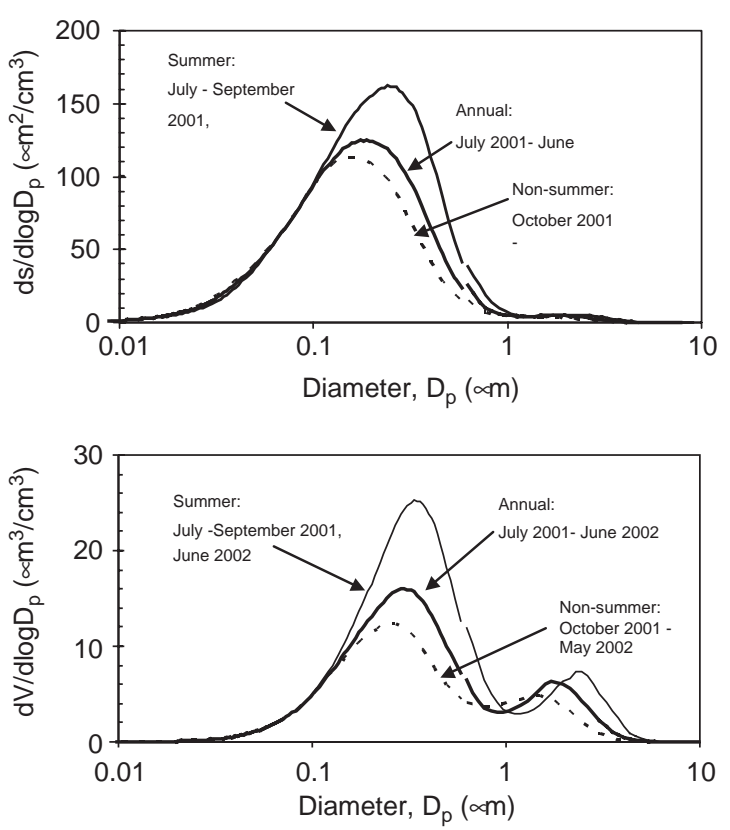

Fig. 8. Monthly average aerosol surface area and volume concentration distribution at the central site from June 2001 to May 2002, as a function of electrical mobility diameter.

\section{Seasonal differences in diurnal patterns at the central site}

Meteorology. Monthly average diurnal profiles were compiled for particular high time resolution measurements for the two intensive periods, July 2001 and January 2002. Diurnal profiles of several key meteorological variables are presented in Fig. 9. Greater than $90 \%$ and $55 \%$ of the days followed these average diurnal patterns in July 2001 and January 2002, respectively.

$\mathrm{PM}_{2.5}$ mass concentration, light scattering, and size distribution. The average diurnal profiles for $\mathrm{PM}_{2.5}$ mass concentration (Fig. 10) did not exhibit a clear diurnal trend. Moreover, less than $25 \%$ of the days followed the corresponding generalized patterns in either month. This behavior can be explained by the fact that the dominant components of the particulate mass (sulfate and organics) were not produced locally but were transported to the area (Tang et al., 2004). The light scattering at ambient RH by aerosols with diameters less than or equal to $2.5 \mu \mathrm{m}$ exhibits a diurnal pattern following that of $\mathrm{RH}$ that is more pronounced during July 2001.

Differences in the diurnal profiles of ambient and dried RH particle size and volume distributions for the intensive months are illustrated in Fig. 11. The number size distributions exhibit diurnal profiles due to diurnal variations in the ultrafine particles, with a maximum

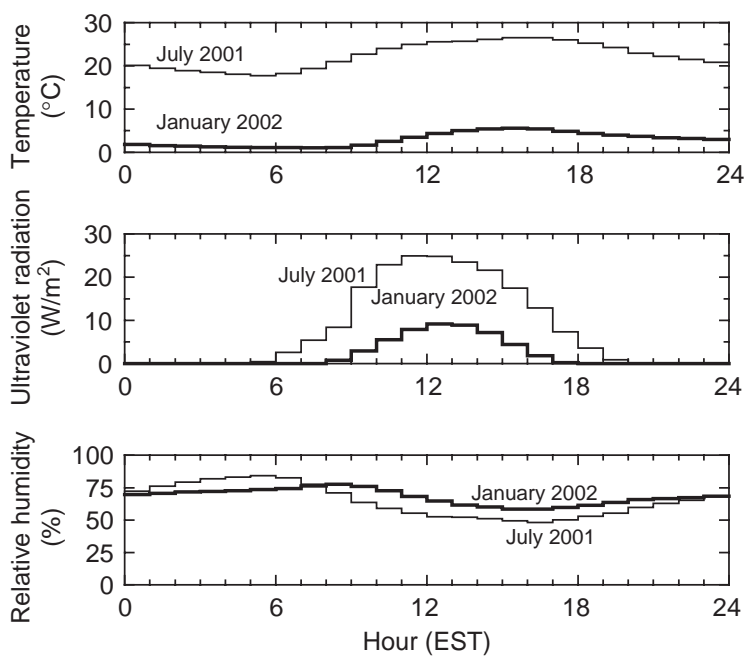

Fig. 9. Average diurnal variation in ambient temperature, ultraviolet radiation, and relative humidity for July 2001 and January 2002 at the central site.
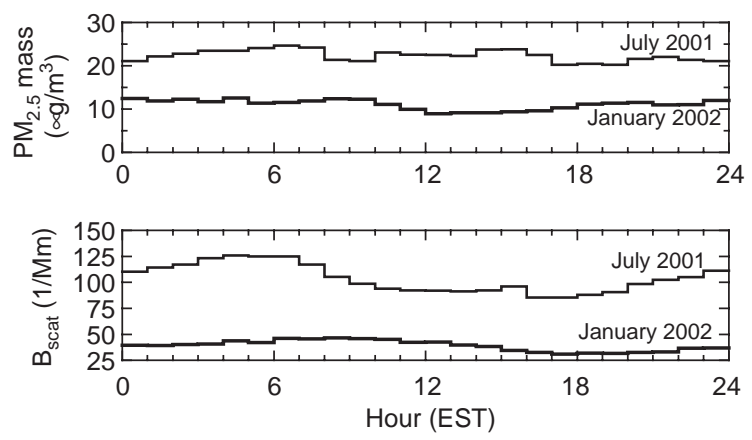

Fig. 10. Diurnal variation in $\mathrm{PM}_{2.5}$ mass and light scattering for July 2001 and January 2002 at the central site. $\mathbf{P M}_{2.5}$ mass was measured using the TEOM and back scatter was measured using the nephelometer.

total number count at midday (Stanier et al., 2004b). The difference between the maximum and minimum number count is more pronounced in the July 2001 profile, when average ambient $\mathrm{PM}_{2.5}$ concentration was higher. Approximately $40 \%$ and $55 \%$ of the days followed these patterns in July 2001 and January 2002, respectively. The dramatic difference between the July 2001 dried and ambient RH volume distributions is due to the substantial water content of the aerosols, attributed to seasonal differences in aerosol acidity.

$P M_{2.5}$ mass composition. Diurnal profiles for specific components of the $\mathrm{PM}_{2.5}$ mass were most evident for nitrate (Fig. 12). Ambient nitrate exhibited a consistent diurnal pattern throughout the study period, with maximum nitrate observed in the early morning and minimum nitrate observed in the late afternoon (Wittig 

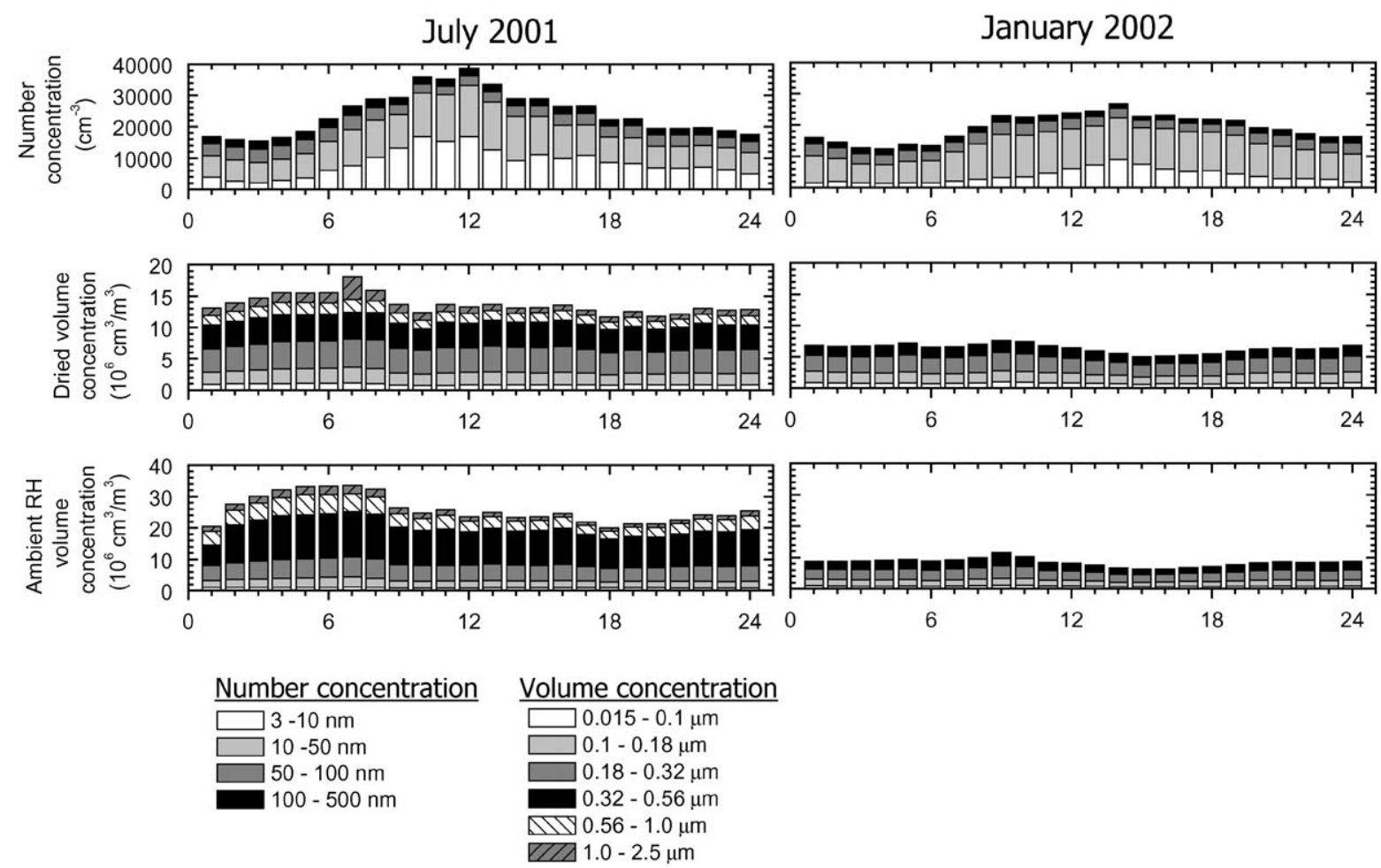

Fig. 11. Average diurnal variation in particle number, and dried and ambient RH volume concentration for July 2001 and January 2002 at the central site. Size distributions were measured using the DAASS and presented as a function of electrical mobility diameter.
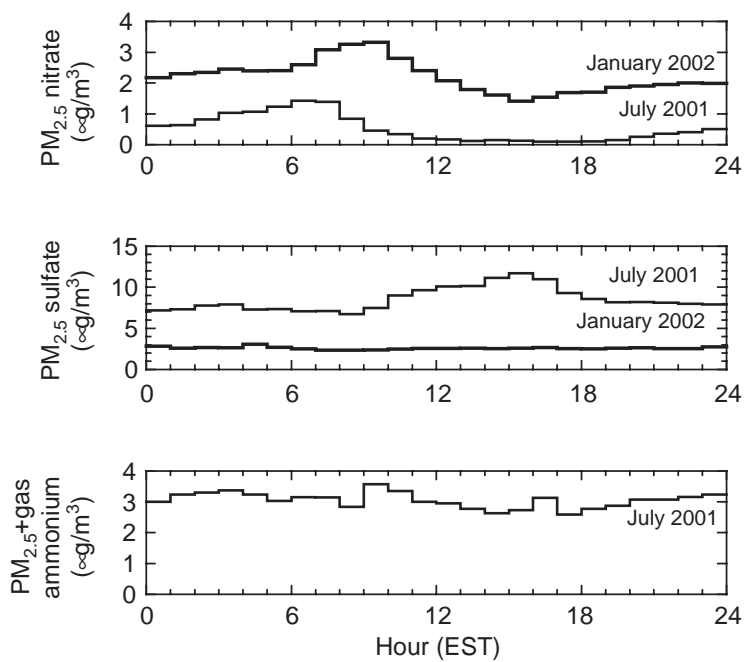

Fig. 12. Average diurnal variation in $\mathrm{PM}_{2.5}$ nitrate, sulfate, and total $\left(\mathrm{PM}_{2.5}\right.$ plus gas) ammonium for July 2001 and January 2002 at the central site. Diurnal values were based on a composite of measurements obtained using the R\&P 8400 instruments, the steam sampler, and the CMU inorganic sampler. et al., 2003). The time of the maximum and minimum nitrate shifted with seasonal changes in ambient temperature and UV radiation. Approximately $90 \%$ and $80 \%$ of the days followed the nitrate patterns in July 2001 and January 2002, respectively. Sulfate concentrations varied diurnally only during the summer, consistent with the gas-phase photochemical production during the day. During the summer, maximum sulfate was observed a couple hours before sunset. Approximately $70 \%$ of the days followed the sulfate pattern in July 2001. During the winter, sulfate concentrations were relatively stable over the course of a day on average. Diurnal profiles for were also evident for $\mathrm{PM}_{2.5}$ organic and total carbon (Fig. 13). Approximately 60\% and $70 \%$ of the days in July 2001 followed the OC and EC patterns, respectively, while around $60 \%$ of the days in January 2002 followed the OC and EC patterns. The features of the profiles varied seasonally, consistent with changes in meteorology.

Gas-phase species. Diurnal profiles for were also evident for several photochemically generated gas-phase species, including $\mathrm{O}_{3}, \mathrm{NO}$, and $\mathrm{NO}_{x}$ (Fig. 14). The features of these particular profiles also varied seasonally. Greater than $75 \%$ and $70 \%$ of the days followed 

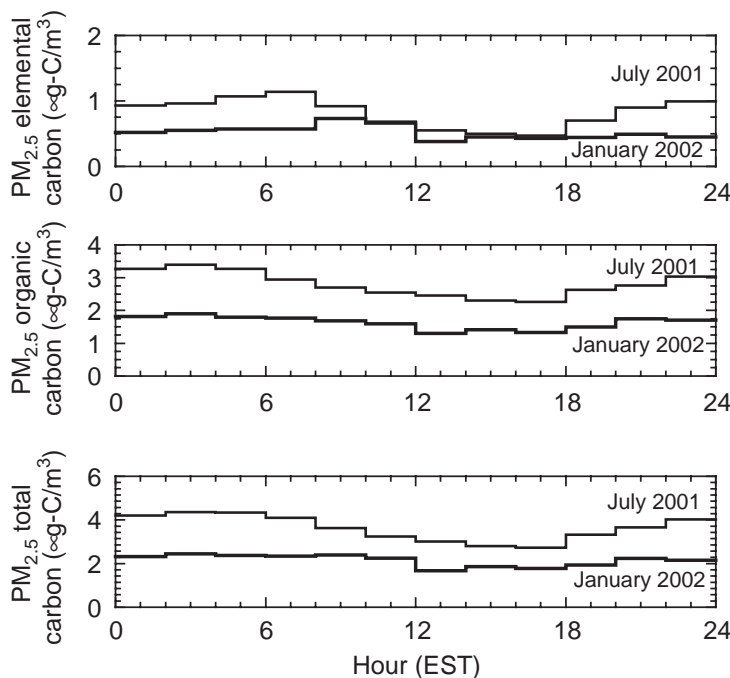

Fig. 13. Average diurnal variation in $\mathbf{P M}_{2.5}$ organic carbon, elemental carbon, and total carbon for July 2001 and January 2002 at the central site. Diurnal values were based on Sunset Labs in-situ carbon analyzer measurements.

these patterns in July 2001 and January 2002, respectively. The $\mathrm{CO}$ pattern is characterized by higher concentrations during the night, a peak during the morning rush hour and a lower concentration during the day. The pattern is more pronounced during the summer because of the stronger atmospheric mixing during the day.

\section{Conclusions}

PAQS is a comprehensive multidisciplinary set of projects in the Pittsburgh region, led by Carnegie Mellon University with contributions from academia, national laboratories, the private sector, and state and local air pollution agencies. The PAQS study design called for measurements to be made at a central site, at several satellite sites, and from two aircraft in order to characterize PM by examination of size, surface area, and volume distribution, chemical composition as a function of size and on a single particle basis, morphology, and temporal and spatial variability in the Pittsburgh region. Measurements made during the 14-month baseline study established the day-to-day character of the meteorology and ambient measurements, while measurements during the 2 intensive periods (ESP01 and ESP02) enhanced the temporal, spatial, and compositional detail of the baseline measurements. These measurements will allow the PAQS hypotheses to be systematically investigated.

Preliminary analyses of the overall dataset were presented in this paper. Nine days of relatively high
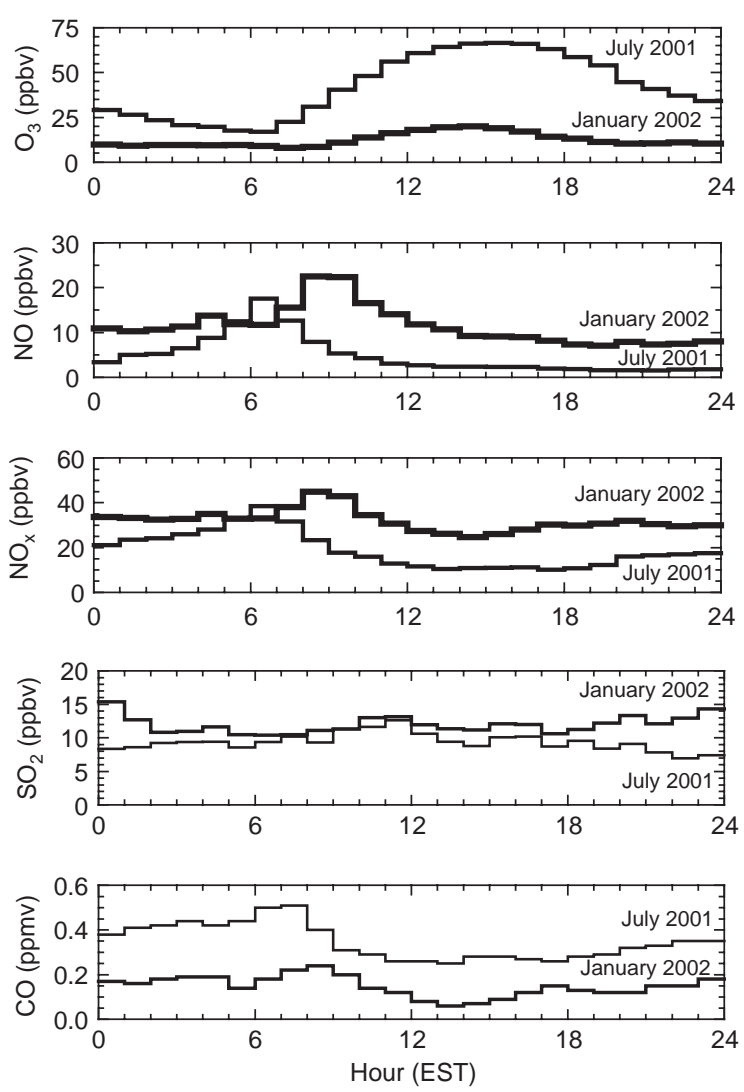

Fig. 14. Average diurnal variation in gas-phase concentrations of $\mathrm{O}_{3}, \mathrm{NO}, \mathrm{NO}_{x}, \mathrm{SO}_{2}$ and $\mathrm{CO}$ for July 2001 and January 2002 at the central site.

$\mathrm{PM}_{2.5}$ mass concentration experienced during PAQS at the central site were discussed, several of which formed multi-day episodes, and all of which occurred during the warmer summer months. Monthly average concentrations and diurnal profiles were also presented, to describe the features of the dataset, to emphasize the processes which affected the observed concentrations, and finally to demonstrate the consistency of the overall dataset. Changes in particular gaseous species or components of the $\mathrm{PM}_{2.5}$ mass were consistent with seasonal differences in average and diurnal patterns in meteorological measurements. On average, some of these patterns were not present or not as dramatic in January 2002 as they were in July 2001. Changes in the overall $\mathbf{P M}_{2.5}$ mass and light scattering were attributed to the major components of the mass, namely the sulfate component.

\section{Acknowledgements}

This research was conducted as part of the Pittsburgh Air Quality Study, which was supported by 
US Environmental Protection Agency under contract R82806101 and the US Department of Energy National Energy Technology Laboratory under contract DEFC26-01NT41017. This paper has not been subject to EPA's peer and policy review, and therefore does not necessarily reflect the views of the Agency. No official endorsement should be inferred.

\section{References}

Blando, J.D., Porcja, R.J., Turpin, B.J., 2001. Issues in the quantitation of functional groups by FTIR spectroscopic analysis of impactor-collected aerosol samples. Aerosol Science and Technology 35, 899-908.

Cabada, J.C., Khlystov, A., Wittig, A.E., Pandis, S.N., 2004a. Fine particle light scattering reconstruction and measurements at PAQS. Journal of Geophysical Research-Atmospheres, submitted for publication.

Cabada, J.C., Pandis, S.N., Subramanian, R., Robinson, A.L., Polidori, A., Turpin, B., 2004b. Estimating the secondary organic aerosol contribution to $\mathrm{PM}_{2.5}$ using the EC tracer method. Aerosol Science and Technology, in press.

Cabada, J.C., Takahama, S., Khlystov, A., Pandis, S.N., Rees, S., Davidson, C.I., Robinson, A.L., 2004c. Mass size distributions and size resolved chemical composition of fine particulate matter at the Pittsburgh Supersite. Atmospheric Environment, this issue, doi: 10.1016/j.atmosenv. 2004.03.004.

Carson, P.G., Johnston, M.V., Wexler, A.S., 1997. Laser desorption ionization of ultra fine aerosol particles. Rapid Communications of Mass Spectroscopy 11, 993-996.

Code of Federal Regulations, 2002. Title 40, Subchapter C Part 50.

Demoz, B., Collett Jr., J.L., Daube Jr., B.C., 1996. On the Caltech active strand cloudwater collectors. Atmospheric Research 41, 47-62.

Eatough, D.J., Eatough, N.L., Obeidi, F., Pang, Y., Modey, W., Long, R., 2001. Continuous determination of $\mathrm{PM}_{2.5}$ mass, including semi-volatile species. Aerosol Science and Technology 34, 1-8.

Erisman, J.W., Otjes, R., Hensen, A., et al., 2001. Instrument development and application in studies and monitoring of ambient ammonia. Atmospheric Environment 35, 1913-1922.

Ge, Z., Wexler, A.S., Johnston, M.V., 1998. Laser desorption/ ionization of single ultra fine multicomponent aerosols. Environmental Science and Technology 32, 3218-3223.

Harris, J.M., Kahl, J.D.W., 1994. An analysis of 10-day isentropic flow patterns for Barrow, Alaska: 1985-1992. Journal of Geophysical Research 99, 25845-25955.

Jaffrezo, J.L., Calas, T., Bouchet, M., 1998. Carboxylic acids measurements with ionic chromatography. Atmospheric Environment 32, 2705-2708.

Jayne, J.T., Leard, D.C., Zhang, X., Davidovits, P., Smith, K.A., Kolb, C.E., Worsnop, D.R., 2000. Development of an aerosol mass spectrometer for size and composition analysis of submicron particles. Aerosol Science and Technology 33, 49-70.

Jimenez, J.L., Jayne, J.T., Shi, Q., Kolb, C.E., Worsnop, D.R., Yourshaw, I., Seinfeld, J.H., Flagan, R.C., Zhang, X.,
Smith, K.A., Morris, J., Davidovits, P., 2003. Ambient aerosol sampling with an aerosol mass spectrometer. Journal of Geophysical Research-Atmospheres (108), ACE 13-1-13-22.

Khlystov, A., Wyers, G.P., Slanina, J., 1995. The steam-jet aerosol collector. Atmospheric Environment 29, 2229-2234.

Khlystov, A., Wittig, A.E., Davidson, C., 2001. Quality assurance project plan for the Pittsburgh air quality study. Report prepared for the U.S.E.P.A., Research Triangle Park, NC.

Khlystov, A., Stanier, C., Pandis, S.N., 2003. An algorithm for combining electrical mobility and aerodynamic size distributions data when measuring ambient aerosol. Aerosol Science and Technology, submitted for publication.

Kidwell, C.B., Ondov, J.M., 2001. Development and evaluation of a prototype system for collecting sub-hourly ambient aerosol for chemical analysis. Aerosol Science and Technology 35, 596-601.

Lazrus, A.L., Kok, G.L., Lind, J.A., Gitlin, S.N., Heikes, B.G., Shetter, R.E., 1986. Automated fluorometric method for hydrogen peroxide in air. Analytical Chemistry 58, 594-597.

Lewis, C.W., Stevens, R.K., Rasmussen, R.A., Caldelino, C.A., Pierce, T.E., 1999. Biogenic fraction of ambient VOC: comparison of radiocarbon, chromatographic, and emission inventory estimates for Atlanta, Georgia. Journal of the Air and Waste Management Association 49, 299-307.

Lipsky, E., Stanier, C.O., Pandis, S.N., Robinson, A.L., 2002. Effects of sampling conditions on the size distribution of fine particulate matter emitted from a pilot-scale pulverizedcoal combustor. Energy and Fuels 16, 302-310.

Malm, W.C., Gebhard, K.A., Molenar, J., Cahill, T., Eldred, R., Huffman, D., 1994a. Examining the relationship between atmospheric aerosols and light extinction at Mount Rainier and North Cascades National Parks. Atmospheric Environment 28, 247-260.

Malm, W.C., Sisler, J.F., Huffman, D., Eldred, R.A., Cahill, T.A., 1994b. Spatial and seasonal trends in particle concentration and optical extinction in the United States. Journal of Geophysical Research 99, 1347-1370.

MSP Corp., Inc., 1998. Operator's Manual for the MicroOrifice Uniform Deposit Impactor. Shoreview, MN.

NIOSH, 1996. Elemental carbon (diesel exhaust). In: NIOSH Manual of Analytical Methods. National Institute of Occupational Safety and Health, Cincinnati, OH.

Slanina, J., Ten Brink, H.M., Otjes, R.P., Even, A., Jongejan, P., Khlystov, A., Waijers-Ijpelaan, A., Hu, M., 2001. The continuous analysis of nitrate and ammonium in aerosols by the Steam Jet Aerosol Collector (SJAC): extension and validation of the methodology. Atmospheric Environment 35, 2319-2330.

Stanier, C.O., Khlystov, A., Chan, R., Mandiro, M., Pandis, S.N., 2004a. A method for the in-situ measurement of fine aerosol water content of ambient aerosols: the Dry-Ambient Aerosol Size Spectrometer (DAASS). Aerosol Science and Technology, in press.

Stanier, C.O., Khlystov, A., Pandis, S.N., 2004b. Nucleation events during the Pittsburgh air quality study: description and relation to key meteorological, gas-phase, and aerosol parameters. Aerosol Science and Technology, in press.

Stolzenburg, M.R., Hering, S.V., 2000. A new method for the automated measurement of atmospheric fine 
particle nitrate. Environmental Science and Technology 34, 907-914.

Subramanian, R., Khlystov, A.Y., Cabada, J.C., Robinson, A.L., 2003. Positive and negative artifacts in particulate organic carbon measurements with denuded and undenuded sampler configurations. Aerosol Science and Technology, submitted for publication.

Takahama, S., Khlystov, A., Wittig, A.E., Davidson, C., Pandis, S.N., 2004. Partitioning of nitrate during the Pittsburgh air quality study and implications of timeaveraging. Journal of Geophysical Research-Atmospheres, submitted for publication.

Tang, W., Raymond, T., Wittig, B., Davidson, C., Pandis, S.N., Robinson, A., Crist, K., 2004. Spatial variations of $\mathrm{PM}_{2.5}$ during the Pittsburgh air quality study. Aerosol Science and Technology, in press.

Thermo Andersen, 1999. Operator's Manual for $\mathrm{PM}_{10}$ High Volume Sampler. Smyrna, GA.

Tolocka, M.P., Solomon, P.A., Mitchell, W., Norris, G.A., Gemmill, D.B., Wiener, R.W., Vanderpool, R.W., Homolya, J.B., Rice, J., 2001. East versus West in the US: chemical characteristics of $\mathrm{PM}_{2.5}$ during the winter of 1999. Aerosol Science and Technology 34, 88-96.

Turpin, B.J., Cary, R.A., Huntzicker, J.J., 1990. An in-situ time-resolved analyzer for aerosol organic and elemental carbon. Aerosol Science and Technology 12, 161-171.

U.S.E.P.A., 1991. EPA Method 200.8 (rev 4.4): Determination of trace elements in waters and wastes by Inductively
Coupled Plasma Mass Spectrometry. Research Triangle Park, NC.

U.S.E.P.A., 1994. EPA Method 6020: Inductively Coupled Plasma Mass Spectrometry. Research Triangle Park, NC.

U.S.E.P.A., 1998. EPA Quality assurance guidance 2.12: Monitoring $\mathrm{PM}_{2.5}$ in ambient air using designated references or Class I equivalence methods. Research Triangle Park, NC.

U.S.E.P.A., 1999. EPA Method 625 (rev R-96/01a): Compendium of methods for the determination of inorganic compounds in ambient air. Research Triangle Park, NC.

Willeke, K., Baron, P.A., 1993. Aerosol Measurement Principles, Techniques, and Applications. Van Nostrand Reinhold, New York.

Wittig, A.E., Davidson, C., 2003. Quality assurance final report for the Pittsburgh Air Quality Study (PAQS) supersite. Report prepared for the U.S.E.P.A., Research Triangle Park, NC.

Wittig, A.E., Takahama, S., Khlystov, A., Pandis, S.N., Hering, S., Kirby, B., Davidson, C., 2003. Semi-continuous $\mathrm{PM}_{2.5}$ inorganic composition measurements during the Pittsburgh Air Quality Study. Atmospheric Environment, submitted for publication.

Zhang, J., Chameides, W.L., Weber, R., Cass, G., Orsini, D., Edgerton, E., Jongejan, P., Slanina, J., 2002. An evaluation of the thermodynamic equilibrium assumption for fine particulate composition: nitrate and ammonium during the 1999 Atlanta supersite experiment. Journal of Geophysicals Research-Atmospheres (108), SOS 2-1-2-11. 


\title{
Fine particle emission profile for a large coke production facility based on highly time-resolved fence line measurements
}

\author{
Emily A. Weitkamp ${ }^{\mathrm{a}}$, Eric M. Lipsky ${ }^{\mathrm{a}}$, Patrick J. Pancras ${ }^{\mathrm{b}}$, John M. Ondov ${ }^{\mathrm{b}}$, \\ Andrea Polidoric ${ }^{\mathrm{c}}$, Barbara J. Turpin ${ }^{\mathrm{c}}$, Allen L. Robinson ${ }^{\mathrm{a}, *}$ \\ ${ }^{a}$ Department of Mechanical Engineering, Carnegie Mellon University, Pittsburgh, PA, USA \\ ${ }^{\mathrm{b}}$ Department of Chemistry and Biochemistry, University of Maryland, College Park, MD, USA \\ ${ }^{c}$ Department of Environmental Sciences, Rutgers University, New Brunswick, NJ, USA
}

Received 23 September 2004; received in revised form 15 June 2005; accepted 15 June 2005

\begin{abstract}
This paper presents a fine particle emission profile for a large metallurgical coke production facility. The profile is developed from highly time-resolved, ambient air quality measurements made at a fence line site adjacent to the plant. A fence line approach was employed because the coke plant has hundreds of stacks and other emission points, making it difficult to develop an integrated, facility-wide emission profile using stack sampling techniques. Continuous or semicontinuous measurements of $\mathrm{PM}_{2.5}$ mass, $\mathrm{PM}_{10}$ mass, $\mathrm{SO}_{2}, \mathrm{NO}_{x}$, organic and elemental carbon (OC and EC), particle size and number, 11 trace metals, wind direction and wind speed were made. Background pollutant levels were also measured. A combination of highly time-resolved meteorology and air quality data were used to determine when the coke facility emissions influenced the sampling site. Concentrations for most pollutants at the fence line site were one to two orders of magnitude higher than background levels when the facility plume heavily influenced the fence line site. Highly time-resolved measurements are essential to resolve these relatively short-duration, large spikes in pollutant concentrations. Simply measuring wind direction is insufficient. From these highly time-resolved measurements an average $\mathrm{PM}_{2.5}$ emission profile for the coke facility was developed. The profile is dominated by OC $\left(40 \% \pm 9 \%\right.$ of $\mathrm{PM}_{2.5}$ mass emissions) and EC (25\% $\pm 5 \%$ of $\mathrm{PM}_{2.5}$ mass emissions). Significant contributions of certain trace metals were also observed, including $\mathrm{As}, \mathrm{Zn}, \mathrm{Se}$, and $\mathrm{Pb}$. The particle emissions are dominated by the fine fraction, with $\mathrm{PM}_{2.5}$ estimated to contribute $84 \% \pm 14 \%$ of the $\mathrm{PM}_{10}$ mass.
\end{abstract}

(C) 2005 Elsevier Ltd. All rights reserved.

Keywords: Metallurgical coke production; Emission profile; $\mathrm{PM}_{2.5}$; Receptor modeling; Coal; Source sampling; Emission characterization; Emission inventory; Primary OC

\section{Introduction}

In 1997 the US EPA promulgated a National Ambient Air Quality Standard for $\mathrm{PM}_{2.5}$ (fine particles

\footnotetext{
*Corresponding author. Tel.: + 14122683657 ; fax: +14122683348

E-mail address: alr@andrew.cmu.edu (A.L. Robinson).
}

with an aerodynamic diameter less than $2.5 \mu \mathrm{m}$ ). Development of regulatory strategies for $\mathrm{PM}_{2.5}$ requires data on fine particle mass emission rates and speciated fine particle emission profiles for a broad range of sources. Stack sampling is a standard technique for measuring emission data from industrial facilities; however stack sampling is not well suited to developing emission profiles from facilities with a 
large number of stacks and/or substantial fugitive emissions.

Another approach to measure emissions is ambient measurements at a fence line site significantly influenced by a source. This basic approach has been used to obtain ammonia emission information for cattle feedlots (McGinn et al., 2003), and the composition of particle emissions from a phosphorus plant (Willis et al., 2001) and a steel mill (Prati et al., 2000). Road tunnel studies have also been widely used to develop emissions data for vehicles, including fine particles (Kristensson et al., 2004).

There are considerable challenges to developing quantitative emission data from ambient fence line measurements. First, transport of pollutants between the emission point and the sampling site depends strongly on local meteorological conditions. Second, one must correct data for background concentrations. These challenges are difficult to overcome using long time-averaged ambient samples such as 12- or 24-h filter samples because of changing meteorological conditions. Unless the meteorological conditions are unchanging, samples with long averaging times combine emissions from many different sources that impact a site. The net effect of this blending is to obscure source-specific variation in a uniform 'background,' greatly complicating source identification.

The challenges associated with meteorology and background corrections can be overcome using highly time-resolved measurements which can reveal short duration spikes in pollutant concentrations at the fence line site. These spikes, in combination with meteorological data, can often be attributed to individual plumes crossing an ambient sampling site (Kidwell and Ondov, 2004). The pollutant concentrations of these spikes are often many times greater than background concentrations, greatly reducing the uncertainty of background corrections. Recent development and commercialization of a number of instruments that provide highly timeresolved measurements of fine particle mass and composition create the possibility of using fence line studies to measure $\mathrm{PM}_{2.5}$ emissions.

This paper presents fence line measurements using a suite of advanced fine particle instruments adjacent to a large, metallurgical coke production facility. Coke production facilities are very large industrial sites that integrate many different processes with associated stacks and numerous locations for fugitive emissions. The primary product, metallurgical coke, is the residue of coal left after removing the volatile material through destructive distillation. It is primarily used in blast furnaces for iron and steel production. At this facility, gaseous byproducts are also recovered and used on site for energy and made into other saleable products. Previous research has examined emissions from specific mechanical processes in coke production, as well as fugitive emissions associated with oven door leaks (USEPA, 2000). Coke oven gases are a complex mixture of organic compounds, such as benzene, naphthalene, and a variety of polycyclic aromatic hydrocarbons (PAHs) (Kirton et al., 1991). They have been the focus of many coke-related studies because of increased risk to plant workers of premature mortality due to cancer, especially respiratory, kidney, and prostate cancer (USEPA, 1984, 1994). Due to these risks, coke oven gases are defined as a hazardous air pollutant (HAP) and are controlled under the Clean Air Act. Particulate matter is emitted from the large number of hightemperature processes used for coke production as well as from the transport and physical processing of coke and coal. Large uncertainties exist regarding the overall fine particle emission rate, composition and size distribution (USEPA, 2000).

This paper presents results from 3 weeks of continuous, semi-continuous, and filter-based air quality measurements made at a site adjacent to a large coking facility near Pittsburgh, PA. A combination of meteorology and pollutant data is used to determine when the fence line site is being impacted by the facility. The data are used to derive an integrated, facility-wide emission profile for $\mathrm{PM}_{2.5}$ for the coke production plant.

\section{Experimental methods}

\subsection{Sampling site}

The sampling site was located at the edge of a plateau and across a river from the coke plant (Figs. 1 and 2) at geo-coordinates 40:18:40-79:52:35. The plant is upwind of the sampling site for wind directions between $175^{\circ}$ and $300^{\circ}$ magnetic (Fig. 1). However, the coke-oven batteries and other major plant operations are primarily located between $240^{\circ}$ and $300^{\circ}$. The sampling site is well above the tallest stacks at the facility. The transit time of emission between the plant and the sampling site is on the order of $5 \mathrm{~min}$ under typical meteorological conditions. The sampling site is surrounded by scrub brush and is approximately $\frac{1}{2} \mathrm{~km}$ from the public access road.

\subsection{Description of facility}

The coking facility is large, annually converting more than 6 million tons of coal into more than 4 million tons of metallurgical coke. As described below, the technology used at this coke facility is quite standard and, therefore, the results from this paper would be applicable to most coking facilities. Easterly et al. (1992) describe the coking process, emissions, and control technologies. The plant operates essentially at full capacity throughout the year. $\mathrm{NO}_{x}$ emission rates from boilers burning coke oven gas suggest normal 


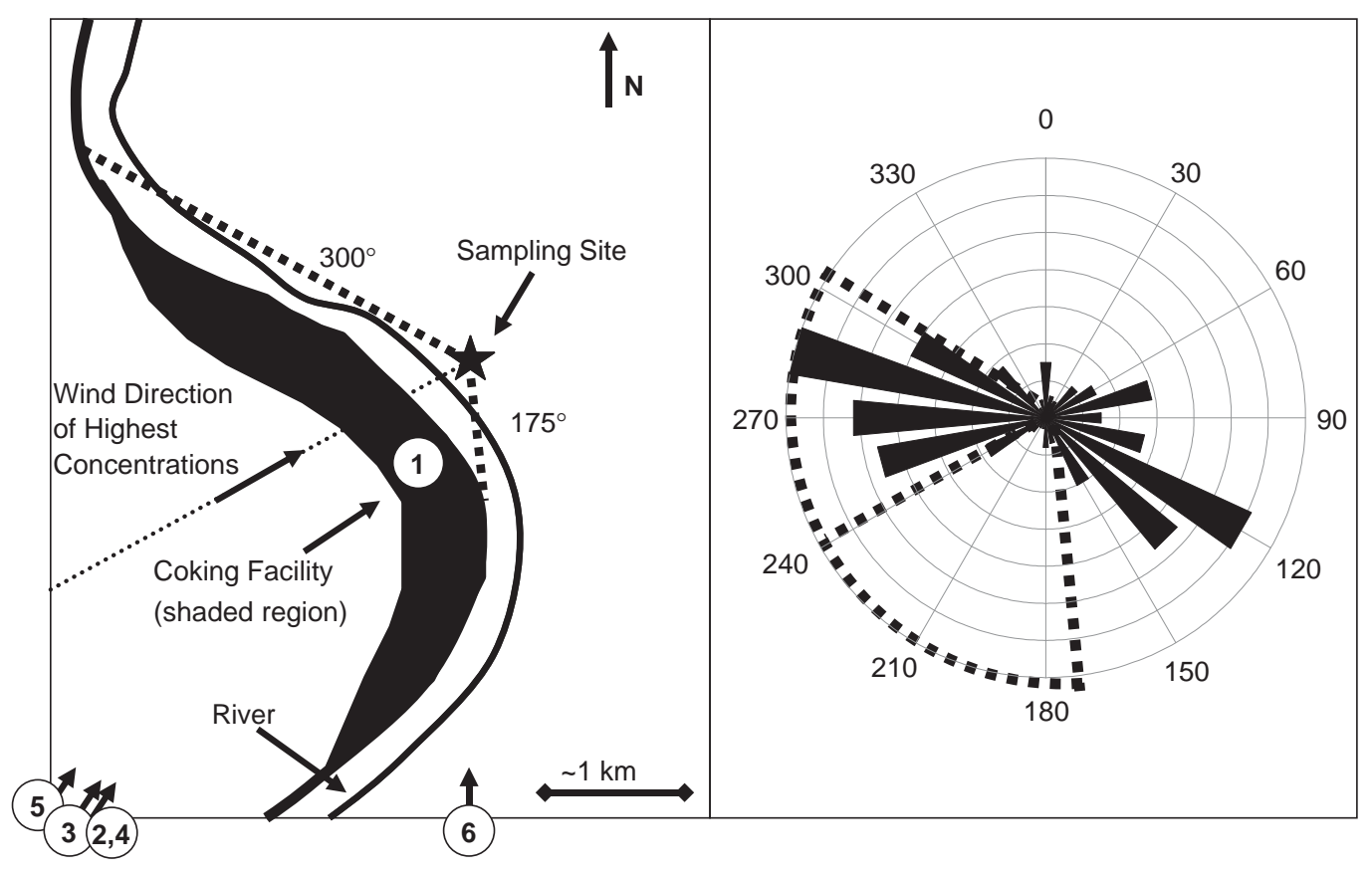

(a)

(b)

Fig. 1. (a) Overhead view of coking facility and sampling site; and (b) wind rose for entire study period. The coking facility influences the sample concentrations for wind directions from $175^{\circ}$ to $300^{\circ}$. The coke batteries and other major operations are located between $240^{\circ}$ and $300^{\circ}$. The circled numbers in (a) and associated arrows indicate bearings from other major upwind sources of $\mathrm{PM}_{2.5}(>10$ tpy) near the coking facility (see Table 1). The length of bars in (b) indicates the fraction of the study period the wind was blowing from the indicated direction. The dashed lines in (b) indicate wind directions when the fence line site is downwind of the coke facility.

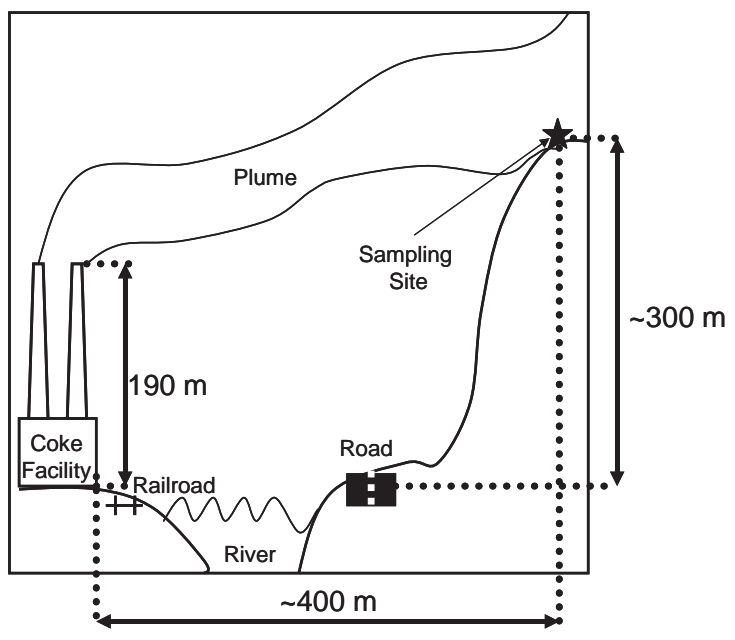

Fig. 2. Side view of coking facility and sampling site.

plant operations throughout the measurement period (USEPA, 2003).

The facility consists of 12 slot oven by-product type coke batteries. Each battery is composed of numerous individual ovens, and the entire facility has more than
850 individual coking ovens. To make coke, coal is tipped into large ovens (charging), heated in a reducing environment to remove the volatile material, removed from the ovens into large bins (pushing) and then cooled with water (quenching). This facility, similar to many modern coke plants, is based on the by-product recovery process in which a portion of the volatiles driven off during the coking process are collected and either burned on site for energy or converted into chemicals. At this plant, the byproduct recovery operations include a tar refinery that converts crude coke oven tars into liquid pitch and other liquid products. The plant uses carbonate scrubbing to remove hydrogen sulfide from the coke oven gas, and an on-site Claus plant converts the hydrogen sulfide to molten sulfur. Finally the ammonia is separated from the coke oven gas and processed into anhydrous ammonia. The balance of the coke oven gas is used for energy, either on site in boilers or at other nearby facilities.

There are numerous points in the coking process when air pollutants are emitted into the atmosphere (Easterly et al., 1992). Significant emissions are associated with the coke ovens. The coke ovens are sealed during most of the coking process, but leaks are not uncommon. This facility is required to visually track and report door, lid, 
and off-take leaks. In 2002, the average percentage of the doors with leaks was $1.3 \%$ and $1.4 \%$ during the study period. The percentage of off-take leaks was $0.6 \%$ for 2002 and $0.7 \%$ for the study period. Reported percentage of lids leaking is low, $0.03 \%$. This information is used to estimate annual emissions from the facility. During pushing, both doors are removed from the coke oven and emissions are difficult to control. This facility has emission control devices installed on the coke-side of the ovens. A traveling canopy hood in conjunction with a bag house is used to control emissions during pushing on 11 of the 12 batteries. The 12th battery uses a coke-side shed and bag house to help control pushing emissions. Sheds are viewed as a much more effective control technology than traveling hoods (Easterly et al., 1992). Though coke production is a batch process, the large number of ovens $(>850)$ dictate that all the steps in the coking process (such as, pushing of an oven) happen every few minutes.

\subsection{Instrumentation and measurement frequency}

Table 2 lists the pollutants measured at the fence line site in addition to instrumentation, measurement frequency and period of operation. The instrumentation and analytical procedures used at the sampling site were identical to those used for the Pittsburgh Supersite (Wittig et al., 2004) to ensure compatibility of the results for source apportionment analysis of ambient data.

A suite of automated instruments was deployed in an air conditioned shelter to provide highly time-resolved measurements of fine particle mass, composition, and number size distribution. Continuous $\mathrm{PM}_{2.5}$ mass was measured using a TEOM (Model 1400a, Rupprecht \& Pataschnick Co., Inc.) operated with a sharp cut $\mathrm{PM}_{2.5}$ cyclone at a controlled temperature of $50^{\circ} \mathrm{C}$. $\mathrm{PM}_{10}$ mass was measured using a TEOM (Model 1400a, Rupprecht $\&$ Pataschnick Co., Inc.) also operating at $50^{\circ} \mathrm{C}$. Particle number and size from 0.013 to $20 \mu \mathrm{m}$ were measured semi-continuously using a standard SMPS (TSI, Inc., Model 3936L10) and Aerodynamic Particle Sizer (APS) (TSI, Inc., Model 3320) (Stanier et al., 2004). The SMPS sheath flow was dried with silica gel; the sample flow was not dried. The $1 \mathrm{~L} \mathrm{~min}^{-1}$ aerosol flow of the APS was dried by a nafion tube dryer; the sheath air of the APS was not dried. $\mathrm{SO}_{2}$ and $\mathrm{NO}_{x}$ concentrations were continuously measured at the site. Wind speed and wind direction were measured at both 10 and $30 \mathrm{~m}$.

Semi-continuous measurements of $\mathrm{PM}_{2.5}$ organic and elemental carbon (OC and EC) were made using a Sunset Laboratory in situ carbon analyzer with an activated carbon parallel plate denuder upstream of the instrument. The instrument used a preliminary version of the STN network analysis protocol (modeled after NIOSH 5040) with a peak helium mode temperature of $870^{\circ} \mathrm{C}$. Details of operation of the carbon instrument are described by Polidori et al. (2005).

Semi-continuous measurements of fine particle elemental composition were made using a Semi-continuous Element in Aerosol Sampler (SEAS) and analyzed offline using Graphite Furnace Atomic Absorption Spectrophotometry (GFAAS) (Kidwell and Ondov, 2004). The SEAS inlet has a size cut of roughly $1.8 \mu \mathrm{m}$ and concentration data are available for 11 elements: $\mathrm{Al}$, $\mathrm{As}, \mathrm{Cd}, \mathrm{Cr}, \mathrm{Cu}, \mathrm{Ni}, \mathrm{Mn}, \mathrm{Fe}, \mathrm{Pb}, \mathrm{Se}$, and $\mathrm{Zn}$. The SEAS sampler was only operated for 2 days because of damage to the sampler control system that occurred during a power failure early in the study period.

Integrated filter samples were collected over 4-6-h periods at night when the plume of the coke facility often strongly influence the monitoring site. Integrated $\mathrm{PM}_{2.5}$ samples were collected on cellulose filters using a high-volume (HIVOL) sampler and analyzed using Inductively Coupled Plasma Mass Spectrometry (ICPMS) to determine concentrations of $\mathrm{Al}, \mathrm{As}, \mathrm{Ba}, \mathrm{Ca}, \mathrm{Cd}$, $\mathrm{Cr}, \mathrm{Cu}, \mathrm{Fe}, \mathrm{Ga}, \mathrm{K}, \mathrm{Mg}, \mathrm{Mn}, \mathrm{Mo}, \mathrm{Na}, \mathrm{Ni}, \mathrm{Pb}, \mathrm{Rb}, \mathrm{Sb}$, Se, Sr, Ti, V and Zn (Pekney and Davidson, 2005). $\mathrm{PM}_{2.5}$ samples were collected with a denuder-filter pack sampler similar to that described in Takahama et al. (2004) for analysis by ion chromatography for $\mathrm{Na}^{+}$, $\mathrm{NH}_{4}^{+}, \mathrm{K}^{+}, \mathrm{Mg}^{2+}, \mathrm{Ca}^{2+}, \mathrm{Cl}^{-}, \mathrm{SO}_{4}^{2-}, \mathrm{NO}_{3}^{-}$and oxalate. Gas-phase concentrations of $\mathrm{NH}_{3}, \mathrm{HCl}$, and $\mathrm{HNO}_{3}$ were obtained by calculating the difference between corresponding ion concentrations in the denuded and undenuded channels.

\subsection{Background concentrations}

Regional background concentrations were measured at the central Pittsburgh Supersite and several other sites in Allegheny County that are not strongly influenced by local sources. Although most of these sites are more than $10 \mathrm{~km}$ away from the fence line site, they provide suitable background data because of the strong regional character of fine particle concentrations in the Pittsburgh region. Tang et al. (2004) found little variation in daily samples collected across the Pittsburgh region supporting the use of regional concentrations as an estimate of background levels. As discussed in the results section, peak concentrations at the coke facility were significantly above this regional background, which minimizes the uncertainty associated with the background corrections.

Background concentrations of $\mathrm{PM}_{2.5}$ mass, organic and elemental carbon were estimated using data collected simultaneously at the Pittsburgh Supersite (Wittig et al., 2004). $\mathrm{PM}_{2.5}$ mass and composition at this site are very similar to other locations within the region (Tang et al., 2004). A $\mathrm{PM}_{2.5}$ TEOM was used to measure $\mathrm{PM}_{2.5}$ mass at the Supersite (Rees et al., 2004). $\mathrm{PM}_{2.5}$ OC and EC were determined from 24-h quartz 
filter samples collected using an undenuded, back-up filter sampler at the Supersite (Subramanian et al., 2004). OC data were corrected for positive sampling artifacts using a quartz backup filter behind a quartz filter (Subramanian et al., 2004). The 24-h concentrations of OC and EC measured at the Supersite were distributed into hourly estimates using the $\mathrm{PM}_{2.5}$ TEOM data and assuming a constant ratio of OC and EC to $\mathrm{PM}_{2.5}$ mass throughout the day and night. These ratios were calculated for each day from the 24-h OC and EC measurements and the 24-h average $\mathrm{PM}_{2.5}$ mass.

Background concentrations of $\mathrm{PM}_{10}$ mass, $\mathrm{SO}_{2}$ and $\mathrm{NO}_{x}$ are estimated using data from several sampling sites operated for compliance monitoring by the Allegheny County Health Department and Pennsylvania Department of Environmental Protection. Background $\mathrm{PM}_{10}$ mass concentrations were estimated using TEOM measurements from sites located in Charleroi, Liberty, Greensburg, Hazelwood, and Pittsburgh. Background $\mathrm{SO}_{2}$ concentrations were estimated using data from sites located in Charleroi, Washington, Holbrook, and Greensburg, Pittsburgh, Liberty, Hazelwood, and Hammerfield. Background $\mathrm{NO}_{x}$ concentrations were estimated using data from sites located in Charleroi, Washington, Holbrook, and Greensburg. Pollutant levels at these sites were compared to identify periods when individual sites were being impacted by obvious site-specific plumes. Periods with site-specific plumes were then removed from the data set, and averages of the remaining data were used as background concentrations.

Background concentrations for trace metals measured with the SEAS sampler were estimated using SEAS data at the fence line site for periods when the fence line site was not downwind of the coke production facility. The validity of this estimate is discussed later in the paper. Background concentrations for elemental composition and major ion concentrations determined from filter samples were estimated using average values for the summer of 2002 measured at the main Pittsburgh Supersite.

\subsection{Calculation of emission profile}

A $\mathrm{PM}_{2.5}$ emission profile for the coke facility was calculated using the measurements at the fence line site and the background values:

$\mathrm{EF}_{i}=\frac{X_{\mathrm{f}, i}-X_{\mathrm{b}, i}}{\mathrm{PM}_{2.5_{\mathrm{f}, i}}-\mathrm{PM}_{2.5 \mathrm{~b}, i}}$,

where $\mathrm{EF}_{i}$ is the contribution of pollutant $X$ to $\mathrm{PM}_{2.5}$ for time period $i, X_{\mathrm{f}, i}$ and $X_{\mathrm{b}, i}$ are the fence line and background concentrations of pollutant $X$ for time period $i$, respectively; and $\mathrm{PM}_{2.5_{\mathrm{f}, i}}$ and $\mathrm{PM}_{2.5_{\mathrm{b}, i}}$ are the fence line and background $\mathrm{PM}_{2.5}$ mass concentrations for time period $i$. The $\mathrm{PM}_{2.5}$ concentrations measured with the TEOM were averaged to correspond to the measurement period of the different instruments; for example, the $10 \mathrm{~min}$ TEOM data were averaged into 30 min blocks for calculation of the emission profile using the SEAS data. Table 2 lists measurement periods for the different pollutants.

Eq. (1) was applied to all measurement periods when the fence line site was downwind of the coke facility. The resulting set of emission profiles was then combined using an uncertainty weighted average to determine an average emission profile for the entire measurement campaign (Taylor, 1997):

$\mathrm{EF}_{\mathrm{wav}}=\frac{\sum w_{i} \mathrm{EF}_{i}}{\sum w_{i}}$.

$\mathrm{EF}_{\mathrm{wav}}$ is the weighted average contribution to $\mathrm{PM}_{2.5}$ of a given species, $w_{i}$ is the weighting factor, $w_{i}=1 / \sigma_{i}^{2} . \sigma_{i}$ is the uncertainty of $\mathrm{EF}_{i}$ calculated for measurement period $i$, and is determined by propagating the measurement uncertainty for each parameter (e.g. $\sigma_{x, \mathrm{f}, i}$, $\left.\sigma_{x, \mathrm{~b}, i,} \sigma_{\mathrm{PM}_{2.5}, \mathrm{f}, i}, \sigma_{\mathrm{PM}_{2.5}, \mathrm{~b}, i}\right)$ through Eq. (1) assuming that the uncertainties for the different measurements are uncorrelated. The overall uncertainty of the average profile is then calculated using error propagation techniques (Taylor, 1997). This approach more heavily weighs periods during which the fence line concentrations are considerably above background levels.

Uncertainty values for each measured parameter were developed as part of the Pittsburgh Supersite (see e.g. Rees et al., 2004). Typical uncertainties are around $10 \%$. Additional uncertainty is assigned to background pollutant concentrations (e.g. $\sigma_{x, \mathrm{~b}, i}, \sigma_{\mathrm{PM}_{2.5} \text { b, } i}$ in Eq. (1)) to account for any differences between the regional measurements used to estimate the background concentrations and actual conditions upwind of the coke plant. Background uncertainties were determined by comparing the estimated background concentrations to measurements at the fence line site when the fence line site was not downwind of the facility. Uncertainties were then defined to account for variation in these data. This approach accounts for both the instrument uncertainty and any biases associated with the background. To illustrate the approach used to define background uncertainties, Fig. 3 compares the hourly $\mathrm{PM}_{10}$ concentrations at the fence line sampling site to the hourly regional average $\mathrm{PM}_{10}$ concentration for periods when the fence line site was not downwind of the coke facility. The dashed lines shown on Fig. 3 bound $90 \%$ of the variation between the regional background and fence line $\mathrm{PM}_{10}$ concentrations for periods when the fence line site is not downwind of the plant. These lines define the $\pm 12 \mu \mathrm{g} \mathrm{m}^{-3}$ uncertainty used for the background $\mathrm{PM}_{10}$ concentrations. Similar comparisons were performed to determine the background uncertainties for the other parameters. Background uncertainties for the filterbased trace metal and inorganic ion concentrations are 


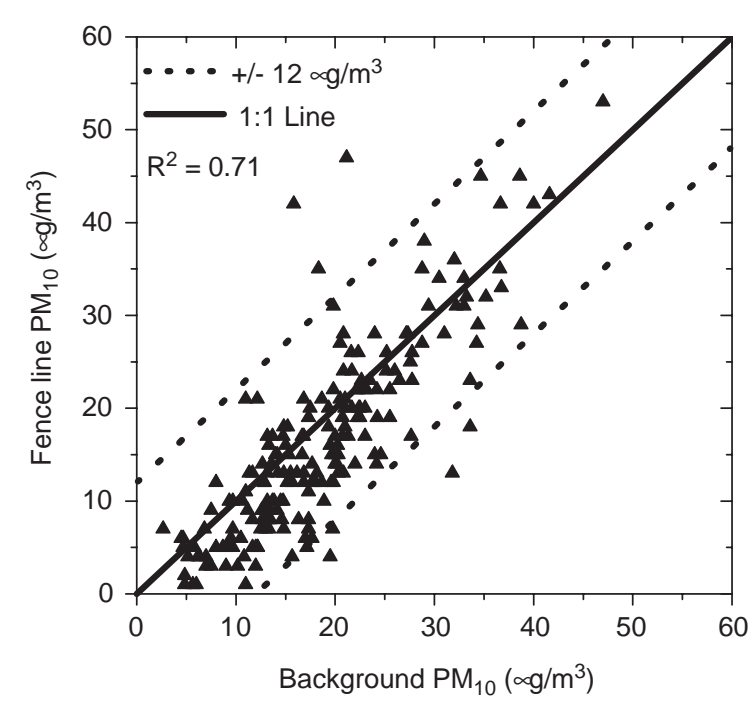

Fig. 3. Comparison of hourly $\mathrm{PM}_{10}$ at the fence line site to background $\mathrm{PM}_{10}$ when the fence line site was not downwind of the coke production plant. Background $\mathrm{PM}_{10}$ is estimated using an average of six $\mathrm{PM}_{10}$ monitoring sites from around the county as described in the text. The uncertainty assigned to the background $\mathrm{PM}_{10}$ concentrations $\left( \pm 12 \mu \mathrm{g} \mathrm{m}^{-3}\right)$ are indicated by the dashed lines. These lines were defined to encompass $90 \%$ of data.

twice the standard deviation of the data used to estimate the background.

\section{Results and discussion}

Figs. 4 and 5 present time-series measurements of fence line site and background air pollutant concentrations and meteorology. Fig. 4 presents wind direction, wind speed, mixing height, $\mathrm{PM}_{2.5}$ mass, $\mathrm{PM}_{10}$ mass, $\mathrm{OC}$, $\mathrm{EC}$, total particle number, $\mathrm{SO}_{2}$, and $\mathrm{NO}_{x}$ data for the 10-day period during which the semi-continuous carbon analyzer was operating. Fig. 5 shows wind direction, $\mathrm{PM}_{2.5}$ mass, and semi-continuous metal concentrations (Al, As, Cd, Cr, Cu, Fe, Mn, Ni, Pb, Se, Zn) for the 2-day period when the SEAS was operated. Breaks in data indicate periods of instrument maintenance or malfunction.

Evidence of the strong influence of emissions from the coke production facility on the fence line site are the "plume events," or intermittent periods when concentrations at the fence line site are high compared to the background. Fig. 4 shows three such plume events: Period 1, a 3-h period around midnight (EST) on August 21, 2002 during which $\mathrm{PM}_{2.5}$ concentrations at the fence line site were $60 \mu \mathrm{g} \mathrm{m}^{-3}$ versus $20 \mu \mathrm{g} \mathrm{m}^{-3}$ in the background; Period 2, a $30-\mathrm{h}$ period that starts around 10:00 EST on August 21 during which the peak $\mathrm{PM}_{2.5}$ concentrations at the fence line site was $160 \mu \mathrm{g} \mathrm{m}^{-3}$ versus $40 \mu \mathrm{g} \mathrm{m}^{-3}$ in the background; and Period 3, a 4-h period around midnight on August 26 during which peak $\mathbf{P M}_{2.5}$ concentrations at the fence line was $60 \mu \mathrm{g} \mathrm{m}^{-3}$ versus $10 \mu \mathrm{g} \mathrm{m}^{-3}$ in the background. The fence line site was downwind of the plant during each of these periods (wind directions range from $175^{\circ}$ to $300^{\circ}$ ). Large spikes in $\mathrm{SO}_{2}, \mathrm{NO}_{x}, \mathrm{OC}, \mathrm{EC}$, and metals concentrations are also observed during these periods. These spikes are significantly greater than background levels, even after accounting for the large uncertainty in the background concentrations.

The fence line site was downwind of the coke facility $46 \%$ of the study period (see Fig. 1), and during $75 \%$ of that time, $\mathrm{PM}_{2.5}$ levels were greater than background levels. Fence line concentrations were 2, 3 and 10 times background concentrations for $37 \%, 20 \%$, and $2 \%$ of the time the sampling site was downwind of the facility, respectively. The plume composition does not significantly change with the strength of a plume event. For example, the OC and EC fraction of $\mathrm{PM}_{2.5}$ is insensitive (within uncertainty) to inclusion or removal of the period on 21 August 2002 during which pollutant levels were $48 \mu \mathrm{g}-\mathrm{C} \mathrm{m}^{-3}$ and $35 \mu \mathrm{g}-\mathrm{C} \mathrm{m}^{-3}$, respectively.

The fact that pollutant levels at the fence line site are not always larger than background levels when the site is downwind of the plant underscores the important role that meteorological conditions play in determining pollutant concentrations at the fence line site. Simply having the correct wind orientation is not sufficient to ensure high pollutant levels compared to background concentrations. For example, Fig. 4 shows that between 0:00 and 17:00 h EST on August 23, 2002, the fence line site was downwind of the coke plant (wind direction between $240^{\circ}$ and $300^{\circ}$ ) but the ratio of fence line to background concentrations of $\mathrm{PM}_{2.5}$ was unity. Periods when the wind direction is from the plant and pollutant concentrations are comparable to background levels supports the regional measurements used to estimate background values at the fence line site.

In addition to wind direction, boundary layer height and wind speed also influence pollutant concentrations at the fence line site. Mixing height data collected at Pittsburgh International Airport located $40 \mathrm{~km}$ to the NW of the fence like site are shown in Fig. 4. The highest concentrations are generally observed at night when the mixing height is lower forcing the core of the plume closer to the ground. During the day when the mixing height is high, the plume from the plant can often be clearly seen passing above the fence line site. Note that during nights when the fence line site was not downwind of the coke facility, elevated pollutant concentrations levels were not observed (e.g. 18:00 EST 24 August to 0:00 EST 25 August in Fig. 4) indicating that the nocturnal pollutant spikes are not regional events. Wind speed is another important factor; 


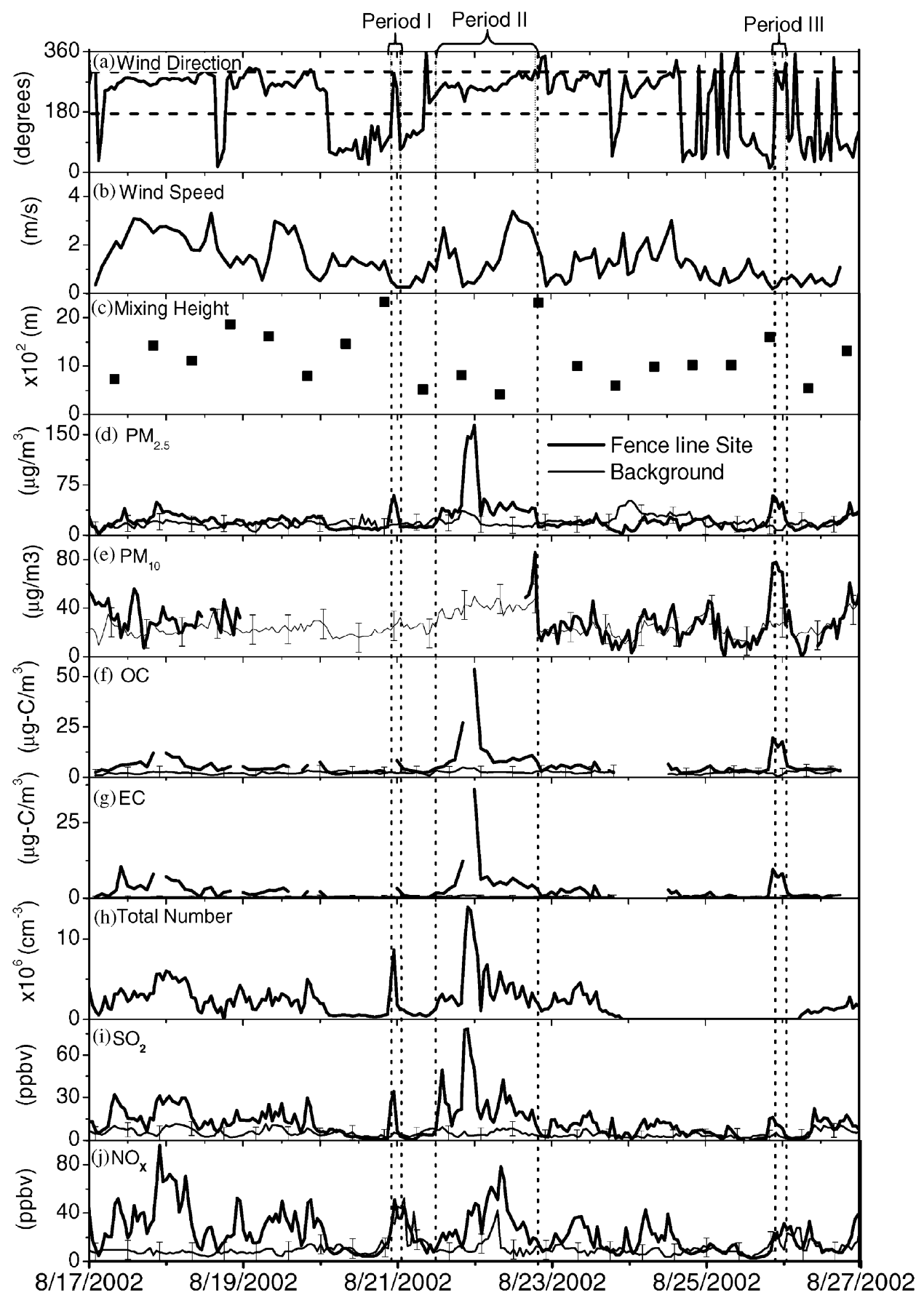

Fig. 4. Time-resolved measurements of air quality and meteorological data. (a) Wind direction, (b) mixing height, (c) wind speed, (d) $\mathrm{PM}_{2.5}$ mass, (e) $\mathrm{PM}_{10}$ mass (f) $\mathrm{PM}_{2.5} \mathrm{OC}$, (g) $\mathrm{PM}_{2.5}$ EC, (h) total particle number, (i) $\mathrm{SO}_{2}$, and (j) $\mathrm{NO}_{x}$. Horizontal dashed lines in (a) indicate wind angles when fence line site is downwind of coke facility. In (d)-(j) thick lines indicate fence line data, and thin lines indicate background values. Error bars indicate uncertainty of background values. Vertical dashed lines indicate three periods of significant impact as discussed in the text.

lower wind speeds reduce pollutant dispersion increasing the strength of a plume event. It should be noted that microscale meteorology caused by the river is likely to influence plume behavior. While this, in combination with the geometry of the site and the distributed nature of the source, complicates application of dispersion 
models to the site, the data strongly support the conclusion that the fence line site is significantly influenced by emissions from the coke production facility.

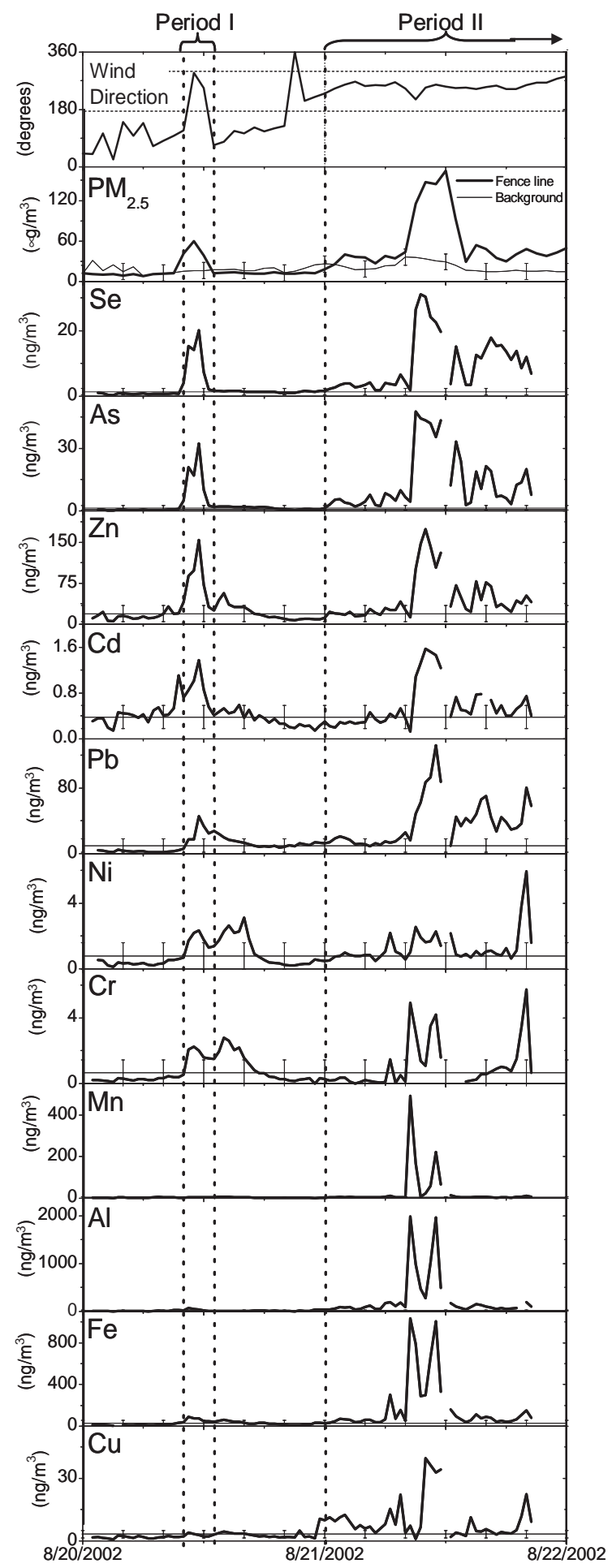

During periods when the wind direction was not from the plant, air pollutant concentrations were, within uncertainty, the same as the regional background. This is illustrated by the $\mathrm{PM}_{10}$ mass data shown in Fig. 3. The regional average $\mathrm{PM}_{10}$ is determined using data from six sites around southwest Pennsylvania. The strong correlation with a 1:1 slope supports the use of regional data for estimating background concentrations at the fence line site.

The short duration of the pollutant spikes shown in Figs. 4 and 5 underscores the importance of highly timeresolved data in combination with meteorological data to identify strong plume events. For example, the 24-h average OC value on 25 August 2002 at the fence line site is $3.5 \mu \mathrm{g}-\mathrm{C} \mathrm{m}^{-3}$, which is comparable to the $24-\mathrm{h}$ background concentration of $2.8 \mu \mathrm{g}-\mathrm{C} \mathrm{m}^{-3}$. However, the peak concentration at the fence line site during this 24-h period was $19.7 \mu \mathrm{g}-\mathrm{Cm}^{-3}$, almost a factor of six higher than the estimated peak background value of $3.3 \mu \mathrm{g}-\mathrm{C} \mathrm{m}^{-3}$. Without the high-time resolution it is difficult to separate the contribution of the coke facility from background.

A potential concern is that the spikes in pollutant concentrations shown in Figs. 4 and 5 are caused by sources other than the coke facility. Several other major point sources are upwind of the site for wind directions between $175^{\circ}$ and $300^{\circ}$ (USEPA, 1999). The distance and bearing of these sources from the fence line site and their $\mathrm{PM}_{2.5}, \mathrm{NO}_{x}$, and $\mathrm{SO}_{2}$ emission rates are listed in Table 1. Arrows indicating the bearings from each of these sources to the fence line site are also shown in Fig. 1.

Several pieces of evidence suggest that these other upwind sources are not responsible for the large spikes in pollutant concentrations at the fence line site. First, the large spikes shown in Fig. 4 occur at wind directions between $240^{\circ}$ and $300^{\circ}$, while the other upwind sources are located between $175^{\circ}$ and $230^{\circ}$. Second, the coke facility is by far the dominant source of $\mathrm{PM}_{2.5}$ mass between the wind angles of $175^{\circ}$ and $300^{\circ}$, estimated to emit more than a factor of two more $\mathrm{PM}_{2.5}$ than the rest of the upwind sources combined. Third, the largest $\mathrm{PM}_{2.5}$ sources listed in Table 1 (e.g., coal-fired power plants) are not major sources of OC and EC and, thus, would not cause the large spikes in those pollutants (Watson et al., 2001; Lipsky et al., 2004). As seen in Figs. 4 and 5, the large spikes in $\mathrm{PM}_{2.5}$ mass are

Fig. 5. Time-resolved meteorology and metals concentrations measured using SEAS. (a) Wind direction and (b) $\mathrm{PM}_{2.5}$ concentration. Heavy lines are measurements at fence line site, thin lines are background concentrations. Horizontal dashed lines in (a) indicate wind angles when fence line site is downwind of coke facility. Error bars indicate uncertainty of background values. Vertical dashed lines correspond to periods of significant impact also indicated in Fig. 4. 
Table 1

Major air pollutant sources upwind of the fence line site for wind directions between $175^{\circ}$ and $300^{\circ}$

\begin{tabular}{|c|c|c|c|c|c|c|}
\hline Number $^{\mathrm{a}}$ & Description & $\begin{array}{l}\text { Distance from } \\
\text { sampling site }(\mathrm{km})\end{array}$ & $\begin{array}{l}\text { Bearing from } \\
\text { site } \\
(\mathrm{deg})\end{array}$ & $\begin{array}{l}\mathrm{PM}_{2.5} \\
\text { Emissions } \\
\text { (tpy) }\end{array}$ & $\begin{array}{l}\mathrm{SO}_{2} \\
\text { Emissions } \\
\text { (tpy) }\end{array}$ & $\begin{array}{l}\mathrm{NO}_{x} \\
\text { Emissions } \\
(\text { tpy })^{\mathrm{b}}\end{array}$ \\
\hline 1 & $\begin{array}{l}\text { Metallurgical coke } \\
\text { production facility }\end{array}$ & 0.4 & 237 & 922 & 2268 & 10335 \\
\hline 2 & Chemical manufacturer & 5.8 & 219 & 10 & 4 & 41 \\
\hline 3 & Float glass plant & 7 & 221 & 60 & 268 & 798 \\
\hline 4 & Coal-fired power plant & 7.5 & 219 & 132 & 4930 & 7023 \\
\hline 5 & Coal-fired power plant & 13 & 227 & 80 & 1031 & 2831 \\
\hline 6 & Fertilizer manufacturer & 13.5 & 183 & 99 & 0 & 377 \\
\hline
\end{tabular}

${ }^{a}$ Number corresponds to those shown in Fig. 1.

${ }^{b}$ tpy $=$ tons per year.

Table 2

Monitoring instrumentation used at fence line sampling site

\begin{tabular}{|c|c|c|c|}
\hline Instrument & Parameter & Frequency & $\begin{array}{l}\text { Period of } \\
\text { operation }\end{array}$ \\
\hline API Model 100A SO $\mathrm{S}_{2}$ Analyzer & $\mathrm{SO}_{2}$ & $5 \mathrm{~min}$ & $8 / 15 / 02-9 / 4 / 02$ \\
\hline API Model 200A NO $\mathrm{NO}_{x}$ Analyzer & $\mathrm{NO}_{x}, \mathrm{NO}$ & $5 \mathrm{~min}$ & $8 / 15 / 02-9 / 4 / 02$ \\
\hline R\&P Model 1400a TEOM ${ }^{\circledR}$ & $\mathrm{PM}_{2.5}$ Mass & $10 \mathrm{~min}$ & $8 / 15 / 02-9 / 5 / 02$ \\
\hline R\&P Model 1400a TEOM ${ }^{\circledR}$ & $\mathrm{PM}_{10}$ Mass & $1 \mathrm{~h}$ & $8 / 15 / 02-9 / 5 / 02$ \\
\hline Meteorological Tower & Wind speed and direction, 10 and $30 \mathrm{~m}$ & $15 \mathrm{~min}$ & $8 / 15 / 02-9 / 5 / 02$ \\
\hline SMPS, APS & Number size distribution from 0.013 to $20 \mu \mathrm{m}$ & $7 \mathrm{~min}$ & $\begin{array}{l}8 / 15 / 02-8 / 24 / 02 \\
8 / 26 / 04-9 / 5 / 02\end{array}$ \\
\hline $\begin{array}{l}\text { Semi-continuous Element in Aerosol } \\
\text { Sampler (SEAS) }\end{array}$ & $\mathrm{Al}, \mathrm{As}, \mathrm{Cd}, \mathrm{Cr}, \mathrm{Cu}, \mathrm{Ni}, \mathrm{Mn}, \mathrm{Fe}, \mathrm{Pb}, \mathrm{Se}, \mathrm{Zn}$ & $30 \mathrm{~min}$ & $8 / 20 / 02-8 / 22 / 02$ \\
\hline $\begin{array}{l}\text { Sunset Laboratory Carbon Analyzer } \\
\text { (Model 2) }\end{array}$ & $\mathrm{OC}, \mathrm{EC}$ & $65 / 90 \mathrm{~min}$ & $8 / 17 / 02-8 / 26 / 02$ \\
\hline $\begin{array}{l}\text { Thermo Anderson High-Volume } \\
\mathrm{PM}_{2.5} \text { Sampler }\end{array}$ & $\begin{array}{l}\mathrm{Al}, \mathrm{As}, \mathrm{Ba}, \mathrm{Ca}, \mathrm{Cd}, \mathrm{Cr}, \mathrm{Cu}, \mathrm{Fe}, \mathrm{Ga}, \mathrm{K}, \mathrm{Mg} \\
\mathrm{Mn}, \mathrm{Mo}, \mathrm{Na}, \mathrm{Ni}, \mathrm{Pb}, \mathrm{Rb}, \mathrm{Sb}, \mathrm{Se}, \mathrm{Sr}, \mathrm{Ti}, \mathrm{V}, \mathrm{Zn}\end{array}$ & $\begin{array}{l}\text { 6-h samples } \\
(N=7)\end{array}$ & $8 / 22 / 02-9 / 5 / 02$ \\
\hline TE 1000 quartz/PUF Sampler & 113 Species & $\begin{array}{l}\text { 6-h samples } \\
(N=5)\end{array}$ & $8 / 21 / 02-9 / 4 / 02$ \\
\hline $\begin{array}{l}\text { CMU Inorganic Sampler, a denuder/ } \\
\text { filter-based speciation }\end{array}$ & $\begin{array}{l}\mathrm{Na}^{+}, \mathrm{NH}_{4}^{+}, \mathrm{K}^{+}, \mathrm{Mg}^{2+}, \mathrm{Ca}^{2+}, \mathrm{Cl}^{-}, \mathrm{SO}_{4}^{2-} \\
\mathrm{NO}_{3}^{-}, \mathrm{C}_{2} \mathrm{H}_{2} \mathrm{O}_{4}, \mathrm{NH}_{3}, \mathrm{HCl}, \mathrm{HNO}_{3}\end{array}$ & $\begin{array}{l}\text { 6-h samples } \\
(N=13)\end{array}$ & $8 / 21 / 02-9 / 4 / 02$ \\
\hline
\end{tabular}

associated with OC. Coal-fired power plants do emit large amounts of metals raising the possibility that emissions from these plants could contribute to the metals concentrations at the fence line site. However, spikes in metals concentration are correlated with spikes of OC, suggesting they are from the same source. Fourth, some of the upwind sources, such as the fertilizer plant, do not have large stacks to create a well-defined plume to impact the fence line site (Table 2).

The highly time-resolved measurements do provide evidence of other sources influencing the fence line site. For example, Fig. 5 shows peaks in $\mathrm{Ni}$ and $\mathrm{Cr}$ but no spike in $\mathrm{PM}_{2.5}$ mass on 21 August 2002 from 1:00 to 4:30 EST. The wind direction during this period varied between $40^{\circ}$ and $140^{\circ}$ and therefore the spikes do not correspond to the coke facility. It is likely these metals are from another source, potentially a metal coating plant located approximately $3 \mathrm{~km}$ from the sampling site at a bearing of $48^{\circ}$.

\subsection{Emission profile for coke production facility}

Under the assumption that the excess pollutant levels observed at the fence line site compared to background 
are due to emissions from the coke facility, an emission profile for the facility can be developed by applying Eq. (1) to the fence line and background data for periods when the site is downwind of the coke facility. Profiles from each measurement period were then combined using a weighted average to determine an average profile for the facility. Tables 3 and 4 list the average emission profile for all of the pollutants measured. The profile accounts for roughly $89 \% \pm 32 \%$ of the $\mathrm{PM}_{2.5}$ mass when using a factor of 1.4 to convert $\mathrm{OC}\left(\mu \mathrm{g}-\mathrm{C} \mathrm{m}^{-3}\right)$ to organic mass (OM, $\left.\mu \mathrm{g} \mathrm{m}^{-3}\right)$.

Table 3

$\mathrm{PM}_{2.5}$ emission profile for coke production facility

\begin{tabular}{lll}
\hline Species & $\% \mathrm{PM}_{2.5}$ mass $(\%)$ & Uncertainty $^{\mathrm{a}}(\%)$ \\
\hline OC $^{\mathrm{b}}$ & 40 & 9 \\
EC $^{\mathrm{b}}$ & 25 & 5
\end{tabular}

SEAS trace metals

\begin{tabular}{lll}
$\mathrm{Al}$ & 0.57 & 0.045 \\
$\mathrm{As}$ & 0.038 & 0.0028 \\
$\mathrm{Cd}$ & 0.0039 & 0.0005 \\
$\mathrm{Cr}$ & 0.0053 & 0.0009 \\
$\mathrm{Cu}$ & 0.023 & 0.0035 \\
$\mathrm{Fe}$ & 0.42 & 0.041 \\
$\mathrm{Mn}$ & 0.092 & 0.010 \\
$\mathrm{Ni}$ & 0.0055 & 0.0011 \\
$\mathrm{~Pb}$ & 0.088 & 0.0084 \\
$\mathrm{Se}$ & 0.026 & 0.0021 \\
$\mathrm{Zn}$ & 0.12 & 0.012 \\
$\mathrm{Hi}-\mathrm{Vol}^{-}$ & \\
$\mathrm{Cs}^{\mathrm{e}}$ & & \\
$\mathrm{Ga}^{\mathrm{e}}$ & 0.0002 & 0.0004 \\
$\mathrm{~K}^{\mathrm{e}}$ & 0.0058 & 0.0032 \\
$\mathrm{Rb}^{\mathrm{e}}$ & 0.075 & 0.21 \\
$\mathrm{Sb}^{\mathrm{e}}$ & 0.0005 & 0.0015 \\
$\mathrm{Sr}^{\mathrm{e}}$ & 0.0031 & 0.0052 \\
$\mathrm{Tl}$ & 0.0022 & 0.0035 \\
$\mathrm{~V}^{\mathrm{e}}$ & 0.0037 & 0.0009 \\
$\mathrm{Inorganics}^{\mathrm{d}}$ & 0.0012 & 0.0021 \\
$\mathrm{Na}^{+\mathrm{e}}$ & & \\
$\mathrm{NH}_{4}^{+\mathrm{e}}$ & 0.28 & 0.31 \\
$\mathrm{Mg}^{2+}$ & 1.8 & 3.9 \\
$\mathrm{Cl}^{-}$ & 7.5 & 5.0 \\
$\mathrm{C}_{2} \mathrm{H}_{2} \mathrm{O}_{4}{ }^{\mathrm{e}}$ & 1.8 & 0.94 \\
\hline
\end{tabular}

${ }^{\mathrm{a}}$ Listed uncertainties are two times the propagated error as a percentage of $\mathrm{PM}_{2.5}$ mass as described in the text.

${ }^{\mathrm{b}} \mathrm{Units}$ of $\mu \mathrm{g}-\mathrm{C} / \mu \mathrm{g} \mathrm{PM}_{2.5}$.

${ }^{\mathrm{c}} \mathrm{Li}, \mathrm{Be}, \mathrm{Na}, \mathrm{Mg}, \mathrm{Ca}, \mathrm{Ti}, \mathrm{Co}, \mathrm{Ag}, \mathrm{Cs}, \mathrm{Ce}$ also measured but fence line concentrations were less than estimated background concentrations.

${ }^{\mathrm{d}} \mathrm{K}^{+}, \mathrm{SO}_{4}^{2-}, \mathrm{NO}_{3}^{-}$, and $\mathrm{HNO}_{3}$ also measured but fence line concentrations were less than estimated background concentrations.

${ }^{\mathrm{e}}$ Values not greater than zero by at least two times propagated error.
Table 4

Gas emission profile for coke production facility

\begin{tabular}{lll}
\hline Species $^{\mathrm{a}}$ & $\mathrm{PM}_{2.5}$ & Ratio uncertainty \\
\hline $\mathrm{SO}_{2}$ & 7.4 & 0.9 \\
$\mathrm{NO}_{x}{ }^{\mathrm{b}}$ & 5.1 & 0.8 \\
$\mathrm{NH}_{3}$ & 0.09 & 0.04 \\
$\mathrm{HCl}^{\mathrm{c}}$ & 0.004 & 0.005 \\
\hline
\end{tabular}

\footnotetext{
${ }^{\mathrm{a}}$ Units are $\mu \mathrm{g}$ of gas per $\mu \mathrm{g}$ of $\mathrm{PM}_{2.5}$ mass.

${ }^{\mathrm{b}}$ Converted to mass using a molecular weight of $46 \mathrm{~g} \mathrm{~mol}^{-1}$.

${ }^{\mathrm{c}}$ Values not greater than zero by at least two times propagated error.
}

Scatter plots of background corrected values for selected pollutant versus background corrected $\mathbf{P M}_{2.5}$ mass are shown in Figs. 6 and 7 to examine the suitability of the average profile. The symbols in these plots indicate data from each measurement period; the solid line is the average emission profile listed in Table 3.

Scatter plots (Fig. 6) show strong correlation of background corrected $\mathrm{OC}$ and $\mathrm{EC}$ versus background corrected $\mathrm{PM}_{2.5}\left(r^{2}=0.82,0.81\right.$, respectively $)$. The single high point strongly influences the $r^{2}$ value, but its inclusion or removal does not significantly change the emission profile value. OC comprises $40 \pm 9 \%$ of the $\mathrm{PM}_{2.5}$ mass, and $\mathrm{EC}$ is $25 \pm 5 \%$ of $\mathrm{PM}_{2.5}$ mass. The high levels of OC and EC in the fine particle emissions are expected given that coke production involves hightemperature processing of coal in a reducing environment. Both coke oven door leaks and pushing likely contribute significantly to these OC and EC emissions. Polycyclic aromatic hydrocarbons (PAHs) dominate the organic fraction from the coke facility, $32 \%$ of total organic mass are PAHs based on the speciated organics data collected at the fence line site. This is expected, as PAHs are known to be a major component of coke oven gas (Kirton and Crisp, 1990; Kirton et al., 1991). The PAH concentrations at the fence line site were often more than two orders of magnitude greater than the background. Further analysis of speciated organics data collected at the fence line site will be presented in a future publication.

The error bars shown in Fig. 6 indicate that not all of the scatter in the data can be explained with uncertainty. The variability in the data is likely due to a combination of changes in plant operations and wind direction. For example, the plant is very large and different wind angles correspond to different parts of the plant. One would expect different emission profiles for different parts and that is a likely cause of some of the variability shown in Fig. 6. Similarly, the composition of emissions is also likely to vary with time.

Fig. 7 shows scatter plots of background-corrected SEAS metals concentrations versus background-corrected 

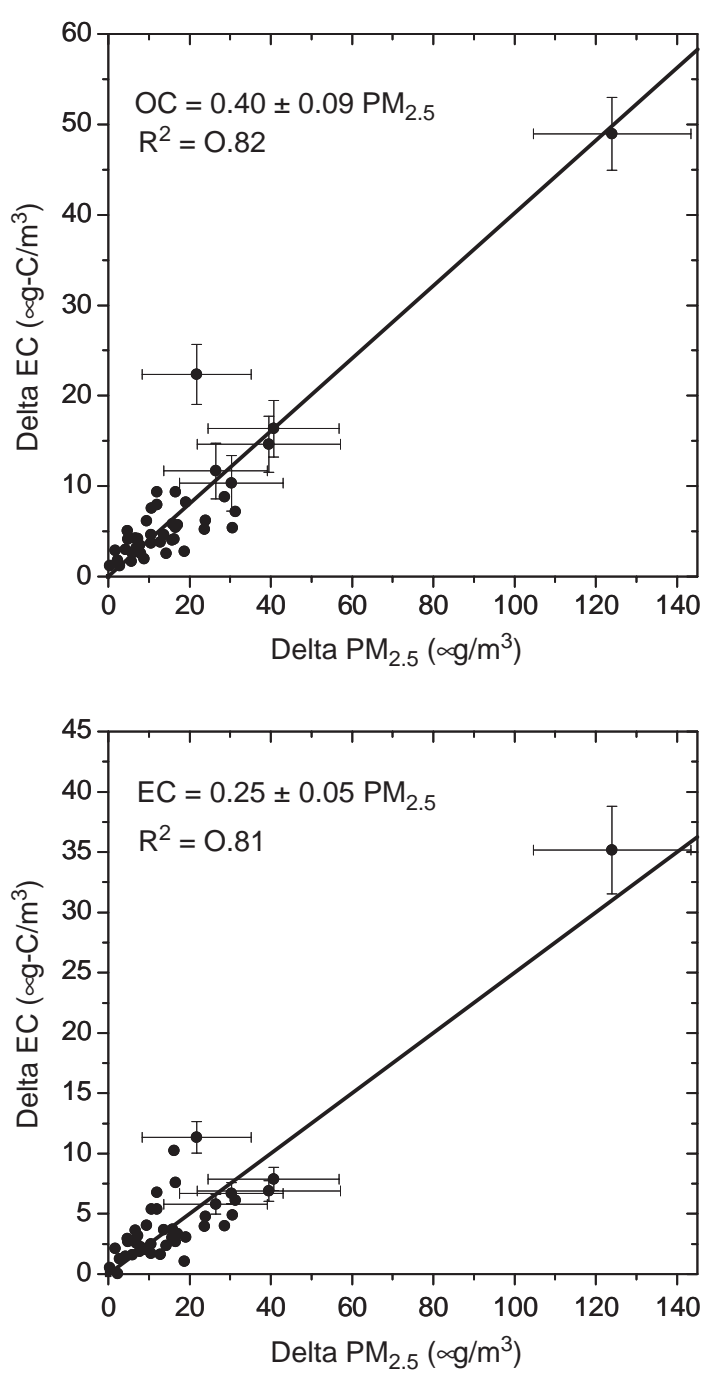

Fig. 6. Background-corrected (a) OC and (b) EC as a function of background-corrected $\mathrm{PM}_{2.5}$ mass ("Delta" indicates background has been subtracted from the fence line measurements). Average emission profile (indicated by solid line) is a weighted average of the data (indicated by the symbols). Error bars, shown only on select points for figure clarity, indicate uncertainty of background-corrected data.

$\mathrm{PM}_{2.5}$ mass. The metals with the strongest correlations with $\mathrm{PM}_{2.5}$ mass are $\mathrm{As}, \mathrm{Zn}, \mathrm{Se}$, and $\mathrm{Pb}\left(r^{2}\right.$ of $0.64,0.65$, 0.47 , and 0.55 , respectively). When the site is downwind of the plant, peak concentrations of these species are all 30-100 times above background and the vast majority of fence line measurements are more than twice background. As and Se are used as tracers for emissions from coal-fired power plants (Harrison et al., 1996) and $\mathrm{Zn}$ and $\mathrm{Pb}$, though not specific to coal combustion, are still present in source profiles from coal-related emissions (Gordon, 1988). The reasonable correlations in combination with the range of wind angles make it unlikely that sources other than the coke facility are contributing to the emission profile for $\mathrm{As}, \mathrm{Pb}, \mathrm{Zn}$ and $\mathrm{Se}$. Again, effects of wind angle and plant operations likely contribute to the variability illustrated in the $\mathrm{As}, \mathrm{Zn}, \mathrm{Se}$, and $\mathrm{Pb}$ data shown in Fig. 7. $\mathrm{Pb}$, and to a lesser extent $\mathrm{Se}$ and $\mathrm{Fe}$, shows distinct bifurcation in the data which could indicate influence from another source. However, there is no discernable correlation between meteorological data and the trends in the data, and therefore no reason to believe there is influence from other sources.

Fig. 7 indicates significant variability in the relationship between $\mathrm{Cd}, \mathrm{Cr}, \mathrm{Cu}, \mathrm{Fe}$, and $\mathrm{Ni}$ and $\mathrm{PM}_{2.5}$ mass. All of these species are much closer to background concentrations indicating lower levels of emissions. For example, $\mathrm{Cr}, \mathrm{Cd}$ and $\mathrm{Ni}$ only have two samples each that are more than twice background concentration. The majority of $\mathrm{Fe}$ and $\mathrm{Cu}$ samples are less than twice background. This results in much larger uncertainties in the emissions of these species. There are also inconsistencies in the data for these metals. For example, there was a single instance when $\mathrm{Cd}$ concentrations were well above the rest of the values. When this point is excluded, the $\mathrm{Cd}$ ratio with $\mathrm{PM}_{2.5}$ becomes $0.0011 \pm 0.0003 \%$, compared to $0.0039 \pm 0.001 \%$ listed in Table 3.

Analysis of filter samples by ICP-MS provides data for 23 metals including some measured by SEAS. However, from these 4-6h samples, it was difficult to separate the signal of the coke facility from the background. For species measured by both samplers, the SEAS data are used to develop the profile because the high temporal resolution of the SEAS clearly resolves periods during which the fence line site is heavily influenced by the plume. Of the remaining species determined by ICP-MS analysis of the filter samples, only $\mathrm{Cs}, \mathrm{Ga}, \mathrm{K}, \mathrm{Rb}, \mathrm{Sb}, \mathrm{Sr}, \mathrm{Tl}$, V were detected in concentrations above background levels. Of these, only $\mathrm{Tl}$ was well above (up to 80 times) background concentrations. For $\mathrm{Cs}, \mathrm{Ga}, \mathrm{K}, \mathrm{Rb}, \mathrm{Sb}, \mathrm{Sr}$, and $\mathrm{V}$, the uncertainties in the plume concentrations and the background estimates resulted in huge uncertainties in the calculated emission profile. For these species, the data only provide a bound for the emission rate.

Inorganic ions may contribute a significant amount to the fine particle mass of the coke emissions, $12 \pm 15 \%$. However, because of the 4-6h sample periods and large uncertainties in background concentrations, the results are highly uncertain.

\subsection{Particle size distribution}

Time-series measurements of total particle number are shown in Fig. 4. The coke production facility is a significant source of particle number. For periods when the fence line site is downwind of the coke facility, the 

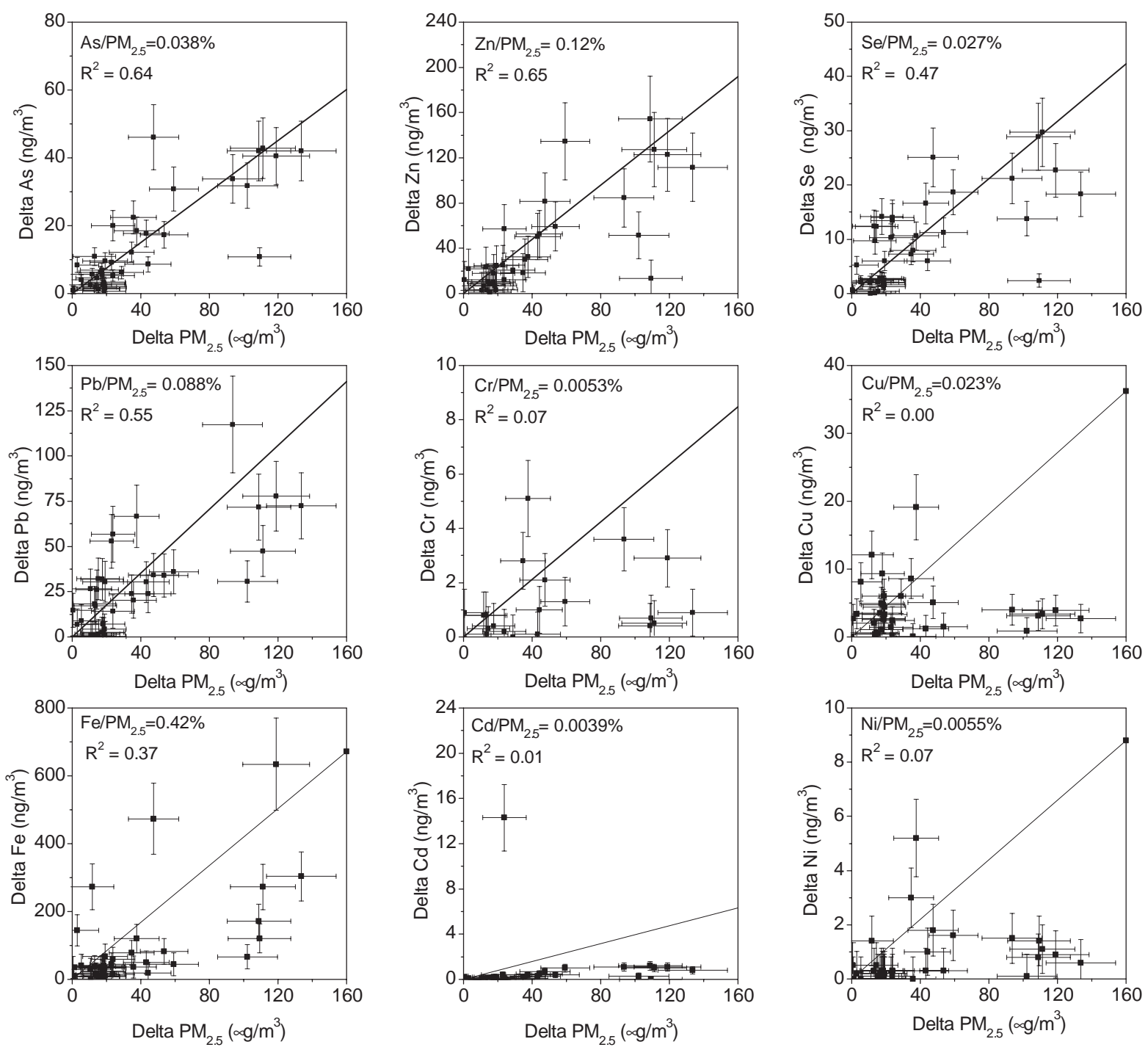

Fig. 7. Background-corrected metals measured with SEAS versus background-corrected $\mathrm{PM}_{2.5}$ mass ("Delta" indicates background has been subtracted from the fence line measurements). Average emission profile (indicated by solid line) is a weighted average of the data (indicated by the symbols). Error bars indicate uncertainty of background-corrected data.

measured number concentration is often more than a factor of 10 higher than the background concentrations.

Fig. 8 shows measured background-corrected number and volume distributions during 20 August 2002 at 23:00 when the wind was coming from $295^{\circ}$ and the fence line was being strongly influenced by emissions from the plant. As a reference, background size distributions measured $2 \mathrm{~h}$ later when the wind shifted to $70^{\circ}$ are also shown. The strongest plume events (total number concentrations $>7,000,000 \mathrm{~cm}^{-3}$ ), such as the one shown in Fig. 8, have a number mode around $45 \mathrm{~nm}$. During weaker plume events such as the one that occurred on 19 August 2002 at 19:00 (total number concentrations $<6,000,000 \mathrm{~cm}^{-3}$ ), the peak number mode is around $35 \mathrm{~nm}$. This ultrafine mode is consistent with fresh emissions from a high temperature or combustion source. Total particle number was also reasonably well correlated with $\mathrm{NO}_{x}$ concentrations, another indicator of a combustion source.

The observed shift in the peak of the number mode as a function of plume event strength may be caused by coagulation of the very high particle number concentrations in the plume. A time scale for coagulation due to Brownian motion can be estimated from the measured size distributions (Seinfeld and Pandis, 1998). Given the very high number concentrations, the coagulation time 

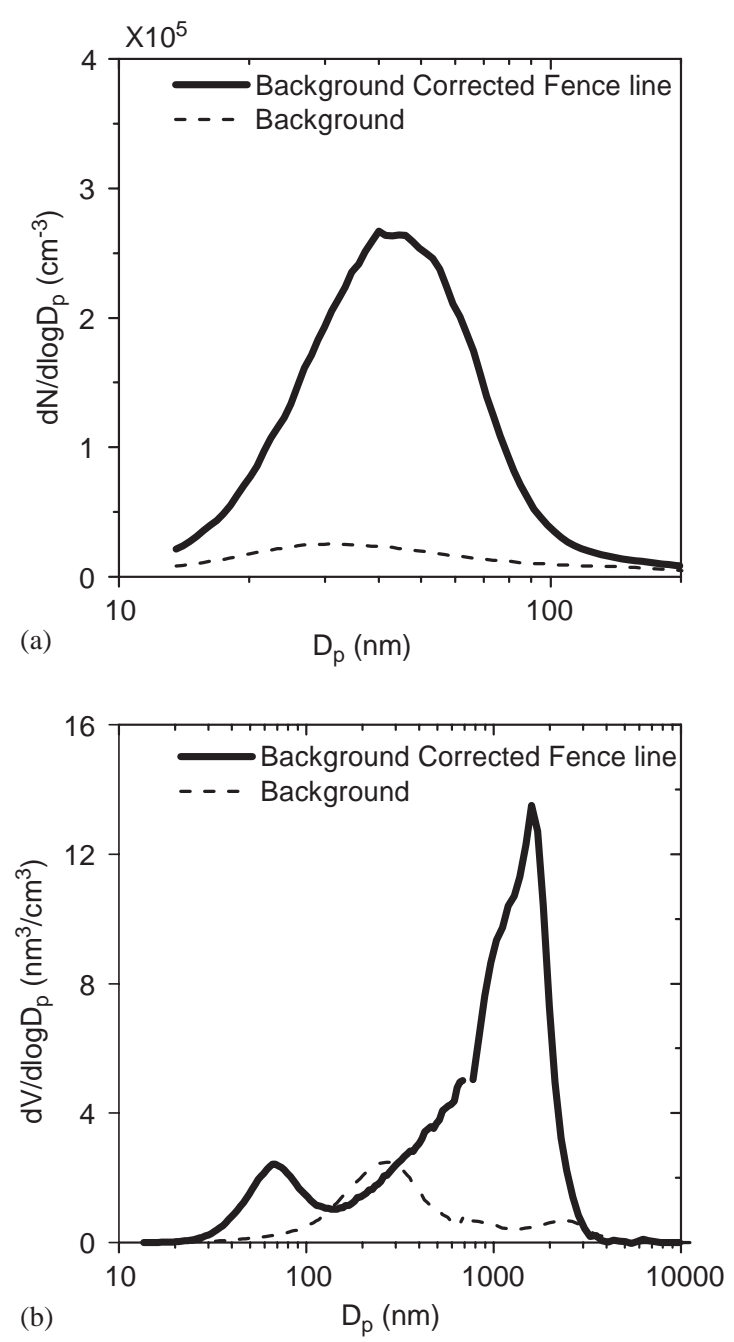

Fig. 8. Background-corrected particle (a) number and (b) volume size distribution measured at the fence line site during a strong impact event that occurred on 20 August 2002 at 23:00 with a wind direction of $295^{\circ}$ (heavy line). The thin line is a background distribution measured $2 \mathrm{~h}$ later when the wind had shifted to $70^{\circ}$ (thin line).

scale is on the order of a minute for the very strong plume events. For the weaker events with lower total number concentrations the coagulation time scales are about 6 times slower. Presumably, the strongest events correspond to periods when the core of the plume impacts the site, while the weaker events correspond to more dilute conditions on the edge of the plume.

Fig. 8 also shows the background corrected particle volume distributions for a strong plume event and a background distribution as a reference. The background corrected volume distribution has two modes, one at around $1300 \mathrm{~nm}$ and a second at $45 \mathrm{~nm}$. The particles in
$1300 \mathrm{~nm}$ mode are likely emitted from the mechanical processes such as pushing, charging, and quenching, while the smaller mode is possibly due to gas to particle conversion inside of the high-temperature processes used to make coke. Note that the background volume distribution has a distinct peak in the accumulation mode around $300 \mathrm{~nm}$ that is not present in the background-corrected plume.

When the site is downwind of the plant there is strong correlation between background-corrected $\mathrm{PM}_{2.5}$ mass measured with the TEOM and integrated particle volume up to $2.5 \mu \mathrm{m}$ measured with the SMPS and APS $\left(r^{2}=0.87\right)$. This comparison yields a particle density of $1.4 \mathrm{~g} \mathrm{~cm}^{-3}$, consistent with expectations for organic particles (Turpin and Lim, 2001). Significant scatter and a poor correlation is observed between $\mathrm{PM}_{10}$ mass measured with the TEOM and the integrated volume up to $10 \mu \mathrm{m}$, possibly due to losses of larger particles in the inlet used for the APS.

A comparison of the mass measurements of the $\mathrm{PM}_{10}$ and the $\mathrm{PM}_{2.5}$ TEOMs at the fence line site indicates emissions from the coke facility are dominated by the fine fraction. Background-corrected $\mathrm{PM}_{2.5}$ contributes $84 \pm 14 \%$ of background-corrected $\mathrm{PM}_{10}$ mass for periods when the fence line site is downwind of the plant $\left(r^{2}=0.71\right)$.

\section{3. $\mathrm{PM}_{2.5}$ mass emission rate for coke production}

The ultimate goal is to convert the $\mathrm{PM}_{2.5}$ emission profile we determine into speciated mass emission rates using an overall plant $\mathrm{PM}_{2.5}$ mass emission factor (e.g. $\mathrm{kg} \mathrm{PM}_{2.5}$ emission per ton of coke produced). Conversations with both plant personnel and local regulators indicate that large uncertainties exist in the $\mathrm{PM}_{2.5}$ emission rate used to develop the emission inventory, while the overall plant $\mathrm{SO}_{2}$ emission rate was felt to be much more certain. Therefore, we estimated the overall plant $\mathrm{PM}_{2.5}$ mass emission rate using the published $\mathrm{SO}_{2}$ emission factor (Hochhauser, 2002) in combination with the average ratio of background-corrected $\mathrm{PM}_{2.5}$ mass to background-corrected $\mathrm{SO}_{2}$ concentration measured during this study when the fence line site was downwind of the plant.

The published emission rate of $\mathrm{SO}_{2}$ for 2002 is $0.3 \pm 0.2 \mathrm{~kg} \mathrm{SO}_{2}$ per ton of coke produced (Hochhauser, 2002). The average ratio between $\mathrm{SO}_{2}$ and $\mathrm{PM}_{2.5}$ concentrations for periods when the fence line site is downwind of the plant (listed in Table 4) is $7.4 \pm 0.9 \mathrm{~kg}$ $\mathrm{SO}_{2}$ per $\mathrm{kg}$ of $\mathrm{PM}_{2.5}$, which is almost three times the $\mathrm{SO}_{2}$ to $\mathrm{PM}_{2.5}$ ratio in the emission inventory $(2.5 \pm 0.8 \mathrm{~kg}$ $\mathrm{SO}_{2}$ per $\mathrm{kg}$ of $\mathrm{PM}_{2.5}$ ) (Hochhauser, 2002). Combining this information, we estimate the $\mathrm{PM}_{2.5}$ mass emission factor to be $40 \pm 20 \mathrm{~g} \mathrm{PM}_{2.5}$ per metric ton of coke produced. 


\section{Conclusions}

Highly time-resolved ambient measurements were made at a fence line site adjacent to a large coke production facility to develop an integrated facility-wide emission profile. The data reveal intermittent spikes of high pollutant concentrations that are associated with emissions from the coke facility. The strength of these plume events depends upon a combination of wind direction, wind speed, and mixing layer height. Simply having the correct wind direction did not guarantee the sampling site was being influenced from the plant. Highly time-resolved measurements greatly simplify separating the contribution of emissions from the coke facility from the background concentrations.

Good correlations were observed between background-corrected $\mathrm{PM}_{2.5}$ mass and background-corrected OC, EC, and several of the trace metals. $\mathrm{PM}_{2.5}$ emissions from the coke facility are predominately organic carbon $(40 \% \pm 9 \%)$ and elemental carbon $(25 \% \pm 5 \%)$. The high levels of OC and EC emissions are consistent with the nature of the coking process, which involves heating of coal in a reducing atmosphere. Trace metals contribute another $1.7 \% \pm 0.8 \%$ of the $\mathrm{PM}_{2.5}$ mass with a distribution of elements similar to fine particle emissions from coal-fired power plants.

The particle emissions from the coke facility are dominated by the fine fraction, with $\mathrm{PM}_{2.5}$ estimated to contribute $84 \pm 14 \%$ of the $\mathrm{PM}_{10}$ mass. The coke production facility is also a large source of particle number, with a number mode at $45 \mathrm{~nm}$ consistent with a combustion/high-temperature source. The particle volume distribution is dominated by larger particles with a mode at $1300 \mathrm{~nm}$ and a smaller mode at $45 \mathrm{~nm}$.

\section{Acknowledgments}

This research was conducted as part of the Pittsburgh Air Quality Study, which was supported by US Environmental Protection Agency under contract R82806101 and the US Department of Energy National Energy Technology Laboratory under contract DEFC26-01NT41017. This paper has not been subject to EPA's required peer and policy review, and therefore does not necessarily reflect the views of the Agency. No official endorsement should be inferred.

The authors acknowledge the contributions of many individuals who provided valuable assistance in this study: Natalie Pekney, Heather Leifeste, R. Subramanian, Juan Cabada Amaya, Andrey Khlystov, Charles Stanier, Leonard Lucas, Satoshi Takahama, and Beth Wittig. We would also like to thank Allegheny County Health Department (ACHD) for access to the sampling site, Darrell Stern at ACHD for coordinating the sampling, and Larry Simmons at E2M for help with site logistics.

\section{References}

Easterly, T.W., Shoup, S.P., Kaegi, D.P., 1992. In: Buonicore, A.J., Davis, W.T. (Eds.), Metallurgical Coke, Air Pollution Engineering Manual. Van Nostrand Reinhold, New York, pp. 606-624.

Gordon, G., 1988. Receptor models. Environmental Science and Technology 22, 1132-1142.

Harrison, R.M., Smith, D.J.T., Luhana, L., 1996. Source apportionment of atmospheric polycyclic aromatic hydrocarbons collected from an urban location in Birmingham, UK. Environmental Science and Technology 30, 825-832.

Hochhauser, P., 2002. Air Quality Program-Point Source Emission Inventory Report for 2002. Allegheny County Health Department, Pittsburgh, PA http://www.achd.net/ airqual/pubs/pdf/2002EIrpt.pdf.

Kidwell, C.B., Ondov, J.M., 2004. Elemental analysis of subhourly ambient aerosol collections. Aerosol Science and Technology 38, 205-218.

Kirton, P.J., Crisp, P.T., 1990. The sampling of coke-oven emissions for polycyclic aromatic-hydrocarbons - a critical review. Fuel 69, 633-638.

Kirton, P.J., Ellis, J., Crisp, P.T., 1991. The analysis of organic matter in coke-oven emissions. Fuel 70, 1383-1389.

Kristensson, A., Johansson, C., Westerholm, R., Swietlicki, E., Gidhagen, L., Wideqvist, U., Vesely, V., 2004. Real-world traffic emission factors of gases and particles measured in a road tunnel in Stockholm, Sweden. Atmospheric Environment 38, 657-673.

Lipsky, E.M., Pekney, N.J., Walbert, G.F., O'Dowd, W.J., Freeman, M.C., Robinson, A.L., 2004. Effects of dilution sampling on fine particle emissions from pulverized coal combustion. Aerosol Science and Technology 38, 574-587.

McGinn, S.M., Janzen, H.H., Coates, T., 2003. Atmospheric ammonia, volatile fatty acids, and other odorants near beef feedlots. Journal of Environmental Quality 32, 1173-1182.

Pekney, N.J., Davidson, C.I., 2005. Determination of trace elements in ambient aerosol samples. Analytica Chimica Acta 540, 269-277.

Polidori, A., Turpin, B.J., Lim, H.J., Cabada, J.C., Subramanian, R., Robinson, A.L., Pandis, S.N., 2005. Local and regional secondary organic aerosol: Insights from a year of semi-continuous carbon measurements at Pittsburgh. Aerosol Science and Technology, submitted for publication.

Prati, P., Zucchiatti, A., Lucarelli, F., Mando, P.A., 2000. Source apportionment near a steel plant in Genoa (Italy) by continuous aerosol sampling and PIXE analysis. Atmospheric Environment 34, 3149-3157.

Rees, S.L., Robinson, A.L., Khlystov, A., Stanier, C.O., Pandis, S.N., 2004. Mass balance closure and the federal reference method for $\mathrm{PM}_{2.5}$ in Pittsburgh, Pennsylvania. Atmospheric Environment 38, 3305-3318.

Seinfeld, J.H., Pandis, S.N., 1998. Atmospheric Chemistry and Physics: From Air Pollution to Climate Change. Wiley, New York.

Stanier, C.O., Khlystov, A.Y., Chan, W.R., Mandiro, M., Pandis, S.N., 2004. A method for the in situ measurement of 
fine aerosol water content of ambient aerosols: the dryambient aerosol size spectrometer (DAASS). Aerosol Science and Technology 38, 215-228.

Subramanian, R., Khlystov, A.Y., Cabada, J.C., Robinson, A.L., 2004. Positive and negative artifacts in particulate organic carbon measurements with denuded and undenuded sampler configurations. Aerosol Science and Technology 38, 27-48.

Takahama, S., Wittig, A.E., Vayenas, D.V., Davidson, C.I., Pandis, S.N., 2004. Modeling the diurnal variation of nitrate during the Pittsburgh Air Quality Study. Journal of Geophysical Research-Atmospheres 109 (D16).

Tang, W., Raymond, T., Wittig, B., Davidson, C., Pandis, S., Robinson, A.L., Crist, K., 2004. Spatial variations of $\mathrm{PM}_{2.5}$ during the Pittsburgh air quality study. Aerosol Science and Technology 38, 80-90.

Taylor, J.R., 1997. An Introduction to Error Analysis: The Study of Uncertainties in Physical Measurements. University Science Books, Sausalito.

Turpin, B.J., Lim, H.J., 2001. Species contributions to $\mathrm{PM}_{2.5}$ mass concentrations: revisiting common assumptions for estimating organic mass. Aerosol Science and Technology 35, 602-610.

USEPA, 1984. Carcinogen assessment of coke oven emissions. Office of Health and Environmental Assessment, Washington, DC. EPA-600/6-82 003F. http://cfpub.epa.gov/ncea/ $\mathrm{cfm} /$ recordisplay.cfm
USEPA, 1994. Health effects notebook for hazardous air pollutants-Draft. EPA-452/D-95-00. http://www.epa.gov/ ttn/atw/hlthef/hapindex.html

USEPA, 1999. AIRData NET Facility Emissions ReportPennsylvania NET Air Pollution Point Sources. AirData. Allegheny County, PA. http://www.epa.gov/air/data/nete mis.html?st $\sim$ PA $\sim$ Pennsylvania

USEPA, 2000. AP-42: Section 12.2: Coke Production. Research Triangle Park. http://www.epa.gov/ttn/chief/ap42/ch12/ final/c12s02.pdf

USEPA, 2003. Acid Rain/OTC Program Hourly Emissions Data. EPA Clean Air Markets. http://www.epa.gov/air markets/emissions/raw/raw023.html\#PA

Watson, J.G., Chow, J.C., Houck, J.E., 2001. PM 2.5 chemical source profiles for vehicle exhaust, vegetative burning, geological material, and coal burning in Northwestern Colorado during 1995. Chemosphere 43, 1141-1151.

Willis, R.D.E., William, D., Conner, Teri, L., 2001. Monitoring and source apportionment of particulate matter near a large phosphorus production facility. Journal of Air \& Waste Management 51, 1142-1166.

Wittig, A.E., Anderson, N., Khlystov, A.Y., Pandis, S.N., Davidson, C., Robinson, A.L., 2004. Pittsburgh air quality study overview. Atmospheric Environment 38, 3107-3125. 


\title{
Simulation of the thermodynamics and removal processes in the sulfate-ammonia-nitric acid system during winter: Implications for $\mathbf{P M}_{2.5}$ control strategies
}

\author{
Dimitris V. Vayenas, ${ }^{1}$ Satoshi Takahama, ${ }^{2}$ Cliff I. Davidson, ${ }^{3,4}$ and Spyros N. Pandis ${ }^{2,4}$ \\ Received 19 May 2004; revised 19 November 2004; accepted 28 December 2004; published 18 March 2005.
}

[1] In the eastern United States, inorganic species account for approximately half of the $\mathrm{PM}_{2.5}$ mass, with sulfate salts comprising the largest fraction. Current strategies for reducing $\mathrm{PM}_{2.5}$ mass concentrations target reducing $\mathrm{SO}_{2}$ to reduce sulfate, but in such a case more ammonium nitrate may form when nitric acid is present. Large-scale chemical transport models suffer from uncertainties associated with emission inventories. To examine how the inorganic $\mathrm{PM}_{2.5}$ concentration responds to changes in emissions, we introduce an observation-based box model, the thermodynamic model with removal (TMR), to estimate responses of $\mathrm{PM}_{2.5}$ to precursor concentrations. TMR assumes that particles are in equilibrium with the gas phase, but the removal rate of total $\left(\mathrm{PM}_{2.5}+\right.$ gas $)$ nitric acid from the system depends on the gas/aerosol partitioning of this species. The model is used to investigate sulfate, total ammonia, and total nitric acid control strategies for western Pennsylvania during the winter using measurements obtained in the Pittsburgh Air Quality Study. Predictions from TMR are compared with observations and predictions of a chemical equilibrium model (GFEMN), where the perturbation of sulfate or total ammonia does not affect the total nitric acid availability. Results show that TMR predicts more aerosol nitrate to form than GFEMN in scenarios where the total ammonia to sulfate ratio is increased, but model results are similar under ammonia-limited conditions. When sulfate is reduced by $50 \%$ during the winter, GFEMN predicts that inorganic $\mathrm{PM}_{2.5}$ mass concentrations will be reduced by $23 \%$, while TMR predicts that there will only be an $8 \%$ reduction. For a $50 \%$ reduction in ammonia availability, inorganic $\mathrm{PM}_{2.5}$ was reduced by $29 \%$, while for a $50 \%$ reduction in total nitric acid a $17 \%$ reduction in inorganic $\mathrm{PM}_{2.5}$ was predicted. The analysis suggests the importance of the phase state of the aerosol for the effectiveness of the emission control strategies.

Citation: Vayenas, D. V., S. Takahama, C. I. Davidson, and S. N. Pandis (2005), Simulation of the thermodynamics and removal processes in the sulfate-ammonia-nitric acid system during winter: Implications for $\mathrm{PM}_{2.5}$ control strategies, J. Geophys. Res., 110, D07S14, doi:10.1029/2004JD005038.

\section{Introduction}

[2] Atmospheric particles have adverse effects on human health and directly affect visibility, air quality and climate change [North American Research Strategy for Tropospheric Ozone (NARSTO), 2003; Intergovernmental Panel on Climate Change (IPCC), 2001]. The U.S. EPA has promulgated regulations regarding mass concentrations of fine particulate matter (PM), which are particles with aerodynamic diameters less than $2.5 \mu \mathrm{m}\left(\mathrm{PM}_{2.5}\right)$, to

\footnotetext{
${ }^{1}$ Department of Environmental and Natural Resources Management, University of Ioannina, Agrinio, Greece.

${ }^{2}$ Department of Chemical Engineering, Carnegie Mellon University, Pittsburgh, Pennsylvania, USA.

${ }^{3}$ Department of Civil and Environmental Engineering, Carnegie Mellon University, Pittsburgh, Pennsylvania, USA.

${ }^{4}$ Also at Department of Engineering and Public Policy, Carnegie Mellon University, Pittsburgh, Pennsylvania, USA.

Copyright 2005 by the American Geophysical Union. 0148-0227/05/2004JD005038\$09.00
}

alleviate the health and economic burdens attributed to high concentrations of particulate matter. $\mathrm{PM}_{2.5}$ consists of many different types of particles covering a broad range of composition and size and can be categorized into primary and secondary. Primary aerosols include automobile exhaust, industrial emissions but also sea spray and dust, and are emitted directly into the atmosphere. Secondary aerosols, which generally have diameters less than $2.5 \mu \mathrm{m}$, are produced in the atmosphere from reactions involving primary and secondary gases [Pandis and Seinfeld, 1998].

[3] In the eastern United States, inorganic species account for approximately half of the $\mathrm{PM}_{2.5}$ mass, with sulfate salts constituting the largest fraction [U.S. Environmental Protection Agency, 1996]. The sources of these particles and precursor gases are known to be primarily anthropogenic, and are obvious targets for emission controls. Oxidation of $\mathrm{SO}_{2}$ is the primary source of sulfate, and current control strategies for reducing $\mathrm{PM}_{2.5}$ target this emission source. Ammonium and nitrate are also significant contributors to inorganic $\mathrm{PM}_{2.5}$ mass, and their sources are primarily 
agricultural sources and $\mathrm{NO}_{\mathrm{x}}$ from traffic and power generation emissions, respectively. Because of the interactions among these species, reduction of sulfate may result in the increase of nitrate [Ansari and Pandis, 1998].

[4] Our objective is to understand how the inorganic $\mathrm{PM}_{2.5}$ mass responds to changes in emissions and provide a means by which alternative emission control strategies can be evaluated. Conceptually, three-dimensional chemical transport models are well suited for this purpose as they link emissions to $\mathrm{PM}_{2.5}$ concentrations directly through detailed descriptions of the physics and chemistry of the atmosphere. However, at this time such models suffer from large uncertainties associated with emission inventories of primary $\mathrm{PM}_{2.5}$ and precursor gases [Seigneur, 2001]. As such, alternative approaches have been proposed, including observation-based approaches or methods combining measurements with models [e.g., Russell and Cass, 1986; Ansari and Pandis, 1998; Pun and Seigneur, 2001].

[5] In particular, aerosol models relying on thermodynamic equilibrium principles to predict the composition and physical state of inorganic atmospheric aerosols have been in development for the past 20 years [Bassett and Seinfeld, 1983; Saxena et al., 1986; Pilinis and Seinfeld, 1987; Kim et al., 1993; Meng et al., 1995; Jacobson et al., 1996; Nenes et al., 1999; Ansari and Pandis, 1999; Wexler and Clegg, 2002]. These have been incorporated as modules in largescale models to predict the partitioning of condensable compounds between the gas and particle phases, but researchers have also used these thermodynamic models alone to conduct investigations of inorganic $\mathrm{PM}_{2.5}$ response to changes in precursor concentrations [Ansari and Pandis, 1998; West et al., 1999; Blanchard and Hidy, 2003]. Precursor species of inorganic $\mathrm{PM}_{2.5}$ include sulfuric acid, ammonia and nitric acid, and are used by the model to calculate the aerosol fraction of these species. Sulfuric acid exists almost entirely in the aerosol phase in a neutralized form, but ammonia and nitric acid can be found in both aerosol and gas phases. Ammonia is the predominant basic gas in the atmosphere and neutralizes sulfuric acid and nitric acid to form aerosols, and small quantities can also be absorbed into liquid aerosols by quantities governed by considerations of vapor-liquid equilibrium. Nitric acid generated in the gas phase can lead to aerosol nitrate formation by two pathways: nitric acid can be neutralized by ammonia to form liquid or solid aerosols, and it can also be incorporated into liquid aerosols by amounts determined by the composition of the particle.

[6] Evaluating emission control strategies with these thermodynamic models, however, makes the implicit assumption that while the concentration of one species is varied, the availability of other precursors remains constant, which may not always be the case. Pandis and Seinfeld [1990] showed that the partitioning of semivolatile species between the gas and aerosol phases can affect their lifetime in the atmosphere. For instance, nitric acid vapor has a much shorter residence time in the atmosphere than aerosol nitrate, and a reduction in sulfate may increase the amount of free ammonia and hence the amount of nitrate partitioned into the aerosol phase. In such a case the concentration of total nitric acid will not remain constant but instead increase, thereby increasing the potential to contribute to the formation of $\mathrm{PM}_{2.5}$.
[7] The present work introduces an Eulerian box model that relies only on observation-based inputs of $\mathrm{PM}_{2.5}$ precursor concentrations and accounts for the variable deposition rates between aerosol nitrate and gas-phase nitric acid. The predictions by this model are evaluated for a winter month in the western Pennsylvania region, where meteorological conditions and atmospheric compositions favored aerosol nitrate formation. The predicted responses of the $\mathrm{PM}_{2.5}$ to emission changes are presented alongside predictions made with the traditional thermodynamic approach of Ansari and Pandis [1998] alone, and the significance of integrating removal processes with an equilibrium module is illustrated.

\section{Measurements From the Pittsburgh Air Quality Study (PAQS)}

[8] Measurements of gases, aerosols, and meteorological conditions were taken between July 2001 to August 2002 for the Pittsburgh Air Quality Study (PAQS). The monitoring site was located atop a hill in Schenley Park, an urban area approximately $6 \mathrm{~km}$ from downtown Pittsburgh, PA, and several hundred meters from any major sources. The city is located in the northeastern United States, in between utility and agricultural emission sources in the Midwest and large urban centers found along the east coast. The average $\mathrm{PM}_{2.5}$ mass concentration as measured by the Federal Reference Method was $12 \mu \mathrm{g} / \mathrm{m}^{3}$ during the winter with sulfate and nitrate constituting $25 \%$ and $17 \%$ of the mass during this period [Rees et al., 2004]. Concentrations of crustal elements in $\mathrm{PM}_{2.5}$ were generally less than $0.2 \mu \mathrm{g} / \mathrm{m}^{3}$ and constituted a small fraction of aerosol mass. Crustal elements in the coarse fraction were also less than $0.2 \mu \mathrm{g} / \mathrm{m}^{3}$ on average. Data for temperature $(\mathrm{T})$, relative humidity $(\mathrm{RH})$, total $\left(\mathrm{PM}_{2.5}+\right.$ gas $)$ ammonia $(\mathrm{TA})$, total $\left(\mathrm{PM}_{2.5}+\right.$ gas) nitric acid (TN), and $\mathrm{PM}_{2.5}$ sulfate (TS) were collected and corrected by the methods described by Wittig et al. [2004a, 2004b] and Takahama et al. [2004].

[9] For this study, hourly measurements made during January 2002 were aggregated to obtain average hourly values of $\mathrm{T}, \mathrm{RH}, \mathrm{TA}, \mathrm{TN}, \mathrm{TS}$, and aerosol nitrate to construct a diurnal profile of these components for an "average day" in January (Figure 1). Error bars, representing the spread of the measurements one standard deviation from the monthly mean in both directions, show that there is a lot of variability over the entire month, but the diurnal trend represented by the mean is preserved. Mean values of the six parameters listed above for the aggregated period in January are as follows: $\mathrm{T}=3.0^{\circ} \mathrm{C}, \mathrm{RH}=68 \%$, $\mathrm{TA}=1.7 \mu \mathrm{g} / \mathrm{m}^{3}, \mathrm{TN}=3.0 \mu \mathrm{g} / \mathrm{m}^{3}, \mathrm{TS}=3.0 \mu \mathrm{g} / \mathrm{m}^{3}$, and $\mathrm{PM}_{2.5}$ nitrate $=2.1 \mu \mathrm{g} / \mathrm{m}^{3}$. The average molar ratio of aerosol ammonium to sulfate for the month was 2.9, indicating that generally, sulfate was fully neutralized and existed as ammonium sulfate. The temperature exhibited a daytime peak and low values in the evening and early morning, and the relative humidity followed an inverted pattern of the temperature. Sulfate in the Pittsburgh region shows on average relatively little variation throughout the day, which is indicative of material transported from outside of the region [Tang et al., 2004]. The total ammonia also shows little variation throughout the day, also indicative of long-range transport, though there is 

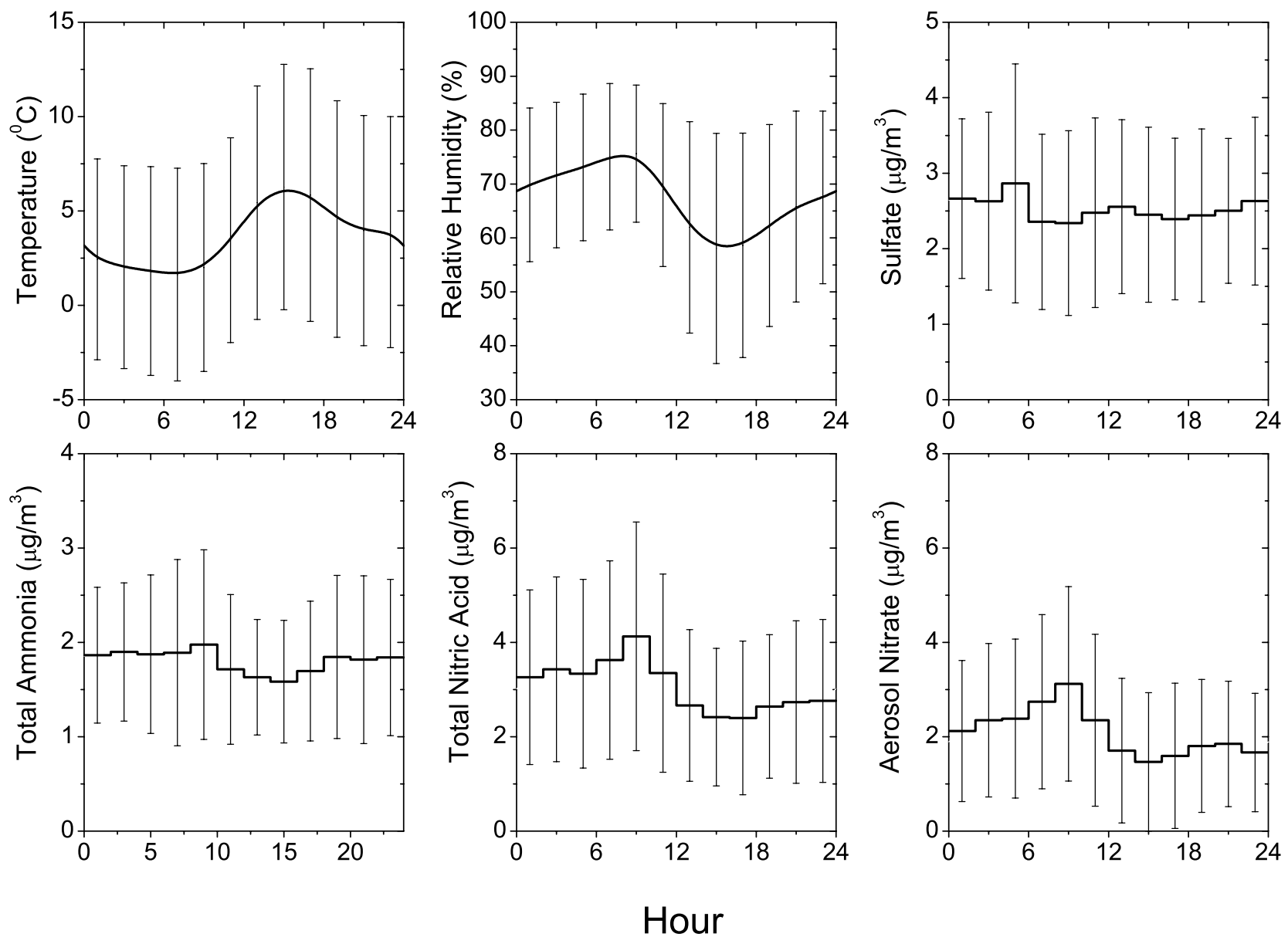

Figure 1. Averages of measured concentrations from the Pittsburgh Air Quality Study during January 2002. Error bars represent \pm one standard deviation of the data.

a slight trend of higher concentrations in the evening and lower concentrations during the day. This small variation can be attributed to vertical mixing by changes in the planetary boundary layer height between morning/evening and daylight hours. Total nitric acid also shows a similar diurnal trend, which can be attributed to chemical production and the change in mixing heights in the morning hours. Nitric acid can be produced during the night by reactions of $\mathrm{N}_{2} \mathrm{O}_{5}$ and water, while limited quantities of ammonia are emitted from local sources. These mechanisms increase the concentrations of these two semivolatile species during the night and early morning while the boundary layer height is low, and the breaking up of the boundary layer after sunrise causes the drop in concentrations during the day. The fraction of nitrate that is in the aerosol phase depends on meteorological conditions, ammonia and nitric acid availability, and also the presence of other compounds in the particle phase. Because of the cold temperatures prevalent in January, a large fraction of the total nitric acid resides in the aerosol phase.

[10] On the basis of aerosol water measurements, Khlystov et al. [2005] concluded that in January 2002 particles were generally liquid above and solid below a threshold relative humidity. The threshold was in the 50$60 \%$ range [Takahama et al., 2004]. A value of $50 \%$ is used as the threshold in this study based on the measurements of Khlystov et al. [2005]. The mutual deliquescence $\mathrm{RH}$ of ammonium nitrate and ammonium sulfate is generally above $70 \%$ for temperatures experienced in January. This suggests that efflorescence and crystallization govern the physical state of the particles. The average RH in January drops to $59 \%$ (Figure 1), and as the average $\mathrm{RH}$ remains above the critical threshold for crystallization, in the base case all modeled particles are assumed to be liquid. The sensitivity of the model results to this assumption will be explored in a subsequent section.

\section{Simulations}

[11] For simplicity, only the sulfate/nitrate/ammonium system is considered in this study. For a region with low concentrations of crustal elements, as is the case in the area of our application, this simplification is reasonable. The potential effect of the crustal species on the phase of the ambient aerosol (solid or liquid) is included by the use of the measured phase state [Khlystov et al., 2005]. Two models are used to assess the system response to changes in current emissions: (1) a thermodynamic model (GFEMN) that partitions total $\left(\mathrm{PM}_{2.5}+\right.$ gas $)$ concentrations into gas and aerosol phases using the sulfate, total ammonia, and 
total nitric acid concentrations, and (2) a new box model (thermodynamic model with removal (TMR)) that simulates partitioning, emission, chemical production and deposition of total nitric acid while sulfate and total ammonia are obtained from ambient measurements. The main difference between these two models is that the phase partitioning of nitric acid affects the lifetime and therefore the availability of total nitric acid in TMR, while in GFEMN the phase change has no such effect.

[12] Emission changes in $\mathrm{SO}_{2}$ and ammonia are reflected by changes in the respective concentrations of sulfate and total ammonia. The average mass concentration of $\mathrm{PM}_{2.5}$ or a particular component over a 24-hour cycle was used to characterize its response to changes in emissions.

[13] Both models, as applied here, use the following assumptions. Since the input of semivolatile components used by the model is the sum of $\mathrm{PM}_{2.5}$ and gas-phase concentrations, the fraction partitioned into coarse mode aerosol is not a consideration for the base case. When the emission changes are simulated, however, we implicitly assume that the fraction partitioned into coarse mode $\mathrm{PM}_{2.5}$ remains small. The error introduced by this assumption is expected to be small for areas with low concentrations of coarse-mode crustal elements that would compete for semivolatile species such as nitric acid. Furthermore, the phase state of the particles is assumed to remain constant as the composition of the aerosol is varied. The possibility of a change in phase after changes in emissions will be addressed in the discussion section. In addition, since these two models employ a bulk equilibrium approach, the traditional assumptions when using this method apply: (1) particles are in equilibrium with the gas phase, and (2) the particles are homogeneous and internally mixed. Also, the influence of organics on inorganic thermodynamics is neglected.

\subsection{GFEMN Model Formulation}

[14] GFEMN [Ansari and Pandis, 1999] is a thermodynamic model that partitions total concentrations between gas and aerosol phases to satisfy chemical equilibrium, which corresponds to the minimum Gibbs free energy of the system subject to mass and charge balance constraints:

$$
\begin{gathered}
\min G=\sum_{i} \mu_{i} n_{i} \\
\text { s.t. } \sum_{a} \nu_{a} n_{a}-\sum_{c} \nu_{c} n_{c}=0 \\
\mathbf{W n}=\mathbf{T},
\end{gathered}
$$

where $\mu$ is the chemical potential, $n$ the number of moles, and $\nu$ the ion charge. The subscript $i$ indicates any chemical species, $a$ indicates anions, and $c$ indicates cations. $\mathbf{W}$ is a matrix vector of stoichiometric coefficients, $\mathbf{n}$ the vector of $n_{i}$ 's, and $\mathbf{T}$ the vector of total concentrations $\{\mathrm{TA}, \mathrm{TN}, \mathrm{TS}\}$.

[15] Takahama et al. [2004] evaluated this model for the case of western Pennsylvania using semicontinuous measurements from PAQS and determined that $\mathrm{PM}_{2.5}$ nitrate prediction errors were on average around $0.5 \mu \mathrm{g} / \mathrm{m}^{3}$, with errors usually lying within the bounds of measurement uncertainties. The measured T, RH, TA, TN, and TS for each hour of the 24-hour cycle are inputs to GFEMN.

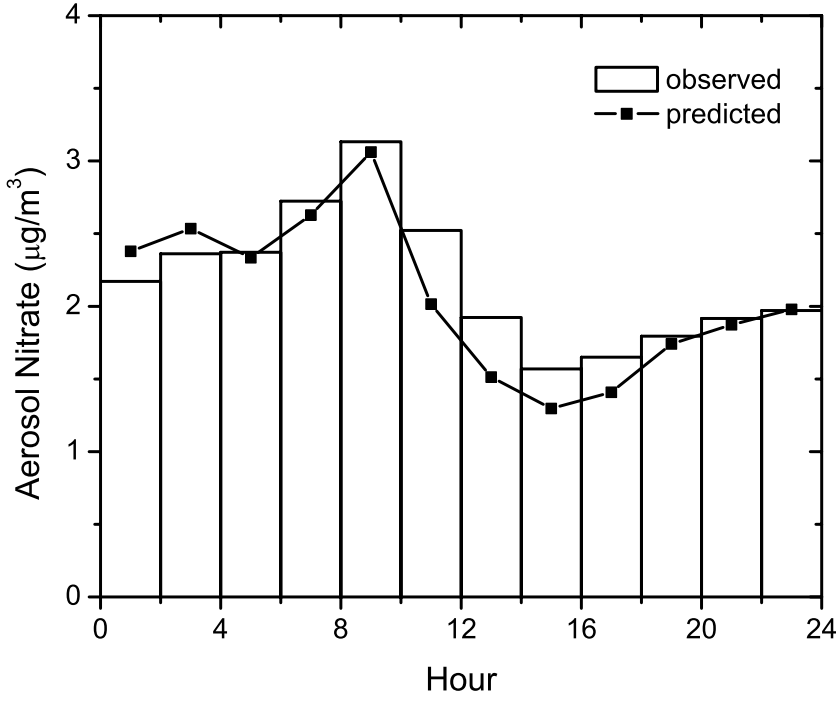

Figure 2. GFEMN prediction versus measurement of aerosol nitrate for January 2002 in Pittsburgh.

[16] Figure 2 shows the comparison between predicted and measured concentrations of aerosol nitrate in the base case simulations; the mean absolute error is $0.2 \mu \mathrm{g} / \mathrm{m}^{3}$ and the model predicts the diurnal variation of aerosol nitrate reasonably well.

\subsection{TMR Model Formulation}

[17] TMR is an extension of the Eulerian box model described by Seinfeld and Pandis [1998]. The concentration of species $i$ in the planetary boundary layer $\left(c_{i}\right)$ is:

$$
\begin{aligned}
\frac{d c_{i}}{d t}= & \left(\frac{\partial c_{i}}{\partial t}\right)_{\text {cond/evap }}-\frac{\nu_{i}}{H(t)} c_{i}+\frac{q_{i}}{H(t)}+R_{i}+\frac{c_{i}^{0}-c_{i}}{\tau_{r}} \\
& +\frac{c_{i}^{a}-c_{i}}{H(t)} \frac{d H}{d t} \Phi\left(\frac{d H}{d t}\right),
\end{aligned}
$$

where $\left(\partial \mathrm{c}_{\mathrm{i}} / \partial \mathrm{t}\right)_{\text {cond/evap }}$ is the rate of mass transfer of the species between the gas and particulate phases, $\nu_{i}$ is its dry deposition velocity, $H$ is the mixing height, $q_{\mathrm{i}}$ is its emission rate, $R_{\mathrm{i}}$ is its production rate, $c^{o}$ is the concentration of the species in the air advected into the box, $\tau_{r}$ is its residence time in the box, and $c^{a}$ is the concentration of the species above the boundary layer. The unit step function, $\Phi$, allows the incorporation of the entrainment term only when the mixing height is increasing [Seinfeld and Pandis, 1998].

[18] Measured concentrations of sulfate and total ammonia are used as inputs by TMR at each time step and the model simulates the evolution of aerosol nitrate and nitric acid. The primary emissions of these species are negligible and therefore $\mathrm{q}_{\mathrm{i}}=0$. For an atmosphere that is relatively homogeneous $\mathrm{c}_{\mathrm{i}} \approx \mathrm{c}_{\mathrm{i}}^{\mathrm{o}} \approx \mathrm{c}_{\mathrm{i}}^{\mathrm{a}}$ and equation 1 is simplified:

$$
\frac{d c_{i}}{d t}=\left(\frac{\partial c_{i}}{\partial t}\right)_{\text {cond/evap }}-\frac{\nu_{i}}{H(t)} c_{i}+R_{i}
$$

Even if the area of this application is relatively homogeneous [Tang et al., 2004], the term $R_{\mathrm{i}}$ in equation 2 is in general the net generation rate with contributions not only from chemistry but also from horizontal and vertical transport. 


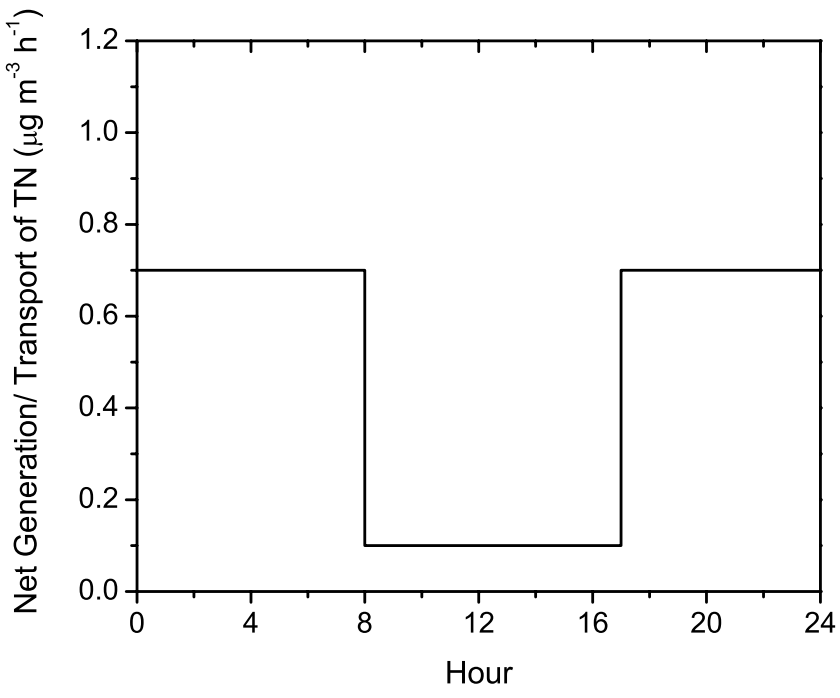

Figure 3. Diurnal profile of nitric acid generation used by TMR for January 2002.

[19] The chemical formation rate can be estimated from other available measurements. Nitric acid is produced primarily by two pathways. In the absence of sunlight, the reaction $\mathrm{NO}_{2}+\mathrm{O}_{3} \rightarrow \mathrm{NO}_{3}+\mathrm{O}_{2}$ is followed by the conversion of $\mathrm{NO}_{3}$ to $\mathrm{N}_{2} \mathrm{O}_{5}$ and eventually to $\mathrm{HNO}_{3}$. Assuming as an upper limit that all of the $\mathrm{NO}_{3}$ produced by this reaction yields $\mathrm{HNO}_{3}$, using $\mathrm{NO}_{2}$ and $\mathrm{O}_{3}$ measurements from PAQS [Wittig et al., 2004a] and a rate constant recommended by DeMore et al. [1997], we estimate a maximum production rate of approximately $1 \mu \mathrm{g} \mathrm{m}^{-3} \mathrm{~h}^{-1}$ by this pathway. Reactions of the $\mathrm{NO}_{3}$ radical with $\mathrm{NO}$ and organic vapors result in a lower production rate. Using the measured concentrations of $\mathrm{NO}, \mathrm{NO}_{2}$, and $\mathrm{O}_{3}$ during the nighttime production rates in the range of $0.3-$ $1 \mu \mathrm{g} \mathrm{m}{ }^{-3} \mathrm{~h}^{-1}$ were estimated. $\mathrm{As}^{\mathrm{NO}_{3}}$ is rapidly photolyzed by UV radiation, during the daytime $\mathrm{NO}_{2}+$ $\mathrm{OH} \rightarrow \mathrm{HNO}_{3}$ becomes the dominant reaction pathway. Measurements of $\mathrm{OH}$ concentrations were not available during PAQS; estimates suggest a range of maximum concentrations of 0.5 to $1 \times 10^{6}$ molecules $/ \mathrm{cm}^{3}$ for the wintertime [Seinfeld and Pandis, 1998]. To calculate the rate of $\mathrm{HNO}_{3}$ production by this mechanism, $\mathrm{OH}$ concentrations in this range were linearly scaled to relative UV intensities for the day [Wittig et al., 2004a] and combined with measurements of $\mathrm{NO}_{2}$ at PAQS [Wittig et al., 2004a] and the reaction rate constant recommended by Dransfield et al. [1999]. By this method we estimate daytime average $\mathrm{HNO}_{3}$ production rates to be in the range of approximately 0.1 to $0.5 \mu \mathrm{g} \mathrm{m}^{-3} \mathrm{~h}^{-1}$.

[20] Using the measured concentrations of total nitric acid as a guide, we selected as a first approximation a constant nitric acid generation rate of $0.7 \mu \mathrm{g} \mathrm{m}^{-3} \mathrm{~h}^{-1}$ for the evening hours and early mornings, and a value of $0.2 \mu \mathrm{g} \mathrm{m}^{-3} \mathrm{~h}^{-1}$ for the daytime (Figure 3). The former value is less than the upper limit of $\mathrm{HNO}_{3}$ production calculated by the $\mathrm{NO}_{2}+\mathrm{O}_{3}$ reaction mechanism and is in the expected range. The daytime generation rate is below the range calculated by the $\mathrm{NO}_{2}+\mathrm{OH}$ reaction using assumed $\mathrm{OH}$ values; it is likely that in this case, entrainment of cleaner air from the free troposphere may be diluting the air in the planetary boundary when the mixing height is rising during the day.

[21] A dry deposition velocity of $6 \mathrm{~cm} \mathrm{~s}^{-1}$ was calculated for $\mathrm{HNO}_{3}$ by the method described by Davidson and $W u$ [1990] assuming aerodynamic resistance to be the only barrier to dry deposition, a mean wind velocity at $10 \mathrm{~m}$ of $2 \mathrm{~m} \mathrm{~s}^{-1}$ as measured at PAQS, and an effective roughness height of $1 \mathrm{~m}$, which is appropriate for western Pennsylvania. The high deposition velocity of $\mathrm{HNO}_{3}$ is the result of its high affinity for surfaces (zero surface resistance). Values in the range of $1-8 \mathrm{~cm} \mathrm{~s}^{-1}$ have been reported [Seinfeld, 1986]. An average deposition velocity of $0.1 \mathrm{~cm} \mathrm{~s}^{-1}$ for aerosols species was taken from a survey of the literature [Davidson and $W u, 1990]$. The single value representing the dry deposition velocity of all $\mathrm{PM}_{2.5}$ is thought to be the average deposition velocity of a polydisperse aerosol population. The sensitivity of the model to relative deposition rates of gas and aerosol phases will be discussed in a later section. The model assumes a mixing height of $1000 \mathrm{~m}$ between 1000 and 1700 EST and $300 \mathrm{~m}$ for all other hours in a 24-hour period, inferred from estimates of twice-daily mixing heights [Holzworth, 1972] (see also http://weather. uwyo.edu) collected for 0700 and 1900 in Pittsburgh, and hours of sunrise and sunset for the modeling period.

[22] Measurements of sulfate and total ammonium are used as inputs to the model, and equation (2) is solved for $\mathrm{PM}_{2.5}$ nitrate and nitric acid. Instead of solving the condensation/evaporation equation to calculate partitioning between gas and aerosol phases, the system is assumed to be in equilibrium. Takahama et al. [2004] argue that this equilibrium assumption is reasonable for $\mathrm{PM}_{2.5}$ in western Pennsylvania. Because of the difference in timescales involved in the processes equation (1), the model is solved by the operator splitting method [Oran and Boris, 2001] where the equilibrium module GFEMN solves the phase partitioning part and the generation and removal processes are solved by the Euler method. To check for problems of convergence and stability, the integration algorithm was tested using increasingly smaller values for the time step until the solutions to the equations no longer changed within a reasonable margin of error. An operator time step of one hour was determined to be sufficiently accurate for this study.

[23] To determine solutions to equation (2) for different emission scenarios that do not depend on the initial conditions of the simulation, the meteorological inputs, sulfate, total ammonia, and total nitric acid generation were assumed to be periodic, with each function assuming values over its 24-hour period as described above. Under such conditions, TMR reaches a dynamic quasi-stationary state in a relatively small number of iterations, in which the diurnal cycle does not change from one simulation day to the next. Figure 4 shows the total nitric acid profile approaching this steady state cycle after 24 hours for the base case scenario. Concentrations from this steady state cycle are used to calculate average $\mathrm{PM}_{2.5}$ and species concentrations.

[24] In the TMR simulations presented, we assumed that the changes in the gas/aerosol phase partitioning of ammonia do not affect significantly its total (gas and aerosol) concentrations. This becomes necessary because of the complex interactions of ammonia with the ground. The 


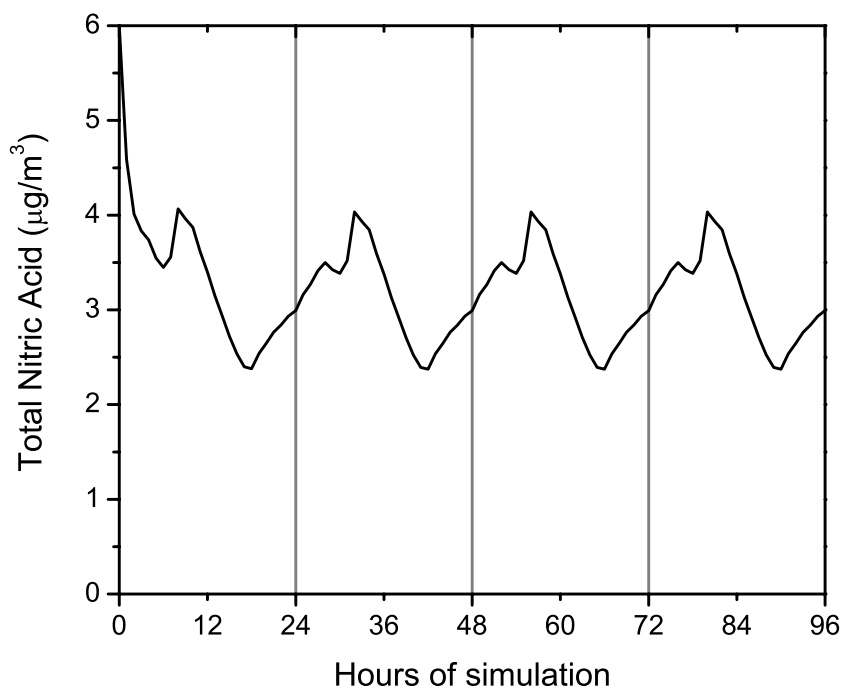

Figure 4. TMR converging on dynamic quasi-stationary state.

surface exchange of ammonia is governed in part by a compensation point [Farquhar et al., 1980], or a local surface concentration below which the direction of ammonia flux changes from deposition to emission, with the ammonia deposition velocity becoming increasingly small as the gas-phase concentrations approach the compensation point [Asman, 1998]. The compensation point reported in the literature varies from 0 to $4 \mu \mathrm{g} / \mathrm{m}^{3}$, and depends on the surface vegetation or surface type as well as meteorological conditions, with winter conditions generally favoring lower compensation points. The mean gas-phase concentrations are less than $0.4 \mu \mathrm{g} / \mathrm{m}^{3}$ for our range of simulations, in the same general range as the expected compensation points.

[25] Figures 5a and 5b show the predicted total nitric acid and aerosol nitrate concentrations by TMR for the base case simulation. The mean absolute error for total nitric acid is

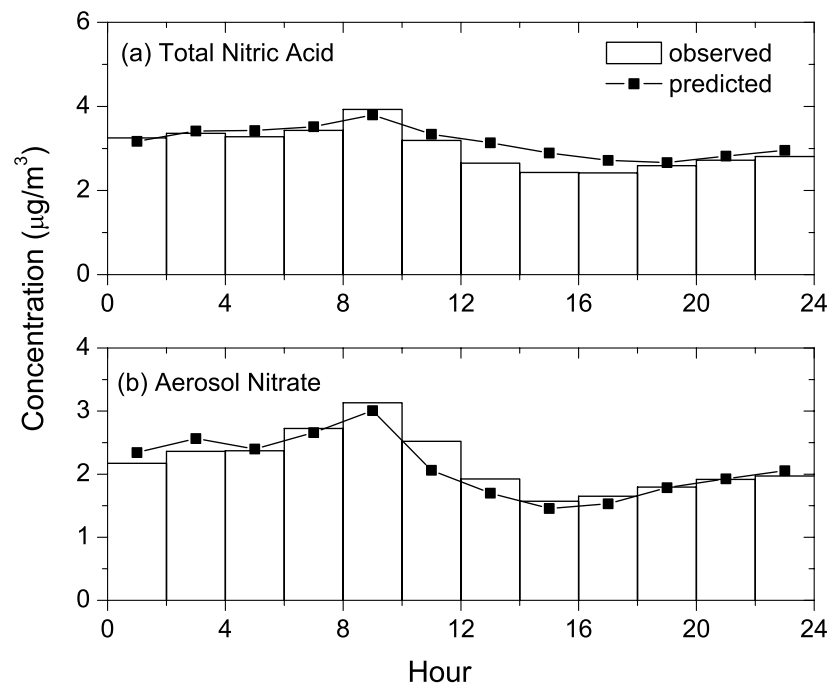

Figure 5. TMR prediction versus measurements of (a) total nitric acid and (b) aerosol nitrate for January 2002 in Pittsburgh.

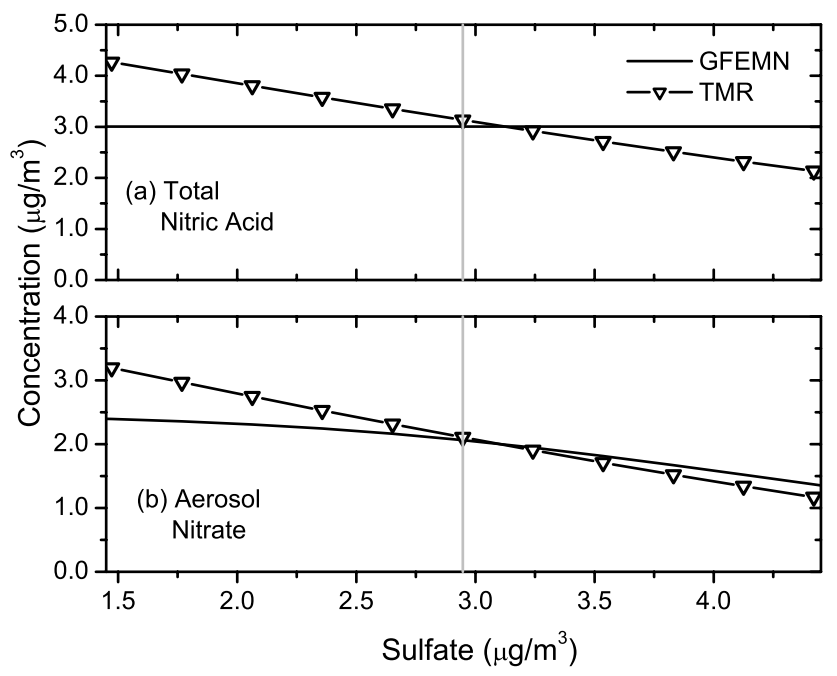

Figure 6. Response of (a) total nitric acid and (b) aerosol nitrate to changes in sulfate concentrations by TMR and GFEMN. Vertical lines indicate conditions for the base case scenario.

$0.3 \mu \mathrm{g} / \mathrm{m}^{3}$. The observed aerosol nitrate is reproduced with a mean error of $0.1 \mu \mathrm{g} / \mathrm{m}^{3}$.

\section{Results}

[26] In the following simulations, concentrations of sulfate and total ammonia and also the total nitric acid generation rate are varied from -50 to $50 \%$ of the original value.

\subsection{Response to Changes in Sulfate Concentrations}

[27] The effect of changes in sulfate concentrations on total nitric acid is shown in Figure 6a. GFEMN assumes that a change in one component has no effect on the others and therefore the average total nitric acid (over the 24-hour steady state cycle) remains constant at $3 \mu \mathrm{g} / \mathrm{m}^{3}$. Meanwhile, TMR predicts that the total nitric acid concentration increases by $0.77 \mu \mathrm{g} / \mathrm{m}^{3}$ for every $\mu \mathrm{g} / \mathrm{m}^{3}$ of sulfate reduced in the system because of the feedback of gas/aerosol partitioning on the total nitric acid availability. Because of the low temperatures observed in Pittsburgh during the winter, when sulfate is reduced and the quantity of ammonia available to react with nitric acid increases, more ammonium nitrate is formed. Figure $6 \mathrm{~b}$ shows that when sulfate concentrations are reduced by $20 \%$, GFEMN predicts that the concentration of aerosol nitrate increases from $2.1 \mu \mathrm{g} / \mathrm{m}^{3}$ from the base case up to $2.3 \mu \mathrm{g} / \mathrm{m}^{3}$. For the same $20 \%$ sulfate reduction, TMR predicts that the aerosol nitrate concentration increases from $2.1 \mu \mathrm{g} / \mathrm{m}^{3}$ in the base case up to $2.5 \mu \mathrm{g} / \mathrm{m}^{3}$. For a $50 \%$ reduction in sulfate, TMR predicts that the aerosol nitrate concentration will be $3.2 \mu \mathrm{g} / \mathrm{m}^{3}$, which is $35 \%$ more than the $2.4 \mu \mathrm{g} / \mathrm{m}^{3}$ that GFEMN predicts.

[28] When sulfate is reduced, TMR generally predicts that more aerosol nitrate will be formed than GFEMN predicts. The increasing fraction of total nitric acid partitioned into the aerosol phase results in higher total nitric acid concentrations; higher partial pressures of nitric acid result in higher aerosol nitrate concentrations. When sulfate is increased, on the other hand, TMR predicts less of both total 


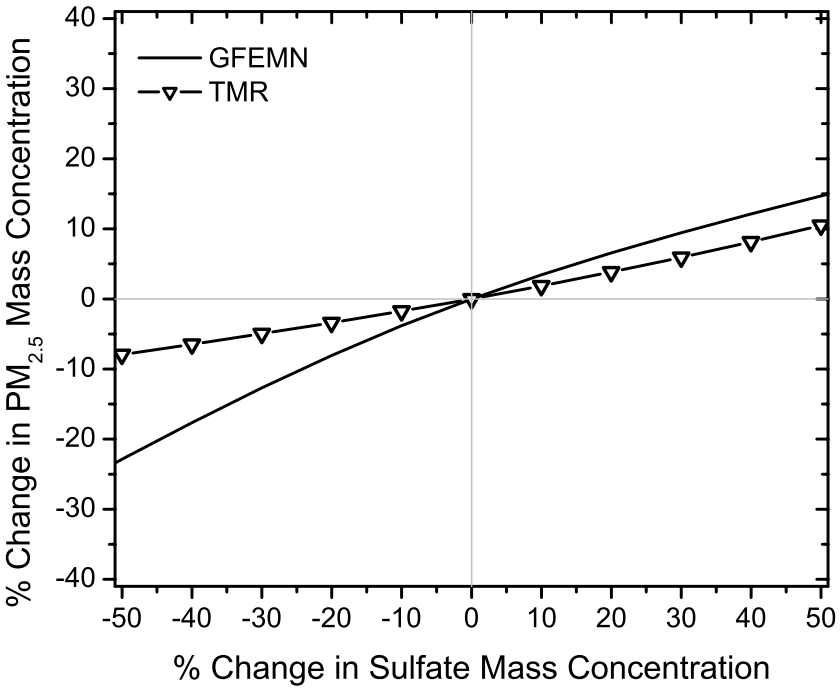

Figure 7. Response of inorganic $\mathrm{PM}_{2.5}$ (plotted as the sum of components without water) to changes in sulfate concentrations.

nitric acid and aerosol nitrate than GFEMN. In this case more ammonia becomes associated with the sulfate and more nitric acid exists in the gas phase. As Figure 6a shows, while total nitric acid concentrations remain constant according to GFEMN, total nitric acid decreases by $0.68 \mu \mathrm{g} / \mathrm{m}^{3}$ for every $\mu \mathrm{g} / \mathrm{m}^{3}$ of sulfate added to the system. The difference between GFEMN and TMR predictions of aerosol nitrate concentrations are not as dramatic for the case of sulfate reductions, as Figure $6 \mathrm{~b}$ shows. The results are somewhat similar because in this concentration regime, the change in sulfate concentrations imposed on both models affects the aerosol nitrate concentration more than the change in total nitric acid. Thus, for a $20 \%$ increase in sulfate, the difference between aerosol nitrate predicted by the two models is $6 \%$, and for a $50 \%$ increase in sulfate the difference is $15 \%$.

[29] The effect of changing sulfate concentrations on inorganic $\mathrm{PM}_{2.5}$ mass concentrations can be seen in Figure 7 . In the ideal case where there is no substitution of nitrate for sulfate in aerosols, $\mathrm{PM}_{2.5}$ would be reduced by $1.34 \mu \mathrm{g} / \mathrm{m}^{3}$ as ammonium sulfate for every $\mu \mathrm{g} / \mathrm{m}^{3}$ of sulfate removed from the system. The PM response is defined as the change in $\mathrm{PM}_{2.5}$ concentration with respect to the change in sulfate concentration, i.e., $\Delta \mathrm{PM} / \Delta \mathrm{TS}$. Ansari and Pandis [1998] defined a PM response between 1.0 and $1.34 \mu \mathrm{gPM}_{2.5} / \mu \mathrm{gTS}$ as being "linear" and PM responses between 0 and $1.0 \mu \mathrm{gPM}_{2.5} / \mu \mathrm{gTS}$ as "nonlinear." We will adapt this convention here. The PM responses predicted by GFEMN for $20 \%$ and $50 \%$ reductions in sulfate are 0.84 and $1.16 \mu \mathrm{gPM}_{2.5} / \mu \mathrm{gTS}$, respectively, indicating that there is a transition from a nonlinear to linear response. This transition occurs because for larger reductions of sulfate there is little nitric acid left to be pulled into the aerosol phase; reductions in $\mathrm{PM}_{2.5}$ then occur mainly as reductions in ammonium sulfate. According to TMR, however, the PM response remains nonlinear (below $0.40 \mu \mathrm{gPM}_{2.5} / \mu \mathrm{gTS}$ ) for the entire range of sulfate reductions (down to 50\%) in our simulations. This is again because of the fact that TMR predicts more nitrate to replace sulfate in the aerosol because of the increased availability of total nitric acid. For reductions in sulfate concentrations of $50 \%$, GFEMN and TMR predict reductions in $\mathrm{PM}_{2.5}$ of $23 \%$ and $8 \%$, respectively. As sulfate concentrations are increased, ammonium sulfate and ammonium bisulfate compete for the available ammonium and drive nitrate into the gas phase. Thus reductions in aerosol nitrate have the effect of offsetting increases in sulfate, and both models predict the PM response to be less than one. For a $50 \%$ increase in sulfate, the $\mathrm{PM}_{2.5}$ mass concentration is predicted to increase by modest amounts of 15 and $10 \%$ by GFEMN and TMR, respectively.

\subsection{Response to Changes in Total Ammonia Concentrations}

[30] The response of total nitric acid to changes in total ammonia concentrations is shown in Figure 8a. GFEMN assumes a constant total nitric acid concentration of $3.0 \mu \mathrm{g} / \mathrm{m}^{3}$, while TMR predicts that on average, the total nitric acid will be reduced by $2.3 \mu \mathrm{g} / \mathrm{m}^{3}$ for every $\mu \mathrm{g} / \mathrm{m}^{3}$ of total ammonia reduced. For 20 and $50 \%$ reductions in ammonia, GFEMN predicts the aerosol nitrate concentrations to be 1.42 and $0.34 \mu \mathrm{g} / \mathrm{m}^{3}$ (Figure $8 \mathrm{~b}$ ), respectively. For corresponding reductions in ammonia, TMR predicts aerosol nitrate concentrations $0.21 \mu \mathrm{g} / \mathrm{m}^{3}$ and $0.12 \mu \mathrm{g} / \mathrm{m}^{3}$ lower than GFEMN. The difference in predictions between the two models is small because when total ammonia is reduced, ammonium nitrate formation becomes limited by ammonia and therefore is more sensitive to changes in total ammonia than total nitric acid. Though by a small amount, TMR consistently predicts concentrations below that of GFEMN because of the feedback of decreasing aerosol nitrate concentrations on the total nitric acid concentrations. When total ammonia concentrations are increased, GFEMN still assumes a constant total nitric acid concentration, while TMR predicts that it will increase by $3.2 \mu \mathrm{g} / \mathrm{m}^{3}$ for every $\mu \mathrm{g} / \mathrm{m}^{3}$ of total ammonia added to the system. For 20 and $50 \%$ increases in total ammonia, GFEMN predicts aerosol nitrate concentrations of 2.45

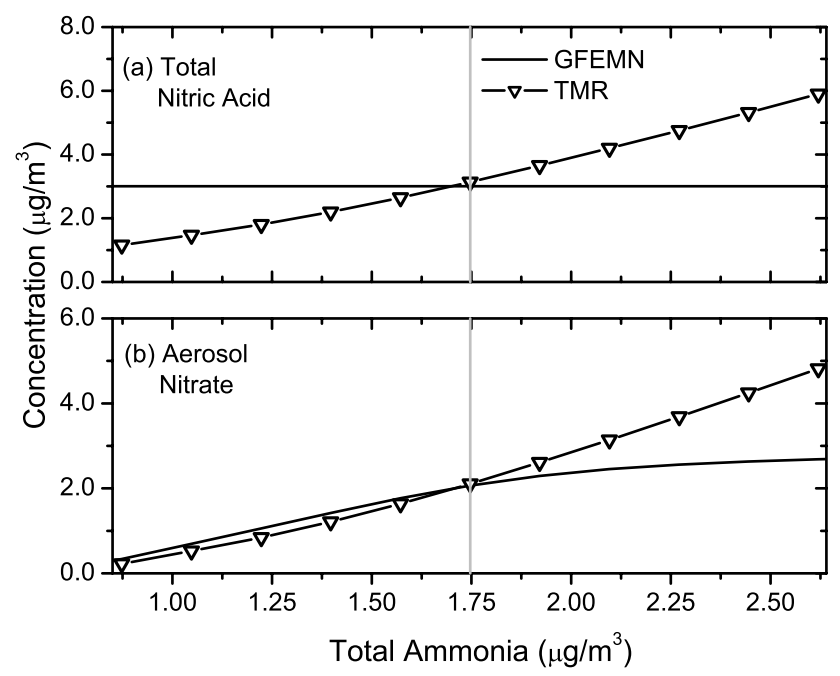

Figure 8. Response of (a) total nitric acid and (b) aerosol nitrate to changes in ammonia concentrations by TMR and GFEMN. Vertical lines indicate conditions for the base case scenario. 


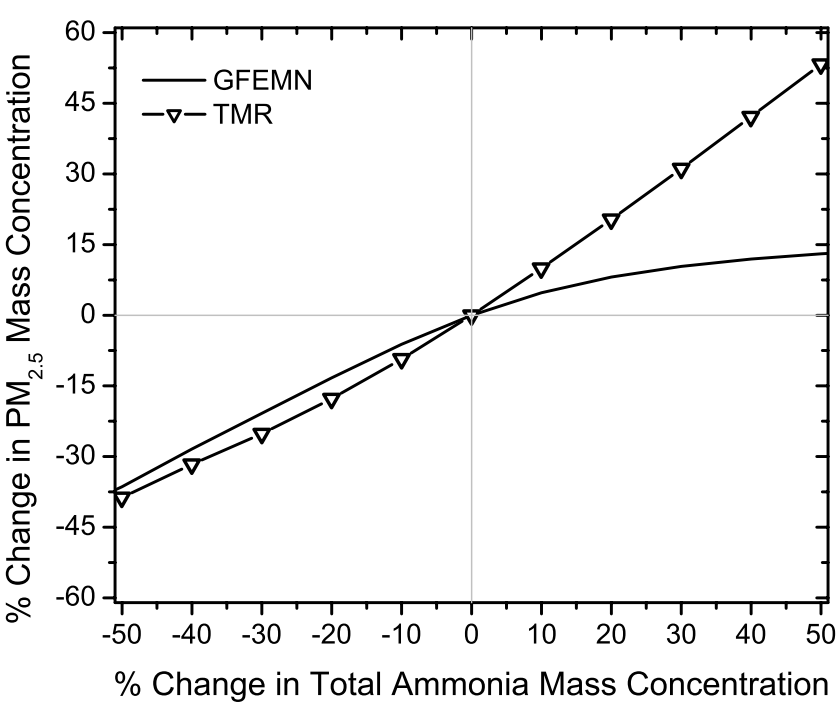

Figure 9. Response of inorganic $\mathrm{PM}_{2.5}$ (plotted as the sum of components without water) to changes in ammonia concentrations.

and $2.68 \mu \mathrm{g} / \mathrm{m}^{3}$, respectively; TMR predicts nitrate concentrations 0.69 and $2.14 \mu \mathrm{g} / \mathrm{m}^{3}$ higher than GFEMN for these cases. At total ammonia concentrations 50\% higher than the base case, $90 \%$ of the total nitric acid is in the aerosol phase in the GFEMN system, while only $83 \%$ of it is in the aerosol phase according to TMR, suggesting that as ammonia concentrations are increased, ammonium nitrate formation is limited by the total nitric acid concentration in simulations with GFEMN more so than with TMR.

[31] Figure 9 shows how $\mathrm{PM}_{2.5}$ concentrations respond to changes in total ammonia concentrations relative to the base case. For reductions in total ammonia of 20 and $50 \%$, the $\mathrm{PM}_{2.5}$ is reduced by 13 and $36 \%$ according to GFEMN and 18 and $39 \%$ according to TMR, respectively. This reduction is mainly achieved through the reduction of ammonium nitrate. When total ammonia is increased by 20 and $50 \%$, the $\mathrm{PM}_{2.5}$ concentration is predicted to increase by only 8 and $13 \%$ by GFEMN but by 20 and $53 \%$ by TMR, respectively. For each model, increasing total ammonia is qualitatively similar to decreasing sulfate concentrations, as both scenarios lead to an increase of the total ammonia to sulfate ratio, which is a metric often used to describe the composition domain for classification into regions of similar thermodynamic responses [Ansari and Pandis, 1999; Zhang et al., 2000]. As Figures $6 \mathrm{~b}$ and $8 \mathrm{~b}$ show, when total ammonia concentrations relative to sulfate increase, the concentration of aerosol nitrate is also increased. For a given set of meteorological conditions and the same total ammonia to sulfate ratio, however, quantitative predictions from each model depend on the difference between the total ammonia and sulfate rather than their ratios, so the actual response of aerosol nitrate and $\mathrm{PM}_{2.5}$ depends on which variable, total ammonia or sulfate, is varied.

\subsection{Response to Changes in Total Nitric Acid Generation Rate}

[32] TMR can also be used to make first-order approximation of how changes in total $\mathrm{HNO}_{3}$ concentrations can impact $\mathrm{PM}_{2.5}$ concentrations by making adjustments in the net generation rate, $R$, in equation (2).

[33] For the same total nitric acid concentrations (and all other variables remaining the same), GFEMN and TMR are identical in every respect except that gas/aerosol partitioning affects the total nitric acid concentration in the TMR system; for a given concentration of total nitric acid, the two models will predict the same $\mathrm{PM}_{2.5}$ concentration. When $R$ is varied from -50 to $50 \%$, total nitric acid concentrations are almost linear in $R$ (correlation coefficient $=0.999$ ) for the range of our changes. Plotted in Figure 10 is the change in $\mathrm{PM}_{2.5}$ mass concentration as a function of the change in $R$ and in total nitric acid. Only one curve is shown because for the same variations in total nitric acid concentrations, GFEMN will trace the same response curve as TMR. For 20 and $50 \%$ reductions in $R$, which correspond to 22 and $60 \%$ reductions in total nitric acid, the $\mathrm{PM}_{2.5}$ mass concentration is reduced by 7 and $21 \%$ from the base case, respectively. The PM response curve is concave because there is more nitric acid than free ammonia initially, and the full extent of total nitric acid reduction is not realized until enough nitric acid is removed such that it becomes the limiting reactant in the formation of ammonium nitrate. For 20 and $50 \%$ increases in $R$, which correspond to 21 and $54 \%$ increases in total nitric acid, the $\mathrm{PM}_{2.5}$ increases by 5 and $9 \%$, respectively. As more nitric acid is introduced into the system, more ammonium nitrate is predicted to form, but this quantity eventually becomes limited by ammonia availability.

\subsection{Changes to Phase State After Emission Changes}

[34] In all of the simulations, the particles were assumed to be liquid because the relative humidities of the average day in January remained above the observed crystallization $\mathrm{RH}$. If the particles were assumed to follow the deliquescence curve, the predictions for TMR would change

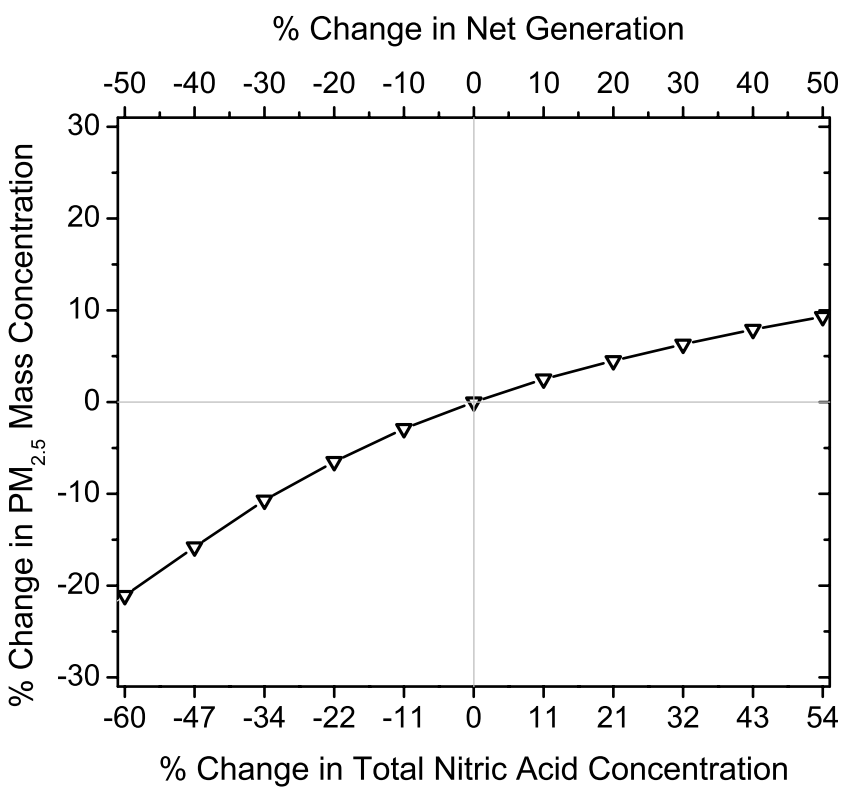

Figure 10. Response of inorganic $\mathrm{PM}_{2.5}$ to changes in total nitric acid generation rate and corresponding total nitric acid concentrations. 
dramatically, though not so much for GFEMN. For instance, in TMR base case simulations, deliquescence predictions of $\mathrm{PM}_{2.5}$ are lower than efflorescence predictions by $29 \%$, and deliquescence predictions of $\mathrm{PM}_{2.5}$ can be as much as $51 \%$ lower in the range of our simulations when the total ammonia to sulfate ratio is increased. With GFEMN the difference is $7 \%$ in the base case and the maximum only $15 \%$ with deliquescence branch simulations consistently predicting lower than efflorescence branch. These differences occur because the mutual deliquescence RH of ammonium sulfate and ammonium nitrate at these temperatures is around $70 \%$, and therefore the phase state predicted is different if deliquescence is assumed. Because the ammonium nitrate dissociation constant is higher for a solid particle than a liquid particle at moderate to high RHs, less ammonium nitrate aerosol is predicted to form in the solid case. In the TMR simulations, this phenomenon is exacerbated by the fact that more nitric acid rapidly deposits when less aerosol is formed; consequently there is less nitric acid available to saturate the gas phase with ammonia to form ammonium nitrate. Since the total nitric acid is constant in the GFEMN simulations, this feedback of nitric acid removal on phase equilibrium is neglected, and therefore the difference is limited to the difference in the dissociation constant between liquid and solid aerosol only and not the quantity of nitric acid in the system.

[35] Measurements of aerosol water content indicate that particles were liquid below the mutual deliquescence point of sulfate and nitrate for January 2002, suggesting that particles were metastable during the winter, and crystallization and not deliquescence governed the phase state of the particles. Furthermore, for the base case simulations, both GFEMN and TMR are able to reproduce the observed concentrations of aerosol nitrate from the partitioning of total nitric acid better when the aerosols are assumed to lie on the efflorescence branch. The prevalence of metastable aerosols in general is also reported by Rood et al. [1989]. Zhang et al. [2003] found that in their evaluation of inorganic measurements during the Atlanta Supersite Experiment, assuming deliquescence returned "unrealistic" partitioning of nitrate and nitric acid.

[36] The change in phase state of the particles when the chemical composition of the system changes is a potentially important issue. The crystallization $\mathrm{RH}$ is a function of temperature and chemical composition of the aerosol. Martin et al. [2003] found that for a $\mathrm{NH}_{4}^{+}-\mathrm{NO}_{3}^{-}-\mathrm{SO}_{4}^{2-}-\mathrm{H}^{+}$ system at $293 \mathrm{~K}$, the crystallization $\mathrm{RH}$ is lowered when (1) the sulfate fraction of anions is reduced, and when (2) the ammonium fraction of cations is reduced. These findings agree with our current knowledge of the hygroscopic properties of single-component particles. For a fixed level of ammonium in the aerosol, as the sulfate fraction of anions is reduced, the chemical character of the aerosol moves away from ammonium sulfate and approaches that of ammonium nitrate. Since the $\mathrm{CRH}$ of ammonium nitrate is extremely low reducing the sulfate fraction of anions lowers the CRH. Furthermore, for a fixed quantity of sulfate, when the ammonium fraction of cations is reduced, the aerosol becomes more acidic, since acidic aerosols are generally more hygroscopic than nonacidic aerosols, so the CRH should decrease. If such trends hold at lower temperatures observed during the winter, the simulated aerosols would remain above the CRH and thus unchanged in physical state when sulfate is reduced; a similar result would be expected for the case of reductions in ammonia. The effect of reducing nitrate is not as clear, as both the sulfate fraction and ammonium fraction would be changed. However, mineral dusts within aqueous particles can increase crystallization RHs by providing sites for heterogeneous nucleation [Martin et al., 2001; Han et al., 2002], and the role of organics in crystallization is uncertain. Therefore we have imposed the assumption that the phase state of the particles remain the same as in the base case during our simulations, but given the sensitivity of our predictions to the assumed phase state, it is apparent that gaining a better understanding of phase transitions of aerosols as they occur in the atmosphere is important.

\subsection{Sensitivity to Relative Deposition Velocities}

[37] The TMR results are fairly robust with respect to the aerosol and nitric acid deposition velocities chosen. The ratio of the nitric acid deposition velocity to that of the aerosol used in the original TMR simulations is 60 . Between the ratios of 30 to $120, \mathrm{PM}_{2.5}$ predictions by TMR differ by no more than $1 \%$, with the largest differences occurring at high total ammonia to sulfate ratios. As this ratio of deposition velocities approaches unity, the TMR predictions approach those of GFEMN, which is the expected outcome as the implicit assumption in the GFEMN simulations is that the deposition velocities of the aerosol and gas phases are the same. These findings are consistent with those of Pandis and Seinfeld [1990] and Seinfeld and Pandis [1998] who found that when the ratio of the gas-phase deposition velocity to that of the aerosol is increased from 10 to 100 , only "minor effects" are observed in their metric of cumulative deposition.

\section{Conclusions}

[38] An observation-based Eulerian box model, TMR, was introduced to evaluate the response of $\mathrm{PM}_{2.5}$ mass concentrations to changes in concentrations of sulfate, total ammonia, and total nitric acid. By using measurements as inputs, TMR avoids the use of uncertain emission inventories. TMR assumes that particles are in equilibrium with the gas phase, but the removal rate of total nitric acid from the system is dependent on its partitioning between the gas and aerosol phases.

[39] Using measurements collected in the Pittsburgh Air Quality Study, an "average" day for January 2002 was constructed and used as the base case scenario for simulations. Predictions of $\mathrm{PM}_{2.5}$ response to changes in precursor concentrations (relative to the base case) were compared with predictions from a chemical equilibrium model, GFEMN, as used in the approach of Ansari and Pandis [1998]. Use of thermodynamic models like GFEMN for simulation of future scenarios implicitly assumes that partitioning has no effect on the total nitric acid concentration.

[40] For scenarios that lead to higher total ammonia to sulfate ratios, i.e., reductions in sulfate and increases in total ammonia, TMR predicts more aerosol nitrate to form than GFEMN because the transformation of gas-phase nitric acid and ammonia to ammonium nitrate increases the lifetime and concentration of total nitric acid in the system. For a 
$50 \%$ decrease in sulfate, GFEMN predicts that the $\mathrm{PM}_{2.5}$ concentration will be reduced by $23 \%$, while TMR predicts that the reduction will be $8 \%$. The results from TMR suggest that when partitioning affects the lifetime of total nitric acid, significant increases in ammonium nitrate may partially offset benefits of sulfate reductions alone in low-temperature conditions as seen in the wintertime in Pittsburgh.

[41] When the total ammonia concentration is increased by $50 \%$, GFEMN predicts a $13 \%$ increase in $\mathrm{PM}_{2.5}$ while TMR predicts a $53 \%$ increase, suggesting that the system as modeled by TMR is more sensitive to levels of total ammonia.

[42] For increases in sulfate and reductions in total ammonia, which lead to ammonia-poor conditions, TMR predicts lower total nitric acid concentrations than GFEMN, but the predictions of aerosol nitrate and $\mathrm{PM}_{2.5}$ concentrations between the two models are similar.

[43] On average, the total nitric acid concentration varies almost linearly with the magnitude of the net generation rate. When the net generation for nitric acid is changed, the $\mathrm{PM}_{2.5}$ concentration responds accordingly. For a 50\% reduction in the net generation, which corresponds to a reduction in total nitric acid concentration of $60 \%$, the $\mathrm{PM}_{2.5}$ decreases by $21 \%$. For the same level of total nitric acid concentrations, GFEMN and TMR predictions are the same because the two models are identical in every respect except the inclusion of feedback of partitioning on total nitric acid concentrations in TMR.

[44] Model predictions of GFEMN and especially TMR are sensitive to the assumed physical state of the aerosol. If particles were allowed to become solid below the mutual DRH during our simulations, the difference in aerosol nitrate predictions could be as great as $15 \%$ for GFEMN and $51 \%$ for TMR in the range of our simulations. How the crystallization $\mathrm{RH}$ will actually change with the composition of the aerosol is very uncertain. Martin et al. [2003] characterized the crystallization RHs for particles composed of ammonium, nitrate, and sulfate at room temperature $(293 \mathrm{~K})$, but the role of organics and other species neglected in partitioning calculations is unclear, and especially at the low temperatures observed during the wintertime in Pittsburgh.

[45] The TMR model predictions are robust with respect to assumed deposition velocities, however. For the base case simulations, the ratio of gas-phase to aerosol-phase deposition velocities used was 60; when this value varies from 30 to 120 the influence on the outcome is no more than $1 \%$.

[46] Acknowledgments. This research was conducted as part of the Pittsburgh Air Quality Study, which was supported by U.S. Environmental Protection Agency under contract R82806101 and the U.S. Department of Energy National Energy Technology Laboratory under contract DE-FC2601NT41017. This paper has not been subject to EPA's peer and policy review and therefore does not necessarily reflect the views of the agency. No official endorsement should be inferred. This work benefited from the comments of Peter J. Adams, Neil M. Donahue, and Andrey Y. Khlystov.

\section{References}

Ansari, A. S., and S. N. Pandis (1998), Response of inorganic $\mathrm{PM}_{2.5}$ to precursor concentrations, Environ. Sci. Technol., 32, 2706-2714.

Ansari, A. S., and S. N. Pandis (1999), Prediction of multicomponent inorganic atmospheric aerosol behavior, Atmos. Environ., 33, 745-757.

Asman, W. A. H. (1998), Factors influencing local dry deposition of gases with special reference to ammonia, Atmos. Environ., 32, 415-421.
Bassett, M., and J. H. Seinfeld (1983), Atmospheric equilibrium model of sulfate and nitrate aerosols, Atmos. Environ., 17, 2237-2252.

Blanchard, C. L., and G. M. Hidy (2003), Effects of changes in sulfate, ammonia, and nitric acid on particulate nitrate concentrations in the southeastern United States, J. Air Waste Manage. Assoc., 53, 283-290.

Davidson, C. I., and Y. L. Wu (1990), Dry deposition of particles and vapors, in Acid Precipitation, vol. 3, edited by S. E. Lindberg, A. L. Page, and S. A. Norton, pp. 103-216, Springer, New York.

DeMore, W. B., S. P. Sanders, D. M. Golden, R. F. Hampson, M. J. Kurylo, C. J. Howard, A. R. Ravishankara, C. E. Kolb, and M. J. Molina (1997), Chemical kinetics and photochemical data for use in stratospheric modeling: Evaluation 14, JPL Publ. 02-25.

Dransfield, T. J., K. K. Perkins, N. M. Donahue, J. G. Anderson, M. M. Sprengnether, and K. L. Demerjian (1999), Temperature and pressure dependent kinetics of the gas-phase reaction of the hydroxyl radical with nitrogen dioxide, Geophys. Res. Lett., 26, 687-690.

Farquhar, G. D., P. M. Firth, R. Wetselaar, and B. Weir (1980), On the gaseous exchange of ammonia between leaves and the environmentDetermination for the ammonia compensation point, Plant Physiol., 66, $710-714$.

Han, J., H. Hung, and S. T. Martin (2002), Size effect of hematite and corundum inclusions on the efflorescence relative humidities of aqueous ammonium nitrate particles, J. Geophys. Res., 107(D10), 4086, doi: $10.1029 / 2001 J D 001054$

Holzworth, G. C. (1972), Mixing heights, wind speeds, and potential for urban air pollution throughout the contiguous United States, Publ. AP101, Off. of Air Programs, U.S. Environ. Protect. Ag., Research Triangle Park, N. C.

Intergovernmental Panel on Climate Change (IPCC) (2001), Climate Change 2001. The Scientific Basis, Contribution of Working Group I to the Third Assessment Report of the Intergovernmental Panel on Climate Change, edited by J. T. Houghton et al., 881 pp., Cambridge Univ. Press, New York.

Jacobson, M. Z., A. Tabazadeh, and R. P. Turco (1996), Simulating equilibrium within aerosols and nonequilibrium between gases and aerosols, J. Geophys. Res., 101, 9079-9091.

Khlystov, A., C. O. Stanier, S. Takahama, and S. N. Pandis (2005), Water content of ambient aerosol during the Pittsburgh Air Quality Study, J. Geophys. Res., 110, D07S10, doi:10.1029/2004JD004651.

Kim, Y. P., J. H. Seinfeld, and P. Saxena (1993), Atmospheric gas-aerosol equilibrium I. Thermodynamic model, Aerosol Sci. Technol., 19, 157181

Martin, S. T., J. H. Han, and H. M. Hung (2001), The size effect of hematite and corundum inclusions on the efflorescence relative humidities of aqueous ammonium sulfate particles, Geophys. Res. Lett., 28, 2601-2604.

Martin, S. T., J. C. Schlenker, A. Malinowski, H. Hung, and Y. Rudich (2003), Crystallization of atmospheric sulfate-nitrate-ammonium particles, Geophys. Res. Lett., 30(21), 2102, doi:10.1029/2003GL017930.

Meng, Z., J. H. Seinfeld, P. Saxena, and Y. P. Kim (1995), Atmospheric gasaerosol equilibrium: IV, Thermodynamics of carbonates, Aerosol Sci. Technol., 23, 131-154.

Nenes, A., C. Pilinis, and S. N. Pandis (1999), Continued development and testing of a new thermodynamic aerosol module for urban and regional air quality models, Atmos. Environ., 33, 1553-1560.

North American Research Strategy for Tropospheric Ozone (NARSTO) (2003), Particulate matter science for policy makers-A NARSTO assessment. Part 2, NARSTO Manage. Off., Pasco, Wash. (Available at http://www.cgenv.com/NARSTO.).

Oran, E. S., and J. P. Boris (2001), Numerical Simulation of Reactive Flow, 2nd ed., Cambridge Univ. Press, New York.

Pandis, S. N., and J. H. Seinfeld (1990), On the interaction between equilibration processes and wet or dry deposition, Atmos. Environ., 24, $2313-$ 2327.

Pilinis, C., and J. H. Seinfeld (1987), Continued development of a general equilibrium model for inorganic multicomponent atmospheric aerosols, Atmos. Environ., 22, 1985-2001.

Pun, B. K., and C. Seigneur (2001), Sensitivity of particulate matter nitrate formation to precursor emissions in the California San Joaquin Valley, Environ. Sci. Technol., 35, 2979-2987.

Rees, S. L., A. L. Robinson, A. Khlystov, C. O. Stanier, and S. N. Pandis (2004), Mass balance closure and the federal reference method for $\mathrm{PM}_{2.5}$ in Pittsburgh, Pennsylvania, Atmos. Environ., 38, 3305-3318.

Rood, M. J., M. A. Shaw, T. V. Larson, and D. S. Covert (1989), Ubiquitous nature of ambient metastable aerosol, Nature, 337, 537-539.

Russell, A. G., and G. R. Cass (1986), Verification of a mathematical model for aerosol nitrate and nitric acid formation and its use for control measure evaluation, Atmos. Environ., 20, 2011-2025.

Saxena, P., A. B. Hudischewskyj, C. Seigneur, and J. H. Seinfeld (1986), A comparative study of equilibrium approaches to the chemical characterization of secondary aerosols, Atmos. Environ., 20, 1471-1483. 
Seigneur, C. (2001), Current status of air quality models for particulate matter, J. Air Waste Manage. Assoc., 51, 1508-1521.

Seinfeld, J. H. (1986), Atmospheric Chemistry and Physics of Air Pollution, John Wiley, Hoboken, N. J.

Seinfeld, J. H., and S. N. Pandis (1998), Atmospheric Chemistry and Physics, John Wiley, Hoboken, N. J.

Takahama, S., A. E. Wittig, D. V. Vayenas, C. I. Davidson, and S. N. Pandis (2004), Modeling the diurnal variation of nitrate during the Pittsburgh Air Quality Study, J. Geophys. Res., 109, D16S06, doi:10.1029/ 2003JD004149.

Tang, W., T. Raymond, A. E. Wittig, C. I. Davidson, S. N. Pandis, A. L. Robinson, and K. Crist (2004), Spatial variations of $\mathrm{PM}_{2.5}$ during the Pittsburgh Air Quality Study, Aerosol Sci. Technol., 38, suppl. 2, $80-90$.

U.S. Environmental Protection Agency (1996), Air Quality Criteria for Particulate Matter, Rep. EPA/600/P-95/001aF, vol. 1, U.S. Govt. Print. Off., Washington, D. C

West, J. J., A. S. Ansari, and S. N. Pandis (1999), Marginal PM2.5: Nonlinear aerosol mass response to sulfate reductions in the eastern United States, J. Air Waste Manage. Assoc., 49, 1415-1424.

Wexler, A. S., and S. L. Clegg (2002), Atmospheric aerosol models for systems including the ions $\mathrm{H}^{+}, \mathrm{NH}_{4}^{+}, \mathrm{Na}^{+}, \mathrm{SO}_{4}^{2-}, \mathrm{NO}_{3}^{-}, \mathrm{Cl}^{-}, \mathrm{Br}^{-}$, and $\mathrm{H}_{2} \mathrm{O}$, J. Geophys. Res., 107(D14), 4207, doi:10.1029/2001JD000451.

Wittig, A. E., N. Anderson, A. Khlystov, S. N. Pandis, C. Davidson, and A. L. Robinson (2004a), Pittsburgh Air Quality Study overview, Atmos. Environ., 38, 3107-3125.
Wittig, A. E., S. Takahama, A. Y. Khlystov, S. N. Pandis, S. Hering, B. Kirby, and C. I. Davidson (2004b), Semi-continuous $\mathrm{PM}_{2.5}$ inorganic composition measurements during the Pittsburgh Air Quality Study, Atmos. Environ., 38, 3201-3213.

Zhang, J., W. L. Chameides, R. Weber, G. Cass, D. Orsini, E. Edgerton, P. Jongejan, and J. Slanina (2003), An evaluation of the thermodynamic equilibrium assumption for fine particulate composition: Nitrate and ammonium during the 1999 Atlanta Supersite Experiment, J. Geophys. Res., 108(D7), 8414, doi:10.1029/2001JD001592.

Zhang, Y, C. Seigneur, J. H. Seinfeld, M. Jacobson, S. L. Clegg, and F. S. Binkowski (2000), A comparative review of inorganic aerosol thermodynamic equilibrium modules: Similarities, differences, and their likely causes, Atmos. Environ., 34, 117-137.

C. I. Davidson, Department of Civil and Environmental Engineering, Carnegie Mellon University, 5000 Forbes Avenue, Pittsburgh, PA 15213, USA.

S. N. Pandis and S. Takahama, Department of Chemical Engineering, Carnegie Mellon University, 5000 Forbes Avenue, Pittsburgh, PA 15213, USA. (spyros@andrew.cmu.edu)

D. Vayenas, Department of Environmental and Natural Resources Management, University of Ioannina, 2 Seferi Street, GR-30100 Agrinio, Greece. 


\title{
Spatial Variations of $\mathrm{PM}_{2.5}$ During the Pittsburgh Air Quality Study
}

\author{
Wei Tang, ${ }^{1}$ Timothy Raymond, ${ }^{2}$ Beth Wittig, ${ }^{2}$ Cliff Davidson, ${ }^{1,3}$ Spyros Pandis, ${ }^{2,3}$ \\ Allen Robinson, ${ }^{3,4}$ and Kevin Crist ${ }^{5}$ \\ ${ }^{1}$ Department of Civil and Environmental Engineering, Carnegie Mellon University, \\ Pittsburgh, Pennsylvania \\ ${ }^{2}$ Department of Chemical Engineering, Carnegie Mellon University, Pittsburgh, Pennsylvania \\ ${ }^{3}$ Department of Engineering and Public Policy, Carnegie Mellon University, Pittsburgh, Pennsylvania \\ ${ }^{4}$ Department of Mechanical Engineering, Carnegie Mellon University, Pittsburgh, Pennsylvania \\ ${ }^{5}$ Ohio University, Athens, Ohio
}

\begin{abstract}
Measurements of $24 \mathrm{~h} \mathrm{PM}_{2.5}$ total mass, sulfate, ammonium, and organic carbon show similar concentrations within experimental error at six sampling locations separated by more than $300 \mathrm{~km}$. The measurements were obtained during summer 2001 in the center of Pittsburgh as well as in less-populated areas upwind and downwind of the city. Measurable differences among the six sites were observed for nitrate and elemental carbon during the same time period. In contrast, measurable differences were observed for total mass and all five chemical species at the same sites during winter 2002. The results suggest that concentrations may be remarkably uniform over large areas due to secondary aerosol production from gases emitted in upwind areas. Meteorological back-trajectories show that concentrations can steadily increase along an airmass trajectory, and that regions downwind of a city such as Pittsburgh may be affected by city emissions; however, $\mathbf{P M}_{2.5}$ levels measured within the city may not be significantly affected by local emissions if background levels are sufficiently high.
\end{abstract}

\section{INTRODUCTION}

Characterizing the spatial variations of aerosol concentration and composition enhances understanding of emission sources,

Received 13 August 2003; accepted 3 February 2004.

This research was conducted as part of the Pittsburgh Air Quality Study, which was supported by US Environmental Protection Agency under contract R82806101 and the US Department of Energy National Energy Technology Laboratory under contract DE-FC26-01NT41017. This article has not been subject to the EPA's peer and policy review, and therefore does not necessarily reflect the views of the Agency. No official endorsement should be inferred.

Address correspondence to Cliff Davidson, Department of Civil and Environmental Engineering, Carnegie Mellon University, 5000 Forbes Avenue, Pittsburgh, PA 15213. E-mail: cliff@cmu.edu secondary PM formation, and transport. Extensive studies have been conducted on the distribution and transport of acid aerosols since the late 1970s (Lioy et al. 1980; Spengler et al. 1990; Keeler et al. 1991; Brook et al. 1997; McCurdy et al. 1999). In North America, ambient concentrations of acid aerosols tend to be regional in nature, with the highest concentrations found in the northeastern United States and southern Ontario (Spengler et al. 1990). It is commonly believed that the source region for most precursors is the Ohio River Valley (Lioy et al. 1980; Hidy 1994). In recent years, more comprehensive characterization of fine particulate matter $\left(\mathrm{PM}_{2.5}\right)$ has drawn increasing interest due to its link to adverse health effects.

During the past several years, programs have been initiated to investigate $\mathrm{PM}_{2.5}$ in Pittsburgh, PA and vicinity by local and state monitoring agencies and the National Energy Technology Laboratory (NETL) (e.g., Anderson et al. 2002; Modey et al. 2004). These studies have focused on Pittsburgh due to its location tens to hundreds of kilometers downwind of several major industrial sources, particularly coal combustion sources. Furthermore, unlike major cities in the Boston-to-Washington corridor where urban plumes often interact in complex ways, the relative importance of sources within the Pittsburgh urban area and those outside the urban area can be more easily determined. Preliminary results provided by past work suggest that the elevated concentrations of $\mathrm{PM}_{2.5}$ in this region may be caused by emissions from Ohio River Valley sources to the west and southwest of the city. Thus $\mathrm{PM}_{2.5}$ episodes in Pittsburgh may not be eliminated by control of source emissions within the city. However, our overall understanding of the sources and characteristics of $\mathrm{PM}_{2.5}$ is lacking. More comprehensive data over all seasons are needed to investigate further the regional nature of $\mathrm{PM}_{2.5}$ and to support the development of strategies for reducing ambient concentrations. 
The Pittsburgh Air Quality Study (PAQS) was organized to characterize airborne particles with respect to size, morphology, and chemical composition, and to identify sources, transport pathways, and atmospheric transformations of these particles (Wittig et al. 2004). In this article, we use data from the PAQS for three specific objectives: (1) to identify the spatial variability in concentration of the different chemical components of $\mathrm{PM}_{2.5}$ across the Pittsburgh region throughout the year, (2) to evaluate the relative contributions of regional and urban sources influencing $\mathrm{PM}_{2.5}$ in summer and in winter, and (3) to explore the role of meteorology in influencing $\mathrm{PM}_{2.5}$ concentrations.

\section{EXPERIMENTAL}

Airborne concentrations and chemical composition of $\mathrm{PM}_{2.5}$ have been measured at the PAQS main site and at 5 additional satellite sites as part of this program. Locations of the six sites are shown in Figure 1. Table 1 lists the latitude, longitude, and sampling periods for each location.

The main site is located in Schenley Park, on top of a grassy hill adjacent to the Carnegie Mellon University (CMU) campus. The site is in the densely populated Oakland neighborhood of Pittsburgh, roughly 6 kilometers east of downtown. This location is more than $500 \mathrm{~m}$ from the nearest heavily traveled road but within a few kilometers of several major roads and industrial sources. Two of the satellite sites are located in the Lawrenceville and Hazelwood neighborhoods of the City of Pittsburgh, maintained by the Allegheny County Health Department (ACHD). The Lawrenceville site is in an older residential area approximately 3 kilometers north of CMU. The Hazelwood location is close to several abandoned industrial plants approximately 3 kilometers south of CMU. Satellite sites are also located in Florence, PA and Greensburg, PA, and these sites are maintained by the Pennsylvania Department of Environmental Protection (PADEP). The Florence site, about 50 kilometers west of the main site, is in a rural area with no major roads or stationary sources within several kilometers, and is typically upwind of Pittsburgh. The Greensburg site, about 55 kilometers east of $\mathrm{CMU}$, is close to a heavily traveled road in a suburban commercial district, but with few major stationary sources nearby and is predominantly downwind of Pittsburgh. The final satellite site is located in Athens, Ohio, about 270 kilometers southwest of Pittsburgh, and is operated by Ohio University.

Measurements were conducted daily over the entire study at the main site, while samples were collected at the satellite sites during intensive runs in summer 2001, winter 2002, and selected days in summer 2002. Additional data were obtained at the satellite sites through routine sampling ( 1 in 3 days or 1 in 6 days) by ACHD, PADEP, and Ohio University. All data presented in this article are for $24 \mathrm{~h}$ integrated filter samples.

At the main site, $\mathrm{PM}_{2.5}$ mass was determined gravimetrically on Teflon filters using a Partisol ${ }^{\circledR}$-federal reference method (FRM) model $2000 \mathrm{PM}_{2.5}$ air sampler (Rupprecht \& Patashnik, Albany, NY, USA). Collection of $\mathrm{PM}_{2.5}$ for anion and cation analysis was accomplished with a sampling system that included citric acid and $\mathrm{MgO}$ denuders, followed by a filterpack with PTFE Teflon (Whatman, catalog no. 7592-104), nylon (Whatman, catalog no. 7410-004), and cellulose fiber (Whatman, catalog no. 1441-047) filters. The cellulose filters were impregnated with citric acid. The airflow through the system was 7.7 liters per minute (LPM) for most of the sampling periods. The filters were analyzed for anions and cations by ion chromatography (IC); details of preparing, sampling, extracting, and analyzing the filters are given by Takahama et al. (2004). In this article, we report data from these filters for sulfate, nitrate, and ammonium.

$\mathrm{PM}_{2.5}$ carbonaceous material was sampled at 16.7 LPM through quartz filters. Some of these filters used activated carbon denuders, while others were undenuded. The filters were analyzed for elemental carbon (EC) and organic carbon (OC) by the thermal optical transmittance (TOT) method as described by Subramanian et al. (2004).

The satellite sites used the MetOne speciation aerosol sampling system (SASS ${ }^{\mathrm{TM}}$; Met One Instruments, Inc., Grants Pass,

Table 1

Monitoring site information of PAQS

\begin{tabular}{llll}
\hline \multicolumn{1}{c}{ Site name } & \multicolumn{1}{c}{ Group } & $\begin{array}{c}\text { Latitude/longitude } \\
\text { (decimal degrees) }\end{array}$ & Sampling period \\
\hline Main site & CMU & $40.4395 /-79.9405$ & $6 / 30 / 01-7 / 31 / 02$ \\
Hazelwood, PA & CMU/ACHD & $40.4124 /-79.9424$ & $6 / 30-8 / 3 / 01,1 / 2-1 / 22 / 02,4 / 2-8 / 7 / / 02^{a, b}$ \\
Lawrenceville, PA & CMU/ACHD & $40.4432 /-79.9595$ & $6 / 30-8 / 3 / 01,1 / 2-1 / 22 / 02,9 / 10-12 / 9 / 01,^{c} 2 / 28-8 / 9 / 02^{b, c}$ \\
Florence, PA & CMU/DEP & $40.4454 /-80.4212$ & $6 / 30-8 / 3 / 01,1 / 2-1 / 22 / 02,9 / 10-12 / 9 / 01,{ }^{a} 2 / 25-8 / 9 / 02^{a, b}$ \\
Greensburg, PA & CMU/DEP & $40.3043 /-79.5059$ & $6 / 30-8 / 3 / 01,1 / 2-1 / 22 / 02,9 / 10-12 / 9 / 01,,^{a} 2 / 25-8 / 9 / 02^{a, b}$ \\
Athens, OH & Ohio Univ. & $39.3283 /-82.9067$ & $6 / 30-8 / 3 / 01,1 / 2-1 / 22 / 02,8 / 29-12 / 21 / 01,{ }^{c} 1 / 23-8 / 29 / 02^{c}$ \\
\hline
\end{tabular}

Groups: CMU, Carnegie Mellon University; ACHD, Allegheny County Health Department; DEP, Pennsylvania Department of Environmental Protection.

${ }^{a} 1$ in 6 days sampling.

${ }^{b}$ Selected days were added during 7/1-8/7/02.

${ }^{c} 1$ in 3 days sampling. 


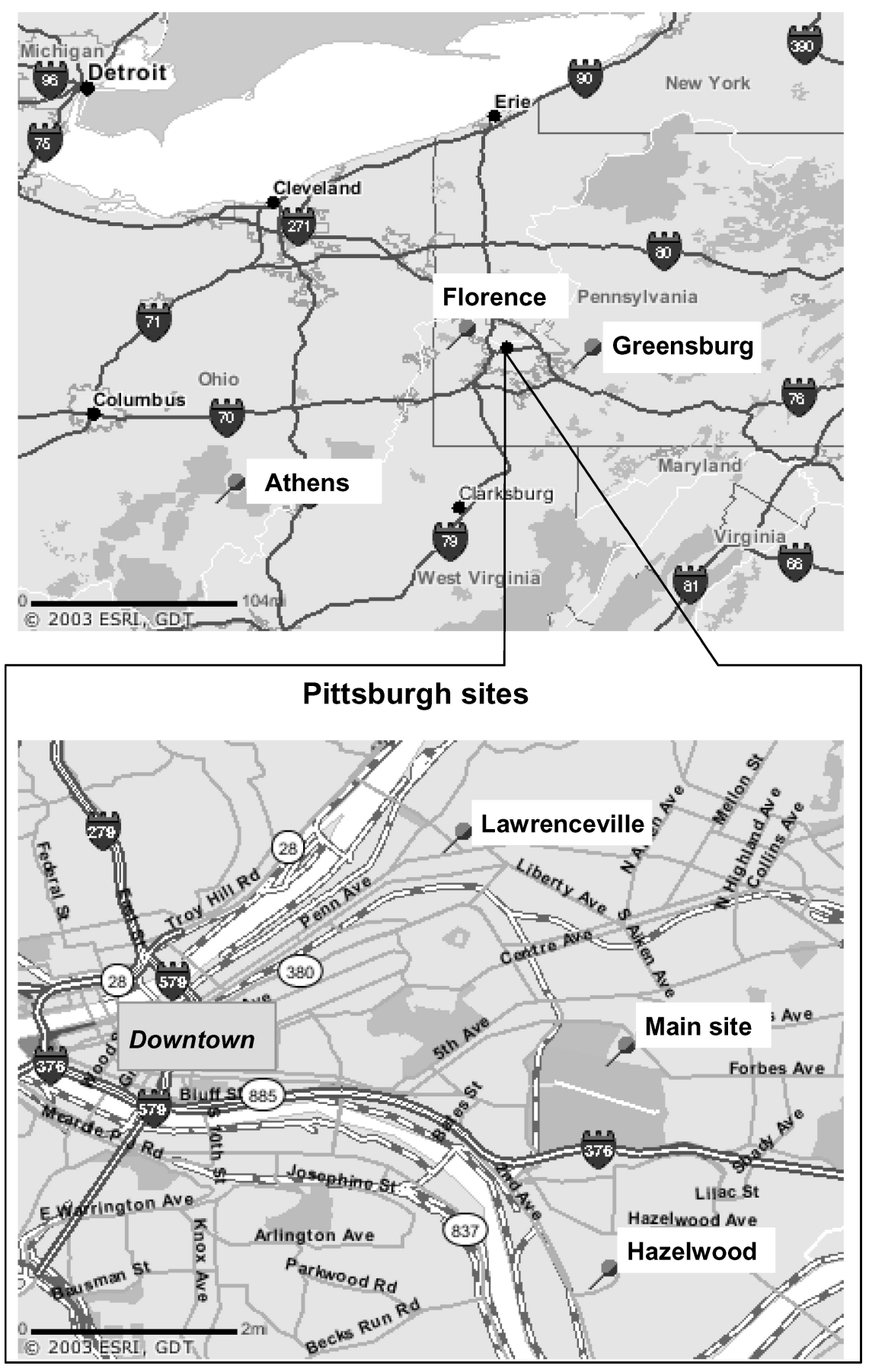

Figure 1. Map of the Pittsburgh region showing the main site and the satellite sites at Lawrenceville, Hazelwood, Florence, Greensburg, and Athens. The enlarged map shows the three sites within the city of Pittsburgh (http://arcweb.esri.com/arcweb_viewer).

OR, USA). The system included an undenuded Teflon filter, an $\mathrm{MgO}$-denuded nylon filter, and an undenuded quartz filter. The Teflon filters were analyzed gravimetrically for $\mathrm{PM}_{2.5}$ mass and for trace elements by x-ray fluorescence; Solomon et al. (2003) note that $\mathrm{PM}_{2.5}$ data from the FRM are directly comparable with data from the MetOne system. The nylon filters were analyzed for anions and cations by IC, and the quartz fil- ters were analyzed for EC and OC by the TOT method. Flow rates through all these filters were 6.7 LPM. Details of the sampling and analysis are given by EPA (1999). All of the satellite sites except Athens are part of the US EPA National $\mathrm{PM}_{2.5}$ Chemical Speciation Network. Preparation of filter packs and sample analyses were conducted by Research Triangle Institute (Research Triangle Park, NC, USA). Preparation of filter 


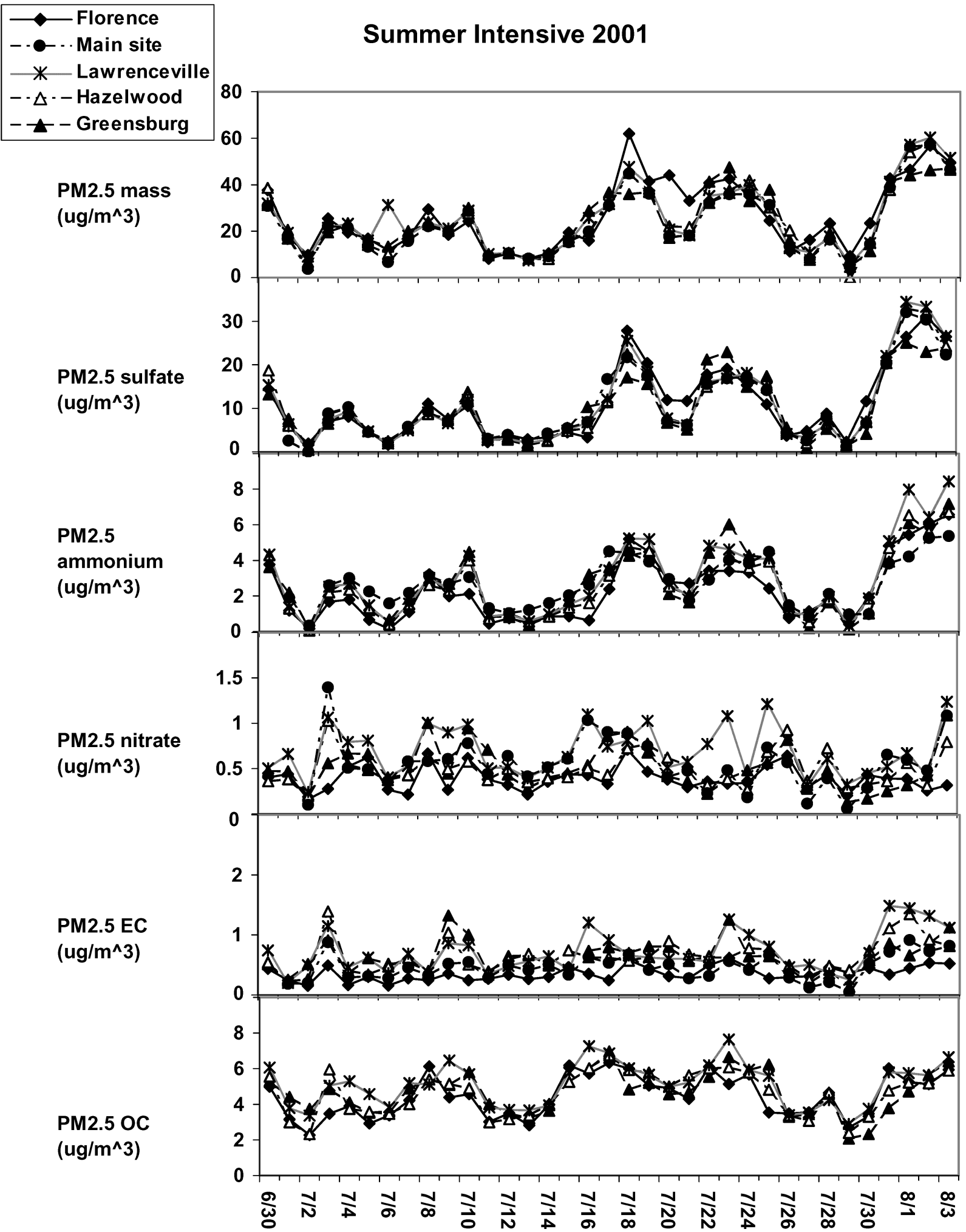

Figure 2. Time series plots of $\mathrm{PM}_{2.5}$ concentrations during intensive sampling periods. (Continued) 


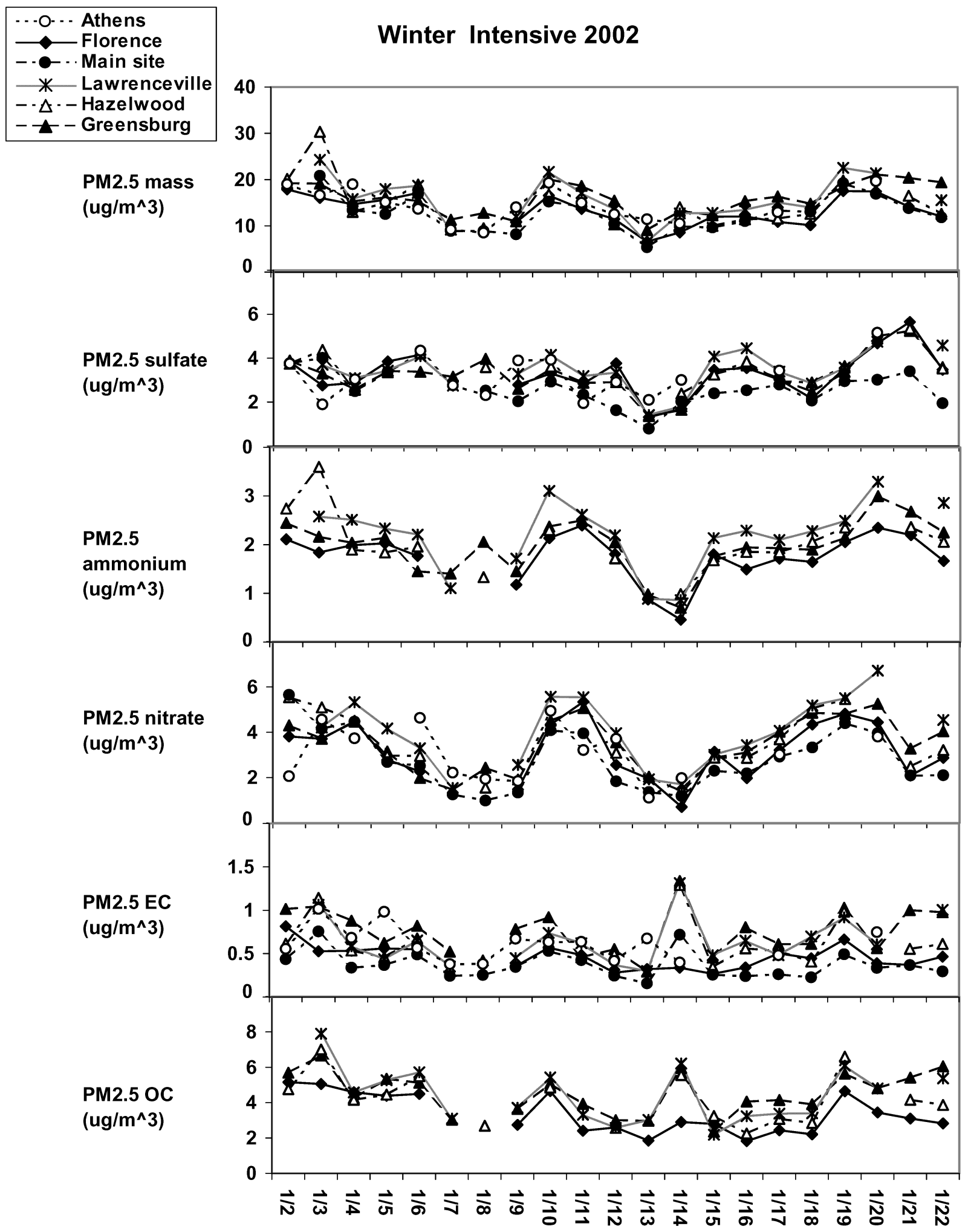

Figure 2. (Continued) 
packs and sample analysis for the Athens site were conducted collaboratively by Ohio University and CMU using similar methods.

\section{RESULTS AND DISCUSSION}

\section{Spatial Variability of $\mathbf{P M}_{2.5}$}

Figure 2 presents time series plots for $\mathrm{PM}_{2.5}$ mass, sulfate, ammonium, nitrate, EC, and OC during the summer 2001 inten- sive and the winter 2002 intensive sampling campaigns. Data from the Athens site during the summer are unavailable, as are the ammonium data for the main site and the Athens site during the winter. There are no OC data shown for the main site because the flow rate was higher than that of the satellite sites, making comparisons difficult due to differences in artifacts. Note that OC values are not corrected for artifacts due to absorption of reactive gases. EC values from the main site are from denuded samplers during the summer and from undenuded

\section{Average PM2.5 concentrations during Summer Intensive 2001}

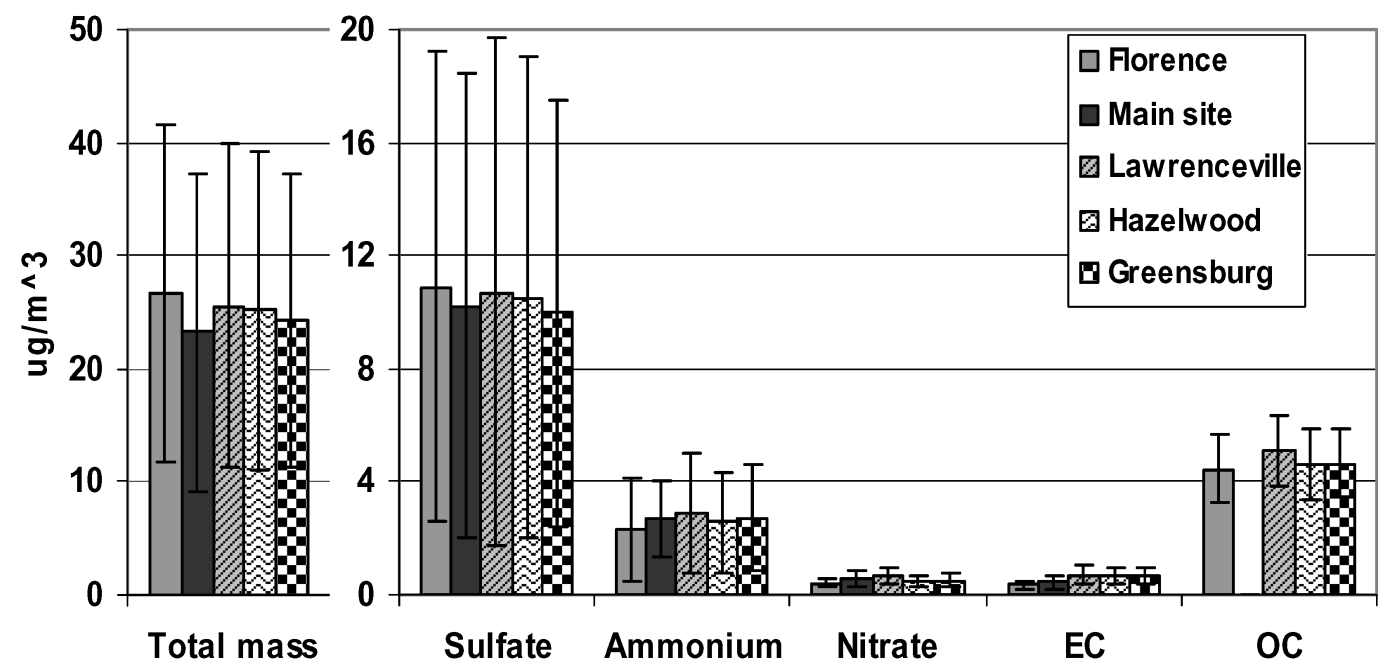

\section{Average PM2.5 concentrations during Winter Intensive 2001}
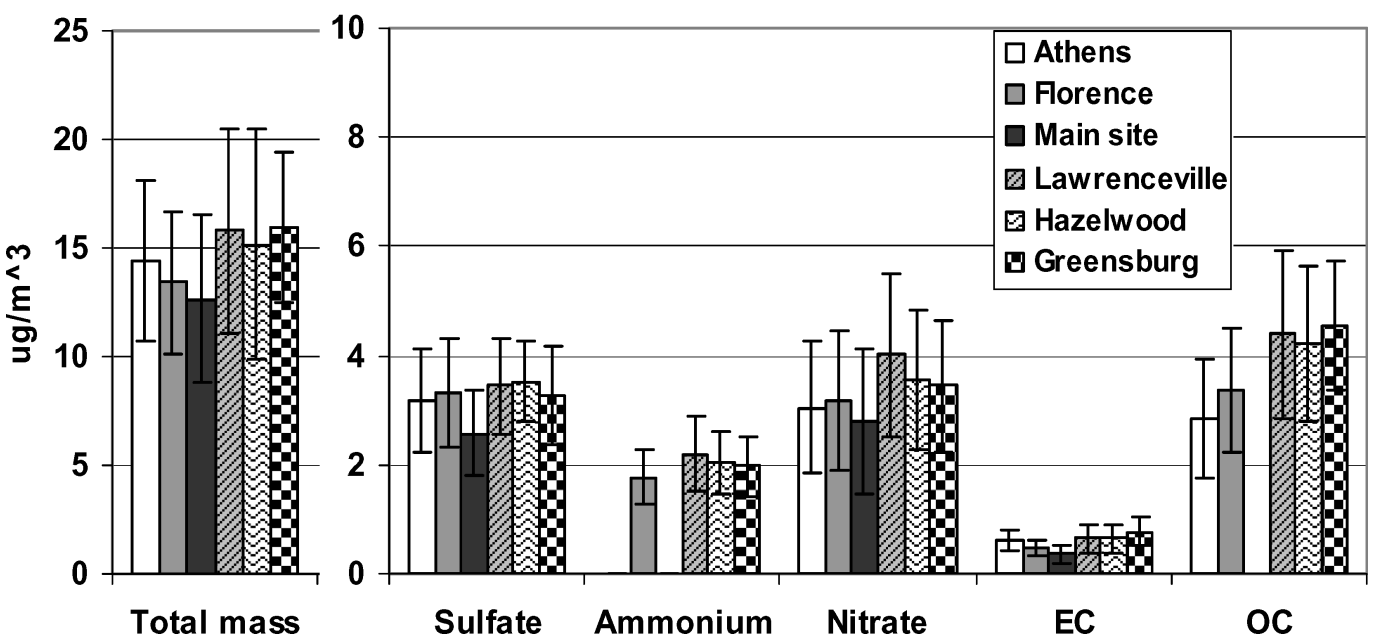

Figure 3. Average $\mathrm{PM}_{2.5}$ concentrations during intensive sampling periods. 
samplers during the winter; the absence of a denuder should not affect EC data.

During summer 2001, sulfate, ammonium, and OC follow the same trends as $\mathrm{PM}_{2.5}$ mass, since these species represent the major components of the PM. The average $\mathrm{PM}_{2.5}$ mass of all sites is $25 \mu \mathrm{g} / \mathrm{m}^{3}$ during the summer intensive, for which sulfate, ammonium, and OC contribute $41 \%, 10 \%$, and $18 \%$, respectively. Furthermore, concentrations of these species at all sites track each other closely, suggesting relatively uniform levels throughout the region. Nitrate and EC have very low concentrations ( $2 \%$ and $3 \%$ of total mass, respectively), and show no clear correlation with mass. The rural Florence site consistently has the lowest EC concentrations, as might be expected since EC is associated with primary particles and these may be partly from local sources, such as diesel engines. The influence of urban sources is more significant for EC than for the other species.

During winter 2002, more scatter is observed for all species among the six sites compared with summer, suggesting less
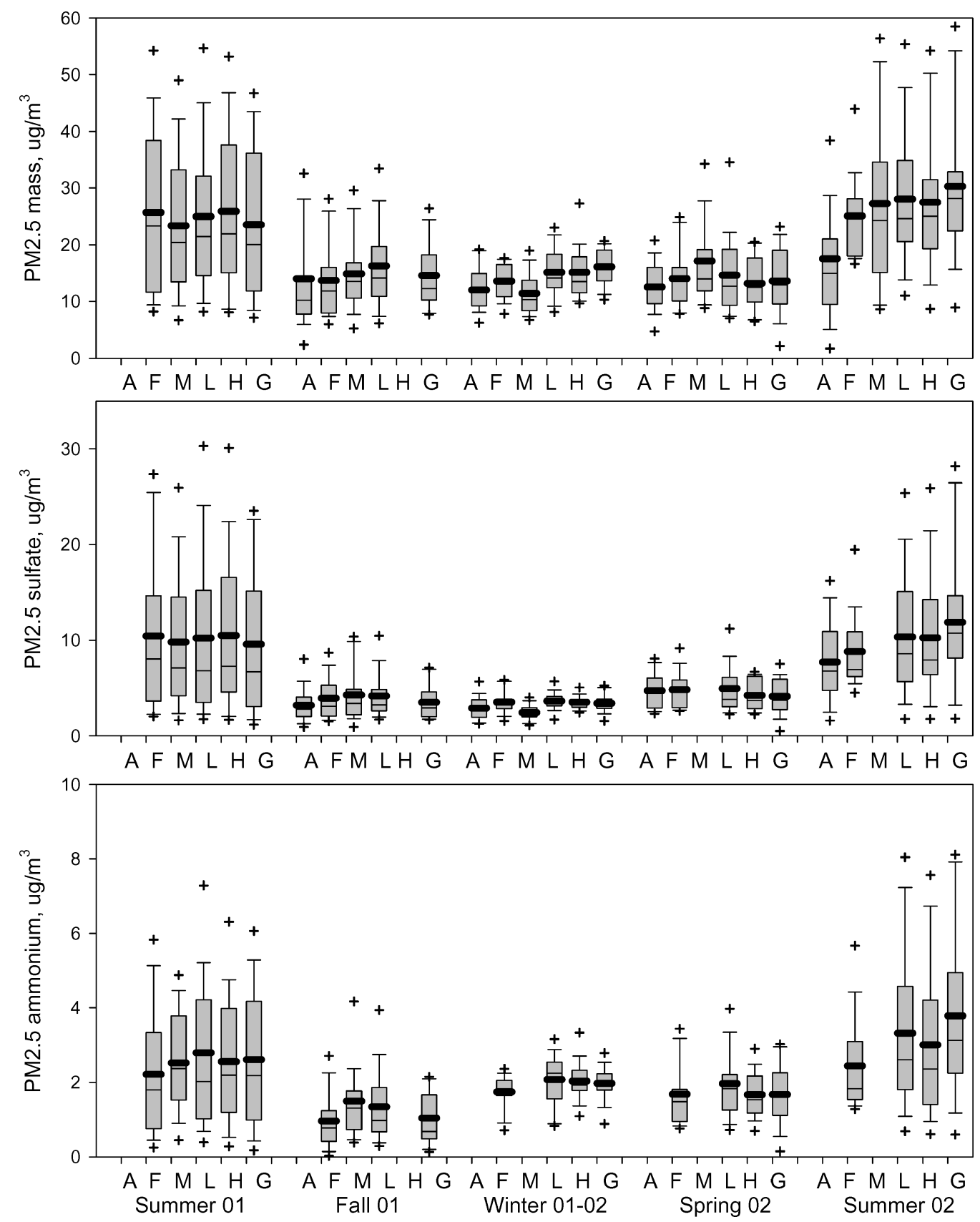

Figure 4. Box plots of $\mathrm{PM}_{2.5}$ concentrations grouped by seasons. A, Athens; F, Florence; M, Main site; L, Lawrenceville; H, Hazelwood; G, Greensburg. (Continued) 

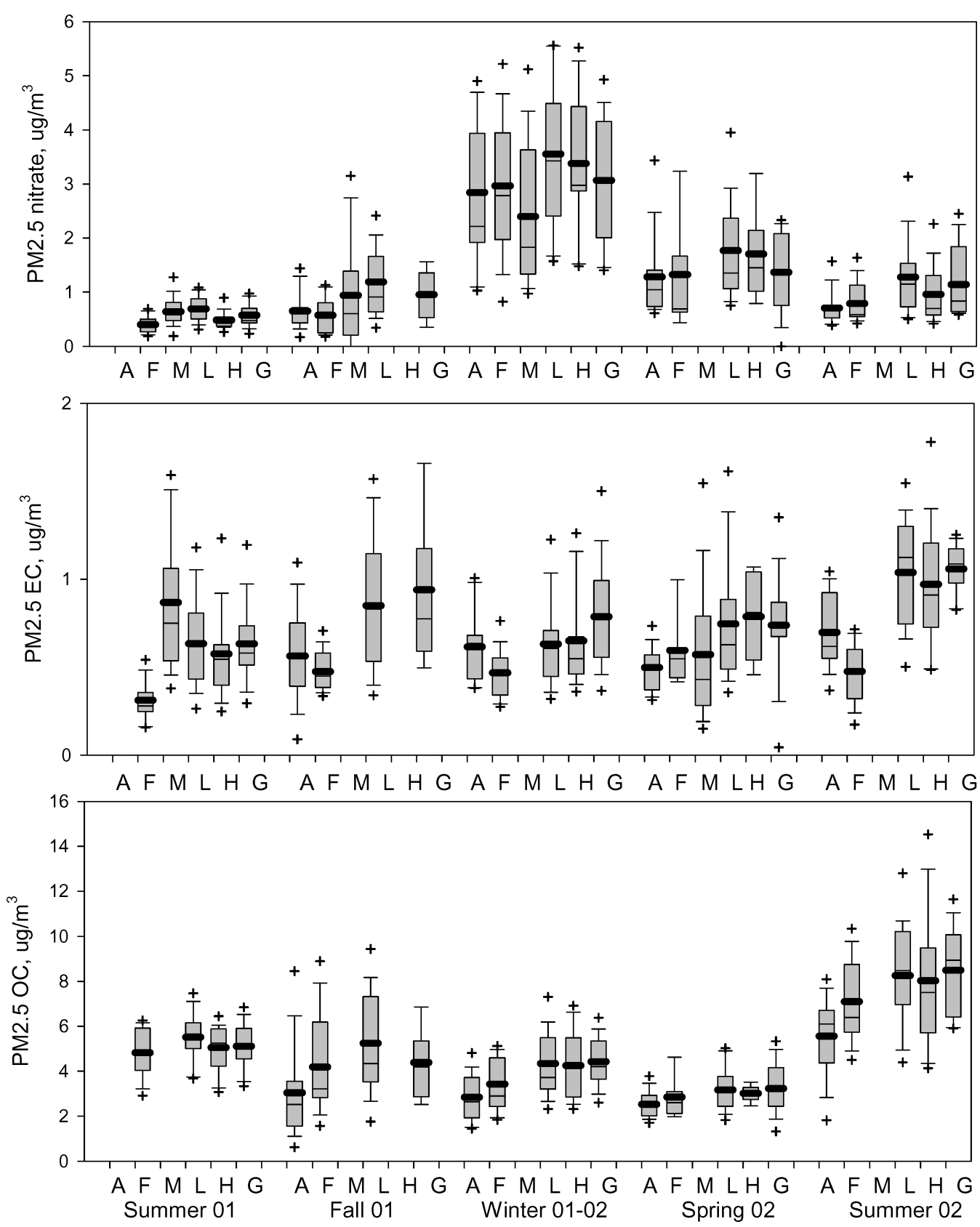

Figure 4. (Continued)

regional uniformity. This may reflect less formation of secondary $\mathrm{PM}$ in winter. The regional average $\mathrm{PM}_{2.5}$ mass is $14 \mu \mathrm{g} / \mathrm{m}^{3}$. Sulfate, ammonium, nitrate, OC, and EC account for $22 \%, 13 \%$, $23 \%, 27 \%$, and $4 \%$ of the $\mathrm{PM}_{2.5}$ mass, respectively. Compared with summer, nitrate and OC represent much greater fractions of total $\mathrm{PM}_{2.5}$ mass, while sulfate decreases by $2 / 3$. Under winter conditions, the conversion of $\mathrm{SO}_{2}$ to sulfate is much slower, and more ammonium is associated with nitrate as modeled by Takahama et al. (2004). The time series plots also show that nitrate and ammonium have better agreement than sulfate and ammonium in winter.
The average $\mathrm{PM}_{2.5}$ concentrations for the two intensive sampling periods are presented in Figure 3. The bars representing the six sites are arranged in order of from west to east, i.e., Athens, Florence, Pittsburgh (Main site, Lawrenceville, Hazelwood), and Greensburg, in order to show possible regional trends. In general, all sites agree reasonably well, especially in the summer, suggesting $\mathrm{PM}_{2.5}$ measurements at all sites are the regional concentrations to a large extent with only limited local contributions. The average concentrations vary somewhat more during the winter intensive. There is a slight increasing trend from west to east, most significantly for OC. 


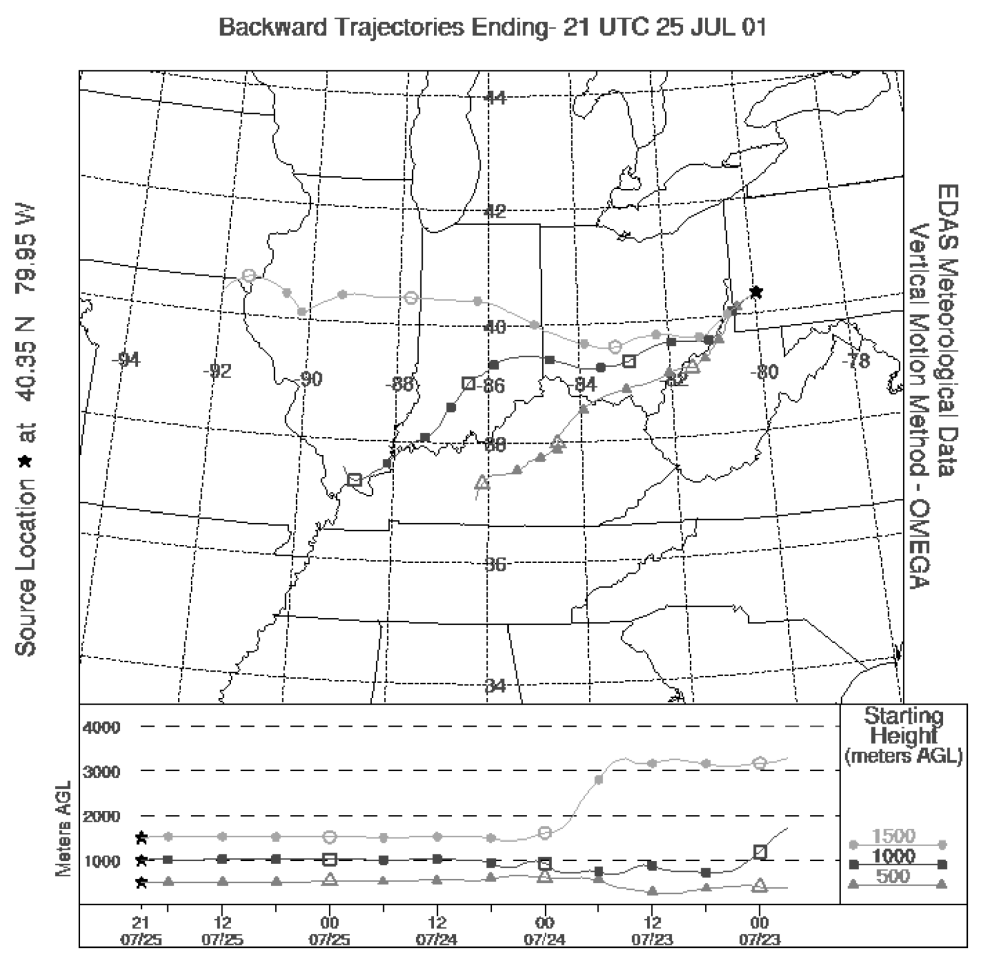

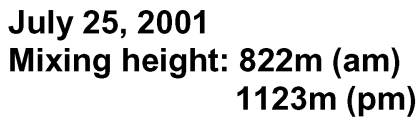

PM2.5 Mass

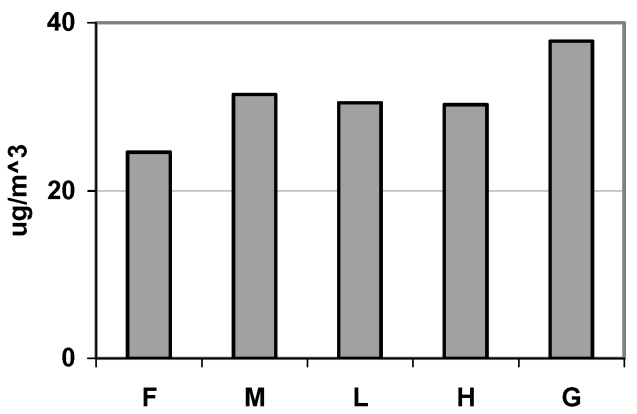

July 18, 2001

Mixing height: $1370 \mathrm{~m}$ (am) $811 \mathrm{~m}(\mathrm{pm})$

PM2.5 Mass

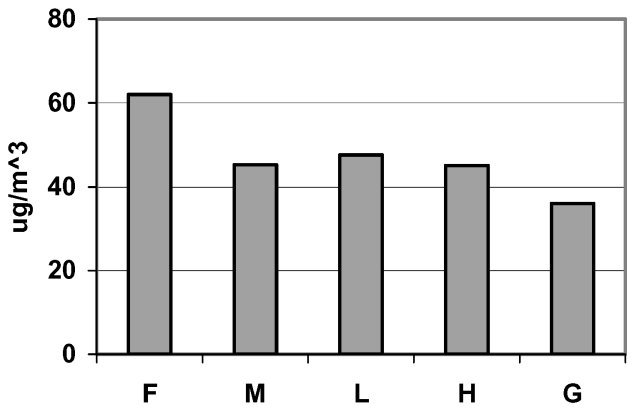

Figure 5. HYSPLIT back trajectories to the main site on three selected days. $\mathrm{PM}_{2.5}$ mass concentrations for 5 sites for each day are plotted on the right. F, Florence; M, Main site; L, Lawrenceville; H, Hazelwood; G, Greensburg. (Continued) 


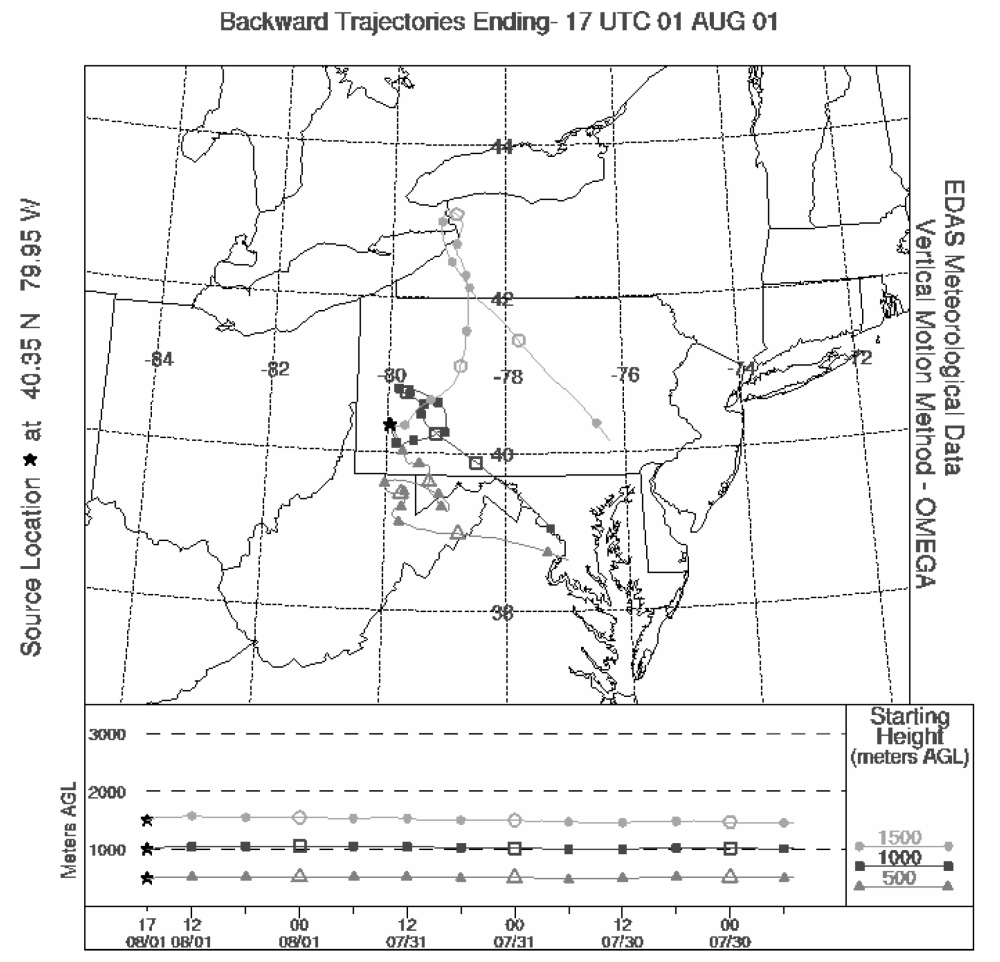

August 1, 2001 Mixing height: $543 \mathrm{~m}(\mathrm{am})$ $566 \mathrm{~m}(\mathrm{pm})$

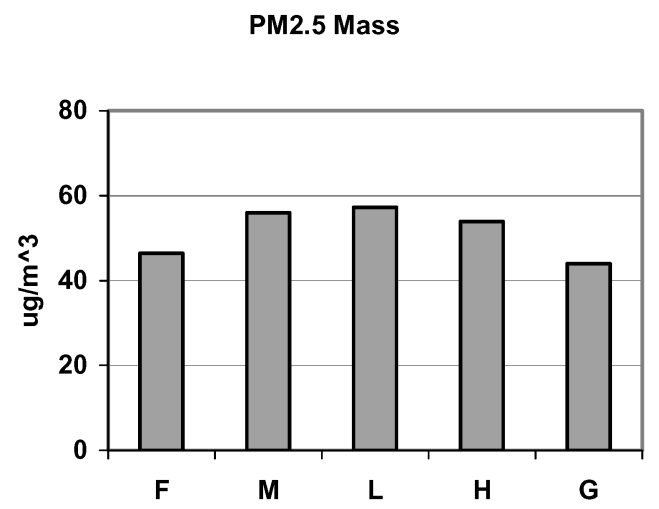

Figure 5. (Continued)

\section{Seasonal Variation in $\mathbf{P} \mathbf{M}_{\mathbf{2 . 5}}$}

The box plots in Figure 4 show the statistics of $\mathrm{PM}_{2.5}$ concentrations measured at the six sites in each season. The boxes stretch from the lower quartile to the upper quartile values. The medians are shown as thin lines across the boxes, while the thick black lines represent the means. The whiskers extending from each end of the box show the 90th percentile and 10th percentile of the data. The crosses show the 95th percentile and 5th percentile values.

A number of trends are evident from these plots. Mass, sulfate, and ammonium have similar seasonal variations, with maximum average concentrations and maximum extreme concentrations occurring in summer. Nitrate follows the opposite trend: the maximum values occur in the winter due to greater partitioning of nitrate to the particulate phase at lower temperatures. No seasonal trends are observed for EC and OC.

Among the six sites there seems to be a net increasing trend from west to east, especially in summer 2002. The predominant wind direction is from the west and southwest, suggesting that pollutants emitted in the Ohio River Valley are transported to the Pittsburgh region, causing elevated $\mathrm{PM}_{2.5}$ concentrations.

\section{Influence of Meteorological Conditions}

Factors affecting the spatial variations of $\mathrm{PM}_{2.5}$ have been studied based on surface meteorological data and estimated back-trajectories using HYSPLIT4 (NOAA 2002). Examples of back-trajectories for three $\mathrm{PM}_{2.5}$ episodes are presented in
Figure 5, along with plots of the mass concentration at the various sites.

Trajectories of the type seen on 25 July 2001 are frequently observed in this region, with winds from the west and southwest. Florence has the lowest mass concentration, while Greensburg has the highest. Trajectories on several other days during the summer intensive show faster winds, more rapid transport, and strong mixing; on such days, the airborne concentrations are similar among all sites.

The trajectories on 18 July 2001 are similar to those that occurred throughout an episode lasting until 21 July 2001. Florence has the highest $\mathrm{PM}_{2.5}$ levels, while Greensburg has the lowest, and concentrations in the city are midway between the two extremes. The increasing concentrations from east to west reflect atypical east-to-west winds.

The trajectories on 1 August 2001 show yet another type of meteorological condition, causing high $\mathrm{PM}_{2.5}$ levels. Due to stagnant air and relatively poor mixing, emissions from local sources accumulate in the area. Note that the trajectories show slowly meandering air movements as opposed to swift transport in a well-defined direction. All sites have mass concentration greater than $40 \mu \mathrm{g} / \mathrm{m}^{3}$, and the city sites have slightly higher values.

\section{CONCLUSIONS}

Sampling with filters for $\mathrm{PM}_{2.5}$ mass, sulfate, ammonium, and OC suggests that these secondary species may be remarkably uniform in concentration over large regions in the summer. 
Species less influenced by secondary production are likely to be more variable in concentration. The influence of sources in the city of Pittsburgh on $\mathrm{PM}_{2.5}$ levels within the city may be small, although regions downwind of the city may experience elevated concentrations resulting from city emissions. Concentrations are observed to increase along airmass trajectories as additional source emissions are added to the airmass and as photochemistry continues.

\section{REFERENCES}

Anderson, R. R., Martello, D. V., Rohar, P. C., Tamilia, J. P., Waldner, K., White, C. M., Modey, W. K., Mangelson, N. L., and Eatough, D. J. (2002). Sources and Composition of $\mathrm{PM}_{2.5}$ at the National Energy Technology Laboratory in Pittsburgh During July and August 2000, Energy Fuels 16:261-269.

Brook, J. R., Wiebe, A. H., Woodhouse, S. A., Audette, C. V., Dann, T. F., Callaghan, S., Piechowski, M., Dabek-Zlotorzynska, E., and Dloughy, J. F. (1997). Temporal and Spatial Relationships in Fine Particle Strong Acidity, Sulphate, $\mathrm{PM}_{10}$, and $\mathrm{PM}_{2.5}$ Across Multiple Canadian Locations, Atmos. Environ. 31:4223-4236.

EPA. (1999). Particulate Matter (PM 2.5$)$ Speciation Guidance. Office of Air Quality Planning and Standards, US EPA, Research Triangle Park, NC.

Hidy, G. M. (1994). Atmospheric Sulfur and Nitrogen Oxides: Eastern North America Source-Receptor Relationships. Academic Press, San Diego, CA.

Keeler, G. J., Spengler, J. D., and Castillo, R. A. (1991). Acid Aerosol Measurements at a Suburban Connecticut Site, Atmos. Environ. 25:681-690.

Lioy, P. J., Samson, P. J., Tanner, R. L., Leaderer, B. P., Minnich, T., and Lyons, W. (1980). The Distribution and Transport of Sulfate "Species" in the New York
Metropolitan Area during the 1977 Summer Aerosol Study, Atmos. Environ. 14:1391-1407.

McCurdy, T., Zelenka, M. P., Lawrence, P. M., Houston, R. M., and Burton, R. (1999). Acid Aerosols in the Pittsburgh Metropolitan Area, Atmos. Environ. 33:5133-5145.

Modey, W. K., Eatough, D. J., Anderson, R. R., Martello, D. V., Takahama, S., Lucas, L. J., and Davidson, C. I. (2004). Meteorological Influence on and Patterns in Ambient Fine Particulate Chemical Composition at Two Sampling Sites in Metropolitan Pittsburgh: A 2001 Intensive Summer Study, Atmos. Environ. 38:3165-3178.

NOAA. (2002). HYSPLIT4 (Hybrid Single-Particle Lagrangian Integrated Trajectory) Model. Available at: http://www.arl.noaa.gov/ready/hysplit4.html

Spengler, J. D., Brauer, M., and Koutrakis, P. (1990). Acid Air and Health, Environ. Sci. Technol. 24:946-956.

Solomon, P., Baumann, K., Edgerton, E., Tanner, R., Eatough, D., Modey, W., Marin, H., Savoie, D., Natarajan, S., Meyer, M. B., and Norris, G. (2003). Comparison of Integrated Samplers for Mass and Composition During the 1999 Atlanta Supersites Project, J. Geophys. Res. B Atmospheres. Special Issue for the Atlanta Supersites Project. 108(D7):8423.

Subramanian, R., Khlystov, A. Y., Cabada, J. C., and Robinson, A. L. (2004). Positive and Negative Artifacts in Particulate Organic Carbon Measurements with Denuded and Undenuded Sampler Configurations, Aerosol Sci. Technol. 38(S1):27-48.

Takahama, S., Wittig, B., Vayenas, D. V., Davidson, C. I., and Pandis, S. N. (2004). Modeling the Diurnal Variation of Nitrate during the Pittsburgh Air Quality Study, JGR-Atmos. Submitted.

Wittig, B., Anderson, N., Khlystov, A., Pandis, S. N., Davidson, C., and Robinson, A. L. (2003). Pittsburgh Air Quality Study Overview and Preliminary Scientific Findings, Atmos. Environ. 38:3107-3125. 


\section{Semicontinuous Measurements of Organic Carbon and Acidity during the Pittshurgh Air Quality Study: Implications for Acid-Catalyzed Organic Aerosol Formation}

\author{
S. TAKAHAMA, ${ }^{\dagger}$ C. I. DAVIDSON,,$\S$ AND \\ S. N. PA N D I S*, $\uparrow, \S$ \\ Departments of Chemical Engineering, Civil and \\ Environmental Engineering, and Engineering and \\ Public Policy, Carnegie Mellon University, 5000 Forbes \\ Avenue, Pittsburgh, Pennsylvania 15213
}

Laboratory evidence suggests that inorganic acid seed particles may increase secondary organic aerosol yields secondary organic aerosol (SOA) through heterogeneous chemistry. Additional laboratory studies, however, report that organic acidity generated in the same photochemical process by which SOA is formed may be sufficient to catalyze these heterogeneous reactions. Understanding the interaction between inorganic acidity and SOA mass is important when evaluating emission controls to meet $\mathrm{PM}_{2.5}$ regulations. We examine semicontinuous measurements of organic carbon $(\mathrm{OC})$, elemental carbon $(\mathrm{EC})$, and inorganic species from the Pittsburgh Air Quality Study to determine if we can detect coupling in the variations of inorganic acidity and $\mathrm{OC}$. We were not able to detect significant enhancements of SOA production due to inorganic acidity in Western Pennsylvania most of the time, but its signal might have been lost in the noise. If we assume a causal relationship between inorganic acidity and $\mathrm{OC}$, reductions in $\mathrm{OC}$ for Western Pennsylvania that might result from drastic reductions in inorganic acidity were estimated to be $2 \pm 4 \%$ by a regression technique, and an upper bound for this geographic area was estimated to be $5 \pm$ $8 \%$ based on calculations from laboratory measurements.

\section{Introduction}

The category of carbonaceous aerosols includes elemental carbon (EC) and organic carbon (OC). Emissions of EC are primarily from fossil fuel combustion or burning of biomass. OC in the atmosphere is either from these same sources or the result of oxidation reactions of volatile organic compounds (VOCs) emitted mainly by motor vehicles and vegetation (1). The latter type of OC is associated with what is called secondary organic aerosol (SOA), and the identification of specific compounds that comprise SOA still poses many challenges (2).

A previously unaccounted pathway for increased SOA formation was proposed by Jang and co-workers $(3,4)$. They proposed that heterogeneous reactions in aerosols, such as

\footnotetext{
* Corresponding author phone: (412)268-3531; fax: (412)268-7139; e-mail: spyros@andrew.cmu.edu.

${ }^{\dagger}$ Department of Chemical Engineering.

₹ Department of Civil and Environmental Engineering.

$\S$ Department of Engineering and Public Policy.
}

acid-catalyzed carbonyl chemistry, can lead to large increases in SOA formation primarily through polymerization mechanisms; a "multifold" increase in organic aerosol yields was observed in the laboratory for heterogeneous reactions of aldehydes and also in SOA yields for acrolein and isoprene reacting with ozone when an acid seed was present, compared with the case where the acid seed was absent (4).

The presence of acid aerosols in the atmosphere is well documented, with anthropogenic emissions of $\mathrm{SO}_{2}$ and $\mathrm{NO}_{x}$ responsible for a large fraction of the acidity in the form of $\mathrm{H}_{2} \mathrm{SO}_{4}$ and $\mathrm{HNO}_{3}$, respectively (5-9). The significant implication of the work of Jang et al. (4) is that if atmospheric acidity is reduced through emission controls of inorganic precursor species (e.g., $\mathrm{SO}_{2}$ ), acid-catalyzed formation of $\mathrm{SOA}$ may also be reduced, thereby resulting in an effective control for $\mathrm{PM}_{2.5}$ mass concentrations.

Since the seminal work by Jang and Kamens (3), other laboratory studies have found that acidic particles can act as catalysts for increased SOA formation. Iinuma et al. (10) reported that in the ozonolysis of $\alpha$-pinene, organic aerosol concentration increased by as much as $40 \%$ when inorganic acid-seed aerosols were present. Jang and co-workers $(11,12)$ observed increased reaction rates and increased yields of SOA over the duration of their studies in the presence of acid catalysts as opposed to their absence. Limbeck et al. (13) found that the presence of sulfuric-acid catalysts increased the formation of polymers resembling humic-like substances when dienes were photooxidized. Tolocka et al. (14) observed oligomeric products in the reaction of $\alpha$-pinene and ozone and proposed reaction pathways for polymerization of organic molecules to proceed in the aerosol phase. Furthermore, Gao et al. (15) observed the presence of more high-molecular weight compounds in the presence of acid seed than with seeds that were not acidic.

Kalberer et al. (16), however, reported that polymerization can proceed without preexisting strong acid seed particles. In a laboratory study of the photooxidation of 1,3,5trimethylbenzene and $\mathrm{NO}_{x}$, organic acids and nitric acid formed from the photooxidation reaction in amounts sufficient to catalyze acetal polymerization reactions. In addition, the polymerization reactions observed continued for more than $25 \mathrm{~h}$, implying that polymerization reactions may occur during the entire lifetime of a tropospheric aerosol. Gao et al. (15) suggested this as a possible explanation for their observation of high molecular weight compounds formed even in the presence of nonacid seeds. In light of the potential implications of the work of Jang et al. (4) and Kalberer et al. (16), it is desirable to know if inorganic acidity is indeed responsible for increased SOA formation in the atmosphere. Current research has focused on laboratory studies, some at particularly high concentrations of VOCs, oxidants, or acidity. At this stage it is difficult to extend conclusions drawn from these studies to the complex atmospheric system.

A synthesis of field studies has likewise produced inconclusive results. From an analysis of 24 -h filter-based measurements from the Speciation Trends Network, Chu (17) reported high levels of both sulfate and organic aerosols accompanied by ammonia deficiency during an episode on July 18th of 2001 on the East Coast. From this observation, the author suggested that SOA formation through heterogeneous reactions may be occurring during the summer. Using higher time-resolution data from the Aerodyne Mass Spectrometer, however, Zhang et al. (18) observed "negligible enhancement" of organics in the presence of acidic aerosols in Pittsburgh during 15 days in September 2002. As Zhang et al. (19) have reported, it may also be possible that the 
periods of high acidity and peak periods of secondary organic aerosol formation do not necessarily coincide in the same period, and 24-h measurements are unable to capture the separate diurnal patterns of these species.

The metric used to quantify inorganic aerosol acidity has also varied across field studies. Sulfate has commonly used as a surrogate, but this may lead to problems because a large fraction of the sulfate may be neutralized by ammonia. Another common metric for acidity is the deficit of ammonia with respect to the quantity required to fully neutralize the strong acids (i.e., sulfate and nitrate) present in the aerosol $(17,19)$. Estimates of acidity from such an ion-balance approach are equivalent to the total extractable acidity obtained from $\mathrm{pH}$ measurements of aqueous extracts of particles (9). Also referred to as the aerosol strong acidity, this metric gives an indication of the total amount of hydrogen that can become available in the particle when removed from its present environment (9). However, the strong acidity often overestimates the actual particle acidity because some of the hydrogen is associated with bisulfate under ambient conditions.

To address these issues, this work examines time-resolved ( $1-4 \mathrm{~h}$ ) field measurements collected during the Pittsburgh Air Quality Study (PAQS) from July 2001 to July 2002 to determine the potential enhancement in OC concentrations during periods of high inorganic acidity in Pittsburgh. Measurements of inorganic species are combined with a thermodynamic model to calculate concentrations of aerosol free acidity, and this analysis is extended over a period of several seasons. In addition, some estimates are made as to the possible reductions in OC that can be achieved as a result of reductions in inorganic acidity.

\section{Experimental Section}

Pittsburgh, PA, is located in the northeastern U.S., between the major agricultural and utility emissions in the Midwest and large urban and coastal areas in the East. Between July 2001 and July 2002, measurements of aerosols, gases, and meteorological conditions were made at the Pittsburgh Air Quality Study monitoring station. The site was located approximately $6 \mathrm{~km}$ east of downtown Pittsburgh, several hundred meters from the closest appreciable emission sources. From July 2001 to March 2002, the mean $\mathrm{PM}_{2.5}$ concentration was $16.1 \mu \mathrm{g} / \mathrm{m}^{3}$, with organic matter contributing on average $30 \%$ of this mass (20).

Continuous measurements of temperature, ultraviolet (UV) radiation intensity, relative humidity $(\mathrm{RH})$, and gasphase concentrations of $\mathrm{O}_{3}, \mathrm{NO}, \mathrm{NO}_{x}$, and $\mathrm{CO}$ were made and documented by Wittig et al. (21). Two to $4 \mathrm{~h}$ measurements of OC and EC were made by an in-situ OC/EC analyzer (22-24). Using measurements of ozone, $\mathrm{NO}_{x}$, CO, and UV radiation collected by collocated samplers at the site, the EC-tracer method was used by Cabada et al. (23) to derive a primary OC/EC ratio for sources affecting the region and then to estimate the SOA concentration.

To estimate the concentration of $\mathrm{H}^{+}$, or free acidity, $1-2-\mathrm{h}$ measurements of sulfate, total nitric acid, and total ammonia were made with semicontinuous instruments and used as inputs to a chemical equilibrium model, GFEMN (25). A steam-jet aerosol collector (26) captured aerosols and gases of these chemical species in a humidified chamber; resulting aqueous droplets were collected and analyzed by ion chromatography (DIONEX models DX-120 and DX-600 for water-soluble cations and anions, respectively) using a method described by Jaffrezo et al. (27) for the anion analysis. Additionally, a R\&P 8400S measured inorganic sulfate by a flash-volatilization technique (28). Concentrations reported by these semicontinuous instruments were calibrated against a filter-based speciation sampler and implemented for simulation as described by Wittig et al. (29) and Takahama

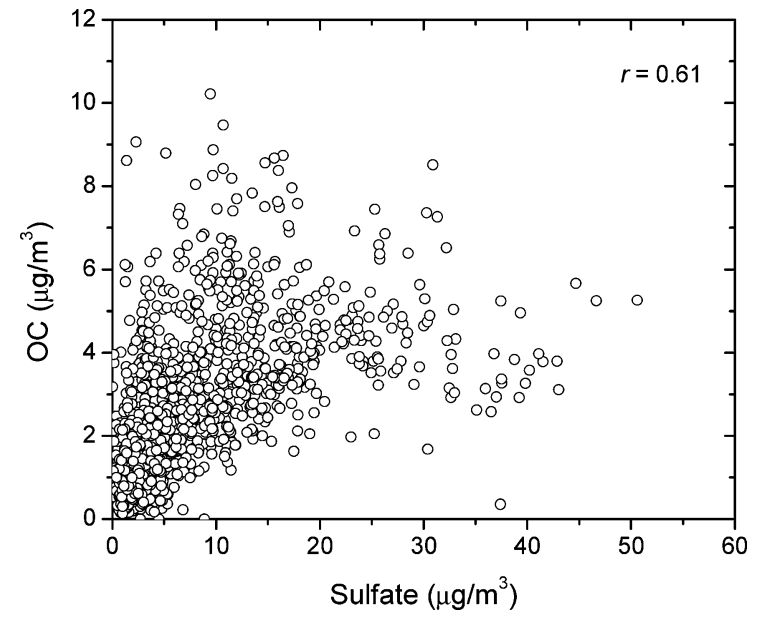

FIGURE 1. Correlation plot of $\mathrm{OC}$ and sulfate concentrations from July 2001 to July 2002. $r$ is the sample correlation coefficient.

et al. (30). The thermodynamic calculations were made assuming particles were liquid, except when the measured growth factor (31) was near unity, indicating that the particles were solid. For these periods, the $\mathrm{H}^{+}$concentrations were set to zero. The inorganic data were then averaged to match the sampling intervals of the in-situ OC/EC analyzer. Takahama et al. (30) showed that aerosol nitrate predictions from GFEMN agreed with observations within measurement uncertainties for most cases, which gives us some measure of confidence in our calculations of acidity.

The observed organic aerosol concentration field is relatively homogeneous in Western Pennsylvania (32) and most of the northeast U.S. Production of SOA takes place during the same time over a large area, and the system can be described by a box model to a first approximation (33). Therefore, the measurements represent the chemical processes taking place over a large area even if they are from a single location. This spatial homogeneity makes the PAQS data set suitable for this analysis because transport in and out of the region is a secondary effect.

\section{Analysis of Results}

In the past, sulfate concentrations have been used as a surrogate for aerosol acidity (8). Figure 1 shows the relationship between OC and sulfate; the sample correlation coefficient $(r)$ of 0.61 indicates a mild correlation. Examining such a graph might suggest that during periods of high acidity, there are significantly larger quantities of organic aerosol that might be attributable to SOA from heterogeneous chemistry (17). However, there may be other possible explanations for this observation. First of all, each parcel of air has its own history of accumulated emissions. Air parcels coming from relatively clean areas tend to have lower pollutant concentrations, while air parcels coming from heavily populated and industrialized areas have higher concentrations. For example, sulfate and primary OC are both derived at least in part from stationary source combustion, which could explain some of the correlation between the two species. In addition, there are meteorological effects, such as the strength of atmospheric mixing, common to both sulfate and OC that may be causing the observed relationship in Figure 1.

The use of free acidity $\left(\mathrm{H}^{+}\right)$calculated from thermodynamic principles has several advantages. Limbeck et al. (13) reported that free acidity and not the quantity of sulfate affects the formation of polymers. Furthermore, Cocker et al. (34) found that the presence of dry $\left(\mathrm{NH}_{4}\right)_{2} \mathrm{SO}_{4}$ or $\mathrm{NH}_{4} \mathrm{HSO}_{4}$ seed particles did not affect the SOA production, but the organic aerosol yields depended on the composition 

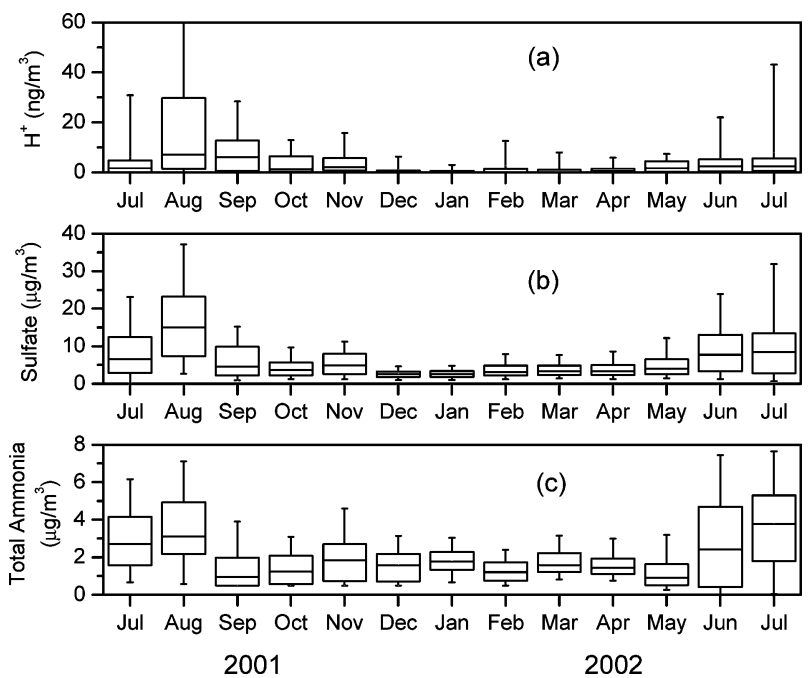

FIGURE 2. Boxplots of (a) $\mathbf{H}^{+}$, (b) sulfate, and (c) total ammonia $\left(\mathrm{PM}_{2.5}+\right.$ gas) illustrating the seasonal variation of these species. Boxes enclose data within the 25th to 75 th percentile range, black line in the box denotes median, and whiskers extend to the 5th and 95th percentile of the data.

of the seed aerosol when wet. Because the estimation of $\mathrm{H}^{+}$ by GFEMN is composition-dependent, the solution properties of the seed aerosol are taken into account when calculating the particle acidity. Sulfate and $\mathrm{H}^{+}$are mildly but not strongly correlated $(r=0.68)$ for our data set $-\mathrm{H}^{+}$is not only a function of the sulfate concentration but also the amount of neutralizing base (predominantly $\mathrm{NH}_{3}$ in this area), the presence of other acidic species (e.g., nitrate), and also the water content of the aerosol. Therefore, we proceed with the analysis as follows: (1) we use the $\mathrm{H}^{+}$concentration as our metric of acidity, (2) we normalize the OC concentrations by the EC concentrations, which takes advantage of the fact that EC is mostly emitted by the same combustion sources as primary OC, and (3) we also examine wind directions in conjunction with the concentrations, as a change in wind direction can indicate a change in combustion source-each of which can have a different ratio of OC to EC emissions.

Acidity. Periods of high acidity $\left(\mathrm{H}^{+}\right)$are generally found during the summer months with fewer instances occurring closer to the winter season (Figure 2a). $\mathrm{H}^{+}$in the aerosol phase calculated by GFEMN results from an aqueous-phase charge balance of primarily $\mathrm{H}_{2} \mathrm{SO}_{4}$, and to a lesser extent $\mathrm{HNO}_{3}$, with $\mathrm{NH}_{3}$. Sulfate concentrations are higher during the summer than in the winter (Figure $2 b$ ) primarily because the conversion rate of $\mathrm{SO}_{2}$ to $\mathrm{H}_{2} \mathrm{SO}_{4}$ is faster during the warm and sunny summer days. Lower sulfate levels in the winter imply that the total ammonia (gas $+\mathrm{PM}_{2.5}$ aerosol) available with respect to $\mathrm{H}_{2} \mathrm{SO}_{4}$ is higher (Figure $2 \mathrm{~b}, \mathrm{c}$ ). In general, this results in a large fraction of bisulfate ions during the summer and more sulfate ions during the winter; the free acidity reflects this difference. During the summer months, the $\mathrm{H}^{+}$ concentration is likely to be higher during the daytime (Figure $3 a$ ), while the opposite is true for the winter (Figure 3b). Diurnal variations in acidity may be caused by many factors. For example, during high periods of photochemical activity in the morning and early afternoon, production of nitric acid and sulfate may exceed their deposition rates, resulting in a rise in the concentrations of these species. It is likely that the strong diurnal pattern in acidity observed during the summer is primarily due to the photochemical production of sulfate. During the winter, however, a combination of aerosol composition and low RHs often induced solid-phase transitions in the aerosols (31), leading to lower concentrations of acidity during the afternoons.

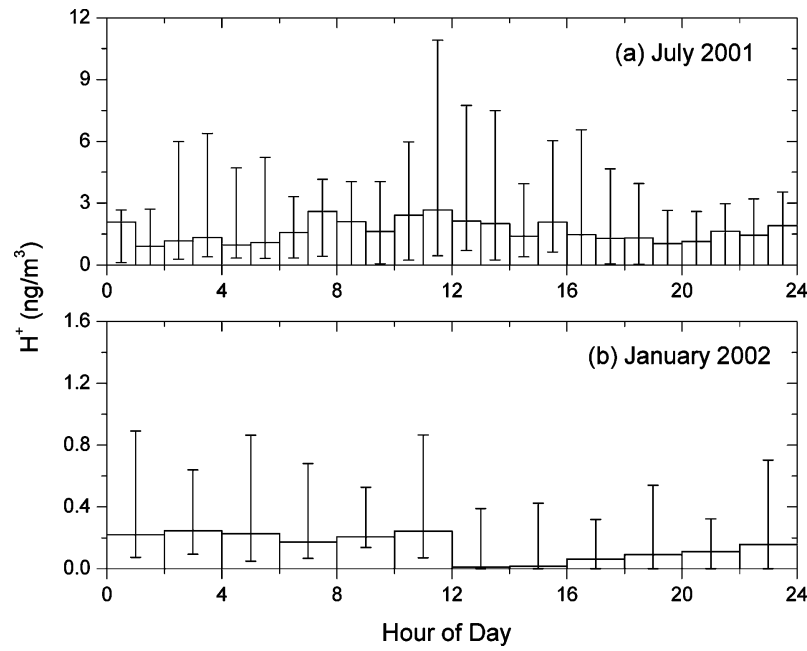

FIGURE 3. Time-series plots of diurnal variations in acidity for (a) July 2001 and (b) January 2002. Bars represent the median and the error bars extend to the 25th and 75th percentile of the monthly data for each sampling period.

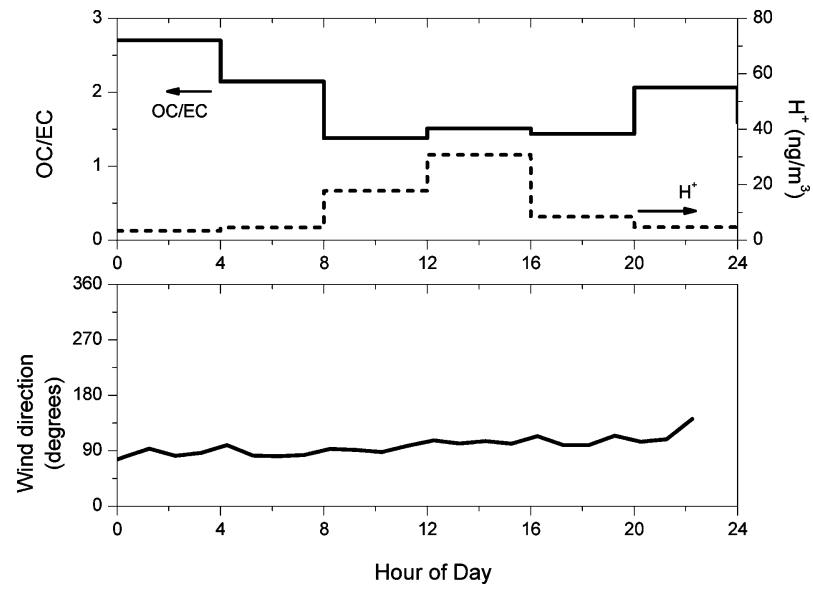

FIGURE 4. Time series of $\mathrm{OC} / \mathrm{EC}, \mathrm{H}^{+}$, and wind direction for March 27, 2002.

OC/EC Ratio, Acidity, and Wind Direction. Measurements of wind direction were used to determine if changes in OC/EC ratios could have occurred as a result of change in source signature. Time series plots of OC/EC ratios, acidity $\left(\mathrm{H}^{+}\right)$, and wind direction were examined for periods in which the aerosol became increasingly acidic. In the examination of these time series from July 2001 to July 2002, 104 occurrences of significant increases in $\mathrm{H}^{+}$were visually identified.

Possible evidence for acid-catalyzed SOA taking place in this region would be illustrated by an increase in the OC/EC ratio accompanying or following a period of high $\mathrm{H}^{+}$ concentrations, when the wind direction does not shift dramatically during this period. We might expect this result if the OC and EC emission source is constant (i.e. characteristic emission ratio of OC to EC remains the same), but OC/EC ratios increase as acid-catalyzed SOA formation proceeds. In general, there was no clear evidence that acidcatalyzed SOA formation was consistently observable in Pittsburgh during our study period. In fact, in some cases the OC/EC decreased when the aerosol became acidic, suggesting possibilities of other processes dominating the change in organic aerosol concentrations or simply the decoupled behavior of organic and inorganic aerosols. Figure 4 shows time-series from March 27, 2002, the wind direction is relatively steady, but between 4 a.m. and 2 p.m., OC/EC decreased from 2.1 to 1.4 at the same time that $\mathrm{H}^{+}$increased 

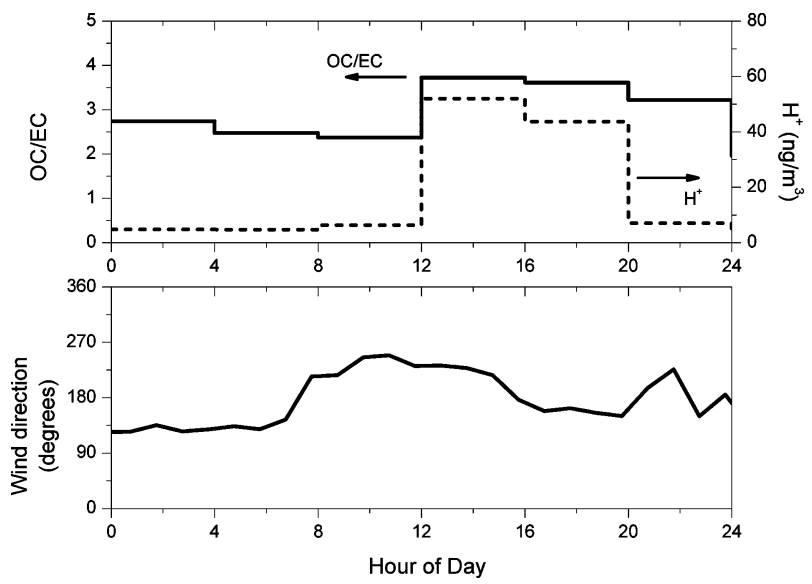

FIGURE 5. Time series of $\mathrm{OC} / \mathrm{EC}, \mathrm{H}^{+}$, and wind direction for June 25, 2002.
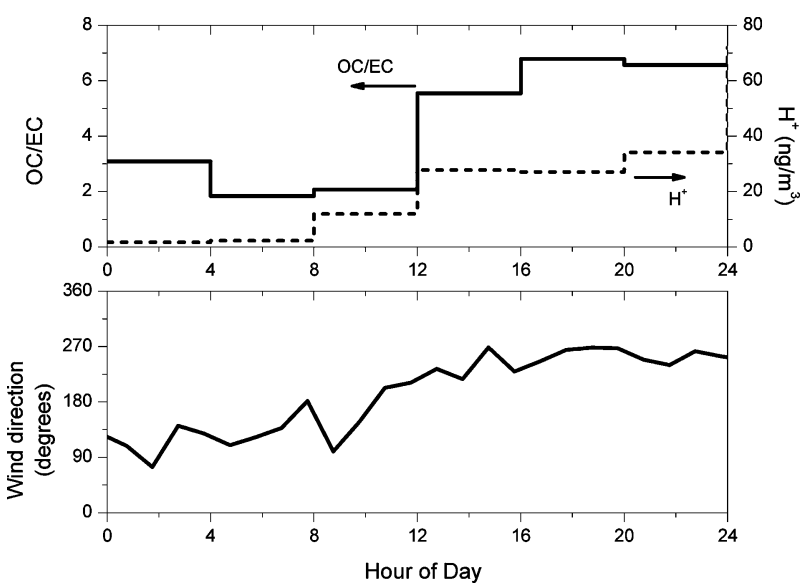

FIGURE 6. Time series of OC/EC, $\mathrm{H}^{+}$, and wind direction for July 17, 2002.

from 5 to $18 \mathrm{ng} / \mathrm{m}^{3}$. Thirteen of the 104 instances of $\mathrm{H}^{+}$ increase exhibited this behavior, where the inorganic acidity increased and the OC/EC decreased simultaneously under conditions of constant wind direction.

Figure 5 shows an example of a case where both the OC/ EC and the acidity increased together, which was observed on June 25,2002 , during midday, and the wind direction was not extremely steady but on average from the same direction. The $\mathrm{H}^{+}$concentration increased from its initial value of 6 $\mathrm{ng} / \mathrm{m}^{3}$ to $52 \mathrm{ng} / \mathrm{m}^{3}$, the latter being one of the highest values attained during the study period. Twenty-two of the 104 accounts of $\mathrm{H}^{+}$increase were of the second type, where inorganic acidity and OC/EC increased together under conditions of constant wind direction.

Changes in OC/EC accompanied by significant changes in wind direction, however, could be indicative of a change in the primary OC to EC ratio emitted by different sources or SOA transported into the region. Figure 6 illustrates this case, which was observed on July 17, 2002. Between 4 a.m. and 12 p.m., the wind direction changed steadily (also with increasing variability), shifting by almost 180 degrees over an 8-h period. At noon, the OC/EC and $\mathrm{H}^{+}$increased together, with $\mathrm{H}^{+}$concentrations again reaching a high value of 28 $\mathrm{ng} / \mathrm{m}^{3}$, which may be possible evidence of acid-enhanced formation of SOA over Pittsburgh, but this may also be indicative of a source region rich in both primary organics and acidic aerosols. Thirty-eight of 104 counts of acidification were of this last type, where the inorganic acidity and OC/EC both increased during the same period but under varying wind direction.

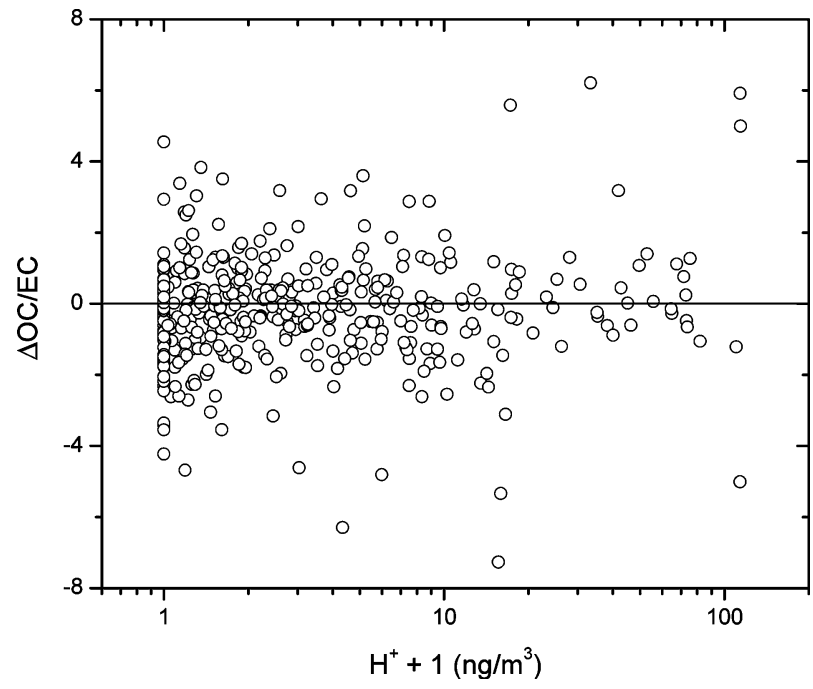

FIGURE 7. Correlation plot of changes in OC/EC between periods $k$ and $k-1$ and acidity at $k$. A constant of $1 \mathrm{ng} / \mathrm{m}^{3}$ is added to the $\mathrm{H}^{+}$for displaying $\mathrm{H}^{+}=\mathbf{0 ~ n g} / \mathrm{m}^{3}$ values on a logarithmic scale.

In the 31 out of 104 cases remaining, there was no detectable change in OC/EC with increase in acidity. These observations may be explained by several means, including the decoupled behavior of inorganics and organics, the acidity perturbing organic aerosol concentrations by an immeasurable amount, or the positive and negative changes in OC/EC due to simultaneous atmospheric processes summing to an amount within measurement uncertainty.

Calculations of air mass back-trajectories with the HYSPLIT (NOAA) software on selected periods generally confirmed that measured wind directions at the site were indicative of source region. For example, 6 -h back-trajectory calculations showed that during June 25, 2002, the air parcels that arrived at Pittsburgh at 10 a.m. and 2 p.m. both originated from the West. During the July 17, 2002, episode, however, the air parcel that arrived in Pittsburgh at 10 a.m. was transported from the Southeast, while the air parcel that arrived at 2 p.m. was transported from the Southwest after following a circuitous path around and over the city.

To formalize this analysis, observed changes in OC/EC and acidity were grouped according to wind directions that were relatively constant and those that were different between two successive sampling intervals. For this analysis, the data were averaged into regularly spaced intervals of $4 \mathrm{~h}$. Wind directions that were different were determined by a test of statistical significance on the mean wind directions between two successive sampling periods. The change in mean wind direction was considered different if rejected by a two-sample $t$-test at an $\alpha=0.05$ significance level and was considered relatively constant otherwise. Though the wind direction data are by no means random or independently sampled, Ott and Mage (35) showed that the mean and variance calculated by the central limit theorem are good approximations when applied to data exhibiting serial correlation; therefore, our simple algorithm is acceptable. Figure 7 shows the change in $\mathrm{OC} / \mathrm{EC}$ as a function of the geometric mean acidity between two successive periods $k$ and $k-1$, for the case in which the change in wind direction between the two periods was relatively constant. If acid-catalyzed SOA formation were detectable in Pittsburgh, we would expect a positive correlation between the two variables, particularly in the region of high $\mathrm{H}^{+}$concentrations. However, as evident in Figure 7, there is little observable correlation $(r=0.10)$ between inorganic acidity and increase in OC/EC.

The change in OC/EC shows a similar lack of correlation with other measures of acidity. One such metric is the $\mathrm{H}^{+}$ 
concentration from the previous time interval-we might expect that this metric is important if the response of increase in OC/EC to availability of $\mathrm{H}^{+}$is on the order of hours; indeed, Kalberer et al. (16) suggest that the OC increase due to acidity could occur over the lifetime of the aerosol. The correlation between $\triangle \mathrm{OC} / \mathrm{EC}$ and $\mathrm{H}^{+}$from the previous time interval is 0.07 , which is lower than the correlation of $\mathrm{H}^{+}$of the current time 4-h interval. In contrast, Czoschke et al. (36) report time scales for polymerization on the order of seconds. In the event that the acid-catalyzed SOA formation is rapid and proceeds with any amount of increase in $\mathrm{H}^{+}$, we might expect a positive correlation between $\Delta \mathrm{OC} / \mathrm{EC}$ and $\Delta \mathrm{H}^{+}$over the same time periods. The sample correlation with changes in OC/EC from this analysis was 0.07.

Other metrics of acidity were also considered. Though the calculation of free acidity takes into the account the chemical composition, ambient $\mathrm{RH}$, and associated water uptake by the aerosol, the $\mathrm{pH}$ provides additional information about the availability of $\mathrm{H}^{+}$in solution as it indicates the quantity of $\mathrm{H}^{+}$relative to the amount of water in solution. The sensitivity of the $\mathrm{pH}$ to the water content of the aerosol provides a means to examine periods when highly concentrated acidic particles exist for short durations, in which case the $\mathrm{H}^{+}$contribution to the total $\mathrm{H}^{+}$burden may not be significant, but ultrafine aerosol solutions highly concentrated in $\mathrm{H}^{+}$may exist. Defining the $\mathrm{pH}$ as $-\log \left[\mathrm{H}^{+}\right]$where $\left[\mathrm{H}^{+}\right]$is the molarity of $\mathrm{H}^{+}$in the solution, the $\mathrm{pH}$ for pure sulfuric acid at $35 \% \mathrm{RH}$ is -1.1 , according to calculations with AIM (37). The mean $\mathrm{pH}$ for all 4 -h periods was 0.8 , with a minimum of -0.9 and 374 periods out of 1715 dropping below 0 , often during the afternoons when $\mathrm{RH}$ was lowest. Despite the low values of $\mathrm{pH}$ attained, the correlation of $\Delta \mathrm{OC} / \mathrm{EC}$ with $\mathrm{pH}$ with the current time period is -0.10 , with the previous time period is -0.07 , and with $\Delta \mathrm{pH}$ is -0.02 . The negative values of correlation are as expected, as lower $\mathrm{pH}$ indicates higher acidity, but the absolute magnitudes of the correlations are not large. When the hydrogen ion activity, considering only nonidealities caused by the inorganic fraction of the aerosol, instead of its concentration is used to define $\mathrm{pH}$, the correlations with $\triangle O C / E C$ increase in some cases but remain small-the correlation of $\triangle \mathrm{OC} / \mathrm{EC}$ and $\mathrm{pH}$ at the current time is -0.16 , with $\triangle \mathrm{OC} / \mathrm{EC}$ and $\mathrm{pH}$ from the previous time is -0.08 , and $\triangle \mathrm{OC} / \mathrm{EC}$ with the $\Delta \mathrm{pH}$ is -0.09 .

There is also a possibility that the polymerization reactions that occur in the presence of acid catalysts are irreversible, or not reversible in atmospherically relevant time scales, and neutralization of the acidity will not lead to reductions in OC. To account for this effect, the relationships examined above were reanalyzed for periods only when the acidity increased. By this method, the strongest correlations observed were between $\triangle \mathrm{OC} / \mathrm{EC}$ and the three metrics of acidity of the current time period. The correlations were $0.17,-0.18$, and -0.28 for $\mathrm{H}^{+}$and $\mathrm{pH}$ defined by the hydrogen ion molarity and activity, respectively. These relationships are stronger than when data points for all changes in acidity are considered, but the correlations are still weak. Zhang et al. (19) showed that during nucleation events in Pittsburgh, unneutralized $\mathrm{H}_{2} \mathrm{SO}_{4}$ existed for short periods in the morning during nucleation events, but rapid SOA formation and condensation occurred several hours after nucleation after the $\mathrm{H}_{2} \mathrm{SO}_{4}$ had been largely neutralized by $\mathrm{NH}_{3}$. Indeed, our analysis suggests that normalizing the airborne $\mathrm{H}^{+}$concentrations by the water content showed little improvement in correlations of acidity with $\triangle \mathrm{OC} / \mathrm{EC}$.

\section{Estimating the Effect of Acidity on OC Concentrations}

Though our method of analysis did not produce evidence of acid-catalyzed heterogeneous chemistry leading to increased SOA concentrations in Pittsburgh, it is possible that the effect of acidity on SOA was masked by the myriad of other
TABLE 1. Sample Correlations of Measurements (with No Lay and Also When Lagged by One 4-h Time Period) with OC/EC

\begin{tabular}{lrr}
\multicolumn{1}{c}{ variable } & lag $=\mathbf{0}$ & lag $=\mathbf{1}$ \\
$\mathrm{H}^{+}$ & 0.30 & 0.25 \\
$\mathrm{NO}$ & -0.25 & -0.21 \\
$\mathrm{NO}_{x}$ & -0.34 & -0.28 \\
$\mathrm{CO}$ & -0.17 & -0.14 \\
$\mathrm{O}_{3}$ & 0.41 & 0.37 \\
$\mathrm{UV}$ & 0.12 & 0.25 \\
temperature & 0.22 & 0.21 \\
sulfate & 0.25 & 0.23 \\
1/NO & 0.44 & 0.32 \\
1/NO & 0.48 & 0.38 \\
1/CO & 0.16 & 0.14 \\
\hline
\end{tabular}

atmospheric processes occurring simultaneously. Therefore, we wish to know what the upper limit of the effect that reducing inorganic acidity might have on OC concentrations over this area in the event that SOA enhancement is still occurring. For this purpose, we use two approaches: (1) predicting changes in OC from a regression model and (2) calculating the reduced OC for an assumed scenario.

Regression Analysis. To determine the contribution of $\mathrm{H}^{+}$in explaining the variability in OC/EC, a regression model was constructed. Candidate regressors for the model were preselected based on our physical understanding of atmospheric chemistry-ozone, ultraviolet radiation, and high temperatures are favorable for SOA production; $\mathrm{CO}, \mathrm{NO}$, and $\mathrm{NO}_{x}$ are tracers of combustion (23). VOC concentrations are also important variables for the formation of SOA, but VOC measurements were not available in the time-resolution necessary so they were not considered. $\mathrm{H}^{+}$was also included as a candidate regressor, as it is the variable of primary interest. Sample correlation coefficients of these variables and their lagged values with OC/EC are presented in Table 1. Because a sampling duration of $2-4 \mathrm{~h}$ is long compared to time scales of many atmospheric processes, we find that correlations of time-integrated OC/EC concentrations are stronger with explanatory variables measured during the same time period (lag $=0$ ), rather than the variable's value at the previous sampling interval. In general, the correlations are consistent with our understanding of atmospheric chemistry. The photochemical cycle ultimately consumes $\mathrm{NO}$ and $\mathrm{NO}_{x}$ in the process of producing ozone, the primary oxidant of reactive organic gases that are precursors to SOA. Therefore, the ozone and the inverse concentrations of NO and $\mathrm{NO}_{x}$ show some correlation with OC/EC. UV, which initiates the photochemical reactions, is the only variable in Table 1 whose previous time value shows a stronger correlation with OC/EC than its present value. The sample correlation coefficient of OC/EC with carbon monoxide is negative, because emissions of $\mathrm{CO}$ indicate an increase in emissions of EC and primary OC, lowering the OC/EC ratio when SOA is present. Though higher temperatures are thought to be favorable for SOA formation, temperature is not strongly correlated with OC/EC. $\mathrm{H}^{+}$shows a stronger correlation than sulfate, and it is the relationship between OC/EC and acidity around which we wish to build our regression model.

First, all variables were averaged to regular intervals of 4 h. Because we do not have knowledge of the functional dependence of the OC/EC ratio on other variables a priori, we began with a subset of candidate regressors from Table 1 with the strongest correlations (i.e. all variables from the current time period except for UV, for which the previous time period was also included) and transformed variables such that the assumption of linearity would approximately hold among the response and predictor variables. Then, we used a stepwise, forward-selection approach to variable 
TABLE 2. Regression Coefficients, Standard Errors, and $t$ - and $p$-Values Calculated from Ordinary Least-Squares Regression

\begin{tabular}{clll} 
coefficient & \multicolumn{1}{c}{ units } & value & std error \\
$\hat{\beta}_{0}$ & & 1.3 & 0.1 \\
$\hat{\beta}_{1}$ & & 0.55 & 0.02 \\
$\hat{\beta}_{2}$ & $\left(\mu \mathrm{g} / \mathrm{m}^{3}\right)^{-1}$ & 3.2 & 0.7 \\
$\hat{\beta}_{3}$ & $(\mathrm{ppb})$ & -0.30 & 0.02 \\
$\hat{\beta}_{4}$ & $\left(\mathrm{~W} / \mathrm{m}^{2}\right)^{-1}$ & 0.0071 & 0.0012 \\
$\hat{\beta}_{5}$ & $\left({ }^{\circ} \mathrm{C}\right)^{-1}$ & 0.0043 & 0.0015 \\
\hline
\end{tabular}

selection with alternatives scored according to the Akaike Information Criterion (AIC). Variables that exhibited strong correlation with variables already included in the model were not considered to avoid problems with multicollinearity, and variables whose regression coefficients were not significant were removed. We obtained a model of the following form

$$
\begin{aligned}
\log (\mathrm{OC} / \mathrm{EC})_{k}=\beta_{0}+ & \beta_{1} \log (\mathrm{OC} / \mathrm{EC})_{k-1}+\beta_{2} \mathrm{H}_{k}^{+}+ \\
& \beta_{3} \log \left(\mathrm{NO}_{x}\right)_{k}+\beta_{4} \mathrm{UV}_{k}+\beta_{5} T_{k}+\epsilon_{k}
\end{aligned}
$$

where OC/EC is the OC/EC ratio, $\mathrm{H}^{+}$is the hydrogen ion concentration in the aerosol and our metric of inorganic acidity, $\mathrm{NO}_{x}$ is the concentration of the oxides of nitrogen, UV is the ultraviolet radiation intensity, $T$ is the temperature, and $\epsilon$ is the disturbance term. The $\beta$ 's are regression coefficients. The subscripts $k$ and $k-1$ denote the time intervals for which the variables are specified, i.e., OC/EC at $k-1$ specifies the OC/EC ratio lagged by one time interval. Lagged values of the dependent variable are introduced as regressors to reduce serial correlation in the residuals that may arise out of unaccounted influence of omitted explanatory variables (38). Even with the lagged dependent variable included the residuals still exhibited serial correlation according to the Breusch-Godfrey test $(39,40)$. Therefore, residuals from ordinary least squares regression were parametrized by a mixed autoregressive moving average process of order $p, q[\operatorname{ARMA}(p, q)]$; an $\operatorname{ARMA}(3,3)$ exhibited an acceptable fit to the residuals. The regression coefficients were then estimated by feasible generalized least squares (41).

The estimated regression coefficients, $\hat{\beta}$ 's, are shown in Table 2. As eq 1 is an empirical construct, it should be mentioned that these regression coefficients are only applicable for the Western Pennsylvania region for conditions experienced during our study period. The correlation of fitted values with observed values of $\log (\mathrm{OC} / \mathrm{EC})_{k}$ is 0.77 , with over 1250 data points included in the regression. The regression coefficients of all regressors included in the model are significant from zero at the $\alpha=0.01$ significance level because variables that were not significant were removed in the model selection process and also because the sample size is large. It is important to point out that statistical significance does not necessarily imply causality. The signs of the regression coefficients are as expected, and they agree with the signs from the sample correlation coefficients of each variable with OC/EC. $\hat{\beta}_{2}$, the regression coefficient for $\mathrm{H}^{+}$, is of particular interest, as this parameter indicates the sensitivity of OC/EC to inorganic aerosol acidity. Because this coefficient relates the $\mathrm{H}^{+}$concentration to the logarithm of OC/EC, its value is difficult to interpret directly unless used in the context of prediction. For instance, assuming that (1) a causal link exists between free acidity and OC/EC in the atmosphere and that (2) the value of all other variables are held fixed while the acidity is perturbed, we can use this statistical model to examine an extreme case where all effects of acidity are removed from additional OC formation by setting $\mathrm{H}^{+}=0$. If EC remains constant, then the ratio of the predicted OC/ $\mathrm{EC}$ to the fitted OC/EC should give an estimate of the decrease
TABLE 3. Estimated Overall Reduction in OC as a Function of the Acidity Threshold and the Threshold's Corresponding Percentile in the Cumulative Distribution Function of $\mathbf{H}^{+}$

$\begin{gathered}\text { acidity threshold, }\left(\mathbf{H}^{+}\right)^{*} \\ \left(\mathbf{n g} / \mathbf{m}^{3}\right)\end{gathered}$
25.0
4.1
0.9
0.1
0.0

$\begin{gathered}\text { percentile of } \mathbf{H}^{+} \\ \text {distribution }\end{gathered}$
96
75
50
25
0

overall reduction in $\mathrm{OC}(\%)$

$0.4 \pm 2.6$

$1.3 \pm 4.0$

$2.3 \pm 4.9$

$3.4 \pm 5.5$ $5.4 \pm 7.9$

in OC concentrations. For our scenario of reduced acidity, the predicted decrease in OC is about $2 \pm 4 \%$ overall. Thus, we find that the sensitivity of $\mathrm{OC}$ to $\mathrm{H}^{+}$is small.

Scenario Analysis. Laboratory studies have cited observations of SOA enhancement by as much as $40 \%$ under conditions of high inorganic acidity $(10,42)$. If such a magnitude of enhancement were occurring in the atmosphere, it is of interest to know what the effect of perturbing the current levels of inorganic acidity might be. For instance, if we assume that the enhancement is occurring above an assumed threshold acidity $\left(\mathrm{H}^{+}\right)^{*}$, reducing the acidity during those periods to values below the threshold would result in a new set of $\mathrm{OC}$ concentrations, $\mathrm{OC}^{\prime}$, in the following way

$$
\mathrm{OC}^{\prime}=\mathrm{OC}_{\mathrm{p}}+\mathrm{SOA}_{0} /(1+\phi)
$$

where

$$
\phi=\left\{\begin{array}{l}
0.4 \text { for } \mathrm{H}^{+} \geq\left(\mathrm{H}^{+}\right)^{*} \\
0 \quad \mathrm{H}^{+}<\left(\mathrm{H}^{+}\right)^{*}
\end{array}\right.
$$

$\mathrm{OC}_{\mathrm{p}}$ is the primary $\mathrm{OC}$ concentration, $\mathrm{SOA}_{0}$ is the original SOA concentration, $\phi$ is the magnitude of enhancement, and $\mathrm{H}^{+}$is the free acidity concentrations calculated for PAQS. Primary OC and SOA were not directly measured during the study, but the EC-tracer method is one approach that is used to estimate their concentrations (23), and we use those estimates here. The functional dependence of SOA enhancement on aerosol acidity has only been recently elucidated by a semiempirical approach (43), but in general the experiments by Limbeck et al. (13) qualitatively suggest a monotonic dependence on free acidity. Here we simply assume a step function, though we are left with the task of arbitrarily defining an acidity threshold to classify aerosols as acidic or not acidic. For our preliminary analysis, we used the reported aerosol volumes for $\mathrm{H}_{2} \mathrm{SO}_{4} /\left(\mathrm{NH}_{4}\right)_{2} \mathrm{SO}_{4}$ seeds and relative humidity conditions reported in the experiments of Jang and Kamens (3) and calculated the $\mathrm{H}^{+}$concentrations using a chemical equilibrium model, AIM (37). The nonacidic seed aerosols $\left(\left(\mathrm{NH}_{4}\right)_{2} \mathrm{SO}_{4}\right)$ were neutral $\left(\mathrm{H}^{+}=0 \mu \mathrm{g} / \mathrm{m}^{3}\right)$, and their acidic seed aerosols (mixed- $\mathrm{H}_{2} \mathrm{SO}_{4}-\left(\mathrm{NH}_{4}\right)_{2} \mathrm{SO}_{4}$ particles) ranged in $\mathrm{H}^{+}$from 0.025 to $0.22 \mu \mathrm{g} / \mathrm{m}^{3}$. Thus we assume a threshold of $\left(\mathrm{H}^{+}\right)^{*}=0.025 \mu \mathrm{g} / \mathrm{m}^{3}$, above which our aerosols are considered acidic.

The observed OC concentration, annually averaged from July 2001 to July 2002 is around $2.5 \mu \mathrm{g} / \mathrm{m}^{3}$. If we assume that emission reductions in precursor gases (e.g., $\mathrm{SO}_{2}$ and $\mathrm{NO}_{x}$ ) to inorganic acidity results in the removal of acid-catalyzed SOA production, the annual average OC would be reduced by approximately $2 \%$ of this amount, though $80 \%$ of the reductions would occur during the summer months of JulyAugust 2001 and June-July 2001, when aerosol acidity concentrations are the highest. Because the enhancement of SOA may actually become significant at acidities below our chosen threshold, we report the \% reduction in average OC as a function of the assumed $\left(\mathrm{H}^{+}\right)^{*}$. Table 3 shows the assumed $\left(\mathrm{H}^{+}\right)^{*}$ and the percentile to which it corresponds 
in the distribution of observed $\mathrm{H}^{+}$concentrations and the calculated percent reduction in annual average OC. The percent reduction is calculated as $\left(1-\mathrm{OC}^{\prime} /\left(\mathrm{OC}_{\mathrm{p}}+\mathrm{SOA}_{0}\right)\right) \times$ $100 \%$ and averaged for the entire study period; the mean and standard deviation of this value is reported. The threshold of $0.025 \mu \mathrm{g} / \mathrm{m}^{3}$ initially selected corresponds to the 96th percentile of the $\mathrm{H}^{+}$distribution, indicating that only in $4 \%$ of our observations, $\mathrm{H}^{+}$concentrations exceeded this threshold. In the extreme case where SOA is reduced during all periods in which the acidity was nonzero (corresponding to the 0th percentile), the overall OC reduction would conservatively be estimated at $5.1 \pm 7.9 \%$, which is only slightly higher than that predicted by the regression model.

\section{Discussion}

We examined the changes observed in OC/EC, acidity, and wind direction from July 2001 to July 2002 using time-series plots and using scatter plots of changes in OC/EC and acidity conditioned on changes in wind direction. Based on this analysis, we observed 104 cases in which the acidity increased noticeably; 22 of which showed possible indications of acidcatalyzed chemistry and 38 of which showed evidence of either acid-catalyzed chemistry or transport and mixing. Chu (17) sites an episode on July 18th, 2001, in which high concentrations of sulfate not fully neutralized by ammonia were accompanied by low UV intensity and high OC concentrations in the Eastern U.S., suggesting acid-catalyzed SOA formation as a possible explanation for this observation. We also observed concurrent increases in acidic aerosols and organic aerosol concentrations at the PAQS monitoring site, but this was a rare phenomenon during our study period. Furthermore, examination of different metrics of acidity and OC/EC ratios showed at most an overall correlation of -0.28 . The implication from this study is that acid-catalyzed SOA is not occurring most of the time, or that the "effect size" of acid-catalyzed SOA formation is not large enough to be observed consistently above the noise of other mechanisms. These mechanisms may include the combination of transport and mixing of primary emissions that can contribute to changes in OC/EC in addition to that caused by SOA formation and the net influence of uncertainties in our measurements.

There are many factors that could contribute to the measurement uncertainty and error, some which are more random and others which confer a systematic bias. One possible error of the latter type is the potential for conversion of polymeric products to light-absorbing carbon which would be misclassified as EC; this could underestimate the contribution of SOA to the OC/EC ratio. However, the peak temperature in the fourth helium mode used by the in-situ carbon analyzer to analyze the samples used in this analysis was $870^{\circ} \mathrm{C}$-this protocol was found to under-report the EC concentration compared to other methods for laboratorygenerated levoglucosan and $\alpha$-pinene SOA samples, possibly through the premature evolution of EC (44). Thus, there may be measurement error resulting from the operational definition of the OC-EC split and the analysis protocol used for the study, but this error may not necessarily bias the OC/EC ratio to be systematically low in the presence of SOA.

The question remains whether this observation is specific to the monitoring site, or if it can be extended to regions outside of Pittsburgh. Stanier et al. (45) observed that nucleation events were regional in nature, which implies that $\mathrm{H}_{2} \mathrm{SO}_{4}$, the main component involved in nucleation of new aerosols in this region (46) and also the primary source of inorganic aerosol acidity, is also generated regionally. During the summer when SOA concentrations are higher (23), the OC concentration is also spatially homogeneous (32), suggesting that primary OC and SOA formation is regionally distributed. Therefore, we expect that our conclu- sion can be extended to the Western Pennsylvania region and that acid-enhancement of SOA would also be unobservable in the area.

Assuming that acid-catalyzed SOA formation has occurred within the noise of our signal, we estimated possible reductions in OC concentrations that might result from a reduction in inorganic acidity using two methods. One was an empirical approach that estimated the possible response of $\mathrm{OC} / \mathrm{EC}$ to $\mathrm{H}^{+}$from field measurements, and the other method was based on preliminary information from laboratory experiments. The regression model, which is the empirical approach, is by design constructed to return an expected value for its predictions. The regression model suffers from the inherent assumption that the relationship derived between $\mathrm{OC} / \mathrm{EC}$ and $\mathrm{H}^{+}$is causal; there may be confounding factors that cause these two variables to show a link that may not exist directly. Therefore, the model cannot tell us that reducing $\mathrm{H}^{+}$will actually reduce the OC concentration. However, if the link is indeed causal, then the expected reduction in OC might be less than $6 \%$, assuming the values of other variables remain unaltered. The second approach is based on laboratory measurements, as the magnitude of SOA enhancement was obtained from laboratory studies examining the effect of inorganic acid aerosols on increases in SOA formation. The second approach returns a more conservative estimate as we chose an upper bound on acid-SOA enhancement observed in the laboratory to calculate the effect of $\mathrm{H}^{+}$reduction on OC. However, the functional dependence of SOA enhancement on acidity had to be assumed, as the actual relationship is not well-known. Furthermore, the SOA estimates used for the calculations have high uncertainty, as a constant OC/EC ratio for primary emissions during all periods was assumed for its calculation (23). However, the methods by which we calculated the response of $\mathrm{OC}$ to $\mathrm{H}^{+}$are rather conservative; despite the high uncertainties in our estimates, the benefit of reducing the inorganic acidity entirely appears to be a reduction in OC concentrations by less than $10 \%$. Recently, Jang et al. (43) have developed more sophisticated models that predict aerosol yields as a result of interaction of carbonyls and free hydrogen, but their model requires carbonyl-specific parameters-in contrast, our calculation is kept sufficiently simple and not specific to any compound such that an estimate of the upper bound for the acid-catalysis effect is captured.

FTIR and mass spectrometry data indicate that polymeric products in atmospheric aerosols appear to be more ubiquitous than initially speculated $(3,15)$, even under the absence of inorganic acidity $(15,16)$. However, Gao et al. (42) also found that increasing seed aerosol acidity results in more rapid polymerization, which may lead to higher SOA yields during certain periods. Thus, how changes in the inorganic aerosol acidity will affect OC concentrations remains an important question. We have shown that for Pittsburgh, this effect may be small, but such conclusions are dependent on the availability of atmospheric acidity and also the contribution of SOA to the total organic aerosol in each location. For the Pittsburgh region, SOA contributes to $\sim 35 \%$ (to a maximum of $50 \%$ ) of organic aerosol (23); Lim and Turpin (47) found that SOA can account for $\sim 46 \%$ of OC in Atlanta, with contributions as high as $88 \%$ during certain periods. As such, the effect of perturbing inorganic acidity may have comparable or larger effects on the OC concentrations in Atlanta. On the other hand, Strader et al. (48) reported that most of the OC in San Joaquin Valley is primary (SOA $<20 \%$ of OC), so reducing the occurrence of acid-catalyzed SOA is likely to have a smaller effect on PM concentrations in that region. Furthermore, the source contribution of inorganic aerosol acidity is different according to region and season. For instance, in the eastern U.S. [where the highest con- 
centrations of aerosol acidity are found (8), sulfate is regionally distributed and is the primary source of inorganic acidity during the summer, with increasing contributions from local production of $\mathrm{HNO}_{3}$ approaching winter. The inorganic acidity in urban regions in southern California, however, originates mainly from vehicular emissions that result in the production of $\mathrm{HNO}_{3}$, but $\mathrm{NH}_{3}$ is also available in sufficient quantities such that much of the acidity is neutralized. As such, the implications of the interaction between inorganic and organic components of the atmospheric aerosol should be evaluated separately for each location.

\section{Acknowledgments}

This research was conducted as part of the Pittsburgh Air Quality Study, which was supported by U.S. Environmental Protection Agency under contract R82806101 and the U.S. Department of Energy National Energy Technology Laboratory under contract DE-FC26-01NT41017. This paper has not been subject to EPA's peer and policy review and therefore does not necessarily reflect the views of the Agency. No official endorsement should be inferred. The authors thank the NOAA Air Resources Laboratory for making the HYSPLIT_4 model available (http://www.arl.noaa.gov/) for back-trajectory calculations. This paper also benefited from the contributions of Mitchell J. Small and Allen L. Robinson.

\section{Literature Cited}

(1) Seinfeld, J. H.; Pandis, S. N. Atmospheric Chemistry and Physics; John Wiley \& Sons: New York, 1998.

(2) Seinfeld, J. H.; Pankow, J. F. Organic atmospheric particulate material. Аnnu. Rev. Phys. Chem. 2003, 54, 121-140.

(3) Jang, M. S.; Kamens, R. M. Atmospheric secondary aerosol formation by heterogeneous reactions of aldehydes in the presence of a sulfuric acid aerosol catalyst. Environ. Sci. Technol. 2001, 35, 4758-4766.

(4) Jang, M. S.; Czoschke, N. M.; Lee, S.; Kamens, R. M. Heterogeneous atmospheric aerosol production by acid-catalyzed particle-phase reactions. Science 2002, 298, 814-817.

(5) Brosset, C.; Ferm, M. Man-Made Airborne Acidity and Its Determination. Atmos. Environ. 1978, 12, 909-916.

(6) Koutrakis, P.; Wolfson, J. M.; Spengler, J. D. An Improved Method for Measuring Aerosol Strong Acidity - Results from a 9-Month Study in St-Louis, Missouri and Kingston, Tennessee. Atmos. Environ. 1988, 22, 157-162.

(7) Tanner, R. L.; Leaderer, B. P.; Spengler, J. D. Acidity of Atmospheric Aerosols. Environ. Sci. Technol. 1981, 15, 11501153.

(8) Spengler, J. D.; Brauer, M.; Koutrakis, P. Acid Air and Health. Environ. Sci. Technol. 1990, 24, 946-956.

(9) Saxena, P.; Mueller, P. K.; Kim, Y. P.; Seinfeld, J. H.; Koutrakis, P. Coupling Thermodynamic Theory with Measurements to Characterize Acidity of Atmospheric Particles. Aerosol Sci. Technol. 1993, 19, 279-293.

(10) Inuma, Y.; Boge, O.; Gnauk, T.; Herrmann, H. Aerosol-chamber study of the alpha-pinene/O-3 reaction: influence of particle acidity on aerosol yields and products. Atmos. Environ. 2004, 38, 761-773.

(11) Jang, M. S.; Carroll, B.; Chandramouli, B.; Kamens, R. M. Particle growth by acid-catalyzed heterogeneous reactions of organic carbonyls on preexisting aerosols. Environ. Sci. Technol. 2003, $37,3828-3837$.

(12) Jang, M.; Lee, S.; Kamens, R. M. Organic aerosol growth by acid-catalyzed heterogeneous reactions of octanal in a flow reactor. Atmos. Environ. 2003, 37, 2125-2138.

(13) Limbeck, A.; Kulmala, M.; Puxbaum, H. Secondary organic aerosol formation in the atmosphere via heterogeneous reaction of gaseous isoprene on acidic particles. Geophys. Res. Lett. 2003, 30.

(14) Tolocka, M. P.; Jang, M.; Ginter, J. M.; Cox, F. J.; Kamens, R. M.; Johnston, M. V. Formation of oligomers in secondary organic aerosol. Environ. Sci. Technol. 2004, 38, 1428-1434.

(15) Gao, S.; Ng, N. L.; Keywood, M.; Varutbangkul, V.; Bahreini, R.; Nenes, A.; He, J. W.; Yoo, K. Y.; Beauchamp, J. L.; Hodyss, R. P.; Flagan, R. C.; Seinfeld, J. H. Particle phase acidity and oligomer formation in secondary organic aerosol. Environ. Sci. Technol. 2004, 38, 6582-6589.
(16) Kalberer, M.; Paulsen, D.; Sax, M.; Steinbacher, M.; Dommen, J.; Prevot, A. S. H.; Fisseha, R.; Weingartner, E.; Frankevich, V.; Zenobi, R.; Baltensperger, U. Identification of polymers as major components of atmospheric organic aerosols. Science 2004, 303, $1659-1662$.

(17) Chu, S. H. PM2.5 episodes as observed in the speciation trends network. Atmos. Environ. 2004, 38, 5237-5246.

(18) Zhang, Q.; Canagaratna, M. R.; Jayne, J. T.; Worsnop, D. R.; Jimenez, J. L. Time- and size-resolved chemical composition of submicron particles in Pittsburgh: Implications for aerosol sources and processes. J. Geophys. Res.-Atmos. 2005, 110.

(19) Zhang, Q.; Stanier, C. O.; Canagaratna, M. R.; Jayne, J. T.; Worsnop, D. R.; Pandis, S. N.; Jimenez, J. L. Insights into the chemistry of new particle formation and growth events in Pittsburgh based on aerosol mass spectrometry. Environ. Sci. Technol. 2004, 38, 4797-4809.

(20) Rees, S. L.; Robinson, A. L.; Khlystov, A.; Stanier, C. O.; Pandis, S. N. Mass balance closure and the federal reference method for PM2.5 in Pittsburgh, Pennsylvania. Atmos. Environ. 2004, 38, 3305-3318.

(21) Wittig, A. E.; Anderson, N.; Khlystov, A. Y.; Pandis, S. N.; Davidson, C.; Robinson, A. L. Pittsburgh air quality study overview. Atmos. Environ. 2004, 38, 3107-3125.

(22) Turpin, B. J.; Saxena, P.; Andrews, E. Measuring and simulating particulate organics in the atmosphere: problems and prospects. Atmos. Environ. 2000, 34, 2983-3013.

(23) Cabada, J. C.; Pandis, S. N.; Subramanian, R.; Robinson, A. L. Polidori, A.; Turpin, B. Estimating the secondary organic aerosol contribution to PM2.5 using the EC tracer method. Aerosol Sci. Technol. 2004, 38, 140-155.

(24) Subramanian, R.; Khlystov, A. Y.; Cabada, J. C.; Robinson, A. L. Positive and negative artifacts in particulate organic carbon measurements with denuded and undenuded sampler configurations. Aerosol Sci. Technol. 2004, 38, 27-48.

(25) Ansari, A. S.; Pandis, S. N. Prediction of multicomponent inorganic atmospheric aerosol behavior. Atmos. Environ. 1999 $33,745-757$.

(26) Khlystov, A.; Wyers, G. P.; Slanina, J. The Steam-Jet Aerosol Collector. Atmos. Environ. 1995, 29, 2229-2234.

(27) Jaffrezo, J. L.; Calas, T.; Bouchet, M. Carboxylic acids measurements with ionic chromatography. Atmos. Environ. 1998, 32, $2705-2708$.

(28) Stolzenburg, M. R.; Hering, S. V. Method for the automated measurement of fine particle nitrate in the atmosphere. Environ. Sci. Technol. 2000, 34, 907-914.

(29) Wittig, A. E.; Takahama, S.; Khlystov, A. Y.; Pandis, S. N.; Hering S.; Kirby, B.; Davidson, C. Semi-continuous PM2.5 inorganic composition measurements during the Pittsburgh air quality study. Atmos. Environ. 2004, 38, 3201-3213.

(30) Takahama, S.; Wittig, A. E.; Vayenas, D. V.; Davidson, C. I.; Pandis, $\mathrm{S}$. N. Modeling the diurnal variation of nitrate during the Pittsburgh Air Quality Study. J. Geophys. Res.-Atmos. 2004, 109.

(31) Khlystov, A.; Stanier, C. O.; Takahama, S.; Pandis, S. N. Water content of ambient aerosol during the Pittsburgh Air Quality Study. J. Geophys. Res. 2005, 110.

(32) Tang, W.; Raymond, T.; Wittig, B.; Davidson, C.; Pandis, S.; Robinson, A.; Crist, K. Spatial variations of PM2.5 during the Pittsburgh air quality study. Aerosol Sci. Technol. 2004, 38, 8090.

(33) Vayenas, D. V.; Takahama, S.; Davidson, C. I.; Pandis, S. N. Simulation of the Thermodynamics and Removal Processes in the Sulfate-Ammonia-Nitric Acid System During Winter: Implications for PM2.5 Control Strategies. J. Geophys. Res. 2005, in press.

(34) Cocker, D. R.; Clegg, S. L.; Flagan, R. C.; Seinfeld, J. H. The effect of water on gas-particle partitioning of secondary organic aerosol. Part I: alpha-pinene/ozone system. Atmos. Environ. 2001, 35, 6049-6072.

(35) Ott, W. R.; Mage, D. T. Measuring Air-Quality Levels Inexpensively at Multiple Locations by Random Sampling. J. Air Pollut. Control Assoc. 1981, 31, 365-369.

(36) Czoschke, N. M.; Jang, M.; Kamens, R. M. Effect of acidic seed on biogenic secondary organic aerosol growth. Atmos. Environ. 2003, 37, 4287-4299.

(37) Wexler, A. S.; Clegg, S. L. Atmospheric aerosol models for systems including the ions $\mathrm{H}+, \mathrm{NH} 4+$, $\mathrm{Na}+$, SO42-, NO3-, $\mathrm{Cl}-$, , Br-, and H2O. J. Geophys. Res.-Atmos. 2002, 107.

(38) Milionis, A. E.; Davies, T. D. Regression and Stochastic-Models for Air-Pollution.1. Review, Comments and Suggestions. Atmos. Environ. 1994, 28, 2801-2810.

(39) Breusch, T. S. Testing for Autocorrelation in Dynamic LinearModels. Aust. Econ. Pap. 1978, 17, 334-355. 
(40) Godfrey, L. G. Testing against General Autoregressive and Moving Average Error Models When Regressors Include Lagged Dependent Variables. Econometrica 1978, 46, 1293-1301.

(41) Judge, G. G.; Griffiths, W. E.; Hill, R. C.; Hutkepohl, H.; Lee, T. C. The Theory and Practice of Econometrics, 2nd ed.; John Wiley \& Sons: New York, 1985.

(42) Gao, S.; Keywood, M.; Ng, N. L.; Surratt, J.; Varutbangkul, V.; Bahreini, R.; Flagan, R. C.; Seinfeld, J. H. Low-molecular-weight and oligomeric components in secondary organic aerosol from the ozonolysis of cycloalkenes and alpha-pinene. J. Phys. Chem. A 2004, 108, 10147-10164.

(43) Jang, M. S.; Czoschke, N. M.; Northcross, A. L. Semiempirical model for organic aerosol growth by acid-catalyzed heterogeneous reactions of organic carbonyls. Environ. Sci. Technol. 2005, 39, 164-174.

(44) Subramanian, R.; Khlystov, A.; Robinson, A. Effect of peak intertmode temperature on ambient Elemental Carbon measured using the Thermal-Optical Transmittance. 2005, submitted for publication.
(45) Stanier, C. O.; Khlystov, A. Y.; Pandis, S. N. Nucleation events during the Pittsburgh air quality study: Description and relation to key meteorological, gas phase, and aerosol parameters. Aerosol Sci. Technol. 2004, 38, 253-264.

(46) Gaydos, T. M.; Stanier, C. O.; Pandis, S. N. Modeling of in situ ultrafine atmospheric particle formation in the eastern United States. J. Geophys. Res.-Atmos. 2005, 110.

(47) Lim, H. J.; Turpin, B. J. Origins of primary and secondary organic aerosol in Atlanta: Results' of time-resolved measurements during the Atlanta supersite experiment. Environ. Sci. Technol. 2002, 36, 4489-4496.

(48) Strader, R.; Lurmann, F.; Pandis, S. N. Evaluation of secondary organic aerosol formation in winter. Atmos. Environ. 1999, 33, $4849-4863$.

Received for review May 4, 2005. Revised manuscript received October 10, 2005. Accepted January 26, 2006.

ES050856+ 


\title{
Modeling the diurnal variation of nitrate during the Pittsburgh Air Quality Study
}

\author{
Satoshi Takahama, ${ }^{1}$ Ann E. Wittig, ${ }^{2}$ Dimitris V. Vayenas, ${ }^{3}$ Cliff I. Davidson, ${ }^{4,5}$ \\ and Spyros N. Pandis ${ }^{1,5}$
}

Received 11 September 2003; revised 9 January 2004; accepted 5 February 2004; published 21 July 2004.

[1] A thermodynamic model, the Gibbs Free-Energy Minimization model (GFEMN), was used to simulate the partitioning of $\mathrm{PM}_{2.5}$ nitrate aerosol and nitric acid using highly timeresolved inorganic measurements collected at the Pittsburgh Air Quality Study during July 2001 and January 2002. Model results were evaluated using independent, high time resolution measurements of aerosol nitrate. The mean observed concentration in July was $0.6 \mu \mathrm{g} / \mathrm{m}^{3}$ and $2.1 \mu \mathrm{g} / \mathrm{m}^{3}$ in January. Model predictions were in agreement with the observations within $0.5 \mu \mathrm{g} / \mathrm{m}^{3}$ on average, with measurement uncertainties often accounting for these discrepancies. The simulations were run assuming particles were liquid in July for all relative humidities (RHs) and solid below $60 \%$ RH in January. For both seasons the assumed physical state did not influence considerably the overall agreement with observations. The assumption of particle mixing state did appear to influence model error; however, assuming that particles were externally mixed during low RH periods in July improved agreement significantly. The exceptional sensitivity of predicted aerosol nitrate to ammonia in western Pennsylvania suggests that reductions in $\mathrm{PM}_{2.5}$ may be assisted by reductions in ammonia emissions. INDEX TERMS: 0305 Atmospheric Composition and Structure: Aerosols and particles $(0345,4801)$; 0345 Atmospheric Composition and Structure: Pollution - urban and regional (0305); 0365 Atmospheric Composition and Structure: Troposphere - composition and chemistry; KEYWORDS: aerosol, nitrate, Pittsburgh Air Quality Study

Citation: Takahama, S., A. E. Wittig, D. V. Vayenas, C. I. Davidson, and S. N. Pandis (2004), Modeling the diurnal variation of nitrate during the Pittsburgh Air Quality Study, J. Geophys. Res., 109, D16S06, doi:10.1029/2003JD004149.

\section{Introduction}

[2] Fine particles in the atmosphere are composed of sulfate, ammonium, nitrate, elemental carbon, organic material, trace metals, crustal elements, and water [Seinfeld and Pandis, 1998]. These aerosols are either primary or secondary in nature, depending on whether they are directly emitted from sources or formed from gas-to-particle conversion processes in the atmosphere. Airborne particulate matter (PM) with aerodynamic diameters less than $2.5 \mu \mathrm{m}$ has been associated with excess mortality [Dockery et al., 1993] and a key component of global change [Seinfeld and Pandis, 1998]. To address these health concerns, EPA has set guidelines on allowable $\mathrm{PM}_{2.5}$ levels based on a mass standard. Understanding the partitioning behavior of semi-

\footnotetext{
${ }^{1}$ Department of Chemical Engineering, Carnegie Mellon University, Pittsburgh, Pennsylvania, USA.

${ }^{2}$ Department of Civil Engineering, City College of New York, New York, USA

${ }^{3}$ Department of Environmental and Natural Resources Management, University of Ioannina, Agrinio, Greece.

${ }^{4}$ Department of Civil and Environmental Engineering, Carnegie Mellon University, Pittsburgh, Pennsylvania, USA.

${ }^{5}$ Department of Engineering and Public Policy, Carnegie Mellon University, Pittsburgh, Pennsylvania, USA.
}

Copyright 2004 by the American Geophysical Union. 0148-0227/04/2003JD004149\$09.00 volatile species between the gas and aerosol phases can help us predict how changes in anthropogenic and biogenic activity will influence formation of aerosols in the atmosphere such that desired goals in particulate matter concentrations can be achieved.

[3] In the eastern United States, $\mathrm{PM}_{2.5}$ particles can be $50 \%$ or more inorganic, with sulfate constituting a significant portion of this inorganic fraction [U.S. Environmental Protection Agency, 1996]. Because sulfate is an oxidation product of $\mathrm{SO}_{2}$, current strategies are targeted at reducing $\mathrm{SO}_{2}$ emissions. However, West et al. [1999] have shown that for the eastern United States, reductions in sulfate may not be as effective as it is often assumed in reducing PM mass, as such reductions may lead to the formation of more aerosol nitrate. This behavior is fairly well understood qualitatively: a portion of atmospheric ammonia is bound in ammonium sulfate, and reductions in sulfate concentrations result in more free ammonia available for reaction with nitric acid to produce ammonium nitrate particles. Such nonlinear chemical interactions between gases and particles suggest the need for a careful investigation of alternative PM control strategies. Evaluation and development of mathematical tools to describe such atmospheric interactions will allow us to examine PM response to changes in concentrations of precursor species, as demonstrated in the work of Ansari and Pandis [1998]. 
[4] One common approach to modeling the partitioning behavior of semivolatile PM components is to assume that the particles are in equilibrium with the gas phase. Several thermodynamic models have been developed for atmospheric simulations [Bassett and Seinfeld, 1983; Saxena et al., 1986; Pilinis and Seinfeld, 1987; Wexler and Seinfeld, 1991; Kim et al., 1993; Meng et al., 1995; Jacobson et al., 1996; Nenes et al., 1999; Ansari and Pandis, 1999]. Generally, these equilibrium models assume that particles can be modeled as a "bulk" phase without consideration for the thermodynamic differences of the particle subpopulations across size and time dimensions. Often, data provided as inputs for these thermodynamic simulations are aggregated measurements obtained through filter and denuderbased sampling methods, which have poor size and time resolution.

[5] To examine the thermodynamic predictions of semivolatile inorganics when particles are not assumed to be the same over all size ranges, Jacobson [1999] and Moya et al. [2002] used a size-resolved chemical equilibrium approach and observed that their model adequately reproduced the size distribution of $\mathrm{PM}_{1}$ nitrate and ammonium measured in southern California. However, these measurements were available only for 24-hour periods. Moya et al. [2001] suggested that in addition to temperature and relative humidity, performance of equilibrium models may be improved by high time resolution measurements.

[6] New semicontinuous instruments at the Pittsburgh Air Quality Study (PAQS) [Wittig et al., 2004a] provided such high time resolution measurements, and this paper will focus on evaluating the performance of a thermodynamic model in predicting nitrate partitioning between the fine particulate matter $\left(\mathrm{PM}_{2.5}\right)$ and the gas phase using these time-resolved data.

\section{Experimental Method}

[7] Inorganic species concentrations used for thermodynamic modeling were collected with semicontinuous instruments employed during the Pittsburgh Air Quality Study (PAQS). $\mathrm{PM}_{2.5}$ sulfate and nitrate aerosol concentrations were measured with Rupprecht and Patashnick (R\&P) models $8400 \mathrm{~S}$ and $8400 \mathrm{~N}$, respectively, which provided resolutions as high as 10 -minutes. For the purposes of this study, the data were averaged to one and two-hour values. Instrument performance in the PAQS is documented in the work of Wittig et al. [2004b]. A steam sampler based on the design of Khlystov et al. [1995] was also used to collect $\mathrm{PM}_{2.5}$ sulfate, total $\left(\mathrm{PM}_{2.5}+\right.$ gas) nitrate $(\mathrm{TN})$, and total $\left(\mathrm{PM}_{2.5}+\right.$ gas) ammonia (TA) for analysis by ion chromatography for one-hour periods in July 2001 and two-hour periods until the end of PAQS.

[8] Additionally, several filter-based instruments provided reference measurements at the site. A Particle Concentrator Brigham Young University Organic Sampling System (PC-BOSS) [Eatough et al., 1999] provided four and sixhour measurements of $\mathrm{PM}_{2.5}$ sulfate and nitrate during July 2001. Another filter-based inorganic speciation sampler at the site supplied four and six-hour measurements in July 2001 and 24-hour measurements from August 2001 to July 2002. This speciation sampler employed multiple filterpacks to collect $\mathrm{PM}_{2.5}$ and $\mathrm{PM}_{10}$ particles after the air stream was passed through a $\mathrm{MgO}$ denuder and citric-acid-coated denuder to remove nitric acid and ammonia gas, respectively. In addition, a separate filterpack was placed upstream of the denuders to measure $\mathrm{PM}_{10}$ and gases, and the nitric acid and ammonia concentrations were determined by denuder difference. Each filterpack consisted of a Teflon filter, a nylon filter, and a citric-acid impregnated cellulose fiber filter in series to measure both stable and semivolatile species. Teflon filters were analyzed for sulfate, nitrate, and ammonium, while nylon filters were used to quantify volatilized nitrate. Citric-acid impregnated filters were analyzed for volatilized ammonium. Analysis for all of these species was by ion chromatography using a method similar to that described by Jaffrezo et al. [1998]. Furthermore, EPA Speciation Network sites at Hazelwood and Lawrenceville, which were located within a $5-\mathrm{km}$ radius from the main site, provided additional 24-hour filter-based measurements of $\mathrm{PM}_{2.5}$ sulfate, nitrate, and ammonium.

[9] Instrument inter-comparisons indicated that filterbased measurements were generally in good agreement with each other, but there appeared to be analytical bias in measurements made by the semicontinuous instruments. Thus the reported concentrations from these instruments were calibrated by regression against the measurements made by the inorganic speciation sampler [Wittig et al., 2004b], which was operated for the entire duration of the study. For 3-5 January 2002, measurements of $\mathrm{PM}_{2.5}$ ammonium from Hazelwood and Lawrenceville were used to calibrate the total ammonia of the steam sampler due to possible instrument difficulty and the unavailability of inorganic speciation sampler measurements during that period.

[10] Instrument inter-comparisons also suggested that a measurement error on the order of $15 \%$ was present for concentrations of total sulfate (TS) measured with the steam sampler, after bias was removed, and similar errors of similar magnitude are expected for other concentrations measured by the steam sampler. Solomon et al. [2001] suggested that the error of the R\&P $8400 \mathrm{~N}$ is around $0.5 \mu \mathrm{g} / \mathrm{m}^{3}$. Though it is more likely that measurement errors are proportional to concentrations, nonetheless this value is considered an estimate of the average error.

[11] The semicontinuous data, after correction, were used for thermodynamic modeling because of their high time resolution. For model inputs, TA and TN measurements were obtained from the steam sampler, and sulfate measurements were averages of the steam sampler and R\&P 8400S for July 2001 and from the R\&P 8400S alone for January 2002. Independent, corrected semicontinuous measurements of aerosol nitrate from the R\&P $8400 \mathrm{~N}$ were used to evaluate the model's performance in predicting nitrate partitioning.

\section{Thermodynamic Modeling}

[12] The Gibbs Free-Energy Minimization model (GFEMN) developed by Ansari and Pandis [1999] is used to simulate the partitioning of ammonia and nitric acid, and the aerosol water concentration. Inputs into the thermodynamic model include temperature $(\mathrm{T})$ and relative humidity (RH), and concentrations of total ammonia (TA), nitrate (TN), and sulfate (TS). Since sulfuric acid has a very low 
vapor pressure at ambient $\mathrm{RH}, \mathrm{PM}_{2.5}$ sulfate concentrations were used for values of TS.

[13] We assume that: (1) equilibrium is achieved between gas and aerosol phases in the atmosphere, (2) all fine particles are thermodynamically similar and can be modeled as a single mixture, and (3) influence of organics on the thermodynamics of the inorganic PM is negligible. In addition, $\mathrm{PM}_{2.5}$ crustal elements were neglected in this study, as their concentrations were very low $\left(<0.2 \mu \mathrm{g} / \mathrm{m}^{3}\right.$ in nearly all cases) in Pittsburgh and had little impact on the results. Nitrate associated with coarse-mode particles is not included in the total nitrate, so the TN used as input into the model is the amount available to partition only between fine-mode particles and the gas phase.

[14] An additional input required for the model is whether the aerosols exist in a state of stable or metastable equilibrium (i.e. whether the aerosols lie on the deliquescence or efflorescence branch), which depends on their history of experienced relative humidities [Ansari and Pandis, 2000]. Experimental evidence suggests that during the summer of 2001, aerosols almost always contained water even at low relative humidities, while during the following winter the aerosols were dry at RH less than 60\% (A. Khlystov et al., Water content of ambient aerosol during the Pittsburgh Air Quality Study, submitted to Journal of Geophysical Research, 2004, hereinafter referred to as Khlystov et al., submitted manuscript, 2004). As such, for the base case simulations, July 2001 data were modeled under the assumption that aerosols were always liquid, while for simulations in January 2002 we assume that particles were liquid above and solid below $60 \% \mathrm{RH}$. Simulations for both months were repeated under the assumption that aerosols lie on the deliquescence branch and compared against the base case.

[15] An important assumption employed in thermodynamic models is that all particles are identical and internally mixed, meaning that the chemical species predicted to be in the aerosol phase interact with each other in the same particle. If the particle population is comprised of particles of different chemical composition (external mixture), the chemistry of nitrate partitioning can be changed significantly. The sensitivity of the aerosol nitrate concentration to the physical state of particles at low RH [Ansari and Pandis, 2000] allows us to investigate the validity of the internal mixture assumption during July. During the summer, the levels of ammonia were often insufficient to fully neutralize the sulfate to form ammonium sulfate, and therefore the particles were often acidic (molar ratios of TA to TS less than 2) on average. However, as described in a later section, there may have been periods when fully neutralized ammonium sulfate particles coexisted with acidic particles in an externally mixed state. If, for instance, both ammonium sulfate and ammonium bisulfate particles coexisted in the atmosphere, it is possible that during periods when the $\mathrm{RH}$ was below $40 \%$, ammonium sulfate would be found in its crystallized form whereas ammonium bisulfate would still exist as a liquid. If the particles are externally mixed, there are infinite combinations of particle composition that are consistent with the observed bulk concentrations. Here, we simulate one rather extreme case of external mixing using the following approach. We first run GFEMN with the original quantities of TA, TN, and TS as inputs, assuming

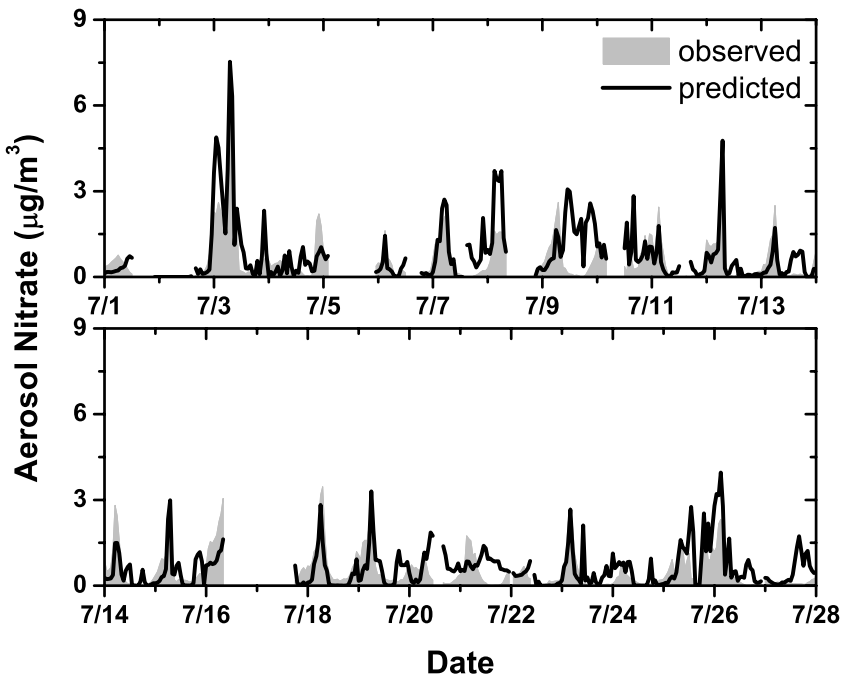

Figure 1. Time series of predicted and observed aerosol nitrate concentrations for July 2001. Missing data either in any of the inputs for the simulation or in the aerosol nitrate measurements are represented by gaps.

that the particles are solid, and calculate the quantity of ammonium sulfate that would form. Then, we remove the ammonium sulfate that has been formed and repeat the simulation with the remaining TN, TS, and TA assuming that the particles are liquid to estimate the amount of acidic aerosol that would form. The sum of aerosol nitrate concentrations associated with each type of particle approximates the total amount of aerosol nitrate that might be found in the aerosol phase if the hypothesized mixing state existed. This approach provides an estimate of a rather extreme case of external mixing in that only two types of particles are considered to exist in the system. The ability of GFEMN to reproduce the observed partitioning behavior in each case is evaluated.

\section{Results}

[16] The observed concentrations of aerosol nitrate in July were relatively low. The average observed concentration was $0.6 \mu \mathrm{g} / \mathrm{m}^{3}$ and reached a maximum of $6 \mu \mathrm{g} / \mathrm{m}^{3}$ on the morning of 3 July. The mean observed nitric acid concentration was $3.4 \mu \mathrm{g} / \mathrm{m}^{3}$ and $73 \%$ of the total nitrate was in the gas phase during July. Aerosol nitrate was observed to consistently peak in the late night and early morning hours when the temperatures were lower and relative humidities higher, but evaporated during the day when temperatures increased, and relative humidity dropped (Figure 1).

[17] Predictions from GFEMN follow the diurnal trend of aerosol nitrate quite well, except during a few daytime periods. During the afternoons of 9, 13, 20, 21, and 27 July, simulations predict that the aerosol nitrate concentrations were rising during the day, when observations indicate that they were close to zero. During these periods, RH was often below $40 \%$ and temperatures were generally above $20^{\circ} \mathrm{C}$ with thermodynamics favoring the existence of nitrate in the gas-phase. In a subsequent section we will test the hypothesis that the ambient aerosols during these periods existed as an external mixture of solid ammonium sulfate particles and 
Table 1. Model Performance Against $\mathrm{PM}_{2.5}$ Measurements at the PAQS for July 2001 and January 2002

\begin{tabular}{clcccc}
\hline Month & \multicolumn{1}{c}{ Scenario } & Observed, $\mu \mathrm{g} / \mathrm{m}^{3}$ & Predicted, $\mu \mathrm{g} / \mathrm{m}^{3}$ & Mean Bias, $\mu \mathrm{g} / \mathrm{m}^{3}$ & $\mathrm{Mean}$ Error, $\mu \mathrm{g} / \mathrm{m}^{3}$ \\
\hline July 2001 & base case & $0.56 \pm 0.73$ & $0.70 \pm 0.89$ & $0.13 \pm 0.67$ & $0.46 \pm 0.50$ \\
& deliquescence & & $0.32 \pm 0.84$ & $-0.25 \pm 0.59$ & $0.42 \pm 0.49$ \\
& external mixture & \multirow{2}{*}{$2.09 \pm 1.65$} & $0.64 \pm 0.89$ & $0.07 \pm 0.61$ & $0.41 \pm 0.46$ \\
January 2002 & base case & $2.15 \pm 1.89$ & $0.06 \pm 0.88$ & $0.64 \pm 0.60$ \\
& deliquescence & & $2.06 \pm 1.90$ & $-0.03 \pm 0.93$ & $0.67 \pm 0.64$ \\
\hline
\end{tabular}

wet, acidic particles. The model predictions for this month had an absolute error of $0.41 \mu \mathrm{g} / \mathrm{m}^{3}$ (Table 1) when the PM during periods with $\mathrm{RH}$ below $40 \%$ is modeled as an external mixture.

[18] The observed average aerosol nitrate concentration during January 2002 was around $2.1 \mu \mathrm{g} / \mathrm{m}^{3}$. As in July, the aerosol nitrate exhibited a diurnal variation with a late night or early morning peak and a minimum in the late afternoon. Figure 2 shows the comparison between predicted and observed aerosol nitrate concentrations for January 2002. In general, there was enough ammonia to neutralize both sulfate and nitrate. During a few periods, for example, 12, $15,16,20$, and 22 January, the model predicts more aerosol nitrate to be present than observed, though in many of these cases measurement uncertainties may account for the discrepancies. For the period 26 to 28 January, no aerosol nitrate is predicted to form, though observations indicate otherwise. The relative humidity was low during these days, and frequently dropped below $40 \%$ during the daytime. The concentrations of ammonia during these periods were such that after neutralizing the sulfate, there was not enough free ammonia remaining to react with the nitric acid vapor to form solid ammonium nitrate particles. Therefore, all of the total nitrate was predicted to remain in the gas phase as nitric acid vapor. Uncertainties and sensitivities of these predictions to measurement error will be discussed in a subsequent section. On average, the model predictions for this month had an absolute error of $0.64 \mu \mathrm{g} / \mathrm{m}^{3}$ (Table 1).

\subsection{Experimental Uncertainty}

[19] Both the predictions and the observations are affected by experimental error. For the predictions, measurement errors in the input variables (TS, TN, TA, T, and RH) affect directly the predicted nitrate. To show how each of these measurement uncertainties can combine to contribute to uncertainties in predictions, a Monte Carlo simulation was performed for two days in July and four days in January. A Latin Hypercube Sampling routine [McKay, 1988] was used considering measurement uncertainties in four of the five input variables (RH, TA, TN, and TS). Normal distributions were chosen for the input distribution of these variables, as uncertainties arising from measurement errors are generally considered to be independent and normally distributed [Ripley and Thompson, 1987] with a mean of zero. A coefficient of variation of 0.15 was assumed for the measured concentrations of TS, TA, and TN, in accordance with our assessment of measurement errors. In addition, a standard deviation of $5 \%$ was assumed for RH. To avoid generating artificial correlations among the different input variables and to allow the statistics (e.g., mean and variance) of the output distribution to converge, a sampling size of 200 was selected. Figure 3 shows the predictions with error bars corresponding to the 5th and 95th percentiles of the simulations' output cumulative distribution function. Combined uncertainties in model inputs can result in uncertain aerosol nitrate concentrations. Furthermore, a standard deviation of $0.5 \mu \mathrm{g} / \mathrm{m}^{3}$ was assumed for uncertainty in aerosol nitrate measurements from the R\&P $8400 \mathrm{~N}$ instrument as reported by Solomon et al. [2001], and 200 random samples for aerosol nitrate concentrations were generated for each period. The shaded band in Figure 3 illustrates the range between the 5 th and 95 th percentiles of the output cumulative distribution function, though concentrations below zero are not shown. In most cases the predictions are within the uncertainties introduced by measurement errors.

\subsection{Sensitivity to Assumed Physical State (Solid or Liquid) of Aerosol}

[20] In three-dimensional chemical transport models simulating atmospheric aerosol thermodynamics it is often assumed that the aerosol growth follows the deliquescence branch of the hysteresis curve [Nenes et al., 1999]. The error introduced by this assumption can be evaluated by comparing the base-case results (the physical state is an input based on the complementary measurements) to the deliquescence branch predictions.

[21] In the base-case January simulations we assumed that the particles were solid below $60 \%$ and aqueous solutions in all other cases, as was observed experimentally (Khlystov et al., submitted manuscript, 2004). The ammonium nitrate deliquescence relative humidity is approxi-

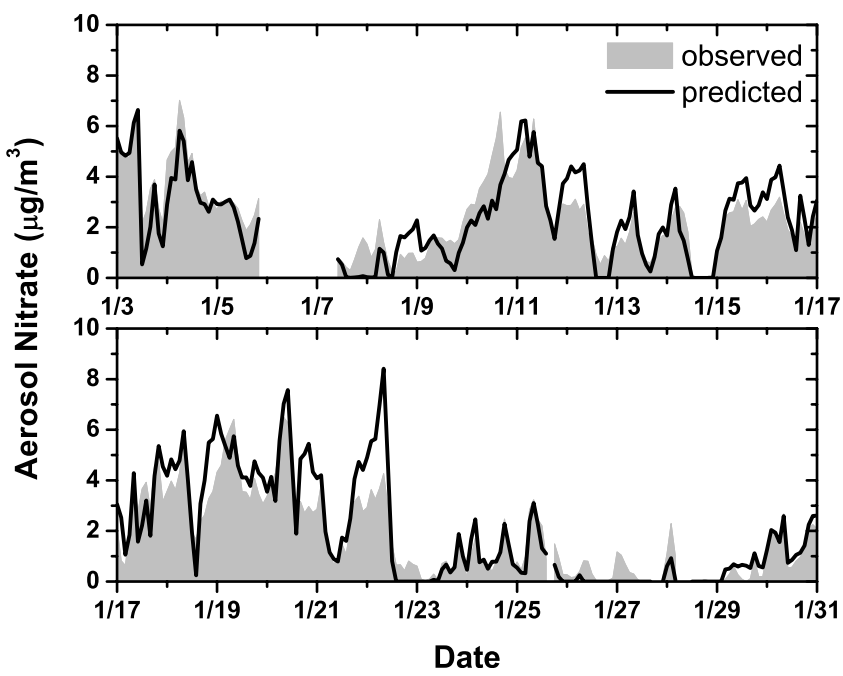

Figure 2. Time series of predicted and observed aerosol nitrate concentrations for January 2002. Missing data either in any of the inputs for the simulation or in the aerosol nitrate measurements are represented by gaps. 

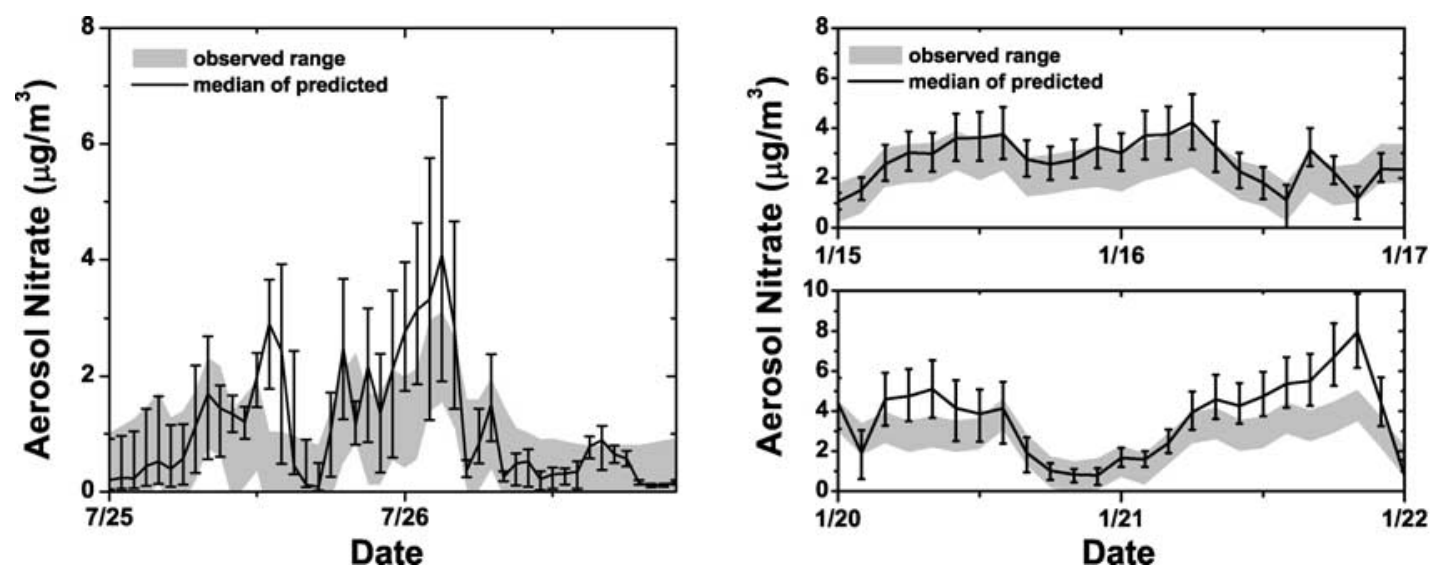

Figure 3. Results from Monte Carlo simulations performed for selected periods in July 2001 and January 2002. Error bars extend to the 5th and 95th percentiles of the cumulative distribution function associated with each prediction. The shaded area bounds the interval between the 5 th and 95 th percentiles of the observed aerosol nitrate cumulative distribution functions, although concentrations below zero are not shown.

mately $60 \%$, so this choice corresponds approximately to the deliquescence branch of the growth curve of an aerosol containing ammonium nitrate. If deliquescence behavior was assumed for January 2002 with GFEMN calculating the deliquescence $\mathrm{RH}$ for each case, particles were predicted to be practically always solid below $66 \% \mathrm{RH}$ (142 cases), while particles were predicted to be always liquid above $80 \%$ RH (79 cases). Out of the 103 remaining cases, the model predicted $57 \%$ to be solid, $18 \%$ liquid, and $25 \%$ a mixture of both. In these instances when the physical state of the particle predicted by deliquescence was different from the base case, the relative difference in mean aerosol nitrate predictions was around $20 \%$. These differences did not have much impact on the overall agreement of predictions and observations of nitrate partitioning, however. Figure 4 shows the model error, defined as the difference between the predicted and observed concentrations of aerosol nitrate, for the 120 cases when the observed and predicted particle states differed: the overall error between the base case and the deliquescence case simulations is not considerably different (Table 1). This indicates that the model's performance using the deliquescence branch of the growth curve is satisfactory during the winter.

[22] For the base case for July 2001, we assumed that particles were liquid at all relative humidities. If deliquescence behavior was assumed for this month, however,
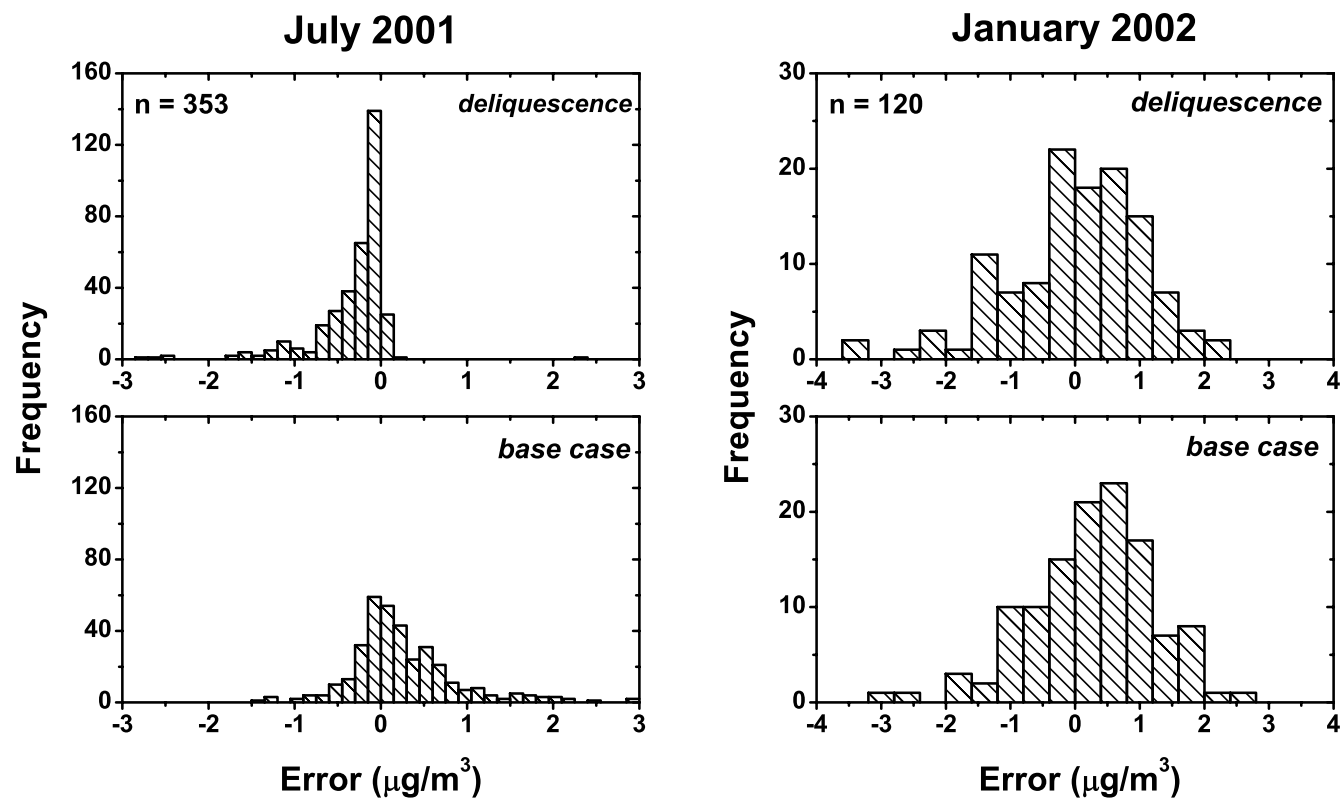

Figure 4. Error distribution assuming different states of particles in July 2001 and January 2002. Errors are calculated as predicted minus observed values of aerosol nitrate. Points included are only those for which the predicted solid or liquid state was different from the one determined based on in situ aerosol water measurements (base case). 


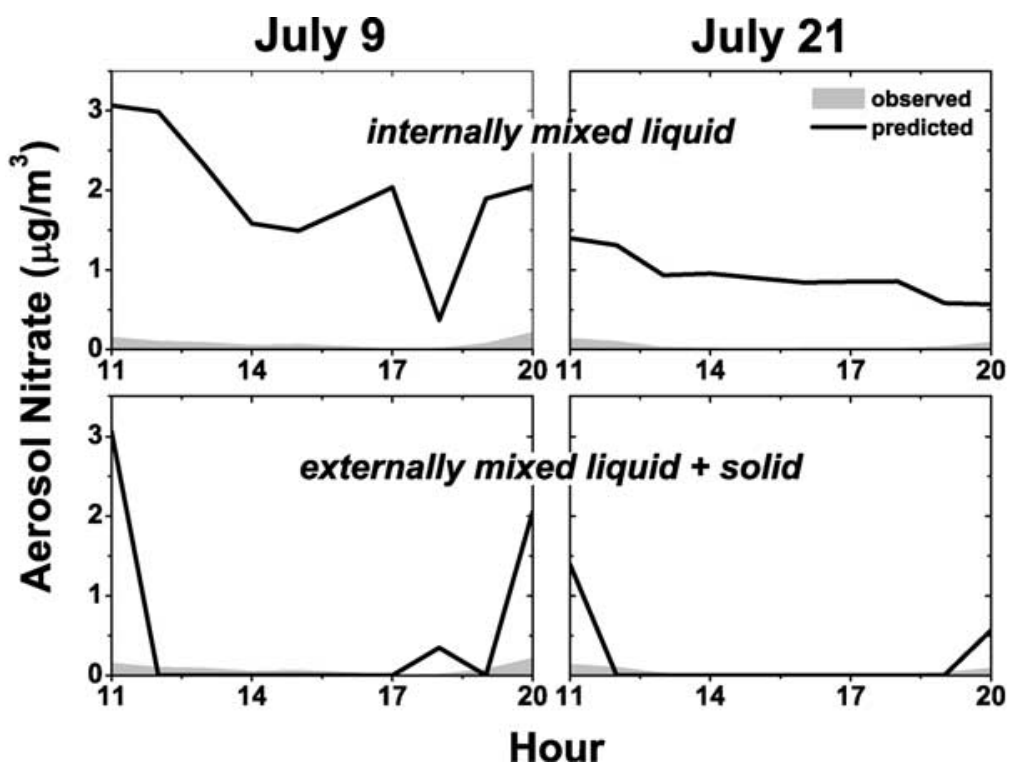

Figure 5. Simulations for 9 and 21 July assuming that particles are (1) internally mixed liquid aerosols, and (2) an external mixture of crystallized ammonium sulfate and wet acidic aerosols when the relative humidity is below $40 \%$.

particles were always predicted to be liquid above $80 \% \mathrm{RH}$ (127 cases), but below this RH, 15\% were predicted to be liquid, $72 \%$ solid, and $13 \%$ a mixture of both (415 cases altogether). In the instances when the physical state of the particle differed between the deliquescence and base case, the relative mean difference in nitrate predictions was almost a factor of two (Table 1). This is consistent with the results of Ansari and Pandis [2000] who suggested that the physical state of the particles affects significantly the aerosol nitrate concentration at low nitrate concentration levels. Figure 4 shows a comparison of the model error for the 353 points when the aerosol is predicted to be solid or a mixture of solid or liquid instead of a pure liquid. The deliquescence case shows a large number of observations have errors close to zero $\mu \mathrm{g} / \mathrm{m}^{3}$ with an overall mean of $-0.32 \mu \mathrm{g} / \mathrm{m}^{3}$, but a consistent underprediction is observed. This underprediction can be explained by the fact that no nitrate was predicted to be in the aerosol phase in 314 out of the 353 simulations for the deliquescence case, while the same was true for only one instance during the same period when particles were simulated as pure liquid. The spread in the error is greater for the base case, but the distribution of these errors is centered about a mean of $0.25 \mu \mathrm{g} / \mathrm{m}^{3}$.

[23] The assumed physical state affected the relative difference in predictions of aerosol nitrate concentrations during July more so than during January because the nitrate concentrations were generally lower during the summer. Prior knowledge of the physical state of the aerosol was a lot more important for the summer months than in the winter (Table 1).

\subsection{Sensitivity to Mixing State (Internal Versus External) During Periods of Acid PM}

[24] We examine here the possibility that the discrepancies during the low RH acidity periods in July were due to the existence of an external mixture of acid and neutral particles. The crystallization $\mathrm{RH}$ for ammonium sulfate is
$40 \%$ and the crystallization $\mathrm{RH}$ for ammonium bisulfate is much lower. As a result the aerosol could consist of solid neutral particles (ammonium sulfate) and liquid acidic particles (ammonium bisulfate) when the RH dropped below $40 \%$. We first examine the impact of this assumption on the nitrate partitioning on the afternoons of 9 and 21 July, when the disagreement between predicted and observed aerosol nitrate is particularly pronounced in the base case. Figure 5 shows that if the particles are modeled as an internally mixed population, the aerosol nitrate concentration is predicted to be $1-2 \mu \mathrm{g} / \mathrm{m}^{3}$ while observations indicate that it is close to zero in the afternoon. However, assuming that the particles are externally mixed and using the algorithm described in the previous section, the predicted aerosol nitrate concentration drops to practically zero, as observed, when the measured $\mathrm{RH}$ drops below $40 \%$ between noon and $7 \mathrm{pm}$ on these two days. Because the RH threshold below which we simulate the particles as an external mixture is set at $40 \%$, the selection of these periods is very sensitive to measurement errors in RH. Figure 6 shows that if a similar approach is used for the other periods in July in which the measured RH fell below $40 \%$, the performance of the model improves significantly during these times. The premise of hypothesizing the existence of this specific external mixture during these low RH periods is that in the base case, the aerosol nitrate concentrations are overestimated because the particles are liquid and sufficiently neutralized to accommodate a fraction of the total nitrate present in the system. If we assume that one type of particle is neutral but devoid of water and the other is wet but significantly more acidic, the partial pressure product of ammonia and nitric acid vapor required to form ammonium nitrate becomes significantly higher than in the base case and hence results in lower aerosol nitrate concentrations, as we observe. While it is possible that the particles do not exist in one extreme condition or the other but a mix of the two, comparisons with observations suggest that particles 


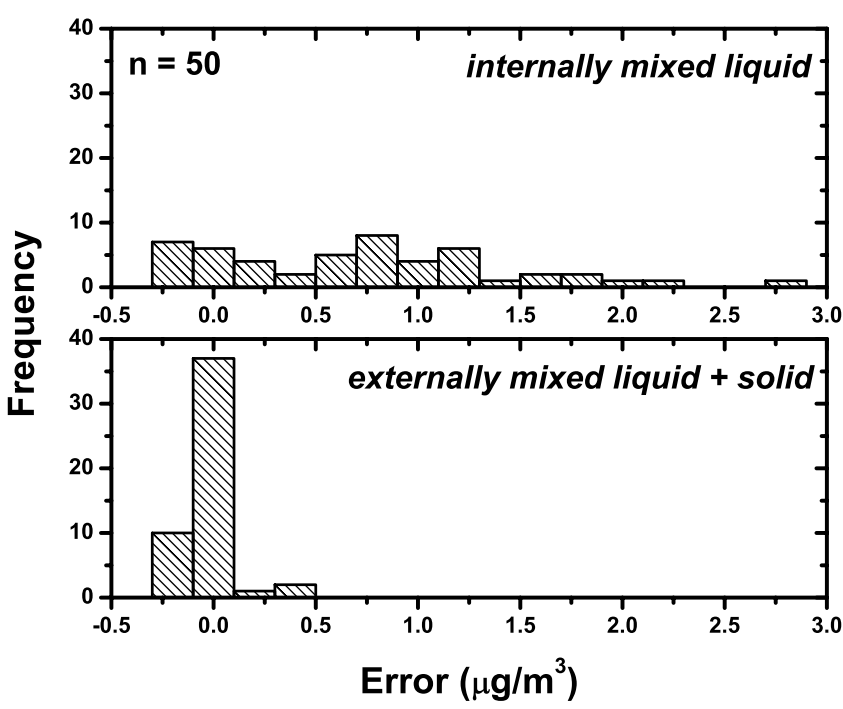

Figure 6. Errors between predictions and observations for periods with $\mathrm{RH}<40 \%$ assuming different states of particles in July 2001. Errors are calculated as predicted minus observed values of aerosol nitrate.

may be closer to the externally mixed state. This simple algorithm is not suitable for running simulations at higher RHs, however, when both neutral and acidic particles are assumed to be liquid. In these cases, the nitric acid and ammonia can be incorporated into both particle types and the algorithm cannot predict the gas-phase vapor pressures that will satisfy equilibrium conditions with the two particle types simultaneously.

[25] Particles can exist in an externally mixed state if their sources and histories are different. For instance, neutralized particles could be transported into the Ohio River Valley and western Pennsylvania from the ammonia-rich midwestern United States while acidic particles are generated locally. The different types of particles could be mixed together as the nighttime boundary layer vanishes in the morning, or some particles could be formed as a result of cloud processing, while others could be generated through condensation and nucleation. Suitable thermodynamic models and further experimental evidence may reveal more information on the existence of different mixing states.

\subsection{Other Sources of Error}

[26] Though predictions of nitrate partitioning capture diurnal trends reasonably well, errors in some periods can be high (Table 1) and explanations other than measurement error are needed. As noted previously, many assumptions are invoked when using thermodynamic models. The equilibrium assumption may not be valid if some particles are in the upper size range of $\mathrm{PM}_{2.5}$. However, data collected at the PAQS suggest that the geometric mean diameters of $\mathrm{PM}_{2.5}$ during the summer were $0.33 \mu \mathrm{m}$ for ammonium, $0.36 \mu \mathrm{m}$ for sulfate, and $0.66 \mu \mathrm{m}$ for nitrate; and during the winter the geometric mean diameters were $0.30 \mu \mathrm{m}$ for ammonium, $0.31 \mu \mathrm{m}$ for sulfate, and $0.34 \mu \mathrm{m}$ for nitrate [Cabada et al., 2004]. As crustal elements are found in low levels in Pittsburgh, salts of nitrate and crustal elements are not expected to be significant. Also, since measurements at reasonably short timescales were used, it is difficult to explain the observed differences by the averaging of different air masses.

[27] Two additional sources of error remain unaccounted for. Though previous studies have shown that on average, the expected influence of organics on partitioning of semivolatile inorganics is low [Koo et al., 2003], their impacts on individual cases are still unknown. In addition, the bulk equilibrium approach used in this study can introduce errors, as nitrate does not partition equally into different particles, and modeling them in bulk can introduce errors.

\subsection{Sensitivity Analysis}

[28] A perturbation analysis was performed to examine the sensitivity of our model to changes in input variables. Such an analysis can strengthen our understanding of the system's chemistry and further elucidate the potential influence of measurement errors. It can also provide a notion of the sensitivity of atmospheric processes to changes in meteorology and chemistry and thus guide our efforts to make preliminary estimates of PM response to changes in concentrations of precursor species. This section will focus primarily on the first objective, but implications for PM control strategies will also be discussed.

[29] The response of the predicted aerosol nitrate concentrations to changes in input parameters depends greatly on the point in the domain space that is examined. Ansari and Pandis [1998] proposed a parameter, the gas ratio (GR), that combines three input variables (TA, TS, and TN) to describe different regions of the domain in terms of the amount of free ammonia relative to the amount of total nitrate:

$$
\mathrm{GR}=\frac{[\mathrm{TA}]-2[\mathrm{TS}]}{[\mathrm{TN}]}
$$

[30] Table 2 summarizes the characteristics of the different regions of ammonia availability parameterized by GR. Dimensionless sensitivity coefficients can be defined by

$$
\mathrm{S}_{\mathrm{x}}=\frac{\mathrm{x}}{\mathrm{NO}_{3}} \frac{\Delta \mathrm{NO}_{3}}{\Delta \mathrm{x}}
$$

where $\mathrm{NO}_{3}$ is the aerosol nitrate concentration and $x$ is an input variable. The sensitivity coefficient can be used to assess the potential impacts of uncertainty in each input variable on the predicted aerosol concentration: $\mathrm{S}_{\mathrm{x}}$ is a measure of the relative change in the predicted aerosol nitrate concentration normalized by the relative change in the input variable $x$ when all other input variables are held fixed. Because coarse nitrate is not included in our calculations, we implicitly assume that the small amount of nitrate associated with particles larger than $2.5 \mu \mathrm{m}$ in the

Table 2. Characterization of GR Regions

\begin{tabular}{llc}
\hline Region Label & Value of GR & Description \\
\hline Low GR & $<0$ & $\begin{array}{c}\text { there is insufficient ammonia to neutralize } \\
\text { all of the sulfate to }\left(\mathrm{NH}_{4}\right)_{2} \mathrm{SO}\end{array}$ \\
Moderate GR & $\geq 0$ and $<1$ & $\begin{array}{c}\text { there is enough ammonia to neutralize } \\
\text { all sulfate but not nitrate } \\
\text { there is a sufficient quantity of ammonia } \\
\text { to neutralize all sulfate and nitrate }\end{array}$ \\
\hline
\end{tabular}




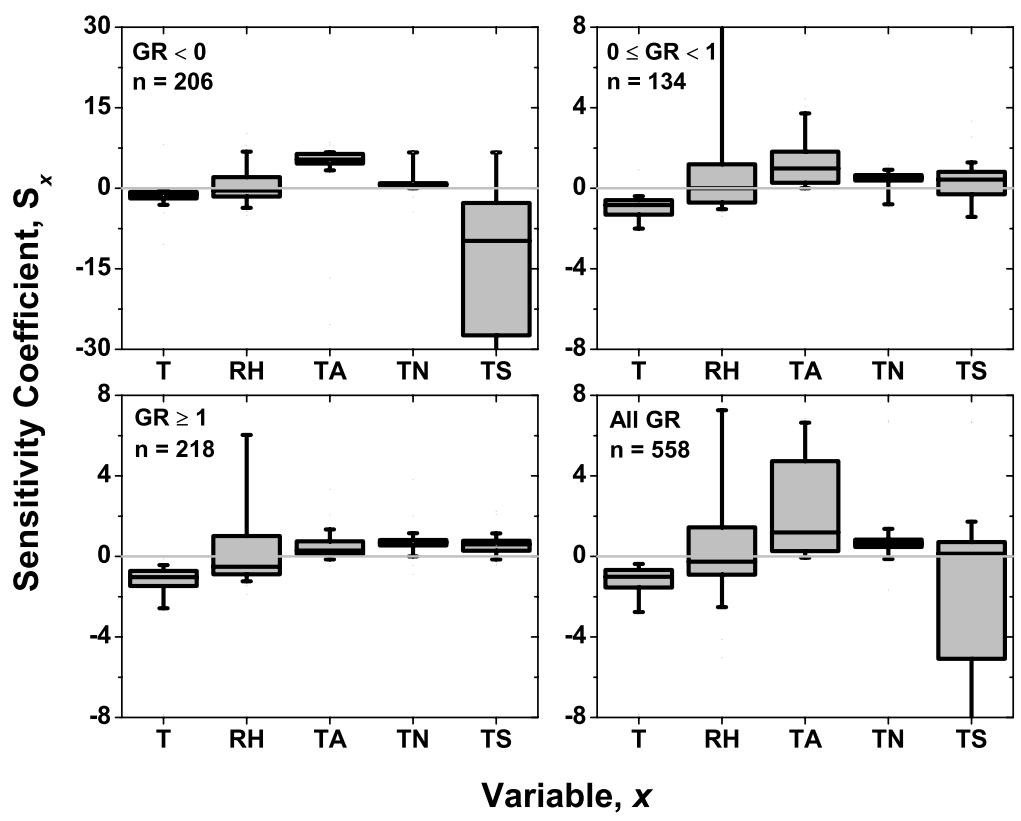

Figure 7. Sensitivity coefficients $\left(\mathrm{S}_{\mathrm{x}}\right)$ of aerosol nitrate in July 2001 in response to model inputs of temperature $\left(-3^{\circ} \mathrm{C}\right), \mathrm{RH}(-5 \%$ absolute $)$, and TA, TN, and TS $(-15 \%$ relative $)$. Separate plots are shown for different regimes of the gas ratio (GR). Shaded boxes cover the interquartile range of $S_{x}$, while whiskers extend to the 5 th and 95 th percentiles of $\mathrm{S}_{\mathrm{x}}$. The black line in the box corresponds to the median.

area [Cabada et al., 2004] will not change during these perturbations. Figures 7 and 8 show values of $\mathrm{S}_{\mathrm{x}}$ when the temperature is perturbed by $-3^{\circ} \mathrm{C}, \mathrm{RH}$ is perturbed by $-5 \%$ (absolute), and TA, TN, and TS are perturbed by $-15 \%$ (relative) for the months of July 2001 and January 2002, respectively. The response of nitrate is approximately linear over a $\pm 15 \%$ range of input concentrations. Overall, the variability in $\mathrm{S}_{\mathrm{x}}$ is much larger during the summer, when $\mathrm{TA}, \mathrm{TN}$, and TS concentrations spanned a wider range than during the winter.

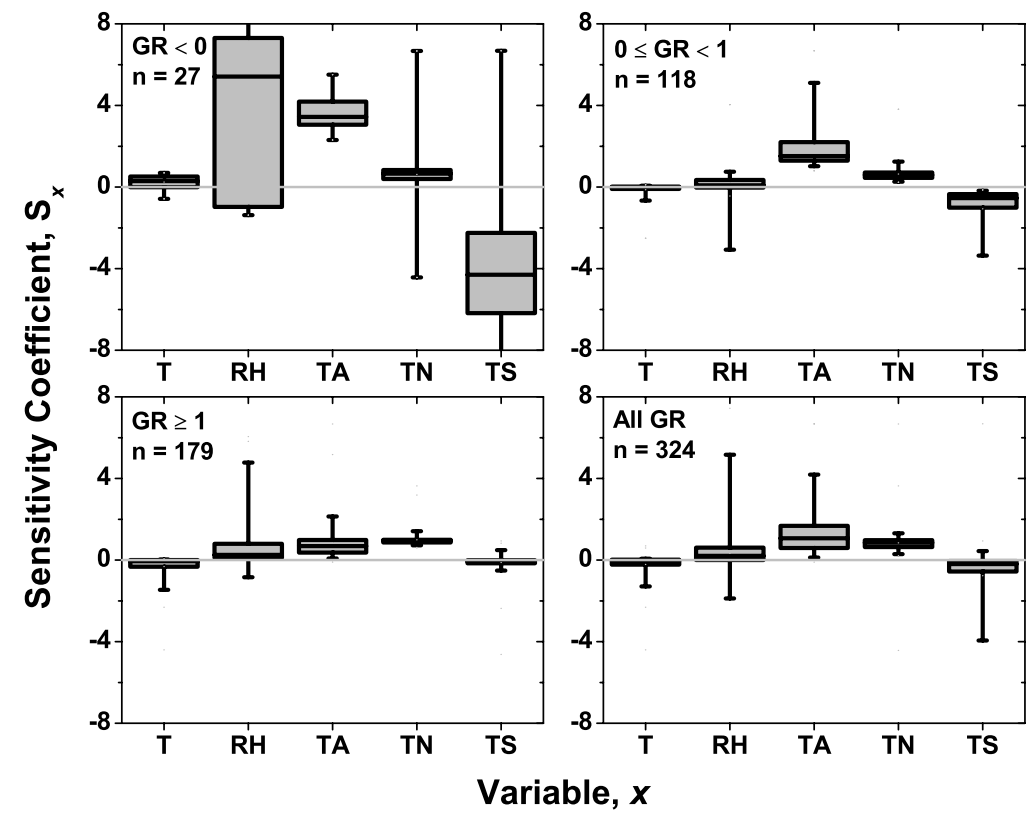

Figure 8. Sensitivity coefficients $\left(\mathrm{S}_{\mathrm{x}}\right)$ of aerosol nitrate in January 2002 in response to model inputs of temperature $\left(-3^{\circ} \mathrm{C}\right), \mathrm{RH}(-5 \%$ absolute $)$, and TA, TN, and TS $(-15 \%$ relative). Separate plots are shown for different regimes of the gas ratio (GR). Shaded boxes cover the interquartile range of $S_{x}$, while whiskers extend to the 5 th and 95 th percentiles of $\mathrm{S}_{\mathrm{x}}$. The black line in the box corresponds to the median. 
[31] During the summer, the sensitivity of nitrate to temperature is relatively small but distinctly negative (median $\mathrm{S}_{\mathrm{x}}=-1.01$ ), because decreasing the temperature lowers the dissociation constant of ammonium nitrate and allows more nitric acid to exist in the aerosol phase. During the winter, nitrate is not as sensitive to this parameter (median $\mathrm{S}_{\mathrm{x}}=-0.03$ ), as most of the nitrate is found in the aerosol phase. The change in nitrate concentrations with respect to $\mathrm{RH}$ is on average close to zero (median $\mathrm{S}_{\mathrm{x}}=$ -0.26 in July and 0.20 in January), but can be significant during certain periods in July 2001 when most particles are liquid, as illustrated by the large variability of $\mathrm{S}_{\mathrm{x}}$ for $\mathrm{RH}$ (Figure 7). At or near crystallization points, the change in nitrate with respect to RH can be even more significant. Since crystallization is a function of $\mathrm{RH}$, changes in $\mathrm{RH}$ by a few percent can change the picture of nitrate partitioning considerably when the predicted aerosol makes a transition from the aqueous to the solid state.

[32] The nitrate response to sulfate can be very large for GRs less than 0 (median $S_{x}=-9.77$ in July and -4.30 in January), as shown in Figures 7 and 8, because removing sulfate from the system can free up ammonia to react with nitric acid, and adding sulfate can remove ammonia gas and prevent nitrate aerosols from being created. At higher GRs, adding sulfate can actually increase nitrate because sulfates are hygroscopic and increase the water content of aerosols, thus allowing more nitric acid to be pulled into the aerosol phase. Since concentrations of sulfate are generally higher than those of other species, small changes relative to the nominal sulfate can elicit a large response in nitrate, and is reflected in the large values of $S_{x}$ for this parameter. $S_{x}$ for TA can be quite large in the case of low and moderate GRs $\left(\mathrm{GR}<0\right.$, median $\mathrm{S}_{\mathrm{x}}=5.34$ in July and 3.44 in January; $0 \leq$ GR $<1$, median $\mathrm{S}_{\mathrm{x}}=1.05$ in July and 1.52 in January), as the formation of nitrate aerosols is sensitive to the availability of ammonia and perturbation in TA directly changes this quantity. This ammonia-responsive condition is observed $61 \%$ of the time in July 2001 and $45 \%$ during January 2002. The magnitude of $S_{x}$ in response to TA can depend quite largely on the sulfate levels if the particles are liquid, as the equilibrium constant of ammonium nitrate is dependent on the ionic strength of the solution, which in turn is influenced by sulfate concentrations [Ansari and Pandis, 1998]. As a result, predictions of aerosol nitrate are generally sensitive to measured concentrations of ammonia as well as sulfate, more so during the summer than in the winter. Since aerosol nitrate formation is dependent on the amount of TN in the system, measurement errors in TN can also influence aerosol nitrate predictions. When there is an excess of ammonia, as reflected by higher GRs, much of the nitrate can be found in the aerosol phase. When this occurs, e.g., at night or during the winter, the predicted aerosol nitrate concentrations are more directly influenced by the measured TN. However, the actual sensitivity is also subject to ambient temperatures and relative humidities.

[33] This sensitivity analysis provides insight as to how measurement errors in input variables can affect predictions in nitrate partitioning according to the domain space, but it also suggests implications for $\mathrm{PM}_{2.5}$ control strategies. For instance, sulfate reduction is an obvious choice for reducing PM mass since reducing sulfate reduces a large component of $\mathrm{PM}_{2.5}$, but this strategy can significantly increase aerosol nitrate concentrations when the formation of nitrate particles is limited by ammonia availability. Our sensitivity analysis shows that aerosol nitrate concentrations are responsive to reductions in ammonia concentrations under such ammonialimited conditions, which were often observed during July 2001. In these cases, reductions in ammonia may be an additionally effective measure in reducing PM mass.

\section{Conclusions}

[34] Nitrate partitioning during the Pittsburgh Air Quality Study was simulated using a chemical equilibrium model, GFEMN, for July 2001 and January 2002. The mean observed aerosol nitrate concentrations for July was $0.56 \mu \mathrm{g} / \mathrm{m}^{3}$, while the model predicted $0.64 \mu \mathrm{g} / \mathrm{m}^{3}$, and for January the observed value was $2.09 \mu \mathrm{g} / \mathrm{m}^{3}$ while the mean predicted value was $2.15 \mu \mathrm{g} / \mathrm{m}^{3}$. GFEMN appears to reproduce observed aerosol nitrate concentrations reasonably well, and errors in predictions are about $0.5 \mu \mathrm{g} / \mathrm{m}^{3}$ on average. Monte Carlo simulations indicate that in many cases experimental uncertainties can explain the discrepancy between model predictions and observations.

[35] For moderate relative humidities $(60-80 \%)$ in January, the agreement between predicted and observed nitrate aerosol concentrations was approximately the same whether the particles were assumed to be in a stable or metastable state. Furthermore, during July, the difference in overall model error between efflorescence branch predictions and deliquescence branch predictions was relatively small.

[36] During periods in July when the relative humidity was low, our modeling results suggest that particles existed not as internally mixed liquids but as an external mixture of crystallized ammonium sulfate and wet, acidic particles. A sensitivity analysis showed that different variables can have varying degrees of influence on the predicted aerosol nitrate, depending on the initial point in the domain space. When temperatures are low and most of the nitrate is in the aerosol phase, as seen in January 2002, errors in temperature measurements do not contribute to model errors as strongly as when the temperatures are higher and nitric acid gas is prevalent. When particles are assumed to be liquid as in July 2001, or when the relative humidity is close to a transition point between solid and liquid aerosol, measurement errors in relative humidity can contribute significantly to model errors for nitrate predictions. Under ammonialimited conditions when aerosol nitrate formation is a strong function of ammonia availability, the predicted amount of nitrate partitioned into the aerosol phase can be sensitive to measurement errors in both sulfate and ammonia concentrations. This sensitivity has an additional implication that while reducing sulfate is a promising strategy for reducing $\mathrm{PM}_{2.5}$ mass, reducing ammonia could also provide additional benefits by inhibiting aerosol nitrate formation for regions where ammonia-limited conditions are frequently observed.

[37] Acknowledgments. This research was conducted as part of the Pittsburgh Air Quality Study, which was supported by U.S. Environmental Protection Agency under contract R82806101 and the U.S. Department of Energy National Energy Technology Laboratory under contract DE-FC2601NT41017. This paper has not been subject to EPA's peer and policy review, and therefore does not necessarily reflect the views of the Agency. No official endorsement should be inferred. This work benefited from the 
contributions of Andrey Y. Khlystov, Charles O. Stanier, and Urmila Diweker.

\section{References}

Ansari, A. S., and S. N. Pandis (1998), Response of inorganic PM to precursor concentrations, Environ. Sci. Technol., 32, 2706-2714.

Ansari, A. S., and S. N. Pandis (1999), Prediction of multicomponent inorganic atmospheric aerosol behavior, Atmos. Environ., 33, 745-757.

Ansari, A. S., and S. N. Pandis (2000), The effect of metastable equilibrium states on the partitioning of nitrate between the gas and aerosol phases, Atmos. Environ., 34, 157-168.

Bassett, M., and J. H. Seinfeld (1983), Atmospheric equilibrium model of sulfate and nitrate aerosols, Atmos. Environ., 17, 2237-2252.

Cabada, J. C., S. Rees, S. Takahama, A. Khlystov, S. N. Pandis, C. I. Davidson, and A. L. Robinson (2004), Mass size distributions and size resolved chemical composition of fine particulate matter at the Pittsburgh Supersite, Atmos Environ, 38, 3127-3141.

Dockery, D. W., A. Pope, X. Xu, J. D. Spengler, J. H. Ware, M. E. Fay, B. G. Ferris, and F. E. Speizer (1993), An association between air pollution and mortality in six U. S. cities, New Engl. J. Med., 329, $1753-1759$

Eatough, D. J., F. Obeidi, Y. Pang, Y. Ding, N. L. Eatough, and W. E. Wilson (1999), Integrated and real-time diffusion denuder samplers for PM2.5 based on BOSS, PC, and TEOM technology, Atmos. Environ., 33 $2835-2844$

Jacobson, M. Z. (1999), Studying the effects of calcium and magnesium on size-distributed nitrate and ammonium with EQUISOLV II, Atmos. Environ., 33, 3635-3649.

Jacobson, M. Z., A. Tabazadeh, and R. P. Turco (1996), Simulating equilibrium within aerosols and nonequilibrium between gases and aerosols, J. Geophys. Res., 101, 9079-9091.

Jaffrezo, J. L., T. Calas, and M. Bouchet (1998), Carboxylic acids measurements with ionic chromatography, Atmos. Environ., 32, $2705-$ 2708 .

Khlystov, A., G. P. Wyers, and J. Slanina (1995), Steam-jet aerosol collector, Atmos. Environ., 29, 2229-2234

Kim, Y. P., J. H. Seinfeld, and P. Saxena (1993), Atmospheric gas-aerosol equilibrium, I. Thermodynamic model, Aerosol Sci. Technol., 19, 157181

Koo, B., A. S. Ansari, and S. N. Pandis (2003), Integrated approaches to modeling the organic and inorganic atmospheric aerosol components, Atmos. Environ., 37, 4757-4768.

McKay, M. D. (1988), Sensitivity and uncertainty analysis using a statistical sample of input values, in Uncertainty Analysis, edited by Y. Ronen, pp. 145-186, CRC Press, Boca Raton, Fla.

Meng, Z., J. H. Seinfeld, P. Saxena, and Y. P. Kim (1995), Atmospheric gasaerosol equilibrium: IV, Thermodynamics of carbonates, Aerosol Sci. Technol., 23, 131-154.
Moya, M., A. S. Ansari, and S. N. Pandis (2001), Partitioning of nitrate and ammonium between the gas and particulate phases during the 1997 IMADA-AVER study in Mexico City, Atmos. Environ., 35, 1791-1804.

Moya, M., S. N. Pandis, and M. Z. Jacobson (2002), Is the size distribution of urban aerosols determined by thermodynamic equilibrium? An application to southern California, Atmos. Environ., 36, 2349-2365.

Nenes, A., C. Pilinis, and S. N. Pandis (1999), Continued development and testing of a new thermodynamic aerosol module for urban and regional air quality models, Atmos. Environ., 33, 1553-1560.

Pilinis, C., and J. H. Seinfeld (1987), Continued development of a general equilibrium model for inorganic multicomponent atmospheric aerosols, Atmos. Environ., 22, 1985-2001.

Ripley, B. D., and M. Thompson (1987), Regression techniques for the detection of analytical bias, Analyst, 112, 377-383.

Saxena, P., A. B. Hudischewskyj, C. Seigneur, and J. H. Seinfeld (1986), A comparative study of equilibrium approaches to the chemical characterization of secondary aerosols, Atmos. Environ., 20, 1471-1483.

Seinfeld, J. H., and S. N. Pandis (1998), Atmospheric Chemistry and Physics, John Wiley, Hoboken, N. J.

Solomon, P., G. Norris, M. Landis, and M. Tolocka (2001), Chemical analysis methods for atmospheric aerosol components, in Aerosol Measurement: Principles, Techniques, and Applications, edited by P. A. Baron and K. Willeke, pp. 261-294, John Wiley, Hoboken, N. J.

U.S. Environmental Protection Agency (1996), Air quality criteria for particulate matter, Rep. EPA/600/P-95/001aF, vol. 1, U.S. Gov. Print. Off., Washington, D. C.

West, J. J., A. S. Ansari, and S. N. Pandis (1999), Marginal $\mathrm{PM}_{2.5}$ : Nonlinear aerosol mass response to sulfate reductions in the eastern United States, J. Air Waste Manage., 49, 1415-1424.

Wexler, A. S., and J. H. Seinfeld (1991), Second-generation inorganic aerosol model, Atmos. Environ. Part A, 25, 2731-2748.

Wittig, A. E., N. Anderson, A. Khlystov, S. N. Pandis, C. Davidson, and A. L. Robinson (2004a), Pittsburgh Air Quality Study overview, Atmos. Environ., 38, 3107-3125.

Wittig, A. E., S. N. Pandis, S. Hering, B. Kirby, A. Y. Khlystov, S. Takahama, and C. I. Davidson (2004b), Semicontinuous $\mathrm{PM}_{2.5}$ inorganic composition measurements during the Pittsburgh Air Quality Study, Atmos. Environ., 38, 3201-3213.

C. I. Davidson, Department of Civil and Environmental Engineering, Carnegie Mellon University, Pittsburgh, PA 15213, USA.

S. N. Pandis and S. Takahama, Department of Chemical Engineering, Carnegie Mellon University, Pittsburgh, PA 15213, USA. (spyros@andrew. cmu.edu)

D. V. Vayenas, Department of Environmental and Natural Resources Management, University of Ioannina, Agrinio GR-30100, Greece.

A. E. Wittig, Department of Civil Engineering, City College of New York, New York, NY 10031, USA 


\title{
Effect of Peak Inert-Mode Temperature on Elemental Carbon Measured Using Thermal-Optical Analysis
}

\author{
Ramachandran Subramanian, ${ }^{1, \dagger}$ Andrey Y. Khlystov, ${ }^{3}$ and Allen L. Robinson ${ }^{1,2}$ \\ ${ }^{I}$ Department of Mechanical Engineering, Carnegie Mellon University, Pittsburgh, Pennsylvania, USA \\ ${ }^{2}$ Department of Engineering and Public Policy, Carnegie Mellon University, Pittsburgh, \\ Pennsylvania, USA \\ ${ }^{3}$ Department of Civil and Environmental Engineering, Duke University, Durham, North Carolina, USA
}

\begin{abstract}
Thermal-optical analysis is a conventional method for classifying carbonaceous aerosols as organic carbon $(O C)$ and elemental carbon (EC). This article examines the effects of three different temperature protocols on the measured EC. For analyses of parallel punches from the same ambient sample, the protocol with the highest peak helium-mode temperature $\left(8^{\circ}{ }^{\circ} \mathrm{C}\right)$ gives the smallest amount of EC, while the protocol with the lowest peak helium-mode temperature $\left(550^{\circ} \mathrm{C}\right)$ gives the largest amount of EC. These differences are observed when either sample transmission or reflectance is used to define the OC/EC split. An important issue is the effect of the peak helium-mode temperature on the relative rate at which different types of carbon with different optical properties evolve from the filter. Analyses of solvent-extracted samples are used to demonstrate that high temperatures $\left(870^{\circ} \mathrm{C}\right)$ lead to premature $\mathrm{EC}$ evolution in the helium-mode. For samples collected in Pittsburgh, this causes the measured EC to be biased low because the attenuation coefficient of pyrolyzed carbon is consistently higher than that of EC. While this problem can be avoided by lowering the peak helium-mode temperature, analyses of wood smoke dominated am-
\end{abstract}

Received 30 June 2005; accepted 28 February 2006.

The authors would like to acknowledge the many useful conversations with B.J. Turpin at Rutgers University. Dr. Eric Lipsky provided the tunnel samples. Erin Casgren-Tindall, Prakash Rao, Leonard Lucas, David Wynne, Michael Quirolo, Mark Prack, Jessica Chiu, and Raymond Obico analyzed the vast majority of the filters for the intercomparison studies. RS thanks Tami Bond for time off to finish this paper. This research was conducted as part of the Pittsburgh Air Quality Study, which was supported by US Environmental Protection Agency under contract R82806101 and the US Department of Energy National Energy Technology Laboratory under contract DE-FC26-01NT41017. This work was supported in part by the Pennsylvania Infrastructure Technology Alliance, a partnership of Carnegie Mellon, Lehigh University, and the Commonwealth of Pennsylvania's Department of Community and Economic Development (DCED). This paper has not been subject to EPA's required peer and policy review, and therefore does not necessarily reflect the views of the Agency. No official endorsement should be inferred.

Address correspondence to Allen L. Robinson, Department of Mechanical Engineering, 5000 Forbes Ave., Carnegie Mellon University, Pittsburgh, PA 15213, USA. E-mail: alr@andrew.cmu.edu

${ }^{\dagger}$ Now at the Department of Civil and Environmental Engineering, University of Illinois, Urbana, IL 61801, USA. bient samples and levoglucosan-spiked filters indicate that too low helium-mode peak temperatures $\left(5^{\circ} 0^{\circ} \mathrm{C}\right)$ allow non-light absorbing carbon to slip into the oxidizing mode of the analysis. If this carbon evolves after the OC/EC split, it biases the EC measurements high. Given the complexity of ambient aerosols, there is unlikely to be a single peak helium-mode temperature at which both of these biases can be avoided.

\section{INTRODUCTION}

The carbonaceous component of fine particulate matter is commonly classified as organic carbon (OC) and elemental carbon (EC). A variety of thermal or thermal-optical measurement techniques have been developed to measure particulate $\mathrm{OC}$ and EC (Malissa, Puxbaum, and Pell 1976; Huntzicker et al. 1982; Chow et al. 1993; Kerminen et al. 1997). While these methods generally measure the same amount of total carbon, the OC/EC split is operationally defined with significant differences in the amount of OC and EC measured by different methods (Chow et al. 2001; Schmid et al. 2001; Fung, Chow, and Watson 2002).

This article largely focuses on the thermal-optical transmission (TOT) technique first developed by Huntzicker et al. (1982), with subsequent developments reported by Birch and Cary (1996). This technique is widely used in the atmospheric science community, including the EPA's Speciation Trends Network (STN). Briefly, a filter sample is exposed to a prescribed temperature protocol first in an inert (helium, He) environment and then in an oxidizing (helium and oxygen mixture, $\mathrm{He} / \mathrm{Ox}$ ) atmosphere. Ideally, OC volatilizes in the He-mode of the analysis, while EC combusts in the oxidizing atmosphere at high temperatures. A major complication is that a fraction of the OC chars or pyrolyzes in the inert mode of the analysis. The residual carbon remaining on the filter after heating in the inert mode is called pyrolyzed organic carbon (PC), and can absorb light and requires an oxidizing atmosphere to evolve off the filter, similar to EC. Not all thermally altered OC absorbs light; however, in this article the term PC is used to refer only to light-absorbing pyrolyzed carbon. The term "non-light absorbing carbon" is used to refer to unaltered OC and thermally altered OC that does not 
absorb light. Not accounting for PC formation can significantly bias the OC/EC split. To account for pyrolysis, the transmission of a laser light through the sample is monitored and used to split the total carbon into OC and EC. A variant on this approach is the thermal-optical reflectance (TOR) method which uses reflected laser light to determine the OC/EC split.

Previous studies have demonstrated that changing the temperature protocol can alter the EC measured by thermal-optical analysis (Chow et al. 2001; Yang and Yu 2002; Conny et al. 2003; Schauer et al. 2003; Chow et al. 2004). Lowering the temperatures of the steps in the He-mode can increase the measured EC; this increase has been attributed to OC not completely evolving or not pyrolyzing into light-absorbing carbon (Conny et al. 2003; Schauer et al. 2003). While previous research has demonstrated that certain types of OC such as wood smoke do not completely evolve or pyrolyze at low inert-mode temperatures (Novakov and Corrigan 1995), the effect of this on the thermaloptical EC is not well understood. Too high of a peak inert-mode temperature is also thought to bias the OC/EC split due to premature EC evolution (Chow et al. 2001). At high temperatures mineral oxides can promote combustion of EC in an inert environment (Fung 1990); however, it has not been demonstrated that the light-absorbing carbon evolving at high temperatures in the He-mode is EC, and why this should alter the measured $\mathrm{EC}$ is unclear. An additional challenge is that the effects of temperature protocol on the measured EC can vary from sample to sample, presumably due to differences in sample composition (Schauer et al. 2003).

While a number of hypotheses have been put forward to explain the observed changes in thermal-optical EC measured with different temperature protocols, the underlying causes of the changes are not well understood. Yang and Yu (2002) indicate that changes in measured EC with temperature protocol are related to the attenuation coefficients of PC and EC. Chow et al. (2004) proposes that the effects of changing temperature protocol on the measured EC may be mitigated by using TOR as opposed to TOT to define the OC/EC split. A critical factor in explaining the variability in the measurements is the highest temperature step in the He-mode (Conny et al. 2003).

This article examines the effect of the peak He-mode temperature (step 4 of the Helium mode, He4) on the OC/EC split defined by thermal-optical analysis. Both ambient and sourcedominated samples were analyzed using three different temperature protocols: two versions of the NIOSH 5040 protocol (NIOSH 1999) and a version of the IMPROVE protocol (Chow et al. 1993). The NIOSH protocols have peak He-mode temperatures of 870 and $700^{\circ} \mathrm{C}$ while the IMPROVE-type protocol has a peak He-mode temperature of $550^{\circ} \mathrm{C}$. A combination of solvent extraction to minimize pyrolysis, analysis of filters doped with model OC mixtures, and quantitative analysis of optical data is used to evaluate the fundamental assumptions behind thermal-optical OC/EC analysis and to investigate the causes of differences in the EC measured using different temperature protocols. While most of the research uses sample transmission
(TOT) to define the OC/EC split, we also report results from a number of analyses based on sample reflectance (TOR).

\section{METHODS}

\section{Sample Collection and Analysis}

Ambient $\mathrm{PM}_{2.5}$ samples were collected at an urban park near the Carnegie Mellon University campus in Pittsburgh, PA as part of the Pittsburgh Air Quality Study (PAQS) (Wittig et al. 2004). Mobile source dominated samples were collected using the same samplers in the ventilation duct of the Squirrel Hill tunnel on Interstate-376 in Pittsburgh (Grieshop et al. 2006). Details of the design and operation of the two organic samplers are provided in Subramanian et al. (2004). Briefly, one sampler used a quartz fiber filter (Gelman Tissuquartz 2500 QAO-UP) downstream of a sharp cut $\mathrm{PM}_{2.5}$ cyclone. The second sampler had a quartz filter downstream of an activated carbon monolith denuder (MastCarbon Ltd, UK) and a sharp-cut $\mathrm{PM}_{2.5}$ cyclone. The majority of the samples were collected for 24 hours, with a limited number of shorter and longer duration samples. All the quartz filters were baked at $550^{\circ} \mathrm{C}$ in air for at least four hours prior to use. The data presented here are not blank-corrected since we compare paired punches from the same samples, and blank correction does not affect the results significantly.

The samples were analyzed using a Sunset Laboratory Thermal/Optical Carbonaceous Aerosol Analyzer. The carbon analyzer was routinely checked for leaks and operated using Ultra-High Purity (Grade 6.0) Helium gas with two oxygen traps installed on the inlet line to avoid problems with oxygen contamination. The instrument is calibrated prior to use with a combination of sucrose standards and manual methane injections. The OC/EC split is defined by the sample transmission measured at $680 \mathrm{~nm}$. Any carbon evolving after the laser signal reaches $97 \%$ of the initial, pre-pyrolysis value is classified as EC. A value of 97\% was used to be consistent with the default value in the Sunset Laboratories data analysis program. The calculation procedures for defining the OC/EC split used the algorithm as described by Sunset Labs (David Smith, personal communication, 2002).

Table 1 lists the three temperature profiles. Two are based on the NIOSH 5040 protocol (NIOSH 1999); they are identical except that one has a peak He-mode temperature (He4) of $870^{\circ} \mathrm{C}$ and the second a peak He-mode temperature of $700^{\circ} \mathrm{C}$ (typical thermograms from these two protocols are shown in Figure 1). The third protocol is an implementation of the IMPROVE protocol, with a peak He-mode temperature of $550^{\circ} \mathrm{C}$ (Chow et al. 1993). We refer to these protocols as the He4-870, He4700 , and $\mathrm{He} 4-550.700^{\circ} \mathrm{C}$ is an intermediate temperature between the NIOSH and IMPROVE protocols. This value was chosen to be more consistent with the original Huntzicker protocol (Huntzicker et al. 1982; Turpin 1989); it is higher $\left(700^{\circ} \mathrm{C}\right.$ versus $650^{\circ} \mathrm{C}$ ) to account for the differences in thermocouple locations in the two analyzers (B.J. Turpin, personal communication). These protocols are based on the temperature measured by a thermocouple located immediately downstream of the sample 
TABLE 1

Temperature protocols used to analyze the samples in this article

\begin{tabular}{|c|c|c|c|c|c|}
\hline \multirow[b]{2}{*}{ Step } & \multicolumn{3}{|c|}{ Based on NIOSH $5040^{a}$} & \multicolumn{2}{|c|}{$\mathrm{He} 4-550^{b}$} \\
\hline & Duration (s) & $\mathrm{He} 4-870 \mathrm{~T}\left({ }^{\circ} \mathrm{C}\right)$ & $\mathrm{He} 4-700 \mathrm{~T}\left({ }^{\circ} \mathrm{C}\right)$ & Duration (s) & $\mathrm{T}\left({ }^{\circ} \mathrm{C}\right)$ \\
\hline Hel & 120 & 340 & 340 & 120 & 120 \\
\hline $\mathrm{He} 2$ & 120 & 500 & 500 & 120 & 250 \\
\hline He3 & 120 & 615 & 615 & 120 & 450 \\
\hline $\mathrm{He} 4$ & 180 & 870 & 700 & 120 & 550 \\
\hline (Helium) & 45 & (cool down) & (cool down) & N/A & N/A \\
\hline $\mathrm{HeOx} 1$ & 45 & 575 & 575 & 300 & 550 \\
\hline $\mathrm{HeO} 2$ & 45 & 650 & 650 & 240 & 700 \\
\hline $\mathrm{HeO} \times 3$ & 45 & 725 & 725 & 240 & 800 \\
\hline $\mathrm{HeOx} 4$ & 45 & 800 & 800 & 180 & 910 \\
\hline $\mathrm{HeO} \times 5$ & 100 & 910 & 910 & N/A & N/A \\
\hline Internal standard & 110 & - & - & 110 & - \\
\hline
\end{tabular}

${ }^{a}$ NIOSH (1999).

${ }^{b}$ Based on IMPROVE protocol (Chow et al. 1993). N/A = not applicable. The IMPROVE protocol does not have a cool-down step or a $\mathrm{HeO} 5$ step.

(the standard design of the Sunset OC/EC analyzer), which may not be exactly equal to the sample temperature. For a limited number of samples, we also lengthened the He/Ox temperature steps (without changing the temperatures listed in Table 1) to investigate the influence of duration of the different temperature steps on the OC/EC split.

We adapted the solvent treatment method of Fung (1990) to extract a portion of the organic carbon before analysis to minimize pyrolysis and thus reduce the PC interference in the optically-defined OC/EC split. Selected filter punches were immersed in a methylene chloride: acetone: hexane mixture (2:4:4 by volume) for an hour (the solvent mixture is replaced after 30 minutes), with periodic gentle stirring. The punches are then rinsed with methylene chloride and dried in an oven at $50^{\circ} \mathrm{C}$ for 30 minutes before OC/EC analysis. The average OC on four pre-baked blanks solvent-treated in this manner was $0.08 \mu \mathrm{g}$ $\mathrm{C} / \mathrm{cm}^{2}$; all four values were below the instrument detection limit for $\mathrm{OC}\left(0.2 \mu \mathrm{g}-\mathrm{C} / \mathrm{cm}^{2}\right)$.

\section{Calculation of the Attenuation Coefficient of Evolved Carbon}

The changes in the laser transmission during OC/EC analysis depend on the mass and the attenuation coefficient of the carbon evolving from the filter. The attenuation coefficient $(\mathrm{k})$ of the carbon that evolves from different segments of the analysis (e.g., the net attenuation coefficient of the $\mathrm{He} / \mathrm{Ox}$ carbon) is calculated using the Lambert-Beer law:

$$
k=\frac{-\ln (I o / I)}{C}
$$

where $I o$ and $I$ are the initial and final laser transmission through the sample and filter for the portion of the analysis of interest and $C$ is the mass of carbon per unit filter area that is lost from (or combusts off) the filter during the analysis segment; thus, Io is the laser transmission through the loaded filter, not the blank. Note that this is the reverse of the more-conventional Aethalometer approach (e.g., Gundel et al. 1984).

When analyzing the optical data, one must be careful to account for potential effects of filter loading. This dependence is illustrated in Figure 2, which shows the net laser attenuation of the He/Ox carbon for samples collected in a highway tunnel as a function of filter loading. For low filter loadings (less than $15 \mu \mathrm{g}-\mathrm{C} / \mathrm{cm}^{2}$ ), the net laser attenuation increases more or less linearly with loading and Equation (1) yields consistent values for the net specific attenuation coefficient of the He/Ox carbon $\left(\sim 20 \mathrm{~m}^{2} / \mathrm{g}-\mathrm{C}\right)$. However, at higher filter loadings, the carbon on heavily loaded filters appears lighter than the carbon on more lightly loaded filters. This effect is referred to as a "shadowing" or "light-pipe" effect (Gundel et al. 1984; Weingartner et al. 2003). The He/Ox carbon loadings of the filters considered in this article are less than $15 \mu \mathrm{g}-\mathrm{C} / \mathrm{cm}^{2}$ to minimize the "shadowing" or "light-pipe" effect.

\section{Instrument Precision}

The precision of the OC/EC analyzer was evaluated by analyzing multiple punches from three 102-mm filter $\mathrm{PM}_{2.5}$ samples collected with a medium-volume sampler and parallel punches from four 47-mm samples using the same protocol. The absolute EC (EC in $\left.\mu \mathrm{g}-\mathrm{C} / \mathrm{cm}^{2}\right)$ measured with the same protocol on our Sunset instrument showed a precision within $7 \%$ (the maximum relative standard deviation (RSD) observed within each of the three analysis protocols with untreated ambient samples); similarly, the TC precision is $3 \%$ across all protocols. The maximum 

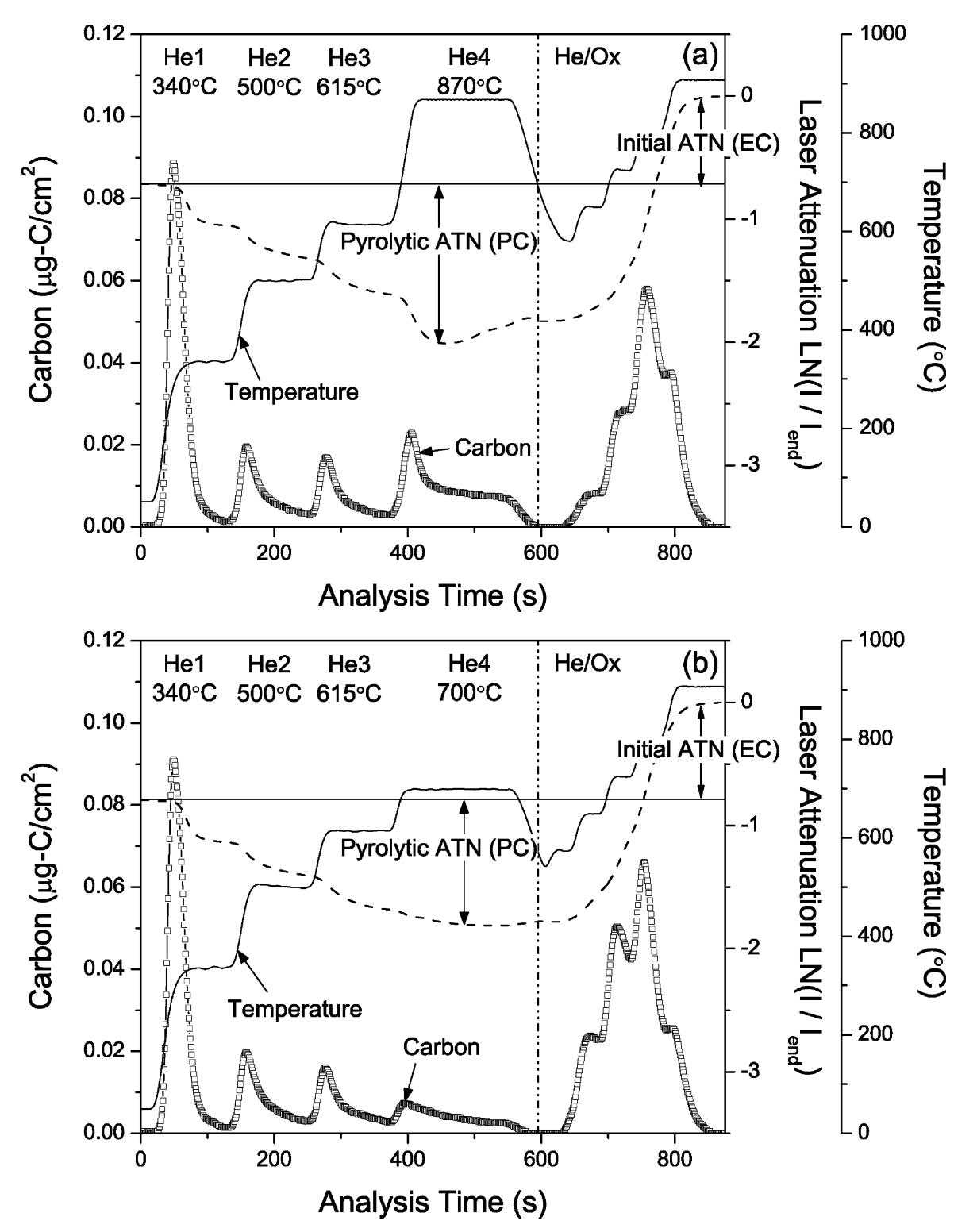

FIG. 1. Thermograms from the analysis of a typical ambient sample with the (a) He4-870 and (b) He4-700 protocols. The thermograms are for parallel punches of the same filter. Dashed line indicates laser attenuation. There is clear loss of light-absorbing carbon in the He-mode of the He4-870 protocol but not in the He-mode of the He4-700 protocol.

RSD in the EC/TC ratios within each protocol was $6 \%$. This performance is consistent with the Sunset Laboratory reported measurement precision of $\pm 5 \%$ for the same instrument, with a minimum uncertainty of $0.2 \mu \mathrm{g}-\mathrm{C} / \mathrm{cm}^{2}$ (Cary 2003). Schauer et al. (2003) report the precision as $4-13 \%$ for OC and between 6-21\% for EC between different instruments running the same temperature-time protocol on parallel punches.

\section{RESULTS}

We first consider results from samples analyzed using the He4-870 protocol. As part of the Supersite study, EPA requested that the He4-870 protocol be used as part of the carbon analysis; therefore, essentially all of the PAQS samples were analyzed with this protocol. We then compare the results from analyses using the He4-870 and He4-700 protocols and critically evaluate the fundamental assumptions underlying TOT analysis in order to explain the differences between the measurements. Finally, we consider the effects of even lower peak He-mode temperatures by comparing results from the He4-700 and He4-550 protocols. Only limited subsets of the PAQS samples were analyzed with the He4-550 and He4-700 protocols for comparison purposes.

A typical thermogram from the analysis of a filter punch using the He4-870 protocol is shown in Figure 1(a). The formation of light-absorbing pyrolyzed carbon (PC) causes the transmission of laser light through the sample (and filter) to decrease 


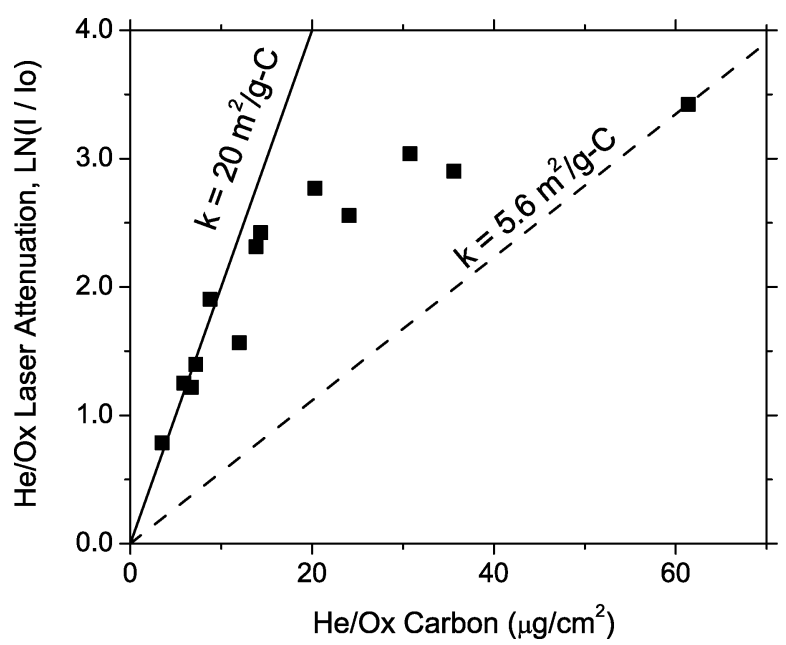

FIG. 2. Net change in laser attenuation over the He/Ox mode of samples collected in a highway tunnel, as a function of filter loading. Lightly loaded filters yield a consistent estimate of the specific attenuation coefficient, $20 \mathrm{~m}^{2} / \mathrm{g}-\mathrm{C}$, indicated by the solid line. At high filter loadings the specific attenuation coefficient appears to decrease, as indicated by the dashed line, due to the "shadowing" or "light-pipe" effect.

through much of the He-mode of the analysis. The transmission reaches a minimum value partway through the $870^{\circ} \mathrm{C}$ temperature step of the He-mode and then begins increasing, indicating premature evolution of light-absorbing carbon. Over 66\% of the almost 500 PAQS samples (both denuded and undenuded) analyzed with the He4-870 protocol exhibited similar loss of light-absorbing carbon in the He4 step. Although there is loss of light-absorbing carbon in the He-mode, the OC/EC split occurred in the $\mathrm{He} / \mathrm{Ox}$ mode for more than $99 \%$ of these samples. The loss of light-absorbing carbon in the inert mode of the analysis is not unique to Pittsburgh samples (e.g., Chow et al. 2001; Peterson and Richards 2002).

In the event of loss of light-absorbing carbon in the He-mode of the analysis, a standard recommendation is to reduce the peak temperature of this mode (NIOSH 1999). Recently, Conny et al. (2003) proposed shortening the duration of the He4 step to avoid the loss of light-absorbing carbon in the He-mode. This is an interesting approach that links the temperature and duration of the $\mathrm{He} 4$ step, essentially by switching to the $\mathrm{He} / \mathrm{Ox}$ mode when the laser reaches its minimum transmission. A complication is that this time will vary with sample loading, sample composition, and He4 temperature, which makes it difficult to use this approach given the large number of samples considered in this study.

Figure 1(b) shows a thermogram from the He4-700 analysis of a second punch from the same filter shown in Figure 1(a). The laser signal reaches its minimum value in the He4 step $\left(700^{\circ} \mathrm{C}\right)$ and does not increase until the He/Ox-mode suggesting no loss of light-absorbing carbon in the He-mode. However, a flat laser signal in the $\mathrm{He} 4$ step does not guarantee that light-absorbing carbon is not lost in the He-mode because the loss of light-absorbing carbon could be offset by continued pyrolysis.

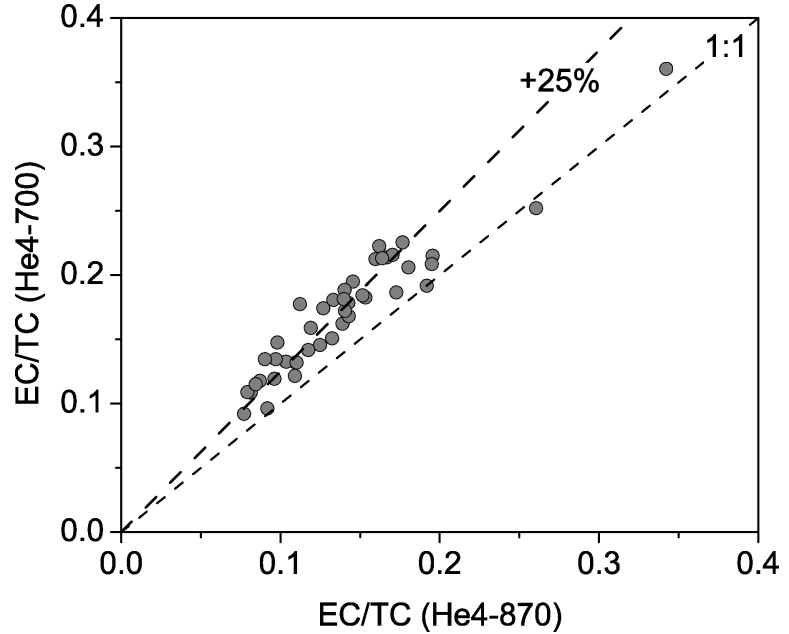

FIG. 3. Scatter plot of the EC (as a fraction of TC) measured by the He4-870 and He4-700 temperature protocols for parallel punches taken from 43 different ambient samples including 24-hour denuded samples and undenuded samples with sampling durations of $8,24,48$, or 96 hours. The He4-700 protocol gives $25 \pm 13 \%$ (average \pm standard deviation) higher EC/TC ratios than the He4870 protocol. The maximum EC loading for these samples is $8.4 \mu \mathrm{g}-\mathrm{C} / \mathrm{cm}^{2}$ (measured by the He4-700 protocol).

Figure 3 compares the TOT-EC measured on parallel punches of 43 different filters analyzed using the He4-700 and He4-870 protocols, the comparison set including both denuded and undenuded samples as well as different sampling durations $(8,24$, 48 , and 96 hours). Comparisons are made in terms of the EC/TC ratio to account for any minor variations in total carbon loadings of the parallel filter punches. During the analysis of all of these samples the laser transmission increased in the He4 step of the He4-870 protocol, indicating premature loss of light absorbing carbon. This rise in the sample transmission during the He4 step is comparatively much smaller with the He4-700 protocol, as the laser reaches a minimum value in the He4 step and remains close to this value till the end of the He-mode for most samples.

The EC/TC ratio of the punches analyzed with the He4-700 protocol is $25 \pm 13 \%$ (average \pm standard deviation) higher than the EC/TC ratio measured on parallel punches from the same filter using the He4-870 protocol. Both the average bias and the variability in the bias are significantly larger than the $6 \%$ instrument precision for EC/TC measurements made on parallel punches from the same sample analyzed with the same protocol. This indicates that the bias is statistically significant and that there is some sample-to-sample variability in the bias, likely associated with sample composition. The maximum bias is 58\%, while the EC/TC ratio measured with the He4-870 protocol is greater than that measured with the He4-700 protocol for only one sample (26\% compared to $25 \%$ ).

The difference between TOT-EC measured with the He4700 and He4-870 protocols is not due to inhomogeneous filter loadings. For the 43 ambient samples included in the intercomparison, parallel punches from the same filter analyzed with the 

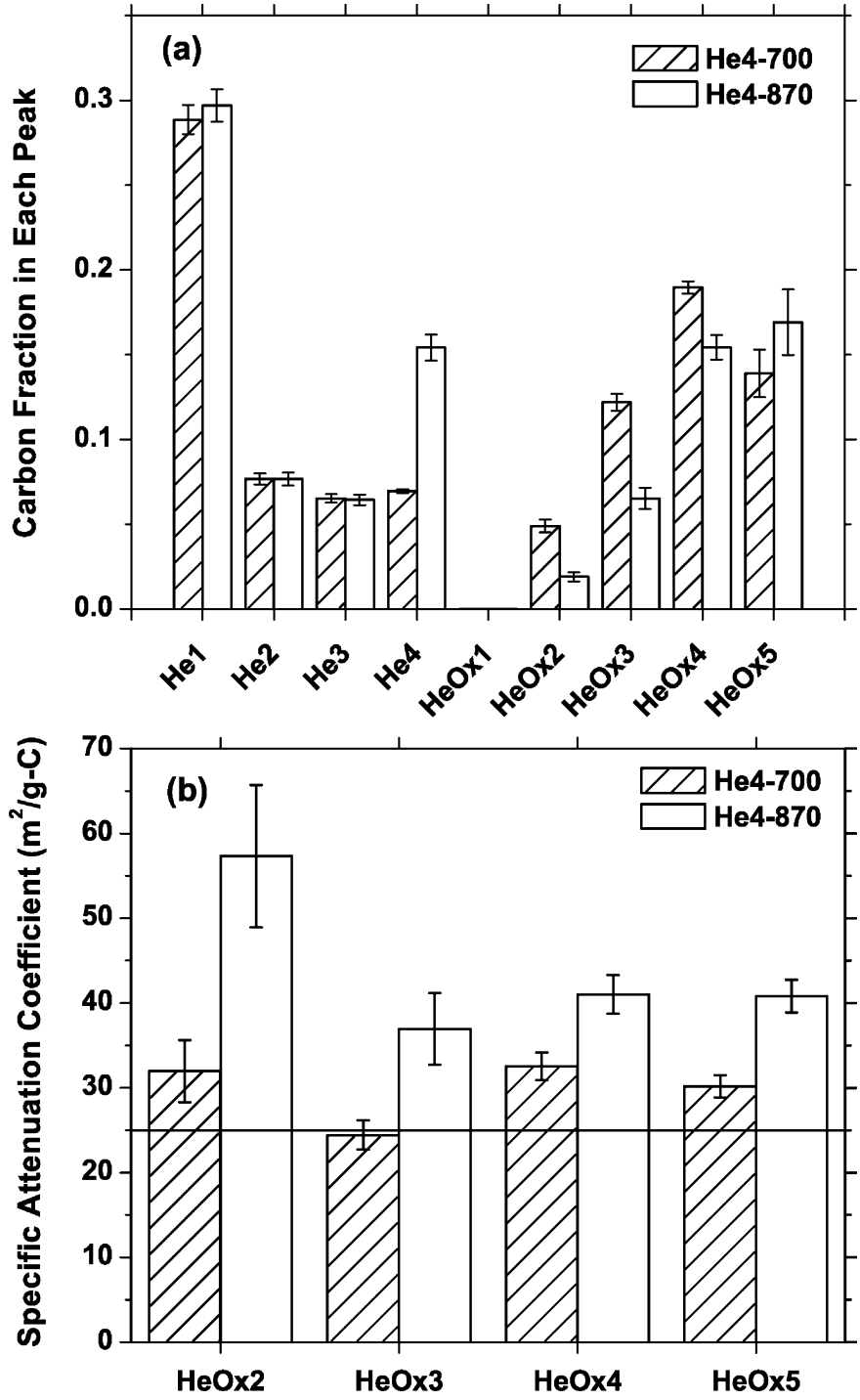

FIG. 4. Average (a) fraction of total carbon that evolves at each temperature step and (b) specific attenuation coefficient of carbon evolving at each temperature step in the He/Ox mode, for twelve undenuded 24-h samples analyzed using both the He4-700 and He4-870 temperature protocols. The horizontal line in (b) indicates an upper bound for $k_{\mathrm{EC}}$, the attenuation coefficient of elemental carbon $\left(25 \mathrm{~m}^{2} / \mathrm{g}-\mathrm{C}\right)$, as discussed in the text. Error bars indicate one standard error of the mean.

H4-870 and He4-700 protocols had TC loadings within 5\% of each other without any systematic differences. A 2-tailed pairedsample t-test of the difference between the final and initial laser transmission indicated that net laser attenuation (i.e., the optical EC loading) was not significantly different between the paired punches analyzed by the two protocols $(p=0.4680)$.

In addition to changing the TOT-EC, there are significant differences in the carbon evolution and laser attenuation of paired punches from the same sample that are analyzed with the He4-870 and He4-700 protocols. Figures 4-6 present averages and scatter plots of carbon evolution pattern and specific attenuation coefficients of samples used in the intercomparison.
Values are shown for different temperature steps of the analysis protocol.

Although there is essentially no difference in the total carbon measured on parallel punches, changing the temperature protocol systematically shifts when carbon evolves from the filter. Figures $4 \mathrm{a}$ and 5 indicate that increasing the peak He-mode temperature shifts carbon from the $\mathrm{He} / \mathrm{Ox}$ to the He-mode. For example, almost twice as much carbon evolves in He4 step of the He4-870 protocol compared to the He4-700 protocol. From the perspective of the He4-700 protocol, more carbon evolves in the lower temperature steps of the He/Ox-mode from the He4700 protocol compared to the He4-870 protocol. The OC/EC split is almost always defined in one of the lower temperature steps of the He/Ox mode, so these shifts in carbon evolution are likely related to the change in the TOT-EC.

Significant differences also exist in the specific attenuation coefficients of the carbon that evolves in the He/Ox mode of samples analyzed with the two protocols. Figure $4 \mathrm{~b}$ shows that, on average, the He/Ox carbon that evolves during the He4870 protocol is significantly darker (i.e., has a higher specific attenuation coefficient) than the carbon coming off the filter in the He/Ox mode of the He4-700 protocol. Figure 6 shows that these trends are consistent across the entire set of samples except for the lower-temperature steps of the heavily loaded multi-day (48-h and 96-h) undenuded samples where we might be seeing the saturation effect illustrated in Figure 2. The changes in sample attenuation may be related to the changes in carbon evolution shown in Figures 4a and 5; for example, if increasing the peak temperature of the He-mode preferentially shifted carbon with a lower attenuation coefficient into the He-mode.

\section{Evaluation of Assumptions Underlying the Optical Correction}

The systematic bias in the TOT-EC measured with the He4870 and He4-700 protocols indicates that we must carefully evaluate the fundamental assumptions underlying the optically defined EC. In order for thermal-optical analysis to correctly define the OC/EC split requires that either (1) PC and non-lightabsorbing carbon evolve completely before EC, or (2) the carbon evolving after the OC/EC split has the same attenuation coefficient as EC (Yang and Yu 2002). If both assumptions are invalid then the TOT method will misclassify the OC/EC split.

To evaluate these assumptions, carbon analyses were performed on filter punches that had been solvent extracted. Figure 7 compares thermograms of extracted and untreated punches taken from the same filter. Figure 7a shows results from a 24-h denuded sample analyzed with the He4-870 protocol, and Figure $7 \mathrm{~b}$ shows results from a $24-\mathrm{h}$ undenuded sample collected on the same day analyzed using the He4-700 protocol.

Figure 7 indicates that solvent extraction removes a large fraction of the carbon from the sample, which, in turn, reduces the formation of PC. Solvent extraction significantly reduces, 


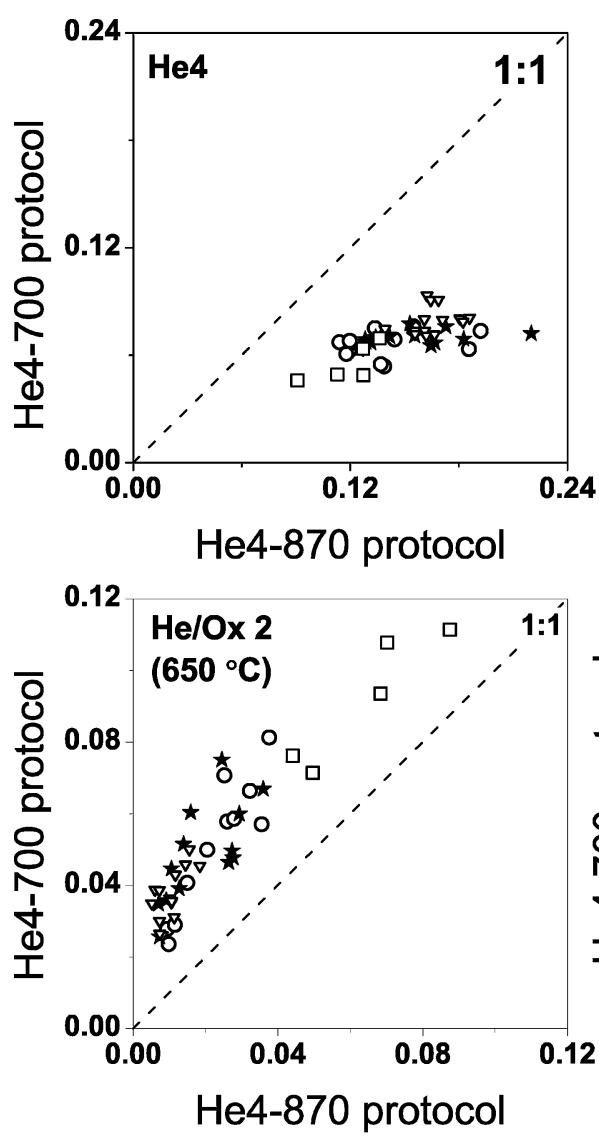

- Denuded 24-h

$\star$ Bare (undenuded) 24-h

$\nabla$ Bare 8-h

Bare 48-h and 96-h

\section{.}
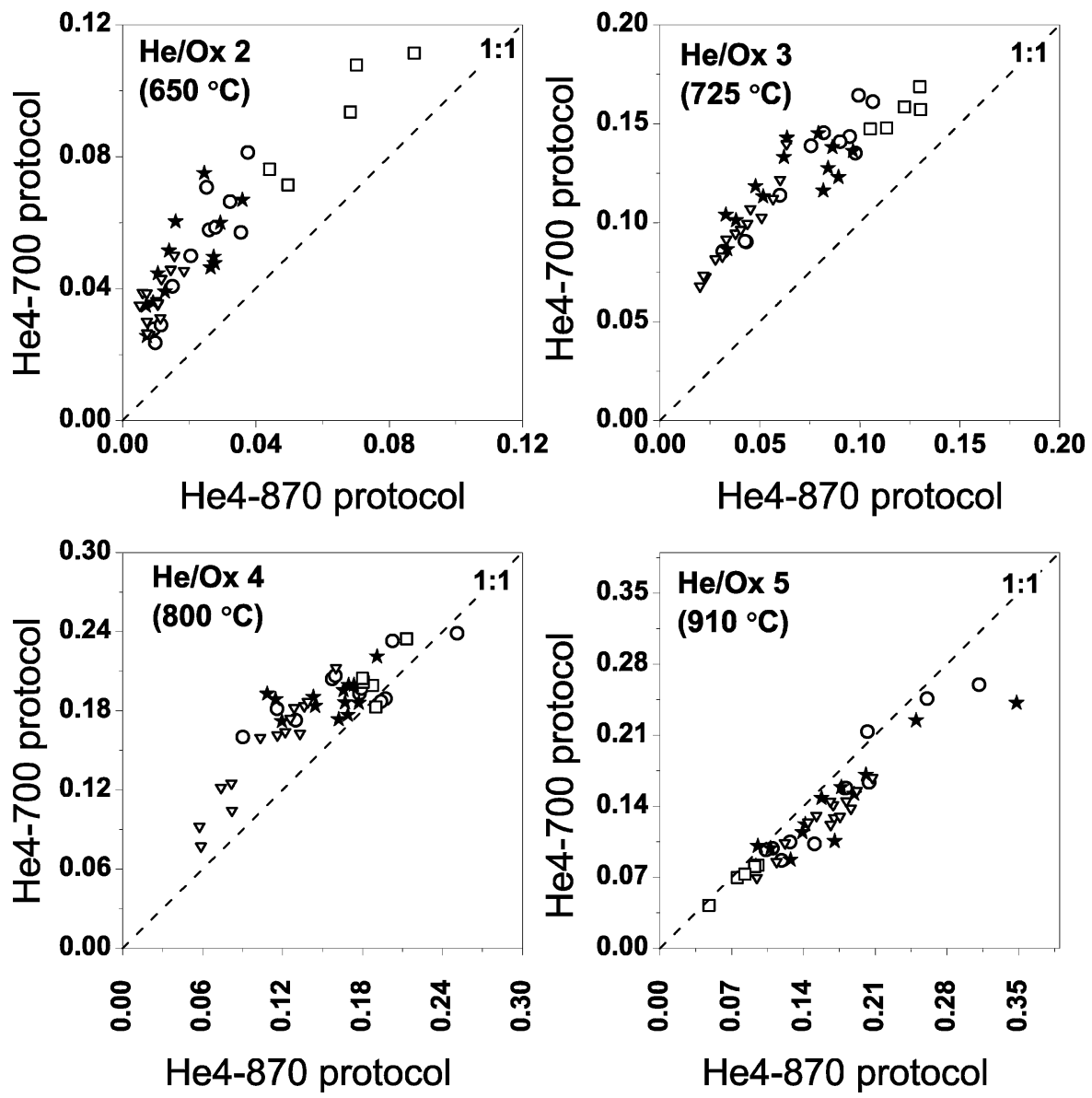

FIG. 5. Scatter plot of carbon peaks (as a fraction of total carbon) for paired filter punches analyzed with both the He4-700 and He4-870 protocols. Lowering the peak He-mode temperature from $870^{\circ} \mathrm{C}$ to $700^{\circ} \mathrm{C}$ shifts carbon from the $\mathrm{He} 4$ step to the lower-temperature steps of the He/Ox mode.

but does not eliminate, PC formation. For example, solvent extraction reduces the dip in laser attenuation associated with PC formation by $88 \%$ for the denuded sample shown in Figure 7 a and by $72 \%$ for the undenuded sample shown in Figure $7 \mathrm{~b}$ compared to the analyses of the untreated punches. Figure $7 \mathrm{~b}$ also shows that the sample transmission increases in the Heliummode as early as the He-3 step, which could be loss of PC (as only $72 \%$ of the pyrolysis is eliminated), or even some EC.

Loss of EC during solvent extraction is a concern. The optical EC loading on the extracted and untreated punches, as indicated by the net change in laser attenuation from the beginning to the end of the analysis, was almost the same (the ratio of net laser attenuation values being $97 \pm 8 \%$ for 14 

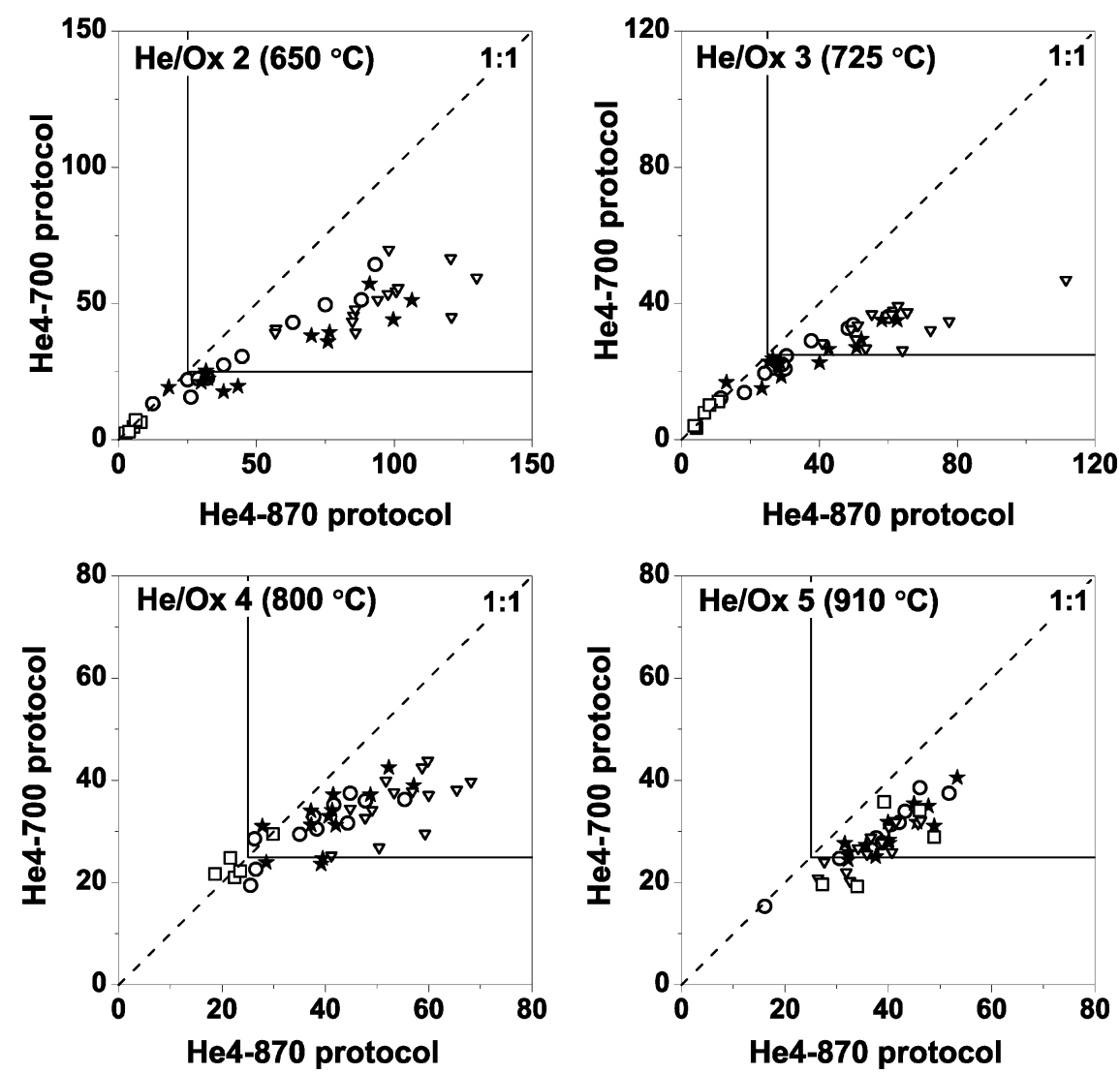

FIG. 6. Scatter plot of the specific attenuation $\left(\mathrm{m}^{2} / \mathrm{g}-\mathrm{C}\right)$ of each carbon peak for paired filter punches analyzed with both the He4-700 and He4-870 protocols. The mixture of EC and PC co-evolving in the He/Ox mode of the He4-870 protocol is more light-absorbing than the carbon coming off the filter in the He/Ox mode of the He4-700 protocol. The solid lines indicate the upper limit for $k_{\mathrm{EC}}\left(25 \mathrm{~m}^{2} / \mathrm{g}-\mathrm{C}\right)$; He4-870 samples to the left of and He4-700 samples below these lines have attenuation coefficients less than this upper bound. Symbols represent the same samples as in Figure 5.

extracted/untreated sample pairs). This indicates that there is minimal loss of EC during the solvent extraction procedure, and differences in carbon loading between the extracted and untreated punches can be attributed to removal of OC. Most of the carbon that evolves from the He/Ox mode of the analysis of the extracted punches is EC and increases in laser transmission during the analysis of extracted punches are primarily due to EC evolving from the sample.

\section{EVOLUTION OF PC AND EC}

A comparison of the carbon evolution from the paired solvent extracted and untreated punches provides strong evidence that PC and EC co-evolve and that this assumption is not valid for the Pittsburgh samples. Figure 7 shows that significantly less carbon evolves from the extracted punch than from the untreated punch in the He/Ox-mode of both protocols (He4-870 and He4-700). Since the extraction procedure did not affect the EC loading, the $\mathrm{He} / \mathrm{Ox}$ carbon removed by the solvent-extraction is PC. Notably, the He/Ox carbon removed by extraction does not just evolve at the beginning of $\mathrm{He} / \mathrm{Ox}$ mode, but throughout the entire mode providing strong evidence that PC and EC co-evolve in both protocols.
The transmission-defined OC/EC split of the solventextracted punches always occurs earlier in the analysis cycle compared to the analysis of untreated punches. For such a shift to occur, some fraction of the native EC must evolve before the transmission-defined OC/EC split of the untreated punch. We refer to this as "pre-split EC." The grey area shown in Figure 7 is an estimate of the pre-split EC; this area is the carbon that evolves from the extracted punch, between the transmissiondefined OC/EC splits of the extracted and untreated punches. This estimate represents an upper-bound for the pre-split EC because the solvent-extraction procedure does not completely eliminate PC formation. For the sample shown in Figure 7a analyzed with the He4-870 protocol, up to $50 \%$ of the native EC evolved before the transmission-defined OC/EC split of the untreated punch. In addition, a large fraction of this pre-split EC evolved in the He4 step. There is less pre-split EC when samples are analyzed with the He4-700 protocol; for the sample shown in Figure 7b up to 35\% of native EC evolved before the transmission-defined OC/EC split of the untreated punch. Increasing the length of the He/Ox steps does not change the conclusion that some fraction of the native $\mathrm{EC}$ evolves before the transmission-defined OC/EC split. 

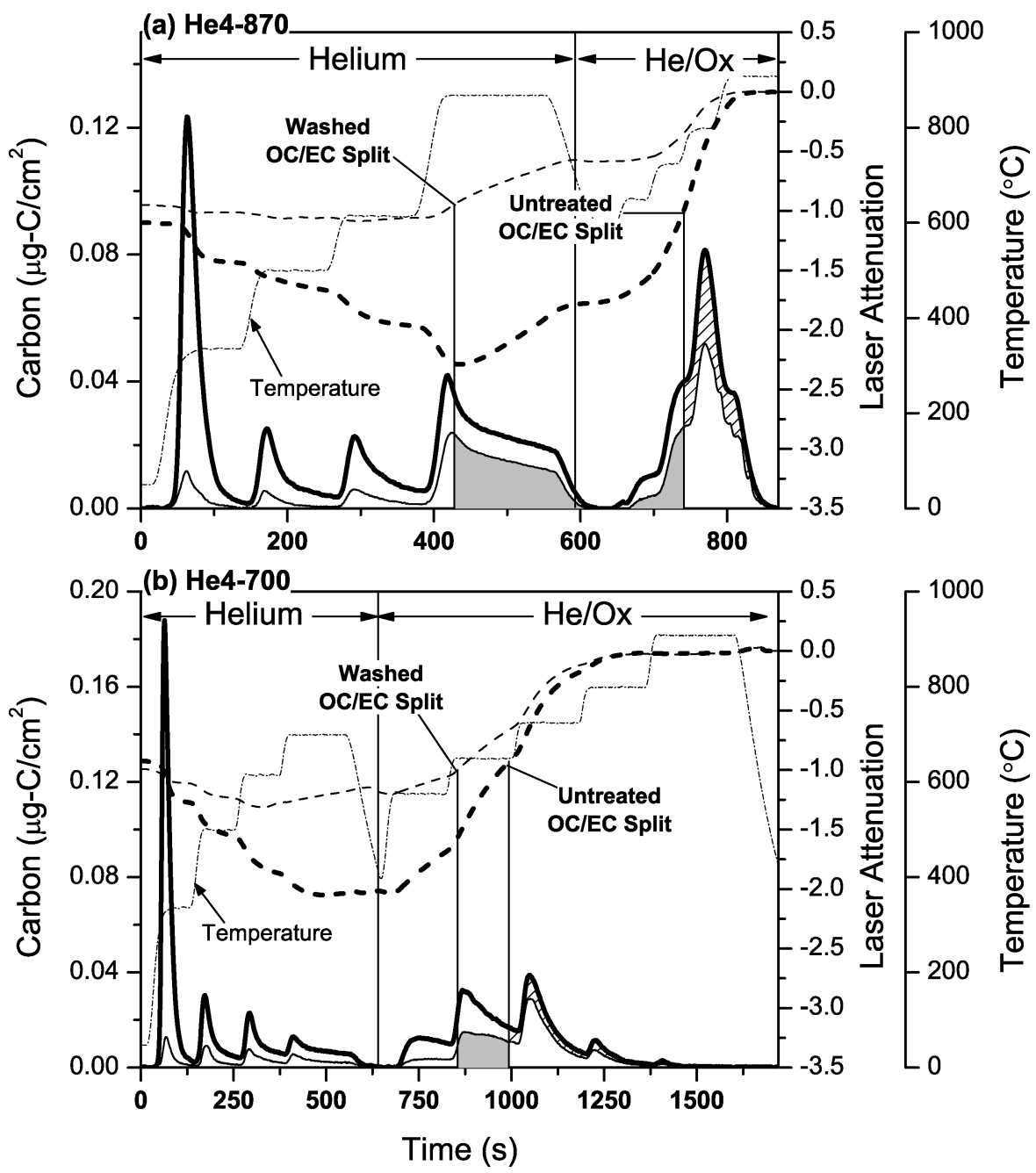

FIG. 7. Thermograms of untreated and solvent-extracted samples collected on April 17, 2002: (a) He4-870 analyses of a denuded sample; (b) He4-700 analyses of an undenuded sample using extended duration He/Ox peaks. The thin lines indicate solvent-extracted or washed punches and thick lines indicate the untreated punches. The solid lines are the carbon data plotted against the left axis, and the dashed lines are the laser attenuation data, $\ln \left(\mathrm{I} / \mathrm{I}_{\text {end }}\right)$, plotted against the right axis. EC and PC co-evolve for samples analyzed with both protocols. The grey area indicates carbon that evolves in the He/Ox mode from the solvent extracted punch before the OC/EC split of the untreated punch. This carbon is native-EC evolving before the OC/EC split. The hashed area is carbon removed by solvent extraction that evolves after the OC/EC split of the untreated punch. This carbon is PC evolving after the OC/EC split. The sample analyzed with the He4-870 protocol shows premature EC loss, causing the OC/EC split of the extracted punch to be defined in the He-mode.

Comparing the solvent-extracted and untreated punches one can also estimate the amount of PC that evolves after the transmission-defined OC/EC split of the untreated filter. For the samples shown in Figures 7a and 7b, solvent extraction removed approximately $30 \%$ of the He/Ox carbon after the transmissiondefined OC/EC split of the untreated filter. This carbon is indicated by the hashed area. It represents a lower-bound estimate of the PC that evolves after the split because solvent extraction did not eliminate $\mathrm{PC}$ formation.

Another issue is the type of light-absorbing carbon that evolves in the He-mode of the analysis-is it PC or native EC? Yu et al. (2002) suggest that it is PC. Figure 7 shows that some light-absorbing carbon evolves in the $\mathrm{He} 4$ temperature step from the solvent-extracted punches analyzed with both protocols. This loss is significantly larger for samples analyzed with the He4-870 protocol compared to those analyzed with the He4-700 protocol. For extracted samples analyzed with the He4-870 protocol (Figure 7a), the transmission-defined OC/EC split always occurs in He-mode, i.e., the sample transmission rises above the initial, pre-pyrolysis value in the He-mode. The only explanation is that native EC is clearly evolving in the $870^{\circ} \mathrm{C}$ step of the He-mode. Notably the split occurs very early in the He4 step, indicating the EC evolves throughout most of the step, making it difficult to simply shorten the He4 step to avoid the premature loss of EC. For solvent-extracted samples analyzed with the He4-700 protocol (Figure 7b), the transmission-defined OC/EC split always occurs in the He/Ox mode; therefore, the modest increase in laser transmission observed in the He-mode 
of extracted samples analyzed with the He4-700 protocol could be due to a minor loss of either native EC or PC. Given that PC and EC co-evolve through the analysis, our results do not contradict Yu et al.'s hypothesis, but complement it; the light-absorbing carbon evolving at the $\mathrm{He} 4 \mathrm{step}\left(870^{\circ} \mathrm{C}\right)$ of the He4-870 analysis of untreated samples is native EC, possibly combined with PC.

Analysis of extracted and untreated pairs of filter punches from fourteen denuded and undenuded samples taken over ten days provided results consistent with those shown in Figure 7. This means that PC and EC co-evolve in the He/Ox mode of the analysis for both protocols. In addition, although our discussion has focused on the He4-700 and He4-870 protocol, similar comparisons (as those shown in Figure 7) for punches analyzed with the He4-550 protocol also show clear evidence of co-evolution of PC and EC. Therefore, the first assumption underlying the thermal-optical analysis method that PC evolves before EC is not valid for the Pittsburgh samples. The co-evolution of PC and EC is consistent with recent results from $\mathrm{Yu}$ and co-workers (Yang and Yu 2002; Yu, Xu, and Yang 2002). Malm et al. (1994) also provides interesting evidence for the co-evolution of PC and $\mathrm{EC}$; in particular, their results suggest that native EC evolves in the lower temperature steps of the He/Ox mode. A consequence of this co-evolution is that it is incorrect to view the post-split carbon as native EC. At best, the mass of the post-split carbon will be equal to native EC. Whether or not this occurs depends on the optical properties of PC and EC, as discussed in the next section.

A second important conclusion from our analysis of the solvent-extracted punches is that increasing the peak He-mode temperature from $700^{\circ} \mathrm{C}$ to $870^{\circ} \mathrm{C}$ systematically shifts when native EC evolves from a sample. Notably, native EC is shifted from the low-temperature steps of the $\mathrm{He} / \mathrm{Ox}$ mode of the He4700 protocol to the He-mode in the He4-870 analysis. Figures 4(a) and 5 indicate that this shift is consistent across a large number of samples. An important consequence of this shift is that increasing the peak $\mathrm{He} 4$ temperature changes the relative rates at which $\mathrm{PC}$ and $\mathrm{EC}$ evolve from the filter. The native EC evolves earlier in the analysis cycle compared to PC when samples are analyzed with the He4-870 protocol. Again, this will only be a problem if PC and EC have different optical properties.

\section{ATTENUATION COEFFICIENTS OF PC AND EC}

The second assumption underlying thermal-optical OC/EC analysis is that $\mathrm{PC}$ and $\mathrm{EC}$ have the same optical properties. If sample transmission is used to define the OC/EC split this means that the specific attenuation coefficients of $\mathrm{PC}\left(k_{\mathrm{PC}}\right)$ and $\mathrm{EC}\left(k_{\mathrm{EC}}\right)$ should be the same. Estimates for $k_{\mathrm{PC}}$ and $k_{\mathrm{EC}}$ can be calculated by applying Equation (1) to the thermograms of the solventextracted and untreated filters. The calculations are based on the measured laser transmission at the beginning and the end of the $\mathrm{He} / \mathrm{Ox}$ mode and the amount of carbon that evolves in the $\mathrm{He} / \mathrm{Ox}$ mode. For the sample shown in Figure $7 \mathrm{a}$, the average attenu- ation coefficients of the $\mathrm{He} / \mathrm{Ox}$ carbon are $16 \mathrm{~m}^{2} / \mathrm{g}-\mathrm{C}$ for the solvent-extracted punch and $31 \mathrm{~m}^{2} / \mathrm{g}-\mathrm{C}$ for the untreated punch. The He/Ox carbon on the solvent-extracted punch is mainly $\mathrm{EC}$, and so we assume $k_{\mathrm{EC}}$ for this sample as $16 \mathrm{~m}^{2} / \mathrm{g}-\mathrm{C}$. The $\mathrm{He} / \mathrm{Ox}$ carbon on the untreated punch is a mixture of PC and EC; therefore $k_{\mathrm{PC}}$ must be greater than $k_{\mathrm{EC}}$. The specific attenuation coefficient of the carbon removed by solvent extraction provides an estimate for $k_{\mathrm{PC}}$. This value can be derived by combining the results from the analysis of the untreated and solvent-extracted punches, and applying Equation (1). This approach yields a $k_{\mathrm{PC}}$ value of $56 \mathrm{~m}^{2} / \mathrm{g}-\mathrm{C}$ for the sample shown in Figure $7 \mathrm{a}$.

To develop a more robust estimate for $k_{\mathrm{EC}}$ and $k_{\mathrm{PC}}$, we repeated the extraction procedure on more ambient samples. A challenge is that the extraction procedure does not eliminate PC formation and therefore does not cleanly separate PC and EC. For six ambient samples collected on different days in which the extraction procedure reduced PC formation by over $70 \%$ (average reduction $81 \%$ ), the specific attenuation of the He/Ox carbon evolving in the solvent-extracted filter, $k_{\mathrm{EC}}$, was $22.9 \pm 5.4 \mathrm{~m}^{2} / \mathrm{g}$ $\mathrm{C}$ (average \pm standard deviation). The average attenuation coefficient of the He/Ox carbon removed by solvent extraction, $k_{\mathrm{PC}}$, was $35.2 \pm 12.7 \mathrm{~m}^{2} / \mathrm{g}-\mathrm{C}$. Therefore, $k_{\mathrm{PC}}$ is consistently higher than $k_{\mathrm{EC}}$, but the large standard deviation suggests significant sample-to-sample variability in $k_{\mathrm{PC}}$. Some of this variability may also be due to changes in extraction efficiency. In addition, systematic differences were observed in the $k_{\mathrm{PC}}$ values for samples analyzed with the different protocols. The $k_{\mathrm{PC}}$ values of samples analyzed with the He4-870 protocol were on average around $40 \%$ greater than those analyzed with the He4-700 protocol. This difference may indicate additional formation and/or darkening of PC at higher temperatures in the He-mode. Finally, these values of $k_{\mathrm{PC}}$ may be at the lower end of the possible range, since for five of the six samples, pyrolysis darkens or reduces the transmission of the untreated samples close to or beyond the limits of linear laser response (as per Gundel et al. [1984], $\ln \left(I_{\text {blank }} / I_{\text {sample }}\right)$ of 2.0) where the "shadowing" or "light-pipe" effect is seen.

Our estimates of $k_{\mathrm{EC}}$ are consistent with literature values for EC deposited on quartz filters, which range from $8.1 \mathrm{~m}^{2} / \mathrm{g}$ to $25.4 \mathrm{~m}^{2} / \mathrm{g}$ for solvent-extracted ambient filters (Gundel et al. 1984; Petzold, Kopp, and Niessner 1997). In comparing different values, one must always keep in mind that $k_{\mathrm{EC}}$ depends on various factors including aging, wavelength, the extraction/thermal methods, and the filter substrate. For example, Bond and Bergstrom (2006) suggests a mass absorption coefficient of $7.5 \pm 1.2 \mathrm{~m}^{2} / \mathrm{g}$ for fresh light absorbing carbon, measured at $550 \mathrm{~nm}$; assuming absorption varies inversely with wavelength gives $6 \mathrm{~m}^{2} / \mathrm{g}-\mathrm{C}$ at $680 \mathrm{~nm}$ (the wavelength of the laser in our Sunset instrument). The aerosol in Pittsburgh is highly aged; aging tends to increase the absorption coefficient of EC due to coating of the EC by non-absorbing species (Fuller, Malm, and Kreidenweis 1999). Finally, collection of EC on a quartz filter enhances the absorption coefficient by about a factor of two (Weingartner et al. 2003). However, although there appears to be 

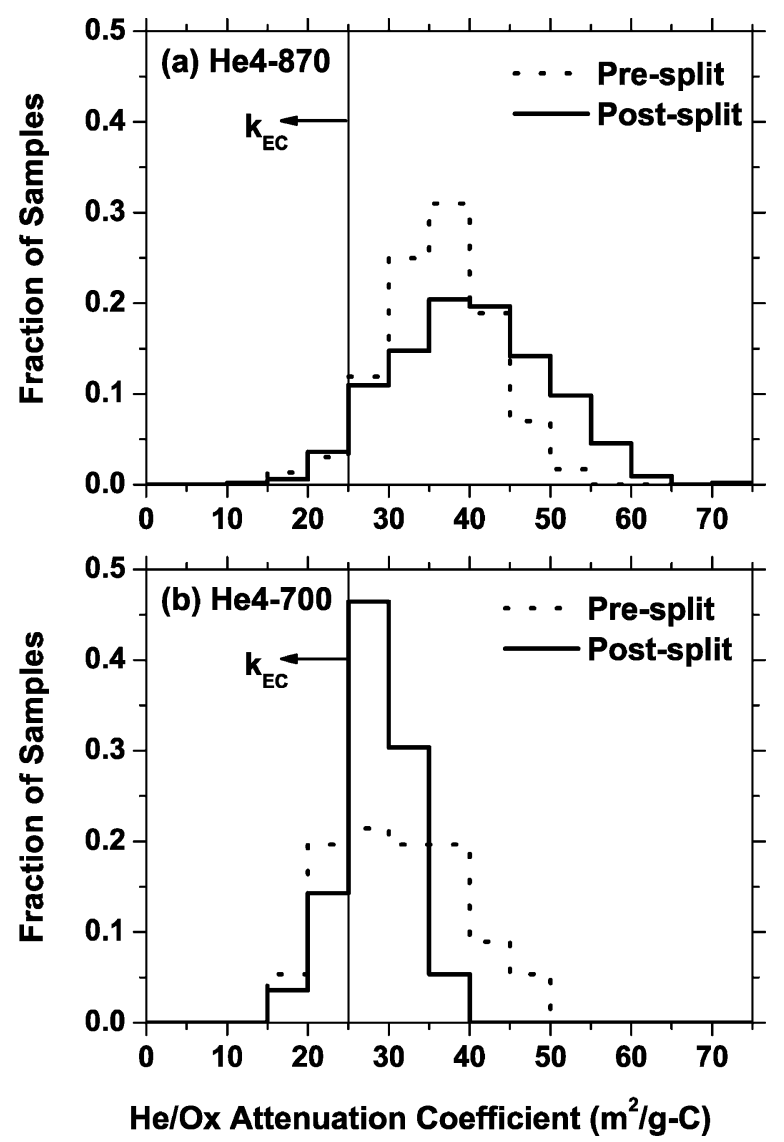

FIG. 8. Histograms of specific attenuation coefficient of pre- and post-split $\mathrm{He} / \mathrm{Ox}$ carbon measured using the (a) He4-870 protocol (number of samples, $\mathrm{N}=529)$, and (b) He4-700 protocol $(\mathrm{N}=56)$. The results are based on the analysis of 24-h denuded and undenuded samples. The vertical line indicates the upper bound for $k_{\mathrm{EC}}\left(25 \mathrm{~m}^{2} / \mathrm{g}-\mathrm{C}\right)$.

no unique value for $k_{\mathrm{EC}}, 25 \mathrm{~m}^{2} / \mathrm{g}-\mathrm{C}$ appears to be a reasonable upper bound (Liousse, Cachier and Jennings 1993). Significantly less research has been published about $k_{\mathrm{PC}}$; Chow et al. (2004) report $k_{\mathrm{PC}}$ values greater than $45 \mathrm{~m}^{2} / \mathrm{g}-\mathrm{C}$.

Additional insight into $k_{\mathrm{EC}}$ and $k_{\mathrm{PC}}$ can be gained by examining histograms of attenuation coefficients of pre- and post-split $\mathrm{He} / \mathrm{Ox}$ carbon for samples analyzed with the He4-700 and He4870 protocols, shown in Figure 8. For the vast majority of the samples, the $\mathrm{He} / \mathrm{Ox}$ carbon attenuation coefficient is greater than $25 \mathrm{~m}^{2} / \mathrm{g}-\mathrm{C}$. This indicates that $k_{\mathrm{PC}}$ is almost always greater than $k_{\mathrm{EC}}$ (assuming that $25 \mathrm{~m}^{2} / \mathrm{g}-\mathrm{C}$ is the upper limit for $k_{\mathrm{EC}}$ ). The $\mathrm{He} / \mathrm{Ox}$ carbon measured with the He4-870 protocol is significantly darker than that measured with the He4-700 protocol. This is consistent with premature evolution of EC in the Hemode of the He4-870 protocol, which means PC contributes a much larger fraction of the He/Ox carbon of samples analyzed with the He4-870 protocol than during analysis with the He4700 protocol. An additional factor is that $k_{\mathrm{PC}}$ values for samples analyzed with the He4-870 protocol are larger than those of samples analyzed with the He4-700 protocol as noted earlier.
By comparing the average post-split carbon attenuation coefficients of the two protocols ( 36 and $28 \mathrm{~m}^{2} / \mathrm{g}-\mathrm{C}$ for the He $4-870$ and He4-700 protocols, respectively), one can estimate the average bias in the TOT-EC defined by the two protocols. The difference in these values means that if the net laser attenuation of the two samples is the same, on average $29 \%$ more carbon evolves after the split (i.e., $29 \%$ more TOT-EC) for a sample analyzed with the He4-700 protocol compared to an analysis with the He4-870 protocol. A $29 \%$ bias is consistent with the intercomparison results shown in Figure 3, which is based on a small subset of the samples shown in Figure 8.

Our examination of the attenuation coefficients of the solventextracted and untreated samples indicates that PC has a significantly higher attenuation coefficient than $\mathrm{EC}\left(k_{\mathrm{PC}}>k_{\mathrm{EC}}\right)$. Therefore the second assumption underlying the thermal optical technique is also not valid. This means that the post-split carbon is not equal to the native EC mass. The bias will depend on the attenuation coefficient of the post-split carbon relative to that of the native EC. For the Pittsburgh samples, $k_{\mathrm{PC}}$ is greater than $k_{\mathrm{EC}}$; combined with co-evolution of $\mathrm{PC}$ and $\mathrm{EC}$, this means the TOT-defined EC is likely biased low compared to the true EC. The extent of this bias depends on the relative rate at which PC and $\mathrm{EC}$ evolve off the filter. An additional complication is that there is no unique value for $k_{\mathrm{EC}}$ or $k_{\mathrm{PC}}$, since optical properties depend on aerosol composition, measuring wavelength, and other parameters.

\section{Effect of Changes in the Relative Rates of EC and PC Evolution on the OC/EC Split}

We now use a model based on the Lambert-Beer law to illustrate quantitatively how changing the relative rate at which EC and $\mathrm{PC}$ evolve from the filter biases the transmission-defined OC/EC split. The model assumes that only two types of carbon, PC and EC, evolve from the sample after laser transmission reaches its minimum value. Under these conditions, the change in laser transmission (once it has passed its nadir) can be defined as,

$$
I(t)=I(t-1) \cdot \exp \left[C(t) \cdot\left(f_{\mathrm{PC}}(t) k_{\mathrm{PC}}+f_{\mathrm{EC}}(t) k_{\mathrm{EC}}\right)\right]
$$

where $I(t-1)$ and $I(t)$ are the laser transmission values at the start and at the end of a discrete time step during which the carbon mass $C(t)$ has evolved from the sample, and $f_{\mathrm{PC}}(t)$ and $f_{\mathrm{EC}}(t)$ are the PC and EC fractions of $C(t)$, with $f_{\mathrm{PC}}(t)+f_{\mathrm{EC}}(t)=1$. The model can easily be generalized to consider additional types of carbon such as non-light-absorbing organic carbon slipping into the He/Ox mode, but two are sufficient to illustrate how changing the relative rate at which different types of carbon with different optical properties evolve changes the TOT-EC. We also assume that $k_{\mathrm{EC}}$ and $k_{\mathrm{PC}}$ are not temperature dependent, but this assumption could also be easily relaxed.

We apply the model using data from the sample shown in Figure 7(a), using the measured carbon evolution during each 
second of the analysis for $C(t)$, and the measured minimum laser transmission as a starting point. These values are from the OC/EC analysis of the untreated punch. The native EC is assumed to be the carbon evolving from the solvent-extracted punch after the minimum laser signal of the untreated sample, $5.6 \mu \mathrm{g}-\mathrm{C} / \mathrm{cm}^{2}$. We assume that PC is the difference of the carbon combusting in the He/Ox mode of the two analyses, $2.2 \mu \mathrm{g}$ $\mathrm{C} / \mathrm{cm}^{2}$, likely an under-estimate since we do not include any PC that evolves in the He-mode (though it could also contain some EC lost from the solvent-extracted sample). We use the specific attenuation coefficient of the He/Ox carbon of the solventextracted sample as $k_{\mathrm{EC}}\left(16 \mathrm{~m}^{2} / \mathrm{g}-\mathrm{C}\right)$, and the specific attenuation coefficient of the carbon removed from the He/Ox mode of the analysis for $k_{\mathrm{PC}}\left(56 \mathrm{~m}^{2} / \mathrm{g}-\mathrm{C}\right)$.

Using these inputs, Figure 9 shows model calculations for two scenarios: (a) "PC-first," the darker PC evolves completely before the EC; and (b) "EC-first," the EC completely evolves before the PC. These scenarios define the minimum and maximum amounts of TOT-EC. Underestimation of PC coupled with some apparent loss of EC in the solvent-extracted sample (indicated by the different net laser attenuation for the untreated and solvent-extracted punches) causes the computed final laser transmission to be slightly less than the measured final value.

The model calculations clearly illustrate how changing the relative rate at which $\mathrm{PC}$ and $\mathrm{EC}$ evolve from the filter alters the OC/EC split. Since $k_{\mathrm{PC}}$ is greater than $k_{\mathrm{EC}}$, the first scenario ("PC-first") yields the maximum amount of TOT-EC. This scenario is consistent with the assumptions underlying the thermal-optical method; therefore it yields a TOT-EC of $5.7 \mu \mathrm{g} / \mathrm{cm}^{2}$, in good agreement with the native EC used as an input for the calculations (a little higher since the split is calculated at $97 \%$ of the initial laser value). The second scenario, "EC-first," defines the minimum value for TOT-EC at $1.7 \mu \mathrm{g} / \mathrm{cm}^{2}$, a factor of three smaller than the native EC. The large difference between these two limits is due to the significantly different attenuation coefficients for PC and EC. Although this second scenario is unlikely to occur during an actual analysis, it provides a useful reference point.

The relative rate at which $\mathrm{PC}$ and $\mathrm{EC}$ evolve (with respect to each other) shifts the OC/EC split between the two limits defined by the model. More premature evolution of EC, the farther the split shifts towards the EC-first limit. Figure 9 shows that the actual analysis of the untreated filter using the He4870 protocol falls between the two calculated limits. This is expected given the co-evolution of PC and EC, especially the significant premature evolution of EC in the $\mathrm{He} 4$ step of the $\mathrm{He} 4-$ 870 protocol. The TOT-EC measured by the He4-870 protocol is $4.4 \mu \mathrm{g}-\mathrm{C} / \mathrm{cm}^{2}$, about $20 \%$ smaller than the value determined by solvent extraction $\left(5.6 \mu \mathrm{g}-\mathrm{C} / \mathrm{cm}^{2}\right)$.

The results illustrated in Figure 9 provide a framework for understanding the systematic biases between the TOT-EC values measured by different temperature protocols. For samples analyzed with the He4-870 protocol, a larger fraction of the EC evolves earlier in the analysis cycle-in the $\mathrm{He} 4$ temperature step-compared to samples analyzed with the He4-700 protocol. This shift alters the TOT-EC because the attenuation coefficients

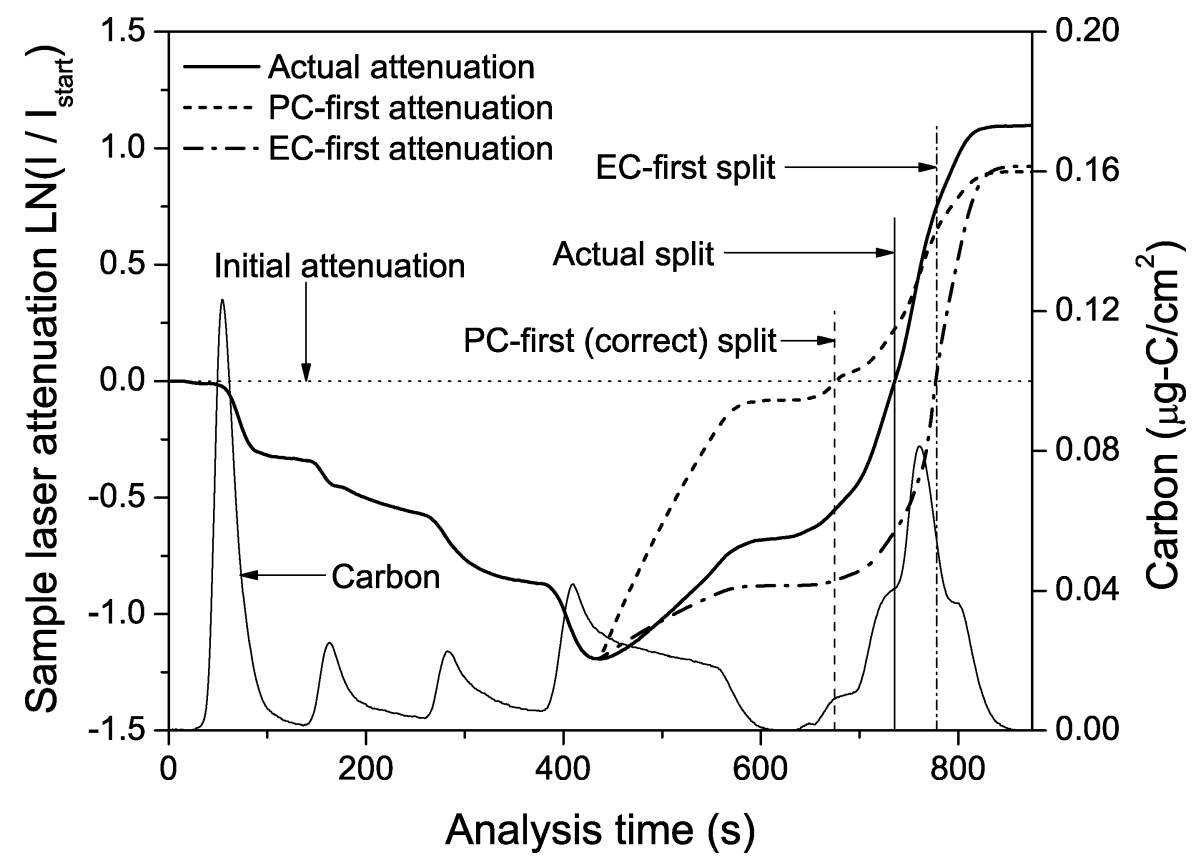

FIG. 9. Predicted change in laser attenuation due to evolution of light-absorbing carbon (PC and EC) off the filter for the sample shown in Figure 7a. The PC-first scenario satisfies the method assumptions, and is the true OC/EC split. Due to the higher specific attenuation coefficient of PC $\left(k_{\mathrm{PC}}>k_{\mathrm{EC}}\right.$ ), the rise in laser transmission in the PC-first scenario is much sharper than is actually observed. The EC-first model is an extreme case which would give the maximum underestimation of EC. In reality, EC and PC co-evolve, and the observed laser transmission falls in between the two extremes. 
of EC and PC are different. In Pittsburgh, $k_{\mathrm{PC}}$ is greater than $k_{\mathrm{EC}}$, so shifting EC evolution to earlier in the analysis cycle (relative to PC) reduces the amount of TOT-EC. Therefore, at least for samples collected in Pittsburgh, the premature evolution of EC causes the TOT-EC defined by the He4-870 protocol to consistently underestimate the native EC.

\section{Peak He-mode Temperature and Non-Light-Absorbing Carbon}

Lowering the peak He-mode temperature to $700^{\circ} \mathrm{C}$ improves the OC/EC split by avoiding premature evolution of EC, and so the question arises: would lowering the peak He-mode temperature further, say to $550^{\circ} \mathrm{C}$ as in the IMPROVE protocol (Chow et al. 1993), give better answers for the OC/EC split? A concern created by lowering the peak He-mode temperature is that not all of the $\mathrm{OC}$ will volatilize or pyrolyze during the inert portion of the analysis resulting in non-light-absorbing carbon evolving in the He/Ox mode (Novakov and Corrigan 1995; Schauer et al. 2003). To examine issues associated with non-light-absorbing carbon we analyzed quartz filters loaded with either levoglucosan (99+\% pure; ACROS Organics) or secondary organic aerosol (SOA). Levoglucosan, a pyrolysis product of cellulose, is a major component of wood smoke (Simoneit et al. 1999; Schauer et al. 2001). The secondary organic aerosol (SOA) was created by reacting $\alpha$-pinene with ozone in the Carnegie Mellon University smog chamber.

Figure 10 shows thermograms of pre-baked blank quartz filters doped with about $10 \mu \mathrm{g}-\mathrm{C} / \mathrm{cm}^{2}$ of levoglucosan analyzed with the He4-700 (Figure 10a) and He4-550 (Figure 10b) protocols. These samples do not contain EC, so all of the He/Ox carbon is either OC or PC. Twenty-three percent of the carbon from the levoglucosan evolves in the He/Ox mode of the He4550 analysis versus only 5\% for the He4-700 analyses. Notably, a significant amount of the carbon evolves in the $615^{\circ} \mathrm{C}$ and $700^{\circ} \mathrm{C}$ steps of the He-mode of the He4-700 protocol. The laser data indicates modest formation of light-absorbing PC during the analysis of levoglucosan-doped filters, with the overall pyrolytic attenuation being a few percent of that typically observed on similarly loaded ambient samples. For analyses using the He4-550 protocol, the average attenuation coefficient of the $\mathrm{He} / \mathrm{Ox}$ carbon was very low, $1.2 \mathrm{~m}^{2} / \mathrm{g}-\mathrm{C}$, which indicates that the bulk of the $\mathrm{He} / \mathrm{Ox}$ carbon is non-light-absorbing. In contrast, the $\mathrm{He} / \mathrm{Ox}$ carbon evolving during analysis with the He4-700 protocol

(a) Levoglucosan analyzed with He4-700

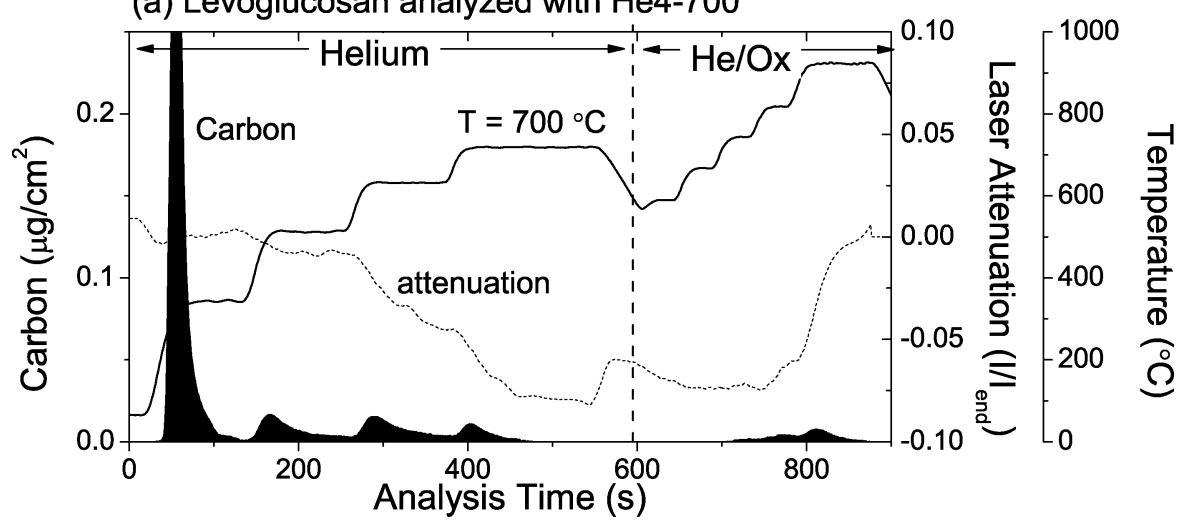

(b) Levoglucosan analyzed with He4-550

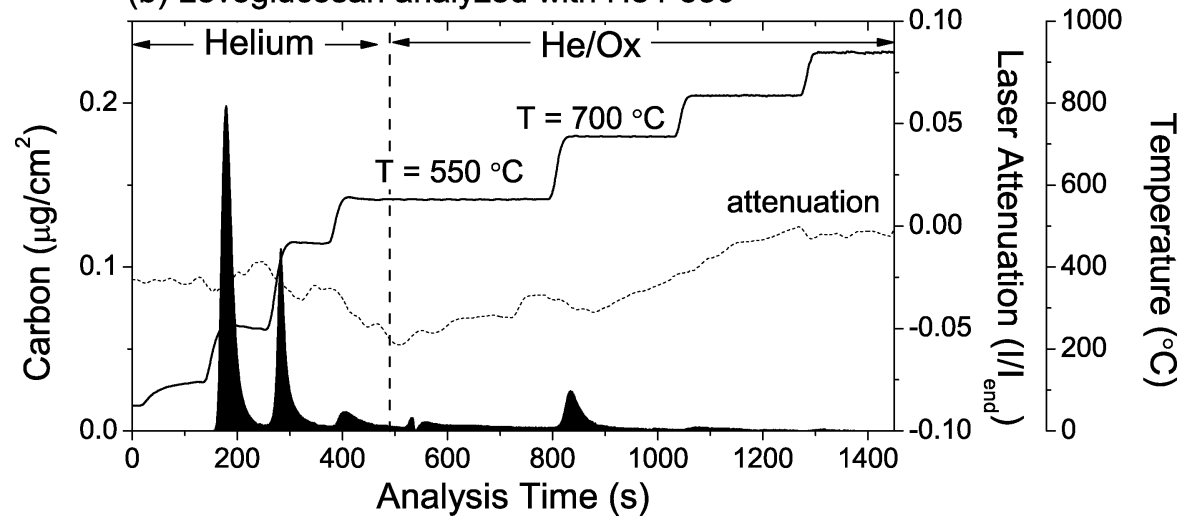

FIG. 10. Thermograms for levoglucosan-doped quartz filters analyzed using the (a) He4-700 and (b) the He4-550 protocols. 23\% of the levoglucosan carbon evolves in the He/Ox mode during analysis with the He4-550 protocol. As discussed in the text, this carbon is largely transparent (not light absorbing). In contrast, just $5 \%$ of the levoglucosan carbon evolves in the He/Ox mode during analysis with the He4-700 protocol. The laser dip due to pyrolysis in these samples is just a few percent of that seen with ambient samples (e.g., Figure 1). 

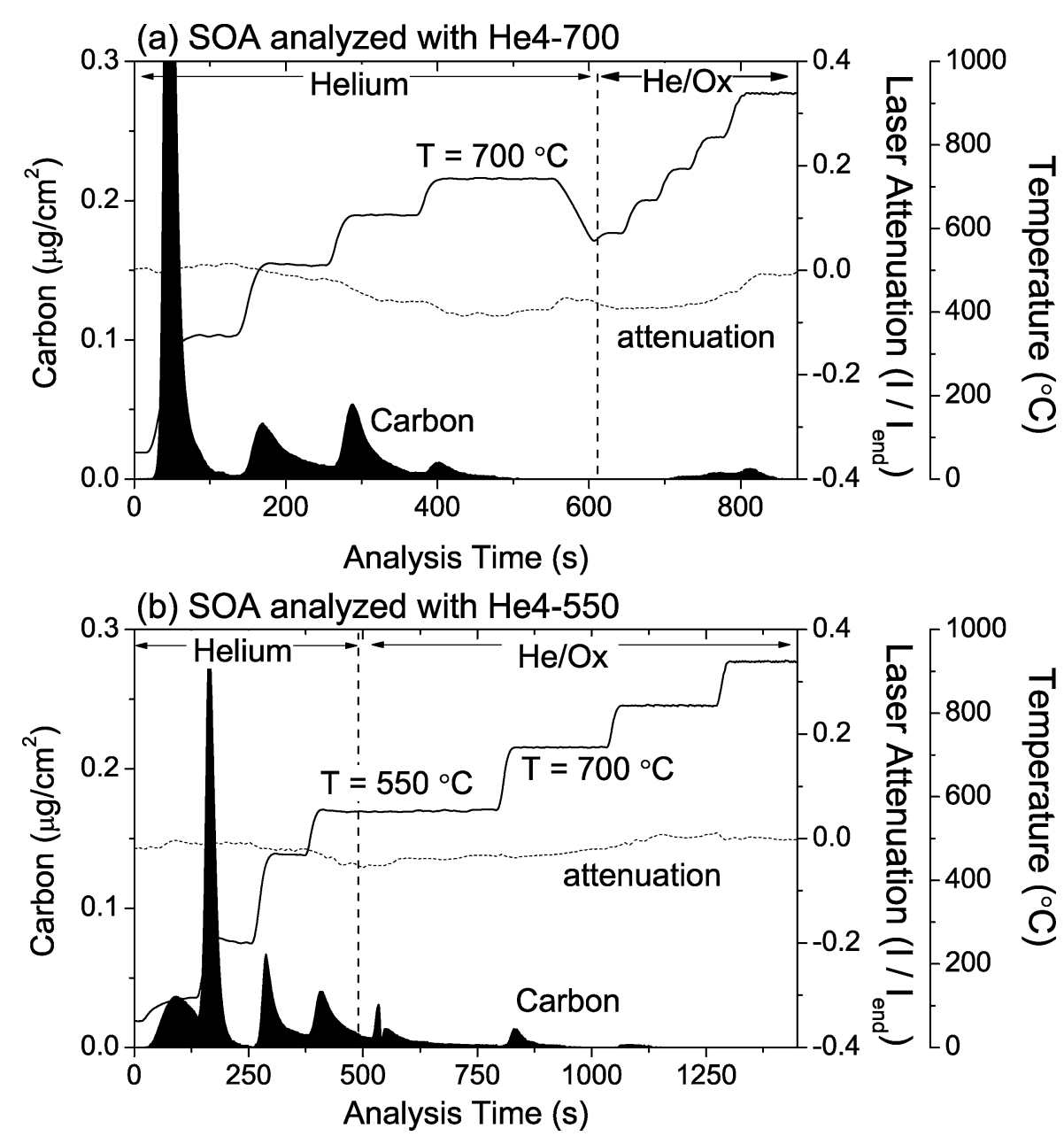

FIG. 11. Thermograms for secondary organic aerosol (SOA) collected on quartz filters analyzed using the (a) He4-700 and (b) the He4-550 protocols. The SOA was formed by the reaction of $\alpha$-pinene with ozone in the Carnegie Mellon University smog chamber.

absorbs light, with an average attenuation coefficient of $18 \mathrm{~m}^{2} / \mathrm{g}-\mathrm{C}$.

Thermograms of SOA analyzed with both the He4-700 protocol and He4-550 protocols are shown in Figure 11. The results for SOA are similar to those of levoglucosan-doped filters. First, some fraction of the SOA carbon evolves in the He/Ox mode with both protocols: $12 \%$ for the $\mathrm{He} 4-550$ protocol versus $5 \%$ for the He4-700 protocol. Minor amounts of PC formation are apparent in the SOA analyses using both protocols; the average attenuation coefficient of the He/Ox carbon evolving from the He4-550 protocol is $2.2 \mathrm{~m}^{2} / \mathrm{g}-\mathrm{C}$ versus $10.5 \mathrm{~m}^{2} / \mathrm{g}$-C for the He4-700 protocol. Finally, a significant fraction of the He/Ox carbon during the SOA analysis evolves in the higher temperature He/Ox steps in both protocols. For the He4-550 protocol, one-third of the $\mathrm{He} / \mathrm{Ox}$ carbon evolved at $700^{\circ} \mathrm{C}$ or higher. Overall, however, the problems associated with SOA appear to be less severe than those associated with levoglucosan for the He4-550 protocol.

Although Figures 10 and 11 show that significant amounts of relatively non-light-absorbing carbon evolve in the $\mathrm{He} / \mathrm{Ox}$ mode during the analysis of levoglucosan and SOA with the He4-550 protocol, this carbon is not misclassified as EC. So, for these simple systems, the transmission defined OC/EC split works. A problem arises only if the non-light-absorbing organics evolve off the filter after the OC/EC split. With ambient samples, which are a complex mixture of many different organics and EC, we cannot isolate this effect.

To illustrate the potential bias created by the slip of nonlight-absorbing carbon on the TOT-EC, Figure 12 presents thermograms from as-collected tunnel samples superimposed on thermograms of levoglucosan-doped tunnel samples, analyzed with both the He4-700 (Figure 12a) and He4-550 (Figure 12b) protocols. Figure 12a shows that negligible levoglucosan carbon evolves in the He/Ox mode of the samples analyzed with the He4-700 protocol. Consequently, the measured TOT-EC remains relatively unchanged despite the addition of levoglucosan (4.9 $\mu \mathrm{g}-\mathrm{C} / \mathrm{cm}^{2}$ for the as-collected tunnel sample compared to $5.1 \mu \mathrm{g}-\mathrm{C} / \mathrm{cm}^{2}$ for the doped sample). However, adding levoglucosan increases the TOT-EC of the tunnel sample analyzed with the He4-550 protocol, from $5.5 \mu \mathrm{g}-\mathrm{C} / \mathrm{cm}^{2}$ for the as-is tunnel sample to $6.5 \mu \mathrm{g}-\mathrm{C} / \mathrm{cm}^{2}$ for the levoglucosan-doped sample. The 
(a) Levoglucosan-doped tunnel sample: He4-700

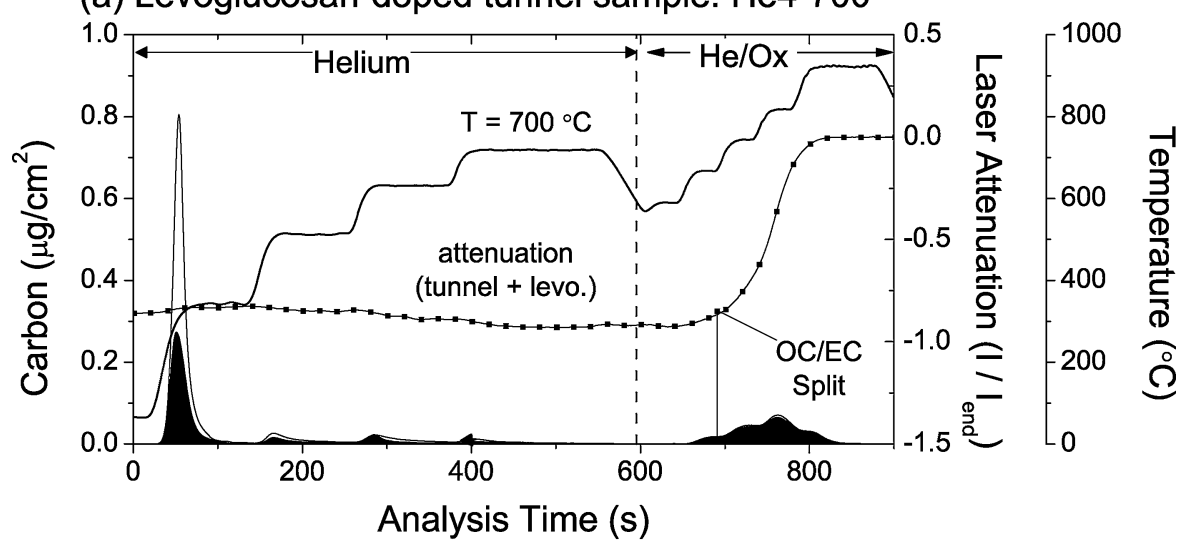

(b) Levoglucosan-doped tunnel sample: He4-550

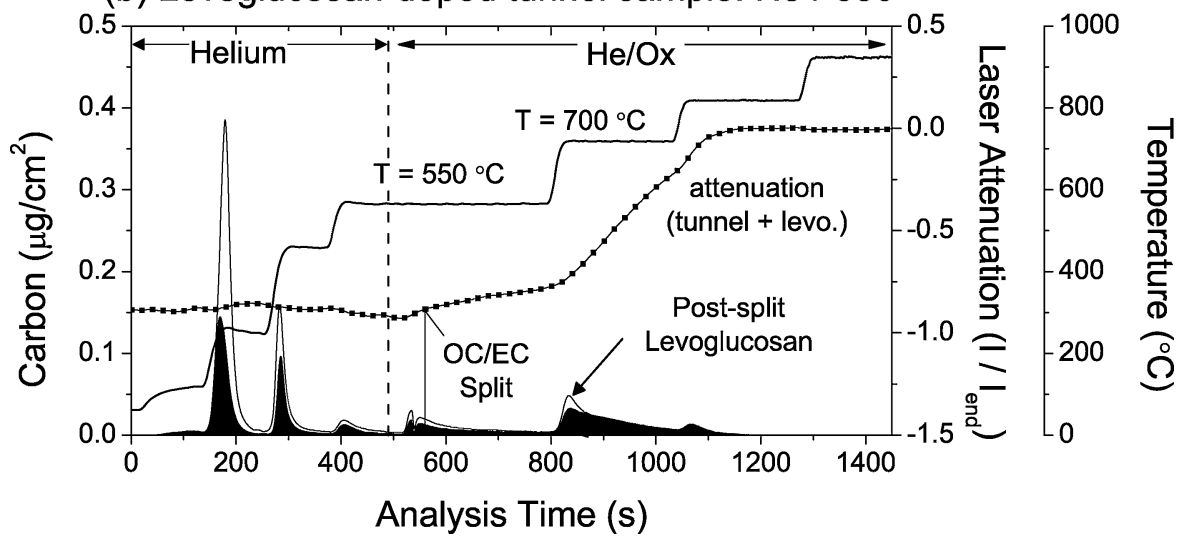

FIG. 12. Thermograms showing the effect of slip of non-light absorbing carbon in the He/Ox mode. Levoglucosan is spiked onto vehicular exhaust-dominated tunnel samples, and analyzed with the (a) He4-700 and (b) He4-550 protocols. As Figure 10 suggests, the He4-700 protocol does not experience significant slip of levoglucosan into the He/Ox mode, while during the He4-550 analysis, a significant fraction of the He/Ox mode levoglucosan comes off at the higher temperature steps, causing a positive bias in EC measurement with the He4-550 protocol relative to the undoped tunnel sample.

attenuation coefficient of the post-split carbon in the He4-550 protocol decreases from $16.9 \mathrm{~m}^{2} / \mathrm{g}-\mathrm{C}$ for the as-is tunnel sample to $13.6 \mathrm{~m}^{2} / \mathrm{g}-\mathrm{C}$ for the doped sample reflecting the effect of non-light-absorbing carbon on the net attenuation coefficient of the carbon evolving after the OC/EC split.

Figure $12 \mathrm{~b}$ provides evidence that other components of the aerosol affect the evolution of OC from the filter. For a clean quartz filter doped with levoglucosan, $23 \%$ of the levoglucosan OC came off in the He/Ox mode (Figure 10b) compared to only $14 \%$ of the levoglucosan OC coming off in the He/Ox mode of the levoglucosan-doped tunnel sample (Figure 12b). This indicates that ambient PM and $\mathrm{OC}$, which are mixtures of emissions from different sources, likely behave differently from pure compounds; results from Yu et al. (2002) also illustrate this effect.

We also compared EC measurements made on parallel punches taken from fourteen 24-hour ambient samples, using the He4-550 and He4-700 protocols. Six of the samples are high-wood-smoke samples (selected based on parallel levoglucosan concentrations). The remaining samples are from periods with low levoglucosan concentrations and moderate to high
OC/EC ratios, which suggest low wood smoke and some SOA. Total carbon and net attenuation for the paired punches were within $10 \%$, indicating that differences between the He4-550 and He4-700 analyses were not due to inhomogeneous filter loadings. Figure 13 compares the EC measurements made using the He4-700 and He4-550 protocols, as a fraction of the total carbon. For the six high wood smoke samples, the EC/TC ratio as per the He4-550 protocol is $1.52 \pm 0.15$ (average \pm standard deviation) times higher than the EC/TC ratio measured with the He4-700 protocol. A smaller, more variable bias is observed for the eight non-wood-smoke samples, for which the $\mathrm{He} 4-550 \mathrm{EC} / \mathrm{TC}$ ratio is $1.21 \pm 0.27$ times He4-700 EC/TC value. For three of the non-wood-smoke samples the agreement between the two protocols is within measurement uncertainty. The large variability in the bias between the two protocols for the non-wood-smoke samples is not unexpected given that the slip of non-light-absorbing carbon likely depends on sample composition and is not limited to wood smoke compounds. Such variability is consistent with the results of Schauer et al. (2003). Given the potential positive bias, it does not appear 


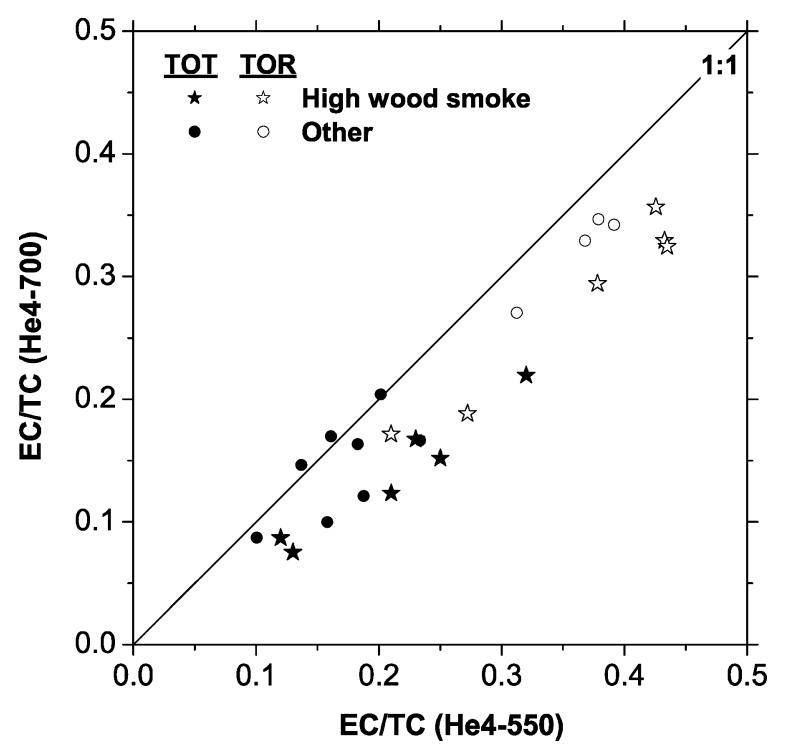

FIG. 13. Scatter plot of EC (as a fraction of TC) measured with He4-550 and He4-700 protocols for paired punches from high wood smoke and other ambient samples. The He4-550 protocol typically measures more EC than the He4-700 protocol, except for the three ambient samples close to the 1:1 line. Results are shown for OC/EC splits defined using transmission (TOT) and reflectance (TOR). TOR EC is not available for some of the samples included in the TOT inter-comparison, including the three TOT samples that show good agreement between the two temperature protocols.

advisable to reduce the peak He-mode temperature from $700^{\circ} \mathrm{C}$ to $550^{\circ} \mathrm{C}$.

\section{Reflectance versus Transmission}

Sample reflectance (TOR) is an alternative approach for defining the OC/EC split (Chow et al. 1993). Chow et al. (2004) found that TOR provides a more consistent OC/EC split than TOT across a wide variety of temperature protocols. Toward the end of this research the Carnegie Mellon University Sunset Laboratory OC/EC analyzer was upgraded to simultaneously measure both TOR and TOT EC. Figure 13 shows TOT- and TOR-EC measured for the set of ten samples used in the He4550 versus $\mathrm{He} 4-700$ comparison.

TOR reduced but did not eliminate the EC bias between the two temperature protocols. As a fraction of total carbon, the TOR EC measured with the He4-550 protocol is $1.23 \pm 0.11$ times higher than the TOR EC measured with the He4-700 protocol (the bias in the TOT EC was $1.48 \pm 0.17$ for the ten samples analyzed by both TOR and TOT). Similar to TOT, a larger bias is observed in the TOR EC of the wood smoke samples $(1.30 \pm 0.09)$ than for the non-wood-smoke samples $(1.13 \pm 0.03)$. These results are in contrast to Chow et al. (2004) who found no bias in TOR EC measured with different temperature protocols. One significant difference between the two studies is the EC loading of the filters; the peak EC loading of our filters was about $8 \mu \mathrm{g}$ $\mathrm{C} / \mathrm{cm}^{2}$, significantly lower than those considered by Chow et al. (2004).
The TOR EC is roughly a factor of two higher than TOT EC for both temperature protocols. This means that the split defined by TOR occurs earlier in the analysis cycle than the TOT split. Chow et al. (2004) also report substantially higher TOR EC than TOT EC. An earlier optical split increases the likelihood that non-light-absorbing carbon evolves after the OC/EC split. However, the ratio of TOR to TOT EC was similar for both temperature protocols (1.9 for He4-550 and 2.2 for He4-700). Therefore, factors other than just slip of non-light-absorbing carbon could contribute to the differences between TOR- and TOT-EC.

\section{DISCUSSION AND CONCLUSIONS}

Different types of carbon evolve during thermal-optical analysis of an ambient aerosol sample. Since the OC/EC split is optically defined, it is important to understand the optical properties of these different types of carbon. In this paper we have classified this carbon into three categories: non-light-absorbing carbon, light-absorbing pyrolyzed carbon (PC), and light-absorbing, native elemental carbon (EC). Our results show that changing the temperature protocol affects the relative rate at which these different types of carbon evolve during thermal-optical analysis. This, in turn, alters the optically defined OC/EC split and the measured EC ("EC"). These ideas provide a framework for understanding why different temperature protocols measure different amounts of EC.

The evolution of different types of carbon must be carefully considered when trying to select an optimum temperature protocol. First, the results presented here suggest $\mathrm{PC}$ and EC co-evolve from the He/Ox mode for any temperature protocol. Therefore, it is incorrect to view the post-split carbon as native EC. For ambient samples analyzed using a protocol with a high peak Hemode temperature (He4-870), a mixture of PC and EC evolves after the split. For ambient samples analyzed with a low peak He-mode temperature (He4-550), a mixture of PC, EC, and nonlight-absorbing carbon can evolve after the split. The optimum protocol will be one in which the amount of post-split carbon is equivalent to the mass of native EC on the filter. This will only occur when the optical properties of the post-split carbon are the same as EC. However, our results show that the optical properties of EC and PC can be significantly different.

For samples analyzed with the He4-870 protocol, a larger fraction of the EC evolves earlier in the analysis cycle-in the He4 temperature step-compared to samples analyzed with the He4-700 protocol. In Pittsburgh, $k_{\mathrm{PC}}$ is greater than $k_{\mathrm{EC}}$, so shifting EC evolution to earlier in the analysis cycle (relative to PC) reduces the measured EC. This explains why the He4870 protocol measures $20-30 \%$ less post-split carbon ("EC") than the He4-700 protocol. Similarly, too low a peak He-mode temperature-as in the He4-550 protocol—can result in nonlight-absorbing carbon evolving in the $\mathrm{He} / \mathrm{Ox}$ mode after the OC/EC split, biasing the EC high relative to the protocols with higher peak He-mode temperatures, (e.g., the He4-700 or 
He4-870 protocols). This effect likely contributes significantly to the He4-550 protocol measuring about 50\% more "EC" than the He4-700 protocol on wood smoke dominated ambient samples. These results are with the transmission-defined split. Similar differences are also seen with a reflectance-based OC/EC split, driven by the same changes in evolution of the different types of carbon (relative to each other) brought about by a changing temperature protocol.

Selection of the temperature protocol does influence relative biases created by either premature evolution of EC (accentuated by higher peak He-mode temperatures) or slip of non-lightabsorbing carbon into the He/Ox mode (possible at lower peak He-mode temperatures). When selecting a temperature protocol, one seeks to balance the two competing effects. The sample composition, both organic and inorganic, can also influence evolution of carbon from filters (Novakov and Corrigan 1995; Yu, $\mathrm{Xu}$, and Yang 2002). Since both premature EC evolution and slip of non-light-absorbing carbon in the He/Ox mode likely occur over a range of temperatures in addition to potentially significant temporal and spatial changes in sample composition, it is unlikely that a single He-mode peak temperature can be specified at which both problems disappear.

Of the three protocols considered here, the He4-700 protocol appears to yield the best estimate of EC for the Pittsburgh samples. Although PC and EC co-evolve in the He/Ox mode of samples analyzed with He4-700 protocol (as they do in all of the protocols considered here), the average attenuation coefficient of the post-split He/Ox carbon for samples analyzed with the He4-700 protocol $\left(28 \mathrm{~m}^{2} / \mathrm{g}-\mathrm{C}\right)$ is only slightly higher than the expected value of $k_{\mathrm{EC}}$ obtained from our washed samples $\left(22.9 \mathrm{~m}^{2} / \mathrm{g}-\mathrm{C}\right)$. Our experiments with levoglucosan and SOA suggest that positive bias in measured EC (due to slip of nonlight-absorbing carbon into the $\mathrm{He} / \mathrm{Ox}$ mode) may not be a significant problem for the He4-700 protocol.

For other locations and sample compositions, one could select a temperature protocol that minimizes loss of EC in the He-mode and slip of non-light-absorbing carbon into the He/Ox mode using repeated analysis and solvent extraction. However, this may be impractical for routine monitoring applications such as the STN. A way out could be to track the laser signal throughout the analysis, which can indicate premature loss of EC. Comparing the attenuation coefficient of the post-split carbon using a reference value for $k_{\mathrm{EC}}$ (or even an upper limit of $k_{\mathrm{EC}}$ ) can also help estimate any bias. For research projects that analyze a small number of samples, a combination of solvent-extraction with thermal/optical analysis is highly recommended.

\section{REFERENCES}

Birch, M. E., and Cary, R. A. (1996). Elemental Carbon-Based Method for Monitoring Occupational Exposures to Particulate Diesel Exhaust, Aerosol Sci. Technol. 25(3):221-241.

Bond, T. C., and Bergstrom, R. W. (2006). Light Absorption by Carbonaceous Particles: An Investigative Review, Aerosol Sci. Technol 40:1-41.
Cary, R. A. (2003). Personal Communication.

Chow, J. C., Watson, J. G., Chen, L. W. A., Arnott, W. P., and Moosmuller, H. (2004). Equivalence of Elemental Carbon by Thermal/Optical Reflectance and Transmittance with Different Temperature Protocols, Environ. Sci. Technol 38(16):4414-4422.

Chow, J. C., Watson, J. G., Crow, D., Lowenthal, D. H., and Merrifield, T. (2001). Comparison of IMPROVE and NIOSH Carbon Measurements, Aerosol Sci. Technol. 34(1):23-34.

Chow, J. C., Watson, J. G., Pritchett, L. C., Pierson, W. R., Frazier C. A., and Purcell, R. G. (1993). The DRI Thermal Optical Reflectance Carbon Analysis System-Description, Evaluation and Applications in United-States Air-Quality Studies, Atmos. Environ Part A-General Topics 27(8):11851201.

Conny, J. M., Klinedinst, D. B., Wight, S. A., and Paulsen, J. L. (2003). Optimizing Thermal-Optical Methods for Measuring Atmospheric Elemental (black) Carbon: A Response Surface Study, Aerosol Sci. Technol. 37(9):703723.

Fuller, K. A., Malm, W. C., and Kreidenweis, S. M. (1999). Effects of Mixing on Extinction by Carbonaceous Particles, J. Geophys. Res.-Atmospheres 104(D13):15941-15954.

Fung, K. (1990). Particulate Carbon Speciation by $\mathrm{MnO}_{2}$ Oxidation, Aerosol Sci. Technol 12(1):122-127.

Fung, K., Chow, J. C., and Watson, J. G. (2002). Evaluation of OC/EC Speciation by Thermal Manganese Dioxide Oxidation and the IMPROVE Method, J. Air \& Waste Manag. Assoc. 52(11):1333-1341.

Grieshop, A. P., Lipsky, E. M., Pekney, N. J., Takahama, S., and Robinson, A. L. (2005). Diesel and Gasoline Vehicle Gaseous and Fine Particle Emission Factors Measured in a Highway Tunnel: Effects of Fleet Composition and Season, Atmos. Environ. in press.

Gundel, L. A., Dod, R. L., Rosen, H., and Novakov, T. (1984). The Relationship Between Optical Attenuation and Black Carbon Concentration for Ambient and Source Particles, Sci. Total Environ 36(JUN):197-202.

Huntzicker, J. J., Johnson, R. L., Shah, J. J., and Cary, R. A. (1982). Analysis of Organic and Elemental Carbon in Ambient Aerosols by a Thermal-Optical Method. In G. T. Wolff and R. L. Klimisch. Particulate Carbon: Atmospheric Life Cycle, New York, Plenum, 79-88.

Kerminen, V. M., Makela, T. E., Ojanen, C. H., Hillamo, R. E., Vilhunen, J. K., Rantanen, L., Havers, N., VonBohlen, A., and Klockow, D. (1997). Characterization of the Particulate Phase in the Exhaust from a Diesel Car, Environ. Sci. Technol. 31(7):1883-1889.

Liousse, C., Cachier H., and Jennings, S. G. (1993). Optical and Thermal Measurements of Black Carbon Aerosol Content in Different EnvironmentsVariation of the Specific Attenuation Cross-Section, Sigma (Sigma), Atmos. Environ. Part A-General Topics 27(8):1203-1211.

Malissa, H., Puxbaum, H., and Pell, E. (1976). Simultaneous RelativeConductometric Determination of Carbon and Sulfur in Dusts. Fresenius Zeitschrift Fur Analytische Chemie (Fresenius' Journal of Analytical Chemistry, and now Analytical and Bioanalytical Chemistry) 282(2):109-113.

Malm, W. C., Sisler, J. F., Huffman, D., Eldred, R. A., and Cahill, T. A. (1994). Spatial and Seasonal Trends in Particle Concentration and Optical Extinction in the United-States, J. Geophys. Res._Atmospheres 99(D1):13471370 .

NIOSH (1999). Elemental Carbon (Diesel Particulate): Method 5040. (http://www.cdc.gov/niosh/nmam/pdfs/5040f3.pdf).

Novakov, T., and Corrigan, C. E. (1995). Thermal Characterization of Biomass Smoke Particles, Mikrochim Acta 119(1-2):157-166.

Peterson, M. R., and Richards, M. H. (2002). Thermal-Optical-Transmittance Analysis for Organic, Elemental, Carbonate, Total Carbon, and OCX2 in PM2.5 by the EPA/NIOSH Method. Symposium on Air Quality Measurement Methods and Technology-2002, San Francisco, Air \& Waste Management Association.

Petzold, A., Kopp, C., and Niessner, R. (1997). The Dependence of the Specific Attenuation Cross-Section on Black Carbon Mass Fraction and Particle Size, Atmos. Environ. 31(5):661-672. 
Schauer, J. J., Kleeman, M. J., Cass, G. R., and Simoneit, B. R. T. (2001). Measurement of Emissions from Air Pollution Sources. 3. C-1-C-29 Organic Compounds from Fireplace Combustion of Wood, Environ. Sci. Technol. 35(9):1716-1728.

Schauer, J. J., Mader, B. T., Deminter, J. T., Heidemann, G. Bae, M. S., Seinfeld, J. H., Flagan, R. C., Cary, R. A., Smith, D., Huebert, B. J., Bertram, T., Howell, S., Kline, J. T., Quinn, P., Bates, T., Turpin, B., Lim, H. J., Yu, J. Z., Yang, H., and Keywood, M. D. (2003). ACE-Asia Intercomparison of a Thermal-Optical Method for the Determination of Particle-Phase Organic and Elemental Carbon, Environ. Sci. Technol. 37(5):993-1001.

Schmid, H., Laskus, L., Abraham, H. J., Baltensperger, U., Lavanchy, V., Bizjak, M., Burba, P., Cachier, H., Crow, D., Chow, J., Gnauk, T., Even, A., ten Brink, H. M., Giesen, K. P., Hitzenberger, R., Hueglin, E., Maenhaut, W., Pio, C., Carvalho, A., Putaud, J. P., Toom-Sauntry, D., and Puxbaum, H. (2001). Results of the "Carbon Conference" International Aerosol Carbon Round Robin Test Stage I, Atmos. Environ. 35(12):21112121.

Simoneit, B. R. T., Schauer, J. J., Nolte, C. G., Oros, D. R., Elias, V. O., Fraser, M. P., Rogge, W. F., and Cass, G. R. (1999). Levoglucosan, a Tracer for Cellulose in Biomass Burning and Atmospheric Particles, Atmos. Environ. 33(2):173-182.
Subramanian, R., Khlystov, A. Y., Cabada, J. C., and Robinson, A. L. (2004). Positive and Negative Artifacts in Particulate Organic Carbon Measurements with Denuded and Undenuded Sampler Configurations, Aerosol Sci. Technol. 38(S1):27-48.

Turpin, B. J. (1989). Secondary Formation of Organic Aerosol: Investigation of the Diurnal Variations of Organic and Elemental Carbon (PhD Thesis). Environmental Science and Engineering, Oregon Graduate Center. Beaverton, Oregon, USA.

Weingartner, E., Saathoff, H., Schnaiter, M., Streit, N., Bitnar, B., and Baltensperger, U. (2003). Absorption of Light by Soot Particles: Determination of the Absorption Coefficient by Means of Aethalometers, J. Aerosol Sci. 34(10): 1445-1463.

Wittig, A. E., Anderson, N., Khlystov, A. Y., Pandis, S. N., Davidson, C. I., and Robinson, A. L. (2004). Pittsburgh Air Quality Study Overview and Initial Scientific Findings, Atmos. Environ. 38(20):3107-3125.

Yang, H., and Yu, J. Z. (2002). Uncertainties in Charring Correction in the Analysis of Elemental and Organic Carbon in Atmospheric Particles by Thermal/Optical Methods, Environ. Sci. Technol. 36(23):5199-5204.

Yu, J. Z., Xu, J. H., and Yang, H. (2002). Charring Characteristics of Atmospheric Organic Particulate Matter in Thermal Analysis, Environ. Sci. Technol. 36(4):754-761. 
In press Atmospheric Environment.

\section{Contribution of motor vehicle emissions to organic carbon and fine particle mass in Pittsburgh, Pennsylvania: Effects of varying source profiles and seasonal trends in ambient marker concentrations}

(Ms. Ref. No.: ATMENV-D-05-00172R2)

R Subramanian $\dagger$

Department of Mechanical Engineering

Carnegie Mellon University, Pittsburgh, PA 15213, USA

Neil M Donahue

Department of Chemistry and Department of Chemical Engineering

Carnegie Mellon University, Pittsburgh, PA 15213, USA

Anna Bernardo-Bricker

Department of Civil and Environmental Engineering

Florida International University, Miami, FL, USA

Wolfgang F Rogge

Department of Civil and Environmental Engineering

Florida International University, Miami, FL, USA

Allen L Robinson ${ }^{1}$

Department of Mechanical Engineering

Carnegie Mellon University, Pittsburgh, PA 15213, USA

\footnotetext{
${ }^{1}$ Corresponding author: alr@andrew.cmu.edu; (412) 268-3657; fax (412) 268-3348. Postal address: Department of Mechanical Engineering, Carnegie Mellon University, 5000 Forbes Ave, Pittsburgh, PA 15213, USA.

$\dagger$ Current address: Department of Civil and Environmental Engineering, University of Illinois at Urbana-Champaign, Urbana, IL 61801, USA
} 


\section{Abstract}

2 We present estimates of the vehicular contribution to ambient organic carbon (OC) and

3 fine particle mass (PM) in Pittsburgh, PA using the Chemical Mass Balance (CMB) model

4 and a large dataset of ambient molecular marker concentrations. Source profiles for CMB

5 analysis are selected using a method of comparing the ambient ratios of marker species with

6 published profiles for gasoline and diesel vehicle emissions. The ambient wintertime data

7 cluster on a hopanes/EC ratio-ratio plot, and therefore can be explained by a large number of

8 different source profile combinations. In contrast, the widely varying summer ambient ratios

9 can be explained by a more limited number of source profile combinations. We present

10 results for a number of different CMB scenarios, all of which perform well on the different

11 statistical tests used to establish the quality of a CMB solution. The results illustrate how

$12 \mathrm{CMB}$ estimates depend critically on the marker-to-OC and marker-to-PM ratios of the source

13 profiles. The vehicular contribution in the winter is bounded between $13 \%$ and $20 \%$ of the

14 ambient OC $\left(274 \pm 56\right.$ to $\left.416 \pm 72 \mathrm{ng}-\mathrm{C} / \mathrm{m}^{3}\right)$. However, variability in the diesel profiles

15 creates uncertainty in the gasoline-diesel split. On an OC basis, one set of scenarios suggests

16 gasoline dominance while a second set indicates a more even split. On a PM basis, all

17 solutions indicate a diesel-dominated split. The summer CMB solutions do not present a

18 consistent picture given the seasonal shift and wide variation in the ambient hopanes-to-EC

19 ratios relative to the source profiles. If one set of source profiles is applied to the entire

20 dataset, gasoline vehicles dominate vehicular $\mathrm{OC}$ in the winter but diesel dominates in the

21 summer. The seasonal pattern in the ambient hopanes-to-EC ratios may be caused by

22 photochemical decay of hopanes in the summer or by seasonal changes in vehicle emission

23 profiles. 
1 Keywords: Chemical Mass Balance (CMB); molecular markers; organic carbon; motor

2 vehicle contribution; gasoline-diesel split.

\section{Introduction}

4 Gasoline and diesel motor vehicles are significant contributors to the ambient organic

5 carbon (OC) and $\mathrm{PM}_{2.5}$ mass in urban environments (Schauer et al. 1996; Watson et al. 1998a;

6 Fraser et al. 2003b). The Chemical Mass Balance (CMB) model with individual organic

7 compounds or molecular markers such as hopanes and polycyclic aromatic hydrocarbons

8 (PAHs) is one way of quantifying the vehicular contribution to ambient OC, as well as the

9 gasoline-diesel split.

10 Selection of source profiles and fitting species requires careful consideration when

11 performing $\mathrm{CMB}$ analysis. More than 20 different source profiles with speciated organics

12 data for gasoline and diesel vehicles have been published. The majority of these source

13 profiles have been developed for use in California, Colorado, and Texas (Rogge et al. 1993;

14 Watson et al. 1998a; Schauer et al. 1999; Fraser et al. 2002; Schauer et al. 2002). The

15 appropriateness of applying these profiles to regions with different climates and vehicle fleets

16 has not yet been established. Further, many parts of the United States are affected by regional

17 transport of fine particulate matter; this transport mixes emissions from large spatial domain

18 which complicates the definition of the vehicular fleet and the selection of source profiles.

19 Each published source profile represents the emissions from a single or a small number of

20 vehicles. A comparison of the published profiles reveals a wide range of emission rates and

21 emission composition. This variability complicates selection of source profiles because the

22 profiles used in CMB need to represent the aggregate emissions from the entire vehicle fleet. 
1 Co-linearity also limits the number of different vehicle profiles that can be simultaneously

2 considered in the $\mathrm{CMB}$ model. For example, previous applications of $\mathrm{CMB}$ have either

3 combined multiple source profiles to construct a fleet-average gasoline profile (Schauer et al.

4 1996; Zheng et al. 2002) or have used a single source profile to represent the gasoline vehicle

5 fleet (Fraser et al. 2003b). The uncertainty estimates considered by CMB are typically based

6 on analytical uncertainties used to develop the data sets without considering the effects of

7 variability in source profiles on the solutions.

8 This paper has two goals. The first is to evaluate the suitability of published motor

9 vehicle source profiles for use in CMB analysis of a large data set of ambient molecular

10 concentrations collected in Pittsburgh, PA. The second is to examine the variability in CMB

11 results due to source profile selection. The paper concludes with a discussion of the factors

12 affecting the source contribution estimates, including vehicle fleet composition and seasonal

13 variations in molecular marker concentrations. This paper is part of a series of papers

14 examining source apportionment of primary organic aerosol in Pittsburgh, PA (Robinson et 15 al. 2006b; c; d).

16 Methods

17 The EPA's CMB8 model (http://www.epa.gov/scram001/) was implemented to estimate

18 the contribution of gasoline and diesel vehicle emissions and other primary sources to ambient

19 OC in Pittsburgh, PA. The analysis uses ambient concentrations of individual organic

20 compounds, $\mathrm{PM}_{2.5}$ organic and elemental carbon, and $\mathrm{PM}_{2.5}$ elemental composition measured

21 on 96 days between June 20, 2001 and July 1, 2002. The data were collected as part of the

22 Pittsburgh Air Quality Study (Wittig et al. 2004). 
1 Samples for organic speciation were collected every day in July 2001 and for much of

2 January 2002, and on a 1-in-6-day schedule during the rest of the study using a $\mathrm{PM}_{2.5}$

3 quartz/PUF sampler operating at $145 \mathrm{lpm}$. Prior to sampling, the quartz fiber filters were

4 baked at $550{ }^{\circ} \mathrm{C}$ for a minimum of four hours to remove any residual organics. The

5 polyurethane foam (PUF) plugs were cleaned thoroughly with a mixture of solvents

6 (dichloromethane, acetone, and hexane), dried in a clean vacuum dessicator, and stored in pre-

7 cleaned glass jars shielded from light, till required for sampling. The relevant sampler parts

8 and sample handling tools were solvent-rinsed before use. All solvents used were high-

9 resolution GC/MS-grade or better. After sampling and prior to analysis, filter-PUF pairs were

10 stored in pre-cleaned glass jars in a freezer $\left(-18^{\circ} \mathrm{C}\right.$ or lower); samples were shipped overnight

11 in coolers packed with dry ice for analysis at Florida International University. Prior to

12 extraction, each sample was spiked with an internal standard consisting of a suite of seven

13 perdeuterated n-alkanes (C12, C16, C20, C24, C28, C32 and C36). The samples were

14 extracted using dichloromethane. The extracts from each filter-PUF pair were combined and

15 the volume was reduced to about $1 \mathrm{ml}$ by rotary evaporation; the volume was further reduced

16 to about $250 \mu \mathrm{L}$ using a gentle stream of pure nitrogen prior to methylation using freshly

17 prepared diazomethane. Methylation converts polar organic acids to their methyl ester

18 analogs enabling their detection by gas chromatography-mass spectrometry (GC/MS). The

19 methylated extracts were analyzed by GC/MS using electron impact ionization. Each analyte

20 was quantified by reference to the internal standard, using a relative response factor

21 determined with authentic standards. Organic and elemental carbon were measured on quartz

22 filters analyzed by a thermal-optical transmission method based on the NIOSH-5040 protocol

23 (Subramanian et al. 2004; Subramanian et al. 2006). Cellulose filter samples were analyzed 
1 by inductively coupled plasma-mass spectrometry (ICP-MS) for $\mathrm{PM}_{2.5}$ elemental composition

2 (Pekney and Davidson 2005).

3 The selection of compounds included in the CMB model is a critical issue; all major

4 sources of each compound must be included in the model and the species should be conserved

5 during transport from source to receptor (Watson et al. 1998b). This paper uses the basic set

6 of compounds and source classes developed by Schauer et al. (1996) and Schauer and Cass

7 (2000). Our focus is the uncertainty associated with the specific motor vehicle profiles

8 included in the model.

9 The following species are included in the CMB model: iron, titanium, elemental carbon

10 (EC), n-heptacosane, n-nonacosane, n-hentriacontane and n-tritriacontane; iso-hentriacontane,

11 anteiso-dotriacontane; hexadecanoic (palmitic) acid, octadecanoic (stearic) acid, 9-

12 hexadecenoic (palmitoleic) acid, cholesterol; syringaldehyde, sum of resin acids (pimaric,

13 sandaracopimaric, dehydroabietic, and 7-oxodehydroabietic acids), acetosyringone,

14 levoglucosan; 17a(H),21b(H)-29-norhopane, 17a(H),21b(H)-hopane, 22R+S-17a(H),21b(H)-

15 30-homohopane, 22R+S, 17a $(\mathrm{H}), 21 \mathrm{~b}(\mathrm{H})-30$-bishomohopane; benzo[e]pyrene, indeno[1,2,3-

$16 \mathrm{~cd}]$ pyrene, benzo[g,h,i]perylene, and coronene. All of these species are included in every

17 calculation. In certain scenarios we also include n-tetracosane and n-hexacosane in the CMB

18 model. Uncertainties for individual compounds are the greater of the relative or absolute

19 uncertainties. Absolute uncertainties are based on multiples of the minimum detection limits,

20 while relative uncertainties were determined by analyzing multiple samples collected in

21 parallel. Relative uncertainties range from $\pm 10 \%$ to $\pm 30 \%$, depending on the species. OC or

22 PM is not included in the model as molecular markers for secondary organic aerosol and

23 potentially other primary sources are not known. 
1 Source profiles for eight source classes are included in the model: diesel vehicles, gasoline

2 vehicles, road dust, biomass combustion, cooking emissions, coke production, vegetative

3 detritus, and cigarette smoke. Source profiles for cooking emissions and biomass smoke are

4 taken from the literature, while a coke-oven emissions profile and Pittsburgh-specific

5 vegetative detritus and road-dust profiles were developed as part of PAQS. The complete list

6 of source profiles used and their references are provided in the Supplementary Material

7 (Table S1). In order to determine the contribution of each source profile to ambient OC,

8 calculations are performed using source profiles normalized by the OC emission rates. The

9 CMB estimates for motor-vehicle emissions are not sensitive to the other source profiles

10 included in the model. An exception is October 7, 2001 - the day with the maximum biomass

11 smoke $\mathrm{OC}$ and $\mathrm{EC}$ - when the motor vehicle estimates are sensitive to the specific biomass

12 smoke profiles. A common set of non-motor vehicle source profiles is used in all simulations.

13 Source profiles for gasoline and diesel vehicles are selected based on comparisons with

14 the ambient concentrations of different molecular markers using scatter and ratio-ratio plots,

15 which allow a visual comparison of source profiles and ambient concentrations. Ratio-ratio

16 plots are constructed using three species; one compound is selected as a reference to

17 normalize the concentrations of the other two compounds (target species). The best reference

18 compounds are relatively abundant, stable, and specific to the sources plotted. Source profiles

19 appear as points on ratio-ratio plots and linear mixing lines can be drawn to visualize the

20 effects of mixing of emissions from different sources. More details on the construction,

21 interpretation, and mathematics of ratio-ratio plots are provided in Robinson et al. (2006a;

22 2006b). 


\section{$1 \quad$ Results and Discussion}

2 Ambient Concentrations of Motor Vehicle Markers: The Pittsburgh dataset includes

3 measurements of a number of compounds that are used as markers for motor vehicle

4 emissions, including hopanes, steranes, polycyclic aromatic hydrocarbons (PAHs), and EC.

5 Hopanes and steranes are associated with unburned lubricating oil and are emitted by both

6 gasoline and diesel vehicles (Zielinska et al. 2004). Certain PAHs are more prevalent in

7 gasoline exhaust while diesel exhaust is relatively enriched in EC; therefore these species

8 have been used to distinguish between emissions from these two source classes (Cadle et al.

9 1999b; Fraser et al. 2003a). However, PAH concentrations in Pittsburgh, PA are dominated

10 by emissions from metallurgical coke production (Robinson et al. 2006b), diminishing their

11 utility as vehicular markers.

12 Figure 1 shows a time series of ambient concentrations of two of the hopanes: norhopane

$13(17 \alpha(\mathrm{H}), 21 \beta(\mathrm{H})-29-N o r h o p a n e)$ and hopane $(17 \alpha(\mathrm{H}), 21 \beta(\mathrm{H})$-Hopane). The 24-hr average

14 concentrations of these compounds are usually less than $0.4 \mathrm{ng} / \mathrm{m}^{3}$. Normalizing the hopanes

15 by EC to account for differences in atmospheric dilution reveals that the hopanes are a factor

16 of two to three higher in the winter than in the summer, as shown in Figure 1c. The other

17 hopanes and the steranes in the Pittsburgh dataset exhibit a similar seasonal pattern.

18 Ambient concentrations of the different hopanes are strongly correlated with linear-

19 regression correlation coefficients greater than 0.95 as illustrated by the scatter plots in

20 Figure 2. From the perspective of $\mathrm{CMB}$, these strong correlations mean that the ambient

21 hopane data can be explained using a single source profile (Robinson et al. 2006b) -

22 presumably one that represents the aggregate emissions from motor vehicles. 
Ambient 24-hr average EC concentrations in Pittsburgh are generally between 0.5 and

$21 \mu \mathrm{g} / \mathrm{m}^{3}$, with occasional large spikes (Figure $1 \mathrm{~b}$ ). Figure $2 \mathrm{~d}$ shows that EC is modestly

3 correlated $\left(\mathrm{R}^{2}=0.65\right)$ with norhopane (and by extension, with the other hopanes), but the $\mathrm{R}^{2}$

4 value drops below 0.3 if the high concentration days (norhopane $>0.4 \mathrm{ng} / \mathrm{m}^{3}$ ) are removed

5 from the dataset. If the data are sorted by season, the $\mathrm{R}^{2}$ values for the lower concentration

6 days improve to about 0.6 , though the summer and winter slopes are different by over a factor

7 of two; this reflects the distinct seasonal pattern in the ratios of the different hopanes to EC

8 shown in Figure 1c.

Ratio-ratio plots comparing ambient data and published source profiles: CMB analysis

10 depends critically on the relative distribution of fitting species included in the model. In this

11 section, we focus on the relative distribution of the five key motor vehicles markers - four

12 hopanes and EC - that are included (fitted) in the CMB model. We do not include steranes in

13 the analysis because not all source profiles report sterane emissions. Ambient data for the

14 five motor vehicle markers can be compared to source profiles using two ratio-ratio plots:

$15 \mathrm{~S}+\mathrm{R}$-homohopanes and $\mathrm{S}+\mathrm{R}-$ bishomohopanes normalized by norhopane (Figures 3a and 3c);

16 and norhopane and hopane normalized by EC (Figures $3 \mathrm{~b}$ and $3 \mathrm{~d})$.

17 The organization of the ambient data in the ratio-ratio plots provides significant insight

18 into potential source profile combinations. In the winter, the ambient data of the five motor

19 vehicle markers largely cluster to a point in the ratio-ratio plots shown in Figures $3 \mathrm{a}$ and $3 \mathrm{~b}$;

20 the modest variability in the wintertime hopanes-to-EC ratios (Figure 3b) can largely be

21 attributed to measurement uncertainty. Therefore, CMB can reproduce the wintertime

22 concentrations of all five motor vehicle markers with a single source profile. In the summer,

23 the hopane data cluster to a point in a ratio-ratio plot (Figure 3c) but the hopanes-to-EC ratios 
1 are distributed along a line (Figure 3d); therefore at least two source profiles are needed that

2 bracket the ambient hopanes-to-EC ratios along the diagonal line defined by the summertime

3 ambient data. A mixing line connecting these two profiles will pass through the summertime

4 data.

5 Source profiles are also shown in the ratio-ratio plots in Figure 3. We consider gasoline

6 and diesel profiles measured in the Denver, $\mathrm{CO}$ area as part of the Northern Front Range Air

7 Quality Study (NFRAQS) (Watson et al. 1998a) and in Southern California (Schauer et al.

8 1999; 2002), as well as diesel profiles measured in Texas (Fraser et al. 2002). We do not

9 consider the oxidation catalyst-fitted metro bus and diesel idling profiles reported by Fraser et

10 al. (2002).

11 The most striking feature of the source profiles is the wide scatter of the different motor

12 vehicle profiles compared to the well-organized ambient data (Figure 3). The fact that the

13 source profiles exhibit much more variability than the ambient data is not surprising. Each

14 source profile represents the emissions from a single or small number of vehicles while the

15 ambient data represent the aggregate emissions of the entire vehicle fleet. The well-organized

16 ambient data indicate that atmospheric mixing averages out much of the vehicle-to-vehicle

17 differences in emissions.

18 When comparing the different profiles (Figure S1, Supporting Information), one must

19 acknowledge the potential effects of differences in testing procedures and analytical

20 techniques used by different studies on the results. For example, the profiles reported by

21 NFRAQS use the IMPROVE method to measure EC (Chow et al. 1993), while the others are

22 based on the NIOSH protocol (NIOSH 1999). Comparison studies have revealed significant

23 differences in EC measurements using these two techniques (Chow et al. 2001), although 
1 more recent research indicates good agreement of EC measured in diesel exhaust samples

2 using both protocols (Fujita et al. 2005). However, analytical issues must be kept in

3 perspective because large variability exists for profiles measured using the same testing

4 procedures and analytical techniques, e.g. the order-of-magnitude difference in the $\mathrm{EC} / \mathrm{OC}$

5 ratios across the subset of profiles measured with either technique (Figure S1). This indicates

6 that actual variability in vehicle-to-vehicle emissions is much larger than differences

7 associated with analytical techniques for either hopanes or EC.

8 The chemical composition of the emissions depends on a number of factors. For example,

9 the relative distribution of the hopanes in emissions depends on the origin of the crude oil or

10 other fossil fuel (Simoneit 1984; Oros and Simoneit 2000). Figures 3a and 3c show that

11 emissions from non-vehicular sources such as low-temperature coal combustion (Oros and

12 Simoneit 2000), fuel oil combustion (Rogge et al. 1997b) and tar pots (Rogge et al. 1997a)

13 can have very different hopane distributions than motor vehicle emissions. This fact coupled

14 with the strong, seasonally-invariant correlation between the ambient hopanes is consistent

15 with a single dominant source for hopanes, presumably motor vehicles. Some of the

16 variability in the source hopanes-to-EC ratios shown in Figures $3 \mathrm{~b}$ and $3 \mathrm{~d}$ is related to engine

17 technology; the diesel vehicle profiles are rich in EC with generally lower hopanes-to-EC

18 ratios than the gasoline vehicle profiles. Notable exceptions are the Schauer et al. (2002)

19 catalytic gasoline profile, which has lower hopanes-to-EC ratios than the most of the diesel

20 profiles, and the Fraser et al. (2002) diesel school bus profile, which has larger hopanes-to-EC

21 ratios than most of the gasoline vehicle profiles. Within the set of NFRAQS gasoline vehicle

22 profiles, smoking vehicles have lower hopanes-to-EC ratios than non-smoking vehicles and

23 winter profiles have lower hopanes-to-EC ratios than summer profiles. 
1 Developing fleet-average profiles: Although the source profiles are highly variable, the

2 available profiles cluster by vehicle type in a manner that is largely consistent with a two-

3 source $\mathrm{CMB}$ solution. In the winter, the hopanes-to-EC ratios of most diesel profiles are

4 smaller than the ambient ratios while the hopanes-to-EC ratios of most gasoline vehicle

5 profiles are larger than the ambient ratios (Figure 3b). Therefore, the winter ambient data can

6 be explained by almost any one of the diesel profiles (with the exception of the Fraser et al.

7 (2002) school bus profile) paired with almost any one of the gasoline vehicle profiles (with

8 the exception of the Schauer et al. (2002) catalytic gasoline and the NFRAQS smoker

9 profiles). The modest day-to-day variability in the wintertime ratios of the different hopanes

10 to EC can then be attributed to changes in the gasoline-diesel split. While a similar

11 combination of gasoline profiles with any one of the NFRAQS diesel profiles can explain the

12 summertime hopanes-to-EC ratios, using the Schauer et al. (1999) and Fraser et al. (2002)

13 diesel profiles for the summer data creates a number of challenges that are considered in a

14 later section.

15 The major challenge for analyzing the winter or summer data with CMB is selecting 16 among the numerous CMB models based on different pairs of individual gasoline and diesel 17 source profiles. The problem is that within the set of CMB models that produce statistically 18 acceptable solutions large differences exist in the amount of OC apportioned to a given source 19 class. These differences are primarily caused by differences in the marker-to-OC and marker20 to-PM ratios of the source profiles included in the model. These ratios vary by more than an 21 order of magnitude across the set of published vehicle profiles (Figure S1, Supplementary 22 Material). 
Marker-to-OC and marker-to-PM ratios are critical because CMB calculates source

2 strengths based on an optimized "best-fit" of the set of marker compounds included in the

3 model. OC or $\mathrm{PM}_{2.5}$ mass is not directly included (or fitted) in the model for CMB analysis

4 with molecular markers because "source profiles" for secondary organic aerosols are not

5 known. CMB models based on source profiles with small marker-to-OC ratios (e.g. the

6 NFRAQS smoker profiles) will apportion more OC to a given source class than profiles with

7 larger marker-to-OC ratios (e.g. the NFRAQS low-emitters). Across the set of NFRAQS

8 profiles the ambient OC apportioned to gasoline vehicles varies by more than a factor of three

9 depending on the specific gasoline vehicle profile included in the model.

10 Another issue associated with using individual profiles is that these profiles are developed

11 for a particular type of vehicle, e.g. low-emitter gasoline, which may not be representative of

12 the emissions from the entire fleet. Therefore we combine the available profiles to create

13 more representative fleet-average profiles. In order to account for any potential issues

14 associated with testing procedures and analytical techniques used by different research groups

15 we develop two sets of profiles: one based on the research by Schauer and Fraser and a

16 second based on the NFRAQS data. We consider only the NFRAQS summer gasoline

17 profiles, since for the winter gasoline profiles many of the compounds were near detection

18 limits (Cadle et al. 1999b).

19 To develop fleet average gasoline vehicle profiles, we use information from the 2001

20 National Household Travel Survey (http://nhts.ornl.gov/2001/), which describes the vehicle

21 fleet in terms of vehicle age and estimated "vehicle miles traveled" (VMT). Although this is a

22 national survey, we assume the results are applicable to the Pittsburgh aerosol given the

23 significance of regional transport that combines emissions across a large spatial domain. 
1 While other factors such as maintenance history likely play a role in emissions, we follow the

2 approach of Cadle et al. (1999a) and assume that vehicle age is a reasonable metric for

3 emissions.

4 Three fleet average gasoline profiles are constructed using the NFRAQS data. The

5 "middle-ground" estimate uses the low-emitter profile to represent vehicles less than five

6 years old ( $43.5 \%$ of the VMT), the medium-emitter profile to represent vehicles between five

7 and fifteen years of age ( $49.6 \%$ of VMT), and an average of the smoker and high-emitter

8 profiles to represent vehicles older than 15 years (6.8\% of VMT). To test the sensitivity of

9 the CMB results, we also use fleet compositions with smokers/high-emitters comprising $1 \%$

10 and $12 \%$ of VMT as two extreme fleet compositions.

11 The second set of fleet average gasoline profiles is based on the Schauer et al. (2002)

12 gasoline profiles. The low and medium-emitters are represented by the Schauer et al. (2002)

13 catalytic gasoline profile and high-emitters/smokers by the Schauer et al. (2002) non-catalytic

14 gasoline profile. In this case, we consider only two vehicle fleets, one with $1 \%$ smokers/high-

15 emitters and a second with $12 \%$ smokers/high-emitters.

16 To estimate the diesel vehicle contribution, we consider two diesel profiles. The Schauer-

17 Fraser average diesel profile consists of the Class 8 truck Fraser et al. (2002) profile (the

18 emission rates from the two reported profiles are averaged into a single profile) and the

19 Schauer et al. (1999) medium-duty profile (a composite sample from two vehicles). We

20 assume that each profile represents an equal fraction of the diesel fleet. The second profile is

21 the NFRAQS heavy-duty diesel profile "N048", a composite of ten vehicles (Watson et al.

22 1998a) - this is the "NFRAQS Heavy-duty 1 (W)" profile shown in Figure S1. 
1 The five fleet-average gasoline and two diesel profiles are plotted with the ambient data

2 on the ratio-ratio plots of Figure 4. Separate plots are shown to illustrate the seasonal shifts in

3 the ambient data relative to the source profiles. These shifts are discussed in a later section.

4 The summer plots also present the Schauer et al. (2002) catalytic and non-catalytic gasoline

5 profiles separately without averaging. The average profiles are listed in Table S2

6 (supplementary material).

CMB analysis of the wintertime data: The five fleet-average gasoline profiles combined

8 with the two different diesel profiles result in 10 different scenarios for CMB analysis of the

9 wintertime data. All of these combinations yield statistically acceptable solutions on all

10 winter days; for example, the median $\mathrm{R}^{2}$ values are 0.92 or 0.93 while median $\chi^{2}$ values range

11 between 2.0 and 2.4 , with 13 to 17 degrees of freedom (the minimum confidence level for any

12 given day is $98 \%$ ). The T-statistics of the source contribution estimate (SCE) for the motor

13 vehicle profiles are greater than 2.0 on over $85 \%$ of the days. Therefore, these statistical

14 parameters provide little guidance for selecting among the ten different statistically-

15 acceptable solutions.

16 Time-series of the ambient OC apportioned to motor vehicle exhaust are shown in

17 Figure 5a. All of the solutions exhibit some day-to-day variability due to changes in ambient

18 marker concentrations. The different solutions are also strongly correlated with one another

19 as illustrated by the scatter plots shown in Figure 6 which compare the daily OC apportioned

20 to all vehicles, diesel vehicles, gasoline vehicles and gasoline-diesel split by two different

21 scenarios.

Figure $5 \mathrm{a}$ indicates that all ten wintertime scenarios apportion a similar amount of ambient

23 OC to motor vehicle emissions. The average total-vehicle OC estimated by the 10 different 
1 scenarios are all within a factor of 1.5 and are not statistically different based on the standard

2 error estimates calculated by CMB (e.g. Figure 6a). The NFRAQS diesel plus the NFRAQS

$31 \%$ high-emitter/smoker gasoline scenario predicts the lowest average total-vehicle OC,

$4274 \pm 56 \mathrm{ng}-\mathrm{C} / \mathrm{m}^{3}$ ( \pm average standard error), while the NFRAQS diesel plus $1 \%$ non-catalytic

5 Schauer gasoline scenario predicts the highest, $416 \pm 72 \mathrm{ng}-\mathrm{C} / \mathrm{m}^{3}$. These predictions

6 correspond to $13.4 \%$ and $20.3 \%$, respectively, of the ambient $\mathrm{OC}$ in the wintertime.

7 Therefore, we conclude that the total contribution of motor vehicles to ambient $\mathrm{OC}$ in the

8 wintertime is well-constrained. However, we must emphasize that the solutions are well-

9 constrained because we are performing analysis with fleet-averaged profiles. As previously

10 discussed one can find a wider range of solutions if one considers individual profiles such as a

11 smoker profile. Therefore, information regarding fleet composition (which profiles are

12 included in the model) provides a critical constraint when considering all statistically-

13 acceptable CMB solutions.

14 Time series of the ambient OC apportioned to diesel vehicles, gasoline vehicles, and the

15 gasoline-diesel split are shown in Figures 5c, 5d, and 5e, respectively. The median

16 wintertime ratio of gasoline vehicle OC to diesel OC estimates varies between 0.63 and 2.90,

17 indicating that some solutions predict diesel emissions dominate while others predict the

18 opposite. The differences in gasoline-diesel split are often greater than the standard errors

19 calculated by CMB, as illustrated by the scatter plot shown in Figure 6c. Therefore, there is

20 little agreement among the solutions regarding the relative contribution of gasoline vehicles

21 and diesel vehicles to ambient OC, or the "gasoline-diesel split".

22 The key factor determining the gasoline-diesel split is which diesel vehicle profile is

23 included in the model. Figure 5e shows that all simulations using the Schauer-Fraser diesel 
1 profile indicate that diesel vehicle emissions are dominant, while gasoline vehicles dominate

2 all simulations based on the NFRAQS diesel profile. The reason for this shift can be

3 understood by closely examining how CMB determines the contribution of gasoline and

4 diesel vehicles to ambient OC.

$5 \quad$ All of the scenarios identify diesel emissions as the dominant source of EC, contributing 6 on average between $67 \%$ and $94 \%$ of the ambient EC (Figure $7 \mathrm{a}$ ). This means that the

7 contribution of diesel emissions is essentially constrained by ambient EC levels, and the

8 diesel $\mathrm{OC}$ depends strongly on the EC/OC ratio of the different diesel profiles. The EC/OC

9 ratio of the NFRAQS diesel profile is 3.7 versus 2.1 for the Schauer-Fraser average profile,

10 which explains why CMB calculations using the Schauer-Fraser profile estimate almost twice

11 as much diesel OC as the NFRAQS diesel profile (Figure 6b).

12 The amount of OC apportioned to gasoline vehicles depends on the hopanes-to-EC ratios

13 of the diesel profile and the hopane-to-OC ratios of the gasoline profile. Since EC constrains

14 the contribution of diesel emissions, the hopanes-to-EC ratios of the diesel profile determine

15 the apportionment of ambient hopanes to the gasoline and diesel profiles. Gasoline-vehicle

16 OC is then determined by the "leftover" hopanes (ambient hopanes minus hopanes

17 apportioned to diesel vehicles) and the hopanes-to-OC ratios of the gasoline vehicle profile.

18 The hopanes-to-EC ratios of the Schauer-Fraser average diesel profile are two to three

19 times higher than the NFRAQS diesel profile. The result is that CMB apportions significantly

20 more of the hopanes to diesel vehicles when the Schauer-Fraser average diesel profile is

21 included in the model, as illustrated in Figure 7b. On average, the Schauer-Fraser diesel

22 profile contributes $50-62 \%$ of the wintertime ambient norhopane compared to $20-26 \%$ by the

23 NFRAQS diesel profile. Hence, given the same gasoline profile, a CMB solution based on 
1 the NFRAQS diesel profile apportions more OC to gasoline vehicles than a solution based on

2 the Schauer-Fraser diesel.

3 The effect of the hopanes-to-OC ratios of the different gasoline profiles can be seen by

4 comparing the predicted gasoline-vehicle OC across a set of calculations performed with the

5 same diesel profile. Fixing the diesel profile means that the amount of hopanes apportioned

6 to gasoline vehicles is essentially constant. Therefore, the factor-of-1.6 variation in the

7 amount of gasoline-vehicle $\mathrm{OC}$ is caused by differences in the hopane-to-OC ratios of the

8 different fleet-average gasoline profiles. As previously discussed, over a factor of three

9 variation is observed if one considers individual gasoline profiles as opposed to the fleet 10 average profiles.

11 CMB analysis of the summertime data: Constructing scenarios to explain the

12 summertime data with its wide range of hopanes-to-EC ratios creates a number of challenges.

13 Robinson et al. (2006a) argues that the seasonal changes in this ratio are caused by

14 photochemical oxidation of hopanes in the regional air mass. If true, the CMB model cannot

15 be used to analyze the summertime data because the assumption that the compounds included

16 in the model are conserved during transport is no longer valid. However the stability of

17 molecular markers in the context of regional transport remains an open question. Therefore,

18 in this section, we apply the CMB model to analyze the summertime data, implicitly assuming

19 that the molecular markers included in the model are conserved.

20 As noted previously, Figures $3 \mathrm{c}$ and $3 \mathrm{~d}$ indicate that the well-organized summertime data

21 are consistent with a two-source solution, but there are few viable source profile combinations

22 that can explain the ambient hopane-to-EC ratios. Notably the Schauer et al. (1999) and

23 Fraser et al. (2002) diesel profiles have larger hopane-to-EC than the summertime data. Only 
1 the NFRAQS diesel profiles and the Schauer et al. (2002) catalytic gasoline profile bracket

2 the low end of the summertime data in Figure 3d. Since non-vehicular sources contribute

3 little EC in the summer (Robinson et al. 2006a; Robinson et al. 2006c), the ambient data

4 require pairing one of the NFRAQS diesel profiles with one of the many gasoline profiles

5 located in the upper right hand corner of Figure 3d.

6 Alternatively, the summertime data can be explained by pairing either the Fraser et al.

7 (2002) or Schauer et al. (1999) diesel profiles with the Schauer et al. (2002) catalytic gasoline

8 profile. However, this combination cannot explain the ambient bishomohopane and

9 homohopane ratios (Figure 4e). One possibility is to include the Schauer et al. (2002) non-

10 catalytic gasoline profile in the model, which would also account for emissions from high-

11 emitting gasoline vehicles. However, Figures $4 \mathrm{e}$ and $4 \mathrm{f}$ indicate that Schauer-Fraser average

12 diesel profile is located on the mixing line connecting the two Schauer et al. (2002) gasoline

13 profiles. To resolve the three motor vehicle profiles requires additional markers, so we add

14 tetracosane (C24) and hexacosane (C26) as fitting species in our CMB model. Schauer et al.

15 (1996) estimate that for Los Angeles, CA in 1982, vehicular emissions contributed about half

16 or more of the lower carbon number n-alkanes ( $\mathrm{C} 23$ to $\mathrm{C} 27)$. We do not fit pentacosane

17 (C25) because there is a marked preference for the odd n-alkanes primarily in the summertime

18 Pittsburgh data, indicative of a strong biogenic influence over the odd n-alkanes (Simoneit 19 1986).

20 The summertime data are analyzed with six scenarios: the NFRAQS diesel profile paired 21 with one of the five fleet-average gasoline vehicle profiles (the NFRAQS scenarios), and the

22 Schauer-Fraser average diesel profile paired with the Schauer et al. (2002) catalytic and non23 catalytic gasoline profiles (the Schauer-Fraser scenario). All six summertime solutions are 
1 statistically acceptable, with median $\mathrm{R}^{2}$ values of 0.93 , median $\chi^{2}$ values of 1.9 or 2.0 and 12 -

218 degrees of freedom (DF) for the five NFRAQS scenarios; and a median $\mathrm{R}^{2}$ value of 0.89 ,

3 median $\chi^{2} 3.9$, and 13-18 degrees of freedom for the Schauer-Fraser model. The minimum

4 confidence level based on the $\chi^{2}$ and DF is $96 \%$ across all scenarios except for two (out of 41)

5 days with the Schauer-Fraser scenario. Therefore the CMB goodness-of-fit parameters

6 provide little guidance in selecting among the different solutions.

7 Time series of results from the summertime solutions are shown in Figure 8. On average,

8 the Schauer-Fraser scenario apportions $31 \%$ of the ambient OC to motor vehicle emissions,

9 five to six times that apportioned by the NFRAQS scenarios (4.9-5.8\%). Figures $8 \mathrm{c}$ and $8 \mathrm{~d}$

10 indicate that the primary difference between the two scenarios is the amount of OC

11 apportioned to gasoline vehicles. The Fraser-Schauer scenario apportions about twenty times

12 more OC to gasoline sources (average $28 \%$ of ambient OC) than the NFRAQS scenarios

13 (average less than $2 \%$ of ambient OC to gasoline sources). This difference is much larger

14 than the standard errors calculated by CMB. Figure $8 \mathrm{c}$ indicates that there is significant day-

15 to-day variation in the amount of gasoline vehicle OC predicted by the Schauer-Fraser

16 scenario; on some days gasoline vehicles contribute almost no OC, while on other days they

17 contribute more than $2 \mu \mathrm{g}-\mathrm{C} / \mathrm{m}^{3}$. Such large variations are not expected given the modest

18 day-to-day variability in vehicle activity.

19 The Fraser-Schauer scenario is dominated by the Schauer et al. (2002) catalytic gasoline

20 profile which contributes $78 \%$ of the vehicular OC, and $27 \%$ of the ambient OC. This occurs

21 for two reasons. First, the ratios of the different hopanes to OC (marker-to-OC ratios) of the

22 Schauer et al. (2002) catalytic gasoline profile are an order of magnitude lower than any of the

23 other vehicle profiles (Figure S1). These extremely low ratios allow this profile to dominate 
1 the OC apportionment while only contributing on average just $4.2 \%$ of the ambient

2 norhopane. Second, this scenario includes the n-alkanes tetracosane and hexacosane as fitting

3 species, which are predominantly apportioned to the catalytic gasoline profile. Note that the

4 Schauer-Fraser scenario cannot be implemented for the summer data without fitting the n-

5 alkanes.

6 The fact that the Schauer-Fraser scenario is dominated by a catalytic gasoline profile with

7 a low emission rate is unexpected because "high emitters" are thought to be the dominant

8 source of emissions, e.g. Beaton et al. (1995). Given its low OC emission rate, the Schauer et

9 al. (2002) catalytic gasoline profile will only influence the fleet average gasoline source

10 profile if it represents a super-majority of the vehicle fleet. Since the very low hopanes-to-OC

11 ratios of the Schauer et al. (2002) catalytic gasoline profile appear to be an outlier compared

12 to all of the other vehicular source profiles (Figure S1), the Schauer-Fraser scenario likely

13 overestimates the actual contribution of gasoline vehicles to the ambient OC.

14 There are also concerns with the summertime NFRAQS solutions; most notably, the large

15 day-to-day changes in the gasoline-diesel split. Figure 8e indicates that on many summertime

16 days diesel vehicles dominate the gasoline diesel split while on others the split is more even.

17 These changes are primarily associated with changes in the amount of gasoline vehicle OC.

18 Previous studies have observed weekday-weekend patterns in the influence of gasoline and

19 diesel vehicles on ambient air quality (Harley et al. 2005); however, the shifts shown in

20 Figure 8e do not follow any discernable pattern. In addition, the summertime shifts in the

21 gasoline-diesel splits are much greater (relative standard deviation of $147 \%$ to $166 \%$,

22 Figure 8e) compared to the winter (RSD $40 \%$ to $62 \%$, Figure $5 \mathrm{e}$ ), as the CMB model has to

23 account for the widely varying summertime hopane-to-EC ratios. 
Seasonal patterns in source-apportionment: CMB models which include the NFRAQS

2 diesel profile paired with a gasoline profile can explain the entire data set; however all of

3 these solutions show strong seasonal changes in the OC apportioned to motor vehicles. The

4 driver for the seasonal changes is the ambient ratios of hopanes-to-EC - the average hopanes-

5 to-EC ratios in the summer are almost a factor of 3 lower than those in the winter (Figure 1c).

6 Therefore, with the NFRAQS diesel-based scenarios, diesel vehicle emissions dominate in the

7 summer (Figure 8e) and gasoline vehicle emissions dominate in the winter (Figure 5e) in

8 order for $\mathrm{CMB}$ to match the observed seasonal patterns in the ambient hopanes-to-EC ratios.

9 A similar seasonal pattern in the gasoline-diesel split has been estimated using CMB and

10 molecular markers in the southeastern US (Zheng et al. 2002).

11 There are a number of potential explanations for seasonally varying ambient hopanes-to-

12 EC ratios. These include seasonal changes in source activity; seasonal changes in the fleet

13 composition; seasonal changes in the source emission factors; and photochemical aging of the

14 hopanes. All of these explanations are discussed in detail in Robinson et al. (2006a) and the

15 first two are relatively easy to dismiss. Some of the scatter in the hopanes-to-EC ratio might

16 be due to issues with EC measurements (Chow et al. 2001; Subramanian et al. 2006);

17 however, there is no evidence that these problems are sensitive to seasonal factors.

18 Seasonal variations in the organic composition of motor vehicle emissions and

19 photochemical decay of hopanes provide the two most likely explanations of the seasonality

20 in the hopanes-to-EC ratios. To explain the ambient data, the motor-vehicle emissions of

21 hopanes relative to EC would need to be higher in the winter compared to the summer -

22 seasonally shifting the location of the source profiles shown in the Figures $3 \mathrm{~b}$ and $3 \mathrm{~d}$.

23 Alternatively the hopanes-to-OC ratio of the emissions could shift seasonally, changing the 
1 amount of OC apportioned to the different sources. However, seasonally shifting profiles

2 would not explain the significant day-to-day variations in the summertime hopanes-to-EC

3 ratio which cause the large day-to-day changes in the gasoline-diesel split shown in Figure 8e.

4 At present little is known about seasonal variability in the organic composition of motor-

5 vehicle emissions. In Pittsburgh, temperatures are much colder in the winter than in the

6 summer; gasoline composition also varies seasonally with reformulated gasoline used in the

7 summer ozone season. Both of these factors likely influence emissions. Two studies have

8 measured warm and cold-weather gasoline-vehicle emission profiles with speciated organics

9 data (Watson et al. 1998a; Zielinska et al. 2004). The cold weather profiles measured by both

10 studies have lower hopanes-to-EC ratios than the warm weather profiles - the opposite of

11 what would be required to explain the ambient data. In fact, Figure $3 b$ indicates that the

12 hopanes-to-EC ratios of the winter NFRAQS gasoline profiles are lower than the winter

13 PAQS ambient data. The result is that CMB cannot find solutions for many of the winter

14 days if the published cold weather profiles are used in the model. As for diesel emissions,

15 there could be seasonal changes in the lubricating oil used in diesel engines. The NFRAQS

16 results do not include heavy-duty diesel tests from the summer, but our CMB solutions

17 already use a winter NFRAQS diesel profile. More source testing is needed to determine if

18 there are significant seasonal variations in the vehicular emission factors of OC, EC, and the

19 hopanes.

20 Photochemical oxidation of the hopanes during long-range transport is another potential

21 explanation for the lower and widely varying hopanes-to-EC ratios in the summer. When

22 considering oxidation as a potential explanation, one must acknowledge the significant

23 differences between Pittsburgh (and other areas in the eastern US) and Los Angeles where the 
1 molecular marker techniques were developed. Air pollution in the Los Angeles basin is

2 dominated by local emissions mixed with fairly clean background air over relatively short

3 transport distances, while air quality in many other areas of the country is dominated by

4 regional transport. Regional transport allows significant time for atmospheric processing of

5 the emissions. Therefore, one might expect hopanes to be depleted in the regional air mass,

6 particularly in summer when oxidant levels are higher. This is exactly what is shown by

7 measurements made in Pittsburgh and at an upwind rural site (Robinson et al. 2006a), and it is

8 difficult to explain this observation with seasonally-varying emission profiles. The widely

9 varying summertime ratios are also consistent with photochemistry; for example,

10 photochemistry is likely to be less important after periods of rain when local emissions are

11 more significant. If oxidation is important, it likely reduces concentrations of all of the

12 reduced organic compounds used as molecular markers in CMB, not just the hopanes.

13 Fall and spring data are shown in Figures $4 \mathrm{c}$ and $4 \mathrm{~d}$. Comparing the different ratio-ratio

14 plots in Figure 4 indicates that the fall and spring data lie in between the summer and winter

15 extremes. In particular, there is more variability in the ratio of the different hopanes to EC in

16 the fall and spring than in the winter but less than the variability in the summer (Robinson et

17 al. 2006a). CMB analysis of the fall and spring data shows some shifting of the gasoline-

18 diesel split towards diesel relative to the winter.

19 Source apportionment of fine particle mass: To estimate the contribution of motor

20 vehicle emissions to fine particle mass, CMB analysis was also performed with source

21 profiles normalized by $\mathrm{PM}_{2.5}$ mass emissions. On a $\mathrm{PM}_{2.5}$ mass basis, the $\mathrm{CMB}$ results

22 exhibit the same variability as the OC apportionment because the source profile marker-to-

$23 \mathrm{PM}_{2.5}$-mass ratios exhibit essentially the same amount of variability as the marker-to-OC 
1 ratios (Figure $\mathrm{S} 1$ ). Normalizing profiles with $\mathrm{PM}_{2.5}$ mass also does not alter the previously

2 discussed seasonality of the solutions; this pattern is driven by the seasonal shift in the

3 ambient hopanes/EC data and not the marker-to-OC or marker-to- $\mathrm{PM}_{2.5}$-mass ratios of the

4 source profiles.

5 In wintertime, the amount of $\mathrm{PM}_{2.5}$ mass apportioned to motor vehicular emissions by the

6 ten different CMB scenarios varies by a factor of 1.4 with an average contribution of

$7 \quad 850 \pm 89 \mathrm{ng} / \mathrm{m}^{3}$ of $\mathrm{PM}_{2.5}$ mass (average \pm standard deviation of the results from the ten

8 scenarios). Therefore, the wintertime CMB estimates of the contribution of vehicular

9 emissions to ambient $\mathrm{PM}_{2.5}$ mass are as well constrained as the estimates to OC shown in

10 Figure 5a. However, the summertime solutions for $\mathrm{PM}_{2.5}$ mass show the same wide

11 divergence as the $\mathrm{OC}$ results shown in Figure 8.

12 There are some interesting differences between the $\mathrm{CMB}$ solutions for $\mathrm{PM}_{2.5}$ mass

13 compared to those for OC, especially related to the gasoline-diesel split. For example, the

14 NFRAQS diesel-based CMB scenarios apportion $449 \pm 184$ to $581 \pm 172 \mathrm{ng} / \mathrm{m}^{3}$ of $\mathrm{PM}_{2.5}$ mass

15 in the wintertime to diesel vehicles versus only $239 \pm 49$ to $500 \pm 108 \mathrm{ng} / \mathrm{m}^{3}$ of $\mathrm{PM}_{2.5}$ mass to

16 gasoline vehicles. The median gasoline-diesel split (the ratio of gasoline contribution to

17 diesel contribution) for the five NFRAQS diesel-based CMB solutions is between 0.42 and

180.93 on a $P M_{2.5}$ mass basis versus between 1.69 and 2.90 on an OC basis (Figure 5e).

19 Therefore, on an OC basis gasoline vehicles dominate the gasoline-diesel split while diesel

20 vehicles dominate the split on a $\mathrm{PM}_{2.5}$ mass basis. Results for the Schauer-Fraser diesel-based

$21 \mathrm{CMB}$ scenarios are even more skewed to diesel dominance over vehicular $\mathrm{PM}_{2.5}$, with median

22 gasoline-diesel splits between 0.23 and 0.54 on a $\mathrm{PM}_{2.5}$ mass basis. This apparent shift in the

23 gasoline-diesel split simply reflects the fact that diesel emissions are dominated by EC and 
1 gasoline vehicle emissions by OC. For example, the NFRAQS average gasoline profile with

$212 \%$ smokers/high-emitters has a $\mathrm{PM}_{2.5}$-mass-to-OC ratio of 1.7 , compared to $\sim 5$ for the

3 NFRAQS diesel profile. This difference is accounted for in the marker-to- $\mathrm{PM}_{2.5}$-mass ratios

4 of the source profiles.

\section{Conclusions}

6 This paper has illustrated some of the strengths and challenges associated with performing

7 CMB analysis with molecular markers in the context of apportioning motor vehicle emissions.

8 A significant strength of the approach is the strong correlations in the ambient molecular

9 marker concentrations. These correlations reflect the high source specificity of certain

10 organic species and imply the existence of well-defined source profiles, even in Pittsburgh, a

11 location strongly influenced by regional transport. However, a major challenge is the

12 variability in the source profiles. The motor-vehicle profiles are much more variable than the

13 ambient data, which creates significant uncertainty in the CMB estimates.

14 The marker-to-OC ratios are a critical parameter in determining the amount of OC

15 apportioned to a source profile. In sum, the molecular markers fitted in the CMB model

16 typically contribute less than $5 \%$ of the OC mass, which means that the marker-to-OC ratios

17 can be highly variable as illustrated by the order-of-magnitude variability in these ratios

18 among the set of published vehicle profiles (Figure S1). This variability and its consequent

19 effects on predicted fleet emissions must be carefully considered when evaluating CMB

20 solutions. In particular the divergence in different solutions caused by this variability is often

21 greater than sampling and analytical uncertainties propagated by CMB (Figure 6). The

22 statistical measures calculated by CMB do not account for the variability in marker-to-OC 
1 ratios; therefore profile-to-profile differences in these ratios can create uncertainties in the

2 source strength estimates that are extremely hard to remove. The same problems exist if one

3 performs $\mathrm{CMB}$ analysis to apportion $\mathrm{PM}_{2.5}$ mass. In this paper we have reduced the effect of

4 marker-to-OC ratios by constructing fleet-average profiles from the set of published profiles.

5 In the winter, the total amount of vehicular OC is well constrained contributing on average

6 between $13 \%$ and $20 \%$ of the ambient OC $\left(274 \pm 56\right.$ to $\left.416 \pm 72 \mathrm{ng} / \mathrm{m}^{3}\right)$. However, source

7 profile variability creates uncertainty in the gasoline-diesel split. On an OC basis, the

8 NFRAQS diesel-based CMB solutions estimate a gasoline-dominated vehicular contribution

9 while the Schauer-Fraser diesel-based CMB models suggest either the opposite or a more

10 even split. On a $\mathrm{PM}_{2.5}$ mass basis, all solutions indicate that diesel emissions tend to

11 dominate the gasoline-diesel split, but there is almost a factor-of-five variability in the split.

12 Variability in the EC and hopanes source emission rates is the main contributor to this

13 uncertainty. Additional source profiles and/or motor vehicle markers are needed to better

14 constrain the gasoline-diesel split.

15 Explaining the summer data with its widely varying hopanes-to-EC ratios is problematic.

16 Although CMB can find statistically acceptable solutions for the summer, there is little

17 agreement among the different scenarios even on the total vehicular contribution (unlike in

18 the winter), the average summer contribution varying from $5 \%$ to $31 \%\left(176 \pm 49 \mathrm{ng} / \mathrm{m}^{3}\right.$ to

$191091 \pm 363 \mathrm{ng} / \mathrm{m}^{3}$ ) of the ambient OC. Therefore, we have little confidence in the CMB

20 solutions for the contribution of motor vehicles to Pittsburgh OC or the gasoline-diesel split

21 during the summer. More research is needed to better understand the seasonal variability of

22 source profiles and the photochemical stability of molecular markers under conditions of

23 significant regional transport. 


\section{Acknowledgements}

This research was conducted as part of the Pittsburgh Air Quality Study, which was supported by US Environmental Protection Agency under contract R82806101 and the US Department of Energy National Energy Technology Laboratory under contract DE-FC2601NT41017. This research was also supported by the EPA STAR program through the National Center for Environmental Research (NCER) under grant R832162. This paper has not been subject to EPA's required peer and policy review, and therefore does not necessarily reflect the views of the Agency. No official endorsement should be inferred.

\section{References}

Beaton, S. P., G. A. Bishop, Y. Zhang, L. L. Ashbaugh, D. R. Lawson and D. H. Stedman (1995). "On-Road Vehicle Emissions - Regulations, Costs, and Benefits." Science 268(5213): 991-993.

Cadle, S. H., P. Mulawa, E. C. Hunsanger, K. Nelson, R. A. Ragazzi, R. Barrett, G. L. Gallagher, D. R. Lawson, K. T. Knapp and R. Snow (1999a). "Light-duty motor vehicle exhaust particulate matter measurement in the Denver, Colorado, area." Journal of the Air \& Waste Management Association 49: 164-174.

Cadle, S. H., P. A. Mulawa, E. C. Hunsanger, K. Nelson, R. A. Ragazzi, R. Barrett, G. L. Gallagher, D. R. Lawson, K. T. Knapp and R. Snow (1999b). "Composition of lightduty motor vehicle exhaust particulate matter in the Denver, Colorado area." Environmental Science \& Technology 33(14): 2328-2339.

Chow, J. C., J. G. Watson, D. Crow, D. H. Lowenthal and T. Merrifield (2001). "Comparison of IMPROVE and NIOSH carbon measurements." Aerosol Science and Technology 34(1): 23-34.

Chow, J. C., J. G. Watson, L. C. Pritchett, W. R. Pierson, C. A. Frazier and R. G. Purcell (1993). "The DRI Thermal Optical Reflectance Carbon Analysis System - Description, Evaluation and Applications in United-States Air-Quality Studies." Atmospheric Environment Part a-General Topics 27(8): 1185-1201.

Fraser, M. P., B. Buzcu, Z. W. Yue, G. R. McGaughey, N. R. Desai, D. T. Allen, R. L. Seila, W. A. Lonneman and R. A. Harley (2003a). "Separation of fine particulate matter emitted from gasoline and diesel vehicles using chemical mass balancing techniques." Environmental Science \& Technology 37(17): 3904-3909.

Fraser, M. P., K. Lakshmanan, S. G. Fritz and B. Ubanwa (2002). "Variation in composition of fine particulate emissions from heavy-duty diesel vehicles." Journal of Geophysical Research-Atmospheres 107(D21): doi:10.1029/2001JD000558.

Fraser, M. P., Z. W. Yue and B. Buzcu (2003b). "Source apportionment of fine particulate matter in Houston, TX, using organic molecular markers." Atmospheric Environment 37(15): 2117-2123.

Fujita, E. M., B. Zielinska, W. P. Arnott and D. E. Campbell (2005). Chemical Characterization of Mobile Source Emissions - Relation to Ambient Measurements in Vehicle Dominated and Urban Background Locations. Particulate Matter Supersites Program and Related Studies: An AAAR International Specialty Conference, Abstract 4A-2, p. 27, Atlanta, GA. 
Harley, R. A., L. C. Marr, J. K. Lehner and S. N. Giddings (2005). "Changes in motor vehicle emissions on diurnal to decadal time scales and effects on atmospheric composition." Environmental Science \& Technology 39(14): 5356-5362.

NIOSH (1999). Elemental Carbon (Diesel Particulate): Method 5040 (http://www.cdc.gov/niosh/nmam/pdfs/5040f3.pdf).

Oros, D. R. and B. R. T. Simoneit (2000). "Identification and emission rates of molecular tracers in coal smoke particulate matter." Fuel 79(5): 515-536.

Pekney, N. J. and C. I. Davidson (2005). "Determination of trace elements in. ambient aerosol samples." Analytica Chimica Acta 540(2): 269-277.

Robinson, A. L., N. M. Donahue and W. F. Rogge (2006a). "Photochemical oxidation and changes in molecular composition of organic aerosol in the regional context." Journal of Geophysical Research-Atmospheres 111(D3): doi:10.1029/2005JD006265.

Robinson, A. L., R. Subramanian, N. M. Donahue, A. Bernardo-Bricker and W. F. Rogge (2006b). "Source apportionment of molecular markers and organic aerosol - 1. Methodology for Visually Comparing Source Profiles and Ambient Data." Environmental Science \& Technology submitted.

Robinson, A. L., R. Subramanian, N. M. Donahue, A. Bernardo-Bricker and W. F. Rogge (2006c). "Source apportionment of molecular markers and organic aerosol - 2. Biomass smoke." Environmental Science \& Technology submitted.

Robinson, A. L., R. Subramanian, N. M. Donahue, A. Bernardo-Bricker and W. F. Rogge (2006d). "Source apportionment of molecular markers and organic aerosol - 3. Food cooking emissions." Environmental Science \& Technology submitted.

Rogge, W. F., L. M. Hildemann, M. A. Mazurek, G. R. Cass and B. R. T. Simoneit (1993). "Sources of Fine Organic Aerosol .2. Noncatalyst and Catalyst-Equipped Automobiles and Heavy-Duty Diesel Trucks." Environmental Science \& Technology 27(4): 636651.

Rogge, W. F., L. M. Hildemann, M. A. Mazurek, G. R. Cass and B. R. T. Simoneit (1997a). "Sources of fine organic aerosol .7. Hot asphalt roofing tar pot fumes." Environmental Science \& Technology 31(10): 2726-2730.

Rogge, W. F., L. M. Hildemann, M. A. Mazurek, G. R. Cass and B. R. T. Simoneit (1997b). "Sources of fine organic aerosol .8. Boilers burning No. 2 distillate fuel oil." Environmental Science \& Technology 31(10): 2731-2737.

Schauer, J. J. and G. R. Cass (2000). "Source apportionment of wintertime gas-phase and particle-phase air pollutants using organic compounds as tracers." Environmental Science \& Technology 34(9): 1821-1832.

Schauer, J. J., M. J. Kleeman, G. R. Cass and B. R. T. Simoneit (1999). "Measurement of emissions from air pollution sources. 2. C-1 through C-30 organic compounds from medium duty diesel trucks." Environmental Science \& Technology 33(10): 15781587.

Schauer, J. J., M. J. Kleeman, G. R. Cass and B. R. T. Simoneit (2002). "Measurement of emissions from air pollution sources. 5. C-1-C-32 organic compounds from gasolinepowered motor vehicles." Environmental Science \& Technology 36(6): 1169-1180.

Schauer, J. J., W. F. Rogge, L. M. Hildemann, M. A. Mazurek and G. R. Cass (1996). "Source apportionment of airborne particulate matter using organic compounds as tracers." Atmospheric Environment 30(22): 3837-3855. 


\section{Simoneit, B. R. T. (1984). "Organic-Matter of the Troposphere .3. Characterization and}

Sources of Petroleum and Pyrogenic Residues in Aerosols over the Western UnitedStates." Atmospheric Environment 18(1): 51-67.

Simoneit, B. R. T. (1986). "Characterization of Organic-Constituents in Aerosols in Relation to Their Origin and Transport - a Review." International Journal of Environmental Analytical Chemistry 23(3): 207-237.

Subramanian, R., A. Y. Khlystov, J. C. Cabada and A. L. Robinson (2004). "Positive and Negative Artifacts in Particulate Organic Carbon Measurements with Denuded and Undenuded Sampler Configurations." Aerosol Science and Technology 38(S1): 27-48.

Subramanian, R., A. Y. Khlystov and A. L. Robinson (2006). "Effect of peak inert-mode temperature on Elemental Carbon measured using Thermal-Optical analysis." Aerosol Science and Technology in press.

Watson, J. G., E. M. Fujita, J. C. Chow and B. Zielinska (1998a). Northern Front Range Air Quality Study Final Report and Supplemental Volumes. DRI Document No. 6580685-8750.1F2. Desert Research Institute, P.O. Box 60220, Reno, NV 89506, http://www.nfraqs.colostate.edu/nfraqs/index $2 . h t m l$

Watson, J. G., N. F. Robinson, E. M. Fujita, J. C. Chow, T. G. Pace, C. Lewis and T. Coulter (1998b). CMB8 Applications and Validation Protocol for PM2.5 and VOCs. US EPA, http://www.epa.gov/scram001/

Wittig, A. E., N. Anderson, A. Y. Khlystov, S. N. Pandis, C. I. Davidson and A. L. Robinson (2004). "Pittsburgh Air Quality Study Overview and Initial Scientific Findings." Atmospheric Environment 38(20): 3107-3125.

Zheng, M., G. R. Cass, J. J. Schauer and E. S. Edgerton (2002). "Source apportionment of PM2.5 in the southeastern United States using solvent-extractable organic compounds as tracers." Environmental Science \& Technology 36(11): 2361-2371.

Zielinska, B., J. Sagebiel, J. D. McDonald, K. Whitney and D. R. Lawson (2004). "Emission rates and comparative chemical composition from selected in-use diesel and gasolinefueled vehicles." Journal of the Air \& Waste Management Association 54(9): 11381150 . 

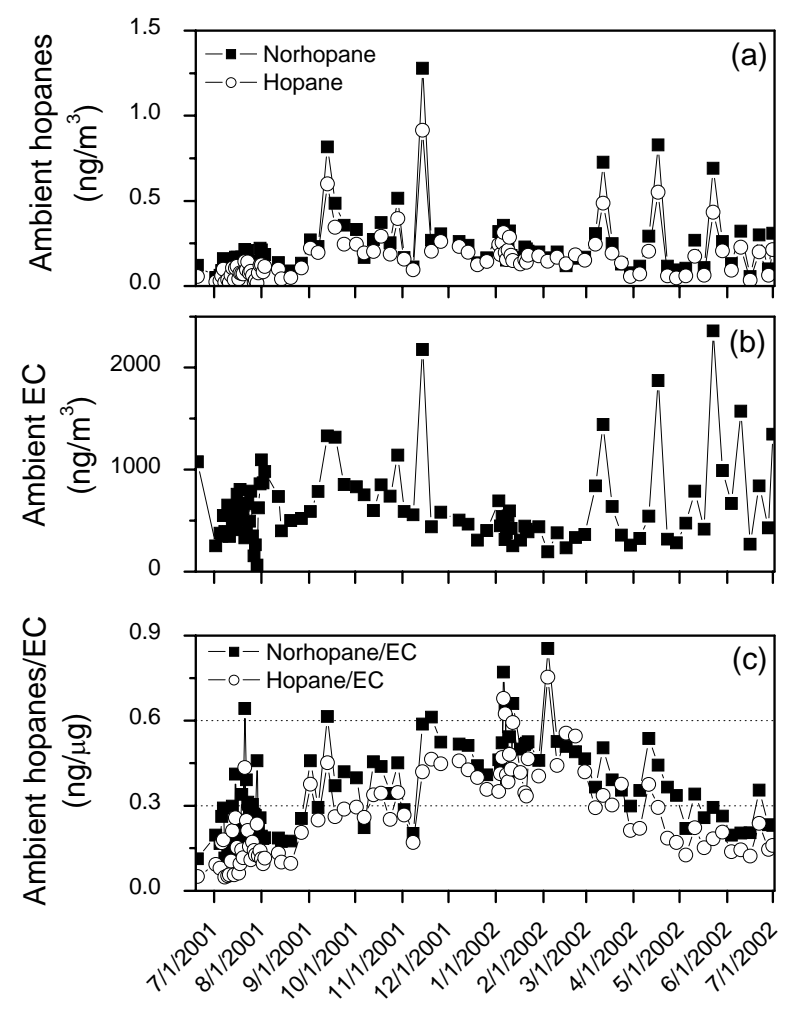

2 Figure 1: Time series of ambient concentrations of (a) norhopane and hopane; (b) EC; and (c)

3 the ratios of the two hopanes to EC. Panel (c) shows the strong seasonal variation in the

4 ambient hopane concentrations relative to EC. 

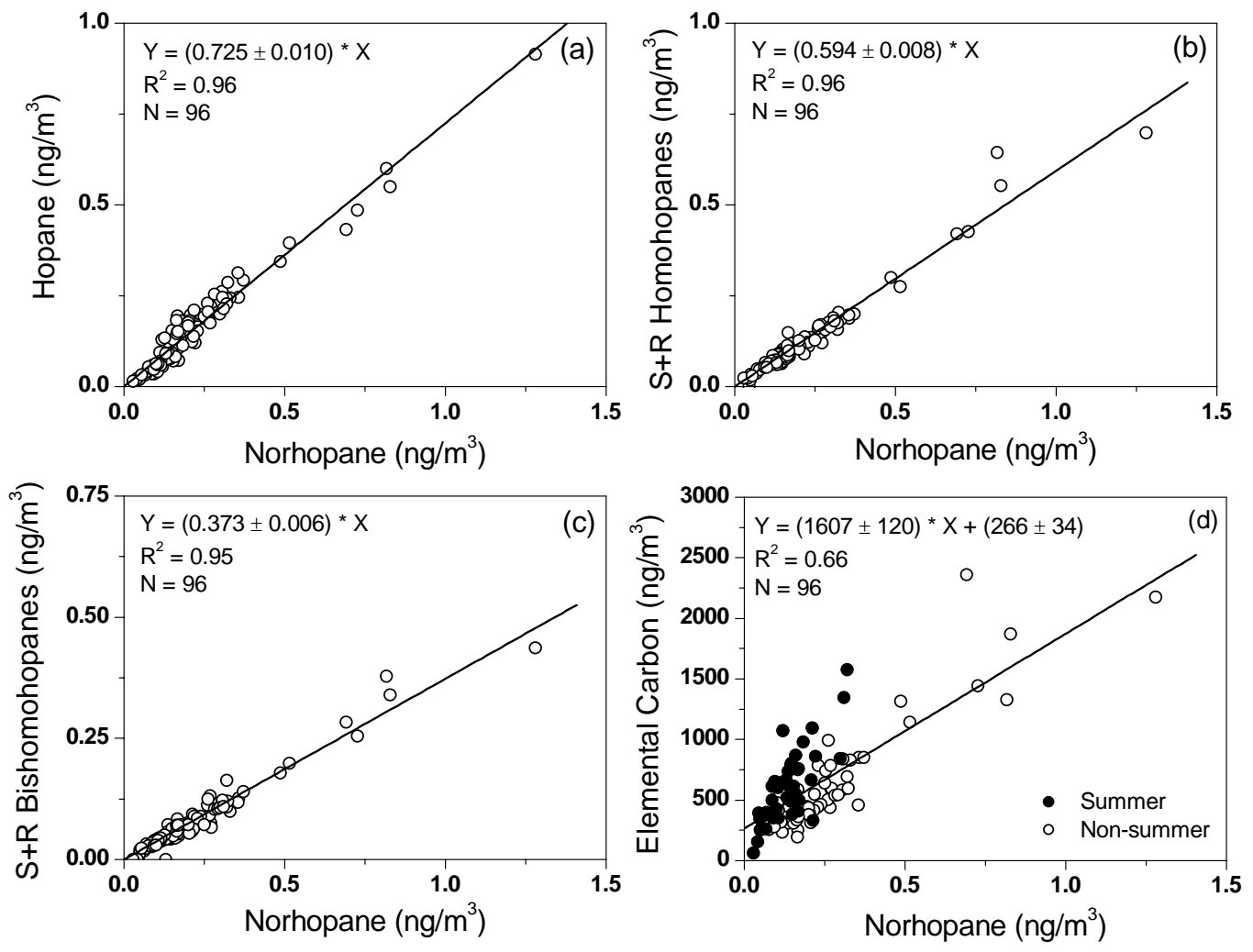

2 Figure 2: Scatter plots of ambient concentrations of selected molecular markers and bulk

3 species versus norhopane. Lines indicate linear regressions. In (d), the filled symbols

4 represent the summer data, while the regression includes all the data points. 

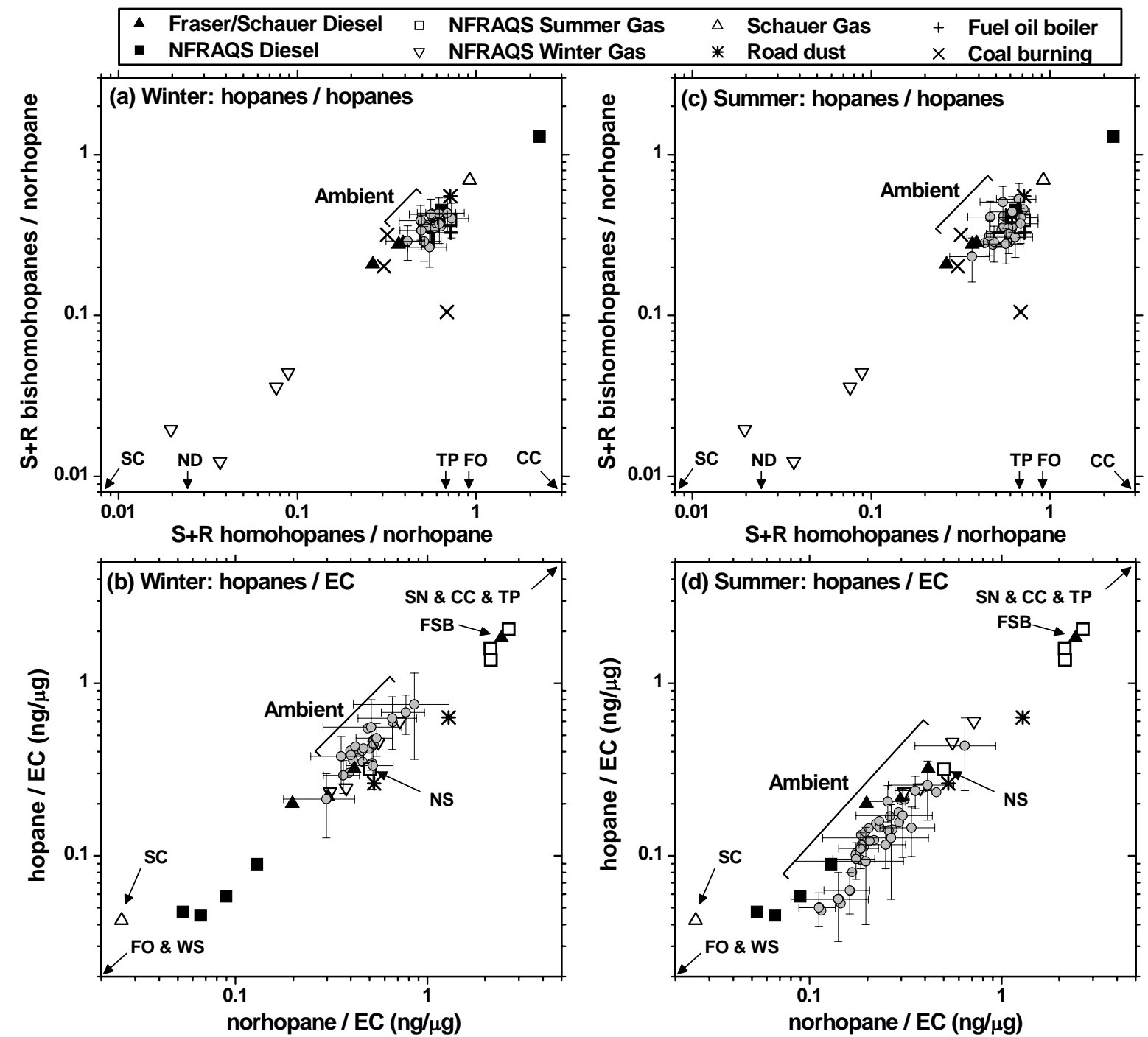

2 Figure 3: Ratio-ratio plots comparing $(a, b)$ winter and (c, d) summer ambient data to

3 published source profiles. Gray circles are ambient data; solid symbols are diesel vehicle

4 profiles, open symbols are gasoline vehicle profiles, and unclosed symbols are non-vehicular

5 sources. Two- and three-character tags are labels for selected source profiles: "SC" is

6 Schauer et al. (2002) catalytic gasoline profile; "FO" is fuel oil boiler (Rogge et al. 1997b);

7 "WS" is wood smoke; "NS" is NFRAQS summer smoker (Watson et al. 1998a); "ND" is

8 NFRAQS diesel profile (Watson et al. 1998a); "FSB" is Fraser et al. (2002) school bus; "SN"

9 is Schauer et al. (2002) non-catalytic gasoline; "CC" is coal combustion (Oros and Simoneit

10 2000); and "TP" is tar pots (Rogge et al. 1997a). Arrows pointing to axes indicate location of

11 profiles located outside bounds of the plots. Propagated analytical and sampling uncertainties

12 are shown as error bars for select ambient data. 

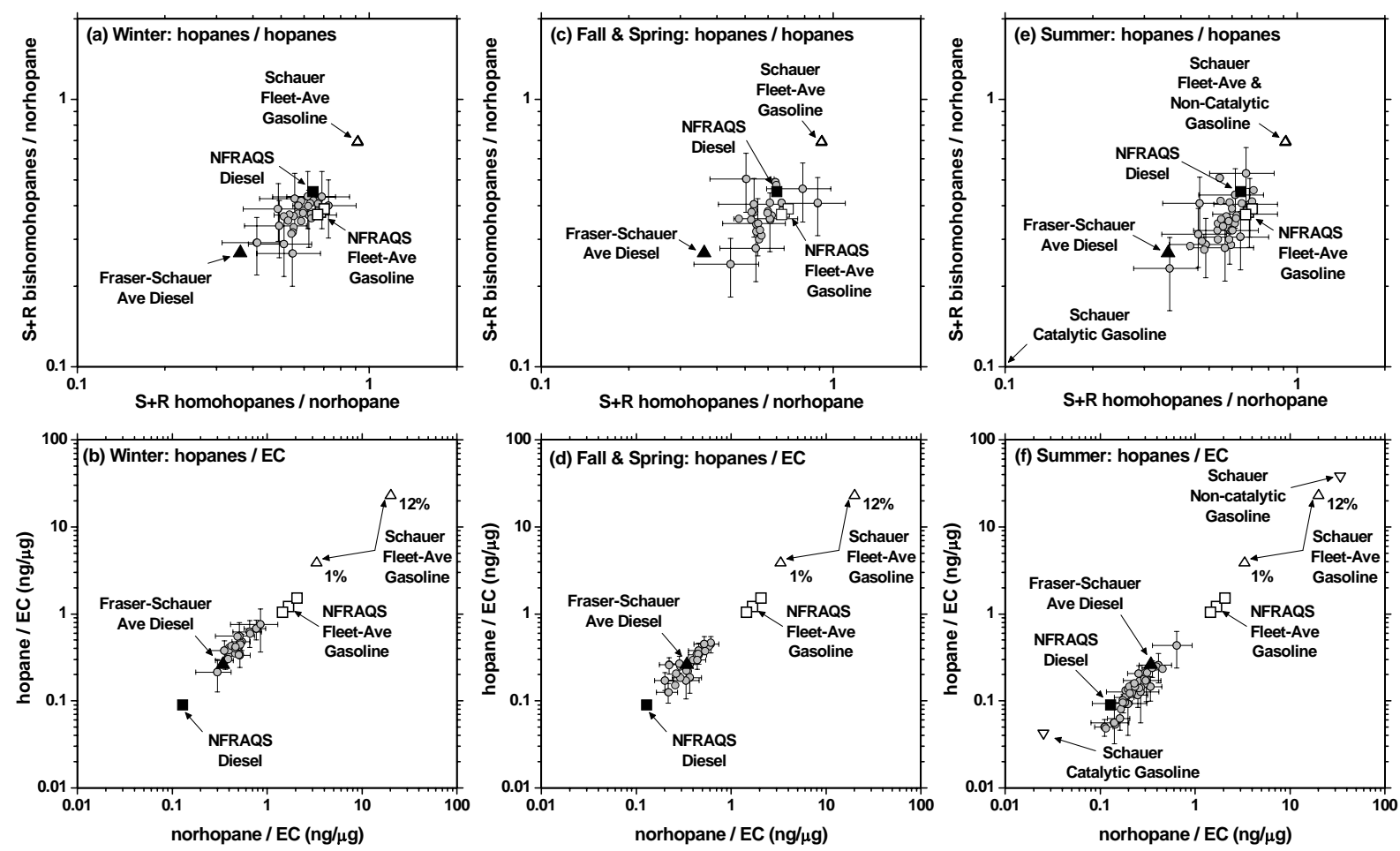

2 Figure 4: Ratio-ratio plots showing different CMB scenarios and ambient data for the

3 (a, b) winter, (c, d) spring and fall, and (e, f) summer. Gray circles are ambient data; solid

4 symbols are diesel vehicle profiles and open symbols are gasoline vehicle profiles. Error bars

5 on select ambient data represent the propagated analytical and sampling uncertainties. 

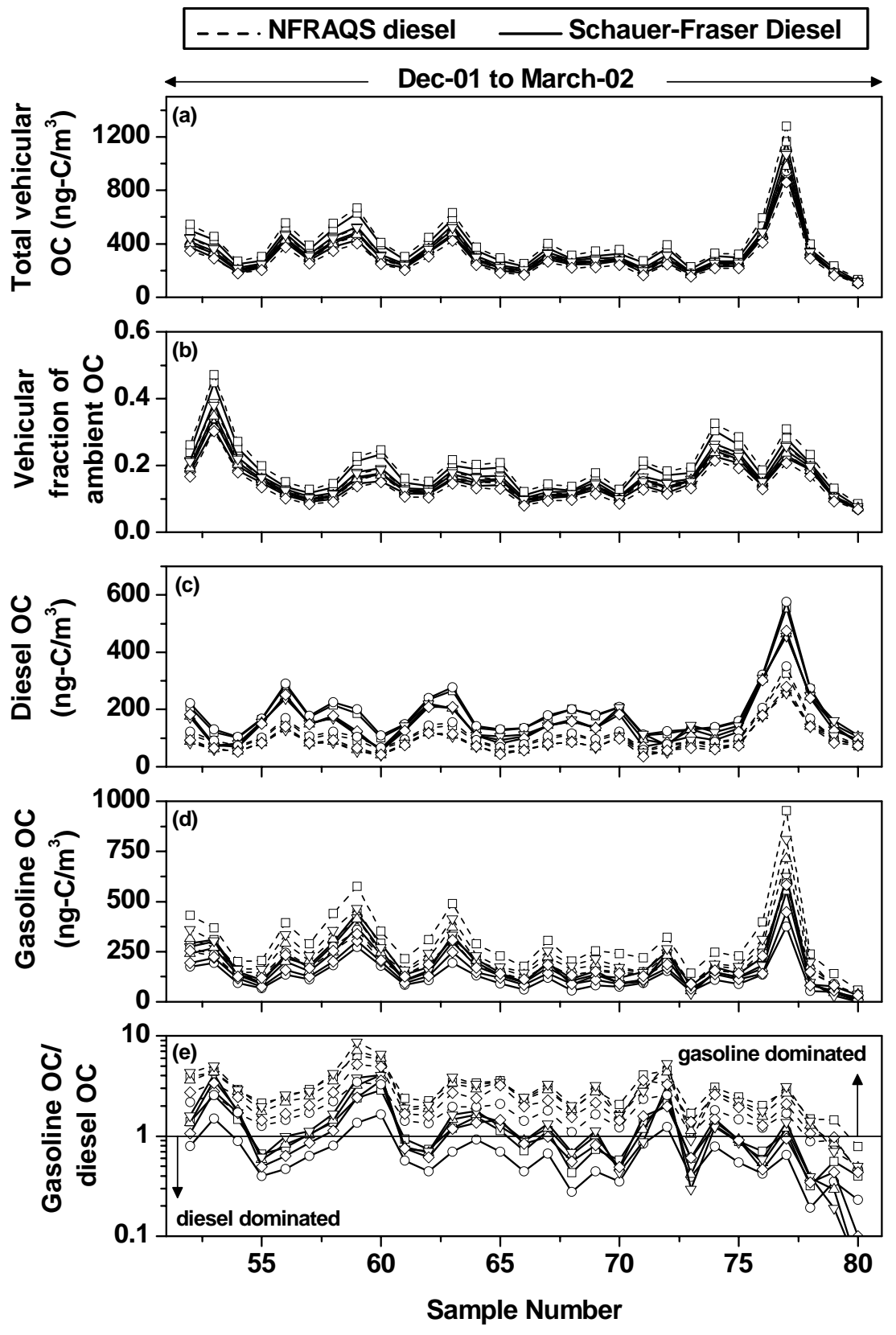

2 Figure 5: Time series of CMB solutions for the winter: (a) total vehicular OC, (b) total

3 vehicular OC as a fraction of ambient OC; (c) gasoline vehicle OC; (d) diesel vehicle OC, and

4 (e) ratio of gasoline OC to diesel OC. Solid lines are calculations using the Schauer- Fraser

5 average diesel profile; dashed lines are calculations using NFRAQS diesel profile. Symbols

6 indicate different fleet-average profiles for gasoline vehicles: $\square$ 1\% high-emitter Schauer

7 gasoline; o 12\% high-emitter Schauer gasoline; $\diamond 1 \%$ high-emitter NFRAQS gasoline; $\Delta 6.8 \%$

8 high-emitter NFRAQS gasoline; and $\nabla$ 12\% high-emitter NFRAQS gasoline. 

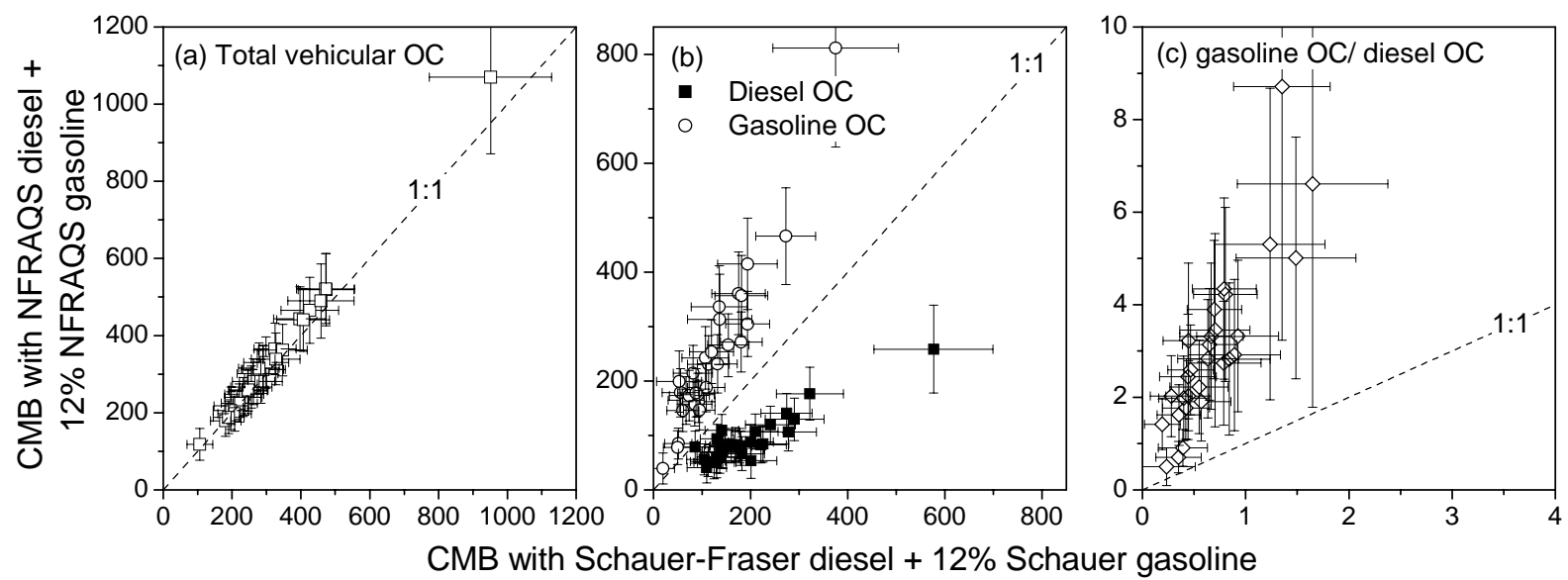

2

3 Figure 6. Scatter plots comparing estimated (a) total vehicular OC (ng-C/m $\left.\mathrm{m}^{3}\right)$, (b) gasoline and

4 diesel OC $\left(\mathrm{ng}-\mathrm{C} / \mathrm{m}^{3}\right)$, and (c) gasoline-diesel split for two different wintertime CMB

5 solutions. " $12 \%$ Schauer gasoline" is the $12 \%$ high-emitter composite profile based on the

6 gasoline profiles of Schauer et al. (2002). "12\% NFRAQS gasoline" is the $12 \%$ high-emitter

7 composite profile based on the NFRAQS data (Watson et al. 1998a). The error bars indicate

8 the standard errors calculated by CMB. 


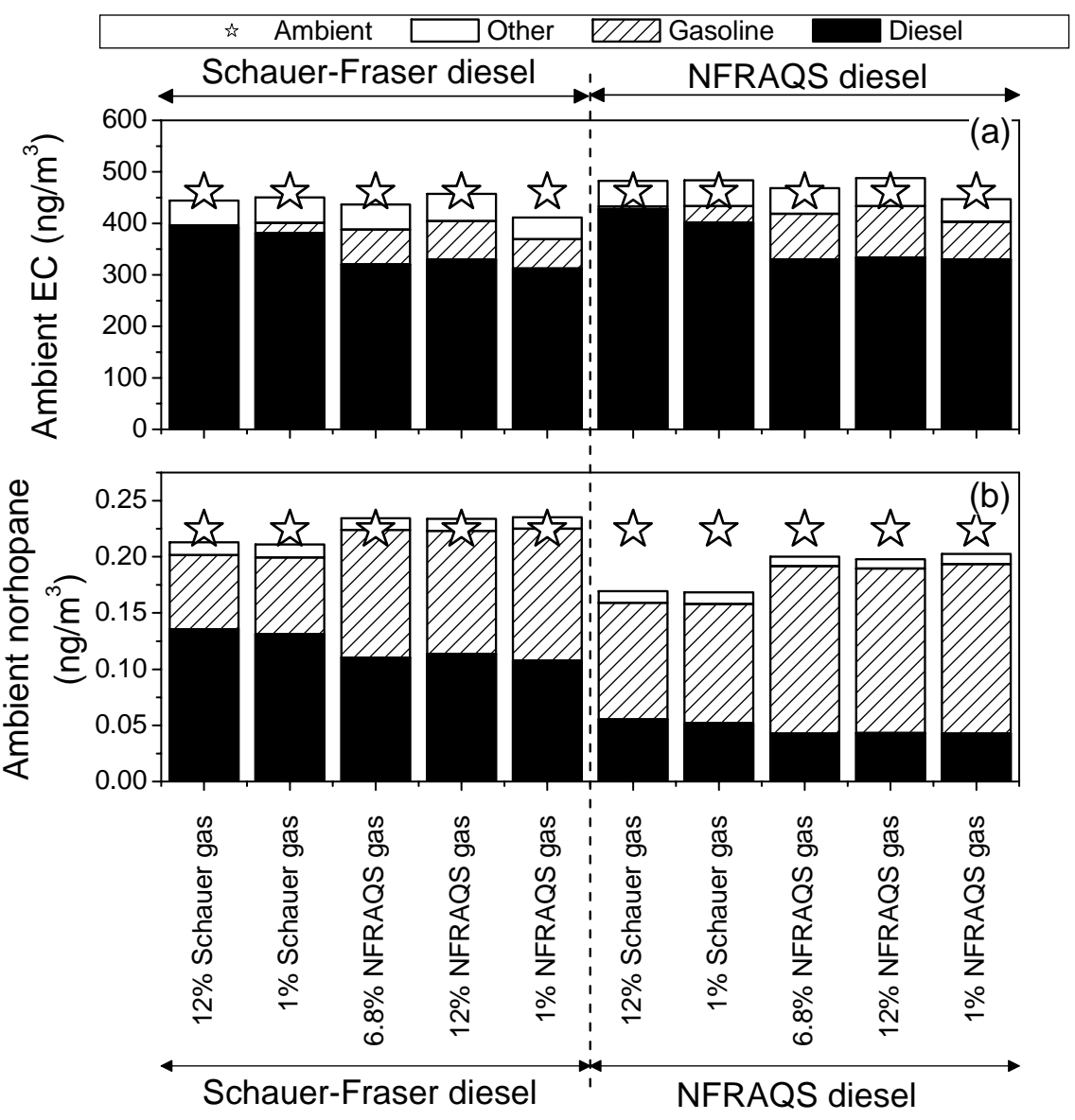

2

3 Figure 7: Average wintertime apportionment of (a) EC and (b) norhopane by the ten different 4 CMB scenarios. 

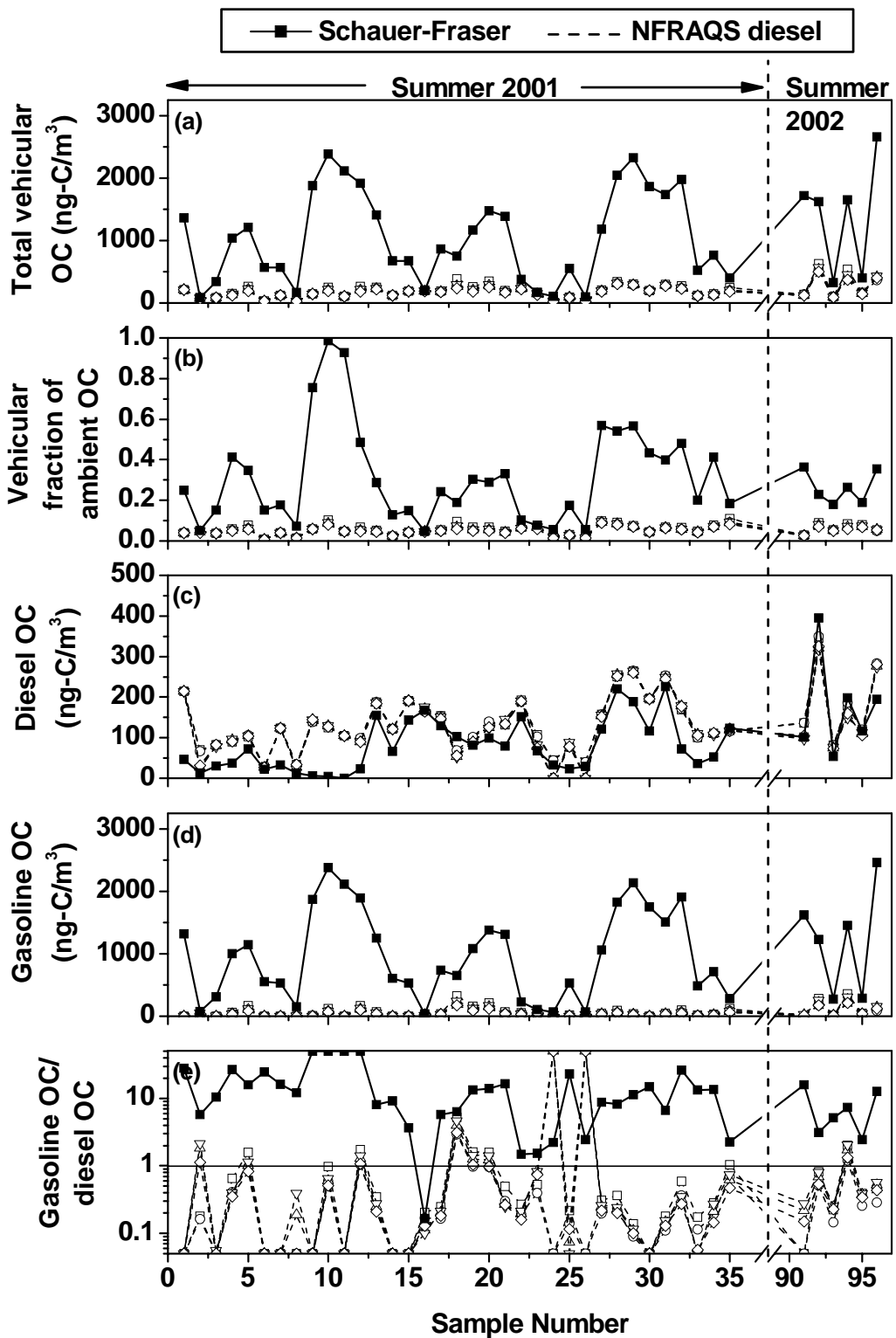

2 Figure 8: Time series of CMB solutions for the summer: (a) total vehicular OC, (b) total

3 vehicular OC as a fraction of ambient OC; (c) gasoline vehicle OC; (d) diesel vehicle OC, and

4 (e) ratio of gasoline OC to diesel OC. Dashed lines are calculations using NFRAQS diesel

5 profile with symbols indicating different fleet-average profiles for gasoline vehicles: $\square 1 \%$

6 high-emitter Schauer gasoline; o 12\% high-emitter Schauer gasoline; $\diamond 1 \%$ high-emitter

$7 \quad$ NFRAQS gasoline; $\Delta 6.8 \%$ high-emitter NFRAQS gasoline; and $\nabla 12 \%$ high-emitter

8 NFRAQS gasoline. In (e) points plotted on axis have a gasoline-diesel split of greater than 50

9 (i.e. diesel OC is zero) or less than 0.05. 


\title{
Evaluation of Measurement Methods
}

\section{Positive and Negative Artifacts in Particulate Organic Carbon Measurements with Denuded and Undenuded Sampler Configurations}

\author{
R. Subramanian, ${ }^{1}$ Andrey Y. Khlystov, ${ }^{2}$ Juan C. Cabada, ${ }^{3}$ and Allen L. Robinson ${ }^{1}$ \\ ${ }^{1}$ Department of Mechanical Engineering, Carnegie Mellon University, Pittsburgh, Pennsylvania \\ ${ }^{2}$ Department of Civil and Environmental Engineering, Duke University, Durham, North Carolina \\ ${ }^{3}$ Earth, Atmospheric and Planetary Sciences, Massachusetts Institute of Technology, \\ Cambridge, Massachusetts
}

Measurement of ambient particulate organic carbon (POC) with quartz filters is prone to positive and negative sampling artifacts. One approach for estimating these artifacts is to sample with a backup quartz filter placed behind either the main quartz filter or a Teflon filter in a parallel line. Another approach is to use a denuder to reduce the positive artifact in combination with a highly adsorbent backup filter to capture any negative artifact. Results obtained using both of these approaches in parallel for over one year in Pittsburgh, PA are presented in this article. A sampler using an activated carbon monolith denuder has been developed and tested extensively. Transmission losses were found to be negligible, and the denuder is on average $94 \%$ efficient at removing gas-phase organics. Denuder breakthrough is corrected for each run using a dynamic blank in parallel with the sample line. Comparisons with the dynamic blank indicate that the denuder almost eliminates the positive artifact on the quartz filter. Negative artifact from the denuded quartz filter is quantified using a carbon-impregnated glass

Received 22 October 2002; accepted 31 March 2003.

This research was conducted as part of the Pittsburgh Air Quality Study, which was supported by US Environmental Protection Agency under contract R82806101 and the US Department of Energy National Energy Technology Laboratory under contract DE-FC26-01NT41017. This article has not been subject to the EPA's required peer and policy review and therefore does not necessarily reflect the views of the Agency. No official endorsement should be inferred.

We are grateful to Professors Spyros Pandis and Cliff Davidson for their valuable inputs over the course of this project. We also thank the Carnegie Mellon University Supersite team, which put a huge amount of work and time into running the PAQS — notably Sarah Rees, Charles Stanier, and Leonard Lucas, who helped us take daily measurements for almost fifteen consecutive months.

Address correspondence to Allen L. Robinson, Department of Mechanical Engineering, Carnegie Mellon University, Pittsburgh, PA 15213.E-mail:alr@andrew.cmu.edu fiber (CIG) backup filter and was found to be small, typically less than $10 \%$ of the ambient POC. Compared to the denuded sampler POC, $24 \mathrm{~h}$ bare quartz samples showed an almost constant positive artifact of $0.5 \mu \mathrm{g}-\mathrm{C} / \mathrm{m}^{3}$ for samples taken throughout the year-long study period. Sampling for shorter durations $(4-6 \mathrm{~h})$ resulted in a larger positive artifact. A quartz filter behind a Teflon filter (QBT) provides a consistent estimate of the positive artifact on the bare quartz filter irrespective of sample duration, though it overcorrects for the positive artifact by $\mathbf{1 6}-\mathbf{- 2 0 \%}$ (attributed to particulate matter volatilizing off the upstream Teflon filter). The quartz behind quartz $(Q B Q)$ approach provides a reasonable estimate of the positive artifact on the bare quartz filter for the $24 \mathrm{~h}$ samples but not for the shorter samples. A slight seasonal variation is observed in the absolute value of the positive artifact, with higher values observed during the summer months.

\section{INTRODUCTION}

Carbonaceous aerosols, commonly classified as organic carbon (OC) and elemental carbon (EC), are a major component of the $\mathrm{PM}_{2.5}$ mass (Shah et al. 1986; Gray et al. 1986; Malm et al. 1994; Turpin et al. 2000). Quartz fiber filters are used to measure particulate organic carbon (POC) and EC since they can be heated to $1000^{\circ} \mathrm{C}$, which is necessary for the usual thermal evolution methods used to determine OC/EC, such as the NIOSH 5040 or IMPROVE protocols (NIOSH Manual of Analytical Methods; Chow et al. 1993). However, sampling with quartz filters is prone to artifacts that result in erroneous measurements of POC. Adsorption of gaseous OC onto the quartz filter leads to overestimation of the POC (positive artifact), while volatilization of the collected PM from the filter results in the underestimation of POC (negative artifact) (Turpin et al. 2000). 
Numerous studies have been conducted to characterize the effect of sampling artifacts on OC/EC measurements, and a recent detailed review is provided by Turpin et al. (2000). Estimates of the OC artifact range from a positive artifact of over $50 \%$ of POC (Kirchstetter et al. 2001; Turpin et al. 1994) to a negative artifact of up to $80 \%$ of POC (Anderson et al. 2002; Eatough et al. 1993; Modey et al. 2001; Ding et al. 2002b). The wide range of reported values reflects the complexity of the problem. Positive and negative artifacts occur simultaneously and hence are difficult to isolate and quantify. A second challenge is that artifacts are strongly influenced by sampler configuration and sampling conditions, which often clouds interpretation and comparison of results from the various published studies. For example, the large negative artifacts reported by Eatough and coworkers occur on a quartz filter behind a denuder, which, as explained later, results in higher volatilization losses. However, the positive artifact appears to dominate samples taken with bare quartz filters (quartz filters without an upstream denuder) (Turpin et al. 1994; Kirchstetter et al. 2001). Experiments have also shown that the artifact is dependent on the face velocity, sampling duration, and location (McDow and Huntzicker 1990; Turpin et al. 1994). Further, Kirchstetter et al. (2001) report a filter lot-to-lot variability in the measured artifact.

One way to account for the positive artifact is to use a backup quartz filter either behind the main quartz filter (the Q-QBQ approach), or in a parallel port behind a Teflon filter (the Q-QBT approach) (McDow and Huntzicker 1990; Turpin et al. 1994; Hart and Pankow 1994; Kim et al. 2001). The OC measured on the backup quartz filter provides an estimate of the positive artifact on the main (or "bare") quartz filter (hence, $\mathrm{POC}=$ bare quartz OC—-backup quartz OC). The Q-QBQ method is simpler in that it requires only one sample line, whereas the Q-QBT requires two lines, one for the bare quartz filter and another for the Teflon/quartz filter pack.

The Q-QBT two-port strategy assumes that the upstream Teflon filter traps only the PM without adsorbing any organic gases, and so the backup filter (quartz behind Teflon, or QBT) is exposed to the same organic vapor concentration as the bare quartz filter, providing a robust estimate of the positive artifact on the bare quartz filter. The Q-QBT approach has been shown to provide a consistent estimate of the POC for samples collected with different face velocities and sample volumes (McDow and Huntzicker 1990; Turpin et al. 1994). However, results from the AUSPEX field study (Chow et al. 1996) suggest that the QBT may overestimate the positive artifact, since this correction resulted in negative POC values (bare quartz-QBT) for 33\% of the 493 samples collected.

For the quartz behind the quartz (QBQ) to provide a good estimate of the positive artifact on a bare quartz filter, both quartz filters should be in equilibrium with the sampled air stream. This may not always be the case, since the backup quartz filter is exposed to lower organic vapor concentrations until the upstream bare quartz filter reaches equilibrium (Mader and Pankow 2001). Hence, the QBT usually provides higher artifact estimates than the QBQ with low sampling volumes (McDow and Huntzicker
1990; Turpin et al. 1994), though for large sample volumes the QBQ and QBT should provide similar estimates of the positive artifact (Hart and Pankow 1994). Kirchstetter et al. (2001) also suggest that longer sampling times with the Q-QBQ combination produce better estimates for the positive artifact.

A second approach to avoid positive artifacts relies on a denuder upstream of the quartz filter to remove organic gases from the incoming air stream (numerous papers by Eatough and coworkers on the BOSS and its variants, including Eatough et al. 1993, 1996; Eatough 1999; Ding et al. 2002a; and other systems by Coutant et al. 1989; Gundel et al. 1995; Gundel and Lane 1999; Mader et al. 2001). This approach can create large negative artifacts because removal of the organic gases alters the gas-particle equilibrium, creating the potential for volatilization of particles collected on the quartz filter. To account for this negative artifact, an adsorbent filter such as a carbon-impregnated filter (Eatough 1999) or a XAD-coated quartz filter (Lewtas et al. 2001) is often installed downstream of the quartz filter. A denuder efficiency of less than $100 \%$ is a serious concern because concentrations of gas-phase organics are typically an order of magnitude greater than the POC, and the adsorbent backup filters capture most of the gas-phase material that escapes the denuder, potentially creating a substantial positive artifact due to the denuder breakthrough.

Although both of these approaches provide important insights into the artifact problem, few comparisons have been published between the denuder-based and quartz backup filter techniques. Such comparisons are important because the magnitude of the artifact can vary with sampler configuration as well as the sampling location. In one of the few available studies, Kirchstetter et al. (2001) compared the Q-QBT, Q-QBQ, and a denuder system, finding the denuded $P O C$ to be slightly higher than either the QBT- or QBQ-corrected bare quartz POC. The higher POC measured by the denuder sampler was attributed to denuder breakthrough. Differences in sampling methodology and use of the backup filters can also make comparisons difficult-for example, Eatough et al. (1996) compare only the denuded quartz data from the BOSS (without the carbon-impregnated backup filter OC) to the bare quartz data of a collocated IMPROVE sampler.

In this article, we compare POC measurements made using both a denuder system and a quartz backup filter-based sampler to examine the effect of artifacts on POC measurements in Pittsburgh. The denuder sampler was extensively characterized using a dynamic blank with each run to estimate any positive artifact associated with denuder breakthrough. Daily and higher frequency measurements were made for over a year. Over seventy-five $24 \mathrm{~h}$ samples were collected with the denuder sampler, and over $30024 \mathrm{~h}$ measurements were made with the quartz backup filter sampler. In addition, high-frequency samples were collected with the quartz backup filter sampler for one month. Such a large data set enables extensive testing and comparison of these different ways of measuring POC and allows a study of temporal variations in artifact. This work is part of the Pittsburgh Air Quality Study (PAQS), one of the EPA Supersites. 


\section{METHODOLOGY}

\section{The Site}

The samplers were operated at the PAQS Supersite, which was located on a hill in an urban park next to the Carnegie Mellon University campus in Pittsburgh, Pennsylvania. The site is not near any major industrial sources and is more than $500 \mathrm{~m}$ from any major road. Additional information and other results from the study are presented by Wittig et al. (2004).

\section{The Samplers}

The quartz backup filter sampler (TQQQ) is a two-port sampler with a double quartz filter pack in one line (QQ) and a Teflon filter followed by a quartz filter in the other (TQ) (Figure 1). The upstream quartz filter is referred to as the "bare quartz," while the two downstream quartz filters are the QBQ and QBT. A PM PM $_{10}$ inlet head (URG, URG-2000-30DBN) is upstream of both filter packs, and a sharp-cut $\mathrm{PM}_{2.5}$ cyclone (URG, URG2000-30EG) is installed upstream of the bare quartz filter, as shown in Figure 1. As listed in Table 1, this sampler provides three estimates of POC: the bare quartz OC, the bare quartz OC minus the QBQ (Q-QBQ); and the bare quartz OC less the QBT (Q-QBT). EC is determined from the analysis of the bare quartz alone. This sampler is similar to that used by McDow and Huntzicker (1990) and Turpin et al. (1994). A set of experiments was performed which showed that the QBT data did not depend on the presence or absence of a $\mathrm{PM}_{2.5}$ cyclone in the TQ line.

The denuder sampler uses an activated carbon monolith denuder (MastCarbon Ltd, UK) with an approximate BET surface area of $900 \mathrm{~m}^{2} / \mathrm{g}$. The denuders are $250 \mathrm{~mm}$ long and $30 \mathrm{~mm}$ in diameter with 230,1 $\mathrm{mm}^{2}$ channels/sq. inch, for a typical residence time of $0.2 \mathrm{~s}$ at $16.7 \mathrm{lpm}$ (assuming plug flow). The denuder is followed by a sharp-cut $\mathrm{PM}_{2.5}$ cyclone (URG, URG-2000-30EG), a quartz filter, and then a carbonimpregnated glass-fiber (CIG) filter, as shown in Figure 2. The denuder removes organic gases from the incoming air stream, while the CIG filter captures volatilization losses from the quartz filter (and also organic gases that escape the denuder). Particulate matter is collected on the sample line, called ACD. Denuder breakthrough is estimated by running a parallel dynamic blank

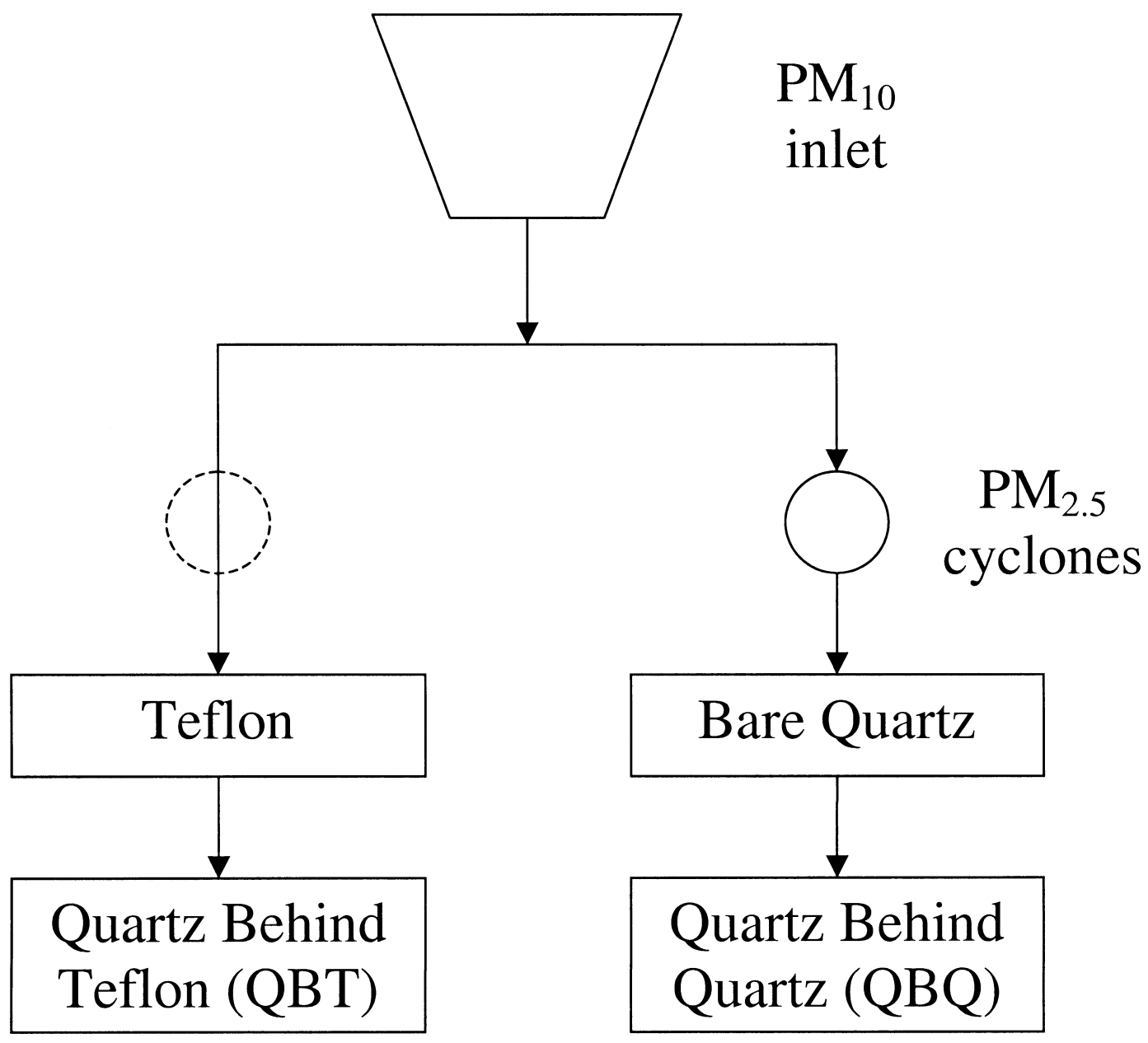

Figure 1. The TQQQ sampler used in the PAQS. This sampler provides three estimates of ambient POC: (a) Bare Quartz, (b) Bare Quartz - QBT, and (c) Bare Quartz - QBQ. A separate set of experiments was performed that showed that the absence or presence of a $\mathrm{PM}_{2.5}$ cyclone in the TQ line (shown by the broken circle above) did not affect the carbon measured on the QBT. 
Table 1

Different particulate organic carbon estimates from the TQQQ and denuder samplers

\begin{tabular}{|c|c|c|c|}
\hline Name & Calculated as & Sampler & Comments \\
\hline Bare Quartz & Bare Quartz & TQQQ & The configuration used by the EPA speciation network \\
\hline Q-QBT & Bare Quartz - QBT & TQQQ & QBT correction for positive artifact on bare quartz filter \\
\hline Q-QBQ & Bare Quartz - QBQ & TQQQ & QBQ correction for positive artifact on bare quartz filter \\
\hline Denuded POC & $\begin{array}{l}\left(\mathrm{Q}_{\mathrm{ACD}}+\mathrm{CIG}_{\mathrm{ACD}}\right) \\
\quad-\left(\mathrm{Q}_{\mathrm{DYN}}+\mathrm{CIG}_{\mathrm{DYN}}\right)\end{array}$ & Denuder & $\begin{array}{l}\text { Reference value of POC used in this study. CIG backup } \\
\text { filter used to measure negative artifact, and dynamic } \\
\text { blank used to correct for denuder slip. }\end{array}$ \\
\hline Denuded Quartz & $\mathrm{Q}_{\mathrm{ACD}}-\mathrm{Q}_{\mathrm{DYN}}$ & Denuder & \\
\hline
\end{tabular}

(DYN), which is similar to the ACD line except that all the $\mathrm{PM}$ is removed by a Teflon filter upstream of the denuder. As indicated in Table 1, the ambient POC level is estimated by subtracting the OC measured by the dynamic blank line (quartz and CIG) from the corresponding sample values, and the net quartz and net CIG OC values are added together to give the ambient POC concentration. The EC is estimated from the ACD quartz filter.

A third line, the undenuded blank (UDB), consisting of a Teflon filter followed by a quartz/CIG combination, was added in September 2001 to measure the gaseous OC that is sorbed onto the quartz and CIG filters in the absence of a denuder. The denuder efficiency is calculated using the UDB-measured ambient gaseous OC concentration as

$$
\begin{aligned}
& \text { Denuder Efficiency } \\
& \quad=1-\left[\left(\mathrm{Q}_{\mathrm{DYN}}+\mathrm{CIG}_{\mathrm{DYN}}\right) /\left(\mathrm{Q}_{\mathrm{UDB}}+\mathrm{CIG}_{\mathrm{UDB}}\right)\right] .
\end{aligned}
$$

Over the course of the study, the denuders were changed once: one set of two denuders was used from 1 July 2001 to 25 October 2001; a second set was used for the remainder of the study period. The denuders of each set were also rotated between the sample and dynamic blank lines to examine potential differences between the denuders, but no significant variations were observed.

\section{Sampling}

The samplers were run in either of two main sampling modes-low frequency and high frequency. In the low-frequency mode, the TQQQ sampler was operated every day for a $24 \mathrm{~h}$ sample starting at approximately midnight, while the denuder sampler was usually run once every six days for a $24 \mathrm{~h}$ sample synchronized with the TQQQ run for that day. Low-frequency samples were collected throughout the study period from June 2001 to July 2002, except for July 2001. High-frequency samples were collected during July 2001, when the TQQQ sampled for 4 or $6 \mathrm{~h}$ on a $0-6,6-10,10-14,14-18$, and 18-24 schedule (all times EST), and the denuder sampler was run every day from midnight to midnight (0-24). In January 2002, the denuder sampler was operated daily ( $24 \mathrm{~h}$ samples) for two weeks. The sampling schedules are summarized in Table 2.
Gelman quartz fiber filters (47 mm, Tissuquartz 2500 QAOUP) and CIG filters (47 mm, Schleicher \& Schuell, GF 3649) were used throughout the study. All of the filters in each set of samples were taken from the same lot, to avoid any problems due to interlot variability (Kirchstetter et al. 2001). Prior to sampling, the quartz filters were baked at $550^{\circ} \mathrm{C}$ in air for a minimum of $4 \mathrm{~h}$; the CIG filters were baked at $360^{\circ} \mathrm{C}$ in UHP Nitrogen for a minimum of $12 \mathrm{~h}$. The prebaked quartz and CIG filters were stored in clean, sealed glass jars at room temperature till further use. In the later part of the study (post-October 2001), the CIG filters were stored in a freezer, but this did not have a significant effect on the handling blanks. The Teflon filters (either Whatman 7592-104 or Pall-Gelman Teflo R2PJ047) were used as provided by the manufacturer without any pretreatment. Filter packs were

Table 2

\begin{tabular}{|c|c|c|}
\hline \multirow[b]{2}{*}{ Month } & \multicolumn{2}{|c|}{ Number of sampling days } \\
\hline & TQQQ & Denuder \\
\hline June 2001 & 23 & 0 \\
\hline July 2001 & $31^{a}$ & 31 \\
\hline August 2001 & $31^{b}$ & 6 \\
\hline September 2001 & 30 & 5 \\
\hline October 2001 & 31 & 5 \\
\hline November 2001 & 30 & 5 \\
\hline December 2001 & 26 & 3 \\
\hline January 2002 & 31 & 13 \\
\hline February 2002 & 28 & 0 \\
\hline March 2002 & 31 & 0 \\
\hline April 2002 & 28 & 4 \\
\hline May 2002 & 31 & 5 \\
\hline June 2002 & 28 & - \\
\hline July 2002 & 30 & - \\
\hline Total (5 samples a day) $)^{a, b}$ & 30 & - \\
\hline Total (24-hour samples) $)^{a, b}$ & 379 & 77 \\
\hline
\end{tabular}

Monthly breakup of TQQQ and denuder sampler runs

${ }^{a}$ July 2001: 27 days of high-frequency sampling and four $24 \mathrm{~h}$ samples.

${ }^{b}$ August 2001: 3 days of high-frequency samples and twenty-eight 24 h samples. 


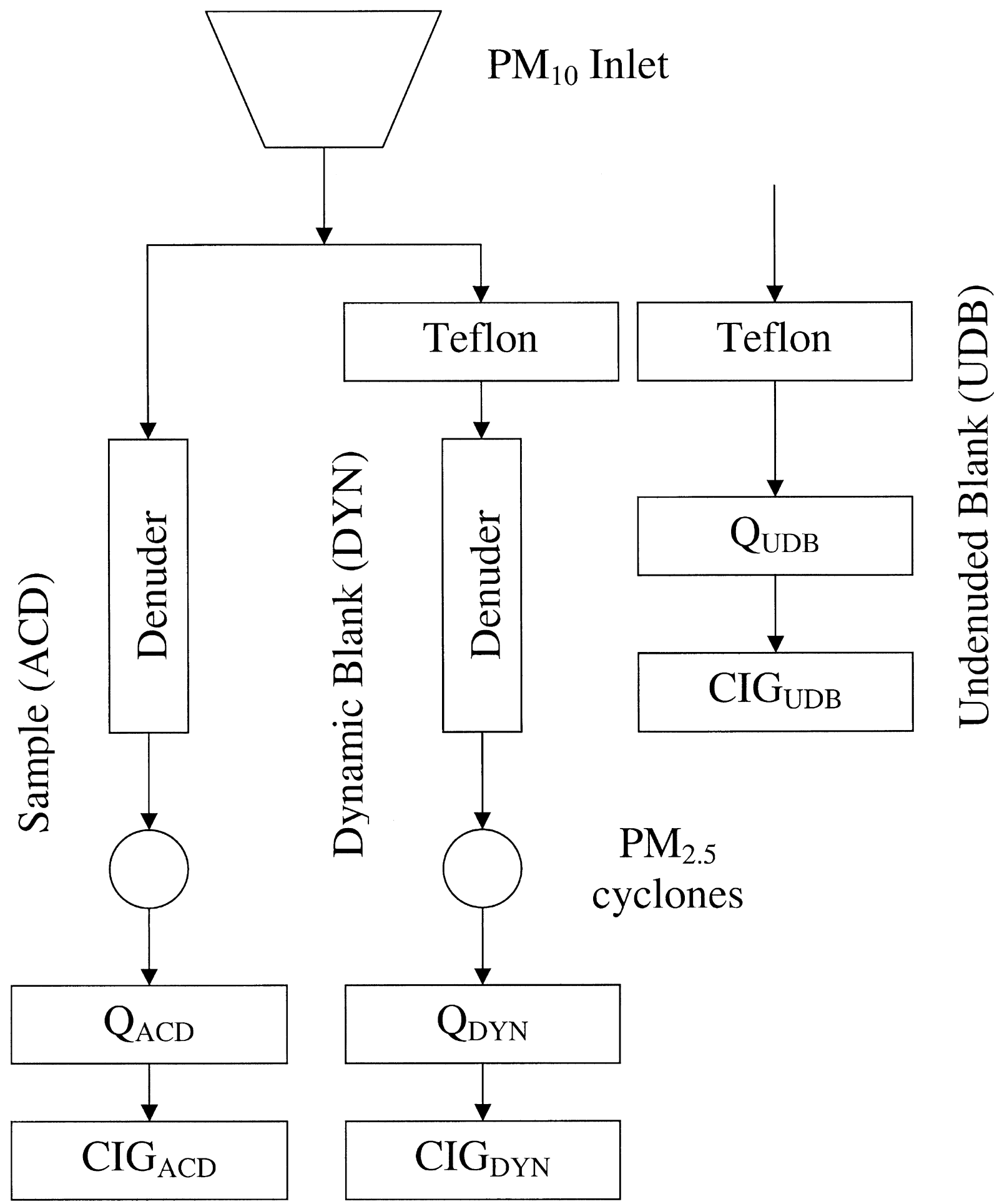

Figure 2. The denuder sampler used in the PAQS. Ambient POC is estimated as $\left[\left(\mathrm{Q}_{\mathrm{ACD}}+\mathrm{CIG}_{\mathrm{ACD}}\right)-\left(\mathrm{Q}_{\mathrm{DYN}}-\mathrm{CIG}_{\mathrm{DYN}}\right)\right]$. The UDB line is used to estimate the denuder efficiency in conjunction with the DYN line, see Equation (1).

loaded and unloaded in a laminar flow hood. After sampling, the filters were stored in polystyrene petridishes at $-20^{\circ} \mathrm{C}$.

All sample lines were operated at a nominal flow rate of $16.7 \mathrm{lpm}$. Gelman 2200 stainless steel filter packs were used, which reduced the effective filter diameter to $35 \mathrm{~mm}$, resulting in a face velocity of $29 \mathrm{~cm} / \mathrm{s}$ at $16.7 \mathrm{lpm}$. The filter packs were modified to allow a double filter set (i.e., Teflon/quartz, quartz/quartz, or quartz/CIG), separating the two filters in each 
Table 3

Study averages of handling blanks for all the filters and the dynamic blank EC for the denuder sampler

\begin{tabular}{lccc}
\hline \multicolumn{1}{c}{ Filter } & $\begin{array}{c}\text { Number of } \\
\text { blanks }\end{array}$ & $\begin{array}{c}\text { OC blank } \\
\text { average }\end{array}$ & $\begin{array}{c}\text { EC blank } \\
\text { average }\end{array}$ \\
\hline QBT & 51 & $0.23 \pm 0.14$ & $0.06 \pm 0.07$ \\
Bare Quartz & 52 & $0.22 \pm 0.15$ & $0.07 \pm 0.08$ \\
QBQ & 52 & $0.23 \pm 0.15$ & $0.08 \pm 0.12$ \\
QACD & 24 & $0.28 \pm 0.18$ & $0.31 \pm 0.26$ \\
CIG $_{\text {ACD }}$ & 24 & $1.79 \pm 0.61$ & NA \\
QDYN $^{\text {DYN }}$ & 77 & NA & $0.32 \pm 0.33$ \\
\hline
\end{tabular}

Values are in $\mu \mathrm{g}-\mathrm{C} / \mathrm{cm}^{2}$. Negative values are taken as zero. Errors are one standard deviation. NA, not applicable.

set by $0.5 \mathrm{~mm}$. During each run, two independent measurements were made of the flow through each sample line using (1) a rotameter in combination with a vacuum gauge and (2) a dry gas meter. The flows measured with the rotameter and the dry gas meter were within $4 \%$ for all runs. The average variability between sampler lines for a given run was $3.2 \%$ for the TQ and QQ lines, and $1.3 \%$ for the ACD and DYN lines. Flow audits were conducted once every seven runs to verify performance of the rotameters against a primary flow measurement device (Gilibrator 2, Sensidyne, Inc.).

One set of handling blanks was taken approximately every ten samples. Handling blanks are filter packs loaded onto the sampler and removed after about a minute, without any air flow. The appropriate study average blank values (shown in Table 3) were subtracted from the sample values (the denuder quartz and CIG OC for each run were corrected using the dynamic blank quartz and CIG values for that day). All the handling blank values for the quartz filters are significantly lower than the EPA specification of $1 \mu \mathrm{g}-\mathrm{C} / \mathrm{cm}^{2}$ used for the speciation network. The EC blank levels of the quartz filters used in the denuder sampler are higher than those of the quartz filters used in the TQQQ sampler, presumably due to contamination from the CIG backup filter. For a typical $24 \mathrm{~h}$ POC concentration of $3 \mu \mathrm{g}-\mathrm{C} / \mathrm{m}^{3}$, a blank OC contribution of $0.3 \mu \mathrm{g}-\mathrm{C} / \mathrm{cm}^{2}$ would be less than $5 \%$ of the filter loading.

\section{Sample Analysis}

All filters were analyzed using a Sunset Laboratories Thermal Optical Transmittance (TOT) OC/EC analyzer, usually within one week of sampling. The CIG filters were analyzed in a Helium atmosphere with a stepped temperature profile ramping to $330^{\circ} \mathrm{C}$ in steps of $10^{\circ} \mathrm{C}$ every $30 \mathrm{~s}$, followed by a $5 \mathrm{~min}$ soak at $330^{\circ} \mathrm{C}$. Typical thermograms from the analysis of CIG filters are shown in Figures $3 a$ and $b$. The carbon level on the filter is determined by integrating the area under the thermogram. Previous studies have reported significant deterioration of carbon-impregnated filters at temperatures around $300^{\circ} \mathrm{C}$ (Modey et al. 2001; Anderson et al. 2002). No evidence of filter degradation was observed
Table 4

The NIOSH protocol used for analyzing the quartz filters on the Sunset Labs OC/EC analyzer

\begin{tabular}{clcc}
\hline Step & Mode (Atmosphere) & Temperature $\left({ }^{\circ} \mathrm{C}\right)$ & Time $(\mathrm{s})$ \\
\hline 1 & Helium & 340 & 120 \\
2 & Helium & 500 & 120 \\
3 & Helium & 615 & 120 \\
4 & Helium & $870(\text { or } 700)^{a}$ & 180 \\
5 & Helium & (cool down) & 45 \\
6 & Helium/Oxygen & 575 & 45 \\
7 & Helium/Oxygen & 650 & 45 \\
8 & Helium/Oxygen & 725 & 45 \\
9 & Helium/Oxygen & 800 & 45 \\
10 & Helium/Oxygen & 910 & 100 \\
11 & Calibration $\left(\mathrm{CH}_{4}\right)$ & - & 110 \\
\hline
\end{tabular}

${ }^{a}$ All the results presented in this article are with the $870^{\circ} \mathrm{C}$ temperature.

during analysis of more than 200 CIG filters. For example, the FID signal on the thermograms of the four different CIG filters shown in Figures $3 \mathrm{a}$ and $\mathrm{b}$ all approach zero during the final 5 min step at $330^{\circ} \mathrm{C}$.

Quartz filters were analyzed with the temperature profile given in Table 4 and shown in Figure 4, which is based on the NIOSH 5040 protocol (NIOSH Manual of Analytical Methods, available at http://www.cdc.gov/niosh/nmam/pdfs/5040f3.pdf). As per the NIOSH protocol, if any EC loss is evident in the fourth Helium temperature step $\left(870^{\circ} \mathrm{C}\right)$, this temperature should be reduced. This was found to be the case, and a number of the filters were analyzed with a modified profile reducing the maximum Helium mode temperature to $700^{\circ} \mathrm{C}$, which appeared to eliminate the loss of light-absorbing carbon in the fourth Helium peak. Reducing the peak temperature of the Helium mode to $700^{\circ} \mathrm{C}$ lowers the OC values by about $5 \%$, but since the first three peaks are the same, it should not affect the OC artifact, which mostly evolves from the filter at the lower temperatures. However, this change in the peak Helium mode temperature did affect the EC (which forms a much smaller component of the carbonaceous aerosol as compared to POC). The EC and corresponding results are discussed in a companion paper (Subramanian et al. 2004).

Based on 101 filters analyzed twice (once with the standard NIOSH protocol used in this study and then a second punch with the modified NIOSH protocol with a peak Helium temperature of $700^{\circ} \mathrm{C}$ ), a linear regression of the total carbon produced a correlation coefficient $\left(\mathrm{R}^{2}\right)$ of 0.99 and a slope of 1.001 (between 0.986 and 1.016 at the $95 \%$ confidence interval), with a negligible intercept. Hence, the precision of the method due to the instrument as well as filter loading can be taken as within $2 \%$ for total carbon. Sunset Laboratory claims the precision due to the instrument and filter loading variability as $\pm 5 \%$ for the same instrument, with a minimum uncertainty of $0.2 \mu \mathrm{g}-\mathrm{C} / \mathrm{cm}^{2}$ 

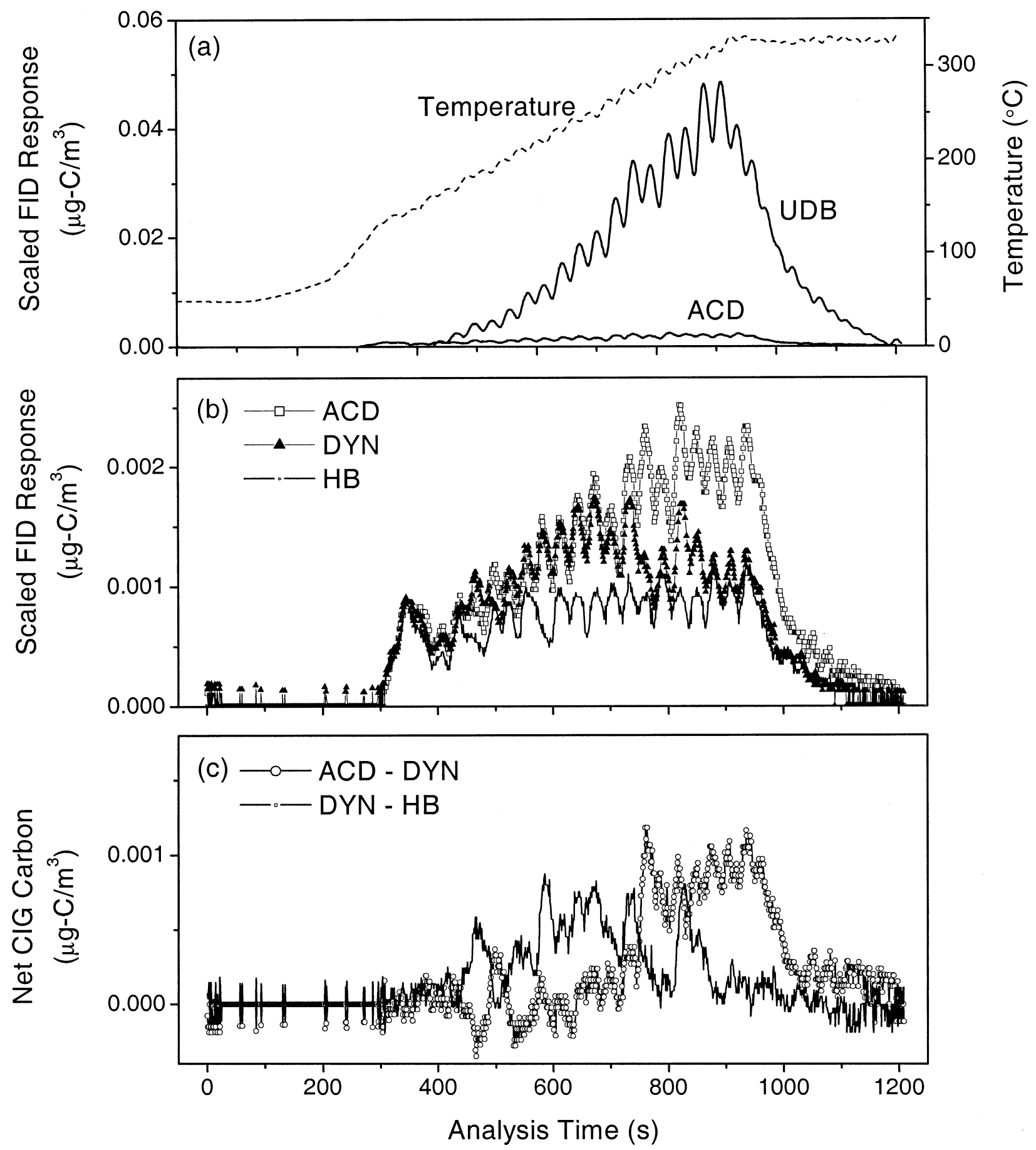

Figure 3. Typical thermograms for the CIG analysis: (a) Temperature profile and FID signals for the $\mathrm{CIG}_{\mathrm{UDB}}$ and $\mathrm{CIG}_{\mathrm{ACD}}$; (b) FID signals for the $\mathrm{CIG}_{\mathrm{ACD}}, \mathrm{CIG}_{\mathrm{DYN}}$, and handling blank (HB) CIG; and (c) Net CIG OC, shown as the (ACD - DYN); and the positive artifact on the CIG due to denuder breakthrough, shown by the (DYN - HB).

(equivalent to $0.08 \mu \mathrm{g}-\mathrm{C} / \mathrm{m}^{3}$ for a normal $24 \mathrm{~h}$ sample) (R. A. Cary 2003, Personal Communication). Schauer et al. (2003) report the precision as $4-13 \%$ for OC and 6-21\% for EC between different instruments running the same temperature-time protocol on parallel punches.

\section{RESULTS AND DISCUSSIONS}

\section{Denuder Performance and Negative Artifact}

The denuder sampler provides the best estimate of POC because (1) the denuder reduces the positive artifact and any 


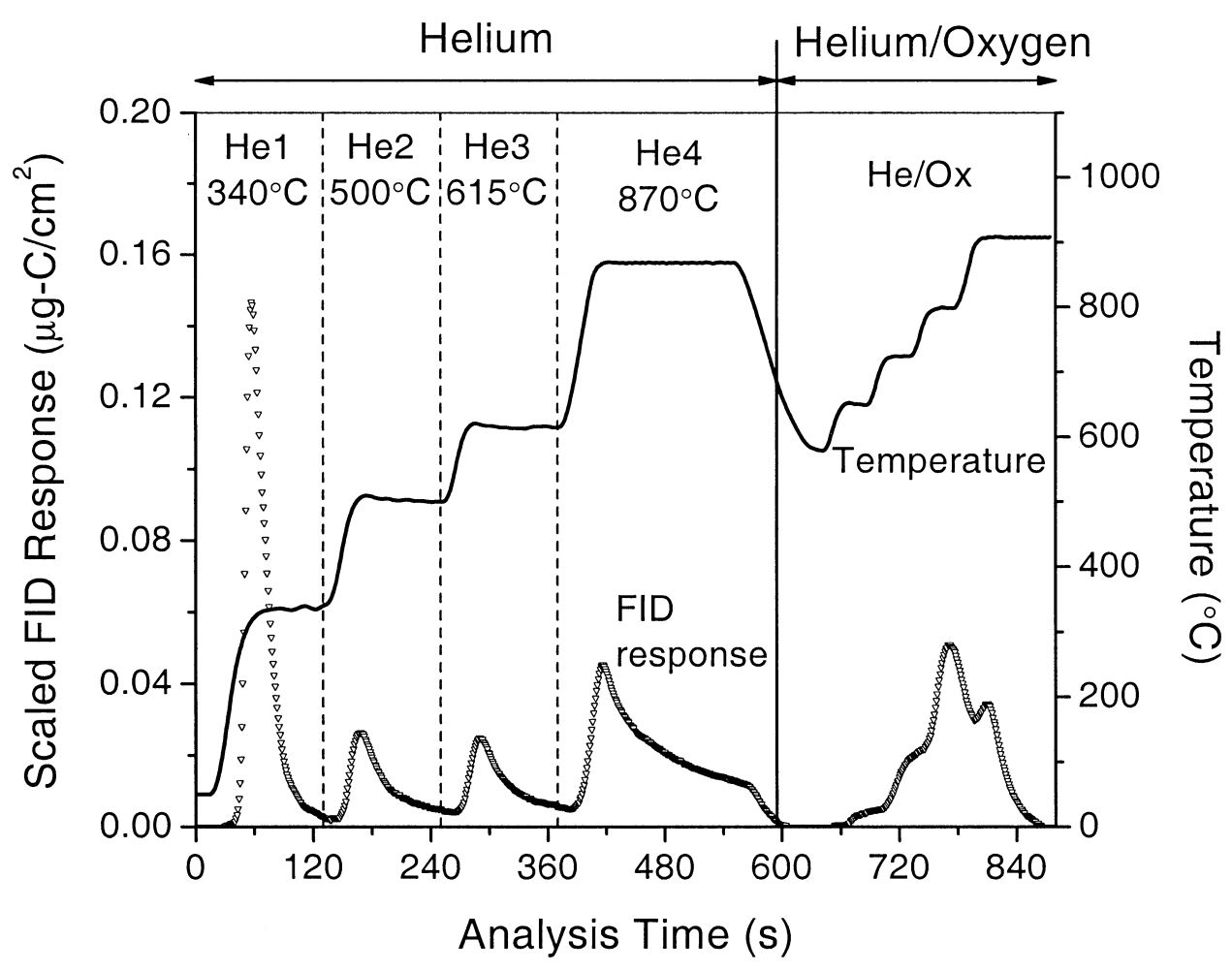

Figure 4. Typical thermogram for a quartz filter from the OC/EC analyzer.

denuder breakthrough is estimated and corrected for using the dynamic blank line, and (2) any negative artifact is quantified with the CIG backup filters. Hence, the denuded POC is taken as the reference to which the POC estimates from the TQQQ sampler are compared. To ensure that the denuder results are reliable, it is essential that the denuder system be properly characterized. Results presented in this section examine three issues with denuder sampler operation, (1) particle losses in and contamination by the denuder; (2) denuder efficiency and positive artifact due to denuder breakthrough; and (3) negative artifacts on the CIG backup filters.

Particle Losses in and Contamination by the Denuder. Two different tests were performed to examine particle losses in the denuder. First, a comparison of the levels of inert species such as EC between the denuded and undenuded quartz filters provides a check on particle losses (Eatough et al. 1993; Ding et al. 2002a). Ambient EC concentrations from the bare quartz and the denuded quartz $24 \mathrm{~h}$ samples are compared in Figure 5. A paired t-test shows that the EC values given by the two are not significantly different at a $95 \%$ level of confidence ( $p=0.547)$. Second, laboratory tests using ammonium sulfate aerosol and an SMPS (Model 3936N25, TSI Inc.) indicated that the transmission efficiency through the denuder is about $95 \%$ or higher for particles larger than $100 \mathrm{~nm}$ in diameter.

As a precaution against contaminating the denuded quartz filters due to shedding of the denuder material (activated carbon), $\mathrm{PM}_{2.5}$ cyclones were installed downstream of the denuders (Figure 2). Significant shedding was observed with an initial set of denuders used for sampler design experiments, but this problem was solved by modifying the manufacturing process of the denuders (curing the "green" denuders after cutting them to the specified length, instead of cutting them after curing the stock). The denuders used for this study and the results presented in this article did not exhibit any shedding. The SMPS experiments showed no increase in particle concentration across the denuder, and the average dynamic blank quartz EC is practically the same as the denuder sampler handling blank EC ( $Q_{D Y N}$ and $\mathrm{Q}_{\mathrm{ACD}}$ in Table 3).

Denuder Efficiency and Positive Artifact Due to Denuder Breakthrough. Comparing the thermograms of the UDB and ACD CIG filters in Figure 3a indicates that the denuder removes a large fraction of the atmospheric gaseous OC that is normally captured by the CIG filter. Figure 6 shows the variation of denuder efficiency over time. The denuders are on average over 94\% efficient (standard deviation, SD 3\%) as calculated with Equation (1), with no noticeable difference between the individual denuders. A comparison of the ACD, DYN, and handling blank (HB) CIG filters shows that the positive artifact on the CIG filters (DYN-HB) violates at temperatures between $150-300^{\circ} \mathrm{C}$ (Figures $3 \mathrm{~b}$ and c), sometimes overlapping with the negative artifact (ACD-DYN, discussed below). No significant deterioration in performance was observed over time for the three denuders, nor does there seem to be any seasonal variability in denuder efficiency. Lower values of denuder efficiency are typically associated with lower gaseous OC concentrations as measured on the UDB line. The first set of denuders was replaced after 45 runs 


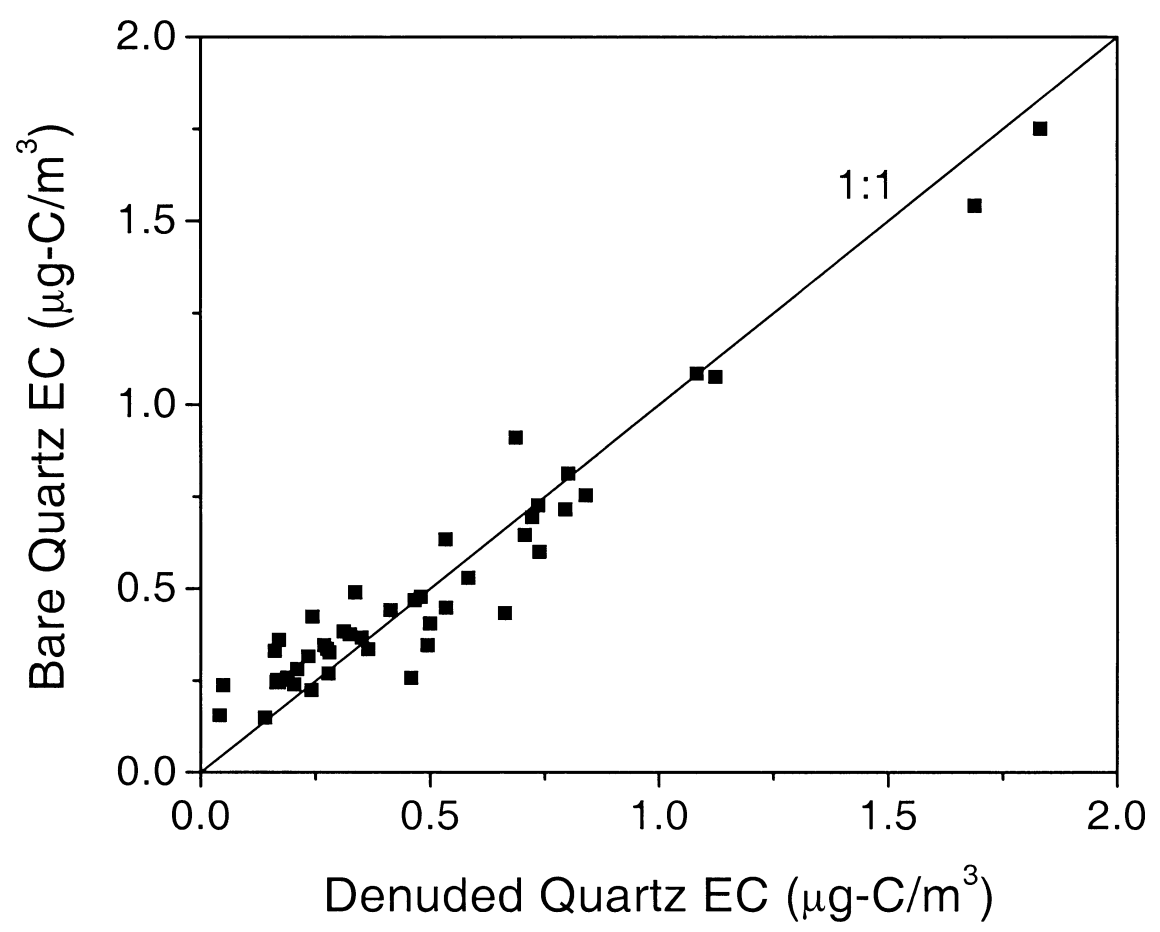

Figure 5. Comparison of EC for the $24 \mathrm{~h}$ runs as an indication that particle losses in the denuder are negligible. At low EC levels, the denuded quartz EC appears lower, probably because the blank correction is higher than that required.

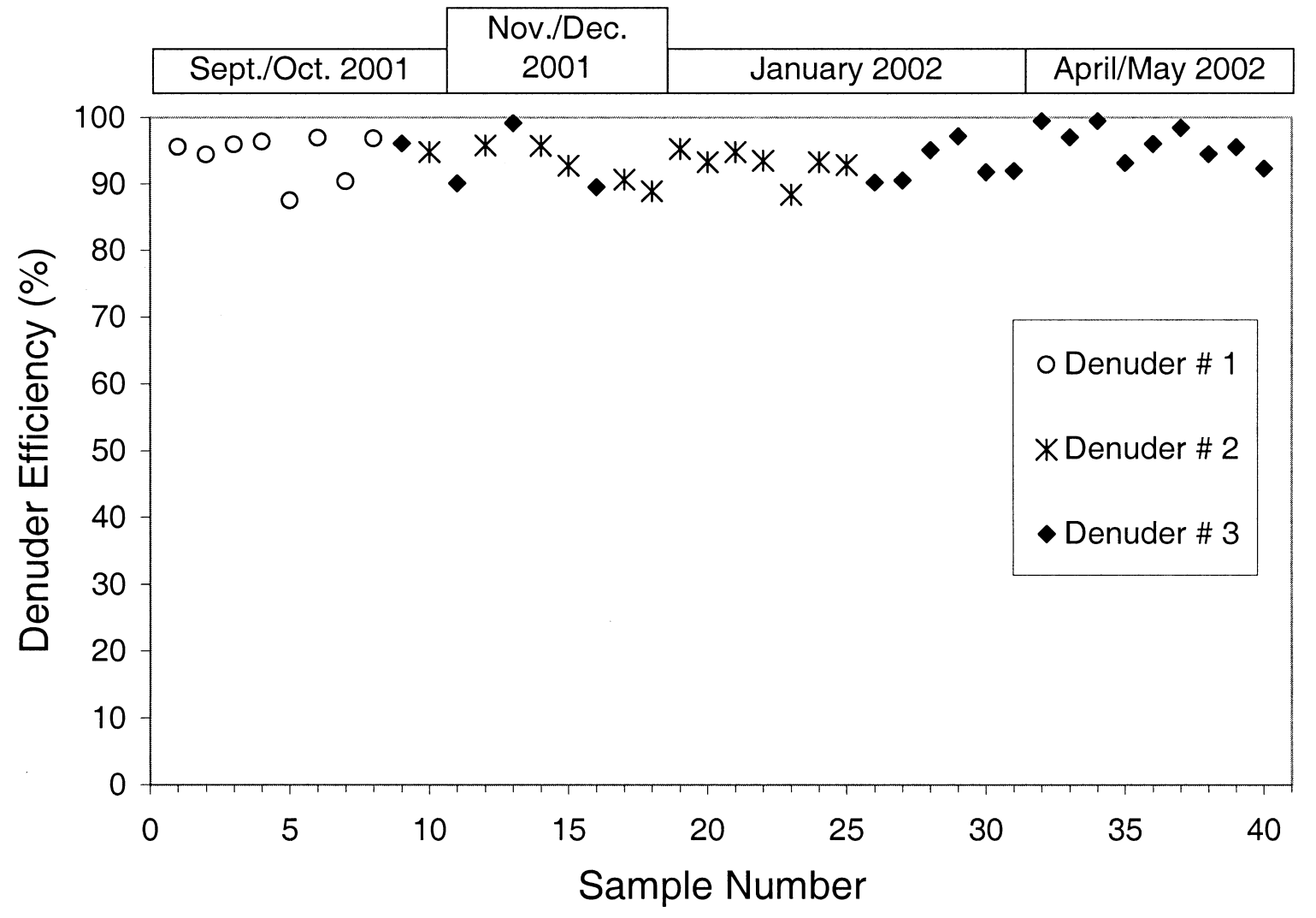

Figure 6. Denuder efficiency calculated using the dynamic blank and undenuded blank lines (Equation (1)). Lower efficiencies were typically associated with lower gaseous OC concentrations as measured by the undenuded blank line. Sample 1 corresponds to 2 September 2001; sample 40 was taken on 29 May 2002. 
as a precaution. Experiments were not performed to determine the potential lifetime of the denuders.

The ratio of the DYN OC to the ACD OC provides an estimate of the positive artifact due to denuder breakthrough. For instance, the DYN quartz OC is just $3.0+/-3.7 \%$ (Average+/-SD) of the ACD quartz $\mathrm{OC}$, indicating negligible positive artifact on the denuded quartz filter. The positive artifact considering both the quartz and CIG filters is on average 18.3\% (SD 12.5\%) of the ACD quartz and CIG OC, necessitating the use of the dynamic blank line in order to correctly interpret the CIG results. This is not unexpected, because the highly sorptive CIG filter is much more susceptible to denuder slip.

Negative Artifact. Comparison of the sample $\left(\mathrm{CIG}_{\mathrm{ACD}}\right)$, dynamic blank $\left(\mathrm{CIG}_{\mathrm{DYN}}\right)$, and handling blank CIG thermograms (Figure 3) provides insight into the negative artifact from the denuded quartz filter $\left(\mathrm{CIG}_{\mathrm{ACD}}-\mathrm{CIG}_{\mathrm{DYN}}\right)$. Figure $3 \mathrm{c}$ shows the net CIG OC (ACD - DYN), which indicates that most of the negative artifact evolves at temperatures over $250^{\circ} \mathrm{C}$, though the negative artifact is also seen to coevolve with the denuder breakthrough at temperatures over $200^{\circ} \mathrm{C}$. This particular sample corresponds to a moderate net (ACD - DYN) negative artifact of $0.28 \mu \mathrm{g}-\mathrm{C} / \mathrm{m}^{3}$, forming $6.2 \%$ of the ambient POC for that day. Both the ACD and DYN CIG OC levels are somewhat higher than the handling blank (HB). Figure $4 \mathrm{c}$ also shows that the dynamic blank accounts for the positive artifact on the CIG due to denuder breakthrough, avoiding an overestimation of the negative artifact, which would occur if only a handling blank correction were to be used for the denuder sampler.

The negative artifact estimates for the entire study are shown in Figure 7. The negative artifact $\left(\mathrm{CIG}_{\mathrm{ACD}}-\mathrm{CIG}_{\mathrm{DYN}}\right)$ is usually less than $10 \%$ of the ambient POC (quartz + CIG), and often zero. The study average of negative artifact was $6.3 \%$ of the POC (quartz + CIG), with a SD of $6.2 \%$. It should be noted that the OC on both the $\mathrm{CIG}_{\mathrm{ACD}}$ and $\mathrm{CIG}_{\mathrm{DYN}}$ is usually less than $10 \%$ of the ambient gaseous OC concentration as measured by the $\mathrm{CIG}_{\mathrm{UDB}}$, and about twice the average handling blank CIG. The difference between the $\mathrm{CIG}_{\mathrm{ACD}}$ and $\mathrm{CIG}_{\mathrm{DYN}}$ organic carbon is often close to the blank variability - the standard deviation of the handling blanks $\left(0.6 \mu \mathrm{g}-\mathrm{C} / \mathrm{cm}^{2}\right.$, Table 3$)$ corresponds to an uncertainty of $0.24 \mu \mathrm{g}-\mathrm{C} / \mathrm{m}^{3}$ in the negative artifact for a typical $24 \mathrm{~h}$ run. This is less than $10 \%$ of the typical ambient OC level of $3.0 \mu \mathrm{g}-\mathrm{C} / \mathrm{m}^{3}$; therefore, the uncertainty due to the $\mathrm{HB}$ variability does not significantly impact the ambient POC estimate from the denuder sampler.

The variability in denuder efficiency could cause errors in determination of the negative artifact. For example, if the DYN denuder was consistently less efficient than the sample line (ACD) denuder, this would result in a systematic underestimation of the negative artifact. The denuders of the ACD and DYN lines were routinely swapped to prevent differences in denuder performance from systematically biasing the estimates of negative artifact. Although the variation in denuder efficiency creates some uncertainty in the estimate of negative artifact for an individual sample, these procedures ensure that the study average values are robust. A maximum estimate of the negative artifact is the sample CIG OC less the handling blank (i.e., assuming a denuder efficiency of $100 \%$ ). In this extreme case (also shown in Figure 7), the negative artifact would be on average $20.2 \%$ (SD $12.2 \%$ ) of the total (quartz $+\mathrm{CIG}$ ) POC; however, this estimate is clearly too large because the denuder efficiency was only $94 \%$.

Many studies have been conducted that indicate a negative artifact (referred to as SVOC by Eatough and coworkers) as much as, or more than, the organic mass retained on the upstream denuded quartz filter (Anderson et al. 2002; Ding et al. 2002b; Modey et al. 2001; Eatough 1999; Eatough et al. 1996). For example, Anderson et al. (2002) used the PC-BOSS at the NETL facility near Pittsburgh in summer 2000 and report negative artifact from the PC-BOSS as $18 \%$ of the $\mathrm{PM}_{2.5}$ mass on average. The negative artifact varied from 0.5 to $7.3 \mu \mathrm{g}-\mathrm{C} / \mathrm{m}^{3}$, which is within the same range as the reported nonvolatile OC concentration of 1.0 to $5.2 \mu \mathrm{g}-\mathrm{C} / \mathrm{m}^{3}$ determined on the denuded quartz filter (correcting their results with the OC multiplication factor as $\mathrm{OC}=0.61 * \mathrm{OM})$. These results are consistent with the negative artifact reported in the other studies with the PC-BOSS cited above. All of these estimates of negative artifact are roughly a factor of 10 higher than that reported here for the PAQS study, and might be due to a variety of reasons such as sampler configuration, location, and time (the NETL study was conducted a year before the PAQS).

\section{TQQQ and Denuder Sampler Inter-Comparison}

Time series plots of POC measured using the different samplers are shown in Figure 8. Figure 8a shows a period of lowfrequency $(24 \mathrm{~h})$ samples from January 2002, and Figure $8 \mathrm{~b}$ shows a two-day period from July 2001 (high-frequency sampling). The results are characteristic of Pittsburgh POC levels in the summer and winter. The average POC concentration in the summer (June to September) is $3.6 \mu \mathrm{g}-\mathrm{C} / \mathrm{m}^{3}$, and in the winter (December to March) it is $2.5 \mu \mathrm{g}-\mathrm{C} / \mathrm{m}^{3}$, based on a bare quartz filter.

Figure 8 illustrates the significant daily and subdaily temporal variability of the POC levels in Pittsburgh. The different estimates of POC generally track each other but with different magnitudes. As expected, the highest POC levels are reported by the bare quartz filter, with the denuder and the two backup corrected levels (from the TQQQ) closer to each other, and less than the bare quartz. The differences between the various measurements are due to sampling artifacts and are explored in detail below.

The low-frequency comparisons are based on forty-seven $24 \mathrm{~h}$ parallel runs of the TQQQ and the denuder sampler. The high-frequency comparisons are based on data from 1 July to 3 August, 2001, which includes thirty $24 \mathrm{~h}$ runs of the denuder sampler made in parallel to the five-samples-a-day schedule of the TQQQ (the TQQQ data are $24 \mathrm{~h}$, sample volume-weighted averages of the five samples in each day). The breakdown of different samples collected each month is shown in Table 2.

Artifacts on the Bare Quartz Filter. Figure 9a compares the OC levels measured with a $24 \mathrm{~h}$ bare quartz filter to the corresponding denuded POC measurements. The bare quartz and denuded POC data show a remarkable fit with an almost 1:1 


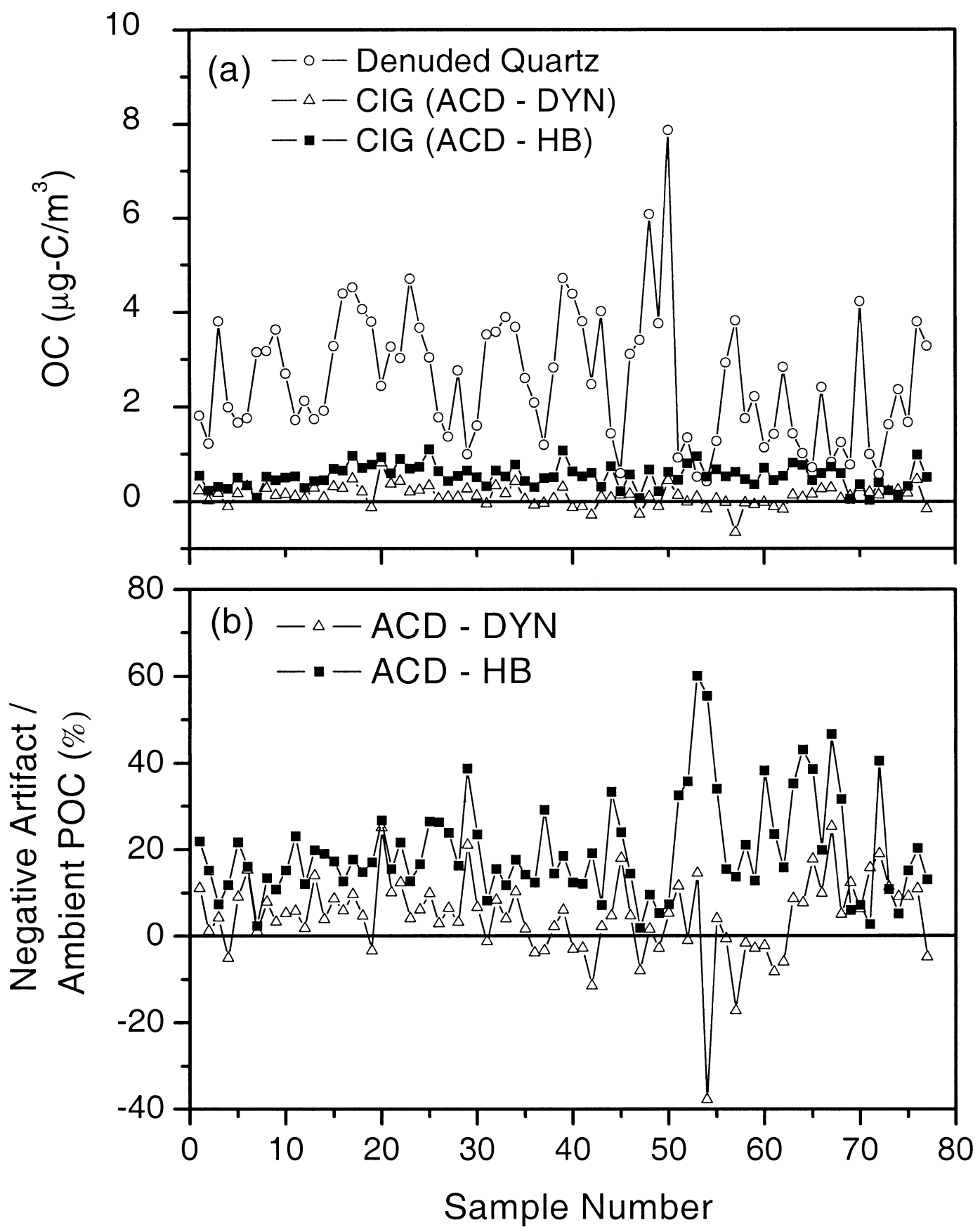

Figure 7. Time series of negative artifact from the denuded quartz filter: (a) absolute values, as compared to the OC on the denuded quartz filter, and (b) negative artifact as a percentage fraction of the ambient POC (Q + CIG). As discussed in the text, $(A C D-D Y N)$ is the best estimate of negative artifact because it accounts for denuder slip. Negative values of the negative artifact correspond to periods when the DYN denuder is less efficient. The (ACD - HB) is a maximum estimate of negative artifact, assuming the denuder is $100 \%$ efficient. Sample 1 corresponds to 1 July 2001; sample 77 was taken on 29 May 2002.

correspondence except for a positive intercept of $0.53 \mu \mathrm{g}-\mathrm{C} / \mathrm{m}^{3}$ (results from a regression analysis are summarized in Table 5), implying a constant positive artifact on the $24 \mathrm{~h}$ bare quartz filter. This fit is based on forty-seven samples taken over all four seasons.
Possible explanations for this reasonably stable artifact are that either the quartz filter is saturated with organic vapors or it is in equilibrium with the gas-phase organic compounds. Figure 10 shows the OC adsorbed on the backup quartz filters as a function of the ambient gaseous OC levels as measured by the UDB 

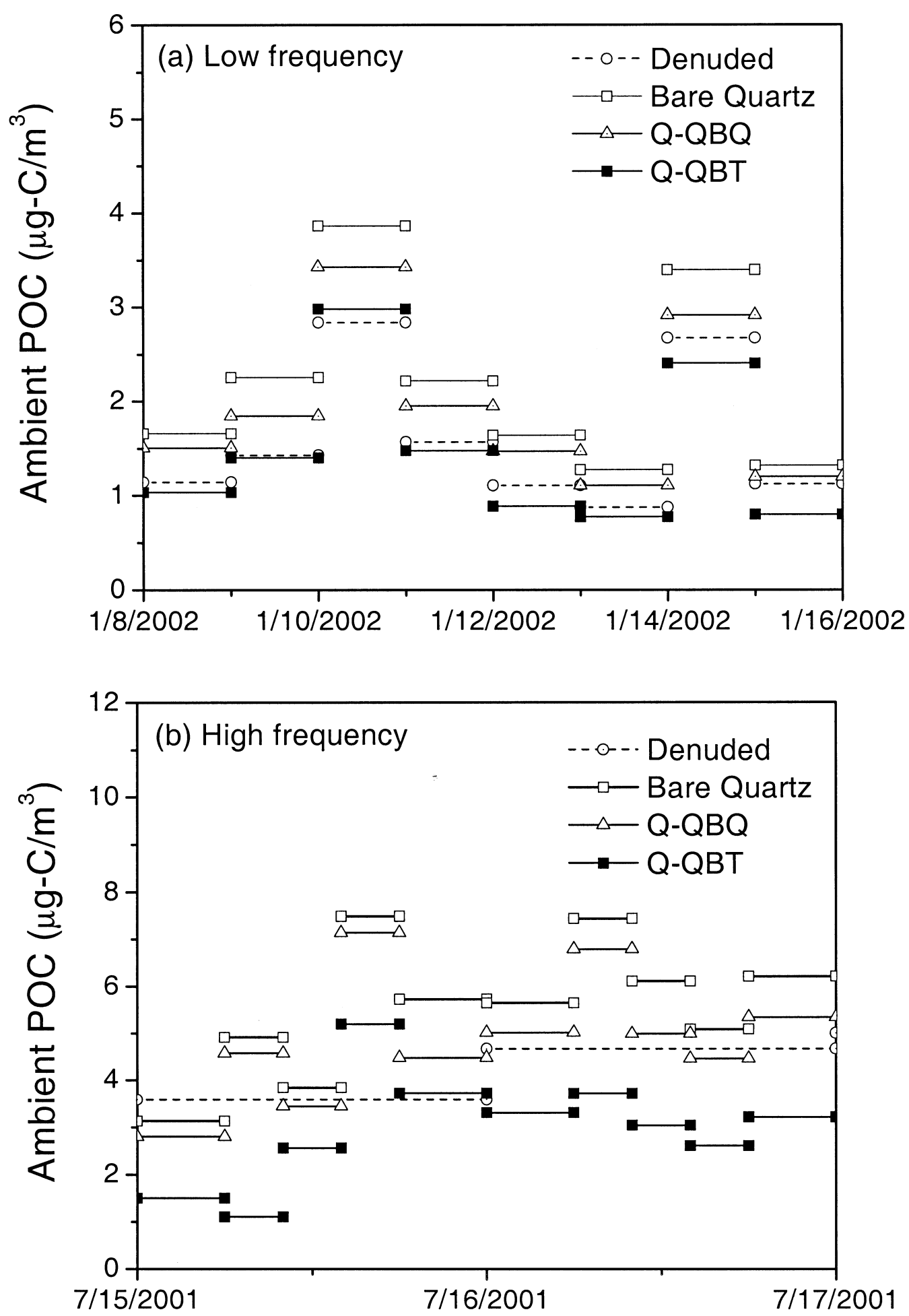

Figure 8. Time series of ambient POC measured by the TQQQ and denuder samplers: (a) a one-week period of low-frequency (24 h) sampling in January 2002, and (b) a two-day period of high-frequency sampling in July 2001.

line (quartz $+\mathrm{CIG})$. A linear relationship is expected between the adsorbed artifact and the gas-phase OC if the quartz filter is in equilibrium with all the gaseous species. Figure 10 indicates that the relationship is generally linear, suggesting the filter is in equilibrium. Applying the framework of Mader and Pankow (2001) suggests a minimum period of $12-14 \mathrm{~h}$ for the quartz filter to reach equilibrium. The scatter can be because not all the gaseous species that are captured on the CIG filter adsorb on the 

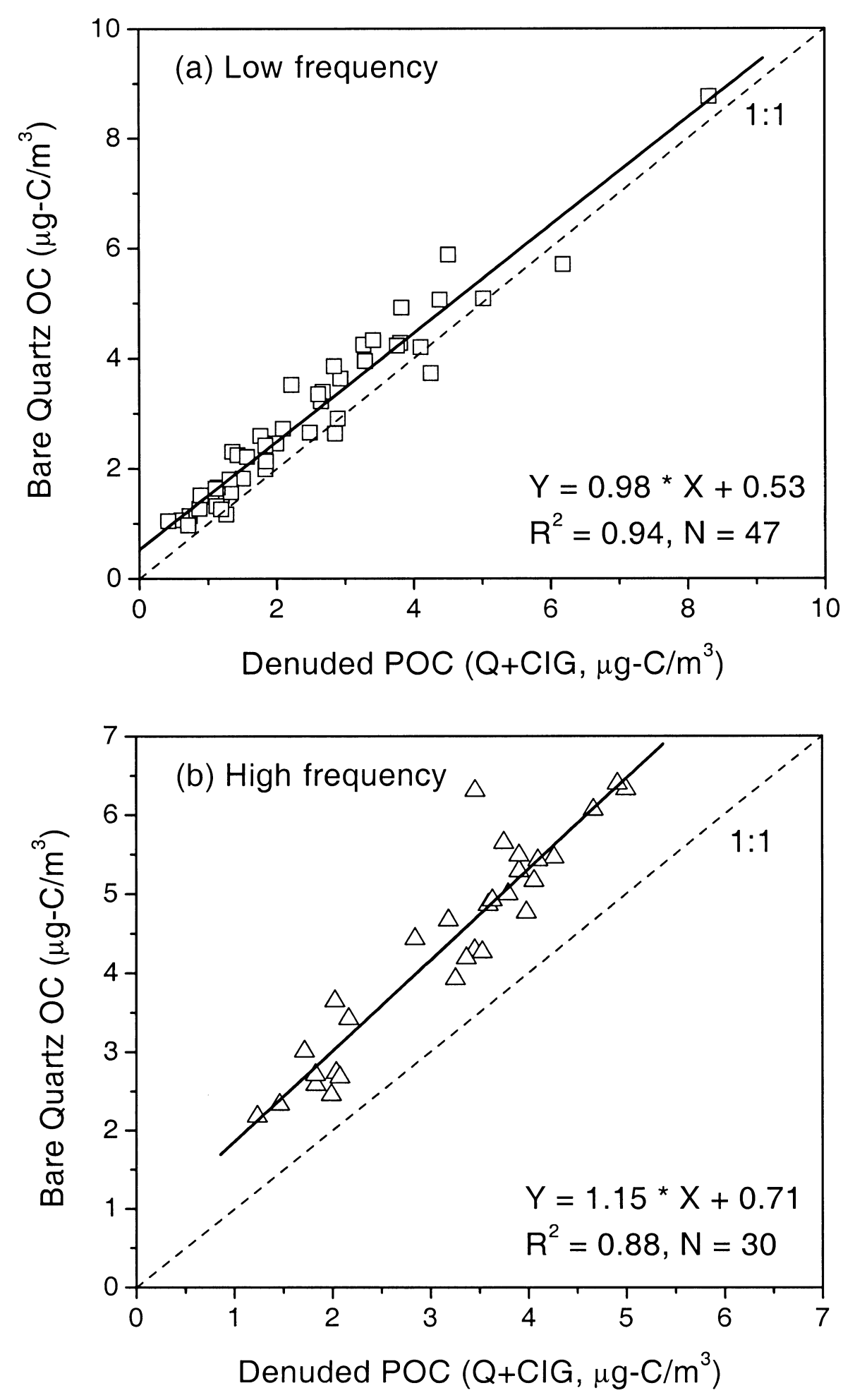

Figure 9. Bare quartz OC compared to the denuded POC: (a) low-frequency, $24 \mathrm{~h}$ samples and (b) high-frequency data ( $24 \mathrm{~h}$ averages of the 4-6 h TQQQ samples). The solid lines show the linear regression indicated in the figures (see Table 5 for regression parameters through the origin). 
Table 5

Results from the linear regressions of the different POC estimates from the TQQQ sampler with the reference POC from the denuder sampler. The errors are one standard error for the coefficients

\begin{tabular}{llcccccc}
\hline & Sampling & Number of & & & & \multicolumn{2}{c}{ Through the origin } \\
\cline { 5 - 8 } POC estimate & $\begin{array}{c}\text { Samples } \\
\text { frequency }\end{array}$ & Slope & Intercept & $\mathrm{R}^{2}$ & Slope & $\mathrm{R}^{2}$ \\
\hline Bare Quartz & High & 30 & $1.15 \pm 0.08$ & $0.71 \pm 0.26$ & 0.88 & $1.35 \pm 0.03$ & 0.85 \\
& Low & 47 & $0.98 \pm 0.04$ & $0.53 \pm 0.11$ & 0.94 & $1.13 \pm 0.02$ & 0.91 \\
Q-QBT & High & 30 & $0.80 \pm 0.06$ & $-0.25 \pm 0.21$ & 0.85 & $0.73 \pm 0.02$ & 0.85 \\
& Low & 47 & $0.84 \pm 0.03$ & $-0.07 \pm 0.09$ & 0.94 & $0.82 \pm 0.02$ & 0.94 \\
Q-QBQ & High & 30 & $1.00 \pm 0.07$ & $0.68 \pm 0.23$ & 0.89 & $1.19 \pm 0.02$ & 0.85 \\
& Low & 47 & $0.92 \pm 0.03$ & $0.31 \pm 0.10$ & 0.95 & $1.01 \pm 0.02$ & 0.93 \\
\hline
\end{tabular}

quartz filter (Lewtas et al. 2001). Figure 10 also suggests there may be a saturation effect at higher gaseous OC levels. Alternative explanations could be (1) the adsorption artifact depends on specific compounds or (2) the vapor collection efficiency of the quartz filter decreases over the sampling period (McDow 1999).
However, further work needs to be done before any definite conclusions can be drawn.

It was seen earlier that the denuded bare quartz filter experienced only a small negative artifact (Figure 7). This indicates negligible volatilization losses from the bare quartz filter because
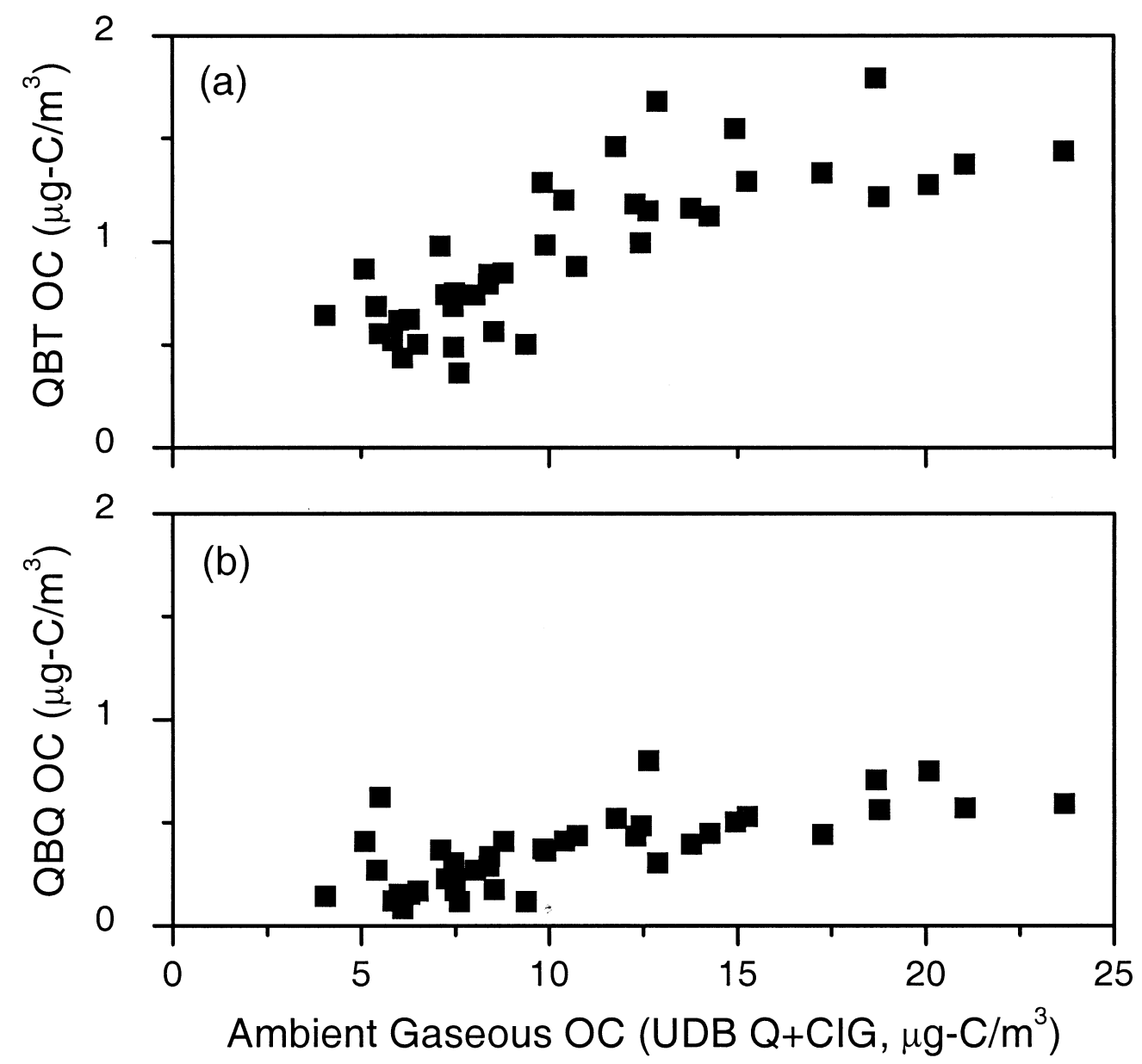

Figure 10. Effect of ambient gaseous OC concentration on the artifact measured on the different filters, (a) QBT OC and (b) QBQ OC. 
the denuded quartz filter should experience a higher negative artifact than a bare (undenuded) quartz filter. Thus, the positive artifact is usually dominant when sampling with a bare quartz filter in the Pittsburgh region, which is consistent with the results of Turpin et al. (1994) for other sites.

A comparison of the high-frequency bare quartz data, averaged by sample volume, with the corresponding $24 \mathrm{hr}$ denuder measurements (Figure 9b and Table 5) shows a larger positive artifact for the shorter duration samples as compared to the $24 \mathrm{~h}$ runs of the low-frequency samples (Figure 9a and Table 5). Again, this is expected since it is unlikely that the quartz filter reaches saturation or equilibrium (with the gaseous OC) within 4-6 h. Once the filter has reached equilibrium, the artifact mass on the quartz filter remains constant and will form a smaller component of the measured POC on a concentration basis with increasing sampling time.

Bare Quartz-QBT Versus the Denuded Sampler. Figure 11 shows that the Q-QBT estimate of POC correlates well, with the denuder POC for both the short-duration and $24 \mathrm{~h}$ samples. However, the QBT overcorrects for the positive artifact on the bare quartz filter by $16 \%$ of the denuded POC for the $24 \mathrm{~h}$ samples (Figure 11a and Table 5). A similar result is obtained for the high-frequency samples where the QBT overestimates the positive artifact on the bare quartz filter by $20 \%$ of the denuded POC (Figure 11b and Table 5). Although the QBT overcorrects for the positive artifact, it does provide a consistent estimate of the positive artifact for both the high- and low-frequency samples. Table 5 shows that the positive artifact on the high-frequency bare quartz filters is significantly larger than on the $24 \mathrm{~h}$ bare quartz filters. The similarity of the regressions of the QBT corrections (Table 5) for both short-duration and $24 \mathrm{~h}$ samples indicates that the QBT accounts for the effects of different sample duration.

Volatilization of organic particulate matter collected on the Teflon filter that is then adsorbed onto the QBT is a likely explanation for the overcorrection of the positive artifact with the Q-QBT approach. Our results are qualitatively similar to Chow et al. (1996), though the PAQS did not see any negative POC after correction by the QBT. The previous section showed negligible volatilization loss from the bare quartz filter. Higher volatilization losses from a Teflon filter than a quartz filter is not unexpected. Quartz filters are thicker and have more surface area than the stretched Teflon membrane filters used in this study. Since the PM is typically trapped in the uppermost part of the quartz filter (McDow and Huntzicker 1990), it is likely that some volatilization does occur for the bare quartz filter, but these vapors are captured downstream on the same quartz filter.

Based on the entire study of $37924 \mathrm{~h}$ samples collected between June 2001 and July 2002, the QBT OC averages 34\% (SD $10 \%$ ) of the bare quartz OC. Figure $12 \mathrm{a}$ shows the QBT OC varying from $11-83 \%$ of the bare quartz $\mathrm{OC}$, with the higher values associated with low OC levels. The PAQS results are consistent with earlier findings, although the absolute magnitude of the QBT artifact is lower, as are the average bare quartz OC levels.
For example, McDow and Huntzicker (1990) reported the QBT OC at about $50 \%$ of the bare quartz OC for low OC values and less than $20 \%$ for bare quartz OC values above $7 \mu \mathrm{g}-\mathrm{C} / \mathrm{m}^{3}$ when sampling in Portland, OR. Kim et al. (2001) also found the QBT OC on an annual average basis to be $30 \%$ of the bare quartz OC based on a year-long study at Rubidoux, CA, with the QBT OC varying between 0.53 to $4.97 \mu \mathrm{g}-\mathrm{C} / \mathrm{m}^{3}$.

Bare Quartz-QBQ Versus the Denuded Sampler. Figure 13a shows that for the 24-hour runs (low-frequency sampling) the Q-QBQ estimate of POC is approximately the same as the denuded POC. However, this is not the case with the shorter duration samples (Figure 13b and Table 5), where considerable positive artifact remains even after applying the QBQ correction to the bare quartz OC. These results suggest that both of the quartz filters are in equilibrium with the vapor phase for the $24 \mathrm{~h}$ TQQQ samples, but they do not adsorb gases in equal measure for a 4-6 h sample. These results are consistent with Kirchstetter et al. (2001), who found that the QBQ method came closest to being a good estimate of the positive artifact on the front quartz filter with a $14 \mathrm{~h}$ sample rather than with samples of 2,4 , or $8 \mathrm{~h}$ duration when sampling at a face velocity of $50 \mathrm{~cm} / \mathrm{s}$. Figure 12b shows the QBQ OC ranging from 4-30\% of the bare quartz OC (except for two values over 50\% at low POC levels). The study average is $13 \%$ with a SD of $5 \%$. However, the trends are less distinct than in the QBT case discussed earlier.

Analysis of the Thermograms of the Different Quartz Filters. Comparing the carbon evolving from the quartz filters at the different temperature steps of the NIOSH protocol (shown in Figure 4) gives additional insight into the artifacts. More volatile compounds (gases and particles) come off at the $340^{\circ} \mathrm{C}$ Helium step, the heavier particulate-phase organics evolve at the subsequent higher temperatures in the Helium mode, and pyrolyzed organic carbon and native black carbon are expected to volatilize in the Helium/Oxygen mode. Therefore, the OC artifact is expected to come off the filter at the lower temperature Heliummode steps.

The average values of the carbon evolving at each Helium temperature step and in the Helium/Oxygen mode are shown in Figure 14 for all the samples (grouped into high-frequency and low-frequency sampling modes). Comparing the size of the peaks from the different filters of the TQQQ sampler with the denuded quartz filter indicates the temperature(s) at which the artifact carbon evolves. For example, comparing the $24 \mathrm{~h}$ bare quartz filter with the denuded quartz filter in Figure 14a indicates that the positive artifact primarily occurs in the first peak (Helium, $340^{\circ} \mathrm{C}$ ). For the high-frequency samples Figure $14 \mathrm{~b}$, appreciable artifact also evolves in the higher Helium stepsthe mass of carbon evolving from the bare quartz filter in each of the higher temperature Helium peaks is significantly greater than the amount of carbon that evolves from the denuded quartz filter in that peak.

The carbon evolution from the backup quartz filters also supports the conclusion that some of the artifact carbon evolves at the higher temperature Helium steps. For example, Figure 14 

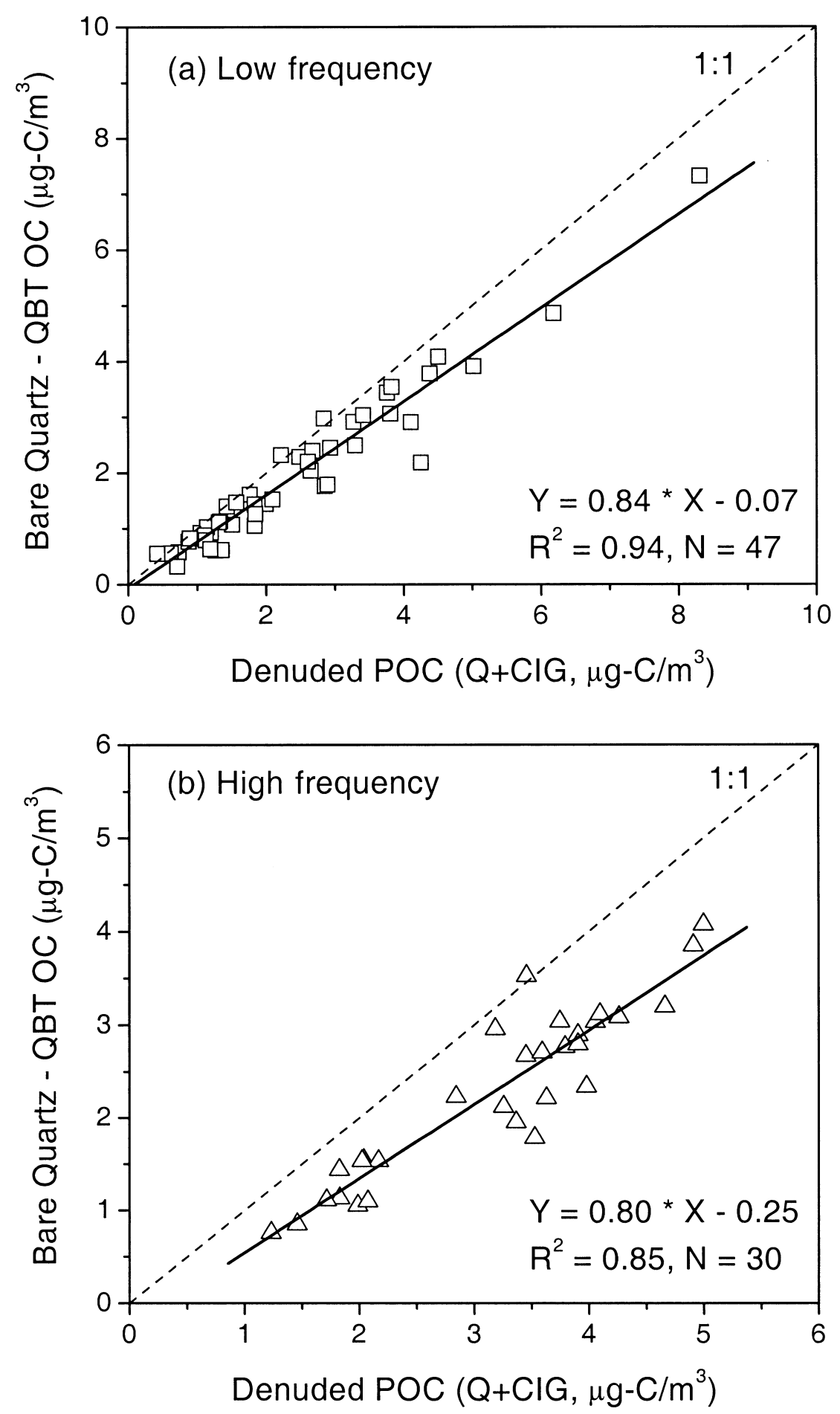

Figure 11. QBT-corrected bare quartz OC compared to the denuded POC for (a) low-frequency, $24 \mathrm{~h}$ samples and (b) highfrequency samples ( $24 \mathrm{~h}$ averages of the 4-6 h TQQQ samples). The solid lines represent the linear regressions indicated in the figures (see Table 5 for the regression parameters through the origin).

shows that significant carbon evolves off the QBT filter in the first two Helium steps $\left(340^{\circ} \mathrm{C}\right.$ and $\left.500^{\circ} \mathrm{C}\right)$ for both the shortand long-duration samples. Again, the shorter duration samples show relatively more carbon evolving at the higher temperatures than the long-duration samples. Finally, the difference between the bare quartz and QBT in the first two peaks is less than the carbon evolving at these peaks from the denuded quartz filter, which is consistent with the hypothesis of volatilization losses 

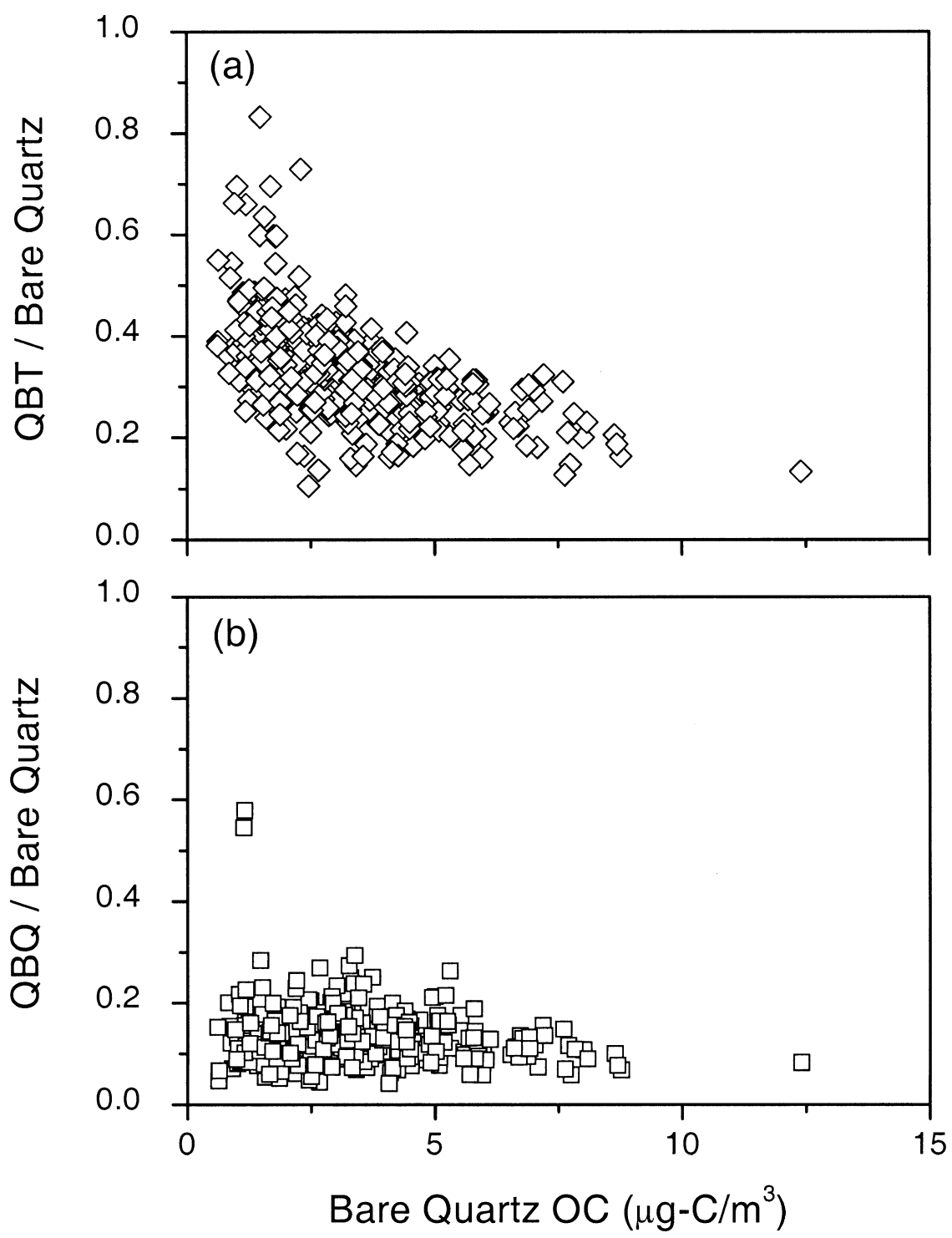

Figure 12. Variation of the backup filter OC, (a) QBT and (b) QBQ, as a fraction of the bare quartz OC. Data shown are for the 37924 h samples between June 2001 and July 2002.

from the Teflon filter proposed earlier. The QBQ short-duration and long samples show similar trends as the corresponding QBT samples, though at much lower magnitudes.

There are two possible explanations for the artifact to evolve at higher temperatures. The artifact can be either physically or chemically bonded to the filter, and the energy needed to evolve the chemisorbed vapors might be higher than that required for the physically sorbed artifact (similar to the conclusions of Kirchstetter et al. 2001). Another possibility is pyrolysis of the positive artifact into compounds that evolve later at higher temperatures. Kirchstetter et al. (2001) dismiss this possibility based on some of their results for single compounds, but Yu et al. (2002) demonstrated that aerosol water extracts (water-soluble organics) can pyrolyze and evolve partly in the Helium mode and also later in the Helium/Oxygen mode. As the sample duration increases, the positive artifact reduces on a concentration basis, and so the higher temperature artifact is less prominent on the low-frequency comparisons than with the shorter-duration samples.

Temporal Variation of Artifact. Figure 15 shows the seasonal variation of the different estimates of the positive artifact, both in absolute terms (Figure 15a), and as a proportion of the bare quartz OC (Figure 15b). For the $24 \mathrm{~h}$ samples, the QBT shows a much greater artifact than the QBQ, forming 30-40\% of the bare quartz OC compared to the 10-20\% for the QBQ. Figure 15a indicates some seasonal variation of the positive artifact on an absolute basis, with higher levels of artifact occurring in the summer. However, there seems to be little variation on 

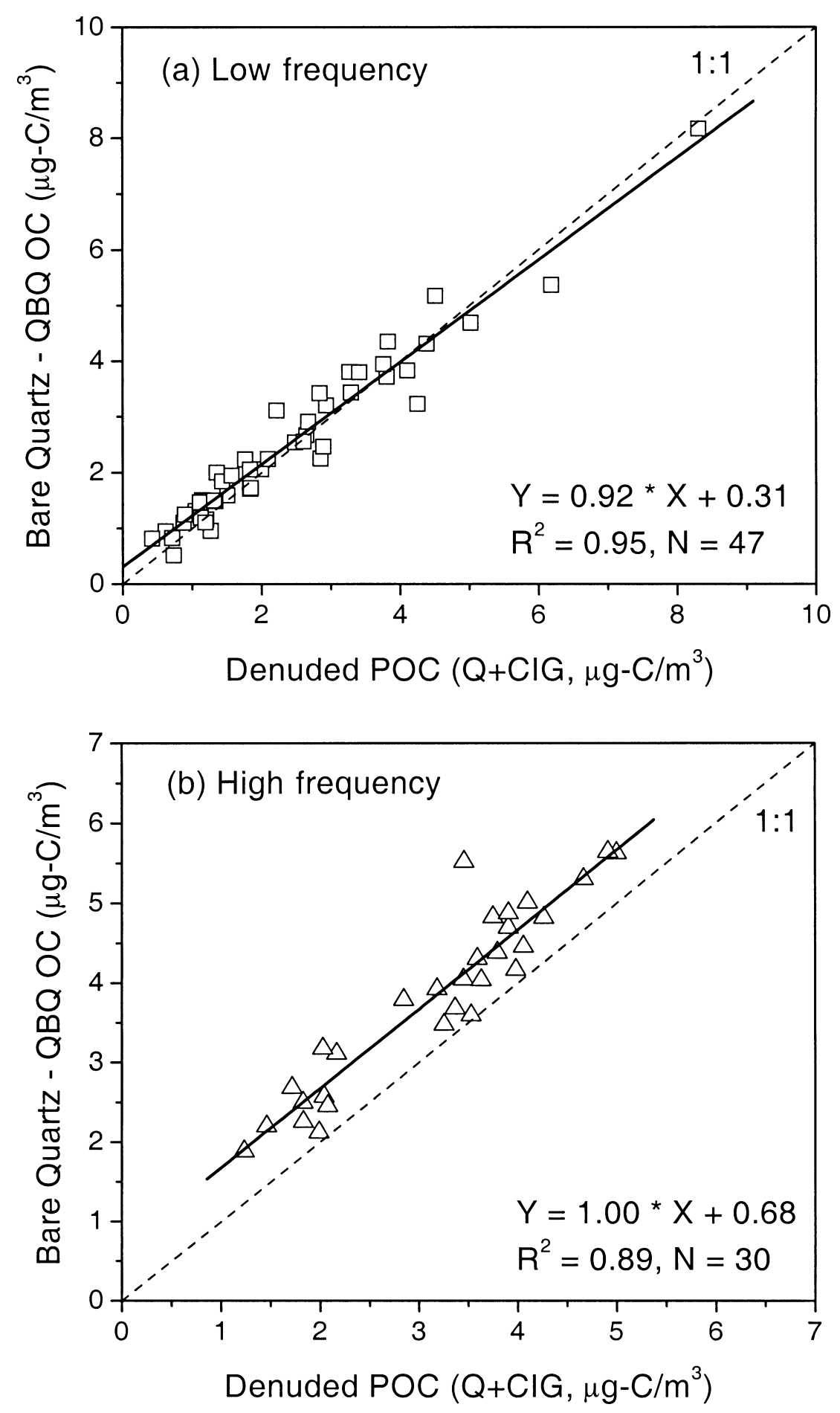

Figure 13. The QBQ corrected bare quartz OC versus the denuder POC for (a) low-frequency, $24 \mathrm{~h}$ samples and (b) highfrequency samples ( $24 \mathrm{~h}$ averages of the 4-6 $\mathrm{h}$ TQQQ samples). The solid lines show the linear regression indicated in the figures (see Table 5 for the regression parameters through the origin).

a relative basis (Figure 15b), as the magnitude of the artifact appears to mirror the seasonal variation in ambient OC levels. Kim et al.'s (2001) results show no significant seasonal trends in the absolute values of the QBT artifact for Rubidoux, CA.
Figure 7 shows that the negative artifact is small. Higher values were seen during the summer months (Figure 7a); however, there does not seem to be any significant seasonal trend in the negative artifact as a fraction of POC (Figure 7b). 

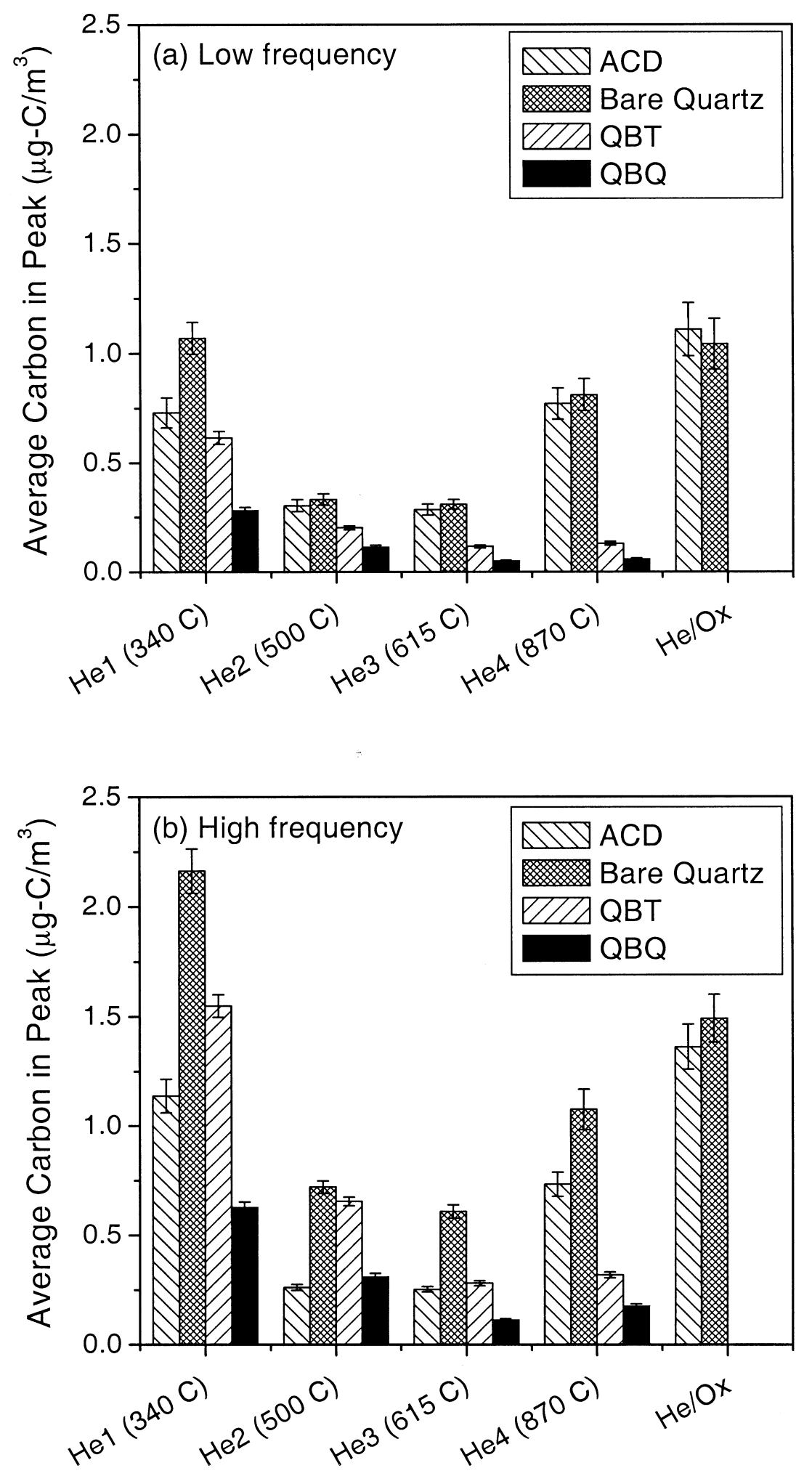

Figure 14. Average carbon that evolves at each step of the NIOSH protocol for (a) forty-seven $24 \mathrm{~h}$ samples and (b) thirty days of high-frequency samples. The data are not corrected for blanks; however, these form a very small fraction of the total carbon. The error bars are one standard error of the study average for each peak in that particular group. 

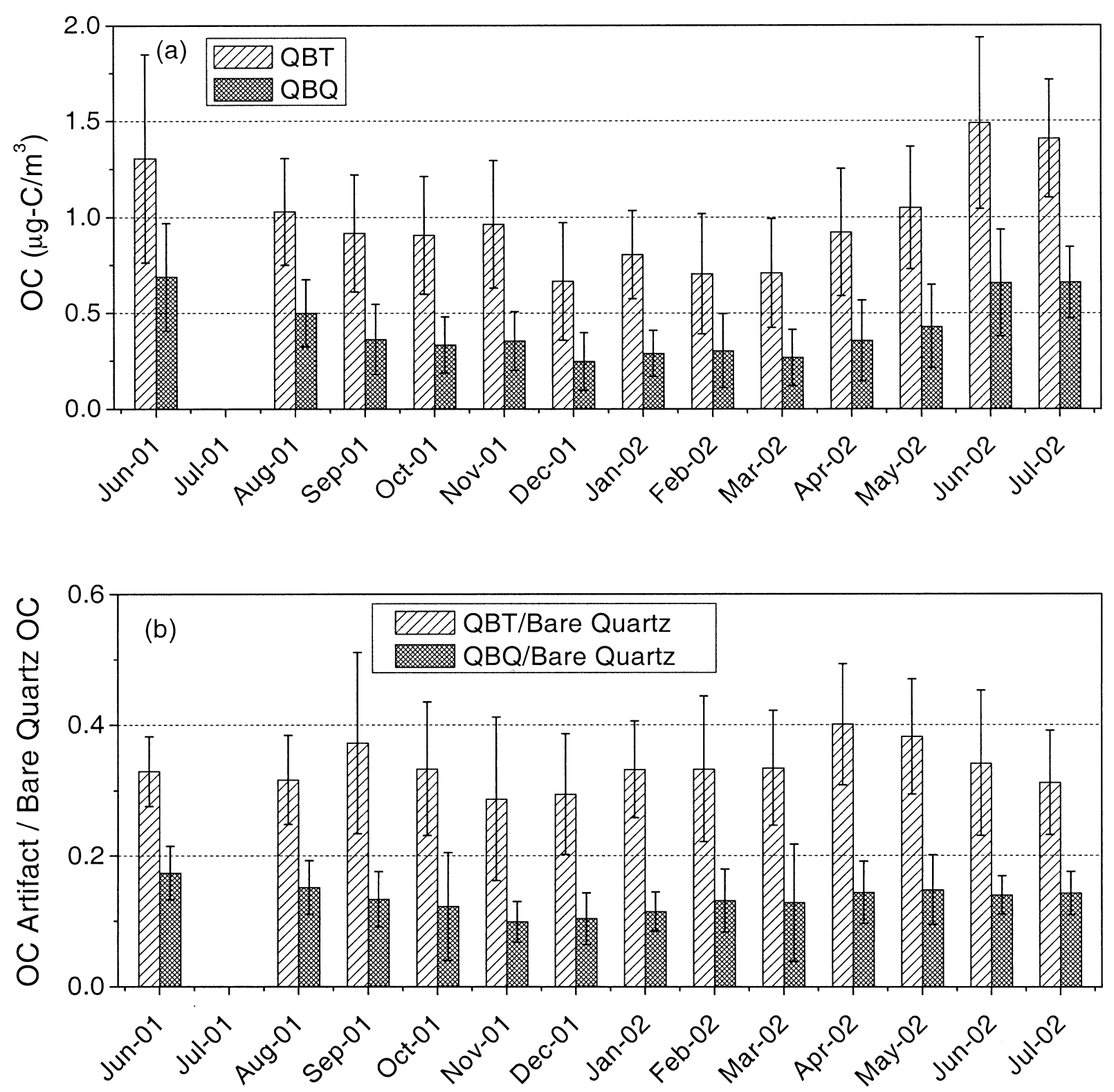

Figure 15. Seasonal variation of artifact using monthly averages of the (a) absolute values of organic carbon on the QBT and QBQ backup filters, and (b) ratios of the OC on the backup filters to the bare quartz OC. The error bars are one standard deviation of the data. Data is not shown for July 2001 since most of the runs were short samples (4-6 h).

\section{CONCLUSIONS}

A comprehensive study comparing the prevalent methods for measurement of ambient particulate organic carbon (POC) has been conducted during the PAQS, and the major conclusions are as follows:

1. An activated carbon monolith denuder sampler has been developed and characterized. The denuder eliminates virtually all of the positive artifact from the quartz filter. The negative artifact due to volatilization of the organic particulate matter from the denuded quartz filter is on average
$6.3 \%$ (SD 6.2\%) of the ambient POC and usually less than $10 \%$ of the ambient POC.

2. With a bare quartz filter sampling for $24 \mathrm{~h}$ at $16.7 \mathrm{lpm}$ (face velocity of $29 \mathrm{~cm} / \mathrm{s}$ ), an almost constant positive artifact of $0.5 \mu \mathrm{g}-\mathrm{C} / \mathrm{m}^{3}$ is found, irrespective of the season. A larger positive artifact occurs on shorter duration samples on a concentration basis because the filter is not in equilibrium. Since the denuded quartz filter experiences only a small negative artifact, negligible volatilization losses can be expected for the bare (undenuded) quartz filter. 
3. The QBT provides a consistent estimate of the positive artifact on a bare quartz filter regardless of sample duration. However, it overestimates the positive artifact by $16-20 \%$, presumably due to volatilization of organic material off the upstream Teflon filter.

4. The QBQ provides a robust estimate of the positive artifact on the bare quartz filter for the $24 \mathrm{~h}$ samples. It does not work for the 4 or $6 \mathrm{~h}$ samples because both of the quartz filters have not achieved equilibrium with the incoming air stream.

5. The positive artifact measured on the quartz backup filters for the $24 \mathrm{~h}$ samples shows a slight seasonal variation on an absolute basis, with higher values during the summer, but there is no temporal variation on a relative basis (compared to the bare quartz OC). The negative artifact from the denuded quartz filter also shows higher values in the summer, but as a fraction of the POC there is no significant seasonal variation.

6. The results of this study are most applicable to regions with similar atmospheric conditions and meteorology. Wittig et al. (2004) and Rees et al. (2004) provide an overview of meteorological and fine particle composition in Pittsburgh. Briefly, sulfate is the dominant component of fine particle mass in Pittsburgh followed by organic material; one third of the $\mathrm{OC}$ is estimated to be secondary in the summer, with smaller fractions in the winter (Cabada et al. 2004). Tang et al. (2004) show that fine particle concentrations in Pittsburgh are dominated by regional transport of pollutants, which allows significant time for atmospheric processing or aging. These conditions are representative of those found in much of the Eastern United States, particularly the Northeast.

\section{REFERENCES}

Anderson, R. R., Martello, D. V., Rohar, P. C., Strazisar, B. R., Tamilia, J. P., Waldner, K., White, C. M., Modey, W. K., Mangelson, N. F., and Eatough, D. J. (2002). Sources and Composition of $\mathrm{PM}_{2.5}$ at the National Energy Technology Laboratory in Pittsburgh During July and August 2000, Energy and Fuels 16(2):261-269.

Cabada, J. C., Pandis, S. N., Subramanian, R., Robinson, A. L., Polidori, A., and Turpin, B. (2004). Estimating the Secondary Organic Aerosol Contribution to $\mathrm{PM}_{2.5}$ Using the EC Tracer Method, Aerosol Science and Technology, 38:140-155.

Chow, J. C., Watson, J. G., Pritchett, L. C., Pierson, W. R., Frazier, C. A., and Purcell, R. G. (1993). The DRI Thermal Optical Reflectance Carbon Analysis System-Description, Evaluation and Applications in United-States Air-Quality Studies, Atmos. Environ. (Part A) 27(8):1185-1201.

Chow, J. C., Watson, J. G., Lu, Z. Q., Lowenthal, D. H., Frazier, C. A., Solomon, P. A., Thuillier, R. H., and Magliano, K. (1996). Descriptive Analysis of $\mathrm{PM}_{2.5}$ and $\mathrm{PM}_{10}$ at Regionally Representative Locations During SJVAQS/AUSPEX, Atmos. Environ. 30(12):2079-2112.

Coutant, R. W., Callahan, P. J., Kuhlman, M. R., and Lewis, R. G. (1989). Design and Performance of a High-Volume Compound Annular Denuder, Atmos. Environ. 23(10):2205-2211.

Ding, Y., Pang, Y., and Eatough, D. J. (2002a). High-Volume Diffusion Denuder Sampler for the Routine Monitoring of Fine Particulate Matter: I. Design and Optimization of the PC-BOSS, Aerosol Sci. Technol. 36(4):369-382.
Ding, Y., Pang, Y., Eatough, D. J., Eatough, N. L., and Tanner, R. L. (2002b). High-Volume Diffusion Denuder Sampler for the Routine Monitoring of Fine Particulate Matter: II. Field Evaluation of the PC-BOSS, Aerosol Sci. Technol. 36(4):383-396.

Eatough, D. J. (1999). BOSS, the Brigham Young University Organic Sampling System: Determination of Particulate Carbonaceous Material Using Diffusion Denuder Sampling Technology. In Gas and Particle Phase Measurements of Atmospheric Organic Compounds, edited by D. A. Lane. Gordon and Breach Science Publishers, Amsterdam, pp. 233-285.

Eatough, D. J., Eatough, D. A., Lewis, L., and Lewis, E. A. (1996). Fine Particulate Chemical Composition and Light Extinction at Canyonlands National Park Using Organic Particulate Material Concentrations Obtained with a Multisystem, Multichannel Diffusion Denuder Sampler, J. Geophys. Res. 101(D14):19515-19531.

Eatough, D. J., Wadsworth, A., Eatough, D. A., Crawford, J. W., Hansen, L. D., and Lewis, E. A. (1993). A Multiple-System, Multichannel Diffusion Denuder Sampler for the Determination of Fine-Particulate Organic Material in the Atmosphere, Atmos. Environ. Part A 27(8):1213-1219.

Gray, H. A., Cass, G. R., Huntzicker, J. J., Heyerdahl, E. K., and Rau, J. A. (1986). Characteristics of Atmospheric Organic and Elemental Carbon Particle Concentrations in Los Angeles, Environmental Sci. Technol. 20(6):580-589.

Gundel, L. A., and Lane, D. A. (1999). Sorbent-Coated Diffusion Denuders for Direct Measurement of Gas/Particle Partitioning by Semi-Volatile Organic Compounds, In Gas and Particle Phase Measurements of Atmospheric Organic Compounds. edited by D. A. Lane. Gordon and Breach Science Publishers, Amsterdam, pp. 287-332.

Gundel, L. A., Lee, V. C., Mahanama, K. R. R., Stevens, R. K., and Daisey, J. M. (1995). Direct Determination of the Phase Distributions of Semi-Volatile Polycyclic Aromatic Hydrocarbons Using Annular Denuders, Atmos. Environ. 29(14):1719-1733.

Hart, K. M., and Pankow, J. F. (1994). High-Volume Air Sampler for Particle and Gas Sampling. 2. Use of Backup Filters to Correct for the Adsorption of Gas-Phase Polycyclic Aromatic-Hydrocarbons to the Front Filter, Environ. Sci. Technol. 28(4):655-661.

Kim, B. M., Cassmassi, J., Hogo, H., and Zeldin, M. D. (2001). Positive Organic Carbon Artifacts on Filter Medium During $\mathrm{PM}_{2.5}$ Sampling in the South Coast Air Basin, Aerosol Sci. Technol. 34(1):35-41.

Kirchstetter, T. W., Corrigan, C. E., and Novakov, T. (2001). Laboratory and Field Investigation of the Adsorption of Gaseous Organic Compounds onto Quartz Filters, Atmos. Environ. 35(9):1663-1671.

Lewtas, J., Pang, Y. B., Booth, D., Reimer, S., Eatough, D. J., and Gundel, L. A. (2001). Comparison of Sampling Methods for Semi-Volatile Organic Carbon Associated with $\mathrm{PM}_{2.5}$, Aerosol Sci. Technol. 34(1):9-22.

Mader, B. T., Flagan, R. C., and Seinfeld, J. H. (2001). Sampling Atmospheric Carbonaceous Aerosols Using a Particle Trap Impactor/Denuder Sampler, Environ. Sci. Technol. 35(24):4857-4867.

Mader, B. T., and Pankow, J. F. (2001). Gas/Solid Partitioning of Semivolatile Organic Compounds (SOCs) to Air Filters. 3. An Analysis of Gas Adsorption Artifacts in Measurements of Atmospheric SOCs and Organic Carbon (OC) When Using Teflon Membrane Filters and Quartz Fiber Filters, Environ. Sci. Technol. 35(17):3422-3432.

Malm, W. C., Sisler, J. F., Huffman, D., Eldred, R. A., and Cahill, T. A. (1994). Spatial and Seasonal Trends in Particle Concentration and Optical Extinction in the United-States, J. Geophy. Res.-Atmos. 99(D1):1347-1370.

McDow, S. R. (1999). Sampling Artifact Errors in Gas/Particle Partitioning Measurements, In Gas and Particle Phase Measurements of Atmospheric Organic Compounds, D. A. Lane, ed., Gordon and Breach Science Publishers, Amsterdam, 105-126.

McDow, S. R., and Huntzicker, J. J. (1990). Vapor Adsorption Artifact in the Sampling of Organic Aerosol-Face Velocity Effects, Atmos. Environ. (Part A) 24(10):2563-2571.

Modey, W. K., Pang, Y., Eatough, N. L., and Eatough, D. J. (2001). Fine Particulate $\left(\mathrm{PM}_{2.5}\right)$ Composition in Atlanta, USA: Assessment of the Particle Concentrator-Brigham Young University Organic Sampling System, PCBOSS, During the EPA Supersite Study, Atmos. Environ. 35:6493-6502. 
NIOSH. NIOSH Manual of Analytical Methods. Available at: http://www.cdc.gov/niosh/nmam/pdfs/5040f3.pdf

Rees, S. L., Robinson, A. L., Khlystov, A. Y., Stanier, C. O., and Pandis, S. N. (2004). Mass Balance Closure and the Federal Reference Method for $\mathrm{PM}_{2.5}$ in Pittsburgh, Pennsylvania, Atmospheric Environment, submitted.

Schauer, J. J., Mader, B. T., DeMinter, J. T., Heidemann, G., Bae, M. S., Seinfeld, J. H., Flagan, R. C., Cary, R. A., Smith, D., Huebert, B. J., Bertram, T., Howell, S., Kline, J. T., Quinn, P., Bates, T., Turpin, B., Lim, H. J., Yu, J. Z., Yang, H., and Keywood, M. D. (2003). ACE-Asia Intercomparison of a Thermal-Optical Method for the Determination of Particle-Phase Organic and Elemental Carbon, Environ. Sci. Technol. 37(5):993-1001.

Shah, J. J., Johnson, R. L., Heyerdahl, E. K., and Huntzicker, J. J. (1986). Carbonaceous Aerosol at Urban and Rural Sites in the United States, J. Air Poll. Control Assoc. 36(3):254-257.

Subramanian, R., Khlystov, A. Y., and Robinson, A. L. (2004). In preparation.
Tang, W., Raymond, T., Wittig, A. E., Davidson, C. I., Pandis, S. N., Robinson, A. L., and Crist, K. (2004). Spatial Variations of $\mathrm{PM}_{2.5}$ During the Pittsburgh Air Quality Study, Aerosol Science and Technology, submitted.

Turpin, B. J., Huntzicker, J. J., and Hering, S. V. (1994). Investigation of Organic Aerosol Sampling Artifacts in the Los Angeles Basin, Atmos. Environ. 28(19):3061-3071.

Turpin, B. J., Saxena, P., and Andrews, E. (2000). Measuring and Simulating Particulate Organics in the Atmosphere: Problems and Prospects, Atmos. Environ. 34(18):2983-3013.

Wittig, A. E., Anderson, N., Khlystov, A. Y., Pandis, S. N., Davidson, C. I., and Robinson, R. L. (2004). Pittsburgh Air Quality Study Overview and Initial Scientific Findings, Atmospheric Environment, submitted.

Yu, J. Z., Xu, J., and Yang, H. (2002). Charring Characteristics of Atmospheric Organic Particulate Matter in Thermal Analysis, Environ. Sci.Technol. 36(4):754-761. 


\title{
Ambient aerosol size distributions and number concentrations measured during the Pittsburgh Air Quality Study (PAQS)
}

\author{
Charles O. Stanier, Andrey Y. Khlystov ${ }^{1}$, Spyros N. Pandis* \\ Department of Chemical Engineering, Carnegie Mellon University, 5000 Forbes Avenue, Pittsburgh, PA 15213-7139, USA
}

\begin{abstract}
Twelve months of aerosol size distributions from 3 to $560 \mathrm{~nm}$, measured using scanning mobility particle sizers are presented with an emphasis on average number, surface, and volume distributions, and seasonal and diurnal variation. The measurements were made at the main sampling site of the Pittsburgh Air Quality Study from July 2001 to June 2002. These are supplemented with 5 months of size distribution data from 0.5 to $2.5 \mu \mathrm{m}$ measured with a TSI aerosol particle sizer and 2 months of size distributions measured at an upwind rural sampling site. Measurements at the main site were made continuously under both low and ambient relative humidity. The average Pittsburgh number concentration $(3-500 \mathrm{~nm})$ is $22,000 \mathrm{~cm}^{-3}$ with an average mode size of $40 \mathrm{~nm}$. Strong diurnal patterns in number concentrations are evident as a direct effect of the sources of particles (atmospheric nucleation, traffic, and other combustion sources). New particle formation from homogeneous nucleation is significant on $30-50 \%$ of study days and over a wide area (at least a hundred kilometers). Rural number concentrations are a factor of 2-3 lower (on average) than the urban values. Average measured distributions are different from model literature urban and rural size distributions.
\end{abstract}

(C) 2004 Elsevier Ltd. All rights reserved.

Keywords: Number concentration; Aerosol size distribution; Urban air quality; Pittsburgh Air Quality Study; PAQS

\section{Introduction}

The size distribution of atmospheric aerosols, together with their composition, sources, and sinks, is a key element in understanding and managing aerosol effects on health, visibility, and climate. A number of epidemiological studies have shown adverse health effects of particulate matter (PM) including respiratory irritation and changes in pulmonary function as well as associations between particulate mass concentrations and mortality (Samet et al., 2000; Wichmann et al., 2000; Lippmann et al., 2000). Recently, there has been an

\footnotetext{
*Corresponding author.

E-mail address: spyros@andrew.cmu.edu (S.N. Pandis).

${ }^{1}$ Currently at Department of Civil and Environmental Engineering, Duke University, Box 90287, Durham, NC 27708, USA.
}

increased interest in the relative health effects of particles of smaller sizes (Oberdorster et al., 1992, 1995; Donaldson and MacNee, 1998). Some laboratory studies have also shown that for a given mass concentration, health effects are larger for smaller particle sizes (Wichmann and Peters, 2000). In addition, the particle size distribution is an important parameter for the estimation of the magnitude of direct and indirect aerosol-climate effects.

There have been many sampling efforts to measure aerosol size distributions in urban, rural, and remote sites around the globe, and nearly all field campaigns now include some measurements of aerosol size distributions. Some recent continental sampling campaigns that measured size distributions include the Atlanta PM Supersite program (Woo et al., 2001), and sampling campaigns in Los Angeles (Kim et al., 2002), Northern Europe (Ruuskanen et al., 2001), Tennessee (Cheng and Tanner, 2002), Brisbane, Australia (Morawska et al., 
2002), England (Harrison et al., 1999), Estonia and Finland (Kikas et al., 1996) and Central Europe (Birmili et al., 2001).

Most of these studies reported 24-h average number concentrations $(10-500 \mathrm{~nm})$ at continental sites ranging from around 5000-25000 $\mathrm{cm}^{-3}$ (Ruuskanen et al., 2001; Kim et al., 2002; Woo et al., 2001; Morawska et al., 2002). Sites that have monitored $3-10 \mathrm{~nm}$ particles find comparable numbers of particles in that size range relative to the $10-500 \mathrm{~nm}$ size range (Woo et al., 2001). Diurnal patterns vary between two extremes: (a) influenced predominantly by meteorology for sites without local particle sources or nucleation; and (b) strongly influenced by local sources such as traffic and nucleation (Kim et al., 2002; Morawska et al., 2002). Little has been reported regarding seasonal patterns in aerosol concentrations due to the lack of long-term monitoring.

Some parameterizations for model size distributions are available for urban and continental aerosols (Whitby, 1978; Jaenicke, 1993). Even if these parameterizations are widely used, their applicability to areas other than the ones for which they were developed has not been investigated.

This paper presents a statistical summary of size distributions measured during $1 \mathrm{yr}$ of continuous monitoring at the Pittsburgh Supersite during the Pittsburgh Air Quality Study (PAQS), forming one of the first longterm data sets for aerosol size distributions in the Northeastern United States. Aerosol size distributions from $3 \mathrm{~nm}$ to $2.5 \mu \mathrm{m}$ were monitored for over 15 months at the central sampling site of the study. Aerosol size distributions were measured at low relative humidity, and at ambient relative humidity to assess the impact of aerosol water on the PM size distribution. Additional aerosol size distributions were collected at an upwind location for approximately 2 months to assess the difference between rural and urban PM size distributions in the airshed. The wide size range, long-term (more than a year) deployment, rural/urban comparison, and dual relative humidity sampling provide advantages in using the size distribution data set to understand and quantify atmospheric aerosol formation, processes, and exposure.

Statistical averages, diurnal and seasonal trends for the urban location, and local sources of particles are discussed. These results are compared to size distributions measured continuously for 6 weeks at a rural site upwind of Pittsburgh to assess the spatial variability and impact of the urban sources on the PM number distribution. While size-resolved aerosol chemistry is not discussed in this work, it was addressed as part of the PAQS. The key findings are summarized in the Results and discussion section, based on Cabada et al. (2004). Finally, the measurement results are compared to other similar investigations and to model size distributions.

\section{Experimental}

The main sampling location was in a park $5 \mathrm{~km}$ east (downwind) of downtown Pittsburgh. Two scanning mobility particle sizer (SMPS) systems (TSI 3936L10 and TSI 3936N25) were operated at the main location continuously from July 2001 to July 2002. These instruments measured the size distribution of particles from 3 to $600 \mathrm{~nm}$. These were augmented by a TSI aerosol particle sizer (APS) 3320 measuring from 0.5 to $20 \mu \mathrm{m}$ for the first 3 months of the sampling period and a TSI APS 3321 measuring in the same size range for the final 2 months of the sampling period. Aerosol measurements at the main site were made at both low relative humidity and ambient relative humidities to assess the importance of relative humidity to the size distribution. This combination of three sizing instruments with RH control, called the dry-ambient aerosol size spectrometer (DAASS) is described in Stanier et al. (2004a). Another SMPS system (TSI 3071/3010) was located at a rural site in Florence, Pennsylvania, $38 \mathrm{~km}$ west (mostly upwind) of the city during 2002. At the Florence site, the aerosol size distribution was measured at slightly sub-ambient relative humidity.

The urban sampling site was relatively far from local sources of primary particles. The nearest major city street was $0.5 \mathrm{~km}$ away, and the nearest highway was $1.1 \mathrm{~km}$ away. Small local sources included the nearby Carnegie Mellon University campus and a local coalfired steam plant that was approximately $1 \mathrm{~km}$ away from the site.

Each of the three sizing instruments acquired an aerosol size distribution eight times per hour, with four of these using the low relative humidity channel, and four of them using the ambient relative humidity inlet. The raw data, and associated temperature and relative humidity information, were acquired using a PC and then processed afterward. Data processing consisted of inversion of the raw size distributions using the TSI SMPS program (version 3.2) and the TSI Aerosol Instrument Manager Program (version 4.3) and corrections for inlet losses and counting efficiencies (see Stanier et al., 2004a, for additional discussion). The size distributions from the individual instruments were then merged into a single size distribution by using the nanoSMPS data through $30 \mathrm{~nm}$, the SMPS data from 30 to $600 \mathrm{~nm}$, and the APS data above $600 \mathrm{~nm}$. The algorithm of Khlystov et al. (2004a) was used to account for the inherent difference between the mobility size measured by the SMPS instruments and the aerodynamic size measured by the APS. The size distributions reported here are a function of mobility diameter for sizes below $600 \mathrm{~nm}$ and as a function of estimated mobility diameter for sizes above $600 \mathrm{~nm}$.

Extensive quality assurance checks were performed prior to instrument deployment and during the field 
study. Pre-study tests are described in Stanier et al. (2004a) and include various counting and sizing precision and accuracy checks using monodisperse aerosols. During the study, a schedule of maintenance activities was adhered to, including daily, weekly, and monthly equipment inspections focusing on maintenance of dry butanol in condensation particle counters, leak checks, and flow calibration.

After initial data reduction, two levels of data validation were performed. The first level focused on internal consistency of the size distribution data between the three instruments that made up the DAASS system, while the second looked for consistency between the DAASS system and other instruments, particularly those that provided continuous measurements of aerosol mass such as the TEOM (Tapered Element Oscillating Microbalance, Rupprecht and Patashnick, Albany, NY). The TEOM is a continuous particulate mass monitor that operated at the PAQS (Wittig et al., 2004) with a sample equilibration system (Meyer et al., 2000) to reduce the interference of water vapor on the sample while retaining as much semivolatile mass as possible. During validation, a small portion of the data was flagged as invalid. Values used for data reduction (sheath and aerosol flowrates and CPC counting efficiencies) were occasionally modified to maintain instrument-to-instrument precision, typically by $\pm 20 \%$ or less. A detailed data quality statement containing the list of corrections (as well as the measured size distributions) is available online in the NARSTO data archive (Stanier, 2003). The data reduction parameters were modified by first examining the time series of the ratio of aerosol mass (TEOM $\mathrm{PM}_{2.5}$ ) to aerosol volume as measured by the SMPS $(30-560 \mathrm{~nm})$, checking particularly for shifts in the ratio corresponding to maintenance (e.g. replacement of sheath blowers, CPCs, and dryers). No significant step changes were found in this ratio throughout the study. Two sets of CPC counting efficiencies (differing by about $15 \%$ for sizes below $30 \mathrm{~nm}$ ) were used for the two different CPC 3010 detectors used during the study. Adjustments to the
UCPC flowrate (and therefore the concentration of particles measured by the nano-SMPS) were required during four separate periods from January 2002 to May 2002 to force agreement in number concentration measured by nano-SMPS and SMPS from 15 to $60 \mathrm{~nm}$. The deviations were most likely caused by poor performance of the NDMA sheath and bypass blowers during this period. The cycling between low and ambient relative humidity caused a higher frequency of failures in sizing equipment than is typical in long-term sampling using SMPS, APS, and optical particle counters.

\section{Results and discussion}

The results are divided into several sections, including (a) summary statistics, (b) size-resolved chemical composition, (c) temporal (seasonal and diurnal) trends, (d) rural versus urban distributions, (e) sources of particles, and (f) comparison to other distribution measurements.

\subsection{Summary statistics}

The grand average number distribution measured during the PAQS (Fig. 1) has a number mode at $40 \mathrm{~nm}$ and a particle count of $22,000 \mathrm{~cm}^{-3}$. The corresponding surface area and volume distributions, calculated from the number distribution assuming spherical particles, are also shown in Fig. 1. The small discontinuity in the surface area and volume distributions are at the point where the SMPS and APS distributions are merged together. These are merged using a procedure described by Khlystov et al. (2004a). The merging procedure removes discontinuities almost completely in the number distribution, but not necessarily in the surface and volume distributions. After the merging procedure, the aerosol mass (TEOM $\mathrm{PM}_{2.5}$ ) and dried aerosol volume through $2.5 \mu \mathrm{m}$ were well correlated $\left(R^{2}\right.$ of 0.95 using a density of $1.52 \mathrm{~g} \mathrm{~cm}^{-3}$ ).

Various descriptive statistics regarding the measured size distributions are included in Table 1. Key points
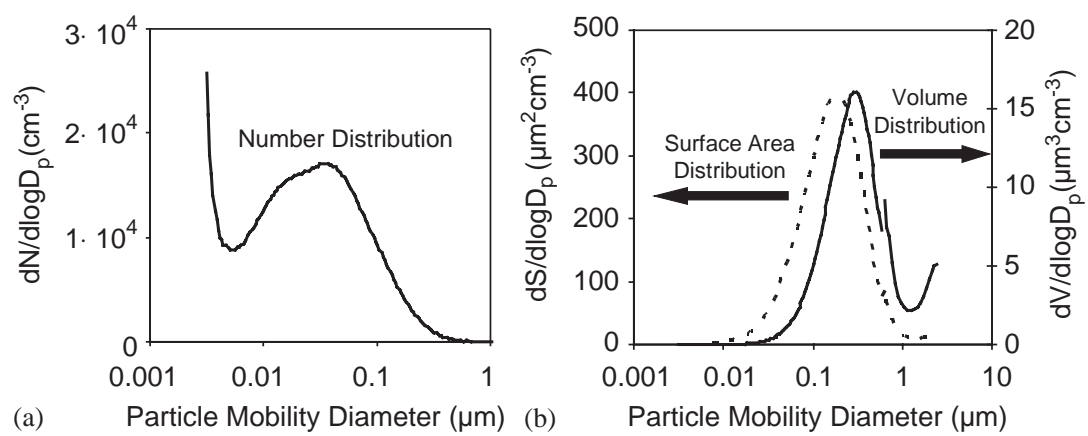

Fig. 1. Grand average (a) number, and (b) surface area and volume distributions from the PAQS (1/7/2001-30/6/2002). 
Table 1

Descriptive statistics of PM size distributions measured during the PAQS (size range $3 \mathrm{~nm}-2.5 \mu \mathrm{m}$ )

Attributes of distributions - grand average for PAQS main site (1/7/2001-30/6/2002)

\begin{tabular}{|c|c|c|c|}
\hline Distribution & Integral & Units & Mode \\
\hline Low $\mathrm{RH}^{\mathrm{a}}$ Number & 22,100 & $\mathrm{~cm}^{-3}$ & 40 \\
\hline Low RH Surface Area & 315 & $\mu \mathrm{m}^{2} \mathrm{~cm}^{-3}$ & 209 \\
\hline Low RH Volume & 11.5 & $\mu \mathrm{m}^{3} \mathrm{~cm}^{-3}$ & 322 \\
\hline Ambient $\mathrm{RH}^{\mathrm{b}}$ Number & 24,500 & $\mathrm{~cm}^{-3}$ & 45 \\
\hline Ambient RH Surface Area & 417 & $\mu \mathrm{m}^{2} \mathrm{~cm}^{-3}$ & 216 \\
\hline Ambient RH Volume & 18.3 & $\mu \mathrm{m}^{3} \mathrm{~cm}^{-3}$ & 414 \\
\hline
\end{tabular}

Descriptive statistics, 24-h averaged $\mathrm{cm}^{-3}$ (low RH channel only)

\begin{tabular}{lllcrrr}
\hline Size bin & Samples & Mean & Median & Min & Max & Standard deviation \\
\hline $3-10 \mathrm{~nm}$ & 307 & 5600 & 4900 & 1380 & 25,000 & 3200 \\
$10-20 \mathrm{~nm}$ & 307 & 4100 & 4100 & 1210 & 8800 & 1420 \\
$20-50 \mathrm{~nm}$ & 336 & 6500 & 6100 & 1880 & 14,000 & 2590 \\
$50-100 \mathrm{~nm}$ & 327 & 3600 & 3400 & 910 & 9700 & 1570 \\
$100-200 \mathrm{~nm}$ & 327 & 1710 & 1510 & 450 & 4550 & 790 \\
$200-500 \mathrm{~nm}$ & 327 & 460 & 361 & 64 & 1632 & 300 \\
$0.5-1 \mu \mathrm{m}$ & 102 & 18 & 11 & 1 & 67 & 17 \\
$1-2.5 \mu \mathrm{m}$ & 103 & 0.59 & 0.56 & 0.10 & 1.5 & 0.27 \\
\hline
\end{tabular}

${ }^{a}$ Low relative humidity distributions were measured at an average relative humidity of $14 \%$.

${ }^{\mathrm{b}}$ Ambient relative humidity distributions were measured at an average relative humidity of $58 \%$.

from Table 1 include (a) the significant difference in surface area and volume between the low RH (14\%) and ambient RH ( $58 \%$ on average) channels of the instrument, (b) the fact that $25 \%$ of the aerosol number is less than $10 \mathrm{~nm}$ and $75 \%$ of the aerosol number is less than $50 \mathrm{~nm}$ in size, and (c) the relatively good data recovery for the SMPS instruments which operated up to $560 \mathrm{~nm}$ $(84-92 \%)$ and relatively poor data recovery for the APS which operated from 0.5 to $2.5 \mu \mathrm{m}(28 \%)$. The APS was removed for service for upgrading and repair early in the study, and not returned to service until late in the study.

Hourly averaged number concentrations from the DAASS are compared in Fig. 2 to hourly averaged $\mathrm{PM}_{2.5}$ values from the TEOM. The highest number concentrations were observed during relatively clean days when the $\mathrm{PM}_{2.5}$ concentrations were less than $25 \mu \mathrm{g} \mathrm{m}^{-3}$. The number concentration is not positively correlated with the mass concentration and there is evidence of a negative correlation.

\subsection{Size-resolved chemical composition}

Measurements of size-resolved aerosol composition taken during the PAQS and reported in Cabada et al. (2004) are summarized here to provide context for interpretation of the size distribution measurements. Average $\mathrm{PM}_{2.5}$ concentrations were $20 \mu \mathrm{g} \mathrm{m}^{-3}$ in summer and $12 \mu \mathrm{g} \mathrm{m}^{-3}$ in winter. The average $\mathrm{PM}_{2.5}$ mass composition in summer (July intensive period) was dominated by sulfate $(\sim 40 \%)$ with significant contribu-

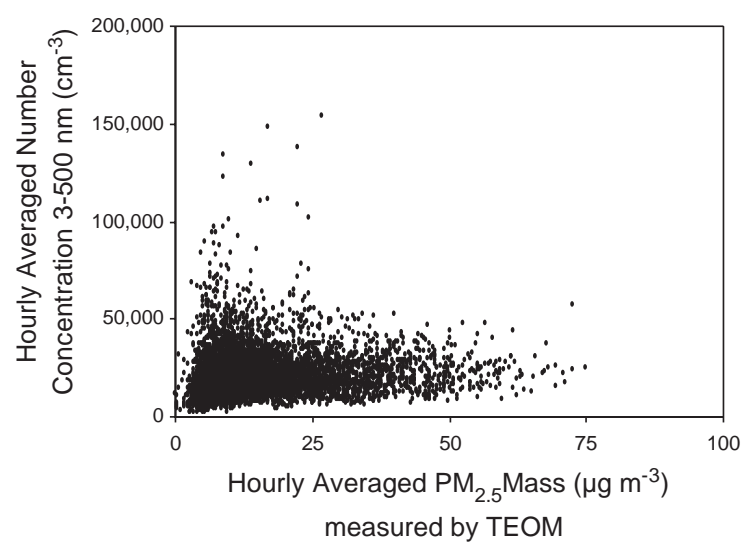

Fig. 2. Aerosol mass versus aerosol number for the PAQS. Hourly average values from the TEOM (mass) are plotted against hourly number integrals from 3 to $500 \mathrm{~nm}$ measured by SMPS.

tions from organic matter $(\sim 30 \%)$ and ammonium $(\sim 15 \%)$ with the balance being made up of elemental carbon, crustal elements, and nitrate. During summer, the sulfate and nitrate had a bimodal mass distribution, with a larger peak at around $0.7 \mu \mathrm{m}$ (from cloud processing) and a smaller peak at around $0.2 \mu \mathrm{m}$. Organic matter and elemental carbon were widely distributed throughout the mass distribution, with geometric mean diameter of $0.3 \mu \mathrm{m}$. During winter, the relative importance of nitrate $(\sim 20 \%)$ and organics 
$(\sim 35 \%)$ increased, while that of sulfate decreased $(\sim 30 \%)$. The mass distribution of the inorganic compounds (sulfate, ammonium, and nitrate) was similar, and showed the same bimodal behavior as the sulfate and ammonium in summer.

Ultrafine chemical composition was also studied by Cabada et al. (2004) and showed a significant seasonal change. Ultrafine $(<100 \mathrm{~nm})$ mass was about $0.6 \mu \mathrm{g} \mathrm{m}^{-3}$ during both summer and winter, making up less than $5 \%$ of the total $\mathrm{PM}_{2.5}$, but representing $90 \%$ of the particle number concentration. The summer ultrafine mass composition was $45 \%$ organic matter and $40 \%$ salts of ammonium and sulfate. During winter, the organics increased to $55 \%$ while the sum of ammonium and sulfate decreased to $35 \%$. This shift is probably caused by higher summertime levels of photochemical activity for oxidation of $\mathrm{SO}_{2}$, and increased wood burning and vehicular organic contributions in wintertime.

\subsection{Temporal trends}

While the aerosol size distributions (especially the number distribution) can exhibit significant variability on the sub-hourly, hourly, and even daily timescales, the monthly average distributions are relatively stable. Figs. 3 and 4 show monthly trends in properties of the size distributions for the low relative humidity and ambient relative humidity instrument channels, respectively. Fig. 3a shows the number mean diameter, surface mode diameter, and volume mode diameter for each month. The monthly averaged number mean diameter was relatively constant, varying from 39 to $52 \mathrm{~nm}$ without a consistent pattern. In contrast, the surface area and volume mode diameters show a clear summer maximum and winter minimum. This behavior is consistent given the summer peak in aerosol volume (and mass) in the Eastern United States. Fig. 3b shows the absence of a clear seasonal trend in average particle number in three different size ranges, while Fig. $3 \mathrm{c}$ shows the strong summertime maximum in aerosol volume, with summer values approximately twice winter levels.

Differences in the ambient and low relative humidity distributions can be seen by comparing Figs. 3 and 4 . While there is negligible difference in ambient and low relative humidity particles counts and number mean diameter, there are significant increases in the surface and volume integrals and mode diameters. For example, the volume mode diameter during summer months (July and August 2001 plus June 2002) was, on average
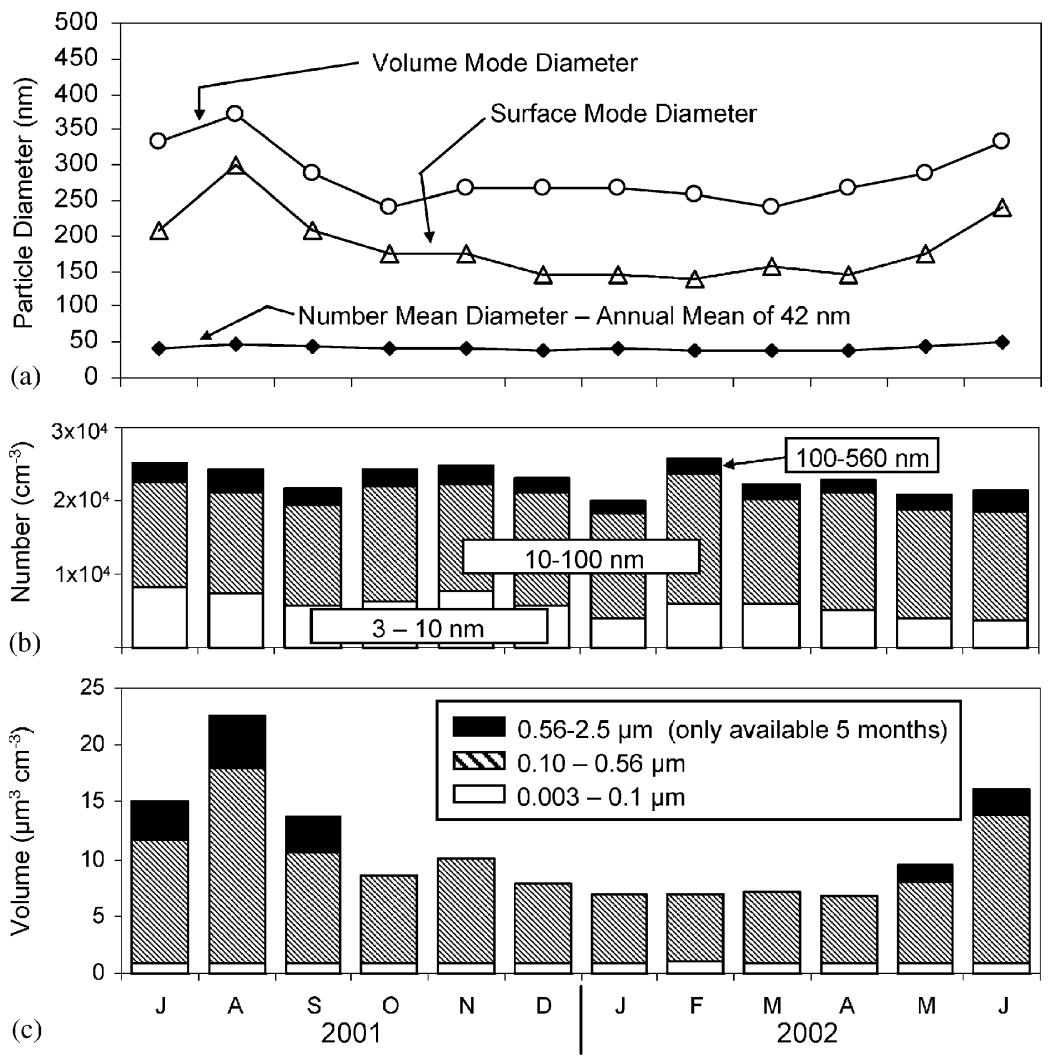

Fig. 3. Monthly patterns in (a) number, (b) surface, and (c) volume distributions for low relative humidity size distributions. 

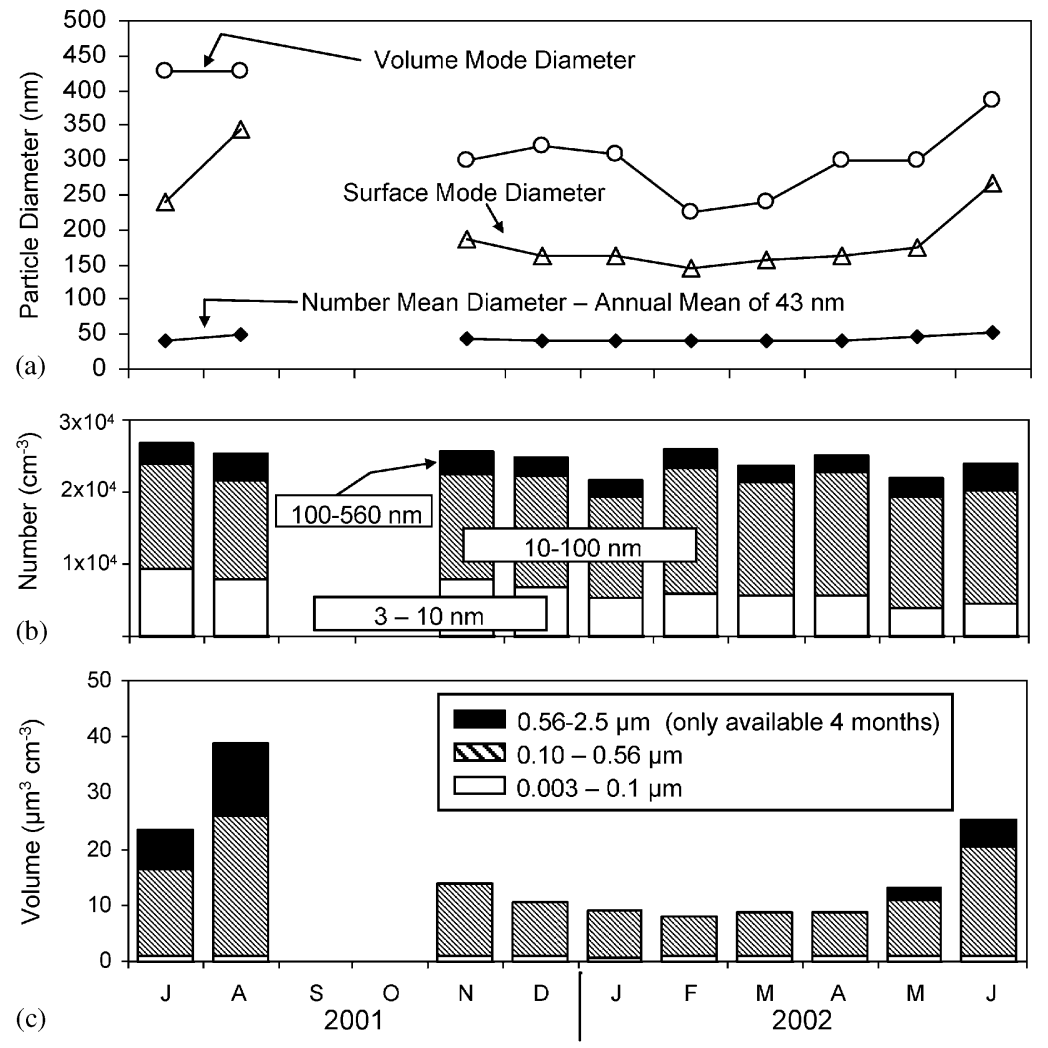

Fig. 4. Monthly patterns in (a) number, (b) surface, and (c) volume distributions for ambient relative humidity size distributions. The DAASS operated in the low relative humidity mode only during September and October 2001.

$415 \mathrm{~nm}$ for the ambient channel and $332 \mathrm{~nm}$ for the low relative humidity channel. The corresponding diameters for winter were $286 \mathrm{~nm}$ for the ambient channel and $256 \mathrm{~nm}$ for the low relative humidity channel. This corresponds to average water content of $38 \%$ (by volume) in measured samples during summer and $23 \%$ (by volume) during winter and average diameter growth factors of 1.16 and 1.09 , respectively. The average ambient summer and winter relative humidities were similar (around 60\%) and therefore the difference in water content is due primarily to seasonal differences in aerosol chemistry. During summer, the higher fraction of inorganic materials in $\mathrm{PM}_{2.5}$, the higher aerosol acidity, and higher levels of water soluble organics increased hygroscopicity and limited crystallization (Khlystov et al., 2004b), leading to higher measured aerosol water contents. These changes in the size distribution due to water uptake need to be considered for chemical and physical processes that rely on aerosol surface area, volume, and chemical composition, including visibility degradation and heterogeneous reactions. The values reported here are difficult to compare to other locations because there are no instances in the literature where simultaneous long-term dried and ambient size distributions are reported.

While seasonal trends were most apparent in the aerosol volume distribution, strong diurnal trends were observed in the aerosol number distribution. The diurnal pattern of aerosol number, averaged over the entire study, is shown in Fig. 5a. The solid line at the top of the figure is the diurnal pattern for total aerosol number, while the other lines in the figure show the diurnal pattern for specific size classes of aerosols, ranging from the nuclei mode $(3-10 \mathrm{~nm})$ up to part of the accumulation mode $(50-100 \mathrm{~nm})$. Figs. $5 \mathrm{~b}$ and $\mathrm{c}$ plot diurnal patterns for specific types of days, rather than for the average day of the entire study. Circled numbers refer to specific diurnal features discussed below in the text.

Total aerosol number, on average, peaked at midday (Fig. 5a-feature 1). Another feature apparent in the grand average diurnal trend is the minimum in $3-20 \mathrm{~nm}$ particles during the early morning, when their sources are at a minimum (Fig. 5a-feature 2). Also apparent is the drop in concentration of $50-100 \mathrm{~nm}$ particles from dilution during the morning when the mixing height is rapidly rising (Fig. 5a-feature 3). 


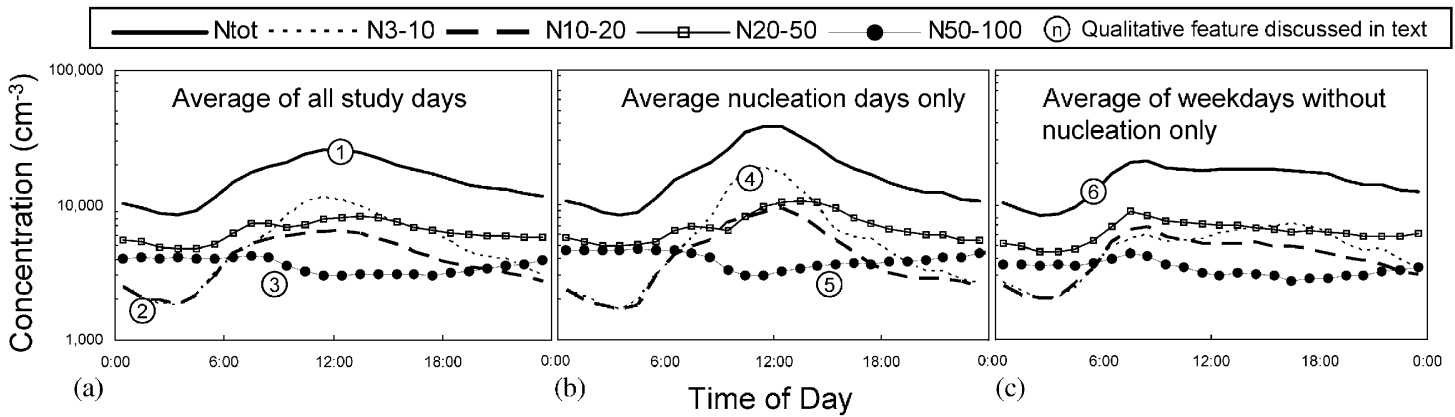

Fig. 5. Diurnal patterns in particle number (a) average of all study days, (b) average of all study days with regional nucleation, and (c) average of all weekdays without significant nucleation, showing influence of traffic. Numbered circles refer to qualitative features discussed in the text. The solid line at top of each profile is the total number, while the other lines are diurnal profile for specific size classifications. The smallest bin plotted is from 3 to $10 \mathrm{~nm}$ while the largest bin plotted is from 50 to $100 \mathrm{~nm}$.

Two of the most representative types of days, in terms of diurnal aerosol size distribution behavior, were days with in situ nucleation and days with a morning rush hour (weekdays) but no apparent nucleation. The former (regional nucleation) occurred on $30 \%$ of the study days and the average diurnal profile of these days is plotted in Fig. 5b. The latter (weekday without nucleation) also occurred on approximately $30 \%$ of the study days, and is plotted in Fig. 5c. Key features of the nucleation diurnal profile include the midday peak in nuclei mode $(3-10 \mathrm{~nm})$ particles (Fig. 5b-feature 4) as well as the slow growth in the number of $50-100 \mathrm{~nm}$ particles during the afternoon as the small particles created earlier in the day grow to larger sizes by coagulation and condensation (Fig. 5b-feature 5). An important feature of the non-nucleation weekday profile is the increase in particle number during the morning rush hour (Fig. $5 \mathrm{c}$ - feature 6). This feature can also be seen in the grand average diurnal profile (Fig. 5a) as a shoulder peak a few hours before the maximum from nucleation. Fig. 5c also shows that there is no afternoon rush hour peak and no midday peak. The former is because the afternoon rush hour is more spread out in time than the morning rush hour, and the mixing height is high during the late afternoon, diluting the emissions. The latter is because the midday peak is primarily from nucleation.

\subsection{Rural Versus urban distributions}

During February and March of 2003, an SMPS system sampled size distributions at a rural site (Florence, PA) $38 \mathrm{~km}$ upwind from the main sampling site. This provided information on the contribution of background aerosols, the spatial homogeneity of aerosol processes (e.g. new particle formation from in situ nucleation), and the difference between the "rural" and "urban" size distributions in the Pittsburgh area. For

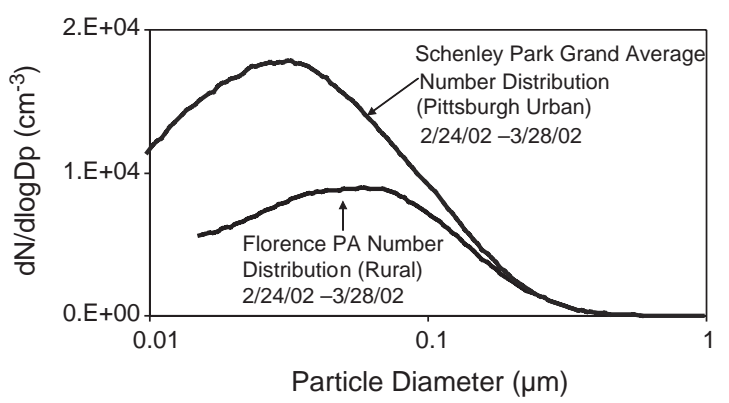

Fig. 6. Average number size distributions from the rural site (Florence, PA) and the urban site (Schenley Park, Pittsburgh, PA).

the remainder of this work, the main site results will be referred to as urban and the Florence site will be referred to as rural. The average size distributions measured during February and March (Fig. 6) show that number concentrations were, on average, 2-3 times higher at the urban site. Furthermore, the rural site had a larger mode size (approximately 62 versus $32 \mathrm{~nm}$ at the urban site). There was little difference in the number distributions above $200 \mathrm{~nm}$, consistent with the regional nature of the fine $\mathrm{PM}$ in the area and the similar $\mathrm{PM}_{2.5}$ mass concentrations measured at the two sites during the study. The rural site was impacted by nucleation very similarly to the urban site (Stanier et al., 2004b), but was not impacted nearly as strongly by traffic. Therefore, the diurnal patterns at the rural site were not as pronounced.

\subsection{Sources of particles}

Nucleation and vehicle emissions were the most important sources of particles impacting the main (urban) sampling site. Fig. 7 shows the evolution of the number size distribution on 2 consecutive days. The 
first day (10 August; top panel) does not exhibit nucleation behavior, while the second day (11 August; bottom panel) does. At 9 AM, there is significant new particle formation on 11 August. The frequency,

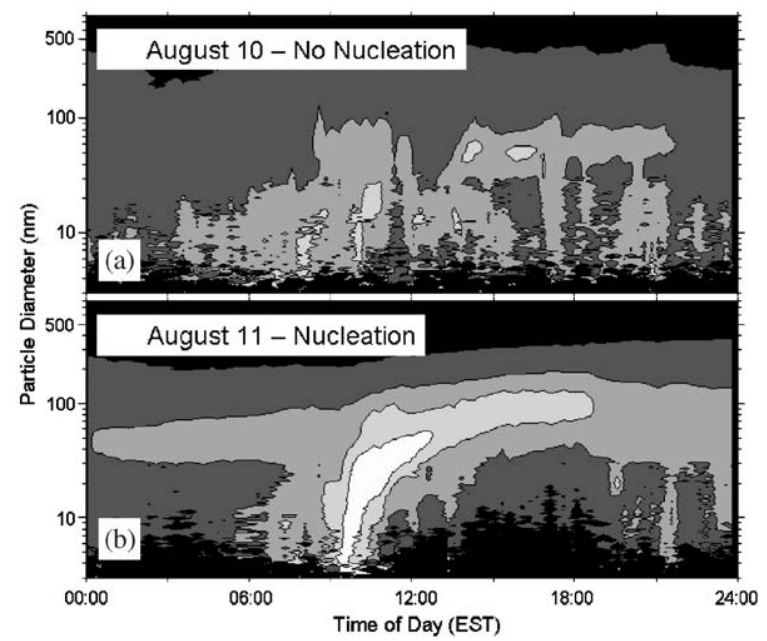

Fig. 7. Evolution of particles size distributions on a day without nucleation (10 August) and a day with nucleation (11 August). The plots show instrument response $\left(\mathrm{d} N / \mathrm{d} \log D_{\mathrm{p}}\right.$ with units of $\mathrm{cm}^{-3}$ ) over all size channels. Light colors are highest number concentrations and dark colors are the lowest number concentrations. Contour lines drawn at $\mathrm{d} N / \mathrm{d} \log \mathrm{D}_{p}$ values of $10^{2}, 10^{3}, 10^{4}, 10^{4.5}$, and $10^{5} \mathrm{~cm}^{-3}$.

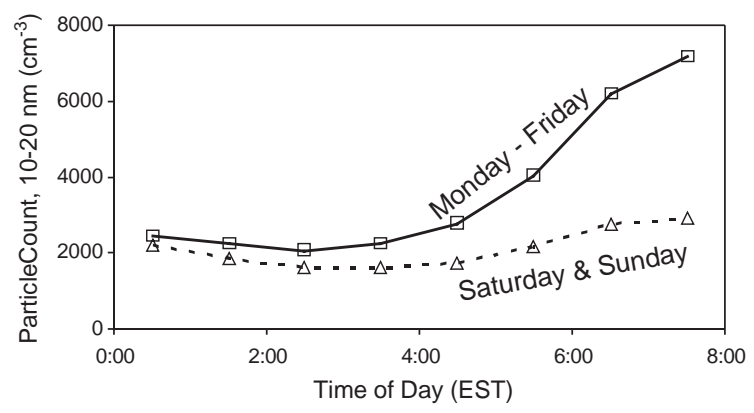

Fig. 8. Traffic as a source of particles. Ten to twenty nanometer particle count as function of time for weekends and weekdays at the urban site. intensity, meteorology, and possible chemistry of such new particle formation has been analyzed (Stanier et al., 2004b), showing that the nucleation events are associated with photochemical sulfuric acid production and occur on approximately $30 \%$ of the study days. The same type of behavior has also been witnessed in St. Louis (Shi, 2003).

The other major particle source impacting the urban site is traffic. On weekdays, there was a pronounced increase in particle concentrations for particles primarily in the size range from 3 to $30 \mathrm{~nm}$ (Fig. 8) beginning at approximately 04:00 EST. On weekends, the increase was much smaller and later in the morning. A similar analysis for daytime traffic is complicated by changes in the mixing height and nucleation activity.

\subsection{Comparison with other distribution measurements}

It is useful to compare the PAQS size distribution measurements to (a) other studies and (b) commonly used "typical" or model size distributions (Whitby, 1978; Jaenicke, 1993). Comparison to the sampling study of Ruuskanen et al. (2001) in three Northern European cities shows very similar results for aerosol number concentration (Table 2).

The comparison with model distributions of Whitby and Jaenicke is shown in Fig. 9, with number distributions in Fig. 9a and volume distributions in Fig. 9b. The model distributions used are Whitby's "urban average" distribution (labeled C-W1), Whitby's “average background" distribution (labeled C-W2), and Jaenicke's rural continental distribution (labeled C-J). Not shown in the figure is Jaenicke's urban distribution, which is very similar to Whitby's urban average distribution. The most general conclusion is that none of the model distributions fit either the urban or rural results from this study well. The model urban size distributions have an order of magnitude higher particle concentrations, with a strong $15 \mathrm{~nm}$ mode not seen in the average distributions from Pittsburgh. The model urban distributions are more appropriate for sites close to (<approximately $100 \mathrm{~m}$ ) to traffic or Los Angeles. Based on these results, it is recommended that caution be exercised in using model distributions.

Table 2

Comparison to other size distribution measurement campaigns

\begin{tabular}{llcl}
\hline Location & Number concentration $\left(\mathrm{cm}^{-3}\right)$ & Source \\
\cline { 2 - 4 } & $10-100 \mathrm{~nm}$ & $100-500 \mathrm{~nm}$ & Ruuskanen et al. (2001) \\
\hline Alkmaar, Netherlands & 18,300 & 2120 & Ruuskanen et al. (2001) \\
Erfurt, Germany & 17,700 & 2270 & Ruuskanen et al. (2001) \\
Helsinki, Finland & 16,200 & 973 & This work \\
Pittsburgh, Urban & 14,300 & 2170 & This work \\
Pittsburgh, Rural & 6500 & 1900 & \\
\hline
\end{tabular}



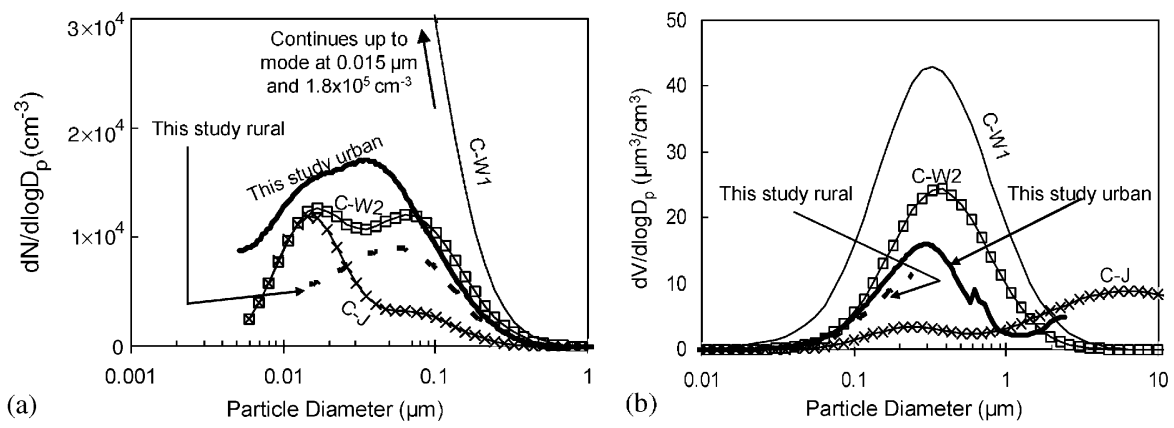

Fig. 9. Comparison of model size distributions to Pittsburgh size distributions on a (a) number basis and (b) volume basis. Model distributions: C-W1 = Whitby (1978) urban average; C-W2 = Whitby (1978) average background; and C-J = Jaenicke (1993) rural continental.

\section{Summary and conclusions}

Twelve months of PM size distributions from the PAQS were summarized. The average Pittsburgh number concentration $(3-500 \mathrm{~nm})$ is $22,000 \mathrm{~cm}^{-3}$ with an average mode size of $40 \mathrm{~nm}$. Seasonal patterns are not very strong for aerosol number, but are clearly evident for the aerosol volume distribution due to the summertime peak in aerosol mass concentrations. Strong diurnal patterns in number concentrations are evident as a direct effect of the sources of particles (atmospheric nucleation, traffic, and other combustion sources). New particle formation from homogeneous nucleation is significant on the $30-50 \%$ of study days and over a wide area (at least a hundred kilometers). Rural number concentrations are a factor of 2-3 lower (on average) than the urban values. Number concentrations are comparable to those found in similar sampling studies in Northern Europe, and model size distributions tested against the PAQS results yield a poor fit between the sampled and model distributions.

The urban and rural PM size distributions measured during the PAQS form an excellent data set for further research on particle sources, nucleation, and aerosol processes. To date, they have been used to address nucleation (Stanier et al., 2004b), aerosol water content (Khlystov et al., 2004b), and source-receptor relationships (Zhou et al., 2004).

\section{Acknowledgements}

This research was conducted as part of the PAQS which was supported by US Environmental Protection Agency under Contract R82806101 and the US Department of Energy National Energy Technology Laboratory under Contract DE-FC26-01NT41017.

\section{References}

Birmili, W., Wiedensolher, A., Heintzenber, J.,Lehmann, K., 2001. Atmospheric particle number size distribution in central Europe: statistical relations to air masses and meteorology. Journal of Geophysical Research 106 (D23), 32,005-32,018.

Cabada, J.C., Takahama, S., Khlystov, A.Y., Wittig, B., Pandis, S., Rees, S., Davidson, C.I., Robinson, A.L., 2004. Mass size distributions and size resolved chemical composition of fine particulate matter at the Pittsburgh Supersite. Atmospheric Environment, this issue, doi:10.1016/j.atmosenv.2004.03.004.

Cheng, M.D., Tanner, R.L., 2002. Characterization of ultrafine and fine particles at a site near the Great Smoky Mountains. Atmospheric Environment 36, 5795.

Donaldson, K., MacNee, W., 1998. The mechanism of lung injury caused by $\mathrm{PM}_{10}$. In: Hester, R.E., Harrison, R.M. (Eds.), Issues in Environmental Science and Technology. The Royal Society of Chemistry, London.

Harrison, R.M., Shi, J.P., Jones, M.R., 1999. Continuous measurements of aerosol physical properties in the urban atmosphere. Atmospheric Environment 33, 1037-1047.

Jaenicke, R., 1993. Tropospheric aerosols. In: Hobbs, P.V. (Ed.), Aerosol-Cloud-Climate Interactions. Academic Press, San Diego, CA, pp. 1-31.

Khlystov, A., Stanier, C., Pandis, S.N., 2004a. An algorithm for combining electrical mobility and aerodynamic size distribution data when measuring ambient aerosol. Aerosol Science and Technology, in press.

Khlystov, A., Stanier, C., Takahama, S., Pandis, S.N., 2004b. Water content of ambient aerosols during the Pittsburgh Air Quality Study. Journal of Geophysical Research, submitted for publication.

Kikas, U., Mirme, A., Tamm, E., Raunemaa, T., 1996. Statistical characteristics of aerosol in Baltic sea region. Journal of Geophysical Research 101 (D14), 19,319-19,327.

Kim, S., Shen, S., Sioutas, C., Zhu, Y., Hinds, W., 2002. Size distributions and diurnal and seasonal trends of ultrafine particles in source and receptor sites of the Los Angeles basin. Journal of the Air and Waste Management 52, 297-307. 
Lippmann, M., Ito, K., Nádas, A., Burnett, R.T., 2000. Association of particulate matter components with daily mortality and morbidity in urban populations. Research Report 95, Health Effects Institute, Cambridge, MA.

Meyer, M.B., Patashnick, H., Ambs, J.L., Rupprecht, E., 2000. Development of a sample equilibration system for the TEOM continuous PM monitor. Journal of the Air and Waste Management 50 (8), 1345-1349.

Morawska, L., Jayarantne, E.R., Mengersen, K., Thomas, S., 2002. Differences in airborne particle and gaseous concentrations in urban air between weekdays and weekends. Atmospheric Environment 36, 4375-4383.

Oberdorster, G., Ferin, J., Gelein, R., Soderholdm, S.C., Finkelstein, J., 1992. Role of alveolar macrophage in lung injury; studies with ultrafine particles. Environmental Health Perspectives 102, 173-179.

Oberdorster, G., Gelein, R.M., Ferin, J., Weiss, B., 1995. Association of particulate air pollution and acute mortality: involvement of ultrafine particles. Inhalation Toxicology 7 , 111-124.

Ruuskanen, J., Tuch, Th., Ten Brink, H., Peters, A., Khystov, A., Mirme, A., Kos, G.P.A., Brunekreef, B., Wichmann, H.E., Buzorius, G., Vallius, M., Kreyling, W.G., Pekkanen, J., 2001. Concentrations of ultrafine, fine and $\mathbf{P M}_{2.5}$ particles in three European cities. Atmospheric Environment 35, 3729-3738.

Samet, J.M., Zeger, S.L., Dominici, F., Curriero, F., Coursac, I., Dockery, D.W., Schwartz, J., Zanobetti, A., 2000. The National Morbidity, Mortality, and Air Pollution Study, part II: morbidity and mortality from air pollution in the United States. Research Report 94, Health Effects Institute, Cambridge, MA.

Shi, Q., 2003. Aerosol size distributions ( $3 \mathrm{~nm}$ to $3 \mu \mathrm{m})$ measured at the St. Louis Supersite (4/1/01-4/30/02). M.S. Thesis, Department of Mechanical Engineering, University of Minnesota, Minneapolis, MN 55455.
Stanier, C. 2003. Dry-ambient aerosol size spectrometer data quality statement. NARSTO Quality Systems Science Center, Website, http://eosweb.larc.nasa.gov/PRODOCS/ narsto/table_narsto.html\#epa.

Stanier, C., Khlystov, A., Chan, W.R., Mandiro, M., Pandis, S.N., 2004a. A method for the in situ measurement of fine aerosol water content of ambient aerosol: the dry-ambient aerosol size spectrometer (DAASS). Aerosol Science and Technology, in press.

Stanier, C., Khlystov, A., Pandis, S.N., 2004b. Nucleation events during the Pittsburgh Air Quality Study: description and relation to key meteorological, gas phase, and aerosol parameters. Aerosol Science and Technology, in press.

Whitby, K.T., 1978. The physical characteristics of sulfate aerosols. Atmospheric Environment 12, 135-159.

Wichmann, H.-E., Peters, A., 2000. Epidemiological evidence of the effects of ultrafine particle exposure. Philosophical Transactions of the Royal Society A 358, 2751-2769.

Wichmann, H.-E., Spix, C., Tuch, T., Wölke, G., Peters, A., Heinrich, J., Kreyling, W.G., Heyder, J., 2000. Daily mortality and fine and ultrafine particles in Erfurt, Germany. Part I: role or particle number and particle mass. Research Report 98, Health Effects Institute, Cambridge, MA.

Wittig, B., Anderson, N., Khlystov, A.Y., Pandis, S.N., Davidson, C., Robinson, A.L., 2004. Pittsburgh Air Quality Study overview. Atmospheric Environment, this issue, doi:10.1016/j.atmosenv.2004.03.003.

Woo, K.S., Chen, D.R., Pui, D.Y.H., McMurry, P.H., 2001. Measurement of Atlanta aerosol size distributions: observations of ultrafine particle events. Aerosol Science and Technology 34, 75-87.

Zhou, L., Kim, E., Hopke, P.K., Stanier, C., Pandis, S.N., 2004. Advanced factor analysis on Pittsburgh particle size distribution data. Aerosol Science and Technology, in press. 


\title{
A Method for the In Situ Measurement of Fine Aerosol Water Content of Ambient Aerosols: The Dry-Ambient Aerosol Size Spectrometer (DAASS)
}

\author{
Charles O. Stanier, Andrey Y. Khlystov, Wanyu R. Chan, Mulia Mandiro, \\ and Spyros N. Pandis \\ Department of Chemical Engineering, Carnegie Mellon University, Pittsburgh, Pennsylvania
}

\begin{abstract}
Hygroscopic growth of atmospheric particles affects a number of environmentally important aerosol properties. Due to the hysteresis exhibited by the aerosol hygroscopic growth, the physical state of particles and the amount of aerosol water are uncertain within a wide range of relative humidities (RHs) found in the troposphere, leading to uncertainties in optical and chemical properties of the aerosol. Here we report the design and tests of an automated system that was built to assess the amount of aerosol water at atmospheric conditions. The system consists of two scanning mobility particle sizers (SMPS) and an aerodynamic particle sizer (APS) that measure the aerosol size distribution between $3 \mathrm{~nm}$ and $10 \mu \mathrm{m}$ in diameter. The inlets of the instruments and their sheath air lines are equipped with computer-controlled valves that direct air through Nafion dryers or bypass them. The Nafion dryers dehydrate the air streams to below $30 \% \mathrm{RH}$ at which point ambient particles are expected to lose most or all water. The switch between the dried and the ambient conditions occurs every $7 \mathrm{~min}$ and is synchronized with the scan times of the aerosol spectrometers. In this way the system measures alternatively dried (below 30\% RH) and ambient aerosol size distributions. A comparison of the ambient RH and the dried RH size distributions and the corresponding integrated volume concentrations provides a measure of the physical state of particles and the amount of aerosol water. The aerosol water content can be treated as a growth factor or as an absolute quantity and can be calculated as a time series or as a function of RH (humidigram). When combined with aerosol composition measurements, the DAASS can be used to compare hygroscopic growth models and measurements.
\end{abstract}

Received 23 October 2002; accepted 22 April 2003.

This research was conducted as part of the Pittsburgh Air Quality Study, which was supported by the US Environmental Protection Agency under contract R82806101 and the US Department of Energy National Energy Technology Laboratory under contract DE-FC26$01 \mathrm{NT} 41017$.

Address correspondence to Spyros Pandis, Department of Chemical Engineering, Carnegie Mellon University, 5000 Forbes Ave., Pittsburgh, PA 15213, USA. E-mail: spyros@andrew.cmu.edu

\section{INTRODUCTION}

The absorption of water by atmospheric aerosols with increasing relative humidity $(\mathrm{RH})$ influences their size, composition, lifetime, chemical reactivity, and light scattering. Water is the most prevalent aerosol component at RHs above $80 \%$ and is often a significant component at lower RHs (Hanel 1976). Accordingly, hygroscopic growth is important in a number of air pollution problems, including visibility impairment, climate effects of aerosols, acid deposition, long-range transport, and the ability of particles to penetrate into the human respiratory system.

A number of laboratory investigations of water uptake by model or smog chamber aerosols have been conducted. Laboratory studies have used single-particle levitation (Tang and Munkelwitz 1993; Chan 1992; Wagner et al. 1996) and hygroscopic tandem DMA (H-TDMA) (Rader and McMurry 1986) to investigate water uptake, deliquescence, and crystalliation of a number of inorganic compounds. More recently, these techniques have been used to investigate hygroscopicity in organic compounds and organic-inorganic mixtures (Xiong et al. 1998; Virkulla et al. 1999; Cruz and Pandis 2000; Peng et al. 2001; Cocker et al. 2001a, b; Brooks et al. 2002).

Most field studies of water uptake in ambient aerosols have used a H-TDMA (McMurry and Stolzenberg 1989; Berg et al. 1998; Dick et al. 2000). Results of H-TDMA studies are reviewed by Cocker et al. (2001c) and typically classify particles into more hygroscopic and less hygroscopic fractions, with the number of fractions, relative size of fractions, and growth factors varying at different sites. Other techniques devised to measure water uptake include RH-conditioned nephelometry (Rood 1987; ten Brink et al. 2000; Day et al. 2000), filter analysis by gravimetry (Vartiainen et al. 1994), filter analysis by chemical analysis (Ohta et al. 1998), filter analysis by beta attenuation (Speer et al. 1997), observation by Fourier transform infrared spectroscopy (Han and Martin 1999; Onasch et al. 1999; Martin et al. 2001; Han et al. 2002), and in situ measurement of 
evaporated water (Lee and Hsu 1998). Kreisberg et al. (2001) used an optical particle counter for in situ automated measurement of dried, humidified, and ambient size distributions.

Several thermodynamic models have been developed that calculate the water uptake of pure and mixed salts (see Ansari and Pandis 1999 and references therein). Recently, these methods have been extended to particles containing organic compounds (Saxena and Hildemann 1997; Ansari and Pandis 2000; Clegg et al. 2001; Ming and Russell 2002).

While this body of experimental, theoretical, and field research has significantly advanced the understanding of water uptake by aerosols, significant uncertainties remain. First, there is a limited amount of information about the liquid water content of "real" particles in the atmosphere. Second, many aerosol compositions exhibit hysteresis in aerosol water content, potentially existing at more than one thermodynamically stable state. Third, field data are required to validate and improve models for mixed organic-inorganic-water models. Finally, a method is needed for direct in situ measurement of this important particulate matter component. Therefore, there is a need for additional field measurements that focus on the in situ aerosol water content, crystallization behavior, comparison with mass-based measurements, and the influence of organic compounds.

This work describes a new field instrument, the dry-ambient aerosol size spectrometer (DAASS), for the in situ measurement of the atmospheric fine aerosol liquid water content. The DAASS was designed and deployed during the Pittsburgh Air Quality Study (PAQS), and its design, calibration, data reduction procedure, and first results are reported in this work.

\section{EXPERIMENTAL}

The DAASS is an automated combination of aerosol-sizing instruments that measures the dried $(<30 \%)$ and ambient $\mathrm{RH}$ aerosol number distribution. The aerosol water content is calculated from the difference of the dried and ambient volume distributions.

The design goals for the DAASS included:

- Measurement of the dried ( $<30 \% \mathrm{RH})$ aerosol size distribution from $3 \mathrm{~nm}-10 \mu \mathrm{m}$ microns for several months with a frequency of at least 4 times per hour.

- Measurement of the ambient aerosol water content up to ambient RH levels of $90 \% \mathrm{RH}$ and on a frequency of at least once per hour.

- Automatic operation and data acquisition with minimal maintenance for field deployment.

- Operation from $-15^{\circ} \mathrm{C}$ to $35^{\circ} \mathrm{C}$ temperatures, and dewpoints up to $25^{\circ} \mathrm{C}$.

The DAASS includes three particle-sizing instruments with associated supporting equipment, as shown in Figure 1. The particle-sizing instruments include two scanning mobility particle sizers (SMPS) and one aerosol particle sizer (APS). The SMPS instruments size particles from 3-80 nm (TSI 3936N25) and 13-680 nm (TSI 3936L10), while the APS (TSI 3320) sizes particles from $0.5-10 \mu \mathrm{m}$. These systems are referred to as the Nano-SMPS, SMPS, and APS systems in this article. Two separate $\mathrm{RH}$ controlled inlets served the aerosol-sizing instruments. One inlet conditioned aerosols for the SMPS systems, while a separate inlet conditioned aerosols for the APS. Supporting these components are a dry air supply system and humidityconditioning systems for the sheath air flows of both SMPS systems and the APS.

All components were housed in a weatherproof plywood enclosure with a volume of $3.6 \mathrm{~m}^{3}$. To meet the goal of measuring the ambient aerosol size distribution at ambient temperature and RH without active temperature and RH control, the instruments, particularly the differential mobility analyzer (DMA) columns, needed be kept at ambient temperature. In the first set of tests, all equipment, including the DMA columns, was placed inside the enclosure, and temperatures were kept near ambient using a large exhaust fan $\left(\sim 35 \mathrm{~m}^{3} \mathrm{~min}^{-1}\right)$. In spite of the large flow of outside air, the DMA column temperatures were elevated about $4{ }^{\circ} \mathrm{C}$ relative to ambient and were therefore drying the ambient samples. The ambient scans reached only approximately $80 \%$ of the ambient RH (e.g., outdoor RH of $95 \%$ yielded an ambient channel RH of around 76\%). To keep the DMA column temperatures closer to ambient, the columns were moved to a protected ledge just outside the enclosure while fans pulled ambient air over them. This configuration improved performance, and the DMAs then achieved greater than $90 \%$ of ambient RH (e.g., outdoor RH of $95 \%$ yielded an ambient channel $\mathrm{RH}$ of greater than $86 \%$ ). The effects of this mismatch are discussed in the data reduction section below.

During winter, the enclosure was maintained at a minimum temperature of $9^{\circ} \mathrm{C}$, which was required for the maintenance of condenser and saturator temperature setpoints in the Condensation Particle Counters (CPCs). This did not significantly affect the final sheath and aerosol relative humidities because the DMA columns were outside the enclosure at (or close to) the outdoor temperature. However, the aerosol flow did pass through this heated enclosure, and the charger was located in the enclosed section of the inlet. This caused the minimum RH encountered by the aerosol flow and the RH (and therefore size) during charging to be different from the final RH at the DMA column. In future deployments of the DAASS, it is recommended that the entire inlet be placed outside of the enclosure in a shaded, ventilated area. The effects of this RH minimum are considered below in the data-reduction section.

Drying of aerosol streams was accomplished using Nafion membrane dryers (Permapure MD-110, Toms River, NJ, USA). Single-tube dryers with stainless steel housings were selected for drying aerosols rather than multitube dryers to limit losses (Woo et al. 2001). For ambient RH measurements, the dryer could be bypassed using automated valves. For the SMPS system, three-way solenoid valves (Alcon U33-43-21-12, Itasca, IL, USA) were used. For the APS inlet, flow selection between the dried and ambient inlet was achieved using full-bore ball 


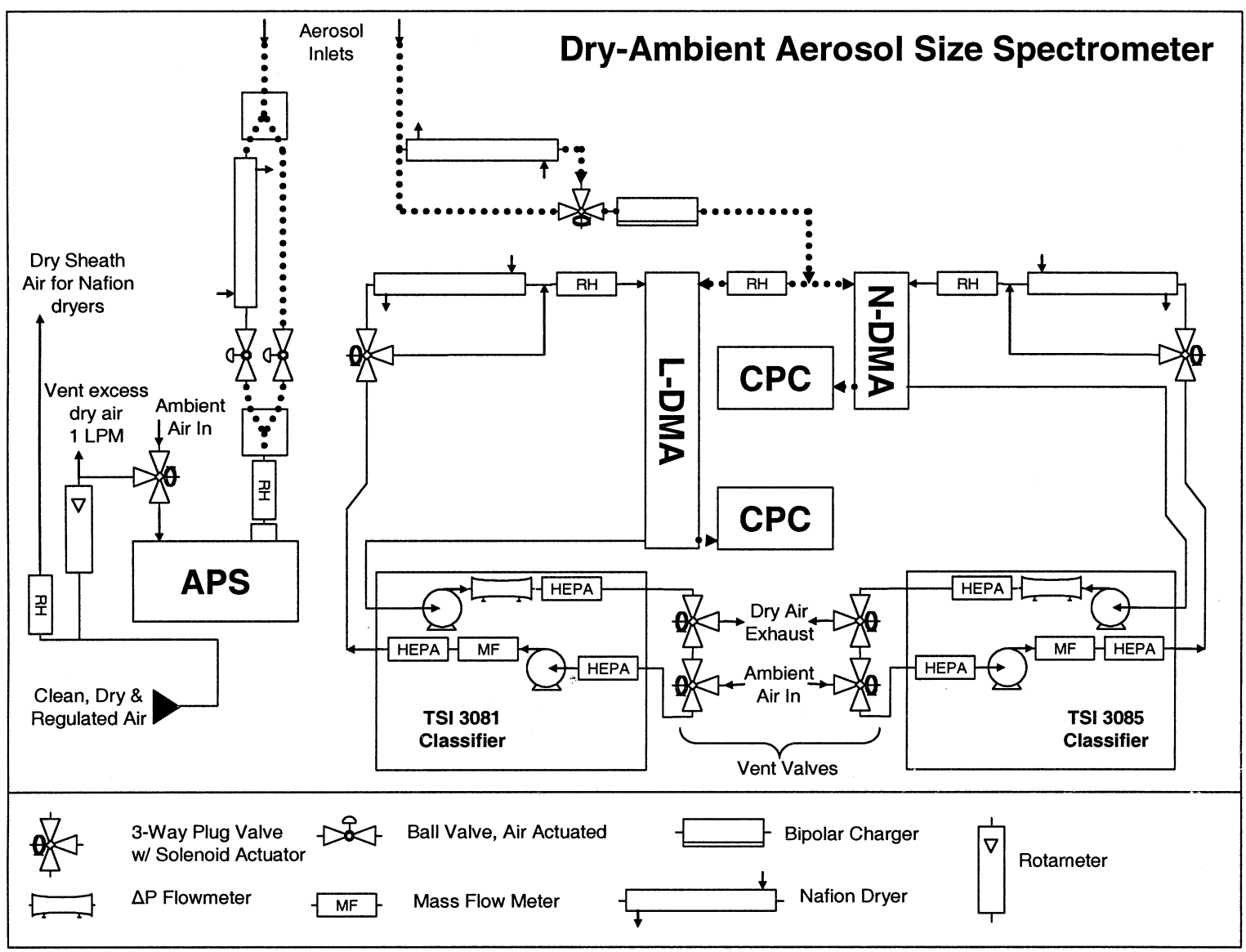

Figure 1. Flow diagram of DAASS. Aerosol streams are shown by dotted lines and other flows are indicated by solid lines.

valves (Swagelock SS-41S2-31DDM) with orifice diameters that matched tubing inner diameters. Copper and stainless steel tubing was used throughout to minimize particle losses.

The APS was modified slightly to allow for drying. With factory settings, the APS samples 5 liters per minute (LPM) and then separates the flow into a 1 LPM aerosol flow and a 4 LPM sheath flow. The sheath flow is filtered and returned in the timeof-flight section of the instrument. In the DAASS system, the dry-ambient inlet sampled 1 LPM and was connected directly to the APS inner nozzle. Particle-free air was supplied at atmospheric pressure directly to the APS sheath air inlet (Figure 1). Depending on the sampling mode, this air was at ambient $\mathrm{RH}$ or dried. The standard APS pump and flow control was used for both the aerosol and sheath flows.

In the SMPS systems, the aerosol is assumed to equilibrate with the sheath flow RH because of the 0.3-6 s exposure time of aerosols to the sheath flow (this assumption is considered in the data-reduction section). In the data analysis, it is the SMPS sheath flow RH that is used to analyze particle size as a function of RH. However, in the APS, the aerosol has a much shorter exposure time to the sheath air $\left(<10^{-4} \mathrm{~s}\right)$. In that case, the aerosol is assumed to be equilibrated at the aerosol stream $\mathrm{RH}$ and unaffected by the sheath RH.
Operation of the dry-ambient SMPS systems required three flow configurations of DMA sheath air: dried, ambient, and vent. The flow configurations for these modes are shown in Figure 2. In dried and ambient modes, the sheath air ran in a closed loop and passed through dryers or bypassed them, depending on the mode. After the dried scans, the system was put in "vent" mode and the sheath flow was switched to a once-through flow configuration. Dry air was exhausted from the system and ambient RH air was drawn into the system.

Drying of sheath air flows for the SMPS systems was accomplished using Nafion membrane dryers (Permapure PD-50T and PD-200T). Multitube dryers were selected to accommodate the flowrates, which were 3.2 and 7.0 LPM. These dryers were rated to achieve dewpoints of $-15^{\circ} \mathrm{C}$ for incoming air at a $20^{\circ} \mathrm{C}$ dewpoint using shell-side utility air supplied at a $-40^{\circ} \mathrm{C}$ dewpoint. This system achieved RHs in sheath air of less than 15\% during the initial summer tests. However, during the winter tests, silica gel desiccant was added to assist in the drying. The winter drop in performance of the membrane dryers was partly due to problems in supplying $-40^{\circ} \mathrm{C}$ dew point utility air during winter, and partly due to the need to reach lower dewpoints in winter than in summer to achieve RHs below 30\% at ambient temperatures. During the coldest periods of the study $\left(-10^{\circ} \mathrm{C}\right)$, DMA 


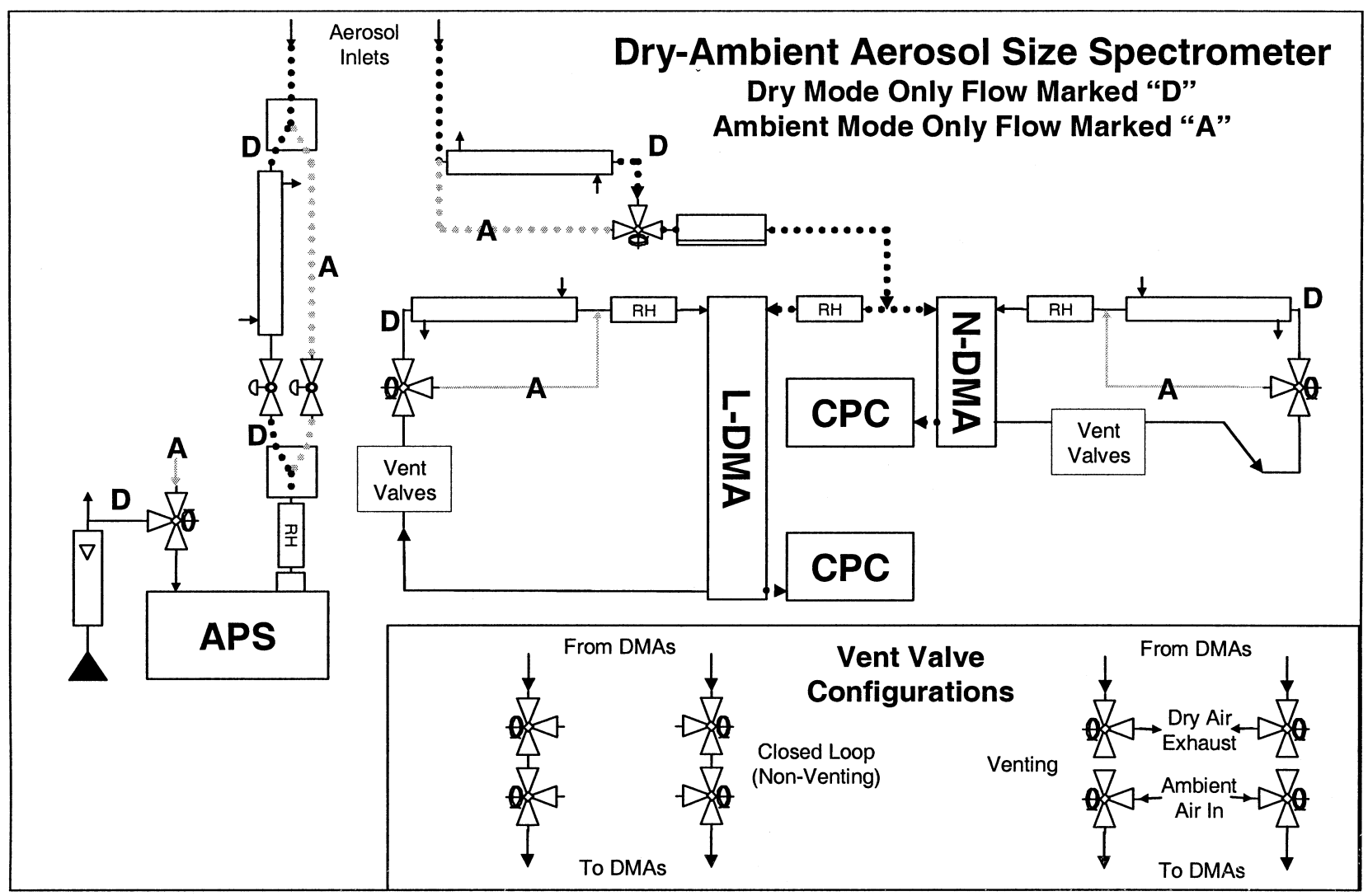

Figure 2. Dry, ambient, and vent configurations of the DAASS System. Dark flow paths labeled with "D" are only active during the dried mode. Light-colored flow paths labeled with "A" are only active in the ambient sampling mode. The third flow configuration is the venting of air from the sheath loops, shown in the lower righthand inset.

sheath air dewpoints of less than $-32^{\circ} \mathrm{C}$ were required. Figures 3 and 4 show examples of the RH cycling achieved in the field for the SMPS systems and the APS, respectively. Two ambient cycles and two dry cycles are shown. The SMPS sheath air responded quickly when the drying cycle started. The venting took longer, and the RH decreased somewhat during the ambient RH scans.

This slight mismatch in outdoor and SMPS ambient channel sheath flow RHs (evident in Figure 3) occurred during the duration of the PAQS. The mismatch was caused by insufficient purging of dry air from the system during the vent mode, which was limited by the strength of the standard sheath and bypass blowers in the TSI 3080 DMA. In future DAASS deployments, it is recommended that a supplemental vacuum be used to assist in purging the system during the vent stage. Other explanations, such as a leak at the three-way solenoid for the dryer bypass, and a positive pressure leak from the sheath- to the tube-side of the dryers, were ruled out. When the vent time was increased from 5 min to 8 min for a test in August 2001, the outdoor-ambient water content mismatch decreased.

Raw particle count data from SMPS systems, temperatures, and RHs were acquired using a PC that also controlled solenoid valves responsible for selecting the operation mode (ambient, dried, or vent). Figure 5 shows an example of dried and wet size distributions measured during $1 \mathrm{~h}$. During that hour, the DAASS measured four dried size distributions at around $14 \% \mathrm{RH}$ and 4 size distributions at $64 \% \mathrm{RH}$.

\section{CALIBRATION AND TESTING}

A number of characterization tests were performed in the laboratory. Goals during the calibration and characterization stage included inlet loss characterization, absolute sizing accuracy, sizing precision between ambient and dried inlet paths, and sizing precision when two instruments measured particles of the same size.

The APS time-of-flight response was calibrated using monodisperse aerosols prior to deployment in the field. This was done using PSL spheres (Duke Scientific, Palo Alto, CA, USA) at diameters equal to $600 \mathrm{~nm}$, and $2.1 \mu \mathrm{m}$ and with monodisperse ammonium sulfate aerosols with aerodynamic diameters from 0.5 to $1.6 \mu \mathrm{m}$. Above $2.1 \mu \mathrm{m}$, the factory time-of-flight calibration curve was used.

After the APS calibration, all three instruments of the DAASS system were checked for sizing accuracy simultaneously with monodisperse aerosols fed through a common inlet. PSL spheres 


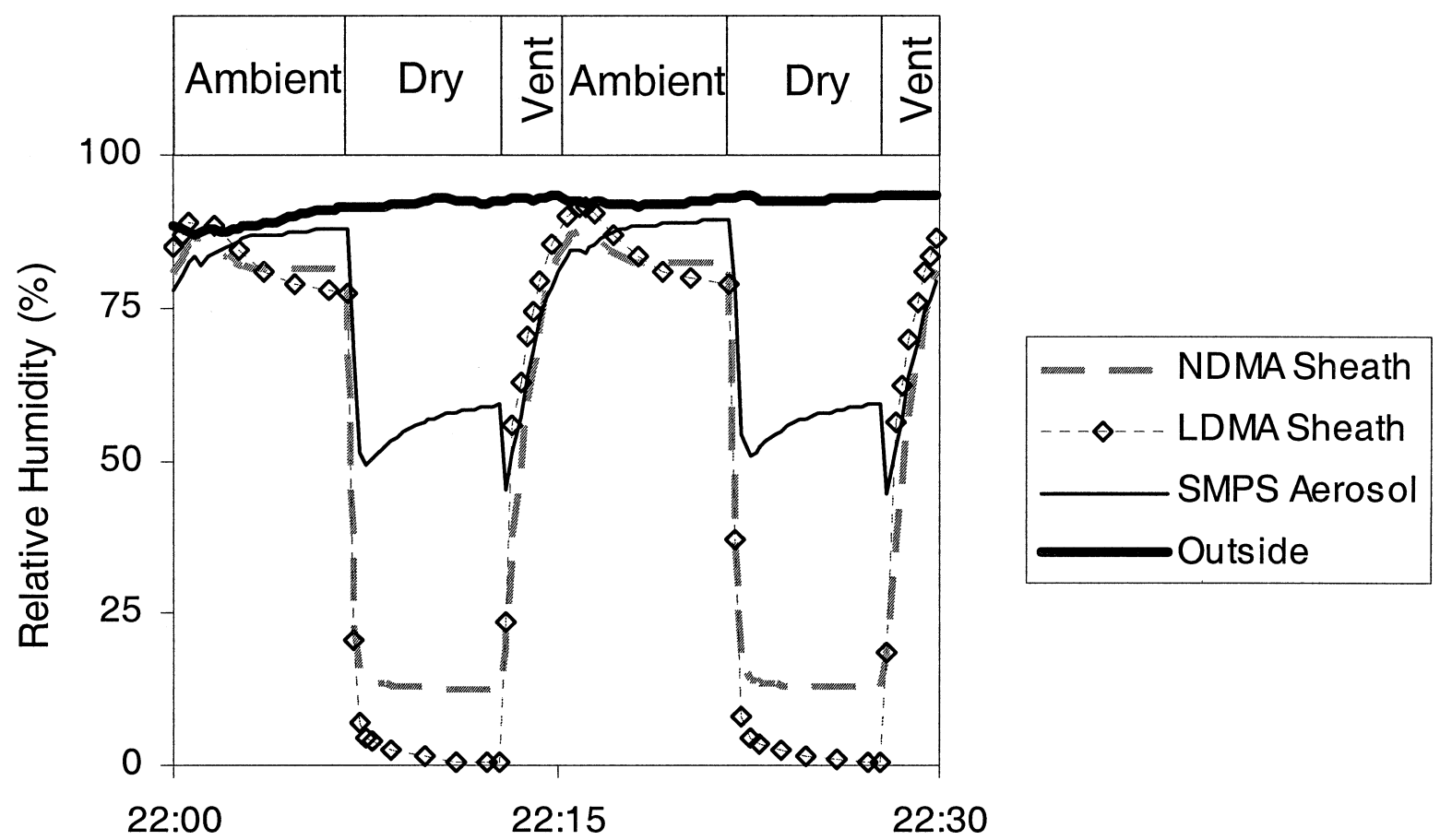

Figure 3. Example of RH time series in the SMPS instruments in DAASS. The SMPS Aerosol RH is the air sample RH before it enters the SMPS and is exposed to the sheath $\mathrm{RH}$ and further dried.

(Duke Scientific, Palo Alto, CA, USA) at diameters equal to $150 \mathrm{~nm}, 500 \mathrm{~nm}, 600 \mathrm{~nm}$, and $2.1 \mu \mathrm{m}$ were used.

The nano-SMPS system and SMPS system measurements overlapped in the diameter range from 13-80 $\mathrm{nm}$. The SMPS and APS systems overlapped from 542-680 nm (mobility equivalent size). Sizing precision between dried and ambient inlets, and sizing from instrument to instrument in overlapping size ranges, were checked by sizing monodisperse ammonium sulfate through a common inlet to the DAASS in 14 different size ranges from $20-900 \mathrm{~nm}$. Differences in particle sizing between instruments and between ambient and drying inlet channels were less than $3 \%$ across the entire size range. Size-dependent inlet losses for the SMPS inlet were determined by measuring the difference in total particle counts across the DAASS inlet (including

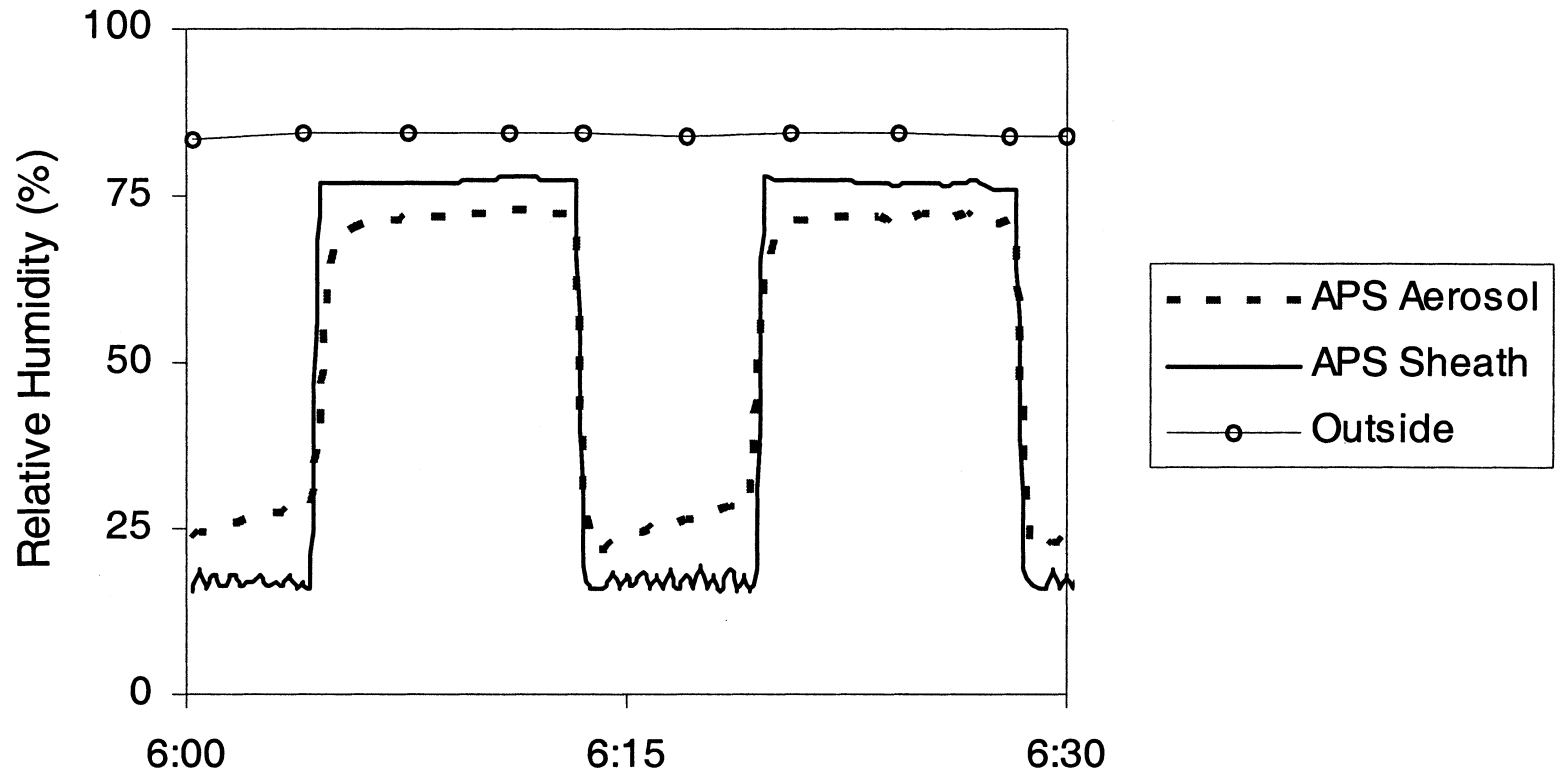

Figure 4. Example of RH time series for APS portion of DAASS. 


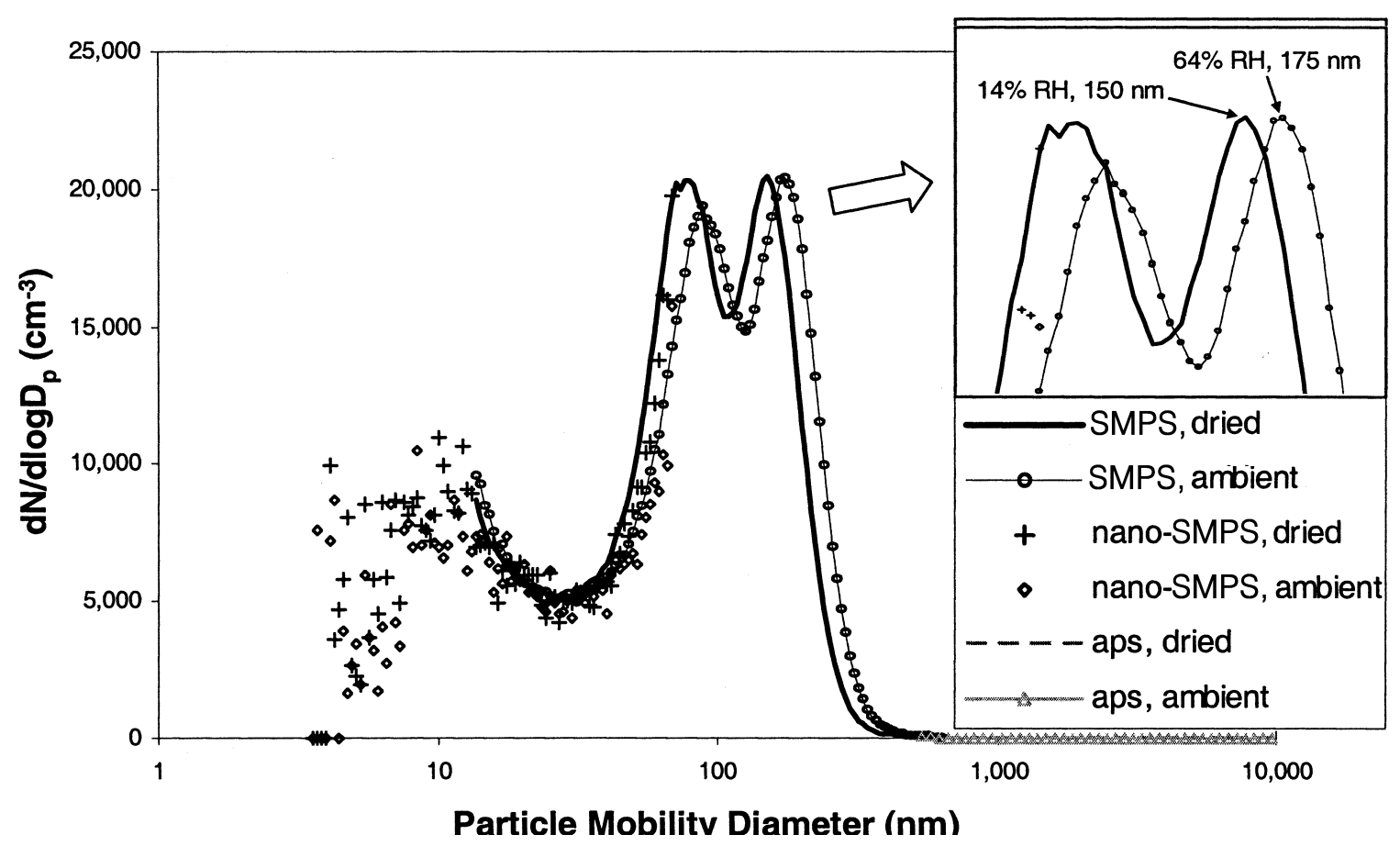

Figure 5. Dried and ambient number size distributions measured during the PAQS. Shown are one hour averages for 22:00-23:00 EST on 3 July 2001. Each of the distributions is the average of 4 ambient and 4 dried distributions.

the aerosol dryer, dryer bypass line, neutralizers, and aerosol RH probe). Particle losses below $20 \mathrm{~nm}$ were estimated using empirical particle loss correlations from Willeke and Baron (1993). Default manufacture-counting efficiencies were used for the CPC 3010 and CPC 3025.

The APS RH-conditioning inlet was designed for maximum possible particle transmission by minimization of tubing restrictions and bends. An inlet transmission efficiency was calculated accounting for turbulent inertial impaction to the inlet tubing walls with enhanced depositions at restrictions and bends (Willeke and Baron 1993). The calculated transmission was nearly $100 \%$ for $0.5 \mu \mathrm{m}$ particles and greater than $90 \%$ through $2.5 \mu \mathrm{m}$, but it fell off to $85 \%$ transmission at $5 \mu \mathrm{m}$.

APS counting efficiencies from Leinert and Weidensohler (2000) were used in data reduction and ranged from $58 \%$ for $512 \mathrm{~nm}$, to $90 \%$ at $1 \mu \mathrm{m}$, to $100 \%$ at $1.33 \mu \mathrm{m}$. The APS 3320 is known to suffer from false counts, or "ghost particles" in sizing channels greater than $2.5 \mu \mathrm{m}$ due to recirculation of small particles (Armendariz and Leith 2002). This behavior could clearly be seen in the APS 3320 data, and the reported size distributions, especially the surface area and volume distributions, are elevated, sometimes significantly, above 2.5 microns.

After all of these basic system characterizations were performed, the DAASS was used to measure diameters of hydrated and dried ammonium sulfate particles. This test was performed at the PAQS central sampling site by filling a $2 \mathrm{~m}^{3}$ teflon bag with polydisperse ammonium sulfate particles. The ammonium sulfate was drawn from the Teflon bag, through a humidifier, and into the DAASS system which sampled alternately at the elevated (hydrated) $\mathrm{RH}$ and a lower RH $(<10 \%$ sheath $\mathrm{RH})$. The results of the test are shown in Figure 6, and they show reasonable agreement with calculated ammonium sulfate growth data (Ansari and Pandis 1999).

\section{DATA REDUCTION: CALCULATION OF WATER CONTENT FROM AEROSOL SIZE DISTRIBUTIONS}

\section{Merging of Separate Size Distributions into One Size Distribution}

The raw SMPS size distributions were inverted by the TSI SMPS program (Version 3.2), and the APS distributions were inverted by the TSI Aerosol Instrument Manager program (Version 4.3). These inverted size distributions were then further corrected for counting efficiencies and inlet losses using the approach outlined in the previous section. The three different instrument distributions (nano-SMPS, SMPS, and APS) were merged to form a single size distribution for each 7.5 min sampling interval. Merging size distributions between the nano-SMPS and SMPS instruments in the overlapping region of $13-80 \mathrm{~nm}$ was accomplished by using nano-SMPS data up to $30 \mathrm{~nm}$ and then SMPS data above $30 \mathrm{~nm}$. This creates a potential discontinuity in the merged size distribution at $30 \mathrm{~nm}$. During most periods of operation, the agreement between the nano-SMPS and SMPS in the overlap region was within $10 \%$. However, when the strength of local sources varied rapidly relative to the $5 \mathrm{~min}$ 


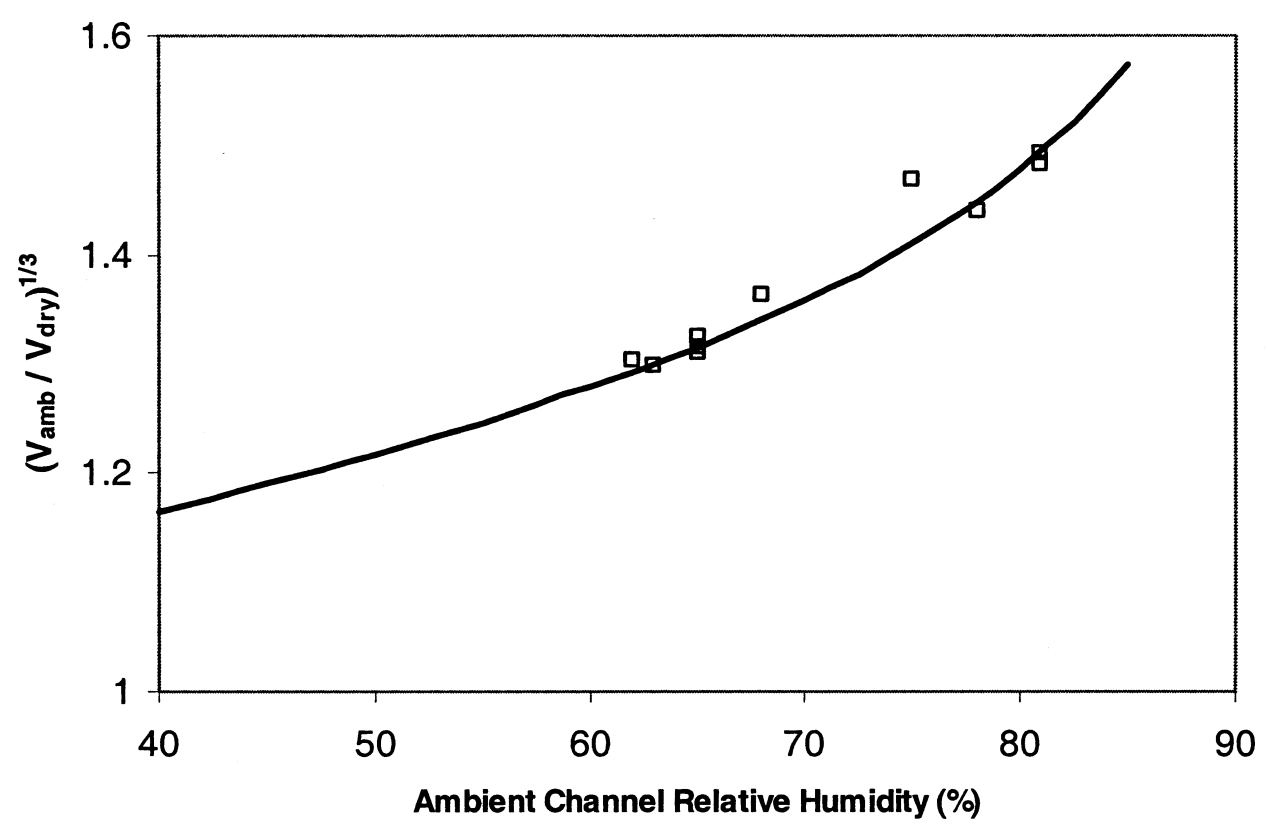

Figure 6. Theoretical (line) and measured (squares) growth factors for ammonium sulfate aerosol. Data for diameter growth factors calculated from Ansari and Pandis (1999). $V_{\mathrm{amb}}$ and $V_{\mathrm{dry}}$ are the aerosol volumes measured by the DAASS system in the ambient and dried configurations.

scan time, the agreement between the two instruments was not good on a distribution-by-distribution comparison. During periods of highly variable particle size distributions, $1 \mathrm{~h}$ averaging reduced but did not always eliminate the discontinuity in the size distribution at $30 \mathrm{~nm}$. This limits the use of the data taken during these periods for applications that depend on the shape of the number distribution between 20 and $40 \mathrm{~nm}$. However, for applications using the aerosol volume distribution, the discontinuity is not a serious issue.

Merging the SMPS and APS data was more involved due to the inherent difference between the electrical mobility measured by the SMPS and the aerodynamic diameter measured by the APS. The procedure used is explained in detail in Khlystov et al. (2004a). Briefly, an apparent density was selected to minimize the difference between the SMPS and APS number distributions in the overlap region. Thus, the shape of the APS distribution was preserved, while the $x$ axis of the APS-measured size distribution was shifted to achieve a good fit with the SMPS-measured size distribution.

\section{Calculation of Aerosol Water Content}

A number of related calculations can be performed with the size distributions measured by the DAASS. The selection among the data reduction methods depends on the application. Described below are calculations for (1) volume growth factors, (2) $\mathrm{PM}_{2.5}$ water content, (3) efflorescence branch humidigrams, and (4) mass growth factors. The first two calculations focus on measuring aerosol water at ambient RH. The third examines water content as a function of $\mathrm{RH}$, and the last is necessary for comparison of DAASS data to mass-based aerosol water correlations and models. The first three calculations are performed with data solely from the DAASS, while the fourth calculation requires aerosol composition data.

As discussed in the experimental section, the ambient channel $\mathrm{RH}$ was slightly lower than the outdoor $\mathrm{RH}$. Therefore, for the calculations presented below, it should be recognized that they are calculated and reported as a function of the ambient channel $\mathrm{RH}$ rather than the outdoor $\mathrm{RH}$.

Calculation of Volume Growth Factor. Once the size distributions are merged, hygroscopic growth factors are calculated for pairs of ambient and dried size distributions using the volume distributions and assuming a single, size-independent growth factor $\mathrm{GF}_{\mathrm{VOL}}$ :

$$
\mathrm{GF}_{\mathrm{VOL}}=\frac{V_{\mathrm{RH} 2}}{V_{\mathrm{RH} 1}}=\frac{\int_{0}^{D_{\mathrm{RH} 2}} D^{3} n_{\mathrm{N}, \mathrm{RH} 2}^{o}(\log D) d \log D}{\int_{0}^{D_{\mathrm{RH} 1}} D^{3} n_{\mathrm{N}, \mathrm{RH} 1}^{o}(\log D) d \log D},
$$

where $D$ is the particle diameter, $V_{\mathrm{RH} 2}$ and $V_{\mathrm{RH} 1}$ are the aerosol volume concentrations measured using the ambient (RH2) and dried (RH1) inlets, $n_{\mathrm{N}, \mathrm{RH} 2}^{o}$ and $n_{\mathrm{N}, \mathrm{RH} 1}^{o}$ are the ambient and dried aerosol size distributions, and $D_{\mathrm{RH} 2}$ and $D_{\mathrm{RH} 1}$ are appropriately selected limits of integration. Figure 7 shows an example of the relationship between hypothetical dried and ambient size distributions (assuming a single size-independent growth factor) and the limits of integration. For this study, focusing on the water content of the fine aerosol, an upper integration limit $D_{\mathrm{RH} 2}$ of $2.5 \mu \mathrm{m}$ was used. This limit is analogous to the size selection performed by the $\mathrm{PM}_{2.5}$ cyclone often employed in 

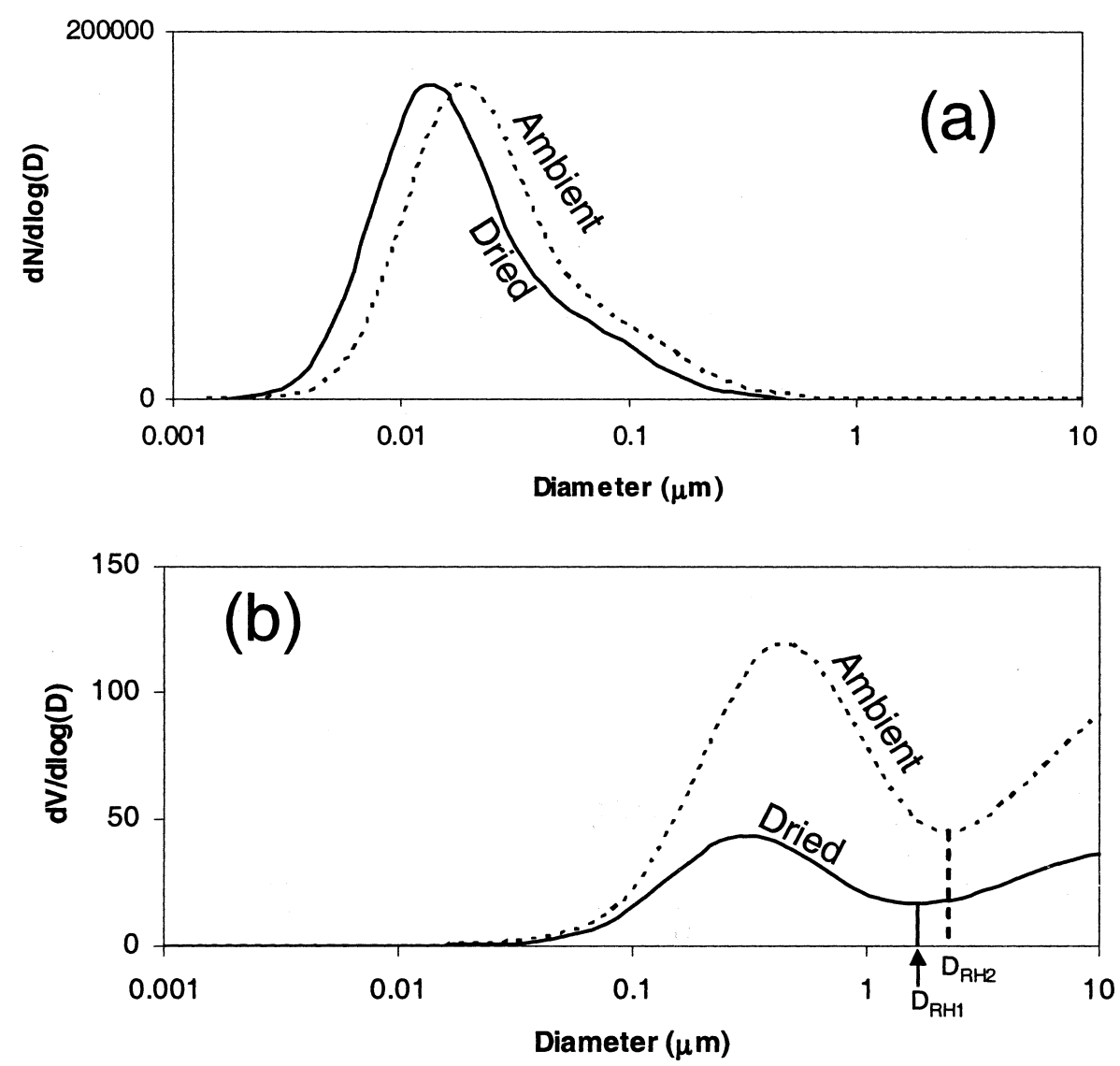

Figure 7. Illustration of the difference between dried and hydrated number and volume distributions. The figure shows a hypothetical trimodal log-normal distribution being shifted by a size-independent growth factor. The volume growth factor calculated in this study is the ratio of the volume integrals. The shifting of the upper volume integration limit with hygroscopic growth (Equation (2)) is shown by the difference between $D_{\mathrm{RH} 2}$ and $D_{\mathrm{RH} 1}$.

aerosol sampling. Using larger diameters was not possible due to the ghost particle artifact in the APS 3320 volume distributions (Armendariz and Leith 2002). The lower limit of integration is theoretically zero, but any value where there is minimal aerosol volume smaller than that size is acceptable. The integration limits for the dried and ambient size distributions are related by the volume (or diameter) growth factor, again assuming a single, size-independent growth factor:

$$
D_{\mathrm{RH} 2}=D_{\mathrm{RH} 1} \sqrt[3]{\mathrm{GF}_{\mathrm{VOL}}}
$$

Equations (1) and (2) can be solved iteratively, given any pair of size distributions to find a volume growth factor $\mathrm{GF}_{\mathrm{VOL}}$ that accounts for the differences between the ambient and dried volume distributions.

A brief discussion of the assumption of a single growth factor is instructive, as several H-TDMA investigations (Cocker et al. 2001b and sources therein) have shown that urban aerosols are usually externally mixed, with two or more populations of aerosols with different hygroscopicities. What is calculated by Equations (1) and (2) is approximately the volume-weighted average growth factor of the various externally mixed aerosol subpopulations. Interpretation of this growth factor (as a volumeweighted average) is relatively straightforward unless the limit of integration $D_{\mathrm{RH} 2}$ is inappropriately selected and a significant fraction of the particle volume lies above the limit. Simulations with log-normal externally mixed aerosol modes of different hygroscopicities show that the growth factor calculated by Equations (1) and (2) may be biased low under this circumstance. This error is expected to be small for the PAQS when an upper integration limit of $2.5 \mu \mathrm{m}$ is used, as most aerosol volume is less than $2.5 \mu \mathrm{m}$, and coarse aerosols are expected to be less hygroscopic than the accumulation mode.

As the dried and ambient aerosol distributions are not measured at the same time but are separated by some sampling interval $\Delta t$, steady increases or decreases in the aerosol volume will lead to biases in the growth factor calculated by Equation (1). This can be corrected for with the following correction factor $\beta$ :

$$
\mathrm{GF}_{\mathrm{VOL}}^{\prime}=\mathrm{GF}_{\mathrm{VOL}} \beta=\mathrm{GF}_{\mathrm{VOL}} \frac{1}{1+\frac{\mathrm{V}_{\mathrm{dry}}^{\prime}}{\mathrm{V}_{\mathrm{dry}}} \Delta t}
$$


where $\Delta t$ is the amount of time by which the dried measurements precede the ambient measurements and $V_{\text {dry }}^{\prime}$ is the rate of change of the dry aerosol volume with respect to time. In the limits where the dried and ambient measurements are performed simultaneously or the dry aerosol volume is stable with respect to time, the correction factor $\beta$ goes to unity. This factor was usually between 0.97 and 1.03 for the PAQS.

As the DAASS method relies on the difference between two aerosol size distributions measured at different times (about 7 min from the start of the ambient scan to the dried scan), variability in the underlying aerosol size distribution on a timescale of shorter than a few minutes can lead to random error in watercontent calculations. Variability in the mean and average rate of change of the dried volume for each hour is calculated and propagated through the water content calculations as uncertainty in the parameters $\mathrm{GF}_{\mathrm{VOL}}$ calculated in Equation (1) and $\beta$ calculated in Equation (2). Calculated growth factors for four days of sampling are shown in Figure 8. The time series shows that growth factors are not a simple function of RH and that they can change rapidly with changing meteorology and composition.

Calculation of PM 2.5 Water Content at Ambient Channel RH. Using the growth factor calculated above in Equation (1), the aerosol water content can be estimated. The method uses only data from the DAASS and relies on two assumptions: (1) water is the only semivolative species causing a volume change; and (2) volume additivity between aerosol water and nonvolatile aerosol components. Applying these assumptions, we can write Equation (4),

$$
V_{\mathrm{H} 2 \mathrm{O}, \mathrm{RH} 2-\mathrm{RH} 1} \cong V_{\mathrm{RH} 2}-V_{\mathrm{RH} 1},
$$

where $V_{\mathrm{H} 2 \mathrm{O}, \mathrm{RH} 2-\mathrm{RH} 1}$ is the volume of evaporated water from the ambient (RH2) channel to the dried (RH1) channel. Combining
Equations (1) and (4),

$$
V_{\mathrm{H} 2 \mathrm{O}, \mathrm{RH} 2-\mathrm{RH} 1}=\left(\mathrm{GF}_{\mathrm{VOL}}-1\right) V_{\mathrm{RH} 1},
$$

where the upper limit for integration of aerosol volume is $2.5 \mu \mathrm{m}$ for the ambient channel $\left(D_{\mathrm{RH} 2}\right)$ and the upper limit for integration of the dried distribution is given by Equation (2). If we further assume minimal residual water content at the dried $\mathrm{RH}$, then $\mathrm{V}_{\mathrm{H} 2 \mathrm{O}, \mathrm{RH} 2-\mathrm{RH} 1}$ is equal to $\mathrm{V}_{\mathrm{H} 2 \mathrm{O}, \mathrm{RH} 2}$, or the amount of aerosol water at ambient $\mathrm{RH}$. Aerosol water contents for $1 \mathrm{July}$ to 7 July 2001 calculated using this method are shown by the filled squares in Figure $9 \mathrm{~b}$ and as a fraction of total dried aerosol mass in Figure 9d. These time series correspond to ambient channel RHs shown in Figure 9a. Figure 9 shows that the DAASS system has good dynamic range in the aerosol water content measurements, ranging from less than $1 \mu \mathrm{g} / \mathrm{m}^{3}$ of water up to $20-30 \mu \mathrm{g} / \mathrm{m}^{3}$ of water and from less than $5 \%$ of the dried aerosol mass to around $100 \%$ of the dried aerosol mass. Furthermore, the time response is excellent, with experimental system and data reduction methods following rapid changes in aerosol volume, aerosol water content, and hygroscopicity.

Calculation of Efflorescence Branch Humidigrams. Aerosol water content is a function of $\mathrm{RH}$, chemical composition, and state of hydration (deliques cence branch or efflorescence branch). When aerosol water content is a smooth function of $\mathrm{RH}$ (e.g., moving on the efflorescence branch without crystallization) growth factors as a function of RH can be fit to simple empirical functions. For significant periods of the PAQS, growth factors were a smooth function of RH, with no apparent deliquescence or crystallization behavior (Khlystov et al. 2004b). Therefore, groups of growth factors representing relatively constant aerosol composition but with different RHs could be fit to an empirical function such as that used by Dick et al.

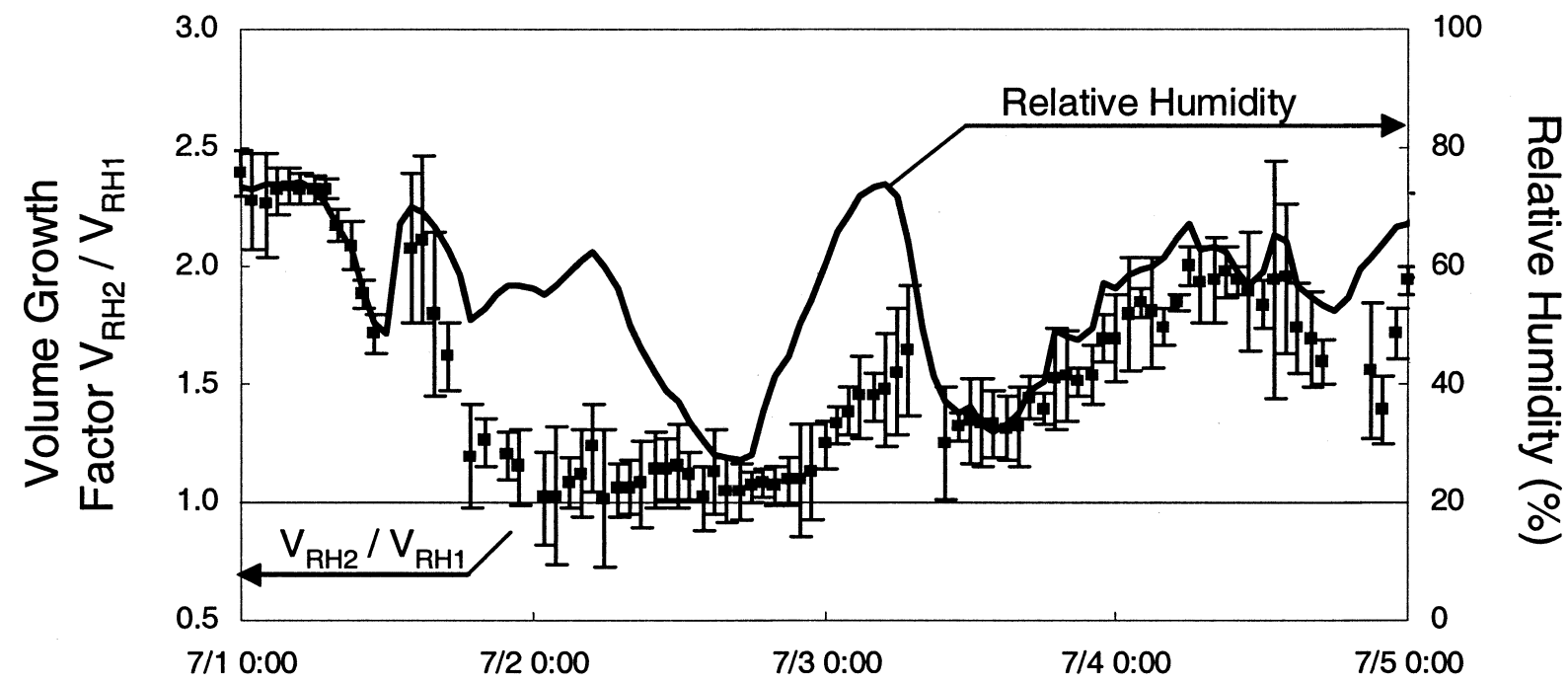

Figure 8. Sample results of volume growth factors as measured by the DAASS for 1 July to 4 July 2001 . The RH trace is the RH of the ambient channel. The RH of the dried samples was $18 \pm 6 \%$. 

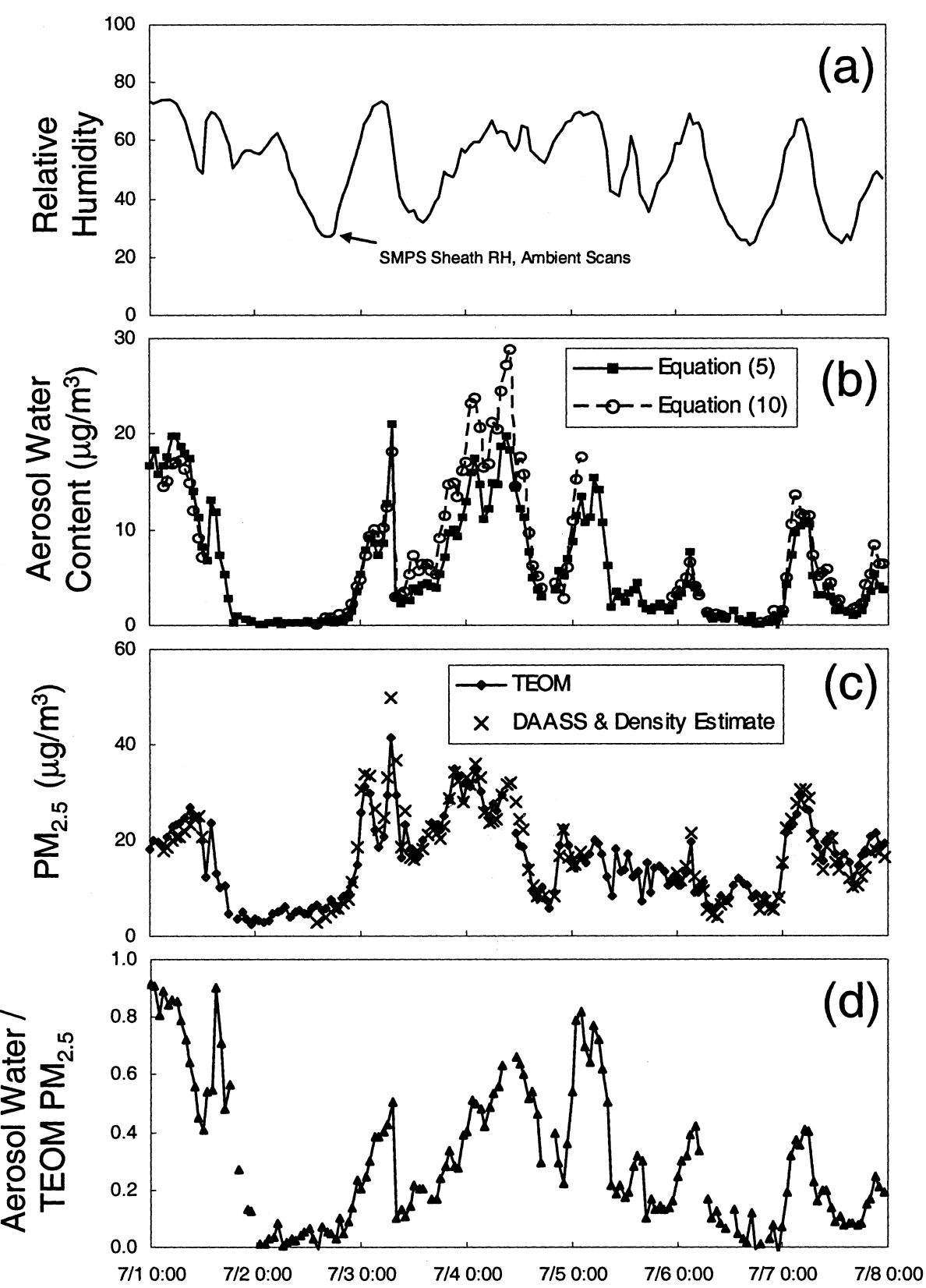

Figure 9. Example of aerosol water content measurement for a 7-day period. (a) ambient channel RH; (b) calculated mass of aerosol water by the difference of ambient and dried volume (Equation (5)) and using an estimated mass growth factor (Equation (10)); (c) $\mathrm{PM}_{2.5}$ measured by TEOM (circles and solid line) and using the DAASS measured volume and compositionbased density estimate ( $\times$ symbols); and (d) ratio of measured water to the TEOM. As noted in the experimental section, these samples are from the time period when the DMA columns were inside the enclosure, and the outdoor RH may be up to $20 \%$ higher than the ambient channel value in the figure.

(2000):

$$
\frac{V(\mathrm{RH})}{V_{\mathrm{dry}}}=1+\left(a+b \mathrm{RH}+c \mathrm{RH}^{2}\right) \frac{\mathrm{RH}}{1-\mathrm{RH}},
$$

where $a, b$, and $c$ are adjustable parameters. In conjunction with DAASS-determined volume growth factors $\mathrm{GF}_{\mathrm{VOL}}$, the param- eters $a, b$, and $c$ could be determined by regression:

$$
\mathrm{GF}_{\mathrm{VOL}}=\frac{1+\left(a+b \mathrm{RH} 2+c \mathrm{RH}^{2}\right) \frac{\mathrm{RH} 2}{1-\mathrm{RH} 2}}{1+\left(a+b \mathrm{RH} 1+c \mathrm{RH}^{2}\right) \frac{\mathrm{RH} 1}{1-\mathrm{RH} 1}} .
$$

Examples of the humidigrams calculated using groups of measured growth factors are shown in Figure 10. The difference in 


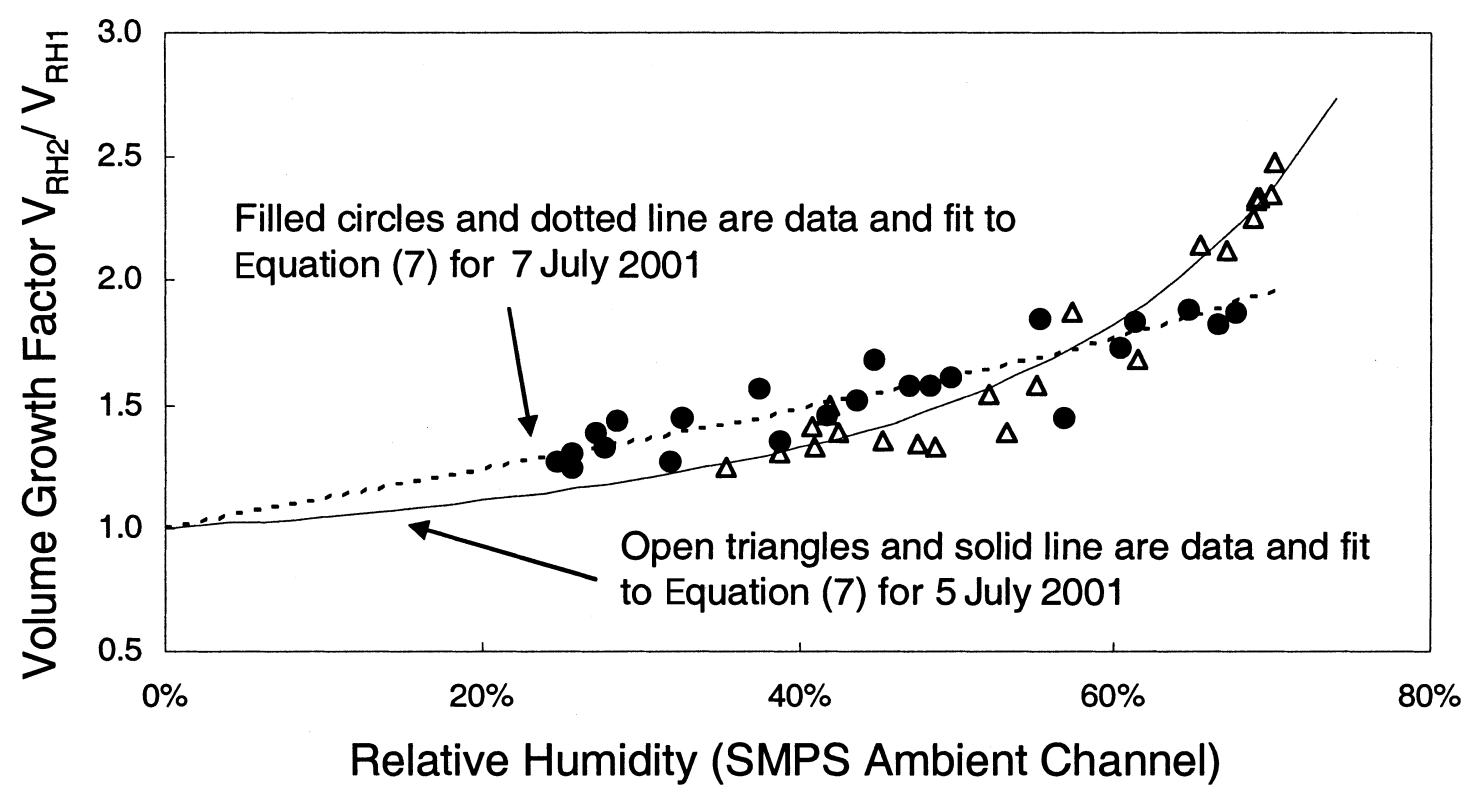

Figure 10. Example of humidigrams for two different days (5 and 7 July 2001). Points are measured hourly volume growth factors, and lines are fits of the hourly data to Equation (7).

the humidigrams between these two time periods is probably related to the aerosol chemistry, which contained more organic matter (52 wt \%) on 7 July than on 5 July (38 wt \%).

In certain applications, it is necessary to calculate aerosol water content at a specific RH. For example, DAASS data has been used to help estimate residual aerosol water in filter-based samples equilibrated at RHs from 15-35\% (Rees et al. 2003). The calculation of residual water is performed using Equation (6), subject to the assumptions discussed above.

Calculation of the Mass Growth Factor. For comparison of volume growth factors to models and correlations involving mass growth factors, it can be useful to convert the DAASSmeasured volume growth factors to mass growth factors. To do this we need to estimate the density of the aerosols as a function of RH using aerosol composition information from sources other than the DAASS instrument. The equations involved in the conversion from the volume to mass growth factor, and a simple method for density estimation during the PAQS, are developed below.

The volume and mass growth factors are related by Equation (8),

$$
\mathrm{GF}_{\mathrm{MASS}}=\mathrm{GF}_{\mathrm{VOL}} \frac{\rho_{\mathrm{RH} 2}}{\rho_{\mathrm{RH} 1}},
$$

where $\rho_{\mathrm{RH} 2}$ and $\rho_{\mathrm{RH} 1}$ are the hydrated (RH2) and dried (RH1) densities.

With this mass-based growth factor in hand, a mass-based estimate of aerosol water content can be made and compared to the volume-based calculation in Equation (5). The remainder of this section first covers the aerosol water mass balance, and then methods for aerosol density estimation for the PAQS are developed.

This definition of the mass growth factor can be used to write the aerosol mass balance (assuming water is the only semivolatile species that partitions as RH changes). If $\mathbf{M}_{\text {dry }}$ is the completely dehydrated aerosol mass, $\mathrm{M}_{\mathrm{H} 2 \mathrm{O}, \mathrm{RH} 2-\mathrm{RH} 1}$ is the water released from the aerosol as it dries from ambient $\mathrm{RH}\left(\mathrm{RH}_{2}\right)$ to the dried channel RH (RH1), and $\mathrm{M}_{\mathrm{H} 2 \mathrm{O}, \mathrm{RH} 1}$ is the residual aerosol water at the dried $\mathrm{RH}(\mathrm{RH} 1)$, then

$$
\begin{aligned}
M_{\mathrm{RH} 2} & =M_{\mathrm{dry}}+M_{\mathrm{H} 2 \mathrm{O}, \mathrm{RH} 2-\mathrm{RH} 1}+M_{\mathrm{H} 2 \mathrm{O}, \mathrm{RH} 1} \\
& =\left(M_{\mathrm{dry}}+M_{\mathrm{H} 2 \mathrm{O}, \mathrm{RH} 1}\right) \mathrm{GF}_{\mathrm{MASS}},
\end{aligned}
$$

where $\rho_{\mathrm{RH} 2}$ and $\rho_{\mathrm{RH} 1}$ are the hydrated (RH2) and dried (RH1) densities. $M_{\mathrm{H} 2 \mathrm{O}, \mathrm{RH} 2-\mathrm{RH} 1}$ is the water released from the aerosol as it dries from ambient $\mathrm{RH}(\mathrm{RH} 2)$ to the dried channel $\mathrm{RH}$ (RH1). Conceptually, there are three possibilities for the behavior of the aerosol water content as the aerosol is dehydrated. At the low RH all particles are dry, or they all have some water, or some of them lose water and some do not. The first case corresponds to $M_{\mathrm{H} 2 \mathrm{O}, \mathrm{RH} 1}$ equal to zero. The other two cases correspond to nonzero values for $M_{\mathrm{H} 2 \mathrm{O}, \mathrm{RH} 1}$. The DAASS instrument gives the measurement of $M_{\mathrm{H} 2 \mathrm{O}, \mathrm{RH} 2-\mathrm{RH} 1}$. Since RH1 is kept as low as possible, $M_{\mathrm{H} 2 \mathrm{O}, \mathrm{RH} 1}$ is expected to be small compared to $M_{\mathrm{H} 2 \mathrm{O}, \mathrm{RH} 2-\mathrm{RH} 1}$. Assuming $M_{\mathrm{H} 2 \mathrm{O}, \mathrm{RH} 1}$ is negligible, $M_{\mathrm{H} 2 \mathrm{O}, \mathrm{RH} 2}$ is then given by Equation (10):

$$
\begin{aligned}
M_{\mathrm{H} 2 \mathrm{O}, \mathrm{RH} 2} & \approx M_{\mathrm{dry}}\left(\mathrm{GF}_{\mathrm{MASS}}-1\right) \\
& =M_{\mathrm{dry}}\left(\mathrm{GF}_{\mathrm{VOL}} \frac{\rho_{\mathrm{RH} 2}}{\rho_{\mathrm{RH} 1}}-1\right) .
\end{aligned}
$$


The density calculations assume that the aerosol is an internal mixture consisting of inorganic matter, organic matter, and elemental carbon fractions, each with a fixed characteristic density. The dry density is calculated assuming volume additivity and that $\mathrm{M}_{\mathrm{H} 2 \mathrm{O}, \mathrm{RH} 1}$ is negligible:

$$
\rho_{\mathrm{RH} 1}=\left(\frac{f_{\mathrm{OM}}}{\rho_{\mathrm{OM}}}+\frac{f_{\mathrm{EC}}}{\rho_{\mathrm{EC}}}+\frac{f_{\text {inorg,dry }}}{\rho_{\text {inorg,dry }}}\right)^{-1},
$$

where $f$ refers to mass fraction: $\rho$ to density; and the subscripts $\mathrm{OM}, \mathrm{EC}$, and inorg, dry refer to organic matter, elemental carbon, and dry inorganic mass, respectively. The ambient humidity density is estimated assuming the aerosol consists of three fractions, a hydrated inorganic fraction, an elemental carbon fraction (assumed not to take up any water), and an organic fraction (assumed not to take up any aerosol water):

$$
\rho_{\mathrm{RH} 2}=\left(\frac{f_{\mathrm{OM}}}{\rho_{\mathrm{OM}}}+\frac{f_{\mathrm{EC}}}{\rho_{\mathrm{EC}}}+\frac{f_{\text {inorg, } \mathrm{dry}}+f_{\mathrm{H} 2 \mathrm{O}, \mathrm{RH} 2}}{\rho_{\text {inorg, wet }}}\right)^{-1},
$$

where $\rho_{\text {inorg, wet }}$ refers to the density of the hydrated inorganics.

For this work the $\mathrm{PM}_{2.5}$ measured by a Tapered Element Oscillating Microbalance (TEOM, Rupprecht \& Patashnick, Albany, NY) at $30^{\circ} \mathrm{C}$ with a sample equilibration system (Meyer et al. 2000) was used for $M_{\text {dry }}$. The mass measured by the instrument was in good agreement with the federal reference method for particulate mass during these tests (Rees et al. 2004). The sample equilibration system is a semipermeable membrane drying inlet for the TEOM that conditions the aerosol sample at $30^{\circ} \mathrm{C}$ and $\sim 15 \% \mathrm{RH}$. The organic matter mass used for calculations in this study was 1.8 times the organic carbon (OC). Elemental carbon (EC) and OC were measured by the thermal optical method (Cabada et al. 2002). The 1.8 multiplier for carbonaceous mass is based on estimates of Turpin and Lim (2001). Densities of 1.2, 1.6, and $1.77 \mathrm{~g} / \mathrm{cm}^{-3}$ were assumed for organic matter, elemental carbon aerosol, and dry inorganic aerosol, respectively. The organic matter density is based on Turpin and Lim (2001) and the dry inorganic mass corresponds to that of ammonium sulfate, the dominant inorganic component in the Pittsburgh area (Anderson et al. 2002). The elemental carbon density is most uncertain, as recent measurements show great variability in the effective density $\left(0.2-1.6 \mathrm{~g} / \mathrm{cm}^{3}\right)$ of soot agglomerates depending on formation conditions (Park et al. 2003 and references therein). However, because of the small contribution of EC to the total aerosol concentration in Pittsburgh, the specific value chosen has little effect on the volume to mass growth factor conversion in this study. The $1.6 \mathrm{~g} / \mathrm{cm}^{3}$ value used in this work is at the upper end of effective densities measured by Park et al. (2003) and at the lower end of the range for the physical density of graphite (Perry et al. 1984). The carbonaceous fraction is assumed to not take up any water in this work, and the hydrated density for ammonium sulfate, calculated using empirical values from Tang (1997), is used for the ambient RH inorganic density.

The main use of the Equations (8)-(12) is for comparison of experimental (volume-based) growth factors with mass-based growth factors from other sources (i.e., thermodynamic models). The comparison can be made on the basis of predicted versus measured aerosol water content or on the basis of predicted versus measured growth factors. In both cases, Equations (8)(12) will be required to make the comparison. While this work does not contain any mass-based water content measurements or model predictions (see Khlystov et al. 2004b for modelmeasurement comparisons), this type of calculation is demonstrated by the open circles in Figure 9b, which are an estimate of aerosol water content from Equation (10) based on the DAASSmeasured volume growth factors, estimates of aerosol density using Equations (11) and (12), and the time series of $\mathrm{M}_{\text {dry }}$ measured independently of the DAASS system. The difference between the water content calculation by Equations (5) and (10) can be attributed to (1) uncertainty in the density calculations, composition, and organic mass multiplier; (2) violations of assumptions such as nonhygroscopic organics and negligible water content in the dried channel; and (3) drifts in the absolute accuracies of the various independent instruments that are involved in making the volume-based aerosol calculations (SMPS \& APS) and the mass-based calculations (TEOM, OC/EC sampler, and inorganic ions sampler). The mismatch is particularly evident during 3 and 4 July. In light of these possible errors and uncertainties, Equation (5) is recommended as a more robust estimate of aerosol water content than Equation (10). A further demonstration of the connection between aerosol mass, aerosol volume, and composition-based density estimates (all measured independently) is shown by the comparison in Figure $9 \mathrm{c}$ of $M_{\text {dry }}$ measured by two independent techniques. The TEOM mass measurement is compared to the DAASS-estimated aerosol mass calculated using the density formula in Equation (11).

\section{Discussion of Some Sources of Error}

The following errors are discussed in this section: (1) charging of the aerosol at different RHs than classification; (2) potential for crystallization during water due to heated zones of inlet; and (3) differences in RH between the SMPS aerosol and sheath flows.

Differences in RH between the bipolar aerosol charger, aerosol flow entering the DMA, and DMA sheath flow can cause changes in the physical state of the particles and errors in particle sizing and/or counting. First, the aerosol may be charged in the bipolar charger while at a different RH (and therefore size) than in the DMA. Second, heated zones of the inlet prior to the classifier (which is at ambient temperature) may induce crystallization of the aerosol. Finally, the difference in the aerosol and sheath flows may cause a size change during size classification. The implications of these errors are discussed below.

Parts of the aerosol inlet (including the bipolar charger) were inside the enclosure, which was usually a few degrees warmer than the DMA column, located outside the enclosure. This caused the RH of the aerosol flow during ambient scans to go through a minimum inside the enclosure, at the time of aerosol charging. In winter, the enclosure (and bipolar charger) was 
Table 1

Estimates in growth factor bias due to charging under dryer conditions than classification

\begin{tabular}{lccc}
\hline \multirow{2}{*}{$\begin{array}{c}\text { Outside RH } \\
(\%)\end{array}$} & $5^{\circ} \mathrm{C}(\%)$ & $0^{\circ} \mathrm{C}(\%)$ & $(\%)-5^{\circ} \mathrm{C}$ \\
\cline { 2 - 4 } 50 & +3 & +3 & +3 \\
70 & +2 & +6 & +6 \\
85 & +4 & +6 & +10 \\
92 & +7 & +10 & +14 \\
\hline
\end{tabular}

maintained at $9^{\circ} \mathrm{C}$, while outdoor temperatures dropped to approximately $-5^{\circ} \mathrm{C}$. As a result of these two effects, in general the aerosol charging was not at the same RH as the size classification in the DMA. For hydrated particles, this means that aerosols may be charged at one size and then classified at another, creating an error in the equilibrium charge distribution assumed during the inversion of the raw SMPS data. This generally leads to an underestimation in the particle number at sizes smaller than about $200 \mathrm{~nm}$, and an overestimation at sizes greater than about $200 \mathrm{~nm}$. The magnitude of this error was estimated using the study average size distribution and an assumed composition of $50 \%$ ammonium sulfate and $50 \%$ organics and other nonhygroscopic material. Volume growth factors are biased high by a factor of 1.00 to 1.14 , with the greatest effects at low temperatures and humid conditions. Our error estimates are shown in Table 1.

The higher inlet temperatures compared to ambient caused temporary drying of the ambient channel aerosol before it was returned to ambient temperature for size classification. During the coldest periods of the study, this drying effect could be important. Therefore, the minimum RH experienced by the aerosol en route to classification is calculated and reported with the PAQS data to allow a more complete interpretation of the results.

Sheath-aerosol stream mismatch should not pose a problem as the thermodynamic equilibration time is expected to be short compared to the transit times in the DMAs $(0.3 \mathrm{~s}$ and $6 \mathrm{~s}$ for the N-SMPS and SMPS, respectively). Characteristic times for equilibration of pure water droplets up to 1 micron in size under the conditions in the SMPS should be of order $10^{-3} \mathrm{~s}$. There is evidence from laboratory studies that coatings of hydrophobic organics over salts (5-60 wt\% organics) may hinder mass transfer (Wagner et al. 1996; Xiong et al. 1998), although the extension of these laboratory studies to atmospheric particles has not been established. To test the assumption that the sheathaerosol RH mismatch did not significantly affect the results, an experiment was performed in 2002 during the PAQS where the SMPS aerosol was dried more thoroughly $(\sim 20 \% \mathrm{RH})$ than usual ( $\sim 50 \% \mathrm{RH})$ while the sheath RH was held at around $5 \%$. The organic fraction of the aerosol was between 30 and $50 \%$ on the day of the experiment, and the outdoor RH was between 85 and $95 \%$. No shift in the dried aerosol size distribution could be detected due to the lower RH and increased time for mass transfer at low RH.

\section{SUMMARY AND CONCLUSION}

A system has been constructed for the in situ measurement of aerosol water content at atmospheric conditions. The system relies on a combination of aerosol sizing instruments and samples the atmospheric aerosol at ambient temperature and at two RHs. The DAASS measures the aerosol volume growth factor (ratio of the ambient and dried aerosol volume concentrations) and aerosol water content every $15 \mathrm{~min}$. A variety of data reduction procedures were presented to convert the raw data to growth factors and aerosol water content, and (when combined with aerosol composition measurements) to facilitate comparison with mass-based growth factors from other sources. DAASS operated for a year during the PAQS.

The system was tested with ammonium sulfate particles equilibrated at different RHs, and its results were in good agreement with the known hygroscopic properties of the particles. The preliminary field results show good response to RH and dry particle mass with the water concentration varying from almost zero to more than $20 \mu \mathrm{g} / \mathrm{m}^{3}$ (0-50\% of the hydrated particle mass). The detailed measurements of water during PAQS and the comparison of these measurements with predictions of theoretical models are discussed in detail in a companion article (Khlystov et al. 2004b).

\section{REFERENCES}

Anderson, R. R., Martello, D. V., Rohar, P. C., Stazisar, B. R., Tamilia, J. P., Waldner, K., and White, C. M. (2002). Sources and Composition of $\mathrm{PM}_{2.5}$ at the National Energy Technology Laboratory in Pittsburgh During July and August 2000, Energy Fuels 16:261-269.

Ansari, A. S., and Pandis, S. N. (1999). Prediction of Multicomponent Inorganic Atmospheric Aerosol Behavior, Atmos. Environ. 33:745-757.

Ansari, A. S., and Pandis, S. N. (2000). Water Absorption by Secondary Organic Aerosol and Its Effect on Inorganic Aerosol Behavior, Environ. Sci. Technol. 34:71-77.

Armendariz, A. J., and Leith, D. (2002). Concentration Measurement and Counting Efficiency for the Aerosol Particle Sizer 3320, J. Aerosol Sci. 33:133-148.

Berg, O. H., Swietlick, E., and Krejci, R. (1998). Hygroscopic Growth of Aerosol Particles in the Marine Boundary Layer Over the Pacific and Southern Oceans During the First Aerosol Characterization Experiment, J. Geophys. Res. 103(D13):16,535-16,545.

Brooks, S., Wise, M., Cushing, M., and Tolbert, M. (2002). Deliquescence Behavior of Organic/Ammonium Sulfate Aerosol, Geophys. Res. Lett. 29:1917, DOI: $10.1029 / 2002$ GL014733.

Cabada, J. C., Pandis, S. N., and Robinson, A. L. (2002). Sources of Atmospheric Carbonaceous Particulate Matter in Pittsburgh, Pennsylvania, J. Air Waste Manage. 52(6):732-741.

Chan, C. K., Flagan, R. C., and Seinfeld, J. H. (1992). Water Activities of Ammonium Nitrate/Ammonium Sulfate Solutions, Atmos. Environ. 26A(9):16611673 .

Clegg, S. L., Seinfeld, J. H., and Brimblecombe, P. (2001). Thermodynamic Modeling of Aqueous Aerosols Containing Electrolytes and Dissolved Organic Compounds, J. Aerosol Sci. 32(6):713-738.

Cocker, D. R., III, Clegg, S. L., Flagan, R. C., and Seinfeld, J. H. (2001a). The Effect of Water on Gas-Particle Partitioning of Secondary Organic Aerosol. Part I: $\alpha$-Pinene/Ozone System, Atmos. Environ. 35:6049-6072. 
Cocker, D. R., III, Mader, B. T., Kalberer, M., Flagan, R. C., and Seinfeld, J. H. (2001b). The Effect of Water on Gas-Particle Partitioning of Secondary Organic Aerosol: II. m-Xylene and 1,3,5-Trimethylbenzene Photooxidation Systems, Atmos. Environ. 35:6073-6085.

Cocker, D. R., III, Whitlock, N. E., Flagan, R. C., and Seinfeld, J. H. (2001c). Hygroscopic Properties of Pasadena, California Aerosol, Aerosol Sci. Technol. 35:637-647.

Cruz, C. N., and Pandis, S. N. (2000). Deliquescence and Hygroscopic Growth of Mixed Inorganic-Organic Atmospheric Aerosol, Environ. Sci. Technol. 34:4313-4319.

Day, D. E., Malm, W. C., and Kreidenweis, S. M. (2000). Aerosol Light Scattering Measurements as a Function of Relative Humidity, J. Air Waste Manage. 50:710-716.

Dick, W. D., Saxena, P., and McMurry, P. H. (2000). Estimation of Water Uptake by Organic Compounds in Submicron Aerosols Measured During the Southeastern Aerosol and Visibility Study, J. Geophys. Res. 105(D1):1471-1479.

Han, J., and Martin, S. T. (1999). Heterogeneous Nucleation of the Efflorescence of $\left(\mathrm{NH}_{4}\right)_{2} \mathrm{SO}_{4}$ Particles Internally Mixed with $\mathrm{Al}_{2} \mathrm{O}_{3}, \mathrm{TiO}_{2}$, and $\mathrm{ZrO}_{2}$, J. Geophys. Res. 104:3543-3554.

Han, J.-H., Hung, H.-M., and Martin, S. T. (2002). Size Effect of Hematite and Corundum Inclusions on the Efflorescence Relative Humidities of Aqueous Ammonium Nitrate Particles, J. Geophys. Res. 107, DOI: 10.1029/2001JD001054.

Hanel, G. (1976). The Properties of Atmospheric Aerosol Particles as Functions of Relative Humidity at Thermodynamic Equilibrium with Surrounding Moist Air. In Advances in Geophysics, Vol. 19, H. E. Landsberg and J. Van Mieghem, eds., Academic Press, New York, pp. 73-188.

Khlystov, A., Stanier, C., and Pandis, S. N. (2004a). An Algorithm for Combining Electrical Mobility and Aerodynamic Size Distribution Data when Measuring Ambient Aerosol, Aerosol Sci. Technol. 38:229-238.

Khlystov, A., Stanier, C., Takahama, S., and Pandis, S. N. (2004b). Water Content of Ambient Aerosols During the Pittsburgh Air Quality Study, J. Geophys. Res. Submitted.

Kreisberg, N. M., Stolzenburg, M. R., and Hering, S. V. (2001). A New Method for Measuring the Dependence of Particle Size Distributions on Relative Humidity, with Application to the Southeastern Aerosol and Visibility Study, J. Geophys. Res. 106:14,935-14,949.

Lee, C. T., and Hsu, W. C. (1998). A Novel Method to Measure Aerosol Water Mass, J. Aerosol Sci. 29(7):827-837.

Leinert, S., and Wiedensohler, A. (2000). APS Counting Efficiency Calibration for Submicrometer Particles, J. Aerosol Sci. 31:S404-S405.

Martin, S. T., Han, J., and Hung, H. M. (2001). The Size Effect of Hematite and Corundum Inclusions on the Efflorescence Relative Humidities of Aqueous Ammonium Sulfate Particles, Geophys. Res. Lett. 28:2601-2604.

Meyer, M. B., Patashnick, H., Ambs, J. L., and Repprecht, E. (2000). Development of a Sample Equilibration System for the TEOM Continuous PM Monitor, J. Air Waste Manage. 50(8):1345-1349.

McMurry, P. H., and Stolzenberg, M. (1989). On the Sensitivity of Particle Size to Relative Humidity for Los Angeles Aerosols, Atmos. Environ. 23(2):497507.

Ming, Y., and Russell, L. M. (2002). Thermodynamic Equilibrium of OrganicElectrolyte Mixtures in Aerosol Particles, AICHE Journal 48:1331-1348.

Ohta, S., Hori, M., Yamagata, S., and Murao, N. (1998). Chemical Characterization of Atmospheric Fine Particles in Sapporo with Determination of Water Content, Atmos. Environ. 32(6):1021-1025.

Onasch, T. B., Siefert, R. L., Brooks, S. D., Prenni, A. J., Murray, B., Wilson, M. A., and Tolbert, M. A. (1999). Infrared Spectroscopic Study of the Deli- quescence and Efflorescence of Ammonium Sulfate Aerosol as a Function of Temperature, J. of Geophys. Res. 104(D17): 21,317-21,326.

Park, K., Cao, F., Kittelson, D., and McMurry, P. H. (2003). Relationship Between Particle Mass and Mobility for Diesel Exhaust, Environ. Sci. Technol. 37:577-583.

Peng, C., Chan, M. N., and Chan, C. K. (2001). The Hygroscopic Properties of Dicarboxylic and Multifunctional Acids: Measurements and UNIFAC Predictions, Environ. Sci. Technol. 35(22):4495-4501.

Perry, R. H., Green, D. W., and Maloney, J. O. (1984). Perry's Chemical Engineering Handbook, 6th ed., McGraw Hill: New York, pp. 3-96.

Rader, D. J., and McMurry, P. H. (1986). Application of the Tandem Differential Mobility Analyzer to Studies of Droplet Growth or Evaporation, J. Aerosol Sci. 17(5):771-787.

Rees, S. L., Robinson A. L., Khlystov, A., Stanier, C. O., Pandis, S. N. (2004). Mass Balance Closure and the Federal Reference Method for $\mathrm{PM}_{2.5}$ in Pittsburgh, Pennsylvania, Atmos. Environ. in press.

Rood, M. J., Covert, D. S., and Larson, T. V. (1987). Temperature and Humidity Controlled Nephelometry: Improvements and Calibration, Aerosol Sci. Technol. 7:57-65.

Saxena, P., and Hildemann, L. M. (1997). Water Absorption by Organics: Survey of Laboratory Evidence and Evaluation of UNIFAC for Estimating Water Activity, Environ. Sci. Technol. 31:3318-3324.

Speer, R. E., Barnes, H. M., and Brown, R. (1997). An Instrument for Measuring the Liquid Water Content of Aerosols, Aerosol Sci. Technol. 27(1): $50-61$.

Tang, I. N., and Munkelwitz, H. R. (1993). Composition and Temperature Dependence of the Deliquescence Properties of Hygroscopic Aerosols, Atmos. Environ. 27A:467-473.

Tang, I. N. (1997). Thermodynamic and Optical Properties of MixedSalt Aerosols of Atmospheric Importance, J. Geophys. Res. 102:18831893.

ten Brink, H. M., Khlystov, A., Kos, G. P. A., Tuch, T., Roth, C., and Kreyling, W. (2000). High-Flow Humidograph for Testing the Water Uptake by Ambient Aerosol, Atmos. Environ. 34:4291-4300.

Turpin, B., and Lim, H. (2001). Species Contributions to $\mathrm{PM}_{2.5}$ Mass Concentrations: Revisiting Common Assumptions for Estimating Organic Mass, Aerosol Sci. Technol. 35:602-610.

Vartiainen, M., McDow, S. R., and Kamens, R. M. (1994). Water Uptake by Aerosol Particles from Automobile Exhaust and Wood Smoke, Chemosphere 29:1661-1669.

Virkkula, A., Van Dingenen, R., Raes, F., and Hjorth, J. (1999). Hygroscopic Properties of Aerosol Formed by Oxidation of Limonene, $\alpha$-Pinene, and $\beta$-Pinene, J. Geophys. Res. 104(D3):3569-3579.

Wagner, J., Andrews, E., and Larson, S. M. (1996). Sorption of Vapor Phase Octanoic Acid Onto Deliquescent Salt Particles, J. Geophys. Res. 101(D14):19,533-19,540.

Willeke, K., and Baron, P. A. (1993). Aerosol Measurement Principles, Techniques, and Applications. Van Nostrand Reinhold: New York.

Woo, K. S., Shi, Q., Sakurai, H., and McMurry, P. H. (2001). A Relative Humidity Conditioner for Atmospheric Sampling. Presented at the American Association of Aerosol Research. Portland, Oregon, October 2001.

Xiong, J. Q., Zhong, C. F., Chen, L. C., and Lippmann, M. (1998). Influence of Organic Films on the Hygroscopicity of Ultrafine Sulfuric Acid Aerosol, Environ. Sci. Technol. 32:3536-3541.

Yi, M., and Russell, L. M. (2002). Thermodynamic Equilibrium of Organic-Electrolyte Mixtures in Aerosol Particles. AIChE J. 48(6):13311348. 


\title{
Characterizing Physical Processes
}

\section{Nucleation Events During the Pittsburgh Air Quality Study: Description and Relation to Key Meteorological, Gas Phase, and Aerosol Parameters}

\author{
Charles O. Stanier, Andrey Y. Khlystov, and Spyros N. Pandis \\ Department of Chemical Engineering, Carnegie Mellon University, Pittsburgh, Pennsylvania
}

During the Pittsburgh Air Quality Study (PAQS) aerosol size distributions between $3 \mathrm{~nm}$ and $680 \mathrm{~nm}$ were measured between July 2001 and June 2002. These distributions have been analyzed to assess the importance of nucleation as a source of ultrafine particles in Pittsburgh and the surrounding areas. The analysis shows nucleation on $50 \%$ of the study days and regional-scale formation of ultrafine particles on $30 \%$ of the days. Nucleation occurred during all seasons, but it was most frequent in fall and spring and least frequent in winter. Regional nucleation was most common on sunny days with below average $\mathbf{P M}_{2.5}$ concentrations. Local nucleation events were usually associated with elevated $\mathrm{SO}_{2}$ concentrations. The observed nucleation events ranged from weak events with only a slight increase in the particle number to relatively intense events with increases of total particle counts between $50,000 \mathrm{~cm}^{-3}$ up to $150,000 \mathrm{~cm}^{-3}$. Averaging all days of the study, days with nucleation events had number concentrations peaking at around noon at about $45,000 \mathrm{~cm}^{-3}$. This is compared to work days without nucleation, when the daily maximum was 8 am at $23,000 \mathrm{~cm}^{-3}$, and to weekends without nucleation, when the daily maximum was at noon at $16,000 \mathrm{~cm}^{-3}$. Twenty-four-hour average number concentrations were approximately $40 \%$ higher on days with nucleation compared to those without. Nucleation was typically observed starting around 9 am EST, although the start of nucleation events was later in winter and earlier in summer. The nucleation events are fairly well correlated with the product of [UV intensity $* \mathrm{SO}_{2}$ concentration] and also depend on the effective area available for condensation.

Received 23 October 2002; accepted 5 March 2003.

This research was conducted as part of the Pittsburgh Air Quality Study, which was supported by the US Environmental Protection Agency under contract R82806101 and the US Department of Energy National Energy Technology Laboratory under contract DE-FC2601NT41017. The authors also wish to thank Hiromu Sakurai, Peter McMurry, and the Minnesota Particle Technology Laboratory for their help with the ultrafine CPC testing.

Address correspondence to Spyros Pandis, Department of Chemical Engineering, Carnegie Mellon University, 5000 Forbes Ave., Pittsburgh, PA 15213, USA. E-mail: spyros@andrew.cmu.edu
This indicates that $\mathrm{H}_{2} \mathrm{SO}_{4}$ is a component of the new particles. Published correlations for nucleation by binary $\mathrm{H}_{2} \mathrm{SO}_{4}-\mathrm{H}_{2} \mathrm{O}$ cannot explain the observed nucleation frequency and intensity, suggesting that an additional component (perhaps ammonia) is participating in the particle formation.

\section{INTRODUCTION}

The creation of new particles by homogenous nucleation of gas-phase atmospheric components is an important atmospheric process. Together with primary particle emission, nucleation is responsible for maintaining the number concentration of particles throughout the atmosphere. Nucleation affects climate and visibility by changing the size distribution of airborne particles (Charlson et al. 1987; Kulmala et al. 2000). The formation of ultrafine particles and the condensation of secondary aerosol components on them may impact human health, as ultrafine particles are likely to cause adverse health effects disproportionate to their mass (Oberdoster et al. 1995; Schwartz et al. 1996; Peters et al. 1997).

Until recently, nucleation was assumed to be limited to clean areas of the atmosphere such as the free troposphere. However, a number of recent studies at both rural and urban sites around the world have reported frequent nucleation events (Allen et al. 1999; Harrison et al. 1999a, b; Harrison et al. 2001; Shi et al. 2001; Woo et al. 2001). Woo et al. (2001) found elevated levels of 3-10 nm particles in Atlanta, with highest frequencies in spring and summer. Birmili et al. (2001) measured elevated ultrafine concentrations in continental Germany in April as well. In both the German and Atlanta studies, nucleation occurred around midday with concentrations of $\mathrm{NO}_{\mathrm{x}}$ elevated prior to many of the nucleation events and $\mathrm{SO}_{2}$ elevated during the events (Woo et al. 2001).

Atmospheric homogeneous nucleation has been the subject of many theoretical and experimental studies. It is recognized 
that there are two important steps to the production of new particles that can grow to detectable size (Zhang and Wexler 2002; Kerminen 1999). The first step is the formation of an initial nucleus, and the second step is the growth of the particles to larger sizes. A number of mechanisms have been proposed as candidates for the initial nucleus formation step based on observations and theoretical considerations, including (1) homogeneous binary nucleation of sulfuric acid and water (Weber et al. 1999); (2) homogeneous ternary nucleation of ammonia-water-sulfuric acid (Eisele and McMurry 1997; Kulmala et al. 2001; O’Dowd et al. 1999); (c) homogenous nucleation of low vapor pressure organic compounds (O'Dowd et al. 2002); (d) ion-induced nucleation (Kim et al. 2002). The second step in forming detectable new particles, growth, is also uncertain. These particles can grow by condensation of sulfuric acid or by self-coagulation. Both of these processes are relatively inefficient, and additional growth mechanisms have been proposed (Kerminen 1999). The limited experimental evidence indicates a potential role for organic compounds (Novakov and Penner 1993; Rivera-Carpio et al. 1996). Recent work considers the potential for heterogenous reactions of $\mathrm{SO}_{2}$ (Kerminen 1999) and organic compounds (Kerminen 1999; Jang and Kamens 2001; Zhang and Wexler 2002) to significantly contribute to growth.

Steps toward a better understanding of tropospheric nucleation include: (a) elucidation of the mechanism responsible for the initial nuclei formation in different environments; (b) identification of the chemical compounds responsible for growth; (c) determination of the geographic scope, frequency, strength, and impact of tropospheric nucleation.

The goal of this work is to describe the nucleation events observed during the Pittsburgh Air Quality Study (PAQS) from July 2001 to June 2002. Analysis of the particle size distributions during this period shows over 100 days with nucleation activity. The gas, aerosol phase concentrations, and meteorological conditions associated with nucleation in Pittsburgh are also discussed.

Identification of initial nuclei and condensing species chemistry is ongoing and will be discussed in a subsequent article.

\section{EXPERIMENTAL}

All measurements were conducted as part of the PAQS, a multidisciplinary air pollution study designed to characterize fine particulate matter around Pittsburgh, evaluate next-generation aerosol monitoring instrumentation, elucidate source-receptor relationships, and improve understanding of atmospheric processes governing aerosol concentrations.

The bulk of the gas and particle measurements discussed in this article were conducted at the main PAQS sampling location in a park $5 \mathrm{~km}$ east (downwind) of downtown Pittsburgh (Figure 1). Two SMPS systems (TSI 3936L10 and TSI 3936N25) were operated at this location continuously. These instruments measured the size distribution of particles from $3 \mathrm{~nm}$ to $680 \mathrm{~nm}$. The SMPS systems were a part of the dry-ambient aerosol sizing system (DAASS) (Stanier et al. 2002) and were specially configured to alternate between ambient RH samples and dried samples. The differential mobility analyzers (DMAs) and most of the inlet tubing were kept at near ambient temperature to avoid volatilization of aerosols. Portions of the inlet were maintained at above $9^{\circ} \mathrm{C}$ at all times because they shared an enclosure with the condensation particle counters (CPCs). This sampling location was $0.5 \mathrm{~km}$ from the nearest major city street and $1.1 \mathrm{~km}$ from the nearest highway. A small coal-fired heating plant operated $0.8 \mathrm{~km}$ from the site. Its plume impacted the sampling site occasionally but did not typically contain nuclei mode particles.

Another SMPS system (TSI 3071/3010) was located $38 \mathrm{~km}$ upwind from the main site in Florence, Pennsylvania during part of winter and spring 2002. The SMPS was sampling dried aerosol size distributions in the size range $12-280 \mathrm{~nm}$. The data from the rural site were used to asses the regional homogeneity of the events.

Other instruments deployed as part of the PAQS and used to understand nucleation in this work include: $\mathrm{O}_{3}, \mathrm{SO}_{2}, \mathrm{NO}_{2} / \mathrm{NO}$, and $\mathrm{CO}$ monitors, a tapered element oscillating microbalance (TEOM), solar radiation, and met station. For PAQS, $10 \mathrm{~min}$ averaged $\mathrm{PM}_{2.5}$ data were used from the TEOM. The TEOM operated at $30^{\circ} \mathrm{C}$ to minimize volatilization of nitrate and organic compounds, and the sample equilibration system was used to dry the aerosol stream prior to the mass measurement. The complete list of instrumentation used to generate the data set for nucleation events is listed in Table 1.

\section{RESULTS AND DISCUSSION}

Around 50\% of the study days (July 2001 to June 2002) were characterized by nucleation events. Of these, about $60 \%$ showed a characteristic growth pattern from the nuclei mode to 30 $100 \mathrm{~nm}$ over the course of several hours. During the other $40 \%$ of the nucleation events the sub-10 nm particles did not appear to grow to larger sizes. The events varied in intensity from weak increases in the ultrafine and nuclei mode particle counts to intense events which increased the overall number concentration from $10,000-20,000$ per $\mathrm{cm}^{3}$ to over 100,000 per $\mathrm{cm}^{3}$ in a few hours. As an example of the contrast between days with and without nucleation, Figure 2 depicts the size distributions measured during 10 August and 11 August 2001. Figures 2a and b show evolution of the size distributions, while Figure $2 \mathrm{c}$ indicates the integrated particle concentration. On 10 August there was no detectable nucleation activity, while an intense nucleation event was observed around 9 AMEST on 11 August, followed by rapid growth of the particles to a size around $100 \mathrm{~nm}$. Missing size distributions in Figure 2 correspond to maintenance of the particlesizing instruments in some cases, and data flagged as invalid in other cases. Data was typically flagged invalid due to occasional communication errors between the data acquisition system and the particle-sizing instruments. 


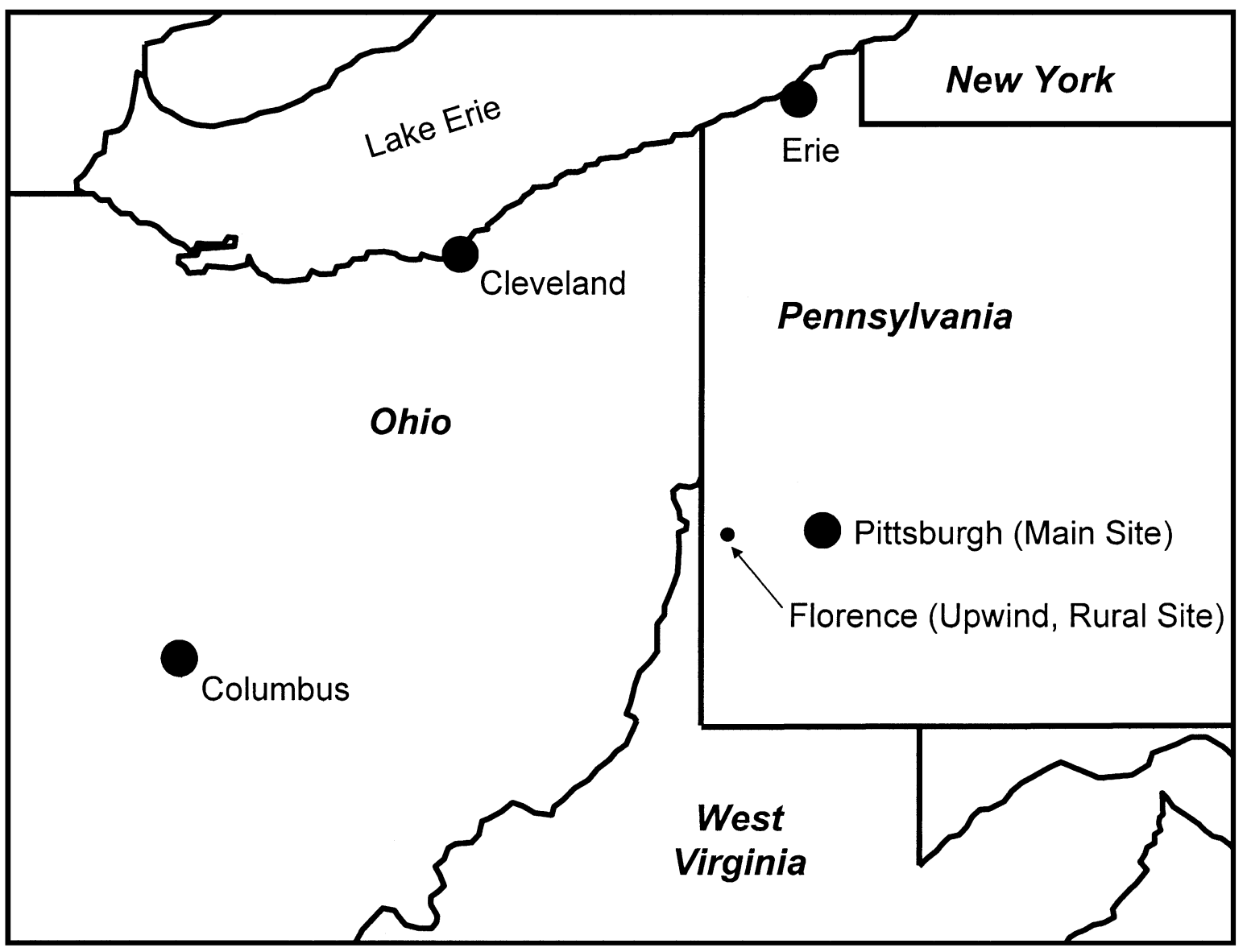

Figure 1. Map of Pittsburgh (main urban site) and Florence (rural upwind site).

\section{Classification of Events}

The size distributions from the study were first analyzed to determine the frequency of nucleation events. The analysis was done by examining the evolution of size distributions on each day of the study and various time series, including those of total number concentration, nuclei mode number concentration, aerosol mass (TEOM), meteorological parameters, and gas phase concentrations of $\mathrm{CO}, \mathrm{NO}, \mathrm{NO}_{2}, \mathrm{O}_{3}$, and $\mathrm{SO}_{2}$.

The most important marker for nucleation was a significant increase in the nuclei mode particle count, defined as particles from the lower detection limit of $3 \mathrm{~nm}$ up to $10 \mathrm{~nm}$, referred to as $N_{10}$ in this work. Once a significant increase in $N_{10}$ was seen in the particle count time series, additional characteristics of the data were examined to rule out primary particle sources, such as vehicular traffic, which also produce particles smaller than $10 \mathrm{~nm}$. Traffic was fairly easy to screen out, as it usually has a weaker signal than most nucleation events. Diurnally averaged number concentrations during nucleation days and nonnucleation weekdays and weekends are shown in Figure 3. The mode of the traffic-related size distribution was consistently $15-20 \mathrm{~nm}$, with most of the contribution to $N_{10}$ between 6 and $10 \mathrm{~nm}$. The traffic influence usually increased $N_{10}$ from a back- ground level of around $2,000 \mathrm{~cm}^{-3}$ to an early morning level of $7,500 \mathrm{~cm}^{-3}$ (Figure 3a). Finally, traffic was usually correlated with $\mathrm{NO}$ and $\mathrm{CO}$ and independent of solar radiation.

Once vehicular traffic was ruled out as the cause of a particular increase in $N_{10}$, the event was classified as a "short-lived" event, or a "regional" event following the approach by Shi (2003). Regional events were characterized by an increase in $N_{10}$ followed by the growth of the nuclei mode to larger sizes, such as that shown in Figure 2 for 11 August. These growth events, lasting several hours, are called regional because throughout the day different air parcels are arriving at the site. If nucleation happened at a given time but was confined to a small area close by the site, the growing mode would disappear once air parcels began arriving at the site from outside the nucleation zone. The other group, classified as "short-lived" events, were characterized by an increase and then a decrease in $N_{10}$, but without the growth of the nuclei mode to larger sizes. These increases in $N_{10}$ were often shorter than $1 \mathrm{~h}$ and correlated with $\mathrm{SO}_{2}$, indicating local plumes. An example of a short-lived event is shown in Figure 4.

Alternately, a regional event interrupted by precipitation, significant change in wind direction, or front would be classified as a short-lived event. 
Table 1

Instruments used to examine nucleation events in this work

\begin{tabular}{|c|c|c|}
\hline Measurement & Instrument & Notes \\
\hline \multicolumn{3}{|c|}{ Main supersite sampling location, Schenley Park, Pittsburgh (urban site)—Sampled 7/1/01-9/30/02 } \\
\hline Particle size distribution, $3-80 \mathrm{~nm}$ & TSI 3085 DMA/TSI 3025A CPC & 5 min upscan; 8 scans per hour \\
\hline Particle size distribution, $13-680 \mathrm{~nm}$ & TSI 3081 DMA/TSI 3010 CPC & 5 min upscan; 8 scans per hour \\
\hline Particle size distribution, $0.5-10 \mu \mathrm{m}$ & TSI 3320 APS & Operated 7/1/01-10/26/01 \\
\hline Particle size distribution, $0.5-10 \mu \mathrm{m}$ & TSI 3321 APS & Operated 5/26/02-9/30/02 \\
\hline $\mathrm{PM}_{2.5}$ mass & $\mathrm{R} \& \mathrm{P} 1400 \mathrm{~A}$ & $\begin{array}{l}\text { Running at } 30^{\circ} \mathrm{C} \text { with sample } \\
\text { equilibration system }\end{array}$ \\
\hline Ozone & API $400 \mathrm{~A}$ & \\
\hline $\mathrm{NO} \& \mathrm{NO}_{\mathrm{x}}$ & API $200 \mathrm{~A}$ & \\
\hline $\mathrm{SO}_{2}$ & API $100 \mathrm{~A}$ & \\
\hline $\mathrm{CO}$ & API $300 \mathrm{~A}$ & \\
\hline Wind speed & MetOne 014A & \\
\hline Wind direction & MetOne 024A & \\
\hline Precipitation & MetOne 370 & \\
\hline Temperature and RH & Campbell HMP45C & \\
\hline Barometric pressure & Campbell CS105 & \\
\hline Downwelling broadband radiation & Kipp \& Zonen CM3 Pyranometer & \\
\hline \multirow[t]{2}{*}{ Downwelling UV radiation } & Kipp \& Zonen CUV3 UV & \\
\hline & Pyranometer & \\
\hline \multicolumn{3}{|c|}{ Upwind sampling location, Florence, PA (rural site) } \\
\hline Particle size dstribution, $12 \mathrm{~nm}-280 \mathrm{~nm}$ & TSI 3071 DMA \& 3010 CPC & Sampled 2/24/02-3/28/02 \\
\hline
\end{tabular}

Regional nucleation events were further classified as "weak," "moderate," and "strong," depending on the net rate of increase in $N_{10}$ during the first hour of the event. The divisions were as follows: $d N_{10} / d t<4,000 \mathrm{~cm}^{-3} \mathrm{~h}^{-1}$ was classified as weak, $d N_{10} / d t$ from $4,000-15,000 \mathrm{~cm}^{-3} \mathrm{~h}^{-1}$ was classified as moderate, and $d N_{10} / d t>15,000 \mathrm{~cm}^{-3} \mathrm{~h}^{-1}$ was classified as strong nucleation. It should be noted that $d N_{10} / d t$ is not the nucleation rate, typically defined as the number of nuclei clusters growing larger than $1 \mathrm{~nm}$. Rather, this is a rough measure of the intensity of the event and also of its impact on the particle number and size distribution in the region.

One final challenge in distinguishing nucleation from primary particle emission was the observation of growth events, beginning with particles larger than $6 \mathrm{~nm}$, rather than the expected situation of nucleation accompanied by particles down to the instrument detection limit of $3 \mathrm{~nm}$. In these cases, the increase in $N_{10}$ was rather modest and difficult to distinguish from the increase in $N_{10}$ associated with traffic. In all other respects, the events look like the other nucleation events, including an increase in $N_{50}$ (number concentration of particles smaller than $50 \mathrm{~nm}$ ) significantly above levels seen on days without nucleation and the characteristic growth of the new mode to 30 $100 \mathrm{~nm}$ in size. An explanation for these events is that nucleation is occurring near the sampling site but not directly at the site, and the particles travel to the site while growing and coagulating. Another possible explanation is that these are primary particles from vehicle emissions that are growing by condensation, and that the increase in $N_{50}$ is explained by an increased lifetime of primary particles due to their larger sizes. Our results support the former explanation for two reasons. First, the sources of primary particles in the 10-20 $\mathrm{nm}$ size range are mainly vehicles in and around the city of Pittsburgh. The size of the grown primary particles as sampled would depend on the source distance from the site, the condensational growth rate, and the wind speed. Since the sources are at a constant distance from the site, and the condensational growth rate varies with photochemical activity, one would expect an initial increase in the size of the grown primary particles, and then a decrease later in the day. Such behavior was not observed. The second reason is that the observed increases in $N_{50}$ for stable wind directions are too rapid to be accounted for by increased lifetimes of particles as they grow in size. Therefore, these events have been classified as nucleation events.

Figure 5 shows the overall frequency of days with nucleation activity from July 2001 to June 2002 according to the classification scheme described above. The overall nucleation frequency, counting all event types, was $53 \%$, or 181 of 345 study days. The regional nucleation events occurred during $31 \%$ of the study days. Regional nucleation seemed to be more frequent during the spring and fall, and less active in summer and winter. Table 2 summarizes key gas phase, meteorological, and aerosol variables during strong, regional nucleation events.

\section{Spatial Scale of Nucleation}

The spatial scale of nucleation was investigated by operating an SMPS in Florence, Pennsylvania (Figure 1) during parts of 


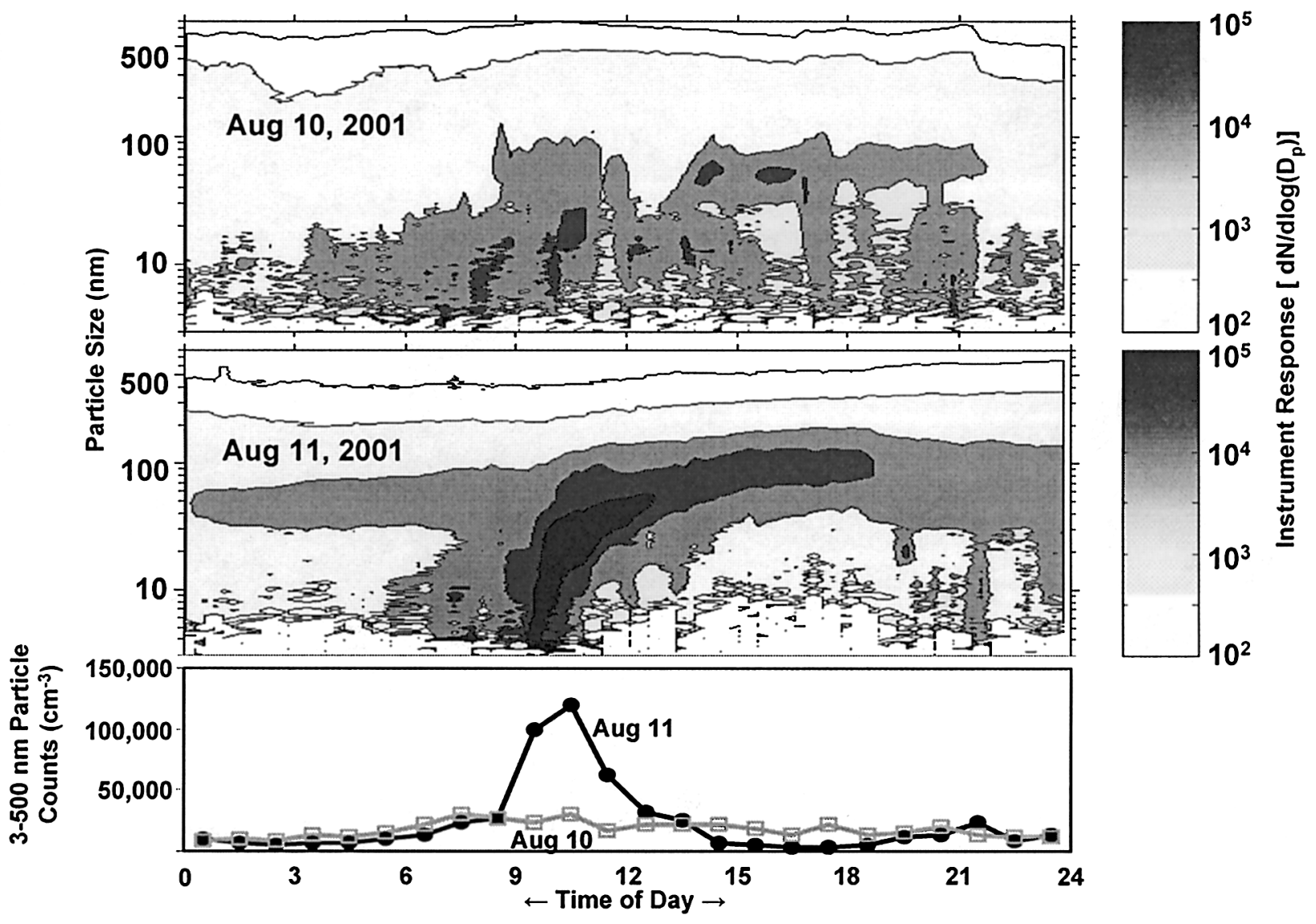

Figure 2. Evolution of particle size distributions and particle concentration on a day without nucleation (10 August) and a day with nucleation (11 August). The top two plots show instrument response over all size channels. The bottom plot shows the integrated particle concentration time series. Nucleation is apparent at 9 am EST on 11 August.

February and March of 2002. This site was $38 \mathrm{~km}$ to the west of the main site. Although the lower limit of the size distributions was at $10 \mathrm{~nm}$, the growth portion of the nucleation events was clearly evident and coincided in time well with the events at the main site (Figure 6). On other days of the study, no nucleation occurred at both sites. Of the 34 days of parallel sampling, all of the stronger nucleation events except one happened at both sites at nearly the same time. Only five days show qualitative disagreement between the two sites, usually with weak nucleation at one site but not the other.

\section{Conditions Favorable to Nucleation}

Nucleation occurred most frequently on sunny days with below-average $\mathrm{PM}_{2.5}$ concentrations (Table 2). This often occurred on days after the passage of a cold front through the area with subsequent high pressure and clear skies. Although this general pattern held, no simple relationship between sunlight, preexisting aerosols, and nucleation was identified. During summer, regional nucleation was mostly associated with light northwesterly winds, while in fall and winter it was mostly associated with stronger southwesterly winds. During spring, the wind direction for nucleation was highly variable.
The hypothesis that these events are due to sulfuric acid nucleation was explored by correlating nucleation activity and $\mathrm{H}_{2} \mathrm{SO}_{4}$ production. This correlation, based on the ideas of Pirjola et al. (1999) and Wexler et al. (1994), was developed to see how well the observed nucleation events, both short-lived and regional, could be explained in terms of condensation and nucleation of $\mathrm{H}_{2} \mathrm{SO}_{4}$. As neither $\mathrm{OH}$ nor $\mathrm{H}_{2} \mathrm{SO}_{4}(\mathrm{~g})$ were measured during PAQS, the product of ultraviolet light and $\mathrm{SO}_{2}$ was used as a surrogate parameter for $\mathrm{H}_{2} \mathrm{SO}_{4}$ production. The condensational sink, CS, was calculated from the measured size distribution at near-ambient relative humidity using the formula

$$
\mathrm{CS}=\int_{3 \mathrm{~nm}}^{500 \mathrm{~nm}} D_{p} \beta\left(D_{p}\right) n\left(D_{p}\right) d D_{p},
$$

where $\beta$ is the transitional correction factor (Fuchs and Sutugin $1970), D_{p}$ is particle diameter, and $n\left(D_{p}\right)$ is the measured number size distribution. The condensational sink is only integrated through $500 \mathrm{~nm}$ because the size distributions extending beyond this size are not available for all time periods of the study. Therefore, this is a low estimate of the actual condensational sink, especially when aged aerosols are sampled. 

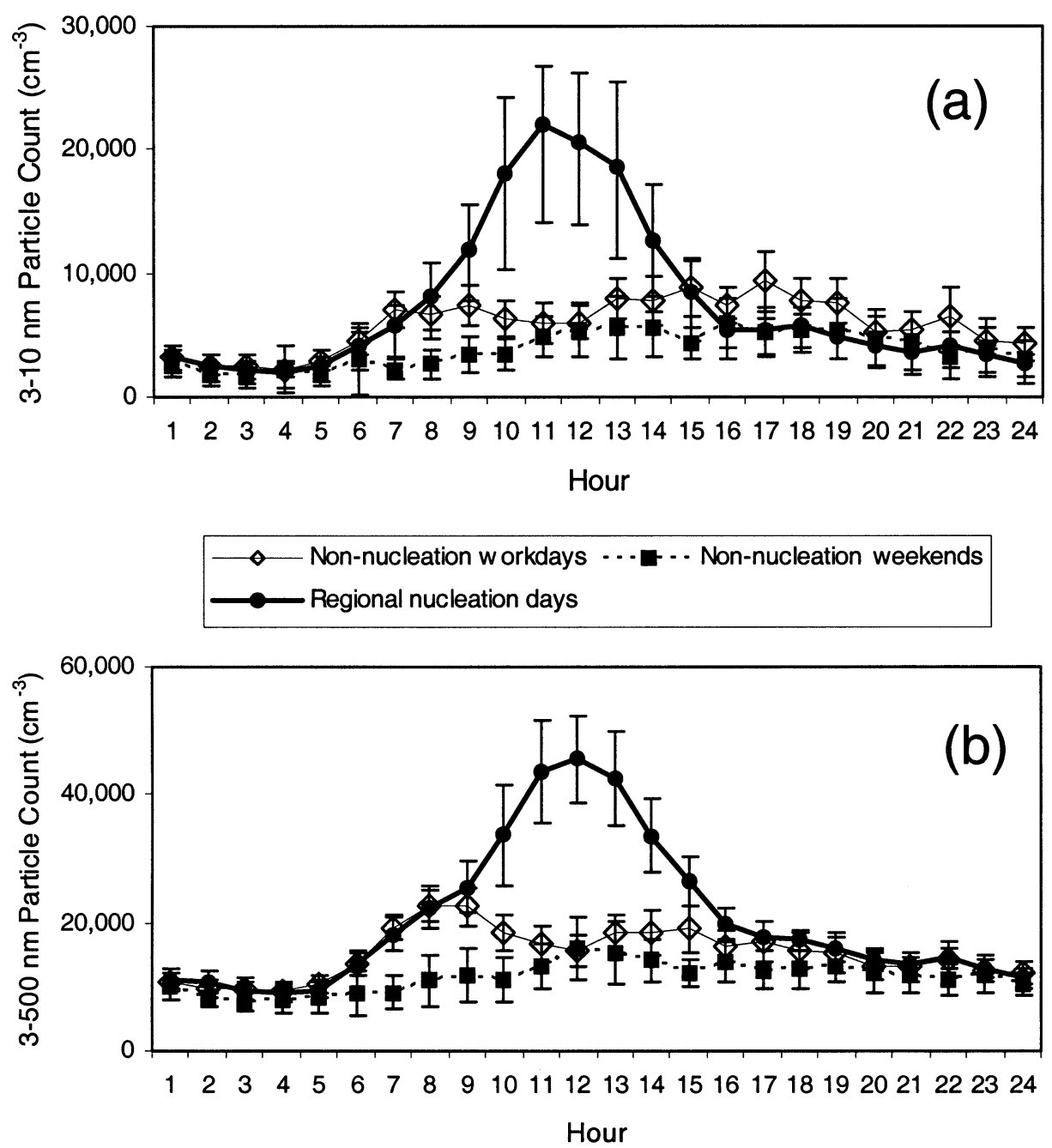

Figure 3. Diurnal averaged number concentrations, July to November 2001. Error bars signify the $95 \%$ confidence interval on the mean: (a) particles $3-10 \mathrm{~nm}, N_{10}$, and (b) particles smaller than $500 \mathrm{~nm}, N_{500}$.

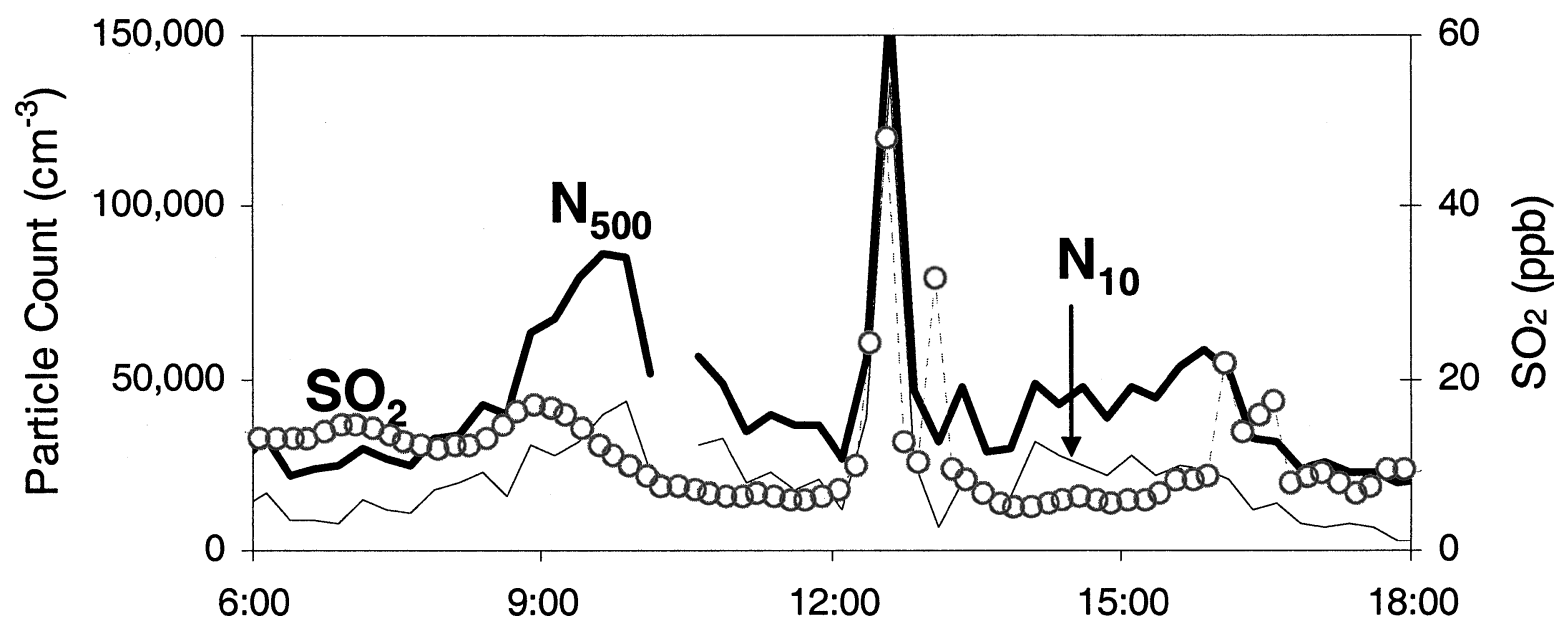

Figure 4. Example of a short-lived nucleation event on 5 July 2001. 


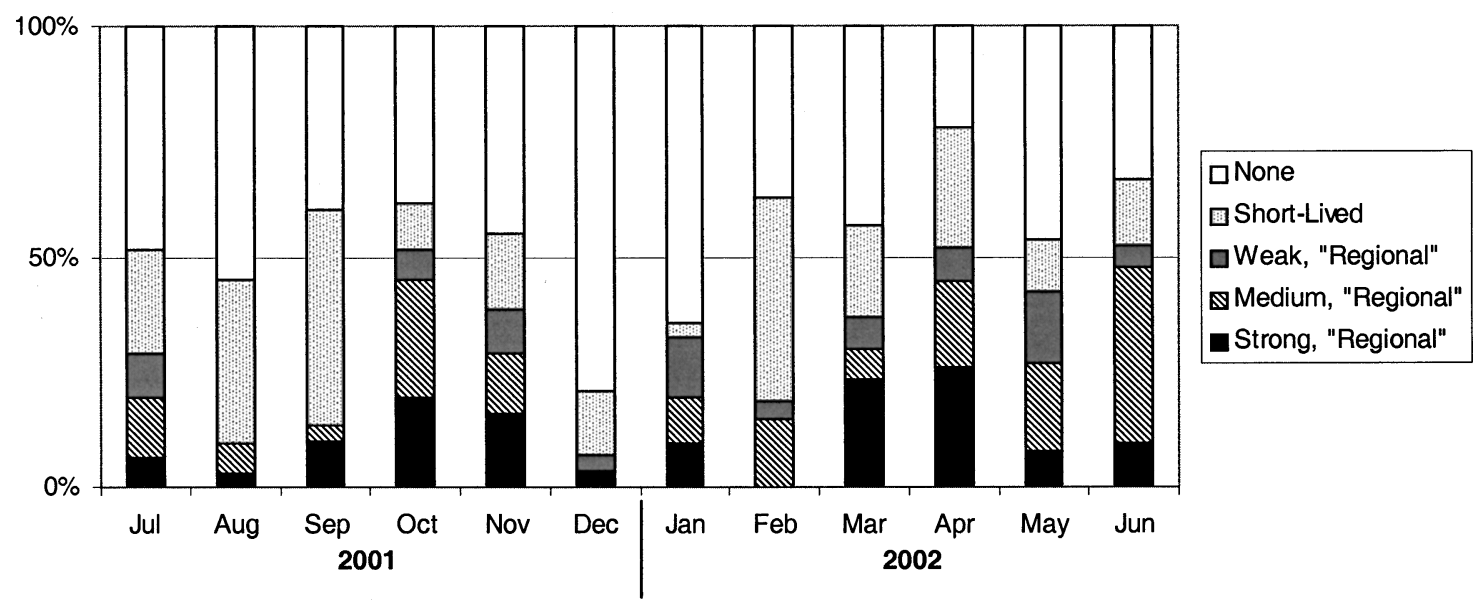

Figure 5. Overall frequency of nucleation. Days are classified by the occurrence of nucleation activity. Weak, medium, and strong refer to the rate of increase of $N_{10}$ during the first hour of the event. $d N_{10} / d t<4,000 \mathrm{~cm}^{-3} \mathrm{~h}^{-1}$ was classified as weak, $d N_{10} / d t$ from 4,000-15,000 $\mathrm{cm}^{-3} \mathrm{~h}^{-1}$ was classified as moderate, and $d N_{10} / d t>15,000 \mathrm{~cm}^{-3} \mathrm{~h}^{-1}$ was classified as strong nucleation. Short-lived events refer to nucleation without growth to larger sizes.

The result of the correlation is shown in Figure 7. The panels of Figure 7 can be divided into 2 qualitative regions. The upper left portion of each figure (Region I), dominated by grey points, is where nucleation is relatively rare, because the sulfuric acid production is too slow, or there is too much area available for condensation. The second region is where the bulk of the black points are, indicating conditions more favorable to nucleation. As the $\mathrm{UV} * \mathrm{SO}_{2}$ product increases and the condensational sink decreases, the ratio of nucleation points (in black) to nonnucleation points (in grey) increases. The nucleating conditions do

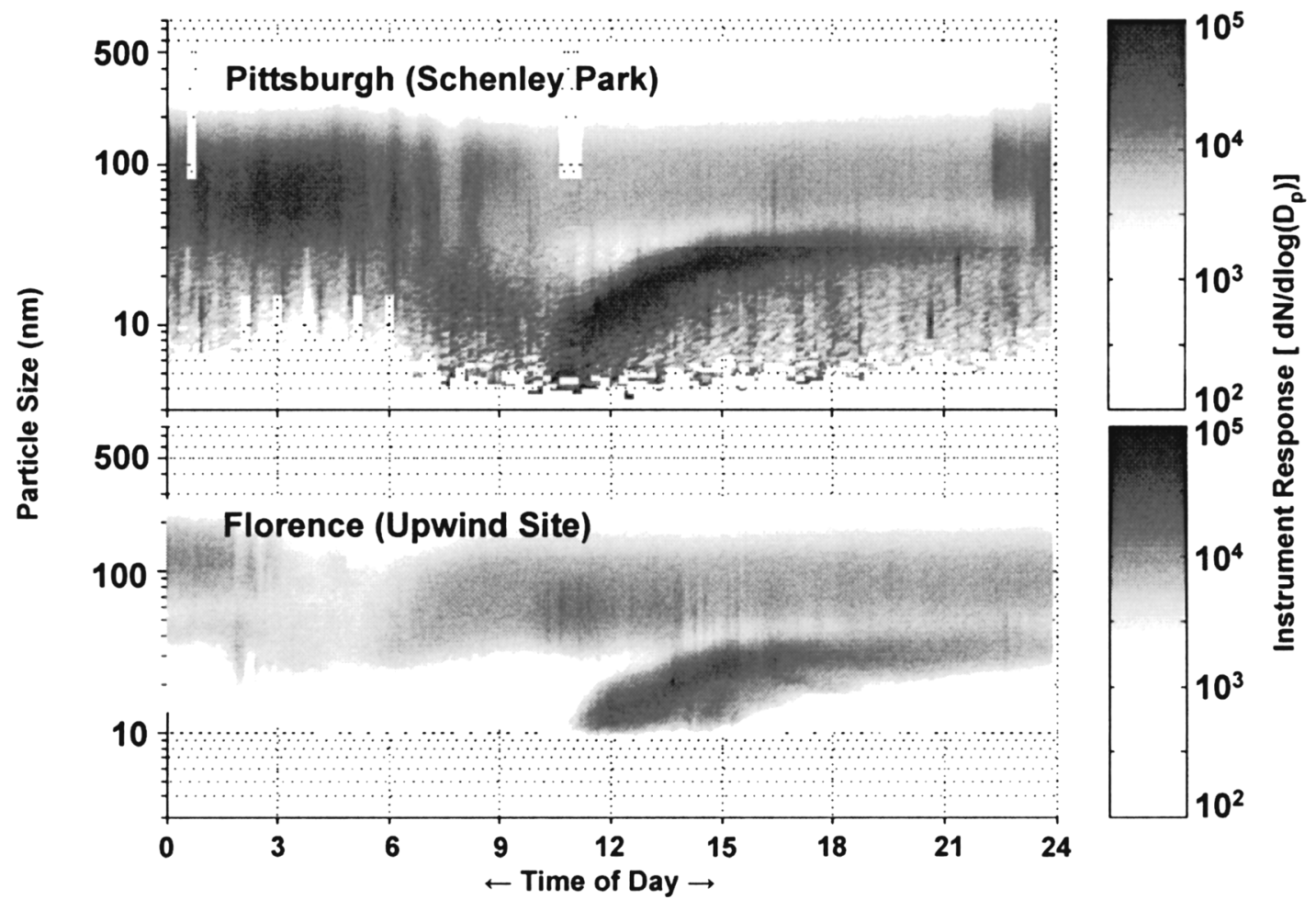

Figure 6. Graphical representation of instrument response for two sites. The top plot shows results at an urban sampling location in Pittsburgh, PA and the bottom graph shows results for the same time period from an upwind, rural site $40 \mathrm{~km}$ away. Nucleation is apparent at around $10 \mathrm{am}$ local time. 


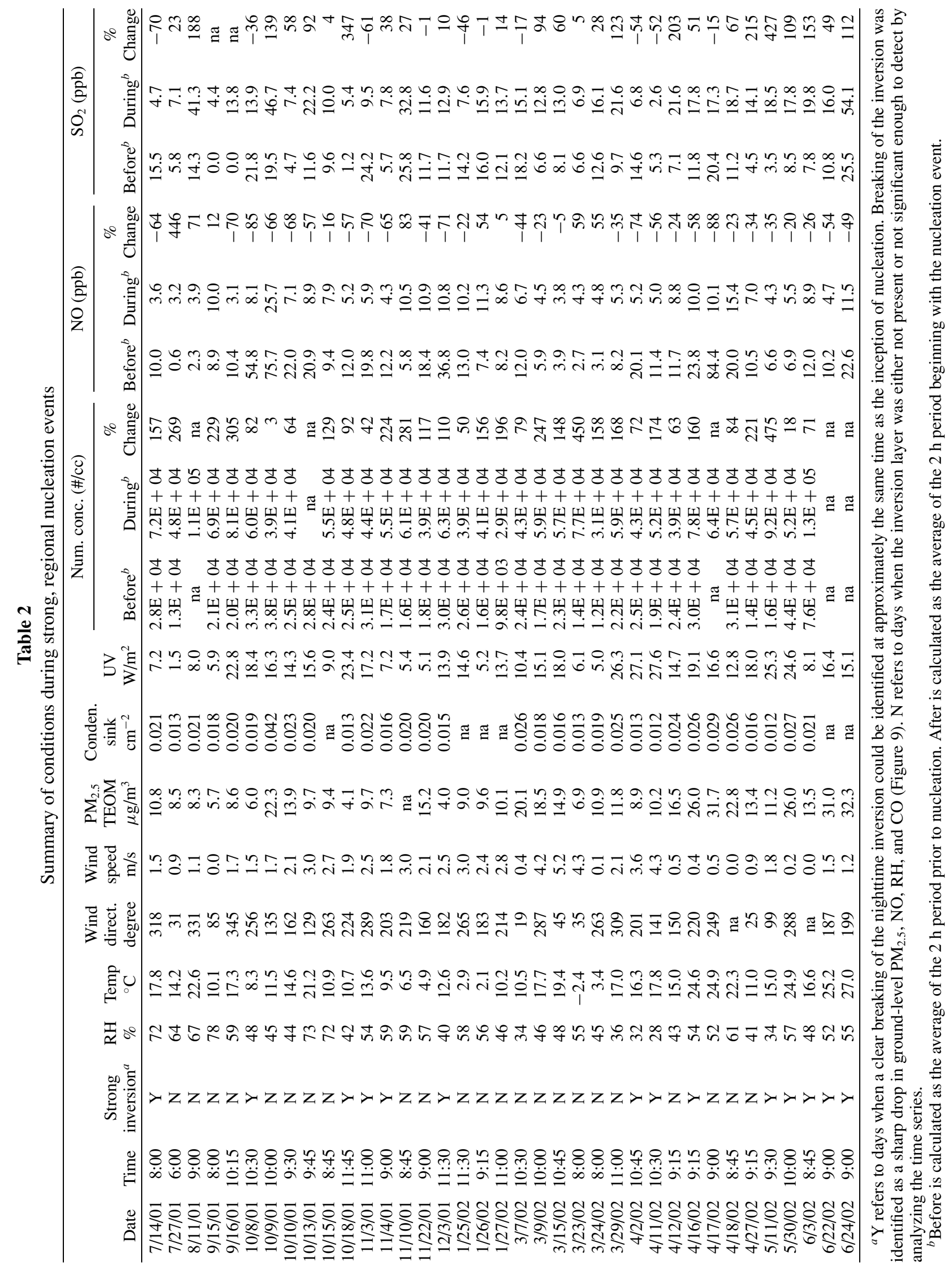




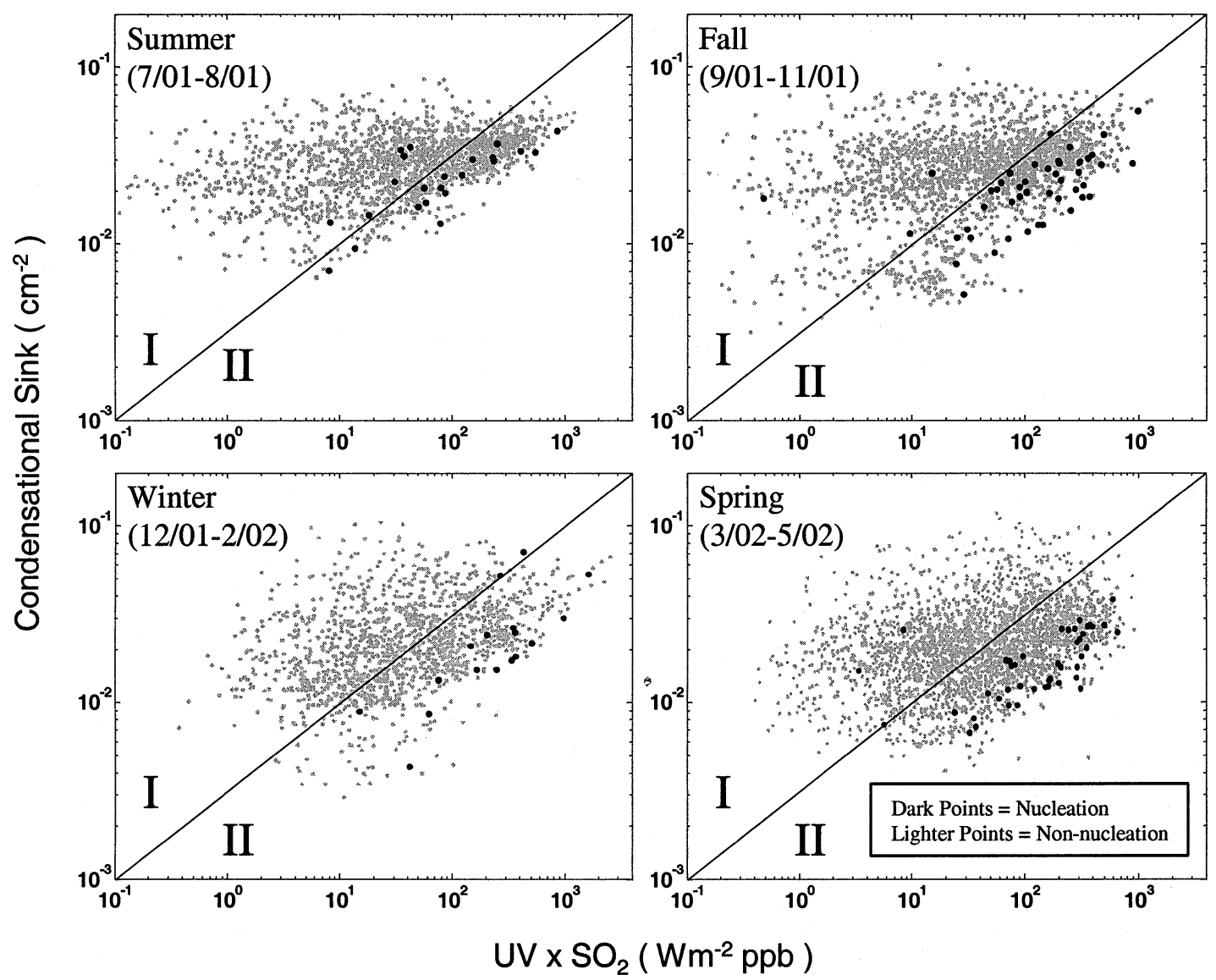

Figure 7. Correlation showing UV * $\mathrm{SO}_{2}$ versus condensational sink for four different seasons. Condensational sink ( $y$ axis) is plotted against the product of ultraviolet light intensity and $\mathrm{SO}_{2}$ concentration ( $x$ axis), a proxy for sulfuric acid production. Fifteenminute averaged values are plotted for all time periods of the study. The black symbols correspond to onset of nucleation. The grey symbols correspond to periods when nucleation is not observed. The 45 degree line roughly divides each plot into two regions- the upper left region I where nucleation is not generally observed, and the lower right region II where nucleation is more common.

not form a sharp line in this plot. While this is partly due to measurement errors and difficulty in assigning a precise start time to nucleation, it is probably mostly due to additional predictive variables that are important to nucleation. During summer, nucleation takes place at higher condensational sink values for the same value of $\mathrm{UV} * \mathrm{SO}_{2}$. Known variables that should be important include temperature and relative humidity.

The nucleation measurements made during this study are compared to model-based correlations of Pirjola et al. (1999) and Wexler et al. (1994) in Figure 8. Condensational sink ( $y$ axis) is plotted against the product of ultraviolet light intensity and $\mathrm{SO}_{2}$ concentration ( $x$ axis), a proxy for sulfuric acid production. These models were designed to predict the required $\mathrm{H}_{2} \mathrm{SO}_{4}$ production rates for nucleation and growth by $\mathrm{H}_{2} \mathrm{SO}_{4}$ as a function of condensational sink, relative humidity, and temperature. The observed nucleation events are plotted as solid black circles, while the black lines are the correlation-based thresholds for $\mathrm{H}_{2} \mathrm{SO}_{4}$ nucleation, with nucleation expected to the right of the lines and not expected to the left. These thresholds are calcu- lated at representative ground-level $\mathrm{RH}$ and temperature values. The lines are calculated using some assumptions, including (1) a proportional relationship between $\mathrm{UV}$ and $\mathrm{OH}$, with full summer sun of $36 \mathrm{Wm}^{-2} \mathrm{UV}$ corresponding to an $\mathrm{OH}$ concentration of $10^{7}$ molec $\mathrm{cm}^{-3}$; and (2) $\mathrm{a} \mathrm{SO}_{2}$ deposition characteristic time of $10^{4} \mathrm{~s}$ (Wexler et al. 1994). With our assumed $\mathrm{OH}$ levels, both of the correlations suggest that the ground-level conditions are several orders of magnitude more polluted than those required to induce binary nucleation and growth of fresh particles by sulfuric acid.

Also plotted are grey points, which are model predictions for the critical condensational sink level at $\mathrm{OH}, \mathrm{SO}_{2}$, temperature, and RH levels matching those of the specific nucleation events. This provides a better comparison of models to observations, because a representative $\mathrm{RH}$ and temperature is not needed. Each observation (black circle) is matched by 4 grey points, at the same point on the $x$ axis but with different condensational sink values. Grey points refer to the correlations of Wexler et al. (1994) (triangles) and Pirjola et al. (1999) (squares). 

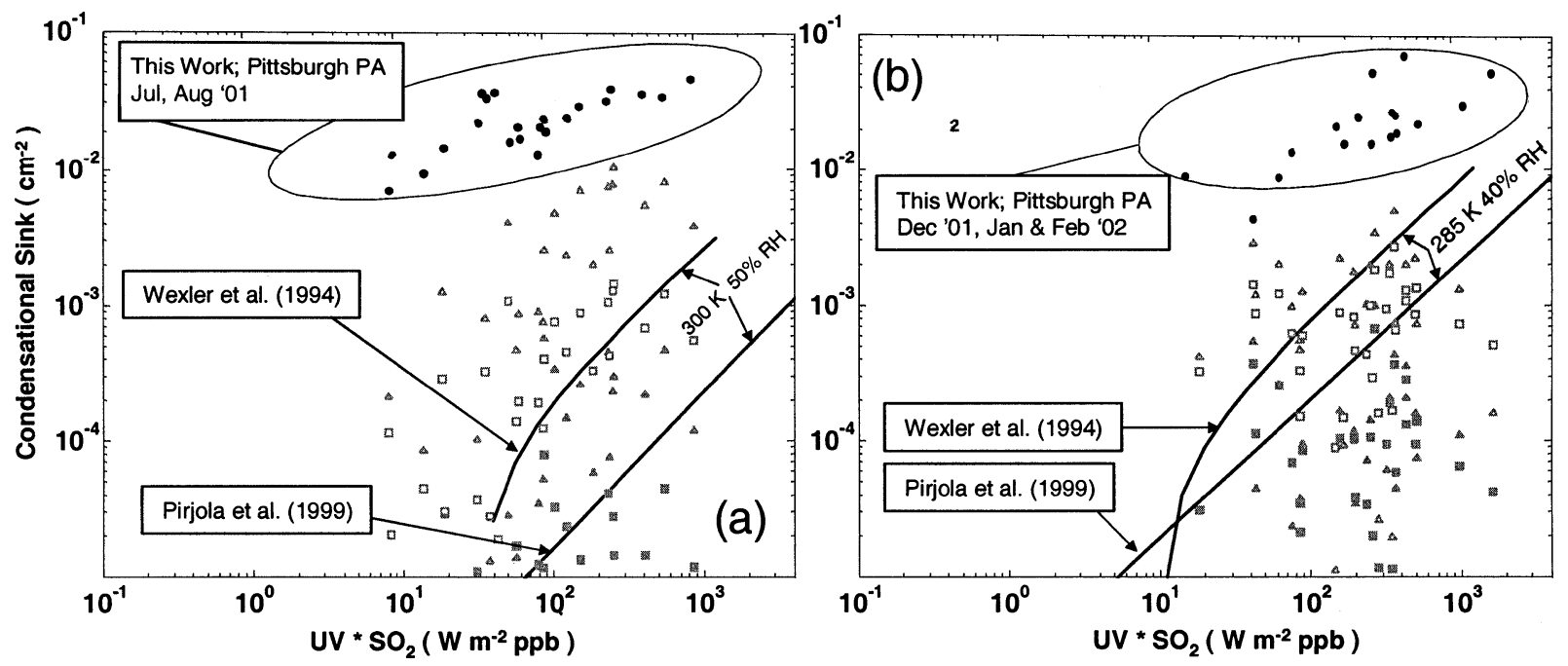

Figure 8. Comparisons of conditions during (a) summer and (b) winter nucleation to model-based correlations for sulfuric acid particle formation from Pirjola et al. (1999) and Wexler et al. (1994). Condensational sink ( $y$ axis) is plotted against the product of ultraviolet light intensity and $\mathrm{SO}_{2}$ concentration ( $x$ axis), a proxy for sulfuric acid production. Measured nucleation events (dark circles) are found at the highest condensational sink values. Solid lines refer to correlation predictions for the nucleation threshold at a representative ground-level RH and temperature (nucleation favored to the right of the lines, and not expected to the left). Additional correlation predictions (grey data points) are plotted to see if data-model agreement improves when specific RH and temperature values for each nucleation event are used in the models (triangles = Wexler et al. (1994); squares = Pirjola et al. (1999)). Filled symbols refer to RH and temperature at ground level; unfilled symbols refer to estimated conditions at the top of the boundary layer.

Filled grey symbols are calculated at ground-level meteorological conditions, while open grey symbols are calculated at reduced temperatures and elevated RHs corresponding to the top of the mixed layer. This is done to check if conditions at the top of the mixed layer would be sufficient for sulfuric-acid-induced nucleation. Key assumptions included constant vertical profiles of dewpoint, $\mathrm{OH}$, and $\mathrm{SO}_{2}$ in the mixed layer, summertime afternoon mixing heights of $2000 \mathrm{~m}$, wintertime afternoon mixing heights of $800 \mathrm{~m}$, and adiabatic cooling of air parcels at a rate of $9.8^{\circ} \mathrm{C} \mathrm{km}^{-1}$. Using these assumptions, the gap between
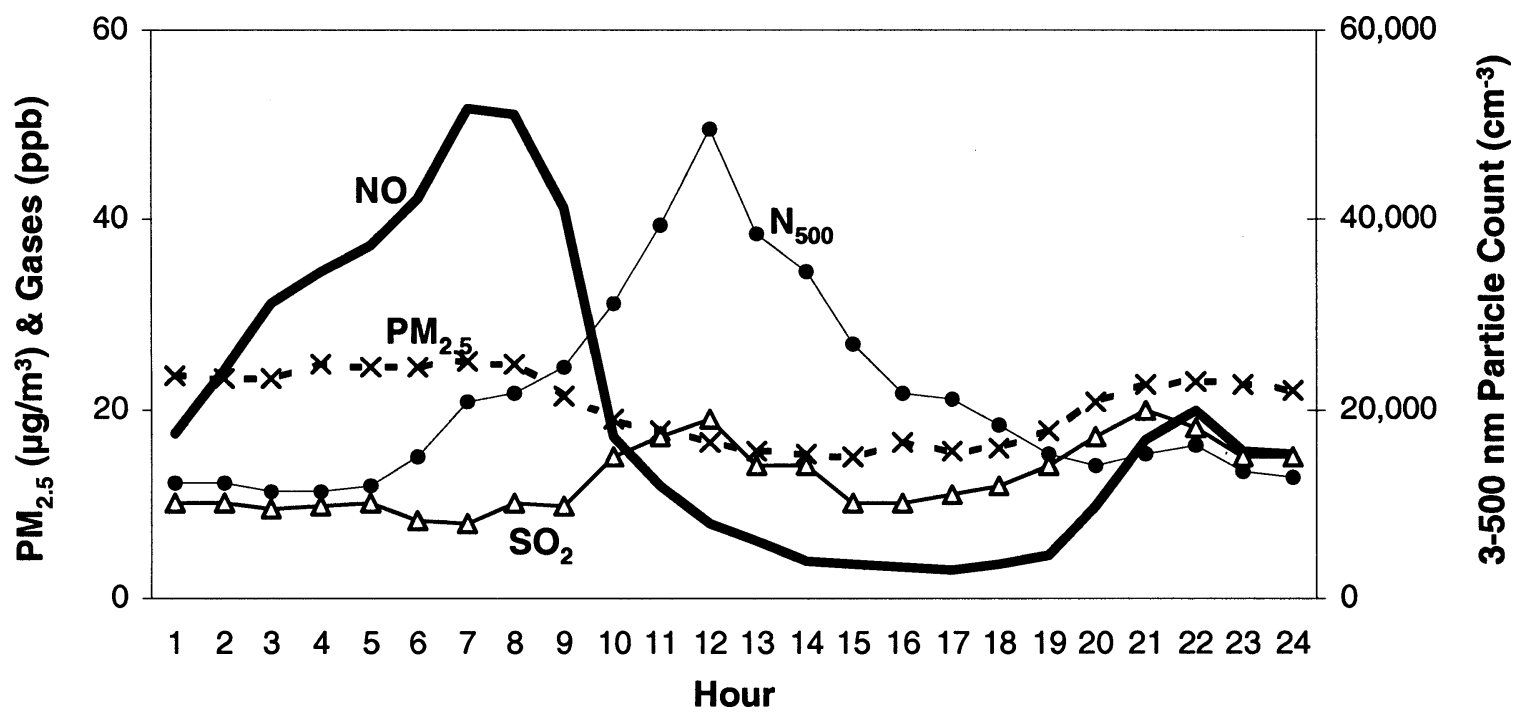

Figure 9. Diurnal profile for inversion-related nucleation. Inversion-related nucleation events from July to November 2001 are averaged. Boundary layer mixing height increases during the mornings, with decreases in $\mathrm{PM}_{2.5}$ and $\mathrm{NO}$, increases in $\mathrm{SO}_{2}$ levels, and nucleation activity. 
model predicted and observed condensational sink levels during nucleation narrowed but did not close.

The conclusions that can be drawn from comparing the nucleation models with the PAQS observations (Figure 8) are as follows: (1) observed nucleation is occurring at significantly higher levels of pre-existing aerosol surface area and/or lower levels of sulfuric acid production than predicted by the models; (2) this gap between models and observations is narrowed, but not removed, when lower temperatures and higher RHs at the top of the mixing layer are taken into account; and (3) the observations are not nearly as dependent on meteorology and sulfuric acid production rate as the models are. These conclusions suggest that additional factors, currently absent from the models, such as ammonia chemistry or growth by organic compounds, are involved in the nucleation events.

Many nucleation events were seen to coincide in time with the breakup of the morning inversion. In Table 2, nucleation events where this was especially prominent are noted in the inversion column. Nucleation concurrent with inversion layer breakup is most common in spring and fall. It is not common in winter, possibly due to higher average wind speeds during nucleation events. Figure 9 shows the average diurnal profile for regional nucleation events associated with inversion breakup. The key features of Figure 9 are the unusually high morning peak of $\mathrm{NO}$, significant decrease in $\mathrm{PM}_{2.5}$ during the morning, and an increase in $\mathrm{SO}_{2}$ and $\mathrm{NO}$ decreases. This indicates the possibility of nucleation occurring aloft in a $\mathrm{SO}_{2}$-enriched and low $\mathrm{PM}_{2.5}$ stable layer, followed by the mixing downward of the nuclei.

\section{SUMMARY AND CONCLUSION}

Continuous particle-size distribution measurements for one year during the PAQS indicate a high frequency of nucleation activity in the region, with nucleation occurring on about $50 \%$ of days and regional nucleation events occurring on $30 \%$ of days on average. Nucleation occurred during all seasons but was most intense during spring and fall. The nucleation events are more probable during bright sunny conditions with elevated $\mathrm{SO}_{2}$ concentrations and low pre-existing aerosol surface area. This relationship was analyzed using a simple correlation of $\mathrm{UV} *$ $\mathrm{SO}_{2}$ versus condensational sink and compared to existing correlations for binary $\mathrm{H}_{2} \mathrm{SO}_{4}-\mathrm{H}_{2} \mathrm{O}$ nucleation. The correlation indicated that nucleation occurs under higher aerosol loading conditions and/or with lower $\mathrm{H}_{2} \mathrm{SO}_{4}$ production rates than expected. A few events that did not follow the overall pattern appear to be influenced by conditions that made classification of the events problematic, such as a weak nucleation rate or a frontal passage. The correlation between $\mathrm{H}_{2} \mathrm{SO}_{4}$ production and nucleation activity provides strong evidence that sulfate plays a major role in new particle formation in Western Pennsylvania. The difference between the model-predicted and observed nucleation frequencies indicates that additional compounds, such as ammonia or organics, are possibly involved in the nuclei formation and/or growth in addition to sulfuric acid.

\section{REFERENCES}

Allen, A. G., Grenfell, J. L., Harrision, R. M., James, J., and Evans, M. J. (1999). Nanoparticle Formation in Marine Airmasses: Contrasting Behaviour of the Open Ocean and Coastal Environments, Atmos. Res. 51:1-14.

Birmili, W., Wiedensohler, A., Heintzenber, J., and Lehmann, K. (2001). Atmospheric Particle Number Size Distribution in Central Europe: Statistical Relations to Air Masses and Meteorology, J. Geophys. Res. 106(D23):32,00532,018 .

Charlson, R. J., Lovelock, J. E., Andreae, M. O., and Warren, S. G. (1987). Ocean Phytoplankton, Atmospheric Sulfur, Cloud Albedo and Climate, Nature 326:655-661.

Eisele, F. L., and McMurry, P. H. (1997). Recent Progress in Understanding Particle Nucleation and Growth, Philos. Trans. R. Soc. London, Ser. B. 352(1350):191-200.

Fuchs, N. A., and Sutugin, A. G. (1970). Highly Dispersed Aerosols. Ann Arbor Science, Ann Arbor, MI.

Harrison, R. M., Grenfell, J. L., Allen, A. G., Clemitshaw, K. C., Penkett, S. A., and Davison, B. (2000). Observations of New Particle Production in the Atmosphere of a Moderately Polluted Site in Eastern England, J. Geophys. Res. 105:17,819-17,832.

Harrison, R. M., Jones, M., and Collins, G. (1999a). Measurements of the Physical Properties of Particles in the Urban Atmosphere, Atmos. Environ. 33:309321.

Harrison, R. M., Shi, J. P., and Jones, M. R. (1999b). Continuous Measurement of Aerosol Physical Properties in the Urban Atmosphere, Atmos. Environ. 33:1037-1047.

Jang, M. S., and Kamens, R. M. (2001). Atmospheric Secondary Aerosol Formation by Heterogeneous Reactions of Aldehydes in the Presence of a Sulfuric Acid Aerosol Catalyst, Environ. Sci. Technol. 35(24):4758-4766.

Kerminen, V. M. (1999). Roles of $\mathrm{SO}_{2}$ and Secondary Organics in the Growth of Nanometer Particles in the Lower Atmosphere, J. Aerosol Sci. 30(8):10691078.

Kim, C. S., Adachi, M., Okuyama, K., and Seinfeld, J. H. (2002). Effect of $\mathrm{NO}_{2}$ on Particle Formation in SO2/H2O/air Mixtures by Ion-Induced and Homogenous Nucleation, Aerosol Sci. Technol. 36(9):941-952.

Kulmala, M., Pirjola, L., and Makela, J. M. (2000). Stable Sulphate Clusters as a Source of New Atmospheric Particles, Nature 404(6773):66-69.

Kulmala, M., Hameri, K., Aalto, P. P., Makela, J. M., Pirjola, L., Nilsson, E. D., Buzorius, G., Rannik, U., Dal Maso, M., Seidl, W., Hoffman, T., Janson, R., Hansson, H. C., Viisanen, Y., Laaksonen, A., and O’Dowd, C. D. (2001). Overview of the International Project on Biogenic Aerosol Formation in the Boreal Forest (BIOFOR), Tellus. Ser. B. 53:324-343.

Novakov, T., and Penner, J. E. (1993). Large Contribution of Organic Aerosols to Cloud Condensation Nuclei Concentrations, Nature 365:823826.

Oberdorster, G., Gelein, R. M., Ferin, J., and Weiss, B. (1995). Association of Particulate Air Pollution and Acute Mortality: Involvement of Ultrafine Particles, Inhal. Toxicol. 7:111-124.

O’Dowd, C. D., Aalto, P., Hmeri, K., Kulmala, M., and Hoffmann, T. (2002). Aerosol Formation: Atmospheric Particles from Organic Vapours, Nature 416:497-498.

O’Dowd, C. D., Lowe, J. A., and Smith, M. H. (1999). Coupling Sea-Salt and Sulphate Interactions and its Impact on Cloud Droplet Concentration Predictions, Geophys. Res. Lett. 26(9):1311-1314.

Peters, A., Wichmann, E., Tuch, T., Heinrich, J., and Heyder, J. (1997). Respiratory Effects are Associated with the Number of Ultrafine Particles, Am. J. Respir. Crit. Care Med. 155:1276-1383.

Pirjola, L., Kulmala, M., Wilck, M., Bischoff, A., Stratmann, F., and Otto, E. (1999). Formation of Sulphuric Acid Aerosols and Cloud Condensation Nuclei: An Expression for Significant Nucleation and Model Comparison, J. Aerosol Sci. 30(8):1079-1094.

Rivera-Carpio, C. A., Corrigan, C. E., Novakov, T., Penner, J. E., Rogers, C. F., and Chow, J. C. (1996). Derivation of Contributions Sulfate and Carbonaceous Aerosols to Cloud Condensation Nuclei from Mass Size Distributions, J. Geophys. Res. 101:19,483-19,493. 
Schwartz, J., Dockery, D. W., and Neas, L. M. (1996). Is Daily Mortality Associated Specifically with Fine Particles? J. Air Waste Manag. Assoc. 46:927-939.

Shi, J. P., Evans, D. E., Khan, A. A., and Harrison, R. M. (2001). Source and Concentration of Nanoparticles ( $<10 \mathrm{~nm}$ diameter) in the Urban Atmosphere, Atmos. Environ. 35:1193-1202.

Shi, Q. (2003). Aerosol Size Distributions (3 $\mathrm{nm}$ to $3 \mu \mathrm{m}$ ) Measured at the St. Louis Supersite (4/1/01-4/30/02), M.S. Thesis, Department of Mechanical Engineering, University of Minnesota, Minneapolis, MN.

Stanier, C., Khlystov, A., Chan, W. R., Mandiro, M., and Pandis, S. N. (2004). A Method for the In-Situ Measurement of Fine Aerosol Water Content of Ambient Aerosols: The Dry-Ambient Aerosol Size Spectrometer (DAASS), Aerosol Sci. Technol. 38:215-228.
Weber, R. J., McMurry, P. H., Mauldin, L., Tanner, D., Eisele, F., Clarke, A. D., and Kapustin, V. N. (1999). New Particle Formation in the Remote Troposphere: A Comparison of Observations at Various Sites, Geophys. Res. Lett. Atmos. Sci. 26:307-310.

Wexler, A. S., Lurmann, F. W., and Seinfeld, J. H. (1994). Modelling Urban and Regional Aerosols-I. Model Development, Atmos. Environ. 28(3):531546

Woo, K. S., Chen, D. R., Pui, D. Y. H., and McMurry, P. H. (2001). Measurement of Atlanta Aerosol Size Distributions: Observations of Ultrafine Particle Events, Aerosol Sci. Technol. 34:75-87.

Zhang, K. M., and Wexler, A. S. (2002). A Hypothesis for Growth of Fresh Atmospheric Nuclei, J. Geophys. Res. 107:4577, DOI: 10.1029/2002JD002180. 


\title{
Photochemical oxidation and changes in molecular composition of organic aerosol in the regional context
}

\author{
Allen L. Robinson, ${ }^{1}$ Neil M. Donahue, ${ }^{2}$ and Wolfgang F. Rogge ${ }^{3}$ \\ Received 23 May 2005; revised 16 September 2005; accepted 8 November 2005; published 7 February 2006.
}

[1] This paper presents evidence that condensed-phase organic compounds are significantly oxidized in regional air masses and in locations affected by regional transport, especially during the summer. The core of the paper examines a large data set of ambient organic aerosol concentrations for removal of reactive compounds relative to less-reactive compounds. The approach allows visualization of both photochemistry and mixing of emissions from multiple sources in order to differentiate between the two phenomena. The focus is on hopanes and alkenoic acids, important markers for motor vehicle and cooking emissions. Ambient data from Pittsburgh, PA and the Southeastern United States contain evidence for significant photochemical oxidation of these compounds in the summertime. There is a strong seasonal pattern in the ratio of different hopanes to elemental carbon consistent with oxidation. In addition, measurements at rural sites indicate that hopanes are severely depleted in the regional air mass during the summer. Alkenoic acids also appear to be photochemically oxidized during the summertime; however, the oxidation rate appears to be much slower than that inferred from laboratory experiments. The significance of photochemistry is supported by rudimentary calculations which indicate substantial oxidation by $\mathrm{OH}$ radicals and ozone on a time scale of a few days or so, comparable to time scales for regional transport. Oxidation is non-linear; therefore it represents a very substantial complication to linear source apportionment techniques such as the Chemical Mass Balance model.

Citation: Robinson, A. L., N. M. Donahue, and W. F. Rogge (2006), Photochemical oxidation and changes in molecular composition of organic aerosol in the regional context, J. Geophys. Res., 111, D03302, doi:10.1029/2005JD006265.

\section{Introduction}

[2] Organic matter is a major component of tropospheric aerosol, contributing between 10 and $70 \%$ of the fine particulate mass [Jacobson et al., 2000; Turpin et al., 2000]. This material consists of thousands of organic compounds, which are either emitted directly from sources (primary organic aerosol) or formed in the atmosphere from low vapor pressure products of the oxidation of anthropogenic and biogenic organic gases (secondary organic aerosol).

[3] There is no question that organic particulate matter is bombarded with oxidants. In addition, collisions involving $\mathrm{OH}$ radical or $\mathrm{O}_{3}$ appear to have a high probability of leading to a reaction. This probability is at least 0.1 for $\mathrm{OH}$ (measured as the overall accommodation coefficient or reaction probability per collision) [Bertram et al., 2001]. In the case of ozone and unsaturated organics the odds are less certain, as discussed below; however, the limits appear

\footnotetext{
${ }^{1}$ Departments of Mechanical Engineering and Engineering and Public Policy, Carnegie Mellon University, Pittsburgh, Pennsylvania, USA.

${ }^{2}$ Departments of Chemical Engineering and Chemistry, Carnegie Mellon University, Pittsburgh, Pennsylvania, USA.

${ }^{3}$ Department of Civil and Environmental Engineering, Florida International University, Miami, Florida, USA.
}

Copyright 2006 by the American Geophysical Union. 0148-0227/06/2005JD006265\$09.00 to be $10^{-3}$ (measured on pure surfaces in the lab [Moise and Rudich, 2000; Morris et al., 2002; Rudich, 2003; Smith et al., 2002; Thornberry and Abbatt, 2004]) and $10^{-6}$ (the gasphase reaction probability). Recent evidence suggests that organic aerosol may be significantly oxidized, even in urban environments [Zhang et al., 2005].

[4] Oxidization alters the chemical composition and physical properties of organic aerosol. Oxidation increases the water solubility and number of polar functional groups of organic aerosol making them more active as cloud condensation nuclei, influencing regional precipitation patterns and climate [Kotzick and Niessner, 1999; Lammel and Novakov, 1995; Saxena et al., 1995; Weingartner et al., 1997]. Changes in molecular-level composition due to photochemical oxidation are also of interest. Individual organic compounds are commonly used as markers for sources of primary organic aerosol. For example, levoglucosan is a marker for wood smoke [Schauer et al., 2001; Simoneit et al., 1999] and hopanes are markers for vehicle emissions [Rogge et al., 1993a; Schauer et al., 1999b, 2002c]. Using these and other compounds, researchers have identified the relative contribution of sources such as automobiles, diesel trucks, meat cooking, and wood smoke to urban organic aerosol [Schauer and Cass, 2000; Schauer et al., 2002a, 1996].

[5] The reduced organic compounds used as molecular markers for source apportionment are assumed to be pho- 
tochemically stable under transport from source to receptor. Schauer et al. [1996] and Rogge et al. [1996] evaluated the stability of organic molecular markers by comparing emission inventories to measured ambient concentrations for the Los Angeles region. Based on this work they classified certain compounds as stable in the context of Los Angeles; even species such as retene, benzo(a)pyrene, and oleic acid all of which have been shown to be at least moderately reactive - passed the test. However, air pollution in the Los Angeles basin is dominated by local emissions mixed with fairly clean background air over relatively short transport distances, while air quality in many other areas of the country is dominated by regional transport. Transport allows for more photochemical processing and mixes local emissions with aged background air. Some of the compounds classified as stable in Los Angeles appeared to have undergone significant chemical processing downwind in Rubidoux [Schauer et al., 1996]. The stability of compounds used as source markers has not been evaluated under conditions of regional transport. At a minimum, photochemical oxidation will reduce the absolute concentration of organic markers, lowering estimated source strengths. If oxidation rates are species dependent, ratios between molecular markers will not be conserved. This would significantly alter the relative contribution of different sources predicted by linear source inversions [ $\mathrm{Li}$ and Kamens, 1993].

[6] Relatively little is known about changes in molecularlevel composition due to photochemical oxidation of organic aerosols. Significant research has examined polycyclic aromatic hydrocarbons (PAH), which are particularly reactive in the atmosphere [Finlayson-Pitts and Pitts, 2000; Kamens et al., 1988; Nielsen, 1988; Schauer et al., 2003]. More recently, laboratory experiments have examined the uptake of oxidants $\left(\mathrm{O}_{3}\right.$ and $\left.\mathrm{OH}\right)$ on carefully prepared organic surfaces or single-component particles [Bertram et al., 2001; de Gouw and Lovejoy, 1998; Moise and Rudich, 2000; Smith et al., 2002; Thomas et al., 2001]. However, significant discrepancies exist between fundamental laboratory measurements made on pure compounds and atmospheric observations. For example, a number of groups have measured the $\mathrm{O}_{3}$ uptake of pure oleic acid; the oleic acid lifetime derived from these measurements is on the order of minutes under polluted conditions [Morris et al., 2002; Worsnop et al., 2002]. Comparisons of measurements with model predictions in Los Angeles suggest that oleic acid is oxidized in the atmosphere [Rogge et al., 1996], but substantial levels of oleic acid are commonly observed in urban environments, indicating that the oxidation of oleic acid in atmospheric particles is orders of magnitude slower than measurements made on pure surfaces in the laboratory. Clearly the complexity of real organic aerosols has significant effects on oxidation rates.

[7] This paper presents evidence that photochemical oxidation significantly alters the molecular-level composition of organic aerosol in the eastern United States, and by extension other areas affected by regional transport. First, basic calculations of the oxidation lifetime as a function of particle size are compared to the age of an aerosol particle in a regional air mass. Next, we present a methodology to examine ambient data for evidence of photochemical oxidation by comparing concentration ratios of condensed- phase organic species. These techniques are applied to large data set of ambient organic $\mathrm{PM}_{2.5}$ recently developed as part of the Pittsburgh Air Quality Study (A. Bernardo-Bricker et al., manuscript in preparation, 2006). The analysis focuses on hopanes and alkenoic acids, important molecular markers for motor vehicle and cooking emissions, respectively. The paper concludes with a discussion of the potential effects of photochemical aging on source apportionment analysis.

\section{Timescales for Photochemical Oxidation}

[8] To gauge the atmospheric significance of photochemical oxidation, we must compare the timescale for oxidation to the average atmospheric age of an aerosol particle. The average particle age will vary with location. For regions with meso-scale pollution transport such as the eastern United States, radioactive tracer measurements indicate an apparent atmospheric residence time for fine aerosols of order 10 days [Gaffney et al., 2004].

[9] The oxidation timescale of an organic aerosol depends on the oxidation rate. Oxidation in a complex condensed phase can be broken up into two components: first, whether the oxidant is taken up by the aerosol and reacts with something, and second, which compound the oxidant reacts with. It is important to separate these because one controls the absolute rate of oxidation and the other the relative behavior of different compounds. In this section, we estimate oxidant uptake and the mass-average lifetime of reduced material in a particle, and then discuss issues associated with non-uniformity in that particle.

[10] To estimate the oxidation time scale associated with the $\mathrm{OH}$ radical and ozone, we consider a model particle comprising a single hydrocarbon; for $\mathrm{OH}$ oxidation we consider n-eicosane, and for ozone oxidation we consider oleic (q-octadecenoic) acid. We expect oxidation by $\mathrm{OH}$ radicals to dominate the aging of organic particles because the vast majority of the identified organic mass is saturated [Rogge et al., 1993d]. However, olefinic compounds are present in ambient aerosol as well, and some, such as oleic acid and cholesterol, are important markers for different sources. These unsaturated compounds may well be oxidized primarily by ozone.

[11] In order to calculate the oxidation timescale, we need to know the rate of oxidant uptake and the number of molecules in a typical particle. The flux of oxidant into the particle of radius $\mathrm{r}$ is [Hanson et al., 1994; Worsnop et al., 2002]

$$
\phi(\mathrm{ox})=\gamma \pi \mathrm{Csr}^{2}
$$

where $\mathrm{C}$ is the oxidant concentration and $\mathrm{s}$ is the oxidant speed, both in the gas phase. This neglects Knudsen-number dependent gas-phase diffusion limitations, which are only important for smaller particles [Worsnop et al., 2002]. This must then be balanced by consumption of reagent in the particles. The number of molecules in a homogeneous spherical particle of radius $r$ is

$$
\mathrm{N}=\frac{4}{3} \pi \mathrm{r}^{3} \frac{\rho \mathrm{Na}}{\mathrm{M}}
$$

where $\rho$ is the particle density (assumed to be $1 \mathrm{~g} \mathrm{~cm}^{-3}$ ) and $\mathrm{M}$ is the reagent molecular weight (for a C20 chain of 


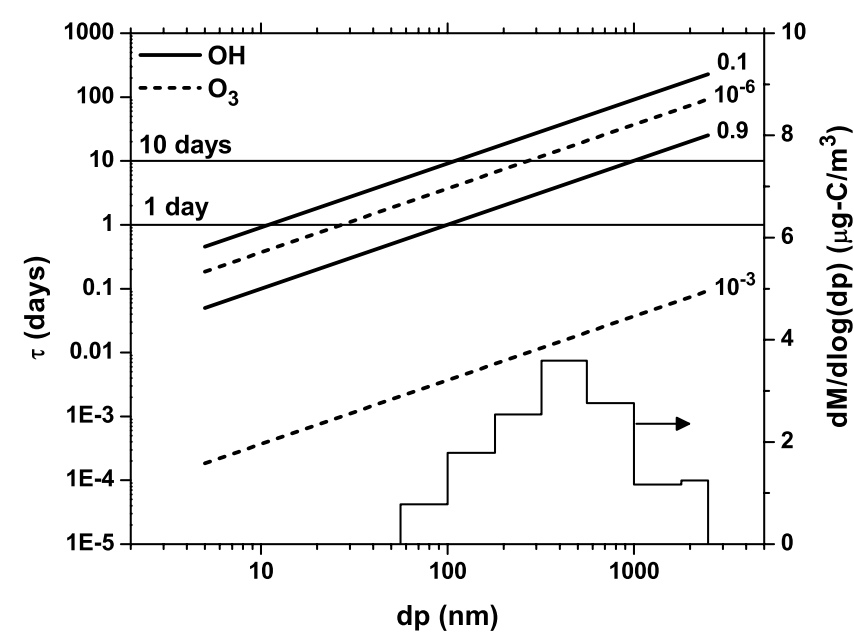

Figure 1. Oxidation lifetime as a function of particle size calculated using equation (3). $\mathrm{OH}$ calculations are for a single component particle of $\mathrm{C} 20$ alkanes, assuming $[\mathrm{OH}]=$ $3 \times 10^{6}$ molecules $/ \mathrm{cm}^{3} . \mathrm{O}_{3}$ calculations are for a single component particle of oleic acid (C18), assuming $50 \mathrm{ppbv}$ $\mathrm{O}_{3}$. Numbers indicate uptake coefficient, $\gamma$, for each calculation. The horizontal lines at 1 and 10 days indicate the range of expected ages in a regional air mass. Also shown is the average organic carbon size distribution measured in Pittsburgh, PA [Cabada et al., 2004]. Compounds in particles with sizes between $100 \mathrm{~nm}$ and $1 \mu \mathrm{m}$ size range may have oxidation lifetimes comparable to time scales associated with regional transport.

methylenes $\mathrm{M}=280 \mathrm{~g} / \mathrm{Mole})$. By simple mass balance, the oxidation timescale is

$$
\tau=\frac{\mathrm{N}}{\phi}=\frac{4}{3} \mathrm{r} \frac{\rho \mathrm{Na}}{\mathrm{M} \gamma \mathrm{Cs}}
$$

In this time $\tau$ the total number of oxidation events will equal the initial number of molecules in the particle; i.e., each molecule in the particle has been oxidized once.

[12] Equation (3) indicates that the oxidation time scale increases linearly with particle size. Organic carbon exists in particles with sizes less than $100 \mathrm{~nm}$ to more than $1 \mu \mathrm{m}$, depending on the source and the extent of atmospheric processing. As a reference, Figure 1 shows the average organic carbon size distribution measured in Pittsburgh, PA; a location significantly influenced by regional transport [Cabada et al., 2004]. The mass median diameter of this organic material is around $0.4 \mu \mathrm{m}$.

\subsection{OH Radical}

[13] Estimates of $\tau$ for oxidation by $\mathrm{OH}$ are shown in Figure 1 as a function of particle size for two uptake coefficients, 0.1 and 0.9 , that span the range measured in the laboratory [Bertram et al., 2001]. These calculations use an $\mathrm{OH}$ concentration of $3 \times 10^{6} \mathrm{~cm}^{-3}$, which is representative of daytime conditions during the summer [Bloss et al., 2005; Heard and Pilling, 2003].

[14] Figure 1 indicates that particles in the size range between 100 and $1000 \mathrm{~nm}$ have $\mathrm{OH}$ oxidation timescales between 1 and 10 days. Therefore, based purely on a mass- balance timescale, we expect significant oxidation due to $\mathrm{OH}$ attack for particles in a regional air mass during the summer. During the winter, $\mathrm{OH}$ levels are 3-5 times lower than during the summer [Bloss et al., 2005], and so we anticipate mild wintertime oxidation, reaching at most the lower bound for summertime $\mathrm{OH}$ oxidation shown in Figure 1 (line labeled 0.1).

[15] While this simple calculation shows that we expect oxidation, the oxidation timescale is on par with other processes such as transport, deposition, and growth. This complicates interpretation of Figure 1 because changing the oxidation timescale by a factor of two either for the bulk particle or individual compounds could have a dramatic effect on their observed behavior. Now we shall explore some assumptions that affect our simple oxidation timescale calculation.

[16] We have assumed that the uptake coefficient $(\gamma)$ for $\mathrm{OH}$ will not change during oxidation. This is one of two possible assumptions; the other being that $\gamma$ evolves as condensed-phase reagents are consumed. Assuming that the $\mathrm{OH}$ uptake remains constant during oxidation seems appropriate given the large number of $\mathrm{CH}_{2}$ groups in typical condensed-phase organic compounds. For example, the model target compound is a C20 alkane (n-eicosane), and until at least 4 carbons are oxidized it is difficult to believe that any extreme substituent effects will radically alter the rate constants or oxidant uptake. However, the reactodiffusive length consistent with these assumptions is of order $1 \mathrm{~nm}$ [Hanson et al., 1994]. Consequently, the oxidation will be confined to an outer shell containing less than $10 \%$ of the particle mass. The compounds in this layer may be multiply oxidized quite rapidly, fresh reagents may diffuse into this layer, or products of oxidation may volatilize to expose fresh reagent [Moise and Rudich, 2001; Molina et al., 2004].

[17] An important consideration may be the internal structure of a particle. For example, are primary organic particles coated by secondary inorganic species that inhibit oxidant uptake shielding the material in the particle core? Almost nothing is known about the in situ structure of ambient particles: the limited available data are for material collected on substrates which could significantly alter the particle structure [Maria et al., 2004]. However, laboratory experiments indicate that it is difficult to create coatings that act as effective shields [Cruz and Pandis, 1998].

[18] Concentration gradients are observed in ambient particles collected on substrates, with evidence that carbonylcontaining compounds favor the particle edges [Maria et al., 2004]. Particles may be inhomogeneous either because of kinetic (slow diffusion) or thermodynamic constraints. The standard diffusion timescale is $\tau=r^{2} / \mathrm{D}$, where $r$ is the particle radius and D is the diffusion constant. We could not find published diffusivities for large organic molecules in realistic substrates, but one can estimate a bound for which transport limitations become significant. The relevant time and length scales are of order 1 day and $1 \mu \mathrm{m}$; therefore diffusivities would need to be smaller than $10^{-13} \mathrm{~cm}^{2} \mathrm{~s}^{-1}$ to sustain concentration gradients within a particle. Teflon (FEP and TFE) permeation devices with $\sim 1-\mathrm{mm}$ thick walls typically equilibrate within 1 day for compounds at least as large as monoterpenes, nicotine, trimethyl benzene 
and the like (Kin Tek literature), which yields diffusion coefficients of order $10^{-8} \mathrm{~cm}^{2} \mathrm{~s}^{-1}$. To the extent Teflon is a reasonable model for an oligomeric organic aerosol, organics within this matrix should diffuse through $1 \mu \mathrm{m}$ on a time scale of seconds. If mass transfer limitations are not sustaining these gradients, then these oxidation products must have a higher surface affinity than the compounds initially present in the particles.

[19] Although there are significant uncertainties in estimating oxidation time scales, we can conclude that substantial summertime oxidation of condensed-phase organics by $\mathrm{OH}$ radicals is plausible. The extent of oxidation is likely sufficient for at least one, and probably several, oxidation events per organic molecule present in each particle. Surface-specific compounds may bear the brunt of this bombardment, but it may also extend to most of the organic mass contained in each particle.

\subsection{Ozone}

[20] Oxidation by ozone is more of a puzzle. In the gas phase, reactions occur every one in ten thousand to ten million collisions of ozone with an alkene. Fundamental laboratory studies on ozone uptake suggest substantially enhanced condensed-phase reactivity [Moise and Rudich, 2000; Morris et al., 2002; Rudich, 2003; Smith et al., 2002; Thornberry and Abbatt, 2004]. For example, the uptake coefficient of ozone on pure oleic acid is about $10^{-3}$, nearly 1000 times the gas-phase reaction probability (a rate constant of $10^{-16} \mathrm{~cm}^{3} /($ molec sec) corresponds to a reaction probability of $10^{-6}$ ). There is a great deal of ozone in the atmosphere, so if the laboratory results apply to atmospheric conditions, unsaturated organics in the condensed phase would not survive long.

[21] Using the expressions developed above, we can calculate a mean timescale for ozone oxidation of a pure oleic acid particle (C18). We assume summertime conditions with a mean $\mathrm{O}_{3}$ mixing ratio of $50 \mathrm{ppbv}$. Estimates are shown in Figure 1 for two uptake coefficients: $10^{-6}$, in line with the gas-phase reaction probability, and $10^{-3}$, consistent with laboratory experiments on pure surfaces. The smaller uptake coefficient results in ozone oxidation of unsaturated organics on a similar timescale to $\mathrm{OH}$ oxidation of saturated organics. As has been pointed out by others [Worsnop et al., 2002], the larger uptake coefficient leads to exceedingly short lifetimes. Therefore, Figure 1 supports the hypothesis that significant ozonolysis occurs on time scales commensurate with regional transport.

[22] Ozone uptake by organic particles is likely to be much more sensitive to conditions than $\mathrm{OH}$ radicals. We argued above that the $\mathrm{OH}$ uptake coefficient is likely to remain high and the reacto-diffusive length small throughout the particle lifetime. However, given the small fraction of olefins in ambient aerosol, ozone may well diffuse entirely through the particles. Under these conditions (Worsnop case 3 [Worsnop et al., 2002]), the uptake coefficient is sensitive to the bulk solubility of ozone, which is likely to change with particle composition (organic polarity, total acidity, etc). It is far less likely that compounds will be able to 'hide' behind some protective surface layer, but the absolute uptake rates must be regarded as highly uncertain. Of course, all organics vulnerable to oxidation by ozone will also be subject to oxidation by $\mathrm{OH}$, though the sheer number of saturated carbons on alkenes such as oleic acid means that the $\mathrm{OH}$ is more likely to engage in $\mathrm{H}$-abstraction than it is to add to the double bond.

\section{Photochemical Aging and Ratio-Ratio Plots}

[23] To find evidence of oxidation in speciated observations, one must observe either the removal of reactive compounds, relative to less reactive compounds, or the emergence of products. Little is known about potential reaction products so we focus on relative removal. This section presents a methodology to examine ambient data for evidence of photochemical oxidation by comparing concentration ratios of condensed-phase organic species. The basic approach has been widely applied to gas phase hydrocarbon data to estimate photochemical age and oxidant concentrations [Blake et al., 1993; Calvert, 1976; McKeen and Liu, 1993; McKeen et al., 1996; McKenna et al., 1995; Parrish et al., 1992; Roberts et al., 1984].

[24] The central idea is that at the point of emission there are characteristic ratios between different compounds, but that these ratios change with distance downwind due to photochemical processing as more reactive compounds are preferentially oxidized. A complication is that mixing of emissions with background air can also influence ambient concentration ratios. One must separate the effects of mixing from oxidation in order to calculate photochemical age; however, our objective is to find evidence of oxidation in the ambient data, not to calculate quantitatively the photochemical age. This requires viewing the ambient data from a slightly different perspective. As emissions are transported downwind from a source, photochemistry affects concentration ratios in two ways: directly, by oxidizing the emissions from the source; and indirectly, by previously oxidizing the background air that is mixed with the emissions. Therefore, we seek to differentiate these two phenomena from the mixing of emissions from different sets of sources that emit the same compounds but in different concentration ratios (source-source mixing). Differentiating source-source mixing from photochemistry is a much easier task then calculating photochemical age.

[25] The core of our technique is to create ratio-ratio plots using ambient concentrations of three species. One compound is selected as a reference to normalize the concentrations of the other two species. In order to interpret these plots one must understand the effects of mixing and chemistry on the organization of ambient data in a ratioratio plot. To help develop this understanding, Figure 2 shows a set of ratio-ratio plots that present different mixing and/or aging scenarios involving three compounds ( $a, b$, and c) that are emitted by one, two, or three different sources. The ratios plotted in Figure 2 are calculated using the equations developed below, three synthetic source profiles $\left(\mathrm{S}_{1}, \mathrm{~S}_{2}\right.$, and $\left.\mathrm{S}_{3}\right)$, and specified kinetic rate constants for each species. The ratio-ratio method is also a powerful tool for visualizing the source apportionment problem (A. L. Robinson et al., Methodology for selecting source profiles for chemical mass balance modeling using organic molecular markers, submitted to Environmental Science and Technology, 2005) (hereinafter referred to as Robinson et al., submitted manuscript, 2005). 

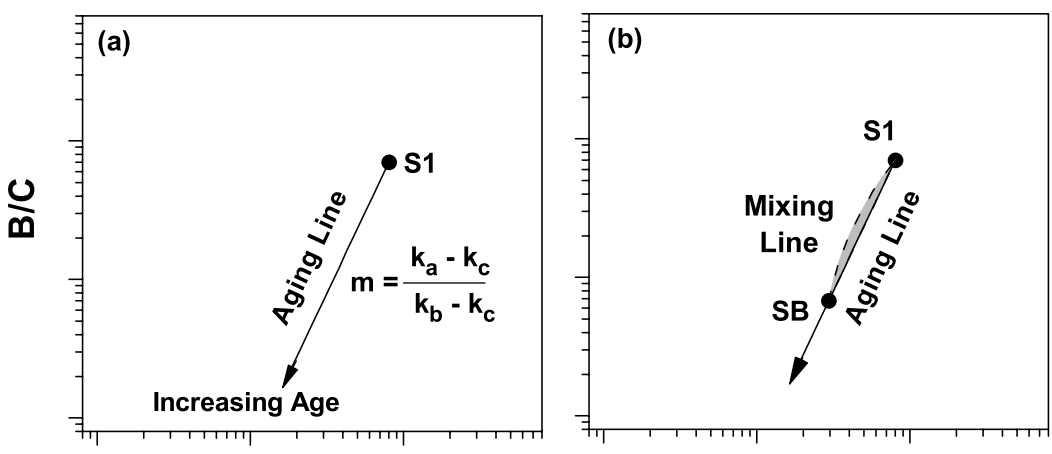

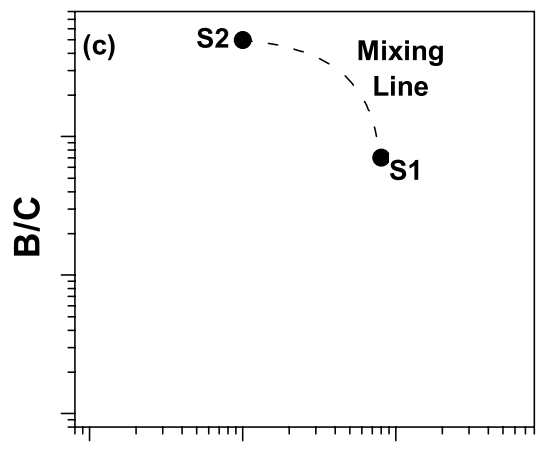

$\mathrm{A} / \mathrm{C}$

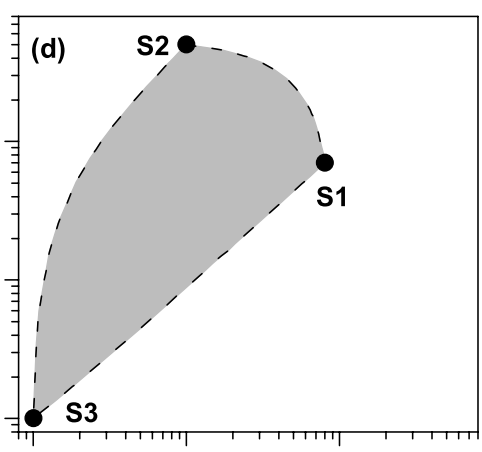

A/C

Figure 2. Ratio-ratio plot constructed from synthetic data for three compounds $\mathrm{A}, \mathrm{B}$, and $\mathrm{C}$ with $\mathrm{C}$ used as the reference. The solid circles indicate source profiles. (a) Emissions from a single source (S1): ambient ratios of non-reactive compounds will fall on point $\mathrm{S} 1$ while ambient ratios of reactive species will distribute along the aging line. (b) Oxidation and mixing of fresh (S1) and aged emissions (SB) from the same source: mixing of fresh and aged emissions is difficult to distinguish from the aging line. (c) Source-source mixing of emissions from two sources (S1 and S2): ambient concentrations of non-reactive compounds will fall on the dashed mixing line. (d) Source-source mixing of emissions from 3 sources (S1, S2 and S3): ambient concentrations of non-reactive compounds will fall inside the grey shaded region bound by the dashed source-source mixing lines. Note that linear mixing lines often appear curved on log-log plots.

[26] We first consider ratios of ambient concentrations for three non-reactive compounds emitted from a single source. Downwind from the source, the concentration of the emissions is reduced by atmospheric mixing and deposition,

$$
\mathrm{C}_{\mathrm{a}}=\mathrm{S}_{1}(\mathrm{t}) \mathrm{a}_{1}
$$

where $\mathrm{S}_{1}(\mathrm{t})$ is the time varying concentration of the emissions from source $\mathrm{S}_{1}$ at the receptor site, and $\mathrm{a}_{1}$ is the fractional abundance of species a in emissions from $\mathrm{S}_{1}$. Ambient concentration ratios of three non-reactive species ( $a, b$, and c) emitted by a single source appear as a point on a ratio-ratio plot,

$\frac{\mathrm{C}_{\mathrm{a}}}{\mathrm{C}_{\mathrm{c}}}=\frac{\mathrm{S}_{1}(\mathrm{t}) \mathrm{a}_{1}}{\mathrm{~S}_{1}(\mathrm{t}) \mathrm{c}_{1}}=\frac{\mathrm{a}_{1}}{\mathrm{c}_{1}}=$ fixed $\quad \frac{\mathrm{C}_{\mathrm{b}}}{\mathrm{C}_{\mathrm{c}}}=\frac{\mathrm{S}_{1}(\mathrm{t}) \mathrm{b}_{1}}{\mathrm{~S}_{1}(\mathrm{t}) \mathrm{c}_{1}}=\frac{\mathrm{b}_{1}}{\mathrm{c}_{1}}=$ fixed

This assumes that the effects of time-varying emission rates, transport, and deposition described by $\mathrm{S}_{1}(\mathrm{t})$ are not species dependent. This is true for condensed species present in internally mixed particles, but likely not valid for mixedphase ratios of particle and gas phase species which have different deposition rates. Equation (5) applies to both emissions from a single source and a spatially distributed set of sources, as long as this set of sources emit compounds a, $\mathrm{b}$ and $\mathrm{c}$ in the same ratios. We refer to this set of sources as a source class.

[27] Both oxidation and source-source mixing can alter the concentration ratios as emissions are transported downwind from a source. We first develop the simplest case, oxidation of emissions from a single source. Under the assumption that the species react with a common oxidant via a bimolecular reaction, ambient concentrations vary as,

$$
\mathrm{C}_{\mathrm{a}}=\mathrm{S}_{1}(\mathrm{t}) \mathrm{a}_{1} \exp \left(-\mathrm{k}_{\mathrm{a}} \mathrm{C}_{\mathrm{ox}} \mathrm{t}\right)
$$

where $\mathrm{k}_{\mathrm{a}}$ is the oxidation rate constant for species a, and $\mathrm{C}_{\mathrm{ox}}$ is the oxidant concentration. Combining similar expressions for compounds $\mathrm{b}$ and $\mathrm{c}$, parametric expressions for the ratioratio pair are

$$
\begin{aligned}
& \ln \left(\frac{\mathrm{C}_{\mathrm{a}}}{\mathrm{C}_{\mathrm{c}}}\right)=\ln \left(\frac{\mathrm{a}_{1}}{\mathrm{c}_{1}}\right)-\left(\mathrm{k}_{\mathrm{a}}-\mathrm{k}_{\mathrm{c}}\right) \mathrm{C}_{\mathrm{ox}} \mathrm{t} \\
& \ln \left(\frac{\mathrm{C}_{\mathrm{b}}}{\mathrm{C}_{\mathrm{c}}}\right)=\ln \left(\frac{\mathrm{b}_{1}}{\mathrm{c}_{1}}\right)-\left(\mathrm{k}_{\mathrm{b}}-\mathrm{k}_{\mathrm{c}}\right) \mathrm{C}_{\mathrm{ox}} \mathrm{t}
\end{aligned}
$$

where the natural logarithm of the ratios varies linearly with the net oxidant exposure $\left(\mathrm{C}_{\mathrm{ox}} \mathrm{t}\right)$ or photochemical age of the 
emissions. The slope of this relationship is defined by the rate constants and the intercept by the species concentration ratio at the point of emissions.

[28] Figure 2a illustrates how ambient concentrations of reactive compounds emitted by a single source organize on a ratio-ratio plot. To avoid singularities the least reactive species is used as the reference compound (e.g. $\mathrm{k}_{\mathrm{a}}$ and $\mathrm{k}_{\mathrm{b}}>\mathrm{k}_{\mathrm{c}}$ ). The point labeled $\mathrm{S}_{1}$ indicates fresh emissions. Oxidation reduces ambient concentration ratios along an aging line that emanates from $\mathrm{S}_{1}$. The length of this line increases with photochemical age. Since the least reactive species is used as the reference compound the aging line will appear as a diagonal with the source located in the upper right hand quadrant of the ratio-ratio plot. The slope of the aging line is defined by the rate constants of the different species,

$$
\frac{\partial \ln y}{\partial \ln x}=\frac{\left(k_{a}-k_{c}\right)}{\left(k_{b}-k_{c}\right)}
$$

[29] In developing this set of equations, we have assumed that reagent concentrations change in an exponential fashion - equation (6). This is true if $\mathrm{C}_{\mathrm{ox}}$ is the local oxidant concentration inside a particle, but is not necessarily true if one considers the spatially averaged oxidant concentration [Donahue et al., 2005]. For example, mass transfer limitations can cause condensed phase concentrations to vary parabolically and not exponentially with gas phase oxidant concentrations [Smith et al., 2002]. Therefore, the rate constants used in these equations are effective rate constants and not true rate constants. This distinction is not important in context of this paper, but must be considered if one seeks to extract quantitative kinetic information from the data. An advantage of considering ratios of condensed species is that these issues involving oxidant uptake are expected to largely cancel out [Donahue et al., 2005].

[30] Unfortunately, no set of compounds is emitted by a single source and we will always need to consider the effects of mixing to properly interpret ratio-ratio plots. At best the set of compounds will be emitted by a single source class that is spatially distributed, which requires accounting for the effects of mixing of fresh and aged emissions. If the compounds are also emitted by multiple source classes then we will also have to account for the effects of source-source mixing.

[31] The mathematics describing mixing can be developed by considering a set of non-reactive compounds emitted by two sources. The ambient concentration of one of these species is

$$
\mathrm{C}_{\mathrm{a}}=\mathrm{S}_{1}(\mathrm{t}) \mathrm{a}_{1}+\mathrm{S}_{2}(\mathrm{t}) \mathrm{a}_{2}
$$

where $\mathrm{S}_{1}(\mathrm{t})$ and $\mathrm{S}_{2}(\mathrm{t})$ are the concentration of emissions from the two sources at the receptor site, and $\mathrm{a}_{1}$ and $\mathrm{a}_{2}$ are the relative abundances of compound a in emissions in each source. Combining similar expressions for compounds $b$ and $\mathrm{c}$, the ratio-ratio pair can be expressed by a linear mixing line connecting the two source profiles,

$$
\frac{\mathrm{C}_{\mathrm{a}}}{\mathrm{C}_{\mathrm{c}}}=\frac{\mathrm{a}_{1}}{\mathrm{c}_{1}} \mathrm{w}+\frac{\mathrm{a}_{2}}{\mathrm{c}_{2}}(1-\mathrm{w}) \quad \frac{\mathrm{C}_{\mathrm{b}}}{\mathrm{C}_{\mathrm{c}}}=\frac{\mathrm{b}_{1}}{\mathrm{c}_{1}} \mathrm{w}+\frac{\mathrm{b}_{2}}{\mathrm{c}_{2}}(1-\mathrm{w})
$$

The exact position along the line depends on a weighting factor that is a function of the relative contribution of each source to the reference compound $\mathrm{c}$ at the receptor site, $\mathrm{w}=$ $\left(1+\mathrm{S}_{2} \mathrm{c}_{2} /\left(\mathrm{S}_{1} \mathrm{c}_{1}\right)\right)^{-1}$. This factor varies between 0 and 1 , so every point will lie between the two sources. Linear mixing lines often appear curved when ratio-ratio plots are displayed with log-log axes.

[32] Before considering source-source mixing, we examine how mixtures of fresh and aged emissions from the same source class organize on a ratio-ratio plot. The simplest scenario is a mixture of fresh emissions with aged background air that contains oxidized emissions of a single age $\left(\mathrm{S}_{\mathrm{B}}\right)$. This mixture will fall somewhere along the mixing line shown in Figure $2 \mathrm{~b}$. Note that the endpoints of this curved mixing line fall on the aging line. The degree of curvature of the mixing line depends on the oxidation rate constants and the age of the background air. If the rate constants of the two target species are similar then the curvature is modest (the rate constant ratio used to generate Figure $2 \mathrm{~b}$ is $0.6: 1$ ).

[33] A more realistic scenario involves mixing of fresh emissions with background air that contains emissions that span a wide range of photochemical ages. This occurs when the sources are spatially distributed. Assuming that $\mathrm{S}_{\mathrm{B}}$ represents the most aged emissions in the background air, the mixture of fresh and aged particles will lie somewhere in the shaded region enclosed by the aging and mixing lines plotted in Figure 2b. Although it is difficult to differentiate between direct oxidation and mixing of fresh and aged emissions using a ratio-ratio plot, we do not need to distinguish between these two phenomena because they are both ultimately due to photochemistry.

[34] In order to help constrain the effects of oxidation, we group compounds by expected oxidation rate when constructing ratio-ratio plots. Ideally the rate constants of the two target compounds $\mathrm{a}$ and $\mathrm{b}$ are similar while the rate constant for the reference compound $\mathrm{c}$ is small or zero. If these compounds are emitted by a single source class, then Figure $2 \mathrm{~b}$ indicates that oxidation will cause ambient data to organize along the diagonal in a ratio-ratio plot with a limit in the upper-right-hand quadrant defined by a source and an almost 1 to 1 slope. Since sources are spatially distributed, the data will likely organize along a curved line on a log-log plot; however, it is unlikely that the ambient data will be sufficiently precise to resolve this curvature.

[35] Many of the compounds of interest are emitted by multiple source classes, each with unique emission ratios; therefore, distinguishing the effects of source-source mixing from photochemistry is essential to avoid misinterpreting ratio-ratio plots. The key distinction is that for sourcesource mixing there is no general restriction on the position of two distinct sources on a ratio-ratio plot; for example, Figure $2 \mathrm{c}$ shows a source-source mixing line that is orthogonal to the expected effects of oxidation. When sources are oriented in this fashion, one can easily differentiate between source-source mixing and photochemistry using ratio-ratio plots.

[36] Source-source mixing presents a challenge when the source pair is organized along the aging line - for example, if $\mathrm{S}_{\mathrm{B}}$ in Figure $2 \mathrm{~b}$ was another source and not aged background air. In this situation, varying the relative contributions of the two sources will cause ambient concen- 
trations of non-reactive compounds to organize in a manner consistent with oxidation. This effect is noteworthy because many appealing reference compounds (the large n-alkanes or elemental carbon) have many sources, some of which are severely depleted in tracer concentrations. In these situations, information about source activity is used to further constrain the effects of source-source mixing.

[37] Mixing of emissions from three or more distinct sources or source-source mixing combined with photochemistry can result in ratio-ratio plots that are difficult to interpret. For example, Figure $2 \mathrm{~d}$ shows a ratio-ratio plot involving three sources. The source-source mixing lines defined by these sources create a convex hull (shaded region in Figure 2d) and mixing of non-reactive compounds can cause ambient data to fall anywhere inside this region. Alternatively $\mathrm{S}_{3}$ in Figure $2 \mathrm{~d}$ could represent aged background air that is mixed with fresh emissions from $\mathrm{S}_{1}$ and $\mathrm{S}_{2}$.

[38] When constructing ratio-ratio plots, we group species to minimize the number of potential source classes. Ideally each plot would consider a set of species emitted by a single source class which would eliminate source-source mixing. In all cases, knowledge of the position of different sources on the ratio-ratio plots is essential to avoid misinterpreting these plots.

\section{Evidence of Photochemical Oxidation in Ambient Data}

[39] In this section we apply the ratio-ratio technique to a large data set of ambient condensed-phase organic species measured as part of the Pittsburgh Air Quality Study (PAQS). The PAQS Supersite was located in the city of Pittsburgh in an urban park. Fine particle concentrations in Pittsburgh are dominated by regional transport. Typically $80-90 \%$ of $\mathrm{PM}_{2.5}$ in the Pittsburgh area comes from outside southwestern Pennsylvania [Tang et al., 2004]. This transport provides significant time for photochemical processing. More information about the site and PAQS are provided by Wittig et al. [2004].

[40] Samples for organic speciation were collected using a $\mathrm{PM}_{2.5}$ sampler consisting of a quartz filter with a downstream polyurethane foam (PUF) plug operated at $140 \mathrm{lpm}$ for 24-hrs. One hundred and three 24-hr samples were collected at the PAQS Supersite between July, 2001 and August, 2002. Daily 24-hr samples were collected during July 2001 and for two weeks in January 2002. During the rest of the study, samples were collected every sixth day. During January 2002 and July 2002, 24-hr samples were also collected at a rural site in Florence, PA in parallel with those at the central Supersite using an identical sampler. Florence is 25 miles west-southwest of Pittsburgh near the West Virginia border. Measurements of organic and elemental carbon were also made at both sites [Subramanian et al., 2004].

[41] The filter and PUF were solvent extracted with dichloromethane and the extract analyzed by GC/MS. Before extraction each filter was spiked with a known amount of an internal standard mix consisting of a suite of seven perdeuterated n-alkanes. The amount of the internal standard spike varied from sample-to-sample, depending on the filter OC loading. The extracts from the filter-PUF pairs were combined; therefore, the reported concentrations are for gas plus particle phase. The overall volume of the combined extracts was reduced to $\sim 100 \mu \mathrm{l}$ and then methylated using freshly prepared diazomethane to convert fatty acids to their methyl ester analogs prior to GC/MS analysis using electron impact ionization. Each analyte was quantified by reference to the internal standard and using a relative response factor determined by the analysis of quantification standards. Procedures such as pre-baking filters and extensive washing of PUFs were used to minimize contamination. Field and laboratory blanks were routinely collected and analyzed to track and correct for any contamination. Samples were stored at $-20{ }^{\circ} \mathrm{C}$ prior to analysis. Additional details of the sample collection and analysis are described elsewhere (A. Bernardo-Bricker et al., manuscript in preparation, 2006).

[42] Although the Pittsburgh data set contains measurements of more than 100 condensed-phase and semi-volatile organic compounds, here we examine two groups of compounds: markers for motor vehicle and cooking emissions. Ambient concentrations of these markers are well correlated, suggesting a single dominant source in each case (R. Subramanian et al., Contribution of motor vehicle emissions to the ambient organic carbon in Pittsburgh, PA: Effects of varying source profiles and seasonal trends in ambient marker concentrations, submitted to Atmospheric Environment, 2006, hereinafter referred to as Subramanian et al., submitted manuscript, 2006; Robinson et al., submitted manuscript, 2005). The resulting well-organized ratioratio plots provide good examples for the current discussion.

\subsection{Hopanes and $\mathrm{OH}$ Oxidation}

[43] First we examine the Pittsburgh data set for evidence of photochemical oxidation of triterpanoid hopanes (hopanes). Hopanes are found in crude oil and are important markers for motor-vehicle exhaust [Schauer et al., 1996; Simoneit, 1984]. Hopanes are saturated, cyclic hydrocarbons and therefore are expected to react with $\mathrm{OH}$ but not $\mathrm{O}_{3}$. For this analysis we consider the two most abundant hopanes in the Pittsburgh data set: $17 \alpha(\mathrm{H}), 21 \beta(\mathrm{H})-29-N o r h o p a n e$ (norhopane) and $17 \alpha(\mathrm{H}), 21 \beta(\mathrm{H})$-Hopane (hopane). As a reference compound we use elemental carbon (EC), which is not reactive. In Pittsburgh and other urban locations, concentrations of EC and hopanes are dominated by mobile source emissions [Schauer et al., 1996; Zheng et al., 2002; Subramanian et al., submitted manuscript, 2006].

[44] Figure 3 shows a ratio-ratio plot of hopane and norhopane, each normalized by EC. The data have been sorted by season. 'Summer' is defined as June-September, 'winter' is December-March, and 'fall/spring' is defined as the remaining four months, giving each of the three periods an equal weighting in time. Because the PAQS intensives were in July and January, summer and winter were disproportionately sampled. In Figure 3, the winter data cluster in the upper right, while the summer data spread from this cluster roughly along a 1:1 line. The data are consistent with photochemical oxidation. Such a scenario implies that there is a relatively stable emissions profile located at the upper extreme of the observed ratios and that oxidation reduces the ratios by different degrees through out the year slightly during the winter and substantially during the summer. 


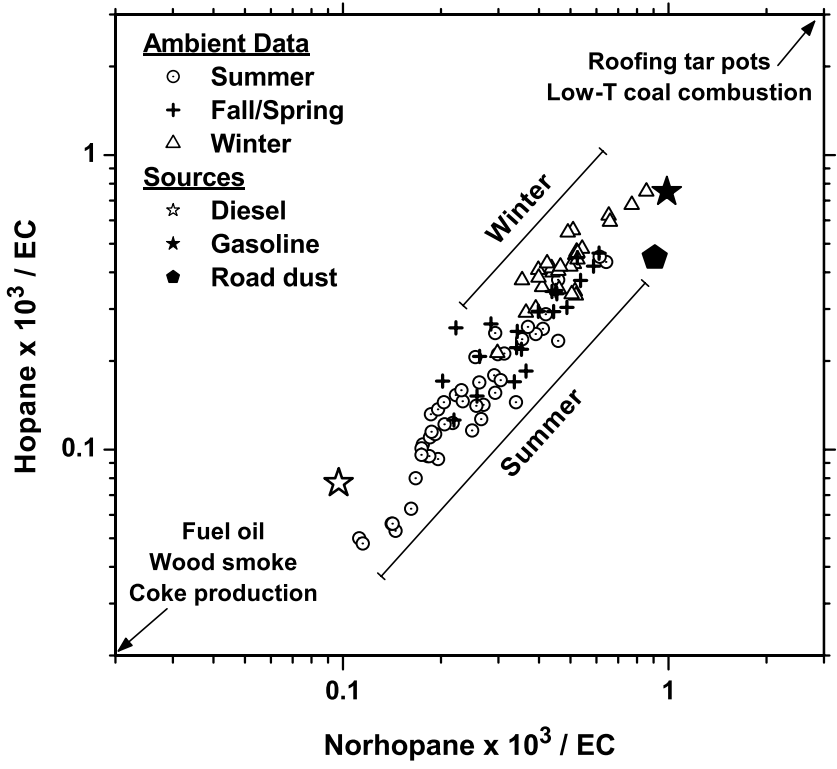

Figure 3. Ratio-ratio plot of two hopanes normalized by EC for 96 daily samples collected in Pittsburgh, PA. Also shown are emission ratios for different sources of hopanes and EC. The gasoline and diesel profiles are averages of a large number of published vehicle profiles [Fraser et al., 2002; Rogge et al., 1993a; Schauer et al., 1999b, 2002c; Watson et al., 1998]. Road dust profile is from Robinson et al. [2005]. Arrows point to sources that do not fall within the bounds of the plot, as discussed in the text. With the exception of a few outliers, the winter data are tightly clustered near $(0.5,0.4)$. Fall/spring data are more spread out, extending to the lower left from the winter cluster along the 1:1 line. Summer data are even more spread along the same line. This is consistent with extensive oxidation during the summer, corresponding to a non-dimensional lifetime $(\mathrm{t} / \tau)$ between 0.5 and 1.5 , relative to wintertime conditions.

[45] One must consider the absolute levels in addition to ratios; time series of monthly average concentrations are shown in Figure 4a. There is a factor-of-four variation in the monthly average hopane, norhopane, and EC levels with no clear seasonal patterns. For example, EC levels are lowest in the winter, but comparably low levels were also observed in July 2001. Within a given season there is significant month-to-month variability; for example monthly average EC in the summer varies between 0.4 and $1 \mu \mathrm{g} / \mathrm{m}^{3}$. Monthly average concentrations of the two hopanes also exhibit significant variability. This variability reflects the considerable influence that meteorological factors such as regional transport patterns, boundary layer height, and deposition rates have upon absolute pollutant concentrations in Pittsburgh.

[46] Figure 4b shows time series of monthly average hopane- and norhopane-to-EC ratios. Unlike the absolute concentrations, these ratios exhibit a strong seasonal pattern, with the monthly average hopane-to-EC ratios in the winter being almost a factor three greater than in the summer. The phase of the signal is consistent with oxidation, as shown by the ozone annual cycle in Figure $4 \mathrm{~b}$. We are not suggesting that ozone itself is the direct cause of oxidation, but rather as one of the source components of $\mathrm{OH}$ radicals it is a reasonable surrogate for $\mathrm{OH}$. There are few direct measurements of $\mathrm{OH}$ in winter, but the evidence suggests that $\mathrm{OH}$ levels drop by at least factor of 3-5 compared with the summer [Bloss et al., 2005].

[47] Figures 3 and 4 present strong evidence for substantial aging of hopanes in the summer. However, there is not a single source of hopanes or EC, so we must consider the potential effects of source-source mixing on the ambient data. Figure 3 compares the ambient data to published emission profiles for road dust, gasoline vehicles, and diesel vehicles. The road dust composition is based on samples collected on urban and rural roads around Pittsburgh [Robinson et al., 2005]. The gasoline and diesel vehicle profiles are emissionweighted averages of published source profiles [Fraser et al., 2002; Rogge et al., 1993a; Schauer et al., 1999b, 2002c; Watson et al., 1998]. The arrows in Figure 3 point to sources that fall outside the bounds of the ratio-ratio plot. Tar pots used in roof construction and low-temperature coal combustion have large hopane- and norhopane-to-EC ratios [Oros and Simoneit, 2000; Rogge et al., 1997a]. Biomass combustion and coke production emit EC but not hopanes, while emissions from fuel oil combustion has a very small hopaneto-EC ratio [Rogge et al., 1997b].

[48] Figure 3 indicates that source-source mixing could explain the ambient concentration ratios of hopanes and EC. However, the strong seasonal pattern in the ambient ratios is a critical constraint; to explain this pattern without aging, the relative contribution of the different sources must vary seasonally, with sources either poor in hopanes or rich in EC gaining relative strength in the summer.

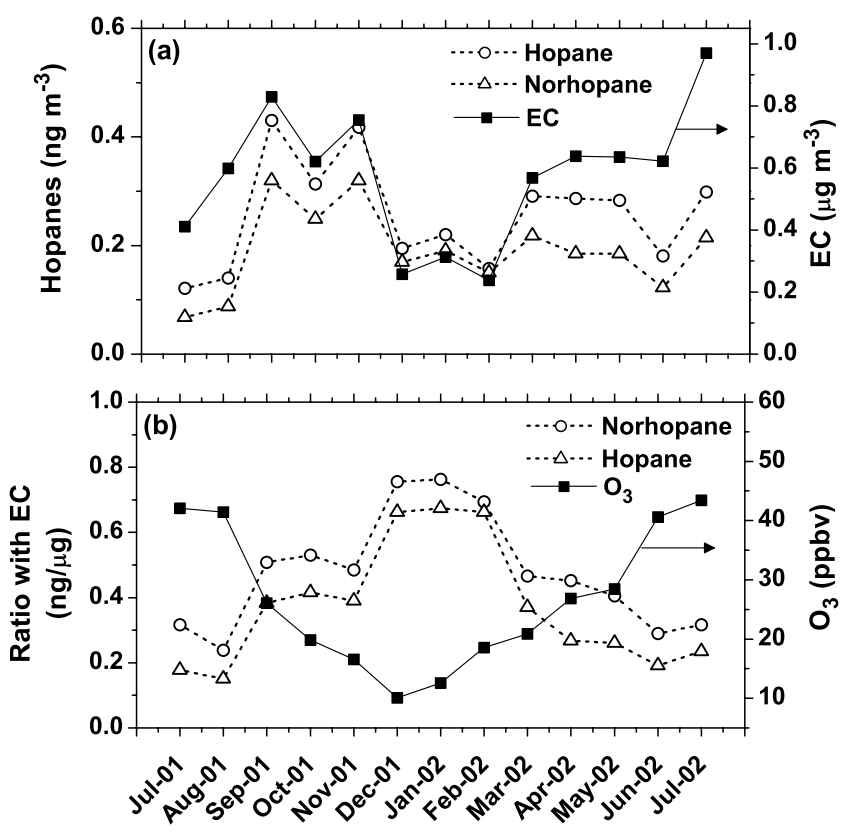

Figure 4. Monthly average (a) hopane, norhopane and elemental carbon (EC) concentrations and (b) ratios with EC ratios measured in Pittsburgh from July 2001 to July 2002. Ozone mixing ratio is also plotted in Figure $4 \mathrm{~b}$ as an indicator of photochemical activity. The hopane-to-EC ratios and $\mathrm{O}_{3}$ levels show strong seasonal patterns with low summer hopane-to-EC ratios consistent with hopane oxidation (presumably by $\mathrm{OH}$ radicals derived from the ozone). 


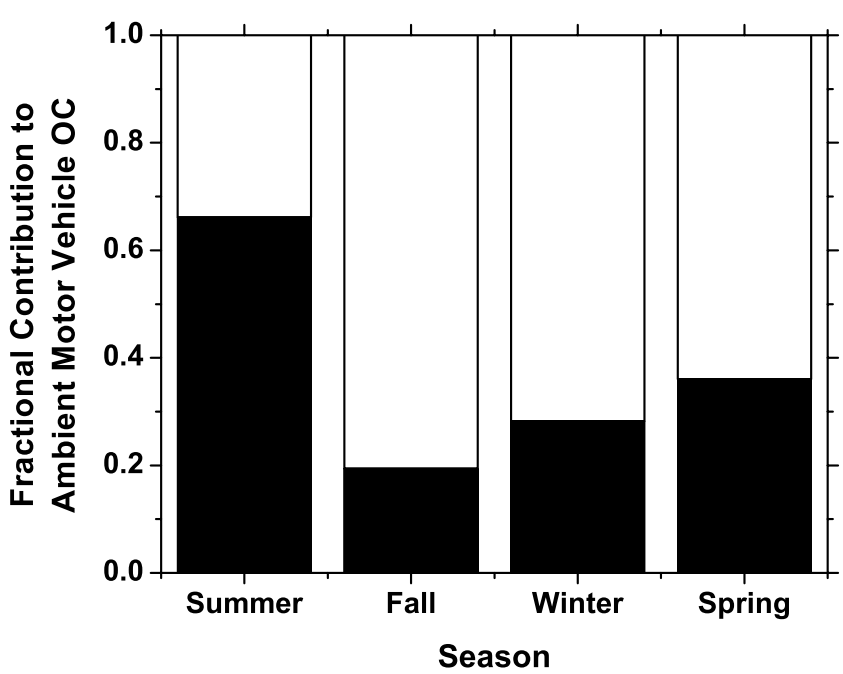

Figure 5. Seasonal average gasoline-diesel split in Pittsburgh predicted using the Chemical Mass Balance (CMB) model. The black bar indicates the fractional contribution from diesel vehicles with the balance from gasoline vehicles. CMB is applied to 96 days of data using 9 sources and 19 fitting species; details of the calculations are provided by Subramanian et al., submitted manuscript, 2006). The strong seasonal pattern in the predicted gasolinediesel split is caused by the seasonal variation in the hopane-to-EC ratios shown in Figure 4.

[49] First we consider potential variability of vehicular sources, the dominant sources of hopanes and EC in Pittsburgh (Subramanian et al., submitted manuscript, 2006). The ambient data are located on the source-source mixing line that connects fleet-average gasoline and diesel vehicle source profiles. Therefore, the ambient data can be explained by seasonally varying contributions of gasoline and diesel vehicles with gasoline vehicles dominant in the winter and diesels in the summer. This large variation is apparent in the Chemical Mass Balance Model (CMB) predictions shown in Figure 5, which are based on the fleet-average profiles shown in Figure 3. These CMB calculations involve 19 species and nine different sources including wood combustion, coke production, and other sources of EC. These calculations are discussed in detail by Subramanian et al., submitted manuscript, 2006.

[50] In order to assess the plausibility of large seasonal variation in the relative contribution of gasoline and diesel vehicles as an explanation for the Pittsburgh data, one must consider two factors: potential seasonal changes in vehicle activity and potential seasonal changes in vehicle emission factors. Fuel consumption data are a reliable measure of vehicle activity. There is a modest seasonal pattern in total on-road fuel usage. However, on-road gasoline and diesel usage follow the same temporal pattern so there is no seasonal variation in the relative amounts of gasoline and diesel fuel consumed by on-road vehicles [EIA, 2002; $F H W A, 2005]$. Non-road vehicles do consume 10-20\% more diesel in the summer than the winter $[E I A, 2002$; $F H W A, 2005]$, which likely explains some of the shift in the ambient data in Figure 3 towards the diesel profile in the summer. However, a 20\% increase in non-road diesel usage only corresponds to a $5 \%$ increase in total diesel consumption because the majority of diesel fuel is used by on-road vehicles. Such a small increase seems an unlikely explanation for the large seasonal pattern in the ambient data shown in Figure 3.

[51] Another potential explanation for the seasonal patterns in the ambient data is seasonal changes in motor vehicle emission factors. For example, the ambient data could be explained if gasoline vehicles emitted a factor of 2 more organic aerosol mass per mile traveled in the winter than in the summer while diesel emissions remained unchanged. Alternatively the hopane-to-EC ratios of vehicle emissions could vary seasonally, shifting the position of the source points in Figure 3. Seasonal changes in temperature and/or fuel composition likely influence vehicle emission rates, but relatively little is known about these effects, especially on speciated emissions. EC emissions from gasoline vehicles are generally higher in colder weather. The only published cold weather profiles with speciated organics data have smaller hopane-to-EC ratios than summertime profiles [Watson et al., 1998; Zielinska et al., 2004; Subramanian et al., submitted manuscript, 2006]. This is opposite to the pattern shown in the ambient data in Figures 3 and 4 . In addition, the daily summer hopane-to-EC ratio varies widely, which is hard to explain with seasonally varying emission factors.

[52] Emissions from non-vehicular sources of hopanes and EC are unlikely to explain the seasonal patterns in the ambient data shown in Figures 3 and 4. For example, the amount of wood, fuel oil, and other fuels used for heating does vary seasonally. These processes emit EC but little or no hopanes, thus pulling the ambient ratios diagonally towards the lower left hand corner of the plot. However, the smallest hopane-to-EC ratios are observed in the summer, not the winter: exactly opposite to the effect that seasonally varying emissions associated with heating should have on ambient concentrations. Significant wood smoke contributions in the summer from forest fires or agricultural burning could explain the ambient data, but summertime concentrations of biomass smoke markers such as levoglucosan in Pittsburgh are extremely low. Other non-vehicular sources of hopanes, such as tar pots, low temperature coal combustion, and coke production, are thought to be very minor sources.

[53] Although we cannot completely rule out the possibility of seasonally varying motor vehicle emissions, a number of additional pieces of evidence strongly support the hypothesis that there is significant oxidation of hopanes in the summer. The first piece of evidence is seasonal differences in ambient concentrations of hopanes in Pittsburgh and a rural site in Florence, PA. This site is almost always upwind of the city and therefore provides data on the pollutants in the regional background air flowing into Pittsburgh [Wittig et al., 2004]. Tang et al. [2004] report that fine particle mass and bulk composition measured at the Pittsburgh, Florence and other sites in Southwestern PA are very similar, underscoring the significant effect that regional transport has on pollutant levels.

[54] Scatterplots comparing 24-hr organic carbon (OC) and hopane concentrations in Pittsburgh and Florence are shown in Figure 6. In January 2002, both OC and hopane concentrations are $\sim 35 \%$ higher in the city than in Florence, 

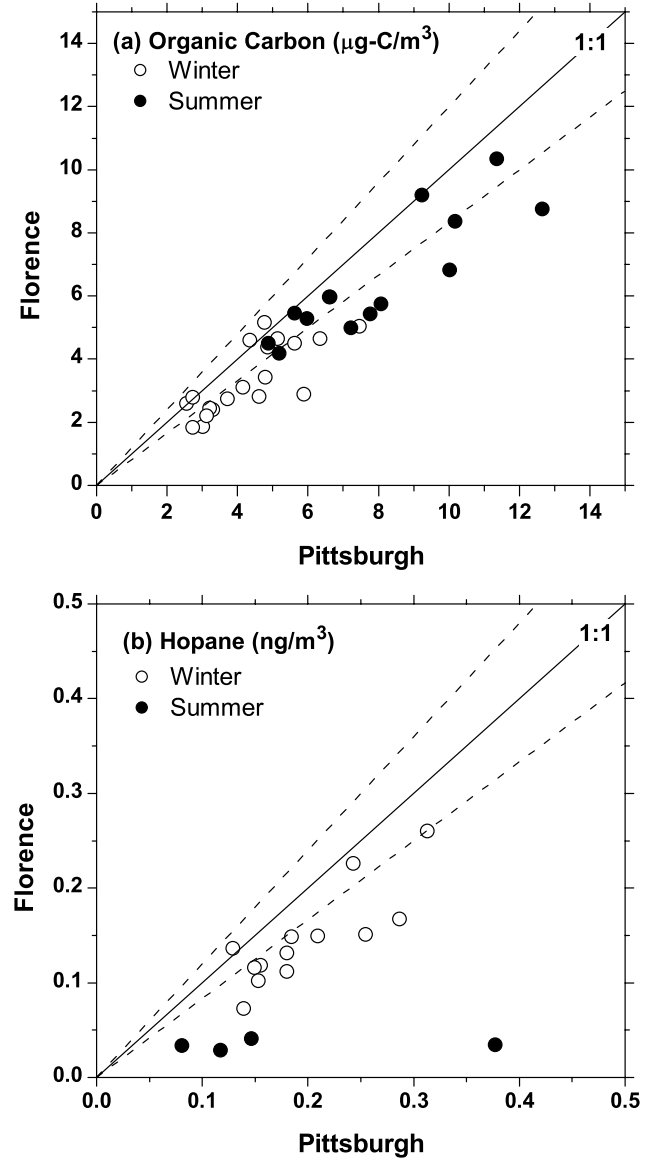

Figure 6. Scatterplots of daily (a) organic carbon (OC) and (b) hopane concentrations measured in Pittsburgh, PA and an upwind rural site in Florence, PA. Given the similarly of the $\mathrm{OC}$ concentrations in the city and the upwind site, a compelling explanation is that there is significant photochemical decay of hopanes during the summer in the regional air mass. The dashed lines indicate points within a factor of 1.2 of the one-to-one line.

reflecting the some contribution of local sources to $\mathrm{OC}$ in the city and the significant contribution of motor vehicle emissions to OC in the regional air mass. In July 2002, the OC concentrations in Pittsburgh are on average 20\% higher than in Florence, but the hopane concentrations in Florence are on average factor of 6 lower than those in the city. Either motor vehicles are not a significant source of fine particles in the summer in Florence or hopanes in the regional air mass are being oxidized during regional transport.

[55] Figure 7 plots paired averages of the Pittsburgh and Florence data on a hopane-to-EC ratio-ratio plot; the averages only include days when measurements were made at both sites. During the winter, the average hopane- and norhopane-to-EC ratios in Pittsburgh and Florence are similar, with the data from both sites falling within wintertime scatter of the complete Pittsburgh data set. However, during the summer, the hopane- and norhopane-to-EC ratios are much smaller in Florence than in Pittsburgh, consistent with significant photochemical oxidation of hopanes in the regional air mass. This regional variation cannot be explained by seasonally varying emission profiles. Even in the winter there may be some oxidation of hopanes in the regional air mass passing through Florence.

[56] Seasonal variations in hopane and EC concentrations in the Southeastern United States also support the conclusion that hopanes are photochemically oxidized in regional air masses and in the summer. Figure 7 also plots hopanesto-EC ratios for Atlanta GA, Birmingham AL, Pensacola FL, and Gulfport MS [Zheng et al., 2002]. In three of these cities the hopane-to-EC ratios are significantly lower in the summer than in the winter. The wintertime ratio in Atlanta is consistent with the Pittsburgh data, while the ambient ratios in Birmingham and Pensacola are somewhat lower. The lone exception is Gulfport, where the summer and winter ratios are the same. The arrow in the lower-left-hand corner of Figure 7 points to data from four rural sites in the Southeast collected in both the summer and the winter. The rural data fall outside the boundaries of the ratio-ratio plot, consistent with the regional air mass in the Southeast being significantly depleted in hopanes relative to EC throughout the entire year. All of the sites in the Southeast should have less pronounced annual cycles in $\mathrm{OH}$ compared to Pittsburgh, so significant regional oxidation throughout the year is not surprising.

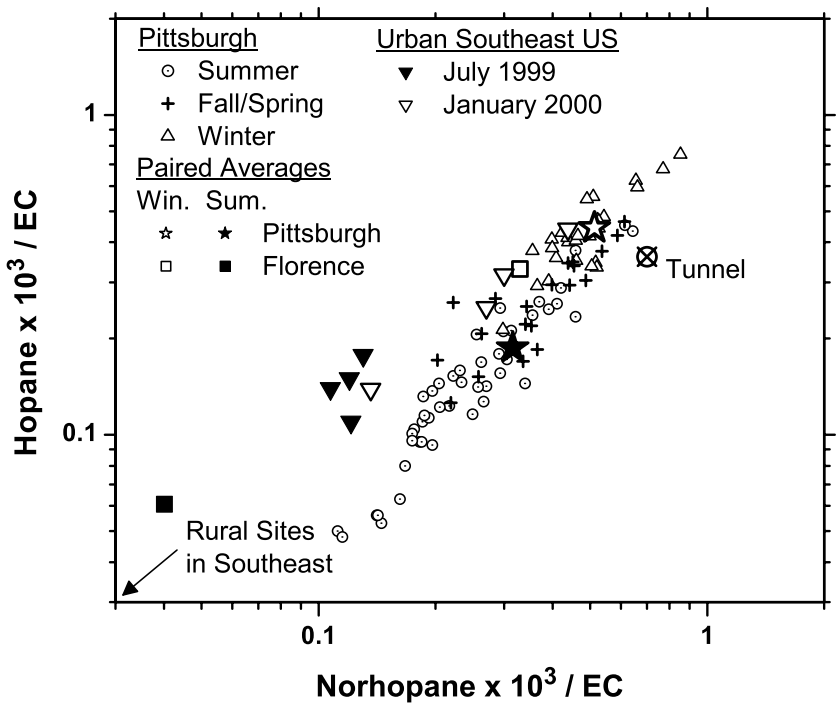

Figure 7. Ratio-ratio plot of two hopanes normalized by EC showing Pittsburgh ambient data. In addition to the complete Pittsburgh data set the figure also plots the summer (open star and square) and winter (solid star and square) paired-averages of measurements made simultaneously in Pittsburgh and an upwind rural site in Florence, PA; measurements made in a highway tunnel in Pittsburgh; and summer and winter measurements made in four cities in the Southeastern United States [Zheng et al., 2002]. The arrow in the lower-left-hand corner points to data from rural sites in the Southeast that fall outside the bounds of the plot. Wintertime hopane-to-EC ratios are similar at all of the sites, expect for Gulfport, MS (open upside-down triangle adjacent to solid upside-down triangles). Summertime hopane-to-EC ratios are lower at all sites suggesting photochemical decay of hopane (again Gulfport, MS is an exception). Data from the Southeastern United States courtesy of Mei Zheng at Georgia Institute of Technology. 


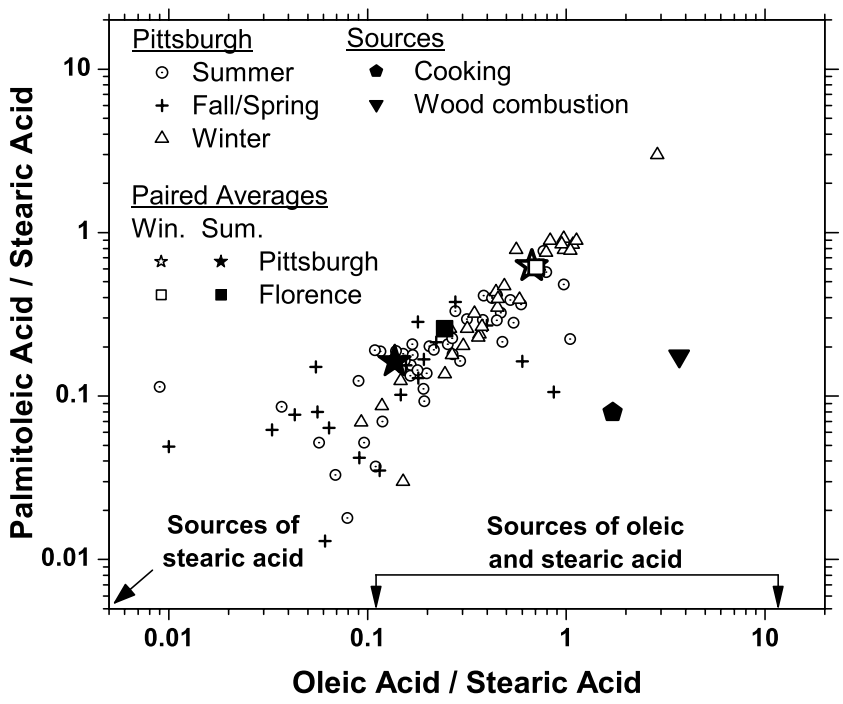

Figure 8. Ratio-ratio plot of two alkenioc acids, palmitoleic (8-hexadecenoic) acid and oleic (9-octadecenoic) acid, normalized by stearic (n-octadecanioc) acid. Also shown are summer and winter paired-averages of measurements made simultaneously in Pittsburgh and an upwind rural site in Florence, PA. The source profiles are averages of a large number of published cooking and wood combustion profiles [Fine et al., 2001, 2002, 2004; Hays et al., 2002; Rogge et al., 1998, 1991; Schauer et al., 1999a, 2001, 2002b]. Arrows point to sources that do not fall within the bounds of the plot. Motor vehicles are an important source of stearic acid but not the unstaturated acids [Rogge et al., 1993a; Schauer et al., 1999b, 2002c]. There are a number of sources of oleic and stearic acids but not palmitoleic acids including certain types of wood combustion, road dust, and vegetative detritus: the oleic-to-stearic acid ratio of these sources range from 0.11 to 13 . The data are suggestive of photochemical decay of the unsaturated acids during the winter.

[57] A complication for interpreting the hopane-to-EC ratios measured in the Southeast is biomass burning. Wood smoke concentrations are much higher in the Southeast than in Pittsburgh [Zheng et al., 2002; A. L. Robinson et al., manuscript in preparation, 2006]. However, wood smoke concentrations in the southeast are lowest in the summer and highest in the winter [Zheng et al., 2002], opposite to the pattern required to explain the trends shown in Figure 7.

[58] Collectively, the ambient data from the Pittsburgh region and the Southeast provide compelling evidence that hopanes are significantly oxidized in the summer. A final piece of evidence is measurements of fresh, in-use vehicle emissions made in a highway tunnel in the Pittsburgh region in November 2002. Figure 7 shows an average of the tunnel data collected for a range of fleet compositions and operating modes (A. P. Grieshop et al., Diesel and gasoline vehicle gaseous and fine particle emission factors measured in a highway tunnel in Pittsburgh, PA, submitted to Atmospheric Environment, 2005). The fresh emissions measured in the tunnel are consistent with ambient ratios observed in the winter, again supporting the hypothesis that hopanes are not significantly oxidized during the winter in Pittsburgh.
[59] Our discussion of vehicle markers has focused on two hopanes and EC. The data sets collected in Pittsburgh and the Southeast contain measurements of a number of additional hopanes and steranes, which are also commonly used as markers for motor vehicle emissions. All of the hopanes and steranes exhibit the same seasonal trends as norhopane and hopane shown in Figures 3-7. Therefore all of these compounds appear to be similarly oxidized in the summer and in the regional air mass, which, given the similarity in the chemical structure of these compounds, is not unexpected.

\subsection{Alkenoic Acids and Ozone Oxidation}

[60] In our second example we look for evidence of oxidation of unsaturated compounds by ozone. Here we are faced with laboratory evidence suggesting that unsaturated acids, such as oleic acid (9-octadecenoic acid), should be oxidized rapidly in the condensed phase $\left(\gamma=10^{-3}\right.$ line in Figure 1). Against this are field observations showing abundant alkenoic acids. Figure 8 shows a ratio-ratio plot of oleic acid and palmitoleic acid (8-hexadecenoic acid) concentrations normalized by stearic acid (n-octadecanoic acid). Cooking, motor vehicles, and biomass combustion are the major anthropogenic sources of these acids in urban environments [Rogge et al., 1996]. Biogenic emission sources of these acids may also be significant [Rogge et al., 1993d; Simoneit and Mazurek, 1982]. Oleic acid is typically emitted together with its saturated homologue, stearic acid [Rogge et al., 1996], which supports using stearic acid as the reference species.

[61] The ambient data in Figure 8 organize along a oneto-one line spanning more than 2 e-folds. Again the data are sorted by season with the winter data nearer to the upper right hand corner and the summer data falling toward the lower end of the range. The seasonal separation of the alkenoic acid data is not as dramatic as with the hopanes shown in Figure 3 because small alkenoic-to-stearic acid ratios were observed on some winter days. Overall, the ratio-ratio plot in Figure 8 is consistent with enhanced oxidation of alkenoic acids in the summer.

[62] Source profiles are also shown in Figure 8 to examine the effects of source-source mixing. The cooking and wood smoke profiles are averages of a large number of published profiles [Fine et al., 2001, 2002, 2004; Hays et al., 2002; Rogge et al., 1998, 1991; Schauer et al., 1999a, 2001, 2002b]. Arrows point to source profiles outside the boundaries of the plot shown in Figure 8. Motor vehicles are an important source stearic acid but not the unstaturated acids [Rogge et al., 1993a; Schauer et al., 1999b, 2002c]. There are a number of sources that emit oleic and stearic acids but not palmitoleic acid including certain types of wood combustion, road dust, and vegetative detritus [Fine et al., 2001, 2002, 2004; Rogge et al., 1993b, 1993c 1998; Schauer et al., 2001]: the oleic-to-stearic acid ratio of these sources range from 0.11 to 13 .

[63] The Pittsburgh data shown in Figure 8 cannot be explained by source-source mixing among the known source profiles. The problem is that the ambient data are rich in palmitoleic acid (or depleted in oleic acid) relative to the reported source profiles. The ambient data have nearly equal loadings of oleic and palmitoleic acids compared to an average oleic-to-palmitoleic acid ratio of 25 across a set of 

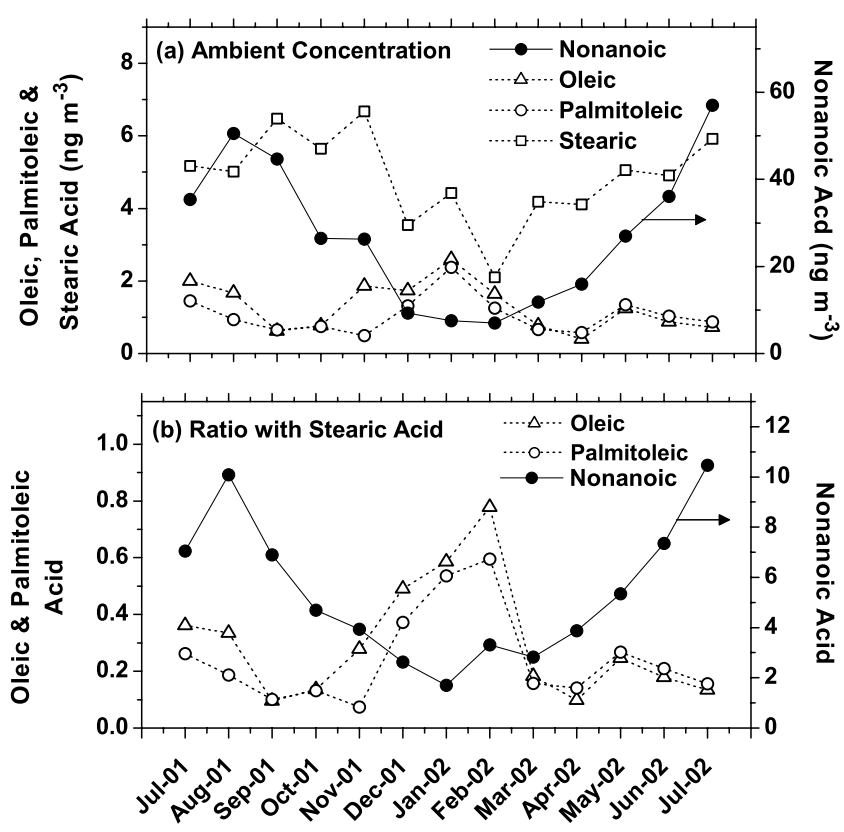

Figure 9. Monthly average (a) alkanoic and alkenoic acid concentrations and (b) ratios with stearic acid measured in Pittsburgh from July 2001 to July 2002. Ratios of oleic and palmitoleic acid with stearic acid show a strong seasonal pattern that is consistent with photo-oxidation during the summertime. Ratio of nonanoic acid with stearic acid shows opposite pattern of ratios involving an unsaturated acid. Nonanoic acid is a product of oleic acid ozonolysis. Monthly average $\mathrm{O}_{3}$ mixing ratio is shown in Figure $4 \mathrm{~b}$.

almost 100 cooking profiles. Although there is significant variability among individual cooking profiles, the profile with the smallest oleic-to-palmitoleic acid ratio (bacon frying with a ratio 4.5 ) is still significantly enriched in oleic acid compared to the ambient data. Almost all of the biomass smoke profiles are similarly enriched in oleic acid compared to palmitoleic acid; the only exceptions are the Fine et al. [2004] Quaking Aspen profile and the Hays et al. [2002] softwood open burn profiles. We do not expect that photochemical processing contributes to the apparent deficit of oleic acid because any aging should equally affect oleic and palmitoleic acids, which have similar chemical structures and sizes. Therefore, an unknown source that is very rich in palmitoleic acid is needed to explain the ambient data. In addition, the emissions from this unknown source need to be highly correlated with cooking and wood smoke contributions in order for the ambient data to organize along the one-to-one line in Figure 8.

[64] Although inconsistencies exist between the known source profiles and the Pittsburgh data, the ambient concentrations do not appear notably depleted in alkenoic acids. If we take the oleic-to-stearic acid ratios from the average cooking and wood smoke source profiles shown in Figure 8 as correct, it is consistent to suggest that all of the data are depleted in oleic acid by up to an e-fold. Therefore, it is clear that the alkenoic acids are not being decimated by ozone oxidation. It is possible that a fraction of these unsaturated acids are oxidized rapidly, after which some unknown mechanism inhibits the kinetics by several orders of magnitude. This sort of behavior has been observed in laboratory experiments using binary mixtures of oleic and stearic acid [Ziemann, 2005].

[65] Figure 8 also plots paired averages of the Pittsburgh and Florence data; the averages only include days when measurements were made at both sites. Absolute concentrations of these acids are similar at both sites in both seasons with concentrations being somewhat higher in the city than in Florence, indicating a modest contribution from local sources. The oleic- and palmitoleic-to-stearic acid ratios at both sites are lower during the summer then the winter, consistent with enhanced oxidation in the summertime. The ratios in Florence are modestly higher than in Pittsburgh, potentially suggesting a local biogenic source of the alkenoic acids. Unlike hopanes (see Figure 7), the alkenoic acid are not more depleted in the regional air mass compared to the city.

[66] When oleic acid reacts with ozone, nonanoic acid is one of the products [Thornberry and Abbatt, 2004]. Monthly average concentrations of the two alkenoic acids, stearic acid, and nonanoic acid are shown in Figure 9. The summer data are absolutely depleted in oleic acid and enriched in nonanoic acid, consistent with oxidation. There could also be significant biogenic sources of nonanoic acid in the summer.

[67] The most plausible explanation of all of the features shown in Figures 8 and 9 is that the source profile of the aggregate emissions influencing Pittsburgh lies near the one-to-one line with alkenoic-to-stearic acid ratios at the upper end of the ambient data (ratios of $\sim 1: 1$ ). The spread from this source profile is consistent with a study average 1 e-fold of oxidation, ranging between no oxidation and at least 2 e-folds, with typically 1.5 e-folds in the summer and 0.5 e-folds in the winter. Varying contributions of sources that emit stearic acid but not alkenoic acids such as motor vehicles will also distribute the ambient data along the one-to-one line, but it is unlikely that variations in source-source mixing would create the strong seasonal patterns shown in Figure 9. All told, the Pittsburgh data suggest an oxidation rate of alkenoic acids by $\mathrm{O}_{3}$ far slower than shown in Figure 1 for $\gamma=10^{-3}$, but generally consistent with the lower estimate $\left(\gamma=10^{-6}\right)$. An initial, rapid period of oxidation, followed by the slower period captured in the data, cannot be ruled out.

[68] While 1.5 e-folds is 'modest oxidation' compared with the potential suggested by absolute uptake measurements, from the perspective of source attribution or the absolute oxidation state of organic aerosol it is very substantial, corresponding to $80 \%$ of the initial alkenoic acids. At this level of oxidation, mass-balance source calculations would be in error by a factor of five, and aggregate measurements (such as aerosol mass spectrometer measurements of oxidized organic aerosol - OOA), would show a large fraction of oxidized material, as indeed they do [McFiggans et al., 2005; Zhang et al., 2005].

\section{Conclusions}

[69] We have presented multiple pieces of evidence suggesting that condensed-phase organic compounds are significantly oxidized in locations impacted by regional transport, especially during the summer. There is no smok- 
ing gun, but collectively the evidence points clearly to this conclusion. Rudimentary calculations suggest that both $\mathrm{OH}$ radicals and ozone should oxidize their respective targets $\left(\mathrm{CH}_{2}\right.$ groups and double bonds) on a time scale of a few days or so, depending on particle size. Ambient data consistently show depletion in reactive compounds during the summer, when oxidation should be fastest. This depletion shows all of the characteristics of oxidation on ratioratio plots designed to isolate oxidation effects. In the one case where an oxidation product has been observed, that product (nonanoic acid) is enriched during the summer when the parent compound (oleic acid) is depleted. While other scenarios can explain each of these observations, only aging during the summer consistently describes the complete suite. Reduced organic compounds are vulnerable to oxidation, and only in extreme cases where local emissions completely dominate the aerosol loading (i.e. Los Angeles) can oxidation be ignored.

[70] The only seemingly contradictory evidence comes from the laboratory where fundamental experiments indicate far more uptake of ozone on oleic acid than the ambient data can support. However, while one must conclude that oleic acid oxidation in atmospheric particles is slower than on pure surfaces in the laboratory, there is no evidence suggesting that oleic acid oxidation is stopped altogether. Indeed, the evidence suggests that oxidation proceeds at a rate approximately 1000 times slower than laboratory uptake on pure oleic acid surfaces.

[71] Oxidation is non-linear, and so it represents a very substantial complication to linear source apportionment techniques such as the Chemical Mass Balance (CMB) or factor analysis. While the assumptions underlying these methods may be valid in the winter when oxidation appears to be relatively slow, during the summer these techniques may lead to gross errors. The CMB predictions shown in Figure 5 illustrate the potential problem. The large seasonal shifts in the predicted gasoline-diesel split are caused by systematic changes in the hopane-to-EC ratio. Although we cannot rule out seasonally varying emissions completely, oxidation provides a compelling explanation for the seasonally varying hopane data. $\mathrm{CMB}$ calculations in the southeast show similar seasonal shifts in the gasoline-diesel split [Zheng et al., 2002], and they may well be subject to the same errors by ignoring chemistry. If aging is significant, then source-apportionment analyses using molecular markers must consider both mixing and oxidation as firstorder processes.

[72] Acknowledgments. The authors acknowledge Mei Zheng at Georgia Tech for sharing the organic aerosol data from the Southeastern United States. This research was conducted as part of the Pittsburgh Air Quality Study, which was supported by the U.S. Environmental Protection Agency under contract R82806101 and the U.S. Department of Energy National Energy Technology Laboratory under contract DE-FC2601NT41017. This research was also supported by the EPA STAR program through the National Center for Environmental Research (NCER) under grant R832162. This paper has not been subject to EPA's required peer and policy review, and therefore does not necessarily reflect the views of the Agency. No official endorsement should be inferred.

\section{References}

Bertram, A. K., A. V. Ivanov, M. Hunter, L. T. Molina, and M. J. Molina (2001), The reaction probability of $\mathrm{OH}$ on organic surfaces of tropospheric interest, J. Phys. Chem. A, 105(41), 9415-9421.
Blake, N. J., S. A. Penkett, K. C. Clemitshaw, P. Anwyl, P. Lightman, A. R. W. Marsh, and G. Butcher (1993), Estimates of atmospheric hydroxyl radical concentrations from the observed decay of many reactive hydrocarbons in well-defined urban plumes, J. Geophys. Res., 98(D2), 28512864.

Bloss, W. J., M. J. Evans, J. D. Lee, R. Sommariva, D. E. Heard, and M. J. Pilling (2005), The oxidative capacity of the troposphere: Coupling of field measurements of $\mathrm{OH}$ and a global chemistry transport model, Faraday Discuss., 130, 425-436.

Cabada, J. C., S. L. Rees, S. Takahama, A. Khlystov, S. N. Pandis, C. I. Davidson, and A. L. Robinson (2004), Mass size distributions and size resolved chemical composition of fine particulate matter at the Pittsburgh supersite, Atmos. Environ., 38, 3127-3141.

Calvert, J. G. (1976), Hydrocarbon involvement in photochemical smog formation in Los Angeles atmosphere, Environ. Sci. Technol., 10(3), $256-262$.

Cruz, C. N., and S. N. Pandis (1998), The effect of organic coatings on the cloud condensation nuclei activation of inorganic atmospheric aerosol, J. Geophy. Res., 103(D11), 13,111-13,123.

de Gouw, J. A., and E. R. Lovejoy (1998), Reactive uptake of ozone by liquid organic compounds, Geophys. Res. Lett., 25(6), 931-934.

Donahue, N. M., A. L. Robinson, K. E. Huff Hartz, A. M. Sage, and E. Weitkamp (2005), Competitive oxidation in atmospheric aerosols: The case for relative kinetics, Geophys. Res. Lett., 32, L16805, doi:10.1029/2005GL022893.

Energy Information Agency (EIA) (2002), Petroleum Marketing Monthly, U. S. Dep. of Energy, Washington, D. C. (Available at http://www.eia.doe. gov/oil_gas/petroleum/data_publications/petroleum_marketing_monthly/ pmm historical.html)

Federal Highway Administration (FHWA) (2005), State motor fuel data reported to FHWA, Off. of Highway Policy Inf., Washington, D. C. (Available at http://www.fhwa.dot.gov/policy/ohpi/qffuel.htm)

Fine, P. M., G. R. Cass, and B. R. T. Simoneit (2001), Chemical characterization of fine particle emissions from fireplace combustion of woods grown in the northeastern United States, Environ. Sci. Technol., 35(13), $2665-2675$.

Fine, P. M., G. R. Cass, and B. R. T. Simoneit (2002), Chemical characterization of fine particle emissions from the fireplace combustion of woods grown in the southern United States, Environ. Sci. Technol., 36(7), $1442-1451$

Fine, P. M., G. R. Cass, and B. R. T. Simoneit (2004), Chemical characterization of fine particle emissions from the wood stove combustion of prevalent United States tree species, Environ. Eng. Sci., 21(6), 705-721.

Finlayson-Pitts, B. J., and J. N. Pitts (2000), Chemistry of the Upper and Lower Atmosphere: Theory, Experiments, and Applications, Elsevier, New York.

Fraser, M. P., K. Lakshmanan, S. G. Fritz, and B. Ubanwa (2002), Variation in composition of fine particulate emissions from heavy-duty diesel vehicles, J. Geophys. Res., 107(D21), 8346, doi:10.1029/ 2001JD000558.

Gaffney, J. S., N. A. Marley, and M. M. Cunningham (2004), Natural radionuclides in fine aerosols in the Pittsburgh area, Atmos. Environ., 38(20), $3191-3200$

Hanson, D. R., A. R. Ravishankara, and S. Solomon (1994), Heterogeneous reactions in sulfuric-acid aerosols: A framework for model-calculations, J. Geophys. Res., 99(D2), 3615-3629.

Hays, M. D., C. D. Geron, K. J. Linna, N. D. Smith, and J. J. Schauer (2002), Speciation of gas-phase and fine particle emissions from burning of foliar fuels, Environ. Sci. Technol., 36(11), 2281-2295.

Heard, D. E., and M. J. Pilling (2003), Measurement of $\mathrm{OH}$ and $\mathrm{HO}_{2}$ in the troposphere, Chem. Rev., 103(12), 5163-5198.

Jacobson, M. C., H. C. Hansson, K. J. Noone, and R. J. Charlson (2000), Organic atmospheric aerosols: Review and state of the science, Rev. Geophys., 38(2), 267-294.

Kamens, R. M., Z. Guo, J. N. Fulcher, and D. A. Bell (1988), Influence of humidity, sunlight, and temperature on the daytime decay of polyaromatic hydrocarbons on atmospheric soot particles, Environ. Sci. Technol., 22(1), 103-108.

Kotzick, R., and R. Niessner (1999), The effects of aging processes on critical supersaturation ratios of ultrafine carbon aerosols, Atmos. Environ., 33(17), 2669-2677.

Lammel, G., and T. Novakov (1995), Water nucleation properties of carbon-black and diesel soot particles, Atmos. Environ., 29(7), 813823.

Li, C. K., and R. M. Kamens (1993), The use of polycyclic aromatichydrocarbons as source signatures in receptor modeling, Atmos. Environ., Part A, 27(4), 523-532.

Maria, S. F., L. M. Russell, M. K. Gilles, and S. C. B. Myneni (2004), Organic aerosol growth mechanisms and their climate-forcing implications, Science, 306(5703), 1921-1924. 
McFiggans, G., et al. (2005), Simplification of the representation of the organic component of atmospheric particulates, Faraday Discuss., 130, $341-362$

McKeen, S. A., and S. C. Liu (1993), Hydrocarbon ratios and photochemical history of air masses, Geophys. Res. Lett., 20(21), $2363-2366$.

McKeen, S. A., S. C. Liu, E. Y. Hsie, X. Lin, J. D. Bradshaw, S. Smyth, G. L. Gregory, and D. R. Blake (1996), Hydrocarbon ratios during PEM-West A: A model perspective, J. Geophys. Res., 101(D1), 2087-2109.

McKenna, D. S., C. J. Hord, and J. M. Kent (1995), Hydroxyl radical concentrations and Kuwait oil fire emission rates for March 1991, J. Geophys. Res., 100(D12), 26,005-26,025.

Moise, T., and Y. Rudich (2000), Reactive uptake of ozone by proxies for organic aerosols: Surface versus bulk processes, J. Geophys. Res., 105(D11), 14,667-14,676.

Moise, T., and Y. Rudich (2001), Uptake of $\mathrm{Cl}$ and $\mathrm{Br}$ by organic surfaces: A perspective on organic aerosols processing by tropospheric oxidants, Geophys. Res. Lett., 28(21), 4083-4086.

Molina, M. J., A. V. Ivanov, S. Trakhtenberg, and L. T. Molina (2004), Atmospheric evolution of organic aerosol, Geophys. Res. Lett., 31, L22104, doi:10.1029/2004GL020910.

Morris, J. W., P. Davidovits, J. T. Jayne, J. L. Jimenez, Q. Shi, C. E. Kolb, D. R. Worsnop, W. S. Barney, and G. Cass (2002), Kinetics of submicron oleic acid aerosols with ozone: A novel aerosol mass spectrometric technique, Geophys. Res. Lett., 29(9), 1357, doi:10.1029/ 2002 GL014692.

Nielsen, T. (1988), The decay of benzo (a)pyrene and cyclopenteno (Cd)pyrene in the atmosphere, Atmos. Environ., 22(10), 2249-2254.

Oros, D. R., and B. R. T. Simoneit (2000), Identification and emission rates of molecular tracers in coal smoke particulate matter, Fuel, 79(5), 515-536.

Parrish, D. D., C. J. Hahn, E. J. Williams, R. B. Norton, F. C. Fehsenfeld, H. B. Singh, J. D. Shetter, B. W. Gandrud, and B. A. Ridley (1992), Indications of photochemical histories of Pacific air masses from measurements of atmospheric trace species at Point Arena, California, J. Geophys. Res., 97(D14), 15,883-15,901.

Roberts, J. M., F. C. Fehsenfeld, S. C. Liu, M. J. Bollinger, C. Hahn, D. L. Albritton, and R. E. Sievers (1984), Measurements of aromatic hydrocarbon ratios and $\mathrm{NO}_{\mathrm{x}}$ concentrations in the rural troposphere: Observation of air-mass photochemical aging and $\mathrm{NO}_{\mathrm{x}}$ removal, Atmos. Environ., 18(11), 2421-2432.

Robinson, A. L., E. M. Lipsky, N. Pekney, W. F. Rogge, A. BernardoBricker, and O. Sevimoglu (2005), Fine particle emission profile for road dust in Pittsburgh, Pennsylvania, paper presented at American Association of Aerosol Research (AAAR) Specialty Conference: Particulate Matter, Supersites Program and Related Studies, Atlanta, Ga.

Rogge, W. F., L. M. Hildemann, M. A. Mazurek, G. R. Cass, and B. R. T. Simonelt (1991), Sources of fine organic aerosol. 1. Charbroilers and meat cooking operations, Environ. Sci. Technol., 25(6), 1112-1125.

Rogge, W. F., L. M. Hildemann, M. A. Mazurek, G. R. Cass, and B. R. T. Simoneit (1993a), Sources of fine organic aerosol. 2. Noncatalyst and catalyst- equipped automobiles and heavy-duty diesel trucks, Environ. Sci. Technol., 27(4), 636-651.

Rogge, W. F., L. M. Hildemann, M. A. Mazurek, G. R. Cass, and B. R. T. Simoneit (1993b), Sources of fine organic aerosol. 3. Road dust, tire debris, and organometallic brake lining dust - Roads as sources and sinks, Environ. Sci. Technol., 27(9), 1892-1904.

Rogge, W. F., L. M. Hildemann, M. A. Mazurek, G. R. Cass, and B. R. T. Simoneit (1993c), Sources of fine organic aerosol. 4. Particulate abrasion products from leaf surfaces of urban plants, Environ. Sci. Technol., 27(13), 2700-2711.

Rogge, W. F., M. A. Mazurek, L. M. Hildemann, G. R. Cass, and B. R. T. Simoneit (1993d), Quantification of urban organic aerosols at a molecularlevel - Identification, abundance and seasonal-variation, Atmos. Environ., Part A, 27(8), 1309-1330.

Rogge, W. F., L. M. Hildemann, M. A. Mazurek, G. R. Cass, and B. R. T. Simoneit (1996), Mathematical modeling of atmospheric fine particleassociated primary organic compound concentrations, J. Geophys. Res., 101(D14), 19,379-19,394.

Rogge, W. F., L. M. Hildemann, M. A. Mazurek, G. R. Cass, and B. R. T. Simoneit (1997a), Sources of fine organic aerosol. 7. Hot asphalt roofing tar pot fumes, Environ. Sci. Technol., 31(10), 2726-2730.

Rogge, W. F., L. M. Hildemann, M. A. Mazurek, G. R. Cass, and B. R. T. Simoneit (1997b), Sources of fine organic aerosol. 8. Boilers burning no. 2 distillate fuel oil, Environ. Sci. Technol., 31(10), 2731-2737.

Rogge, W. F., L. M. Hildemann, M. A. Mazurek, G. R. Cass, and B. R. T. Simoneit (1998), Sources of fine organic aerosol. 9. Pine, oak and synthetic log combustion in residential fireplaces, Environ. Sci. Technol., $32(1), 13-22$.
Rudich, Y. (2003), Laboratory perspectives on the chemical transformations of organic matter in atmospheric particles, Chem. Rev., 103(12), 50975124 .

Saxena, P., L. M. Hildemann, P. H. McMurry, and J. H. Seinfeld (1995), Organics alter hygroscopic behavior of atmospheric particles, J. Geophys. Res., 100(D9), 18,755-18,770.

Schauer, C., R. Niessner, and U. Poschl (2003), Polycyclic aromatic hydrocarbons in urban air particulate matter: Decadal and seasonal trends, chemical degradation, and sampling artifacts, Environ. Sci. Technol., $37(13), 2861-2868$.

Schauer, J. J., and G. R. Cass (2000), Source apportionment of wintertime gas-phase and particle-phase air pollutants using organic compounds as tracers, Environ. Sci. Technol., 34(9), 1821-1832.

Schauer, J. J., W. F. Rogge, L. M. Hildemann, M. A. Mazurek, and G. R. Cass (1996), Source apportionment of airborne particulate matter using organic compounds as tracers, Atmos. Environ., 30(22), 3837-3855.

Schauer, J. J., M. J. Kleeman, G. R. Cass, and B. R. T. Simoneit (1999a), Measurement of emissions from air pollution sources. 1. C-1 through C-29 organic compounds from meat charbroiling, Environ. Sci. Technol., 33(10), 1566-1577.

Schauer, J. J., M. J. Kleeman, G. R. Cass, and B. R. T. Simoneit (1999b), Measurement of emissions from air pollution sources. 2. C-1 through C-30 organic compounds from medium duty diesel trucks, Environ. Sci. Technol., 33(10), 1578-1587.

Schauer, J. J., M. J. Kleeman, G. R. Cass, and B. R. T. Simoneit (2001), Measurement of emissions from air pollution sources. 3. C-1-C- 29 organic compounds from fireplace combustion of wood, Environ. Sci. Technol., 35(9), 1716-1728.

Schauer, J. J., M. P. Fraser, G. R. Cass, and B. R. T. Simoneit (2002a), Source reconciliation of atmospheric gas-phase and particle- phase pollutants during a severe photochemical smog episode, Environ. Sci. Technol., 36(17), 3806-3814.

Schauer, J. J., M. J. Kleeman, G. R. Cass, and B. R. T. Simoneit (2002b), Measurement of emissions from air pollution sources. 4. C-1-C- 27 organic compounds from cooking with seed oils, Environ. Sci. Technol., $36(4), 567-575$.

Schauer, J. J., M. J. Kleeman, G. R. Cass, and B. R. T. Simoneit (2002c), Measurement of emissions from air pollution sources. 5. C-1-C- 32 organic compounds from gasoline-powered motor vehicles, Environ. Sci. Technol., 36(6), 1169-1180.

Simoneit, B. R. T. (1984), Organic-matter of the troposphere. 3. Characterization and sources of petroleum and pyrogenic residues in aerosols over the western United States, Atmos. Environ., 18(1), 51-67.

Simoneit, B. R. T., and M. A. Mazurek (1982), Organic-matter of the troposphere. 2. Natural background of biogenic lipid matter in aerosols over the rural western United States, Atmos. Environ., 16(9), 2139-2159.

Simoneit, B. R. T., J. J. Schauer, C. G. Nolte, D. R. Oros, V. O. Elias, M. P. Fraser, W. F. Rogge, and G. R. Cass (1999), Levoglucosan, a tracer for cellulose in biomass burning and atmospheric particles, Atmos. Environ., 33(2), 173-182.

Smith, G. D., E. Woods, C. L. DeForest, T. Baer, and R. E. Miller (2002), Reactive uptake of ozone by oleic acid aerosol particles: Application of single-particle mass spectrometry to heterogeneous reaction kinetics, J. Phys. Chem. A, 106(35), 8085-8095.

Subramanian, R., A. Y. Khlystov, J. C. Cabada, and A. L. Robinson (2004), Positive and negative artifacts in particulate organic carbon measurements with denuded and undenuded sampler configurations, Aerosol Sci. Technol., 38(S1), 27-48.

Tang, W., T. Raymond, B. Wittig, C. I. Davidson, S. N. Pandis, A. L. Robinson, and K. Crist (2004), Spatial variations of PM2.5 during the Pittsburgh Air Quality Study, Aerosol Sci. Technol., 38(S2), 80-90.

Thomas, E. R., G. J. Frost, and Y. Rudich (2001), Reactive uptake of ozone by proxies for organic aerosols: Surface-bound and gas-phase products, J. Geophys. Res., 106(D3), 3045-3056.

Thornberry, T., and J. P. D. Abbatt (2004), Heterogeneous reaction of ozone with liquid unsaturated fatty acids: Detailed kinetics and gas-phase product studies, Phys. Chem. Chem. Phys., 6(1), 84-93.

Turpin, B. J., P. Saxena, and E. Andrews (2000), Measuring and simulating particulate organics in the atmosphere: Problems and prospects, Atmos. Environ., 34(18), 2983-3013.

Watson, J. G., E. M. Fujita, J. C. Chow, and B. Zielinska (1998), Northern Front Range Air Quality Study final report and supplemental volumes, Desert Res. Inst., Reno, Nev.

Weingartner, E., H. Burtscher, and U. Baltensperger (1997), Hygroscopic properties of carbon and diesel soot particles, Atmos. Environ., 31(15), $2311-2327$

Wittig, A. E., N. Anderson, A. Y. Khlystov, S. N. Pandis, C. I. Davidson, and A. L. Robinson (2004), Pittsburgh Air Quality Study overview and initial scientific findings, Atmos. Environ., 38(20), 3107-3125. 
Worsnop, D. R., J. W. Morris, Q. Shi, P. Davidovits, and C. E. Kolb (2002), A chemical kinetic model for reactive transformations of aerosol particles, Geophys. Res. Lett., 29(20), 1996, doi:10.1029/ 2002GL015542.

Zhang, Q., M. R. Alfarra, D. R. Worsnop, J. D. Allan, H. Coe, M. R. Canagaratna, and J. L. Jimenez (2005), Deconvolution and quantification of hydrocarbon-like and oxygenated organic aerosols based on aerosol mass spectrometry, Environ. Sci. Technol., 39(13), 4938-4952.

Zheng, M., G. R. Cass, J. J. Schauer, and E. S. Edgerton (2002), Source apportionment of PM2.5 in the southeastern United States using solventextractable organic compounds as tracers, Environ. Sci. Technol., 36(11), $2361-2371$

Zielinska, B., J. Sagebiel, J. D. McDonald, K. Whitney, and D. R. Lawson (2004), Emission rates and comparative chemical composition from selected in-use diesel and gasoline-fueled vehicles, J. Air Waste Manage. Assoc., 54(9), 1138-1150.
Ziemann, P. J. (2005), Aerosol products, mechanisms, and kinetics of heterogeneous reactions of ozone with oleic acid in pure and mixed particles, Faraday Discuss., 130, 469-490.

N. M. Donahue, Departments of Chemical Engineering and Chemistry, Carnegie Mellon University, Pittsburgh, PA 15213, USA. (nmd@andrew. cmu.edu)

A. L. Robinson, Departments of Mechanical Engineering and Engineering and Public Policy, Carnegie Mellon University, Pittsburgh, PA 15213, USA. (alr@andrew.cmu.edu)

W. F. Rogge, Department of Civil and Environmental Engineering, Florida International University, Miami, FL 33199, USA. (rogge@eng. fiu.edu) 


\title{
Source apportionment of molecular markers and organic aerosol -
}

\section{Methodology for Visually Comparing Source Profiles and}

\author{
Ambient Data \\ Allen L Robinson*, R Subramanian† \\ Department of Mechanical Engineering, Carnegie Mellon University, Pittsburgh, PA 15213, \\ USA \\ Neil M Donahue \\ Department of Chemistry and Department of Chemical Engineering, Carnegie Mellon \\ University, Pittsburgh, PA 15213, USA \\ Anna Bernardo-Bricker \\ Department of Civil and Environmental Engineering, Florida International University, \\ Miami, FL, USA \\ Wolfgang F Rogge \\ Department of Civil and Environmental Engineering, Florida International University, \\ Miami, FL, USA
}

* Corresponding author. e-mail: alr@andrew.cmu.edu; phone: 412-268-3657; fax 412-2683348.

† Currently at: Department of Civil and Environmental Engineering, University of Illinois, Urbana, IL 61801, USA 


\section{Abstract}

Individual organic compounds or molecular markers are often used in conjunction with the chemical mass balance model (CMB) to apportion sources of primary organic aerosol. This paper is first in a series of papers that critically examines issues associated with selection of source profiles and fitting species for CMB analysis with molecular markers. This paper presents a methodology to visualize the organization of ambient molecular marker data, to compare the data to source profiles, and to better understand CMB solutions. The technique can also be used to assess chemical stability and aging. The core of the technique involves construction of plots of ratios of species concentrations (ratio-ratio plots) in which source profiles appear as points connected by linear mixing lines. The approach is illustrated using ambient measurements made in Pittsburgh, Pennsylvania over a one-year period of five, large polycyclic aromatic hydrocarbons (PAH) commonly used as molecular markers in CMB: benzo(b+j+k)fluoranthene, benzo(e)pyrene, benzo[g,h,i]perylene, coronene, and indeno(1,2,3-cd)pyrene. In Pittsburgh, the ambient concentrations of these PAH are strongly correlated suggesting a single dominant source. These correlations underscore the significant source information contained in molecular marker concentrations. Ratio-ratio plots are then used to evaluate the potential contribution of gasoline exhaust, diesel exhaust, wood smoke, and coke production emissions to the ambient concentrations of the target PAHs. Coke production is the dominant source of these large PAHs in Pittsburgh. Ambient concentrations of these large PAH provide little information on the gasoline-diesel split because of the strong influence of local emissions from coke production combined with evidence of photochemical decay of PAH in the regional air mass. 


\section{Introduction}

Molecular markers are reduced organic species that have been used in conjunction with the Chemical Mass Balance (CMB) to identify sources of primary organic carbon (OC) and fine particle mass (1). Examples of molecular markers include hopanes for vehicular exhaust (2) and levoglucosan for biomass smoke (3). Their high-degree of source specificity makes them excellent for use in source apportionment analysis. Unfortunately measuring ambient molecular marker concentrations requires complex and costly sampling and analytical procedures. Therefore, source apportionment analyses using molecular markers have only been performed in only a limited number of locations with a relatively small number of samples. This has limited our ability to fully evaluate the utility of molecular markers for source apportionment analysis.

The ideal molecular marker would be (a) unique to a given source class (i.e. gasoline powered cars), (b) emitted at a constant ratio with respect to other markers for that class (have a well-defined source profile), (c) emitted at a constant ratio with respect to the total OC or fine particle mass from that class (have a constant marker-to-OC ratio), and (d) stable with respect to OC in the atmosphere (not decay in the atmosphere). Unfortunately, none of these ideals is met in reality: all markers are emitted by at least several source classes, published source profiles for a given source class exhibit significant variability, and all reduced organic compounds are vulnerable to oxidation. The first issue of multiple sources can be solved by linear inversion using a model such as CMB, provided that the second and third issues can be constrained. Photochemical decay is potentially a very challenging problem (4).

The CMB model solves a system of equations in which the ambient concentrations of specific chemical constituents are described using a linear combination of a set of source profiles (5). Given the substantial resources required for source characterization, a common practice is to 
use published source profiles, with the usually implicit assumption that literature profiles are appropriate for a given dataset. A challenge is that a reasonably large number of source profiles with speciated organics data have been published; for example, more than 35 different biomass smoke profiles have been published. In addition, source profiles for individual sources in the same source class often show considerable differences from each other. Co-linearity limits the number of source profile that can be simultaneously included in the model, forcing the analyst to choose among sets of profiles.

To help the analyst assess the quality of the solution, CMB calculates a number of fitting statistics that describe how well the sources included in the model can (a) explain the ambient data; and (b) whether the separation of the sources is statistically significant given the set of fitting species (5). However, one can encounter solutions based on different sets of profiles with significantly different results but comparable fitting statistics, even when considering only a subset of the available source profiles $(6,7)$. Including all the possible source profiles and fitting species magnifies the problem faced by the analyst: how do we select the most appropriate solution given similar fitting statistics?

The goal of CMB analysis is to quantify the absolute and relative contributions of different source classes such as motor vehicles or wood combustion to ambient organic carbon (OC) or fine particle mass. Each source class is typically made up of a very large number of individual sources. Although there may be significant differences in emissions from individual sources, we are interested in predicting aggregate contribution of emissions from all the sources in a given source class. Therefore, the source profiles used in the CMB model need to represent the aggregate emissions of set of individual sources that influence a receptor site. Each published profile is based on a single or at most a small number of source tests and therefore may only 
represent a small fraction of the sources in a given source class. Using activity data is one approach for selecting and compositing of source profiles (1).

Factor analysis-based approaches such as UNMIX and PMF offer the possibility to directly recover "unknown” source profiles from ambient data. These are complex and powerful tools, but are subject to a number of well-recognized limitations (8). The approaches require large datasets and therefore have not been applied using molecular markers. These techniques reveal substantial information about the co-location of various sources in time and space but do not reveal specific sources per-se. While there are implementations of factor analysis methods which utilize target source profiles (9), they still do not provide a direct test of the source vectors themselves. Finally, the solutions are not unique, and therefore again subject to considerable analyst judgment.

Visualizing the solution space can help the analyst evaluate potential source profile combinations and to better understand how different profile combinations influence the CMB results. For example, scatter plots of species concentrations are used to find "edges” that correspond to factors, e.g. (10). However, the highly dimensional nature of receptor modeling makes visualizing the solution difficult. In addition, techniques are needed that consider both mixing of emissions of different source classes as well as photochemical stability.

This paper is the first in a series of papers that examines the use of molecular markers to apportion ambient organic aerosol to primary sources. The papers utilize a large data set of ambient molecular marker concentrations recently developed as part of the Pittsburgh Air Quality Study (11). Each paper seeks to answer a number of questions for small groups of compounds associated with specific source classes (e.g. levoglucosan, resin acids, and syringols as markers for biomass combustion). First, are the ambient molecular marker data organized in a 
fashion that implies the existence of a well-defined source profile or set of profiles? Second, which published profiles or combinations of published profiles can explain the ambient data? Third, how well is the amount ambient OC apportioned to a source class constrained by CMB analysis given the set of viable profile combinations? Finally, what causes differences between different CMB solutions?

To address these questions, this paper develops a methodology based on the linear mixing assumption of $\mathrm{CMB}$ and other receptor models that facilitates visual assessment of the organization of ambient molecular marker data and the consistency between ambient data and source profiles. To illustrate the approach, we compare ambient concentrations of five large polycyclic aromatic hydrocarbons (PAH) and elemental carbon to source profiles for motor vehicle emissions, wood smoke, and coke production. The analysis explicitly examines the suitability of using PAH to determine the gasoline-diesel split. Companion papers apply this approach to source profiles and ambient marker data for meat cooking emissions (12), biomass smoke (13), and motor vehicle emissions (7).

\section{Methodology for visualizing ambient data and source profiles}

In this section we present a graphical approach to visualize the organization of ambient molecular marker data and to compare these data to source profile which helps the analyst better understand CMB solutions. It also can be used to assess chemical stability and aging. Simultaneous consideration of mixing and aging is especially important for receptor sites where regional emissions dominate the ambient concentrations, such as Pittsburgh or other locations in the Eastern U.S. The technique is intended to complement the quantitative CMB or factor analysis based approaches. 
To illustrate our approach, Figure 1 shows simulated ambient data for three compounds (A, $B, C)$ constructed from mixing of emissions from three source classes $\left(S_{1}, S_{2}, S_{3}\right)$. A source class is a set of sources whose emissions can be represented using the same source profile. The compounds are assumed to be non-reactive and to have the same atmospheric lifetime - core assumptions of CMB and other linear receptor models.

Scatter plots of two species (Figures 1a, 1c, 1e) are the simplest way to visually compare ambient data and source profiles. On a scatter plot, a source profile appears as a straight line with a slope corresponding to the ratio of the emission rates of the two compounds. If the two compounds are emitted by a single source class, then the ambient concentrations will fall on top of the source profile (Figure 1a). If two source classes emit the compounds, then two source lines appear on the scatter plot and the ambient data will fall between these two lines under the assumption of linear mixing (Figure 1c). In practice multiple source classes emit each compound; therefore, multiple source lines will appear on a scatter plot (Figure 1e). A further complication is that multiple source profiles exist for many source classes, such as diesel trucks. The result is that simple scatter plots rapidly fill up with source profiles, making visual assessment difficult.

A more powerful approach for visually comparing ambient data to source profiles is a ratioratio plot, which considers three compounds, adding a third dimension to the visual analysis. Although three species only represents a small fraction of compounds considered by CMB, molecular markers are often highly source specific. Therefore, through the judicious groupings of compounds, ratio-ratio plots can often provide significant insight into the suitability of source profiles as illustrated by the example below. 
To construct a ratio-ratio plot, one compound is selected as a reference to normalize the concentrations of the other two compounds, which are called target species. The best reference compounds are relatively abundant and specific to the sources plotted. In this situation, the ratios account for the effects of atmospheric dilution. Typically, logarithmic axes are used to visualize the wide dynamic range of emission profiles. The mathematics underlying the ratio approach are described in Robinson et al. (4).

There are many advantages to using ratio-ratio plots to evaluate source profiles compared to simple scatter plots. First, source profiles appear as individual points on a ratio-ratio plot (Figures 1b, 1d, 1f), which allows efficient comparison of ambient data with numerous source profiles. Also, ambient concentration ratios of compounds emitted by a single source class cluster to a point on a ratio-ratio plot corresponding to the source profile (Figure 1b). This assumes that the effects of time-varying emission rates, transport, and deposition are not species dependent (4).

If two source classes emit the compounds of interest then a linear mixing line can be drawn between the two profiles, and the ambient data are expected to fall on this mixing line, as in Figure 1(d). This allows one to visualize the effects of mixing of emissions from different sources (source-source mixing) on ambient concentrations. The exact position along the line depends on a weighting factor (4); however, the key point is that this line defines the potential atmospheric states assuming linear mixing. Linear mixing lines often appear curved on a log-log plot, so we typically draw them to assist with visualization and interpretation.

For compounds emitted by multiple source classes, one simply defines mixing lines between the different source profile pairs; the area enclosed by these mixing lines (mixing region) is the 
space in which ambient concentrations are expected if they are governed by mixing. This scenario is illustrated in Figure 1(f) which shows a 3-source ratio-ratio plot.

The power of ratio-ratio plots is that they allow visual identification of inconsistencies between the ambient data and source profiles. If the ambient data fall outside of the mixing lines created by the source profiles then there could be problems with the source profiles, an unknown source of the compounds, or the compounds could have different atmospheric lifetimes (e.g. one species is being oxidized). Under these conditions, linear source inversions such as CMB will fail. Ratio-ratio approach can provide insight into the characteristics of missing sources and/or the possibility of photochemistry. Finally, ratio-ratio plots allow the analyst to visually compare multiple profiles for a given source class to help guide development of aggregate source profiles.

Another advantage of the ratio-ratio approach is the uncertainty of concentration ratios of species measured using the same instrument, for example, GC-MS. In these situations the uncertainties of the individual species are typically highly correlated - even instrumentation calibration uncertainties are typically correlated for multiple species - and so measurements of source ratios are more precise than simple error propagation suggests. To take advantage of this substantial advantage requires a careful review of the speciated data.

\section{Results and Discussion}

To illustrate the use of ratio-ratio plots, we examine the contribution of different sources to ambient concentrations of EC and five large polycyclic aromatic hydrocarbons (PAH) in Pittsburgh, PA: benzo(b+j+k)fluoranthene, benzo(e)pyrene, benzo[g,h,i]perylene, coronene, and indeno(1,2,3-cd)pyrene. In this paper, we use "PAH" to refer specifically to these five compounds and not to the broader compound class. Ambient PAH and EC concentrations were 
measured on 103 days between July, 2001 and August, 2002 in an urban park in Pittsburgh (11). Daily measurements were made in July 2001 and most of January 2002; during other periods 24hr samples were collected on a 1 in 6 day schedule. Additional details on the data set are provided in Supporting Information.

The Pittsburgh PAH data provide an excellent illustration of the ratio-ratio methodology because PAHs are emitted by multiple source classes (e.g. motor vehicles, biomass combustion, industrial processes) and many different source profiles are available for each source class; in addition, there is also strong evidence for atmospheric decay of PAHs (14-16). In the context of CMB modeling of ambient OC, PAHs have been used in conjunction with EC to differentiate between emissions of gasoline and diesel vehicles $(17,18)$. However, biomass combustion and industrial processes can also be important sources of PAH (19). Photochemistry is especially a concern in Pittsburgh where fine particle concentrations are strongly influenced by regional transport which allows for significant time for photochemical processing (4).

Scatter plots of ambient PAH concentrations versus indeno[1,2,3-cd]pyrene for Pittsburgh are shown in Figure 2a. The Pittsburgh PAH data are highly correlated $\left(\mathrm{R}^{2}>0.95\right)$, and only coronene and benzo[g,h,i]perylene show a small, but statistically significant, intercept. These strong correlations suggest a single dominant source for these compounds. On most days the ambient PAH concentrations are less than a few $\mathrm{ng} / \mathrm{m}^{3}$; however, greatly elevated concentrations were intermittently observed in non-winter months (April - November). Removing the high concentration days from the dataset does not alter the strong correlations among the PAHs. On average, the ambient PAH concentrations in Pittsburgh are comparable to those measured in Birmingham, AL, but two to six times higher than the PAH concentrations measured in Atlanta, GA (20). 
Figure $2 \mathrm{~b}$ shows a scatter plot of benzo[e]pyrene versus indeno[1,2,3-cd]pyrene that compares ambient data to source profiles for wood smoke, coke production, and gasoline and diesel vehicles. The ambient data can be explained by mixing of any two profiles that bracket the data; the large number of viable source profile combinations makes it difficult to evaluate potential scenarios.

A ratio-ratio plot is a more effective tool for visualizing the ambient data and to compare them to source profiles. Figure 3 shows a ratio-ratio plot of 3 PAHs; as expected from the strong correlations shown in Figure 2a, the ambient PAH data tightly cluster around a point in the ratioratio plot.

Figure 3 shows that the ambient distribution of the three PAHs is similar to the coke production source profile and a number of the biomass smoke profiles. Notably, the ambient PAH data cannot be explained by mixing of gasoline and diesel emissions. For example, Figure 3 indicates that the indeno(1,2,3-cd)pyrene-to-benzo(e)pyrene ratio of many of gasoline vehicle profiles is similar to the ambient data, but that these profiles are substantially enriched in benzo(g,h,i)perylene compare to the ambient data. Many of the biomass smoke source profiles cluster together in Figure 3 as do many of the gasoline vehicle source profiles; this indicates that the there are characteristic distributions of these PAHs for different source class. This clustering reduces issues associated with source profile variability and supports averaging of profiles to create aggregate profiles for a given source class.

A CMB analysis was performed on the Pittsburgh data. The model considers four PAHs: benzo[e]pyrene, indeno[1,2,3-cd]pyrene, benzo[g,h,i]perylene and coronene. Additional species in the model are four n-alkanes, iso-hentriacontane, anteiso-dotriacontane, four hopanes, two alkanoic acids, palmitoleic acid, cholesterol, levoglucosan, resin acids, syringaldehyde, 
acetosyringone, titanium, iron, and elemental carbon. Source profiles for eight source classes are included in the model: diesel vehicles, gasoline vehicles, road dust, biomass combustion, cooking emissions, coke production, vegetative detritus, and cigarettes. Additional details of the CMB calculations are provided in the Supporting Information.

The CMB model apportions more than $80 \%$ of the four large PAHs included in the model on all study days to coke production. This is not unexpected given the clustering of the ambient data around the coke production source profile shown in Figure 3. There are two large coke production facilities located within $15 \mathrm{~km}$ of the monitoring site, one to the northwest and the other to the southeast. Not including the coke production source profile in the model results in large fractions of the ambient PAH being unapportioned (model-to-measured PAH ratios much less than 0.5) and alters the relative contribution of other sources of PAHs.

On most days coke production is only a minor source of OC and fine particle mass. CMB estimates a median daily contribution of coke production emissions to ambient OC of $18 \pm 2 \mathrm{ng}$ $\mathrm{C} \mathrm{m}{ }^{-3}$. Its median daily contribution to $\mathrm{PM}_{2.5}$ mass is $45 \pm 5 \mathrm{ng} \mathrm{m}^{-3}$ to $\mathrm{PM}_{2.5}$ mass. Although PAH concentrations in Pittsburgh are higher than in other locations, coke is only a minor source of OC and fine particle mass because the emissions are so rich in PAHs. On 9\% of the study days, the CMB model apportions more than $200 \mathrm{ng} \mathrm{C} \mathrm{m}^{-3}$ of ambient OC to coke production with a maximum daily contribution of $715 \pm 81 \mathrm{ng} \mathrm{C} \mathrm{m}^{-3}$. Its maximum daily contribution to ambient $\mathrm{PM}_{2.5}$ mass is $1785 \pm 202 \mathrm{ng} \mathrm{m}^{-3}$. These spikes occur on days when PAH concentrations were high and the meteorology is consistent with transport from one of the local coke production facilities.

Gasoline-diesel split: Although emissions from coke production dominate PAH concentrations in Pittsburgh, PAHs in combination with EC have been used to differentiate 
between gasoline and diesel vehicle OC emissions $(17,18)$. Diesel vehicles are the dominant source of EC in urban environments while gasoline vehicle emissions are significantly enriched in PAHs compared to diesel emissions. The feasibility of separating gasoline and diesel emissions using PAHs and EC can be evaluated using the ratio-ratio plots shown in Figure 4, which presents the indeno[1,2,3-cd]pyrene and benzho[g,h,i]perylene data normalized by EC. The ambient PAH and EC concentrations are only weakly correlated, which causes the data to be distributed along the diagonal in the ratio-ratio plot.

PAH-to-EC ratios of many different source profiles are compared to the ambient data in Figure 4a. The biomass smoke profiles show a similar distribution of PAH-to-EC ratios as the ambient data indicating that biomass smoke, by itself, can explain both the ambient PAH and EC data. The diesel profiles with a relatively low PAH-to-EC ratios and the coke production profile with a relatively high PAH-to-EC ratio bracket the ambient data. Figure 4a indicates that the gasoline profiles are either depleted in indeno[1,2,3-cd]pyrene or enriched in benzho[g,h,i]perylene compared to the ambient data.

To facilitate consideration of different mixing scenarios, Figure 4 shows arithmetic average source profiles for each source class (profiles that do not report for certain species, e.g. diesel profiles with no indeno[1,2,3-cd]pyrene, are included in the average as a zero). A mixing line connecting the average diesel profile with the coke production profile passes through the ambient data; therefore mixing of emissions from these two source classes can also explain the ambient PAH and EC data. The location of this mixing line in the region of the ambient data is insensitive to the approach used to calculate the average diesel profile.

To better understand the potential contributions of gasoline and diesel vehicles to ambient PAH and EC concentrations, Figure 4b shows a ratio-ratio of data on days when ambient 
concentrations of wood smoke markers (levoglucosan, resin acids, syringols) were low. On these days motor vehicles and coke production are expected to be the dominant sources of PAH and EC, eliminating any complications due to wood smoke.

Figure $4 \mathrm{~b}$ compares the low wood smoke ambient data to different mixing scenarios defined by the average gasoline, average diesel and coke production source profiles. The mixing lines represent four of the many possible mixing scenarios. The two solid lines indicate $100 \%$ gasoline or 100\% diesel emissions mixed with coke production emissions. The two dashed lines are mixtures of emissions from two different mixed vehicle fleets with coke production emissions. The composition of the mixed vehicle fleet is defined by the fraction of motor vehicle OC contributed by each vehicle type -- in a 2:1 diesel:gasoline fleet, diesel vehicles emit twice as much $\mathrm{OC}$ as gasoline vehicles. The relative contribution of coke production varies along each line with the lower and upper ends of each line correspond to coke production contributing $0 \%$ and $100 \%$ of the combined OC, respectively.

The mixing line defined by average diesel profile and the coke production profile plotted in Figure 4 passes through the entire ambient data set. However, other mixing lines collapse along the diagonal defined by the ambient data when coke production contributes just $5 \%$ of the combined OC from vehicle emissions and coke production. For example, Figure $4 \mathrm{~b}$ indicates that ambient data with PAH-to-EC ratios greater than 1 can correspond to vehicle fleets ranging from $100 \%$ diesel to a 2:1 diesel-gasoline split. This occurs because emissions from coke production are much richer in PAHs compared to the other sources and illustrates how a large, but unexpected source (coke production), can significantly influence the results. This severely limits the feasibility of using the PAH and EC data to specify the gasoline diesel split. 
Figure $4 \mathrm{~b}$ does suggest that on a reasonable number of days diesel emissions dominate gasoline emissions because a large fraction of the data with low PAH-to-EC ratios falls below even the 2:1 diesel:gasoline mixing line. Notably there is not even a hint of spread towards the average gasoline source profile in Figure 4b, suggesting minimal contribution of gasoline vehicles to ambient OC on many study days. However, this conclusion is based on the assumption that PAHs are stable in the atmosphere.

Photochemical aqing and reqional transport: Ratio-ratio plots also provide insight into photochemical aging (4). PAHs are known to photochemically decay (14-16) while EC is stable (except for deposition, which we assume affects PAHs and EC equally). Therefore, photochemical degradation of PAH will reduce the PAH-to-EC ratio in the ambient air compared to fresh emissions. In this section we examine the ambient data for evidence of photochemical aging of PAHs and discuss implications of this aging on source apportionment estimates.

Photochemical aging should create a seasonal pattern in the ambient data. Assuming constant source strengths, PAH-to-EC ratios are expected to be lower in the summer than in the winter due to seasonal changes in photochemical activity. Another important issue is the spatial distribution of sources. Regional transport has a significant influence on fine particle concentrations in Pittsburgh (21). Photochemistry will have a much larger effect on PAH concentrations in the regional air mass compared to those emitted locally because of the differences in transport times. Therefore, PAH-to-EC ratios are expected to be lower in the regional air mass than in locations strongly influenced by local emissions.

We examined the data from the Pittsburgh site for a seasonal pattern in the PAH-to-EC ratio. However, the large, intermittent spikes in PAH concentrations observed at the Pittsburgh site is a clear sign of variable source strengths; this is not unexpected given the contribution of the local 
point source (coke production) is strongly dependent on local meteorology. If one removes the large spikes in PAH concentration (which are presumably due to strong influence of local coke production) then the PAH-to-EC ratios measured at the Pittsburgh site exhibit a strong seasonal pattern consistent with aging.

To characterize the PAH concentrations in the regional air mass, measurements were made at a rural site in Florence, PA, 40 km west-southwest of Pittsburgh (11). Florence is almost always upwind of the city and fine particle mass and bulk composition measured at the Pittsburgh, Florence and other sites in Southwestern PA are very similar (21). Thirteen paired sets of samples for organic speciation were collected from both sites during January 2002 and four paired sets during July 2002.

Figure 5 presents a ratio-ratio plot that compares the data collected in Florence and Pittsburgh. The summertime PAH-to-EC ratios in Florence are smaller than those measured in Pittsburgh, particularly on two of the days. The summertime Florence PAH-to-EC ratios are comparable to the lowest values observed in Pittsburgh during the entire study. During the wintertime, the PAH-to-EC ratios in Pittsburgh and Florence are essentially the same, with a range of values indicated by the arrow in Figure 5. The winter the PAH-to-EC ratios in Florence are, on average, a factor of 4 higher than the summer ratio. Therefore, the data are consistent with photochemical degradation of PAHs in the regional air mass during the summer.

The Florence data shown in Figure 5 also fall on of the 100\% diesel-coke production mixing line, suggesting that emissions from diesel vehicles dominate the gasoline-split at this site and that coke production emissions also contribute to PAH concentrations. There is a large coke production facility in Follansbee, WV, which is roughly $25 \mathrm{~km}$ west of the Florence site. The presence of coke facility upwind of Florence potentially complicates the interpretation of the 
PAH data from the site. For example, is the seasonal pattern in the Florence data due to a stronger wintertime influence of the Follansbee coke production facility at this site during the winter? This seems unlikely given the large number of wintertime data points combined with the available meteorology data -- on some sampling days the wind direction measured at the Florence site does not point towards the Follansbee plant.

Photochemical degradation of PAHs has important implications for using PAH data to help infer the gasoline-diesel split in locations such as Pittsburgh where emissions from local sources are mixed regional transport. As previously discussed, one interpretation of Figure $4 \mathrm{~b}$ is that the gasoline-diesel split is dominated by diesel emissions. However, the organization of the ambient data in the PAH-to-EC ratio-ratio plots is also entirely consistent with mixing of relatively fresh emissions from coke production with photochemically aged, PAH-depleted vehicle emissions in the regional air mass. Such mixing will disperse the data along a diagonal line extending from the coke production source profile towards the lower-left hand corner of the ratio-ratio plot exactly as the heavy, solid mixing line shown in Figure 5. This mixing line connects the coke production source profile and the Florence data with the lowest PAH-to-EC ratio. The small PAH-to-EC ratios observed in the Florence during the summer are consistent with the regional air mass being depleted in PAH during periods of high photochemical activity.

A more complete analysis of the contribution of gasoline and diesel vehicles emissions using additional molecular markers such as hopanes indicates that in the winter (when photochemical activity is low), the vehicular OC contribution could be dominated by gasoline vehicle emissions (7). Therefore, inferences regarding gasoline-diesel split based on ambient PAH concentrations are likely incorrect in locations like Pittsburgh where strong local sources (coke production) are coupled significant regional transport. 


\section{Acknowledgments}

This research was conducted as part of the Pittsburgh Air Quality Study, which was supported by US Environmental Protection Agency under contract R82806101 and the US Department of Energy National Energy Technology Laboratory under contract DE-FC2601NT41017. This research was also supported by the EPA STAR program through the National Center for Environmental Research (NCER) under grant R832162. This paper has not been subject to EPA's required peer and policy review, and therefore does not necessarily reflect the views of the Agency. No official endorsement should be inferred.

\section{References}

(1) Schauer, J. J.; Rogge, W. F.; Hildemann, L. M.; Mazurek, M. A.; Cass, G. R., Source apportionment of airborne particulate matter using organic compounds as tracers. Atmospheric Environment 1996, 30, 3837-3855.

(2) Simoneit, B. R. T., Organic-Matter of the Troposphere .3. Characterization and Sources of Petroleum and Pyrogenic Residues in Aerosols over the Western United-States. Atmospheric Environment 1984, 18, 51-67.

(3) Simoneit, B. R. T.; Schauer, J. J.; Nolte, C. G.; Oros, D. R.; Elias, V. O.; Fraser, M. P.; Rogge, W. F.; Cass, G. R., Levoglucosan, a tracer for cellulose in biomass burning and atmospheric particles. Atmospheric Environment 1999, 33, 173-182.

(4) Robinson, A. L.; Donahue, N. M.; Rogge, W. F., Photochemical oxidation and changes in molecular composition of organic aerosol in the regional context. Journal of Geophysical Research-Atmospheres 2006, 111, doi:10.1029/2005JD006265.

(5) Watson, J. G.; Robinson, N. F.; Fujita, E. M.; Chow, J. C.; Pace, T. G.; Lewis, C.; Coulter, T. "CMB8 Applications and validation protocol for PM2.5 and VOCs," Desert Research Institute, 1998.

(6) Lee, S.; Baumann, K.; Schauer, J. J.; Sheesley, R. J.; Naeher, L. P.; Meinardi, S.; Blake, D. R.; Edgerton, E. S.; Russell, A. G.; Clements, M., Gaseous and particulate emissions from prescribed burning in Georgia. Environmental Science \& Technology 2005, 39, 9049-9056.

(7) Subramanian, R.; Donahue, N. M.; Bernardo-Bricker, A.; Rogge, W. F.; Robinson, A. L., Contribution of motor vehicle emissions to the ambient organic carbon in Pittsburgh, PA: Effects of varying source profiles and seasonal trends in ambient marker concentrations. Atmospheric Environment 2006, submitted.

(8) Seinfeld, J. H.; Pandis, S. N. Atmospheric chemistry and physics: From air pollution to climate change; John Wiley \& Sons Inc.: New York, 1998.

(9) Hopke, P. K., Target Transformation Factor Analysis As An Aerosol Mass Apportionment Method: A Review and Sensitivity Study. Atmospheric Environment 1988, 22, 1777-1792.

(10) Henry, R. C., Multivariate receptor modeling by N-dimensional edge detection. Chemometrics and Intelligent Laboratory Systems 2003, 65, 179-189.

(11) Wittig, A. E.; Anderson, N.; Khlystov, A. Y.; Pandis, S. N.; Davidson, C.; Robinson, A. L., Pittsburgh air quality study overview. Atmospheric Environment 2004, 38, 3107-3125.

(12) Robinson, A. L.; Subramanian, R.; Donahue, N. M.; Bernardo-Bricker, A.; Rogge, W. F., Source apportionment of molecular markers and organic aerosol - 3. Food cooking emissions. Environmental Science \& Technology 2006, submitted. 
(13) Robinson, A. L.; Subramanian, R.; Donahue, N. M.; Bernardo-Bricker, A.; Rogge, W. F., Source apportionment of molecular markers and organic aerosol - 2. Biomass smoke. Environmental Science \& Technology 2006, submitted.

(14) Kamens, R. M.; Guo, Z.; Fulcher, J. N.; Bell, D. A., Influence of Humidity, Sunlight, and Temperature on the Daytime Decay of Polyaromatic Hydrocarbons on Atmospheric Soot Particles. Environmental Science \& Technology 1988, 22, 103-108.

(15) Finlayson-Pitts, B. J.; Pitts, J. N. Chemistry of the upper and lower atmosphere : theory, experiments, and applications; Academic Press: San Diego, CA, 2000.

(16) Schauer, C.; Niessner, R.; Poschl, U., Polycyclic aromatic hydrocarbons in urban air particulate matter: Decadal and seasonal trends, chemical degradation, and sampling artifacts. Environmental Science \& Technology 2003, 37, 2861-2868.

(17) Fraser, M. P.; Buzcu, B.; Yue, Z. W.; McGaughey, G. R.; Desai, N. R.; Allen, D. T.; Seila, R. L.; Lonneman, W. A.; Harley, R. A., Separation of fine particulate matter emitted from gasoline and diesel vehicles using chemical mass balancing techniques. Environmental Science \& Technology 2003, 37, 3904-3909.

(18) Cadle, S. H.; Mulawa, P. A.; Hunsanger, E. C.; Nelson, K.; Ragazzi, R. A.; Barrett, R.; Gallagher, G. L.; Lawson, D. R.; Knapp, K. T.; Snow, R., Composition of light-duty motor vehicle exhaust particulate matter in the Denver, Colorado area. Environmental Science \& Technology 1999, 33, 2328-2339.

(19) Schauer, J. J.; Cass, G. R., Source apportionment of wintertime gas-phase and particle-phase air pollutants using organic compounds as tracers. Environmental Science \& Technology 2000, 34, 1821-1832.

(20) Zheng, M.; Cass, G. R.; Schauer, J. J.; Edgerton, E. S., Source apportionment of PM2.5 in the southeastern United States using solvent-extractable organic compounds as tracers. Environmental Science \& Technology 2002, 36, 2361-2371.

(21) Tang, W.; Raymond, T.; Wittig, A. E.; Davidson, C. I.; Pandis, S. N.; Robinson, A. L.; Crist, K., Spatial Variations of PM2.5 during the Pittsburgh Air Quality Study. Aerosol Science \& Technology 2004, 38, 80-90.

(22) Watson, J. G.; Fujita, E. M.; Chow, J. C.; Zielinska, B. "Northern Front Range Air Quality Study Final Report and Supplemental Volumes," 1998.

(23) Schauer, J. J.; Kleeman, M. J.; Cass, G. R.; Simoneit, B. R. T., Measurement of emissions from air pollution sources. 5. C-1-C-32 organic compounds from gasoline-powered motor vehicles. Environmental Science \& Technology 2002, 36, 1169-1180.

(24) Schauer, J. J.; Kleeman, M. J.; Cass, G. R.; Simoneit, B. R. T., Measurement of emissions from air pollution sources. 2. C-1 through C-30 organic compounds from medium duty diesel trucks. Environmental Science \& Technology 1999, 33, 1578-1587.

(25) Rogge, W. F.; Hildemann, L. M.; Mazurek, M. A.; Cass, G. R.; Simoneit, B. R. T., Sources of Fine Organic Aerosol .2. Noncatalyst and Catalyst-Equipped Automobiles and Heavy-Duty Diesel Trucks. Environmental Science \& Technology 1993, 27, 636-651.

(26) Fraser, M. P.; Lakshmanan, K.; Fritz, S. G.; Ubanwa, B., Variation in composition of fine particulate emissions from heavy-duty diesel vehicles. Journal of Geophysical Research-Atmospheres 2002, 107.

(27) Fine, P. M.; Cass, G. R.; Simoneit, B. R. T., Chemical characterization of fine particle emissions from fireplace combustion of woods grown in the northeastern United States. Environmental Science \& Technology 2001, 35, 2665-2675. 
(28) Fine, P. M.; Cass, G. R.; Simoneit, B. R. T., Chemical characterization of fine particle emissions from the fireplace combustion of woods grown in the southern United States. Environmental Science \& Technology 2002, 36, 1442-1451.

(29) Fine, P. M.; Cass, G. R.; Simoneit, B. R. T., Chemical characterization of fine particle emissions from the wood stove combustion of prevalent United States tree species. Environmental Engineering Science 2004, 21, 705-721.

(30) Rogge, W. F.; Hildemann, L. M.; Mazurek, M. A.; Cass, G. R.; Simoneit, B. R. T., Sources of fine organic aerosol. 9. Pine, oak and synthetic log combustion in residential fireplaces. Environmental Science \& Technology 1998, 32, 13-22.

(31) Schauer, J. J.; Kleeman, M. J.; Cass, G. R.; Simoneit, B. R. T., Measurement of emissions from air pollution sources. 3. C-1-C-29 organic compounds from fireplace combustion of wood. Environmental Science \& Technology 2001, 35, 1716-1728.

(32) Hays, M. D.; Geron, C. D.; Linna, K. J.; Smith, N. D.; Schauer, J. J., Speciation of gas-phase and fine particle emissions from burning of foliar fuels. Environmental Science \& Technology 2002, 36, 2281-2295.

(33) Hays, M. D.; Fine, P. B.; Gerona, C. D.; Kleeman, M. J.; Gulletta, B. K., Open Burning of Agricultural Biomass: Physical and Chemical Properties of Particle-Phase Emissions. Atmospheric Environment 2005, 39, 6747-6764. 


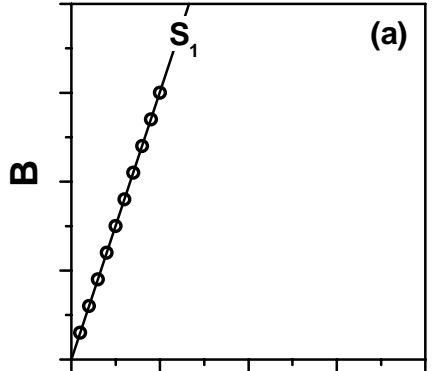

A

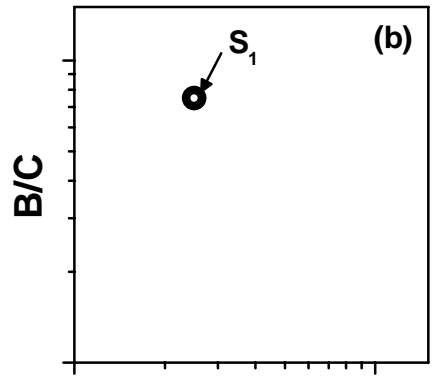

A/C

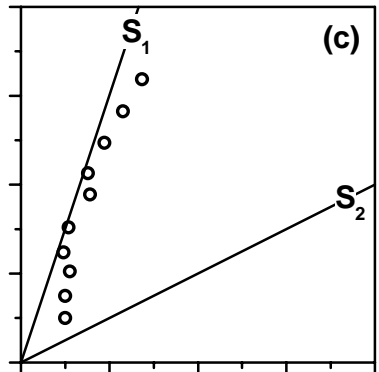

A

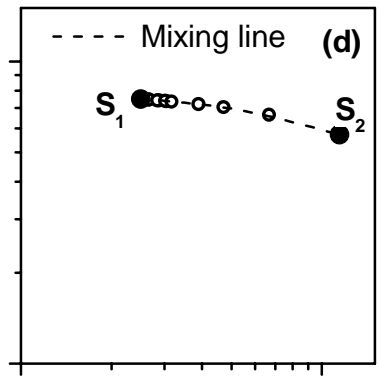

A/C

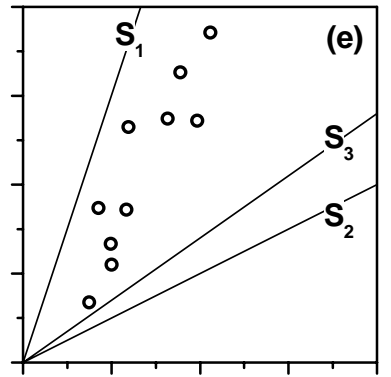

A

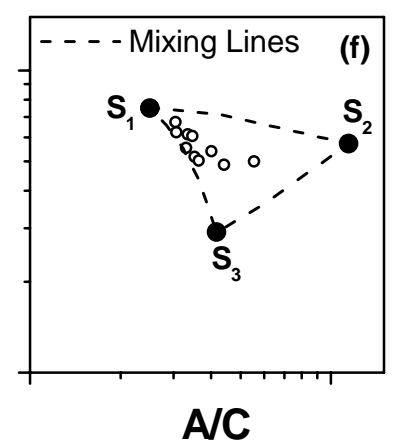

Figure 1. Scatter and ratio-ratio plots of simulated ambient data (indicated by open circles). The simulated data are based on mixing of emissions from up to three different sources: $S_{1}, S_{2}$, and $\mathrm{S}_{3}$. Plots (a) and (b) are a one source scenario; plots (c) and (d) are a two source scenario; and plots (e) and (f) are a three source scenario. 

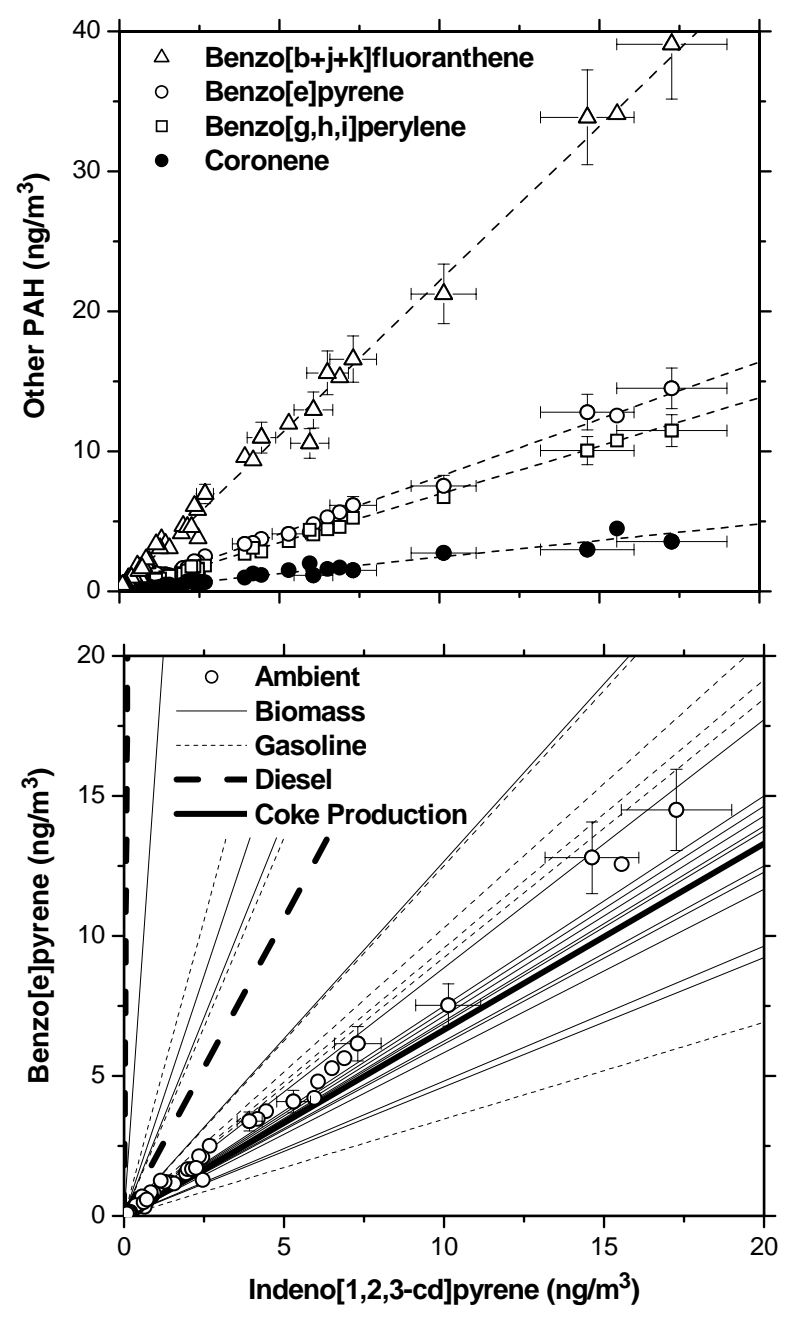

Figure 2. Scatter plots of different PAHs. The dashed lines in (a) are linear regressions $\left(\mathrm{R}^{2}>\right.$ 0.95). The lines in (b) are different source profiles taken from the literature (18,22-33). Error bars indicate measurement uncertainty of selected points. Uncertainty of low concentration and coronene data is comparable to the marker size. 


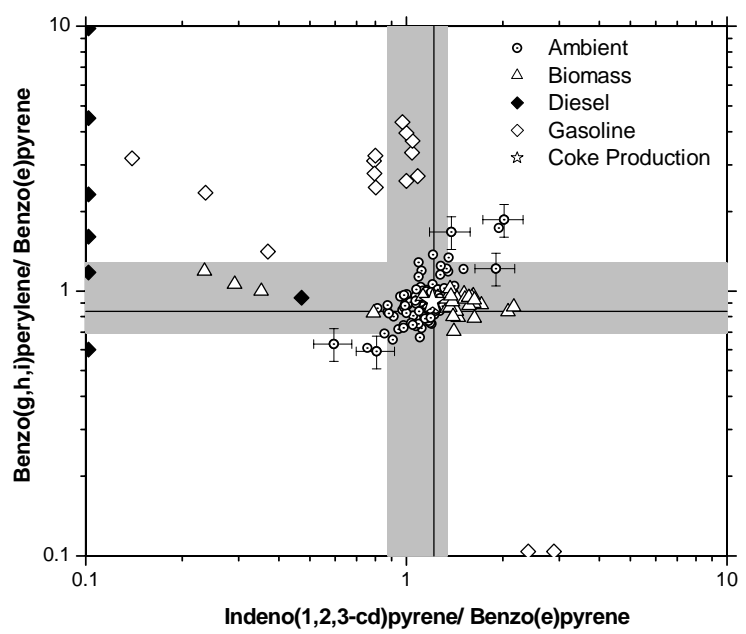

Figure 3. Ratio-ratio plot comparing ambient PAH to source profiles. The lines indicate the slopes of linear regressions shown in Figure 2a and the gray areas indicate fifth and ninety-fifth percentiles of the data. Error bars are shown a limited number of points to indicate typical level of measurement uncertainty. 

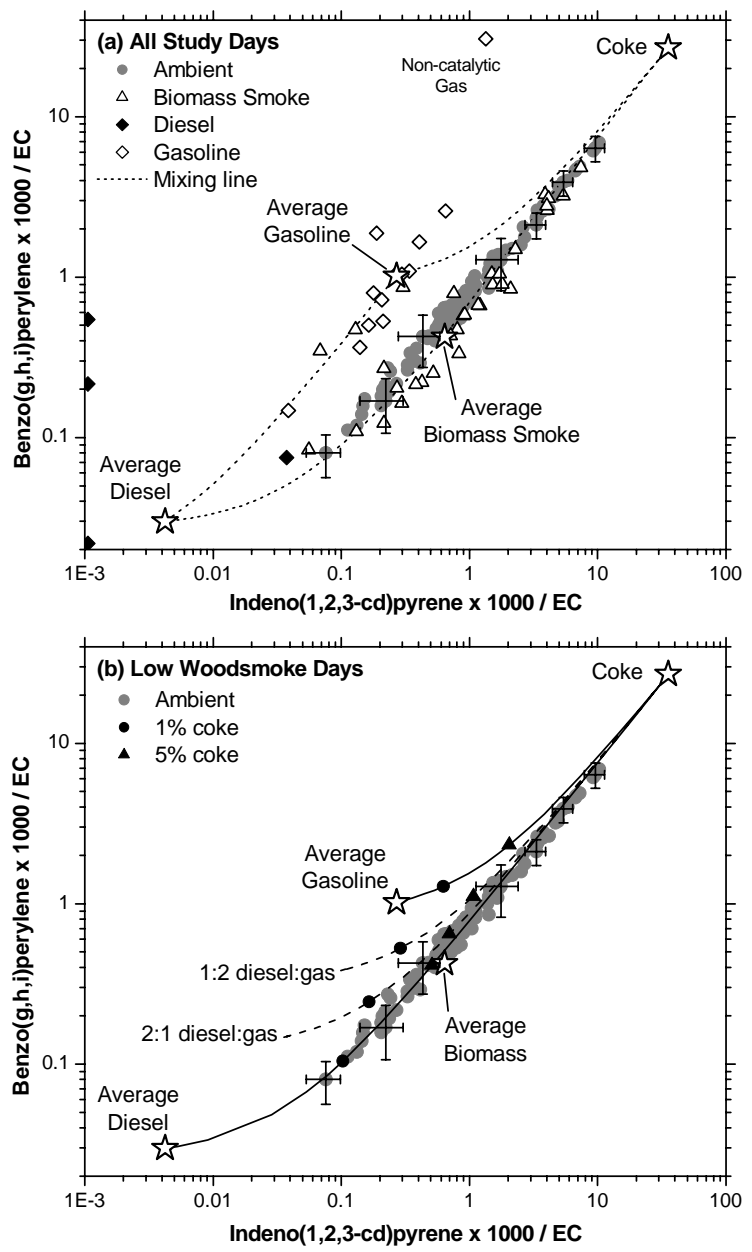

Figure 4. Ratio-ratio plots focused on using PAH and EC data to determine the gasolinediesel split: (a) complete ambient dataset; (b) 64 days with low biomass smoke concentrations. The stars indicate average emission profiles. The lines in (b) indicate mixing scenarios as described in the text, and the filled symbols in (a) indicate mixtures in which coke production contributes $1 \%$ and $5 \%$ of the OC from vehicle emissions plus coke production. Profiles plotted on the $y$-axis and/or $\mathrm{x}$-axis do not report or have indeno(1,2,3-cd)pyrene and/or benzo(g,h,i)perylene emissions outside of the range of the plot. Source profiles are from (18,2233). Error bars are shown a limited number of points to indicate typical level of measurement uncertainty. 


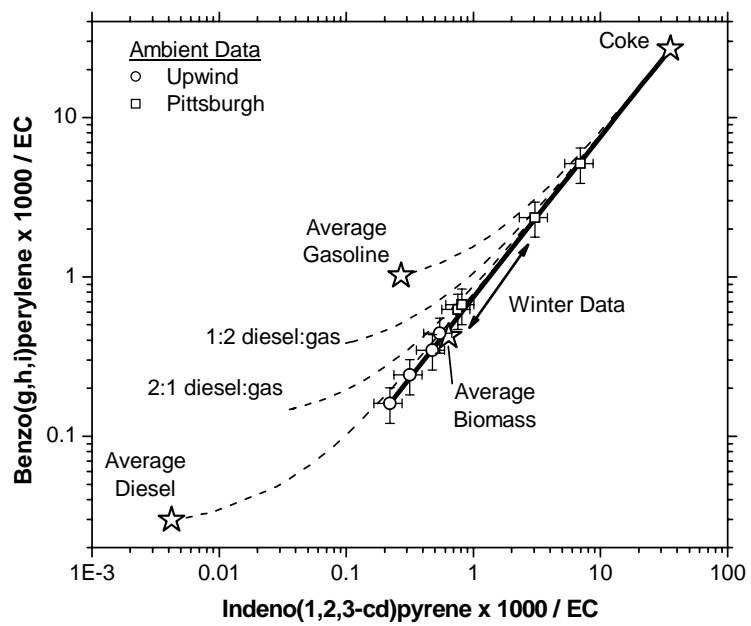

Figure 5. Ratio-ratio plot comparing Pittsburgh data and regional background air measured at an upwind site in Florence, PA. Stars are the average source profiles shown in Figure 4. The dashed lines correspond to the mixing scenarios shown in Figure 4b. The heavy solid line indicates mixing between the coke production source profile and aged regional background air, as described in the text. 


\title{
Source apportionment of molecular markers and organic aerosol -3 . Food cooking emissions
}

\author{
Allen L Robinson ${ }^{*}$, R Subramanian $\dagger$ \\ Department of Mechanical Engineering, Carnegie Mellon University, Pittsburgh, PA 15213, \\ USA
}

Neil M Donahue

Department of Chemistry and Department of Chemical Engineering, Carnegie Mellon University, Pittsburgh, PA 15213, USA

Anna Bernardo-Bricker

Department of Civil and Environmental Engineering, Florida International University, Miami, FL, USA

Wolfgang F Rogge

Department of Civil and Environmental Engineering, Florida International University, Miami, FL, USA

*Corresponding author: alr@andrew.cmu.edu; (412) 268-3657; (412) 268-3348 


\section{Abstract}

A large dataset of ambient cholesterol, palmitoleic acid, oleic acid, palmitic acid, and stearic acid concentrations is used to apportion ambient organic aerosol to food cooking emissions in Pittsburgh, Pennsylvania. Ambient concentrations of these species are well-organized which implies the existence of well-defined source profiles; however, significant inconsistencies exist between the ambient data and published source profiles. Most notably, the palmitoleic acid to oleic acid ratio is roughly a factor of ten greater than the ratio in essentially all published source profiles. The reason for this discrepancy is not known, but means that the fitting statistics calculated by the Chemical Mass Balance (CMB) model are poor if both species are included in the model at the same time. CMB analysis is performed using three different combinations of food cooking source profiles and molecular makers. The amount of OC apportioned to food cooking emissions by these different scenarios varies by factor 8 . Differences in the marker-toorganic-carbon ratios of the source profiles cause the large systematic biases between the different solutions. The best CMB solution is based on three profiles, two meat cooking profiles with widely divergent marker to OC ratios and a seed oil cooking profile. This CMB model also includes two alkanoic acids as fitting species, which helps constrain the estimates of meat cooking emissions. This solution explains much of the variability in the ambient molecular data and apportions an average of $320 \pm 140 \mathrm{ng}-\mathrm{C} \mathrm{m}^{-3}$ or $10 \%$ of ambient organic carbon in Pittsburgh to food cooking emissions. Although these results illustrate the significant challenges created by source profile variability, the strong correlations in the ambient dataset underscore the significant promise that molecular markers hold for source apportionment analysis. 


\section{Introduction}

Food cooking has been shown to be an important source of the ambient fine organic aerosol in urban environments (1-5). Organic molecular markers such as oleic acid, palmitoleic acid, and cholesterol have been used to predict the contribution of meat-cooking emissions to primary organic aerosol with the chemical mass balance (CMB) model (1,5-7). Cooking emissions are also a major source of palmitic and stearic acids $(1,6,8)$.

Selection of source profiles and fitting species requires careful consideration when performing CMB analysis. Previous CMB analyses have used different combinations of source profiles and molecular markers to estimate the contribution of food cooking emissions to ambient particle concentrations (1-5). More than 10 food cooking source profiles have been published with speciated organics data (7-11); a comparison of these profiles reveals of wide range in emission rates and emission composition depending on food type and preparation technique.

This paper is part of a series of papers that examines issues associated with CMB analysis of molecular marker data in Pittsburgh, PA (12-14). The goal of this paper is to examine uncertainties associated with estimating the contribution of food cooking emissions to organic aerosol in Pittsburgh using molecular markers. First, a large data set of ambient concentrations of oleic acid, palmitoleic acid, palmitic acid, stearic acid, and cholesterol are examined for correlations among different compounds emitted by food cooking. Next, the data are compared to the available cooking source profiles using the approach described in Robinson et al. $(12,15)$. Based on these comparisons, different scenarios for CMB analysis are defined and the paper concludes with a discussion of the CMB results. 


\section{Methods}

CMB analysis was performed to apportion ambient OC in Pittsburgh, PA to sources of primary organic aerosol. The analysis uses ambient concentrations of individual organic compounds, $\mathrm{PM}_{2.5}$ organic and elemental carbon, and $\mathrm{PM}_{2.5}$ elemental composition measured on 103 days between July, 2001 and August, 2002 (16). Daily measurements were made in July 2001 and most of January 2002; during other periods 24-hr samples were collected on a 1 in 6 day schedule. Additional details of the data set are provided in Supporting Information.

Source contributions to ambient OC are determined by calculating the linear combination of source emissions needed to reproduce the ambient concentrations of a set of fitting species. The selection of compounds included in the CMB model is a critical issue; all major sources of each compound must be included in the model and the species should be conserved during transport from source to receptor (17). This work uses the basic set of compounds and source classes developed by Schauer et al. $(1,5)$. In addition to the cooking related markers discussed below, all calculations include four n-alkanes, iso-hentriacontane, anteiso-dotriacontane, syringaldehyde, sum of resin acids, acetosyringone, levoglucosan, four hopanes, four PAHs, titanium, iron, and elemental carbon. Source profiles for eight source classes are included in the model: diesel vehicles, gasoline vehicles, road dust, biomass combustion, cooking emissions, coke production, vegetative detritus, and cigarettes. The calculations were performed using the computer program CMB8 distributed by the US EPA. Lists of source profiles and fitting species for each scenario are contained in Supporting Information.

This paper focuses on five important markers for cooking emissions: n-hexadecanoic (palmitic) acid, n-octadecanoic (stearic) acid, 9-hexadecenoic (palmitoleic) acid, 9-octadecenoic (oleic) acid, and cholesterol. CMB analysis is performed using different combinations of these 
species with different food cooking source profiles (in addition to a standard set of non-food cooking source profiles and molecular markers). Experimental uncertainties are estimated as $\pm 30 \%$ for cholesterol and as $\pm 20 \%$ for the alkenoic and alkanoic acids; these values were determined by comparing results for samples collected in parallel. These uncertainties are applied to both the source profiles and the ambient data. Although some of the food cooking markers are present in source profiles for non-cooking source classes included in the model, CMB apportions the vast majority of the ambient concentrations of these give compounds to the food cooking profiles. Therefore, the specific combination of non-food cooking profiles has little influence on the OC apportioned to food cooking emissions.

The specific combinations of food cooking source profiles and fitting species used for CMB analysis are selected based on comparisons made using scatter and ratio-ratio plots of ambient concentrations of different molecular markers. These plots allow one to visually evaluate relationships between ambient concentrations of different markers and to compare source profiles to ambient concentrations. Ratio-ratio plots are constructed using three species; one compound is selected as a reference to normalize the concentrations of the other two compounds, called target species. The best reference compounds are relatively abundant, stable, and specific to the sources plotted. Source profiles appear as points on ratio-ratio plots and linear mixing lines can be drawn to visualize the effects of mixing of emissions from different sources. More details on the construction, interpretation, and mathematics of ratio-ratio plots are provided in Robinson et al. $(12,15)$.

\section{Results}

Time series of ambient concentrations of cholesterol, palmitoleic acid, and palmitic acid are shown in Figure 1. The results are presented using box-plots constructed by grouping the data 
into one or two month periods, depending on the number of samples. Ambient palmitoleic and oleic acid concentrations are well correlated (Figure 2a) so time series for oleic acid appears similar to the palmitoleic acid data shown in Figure 1b. Similarly ambient palmitic and stearic acid concentrations are well correlated (Figure 2d) therefore time series of stearic appear similar to the palmitic acid data shown in Figure 1c.

The height of the boxes in Figure 1 reflects the significant day-to-day variability in the ambient cooking marker concentrations. The median ambient concentrations of cholesterol and the two alkenoic acids are around $1 \mathrm{ng} \mathrm{m}^{-3}$. There is no seasonal pattern in the concentrations of cholesterol or the two alkenoic acids; however, only a few high concentration days were observed in the spring and the fall. This may be due in part to only $20 \%$ of the samples being collected in the fall and spring versus 50\% in summer and 30\% in winter. Concentrations of the two alkanoic acids exhibit a distinct seasonal pattern, with wintertime concentrations being on average a factor of 3 smaller than summertime concentrations.

The ambient concentrations of the different cooking markers measured in Pittsburgh are similar to other locations in the United States. For example, the Pittsburgh oleic acid levels are comparable to the "seasonal composite" concentrations in Houston, TX (18) but a factor of two or three lower than in Birmingham, AL and Atlanta, GA (3) The Pittsburgh palmitoleic acid levels are a factor of two or three higher than other locations. The cholesterol levels in Pittsburgh are similar to average concentrations in Los Angeles (19).

CMB analysis seeks to describe ambient concentrations using a linear combination of source profiles; therefore, a key consideration is the relative distribution of different species in the ambient data set. Scatter plots provide one tool for visualizing relationships among species (12); Figure 2 shows scatter plots of ambient daily concentrations of the different cooking markers. 
Species whose ambient concentrations exhibit strong linear correlations are likely emitted by a single dominant source class with the slope of the regression indicating the emission ratio of the two species (12).

Ambient oleic and palmitoleic acid concentrations are well correlated with a slope of roughly 1 (Figure 2a). In addition, except for three possible outliers, most of the scatter in data can be attributed to measurement uncertainty. This implies a single dominant source for the two alkenoic acids, presumably meat cooking. Cholesterol is also an important marker for meat cooking emissions (7). Figure 2b indicates only modest correlation between ambient palmitoleic and cholesterol data; however, a reasonable amount of the scatter between the alkenoic acid and cholesterol data can be attributed to measurement uncertainty. Therefore, ambient concentrations of these three compounds are likely dominated by the same source. Ambient palmitic and stearic acid concentrations are well correlated (Figure 2c), but little correlation is observed between the two alkenoic and the two alkanoic acids (Figure 2d). This implies that these saturated and unsaturated acids have different dominant sources.

Ratio-ratio plots provide another tool for visualizing relationships in ambient datasets $(12,15)$. Figure 3a plots the two alkenoic acids normalized by cholesterol, while Figure 3b plots the two alkanoic acids normalized by cholesterol. The strong correlations among the different cooking markers in the ambient data result in well organized ratio-ratio plots from which one can infer potential source profiles of the aggregate emissions influencing Pittsburgh.

The organization of the data in Figure 3 suggests a number of viable hypotheses to explain the ambient data. First, we consider the ambient alkenoic acid and cholesterol data plotted in Figure 3a. Within experimental uncertainty, the large majority of these data can be explained by a source profile that is approximately 1:1:1 in palmitoleic acid:oleic acid:cholesterol. The 
dispersion along the one-to-one line indicates some variation in the relative amount of cholesterol in the emissions; however, the somewhat higher uncertainty of the cholesterol data may also contribute to this dispersion. Another possible scenario includes an average source profile that is approximately 5:5:1 (1 being cholesterol), with photochemical aging causing up to an order of magnitude depletion of the alkenoic acids (15). A final viable explanation of the data in Figure 3a is two sources that bracket the ambient data along the diagonal, one of at least 5:5:1 and one below 0.2:0.2:1, each with variable strengths.

Explaining the ambient alkanoic acid and cholesterol data in Figure 3b requires at least two source profiles; a profile rich in cholesterol located in the lower left hand corner of the plot and a second source that emits alkanoic acids but little or no cholesterol (such as seed oil cooking or even motor vehicles) that is located in the upper right hand corner of the graph. A mixing line between these two sources passes through the ambient data.

Comparison of Ambient Data with Source Profiles: Relatively few cooking source profiles have been published that report alkanoic acid, alkenoic acid, and cholesterol data. The majority of the available cooking profiles are for commercial-scale frying and charbroiling of hamburger meat $(7,9)$. Profiles have also been published for frying of vegetables in oil, which emits the different acids but no cholesterol (8). There are also non-cooking related sources of these compounds. Biomass smoke, motor vehicle exhaust and road dust all contain the four acids $(1,6)$. Cholesterol is thought to be a good marker for meat cooking emissions in urban environments (7); although recent papers have reported unexpectedly high levels of cholesterol in a remote natural area (20) and in emissions from prescribed burns (21). All of these compounds are important constituents of plant and/or animal tissues, but the potential contribution of biogenic sources is not well defined. 
Figure 3 compares published cooking source profiles to the ambient data. Source profiles appear as points in the ratio-ratio plots, which are connected by linear mixing lines to define regions with the plot that can be described by linear mixing (12,15). Also shown on Figure 3 are some new, unpublished residential cooking profiles for frying steaks, hamburgers, and breaded fish fillets using both electric and natural gas stoves. These new profiles fall within the scatter of the published profiles. Arrows in Figure 3 point towards sources of the different acids that do not emit cholesterol.

Cooking emissions depend on type of food and cooking technique (7-9). The available profiles organize along a diagonal line in the ratio-ratio plots. This occurs because there is only modest profile-to-profile variability in the palmitoleic acid: oleic acid and palmitic acid: stearic acid ratios of the different profiles. The biggest difference between profiles is the almost two order of magnitude variability in relative emissions of cholesterol. Figure 3 indicates that the variability across the set of cooking profiles is similar to that exhibited by the ambient data.

The most striking aspect of a comparison between the ambient data and the source profiles is the large differences in the palmitoleic to oleic acid ratios. Figure 3a indicates that the ambient data have nearly equal loadings of these two acids compared to an average 20:1 dominance for oleic acid in the published meat cooking source profiles. Although there is some profile-toprofile variability in the ratio of these two acids, even the profile with the smallest oleic-topalmitoleic acid ratio is still enriched by almost a factor in ten in oleic acid compared to the ambient data.

Palmitoleic and oleic acid are also emitted by a number of other non-meat cooking sources. Almost all of the available profiles for these other source classes are also significantly enriched in oleic acid and therefore cannot explain the ambient oleic-to-palmitoleic acid ratios. The only 
profiles we have found that report a palmitoleic acid to oleic acid ratio greater than one are the Fine et al. (22) Quaking Aspen and softwood open burn profiles (23). We explored including these profiles in CMB to explain the ambient data, but even on the limited number of days with significant biomass smoke, only a small fraction of the ambient alkenoic acids are apportioned to the non-cooking profiles. Consequently, CMB solutions that simultaneously try to fit both oleic and palmitoleic acid data have poor fitting statistics.

The unexpectedly small ambient ratios of oleic to palmitoleic acid relative to the known source profiles are not unique to the Pittsburgh. Ratios of 1:1 to 3:1 (oleic:palmitoleic) have been reported for rural sites in the Southeastern US (3), Houston, TX (18), and Los Angeles (24). Urban locations in the Southeastern US have larger ratios that are more consistent with available cooking source profiles (3).

Explaining the ambient alkenoic acid data requires a source rich in palmitoleic acid that is mixing with emissions from the known sources. In addition, the strong correlation shown in Figure 2a requires that the emissions from this unknown source co-vary with the emissions from the known important sources of oleic acid. This constraint makes it very unlikely that this source is a completely different (i.e. non-cooking) activity. Rather, it seems likely that the aggregate cooking profile characteristic of Pittsburgh differs from the published profiles, which points to need to expand our knowledge of cooking emissions beyond the limited number of available profiles. An alternative explanation is that the concentrations are in some way altered between the sources and the receptor. Although laboratory studies observe rapid oxidation of oleic acid (25), oleic acid would have to be oxidized at a much higher rate than palmitoleic acid. More recent data indicate that in model meat smoke aerosols that the two alkenoic acids oxidize at essentially same rate (26). 
$\underline{\text { CMB analysis: }}$ In this section we develop scenarios for CMB analysis focusing on the contribution of meat cooking emissions to ambient OC. First we consider CMB models in which the key markers for meat cooking emissions are either cholesterol and oleic acid or cholesterol and palmitoleic acid. We do not fit both alkenoic acids at the same time because of the significant inconsistencies between the palmitoleic acid-to-oleic acid ratios of the source profiles and the Pittsburgh ambient data. Interestingly, almost all previous CMB analyses of molecular marker data have only fit one of the two alkenoic acids. Initial analyses used oleic acid (1), which was subsequently replaced with palmitoleic acid because of concerns over emissions of oleic acid from seed oil cooking (5).

In order to fit the ambient cholesterol and oleic acid data the ratio of these species in the cooking source profile must the same as the ambient data. Graphically, this profile needs to be somewhere in the horizontal grey band shown in Figure 3a. Within experimental uncertainty, the red meat frying profiles can explain the ambient cholesterol and oleic acid data. Therefore, for the first CMB scenario, we fit cholesterol and oleic acid using the average frying profile indicated by the open star in Figure 3a; this profile is the arithmetic average of the individual red meat frying profiles. In order to fit the ambient cholesterol and palmitoleic acid data the cooking source profile should lie within the vertical gray band shown in Figure 3a. For this second case we use an average red meat charbroiling profile in CMB, which is indicated by the filled star in Figure 3a.

Although seeking to explain the ambient alkenoic acid and cholesterol data using a red meat frying or charbroiling profiles seems problematic given the diversity of cooking operations, the limited number of published profiles makes it impossible to construct a more realistic aggregate cooking profile based on food consumption and cooking data. Essentially all previous CMB 
analyses of molecular marker data have represented aggregate cooking emissions using a single are most two red meat cooking profiles $(1-3,5,20)$. One contribution of this work is to investigate the effects of combinations of different profiles and fitting species on the amount of ambient OC apportioned to cooking by the CMB model.

Figure 4 plots the monthly average ambient OC apportioned to meat cooking emissions by two CMB scenarios which fit one alkenoic acid and cholesterol. Monthly average concentrations are determined by arithmetically averaging the CMB results calculated for individual days. On almost all days, the statistical quality of both solutions based on the performance measures calculated by CMB $\left(\mathrm{R}^{2}, \chi^{2}\right.$, calculated over measured values, T-statistics, etc.) are within the guidelines described in the CMB manual (17). For example, both solutions have average $\mathrm{R}^{2}$ and $\chi^{2}$ values 0.92 and 2.0 respectively and both solutions have confidence levels greater than $96 \%$ based on the $\chi^{2}$ values and number of degrees of freedom. Therefore, one cannot use the statistical measures calculated by CMB to differentiate between the two solutions. Additional information on the statistical quality of the solutions is presented in the Supporting Information.

Figure 4 shows that the CMB analysis of the palmitoleic acid and cholesterol scenario (which fits the average charbroiling profile) apportions significantly more ambient OC to meat cooking than the oleic acid and cholesterol scenario (which fits the average frying profile). Both scenarios also exhibit strong seasonal patterns with peak meat cooking contributions in the winter and lower contributions in the summer. The daily OC apportioned to meat cooking by the two scenarios is highly correlated $\left(\mathrm{R}^{2}=0.84\right.$ for the linear regression), but the palmitoleic acid and cholesterol scenario apportions eight times more OC to meat-cooking than the model fitting oleic acid and cholesterol. 
The large systematic bias between these two scenarios is caused by differences in the markerto-OC ratios of the source profiles. This ratio is a critical parameter in determining the amount of OC apportioned to a source class because CMB only fits the ambient concentrations of the species included in the model. OC is not included in the model because source profiles do not exist for important contributors to OC such as secondary organic aerosol (SOA). After determining the optimum fit of the target species, $\mathrm{CMB}$ apportions $\mathrm{OC}$ to each source using the marker-to-OC ratio of the different source profiles. This second step is built into the analysis because the sources profiles have been normalized by the OC.

The marker-to-OC ratios for the published meat cooking source profiles can vary by more than an order of magnitude. For example, the average charbroiling profile used in the palmitoleic acid and cholesterol scenario has a cholesterol-to-OC ratio that is 5.3 times smaller than the average frying profile used for the other scenario. This means that, for a given ambient cholesterol concentration, the average charbroiling apportions 5.3 times more meat cooking OC than the average frying profile. Even larger differences exist between ratios of the two alkenoic acids with OC of the average charbroiling and average frying profiles. The net effect of these differences is an average bias of a factor of 8 between the two scenarios. Figure 4 indicates that this bias is much larger than the standard errors calculated by CMB.

The fact that source profiles typically have small marker-to-OC ratio can result in an unrealistically large fraction of the ambient OC apportioned to a given profile. For example, on the six days with the highest cooking marker concentrations, more than $100 \%$ of the ambient OC is apportioned to the average charbroiling profile by the palmitoleic acid and cholesterol scenario. Similarly, during the winter when cooking markers were high, this scenario 
apportions, on average, more than $60 \%$ of the ambient OC to meat, leaving little room for the contribution of other, non-cooking primary sources such as motor vehicles and biomass burning.

Profile variability is also a problem if one normalizes the marker emissions with $\mathrm{PM}_{2.5}$ mass emissions as opposed to $\mathrm{OC}$-- the marker-to- $\mathrm{PM}_{2.5}$-mass ratios of the difference source profiles exhibit as much variability as the marker-to-OC ratios. We have performed CMB analysis using source profiles normalized with $\mathrm{PM}_{2.5}$ mass and the results look essentially the same as those shown for OC in Figure 4.

Additional insight into the quality of the CMB solutions is provided by examining results for species not included as fitting species in the model. CMB calculates the ambient concentrations of these species using the source profiles and the optimum solution based on the specified set of target compounds. Mass balance requires that these calculated concentrations be less than or equal to the observed concentrations, but some modest overprediction is acceptable given the uncertainty in the analysis. One of the target criteria for CMB analysis is that calculated concentrations of the fitted species should not be more than a factor of two greater than ambient data (17). This seems like a reasonable criterion for the non-fitted species as well.

The CMB results for the non-fitting species raise a number of additional concerns for the palmitoleic acid and cholesterol solution. On winter days, this scenario overpredicts the ambient stearic acid concentrations by more than a factor of 3 . On essentially all days this scenario overpredicts the ambient oleic acid concentrations by almost a factor of 10 . These problems are clearly apparent in the ratio-ratio plots presented in Figure 3; for example, Figure 3a shows that a reasonable fraction of the ambient data having alkanoic-acid-to-cholesterol ratios that are much smaller than the average charbroiling profile. The oleic acid and cholesterol scenario does not have problems with overpredicting non-fitted species. 
Although the oleic acid and cholesterol CMB solution does not overapportion OC or other species not explicitly included in the model, it apportions only $92 \pm 24$ ng-C $\mathrm{m}^{-3}$ ( \pm standard error) or 3\% of the study average OC to meat cooking. This is much less than previous CMB analyses using molecular marker data (1-3,5). Another potential problem with the oleic acid and cholesterol scenario is that Figure 4 indicates that this scenario apportions almost no ambient OC to meat cooking in the fall and spring months. Strong seasonal changes are not expected for cooking emissions.

The results from the first two CMB scenarios clearly indicate that more information is needed to constrain the contribution of meat cooking emissions to ambient OC. Stearic and palmitic acid are important constituents of meat cooking emissions (7). The organization of the ambient data relative to the source profiles in Figure 3 provides insight into potential CMB scenarios that include cholesterol, palmitoleic acid, palmitic acid, and stearic acid as fitting species. The average red meat frying profile and the Schauer et al. (9) charbroiling profile bracket both the ambient palmitoleic acid to cholesterol ratios (Figure 3a) and the ambient palmitic and stearic acid to cholesterol ratios (Figure 3b). The mixing line connecting these profiles passes through the vast majority of the ambient data in Figure 3b. The only problem with this solution is that it requires substantially higher ambient oleic acid concentrations than were actually measured as illustrated by the mixing line drawn in Figure 3a.

Palmitic and stearic acids are typically not included as fitting species in CMB because of concerns with unidentified sources (1). Although meat cooking is an important source of these acids, it is predicted to contribute less than $50 \%$ of the n-alkanoic acids in Los Angeles (6). The poor correlation between the alkenoic and alkanoic acids (Figure 2d) implies that meat cooking is not the dominant source of alkanoic acids in Pittsburgh. Other sources in the model such as 
biomass smoke are only minor contributors of the two alkanoic acids. The strong correlation of the ambient palmitic and stearic acid suggests there is a single dominant source of these acids. To account for the emissions from this unknown source we also include an average seed oil cooking profiles in the model. This profile is the arithmetic average of the three profiles in Schauer et al. (8).

CMB analysis was performed including palmitoleic acid, cholesterol, palmitic acid and stearic acid in the model and three cooking profiles - average red meat frying, Schauer et al. (9) charbroiling, and average seed oil cooking. The statistical performance of this solution is strong on almost all days, with an average $\chi^{2}$ and $\mathrm{R}^{2}$ values of 0.92 and 2.1 respectively. The minimum confidence level is $96 \%$ based on the $\chi^{2}$ and number of degrees of freedom. Therefore, on the vast majority of the days, one cannot use these parameters to differentiate between the different solutions.

Time series of the OC apportioned to meat and seed oil cooking are shown in Figures 4 and 5a. The amount of OC apportioned by the three source scenarios falls between the other two CMB solutions. In addition, Figure 4 indicates there is much less month-to-month variability in the amount of ambient OC apportioned to food cooking compared to the previous cooking scenarios. On a study average basis, the three source scenario apportions $320 \pm 140 \mathrm{ng}-\mathrm{C} \mathrm{m}^{-3}$ or $10 \%$ of ambient OC to food cooking emissions. On a $\mathrm{PM}_{2.5}$ mass basis, CMB apportions $560 \pm$ $240 \mathrm{ng} \mathrm{m}^{-3}$ or $3 \%$ of the study average ambient $\mathrm{PM}_{2.5}$ mass to cooking emissions. These estimates are comparable to results from CMB analyses with molecular performed in the Southeastern US (3), but a factor of two or more smaller than results from Houston (2).

Figure 5 presents the daily time series of the apportionment of ambient OC (Figure 5a), cholesterol (Figure 5b), and palmitic acid (Figure 5c) to the different cooking profiles. These 
plots provide insight into the role that each food cooking profile plays in the CMB solution. Of the three cooking profiles, the Schauer et al. (9) charbroiling profile is the dominant contributor to ambient OC (Figure 5a), the average frying profile is the dominant contributor to cholesterol (Figure 5b), and all three profiles contribute significantly to ambient alkanoic acid concentrations (Figure 5c). These differences can be attributed to the marker-to-OC ratios of the food cooking profiles. Of the three profiles, the Schauer et al. (9) charbroiling profile has by far the smallest marker-to-OC ratios which enables this profile to contribute lots of OC while only contributing a relatively small amount of the different fitting species. In contrast, the cholesterol-to-OC ratio of the average frying profile is a factor of 25 greater than the Schauer et al. (9) charbroiling profile. Therefore, the frying profile dominates the cholesterol apportionment, while only contributing only modestly to the ambient OC. Almost no ambient OC is apportioned to the seed oil profile the maximum monthly average contribution is $28 \pm 12 \mathrm{ng}-\mathrm{C} \mathrm{m}^{-3}$. Its contribution is so small because the emissions from seed oil cooking are dominated by alkanoic acids; for example, the palmitic-acid-to-OC ratio of the average seed oil cooking profile is 0.23 . Even if all the ambient alkanoic acids are apportioned to seed oil cooking profile, it would contribute, on average, only $60 \mathrm{ng}-\mathrm{C} \mathrm{m}{ }^{-3}$ of OC.

A key feature of the palmitic and stearic acid CMB scenario is that it includes two meat cooking profiles with very different marker-to-OC ratios in model. Figure 5 shows that, as the ambient concentrations of the cooking markers increase, the frying profile becomes more important in the model. This allows the model to explain the ambient cooking marker data on high concentration days without apportioning unrealistically large amounts of ambient OC to meat cooking (e.g. wintertime apportionment by the palmitoleic acid and cholesterol scenario shown in Figure 4). Conversely on days when the ambient concentrations of the meat cooking 
markers are low the Schauer et al. (9) charbroiling profile dominates, which avoids the unrealistically small amounts of meat cooking OC apportioned by the oleic acid and cholesterol scenario in the fall and spring (Figure 4). These shifts are due to the variation in the ambient data shown in Figure 3.

Fitting the two meat cooking profiles requires including the two alkanoic acids as fitting species in the model. These acids force the CMB model to apportion the core cooking markers, cholesterol and palmitoleic acid, to the frying profile. Without these acids, CMB analysis with the two meat cooking profiles finds essentially the same solution as the previously discussed palmitoleic acid and cholesterol scenario fit with the average charbroiling profile.

Although little ambient OC is apportioned to the seed oil profile, this profile plays an important role in the CMB model by serving as a sink for the alkanoic acids. This prevents the alkanoic acids driving up the amount of OC apportioned to meat cooking on days when ambient alkanoic acid concentrations are high but the levels of other meat cooking markers such as cholesterol are low. The role of the seed oil profile in the CMB model is reflected by the amount of alkanoic acids apportioned to seed oil cooking varying seasonally from roughly $25 \%$ in the summer to essentially none in the winter (Figure 5c), matching the seasonal pattern in the ambient data shown in Figure 1c. One does not expect a strong seasonal pattern in cooking emissions; therefore the acids apportioned by CMB to the seed oil profile may come from some other source, with a biogenic source being a likely candidate. However, in our view, the benefits of including alkanoic acids in the model as a constraint for meat cooking significantly outweigh concerns over unidentified sources.

There are a number of problems with the CMB scenario that fits the two alkanoic acids, palmitoleic acid, and cholesterol with the three cooking profiles. First, it overpredicts the 
ambient concentrations of oleic acid by about a factor of 10 . Oleic acid was not included in the model because of the large discrepancy between the palmitoleic to oleic acid ratio between the source profiles and the ambient data illustrated in Figure 3a. The cause of this discrepancy is not known. Second, on nine days a significant amount of ambient cholesterol is not being accounted for by the model - see, for example, the first few winter days in Figure 5b. These are all high cholesterol days, on which the fit of the meat cooking profiles is being constrained by the alkanoic acids. Similar problems are apparent for palmitoleic acid in the three cooking source solution.

\section{Discussion}

One of the most important findings of this paper is the strong correlations and resulting wellorganized ratio-ratio plots among different molecular markers associated with food cooking emissions in a very large ambient dataset. The organization of the ambient data reflects the source specificity of these compounds and implies the existence of well-defined source profiles. These sorts of relationships are essentially never observed among bulk species (metals, ions, OC/EC) commonly used for source apportionment analysis. This underscores the utility of using molecular markers for source apportionment analysis. Although previous studies have measured molecular markers, the datasets have been much too small to directly probe for relationships between ambient marker concentrations.

This paper also describes a number of challenges associated with performing CMB analysis with molecular markers. Source profile variability can create significant uncertainty in the CMB results. For example, the factor of 8 difference in the amount of ambient OC apportioned to meat cooking by the different CMB scenarios is caused by differences in source profile markerto-OC ratios. Selecting profiles with the smallest ratios maximizes the amount of OC 
apportioned to a source class. This ratio can introduce uncertainties in the source strength estimates that are difficult to remove because it is not explicitly considered by statistical measured calculated by CMB. The problem of variable marker-to-OC ratios is not unique to cooking $(13,14)$. In this paper we have illustrated how adding alkanoic acids to the CMB model helps constrain CMB results for meat cooking, but more research is needed to address the problem of source profile variability.

Given the central role of the marker-to-OC ratio in determining the amount of OC apportioned to a source class, these ratios must be carefully consider when performing and interpreting CMB analysis with molecular markers. For example, many recent CMB analyses with molecular markers have used the Schauer et al. (9) charbroiling profile to represent the aggregate emissions from meat cooking operations $(2,3,5,20,27)$. This profile has a cholesterolto-OC ratio that is a factor of 10 smaller than many other published profiles (see Figure S-1 in supporting information). Similar variation is observed in the cholesterol-to- $\mathrm{PM}_{2.5}$ mass ratios of the different meat cooking profiles. Therefore, CMB analysis using this profile and cholesterol data will apportion significantly more $\mathrm{OC}$ (or $\mathrm{PM}_{2.5}$ mass) to meat cooking emissions than $\mathrm{CMB}$ analysis based on other profiles. Unfortunately, assessing these issues is difficult because many papers do not clearly specify the set of fitting species and source profiles. Cholesterol has not been routinely used as a fitting species in CMB analysis with molecular makers, but problems of apportioning more than $100 \%$ OC are reported in one paper that did fit ambient cholesterol data with the Schauer et al. (9) charbroiling profile (20). Sheesley et al. (20) attributed the problem to an unknown source of cholesterol, but a significant factor may have been the very small cholesterol-to-OC ratio the Schauer et al. (9) charbroiling profile. 
A second challenge for CMB analysis is inconsistencies between the published cooking source profiles and the ambient data, most notably for the oleic and palmitoleic acid data. Such inconsistencies points to the need to develop more source profiles, especially for source classes such as cooking for which fewer available profiles are available compared to source classes such as motor vehicles and biomass combustion.

\section{Acknowledgments}

This research was conducted as part of the Pittsburgh Air Quality Study, which was supported by US Environmental Protection Agency under contract R82806101 and the US Department of Energy National Energy Technology Laboratory under contract DE-FC2601NT41017. This research was also supported by the EPA STAR program through the National Center for Environmental Research (NCER) under grant R832162. This paper has not been subject to EPA's required peer and policy review, and therefore does not necessarily reflect the views of the Agency. No official endorsement should be inferred.

\section{References}

(1) Schauer, J. J.; Rogge, W. F.; Hildemann, L. M.; Mazurek, M. A.; Cass, G. R., Source apportionment of airborne particulate matter using organic compounds as tracers. Atmospheric Environment 1996, 30, 3837-3855.

(2) Fraser, M. P.; Yue, Z. W.; Buzcu, B., Source apportionment of fine particulate matter in Houston, TX, using organic molecular markers. Atmospheric Environment 2003, 37, 2117-2123.

(3) Zheng, M.; Cass, G. R.; Schauer, J. J.; Edgerton, E. S., Source apportionment of PM2.5 in the southeastern United States using solvent-extractable organic compounds as tracers. Environmental Science \& Technology 2002, 36, 2361-2371.

(4) Watson, J. G.; Fujita, E. M.; Chow, J. C.; Zielinska, B. "Northern Front Range Air Quality Study Final Report and Supplemental Volumes," 1998.

(5) Schauer, J. J.; Cass, G. R., Source apportionment of wintertime gas-phase and particle-phase air pollutants using organic compounds as tracers. Environmental Science \& Technology 2000, 34, 1821-1832. 
(6) Rogge, W. F.; Hildemann, L. M.; Mazurek, M. A.; Cass, G. R.; Simoneit, B. R. T., Mathematical modeling of atmospheric fine particle-associated primary organic compound concentrations. Journal of Geophysical Research-Atmospheres 1996, 101, 19379-19394.

(7) Rogge, W. F.; Hildemann, L. M.; Mazurek, M. A.; Cass, G. R.; Simonelt, B. R. T., Sources of Fine Organic Aerosol .1. Charbroilers and Meat Cooking Operations. Environmental Science \& Technology 1991, 25, 1112-1125.

(8) Schauer, J. J.; Kleeman, M. J.; Cass, G. R.; Simoneit, B. R. T., Measurement of emissions from air pollution sources. 4. C-1-C-27 organic compounds from cooking with seed oils. Environmental Science \& Technology 2002, 36, 567-575.

(9) Schauer, J. J.; Kleeman, M. J.; Cass, G. R.; Simoneit, B. R. T., Measurement of emissions from air pollution sources. 1. C-1 through C-29 organic compounds from meat charbroiling. Environmental Science \& Technology 1999, 33, 1566-1577.

(10) McDonald, J. D.; Zielinska, B.; Fujita, E. M.; Sagebiel, J. C.; Chow, J. C.; Watson, J. G., Emissions from charbroiling and grilling of chicken and beef. Journal of the Air \& Waste Management Association 2003, 53, 185-194.

(11) Nolte, C. G.; Schauer, J. J.; Cass, G. R.; Simoneit, B. R. T., Highly polar organic compounds present in meat smoke. Environmental Science \& Technology 1999, 33, 3313-3316.

(12) Robinson, A. L.; Subramanian, R.; Donahue, N. M.; Bernardo-Bricker, A.; Rogge, W. F., Source apportionment of molecular markers and organic aerosol - 1. Methodology for Visually Comparing Source Profiles and Ambient Data. Environmental Science \& Technology 2006, submitted.

(13) Robinson, A. L.; Subramanian, R.; Donahue, N. M.; Bernardo-Bricker, A.; Rogge, W. F., Source apportionment of molecular markers and organic aerosol - 2. Biomass smoke. Environmental Science \& Technology 2006, submitted.

(14) Subramanian, R.; Donahue, N. M.; Bernardo-Bricker, A.; Rogge, W. F.; Robinson, A. L., Contribution of motor vehicle emissions to the ambient organic carbon in Pittsburgh, PA: Effects of varying source profiles and seasonal trends in ambient marker concentrations. Atmospheric Environment 2006, submitted.

(15) Robinson, A. L.; Donahue, N. M.; Rogge, W. F., Photochemical oxidation and changes in molecular composition of organic aerosol in the regional context. Journal of Geophysical Research-Atmospheres 2006, 111, doi:10.1029/2005JD006265.

(16) Wittig, A. E.; Anderson, N.; Khlystov, A. Y.; Pandis, S. N.; Davidson, C.; Robinson, A. L., Pittsburgh air quality study overview. Atmospheric Environment 2004, 38, 3107-3125.

(17) Watson, J. G.; Robinson, N. F.; Fujita, E. M.; Chow, J. C.; Pace, T. G.; Lewis, C.; Coulter, T. "CMB8 Applications and validation protocol for PM2.5 and VOCs," Desert Research Institute, 1998.

(18) Fraser, M. P.; Yue, Z. W.; Tropp, R. J.; Kohl, S. D.; Chow, J. C., Molecular composition of organic fine particulate matter in Houston, TX. Atmospheric Environment 2002, 36, 5751-5758.

(19) Rogge, W. F.; Mazurek, M. A.; Hildemann, L. M.; Cass, G. R.; Simoneit, B. R. T., Quantification of Urban Organic Aerosols at a Molecular-Level - Identification, Abundance and Seasonal-Variation. Atmospheric Environment Part a-General Topics 1993, 27, 1309-1330.

(20) Sheesley, R. J.; Schauer, J. J.; Bean, E.; Kenski, D., Trends in secondary organic aerosol at a remote site in Michigan's upper peninsula. Environmental Science \& Technology 2004, 38, 6491-6500. 
(21) Lee, S.; Baumann, K.; Schauer, J. J.; Sheesley, R. J.; Naeher, L. P.; Meinardi, S.; Blake, D. R.; Edgerton, E. S.; Russell, A. G.; Clements, M., Gaseous and particulate emissions from prescribed burning in Georgia. Environmental Science \& Technology 2005, 39, 9049-9056.

(22) Fine, P. M.; Cass, G. R.; Simoneit, B. R. T., Chemical characterization of fine particle emissions from the wood stove combustion of prevalent United States tree species. Environmental Engineering Science 2004, 21, 705-721.

(23) Hays, M. D.; Geron, C. D.; Linna, K. J.; Smith, N. D.; Schauer, J. J., Speciation of gas-phase and fine particle emissions from burning of foliar fuels. Environmental Science \& Technology 2002, 36, 2281-2295.

(24) Fraser, M. P.; Cass, G. R.; Simoneit, B. R. T., Air quality model evaluation data for organics. 6. C-3-C-24 organic acids. Environmental Science \& Technology 2003, 37, 446453.

(25) Rudich, Y., Laboratory perspectives on the chemical transformations of organic matter in atmospheric particles. Chemical Reviews 2003, 103, 5097-5124.

(26) Huff-Hartz, K. E.; Weitkamp, E. A.; Sage, A. M.; Donahue, N. M.; Robinson, A. L., Laboratory Measurements of the Oxidation Kinetics of Organic Aerosol Mixtures using a Relative Rate Constants Approach. Journal of Geophysical Research 2006, in preparation.

(27) Schauer, J. J.; Fraser, M. P.; Cass, G. R.; Simoneit, B. R. T., Source reconciliation of atmospheric gas-phase and particle-phase pollutants during a severe photochemical smog episode. Environmental Science \& Technology 2002, 36, 3806-3814. 

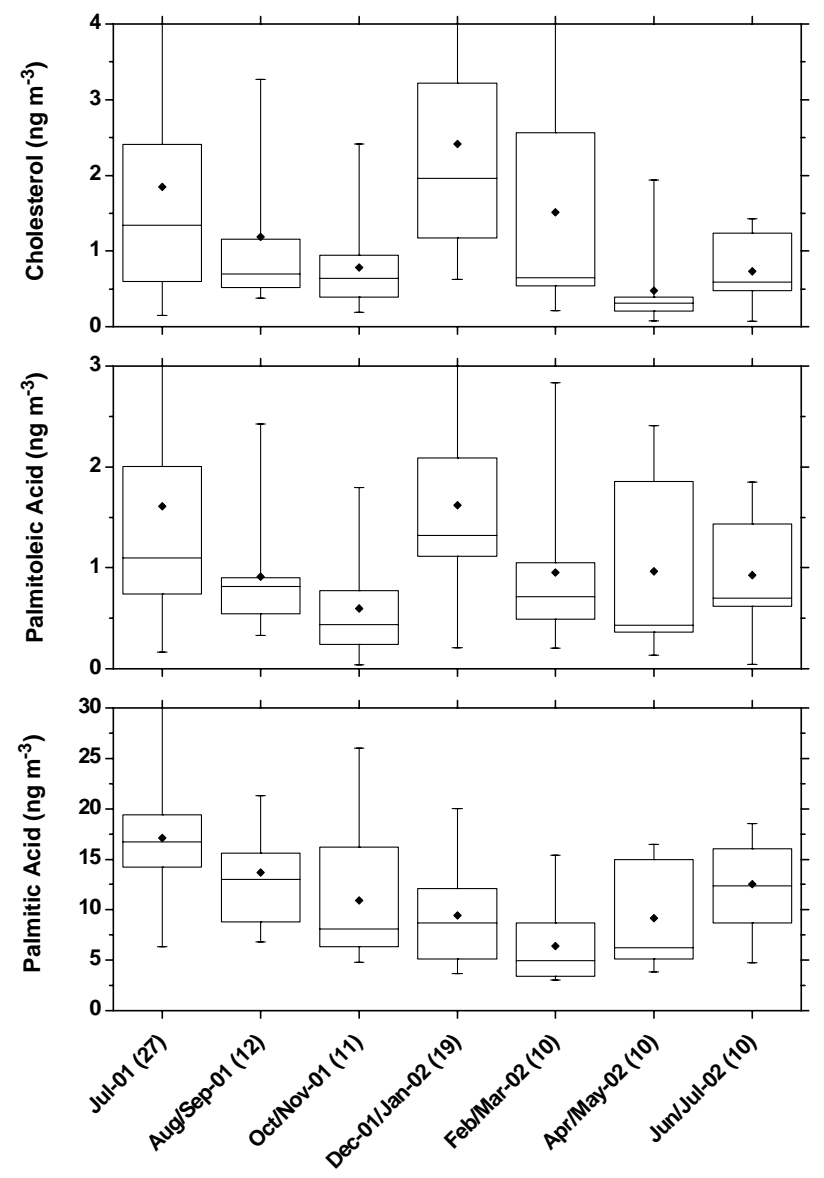

Figure 1. Box plots constructed from time series ambient data for (a) cholesterol, (b) palmitoleic acid, and (c) palmitic acid. The boxes stretch from the lower quartile to the upper quartile values; median values are shown as lines across the boxes; filled diamonds indicate average values. The whiskers indicate the maximum and minimum values; whiskers that intersect upper boundary of the plot indicate that the maximum value is beyond the scale of the graph. The numbers in parentheses in $\mathrm{x}$-axis labels indicate number of samples for specified period. 

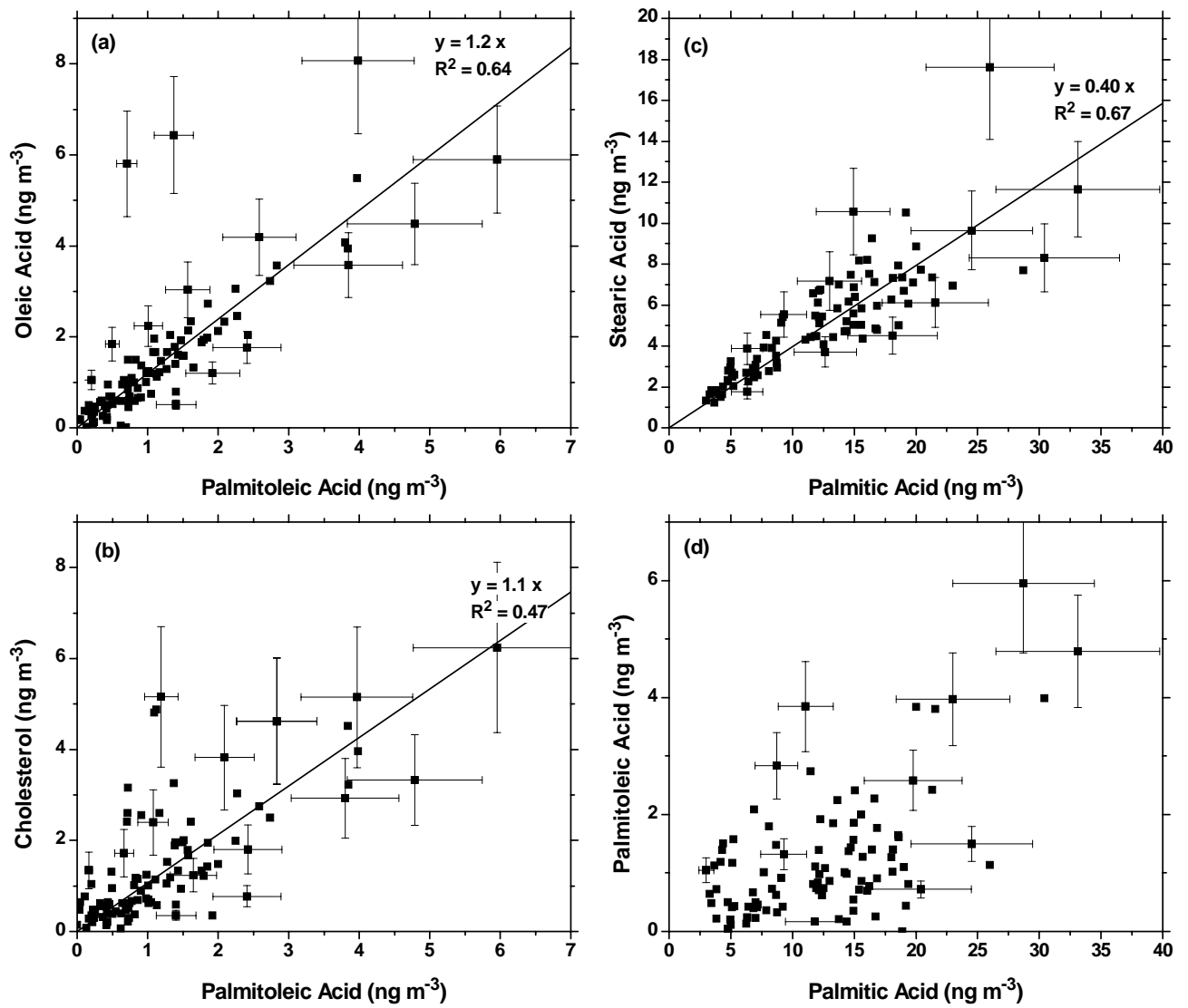

Figure 2. Scatter plots of the Pittsburgh ambient concentrations of the five cooking markers. Straight lines in (a) - (c) are linear regressions of the data which have been forced through zero. Excluding the three potential outliers in panel (a) improves the $\mathrm{R}^{2}$ value of the regression to 0.92 and minimally influences the slope. Measurement uncertainty indicated for selected points, uncertainty on other points is comparable. 

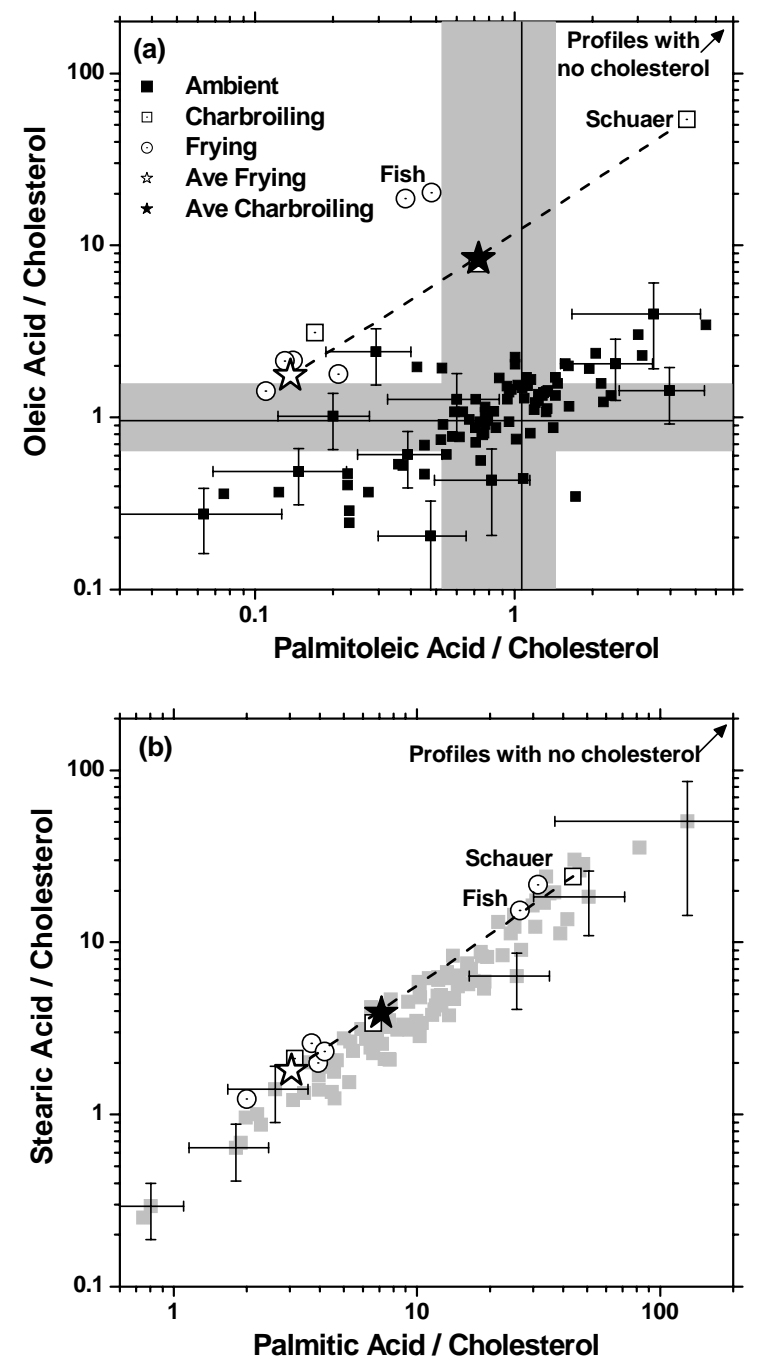

Figure 3. Ratio-ratio plots comparing ambient data for the five cooking markers to source profiles. The dashed line is a linear mixing line connecting the average frying and the Schauer et al. (9) charbroiling profile. The horizontal and vertical lines in (a) indicate the slopes of linear regressions the oleic acid and palmitoleic acid data versus cholesterol (e.g. Figure 2b). The gray areas in (a) indicate twentieth and eightieth percentiles of the data. The arrow in the upper righthand corner of the plot points towards sources such as biomass combustion, motor vehicles, road dust, and seed oil coil cooking that emit the different acids but not cholesterol. Error bars are shown for a limited number of points to indicate typical level of measurement uncertainty. 


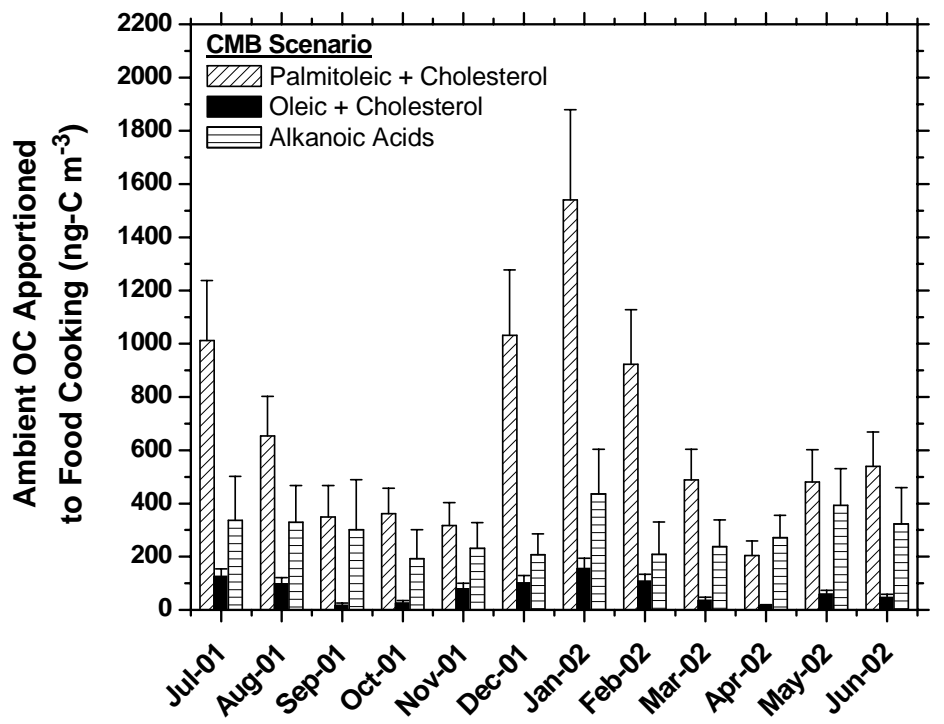

Figure 4. Time series of monthly average ambient OC apportioned to food cooking emissions by different CMB scenarios. As described in the text, the "palmitoleic + cholesterol" scenario uses an average red meat charbroiling profile; the "oleic + cholesterol" scenario uses an average red meat frying profile; and the "alkanoic acids” scenario uses the Schauer et al. (9) charbroiling profile, an average frying profile, and an average seed oil cooking profile. 


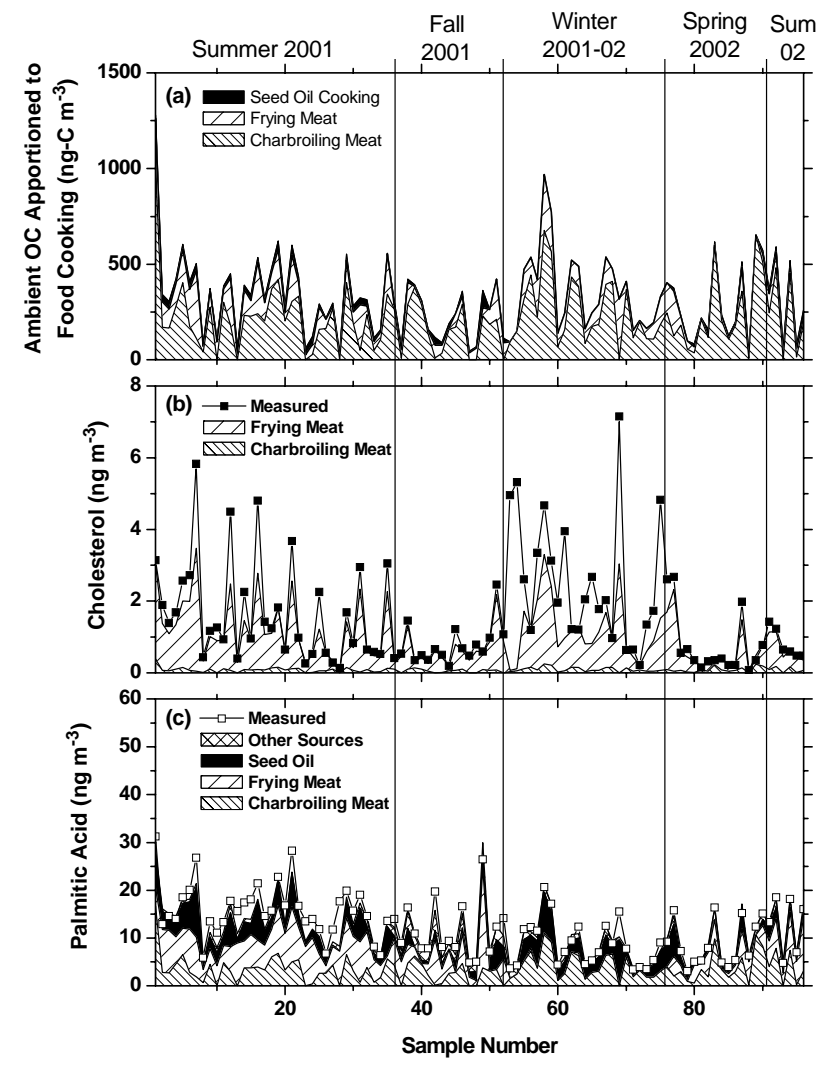

Figure 5. Time series of (a) food cooking OC, (b) measured and calculated cholesterol, and (c) measured and calculated palmitic acid for the CMB scenario that fits the two alkanoic acids, palmitoleic acid, and cholesterol using the Schauer et al. (9) charbroiling profile, an average frying profile, and an average seed oil cooking profile. 


\title{
Source apportionment of molecular markers and organic aerosol - 2. Biomass smoke
}

\author{
Allen L Robinson ${ }^{1}$, R Subramanian $\dagger$ \\ Department of Mechanical Engineering, Carnegie Mellon University, Pittsburgh, PA 15213, \\ USA
}

\begin{abstract}
Neil M Donahue
Department of Chemistry and Department of Chemical Engineering, Carnegie Mellon University, Pittsburgh, PA 15213, USA
\end{abstract}

Anna Bernardo-Bricker, Wolfgang F Rogge

Department of Civil and Environmental Engineering, Florida International University, Miami, FL, USA

\footnotetext{
${ }^{1}$ Corresponding author: alr@andrew.cmu.edu; (412) 268-3657; (412) 268-3348
} 


\section{Abstract}

Chemical Mass Balance analysis is performed using a large dataset of molecular marker concentrations to estimate the contribution of biomass smoke to ambient organic carbon (OC) in Pittsburgh, PA. Detailed comparisons are made between the ambient data and a large number of published source profiles. The fall and winter data are analyzed with fireplace and woodstove source profiles while open burning profiles were used to analyze the spring and summer data. At the upper limit, biomass smoke is estimated to contribute $520 \pm 180 \mathrm{ng}-\mathrm{C} / \mathrm{m}^{3}$ or $14.5 \%$ of the total OC in the fall, $210 \pm 100 \mathrm{ng}-\mathrm{C} \mathrm{m}^{3}$ or $10 \%$ of the total OC in the winter, and $65 \pm 29 \mathrm{ng}$ $\mathrm{C} / \mathrm{m}^{3}$ or $2 \%$ of the total OC in the summer. In the fall and winter, there is large day-to-day variability in the amount of OC apportioned to biomass smoke. The levels of biomass smoke in Pittsburgh are much lower than some other areas of the United States, indicating that there is significant regional variability in the importance of biomass combustion as a source of fine particulate matter. The calculations face two major sources of uncertainty. First, the ambient data for levoglucosan, resin acids, and syringhaldehyde are relatively disorganized, showing that numerous sources with distinct source profiles contribute to marker concentrations. Second, the marker-to-OC ratio of available biomass smoke profiles is highly variable. This variability creates a bias of more than a factor of two in the amount of ambient OC apportioned to biomass smoke by different statistically acceptable CMB solutions. The marker-to-OC ratio is a critical parameter to consider when evaluating CMB solutions. In addition to the specific application, this paper demonstrates a general methodology for addressing a source class with multiple, highly variable contributors. 


\section{Introduction}

Emissions from biomass combustion can be an important source of organic aerosol in urban environments (1-4). Organic molecular markers have been used in conjunction with the chemical mass balance (CMB) model to apportion ambient organic aerosol to biomass smoke and other sources of primary organic aerosol (1-4). Simoneit (5) reviews molecular markers for emissions from incomplete biomass combustion. In this paper we focus on some of the most commonly-used markers discussed in that review: levoglucosan, syringols, and resin acids. Levoglucosan is a general marker for biomass combustion emissions (6). It is a cellulose pyrolysis product; since cellulose is the dominant natural biopolymer, levoglucosan is emitted during the combustion of most biomaterials. Additional molecular markers have been identified to differentiate between emissions from softwood and hardwood combustion $(3,7)$. Softwood smoke contains resin acids from gymnosperms (conifers), while emissions from hardwood combustion are enriched in syringols compared to emissions from softwood burning $(7,8)$.

Using CMB with molecular marker data to apportion ambient OC to different source classes relies heavily on two implicit assumptions: first, emissions from a given source class are well represented by an average source profile; second, the ratio of marker levels to total organic carbon in that source profile are stable and well known (9). These assumptions are challenged by the highly variable nature of biomass combustion. Currently, more than 35 source profiles with speciated condensed and semi-volatile organics have been published for biomass smoke (10-15). Emissions from biomass combustion can vary widely depending on fuel type and combustion conditions (16). For instance, the formation of levoglucosan depends strongly on combustion temperature (6) and therefore can vary widely, especially in uncontrolled exterior fires such as wildfires and agricultural burns (15). Most CMB analyses of molecular marker data 
do not consider the effects of source profile variability, but recent results indicate that source profile selection can significantly alter the CMB results (15).

This paper is one of a series of papers that examines issues associated with CMB analysis of molecular marker data in Pittsburgh, PA (9,17-19). The goal of this paper is to estimate the contribution of biomass smoke to organic aerosol in Pittsburgh using the CMB model, taking into consideration the issues associated with source profile variability. First, ambient concentrations of biomass smoke markers are examined for seasonal patterns and for correlations between different makers. Next, the data are compared to the available source profiles using the approach described in Robinson et al. $(9,20)$. Finally, different scenarios for CMB analysis are defined and the paper concludes with a discussion of the CMB results.

\section{Methods}

CMB analysis was performed to apportion ambient OC in Pittsburgh, PA to sources of primary organic aerosol. The analysis uses ambient concentrations of individual organic compounds, $\mathrm{PM}_{2.5}$ organic and elemental carbon, and $\mathrm{PM}_{2.5}$ elemental composition measured on 103 days between July, 2001 and August, 2002 (21). Daily measurements were made in July 2001 and most of January 2002; during other periods 24-hr samples were collected on a 1 in 6 day schedule. Additional details of the data set are provided in Supporting Information.

Source contributions to ambient OC are determined by calculating the linear combination of source emissions needed to reproduce the ambient concentrations of a set of fitting species. The selection of compounds included in the CMB model is a critical issue; all major sources of each compound must be included in the model and the species should be conserved during transport from source to receptor (22). This work uses the basic set of compounds and source classes developed by Schauer et al. (1,3). In addition to the biomass smoke related markers discussed 
below, all calculations include four n-alkanes, iso-hentriacontane, anteiso-dotriacontane, four hopanes, four PAHs, two alkanoic acids, palmitoleic acid, cholesterol, titanium, iron, and elemental carbon. Source profiles for eight source classes are included in the model: diesel vehicles, gasoline vehicles, road dust, biomass combustion, cooking emissions, coke production, vegetative detritus, and cigarettes. The calculations were performed using the computer program CMB8 distributed by the US EPA. Additional details on the CMB analysis are contained in Supporting Information.

This paper focuses on levoglucosan, resin acids, syringaldehyde, and acetosyringone, species which are commonly used as markers for biomass smoke. CMB analysis is performed using different combinations of these species with different biomass smoke source profiles (in addition to a standard set of non-biomass smoke profiles and molecular markers). Biomass smoke also contributes to other species included in model such as polycyclic aromatic hydrocarbons, elemental carbon, palmitoleic acid, alkanoic acids, and n-alkanes, but CMB apportions the vast majority of these markers to non-biomass-smoke source profiles. Of these species, only the two alkanoic acids (palmitic acid and stearic acid) significantly influence the biomass smoke contributions. These acids are included in the CMB model to help constrain the contribution of meat cooking emissions (17). Including them as fitting species reduces the amount of ambient OC apportioned to biomass smoke by $10 \%$ relative to solutions that do not fit the two alkanoic acids.

There are a number of issues associated with including resin acids in $\mathrm{CMB}$ as markers for softwood smoke. First, the distribution of individual resin acids in wood smoke is highly variable, and depends on combustion conditions and the type of wood $(11,12,23)$. Second, resin 
acids interconvert in the atmosphere; abietic- and pimaric-type resin acids are converted to dehydroabietic acid and ultimately to 7-oxodehydroabietic acid $(5,24,25)$.

The Pittsburgh samples were analyzed for 11 different resin acids, but only four of these acids (pimaric acid, sandaracopimaric acid, dehydroabietic acid and 7-oxodehydroabietic acid) were above the detection limit. Dehydroabietic and 7-oxodehydroabietic were the dominant resin acids, contributing $86 \pm 10 \%$ (average \pm standard deviation) of the total resin acid concentrations. In contrast fresh emissions are often enriched in unaltered acids $(11,12,23,26)$; therefore, the Pittsburgh data are consistent with substantial conversion of unaltered acids to dehydroabietic and then, ultimately, to 7-oxodehydroabietic acid. In addition the ratio of dehydroabietic acid to 7-oxodehydroabietic acid varied seasonally (average wintertime ratio of 4.7 versus 0.7 in the summer), while the total resin acid concentrations in both seasons were comparable. The result is that ambient concentrations of dehydroabietic and 7oxodehydroabietic acid were inversely correlated (linear regression yields a slope of -0.93 and $\mathrm{R}^{2}$ 0.71), which is consistent with photochemistry. For CMB analysis, we assume that resin acids are conserved as a compound class, but that individual resin acids are not conserved. Therefore, we add all of the resin acids together in both the ambient data and the source profiles.

Source profiles for biomass combustion are selected based on comparisons made using scatter and ratio-ratio plots of ambient concentrations of different molecular markers. These plots allow one to visually compare source profiles to ambient concentrations. Ratio-ratio plots are constructed using three species; one compound is selected as a reference to normalize the concentrations of the other two compounds, called target species. The best reference compounds are relatively abundant, stable, and specific to the sources plotted. Source profiles appear as points on ratio-ratio plots and linear mixing lines can be drawn to visualize the effects of mixing 
of emissions from different sources. More details on the construction, interpretation, and mathematics of ratio-ratio plots are provided in Robinson et al. $(9,20)$.

\section{Results}

Figure 1 presents time series of ambient data for levoglucosan, total resin acids, and syringaldehyde. The results are presented using box-plots constructed by grouping the data into one or two month periods, depending on the number of samples. The highest levoglucosan and resin acid concentrations were observed in the fall; the peak syringaldehyde and elevated levoglucosan concentrations were observed in the winter. During the rest of the year (AprilMay) ambient concentrations of the biomass smoke markers are low, with only an occasional modest spike. On average, the levoglucosan concentrations in Pittsburgh appear comparable to those in Houston, TX (27), but are a factor of 5-or-more lower than those in the Southeast (4).

Biomass combustion encompasses a diverse set of sources, the distribution of which varies seasonally. The ambient temperature provides one indicator of the types of biomass sources influencing Pittsburgh. When the weather is warm, forest fires, structural fires, and agricultural burns are likely the dominant sources of biomass smoke. During colder weather, wood combustion in fireplaces and woodstoves used for space heating may be an important source. We classified each sampling day as "warm” or "cold” based on the average ambient temperature; days with an average temperature less than $12{ }^{\circ} \mathrm{C}$ are defined as cold. Thirty eight percent of the sampled days are classified as cold.

Winters in Pittsburgh are cold with typical average daily temperatures between -5 and $5{ }^{\circ} \mathrm{C}$; therefore, wood combustion for space heating likely contributes to wintertime biomass smoke marker concentrations. Comparing the winter and summer resin acid and syringaldehyde data provides an indication of the type of wood used for space heating. Syringaldehyde 
concentrations were, on average, seven times higher on cold days $\left(2.8 \mathrm{ng} / \mathrm{m}^{3}\right)$ than on warm days $\left(0.4 \mathrm{ng} / \mathrm{m}^{3}\right)$, while resin acid concentrations in the winter and summer were comparable. This indicates that hardwood is burned in the winter for space heating; a survey of wood distributors found that $90 \%$ of fuel wood sold in the Pittsburgh region is hardwood.

The most striking feature of the ambient wintertime data is the significant day-to-day variability in the biomass smoke marker concentrations. For example, wintertime (December through February) levoglucosan levels ranged from 0.3 to $120 \mathrm{ng} \mathrm{m}^{-3}$. On two-thirds of the wintertime sampling days, levoglucosan levels were less than $5 \mathrm{ng} \mathrm{m}^{-3}$. The other biomass smoke markers exhibited similar variability. Ambient temperatures were consistently low throughout the winter, indicating a reasonably constant demand for space heating. The large number of cold days with very low levoglucosan levels suggest that wood is not an important fuel for space-heating. Figure 1 shows that the December and January levoglucosan and syringaldehyde data exhibit a pronounced weekend-weekday pattern. The greatly elevated weekend concentrations are consistent with residential wood burning for pleasure - enjoying a weekend evening around the fire.

In the fall (October and November), the average daily temperature in Pittsburgh varies widely, ranging from 3 to $21^{\circ} \mathrm{C}$ on the days on which samples were collected. The peak levoglucosan and resin acid concentrations were observed on cold fall days, but high concentrations of these markers were also observed on warm fall days. High concentrations on warm days are unlikely to be caused by local wood combustion for space heating; more likely explanations include long-range transport of emissions and/or leaf and agricultural burning. There are state and local regulations that limit open burning, but it is not uncommon to see 
burning of yard waste in rural areas, particularly in the fall. The high resin acid concentrations in the fall indicate a significant contribution of softwood smoke.

Concentrations of biomass smoke markers were consistently low on non-fall warm days, indicating minimal influence of summertime wildfire emissions during the study. For example, modestly elevated levels of levoglucosan ( 36 to $47 \mathrm{ng} \mathrm{m}^{-3}$ ) were observed on only three of the forty-eight summer samples. On these days, resin acid concentrations (but not syringaldehyde) also spiked, indicating influence of softwood smoke.

Scatter and ratio-ratio plots for the biomass smoke markers are shown in Figures 2 and 3. These plots are useful for identifying correlations in the ambient concentrations and to compare the ambient data to the published source profiles. Figure 2 shows scatter plots of resin acids and syringaldehyde versus levoglucosan. Figure 3 shows ratio-ratio plots of the four biomass smoke markers, with levoglucosan as the normalizing compound. Figure 3 divides the ambient data into two groups to facilitate the development of scenarios for CMB analysis discussed later in the paper. Data from the fall and winter are shown in Figures 3a and 3b, and data from the nonwinter months are shown in Figure 3c and 3d.

There is only modest correlation among the biomass smoke markers in the Pittsburgh data set, with linear regressions yielding $\mathrm{R}^{2}$ values between 0.5 and 0.6 (if one excludes the few very high concentration points from the dataset). The notable exception is that on cold days syringaldehyde and acetosyringone concentrations are strongly correlated, with a linear regression yielding a slope of 4.2 , zero intercept, and an $R^{2}$ of 0.94 . Somewhat stronger correlations are observed if one restricts the analysis to subsets of the data such as cold, warm, winter, or fall days. 
The modestly correlated ambient data create relatively disorganized ratio-ratio plots. For example, Figure 3 indicates that the ratios of the ambient concentrations of the different biomass smoke markers vary by at least one order of magnitude. The greatest variability was observed in the winter, when the syringaldehyde-to-levoglucosan ratios varied by almost two orders of magnitude. Although ratios on some low concentration days are highly uncertain (e.g. point in upper right hand corner of Figure 3a), the overall variability is much greater than the measurement uncertainty. The poorly organized ratio-ratio plots imply that a diverse set of sources contributes to the biomass smoke marker concentrations in Pittsburgh.

Comparison with source profiles: More than 35 different biomass-combustion source profiles with levoglucosan, syringols, and resin acids have been published (10-15). The vast majority of these profiles are for soft or hardwood combustion in either residential fireplaces or woodstoves $(10-12,23)$ with more limited data for open- or prescribed-burns (13-15). Source profiles appear as lines in the scatter plots presented in Figure 2 and as points in the ratio-ratio plot shown in Figure 3 (9). To reduce clutter, only a few profiles are shown in the scatter plots. When using hardwood profiles, we assume that hardwood smoke contains no resin acids because common hardwoods do not have resin acid ducts. A few of the published hardwood profiles report resin acid emissions, which have been attributed to contamination from earlier experiments using softwood (10).

Figure 3 indicates that there is significant variability between the different source profiles. For example, resin-acid-to-levoglucosan ratios of the published softwood profiles span three orders of magnitude. Each profile represents the emissions from a single or, at best, a small number of experiments; therefore, the significant variability of the published profiles simply reflects the heterogeneous nature of wood combustion. Note that significant variability is 
observed across the set of source profiles reported by a single study, so this variability is not due to difference in analytical methods used by different groups. Profile-to-profile variability is not unique to the biomass smoke source profiles $(9,17,18)$.

The ratio-ratio plots presented in Figure 3 allow one to easily compare the ambient data to the entire set of source profiles. Much of the ambient data fall within regions of the plot that can be explained by linear mixing of different combinations of the available source profiles; however, at least three source profiles are required in order to account for the variability in the ambient dataset. This indicates that there is day-to-day variability in the aggregate biomass smoke profile. For example, Figure 2 indicates that on winter days with the highest concentrations of syringaldehyde and levoglucosan, the ambient data are most consistent with the Fine et al. (23) Black Cherry profile, while data on lower concentration winter days are more similar to Fine et al. (23) American Beech profile.

A subset of the ambient data appears to be inconsistent with any combination of the published profiles. For example, there are 10 winter days with syringaldehyde-to-levoglucosan ratios that are significantly larger than any of the published source profiles. These data appear in the upper right hand corner of the ratio-ratio plot in Figure 3a, and to the left of the Fine et al. (23) American Beech profile in Figure 2b, the profile with the highest syringaldehyde-tolevoglucosan ratio. Ambient concentrations on many of these days were low, resulting in highly uncertain marker ratios; therefore, within experimental uncertainty, these low concentration data overlap with potential mixing scenarios. However, on some inconsistent days ambient concentrations of syringaldehyde were above average, and the error bars do not overlap any possible source profile combinations. A likely explanation for these points is that the available source profiles do not capture the actual variability in biomass smoke composition. 
CMB analysis: CMB analysis was performed using different combinations of biomass smoke source profiles, referred to as mixing scenarios, to constrain the potential contribution of biomass smoke to OC in Pittsburgh. To account for the expected seasonal variation in the biomass combustion sources, the winter data (Dec-Mar) are analyzed with woodstove and fireplace (space-heating) profiles, while open burning profiles are used in the analysis of spring and summer data (Apr-Sep). We analyze the fall data (Oct-Nov) with both sets of profiles because of the large number of cold days as well as the possibility of significant open burning during that period.

We consider two space heating scenarios, constructed from fireplace and woodstove profiles. Space Heating \#1 combines the Schauer et al. (10) pine and oak profiles with the Fine et al. (23) American Beech profile. Space Heating \#2 combines the Fine et al. (11) Eastern Hemlock, Eastern White Pine, and Red Maple source profiles. Although other combinations of space heating profiles will also yield statistically acceptable solutions, we have chosen these two scenarios because they bracket the potential biomass-smoke contribution to ambient OC. Given the limited number of published profiles, we only consider one open burn scenario: the Hays et al. (13) MHFF and Florida Palmetto \& Slash Pine profiles with the Hays et al. (14) wheat straw profile.

Ratio-ratio plots comparing these scenarios to the ambient data are shown in Figure 3. Given the scatter in the ambient biomass smoke markers, none of these scenarios can describe all of the data. Figures $3 \mathrm{a}$ and $3 \mathrm{~b}$ indicate that the mixing region defined by the Space Heating \#2 scenario encapsulates a larger fraction of the ambient data than the Space Heating \#1 scenario; especially if one considers the acetosyringone data plotted in Figure 3b. As previously discussed, a number 
of fall and winter points in the upper right hand quadrant of the Figures 3a and 3b are inconsistent with any scenario.

Figure 3c shows good agreement between the non-winter ambient data and the open burn scenario for resin acids, syringaldehyde and levoglucosan; however, the ambient acetosyringoneto-levoglucosan ratios are smaller than any of the open burn profiles (Figure 3d). Almost all of the non-winter ambient acetosyringone data can be satisfactorily explained if the Schauer et al. (10) pine fireplace profile is added to the model; this improves the fitting statistics but does not change the amount of OC apportioned to biomass smoke. Given the uncertainty in the data, CMB finds statistically acceptable solutions even when acetosyringone is included as a fitting species. Eliminating acetosyringone improves the statistics of the CMB solution, and increases the amount of OC apportioned to biomass smoke by the warm scenario.

The CMB model using the open burn scenario does not converge on the fall day with the highest biomass smoke marker concentrations (240 $\mathrm{ng} \mathrm{m}^{-3}$ of levoglucosan). The problem is not the biomass smoke markers but the higher odd n-alkanes (C27, C29, C31, C33) commonly associated with vegetative detritus (28). These compounds are found in appreciable levels in the Hays et al. (13) MHFF and the Hays et al. (14) agricultural residue burning source profiles. CMB analyses using these profiles cause CMB to significantly over-apportion higher odd nalkanes on days with high biomass smoke marker concentrations.

CMB calculates a number of statistical parameters used to evaluate the quality of a solution. Watson et al. (22) describes the target ranges for these parameters. Based on these guidelines, all of the scenarios considered yield solutions that are statistically acceptable. For example, the average $\mathrm{R}^{2}$ value for both space heating solutions is 0.91 with confidence levels greater than $90 \%$ on all days based on the $\chi^{2}$ and number of degrees of freedom. In addition, the statistical quality 
of the solutions is essentially the same; therefore, these performance measures do not provide a basis for selecting one solution over the other. Additional details on the statistical quality of the solutions are provided in Supporting Information

Although the statistical quality of the two CMB solutions is comparable, the amount ambient OC apportioned to biomass smoke by the two space heating scenarios is very different. This is illustrated in Figure 4, which presents a scatter plot of ambient OC apportioned to biomass smoke by the two space heating scenarios for the October through March period. The results are highly correlated with a linear regression through the origin calculating a slope of 2.4 (Space Heating \#2 greater than Space Heating \#1) and an $\mathrm{R}^{2}$ value of 0.96 . On the high concentration days, the bias between the two solutions is significantly greater than the standard errors calculated by CMB. In the fall when calculations were made with all three scenarios, all three solutions are strongly correlated. The amount of ambient OC apportioned by the open burn scenario falls to biomass smoke between the two Space Heating scenarios.

The systematic biases between different solutions are caused by differences in the marker-toOC ratios of the biomass smoke source profiles. CMB fits the ambient concentrations of the species included in the model; OC is not included in the model because source profiles do not exist for important contributors to OC such as Secondary Organic Aerosol (SOA). After determining the optimum fit of these species, CMB apportions the ambient OC to each source using the marker-to-OC ratio of the different source profiles. This second step is built into the analysis because the sources profiles used in CMB have been normalized by the OC. A figure comparing these ratios for a large number of published profiles is presented in the Supporting Information. 
Differences in the levoglucosan-to-OC ratios of the source profiles explain much of the bias in the amount of OC apportioned to biomass smoke by the two space heating scenarios. Space Heating \#1 includes two Schauer et al. (10) profiles with large levoglucosan-to-OC ratios while the Space Heating \#2 scenario is based on three profiles with relatively small levoglucosan-toOC ratios. The arithmetic average levoglucosan-to-OC ratio of the three profiles used in Space Heating \#1 is 0.19 versus 0.085 for Space Heating \#2. Therefore, assuming equal weighting to each profile, Space Heating \#2 will apportion 2.2 times more biomass smoke OC than Space Heating \#1 for a given ambient levoglucosan concentration, which is comparable to the actual bias shown in Figure 5.

An important benefit of including three biomass smoke profiles in the CMB model is to reduce the effects of profile-to-profile variability in the marker-to-OC ratios on the solution. For example, the marker-to-OC ratios of the biomass smoke profiles span more than an order of magnitude, which can cause CMB solutions that use only a single biomass profile to vary by a similar amount. In contrast, only a factor of 2.4 difference was observed in the OC apportioned by two CMB models involving three different biomass smoke source profiles.

Figure 5 shows a time series of the ambient OC apportioned by CMB to biomass smoke. For the October through March period, results are plotted for the maximum space heating scenario (Space Heating \#2) and for the open burn scenario for the remainder of the study. On a study average basis, this combination apportions of $160 \pm 66 \mathrm{ng}-\mathrm{C} / \mathrm{m}^{3}$ ( \pm standard error) or $5 \%$ of the ambient OC to biomass smoke. The peak contribution is in the fall when an average of $520 \pm$ $180 \mathrm{ng}-\mathrm{C} / \mathrm{m}^{3}$ or $14.5 \%$ of the total OC is apportioned to biomass smoke. In the fall and winter, the variability in the ambient marker concentrations causes significant day-to-day variability in the amount of OC apportioned to biomass smoke. On only $28 \%$ of the fall and winter days (and 
no days in the other periods) is more than $10 \%$ of the ambient OC apportioned to biomass smoke with a maximum relative contribution of $43 \%$.

CMB analysis was also performed to estimate the contribution of each source profile to ambient fine particle mass. The difference between the OC and $\mathrm{PM}_{2.5}$ mass results depends on the $\mathrm{PM}_{2.5}$-mass-to-OC ratio of the different source profiles. The average $\mathrm{PM}_{2.5}$-mass-to-OC ratio of the three profiles used in the Space Heating \#2 scenario is only 1.17. Therefore, this scenario apportions only slightly more $\mathrm{PM}_{2.5}$ mass than OC to biomass smoke -- for example, on average

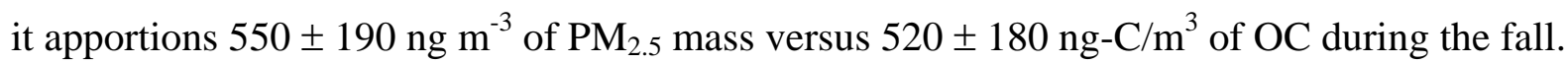
On a $\mathrm{PM}_{2.5}$ mass basis, the average bias between the Space Heating \#2 and Space Heating \#1 solution is reduced to a factor of 1.6 versus the factor of 2.4 between the two solutions on an OC basis shown in Figure 4. This reduction is due to the average $\mathrm{PM}_{2.5}$-mass-to-OC ratio of the three profiles used in the Space Heating \#1 scenario being 1.61 versus 1.17 for the Space Heating \#2 scenario.

The CMB results for $\mathrm{PM}_{2.5}$ mass provide insight into the potential effects of organic sampling artifacts on the CMB apportionment of ambient OC to the biomass smoke profiles. Adsorption of organic vapors can create a significant positive artifact when quartz filters are used to sample wood smoke emissions (29). The $\mathrm{PM}_{2.5}$-mass-to-OC ratios of the biomass smoke profiles used in CMB suggest that sampling artifacts contributed significantly to the OC emission rate, especially for the Space Heating \#2 scenario. Wood smoke is expected to have $\mathrm{PM}_{2.5}$-mass-to-OC ratios of around two are expected given the relatively polar nature of the organic species (30) and the contribution elemental carbon and other non-carbonaceous species to the emissions. The ratios of the profiles used by the Space Heating \#2 are much smaller than two. Correcting for a positive sampling artifact will reduce the amount OC apportioned by the 
CMB model; for example, if $50 \%$ of the OC emissions are positive sampling artifact, then correcting for that artifact will reduce the contribution of that source to ambient particulate OC by $50 \%$.

\section{Discussion}

On most days biomass smoke is only a minor source of ambient OC and fine particulate matter in Pittsburgh. The amount of OC apportioned to biomass smoke in Pittsburgh is comparable to that in Houston (31) but a factor of 5 to 20 less than that in the Southeastern US (4). This indicates that there are large regional differences in the importance of biomass combustion as a source of fine particles, even for locations with cold climates. Only on a few peak days were biomass-smoke OC levels in Pittsburgh comparable to the monthly average levels in the wintertime in the Southeast. Variability in source profiles creates a factor of two uncertainty in the amount of ambient OC apportioned to biomass smoke; this variability is especially important to consider in regions which experience high levels of biomass smoke.

A key challenge for source apportionment analysis using molecular markers for biomass smoke is the variability in the ambient data. This variability is reflected in the modest correlations between the biomass smoke markers (Figure 2) and by the relatively disorganized ratio-ratio plots (Figure 3). In comparison, ambient data of molecular markers for other important source classes such as motor vehicles and meat cooking create relatively organized scatter and ratio-ratio plots $(9,17,18)$. Well-organized ambient data implies the existence of well-defined source profile -- atmospheric mixing averages out the source-to-source variability in emissions. The disorganized biomass smoke marker data implies significant day-to-day variability in the aggregate source profile. This variability likely reflects the heterogeneous 
nature of biomass combustion. To account for the variability in the Pittsburgh data, three different biomass smoke source profiles had to be simultaneously included in the CMB model.

\section{Acknowledgments}

This research was conducted as part of the Pittsburgh Air Quality Study, which was supported by US Environmental Protection Agency under contract R82806101 and the US Department of Energy National Energy Technology Laboratory under contract DE-FC2601NT41017. This research was also supported by the EPA STAR program through the National Center for Environmental Research (NCER) under grant R832162. This paper has not been subject to EPA's required peer and policy review, and therefore does not necessarily reflect the views of the Agency. No official endorsement should be inferred.

\section{References}

(1) Schauer, J. J.; Rogge, W. F.; Hildemann, L. M.; Mazurek, M. A.; Cass, G. R., Source apportionment of airborne particulate matter using organic compounds as tracers. Atmospheric Environment 1996, 30, 3837-3855.

(2) Watson, J. G.; Fujita, E. M.; Chow, J. C.; Zielinska, B. "Northern Front Range Air Quality Study Final Report and Supplemental Volumes," 1998.

(3) Schauer, J. J.; Cass, G. R., Source apportionment of wintertime gas-phase and particle-phase air pollutants using organic compounds as tracers. Environmental Science \& Technology 2000, 34, 1821-1832.

(4) Zheng, M.; Cass, G. R.; Schauer, J. J.; Edgerton, E. S., Source apportionment of PM2.5 in the southeastern United States using solvent-extractable organic compounds as tracers. Environmental Science \& Technology 2002, 36, 2361-2371.

(5) Simoneit, B. R. T., Biomass burning - a review of organic tracers for smoke from incomplete combustion. Applied Geochemistry 2002, 17, 129-162.

(6) Simoneit, B. R. T.; Schauer, J. J.; Nolte, C. G.; Oros, D. R.; Elias, V. O.; Fraser, M. P.; Rogge, W. F.; Cass, G. R., Levoglucosan, a tracer for cellulose in biomass burning and atmospheric particles. Atmospheric Environment 1999, 33, 173-182.

(7) Simoneit, B. R. T.; Rogge, W. F.; Mazurek, M. A.; Standley, L. J.; Hildemann, L. M.; Cass, G. R., Lignin Pyrolysis Products, Lignans, and Resin Acids as Specific Tracers of Plant Classes in Emissions from Biomass Combustion. Environmental Science \& Technology 1993, 27, 2533-2541. 
(8) Hawthorne, S. B.; Miller, D. J.; Barkley, R. M.; Krieger, M. S., Identification of Methoxylated Phenols as Candidate Tracers for Atmospheric Wood Smoke Pollution. Environmental Science \& Technology 1988, 22, 1191-1196.

(9) Robinson, A. L.; Subramanian, R.; Donahue, N. M.; Bernardo-Bricker, A.; Rogge, W. F., Source apportionment of molecular markers and organic aerosol - 1. Methodology for Visually Comparing Source Profiles and Ambient Data. Environmental Science \& Technology 2006, submitted.

(10) Schauer, J. J.; Kleeman, M. J.; Cass, G. R.; Simoneit, B. R. T., Measurement of emissions from air pollution sources. 3. C-1-C-29 organic compounds from fireplace combustion of wood. Environmental Science \& Technology 2001, 35, 1716-1728.

(11) Fine, P. M.; Cass, G. R.; Simoneit, B. R. T., Chemical characterization of fine particle emissions from fireplace combustion of woods grown in the northeastern United States. Environmental Science \& Technology 2001, 35, 2665-2675.

(12) Fine, P. M.; Cass, G. R.; Simoneit, B. R. T., Chemical characterization of fine particle emissions from the fireplace combustion of woods grown in the southern United States. Environmental Science \& Technology 2002, 36, 1442-1451.

(13) Hays, M. D.; Geron, C. D.; Linna, K. J.; Smith, N. D.; Schauer, J. J., Speciation of gas-phase and fine particle emissions from burning of foliar fuels. Environmental Science \& Technology 2002, 36, 2281-2295.

(14) Hays, M. D.; Fine, P. B.; Gerona, C. D.; Kleeman, M. J.; Gulletta, B. K., Open Burning of Agricultural Biomass: Physical and Chemical Properties of Particle-Phase Emissions. Atmospheric Environment 2005, 39, 6747-6764.

(15) Lee, S.; Baumann, K.; Schauer, J. J.; Sheesley, R. J.; Naeher, L. P.; Meinardi, S.; Blake, D. R.; Edgerton, E. S.; Russell, A. G.; Clements, M., Gaseous and particulate emissions from prescribed burning in Georgia. Environmental Science \& Technology 2005, 39, 9049-9056.

(16) Fine, P. M.; Cass, G. R.; Simoneit, B. R. T., Organic compounds in biomass smoke from residential wood combustion: Emissions characterization at a continental scale. Journal of Geophysical Research-Atmospheres 2002, 107.

(17) Robinson, A. L.; Subramanian, R.; Donahue, N. M.; Bernardo-Bricker, A.; Rogge, W. F., Source apportionment of molecular markers and organic aerosol - 3. Food cooking emissions. Environmental Science \& Technology 2006, submitted.

(18) Subramanian, R.; Donahue, N. M.; Bernardo-Bricker, A.; Rogge, W. F.; Robinson, A. L., Contribution of motor vehicle emissions to the ambient organic carbon in Pittsburgh, PA: Effects of varying source profiles and seasonal trends in ambient marker concentrations. Atmospheric Environment 2006, submitted.

(19) Subramanian, R.; Donahue, N. M.; Bernardo-Bricker, A.; Rogge, W. F.; Robinson, A. L., Source Apportionment of Primary Organic Aerosol in Pittsburgh, PA using organic molecular markers. Environmental Science \& Technology 2006, in preparation.

(20) Robinson, A. L.; Donahue, N. M.; Rogge, W. F., Photochemical oxidation and changes in molecular composition of organic aerosol in the regional context. Journal of Geophysical Research-Atmospheres 2006, 111, doi:10.1029/2005JD006265.

(21) Wittig, A. E.; Anderson, N.; Khlystov, A. Y.; Pandis, S. N.; Davidson, C.; Robinson, A. L., Pittsburgh air quality study overview. Atmospheric Environment 2004, 38, 3107-3125. 
(22) Watson, J. G.; Robinson, N. F.; Fujita, E. M.; Chow, J. C.; Pace, T. G.; Lewis, C.; Coulter, T. "CMB8 Applications and validation protocol for PM2.5 and VOCs," Desert Research Institute, 1998.

(23) Fine, P. M.; Cass, G. R.; Simoneit, B. R. T., Chemical characterization of fine particle emissions from the wood stove combustion of prevalent United States tree species. Environmental Engineering Science 2004, 21, 705-721.

(24) Corin, N. S.; Backlund, P. H.; Kulovaara, M. A. M., Photolysis of the resin acid dehydroabietic acid in water. Environmental Science \& Technology 2000, 34, 2231-2236.

(25) Egenberg, I. M.; Holtekjølen, A. K.; Lundanes, E., Characterisation of naturally and artificially weathered pine tar coatings by visual assessment and gas chromatography-mass spectrometry. Journal of Cultural Heritage 2203, 4, 221-241.

(26) Rogge, W. F.; Hildemann, L. M.; Mazurek, M. A.; Cass, G. R.; Simoneit, B. R. T., Sources of fine organic aerosol. 9. Pine, oak and synthetic log combustion in residential fireplaces. Environmental Science \& Technology 1998, 32, 13-22.

(27) Fraser, M. P.; Yue, Z. W.; Tropp, R. J.; Kohl, S. D.; Chow, J. C., Molecular composition of organic fine particulate matter in Houston, TX. Atmospheric Environment 2002, 36, 5751-5758.

(28) Rogge, W. F.; Hildemann, L. M.; Mazurek, M. A.; Cass, G. R.; Simoneit, B. R. T., Sources of Fine Organic Aerosol .4. Particulate Abrasion Products from Leaf Surfaces of Urban Plants. Environmental Science \& Technology 1993, 27, 2700-2711.

(29) Lipsky, E. M.; Robinson, A. L., Effects of Dilution on Fine Particle Mass and Partitioning of Semivolatile Organics in Diesel Exhaust and Wood Smoke. Environmental Science \& Technology 2006, 40, 155-162.

(30) Turpin, B. J.; Lim, H. J., Species contributions to PM2.5 mass concentrations: Revisiting common assumptions for estimating organic mass. Aerosol Science and Technology 2001, 35, 602-610.

(31) Fraser, M. P.; Yue, Z. W.; Buzcu, B., Source apportionment of fine particulate matter in Houston, TX, using organic molecular markers. Atmospheric Environment 2003, 37, 2117-2123. 

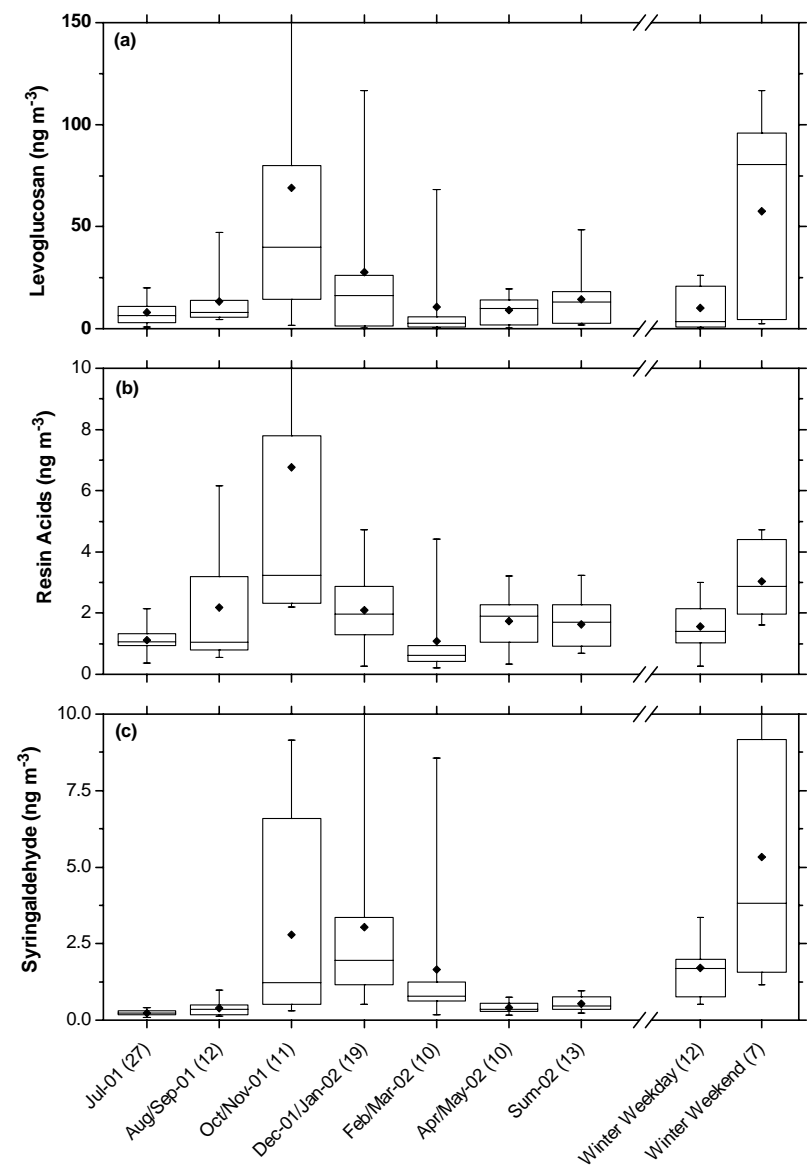

Figure 1. Box plots constructed from time series ambient data for (a) levoglucosan, (b) total resin acids, and (c) syringaldehyde. The boxes stretch from the lower quartile to the upper quartile values; median values are shown as lines across the boxes; the filled diamonds indicate average values. The whiskers indicate the maximum and minimum values; whiskers that intersect upper boundary of the plot indicate that the maximum value is beyond the scale of the graph. The numbers in parentheses in $\mathrm{x}$-axis labels indicate number of samples for specified period. Winter weekend and weekdays are taken from the Dec-01 and Jan-02. 

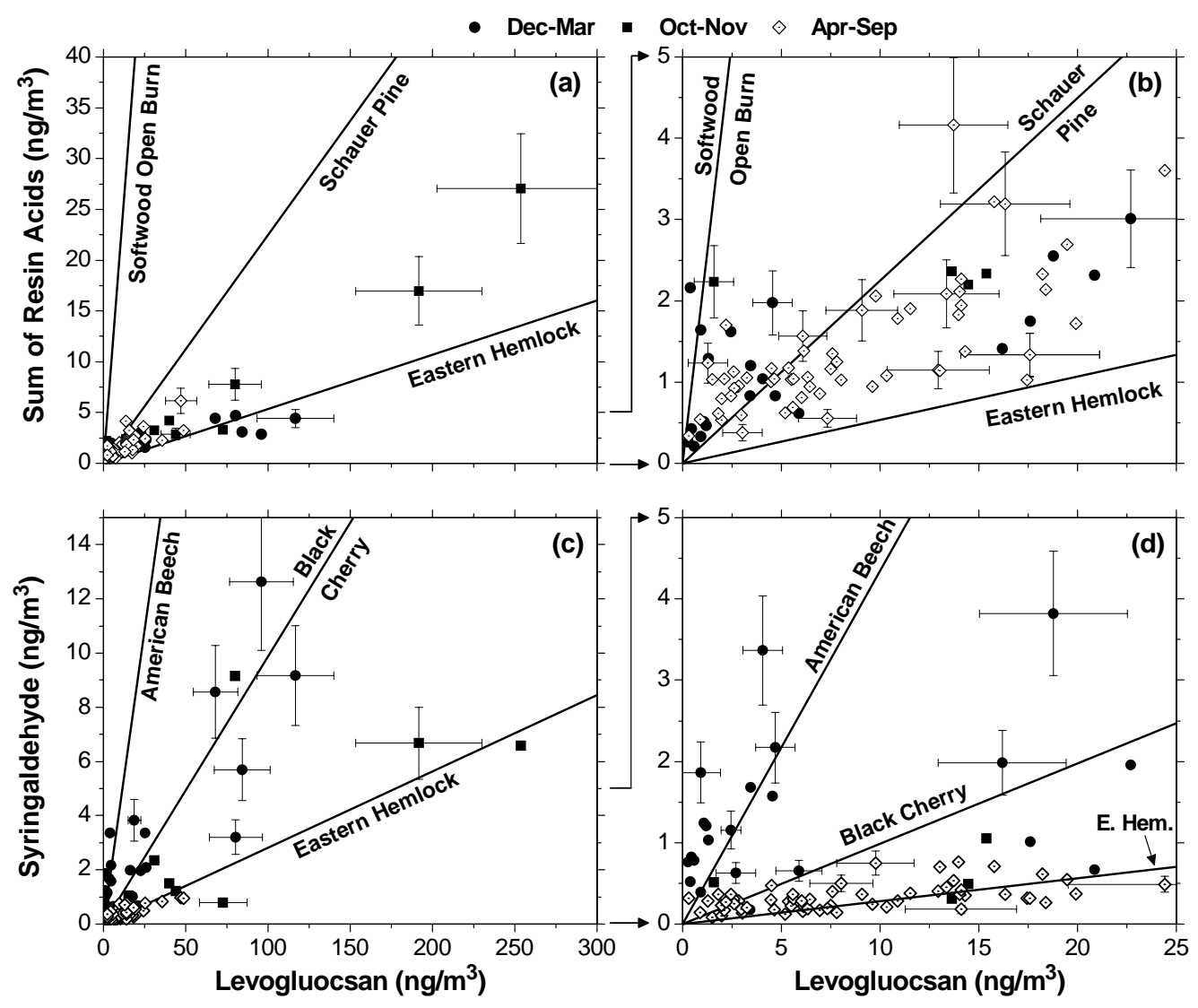

Figure 2. Scatter plots of ambient concentrations of biomass smoke markers. Panels (a) and (b) plot resin acids versus levoglucosan and panels (c) and (d) plot syrignaldehyde against levoglucosan in Pittsburgh. The lines indicate different source profiles reported by $(10-13,23)$. Measurement uncertainty indicated for selected points, uncertainty on other points is comparable. 

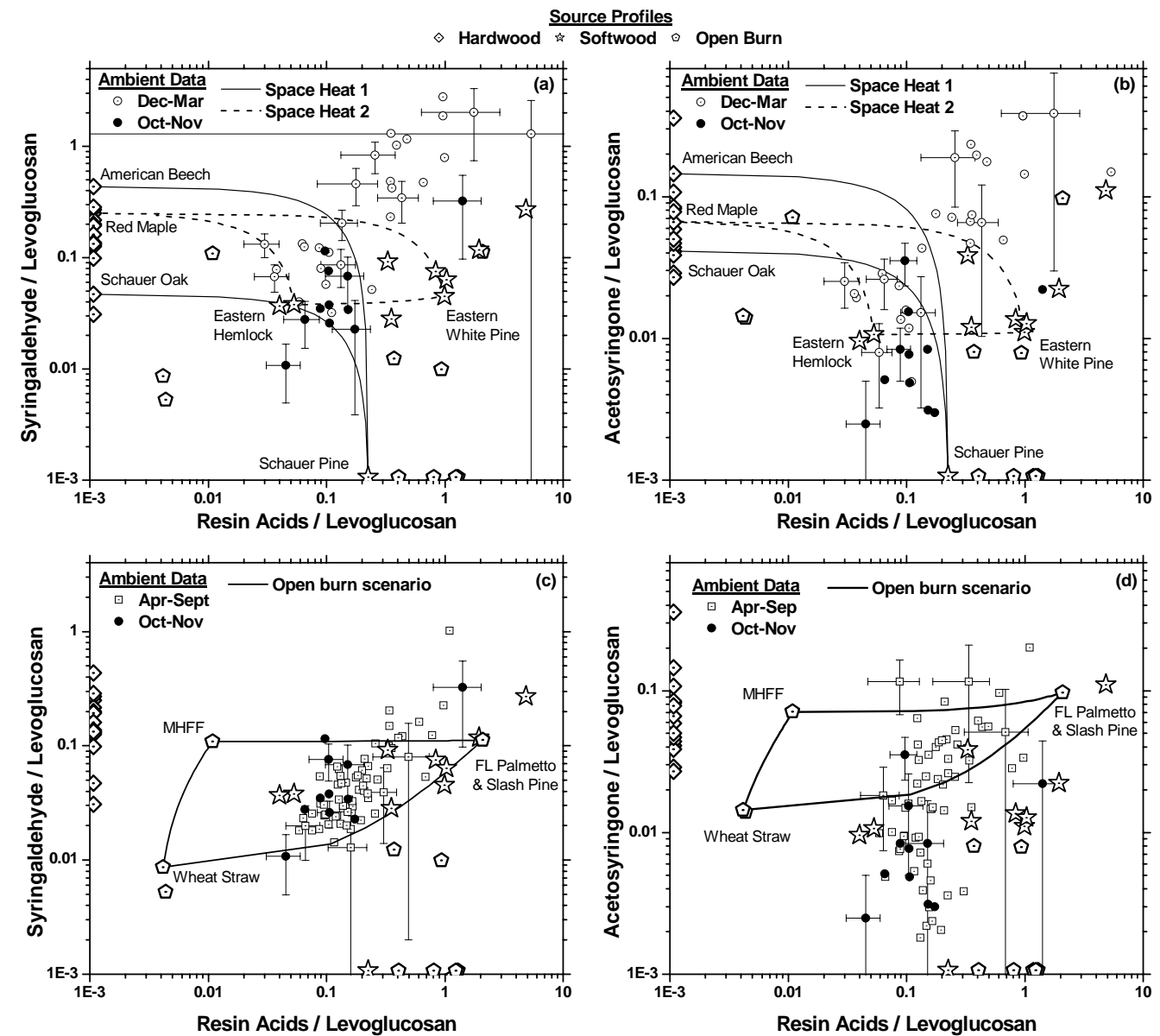

Figure 3. Ratio-ratio plots of biomass smoke markers: (a) \& (b) ambient data on fall and winter days compared to space heating mixing scenarios; and (c) \& (d) ambient data on non-winter days compared to open burn scenario. "Softwood" and "Hardwood" are fireplace or woodstove profiles (10-12,23). “Open burn” includes emissions from prescribed and simulated open-burns of hardwood, softwood, and agricultural residues (13-15). Measurement uncertainty indicated for selected points, uncertainty on other points is comparable. 


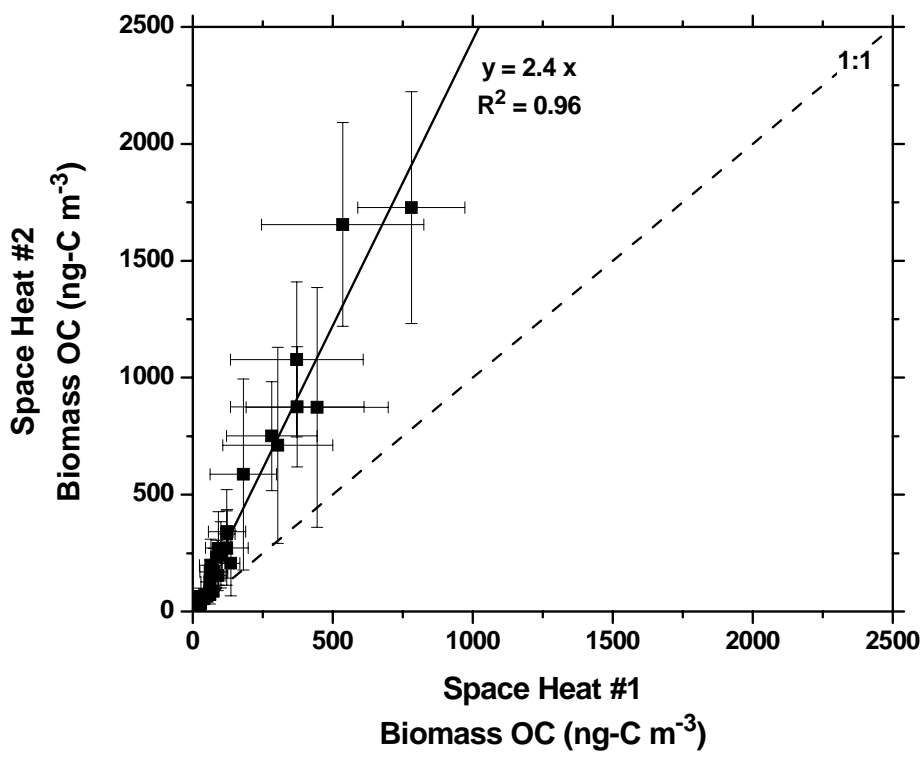

Figure 4. Scatter plot of ambient OC apportioned to biomass by the Space Heat \#1 and Space Heat \#2 scenarios on for October through March period. Error bars indicate one standard error as calculated by CMB. Solid line is linear regression forced through the origin. 


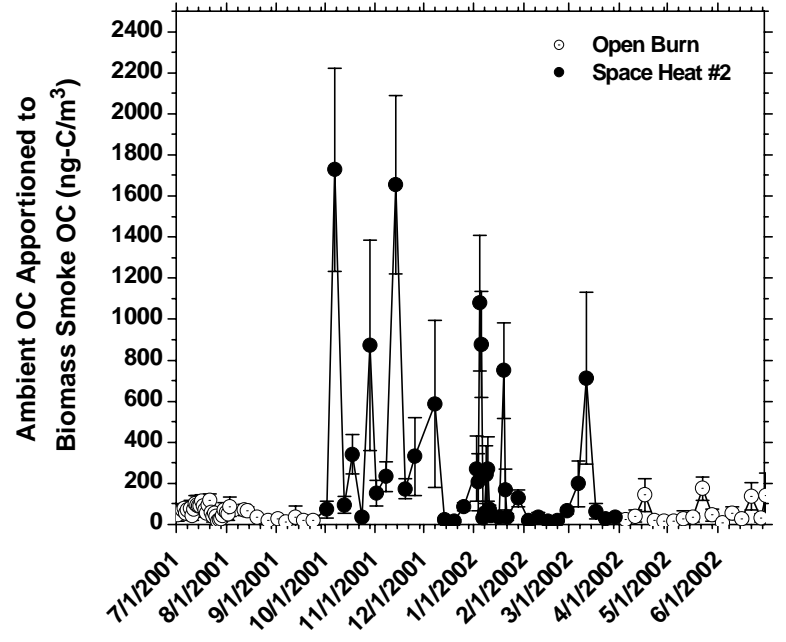

Figure 5. Time series of ambient OC apportioned to biomass smoke by CMB. Error bars are one standard error as calculated by CMB. 


\title{
Mass balance closure and the Federal Reference Method for $\mathrm{PM}_{2.5}$ in Pittsburgh, Pennsylvania
}

\author{
Sarah L. Rees ${ }^{\mathrm{a}, \mathrm{b}}$, Allen L. Robinson ${ }^{\mathrm{b}, \mathrm{c}, *}$, Andrey Khlystov ${ }^{\mathrm{d}}$, \\ Charles O. Stanier ${ }^{\mathrm{d}}$, Spyros N. Pandis ${ }^{\mathrm{b}, \mathrm{d}}$ \\ ${ }^{a}$ Department of Civil and Environmental Engineering, Carnegie Mellon University, Pittsburgh, PA 15213, USA \\ ${ }^{\mathrm{b}}$ Department of Engineering and Public Policy, Carnegie Mellon University, Pittsburgh, PA 15213, USA \\ ${ }^{\mathrm{c}}$ Department of Mechanical Engineering, Carnegie Mellon University, 5000 Forbes Ave., Pittsburgh, PA 15213, USA \\ ${ }^{\mathrm{d}}$ Department of Chemical Engineering, Carnegie Mellon University, Pittsburgh, PA 15213, USA
}

\begin{abstract}
Daily ambient aerosol samples were taken in Pittsburgh, Pennsylvania from the summer 2001 to the winter 2002 as part of the Pittsburgh Air Quality Study (PAQS). The study measured PM $_{2.5}$ mass by the Federal Reference Method (FRM) and the $\mathrm{PM}_{2.5}$ chemical composition by a variety of filter-based and continuous instruments. This paper examines the mass balance between the FRM-measured mass and the sum of the aerosol chemical components. For the 7 -month study period, the average FRM-measured mass is $11 \%$ greater than the sum of the mass of the aerosol chemical components. This mass balance discrepancy varies seasonally, with the average FRM-measured mass $17 \%$ greater than the sum of the chemical components for the summer months, with discrepancies as large as $30 \%$ during certain periods. Meanwhile, the FRM-measured mass was at or slightly below the sum of the chemical components for the winter months.

The mass balance discrepancy and its seasonal shift cannot be explained by measurement uncertainty; instead the discrepancy is due to combination of retained aerosol water on the conditioned FRM filters and volatilization losses. The relative importance of these different effects varies with aerosol composition and causes the observed seasonal variation in the mass balance. The contribution of the aerosol water to the FRM-measured mass is estimated using continuous measurements of aerosol water at the site; volatilization losses are estimated from other filter-based instruments. Water contributes $16 \%$ of the FRM mass in the summer, and $8 \%$ of the FRM mass in the winter; it also appears responsible for episodes where the FRM-measured mass is significantly greater than the sum of components. Retention of water is greatest during acidic conditions, which commonly occur during the summer months. Volatilization losses are estimated at 5\% of the FRM mass during the summer, and $9 \%$ for the winter. Volatilization losses appear to be most significant on days dominated by organic aerosol, or winter days with relatively high nitrate concentration. Accounting for the effects of water and volatilization losses closes the mass balance between the FRM and the sum of the chemical components, providing insight into the FRM measurements.
\end{abstract}

(C) 2004 Elsevier Ltd. All rights reserved.

Keywords: Federal Reference Method; Mass balance; Aerosol water; Aerosol sampling; Aerosol composition; Aerosol volatilization

\section{Introduction}

*Corresponding author. Department of Mechanical Engineering, Carnegie Mellon University, 5000 Forbes Ave., Pittsburgh, PA 15213, USA. Tel.: +1-412-268-3657; fax: + 1412-268-3348.

E-mail address: alr@andrew.cmu.edu (A.L. Robinson).
Fine particulate matter is a cause for concern because of its impact on human health and the environment. The US Environmental Protection Agency (EPA) promulgated standards regulating particulate matter with an aerodynamic diameter of $2.5 \mu \mathrm{m}$ or less in July 1997 
$\left(\mathrm{PM}_{2.5}\right)$, setting the $24 \mathrm{~h}$ standard at $65 \mu \mathrm{g} \mathrm{m}^{-3}$, and the annual average standard at $15 \mu \mathrm{g} \mathrm{m}^{-3}$. The new standards define $\mathrm{PM}_{2.5}$ as the mass measured by the Federal Reference Method (FRM). These instruments use gravimetric analysis of filters sampled over a $23-25 \mathrm{~h}$ period to determine $\mathrm{PM}_{2.5}$ mass concentration. EPA also specified operating and handling conditions for the FRM to minimize effects that could impact measurement accuracy, and to provide a consistent measure for regulatory standards. These effects include volatilization of species from sample filters, errors in filter tare and sample weights, and the presence of water on filters.

As a filter-based measurement, the FRM measures accumulated mass on a filter for a 24-h period, and therefore may not be truly reflective of atmospheric $\mathrm{PM}_{2.5}$ mass. The effects of emission reductions are estimated using chemical transport models and other analytical tools that are based on a scientific definition of $\mathrm{PM}_{2.5}$ behavior in the atmosphere, not FRM measurements. It is therefore important to understand the relationship between what the FRM measures and different definitions of $\mathrm{PM}_{2.5}$ mass in the atmosphere to ensure alignment in regulatory development.

One frequently used method of quality assurance of PM measurements is comparison of the gravimetrically measured mass to the sum of the aerosol chemical components as measured by collocated samplers. Many previous studies have used this method to evaluate aerosol measurements often finding gaps in mass closure (e.g., Chow et al., 1993; Malm et al., 1994a, b; Turpin et al., 1997; Andrews et al., 2000; Tanner and Parkhurst, 2000; Tolocka et al., 2001; Jansen et al., 2002). Mass closure gaps have exceeded $40 \%$ (Jansen et al., 2002) and include both instances where gravimetric-measured mass exceeds the sum of the chemical components ("positive discrepancy") and where it is less than the sum of the chemical components ("negative discrepancy"). Sometimes the mass discrepancy observed in these studies can be explained by uncertainties stemming from analytic measurements (Tolocka et al., 2001). In other studies measurement uncertainty accounts for only a portion of mass discrepancy, the remainder believed attributable to the presence of aerosol water (positive artifact) (Meng et al., 1995), volatilization of organics and nitrates (negative artifact) (Anderson et al., 2002), and uncertainty in the assumptions used to estimate chemical species not directly measured, or a combination thereof (Andrews et al., 2000).

This paper presents FRM data from the Pittsburgh Air Quality Study (PAQS), and uses these data and other measurements from PAQS to investigate FRM mass balance closure. First, the FRM-measured mass is compared to mass measured by a Tapered Element Oscillating MicroBalance (TEOM) and a dichotomous sampler to provide a measure of validation of the mass results. A daily mass balance is constructed for a 7-month period beginning in July 2001, capturing both summer and winter periods. The mass balance compares the sum of major chemical components (sulfate, nitrate, ammonium, elemental carbon (EC), organic carbon (OC), and crustal) measured by a variety of filter-based and continuous instruments at PAQS to FRM-measured mass. We then discuss several hypotheses explaining the mass balance discrepancy observed, including the impact of measurement uncertainty, the presence of water, and volatilization effects.

\section{Experimental}

The main PAQS ambient monitoring station was located in Schenley Park within the city of Pittsburgh (Wittig et al., 2004b). This location was not significantly impacted by local sources. Aerosol samples were taken with both filter-based and continuous instruments; sampling times for filter-based instruments were coordinated to run concurrently, with filter changes scheduled between 22:00 and 00:00 daily.

\subsection{PM 2.5 mass measurements}

$\mathrm{PM}_{2.5}$ mass was measured using three different instruments collocated at the PAQS station; a Partisol ${ }^{\mathbb{R}}$-FRM Model $2000 \mathrm{PM}_{2.5}$ Air Sampler (Rupprecht \& Pataschnick Co., Inc.), a Series 241 Dichotomous Sampler for $\mathrm{PM}_{10} / \mathrm{PM}_{2.5}$ (Thermo Andersen) ("Dichot") and a Model 1400a "TEOM ${ }^{\mathbb{R}}$ " (Rupprecht \& Pataschnick Co., Inc.). All three instruments were operated continuously for the duration of the study; the FRM and Dichot were operated daily to collect 24-h samples. Both the FRM and Dichot were operated according to the specifications provided by the manufacturer, and the EPA guidelines (40 C.F.R. Part 51 and the EPA Quality Assurance Manual). The TEOM operated at $30^{\circ} \mathrm{C}$ with a Nafion dryer on the inlet. Validated data from all three mass instruments are available for $92 \%$ of sampling time.

The FRM used $47 \mathrm{~mm}$ Teflon filters (Whatman No. 7592-104), while the Dichot used $37 \mathrm{~mm}$ Teflon filters (Pall Gelman No. R2PJ037). Prior to sampling, the filters for both of the instruments were conditioned for $24 \mathrm{~h}$ and then weighed in a controlled-environment chamber maintained at a relative humidity of $35 \pm 2 \%$ and a temperature of $22 \pm 2^{\circ} \mathrm{C}$. Filters were weighed on a UTM50 microbalance (Toledo Instruments). Strips of Polonium (Staticmaster) were used to minimize weighing errors induced by electrostatic charge. Filters were loaded into the filter cartridges for both the FRM and Dichot in the laboratory under a HEPA-filtered laminar flow hood to minimize contamination, and brought to the PAQS station at the time of the scheduled filter change. After the filter change, the exposed filters from 
both instruments were returned to the laboratory, and stored in a freezer to minimize loss of semi-volatile species. Exposed filters were typically weighed within a day or two of collection, which involved returning the filters to the controlled-environment chamber, conditioning the filters for $24 \mathrm{~h}$, and then weighing the filters to determine sample weight.

\subsection{Intercomparison of $P M_{2.5}$ mass measurements}

$\mathrm{PM}_{2.5}$ mass measurements of all three instruments compare well to each other, with linear regression $r^{2}$ values of 0.95 , and the regression lines within $10 \%$ of the 1:1 line for each comparison (Figs. 1 and 2). The timeaveraged TEOM is on average $1.5 \%$ higher than the FRM, and the FRM about 3\% higher than the Dichot

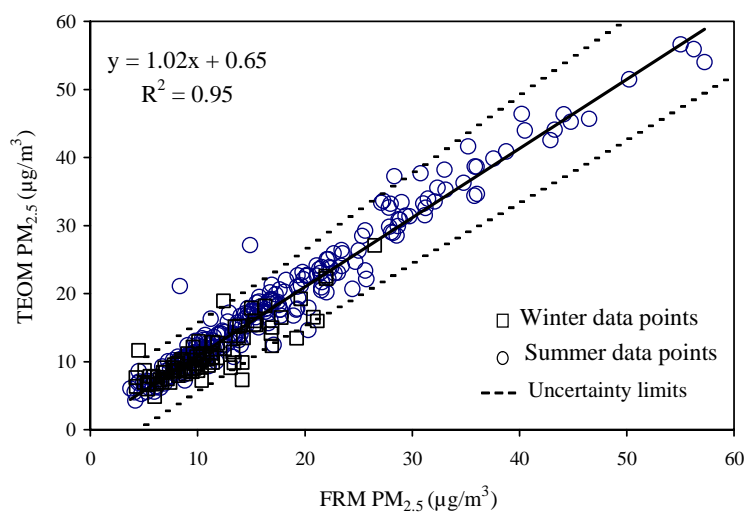

Fig. 1. FRM versus TEOM $\mathrm{PM}_{2.5}$ mass for the period of July 2001-March 2002. "Summer data points" are from 1 July to 31 October 2001; "winter data points" are from 1 November 2001 to 31 March 2002. TEOM data averaged to $24-\mathrm{h}$ periods to correspond to FRM measurements.

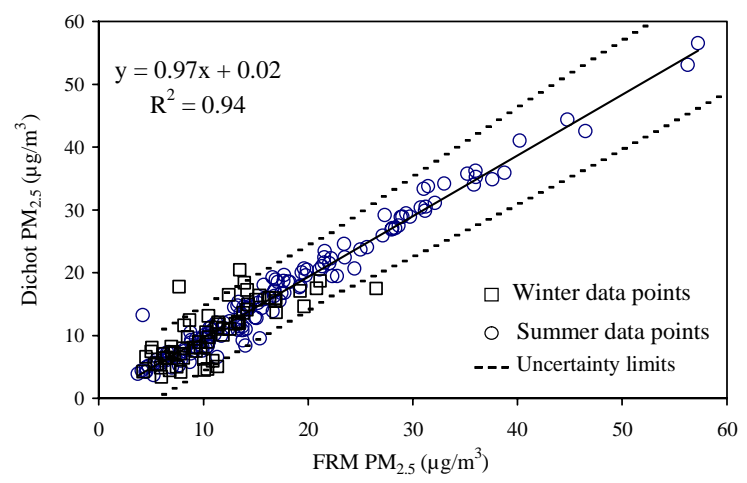

Fig. 2. FRM versus Dichot for the period of July 2001-March 2002. "Summer data points" are from July to November 2001; "winter data points" are from November 2001 to March 2002. for the study period. However, the scatter shown in Fig. 1 indicates that there are some periods of greater discrepancy, mostly occurring when $\mathrm{PM}_{2.5}$ concentrations are $<20 \mu \mathrm{g} \mathrm{m}^{-3}$ and during the winter months.

The TEOM has been reported to under-measure mass due to volatilization, particularly during the winter months (Allen et al., 1997). In December 2001 and January 2002, there are about 10 days where the TEOM measurements are significantly lower than those measured by the FRM and Dichot-up to $50 \%$ lower. This behavior is not unexpected, given the elevated temperature of the TEOM compared to the other instruments. The TEOM used in PAQS is a $30^{\circ} \mathrm{C}$ TEOM, not the $50^{\circ} \mathrm{C}$ TEOM that has typically been used in previous studies. Because we only observed the effect on a handful of exceptionally cold (ambient temperatures $<-6^{\circ} \mathrm{C}$ ) days when the $\mathrm{PM}_{2.5}$ mass was relatively low, volatilization did not significantly impact TEOM performance during days where $\mathrm{PM}_{2.5}$ mass exceeded $20-30 \mu \mathrm{g} \mathrm{m}^{-3}$. However, the winter of 2002 was relatively mild compared to those typically experienced in Pittsburgh, so TEOM volatilization may be more significant in colder regions or during a more typical Pittsburgh winter.

\subsection{Sulfate, nitrate, and ammonium}

$\mathrm{PM}_{2.5}$ sulfate, nitrate, and ammonium were measured using both continuous instruments and filter-based samplers. To evaluate the mass balance, we used data from the CMU inorganic sampler, a denuder/filterbased speciation sampler (Takahama et al., 2004). The $\mathrm{CMU}$ inorganic sampler yielded daily inorganic $\mathrm{PM}_{2.5}$ composition on a 24-h basis for the entire sampling period, except for July 2001, when it provided five measurements per day.

R\&P sulfate (Model 8400S) and nitrate (Model $8400 \mathrm{~N}$ ) analyzers were run concurrently with the CMU inorganic sampler to provide high time resolution data. We used the data from the R\&P instruments in conjunction with TEOM data to evaluate the $\mathrm{PM}_{2.5}$ mass balance on an hourly basis. Wittig et al. (2004a) discuss in detail the comparison between the R\&P instruments and the traditional samplers, as well as the approach used for the calibration of the continuous instruments. The calibrated final values of Wittig et al. (2004a) are used for the analysis here.

A steam sampler (Khlystov et al., 1995) was used to provide high time resolution data for ammonium and data for intercomparison. To construct the mass balance, we used $\mathrm{PM}_{2.5}$ ammonium measurements from the CMU inorganic sampler for the summer months. Because data indicate that atmospheric conditions are neutral in the winter (December 2001-March 2002), we estimated winter $\mathrm{PM}_{2.5}$ ammonium concentrations by 
assuming ammonium was present in sufficient concentrations to result in neutral particles.

\subsection{Organic carbon/elemental carbon}

$\mathrm{PM}_{2.5} \mathrm{OC}$ and EC were measured by the CMUdesigned Teflon-Quartz, Quartz-Quartz sampler (TQQQ). The TQQQ is a two-port sampler with a double quartz filter pack in one line and a Teflon filter followed by a quartz filter in the second. The $\mathrm{OC}$ is estimated as a value between $\mathrm{OC}$ from the upstream quartz filter ('bare quartz') of the double quartz filter pack, and that obtained by subtracting the OC of the quartz behind the quartz from the bare quartz OC. Comparison with a denuder-based sampler showed that this configuration provides robust estimate of particulate OC (Subramanian et al., 2004). EC is estimated from the bare quartz alone. Each quartz filter is analyzed for OC/EC using a Sunset Labs ThermalOptical Transmittance analyzer with the NIOSH 5040 protocol (NIOSH, 1998) as implemented by Subramanian et al. (2004). A Sunset Laboratories semi-continuous $\mathrm{OC} / \mathrm{EC}$ analyzer using the same analysis protocol (Cabada et al., 2004) was used to provide higher time resolution $\mathrm{OC} / \mathrm{EC}$ data.

Most mass balance studies use a value of 1.4 for the OC multiplier. Recent work by Turpin and Lim (2001) examines this factor, recommending values ranging from 1.1 for fresh emissions to 1.6-2.1 for an aged aerosol. Comparison of PAQS main site data with satellite sites indicates that the air quality in Pittsburgh is dominated by regional transport (Tang et al., 2004). We therefore used a multiplication factor of 1.8 which is representative of an aged, regional aerosol to estimate total organic mass from OC measurements. The sensitivity of the results to this factor will be discussed in a subsequent section.

\subsection{Crustal}

We estimated the crustal component of $\mathrm{PM}_{2.5}$ using metals data from XRF analysis of filter-based samples taken daily from the PAQS sites during July 2001 and January 2002. Elemental composition was also measured using ICP-MS analysis of high volume filter samples taken at the main PAQS site. The trace metal composition data do not vary substantially from site to site, indicating that Pittsburgh aerosol is of a regional nature and that none of the sampling sites were heavily impacted by local sources.

We used the sum of oxides algorithm (Malm et al., $1994 a, b)$ to estimate the daily crustal $\mathrm{PM}_{2.5}$ for July 2001 and January 2002 using the XRF data. The average crustal contribution was $1 \mu \mathrm{g} \mathrm{m}^{-3}$ (approximately $3-6 \%$ of total $\mathrm{PM}_{2.5}$ mass) with a standard deviation of $0.4 \mu \mathrm{g} \mathrm{m}^{-3}$. This estimate compares well with crustal estimates from similar studies in the Eastern US (Andrews et al., 2000; Tolocka et al., 2001). The ICPMS data suggest that the crustal contribution remains fairly constant throughout the year; however, these data were not used to estimate the crustal contribution because the ICP-MS data quality for $\mathrm{Si}$, an important crustal species, are poor due to analytical interferences. Therefore, for the remaining months, we assume that crustal material contributes $1 \mu \mathrm{g} \mathrm{m}^{-3}$, or $4 \%$ of the average $\mathrm{PM}_{2.5}$ mass. The potential effects of this assumption on the mass balance are discussed below.

\subsection{Water}

Aerosol water content at ambient relative humidity (RH) was measured by the Dry and Ambient Aerosol Size Spectrometer (DAASS), an automatic in situ system, built to measure ambient aerosol size and distributions at both ambient and low RH conditions (Khlystov et al., 2004). The system consists of two Scanning Mobility Particle Sizers (SMPS, TSI, Inc.) and an Aerodynamic Particle Sizer (APS, TSI, Inc.). The instruments measure the aerosol size distribution between $5 \mathrm{~nm}$ and $10 \mu \mathrm{m}$ in diameter. By measuring alternatively "wet" and "dry" size distributions and the corresponding integrated volume concentrations, the aerosol water content of ambient aerosol can be estimated. The water content at $35 \% \mathrm{RH}$, the $\mathrm{RH}$ at which FRM filters were conditioned, was then estimated according to the method described later in this paper.

Aerosol water content at $35 \% \mathrm{RH}$ was also calculated directly using the GFEMN aerosol thermodynamic model (Ansari and Pandis, 1999). GFEMN predicts inorganic aerosol composition, including aerosol water, based on chemical thermodynamic principles. Using PAQS chemical speciation data (sulfate, nitrate, ammonium, etc.), temperature and $\mathrm{RH}$, the model estimates the PM water concentration at the $\mathrm{RH}$ of the FRM measurements.

\section{Results and discussion}

Fig. 3 shows the $\mathrm{PM}_{2.5}$ FRM daily time series for July 2001-March 2002. The average $\mathrm{PM}_{2.5}$ mass measured with the FRM was $16.1 \mu \mathrm{g} \mathrm{m}^{-3}$. There is significant seasonal variation in the $\mathrm{PM}_{2.5}$ mass levels, with an average mass concentration of $24.1 \mu^{-3}$ for the summer of 2001 and $12.2 \mu \mathrm{g} \mathrm{m}^{-3}$ for the winter of 2002. Fig. 3 also illustrates the episodic nature of $\mathrm{PM}_{2.5}$ concentrations in the Pittsburgh area, with high peaks and low valleys of $\mathrm{PM}_{2.5}$ levels occurring over periods of several days due to the effect of meteorological conditions on ambient air quality. This behavior occurs throughout the sampling period, but is more pronounced in the summer than in the winter months. 


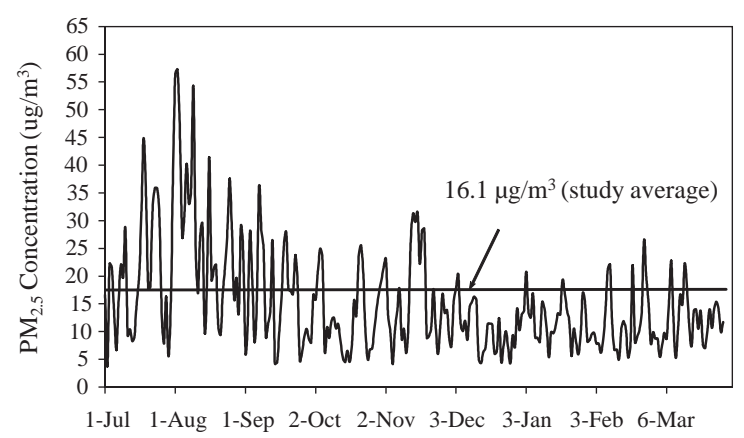

Fig. 3. Daily $\mathrm{PM}_{2.5}$ mass measured with an FRM sampler from July 2001 to March 2002. Horizontal line is the average $\mathbf{P M}_{2.5}$ for this period.

\subsection{Comparison of FRM mass to sum of chemical components}

Fig. 4 presents the monthly average $\mathrm{PM}_{2.5}$ mass and composition. The height of each bar represents the FRM mass. The component labeled "missing" is the difference between the FRM-measured mass and the sum of chemical components; it is positive when the FRM-measured mass exceeds the sum of components, and negative when it is less than the sum of chemical components. During the summer, sulfate comprises over $45 \%$ of the FRM-measured $\mathrm{PM}_{2.5}$ mass, while nitrate comprises $<3 \%$. In the winter months, the sulfate contribution drops to $35 \%$, while the nitrate contribution approaches $15 \%$ of FRM-measured $\mathrm{PM}_{2.5}$ mass. The organic mass varies slightly through the year, contributing approximately $20 \%$ of total mass in the summer, $30 \%$ in the fall, and $20 \%$ in the winter, for a study average contribution of $23 \%$ of the FRMmeasured $\mathrm{PM}_{2.5}$ mass. For the period of July 2001March 2002, the average FRM-measured mass is $11 \%$ greater than the average mass of the sum of the components (Fig. 5).

Examination of the mass balance on a monthly average basis (Fig. 4) indicates that there is a significant seasonal dependence of the mass balance discrepancy. From July to November 2001, the FRM mass exceeded the sum of the chemical components with an average missing mass from the sum of the chemical components of $2.9 \mu \mathrm{g} \mathrm{m}^{-3}$, or $15 \%$ of the FRM-measured mass. For the period of December 2001-March 2002, the sum of the chemical components slightly exceeds the FRMmeasured mass, with an average excess mass of $0.2 \mu \mathrm{g} \mathrm{m}^{-3}$ or $2 \%$ of the FRM-measured mass. As discussed below, the average discrepancy for the summer is greater than the measurement uncertainty, while the average discrepancy for the winter is within the range of measurement uncertainty.

Fig. 6 shows the daily mass balance for August 2001 and February 2002, months with the largest positive and

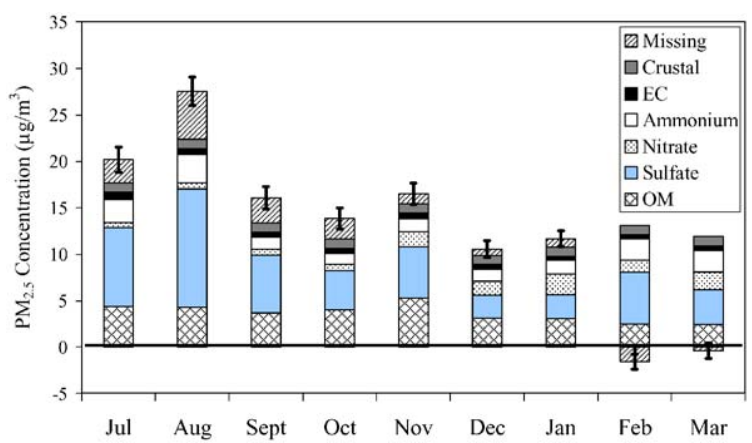

Fig. 4. Monthly average $\mathrm{PM}_{2.5}$ mass and composition calculated from daily 24-h measurements. The "missing" component is the difference between the FRM measurement and the sum of the chemical components. The FRM mass is greater than the sum of the chemical components for July-December, and less than the sum of the chemical components in February and March. The error bars represent uncertainty in the mass balance on a monthly average basis. $\mathrm{OM}$ is defined as $1.8 \times \mathrm{OC}$.

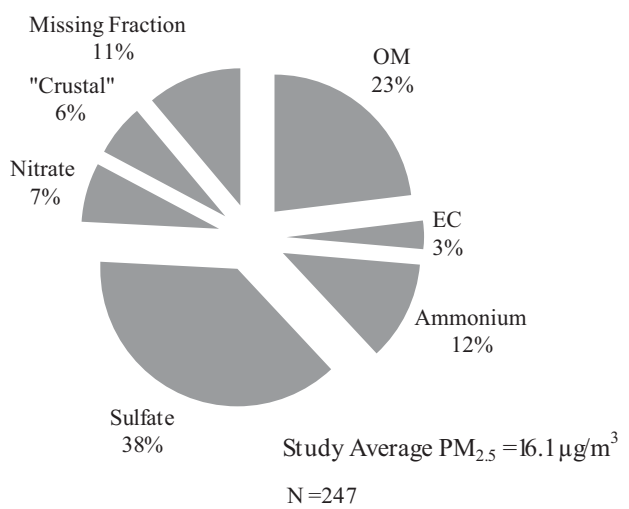

Fig. 5. Average $\mathrm{PM}_{2.5}$ composition July 2001-March 2002 calculated from 247, 24-h measurements.

negative discrepancies, respectively. Overall for August $2001,19 \%\left(5.2 \mu \mathrm{g} \mathrm{m}^{-3}\right)$ of the FRM-measured mass was unaccounted for by the sum of the chemical components indicating a positive discrepancy. The positive discrepancies in August are episodic, occurring periodically and lasting for several days at a time, interdispersed with short episodes of mass closure. Similar behavior was observed for the other months when the FRM mass was greater than the sum of the chemical components. In February, the FRM-measured mass was $12 \%$ $\left(1.4 \mu \mathrm{g} \mathrm{m}^{-3}\right)$ less than the sum of the chemical components indicating a negative discrepancy. For the other winter months, there are periods of both slight positive and negative discrepancy, but the periods of positive discrepancy observed are far fewer than those observed in the summer. The overall effect in the winter is that the periods of positive discrepancy approximately equal the 

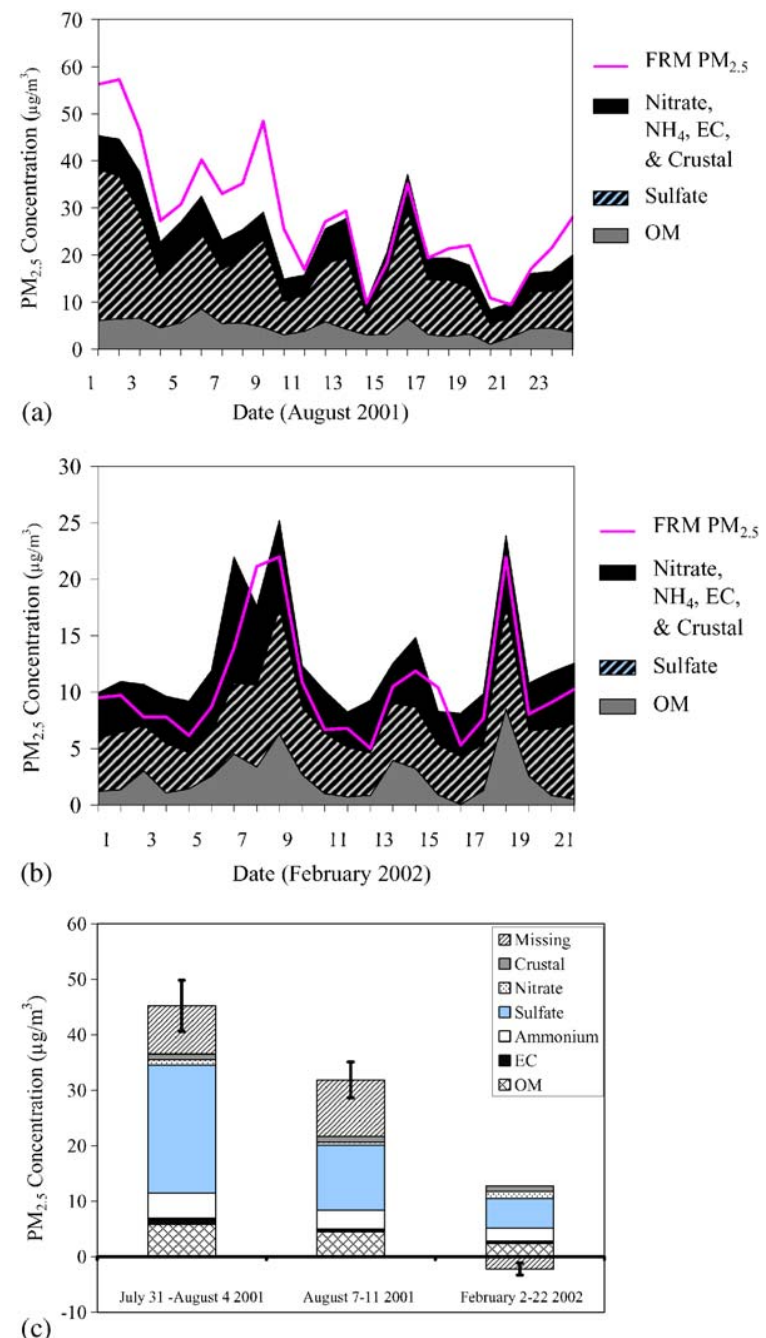

Fig. 6. (a) Time series mass balance for August $2001\left(\mu \mathrm{g} \mathrm{m}^{-3}\right)$. (b) Time series mass balance for February $2002\left(\mu \mathrm{g} \mathrm{m}^{-3}\right)$. Data in (c) are averages of daily $24-\mathrm{h}$ data over the indicated period. The error bars in (c) represent average mass balance uncertainty for the period. $\mathrm{OM}$ is defined as $1.8 \times \mathrm{OC}$.

negative discrepancy, resulting in mass balance closure on the average.

The positive mass discrepancy can be further explored by evaluating the average mass balance for select episodes in August 2001 shown in Fig. 6c. The average FRM-measured mass exceeded the sum of chemical components by $19 \%$ or $8.7 \mu \mathrm{g} \mathrm{m}^{-3}$ for the period between 31 July and 4 August 2001, and by $32 \%$ or $10.2 \mu \mathrm{g} \mathrm{m}^{-3}$ for the period between 7 and 11 August 2001. Both of these periods occurred during episodes of hot, humid weather with relatively high $\mathrm{PM}_{2.5}$ concentrations. Fig. $6 \mathrm{c}$ also shows data for a winter period with significant negative discrepancy; the average
FRM-measured mass for 1-22 February 2002 was $21 \%$ or $2.2 \mu \mathrm{g} \mathrm{m}^{-3}$ below the sum of the chemical components.

Fig. 7 presents the ratio of the FRM-measured mass to the sum of chemical components as a function of FRM mass. For this analysis the data have been averaged into periods with similar $\mathrm{PM}_{2.5}$ mass concentrations. If the mass balance is closed, the data points will fall close to 1 within measurement uncertainty. The results indicate that the mass discrepancy varies with mass concentration. When the FRM-measured mass is less than about $10 \mu \mathrm{g} \mathrm{m}^{-3}$, there is a consistent negative discrepancy. This observation reflects the negative mass balance discrepancy observed during the winter months, when the $\mathrm{PM}_{2.5}$ concentration averages around 10$12 \mu \mathrm{g} \mathrm{m}^{-3}$ with large fractions of nitrates and organics. Positive discrepancy occurs at $\mathrm{PM}_{2.5}$ levels $>10 \mu \mathrm{g} \mathrm{m}^{-3}$, which primarily occur during the warmer months of the summer and fall.

\subsection{Hypotheses explaining the mass balance}

Several hypotheses may explain the periods of mass balance discrepancy observed:

(1) the discrepancy could be due to uncertainty in the measurements of chemical composition and $\mathrm{PM}_{2.5}$ mass;

(2) periods of significant positive mass discrepancy could be explained by the presence of bound water on the FRM filter post-conditioning;

(3) periods of negative mass discrepancy could be due to volatilization of organic compounds and nitrates;

(4) the discrepancy could result from the use of an incorrect OC multiplication factor; and

(5) inaccuracies in estimates for the crustal component of $\mathrm{PM}_{2.5}$ mass could impact mass balance closure.

We discuss each of these hypotheses below.

\subsubsection{Impact of measurement uncertainty}

In order to evaluate whether the mass balance discrepancy is significant, it is important to quantify the uncertainty in the individual measurements used evaluate the mass balance. The uncertainties for the individual measurements are listed in Table 1. These conservative estimates were derived from intercomparisons of data from different samplers and instruments used during PAQS (Wittig et al., 2004b), and therefore account for both stochastic and systematic uncertainties. To minimize potential biases due to systematic uncertainties, we eliminated the small number of data points for which large unexplained discrepancies exist between the different measurements of the same parameter. For the remaining data we are confident that the systematic uncertainties are reasonably small because it 


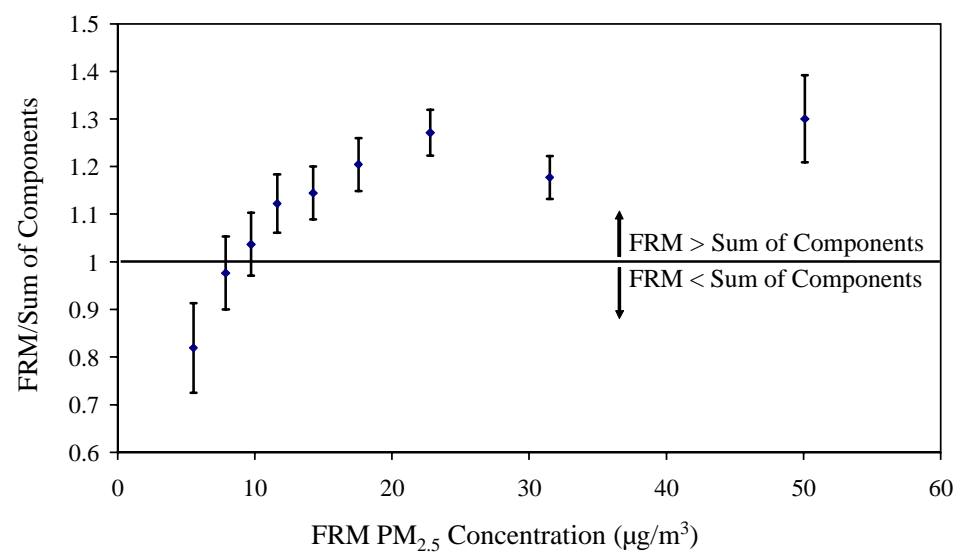

Fig. 7. Mass discrepancy ratio as a function of FRM PM2.5. The mass balance discrepancy ratio is the FRM mass divided by the sum of the chemical components. The FRM measurement exceeds the sum of the chemical components when the ratio $>1$, indicating a positive mass discrepancy. When the ratio $<1$, the FRM measurement is less than the sum of the chemical components, indicating a negative mass discrepancy. The data in this figure are averages computed by sorting the FRM data into bins as a function of $\mathrm{PM}_{2.5}$ mass concentrations and then calculating the average mass discrepancy ratio and average mass balance uncertainty (Eq. (2)) for each bin. Approximately 30 days of data are in each bin.

Table 1

Average estimated uncertainty of individual instrument measurements (relative and absolute)

\begin{tabular}{lll}
\hline Parameter & $\begin{array}{l}\text { Relative uncertainty } \\
(\%)\end{array}$ & $\begin{array}{l}\text { Absolute uncertainty } \\
\left(\mu \mathrm{g} \mathrm{m}^{-3}\right)\end{array}$ \\
\hline FRM PM & & \pm 2 \\
mass & \pm 15 & \pm 2 \\
$\mathrm{SO}_{4}$ & \pm 25 & \pm 1.5 \\
$\mathrm{NO}_{3}$ & \pm 40 & \pm 0.5 \\
$\mathrm{NH}_{4}$ & \pm 30 & \pm 0.5 \\
$\mathrm{OM}$ & \pm 25 & \pm 0.9 \\
EC & \pm 50 & \pm 0.25 \\
Crustal & \pm 50 & \\
\hline
\end{tabular}

Note: The higher of the relative or absolute value was used to calculate daily uncertainty for each parameter. As described in the text, these values were determined based on intercomparison of different samplers and instruments at the site.

${ }^{\text {a }}$ Crustal uncertainty is assumed at $50 \%$ based on estimates from previous work (Andrews et al., 2000).

is extremely unlikely that different samplers and instruments operating on different physical principles and by different groups would produce consistent results if significant systematic errors were present.

The approach used to determine the measurement uncertainty is illustrated in Figs. 1 and 2 using the intercomparisons of the $\mathrm{PM}_{2.5}$ mass measurements. During PAQS, the $\mathrm{PM}_{2.5}$ mass was measured using two filter samplers (FRM and DICHOT) and a TEOM. The high degree of correlation between the $\mathrm{PM}_{2.5}$ measurements provides confidence in the precision of the measurements and indicates that any systematic biases are small. The estimated uncertainty of the $\mathrm{PM}_{2.5}$ mass measurements is shown by the dashed lines in Figs. 1 and 2, and accounts for the variation among the different measurements of $\mathrm{PM}_{2.5}$ mass at the site. These uncertainty limits were defined to include $>95 \%$ of the data lie within these limits and therefore they can be viewed as $95 \%$ confidence limits. Uncertainty estimates for the aerosol chemical components were derived using similar intercomparisons: Subramanian et al. (2004) provide a detailed intercomparison of carbon measurements, and Wittig et al. (2004a) provide a detailed intercomparison of the inorganic measurements.

The uncertainty in the mass balance was estimated by combining the uncertainties of the individual measurements using standard analysis procedures assuming that the uncertainties of the individual measurements are uncorrelated:

$\sigma_{\mathrm{T}}=\sqrt{\sigma_{\mathrm{SO}_{4}}^{2}+\sigma_{\mathrm{NO}_{3}}^{2}+\sigma_{\mathrm{NH}_{4}}^{2}+\sigma_{\mathrm{OM}}^{2}+\sigma_{\mathrm{EC}}^{2}+\sigma_{\mathrm{Crustal}}^{2}}$,

where $\sigma_{\mathrm{T}}$ is the overall uncertainty, and $\sigma_{\mathrm{SO}_{4}}, \sigma_{\mathrm{NO}_{3}}$, $\sigma_{\mathrm{NH}_{4}}, \sigma_{\mathrm{OM}}, \sigma_{\mathrm{EC}}$, and $\sigma_{\mathrm{Crustal}}$ are the uncertainties of each individual measurement (sulfate, nitrate, ammonium, organic matter (OM), EC, and crustal, respectively). The uncertainty of averaged data (e.g., monthly average) is estimated using

$\sigma_{\mathrm{Ave}}=\frac{\text { Average } \sigma_{n}}{\sqrt{n}}$

where Average $\sigma_{n}$ is the average uncertainty in the mass balance (Eq. (1)) for the given set of data, and $n$ is the number of data points. Eq. (2) is strictly valid for averaged data when only stochastic errors are present. 
The measurement intercomparisons (e.g., Figs. 1 and 2) indicate that the variations in the data between the different samplers are largely stochastic (there are no significant systematic biases in the data). Therefore, Eq. (2) should provide a reasonable estimate of uncertainty for averaged data.

A statistically significant discrepancy in the daily mass balance was observed on 82 study days. A statistically significant discrepancy is larger than the uncertainty defined by Eq. (1). The data support the conclusion that the mass balance discrepancy varies seasonally in Pittsburgh. In the summer months, the FRM mass is consistently greater than the sum of the chemical components with 17 days exhibiting a significant positive discrepancy, and only 1 day with a significant negative discrepancy. In the winter months, there are a large number of days with either a positive or a negative discrepancy: 16 winter days had a significant negative discrepancy, while 10 had a significant positive discrepancy. The remaining 38 days of significant mass discrepancy were spread from September to November 2001, with 27 days of positive discrepancy, and 11 days of negative discrepancy. It is clear from these observations that positive and negative mass discrepancies occur during both seasons, but that the positive discrepancy is dominant in the summer, and that the negative discrepancy is more prevalent in the winter.

Evaluating select periods with significant mass balance discrepancy further supports the conclusion that the observed discrepancy cannot be explained by measurement uncertainty. Measurement uncertainty was approximately $\pm 8 \%$ for the periods of 31 July -4 August and 7-11 August (Fig. 6c), much smaller than the observed mass discrepancy of $19 \%$ and $32 \%$, respectively. For the period 1-22 February 2002, the FRM mass was $21 \%$ greater than the sum of the chemical components; a significantly larger difference than the average measurement uncertainty for that period, $\pm 14 \%$. On a monthly average basis the mass balance discrepancy was statistically significant for the months of July, August, September, October, and November 2001 and February 2002 (Fig. 4).

\subsubsection{Retention of water on conditioned FRM filters}

Retention of water on the conditioned FRM filters is a concern in Pittsburgh and other areas of the Eastern US because particles are often acidic in the summer, containing substantial amounts of ammonium bisulfate $\left(\mathrm{NH}_{4} \mathrm{HSO}_{4}\right) . \mathrm{NH}_{4} \mathrm{HSO}_{4}$ retains significant water at $35 \%$ $\mathrm{RH}$, the $\mathrm{RH}$ at which FRM filters are conditioned (Speer et al., 1997; Hand et al., 2000). The presence of $\mathrm{NH}_{4} \mathrm{HSO}_{4}$ could therefore cause aerosol on FRM filters to retain water post-conditioning, potentially explaining the observed mass balance discrepancy.

Two types of evidence are available to examine the potential contribution of particle bound water to the
FRM mass measurements. First, data are presented to identify periods when $\mathrm{NH}_{4} \mathrm{HSO}_{4}$ is likely present. Second, direct measurements of ambient aerosol water content are used to estimate the contribution of water to the conditioned FRM filters. We examine each of these types of evidence below.

In Pittsburgh, a significant fraction of the $\mathrm{PM}_{2.5}$ mass is sulfate. During the summer, the aerosol is periodically acidic because insufficient ammonium is available to neutralize the sulfate aerosol present. Under these conditions, some of the sulfate will be present in the form of bisulfate $\left(\mathrm{NH}_{4} \mathrm{HSO}_{4}\right)$; the more acidic the aerosol, the larger the fraction of bisulfate present. Since bisulfate retains water at a low $\mathrm{RH}$, we expect that the positive mass balance discrepancy will be present on acidic days. To examine this hypothesis, estimates of aerosol acidity are compared to mass balance discrepancy. Aerosol acidity can be estimated using the ratio of anions to cations (acidity ratio). In the summer in Pittsburgh this ratio is largely determined by ammonium and sulfate levels because nitrates and crustal material are minor $\mathrm{PM}_{2.5}$ components. For the summer periods we calculate the acidity ratio as

acidity ratio $=\frac{2\left[\mathrm{SO}_{4}\right]}{\left[\mathrm{NH}_{4}\right]}$,

where $\left[\mathrm{SO}_{4}\right]$ is the measured molar concentration of aerosol sulfate, and $\left[\mathrm{NH}_{4}\right]$ is the measured molar concentration of ammonium. A ratio of approximately 1 indicates neutral conditions; ratios $>1$ indicates acidic conditions when some bisulfate is likely present; and ratios of 2 or greater indicate that all of the sulfate present is likely in the form of bisulfate.

Time series of the acidity ratio (Eq. (3)) indicates that the aerosol is periodically acidic, and that specific episodes of positive discrepancy are correlated with acidity. For example, during the August mass discrepancy episodes shown in Fig. 6c, the atmospheric acidity ratio averaged 2.0 for the 31 July -4 August period, and 1.33 for the 7-11 August period, indicating acidic conditions. Meanwhile, the average acidity ratio for periods in August with no mass discrepancy was 1.08, indicating conditions close to neutral.

High time resolution data provide further evidence of the relationship between periods of positive mass discrepancy and aerosol acidity. For this analysis, the TEOM is used to measure mass, the R\&P continuous instruments are used for sulfate and nitrate, the steam sampler is used for ammonium, and the semi-continuous $\mathrm{OC} / \mathrm{EC}$ analyzer is used for OC and EC measurements. Fig. 8 shows an interesting 5-day period during July 2001, which includes episodes of both positive and negative mass balance discrepancies. There are large variations in atmospheric acidity, and periods of high atmospheric acidity correlate well with periods of positive discrepancy. 


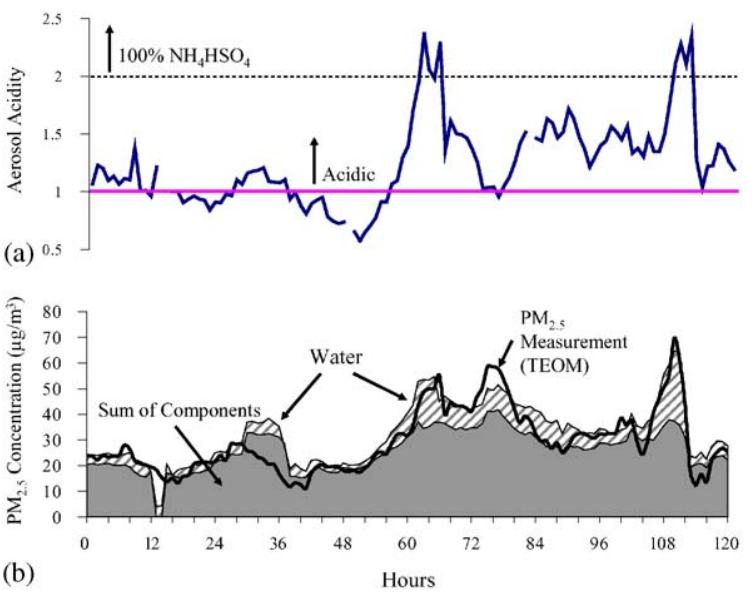

Fig. 8. Hourly atmospheric (a) acidity (Eq. (3)) and (b) $\mathbf{P M}_{2.5}$ mass balance for 20-25 July 2001. Data are from continuous instruments. Aerosol acidity $>1$ indicates acidic conditions, aerosol acidity $>2$ indicates $100 \% \mathrm{NH}_{4} \mathrm{HSO}_{4}$. Water estimates are from DAASS measurements. The data illustrate that mass balance discrepancy is accounted for by measured aerosol water and generally corresponds to acidic conditions.

These data support the conclusion that the periods when the FRM mass exceeds the sum of the chemical components correspond to acidic conditions. This relationship appears most strongly during episodes of significant positive discrepancy, when the acidity ratio indicates the presence of $\mathrm{NH}_{4} \mathrm{HSO}_{4}$. It is also observed on a monthly basis; months with positive mass discrepancy tend to be acidic. However, acidity and the positive mass discrepancy are not always correlated, indicating that other factors besides acidity influence the mass balance. For example, a significant positive mass discrepancy was observed on the morning of 23 July ( $~ 76 \mathrm{~h}$ in Fig. 8) even though the atmosphere was not especially acidic. Most of these events appeared to be "triggered" by a preceding high acidity event, and are probably related to the history of the air mass and the hysteresis behavior of inorganic PM.

The second piece of evidence of the role of aerosol water in the mass balance uses measurements of ambient aerosol water content. The amount of water at $35 \% \mathrm{RH}$ was estimated from the DAASS growth factor data $\left(V_{\text {wet }} / V_{\text {dry }}\right)$ by assuming that the aerosol water content is proportional to its water-soluble mass as follows from Raoult's law:

$M_{\mathrm{w}}(\mathrm{RH})=a M_{\mathrm{s}} \frac{\mathrm{RH}}{1-\mathrm{RH}}=A \frac{\mathrm{RH}}{1-\mathrm{RH}}$,

where $M_{\mathrm{w}}$ is the amount of water in the aerosol, $M_{\mathrm{s}}$ is the mass of water-soluble aerosol components, $a$ and $A$ are proportionality coefficients, and $\mathrm{RH}$ is the relative humidity expressed as a fraction of 1 . The aerosol water content measured with the DAASS at both wet and dry
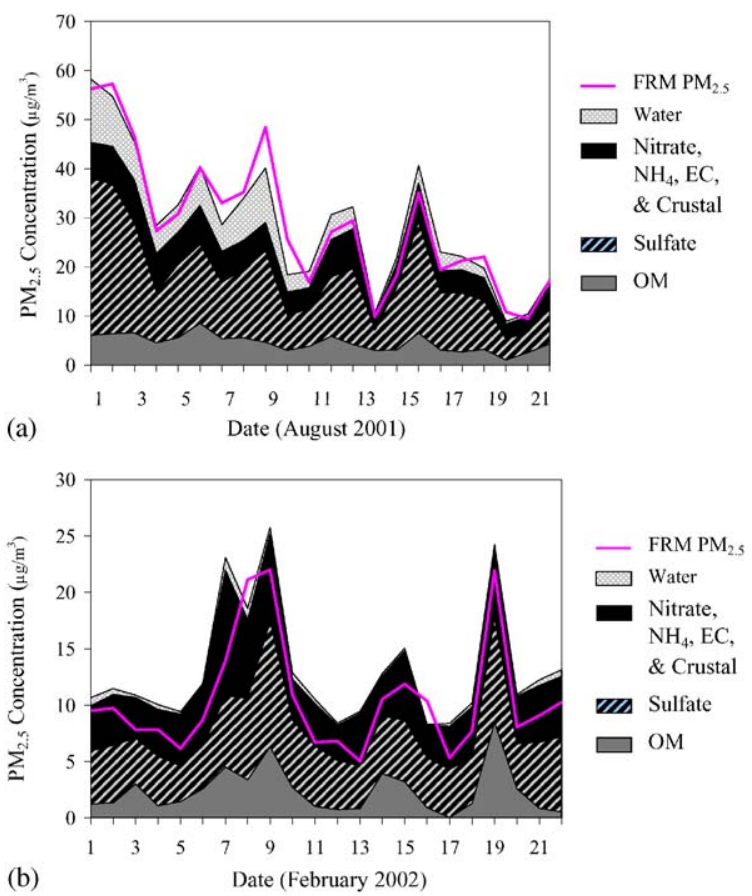

Fig. 9. Time series daily mass balances with estimated water content for (a) August 2001, and (b) February 2002. Water estimated from DAASS measurements extrapolated to a $35 \%$ $\mathrm{RH}$ to reflect FRM filter conditioning. OM is defined as $1.8 \times$ OC.

RH points was fitted using Eq. (4) by plotting all DAASS growth factor data to find coefficient $A$. The amount of water at $35 \% \mathrm{RH}$ was then estimated using Eq. (4) on an hourly basis.

It should be noted that the water content estimated by Eq. (4) is an upper limit estimate for the amount of water that could be retained on the filter after conditioning. The DAASS measures the water content of particles that are suspended in air. At low RH, such as that during filter weighing, any amount of water associated with the aerosol is in a state of a supersaturated salt solution. Contact with the filter surface or with an insoluble particle collected on the filter may initiate crystallization which would lead to a partial or a complete loss of water from the filter.

Water estimates from the DAASS measurements are included in daily and high time resolved mass balances shown in Figs. 8 and 9. The estimated aerosol water content fits well with periods of positive mass balance discrepancy on an hourly basis (Fig. 8). The fit is not perfect, for example the morning of 21 July $(\sim 30 \mathrm{~h}$ in Fig. 8) when there is a significant negative mass balance discrepancy; however, these periods correspond to times with relatively high OC concentrations, during which volatilization losses may be more significant than 
aerosol water. Fig. 9a shows that there is a significant amount of estimated water present in August, which fills in the periods of mass discrepancy. This estimate of water, at an average of $3.9 \mu \mathrm{g} \mathrm{m}^{-3}(16 \%)$, closes the mass balance within measurement uncertainty during the summer months. Fig. 9b shows that there is almost no water present in February, corresponding to the overall negative mass discrepancy observed during that month.

The data indicate a seasonal variation in the contribution of water to the mass balance discrepancy. The positive discrepancy due to water is the largest during acidic conditions in the summer, and relatively small during the neutral conditions found in the winter. The lower water content of the winter aerosol is thermodynamically expected because atmospheric conditions tend to be more neutral in Pittsburgh during the winter months.

\subsubsection{Sampling losses of semi-volatile species}

Loss of semi-volatile aerosol species due to evaporation from FRM filters during sampling and postsampling filter handling can cause a negative mass discrepancy. The two primary species of issue are nitrate and organics. Volatilization of organic material is a concern throughout the year, whereas volatilization of nitrate tends to be more significant in the winter months, when nitrate constitutes a greater proportion of $\mathrm{PM}_{2.5}$ mass.

The volatilization loss of nitrates was estimated using data from the CMU inorganic sampler. The sampler consists of a denuder followed by a filter pack containing a Teflon filter followed by a nylon filter. The nylon filter captures the nitrate that volatilizes off the Teflon filter during sampling. Since the filter pack is downstream of a denuder, the nitrate volatilization estimate represents an upper bound for losses during sampling. However, this estimate for nitrate volatilization does not account for nitrate losses that occur after sampling, such as losses during filter handling and conditioning, which are likely to be significant.

During the summer months, $80-90 \%$ of the particulate nitrate present evaporated from the Teflon filter used in the CMU inorganic sampler. This loss represents approximately $0.5 \mu \mathrm{g} \mathrm{m}^{-3}$ or $2 \%$ of FRM mass and therefore does not significantly impact the mass balance discrepancy. During the winter months, $20-30 \%$ of particulate nitrate volatilized from the Teflon filter used in the CMU inorganic sampler. Because nitrates contribute significantly to $\mathrm{PM}_{2.5}$ mass in the winter months in Pittsburgh, this loss, approximately $1.8 \mathrm{\mu g} \mathrm{m}^{-3}$ or $15 \%$ of FRM mass, is a significant contributor to the negative discrepancy observed in the winter.

Evaporation of organic material tended to occur consistently throughout the year. Subramanian et al.
(2004) estimated that on average $19 \%$ of the OC volatilized from Teflon filters during sampling at the PAQS site. Again, this volatilization estimate represents only that which occurs during sampling, not accounting for additional losses expected during filter handling and conditioning. Over the 7-month period evaluated, this volatilization artifact amounts to $0.7 \mu \mathrm{g} \mathrm{m}^{-3}$, or $4 \%$ of FRM $\mathrm{PM}_{2.5}$ mass, and remained relatively constant throughout the year. The largest OC volatilization artifact was observed in Fall 2001, where the artifact was approximately $1 \mu \mathrm{g} \mathrm{m}^{-3}$, or just over $6 \%$ of the FRM mass.

These results illustrate seasonal variation in the volatilization losses. In the summer months, OC losses are more important than nitrate losses, with the total volatilization losses amounting to $5 \%$ of FRM mass. In the winter months, nitrate losses rise to $15 \%$ of FRM mass which in combination with the OC losses (3\% of FRM mass) representative a significant negative discrepancy. Therefore, there is a strong seasonal variation to the relative importance of the volatilization losses.

\subsubsection{Uncertainty from estimation of the organic $P M$ concentrations from the OC measurements}

Uncertainty in the $\mathrm{OC}$ multiplier used to estimate organic mass alone cannot explain the observed mass balance discrepancy. For example, during the episodes of significant positive discrepancy in August 2001, the OC multiplication factor required to close the mass balance is 3.5 for the 31 July -4 August period, and 4.4 for the 7-11 August period. Accounting for expected losses of nitrate and organics due to volatilization, increases the $\mathrm{OC}$ multiplication factor required to close the mass balance during those periods to 4.1 and 5.2, respectively. These values are significantly above the range considered appropriate for urban aerosol (Turpin and Lim, 2001); therefore, the OC multiplier value is the not the cause the periods of significant positive mass balance discrepancy. (In the winter the OC multiplication factor is within the upper range of acceptable values, after accounting for volatilization.)

\subsubsection{Uncertainty from estimation of crustal components}

The sum of the oxides method is the most common method used to estimate the crustal component of $\mathrm{PM}_{2.5}$ mass (Andrews et al., 2000). The actual crustal contribution can deviate from this estimate due to underlying assumptions for the estimate or analytical uncertainty; for example, all the metals may not be in the form of oxides. Andrews et al. (2000) concluded that the estimate from the sum of component method represents the lower bound of the crustal estimate, underestimating the crustal component by as much as $50 \%$. 
A second issue is that we only have data to estimate the daily crustal contribution for July 2001 and January 2002. As previously discussed, for the other periods we assumed a crustal contribution of $1 \mu \mathrm{g} \mathrm{m}^{-3}$, the average value for the periods for which we have complete set of trace element data. Using an assumed, constant value does not cause the mass balance discrepancy for the periods for which we do not have crustal data. First, the day-to-day variations in the crustal $\mathrm{PM}_{2.5}$ were relatively small; the standard deviation of $\mathrm{PM}_{2.5}$ crustal for the 54 days for which we have a complete set of data is $0.4 \mu \mathrm{g} \mathrm{m}^{-3}$, less than the uncertainty assigned to the crustal component. Second, even the largest measured

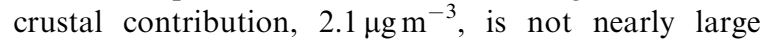
enough to close the mass balance for periods such as that shown in Fig. 6. Finally, it is unlikely that using an average value for crustal would cause the persistent negative mass balance discrepancy observed in February 2002 (see Fig. 6).

There are clearly large uncertainties regarding the potential contribution of the crustal component; however, the crustal material in Pittsburgh is a relatively small fraction of overall $\mathrm{PM}_{2.5}$ mass. This large uncertainty has been incorporated into the uncertainty analysis (Table 1), and the uncertainty in the crustal estimate alone would not be sufficient to close the observed mass balance discrepancy on days with significant positive discrepancies.

\subsection{Estimation of bound water by aerosol thermodynamic models}

Thermodynamic aerosol models are available that estimate ambient aerosol water using aerosol composition data, ambient temperature and RH. Since aerosol composition data are often available modeling aerosol water could provide a way to estimate aerosol water effects in other areas. Evaluating the suitability of these models to estimate the aerosol bound water is important because many monitoring networks do not have the sophisticated instrumentation like the DAASS.

We used the GFEMN model (Ansari and Pandis, 1999) with PAQS speciation data to compare modeled aerosol water results to those measured by the DAASS (Fig. 10). The GFEMN estimates compare well with the DAASS measured water. Overall for the summer, the DAASS measured an average of $3.8 \mu \mathrm{g} \mathrm{m}^{-3}$, while the GFEMN results were $13 \%$ lower at $3.3 \mu \mathrm{g} \mathrm{m}^{-3}$ of water. In January, modeled results are a little greater than those measured, averaging $1.3 \mu \mathrm{g} \mathrm{m}^{-3}$ while measured results average $0.8 \mu \mathrm{g} \mathrm{m}^{-3}$. Based on these results, the GFEMN model provides reasonable estimates of bound aerosol water. Using this model in conjunction with measured volatilization effects allows further examination of the mass discrepancy effect in other areas.
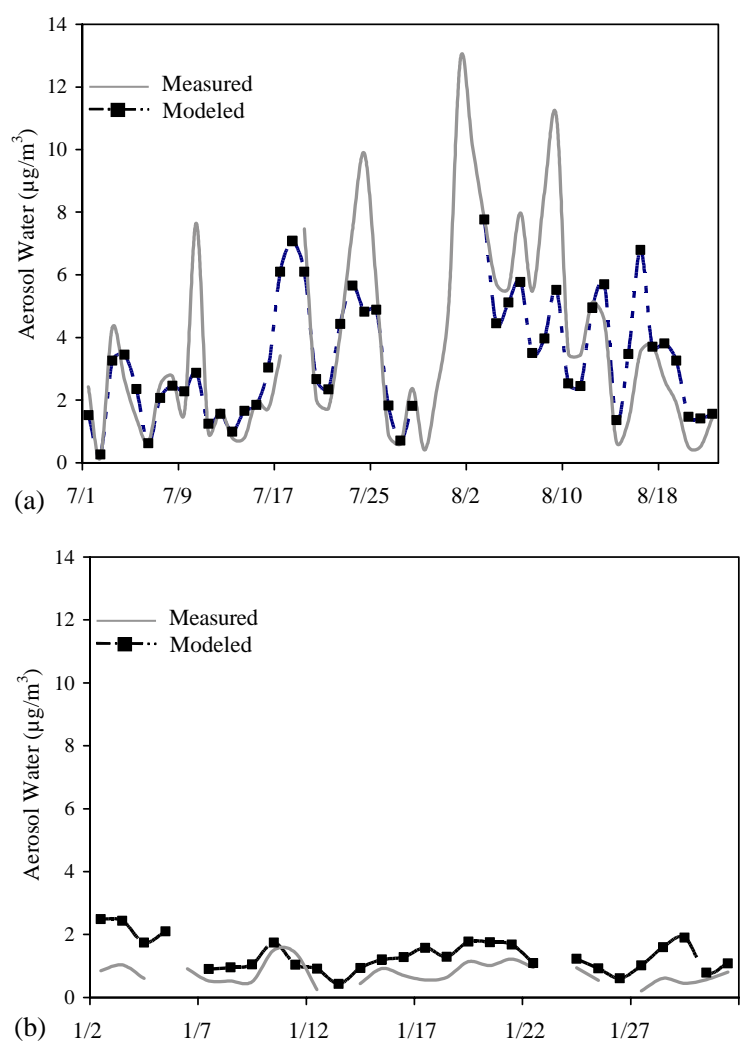

Fig. 10. Comparison of measured and predicted daily average aerosol water for (a) summer 2001 and (b) January 2002. Measured water results are based on DAASS measurements extrapolated to $35 \% \mathrm{RH}$, and the predicted results are from the GFEMN model using aerosol composition data. Results shown are at $35 \% \mathrm{RH}$.

\subsection{Closing the mass balance}

Our analysis suggests that the FRM mass can be estimated by summing measurements of individual chemical components, adding estimated aerosol water and subtracting volatilization effects. Using this approach we can reconstruct the FRM mass based on measurements of the individual $\mathrm{PM}_{2.5}$ components in the atmosphere. Results for the August episodes are summarized in Fig. 11a. Including water brings the mass balance within measurement uncertainty for the August episodes; eliminating the $19 \%$ positive discrepancy $\left(8.7 \mu \mathrm{g} \mathrm{m}^{-3}\right)$ for the 31 July-4 August period and reducing the $32 \%$ positive discrepancy $\left(10.5 \mu \mathrm{g} \mathrm{m}^{-3}\right)$ to an $8 \%$ positive discrepancy $\left(2.6 \mu \mathrm{g} \mathrm{m}^{-3}\right)$ for the $7-11$ August period. Water accounted for $8.3 \mu \mathrm{g} \mathrm{m}^{-3}$ or $18 \%$ of the FRM mass for the 31 July-4 August period, and $6.4 \mu \mathrm{g} \mathrm{m}^{-3}$ or $20 \%$ of the FRM mass for the $7-11$ August period. Volatilization losses had substantially less impact on the mass balance than aerosol water 

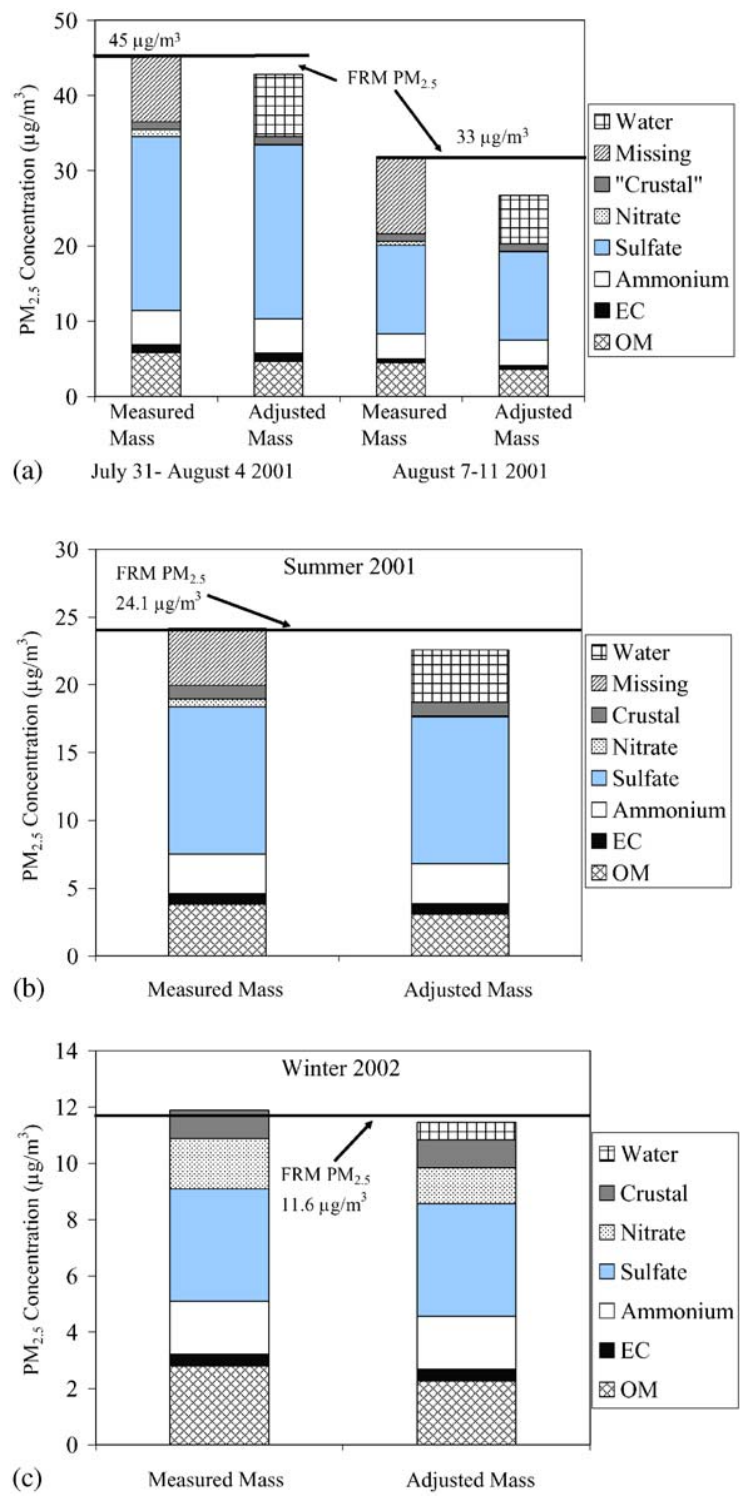

Fig. 11. Mass balances adjusted for water and volatilization. In the column labeled "adjusted mass", estimated water is added to sum of the chemical components, and measured nitrate and $\mathrm{OM}$ are corrected for volatilization losses. (a) Average data for two episodes in the summer of 2001. (b) Average daily mass balance for July and August 2001. (c) Average daily mass balance for January and February 2002. OM is defined as $1.8 \times$ OC.

during these periods, impacting $<2 \%$ of the FRMmeasured mass for these periods.

Closure of the mass balance for the summer averaged data is shown in Fig. 11b. The $17 \%$ positive discrepancy $\left(4.5 \mu \mathrm{g} \mathrm{m}^{-3}\right)$ disappears and the mass balance is within measurement uncertainty when adjusted for water and volatilization. Water accounts for $3.9 \mu \mathrm{g} \mathrm{m}^{-3}$ or $16 \%$ of the FRM mass, while volatilization losses account for $1.3 \mu \mathrm{g} \mathrm{m}^{-3}$ or $5 \%$ of total mass.

Fig. 11c shows similar success in accounting for the mass balance in the winter months. During the winter the average mass balance between the FRM and the sum of the chemical components was closed. In the winter, the loss of mass due to volatilization is offset by the gain of mass due to water; on average, volatilization losses were $1 \mu \mathrm{g} \mathrm{m}^{-3}(8.6 \%)$ and water contributed $0.9 \mu \mathrm{g} \mathrm{m}^{-3}$ $(7.8 \%)$ to the FRM mass.

Many studies that reconstruct an FRM mass balance do not observe the positive artifact in the summer months, and instead find a consistent negative artifact (e.g., Tanner and Parkhurst, 2000; Modey et al., 2001; Pang et al., 2002a,b). The presence of a significant negative discrepancy is dependent on aerosol composition. Studies conducted in the western US, where aerosol composition is dominated by nitrates and organics, suggest that FRM $\mathrm{PM}_{2.5}$ measurements may be up to $30 \%$ less than the reconstructed aerosol mass, with the losses resulting from volatilization of nitrates and organic species (Hering and Cass, 1999; Pang et al., $2002 \mathrm{a}, \mathrm{b})$. As previously discussed, we also observe significant volatilization losses of these species. However, the aerosol in Pittsburgh is dominated by sulfates, and in the summer it is often subjected to acidic atmospheric conditions, inducing hygroscopic behavior at low RH. These conditions result in a significant positive discrepancy between the FRM and the sum of the chemical components caused by water retained on the FRM filter after conditioning. This positive artifact due to water may be more of a regional phenomenon, more prevalent in the eastern US which is characterized by high sulfate levels and acidic conditions, than the western US. Indeed, data from the 1995 SEAVS experiment in Tennessee and from IMPROVE data taken in the southeast US show that up to $42 \%$ of $\mathrm{PM}_{2.5}$ mass was not accounted for by the measured chemical components, and that aerosol water was suspected to contribute significantly to that discrepancy (Andrews et al., 2000; Jansen et al., 2002).

\section{Summary and conclusions}

FRM measurements of $\mathrm{PM}_{2.5}$ mass are used for determining compliance with the recently revised National Ambient Air Quality Standards. In this context, it is important to understand what the FRM is measuring and how this relates to atmospheric $\mathbf{P M}_{2.5}$ concentrations. Episodes of high $\mathrm{PM}_{2.5}$ mass in August 2001 left around $19-32 \%$ of the mass measured by the FRM unaccounted for by summing the mass of the chemical components. Overall for the summer 2001, approximately $17 \%$ of the mass measured by the FRM 
was not accounted for by the sum of the mass of the chemical components. This positive mass discrepancy observed is greater than the estimated measurement uncertainty. The winter average FRM mass was $11.6 \mu \mathrm{g} \mathrm{m}^{-3}$, roughly equal to the mass of the sum of the aerosol chemical components.

Bound water remaining on the FRM filter can explain most of the observed positive discrepancy in the summer. Water is present when the inorganic PM component is dominated by ammonium bisulfate, which does not effloresce at the $\mathrm{RH}$ of filter conditioning. The presence of bound water occurs simultaneously with losses of nitrates and organics from sampling volatilization. Accounting for these positive and negative artifacts closes the mass balance.

Both water and volatilization effects occur simultaneously throughout the year in western Pennsylvania, with relative importance of each effect varying seasonally. In the summer months, contributions of water dominate, resulting in an overall positive discrepancy. In the winter, volatilization effects become more pronounced, resulting in a negative or net balance. In the summer months, the FRM measured on average $4.5 \mathrm{~g} \mathrm{~m}^{-3}(17 \%)$ more mass than the sum of the chemical components. Water accounted for $3.9 \mu \mathrm{g} \mathrm{m}^{-3}$ $(16 \%)$ of the FRM mass, while volatilization losses are estimated to reduce the FRM mass by $1.3 \mu \mathrm{g} \mathrm{m}^{-3}(5 \%)$. Accounting for these artifacts closes the mass balance between the FRM and the sum of the chemical components, and the large contribution of water during the summer appears related to acidic conditions that favor retention of water at low relative humidity. In the winter months there is on average no mass balance discrepancy; with the contribution of water $\left(0.9 \mu \mathrm{g} \mathrm{m}^{-3}\right)$ offsetting volatilization losses $\left(1 \mu \mathrm{g} \mathrm{m}^{-3}\right)$. Although the mass balance closed on average in the winter, the negative discrepancy occurred more frequently than the positive discrepancy. The seasonal variation in the mass balance discrepancy is directly attributable to changes in aerosol composition. The application of aerosol thermodynamic models, such as GFEMN, provides a method to estimate bound aerosol water from chemical speciation data.

\section{Acknowledgements}

This research was conducted as part of the Pittsburgh Air Quality Study which was supported by US Environmental Protection Agency under contract R82806101 and the US Department of Energy National Energy Technology Laboratory under contract DEFC26-01NT41017. This paper has not been subject to EPA's peer and policy review, and therefore does not necessarily reflect the views of the Agency. No official endorsement should be inferred.

\section{References}

Allen, G., Sioutas, C., Koutrakis, P., Reiss, R., Lurmann, F., Roberts, T., 1997. Evaluation of the TEOM ${ }^{\circledR}$ method for measurement of ambient particulate mass in urban areas. Journal of the Air and Waste Management Association 47, 682-689.

Anderson, R.R., Martello, D.V., Rohar, P.C., Starzisar, B.R., Tamilia, J.P., Waldner, K., White, C.M., Modey, W.K., Mangelson, N.F., Eatough, D.J., 2002. Sources and Composition of $\mathrm{PM}_{2.5}$ at NETL in Pittsburgh during July and August 2000. Energy and Fuels 16, 261-269.

Andrews, E., Saxena, P., Musarra, S., Hildemann, L.M., Koutrakis, P., McMurray, P.H., Olmez, I., White, W.H., 2000. Concentration and composition of atmospheric aerosols from the 1995 SEAVS and a review of the closure between chemical and gravimetric measurements. Journal of the Air and Waste Management Association 50, 648-664.

Ansari, A.S., Pandis, S.N., 1999. Prediction of multicomponent inorganic atmospheric aerosol behavior. Atmospheric Environment 33, 745-757.

Cabada, J.C., Pandis S.N., Robinson, A.L., Subramanian, R., Polidori, A., Turpin, B.J., 2004. Estimating the secondary organic aerosol contribution to $\mathrm{PM}_{2.5}$ using the $\mathrm{OC}$ to EC ratio method. Aerosol Science and Technology, in press.

Chow, J.C., Watson, J.G., Lowenthal, D.H., Solomon, P.A., Magliano, K.L., Ziman, S.D., Richards, L.W., 1993. PM 10 and $\mathrm{PM}_{2.5}$ compositions in California's San Joaquin valley. Aerosol Science and Technology 18, 105-128.

Hand, J.L., Ames, R.B., Kreidenweis, S.M., 2000. Estimates of particle hygroscopicity during the southeastern aerosol and visibility study. Journal of the Air and Waste Management Association 50, 677-685.

Hering, S., Cass, G., 1999. The magnitude of bias in measurement of $\mathrm{PM}_{2.5}$ arising from volatilization of particulate nitrate from Teflon filters. Journal of the Air and Waste Management Association 49, 725-733.

Jansen, J.J., Edgerton, E.S., Hansen, D.A., Hartsell, B.E., 2002. Sampling artifacts in the Federal Reference Method for $\mathrm{PM}_{2.5}$. www.atmospheric-research.com.

Khlystov, A., Wyers, G.P., Slanina, J., 1995. The steam-jet aerosol collector. Atmospheric Environment 29, 2229-2234.

Khlystov, A., Stanier, C., Pandis, S., 2004. Water content of ambient aerosol during Pittsburgh Air Quality Study. Journal of Geophysical Research, submitted for publication.

Malm, W.C., Gebhard, K.A., Molenar, J., Cahill, T., Eldred, R., Huffman, D., 1994a. Examining the relationship between atmospheric aerosols and light extinction at Mount Rainier and North Cascades National Parks. Atmospheric Environment 28, 247-260.

Malm, W.C., Sisler, J.F., Huffman, D., Eldred, R.A., Cahill, T.A., 1994b. Spatial and seasonal trends in particle concentration and optical extinction in the United States. Journal of Geophysical Research 99, 1347-1370.

Meng, Z., Seinfeld, J.H., Saxena, P., Kim, Y.P., 1995. Contribution of water to particulate mass in the South Coast Air Basin. Aerosol Science and Technology 22, 111-123.

Modey, W.K., Pang, Y., Eatough, N.L., Eatough, D.J., 2001. Fine particulate $\left(\mathrm{PM}_{2.5}\right)$ composition in Atlanta, USA: 
assessment of the particle concentrator-Brigham Young University organic sampling system, PC-BOSS, during the EPA supersite study. Atmospheric Environment 35, 6493-6502.

NIOSH, 1998. Method 5040 Issue 3 (Interim): elemental carbon (diesel exhaust). In: NIOSH Manual of Analytical Methods. National Institute of Occupational Safety and Health, Cincinnati, OH; http://www.cdc.gov/niosh/nmam/pdfs/ 5040f3.pdf.

Pang, Y., Eatough, N.L., Eatough, D.J., 2002a. PM 2.5 semivolatile organic material at riverside, California: implications for the $\mathrm{PM}_{2.5}$ Federal Reference Method sampler. Aerosol Science and Technology 36, 277-278.

Pang, Y., Eatough, N.L., Wilson, J., Eatough, D.J., 2002 b. Effect of semivolatile material on $\mathrm{PM}_{2.5}$ measurement by the $\mathrm{PM}_{2.5}$ Federal Reference Method sampler at Bakersfield, California. Aerosol Science and Technology 36, 289-299.

Speer, R.E., Barnes, H.M., Brown, R., 1997. An instrument for measuring the liquid content of aerosols. Aerosol Science and Technology 27, 50-61.

Subramanian, R., Khlystov, A.Y., Cabada, J.C., Robinson, A.L., 2004. Positive and negative artifacts in particulate organic carbon measurements with denuded and undenuded sampler configurations. Aerosol Science and Technology, in press.

Takahama, S., Wittig, B., Vayenas, D., Davidson, C.I., Pandis, S.N., 2004. Modeling the diurnal variation of nitrate during the Pittsburgh Air Quality Study. Journal of Geophysical Research, in press.
Tang, W., Raymond, T., Wittig, B., Davidson, C.I., Pandis, S.N., Robinson, A.L., Crist, K., 2004. Spatial variations in $\mathrm{PM}_{2.5}$ during the Pittsburgh Air Quality Study. Aerosol Science and Technology, in press.

Tanner, R.L., Parkhurst, W.J., 2000. Chemical composition of fine particles in the Tennessee Valley Region. Journal of the Air and Waste Management Association 50, 1299-1307.

Tolocka, M.P., Solomon, P.A., Mitchell, W., Norris, G.A., Gemmill, D.B., Wiener, R.W., Vanderpool, R.W., Homolya, J.B., Rice, J., 2001. East versus west in the US: chemical characteristics of $\mathrm{PM}_{2.5}$ during the winter of 1999. Aerosol Science and Technology 34, 88-96.

Turpin, B., Lim, H., 2001. Species contributions to $\mathrm{PM}_{2.5}$ mass concentrations: revisiting common assumptions for estimating organic mass. Aerosol Science and Technology 35, 602-610.

Turpin, B., Saxena, P., Allen, G., Koutrakis, P., McMurray, P.H., Hildemann, L., 1997. Characterization of the southwestern desert aerosol, Meadview, AZ. Journal of the Air and Waste Management Association 47, 344-356.

Wittig, B., Takahama, S., Khylstov, A., Pandis, S.N., Hering, S., Kirby, B., Davidson, C., 2004a. Semi-continuous PM $_{2.5}$ inorganic composition measurements during the Pittsburgh Air Quality Study. Atmospheric Environment, this issue, doi:10.1016/j.atmosenv.2004.03.002.

Wittig, B., Anderson, N., Khlystov, A.Y., Pandis, S.N., Davidson, C.I., Robinson, A.L., 2004b. Pittsburgh Air Quality Study overview. Atmospheric Environment, this issue, doi:10.1016/j.atmosenv.2004.03.003. 


\title{
Local and Regional Secondary Organic Aerosol: Insights from a Year of Semi-Continuous Carbon Measurements at Pittsburgh
}

\author{
Andrea Polidori, ${ }^{1}$ Barbara J. Turpin,, ${ }^{1,2}$ Ho-Jin Lim,,${ }^{1, \dagger}$ Juan C. Cabada,,${ }^{3, \dagger}$ \\ Ramachandran Subramanian, ${ }^{4, \dagger \dagger}$ Spyros N. Pandis, ${ }^{3, \dagger \dagger \dagger \dagger}$ and Allen L. Robinson ${ }^{4}$ \\ ${ }^{1}$ Department of Environmental Sciences, Rutgers University, New Brunswick, New Jersey, USA \\ ${ }^{2}$ Rutgers Cooperative Extension, New Brunswick, New Jersey, USA \\ ${ }^{3}$ Department of Chemical Engineering, Carnegie Mellon University, Pittsburgh, Pennsylvania, USA \\ ${ }^{4}$ Department of Mechanical Engineering, Carnegie Mellon University, Pittsburgh, Pennsylvania, USA
}

During the Pittsburgh Air Quality Study (PAQS) an automated semi-continuous thermal-optical transmittance (TOT) carbon analyzer was used to measure $2-4 \mathrm{~h}$ average particulate organic (OC) and elemental carbon (EC) concentrations from July 1, 2001 to August 13, 2002. To minimize the adsorption of vapor-phase organics, the sample air was drawn through a multi-channel parallelplate diffusion denuder placed upstream of the carbon analyzer. Particulate $\mathrm{OC}$ and EC in the sample air were then collected on a quartz fiber filter (QFF) mounted inside the carbon analyzer, and analyzed immediately after collection. To account for any remaining organic vapors not retained by the denuder and collected on the sampling filter (positive artifact) a dynamic blank was run every two weeks. An upper-bound estimate of volatilization induced by the presence of the denuder upstream of the sampling filter (negative artifact) was also made. A detailed description of the operating protocol and quality assurance measurements is provided. The contributions of primary and secondary organic aerosol (SOA) to particulate OC were calculated using an "EC tracer method," which is

Received 1 June 2005; accepted 20 March 2006.

We gratefully acknowledge the hospitality and assistance of our Carnegie Mellon University hosts. We appreciated the encouragement of PAQS' neighbor, Mr. Fred Rogers, and wish to thank David Smith and Bob Cary at Sunset Labs for their continued assistance. This work was supported in part by the US Department of Energy, US Environmental Protection Agency, and the NJ Agricultural Experiment Station. This research has not been subjected to Agency review and therefore does not necessarily reflect the views of DOE or EPA; no official endorsement should be inferred.

${ }^{\dagger}$ Currently at: Department of Environmental Engineering, Kyungpook National University, Korea.

${ }^{\dagger \dagger}$ Currently at: Department of Chemical Engineering, Tecnológico de Monterrey, Monterrey, Mexico.

${ }^{\dagger \dagger}$ Currently at: Department of Civil and Environmental Engineering, University of Illinois, Urbana-Champaign, IL.

${ }^{\dagger \dagger \dagger}$ Currently at: Department of Chemical Engineering, University of Patras, Patras, Greece.

Address corresponding to Barbara J. Turpin, Department of Environmental Sciences, 14 College Farm Rd., New Brunswick, NJ 08901, USA. E-mail: turpin@aesop.rutgers.edu codified herein. Annual average SOA accounted for $33 \%$ of particulate $\mathrm{OC}$. SOA accounted for 30-40\% of monthly average $\mathrm{OC}$ from June to November in Pittsburgh, similar to previous summertime estimates for Atlanta (Lim and Turpin 2002) and much larger than previous estimates of SOA in the Los Angeles Basin (Turpin and Huntzicke 1995). Examination of concentration dynamics suggests that multi-day formation and regional transport is an important contributor to the higher SOA contributions to OC in Pittsburgh and suggests that SOA is likely to be a particularly important contributor to particulate $\mathrm{OC}$ in locations that are recipients of long distance transport, such as the eastern United States.

\section{INTRODUCTION}

Carbonaceous material (total carbon; TC) is a major constituent (10-70\%) of atmospheric particulate matter (PM) and a substantial contributor to visibility reduction, climate forcing, and adverse health effects (IPCC 2004; U.S. EPA 2004). Atmospheric carbonaceous PM consists of organic (OC) and elemental carbon (EC) and includes hundreds of organic species with widely varying chemical and physical properties (Turpin et al. 2000). OC is directly emitted in particulate form (primary) and formed in the atmosphere from semi- and low-volatility products of chemical reactions involving reactive organic gases (secondary; Seinfeld and Pandis 1998; Turpin et al. 2000). EC is produced during incomplete combustion and emitted directly in the particle phase.

Development of effective control strategies for ambient PM requires an understanding of the contributions of primary and secondary OC. Smog chamber experiments have extended our understanding of secondary organic aerosol (SOA) formation mechanisms (Odum et al. 1997; Jang and Kamens 2001). However, there remains a need to estimate primary and secondary OC from field measurements, to test evolving predictive models and to quantify contributions in locations where key model inputs are not available. SOA concentrations have been estimated from 
measured OC and EC concentrations using EC as a tracer for primary OC ("EC tracer method"; e.g., Turpin and Huntzicker 1995; Strader et al. 1999; Cabada et al. 2004). SOA concentrations have also been predicted using models that couple the formation, transport, and deposition of SOA with atmospheric dynamics (e.g., Strader et al. 1999), and estimated from receptor model results by subtracting the sum of primary source contributions from measured OC concentrations (e.g., Schauer et al. 1996; Schauer and Cass 2000). These three approaches have provided reasonably comparable estimates of SOA in the few locations where comparisons have been made (Turpin and Huntzicker 1995; Strader et al. 1999). However, in most locations outside of southern California the primary and secondary contributions to OC are still largely unknown.

Previous studies have shown that Pittsburgh is a location influenced by SOA production in every period of the year and one where atmospheric conditions and SOA precursor emissions are substantially different from those in southern California (Cabada et al. 2002). This article analyzes in detail semi-continuous OC and EC measurements obtained during the Pittsburgh Air Quality Study (PAQS), provides a codified "EC tracer method," and uses this method to estimate the primary and secondary contributions to OC at Pittsburgh. Because SOA is best estimated with time-resolved measurements and semi-continuous carbon measurement is only now becoming routine, detailed carbon measurement and quality control protocols are provided and discussed. Concentration dynamics are used to provide insights regarding local and regional sources and atmospheric processing.

\section{EXPERIMENTAL}

An automated semi-continuous thermal-optical transmittance (TOT) carbon analyzer (Sunset Laboratory, Beaverton, $\mathrm{OR}$ ) was used to measure $2-4 \mathrm{~h}$ average particulate organic (OC) and elemental (EC) carbon concentrations during the Pittsburgh Air Quality Study (PAQS), from July 1, 2001 to August 13, 2002. This instrument is based on the OGI/Rutgers instrument described elsewhere (Turpin et al. 1990). Instrument performance was previously characterized by Lim et al. (2003a). The field instrument was located inside a climate-controlled trailer on the top of a hill in Schenley Park, near the Carnegie Mellon University campus and about three miles east of downtown Pittsburgh (Wittig et al. 2004). The site was more than $500 \mathrm{~m}$ from any major road and was not near any major industrial sources.

During sample collection, ambient air (16.7 L/min) was drawn through a $2.5 \mu \mathrm{m}$ aerodynamic diameter cut point cyclone and the flow was isokinetically split between the semicontinuous carbon analyzer $(8.7 \mathrm{~L} / \mathrm{min})$ and two auxiliary sampling lines, each pulling $4 \mathrm{~L} / \mathrm{min}$. During July 2001 each auxiliary sampling line contained a baked $47 \mathrm{~mm}$ diameter quartz fiber filter (QFF; QAT-UP, Pall Gelman, Ann Arbor, MI); these were used to conduct volatilization artifact experiments described below. A multi-channel parallel-plate diffusion denuder was placed horizontally upstream of the semi-continuous carbon analyzer to reduce the adsorption of vapor-phase organics on the instrument's sampling filter. The denuder contained 14 strips $\left(8^{\prime \prime} \times 1.25^{\prime \prime}\right)$ of carbon-impregnated filter (CIF; Schleicher Schuell, Keene, NH) spaced at $2 \mathrm{~mm}$ intervals inside an aluminum housing. The semi-continuous carbon analyzer collects particles on a $16 \mathrm{~mm}$ diameter QFF punch mounted inside the instrument and analyzes each sample automatically immediately after collection with no sample handling. Eliminating sample handling and storage lowers the instrument detection limits and allows for better time resolution. The carbon analyzer sample collection time was 1.5-3.5 h, yielding collection/analysis cycles of 2-4 h.

Thermal-optical analysis defines $\mathrm{OC}$ as the material that volatilizes in the absence of oxygen, whereas EC requires oxygen to combust. Table 1 provides the temperature protocol for semi-continuous carbon analysis during PAQS, and Figure 1 shows a typical thermogram. The protocol was designed to be comparable to the proposed EPA Speciation Trends Network (STN) protocol. The same protocol was used during the Aerosol Characterization Experiment in Asia (ACE-ASIA study; Lim et al. 2003a). In a typical analysis, air is purged and the filter is heated in a helium (He) environment, stepwise, to $870^{\circ} \mathrm{C}$ to volatilize OC. The main oven temperature is then reduced; the carrier gas is changed to $2 \%$ oxygen $\left(\mathrm{O}_{2}\right)$ in $\mathrm{He}$, and the temperature is increased stepwise to $880^{\circ} \mathrm{C}$ to combust EC. In the final step of the analysis, a calibration gas ( $2 \%$ methane in $\mathrm{He})$ is automatically injected for quantitation. All carbon is converted to methane $\left(\mathrm{CH}_{4}\right)$ and detected with a flame ionization detector (FID). OC and EC are automatically quantified by dividing their peak areas by the internal calibration peak area and multiplying by the amount of carbon in the calibration peak. After analysis, the QFF is cooled to room temperature and sampling begins again.

While heating a sample in an oxygen-free environment some OC is pyrolitically converted to EC, reducing the transmittance

\section{TABLE 1}

Temperature protocol used by the semi-continuous carbon analyzer for the analysis of particulate OC and EC during PAQS

\begin{tabular}{lcc}
\hline Carrier gas & Temperature $\left({ }^{\circ} \mathrm{C}\right)$ & Duration $(\mathrm{sec})$ \\
\hline $\mathrm{He}$ & 340 & 60 \\
$\mathrm{He}$ & 500 & 60 \\
$\mathrm{He}$ & 615 & 60 \\
$\mathrm{He}$ & 870 & 90 \\
$\mathrm{He} / \mathrm{O}_{2}$ & 575 & 45 \\
$\mathrm{He} / \mathrm{O}_{2}$ & 650 & 45 \\
$\mathrm{He} / \mathrm{O}_{2}$ & 725 & 45 \\
$\mathrm{He} / \mathrm{O}_{2}$ & 800 & 45 \\
$\mathrm{He} / \mathrm{O}_{2}$ & 880 & 100 \\
\hline
\end{tabular}




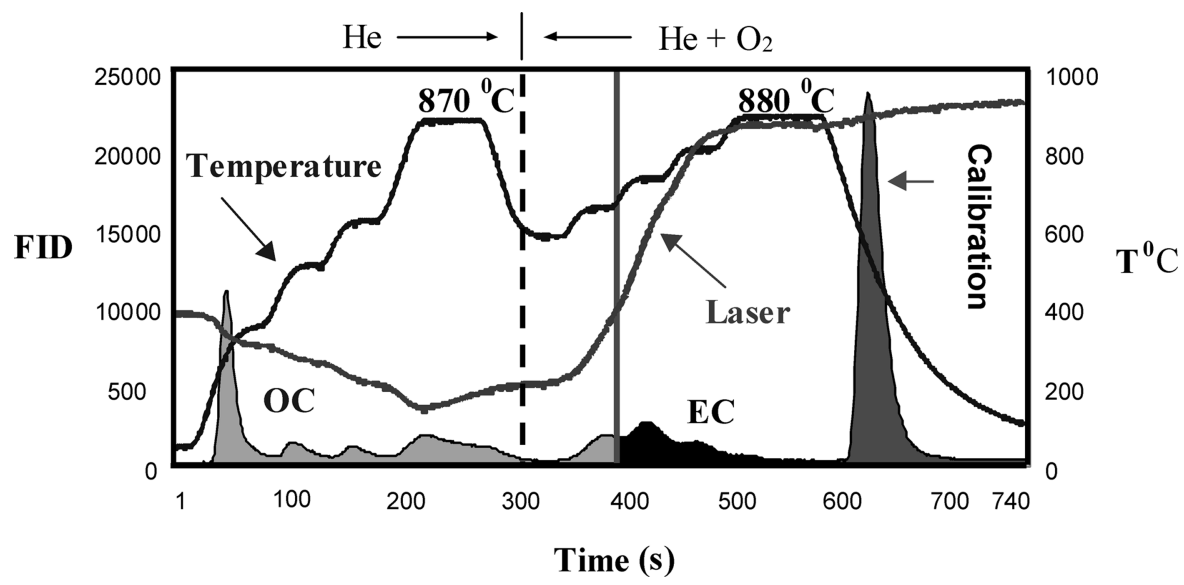

FIG. 1. Typical carbon analysis thermogram obtained by the semi-continuous carbon analyzer using the analysis protocol employed during PAQS. Shown are oven temperature $\left({ }^{\circ} \mathrm{C}\right.$ ), flame ionization detector (FID) signal (shaded area), and laser transmittance. OC, EC, and calibration segments of the FID signal are labeled. The dashed line indicates the introduction of $\mathrm{O}_{2}$ and the solid vertical line identifies the point at which the laser transmittance regains its pre-pyrolysis value (adjusted for the transit time). Material evolved before this point is considered OC and after, EC.

through the filter. Left uncorrected, pyrolysis can lead to substantial bias in $\mathrm{OC}$ and $\mathrm{EC}$ (but not $\mathrm{TC} ; \mathrm{TC}=\mathrm{OC}+\mathrm{EC}$ ). Therefore a diode laser and photodetector monitor the transmittance of light through the QFF during analysis to correct for the pyrolysis of OC. When oxygen is added, EC evolves increasing the transmittance. The carbon removed to bring the transmittance back to its pre-pyrolysis level is considered to be equal to the pyrolytically-generated EC. Thus, all carbon evolved before this point is reported as OC, and after is EC. The pyrolysis correction assumes either (1) that the original and pyrolytically generated EC have the same absorptivity or (2) that pyrolytically generated EC evolves first. While neither assumption is likely to be completely true, the error introduced is likely to be small relative to the size of the pyrolysis correction.

High purity gases and zero grade air (Matheson Gas Products, Montgomeryville, PA) were used. To remove trace amounts of $\mathrm{O}_{2}$, the $\mathrm{He}$ gas was purified through a series of two $\mathrm{O}_{2}$ traps (4002, 4004; Alltech, Deerfield, IL) before use. The $\mathrm{O}_{2}$ trap color indicator showed no color change during the study. All QFFs were pre-baked in a muffle furnace at $550^{\circ} \mathrm{C}$ for at least $2 \mathrm{~h}$ (auxiliary filters) or inside the main oven of the instrument at $870^{\circ} \mathrm{C}$ for about 4 minutes before collection. The semi-continuous carbon analyzer QFF was replaced every 2-3 days.

\section{QUALITY CONTROL}

\section{Quality Control Analyses}

Between July 1, 2001 and August 13, 2002 the semicontinuous carbon analyzer was collecting or analyzing samples $80 \%$ of the time. (No data were collected September 4-October 11, 2001 due to instrument malfunction and an air traffic moratorium after September 11). Quality control measurements performed during PAQS are summarized in Table 2 and described below.
An instrument blank was run daily to check for system contamination and any dependence of the laser signal on oven temperature. The instrument blank analytical settings were identical to those used for normal sample analyses, except the instrument was operated with a "zero-second" sampling time. The average instrument blank was $0.1 \pm 0.11(1 \sigma)$ and $0.01 \pm 0.03 \mu \mathrm{g} \mathrm{C}$ for OC and EC, respectively.

A dynamic blank was run every two weeks to quantify adsorption of any remaining organic vapors (i.e., not retained by the denuder) on the sampling filter (positive artifacts; Turpin et al. 2000). To collect a dynamic blank, a $47 \mathrm{~mm}$ Teflon filter ( $2 \mu \mathrm{m}$ pore, PallGelman, Ann Arbor, MI) was placed upstream of the denuder so that particle-free ambient air was sampled and analyzed (standard analysis protocol) on a 2 or $4 \mathrm{~h}$ collection/analysis cycle. Teflon filters employed for the dynamic blank analyses were used as provided by the manufacturer without any pretreatment. The study-average dynamic blank was $0.33 \pm 0.11 \mu \mathrm{g} / \mathrm{m}^{3}$ (mean $\pm 1 \sigma$ ), which corresponds to $16 \%$ of measured OC. Correction was made for this artifact (see Sampling Artifacts). The denuder material was replaced three

TABLE 2

Schedule of quality control (QC) activities performed during PAQS

\begin{tabular}{llc}
\hline QC activity & Frequency & Duration (min) \\
\hline Instrument blank & Daily & 25 \\
Dynamic blank & Biweekly & 35 \\
FID relative response & Biweekly & 25 \\
Standard injection & Monthly & 25 \\
Filter change & 2-3 Days & 15 \\
Flow rate check & Monthly & 25 \\
Denuder change & 4 months & 20 \\
\hline
\end{tabular}


times, approximately every four months. No significant change in dynamic blank OC was observed after replacing the denuder material.

To check for variability in the FID response over the course of an analysis a relative response factor analysis was run approximately every 2 weeks. During this analysis a known amount of calibration gas $\left(2 \% \mathrm{CH}_{4}\right.$ in $\left.\mathrm{He}\right)$ is automatically injected upstream of the $\mathrm{MnO}_{2}$ oven during the $\mathrm{He}$ and the $\mathrm{He}-\mathrm{O}_{2}$ analyses segments as well as during the usual calibration segment. The variability of FID sensitivity across an analysis was less than 5\% for more than $95 \%$ of the study (calibration peak/peak in Helium $=0.97 \pm 0.07$; calibration peak/peak in $\mathrm{He}-\mathrm{O}_{2}=0.98 \pm$ 0.08). For a small subset of data collected in March 2002, the responses in $\mathrm{He}$ and $\mathrm{He}-\mathrm{O}_{2}$ were 90 and $95 \%$, respectively, of the calibration response. These data were corrected for the change in FID response with carrier gas. The quality control measurements above suggest that instrument blank, dynamic blank and relative response factor analyses could have been performed as infrequently as monthly and still meet long term monitoring objectives. However, daily instrument blanks and relative response factor analyses can easily be programmed into the software and performed automatically to provide rapid feedback on instrument performance.

Internal calibration is performed as follows. A certified mixture of $\mathrm{CH}_{4}$ in He flows continuously through a loop of Teflon tubing inside the analyzer; this loop is switched on line for internal calibration. Therefore, in order to calculate the exact amount of methane injected it is important to know the calibration loop volume. This was measured right before the beginning of PAQS and in advance of the ACE-Asia Study (Lim et al. 2003a) by manually injecting different known volumes of calibration gas $\left(2 \% \mathrm{CH}_{4}\right.$ in $\mathrm{He}$ ) into the main oven with a $2 \mathrm{ml}$ gas-tight syringe during a standard analysis after "zero-second" collection. The loop volume was calculated by integrating the areas of the resulting peaks and comparing them to the internal calibration peak area. For this instrument the loop volume is $1.63 \mathrm{ml}$. For a calibration gas containing $2.1 \% \mathrm{CH}_{4}$ in $\mathrm{He}$ this corresponds to $17.08 \mu \mathrm{g}$ of carbon $(\mu \mathrm{g} \mathrm{C})$ in the internal calibration peak. Note, efficient conversion of carbon to $\mathrm{CO}_{2}$ and subsequently to $\mathrm{CH}_{4}$ is validated on set-up and would have been checked during trouble-shooting if deterioration of peak areas had been observed.

The laser signal responds immediately to the removal of EC from the filter, whereas the FID signal is delayed by the transit time between the sampling filter and the FID detector. Proper alignment of these two signals is essential for the determination of the correct OC-EC split. Changes in an instrument's transit time can occur, for example with replacement of packed columns or changes in plumbing. The transit time was determined prior the beginning of PAQS as the time interval between a rapid increase in optical transmittance with EC evolution and the corresponding increase in the FID signal with arrival of this material (now $\mathrm{CH}_{4}$ ) in the FID. The transit time was 5 seconds and did not change perceptibly over the course of the study.

\section{Measurement Uncertainties}

A manual injection of calibration gas $\left(2 \% \mathrm{CH}_{4}\right.$ in $\mathrm{He}$, main oven) was performed monthly, and actual and measured carbon mass were always within $\pm 5 \%$ (analytical accuracy). Measurement uncertainty for particulate $\mathrm{OC}$ and $\mathrm{EC}$ is also impacted by the accuracy of the sampling artifact correction, the accuracy of the pyrolysis correction, and flow rate variability. The flow rates of sample air and analysis gases were checked approximately monthly with a primary flow measurement device (Gilibrator). Measured and actual flow rates were always within $5 \%$. Since each analysis uses the entire sample, determination of the analytical precision by replicate analysis was not possible. However, the analytical precision is expected to be better than that of a laboratory carbon analyzer, which is roughly 4-13\% for OC and 6-21\% for EC (e.g., Schauer et al. 2003).

During PAQS, the detection limit for OC was $0.43 \mu \mathrm{gC}$ $\left(\sim 0.32 \mu \mathrm{gC} / \mathrm{m}^{3}\right)$, calculated as three times the standard deviation $(3 \sigma)$ of the dynamic blank OC. Since no dynamic blank EC was ever detected throughout the study, the detection limit for EC was calculated as $3 \sigma$ of the instrument blank EC, and was $0.15 \mu \mathrm{gC}\left(\sim 0.11 \mu \mathrm{gC} / \mathrm{m}^{3}\right)$. The OC detection limit for the study is higher than the instrument detection limit, i.e., despite the use of the denuder the detection limit is still dictated by variability in the dynamic blank. The OC detection limit could be reduced further by collecting a dynamic blank concurrently with each sample.

\section{Laser Transmittance}

The semi-continuous carbon-analyzer sampling filter is mounted inside the instrument and is typically used for multiple collection-analysis cycles. However, after about 12 hours of operation in Pittsburgh the accumulation of refractory materials such as iron oxide on the filter began to give the filter a red color, decreased the transmittance through the unloaded filter, and caused a temperature-dependent decrease in the transmittance through the filter at the highest temperature steps of the analysis $\left(870\right.$ and $\left.880^{\circ} \mathrm{C}\right)$. In some samples this temperaturedependent decrease in transmittance caused a delay in the time at which the transmittance regained its pre-pyrolysis value, overestimating the pyrolysis correction and, hence, OC. This phenomenon was also observed during the ACE-Asia study after more than 7 days of continuous operation (Lim et al. 2003a) but not during the Southern California Air Quality Study (SCAQS; Turpin and Huntzicker 1995). During SCAQS deposits of refractory materials were only sufficient to warrant a filter change after approximately one month of continuous operation. To prevent the introduction of errors due to this temperature dependence we normalized the laser signal intensity for each sample analysis (I) by that of the corresponding instrument blank $\left(\mathrm{I}_{\mathrm{o}}\right)$ and recalculated the OC-EC split for all PAQS measurements (i.e., in accordance with Beer's law). This resulted in an average $( \pm 1 \sigma)$ correction equivalent to $10 \pm 8 \%$ of $\mathrm{OC}$ when the filter was changed every three days. When analyses were conducted at 
PAQS with a top OC temperature of $700^{\circ} \mathrm{C}$ (rather than $870^{\circ} \mathrm{C}$ ), as was done for 40 study days, the temperature dependence was substantially reduced yielding an average OC correction of only $5 \pm 7 \%$. Thus, differences in behavior between locations are likely to be due both to differences in the concentration of refractory materials in fine particles and differences in carbon analysis temperature protocols. (The top OC temperature for SCAQS measurements was $700^{\circ} \mathrm{C}$.)

A more recent version of the Sunset laboratory calculation program now automatically corrects for the laser temperature dependence (version RT-Calc 114 or newer); PAQS OC and EC data corrected as described above are in excellent agreement with those recalculated using the Sunset laboratory automated correction software $\left(\mathrm{OC}: \mathrm{R}^{2}=0.99, \mathrm{Y}=1.01 \mathrm{X}+0.02\right.$; EC: $\mathrm{R}^{2}=0.98, \mathrm{Y}=0.96 \mathrm{X}-0.03$; where $\mathrm{Y}$ is the concentration calculated by RT_Calc114 and X is the concentration calculated manually).

The refractory loading combined with OC analytical temperatures higher than historically used in thermal-optical carbon analysis (i.e., $>700-750^{\circ} \mathrm{C}$ ) also appears to enable some EC to evolve at the highest temperature of the helium analysis segment $\left(870^{\circ} \mathrm{C}\right.$ ), prior to the intentional introduction of oxygen (Chow et al. 2001). The premature evolution of EC could affect the pyrolysis correction and the OC-EC determination, but only if the OC-EC split point were to occur before all OC was able to volatilize or if these conditions somehow affect the accuracy of the pyrolysis assumptions (Subramanian et al. 2006).

\section{Sampling Artifacts}

Organic vapors readily adsorb onto QFFs. Unless reduced or corrected for, this positive artifact can cause particulate $\mathrm{OC}$ to be substantially overestimated (i.e., 30-40\% in urban areas; Turpin et al. 1994, 2000; Mader and Pankow 2001). In this study the adsorption artifact was substantially reduced by placing a denuder upstream of the semi-continuous carbon analyzer. The diffusion of gas-phase material to the denuder carbon strips removes adsorbable vapors but also disturbs the gas-particle equilibrium of semi-volatile species. This could induce volatilization of organic particulate material from the sampling QFF. Therefore, two main issues must be considered: positive artifacts due to denuder breakthrough (estimated by dynamic blank analysis described above) and negative artifacts due to particulate matter volatilization from the sampling QFF. No EC was ever detected in a PAQS dynamic blank analysis (i.e., when sampling particlefree ambient air) ruling out EC contamination from the denuder's carbon strips.

Figure 2 shows the percentage of measured OC that was adsorbed vapor $[\%$ of adsorbed vapor $=($ dynamic blank OC/sample OC) $\times 100)$ ] for the semi-continuous carbon analyzer measurements made during PAQS, ACE-Asia (Lim et al. 2003a), and the Atlanta supersite study (Lim and Turpin 2002). The use of a denuder upstream of the carbon analyzer during PAQS and ACE-ASIA substantially reduced the adsorption

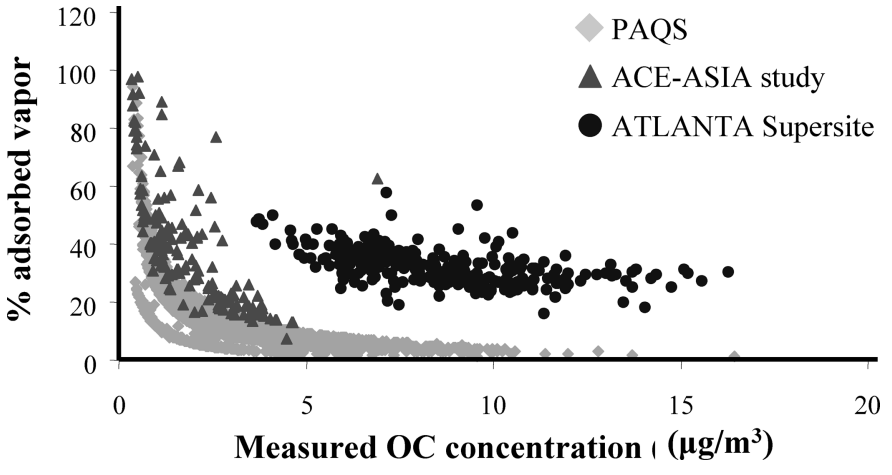

FIG. 2. Percentage of measured OC that is adsorbed vapor (\% adsorbed vapor $=$ dynamic blank OC / sample OC) and OC concentrations $\left(\mu \mathrm{gC} / \mathrm{m}^{3}\right)$ from the semi-continuous carbon analyzer. Shown are measurements made during the Pittsburgh Air Quality Study (PAQS), Aerosol Characterization Experiment (ACE-Asia), and the Atlanta Supersite study. ACE-Asia and PAQS used a denuder to reduce adsorption.

artifact in comparison to the undenuded measurements made in Atlanta. (Note that dynamic blanks were collected concurrently with each sample in Atlanta and subtracted on a sample-bysample basis to correct for the adsorption artifact.) For both denuded and undenuded systems the adsorption artifact is a larger percentage of measured $\mathrm{OC}$ for low OC loadings/concentrations than for high loadings/concentrations. During PAQS, the average adsorption artifact (dynamic blank) was $0.33 \pm 0.11 \mu \mathrm{g} / \mathrm{m}^{3}$ $(1 \sigma)$ or $16 \%$ of the OC measured on the carbon analyzer QFF. It was $33 \%$ for the Atlanta study, where an undenuded system was used, even though ambient $\mathrm{OC}$ concentrations were higher.

In previous organic artifact experiments conducted without a denuder we have observed an increase in the magnitude of the adsorption artifact with increased loading/concentration of filter-collected OC (e.g., Turpin et al. 1994), even though the percentage of the filter-collected OC that is artifact decreases with increased OC loading/concentration. This makes sense because (1) adsorption is expected to increase with increasing concentrations of organic gases based on equilibrium partitioning and (2) because atmospheric concentrations of organic gases and organic PM are likely to be covariant. However, in this study the magnitude and variability of the adsorption artifact was small and showed no dependence on measured OC. This is presumably because of the use of a denuder that removed adsorbable vapors with high efficiency.

Thus, PAQS OC measurements were corrected for the adsorption artifact to yield particulate OC concentrations as follows. Seasonal average $2 \mathrm{~h}$ and $4 \mathrm{~h}$ dynamic blank values were subtracted from OC measured on 2 and $4 \mathrm{~h}$ sampling/analysis cycles, respectively, to yield particulate OC concentrations. The adsorption estimate for the small number of $\mathrm{OC}$ measurements made on a $3 \mathrm{~h}$ cycle was estimated by interpolation.

Volatilization of particulate OC from the sampling QFF (negative artifact) could be induced by removal of vapor-phase OC with a denuder. During the first intensive period of PAQS a set 
of experiments was conducted to provide an upper bound estimate of the magnitude of this type of loss. Six pairs of $24 \mathrm{~h}$ integrated samples were collected on QFFs in two auxiliary sampling lines. Six "blow-off" experiments were conducted thereafter by placing a Teflon filter upstream of the denuder inlet, one $47 \mathrm{~mm}$ QFF loaded with particulate matter ("loaded" QFF) between the denuder and the semi-continuous carbon analyzer, and operating the instrument in "sampling mode" for 60 to 240 min. The Teflon filter and denuder remove particulate matter and most adsorbable organic vapors present in the sample air, allowing "clean" air to pass through the "loaded" QFF for time periods and at loadings comparable to those used for standard semi-continuous carbon analyzer sample collection. The QFF exposed to "clean" air and the concurrently collected "unexposed" QFF were both analyzed in a laboratory carbon analyzer (Sunset Laboratory, Beaverton, OR). The gaseous OC lost from the "loaded" QFF during these experiments evolved during the first temperature step of the OC-EC analysis (between 25 and $340^{\circ} \mathrm{C}$ ). Volatile losses from the "loaded" QFF were $10 \pm 6 \%$ $(1 \sigma)$ of the OC loading with no discernable trend with OC loading. Interestingly Subramanian et al. (2004) reported that volatile losses on an integrated denuded PAQS sampler were on the order of $10 \%$. The results from these experiments represent an upper-limit estimate of volatile losses from the semi-continuous carbon analyzer since the loading of particle-phase organics on the carbon analyzer filter normally increases from zero at $\mathrm{t}=0$, and the amount of OC volatilized from a "loaded" QFF is maximized when the filter is impacted by particle-free air. These findings support the assumption that during PAQS volatilization losses of OC from the semi-continuous carbon analyzer QFF (negative artifacts) were small.

\section{Assessment of Measurement Errors}

In summary, the adsorption artifact and the pyrolytic conversion of OC to EC during analysis can introduce substantial errors in the determination of OC and EC by thermal carbon analysis. Optical correction for pyrolysis (thermal-optical carbon analysis) and the use of a denuder reduce these errors substantially. The remaining adsorption artifact at PAQS was $16 \pm$ $13 \%\left(0.33 \pm 0.11 \mu \mathrm{g} / \mathrm{m}^{3}\right)$ and correction for this artifact could easily be made using periodic dynamic blank analyses. Volatile losses appear to be small $(<10 \pm 6 \%)$ but not well enough quantified to enable correction. Errors introduced into the pyrolysis correction by development of a temperature-dependent laser signal can be large in locations with high concentrations of refractory materials (e.g., Pittsburgh) unless the sample filter is changed every few days. At PAQS this error was reduced from $10 \pm 8 \%$ (mean $\pm 1 \sigma$ ) to $5 \pm 7 \%$ when the top OC analysis temperature was reduced to $700^{\circ} \mathrm{C}$. Errors due to flow rate variations and variations in FID response within an analysis can easily be held below 5\%. Filter change is the only maintenance activity that requires a technician to visit the site more than monthly.

\section{RESULTS}

\section{Organic and Elemental Carbon Concentrations}

The study mean OC, EC, and TC concentrations were 2.75 (0.23-15.70), 0.89 (0.20-7.98), and $3.64(0.43-22.32) \mu \mathrm{gC} / \mathrm{m}^{3}$, respectively. Ranges of 2-4 h measurements are given in parentheses. The highest monthly average concentrations were measured in July $2002\left(\mathrm{OC}=3.88, \mathrm{EC}=1.13, \mathrm{TC}=5.01 \mu \mathrm{gC} / \mathrm{m}^{3}\right)$, while the lowest monthly average concentrations were recorded in January $\left(\mathrm{OC}=1.89, \mathrm{EC}=0.64, \mathrm{TC}=2.53 \mu \mathrm{gC} / \mathrm{m}^{3}\right)$ and April $2002\left(\mathrm{OC}=1.61, \mathrm{EC}=0.69, \mathrm{TC}=2.30 \mu \mathrm{gC} / \mathrm{m}^{3}\right)$. The highest time-resolved $\mathrm{OC}$ and TC concentrations measured during PAQS (15.70 and $22.32 \mu \mathrm{gC} / \mathrm{m}^{3}$, respectively) were recorded 08 July 2002 (00:00-03:30 EST). The highest EC concentration $\left(7.98 \mu \mathrm{gC} / \mathrm{m}^{3}\right)$ was recorded April 172002 (04:00-07:30 EST). The concentrations of OC, EC and TC were unexpectedly high in November 2001 and low in April-May 2002.

\section{EC Tracer Method}

Using EC as a tracer for primary combustion-generated OC, primary $\mathrm{OC}\left(\mathrm{OC}_{\mathrm{pri}}\right)$ can be described as follows (Turpin and Huntzicker 1995):

$$
\mathrm{OC}_{\mathrm{pri}}=a E C+b
$$

where $a E C$ and $b$ are combustion and non-combustion primary $\mathrm{OC}$, respectively. The difference between measured particulate $\mathrm{OC}\left(\mathrm{OC}_{\text {tot }}\right)$ and estimated $\mathrm{OC}_{\text {pri }}$ provides an estimate of secondary $\mathrm{OC}\left(\mathrm{OC}_{\mathrm{sec}}\right)$ :

$$
\mathrm{OC}_{\mathrm{sec}}=\mathrm{OC}_{\mathrm{tot}}-\mathrm{OC}_{\mathrm{pri}}
$$

An ambient OC to EC ratio that is elevated above the ratio representing the mix of primary combustion sources can result when there is a substantial contribution of secondary OC (SOA) or when EC is very low so that primary non-combustion OC (the intercept, $b$, in Equation [1]) contributes substantially to the OC to $\mathrm{EC}$ ratio.

If a large data set is available and includes times when secondary formation is unlikely, $a$ and $b$ can be determined from a subset of ambient measurements (Turpin and Huntzicker 1995; Strader et al. 1999). The EC tracer method can also be applied to an emission inventory of the principal sources developed for the area of interest (Gray 1986; Cabada et al. 2002). The slope of Equation (1) represents the ratio of primary OC to EC for all contributing combustion sources. It should be noted that primary $\mathrm{OC} / \mathrm{EC}$ varies with source type. For example the primary OC/EC ratio for biomass burning is much higher than that of diesel PM (Hays et al. 2002). For this reason Equation (1) could vary with location, season, or time of day because of changes in the source mix. Variations in the source mix that have seasonal or diurnal regularity can be identified and accommodated by the EC-tracer method (see below). Occasional irregular contributions from a 


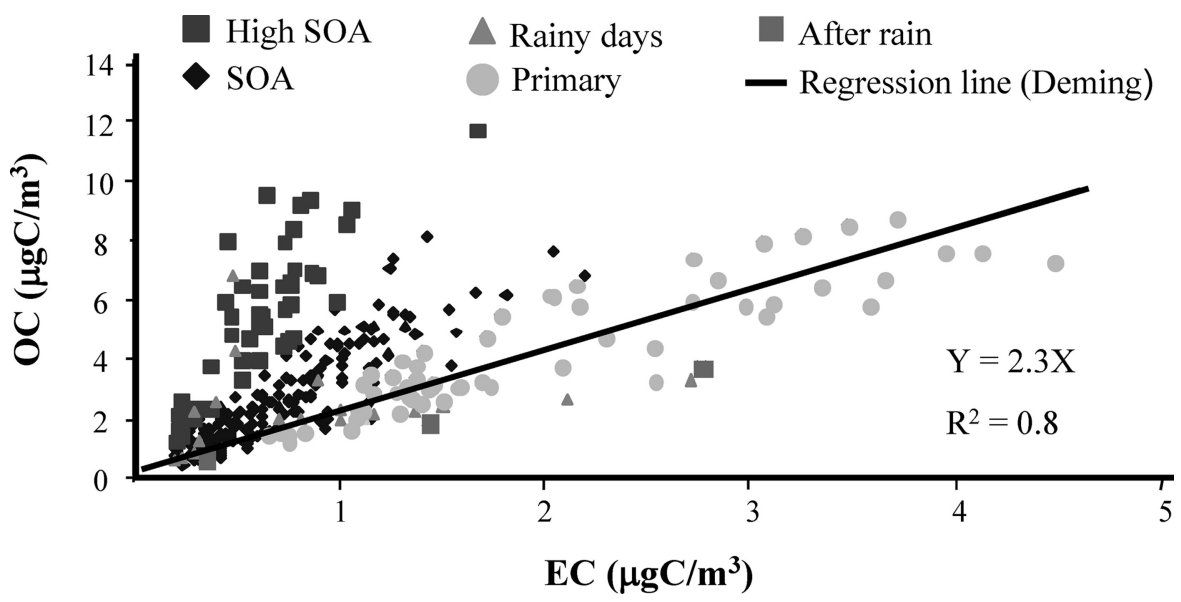

FIG. 3. Particulate OC and EC semi-continuous carbon measurements made during November 2001. High SOA: measurements with a high probability of SOA formation; SOA: measurements with a moderate probability of SOA formation; Primary: measurements dominated by primary emissions; Rainy days and After rain: measurements impacted by rain events. The regression line, equation, and coefficient of determination $\left(\mathrm{R}^{2}\right)$ were obtained by Deming regression of measurements labeled "Primary." The intercept was 0 in this case. Similar scatter plots were obtained for each month of PAQS.

source with a primary $\mathrm{OC}$ to EC ratio vastly different from the usual mix of sources could cause errors in estimated $\mathrm{OC}_{\mathrm{pri}}$ and $\mathrm{OC}_{\mathrm{sec}}$ during the effected time period(s), but such events are unlikely to affect monthly averages when high frequency measurements are used. If $a$ and $b$ are determined from a data set that is always impacted by SOA, the resulting SOA estimates will be lower bounds.

\section{Estimation of $a, b, S O A$, and Uncertainties}

Time periods when the ambient concentrations of particulate OC were likely to be predominantly primary were identified and used to establish a relationship between primary OC and EC (i.e., to estimate $a$ and $b$ in Equation [1]). For this purpose, hourly concentrations of carbon monoxide (CO) and nitrogen monoxide (NO) were used as indicators of regional and local combustion-related primary emissions, respectively. Hourly ozone $\left(\mathrm{O}_{3}\right)$ concentrations and the ratio between nitrogen oxides $\left(\mathrm{NO}_{\mathrm{x}}\right)$ and $\mathrm{NO}$ concentrations were used as indicators of photochemical activity. Time periods were defined as dominated by primary emissions when their 1-h CO and NO concentrations both exceeded the corresponding monthly average and their 1-h $\mathrm{O}_{3}$ concentration and $\mathrm{NO}_{\mathrm{x}} / \mathrm{NO}$ concentration ratio were lower than the corresponding monthly averages. Periods with a high probability of SOA formation were defined as times when the 1-h $\mathrm{O}_{3}$ concentration and $\mathrm{NO}_{\mathrm{x}} / \mathrm{NO}$ concentration ratio were higher than the corresponding monthly averages. To account for the time necessary for photochemical processes to form SOA, this comparison was made with $\mathrm{O}_{3}$ and $\mathrm{NO}_{\mathrm{x}} / \mathrm{NO}$ measurements 0 $2 \mathrm{~h}$ prior to the time of interest. All other time periods were considered to have a moderate probability of SOA formation. Sampling periods effected by rain were separately identified and were not used in the estimation of $a$ and $b$ because of the possibility of differential wet scavenging (Lim et al. 2003a). (Periods "effected by rain" were identified based on visual observations recorded at the site; "rainy days" means it was raining during sample collection; "after rain" refers to the first sample collected after the rain stopped.)

Figure 3 shows particulate $\mathrm{OC}$ and $\mathrm{EC}$ for all semi-continuous carbon analyzer measurements made during November 2001. Labels segregate data into measurements with a high probability of SOA formation (high SOA), measurements with a moderate probability of SOA formation (SOA), measurements dominated by primary emissions (primary), and measurements impacted by rain events (rainy days; after rain) using the procedure described above. The slope $(a)$ and the intercept $(b)$ in Equation (1) were calculated by regressing OC on EC using only data dominated by primary emissions (circles, Figure 3 ).

To evaluate the impact of different reasonable approaches these regressions were performed on a monthly, seasonal, and annual basis to provide values for $a$ and $b$ in Equation (1). To evaluate whether the primary OC-EC relationship varied because of diurnal variations in the source mix, $a$ and $b$ were also calculated after dividing periods dominated by primary emissions into four different time periods (00:00-06:00; 06:0012:00; 12:00-18:00; 18:00-00:00 EST). In all cases a Deming linear least-squares regression was used (Deming 1943; Cornbleet and Gochman 1979) and the uncertainties in OC and EC were assumed to be equal. (Conventional linear least-squares regression assumes that there are uncertainties only in the dependent variable.)

Resulting $a$ and $b$ values are provided in Table 3. Month-tomonth variations in $a$ and $b$ are not systematic, suggesting that the data could be adequately represented by annually derived values $\left(R^{2}=0.81\right)$. When the data were divided by time-ofday a higher $a$ value (slope) was obtained for the 1200-1800 $\mathrm{h}$ time period. This larger value could occur if the mix of primary sources during that time of day typically had a higher primary OC/EC ratio (e.g., due to a reduced contribution from 


\section{TABLE 3}

Primary combustion-generated OC/EC (the slope, $a$, in Equation 1) and primary non-combustion OC (in intercept, $b$, in Equation 1) calculated by month, season, time-of-day, and annually from a Deming linear least squares regression of OC on EC for data dominated by primary emissions. Shown also are the standard error (SE) of $a$ and $b$ and the coefficient of determination $\left(\mathrm{R}^{2}\right)$

\begin{tabular}{|c|c|c|c|c|c|}
\hline Time period & $\begin{array}{c}\text { Primary } \\
\text { combustion } \\
\text { OC/EC }(a)\end{array}$ & $\begin{array}{c}\text { Primary } \\
\text { non-combustion } \\
\text { OC }(b)\end{array}$ & $\mathrm{R}^{2}$ & $\begin{array}{l}\mathrm{SE} \\
(a)\end{array}$ & $\begin{array}{l}\text { SE } \\
(b)\end{array}$ \\
\hline Jul. 2001 & 2.51 & 0.00 & 0.83 & 0.21 & 0.36 \\
\hline Aug. 2001 & 1.64 & 0.32 & 0.84 & 0.13 & 0.29 \\
\hline Oct. 2001 & 2.64 & 0.00 & 0.82 & 0.28 & 0.39 \\
\hline Nov. 2001 & 2.30 & 0.00 & 0.80 & 0.15 & 0.34 \\
\hline Dec. 2001 & 1.89 & 0.00 & 0.92 & 0.07 & 0.12 \\
\hline Jan. 2002 & 2.46 & 0.07 & 0.85 & 0.14 & 0.15 \\
\hline Feb. 2002 & 2.11 & 0.18 & 0.86 & 0.15 & 0.23 \\
\hline Mar. 2002 & 1.81 & 0.12 & 0.82 & 0.13 & 0.21 \\
\hline Apr. 2002 & 1.24 & 0.62 & 0.88 & 0.07 & 0.13 \\
\hline May 2002 & 1.24 & 0.66 & 0.81 & 0.09 & 0.15 \\
\hline Jun. 2002 & 2.38 & 0.00 & 0.80 & 0.20 & 0.42 \\
\hline Jul. 2002 & 2.25 & 0.04 & 0.85 & 0.14 & 0.35 \\
\hline Summer 2001 & 1.91 & 0.03 & 0.79 & 0.13 & 0.25 \\
\hline Fall 2001 & 2.20 & 0.00 & 0.86 & 0.08 & 0.14 \\
\hline Winter 2002 & 2.08 & 0.14 & 0.81 & 0.09 & 0.12 \\
\hline Spring 2002 & 1.74 & 0.32 & 0.74 & 0.09 & 0.17 \\
\hline 00:00 to $06: 00$ & 1.99 & 0.15 & 0.76 & 0.09 & 0.20 \\
\hline $06: 00$ to $12: 00$ & 2.01 & 0.00 & 0.85 & 0.06 & 0.11 \\
\hline $12: 00$ to $18: 00$ & 3.06 & 0.00 & 0.75 & 0.24 & 0.23 \\
\hline $18: 00$ to $00: 00$ & 2.49 & 0.00 & 0.82 & 0.12 & 0.17 \\
\hline Year & 2.07 & 0.01 & 0.81 & 0.04 & 0.08 \\
\hline
\end{tabular}

diesel trucks) or if more secondary OC was typically present in the "primary-dominated" data at that time of day. Coefficients of determination $\left(\mathrm{R}^{2}\right)$ for monthly regressions were all greater than 0.80 . Coefficients of determination by season and by time-of-day ranged from $0.74-0.86$. Some scatter in primary-dominated data is expected due to variations in the primary source mix. While it is possible that some measurements identified as primarydominated could have some secondary contribution, selecting the primary ratio based on the lowest OC/EC would ignore the variability in the primary source mix. The fact that the "primary dominated" data resemble a normal distribution around the regression line is reassuring.

Using Equations (1) and (2) and $a$ and $b$ values from each of the 4 approaches in Table 3, primary and secondary OC concentrations were estimated for the duration of PAQS. SOA concentrations estimated from $a$ and $b$ values derived on a monthly, seasonal, annual and time-of-day basis were $0.92 \pm$ $1.11,1.00 \pm 1.16,1.01 \pm 1.14$, and $0.85 \pm 1.09 \mu \mathrm{g} / \mathrm{m}^{3}$ (mean $\pm 1 \sigma$ ), respectively. The lowest mean SOA concentration was obtained when $a$ and $b$ were calculated separating the data by time-of-day, due to the larger $a$ value obtained for the 1200-1800 $\mathrm{h}$ time period. Despite this, the variability in calculated primary and secondary OC concentrations across these four approaches is small, $7 \%$ and $11 \%$ (pooled coefficient of variation), respectively. This provides confidence in the results and provides one estimate of precision in primary and secondary OC estimates. (This estimate of precision includes "model" uncertainties but does not include measurement uncertainties.) Propagation of error (i.e., including a $10 \%$ uncertainty in OC and EC measurements and using the standard error of $a$ and $b$ values) suggests uncertainties in 2-4 h primary and secondary OC estimates are on the order of $10 \%$ and $40 \%$, respectively. Below, results using $a$ and $b$ values derived on a monthly basis are reported.

\section{Primary and Secondary OC}

The annual average SOA concentration was $0.92 \mu \mathrm{g} / \mathrm{m}^{3}$, which represents $33 \%$ of the annual average particulate OC. (On a percentage basis 29\%, on average, of particulate OC was SOA.) This is in good agreement with the results obtained by Cabada et al. (2002), who developed an emission inventory of primary OC and EC in the Pittsburgh area, applied the EC tracer method to this reconstructed dataset, and concluded that in 1995 the annual SOA contribution to particulate OC was at least $25 \%$. Cabada et al. (2004) independently applied the EC-tracer method to PAQS semi-continuous OC and EC measured from July 1 to August 4, 2001. The same tracers of primary and secondary emissions, but a different decision strategy was used to identify time periods dominated by primary OC. Also Equation (1) was obtained by standard rather than Deming linear least squares regression. For this time period, SOA concentrations of $0.92 \pm$ 0.98 and $1.18 \pm 1.05$ (mean $\pm 1 \sigma$ of time-resolved SOA estimates) were obtained by Cabada et al. (2004) and in the current work, respectively.

During the summer of 2001 and July 2002, SOA was on average $38 \%$ of measured OC ( $\mathrm{SOA}=1.30 \mu \mathrm{gC} / \mathrm{m}^{3}$ June-August $2001 ; 1.44 \mu \mathrm{gC} / \mathrm{m}^{3}$ July 2002). This agrees quite well with the contribution of SOA (44\%) to particulate OC obtained in Atlanta during August 1999 (Lim and Turpin 2002). The percentage contribution of SOA to particulate OC was much greater for Pittsburgh than for Claremont, CA, where the SOA concentration exceeded $40 \%$ of the daily OC concentration only in the afternoon hours of summertime photochemical smog episodes (SCAQS; Turpin and Huntzicker 1995). It should be noted that OC concentrations were much higher in Claremont.

The typical summertime diurnal pattern of carbonaceous species measured/ estimated during PAQS is illustrated in Figure 4. OC and EC peaked between 00:00 and 08:00 EST. Similarly, NO and CO peaked during 00:00-08:00 EST; NO and $\mathrm{CO}$ are often used as local and regional combustion tracers. Typically, the summer periods were characterized by high photochemical activity in the afternoon, which resulted in $\mathrm{O}_{3}$ and SOA concentrations that peaked together between 13:00 and 


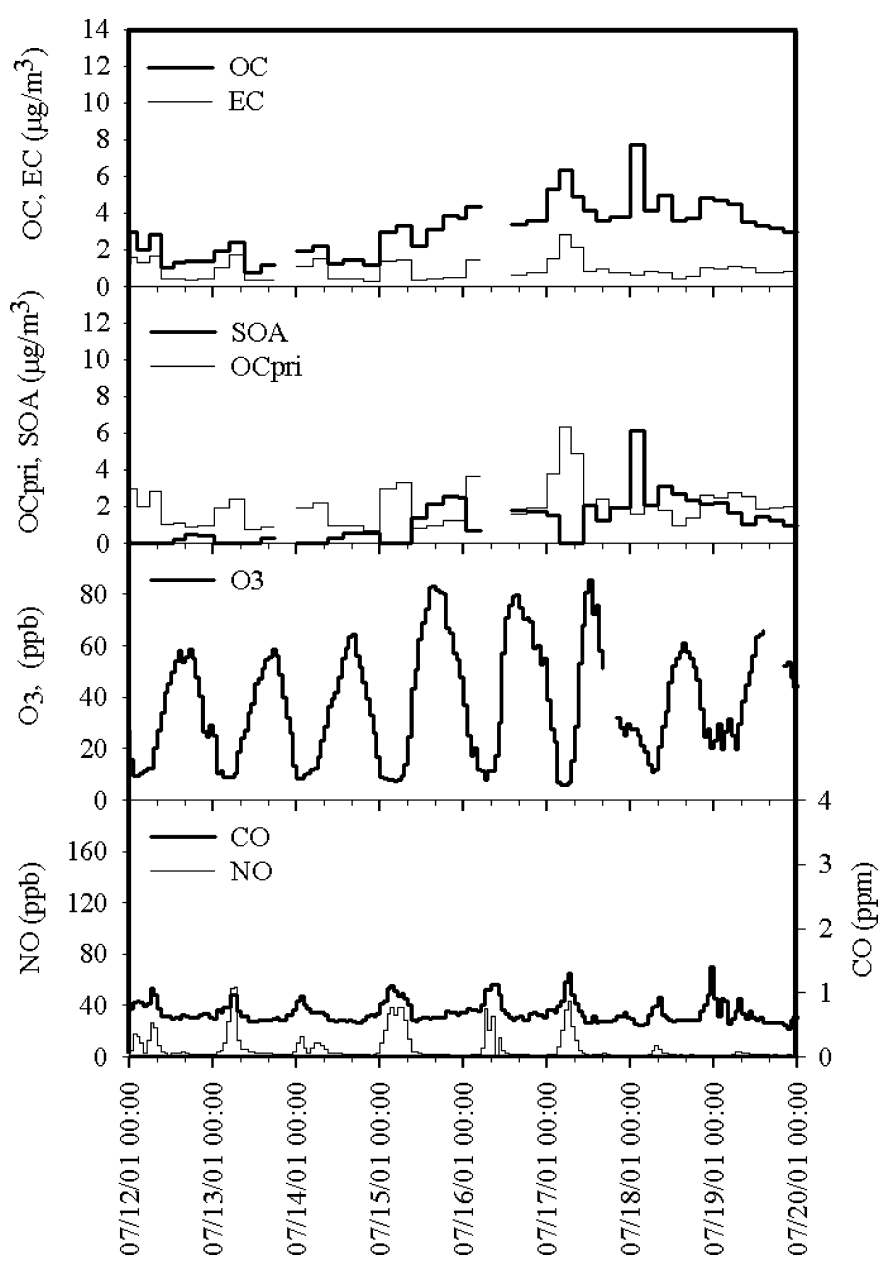

FIG. 4. Particulate $\mathrm{OC}$ and EC, primary $\mathrm{OC}\left(\mathrm{OC}_{\mathrm{pri}}\right)$, secondary $\mathrm{OC}(\mathrm{SOA})$, ozone $\left(\mathrm{O}_{3}\right), \mathrm{NO}$, and $\mathrm{CO}$ concentrations measured/estimated July 12-19, 2001. This period illustrates typical summertime concentration dynamics.

19:00 EST. This temporal behavior is expected when SOA is formed locally that afternoon. This diurnal pattern was also typical of that observed in the summertime in Atlanta (Supersite; Lim and Turpin 2002). A more pronounced afternoon OC peak was found in Claremont during summertime smog episodes (SCAQS; Turpin and Huntzicker 1995).

Interestingly, on July 17-19 SOA remained elevated over a 3 -day period, even at night. While on previous days the $\mathrm{O}_{3}$ concentration decreased rapidly at night (perhaps with the aid of NO scavenging), at 00:00 EST, July 18 and July 19 it remained considerably elevated (30 ppb, $45-50 \%$ of the peak $\mathrm{O}_{3}$ concentrations) suggesting either replacement of air from aloft or from upwind. When SOA remains elevated over several days, it is likely that it has been formed through multi-day transport and transformation (i.e., regionally). Regional SOA formation is especially likely aloft where transport distances are great and conditions for photochemistry are favorable. Vertical transport from aloft has been shown to yield elevated nighttime $\mathrm{O}_{3}$ concentrations (Corsmeier et al. 1997) and would bring SOA formed

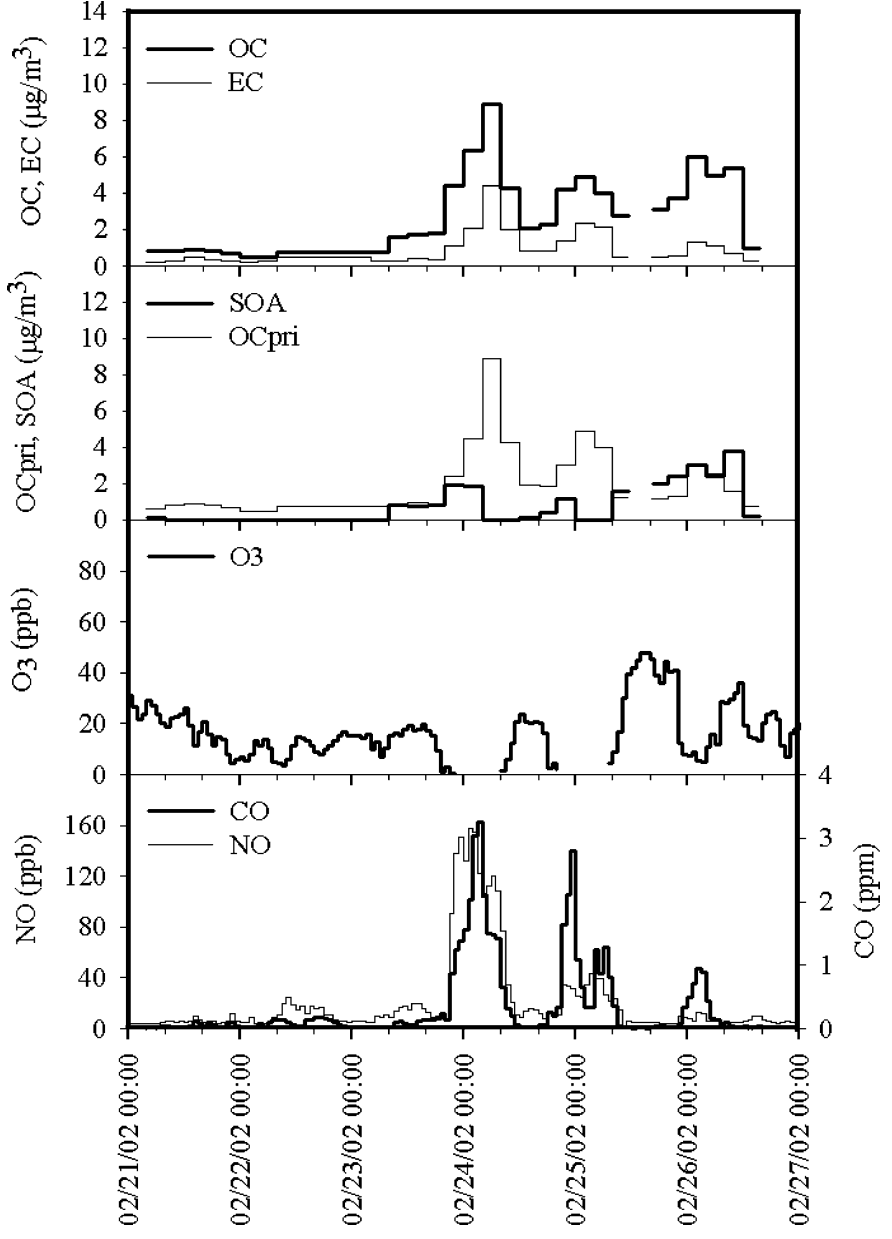

FIG. 5. Particulate $\mathrm{OC}$ and EC, primary $\mathrm{OC}\left(\mathrm{OC}_{\mathrm{pri}}\right)$, secondary $\mathrm{OC}(\mathrm{SOA})$, ozone $\left(\mathrm{O}_{3}\right), \mathrm{NO}$, and $\mathrm{CO}$ concentrations measured/estimated February 21-26, 2002. This period illustrates typical wintertime concentration dynamics.

aloft to ground level as well. Lower nighttime temperatures also contribute to increase the SOA concentration through the favorable partitioning of oxidation products into the particle phase. It was not uncommon for $\mathrm{O}_{3}$ and SOA concentrations to remain elevated at night. This occurred on approximately 11 nights in the summer and 7 nights in the winter.

A characteristic wintertime period is illustrated in Figure 5. Typically, the concentrations of OC and EC closely tracked those of $\mathrm{NO}$ and $\mathrm{CO}$ across the day, suggesting that primary combustion sources dominated $\mathrm{OC}$ and $\mathrm{EC}$ concentrations in the winter. $\mathrm{OC}$ and $\mathrm{EC}$ concentrations were usually quite low (e.g., February 21-22) but occasionally strong peaks were observed between 02:00 and 08:00 EST, with concentrations substantially higher than those recorded during the summertime, probably because of increased stability and low mixing heights. On February 24, 2002 the OC and EC concentrations peaked between 04:00 and 07:30 EST, reaching values of 8.9 and $4.4 \mu \mathrm{gC} / \mathrm{m}^{3}$, respectively, the highest measured winter carbon concentrations. 
Wintertime SOA and $\mathrm{O}_{3}$ concentrations measured during PAQS were small compared to the summertime values, but there were times when the EC-tracer method suggested SOA formation. For example, between February 24 and February 26, 2002 peak concentrations of $\mathrm{NO}, \mathrm{CO}$, and $\mathrm{EC}$ decreased. OC decreased initially and then increased during this period. While the $\mathrm{O}_{3}$ concentration is still reasonably low, it reached a maximum for this February time period (36 ppb) on February 25, just as the calculated SOA concentration began to increase. It is likely that the elevated ozone peak on February 25 occurs as a result of down-mixing of air from aloft rather than local photochemical formation. This air mass had an elevated OC/EC ratio, suggesting either regional secondary formation or regional transport of a primary carbon source with a high OC/EC ratio (e.g., biomass burning). OC/EC ratios from open burning of biomass can be quite high; transport to Pittsburgh from the southeastern US commonly occurs, and prescribed burning is practiced in the Southeast in the fall/winter (Hays et al. 2002). Thus, the later possibility cannot be ruled out, and illustrates a potential limitation in the EC-tracer method in locations where regional transport is substantial. It should be noted that the decrease in peak $\mathrm{CO}$ on February 26 does not support a primary regional combustion origin for the additional OC. The quantity of SOA formed regionally over several days is likely to depend in a complicated way on the integrated exposure of precursors to oxidants (e.g., ozone and hydroxyl radical) during transport (e.g., from the southeastern U.S.). Regional formation could occur as a result of gas-phase, surface, or aqueous-phase reactions in clouds or aerosols (Odum et al. 1997; Jang and Kamens 2001; Lim et al. 2005). On February 26 the calculated primary and secondary OC concentrations were roughly equal. SOA formation was reported to account for $20 \%$ of wintertime OC on average with short peaks as great as $60 \%$ in the San Joaquin Valley, CA (1995-96; Strader et al. 1999). The average wintertime (2002, Pittsburgh) SOA concentration calculated herein was $24 \%$ of measured OC $\left(0.56 \mu \mathrm{gC} / \mathrm{m}^{3}\right)$.

Fall 2001 and spring 2002 were characterized by an average SOA concentration of 0.92 and $0.61 \mu \mathrm{gC} / \mathrm{m}^{3}$, corresponding to 28 and $20 \%$ of OC, respectively. Daily OC and EC concentration dynamics in the fall were more similar to those observed in the summer, but OC, EC, and SOA maxima were generally smaller. Spring concentration dynamics were more similar to those observed in the winter. March-May had many precipitation events (a total of $144 \mathrm{~h}$ with greater than $25 \mathrm{~mm}$ of rain), which contributed to the low springtime PM concentrations. The lowest monthly mean SOA concentration $\left(0.27 \mu \mathrm{gC} / \mathrm{m}^{3} ; 14 \%\right.$ of OC) occurred in April, probably as a result of both low photochemical activity and considerable precipitation.

November 2001 had three time periods when EC dropped or remained low while OC increased dramatically. These are shown in Figure 6 as Episodes A, B, and C. Episode A occurred on 01-03 November. During that period $\mathrm{O}_{3}$ and $\mathrm{OC}$ increased and remained elevated for almost $48 \mathrm{~h}$ with no increase in the primary combustion tracer, EC. Then, at the onset of precipitation,

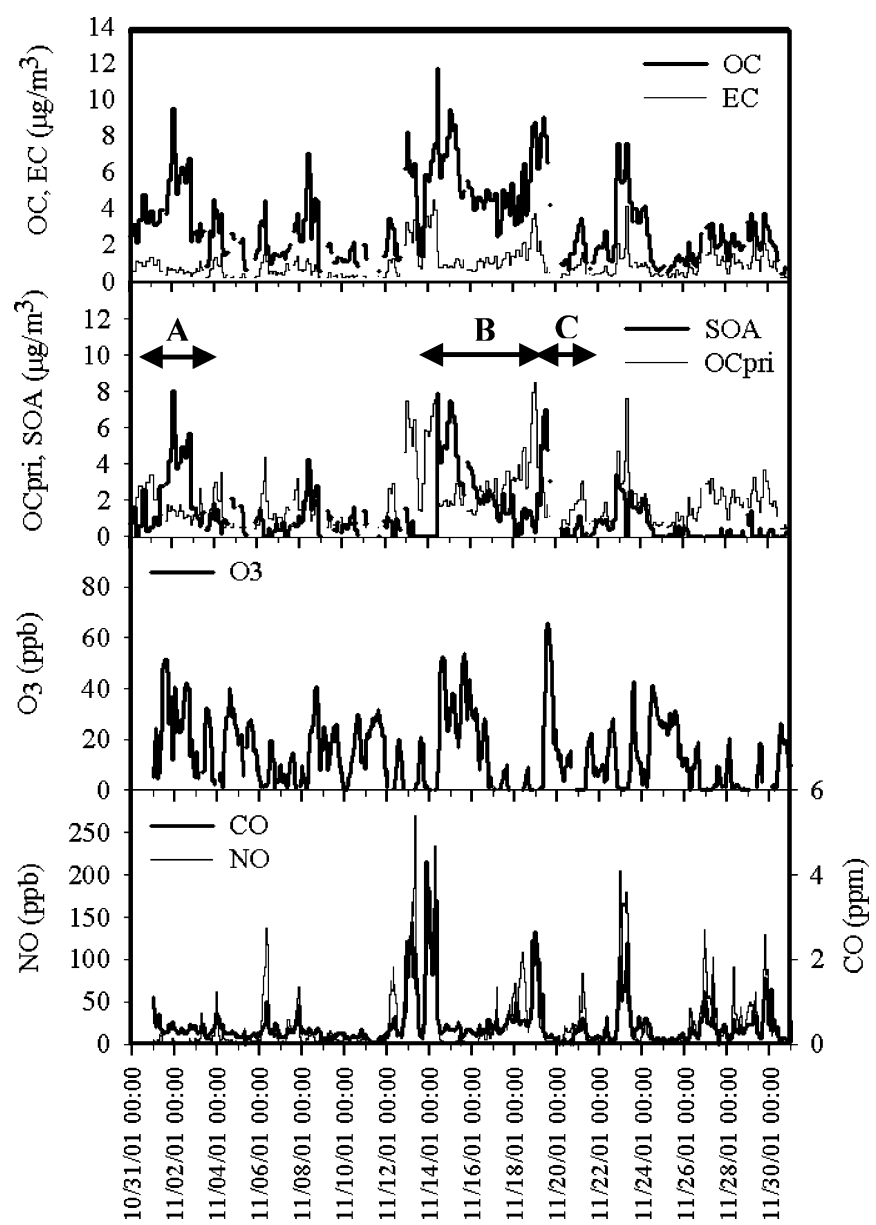

(a)

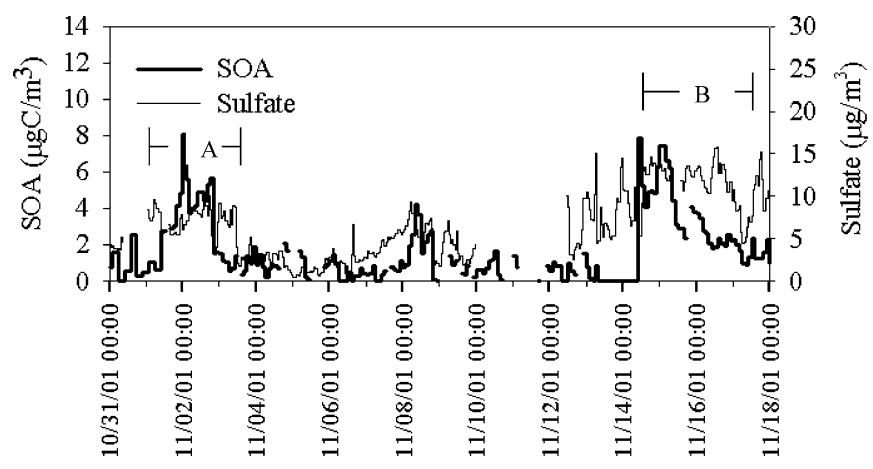

(b)

FIG. 6. (a) Particulate $\mathrm{OC}$ and EC, primary $\mathrm{OC}\left(\mathrm{OC}_{\text {pri }}\right)$, secondary $\mathrm{OC}$ (SOA), ozone $\left(\mathrm{O}_{3}\right), \mathrm{NO}$ and $\mathrm{CO}$ concentrations measured/estimated October 31-November 30, 2001. High average SOA in November was mostly due to three SOA formation episodes: A (November 1-3), B (November 14-18), and $\mathrm{C}$ (November 19). (b) Secondary organic aerosol ( $\mathrm{SOA} ; \mu \mathrm{gC} / \mathrm{m}^{3}$ ) and sulfate $\left(\mu \mathrm{g} / \mathrm{m}^{3}\right)$, October 31-November 17, 2001. Episode A: November 1-3; Episode B: November 14-18.

$\mathrm{OC}$ and $\mathrm{O}_{3}$ decreased rapidly. Episode $\mathrm{B}$ occurred on November 14-18, to following 2-3 days of very high concentrations of the primary tracers (EC, CO, and NO), as well as high OC concentrations. Rapid decreases in EC, $\mathrm{CO}$, and $\mathrm{NO}$ were followed by 
a 2-3 day period of continuously elevated $\mathrm{O}_{3}$ accompanied by a peak and a gradual decrease in OC. Episode $\mathrm{C}$ occurred on November 19 and was characterized by increasing $\mathrm{OC}$ and $\mathrm{O}_{3}$ and decreasing $\mathrm{EC}, \mathrm{CO}$, and $\mathrm{NO}$.

The ozone concentrations during these episodes were not high by summertime standards and might not provide sufficient oxidant concentrations to form SOA locally. Ground level ozone concentrations are often quite low due to titration by primary pollutants, such as NO. The elevated ozone concentrations likely occur because ozone is being mixed down to the surface from aloft (or perhaps transported in from an upwind location). What is apparent in Episodes A and B is that when ozone is replenished it brings an air mass elevated in OC but not in EC. Pollutants can be transported long distances aloft under conditions that are conducive to photochemistry. Thus it is quite plausible that this additional OC is SOA. During Episode A, the EC tracer method calculated SOA concentrations that remained between 4.0 and $8.0 \mu \mathrm{gC} / \mathrm{m}^{3}$ (60-85\% of OC) over a 24-h period. During Episode $\mathrm{B}$, the calculated SOA maximum was $7.5 \mu \mathrm{gC} / \mathrm{m}^{3}$ (79\% of OC) and occurred at 00:00-02:00 EST on November 15. During both Episodes A and B, sulfate (a secondary species formed regionally through gas and aqueous phase reactions) also remained elevated (see Figure 6b). SOA and $\mathrm{O}_{3}$ concentrations that increased and remained elevated over a few days were observed many times during PAQS, during the winter and summer.

It is possible that such concentration dynamics could occur due to the influence of a large primary source with a high OC/EC ratio and no temporal regularity (e.g., wildfires or prescribed burning) located distant from Pittsburgh (e.g., the southeastern U.S.). Down-mixing of these pollutants from aloft could cause a sudden increase in OC without a concurrent increase in EC. $\mathrm{OC} / \mathrm{EC}$ ratios from biomass burning can be greater than 10 (Hays et al. 2002). The possibility that calculated SOA is really primary OC from biomass combustion cannot be entirely ruled out for these two November episodes. However, the similar SOA and sulfate dynamics supports secondary formation. Additionally, the regional combustion tracer, $\mathrm{CO}$, was highly correlated with primary OC $\left(\mathrm{R}^{2}=0.82\right)$ and uncorrelated with calculated SOA.

The largest limitation of the analysis above occurs because of the potential for confounding by biomass burning. While we did not identify any local or more distant prescribed burns that would bias the assessment of SOA, it would be quite difficult to rule out such confounding based on emissions information, which is rarely complete. However, tracer compounds can provide useful insights. Molecular source tracers, including biomass smoke tracers (i.e., levoglucosan, syringols, and resin acids) were measured at PAQS daily in July and every 6th day the remainder of the year. An upper limit of 2\%, 14.5\%, and 10\% of OC was attributed to biomass burning during the summer, fall and winter, respectively, when molecular source tracers were used in chemical mass balance source apportionment (Robinson et al. 2006). Biomass smoke tracer concentrations were quite low during July. Using the average levoglucosan concentration $\left(10 \mathrm{ng} / \mathrm{m}^{3}\right)$ during the July 12-20 time period (Figure 4) and the levoglucosan to OC ratio (0.094) of Lee et al. (2005), biomass smoke contributed only $0.1 \mu \mathrm{gC} / \mathrm{m}^{3}$, with a peak of $0.17 \mu \mathrm{gC} / \mathrm{m}^{3}$ on 17 July. The highest biomass smoke marker concentrations were measured in October and November, with levoglucosan reaching 73 and $192 \mathrm{ng} / \mathrm{m}^{3}$ on November 2 and 14, respectively. This corresponds to roughly 0.7 and $2.0 \mu \mathrm{gC} / \mathrm{m}^{3}$ of biomass OC (SOA ranged from $4-8 \mu \mathrm{gC} / \mathrm{m}^{3}$ on these days). Thus, biomass burning could contribute to, but does not appear to explain completely, the elevated OC concentrations observed in November. Biomass markers were not measured during the February 24-26 period, although the PAQS molecular marker data show surprisingly little influence of biomass smoke in the winter (Robinson et al. 2006).

\section{CONCLUSIONS}

During the Pittsburgh summertime, SOA was a much more substantial contributor to particulate OC than in the Los Angeles Basin (Claremont, CA; 1987 SCAQS; Turpin and Huntzicker 1995), although Claremont concentrations were greater. In Claremont, SOA only exceeded $40 \%$ of OC during the afternoon hours of summertime photochemical smog episodes. In contrast, the summertime average SOA contribution was nearly that great in Pittsburgh (38\% of particulate OC). Summer periods in Pittsburgh were often characterized by early morning peaks in OC and $\mathrm{EC}$ and late afternoon peaks in $\mathrm{O}_{3}$ and SOA. This pattern is consistent with local formation of SOA. However, the EC tracer method produced the highest SOA concentration estimates at times when $\mathrm{O}_{3}$ and $\mathrm{OC}$ increased and remained elevated over several days. This ozone pattern suggests down-mixing of ozone and other species from aloft, where pollutants can be transported long distances and are exposed to conditions conducive to photochemistry. The elevated OC relative to EC combined with low $\mathrm{CO}$ and complementary sulfate dynamics during these episodes is consistent with multi-day regional SOA formation, although regional transport of primary $\mathrm{OC}$ from biomass combustion cannot be completely ruled out.

These findings suggest that SOA could be an important contributor to particulate $\mathrm{OC}$ in locations that are recipients of long distance transport (e.g., the eastern United States) and regional sulfate formation. Neither in-cloud formation (Ervens et al. 2004; Lim et al. 2005) nor acid-catalyzed surface reactions (Jang and Kamens 2001) are currently included in predictive models and are likely to contribute to regional SOA formation.

\section{REFERENCES}

Cabada, J. C., Pandis, S. N., and Robinson, A. L. (2002). Sources of atmospheric carbonaceous particulate matter in Pittsburgh, Pennsylvania, J. Air Waste Manag. Assoc. 52:732-741.

Cabada, J. C., Pandis, S. N., Subramanian, R., Robinson, A. L., Polidori, A., and Turpin, B. J. (2004). Estimating the secondary organic aerosol contribution to PM2.5 using the EC tracer method, Aerosol Sci. Technol., 38:140-155.

Chow, J. C., Watson, J. G., Crow, D., Lowenthal, D. H., and Merrifield, T. (2001). Comparison of IMPROVE and NIOSH carbon measurements, Aerosol Sci. Technol. 34:23-34. 
Cornbleet, P. J., and Gochman, N. (1979). Incorrect least-squares regression coefficients in method-comparison analysis, Clin. Chem. 25:432-438.

Corsmeier, U., Kalthoff, N., Kolle, O., Kotzian, M., and Fiedler, F. (1997). Ozone concentration jump in the stable nocturnal boundary layer during a LLJ-event, Atmos. Environ. 31:1977-1989.

Deming, W. E. (1943). Statistical Adjustment of Data. Wiley, New York, NY.

Ervens, B., Feingold, G., Frost, G. J., and Kreidenweis, S. M. (2004). A modeling study of aqueous production of dicarboxylic acids: 1 . Chemical pathways and speciated organic mass production, J. Geophys. Res. 109, doi:10.1029/2003JD004387.

Gray, H. A. (1986). Control of atmospheric fine primary carbon particle concentrations. EQL Report No. 23, Environmental Quality Laboratory, California Institute of Technology, Pasadena, California, pp. 103-108.

Hays, M. D., Geron, C. D., Linna, K. J., Smith, N. D., and Schauer, J. J. (2002). Speciation of Gas-Phase and Fine Particle Emissions from Burning of Foliar Fuels, Environ. Sci. Technol. 36:2281-2295.

Intergovernmental Panel on Climate Change (IPCC). (2001). Climate Change 2001: The Scientific Basis. Cambridge University Press, UK. pp. 944.

Jang, M. S., and Kamens, R. M. (2001). Atmospheric secondary aerosol formation by heterogeneous reactions of aldehydes in the presence of a sulfuric acid aerosol catalyst, Environ. Sci. Technol. 24:4758-4766.

Lee, S. Baumann, K., Schauer, J. J., Sheesley, R. J., Naeher, L. P., Meinardi, S., Blake, D. R., Edgerton, E. S., Russell, A. G., and Clements, M. (2005) Gaseous and particulate emissions from prescribed burning in Georgia, Environ. Sci. Technol. 39:9049-9056.

Lim, H. J., Carlton, A. G., and Turpin, B. J. (2005). Isoprene forms secondary organic aerosols through cloud processing: Model simulations, Environ. Sci. Technol. 39:4441-4446.

Lim, H. J., and Turpin, B. J. (2002). Origins of primary and secondary organic aerosol in Atlanta: Results of time-resolved measurements during the Atlanta supersite experiment, Environ. Sci. Technol. 36:4489-4496.

Lim, H. J., Turpin, B. J., Russell, L. M., and Bates, T. S. (2003a). Organic and elemental carbon measurements during ACE-Asia suggest a longer atmospheric lifetime for elemental carbon, Environ. Sci. Technol. 14:30553061.

Lim, H. J., Turpin, B. J., Edgerton, E., Hering, S. V., Allen, G., Maring, H., and Solomon, P. (2003b). Semicontinuous aerosol carbon measurements: Comparison of Atlanta Supersite measurements, J. Geophys. Res. 108 (D7): Art. No. 8419 .

Mader B. T., and Pankow, J. F. (2001). Gas/solid partitioning of semivolatile organic compounds (SOCs) to air filters. 3. An analysis of gas adsorption artifacts in measurements of atmospheric SOCs and organic carbon (OC) when using Teflon membrane filters and quartz fiber filters, Environ. Sci. Technol. 35:3422-3432.

Odum, J. R., Jungkamp, T. P. W., Griffin, R. J., Flagan, R. C., and Seinfeld, J. H. (1997). The atmospheric aerosol-forming potential of whole gasoline vapor, Science 276:96-99.
Robinson, A. L., Subramanian, R., Donahue, N. M., Bernardo-Bricker, A., Rogge, W. F. (2006) Source apportionment of molecular markers and organic aerosol-2. Biomass smoke, Environ. Sci. Technol. submitted.

Schauer, J. J., Rogge, W. F., Hildemann, L. M., Mazurek, M. A., and Cass, G. R. (1996). Source apportionment of airborne particulate matter using organic compounds as tracers, Atmos. Environ. 30:3837-3855.

Schauer, J. J., and Cass, G. R. (2000). Source apportionment of wintertime gasphase and particle-phase air pollutants using organic compounds as tracers, Environ. Sci. Technol. 34:1821-1832.

Schauer, J. J., Mader, B. T., Deminter, J. T., Heidemann, G., Bae, M. S., Seinfeld, J. H., Flagan, R. C., Cary, R. A., Smith, D., Huebert, B. J., Bertram, T., Howell, S., Quinn, P., Bates, T., Turpin, B., Lim, H. J., Yu, J., and Yang, C. H. (2003). ACE-Asia intercomparison of a thermal-optical method for the determination of particle-phase organic and elemental carbon, J. Geophys. Res. 37:993-1001.

Seinfeld, J. H., and Pandis, S. N. (1998). Atmospheric Chemistry and Physics: From Air Pollution to Global Change. John Wiley and Sons Inc., New York.

Strader, R. L., Lurmann, F., and Pandis, S. N. (1999). Evaluation of secondary organic aerosol formation in winter, Atmos. Environ. 33:4849-4863.

Subramanian, R., Khlystov, A. Y., Cabada, J. C., and Robinson, A. L. (2004). Positive and negative artifacts in particulate organic carbon measurements with denuded and undenuded sampler configurations, Aerosol Sci. Technol. 38:27-48

Subramanian, R., Khlystov, A. Y., and Robinson, A. L. (2006). Effect of peak inert-mode temperature on elemental carbon using thermal-optical transmittance, Aerosol Sci. Technol. in press.

Turner, J. R., and Hering, S. V. (1994). The additivity and stability of carbon signatures obtained by evolved gas analysis, Aerosol Sci. Technol. 21:294305 .

Turpin, B. J., Cary, R. A., and Huntzicker, J. J. (1990). An in situ, time-resolved analyzer for aerosol organic and elemental carbon, Aerosol Sci. Technol. 12:161-171.

Turpin, B. J., Huntzicker, J. J., and Hering, S. V. (1994). Investigation of organic aerosol sampling artifacts in the Los Angeles basin, Atmos. Environ. 28: 30613071.

Turpin, B. J., and Huntzicker, J. J. (1995). Identification of secondary organic aerosol episodes and quantification of primary and secondary organic aerosol concentration during SCAQS, Atmos. Environ. 29:3527-3544.

Turpin, B. J., Saxena, P., and Andrews, E. (2000). Measuring and simulating particulate organics in the atmosphere: problems and prospects, Atmos. Environ. 34:2983-3013.

U.S. Environmental Protection Agency [U.S. EPA] 2004). Air Quality Criteria for Particulate Matter; Office of Research and Development: Research Triangle Park, NC; Vols. 1 and 2.

Wittig, A. E., Anderson, N., Khlystov, A. Y., Pandis, S. N., Davidson, C., and Robinson, A. L. (2004). Pittsburgh air quality study overview, Atmos. Environ. 38:3107-3125. 


\title{
Application of PSCF and CPF to PMF-Modeled Sources of $\mathrm{PM}_{2.5}$ in Pittsburgh
}

\author{
Natalie J. Pekney, ${ }^{1}$ Cliff I. Davidson, ${ }^{2}$ Liming Zhou, ${ }^{3}$ and Philip K. Hopke ${ }^{3}$ \\ ${ }^{1}$ Department of Civil and Environmental Engineering, Carnegie Mellon University, Pittsburgh, \\ Pennsylvania, USA \\ ${ }^{2}$ Department of Civil and Environmental Engineering and Engineering and Public Policy, \\ Carnegie Mellon University, Pittsburgh, Pennsylvania, USA \\ ${ }^{3}$ Center for Air Resources Engineering and Science, and Department of Chemical Engineering, \\ Clarkson University, Potsdam, New York, USA
}

\begin{abstract}
Ambient $\mathbf{P M}_{2.5}$ composition data in Pittsburgh, PA have been used with Positive Matrix Factorization (PMF) to determine the major sources of $\mathbf{P M}_{2.5}$ sampled. This paper describes the use of the potential source contribution function (PSCF) with the PMF-modeled source contributions to locate the sources in a grid of $0.1^{\circ} \times 0.1^{\circ}$ cells. The domain extends from the Pittsburgh Supersite at $40.44^{\circ} \mathrm{N}, 79.94^{\circ} \mathrm{W}$ over the range $35^{\circ}-50^{\circ}$ north latitude and $75^{\circ}-90^{\circ}$ west longitude. Six-hour back trajectories have been obtained from HYSPLIT four times each day for the 13 months of the study for use with PSCF. Using the results, higher probability locations are compared with known locations of specific source types, based on information from the EPA Toxic Release Inventory (TRI) and the EPA AIRS Database. PSCF results for several sources are compared to the conditional probability function (CPF) analysis, which uses 15-minute wind direction data to determine the most probable direction of a source. Using PSCF and CPF together aids in interpretation of potential source regions. The selenium and sulfate factor source locations are regional, while the lead, cadmium, and specialty steel factor source locations are local. The galliumrich and $\mathrm{Fe}, \mathrm{Mn}$, and $\mathrm{Zn}$ factor source locations are potentially both local and regional. The nitrate, vehicle emissions and road dust, wood combustion, vegetative detritus and cooking, and crustal material factor CPF and PSCF results were inconclusive as sources of these factors exist in all directions from the site and therefore one would not expect a clear probability field in any one direction.
\end{abstract}

\section{INTRODUCTION}

The concentrations of atmospheric fine particulate matter in Pittsburgh, Pennsylvania are largely affected by regional transport of $\mathrm{PM}_{2.5}$ as well as local source emissions (Tang et al. 2004). Positive Matrix Factorization (PMF), a source-receptor model, was used with data collected during the Pittsburgh Air Quality Study (PAQS) to determine the most significant sources of

Received 24 February 2005; accepted 28 December 2005.

Address correspondence to Natalie J. Pekney, Department of Civil and Environmental Engineering, Carnegie Mellon University, Pittsburgh, PA 15213, USA. E-mail: Natalie.Pekney@or.netl.doe.gov
$\mathrm{PM}_{2.5}$ in the Pittsburgh region. Details of PMF use with these data are reported in Pekney et al. (2005a). The current paper uses the PMF-modeled source contributions with the potential source contribution function (PSCF) and conditional probability function $(\mathrm{CPF})$ to locate the sources and to examine each source as being regional or local.

From July 2001 through July 2002, daily 24-hour filter-based samples were collected at the Pittsburgh Supersite, located in a park near the Carnegie Mellon campus approximately $6 \mathrm{~km}$ east of downtown Pittsburgh (Wittig et al. 2004). Filters were analyzed for $\mathrm{PM}_{2.5}$ sulfate, nitrate, organic carbon (OC), elemental carbon (EC), and trace elements. Samples collected on Teflon filters were analyzed by IC analysis for sulfate and nitrate concentrations (Takahama et al. 2004). OC and EC were determined by the Thermal Optical Transmittance method and the NIOSH thermal evolution protocol, which used quartz filters for sample collection (NIOSH 1996; Cabada et al. 2004). A multiplier of 1.8 was used to approximate total organic carbon mass from the OC measurements (Polidori et al. 2005; Turpin and Lim 2001). Samples collected on cellulose filters using a high volume sampler were analyzed for trace elements by ICP-MS after microwave-assisted acid digestion (Pekney and Davidson 2005). A TEOM recorded continuous total $\mathrm{PM}_{2.5}$ mass concentrations, which were averaged over 24 hours for use in this study. To determine the specific chemical components of OC, a cyclone/filterpack sampler collected 24-hour samples on a quartz filter followed by a polyurethane foam (PUF) plug. This sampler operated on a one-in-six day schedule for speciated OC analysis. Several of the OC species were used in the PMF analysis, including hopanes, resin acids, PAHs, syringols, and n-alkanes.

\section{Positive Matrix Factorization}

PMF is a source-receptor model that solves the equation:

$$
x_{i j}=\sum_{i=1}^{p} g_{i k} f_{k j}+e_{i j}
$$


where $x$ is the matrix of ambient data collected at the receptor site, consisting of the species in columns and dates in rows, $g$ is the matrix of source contributions, where each source $k$ contributes to each sample $i$, and $f$ is the mass of each element $j$ in each source $k$ (Paatero and Tapper 1993, 1994; Paatero 1997). A matrix of residual error, $e$, accounts for error in the model fit. PMF seeks to minimize the sum of squares:

$$
Q=\sum_{i=1}^{n} \sum_{j=1}^{m}\left(\frac{e_{i j}}{s_{i j}}\right)^{2}
$$

where $s_{i j}$ are the error estimates for the elements in $x$ described above. The value of $\mathrm{Q}$ should approach the number of degrees of freedom, $n \times m$, or the number of entries in the data matrix. Because uncertainty in the data is taken into account by the model, PMF can accommodate data sets with missing data by assigning an average value to the concentration with a large uncertainty such that the missing data will not significantly weight the results. This characteristic allowed use of the speciated OC data, which was only available for one out of every six days.

With the Pittsburgh Supersite data, the PMF-modeled factors were identified as vehicle emissions and road dust; sulfate; nitrate; selenium; cadmium; lead; wood combustion, vegetative detritus and cooking; gallium-rich; $\mathrm{Fe}, \mathrm{Mn}$, and $\mathrm{Zn}$; specialty steel production and processing; and crustal material. Source compositions and contributions to ambient $\mathrm{PM}_{2.5}$ levels are given and discussed in Pekney et al. (2005a).

The sulfate, nitrate, crustal material, vehicle emissions and road dust, and wood combustion, vegetative detritus and cooking factors all describe regional sources. The sulfate factor contributes the most to $\mathrm{PM}_{2.5}$ on an annual average, $33 \%$. This factor has high concentrations of sulfate as well as a fraction of the OC. This is a secondary source of $\mathrm{PM}_{2.5}$, with particle formation from reactions of gases, with higher concentrations in summer due to an increase in photochemistry. The nitrate, crustal material, and vehicle emissions and road dust factors make smaller contributions to $\mathrm{PM}_{2.5}$, with $8 \%, 12 \%$, and $11 \%$ annual average contributions, respectively. The nitrate factor, with high concentrations of nitrate and some OC, shows lower concentrations in summer due to the temperature sensitivity of nitrate to volatilization. The crustal material contribution, with calcium and titanium tracers, is slightly higher during the first three months of the study with high variability. The vehicle emissions and road dust factor (total OC, EC, hopanes, PAHs, and $\mathrm{n}$-alkanes as tracers) is also highly variable but with no seasonal trend. The wood combustion, vegetative detritus, and cooking factor, with total OC, EC, n-alkanes, syringols, levoglucosan, and resin acid tracers, shows lower contributions during the first three months, with increases in contribution during winter when there is increased wood burning. This factor is approximately $4 \%$ of the $\mathrm{PM}_{2.5}$ mass concentration on an annual average.
The gallium-rich factor contributions correlate very strongly with ambient gallium concentrations, with significant contributions to this factor from arsenic, vanadium, nickel, and copper. While gallium is infrequently used in source-receptor modeling studies and known sources of gallium are few, the latter four elements can be emitted from a variety of sources. However, all of these elements present in trace amounts in coal and therefore this factor could represent primary emissions from coal combustion.

The Fe, Mn, and Zn, specialty steel production and processing, selenium, cadmium, and lead factors are probably local sources rather than regional. These factors are minor in their contribution to $\mathrm{PM}_{2.5}, 1-3 \%$ on an annual average. Despite the minor contribution, importance of these factors can be explained by the possibility of adverse health effects due to $\mathrm{PM}_{2.5}$ composition rather than $\mathrm{PM}_{2.5}$ mass (Carter et al. 1997; Monn and Becker 1999). The source contributions are distinguished by low concentrations for much of the study with peaks on some days. Iron, manganese, and zinc tracers mark the steel production factor. Specialty steel production and processing tracers are molybdenum and chromium, which are both alloying elements in steel. Selenium, cadmium, and lead, are all factors with a unique elemental tracer. Selenium is associated with coal combustion, but this factor is identified by PMF as separate from the sulfate factor as it represents primary and not secondary emissions. Cadmium and lead can be emitted from a variety of industrial activities.

\section{Conditional Probability Function}

The Conditional Probability Function (CPF) calculates the probability that a source is located within a particular wind direction sector, $\Delta \Theta$, or:

$$
\mathrm{CPF}=\frac{m_{\Delta \Theta}}{n_{\Delta \Theta}}
$$

where $n_{\Delta \Theta}$ is the number of times that the wind passed through direction sector $\Delta \Theta$, and $m_{\Delta \Theta}$ is the number of times that the source contribution peaked while the wind passed through sector $\Delta \Theta$ Ashbaugh 1985). To use CPF with the Pittsburgh Supersite data, the 24-hour averaged source contribution data have been applied to all 15-minute wind direction averages measured at the site for each date. All time periods of wind speed less than $1 \mathrm{~m} / \mathrm{s}$ have been removed from the dataset. The angular interval $\Delta \Theta$ was set at $10^{\circ}$. To calculate $m_{\Delta \Theta}$, the highest $25 \%$ of source contribution concentrations were counted. CPF is useful in determining the direction of a source from a receptor site; however, it cannot determine the actual location of the source. To estimate source locations, the Potential Source Contribution Function (PSCF) is used.

\section{Potential Source Contribution Function}

PSCF calculates the probability that a source is located at latitude $i$ and longitude $j$. The basis of PSCF is that if a source is 
located at $(i, j)$, an air parcel back trajectory passing through that location indicates that material from the source can be collected and transported along the trajectory to the receptor site. PSCF solves

$$
\mathrm{PSCF}=\frac{m_{i j}}{n_{i j}}
$$

where $n_{i j}$ is the number of times that the trajectories passed through the cell $(i, j)$ and $m_{i j}$ is the number of times that a source concentration was high when the trajectories passed through the cell $(i, j)$ (Malm et al. 1986). The criteria for determining $m_{i j}$ were either the 75th or 90th percentile highest source contributions, depending on the structure of the source contribution time series for each factor. For example, the time series for the cadmium factor was characterized by relatively low concentrations for most of the study with high peaks much greater than the background concentrations on some days, while the time series for the sulfate factor had many more dates of peak concentrations with not as much difference from the background concentrations. The 75th percentile was used for the sulfate, selenium, lead, gallium-rich, and $\mathrm{Fe}, \mathrm{Zn}$, and $\mathrm{Mn}$ factors while the 90th percentile was used for the specialty steel and cadmium factors. A cell $(i, j)$ with a PSCF value close to one indicates a probable source location. A probability field is constructed for the domain covered by the back trajectories such that locations from which transport to the receptor site results in high concentrations of a source material are most likely locations of sources of that material.

The Hybrid Single-Particle Lagrangian Integrated Trajectory (HYSPLIT) model was used to calculate air mass backtrajectories in 1-hour sequential increments ending at the receptor site at $40.44^{\circ} \mathrm{N}$ latitude and $79.94^{\circ} \mathrm{W}$ longitude (Draxler 1999). HYPLIT was run four times a day for every day of the study, or every six hours starting at midnight EST from July 11, 2001 to July 31, 2002. A starting height of $500 \mathrm{~m}$ AGL was used. Six-hour back trajectories were selected because this time is sufficient to determine probable locations of both local and regional sources, and increasing the time of trajectories also increases the uncertainty, resulting in unrealistic source locations. However, this trajectory time is not sufficient to locate very distant sources that may be hundreds of kilometers away.

The trajectory domain extends from $35^{\circ}-50^{\circ}$ north latitude and $75^{\circ}-90^{\circ}$ west longitude, and is divided into $0.1^{\circ} \times 0.1^{\circ}$ cells, resulting in 22,500 total cells. The maximum $n_{i j}$ is 1570 , although many cells on the outer edges of the grid have $n_{i j}$ less than 10. These outer-edge cells could have misleading large PSCF values if a trajectory passed through them during a source contribution peak, resulting in mistaken attribution of a source to an area. To reduce the effect of spurious large ratios in grid cells with low $n_{i j}$, a weighting function $W_{i j}$ is multiplied by the
PSCF value:

$$
W_{i j}=\left\{\begin{array}{rr}
1.0 & 9<n_{i j} \\
0.9 & 8<n_{i j} \leq 9 \\
0.8 & 7<n_{i j} \leq 8 \\
0.7 & 8<n_{i j} \leq 7 \\
0.6 & 7<n_{i j} \leq 6 \\
0.5 & 6<n_{i j} \leq 5 \\
0.4 & 5<n_{i j} \leq 4 \\
0.3 & 4<n_{i j} \leq 3 \\
0.2 & 3<n_{i j} \leq 2 \\
0.1 & 2<n_{i j} \leq 1
\end{array}\right\}
$$

\section{RESULTS AND DISCUSSION}

Wind direction in the Pittsburgh region is primarily from the southwest to northwest. Figure 1 shows the frequency of trajectories passing through the grid cells in the PSCF grid domain, or $n_{i j}$. There are relatively few occurrences of wind direction from the east during the study period. The results of the PSCF analysis for seven of the eleven PMF-odeled source contributions are shown in Figures 2-8. Cells are shaded according to the PSCF value, with the darkest cells indicating the highest probability of a source in that location. CPF results for six of the eleven sources are shown in Figures 1-5, 7, and 8.

To compare the PSCF results with actual source locations in the region, the EPA Toxics Release Inventory (TRI) is used to identify locations of facilities that emit various elements, specifically the tracer elements for each source category, when available (EPA 2002). The TRI lists toxic chemical releases and other waste management activities reported annually by certain industry groups and federal facilities in a public database. In this application, reported fugitive and point source releases to the air in 2002 are used. Source locations are shown in Figures 3-8, with symbols sized according to the emissions reported.

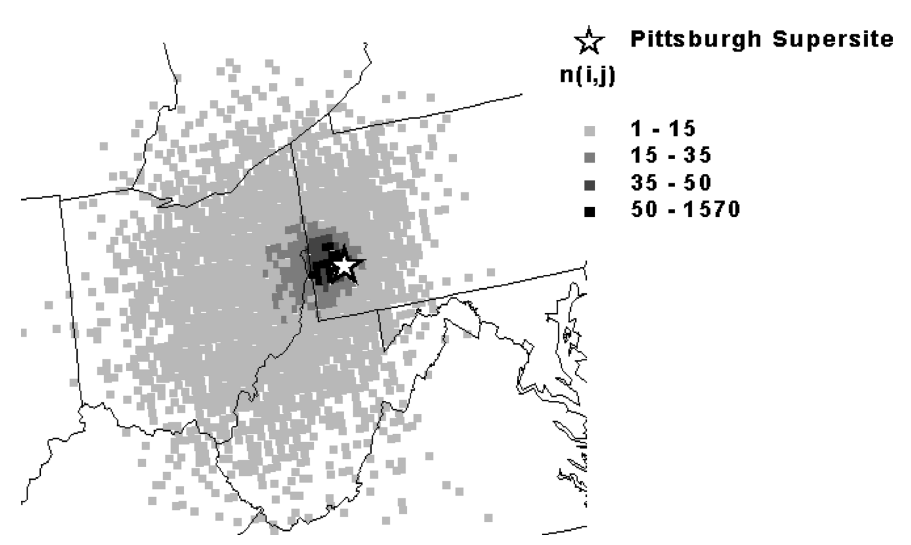

FIG. 1. The values of $n_{i j}$ from Equation (4) for application of PSCF to this study. 


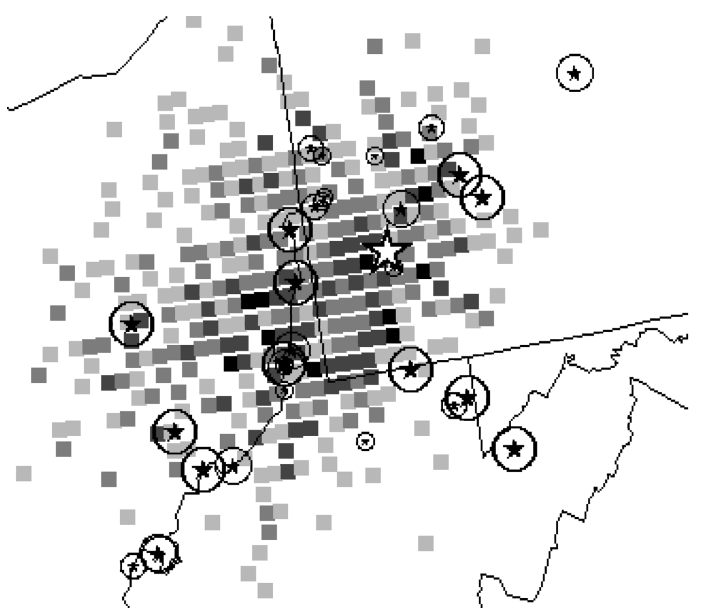

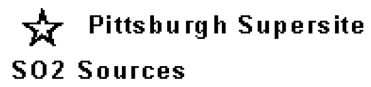

○ $2438-10010$

@ $10010-27596$

(ה) $27596-67139$

(*) $67139-163462$

Sulfate PSCF Values

$0.111-0.25$

$0.25-0.378$

$0.378-0.5$

$0.5-0.667$

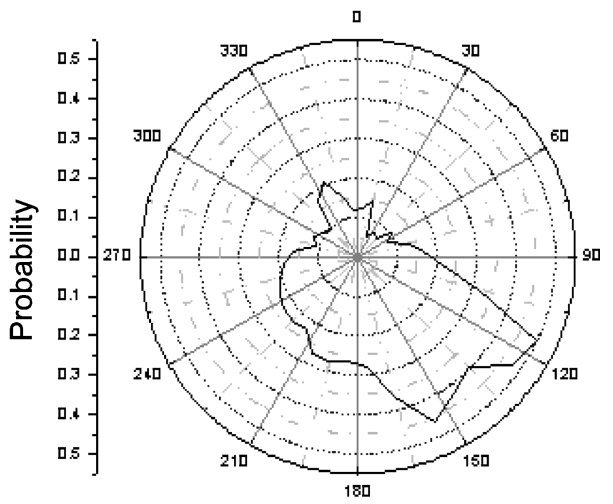

FIG. 2. PSCF and CPF results for the PMF-modeled sulfate source contributions. The top $25 \%$ of source contributions were used for $m_{\Delta \theta}$ and $m_{i j}$. Sulfur dioxide sources are from the AIRS Database (EPA 1999) given in tons emitted in 1997.

Sulfate is a major component of $\mathrm{PM}_{2.5}$ in Pittsburgh, comprising $38 \%$ on an annual average (Rees et al. 2004). The main source of sulfate in the area is from oxidation of the sulfur dioxide emitted from coal-fired power plants. The major sources of electricity in the region are from coal-fired power plants, and there are a large number of these sources in the area. The highest 25\% factor contribution dates are used for determining $m_{i j}$. PSCF results for the PMF-modeled sulfate factor contributions are shown in Figure 2. The region of higher probability of sulfate factor locations is SW of the site in the Ohio River Valley. Sulfate is not reported in the TRI. However, sulfur dioxide is a criteria air pollutant and is listed in the AIRS Database (EPA 1999) pro- viding the source data shown in Figure 2. With the exception of a petroleum and coal products facility in West Virginia, nearly all major sulfur dioxide sources are power plants, and most fall into the area of highest probability of source locations. The most probable direction of the sulfate factor as determined by CPF, also in Figure 2, is to the SE of the site, rather than the Ohio River Valley. The reasons for the discrepancy are unknown. The indication that the Ohio River Valley area is a major source of secondary sulfate has been reported in other studies using PMF and PSCF (Polissar et al. 2001; Lee et al. 2002; Liu et al. 2003), influencing regions as far north as upstate New York, New Jersey, and Vermont.
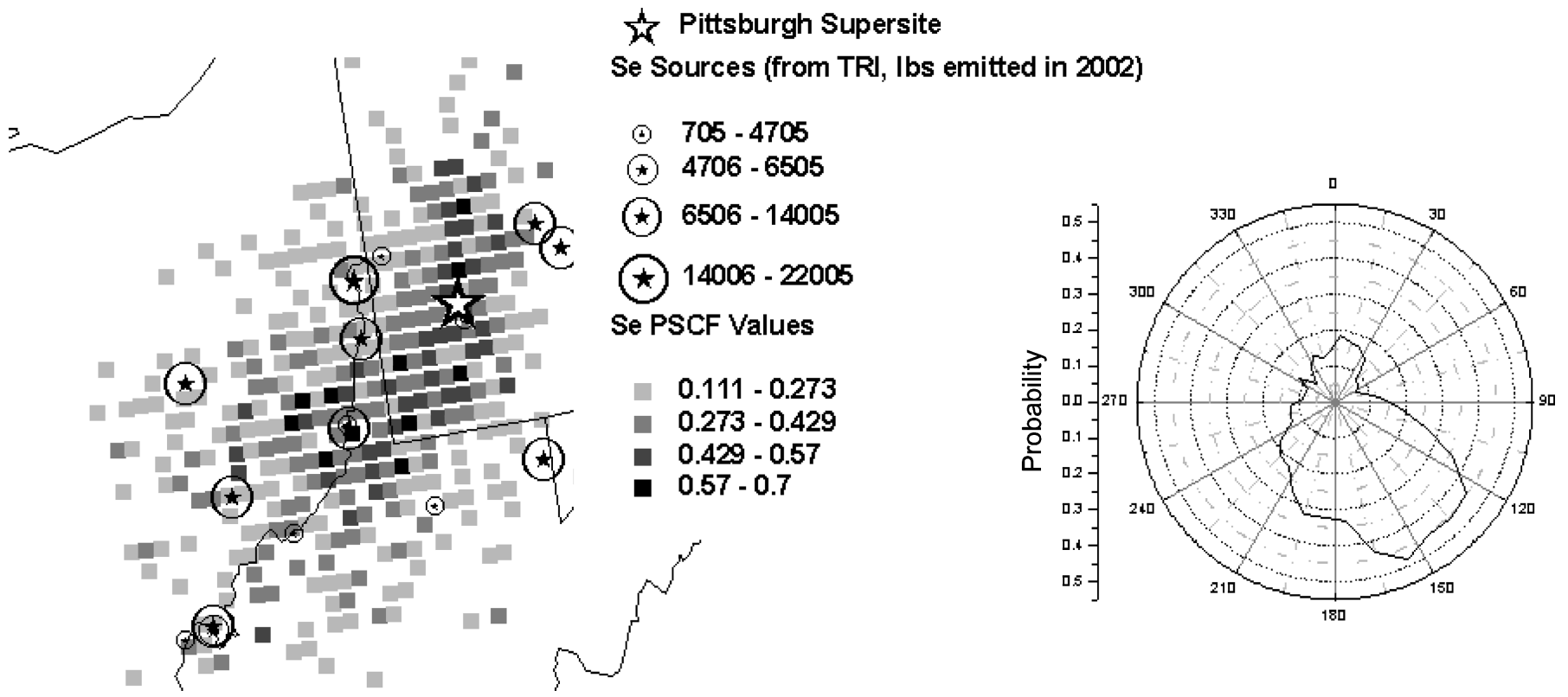

FIG. 3. PSCF and CPF results for the PMF-modeled selenium source contributions. The top $25 \%$ of source contributions were used for $m_{\Delta \theta}$ and $m_{i j}$. 

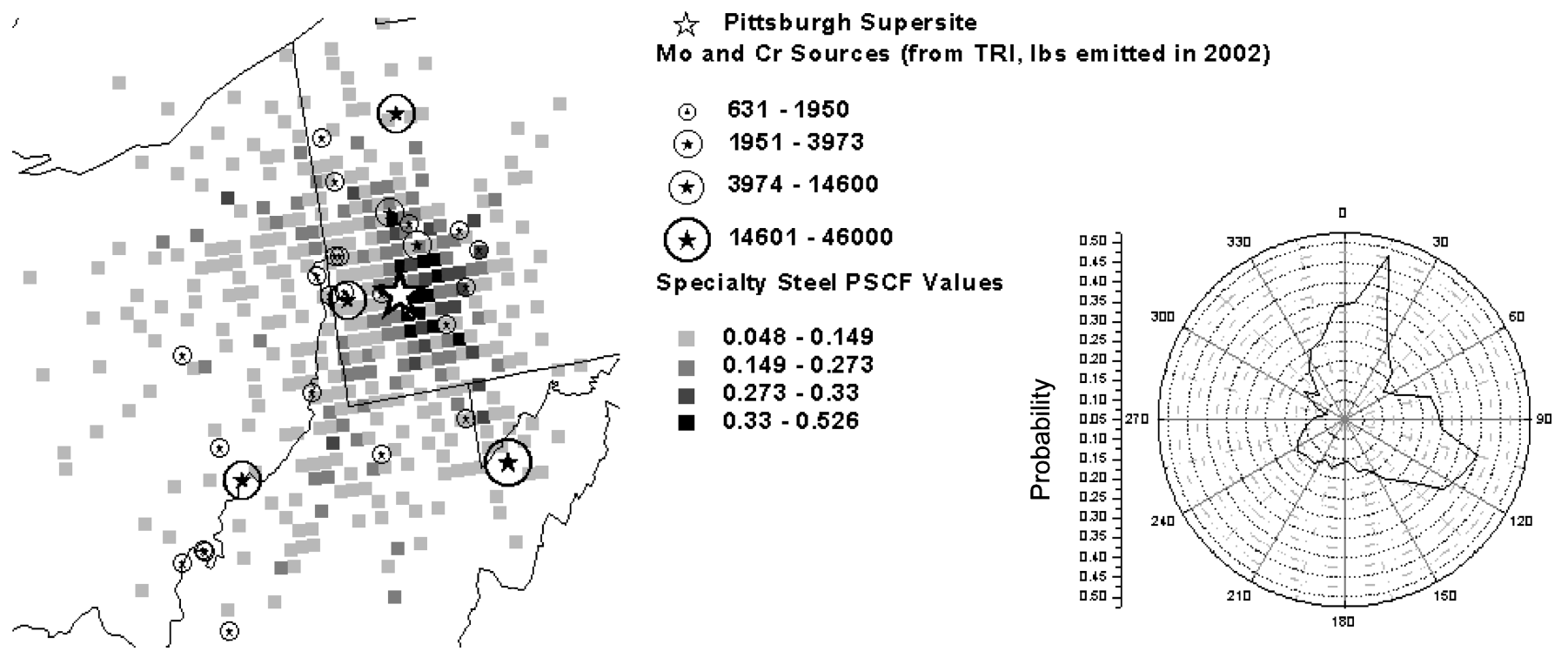

FIG. 4. PSCF and CPF results for the PMF-modeled specialty steel source contributions. The top $25 \%$ of source contributions were used for $m_{\Delta \theta}$ and the top $10 \%$ were used for $m_{i j}$. Symbols for the sizes of the sources are based on the sum of Mo and Cr emissions given in the TRI.

Selenium factor PSCF results are shown in Figure 3. Selenium in $\mathrm{PM}_{2.5}$ is typically associated with combustion of coal. Selenium is a primary pollutant released from burning coal, while sulfate is a secondary pollutant, so it is not surprising that these two factors are defined separately by the PMF analysis. Furthermore, most of the individual point sources of sulfur dioxide in Figure 2 are the same as those shown in Figure 3 for selenium. Selenium factor contributions are usually a low background with occasional high peaks, many more than the cadmium factor contributions, so the $25 \%$ highest factor contribution dates are used rather than $10 \%$ highest. The selenium factor shows highest probabilities of being located SW of the site, following the Ohio River, and to a lesser extent NE of the site. This is in good agreement with sources identified in the TRI as shown in Figure 3. Highest probabilities of a selenium source in a particular cell reach 0.70 . TRI emissions of selenium and selenium compounds in the region are all from electric services, known to be coal-fired power plants, as well as one flat glass production facility and one refuse system facility. Like the sulfate factor, CPF results for this factor (Figure 3) indicate a most probable direction to the SE rather than SW, with a maximum probability of 0.45 . As for sulfate, the reasons for this discrepancy are unknown.

The specialty steel production and processing factor is marked by molybdenum and chromium tracers, as both are alloying elements in specialty steels. The highest $10 \%$ factor

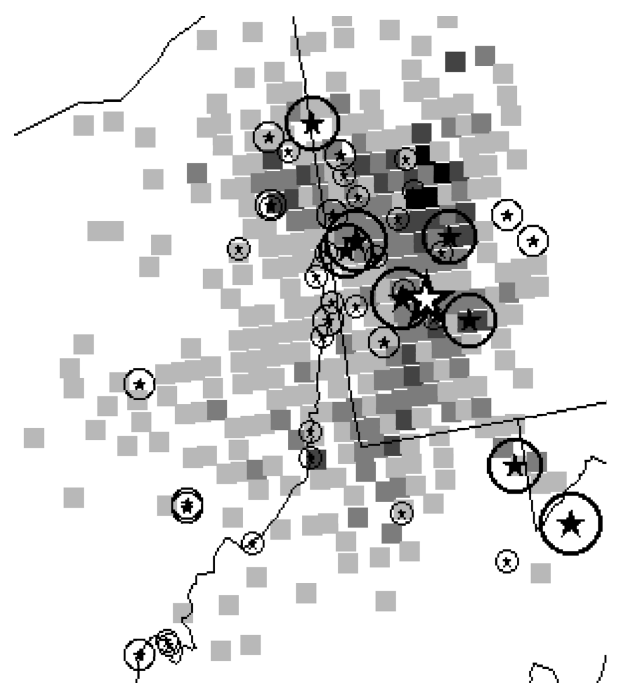

h Pittsburgh Supersite

Lead Sources (from TRI, lbs emitted in 2002)

(-) $151-531$

( $532-1417$

(ऋ) $1418-3490$

(Æ) $3491-10394$

Pb PSCF Values
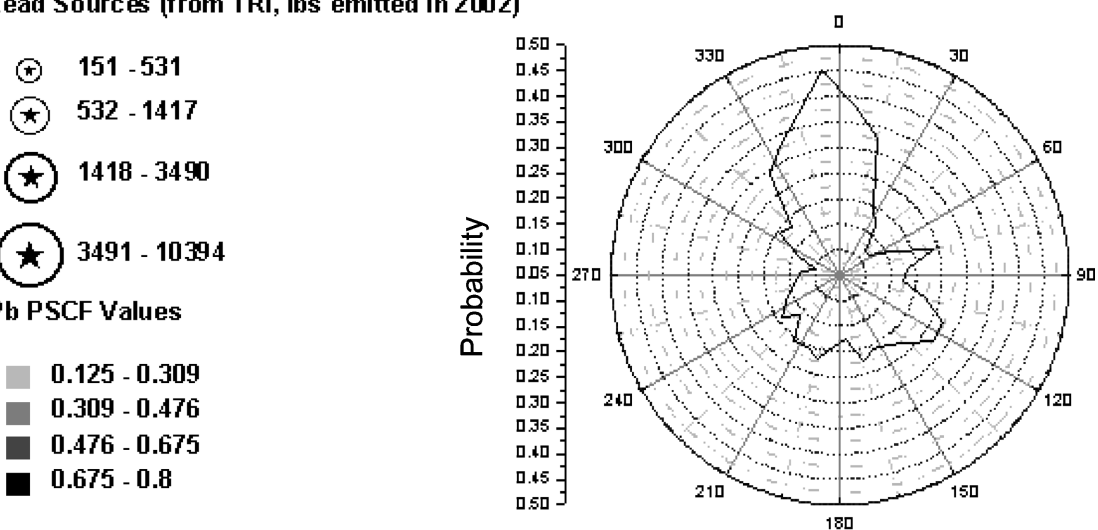

FIG. 5. PSCF and CPF results for the PMF-modeled lead source contributions. The top $25 \%$ of source contributions were used for $m_{\Delta \theta}$ and $m_{i j}$. 

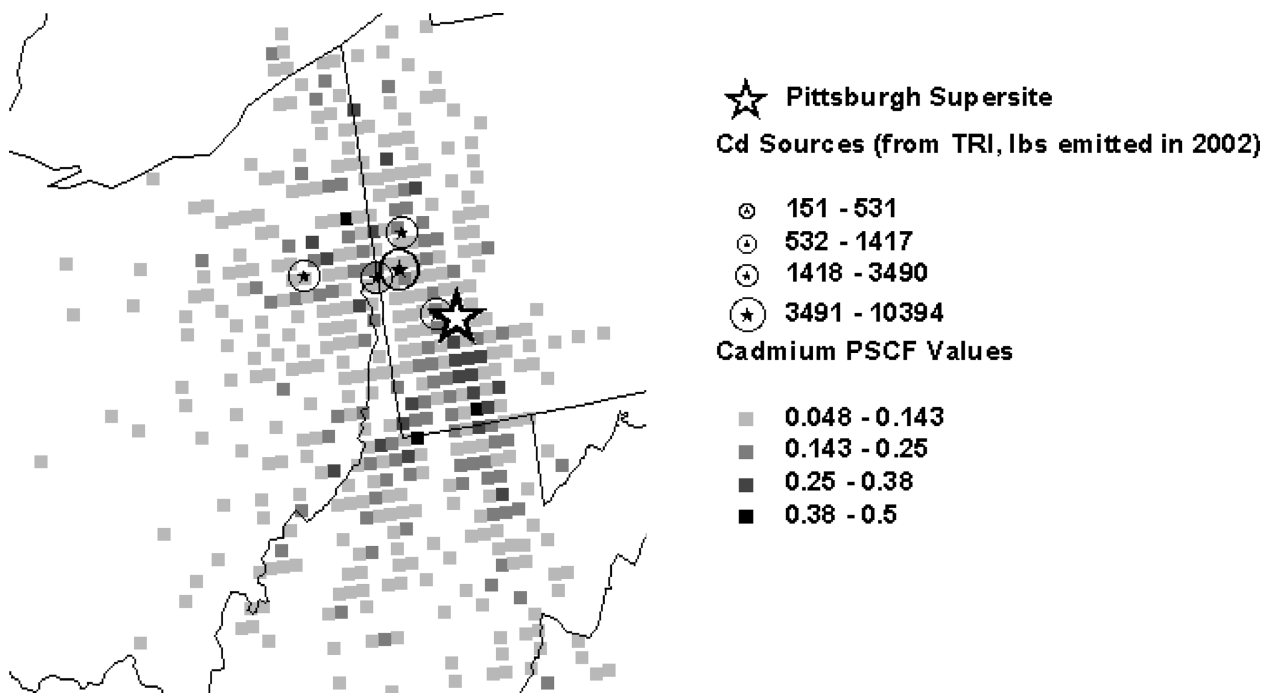

FIG. 6. PSCF results for the PMF-modeled cadmium source contributions. The top $25 \%$ of source contributions were used for $m_{\Delta \theta}$ and the top $10 \%$ were used for $m_{i j}$.

contribution dates are used for $m_{i j}$ as there are fewer dates of high concentrations for this factor. PSCF results in Figure 4 show highest probability of source locations clustered nearest the site, indicating that this is most likely a local source. There are many specialty steel production and processing facilities in the greater Pittsburgh region.

There are only seven known sources of molybdenum in the region (molybdenum trioxide as reported in the TRI) with industry types listed in Table 1 . The molybdenum sources are located to the $\mathrm{N}$ and $\mathrm{W}$ of the site. There are many more sources of chromium in the area. Industry types include the same listed for molybdenum trioxide emissions as well as others shown in Table 1. Chromium sources are located in all directions from the Supersite. PSCF shows highest probability of specialty steel source locations immediately N, E, and S of the site. These locations correspond to many of the chromium and molybdenum sources. CPF corroborates these findings, with most probable directions to the NNE and ESE (Figure 4).

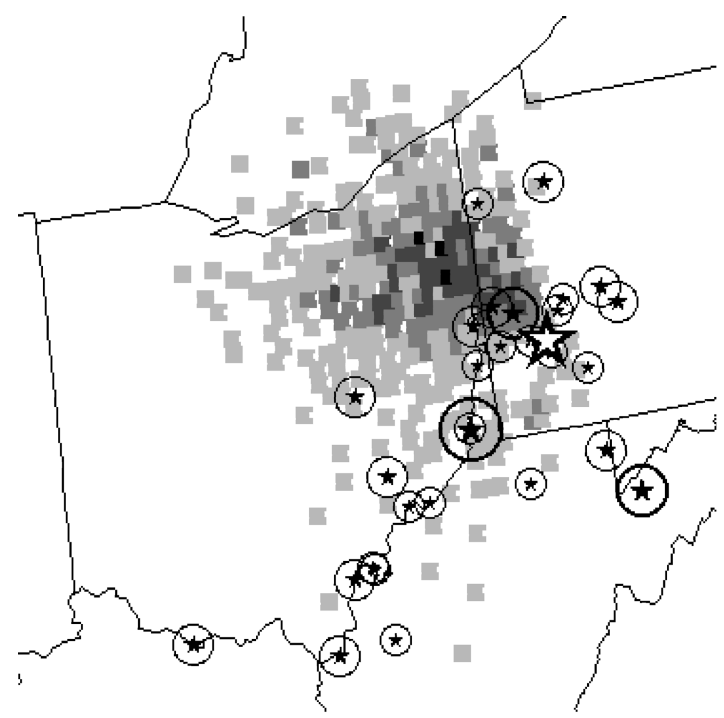

2. Pittsburgh Supersite

$\mathrm{Ni}, \mathrm{V}, \mathrm{Cu}$, and As Sources (from TRI, Ibs emitted in 2002)

$\oplus 1106-3860$

(大) $3861-12480$

(₫) $12481-36700$

( $36701-154400$

Gallium-rich Factor PSCF Values

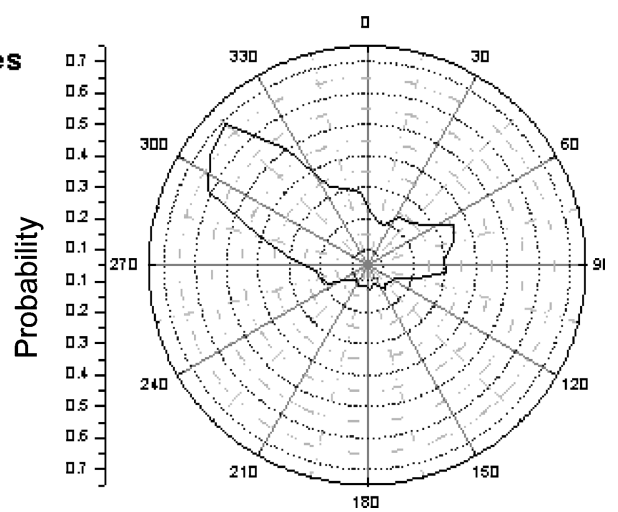

FIG. 7. PSCF and CPF results for the PMF-modeled gallium-rich source contributions. The top $25 \%$ of source contributions were used for $m_{\Delta \theta}$ and $m_{i j}$. Symbols for the sizes of the sources are based on the sum of $\mathrm{Ni}, \mathrm{V}, \mathrm{Cu}$, and $\mathrm{As}$ emissions given in the TRI. 


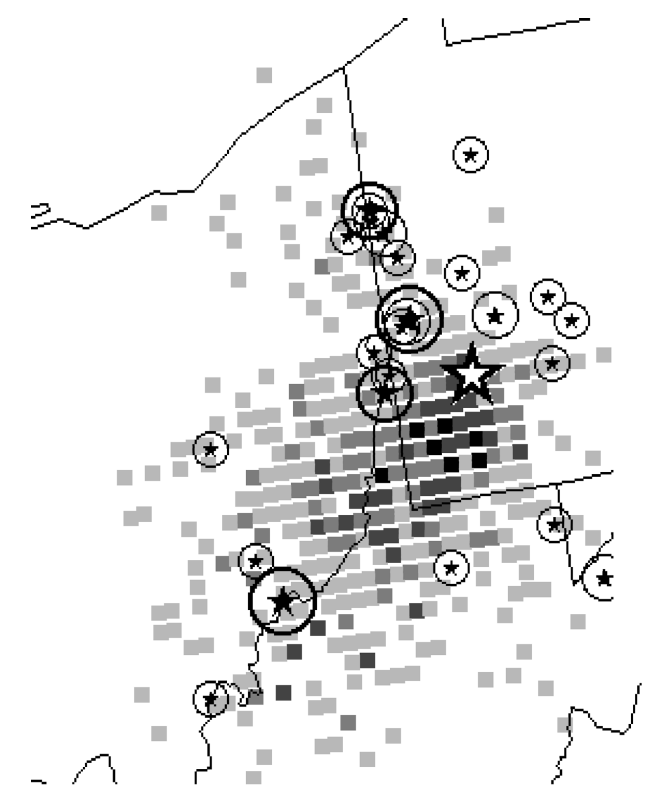

\section{ל Pittsburgh Supersite \\ Zn and Wh Sources (from TRI, Ibs emitted in 2002)}

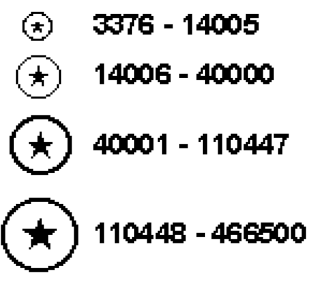

$\mathrm{Fe}, \mathrm{Mn}$, and $\mathrm{Zn}$ PSCF Values

$0.125-0.308$

$0.308-0.474$

- $0.474-0.6$

- $0.6-0.733$
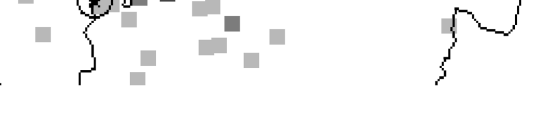

FIG. 8. PSCF and CPF results for the PMF-modeled Fe, Mn, and Zn source contributions. The top $25 \%$ of source contributions were used for $m_{\Delta \theta}$ and $m_{i j}$. Symbols for the sizes of the sources are based on the sum of $\mathrm{Zn}$ and $\mathrm{Mn}$ emissions given in the TRI.

Source locations of the lead factor are difficult to locate using PSCF and CPF, because there are many small point sources of lead in this region. Resuspension of lead particles that were initially emitted during the era of leaded gasoline use can be distributed across a large area, making this source possibly regional as well as local. Industries reporting lead emissions in the TRI are listed in Table 1. In Figure 5, the PSCF results show a higher probability that the lead factor is located mostly to the $\mathrm{N}$ and also $\mathrm{S}$ of the site. The highest $25 \%$ of factor contribution dates were used for $m_{i j}$. The results agree reasonably well with the location of many lead point sources to the $\mathrm{N}$, but reasons for the higher probabilities $\mathrm{S}$ of the site are less clear. $\mathrm{CPF}$ results are somewhat consistent with the PSCF results with $\mathrm{N}$ as the most probable direction (Figure 5).

Figure 6 shows the results for the cadmium factor PSCF. Cadmium source contributions are mostly at background levels, but with a few days of concentration 10-100 times the background. These high concentrations are indicative of local sources that impact the receptor site under the right meteorological conditions. Therefore, to determine $m_{i j}$, the $10 \%$ highest source contribution dates are used. The cadmium factor shows a higher probability of being located to the NW, SW, and S directions from the site. However, the locations of cadmium sources in the region according to the TRI are mostly $\mathrm{N}$ and $\mathrm{NW}$, but none $\mathrm{S}$ of the site. It is possible that there are sources of cadmium in that direction, but the facilities do not report emissions to the TRI. The highest probabilities for the cadmium factor reach 0.50 . Several industry types are responsible for the $\mathrm{Cd}$ emissions shown in Figure 6; those given in the TRI are listed in Table 1. In using CPF (results not shown), the probability of a cadmium source in any direction is less than 0.25 . This low probability renders the CPF results inconclusive.

The gallium-rich factor shows surprising results with the PSCF analysis (Figure 7). The highest 25\% factor contribution dates were used for $m_{i j}$. Gallium ambient concentrations correlate strongly with this factor, but there are also high contributions from arsenic, vanadium, nickel, and copper. Therefore, this factor was assumed to be related to coal combustion, as all of these elements are present in trace amounts in coal. Major coal-fired power plants are located $\mathrm{W}$ and $\mathrm{SW}$ of the site with some sources identical to those shown for selenium and sulfate. In contrast, PSCF results show a clear directional preference to the NW, with very high probabilities of $0.75-0.91$. CPF results agree well with PSCF results for this factor, with a high probability of 0.70 that the source is located to the NW (Figure 7). Gallium is not a toxic chemical and thus is not listed in the TRI; however, all of the other elements are listed. Besides being found in coal fly ash, gallium is a by-product of bauxite processing, as it is associated with aluminosilicates. Gallium and arsenic are used in the microelectronics industry to make GaAs wafers. There are several microelectronics, optics, and photonics companies in northeast Ohio that have foundries for processing aluminum and list GaAs wafers as one of their products. While there are a couple coal-fired power plants NW of the site, PMF distinguished this gallium-rich factor as separate from the sulfate and selenium factors associated with all coal-fired power plants in the region due to the influence of gallium emissions from unknown NW sources.

PSCF results for the Fe, Mn, and Zn factor, shown in Figure 8, are inconclusive as the highest probability areas identified by 


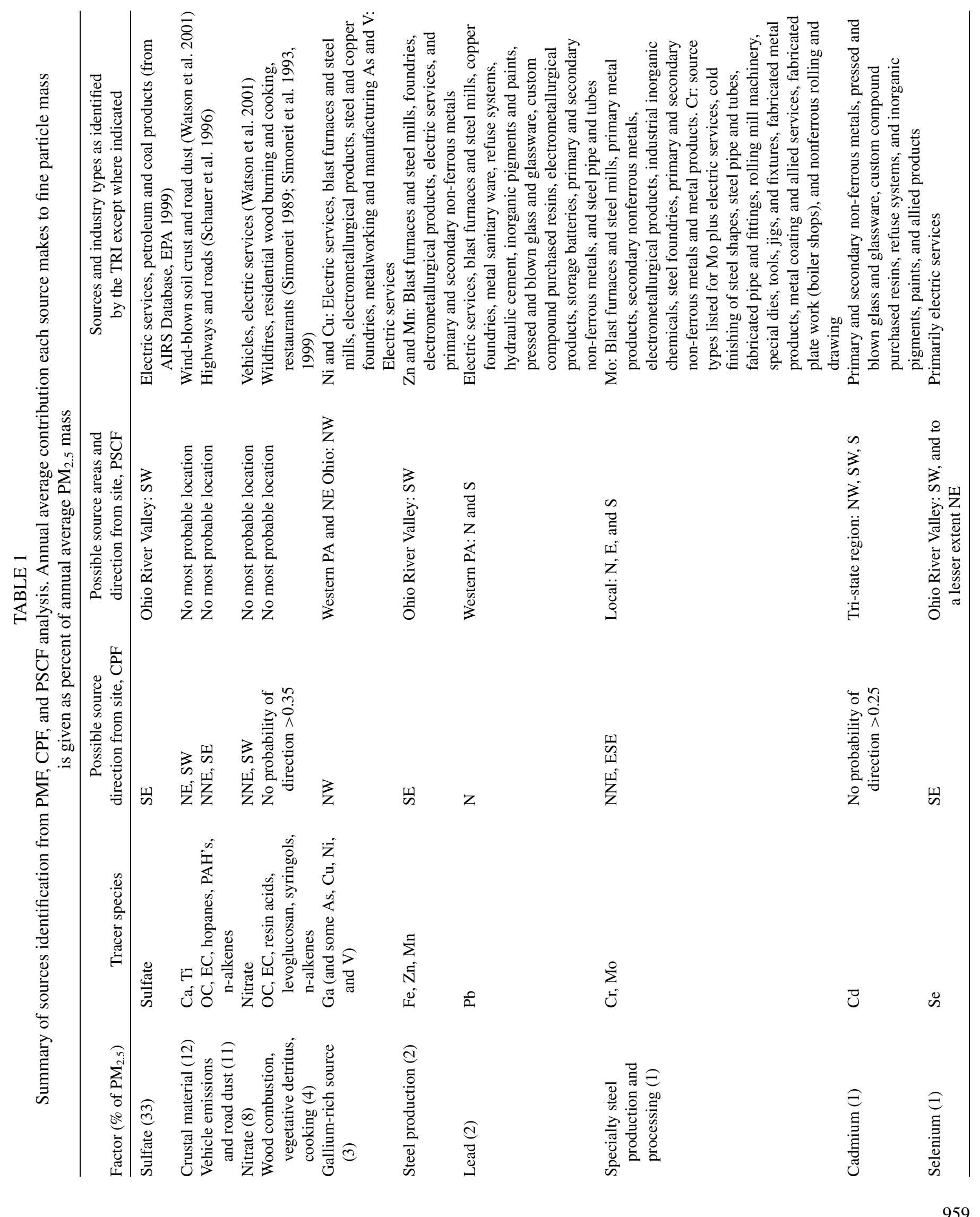


PSCF do no coincide with zinc and manganese sources as reported in the TRI. PSCF, using the highest $25 \%$ factor contribution dates, shows highest probability of this factor being located to the SW. However, zinc sources are mostly located to the NW and manganese sources are in every direction. Iron, also a tracer species for this factor, is not a toxic chemical listed in the TRI and hence the emissions and their locations are unknown. Industry types that report emissions of zinc and manganese in the TRI are listed in Table 1. CPF results (Figure 8) indicate SE as the most probable direction, which further complicates determining the location of sources of this factor.

There are several factors determined by PMF that do not yield conclusive results from the PSCF or CPF analyses: nitrate; wood combustion, vegetative detritus and cooking; crustal material; and vehicle emissions and road dust. These source categories exist in all directions from the Supersite and therefore one would not expect a clear probability field in any one direction. Therefore, PSCF and CPF results are not shown for these sources. Brief summaries are listed for all factors in Table 1.

\section{CONCLUSIONS}

PSCF and CPF results for the PMF factors presented can be grouped into three different categories: regional sources, local sources, or potentially both regional and local sources. The sulfate and selenium factors represent regional sources identified as coal fired power plants located regionally. For these factors, the PSCF results show most probable source locations in agreement with TRI or AIRS Database source locations while the CPF results show a slightly offset most probable direction that does not agree well with the PSCF results. However, for the specialty steel and lead factors, which represent local sources, CPF results show clear most probable directions that agree well with both the PSCF results, which show a clustering of most probable locations nearest the site, and the known locations of molybdenum, chromium, and lead sources by TRI. Although the cadmium factor is assumed to also represent a local source or sources, the CPF results were inconclusive, with no direction showing a strongly significant probability. The gallium-rich and $\mathrm{Fe}, \mathrm{Mn}$, and $\mathrm{Zn}$ factors could represent either local or regional sources, or both. The gallium-rich factor PSCF and CPF results show very good agreement but as this source cannot be identified, a conclusion as to its being regional or local cannot be drawn. And while the Fe, Mn, and Zn factor PSCF and CPF results are similar to results for the sulfate and selenium factors, the difference that there are known local $\mathrm{Mn}$ and $\mathrm{Zn}$ sources is the direction indicated as most probable by CPF. Therefore, this factor can be considered both regional and local. In conclusion, PSCF appears to better predict locations of regional sources while CPF is useful in pinpointing directions of local sources.

Table 1 presents a summary of the findings presented in this paper. PMF-modeled factor names are given with their tracer species, annual average contribution to $\mathrm{PM}_{2.5}$ mass concentra- tions in Pittsburgh, source regions as determined by the PSCF and CPF analyses, and possible sources as identified by the tracer species and the TRI. Overall, the results for several of the source categories are consistent with single particle composition data from the PAQS (Pekney et al. 2005b). PMF, CPF, and PSCF are powerful tools for determining the major sources of particulate pollution in an urban area like Pittsburgh, but each model has its limitations. Both models rely on counting statistics and therefore require large amounts of data such that results are statistically significant. PMF cannot distinguish between sources in the same area that impact the receptor location. This may lead to a mixing of different types of sources that makes it difficult to determine the apportionment of source contributions to $\mathrm{PM}_{2.5}$ mass concentrations. CPF can provide the probability that a source is located in a particular direction, but cannot provide any information as to the proximity of the source. PSCF, however, provides probability that a source is located in a particular grid cell, but is dependent upon the accuracy of the HYSPLIT model. HYSPLIT trajectory accuracy decreases with increasing trajectory time, and effects of the boundary layer are not considered. In an urban area like the Pittsburgh, where there are many industrial activities in and near the city as well as in the surrounding heavily industrialized region, clearly identifying source locations using any of these models is difficult. High concentrations of some pollutant species can occur for a wide range of wind directions. Furthermore, mixing height as well as wind direction can affect airborne concentrations of the tracer species, although only the latter is used in these models. When 24-hour data are used, source plumes that occur on the order of minutes are masked due to the variability of wind direction over a day. Despite these limitations, using PMF, CPF, and PSCF together determined probable locations for several of the modeled sources that have been shown to correlate with the locations of known sources as reported to the TRI. This study demonstrated that CPF and PSCF aided in determining PM source locations to significantly improve source apportionment study.

\section{REFERENCES}

Ashbaugh, L., Malm, W., and Sadeh, W. (1985). A Residence Time Probability Analysis of Sulfur Concentrations at Ground Canyon National Park, Atmos. Environ. 19(8):1263-1270.

Cabada, J. C., Khlystov, A., Wittig, E., Pilinis, C., and Pandis, S. (2004). Light Scattering by Fine Particles During the Pittsburgh Air Quality Study: Measurements and Modeling, J. Geophys. Res. 109:D16S03.

Carter, J. D., Ghio, A. J., Samet, J. M., and Devlin, R. B. (1997). Cytokine Production by Human Airway Epithelial Cells After Exposure to an Air Pollution Particle Is Metal-Dependent, Toxicol. Appl. Pharmacol. 146:180188.

Draxler, R. R. (1999). HYSPLIT_4 User's Guide, NOAA Technical Memorandum ERL ARL-230, Air Resources Laboratory, Silver Spring, MD.

Malm, W. C., Johnson, C. E., and Bresch, J. F. (1986). Application of Principal Component Analysis for Purposes of Identifying Source-Receptor Relationships. In Receptor Methods for Source Apportionment (edited by T. G. Pace), Publication TR-5. Air Pollution Control Association, Pittsburgh, PA.

Monn, C. and Becker, S. (1999). Cytotoxicity and Induction of Proinflammatory Cytokines from Human Monocytes Exposed to Fine $\left(\mathrm{PM}_{2.5}\right)$ and Coarse 
Particles $\left(\mathrm{PM}_{10-2.5}\right)$ in Outdoor and Indoor Air, Toxicol. Appl. Pharmacol. 155:245-252.

Lee, J. H., Yoshida, Y., Turpin, B., Hopke, et al. (2002). Identification of Sources Contributing to Mid-Atlantic Regional Aerosol, J. Air and Waste Manag. Assoc. 52:1186-1205.

Liu, W., Hopke, P., Han, Y., Yi, S., et al. (2003). Appliation of Receptor Modeling to Atmospheric Constituents at Potsdam and Stockton. NY, Atmos. Environ. 37:4997-5007.

NIOSH. (1996). Elemental Carbon (Diesel Exhaust). In: NIOSH Manual of Analytical Methods. National Institute of Occupational Safety and Health, Cincinnati, $\mathrm{OH}$.

Paatero, P. and Tapper, U. (1993). Analysis of Different Modes of Factor Analysis as Least Square Fit Problems, Chemometrics and Intelligent Laboratory Systems. 18:183-194.

Paatero, P. and Tapper, U. (1994). Positive Matrix Factorization: A Non-Negative Factor Model with Optimal Utilization of Error Estimates of Data Values, Environmetrics 5:111-126.

Paatero, P. (1997). Least Squares Formulation of Robust Non-Negative Factor Analysis, Chemometrics and Intelligent Laboratory Systems 37:15-35.

Pekney, N., Davidson, C., Robinson, A., Zhou, L. et al. (2005a). Identification of Major Sources of $\mathrm{PM}_{2.5}$ in Pittsburgh using PMF and Unmix, Aerosol Sci. Technol. in press.

Pekney, N., Davidson, C., Bein, K., Wexler, A., and Johnston, M. (2005b). Identification of Sources of Atmospheric PM at the Pittsburgh Supersite Part I: Single Particle Analysis and Filter-Based Positive Matrix Factorization, Atmos. Environ. in press.

Pekney, N. and Davidson, C. I. (2005). Determination of Trace Elements in Ambient Aerosol Samples, Analytica Chimica Acta. 540:269-277.

Polidori, A., Turpin, B., Lim, H., Totten, L., and Davidson, C. (2005). Polarity and Molecular Weight/Carbon Weight of the Pittsburgh Organic Aerosol. Manuscript in preparation.

Polissar, A., Hopke, P., and Poirot, R. (2001). Atmospheric Aerosol over Vermont: Chemical Composition and Sources, Environ. Sci. Technol. 35:46044621.
Rees, S. L., Robinson, A. L., Khylstov, A., Stanier, C. O., and Pandis, S. (2004). Mass Balance Closure and the Federal Reference Method for $\mathrm{PM}_{2.5}$ in Pittsburgh, Pennsylvania, Atmos. Environ. 38:3305-3318.

Schauer, J. J., Rogge, W. F., Hildermann, L. M., Mazurek, M. A., and Cass, G. R. (1996). Source Apportionment of Airborne Particulate Matter using Organic Compounds as Tracers, Atmos. Environ. 30:3837-3855.

Simoneit, B. R. T. (1989). Organic Matter of the Troposphere. 5. Application of Molecular Marker Analysis to Biogenic Emissions into the Troposphere for Source Reconciliations, J. Atmos. Chem. 8:251-275.

Simoneit, B. R. T., Rogge, W. F., Mazurek, M. A., Standley, L. J., Hildemann, L. M., and Cass, G. R. (1993). Lignin Pyrolosis Products, Lignans, and Resin Acids as Specific Tracers of Plant Classes in Emissions from Biomass Combustion, Environ. Sci. Technol. 27:2533-2541.

Simoneit, B. R. T., Schauer, J. J., Nolte, C. G., Oros, D. R. et al. (1999). Levoglucosan, A Tracer for Cellulose in Biomasa Burning and Atmospheric Particles, Atmos. Environ. 33:173-182.

Takahama, S., Wittig, B., Vayenas, D., and Davidson, C. I. et al. (2004). Modeling the Diurnal Variation of Nitrate During the Pittsburgh Air Quality Study, J. Geophys. Res.—Atmos. 109(D16):Art. No. D16S06.

Tang, W., Raymond, T., Wittig, E., Davidson, C. I. et al. (2004). Spatial Variations in $\mathrm{PM}_{2.5}$ During the Pittsburgh Air Quality Study, Aerosol Sci. Technol. 38(2):80-90.

Turpin, B. and Lim, H. (2001). Species Contributions to $\mathrm{PM}_{2.5}$ Mass Concentrations: Revisiting Common Assumptions for Estimating Organic Mass, Aerosol Sci. Technol. 35:602-610.

U.S. EPA. (1999). AirData Website (www.epa.gov/air/data/index.html). 1999 data used, accessed 2004.

U.S. EPA. (2002). 2002 TRI Data Release, www.epa.gov/tri. Accessed 2004

Watson, J. G., Chow, J. C., and Houck, J. E. (2001). PM 2.5 Chemical Source Profiles for Vehicle Exhaust, Vegetative Burning, Geological Material, and Coal Burning in Northwestern Colorado During 1995, Chemosphere 43:11411151

Wittig, A.E., Anderson, N. J., Khlystov, A. Y., Pandis, S. N. et al. (2004). Pittsburgh Air Quality Study overview, Atmos. Environ. 38:3170-3125. 


\title{
Major Source Categories for $\mathbf{P M}_{2.5}$ in Pittsburgh using PMF and UNMIX
}

\author{
Natalie J. Pekney, ${ }^{1}$ Cliff I. Davidson, ${ }^{2}$ Allen Robinson, ${ }^{3}$ Liming Zhou, ${ }^{4}$ \\ Philip Hopke, ${ }^{4}$ Delbert Eatough, ${ }^{5}$ and Wolfgang F. Rogge ${ }^{6}$ \\ ${ }^{1}$ Department of Civil and Environmental Engineering, Carnegie Mellon University, Pittsburgh, \\ Pennsylvania, USA \\ ${ }^{2}$ Department of Civil and Environmental Engineering and Engineering and Public Policy, \\ Carnegie Mellon University, Pittsburgh, Pennsylvania, USA \\ ${ }^{3}$ Department of Mechanical Engineering and Engineering and Public Policy, Carnegie Mellon \\ University, Pittsburgh, Pennsylvania, USA \\ ${ }^{4}$ Center for Air Resources Engineering and Science, and Department of Chemical Engineering, \\ Clarkson University, Potsdam, New York, USA \\ ${ }^{5}$ Department of Chemistry and Biochemistry, Brigham Young University, Provo, Utah, USA \\ ${ }^{6}$ Department of Civil and Environmental Engineering, Florida International University, \\ Engineering Center, Miami, Florida, USA
}

An objective of the Pittsburgh Air Quality Study was to determine the major sources of $\mathbf{P M}_{2.5}$ in the Pittsburgh region. Daily 24-hour averaged filter-based data were collected for 13 months, starting in July 2001, including sulfate and nitrate data from IC analysis, trace element data from ICP-MS analysis, and organic and elemental carbon from the thermal optical transmittance (TOT) method and the NIOSH thermal evolution protocol. These data were used in two source-receptor models, Unmix and PMF. Unmix, which is limited to a maximum number of seven factors, resolved six source factors, including crustal material, a regional transport factor, secondary nitrate, an iron, zinc and manganese factor, specialty steel production and processing, and cadmium. PMF, which has no limit to the number of factors, apportioned the $\mathbf{P M}_{2.5}$ mass into ten factors, including crustal material, secondary sulfate, primary $O C$ and EC, secondary nitrate, an iron, zinc and manganese factor, specialty steel production and processing, cadmium, selenium, lead, and a gallium-rich factor. The Unmix and PMF common factors agree reasonably well, both in composition and contributions to $\mathbf{P M}_{2.5}$. To further identify and apportion the sources of $\mathbf{P M}_{2.5}$, specific OC compounds that are known markers of some sources were added to the PMF analysis. The results were similar to the

Received 8 January 2005; accepted 27 September 2005.

This research was conducted as a part of the Pittsburgh Air Quality Study which was supported by the US Environmental Protection Agency under contract R82806101 and the US Department of Energy National Energy Technology Laboratory under contract DE-FC2601NT41017. This paper has not been subject to EPA's peer and policy review, and therefore does not necessarily reflect the views of the Agency. No official endorsement should be inferred.

Address correspondence to Cliff I. Davidson, Department of Civil and Environmental Engineering, Carnegie Mellon University, 5000 Forbes Avenue, Pittsburgh, PA 15213, USA. E-mail: cliff@cmu.edu original solution, except that the primary $\mathrm{OC}$ and $\mathrm{EC}$ factor split into two factors. One factor was associated with vehicles as identified by the hopanes, PAH's, and other OC compounds. The other factor had strong correlations with the $\mathrm{OC}$ and $\mathrm{EC}$ ambient data as well as wood smoke markers such as levoglucosan, syringols, and resin acids.

\section{INTRODUCTION}

Elevated levels of $\mathrm{PM}_{2.5}$ have been linked to human health effects (e.g., Dockery et al. 1993; Pope et al. 2002). Upon establishment of a National Ambient Air Quality Standard for $\mathrm{PM}_{2.5}$ in 1997 (US EPA 1997a), many urban areas find themselves to be in non-attainment of the $\mathrm{PM}_{2.5}$ standard. Effective air pollution control strategies for $\mathrm{PM}_{2.5}$ require investigation into its sources, composition, and spatial and temporal variations. The Pittsburgh Air Quality Study (PAQS) was a multidisciplinary set of projects in the Pittsburgh region designed to address these issues. PAQS program objectives, hypotheses, site description, and measurement methods are described in Wittig et al. (2004). In this paper, data collected during the PAQS are analyzed with two commonly used source-receptor models, Unmix and Positive Matrix Factorization (PMF), to determine the major source categories and their contributions to ambient concentrations of $\mathrm{PM}_{2.5}$.

The main contributions of this paper are (1) a source apportionment for Pittsburgh $\mathrm{PM}_{2.5}$, (2) a comparison of the PMF and Unmix models, and (3) evaluation of PMF results upon addition of individual organic markers. Several studies of the sources of $\mathrm{PM}_{2.5}$ in Pittsburgh have been conducted as a part of the PAQS; 
however, they have been focused on measurements from one instrument or analysis technique or a small set of the components of $\mathrm{PM}_{2.5}$. Source apportionment of the carbon fraction of $\mathrm{PM}_{2.5}$ has been reported for Pittsburgh (Subramanian 2004). Sourcereceptor modeling based on one month or less of sampling has been conducted (Modey et al. 2005; Zhou et al. 2004). And composition of single particles in an effort to identify sources of the particles has been explored (Lithgow et al. 2004). This paper describes source-receptor modeling with a larger dataset such that more factors and their seasonal variations can be identified. PMF and Unmix comparisons have been conducted in other studies (Maykut et al. 2003; Kim et al. 2004; Poirot et al. 2001; Larsen and Baker 2003; Eatough et al. 2005) with varying results. Confidence in using these two receptor modeling tools is gained with convergent results, whereas divergent results signify limitations in the data or models. Trace elements have been used in many receptor modeling studies due to the assumption that they can serve as tracers for some sources. However, most elements can be emitted from multiple types of sources, and thus identifying factors based on their correlation with elemental data is difficult. Molecular markers have been shown to be good tracers of certain sources (Schauer and Cass 2000; Schauer et al. 1996; Simoneit et al. 1999; Subramanian et al. 2005b). This paper discusses the use of both elemental species and molecular markers in a PMF analysis in an effort to improve the interpretation of factors as well as to resolve more factors than a PMF analysis using elemental tracers alone.

\section{EXPERIMENTAL AND METHODOLOGY SECTION}

\section{Site Description}

All samples in this study were collected at the PAQS main monitoring station. The station was located approximately $6 \mathrm{~km}$ east of downtown Pittsburgh in a park, where impact from local sources was minimized. Sampling equipment was housed on the roof of a trailer, approximately $5 \mathrm{~m}$ from the ground. Samples were collected daily from July 2001 through September 2002 although only data collected from July 2001 to July 2002 have been used in this study. Site and project details are described more completely in Wittig et al. (2004).

\section{Sampling and Analysis}

Ambient concentration data used in the source-receptor modeling include total ambient $\mathrm{PM}_{2.5}$ mass, sulfate, nitrate, total organic carbon (OC), elemental carbon (EC), and trace elements. These species represent the major constituents of $\mathrm{PM}_{2.5}$ in Pittsburgh with the exception of ammonium (Rees et al. 2004). Total number of missing and below detection limit values are summarized in Table 1 for each species.

\section{PM 2.5 Mass}

Samples were collected on $47 \mathrm{~mm}$ Teflon filters (Whatman No: 7592-104) using a Partisol ${ }^{\circledR}$-FRM Model $2000 \mathrm{PM}_{2.5}$ Air
TABLE 1

Summary of the dataset for PMF and Unmix use. All considered species are listed; not all species were used in the two models. Number of values below the minimum detection limit (MDL) and number of missing values are given out of a total of 386 sampling dates

\begin{tabular}{|c|c|c|}
\hline $\mathrm{PM}_{2.5}$ species & $\begin{array}{l}\text { Number of values } \\
\text { below MDL }\end{array}$ & $\begin{array}{c}\text { Number of missing } \\
\text { values }\end{array}$ \\
\hline Total $\mathrm{PM}_{2.5}$ & 0 & 45 \\
\hline Sulfate & 0 & 20 \\
\hline Nitrate & 0 & 9 \\
\hline $\mathrm{OC}$ & 0 & 1 \\
\hline $\mathrm{EC}$ & 0 & 1 \\
\hline $\mathrm{Ag}$ & 230 & 5 \\
\hline As & 5 & 5 \\
\hline $\mathrm{Ba}$ & 69 & 5 \\
\hline $\mathrm{Be}$ & 278 & 5 \\
\hline $\mathrm{Ca}$ & 191 & 5 \\
\hline $\mathrm{Cd}$ & 45 & 5 \\
\hline $\mathrm{Ce}$ & 260 & 57 \\
\hline Co & 175 & 5 \\
\hline $\mathrm{Cr}$ & 63 & 5 \\
\hline Cs & 295 & 5 \\
\hline $\mathrm{Cu}$ & 122 & 5 \\
\hline $\mathrm{Fe}$ & 31 & 5 \\
\hline $\mathrm{Ga}$ & 95 & 5 \\
\hline K & 41 & 5 \\
\hline $\mathrm{Li}$ & 189 & 5 \\
\hline $\mathrm{Mg}$ & 115 & 5 \\
\hline $\mathrm{Mn}$ & 5 & 5 \\
\hline Мo & 42 & 5 \\
\hline $\mathrm{Ni}$ & 72 & 5 \\
\hline $\mathrm{Pb}$ & 5 & 5 \\
\hline $\mathrm{Rb}$ & 124 & 5 \\
\hline $\mathrm{Sb}$ & 14 & 5 \\
\hline $\mathrm{Se}$ & 4 & 5 \\
\hline $\mathrm{Sr}$ & 95 & 5 \\
\hline $\mathrm{Ti}$ & 30 & 5 \\
\hline $\mathrm{Tl}$ & 238 & 5 \\
\hline V & 22 & 5 \\
\hline $\mathrm{Zn}$ & 38 & 5 \\
\hline
\end{tabular}

Sampler (Rupprecht \& Pataschnick Co., Inc.). Semi-continuous $\mathrm{PM}_{2.5}$ mass was measured using a Tapered Element Oscillating Microbalance (TEOM) monitor with a Sample Equilibration System (SES) (Rupprecht and Patashnick, Model 1400A). Data from the TEOM were averaged to get 24-hour measurements. The 24-hour averaged data from the two $\mathrm{PM}_{2.5}$ mass measurement methods agree well with a linear regression $\mathrm{R}^{2}$ value of 0.95 , a slope of 1.02 and an intercept of 0.65 (Rees et al. 2004). However, the TEOM recorded fewer missing dates, 
hence the data collected by the TEOM will be used in this study.

\section{Sulfate and Nitrate}

A $\mathrm{PM}_{2.5}$ sampler with cyclones, denuders and filter packs containing PTFE Teflon, nylon, and cellulose filters was used to collect daily 24-hour averaged samples. The material collected on the filters was extracted in deionized water and the solution was analyzed for nitrate, nitrite, sulfate, chloride, ammonium, and sodium by Ion Chromatography (IC) (Takahama et al. 2004).

\section{$O C$ and $E C$}

$\mathrm{PM}_{2.5}$ samples were collected on quartz filters using a cyclone/filter pack sampler (Subramanian et al. 2004). The quartz filters were analyzed for OC and EC using the Thermal Optical Transmittance (TOT) method and the NIOSH thermal evolution protocol (NIOSH 1996; Cabada et al. 2004). Because the OC data are used in a mass balance application, an OC multiplier is applied to the measurements to approximate total organic mass. Most mass balance studies use a value of 1.4 for the OC multiplier. Based on recent work by Polidori et al. (2005) and Turpin and Lim (2001) and the hypothesis that the air quality in Pittsburgh is dominated by regional transport (Tang et al. 2004; Anderson et al. 2004), a multiplication factor of 1.8, which is representative of an aged, regional aerosol, was used to estimate total organic mass from OC measurements.

\section{Trace Elements}

A Thermo Andersen $\mathrm{PM}_{2.5}$ high volume sampler (hi vol) was used to collect daily 24-hour averaged filter-based samples from July 11, 2001 through July 31, 2002. Samples collected on Whatman 41 cellulose filters were cut into sections, and digested in a laboratory microwave in closed vessels containing a solution of nitric/hydrofluoric acid and hydrogen peroxide. The sample preparation and subsequent analysis by ICP-MS were verified to be accurate by testing recovery of a NIST standard reference material (Pekney and Davidson 2005). Measured elements include $\mathrm{Ag}$, As, Ba, Be, Ca, Cd, Ce, Co, Cr, Cs, Cu, Fe, Ga, K, Li, Mg, Mn, Mo, Ni, Pb, Rb, Sb, Se, Sr, Ti, Tl, V, and Zn. Minimum detection limits (MDLs), calculated for each sample, were defined as $3.3 \sigma_{\mathrm{B}} / \mathrm{V}$, where $\sigma_{\mathrm{B}}$ is the uncertainty in the field blank measurements and V is the volume of air sampled (US EPA 1997b).

\section{The Unmix Model}

Unmix is a commonly used source-receptor model (Henry 1997, 2001) based on the assumption that each source of unknown composition contributes an unknown amount to each sample collected. The model uses a principal component analysis to solve the general equation

$$
x_{i j}=\sum_{i=1}^{p} g_{i k} f_{k j}+e_{i j}
$$

where $x$ is the matrix of ambient data collected at the receptor site, consisting of the species in columns and dates in rows. The matrix $f$ is the gravimetric mass of each element $j$ per unit PM mass emitted from each source $k$, or source compositions, and $g$ is the matrix of the fraction of total $\mathrm{PM}_{2.5}$ ambient concentration from source $k$ in sample $i$, or source contributions. The goal is to find $f$ and $g$ by minimizing the residual error, $e$, which accounts for error in the model fit. Uncertainty in the data is not considered by the model, which implicitly assumes a certain standard of accuracy in the data for a good model fit. There are an infinite number of solutions to Equation (1), so the model restricts solutions to those that are physically meaningful in that factor compositions and contributions are non-negative. Also, the sum of the concentrations of an element from all factors in one day cannot be greater than the measured mass concentration of the element for that day. The model operates under the assumption that the data form well-defined "edges," meaning that each factor, when plotted against another, will have some dates during which the contribution is zero for one factor and non-zero for the other factor, creating an edge in a multi-dimensional space. The results are limited to a maximum of seven factors but the user must select which measured species to use in the model. The model does not use data from any date from which one or more species concentrations are missing.

\section{PMF Model}

The 2-dimensional PMF model, PMF2, solves Equation (1) by a weighted least-squares fit with the known error estimates of the elements of matrix $x$ used to derive the weights (Paatero and Tapper 1993, 1994; Paatero 1997). PMF minimizes $Q$, defined as

$$
Q=\sum_{i=1}^{n} \sum_{j=1}^{m}\left(\frac{e_{i j}}{s_{i j}}\right)^{2}
$$

where $s_{i j}$ are the error estimates for the elements in $x$ described above. Ideally $Q$ would have $e_{i j}$ approximately equal to $s_{i j}$, producing a value of $n \times m$, the number of values in the data matrix $x$ or the number of degrees of freedom. The same non-negativity constraints from Unmix apply to PMF. Because PMF has provision to incorporate uncertainty in the data into the model, data sets with missing values can be used by applying a large uncertainty so that the missing data will have negligible influence on the model results. Missing data can be approximated as the geometric mean of the corresponding element concentration with an uncertainty of 4 times the geometric mean (Polissar et al. 1998).

Uncertainty estimates for the $s$ matrix were determined as follows for each of the species included in the model. For trace element data above the minimum detection limit (MDL), 
uncertainty in the ambient concentrations was determined by compounding errors from the most uncertain components: the volume of air sampled, contamination as reflected in the field blanks, and the variation in element mass measured on two or three different sections from each filter. For data below the MDL, the concentration was approximated as one half the sample MDL, with an assigned uncertainty of one half the sample MDL plus one-third the study-average MDL for the corresponding element according to Polissar et al. (1998). Uncertainty in the sulfate and nitrate data was calculated from propagation of error in the volume of air sampled, the variation in the field blanks, the extraction volume, and the variation in replicate analyses. Uncertainty in the OC and EC data was calculated from propagation of error in the volume of air sampled and the variation in the replicate analyses of collected samples. In this study, a third of the MDL was added to the analytical uncertainty for each value above the MDL (Polissar et al. 1998).

The non-negativity constraint decreases the rotational freedom, but with many datasets, more than one solution still exists. The parameter FPEAK allows manipulation of the $g$ and $f$ matrices (Equation (1)) such that rotational freedom in the model can be examined. Selection of the number of factors, or sources, requires some subjectivity. The user must select a maximum number of factors that can adequately describe the total $\mathrm{PM}_{2.5}$ mass while excluding factors that do not make physical sense, such as duplicate factors or factors with unrealistic compositions or contributions. This requires some knowledge of the quality of the data and the source types in the area. Evaluating multiple solutions within the range of FPEAK values that yield an acceptable $Q$ value and assessing the edge plots are more objective ways to evaluate the model results.

\section{RESULTS AND DISCUSSION}

\section{Unmix Model Results}

The Unmix model created a six-factor solution with the source compositions shown in Figure 1 and source contributions shown in Figure 2. Some combinations of the species considered resulted in a solution that was not feasible. The combination of species that provided the best solution included $\mathrm{Ca}, \mathrm{Ti}, \mathrm{Cr}, \mathrm{Mn}$, $\mathrm{Fe}, \mathrm{Zn}, \mathrm{Mo}, \mathrm{Cd}$, sulfate, nitrate, OC, and EC. Three parameters designed to evaluate the model results are the minimum $R^{2}$, the signal-to-noise ratio, and the strength. The $\mathrm{R}^{2}$ value is related to the proportion of variance of each species explained by the factors. For all species, the minimum $\mathrm{R}^{2}$ value is recommended to be greater than 0.8 . For the selected species, the minimum $\mathrm{R}^{2}$ value was 0.86 . The minimum signal-to-noise ratio is the smallest estimated signal-to-noise ratio for any of the factors in the model, recommended to be greater than 2. A value of 2.32 was obtained using this dataset. The strength is a measure of the confidence in the model. Strength is recommended to be greater than 3 , but with some datasets this is unachievable and thus a strength less than 3 may still be acceptable (Henry, personal

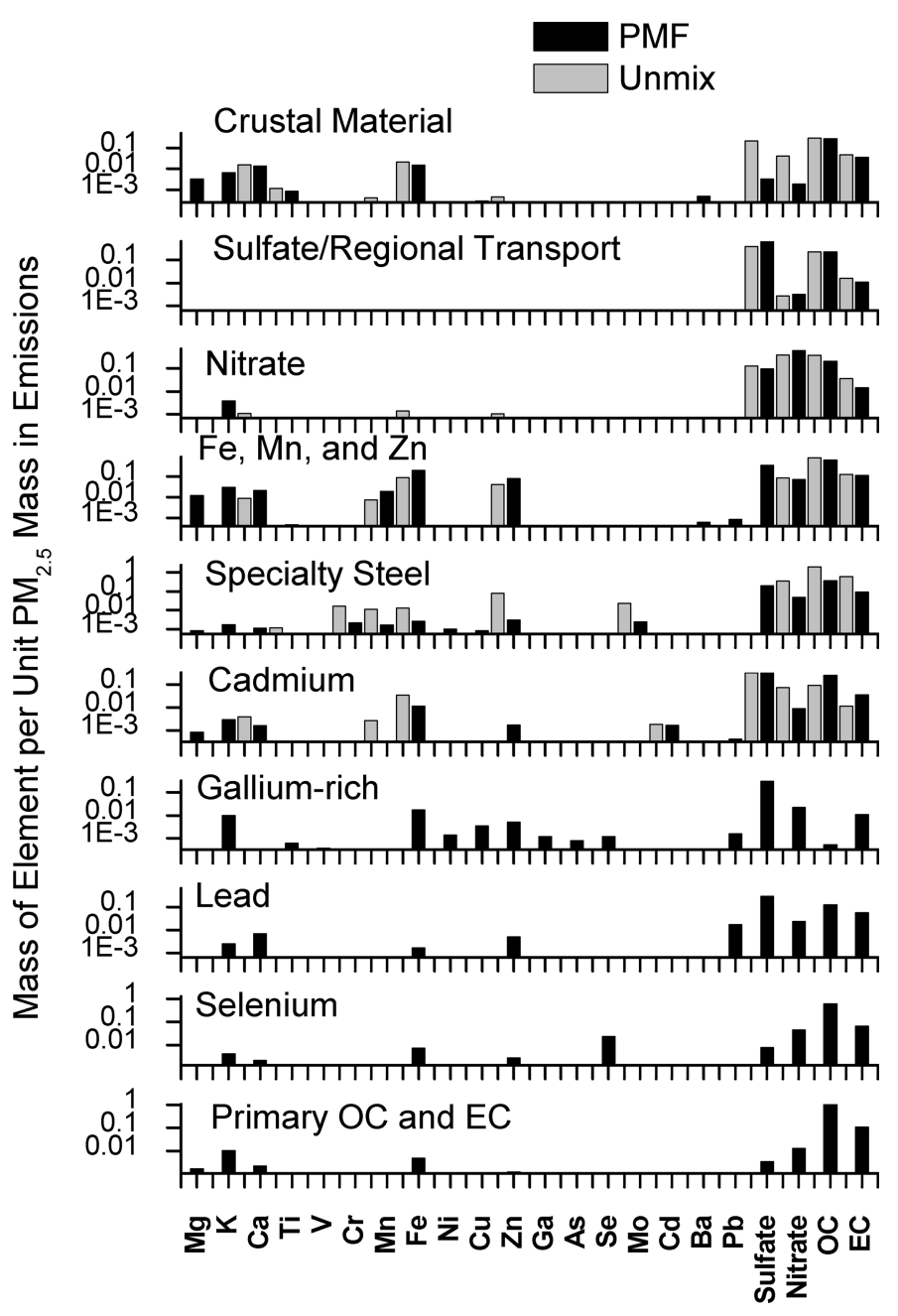

FIG. 1. Unmix source compositions apportioned by $\mathrm{PM}_{2.5}$ mass. All ten factors are outputs of PMF, while only the first six factors are outputs of Unmix. Although the Unmix regional transport factor includes primary OC and EC, it is graphed for comparison with the PMF sulfate factor.

communication). For this dataset, it was impossible to find a combination of species yielding a strength greater than 3 , and the final solution had a strength of 1.41 .

Species for which the ambient data correlated strongly with the source contributions (correlation coefficient greater than 0.7) allow determination of the source types. The six factors in the model have been designated a crustal material factor, a regional transport factor, a nitrate factor, an $\mathrm{Fe}, \mathrm{Mn}$, and $\mathrm{Zn}$ factor, a specialty steel production factor, and a cadmium factor. Descriptions of the nature of the factors, such as their contributions to $\mathrm{PM}_{2.5}$ mass on a seasonal basis as well as a yearly average, are given below. To determine the mass contribution to $\mathrm{PM}_{2.5}$, the total $\mathrm{PM}_{2.5}$ mass was included as a species in the model, and the calculated source compositions and contributions were normalized by the Unmix-apportioned $\mathrm{PM}_{2.5}$ mass. Because $\mathrm{PM}_{2.5}$ is used as a fitting species, together these six factors account for all of the $\mathrm{PM}_{2.5}$ mass. Figure 3 shows 


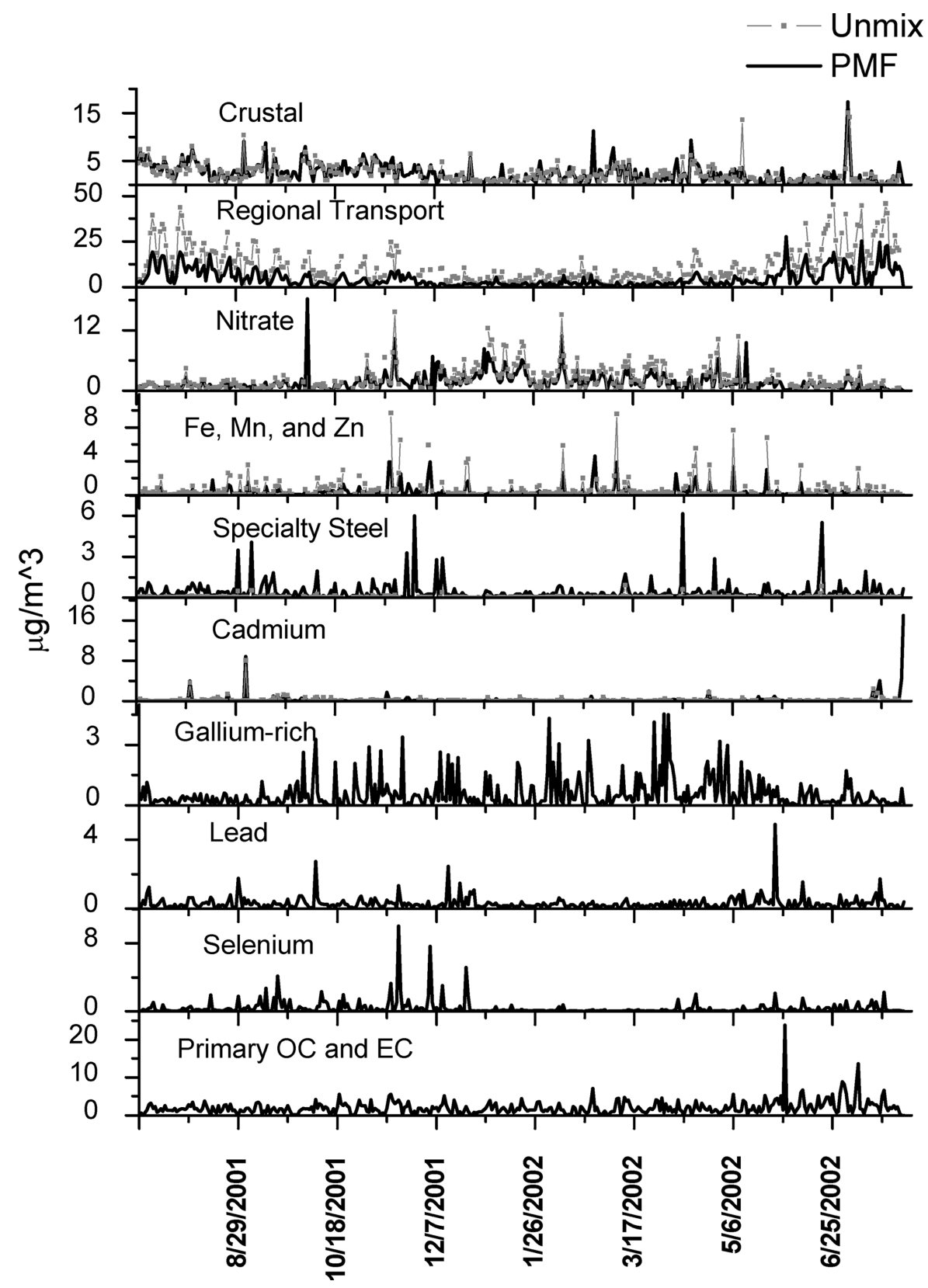

FIG. 2. PMF and Unmix source contributions apportioned by $\mathrm{PM}_{2.5}$ mass. All ten factors are outputs of PMF, while only the first six factors are outputs of Unmix. Note that for comparison purposes, the Unmix regional transport factor is compared to the PMF sulfate plus primary OC and EC factors.

the Unmix source contributions normalized by the Unmixapportioned $\mathrm{PM}_{2.5}$ mass, averaged monthly and for the entire study.

\section{Crustal Material Factor}

The crustal material factor, with $\mathrm{Ca}$ and Ti tracers, contributes $13 \%$ to the $\mathrm{PM}_{2.5}$ on an annual average. The factor is composed of $46 \%$ OC, $34 \%$ sulfate, $7 \% \mathrm{EC}, 6 \%$ nitrate, $3 \% \mathrm{Fe}$, and $2 \% \mathrm{Ca}$. The high concentrations of sulfate and $\mathrm{OC}$ in the factor indicate the resuspended crustal material includes noncrustal deposited material, presumably of anthropogenic origin. Seventy-three percent of the Ca mass, $71 \%$ of the Ti mass, and $36 \%$ of the Fe mass is explained by this factor. Crustal material is present in $\mathrm{PM}_{2.5}$ from weathering of rocks and soil. Typically most of the mass of crustal elements such as calcium and titanium is found in larger particles, but Unmix can still identify this factor using only $\mathrm{PM}_{2.5}$ data. Seasonally, the crustal material source contribution is slightly higher during the first five months of the study in the summer/fall, but overall no consistent seasonal trend was observed. 


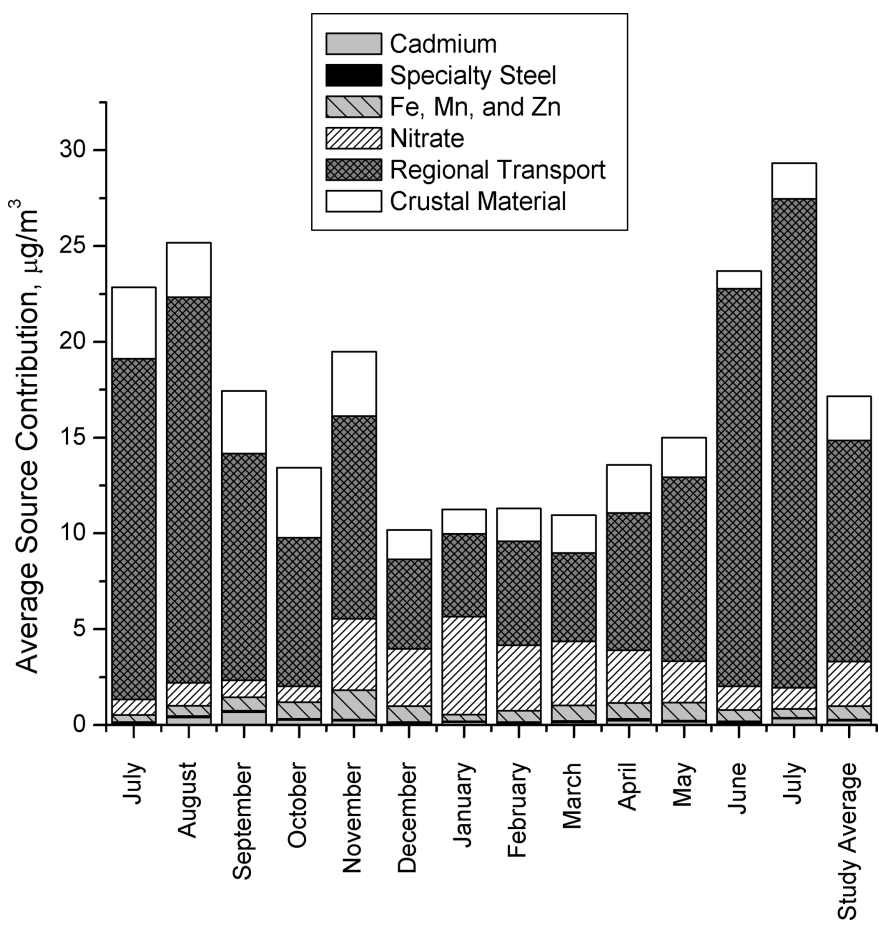

FIG. 3. Monthly average Unmix source contributions. Height of the bars corresponds to the monthly average $\mathrm{PM}_{2.5}$ mass measured with a TEOM. The study average represents the average source contributions from July 11, 2001 through July 31,2002 . Unmix uses $\mathrm{PM}_{2.5}$ mass as a fitting species so the mass of $\mathrm{PM}_{2.5}$ unexplained by Unmix is less than $1 \%$.

\section{Regional Transport Factor}

This factor, with sulfate, OC, and EC tracer species, contributes $68 \%$ of the $\mathrm{PM}_{2.5}$ mass on an annual average, making it by far the largest source category. The factor is mostly composed of $61 \%$ sulfate, $36 \% \mathrm{OC}$, and $2 \% \mathrm{EC}$, and accounts for $84 \%$ of the sulfate mass, $54 \%$ of the OC mass, and $37 \%$ of the EC mass. Sulfate is a major component of ambient $\mathrm{PM}_{2.5}$ in the Pittsburgh region, comprising $38 \%$ on an annual average (Rees et al. 2004). Many coal-fired power plants are located in the Ohio River Valley south and southwest of the monitoring station. These sources emit large amounts of $\mathrm{SO}_{2}$ as well as some coal fly ash. The $\mathrm{SO}_{2}$ is oxidized to eventually form particulate sulfate, making this category predominantly secondary material. Because Pittsburgh is frequently downwind, these sources are suspected to contribute significantly to the regional nature of Pittsburgh's air pollution (Tang et al. 2004; Anderson et al. 2004). Based on the available data Unmix is unable to separate the secondary sulfate, usually associated with some secondary OC, from the primary OC and EC, associated with many different sources, including vehicular emissions. EC is emitted from diesel engines, but because of the same location of the vehicular sources (gas and diesel) and the same meteorology affecting the concentrations at the receptor site, Unmix is unable to separate the gasoline engine source profile from the diesel engine profile. Application of Unmix to multi-sample/day data from Pittsburgh for July 2001 did allow separation of the sulfate, diesel and gasoline sources
(Modey et al. 2005). The information on the diurnal data probably aided in this separation of sources. EC may also be associated with biomass burning, which is not a major source of $\mathrm{PM}_{2.5}$ in the Pittsburgh region (Cabada et al. 2002). Therefore this factor is dominated by regionally transported secondary sulfate and $\mathrm{OC}$ and includes primary OC and EC emissions as well. The contribution of this factor to total $\mathrm{PM}_{2.5}$ is higher in the summer than in the winter due to the increase in photochemistry that favors formation of particulate sulfate.

\section{Nitrate Factor}

This factor is also attributed to a secondary source, as the tracer, nitrate, is a secondary pollutant. The factor is mostly composed of $41 \%$ nitrate and $40 \%$ OC, and accounts for $80 \%$ of the nitrate mass and $17 \%$ of the OC mass. The major source of nitrate is conversion of $\mathrm{NO}_{\mathrm{x}}$ from high-temperature combustion, such as internal combustion engines in cars. The significant OC associated with this factor could reflect secondary organic material formed at the same time as the nitrate. The source contribution is highest in the winter months as expected, when lower temperatures favor nitrate particles rather than nitric acid vapor. The higher winter contribution could also reflect lower mixing heights and the increased importance of local sources. The nitrate factor is second largest, with an annual average contribution of $14 \%$ to $\mathrm{PM}_{2.5}$.

\section{Iron, Manganese, and Zinc Factor}

This factor has tracer species of Fe, Mn, and $\mathrm{Zn}$, while the bulk of the factor $(70 \%)$ is composed of OC. Most of the Fe, $\mathrm{Mn}$, and $\mathrm{Zn}$ masses are attributed to this factor $(49 \%, 67 \%$, and $78 \%$, respectively). These elements are quite common in a number of source categories, including steel mills, vehicles, waste incinerators $(\mathrm{Zn})$, soil dust, and even coal combustion (Parekh 1990; Olmez et al. 1988; Watson et al. 2001). Blast furnaces and steel mills produce emissions of iron, manganese, and zinc and there are several in the area (US EPA 2002). Iron ore is a raw material used for making steel, manganese is an alloying element, and zinc is used for coating and galvanizing steel (AISI 2004). This factor is likely to represent emissions from the steel production industry. The source contribution does not show a seasonal trend, and annual average contribution to $\mathrm{PM}_{2.5}$ is $4 \%$ for this factor.

\section{Specialty Steel Factor}

Mo and $\mathrm{Cr}$ are the tracer species for this factor, most of which is composed of $65 \%$ OC and $19 \%$ EC. Both chromium and molybdenum are used as alloying elements in specialty steel; together these elements enhance the corrosion resistance of stainless steel (AISI 2004). The Pittsburgh region has many specialty steel processing and finishing facilities. The Unmix factor marked by molybdenum and chromium is assumed to be associated with these facilities; however, it is a minor factor, contributing less than $1 \%$ to the annual average $\mathrm{PM}_{2.5}$. No seasonal trend in contribution is observed. 


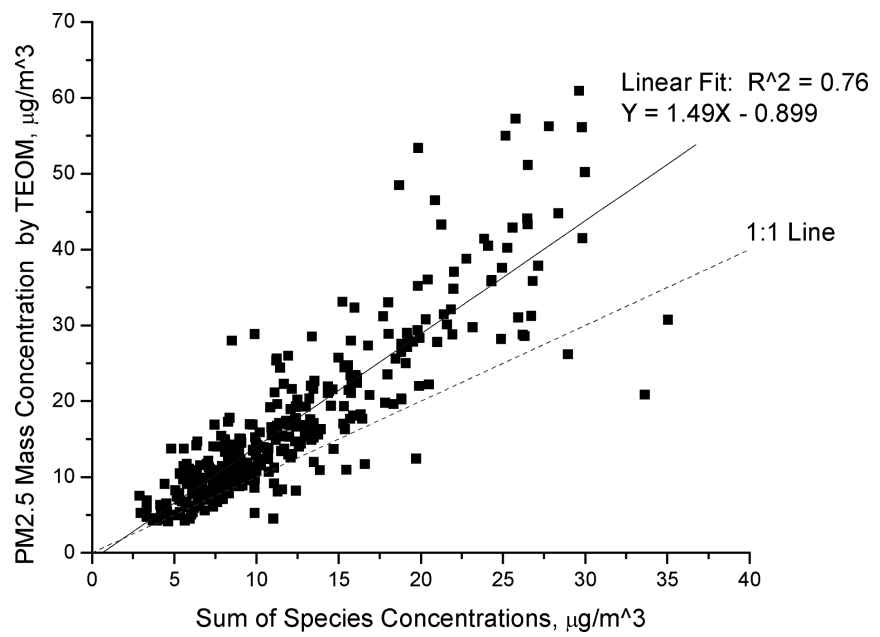

FIG. 4. Mass balance. Sum of the species concentrations used in the PMF analysis vs. the total $\mathrm{PM}_{2.5}$ mass as measured by the TEOM. A linear regression of the data shows an $\mathrm{R}^{2}$ value of 0.76 . The regression slope of 1.49 shows that the line is biased high as expected, since PMF could not identify the sources of all of the $\mathrm{PM}_{2.5}$ mass.

\section{Cadmium Factor}

This factor, with a Cd tracer, is minor in $\mathrm{PM}_{2.5}$ contribution, approximately $1 \%$ on an annual average. Factor composition is $60 \%$ sulfate, $17 \%$ OC, and $13 \%$ nitrate, and $82 \%$ of the Cd mass is explained by this factor. The contribution of the cadmium factor is almost always quite low except for a few large peaks. This may indicate a local source, possibly a point source whose plumes only impact the site on a few days out of a year. Other source-receptor studies that include cadmium generally show this element in association with other species rather than by itself, suggesting that Pittsburgh has a different mix of cadmium sources than other locations.

\section{PMF Model Results}

The robust mode, rather than the default mode, was selected to handle outliers in the data. The robust factorization iteratively reweighs individual values such that the minimization of $Q$ now becomes

$$
Q=\sum_{i=1}^{n} \sum_{j=1}^{m}\left(\frac{e_{i j}}{h_{i j} s_{i j}}\right)^{2}
$$

where

$$
h_{i j}= \begin{cases}1 & \text { if }\left|e_{i f} / s_{i f}\right| \leq \alpha \\ \left|e_{i j} / s_{i j}\right| / \alpha & \text { otherwise }\end{cases}
$$

A typical value of $\alpha=4$ was chosen. To apportion each factor by mass, typically a scaling constant $S$ normalizes the source contributions. The value of $S$ is determined by a multiple linear regression of the sum of concentrations of the component species in a sample versus the sum of modeled source contributions for that sample, or the observed versus the predicted $\mathrm{PM}_{2.5}$. However, as shown in Figure 4, the sum of species concentrations plotted against the total $\mathrm{PM}_{2.5}$ mass concentration as measured by the TEOM shows agreement that worsens at higher concentrations. The sums of species concentrations, including $\mathrm{PM}_{2.5}$ sulfate, nitrate, $\mathrm{OC}$ (multiplied by 1.8 ), EC, and the trace elements used in the PMF analysis (see Figure 5), are on average lower than the total $\mathrm{PM}_{2.5}$ mass concentration. Reasons for this discrepancy have been explored in detail in Rees et al. (2004). Because of the lack of mass balance closure, total $\mathrm{PM}_{2.5}$ measured mass concentration was included as a species in the model so that PMF could apportion the mass directly. $\mathrm{PM}_{2.5}$ uncertainty was set at 4 times the concentration to decrease its weight in the model fit (Kim et al. 2003). The source contributions and compositions were then normalized by the $\mathrm{PM}_{2.5}$

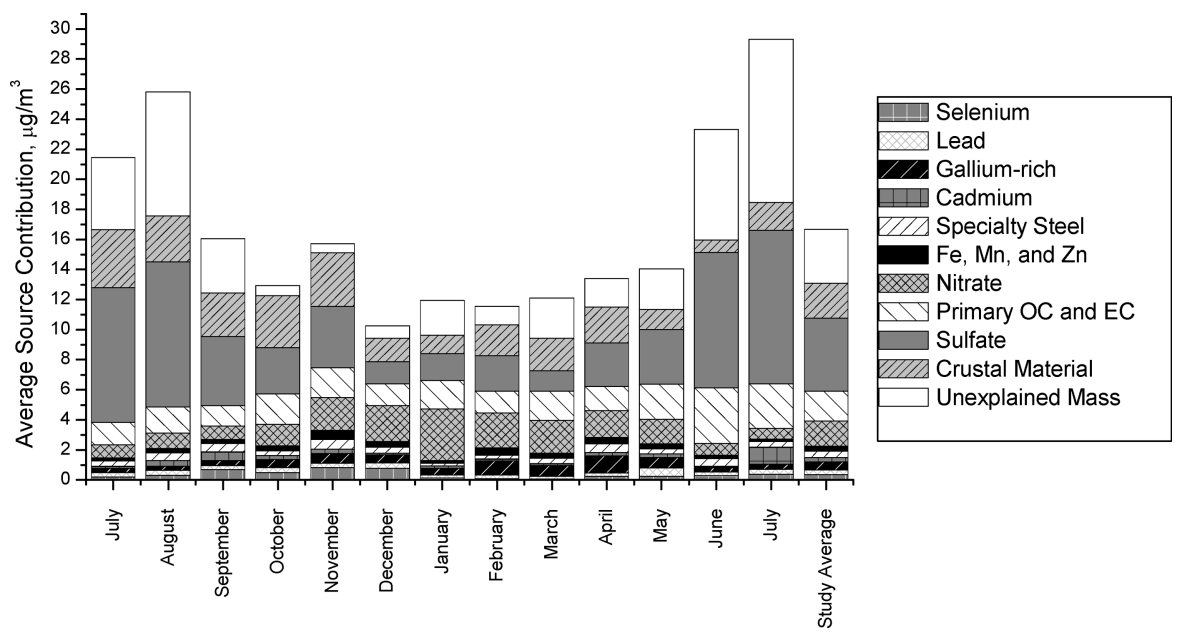

FIG. 5. Monthly average PMF source contributions. Height of the bars corresponds to the monthly average PM 2.5 mass measured with a TEOM. The study average represents the average source contributions from July 11, 2001 through July 31, 2002. The unexplained mass is the difference between the monthly average $\mathrm{PM}_{2.5}$ mass and the sum of the monthly averaged source contributions from each factor. 
mass concentration apportioned to each factor to get quantitative results.

A detailed examination of model results and goodness-of-fit were carried out for models with 8-12 solutions. An analysis of the effects of changing the FPEAK value from -0.8 to +0.8 showed that the change in rotation did not have a very significant effect on the results. A 10 -factor model provided the most realistic results. The actual $Q$ value was 8884 as compared to the theoretical value of 8878 .

G-space plotting, similar to the edge plots from the Unmix model, involves forming scatter plots of pairs of source contribution factors (Paatero et al. 2005). The solution can be rotated to achieve the optimal solution, which has edges that lie on or parallel to the two axes for all scatter plots. A value of -0.2 was chosen for FPEAK: although the $Q$-value did not change significantly from the no-rotation solution, several of the edges in the edge plots improved with this slight rotation.

PMF allows inclusion of more species in the model due to the consideration of uncertainties that enables handling of missing and below detection limit data. Species included in the PMF solution are $\mathrm{PM}_{2.5}$ sulfate, nitrate, $\mathrm{OC}, \mathrm{EC}, \mathrm{Mg}, \mathrm{K}, \mathrm{Ca}, \mathrm{Ti}, \mathrm{V}$, $\mathrm{Cr}, \mathrm{Mn}, \mathrm{Fe}, \mathrm{Ni}, \mathrm{Cu}, \mathrm{Zn}, \mathrm{Ga}$, As, Se, Mo, Cd, Ba, and Pb. We can identify a tracer species for each factor based on the source compositions shown in Figure 1. However, a better indication is the correlation of the species ambient concentration with the PMFmodeled source contribution. A correlation greater than 0.7 is a good indication of a tracer species. Based on the tracer species for each factor, the factors were defined as crustal material (Ca and Ti tracers), sulfate, nitrate, an Fe, $\mathrm{Zn}$, and Mn factor, specialty steel (Mo and $\mathrm{Cr}$ tracers), cadmium, a gallium-rich factor, lead, selenium, and primary OC and EC (OC and EC tracers).

\section{Description of PMF Sources}

A comparison of compositions and contributions for factors found by both Unmix and PMF are shown in Figures 1 and 2. These source categories include crustal material, sulfate, nitrate, steel production, specialty steel, and cadmium. The factors not found by Unmix but found by PMF are described below, and their source compositions and contributions are also shown in Figures 1 and 2. Figure 5 shows the average PMF source contributions apportioned by average $\mathrm{PM}_{2.5}$ mass concentration. A total of $22 \%$ of the $\mathrm{PM}_{2.5}$ measured with the TEOM is not apportioned to any source by PMF. This missing mass could be explained by species not included in the model, such as particulate ammonium, or the presence of water in the particles that was measured as $\mathrm{PM}_{2.5}$. If all of the sulfate is assumed to be ammonium sulfate, the missing mass fraction decreases to $13 \%$. Assuming that all of the nitrate is ammonium nitrate as well decreases the missing mass fraction to $9 \%$.

\section{Gallium-Rich Factor}

The gallium-rich factor is mainly composed of $79 \%$ sulfate and $6 \%$ nitrate, and $92 \%$ of the tracer Ga mass is explained by this factor. Ga is not frequently used in source-receptor modeling studies, nor is it measured in many source profiles as it is not an air toxic. Ga is obtained for use as a substrate material for electronics devices by extraction from bauxite and aluminum processing, zinc refinery residues, and coal fly ash (Bautista 2003). Therefore, the Ga in $\mathrm{PM}_{2.5}$ in Pittsburgh could be emitted from these industries, including coal-fired power plants. Other elements such as $\mathrm{As}, \mathrm{V}, \mathrm{Cu}$, and $\mathrm{Ni}$ show significant fractions in this source category but with correlations less than 0.7 . While secondary pollutants from coal combustion, such as sulfate, are significant in their contribution to $\mathrm{PM}_{2.5}$, this factor, which includes primary and secondary material, contributes only $3 \%$ to the $\mathrm{PM}_{2.5}$ mass.

\section{Lead Factor}

The lead factor is composed mostly of sulfate (55\%), OC (23\%), and EC (11\%), and explains $68 \%$ of the Pb mass. Due to years of using leaded gasoline, batteries, paint, soldered cans, and other products, lead is ubiquitous in the environment. Resuspension of lead particles, which occurs on a regional scale, is expected to contribute to ambient lead concentrations, resulting in an area source. There are also several point sources of lead emissions in the Pittsburgh region, such as waste incinerators, a battery manufacturing plant, power plants, blast furnaces and steel mills. Overall concentrations of lead are low and therefore this factor makes only a small contribution (2\%) to the total $\mathrm{PM}_{2.5}$ mass.

\section{Selenium Factor}

The selenium factor is composed of mostly OC (79\%) with some EC (9\%) and nitrate (6\%), and explains $87 \%$ of the Se mass. Ambient Se concentrations have a distinct profile of very low background levels accentuated by occasional large peaks. Selenium is typically associated with coal combustion as there are trace amounts of this element in coal, but selenium is more volatile than some of the other coal constituents. Hence this species behaves differently in the atmosphere, which may be responsible for PMF isolating this element into its own factor. This is a minor category, contributing only $2 \%$ to the $\mathrm{PM}_{2.5}$ mass.

\section{Primary OC and EC Factor}

The primary OC and EC factor is composed of OC (88\%) and EC (9\%). Forty-six percent of the OC mass and $44 \%$ of the EC mass is explained by this factor. Primary OC and EC are emitted from many sources including vehicles, mostly from fuel combustion as well as lubricating oil. EC is often used as a tracer for diesel engines. PMF was able to separate a vehicle emissions factor from the combined sulfate/EC/OC factor associated with regional transport as identified from the Unmix analysis. However, PMF did not discern between the diesel and gasoline engines. The source contributions do not show a seasonal trend, with concentrations being somewhat steady throughout the year. Overall, this factor is significant in its contribution to $\mathrm{PM}_{2.5}$ in Pittsburgh, which is $12 \%$ on average. 


\section{Discussion}

\section{Comparison of Unmix and PMF Results}

Agreement between the two models is generally quite good, both in composition of sources and source contribution trends. However, there are a few significant differences. While source contributions track well for the two models, showing similar trends in concentration with time, the magnitude of the contribution does not agree for some factors. Figure 6 shows the results of a linear regression of the Unmix source contributions against the same factors found by PMF. For this comparison, the PMF sulfate and primary $\mathrm{OC}$ and EC factors were added together for comparison with the Unmix regional transport factor.

The $\mathrm{R}^{2}$ values for all factors are reasonable and statistically significant, ranging from 0.64 for crustal material to 0.99 for the cadmium factor. The slope of the regression line, however, ranges from 0.41 for the $\mathrm{Fe}, \mathrm{Mn}$, and $\mathrm{Zn}$ factor to 6.2 for the specialty steel factor. The crustal material and the cadmium factors are within $20 \%$ in source contribution magnitude, suggesting that results are robust for these factors. PMF apportions more mass to the specialty steel factor due to the inclusion of $2 \%$ of the sulfate mass. PMF apportions less mass to the sulfate and primary OC and EC factors, the nitrate factor, and the Fe, Mn, and $\mathrm{Zn}$ factor. The apportionment of less mass to the sulfate and primary OC and EC factors by PMF as compared to the Unmix regional transport factor is likely due to Unmix fitting the model to total $\mathrm{PM}_{2.5}$, while PMF has a significant fraction of unexplained mass. For the nitrate and Fe, $\mathrm{Zn}$, and Mn factors, the difference is due to the apportionment of OC and EC. Unmix apportions $17 \%$ of the OC mass and $17 \%$ of the EC mass to the nitrate factor whereas PMF apportions $7 \%$ OC mass and
$5 \% \mathrm{EC}$ mass to the nitrate factor. For the Fe, Mn, and $\mathrm{Zn}$ factor, the apportionment is $12 \%$ of the OC mass and $18 \%$ of the EC mass explained by Unmix, but only $4 \%$ of the OC mass and $7 \%$ of the EC mass is explained by the same PMF factor. Results from previous comparisons of PMF and Unmix show similar conclusions: convergence for some factors but poor agreement for others (Poirot et al. 2001; Maykut et al. 2003).

In comparing Figures 3 and 5, the average source contributions as a percent of average $\mathrm{PM}_{2.5}$ mass are within a few percent for the two models for all factors, with the exception of the Unmix regional transport factor $(68 \%)$ and the sulfate and primary OC and EC factor for PMF (total 40\%). Unmix apportions all the mass, while on average PMF apportions only $78 \%$ of the mass, so some discrepancy is expected. PMF is more effective at discerning between primary and secondary OC; Unmix does not distinguish between the two and therefore can only give a large factor that is general regionally transported material and is not very informative from a policy-making perspective.

\section{Adding Individual Organic Markers into Model}

In an effort to further identify the sources apportioned by PMF, selected species of OC were added to the dataset. Individual organic compounds have been used as markers for specific sources; for example, hopanes are good tracers for vehicle exhaust, both gasoline and diesel engines (Simoneit 1984; Rogge et al. 1993a; Schauer et al. 1996). Organic molecular markers have been identified through a combination of source testing and analysis of ambient data (see, e.g., Rogge et al. 1996; Schauer et al. 1996; Simoneit 1999, 2002 and references therein). Table 2 lists the organic species included in the PMF model and their
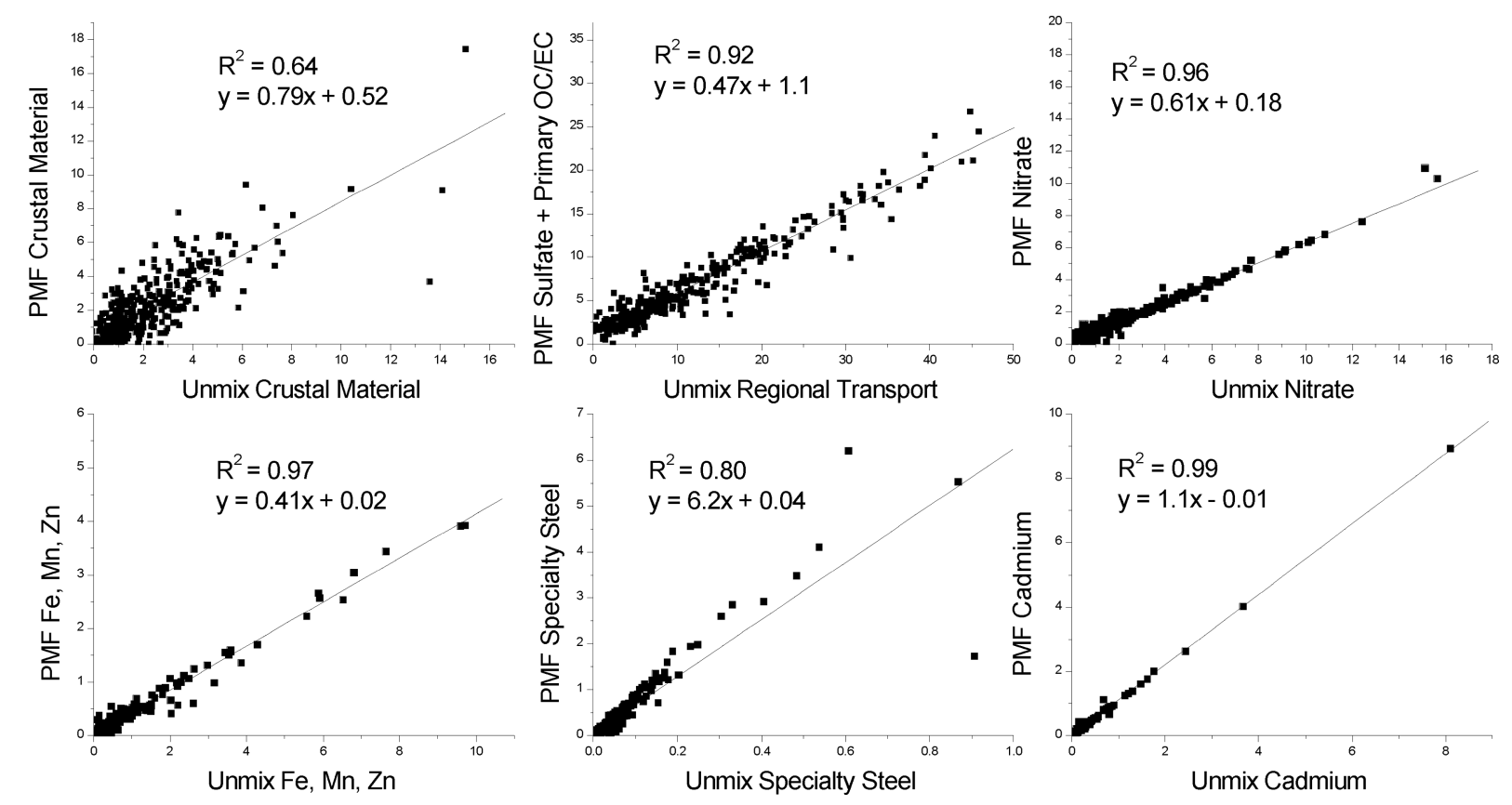

FIG. 6. Linear regression results of PMF and Unmix source contributions. 
TABLE 2

Species of organic carbon that are included in the PMF model. The abbreviations in all capital letters correspond to the $\mathrm{x}$-axis labels in Figure 7

\begin{tabular}{|c|c|c|}
\hline Compound (abbreviation) & Sources & References \\
\hline n-Nonacosane (NAL09) & Vegetative detritus, road-dust, tire wear & $\begin{array}{l}\text { (Simoneit and Mazurek 1982, Rogge } \\
\text { et al. 1993b, 1993c) }\end{array}$ \\
\hline n-Hentriacontane (NAL11) & Vegetative detritus, road-dust, tire wear & $\begin{array}{l}\text { (Simoneit and Mazurek 1982; Rogge } \\
\text { et al. 1993b, 1993c) }\end{array}$ \\
\hline n-Tritriacontane (NAL13) & Vegetative detritus, road-dust, tire wear & $\begin{array}{l}\text { (Simoneit and Mazurek 1982; Rogge } \\
\text { et al. 1993b, 1993c) }\end{array}$ \\
\hline $\begin{array}{l}\text { Hexadecanoic acid (palmitic acid) } \\
\quad \text { (NACD6) }\end{array}$ & Cooking & $\begin{array}{l}\text { (Rogge et al. 1991; Schauer et al. } \\
\text { 1999, 2002b) }\end{array}$ \\
\hline Syringaldehyde (SYALD) & Hardwood combustion & (Rogge et al. 1998; Simoneit 2002) \\
\hline Acetosyringone (ACSYN) & Hardwood combustion & (Rogge et al. 1998; Simoneit 2002) \\
\hline Syringic Acid (SYNCD) & Hardwood combustion & (Rogge et al. 1998; Simoneit 2002) \\
\hline Levoglucosan (LEVOG) & Wood combustion (both types) & (Simoneit et al. 1999) \\
\hline 22,29,30-Trisnorneohopane (HOP1) & Vehicular exhaust & (Simoneit 1985; Rogge et al. 1993a) \\
\hline $\begin{array}{l}\text { 17@(H),21A(H)-29-Norhopane } \\
\quad(\mathrm{HOP} 2)\end{array}$ & Vehicular exhaust & (Simoneit 1985; Rogge et al. 1993a) \\
\hline 17@(H),21A(H)-Hopane (HOP3) & Vehicular exhaust & (Simoneit 1985; Rogge et al. 1993a) \\
\hline $\begin{array}{l}\text { 22S+R-17@(H),21A(H)-30-Homo- } \\
\text { hopane (HOP4,5) }\end{array}$ & Vehicular exhaust & (Simoneit 1985; Rogge et al. 1993a) \\
\hline Indeno[1,2,3-cd]pyrene (INDCDP) & $\begin{array}{l}\text { For Pittsburgh, mainly: Vehicular } \\
\text { exhaust, wood combustion, } \\
\text { coke-production }\end{array}$ & $\begin{array}{l}\text { (Simoneit 1985; Rogge et al. 1993a; } \\
\text { Schauer and Cass 2000; Robinson } \\
\text { et al. 2006a, b, c; Subramanian et al. } \\
\text { 2005a, b) }\end{array}$ \\
\hline Benzo[g,h,i]perylene (BNZGHI) & $\begin{array}{l}\text { For Pittsburgh, mainly: Vehicular } \\
\text { exhaust, wood combustion, } \\
\text { coke-production }\end{array}$ & $\begin{array}{l}\text { (Simoneit 1985; Rogge et al. 1993a; } \\
\text { Schauer and Cass 2000; Robinson } \\
\text { et al. 2006a, b, c; Subramanian et al. } \\
\text { 2005a, b) }\end{array}$ \\
\hline Coronene (CORON) & $\begin{array}{l}\text { For Pittsburgh, mainly: Vehicular } \\
\text { exhaust, wood combustion, } \\
\text { coke-production }\end{array}$ & $\begin{array}{l}\text { (Simoneit 1985; Rogge et al. 1993a; } \\
\text { Schauer and Cass 2000; Robinson } \\
\text { et al. 2006a, b, c; Subramanian et al. } \\
\text { 2005a, b) }\end{array}$ \\
\hline $\begin{array}{l}\text { Benzo }(b+j+k) \text { fluoranthenes } \\
\quad(\text { BNBJKF })\end{array}$ & $\begin{array}{l}\text { For Pittsburgh, mainly: Vehicular } \\
\text { exhaust, wood combustion, } \\
\text { coke-production }\end{array}$ & $\begin{array}{l}\text { (Simoneit 1985; Rogge et al. 1993a; } \\
\text { Schauer and Cass 2000; Robinson } \\
\text { et al. 2006a, b, c; Subramanian et al. } \\
\text { 2005a, b) }\end{array}$ \\
\hline
\end{tabular}


major sources (Rogge et al. 1996; Schauer et al. 1996; Robinson et al. 2006a, b, c; Subramanian et al. 2005a, b). These are the set of species most commonly used for chemical mass balance modeling of sources of primary organic aerosol (Schauer et al. 1996; Watson et al. 1998; Schauer and Cass 2000; Schauer et al. 2002a; Zheng et al. 2002; Fraser et al. 2003; Robinson et al. 2006a, b, c; Subramanian et al. 2005a, b). Adding these compounds presents a new level of difficulty, however, since compounds can experience transformations during transport from source to receptor. Schauer et al. (1996) and Rogge et al. (1996) examined issues associated with chemical stability and classified certain compounds as stable in the context of Los Angeles. Here, as in previous source apportionment analysis using molecular markers, we assume that mass is conserved and the chemical transformation of tracer species during transport to the receptor site is minimal. Robinson et al. (2005) examines the Pittsburgh data set for evidence of photochemical oxidation of molecular markers.

For organic compound speciation of $\mathrm{PM}_{2.5}$, a total of 133 samples, including field handling blanks, were collected using a $\mathrm{PM}_{2.5}$ sampler (Tisch Environmental, Inc. Model TE-1000 PUF) equipped with an URG cyclone (URG-2000-30AE, URG Corp.) to remove particles with an aerodynamic diameter larger than $2.5 \mu \mathrm{m}$. The particulate matter was collected on a sampling module consisting of a quartz fiber filter $(102 \mathrm{~mm}$ diameter, Pall Lifesciences, Tissuquartz 2500 QAT-UP) followed by a polyurethane foam (PUF) plug ( $7.5 \mathrm{~cm}$ long, Tisch Environmental, Inc. TE-1010) installed downstream of the filter to trap semi-volatile organic compounds that are associated with particulate matter and volatilize off the filter during the sampling process. The sampler was operated at $145 \mathrm{lpm}$. Samples were collected for 24-hour periods starting at midnight daily during July 2001 and several days in January 2002, and every sixth day during the baseline study (August 12 to December 26, 2001; January 29 to July 1, 2002). Details of the quartz and PUF plug preparation and the subsequent GC/MS analysis and ambient concentrations of individual organic compounds are reported by Bernardo-Bricker et al. (2005). While the trace element, sulfate, nitrate, $\mathrm{OC}$ and EC data are available as daily 24-hour averages for a total of 386 days, the speciated OC sampling and analysis occurred on a more intermittent schedule, with 24-hour averages for a total of only 97 days. However, PMF is capable of providing reasonable results for datasets containing elements with as much as $27-98 \%$ of the data missing or below the detection limit (Polissar et al. 2001; Polissar et al. 1998). As with the other species, missing data for the OC species were replaced by the geometric mean concentration and assigned an uncertainty of four times that average. The missing data were assumed to have sufficiently high uncertainty so as not to give weight to the model results.

In including the speciated OC data in the PMF model, groups of similar species, such as the hopanes, were added individually to determine the effect on the model results. With positive results, more species were added and many combinations were tested until an optimum solution was reached. Some species showed very strong correlations with existing PMF-modeled factors. All of the hopane species showed strong correlation with the primary $\mathrm{OC}$ and EC factor, further reinforcing the assumption that this factor is related to vehicle emissions. Some species did not add any new information to the model as they did not correlate with any of the 10 existing factors, or did not form a new factor. The cigarette smoke tracers and some food cooking tracers are examples of this situation and therefore they were not included in the model. It is possible that these sources are relatively small as compared to the other identified factors and therefore could not be identified by PMF. It is also possible that with so much missing data, the quality of the data is insufficient to form a recognizable source signature.

The best solution is presented in Figures 7-9. PMF determined 11 factors in a result that is similar to the solution described by Figures 1, 2, and 5, but with one factor changing significantly. This is the primary OC and EC factor, which has split into two different factors, one associated with vehicles and road dust, and the other associated with wood combustion, cooking, and vegetative detritus. Although there are separate tracers for hardwood and softwood combustion, the model could not separate the two, and therefore the factor is termed "wood combustion." The partitioning of the OC fraction in the secondary sulfate and nitrate factors changed slightly, with the fraction of OC in the sulfate factor decreasing from $19 \%$ to $5 \%$, and the fraction of $\mathrm{OC}$ in the nitrate factor decreasing from $7 \%$ to $6 \%$. This could suggest that adding the molecular markers to the PMF analysis enables further identification of primary sources in such a manner that the secondary contribution of OC

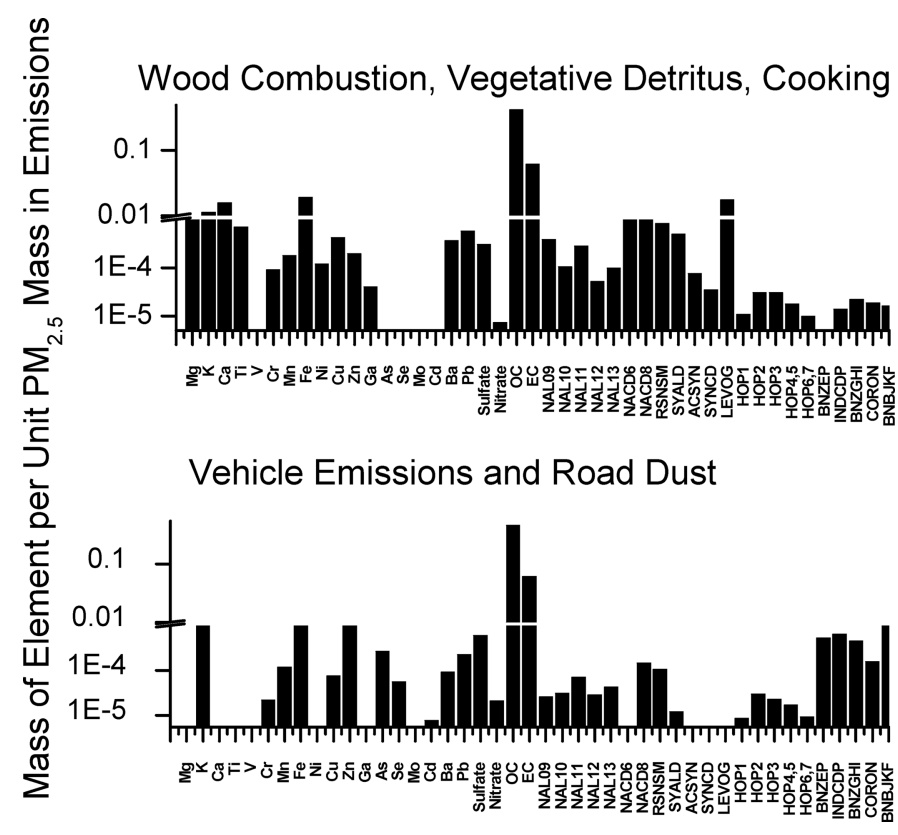

FIG. 7. PMF with speciated OC source composition for the wood combustion, vegetative detritus and cooking factor and the vehicle emissions and road dust factor. 


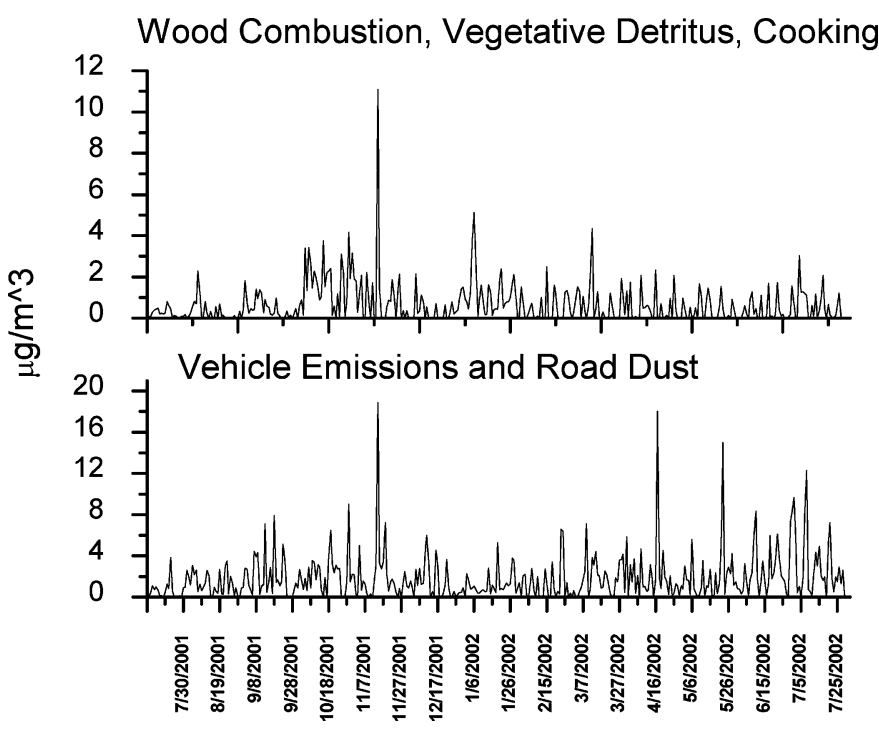

FIG. 8. PMF with speciated OC source contribution for the wood combustion, vegetative detritus and cooking factor and the vehicle emissions and road dust factor.

is reduced. Because all other factors are approximately the same as shown in Figures 1, 2, and 5, Figures 7 and 8 only show the results for the wood combustion, cooking, and vegetative detritus factor and the vehicle emissions and road dust factor. The theoretical $Q$ value for this dataset was 17,370 and the actual $Q$ value was 17,711 . As was the case for the original solution, the result with speciated $\mathrm{OC}$ was not sensitive to rotation, but Gspace plotting showed the best solution occurred with FPEAK of -0.2 .

Figure 9 is consistent with Figure 5, showing a maximum 5\% difference in study average contributions for comparable source categories. Note that the primary $\mathrm{OC}$ and EC factor in Figure 5 is now represented by two factors in Figure 9: (1) vehicles and road dust and (2) wood combustion, vegetative detritus, and cooking. These minor differences are mostly due to the distribution of OC and EC in the two models. For example, the OC fraction in the sulfate source went from $25 \%$ in the first PMF solution to $19 \%$ in the solution that includes molecular markers.

\section{Vehicles and Road Dust Factor}

This factor accounts for $11 \%$ of the $\mathrm{PM}_{2.5}$ mass and is primarily composed of $86 \%$ OC and $11 \%$ EC. OC, EC, hopanes, PAH's, and n-alkanes are important components of this factor. For OC, EC, hopanes, and PAH's, correlations between species concentrations and source contributions were 0.6 or greater, while the correlations for the larger n-alkanes were 0.4-0.5. These compounds are molecular markers for motor vehicle exhaust (Simoneit 1985; Rogge et al. 1993a, b, c; Simoneit and Mazurek 1982; Schauer and Cass 2000; Robinson et al. 2006a, b, c; Subramanian et al. 2005a, b). The lower correlation for the n-alkanes, less than the 0.7 desired, is likely due to the fact that they are present in emissions from many sources and not just motor vehicles. But their appearance as markers for this factor suggests that a fraction of the larger n-alkanes is attributed to road dust that is redistributed by vehicular traffic. It is assumed that the fraction of the larger n-alkanes in vegetative detritus would be better correlated with wood combustion. No seasonal trend is observed for this factor.

\section{Wood Combustion, Vegetative Detritus and Cooking Factor}

Levoglucosan, resin acids, syringols, stearic acid, palmitic acid, and the n-alkanes are important markers for this factor, composed of $75 \%$ OC, $11 \%$ EC, $3 \%$ Levoglucosan, $3 \%$ Ca, $3 \%$ $\mathrm{Fe}$, and $2 \% \mathrm{~K}$. These compounds, as listed in Table 2, are markers of wood smoke, vegetative detritus, and cooking and therefore this factor is labeled as a composite of these three sources. Correlations of these species concentrations with source contributions are between 0.5-0.6, with the exception of stearic acid (0.3). The source contribution is highest during the autumn months of October and November, presumably from residential wood burning. Although PAH's can also be markers of wood smoke, these species do not correlate well with this factor, suggesting that PAH emissions in the Pittsburgh region are dominated by other sources listed in Table 2 , such as coke production and vehicle exhaust (Robinson et al. 2006a, b, c). Potassium is often considered to be a tracer of biomass combustion. Although there is a significant amount of potassium in this factor $(2 \%)$, the correlation is low and it is therefore not considered a tracer here.

\section{CONCLUSIONS}

With the application of the Unmix and PMF models to the data collected during the PAQS, the contributions of a broad range of sources to the local airborne particles in the region have been determined. Comparison of the two models shows similar source composition and contribution for five factors: crustal material, nitrate, an $\mathrm{Fe}, \mathrm{Mn}$, and $\mathrm{Zn}$ factor, specialty steel production, and a cadmium factor. PMF found several additional factors: a gallium-rich factor, a lead factor, and a selenium factor assumed to be related to coal combustion. The PMF model found a sulfate factor separate from the OC and EC associated with primary emissions, while Unmix grouped these three species together into a single factor. Comparison between source contributions for the similar factors shows reasonable agreement between the two models. The sulfate factor shows the highest contribution to local $\mathrm{PM}_{2.5}$ with an annual average contribution of approximately $28 \%$ (from PMF). The nitrate, crustal material, and primary OC and EC factors also show significant contributions on the order of $10-14 \%$. The sulfate factor is affected by photochemistry and therefore shows maximum values in summer. The nitrate factor is temperature sensitive due to the volatility of nitrate; maximum values of particulate nitrate occur in winter. The crustal material and vehicle sources somewhat more constant contributions throughout the year. The remaining factors contribute on a smaller scale and are defined by plume events, with peaks in concentration distinctly higher than average concentration. 


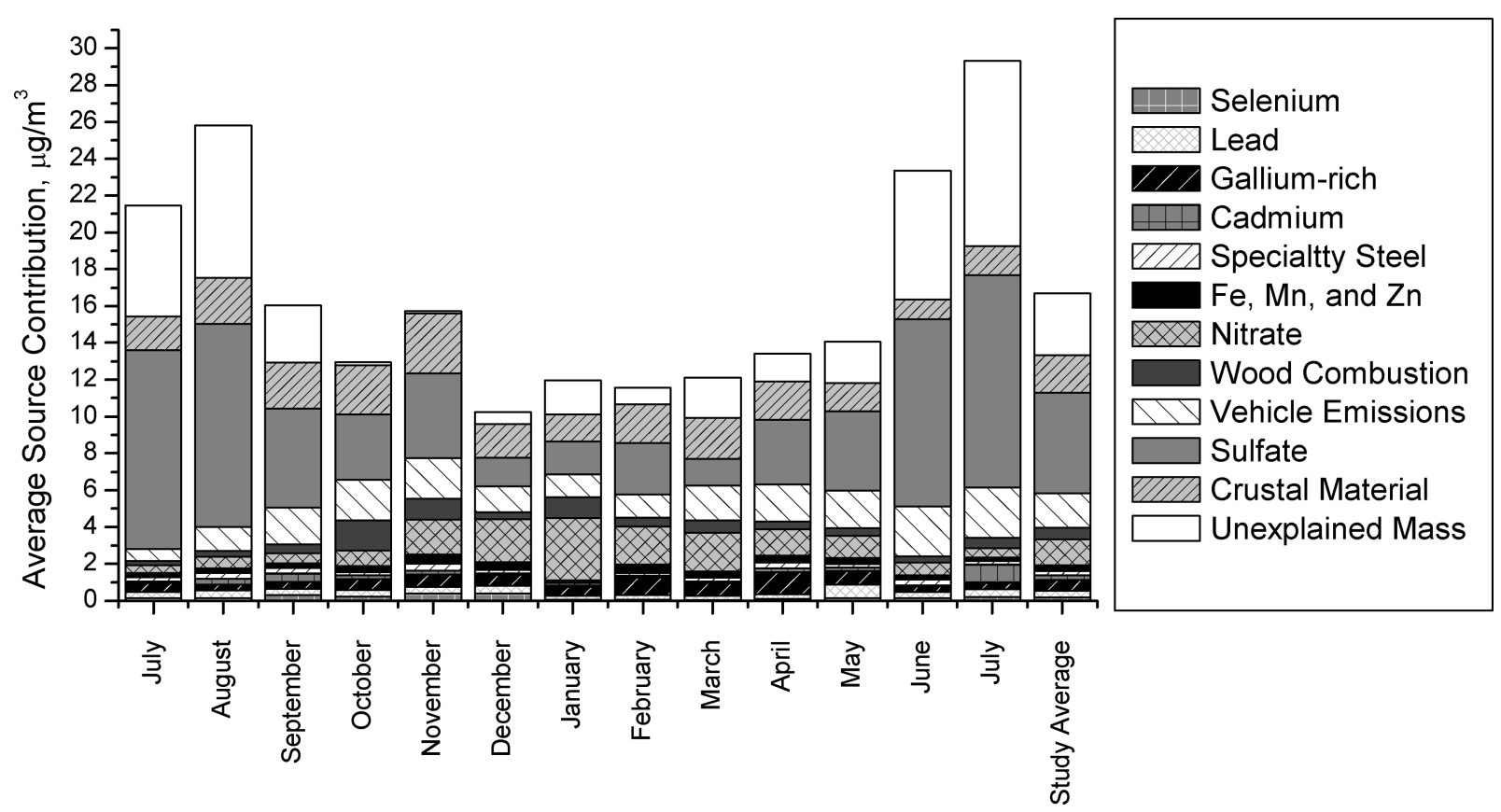

FIG. 9. Monthly average PMF source contributions for the PMF solution containing molecular markers. Height of the bars corresponds to the monthly average $\mathrm{PM}_{2.5}$ mass measured with a TEOM. The study average represents the average source contributions from July 11, 2001 through July 31 , 2002. The unexplained mass is the difference between the monthly average $\mathrm{PM}_{2.5}$ mass and the sum of the monthly averaged source contributions from each factor.

Adding speciated OC to the existing PMF model separates the primary OC and EC into two factors: vehicle emissions and road dust, and wood combustion, vegetative detritus and cooking. The hopanes, PAH's, and wood smoke tracers, despite a large percentage of missing data, showed good correlations with their respective factors, illustrating the power of PMF to accommodate datasets with large quantities of missing data. The wood combustion, cooking, and vegetative detritus factor contributes a small amount to ambient $\mathrm{PM}_{2.5}$ in Pittsburgh, approximately $4 \%$. There were several molecular markers considered or used in the model that did not yield a resolved factor based on the source of the molecular marker in Table 2. Meat cooking and cigarette smoking factors were not resolved, and distinctions between hardwood and softwood combustion factors were not made. Some molecular markers may prove better tracers than others and therefore the lack of further resolution of factors may be due to the large amount of missing data.

A future paper will discuss identifying the locations of sources of the observed species, Pekney et al. (2005). The conditional probability function (CPF) and the potential source contribution function (PSCF) will use the modeled source contributions and wind direction data or back trajectories to find most probable location of the sources.

\section{REFERENCES}

American Iron and Steel Institute (AISI). (2004). http://www.steel.org. Anderson, R., Martello, D., White, C., Crist, K., John, K., Modey, W., and Eatough, D. J. (2004). The Regional Nature of $\mathrm{PM}_{2.5}$ Episodes in the Upper Ohio River Valley, J. Air \& Waste Manage. Assoc. 54:971984.

Bautista, R. G. (2003). Processing to Obtain High-Purity Gallium, JOM. 55:2326.

Bernardo-Bricker, A., and Rogge, W. (2005). Ambient Organic $\mathrm{PM}_{2.5}$ at the Pittsburgh Air Quality Study: Seasonal Variations and Regional Contributions. To be submitted to Atmospheric Environment.

Cabada, J. C., Khlystov, A., Wittig, E., Pilinis, C., and Pandis, S. (2004). Light Scattering by Fine Particles During the Pittsburgh Air Quality Study: Measurements and Modeling, J. Geophys. Res. 109, D16S03.

Cabada, J. C., Pandis, S. N., and Robinson, A. L. (2002). Sources of Atmospheric Carbonaceous Particulate Matter in Pittsburgh, Pennsylvania, J. Air \& Waste Manage. Assoc. 52:732-741.

Dockery, D. W., Pope, C. A., Xu, X. P., Spengler, J. D., Ware, J. H., Fay, M. E., and Ferris, B. G. (1993). An Association Between Air Pollution and Mortality in Six United States Cities, New Engl. J. Med. 329:1753-1759.

Eatough, D. J., Modey, W. K., Mangelson, N. F., Anderson, R. R., and Martello, D. V. (2005). Apportionment of Ambient Primary and Secondary $\mathrm{PM}_{2.5}$ During a 2001 Summer Study in the NETL Pittsburgh Site Using PMF2 and EPA UNMIX, Aerosol Sci. Technol. in press.

Fraser, M. P., Yue, Z. W., and Buzcu, B. (2003). Source Apportionment of Fine Particulate Matter in Houston, TX, Using Organic Molecular Markers, Atmos. Environ. 37:2117-2123.

Henry, R. C. (1997). History and Fundamentals of Multivariate Air Quality Models, Chemometrics and Intelligent Laboratory Systems 37:37-42.

Henry, R. C. (2001). Unmix Version 2.4 Manual. Available with Unmix software (rhenry@usc.edu).

Henry, R. C. Personal communication.

Kim, E., Hopke, P. K., and Edgerton, E. (2003). Source Identification of Atlanta Aerosol by Positive Matrix Factorization, J. Air \& Waste Manage. Assoc. 53:731-739.

Kim, E., Hopke, P. K., Larson, T. V., and Covert, D. S. (2004). Analysis of Ambient Particle Size Distributions using Unmix and Positive Matrix Factorization, Environ. Sci. Technol. 38:202-209. 
Larsen, R. K., and Baker, J. E. (2003). Source Apportionment of Polycyclic Aromatic Hydrocarbons in the Urban Atmosphere: A Comparison of Three Methods, Environ. Sci. Technol. 37:1873-1881.

Lithgow, G., Robinson, A., and Buckley, S. (2004). Ambient Measurements of Metal-Containing $\mathrm{PM}_{2.5}$ in an Urban Environment Using Laser-Induced Breakdown Spectroscopy, Atmos. Environ. 38:3319-3328.

Maykut, N., Lewtas, J., Kim, E., and Larson, T. (2003). Source Apportionment of $\mathrm{PM}_{2.5}$ at an Urban IMPROVE Site in Seattle, Washington, Environ. Sci. Technol. 37:5135-5142.

Modey, W., Eatough, D. J., Anderson, R., Martello, D., Takahama, S., Lucas, L., and Davidson, C. I. (2005). Apportionment of Ambient Primary and Secondary Pollutants During a 2001 Summer Study in Pittsburgh Using EPA UNMIX, J. of the Air \& Waste Manage. Assoc. Submitted.

NIOSH. (1996). Elemental Carbon (Diesel Exhaust). In: NIOSH Manual of Analytical Methods. National Institute of Occupational Safety and Health, Cincinnati, $\mathrm{OH}$.

Olmez, I., Sheffield, A., Gordon, G., Houck, J., Pritchett, L., Cooper, J., Dzubay, T., and Bennett, R. (1998). Compositions of Particles from Selected Sources in Philadelphia for Receptor Modeling Applications, JAPCA. 38:1392-1402.

Paatero, P., and Tapper, U. (1993). Analysis of Different Modes of Factor Analysis as Least Square Fit Problems, Chemometrics and Intelligent Laboratory Systems. 18:183-194.

Paatero, P., and Tapper, U. (1994). Positive Matrix Factorization: A NonNegative Factor Model with Optimal Utilization of Error Estimates of Data Values, Environmetrics. 5:111-126.

Paatero, P. (1997). Least Squares Formulation of Robust Non-Negative Factor Analysis, Chemometrics and Intelligent Laboratory Systems. 37:15-35.

Paatero, P., Hopke, P. K., Begum, B. A., and Biswas, S. K. (2005). A Graphical Diagnostic Method for Assessing the Rotation in Factor Analytical Models of Atmospheric Pollution, Atmos. Environ. 39:193-201.

Parekh, P. (1990). Study of Manganese from Anthropogenic Emissions at a Rural Site in the Eastern United States, Atmos. Environ. 24A:415-421.

Pekney, N. J., and Davidson, C. I. (2005). Determination of Trace Elements in Ambient Aerosol Samples, Analytica Chimica Acta. 540:269-277.

Pekney N. J., Davidson, C. I., Zhou, L., and Hopke, P. K. (2005) Application of PSCF and CPF to PMF-Modeled Sources of $\mathrm{PM}_{2.5}$ in Pittsburgh, Aerosol Sci. \& Technol. in press.

Poirot, R. L., Wishinski, P. R., Hopke, P. K., and Polisar, A. V. (2001). Comparative Application of Multiple Receptor Methods to Identify Aerosol Sources in Northern Vermont, Environ. Sci. Technol. 35:4622-4636.

Polidori, A., Turpin, B., Lim, H., Totten, L., and Davidson, C. (2005). Polarity and Molecular Weight/Carbon Weight of the Pittsburgh Organic Aerosol. Manuscript in preparation.

Polissar, A. V., Hopke, P. K., Malm, W. C., and Sisler, J. F. (1998). Atmospheric Aerosol over Alaska: 2. Elemental Composition and Sources, J. Geophys. Res. 103:19,045-19,057.

Polissar, A. V., Hopke, P. K., and Poirot, R. L. (2001). Atmospheric Aerosol over Vermont: Chemical Composition and Sources, Environ. Sci. Technol. 35:4604-4621.

Pope, C. A., Burnett, R. T., Thun, M. J., Calle, E. E., Krewski, D., Ito, K., and Thurston, G. D. (2002). Lung Cancer, Cardiopulmonary Mortality and LongTerm Exposure to Fine Particulate Air Pollution, JAMA 287:1132-1141.

Rees, S. L., Robinson, A. L., Khylstov, A., Stanier, C. O., and Pandis, S. (2004). Mass Balance Closure and the Federal Reference Method for $\mathrm{PM}_{2.5}$ in Pittsburgh, Pennsylvania, Atmos. Environ. 38:3305-3318.

Robinson, A. L., Donahue, N. M., and Rogge, W. F. (2005). Photochemical Oxidation and Changes in Molecular Composition of Organic Aerosol in the Regional Context, J. Geophys. Res.-Atmos. Submitted.

Robinson, A. L., Subramanian, R., Donahue, N. M., Bernardo-Bricker, A., and Rogge, W. F. (2006a). Source Apportionment of Molecular Markers and Organic Aerosol-1. Methodology for Visually Comparing Source Profiles and Ambient Data, Environ. Sci. Technol. Submitted.

Robinson, A. L., Subramanian, R., Donahue, N. M., Bernardo-Bricker, A., and Rogge, W. F. (2006b). Source Apportionment of Ambient Organic Aerosol2. Biomass Smoke, Environ. Sci. Technol. Submitted.
Robinson, A. L., Subramanian, R., Donahue, N. M., Bernardo-Bricker, A., and Rogge, W. F. (2006c). Source Apportionment of Ambient Organic Aerosol3. Food Cooking Emissions, Environ. Sci. Technol. Submitted.

Rogge, W. F., Hildemann, L. M., Mazurek, M. A., Cass, G. R., and Simoneit, B. R. T. (1993a). Sources of Fine Organic Aerosol 2. Noncatalyst and CatalystEquipped Automobiles and Heavy-Duty Diesel Trucks. Environ. Sci. Technol. 27:636-651.

Rogge, W. F., Hildemann, L. M., Mazurek, M. A., Cass, G. R., and Simoneit, B. R. T. (1993b). Sources of Fine Organic Aerosol .3. Road Dust, Tire Debris, and Organometallic Brake Lining Dust—Roads as Sources and Sinks, Environ. Sci. Technol. 27:1892-1904.

Rogge, W. F., Hildemann, L. M., Mazurek, M. A., Cass, G. R., and Simoneit, B. R. T. (1993c). Sources of Fine Organic Aerosol .4. Particulate Abrasion Products from Leaf Surfaces of Urban Plants, Environ. Sci. Technol. 27:27002711.

Rogge, W. F., Hildemann, L. M., Mazurek, M. A., Cass, G. R., and Simoneit, B. R. T. (1996). Mathematical Modeling of Atmospheric Fine ParticleAssociated Primary Organic Compound Concentrations. J. Geophys. Res. Atmospheres. 101(D14):19379-19394.

Rogge, W. F., Hildemann, L. M., Mazurek, M. A., Cass, G. R., and Simonelt, B. R. T. (1991). Sources of Fine Organic Aerosol .1. Charbroilers and Meat Cooking Operations, Environ. Sci. Technol. 25:1112-1125.

Schauer, J. J., and Cass, G. R. (2000). Source Apportionment of Wintertime Gas-Phase and Particle-Phase air Pollutants Using Organic Compounds as Tracers, Environ. Sci. Technol. 34:1821-1832.

Schauer, J. J., Fraser, M. P., Cass, G. R., and Simoneit, B. R. T. (2002a). Source Reconciliation of Atmospheric Gas-Phase and Particle-Phase Pollutants During a Severe Photochemical Smog Episode, Environ. Sci. Technol. 36:38063814.

Schauer, J. J., Kleeman, M. J., Cass, G. R., and Simoneit, B. R. T. (2002b). Measurement of Emissions from Air Pollution Sources. 4. C-1-C-27 Organic Compounds from Cooking with Seed Oils, Environ. Sci. Technol. 36:567-575.

Schauer, J. J., Kleeman, M. J., Cass, G. R., and Simoneit, B. R. T. (1999). Measurement of Emissions from Air Pollution Sources. 1. C-1 Through C-29 Organic Compounds from Meat Charbroiling, Environ. Sci. Technol. 33:15661577.

Schauer, J. J., Rogge, W. F., Hildemann, L. M., Mazurek, M. A., and Cass, G. R. (1996). Source Apportionment of Airborne Particulate Matter Using Organic Compounds as Tracers, Atmos. Environ. 30:3837-3855.

Simoneit, B. R. T. (1984). Organic-Matter of the Troposphere .3. Characterization and Sources of Petroleum and Pyrogenic Residues in Aerosols over the Western United-States, Atmos. Environ. 18:51-67.

Simoneit, B. R. T. (1985). Application of Molecular Marker Analysis to Vehicular Exhaust for Source Reconciliations, Int. J. Environ. Anal. Chem. 22:203233.

Simoneit, B. R. T. (1999). A Review of Biomarker Compounds as Source Indicators and Tracers for Air Pollution, Environ. Sci. Pollution Res. 6:159169.

Simoneit, B. R. T. (2002). Biomass Burning-A Review of Organic Tracers for Smoke from Incomplete Combustion, Appl. Geochem. 17:129-162.

Simoneit, B. R. T., and Mazurek, M. A. (1982). Organic-Matter of the Troposphere. 2. Natural Background of Biogenic Lipid Matter in Aerosols over the Rural Western United-States, Atmos. Environ. 16:2139-2159.

Simoneit, B. R. T., Schauer, J. J., Nolte, C. G., Oros, D. R., Elias, V. O., Fraser, M. P., Rogge, W. F., and Cass, G. R. (1999). Levoglucosan, A Tracer for Cellulose in Biomass Burning and Atmospheric Particles, Atmos. Environ. 33:173-182.

Subramanian, R., Robinson, A. L., Donahue, N. M., Bernardo-Bricker, A., and Rogge, W. F. (2005a). Source Apportionment of Gasoline and Diesel Vehicles using the Chemical Mass Balance Model and Molecular Markers, Atmos. Environ. Submitted.

Subramanian, R., Donahue, N. M., Bernardo-Bricker, A., Rogge, W. F., and Robinson, A. L. (2005b). Source Apportionment of Primary Organic Aerosol in Pittsburgh, PA Using Organic Molecular Markers. Atmos. Environ. Manuscript in preparation. 
Subramanian, R. (2004). Sampling, Analysis, and Source-Apportionment of Ambient Carbonaceous Aerosols. Ph.D Dissertation. Carnegie Mellon University, Pittsburgh, PA.

Subramanian, R., Khlystov, A. Y., Cabada, J. C., and Robinson, A. L. (2004). Positive and Negative Artifacts in Particulate Organic Carbon Measurements with Denuded and Undenuded Sampler Configurations, Aerosol Sci. Technol. 38(S1):27-48.

Takahama, S., Wittig, B., Vayenas, D., Davidson, C., and Pandis, S. N. (2004). Modeling the Diurnal Variation of Nitrate During the Pittsburgh Air Quality Study, J. Geophys. Res.-Atmos. 109(D16): Art. No. D16S06.

Tang, W., Raymond, T., Wittig, B., Davidson, C. I., Pandis, S. N., Robinson, A. L., and Crist, K. (2004). Spatial Variations in $\mathrm{PM}_{2.5}$ During the Pittsburgh Air Quality Study, Aerosol Sci. Technol. 38(S2):80-90.

Turpin, B., and Lim, H. (2001). Species Contributions to $\mathrm{PM}_{2.5}$ Mass Concentrations: Revisiting Common Assumptions for Estimating Organic Mass, Aerosol Sci. Technol. 35:602-610.

U.S. Department of Energy (2002). Energy Information Administration, State Electricity Profiles. http://www.eia.doe.gov/cneaf/electricity/st_profiles/ pennsylvania.pdf. Accessed 2004.

U.S. Environmental Protection Agency. (1997a). National Ambient Air Quality Standards for Particulate Matter-Final Rule, 40 CFR part 50. Federal Register 62(138), 38,651-38,760, July 18.
U.S. Environmental Protection Agency. (1997b). Compendium of Methods for the Determination of Inorganic Compounds in Ambient Air. EPA/625/R96/010a. January 1997.

U.S. Environmental Protection Agency. (2002). Toxics Release Inventory Explorer version 4.3, http://www.epa.gov/triexplorer. Accessed 2004.

Watson, J. G., Fujita, E. M., Chow, J. C., and Zielinska, B. (1998). Northern Front Range Air Quality Study Final Report and Supplemental Volumes, http://www.nfraqs.colostate.edu/nfraqs/index2.html. Accessed 2006.

Watson, J., Chow, J., and Houck, J. (2001). PM $_{2.5}$ Chemical Source Profiles for Vehicle Exhaust, Vegetative Burning, Geological Material, and Coal Burning in Northwestern Colorado During 1995, Chemosphere. 43:11411151.

Wittig, A. E., Anderson, N. J., Khlystov, A. Y., Pandis, S. N., Davidson, C. I., and Robinson, A. L. (2004). Pittsburgh Air Quality Study Overview, Atmos. Environ. 38:3107-3125.

Zheng, M., Cass, G. R., Shauer, J. J., and Edgerton, E. S. (2002). Source Apportionment of PM2.5 in the Southeastern United States Using SolventExtractable Organic Compounds as Tracers, Environ. Sci. Technol. 36:23612371.

Zhou, L., Hopke, P. K., Paatero, P., Ondov, J., Pekney, N., and Davidson, C. I. (2004). Advanced Factor Analysis for Multiple Time Resolution Aerosol Composition Data, Atmos. Environ. 38:4909-4920. 


\title{
Identification of sources of atmospheric PM at the Pittsburgh supersite, Part I: Single particle analysis and filter-based positive matrix factorization
}

\author{
Natalie J. Pekney ${ }^{\mathrm{a}}$, Cliff I. Davidson ${ }^{\mathrm{a}, \mathrm{b}, *}$, Keith J. Bein ${ }^{\mathrm{c}}$, \\ Anthony S. Wexler ${ }^{\mathrm{c}, \mathrm{d}}$, Murray V. Johnston ${ }^{\mathrm{e}}$ \\ ${ }^{a}$ Department of Civil and Environmental Engineering, Carnegie Mellon University, Pittsburgh, PA 15213, USA \\ ${ }^{\mathrm{b}}$ Department of Engineering and Public Policy, Carnegie Mellon University, Pittsburgh, PA 15213, USA \\ ${ }^{\mathrm{c}}$ Department of Land, Air and Water Resources, University of California, Davis, CA 95616, USA \\ ${ }^{\mathrm{d}}$ Department of Mechanical and Aeronautical Engineering, University of California, Davis, CA 95616, USA \\ ${ }^{\mathrm{e}}$ Department of Chemistry and Biochemistry, University of Delaware, Newark, DE 19716, USA
}

Received 30 July 2005; received in revised form 29 November 2005; accepted 19 December 2005

\begin{abstract}
During the Pittsburgh Air Quality Study (PAQS), July 2001-September 2002, three co-located instruments analyzed the composition of ambient particulate matter (PM): (1) A single particle mass spectrometer, RSMS-3, was deployed to obtain high-temporal-resolution measurements of single particle size $(>1.1 \mu \mathrm{m})$ and composition which were correlated with meteorological data to identify sources; (2) $\mathrm{PM}_{2.5}$ and $\mathrm{PM}_{10}$ were collected on cellulose filters using high-volume (hi-vol) samplers, followed by microwave-assisted digestion and analysis by inductively coupled plasma-mass spectrometry (ICP-MS). Positive matrix factorization (PMF) was used to identify possible source categories; and (3) a micro-orifice uniform-deposit impactor (MOUDI) obtained size-distributed samples of PM. Several days of MOUDI filters were selected for microwave-assisted digestion and analysis by ICP-MS.

In this paper, sources identified using the single particle data were compared to the PMF results for the hi-vol/ICP-MS data. The strengths of each method were combined to hypothesize the most likely sources of various elements in ambient PM in Pittsburgh. In the final results, $\mathrm{Mo}$ and $\mathrm{Cr}$ are attributed to local specialty steel facilities; $\mathrm{Fe}, \mathrm{Mn}, \mathrm{Zn}$, and $\mathrm{K}$ are attributed to a steel mill $\mathrm{SE}$ of the monitoring station; internally mixed $\mathrm{Pb}$-containing particles are attributed to a major source to the $\mathrm{NW}$; and Ga is attributed to coal combustion sources to the NW. There is a notable lack of oil combustion sources.

The MOUDI data were used to resolve discrepancies between the single particle and hi-vol/ICP-MS data concerning the detection of Ti and Se. The hi-vol data showed appreciable Ti and Se masses, but RSMS-3 was unable to detect significant numbers of Ti-containing particles because of their large size, while we hypothesize that the volatility of Se caused it to be distributed more evenly over all emitted particles such that the amount of Se in any individual particle is below the limit of detection.
\end{abstract}

(C) 2006 Elsevier Ltd. All rights reserved.

Keywords: Real-time single particle mass spectrometry; Positive matrix factorization; Ambient aerosols; Trace elements; Particle size-composition

*Corresponding author. Tel.: + 14122682951.

E-mail address: cliff@cmu.edu (C.I. Davidson). 


\section{Introduction}

Several methods of identifying source types and specific sources of airborne contaminants have been developed over the past few decades. Beginning with the chemical mass balance first proposed by Friedlander and coworkers (Miller et al., 1972) and later developed by Watson et al. (1989), Hopke (1985), and others, techniques developed more recently can now incorporate a wide variety of data on airborne species in particulate and gaseous form, and also meteorological information (Zhou et al., 2004; Lewis et al., 2003; Kidwell and Ondov, 2004). Improvements in sampling and chemical analysis as well as data handling have also enhanced our ability to identify sources. Nevertheless, our overall capabilities are still very limited. One approach for obtaining better source attribution is to combine ambient data collected with a quantitative lowtemporal-resolution instrument and semi-quantitative high-temporal-resolution instrument, and to use these data with complementary data analysis techniques. In this paper, we use this approach to identify sources from data collected during the Pittsburgh Air Quality Study (PAQS). The second part of this paper (Part II: Quantitative Comparisons of Single Particle, Particle Number, and Particle Mass Measurements, Bein et al., 2005b) describes a more detailed analysis of data from different techniques used during the PAQS on two dates, 27 October 2001 and 14 March 2002.

\section{Experimental}

The PAQS was conducted from July 2001 to September 2002. The study included more than 20 different types of analyses of gas and aerosol samples, including analysis of trace elements in aerosol particles. Most samples were collected at the main monitoring station in Schenley Park, adjacent to the Carnegie Mellon University campus (Wittig et al., 2004). This location is approximately $6 \mathrm{~km}$ east of downtown Pittsburgh in the densely populated neighborhood of Oakland.

Three methods were used to collect airborne particle data during PAQS.

- Single particle measurements were obtained using RSMS-3, a laser ablation time-of-flight single particle mass spectrometer described by Lake et al. (2003) and Bein et al. (2005a). RSMS3 sampled on a semi-continuous basis with sampling intervals starting every $3 \mathrm{~h}$ for the first 4 months (September-December 2001) and every $2 \mathrm{~h}$ for the remainder of the study (January-September, 2002). Each sampling interval consisted of cycling through nine flow-limiting orifices, corresponding to nine different particle sizes transmitted into the instrument ranging from about $30 \mathrm{~nm}$ to $1.1 \mu \mathrm{m}$. The instrument was operated at each orifice until either $10 \mathrm{~min}$ expired or 30 particles were sampled, whichever came first. A positive and negative ion mass spectrum, as well as particle size and time of detection, were obtained for each particle sampled. Further information on the operation of RSMS-3 at the PAQS can be found in Bein et al. (2005a).

- $\mathrm{PM}_{10}$ and $\mathrm{PM}_{2.5}$ high-volume (hi-vol) samplers (Andersen Instruments, Inc., Smyrna, GA), specially equipped with brushless motors to minimize contamination, operated from July 2001 through July 2002 at a flow rate of $1.13 \mathrm{~m}^{3} \mathrm{~min}^{-1}$. The 24-h samples employed $20 \times 25 \mathrm{~cm}$ Whatman 41 (Whatman Inc., Clifton, NJ) cellulose filters. After sample collection, filters were cut into six or seven equal-sized strips. The two or three strips selected for analysis were microwave-digested in a solution of nitric acid, hydrogen peroxide, and trace hydrofluoric acid, and analyzed by inductively coupled plasma-mass spectrometry (ICP-MS). Concentrations of $\mathrm{PM}_{10}$ and $\mathrm{PM}_{2.5}$ are available for the following elements: Ag, As, Ba, Be, Ca, $\mathrm{Cd}, \mathrm{Ce}, \mathrm{Co}, \mathrm{Cr}, \mathrm{Cs}, \mathrm{Cu}, \mathrm{Fe}, \mathrm{Ga}, \mathrm{K}, \mathrm{Li}, \mathrm{Mg}, \mathrm{Mn}$, $\mathrm{Mo}, \mathrm{Ni}, \mathrm{Pb}, \mathrm{Rb}, \mathrm{Sb}, \mathrm{Se}, \mathrm{Sr}, \mathrm{Ti}, \mathrm{Tl}, \mathrm{V}$, and $\mathrm{Zn}$. Details of sample collection, preparation, and analysis are given by Pekney and Davidson (2005).

- A micro-orifice uniform-deposit impactor (MOUDI, Model 110, MSP Co., Minneapolis, $\mathrm{MN}$ ), operating at a flow rate of $30 \mathrm{~L} \mathrm{~min}^{-1}$, collected daily size-resolved aerosol samples from July 2001 through June 2002. The Teflon filters were weighed using a high-precision microbalance (UMX2, Mettler-Toledo, Columbus, $\mathrm{OH}$ ) in a temperature- and humidity-controlled environment (Cabada et al., 2004). Size cuts for the MOUDI stages were $0.056,0.10,0.18,0.32,0.56$, $1.0,1.8,2.5,5.6$, and $10 \mu \mathrm{m}$. For elemental analysis, the MOUDI filters were digested and analyzed according to the procedure described for the hi-vol cellulose filters above, with the exception that the polypropylene support rings 
on the Teflon filters were removed prior to digestion. Because of the lower flow rate and size distributed sampling, fewer elements had detectable concentrations on the MOUDI filters than from the hi-vol cellulose filters. MOUDI samples from only a few selected days of high PM concentration during the year of sampling were analyzed by ICP-MS. Size-resolved trace element ambient concentrations are available for 27 October 2001; 14 March 2002; and 17 April 2002 for As, Ba, Ca, Cd, Ce, Co, Cr, Cs, Cu, Fe, $\mathrm{Ga}, \mathrm{K}, \mathrm{Mg}, \mathrm{Mn}, \mathrm{Mo}, \mathrm{Ni}, \mathrm{Pb}, \mathrm{Rb}, \mathrm{Se}, \mathrm{Sr}, \mathrm{Ti}, \mathrm{V}$, and $\mathrm{Zn}$.

\section{Data analysis}

There are several differences between these methods of particle analysis that provide complementary information on sources. The RSMS yields data on the composition of individual particles from which a classification scheme may be constructed; particles are grouped together into classes based on the similarity in their composition. Each class of particles is believed to be attributable to a particular source or source type. The composition profile of the class, as well as the distribution of particle sizes within the class, provides a signature for that source. In addition, the high temporal resolution makes it possible to associate each particle with a specific wind speed and direction. The predominant wind direction is then determined for each class of particles. In situations where specific wind signatures can be resolved, knowledge of the local industry may be used to connect the particle class to a particular point source. Results from the application of this source attribution technique to the entire single particle PAQS data set are presented in detail by Bein et al. (2005a).

In contrast to the single particle instrument, which is not considered quantitative, the hi-vol/ ICP-MS data provide bulk quantitative airborne mass concentrations of each element of interest. However, the hi-vol data reveal very little about particle size. Pekney et al. (2006a) describe calculations of ambient concentrations from the hi-vol filters for use with source-receptor modeling. Positive matrix factorization, or PMF (Paatero and Tapper 1993, 1994; Paatero, 1997) was run with sulfate, nitrate, organic carbon (OC), elemental carbon (EC), several trace elements, and OC species to identify potential sources of $\mathrm{PM}_{2.5}$. An 11-source PMF model included the following identified factors with tracer species in parentheses where needed: sulfate; nitrate; crustal material $(\mathrm{Ca}, \mathrm{Ti})$; vehicle emissions and road dust (OC, EC, hopanes, PAHs, n-alkanes); gallium-rich (Ga); wood combustion, vegetative detritus and cooking (OC, EC, syringols, resin acids, levoglucosan, $n$-alkanes); steel production ( $\mathrm{Fe}, \mathrm{Mn}, \mathrm{Zn})$; specialty steel production and processing (Mo, Cr); cadmium; selenium; and lead. Factor contributions are given by Pekney et al. (2006a). The potential source contribution function (PSCF) and conditional probability function (CPF) were used with the PMF-modeled factor contributions to determine the most probable locations of the sources. PSCF uses HYSPLIT back trajectories and CPF uses wind direction. Descriptions of these methods and results are presented by Pekney et al. (2006b).

Similar to the hi-vol filter analysis, the MOUDI/ ICP-MS analysis provides size-resolved quantitative airborne mass concentrations, but only for a limited number of elements. To calculate ambient concentrations from the MOUDI filters, the mass of an element measured on each filter was first corrected by subtracting the average mass from four lab blanks obtained on different sampling days. If the sample mass measured on each filter was less than the established detection limit of $2 \sigma B$, where $\sigma B$ is the standard deviation of the four lab blanks, then the detection limit was used to represent an upper limit. The standard deviation was calculated by compounding the variability in the lab blanks and the variability in the sample concentration from three replicate ICP-MS analyses. The mass concentration distribution function was then calculated as $\left(\mathrm{d} M /\left(d \log D_{\mathrm{p}}\right)\right)$ for each impactor stage, where $\mathrm{d} M$ is the mass concentration of an element in size range $d \log D_{\mathrm{p}}$. For situations in which the single particle and PMF analyses disagree, the MOUDI data are used to resolve whether the discrepancy is due to the offset between the upper size limit of RSMS-3 and the hi-vol cut points.

\section{Hypotheses tested}

Several hypotheses were tested through the comparisons of data from the three PM sampling instruments.

1. $M o$ and $C r$ are consistently found together in both the ICP-MS/PMF results and the RSMS data 
because both elements are emitted by steel processing and alloying sources.

The RSMS identified a minor particle class containing $\mathrm{Cr}$, Mo, MoO, and $\mathrm{W}$ that comprised an average over all dates of $\sim 0.13 \%$ of the total number of particles analyzed. $\mathrm{Cr}, \mathrm{Mo}$, and $\mathrm{W}$ are common alloying elements in the production of stainless steels. Only $8.9 \%$ of the positive ion mass spectra collected for the particles in this class had complementary negative ion mass spectra. This suggests a local source was responsible, since the existence of negative ion mass spectra typically indicates the presence of secondary PM from atmospheric processing (Kane et al., 2002). This class was most frequently observed for SW winds at $\sim 235^{\circ}$, with another significant direction pointing SE toward a steel mill, which is $8.7 \mathrm{~km}$ and $129^{\circ}$ from the monitoring station. There are several facilities related to the production of steel to the SW of the monitoring station, with the closest ones being a specialty steel alloying plant $16 \mathrm{~km}$ away at $237^{\circ}$, a steel mill $32 \mathrm{~km}$ away at $228^{\circ}$, and a coke works $13 \mathrm{~km}$ away at $297^{\circ}$.

One of the 11 factors identified in the PMF analysis (Pekney et al., 2006a) was a specialty steel production and processing factor with $\mathrm{Mo}$ and $\mathrm{Cr}$ tracers. Ambient $\mathrm{W}$ concentrations were not measured by ICP-MS. The CPF analysis shows a most probable direction of this source to the SE. The probability of a specialty steel source in this direction was $45 \%$ and is most likely the steel mill at $129^{\circ}$. Another probable direction was NNE with a $35 \%$ probability. There are several specialty steel sources in this direction, which are located $26 \mathrm{~km}$ and $43^{\circ}$ from the station, $45 \mathrm{~km}$ away at $2^{\circ}$, $51 \mathrm{~km}$ away at $11^{\circ}, 112 \mathrm{~km}$ away at $10^{\circ}$, and $11 \mathrm{~km}$ away at $44^{\circ}$. The probability of a SW direction is approximately $20 \%$. PSCF corroborates these directions, with highest probability that the sources are located locally, within Allegheny County. The agreement between RSMS and $\mathrm{PMF} / \mathrm{CPF} / \mathrm{PSCF}$ analyses indicates that the results are robust and although multiple source directions were identified, the directions of highest probability were all coincident with the location of local steel mills or specialty steel sources. This source type has a distinct profile that allows identification from both methods, and the proximity to the monitoring station allows identification with wind direction, even with the 24-h averaged filter-based samples.
2. Although $\mathrm{Fe}, \mathrm{Mn}$, and $\mathrm{Zn}$ are found together in the ICP-MS/PMF results, $\mathrm{Fe}-$-, $\mathrm{Mn}$-, and $\mathrm{Zn}$-containing particles, in general, are externally mixed and result from either steel production or the metal refining/ forging industry.

The PMF results show a factor identified by the $\mathrm{Fe}, \mathrm{Mn}$, and $\mathrm{Zn}$ tracers (Pekney et al., 2006a). This factor is probably related to steel production since all three metals are used in the steelmaking process. PSCF results were inconclusive for this source, but $\mathrm{CPF}$ results show a most probable SE direction ( $45 \%$ probability for the $115-145^{\circ}$ interval).

Interpretation of $\mathrm{Mn}$ and $\mathrm{Fe}$ in single particle mass spectra is difficult because the Mn peak can be easily obscured by nearby Fe peaks. Since Mn was seen almost exclusively in Fe-containing particles during the PAQS, this interference is of significant importance in the discussions that follow and it is strongly believed that manganese was present in $\mathrm{Fe}$ containing particles more often than was detected.

According to the single particle data, $\mathrm{Fe}$ was one of the most frequently observed metals throughout the PAQS, and numerous particle classes were identified which contain Fe. In fact, almost every metal-containing particle class that was identified during this experiment had a sub-population of particles that also contained Fe. This implies that there are many sources of iron in the Pittsburgh area, both major and minor, ranging from combustion to wind-blown dust. The three major particle classes containing $\mathrm{Fe}$ are the $\mathrm{Si} / \mathrm{K} / \mathrm{Fe} / \mathrm{Ga}$ class, which is associated with coal combustion, a class of particles dominated by $\mathrm{Fe}$, and the $\mathrm{Li} / \mathrm{Na} / \mathrm{K} / \mathrm{Fe}$ class. The last two classes have both been attributed to steel processing and are of significant interest here since they appear to correlate best with the $\mathrm{PMF} \mathrm{Fe}, \mathrm{Mn}$, and $\mathrm{Zn}$ factor.

The $\mathrm{Fe}$ class comprises $\sim 1.2 \%$ of the total number of RSMS-3 particle hits and was most frequently observed when the wind was blowing from $\sim 125^{\circ}$, which agrees well with the CPF results and is in the direction of the major steel mill located at $129^{\circ}$. Although $24 \%$ of the mass spectra contained negative ions, a majority of these were $\mathrm{Cl}$ - and F-ions and have been attributed to the sequestration and conversion to salts of the acids used in steel production, such that the source is still considered to be local. In addition, since these particles were composed primarily of iron, the signal intensity of the iron peaks within the mass spectra was consistently large and poorly resolved such that 
interference in the detection of $\mathrm{Mn}$ was most significant for this class of particles as compared to any other particle class. Despite this, Mn was detected far more frequently in this class of particles than all others combined. In conjunction with the strong correlation between the results of the single particle and $\mathrm{PMF} / \mathrm{CPF}$ analyses, this is evidence that $\mathrm{Mn}$ went largely undetected in the Fe class and that a significantly larger fraction of the particles in this class contained Mn, suggesting the existence of a separate $\mathrm{Fe} / \mathrm{Mn}$ class.

Similar to the $\mathrm{Fe}$ class, the $\mathrm{Li} / \mathrm{Na} / \mathrm{K} / \mathrm{Fe}$ class comprises $\sim 1.3 \%$ of the total number of particle hits, and was most frequently observed for SE winds $\left(\sim 120^{\circ}\right)$. This class is probably local, since only $11.6 \%$ of the spectra had negative ions associated with them. $\mathrm{Li}$ and $\mathrm{Na}$ were not included in the PMF analysis, as ICP-MS data were unreliable for these two elements. The correlation coefficient for $\mathrm{K}$ versus the $\mathrm{Fe}, \mathrm{Mn}$, and $\mathrm{Zn}$ factor was well below the value of 0.7 needed to establish $\mathrm{K}$ as a tracer for this source (correlation coefficient of 0.53). However, the highest mass fraction of $\mathrm{K}, 39 \%$, was present in this factor. In addition, according to the single particle data, potassium was the most frequently observed metal in individual particles and was present in almost every metal-containing particle class identified. This is consistent with the PMF results showing $\mathrm{K}$ distributed over many types of sources.

In contrast to the case for $\mathrm{K}$ and $\mathrm{Fe}, \mathrm{Zn}$, the last metal present in the PMF factor, was found almost exclusively in one class of particles throughout the PAQS, the $\mathrm{Na} / \mathrm{K} / \mathrm{Zn} / \mathrm{Pb}$ class. This class represents $\sim 2.9 \%$ of the total number of particle hits. About $19 \%$ of the spectra had negative ions associated with them, suggesting a local source, and was most frequently observed from two dominant wind directions. One centered at $\sim 300^{\circ}$, in the direction of a zinc smelter $\left(307^{\circ}\right)$, and the other at $\sim 130^{\circ}$, in the direction of the steel mill. Closer inspection of the $\mathrm{Na} / \mathrm{K} / \mathrm{Zn} / \mathrm{Pb}$ class reveals several sub-classes which contain iron. In fact, $36 \%$ of the particles within this class belong to the $\mathrm{Na} / \mathrm{K} / \mathrm{Fe} / \mathrm{Zn} / \mathrm{Pb}$ subclass. This sub-class was originally placed within the $\mathrm{Na} / \mathrm{K} / \mathrm{Zn} / \mathrm{Pb}$ class due to the similarity in composition. However, in light of the results of the PMF analysis, it appears that this categorization was incorrect and the $\mathrm{Na} / \mathrm{K} / \mathrm{Fe} / \mathrm{Zn} / \mathrm{Pb}$ class should be considered a separate class of particles originating from an entirely different source. Re-analysis of the wind data for the case of separating these two classes supports this in that the $\mathrm{Na} / \mathrm{K} / \mathrm{Fe} / \mathrm{Zn} / \mathrm{Pb}$ class is more strongly correlated with the $130^{\circ}$ direction, and the $\mathrm{Na} / \mathrm{K} / \mathrm{Zn} / \mathrm{Pb}$ class is more strongly correlated with the $300^{\circ}$ direction. The presence of $\mathrm{K}, \mathrm{Fe}$, and $\mathrm{Zn}$ together in individual particles, along with a dominant wind direction of $130^{\circ}$, suggests that the $\mathrm{Na} / \mathrm{K} / \mathrm{Fe} / \mathrm{Zn} / \mathrm{Pb}$ class, similar to the other two iron-containing classes, is strongly correlated to the PMF Fe, Mn, and $\mathrm{Zn}$ factor.

Altogether, the data presented above reveal good agreement between the RSMS and PMF/CPF results and show strong evidence that the steel mill at $129^{\circ}$ is the source of several different types of $\mathrm{Mn}-, \mathrm{Fe}-$, and $\mathrm{Zn}$-containing particles. The combination of the two source attribution techniques resulted in a more complete description of Fe-, $\mathrm{Mn}$-, and $\mathrm{Zn}$-containing particles than either technique alone. More specifically, the isolation of a $\mathrm{Fe} / \mathrm{Mn}$ particle class, the distinction between the $\mathrm{Na} / \mathrm{K} / \mathrm{Zn} /$ $\mathrm{Pb}$ and $\mathrm{Na} / \mathrm{K} / \mathrm{Fe} / \mathrm{Zn} / \mathrm{Pb}$ classes, an explanation for the absence of $\mathrm{K}$ as a tracer in the $\mathrm{Fe}, \mathrm{Mn}$, and $\mathrm{Zn}$ factor and association of this factor with several different classes of particles were possible.

3. Although $\mathrm{Pb}$ is found in its own source category with no other elements according to the ICP-MS/ $P M F$ results, $P b$-containing particles, in general, are internally mixed and result from a variety of different sources throughout the Pittsburgh area.

$\mathrm{Pb}$ in atmospheric $\mathrm{PM}$ can originate from point sources or can represent area sources, like the resuspension of road dust containing $\mathrm{Pb}$ deposited from previous years or the continued use of leaded fuel in general avionics (Harris and Davidson, 2005). Many types of point sources emit Pb, including coal and oil combustion, steel mills, foundries, incinerators, battery manufacturing, and glass manufacturing. This is clearly evident in the single particle data in that, similar to $\mathrm{Fe}, \mathrm{Pb}$ was one of the most frequently observed metals in Pittsburgh PM. Although there were only a few major particle classes containing $\mathrm{Pb}$, almost every metal-containing particle class had a sub-population of particles that also contained $\mathrm{Pb}$. It is clear that ambient $\mathrm{Pb}$ concentrations do not solely originate from one point source.

4. Gallium in atmospheric PM originates from coal combustion. Both RSMS and PMF/CPF analyses identified a source of gallium-containing particles $N W$ of the site. Although there are major coal 
combustion sources in both the $N W$ and $S W$ quadrants, the RSMS and PMF analyses isolated the $N W$ quadrant because the measurement site is most heavily influenced by the sources of $G a$ in that direction.

The PMF analysis identified a gallium-rich factor with a $\mathrm{Ga}$ tracer that also showed significant contributions from $\mathrm{As}, \mathrm{Cu}, \mathrm{V}$, and $\mathrm{Ni}$. The PSCF and $\mathrm{CPF}$ analyses show that NW is the most probable direction. CPF shows a $70 \%$ probability of a source at $315^{\circ}$, and PSCF shows highest probability of a location in NW Pennsylvania or NE Ohio. Similarly, the single particle analysis identified a class of particles composed primarily of $\mathrm{Si}, \mathrm{K}$, $\mathrm{Fe}$, and $\mathrm{Ga}$. This was the single largest class of metal-containing particles detected throughout the PAQS and was observed almost exclusively when the wind was blowing from the NW at $\sim 302^{\circ}$. Numerous other metals were observed in these particles, including those associated with the gallium factor from the PMF analysis, but their appearance was highly variable and only $\mathrm{Si}, \mathrm{K}$, $\mathrm{Fe}$, and $\mathrm{Ga}$ were consistently observed. In addition, a majority of the particles within this class fell in the size range of $100-300 \mathrm{~nm}$, implicating combustion or a high-temperature furnace as their source.

Fig. (1) shows the location of all of the major coal combustion sources within $150 \mathrm{~km}$ of the measurement site as well as the frequency of observation versus wind direction for all wind observations obtained during the PAQS with wind speeds greater than $2 \mathrm{~m} \mathrm{~s}^{-1}$, which is plotted in the background as a continuous curve. The distance and direction from source to receptor, as well as $\mathrm{PM}_{2.5}$ emission rates in metric tons per year, are listed for each source in Table 1. $\mathrm{PM}_{2.5}$ emission rates were obtained from the National Emissions Inventory database (US EPA, 1999). The figure and table indicate that: (1) a large majority of the coal combustion sources are in the NW and SW quadrants, (2) the largest sources are coal-fired power plants and are located at distances greater than $20 \mathrm{~km}$ from the site, (3) the wind is most frequently NW, followed by the SW and SE, and (4) there is a cluster of four facilities (site \#s 1-4) to the NW, all of which use coal fired boilers to generate steam and are within $10 \mathrm{~km}$ of the site.

Although valuable as a starting point, simply using emission rates and distances between source and receptor to determine which sources are most important can be misleading. The Gaussian plume

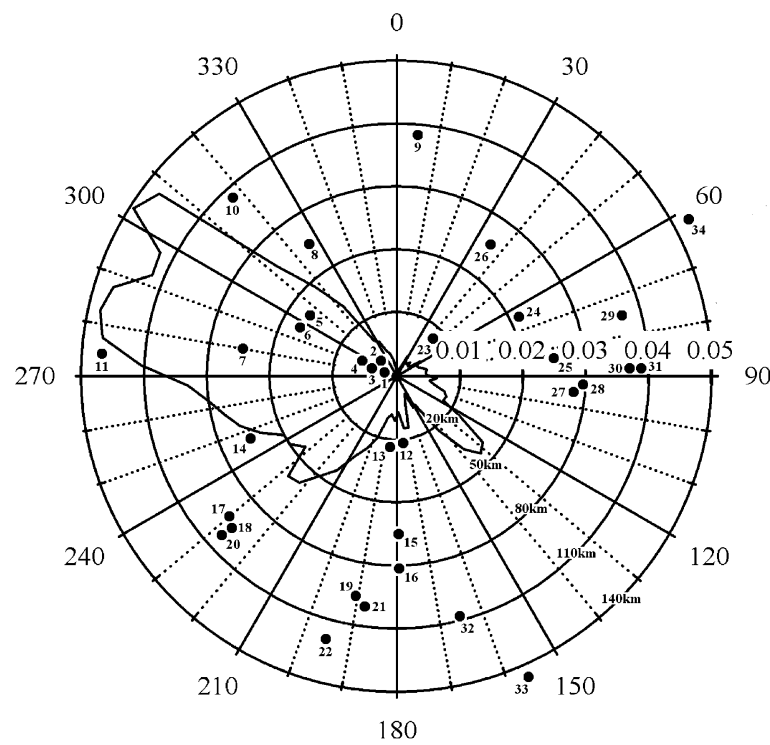

Fig. 1. The location of the major coal combustion sources within $150 \mathrm{~km}$ relative to the Schenley Park measurement site. The frequency of observation versus wind direction for all wind observations obtained during the PAQS that have wind speeds greater than $2 \mathrm{~m} \mathrm{~s}^{-1}$ is plotted in the background as a continuous curve.

dispersion model can be used to combine all of the available data into a first-order approximation of the relative impact of each source, and to suggest how the impact of each source changes as a function of meteorology. In general, the Gaussian plume dispersion equation gives the time averaged concentration of a particular species, $\langle C(x, y, z)\rangle_{\mathrm{S}}$, emitted from source $S$, at a receptor location. If the coordinate axis is aligned with its $x$-axis parallel to the mean wind vector and its origin at the source (ground level), and it is assumed that the receptor site lies along the $x$-axis, i.e. the plume centerline, then $\langle C(x, y, z)\rangle_{\mathrm{S}}$ may be written as

$\langle C(x, z)\rangle_{\mathrm{S}}=\frac{q_{\mathrm{S}}}{2 \pi \bar{u} \sigma_{y} \sigma_{z}} \Psi\left(z, h_{\mathrm{s}}, \Delta h, H, \sigma_{z}\right)$,

where $q_{\mathrm{s}}$ is the emission rate of source $S, \bar{u}$ is the average wind speed at the height of the emissions stack $\left(h_{\mathrm{s}}\right), \sigma_{y}$ and $\sigma_{z}$ are the Gaussian plume dispersion parameters for the $y$ and $z$ directions, respectively, and $\Psi\left(z, h_{\mathrm{s}}, \Delta h, H, \sigma_{z}\right)$ describes the influence of the boundaries on the dispersion of the plume, which is a function of $z, h_{\mathrm{s}}$ and $\sigma_{z}$, as well as the plume rise, $\Delta h$, and the boundary layer height, $H$. For the purposes currently being 
Table 1

$\mathrm{PM}_{2.5}$ emission rate and distance and direction from receptor to source for coal combustion sources within $100 \mathrm{~km}$ of the measurement site

\begin{tabular}{|c|c|c|c|c|c|c|c|c|}
\hline & Quadrant & Company & Facility & State & $\begin{array}{l}\text { Process } \\
\text { (coal fired) }\end{array}$ & $\mathrm{PM}_{2.5} \mathrm{mtpy}^{\mathrm{a}}$ & $\begin{array}{l}\text { Distance } \\
\text { from site } \\
(\mathrm{km})\end{array}$ & $\begin{array}{l}\text { Bearing } \\
\text { from site } \\
\text { (true north) }\end{array}$ \\
\hline 1 & NW & Bellefield Boiler Plant & $\mathrm{N} / \mathrm{A}^{\mathrm{b}}$ & PA & Steam & 10.6 & 0.9 & 286 \\
\hline 2 & NW & Pittsburgh Brewing Co. & $\mathrm{N} / \mathrm{A}$ & PA & Steam & 4.4 & 3.4 & 316 \\
\hline 3 & NW & H.J. Heinz Co. & Pittsburgh Plant & PA & Steam & 37.7 & 4.1 & 292 \\
\hline 4 & NW & $\mathrm{N} / \mathrm{A}$ & $\begin{array}{l}\text { State Correctional } \\
\text { Inst.-Pgh. }\end{array}$ & PA & Steam & 0.4 & 9.3 & 293 \\
\hline 5 & NW & AES BV Partners & $\begin{array}{l}\text { Beaver Valley Power } \\
\text { Plant }\end{array}$ & PA & Power & 77.6 & 42.4 & 304 \\
\hline 6 & NW & Pennsylvania Power Co. & $\begin{array}{l}\text { Bruce Mansfield Power } \\
\text { Plant }\end{array}$ & PA & Power & 2410 & 45.9 & 298 \\
\hline 7 & NW & Ohio Edison Co. & $\begin{array}{l}\text { W.H. Sammis Power } \\
\text { Plant }\end{array}$ & $\mathrm{OH}$ & Power & 7300 & 59.5 & 280 \\
\hline 8 & NW & $\begin{array}{l}\text { Orion Power MidWest, } \\
\text { L.P. }\end{array}$ & New Castle Power Plant & PA & Power & 1140 & 66.1 & 327 \\
\hline 12 & SE & $\begin{array}{l}\text { Orion Power MidWest, } \\
\text { L.P. }\end{array}$ & Elrama Power Plant & PA & Power & 370 & 20.8 & 175 \\
\hline 13 & SW & West Penn Power Co. & Mitchell Power Plant & PA & Power & 217 & 24.4 & 186 \\
\hline 14 & SW & Cardinal Operating Co. & Cardinal Power Plant & $\mathrm{OH}$ & Power & 5680 & 64.3 & 248 \\
\hline 15 & SE & West Penn Power Co. & Hatfields Power Plant & PA & Power & 7820 & 64.9 & 179 \\
\hline 16 & SE & Monongahela Power Co. & Fort Martin Power Plant & WV & Power & 5320 & 82.2 & 179 \\
\hline 17 & SW & Ohio Edison Co. & R.E. Burger Power Plant & $\mathrm{OH}$ & Power & 2560 & 94.1 & 230 \\
\hline 18 & SW & Ohio Power Co. & Kammer Power Plant & WV & Power & 5400 & 99.3 & 228 \\
\hline 19 & SW & $\begin{array}{l}\text { American Bituminous } \\
\text { Power Partners, L.P. }\end{array}$ & Grant Town Power Plant & WV & Power & 19.9 & 99.6 & 191 \\
\hline 20 & SW & Ohio Power Co. & Mitchell Power Plant & WV & Power & 2420 & 99.9 & 228 \\
\hline 23 & $\mathrm{NE}$ & $\begin{array}{l}\text { Orion Power MidWest, } \\
\text { L.P. }\end{array}$ & Cheswick Power Plant & PA & Power & 2440 & 16.5 & 49 \\
\hline 24 & $\mathrm{NE}$ & Sithe Energies Inc. & Keystone Power Plant & PA & Power & 8750 & 56.2 & 64 \\
\hline 25 & NE & $\begin{array}{l}\text { Edison Mission Energy } \\
\text { Co. }\end{array}$ & Homer City Power Plant & PA & Power & 7810 & 63.3 & 83 \\
\hline 26 & NE & West Penn Power Co. & Armstrong Power Plant & PA & Power & 1530 & 67.5 & 36 \\
\hline 27 & SE & Sithe Energies Inc. & Conemaugh Power Plant & PA & Power & 1370 & 74.5 & 95 \\
\hline 28 & SE & Sithe Energies Inc. & Seward Power Plant & $\mathrm{PA}$ & Power & 533 & 76.6 & 93 \\
\hline
\end{tabular}

Sources are shown by site number in Fig. 1. $\mathrm{PM}_{2.5}$ emission rates were obtained from the National Emissions Inventory database (US EPA, 1999).

${ }^{\mathrm{a}}$ mtpy, metric tons per year.

${ }^{\mathrm{b}} \mathrm{N} / \mathrm{A}$, not applicable.

considered, the application of Eq. (1) requires some manipulation. First, the annual $\mathrm{PM}_{2.5}$ emission rates of the various coal combustion sources, as listed in Table (1), are represented by $q_{\mathrm{s}}$. Second, the right-hand side of Eq. (1) was multiplied by the wind direction frequency depicted in Fig. (1). Last, it was assumed that $z \approx h_{\mathrm{s}}$ and $\bar{u}$ is represented by the wind speed measured at the site, as averaged over all wind observations made during the PAQS for which the wind was determined to be blowing from the direction of the source. The relative contribution of source $S$ to the total coal combustion $\mathrm{PM}_{2.5}$ measured at the site, $\Phi_{\mathrm{S}}$, was then estimated from

$\Phi_{\mathrm{S}}=\frac{\langle C(x, z)\rangle_{\mathrm{S}}}{\sum\langle C(x, z)\rangle_{\mathrm{S}}}$,

where the summation in the denominator is over all coal combustion sources. Since the values of $\sigma_{y}, \sigma_{z}$ and $\Psi\left(z, h_{\mathrm{s}}, \Delta h, H, \sigma_{z}\right)$ in Eq. (1) depend strongly on the stability of the atmosphere, and since the stability of the atmosphere was continually fluctuating throughout the PAQS, the value of $\Phi_{\mathrm{S}}$ in Eq. (2) was calculated for each of the six Pasquill stability classes (A-F; extremely unstable-moderately stable). For the cases in which the atmosphere 
was unstable $(\mathrm{A}-\mathrm{C})$, it is most likely that there was a well-developed turbulent boundary layer with an elevated inversion layer at a height $H$ such that, remembering $z \approx h_{\mathrm{s}}$ and assuming reflection at the ground, $\Psi\left(z, h_{\mathrm{s}}, \Delta h, H, \sigma_{z}\right)$ was calculated using

$$
\begin{aligned}
\Psi\left(h_{\mathrm{s}}, \Delta h, H, \sigma_{z}\right)= & \sum_{j=-\infty}^{j=+\infty}\left[\exp \left[-\frac{(2 j H-\Delta h)^{2}}{2 \sigma_{z}^{2}}\right]\right. \\
& \left.+\exp \left[-\frac{\left(2 h_{\mathrm{s}}+\Delta h+2 j H\right)}{2 \sigma_{z}^{2}}\right]\right],
\end{aligned}
$$

assuming that $H \approx 3 \mathrm{~km}$ for stability class $\mathrm{A}, H \approx$ $2 \mathrm{~km}$ for stability class $\mathrm{B}$, and $H \approx 1 \mathrm{~km}$ for stability class $\mathrm{C}$. For the situations in which the atmosphere was neutral or stable (D-F), it is most likely that there was little to no turbulence, and no elevated inversion layer, such that, making the same assumptions as above, $\Psi\left(z, h_{\mathrm{s}}, \Delta h, H, \sigma_{z}\right)$ was calculated using

$\Psi\left(h_{\mathrm{s}}, \Delta h, H, \sigma_{z}\right)=\exp \left[-\frac{\Delta h^{2}}{2 \sigma_{z}^{2}}\right]+\exp \left[-\frac{\left(2 h_{\mathrm{s}}+\Delta h\right)^{2}}{2 \sigma_{z}^{2}}\right]$.

For all six stability classes and all coal combustion sources considered, it was assumed that $h_{\mathrm{s}} \approx$ $80 \mathrm{~m}$ and $\Delta h \approx 200 \mathrm{~m}$. In addition, the values of $\sigma_{y}$ and $\sigma_{z}$, appropriate to each source and stability class, were determined using the formulations outlined by Turner (1969). Combining all of the data above, $\Phi_{\mathrm{S}}$ was calculated for all of the sources found in the NW and SW quadrants - site numbers 1-8 and 12-20, respectively, in Fig. (1) - and for each of the stability classes. Sites 12,15 , and 16 have been included in the SW quadrant for calculating the relative contribution from the SW sources as well as the NW/SW ratios. The results are shown in Fig. (2). The dispersion modeling calculations for $\langle C(x, y, z)\rangle_{\mathrm{S}}$ resulted in a range of $0.07-2.9 \mu \mathrm{g} \mathrm{m}^{-3}$ from stability classes $\mathrm{A}-\mathrm{F}$ for the concentrations at the receptor site from all of the coal combustion sources in Table 1. The average Ga-rich factor concentration from the PMF analysis was $0.59 \mu \mathrm{g} \mathrm{m}^{-3}$ with a range of $0.00003-4.8 \mu \mathrm{g} \mathrm{m}^{-3}$. Given all the sources of uncertainty and assumptions made in the dispersion modeling, this comparison is quite reasonable.

There are several interesting things to note in Fig. (2). (1) The impact of the NW sources on the measurement site is more significant than the SW sources for all stability classes, and the extent to which this is true increases with decreasing atmo- spheric stability. (2) Considering only coal-fired power production, Bruce Mansfield (site \#6 at $\sim 300^{\circ}$ ) and W.H. Sammis (site \#7 at $\sim 280^{\circ}$ ) are the largest contributors of $\mathrm{PM}_{2.5}$ to the site for all stability classes. (3) Considering only the sources in the SW quadrant, Cardinal (site $\# 14$ at $\sim 251^{\circ}$ ) has the largest impact for stability classes B-E, and Elrama (site $\# 12$ at $\sim 175^{\circ}$ ) has the largest impact for stability class A. (4) In the NW quadrant, the extent to which the local coal-fired steam generation facilities (site \#s 1-4) impact the site, relative to the coal-fired power plants, increases with decreasing atmospheric stability. (5) For stability class A, the contributions from the Bellefield Boiler Plant (site $\# 1$ at $\sim 286^{\circ}$ ) and the H.J. Heinz Pittsburgh Factory (site \#3 at $\sim 292^{\circ}$ ) are two orders of magnitude larger than all the coal-fired power plants combined.

Although many of the larger coal-fired power plants are located to the SE, SW, and NE of the site and not NW, the results suggest that the coal-fired sources to the NW can indeed have a significant impact on the receptor site, and the assumption that the Ga-containing particles coming from the NW originated from coal combustion becomes more likely. In addition, the results also suggest the potential for the impact of numerous coal-fired steam-generating facilities, which are small $\mathrm{PM}_{2.5}$ sources located close to the site $(<10 \mathrm{~km})$, to be comparable to, if not larger than, the impact of the major coal-fired power plants, which are located in the surrounding area $(20-100 \mathrm{~km})$, under certain atmospheric conditions. This can be substantiated by considering work presented by Bein et al. (2005b) in which an isolated plume of Ga-containing particles was detected and the particles within the plume fully characterized using the combination of single particle data and MOUDI/ICP-MS data.

5. Ni and V are tracers for oil combustion. A particle originating from this type of source should contain both elements; however, the RSMS did not identify a particle class containing both $\mathrm{Ni}$ and $V$, and the PMF analysis did not yield an oil-combustion factor as identified by $\mathrm{Ni}$ and $V$ tracers.

Commercial fuel oil combustion has not historically been a major source of $\mathrm{PM}_{2.5}$ in Pittsburgh. Currently, the fraction of ambient $\mathrm{PM}_{2.5}$ primary $\mathrm{OC}$ from commercial distillate and residual fuel is estimated at approximately $0.2 \%$, and the fraction of EC from these sources is estimated at $1.2 \%$ (Cabada et al., 2002). Such minor sources cannot be explained by PMF. Ni and $\mathrm{V}$ are present in other 


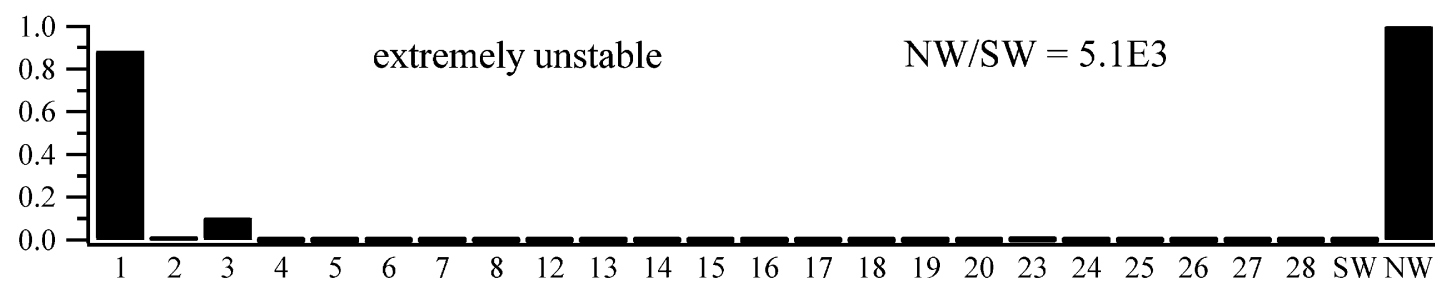

(A) Site Number

论

(B)

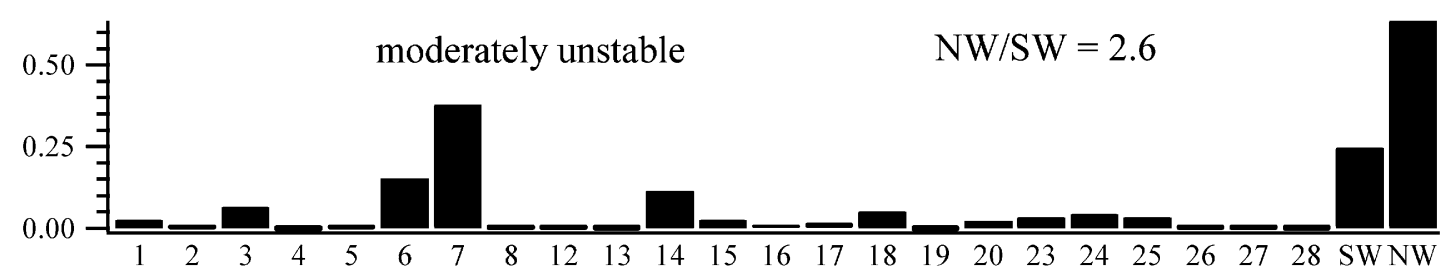

Site Number

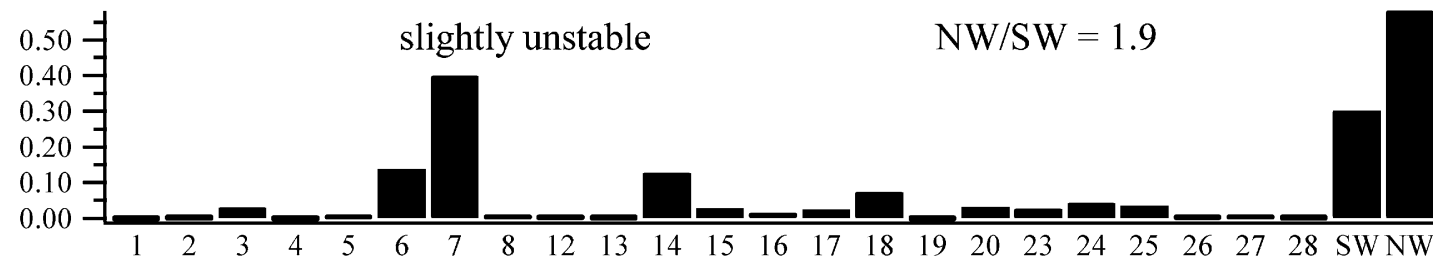

(C) Site Number

(D)

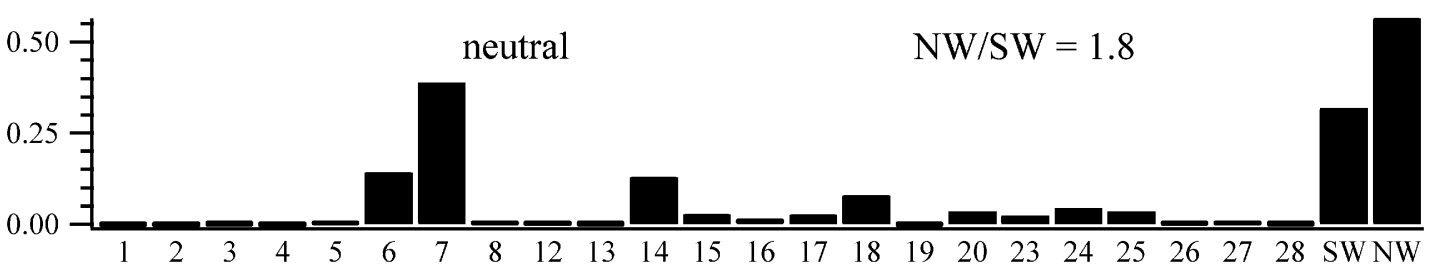
Site Number

(E)

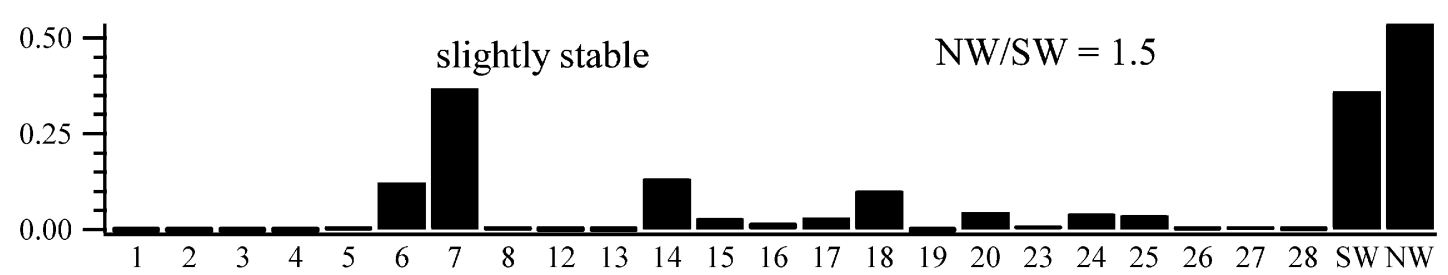
Site Number

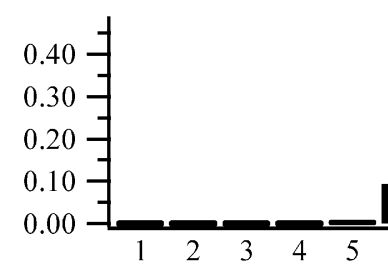

moderately stable

$\mathrm{NW} / \mathrm{SW}=1.2$

(F)

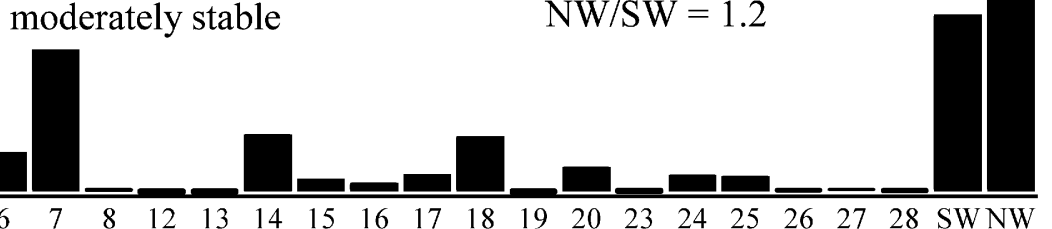

Site Number

Fig. 2. The relative contribution of each coal combustion source, for all coal combustion sources located in either the NW or SW quadrant, to the total coal combustion related $\mathrm{PM}_{2.5}$ potentially impacting the Schenley Park site, as determined for all six Pasquill stability classes. Sites 1-8 are in the NW quadrant, sites 12-20 are in the SW quadrant or nearly due south, and sites 23-28 are in the NE quadrant or nearly due east. The last two categories are the relative contributions of the sum of the SW sources and the sum of the NW sources, respectively, and the ratio of the two, NW/SW, is listed at the top of each plot. 
sources, however. Most Ni mass is explained by the gallium-rich factor $(39 \%)$ and the specialty steel factor $(52 \%)$. Most $\mathrm{V}$ mass is explained by the gallium-rich factor $(45 \%)$ and the crustal material factor $(26 \%)$.

Particles from oil combustion are expected to contain OC and EC as well as both $\mathrm{Ni}$ and V. The RSMS data show a class of Ni-containing particles without detectable amounts of $\mathrm{V}$, and without $\mathrm{OC}$ or EC. In addition, the particles were larger than typical combustion-sized aerosol, with size distributions peaking in the $400-1100 \mathrm{~nm}$ range rather than $50-200 \mathrm{~nm}$. The observation of this particle class was a completely isolated event occurring over a 4day period in July 2001. We thus conclude that none of the major particle classes identified by the RSMS, including the Ni particle class, are associated with oil combustion.
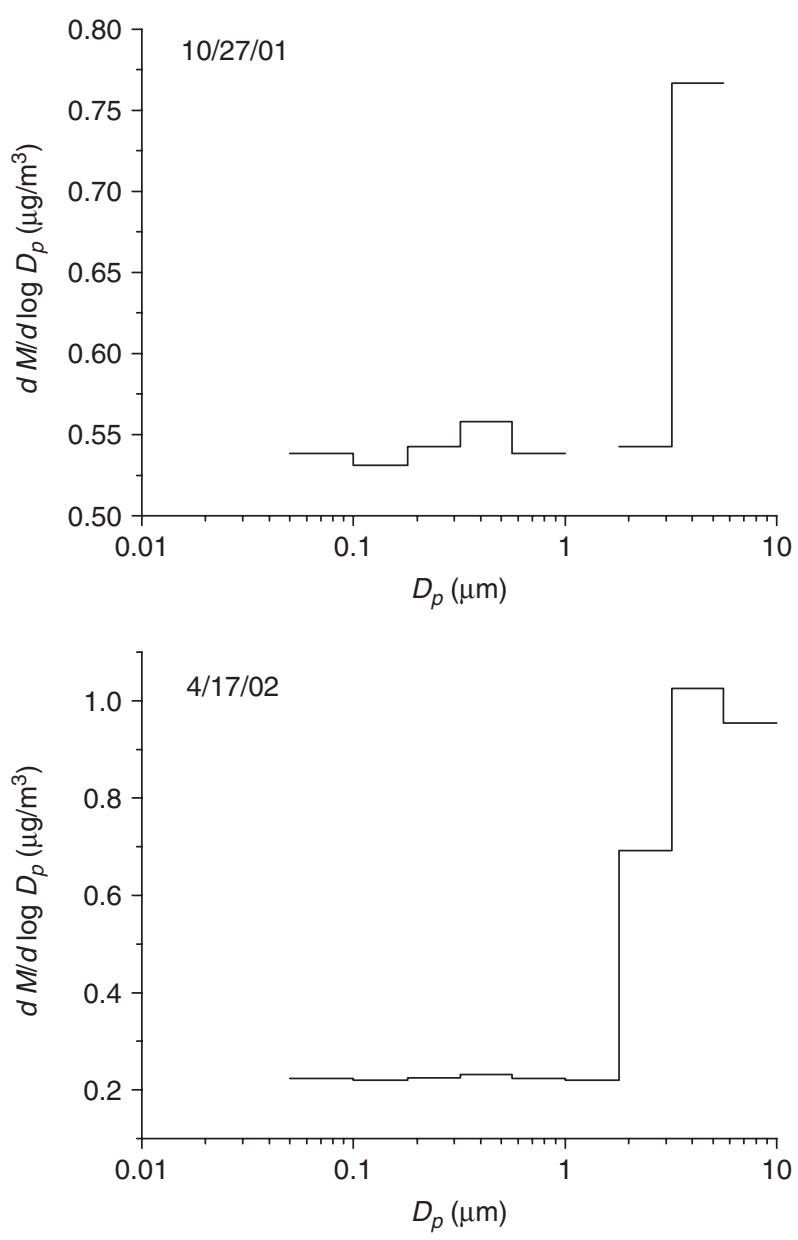

6. The lack of Ti in RSMS data compared with appreciable Ti concentrations in the ICP-MS data reflects a significant amount of Ti between 1.1 and $2.5 \mu \mathrm{m}$.

Size distributions of $\mathrm{Ti}$, seen in Fig. (3), show virtually all of the mass associated with particles larger than $1.8 \mu \mathrm{m}$ in diameter. Although $\mathrm{Ti}$ was occasionally detected in individual particles, significant amounts were not observed because the maximum particle size measured by the RSMS is $1.1 \mu \mathrm{m}$. The average ratio $\mathrm{PM}_{2.5}: \mathrm{PM}_{10}$ for $\mathrm{Ti}$ averaged over the entire data set is $0.23 \pm 0.10$. Titanium is typically associated with crustal material but has uses as a white pigment for paints, papers, and other materials, and is also occasionally used in its metallic form and has been observed in coal fly ash. The PMF results show a factor whose

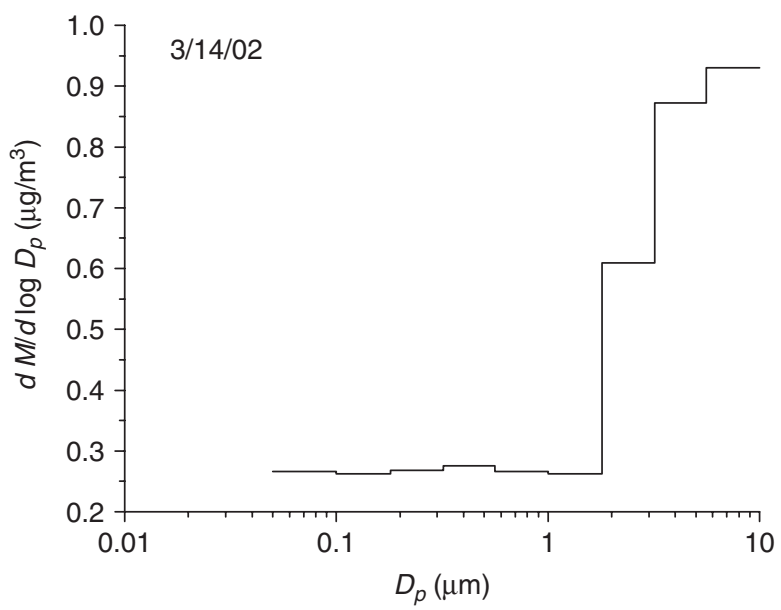

Fig. 3. Ti size distribution as measured by MOUDI filter analysis for three 24-h samples. Values shown for $D_{\mathrm{p}}$ less than $1.8 \mu \mathrm{m}$ represent detection limits; concentrations measured on these stages were below these detection limits. 
contributions correlate strongly with the $\mathrm{Ca}$ and $\mathrm{Ti}$ ambient data and therefore this was identified as a crustal material factor. However, while $14 \%$ of the Ti mass is in the crustal material factor, $21 \%$ is in the $\mathrm{Fe}, \mathrm{Mn}$, and $\mathrm{Zn}$ factor, $15 \%$ is in the wood combustion, vegetative detritus and cooking factor, $15 \%$ is in the gallium-rich factor, and $19 \%$ is in the specialty steel factor. The remaining factors were composed of less than $5 \%$ of the Ti mass.

7. Se, typically associated with coal combustion, is a semi-volatile metal such that the mass of selenium emitted from a particular coal combustion source will tend to be spread thinly over a large number of particles, which obscures its detection on a particle-byparticle basis, but is sufficient enough over the ensemble of particles to be detected by ICP-MS analysis.

The PMF analysis shows a factor marked by a Se tracer. Se is typically associated with coal combustion. The PSCF/CPF analyses conclude that the Ohio River Valley, SW from the monitoring station, is the most probable location of the Se sources. There are indeed several large coal-fired power plants in this region. Surprisingly, RSMS-3 did not detect any particles containing a significant amount of Se. Unlike the issue of Ti detection described in hypothesis 6 , Se mass is almost completely contained in particles less than $2.5 \mu \mathrm{m}$ in diameter. The average ratio of $\mathrm{PM}_{2.5}: \mathrm{PM}_{10}$ for the hi-vol samples is $0.97 \pm 0.2$. In addition, MOUDI/ICP-MS data for individual days show Se mass above the method detection limit for MOUDI stages, which correspond to diameters in the fine particle size range. Therefore, lack of detection of Se by the RSMS is not due to particle size. Ambient concentrations of Se as measured by hi-vol shown in Fig. (4) indicate that relatively high peaks occur in September,
November, and December 2001, with lower concentrations for the remainder of the study.

Why does RSMS-3 miss the Se? There is a possibility that Se peaks were obscured by ions of OC fragments nearby in the spectrum. However, since $\mathrm{Se}$ is considered to originate from coal combustion and $\mathrm{OC}$ is not, it is unlikely that they would consistently appear together in individual particles. In addition, the results of the PMF analysis do not show any OC tracers in the selenium factor.

The most likely explanation is that Se was below the limit of detection of the RSMS. Because the instrument is significantly more sensitive to detection of some other metal species, such as $\mathrm{K}, \mathrm{Fe}, \mathrm{Si}$, and $\mathrm{Al}$, the presence of these species within the particle would tend to amplify this affect by further obscuring Se detection. However, over a large ensemble of Se-containing particles and for a long sampling interval, the total Se mass was sufficient to be detected by ICP-MS analysis. A possible explanation for the presence of Se in trace amounts within individual particles is that because $\mathrm{Se}$ is a semi-volatile metal, the original mass of Se vaporized during coal combustion is scavenged by a large number of pre-existing particles. Selenium emitted from coal combustion exists predominantly in the vapor phase (Andren and Klein, 1975, Germani and Zoller, 1988). The selenium would thus be spread thinly over the entire particle population and the amount of selenium in any individual particle would be small.

\section{Conclusions}

From analysis of the entire Pittsburgh Supersite data set, a comparison of results from the single

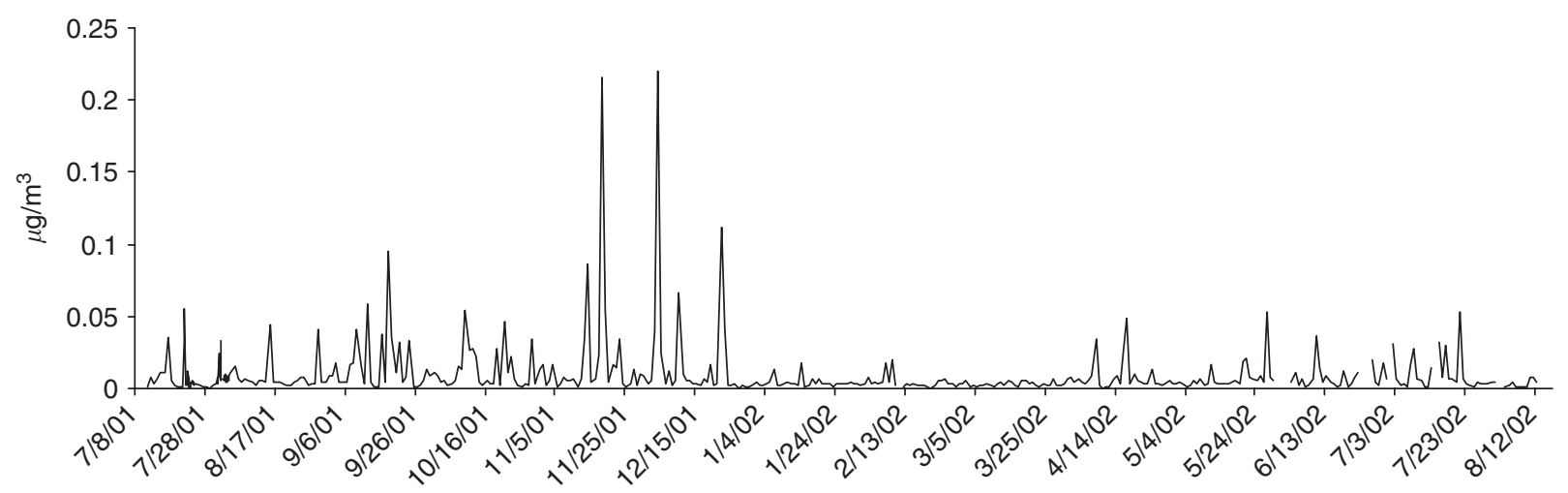

Fig. 4. Ambient $\mathrm{PM}_{2.5}$ Se concentrations as measured by hi-vol sampling and ICP-MS analysis. 
particle source attribution to results from the ICP-MS/PMF analysis revealed converging conclusions about several hypotheses addressed in this work.

Factor analysis methods such as PMF attempt to group individual species measurements into factors that explain the data set in a more compact way. Particles originating from a source that does not explain the data set in a statistically significant manner are not identified in a factor analysis. Despite this limitation, the comparison of the RSMS data and the ICP-MS/PMF analysis for source identification shows clear correlations between some particle classes and PMF factors. Convergent results include $\mathrm{Mo}$ and $\mathrm{Cr}$ attributed to local specialty steel facilities, Fe, Mn, Zn, and $\mathrm{K}$ attributed to a steel mill SE of the monitoring station, internally mixed $\mathrm{Pb}$-containing particles attributed to major source(s) to the NW, Ga associated with coal combustion sources to the NW, and the lack of significant particles from oil combustion. In addition, the combination of the two source attribution techniques resulted in a more complete description of the sources of Pittsburgh PM than either technique alone and allowed for new information about the nature of each technique. The two most significant conclusions from this are: (1) small differences in the composition of individual particles can potentially play a large role in determining the sources of those particles such that understanding the limitations of the algorithm used to classify single particle data, as well as knowing the variability in the data itself, are both important, and (2) unless the metal is rare, like Ga and Se, it is hard to use an individual metal alone as a tracer for a specific source type.

Finally, divergent results between the two source attribution techniques revealed new information about the detection of $\mathrm{Ti}$ and $\mathrm{Se}$ in individual particles within the Pittsburgh area. The Ti size distribution obtained from MOUDI filter analysis showed that RSMS-3 was unable to detect significant numbers of Ti-containing particles, since they were outside the fine particle size range of the single particle instrument. Se was detected in abundance by ICP-MS but was not detected in individual particles using RSMS-3 even though the MOUDI data showed Se mass in the fine mode. Since Se is a semi-volatile metal, the original mass of Se may have vaporized during coal combustion and is believed to have spread over a large population of pre-existing particles such that the amount of Se in any individual particle is very small. Continued examination of these converging and diverging results from comparative studies such as this will result in better understanding of each technique.

\section{Acknowledgments}

This research was conducted as a part of the Pittsburgh Air Quality Study, which was supported by the US Environmental Protection Agency under Contract R82806101 and the US Department of Energy National Energy Technology Laboratory under Contract DE-FC26-01NT41017. This paper has not been subject to EPA's peer and policy review, and therefore does not necessarily reflect the views of the Agency. No official endorsement should be inferred.

\section{References}

Andren, A.W., Klein, D.H., 1975. Selenium in coal-fired steam plant emissions. Environmental Science and Technology 9, 856-858.

Bein, K.J., Zhao, Y., Wexler, A.S., Johnston, M.V., 2005 a. Speciation of size-resolved individual ultrafine particles in Pittsburgh, Pennsylvania. Journal of Geophysical Research 110, D07S05.

Bein, K.J., Zhao, Y., Pekney, N.J., Davidson, C.I., Johnston, M.V., Wexler, A.S., 2005b. Identification of sources of atmospheric PM at the Pittsburgh Supersite. Part II: Quantitative comparisons of single particle, particle number, and particle mass measurements. Atmospheric Environment, in press, doi:10.1016/j.atmosenv.2006.01.064.

Cabada, J.C., Pandis, S.N., Robinson, A.L., 2002. Sources of atmospheric carbonaceous particulate matter in Pittsburgh, Pennsylvania. Journal of the Air \& Waste Management Association 52, 732-741.

Cabada, J.C., Rees, S., Takahama, S., Khlystov, A., Pandis, S.N., Davidson, C.I., Robinson, A.L., 2004. Mass size distributions and size resolved chemical composition of fine particulate matter at the Pittsburgh Supersite. Atmospheric Environment 38, 3127-3141.

Germani, M.S., Zoller, W.H., 1988. Vapor-phase concentrations of arsenic, selenium, bromine, iodine, and mercury in the stack of a coal-fired power plant. Environmental Science and Technology 22, 1079-1085.

Harris, A.R., Davidson, C.I., 2005. The role of resuspended soil in lead flows in the California South Coast Air Basin. Environmental Science and Technology 39, 7410-7415.

Hopke, P.K., 1985. Receptor Modeling in Environmental Chemistry. Wiley, New York.

Kane, D.B., Wang, J.J., Frost, K., Johnston, M.V., 2002. Detection of negative ions from individual ultrafine particles. Analytical Chemistry 74, 2092-2096.

Kidwell, C.B., Ondov, J.M., 2004. Elemental analysis of subhourly ambient aerosol collections. Aerosol Science and Technology 38, 205-218. 
Lake, D.L., Tolocka, M.P., Wexler, A.S., Johnston, M.V., 2003. Mass spectrometry of individual particles between 50 and $750 \mathrm{~nm}$ in diameter at the Baltimore Supersite. Environmental Science and Technology 37, 3268-3274.

Lewis, C.W., Norris, G.A., Conner, T.L., Henry, R.C., 2003. Source apportionment of Phoenix $\mathrm{PM}_{2.5}$ aerosol with the Unmix receptor model. Journal of the Air \& Waste Management Association 53, 325-338.

Miller, M.S., Friedlander, S.K., Hidy, G.M., 1972. A chemical element balance for the Pasadena aerosol. Journal of Colloid and Interface Science 39, 165-176.

Paatero, P., 1997. Least squares formulation of robust nonnegative factor analysis. Chemometrics and Intelligent Laboratory Systems 37, 15-35.

Paatero, P., Tapper, U., 1993. Analysis of different modes of factor analysis as least square fit problems. Chemometrics and Intelligent Laboratory Systems 18, 183-194.

Paatero, P., Tapper, U., 1994. Positive matrix factorization: a non-negative factor model with optimal utilization of error estimates of data values. Environmetrics 5, 111-126.

Pekney, N.J., Davidson, C.I., 2005. Determination of trace elements in ambient aerosol samples. Analytica Chimica Acta 540, 269-277.
Pekney, N.J., Davidson, C.I., Robinson, A.L., Zhou, L., Hopke, P.K., Eatough, D., 2006a. Major source categories for $\mathrm{PM}_{2.5}$ in Pittsburgh using PMF and Unmix. Aerosol Science and Technology 40, 910-924.

Pekney, N.J., Davidson, C.I., Zhou, L., Hopke, P.K., 2006b. Application of PSCF and CPF to PMF-modeled sources of $\mathrm{PM}_{2.5}$ in Pittsburgh. Aerosol Science and Technology 40, 952-961.

Turner, D.B., 1969. Workbook of Atmospheric Diffusion Estimates, USEPA 999-AP-26. US Environmental Protection Agency, Washington, DC.

US EPA, 1999. National Emission Inventory Database. $\langle$ www.epa.gov/air/data.

Watson, J.G., Robinson, N.F., Chow, J.C., Henry, R.C., Kim, B., Nguyen, Q.T., Meyer, E.L., Pace, T.G., 1989. Receptor modeling technical series, vol. III (1989 revision). CMB7 User's Manual. EPA-450/4-90-004.

Wittig, A.E., Anderson, N.J., Khlystov, A.Y., Pandis, S.N., Davidson, C.I., Robinson, A.L., 2004. Pittsburgh air quality study overview. Atmospheric Environment 38, 3107-3125.

Zhou, L., Hopke, P.K., Paatero, P., Ondov, J.M., Pancras, J.P., Pekney, N.J., Davidson, C.I., 2004. Advanced factor analysis for multiple time resolution aerosol composition data. Atmospheric Environment 38, 4909-4920. 


\title{
Determination of trace elements in ambient aerosol samples
}

\author{
Natalie J. Pekney ${ }^{\mathrm{a}}$, Cliff I. Davidson ${ }^{\mathrm{a}, \mathrm{b}, *}$ \\ ${ }^{a}$ Department of Civil and Environmental Engineering, Carnegie Mellon University, 5000 Forbes Ave., Pittsburgh, PA 15213, USA \\ ${ }^{\mathrm{b}}$ Engineering and Public Policy, Carnegie Mellon University, 5000 Forbes Ave., Pittsburgh, PA 15213, USA
}

Received 6 December 2004; received in revised form 19 March 2005; accepted 22 March 2005

Available online 25 April 2005

\begin{abstract}
A microwave-assisted digestion procedure using $\mathrm{HNO}_{3}, \mathrm{HF}$, and $\mathrm{H}_{2} \mathrm{O}_{2}$ has been developed for analysis of elements in ambient particulate matter (PM). The samples are collected on cellulose filters and analyzed by inductively coupled plasma mass spectrometry (ICP-MS). The ICP-MS is calibrated with external standards, and recovery of analytes is tested with NIST SRM 1648 Urban Dust. This method has been used to quantify the airborne concentrations of a large number of elements, including $\mathrm{Ag}, \mathrm{As}, \mathrm{Ba}, \mathrm{Be}, \mathrm{Ca}, \mathrm{Cd}, \mathrm{Ce}, \mathrm{Co}, \mathrm{Cr}, \mathrm{Cs}, \mathrm{Cu}, \mathrm{Fe}, \mathrm{Ga}, \mathrm{K}$, $\mathrm{Li}, \mathrm{Mg}, \mathrm{Mn}, \mathrm{Mo}, \mathrm{Ni}, \mathrm{Pb}, \mathrm{Rb}, \mathrm{Se}, \mathrm{Sb}, \mathrm{Sr}, \mathrm{Ti}, \mathrm{Tl}, \mathrm{V}$, and $\mathrm{Zn}$. For the majority of these elements, recovery of the NIST SRM is within $15 \%$ of the certified values.
\end{abstract}

(C) 2005 Elsevier B.V. All rights reserved.

Keywords: Trace elements; Atmospheric aerosols; Inductively coupled plasma-mass spectrometry; Microwave digestion; Cellulose filter

\section{Introduction}

Trace element concentrations in atmospheric aerosols are now widely used in source-receptor modeling studies. However, obtaining such data requires collection of a large number of samples and a cost- and time-effective method of analysis. Sample preparation varies, but one popular method is microwave-assisted digestion of filter-based samples. This method is appealing for analyses that require liquid samples, due to the ease and low contamination levels with which samples can be prepared. Analysis methods also vary; inductively coupled plasma-mass spectrometry (ICP-MS) as used here has low detection limits, large linear dynamic range and simultaneous multi-element output data. However, because ICP-MS requires a liquid sample, accurate measurements are dependent on the sample extraction efficiency. Other analysis techniques include graphite furnace atomic absorption spectrometry (GFAAS), proton-induced X-ray emission spectrometry (PIXE), and X-ray fluorescence (XRF). GFAAS has comparable detection limits to those of ICP-MS, but does not

\footnotetext{
* Corresponding author. Tel.: +1 4122682951.

E-mail address: cliff@cmu.edu (C.I. Davidson).
}

have the capability of simultaneous multiple element measurement and therefore is not time-effective. PIXE and XRF are both non-destructive analysis techniques but detection limits are much higher, two orders of magnitude for some elements, as compared to ICP-MS.

The choice of digestion reagents is an important consideration for measurement of multiple elements in ambient aerosols. Atmospheric particulate matter (PM) includes primary emissions of carbonaceous and metallic particles from combustion and industrial processes, crustal material from erosion of soil and rock, and secondary pollutants such as sulfates, nitrates and organic aerosols, among other species. The reagents chosen must digest the sample as completely as possible and keep the elements stable in solution. Nitric acid can accomplish this for many elements; however, it cannot completely digest silicon-containing compounds and the elements bonded to siliceous material. Previous groups have used relatively large amounts of hydrofluoric acid (HF) to break the silica bonds despite the fact that HF is extremely hazardous and therefore difficult to work with. Jalkanen and Hasanen [1] used a 3:1 mixture of $\mathrm{HNO}_{3}$ and $\mathrm{HF}$ in a total volume of $2.0 \mathrm{~mL}$. Yang et al. [2] used a digestion solution of $5 \mathrm{~mL} \mathrm{HNO}_{3}, 4 \mathrm{~mL} \mathrm{H}_{2} \mathrm{O}_{2}, 0.5 \mathrm{~mL}$ of $\mathrm{HF}$, and $5 \mathrm{~mL}$ of $\mathrm{H}_{3} \mathrm{BO}_{3}$. 
If the silica content of the samples can be measured or estimated, the use of HF can be minimized. In this paper, we examine the use of trace amounts of HF in digesting aerosol filter samples for trace element analysis.

We begin by using a certified NIST standard reference material (SRM) to test the recovery of trace elements using the digestion procedure developed here. Next, we apply the digestion procedure to ambient $\mathrm{PM}_{2.5}$ and $\mathrm{PM}_{10}$ collected in Pittsburgh, PA on cellulose filters with analysis by ICP-MS. We then compare SRM samples digested with and without HF to quantify the influence of using this acid. Finally, we compare the ambient PM analysis by ICP-MS using the new digestion procedure with non-destructive analysis by X-ray fluorescence (XRF) for samples collected at other sites in the Pittsburgh region.

\section{Experimental}

The Pittsburgh Air Quality Study (PAQS) took place during July 2001-September 2002. The study included more than 20 different types of analyses of gas and aerosol samples, including analysis of trace elements in aerosol particles. Most samples were collected at the main monitoring station in Schenley Park, adjacent to the Carnegie Mellon University campus [3]. This location is approximately $6 \mathrm{~km}$ east of downtown Pittsburgh in the densely populated neighborhood of Oakland. Ambient air was sampled for concentrations of elements using $\mathrm{PM}_{10}$ and $\mathrm{PM}_{2.5}$ high-volume (hi vol) samplers from Andersen Instruments Inc. (Smyrna, Georgia) using $20 \mathrm{~cm} \times 25 \mathrm{~cm}$ filters. The hi vols operated at a flow rate of $1.13 \mathrm{~m}^{3} / \mathrm{min}$, and they were specially equipped with brushless motors to minimize contamination.

Many types of Teflon filters are commercially available, and these filters are useful for non-destructive analysis methods such as XRF or proton induced X-ray emission. In the current study, cellulose filters (Whatman 41, Whatman Inc., Clifton, NJ) were used as they completely digest in the acids, leaving a liquid solution for injection into the ICPMS. Retention efficiency of the Whatman 41 filters varies with particle size, with a minimum value at $0.3 \mu \mathrm{m}$ diameter of $92 \%$ at a face velocity of $45.2 \mathrm{~cm} / \mathrm{s}$. [4]. This is admittedly somewhat lower than the minimum $99.7 \%$ retention efficiency for Teflon filters [5]. However, the efficiency is over $99 \%$ for particles larger than $1.0 \mu \mathrm{m}$ diameter [4].

\subsection{Reagents}

All reagents were purchased in the purest form available: redistilled nitric acid (70\%) and custom redistilled 70\% nitric/0.5\% hydrofluoric acid blend from GFS Chemicals (Columbus, $\mathrm{OH}$ ), as well as semiconductor grade hydrogen peroxide (30-32\%) from Sigma Aldrich (St. Louis, MO). Distilled, deionized water from a NANOpure ultrapure water system was used (Barnstead/Thermolyne, Dubuque, IA).

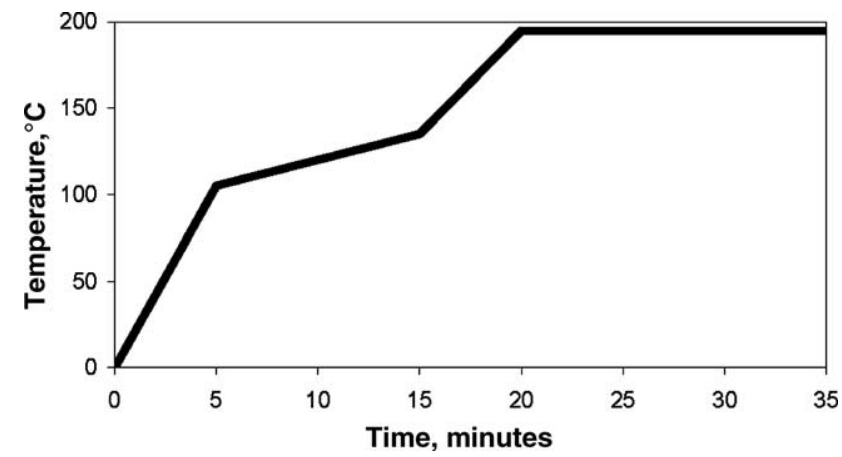

Fig. 1. Temperature-controlled program for the MARS 5 digestion method.

\subsection{Microwave description}

The microwave used was a MARS 5 (CEM Corp., Matthews, NC) equipped with an EST-300 Plus temperature sensor and an ESP-1500 Plus pressure sensor. The microwave digestion vessels, HP500 Plus, had working maximum pressure and temperature of $2.4 \times 10 \mathrm{~N} / \mathrm{m}$ and $195^{\circ} \mathrm{C}$, respectively. The microwave could accommodate digestion of up to 14 samples simultaneously. For sample preparation, Standard EPA Method 3052 was followed with some adaptations [6]. The microwave method developed for sample digestion was controlled by temperature and is illustrated in Fig. 1. The slower ramp time between 105 and $135^{\circ} \mathrm{C}$ allowed for slower decomposition of the cellulose filter material without a very large or very rapid increase in pressure.

\subsection{ICP-MS description}

Analyses were performed at Duquesne University using an Agilent 4500 ICP-MS (Agilent Technologies, Palo Alto, CA). The ICP-MS was equipped with a concentric nebulizer and a quartz torch. Operating parameters are listed in Table 1. All other parameters, such as torch position, sample depth, extraction, and ion focusing lenses voltages, Omega lenses voltages, quadrupole settings, and carrier and blend gas flow rates were tuned at the start of each analysis session such that the sensitivity was optimized. Oxides and doubly charged ion ratios were kept below $2 \%$.

Table 1

ICP-MS operating parameters

\begin{tabular}{ll}
\hline RF power $(\mathrm{kW})$ & $1.4-1.5$ \\
RF matching $(\mathrm{V})$ & 1.99 \\
Plasma gas flow rate $(\mathrm{L} / \mathrm{min})$ & 15 \\
Auxiliary gas flow rate $(\mathrm{L} / \mathrm{min})$ & 1 \\
Peristaltic pump flow (rps) & 0.10 \\
Measurement mode & Spectrum analysis mode \\
Number of measurements per peak & 3 (full quant. mode) \\
Integration time & $0.10 \mathrm{~s} /$ point \\
Number of repetitions & 3 \\
Time per sample measurement (min) & $2-3$ \\
Rinse time (min) & 1 \\
\hline
\end{tabular}




\subsection{Filter handling}

Filters were handled in ways to minimize contamination. Blank filters were handled identically to the samples, but were run on the hi vol for only 1 min. For the first two months of the study, field blanks were taken once per filter change. Subsequently, field blanks were taken once weekly. For the majority of the study period, 24-h samples were taken.

After collection of the samples, filter cassettes were delivered to the laboratory in clean, sealed bags. Filters were removed from the cassettes under a laminar flow hood and cut into strips using a polypropylene template and disposable stainless steel knife blades. Cutting the filter into smaller sections was necessary to get the sample mass below $0.5 \mathrm{~g}$, which is the working limit of the microwave vessels, and also allowed for comparison of analysis results from multiple strips per filter. For the first two months of the study, the filter-cutting template made seven strips per filter, each sized $2.5 \mathrm{~cm} \times 17.8 \mathrm{~cm}$. Subsequent filters were cut with a larger template, which made six strips per filter, sized $3.2 \mathrm{~cm} \times 17.8 \mathrm{~cm}$. Individual filter strips were transferred to sterilized polypropylene centrifuge tubes (tested to be clean for metals) and labeled, sealed in a clean plastic bag (KNF Clean Room Products Corp., Ronkonkoma, NY) and stored in a freezer to minimize biological activity until digestion and analysis. With the exception of the filter loading and removal at the sampling site, all work was conducted under HEPA-filtered laminar flow hoods.

\subsection{Sample preparation}

We can estimate the amount of HF needed to dissolve all of the silicate material in a typical sample. Based on Rees et al. [7], the crustal component of the samples is estimated to account for about $4 \%$ of the $\mathrm{PM}_{2.5}$ mass. For the hi vol samples collected at the main site, the annual average 24-h air volume was $1630 \mathrm{~m}^{3}$ and the annual average $\mathrm{PM}_{2.5}$ mass concentration was $17 \mu \mathrm{g} / \mathrm{m}^{3}$. If we assume conservatively that all crustal mass was $\mathrm{SiO}_{2}$, the typical amount of $\mathrm{Si}$ in a strip comprising $1 / 6$ of the total filter would be $1.8 \times 10^{-4} \mathrm{~g}$. The amount of HF necessary for complete digestion of the $\mathrm{SiO}_{2}$ can be determined from the following reaction:

$\mathrm{SiO}_{2}+4 \mathrm{HF} \stackrel{\text { Heat }}{\longrightarrow} \mathrm{SiF}_{4}(\mathrm{~g})+2 \mathrm{H}_{2} \mathrm{O}$

Results show that $1.2 \times 10^{-5}$ moles of $\operatorname{HF}\left(2.5 \times 10^{-4} \mathrm{~g}\right)$, or less than $0.1 \% \mathrm{HF}$ in the digestion solution, completely digests all of the crustal material in an average sample. A concentration of $0.5 \% \mathrm{HF}$ was therefore chosen to digest the crustal material in all the samples. HF at this low concentration can be purchased already blended with nitric acid, and thus it is not necessary to use the stringent precautions invoked for more concentrated HF. Also, the sample introduction system for the ICP-MS does not have to be replaced with an expensive inert system needed for more concentrated HF. Only one quartz torch was used for the duration of this study that involved analysis of over 3200 samples during the course of one year.

Hydrogen peroxide can reduce NO fumes as well as pressure in the closed microwave vessel. The pressure-reducing mechanism is described by the following chemical reactions [8]:

$$
\begin{aligned}
& 2 \mathrm{H}_{2} \mathrm{O}_{2} \stackrel{\text { Heat }}{\longrightarrow} 2 \mathrm{H}_{2} \mathrm{O}+\mathrm{O}_{2}(\mathrm{~g}) \\
& 2 \mathrm{NO}(\mathrm{g})+\mathrm{O}_{2}(\mathrm{~g}) \rightarrow 2 \mathrm{NO}_{2}(\mathrm{~g}) \\
& 2 \mathrm{NO}_{2}(\mathrm{~g})+\mathrm{H}_{2} \mathrm{O} \rightarrow \mathrm{HNO}_{3}+\mathrm{HNO}_{2}
\end{aligned}
$$

Boric acid is sometimes used to complex the HF at higher concentrations but was not used here. Other reagents sometimes used include perchloric and hydrochloric acid, neither of which was used in this study since $\mathrm{ClO}, \mathrm{ClOH}$, and $\mathrm{ArCl}$ can cause interference problems in the measurement of As, Se, V, and Cr by ICP-MS [9].

Using Teflon-coated forceps, filter strips were transferred to the digestion vessels, the solution was added, and the vessels were capped. The digestion solution consisted of $9 \mathrm{~mL}$ custom redistilled $70 \%$ nitric/ $0.5 \%$ hydrofluoric acid blend and $2 \mathrm{~mL}$ hydrogen peroxide. After digestion in the microwave, the vessels were transferred to the CEM Microvap assembly, connected to a vacuum pump, and heated in the microwave until the volume of the sample decreased to approximately $0.5 \mathrm{~mL}$. This allowed evaporation of the acid without loss of the analytes [10]. The samples were then removed and poured into sterilized, graduated sample cups already containing the internal standard solution. Deionized water was added to bring the total volume to $10 \mathrm{~mL}$. Each sample contained $50 \mathrm{ppb}$ of the internal standard solution because this concentration level was approximately mid-calibration range for most elements. The final acid concentrations in the samples were approximately $4 \%$ nitric acid and $0.02 \%$ hydrofluoric acid.

\subsection{Analysis}

Samples were transported to the Duquesne University Laboratory clean room in airtight plastic containers. The ICPMS was tuned and calibrated. A check standard was analyzed after the initial calibration and subsequently after every 10 samples. If the measured check standard concentrations were not within $10 \%$ of actual concentrations, the instrument was recalibrated and the previous 10 samples were re-analyzed. If a sample concentration was higher than the highest calibration standard for an element, the sample was diluted and re-analyzed for that element.

External ICP-MS calibration standards were custommade from SCP Science (Champlain, NY). Because of the great difference in concentrations of various elements in each sample, a standard stock solution was made to reflect these differences, and five calibration standards were mixed from the stock solution. Maximum standard concentration for elements of lowest concentrations in the samples was $200 \mathrm{ppb}$, 
while maximum for elements of highest concentrations was $8000 \mathrm{ppb}$.

The multi-element internal standard solution, also from SCP Science, contained Sc, In, Tb, Ho, Y, and Bi. The internal standard solution was added to every external calibration standard and sample. Addition of the internal standard helped to account for matrix effects. Selection of the appropriate internal standard element for each sample element depends on similarities in mass number, ionization potential, and chemical characteristics. Table 2 shows selected internal standard elements for each analyte.

\section{Results and discussion}

\subsection{Recovery of NIST standard reference material}

Success of the digestion procedure was measured by the recovery of elements in the NIST SRM 1648, Urban Dust, and also the agreement between two different strips per filter. For each batch of samples digested, one microwave vessel contained a measured mass of the SRM, approximately $1.0 \mathrm{mg}$ to represent sample mass, in the same digestion solution. Also, one microwave vessel per digestion batch contained only reagents to examine contamination from the reagents. The reagent blank sample concentrations (RGB) were subtracted from the NIST SRM samples $\left(\mathrm{SRM}_{\text {measured }}\right)$ prior to calculating recovery. Percent recovery, $\% R$, was calculated as:

$\% R=\frac{\mathrm{SRM}_{\text {measured }}-\mathrm{RGB}}{\mathrm{SRM}_{\text {certified }}} \times 100 \%$

where $\mathrm{SRM}_{\text {certified }}$ is the concentration of the element in the SRM as certified by NIST. Minimum detection limits (MDLs) were established as per EPA Method 200.8 [9] and are listed for each element in Table 2. Where concentrations of elements in either the SRM or the RGB samples were below the MDL, they were replaced with the MDL as a conservative upper limit of the concentration, and were listed as an upper limit. For blank correction, typically either the SRM was many times the RGB or both the SRM and RGB were below the MDL; there were not a significant number of cases in which the subtraction of a RGB replaced by the MDL for an element resulted in a substantial decrease from the true concentration that would therefore render the recovery unacceptable. Elements in a sample for which either the measured concentration or the NIST certified concentration were less than two times the reagent blank concentration were flagged as below detection. Also in Table 2 is the number of samples analyzed that were above detection out of a possible total of 139. This total does not include values deleted due to a malfunction of the balance. Note that early tests of this method included a blank filter strip along with the NIST SRM in some samples; the presence of the filter strip did not affect the results and hence further work with the SRM samples did not incorporate blank filter strips.

Recoveries for each element are shown in Table 2. Data for $\mathrm{Na}$ and $\mathrm{Al}$ are unreliable and therefore are not given. All other elements are within the target recovery of $100 \pm 15 \%$ with the exception of $\mathrm{Cr}(59 \%)$ and $\mathrm{Cs}(82 \%)$. The precision and accuracy of the results of this study compare well with recoveries of the NIST SRM 1648 reported by others, despite much higher concentrations of HF used in their studies. Jalkanen and Hasanen [1], using a 3:1 mixture of $\mathrm{HNO}_{3}$ and $\mathrm{HF}$, report recoveries in the range of $80-98 \%$ for several elements in 10 samples with the exception of a very low recovery of $\mathrm{Cr}(28 \%)$. Yang et al. [2] and Wu et al. [11], both using a digestion solution of $\mathrm{HNO}_{3} / \mathrm{H}_{2} \mathrm{O}_{2} / \mathrm{HF} / \mathrm{H}_{3} \mathrm{BO}_{3}$ report recoveries of $90-110 \%$ for several elements, with lower recoveries of 70\% and $77 \%$ for Se and Cr, respectively, reported by Yang et al. Swami et al. [12] find similar recoveries for most elements at varying amounts of $\mathrm{HF}$ in a $\mathrm{HNO}_{3} / \mathrm{H}_{2} \mathrm{O}_{2}$ digestion solution. Precision and recovery of Se decreased with increasing amounts of $\mathrm{HF}$, although recovery of Cr improved. The low recovery of chromium in the NIST SRM 1648 is a documented problem [13]. It has been hypothesized that the high soot content of NIST SRM 1648 signifies the presence of organic material that somehow inhibits dissolution of all the chromium [1]. Most of the samples had concentrations below detection for Cs. Therefore, the low recovery of Cs is likely due to detection limit problems. The SRM did not have certified concentrations for several elements analyzed, namely $\mathrm{Li}, \mathrm{Be}, \mathrm{Ca}, \mathrm{Ga}, \mathrm{Sr}, \mathrm{Mo}$, and Tl.

\subsection{Trace elements in ambient $P M$}

Data from either two or three filter strips have been averaged for every filter used in this study to report airborne concentrations [14]. However, agreement between filter strips is discussed here only for two typical months, October 2001 (two strips per filter) and January 2002 (three strips per filter). Ambient concentrations were determined by subtracting the average of the field blank filter mass from the filter sample mass, and dividing by the volume of air sampled. For a sample to be considered above detection, concentration must be greater than 3.3 times the standard deviation of the field blank concentrations [9]. Field blank concentrations varied by element, ranging from 0.001 to $4 \mathrm{ng} / \mathrm{m}^{3}$. Table 3 shows the average difference between concentrations from the two strips divided by the daily average mass concentration for each element for the month of October 2001, termed the percent error. For both $\mathrm{PM}_{10}$ and $\mathrm{PM}_{2.5}$, the average errors are almost always less than 30\%. For the January 2002 filters, the relative standard deviation of the data from three strips was smaller than the values of the average percent error given in Table 3 for nearly all elements. These levels of error are acceptable for using the data with such source-receptor models as positive matrix factorization (PMF) and Unmix [14]. 
Table 2

Percent recovery for each element

\begin{tabular}{|c|c|c|c|c|}
\hline Element & Internal standard element & Minimum detection limit (ppb) & $\begin{array}{l}\text { Number of samples above } \\
\text { detection (139 total samples) }\end{array}$ & $\begin{array}{l}\text { Recovery (\%) (S.D., \%) } \\
\text { of NIST SRM } 1648\end{array}$ \\
\hline $\mathrm{Li}$ & $\mathrm{Sc}$ & 0.076 & & \\
\hline $\mathrm{Be}$ & $\mathrm{Sc}$ & 0.048 & & \\
\hline $\mathrm{Mg}$ & $\mathrm{Sc}$ & 0.439 & 125 & $96(18)$ \\
\hline $\mathrm{K}$ & $\mathrm{Sc}$ & 66.1 & 139 & $96(18)$ \\
\hline $\mathrm{Ca}$ & $\mathrm{Sc}$ & 5.26 & & \\
\hline $\mathrm{Ti}$ & $\mathrm{Sc}$ & 0.506 & 139 & $86(12)$ \\
\hline V & $\mathrm{Sc}$ & 0.155 & 139 & $103(21)$ \\
\hline $\mathrm{Cr}$ & $\mathrm{Sc}$ & 0.358 & 139 & $59(17)$ \\
\hline $\mathrm{Mn}$ & $\mathrm{Sc}$ & 0.372 & 139 & $95(22)$ \\
\hline $\mathrm{Fe}$ & $\mathrm{Sc}$ & 50.5 & 139 & $102(17)$ \\
\hline Co & $\mathrm{Sc}$ & 0.070 & 118 & $91(33)$ \\
\hline $\mathrm{Ni}$ & $\mathrm{Y}$ & 0.268 & 137 & $96(34)$ \\
\hline $\mathrm{Cu}$ & $\mathrm{Y}$ & 0.129 & 139 & $93(17)$ \\
\hline $\mathrm{Zn}$ & $\mathrm{Y}$ & 0.340 & 139 & $97(19)$ \\
\hline $\mathrm{Ga}$ & $\mathrm{Y}$ & 0.031 & & \\
\hline As & $\mathrm{Y}$ & 0.039 & 139 & $89(15)$ \\
\hline $\mathrm{Se}$ & $\mathrm{Y}$ & 0.536 & 121 & $88(48)$ \\
\hline $\mathrm{Rb}$ & $\mathrm{Y}$ & 0.039 & 136 & $85(21)$ \\
\hline $\mathrm{Sr}$ & $\mathrm{Y}$ & 0.037 & & \\
\hline Mo & $\mathrm{Y}$ & 0.115 & & \\
\hline $\mathrm{Ag}$ & In & 0.060 & 86 & $85(35)$ \\
\hline $\mathrm{Cd}$ & In & 0.071 & 139 & $95(14)$ \\
\hline $\mathrm{Sb}$ & In & 0.092 & 129 & $87(22)$ \\
\hline Cs & In & 0.083 & 28 & $82(33)$ \\
\hline $\mathrm{Ba}$ & In & 0.312 & 139 & 105 (15) \\
\hline $\mathrm{Ce}$ & In & 0.101 & 69 & $97(32)$ \\
\hline $\mathrm{Tl}$ & $\mathrm{Bi}$ & 0.025 & & \\
\hline $\mathrm{Pb}$ & $\mathrm{Bi}$ & 0.032 & 130 & 95 (19) \\
\hline
\end{tabular}

For Ag, Ce, and Cs, a large fraction of the NIST samples were below detection because of significant reagent blanks. For all other elements, the NIST sample concentrations exceeded the reagent blanks by a factor of six or more except in two instances (Co and Rb). Certified concentrations were not given by NIST for some elements.

\subsection{Comparison of recoveries from the SRM with and without $\mathrm{HF}$}

To show that the addition of a trace amount of HF to the digestion solution improved recovery of the elements in NIST SRM samples, a batch of seven NIST samples, three with trace HF and four without HF, were prepared and analyzed according to the procedure described above. Table 4 presents the results of this comparison. The improved recoveries of the elements in the NIST SRM samples for $\mathrm{K}, \mathrm{Ti}, \mathrm{Cr}, \mathrm{Rb}$, $\mathrm{Sb}, \mathrm{Cs}$, and Ba were statistically significant with the addition of trace $\mathrm{HF}$ at the $P=0.05$ level of significance. The other elements showed recoveries that were not statistically different between the two sets of samples with the exception of $\mathrm{Mg}$. Recovery of Mg in these samples significantly decreased with the addition of $\mathrm{HF}$, but recoveries of $\mathrm{Mg}$ as reported in Table 2 are quite good. The reason for the low recovery of $\mathrm{Mg}$ in the three samples digested with HF presented in Table 4 as compared to the good recovery of $\mathrm{Mg}$ in the samples reported in Table 2 is unknown.

\subsection{Comparison of ambient PM analyzed by ICP-MS and $X R F$}

During July 2001 and January 2002, daily Teflon filter samples were collected at four satellite sites surrounding the main Pittsburgh sampling site, and these filters were analyzed by XRF by Research Triangle Institute, Triangle Park, NC. The main Pittsburgh sampling site, the Lawrenceville site, and the Hazelwood site are located within the Pittsburgh city limits. The rural Florence site is located $50 \mathrm{~km}$ west of Pittsburgh, which is typically upwind of the city, and the Greensburg site is located $55 \mathrm{~km}$ east of Pittsburgh, typically downwind. More information about the locations of the four satellite sites and details of sample collection are given in Tang et al. [15].

Ambient concentrations of many elements analyzed by XRF appear noisy as compared to the ICP-MS results for the main site due to the smaller air volumes sampled for XRF analysis and the higher detection limits of XRF as compared to ICP-MS. Nevertheless, several elements with high concentrations show reasonable agreement, namely $\mathrm{K}, \mathrm{Ti}, \mathrm{V}, \mathrm{Mn}, \mathrm{Fe}$, $\mathrm{Zn}, \mathrm{As}, \mathrm{Se}$, and $\mathrm{Pb}$. The correlation coefficient $\rho$ and the number of valid samples for comparison $n$ are shown in Table 5 . Note that sampling at the Pittsburgh main site began on July 11, 2001, while sampling at the four satellite sites was continuous beginning on June 30 until July 31, 2001. Based on a two-tailed $t$-test with $n-2$ degrees of freedom, the correlation coefficients were tested against the hypothesis that they were significantly greater than zero. The level of significance of the correlation is given in Table 5 as well. Figs. 2 and 3 
Table 3

A comparison of the concentrations measured on two different strips for samples collected in October 2001

\begin{tabular}{|c|c|c|c|c|}
\hline \multirow[t]{2}{*}{ Element } & \multicolumn{2}{|l|}{$\mathrm{PM}_{10}$} & \multicolumn{2}{|l|}{$\mathrm{PM}_{2.5}$} \\
\hline & $\begin{array}{l}\text { Number of samples above } \\
\text { detection ( } 31 \text { total samples) }\end{array}$ & Percent error & $\begin{array}{l}\text { Number of samples above } \\
\text { detection ( } 31 \text { total samples) }\end{array}$ & Percent error \\
\hline $\mathrm{Li}$ & 22 & 7.4 & 20 & 6.6 \\
\hline $\mathrm{Be}$ & 22 & 50.2 & 19 & 16.5 \\
\hline $\mathrm{Mg}$ & 31 & 13.1 & 31 & 8.7 \\
\hline K & 31 & 23.2 & 31 & 9.5 \\
\hline $\mathrm{Ca}$ & 31 & 14.7 & 30 & $14.1^{\mathrm{b}}$ \\
\hline $\mathrm{Ti}$ & 31 & 16.5 & 29 & 19.2 \\
\hline V & 31 & 25.8 & 29 & 7.9 \\
\hline $\mathrm{Cr}$ & 31 & 20.2 & 28 & 34.4 \\
\hline $\mathrm{Mn}$ & 30 & $28.0^{\mathrm{a}}$ & 30 & $21.7^{\mathrm{b}}$ \\
\hline $\mathrm{Fe}$ & 31 & 7.1 & 31 & 21.3 \\
\hline Co & 21 & $37.0^{\mathrm{a}}$ & 13 & 21.9 \\
\hline $\mathrm{Ni}$ & 26 & 12.6 & 22 & 21.8 \\
\hline $\mathrm{Cu}$ & 28 & 14.0 & 14 & 17.6 \\
\hline $\mathrm{Zn}$ & 31 & 15.9 & 26 & 9.4 \\
\hline $\mathrm{Ga}$ & 31 & 16.5 & 23 & 36.8 \\
\hline As & 30 & 9.3 & 30 & 8.3 \\
\hline $\mathrm{Se}$ & 31 & 26.1 & 31 & 9.6 \\
\hline $\mathrm{Rb}$ & 29 & 14.2 & 25 & 4.6 \\
\hline $\mathrm{Sr}$ & 31 & 4.9 & 26 & $19.9^{\mathrm{b}}$ \\
\hline Мo & 28 & 11.0 & 19 & 11.9 \\
\hline $\mathrm{Ag}$ & 15 & 26.1 & 14 & 32.1 \\
\hline $\mathrm{Cd}$ & 30 & $11.0^{\mathrm{a}}$ & 28 & $6.4^{\mathrm{b}}$ \\
\hline $\mathrm{Sb}$ & 30 & 16.6 & 26 & 35.9 \\
\hline Cs & 23 & 24.6 & 17 & 6.0 \\
\hline $\mathrm{Ba}$ & 31 & 9.1 & 31 & 29.0 \\
\hline $\mathrm{Ce}$ & 29 & 10.8 & 13 & 13.9 \\
\hline $\mathrm{Pb}$ & 31 & 12.1 & 30 & 5.6 \\
\hline
\end{tabular}

All Tl concentrations were below detection in October 2001.

${ }^{\text {a }}$ One outlier pair was removed from the full dataset for each of these elements (total 3 outlier pairs out of 769 pairs).

b One outlier pair was removed from the full dataset for each of these elements (total 4 outlier pairs out of 670 pairs).

Table 4

A comparison of samples digested with and without trace $\mathrm{HF}$

\begin{tabular}{|c|c|c|c|c|c|c|}
\hline & \multicolumn{2}{|c|}{ Samples digested without HF $(n=4)$} & \multicolumn{2}{|c|}{ Samples digested with HF $(n=3)$} & \multirow[t]{2}{*}{$t_{\mathrm{obs}}$} & \multirow[t]{2}{*}{$P$-value } \\
\hline & $\mu$ & $\sigma$ & $\mu$ & $\sigma$ & & \\
\hline $\mathrm{Mg}$ & 0.831 & 0.126 & 0.693 & 0.051 & -1.99 & 0.05 \\
\hline K & 0.517 & 0.055 & 0.716 & 0.052 & 4.87 & 0.003 \\
\hline $\mathrm{Ca}$ & 0.845 & 0.138 & 0.717 & 0.06 & -1.66 & 0.08 \\
\hline $\mathrm{Ti}$ & 0.408 & 0.062 & 0.605 & 0.093 & 3.17 & 0.012 \\
\hline V & 0.829 & 0.133 & 0.841 & 0.067 & 0.16 & 0.44 \\
\hline $\mathrm{Cr}$ & 0.421 & 0.018 & 0.626 & 0.038 & 8.66 & 0.0002 \\
\hline $\mathrm{Mn}$ & 0.864 & 0.153 & 0.788 & 0.067 & -0.89 & 0.21 \\
\hline $\mathrm{Fe}$ & 0.793 & 0.123 & 0.734 & 0.062 & -0.84 & 0.22 \\
\hline Co & 0.754 & 0.131 & 0.751 & 0.136 & -0.03 & 0.49 \\
\hline $\mathrm{Ni}$ & 0.781 & 0.116 & 0.724 & 0.099 & -0.69 & 0.26 \\
\hline $\mathrm{Cu}$ & 0.720 & 0.126 & 0.600 & 0.137 & -1.19 & 0.14 \\
\hline $\mathrm{Zn}$ & 0.689 & 0.108 & 0.903 & 0.610 & 0.60 & 0.29 \\
\hline As & 0.722 & 0.105 & 0.614 & 0.120 & -1.25 & 0.13 \\
\hline $\mathrm{Se}$ & 0.637 & 0.096 & 0.533 & 0.113 & -1.23 & 0.13 \\
\hline $\mathrm{Rb}$ & 0.411 & 0.052 & 0.590 & 0.118 & 2.45 & 0.029 \\
\hline $\mathrm{Ag}$ & 1.126 & 0.181 & 1.024 & 0.034 & -1.10 & 0.16 \\
\hline $\mathrm{Cd}$ & 1.010 & 0.124 & 0.910 & 0.042 & -1.50 & 0.10 \\
\hline $\mathrm{Sb}$ & 0.463 & 0.042 & 0.939 & 0.053 & 12.75 & 0.00003 \\
\hline Cs & 0.609 & 0.140 & 1.041 & 0.033 & 5.95 & 0.001 \\
\hline $\mathrm{Ba}$ & 0.849 & 0.128 & 1.003 & 0.048 & 2.20 & 0.04 \\
\hline $\mathrm{Pb}$ & 0.867 & 0.139 & 0.806 & 0.071 & -0.75 & 0.24 \\
\hline
\end{tabular}

Average recovery, $\mu$, is the measured concentration divided by the certified concentration and $\sigma$ is the standard deviation of the recoveries for each element. The test statistic $t_{\mathrm{obs}}$, based on a student's $t$-test, is given for each element and $P$-values are given for all elements. 
Table 5

Comparison of ICP-MS data from the Pittsburgh Supersite and XRF data from the Lawrenceville, Hazelwood, Greensburg, and Florence sites

\begin{tabular}{|c|c|c|c|c|c|c|c|c|c|}
\hline & K & $\mathrm{Ti}$ & $\mathrm{V}$ & $\mathrm{Mn}$ & $\mathrm{Fe}$ & $\mathrm{Zn}$ & As & $\mathrm{Se}$ & $\mathrm{Pb}$ \\
\hline \multicolumn{10}{|l|}{ July 2001} \\
\hline Pittsburgh-Lawrenceville & $0.65 / 20 / 1 \%$ & $0.70 / 20 / 1 \%$ & $0.69 / 20 / 1 \%$ & $0.16 / 20 / \mathrm{NC}$ & $0.46 / 20 / 5 \%$ & $0.46 / 19 / 5 \%$ & $-0.14 / 19 / \mathrm{NC}$ & $0.65 / 19 / 1 \%$ & $0.65 / 19 / 1 \%$ \\
\hline Pittsburgh-Hazelwood & $0.53 / 19 / 2 \%$ & $0.36 / 19 / \mathrm{NC}$ & $0.76 / 19 / 1 \%$ & $0.10 / 19 / \mathrm{NC}$ & $0.28 / 19 / \mathrm{NC}$ & $0.09 / 19 / \mathrm{NC}$ & $-0.22 / 19 / \mathrm{NC}$ & $0.49 / 19 / 5 \%$ & $0.73 / 19 / 1 \%$ \\
\hline Pittsburgh-Greensburg & $0.77 / 20 / 1 \%$ & $0.35 / 20 / \mathrm{NC}$ & $0.45 / 20 / 5 \%$ & $0.16 / 20 / \mathrm{NC}$ & $0.48 / 20 / 5 \%$ & $-0.10 / 19 / \mathrm{NC}$ & $-0.04 / 19 / \mathrm{NC}$ & $0.47 / 19 / 5 \%$ & $0.35 / 19 / \mathrm{NC}$ \\
\hline Pittsburgh-Florence & $0.63 / 20 / 1 \%$ & $0.35 / 20 / \mathrm{NC}$ & $0.48 / 20 / 5 \%$ & $0.05 / 20 / \mathrm{NC}$ & $0.40 / 20 / 10 \%$ & $-0.06 / 19 / \mathrm{NC}$ & $0.32 / 19 / \mathrm{NC}$ & $0.05 / 19 / \mathrm{NC}$ & $0.64 / 19 / 1 \%$ \\
\hline Lawrenceville-Hazelwood & $0.97 / 30 / 1 \%$ & $0.73 / 30 / 1 \%$ & $0.67 / 30 / 1 \%$ & $-0.04 / 30 / \mathrm{NC}$ & $0.11 / 30 / \mathrm{NC}$ & $0.24 / 30 / \mathrm{NC}$ & $-0.32 / 30 / \mathrm{NC}$ & $0.70 / 30 / 1 \%$ & $0.42 / 30 / 2 \%$ \\
\hline Lawrenceville-Greensburg & $0.93 / 31 / 1 \%$ & $0.59 / 31 / 1 \%$ & $0.70 / 31 / 1 \%$ & $0.00 / 31 / \mathrm{NC}$ & $0.73 / 31 / 1 \%$ & $0.06 / 31 / \mathrm{NC}$ & $0.23 / 31 / \mathrm{NC}$ & $0.47 / 31 / 1 \%$ & $0.51 / 31 / 1 \%$ \\
\hline Lawrenceville-Florence & $0.78 / 31 / 1 \%$ & $0.26 / 31 / \mathrm{NC}$ & $0.09 / 31 / \mathrm{NC}$ & $0.47 / 31 / 1 \%$ & $0.42 / 31 / 2 \%$ & $0.22 / 31 / \mathrm{NC}$ & $-0.35 / 31 / \mathrm{NC}$ & $-0.08 / 31 / \mathrm{NC}$ & $0.44 / 31 / 2 \%$ \\
\hline Hazelwood-Greensburg & $0.87 / 30 / 1 \%$ & $0.60 / 30 / 1 \%$ & $0.10 / 30 / \mathrm{NC}$ & $-0.18 / 30 / \mathrm{NC}$ & $0.00 / 30 / \mathrm{NC}$ & $0.07 / 30 / \mathrm{NC}$ & $0.09 / 30 / \mathrm{NC}$ & $0.08 / 30 / \mathrm{NC}$ & $-0.12 / 30 / \mathrm{NC}$ \\
\hline Hazelwood-Florence & $0.67 / 30 / 1 \%$ & $0.35 / 30 / 10 \%$ & $0.26 / 30 / \mathrm{NC}$ & $-0.20 / 30 / \mathrm{NC}$ & $-0.10 / 30 / \mathrm{NC}$ & $-0.07 / 30 / \mathrm{NC}$ & $-0.06 / 30 / \mathrm{NC}$ & $-0.04 / 30 / \mathrm{NC}$ & $0.27 / 30 / \mathrm{NC}$ \\
\hline Greensburg-Florence & $0.87 / 31 / 1 \%$ & $0.37 / 31 / 5 \%$ & $0.18 / 31 / \mathrm{NC}$ & $0.14 / 31 / \mathrm{NC}$ & $0.37 / 31 / 5 \%$ & $0.23 / 31 / \mathrm{NC}$ & $0.01 / 31 / \mathrm{NC}$ & $-0.13 / 31 / \mathrm{NC}$ & $0.44 / 31 / 2 \%$ \\
\hline \multicolumn{10}{|l|}{ January 2002} \\
\hline Pittsburgh-Lawrenceville & $0.66 / 18 / 1 \%$ & $0.10 / 18 / \mathrm{NC}$ & $0.03 / 18 / \mathrm{NC}$ & $0.91 / 18 / 1 \%$ & $0.92 / 18 / 1 \%$ & $0.86 / 18 / 1 \%$ & $0.73 / 18 / 1 \%$ & $0.86 / 18 / 1 \%$ & $0.47 / 18 / 5 \%$ \\
\hline Pittsburgh-Hazelwood & $0.79 / 18 / 1 \%$ & $0.31 / 18 / \mathrm{NC}$ & $0.19 / 18 / \mathrm{NC}$ & $0.55 / 18 / 2 \%$ & $0.89 / 18 / 1 \%$ & $0.95 / 18 / 1 \%$ & $0.30 / 18 / \mathrm{NC}$ & $0.93 / 18 / 1 \%$ & $0.60 / 18 / 1 \%$ \\
\hline Pittsburgh-Greensburg & $0.79 / 21 / 1 \%$ & $0.53 / 21 / 2 \%$ & $0.09 / 21 / \mathrm{NC}$ & $0.73 / 20 / 1 \%$ & $0.83 / 20 / 1 \%$ & $0.89 / 21 / 1 \%$ & $0.11 / 21 / \mathrm{NC}$ & $-0.10 / 21 / \mathrm{NC}$ & $0.81 / 21 / 1 \%$ \\
\hline Pittsburgh-Florence & $0.57 / 19 / 2 \%$ & $0.23 / 19 / \mathrm{NC}$ & $0.08 / 19 / \mathrm{NC}$ & $-0.31 / 19 / \mathrm{NC}$ & $0.10 / 19 / \mathrm{NC}$ & $0.16 / 19 / \mathrm{NC}$ & $0.60 / 19 / 1 \%$ & $-0.07 / 19 / \mathrm{NC}$ & $0.52 / 19 / 5 \%$ \\
\hline Lawrenceville-Hazelwood & $0.82 / 16 / 1 \%$ & $-0.29 / 16 / \mathrm{NC}$ & $0.09 / 16 / \mathrm{NC}$ & $0.72 / 16 / 1 \%$ & $0.90 / 16 / 1 \%$ & $0.91 / 16 / 1 \%$ & $0.32 / 16 / \mathrm{NC}$ & $0.87 / 16 / 1 \%$ & $0.43 / 16 / 10 \%$ \\
\hline Lawrenceville-Greensburg & $0.74 / 18 / 1 \%$ & $0.43 / 18 / 10 \%$ & $0.20 / 18 / \mathrm{NC}$ & $0.70 / 18 / 1 \%$ & $0.88 / 18 / 1 \%$ & $0.92 / 18 / 1 \%$ & $0.32 / 18 / 10 \%$ & $0.00 / 18 / \mathrm{NC}$ & $0.46 / 18 / 10 \%$ \\
\hline Lawrenceville-Florence & $0.63 / 18 / 1 \%$ & $0.21 / 18 / \mathrm{NC}$ & $0.35 / 18 / 10 \%$ & $-0.22 / 18 / \mathrm{NC}$ & $0.02 / 18 / \mathrm{NC}$ & $0.22 / 18 / \mathrm{NC}$ & $0.67 / 18 / 1 \%$ & $0.01 / 18 / \mathrm{NC}$ & $0.48 / 18 / 5 \%$ \\
\hline Hazelwood-Greensburg & $0.97 / 16 / 1 \%$ & $0.31 / 16 / \mathrm{NC}$ & $-0.22 / 16 / \mathrm{NC}$ & $0.36 / 16 / 10 \%$ & $0.86 / 16 / 1 \%$ & $0.93 / 16 / 1 \%$ & $0.47 / 16 / 10 \%$ & $-0.03 / 16 / \mathrm{NC}$ & $0.66 / 16 / 1 \%$ \\
\hline Hazelwood-Florence & $0.62 / 16 / 1 \%$ & $0.25 / 16 / \mathrm{NC}$ & $-0.39 / 16 / \mathrm{NC}^{\mathrm{a}}$ & $-0.21 / 16 / \mathrm{NC}$ & $0.02 / 16 / \mathrm{NC}$ & $0.18 / 16 / \mathrm{NC}$ & $0.66 / 16 / 1 \%$ & $-0.23 / 16 / \mathrm{NC}$ & $0.25 / 16 / \mathrm{NC}$ \\
\hline Greensburg-Florence & $0.66 / 19 / 1 \%$ & $0.40 / 19 / 10 \%$ & $0.39 / 19 / 10 \%$ & $-0.06 / 19 / \mathrm{NC}$ & $0.09 / 19 / \mathrm{NC}$ & $0.32 / 19 / 10 \%$ & $0.60 / 19 / 1 \%$ & $-0.08 / 19 / \mathrm{NC}$ & $0.34 / 19 / 10 \%$ \\
\hline
\end{tabular}

Given in each cell are: correlation coefficient, $\rho$; number of valid datapoints to compare, $n$; level of significance of correlation, tested at the $1 \%, 2 \%, 5 \%$, and $10 \%$ level. Correlations not significant at the $10 \%$ level or better are listed as not correlated, NC.

${ }^{a}$ Correlation is negative at the $10 \%$ significance level. 


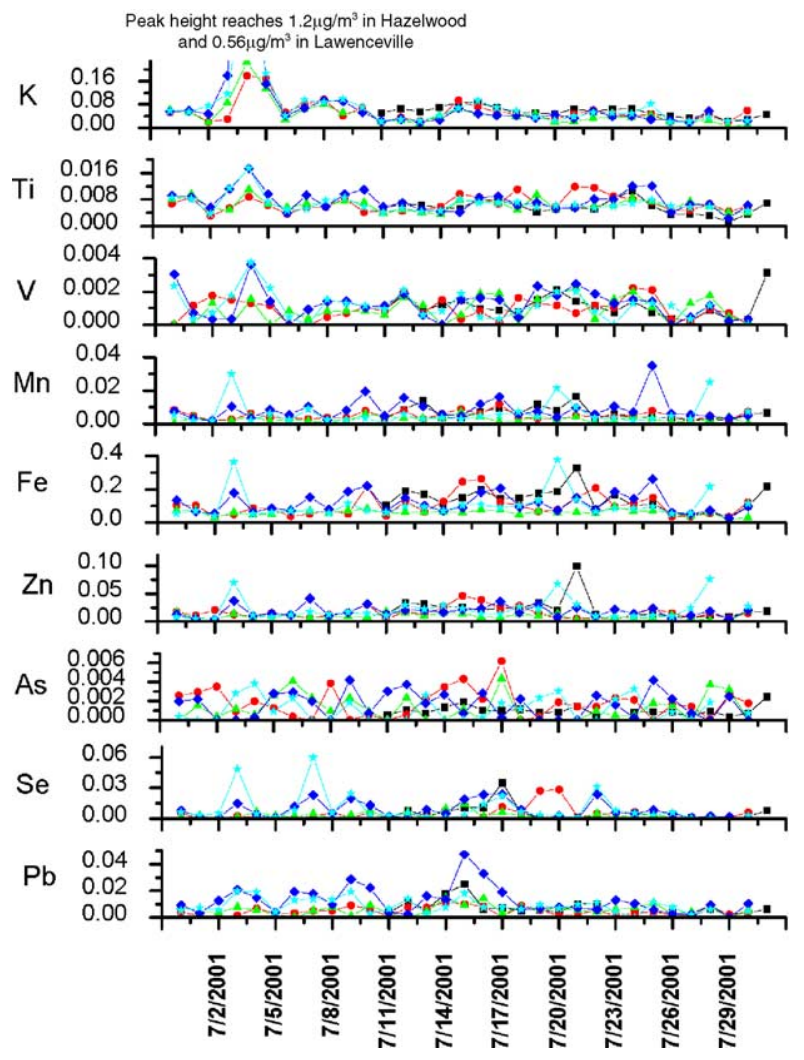

Fig. 2. Airborne concentrations during June 30-July 31, 2001 for the Pittsburgh Supersite and XRF data for four satellite sites in the Pittsburgh area.

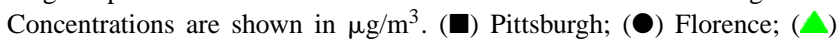
Greensburg; ( $)$ Lawrenceville; (*) Hazelwood.

show time series graphs of these elements for the two months.

Potassium correlation coefficients are high among all sites for both months. The large peak in early July 2001 is likely due to Fourth of July fireworks, as most fireworks contain potassium salts for ignition. Titanium concentrations are significantly correlated among the sites for July, but there is more scatter in the January XRF data and not as many of the site pairs are correlated. Concentrations of titanium are slightly higher on average during July as compared to January, so poorer correlations in January may be due to decreased detection capability of the XRF.

Most site-to-site correlations of vanadium concentrations are insignificant in January. There is more day-to-day variability in the concentration data in July, and correlations are in general higher.

Concentrations of manganese, iron, and zinc tend to be higher at the city sites, suggesting that there are significant sources of these elements within the city. Manganese and zinc concentrations show poor correlations between the sties in July. Correlations of iron data for July are significant for many of the site pairs. In January, the rural Florence site data do not correlate well with any other site for manganese, iron, and zinc, but the other sites are significantly correlated.

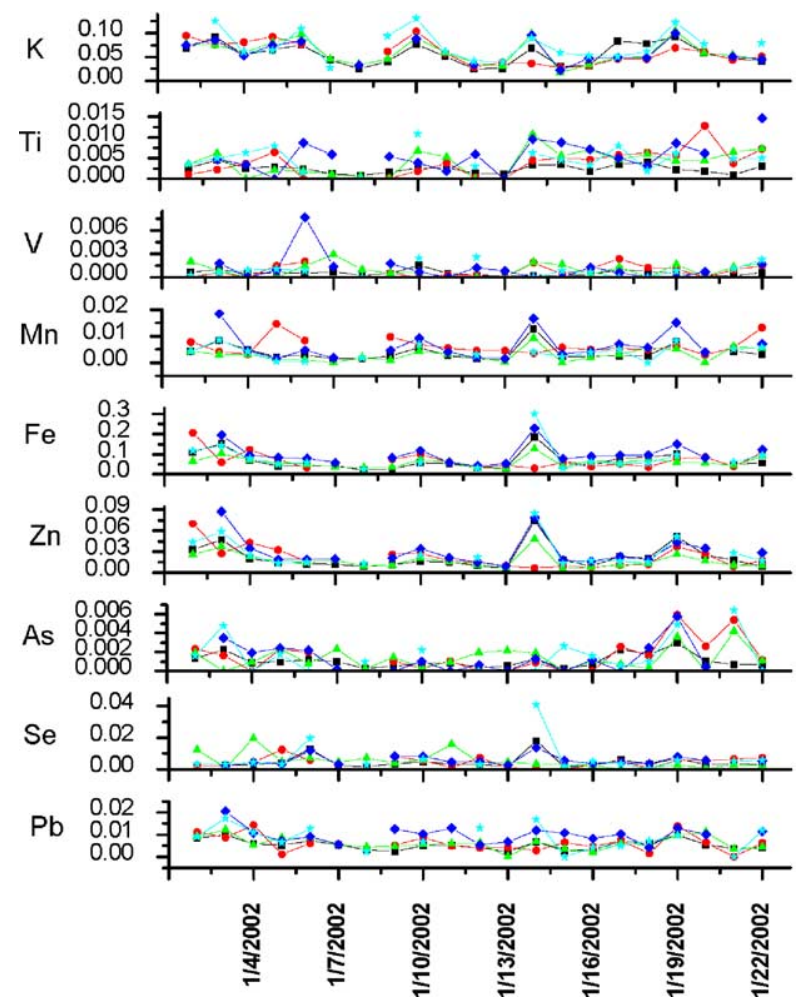

Fig. 3. Airborne concentrations during January 2-22, 2002 at the same sites as in Fig. 2. Concentrations are shown in $\mu \mathrm{g} / \mathrm{m}$. ( $\mathbf{\square})$ Pittsburgh; ( $)$ Florence;

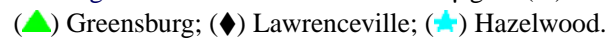

Arsenic shows reasonable agreement among all sites in January with most site-to-site correlations being significant, but there is more scatter in the XRF data in July where none of the site pairs correlate significantly. Concentrations on average are approximately equal for the two months. Selenium concentrations show some significant correlations but also several poorly correlated site pairs, especially in January. Concentrations of arsenic and selenium are relatively low in $\mathrm{PM}_{2.5}$, making these elements more difficult to detect by XRF.

Lead data show most sites significantly correlated for both months with the concentrations at the Lawrenceville site somewhat elevated on some dates. It is possible that there is a local source of lead near this site.

Overall, there are many significant correlations between the Supersite and the satellite site data for July 2001 and January 2002. Instances of uncorrelated data could be due to differences in emissions at the five sites, not just analytical differences. The method developed here for measurement of trace element concentrations in ambient PM and analysis by ICP-MS compares reasonably well with non-destructive XRF analysis using different filter media.

\section{Conclusions}

The method described in this paper for digestion and analysis of trace elements in ambient aerosol samples is suitable 
for routine analysis of the large number of samples commonly generated in air quality monitoring studies. Sample preparation by microwave digestion minimizes contamination and allows for digestion of silicate materials with the use of nitric/hydrofluoric acid blend and hydrogen peroxide solution. Results suggest that only a trace amount of HF is required for essentially complete digestion. Recovery of the NIST SRM 1648 Urban Dust is acceptable using this method, with recoveries within $15 \%$ of certified values for all analyzed elements except $\mathrm{Na}, \mathrm{Al}, \mathrm{Cr}$, and $\mathrm{Cs}$. Comparison of element concentrations measured on two or three different strips from the same filter allows a quantitative examination of uncertainty, which was within $20 \%$ for $\mathrm{PM}_{10}$ and $\mathrm{PM}_{2.5}$ for nearly all elements. The method developed here for digestion of cellulose filter samples and analysis by ICP-MS shows results that compare well with non-destructive XRF analysis of Teflon filters exposed simultaneously to ambient PM at other sites in the Pittsburgh area.

\section{Acknowledgments}

This research was conducted as part of the Pittsburgh Air Quality Study, which was supported by US Environmental Protection Agency under contract R82806101 and the US Department of Energy National Energy Technology Laboratory under contract DE-FC26-01NT41017. Support was also provided by the US National Science Foundation under grant BES-9714162. This paper has not been subject to EPA's peer and policy review, and therefore does not necessarily reflect the views of the Agency. No official endorsement should be inferred.

We would like to thank Dr. H.M. "Skip" Kingston and his group at Duquesne University for guidance in microwave digestion method development and instruction and use of their ICP-MS. The analyses provided by R.K.M. Jayanty at Research Triangle Institute are greatly appreciated. We would also like to thank Carnegie Mellon University students and researchers Dr. Andrey Khlystov, Dr. Wei Tang, Satoshi Taka- hama, Lenny Lucas, Sam Byun, Emily Lauffer, Lin Tian, Nick McCullar, Lisa Branden, David Carothers, Meredith Fry, Emma Goodson, Mackenzie Dilts, Serge LiHoiFooGregory, Kseniya Shabanova, Gustavo Fimbres, Mithun Shenoi, Dimitris Vayenas, and Elizabeth Higgins for their assistance in collecting and preparing the samples.

\section{References}

[1] L.M. Jalkanen, E.K. Hasanen, J. Anal. At. Spectrom. 11 (1996) 365-369.

[2] K.X. Yang, K. Swami, L. Husain, Spectrochim. Acta Part B 57 (2002) 73-84.

[3] E.A. Wittig, N.J. Anderson, A. Khlystov, S.N. Pandis, C.I. Davidson, A.L. Robinson, Atmos. Environ. 38 (2004) 3107-3125.

[4] B.Y.H. Liu, K.L. Rubow, in: V.A. Marple, B.Y.H. Liu (Eds.), Aerosols in the Mining and Industrial Work Environments, vol. 3, Ann Arbor Science Publishers, Ann Arbor, Michigan, 1983.

[5] Whatman Inc., Whatman PM2.5 Filters Statement of Conformance, http://www.whatman.com, accessed July 2004.

[6] U.S. Environmental Protection Agency, Microwave Assisted Acid Digestion of Siliceous and Organically Based Matrices, http://www. epa.gov/epaoswer/hazwaste/test/pdfs/3052.pdf.

[7] S. Rees, A.L. Robinson, A. Khlystov, C.O. Stanier, S.N. Pandis, Atmos. Environ. 38 (2004) 3305-3318.

[8] H.M. Kingston, S. Haswell (Eds.), Microwave Enhanced Chemistry, ACS Professional Reference Series, ACS, Washington, DC, 1997.

[9] U.S. Environmental Protection Agency. Determination of Trace Elements in Waters and Wastes by Inductively Coupled Plasma-Mass Spectrometry, revision 4.4, EPA-600-R-94-111, April 1991.

[10] CEM Corp., Technical Note 003, Temperature-Controlled Microwave Concentration Using Vacuum-Assisted Evaporation, CEM Corporation, Matthews, NC, www.cem.com, accessed Fall 2003.

[11] S. Wu, Y. Zhao, X. Feng, A. Wittmeier, J. Anal. At. Spectrom. 11 (1996) 287-296.

[12] K. Swami, C. Judd, J. Orsini, K. Yang, L. Husain, Fresenius J. Anal. Chem. 369 (2001) 63-70.

[13] T. Yamashige, M. Yamamoto, H. Sunahara, Analyst 114 (1989) 1071.

[14] N.J. Pekney, C.I. Davidson, A.L. Robinson, L. Zhou, P.K. Hopke, D. Eatough, Aerosol Sci. Technol., submitted for publication.

[15] W. Tang, T. Raymond, E.A. Wittig, C.I. Davidson, S.N. Pandis, A.L. Robinson, K. Crist, Aerosol Sci. Technol. 38 (Suppl. 2) (2004) 80-90. 


\title{
Application of the Pseudo-Deterministic Receptor Model to Resolve Power Plant Influences on Air Quality in Pittsburgh
}

\author{
Seung Shik Park, ${ }^{1,2}$ J. Patrick Pancras, ${ }^{1}$ John M. Ondov, ${ }^{1}$ and Allen Robinson ${ }^{3}$ \\ ${ }^{1}$ Chemistry and Biochemistry, University of Maryland, College Park, Maryland, USA \\ ${ }^{2}$ Department of Environmental Engineering, Chonnam National University, Buk-ku, Gwangju 500-757, \\ Republic of Korea \\ ${ }^{3}$ Mechanical Engineering, Carnegie Mellon University, Pittsburgh, Pennsylvania, USA
}

\begin{abstract}
A multivariate pseudo-deterministic receptor model was applied to determine emission and ambient source contributions rates of $\mathrm{SO}_{2}$ and elements from four small coal-fired boilers influencing air quality at the Carnegie Mellon University (CMU) Supersite. The model was applied to ambient $\mathrm{SO}_{2}$ and particle measurements, the latter, made every 30-min for 10 elements ( $\mathrm{Al}, \mathrm{As}, \mathrm{Cr}, \mathrm{Cu}, \mathrm{Fe}$, $\mathrm{Mn}, \mathrm{Ni}, \mathrm{Pb}$, Se, and $\mathrm{Zn}$ ) during a 12.5-h period on April 1, when winds blew from between $290-330^{\circ}$ in which the four coal boilers are situated. Agreement between predicted and observed $\mathrm{SO}_{2}$ concentrations was excellent ( $\mathrm{r}$ of 0.92 ; and their ratio, $1.09 \pm 0.22$ ) when 4 emission sources were used in the model. Average ratios of predicted and observed concentrations for $\mathrm{As}, \mathrm{Cr}, \mathrm{Cu}, \mathrm{Ni}, \mathrm{Pb}, \mathrm{Se}$, and $\mathrm{Zn}$ varied from $0.97 \pm \mathbf{0 . 2 0}$ for $\mathrm{Cr}$ to $1.07 \pm \mathbf{0 . 4 4}$ for As. Performance indices for these elements were all well within acceptable ranges. Emission rate ratios of various metal species to Se predicted are similar for the three of the coal boilers, but differed substantially for the fourth, as expected for a boiler with minimal particle control technology. All are within the range derived from previous PDRM results and in-stack measurements (except $\mathrm{Al}$ ) at 7 Eastern U.S. coal-fired power plants. The results suggest that the PDRM approach is applicable to a city encompassing complex topography and may successfully be applied using commonly available meteorological data.
\end{abstract}

\section{INTRODUCTION}

Source apportionment, that is quantitative determination of the contributions of pollutants from their sources to ambient at-

Received 29 April 2005; accepted 18 April 2006.

This work was funded in part by United States Environmental Protection Agency under contract R82806101 as part of the Pittsburgh Air Quality Study (PAQS), and in part by the United States Environmental Protection Agency through grant/cooperative agreement (BSS R82806301) to the University of Maryland, College Park (UMCP). Nevertheless it has not been subjected to the Agency's required peer and policy review and therefore does not necessarily reflect the views of the Agency and no official endorsement should be inferred.

Address correspondence to John M. Ondov, Department of Chemistry and Biochemistry, University of Maryland, College Park, MD 20742, USA. E-mail: jondov@umd.edu mospheric levels, is necessary for developing emission control strategies that effectively reduce exposures and health risks, and prevent degradation of air quality (Gordon 1988). Source apportionment may be accomplished with either source- or receptorbased models, however, applications of the former are often limited because of the lack of emission rate data. In the latter, source contributions are determined using observations at sampling sites. Until recently, most of the data obtained for receptor modeling has been derived from samples collected over time scales far longer than those for changes in source strengths and important meteorological parameters, e.g., wind direction and mixing height. The accompanying homogenization of source signals by this practice severely reduces the resolving power achievable with factor analytical methods (Lioy et al. 1989). Hourly resolved elemental data were used to develop a source profile for motor vehicles in the Baltimore Harbor Tunnel (Ondov et al. 1982). At 2-h resolution, Rheingrover and Gordon (1988) demonstrated that plumes of stationary sources in St. Louis are readily observed as excursions in time series profiles of the concentrations of the various marker elements and that the excursions could be correlated with wind direction to identify sources. More recently, Kidwell and Ondov (2001, 2004) developed a system for measuring elemental constituents at 30min intervals and were able to identify influences of individual sources at this improved resolution (Ondov et al. 2003). It is now well demonstrated that wind directions corresponding to concentration peaks are consistent with the locations of known sources when wind direction is relatively constant during the time required for plume transport. Additionally, information on source distances can be inferred from the width of their plumes (US EPA 1980).

To better exploit the directionality and plume dispersion information inherent in highly time-resolved ambient data, Yamartino (1982) proposed a mass-balance model, wherein ambient concentrations were expressed as the products of source emission and plume dilution rates derived deterministically using Gaussian plume dispersion equations for individual sources 
of $\mathrm{SO}_{2}$. Cooper (1982) reported a similar approach to ambient aerosol particle mass data. However, these attempts met with limited success, owing largely due to the fundamental inaccuracies in the Gaussian plume model. More recently, Park et al. (2005) devised a multivariate pseudo-deterministic receptor model (PDRM), in which a Gaussian plume model is used to constrain the solutions to the basic mass-balance model, rather than applying it in a deterministic manner. Unlike traditional receptor models, the PDRM (as well as those by Yamartino 1982 and Cooper 1982) exploits knowledge of the number and locations of major stationary sources, source and transport wind directions and distances, stack gas emission parameters, and meteorological plume dispersion during sample collections. Furthermore, emission rates are predicted for specific, individual sources, rather than generic source categories. The PDRM was applied with good success to $30-\min \mathrm{SO}_{2}$ and aerosol metals data collected at a site in Tampa, Florida, during a period of moderate winds and good mixing, using micrometeorological parameters derived from $10-\mathrm{Hz} u, v, w$ measurements made at the site. The Tampa modeling domain is relatively flat, and encompassed 6 sources, including 4 utility power plants and 2 much smaller industrial sources, lying in a $90^{\circ}$ sector at distances ranging from 15 to $41 \mathrm{~km}$ from the air monitoring site. Sulfur dioxide emission rates predicted by the model were in excellent agreement with emission rates derived from continuous emission monitors (CEMs) available for the 4 power plants and ambient concentration versus time profiles were well fit for both $\mathrm{SO}_{2}$ and the major marker species (especially Se for coal-boilers and $\mathrm{Ni}$ for oil).

Herein, we describe the application of the PDRM to an otherwise similar data set collected in Pittsburgh, PA, an area characterized by rough Appalachian Mountain terrain, during the Pittsburgh Air Quality Study (PAQS), and for which only standard meteorological data were available.

\section{EXPERIMENTAL DATA}

\subsection{Description of Measurement Site and Modeling Period}

The sampling site (312.4 m ASL, Figure 1) was located in Schenley Park on the top of a hill adjacent to the Carnegie Mellon University campus, approximately $6 \mathrm{~km}$ east of downtown Pittsburgh. There are roughly two million people living in the Pittsburgh Metropolitan Statistical Area, and elderly people represent a significant fraction of the population. The area is located between the utilities and agricultural sources of the Midwest and the large urban centers of the East. The site is more than several hundred meters from any major source of air pollution, and roughly one kilometer of parkland exists between the site and the city in the predominant upwind direction (south and west). Sampling equipment was housed in a $33 \mathrm{~m}^{2}$ air pollution monitoring station. Our modeling was performed on ambient data collected between 06:00 and 18:30 h on April 1, 2002, during which time wind angles (measured from true north) ranged from $290-330^{\circ}$ and 30-min averaged wind speeds ranged from 2.5 to $5.2 \mathrm{~m} \mathrm{sec}^{-1}$ (Figure 2) with maximum ranges $5.0-8.2 \mathrm{~m} \mathrm{sec}^{-1}$. This sector contains two small-scale coal-fired boilers within $45 \mathrm{~km}$ from the site, which are used for supplying process steam, specifically, the Bellefield boiler plant and Pittsburgh brewing plant; and two industrial sources (Shenango coke works and Zinc Corp. Amer). Source-receptor distances and station angles (measured at Schenley Park from due North), and annual emissions data for $\mathrm{PM}_{2.5}$ and $\mathrm{SO}_{2}$ (NEI 1999 annual emission inventory data) are listed in Table 1. As indicated, the Bellefield boiler plant is located at an angle of $286^{\circ}$ and $0.8 \mathrm{~km}$ away from the site. The Bellefield boiler plant provides steam heat to the hospital complex, museums and universities in the Oakland district of Pittsburgh, and burns bituminous/sub-bituminous coal to produce steam in an over- and under-feed stoker boiler with a cyclone separator to control particle emissions. The Pittsburgh brewing coal-fired boiler (station angle, $316^{\circ}$; distance, 3.4 $\mathrm{km}$ ) uses bituminous/sub-bituminous coal in an overfeed stoker boiler to produce process steam. The Shenango coke works, located on Neville Island on the Ohio River (station angle of $297^{\circ}$; distance $13.0 \mathrm{~km}$ ) operates a coke battery, a by-product facility, and a steam and power plant, and produces approximately 350,000 tons of blast furnace coke and related by-products a year. Zinc Corporation of America (ZCA) operates its multiproduct zinc manufacturing plant. The ZCA is the largest zinc producer (zinc oxide, zinc metal, zinc dust) in the USA, and the plant site, powered by its own 110-MW power station, is located $41.9 \mathrm{~km}$ northwest of the site along the Ohio River and at an angle of $307^{\circ}$.

\subsection{Meteorological Measurements}

Ten-minute averaged surface wind speed/direction, temperature, relative humidity, solar radiation, and pressure were recorded during the PAQS by Carnegie Mellon University (CMU). 30-min averages of the wind speed and direction measurements made during the study period on April 1, 2002 are shown in Figure 2. During the modeling period, the temperature and relative humidity ranged from $3.4-10.1^{\circ} \mathrm{C}$ and $34-93 \%$, respectively, with moderate winds between $2.5-5.5 \mathrm{~m} / \mathrm{sec}$ over a narrow range of wind directions $\left(280-330^{\circ}\right)$. On that day the mixing height remained almost constant (1960-2010 m) through the day because of the persistence of a strong inversion. Sunrise was at 6:03 eastern standard time (EST), and sunset was at 18:44 EST. Light precipitation $(0.1 \mathrm{~cm})$ was recorded across the study area between 02:00 and 05:00 $\mathrm{h}$.

\section{3. $\mathrm{SO}_{2}$ and Elemental Measurements}

2.3.1. $\mathrm{SO}_{2}$ Data

Ambient $\mathrm{SO}_{2}$ mixing rations (ppb) were measured with a pulsed fluorescence analyzer (API 100A model) at 10-minute intervals during the study period. These were converted to $\mu \mathrm{g} \mathrm{m}^{-3}$ using ambient temperature and pressure data and used to construct 30-minute averages for use in the model. Examination of 


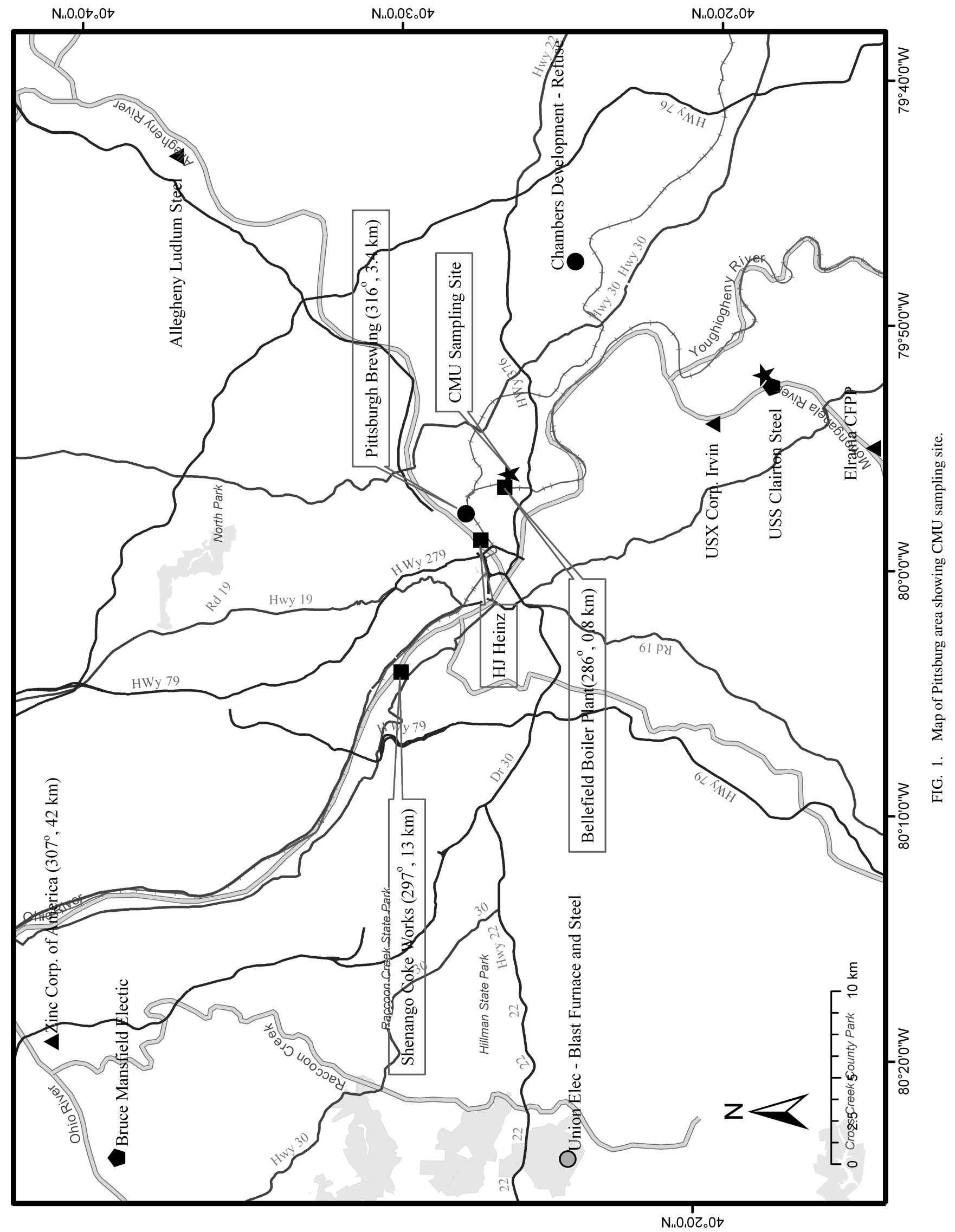




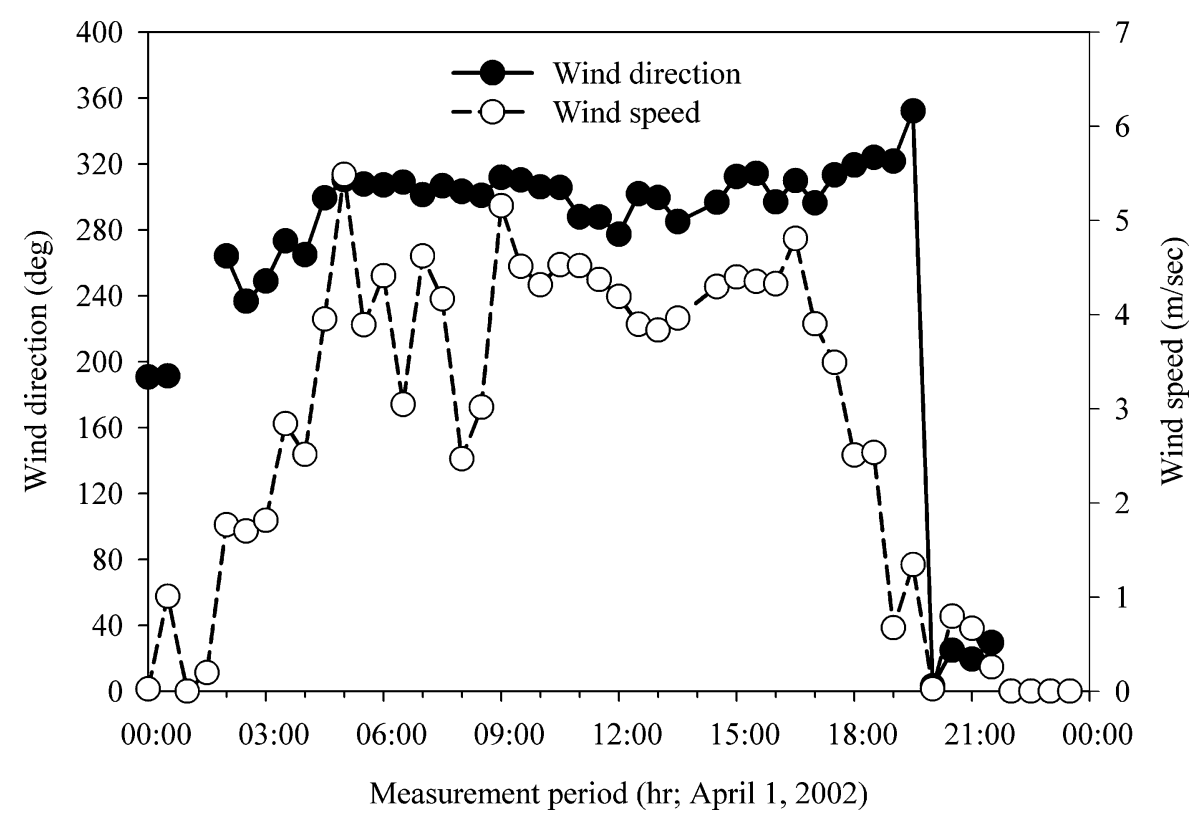

FIG. 2. Wind speed and direction during the modeling period on April 1, 2002.

the $\mathrm{SO}_{2}$ data reveals that six different excursions (04:30-06:00, 07:30, 09:00-11:00, 12:00-13:00, 14:00-15:30, and 17:00 h) were observed during the study period (see Figure 3 ). The highest 30-min average $\mathrm{SO}_{2}$ concentration, $75.7 \mathrm{ppb}\left(205.0 \mu \mathrm{g} \mathrm{m}^{-3}\right)$, was observed at 09:30 on April 1.

\subsection{2. $\quad P M_{2.5}$ Sampling and Elemental Analyses}

Approximately 800 ambient aerosol samples were collected continuously at 30-min intervals at the Pittsburgh CMU Supersite between March 31 and April 17, 2002 using the University of Maryland Semi-continuous Elements in Aerosol Sampler (SEAS). The SEAS employs a state-of-the art dynamic aerosol concentrator to extract particles as small as $80 \mathrm{~nm}$ after steaminjection and subsequent cooling to induce particles to grow to a size $>0.7 \mu \mathrm{m}$. The resulting droplets are collected in a real impactor and then transferred to a fraction collector for stor- age in clean vials, every $30 \mathrm{~min}$, providing 48 samples a day. Within $24 \mathrm{~h}$ of collection, the samples were capped and transferred to storage at $\mathrm{CMU}$ at $-14^{\circ} \mathrm{C}$. Detailed descriptions of the SEAS sampler have been presented elsewhere (Kidwell and Ondov 2001, 2004). A subset of these, including 28 of those collected for the April 1, 2002 study period were shipped to the University of Maryland at dry-ice temperature and analyzed in triplicate for 11 elements ( $\mathrm{Al}, \mathrm{As}, \mathrm{Cd}, \mathrm{Cr}, \mathrm{Cu}, \mathrm{Fe}, \mathrm{Mn}, \mathrm{Ni}, \mathrm{Pb}$, $\mathrm{Se}$, and $\mathrm{Zn}$ ) by simultaneous multielement Graphite Furnace Atomic Absorption Spectroscopy (GFAAS) with Zeeman background correction (SIMMA 6000, Perkin Elmar Corp., Danbury, CT) by methods developed by Pancras et al. (2005). The coefficient of variation for the three replicate measurements was $<7 \%$, except for $\mathrm{Ni}, \mathrm{Al}$ and $\mathrm{Cr}$ for which it was generally $\leq 10 \%$. From 91 to $100 \%$ of all values measured were above their MDLs in samples comprising the study period. Except $\mathrm{Ni}$

TABLE 1

Emission information for stationary sources

\begin{tabular}{|c|c|c|c|c|c|c|c|}
\hline Facility name & $\begin{array}{l}\text { Control } \\
\text { devices }\end{array}$ & $\begin{array}{l}\text { Distance } \\
\quad(\mathrm{km})\end{array}$ & $\begin{array}{l}\text { Station } \\
\text { angle } \\
\text { (deg) }\end{array}$ & $\begin{array}{c}\mathrm{PM}_{2.5} \\
\text { (ton } \mathrm{yr}^{-1} \text { ) }\end{array}$ & $\begin{array}{c}\mathrm{SO}_{2} \\
\text { (ton } \mathrm{yr}^{-1} \text { ) }\end{array}$ & Industry type & Category \\
\hline Bellefield boiler plant & Cyclone & 0.8 & 286 & 94 & 745 & Steam supply & Coal fired steam \\
\hline Pittsburgh brewing co. & - & 3.4 & 316 & 9 & 106 & $\begin{array}{l}\text { Food, agricultural, } \\
\text { and beer }\end{array}$ & Coal fired steam \\
\hline Shenango Coke Works & Fabric filter & 13.0 & 297 & 79 & 2450 & $\begin{array}{l}\text { Blast furnace and } \\
\text { steel mill }\end{array}$ & Coke \\
\hline Zinc Corp of America & - & 41.9 & 307 & 678 & 8641 & $\begin{array}{l}\text { Coal-fired boiler/ } \\
\text { industrial } \\
\text { processes }\end{array}$ & $\begin{array}{l}\text { Primary non-ferrous } \\
\text { metals }\end{array}$ \\
\hline
\end{tabular}




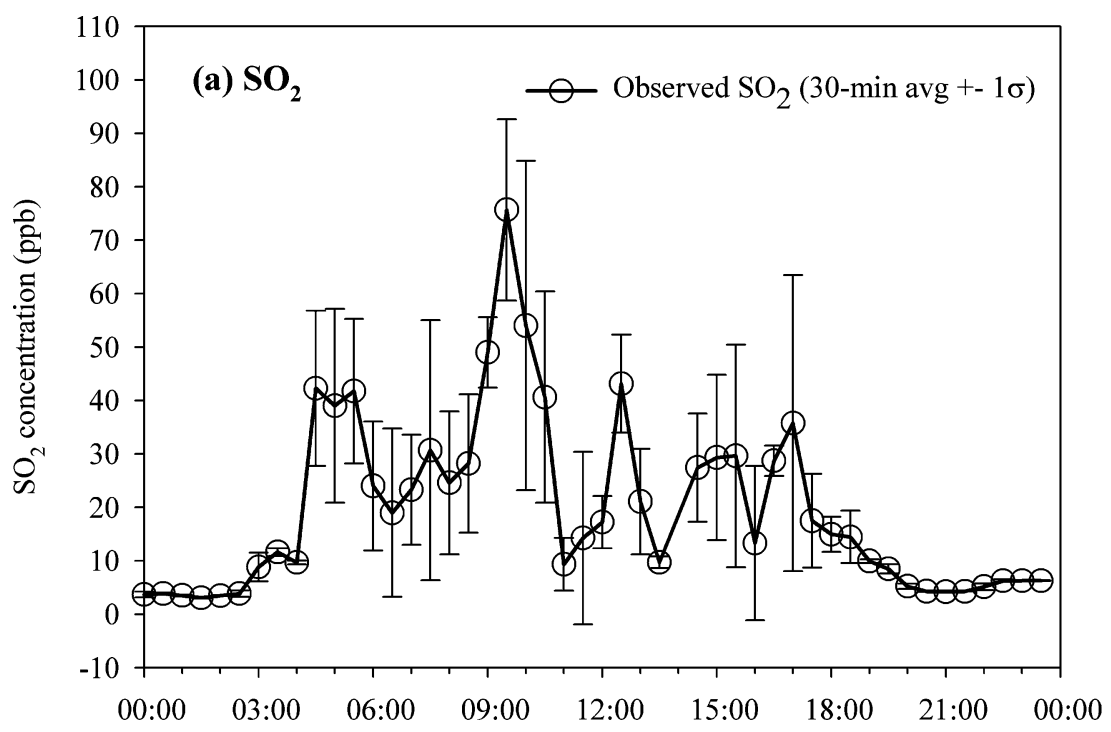

Measurement period (hr; April 1, 2002)

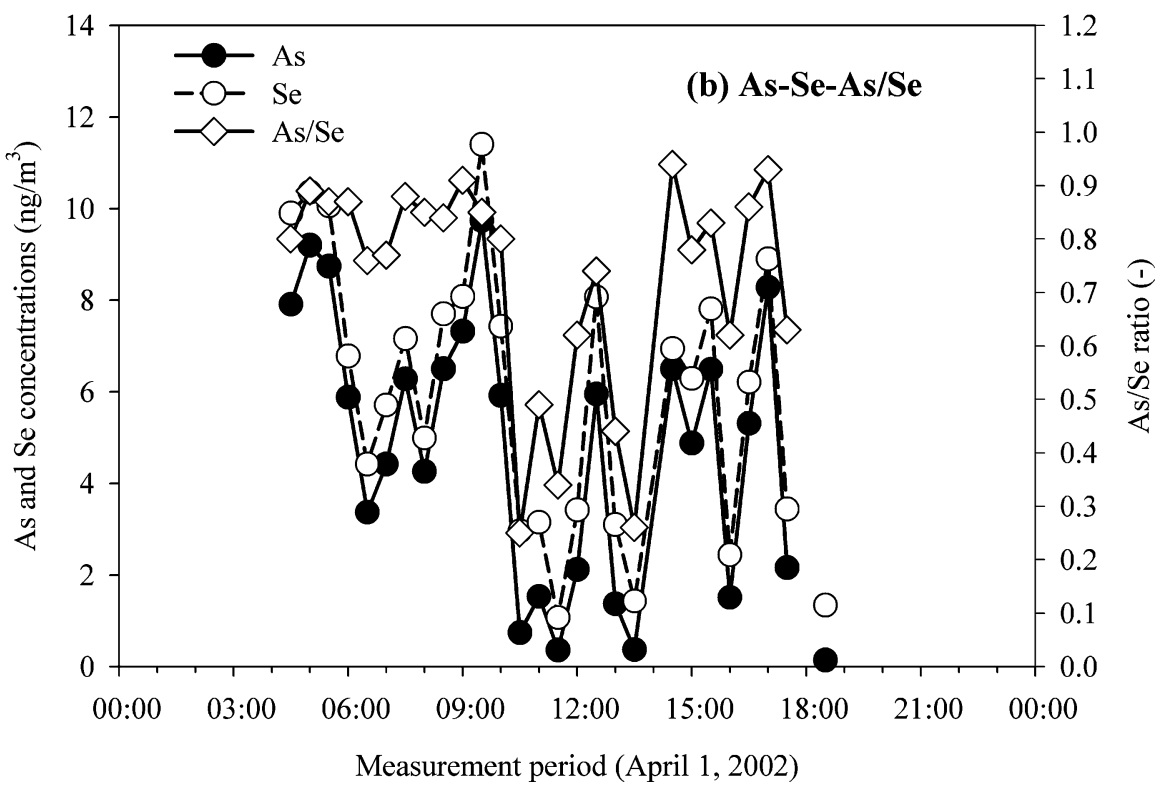

FIG. 3. Temporal profile of 30-min averaged $\mathrm{SO}_{2}$ concentrations (a); and As and Se concentration profiles, and As/Se ratios (b).

$(81 \%)$ and $\mathrm{Cd}(19 \%)$. Because $\mathrm{Cd}$ was at or below the detection limit in $81 \%$ of the samples, it could not be used in the model.

The PDRM is designed specifically to apportion contributions from stationary sources whose plumes induce excursions in concentrations above the background levels induced by dispersed and very distant sources. Therefore, background concentrations were evaluated as the concentrations of $\mathrm{SO}_{2}$ or metal species measured at intervals prior to the onset of an excursion and immediately after the excursion, and were subtracted prior to use in the PDRM. These were as follows: $\mathrm{SO}_{2}, 3.5$ $\mathrm{ppb}$; and metals in $\mathrm{ng} \mathrm{m}^{-3}, \mathrm{Al}, 2.64$; As, 0.66; $\mathrm{Cr}, 0.13$; $\mathrm{Cu}$, 2.71; $\mathrm{Fe}, 6.03 ; \mathrm{Mn}, 0.52 ; \mathrm{Ni}, 0.41 ; \mathrm{Pb}, 1.36 ; \mathrm{Se}, 0.64$; and
$\mathrm{Zn}$, 4.24. Background-corrected concentrations for $\mathrm{SO}_{2}$ and each of the 10 elemental particle constituents are shown in Figure 4.

\subsection{Model Description}

The basis of the model is a mass balance equation wherein the ambient contributions of each of the sources to each species are expressed as products of emission rates $\left(E R_{i, j}, \mathrm{~g} \mathrm{~s}^{-1}\right)$ and meteorological dispersion factors $\left(\chi / Q_{j, t}, \mathrm{~s} \mathrm{~m}^{-3}\right)$ appropriate for each sampling period, $t$, i.e.,

$$
\left[E_{i}\right]_{t}=\sum_{j=1}^{n} E R_{i, j} \cdot(\chi / Q)_{j, t}
$$


where $\left[E_{i}\right]_{t}$ are ambient concentrations of species of interest (i, in $\mathrm{ng} \mathrm{m}^{-3}$ ) measured in sample, $t$, and $E R_{i, j}$ s are emission rates of species, $i$, from $j$ stationary sources and represent averages for the period during which the samples (time intervals) used in model were collected. To solve the model, $\chi / Q_{j, t}$ 's are calculated for each sampling interval using a simple Gaussian plume model,

$$
\begin{aligned}
(\chi / Q)_{j, t}= & \frac{1}{2 \pi \sigma_{y} \sigma_{z} u} \exp \left[-\frac{1}{2} \frac{y^{2}}{\sigma_{y}^{2}}\right]\left\{\exp \left[-\frac{1}{2}\left(\frac{z-h}{\sigma_{z}}\right)^{2}\right]\right. \\
& \left.+\exp \left[-\frac{1}{2}\left(\frac{z+h}{\sigma_{z}}\right)^{2}\right]\right\},
\end{aligned}
$$

where $\chi$ is the concentration $\left(\mathrm{g} \mathrm{m}^{-3}\right), Q$ is the continuous mass emission rate $\left(\mathrm{g} \mathrm{s}^{-1}\right)$, and $u$ is the transport speed $\left(\mathrm{m} \mathrm{s}^{-1}\right)$ of the plume over its trajectory. Dispersion parameters, $\sigma_{y}$ and $\sigma_{z}$, are the standard deviations of the concentration distributions in the lateral $(y)$ and vertical $(z)$ directions, and increase with downwind distance $(x)$, from the source. $h$ is the height of the plume centerline when it becomes essentially level, and is the sum of actual stack height $\left(h_{s}\right)$ and the plume rise $(\Delta h)$. The calculated plume rises for the Bellefield boiler plant, Pittsburgh brewing co., Shenango Coke Works, and Zinc Corp of America applied in the modeling study are 84, 53, 95, and $174 \mathrm{~m}$, respectively. Gaussian plume models are commonly used to explain (a)
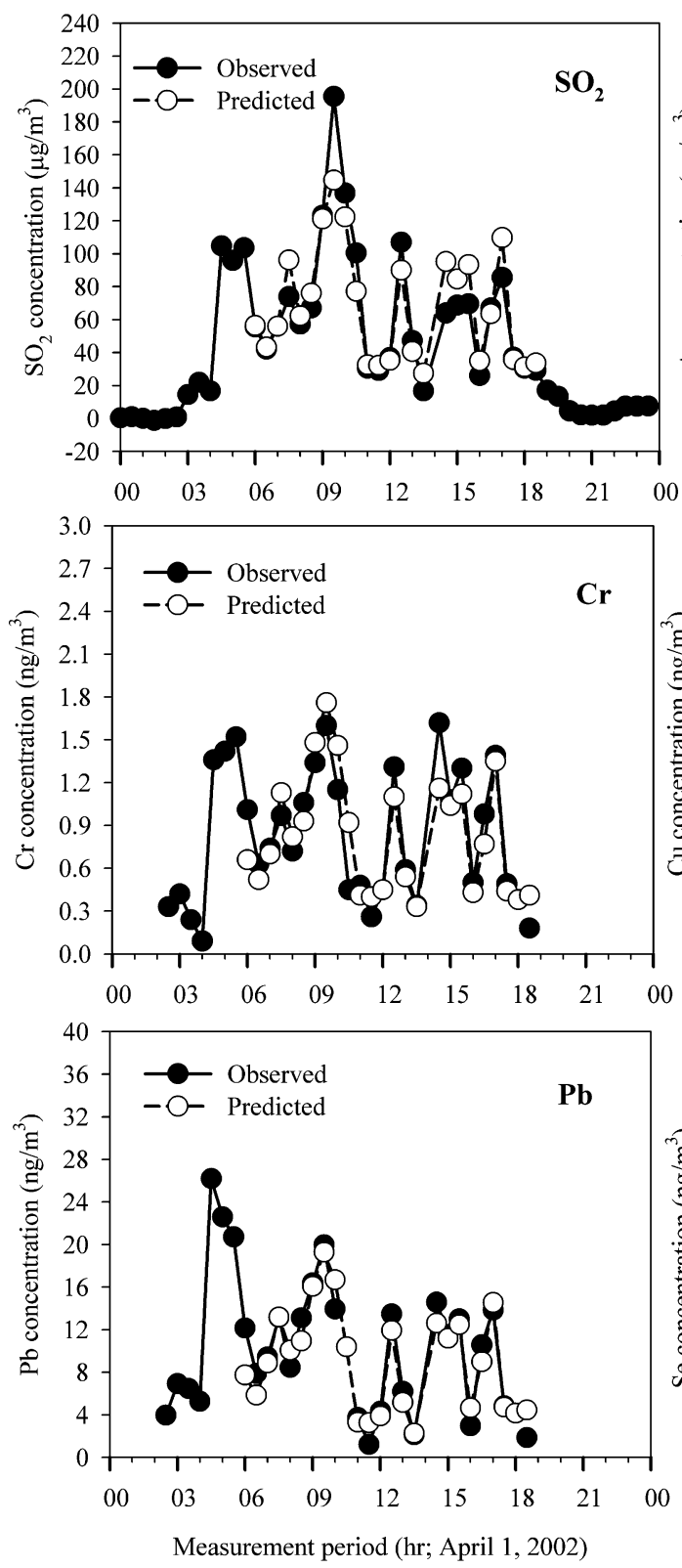

(b)
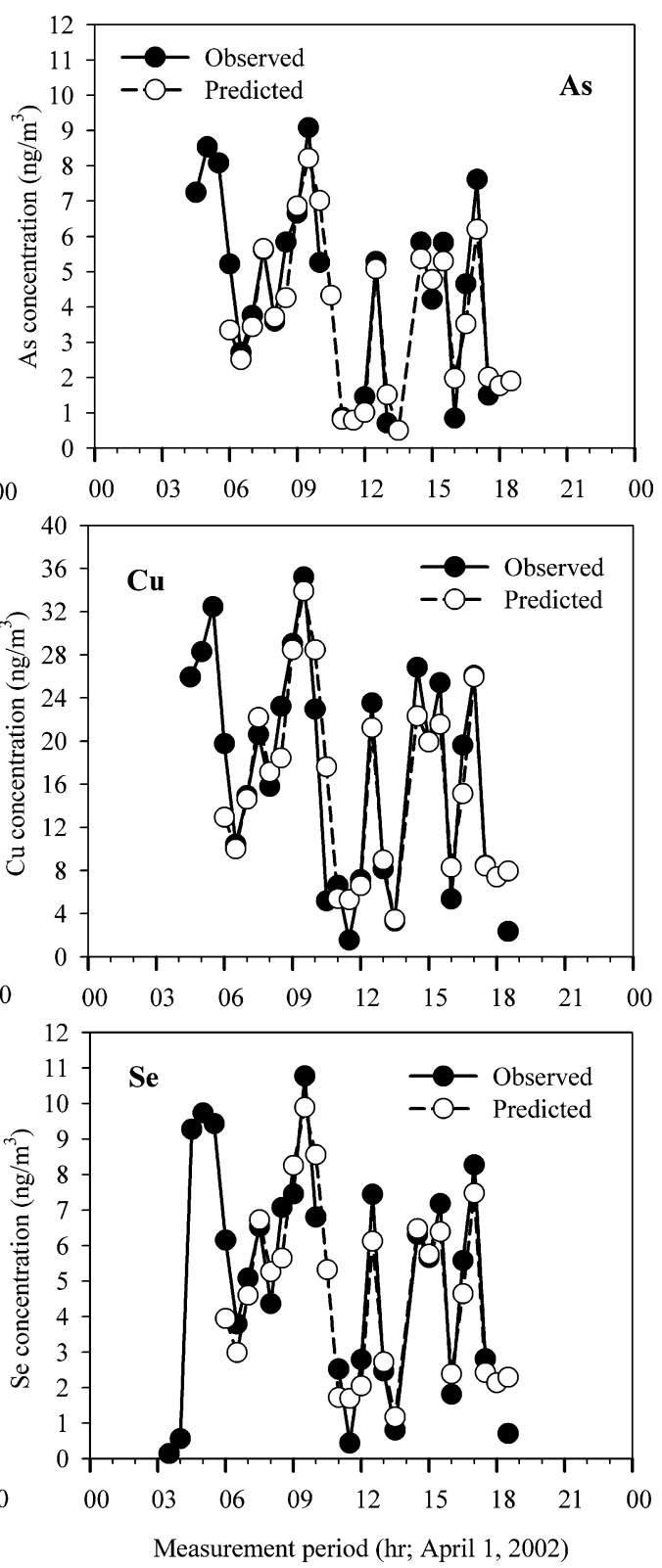

FIG. 4. (a) PDRM-predicted and observed concentrations of modeled species. (b). PDRM-predicted and observed concentrations of modeled species. (Continued) 

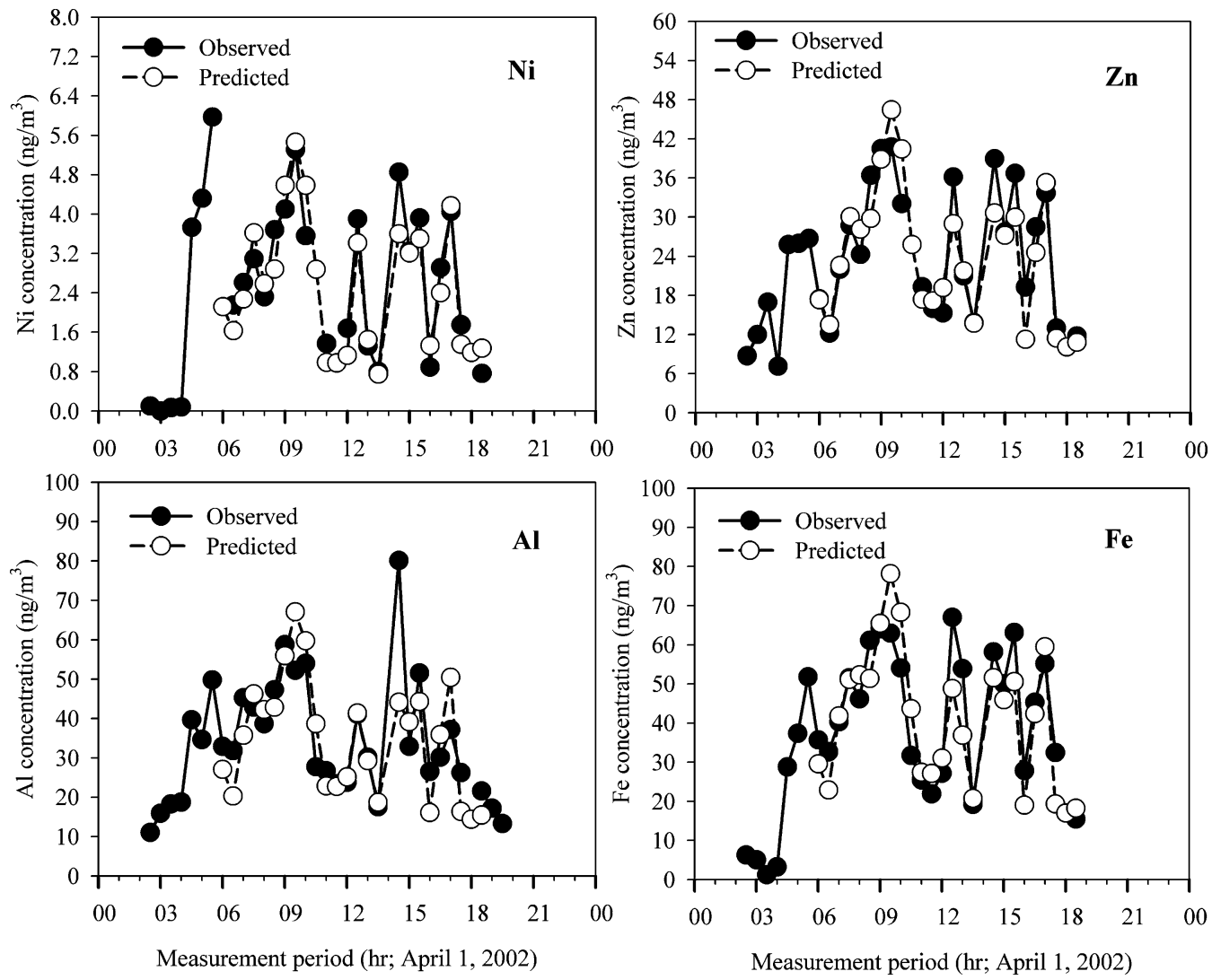

FIG. 4. Continued.

the dispersion of a species emitted from a point source and used because of their simplicity (US EPA 1980). In the plume model (Equation 2), the effects of gravitational settling and dry deposition on the ambient gases and particles are neglected, as are air pollutant removal by physical or chemical processes, and horizontal and vertical turbulence is assumed to be homogeneous. Mathematical expressions to calculate $\sigma_{y}$ and $\sigma_{z}$, mean $u$, displacement $(y)$ and $h$ are described previously (Park et al. 2005; Seinfeld and Pandis 1998).

Equations (1) and (2) are solved, simultaneously, using a nonlinear least squares solver ("lsqcurvefit") in MATLAB (MathWorks, Inc., version 6.5). The MATLAB program provides a solution that minimizes an object function, FUN, which we define as follows,

$$
\mathrm{FUN}=\sum_{i=1}^{l} \sum_{t=1}^{m} \sum_{j=1}^{n}\left(E R_{i, j} \cdot(\chi / Q)_{j, t}^{\mathrm{PDRM}}-[E]_{i, t}\right)^{2}
$$

to which we apply the constraint that:

$$
\mathrm{LB}(\chi / Q)_{j, t}^{\mathrm{Met}} \leq(\chi / Q)_{j, t}^{\mathrm{PDRM}} \leq \mathrm{UB}(\chi / Q)_{j, t}^{\mathrm{Met}}
$$

where LB and UB are upper and lower bounds within which the solver is directed to find solutions. Setting up constraints is essential to the model because the number of solutions for a product of unknowns is infinite and the Gaussian plume model is inaccurate. Once configured, the constrained model was applied to estimate the emission rates of $\mathrm{SO}_{2}$ and elemental constituents of primary particles. Solutions for $(\chi / Q)_{j, t}^{\mathrm{PDRM}}$ were constrained to the range $0.1-2.5$, consistently, for all four sources. This choice was derived from information reported for an intentional tracer study (Ondov et al. 1992) conducted at a coal-fired power plant $20 \mathrm{~km}$ from an arc of samplers in Maryland, in which $\chi / Q$ 's calculated with two different parameterizations of a Gaussian plume model differed by factors ranging from 5-10.

Input variables used in the hybrid receptor model are as follows; (1) 30-min ambient concentrations of $\mathrm{SO}_{2}, \mathrm{Al}, \mathrm{As}, \mathrm{Cr}, \mathrm{Cu}$, $\mathrm{Fe}, \mathrm{Mn}, \mathrm{Ni}, \mathrm{Pb}, \mathrm{Se}$, and $\mathrm{Zn}$, (2) derived and measured meteorological parameters, as described below, (3) stack data (physical stack height, stack inside diameter, exit gas velocity, and exit gas temperature (see Table 2), and (4) station angles for the emission sources. Herein, the best fits were obtained with a four-source model.

\subsection{Estimation of Micrometeorological Parameters}

Horizontal and vertical dispersion parameters $\left(\sigma_{y}\right.$ and $\left.\sigma_{z}\right)$ and atmospheric turbulence components were derived from micrometeorological parameters (i.e., friction velocity, $u^{*}$; Monin-Obukhov length, $L$; and convective velocity scale, $w^{*}$ ) via similarity theory as described previously (Park et al. 2005). 
TABLE 2

Stack information

\begin{tabular}{lccccc}
\hline Plant & $\begin{array}{c}\text { Base } \\
\text { Elevation }\left(\mathrm{m} \mathrm{ASL}^{1}\right)\end{array}$ & Height $(\mathrm{m})$ & Inside diameter $(\mathrm{m})$ & Exit temp $(\mathrm{K})$ & Exit velocity $\left(\mathrm{m} \mathrm{s}^{-1}\right)$ \\
\hline Bellefield boiler plant & 267.4 & $59.4^{2}$ & 2.74 & 565.0 & 7.70 \\
& & $77.7^{3}$ & 3.35 & 426.5 & 5.90 \\
Pittsburgh brewing co. & 234.4 & 64.0 & 2.44 & 478.0 & 4.57 \\
Shenango Coke Works & 223.4 & 76.2 & 3.05 & 584.3 & 6.08 \\
Zinc Corp of America & 238.4 & $84.0^{4}$ & 3.10 & 422.0 & 9.04 \\
& & $121.9^{5}$ & 5.40 & 365.0 & 12.00 \\
\hline
\end{tabular}

Note: ${ }^{1}$ ASL means above sea level; ${ }^{2}$ Stack \#1 spec of the Bellefield plant; ${ }^{3}$ stack \#2 spec (used in the modeling study); ${ }^{4}$ stack spec of boiler plants; ${ }^{5}$ stack spec of primary non-ferrous metal production plant (used in the modeling study). Respective plume rises, for Bellefield, Pittsburgh Brewing Co., Shenango Coke, and Zinc Corp, were calculated to be 84, 53, 95, and $174 \mathrm{~m}$ during peak concentration periods.

Calculation of the micrometeorological parameters is described below as are atmospheric stability Class and mixing layer heights. Also, because 3-D sonic anemometer data were not available, sensible heat fluxes $(\mathrm{H})$ and friction velocities $\left(u^{*}\right)$ were obtained from a 3-hourly, $80 \mathrm{~km}$ NCEP's EDAS dataset (http://www.arl.noaa.gov/ready/ametus.html) from which hourly data were then interpolated. Kleissl (2004) observed that values of $u^{*}$ and $\mathrm{H}$ deived from the $80 \mathrm{~km}$ NCEP's EDAS data corresponded fairly well to those derived from 3-D sonic anemometer measurements at the Baltimore Supersite.

\subsubsection{Atmospheric Stability}

Stability class was estimated based on Turner's method (1964), which considers the effects of solar altitude (a function of time of day and time of year), total cloud cover, and ceiling height. The net radiation index is related to the solar altitude and is determined from the procedure described by the US EPA (1999). Solar altitude can be determined from the Smithsonian Meteorological Tables (ref. 17). The solar altitude angle $(\alpha)$ is calculated as follows:

$$
\alpha=\sin ^{-1}\left[\left\{\cos (L)^{*} \cos (\delta)^{*} \cos \left(h_{s}\right)\right\}+\{\sin (L) * \sin (\delta)\}\right]
$$

where $L$ is local latitude $\left(40.44^{\circ}\right), \delta$ is solar declination (List 1966), and $h_{s}$ is hour angle, describing the difference between local solar time and solar noon. Accordingly, the atmospheric stability was estimated to be slightly unstable or neutral during the study period.

\subsubsection{Mixing Layer Height ( $\mathrm{z}$ )}

Hourly mixing heights were determined from (1) morning and afternoon estimates of mixing heights; (2) the local standard time of sunrise and sunset; and (3) hourly estimates of stability. Morning and afternoon mixing height estimates are based on Holzworth's method (Holzworth 1972) using radiosonde data from the Pittsburgh airport. The morning mixing height is estimated as the height above ground at which the dry adiabatic extension of the morning minimum surface temperature (i.e., between 02:00 and 06:00 local standard time) plus 5 intersects the vertical temperature profile observed at 12:00 GMT. A similar computation for the afternoon mixing height is made using the maximum surface temperature observed from 12:00 through 16:00 LST, except that the surface temperature is not adjusted. Hourly mixing heights are interpolated from these twice per day estimates, as described in the user's guide for the ISC dispersion model (19). The procedure uses the maximum mixing height $\left(z_{\max }\right)$ from the previous day $(i-1$; March 31$)$, the computation day ( $i$; April 1$)$ and the following day $(i+1$; April 2) and the minimum mixing height $\left(z_{\min }\right)$ for days April 1 and 2. If the boundary layer was classified as stable in the hour before sunrise, the value for the minimum mixing height is used as the mixing heights between midnight and sunrise. Between sunrise and 14:00 LST, the interpolation is between $z_{\min , i}$ and $z_{\max , i}$. For the period 14:00 LST and sunset, the value for $z_{\max , i}$ is used. During the hours between sunset and midnight under stable stability, the interpolation is between $z_{\max , i}$ at sunset and $z_{\min , i+1}$ at midnight.

\subsubsection{Monin-Obukhov Length ( $L$ ) and Convective Velocity Scale $\left(\mathrm{w}^{*}\right)$}

The Monin-Obukhov length (L), a stability parameter relating $u^{*}$ and $\mathrm{H}$, was computed from the equation suggested by Venkatram (1996)

$$
L=-\frac{\rho \cdot C_{p} \cdot T u_{*}^{3}}{K \cdot g \cdot H}
$$

where $\rho$ is the density of dry air $\left(1.25 \mathrm{~kg} \mathrm{~m}^{-3}\right), \mathrm{C}_{p}$ is the specific heat capacity of air $\left(1004 \mathrm{~J} \mathrm{~kg}^{-1} \cdot \mathrm{K}^{-1}\right), T$ is ambient temperature $(\mathrm{K}), \mathrm{u}^{*}$ is the surface friction velocity $\left(\mathrm{m} \mathrm{s}^{-1}\right), K$ is the von Karman constant (0.4), $\mathrm{g}$ is the acceleration due to gravity $\left(9.81 \mathrm{~m} \mathrm{~s}^{-2}\right)$, and $\mathrm{H}$ is the surface sensible heat flux $\left(\mathrm{W} \mathrm{m}^{-2}\right)$; the negative sign is used by convention to distinguish stable from unstable conditions. 
TABLE 3

Estimation of emission rates for $\mathrm{SO}_{2}$ and metal species for each of 4 sources (units: $\mathrm{g} \mathrm{s}^{-1}$ )

Bellefield Pittsburgh Shenango Zinc Corp Species boiler plant brewing plant Coke Works of America

\begin{tabular}{lcccc}
\hline $\mathrm{SO}_{2}$ & 48 & 176 & 123 & 176 \\
$\mathrm{Al}$ & 0.0336 & 0.0800 & 0.1203 & 0.1453 \\
$\mathrm{As}$ & 0.0012 & 0.0105 & 0.0046 & 0.0009 \\
$\mathrm{Cr}$ & 0.0006 & 0.0021 & 0.0017 & 0.0020 \\
$\mathrm{Cu}$ & 0.0007 & 0.0408 & 0.0402 & 0.0480 \\
$\mathrm{Fe}$ & 0.0365 & 0.0926 & 0.1610 & 0.1130 \\
$\mathrm{Mn}$ & 0.0038 & 0.0032 & 0.0038 & 0.0010 \\
$\mathrm{Ni}$ & 0.0016 & 0.0065 & 0.0062 & 0.0070 \\
$\mathrm{~Pb}$ & 0.0010 & 0.0232 & 0.0236 & 0.0369 \\
$\mathrm{Se}$ & 0.0005 & 0.0119 & 0.0126 & 0.0184 \\
$\mathrm{Zn}$ & 0.0299 & 0.0554 & 0.0807 & 0.0633 \\
\hline
\end{tabular}

Convective velocity scale $\left(\mathrm{w}^{*}\right)$ is computed from the following definition (Wyngaard, 1988) as:

$$
w_{*}=\left(\frac{g \cdot H \cdot z_{i}}{\rho \cdot C_{p} \cdot T}\right)^{1 / 3}
$$

where $z_{i}$ denotes mixing layer height $(m)$.

\section{RESULTS AND DISCUSSION}

Ambient concentrations of $\mathrm{SO}_{2}, \mathrm{As}$, and $\mathrm{Se}$ are plotted in Figure 3. Herein, the model was solved for a set of 44 emission rates and 100 dispersion factors $(\chi / Q)^{\mathrm{PDRM}}$. The $\chi / Q s$ predicted by both the PDRM and Gaussian plume model (Equation 2) for each of the four sources are plotted as a function of time of day in Figure 5. Predicted emission rates (averages for the study period) are listed in Table 3 and emission rate ratios (to $\mathrm{Se})$ predicted for the four sources are listed in Table 4. Analogous ratios derived previously from application of the PDRM as well as those derived from in-stack measurements reported for coal-fired boilers/power plants in the Eastern U.S. are listed in Table 5. Average concentrations induced by each of the sources during the study period were calculated as the product of the

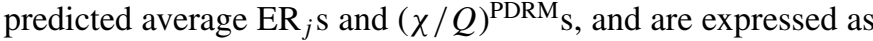
percentages of total elemental concentrations observed for the study period in Table 6 . Results of a sensitivity analysis to the choice of constraints applied to solutions for $(\chi / Q)^{\mathrm{PDRM}}$ are shown in Figure 6. Last, Observed and predicted concentrations are compared in Figure 4, and model performance statistics are listed in Table 7.

\subsection{Dispersion Factors for Each of $\mathbf{4}$ Stationary Sources}

As shown in Table 1, all four boilers were quite small, the largest being the $110 \mathrm{MW}$ boiler at ZCA, $42 \mathrm{~km}$ from the site. Nevertheless, $\mathrm{SO}_{2}$ concentrations observed at the CMU site during the study period were quite high, 20 to $80 \mathrm{ppb}$, during the periods of plume influence (see Figure 3). We attribute this to the proximity of the Bellefield and $\mathrm{PBC}$ boilers and the elevation of the CMU site which reduces distance between the ground and the plume centerlines. As shown in Figure 5, $\chi / Q$ maxima predicted by the Met model for these sources were large, i.e., $\sim 10^{-6} \mathrm{~s} \mathrm{~m}^{-3}$. The largest value predicted for the PBC corresponds to the study period maximum $\mathrm{SO}_{2}$ (30-min average) of $80 \mathrm{ppb}\left(205 \mu \mathrm{g} \mathrm{m}^{-3}\right)$, which occurred at 09:30 h.

As is evident from Figure 5, the $\chi / Q$ s, plumes of each of the sources are predicted to have influenced air at the site on multiple occasions, as winds shifted them towards and away from

TABLE 4

Emission rate ratios and regression statistics for ambient concentrations.

\begin{tabular}{llccccc}
\hline & & & \multicolumn{3}{c}{ ER_ratio from modeling results } \\
\cline { 3 - 7 } & \multicolumn{1}{c}{$\begin{array}{c}\text { Regression relationship } \\
\text { Species }\end{array}$} & \multicolumn{1}{c}{$\begin{array}{c}\text { Bellefield boiler } \\
\text { plant }\end{array}$} & $\begin{array}{c}\text { Pittsburgh brew. } \\
\text { plant }\end{array}$ & $\begin{array}{c}\text { Shenango coke } \\
\text { works }\end{array}$ & $\begin{array}{c}\text { Zinc Corp } \\
\text { America }\end{array}$ \\
\hline $\mathrm{SO}_{2}$ & $\mathrm{SO}_{2}^{1}=(11.2 \pm 1.9)^{* * *} \mathrm{Se}+(12 \pm 13)$ & 0.772 & $96.0^{4}$ & $14.8^{4}$ & $9.76^{4}$ & $9.57^{4}$ \\
$\mathrm{Al}$ & $\mathrm{Al}=(3.0 \pm 0.7)^{* *} \mathrm{Se}+(23 \pm 5)^{* *}$ & 0.635 & 67.2 & 6.7 & 9.55 & 7.9 \\
$\mathrm{As}$ & $\mathrm{As}=(0.99 \pm 0.03)^{* * *} \mathrm{Se}+(-1.1 \pm 0.2)^{* * *}$ & 0.987 & 2.4 & 0.88 & 0.36 & 0.049 \\
$\mathrm{Cr}$ & $\mathrm{Cr}=(0.14 \pm 0.01)^{* * *} \mathrm{Se}+(0.23 \pm 0.07)^{*}$ & 0.944 & 1.2 & 0.18 & 0.13 & 0.11 \\
$\mathrm{Cu}$ & $\mathrm{Cu}=(3.31 \pm 0.16)^{* * *} \mathrm{Se}+(0.35 \pm 1.1)$ & 0.973 & 1.4 & 3.4 & 3.2 & 2.6 \\
$\mathrm{Fe}$ & $\mathrm{Fe}=(3.3 \pm 0.8)^{* *} \mathrm{Se}+(30 \pm 5)^{* * *}$ & 0.655 & 73.0 & 7.8 & 12.8 & 6.1 \\
$\mathrm{Mn}$ & $\mathrm{Mn}=(0.029 \pm 0.033) \mathrm{Se}+(1.9 \pm 0.2)^{* * *}$ & 0.177 & 7.6 & 0.27 & 0.30 & 0.05 \\
$\mathrm{Ni}$ & $\mathrm{Ni}=(0.48 \pm 0.04)^{* * *} \mathrm{Se}+(0.42 \pm 0.26)$ & 0.939 & 3.2 & 0.55 & 0.49 & 0.38 \\
$\mathrm{~Pb}$ & $\mathrm{~Pb}=(2.2 \pm 0.14)^{* * *} \mathrm{Se}+(-0.8 \pm 0.9)$ & 0.956 & 2.0 & 1.95 & 1.87 & 2.00 \\
$\mathrm{Zn}$ & $\mathrm{Zn}=(2.5 \pm 0.5)^{* * *} \mathrm{Se}+(15.0 \pm 3.2)^{* * *}$ & 0.726 & 59.8 & 4.7 & 6.4 & 3.4 \\
\hline
\end{tabular}

Note: ${ }^{*} p<0.01,{ }^{* *} p<0.001 ;{ }^{* * *} \mathrm{p}<0.0001$

${ }^{1}$ Unit is in $\mu \mathrm{g} \mathrm{m}^{-3} ;{ }^{2} r$ indicates correlation coefficient; ${ }^{3} \mathrm{ER}$ ratio means ratio of emission rate for each species to emission rate of Se; ${ }^{4}$ indicates the ratio divided by 1000 . 

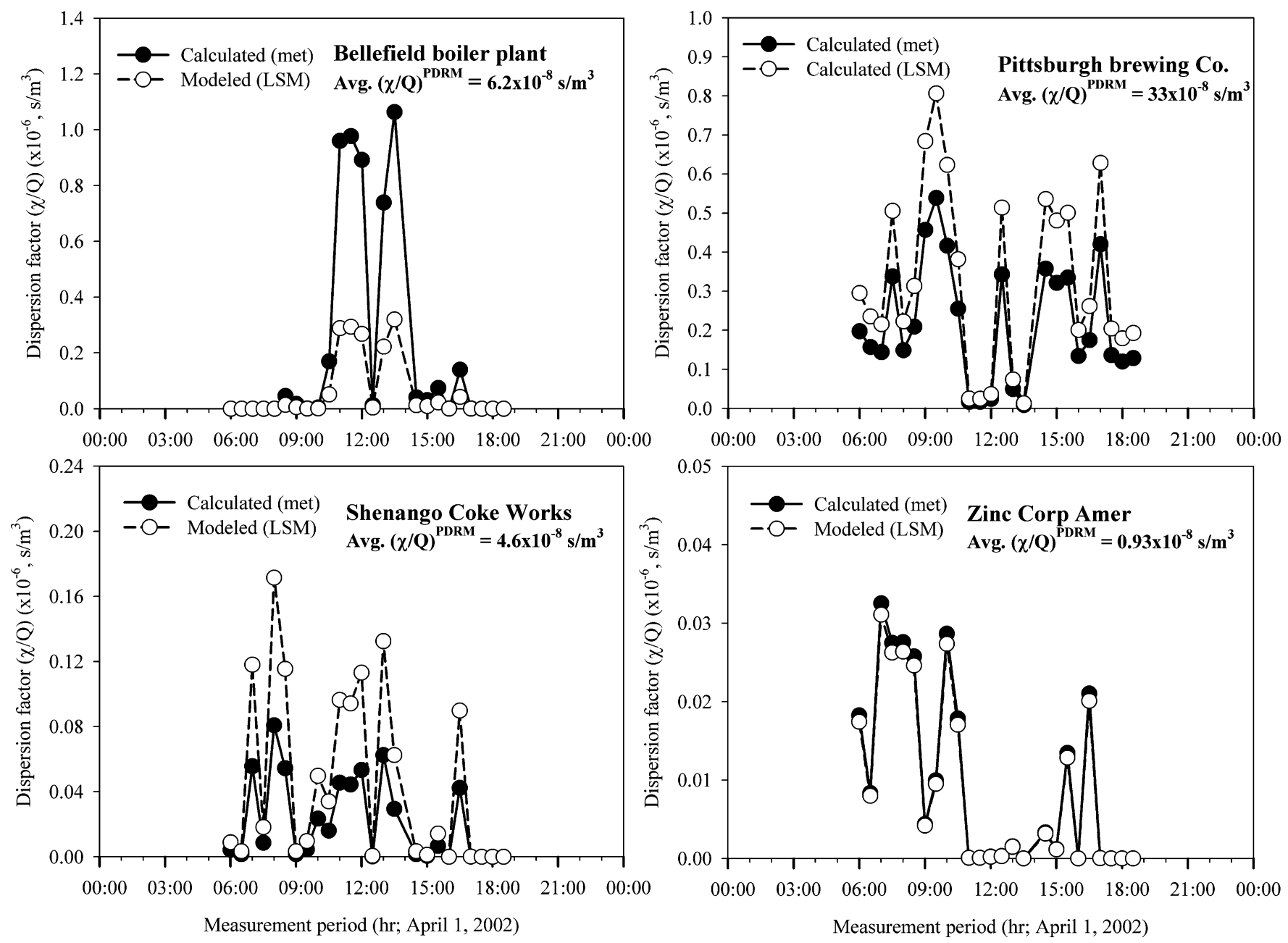

FIG. 5. Calculated and PDRM-derived dispersion factor $(\chi \mathrm{Q}) \mathrm{j}, \mathrm{t}$ for each of 4 emission sources.

their station angles. On this basis, plumes from the Bellefield boiler plant $\left(286^{\circ}, 0.8 \mathrm{~km}\right.$ distant), influenced the site between $10: 30$ and $13: 30 \mathrm{~h}$, and $(\chi / Q)^{\mathrm{PDRM}_{\mathrm{S}}}$ for this source were in the range $0.05-0.32 \times 10^{-6} \mathrm{~s} \mathrm{~m}^{-3} \cdot \chi / Q$ 's calculated with the meteorological model (Equation 2) were substantially less than those predicted by the PDRM, presumably due to the effects of buildings and roughness elements on the CMU campus which tend to increase dispersion, and our application of the larger of the two stack heights reported for this source, which also leads to greater dispersion estimates. Bellefield's $\mathrm{SO}_{2}$ plume is predicted to have influenced the site between 10:30-12:00 h and again between 12:30-13:00 $\mathrm{h}$ and as indicated in Table 3, its average $\mathrm{SO}_{2}$ emission rate was $48 \mathrm{~g} \mathrm{~s}^{-1}$.

$\chi / Q$ 's for the PBP (station angle, $316^{\circ}$; distance, $3.4 \mathrm{~km}$ ) suggest that its plume was influential on five occasions (i.e., at 07:30, 09:30, 12:30, 14:30-15:30 and 17:00 h). Maximum predicted influence occurred at 09:30 h, which as mentioned above, precisely corresponds to the time at which the period maximum $\mathrm{SO}_{2}$ concentration (195 $\mu \mathrm{g} \mathrm{m}^{-3}$, after background correction) was observed at the site. The predicted $\mathrm{SO}_{2}$ emission rate average (176 $\mathrm{g} \mathrm{s}^{-1}$, Table 3$)$ for this source is 3-fold larger

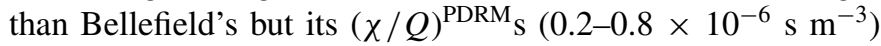
were comparable to Bellefield's. Thus, its influence was greater than that of Bellefield. In contrast to the case for Bellefield, $(\chi / Q)^{\text {Met }} \mathrm{s}$ for the PBP were less than those predicted by the PDRM. This is not surprising, given the uncertainties in the inputs to the plume model as well as its simplicity. This was also the case for the SCW (station angle $297^{\circ}$; distance $13.0 \mathrm{~km}$ ).

The plumes from the SCW were predicted to influence the site at 07:00, 08:30, 10:30-12:00, 13:00-13:30, and 16:30 h,

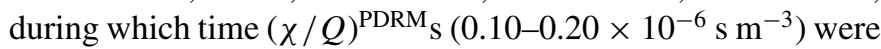
$\sim 5$-fold less than those for Bellefield and the predicted $\mathrm{SO}_{2}$ emission rate average ( $\left.123 \mathrm{~g} \mathrm{~s}^{-1}\right)$ is comparable to that predicted for the PBC. As also indicated in Figure 4, the plumes from ZCA (station angle, $307^{\circ}$; distance, $41.9 \mathrm{~km}$ ), arrived at the measurement site at 07:00-08:30, 09:30, 15:30, and 16:30 h, dur-

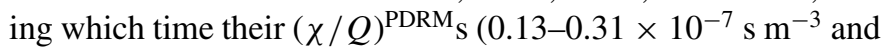
nearly identical to their $(\chi / Q)^{\mathrm{Met}} \mathrm{s}$ were 10 - to 100 -fold smaller 
TABLE 5

Fine-particle metal: Se ratios reported for various Eastern coal-fired power plants with ESPs

\begin{tabular}{|c|c|c|c|c|c|c|c|c|}
\hline & \multirow{2}{*}{$\begin{array}{l}\text { Tampa } \\
\text { Park et al. } \\
(2005)^{1}\end{array}$} & \multirow{2}{*}{$\begin{array}{l}\text { Chalk Pt, } \\
\text { MD Gladney } \\
\text { et al. }(1976)^{2}\end{array}$} & \multirow{2}{*}{$\begin{array}{c}\text { Mt. Tom } \\
\text { Kowalczyk } \\
\text { et al. }[1984]^{2}\end{array}$} & \multirow{2}{*}{$\begin{array}{l}\text { Dickerson, MD } \\
\text { Small }(1974)^{2}\end{array}$} & \multirow{2}{*}{$\begin{array}{c}\text { Eddystone, PA } \\
\text { Olmez et al. }(1988)^{2}\end{array}$} & \multicolumn{3}{|c|}{$\begin{array}{l}\text { Ohio River Valley } \\
\text { Power Plants } \\
(\text { Baker et al. 1983) }\end{array}$} \\
\hline & & & & & & "A" & "B" & "C" \\
\hline $\mathrm{SO}_{2}$ & 79000 & & & & & & & \\
\hline $\mathrm{Al}$ & 30 & 970 & 635 & 147 & 568 & 726 & 402 & 2154 \\
\hline As & 0.91 & 20 & 1.55 & 1.17 & 2.59 & 4.50 & 1.50 & 6.10 \\
\hline $\mathrm{Cr}$ & 0.73 & 1.77 & 1.97 & 0.48 & 1.52 & 4.67 & 1.50 & 7.40 \\
\hline $\mathrm{Cu}$ & 0.55 & & 0.68 & 0.31 & 1.24 & 1.67 & 1.50 & 4.90 \\
\hline $\mathrm{Fe}$ & 33 & 272 & 377 & 80 & 307 & 169 & 94 & 482 \\
\hline $\mathrm{Mn}$ & 0.33 & 3.09 & 2.39 & 0.66 & 1.18 & 1.67 & 1.50 & 5.00 \\
\hline $\mathrm{Ni}$ & 0.30 & 2.10 & 0.55 & & 1.59 & 2.00 & 1.00 & 3.70 \\
\hline $\mathrm{Pb}$ & 1.00 & 3.20 & 0.35 & & 1.76 & 1.17 & & 1.70 \\
\hline $\mathrm{Zn}$ & 1.76 & 5.66 & & 1.39 & 1.67 & 2.83 & 1.50 & 5.10 \\
\hline
\end{tabular}

${ }^{1}$ Derived from deconvolution of ambient concentration measurements near Tampa, FL, using the Pseudo-Deterministic Receptor Model.

${ }^{2}$ Derived from analysis of in-stack sampling of fine "fine" particles, except Mt. Tom ratios, which were drived from analyses of all in-stack particles.

${ }^{3}$ Electrostatic Precipitator.

than maxima predicted for the other sources, while their average $\mathrm{SO}_{2}$ emission rate was estimated to have been only $176 \mathrm{~g} \mathrm{~s}^{-1}$, i.e., precisely that estimated for PBC. Consequently, their influence on the site during the study period was shown to be small. As indicated in Table 6,70-80\% of the ambient concentration excesses over background during the study period are predicted to have been induced the PBP, i.e., for all species listed. Despite its proximity, predicted contributions for Bellefield were $<10 \%$ for all species except Mn (15\%); those for the SCW and ZCA were $11-20 \%$ and $<3.5 \%$, respectively.

\subsection{Performance Statistics}

The overall performance of the model was evaluated for the observed and predicted $\mathrm{SO}_{2}$ and metal concentrations using the following statistical measures (Hana 1988; Kumar et al. 1993; Patel and Kumar 1998) which they applied to modeling results

TABLE 6

Predicted contribution averages for the study period, $\%$ of measured concentration attributed to indicated source

\begin{tabular}{lcccc}
\hline & $\begin{array}{c}\text { Bellefield } \\
\text { boiler } \\
\text { plant }\end{array}$ & $\begin{array}{c}\text { Pittsburgh } \\
\text { brewing } \\
\text { plant }\end{array}$ & $\begin{array}{c}\text { Shenango } \\
\text { Coke } \\
\text { Works }\end{array}$ & $\begin{array}{c}\text { Zinc Corp } \\
\text { of } \\
\text { America }\end{array}$ \\
Species & $6-10$ & $70-80$ & $11-19$ & $0.2-3.4$ \\
\hline $\mathrm{SO}_{2}, \mathrm{Zn}, \mathrm{Cr}, \mathrm{Ni}$, & 6 & & & \\
$\quad \mathrm{Al}, \mathrm{Fe}, \mathrm{As}$ & & & 20 & 3.2 \\
$\mathrm{~Pb}, \mathrm{Se}$ & 1.8 & 75 & 20 & 2.5 \\
$\mathrm{Cu}$ & 0.8 & 76 & 12 & 0.6 \\
$\mathrm{Mn}$ & 15 & 72 & & \\
\hline
\end{tabular}

for ambient $\mathrm{SO}_{2}$ concentrations: mean bias (MB), mean normalized bias (MNB), root mean square error (RMSE), normalized mean square error (NMSE), correlation coefficients (CC), and the fraction of predicted concentrations lying within a factor of 2 (Fa2) (i.e., $0.5 \leq C_{\text {pred }} / C_{\text {obs }} \leq 2.0$ ) of the measured ambient concentrations; all as defined in Table 7. According to Kumar et al. (1993), model performance is deemed acceptable if NMSE $\leq 0.5(50 \%)$ and $\mathrm{Fa} 2 \geq 0.8(80 \%)$.

For $\mathrm{SO}_{2}$, the average ratio of the predicted and observed concentrations (P:O) was $1.09 \pm 0.22$. As indicated in Figure 5, the $\mathrm{SO}_{2}$ concentration profile predicted by the 4 -source model is

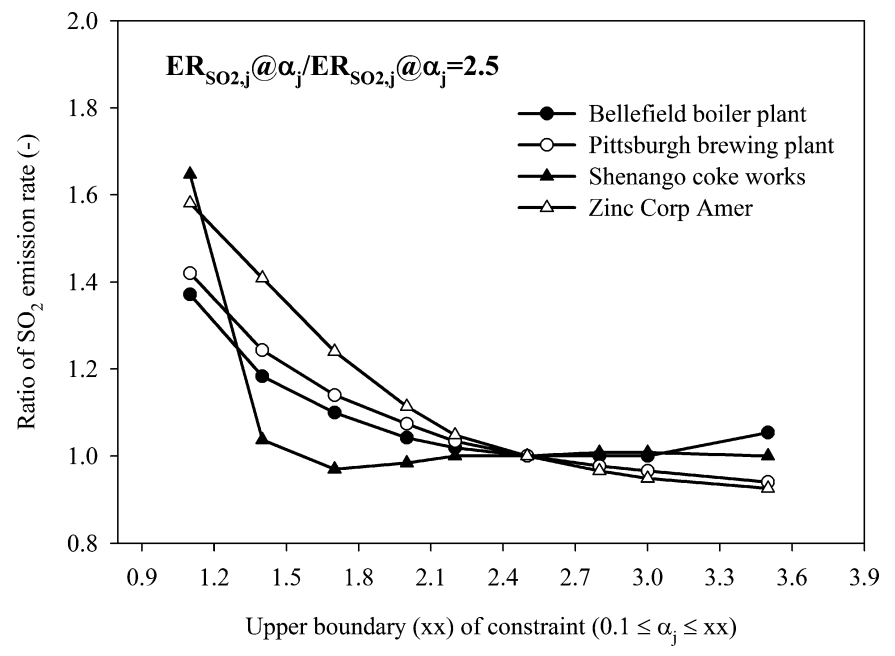

FIG. 6. Normalized $\mathrm{SO}_{2}$ emission rates predicted as a function of the value of the base upper-bound constraint. 


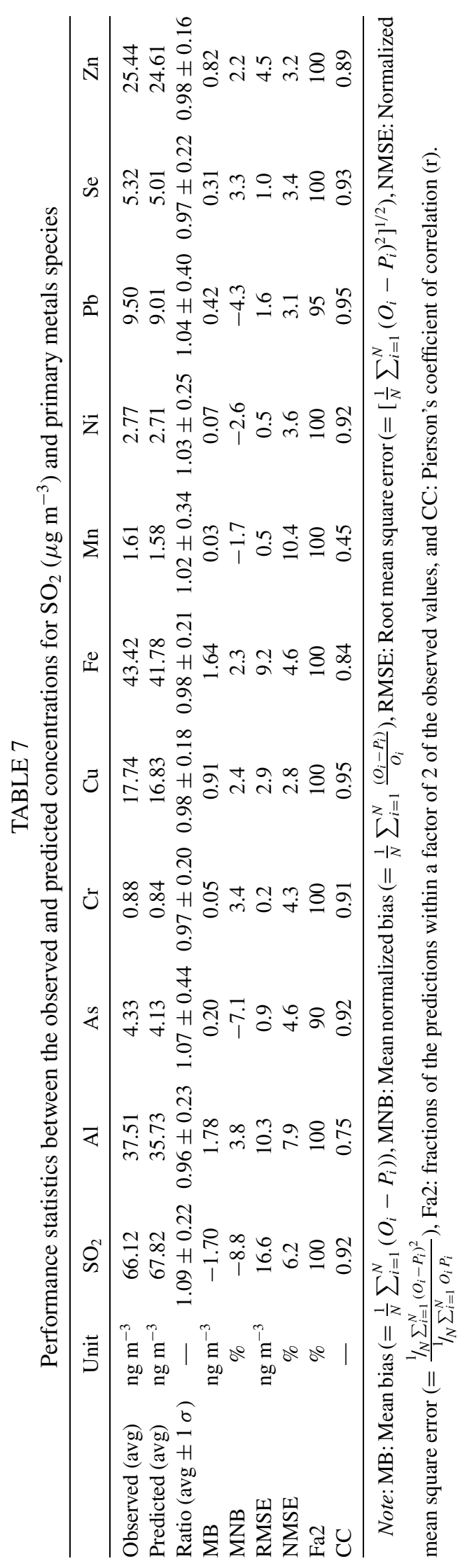


in excellent agreement with the observed $\mathrm{SO}_{2}$ concentrations except at 09:30 and between 3:30-16:00 hr. Underestimates (by $26 \%$ ) occurred at 09:30 $\mathrm{hr}$ when the highest concentration was observed, whereas overestimates ranging from $23-64 \%$ were observed between 14:30-16:00 h, i.e., when the plume from PBC is predicted to have been its main source. During this period, the maxima in $(\chi / Q)^{\mathrm{PDRM}}$ exceeded $(\chi / Q)^{\mathrm{Met}}$ by 2 , i.e., close to, but not in excess of the limiting factor of $2.5 \mathrm{im}$ posed by our constraint (which, as indicated below, was the global optimum value). Nevertheless, the average observed $\mathrm{SO}_{2}$ concentration $\left(66 \mu \mathrm{g} \mathrm{m}^{-3}\right)$ is nearly identical to the predicted average $\left(68 \mu \mathrm{g} \mathrm{m}^{-3}\right)$, and the MNB, which is sensitive to small observed concentrations, is only $-8.8 \%$. Additionally, RMSE and NMSE for $\mathrm{SO}_{2}$ are $16.6 \mathrm{ng} \mathrm{m}^{-3}$ and $6.2 \%$, respectively, and all predictions were within a factor of 2 . All are within the acceptable ranges suggested by Kumar et al. (1993).

For those metals having temporal concentration profiles similar to that of $\mathrm{SO}_{2}$, i.e., $\mathrm{As}, \mathrm{Cr}, \mathrm{Cu}, \mathrm{Ni}, \mathrm{Pb}, \mathrm{Se}$, and $\mathrm{Zn}$, agreement between observed and predicted concentrations was excellent (see Figure 5 and Table 7). The average P:O ratio was $1.07 \pm$ $0.44(0.64 \sim 2.33)$ for As, $0.97 \pm 0.20(0.65 \sim 1.54)$ for $\mathrm{Cr}, 0.98$ $\pm 0.18(0.65 \sim 1.54)$ for $\mathrm{Cu}, 1.03 \pm 0.25(0.73 \sim 1.65)$ for $\mathrm{Ni}$, $1.04 \pm 0.40(0.64 \sim 2.65)$ for $\mathrm{Pb}, 0.97 \pm 0.22(0.64 \sim 1.47)$ for $\mathrm{Se}$, and $0.98 \pm 0.16(0.58 \sim 1.26)$ for $\mathrm{Zn}$, respectively. (Values in parentheses are uncertainties in the ratio expressed as 1 standard deviation.) The largest deviations (from 1) were mostly observed at times when their concentrations were very near background levels, e.g., at 11:30, 13:00, or 16:00 hr, and therefore, have small effects on the mean contributions predicted for the various sources. Residuals ranged from -1.8 to $1.9 \mathrm{ng} \mathrm{m}^{-3}$ (mean bias: $0.20 \mathrm{ng} \mathrm{m}^{-3}$, see Table 7) for As, from -0.3 to $0.5 \mathrm{ng}$ $\mathrm{m}^{-3}$ (mean bias: $0.05 \mathrm{ng} \mathrm{m}^{-3}$ ) for $\mathrm{Cr}$, from -5.5 to $6.8 \mathrm{ng} \mathrm{m}^{-3}$ (mean bias: $0.91 \mathrm{ng} \mathrm{m}^{-3}$ ) for $\mathrm{Cu}$, from -0.5 to $1.3 \mathrm{ng} \mathrm{m}^{-3}$ (mean bias: $0.07 \mathrm{ng} \mathrm{m}^{-3}$ ) for $\mathrm{Ni}$, from -2.8 to $4.4 \mathrm{ng} \mathrm{m}^{-3}$ (mean bias: $0.42 \mathrm{ng} \mathrm{m}^{-3}$ ) for $\mathrm{Pb}$, from -1.8 to $2.2 \mathrm{ng} \mathrm{m}^{-3}$ (mean bias: $0.31 \mathrm{ng} \mathrm{m}^{-3}$ ) for $\mathrm{Se}$, and from -8.4 to $8.3 \mathrm{ng} \mathrm{m}^{-3}$ (mean bias: $0.82 \mathrm{ng} \mathrm{m}^{-3}$ ) for Zn. As indicated in the Table 7, MNB ranged from $-7.1 \%$ for As to $3.4 \%$ for Cr. The performance indices, NMSE and $\mathrm{Fa} 2$, are also quite reasonable for these elements described above, ranging from 4.6 and $90 \%$ for As to 3.4 and $100 \%$ for Se. The RMSE ranged from $0.9 \mathrm{ng} \mathrm{m}^{-3}$ for As to 4.5 for $\mathrm{Zn}$.

Quite reasonable agreement also was observed for $\mathrm{Al}$ and $\mathrm{Fe}$, even though their concentrations might be expected to be influenced by inopportune fluctuations in local dust concentrations. Indeed, $\mathrm{Al}$ (but not $\mathrm{Fe}$ ) was severely under predicted (i.e., by nearly 2 -fold) in one sample, i.e., at 14:30, possibly, due to this reason. Nonetheless, the average P:Os were $0.96 \pm 0.23$ $(0.55 \sim 1.39)$ and $0.98 \pm 0.21(0.60 \sim 1.38)$ for $\mathrm{Al}$ and $\mathrm{Fe}$, respectively, and their respective MNBs were only 3.8 and 2.3\%. Clearly, both errors and performance indices (NMSE and Fa2) associated with the model predictions are all within the acceptable ranges.

\subsection{Sensitivity to Constraints}

A sensitivity analysis was performed by varying the upper bound of $\alpha_{j}$ (UB) from 1.0 to 5.0. Results are shown in Figure 6 wherein the $\mathrm{P}: \mathrm{O}$ ratio for average $\mathrm{SO}_{2}$ emission rates are plotted against UB. In this figure, the predicted emission rates are normalized to those calculated for the base UB constraint $=2.5$. As shown in the Figure 6, there is little change in ratio of the observed and predicted $\mathrm{SO}_{2}$ emission rates for UBs exceeding 2.5. Ratios for UB $<2.5$ increased gradually, varying from $0.97-1.65$ depending on the source. Quite similar behavior was observed in our Tampa study (Park et al., 2005). For these reasons, results reported herein are those obtained with $\mathrm{UB}=2.5$.

\subsection{Comparison of emission profiles}

Emission rates predicted for 9 elements from each of the 4 sources are also listed in Table 3. Unfortunately, neither $\mathrm{SO}_{2}$ nor particle-borne elements measurements, against which these results could be substantiated, were available. Nevertheless, a test of "reasonableness" can be made by comparing the ratios of predicted concentrations (i.e., source profiles) with those reported for other coal-burning plants. Coal combustion is the largest source of $\mathrm{SO}_{2}$ emissions in the Pittsburgh area. While there are numerous coal boilers and coking plants, there are no primary metal smelters in the modeling domain. Consequently, $\mathrm{SO}_{2}$ and $\mathrm{Se}$ (a sulfur analog) are themselves, good indicators of coal or coke emissions. Moreover, ambient concentrations (uncorrected) of As and Se were highly correlated $(r=0.987$, see Table 4), as were those of $\mathrm{Pb}, \mathrm{Ni}, \mathrm{Cr}$, and $\mathrm{Cu}$; and their regression equation intercepts were generally small or insignificant with respect to ambient concentrations, suggesting (1) great similarity in the sources of particles affecting air quality at different times of the day and (2) little influence from non-modeled sources.

Moreover, the As/Se ratio is reported to be approximately 1 in air sheds influenced by coal combustion and has been used to distinguish between the influences of coal combustion and other anthropogenic sources (Olmez et al., 1998). Six SO $\mathrm{S}_{2}$ excursions are evident in Figure 3 (i.e., at 04:30-05:30, 07:30, 09:30, 12:30, 14:30-15:30, and 17:00 h) and each corresponds to excursions in Se and As. As shown in Figure 3(b), Se (As) concentrations ranged from 4.4-11.4 $\mathrm{ng} \mathrm{m}^{-3}$ (3.4-9.7 $\mathrm{ng} \mathrm{m}^{-3}$ ) between 04:30-10:00 $\mathrm{h}$ and from 2.4-8.9 $\mathrm{ng} \mathrm{m}^{-3}(1.5-8.3 \mathrm{ng}$ $\mathrm{m}^{-3}$ ) between 14:30-17:30 $\mathrm{h}$, and were substantially elevated over their regional background concentrations. During these periods, As/Se ratios were between 0.8-0.9 (04:30-10:00 h), and 0.63-0.94 (14:30-17:30 h), i.e., in reasonable agreement with a coal combustion source. For periods when impacts of As, Se, and $\mathrm{SO}_{2}$ were minimal, the As/Se ratio was $<0.4$, i.e., substantially different, as could be expected during times when air was more affected by a more diverse mixture of sources.

Comparison of entries in Tables 4 and 5 shows that our PDRM-derived ratios are well within the ranges reported for other Eastern U.S. coal-fired boilers (Gladney 1974; Gladney et al. 1976; Baker et al. 1983; Kowalczyk 1984; Olmez et al. 1988; Park et al. 2005), especially PDRM-derived ratios for 
a coal-fired power plant in Tampa. Such ratios depend on the composition of coal, boiler and exhaust gas residence times and temperatures, and on the type and efficiency of emission control devices (Ondov et al. 1979). Relative to bulk ash composition and its major/minor constituents ( $\mathrm{Si}, \mathrm{Al}$, and $\mathrm{Fe}$ ), concentrations of $\mathrm{Se}, \mathrm{As}, \mathrm{Ni}, \mathrm{Pb}$, and $\mathrm{Zn}$ become enriched in submicrometer coal-combustion particles as a result of differences in volatility and the physics of nucleated condensation (Ondov and Biermann 1980) and these tend to be less efficiently removed by electrostatic precipitators (ESP), further contributing to the "enrichments" observed for emitted particles. Lower enrichments are observed for boilers with inefficient particle control devices, as a greater proportion of larger, less-enriched, particles are emitted. Thus, it is not surprising that $\mathrm{Fe} / \mathrm{Se}$ and $\mathrm{Al} / \mathrm{Se}$ ratios attributed to Bellefield, a plant equipped only with a cyclone, are $\sim 10$ times those attributed to the other Pittsburgh boilers. Such differences are emphasized by normalizing to Se. This is because substantial fractions of Se are present in the gas phase at flue gas temperatures (typically these are in excess of $100^{\circ} \mathrm{C}$ ). The gaseous $\mathrm{Se}$ is not removed by ESPs, but condenses on particles upon cooling after release into the atmosphere (Ondov et al., 1989), and thus leading to smaller element:Se ratios for boilers with highly efficient ESPs. Despite the potential for much variability, some ratios are remarkably consistent in emitted fine particles (see Table 5 footnotes), most notably those for $\mathrm{Pb}, \mathrm{Zn}, \mathrm{Cu}$, and $\mathrm{As}$. The good agreement for this substantial number of components of the PDRM-derived profiles is reassuring.

\section{CONCLUDING REMARKS}

Unlike factor analysis and chemical mass balance models, the hybrid multivariate receptor model used in this study, directly utilizes the numbers and locations of known sources, their geographic relation to the receptor site, and wind direction during sampling, in a Gaussian filter to reduce unwanted contributions. Furthermore, results for individual sources are obtained. These encompass emission rates of primary pollutants from specific sources, meteorological dispersion factors $(\chi / Q$ s $)$ for each source, and the ambient concentrations induced at the receptor site by the modeled sources. Emission rates determined for individual sources the can be readily tested against in-stack measurements. In this study we have obtained highly credible results using ambient concentration data for only 25-30-min measurement periods and commonly available meteorological data, for a modeling domain encompassing complex terrain. The performance measures reported suggest that the PDRM, coupled with highly time-resolved ambient measurements, might be used as a tool to monitor emission rates of $\mathrm{SO}_{2}$ and estimate emission rates of toxic and other non-criteria pollutants without expensive in-stack monitoring.

\section{REFERENCES}

Baker, G., Clarke, P., Gerstle, R., Mason, W., and Phillips, M. (1983). Emission Characterization of Major Fossil Fuel Power Plants in the Ohio
River Valley, Contract No. 68-02-3271, K.T. Knapp, Project Officer, Environmental Sciences Research Laboratory, Office of Research and Development, U.S. Environmental Protection Agency, Research Triangle Park, NC.

Cooper, D. W. (1982). Receptor-Oriented Source-Receptor Analysis. In Proceedings of the Specialty Conference on Receptor Models Applied to Contemporary Pollution Problems; The Air Pollution Control Association, Ed.; Northeast Atlantic International Section of the Air Pollution Control Association, Danvers, MA, pp. 296-316.

Gladney, E. S. (1974). Trace Element Emissions of Coal-Fired Power Plants: A Study of the Chalk Point Electric Generating Station, Ph.D Thesis, University of Maryland, College Park, MD.

Gladney, E. S., Small, J. A., Gordon, G. E., and Zoller, W. H. (1976). Composition and Size Distribution of In-stack Particulate Material at a Coal-Fired Power Plant, Atmos. Environ. 10:1071-1077.

Gordon, G. E. (1982). Receptor Models, Environ. Sci. Technol. 22:1132-1142. Hanna, S. R. (1988). Air Quality Model Evaluation and Uncertainty, JAPCA 38:406-412.

Holzworth, G. C. (1972). Mixing heights, wind speeds, and potential for urban air pollution throughout the contiguous; United States. AP-101; U.S. EPA: Research Triangle Park, NC.

Kidwell, C. B., and Ondov, J. M. (2001). Development and Evaluation of a Prototype System for Collecting Sub-Hourly Ambient Aerosol for Chemical Analysis, Aerosol Sci. Technol. 35:596-601.

Kidwell, C. B., and Ondov, J. M. (2004). Elemental Analysis of Sub-Hourly Ambient Aerosol Collections, Aerosol Sci. Technol. 38:205-218.

Kleissl, J. (2004). Personal communication, March 24.

Kowalczyk, G. S. (1984). Emission and Atmospheric Impact of Trace Elements from a Reconverted Coal-Fired Power Plant, presented at the 77th Annual Meeting of the Air Pollution Control Association, San Francisco, CA, June 24-29.

Kumar, A., Luo, J., and Bennett, G. (1993). Statistical Evaluation of Lower Flammability Distance (LFD) using four Hazardous Release Models, Process Safety Prog. 12:1-11.

Lioy, P. J., Zelenka, M. P., Cheng, M. D., Reiss, N. M., and Wilson, W. E. (1989). The Effects of Sampling Duration of the Ability to Resolve Source Types using Factor Analysis, Atmos. Environ. 23:239-254.

List, R. J. (1966). Smithsonian Meteorological Tables; Sixth Revised Editions (Third reprint); Smithsonian Institution: Washington, DC.

Olmez, I., Ames, M. R., and Gullu, G. (1998). Environ. Sci. Technol. 32:30483054.

Olmez, I., Schefield, A. E., Gordon, G. E., Houck, J. E., Pritchett, L. C., Cooper, J. A., Dzubay, T. G., and Bennett, R. L. (1988). Compositions of Particles from Selected Sources in Philadelphia for Receptor Modeling Applications, JAPCA 38:1392-1402.

Ondov, J. M. et al. (2003). Highly time-resolved measurements of elemental composition at the Baltimore, St. Louis, Pittsburgh, and Tampa Supersites using the UM high-frequency aerosol slurry sampler: Unprecedented resolution of the sources of primary atmospheric aerosol; 2003 PM AAAR meeting; AAAR: Pittsburgh, PA.

Ondov, J. M., Ragaini, R. C., and Biermann, A. H. (1979). Elemental Emissions from a Coal-Fired Power Plant: Comparison of a Ventrui Wet Scrubber System with a Cold-side Electrostatic Precipitator, Environ. Sci. Technol. 13:598607.

Ondov, J. M., and Biermann, A. H. (1980). Application of Surface-Deposition Models to Size-Fractionated Coal Fly Ash, Atmos. Envrion. 14:289295.

Ondov, J. M., Zoller, W. H., and Gordon, G. E. (1982). Trace Element Emissions on Aerosols from Motor Vehicles, Environ. Sci. Technol. 16:318328.

Ondov, J. M., Choquette, C. E., Zoller, W. H., Gordon, G. E., Biermann, A. H., and Heft, R. E. (1989). Atmospheric Behavior of Trace Elements on Particles Emitted from a Coal-Fired Power Plant, Atmos. Environ. 23:21932204. 
Ondov, J. M., Kelly, W. R., Holland, J. Z., Lin, Z., and Wight, S. A. (1992). Tracing Fly Ash Emitted from a Coal-Fired Power Plant with Enriched RareEarth Isotopes, Atmos. Environ. 26B:453-462.

Patel, V. C., and Kumar, A. (1998). Evaluation of Three Air Dispersion Models: ISCT2, ISCLT2, and SCREEN2 for Mercury Emissions in an Urban Area, Environ. Monitor. Assess. 53:259-277.

Pancras, J. P., Ondov, J. M., and Zeisler, R. (2005). Multi-Element Electrical AAS Determination of 11 Marker Elements in Fine Ambient Aerosol Slurry Samples Collected with SEAS-II, Anal. Chimica Acta 538:303-312.

Park, S. S., Pancras, J. P., Ondov, J. M., and Poor, N. J. (2005). A New Pseudodeterministic Multivariate Receptor Model for Individual Source Apportionment using Highly Time-Resolved Ambient Concentration Measurements, J. Geophys. Res. 110:D07S15, doi:10.1029/2004JD004664.

Rheingrover, S. W., and Gordon, G. E. (1988). Wind-Trajectory Method for Determining Compositions of Particles from Major Air Pollution Sources. Aerosol Sci. Technol. 8:29-61.

Seinfeld, J. H., and Pandis, S. N. (1998). Atmospheric Chemistry and Physics: From Air Pollution to Climate Change; John Wiley and Sons: New York.
U.S. EPA (1999). PCRAMMET User's Guide. U.S. EPA Report EPA-454/B-96001. U.S. Government Printing Office: Research Triangle Park, NC.

U.S. EPA. (1995). User's Guide for the Industrial Source Complex (ISC3) Dispersion Models. vol II.-Description of Model Algorithms. Research Triangle Park, NC.

U.S. EPA. (1980). OAQPS Guideline Series. Research Triangle Park, NC.

Turner, D. B. (1964). A Diffusion Model for an Urban Area, J. Appl. Meteor. 3:83-91.

Venkatram, A. (1996). An Examination of the Pasquill-Gifford-Turner Dispersion Scheme, Atmos. Environ. 30:1283-1290.

Wyngaard, J. C. (1988). In Lectures on Air-Pollution Modeling. Venkatram, A., and Wyngaard, J. C., Eds. American Meteorological Society, Boston, pp. 9-61.

Yamartino, R. J. (1982). Formulation and Application of a Hybrid Receptor Model. In Proceedings of the Specialty Conference on Receptor Models Applied to Contemporary Pollution Problems. Northeast Atlantic International Section of the Air Pollution Control Association, Danvers, MA, pp. 285-295, October 17-20. 


\title{
Preface to special section on Particulate Matter Supersites
}

\author{
Spyros Pandis \\ Department of Chemical Engineering, Carnegie Mellon University, Pittsburgh, Pennsylvania, USA
}

Paul A. Solomon

Office of Research and Development, U.S. Environmental Protection Agency, Las Vegas, Nevada, USA

Richard Scheffe

Office of Air Quality Planning and Standards, U.S. Environmental Protection Agency, Research Triangle Park, North Carolina, USA

Published 13 April 2005.

Citation: Pandis, S., P. A. Solomon, and R. Scheffe (2005), Preface to special section on Particulate Matter Supersites, J. Geophys. Res., 110, D07S01, doi:10.1029/2005JD005983.

[1] In July 1997, the U.S. Environmental Protection Agency (EPA) issued new National Ambient Air Quality Standards (NAAQS) for fine particulate matter $\left(\mathrm{PM}_{2.5}\right.$, atmospheric particles with aerodynamic diameters less than $2.5 \mu \mathrm{m})$. The $\mathrm{PM}_{2.5}$ NAAQS was developed by the EPA based on the results of a series of epidemiological studies that found persistent associations between outdoor concentrations of particulate matter (PM) and significant adverse health effects. However, considerable uncertainty remained concerning mechanisms by which various classes of particles might cause adverse health effects, as well as more detailed information on the composition and concentrations of ambient fine PM, that would be critical in implementing the new standards.

[2] An improved understanding of the key sources, development of the most cost-effective control strategies, and health risks associated with $\mathrm{PM}_{2.5}$ requires high-quality measurements of $\mathrm{PM}_{2.5}$ composition, size, and concentration over a variety of spatial and temporal scales. However, many of the measurement methods for $\mathrm{PM}_{2.5}$, capable of resolving size and composition, had not been thoroughly evaluated nor previously deployed in regulatory monitoring networks. In response to this need, EPA embarked on an ambient monitoring research program, commonly referred to as the Particulate Matter Supersites Program [EPA, 2000]. The primary goals of this program are as follows.

[3] 1. Ambient particulate matter and related species at several locations throughout the United States with differing atmospheric environments will be characterized to elucidate source-receptor relationships and atmospheric processes. This will provide the scientific foundation for modeling and data analysis efforts that will support the development of state implementation plans (SIPs) and will develop more effective risk management approaches.

[4] 2. Health effects and exposure research will be supported by providing enhanced atmospheric measurements at ambient sites to obtain an improved understanding of the physical and chemical characteristics of aerosols. This information will help to reduce uncertainty in defining

Copyright 2005 by the American Geophysical Union. 0148-0227/05/2005JD005983\$09.00 and implementing the NAAQS and improve the healthexposure research community's ability to address research questions and scientific uncertainties about PM sourcereceptor-exposure-effects relationships.

[5] 3. Methods testing will be conducted to compare, evaluate, and help validate emerging and routine methods for characterizing PM. Results will help to define and quantify methods uncertainty, to identify methods suitable for routine monitoring, and to further support the first two goals.

[6] The Supersites Program combines a mix of intensive or advanced measurements at a central location combined with other monitoring sites. The program consists of three phases. In Phase I, EPA selected two initial sites: Atlanta, Georgia, and Fresno, California. These sites were selected by virtue of (1) ongoing and planned research activities that closely aligned with those of the PM Supersites Program and (2) distinctly different airsheds (e.g., atmospheric chemistry, sources, etc.). In Phase II, seven additional sites were established, located in Baltimore, Maryland; Fresno, California; Houston, Texas; Los Angeles, California; New York, New York; Pittsburgh, Pennsylvania; and St. Louis, Missouri. Detailed descriptions of the Supersites projects are available at the EPA Supersites Program web page (http://www.epa.gov/ttn/amtic/supersites.html). Phase III, now underway, includes the development of an integrated relational database containing air quality and meteorological data nationally from the end of June 2001 to August 2002 (http://supersitesdata.umd.edu/), the completion of the NARSTO data archive (http://eosweb.larc.nasa. gov/PRODOCS/narsto/table narsto.html), and the development of a key and policy-relevant findings document.

[7] This collection of papers, which is the third coordinated publication of results from the Phase II Supersites Program, reflects the objectives of the program: to characterize particulate matter, to provide information supporting health effects and exposure research, and to develop new methods for characterizing fine particulate matter. Papers describe results from Supersites projects in Pittsburgh, Pennsylvania; Los Angeles, California; Baltimore, Maryland; Houston, Texas; Atlanta, Georgia; the NorthEast Oxidant and Particle Study (NE-OPS); modeling 
papers using data from Pittsburgh and Atlanta, and a paper examining continuous PM mass methods in Los Angeles, California, and Lindon, Utah.

[8] A Phase I Supersites Program special issue describing results from Atlanta is published [Solomon et al., 2003]. The first Phase II Supersites Program special issue is published in Aerosol Science and Technology [Solomon and Allen, 2004], and the second is published in Atmospheric Environment [Solomon, 2004]. Additional coordinated publications of research results are planned to continue to allow the science and policy communities interested in airborne PM to effectively monitor the advances in understanding PM in air made through the Supersites Program.

[9] One of the major objectives of the Supersites Program is to better understand PM accumulation on urban and regional scales, including links between sources and receptors. By addressing this objective, results from the program should improve our approaches to developing efficient and effective emissions management strategies for reducing ambient levels of PM that may adversely impact human heath and welfare. The following policyrelevant findings derive from the measurement and modeling results presented within this special issue and support this objective.

[10] 1. Aerosol composition is different in different particle size modes (ultrafine, accumulation, and coarse), and the composition within these modes varies considerably spatially and temporally, including between source and receptor sites within the same air basin and on timescales ranging from minutes to days as well as by season. These differences reflect the impact of different sources, atmospheric chemical processing, transport, and meteorological conditions both on local and regional scales. These results suggest that different control strategies may be needed in the future depending on location and time of the year.

[11] 2. In the eastern United States, especially during the summer, the aerosol is often acidic. During these conditions the particles remain wet even at low relative humidity. Results presented here clearly indicate that some of the mass associated with the acidic aerosols during these periods is water, even down to $30 \% \mathrm{RH}$.

[12] 3. To account for losses of semi-volatile species, improved estimates of $\mathrm{PM}_{2.5}$ mass can now be obtained using commercially available methods (e.g., FDMS) that measure mass continuously in near-real time with little bias from retention of water or volatilization of semi-volatile species as is observed with the FRM under a variety of conditions (Federal Register, 40 CFR Part 50, vol. 62(138), July 18, 1997).

[13] 4. Primary and secondary organic aerosols contribute to PM concentrations in urban areas in the United States. (1) Primary aerosols dominate during the winter in the east, while secondary organic aerosols (SOA) are important contributors during the summer (approaching $40 \%$ of the OC) with higher contributions observed during ozone episodes (approaching $80 \%$ of the OC). On the basis of results presented within, long-range transport of primary and secondary organic aerosols appear to be important in the eastern United States, suggesting that regional controls on primary organic aerosols may be more effective than local reductions in reducing the organic carbon portion of
PM in this part of the country. There should be expected co-benefits on regional SOA derived from ozone reduction strategies, recognizing that much of the summertime SOA is derived from biogenic sources, and subject to direct emission abatement. (2) In the Houston area, previous results indicate that primary and secondary carbon comprise about equal portions of $\mathrm{PM}_{2.5}$ [Russell and Allen, 2004]. Of the secondary component, most appears to be from biogenic precursors, formed as oxidants created in the Houston urban areas mix with air masses rich in biogenic VOCs emitted to the north of the urban core. However, close to the urban and industrial areas of Houston, aromatic VOCs are significant SOA precursors. This result suggests ambient concentrations of ozone and other oxidants within the greater Houston area may need to be reduced to decrease levels of SOA. (3) Highly time-resolved measurements of aerosol composition (major component and trace elements), size distributions, single particle composition, and VOC concentrations coupled with newly developed mathematical approaches provide unprecedented ability to identify the contribution of primary sources to the observed $\mathrm{PM}_{2.5}$ concentrations in the measurement area. This capability will help guide the refinement of urban based strategies that address the "urban carbon excess" remaining after national rules are implemented that target inorganic precursor gases.

[14] 5. Forest fires in both the eastern and the western United States can lead to high $\mathrm{PM}_{2.5}$ concentrations over large areas. These natural events cannot be controlled, and their impact needs to be considered for areas near attainment of the NAAQS for PM. The integration of satellite data showing plumes from these major natural pollution events will be beneficial to air quality managers by illustrating their spatial and temporal dimensions and identify periods and locations that should be examined for their influence on PM levels.

[15] 6. In the east, ammonia often appears to be the limiting reagent in the formation of nitrate, at least during the winter, when nitrate concentrations are the highest. (1) Significant substitution of sulfate by nitrate is predicted for the winter months in Pittsburgh if $\mathrm{SO}_{2}$ emissions are reduced. This increase in nitrate can be prevented by reductions in $\mathrm{NO}_{\mathrm{x}}$ or $\mathrm{NH}_{3}$ emissions, although $\mathrm{NH}_{3}$ reductions seem to provide the largest benefit. This substitution is predicted to be small during the summer months for the same area. (2) The frequent nucleation bursts observed during clean days in the northeastern United States are due to the sulphuric acid-ammonia-water ternary system. Modeling results indicate that controls of only $\mathrm{SO}_{2}$ emissions by up to $50 \%$ are predicted to increase the frequency of these events in the northeastern United States during the summer and to reduce it during the winter. Controls of $\mathrm{NH}_{3}$ emissions are predicted to decrease the frequency of these events during both the summer and winter. A combination of $\mathrm{SO}_{2}$ and $\mathrm{NH}_{3}$ emission controls can decrease the frequency of nucleation events and the $\mathrm{PM}_{2.5}$ concentration without increasing atmospheric acidity.

[16] 7. Three-dimensional (3-D) chemical transport models (e.g., CMAQ) and modules as well as observationally based models are described and evaluated for performance or process understanding. (1) The available inorganic aerosol thermodynamic models and 3-D chemical transport 
models (e.g., CMAQ) can reproduce the observed partitioning of the major semi-volatile inorganic aerosol components (ammonium, nitrate, and water) within about a factor of 1.5 to 2 on $50-90 \%$ of the data points. However, in many cases the observed discrepancies can be explained by experimental errors. Uncertainty analysis of the predictions of these models is likely needed to have more confidence in future control strategy results. (2) Measurements of the total available (gas and aerosol) ammonia and total nitric acid are important for both the evaluation of the available modeling tools and for use as input to observation-based models that can be used for the design of emission control strategies. For example, nitrate substitution as sulfate levels are reduced resulting in a nonlinear decrease of $\mathrm{PM}_{2.5}$ mass was described by an observationally based model in this special issue. The air quality model and emissions inventory evaluation process would be markedly enhanced with the deployment of ammonia measurements in the national ambient monitoring networks.

[17] Acknowledgments. The United States Environmental Protection Agency through its Office of Research and Development partially funded and collaborated in the research described here under Assistance agreements CR8280257-01 (Desert Research Institute, Reno, Nevada), CR8280258-01-0 (Washington University, St. Louis, Missouri), CR8280259-01-0 (University of California, Los Angeles, California), CR8280260-01-0 (State University of New York, New York, New York), CR8280261-01-0 (Carnegie Mellon University, Pittsburgh, Pennsylvania), CR8280262-01-0 (University of Texas, Austin, Texas), and CR8280263-01-0 (University of Maryland, Baltimore, Maryland). It has been subjected to Agency review and approved for publication. Mention of trade names or commercial products does not constitute endorsement or recommendation for use by EPA.

\section{References}

Environmental Protection Agency (2000), PM Supersites Program background, report, Washington, D. C. (Available at: http://www.epa.gov/ ttn/amtic/files/ambient/super/ssback.pdf 07-01-00)

Russell, M., and D. T. Allen (2004), Seasonal and spatial trends in primary and secondary organic carbon concentrations in southeast Texas, Atmos. Environ., 30(20), 3225-3239.

Solomon, P. A. (2004), Preface: Special issue of Atmospheric Environment on findings from EPA's Particulate Matter Supersites Program, Atmos. Environ., 38(20), 3101-3106.

Solomon, P. A., and D. Allen (2004), Preface: Special issue of Aerosol Science and Technology on findings from the Fine Particulate Matter Supersites Program, Aerosol Sci. Technol., 38(S1), 1-4.

Solomon, P. A., E. Cowling, and R. Weber (Eds.) (2003), Preface to special section: Southern Oxidants Study 1999 Atlanta Supersite Project (SOS3), J. Geophys. Res., 108(D7), 8428, doi:10.1029/2003JD003536. 


\title{
Ambient fine particulate concentrations and chemical composition at two sampling sites in metropolitan Pittsburgh: a 2001 intensive summer study
}

\author{
William K. Modey ${ }^{\mathrm{a}}$, Delbert J. Eatough ${ }^{\mathrm{a}, *}$, Richard R. Anderson ${ }^{\mathrm{b}}$, Donald \\ V. Martello ${ }^{\text {, Satoshi Takahama }}{ }^{\mathrm{c}}$, Leonard J. Lucas ${ }^{\mathrm{c}}$, Cliff I. Davidson ${ }^{\mathrm{c}}$ \\ a Department of Chemistry \& Biochemistry, Brigham Young University, Provo, UT 84602, USA \\ ${ }^{\mathrm{b}}$ National Energy Technology Laboratory, US Department of Energy, Pittsburgh, PA, USA \\ ${ }^{\mathrm{c} C i v i l}$ \& Environmental Engineering, Carnegie Mellon University, Pittsburgh, PA, USA
}

\begin{abstract}
The concentration and chemical composition of ambient fine particulate material $\left(\mathrm{PM}_{2.5}\right)$ is reported for two sampling sites in the Pittsburgh, Pennsylvania metropolitan area: the Department of Energy, National Energy Technology Laboratory (NETL) PM study site south of the city center, and the Carnegie Mellon Pittsburgh Air Quality Study (PAQS) site $5 \mathrm{~km}$ east of central Pittsburgh established with funding by the EPA Supersites Program and by DOE-NETL. Data from these sampling sites were characterized by one to three-day episodes with $\mathrm{PM}_{2.5}$ concentrations (constructed from the sum of the chemical components) exceeding $40.0 \mu \mathrm{g} \mathrm{m}^{-3}$. The episodes were dominated by high concentrations of ammonium sulfate. The fine particle concentrations were compared with meteorological data from surface weather maps and a Hybrid Single Particle Lagrangian Integrated Trajectory model (HYSPLIT model), with back-trajectories estimated over $24 \mathrm{~h}$. High $\mathrm{PM}_{2.5}$ concentrations were associated with transition from a high pressure to a low pressure regime in advance of an approaching frontal system indicating longrange transport of pollutants. In contrast, fine particulate organic material appeared to be dominated by nearby sources. Distinct differences were observed in the diurnal variations in concentration between the two sites. The NETL site showed clear maximum concentrations of semi-volatile organic material (SVOM) during midday, and minimum concentrations of nonvolatile organic compounds in the afternoon. In contrast, the Carnegie Mellon PAQS site showed an absence of diurnal variation in SVOM, but still with minimum concentrations of nonvolatile organic compounds in the afternoon and evening. Neither site showed significant diurnal variation in ammonium sulfate.
\end{abstract}

(C) 2004 Elsevier Ltd. All rights reserved.

Keywords: $\mathrm{PM}_{2.5}$ episode; $\mathrm{PM}_{2.5}$ composition; Pittsburgh; Local emissions; Long-range transport

\section{Introduction}

Fine particulate matter is a ubiquitous component of the atmosphere, and has become a persistent and pervasive environmental problem that imposes significant health risks (Schwartz, 2001; Pope, 2000) and economic costs (Hughes and Lovei, 1999) on society. In 1997, the United States Environmental Protection

\footnotetext{
*Corresponding author. Tel.: + 1-801-422-6040; fax: + 1-801422-0153.

E-mail address: delbert_eatough@byu.edu (D.J. Eatough).
}

Agency (EPA) revised the National Ambient Air Quality Standards for fine particulate matter $\left(\mathrm{PM}_{2.5}\right)$, establishing a 24-h average concentration limit of $65 \mu \mathrm{g} \mathrm{m}^{-3}$, and an annual average concentration of $15 \mu \mathrm{g} \mathrm{m}^{-3}$. Achieving these standards will require strategies for modeling and monitoring airborne concentrations so that appropriate emission reductions can be achieved. This demands an understanding of the factors responsible for current airborne particle levels.

In July 2001, researchers from several universities, research laboratories, and government agencies met to participate in the Pittsburgh Air Quality Study (PAQS), 
a research program funded by the Department of Energy (DOE) and the Supersite Program of the EPA. This was a 1-month intensive measurement campaign to test and compare innovative particle measurement methods, and to improve our scientific understanding of atmospheric particles. Several sites were included in PAQS, with the main site about $5 \mathrm{~km}$ east of downtown Pittsburgh. That location was adjacent to the Carnegie Mellon University (CMU) campus in Schenley Park. One of the additional sampling sites was located at the National Energy Technology Laboratory (NETL), located in the suburban area about $18 \mathrm{~km}$ south of downtown Pittsburgh. This paper compares the diurnal patterns in $\mathrm{PM}_{2.5}$ concentration and chemical composition (including semi-volatile components lost from particles during sampling) at the PAQS site and the NETL site for samples collected using the Particle Concentrator-Brigham Young University Organic Sampling System (PC-BOSS) (Modey et al., 2001; Lewtas et al., 2001).

\section{Experimental}

\subsection{The NETL sampling site}

This site is located at $40.31 \mathrm{~N}$ latitude and $79.98 \mathrm{~W}$ longitude on an open hill at the NETL facility. The site is in a suburban portion of Pittsburgh, $18 \mathrm{~km}$ south of the downtown Pittsburgh area, Fig. 1. The location of the sampling station was chosen to take advantage of an existing meteorological tower which supplies full weather data for the program. Two experimental combustors, located at the NETL facility just $200 \mathrm{~m}$ east of the site, occasionally burn a variety of fuels $\left(230 \mathrm{~kg} \mathrm{~h}^{-1}\right.$ of coal and about $20 \mathrm{kgh}^{-1}$ of various other fuels). About $600 \mathrm{~m}$ ESE is a coal-fired steam plant which burns up to $900 \mathrm{~kg} \mathrm{~h}^{-1}$ to provide heating for the NETL facility. Considering the infrequent occurrence of winds from the direction of these two facilities, and the short time period when these winds are present, the contribution of these nearby sources to $\mathrm{PM}_{2.5}$ at the NETL site appears to be negligible (Anderson et al., 2002). High concentrations of $\mathrm{NO}_{x}$ and $\mathrm{CO}$ identify when these sources impact the NETL site. This occurs less than $0.2 \%$ of the time and the $\mathrm{PM}_{2.5}$ concentration during these time periods changes less than $2 \mu \mathrm{g} \mathrm{m}^{-3}$. Major coal-fired power plants, iron and coke processing facilities, and chemical manufacturing plants are located along the Ohio River Valley to the west and southwest of the NETL site. Some coke and iron processing facilities are also located along the Monongahela River to the east of the facility and on the Ohio River to the north of the facility. These are all potential sources of transported pollutants to the site.

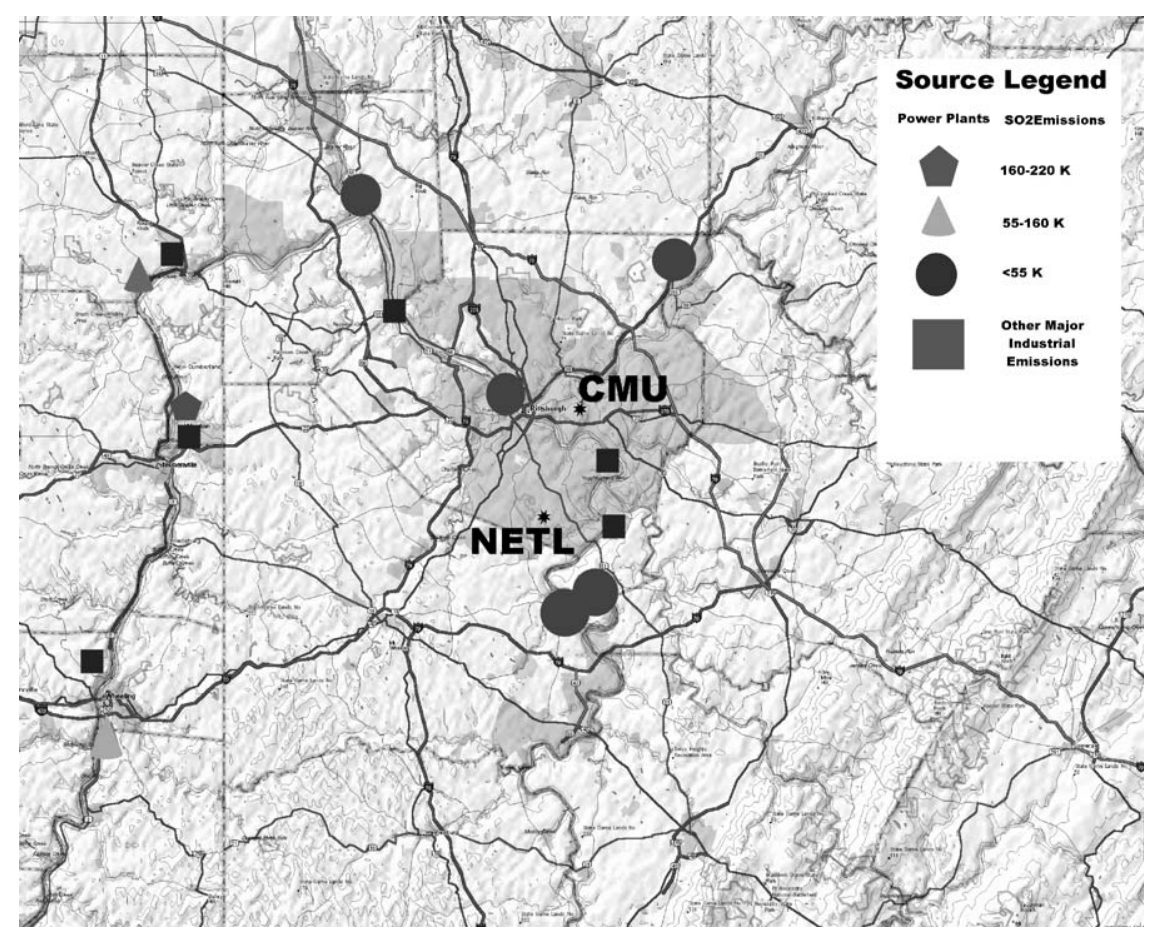

Fig. 1. Map showing location of the CMU and NETL sites in the metropolitan Pittsburgh area (gray shaded in center of figure), main point sources and $100 \mathrm{~m}$ contour lines in the region around Pittsburgh. 


\subsection{The Carnegie Mellon University PAQS site}

The CMU PAQS site is located at $40.44 \mathrm{~N}$ latitude and $79.94 \mathrm{~W}$ longitude in the middle of the Pittsburgh urban area. The site is about $2 \mathrm{~km}$ from the heavily populated and trafficked area of Pittsburgh known as Oakland and $5 \mathrm{~km}$ east of the downtown area, Fig. 1. The Bellefield boiler, a small steam heating plant that burns a mixture of bituminous coal and natural gas, is located about $1 \mathrm{~km}$ to the west. The site is also influenced by minor emissions from the CMU campus. Like the NETL site, the prevailing winds at the CMU site are frequently from the southwest to northwest directions. Many of the same distant industrial sources influencing the NETL site are also likely to impact the CMU site.

Fig. 1 also contains $100 \mathrm{~m}$ topographical contours. These data emphasize that the region to the southwest becomes quite hilly, as the foothills of the central Pennsylvania mountains appear. In addition, the regions surrounding the city to the southwest and north have significant hills (on a $100 \mathrm{~m}$ contour basis). However, the Pittsburgh metropolitan area is much less influenced by hills. While the city is hilly, these height variations are not significant at the $100 \mathrm{~m}$ level.

\subsection{Sampling protocols}

Five sets of PC-BOSS samples were collected each day at the CMU site. The time periods (EST) were: 12:00 a.m. to 6:00 a.m.; 6:00 a.m. to 10:00 a.m.; 10:00 a.m. to 2:00 p.m.; 2:00 p.m. to 6:00 p.m.; and 6:00 p.m. to $12: 00$ a.m. Because of limitations on site access each day, only four sample sets were collected each day at the NETL site using a nearly identical PC-BOSS. The sampling time periods (EST) at this site were: 5:00 a.m. to 10:00 a.m.; 10:00 a.m. to 2:00 p.m.; 2:00 p.m. to 7:00 p.m.; and 7:00 p.m. to 5:00 a.m. the next day. The differences in sampling times between the two sites thus include only one sample collected at night at the NETL site versus two night samples at the CMU site and a 1-h earlier start and end time for the NETL early morning and late evening samples. The latter differences were chosen to match the expected earlier start and end of the morning and evening commutes at the NETL site.

\subsection{The PC-BOSS Samplers}

Details of the PC-BOSS sampler used at both sampling sites have been previously published (Lewtas et al., 2001; Modey and Eatough, 2003). Ambient aerosols were drawn through an inlet that removed particles greater than $\sim 2.5 \mu \mathrm{m}$ in aerodynamic diameter from the air stream. The inlet on the CMU PC-BOSS was an MSP Corporation $\mathrm{PM}_{2.5}$ virtual impactor (MSP Corporation, Minneapolis, MN, USA) with a $2.5 \mu \mathrm{m}$ cut, and that on the NETL PC-BOSS was a Bendix cyclone with a $2.4 \mu \mathrm{m}$ cut (Chan and Lippmann, 1977). Inlet flows were $140-1501 \mathrm{~min}^{-1}$.

After the inlet and a small mixing chamber, $151 \mathrm{~min}^{-1}$ was removed into a side flow manifold which held a single filter pack containing a $47-\mathrm{mm}$ diameter Whatman Nuclepore filter $(0.4 \mu \mathrm{m}$ pore, Whatman Inc., Clifton, NJ) for sulfate determination, and a $47-\mathrm{mm}$ diameter charcoal-impregnated glass fiber filter (CIG) (Schleicher and Schuell, Dassel, Germany) for volatile organic material (VOM) determination. Samples collected on the Nuclepore filters were concentrated in a $4-\mathrm{cm}^{2}$ area on the filter using a stainless steel masking disk, positioned underneath the Nuclepore filter. The remainder of the sampled air stream entered a virtualimpactor particle concentrator whose design and evaluation have been described (Sioutas et al., 1994; Ding et al., 2002). The particle concentrator separated $75 \%$ of the gas phase material into the major flow and left particles larger than the cut point $(>0.1 \mu \mathrm{m})$ along with a significantly reduced fraction of the gas phase material in the minor flow channel. The minor flow stream containing concentrated particles entered a diffusion denuder comprised of parallel strips of charcoalimpregnated cellulose fiber filter (CIF, Schleicher and Schuell, Keene, $\mathrm{NH}$ ) which were separated at the long edges by $2-\mathrm{cm}$ diameter glass rods. With most of the gas phase species removed through the particle concentrator prior to passage of aerosol through the denuder, efficiency of the denuder for removal of gas phase $\mathrm{SO}_{2}, \mathrm{HNO}_{3}$, and organic compounds is expected to equal or exceed $99 \%$ (Lewtas et al., 2001; Eatough et al., 1999; Ding et al., 2002).

The denuder was followed by two parallel filter packs. One contained a 47-mm Pallflex quartz filter (Gelman Sciences, Ann Arbor, MI) followed by a $47-\mathrm{mm}$ diameter CIG filter. The first filter was used to determine fine particulate elemental carbon (EC), and nonvolatile organic material (NVOM), sulfate and nonvolatile nitrate concentrations. The CIG filter was used to capture semi-volatile organic material (SVOM) evolved from the quartz filter. Particulate samples collected on both of these filters were concentrated on a $4-\mathrm{cm}^{2}$ area on the filters using the mask described earlier for the Nuclepore filter. The other parallel filter pack contained a 47-mm diameter ring-supported Teflon filter (Whatman Inc.) followed by a 47-mm diameter Nylasorb filter (Gelman Sciences). Unlike sample collection on the quartz and CIG filter pack, collection on the Teflon and Nylasorb filters was over the entire filter surfaces. The Teflon filters were analyzed for sulfate and nonvolatile nitrate, and the Nylasorb filters were analyzed for nitrate lost from particles during sampling. With these combinations of sample analysis techniques on both parallel channels of the minor flow of the sampler, negative and positive sampling artifacts were minimized. 
The sulfate determination on the side flow Nuclepore filter allowed for the direct determination of the particle concentrator efficiency and losses (Lewtas et al., 2001; Modey et al., 2001) when regressed against sulfate determinations on the minor flow quartz and Teflon filters. Previous studies in similar environments in the eastern United States (Pang et al., 2002; Modey et al., 2001) have shown that efficiencies calculated from sulfate, OM or EC data are all similar. However, only sulfate data were available for this calculation in the data reported here. Particle size dependence of the fine particle components indicated that the mass median diameters for sulfate and carbon materials are similar and also, not close to the lower cut point of the particle concentrator, indicating efficiencies for all components should be comparable (Cabada et al., 2004).

\subsection{Filter pre-treatment}

All quartz filters were baked in an oven at $800^{\circ} \mathrm{C}$ for $10 \mathrm{~h}$ before use. Such treatment is likely to alter the sorption sites for SVOM, and cause higher SVOM losses from the quartz filters. This may account for differences in the split between NVOM and SVOM determined with the PC-BOSS, compared to the simultaneous sampling with a diffusion denuder system (Subramanian et al., 2004). However, the sum of EC, NVOM and SVOM determined by the two systems in 24-h sampling periods agreed $\left(n=14, \quad R^{2}=0.92, \quad\right.$ regression slope $=1.01)$. This is a further indication that the particle concentrator efficiency based on sulfate measurements was also valid for the carbonaceous material. The CIG sorbent filters used in the study were baked in an oven at $340^{\circ} \mathrm{C}$ for $24 \mathrm{~h}$ in an atmosphere of nitrogen. Such treatments were to ensure that the filters contained minimal residual organic material prior to being used for sampling. The Nuclepore, Teflon, and Nylasorb filters and the denuder CIF strips were used as received from the manufacturers without any pre-treatment.

\subsection{Analysis of collected carbonaceous material}

A $2-\mathrm{cm}^{2}$ portion from the center of each quartz filter were analyzed for elemental carbon (EC) and nonvolatile organic material (NVOM) by thermal desorption of the collected materials using temperature programmed volatilization (Eatough et al., 1993; Ellis and Novakov, 1982). Similarly sized portions from the centers of the CIG filters were analyzed for semi-volatile organic material (SVOM). Both EC and NVOM or SVOM were converted to $\mathrm{CO}_{2}$ over a barium chromate combustion catalyst (UIC Inc., IL) at $800^{\circ} \mathrm{C}$ and the $\mathrm{CO}_{2}$ concentration was then determined using nondispersive infrared (NDIR) detection on an ULTRAMAT 3 - Gas Analyzer (Siemens Inc., GA). The NDIR detector was calibrated using three certified $\mathrm{CO}_{2}$ standards which spanned the analysis concentration range. The CIG filters were heated from $50^{\circ} \mathrm{C}$ to $340^{\circ} \mathrm{C}$ at a ramp rate of $15^{\circ} \mathrm{C} \mathrm{min}^{-1}$ in a stream of nitrogen. The maximum temperature for the CIG analysis was imposed by the fact that degradation of the filter charcoal began $10-20^{\circ} \mathrm{C}$ below the maximum analysis temperature. Collected SVOM lost from the particles were evolved from the CIG between $200^{\circ} \mathrm{C}$ and $300^{\circ} \mathrm{C}$. The quartz filters were heated from $50^{\circ} \mathrm{C}$ to $800^{\circ} \mathrm{C}$ at a ramp rate of $28^{\circ} \mathrm{C} \mathrm{min}^{-1}$ in a stream of $\mathrm{N}_{2} / \mathrm{O}_{2}(70: 30 \%$ $\mathrm{v} / \mathrm{v})$. Soot was estimated from the high temperature (usually above $440^{\circ} \mathrm{C}$ ) peak (Ellis and Novakov, 1982) on the thermogram obtained from the quartz filter analysis. Because of the frequent presence of high concentrations of nonvolatile organic material that evolve at a temperature just below that for EC, the uncertainty in this estimate varied from about $30-70 \%$. However, EC was a small fraction of the total carbonaceous material. The shape of the thermograms indicated that all collected organic compounds and soot were evolved from the collection matrix at the maximum temperatures used. No evidence for the high-temperature evolution of $\mathrm{CO}_{2}$ from inorganic carbonates was seen. Field handled blank determinations were made for both quartz and CIG filters. The blank filter thermograms were used to correct the sampled filter thermograms for blank filter material. The variability in blank filters was less than $\pm 3 \mu \mathrm{gC}$ filter $^{-1}$, corresponding to an uncertainty in the blank correction of less than $0.4 \mu \mathrm{g} \mathrm{C} \mathrm{m}^{-3}$.

\subsection{Analysis for nitrate and sulfate}

Sulfate and nitrate analysis was by ion chromatography. All ion chromatographic analyses were performed using a Dionex LC20 Chromatograph equipped with a CD20 conductivity detector. The eluant was a $3.5 \mathrm{mM}$ $\mathrm{Na}_{2} \mathrm{CO}_{3} / 1.0 \mathrm{mM} \mathrm{NaHCO}$ solution used at a flow rate of $1.2 \mathrm{ml} \mathrm{min}^{-1}$. An anion self-generating suppressor was used. One $2-\mathrm{cm}^{2}$ punch of the quartz filters was extracted by ultrasonication with deionized water in a monovette (Sarstedt, Newton, NC). The extracted solution was analyzed for sulfate and nitrate. Whole Teflon filters and half of each Nuclepore filter were also extracted by ultrasonication in deionized water in a monovette and analyzed for sulfate and nitrate. Whole Nylasorb filters were extracted ultrasonically in the ion chromatography eluant solution and analyzed for nitrate. Field handled blank determinations were made for all filters.

\section{8. $\mathrm{O}_{3}, \mathrm{NO}_{x}$ and $\mathrm{NO}_{2}$ Data}

Hourly averaged concentrations of $\mathrm{O}_{3}, \mathrm{NO}_{x}$ and $\mathrm{NO}_{2}$ were determined by NETL (Anderson et al., 2004) and 
CMU (Wittig et al., 2004) using continuous gas monitors. These data were combined to give average concentrations of each gas phase species for the various PC-BOSS sampling times.

\section{Results and discussion}

Results for the studies conducted at both sites showed periods lasting over $24 \mathrm{~h}$ when peak constructed $\mathrm{PM}_{2.5}$

July, 2001 at NETL
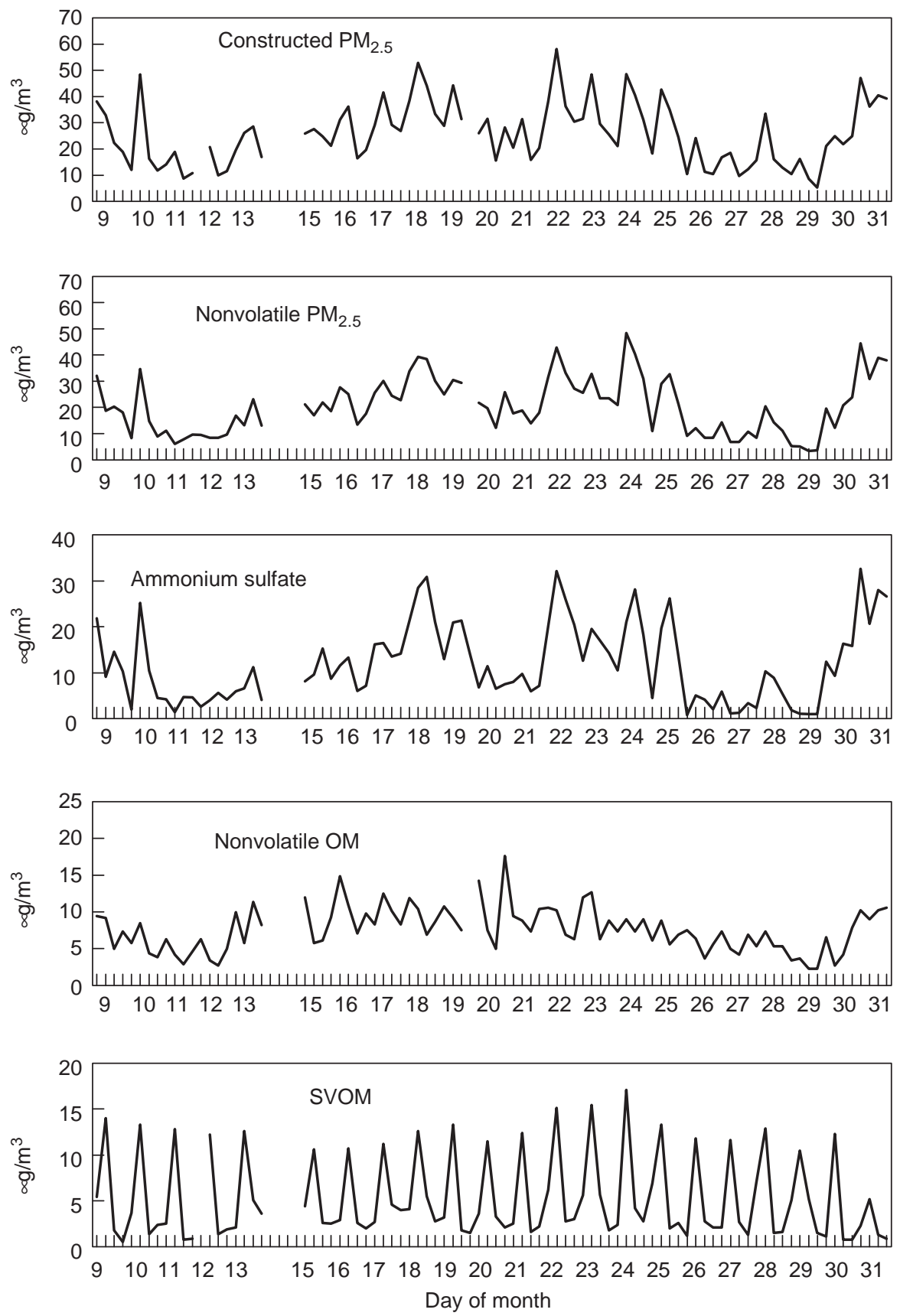

Fig. 2. $\mathrm{PM}_{2.5}$ concentrations at the NETL and Carnegie Mellon University PAQS sites from 9-31 July, 2001. At the NETL site, the first tick mark for each day on the horizontal axes is for the first sampling period starting from 5:00 a.m. to 10:00 a.m. At the PAQS site the first tick mark for each day on the horizontal axes is for the first sampling period starting from 12:00 a.m. to 6:00 a.m. 

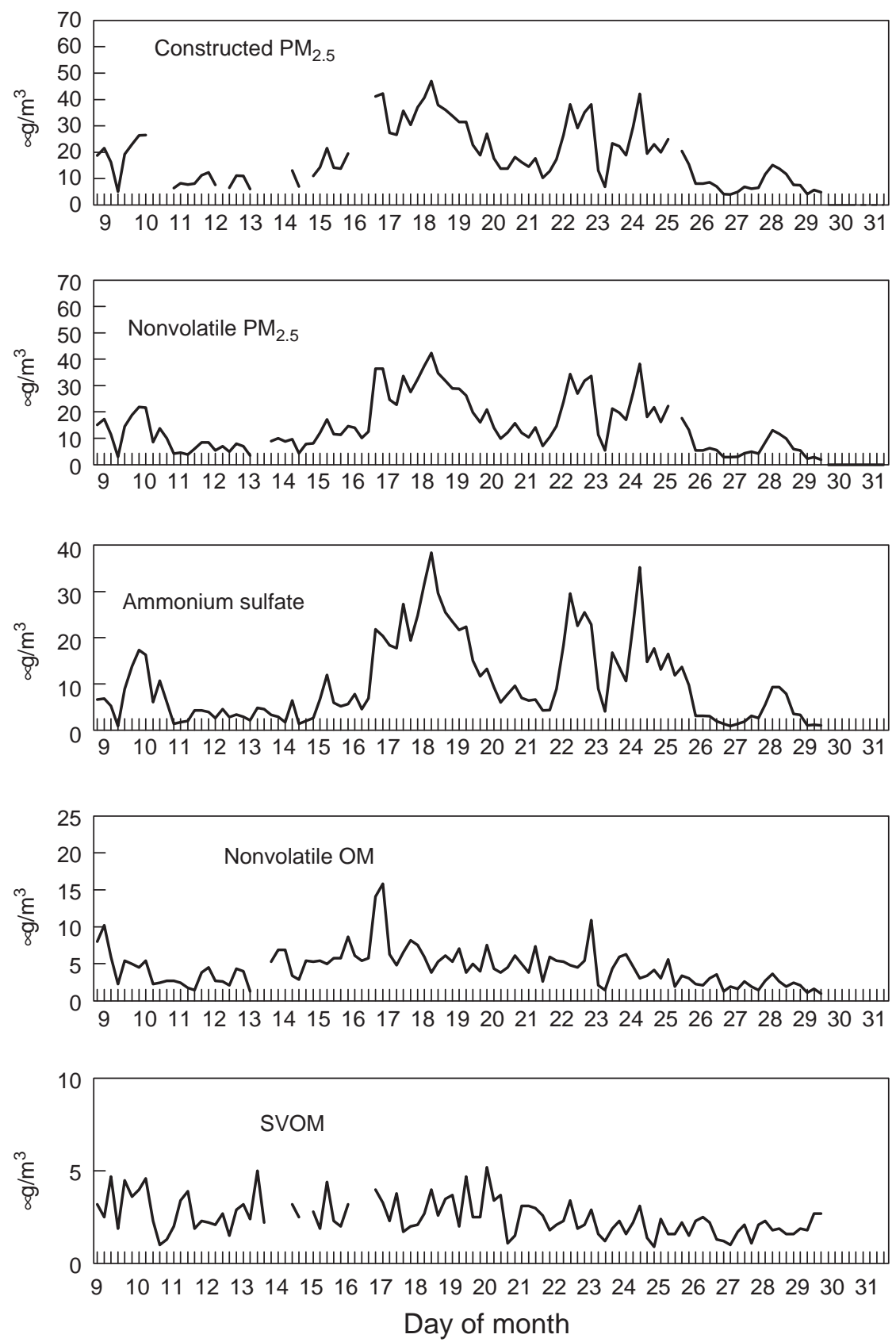

Fig. 2 (continued).

concentrations exceeded $30.0 \mu \mathrm{g} \mathrm{m}^{-3}$, which is considered to be an episode. Constructed nonvolatile $\mathbf{P M}_{2.5}$ was calculated as the sum of the concentrations of ammonium sulfate and ammonium nitrate determined on the Teflon filter, and EC, and nonvolatile organic material (NVOM) determined on the quartz filter. Constructed $\mathrm{PM}_{2.5}$ was then calculated as the sum of the constructed nonvolatile $\mathrm{PM}_{2.5}$ concentration, and lost ammonium nitrate and SVOM determined on the Nylasurb and CIG filters, respectively. It was assumed that gas phase VOM and particulate nonvolatile NVOM and SVOM were $61 \%$ carbon by mass (Turpin and Lim, 2001). The same factor was used to convert OC to OM for the VOM, NVOM and SVOM fractions, since the 
Table 1

Diurnal concentrations in $\mu \mathrm{g} \mathrm{m}^{-3}$ for constructed $\mathrm{PM}_{2.5}$ and the three major $\mathrm{PM}_{2.5}$ components and for gas phase VOM $\left(\mu \mathrm{g} \mathrm{m}{ }^{-3}\right.$ ) and $\mathrm{NO}_{x}(\mathrm{ppb})$

\begin{tabular}{|c|c|c|c|c|c|c|c|}
\hline Period of day & Category & Constructed $\mathrm{PM}_{2.5}$ & $\left(\mathrm{NH}_{4}\right)_{2} \mathrm{SO}_{4}$ & NVOM & SVOM & VOM & $\mathrm{NO}_{x}$ \\
\hline \multicolumn{8}{|c|}{ (a) NETL site in Pittsburgh } \\
\hline \multirow[t]{3}{*}{ 5:00 a.m. to $10: 00$ a.m } & Average & 24.4 & 10.2 & 8.9 & 3.7 & 13.9 & 17.2 \\
\hline & Maximum & 47.1 & 32.6 & 14.9 & 7.1 & 23.1 & 32.4 \\
\hline & Minimum & 10.4 & 0.8 & 3.3 & 1.1 & 5.6 & 6.8 \\
\hline \multirow[t]{3}{*}{ 10:00 a.m. to $2: 00$ p.m. } & Average & 34.9 & 13.0 & 7.8 & 12.4 & 13.5 & 8.7 \\
\hline & Maximum & 58.1 & 32.2 & 12.7 & 17.1 & 25.2 & 18.3 \\
\hline & Minimum & 16.2 & 1.1 & 2.8 & 5.2 & 7.1 & 4.1 \\
\hline \multirow[t]{3}{*}{ 2:00 p.m. to $7: 00$ p.m. } & Average & 23.3 & 13.8 & 6.0 & 2.8 & 10.6 & 6.0 \\
\hline & Maximum & 44.0 & 30.9 & 11.4 & 5.7 & 17.2 & 8.9 \\
\hline & Minimum & 8.6 & 1.0 & 2.2 & 0.8 & 4.1 & 2.9 \\
\hline \multirow[t]{3}{*}{ 7:00 p.m. to 5:00 a.m. } & Average & 20.7 & 10.4 & 7.7 & 2.0 & 15.9 & 12.4 \\
\hline & Maximum & 39.1 & 26.6 & 17.6 & 4.0 & 24.2 & 26.0 \\
\hline & Minimum & 5.2 & 1.0 & 2.3 & 0.5 & 11.1 & 9.1 \\
\hline \multicolumn{8}{|c|}{ (b) Carnegie Mellon University PAQS site in Pittsburgh } \\
\hline \multirow[t]{3}{*}{ 12:00 a.m. to $6: 00$ a.m. } & Average & 22.1 & 11.4 & 5.9 & 2.6 & 24.0 & 24.4 \\
\hline & Maximum & 48.9 & 36.0 & 14.1 & 5.0 & 53.8 & 46.8 \\
\hline & Minimum & 4.0 & 1.4 & 1.3 & 0.9 & 8.2 & 6.6 \\
\hline \multirow[t]{3}{*}{ 6:00 a.m. to $10: 00$ a.m. } & Average & 21.3 & 10.5 & 6.5 & 3.1 & 28.1 & 28.6 \\
\hline & Maximum & 42.1 & 24.8 & 15.8 & 6.2 & 68.3 & 68.5 \\
\hline & Minimum & 3.9 & 0.9 & 1.9 & 1.0 & 5.3 & 5.9 \\
\hline \multirow[t]{3}{*}{ 10:00 a.m. to $2: 00$ p.m. } & Average & 20.6 & 12.0 & 4.9 & 3.0 & 24.3 & 10.7 \\
\hline & Maximum & 43.2 & 31.7 & 10.8 & 8.4 & 59.6 & 24.2 \\
\hline & Minimum & 4.0 & 1.0 & 1.1 & 1.6 & 5.2 & 5.1 \\
\hline \multirow[t]{3}{*}{ 2:00 p.m. to $6: 00$ p.m } & Average & 22.3 & 11.8 & 4.2 & 3.7 & 18.7 & 7.7 \\
\hline & Maximum & 65.1 & 49.6 & 12.9 & 7.9 & 44.4 & 13.6 \\
\hline & Minimum & 5.0 & 0.9 & 1.4 & 1.1 & 2.1 & 5.0 \\
\hline \multirow[t]{3}{*}{ 6:00 p.m. to $12: 00$ a.m. } & Average & 16.4 & 10.0 & 3.6 & 2.0 & 20.5 & 14.3 \\
\hline & Maximum & 37.9 & 29.5 & 6.5 & 4.5 & 48.3 & 24.2 \\
\hline & Minimum & 4.7 & 0.9 & 0.8 & 1.0 & 4.0 & 6.3 \\
\hline
\end{tabular}

Maximum concentrations given are for the entire period for the given sampling period.

relative importance of primary and secondary organic material in these fractions is not identifiable from the data given here. However, if the SVOM is dominantly secondary in nature, as suggested in the discussion, the factor used to convert from SVOC to SVOM may be too small and the SVOM concentrations may be relatively somewhat higher than given here (Turpin and Lim, 2001). Sulfate and nitrate determined by ion chromatography were assumed to be present as ammonium sulfate and ammonium nitrate, respectively. These same assumptions and calculations were used for both the NETL and Carnegie Mellon PAQS site PC-BOSS data. The resulting concentrations are shown in Fig. 2 with average, maximum and minimum concentrations given in Table 1.

\subsection{Gas phase $N O_{x}$ and VOM diurnal patterns}

The diurnal variations in $\mathrm{NO}_{x}$ and VOM gas phase concentrations are given in Figs. 3 and 4, respectively.
Sampling period average, maximum and minimum concentrations of these species are given in Table 1 . The diurnal variations in the concentrations of both these species will be dependent on a combination of diurnal variations in emission and in meteorology. For species which are emitted in the metropolitan area, if emission rates are constant, concentrations will be the highest during the night-time, with the presence of a stable night-time mixed layer, and lowest during midday and afternoon when the atmosphere is well mixed. For species associated with mobile emissions, concentrations will be high during the early morning commute, and low during the middle of the day when traffic density is lower and the atmosphere is well mixed. Concentrations would also be expected to be low during the evening commute because of the well-mixed atmosphere. However, even though traffic is reduced during the night, concentrations may increase due to the presence of a relatively shallow, stable mixed layer. Examination of the data in Table 1 and in Fig. 3 both 

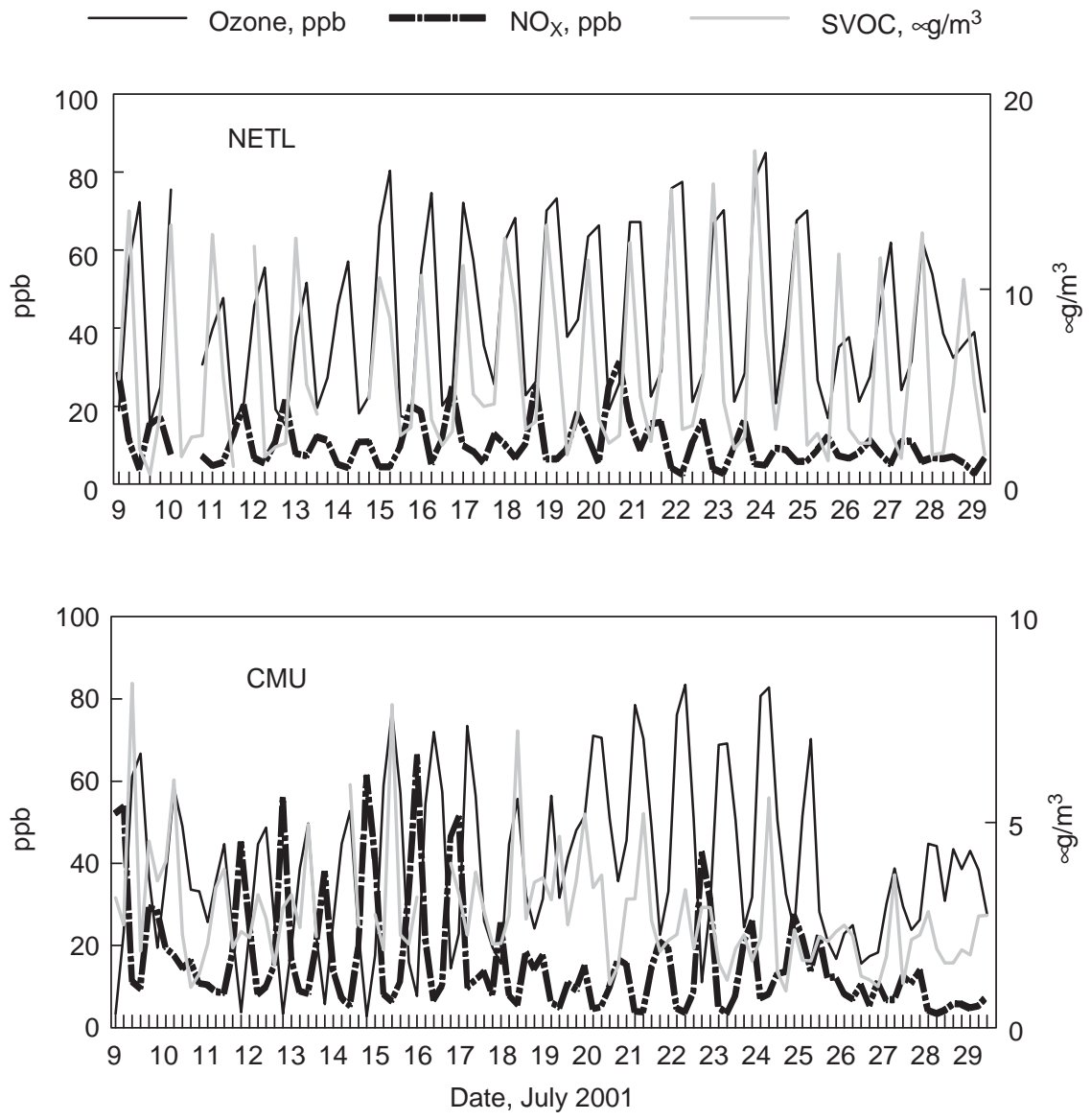

Fig. 3. Diurnal $\mathrm{NO}_{x}$, Ozone and SVOM concentrations at the two sampling sites.

suggest this is the case for $\mathrm{NO}_{x}$ at both the NETL and CMU sites, indicating mobile emissions are a significant contributor to $\mathrm{NO}_{x}$ at both sites. Furthermore, the concentrations of $\mathrm{NO}_{x}$ are consistently higher at the CMU, compared to the NETL site, especially for the early morning time period, consistent with the higher traffice density in the downtown region close to the CMU site, compared to the more suburban NETL site, Fig. 1. The picture is similar for VOM with highest concentrations always present at the CMU site, compared to the NETL site. However, the significant drop in concentrations of $\mathrm{NO}_{x}$ seen in the mid-day, compared to early morning samples, is less pronounced for VOM. This suggests other mid-day VOM sources may be present, for example vegetative emissions, or increased emissions of VOM compared to $\mathrm{NO}_{x}$ from combustion sources, including mobile sources as the day progresses. There is, however, no reason to believe vegetative sources will be more important at either one of the two sites. The increased importance of VOM, relative to $\mathrm{NO}_{x}$, may reflect a changing composition of mobile sources as the morning commute passes.

\section{2. $P M_{2.5}$ diurnal distribution}

Fig. 2 shows the diurnal patterns for constructed $\mathrm{PM}_{2.5}$ mass concentrations and composition at the two sampling sites. Highest constructed $\mathrm{PM}_{2.5}$ concentrations at the NETL site were observed during the sample collection period each day from 10.00 a.m. to 2.00 p.m. (indicated by the second and third vertical tick marks each day). These maxima are greatly influenced by the striking pattern in SVOM concentrations shown at the bottom of Fig. 2. In contrast, the constructed $\mathbf{P M}_{2.5}$ concentrations at the CMU site do not show a strong diurnal variation. Averages, maxima and minima in the different patterns between the two sites are given in Table 1. The NETL site shows an average SVOM concentration of $12.4 \mu \mathrm{g} \mathrm{m}^{-3}$ and an average constructed $\mathrm{PM}_{2.5}$ mass concentration of $34.9 \mu \mathrm{g} \mathrm{m}^{-3}$ during 10:00 a.m. $-2: 00$ p.m., both values well above the averages for any of the other time periods. The CMU site shows very similar average SVOM and constructed $\mathrm{PM}_{2.5}$ concentrations in all five time periods. Table 1 indicates that ammonium sulfate is the largest single component 

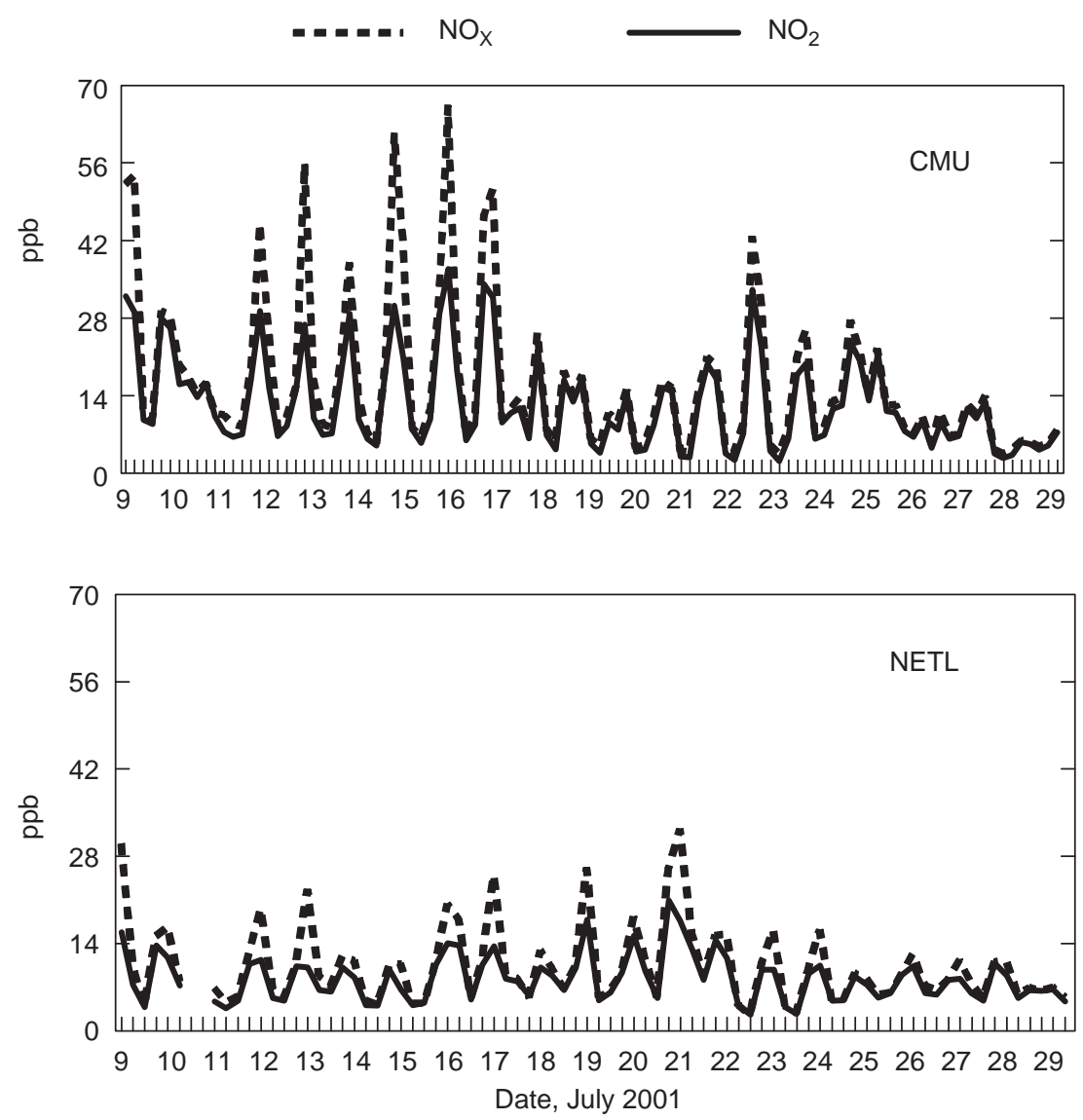

Fig. 4. Diurnal concentrations of $\mathrm{NO}_{x}$ and $\mathrm{NO}_{2}$ at the two sampling sites.

affecting constructed $\mathrm{PM}_{2.5}$ concentrations at both sites. The high SVOM concentration at the NETL site during the peak time period is also a significant contributor to constructed $\mathrm{PM}_{2.5}$ at that site. A comparison of the diurnal variations in SVOM, ozone (Fig. 3), and $\mathrm{NO}_{x}$ at the two sampling sites shows that maximum SVOM concentrations coincide with high ozone concentrations during mid-day, possibly indicating a high occurrence of secondary SVOM. It is clear that this high SVOM is not associated with the morning commute since maximum ozone and SVOM concentrations are observed hours after maximum $\mathrm{NO}_{x}$ concentrations as shown in Fig. 3 and Table $1 . \mathrm{NO}_{x}$ is principally associated with fresh or primary emissions, and maximum concentrations are observed during early morning and night-time periods as discussed above. The lack of a continued rise in SVOM, with continued high ozone concentrations, during the late afternoon suggests that the apparent conversion of VOM to SVOM at the NETL site is rapid, and does not involve VOM emitted later in the day, e.g. from vegetative sources. Fig. 4 (trends in $\mathrm{NO}_{x}$ at the two sampling sites) and Fig. 5 (trends in VOM, and SVOM at the two sampling sites) in combination with Fig. 3 suggest the importance of $\mathrm{NO}_{x}$, compared to SVOM chemistry at the CMU site but a shift to more SVOM chemistry at the NETL site. A connection between the two phenomena is not obvious, and it is not expected that the two different phenomena would occur in the same city. The observed differences are, presumably, linked to the differences in the importance of organic versus $\mathrm{NO}_{x}$ emissions by nearby sources at the two sampling sites. For example, higher concentrations of $\mathrm{NO}_{x}$ at the CMU site may favor $\mathrm{NO}_{x}$ over organic compound chemistry. The high concentrations and regular diurnal pattern of SVOM at the NETL site may be due to a suburban source, but the nature of this source is presently unknown.

High concentrations of NVOM were frequently observed during the time period from 5.00 a.m. to 10.00 a.m. (9, 11, 12, 15, 16, 18, 19, 26, 27 July), and occasionally in the night-time (20, 21, 31 July). As discussed above, the NVOM measured at these times is likely to be associated with local sources. But Fig. 6 shows that concentrations at the NETL site were 

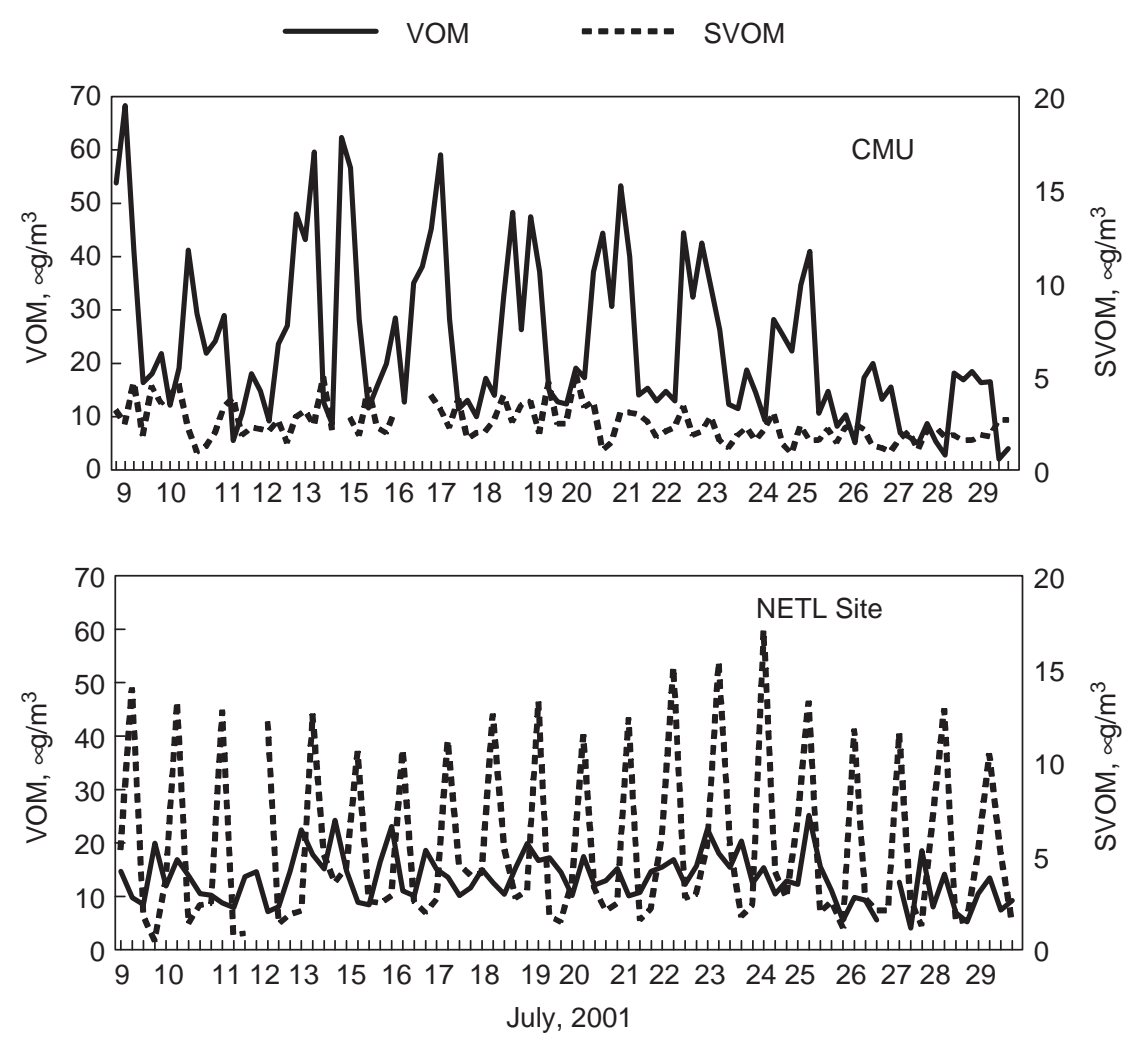

Fig. 5. Diurnal concentrations of gas phase volatile organic material (VOM) and SVOM at the two sampling sites.

consistently higher than those at CMU for all sampling time periods. Therefore, it is possible that $\mathrm{OM}$ emission sources near the NETL site may be lower in $\mathrm{NO}_{x}$ content, or that organic material impacting the NETL site includes more secondary material. This would be consistent with the increased importance of secondary SVOM at the NETL site as noted above.

Fig. 6 shows that ammonium sulfate concentrations during the midday and late afternoon sampling periods at both sites were comparable for most of the samples, suggesting similar concentrations over a large region which is probably due to long-range transport. The sulfate formed during long-range transport will be dominated by homogeneous chemistry. The transported sulfate observed at the site is formed during and is present at the site in clear sky conditions, in advance of, not associated with a frontal passage. Thus, homogeneous chemistry would be expected to dominate in the formation of sulfate. This expectation is also consistent with the low MMD observed for sulfate under these conditions (Cabada et al., 2004). However, during the early morning sampling period, differences in ammonium sulfate concentrations at the two sites were observed, consistent with the decoupling of surface air from elevated layers and the importance of varying low concentrations of sulfate from local sources. When pollutants are locally generated, they are likely to be non-uniformly distributed at the two sites because the source contributions impacting the sites may be diverse in nature.

\subsection{Meteorological influence on $P M_{2.5}$ distribution and episode occurrence}

Previous work has explored the relationship between meteorology and the occurrence of fine particle episodes in the Pittsburgh area (Anderson et al., 2002). The use of surface weather maps (UNISYS, 2002) and the HYSPLIT back-trajectory model (NOAA, 2002) indicated that high $\mathrm{PM}_{2.5}$ episodes in Pittsburgh were associated with transitions from locally high pressure to low pressure regimes, and that fine particle episodes were most commonly associated with pollutant transport from the west, southwest, and northwest (Anderson et al., 2002; Modey and Eatough, 2002). Similar transport patterns were observed in the present study. For example, the high concentration of particulate matter observed at both sampling sites on 18 July was preceded by a period of high pressure on 16 and 

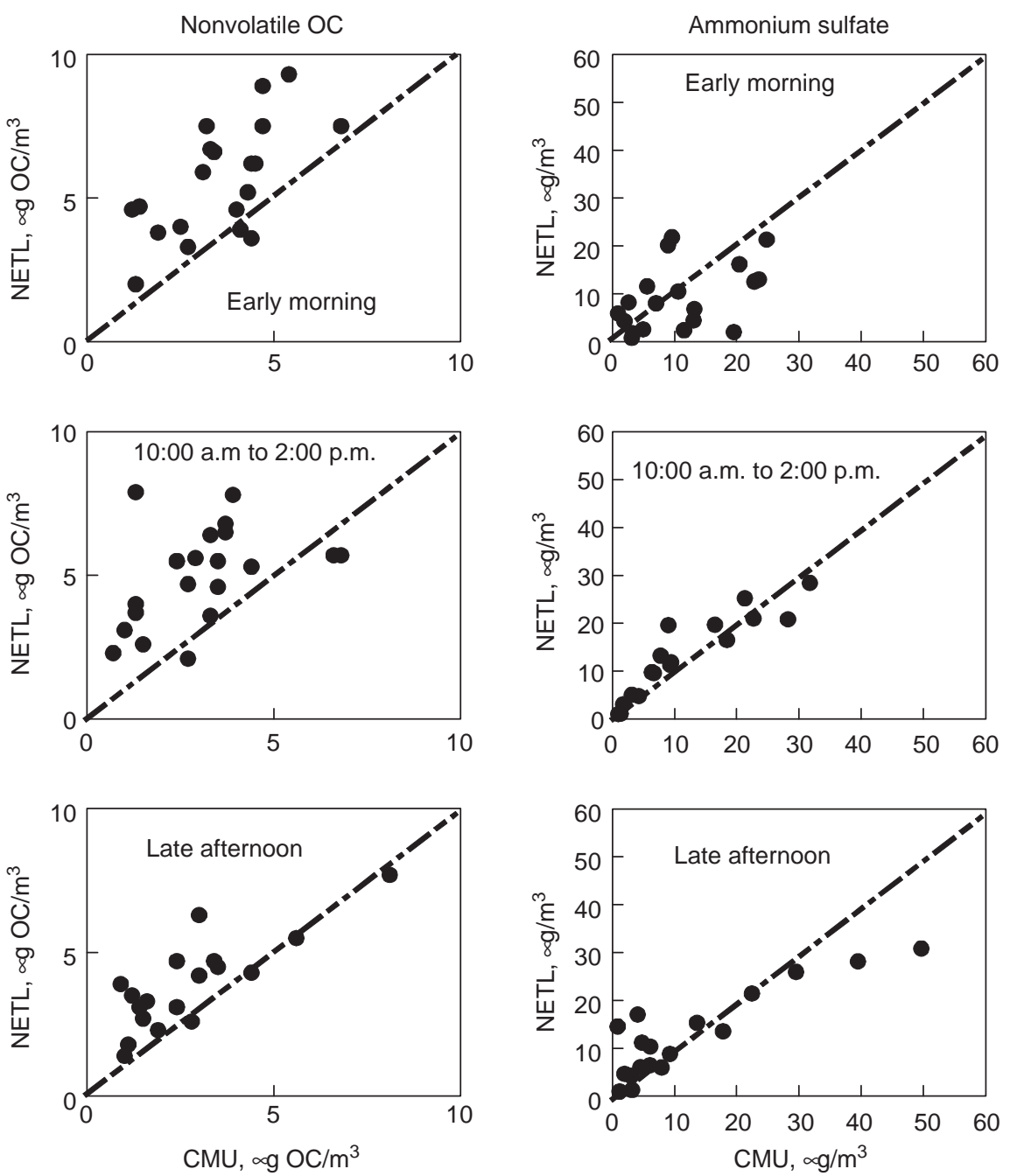

Fig. 6. Plots showing the diurnal distribution of ammonium sulfate and NVOM at both sampling sites. The 1:1 line represents data from Carnegie Mellon University (CMU) PAQS site, and data points in closed circles represent data from the NETL site.

17 July. Pollutant concentrations increased with the approach of a frontal system and the development of low pressure on 18 July. In the previous studies (Anderson et al., 2002; Modey and Eatough, 2003), high concentrations similar to that reported for 18 July were generally shown to be associated with transport from the southwest and the west. In contrast, transport at the start of the episode on 18 July was from the east, with short-term $\mathrm{PM}_{2.5}$ concentrations exceeding $50 \mu \mathrm{g} \mathrm{m}^{-3}$ at both sites. Peak concentrations of ammonium sulfate exceeded $30 \mu \mathrm{g} \mathrm{m}^{-3}$ (Fig. 2), with 24-h averages above $24 \mu \mathrm{g} \mathrm{m}^{-3}$ (Table 2). Transport from the east may include emissions from the Monongahela River coke and steel industries (Fig. 1) as well as from coal-fired power plants east of Pittsburgh.
In this study, comparably high concentrations of ammonium sulfate were also observed at both sampling sites during pollutant transport from the west $(9,10,17$, 24, 25 July) and southwest (16, 22, and 23 July) (Table 2). Transport from these directions may include emissions from coal-fired power plants and the various coke processing and steel manufacturing plants located along the Ohio River valley to the west of both sampling sites, Fig. 1. Much lower pollutant concentrations were seen with transport from the NW $(12,13,15$, and 26 July) as shown in Table 2, and from the SE (19, 20, 27 July). Similarly, low ammonium sulfate concentrations were also associated with some incidences of transport from the southwest ( $16 \mathrm{July}$ ), and the west (9 July).

In contrast to episodes of long-range transported pollutants, the episode on 31 July was locally influenced. 
Table 2

Relationship between the frequent meteorological transport pathways and composition of constructed $\mathrm{PM}_{2.5}$ and the major components

Dates 24-h Back-trajectory Concentration

(a) NETL site in Pittsburgh

July 9, 10, 17, 24, 25

July 16, 22, 23

July $12,13,15,26$

July 18

(b) Carnegie mellon PAQS site in Pittsburgh July 9, 10, 17, 24, 25

July 16, 22, 23

July $12,13,15,26$

July 18
East

Constructed $\mathrm{PM}_{2.5}$

Ammonium sulfate

Nonvolatile OM

Southwest

\section{SVOM}

Constructed $\mathrm{PM}_{2.5}$

Ammonium sulfate

Nonvolatile OM

SVOM

Northwest

Constructed $\mathrm{PM}_{2.5}$

Ammonium sulfate

Nonvolatile OM

SVOM

East

Constructed $\mathrm{PM}_{2.5}$

Ammonium sulfate

Nonvolatile OM

SVOM

West

Constructed $\mathrm{PM}_{2.5}$

Ammonium sulfate

Nonvolatile OM

SVOM

Southwest

Constructed $\mathrm{PM}_{2.5}$

Ammonium sulfate

Nonvolatile OM

SVOM

Northwest

Constructed $\mathrm{PM}_{2.5}$

Ammonium sulfate

Nonvolatile OM

SVOM

Constructed $\mathrm{PM}_{2.5}$

Ammonium sulfate

Nonvolatile OM

SVOM
27.4

14.2

7.4

4.8

31.1

15.9

9.3

5.0

17.8

5.8

6.9

4.2

39.8

24.4

9.3

5.3

24.4

15.1

5.9

2.9

21.5

12.9

5.5

2.3

11.7

4.3

3.9

2.7

40.8

29.9

6.8

3.2

Average daily species concentration for each type of transport is given in $\mathrm{g} \mathrm{m}^{-3}$.

Prior to this date, transport from the southeast to the NETL site occurred at very low altitude. But a stable air mass with little wind was present at the NETL site throughout 31 July, leading to high $\mathrm{PM}_{2.5}$ concentrations, with average 24-h constructed $\mathrm{PM}_{2.5}$, ammonium sulfate, and nonvolatile organic material (NVOM) concentrations reaching $40 \mu \mathrm{g} \mathrm{m}^{-3}, 27 \mu \mathrm{g} \mathrm{m}^{-3}$, and $10 \mu \mathrm{g} \mathrm{NVOM} \mathrm{m}^{-3}$, respectively. However, the locally dependent pollutant concentration observed on 21 July was characterized by lower $\mathrm{PM}_{2.5}$ concentrations.

\section{Conclusions}

The concentration and composition of $\mathrm{PM}_{2.5}$ impacting the NETL PM characterization site and Carnegie
Mellon University EPA PAQS site were dependent on meteorological conditions. High-pressure conditions were characterized by low concentrations of ammonium sulfate and high concentrations of nonvolatile organic material. High concentrations of ammonium sulfate were generally associated with transport of pollutants to the sampling sites during transition from high pressure to low pressure regimes, thus suggesting the transport of sulfur oxide emissions from distant sources. Therefore, such pressure transitions could be used to predict the occurrence of fine particulate $\mathrm{PM}_{2.5}$ episodes around the Pittsburgh area. Trends in VOM and SVOM at the two sampling sites in combination with trends in $\mathrm{NO}_{x}$ suggested the importance of $\mathrm{NO}_{x}$ chemistry at the CMU site but organic chemistry at the NETL site. This was reflected in higher concentrations of NVOM and SVOM 
at the NETL site. The details of differences in emissions and atmospheric chemistry which lead to these observed differences in fine particulate organic material at the two sites are not yet known.

\section{Acknowledgements}

The participation of Brigham Young University personnel in the NETL sampling program was funded under a University Coal Research Grant \# DE-FG2699FT40581 from the United States Department of Energy, National Energy Technology Laboratory. The research by Carnegie Mellon University was conducted as part of the Pittsburgh Air Quality Study, which was supported by the US Environmental Protection Agency under contract R82806101 and the US Department of Energy National Energy Technology Laboratory under contract DE-FC26-01NT41017. This paper has not been subject to EPA's required peer and policy review, and therefore does not necessarily reflect the views of the Agency. No official endorsement should be inferred. We express our appreciation for the following NETL staff, Paul C. Rohar, and Karl Waldner for their field support. We also express appreciation to the United States Department of Energy for financial support for Brigham Young University's participation in the CMU PAQS site study. Sincere thanks is also expressed to the Air Resources Laboratory of NOAA for permission to access the HYSPLIT transport and dispersion model on the NOAA Air Resources Laboratory's READY web server. Helpful discussions with Juan Cabada, R. Subramanian, Andrey Khlystov, Beth Wittig, Allen Robinson and Spyros Pandis are appreciated.

\section{Disclaimer}

Reference to this report to any specific commercial product, process or service is to facilitate understanding and does not necessarily imply its endorsement or favoring by the United States Department of Energy nor by the United States Environmental Protection Agency.

\section{References}

Anderson, R.R., Martello, D.V., Rohar, P.C., Strazisar, B.R., Tamilia, J.P., Waldner, K., White, C.M., Modey, W.K., Mangelson, N.F., Eatough, D.J., 2002. Sources and Composition of $\mathrm{PM}_{2.5}$ at the National Energy Technology Laboratory in Pittsburgh during July and August 2002. Energy and Fuels 16, 261-269.

Anderson, R.R., Irdi, G., Lynn, R., Martello, D.V., Rohar, P.C., Tamilia, J.P., Veloski, G.A., Waldner, K., White,
C.M., Eatough D.J., 2004. The DOE NETL OST PM 2.5 Characterization Program. Environmental Science and Technology, submitted for publication.

Cabada, J.C., Takahama, S., Khlystov, A., Pandis, S.N., Rees, S., Davidson, C.I., Robinson, A.L., 2004. Mass size distributions and size resolved chemical composition of fine particulate matter at the Pittsburgh Supersite. Atmospheric Environment, this issue, doi:10.1016/ j.atmosenv.2004.03.004.

Chan, T., Lippmann, M., 1977. Particle collecting efficiencies of air sampling cyclones: an empirical theory. Environmental Science and Technology 11, 377-382.

Ding, Y., Pang, Y., Eatough, D.J., Eatough, N.L., Tanner, R.L., 2002. High-volume diffusion denuder sampler for the routine monitoring of fine particulate matter: II. Field evaluation of the PC-BOSS. Aerosol Science and Technology 36, 383-396.

Eatough, D.J., Wadsworth, A., Eatough, D.A., Crawford, J.W., Hansen, L.D., Lewis, E.A., 1993. A multi-system, multi-channel diffusion denuder sampler for the determination of fine particulate organic material in the atmosphere. Atmospheric Environment 27A, 1213-1219.

Eatough, D.J., Pang, Y., Eatough, N.L., 1999. Determination of $\mathrm{PM}_{2.5}$ sulfate and nitrate with PC-BOSS designed for routine sampling for semi-volatile particulate matter. Journal of the Air and Waste Management Association $49,69-75$.

Ellis, E.C., Novakov, T., 1982. Application of thermal analysis to the characterization of organic aerosol particles. The Science of the Total Environment 23, 227-238.

Hughes, G., Lovei, M., 1999. Economic reform and environmental performance in transition economies. World Bank Technical Paper 446, Washington, DC.

Lewtas, J., Booth, D., Pang, Y., Reimer, S., Eatough, D.J., Gundel, L., 2001. Comparison of sampling methods for semi-volatile organic carbon (SVOC) associated with $\mathrm{PM}_{2.5}$. Aerosol Science and Technology 34, 9-22.

Modey, W.K., Eatough, D.J., 2003. Trends in $\mathrm{PM}_{2.5}$ Composition at the Department of Energy OST NETL PM 2.5 characterization site in Pittsburgh, Pennsylvania, USA. Advances in Environmental Research 7, 859-869.

Modey, W.K., Pang, Y., Eatough, N.L., Eatough, D.J., 2001. Fine particulate $\left(\mathrm{PM}_{2.5}\right)$ composition in Atlanta, USA: assessment of the Particle Concentrator-Brigham Young University Organic Sampling System, PC-BOSS, during the EPA supersite study. Atmospheric Environment 35, 6493-6502.

NOAA, 2002. http://www.arl.noaa.gov/ready/hysplit4.html.

Pang, Y., Eatough, N.L., Modey, W.K., Eatough, D.J., 2002. Evaluation of the performance of annular denuder samplers. Aerosol Science and Technology 36, 790-798.

Pope, C.A. III., 2000. Epidemiology of fine particulate air pollution and human health: biologic mechanisms and who's at risk. Environmental Health Perspectives 108, 713-723.

Schwartz, J., 2001. Is there harvesting in the association of airborne particles with daily deaths and hospital admissions? Epidemiology 12, 55-61.

Sioutas, C., Koutrakis, P., Olson, B.A., 1994. Development and evaluation of a low cutpoint virtual impactor. Aerosol Science and Technology 21, 223-235. 
Subramanian, R., Khlystov, A.Y., Cabada, J.C., Robinson, A.L., 2004. Positive and negative artifacts in particulate organic carbon measurements with denuded and undenuded sampler configurations, Aerosol Science and Technology, in press.

Turpin, B.J., Lim, H.-J., 2001. Species contributions to $\mathbf{P M}_{2.5}$ mass concentrations: revisiting common assumptions for estimating organic mass. Aerosol Science and Technology $35,602-610$.
UNISYS, 2002. http://www.weather.unisys.com/surface/index. html.

Wittig, B., Anderson, N., Khlystov, A.Y., Pandis, S.N., Davidson, C., Robinson, A.L., 2004. Pittsburgh air quality study overview and preliminary findings. Aerosol Science and Technology, in press. 


\title{
Atmospheric volatile organic compound measurements during the Pittsburgh Air Quality Study: Results, interpretation, and quantification of primary and secondary contributions
}

\author{
Dylan B. Millet, ${ }^{1,2}$ Neil M. Donahue, ${ }^{3}$ Spyros N. Pandis, ${ }^{3}$ Andrea Polidori, ${ }^{4}$ \\ Charles O. Stanier, ${ }^{2,5}$ Barbara J. Turpin, ${ }^{4}$ and Allen H. Goldstein ${ }^{1}$ \\ Received 3 February 2004; revised 7 April 2004; accepted 22 April 2004; published 25 January 2005.
}

[1] Primary and secondary contributions to ambient levels of volatile organic compounds (VOCs) and aerosol organic carbon (OC) are determined using measurements at the Pittsburgh Air Quality Study (PAQS) during January-February and July-August 2002. Primary emission ratios for gas and aerosol species are defined by correlation with species of known origin, and contributions from primary and secondary/biogenic sources and from the regional background are then determined. Primary anthropogenic contributions to ambient levels of acetone, methylethylketone, and acetaldehyde were found to be $12-23 \%$ in winter and $2-10 \%$ in summer. Secondary production plus biogenic emissions accounted for $12-27 \%$ of the total mixing ratios for these compounds in winter and $26-34 \%$ in summer, with background concentrations accounting for the remainder. Using the same method, we determined that on average $16 \%$ of aerosol OC was secondary in origin during winter versus 37\% during summer. Factor analysis of the VOC and aerosol data is used to define the dominant source types in the region for both seasons. Local automotive emissions were the strongest contributor to changes in atmospheric VOC concentrations; however, they did not significantly impact the aerosol species included in the factor analysis. We conclude that longer-range transport and industrial emissions were more important sources of aerosol during the study period. The VOC data are also used to characterize the photochemical state of the atmosphere in the region. The total measured $\mathrm{OH}$ loss rate was dominated by nonmethane hydrocarbons and $\mathrm{CO}(76 \%$ of the total) in winter and by isoprene, its oxidation products, and oxygenated VOCs (79\% of the total) in summer, when production of secondary organic aerosol was highest.

Citation: Millet, D. B., N. M. Donahue, S. N. Pandis, A. Polidori, C. O. Stanier, B. J. Turpin, and A. H. Goldstein (2005), Atmospheric volatile organic compound measurements during the Pittsburgh Air Quality Study: Results, interpretation, and quantification of primary and secondary contributions, J. Geophys. Res., 110, D07S07, doi:10.1029/2004JD004601.

\section{Introduction}

[2] Airborne particulate matter (PM) can adversely affect human and ecosystem health, and exerts considerable influence on climate. Effective PM control strategies require an understanding of the processes controlling PM concentration and composition in different environments. The

\footnotetext{
${ }^{1}$ Division of Ecosystem Sciences, University of California, Berkeley, California, USA.

${ }^{2}$ Now at Department of Earth and Planetary Sciences, Harvard University, Cambridge, Massachusetts, USA.

${ }^{3}$ Department of Chemical Engineering, Carnegie Mellon University, Pittsburgh, Pennsylvania, USA.

${ }^{4}$ Department of Environmental Sciences, Rutgers University, New Brunswick, New Jersey, USA.

${ }^{5}$ Now at Department of Chemical and Biochemical Engineering, University of Iowa, Iowa City, Iowa,USA.
}

Copyright 2005 by the American Geophysical Union. 0148-0227/05/2004JD004601\$09.00
Pittsburgh Air Quality Study (PAQS) is a comprehensive, multidisciplinary project directed at understanding the processes governing aerosol concentrations in the Pittsburgh region [e.g., Wittig et al., 2004a; Stanier et al., 2004a, 2004b]. Specific objectives include characterizing the physical and chemical properties of regional PM, its morphology and temporal and spatial variability, and quantifying the impacts of the important sources in the area.

[3] Volatile organic compounds (VOCs) can directly influence aerosol formation and growth via condensation of semivolatile oxidation products onto existing aerosol surface area [Odum et al., 1996; Jang et al., 2002; Czoschke et al., 2003], and possibly via the homogeneous nucleation of new particles [Koch et al., 2000; Hoffmann et al., 1998]. They also have strong indirect effects on aerosol via their control over ozone production and $\mathrm{HO}_{\mathrm{x}}$ cycling, which in turn dictate oxidation rates of organic and inorganic aerosol precursor species. Comprehensive and high time resolution VOC measurements in conjunction with particle measurements thus aid in characterizing chemical conditions con- 


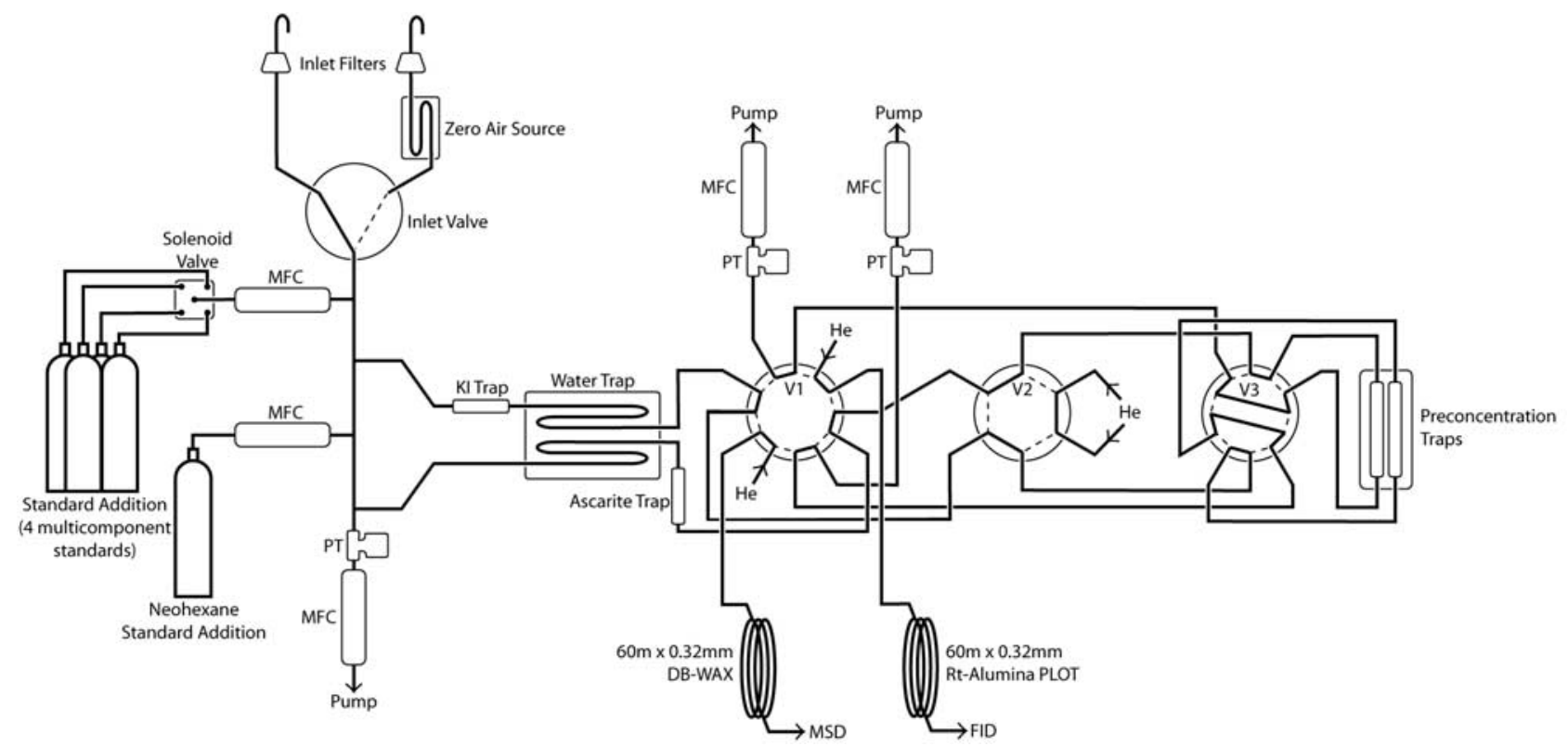

Figure 1. Schematic of the VOC sampling system. MFC, mass-flow controller; V1-V3, valves 1-3; MSD, mass selective detector; FID, flame ionization detector; PT, pressure transducer.

ducive to particle formation and growth. VOC data can also yield information on the nature of source types impacting the study region [Goldstein and Schade, 2000], photochemical aging and transport phenomena [Parrish et al., 1992; McKeen and Liu, 1993], and estimates of regional emission rates [Barnes et al., 2003; Bakwin et al., 1997], all of which can be useful in interpreting other gas and particle phase measurements.

[4] This paper describes the results from two field deployments, during January-February 2002 and July-August 2002, in which we made in situ VOC measurements alongside the comprehensive aerosol measurements at the PAQS site, with the aim of specifically addressing the connection between atmospheric trace gases and particle formation and source attribution. The data set provides an opportunity to examine aerosol formation and chemistry in the context of high time resolution speciated VOC measurements.

[5] The specific goals of this paper include: characterizing the dominant source types impacting the Pittsburgh region, their composition and variability; assessing the relative importance of different types of VOCs to regional photochemistry, and the relationship between aerosol concentrations and the chemical state of the atmosphere; and quantifying the relative importance of primary and secondary sources in determining organic aerosol and oxygenated VOC (OVOC) concentrations. For the latter we quantify the primary emission ratios for species with multiple source types, by correlation with combustion and photochemical marker compounds.

\section{Experimental}

\subsection{Pittsburgh Air Quality Study (PAQS)}

[6] The field component of the Pittsburgh Air Quality Study was carried out from July 2001 through August 2002. Measurement platforms consisted of a main sampling site located in a park about $6 \mathrm{~km}$ east of downtown Pittsburgh, as well as a set of satellite sites in the surrounding region.
For details on the PAQS study, see Wittig et al. [2004a] and the references cited therein. Measurements described here were made at the main sampling site.

\subsection{VOC Measurements}

[7] A schematic of the VOC measurement setup is shown in Figure 1. To provide information on as wide a range of compounds as possible, two separate measurement channels were used, equipped with different preconditioning systems, preconcentration traps, chromatography columns, and detectors. Channel 1 was designed for preconcentration and separation of $\mathrm{C}_{3}-\mathrm{C}_{6}$ nonmethane hydrocarbons, including alkanes, alkenes and alkynes, on an Rt-Alumina PLOT column with subsequent detection by FID. Channel 2 was designed for preconcentration and separation of oxygenated, aromatic, and halogenated VOCs, NMHCs larger than $\mathrm{C}_{6}$, and some other VOCs such as acetonitrile and dimethylsulfide, on a DB-WAX column with subsequent detection by quadropole MSD (HP 5971).

[8] Air samples were drawn at $4 \mathrm{sl} / \mathrm{min}$ through a 2 micron Teflon particulate filter and $1 / 4^{\prime \prime}$ OD Teflon tubing (FEP fluoropolymer, Chemfluor) mounted on top of the laboratory container. Two $15 \mathrm{scc} / \mathrm{min}$ subsample flows were drawn from the main sample line, and through pretreatment traps for removal of $\mathrm{O}_{3}, \mathrm{H}_{2} \mathrm{O}$ and $\mathrm{CO}_{2}$. For $30 \mathrm{~min}$ out of every hour, the valve array (V1, V2, and V3; valves from Valco Instruments) was switched to sampling mode (Figure 1, as shown) and the subsamples flowed through $0.03^{\prime \prime}$ ID fused silica-lined stainless steel tubing (Silcosteel, Restek Corp) to the sample preconcentration traps where the VOCs were trapped prior to analysis. When sample collection was complete, the preconcentration traps and downstream tubing were purged with a forward flow of UHP helium for $30 \mathrm{~s}$ to remove residual air. The valve array was then switched to inject mode, the preconcentration traps heated rapidly to $200^{\circ} \mathrm{C}$, and the trapped analytes desorbed into the helium carrier gas and transported to the $\mathrm{GC}$ for separation and quantification. 
[9] As noninert surfaces are known to cause artifacts and compound losses for unsaturated and oxygenated species, all surfaces contacted by the sampled airstream prior to the valve array were constructed of Teflon (PFA or FEP). All subsequent tubing and fittings, except the internal surfaces of the Valco valves V1, V2, and V3, were Silcosteel. The valve array, including all silcosteel tubing, was housed in a temperature controlled box held at $50^{\circ} \mathrm{C}$ to prevent compound losses through condensation and adsorption. All flows were controlled using Mass-Flo Controllers (MKS Instruments), and pressures were monitored at various points in the sampling apparatus using pressure transducers (Data Instruments).

[10] In order to reduce the dew point of the sampled airstream, both subsample flows passed through a loop of $1 / 8^{\prime \prime}$ OD Teflon tubing cooled thermoelectrically to $-25^{\circ} \mathrm{C}$. Following sample collection, the water trap was heated to $105^{\circ} \mathrm{C}$ while being purged with a reverse flow of dry zero air to expel the condensed water prior to the next sampling interval. A trap for the removal of carbon dioxide and ozone (Ascarite II, Thomas Scientific) was placed downstream of the water trap in the Rt-Alumina/FID channel. An ozone trap (KI-impregnated glass wool, following Greenberg et al. [1994]) was placed upstream of the water trap in the other channel leading to the DB-WAX column and the MSD (Figure 1).

[11] Sample preconcentration was achieved using a combination of thermoelectric cooling and adsorbent trapping. The preconcentration traps consisted of three stages (glass beads/Carbopack B/Carboxen 1000 for the Rt-Alumina/FID channel, glass beads/Carbopack B/Carbosieves SIII for the DB-WAX/MSD channel; all adsorbents from Supelco), held in place by DMCS-treated glass wool (Alltech Associates) in a $9 \mathrm{~cm}$ long, $0.04^{\prime \prime}$ ID fused silica-lined stainless steel tube (Restek Corp). A nichrome wire heater was wrapped around the preconcentration traps, and the trap/heater assemblies were housed in a machined aluminum block that was thermoelectrically cooled to $-15^{\circ} \mathrm{C}$. After sample collection and the helium purge, the preconcentration traps were isolated via V3 (see Figure 1) until the start of the next chromatographic run. The traps were small enough to permit rapid thermal desorption $\left(-15^{\circ} \mathrm{C}\right.$ to $200^{\circ} \mathrm{C}$ in $\left.10 \mathrm{~s}\right)$ eliminating the need to cryofocus the samples before chromatographic analysis (following Lamanna and Goldstein [1999]). The samples were thus introduced to the individual GC columns, where the components were separated and then detected with the FID or MSD.

[12] Chromatographic separation and detection of the analytes was achieved using an HP 5890 Series II GC. The temperature program for the GC oven was: $35^{\circ} \mathrm{C}$ for $5 \mathrm{~min}, 3^{\circ} \mathrm{C} / \mathrm{min}$ to $95^{\circ} \mathrm{C}, 12.5^{\circ} \mathrm{C} / \mathrm{min}$ to $195^{\circ} \mathrm{C}$, hold for $6 \mathrm{~min}$. The oven then ramped down to $35^{\circ} \mathrm{C}$ in preparation for the next run. The carrier gas flow into the MSD was controlled electronically and maintained constant at $1 \mathrm{~mL} / \mathrm{min}$. The FID channel carrier gas flow was controlled mechanically by setting the pressure at the column head such that the flow was $4.5 \mathrm{~mL} / \mathrm{min}$ at an oven temperature of $35^{\circ} \mathrm{C}$. The carrier gas for both channels was UHP $(99.999 \%)$ helium which was further purified of oxygen, moisture and hydrocarbons (traps from Restek Corp.).

[13] Zero air for blank runs and calibration by standard addition was generated by flowing ambient air over a bed of platinum heated to $370^{\circ} \mathrm{C}$. This system passes ambient humidity, creating VOC free air in a matrix resembling real air as closely as possible. Zero air was analyzed daily to check for blank problems and contamination for all measured compounds.

[14] Compounds measured on the FID channel were quantified by determining their weighted response relative to a reference compound (see Goldstein et al. [1995a] and Lamanna and Goldstein [1999] for details). Neohexane (5.15 ppm, certified NIST traceable $\pm 2 \%$; Scott-Marrin Inc.) was employed as the internal standard for the FID channel, and was added by dynamic dilution to the sampling stream. Compound identification was achieved by matching retention times with those of known standards for each compound (Scott Specialty Gases, Inc.).

[15] The MSD was operated in single ion mode (SIM) for optimum sensitivity and selectivity of response. Ionmonitoring windows were timed to coincide with the elution of the compounds of interest. Calibration curves for all of the individual compounds were obtained by dynamic dilution of multicomponent low-ppm level standards (ApelRiemer Environmental Inc.) into zero air to mimic the range of ambient mixing ratios. A calibration or blank was performed every 6th run.

[16] The system was fully automated for unattended operation in the field. The valve array (V1, V2 and V3) and the preconcentration trap resistance heater circuit were controlled through the GC via auxiliary output circuitry. The PC controlling the GC was also interfaced with a CR10X data logger (Campbell Scientific Inc.), which was triggered at the outset of each analysis run. The inlet valve, the standard addition solenoid valve and the water trap cooling, heating and valve circuitry were switched at the appropriate times during the sampling cycle by a relay module (SDMCD16AC, Campbell Scientific) controlled by the data logger. Relevant engineering data (time, temperatures, flow rates, pressures, etc.) for each sampling interval were recorded by the CR10X data logger with a AM416 multiplexer (Campbell Scientific Inc.), then uploaded to the PC and stored with the associated chromatographic data. Chromatogram integrations were done using HP Chemstation software. All subsequent data processing and QA/QC was performed using routines created in S-Plus (Insightful Corp.). Instrumental precision, detection limits, and accuracy for each measured compound during this experiment, along with the $0.25,0.50$, and 0.75 quantiles of the data, are given in Table 1.

\subsection{Aerosol, Trace Gas, and Meteorological Measurements}

[17] Additional measurements which are used in this paper are described briefly below. For a more thorough overview of the gas and particle measurement methods and results from PAQS, the reader is directed to Wittig et al. [2004a] and the references cited therein.

[18] Semicontinuous measurements of PM 2.5 (i.e., $<2.5 \mu \mathrm{m}$ diameter) particulate mass were made using a tapering element oscillating microprobe (TEOM) instrument (Model 1400a, Rupprecht \& Patashnick Co., Inc.). PM 2.5 nitrate and sulfate were also measured on a semicontinuous basis using Integrated Collection and Vaporization Cell (ICVC) instruments (Rupprecht \& Patashnick Co., Inc.) 
Table 1. Concentration Quantiles and Figures of Merit for Measured VOCs

\begin{tabular}{|c|c|c|c|c|c|c|c|}
\hline \multirow[b]{2}{*}{ Compound } & \multirow{2}{*}{$\begin{array}{c}\text { Precision }^{\mathrm{a}} \\
\%\end{array}$} & \multirow{2}{*}{$\begin{array}{l}\text { Detection } \\
\text { Limit, ppt }\end{array}$} & \multirow{2}{*}{$\begin{array}{c}\text { Accuracy, } \\
\% \\
\end{array}$} & \multicolumn{2}{|c|}{ Winter $^{\mathrm{b}}$} & \multicolumn{2}{|c|}{ Summer $^{\mathrm{c}}$} \\
\hline & & & & Median, ppt & $\mathrm{IQR}^{\mathrm{d}}{ }^{\mathrm{p}} \mathrm{ppt}$ & Median, ppt & $\mathrm{IQR}^{\mathrm{d}}{ }^{\mathrm{d}}$ \\
\hline Propane & 2.5 & 1.6 & 7.6 & 2960 & $2087-4307$ & 1787 & $992-3540$ \\
\hline Isobutane & 2.5 & 1.2 & 7.6 & 668 & $479-953$ & 323 & $212-634$ \\
\hline Butane & 2.5 & 1.2 & 7.6 & 1333 & $978-1799$ & 632 & $375-1106$ \\
\hline Isopentane & 2.5 & 0.9 & 7.6 & 575 & $448-809$ & 649 & $409-1139$ \\
\hline Pentane & 2.5 & 0.9 & 7.6 & 355 & $279-493$ & 352 & $213-613$ \\
\hline Methylpentanes & 2.5 & 0.8 & 7.6 & 268 & $203-368$ & 276 & $183-506$ \\
\hline Propene & 2.5 & 1.5 & 7.6 & 214 & $147-306$ & 219 & $159-336$ \\
\hline t-2-butene & 2.5 & 1.1 & 7.6 & 30 & $19-52$ & 11 & $8-18$ \\
\hline 1-butene & 2.5 & 1.1 & 7.6 & 57 & $40-83$ & 62 & $44-88$ \\
\hline 2-methylpropene & 2.5 & 1.1 & 7.6 & 38 & $32-51$ & $N Q^{f}$ & $N^{f}$ \\
\hline Cyclopentane & 2.5 & 0.9 & 7.6 & 53 & $35-92$ & 47 & $36-72$ \\
\hline c-2-butene & 2.5 & 1.1 & 7.6 & 27 & $18-44$ & 20 & $15-28$ \\
\hline Cyclopentene & 2.5 & 1.0 & 7.6 & $N^{f}$ & $N Q^{f}$ & 3 & $0-8$ \\
\hline Propyne & 2.5 & 1.4 & 7.6 & 29 & $22-40$ & 7 & $5-12$ \\
\hline t-2-pentene & 2.5 & 0.9 & 7.6 & 19 & $12-33$ & 44 & $33-62$ \\
\hline 1-pentene & 2.5 & 0.9 & 7.6 & 36 & $24-56$ & 20 & $14-32$ \\
\hline 2-methyl-1-butene & 2.5 & 0.9 & 7.6 & 16 & $11-25$ & 42 & $22-74$ \\
\hline Benzene & 4.4 & 26 & 10 & 279 & $231-355$ & 215 & $143-405$ \\
\hline Perchloroethylene & 5.4 & 0.6 & 10 & 18 & $12-25$ & 22 & $13-41$ \\
\hline Ethylbenzene & 5.8 & 1.6 & 10 & 47 & $34-69$ & 71 & $44-141$ \\
\hline Isoprene & 4.3 & 3.1 & 10 & $<\mathrm{DL}^{\mathrm{g}}$ & $<\mathrm{DL}^{\mathrm{g}}$ & 619 & $153-1475$ \\
\hline Methyl-t-butyl ether & 4.2 & 1.7 & 10 & 10 & $7-14$ & 31 & $19-61$ \\
\hline Acetaldehyde & 7.2 & 82 & 10 & 538 & $403-729$ & 1559 & $1103-2150$ \\
\hline Dimethylsulfide & 5.6 & 3.2 & 10 & $N^{f}$ & $N Q^{f}$ & 7 & $5-10$ \\
\hline Acetone & 4.0 & 47 & 10 & 943 & $655-1385$ & 4031 & $3128-4894$ \\
\hline Butanal & 6.0 & 28 & 10 & $N Q^{f}$ & $N Q^{f}$ & 91 & $64-122$ \\
\hline Methacrolein & 5.6 & 11 & 10 & $<\mathrm{DL}^{\mathrm{g}}$ & $<\mathrm{DL}^{\mathrm{g}}$ & 266 & $178-366$ \\
\hline 3-methylfuran & 4.2 & 2.2 & 10 & $<\mathrm{DL}^{\mathrm{g}}$ & $<\mathrm{DL}^{\mathrm{g}}$ & 10 & $6-16$ \\
\hline Methanol & 8.2 & 370 & 11 & 3760 & $2347-5773$ & 10717 & $7122-14601$ \\
\hline Methylethylketone & 5.1 & 10 & 10 & 215 & $153-299$ & 559 & $408-674$ \\
\hline Methylene chloride & 7.1 & 22 & 10 & $N Q^{f}$ & $N Q^{f}$ & 79 & $48-145$ \\
\hline Methylvinylketone & 3.5 & 6.8 & 10 & $<\mathrm{DL}^{\mathrm{g}}$ & $<\mathrm{DL}^{\mathrm{g}}$ & 463 & $273-665$ \\
\hline Pentanal & 8.3 & 19 & 11 & $N^{f}$ & $N Q^{f}$ & 137 & $98-193$ \\
\hline Acetonitrile & 13 & 38 & 14 & $N^{f}$ & $\mathrm{NQ}^{\mathrm{f}}$ & 131 & $105-155$ \\
\hline Chloroform & 3.6 & 1.2 & 10 & 11 & $10-13$ & 17 & $13-30$ \\
\hline$\alpha$-pinene & 5.9 & 0.6 & 10 & $<\mathrm{DL}^{\mathrm{g}}$ & $<\mathrm{DL}^{\mathrm{g}}$ & 16 & $10-29$ \\
\hline Toluene & 2.9 & 22 & 10 & 331 & $248-494$ & 443 & $274-902$ \\
\hline Hexanal & 11 & 25 & 13 & 34 & $22-52$ & $N Q^{f}$ & $N Q^{f}$ \\
\hline p-xylene & 5.8 & 3.4 & 10 & 62 & $42-95$ & 91 & $51-173$ \\
\hline m-xylene & 5.8 & 5.3 & 10 & 113 & $76-176$ & 163 & $89-306$ \\
\hline o-xylene & 5.8 & 2.4 & 10 & 60 & $41-89$ & 52 & $29-93$ \\
\hline
\end{tabular}

${ }^{a}$ Defined as the relative standard deviation of the calibration fit residuals.

${ }^{b}$ Dates of 9 January to 12 February 2002.

${ }^{\mathrm{c}}$ Dates of 9 July to 10 August 2002

${ }^{\mathrm{d}} \mathrm{IQR}$, interquartile range.

'The sum of 2-methylpentane and 3-methylpentane, which coelute.

${ }^{\mathrm{f}} \mathrm{NQ}$, not quantified, due to inadequate resolution, unavailability of standard or other reason.

$\mathrm{g}_{<\mathrm{DL}}$, below detection limit.

[Wittig et al., 2004b]. Aerosol number size distributions $(0.003-10 \mu \mathrm{m})$ were quantified using an array of particle sizing measurements: a nano scanning mobility particle sizer (SMPS) (TSI, Inc., Model 3936N25), standard SMPS (TSI, Inc., Model 3936L10), and Aerodynamic Particle Sizer (APS) (TSI, Inc., Model 3320). Aerosol number size distribution measurements were made semicontinuously throughout the PAQS campaign [Stanier et al., 2004a]. Aerosol organic carbon (OC) and elemental carbon (EC) were quantified in situ throughout the study with 2-4 hour time resolution using a Sunset Labs in situ carbon analyzer (A. Polidori et al., manuscript in preparation, 2005).

[19] $\mathrm{O}_{3}, \mathrm{NO}, \mathrm{NO}_{2}, \mathrm{CO}$ and $\mathrm{SO}_{2}$ were measured continuously with commercial gas analyzers (Models 400A,
200A, 300 and 100A, Teledyne Advanced Pollution Instrumentation). Measurements of relevant meteorological parameters (incoming radiation, air temperature, wind speed and direction, precipitation, and relative humidity) were also made continuously throughout the experiment.

\section{Results and Discussion}

\subsection{Meteorological Conditions}

[20] Observed wind speed and direction for the two study periods (9 January to 12 February and 9 July to 10 August 2002) are shown as a wind rose plot in Figure 2. Throughout this paper, data collected during the January-February 2002 deployment will be referred to as "winter" data and 


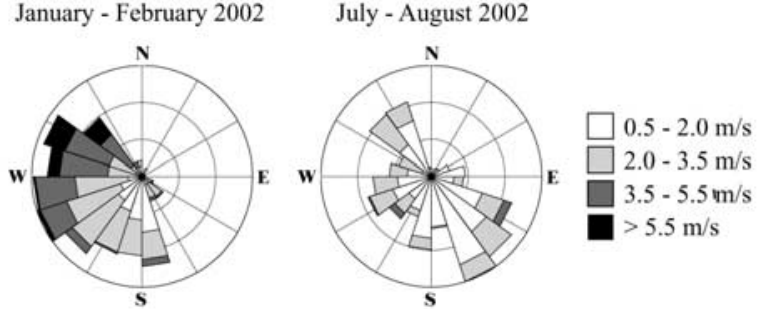

Figure 2. Wind rose plots for the winter and summer experiments. The lengths of the wedges are proportional to the frequency of observation.

that collected during July-August 2002 as "summer" data. Winds in the winter were predominantly out of the west (south to northwest), whereas in the summer southeasterly and northwesterly winds were most common (Figure 2). There was a diurnal cycle in wind speed in both seasons, with stronger winds during the day and weaker winds at night (not shown).

\subsection{Factor Analysis}

[21] Factor analysis can be used to categorize measured compounds into distinct source groups based on the covariance of their concentrations, creating an understanding of the variety of sources contributing to a broad range of measured species [Sweet and Vermette, 1992; Thunis and Cuvelier, 2000; Lamanna and Goldstein, 1999]. In this section we characterize the dominant source types impacting the Pittsburgh region in summer and winter, based on a factor analysis of the VOC data set, combined with other available trace gas and high temporal resolution aerosol data. Compounds are grouped into factors according to their covariance, and the strength of association between compounds and factors is expressed as a loading matrix. Each factor is a linear combination of the observed variables and in theory represents an underlying process which is causing certain species to behave similarly. Prior knowledge of source types for the dominant compounds is then used to assign source categories to the statistically identified factors.

[22] The analysis was performed using principal components extraction and varimax rotation (S-Plus 6.1, Lucent Technologies Inc.). Species having a significant amount $(>8 \%)$ of missing data were excluded from the analysis. Results for the winter and summer data sets are presented in Tables 2 and 3, respectively, and discussed in detail below. Compounds not loading significantly on any of the factors are omitted from the loadings tables.

\subsubsection{Winter Trace Gas and Aerosol Data Set}

[23] Six factors were extracted from the winter data set, which accounted for a total of $83 \%$ of the cumulative variance (Table 2). Each of the six factors accounted for a statistically significant portion of the variance $(\mathrm{P}<0.01$, where $\mathrm{P}$ is the statistical probability of incorrectly attributing a nonzero fraction of the variance to a given factor). The analysis was limited to six factors since including more factors failed to account for more than an additional $2 \%$ of the variance in the data set.

[24] Factor 1, explaining 44\% of the total variability in the data set, was associated most strongly with short-lived combustion-derived pollutants, such as the anthropogenic alkenes and aromatic species, in addition to $\mathrm{NO}_{\mathrm{x}}$ and the gasoline additive methyl-t-butyl ether (MTBE). We attribute this factor to local automobile emissions. The diurnal cycle exhibited by this factor (Figure 3a) showed a clear pattern, higher during the day than at night, and with prominent peaks during the morning and evening rush hours. Note that factor 1 accounted for $44 \%$ of the data set variability, indicating that automobile exhaust was most strongly responsible for changes in atmospheric VOC concentrations in Pittsburgh in the winter. Note also, however, that none of the aerosol parameters included in the factor analysis (PM 2.5 mass, aerosol sulfate and nitrate mass, and aerosol number density) loaded significantly on this factor, suggesting that this source was a relatively minor contributor to these components of regional PM.

[25] Factor 2, accounting for $10 \%$ of the variance, was associated exclusively with the anthropogenic alkanes (Table 2), most strongly with propane, and probably represents leaks of propane fuel or natural gas. None of the aerosol measurements loaded on this factor. Factor 2 was on average highest with winds out of the south, and the diurnal pattern showed a maximum in the early morning before dawn (Figure 3b), with a minimum in the afternoon.

[26] The third factor, accounting for $9 \%$ of the data set variance, like factor 1 was associated with some gas-phase combustion products (such as $\mathrm{CO}$, benzene and propyne). Unlike factor 1 , however, it also contained a significant aerosol component, in particular sulfate and PM 2.5 mass. The diurnal cycle of factor 3 (Figure 3c) was distinct from that of factor 1 , with higher concentrations at night, and no noticeable rush hour contribution. The highest levels of factor 3 were seen with winds out of the south-southeast. We attribute this factor to industrial emissions from point sources in the region. In particular, the U.S. Steel Clairton Works, which is the largest manufacturer of coke and coal chemicals in the United States, and is located 11 miles to the south-southeast of Pittsburgh, may have been a significant contributor to this factor.

[27] Factor 4 was composed of species (acetone, acetaldehyde, methylethylketone (MEK)) that are both emitted directly and produced photochemically. Acetone and acetaldehyde are also known to have significant biogenic sources [Schade and Goldstein, 2001]; however, biogenic emissions are unlikely to be a dominant source of these compounds in the Pittsburgh winter. PM 2.5 mass was also associated with this category, consistent with the importance of both primary emissions and secondary production of regional aerosol. The diurnal cycle of factor 4 (Figure $3 \mathrm{~d}$ ) showed evidence of both primary and secondary influence. Daytime concentrations were slightly higher than at night, and there was a marked increase in the morning which was coincident with sunrise. Unlike factor 1, this factor did not show the distinct morning and evening peaks coinciding with rush hour. The day-night difference was much less than in summer (see following section), likely reflecting weak wintertime photochemistry and a consequently greater relative impact from direct emissions. The relative importance of primary and photochemical sources for these compounds is explored further in section 3.3.

[28] Factor 5, which explained a further $6 \%$ of the variance, was negatively associated with ozone and nuclei mode aerosol number density, and positively associated with total PM 2.5 mass, aerosol nitrate and accumulation 
Table 2. Factor Analysis Results: Winter Data ${ }^{a}$

\begin{tabular}{|c|c|c|c|c|c|c|}
\hline \multirow[b]{2}{*}{ Compound } & \multicolumn{6}{|c|}{ Loadings } \\
\hline & $\begin{array}{c}\text { Factor 1: } \\
\text { Local Auto }\end{array}$ & $\begin{array}{c}\text { Factor 2: } \\
\text { Natural Gas }\end{array}$ & $\begin{array}{l}\text { Factor 3: } \\
\text { Industrial }\end{array}$ & $\begin{array}{c}\text { Factor 4: } \\
1^{\circ}+2^{\circ}\end{array}$ & $\begin{array}{l}\text { Factor 5: } \\
2^{\circ}+\text { Mix }\end{array}$ & $\begin{array}{c}\text { Factor 6: } \\
\text { Coal } \\
\end{array}$ \\
\hline Propane & & 0.87 & & & & \\
\hline Isobutane & 0.64 & 0.66 & & & & \\
\hline Butane & 0.63 & 0.63 & & & & \\
\hline t-2-butene & 0.90 & & & & & \\
\hline Isopentane & 0.76 & 0.49 & & & & \\
\hline Pentane & 0.63 & 0.62 & & & & \\
\hline Methylpentanes ${ }^{\mathrm{b}}$ & 0.77 & 0.44 & & & & \\
\hline Hexane & 0.65 & 0.54 & & & & \\
\hline Propene & 0.76 & & 0.47 & & & \\
\hline 1-butene & 0.86 & & & & & \\
\hline 2-methylpropene & 0.60 & & 0.49 & & & \\
\hline Cyclopentane & 0.57 & & & & & \\
\hline c-2-butene & 0.91 & & & & & \\
\hline Propyne & 0.64 & & 0.53 & & & \\
\hline 3-methyl-1-butene & 0.90 & & & & & \\
\hline t-2-pentene & 0.90 & & & & & \\
\hline 1-pentene & 0.91 & & & & & \\
\hline 2-methyl-1-butene & 0.91 & & & & & \\
\hline Benzene & 0.42 & & 0.63 & & & \\
\hline $\mathrm{C}_{2} \mathrm{Cl}_{4}$ & 0.68 & & & & & \\
\hline Ethylbenzene & 0.89 & & & & & \\
\hline MTBE & 0.74 & & & & & \\
\hline Acetaldehyde & 0.41 & & & 0.58 & & \\
\hline Acetone & & & & 0.82 & & \\
\hline MEK & 0.47 & & & 0.64 & & \\
\hline Chloroform & 0.52 & & & & & \\
\hline Toluene & 0.80 & & & & & \\
\hline Hexanal & 0.61 & & & & & \\
\hline p-xylene & 0.90 & & & & & \\
\hline m-xylene & 0.91 & & & & & \\
\hline o-xylene & 0.90 & & & & & \\
\hline $\mathrm{O}_{3}$ & & & & & -0.68 & \\
\hline $\mathrm{NO}_{\mathrm{x}}$ & 0.76 & & & & & \\
\hline $\mathrm{SO}_{2}$ & & & & & & 0.75 \\
\hline $\mathrm{CO}$ & 0.52 & & 0.59 & & & \\
\hline PM 2.5 & & & 0.50 & 0.42 & 0.44 & 0.40 \\
\hline Aerosol $\mathrm{SO}_{4}^{2-}$ & & & 0.54 & & & 0.54 \\
\hline Aerosol $\mathrm{NO}_{3}^{-}$ & & & & & 0.62 & \\
\hline $\mathrm{N}_{\text {nuc }}{ }^{\mathrm{c}}$ & & & & & -0.42 & \\
\hline $\mathrm{N}_{\mathrm{acc}}^{\mathrm{nuc}} \mathrm{c}$ & & & 0.41 & & 0.45 & 0.59 \\
\hline Importance of factors & & & & & & \\
\hline Fraction of variance & 0.44 & 0.10 & 0.09 & 0.08 & 0.06 & 0.06 \\
\hline Cumulative variance & 0.44 & 0.54 & 0.63 & 0.71 & 0.77 & 0.83 \\
\hline
\end{tabular}

${ }^{\mathrm{a}}$ The degree of association between measured compounds and each of the six factors is indicated by a loading value, with the maximum loading being 1 . Loadings of magnitude $<0.4$ omitted.

${ }^{\mathrm{b}}$ The sum of 2-methylpentane and 3-methylpentane, which coelute.

${ }^{c} \mathrm{~N}_{\text {nuc }}$ and $\mathrm{N}_{\mathrm{acc}}$ refer to aerosol number densities in the nuclei $(3-10 \mathrm{~nm})$ and accumulation $(100-500 \mathrm{~nm})$ modes.

mode number density. This factor may represent the combined influences of photochemical activity and mixed layer dynamics. Production of ozone and nucleation mode particles is driven by sunlight, and owing to their relatively short lifetimes their concentrations were highest during the day and lower at night. By contrast, longer lived pollutants less strongly impacted by photochemistry exhibited higher concentrations at night when winds were calmer and vertical mixing limited. In addition, partitioning of semivolatile species such as nitrate into the particle phase is thermodynamically favored by the colder temperatures and higher relative humidity at night.

[29] The 6th factor, accounting for $6 \%$ of the variability, was associated with gas phase $\mathrm{SO}_{2}$, aerosol sulfate, PM 2.5 mass, and accumulation mode number density. Factor 6 showed a diurnal pattern with higher impact during the day than at night, consistent with a photochemically driven process (Figure 3f). However, nucleation mode number density did not load significantly on this factor. This factor may reflect regional coal burning power plant emissions of gases and particles, and the subsequent photochemical aging of those emissions.

\subsubsection{Summer Trace Gas and Aerosol Data Set}

[30] Six factors were extracted from the summer data set, which together accounted for $77 \%$ of the variability in the observations (Table 3). Each of the six factors accounted for a statistically significant portion of the variance $(\mathrm{P}<0.01)$. Including additional factors explained less than $2 \%$ of the remaining variance. The PM 2.5 measurements had a large number $(19 \%)$ of missing values, and as there was a strong correlation $\left(r^{2}=0.92\right)$ between PM 2.5 mass and aerosol volume measured with the SMPS, missing PM 2.5 concentrations were estimated by scaling to aerosol volume prior to performing the factor analysis. 
Table 3. Factor Analysis Results: Summer Data ${ }^{a}$

\begin{tabular}{|c|c|c|c|c|c|c|}
\hline \multirow[b]{2}{*}{ Compound } & \multicolumn{6}{|c|}{ Loadings } \\
\hline & $\begin{array}{l}\text { Factor 1: } \\
\text { Local Auto }\end{array}$ & $\begin{array}{l}\text { Factor 2: } \\
2^{\circ}+\text { Bio }\end{array}$ & $\begin{array}{l}\text { Factor 3: } \\
\text { Transport }\end{array}$ & $\begin{array}{l}\text { Factor 4: } \\
\text { Industrial }\end{array}$ & $\begin{array}{c}\text { Factor 5: } \\
\text { Isopentane } \mathrm{Ox}\end{array}$ & $\begin{array}{c}\text { Factor 6: } \\
\text { Natural Gas }\end{array}$ \\
\hline Propane & 0.59 & & & & & 0.58 \\
\hline Isobutane & 0.74 & & & & & 0.54 \\
\hline Butane & 0.78 & & & & & 0.52 \\
\hline Isopentane & 0.91 & & & & & \\
\hline Pentane & 0.89 & & & & & \\
\hline Methylpentanes ${ }^{\mathrm{b}}$ & 0.93 & & & & & \\
\hline Hexane & 0.90 & & & & & \\
\hline Propene & 0.71 & & & 0.45 & & \\
\hline t-2-butene & 0.89 & & & & & \\
\hline 1-butene & 0.57 & 0.66 & & & & \\
\hline Cyclopentane & 0.66 & & & 0.49 & & \\
\hline c-2-butene & 0.80 & & & & & \\
\hline Propyne & 0.88 & & & & & \\
\hline 3-methyl-1-butene & 0.95 & & & & & \\
\hline t-2-pentene & 0.94 & & & & & \\
\hline 1-pentene & 0.93 & & & & & \\
\hline 2-methyl-1-butene & 0.82 & & & & & \\
\hline Benzene & & & & 0.68 & & \\
\hline $\mathrm{C}_{2} \mathrm{Cl}_{4}$ & 0.48 & & & & & \\
\hline Ethylbenzene & 0.89 & & & & & \\
\hline Isoprene & & 0.44 & & & & \\
\hline MTBE & 0.91 & & & & & \\
\hline Acetaldehyde & & 0.88 & & & & \\
\hline Acetone & & 0.64 & 0.64 & & & \\
\hline Butanal & & 0.85 & & & & \\
\hline MACR & & & & & 0.90 & \\
\hline 3-methylfuran & & 0.45 & & & 0.53 & \\
\hline MEK & 0.44 & 0.44 & 0.40 & & & \\
\hline Isopropanol & 0.47 & & & & & \\
\hline MVK & & & & & 0.89 & \\
\hline Pentanal & 0.55 & 0.72 & & & & \\
\hline Acetonitrile & & & & 0.43 & & \\
\hline Chloroform & 0.67 & & & & & \\
\hline$\alpha$-pinene & 0.57 & & & & & \\
\hline Toluene & 0.80 & & & 0.47 & & \\
\hline p-xylene & 0.90 & & & & & \\
\hline m-xylene & 0.90 & & & & & \\
\hline o-xylene & 0.84 & & & & & \\
\hline $\mathrm{O}_{3}$ & -0.51 & & & & & -0.43 \\
\hline $\mathrm{NO}_{\mathrm{x}}$ & 0.52 & & & 0.44 & & \\
\hline $\mathrm{SO}_{2}$ & & & 0.42 & & & \\
\hline $\mathrm{CO}$ & 0.50 & & & 0.44 & & \\
\hline PM 2.5 & & & 0.88 & & & \\
\hline Aerosol $\mathrm{SO}_{4}^{2-}$ & & & 0.85 & & & \\
\hline $\mathrm{N}_{\mathrm{acc}} \mathrm{c}$ & & & 0.70 & & & \\
\hline Importance of factors & & & & & & \\
\hline Fraction of variance & 0.42 & 0.10 & 0.08 & 0.07 & 0.05 & 0.04 \\
\hline Cumulative variance & 0.42 & 0.53 & 0.60 & 0.67 & 0.73 & 0.77 \\
\hline
\end{tabular}

${ }^{a}$ The degree of association between measured compounds and each of the six factors is indicated by a loading value, with the maximum loading being 1 . Loadings of magnitude $<0.4$ omitted.

${ }^{\mathrm{b}}$ The sum of 2-methylpentane and 3-methylpentane, which coelute.

${ }^{c}$ Accumulation mode $(100-500 \mathrm{~nm})$ aerosol number density.

[31] As with the winter data, the dominant factor, explaining $42 \%$ of the total variance, was associated with anthropogenic alkenes, aromatics, MTBE and other markers of tailpipe emissions (Table 3). The diurnal cycle of this source type (Figure 4a), however, with a sharp early morning maximum at sunrise and a broad afternoon minimum, was markedly different than in the winter, when traffic patterns determined the diurnal pattern. In summer, a deeper daytime mixed layer and more rapid photooxidation combined to give rise to the observed temporal pattern. The fact that benzene is not associated with factor 1 is due to the influence of a nearby source (not associated with other tailpipe compounds or solvents), which resulted occasionally in extremely elevated benzene levels. If the factor analysis is repeated after remov- ing the highest ( $>0.9$ quantile) benzene values, benzene in fact loads most strongly on this automotive factor.

[32] Factor 2 encompassed compounds, such as acetone, acetaldehyde, and isoprene, known to have photochemical sources, sunlight dependent biogenic sources, or both. We thus interpret this factor as representing a combination of these radiation-driven source types. The clear diurnal pattern for this source category (Figure $4 \mathrm{~b}$ ) reflected its light dependent nature, and suggests, for the associated OVOCs, that photochemical and/or biogenic production were more important than direct combustion emissions. The association of 1-butene with factor 2 suggests a regional lightdriven biogenic 1-butene source, as has been reported for other locations [Goldstein et al., 1996]. 


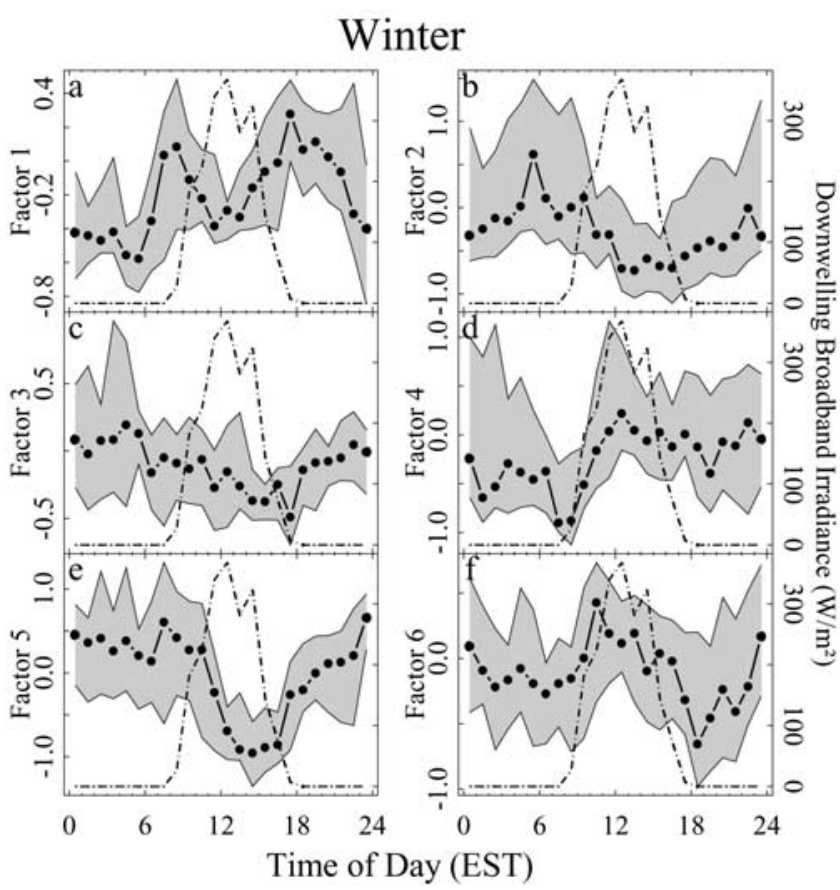

Figure 3. Median diurnal cycles in factor scores (circles) for the winter data set. Banded gray areas show the interquartile range. Incoming solar radiation is also shown (dot-dash line).

[33] Factor 3, consisting of fine particle number (accumulation mode only; nuclei and aitken mode number densities were not included in the analysis as they contained too many missing values), PM 2.5 mass, sulfur dioxide, and particle sulfate, had a weak diurnal pattern containing a maximum at midday (Figure 4c). The correlation of acetone and MEK with the other species associated with this factor may arise from distinct sources which lie along the same transport trajectory, or may reflect long-range transport of pollution with concurrent photochemical production.

[34] The fourth factor, which explained 7\% of the cumulative variance, associated with combustion markers such as benzene, $\mathrm{NO}_{\mathrm{x}}$ and $\mathrm{CO}$, is analogous to the source represented by the third factor extracted from the winter data set. The two factors both exhibited diurnal patterns with concentrations elevated at night and early morning (Figures $3 \mathrm{c}$ and $4 \mathrm{~d}$ ), and in both cases the highest levels were associated with winds from the south-southeast. Again, we attribute this factor to industrial emissions. PM 2.5 loaded on the analogous factor in the winter data set, but was not significantly associated with this factor in the summer. This may be due to the fact that concentrations of all measured PM components increased significantly during the summer, and so the contribution of this local source to the total PM 2.5 mass was less important during this time. The fifth factor accounted for a further $5 \%$ of the data set variance and was associated exclusively with oxidation products of isoprene: methacrolein (MACR), methylvinylketone (MVK) and 3-methylfuran.

[35] Propane, isobutane and butane grouped together on factor 6 , which likely represents propane fuel or natural gas leakage. The diurnal pattern for this factor (Figure 4f) was similar to that of factor 1 , with a strong predawn maximum and afternoon minimum. There was also a weak negative association with ozone, as there was with factor 1, owing to the co-occurrence of the maximum mixed layer depth (and lowest levels of factor 1 and factor 6 compounds) with the maximum daily ozone concentrations.

\subsubsection{Summary of Factor Analysis Results}

[36] The results of the factor analyses provide a context from which to interpret the combined VOC and fine particle data sets. In both seasons, local tailpipe emissions formed a substantial component of the ambient VOC concentrations. They did not, however, significantly impact the aerosol species that were included in the factor analysis. Nonautomotive combustion emissions, probably from industrial point sources in the area, were an important source of aerosol mass, as well as of $\mathrm{CO}, \mathrm{NO}_{\mathrm{x}}$ and several unsaturated hydrocarbons. There was pronounced photochemical production of OVOCs such as acetone, MEK, and acetaldehyde in summer. Diurnal concentration patterns indicated that this source was more important than primary combustion emissions. In winter this was not the case, although secondary production was still evident. Along with isoprene, 1-butene showed evidence of a local light-driven biogenic source. There was a distinct source of alkanes that did not appear to be a significant source of other compounds, which was likely leakage of propane fuel or natural gas. Finally, ambient PM showed evidence of a significant secondary component even in winter. The importance of primary and secondary sources to OVOC and OC levels is explored in detail in the following section.

\subsection{Source Apportionment of OVOCs and Aerosol Organic Carbon}

\subsubsection{OVOC Source Apportionment}

[37] Oxygenated VOCs can make up a sizable and even dominant fraction of the total VOC abundance and reactiv-

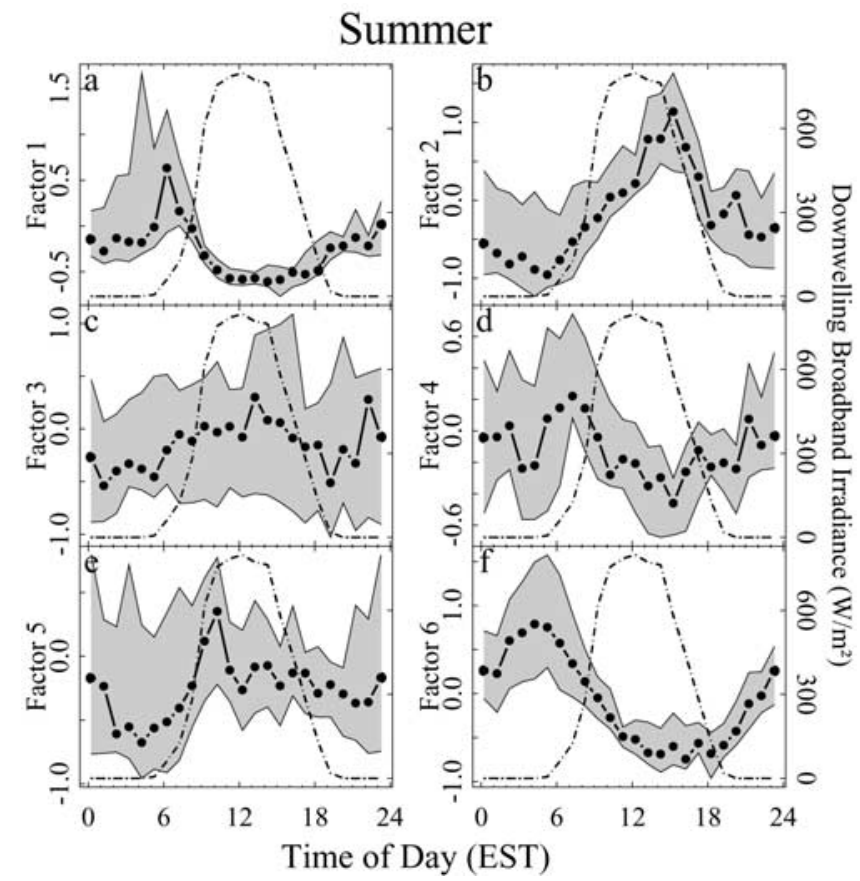

Figure 4. Median diurnal cycles in factor scores (circles) for the summer data set. Banded gray areas show the interquartile range. Incoming solar radiation is also shown (dot-dash line). 
ity, in the urban [Grosjean, 1982; Goldan et al., 1995a], rural [Goldan et al., 1995b; Riemer et al., 1998], and even remote marine atmosphere [Singh et al., 1995, 2001]. Many OVOCs, such as acetone, MEK and acetaldehyde, are known to have a diversity of sources, including combustion emissions, photochemical production from both anthropogenic and biogenic precursor species, and direct biogenic emissions. Understanding the magnitudes of these sources in different environments is prerequisite to an accurate representation of odd hydrogen cycling and ozone chemistry in models of atmospheric chemistry and air quality from the local to global scale.

[38] Here we present a new approach to unraveling source contributions to such species. We define the ambient concentrations of VOC species $\mathrm{Y}\left(\mathrm{X}_{\mathrm{y}}\right.$, in ppt) as being the sum of direct combustion $\left(\chi_{y c}\right)$ and other components $\left(\chi_{y o}\right)$, which could represent secondary or biogenic sources, as well as a background concentration $\left(\mathrm{X}_{\mathrm{a}}\right)$,

$$
\chi_{y}=\chi_{y c}+\chi_{y o}+\chi_{a} .
$$

[39] For relatively long lived species, such as acetone, $\chi_{a}$ may be considered to represent a regional background level. In this case, $\chi_{a}$ will presumably include contributions from both combustion and secondary/biogenic production that has taken place elsewhere and been integrated into the regional background. For acetaldehyde, a compound with an atmospheric lifetime of only a few hours, there was nonetheless a nonzero observed minimum concentration in both summer and winter. Here, the parameter $\chi_{a}$ may represent a relatively invariant area source that maintains ambient levels of acetaldehyde above a certain threshold. In either case, we operationally define the background concentration of each species as the 0.1 quantile of the measured concentrations [Goldstein et al., 1995b].

[40] If $\mathrm{Y}$ and a combustion tracer, such as toluene, are emitted in a relatively consistent ratio from different types of combustion sources, then $\chi_{y c}$ can be estimated as

$$
\chi_{\mathrm{yc}}=\chi_{\mathrm{tol}}\left(\frac{Y}{T O L}\right)_{\mathrm{E}},
$$

where $(Y / T O L)_{\mathrm{E}}$ is the primary emission ratio of $\mathrm{Y}$ relative to toluene, and $\chi_{\text {tol }}$ represents toluene enhancements above background (ppt; see the following section for a discussion of the choice of combustion marker). $\chi_{y_{0}}$ is then given by

$$
\chi_{\text {yo }}=\chi_{\mathrm{y}}-\chi_{\text {tol }}\left(\frac{Y}{T O L}\right)_{\mathrm{E}}-\chi_{\mathrm{a}} .
$$

In (3), $\chi_{\text {tol }}, \chi_{y}$, and $\chi_{a}$ are known quantities. All that is required to calculate the combustion $\left(\chi_{y c}\right)$ and secondary plus biogenic $\left(\mathrm{X}_{\mathrm{yo}}\right)$ components of species $\mathrm{Y}$ is the primary emission ration $(Y / T O L)_{\mathrm{E}}$.

[41] To determine $(Y / T O L)_{\mathrm{E}}$ for each species $\mathrm{Y}$, we make use of the combustion tracers associated with the first factor in the factor analyses (Tables 2 and 3). For a given value of $(Y / T O L)_{\mathrm{E}}$, we can calculate a $\chi_{\text {yo }}$ vector, and the coefficient of determination $\left(\mathrm{r}^{2}\right)$ between $\chi_{\mathrm{yo}}$ and each of our combustion tracers. By varying $(Y / T O L)_{\mathrm{E}}$ over a range of possible values and repeating this calculation, we can derive $r^{2}$

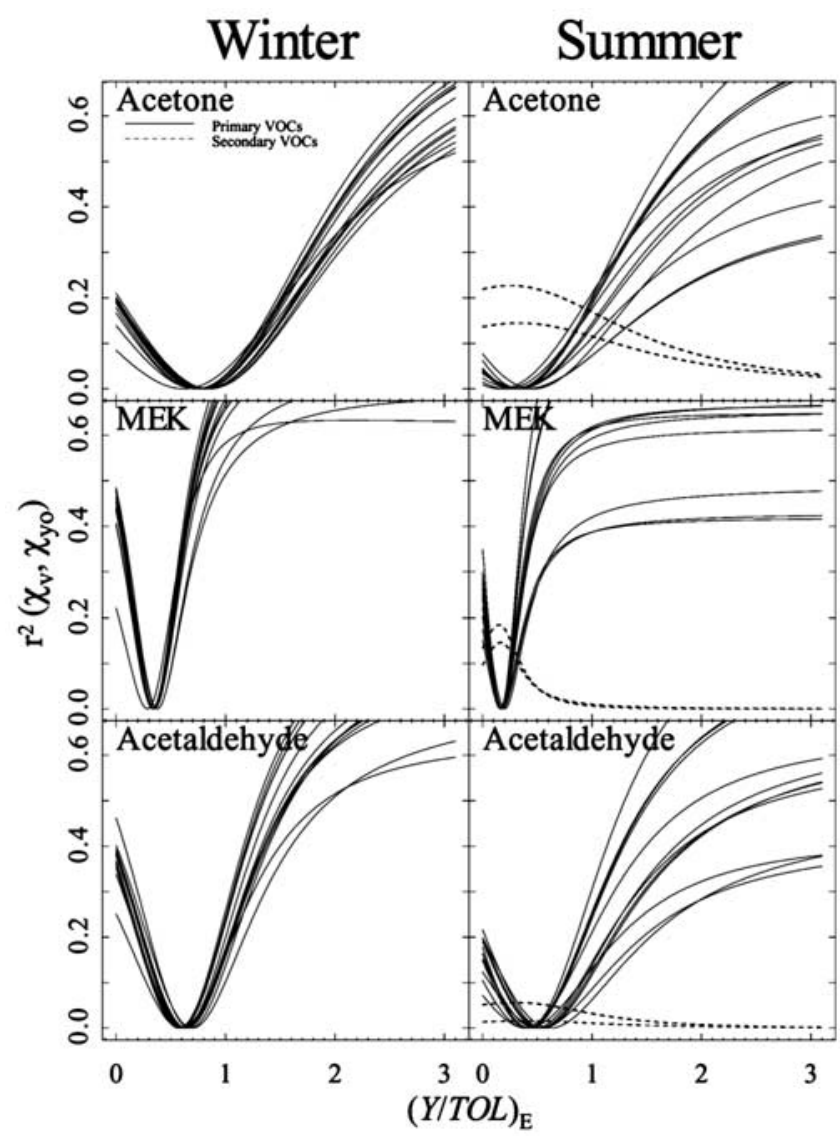

Figure 5. Coefficient of determination between combustion or photochemically derived VOCs and the residual term $\chi_{\text {yo }}$, representing photochemical and biogenic OVOC sources, as a function of the primary emission ratio $(Y / T O L)_{\mathrm{E}}$. Each solid (dashed) line represents a separate combustion (photochemical) marker compound (V, with mixing ratio $\chi_{\mathrm{v}}$, for $\mathrm{V}=$ propyne, 2-methylpropene, t-2-butene, c-2-butene, 2-methyl-1-butene, 3-methyl-1butene, t-2-pentene, benzene, ethylbenzene, p-xylene, m-xylene, o-xylene, $\mathrm{NO}_{\mathrm{x}}, \mathrm{MACR}$, or MVK). The critical point in the curves gives the combustion emission ratio for species Y (acetone, MEK, or acetaldehyde) relative to toluene.

between the calculated $\chi_{\text {yo }}$ and each of our combustion tracers, as a function of $(Y / T O L)_{\mathrm{E}}$. At low values of $(Y / T O L)_{\mathrm{E}}$, the calculated $\chi_{\text {yo }}$ will still contain a significant combustion component. At high values of $(Y / T O L)_{\mathrm{E}}, \chi_{\text {yo }}$ will become dominated by the $\chi_{\text {tol }}$ term. At the correct value for $(Y / T O L)_{\mathrm{E}}$ all contributions of combustion emissions should be removed from $\chi_{y o}$, and hence correlation of $\chi_{y o}$ with a pure combustion parameter should be at a minimum. Conversely, if the noncombustion sources of Y are dominantly photochemical, then the correlation between $\chi_{y o}$ and a photochemically derived VOC should reach a maximum at that same point.

[42] The results of performing this analysis for $\mathrm{Y}=$ acetone, MEK and acetaldehyde are shown in Figure 5. Each solid line shows the coefficient of determination between an individual combustion marker and $\chi_{\text {yo }}$, as a function of the value of $(Y / T O L)_{\mathrm{E}}$ that was used to calculate $\chi_{\text {yo }}$. The compounds used as markers of combustion (V, with mixing ratios $\chi_{v}$ ) were those VOCs thought to 
Table 4. OVOC Combustion Emission Ratios and Source Contributions ${ }^{\mathrm{a}}$

\begin{tabular}{|c|c|c|c|c|c|c|c|c|c|c|c|c|c|c|c|}
\hline \multirow[b]{3}{*}{ Species (Y) } & \multirow{2}{*}{\multicolumn{2}{|c|}{$\begin{array}{c}\text { Ambient } \\
\text { Concentration } \\
\chi_{y}, p p t \\
\end{array}$}} & \multirow{2}{*}{\multicolumn{2}{|c|}{$\begin{array}{c}\text { Primary Emission } \\
\text { Ratio } \\
(Y / T O L)_{\mathrm{E}} \\
\end{array}$}} & \multirow{2}{*}{\multicolumn{3}{|c|}{$\begin{array}{c}\begin{array}{c}\text { Background } \\
\text { Concentration }\end{array} \\
\chi_{\mathrm{a}} / \chi_{\mathrm{y}}\end{array}$}} & \multicolumn{4}{|c|}{ Combustion Emissions } & \multicolumn{4}{|c|}{ Other Sources } \\
\hline & & & & & & & & \multicolumn{2}{|c|}{$\chi_{y c}, p p t$} & \multicolumn{2}{|c|}{$\chi_{y c} / \chi_{y}$} & \multicolumn{2}{|c|}{$\chi_{\text {yo }}, \mathrm{ppt}$} & \multicolumn{2}{|c|}{$\chi_{y o} / \chi_{y}$} \\
\hline & Median & $\mathrm{IQR}^{\mathrm{b}}$ & Median & $\mathrm{IQR}^{\mathrm{b}}$ & ppt & Median & $\mathrm{IQR}^{\mathrm{b}}$ & Median & $\mathrm{IQR}^{\mathrm{b}}$ & Median & $\mathrm{IQR}^{\mathrm{b}}$ & Median & $\mathrm{IQR}^{\mathrm{b}}$ & Median & $\mathrm{IQR}^{\mathrm{b}}$ \\
\hline \multicolumn{16}{|c|}{ Winter } \\
\hline Acetone & 943 & $655-1390$ & 0.78 & $0.74-0.82$ & 526 & 0.56 & $0.38-0.80$ & 114 & $49-241$ & 0.12 & $0.05-0.21$ & 237 & $23-624$ & 0.24 & $0.04-0.48$ \\
\hline MEK & 215 & $153-299$ & 0.34 & $0.34-0.34$ & 120 & 0.56 & $0.40-0.79$ & 50 & $21-105$ & 0.23 & $0.10-0.39$ & 24 & $0-92$ & 0.12 & $0.00-0.35$ \\
\hline Acetaldehyde & 538 & $403-729$ & 0.62 & $0.60-0.64$ & 289 & 0.54 & $0.40-0.72$ & 91 & $39-192$ & 0.17 & $0.07-0.31$ & 146 & $24-290$ & 0.27 & $0.05-0.40$ \\
\hline \multicolumn{16}{|c|}{ Summer } \\
\hline Acetone & 4030 & $3130-4890$ & 0.32 & $0.29-0.34$ & 2650 & 0.66 & $0.54-0.85$ & 81 & $29-224$ & 0.02 & $0.01-0.06$ & 1200 & $353-1940$ & 0.29 & $0.12-0.41$ \\
\hline MEK & 559 & $408-674$ & 0.17 & $0.16-0.18$ & 319 & 0.57 & $0.47-0.78$ & 45 & $16-123$ & 0.10 & $0.03-0.23$ & 138 & $29-257$ & 0.26 & $0.06-0.40$ \\
\hline Acetaldehyde & 1560 & $1100-2150$ & 0.43 & $0.40-0.52$ & 798 & 0.51 & $0.37-0.72$ & 113 & $40-310$ & 0.09 & $0.03-0.20$ & 542 & $126-1050$ & 0.34 & $0.11-0.50$ \\
\hline
\end{tabular}

be solely or predominantly derived via combustion processes (propyne, 2-methylpropene, t-2-butene, c-2-butene, 2-methyl-1-butene, 3-methyl-1-butene, t-2-pentene, benzene, ethylbenzene, p-xylene, m-xylene, o-xylene) and $\mathrm{NO}_{\mathrm{x}}$. Dashed lines show $\mathrm{r}^{2}$ between $\chi_{\mathrm{yo}}$ and VOCs thought to be solely photochemically produced (MACR and MVK, which were present above detection limit in the summer experiment only), as a function of $(Y / T O L)_{\mathrm{E}}$.

[43] There is a well defined minimum in the curve for the combustion markers, the location of which, for a given oxygenated VOC species $\mathrm{Y}$, is consistent across all marker compounds. For the summer data, the location of this minimum coincides with the maximum $r^{2}$ value for the photochemically produced tracer species. We interpret the location of the critical value of $\mathrm{r}^{2}$ as the representative ( $Y$ / $T O L)_{\mathrm{E}}$ value for that time of year (Table 4).

[44] Primary emission ratios, relative to toluene, for acetone, MEK and acetaldehyde were all substantially (1.4-2.4 times) higher in January-February 2002 than in July-August 2002. Since the emission ratio depends on the toluene as well as OVOC emission strength, seasonal changes in the emission ratio can be due to changes in the numerator, denominator or both. This issue is discussed further in the following section. The primary emission ratios calculated in this section are averages over the sources impacting the air masses that were sampled during the course of the study. They therefore represent integrated regional emission ratios for Pittsburgh in January-February and July-August 2002.

[45] Urban and industrial VOC emission ratios depend on a number of factors, in particular vehicle fleet and fuel characteristics as well as types of industrial activity in the region. Such variability complicates efforts to construct reliable emission inventories for use in air quality modeling, and emphasizes the utility of the approach developed here, which provides top-down observational constraints on regional pollutant emission ratios. On-road studies of motor vehicle exhaust in the U.S. (generally carried out during summer) report emission ratios for acetone, MEK and acetaldehyde relative to toluene ranging from $2-4 \%, 2-$ $12 \%$, and $<1-8 \%$ (molar basis) respectively for light-duty vehicles [Kirchstetter et al., 1999; Fraser et al., 1998; Zielinska et al., 1996; Kirchstetter et al., 1996]. Heavy-duty or diesel vehicles emit substantially higher amounts of these OVOCs relative to toluene, with emission ratios frequently greater than unity [Zielinska et al., 1996; Staehelin et al., 1998]. Inventory estimates (including mobile, point and nonpoint sources) of annual acetaldehyde and MEK emissions in Allegheny County are $14 \%$ and $10 \%$ those of toluene respectively on a molar basis (see http://www.epa. gov/ttn/chief/net/index.html), substantially lower than the values determined here (Table 4). If inventory estimates of toluene emissions are accurate, this suggests that acetaldehyde and MEK emissions are underestimated by factors of approximately 3.8 and 2.6 (from the average of the summer and winter ratios, Table 4).

[46] For the summer data, $\chi_{\text {yo }}$ for both acetone and MEK exhibited a well-defined maximum correlation with MACR and MVK (Figure 5), indicating that the other, noncombustive, source represented by $\chi_{\text {yo }}$ is likely to be largely photochemical. For acetaldehyde, the poor correlation of $\chi_{\text {yo }}$ with MACR and MVK suggests that $\chi_{\text {yo }}$ is not exclusively photochemical in nature, and may contain another significant component such as biogenic emissions.

[47] For comparison, Figure 6 shows results of the same analysis for $\mathrm{Y}=$ MACR and MVK, species whose only significant known source is from photochemical oxidation of isoprene. In this case, the minimum correlation of $\chi_{\text {yo }}$ with combustion derived VOCs (and maximum correlation with MVK or MACR) occurs at a combustion emission ratio $(Y / T O L)_{\mathrm{E}}$ of zero, showing that there are no significant primary emissions of these compounds.

[48] With $(Y / T O L)_{\mathrm{E}}$ determined by the critical points in Figure 5, the contributions to the concentration of species $\mathrm{Y}$ from background $\left(\chi_{\mathrm{a}}\right)$, combustion emissions $\left(\chi_{y c}\right)$, and other sources $\left(\chi_{y_{0}}\right)$ as a function of time can then be calculated from (2) and (3). Contributions of $\chi_{a}, \chi_{y c}$, and $\chi_{\text {yo }}$ to the ambient levels of acetone, MEK, and acetaldehyde in summer and winter are summarized in Table 4. Negative values of $\chi_{\text {yo }}$ were assumed to contain no secondary or biogenic material and were set to zero.

[49] Ambient concentrations of acetone, MEK and acetaldehyde during summer were on average 3-4 times higher than winter (Table 4). Increases in background concentrations were responsible for a significant portion of this winter to summer difference, with summer background levels on average $2.5-5$ times higher than in the winter. However, the fraction of the total concentration due to the background was comparable in summer and winter. In both seasons, the background made up, on average, slightly over half of the 


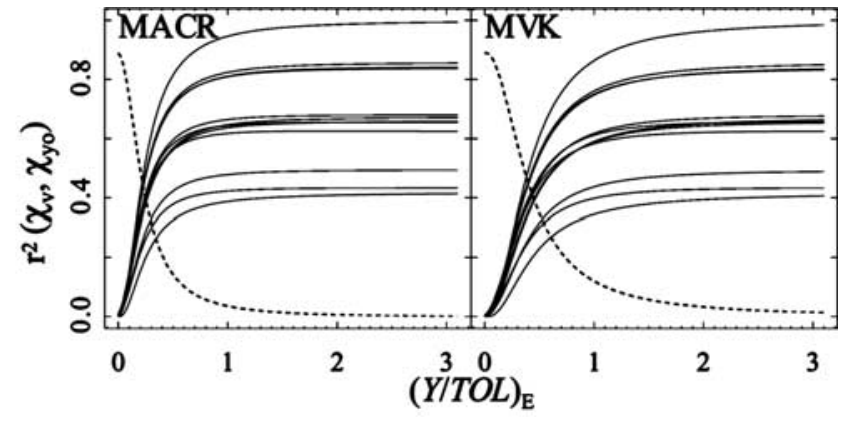

Figure 6. Same as Figure 5, except for $\mathrm{Y}=$ methacrolein (MACR) and methylvinylketone (MVK). The minimum correlation of $\chi_{\text {yo }}$ with combustion derived VOCs (and maximum correlation with photochemical VOCs) occurs at an emission ratio $(Y / T O L)_{\mathrm{E}}$ of zero, showing that there are no significant primary emissions of these compounds.

overall abundance for all three compounds (Table 4). The higher summer background concentrations for these species were probably due to increased nonlocal photochemical production and biogenic emission during that time of year.

[50] The absolute contribution from combustion to atmospheric mixing ratios was very similar in summer and winter, despite the large changes in emission ratios (which were higher in winter by factors of $2.4,2.0$ and 1.4 for acetone, MEK and acetaldehyde; see discussion in following section). However, total concentrations were substan- tially higher in summer, and combustion emissions were a significantly smaller fraction of the total source (Table 4).

[51] Other sources, which we assume to be predominantly photochemical but which also likely include some biogenic emissions in summer, were substantially higher in summer for all three compounds. Median summer values of $\chi_{\text {yo }}$ were over 5 times higher than in winter for acetone and MEK and nearly 4 times higher for acetaldehyde.

[52] With the exception of MEK, combustion was not the major source of these compounds, even in winter. For MEK, combustion emissions were more important than other sources $\left(\chi_{y o}\right)$ in the winter (a median of $23 \%$ versus $13 \%$ ). This was not the case in the summer, however, nor was it true for acetone or acetaldehyde in either season. For acetone, other sources were twice as important as combustion emissions in the winter and ten times as important in the summer. For acetaldehyde, other sources were 50\% larger than combustion emissions in winter and 4 times larger in summer.

[53] Diurnally averaged OVOC source contributions, overlaid with ozone concentrations, in winter and summer are shown in Figure 7. For the summer data set, the other OVOC sources $\left(\chi_{\text {yo }}\right)$ showed a strong photochemical signature: low at night, increasing after sunrise and peaking in the afternoon. For each compound, acetone, MEK and acetaldehyde, the $\chi_{\text {yo }}$ term tracked ozone quite closely. For the winter data set, the $\chi_{y o}$ terms for each OVOC showed a much weaker photochemical signal, and the relative contribution from combustion was substantially larger than in the summer. Note that since $\chi_{y c}$ for each
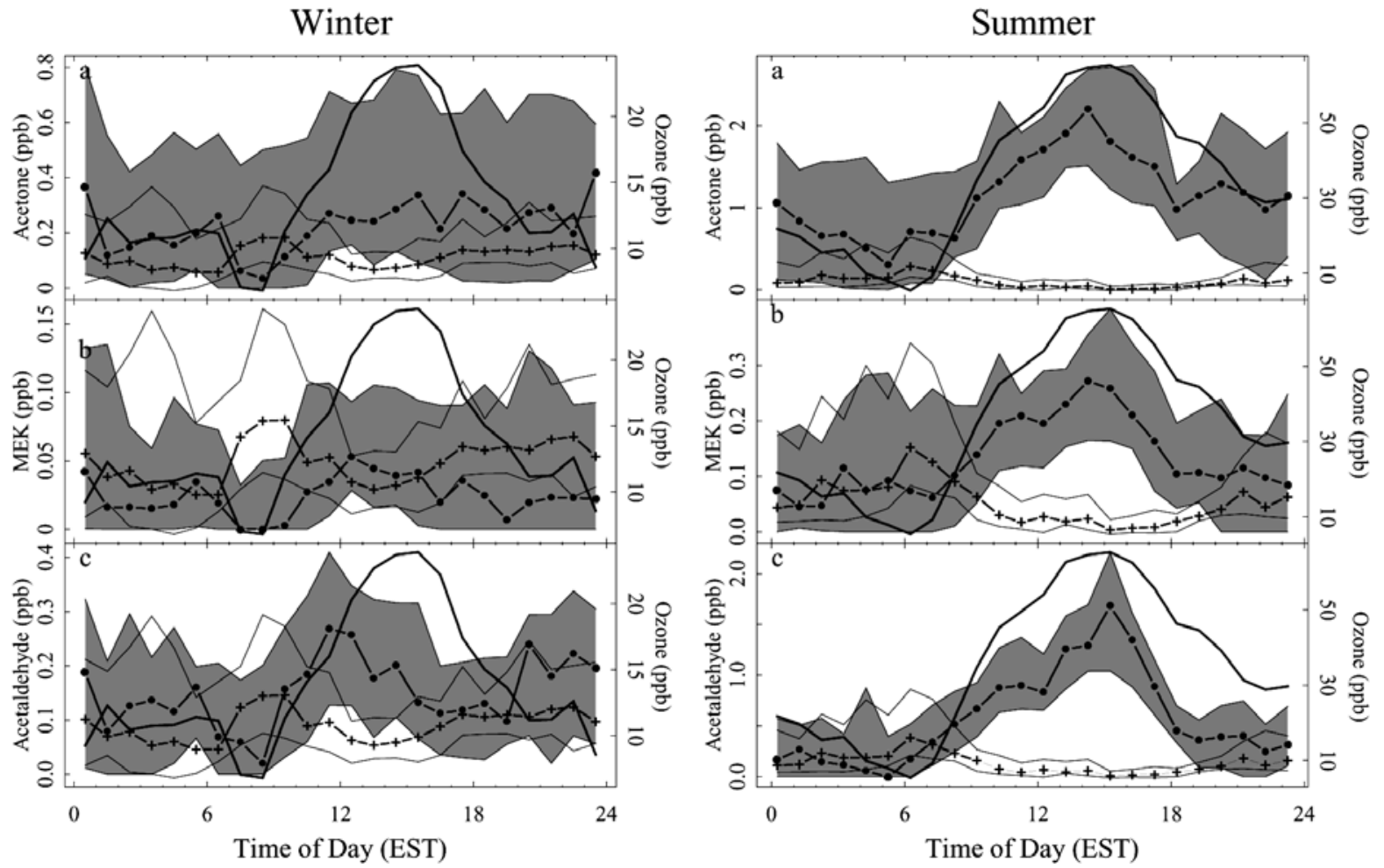

Figure 7. Diurnal patterns in OVOC source contributions (winter and summer data). Combustion source $\left(\chi_{y c}, p p b\right)$, pluses and unshaded region; photochemical and biogenic sources ( $\left.\chi_{\text {yo }}, p p b\right)$, circles and surrounding gray area. Ozone is also shown (solid dark line). Points show median values; banded areas show the interquartile range. Note different $y$ axis scales for winter and summer. 
OVOC is defined as $\chi_{\text {tol }}(Y / T O L)_{\mathrm{E}}$, i.e. the observed toluene enhancements multiplied by a primary emission ratio, diurnal patterns in $\chi_{y c}$ shown in Figure 7 reflect that of toluene.

\subsubsection{Choice of Combustion Marker and Seasonal Patterns in Emission Ratios}

[54] Repeating the above analysis using other combustion derived compounds instead of toluene as the primary emission tracer resulted in only minor changes to the calculated OVOC partitioning (Table 4) and did not alter any of the conclusions. This gives us confidence that this approach to partitioning VOC source contributions is robust. For a given primary emission tracer, the calculated OVOC emission ratios, given by the critical $r^{2}$, were consistent using compounds that are solely combustion derived (e.g. alkenes and alkynes) and compounds that have additional anthropogenic noncombustion sources, such as evaporative losses and chemical processing (e.g. benzene and toluene). Hence the approach is not sensitive to slight differences in source profiles for the marker compounds. We conclude that the calculated emission ratios represent an integrated regional primary pollution signal, rather than one specific source type.

[55] In addition, we note that the combustion markers employed to calculate the OVOC primary emission ratios relative to toluene (Figure 5) have lifetimes that vary by nearly a factor of 50, yet they give consistent emission ratio estimates. This may indicate that much of the variability observed is relatively local and not driven by photochemical lifetime or by the different sampling footprints for species of different lifetimes.

[56] Seasonal differences in the OVOC primary emission ratios, however, calculated relative to the tracer compound, changed dramatically depending on the tracer used. This is to be expected since the primary emission ratios are sensitive to changes in both the numerator and denominator, and different combustion tracers do not necessarily have identical seasonal patterns in emission strength. While the primary OVOC emission ratios relative to toluene were all higher in the winter, OVOC emission ratios calculated relative to alkenes and alkynes were generally 2-3 times higher in the summer. It is possible that noncombustion toluene sources, i.e. evaporative emissions, are higher in summer which would decrease the OVOC emission ratio for that time of year. However, the short-lived alkenes are oxidized much more rapidly in the summer months due to higher concentrations of $\mathrm{OH}$ and ozone. Over a given source-receptor distance, then, the alkenes would be more depleted relative to the OVOCs in the summer than in the winter. This would lead to higher OVOC:alkene emission ratios in the summer, as observed. While this effect would also occur with toluene, either the effect was small due to toluene's longer lifetime (10 times that of t-2-butene) and/or it was offset by increased emissions.

\subsubsection{Quantification of Secondary Organic Aerosol}

[57] Organic carbon (OC) constitutes a significant fraction of atmospheric aerosol [Lim and Turpin, 2002; Cabada et al., 2002, 2004; Tolocka et al., 2001]; however, its origin and composition remain poorly understood. OC consists of hundreds or thousands of individual organic compounds. Both anthropogenic sources (e.g. combustion) and biogenic sources (e.g. plants) can contribute to aerosol organic carbon via direct emission of particles (primary OC), and via emission of gas-phase precursor compounds that partition into the aerosol phase upon oxidation (secondary OC). Clarifying the roles of primary and secondary OC production is an important step toward an improved understanding and modeling of the sources, morphology and effects of aerosol OC. The technique of minimizing (maximizing) the correlation between combustion (photochemical) tracer compounds and the photochemical component of a species of interest, developed in the previous section, also has utility in determining the primary emission ratio for pollutants other than VOCs. Here, we apply the method to quantify the relative importance of primary and secondary OC sources in the study region.

[58] As above, aerosol organic carbon concentrations $\left(M_{\mathrm{oc}}\right.$, in $\mu \mathrm{g}$ of carbon per cubic meter, $\left.\mu \mathrm{gC} / \mathrm{m}^{3}\right)$ are defined as being composed of combustion $\left(M_{\mathrm{c}}\right)$ and other $\left(M_{\mathrm{o}}\right)$ components, plus a regional background $\left(M_{\mathrm{a}}\right)$ [Turpin and Huntzicker, 1995]:

$$
M_{\mathrm{oc}}=M_{\mathrm{c}}+M_{\mathrm{o}}+M_{\mathrm{a}} .
$$

[59] Elemental carbon (EC, or soot) is an aerosol component whose only source is direct emission from combustion. If both $\mathrm{OC}$ and $\mathrm{EC}$ are emitted from primary sources according to a characteristic averaged OC:EC emission ratio $(O C / E C)_{\mathrm{E}}$, then the combustion-derived organic carbon can be estimated as

$$
M_{\mathrm{c}}=M_{\mathrm{ec}}\left(\frac{O C}{E C}\right)_{\mathrm{E}},
$$

where $M_{\text {ec }}$ represents elemental carbon enhancements above background (in $\mu \mathrm{gC} / \mathrm{m}^{3}$ ), and $M_{\mathrm{o}}$ is given by

$$
M_{\mathrm{o}}=M_{\mathrm{oc}}-M_{\mathrm{ec}}\left(\frac{O C}{E C}\right)_{\mathrm{E}}-M_{\mathrm{a}}
$$

[60] The background term, $M_{\mathrm{a}}$, represents noncombustion primary $\mathrm{OC}$ (e.g. from biogenic sources) as well as any regional aerosol organic carbon background. As above, we estimate $M_{\mathrm{a}}$ as the 0.1 quantile of the measured $\mathrm{OC}$ concentrations. $M_{\mathrm{o}}$ is then assumed to be exclusively secondary OC. It should be pointed out, however, that if there exist significant sources of primary OC which do not correlate with EC and are highly variable through time (and thus are not entirely captured by the $M_{\mathrm{a}}$ parameter), then $M_{\mathrm{o}}$ may also contain some primary influence.

[61] One challenge associated with the EC tracer method as it has been applied in the past involves defining the OC:EC ratio of primary emissions, as this can vary significantly between sources and consequently as a function of time. In addition, defining $(O C / E C)_{\mathrm{E}}$ from ambient $\mathrm{OC}$ and $\mathrm{EC}$ concentration data requires that there be a subset of data with no significant secondary contributions to the measured OC concentrations. The typical approach is to qualitatively eliminate data points that are likely to be impacted by significant secondary production or other factors such as rain events, and regress $\mathrm{OC}$ on $\mathrm{EC}$ for that subset of data dominated by primary OC [Turpin and Huntzicker, 1995; Cabada et al., 2004]. This then gives a regression slope that is in theory reflective solely of primary emissions. The 


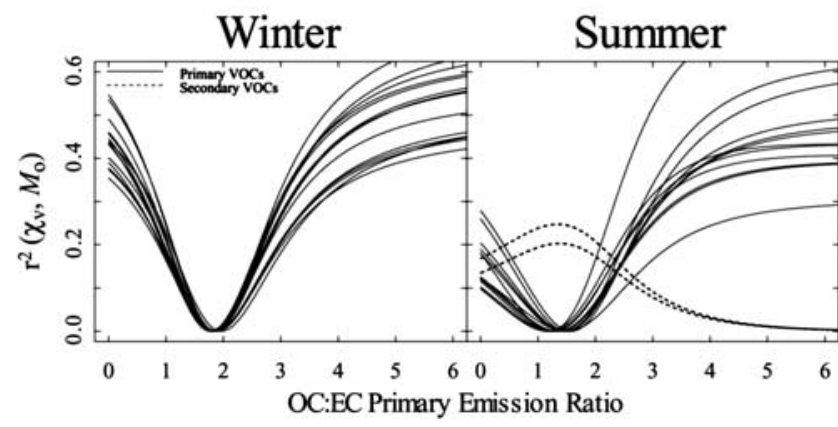

Figure 8. Coefficient of determination between combustion or photochemically derived VOCs and the residual term $M_{\mathrm{o}}$, representing secondary $\mathrm{OC}$, as a function of the primary emission ratio $(O C / E C)_{\mathrm{E}}$. Each solid (dashed) line represents a separate combustion (photochemical) marker compound. The critical point in the curves gives the primary emission ratio $(O C / E C)_{\mathrm{E}}$.

parameter $M_{\mathrm{a}}$, reflecting primary noncombustion $\mathrm{OC}$, is then assumed to be constant and given by the intercept, enabling the calculation of $M_{\mathrm{o}}$. In the event of significant temporal variability in the primary OC:EC ratio impacting the sampling site, this process may be repeated on subsets of the data.

[62] Here we employ the technique developed in the previous section, using the range of markers for primary and secondary processes provided by the VOC data set to define the characteristic OC:EC primary emission ratio for the Pittsburgh region in summer and winter. This approach avoids the need to carefully select time periods that will yield the "correct" value of $(O C / E C)_{\mathrm{E}}$. In addition, the suite of primary and secondary VOCs available provides bounds on the value of $(O C / E C)_{\mathrm{E}}$ appropriate to a given time period. The secondary organic aerosol is then calculated according to (6).

[63] The coefficient of determination between $M_{\mathrm{o}}$ and combustion and photochemically derived VOCs is shown in Figure 8 as a function of $(O C / E C)_{\mathrm{E}}$ for winter and summer. Again, the critical point of the curves gives the representative value of $(O C / E C)_{\mathrm{E}}$ for that time of year.

[64] The median value of $(O C / E C)_{\mathrm{E}}$ determined for the winter data set was 1.85 (IQR: $1.82-1.86$ ), whereas that for the summer data set was lower with a median of 1.36 (IQR: 1.27-1.48) (Table 5). Substantially higher particulate concentrations of levoglucosan were observed in the winter, indicative of increased wood combustion. More widespread wood burning is a likely cause of the higher primary OC:EC emission ratio at that time of year. Colder engines and less efficient combustion may have also contributed to the higher wintertime ratio.
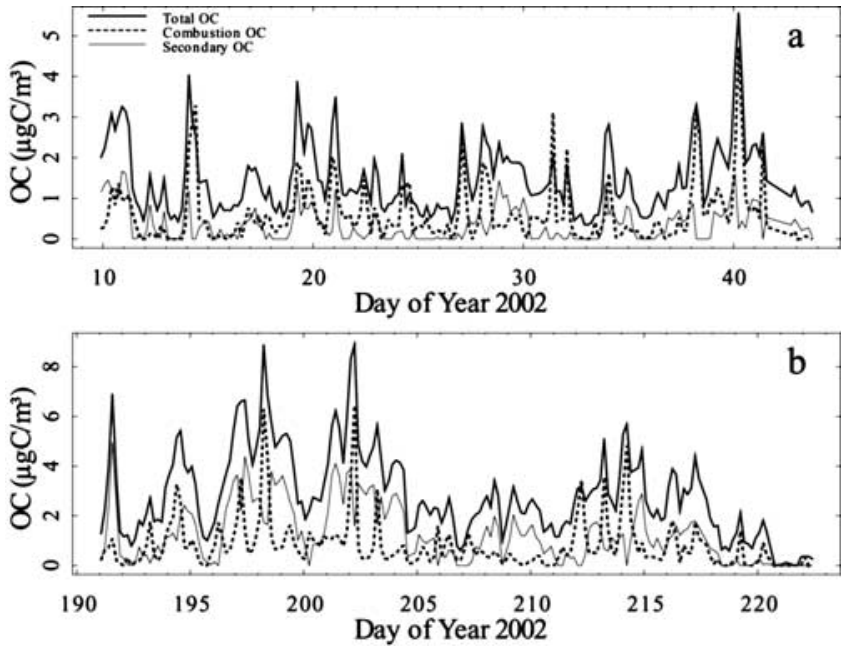

Figure 9. Timelines of total $\mathrm{OC}\left(M_{\mathrm{oc}}, \mu \mathrm{gC} / \mathrm{m}^{3}\right)$, dark solid line; combustion $\mathrm{OC}\left(M_{\mathrm{c}}, \mu \mathrm{gC} / \mathrm{m}^{3}\right)$, dashed line; secondary OC $\left(M_{\mathrm{o}}, \mu \mathrm{gC} / \mathrm{m}^{3}\right)$, light solid line. Data are shown for the (a) winter and (b) summer deployments.

[65] Using the derived values of $(O C / E C)_{\mathrm{E}}$ for summer and winter, we can then calculate $M_{\mathrm{o}}$, the secondary $\mathrm{OC}$, according to (6). Timelines of the total $\left(M_{\mathrm{oc}}\right)$, combustion $\left(M_{\mathrm{c}}\right)$, and secondary $\left(M_{\mathrm{o}}\right)$ aerosol organic carbon concentrations (in $\mu \mathrm{gC} / \mathrm{m}^{3}$ ) for winter and summer 2002 are plotted in Figure 9, and quantiles of these quantities are given in Table 5. Note that since $M_{\mathrm{c}}$ is defined as $M_{\mathrm{ec}}(O C / E C)_{\mathrm{E}}$, there are occasional episodes where $M_{\mathrm{c}}>M_{\mathrm{oc}}$. Negative values of $M_{\mathrm{o}}$ were assumed to contain no secondary material and were set to zero.

[66] Ambient concentrations of aerosol organic carbon in the summer experiment were on average twice as high as in the winter (Table 5). Background levels $\left(M_{\mathrm{a}}\right)$ made up a significant fraction of the total ambient aerosol OC concentrations in both seasons. Background aerosol OC concentrations were slightly higher in summer but a larger fraction of the total in winter (median of $49 \%$ versus 35\%). Similarly, combustion OC was slightly higher in the summer, however, it made up a larger fraction of the total OC in winter (median of 30\% versus 19\%). Secondary organic carbon $\left(M_{\mathrm{o}}\right)$ accounted for a median of $16 \%$ (IQR: $0-35 \%$ ) of the aerosol OC in winter, and 37\% (IQR: $15-56 \%$ ) in summer (Table 5).

[67] A. Polidori et al. (manuscript in preparation, 2005) carried out an analysis of the primary and secondary components of $\mathrm{OC}$ in Pittsburgh during the PAQS study

Table 5. OC Combustion Emission Ratios and Source Contributions ${ }^{\mathrm{a}}$

\begin{tabular}{|c|c|c|c|c|c|c|c|c|c|c|c|c|c|c|}
\hline \multirow[b]{3}{*}{ Season } & \multirow{2}{*}{\multicolumn{2}{|c|}{$\begin{array}{c}\begin{array}{c}\text { Ambient } \\
\text { Concentration }\end{array} \\
M_{\mathrm{oc}} \\
\mu \mathrm{gC} / \mathrm{m}^{3} \\
\end{array}$}} & \multirow{2}{*}{\multicolumn{2}{|c|}{$\begin{array}{c}\begin{array}{c}\text { Primary } \\
\text { Emission } \\
\text { Ratio }\end{array} \\
(O C / E C)_{\mathrm{E}} \\
\end{array}$}} & \multirow{2}{*}{\multicolumn{3}{|c|}{$\begin{array}{c}\begin{array}{c}\text { Background } \\
\text { Concentration }\end{array} \\
M_{\mathrm{a}}, \quad M_{\mathrm{a}} / M_{\mathrm{oc}}\end{array}$}} & \multicolumn{4}{|c|}{ Combustion Emissions } & \multicolumn{3}{|c|}{ Secondary Production } \\
\hline & & & & & & & & & $\begin{array}{l}M_{\mathrm{c}} \\
\mathrm{C} / \mathrm{m}^{3}\end{array}$ & & $\mathrm{~d} / M_{\mathrm{oc}}$ & & $\begin{array}{l}M_{\mathrm{o}} \\
\mathrm{C} / \mathrm{m}^{3}\end{array}$ & $M_{\mathrm{o}} / M_{\mathrm{oc}}$ \\
\hline & Median & $\mathrm{IQR}^{\mathrm{b}}$ & Median & $\mathrm{IQR}^{\mathrm{b}}$ & $\mu \mathrm{gCC} / \mathrm{m}^{3} \mathrm{I}$ & Median & $\mathrm{IQR}^{\mathrm{b}}$ & Median & $\mathrm{IQR}^{\mathrm{b}}$ & Median & $\mathrm{IQR}^{\mathrm{b}}$ & Median & $\mathrm{IQR}^{\mathrm{b}}$ & Median $\mathrm{IQR}^{\mathrm{b}}$ \\
\hline Winter & 1.2 & $0.83-2.0$ & 1.85 & $1.82-1.86$ & 0.60 & 0.49 & $0.30-0.72$ & 0.37 & $0.13-0.75$ & 0.30 & $0.13-0.48$ & 0.20 & $0.00-0.59$ & $0.16 \quad 0.00-0.35$ \\
\hline Summer & 2.5 & $1.6-3.9$ & 1.36 & $1.27-1.48$ & 0.87 & 0.35 & $0.22-0.54$ & 0.50 & $0.20-1.1$ & 0.19 & $0.10-0.32$ & 0.99 & $0.27-1.9$ & $0.15-0.56$ \\
\hline
\end{tabular}


using the Turpin and Huntzicker [1995] EC tracer method. For overlapping time periods (10 January to 12 February and $10-31$ July 2002), they calculate median secondary OC concentrations of $0.21 \mu \mathrm{gC} / \mathrm{m}^{3}(15 \%$ of total OC) and $1.04 \mu \mathrm{gC} / \mathrm{m}^{3}$ (47\% of total OC) respectively. These values are in good agreement with those calculated here for the same periods: $0.20 \mu \mathrm{gC} / \mathrm{m}^{3}(16 \%$ of total OC) and $1.15 \mu \mathrm{gC} / \mathrm{m}^{3}$ ( $43 \%$ of total OC) (note that these values differ slightly from those in Table 5 since they do not reflect identical time periods).

\subsection{Characterization of the Chemical State of the Atmosphere: VOC Contributions to $\mathrm{OH}$ Loss}

[68] Photochemical production of secondary organic aerosol (SOA) depends on the chemical state of the atmosphere, both in terms of oxidative capacity, and in term of the quantity and nature of gas phase organic material that is present to form aerosol. In this section we describe the relative importance of different classes of VOCs to tropospheric photochemistry in the Pittsburgh region in summer and winter, and show that higher levels of photochemically active compounds are present in summer, when SOA levels are highest, due to biogenic emissions and photochemical production of OVOCs.

[69] A useful measure of air mass chemical reactivity is the $\mathrm{OH}$ loss rate $\left(\mathrm{L}_{\mathrm{OH}}, \mathrm{s}^{-1}\right)$, defined as

$$
\mathrm{L}_{\mathrm{OH}}=\sum_{\mathrm{i}} \mathrm{k}_{\mathrm{i}} \chi_{\mathrm{i}}
$$

where $\mathrm{k}_{\mathrm{i}}$ is the reaction rate constant for species $\mathrm{i}$ with the hydroxyl radical [Atkinson, 1994], and $\chi_{i}$ is the concentration of $\mathrm{i}$ in molec $/ \mathrm{cm}^{3}$. $\mathrm{L}_{\mathrm{OH}}$ has units of $\mathrm{s}^{-1}$ and represents the inverse lifetime of the hydroxyl radical with respect to reaction with the measured compounds.

[70] Daytime (1000-1600 EST) values of $\mathrm{L}_{\mathrm{OH}}$ were calculated for the following groups of compounds: total (all measured VOCs plus CO); alkanes; alkenes + alkynes; aromatics; OVOCs; isoprene plus its oxidation products methacrolein, methylvinylketone, and 3-methylfuran; and CO (Figure 10; Tables 6 and 7).

[71] Due to analytical challenges, VOC measurements in many field studies of air quality and atmospheric chemistry comprise only the anthropogenic nonmethane hydrocarbons (NMHCs; alkanes, alkenes, alkynes and aromatics). In Pittsburgh during January and February 2002, these species accounted for a substantial portion (approximately $60 \%$ ) of the total measured $\mathrm{OH}$ loss rate. However, while their collective $\mathrm{OH}$ reactivity was only slightly less in summer $\left(0.68 \mathrm{~s}^{-1}\right.$ versus $\left.0.89 \mathrm{~s}^{-1}\right)$, their importance relative to other VOCs was dramatically lower, as they accounted for only $11 \%$ on average of total $\mathrm{L}_{\mathrm{OH}}$ during summer. Similarly, the $\mathrm{CO}$ reactivity was comparable in both seasons (median of $0.49 \mathrm{~s}^{-1}$ in winter and $0.53 \mathrm{~s}^{-1}$ in summer), but its relative contribution to the total measured $\mathrm{OH}$ loss rate was much greater in winter (median of $23 \%$ versus $7 \%$ in the summer). It should be pointed out that these calculations do not include the $\mathrm{C}_{2}$ hydrocarbons ethane, ethene and ethyne, which were not measured. Based on published ratios of these compounds to other species [Parrish et al., 1998], we estimate that they would cause an $\mathrm{OH}$ loss rate of approximately $0.05 \mathrm{~s}^{-1}$ and $0.13 \mathrm{~s}^{-1}$ for summer and winter.

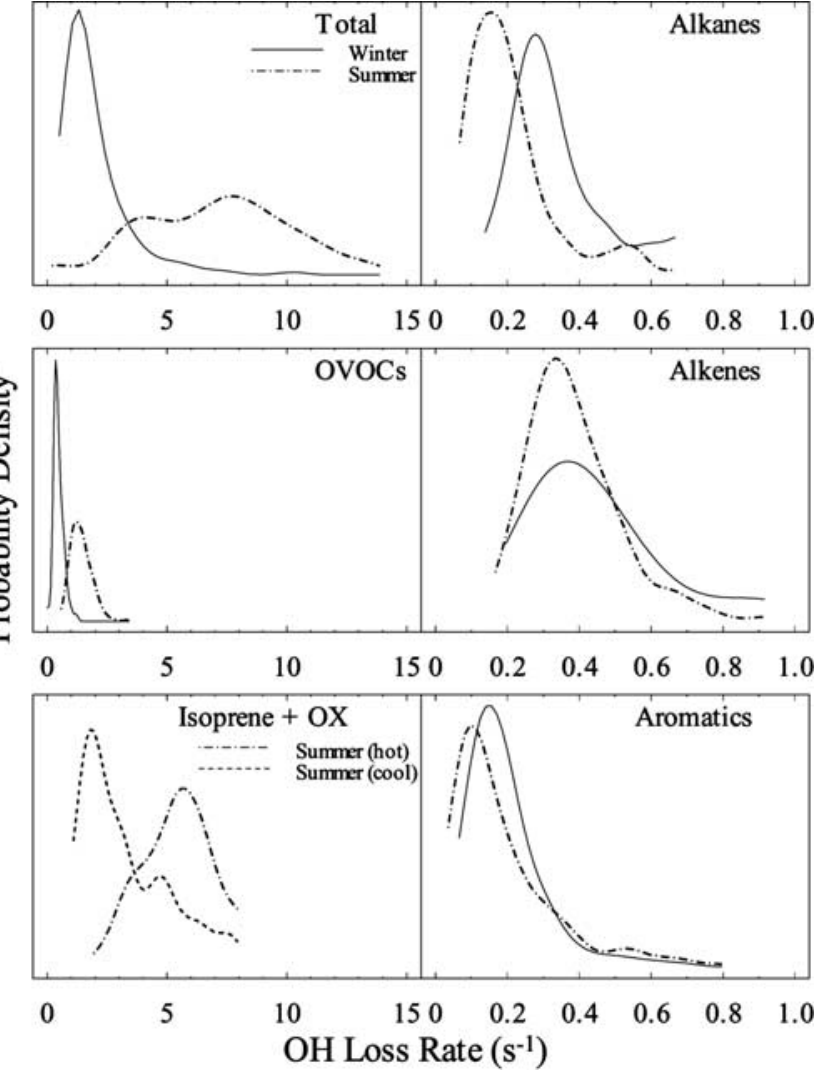

Figure 10. Probability density curves of measured daytime (1000-1600 EST) VOC OH loss rate by compound class for winter and summer 2002. Measured $\mathrm{OH}$ loss rate for isoprene plus its oxidation products methacrolein, methylvinylketone, and 3-methylfuran is shown for both hot (maximum air temperature $\geq 29^{\circ} \mathrm{C}$ ) and cool (maximum air temperature $<29^{\circ} \mathrm{C}$ ) days in the summer. These compounds were not present above detection limit in the winter. Note the different scales for the $\mathrm{x}$ axes in the leftand right-hand columns.

[72] Despite the comparable NMHC and CO reactivity in the two seasons, the total measured daytime $\mathrm{OH}$ loss rate underwent a more than fourfold increase from winter (median $=1.42 \mathrm{~s}^{-1}$; IQR: $1.12-2.30 \mathrm{~s}^{-1}$ ) to summer (median $=7.25 \mathrm{~s}^{-1}$; IQR: $4.60-9.38 \mathrm{~s}^{-1}$ ). This was due to the presence of high levels of isoprene and its oxidation products in summer, and also to the three-fold increase in oxygenated VOC concentration and reactivity from winter to summer (Tables 6,7). Isoprene plus its oxidation products accounted for a median of $62 \%$ (IQR: $52-70 \%$ ) of the daytime $\mathrm{OH}$ loss rate in summer, with the OVOCs accounting for an additional 20\% (IQR: 15-26\%). Formaldehyde measurements were not made during the PAQS study, and including the effects of this compound would result in an increased contribution to the calculated $\mathrm{OH}$ loss rate from the OVOCs in both seasons.

[73] The PAQS sampling site was located at the north end of Schenley Park, a 456 acre urban park with substantial tree cover. To test whether the observed isoprene concentrations were biased by the presence of a large nearby source, the daytime $\mathrm{OH}$ loss due to isoprene and its 
Table 6. Quantiles of Daytime OH Loss Rate: Winter Data ${ }^{a}$

\begin{tabular}{|c|c|c|c|c|c|c|c|c|}
\hline \multirow[b]{3}{*}{ Category } & \multicolumn{4}{|c|}{ All Days } & \multicolumn{4}{|c|}{ High Ozone Days ${ }^{c}$} \\
\hline & \multicolumn{2}{|c|}{$\mathrm{L}_{\mathrm{OH}}, \mathrm{s}^{-1}$} & \multicolumn{2}{|c|}{$\begin{array}{c}\text { Fraction of Total VOC } \\
\mathrm{L}_{\mathrm{OH}} \\
\end{array}$} & \multicolumn{2}{|c|}{$\mathrm{L}_{\mathrm{OH}}, \mathrm{s}^{-1}$} & \multicolumn{2}{|c|}{$\begin{array}{c}\text { Fraction of Total VOC } \\
\mathrm{L}_{\mathrm{OH}}\end{array}$} \\
\hline & Median & $\mathrm{IQR}^{\mathrm{e}}$ & Median & $\mathrm{IQR}^{\mathrm{e}}$ & Median & $\mathrm{IQR}^{\mathrm{e}}$ & Median & $\mathrm{IQR}^{\mathrm{e}}$ \\
\hline Total & 1.42 & $1.12-2.30$ & & & 1.00 & $0.91-1.27$ & & \\
\hline Alkanes ${ }^{\mathrm{f}}$ & 0.31 & $0.25-0.41$ & 0.20 & $0.17-0.25$ & 0.27 & $0.17-0.32$ & 0.20 & $0.18-0.26$ \\
\hline Alkenes + Alkynes $^{\mathrm{f}}$ & 0.41 & $0.32-0.57$ & 0.27 & $0.23-0.32$ & 0.29 & $0.23-0.40$ & 0.25 & $0.22-0.30$ \\
\hline Aromatics & 0.17 & $0.12-0.23$ & 0.11 & $0.09-0.13$ & 0.12 & $0.09-0.19$ & 0.10 & $0.09-0.12$ \\
\hline OVOCs & 0.41 & $0.33-0.57$ & 0.29 & $0.23-0.35$ & 0.45 & $0.31-0.54$ & 0.38 & $0.29-0.45$ \\
\hline \multirow[b]{4}{*}{ Category } & 0.49 & $0.21-1.05$ & 0.23 & $0.17-0.36$ & 0.19 & $0.19-0.19$ & 0.20 & $0.20-0.22$ \\
\hline & \multicolumn{4}{|c|}{ High OC Days $^{\mathrm{c}}$} & \multicolumn{4}{|c|}{ Nucleation Days $^{\mathrm{d}}$} \\
\hline & \multicolumn{2}{|c|}{$\mathrm{L}_{\mathrm{OH}}, \mathrm{s}^{-1}$} & \multicolumn{2}{|c|}{$\begin{array}{c}\text { Fraction of Total VOC } \\
\mathrm{L}_{\mathrm{OH}}\end{array}$} & \multicolumn{2}{|c|}{$\mathrm{L}_{\mathrm{OH}}, \mathrm{s}^{-1}$} & \multicolumn{2}{|c|}{$\begin{array}{c}\text { Fraction of Total VOC } \\
\mathrm{L}_{\mathrm{OH}}\end{array}$} \\
\hline & Median & $\mathrm{IQR}^{\mathrm{e}}$ & Median & $\mathrm{IQR}^{\mathrm{e}}$ & Median & $\mathrm{IQR}^{\mathrm{e}}$ & Median & $\mathrm{IQR}^{\mathrm{e}}$ \\
\hline Total & 2.70 & $1.64-3.35$ & & & 1.04 & $0.98-1.20$ & & \\
\hline Alkanes ${ }^{\mathrm{f}}$ & 0.40 & $0.33-0.58$ & 0.18 & $0.14-0.20$ & 0.26 & $0.24-0.30$ & 0.27 & $0.21-0.29$ \\
\hline Alkenes + alkynes $^{\mathrm{f}}$ & 0.57 & $0.44-0.84$ & 0.24 & $0.20-0.30$ & 0.28 & $0.26-0.35$ & 0.28 & $0.25-0.31$ \\
\hline Aromatics & 0.25 & $0.14-0.31$ & 0.09 & $0.07-0.11$ & 0.12 & $0.10-0.16$ & 0.11 & $0.09-0.13$ \\
\hline OVOCs & 0.69 & $0.51-0.78$ & 0.26 & $0.21-0.32$ & 0.37 & $0.34-0.45$ & 0.35 & $0.32-0.38$ \\
\hline $\mathrm{CO}$ & 0.74 & $0.45-1.56$ & 0.28 & $0.16-0.36$ & $<\mathrm{DL}^{\mathrm{g}}$ & $<\mathrm{DL}^{\mathrm{g}}$ & $<\mathrm{DL}^{\mathrm{g}}$ & $<\mathrm{DL}^{\mathrm{g}}$ \\
\hline
\end{tabular}

${ }^{a}$ Note that the median values of the components do not necessarily add up to the median of the total as the median is not a distributive property.

baytime: $1000-1600$ EST.

${ }^{c}$ High ozone and high OC days are defined as days when the daily maximum concentration was above the 0.8 quantile for all daily maxima.

${ }^{\mathrm{d}}$ Days on which moderate to strong nucleation events occurred [Stanier et al., 2004b].

${ }^{\mathrm{e}} \mathrm{IQR}$, interquartile range

${ }^{\mathrm{f}}$ Note that ethane, ethene, and ethyne were not measured. See text for discussion.

${ }^{\mathrm{g}} \mathrm{CO}$ concentrations during these periods were below the instrumental detection limit of $0.1 \mathrm{ppm}$.

oxidation products was calculated for time periods when the wind was only from the northern sector and the wind speed was greater than $1 \mathrm{~m} / \mathrm{s}$. The resulting $\mathrm{OH}$ loss rate (median $4.55 \mathrm{~s}^{-1}$; IQR $2.85-5.73 \mathrm{~s}^{-1}$ ) was not significantly different from that calculated using all of the daytime data (median $4.71 \mathrm{~s}^{-1}$; IQR 2.77-6.20 $\mathrm{s}^{-1}$ ). Hence we find that in winter, the total daytime $\mathrm{OH}$ loss rate is dominated by the nonmethane hydrocarbons and $\mathrm{CO}$, whereas in summer it is dominated by isoprene, its oxidation products, and oxygenated VOCs. The above calculations do not include methane,

Table 7. Quantiles of Daytime OH Loss Rate: Summer Data ${ }^{a, b}$

\begin{tabular}{|c|c|c|c|c|c|c|c|c|}
\hline \multirow[b]{3}{*}{ Category } & \multicolumn{4}{|c|}{ All Days } & \multicolumn{4}{|c|}{ High Ozone Days ${ }^{c}$} \\
\hline & \multicolumn{2}{|c|}{$\mathrm{L}_{\mathrm{OH}}, \mathrm{s}^{-1}$} & \multicolumn{2}{|c|}{$\begin{array}{c}\text { Fraction of Total VOC } \\
\mathrm{L}_{\mathrm{OH}}\end{array}$} & \multicolumn{2}{|c|}{$\mathrm{L}_{\mathrm{OH}}, \mathrm{s}^{-1}$} & \multicolumn{2}{|c|}{$\begin{array}{c}\text { Fraction of Total VOC } \\
\mathrm{L}_{\mathrm{OH}}\end{array}$} \\
\hline & Median & $\mathrm{IQR}^{\mathrm{e}}$ & Median & $\mathrm{IQR}^{\mathrm{e}}$ & Median & $\mathrm{IQR}^{\mathrm{e}}$ & Median & $\mathrm{IQR}^{\mathrm{e}}$ \\
\hline Total & 7.25 & $4.60-9.38$ & & & 8.53 & $7.17-9.77$ & & \\
\hline Alkanes ${ }^{\mathrm{f}}$ & 0.17 & $0.12-0.24$ & 0.03 & $0.02-0.04$ & 0.19 & $0.17-0.30$ & 0.03 & $0.02-0.04$ \\
\hline Alkenes + alkynes $^{\mathrm{f}}$ & 0.36 & $0.30-0.45$ & 0.06 & $0.04-0.07$ & 0.42 & $0.36-0.51$ & 0.05 & $0.04-0.06$ \\
\hline Aromatics & 0.14 & $0.08-0.24$ & 0.02 & $0.01-0.04$ & 0.13 & $0.08-0.24$ & 0.02 & $0.01-0.03$ \\
\hline OVOCs & 1.35 & $1.09-1.64$ & 0.20 & $0.15-0.26$ & 1.80 & $1.53-1.97$ & 0.21 & $0.16-0.24$ \\
\hline Isop $+O x^{f}$ & 4.71 & $2.77-6.20$ & 0.62 & $0.52-0.70$ & 5.69 & $3.75-6.67$ & 0.63 & $0.58-0.71$ \\
\hline \multirow[t]{3}{*}{$\mathrm{CO}$} & 0.53 & $0.26-0.86$ & 0.07 & $0.04-0.12$ & 0.60 & $0.24-0.83$ & 0.06 & $0.03-0.09$ \\
\hline & \multicolumn{4}{|c|}{ High OC Days $^{\mathrm{c}}$} & \multicolumn{4}{|c|}{ Nucleation Days $^{\mathrm{d}}$} \\
\hline & \multicolumn{2}{|c|}{$\mathrm{L}_{\mathrm{OH}}, \mathrm{s}^{-1}$} & \multicolumn{2}{|c|}{$\begin{array}{c}\text { Fraction of Total VOC } \\
\mathrm{L}_{\mathrm{OH}}\end{array}$} & \multicolumn{2}{|c|}{$\mathrm{L}_{\mathrm{OH}}, \mathrm{s}^{-1}$} & \multicolumn{2}{|c|}{$\begin{array}{c}\text { Fraction of Total VOC } \\
\mathrm{L}_{\mathrm{OH}}\end{array}$} \\
\hline Category & Median & $\mathrm{IQR}^{\mathrm{e}}$ & Median & $\mathrm{IQR}^{\mathrm{e}}$ & Median & $\mathrm{IQR}^{\mathrm{e}}$ & Median & $\mathrm{IQR}^{\mathrm{e}}$ \\
\hline Total & 9.26 & $8.23-10.43$ & & & 4.96 & $3.37-6.47$ & & \\
\hline Alkanes ${ }^{\mathrm{f}}$ & 0.21 & $0.16-0.27$ & 0.03 & $0.02-0.04$ & 0.11 & $0.09-0.20$ & 0.03 & $0.02-0.03$ \\
\hline Alkenes + alkynes $^{\mathrm{f}}$ & 0.38 & $0.31-0.48$ & 0.04 & $0.04-0.06$ & 0.35 & $0.25-0.43$ & 0.07 & $0.06-0.07$ \\
\hline Aromatics & 0.27 & $0.19-0.40$ & 0.03 & $0.02-0.05$ & 0.12 & $0.08-0.14$ & 0.02 & $0.02-0.03$ \\
\hline OVOCs & 1.54 & $1.35-1.84$ & 0.17 & $0.14-0.20$ & 1.30 & $1.05-1.59$ & 0.26 & $0.23-0.30$ \\
\hline Isop $+\mathrm{Ox}^{\mathrm{g}}$ & 5.71 & $4.58-6.75$ & 0.64 & $0.57-0.70$ & 3.16 & $1.91-3.92$ & 0.59 & $0.52-0.62$ \\
\hline $\mathrm{CO}$ & 0.71 & $0.57-1.04$ & 0.09 & $0.07-0.11$ & 0.22 & $0.16-0.54$ & 0.07 & $0.02-0.09$ \\
\hline
\end{tabular}

${ }^{a}$ Note that the median values of the components do not necessarily add up to the median of the total as the median is not a distributive property.

${ }^{b}$ Daytime: $1000-1600$ EST.

${ }^{c}$ High ozone and high OC days are defined as days when the daily maximum concentration was above the 0.8 quantile for all daily maxima.

${ }^{\mathrm{d}}$ Days on which moderate to strong nucleation events occurred [Stanier et al., 2004b].

${ }^{\mathrm{e}} \mathrm{IQR}$, interquartile range.

${ }^{\mathrm{f}}$ Note that ethane, ethene, and ethyne were not measured. See text for discussion.

${ }^{\mathrm{g}}$ Isoprene plus its oxidation products MACR, MVK, and 3-methylfuran. 
which was not measured during the experiment. Based on background concentrations of methane at this latitude (see http://www.cmdl.noaa.gov/info/ftpdata.html), we estimate the $\mathrm{OH}$ loss rate in Pittsburgh due to methane at approximately $0.21 \mathrm{~s}^{-1}$ during the winter and $0.32 \mathrm{~s}^{-1}$ during the summer.

[74] Daytime $\mathrm{OH}$ loss rates were also calculated on the following subsets of the data: high ozone days, high aerosol OC days, and days in which moderate to strong nucleation events were observed [Stanier et al., 2004b] (Tables 6 and 7). High ozone and high OC days were defined as days in which the maximum value of these quantities was above the 0.8 quantile for all observed daily maxima.

[75] For July and August, the 0.8 concentration quantile for the daily maximum ozone was $84 \mathrm{ppb}$. On high ozone days, the total measured $\mathrm{OH}$ loss rate was higher (median: $8.53 \mathrm{~s}^{-1}$ ) than otherwise (days not exceeding this threshold had a median $\mathrm{OH}$ loss rate of $6.74 \mathrm{~s}^{-1}$ ), and this increase was distributed relatively evenly among the different compound classes (Table 7). During January and February, the 0.8 quantile for daily ozone maxima was only $30 \mathrm{ppb}$. Days during which ozone exceeded this amount had a lower overall $\mathrm{OH}$ loss rate (median $1.00 \mathrm{~s}^{-1}$ ) than days on which it did not (median $1.57 \mathrm{~s}^{-1}$ ). These higher ozone days in the winter may have occurred during periods of enhanced vertical mixing. Comrie and Yarnal [1992] analyzed the dependence of surface ozone in Pittsburgh on synoptic climatology. They concluded that high ozone levels in summer developed under stagnant anticyclonic conditions, whereas in winter high ozone concentrations were associated with tropopause folding and vertical transport of stratospheric ozone.

[76] In both seasons, days with high OC loadings were associated with higher levels of all VOC compound categories and CO. In January-February, the median $\mathrm{OH}$ loss rate was $2.70 \mathrm{~s}^{-1}$ on days with high OC versus $1.28 \mathrm{~s}^{-1}$ on days without. In July-August, the median daytime $\mathrm{OH}$ loss rate was $9.26 \mathrm{~s}^{-1}$ on high OC days and $6.81 \mathrm{~s}^{-1}$ on other days. By contrast, days on which nucleation events occurred had lower overall $\mathrm{OH}$ loss rates (medians of $1.04 \mathrm{~s}^{-1}$ and $4.96 \mathrm{~s}^{-1}$ in winter and summer, respectively) than days without nucleation events (medians of $1.54 \mathrm{~s}^{-1}$ and $7.93 \mathrm{~s}^{-1}$ in winter and summer). Stanier et al. [2004b] found that the occurrence of nucleation events during PAQS was dependent on the preexisting aerosol surface area available for condensation. The lower $\mathrm{OH}$ loss rates on nucleation days may be due to a positive correlation between gas phase reactivity and aerosol surface area $\left(\mathrm{r}^{2}=0.48\right.$ in winter and 0.28 in summer); i.e. less polluted days with low $\mathrm{OH}$ loss rates also had lower particle surface area available for condensation of semivolatile aerosol precursors, which increased the likelihood of new particle nucleation.

\section{Conclusions}

[77] High temporal density speciated VOC measurements provide a useful framework for interpreting aerosol measurements. Statistical analyses such as factor analysis on combined VOC-aerosol data sets can test precepts used in source-receptor modeling. The range of combustion and photochemical markers in the VOC data set also enables us to deconvolve the relative contributions to ambient levels of
OVOCs and aerosol OC from different source types. We calculate that secondary plus biogenic sources accounted for $24 \%, 12 \%$ and $27 \%$ of the ambient concentrations of acetone, MEK and acetaldehyde respectively in the winter and $29 \%, 26 \%$ and $34 \%$ respectively in the summer. Aerosol OC was found to be composed of $16 \%$ secondary carbon in the winter and $37 \%$ secondary carbon in the summer. The importance of the background contribution to observed concentrations of both OVOCs and aerosol OC emphasizes the role of longer-range transport and the need for a regional perspective in addressing air quality concerns. While local automotive emissions were the primary factor driving changes in VOC concentrations in Pittsburgh, they did not contribute significantly to variability in the aerosol species included in the factor analysis (PM 2.5 mass, aerosol sulfate and nitrate mass, and aerosol number density).

[78] VOC concentration data can also help define chemical conditions that are conducive to particle formation and growth. We find that while aerosol OC loadings are highest when VOC concentrations and reactivities are high, nucleation events tended to occur on days when levels of VOCs and $\mathrm{CO}$ were low. High ozone days in the summer were associated with high $\mathrm{OH}$ loss rates due to the VOCs and $\mathrm{CO}$, whereas in the winter the highest ozone levels occurred on days with low levels of $\mathrm{CO}$ and nonmethane hydrocarbons but slightly higher OVOC concentrations.

[79] One of the overall objectives of the PAQS study is to develop the ability to predict changes in PM characteristics and atmospheric composition due to proposed changes in emissions. Reaching this objective will require accurate modeling of the chemical and dynamical processes controlling atmospheric composition in the Pittsburgh region. The results presented here should help to provide a basis upon which to test mechanisms included in such models.

[80] Acknowledgments. This research was conducted as part of the Pittsburgh Air Quality Study, which was supported by the US EPA under contract R82806101 and the US DOE National Energy Technology Laboratory under contract DE-FC26-01NT41017. DBM thanks the DOE for a GREF fellowship. The authors thank all of the PAQS researchers for their help; in particular, Allen Robinson, Beth Wittig, and Andrey Khlystov. Thanks also to Megan McKay for her considerable help.

\section{References}

Atkinson, R. (1994), Gas phase tropospheric chemistry of organic compounds, J. Phys. Chem. Ref. Data Monogr., 2, 1-216.

Bakwin, P. S., D. F. Hurst, P. P. Tans, and J. W. Elkins (1997), Anthropogenic sources of halocarbons, sulfur hexafluoride, carbon monoxide, and methane in the southeastern United States, J. Geophys. Res., 102(D13), 15,915-15,925

Barnes, D. H., S. C. Wofsy, B. P. Fehlau, E. W. Gottlieb, J. W. Elkins, G. S. Dutton, and S. A. Montzka (2003), Urban/industrial pollution for the New York City-Washington, D. C., corridor, 1996-1998: 1. Providing independent verification of $\mathrm{CO}$ and PCE emissions inventories, J. Geophys. Res., 108(D6), 4185, doi:10.1029/2001JD001116.

Cabada, J. C., S. N. Pandis, and A. L. Robinson (2002), Sources of atmospheric carbonaceous particulate matter in Pittsburgh, Pennsylvania, J. Air Waste Manage., 52(6), 732-741.

Cabada, J. C., S. Takahama, A. Khlystov, S. N. Pandis, S. Rees, C. I. Davidson, and A. L. Robinson (2004), Mass size distributions and size resolved chemical composition of fine particulate matter at the Pittsburgh Supersite, Atmos. Environ., 38(20), 3127-3141.

Comrie, A. C., and B. Yarnal (1992), Relationships between synopticscale atmospheric circulation and ozone concentrations in metropolitan Pittsburgh, Pennsylvania, Atmos. Environ., 26(3), 301-312.

Czoschke, N. M., M. Jang, and R. M. Kamens (2003), Effect of acidic seed on biogenic secondary organic aerosol growth, Atmos. Environ., 37(30), 4287-4299. 
Fraser, M. P., G. R. Cass, and B. R. T. Simoneit (1998), Gas-phase and particle-phase organic compounds emitted from motor vehicle traffic in a Los Angeles roadway tunnel, Environ. Sci. Technol., 32(14), 2051-2060.

Goldan, P. D., M. Trainer, W. C. Kuster, D. D. Parrish, J. Carpenter, J. M. Roberts, J. E. Yee, and F. C. Fehsenfeld (1995a), Measurements of hydrocarbons, oxygenated hydrocarbons, carbon monoxide, and nitrogen oxides in an urban basin in Colorado: Implications for emission inventories, J. Geophys. Res., 100(D11), 22,771-22,783.

Goldan, P. D., W. C. Kuster, F. C. Fehsenfeld, and S. A. Montzka (1995b), Hydrocarbon measurements in the southeastern United States: The Rural Oxidants in the Southern Environment (ROSE) program 1990, J. Geophys. Res., 100(D12), 25,945-25,963.

Goldstein, A. H., and G. W. Schade (2000), Quantifying biogenic and anthropogenic contributions to acetone mixing ratios in a rural environment, Atmos. Environ., 34(29-30), 4997-5006.

Goldstein, A. H., B. C. Daube, J. W. Munger, and S. C. Wofsy (1995a), Automated in-situ monitoring of atmospheric non-methane hydrocarbon concentrations and gradients, J. Atmos. Chem., 21(1), 43-59.

Goldstein, A. H., S. C. Wofsy, and C. M. Spivakovsky (1995b), Seasonal variations of nonmethane hydrocarbons in rural New England: Constraints on $\mathrm{OH}$ concentrations in northern midlatitudes, J. Geophys. Res., 100(D10), 21,023-21,033.

Goldstein, A. H., S. M. Fan, M. L. Goulden, J. W. Munger, and S. C. Wofsy (1996), Emissions of ethene, propene, and 1-butene by a midlatitude forest, J. Geophys. Res., 101(D4), 9149-9157.

Greenberg, J. P., B. Lee, D. Helmig, and P. R. Zimmerman (1994), Fully automated gas chromatograph-flame ionization detector system for the in situ determination of atmospheric nonmethane hydrocarbons at low parts per trillion concentration, J. Chromatogr. A, 676(2), 389-398.

Grosjean, D. (1982), Formaldehyde and other carbonyls in Los Angeles ambient air, Environ. Sci. Technol., 16(5), 254-262.

Hoffmann, T., R. Bandur, U. Marggraf, and M. Linscheid (1998), Molecular composition of organic aerosols formed in the alpha-pinene/ $\mathrm{O}_{3}$ reaction: Implications for new particle formation processes, J. Geophys. Res., 103(D19), 25,569-25,578.

Jang, M. S., N. M. Czoschke, S. Lee, and R. M. Kamens (2002), Heterogeneous atmospheric aerosol production by acid-catalyzed particle-phase reactions, Science, 298(5594), 814-817.

Kirchstetter, T. W., B. C. Singer, R. A. Harley, G. B. Kendall, and W. Chan (1996), Impact of oxygenated gasoline use on California light-duty vehicle emissions, Environ. Sci. Technol., 30(2), 661-670.

Kirchstetter, T. W., B. C. Singer, R. A. Harley, G. R. Kendall, and J. M. Hesson (1999), Impact of California reformulated gasoline on motor vehicle emissions. 2. Volatile organic compound speciation and reactivity, Environ. Sci. Technol., 33(2), 329-336.

Koch, S., R. Winterhalter, E. Uherek, A. Kolloff, P. Neeb, and G. K Moortgat (2000), Formation of new particles in the gas-phase ozonolysis of monoterpenes, Atmos. Environ., 34(23), 4031-4042.

Lamanna, M. S., and A. H. Goldstein (1999), In situ measurements of $\mathrm{C}_{2}-$ $\mathrm{C}_{10}$ volatile organic compounds above a Sierra Nevada ponderosa pine plantation, J. Geophys. Res., 104(D17), 21,247-21,262.

Lim, H. J., and B. J. Turpin (2002), Origins of primary and secondary organic aerosol in Atlanta: Results of time-resolved measurements during the Atlanta Supersite experiment, Environ. Sci. Technol., 36(21), 44894496.

McKeen, S. A., and S. C. Liu (1993), Hydrocarbon ratios and photochemical history of air masses, Geophys. Res. Lett., 20(21), 2363-2366.

Odum, J. R., T. Hoffmann, F. Bowman, D. Collins, R. C. Flagan, and J. H. Seinfeld (1996), Gas/particle partitioning and secondary organic aerosol yields, Environ. Sci. Technol., 30(8), 2580-2585.

Parrish, D. D., C. J. Hahn, E. J. Williams, R. B. Norton, F. C. Fehsenfeld, H. B. Singh, J. D. Shetter, B. W. Gandrud, and B. A. Ridley (1992) Indications of photochemical histories of Pacific air masses from measurements of atmospheric trace species at Point Arena, California, J. Geophys. Res., 97(D14), 15,883-15,901.
Parrish, D. D., et al. (1998), Internal consistency tests for evaluation of measurements of anthropogenic hydrocarbons in the troposphere, J. Geophys. Res., 103(D17), 22,339-22,359.

Riemer, D., et al. (1998), Observations of nonmethane hydrocarbons and oxygenated volatile organic compounds at a rural site in the southeastern United States, J. Geophys. Res., 103(D21), 28,111-28,128.

Schade, G. W., and A. H. Goldstein (2001), Fluxes of oxygenated volatile organic compounds from a ponderosa pine plantation, J. Geophys. Res., 106(D3), 3111-3123

Singh, H. B., M. Kanakidou, P. J. Crutzen, and D. J. Jacob (1995), High concentrations and photochemical fate of oxygenated hydrocarbons in the global troposphere, Nature, 378(6552), 50-54.

Singh, H., Y. Chen, A. Staudt, D. Jacob, D. Blake, B. Heikes, and J. Snow (2001), Evidence from the Pacific troposphere for large global sources of oxygenated organic compounds, Nature, 410(6832), 1078-1081.

Staehelin, J., C. Keller, W. Stahel, K. Schlapfer, and S. Wunderli (1998), Emission factors from road traffic from a tunnel study (Gubrist tunnel, Switzerland). part III: Results of organic compounds, $\mathrm{SO}_{2}$ and speciation of organic exhaust emission, Atmos. Environ., 32(6), 999-1009.

Stanier, C. O., A. Y. Khlystov, and S. N. Pandis (2004a), Ambient aerosol size distributions and number concentrations measured during the Pittsburgh Air Quality Study (PAQS), Atmos. Environ., 38(20), 3275-3284.

Stanier, C. O., A. Y. Khlystov, and S. N. Pandis (2004b), Nucleation events during the Pittsburgh Air Quality Study: Description and relation to key meteorological, gas phase, and aerosol parameters, Aerosol Sci. Technol., $38(21), 253-264$.

Sweet, C. W., and S. J. Vermette (1992), Toxic volatile organic compounds in urban air in Illinois, Environ. Sci. Technol., 26(1), 165-173.

Thunis, P., and C. Cuvelier (2000), Impact of biogenic emissions on ozone formation in the Mediterranean area-A BEMA modelling study, Atmos. Environ., 34(3), 467-481.

Tolocka, M. P., P. A. Solomon, W. Mitchell, G. A. Norris, D. B. Gemmill, R. W. Wiener, R. W. Vanderpool, J. B. Homolya, and J. Rice (2001), East versus west in the US: Chemical characteristics of PM2.5 during the winter of 1999, Aerosol Sci. Technol., 34(1), 88-96.

Turpin, B. J., and J. J. Huntzicker (1995), Identification of secondary organic aerosol episodes and quantitation of primary and secondary organic aerosol concentrations during SCAQS, Atmos. Environ., 29(23), 3527-3544.

Wittig, B., N. Anderson, A. Y. Khlystov, S. N. Pandis, C. Davidson, and A. L. Robinson (2004a), Pittsburgh Air Quality Study overview and initial scientific findings, Atmos. Environ., 38(20), 3107-3125.

Wittig, B., S. Takahama, A. Khlystov, S. N. Pandis, S. Hering, B. Kirby, and C. Davidson (2004b), Semicontinuous PM2.5 inorganic composition measurements during the Pittsburgh Air Quality Study, Atmos. Environ., $38(20), 3201-3213$

Zielinska, B., J. C. Sagebiel, G. Harshfield, A. W. Gertler, and W. R. Pierson (1996), Volatile organic compounds up to $\mathrm{C}_{20}$ emitted from motor vehicles: Measurement methods, Atmos. Environ., 30(12), $2269-2286$

A. H. Goldstein, Department of ESPM-Ecosystem Sciences, University of California, Berkeley, 151 Hilgard Hall, Berkeley, CA 94720, USA. (ahg@nature.berkeley.edu)

N. M. Donahue and S. N. Pandis, Department of Chemical Engineering, Carnegie Mellon University, 5000 Forbes Ave, Pittsburgh, PA 15213, USA.

D. B. Millet, Department of Earth and Planetary Sciences, Harvard University, Pierce Hall, 29 Oxford St., Cambridge, MA 02138, USA. (dbm@io.harvard.edu)

A. Polidori and B. J. Turpin, Department of Environmental Sciences, Rutgers University, 14 College Farm Rd., New Brunswick, NJ 08901, USA.

C. O. Stanier, Department of Chemical and Biochemical Engineering, University of Iowa, 4122 Seamens Center, Iowa City, IA 52242, USA. 


\title{
Ambient measurements of metal-containing $\mathrm{PM}_{2.5}$ in an urban environment using laser-induced breakdown spectroscopy
}

\author{
G.A. Lithgow ${ }^{\mathrm{a}}$, A.L. Robinson ${ }^{\mathrm{b}}$, S.G. Buckley ${ }^{\mathrm{a}, *}$ \\ ${ }^{a}$ Department of Mechanical and Aerospace Engineering, University of California, San Diego, La Jolla, CA 92093, USA \\ ${ }^{\mathrm{b}}$ Department of Mechanical Engineering, Carnegie Mellon University, Pittsburgh, PA 15213, USA
}

\begin{abstract}
Laser-induced breakdown spectroscopy (LIBS) was used to measure the distribution of seven species in individual ambient aerosol particles during an 8-day period from 26 August to 2 September 2002 at the Pittsburgh Aerosol Supersite. Particle hit rates were on the order of $10^{-4}-10^{-5}$ for $\mathrm{Al}, \mathrm{Ca}, \mathrm{Cr}, \mathrm{Cu}, \mathrm{Mg}, \mathrm{Mn}$, and $\mathrm{Na}$. Weekly average concentrations between 29 and $720 \mathrm{ng} \mathrm{m}^{-3}$ are reported along with conservative threshold detection limits for individual particles between 15 and $184 \mathrm{fg}$, depending on the element. Hourly concentrations are reported for $\mathrm{Ca}, \mathrm{Mg}$, and $\mathrm{Na} ; \mathrm{Mg}$ concentrations are found to be somewhat correlated with both $\mathrm{Ca}$ and $\mathrm{Na}$, while $\mathrm{Ca}$ and $\mathrm{Na}$ appear uncorrelated. A representative example of measured $\mathrm{Mg}$ particle masses illustrates that the detection threshold poses a limitation in this data set, which could be rectified in future implementations. Finally, the presence of multi-element particles in the data set suggest the use of high-sensitivity, wide-range echelle spectrometers for particle source apportionment and determination of associations between elements.
\end{abstract}

(C) 2004 Elsevier Ltd. All rights reserved.

Keywords: Particulate matter; Aerosol composition; Laser-induced breakdown spectroscopy; LIBS; $\mathrm{PM}_{2.5}$

\section{Introduction}

The composition of aerosol particles in an urban environment is of interest from the standpoint of environmental impacts and potential human health effects. Aerosol particles in the US are regulated on the basis of $\mathrm{PM}_{10}$ and $\mathrm{PM}_{2.5}$ mass, which are the fraction of particles smaller than 10 and $2.5 \mu \mathrm{m}$, respectively. These mass or volume-based regulations are convenient to measure, relative to number or composition, but likely do not capture all of the information that is relevant to health effects. Recent studies have suggested that the number, size, and composition of particulate matter (PM) may all have an important role in health effects attributed to PM

\footnotetext{
*Corresponding author. Fax: +1-858-534-5354.

E-mail address: buckley@ucsd.edu (S.G. Buckley).
}

(Dockery and Pope, 1994). In particular, although the mechanisms remain unclear, the presence of toxic metals, polycyclic aromatic hydrocarbons, and other known carcinogens in the respirable fraction of PM are suspected to contribute to measurable health effects (Brook et al., 2003; Salvi and Holgate, 1999), and the metal fraction may represent a substantial portion of the toxicity of aerosol particles (Linak and Wendt, 1993).

For this reason, methods for measuring composition of aerosol particles are of interest. Static filter-based methods such as the US Environmental Protection Agency (EPA) Method 29 are useful for bulk measurements of metals in PM, and similar analysis may be performed on impactor size-segregated aerosol particles to yield an understanding of the distribution of metals as a function of size (Davison et al., 1974) and the elemental and organic carbon fractions (Chow et al., 1993). However, the urban environment is far from a 
steady-state system. Shifting meteorological patterns coupled with temporally and spatially varying emissions result in exposure patterns that may change significantly over time scales of minutes. Understanding human PM exposure patterns thus requires a degree of temporal resolution not afforded by simple filter-based methods.

A number of near-real-time and real-time methods are under investigation for measurement of particle composition, applicable either to the effluent of thermal processes or to ambient measurements. Seltzer and coworkers have used a customized ICP system to monitor metal emissions from an incinerator (Seltzer, 2000); this process has met the EPA relative accuracy requirements and is commercially available. However, the instrument is unable to measure individual particles. Similar investigations on another continuous metals emissions monitor based on an atmospheric microwave plasma are underway by Woskov and co-workers (Woskov et al., 2000).

Ondov's group at the University of Maryland has developed a wet chemistry technique called Semicontinuous Elements in Aerosol System (SEAS), in which particles of all sizes entering the instrument are grown by condensation in supersaturated water vapor, and then separated using a virtual impactor (Kidwell and Ondov, 2001). The particle-laden stream is collected in impingers, and the metal content of the wash is analyzed using an ICP or other elemental analysis method. Time-resolved samples can be collected along with corresponding meteorological data to perform source apportionment (Suarez and Ondov, 2002). However, there is a substantial time lag between data collection and analysis using this technique as currently configured.

Single-particle mass spectrometry, or aerosol time-offlight mass spectrometry (ATOFMS) has been under development for some time as a real-time method for measurement of a range of atmospheric particles (Prather et al., 1994). Aerosol particles are sampled into a low pressure inlet, aerodynamically focused, and their velocity is measured using a pair of lasers. A third laser is thus synchronized and fires to ablate the particle and create an ion signal. Both positive and negative ions can be measured, yielding both inorganic species and molecular fragment ion mass spectra, positive and negative. These instruments can measure both metals and atmospherically important fragments such as sulfates and nitrates on particles as small as a few hundred nanometers. Recent developments include both wide-range calibrations based on multivariate methods (Fergenson et al., 2001), and on extending the technique to particles in the tens of $\mathrm{nm}$, eliminating the timing lasers and using a free-firing ablation laser (Phares et al., 2002). The instrument developed by Prather's group is under commercial development by TSI, Inc. This method, however, is still largely qualitative.
Laser-induced breakdown spectroscopy (LIBS) has been used as an analytical technique for gases, liquids, and solids for some time, and has been extensively reviewed (Radziemski, 1994; Schechter, 1997; Sneddon and Lee, 1999; Song et al., 1997). Applications of LIBS typically employ a pulsed laser with a high peak power to form a spark (breakdown) in the medium to be examined. The temperature of the resulting plasma at short times $(<10 \mu \mathrm{s})$ is in the range of 10,000-25,000 K (Yalcin et al., 1999), hot enough to dissociate molecules into their constituent atoms, and to excite the electrons in the neutral atoms and ions formed in the plasma out of the ground state and into excited electronic states. As the plasma cools, excited electrons and ions relax back into their ground states, emitting light at characteristic atomic frequencies. Identification of the atoms present in the sample volume occurs using well-known atomic emission lines, and quantification of the elemental species concentration occurs via measurement of the intensity of the emission lines.

LIBS has been applied to the measurement of aerosol particles emitted from combustion process exhaust streams and ambient particles. Ottesen and co-workers (Ottesen et al., 1991) used LIBS to measure the composition of individual coal particles during and after combustion. Absolute elemental analysis of aerosol particles was suggested by Schechter's group in 1997 (Xu et al., 1997); almost simultaneously Hahn developed the method of conditional analysis of individual aerosol particles to increase sensitivity over averaged measurements in dilute aerosol flows and for single particle analysis (Hahn et al., 1997; Hahn, 1998).

In combustion process exhaust streams, LIBS has primarily been used to measure inorganic species such as toxic metals, which typically occur in particulate form. Groups from Sandia National Laboratories (Buckley et al., 2000; Hahn et al., 1997) and Mississippi State University (Zhang et al., 1999) have applied LIBS to quantification of emissions from incinerators and thermal processes. Recent work has been focused on increasing the sensitivity and accuracy of LIBS via optimization of the detection timing (Fisher et al., 2001) and statistical treatment of the detected signals (Carranza and Hahn, 2002). Hahn and co-workers have also recently measured ambient particles from 4 th of July fireworks in the relatively rural environment around Gainesville, Florida, detecting $\mathrm{Al}, \mathrm{Ca}$, $\mathrm{Mg}$, and $\mathrm{Na}$ (Carranza et al., 2001). LIBS systems that have been deployed in the field have been relatively compact and simple compared with other instruments; LIBS systems have been deployed, for example, 90 feet above the ground on $90 \mathrm{~cm}$ wide scaffolding for incinerator measurements (Buckley et al., 2000). 


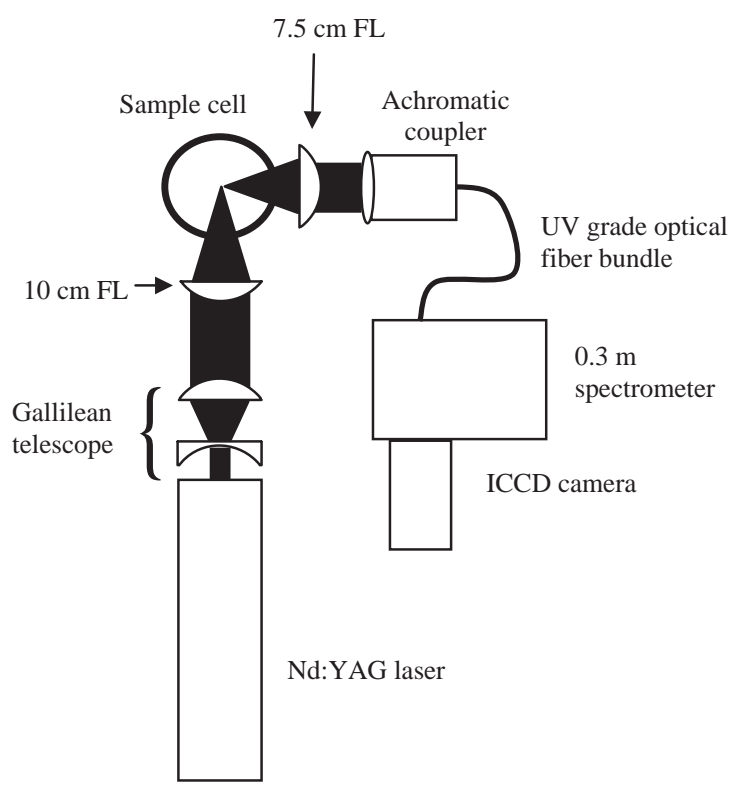

Fig. 1. Experimental apparatus.

\section{Experimental methods}

\subsection{LIBS setup}

A schematic of the LIBS system is shown in Fig. 1. The excitation source for the plasma was a Q-switched $\mathrm{Nd}$ :YAG laser operating at the fundamental wavelength $(1064 \mathrm{~nm})$. The laser repetition rate was $20 \mathrm{~Hz}$, with pulse energy of nominally $40 \mathrm{~mJ}$ and nominal pulse width of $6 \mathrm{~ns}$. The beam was expanded from its initial diameter of $6 \mathrm{~mm}$ to a diameter of nearly $25 \mathrm{~mm}$, then focused with a $100 \mathrm{~mm}$ focal length, $50 \mathrm{~mm}$ diameter lens. The laser pulse energy is only slightly above the breakdown threshold of approximately $25 \mathrm{~mJ}$ measured under these conditions in ambient air.

This low laser pulse energy was chosen because previous, unpublished experiments by the authors suggested that single-particle detection limits were optimized at low energy for highly dispersed particles. This could possibly be due to the dependence of plasma size on excitation energy, particularly if the plasma were optically thick under some conditions. The background light from the plasma scales with laser pulse energy, while emission from an individual particle in a plasma at local thermodynamic equilibrium is relatively fixed. Signal from a small particle could potentially be overwhelmed by emissions from a large, bright plasma, particularly if the particle is located such that an optically thick plasma screens the particle emission from the collection optics. However, a subsequent study by Hahn's group (Carranza and Hahn, 2002) demon- strated a drawback of lower pulse energies; the amount of energy absorbed by the plasma fluctuates greatly from shot to shot of the laser, resulting in reduced precision of the LIBS signal.

Emissions from the plasma were collected at a right angle to the incident laser beam. Plasma emissions were first collimated with a $75 \mathrm{~mm}$ focal length, $50 \mathrm{~mm}$ diameter fused silica lens, then coupled to a fiber optic bundle with a Multichannel Instruments CC52 collector. The light was dispersed with a $0.3 \mathrm{~m}$ spectrometer with a 1200 groove $\mathrm{mm}^{-1}$ grating. Light was detected with a time gated, intensified charge-coupled device (ICCD) detector array. The effective linear dispersion was approximately $0.07 \mathrm{~nm}$ per pixel. Spectra were collected in four separate spectral windows, centered at 270, 340, 413 , and $590 \mathrm{~nm}$. The signal was integrated over different time gates for each window. The delay with respect to the laser pulse, and the gate width, were optimized for the elements of interest in each spectral region. In the $270 \mathrm{~nm}$ window, the delay was $1 \mu$ s and gate width was $5 \mu \mathrm{s}(1 / 5)$. For the other windows, the times were $15 / 20,15 / 40$, and 20/40, respectively.

\subsection{LIBS calibration}

Elemental concentrations are determined from LIBS spectra by measuring the peak-to-base $(\mathrm{P} / \mathrm{B})$ ratio and comparing it to a calibration. The $\mathrm{P} / \mathrm{B}$ ratio is defined as the integral of the atomic emission peak normalized by the continuum emission of the plasma in the spectral region adjacent to the atomic peak. This method is used, rather than absolute elemental emission, to reduce the effect of shot to shot variations in plasma energy (Xu et al., 1997). Calibrations are performed by sampling particle-laden streams of known metal concentration, and measuring the $\mathrm{P} / \mathrm{B}$ ratio. Streams of known concentration are generated by nebulizing reference standard metal solutions using a constant output atomizer (TSI model 3075). To control the concentration of the stream, the output of the atomizer is diluted with filtered, dry air, and for $\mathrm{Na}, \mathrm{Ca}$, and $\mathrm{Mg}$ the concentration of the solution is also varied to increase the range of the calibration. The total flow passes through a diffusion dryer to ensure that the particles are dry.

The metal concentration in the output stream of the atomizer is determined by sampling with a scanning mobility particle sizer (SMPS). The SMPS calculates the total volume of PM per unit volume of the carrier gas, based on a measured electrical mobility diameter and the assumption of spherical particles. This procedure should be relatively accurate for dry particles. Following common practice, the composition of the particles is assumed, to deduce density and mass fraction of the metal species. For most of the calibrations, particles are assumed to be composed of the most common oxide of 


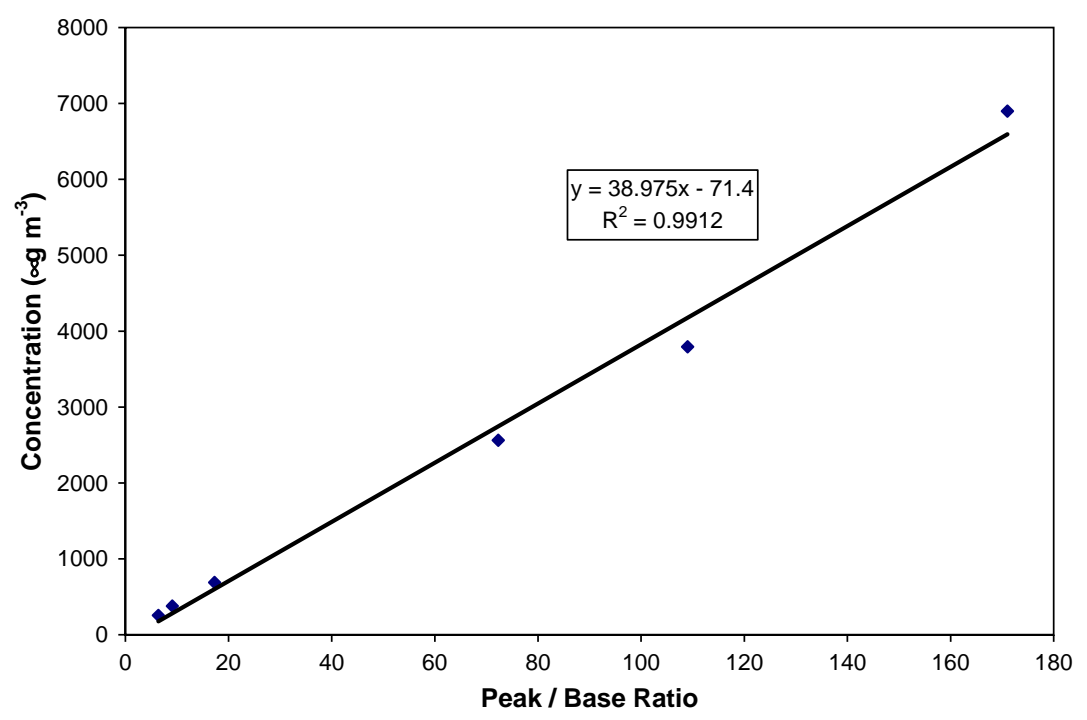

Fig. 2. Calibration for $\mathrm{Ca}(\mathrm{II})$ line at $422.0 \mathrm{~nm}$.

the metal. For sodium, a sodium chloride solution is used, which is assumed to form $\mathrm{NaCl}$ particles. The reported total particulate volume is then multiplied by the density of the particle and the mass fraction of the element of interest to yield the mass of the element per volume of gas.

Ensemble averages of 1000 laser shots were used to determine the $\mathrm{P} / \mathrm{B}$ ratio for a given concentration. Data points were fit with a linear regression, and regression coefficient $\left(R^{2}\right)$ values ranged from 0.97 to 0.99 for the seven calibrations. The calibration curve for the Ca (II) line at $422 \mathrm{~nm}$ is shown as a representative example (Fig. 2).

\subsection{Data acquisition and analysis}

Data was acquired during a one week period from 26 August 2002 to 2 September 2002. The system was run continuously for most of the period, with occasional breaks taken to perform minor maintenance and data backup. The system continuously cycled through four spectral windows, acquiring 2000 shots in just under two minutes per window. The spectrometer position and the timing of the camera were controlled by computer, allowing the system to be fully automated.

The concentrations of seven metals $(\mathrm{Al}, \mathrm{Ca}, \mathrm{Cr}, \mathrm{Cu}$, $\mathrm{Mg}, \mathrm{Mn}, \mathrm{Na}$ ) were measured during the week long sampling period. A spectral region of approximately $40 \mathrm{~nm}$ can be monitored at any given time, so elements can be measured simultaneously only if they have emission lines close to each other. To monitor a larger number of elements, spectral regions must be scanned, resulting in a loss of temporal resolution. The choice of metals was based on their prevalence in the atmosphere, the strength of their LIBS signal, and the location of their emission lines, in an attempt to acquire the greatest amount of useful data in a limited time period.

Time averaged element concentrations were determined using a conditional averaging technique (Hahn, 1998). For each laser shot, the $\mathrm{P} / \mathrm{B}$ ratio is calculated. If the $\mathrm{P} / \mathrm{B}$ ratio is greater than the detection threshold, the spectrum is considered a "hit," meaning there was a particle present in the plasma. The concentration of the element within the sample volume of the plasma is calculated for that shot and saved. If the $\mathrm{P} / \mathrm{B}$ ratio is below the detection limit, it is considered to contain no particle. In a given time period, the average concentration of the hits times the number of hits is divided by the total number of shots to determine the average concentration.

In this study, the hit rates were very low, so even a small rate of false hits would significantly influence the calculated concentration. For this reason a relatively high threshold was used to determine hits. The threshold for each element was set by taking several thousand spectra in filtered air, and increasing the threshold for a particular line until no hits were recorded. This yielded a higher selectivity than the standard $3 \sigma$ criterion for signal-to-noise. The resulting thresholds correspond to signal-to-noise ratios of about 4-5, and thus the probability of false hits is minimal.

Along with elemental concentrations, the LIBS technique can provide information about particle mass distributions. The LIBS signal is proportional to the concentration of analyte within the plasma volume. If the concentration is multiplied by the plasma volume, the mass of analyte can be calculated. When a single particle is hit the mass of analyte within the plasma is 
equal to the mass of analyte in the sampled particle. In this manner, the particle mass distribution of a given element can be determined. Given a dilute particle phase, the probability of a multiple particle hit is roughly the square of the single-particle hit rate. As discussed below, the hit rate in these experiments is sufficiently low that the single-particle assumption is justified.

\subsection{Aerosol sampling}

Ambient PM was sampled at the Pittsburgh Air Quality Study Supersite in Pittsburgh, PA. The equipment was housed in a trailer located at the top of a hill in Schenley Park, adjacent to the campus of Carnegie Mellon University. Air was sampled several meters above the roof of the trailer, using a particle concentrator from Eatough's group at Brigham Young. The particle concentrator consists of a $\mathrm{PM}_{2.5}$ cyclone inlet and a virtual impactor. The virtual impactor removes excess air, producing a flow stream with an increased concentration of fine particles. The design and characterization of the system have been previously described by Ding et al. (2002) (note: the diffusion denuder and filter packs were removed for this study). In these experiments, the concentrator runs at a minor to total flow ratio of $20 \%$ and a total flow of $2.5 \times 10^{-3} \mathrm{~m}^{3} \mathrm{~s}^{-1}$ $\left(1501 \mathrm{~min}^{-1}\right)$. This results in approximately a five-fold concentration of particles and a low cutoff of approximately $0.1 \mu \mathrm{m}$. The detection limit of the LIBS system is above $100 \mu \mathrm{m}$, therefore the concentrator losses should not significantly affect the LIBS performance.

After passing through the particle concentrator, the sample air is carried approximately three meters to the sampling cell of the LIBS system in $1.2 \mathrm{~cm}$ diameter Teflon $^{\text {TM }}$ lines. The sampling cell is a sealed $6 \mathrm{~cm}$ diameter cylindrical Teflon chamber with planar windows allowing optical access. The choice of Teflon ${ }^{\mathrm{TM}}$ may have adversely influenced our small particle hit rate due to electrostatic deposition of particles in the line; this should be avoided in future efforts of this kind. Because the repetition rate of the laser is fixed at $20 \mathrm{~Hz}$ and the flow rate from the concentrator was set at $5 \times 10^{-4} \mathrm{~m}^{-3} \mathrm{~s}^{-1}\left(301 \mathrm{~min}^{-1}\right)$ in these experiments, a section of $5 / 8 \mathrm{~cm}$ stainless-steel tube was used to accelerate the flow and introduce it to the plasma. The tube was positioned so that the plasma was approxi- mately $1 \mathrm{~cm}$ from the opening of the tube. This ensured that the velocity was sufficiently high that fresh gas was sampled with each laser shot.

\section{Results and discussion}

\subsection{Particle hit rates}

During the week, the system ran 4552 acquisition cycles, recording 2,276,000 spectra in each window. Of the seven elements, $\mathrm{Ca}, \mathrm{Na}$, and $\mathrm{Mg}$ were the most common, with hit rates on the order of several hits per 10,000 shots. For the other elements, the frequencies were on the order of several hits per 100,000 shots. The number of hits and the hit rates for all elements are shown in Table 1. Presumably, the use of the particle concentrator helped to increase the sampling efficiency of the LIBS system. Unfortunately, due to time limitations, data was not acquired without the concentrator to determine its effect.

\subsection{Ambient metal concentrations}

The combination of the measured hits for each element allows the construction of an ensemble-averaged spectrum, shown for the $932 \mathrm{Ca}$ hits in Fig. 3. Ca (II) peaks are visible at $393.4,396.8$, and $422.0 \mathrm{~nm}$. The average concentration of each element is calculated for the entire week, as discussed above (Table 2). The concentrations ranged from tens of nanograms per cubic meter for four minor elements to hundreds of nanograms per cubic meter for $\mathrm{Na}, \mathrm{Mg}$, and $\mathrm{Ca}$. Hourly concentrations of the three major elements are plotted in Figs. $4 \mathrm{a}-\mathrm{c}$.

Relationships between element concentrations were measured by determining the Pearson correlation coefficients between of sets of time averaged elemental concentrations. The Pearson correlation coefficient is defined as

$R=\frac{n\left(\sum X Y\right)-\left(\sum X\right)(\Sigma Y)}{\sqrt{\left[n \Sigma X^{2}-(\Sigma X)^{2}\right]\left[n \Sigma Y^{2}-(\Sigma Y)^{2}\right]}}$.

The correlation coefficients between $\mathrm{Na}, \mathrm{Mg}$, and $\mathrm{Ca}$ are given for 1-, 4- and 12-h averages in Table 3. Interestingly, magnesium shows a moderate correlation with both sodium and calcium, but sodium and calcium

Table 1

Number of recorded hits and particle hit rates for experiments conducted from 26 August to 2 September 2002

\begin{tabular}{llllllll}
\hline Element & $\mathrm{Ca}$ & $\mathrm{Na}$ & $\mathrm{Mg}$ & $\mathrm{Cr}$ & $\mathrm{Al}$ & $\mathrm{Cu}$ & $\mathrm{Mn}$ \\
\hline Total hits & 932 & 836 & 327 & 52 & 40 & 56 & 70 \\
Total shots & 2276000 & 2276000 & 2276000 & 2276000 & 2276000 & 2276000 & 2276000 \\
Hit rate & 0.0004407 & 0.0002869 & 0.000157 & $1.8 \mathrm{E}-05$ & $1.757 \mathrm{E}-05$ & $3.207 \mathrm{E}-05$ & $3.08 \mathrm{E}-05$ \\
\hline
\end{tabular}




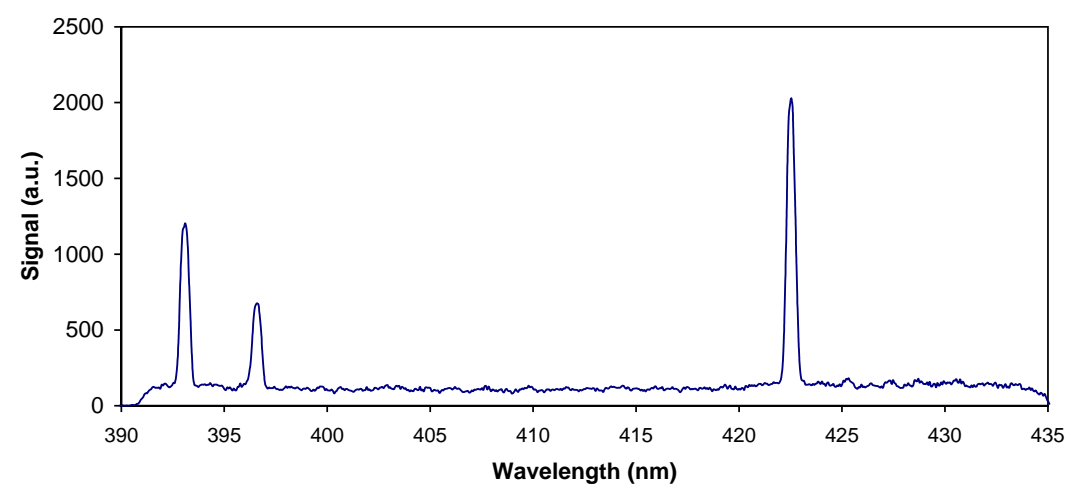

Fig. 3. Averaged spectrum of $932 \mathrm{Ca}$ particle hits.

Table 2

Weekly average concentrations for measured elements $\left(\mathrm{ng} \mathrm{m}^{-3}\right)$ and threshold mass detection limits (fg)

\begin{tabular}{lrrrrrrr}
\hline & $\mathrm{Ca}$ & $\mathrm{Na}$ & $\mathrm{Mg}$ & $\mathrm{Cu}$ & $\mathrm{Al}$ & $\mathrm{Mn}$ & $\mathrm{Cr}$ \\
\hline Weekly avg. & 304 & 716 & 225 & 32 & 30 & 29 & 37 \\
Mass detection limit & 50 & 143 & 53 & 15 & 184 & 176 & 166 \\
\hline
\end{tabular}

show essentially zero correlation. This behavior may be explained by considering the volatilities of the metals: in general, the volatility of $\mathrm{Na}>\mathrm{Mg}>\mathrm{Ca}$. Thus for combustion-generated particles, which are the bulk of the particles containing alkali and alkaline earth elements smaller than $1 \mu \mathrm{m}$, one might expect $\mathrm{Mg}$ to share some commonality with both $\mathrm{Na}$ and $\mathrm{Ca}$. It is also interesting that both the $\mathrm{Mg}-\mathrm{Na}$ and $\mathrm{Mg}-\mathrm{Ca}$ correlations get stronger for longer averaging intervals. Further study is needed to determine whether this is due to actual short time-scale fluctuations of element concentrations, or if the hit frequencies are too low to obtain representative samples at the short time scale.

Twelve-hour Micro-Orifice Uniform Deposit Impactor (MOUDI) samples were acquired concurrently to the LIBS measurements. Those samples could be used to provide a reference for verification of the LIBS data. At the time of publication, however, the MOUDI samples had yet to be analyzed, illustrating the advantage of the real-time capability of LIBS.

\subsection{Particle mass distributions}

Mass distributions were determined for the elements based on particle hits. Single-shot element concentrations were multiplied by a plasma volume of $0.25 \mathrm{~mm}^{3}$. The $\mathrm{Mg}$ distribution is shown in Fig. 5, other distributions were similar. The distributions of $\mathrm{Na}, \mathrm{Mg}$, and $\mathrm{Ca}$ all show that the distributions are dominated by particles near the detection limit. The distributions are truncated at the mass corresponding to the conservatively determined detection threshold of the LIBS system. The mass detection limit for each element is shown in Table 2. The location of the mode of the full distribution, and how far below the detection limit it would be, cannot be determined. It is likely that the LIBS system is missing a significant number of the smallest particles and thus under predicts the mass concentrations of elements. This fact is ameliorated somewhat by the fact that the elemental mass concentrations are a weighted average of the mass number distribution, and hence the larger particles contribute the most to the mass determination.

While ambient particles are typically comprised of multiple compounds, as discussed below, it may be useful to determine an equivalent size based on a pure particle of assumed composition. For example, the mass detection limits in Table 2 for $\mathrm{Ca}$ and $\mathrm{Mg}$ would correspond to $342 \mathrm{~nm} \mathrm{CaO}$ and $360 \mathrm{~nm} \mathrm{MgO}$ particles. The ultimate mass concentration detection limit, which would be expressed in $\mu \mathrm{g} \mathrm{m}^{-3}$, is difficult to define for a LIBS monitor, due to the fact that discrete particle hits may be followed by an arbitrary number of non-hits. Each "miss" acts to lower the effective mass concentration detection limit by increasing the volume sampled. Hence the mass concentration detection limit depends both upon the particle size distribution and the mass detection limit.

\subsection{Multiple-element spectra}

Several individual spectra contained signal from more than one element. These multi-element spectra illustrate the potential for LIBS to determine complete particle composition and associations between elements. Fig. 6 shows a single particle containing $\mathrm{Ca}, \mathrm{Mn}$, and $\mathrm{Cr}$. Several other particle types were observed, including predominantly $\mathrm{Mg}$ particles with several additional species such as $\mathrm{Si}, \mathrm{Ca} / \mathrm{Al}$ particles, and $\mathrm{Fe} / \mathrm{Cu} / \mathrm{Cr}$ 

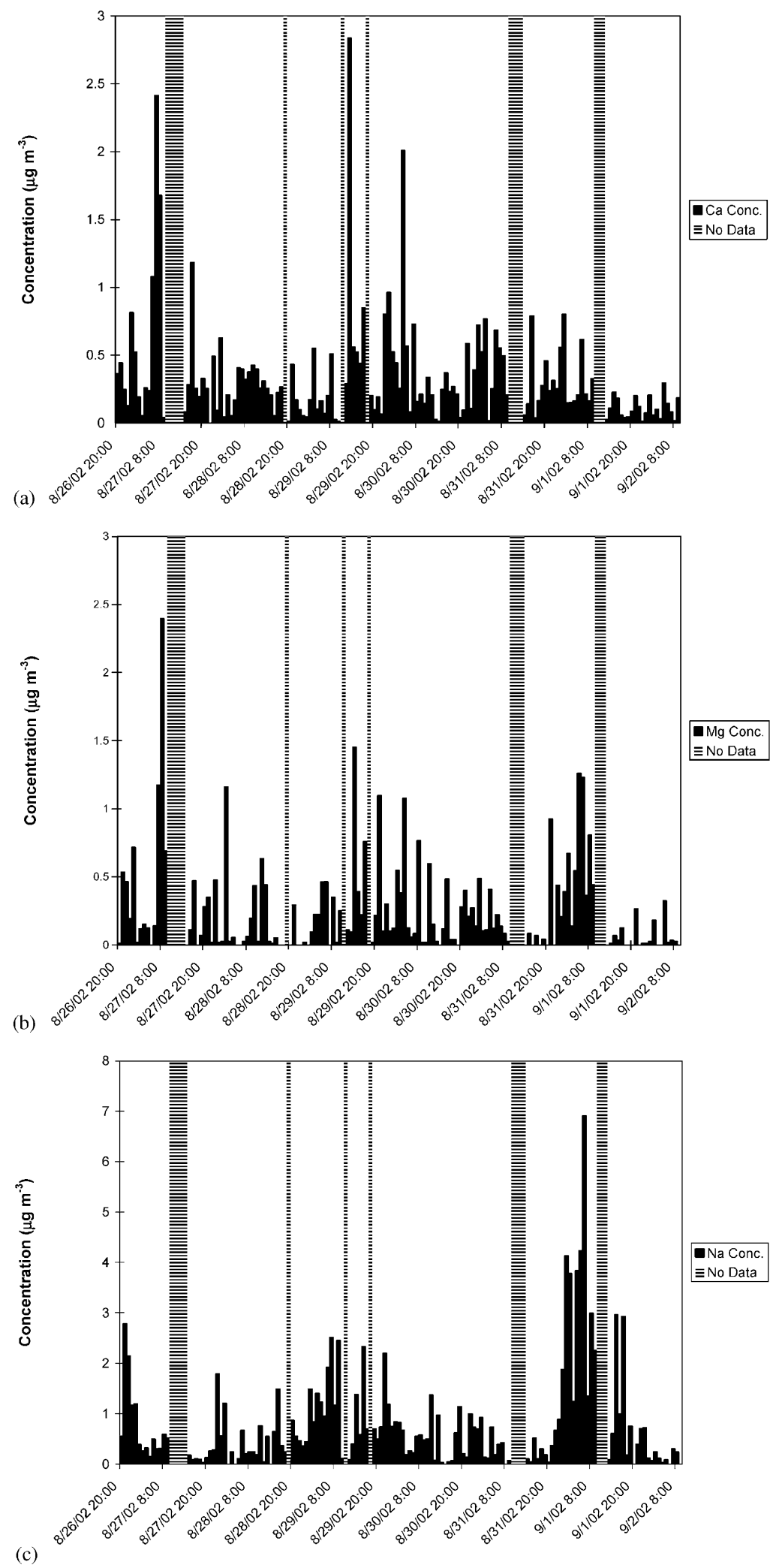

Fig. 4. Measured hourly concentrations for (a) $\mathrm{Ca}$, (b) $\mathrm{Mg}$ and (c) $\mathrm{Na}$. 
Table 3

Correlation of $\mathrm{Ca}, \mathrm{Mg}$, and $\mathrm{Na}$ measurements at 1-, 4-, and 12$\mathrm{h}$ time scales, measured using the Pearson correlation coefficient

\begin{tabular}{rlll}
\hline & $\mathrm{Na}: \mathrm{Mg}$ & $\mathrm{Na}: \mathrm{Ca}$ & $\mathrm{Mg}: \mathrm{Ca}$ \\
\hline 1 & 0.336111 & 0.010386 & 0.392389 \\
3 & 0.468767 & -0.04872 & 0.609318 \\
12 & 0.512869 & -0.10412 & 0.696781 \\
\hline
\end{tabular}

particles. Echelle spectrometers providing spectral coverage from UV to NIR are recently available. Successful implementation of such a device in a LIBS system could greatly improve the usefulness of LIBS for measurement of atmospheric PM. In fact, an initial attempt was made by the authors to measure ambient PM with an echelle spectrometer at the Supersite. Unfortunately the optical efficiency of this particular spectrometer was very poor,

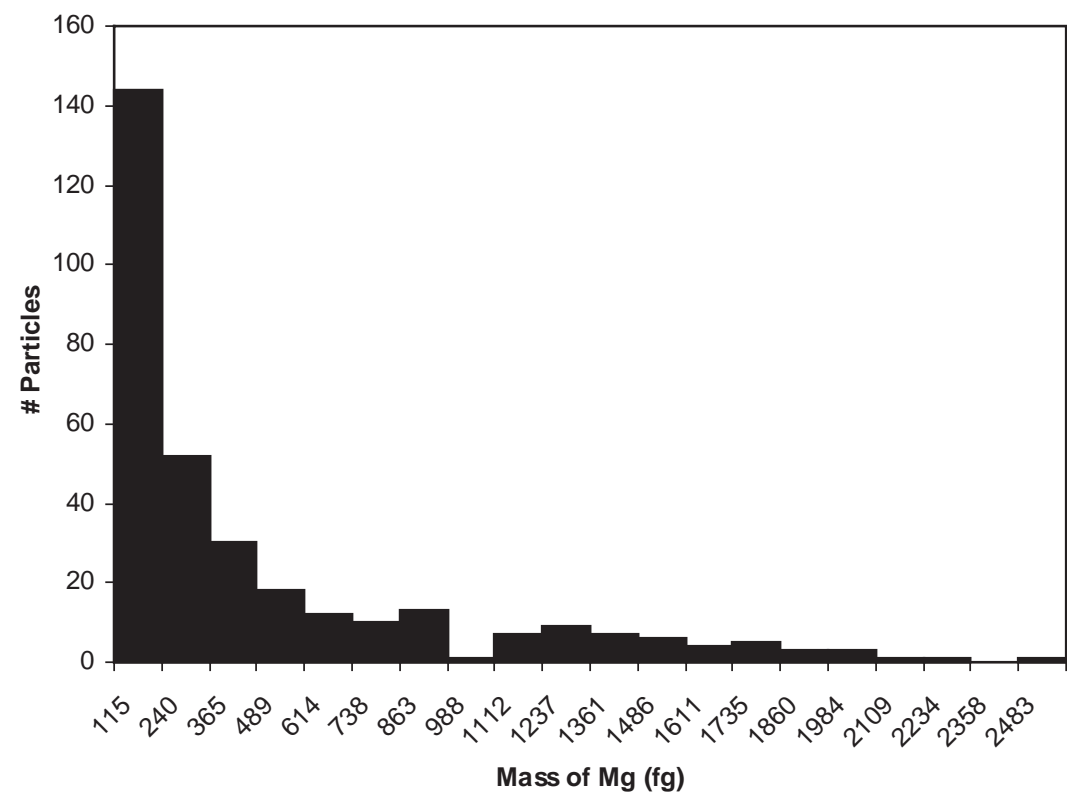

Fig. 5. Distribution of $\mathrm{Mg}$ mass in measured particles.

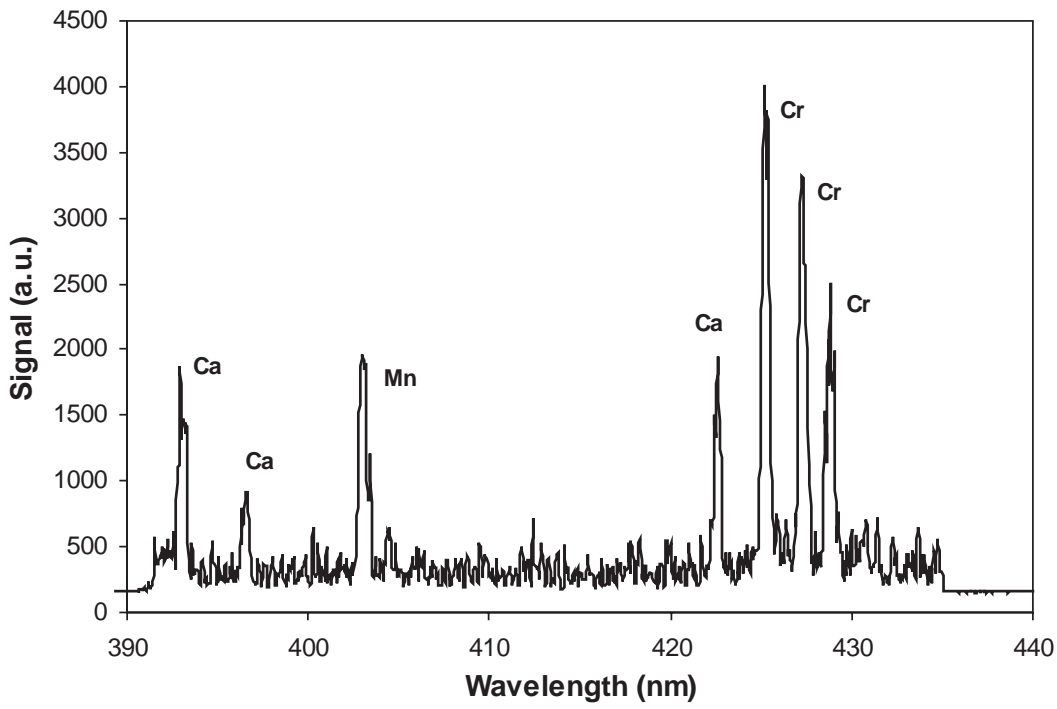

Fig. 6. A multi-element particle containing $\mathrm{Ca}, \mathrm{Mn}$, and $\mathrm{Cr}$. 
and single particles could not be detected. As the sensitivity of the LIBS technique improves, and with the use of large bandwidth echelle spectrometers, useful estimates of total composition and size (requiring assumptions about density and molecular composition) of single particles could potentially emerge, and source apportionment using the methods of Hopke and co-workers, e.g. (Fergenson et al., 2001) could be implemented.

\subsection{Discussion}

To our knowledge, the only previous measurement of ambient particles using LIBS was performed by Hahn and co-workers (Carranza et al., 2001). Their experiments demonstrated that LIBS could detect increased levels of $\mathrm{Mg}$ and $\mathrm{Al}$ introduced by fireworks displays during the 4th July holiday. They measured the daily concentrations of $\mathrm{Mg}, \mathrm{Na}, \mathrm{Al}$, and $\mathrm{Ca}$ for several weeks around the fourth of July, sampling roughly $4 \mathrm{hday}^{-1}$. This current study seeks to demonstrate the capability of LIBS to characterize ambient PM in greater detail, by measuring an extended list of elements at greater continuous temporal resolution. The data from the current study will also provide benchmark accuracy data for verification once the corresponding MOUDI data becomes available.

It is important to note that Hahn et al. (Carranza et al., 2001) reported particle hit rates one to two orders of magnitude greater than those reported here. This could be due to several factors, including either sampling issues or issues surrounding the detection limits of the system. One obvious sampling issue is that smaller particles may be trapped in the Teflon ${ }^{\mathrm{TM}}$ lines used in our experiments. In addition, environmental differences between Hahn's Florida location and our Pittsburgh location may result in substantial differences in elemental concentrations. Finally, the spark volume is expected to be roughly proportional to the laser pulse energy, and as a consequence Hahn's analytical volume should be significantly larger, as their energy is $\sim 9$ times greater than ours.

Considering factors influencing detection limits, the higher pulse energy used in Hahn's work results in a more repeatable plasma. Increased variability in the background signal necessitates more conservative detection thresholds. Hahn et al. also could have used a slightly more efficient optical setup for collecting light from the plasma or more optimal detection timing. In addition, they did use a different data processing technique, triggering on one emission line and quantifying using a second line. This method minimizes the influence of false hits, allowing lower thresholds, but can only be applied when more than one line is visible.

Overall, increases in sensitivity yield a greater ability to determine a complete picture of the particle mass distribution, and comparisons indicate that there is room for improvement in our current system. With better detection limits, and correspondingly higher hit rates, improved measurements of the particle mass distribution are certainly possible. However, given the fact that the mass is predominantly in the larger particles, the mass concentrations determined here, based on particles of roughly $300-400 \mu \mathrm{m}$ in diameter and larger, are likely to be close to the actual values.

\section{Conclusions}

This work has illustrated the usefulness of LIBS for long-term measurements of multiple elements in ambient air. These methods are equally applicable to thermal process streams or other situations where dilute particle suspensions mass and composition are of interest. Weekly and hourly mass concentrations illustrate the efficacy of the LIBS technique for temporal measurements, and the potential for LIBS to reveal elemental associations that may be important for understanding chemistry or for source apportionment. Particle mass distributions were limited in this work by the threshold detection limit, illustrating the importance of optimizing laser pulse energy, detection timing, and optical design to lower the detection threshold. However, mass concentrations determined here are likely to be largely correct, due to the preponderance of the mass in the larger size particles. Multi-element spectra suggest the potential of LIBS as a means for understanding particle formation chemistry through observed elemental affinities, and for source apportionment.

\section{Acknowledgements}

This research was conducted as part of the Pittsburgh Air Quality Study, which was supported by US Environmental Protection Agency under contract R82806101 and the US Department of Energy National Energy Technology Laboratory under contract DEFC26-01NT41017. This paper has not been subject to EPA's peer and policy review, and therefore does not necessarily reflect the views of the Agency. No official endorsement should be inferred.

The Office of Naval Research Grant \#N000140110698 and National Science Foundation Grant \#BES-0093853 provide general funding for LIBS development. The authors thank Francesco Ferioli, Jaykumar Patil, Sam Krehnbrink, Kyle Kratszch, Eric Lipsky, and Charles Stanier for their valuable help with the experiments. We thank Delbert Etough for the use of the particle concentrator. 


\section{References}

Brook, R., et al., 2003. Air pollution: the heart of the problem. Current Hypertension Reports 5 (1), 32-39.

Buckley, S.G., et al., 2000. Laser-induced breakdown spectroscopy as a continuous emission monitor for toxic metals in thermal treatment facilities. Waste Management 20, 455-462.

Carranza, J.E., Hahn, D.W., 2002. Sampling statistics and considerations for single-shot analysis using laser-induced breakdown spectroscopy. Spectrochimica Acta B 57, 779-790.

Carranza, J., et al., 2001. On-line analysis of ambient air aerosols using laser-induced breakdown spectroscopy. Spectrochimica Acta B 56, 851-864.

Chow, J., et al., 1993. The DRI thermal optical reflectance carbon analysis system - description, evaluation, and applications in United States Air Quality Studies. Atmospheric Environment Part A 27 (8), 1185-1201.

Davison, R.L., et al., 1974. Trace elements in fly ash. Environmental Science and Technology 8 (13), 1107-1113.

Ding, Y., et al., 2002. High-volume diffusion denuder sampler for the routine monitoring of fine particulate matter: I. Design and optimization of the PC-boss. Aerosol Science and Technology 36, 369-382.

Dockery, D.W., Pope, C.A.I., 1994. Acute respiratory effects of particulate air pollution. Annual Review of Public Health 15, 107-132.

Fergenson, D., et al., 2001. Quantification of ATOFMS data by multivariate methods. Analytical Chemistry 73, 3535-3541.

Fisher, B., et al., 2001. Temporal gating for the optimization of laser-induced breakdown spectroscopy detection and analysis of toxic metals. Applied Spectroscopy 55 (10), 1312-1319.

Hahn, D.W., 1998. Laser-induced breakdown spectroscopy for sizing and elemental analysis of discrete aerosol particles. Applied Physics Letters 72 (23), 2960-2962.

Hahn, D.W., et al., 1997. Discrete particle detection and metal emissions monitoring using laser-induced breakdown spectroscopy. Applied Spectroscopy 51 (12), 1836-1844.

Kidwell, C., Ondov, J., 2001. Development and evaluation of a prototype system for collecting sub-hourly ambient aerosol for chemical analysis. Aerosol Science and Technology 35 (1), 596-601.
Linak, W.P., Wendt, J.O.L., 1993. Toxic metal emissions from incineration: mechanisms and control. Progress in Energy and Combustion Science 19 (2), 145-185.

Ottesen, D.K., et al., 1991. Laser spark emission spectroscopy for in situ, real-time monitoring of pulverized coal combustion. Energy and Fuels 5, 304.

Phares, D., et al., 2002. Performance of a single ultrafine particle mass spectrometer. Aerosol Science and Technology 36, 583-592.

Prather, K., et al., 1994. Real-time characterization of individual aerosol particles using time-of-flight mass spectrometry. Analytical Chemistry 66, 1403-1407.

Radziemski, L.J., 1994. Review of selected analytical applications of laser plasmas and laser ablation, 1987-1994. Microchemical Journal 50, 218-234.

Salvi, S., Holgate, S., 1999. Mechanisms of particulate matter toxicity. Clinical and Experimental Allergy 29 (9), 1187-1194.

Schechter, I., 1997. Laser induced plasma spectroscopy. A review of recent advances. Reviews in Analytical Chemistry 16 (3), 173-298.

Seltzer, M., 2000. Performance testing of a multimetals continuous emissions monitor. Journal of the Air and Waste Management Association 50 (6), 1010-1016.

Sneddon, J., Lee, Y.-I., 1999. Novel and recent applications of elemental determination by laser-induced breakdown spectrometry. Analytical Letters 32 (11), 2143-2162.

Song, K., et al., 1997. Applications of laser-induced breakdown spectroscopy. Applied Spectroscopy Reviews 32 (3), $183-235$.

Suarez, A., Ondov, J., 2002. Ambient aerosol concentrations of elements resolved by size and by source: contributions of some cyokine-active metals from coal- and oil-fired power plants. Energy and Fuels 16 (3), 562-568.

Woskov, P.P., et al., 2000. Accurate and sensitive metals emissions monitoring with an atmospheric microwaveplasma having a real-time span calibration. Waste Management 20 (5-6), 395-402.

$\mathrm{Xu}, \mathrm{L}$., et al., 1997. Absolute analysis of particulate materials by laser-induced breakdown spectroscopy. Analytical Chemistry 69, 2103-2108.

Yalcin, S., et al., 1999. Influence of ambient conditions on the laser air spark. Applied Physics B 68 (1), 121-130.

Zhang, H., et al., 1999. Laser-induced breakdown spectrometry as a multimetal continuous emissions monitor. Applied Optics 38 (9), 1459-1466. 


\section{Effects of Dilution on Fine Particle Mass and Partitioning of Semivolatile Organics in Diesel Exhaust and Wood Smoke}

ERIC M. LIPSKY ${ }^{\dagger}$ A N D

A L L E L . ROB I N S N*

Department of Mechanical Engineering, Carnegie Mellon University, Pittsburgh, Pennsylvania, 15213

Experiments were conducted to examine the effects of dilution on fine particle mass emissions from a diesel engine and wood stove. Filter measurements were made simultaneously using three dilution sampling systems operating at dilution ratios ranging from 20:1 to 510:1. Denuders and backup filters were used to quantify organic sampling artifacts. For the diesel engine operating at low load and wood combustion, large decreases in fine particle mass emissions were observed with increases in dilution. For example, the $\mathrm{PM}_{2.5}$ mass emission rate from a diesel engine operating at low load decreased by $50 \%$ when the dilution ratio was increased from 20:1 to 350:1. Measurements of organic and elemental carbon indicate that the changes in fine particle mass with dilution are caused by changes in partitioning of semivolatile organic compounds. At low levels of dilution semivolatile species largely occur in the particle phase, but increasing dilution reduces the concentration of semivolatile species, shifting this material to the gas phase in order to maintain phase equilibrium. Emissions of elemental carbon do not vary with dilution. Organic sampling artifacts are shown to vary with dilution because of the combination of changes in partitioning coupled with adsorption of gas-phase organics by quartz filters. The fine particle mass emissions from the diesel engine operating at medium load did not vary with dilution because of the lower emissions of semivolatile material and higher emissions of elemental carbon. To measure partitioning of semivolatile materials under atmospheric conditions, partitioning theory indicates that dilution samplers need to be operated such that the diluted exhaust achieves atmospheric levels of dilution. Too little dilution can potentially overestimate the fine particle mass emissions, and too much dilution (with clean air) can underestimate them.

\section{Introduction}

Many combustion and other high-temperature sources emit compounds that are semivolatile or volatile at exhaust temperatures but undergo gas-to-particle conversion as the combustion products mix with ambient air. Dilution sampling is a technique developed to simulate these processes in order to better characterize fine particle emissions (1).

\footnotetext{
* Corresponding author phone: (412)268-3657; fax: (412)268-3348; e-mail: alr@andrew.cmu.edu.

${ }^{\dagger}$ Present address: Department of Engineering, Penn State McKeesport, McKeesport, PA 15132.
}

Previous research has shown that fine particle emissions are sensitive to dilution conditions. A major focus has been on nucleation and the particle size distribution, both of which can be extremely sensitive to dilution conditions (2). However, existing emission and ambient standards are based on fine particle mass. Fine particle mass emissions depend on the phase partitioning of semivolatile compounds in the exhaust. For sources with significant organic aerosol emissions, dilution samplers measure higher fine particle mass emission rates compared to filters collected at exhaust temperatures $(1,3)$. This well-recognized effect is due to gas-to-particle conversion of semivolatile species as the exhaust is cooled during dilution. Cooling reduces the saturation pressures of the semivolatile compounds in the exhaust.

Dilution samplers are typically operated at dilution ratios between 20:1 and 200:1. A dilution ratio of 100:1 is generally high enough to reduce the exhaust temperature to ambient levels, but the median dilution ratio of vehicle exhaust in an urban atmosphere is around $10000: 1$ (20). Therefore, the concentration of semivolatile species inside a dilution sampler can be orders of magnitude higher than typical atmopsheric conditions. One also needs to account for effects of background pollution on the emissions because dilution samplers mix exhaust with particle- and organics-free air.

Dilution reduces the concentrations of semivolatile species; partitioning theory indicates that, under isothermal conditions, this should reduce the amount of semivolatile material in the particle phase $(5,6)$. However, the significance of this effect is not well understood as illustrated by a recent review concluding that fine particle mass tends to be conserved upon dilution (2). Experiments with diesel exhaust report modest decreases in fine particle mass emissions at higher dilution ratios $(3,7)$. These changes were attributed to changes in partitioning of semivolatile organics. However, both studies only considered dilution ratios smaller than 100:1. In addition, one study performed the experiments with a constant filter temperature of $52^{\circ} \mathrm{C}(7)$ while the other only observed a decrease in emissions at one dilution ratio (3). In contrast, measurements by Hildemann et al. (1) made on a fuel oil boiler suggest increasing emissions with higher dilution ratio. Interpretation of these data is potentially complicated by organic sampling artifacts (8).

This paper examines the effects of dilution sampling on the fine particle mass emissions from a diesel engine and a wood stove. Measurements of $\mathrm{PM}_{2.5}$ mass, organic carbon, and elemental carbon emissions were made at dilution ratios between 20:1 and 510:1. Backup and denuded filters were collected to estimate organic sampling artifacts. Partitioning theory is used to discuss the data in the context of real-world dilution.

\section{Experimental Setup and Procedure}

Experiments were performed to measure the effects of dilution on fine particle mass emissions from a diesel engine and a wood stove. Figure 1 shows a schematic of the experimental setup. Filter samples were collected using three completely independent dilution sampling systems operated simultaneously at different dilution ratios. This approach of simultaneous sampling with multiple samplers minimizes the effects of temporal variations in emissions on the results.

Detailed descriptions of the design, characterization, and operation of the dilution samplers can be found in Lipsky and co-workers $(9,10)$. Briefly, each sampler isokinetically collects exhaust through separate, heated inlet lines that are maintained at a temperature slightly above the exhaust temperature to minimize thermophoretic losses. The sampled 


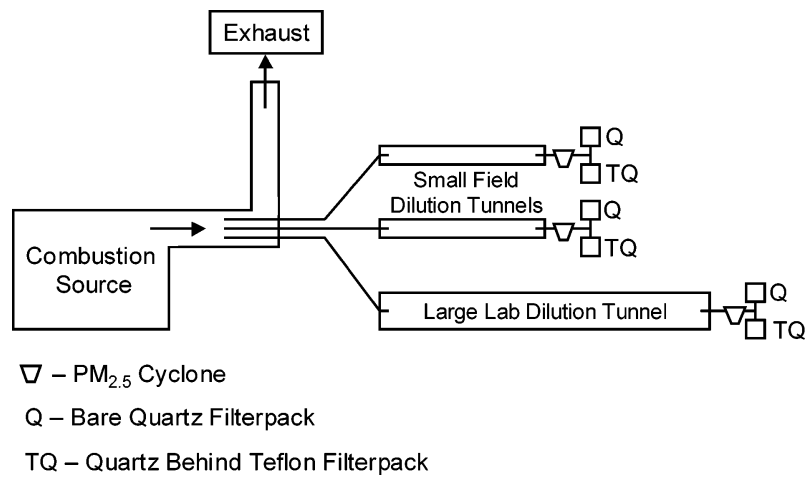

FIGURE 1. Experimental setup of simultaneous sampling with three dilution tunnels. Each sampler has a separate heated inlet line.

exhaust is then rapidly mixed by turbulence with filtered (HEPA and activated carbon) dilution air inside a dilution tunnel. Filter trains are connected to the end of the dilution tunnel. Each tunnel provides about $2.5 \mathrm{~s}$ of residence time after mixing but before filter collection. Previous research has shown that additional residence time beyond that provided by the tunnel does not affect filter measurements under the conditions of these experiments (10). All samplers are constructed out of stainless steel with Teflon gaskets to minimize sample contamination.

Two different dilution tunnel designs were used for these experiments. To establish the comparability of the different designs, separate experiments were conducted while operating all three samplers simultaneously at the same dilution ratio (10). These intercomparison experiments show excellent agreement between the different samplers across the range of dilution ratios considered here; for example, the average relative bias in the $\mathrm{PM}_{2.5}$ mass emissions measured using the two designs is $1 \% \pm 17 \%$ (average \pm standard deviation).

The dilution ratio within each sampler is determined by simultaneously measuring exhaust and dilution tunnel $\mathrm{CO}_{2}$ levels:

$$
\mathrm{DR}=\frac{\left(\mathrm{CO}_{2}\right)_{\mathrm{ex}}-\left(\mathrm{CO}_{2}\right)_{\mathrm{bck}}}{\left(\mathrm{CO}_{2}\right)_{\mathrm{tun}}-\left(\mathrm{CO}_{2}\right)_{\mathrm{bck}}}
$$

where $\left(\mathrm{CO}_{2}\right)_{\mathrm{ex}},\left(\mathrm{CO}_{2}\right)_{\text {bck }}$, and $\left(\mathrm{CO}_{2}\right)_{\text {tun }}$ are the $\mathrm{CO}_{2}$ mixing ratios in the exhaust, dilution air, and dilution tunnel, respectively. Separate $\mathrm{CO}_{2}$ monitors are used to continuously monitor $\left(\mathrm{CO}_{2}\right)_{\text {tun }}$ at the end of each tunnel. The dilution air $\mathrm{CO}_{2}$ mixing ratio is measured both before and after each day of tests; the small variations in $\left(\mathrm{CO}_{2}\right)_{\text {bck }}$, are insignificant compared to the relatively high tunnel and exhaust $\mathrm{CO}_{2}$ levels. No dilution corresponds to a dilution ratio of one.

To compare measurements made at different dilution ratios, emissions are reported as fuel-based emission factors (e.g., g $\mathrm{PM}_{2.5} / \mathrm{kg}$ fuel):

$$
\mathrm{EF}=\frac{[\mathrm{P}]}{[\mathrm{C}]} C_{\mathrm{f}}
$$

where $[\mathrm{P}]$ and $[\mathrm{C}]$ are the background-corrected pollutant and carbon concentrations inside the dilution tunnel, respectively, and $C_{\mathrm{f}}$ is the mass fraction of carbon in the fuel. $[\mathrm{C}]$ is determined from the measured exhaust gas composition, assuming all of the fuel carbon is emitted as CO and $\mathrm{CO}_{2}$. Small amounts of fuel carbon are emitted as either gasor condensed-phase organic compounds; however this organic material contributes negligibly to the overall carbon balance. We use a value of $C_{\mathrm{f}}$ of 0.87 for the diesel fuel and 0.40 for the wood fuel. Unless otherwise noted, the word "emission" or "emission rate" refers to fuel-based emissions.
Aerosol Characterization. Filter samples were collected using a sampling train that consists of a sharp-cut $\mathrm{PM}_{2.5}$ cyclone operating at 24 liters per min (lpm) (Figure 1). Downstream of the cyclone the flow is split and passed through two filter packs each operating at $12 \mathrm{lpm}$. One filter pack contains a single quartz filter (Bare-Q); the second filter pack contains a Teflon filter followed by a backup quartz filter (quartz behind Teflon or QBT). The QBT filter is used as an estimate of the positive sampling artifact from gas adsorption of semivolatile organic material (8). Identical filter trains were attached to the end of each dilution tunnel.

The Teflon filter is used for quantifying total $\mathrm{PM}_{2.5}$ mass emissions. Teflon filters are weighed before and after sampling using a microbalance in a temperature and relative humidity controlled environment $\left(30-40 \% \mathrm{RH}\right.$ at $\left.21-23^{\circ} \mathrm{C}\right)$. The filters are equilibrated for $24 \mathrm{~h}$ before gravimetric analysis. Samples on quartz filters are used to quantify organic and elemental carbon (OC and EC) emissions using a Sunset Laboratories laboratory thermal-optical transmission OC/ EC analyzer. The temperature protocol is a modified version of the NIOSH 5040 protocol (11). Quartz filters are prepared before sampling by baking them at $500{ }^{\circ} \mathrm{C}$ in air for at least $6 \mathrm{~h}$ to remove any residual carbon. Both quartz and Teflon filters are stored in a freezer $\left(-18{ }^{\circ} \mathrm{C}\right)$ between sample collection and analysis. All filter measurements are blank corrected (10).

A second filter train was occasionally operated in parallel with the first train to further investigate organic sampling artifacts. This train consists of a sharp-cut $\mathrm{PM}_{2.5}$ cyclone, a carbon monolith denuder (MastCarbon Ltd., U.K.), followed by a filter pack with a quartz fiber filter and a carbon impregnated glass-fiber (CIG) filter ( $47 \mathrm{~mm}$, Schleicher \& Schuell, GF 3649) (11). The flow rate through the denuded filter train was $12 \mathrm{lpm}$ to match the filter face velocity of the standard train. The CIG filter was analyzed with the Sunset OC/EC analyzer in a helium atmosphere using a stepped temperature profile ramping to $330^{\circ} \mathrm{C}$ (11).

Sources. Emissions tests were performed using a small diesel generator and a wood stove. The diesel generator is a single-cylinder Yanmar L70AE air-cooled diesel engine connected to a $4.5 \mathrm{~kW}$ generator. The engine is EPA and CARB exhaust emission compliant. Experiments were performed at constant load: low load ( $25 \%$ of rated capacity) or medium load (55\% of rated capacity). Exhaust temperatures at the sampling location were in the range of 230-260 ${ }^{\circ} \mathrm{C}$ for low-load tests and $260-290{ }^{\circ} \mathrm{C}$ for medium-load tests. The engine was operated at the specified load setting for a minimum of 45 min before filter sampling to allow all components to achieve a steady-state temperature. Diesel fuel was purchased at a local gas station.

The wood stove is an EPA approved Jøtul "602 CB Classic” wood stove. The wood fuel was a mixture of oak, cherry, and some ash. Wood-burning experiments involved starting the fire with a small amount of wood. After the fire was established, the stove was loaded to capacity and the wood was allowed to burn down for 45-75 min until the combustion stabilized and the exhaust temperature at the sampling location was in the range of $130-150^{\circ} \mathrm{C}$. Sampling was then started, and stable flaming combustion conditions were maintained by adjusting the vents on the door of the stove. Exhaust temperatures and $\mathrm{O}_{2}$ and $\mathrm{CO}$ levels were reasonably constant during sampling.

\section{Results}

Fuel-based emissions of $\mathrm{PM}_{2.5}$ mass, total carbon (TC), and organic and elemental carbon (OC \& EC) as a function of dilution ratio are shown in Figure 2 for low- and medium- 

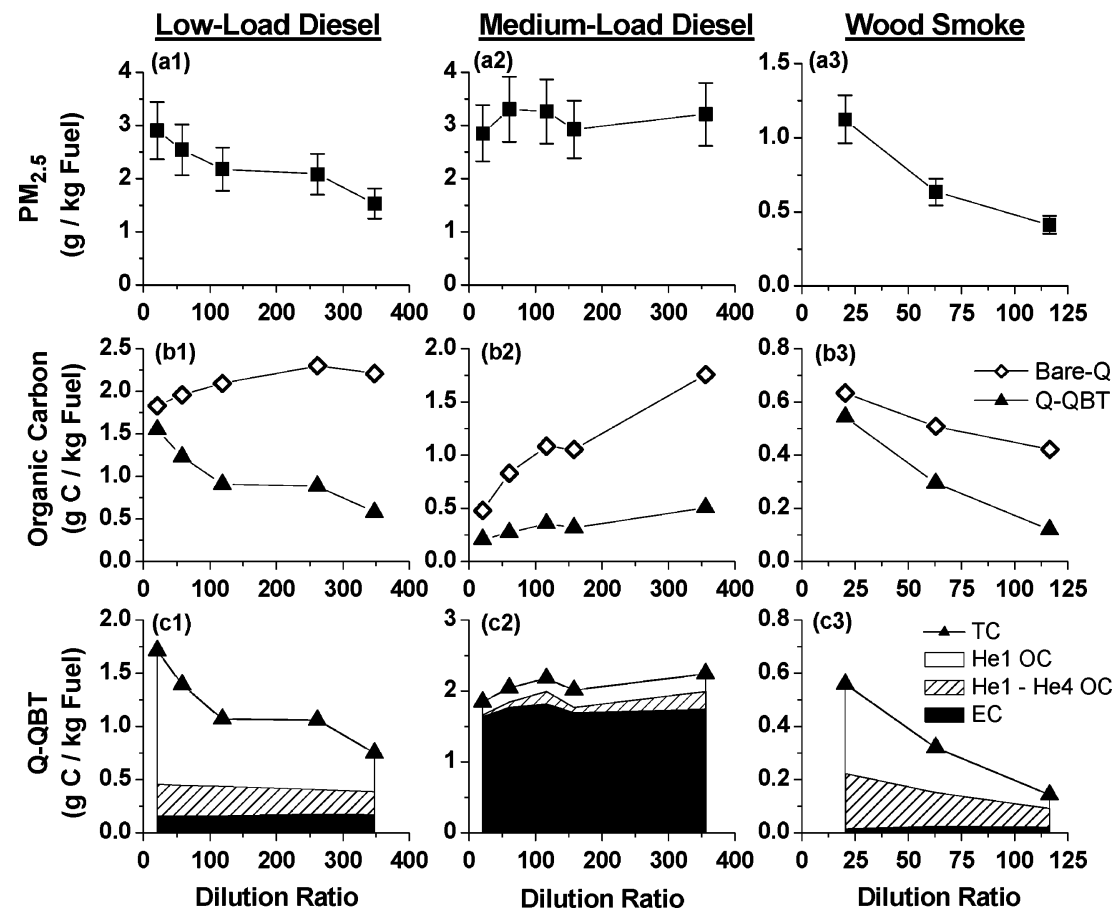

FIGURE 2. Fuel-based emissions from low-load diesel (left column), medium-load diesel (middle column), and wood smoke (right column) experiments. Plots a1-a3 show $\mathrm{PM}_{2.5}$ mass emission rate; plots b1-b3 compare organic carbon emission factors measured with the Bare-0 and 0 - OBT approaches; plots c1-c3 show particulate organic carbon (OC) and elemental carbon (EC) emission factors measured using the $\mathbf{0}-\mathbf{0 B T}$ approach. Bare- $\mathbf{0}$ is carbon measured with a quartz filter; $\mathbf{0}-\mathbf{O B T}$ is carbon measured with a backup-corrected quartz filter. $\mathbf{O}-$ OBT is particulate OC, while Bare- 0 has substantial positive artifact, as discussed in the text. In plots $\mathrm{C1}-\mathrm{c} 3$ symbols indicate total carbon emissions and shading indicates the contribution of $\mathrm{OC}$ and EC to the emissions. Plots c1-c3 also show the carbon fractions from the $\mathrm{OC} / \mathrm{EC}$ analysis: $\mathrm{He} 1 \mathrm{OC}$ is the $\mathrm{OC}$ that evolves at $340^{\circ} \mathrm{C}$, and $\mathrm{He} 2-\mathrm{He} 4 \mathrm{OC}$ is the $\mathrm{OC}$ that evolves at temperatures greater than $340{ }^{\circ} \mathrm{C}$ during the $\mathrm{OC} / \mathrm{EC}$ analysis. The diesel plots combine data from back-to-back experiments conducted on the same day. Lines are intended as a visual aid. Vertical bars in (a) are experimental uncertainties determined from intercomparison experiments (10).

load diesel operations and a wood smoke experiment. The emissions are presented on a fuel basis in order to compare measurements made at different dilution ratios on a consistent basis; fuel-based emissions of inert pollutants such as EC should be independent of dilution ratio, appearing as a horizontal line in Figure 2.

The fuel-based $\mathrm{PM}_{2.5}$ mass emissions decrease with increasing dilution ratio during low-load diesel and wood smoke experiments. For example, Figure 2 shows that increasing the dilution ratio from 20:1 to 350:1 decreased the $\mathrm{PM}_{2.5}$ mass emissions from the diesel engine operating at low load by $55 \%$. Increasing the dilution ratio from $20: 1$ to 120:1 decreased the $\mathrm{PM}_{2.5}$ mass emissions from the wood stove by over $60 \%$. Within experimental uncertainty, the $\mathrm{PM}_{2.5}$ mass emissions measured during medium-load operation of the diesel engine are constant (Figure 2). Trends similar to those shown in Figure 2 were observed across the entire set of experiments; however, combining results from different experiments is difficult because of day-to-day variability in engine emissions and experiment-to-experiment variation in wood smoke emissions.

Changes in the phase partitioning of the semivolatile organic material with dilution is the likely explanation for the changes in the $\mathrm{PM}_{2.5}$ mass shown in Figure 2. Although dilution does not alter the total (gas + particle) emission rate of semivolatile species, dilution does affect the phase partitioning of this material. Gas-particle partitioning occurs via absorption with an organic solution or adsorption to soot and mineral surfaces $(5,6)$. Therefore, phase partitioning depends on the concentration and saturation pressure of the semivolatile species and the concentration and composition of the sorptive material $(5,6)$. Under the conditions of these experiments, changes in concentration of the semivolatile and sorptive material with dilution likely cause the changes in partitioning. The changes are not caused by changes in saturation pressure because the temperature of the diluted exhaust at the filter holder was essentially constant across this entire set of experiments $\left(27 \pm 2{ }^{\circ} \mathrm{C}\right)$. (Note that mixing inside the dilution tunnel is not adiabatic; at the lowest dilution ratios there was some heat transfer to the surroundings).

Dilution reduces the concentration of both semivolatile species and the sorptive material which, under constant temperature conditions, requires semivolatile species to transfer from the particle to the gas phase to maintain phase equilibrium. This reduces the $\mathrm{PM}_{2.5}$ mass emission rate. $\mathrm{A}$ complication is the fact that dilution samplers are operated using cleaned air, while real-world dilution mixes emissions with polluted air. This issue is addressed in the discussion section.

If changes in phase partitioning of semivolatile organics are responsible for decrease in $\mathrm{PM}_{2.5}$ mass, then these changes should be apparent in measurements of particulate OC. The simplest approach for measuring OC emissions is to use a quartz filter (Bare-Q). Figure 2 shows that the trends in Bare-Q OC with dilution are different than the changes in $\mathrm{PM}_{2.5}$ mass. For example, at low load the Bare-Q OC increase with dilution ratio while the $\mathrm{PM}_{2.5}$ mass decreases. However, OC measurements can be significantly impacted by sampling artifacts; these artifacts are carefully considered in the next section.

Dilution-ratio-dependent losses are another potential explanation for the trends shown in Figure 2. Fuel-based EC emissions measured by the different samplers were within $\pm 12 \%$ for a given experiment, indicating consistent collection of a nonvolatile component of $\mathrm{PM}_{2.5}$ (Figure 2c). Furthermore, there were no consistent trends in the EC emission factor with dilution ratio. 


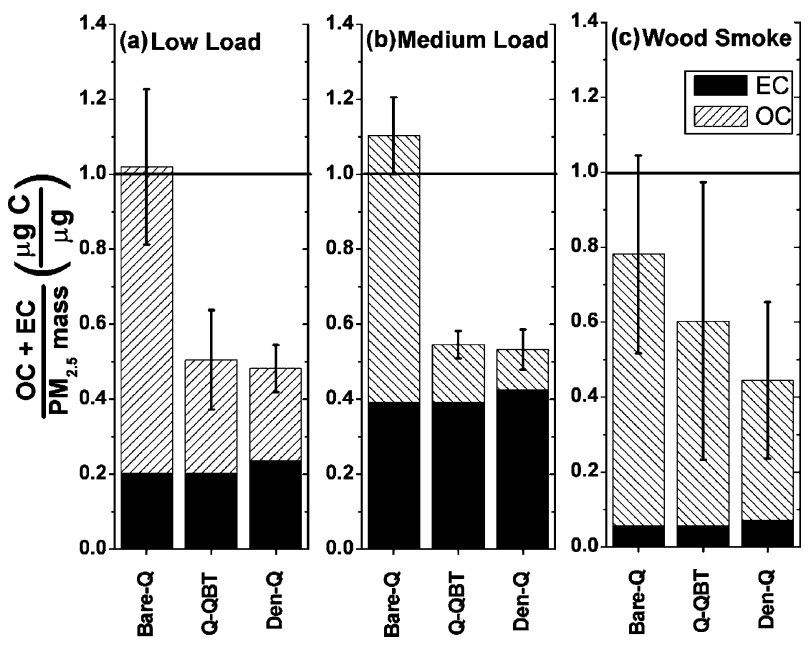

FIGURE 3. Average ratio of total carbon (OC $+E C$ ) to $\mathbf{P M}_{2.5}$ mass for (a) low-load diesel, (b) medium-load diesel, and (c) wood smoke experiments. Three different estimates of $\mathrm{OC}$ are shown: bare quartz filter (Bare-0), backup-corrected quartz filter ( $0-0 B T)$, and denuded quartz filter (Den0). There are two different estimates of EC ( 0 OBT EC and Bare-0 EC are the same). Error bars are standard deviations of the ratio of $\mathrm{OC}+\mathrm{EC}$ to $\mathrm{PM}_{2.5}$ mass across the set of experiments. This ratio is expected to be less than one because of the contribution of elements other than carbon to the $\mathrm{PM}_{2.5}$ mass.

Organic Sampling Artifacts. Both positive and negative artifacts can significantly affect quartz-filter measurements of particulate OC (8). Positive artifact is caused by organic vapors adsorbing onto the quartz filter resulting in an overestimate in the amount of organic aerosol. Negative artifact is caused by particle-phase organic compounds volatilizing after collection resulting in an underestimate of the organic aerosol mass. Organic sampling artifacts are commonly accounted for using a combination of denuders and/or backup filters (8). We employed both approaches in this study.

The OC collected on a backup quartz filter behind a Teflon filter (QBT) is a standard estimate of the positive artifact (8). The approach assumes that the inert Teflon filter only collects particles, allowing gas-phase organics to pass through to the backup quartz filter. The particulate OC emissions are then estimated by subtracting the OC collected on the QBT from that collected on the Bare-Q filter $(\mathrm{Q}-\mathrm{QBT})$.

The denuded filter train provides a second estimate of the particulate OC emissions. The denuder removes most of the organic vapor from the sample upstream of the quartz filter, minimizing the positive artifact. However, the depletion of semivolatile organic vapor from the air stream may cause volatilization of semivolatile organic material collected on the quartz filter resulting in a negative artifact (8). This negative artifact is estimated using the carbon impregnated glass-fiber (CIG) filter downstream of the denuded quartz filter. Therefore, the particulate OC emissions are estimated by adding the OC measured on the CIG filter and the denuded quartz filter (DenQ + CIG).

Figure 3 compares the average OC emissions measured using the three different approaches: Bare-Q OC, backupcorrected (Q - QBT) OC, and denuded OC. To combine data from different experiments, the OC and EC data are normalized by $\mathrm{PM}_{2.5}$ mass before averaging. The ratio of TC to $\mathrm{PM}_{2.5}$ mass should be less than 1 because non-carbon organic components and inorganic species also contribute to $\mathrm{PM}_{2.5}$ mass. Figure 3 combines data from filters collected at different dilution ratios.

When the diesel engine is operated at low and medium loads (Figure 3, parts a and b), the TC measured with a Bare-Q filter is, on average, slightly greater than the $\mathrm{PM}_{2.5}$ mass consistent with a significant positive artifact on the Bare-Q filter. For the wood smoke experiments (Figure 3c), the TC measured by a Bare-Q filter is smaller than the Teflon mass. Assuming wood smoke is dominated by carbonaceous species, an organic-mass-to-organic-carbon ratio (OM/OC) of 1.3 is required to close the mass balance between the Teflon and Bare-Q filters. This value is smaller than expected given the relatively polar composition of wood smoke (12), indicating that the wood smoke also creates a net positive artifact on a quartz filter. Other researchers report that the positive artifact is dominant when sampling both diesel exhaust and wood smoke (13-17).

Figure 3 indicates that the artifact-corrected estimates of particulate $\mathrm{OC}$ are less than the $\mathrm{PM}_{2.5}$ mass for all three experimental conditions. For diesel exhaust, the backup and denuder approaches measure the same OC and EC emission rates and the majority of the Bare-Q OC appears to be positive artifact. On average, particulate OC contributes only $35 \%$ and $20 \%$ of the Bare-Q OC at low and medium loads, respectively. In contrast, other papers report that particulate OC contributes a majority of Bare-Q OC (13-15). Differences in engine technology and operating conditions likely influence sampling artifacts; in addition, the larger positive artifacts reported here may be due, in part, to the effects of dilution on artifacts discussed below. The CIG filter indicates that there was little negative artifact from the denuded quartz filter.

For wood smoke, the EC emissions measured by the different approaches are comparable, but OC emissions measured using the denuded filters are somewhat smaller than the Q - QBT OC. However, the wood smoke emissions are highly variable as indicated by the large error bars in Figure 3. Positive artifact is estimated to contribute between $25 \%$ and $50 \%$ of wood smoke Bare-Q OC, which is consistent with results from Fine et al. $(16,17)$.

The Teflon mass minus EC divided by the artifactcorrected, particulate OC can be used as a crude consistency check for the artifact corrections. Under the assumption that carbonaceous materials dominate the fine particle mass, this value is the OM/OC ratio. For wood smoke, the $\mathrm{Q}-\mathrm{QBT}$ OC requires an $\mathrm{OM} / \mathrm{OC}$ ratio of 1.8 for mass balance closure, which is within the range of expected values (12) suggesting the Q - QBT approach provides a reasonable estimate of particulate OC. However, this approach yields estimated OM/ OC ratios that are much larger than expected for diesel exhaust; for example, the low-load data require a ratio of 2.6 for mass balance closure versus expected values in the range of 1.2-1.4 (12). This indicates that, for the diesel exhaust, the Q - QBT (or denuder) approaches are underestimating the particulate OC emissions and/or that a significant fraction of the emissions are inorganic. Mass balance problems between sum of aerosol species and Teflon mass are not uncommon for measurements of diesel exhaust (13). A linear regression of the Teflon mass minus $\mathrm{EC}$ versus $\mathrm{Q}-\mathrm{QBT}$ OC yields a slope of $1.4 \mu \mathrm{g} / \mu \mathrm{g}-\mathrm{C}$ and an intercept of $0.5 \mathrm{~g} / \mathrm{kg}$ fuel with an $R^{2}$ value of 0.85 (4). This suggests an OM/OC ratio of 1.4, in line with expectations, and that there are significant inorganic emissions from the diesel engine (water or sulfate seem to be the most obvious candidates).

Effects of Dilution on Organic Particulate Emissions. Figure 2 indicates that the trends in artifact-corrected, particulate OC match those of the $\mathrm{PM}_{2.5}$ mass, which is consistent with the changes emissions with dilution being caused by changes in phase partitioning of semivolatile organic compounds. Increasing the dilution ratio from 20:1 to $120: 1$ decreased the Q - QBT OC by 75\% during the wood smoke experiment shown in Figure 2. At low load, the diesel particulate OC decreased by almost $70 \%$ when the dilution 


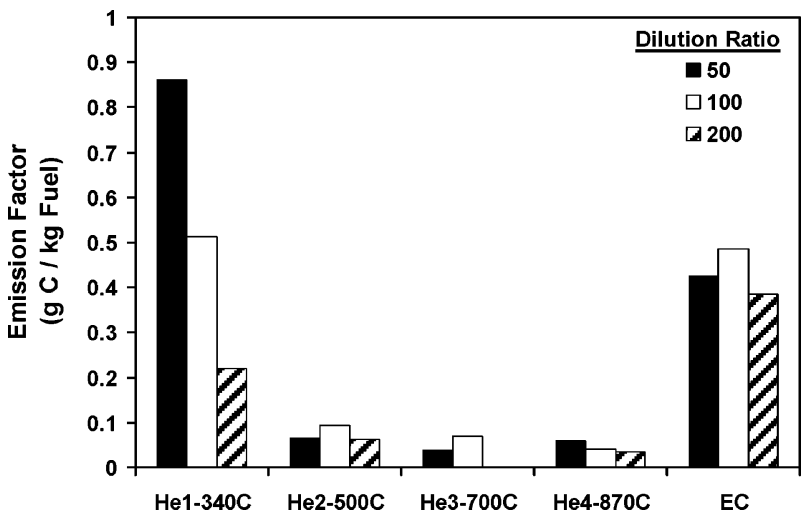

FIGURE 4. Evolution of particulate carbon during OC/EC analysis of quartz filters collected at three dilution ratios during a low-load diesel experiment. Particulate carbon is defined as backupcorrected carbon ( $0-0 B T)$. The first four groups of bars indicate the amount of carbon that evolves from the four different temperature steps of the He mode of the analysis $\left(340,500,700\right.$, and $\left.870^{\circ} \mathrm{C}\right)$. The final group of bars indicates the amount of EC that evolves during the analysis. Increasing the dilution ratio substantially reduces the amount of carbon that evolves at the lowest temperature step of the He mode, while the carbon at the higher temperature steps remains relatively constant. Backup and Bare- 0 levels at the He3$700 C$ peak were the same at $D R=200$.

ratio was increased from 20:1 to 350:1. For the medium-load diesel experiments, the Q - QBT OC is constant.

Dilution also alters the composition of the particle-phase emissions. The ramping temperature protocol used for OC/ EC analysis crudely classifies the OC by volatility; the most volatile species evolving in the lowest temperature step of the helium mode $\left(340{ }^{\circ} \mathrm{C}\right)$ and progressively less volatile compounds evolving in the higher temperature steps.

Figure 4 plots a thermogram of particulate OC $(\mathrm{Q}-\mathrm{QBT})$ measured during a low-load diesel experiment. Increasing the dilution ratio dramatically decreases the amount of OC evolving in the lowest temperature step of the OC/EC analysis, while the amount of $\mathrm{OC}$ evolving in the higher temperature steps ( $500{ }^{\circ} \mathrm{C}$ or higher) remains relatively constant. At low dilution ratios, carbon that evolves at $340^{\circ} \mathrm{C}$ contributes the majority of the particulate OC emissions; at high dilution ratios the higher temperature (less volatile) fractions dominate the particulate emissions. Figure 2, part c3, indicates that similar trends with dilution are observed in the wood smoke particulate OC with dilution. The modest decrease in particulate wood smoke OC with dilution in the higher temperature steps may be related to pyrolysis of semivolatile organics during OC/EC analysis.

Low- versus Medium-Load Diesel Operations. Although the overall $\mathrm{PM}_{2.5}$ mass emission factors of the diesel engine at low and medium loads are similar, at low load the $\mathrm{PM}_{2.5}$ mass emission factor is a strong function of dilution ratio, but independent of dilution ratio at medium load. This difference appears related to the composition of the emissions.

At low load the $\mathrm{PM}_{2.5}$ emissions are dominated by OC (average OC/EC ratio of 7) while at medium load emissions are dominated by EC (average OC/EC ratio of 0.4). This likely influences the relative importance of the different sorption mechanisms controlling gas-particle partitioning. At higher EC loadings adsorption to EC is likely more important than absorption in organic matter. This effect is illustrated by changes in thermal desorption profiles of diesel nanoparticles with soot loading (18).

Another difference between the low- and medium-load diesel exhaust appears to be the composition of the OC emission. Carbon that evolves in the $340{ }^{\circ} \mathrm{C}$ temperature

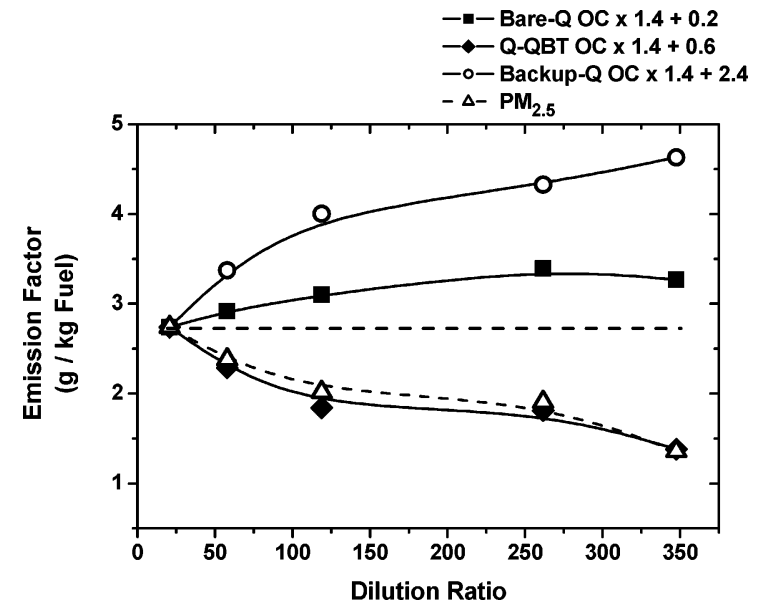

FIGURE 5. Changes in fuel-based $\mathrm{PM}_{2.5}$ and organic mass emissions of low-load diesel exhaust as a function of dilution ratio. Organic mass is defined as $\mathrm{OC}$ multiplied by 1.4. To easily compare the changes in the different measurements with dilution ratio, the quartz filter data have been shifted to match the $\mathbf{P M}_{2.5}$ mass data at a dilution ratio of 20 . This is done by adding the number indicated in the legend to each measurement. The changes in particulate organic mass $(\mathbf{0}-\mathbf{0 B T})$ match those of the overall $\mathrm{PM}_{2.5}$ mass. The backup- 0 filter provides a measure of the gas-phase semivolatile organic carbon which, on a fuel basis, increases with dilution due to changes in partitioning.

step of the OC/EC analysis contributes $60-70 \%$ of the lowload Bare-Q OC versus only $25-35 \%$ at the medium load. Therefore, less semivolatile carbon may be emitted under medium load.

Sampling Artifacts and Dilution. Data presented in Figure 5 suggest that the individual quartz filters provide a surprisingly consistent picture of the changes in phase partitioning of the semivolatile organics. The measurements are from a low-load diesel experiment; to make direct comparisons with $\mathrm{PM}_{2.5}$ mass, organic mass and not $\mathrm{OC}$ is plotted using an $\mathrm{OM} / \mathrm{OC}$ ratio of 1.4. Figure 5 indicates that the changes in particulate organic mass, $(\mathrm{Q}-\mathrm{QBT}) \times 1.4$, with dilution ratio exactly match the decrease of $\mathrm{PM}_{2.5}$ mass.

Compared to the changes observed with the other filters, the Bare-Q OC reported on a fuel basis is relatively constant, varying by less than $\pm 25 \%$ across the range dilution ratio for the set of filters collected during a given experiment (Figure $2 \mathrm{~b}$ and Figure 5). The Bare-Q OC modestly increased with dilution ratio during the diesel experiments and decreased modestly during the wood smoke experiments. In contrast, for experiments in which there was a decreasing trend in $\mathrm{PM}_{2.5}$ mass with dilution, the OC measured by the QBT filter increases dramatically with dilution ratio. Figure 5 illustrates this trend for a low-load diesel experiment.

One explanation of the Bare-Q and QBT OC data in Figure 5 is that the quartz filters provide a consistent measure of the gas-phase semivolatile organics. The Bare-Q OC emissions are relatively constant because the filter is collecting both gas- and particle-phase OC and the total OC emissions (gas + particle) do not change with dilution. At low dilution ratios the Bare-Q filter collects the majority of the semivolatile organics as particles while at higher dilution ratios it collects them as gases. In contrast, the QBT OC emissions increase with dilution because these filters are only exposed to the gas-phase compounds whose relative concentration increases at higher dilution ratios due to changes in partitioning.

This interpretation of the quartz-filter data depends on the filters not being in equilibrium (or saturated) with the gas-phase organics (19). Figure 6 plots the mass of OC collected on the QBT filter as a function of the volume of exhaust passed through the filter during low-load diesel 


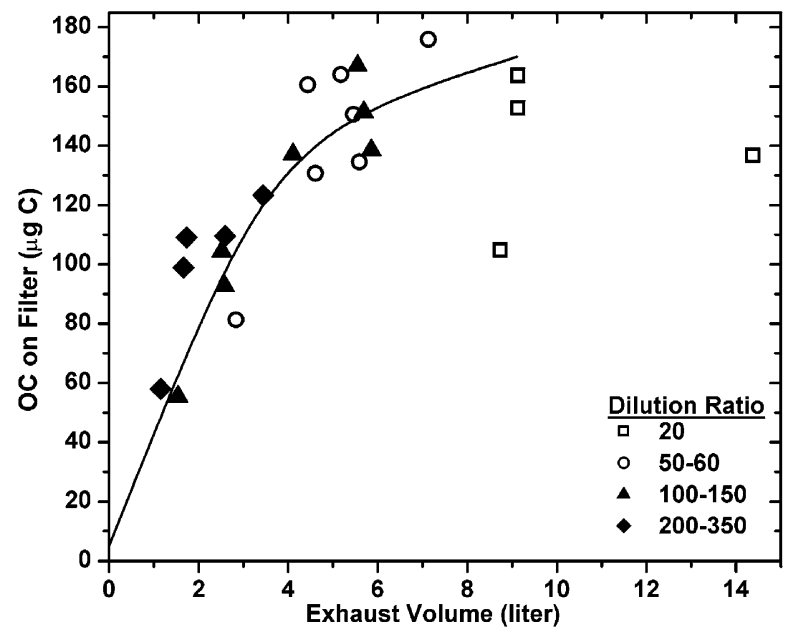

FIGURE 6. Organic carbon collected on a backup quartz filter behind a Teflon filter (OBT) as a function of volume of sampled exhaust. Data are from the low-load diesel experiments. Volume of sampled exhaust is used as a proxy for the total mass of organics to which the filter is exposed. The data show that quartz filters do not reach equilibrium with the gas-phase organics under the conditions of the experiments. The roll over at high volumes of sample exhaust may indicate the filters are approaching equilibrium but also may be caused by changes in gas-to-particle partitioning of semivolatile organics. Higher exhaust volumes correspond to lower dilution ratio experiments.

experiments. The volume of exhaust is a proxy for the mass of gas-phase organics to which the filter is exposed. Except for a couple of low dilution ratio points, the amount of carbon collected by the backup quartz filter increases with the volume of sampled exhaust indicating the quartz filters are not in equilibrium with the gas phase. At the highest volumes of sampled exhaust, there is evidence of the mass of carbon collected starting to roll over consistent with the quartz filters approaching equilibrium. However, Figure 6 is based on measurements made at different dilution ratios. At low dilution ratios a larger fraction of the semivolatile OC exists in the particle phase; therefore some of the roll over shown in Figure 6 is likely due to changes in partitioning and not equilibrium effects.

Although Figure 6 indicates that the quartz filters are clearly not in equilibrium with the exhaust, we attribute the increases in dilution-corrected OC measured by the Bare-Q filters in the diesel experiments to the effects of equilibrium. As a filter approaches equilibrium the collection efficiency of gas-phase OC decreases, reducing the amount of gasphase OC collected per unit volume of exhaust. Under the conditions of these experiments (variable dilution ratio coupled with fixed filter sampling times), this change in collection efficiency will cause an apparent increase in the fuel-based OC emissions as a function of dilution ratio consistent with the diesel Bare-Q OC data shown in Figure 2. The fact that the ratio of $\mathrm{PM}_{2.5}$ mass to the backup-corrected OC (Q - QBT) remains constant across an experiment indicates that the Bare-Q and QBT filters are approaching equilibrium at the same rate.

An interesting consequence of the changes in phase partitioning with dilution ratio coupled with the efficient collection of gas-phase semivolatile organics by quartz filters is that the positive artifact on a Bare-Q filter increases with dilution ratio. This effect is illustrated in Figure 7 which plots the ratio of TC to $\mathrm{PM}_{2.5}$ mass as a function of dilution ratio for the two diesel load conditions. Across the entire range of dilution ratios, the ratios of Q - QBT and the DenQ TC to $\mathrm{PM}_{2.5}$ mass are essentially constant indicating that both approaches provide a consistent correction of the positive
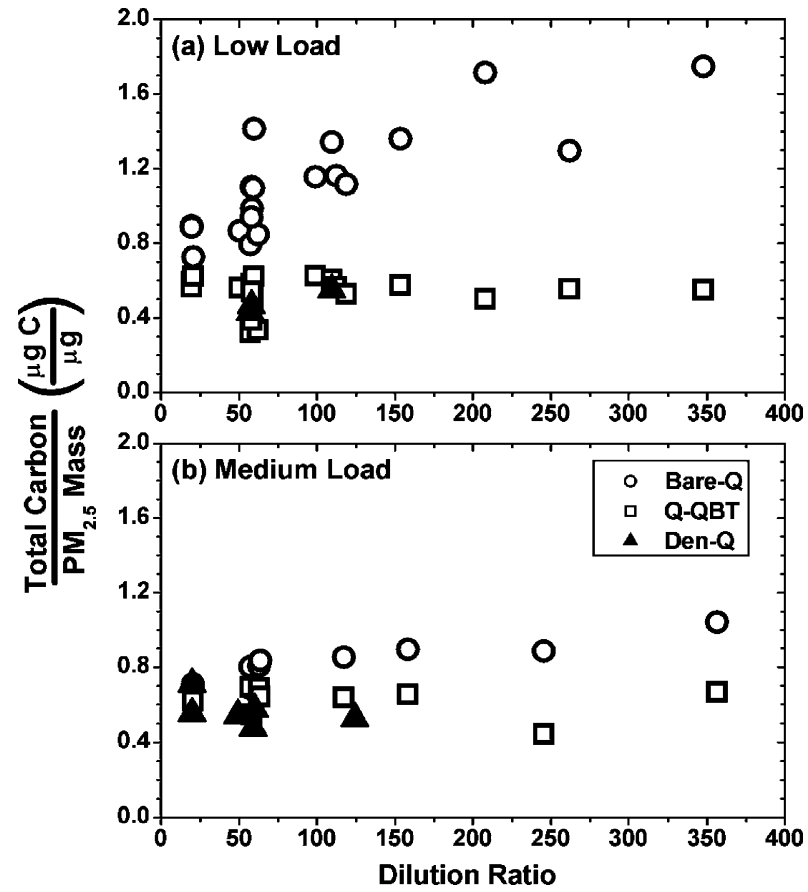

FIGURE 7. Ratio of total carbon (OC + EC) to $\mathrm{PM}_{25}$ mass measured using three different filter configurations while sampling from the diesel engine operating at (a) low and (b) medium load. The results show a substantial increase in the positive artifact on a Bare- 0 filter with dilution ratio. Both the denuded quartz and backupcorrected quartz approaches provide a consistent correction for this positive artifact.

artifact. In contrast, the ratio of Bare-Q TC to $\mathrm{PM}_{2.5}$ mass increases with dilution ratio indicating increasing positive artifact with dilution ratio. This increase is particularly dramatic for low-load operations where the Bare-Q TC to $\mathrm{PM}_{2.5}$ mass ratio increases from 0.8 at a dilution ratio of 20:1 to a value of 1.7 at a dilution ratio of 350:1.

The trends in Figure 7 can be explained by a combination of changes in partitioning and effects of the quartz filter approaching equilibrium. Under low-load conditions, the relative amount of gas-phase semivolatile organics increases with dilution due to changes in phase partitioning, which, in turn, increases the positive artifact. Under medium-load conditions, changes in partitioning were not observed and the increase in the positive artifact is likely associated with the previously discussed equilibrium effects. At high dilution ratios, the positive artifact dominates the OC measured with the Bare-Q filter. At lower dilution ratios, the relative amount of positive artifact reported here is consistent with results of previous studies of diesel exhaust (13-15)

\section{Discussion}

The major finding of this paper is that increasing dilution after the temperature of the exhaust has reached ambient levels can dramatically reduce the $\mathrm{PM}_{2.5}$ mass emission rate from a diesel engine and wood stove. For example, the measured $\mathrm{PM}_{2.5}$ mass emission factor for the diesel engine operating under low load is $2.9 \mathrm{~g} / \mathrm{kg}$ fuel at a dilution ratio of $20: 1$ versus $1.4 \mathrm{~g} / \mathrm{kg}$ fuel at a dilution ratio of 350:1. This decrease is caused by changes in partitioning of semivolatile organic compounds in the emissions. From a scientific perspective, there is not a unique value for the fine particle mass emission rate for sources that emit semivolatile species because the partitioning of these species varies continuously with dilution and temperature. One needs to understand these effects in order to correctly interpret measurements made with dilution samplers. For the two sources considered 
here, measurements at dilution ratios less than 100:1 likely substantially overestimate the emitted fine particle mass relative to the more dilute conditions encountered in the real world. This has obvious implications for using emission measurements in air quality models that mix emissions directly into large grid cells or use source profiles with species concentrations that are normalized by $\mathrm{OC}$ or $\mathrm{PM}_{2.5}$ mass.

Partitioning theory provides a context for understanding the results of these experiments. Following Pankow $(5,6)$, the concentration of a semivolatile compound in the aerosol phase is as follows:

$$
C_{\mathrm{a}, i}=\frac{1}{1+\frac{1}{K_{\mathrm{p}, i} C_{\mathrm{PM}}}} C_{\mathrm{tot}, i}
$$

where $K_{\mathrm{p}, i}$ is the partitioning coefficient for compound $i, C_{\mathrm{PM}}$ is the concentration of particulate matter (a proxy for the amount of sorptive material), and $C_{\text {tot }, i}$ is the total (gas + particle) concentration of species $i$ in the system. $K_{\mathrm{p}, i}$ depends on the saturation pressure of the species $i$, the type of sorptive process (adsorptive vs absorptive), and the properties of sorptive material $(5,6)$. Under the conditions of these experiments (isothermal dilution), $K_{\mathrm{p}, i}$ will be independent of dilution ratio (if one assumes it does not change as the composition of the particle phase changes with dilution). Therefore, $C_{\mathrm{PM}}$ and $C_{\mathrm{tot}, i}$ determine the partitioning of the emissions; these concentrations are determined by the overall emission rate, the composition of emissions, and the amount of dilution. To predict the overall change in semivolatile organic mass one needs to apply eq 3 to each semivolatile compound in the system. This is a challenging task given the complexity of the emissions.

Dilution samplers are typically operated with particleand organics-free air. This means that, inside the dilution sampler, $C_{\mathrm{PM}}$ and $C_{\mathrm{tot}, i}$ will both scale as $1 / \mathrm{DR}\left(C_{\mathrm{PM}}\right.$ may actually decrease faster than $1 / D R$ due to changes in partitioning). Increasing the dilution ratio will reduce the semivolatile mass in the particle phase. However, in the real world, emissions are mixed with polluted background air, not particle- and organic-free air. This background pollution contributes to $C_{\mathrm{PM}}$ and $C_{\mathrm{tot}, i}$. As the exhaust becomes very dilute, the background concentrations will strongly influence the partitioning of the semivolatile material.

To measure the partitioning that occurs under atmospheric conditions, eq 3 indicates that one needs to operate a dilution sampler such that $C_{\mathrm{PM}}$ and $C_{\mathrm{tot}, i}$ of the diluted exhaust are at typical atmospheric levels. If the concentrations inside the dilution sampler are significantly greater than atmospheric levels, then too much of the semivolatile material will partition into the particle phase and the measurements will overestimate fine particle emissions relative to atmospheric levels of dilution. Conversely, if the diluted concentrations are significantly smaller than atmospheric levels, then one can underestimate fine particle emissions. These ideas are quantitatively explored in a companion paper that applies absorptive partitioning theory to the low-load diesel and wood smoke data (4). To assist in interpretation of measurements made with dilution samplers, researchers should report the PM or OC concentration (and temperature) of the diluted exhaust.

The amount of dilution required to reach atmospherically relevant concentrations depends on the emission rate of semivolatile and sorptive material. For the sources considered here, the minimum OC concentrations of the diluted exhaust were between 100 and $200 \mu \mathrm{g}-\mathrm{C} / \mathrm{m}^{3}$ at a dilution ratio of 350:1 for the low-load diesel and 510:1 for the wood smoke experiments. These concentrations are an order of magnitude higher than typical ambient OC levels, indicating that a dilution ratio of $\sim 5000: 1$ is needed to approach typical atmospheric levels for these sources. The amount of fine particle mass at higher levels of dilution depends on the composition of the emissions. Figure 2 indicates that the changes in fine particle mass are most pronounced at low dilution ratios but that the $\mathrm{PM}_{2.5}$ mass emission rate may still be decreasing at a dilution ratio of 350:1.

The fine particle emission rate of our diesel engine emissions is somewhat higher than other engines $(13,15)$, while our wood combustion emissions were somewhat lower (15-17). Dilution ratios less than 100:1 may be adequate to reach atmospheric levels for low-emitting sources such as natural gas combustion; conversely dilution ratios significantly larger than 10,000:1 may be needed for smoking cars (4). Although not considered in these experiments, the diluted exhaust also needs to be at ambient temperatures. Emission measurements at typical atmospheric concentrations are experimentally challenging; a more attractive approach may be to characterize the composition of the semivolatile species and then use a partitioning model to predict emissions at atmospheric conditions (4).

The effects of dilution on partitioning also depend on the composition of the emissions. The exhaust must have significant levels of semivolatile material in order for partitioning to be important. For the sources considered here, the highest fine particle concentrations were several thousand micrograms per cubic meter at a dilution ratio of 20. Given these levels of emissions, eq 3 indicates that compounds with partitioning coefficients such that $1 / K_{\mathrm{p}, i}$ is in range of 1 and $5000 \mu \mathrm{g} / \mathrm{m}^{3}$ at ambient temperature will undergo phase transition as the exhaust is diluted to typical atmospheric concentrations. C-17 through C-29 $n$-alkanes are compounds commonly found in combustion exhaust with partitioning coefficients in this range; however, the vast majority of the semivolatile material has never been identified on a compound-by-compound basis (13). In order for the changes in partitioning to measurably alter the overall fine particle mass emissions, the total concentration of these semivolatile species $\left(\Sigma C_{\text {tot }, i}\right)$ must be comparable to the fine particle mass. Composition effects likely explain why dilution did not effect the fine particle mass emissions of the diesel engine operating under medium load even though the concentrations of PM in the diluted exhaust were a factor of 10 or more greater than typical ambient levels.

\section{Acknowledgments}

The authors acknowledge Emily Weitkamp, Andy Grieshop, Mark Prack, Jessica Chiu, Neal Shyam, and R. Subramanian at Carnegie Mellon University for their assistance in conducting this research and an anonymous reviewer whose comments greatly improved the manuscript. This research was supported by the U.S. Department of Energy National Energy Technology Laboratory under Contract DE-FC26$01 N T 41017$.

\section{Literature Cited}

(1) Hildemann, L. M.; Cass, G. R.; Markowski, G. R. A dilution stack sampler for collection of organic aerosol emissions-Design, characterization and field-tests. Aerosol Sci. Technol. 1989, 10, 193-204.

(2) Kittelson, D. B. Engines and nanoparticles: A review. J. Aerosol Sci. 1998, 29, 575-588.

(3) Frisch, L. E.; Johnson, J. H.; Leddy, D. G. Effect of Fuels and Dilution Ratio on Diesel Particulate Emissions; SAE Paper 790417; Society of Automotive Engineers: Warrendale, PA, 1980.

(4) Shrivastava, M. K.; Stanier, C. O.; Lipsky, E. M.; Robinson, A. L. Modeling semi-volatile organic aerosol mass emissions from combustion systems. Environ. Sci. Technol., submitted.

(5) Pankow, J. F. An absorption-model of gas-particle partitioning of organic-compounds in the atmosphere. Atmos. Environ. 1994, $28,185-188$.

VOL. 40, NO. 1, 2006 / ENVIRONMENTAL SCIENCE \& TECHNOLOGY • 161 
(6) Pankow, J. F. Review and comparative-analysis of the theories on partitioning between the gas and aerosol particulate phases in the atmosphere. Atmos. Environ. 1987, 21, 2275-2283.

(7) MacDonald, J. S.; Plee, S. L.; D'Arcy, J. B.; Schreck, R. M. Experimental Measurements of the Independent Effects of Dilution Ratio and Filter Temperature on Diesel Exhaust Particulate Samples; SAE Paper 800185; Society of Automotive Engineers: Warrendale, PA, 1981.

(8) Turpin, B. J.; Saxena, P.; Andrews, E. Measuring and simulating particulate organics in the atmosphere: Problems and prospects. Atmos. Environ. 2000, 34, 2983-3013.

(9) Lipsky, E.; Stanier, C. O.; Pandis, S. N.; Robinson, A. L. Effects of sampling conditions on the size distribution of fine particulate matter emitted from a pilot-scale pulverized-coal combustor. Energy Fuels 2002, 16, 302-310.

(10) Lipsky, E. M.; Robinson, A. L. Design and evaluation of a portable dilution sampling system and the effects of residence time on mass emission rates. Aerosol Sci. Technol. 2005, 39, 542-553.

(11) Subramanian, R.; Khlystov, A. Y.; Cabada, J. C.; Robinson, A. L. Positive and negative artifacts in particulate organic carbon measurements with denuded and undenuded sampler configurations. Aerosol Sci. Technol. 2004, 38, 27-48.

(12) Turpin, B. J.; Lim, H. J. Species contributions to PM2.5 mass concentrations: Revisiting common assumptions for estimating organic mass. Aerosol Sci. Technol. 2001, 35, 602-610.

(13) Schauer, J. J.; Kleeman, M. J.; Cass, G. R.; Simoneit, B. R. T. Measurement of emissions from air pollution sources. 2. C-1 through C-30 organic compounds from medium duty diesel trucks. Environ. Sci. Technol. 1999, 33, 1578-1587.

(14) Shah, S. D.; Cocker, D. R.; Miller, J. W.; Norbeck, J. M. Emission rates of particulate matter and elemental and organic carbon from in-use diesel engines. Environ. Sci. Technol. 2004, 38, 25442550 .
(15) Hildemann, L. M. Markowski, G. R · Jones, M. C. Cass, G. R Submicrometer aerosol mass distributions of emissions from boilers, fireplaces, automobiles, diesel trucks, and meat-cooking operations. Aerosol Sci. Technol. 1991, 14, 138-152.

(16) Fine, P. M. Cass, G. R. Simoneit, B. R. T. Chemical characterization of fine particle emissions from fireplace combustion of woods grown in the northeastern United States. Environ. Sci. Technol. 2001, 35, 2665-2675.

(17) Fine, P. M.; Cass, G. R.; Simoneit, B. R. T. Chemical characterization of fine particle emissions from the fireplace combustion of woods grown in the southern United States. Environ. Sci. Technol. 2002, 36, 1442-1451.

(18) Sakurai, H.; Tobias, H. J.; Park, K.; Zarling, D.; Docherty, S.; Kittelson, D. B.; McMurry, P. H.; Ziemann, P. J. On-line measurements of diesel nanoparticle composition and volatility. Atmos. Environ. 2003, 37, 1199-1210.

(19) Mader, B. T.; Pankow, J. F. Gas/solid partitioning of semivolatile organic compounds (SOCs) to air filters. 3. An analysis of gas adsorption artifacts in measurements of atmospheric SOCs and organic carbon (OC) when using Teflon membrane filters and quartz fiber filters. Environ. Sci. Technol. 2001, 35, 34223432 .

(20) Zhang, K. M.; Wexler, A. S. Evolution of particle number distribution near roadways. Part I: Analysis of aerosol dynamics and its implications for engine emission measurement. Atmos. Environ. 2004, 38, 6643-6653.

Received for review February 16, 2005. Revised manuscript received August 5, 2005. Accepted October 14, 2005.

ES050319P 


\title{
Design and Evaluation of a Portable Dilution Sampling System for Measuring Fine Particle Emissions from Combustion Systems
}

\author{
Eric M. Lipsky and Allen L. Robinson \\ Department of Mechanical Engineering, Carnegie Mellon University, Pittsburgh, PA, USA
}

\begin{abstract}
The size and complexity of current dilution samplers is a major barrier to more wide-spread application of these systems for source characterization. A new, more portable dilution sampler has been designed to provide measurements consistent with the widely cited Caltech dilution sampler. Intercomparison experiments were performed using a diesel engine and wood stove to evaluate the comparability of the new design with a sampler based on the Caltech design. These experiments involved simultaneous operation of multiple dilution samplers from the same source. Filter based measurements included $\mathbf{P M}_{2.5}$ mass, organic carbon, and elemental carbon emissions. Particle size distributions in the range from $10-480 \mathrm{~nm}$ were measured using a scanning mobility particle sizer. The filterbased and integrated-total volume measurements made with the two designs are in good agreement. For example, the average relative bias between the two samplers of $\mathbf{P M}_{2.5}$ mass emission rate measured with Teflon filters is $1 \%$. Nucleation was intermittently observed in the sampler based on the Caltech design, but rarely observed in the new design. Significant discrepancies in total number emissions between the two samplers occurred during periods of nucleation. Experiments were also conducted to examine the effects of residence time on the diluted emissions. No changes in the filterbased or integrated volume measurements were observed with an additional 40-s residence time, indicating that phase equilibrium is established in the $2.5 \mathrm{~s}$ of residence time provided by the dilution tunnel. This conclusion is consistent with theoretical analysis. These results provide new insight into the effects of dilution sampling on measurements of fine particle emissions, providing important data for the ongoing effort of the EPA and ASTM to define a standardized dilution sampling methodology for characterizing emissions from stationary combustion sources.
\end{abstract}

\section{INTRODUCTION}

Dilution sampling is used to characterize fine particle emissions from combustion and other high-temperature sources because it simulates the rapid cooling and dilution that occurs as

Received 10 December 2004; accepted 13 April 2005.

Address correspondence to Allen L. Robinson, Department of Mechanical Engineering, Carnegie Mellon University, Pittsburgh, PA 15213, USA. E-mail: alr@andrew.cmu.edu exhaust mixes with the atmosphere. Cooling and dilution influence the gas-particle partitioning of semivolatile material in the exhaust, which in turn impacts the overall fine particle emissions. Dilution techniques can also be used to "freeze" the size distribution, when properly applied, to avoid or at least reduce unwanted nucleation, coagulation, and condensation. EPA requires dilution sampling for testing of diesel engines (EPA 1999). Efforts are underway by EPA and ASTM to develop a formal method for dilution sampling for stationary sources (ASTM 2003; EPA 2004). England et al. (1998) have reviewed the design criteria and the designs of different samplers that have been developed for stack testing of stationary sources.

Hildemann et al. (1989) outline a set of design requirements for dilution sampling systems. Briefly, a dilution sampler should simulate atmospheric dilution, provide adequate residence time for aerosol processes to occur, and minimize contamination and sampling losses. Using these criteria, Hildemann et al. (1989) developed the Caltech dilution sampler. This sampler has been widely used in source characterization studies and refined versions of the design have been used to construct other dilution sampling systems (England et al. 1998; Lipsky et al. 2002).

Portability is a major barrier to more widespread application of dilution samplers for source testing. Current dilution samplers are large, complex machines compared to traditional stack sampling techniques. For example, the widely cited Caltech design consists of a 1.5-m-long mixing tunnel, a large residence time tank, and collection of pumps and accessories (Hildemann et al. 1989). More recent research has examined the development of smaller dilution systems (England 2004; Lyyranen et al. 2004). Given the complexity of the dilution process, an important need is the intercomparison of different sampler designs. The few published intercomparisons indicate that sampler design can significantly effect measurements (Maricq et al. 1999; Lyyranen et al. 2004).

This paper discusses the design and performance of a new, smaller dilution sampler that is designed to simulate atmospheric dilution. The performance of the new sampler is evaluated through comparisons with a dilution sampler based on the Caltech design (Hildemann et al. 1989). Simultaneous testing 
with both designs is performed on emissions from a wood stove and a small diesel engine. Residence time tests were performed to assess the effects of additional residence time on fine particle mass emission rates. The elimination of a residence time tank greatly reduces the size and complexity of a dilution system.

\section{METHODS}

\section{Dilution Sampler Design}

The primary design objective for the new dilution sampler was to create a system that reproduces the results of the widely cited Caltech design but that is simpler and more portable. Before describing the new design, it is useful to outline the two major design changes. First, the Caltech sampler operates at a high total flow rate $(\sim 1000 \mathrm{lpm})$, but only a relatively small fraction of this flow is used for source characterization with the balance of the diluted exhaust vented to the atmosphere. In developing the new design we seek to optimize the total flow rate of diluted exhaust based on the required combination of filter packs and other instrumentation to be used during source characterization in order to minimize the amount of diluted exhaust vented to the atmosphere. This reduces the size of the dilution tunnel and associated flow control system. Although the new dilution tunnel operates at a much lower flow rate, the total residence time within the new tunnel is similar to the Caltech tunnel, both around $2.5 \mathrm{~s}$.
The second major design change was to eliminate the residence time tank. In the Caltech design and its variants, diluted exhaust passes flows through a large residence time tank before filter sampling or aerosol characterization. Eliminating the residence time tank significantly reduces the size and complexity of the new dilution sampler. The purpose of the residence time tank is to provide adequate time after dilution for microphysical processes such as condensation. As part of the evaluation of the new design, experiments were performed to assess the effects of additional residence time on the measured fine particle concentrations.

Figure 1 shows a schematic of the new dilution sampling system, which we refer to as the field sampler. Similar to other dilution samplers, exhaust is sampled isokinetically into a heatedinlet line that is maintained at a temperature slightly $\left(\sim 10^{\circ} \mathrm{C}\right)$ above the exhaust temperature to minimize thermophoretic losses. A stack-sampling cyclone can be installed on the inlet line to prevent large particles from entering the system. The sampled exhaust is then rapidly mixed by turbulence with filtered (HEPA and activated carbon) dilution air inside of a 0.9-m-long, 0.15$\mathrm{m}$-diameter stainless dilution tunnel. The total flow rate through the dilution sampler is $174 \mathrm{lpm}$, and the dilution ratio is varied by changing the relative amount of exhaust and dilution air flow. Filter trains and other aerosol characterization instrumentation are connected to ports at the end of the dilution tunnel. The sampler is constructed out of stainless steel to minimize contamination.

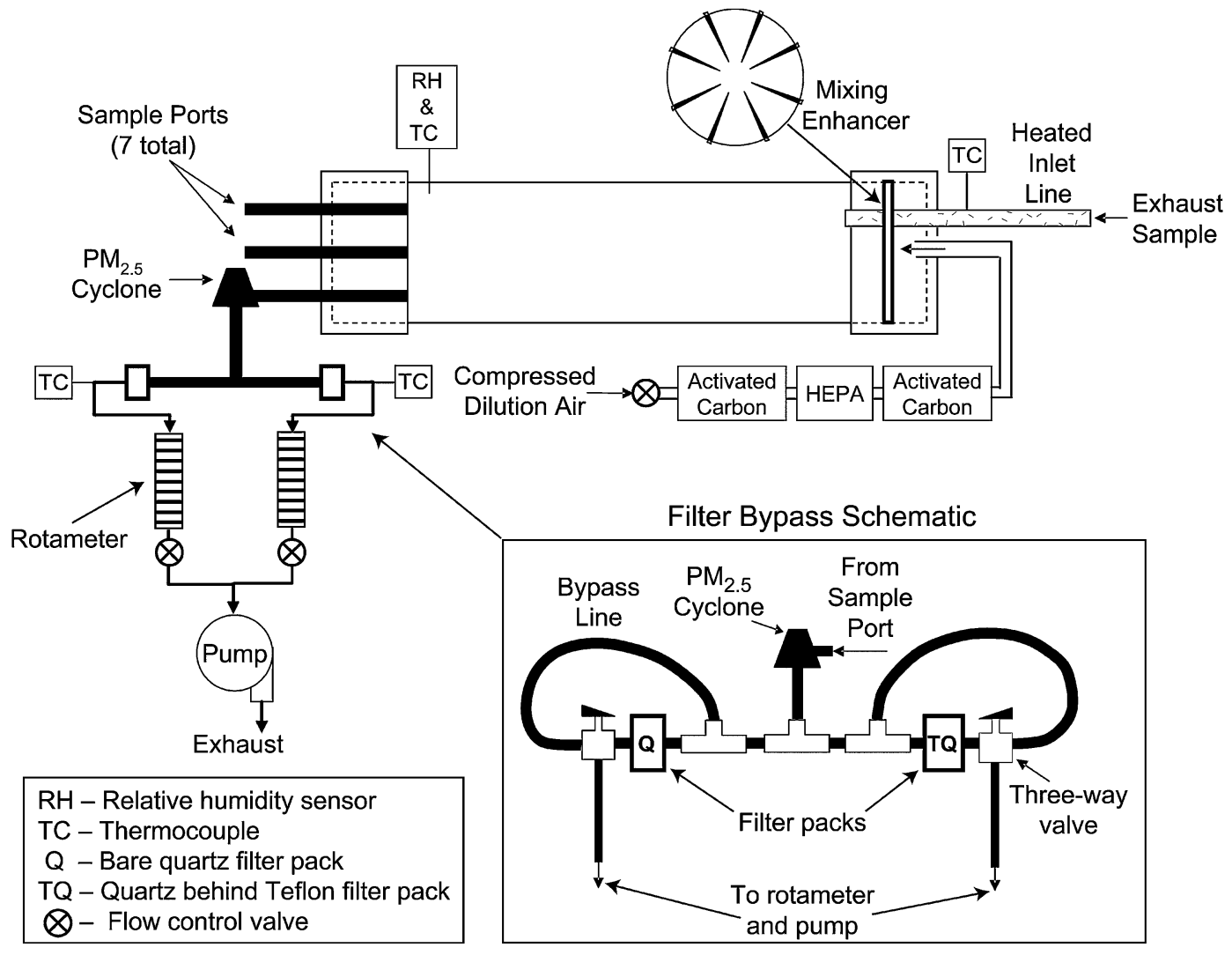

FIG. 1. Schematic of field tunnel sampler. Not drawn to scale. 
To promote mixing, the dilution air is passed through a fan shaped mixing enhancer immediately upstream of where the dilution air and exhaust sample gas streams meet. The mixing enhancer breaks up the dilution air flow causing rapid and thorough mixing within the dilution tunnel. Mixing experiments were conducted using $\mathrm{CO}_{2}$ as a tracer to verify that the exhaust and dilution air are completely mixed at the end of the smaller tunnel across the entire operational range. The mixing rate in the smaller dilution tunnel was not characterized, only verified that the conditions were well mixed at the sampling ports. With the addition of a mixing enhancer, we expect more rapid mixing in the new dilution tunnel compared to the Caltech design.

Since the new dilution sampler has been designed to maximize the use of diluted exhaust for sampling and analysis, a challenge in operating the field-tunnel is stabilizing the flows before starting to collect filter samples. Flow bypasses are installed around each filter-pack in order to stabilize flow through the tunnel. A schematic of the bypass arrangement is shown in Figure 1, which consists of a tee upstream of the filter pack and a three-way valve after the filter-pack. To help minimize the effects of varying stack pressure on exhaust sample flow rate, a tank can be used in-line between the incoming control valve and the tunnel. This volume acts as a buffer for small changes in pressure.

Two changes have been made in the new design compared to the original Caltech design to reduce particle losses. First we measure the $\mathrm{CO}_{2}$ mixing ratios of the undiluted and diluted exhaust to determine dilution ratio. This eliminates the need for a venturi flow meter on the inlet line, which has been shown to be a significant cause of particle losses (Hildemann et al. 1989). Second, we changed the geometry at which the exhaust sample and dilution air are introduced inside the dilution tunnel. As shown in Figure 1, the exhaust sample passes through the end of the dilution tunnel flowing in parallel with the dilution air. In the Caltech design, the exhaust sample is introduced through the side of the tunnel, perpendicular to the dilution air flow. Visual inspection of the inside of the large tunnel indicates that losses occur on the opposite side of the dilution tunnel from the exhaust port and just downstream of the initial mixing point. The new flow orientation appears to reduce these losses.

\section{Experimental Procedure}

Experiments were performed to compare the performance of the new sampler design with the Caltech design using the experimental set up shown in Figure 2. Experiments were conducted using two different sources: a small diesel engine and a wood stove. An enclosed exhaust duct system was installed on each source so that all of the inlets were at the same location.

The diesel generator is a single-cylinder Yanmar L70AE aircooled diesel engine connected to a $4.5 \mathrm{~kW}$ generator. The diesel engine was operated at constant load during a test on fuel purchased at a local filling station. Separate tests were conducted at a low load (25\% of full capacity) and a medium load (55\% of full capacity). The engine was operated for at least an hour before beginning filter sampling to ensure steady state conditions.

The wood stove is an EPA approved Jøtul "602 CB Classic" wood stove and was operated on a blend of hardwoods including oak, cherry, and some ash. Wood smoke sampling was conducted after lighting the wood and allowing for a period of time for the combustion to reach steady state as indicated by relatively constant exhaust temperature and $\mathrm{CO}_{2}$ levels. During the wood smoke experiments, the wood combustion was controlled by adjusting a vent in the door of the stove, maintaining an exhaust duct temperature of around $150^{\circ} \mathrm{C}$ at the sampling location. More details of the operation of the source are provided by Lipsky et al. (2005).

The intercomparison experiments involved simultaneous sampling with the different dilution samplers from the same

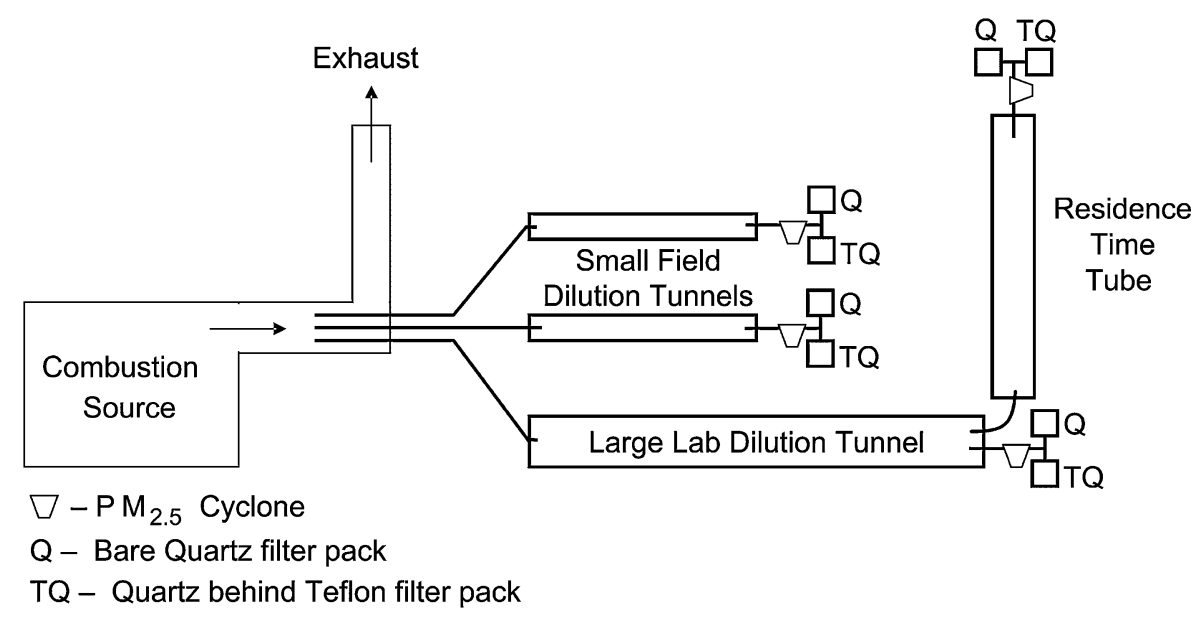

FIG. 2. Setup for intercomparison and residence time experiments. Intercomparison experiments involved simultaneous sampling with two or three dilution tunnels operating at the same dilution ratio. The two small field systems are of the same design. Each sampler has a separate heated inlet line. Experiments to examine effects of residence time used a residence time tube installed on the larger lab tunnel, which provided an additional $40 \mathrm{~s}$ of aging time for the diluted exhaust. 
location of an enclosed exhaust duct. Each sampler has separate heated inlet lines; cyclones were not installed on any of the inlet lines. Two or three samplers were operated simultaneously during a given experiment: one large sampler based on the Caltech design, and one or two of the new, more compact design. In this paper we refer to the dilution sampler based on the Caltech design as the laboratory sampler, and the new, more portable sampler as the field sampler. Although relatively few intercomparison experiments were conducted using the two field samplers, the data provide additional statistical confidence and insight into experimental variability. For the intercomparison experiments, all of the samplers were operated at the same dilution ratio, and separate tests were performed at different dilution ratios ranging from 20-350. The dilution ratio was determined using $\mathrm{CO}_{2}$ as a tracer; venturi flow meters were not installed on any of the inlet lines.

Thermocouples are used to measure gas temperatures at the different locations shown in Figure 1. For a given experiment, the measured temperature profile within each sampler was essentially the same. For example, the temperature of the exhaust in the sampler inlet lines varied by less than $10^{\circ} \mathrm{C}$ and the temperature of the diluted exhaust varied by less than $3^{\circ} \mathrm{C}$. The temperature of the exhaust at the sampling location was $\sim 250^{\circ} \mathrm{C}$ for low load diesel experiments, $\sim 300^{\circ} \mathrm{C}$ for medium load diesel experiments, and $\sim 150^{\circ} \mathrm{C}$ for wood smoke experiments. The diluted exhaust was typically at room temperature, $\sim 27^{\circ} \mathrm{C}$.

Experiments to investigate the effects of additional residence time beyond that provided by the tunnel were conducted using the large dilution tunnel as shown in Figure 2. For these experiments, a slipstream of diluted exhaust was drawn from the end of the dilution tunnel and continuously passed through a 0.9$\mathrm{m}$-long, 10-cm-diameter stainless tube called the residence time tube. Filter samples were collected simultaneously from the end of the dilution tunnel and the end of the residence time tube; simultaneous sampling at both locations minimizes the effects of variations in source operations on the results. All residence time experiments were performed with a slipstream flow rate of $10 \mathrm{lpm}$, which, under plug flow conditions, allows for 40 seconds of residence time in the residence time tube.

Our implementation of the Caltech design is described in detail by Lipsky et al. (2002). For the intercomparison experiments this sampler was operated without the residence time tank-all measurements are made at the end of the dilution tunnel. The laboratory sampler was operated at a relatively constant dilution air flow rate of $1000 \mathrm{lpm}$ to maintain a residence time of $\sim 2.5 \mathrm{~s}$ inside the tunnel and standardize mixing conditions across all experiments. The dilution air and exhaust mix by turbulence inside the dilution tunnel; the Reynolds number of the flow inside the dilution tunnel of the laboratory sampler was 10,000 . The dilution ratio is changed by varying the exhaust flow rate into the sampler.

The dilution ratio of each sampler was determined by simultaneously measuring exhaust gas and dilution tunnel $\mathrm{CO}_{2}$ levels.
The dilution ratio is defined as:

$$
\mathrm{DR}=\frac{\left(\mathrm{CO}_{2}\right)_{\mathrm{ex}}-\left(\mathrm{CO}_{2}\right)_{\mathrm{amb}}}{\left(\mathrm{CO}_{2}\right)_{\mathrm{tun}}-\left(\mathrm{CO}_{2}\right)_{\mathrm{amb}}}
$$

where $\left(\mathrm{CO}_{2}\right)_{\mathrm{ex}}$ is the exhaust $\mathrm{CO}_{2}$ mole fraction, $\left(\mathrm{CO}_{2}\right)_{\mathrm{amb}}$ is the $\mathrm{CO}_{2}$ mole fraction of the dilution air, and $\left(\mathrm{CO}_{2}\right)_{\mathrm{tun}}$ is the mole fraction of $\mathrm{CO}_{2}$ of the diluted exhaust. The $\mathrm{CO}_{2}$ mixing ratio of the exhaust is measured at the sample inlet location. The diluted exhaust $\mathrm{CO}_{2}$ mixing ratio is monitored at the end of the tunnel after the sample is well mixed. Separate $\mathrm{CO}_{2}$ monitors were used on each tunnel to continuously monitor dilution ratio in each system. The $\mathrm{CO}_{2}$ mixing ratio of dilution air was measured both before and after each day of testing. Variations of $\mathrm{CO}_{2}$ levels in the dilution air were small and negligibly impacted dilution ratios calculated using Equation (1). Zero and span calibrations were performed on all gas analyzers before every day of testing. In order to account for small variations in dilution ratio between the systems, all measured emissions rates were multiplied by the dilution ratio to convert the results to equivalent exhaust concentrations.

The performance of the different samplers was quantitatively evaluated by calculating the relative bias errors between the two systems for a given experiment,

$$
\text { Rel Error }=\frac{\mathrm{P}_{\mathrm{FT}}-\mathrm{P}_{\mathrm{LT}}}{\mathrm{P}_{\mathrm{LT}}}
$$

where $\mathrm{P}_{\mathrm{FT}}$ is the measured quantity (e.g. $\mathrm{PM}_{2.5}$ mass) in the field tunnel and $\mathrm{P}_{\mathrm{LT}}$ is the measured quantity in the lab tunnel. A negative bias indicates that the field tunnel measures less than the lab tunnel. The average and standard deviation of the relative bias are then calculated across a specified set of experiments.

\section{Aerosol Characterization}

The sampling train used to collect $\mathbf{P M}_{2.5}$ filter samples of diluted exhaust is shown in Figure 2. This train consisted of a sharp cut $\mathrm{PM}_{2.5}$ cyclone operated at $24 \mathrm{lpm}$ followed by a flow splitter and two filter packs operated in parallel at $12 \mathrm{lpm}$. One filter pack contained a quartz filter (Gelman $47 \mathrm{~mm}$, Tissuquartz 2500 QAO-UP). This filter is referred to as the bare quartz filter or Bare-Q. The second filter pack contained a Teflon membrane filter (47-mm, 2- $\mu \mathrm{m}$ Teflo filters, Gelman Corp) followed by a quartz filter. The back up quartz filter provides an estimate of the positive sampling artifact (Turpin et al. 1994) and is referred to as the quartz behind Teflon or QBT. Separate, identical sampling trains were connected to the end of each dilution tunnel for the intercomparison experiments. The same train was used at the end of the residence time tube except with a cyclone that provides $\mathrm{PM}_{2.5}$ cut at $10 \mathrm{lpm}$.

The Teflon filters are used to quantify mass emission rates using gravimetric analysis. The filters are weighed pre- and post-sampling using a microbalance inside an environmentally 
controlled glove box at a standard temperature $\left(21-23^{\circ} \mathrm{C}\right)$ and relative humidity (30-40\%). Filters are equilibrated for $24 \mathrm{~h}$ before being weighed.

The quartz filters are analyzed to determine organic and elemental carbon (OC and EC) loadings using a Sunset Laboratories Laboratory Thermal-Optical Transmission OC/EC Analyzer using a modified version of the NIOSH 5040 protocol (NIOSH 1996 ) with a peak He-mode temperature of $870^{\circ} \mathrm{C}$. A complete listing of the times and temperature steps of the analysis protocol is given by Subramanian et al. (2004). EC emissions are determined from the analysis of the Bare-Q filter. OC emissions are determined from the Bare-Q filter, and the OC measured on the backup-Q filter (QBT) is used as an estimate of the positive artifact. The quartz filters are prepared before sampling by baking them at $500^{\circ} \mathrm{C}$ in air for $6 \mathrm{~h}$ to remove any organic material that may have been adsorbed onto the filter ensuring low blank levels. Both quartz and Teflon filters are stored in a freezer $\left(-18^{\circ} \mathrm{C}\right)$ between sample collection and analysis.

Size distributions are measured using a TSI Scanning Mobility Particle Sizer (SMPS), either a TSI 3081 DMA with the model 3010 CPC or a TSI 3070 DMA with the model 3025 CPC. Two neutralizers are used in series before the SMPS analyzers to ensure a known charge on the particles. Scans are made from $10 \mathrm{~nm}$ to $460 \mathrm{~nm}$ using a 120 -s up-scan and a 30-s down scan. Only one SMPS is used for the experiments; when sampling with multiple samplers or with the residence time tube, the inlet to the SMPS is rotated between samplers or between the end of the dilution tunnel and the end of the residence time tube to measure size distributions at each location. The SMPS is rotated between sampling locations every few minutes, and a complete set of size distribution measurements on all of the samplers required around $10 \mathrm{~min}$. Emissions from the diesel engine are stable enough to allow comparison of size distribution measurements made sequentially on different samplers, but the wood smoke emissions are too variable for comparison of sequential measurements.

\section{Blanks}

Both handling and dynamic system blanks were collected to quantify contamination associated with each dilution system. Handling blanks were low (for example, $<1 \mu \mathrm{g} \mathrm{C} / \mathrm{cm}^{2}$ on a quartz filter), and much less than $5 \%$ of sample collected during an actual experiment.

Dynamic system blanks were performed by operating the sampler only on filtered air (HEPA and activated carbon), and measuring contamination levels using the SMPS and the previously described filter train. Dynamic system blanks were typically run after the system had been cleaned, which involved wiping all surfaces with acetone, reassembly and leak testing, wrapping the dilution tunnel with heat tape, and operation of the system on heated filtered air for at least an hour to remove any residual semivolatile organics from the system. The system was cleaned after one to five days of experiments and before switching between the diesel and wood smoke sources.
Teflon mass and EC measured during dynamic system blanks were comparable to handling blanks indicating particle free dilution air. Similarly, SMPS measurements made during system blank test show total number and volume to be less than $0.01 \%$ of typical diluted exhaust levels. OC levels on dynamic system blanks were a factor of two higher than handling blanks, but still less than $10 \%$ of typical tunnel concentrations. During a dynamic system blank, equal amounts of OC were measured on both the Bare-Q and QBT filters indicating that the dynamic blank OC is organic vapor adsorbed onto the filters. In addition, all of the $\mathrm{OC}$ collected during a dynamic system blank evolves in the lowest He-mode temperature step $\left(340^{\circ} \mathrm{C}\right)$ of the carbon analysis consistent with relatively volatile species. Dynamic system blank levels measured using all three samplers were comparable.

A limited number of dynamic system blanks were collected after completing a series of experiments but before cleaning the system. These dynamic blanks indicated particle free air (Teflon mass and EC at handling blank levels), but modestly higher levels of OC compared to dynamic blanks collected immediately after cleaning $\left(2.7 \mathrm{ug} \mathrm{C} / \mathrm{cm}^{2}\right.$ before cleaning versus $0.65 \mathrm{ug} \mathrm{C} / \mathrm{cm}^{2}$ after cleaning). Again, the same amount of OC was measured on the Bare-Q and QBT filters indicating that the dynamic blank OC is organic gases adsorbing to the quartz filters. The higher OC dynamic system blanks measured after a series of experiments but before cleaning indicates some build up of semivolatile material on the walls of the sampler during the experiments that then desorbs and is collected on the quartz filters during the dynamic blank measurements.

All filter measurements are blank corrected using one set of blank values (e.g., OC, EC, Teflon mass) that is the average of all of the dynamic system blank data collected from all the samplers. Therefore, the same set of blank values is used for each sampler. Blank corrections for Teflon mass and EC were less than $1 \%$ of filter measurements, while blank corrections for OC were less than $10 \%$ of the OC collected on a given filter.

\section{RESULTS AND DISCUSSION}

\section{Sampler Intercomparison}

A series of intercomparison experiments were conducted by simultaneously sampling exhaust from a diesel engine and a wood stove with different dilution samplers operating at the same dilution ratio. Scatter plots of the filter measurements from the parallel intercomparison experiments are shown in Figure 3. Filter samples were collected simultaneously on each system and corrected for any slight variations in dilution ratio between the different systems. For tests performed using two field-tunnels, each is compared with the lab tunnel individually. Measurements on the 1:1 line indicate perfect agreement between the samplers; points within $\pm 30 \%$ are defined as acceptable by the EPA Validation Protocol in Method 301 (EPA 1992).

Good agreement is generally observed between the two systems for $\mathrm{PM}_{2.5}$ mass (Figure 3a), OC (Figures $3 \mathrm{~b}$ and $3 \mathrm{~d}$ ), and EC (Figure 3c), with dilution ratios ranging from 20 and 120. 

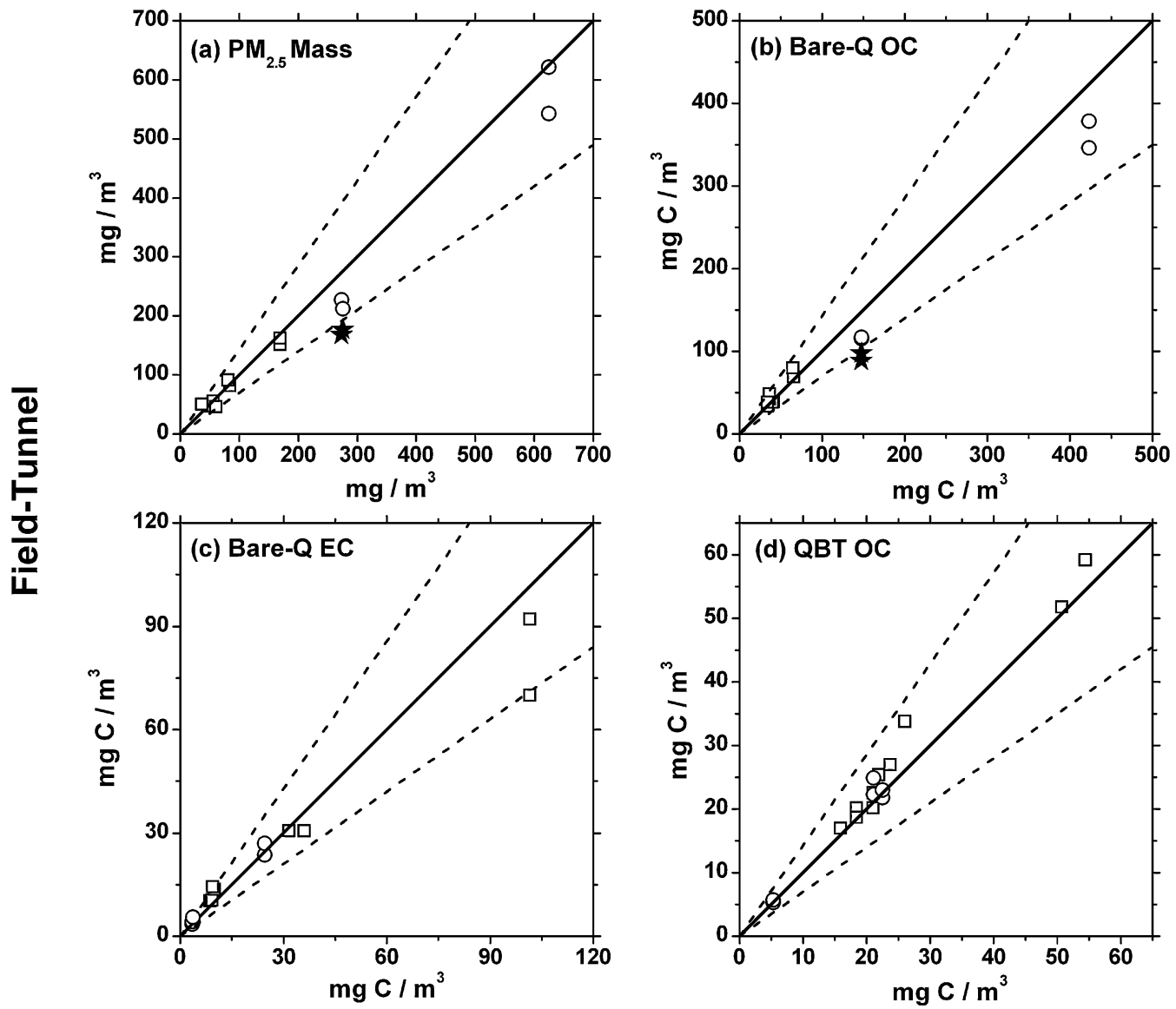

Lab-Tunnel

FIG. 3. Scatter plots of simultaneous filter measurements made using the field tunnel sampler versus the lab tunnel sampler. Data are dilution corrected exhaust concentrations at ambient temperature and pressure. Experiments considered dilution ratios from 20 and 120. Heavy line indicates one-to-one relationship with $\pm 30 \%$ indicated by dashed lines. Squares indicate exhaust concentrations measured from diesel exhaust experiments. Circles indicate exhaust concentrations measured from wood smoke experiments. Stars indicate data from final wood smoke intercomparison tests which are considered outliers, as discussed in text.

The intercomparison experiments considered a wide range of $\mathrm{PM}_{2.5}$ emissions rates from the diesel engine and wood stove. Emissions from the wood smoke are 2-6 times higher than the diesel, and there is roughly a factor a 4 difference in emission rates from the diesel engine for the two different load conditions considered here. Emissions from both systems are dominated by $\mathrm{OC}$ and EC.

Filter measurements made with the different sampler designs are generally within the $\pm 30 \%$. Larger discrepancies were observed during the last two of the wood smoke experiments with one of the field tunnel samplers collecting significantly less mass $(30-40 \%)$ than the lab tunnel sampler. It is suspected that these larger discrepancies were caused by build up of material on the exhaust inlet lines over the series of wood smoke experiments. This build up was discovered when the systems were disassembled for cleaning after the experiments.

Quantitative comparisons of the filter measurements made using the laboratory and field samplers are summarized in Table 1. For each experiment, we calculated the relative error between the measurements made with the different samplers using Equation (2). Table 1 lists the average and standard deviation of the relative error values separately for the diesel data, wood smoke data, and the entire data set. The average relative error is a measure of any systematic bias between the two designs with a negative value indicating that the field sampler measures less than the laboratory sampler. The standard deviation of the relative error values is a measure of the variability across a specified set of experiments. Table 1 compares sampler performance in terms of both absolute emissions rates and emission ratios (e.g., ratio of OC-to-PM $\mathrm{PM}_{2.5}$ mass). Emission ratios form the core of source profiles used in receptor modeling; comparing ratios measured by the different samplers provides insight into species dependent losses.

First we consider the performance of the samplers across the entire set of experiments - the columns labeled "All data" in Table 1. On average there is little systematic bias between the laboratory and field samplers, but some experiment-toexperiment variability. For example, the field sampler measures 
TABLE 1

Relative error between laboratory and field sampler

\begin{tabular}{|c|c|c|c|c|c|c|}
\hline & \multicolumn{2}{|c|}{$\begin{array}{l}\text { Diesel } \\
(\mathrm{n}=7)\end{array}$} & \multicolumn{2}{|c|}{$\begin{array}{l}\text { Wood }^{1} \\
(\mathrm{n}=4)\end{array}$} & \multicolumn{2}{|c|}{$\begin{array}{l}\text { All data } \\
(\mathrm{n}=11)\end{array}$} \\
\hline & Average & SD & Average & SD & Average & SD \\
\hline $\mathrm{PM}_{2.5}$ mass & 0.05 & 0.17 & -0.13 & 0.09 & -0.01 & 0.17 \\
\hline Bare $\mathrm{TC}^{2}$ & 0.03 & 0.15 & -0.17 & 0.05 & -0.04 & 0.16 \\
\hline Bare $\mathrm{OC}^{3}$ & 0.08 & 0.11 & -0.18 & 0.05 & -0.02 & 0.16 \\
\hline $\mathrm{EC}^{4}$ & 0.02 & 0.24 & 0.18 & 0.21 & 0.08 & 0.23 \\
\hline Backup OC & 0.09 & 0.05 & 0.06 & 0.08 & 0.08 & 0.06 \\
\hline $\mathrm{OC} / \mathrm{EC}^{5}$ & 0.10 & 0.26 & -0.29 & 0.14 & -0.04 & 0.29 \\
\hline $\mathrm{OC} / \mathrm{TC}^{5}$ & 0.05 & 0.12 & -0.01 & 0.00 & 0.03 & 0.10 \\
\hline $\mathrm{EC} / \mathrm{TC}^{5}$ & -0.02 & 0.14 & 0.42 & 0.29 & 0.14 & 0.30 \\
\hline $\mathrm{TC} / \mathrm{PM}_{2.5}$ mass $^{5}$ & -0.02 & 0.06 & -0.04 & 0.06 & -0.02 & 0.06 \\
\hline $\mathrm{OC} / \mathrm{PM}_{2.5}$ mass $^{5}$ & 0.03 & 0.12 & -0.05 & 0.05 & 0.00 & 0.10 \\
\hline $\mathrm{EC} / \mathrm{PM}_{2.5}$ mass $^{5}$ & -0.03 & 0.15 & 0.38 & 0.37 & 0.12 & 0.31 \\
\hline Total number ${ }^{6}$ & -0.28 & 0.17 & & & -0.28 & 0.17 \\
\hline Integrated volume ${ }^{6}$ & -0.06 & 0.09 & & & -0.06 & 0.09 \\
\hline
\end{tabular}

${ }^{1}$ Not including the two "outliers" indicated by the stars in Figure 3; ${ }^{2} \mathrm{TC}=$ total carbon; ${ }^{3} \mathrm{OC}=$ organic carbon; ${ }^{4} \mathrm{EC}=$ elemental carbon; ${ }^{5}$ Ratios using data from bare quartz filter; ${ }^{6}$ Measured with SMPS.

$1 \% \pm 17 \%$ (average \pm standard deviation) less mass than the laboratory sampler. Of the filter data, EC shows the largest bias and variability with the field sampler measuring $8 \% \pm 23 \%$ more EC than the laboratory sampler. For most measured parameters, the average relative error between the two designs is less than $\pm 5 \%$ with standard deviations between $10 \%$ and $20 \%$. All of the filter-based measurements of absolute emissions rates have average bias errors of $10 \%$ or less as required by EPA to establish method comparability (EPA 1992). Ratios of EC to total carbon (TC) and $\mathrm{EC}$ to $\mathrm{PM}_{2.5}$ mass have the largest average biases; problems with EC are primarily associated with the wood smoke experiments.

Examining the diesel and wood smoke data separately provides more insight into the performance of the two systems and the challenges associated with dilution sampling. For the diesel experiments, Table 1 indicates excellent agreement between the two designs across the entire set of measured parameters except for particle number. The filter data from the wood smoke experiments show significantly larger systematic errors than the diesel data. In addition, the average relative error is larger than the experiment to experiment variation in the errors; for example, the average $\mathrm{PM}_{2.5}$ mass measured by the field sampler was $13 \% \pm$ $9 \%$ less than that measured by the laboratory tunnel. Table 1 indicates similar biases exist in the wood smoke TC and OC data. For wood smoke, emissions ratios are more consistent than the absolute emission rates (except for ratios involving EC).

We attribute the bias in the wood smoke data to losses in the sampler inlet lines, which were discovered when the samplers were disassembled for cleaning. The discrepancy between the two systems increased with time, consistent with a gradual build up of material in the inlet lines. For example, the smallest error in the $\mathrm{PM}_{2.5}$ mass data (1\%) was observed from the first wood smoke intercomparison experiment right after the system was cleaned and the largest error in the final experiment before cleaning. Build up of material was observed in the inlet of all three samplers; however, Table 1 suggests that each system was affected differently. The geometry of the sampler inlets were the same, but the flow rate of exhaust through the field sampler inlet was much lower than that of the laboratory sampler. In addition, the problems appear primarily associated with one of the two field samplers. Little evidence of losses was observed during cleaning of the systems after the diesel experiments. Fine particle concentrations in the wood smoke were significantly higher than for the diesel exhaust (up to a factor of 6). Wood smoke emissions are also highly variable, with extremely smoky conditions often observed soon after lighting the fire when the sampler was operating, but before starting filter collection. Wood smoke is also stickier than diesel exhaust, making the samplers more difficult to clean. All of these factors likely explains the build up of material in the sampler inlet lines during the wood smoke but not the diesel experiments. Clearly more frequent cleaning is needed for wood smoke sampling; this was implemented on subsequent experiments.

Table 1 indicates more variability and bias in the EC data compared to other measurements. This is particularly true for the wood smoke experiments. The large errors in wood smoke EC are primarily attributable to experiments in which EC contributed less than $1 \%$ of the $\mathrm{PM}_{2.5}$ mass. In our view, the poor wood smoke EC comparison is most likely due to problems associated with thermal-optical OC/EC analysis of heavily loaded filters, with little EC, and substantial pyrolysis. These factors create substantial uncertainty in the operationally defined OC/EC split. For example, during OC/EC analysis of the wood smoke filters, the change in laser transmittance due to pyrolysis was significantly larger than the change associated with EC. Another issue is that the results errors are presented on a relative basis; on an absolute basis, the errors in experiments with low EC loadings were comparable or smaller than experiments with higher EC loadings. We do not view the EC discrepancy as an indication of a fundamental design problem associated with measuring trace species emissions. We expect better performance for other, better defined trace species such as individual ions or metals. For diesel, the EC comparisons show good agreement between the systems but with substantial variation; EC levels in diesel exhaust are much higher in wood smoke, and there is little pyrolysis of diesel samples during OC/EC analysis which reduces problems associated with thermal-optical analysis.

Quantitative comparisons between the two field samplers are listed in Table 2. Only 5 field sampler intercomparison experiments were performed, 3 of which were done with wood smoke. Table 2 indicates that the emissions measured by one field tunnel were $16 \%$ to $18 \%$ higher than the other field-tunnel sampler. We attribute this bias largely to the fact that the comparisons were 
TABLE 2

Relative error between two field samplers

\begin{tabular}{|c|c|c|}
\hline & \multicolumn{2}{|c|}{ All data $(\mathrm{n}=5)$} \\
\hline & Average & SD \\
\hline $\mathrm{PM}_{2.5}$ mass & 0.13 & 0.09 \\
\hline Bare $\mathrm{TC}^{1}$ & 0.17 & 0.06 \\
\hline Bare $\mathrm{OC}^{2}$ & 0.15 & 0.06 \\
\hline $\mathrm{EC}^{3}$ & 0.19 & 0.04 \\
\hline Backup OC & 0.00 & 0.10 \\
\hline $\mathrm{OC} / \mathrm{EC}^{4}$ & -0.04 & 0.08 \\
\hline $\mathrm{OC} / \mathrm{TC}^{4}$ & -0.02 & 0.04 \\
\hline $\mathrm{EC} / \mathrm{TC}^{4}$ & 0.02 & 0.05 \\
\hline $\mathrm{TC} / \mathrm{PM}_{2.5}$ mass $^{4}$ & 0.04 & 0.09 \\
\hline $\mathrm{OC} / \mathrm{PM}_{2.5} \mathrm{mass}^{4}$ & 0.03 & 0.08 \\
\hline $\mathrm{EC} \mathrm{PM}_{2.5}$ mass $^{4}$ & 0.06 & 0.11 \\
\hline Total number ${ }^{5}$ & 0.30 & 0.32 \\
\hline Integrated volume ${ }^{5}$ & 0.14 & 0.12 \\
\hline
\end{tabular}

${ }^{1} \mathrm{TC}=$ total carbon; ${ }^{2} \mathrm{OC}=$ organic carbon; ${ }^{3} \mathrm{EC}=$ elemental carbon; ${ }^{4}$ Ratios using data from bare quartz filter; ${ }^{5}$ Measured with SMPS.

primarily made with wood smoke as opposed to diesel exhaust. Good agreement was observed for the two diesel intercomparison experiments; for example, the average relative bias in $\mathrm{PM}_{2.5}$ mass measurements between the two field samplers was $5 \% \pm$ $2 \%$ versus $18 \% \pm 7 \%$ for the wood smoke data. Table 2 also indicates little bias or variation in the emission ratios measured with the two field samplers, including ratios involving EC, suggesting losses are not species dependent. Given the issues associated with the wood smoke experiments the data suggest comparable performance between the two sampler designs for filter-based measurements.

Volume and number size distributions measured using the SMPS for two diesel experiments are shown in Figure 5. The distributions show a volume and number mode around $100 \mathrm{~nm}$. Occasionally a large ultrafine number mode at around $20 \mathrm{~nm}$ was observed, indicating nucleation. SMPS data for the wood smoke intercomparison experiments are not shown because the wood stove emissions are highly temporally variable, making it difficult to separate the effects of this variability from the differences between the samplers using sequential measurements made with one SMPS system.

Scatter plots comparing integrated volume and total particle number emissions measured using the two sampler designs for the diesel intercomparison experiments are shown in Figure 4. There is excellent agreement in the integrated particle volume measured by the two samplers, with an average relative bias of $-5.5 \%$ and a standard deviation of $9 \%$ (Table 1).

A scatter plot comparison of total number measured using the two sampler designs is shown in Figure $4 \mathrm{~b}$. Higher total particle numbers were consistently measured in the lab tunnel,
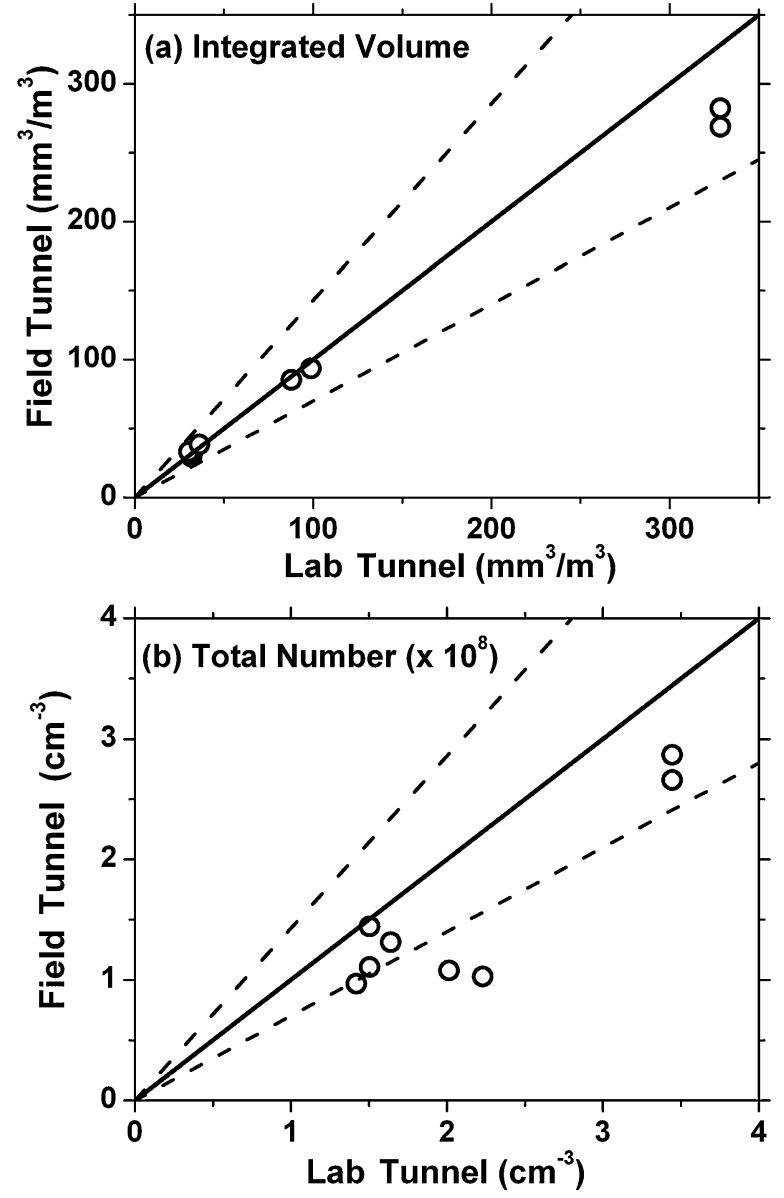

FIG. 4. Comparison of (a) integrated volume and (b) total number measured using the SMPS system during the intercomparison experiments performed with the diesel engine. Heavy line indicates one-to-one relationship with $\pm 30 \%$ indicated by dashed lines.

with several tests showing significantly higher totals in the lab tunnel. Occasionally there were large discrepancies between the samplers; this occasional poor agreement in total number was due to intermittent nucleation in the laboratory tunnel. Figure 5 shows size distributions measured on a day with nucleation (labeled "Nucleation") and a day without nucleation (labeled "No Nucleation"). Volume distributions measured during these tests with the different samplers agree, but nucleation dramatically increases the total number emissions in the laboratory tunnel relative to the field tunnel.

Nucleation was observed during approximately half the experiments performed using the lab tunnel, but rarely in the field tunnel. Nucleation in the lab tunnel appeared random with no obvious correlations with parameters such as dilution ratio, relative humidity, and engine load. For experiments with evidence of nucleation in both samplers, the peak of the nucleation mode in the field sampler was much smaller than that in the lab tunnel sampler suggesting weaker nucleation in the field tunnel. The difference in nucleation frequency between the two tunnels may 


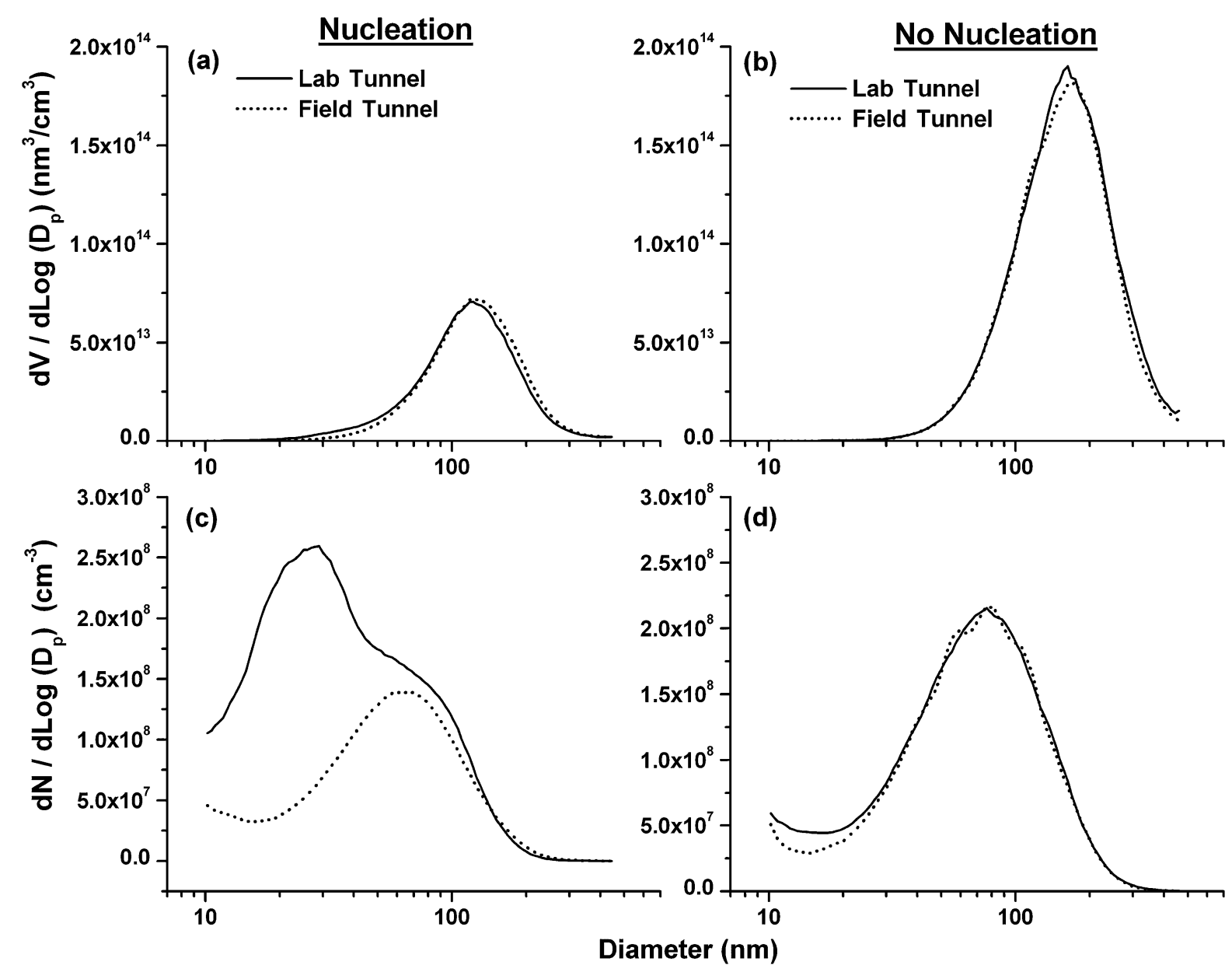

FIG. 5. Dilution-corrected volume and number size distributions measured during two diesel intercomparison experiments. Panels (a) and (c) correspond to experiment during which nucleation occurred (dilution ratio $=20$ ). Panels $(b)$ and $(d)$ correspond to an experiment during which nucleation did not occur (dilution ratio $=125$ ). Measurements were made sequentially on the different samplers using a single SMPS system while the diesel engine was operated at a constant load.

be due to differences in mixing rates. The more rapid mixing in the field tunnel may not allow sufficient time at higher supersaturations to enable nucleation to occur. The mixing rate also affects the time-temperature profile of the dilution. The relative humidity of the dilution air was another difference between the two samplers; the dilution air used in the field sampler was typically much drier than the laboratory sampler. However, there was no obvious relationship between relative humidity and nucleation. Previous research has shown that nucleation, particle size distributions, and total particle number in diesel exhaust are very sensitive to sampling conditions (Abdul-Khalek et al. 1998; Abdul-Khalek et al. 1999).

Apparent particle density can be estimated using the integrated volume measured with the SMPS and $\mathrm{PM}_{2.5}$ mass measured with the Teflon filter. A major complication in making such comparisons is the nonsphericity of diesel particles and the effect of this nonsphericity on SMPS measurements (Park et al. 2003). In this work, we are interested in using the apparent density as an indicator for changes in particle shape with dilution ratio.
Figure 6 shows the apparent density of particle emissions from the diesel engine as a function of dilution ratio. The apparent density measured at low load is $\sim 1.5 \mathrm{~g} / \mathrm{cm}^{3}$ with no trend with dilution ratio. The fine particle emissions under low load are dominated by $\mathrm{OC}$ with less than $25 \%$ of the $\mathrm{PM}_{2.5}$ mass emission being EC. Under these conditions particles are expected to be relatively spherical, and the estimated apparent density should be consistent with the actual density of the particles. A particle density of $1.5 \mathrm{~g} / \mathrm{cm}^{3}$ is consistent with expectations for organic particles.

The medium load experiments show lower apparent densities in the range of $0.5-1.0 \mathrm{~g} / \mathrm{cm}^{3}$. The lower calculated apparent densities reflect the nonspherical nature of the fine particle emissions; at medium load, the fine particle emissions are dominated by EC-5 to $60 \%$ of the $\mathrm{PM}_{2.5}$ mass emissions is EC. For medium load operations, there also appears to be a trend in apparent density with dilution ratio; at a low dilution ratio of 20 , the apparent density is $\sim 0.5 \mathrm{~g} / \mathrm{cm}^{3}$ and increases to a maximum at around $1.0 \mathrm{~g} / \mathrm{cm}^{3}$ at a dilution ratio of 160 followed 


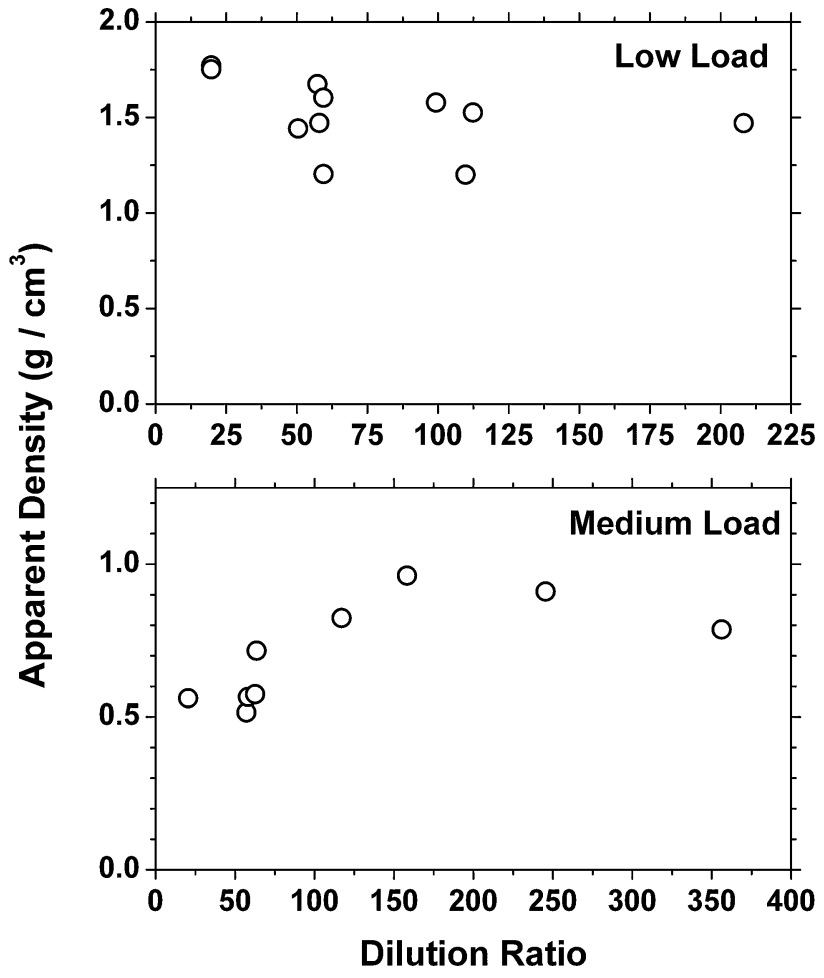

FIG. 6. Apparent density calculated from the $\mathrm{PM}_{2.5}$ filter mass and SMPS integrated volume plotted against dilution ratio for low and medium load experiments. Measurements were made on a small, single cylinder diesel engine.

by some decrease. This suggests changes in particle shape with dilution ratio. We expect that these changes are due to restructuring of the fractal carbon soot chains that contribute significantly to the fine particle emissions at medium load (high EC). Previous studies have shown that changes in relative humidity can alter the fractal structure of carbon chains changing the mobility diameter (Weingartner et al. 1995). The particle structure could depend on the amount of semi-volatile material in the condensed phase, which can depend strongly on dilution ratio (Lipsky and Robinson 2005). The relative humidity of the diluted exhaust varied between $10 \%$ and $60 \%$ across the set of experiments, but no trend in apparent density with relative humidity was observed.

\section{Effects of Residence Time}

The results of the intercomparison shown in Figure 3 indicate good agreement between measurements made with the field and laboratory samplers without a residence time tank. A residence time tank is not part of the new field sampler design, but the original Caltech sampler included a large residence time tank (Hildemann et al. 1989). An important design question is how much residence time is needed before aerosol characterization? Eliminating the residence time tank simplifies the design and operation of a dilution sampler.

The effects of residence time were evaluated by comparing simultaneous measurements made at the end of the dilution tun-

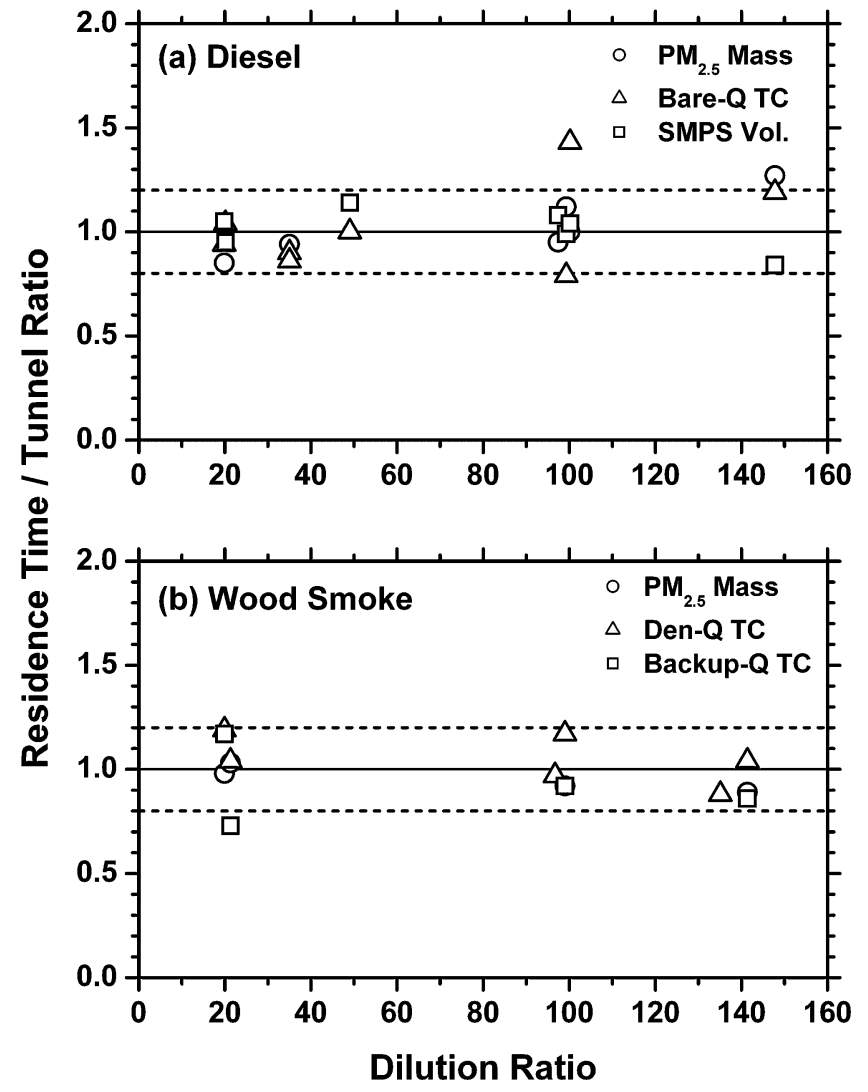

FIG. 7. Ratios of measurements made at the end of the residence time tube with measurements made at the end of the dilution tunnel for (a) diesel and (b) wood smoke experiments. Experimental schematic is shown in Figure 2. The horizontal solid line indicates a ratio of one, perfect agreement between the two measurements. The dashed horizontal lines indicate agreement within $\pm 20 \%$. TC $=$ total carbon. Den-Q TC $=$ TC measurements made using a quartz filter behind a carbon monolith denuder.

nel of the laboratory sampler and the residence time tube (see Figure 2). For the conditions of these experiments, the residence time inside the dilution tunnel is around 2.5 seconds, and the residence time tube provided an additional 40 seconds of time for aging. Figure 7 presents ratios of measurements made simultaneously at the beginning and end of the residence time tube. Data are shown for both diesel and wood smoke experiments as a function of dilution ratio.

Figure 7 indicates that additional residence time beyond the roughly 2.5 seconds provided by the dilution tunnel does not affect filter measurements or the integrated volume measured with the SMPS. The majority of the measurements made at the beginning and end of the residence tube are within $\pm 20 \%$, with a few outliers. These results indicate that the system has reached phase equilibrium by the end of the dilution tunnel. This conclusion is consistent with measurements made on diluted coal exhaust which show additional residence time beyond that provided by the tunnel did not affect filter based measurements of fine particle mass and composition (Lipsky et al. 2004). 
Additional residence time did alter the measured number size distribution and reduce total number emissions. These changes are due to microphysical processes such as coagulation occurring inside the residence time tube (Lipsky et al. 2002). Since the integrated particle volume did not change there was no addition of fine particle mass due to condensation inside the residence time tube. However it is not clear that the changes in particle size distribution that occur inside of a residence time tank are representative of those that occur in the actual atmosphere because a residence time tank ages exhaust at a modest dilution ratio (e.g., 100) while in the atmosphere exhaust is rapidly mixed with ambient air to much higher dilution ratios (e.g., 1000) in a few seconds.

The finding that integrated-volume and filter measurements do not depend on residence time is consistent with theoretical estimates of the characteristic times for phase equilibrium inside the dilution tunnel. Seinfeld and Pandis (1998) present a theoretical framework to estimate this equilibration time based on mass transfer in the gas phase,

$$
\tau_{\mathrm{s}}=\frac{1}{4 \pi \mathrm{D}_{\mathrm{A}} \int_{0}^{\infty} \mathrm{n}\left(\mathrm{R}_{\mathrm{p}}\right) \mathrm{R}_{\mathrm{p}} \mathrm{f}(\mathrm{Kn}, \alpha) \mathrm{dR}_{\mathrm{p}}}
$$

where $D_{A}$ is gas-phase diffusivity (assumed to be $0.06 \mathrm{~cm}^{2} / \mathrm{s}$ ), $\mathrm{n}\left(\mathrm{R}_{\mathrm{p}}\right)$ is the number of particles at radius $\mathrm{R}_{\mathrm{p}}$, and $\mathrm{f}(\mathrm{Kn}, \alpha)$ corrects the mass flux for noncontinuum effects and imperfect accommodation. To evaluate this equation we use the number size distribution measured with the SMPS, the Fuchs and Sutugin approach to evaluate $\mathrm{f}(\mathrm{Kn}, \alpha)$ and an accommodation coefficient $(\alpha)$ of 0.1 (Seinfeld and Pandis 1998).

Equation (3) predicts equilibration timescales ranging from $0.1-0.55$ seconds for the conditions of these experiments. The longest equilibration times correspond to the high dilution ratio experiments with the lowest tunnel concentrations. The theoretical equilibration times are at least a factor of 4 shorter than the residence time inside either dilution tunnel supporting the conclusion that the aerosol is in phase equilibrium by the end of the dilution tunnel.

Using Equation (3) one can predict under what conditions an aerosol might not be in phase equilibrium at the end of the dilution time tunnel (after a residence time of $2.5 \mathrm{~s}$ ). For the size distributions of particles considered here, noncontinuum effects are not that important; therefore total surface area is the key parameter in determining equilibration times. If the total particle surface area is greater than $0.01 \mathrm{~m}^{2} / \mathrm{m}^{3}$, then the equilibration time is less than $2 \mathrm{~s}$. For the sources tested here, these conditions were only encountered at high dilution ratios $(>300)$. Not achieving phase equilibrium within the short residence time provided by the dilution tunnel is also more of a concern for cleaner sources.

Although Equation (3) indicates that under some conditions (high dilution ratio, low emitting source) the diluted exhaust may not always be in phase equilibrium at the end of the dilution tunnel, this is not likely a concern for measurements made using filters. The fundamental issue is the difference between filter collection times and the time scale required to achieve phase equilibrium. For the experiments described here, the filter collection times ranged from 15-40 min, much longer than the equilibration time scales estimated using Equation (3). Phase equilibrium depends on the composition of the gas and condensed phases. When filter sampling, particles collected on the filter at the beginning of a test are continuously exposed to gasphase emissions throughout the entire test. Therefore changes in composition of the gas-phase passing through the filter can alter the phase partitioning of semivolatile material already collected on the filter. This will occur regardless of whether or not the aerosol achieved phase equilibrium as it passed through the dilution sampler. If gas phase emissions are constant then postcollection changes in partitioning will only occur if the aerosol did not reach phase equilibrium inside the dilution tunnel. If gas phase emissions are changing throughout an experiment then postcollection changes in phase partitioning may be significant. A wood stove or vehicles undergoing a test cycle have highly variable emissions. The effect of variable emissions on phase partitioning depends on the equilibration time scale for material collected on a filter compared to the filter collection time.

The residence time experiments considered both stable and highly variable emissions. The diesel experiments were conducted under steady load conditions, which presumably correspond to constant emission rates and particles collected on the filter being exposed to the same gaseous emissions throughout an experiment. In contrast, wood smoke emissions varied significantly throughout a given experiment because of the relatively uncontrolled nature of combustion in a wood stove. Therefore, particles collected on the filter at the beginning of an experiment will likely not be in equilibrium with the exhaust passing through the filter later in the experiment. Figure 7 indicates that additional residence time did not influence the measurements under either of these conditions.

In this paper we have examined the effect of residence time on bulk $\mathrm{PM}_{2.5}$ emissions, not the phase partitioning of individual organic species or potential chemical interactions within the aerosol after emission. Although the data indicate that the bulk $\mathrm{PM}_{2.5}$ achieved phase equilibrium, individual, trace-level semivolatile organics may not achieve equilibrium in the 2.5 seconds of aging time provided by the tunnel if they have mass accommodation coefficients much smaller than the value of 0.1 used to evaluate Equation (3). In this situation, a dilution sampler with $2.5 \mathrm{~s}$ of residence time might underestimate the particle phase emissions of these semivolatile species. However, as just discussed, residence time of the system may not matter if the species are measured using filters with long collection times. A second issue is that the partitioning of semivolatile material depends on dilution conditions, including temperature and dilution ratio. Dilution samplers are operated at much lower dilution ratios than atmospheric conditions, which can significantly bias the phase partitioning of semivolatile species (Lipsky and Robinson 2005). Therefore, additional residence time is unlikely to provide more atmospherically relevant data 
on the phase partitioning of semivolatile species. In order to avoid artifacts associated with phase partitioning in dilution samplers, one must simultaneously measure both gas and particle phase emissions of semivolatile species. Dilution samplers are designed to measure emissions before they have reacted chemically with the surrounding atmosphere; reactions between different components of the emissions on time scales of a few minutes are not expected to be significant.

\section{CONCLUSIONS}

The size and complexity of current dilution samplers is a major barrier to more widespread application of these systems for source characterization. A new, more portable dilution sampler was designed to provide measurements consistent with the widely cited Caltech dilution sampler (Hildemann et al. 1989). Both of these samplers are designed to simulate atmospheric dilution. Intercomparison experiments were performed using emissions from diesel generator and a wood stove. Good agreement was observed between the new field tunnel sampler and the sampler based on the Caltech design for $\mathrm{PM}_{2.5}$ total mass, $\mathrm{OC}$, and EC measured with filters, and integrated volume measured with an SMPS. All filter-based measurements have average bias errors of $10 \%$ or less as required by EPA to establish method comparability (EPA 1992). The larger variability in EC between the two samplers is attributed to the low levels of EC in the wood smoke and the significant pyrolysis of wood smoke OC during thermal-optical OC/EC analysis.

During certain experiments large differences in total particle number were observed between the two samplers. These differences corresponded to experiments during which nucleation occurred in the larger, laboratory sampler tunnel but not in the smaller, field sampler. More frequent nucleation in the laboratory system is attributed to differences in mixing rates between the two systems.

The new dilution sampler does not have a residence time tank. Experiments were conducted to examine the effects of residence time on diluted exhaust. No difference was observed in simultaneous measurements made 2.5 and 40 seconds after dilution. Theoretical analysis indicates that, under the conditions of these experiments, the aerosol should achieve phase equilibrium in less than 1 second. Eliminating the residence time tank greatly simplifies the design and operation of dilution samplers.

\section{ACKNOWLEDGMENTS}

The authors wish to acknowledge the assistance of Emily Weitkamp, Andy Grieshop, Mark Prack, Jessica Chiu, Neal Shyam, and R. Subramanian at Carnegie Mellon for their assistance in conducting this research. This research was supported by the US Department of Energy National Energy Technology Laboratory under contract DE-FC26-01NT41017.

\section{REFERENCES}

Abdul-Khalek, I., Kittelson, D. B., and Brear, F. (1999). The Influence of Dilution Conditions on Diesel Exhaust Particle Size Distribution Measurements, SAE Technol. Pap. Ser. No. 1999-01-1142: 563-571.
Abdul-Khalek, I., Kittelson, D. B., Graskow, B. R., Wei, Q., and Brear, F. (1998). Diesel Exhaust Particle Size: Measurement Issues and Trends, SAE Paper \#980525.

ASTM (2003). WK752 Test Method for Determination of $\mathrm{PM}_{2.5}$ Mass and Species Emissions from Stationary Combustion Sources by Dilution Sampling, ASTM International.

England, G. C. (2004). Development of Fine Particulate Emission Factors and Speciation Profiles for Oil- and Gas-Fired Combustion Systems, Final Report, NYSERDA Rep. No. 04-05, New York State Energy Research and Development Authority (NYSERDA), Albany, New York. Retrieved from http:// www.nyserda.org/programs /Environment/emepreports/13_FinalReport_R1V0.pdf

England, G., Toby, B., and Zielinska, B. (1998). Critical review of source sampling and analysis methodologies for characterizing organic aerosol and final particulate source emission profiles. Report \# API 344, American Petroleum Institute, Washington, DC.

Environmental Protection Agency. (1992). Method 301-Field Validation of Pollutant Measurement Methods from Various Waste Media. US Environmental Protection Agency. Retrieved from http://www.epa.gov/ttn/emc/promgate/ m-301.pdf.

Environmental Protection Agency. (1999). Control of emissions from new and in-use highway vehicles and engines. CFR Title 40, Part 86.

Environmental Protection Agency. (2004). (CTM-039) Measurement of $\mathrm{PM}_{2.5}$ and $\mathrm{PM}_{10}$ Emissions by Dilution Sampling (Constant Sampling Rate Procedures) Revised 7/04, US Environmental Protection Agency, Retrieved from http://www.epa.gov/ttn/emc/ctm.html.

Hildemann, L. M., Cass, G. R., and Markowski, G. R. (1989). A Dilution Stack Sampler for Collection of Organic Aerosol Emissions-Design, Characterization and Field-Tests, Aerosol Sci. Technol. 10:193-204.

Lipsky, E., Stanier, C. O., Pandis, S. N., and Robinson, A. L. (2002). Effects of Sampling Conditions on the Size Distribution of Fine Particulate Matter Emitted from a Pilot-Scale Pulverized-Coal Combustor, Energy \& Fuels 16:302-310.

Lipsky, E. M., Pekney, N. J., Walbert, G. F., O’Dowd, W. J., Freeman, M. C., and Robinson, A. L. (2004). Effects of Dilution Sampling on Fine Particle Emissions from Pulverized Coal Combustion, Aerosol Sci. Technol. 38:574587.

Lipsky, E. M., and Robinson, A. L. (2005). Effects of dilution on fine particle mass and partitioning of semi-volatile organics in diesel exhaust and wood smoke, Environ. Sci. Technol. under review.

Lyyranen, J., Jokiniemi, J., Kauppinen, E. I., Backman, U., and Vesala, H. (2004). Comparison of different dilution methods for measuring diesel particle emissions. Aerosol Sci. Technol. 38:12-23.

Maricq, M. M., Chase, R. E., Podsiadlik, D. H., and Vogt, R. (1999). Vehicle Exhaust Particle Size Distributions: A Comparison of Tailpipe and Dilution Tunnel Measurements. SAE Paper 1999-01-1461.

NIOSH (1996). Elemental Carbon (Diesel Particulate): Method 5040. NIOSH Manual of Analytical Methods. National Institute of Occupational Safety and Health, Cincinnati, OH, http://www.cdc.gov/niosh/nmam/pdfs/5040f3. pdf.

Park, K., Cao, F., Kittelson, D. B., and McMurry, P. H. (2003). Relationship between Particle Mass and Mobility for Diesel Exhaust Particles, Environ. Sci. Technol. 37:577-583.

Seinfeld, J. H., and Pandis, S. N. (1998). Atmospheric chemistry and physics: From air pollution to climate change. John Wiley, New York.

Subramanian, R., Khlystov, A. Y., Cabada, J. C., and Robinson, A. L. (2004). Positive and Negative Artifacts in Particulate Organic Carbon Measurements with Denuded and Undenuded Sampler Configurations, Aerosol Sci. Technol. 38:27-48

Turpin, B. J., Huntzicker, J. J., and Hering, S. V. (1994). Investigation of Organic Aerosol Sampling Artifacts in the Los-Angeles Basin, Atmos. Environ. 28:3061-3071.

Weingartner, E., Baltensperger, U., and Burtscher, H. (1995). Growth and Structural-Change of Combustion Aerosols At High Relative-Humidity, Environ. Technol. 29:2982-2986. 
Submitted to Atmospheric Environment

\title{
Source contributions to primary organic aerosol; Comparison of the results of a source- resolved model and the Chemical Mass Balance approach
}

Timothy E. Lane ${ }^{\mathrm{a}}$, Robert W. Pinder ${ }^{\mathrm{b}}$, Manish Shrivastava ${ }^{\mathrm{c}}$, Allen L. Robinson ${ }^{\mathrm{b}, \mathrm{c}}$, and Spyros N. Pandis ${ }^{\mathrm{a}, \mathrm{b}, \mathrm{d}, *}$

${ }^{a}$ Department of Chemical Engineering, Carnegie Mellon University, Pittsburgh, PA 15213, USA

${ }^{\mathrm{b}}$ Department of Engineering and Public Policy, Carnegie Mellon University, Pittsburgh, PA 15213, USA

c Department of Mechanical Engineering, Carnegie Mellon University, Pittsburgh, PA 15213, USA

${ }^{d}$ Department of Chemical Engineering, University of Patras, Patra, 26500, Greece

\begin{abstract}
A source-resolved model has been developed to predict the contribution of eight different sources to primary organic aerosol concentrations. The model was applied to the eastern United States during a seventeen day pollution episode beginning on July 12, 2001. Primary organic matter (OM) and elemental carbon (EC) concentrations are tracked for eight different sources: gasoline vehicles, non-road diesel vehicles, on-road diesel vehicles, biomass burning, wood burning, natural gas combustion, road dust, and all other sources. Individual emission inventories are developed for each source and a three-dimensional chemical transport model $(\mathrm{PMCAMx}+)$ is used to predict the primary OM and EC concentrations from each source. The source-resolved model is simple to implement and is faster than the existing source-oriented models. The results of the source-resolved model are compared to the results of chemical mass balance models (CMB) for Pittsburgh (Subramanian et al., 2005a,b,c) and Atlanta (Zheng et al., 2002). Significant discrepancies exist between the source-resolved model predictions and the CMB model predictions for some of the sources. There is strong evidence that the organic PM emissions from natural gas combustion are overestimated. It also appears that the OM and EC emissions from wood burning and off-road diesel are overwhelming the Northeastern US. Other similarities and discrepancies between the source-resolved model and the chemical mass balance
\end{abstract}

\footnotetext{
Corresponding author phone:

(412) 268-3531; fax:

(412) 268-7139; email: spyros@andrew.cmu.edu.
} 
model for primary OM and EC are discussed along with problems in the current emission inventory for certain sources.

Keywords: source apportionment, organic matter and elemental carbon, $\mathrm{PMCAMx}+$, eastern United States

\section{Introduction}

Atmospheric particles with diameters less than $2.5 \mu \mathrm{m}\left(\mathrm{PM}_{2.5}\right)$ have adverse effects on human health and visibility and contribute to global climate changes by scattering light and serving as cloud condensation nuclei. To limit the effects on human health and visibility, the United States Environmental Protection Agency (US EPA) has set a National Ambient Air Quality Standard (NAAQS) for $\mathrm{PM}_{2.5}$ at $15 \mu \mathrm{g} \mathrm{m} \mathrm{m}^{-3}$ (3 year average concentrations) and $65 \mu \mathrm{g} \mathrm{m}{ }^{-3}$ (24 hour average concentrations). According to the US EPA, all or part of 225 counties nationwide, many of which are in the eastern US, are not in attainment of the standards. In order for these areas to meet the NAAQS, control strategies must be implemented to reduce the $\mathrm{PM}_{2.5}$ concentrations.

$\mathrm{PM}_{2.5}$ is comprised of sulfate, nitrate, ammonia, organic matter, elemental carbon (EC), crustal species, and other compounds. Organic matter represents a major fraction of the total $\mathrm{PM}_{2.5}$ concentrations across the United States. Organic matter has both primary and secondary components while EC is only emitted from sources. Primary OM is emitted from various sources such as gasoline and diesel vehicles, biomass burning, industrial sources, and other forms of combustion. Dominant sources of EC are on-road and off-road diesel vehicles and biomass burning. Implementation of effective organic $\mathrm{PM}_{2.5}$ control strategies requires the quantification of the contribution of each source to the ambient OM and EC concentrations.

Chemical mass balance (CMB) receptor modeling methods can determine the source contributions to primary OM concentrations using organic compounds as tracers (Rogge et al., 1993a; Schauer et al., 1996). In the CMB method, the total concentration of each organic compound tracer in the ambient sample is reconstructed from a linear combination of the source emissions profiles. A CMB receptor model has been used to determine the source apportionment of $\mathrm{PM}_{2.5}$ primary OM in Los Angeles (Schauer et al., 1996), the San Joaquin Valley (Schauer and Cass, 2000), the southeastern United States (Zheng et al., 2002), and Pittsburgh 
(Subramanian et al. 2005a,b,c). The results of the CMB modeling suggest that gasoline and diesel vehicles along with wood burning are the major primary OM emission sources in the investigated areas.

A second approach to source apportionment is source-oriented modeling. Unlike receptor-oriented modeling, source-oriented models predict the pollutant concentrations by using a regional chemical transport model and emissions data as input. Source-oriented modeling separately tracks PM emissions from different source categories in the model instead of combining them into a single species. Both a Lagrangian and a 3D Eulerian source-oriented model have been applied to Los Angeles and the San Joaquin Valley (Kleeman et al., 1997; Kleeman and Cass, 1998; Kleeman et al., 1996; Kleeman and Cass, 2001; Held et al., 2004) predicting the source contribution to the size and composition distribution of $\mathrm{PM}_{10}$. In the 3D Eulerian model, Kleeman and Cass (2001) separately tracked the particles emitted by ten different source classes. For example, instead of having one primary OM species Kleeman and Cass (2001) used ten different primary OM species, one for each source class. Although the model is numerically accurate, the simulations require considerable CPU time.

The difference between the source-oriented models used in the previous studies and the source-resolved model used here is that the source-resolved model simulates the primary OM and EC for one source at a time, neglecting the rest of the sources. The source-resolved model only has one primary OM and EC species; therefore it uses less CPU time because there are fewer differential equations that must be solved simultaneously. Although the source-resolved model must be run for each source, the model is still faster than the source-oriented models.

The current study applies the source-resolved three-dimensional chemical transport model to the eastern United States in order to critically evaluate the primary OM and EC emission inventories. The overall performance of PMCAMx+ for the July 2001 episode using the current emission inventory has been evaluated by Gaydos et al. (2005). PMCAMx+ is used to predict contributions of eight different source categories to primary OM and EC: on-road diesel, off-road diesel, gasoline engines, natural gas, wood burning, biomass burning, dust, and all other sources. The primary organic aerosol from each source category is modeled individually by splitting the emissions inventories into the sub-categories. By having individual source emission inventories, only one primary OM and EC species are required in the model instead of eight species for both primary OM and EC. 
The predicted primary OM and EC concentrations for each source category are then compared to published CMB results for Atlanta and Pittsburgh. Agreement between the sourceresolved inventory results and the CMB results can increase our confidence in the source apportionment for primary OM and EC. Disagreement can point to problems in individual source emission inventories.

\section{Description of PMCAMx+}

PMCAMx+ is a three-dimensional chemical transport model which uses the framework of CAMx (Environ, 2003) to simulate horizontal and vertical advection, horizontal and vertical dispersion, wet and dry deposition, and gas-phase chemistry. Three aerosol modules have been implemented in PMCAMx+ to describe inorganic and organic aerosol dynamics and aerosolcloud interactions using an operator-splitting approach (Gaydos et al., 2005). The order of the processes is: emission, horizontal advection, vertical advection, vertical dispersion, horizontal dispersion, wet deposition, gas-phase chemistry, aerosol processes (nucleation, coagulation, inorganic aerosol condensation/evaporation), secondary organic aerosol formation, and aqueous phase-chemistry. This approach is further described in Gaydos et al. (2005). PMCAMx+ tracks thirteen different aerosol species: sulfate, nitrate, ammonium, aerosol water content, four secondary organic aerosol species, sodium, chloride, primary organic aerosol, primary elemental carbon, and all primary inert material. Each aerosol species has ten size sections, ranging from $40 \mathrm{~nm}$ to $40 \mu \mathrm{m}$.

For this study, PMCAMx+ is applied to a seventeen day pollution episode in the eastern United States starting on July 12, 2001. The modeling domain covers a 3492x3240 km region in the eastern United States with 36x36 km grid resolution with 14 different levels up to $6 \mathrm{~km}$ (Gaydos et al., 2005). The initial and boundary conditions for primary $\mathrm{PM}_{2.5} \mathrm{OM}$ are 2.0 and 1.0

$\mu \mathrm{g} \mathrm{m}^{-3}$, respectively, while zero initial and boundary conditions are used for EC. Inputs to the model include horizontal wind components, temperature, pressure, water vapor, vertical diffusivity, clouds, and rainfall, all created using the meteorological model MM5 (Gaydos et al., 2005; Grell et al., 1995).

Eight source-resolved inventories are used as the emissions inputs in PMCAMx+ when applied to the eastern United States. The primary OM and EC concentrations for each of the eight sources over the eastern US are simulated for the seventeen-day episode. For each source- 
resolved simulation, the boundary conditions for primary OM and EC are set to zero and the initial conditions are set to a very small non-zero value to avoid numerical problems (0.006 $\mu \mathrm{g}$

$\mathrm{m}^{-3}$ ). There are two more cases simulated as well: the initial conditions case and the boundary conditions case. For these two cases, the emissions for primary OM and EC within the domain are set at zero. For the boundary condition case, the initial conditions were set to $0.006 \mu \mathrm{g} \mathrm{m}^{-3}$; similarly, the boundary conditions are set to zero for the initial conditions case. The entire emission inventory was also simulated so that the sum of the primary OM and EC for the subinventories can be compared to primary OM and EC from the inventory to evaluate the quality of the solution.

\section{Emission Inventories}

The LADCO BaseE inventory generated using EMS-2003 (LADCO, 2003), is used to generate source-resolved inventories. Emissions are derived primarily from the U.S. EPA's National Emission Inventory (NEI) 1999 Version 2.0 (U.S. EPA, 2002a), with the following changes: on-road transportation sources are from the U.S. EPA's MOBILE6 (U.S. 2002b); nonroad sources are from U.S. EPA's NONROAD (U.S. EPA 2002c). The temporal profiles for electric power utility point sources are from an analysis of Continuous Emission Monitors (Janssen, 2003). Ammonia emissions are from the CMU Ammonia Emission Inventory (Goebes et al., 2003; Pinder et al., 2004). Biogenic emissions are from BIOME3 (Wilkinson and Janssen, 2001). A different emission inventory is used for weekdays, Saturdays, and Sundays.

From the overall emission inventory described above, eight source-resolved emission inventories have been developed. The primary OM and EC in each source-resolved inventory consist of the carbonaceous aerosol emissions from that source, while the rest of the inventory remains the same. Table 1 lists the emission source categories and the fraction of the total emissions of elemental carbon and organic carbon that each category contributes. The sources are sorted based on Source Classification Codes from the NEI database. The "other" source category consists of all source codes not mapped to one of the other seven source categories.

Table 1 shows the contribution of each source to the overall inventory for each day. The total emissions for the entire domain decrease on the weekend; for example, the EC emissions decrease from $1.09 \mathrm{ktons} \mathrm{day}^{-1}$ on a weekday to $0.89 \mathrm{ktons}^{-1} \mathrm{day}^{-1}$. There is also a change in the distribution of sources. For instance, the major difference between weekdays and weekends is 
that gasoline exhaust increases from $13 \%$ of the overall primary OM emissions to $22 \%$ of the primary OM on the weekends. Figure 1 shows the total emissions for primary OM and EC on a weekday across the eastern US. As expected, there are higher emission rates for OM and EC in urban areas. The highest daily emission rate for $\mathrm{OM}$ in a $36 \times 36 \mathrm{~km}$ cell is 19 tons day ${ }^{-1}$ over southern New Jersey, east of Philadelphia, and the highest emission rate for EC is 19.5 tons day $^{-1}$ located over Nashville, TN.

\section{Model Results}

\subsection{LADCO BaseE comparison with measurements}

Figure 2 shows the predicted ground-level average $\mathrm{PM}_{2.5}$ total carbonaceous aerosol, secondary organic aerosol (SOA), primary organic matter, and EC concentrations for the July 2001 episode from PMCAMx+ when using the base emission inventories. The first four simulation days are not included in the average so that the initial conditions do not have a significant impact on the average concentrations. The maximum average concentrations are: $13.4 \mathrm{\mu g} \mathrm{m}^{-3}$ over New York City for total carbonaceous aerosols, $2.9 \mu \mathrm{g} \mathrm{m}^{-3}$ over Arkansas for SOA, $8.3 \mu \mathrm{g} \mathrm{m}^{-3}$ over southern New Jersey for primary OM, and $5.4 \mu \mathrm{g} \mathrm{m}^{-3}$ found over Boston for EC. The domain average concentrations for total carbonaceous aerosol, SOA, primary OM, and EC predicted over the eastern United States are $2.01 \mu \mathrm{g} \mathrm{m}^{-3}, 0.73 \mu \mathrm{g} \mathrm{m}^{-3}, 1.03 \mu \mathrm{g} \mathrm{m} \mathrm{m}^{-3}$, and $0.25 \mu \mathrm{g} \mathrm{m}^{-3}$. On average, the predicted primary OM and EC concentrations represent $51.2 \%$ and $12.4 \%$, respectively, of the total carbonaceous aerosol across the eastern United States.

Primary OM and EC concentrations are highest in urban areas. For example, there are high average primary OM concentrations predicted in cities such as Atlanta $\left(2.7 \mu \mathrm{g} \mathrm{m}^{-3}\right)$, Baton Rouge (2.2 $\mu \mathrm{g} \mathrm{m}^{-3}$ ), Boston (4.3 $\left.\mu \mathrm{g} \mathrm{m}^{-3}\right)$, Chicago $\left(5.4 \mu \mathrm{g} \mathrm{m}^{-3}\right)$, Detroit (5.1 $\left.\mu \mathrm{g} \mathrm{m}^{-3}\right)$, Houston (3.3

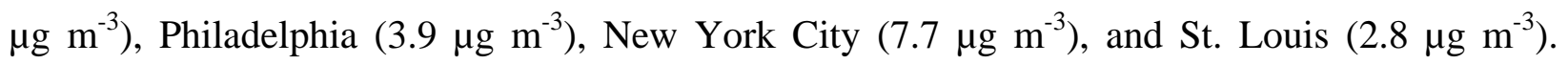
Similarly, high average EC concentrations are predicted in urban areas such as: Atlanta $(2.2 \mu \mathrm{g}$ $\left.\mathrm{m}^{-3}\right)$, Baton Rouge $\left(1.9 \mu \mathrm{g} \mathrm{m}^{-3}\right)$, Chicago $\left(2.6 \mu \mathrm{g} \mathrm{m}^{-3}\right)$, Detroit $\left(1.7 \mu \mathrm{g} \mathrm{m}^{-3}\right)$, Houston $\left(2.9 \mu \mathrm{g} \mathrm{m}^{-3}\right)$, Philadelphia $\left(2.2 \mu \mathrm{g} \mathrm{m}^{-3}\right)$, Portland $\left(2.8 \mu \mathrm{g} \mathrm{m}^{-3}\right)$, and New York City $\left(4.4 \mu \mathrm{g} \mathrm{m}^{-3}\right)$. For many of these locations, the EC is almost as great as the primary OM.

The 24-hour average model predictions are compared to the measurements from the EPA Speciation Trends Network (STN) and the Interagency Monitoring of Protected Visual Environments (IMPROVE). As described by Gaydos et al. (2005), the predicted primary OM 
was divided by 1.2 to convert to primary OC and the predicted SOA is divided by 1.8 to convert to secondary organic carbon. Figure 3 shows the comparison of the predicted total carbon (TC), OC, and EC concentrations with STN and IMPROVE. The TC predictions appear to have little bias but with significant scatter, with concentrations in many locations greatly over or underpredicted. As discussed in Gaydos et al. (2005), the mean bias between the predicted total carbonaceous aerosol and IMPROVE and STN measurements is $0.11 \mu \mathrm{g} \mathrm{C} \mathrm{m}^{-3}$ and $-0.92 \mu \mathrm{g}$ $\mathrm{m}^{-3}$, respectively.

There is better agreement with the IMPROVE measurements because the inventories for most of the major sources of carbonaceous material were constructed using the TOR protocol (Bhave, 2004; Gaydos et al., 2005). The STN measurements used the NIOSH TOT protocol for measuring EC and OC (NIOSH, 1999); while the IMPROVE measurements used the TOR protocol to analyze the filters (Chow et al., 1993). The STN data in Figure 3 are not blank corrected while the IMPROVE measurements already include this correction. Without a blank correction, the carbon measurements from the two networks are in poor agreement. With a blank correction of $\sim 1 \mu \mathrm{g} \mathrm{m}^{-3}$ needed to bring the STN measurements into agreement with the IMPROVE measurements, the model predictions for total carbon will have better agreement with the STN measurements (Graham et al., 2004; Gaydos et al., 2005).

For OC concentrations, the model overpredicts at low observed levels of OC and underpredicts at high observed levels of OC. Observed OC concentrations of less than $1.0 \mu \mathrm{C}$ $\mathrm{m}^{-3}$ are consistently overpredicted by the model. Overall, the model predictions compared to IMPROVE for OC have little bias, yet many sites are over or underpredicted. However, the model consistently underpredicts the STN measurements. This may be due in part because the STN sites are located in more populated areas than the IMPROVE sites and therefore generally have higher OC levels. The mean bias for the predicted OC compared to STN is $-1.8 \mu \mathrm{g} \mathrm{C} \mathrm{m}^{-3}$ (Gaydos et al., 2005). Even assuming a blank correction of $\sim 1 \mu \mathrm{g} \mathrm{m}^{-3}$, the STN measurements for OC are still underpredicted by the model.

Figure 3 also shows that the predicted EC concentrations are about 3 times higher than the observed EC concentrations from STN and have little bias when compared to the IMPROVE measurements (Gaydos et al., 2005). Again the IMPROVE EC levels are much lower than STN because they are rural. This suggests that the EC emissions in cities are overestimated. For example, the model predicts over $5 \mu \mathrm{g} \mathrm{m}^{-3}$ of EC in New York City which is significantly greater 
than $\sim 0.2-1.5 \mu \mathrm{g} \mathrm{m}^{-3}$ measured at STN sites in Queens and the Bronx. There are a few IMPROVE sites that are greatly overpredicted, such as Casco Bay, ME and Cape Cod, MA, where the predicted EC concentration is over $2.0 \mu \mathrm{g} \mathrm{m}^{-3}$ versus the measured concentrations of less than $\sim 1.0 \mu \mathrm{g} \mathrm{m}^{-3}$. The mean bias for IMPROVE and STN when compared to the model predictions are $0.17 \mu \mathrm{g} \mathrm{m}^{-3}$ and $0.88 \mu \mathrm{g} \mathrm{m} \mathrm{m}^{-3}$, respectively (Gaydos et al., 2005). The model overprediction probably indicates significant problems with the EC emission inventory. Although the predicted primary OC and EC concentrations do not match the observed concentrations, the source-resolved model will help to predict which sources contribute the most to primary OC and EC and will also provide insight into which source's emission inventory needs improvement.

\subsection{Source-resolved mass balance test}

The sum of the predicted primary OM and EC concentrations from each source resolved subcategory should equal the primary OM and EC concentration predicted from the base case. However, because of the previously discussed effects of deposition, there are slight discrepancies. Throughout the simulation, the sum of the primary OM form each source category is on average $0.001 \mu \mathrm{g} \mathrm{m}^{-3}$ (an average of $0.6 \%$ more than the base case) greater than the predicted base case primary OM over the whole domain. The maximum absolute error and relative error of any grid cell at any time step is $0.78 \mu \mathrm{g} \mathrm{m}^{-3}$ (relative error of $10 \%$ over New York City) and $24 \%$ (absolute error of $9.7 * 10^{-7} \mu \mathrm{g} \mathrm{m}^{-3}$ over the Atlantic Ocean north of Miami), respectively. Overall, the source-resolved method works well in predicting the source contributions for primary OM.

On average, the sum of the EC concentrations from each source is also $0.001 \mu \mathrm{g} \mathrm{m}{ }^{-3}$ greater than the base case EC concentration over the whole domain. The average relative error is $9 \%$ for areas with a predicted base EC concentration greater than $0.005 \mu \mathrm{g} \mathrm{m}^{-3}$. Larger relative errors exist for grid cells with very low predicted EC concentrations, for example cells over the Atlantic Ocean. Due to the precision of PMCAMx+, the lower limit for the EC concentrations is near $1 * 10^{-8} \mu \mathrm{g} \mathrm{m}^{-3}$. Although the absolute error is very small, the relative error is large in areas where the EC concentration is near the lower limit for each source category and the base case. At high predicted base case EC concentrations, the source-resolved model performs similarly as with the primary OM concentrations. 


\subsection{Source resolved OM}

In order to correctly predict the primary OM concentration for each source, the primary OM concentrations predicted from the boundary and initial condition cases must be factored into the source-resolved predictions for each source. Figure 4 shows the average contributions of the initial and boundary conditions for the last thirteen days of the episode for the primary OM concentrations. The boundary conditions have a major impact on areas with low OM emissions, which are located over the oceans and along the boundaries in the Northern Plains and Canada. In these areas, the predicted average $\mathrm{OM}$ concentrations are $1.0 \mu \mathrm{g} \mathrm{m}^{-3}$ with the boundary condition contributing almost $100 \%$ of the primary OM. Away from the boundary in the eastern United States, the boundary conditions contribute between $0.1-0.6 \mu \mathrm{g} \mathrm{m}^{-3}(\sim 10-20 \%$ in urban areas and $\sim 30-50 \%$ in rural areas) to the total primary OM concentrations. Excluding the initial four day spin up, the initial conditions on average only contribute $8 \%$ of the total primary OM in the eastern US. Over Arkansas, northern and eastern Louisiana, northeast Texas, and southern Missouri, the initial conditions contribute $\sim 0.26 \mu \mathrm{g} \mathrm{m}^{-3}$ (less than $30 \%$ ) to the primary OM concentrations. The initial conditions have little impact on the primary OM in other areas of the eastern United States after the first four simulation days.

The OM contributions from the aerosol that pre-existed in the model domain before the beginning of the simulation (initial conditions) and from sources outside the modeling domain (boundary conditions) are split into the eight source categories by assuming the same source distribution of the weekday inventory. For example, in areas where the boundary condition contribution is close to 100 percent of the total primary OM, the source contributions to the primary OM are: gasoline $13 \%$, on-road diesel $7 \%$, off-road diesel $14 \%$, natural gas $23 \%$, wood $15 \%$, biomass $6 \%$, dust $4 \%$, and other $18 \%$. The total contribution of each source is calculated by combining concentrations predicted using the source-resolved inventory plus the contribution of the boundary and initial condition concentrations.

As expected, the domain average primary OM contribution predicted for each source is similar to the domain average fraction of the total emissions for each source. The domain average primary OM concentration across the eastern US is $1.03 \mu \mathrm{g} \mathrm{m}^{-3}$. The predicted domain average primary $\mathrm{OM}$ concentrations over the thirteen day period for each category are: gasoline $14 \%$; on-road diesel 7\%; non-road diesel $13 \%$; natural gas $22 \%$; wood $16 \%$; biomass $6 \%$; dust 
4\%; and other $18 \%$. These domain average contributions are similar to the emissions shown in Table 1; however, there are temporal and spatial variations in the contribution of each source category.

Temporal variations during the 17 day pollution episode for Pittsburgh, PA are shown in Figures 5 and 6. Figure 5 shows peak primary OM concentrations occur during morning rush hour and then decrease to a minimum in the early afternoon before the evening traffic increases the OM concentrations. This cycle can be seen in all urban areas across the whole domain. In Pittsburgh, the predicted peak hourly primary OM concentration is $5.3 \mu \mathrm{g} \mathrm{m}^{-3}$, whereas the low is $0.9 \mu \mathrm{g} \mathrm{m}^{-3}$. The average predicted primary OM concentration for Pittsburgh during the episode is $2.6 \mu \mathrm{g} \mathrm{m}^{-3}$. The day-to-day variability in the 24-hour average source contributions for Pittsburgh are shown in Figure 6. The small fluctuations in the source contributions for Pittsburgh are due to the change in wind patterns, temperature, and emission inventories from weekdays to weekends. Across the entire domain, temporal variations are not significant because of the small temporal variability of the inventory. The focus will be on the average contribution of each source and its spatial variations.

Figure 7 shows the spatially resolved thirteen day average (the first four days are not included in the average) contribution of each source category to the total primary $\mathrm{PM}_{2.5} \mathrm{OM}$ concentrations. The scale $\left(0.0\right.$ to $\left.0.4 \mu \mathrm{g} \mathrm{m}^{-3}\right)$ is the same for each source to allow for comparisons of relative contributions of the different categories. The source that surprisingly contributes the most primary OM in the eastern US according to the inventory is the natural gas category. The maximum average natural gas contribution is $3.4 \mu \mathrm{g} \mathrm{m}^{-3}$ for New York City. This represents $42 \%$ of the total predicted primary OM in New York City (Figure 8). Other areas that have high predicted primary OM concentrations from natural gas are Baton Rouge with $0.68 \mu \mathrm{g}$ $\mathrm{m}^{-3}$ (29\% of the total primary OM in Baton Rouge), Chicago with $1.8 \mu \mathrm{g} \mathrm{m}^{-3}$ (30\%), Detroit with $1.2 \mathrm{\mu g} \mathrm{m}^{-3}$ (22\%), Houston with $1.2 \mathrm{\mu g} \mathrm{m}^{-3}$ (34\%), and Philadelphia with $0.80 \mu \mathrm{g} \mathrm{m} \mathrm{m}^{-3}$ (20\%).

Diesel exhaust has a significant impact on primary OM concentrations as well. Overall, diesel exhaust is predicted to contribute an average of $20 \%$ of the total primary $\mathrm{OM}$ in the eastern US. Non-road diesel contributes more primary OM to the Midwest and North Plains associated with farming activities. There are also high contributions of non-road diesel ( 2025\%) to primary OM along the Mississippi river caused by river boats. Non-road diesel also contributes significant amounts of primary OM in eastern cities (Figure 7). On-road diesel 
contributes mostly to urban primary OM. The maximum primary OM concentrations for on and off-road diesel exhaust are $0.35 \mu \mathrm{g} \mathrm{m}^{-3}$ northeast of New York City (7\%) and $1.4 \mu \mathrm{g} \mathrm{m}^{-3}$ over Boston (31\%).

Gasoline exhaust, similar to on-road diesel exhaust, contributes large amounts of primary OM in cities across the eastern US. Gasoline contributes a maximum primary OM concentration above Detroit of $2.7 \mathrm{\mu g} \mathrm{m}^{-3}$, which represents $50 \%$ of the total primary OM in Detroit. As shown in Figure 5, other high areas of primary OM from gasoline exhaust include: Atlanta (0.66 $\mu \mathrm{g} \mathrm{m}^{-}$ ${ }^{3}$, 25\%), Boston (0.73 $\left.\mu \mathrm{g} \mathrm{m}^{-3}, 17 \%\right)$, Chicago (0.78 $\left.\mu \mathrm{g} \mathrm{m}^{-3}, 15 \%\right)$, Houston (0.52 $\left.\mu \mathrm{g} \mathrm{m}^{-3}, 16 \%\right)$, Miami (0.64 $\left.\mathrm{g} \mathrm{m} \mathrm{m}^{-3}, 26 \%\right)$, New York City (0.63 $\left.\mu \mathrm{g} \mathrm{m}^{-3}, 9 \%\right)$, and St. Louis $\left(0.47 \mu \mathrm{g} \mathrm{m}^{-3}, 17 \%\right)$. Gasoline exhaust is more of an urban problem than a rural problem, as seen in Figures 7 and 8.

Wood burning is predicted to contribute a domain average of $15 \%$ of the total primary $\mathrm{OM}$, with most of the primary OM from wood burning located in the North, especially the Northeast. Above Portland ME, the primary OM concentration from wood burning is predicted to be $1.5 \mu \mathrm{g} \mathrm{m}^{-3}$ (55\%). Atlanta (0.69 $\left.\mu \mathrm{g} \mathrm{m}^{-3}, 26 \%\right)$, Boston (0.93 $\mu \mathrm{g} \mathrm{m}^{-3}$, 22\%), Chicago (0.65 $\left.\mu \mathrm{g} \mathrm{m}^{-3}, 12 \%\right)$, New York City (0.95 $\left.\mu \mathrm{g} \mathrm{m}^{-3}, 12 \%\right)$, Philadelphia $\left(0.67 \mu \mathrm{g} \mathrm{m}^{-3}, 17 \%\right)$, and St. Louis $\left(0.75 \mu \mathrm{g} \mathrm{m}^{-3}, 26 \%\right)$ are all areas with high primary OM contributions from wood burning.

Biomass burning according at least to the inventory only has a significant impact in Texas. In northern and east Texas, the predicted primary OM concentrations from biomass burning range from 0.4 to $1.0 \mu \mathrm{g} \mathrm{m}^{-3}$ ( 30-50\%). The maximum OM concentration is found east of Houston and has a concentration of $1.18 \mu \mathrm{g} \mathrm{m}^{-3}$ (50\%). In central Texas, the primary OM concentration ranges between 0.15 and $0.3 \mu \mathrm{g} \mathrm{m}^{-3}$ (20-30\%). Over the whole domain, there are relatively little contributions ( $\sim \%)$ from biomass burning (Figure 8 ).

Road dust contributes little primary OM. The maximum concentration predicted for road dust is $0.22 \mu \mathrm{g} \mathrm{m}^{-3}$ northeast of New York City, which only represents $4 \%$ of the primary OM. Overall, the average primary OM concentration from road dust is $0.04 \mu \mathrm{g} \mathrm{m}^{-3}$ or $\sim 4 \%$ of the total primary OM.

The others source category has high concentrations around Chicago, Philadelphia, and Canada (northwest of Ottawa and Montreal). The highest predicted others concentration for primary OM is east of Philadelphia in southern New Jersey (5.4 $\mu \mathrm{g} \mathrm{m}^{-3}, 59 \%$ of the baseE case). Over Chicago, the contribution is predicted to be $21 \%\left(1.1 \mu \mathrm{g} \mathrm{m}^{-3}\right)$ of the primary OM. In Canada, the concentration is predicted to be $0.77 \mu \mathrm{g} \mathrm{m}^{-3}$ (42\%) for the others source category. 
However, the mobile gasoline and diesel emissions are included in the others source category in Canada. Although it appears the others concentrations in Canada are high, the gasoline and diesel exhaust are the sources contributing significant amounts of OM in Canada.

\subsection{Source resolved EC}

Since EC has no initial concentration or boundary condition, the sum of the subcategories is the sum of the eight sources. The domain average EC concentration for the eastern United States is predicted to be $0.25 \mu \mathrm{g} \mathrm{m}^{-3}$. The average source contributions for the whole domain are: gasoline $4 \%$, non-road diesel $54 \%$, on-road diesel $24 \%$, natural gas $0 \%$, wood burning $8 \%$, biomass burning $4 \%$, road dust $0 \%$, and other $6 \%$. Similar to the primary OM, the domain averages for EC are comparable to the emissions of EC shown in Table 1.

The daily fluctuations in the EC concentrations follow the same pattern as the OM concentrations, shown in Figure 5 for Pittsburgh, PA. On average, the predicted EC concentrations are a factor of two higher than the measurements in Pittsburgh (Gaydos et al., 2005). The discrepancies are larger at night, with the model considerably overpredicting the observed concentrations by $\sim 1 \mu \mathrm{g} \mathrm{m}^{-3}$ for most nights. Similar to primary OM, the EC source contributions exhibit little day-to-day variability. The day-to-day variations in source contributions to ambient EC are due to changes in wind patterns and temperature along with the difference in emissions from weekdays and weekends (Figure 6). Therefore, the focus will be on the thirteen day average contribution of each source and the spatially variations.

Figure 9 shows the average contribution of each source to the total EC predicted from the base case. Diesel exhaust is predicted to contribute an average of $75 \%$ of the EC in the eastern United States. In the Midwest/Northern Plains the EC concentrations are dominated by non-road diesel emissions, which contribute $60-100 \%$ (Figure 10), which represents $\sim 0.3-0.6 \mu \mathrm{g} \mathrm{m}^{-3}$. As with the primary $\mathrm{OM}$, non-road diesel contributes $0.3-0.4 \mu \mathrm{g} \mathrm{m}^{-3}(\sim 70 \%)$ of EC along the Mississippi river. In the southeast, non-road diesel emissions contribute $30-50 \%$ of the EC (0.1$\left.0.2 \mu \mathrm{g} \mathrm{m}^{-3}\right)$. Areas with high EC concentrations predicted from non-road diesel exhaust are Boston (4.3 $\mu \mathrm{g} \mathrm{m}^{-3}, 77 \%$ of total EC), Houston (2.0 $\left.\mu \mathrm{g} \mathrm{m}^{-3}, 70 \%\right)$, Nashville (2.1 $\left.\mu \mathrm{g} \mathrm{m}{ }^{-3}, 92 \%\right)$, and New York City $\left(2.5 \mu \mathrm{g} \mathrm{m} \mathrm{m}^{-3}, 55 \%\right)$. Non-road diesel exhaust is both an urban and rural problem for EC concentrations. 
On-road diesel exhaust concentrations are $\sim 0.02 \mu \mathrm{g} \mathrm{m}^{-3}$ (5-15\%) of EC in the Midwest/Northern Plains and $\sim 0.1-0.2 \mu \mathrm{g} \mathrm{m}^{-3}$ (30-50\%) of EC in the Southeast. Urban areas with high predicted EC contributions from on-road diesel exhaust are Atlanta $\left(0.86 \mu \mathrm{g} \mathrm{m}^{-3}\right.$, $38 \%$ ), Boston (0.56 $\left.\mathrm{gg} \mathrm{m}^{-3}, 11 \%\right)$, Chicago (0.65 $\left.\mu \mathrm{g} \mathrm{m}^{-3}, 25 \%\right)$, Houston (0.58 $\left.\mu \mathrm{g} \mathrm{m}^{-3}, 21 \%\right)$, Philadelphia (0.51 $\left.\mu \mathrm{g} \mathrm{m}^{-3}, 24 \%\right)$, New York City $\left(0.58 \mu \mathrm{g} \mathrm{m} \mathrm{m}^{-3}, 14 \%\right)$, and St. Louis $\left(0.53 \mu \mathrm{g} \mathrm{m}^{-3}\right.$, 26\%). The maximum EC predicted from on-road diesel is found outside of New York City (1.0 $\left.\mu g \mathrm{~m}^{-3}, 20 \%\right)$.

A large fraction of the predicted EC concentration over Maine comes from wood burning (60-80\%), similar to primary OM. Above Portland, ME wood burning contributes $2.0 \mu \mathrm{g} \mathrm{m}^{-3}$ (70\%) to the EC concentration. Over the coast of Maine, the EC concentration from wood burning contributes $0.5-1.8 \mu \mathrm{g} \mathrm{m}^{-3}$. Other areas with relatively high EC concentrations from wood burning emissions are northern Illinois $\left(\sim 0.2 \mu \mathrm{g} \mathrm{m}^{-3}, 20 \%\right)$ and Boston $\left(0.31 \mu \mathrm{g} \mathrm{m}{ }^{-3}, 6 \%\right)$. Wood burning also contributes $\sim 10-15 \%$ of the EC in the Northeast and $\sim 2-5 \%$ of the EC in the Southeast.

The maximum EC concentration predicted from gasoline is found over Detroit ( $0.31 \mu \mathrm{g}$ $\left.\mathrm{m}^{-3}, 18 \%\right)$. Other areas with high gasoline EC concentrations are Atlanta $\left(0.14 \mathrm{\mu g} \mathrm{m}^{-3}, 6 \%\right)$, Boston (0.12 $\left.\mu \mathrm{g} \mathrm{m}^{-3}, 2 \%\right)$, Chicago $\left(0.14 \mu \mathrm{g} \mathrm{m}^{-3}, 5 \%\right)$, Houston $\left(0.09 \mu \mathrm{g} \mathrm{m}^{-3}, 3 \%\right)$, Miami $(0.11$ $\left.\mu \mathrm{g} \mathrm{m}^{-3}, 6 \%\right)$, New York City $\left(0.11 \mu \mathrm{g} \mathrm{m}^{-3}, 3 \%\right)$, and St. Louis $\left(0.10 \mu \mathrm{g} \mathrm{m}^{-3}, 5 \%\right)$. As shown in Figure 10, gasoline exhaust contributed roughly $5-10 \%$ of the EC across the eastern United States.

Except in the Northern Plains ( $\sim \%)$ and Texas, biomass burning contributes $\sim 5 \%$ of the EC which represents around 0.02-0.05 $\mu \mathrm{g} \mathrm{m}^{-3}$ of the EC across the eastern US. Biomass burning contributes about $20 \%$ of the EC in Texas, with on-road and off-road each adding around 30$40 \%$. In northern and eastern Texas, biomass burning contributes $\sim 0.1 \mu \mathrm{g} \mathrm{m}^{-3}$ which is $25-35 \%$ of the total predicted EC. The maximum EC concentration from biomass burning is around Atlanta (0.16 $\left.\mu \mathrm{g} \mathrm{m}^{-3}, 12 \%\right)$.

The road dust and natural gas sources are predicted to have little impact on the EC concentrations. Combined, these sources contribute less than 1\% of the EC in the eastern United States.

The others source category predicts the domain average contribution of EC to be $6 \%$. As seen with primary OM, the others category predicts high concentrations of EC in three main 
areas: Chicago, Philadelphia, and Canada. East of Philadelphia, the others concentration is predicted to be $0.5 \mu \mathrm{g} \mathrm{m}^{-3}$ (26\%) and in northern Illinois the others concentration of EC is $0.1 \mu \mathrm{g}$ $\mathrm{m}^{-3}(5 \%)$. Over parts of Canada, the EC concentration predicted from the others category is 1.2 $\mu \mathrm{g} \mathrm{m}^{-3}$. As mentioned with the primary $\mathrm{OM}$, a large fraction of the others source emissions is actually the mobile gasoline and diesel exhaust. So a majority of the predicted EC in Canada is actually from mobile gasoline and diesel emissions.

\subsection{Comparison with CMB}

Figure 11 shows the source apportionment predicted by the source-resolved model compared to the chemical mass balance (CMB) results for Pittsburgh in July 2001 (Subramanian et al. 2005a,b,c). The high and low CMB results represent the source apportionment calculated using two different models, each with a different sets of source profiles.

In Pittsburgh, the predicted primary OC concentration from the BaseE case, which was calculated by dividing the primary OM by 1.4, is higher than the primary OC predicted by CMB. Looking at individual source categories, large discrepancies exist for natural gas, wood combustion, and diesel exhaust when compared to the CMB results. Diesel does not look to bad. The gasoline and road dust sources fall within the predicted high and low CMB predictions; while the others source is slightly less than the low CMB result. Although, the others category for PMCAMx+ is mainly from industrial sources whereas the CMB others category is largely meat cooking emissions.

The predicted EC concentrations in Pittsburgh are on average two times higher than the measurements taken during the Pittsburgh Air Quality Study, which are comparable to the summed CMB predictions (Gaydos et al., 2005). This is mainly due to the diesel emission inventory being overestimated. In Pittsburgh, the source-resolved model predicts that non-road diesel contributes $0.93 \mu \mathrm{g} \mathrm{m}^{-3}$ of EC and on-road diesel contributes $0.43 \mu \mathrm{g} \mathrm{m}^{-3}$. Clearly the nonroad diesel emissions are too high; however, the on-road diesel emissions may also be overestimated.

Figure 12 shows the comparison of the predicted EC concentrations in Atlanta for the source-resolved model and the CMB model (Zheng et al., 2002). The CMB results are from 1999, whereas the source-resolved results are for 2001. It is also important to note that the Atlanta supersite was located near a bus depot (Solomon et al., 2003). The predicted primary 
OC concentrations for wood combustion, road dust, and others are similar to the CMB results. The primary OC from gasoline and natural gas appear to be overpredicted in Atlanta. Although the source-resolved EC concentrations are comparable to the CMB results for Atlanta, both the $\mathrm{CMB}$ and the source-resolved model results are most likely overpredicting the EC concentrations in Atlanta. The CMB is too high because the site was located near a bus depot; while the sourceresolved EC concentrations are on average around three times higher than observed STN measurements.

\section{Conclusions}

The source-resolved model for primary OM and EC is simple to implement compared to the source-oriented model developed by Kleeman and Cass (2001). No species need to be added to $\mathrm{PMCAMx}+$ in order to separately track the concentration of primary OM and EC from different sources. Splitting the emission inventory for primary OM and EC and running each source inventory in PMCAMx+ is a faster way to accomplish the source apportionment of primary components.

Even in cases where the OM results from PMCAMx+ for the BaseE inventory case are in reasonable agreement with the results from IMPROVE and STN, the source-resolved model and the CMB results still have significant differences. For EC, the model predicts EC concentrations 3 times higher than measurements from STN. Non-road diesel, according to the emission inventory, is predicted to contribute more to EC in urban areas than on-road diesel. This overprediction suggests that the non-road diesel emission inventory is currently too high. While the non-road diesel inventory should be reduced, the on-road diesel emission inventory may also need to be reduced.

Natural gas, wood burning and biomass burning are other sources that have emission inventory problems. Clearly the natural gas is overpredicted in the model. Rogge et al. (1993b) found that the emission of OM from natural gas combustion is low (48.5 ng kJ${ }^{-1} \pm 17.4$ ) and that natural gas contributed only around $0.1 \%$ of the total primary OM concentrations over Los Angeles in 1982 (Hildemann et al. 1991). The primary OM emission inventory for natural gas should be reduced by at least 50 times the current value. By lowering the natural gas contribution to primary OM, the source-resolved model results will compare better with the CMB results. 
The contribution of primary OM and EC from wood burning is too high along the northeastern coast, predicting average primary $\mathrm{OM}$ concentrations greater than $0.5 \mu \mathrm{g} \mathrm{m}^{-3}$. In July, there should not be much residential wood burning. By lowering the wood burning emission rates, both the predicted primary OM and EC will decrease in Maine and the rest of the northeastern coast.

Primary OM contributions from biomass burning are over estimated in Texas. The primary $\mathrm{OM}$ concentration across Texas ranges from 0.1 to $0.9 \mu \mathrm{g} \mathrm{m}^{-3}$; whereas the concentrations across the rest of the eastern United States are $\sim 0.06 \mu \mathrm{g} \mathrm{m}^{-3}$. The biomass burning inventory should be reduced in Texas so that similar concentrations of primary OM would be predicted across the entire eastern US. The biomass burning emissions inventory does a better job predicting the contribution of EC. The EC contribution from biomass burning is more uniform throughout the eastern United States (5-15\%).

If the natural gas inventory is lowered by $95 \%$ and the wood burning emissions are also reduced in July for primary OM, then predicted OC concentrations from PMCAMx+ would be even less than the STN and IMPROVE measurements. If the underprediction of the OC is due to the primary $\mathrm{OM}$ emissions, then one or more of the remaining source emission inventories would need to be increased. The primary OM emissions would likely need to be increased for the gasoline or diesel inventories in order to offset the reduction of the natural gas and wood burning inventories.

The model predicts reasonable values for road dust and others for both primary OM and EC. The predicted primary OM and EC concentrations for these source categories in Pittsburgh and Atlanta are in agreement with the CMB results.

Overall, the source-resolved model is a quick and easy technique to predict the source contributions of primary OM and EC. Clearly the model has shown that there are significant problems in the emission inventories.

\section{Acknowledgments}

The authors would like to thank the Lake Michigan Air Directors Consortium for providing the meteorological files, the area and point emission files, and the initial and boundary condition files for PMCAMx+. This research was conducted as part of the Pittsburgh Air Quality Study, which was supported by US Environmental Protection Agency under contract 
R82806101 and the US Department of Energy National Energy Technology Laboratory under contract DE-FC26-01NT41017. This research was also supported by the EPA STAR program through the National Center for Environmental Research (NCER) under grant R832162. This paper has not been subject to EPA's required peer and policy review, and therefore does not necessarily reflect the views of the Agency. No official endorsement should be inferred.

\section{References}

Bhave, P.V., 2004. Measurement Needs for Evaluation Model Calculations of Carbonaceous Aerosol. Presented at the EMEP Workshop on Particulate Matter Measurement \& Modeling, April 20-23, New Orleans, LA.

Chow, J.C.; Watson, J. G.; Pritchett, L. C.; Pierson, W. R.; Frazier, C. A.; Purcell, R. G., 1993. The DRI Thermal/Optimal Reflectance Carbon Analysis System: Description, Evaluation and Applications in United States Air Quality Studies, Atmospheric Environment 27A (8), 1185-1201.

Environ, 2003. User's Guide to the Comprehensive Air Quality Model With Extensions (CAMx). Version 4.02. Report prepared by ENVIRON International Corporation, Novato, CA.

Gaydos, T. M.; Pinder, R. W.; Koo, B.; Fahey, K. M.; Pandis, S. N., 2005. Development and Application of a Three-Dimensional Aerosol Chemical Transport Model, PMCAMx+, Not yet published.

Goebes, M.D., Strader R. and Davidson C., 2003. An Ammonia Emission Inventory For Fertilizer Application in the United States, Atmospheric Environment 37 (18), 25392550.

Graham, J., 2004. IMPROVE/STN Comparison \& Implications for Visibility and $\mathrm{PM}_{2.5}$. Presented at the MANE-VU/MARAMA 2004 Science Meeting, January 27-29, Baltimore, MD.

Grell, G.A.., Dudhia, J. and Stauffer, D. R., 1995. A Description of the Fifth-Generation Penn State/NCAR Mesoscale Model (MM5). NCAR/TN-398+STR. http://www.mmm.ucar.edu/mm5/documents/mm5-desc-doc.html.

Hildemann, L. M., Markowski, G. R. and Cass, G. R., 1991. Chemical Composition of Emissions From Urban Sources of Fine Organic Aerosol. Environmental Science and Technology 25 (4), 744-759.

Held, T.; Ying, Q.; Kaduwela, A.; Kleeman, M., 2004. Modeling Particulate Matter in the San Joaquin Valley With a Source-Oriented Externally Mixed Three-Dimensional Photochemical Grid Model. Atmospheric Environment 38 (22), 3689-3711.

Janssen, M., 2003. CEM Data Analysis and Use. Presented at the RPO National Technical Workgroup Meeting. St. Louis, MO. November 4-6, 2003. 
Kleeman, M. J., Cass, G. R. and Eldering, A., 1997. Modeling the Airborne Particle as a SourceOriented External Mixture. Journal of Geophysical Research-Atmospheres 102 (17), 21355-21372.

Kleeman, M. J. and Cass, G. R., 1998. Source Contribution to the Size and Composition Distribution of Urban Particulate Air Pollution. Atmospheric Environment 32 (16), 28032816.

Kleeman, M. J.; Hughes, L. S.; Allen, J. O.; Cass, G. R., 1999. Source Contributions to the Size and Composition Distribution of Atmospheric Particles: Southern California in September 1996. Environmental Science and Technology 33 (23), 4331-4341.

Kleeman, M.J. and Cass, G.R., 2001. A 3D Eulerian Source-Oriented Model For an Externally Mixed Aerosol. Environmental Science and Technology 35 (24), 4834-4848.

LADCO, 2003. Base E Modeling Inventory. Midwest Regional Planning Organization, Des Plaines, IL, September 16, 2003. http://www.ladco.org/tech/emis/BaseE/baseEreport.pdf

Pinder, R. W.; Pekney, N. J.; Davidson, C. I.; Adams, P. J., 2004. A Process Based Model of Ammonia Emissions from Dairy Cows: Improved Temporal and Spatial Resolution. Atmospheric Environment 38 (9),1357-1365.

Rogge, W. F.; Mazurek, M. A.; Hildemann, L. M.; Cass, G. R.; Simoneit, B. R. T., 1993a. Quantification of Urban Organic Aerosols at a Molecular Level: Identification, Abundance and Seasonal Variation. Atmospheric Environment 27A (8), 1309-1330.

Rogge, W. F.; Hildemann, L. M.; Mazurek, M. A.; Cass, G. R.; Simoneit, B. R. T., 1993 b. Sources of Fine Organic Aerosol. 5. Natural Gas Home Appliances. Environmental Science and Technology 27, 2736-2744.

Schauer, J. J.; Rogge, W. F.; Hildemann, L. M.; Mazurek, M. A.; Cass, G. R.; Simoneit, B. R. T., 1996. Source Apportionment of Airborne Particulate Matter Using Organic Compounds as Tracers. Atmospheric Environment 30 (22), 3837-3855.

Schauer, J. J. and Cass, G. R., 2000. Source Apportionment of Wintertime Gas-Phase and Particle-Phase Air Pollutants Using Organic Compounds as Tracers. Environmental Science and Technology 34 (9), 1821-1832.

Solomon, P. A.; Chameides, W.; Weber, R.; Middlebrook, A.; Kiang, C. S.; Russell, A. G.; Bulter, A.; Turpin, B.; Mikel, D.; Scheffe, R.; Cowling, E.; Edgerton, E.; St. John, J.; Jansen, J.; McMurry, P.; Hering, S.; Bahadori, T., 2003. Overview of the 1999 Atlanta Supersite Project. Journal of Geophysical Research 108 (7), SOS1/1-SOS 1/24.

Subramanian, R.; Donahue, N. M.; Bernardo-Bricker, A.; Rogge, W. F.; Robinson, A. L., 2005a. Selection of Source Profiles for Chemical Mass Balance Modeling using Organic Molecular Markers. In preparation.

Subramanian, R.; Donahue, N. M.; Bernardo-Bricker, A.; Rogge, W. F.; Robinson, A. L., $2005 b$. Source Apportionment of Gasoline and Diesel Vehicles using the Chemical Mass Balance Model and Molecular Markers. To be submitted to ES\&T.

Subramanian, R.; Donahue, N. M.; Bernardo-Bricker, A.; Rogge, W. F.; Robinson, A. L., 2005c. Source Apportionment of Primary Organic Aerosol in Pittsburgh, PA using organic molecular markers. In preparation. 
U.S. EPA, 2002a. 1999 National Emission Inventory Documentation and Data, Office of Air Quality Planning and Standards, U.S. Environmental Protection Agency Research Triangle Park, NC. http://www.epa.gov/ttn/chief/net/1999inventory.html

U.S. EPA, 2002b. NONROAD Model. U.S. Environmental Protection Agency, Office of Transportation and Air Quality: Ann Arbor, MI, 2002. http://www.epa.gov/oms/nonrdmdl.htm

U.S. EPA, 2002c. User's Guide to MOBILE6.1 and MOBILE6.2: Mobile Source Emission Factor Model, Office of Transportation and Air Quality, Ann Arbor, MI, October 2002; Report No. EPA420-R-02-028. http://www.epa.gov/otaq/m6.htm

Watson, J.G.; Robinson, N.F.; Chow, J.C.; Henry, R.C.; Kim, B.M.; Pace, T.G.; Meyer, E.L.; Nguyen, Q., 1990. The USEPA/DRI Chemical Mass Balance Receptor Model, CMB 7.0. Environmental Software 5, 38-49.

Wilkinson, J. and Janssen, M., 2001. BIOME3. Proceedings from the 10th International Emission Inventory Conference. Denver, CO, April 30, 2001.

Zheng, M.; Cass, G. R.; Schauer, J. J.; Edgerton, E. S., 2002. Source Apportionment of PM2.5 in the Southeastern United States Using Solvent-Extractable Organic Compounds as Tracers. Environmental Science and Technology 36 (11), 2361-2371. 


\section{Figure Captions}

Figure 1. Daily emission rates [tons day $^{-1}$ ] for primary OM and EC from the weekday emission inventory.

Figure 2. Predicted average ground-level $\mathrm{PM}_{2.5}$ total carbonaceous aerosol, SOA, primary OM, and EC concentrations [ $\mu \mathrm{g} \mathrm{m}^{-3}$ ] in the eastern US for the period from July 16 to July 282001. Values above the maximum of the colorbar are indicated with the same color.

Figure 3. Comparison of predicted $\mathrm{PM}_{2.5}$ total carbon, organic carbon (primary OM divide by 1.2 and SOA divided by 1.8), and elemental carbon concentrations to STN and IMPROVE measurements. Dashed lines represent $\pm 30 \%$.

Figure 4. Predicted average ground-level $\mathrm{PM}_{2.5}$ primary $\mathrm{OM}$ concentrations $\left[\mu \mathrm{g} \mathrm{m}^{-3}\right.$ ] from the boundary and initial conditions for the eastern US between July 16 and July 282001.

Figure 5. Predicted ground-level $\mathrm{PM}_{2.5}$ primary $\mathrm{OM}$ and EC concentrations during the simulation for Pittsburgh, PA.

Figure 6. Predicted daily average source contribution of primary OM and EC in the eastern US for July 12 to July 282001.

Figure 7. Predicted average $\mathrm{PM}_{2.5}$ primary OM concentrations $\left[\mu \mathrm{g} \mathrm{m}^{-3}\right.$ ] from each source for the eastern US between July 16 and July 282001.

Figure 8. Predicted average contribution of each source to the total $\mathrm{PM}_{2.5}$ primary OM concentrations for the eastern US between July 16 and July 282001.

Figure 9. Predicted average $\mathrm{PM}_{2.5} \mathrm{EC}$ concentrations $\left[\mu \mathrm{g} \mathrm{m}^{-3}\right.$ ] from each source for the eastern US between July 16 and July 282001.

Figure 10. Predicted average contribution of each source to the total $\mathrm{PM}_{2.5} \mathrm{EC}$ concentrations for the eastern US between July 16 and July 282001.

Figure 11. Predicted average primary OC and EC concentrations from each source in Pittsburgh for July 16 to July 282001 compared to CMB results. Primary OC calculated by dividing OM concentrations by 1.4. Diesel = Onroad and Offroad Diesel; and Wood Combustion = Biomass + Wood. For EC only: Others = Others + Natural Gas.

Figure 12. Predicted average primary OC and EC concentrations from each source in Atlanta for July 16 to July 282001 compared to CMB results. Primary OC calculated by dividing OM concentrations by 1.4. Diesel $=$ Onroad and Offroad Diesel; and Wood Combustion $=$ Biomass + Wood. For EC only: Others = Others + Dust + Gasoline + Natural Gas. 
Table 1. Contribution of source categories to primary OM and EC emissions across the entire domain

\begin{tabular}{|c|c|c|c|c|c|c|c|}
\hline \multirow[t]{2}{*}{ Name } & \multirow[t]{2}{*}{ Description } & \multicolumn{3}{|c|}{$\begin{array}{c}\text { Elemental } \\
\text { Carbon (\%) * }\end{array}$} & \multicolumn{3}{|c|}{$\begin{array}{l}\text { Primary Organic } \\
\text { Matter }(\%) * \\
\end{array}$} \\
\hline & & $\mathrm{W}$ & $\mathrm{Sa}$ & $\mathrm{Su}$ & $\mathrm{W}$ & $\mathrm{Sa}$ & $\mathrm{Su}$ \\
\hline Gasoline & $\begin{array}{l}\text { On-road, non-road, and } \\
\text { stationary gasoline combustion }\end{array}$ & 4 & 5 & 5 & 13 & 22 & 22 \\
\hline $\begin{array}{l}\text { On-road } \\
\text { diesel }\end{array}$ & $\begin{array}{l}\text { On-road transportation diesel } \\
\text { combustion }\end{array}$ & 26 & 25 & 24 & 7 & 6 & 5 \\
\hline $\begin{array}{l}\text { Off-road } \\
\text { diesel }\end{array}$ & $\begin{array}{l}\text { Non-road and stationary diesel } \\
\text { combustion }\end{array}$ & 53 & 51 & 53 & 14 & 11 & 12 \\
\hline Natural gas & Natural gas combustion & 0 & 0 & 0 & 23 & 21 & 21 \\
\hline Wood & $\begin{array}{l}\text { Residential fireplaces, wood } \\
\text { stoves, wood-fired boilers }\end{array}$ & 7 & 8 & 8 & 15 & 15 & 15 \\
\hline Biomass & $\begin{array}{l}\text { Open agricultural burning, } \\
\text { land-clearing, residential yard } \\
\text { waste }\end{array}$ & 4 & 4 & 4 & 6 & 6 & 6 \\
\hline Dust & Soil and road dust & 0 & 0 & 0 & 4 & 4 & 4 \\
\hline Other & All other sources & 6 & 7 & 6 & 18 & 15 & 15 \\
\hline Total & $\begin{array}{l}\text { Total emissions for entire } \\
\text { modeling domain [ktons/day] }\end{array}$ & 1.09 & 0.93 & 0.89 & 1.28 & 1.27 & 1.22 \\
\hline
\end{tabular}


Primary OM Emissions

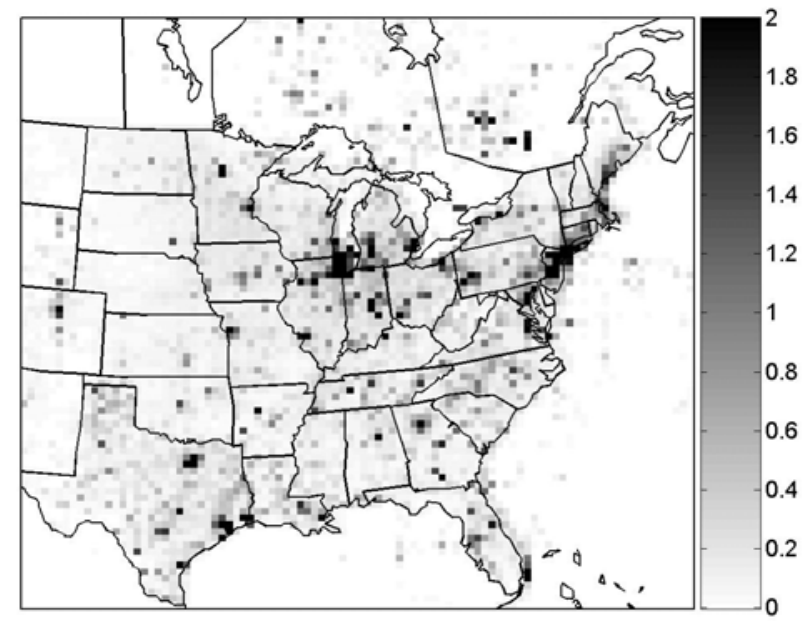

Elemental Carbon Emissions

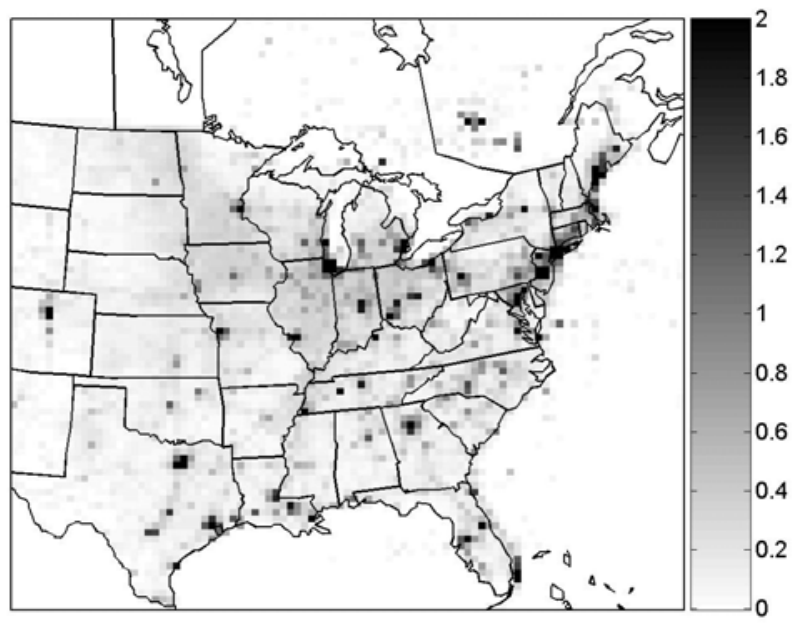

Figure 1. Daily emission rates [tons day ${ }^{-1}$ ] for primary OM and EC from the weekday emission inventory. 
Total Carbon
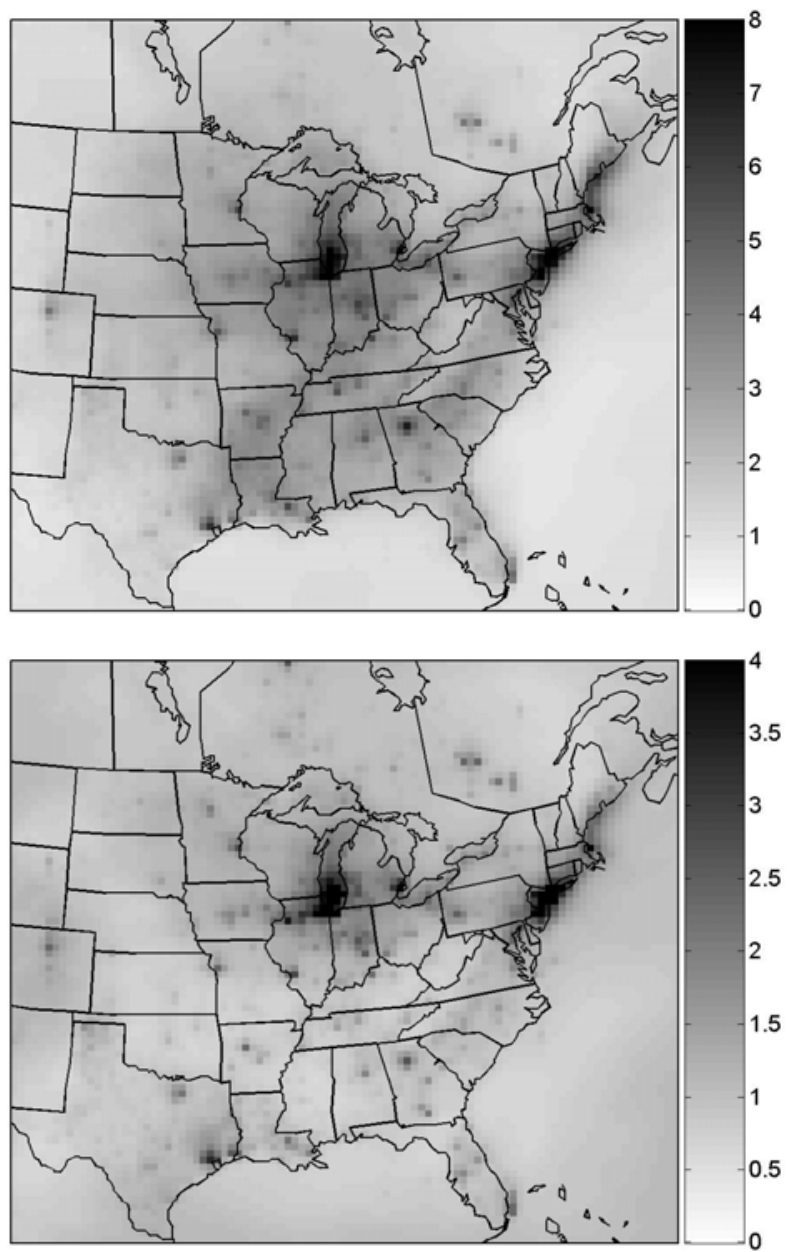

Primary Organic Carbon
Secondary Organic Carbon
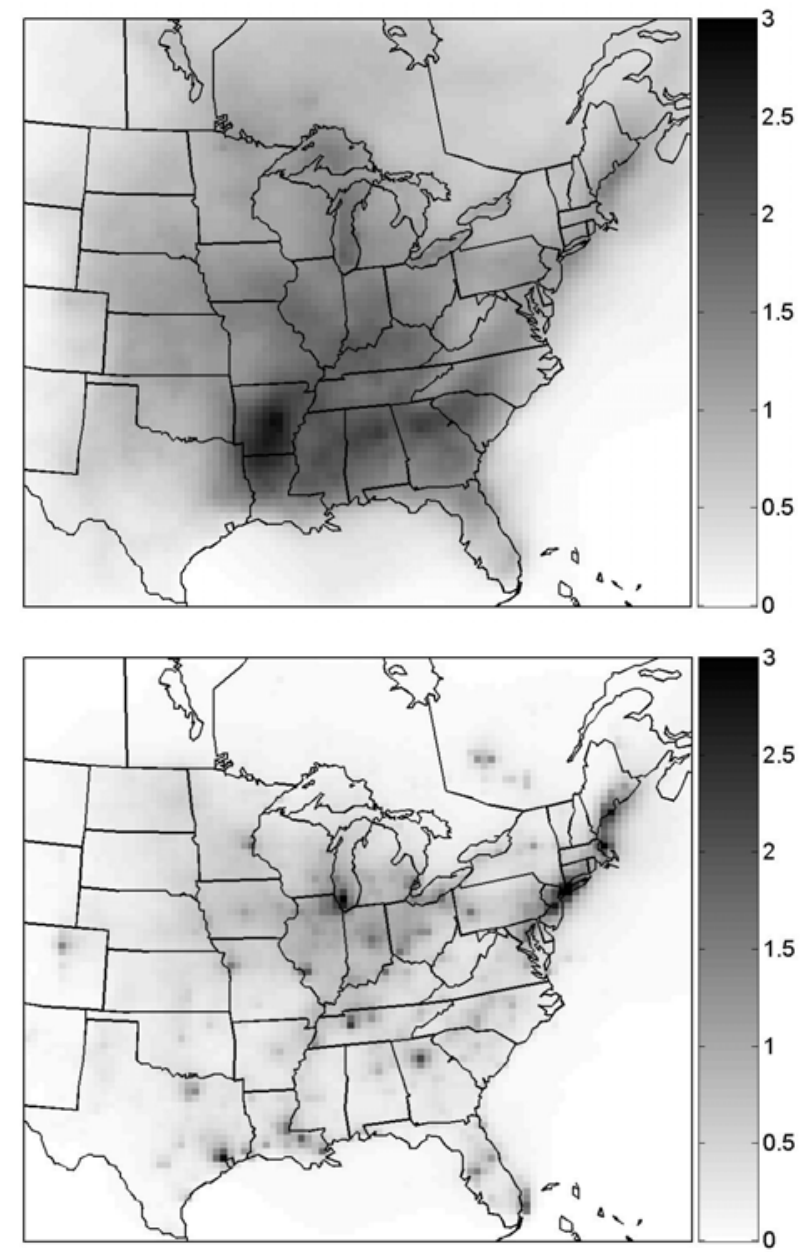

Elemental Carbon

Figure 2. Predicted average ground-level $\mathrm{PM}_{2.5}$ total carbonaceous aerosol, SOA, primary OM, and EC concentrations [ $\mu \mathrm{g} \mathrm{m}^{-3}$ ] in the eastern US for the period from July 16 to July 282001. Values above the maximum of the colorbar are indicated with the same color. 

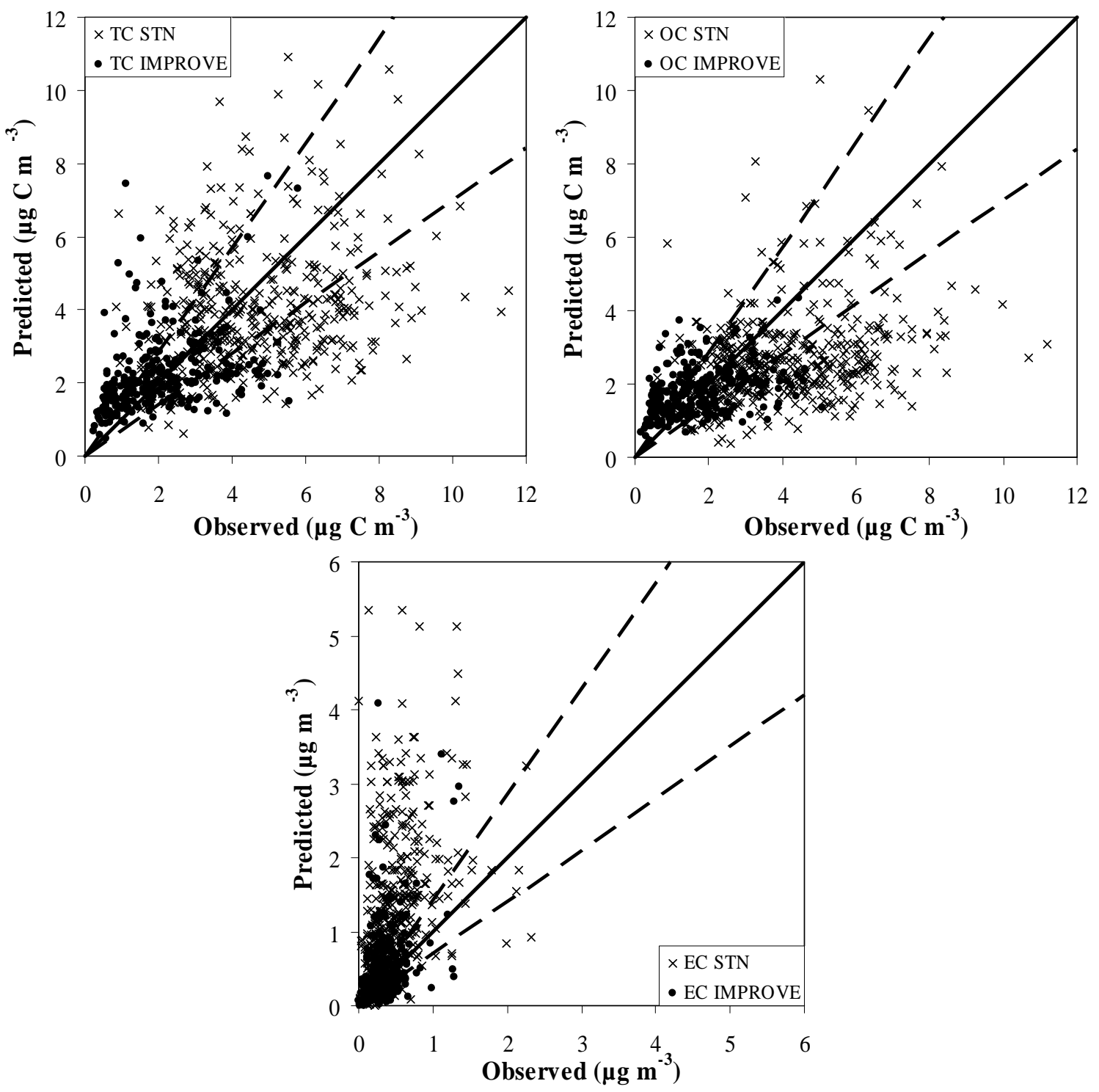

Figure 3. Comparison of predicted $\mathrm{PM}_{2.5}$ total carbon, organic carbon (primary OM divide by 1.2 and SOA divided by 1.8), and elemental carbon concentrations to STN and IMPROVE measurements. Dashed lines represent $\pm 30 \%$. 
Primary OM Boundary Conditions

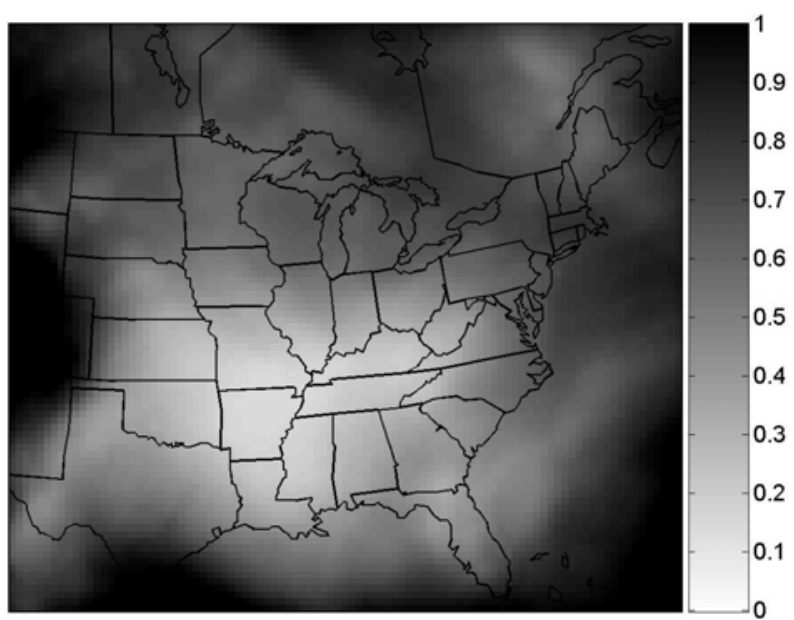

Primary OM Initial Conditions

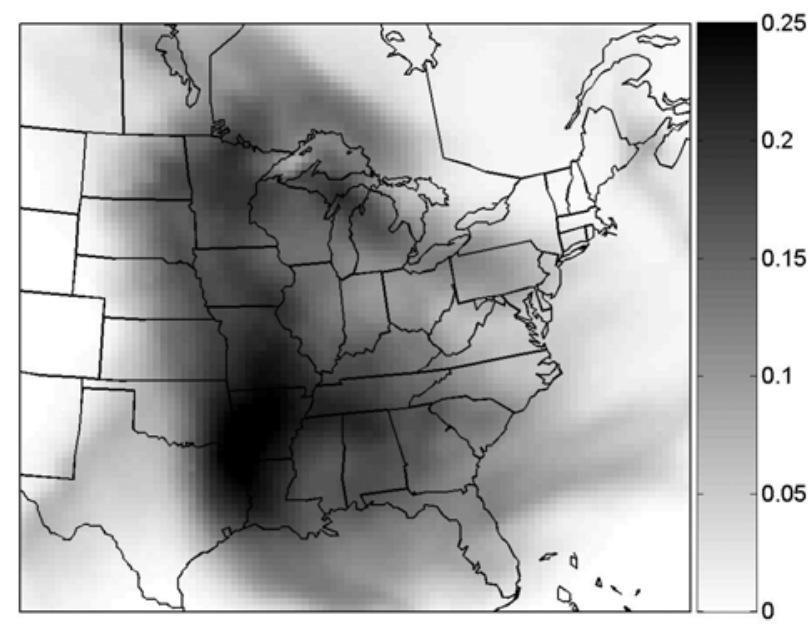

Figure 4. Predicted average ground-level $\mathrm{PM}_{2.5}$ primary OM concentrations $\left[\mu \mathrm{g} \mathrm{m}^{-3}\right.$ ] from the boundary and initial conditions for the eastern US between July 16 and July 282001. 

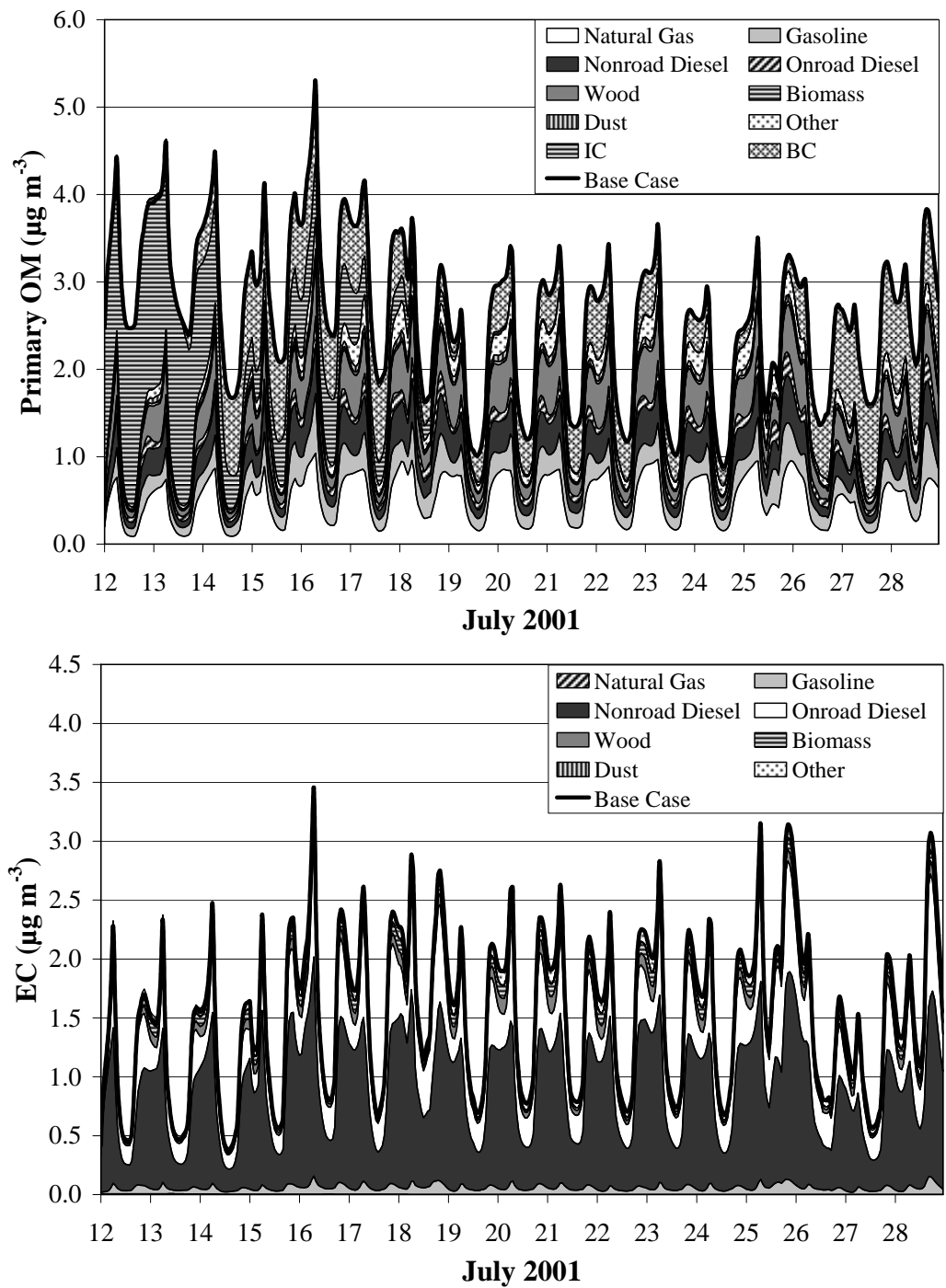

Figure 5. Predicted ground-level $\mathrm{PM}_{2.5}$ primary $\mathrm{OM}$ and EC concentrations during the simulation for Pittsburgh, PA. 


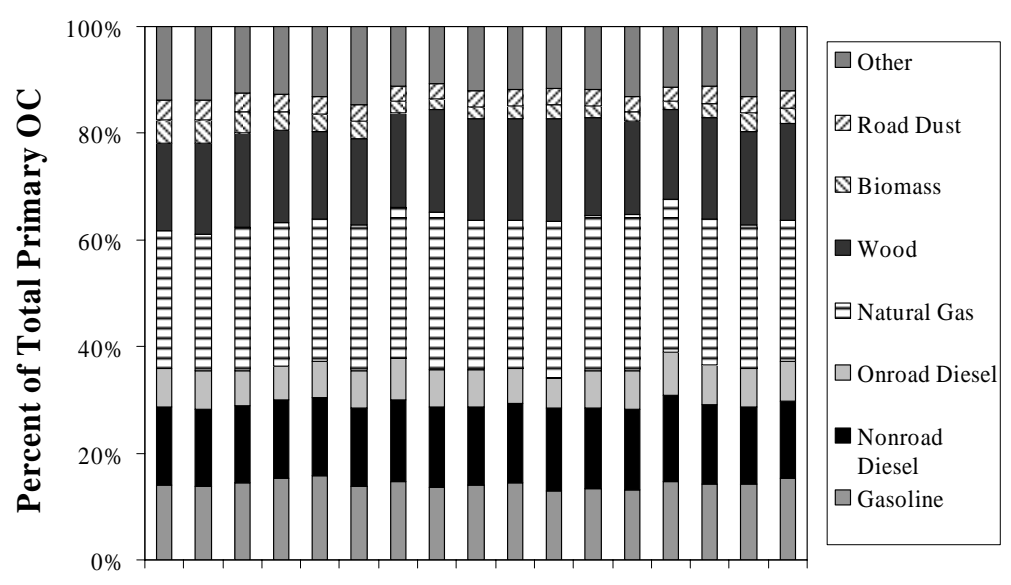

$\begin{array}{lllllllllllllllll}12 & 13 & 14 & 15 & 16 & 17 & 18 & 19 & 20 & 21 & 22 & 23 & 24 & 25 & 26 & 27 & 28\end{array}$ Julv 2001

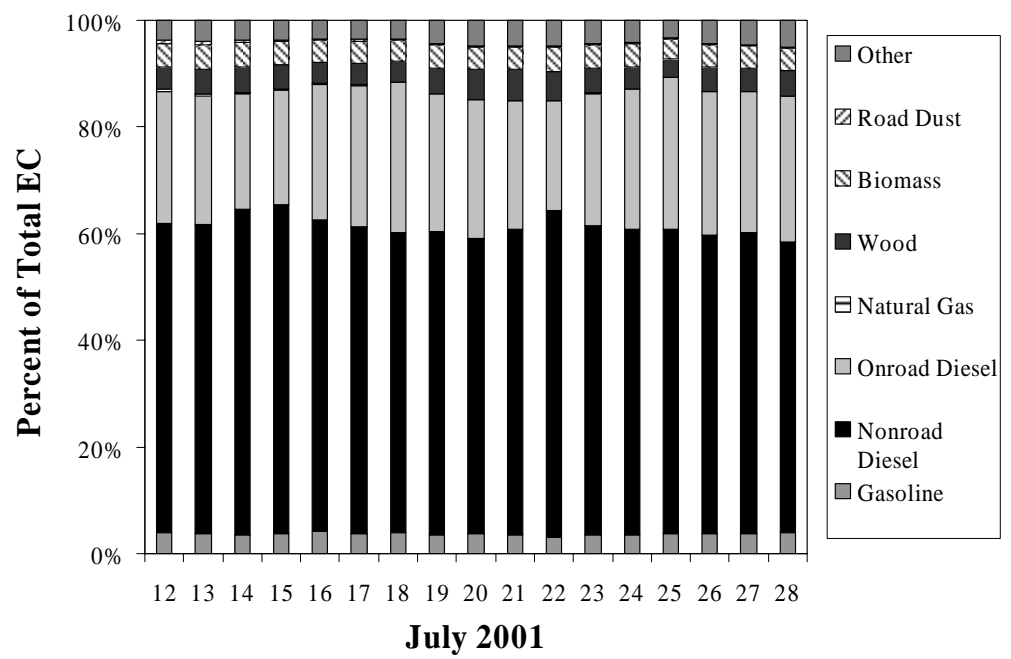

Figure 6. Predicted daily average source contribution of primary OM and EC in the eastern US for July 12 to July 282001. 

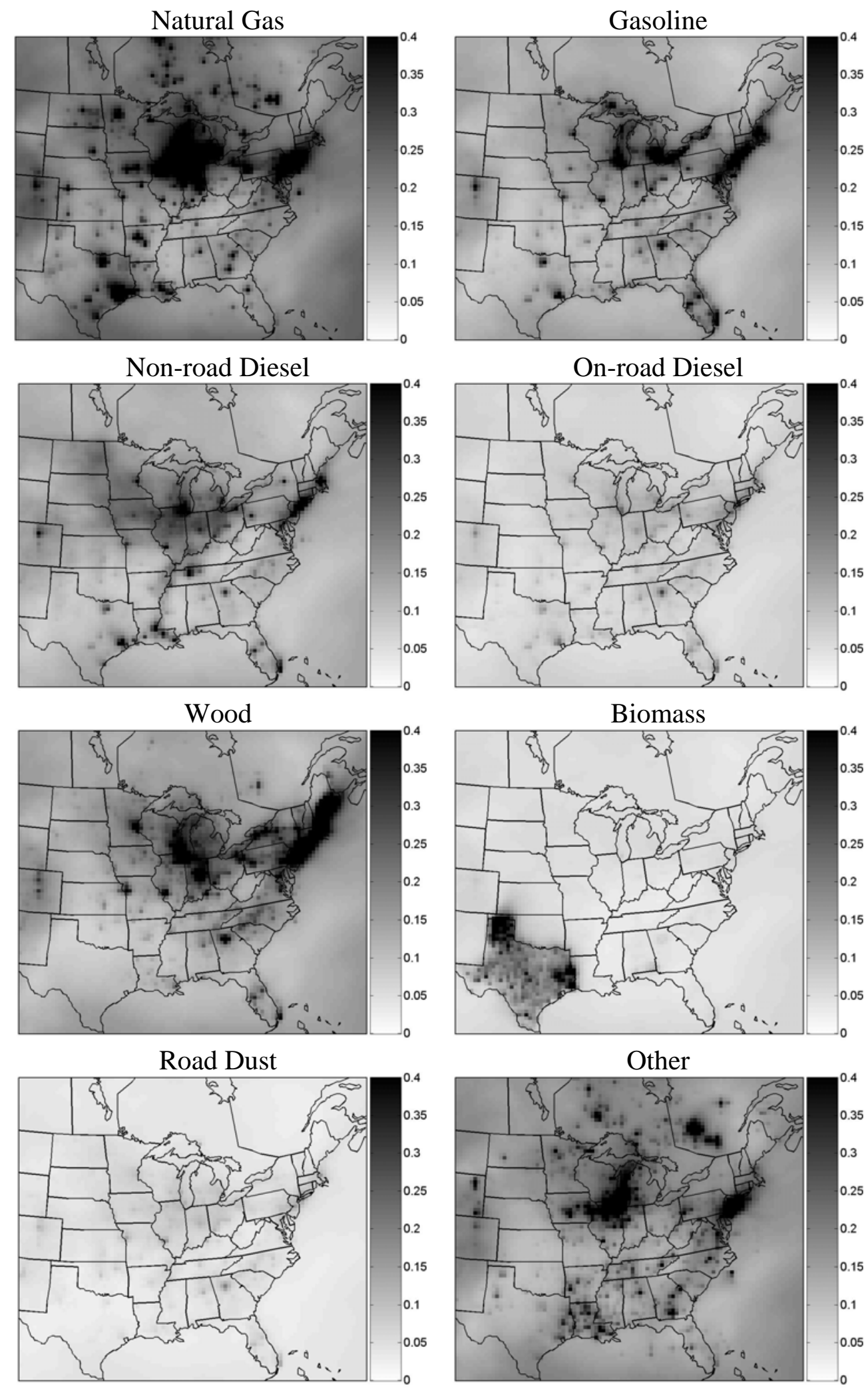

Figure 7. Predicted average $\mathrm{PM}_{2.5}$ primary OM concentrations $\left[\mu \mathrm{g} \mathrm{m}^{-3}\right]$ from each source for the eastern US between July 16 and July 282001. 
Natural Gas

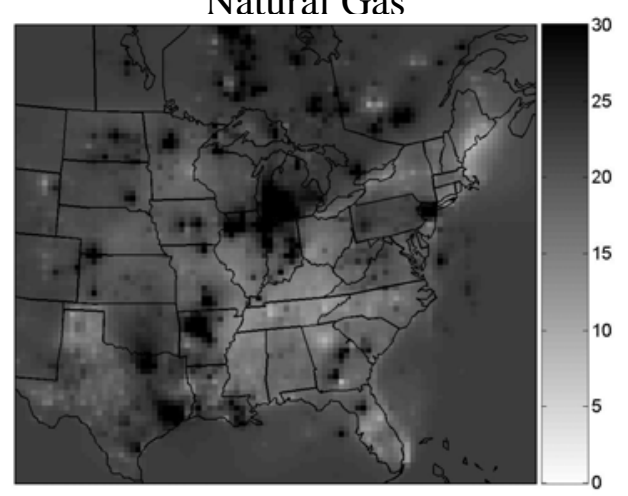

Non-road Diesel

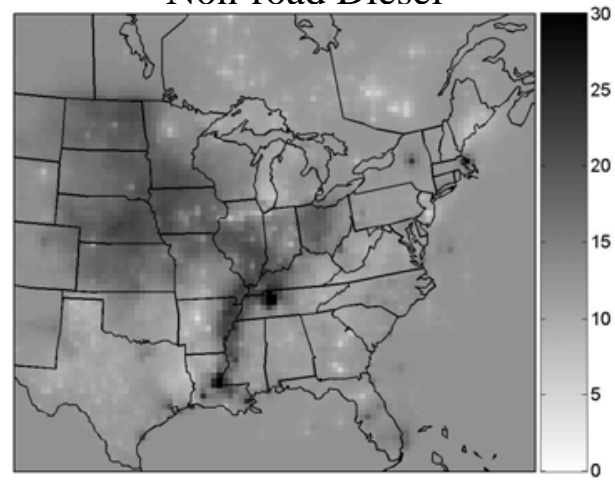

Wood

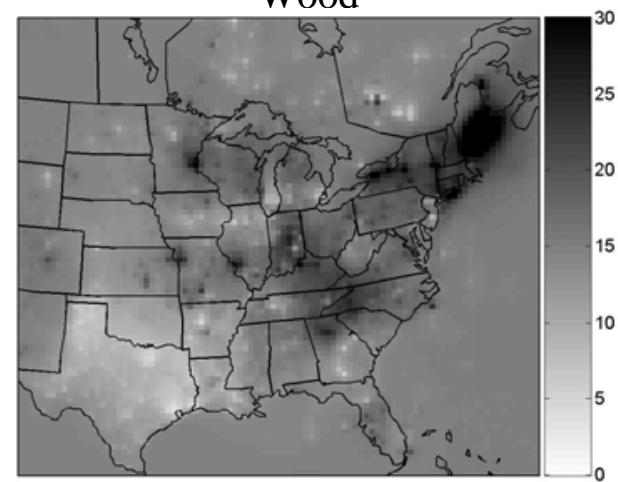

\section{Road Dust}

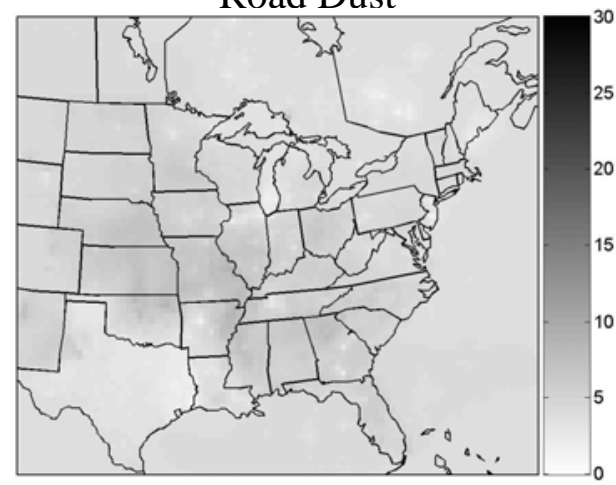

Gasoline
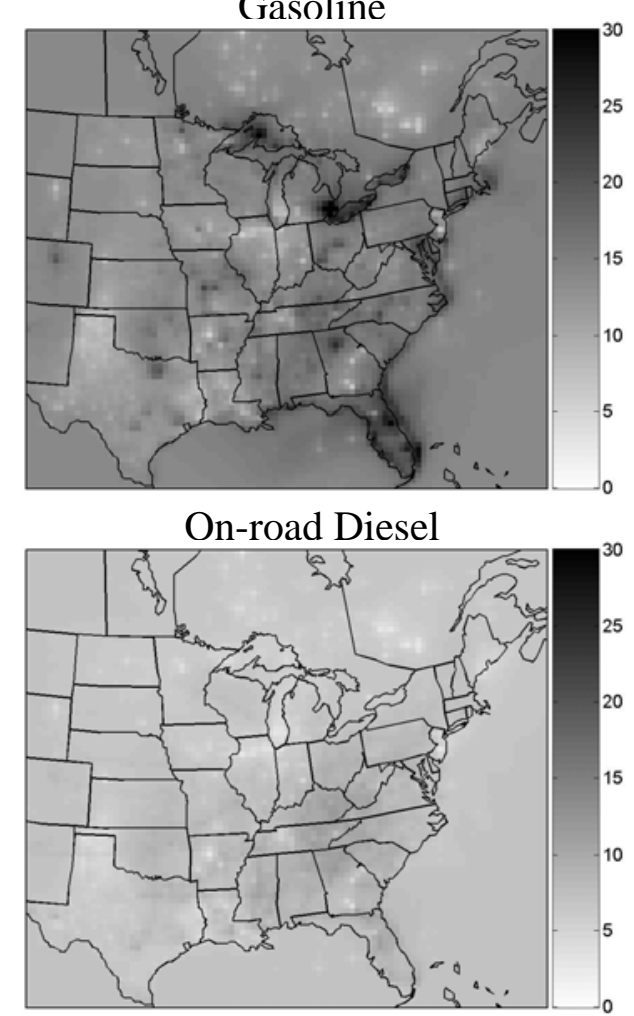

Biomass

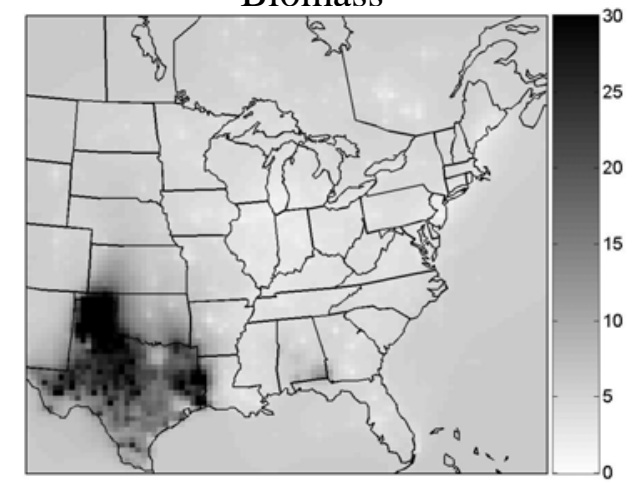

Other

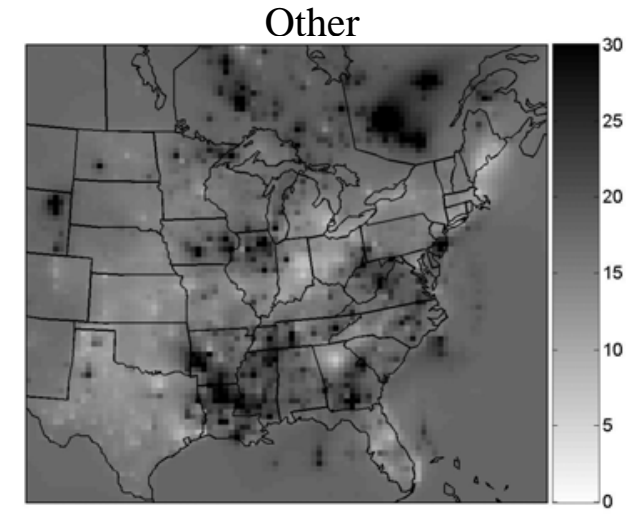

Figure 8. Predicted average contribution of each source to the total $\mathrm{PM}_{2.5}$ primary OM concentrations for the eastern US between July 16 and July 282001. 

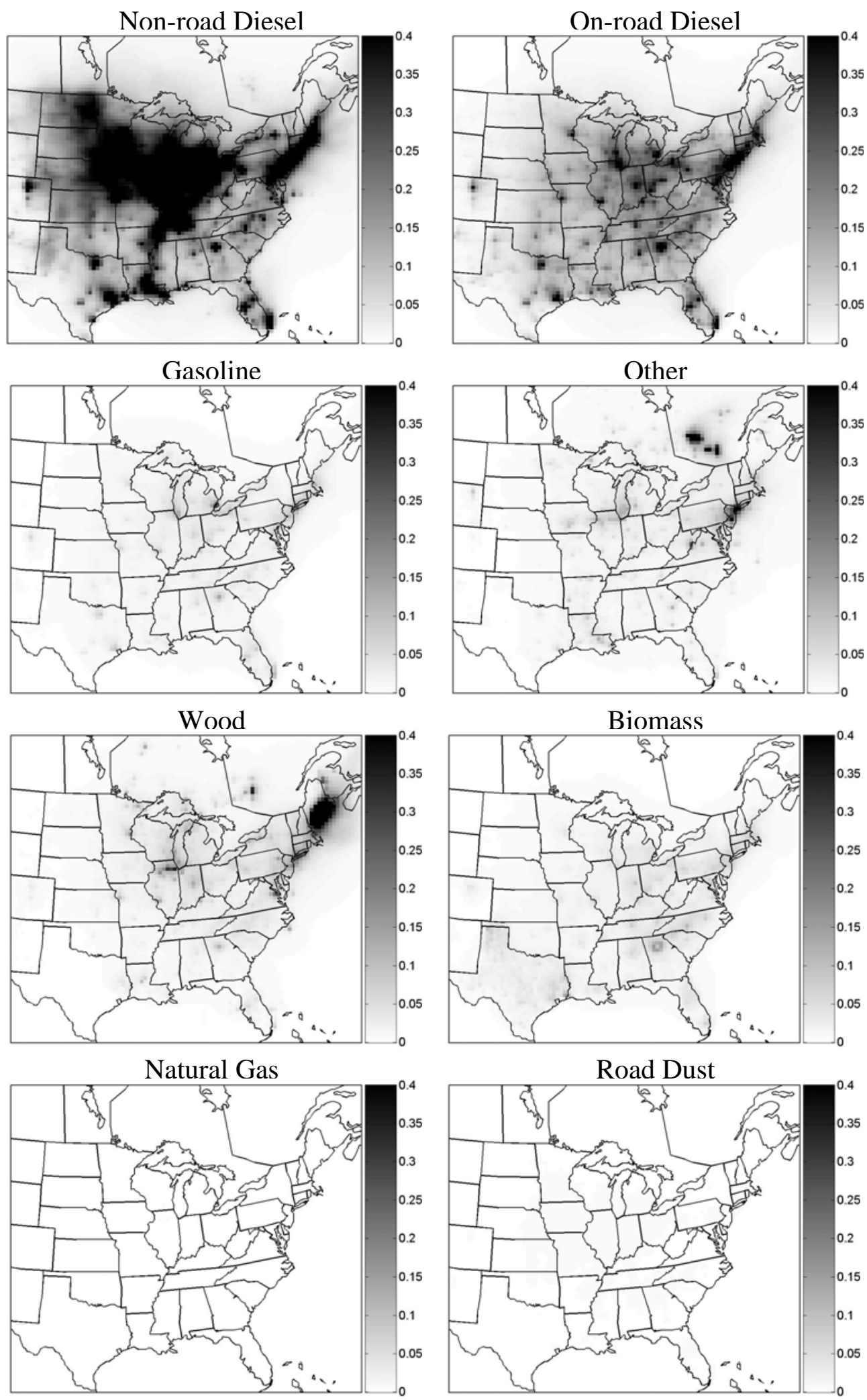

Figure 9. Predicted average $\mathrm{PM}_{2.5}$ EC concentrations $\left[\mu \mathrm{g} \mathrm{m}^{-3}\right.$ ] from each source for the eastern US between July 16 and July 282001. 

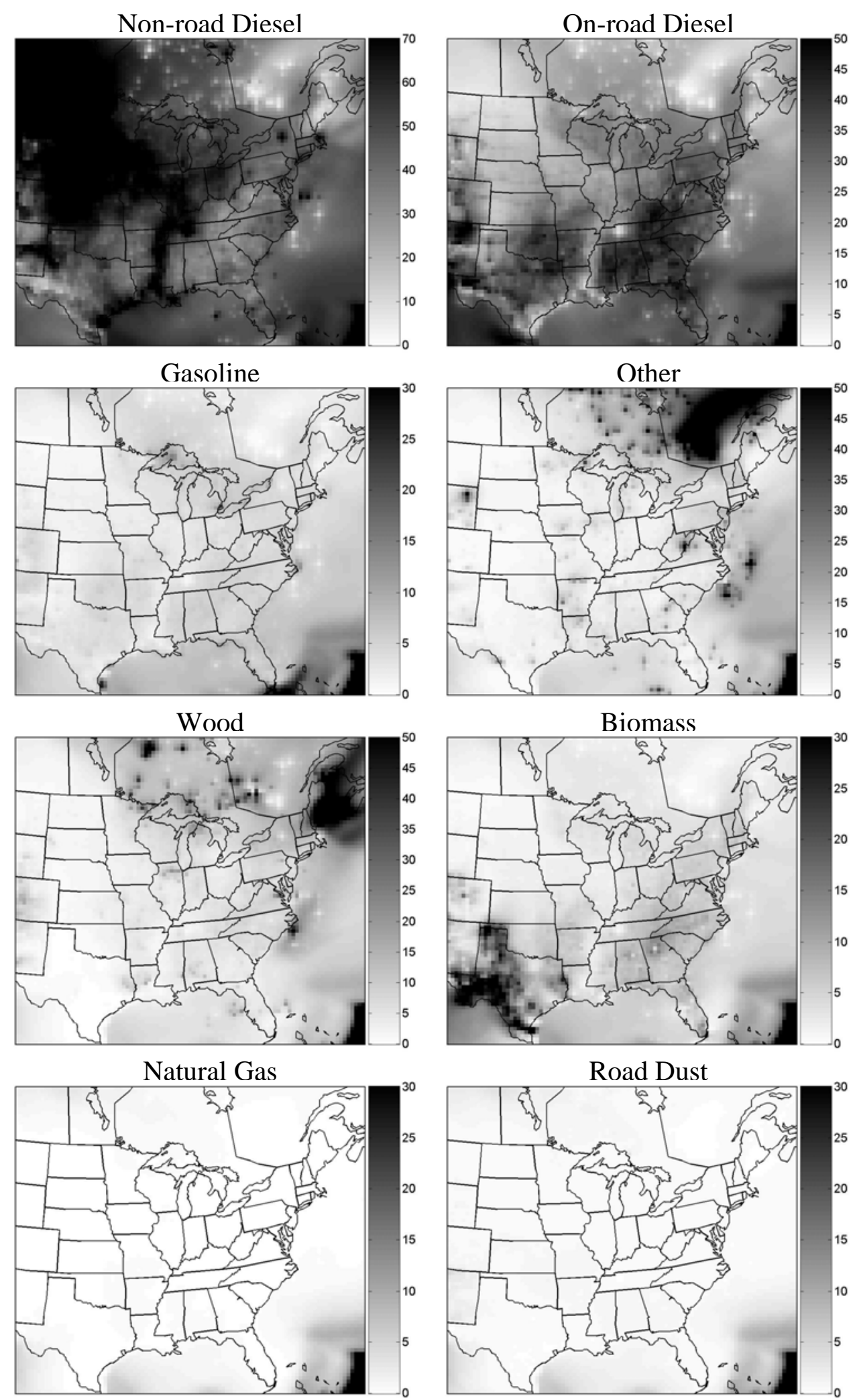

Figure 10. Predicted average contribution of each source to the total $\mathrm{PM}_{2.5} \mathrm{EC}$ concentrations for the eastern US between July 16 and July 282001. 

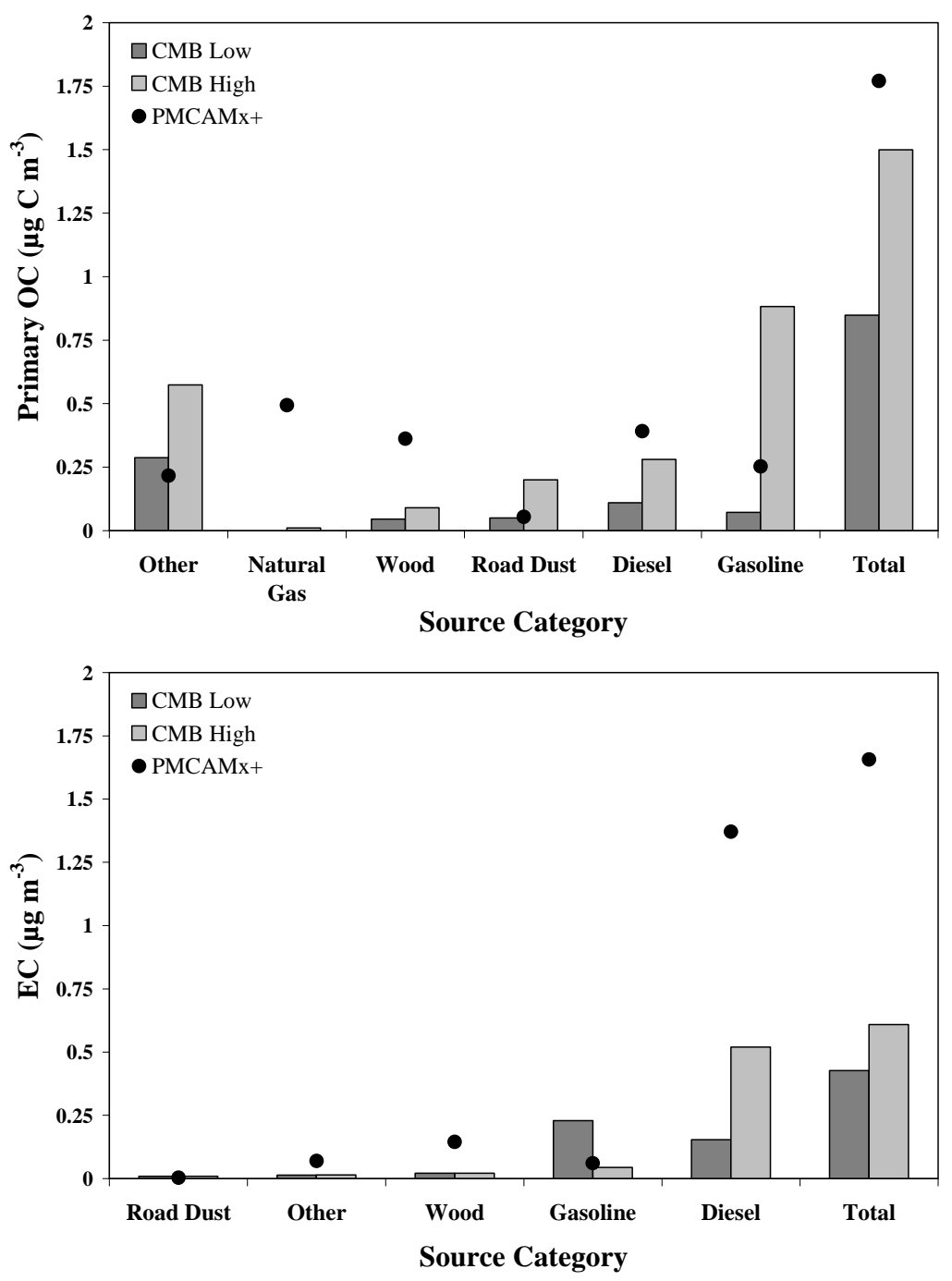

Figure 11. Predicted average primary OC and EC concentrations from each source in Pittsburgh for July 16 to July 282001 compared to CMB results. Primary OC calculated by dividing OM concentrations by 1.4. Diesel $=$ Onroad and Offroad Diesel; and Wood Combustion = Biomass + Wood. For EC only: Others = Others + Natural Gas. 

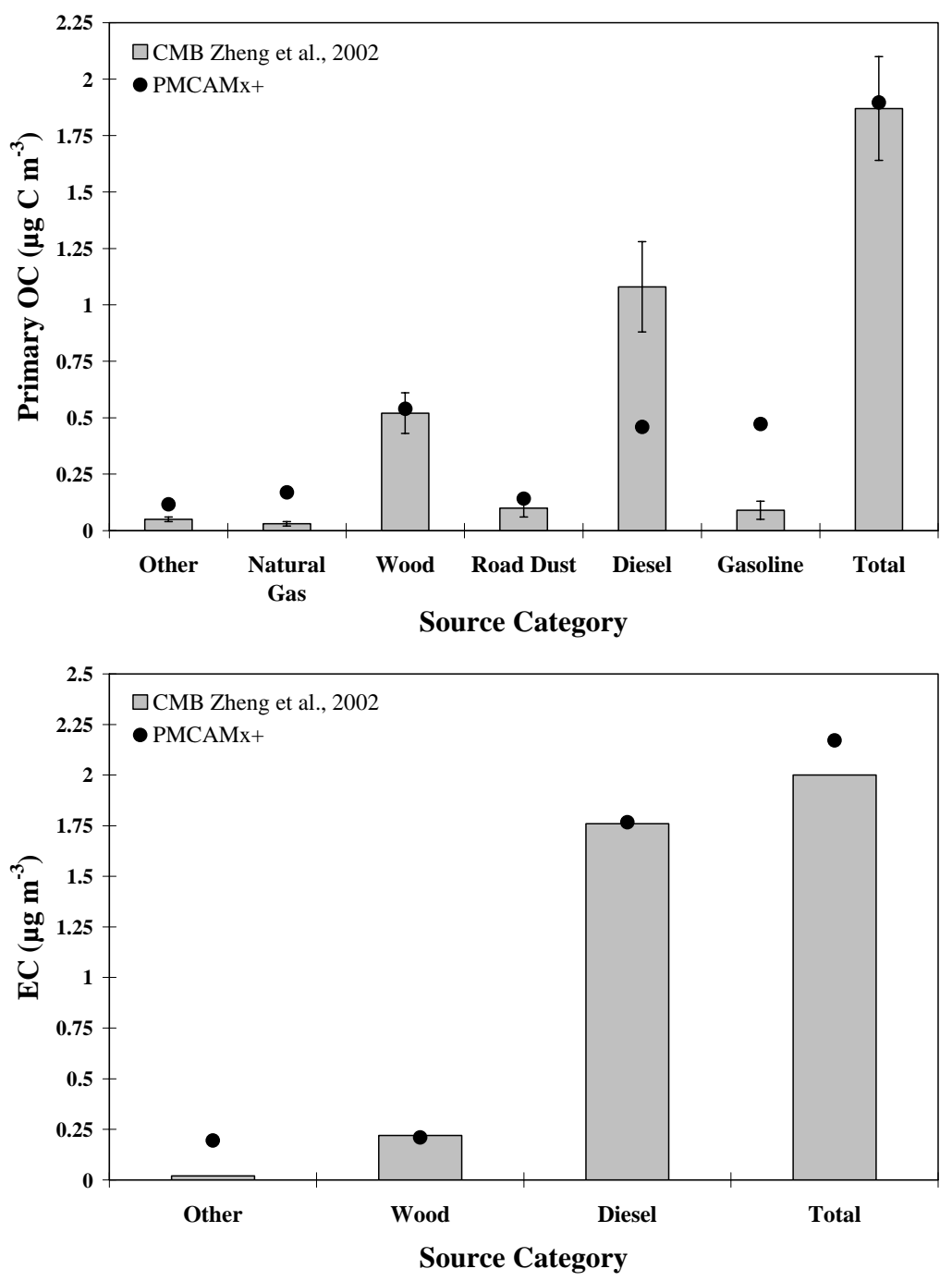

Figure 12. Predicted average primary OC and EC concentrations from each source in Atlanta for July 16 to July 282001 compared to CMB results. Primary OC calculated by dividing OM concentrations by 1.4. Diesel $=$ Onroad and Offroad Diesel; and Wood Combustion $=$ Biomass + Wood. For EC only: Others = Others + Dust + Gasoline + Natural Gas. 


\title{
Water content of ambient aerosol during the Pittsburgh Air Quality Study
}

\author{
Andrey Khlystov \\ Department of Civil and Environmental Engineering, Duke University, Durham, North Carolina, USA
}

Charles O. Stanier, Satoshi Takahama, and Spyros N. Pandis

Department of Chemical Engineering, Carnegie Mellon University, Pittsburgh, Pennsylvania, USA

Received 16 February 2004; revised 4 June 2004; accepted 22 June 2004; published 3 March 2005.

[1] The aerosol water content and volumetric growth factors of fine particulate matter were measured during July-August 2001 and January-June 2002 in an urban park about $6 \mathrm{~km}$ from downtown Pittsburgh, Pennsylvania. Most of the aerosol during the study was transported to the region from other areas, and its composition and concentration were characteristic of the regional particulate matter in the northeastern United States. During the summer months the ambient aerosol practically always contained water even when the relative humidity $(\mathrm{RH})$ was as low as $30 \%$. In contrast, during the winter the aerosol was dry below $60 \% \mathrm{RH}$. The spring months were characterized by a transitional behavior between these two states. The observed seasonal behavior can be explained by the aerosol acidity. The summer aerosol was acidic and retained water at low RH. The winter aerosol was neutral and became wet when the relative humidity reached the deliquescence point

of ammonium nitrate. The observations during July 2001 were compared with the predictions of the thermodynamic Gibbs Free Energy Minimization (GFEMN) model and the aerosol inorganics model (AIM), neglecting the organic aerosol contribution to water absorption. The models under-predicted water concentrations by about $35 \%$, but no clear correlation between organic mass and the excess water was observed. On average, the contribution of the organics to water absorption appeared to be higher during the afternoon hours and when the aerosol was presumably more oxidized.

Citation: Khlystov, A., C. O. Stanier, S. Takahama, and S. N. Pandis (2005), Water content of ambient aerosol during the Pittsburgh Air Quality Study, J. Geophys. Res., 110, D07S10, doi:10.1029/2004JD004651.

\section{Introduction}

[2] Absorption of water by aerosol particles has a major effect on their physical and chemical properties. Water increases particle size and thus affects its lifetime. The light scattering and, consequently, the visibility reduction and direct climate forcing by the aerosol particles depend strongly on their water content [Ramaswamy, 2001]. The presence of water changes the partitioning of semivolatile species between the gas and aerosol phase and affects the particle composition [Ansari and Pandis, 2000]. In addition, aerosol water provides a medium for heterogeneous chemical reactions in the atmosphere.

[3] The hygroscopic behavior (absorption of water) of aerosol particles exhibits a hysteresis. A dry single-salt particle becomes wet at a certain relative humidity, the so-called deliquescence relative humidity. For example, the deliquescence point of ammonium sulfate at $25^{\circ} \mathrm{C}$ is at approximately $80 \% \mathrm{RH}$ [Tang and Munkelwitz, 1994]. After becoming wet, the particle will absorb more water if the relative humidity is increased. If the relative humidity is

Copyright 2005 by the American Geophysical Union. 0148-0227/05/2004JD004651\$09.00 then decreased, the particle will release some of the absorbed water to equilibrate with the new conditions. However, if the relative humidity is decreased below the deliquescence point, the particle will not crystallize but it will remain wet until a substantially lower RH, $35-40 \%$ for ammonium sulfate. Most other inorganic species present in the ambient aerosol have a similar behavior [Tang, 1997]. Because of the hysteresis effect even for ammonium sulfate particles there is a wide range of relative humidity (40$80 \%$ ) within which the physical state (dry or wet) of the ambient particles is not known. The crystallization point of the ambient aerosol is also uncertain. It has been shown that the presence of other components such as minerals can affect the crystallization relative humidity [Han et al., 2002; Martin et al., 2001]. Organic compounds may also affect deliquescence and crystallization points of inorganic salts [Choi and Chan, 2002]. All of this leads to uncertainties in predicting aerosol properties that depend on absorbed water, including partitioning of semivolatile compounds and light scattering.

[4] In addition to the uncertainty in the physical state of the particles, there is difficulty in predicting the amount of water that would be present in the aerosol phase at given atmospheric conditions. The difficulty arises from the 
multicomponent nature of the aerosol and high concentrations of dissolved species. The role of the organic compounds in water absorption is especially uncertain, with a number of recent investigations reaching differing conclusions regarding the role of organics in water uptake. Saxena et al. [1995] reported that organics at a remote continental location increased water absorption by $30 \%$ or so at $85 \%$ relative humidity, while at an urban location, organics decreased overall water absorption by approximately $30 \%$ at $85 \%$ RH. Dick et al. [2000] reported, for a rural continental site, organics contributed significantly to water uptake at relative humidity below $50 \%$ and less significantly at higher relative humidity. In a modeling study, Ansari and Pandis [2000] estimated that a high loading (35\% by mass) of secondary organic compounds increased water content by about $20 \%$ at $50 \%$ relative humidity, with smaller effects at higher relative humidity. Laboratory studies by Cruz and Pandis [2000] and Choi and Chan [2002] have demonstrated that the presence of organic substances in the mixed particles may either reduce or enhance water absorption by inorganic salts.

[5] The hygroscopic aerosol growth is usually studied with humidified tandem differential mobility analyzers (H-TDMAs). H-TDMAs select a narrow size range of particles and subject it to a certain relative humidity, after which the sizes of the grown particles are measured [Cocker et al., 2001; Rader and Mcmurry, 1986; Swietlicki et al., 1999]. The H-TDMA studies show that particles are often externally mixed with respect to their water absorption properties, i.e., there are more hygroscopic and less hygroscopic fractions. The number of fractions and their behavior varies with time, place and size [Cocker et al., 2001]. The H-TDMA studies, however, can provide information on the hygroscopic properties of only a few size classes and they do not measure directly the aerosol water concentration. Nessler et al. [2003] have measured aerosol size distributions at ambient and indoor (heated and dry) conditions to investigate size changes due to the hygroscopic growth of the entire size spectrum. The observed shift of the distribution was consistent with the measured growth factors using an H-TDMA. However, due to the heating, considerable losses of particles smaller than $100 \mathrm{~nm}$ were observed.

[6] In this study we have used the recently developed dry and ambient aerosol size spectrometer (DAASS) that measures aerosol size distributions at ambient and low $(<35 \%$ $\mathrm{RH})$ relative humidity conditions without substantial changes in sample temperature [Stanier et al., 2004]. The DAASS provides information on the effect of ambient humidity on the whole size distribution, the volumetric growth factors and the amount of water present in the aerosol phase. Here we report our observations using the DAASS during the Pittsburgh Air Quality Study (PAQS).

\section{Experiment}

\subsection{Sampling Site}

[7] The measurements were carried out continuously during July-August 2001 and January-October 2002 at the central monitoring site of the PAQS located in an urban park, approximately $6 \mathrm{~km}$ from downtown Pittsburgh, PA. The city is located between the utilities and agricultural sources of the Midwest and the large urban centers of the eastern United States. Roughly one kilometer of parkland exists between the site and the residential areas in the predominant upwind direction (south and west). The site was several hundred meters from any major sources.

[8] The average $\mathrm{PM}_{2.5}$ mass concentration in the summer was $18.2 \mu \mathrm{g} / \mathrm{m}^{3}$ and $12.2 \mu \mathrm{g} / \mathrm{m}^{3}$ in the winter. Sulfate was the major aerosol constituent comprising over $45 \%$ of the $\mathrm{PM}_{2.5}$ mass in the summer and $35 \%$ during the winter, while the organic material contributed approximately $20 \%$ and ammonium $15-17 \%$. The contribution of nitrate during summer was small (less than 3\%). However, during the winter it contributed $15 \%$ of $\mathrm{PM}_{2.5}$ mass. The crustal material contributed 3-6\% to $\mathrm{PM}_{2.5}$ mass [Rees et al., 2004].

[9] During the summer, long-range transport was the major secondary aerosol source. For example, concentrations of $\mathrm{PM}_{2.5}$ mass, sulfate, ammonium, and organic carbon were measured to be the same within experimental error at six sampling locations in and around Pittsburgh separated by more than $300 \mathrm{~km}$ [Tang et al., 2004]. However, less abundant species such as nitrate and elemental carbon were more affected by the local emissions. The contribution of local sources was stronger during winter than in summer.

\subsection{DAASS}

[10] Measurements of water content of ambient aerosol and its hygroscopic growth were made using a dry and ambient aerosol size spectrometer (DAASS), which is described in detail by Stanier et al. [2004]. The DAASS measures alternatively the aerosol size distribution at ambient and at low RH $(18 \pm 5 \%)$ conditions (referred to as ambient and dried, respectively). A comparison of the dried and ambient distributions provides information on both the amount of absorbed water and the change in aerosol size with RH. The amount of absorbed water can be calculated from the difference in the aerosol volumes at ambient conditions and at the low RH state. The changes in size can be deduced from the mass or number mean diameters, shifts in the position of different modes in the distribution, etc.

[11] The DAASS consists of two SMPS (TSI 3936N25 and TSI 3936L10, TSI Inc.) and an APS system (TSI 3320, TSI Inc.) that measure the aerosol size distribution in the range of $3 \mathrm{~nm}$ to $10 \mu \mathrm{m}$. The APS and the SMPS data were merged using the algorithm of Khlystov et al. [2004]. During the dried measurements the sample flow and the sheath flows of the instruments were directed through a set of nafion driers (Permapure MD-110, Toms River, NJ) such that the relative humidity was reduced below $25 \%$ while the temperature remained virtually unchanged. Multichannel dryers (Permapure PD-50T and PD-200T) were used in the sheath lines of the instruments. During the ambient measurements the dryers were bypassed and the aerosol was directed to the instruments at conditions close to the ambient. The switching between the dried and ambient measurements was accomplished by means of computercontrolled 3-way solenoid valves. Four alternating dried and ambient measurements each were made during one hour.

[12] To avoid large deviations from the ambient conditions in temperature and, thus, relative humidity the DAASS was placed in a $3.6 \mathrm{~m}^{3}$ plywood enclosure on the roof of the central sampling station of the PAQS in Pittsburgh, PA. The 
enclosure was actively ventilated with a large $35 \mathrm{~m}^{3} / \mathrm{min}$ fan. Despite the precautions taken, the temperature of the system was about $4^{\circ} \mathrm{C}$ higher than the ambient during July 2001. During that time the ambient scans reached only approximately $80 \%$ of the ambient relative humidity (e.g., outdoor relative humidity of $95 \%$ yielded an ambient channel RH of around 76\%). Starting August 2001, the DAASS was modified by placing the DMA columns of the SMPS systems outside the enclosure with fans pulling ambient air over them. This way aerosol classification was done very close to the ambient temperature and the system achieved greater than $90 \%$ of ambient relative humidity (e.g., outdoor relative humidity of $95 \%$ yielded an ambient channel RH of greater than $86 \%$ ). The discrepancy in RH was due to the inlet and sheath lines being still inside the enclosure. The RH of the measurements will be used for the reporting of the data and the analysis in the rest of the paper.

[13] During winter, the enclosure was maintained at a minimum temperature of $9^{\circ} \mathrm{C}$ which was required for the correct operation of the condensation particle counters. This did not significantly affect the final sheath and aerosol relative humidity because the DMA columns were outside the enclosure at (or close to) the outdoor temperature. However, the aerosol flow did pass through the heated enclosure, which exposed the aerosol to a lower than ambient RH. Even though this did not affect the RH of the measurements, it might induce crystallization of aerosol at low RH. For this reason, the lowest RH encountered by the aerosol within the system is taken into account during the analysis of the data.

[14] Uncertainties in the DAASS measurements are described in detail by Stanier et al. [2004]. For an accurate determination of the water amount in the aerosol phase it is important that there is no significant bias between the dried and ambient channels of the DAASS. It was shown that no such significant bias exists and the number concentrations of the channels are within $5 \%$ of each other. The other sources of error are associated with the differences in temperature between aerosol charging and actual measurements during winter months when the ambient temperature was below $9^{\circ} \mathrm{C}$. The magnitude of this error was estimated using the study average size distribution and a composition of $50 \%$ ammonium sulfate and $50 \%$ organics and other nonhygroscopic aerosols. It was shown that at low ambient RH (around 50\%) the volume growth factor is biased positively by about $3 \%$. The maximum error is expected at low temperatures and high relative humidity conditions. For example, in the worst-case scenario, at $-5^{\circ} \mathrm{C}$ and $92 \%$ $\mathrm{RH}$, the error in the wet aerosol state is estimated to be $14 \%$ [Stanier et al., 2004].

\subsection{DAASS Data Reduction and Interpretation}

[15] The DAASS data reduction is reported in detail by Stanier et al. [2004]. For this work we have calculated the amount of water in the aerosol phase and the volume-based growth factors. These calculations are described briefly below.

[16] The amount of aerosol water can be found from the comparison of integrated dried and ambient aerosol volumes. Assuming volume additivity, the increase in volume between the ambient and the dried measurements is proportional to the mass of absorbed water (the proportionality factor being the density of liquid water). Consequently, the amount of absorbed water can be calculated as:

$$
m_{w}=\rho_{w}\left(V_{\text {wet }}-V_{\text {dried }}\right)
$$

in which $m_{\mathrm{w}}$ is the mass concentration of water, $\rho_{\mathrm{w}}$ is the density of liquid water, $V_{\text {wet }}$ and $V_{\text {dried }}$ are, respectively, the ambient and dried integrated volume concentrations. The assumption of volume additivity may introduce a small error, because the actual solution may deviate from ideal. However, the error due to the assumption of additivity is negligibly small [Dick et al., 2000].

[17] When integrating the volume distributions to obtain $V_{\text {wet }}$ and $V_{\text {dried, }}$, the shift in aerosol size distribution due to the hygroscopic growth needs to be accounted for. This is done by iteration, searching for the size boundaries that satisfy the following criteria:

$$
\begin{gathered}
V_{w e t}=\frac{\pi}{6} \int_{D w 1}^{D w 2} D^{3} n_{w e t}(D) d D, \\
V_{d r y}=\frac{\pi}{6} \int_{D d 1}^{D d 2} D^{3} n_{d r y}(D) d D, \\
G F_{v}=\frac{V_{w e t}}{V_{d r y}}, \\
D_{w 2}=D_{d 2} \sqrt[3]{G F_{v}},
\end{gathered}
$$

in which $G F_{\mathrm{v}}$ is the volumetric growth factor, $D$ is the particle size, $n_{\text {wet }}(D)$ and $n_{\text {dry }}(D)$ are the number-based ambient and dried size distributions, respectively, subscripts $w$ and $d$ correspond to the ambient and dried integration limits. To simplify calculations the lower integration limits $\left(D_{d 1}\right.$ and $\left.D_{w 1}\right)$ are set to be both equal to $5 \mathrm{~nm}$. A more accurate calculation would require correcting the lower boundaries using equation (5), however, because of the negligible amount of aerosol mass below $10 \mathrm{~nm}$, the error introduced by this simplification is insignificant.

[18] The amount of absorbed water was calculated from the DAASS measurements for the wet size range up to $2.5 \mu \mathrm{m}$ (i.e., $D_{w 2}=2.5 \mu \mathrm{m}$ ). This was done to allow a direct comparison of the amount of water to measured $\mathrm{PM}_{2.5}$ concentrations of individual chemical species. However, due to the frequent malfunction of the Aerosol Particle Sizer in the DAASS, consistent measurements of water content in the $\mathrm{PM}_{2.5}$ range are only available for July and August 2001. For the rest of the study the measurements extended only up to $0.5 \mu \mathrm{m}$ in diameter (ambient size) and the comparison with $\mathrm{PM}_{2.5}$ measurements was not possible.

\subsection{Complementary Measurements}

[19] The amount of absorbed water as a function of ambient RH was related to the amount of water-soluble inorganic constituents and the amount of organic matter present in the $\mathrm{PM}_{2.5}$ aerosol. These aerosol constituents were measured in parallel to and at the same location as the 
DAASS measurements. The measurements of chemical constituents are described by Wittig et al. [2004]. Aerosol sulfate and nitrate were measured with a denuder filter-pack system and at a higher time resolution with Rupprecht and Patashnick (R\&P) models 8400S and 8400N (R\&P Co., Albany, NY). The $\mathrm{PM}_{2.5}$ aerosol nitrate and ammonium and the gas phase nitric acid and ammonia were measured with a steam sampler [Khlystov et al., 1995]. Organic and elemental carbon content in the aerosol was measured with a denuder-filter pack system described by Subramanian et al. [2004] and at a higher frequency with a Sunset Labs in situ analyzer (Sunset Labs Co., Portland, Oregon). In addition to the measurements of the aerosol chemical composition, $\mathrm{PM}_{2.5}$ aerosol mass was measured with a TEOM (Model 1400a, R\&P Co, Albany, NY) that was operated at $30^{\circ} \mathrm{C}$ and with a naphion dryer to minimize losses of volatile material.

[20] Data from the different instruments were combined to form a best estimate of the corresponding concentration [Takahama et al., 2004]. The instruments measuring the same component were intercompared and the outliers identified. After the outliers were removed, the high time resolution data were corrected such that their average over a sampling period produced the same value as the filter pack measurements. This was done because the denuder filterpack techniques are generally considered to be the reference for newer and less widely tested high time resolution methods.

\subsection{Estimation of Water Absorption by Organic Aerosol Fraction}

[21] The observations during July 2001 (as a representative month for summer) were compared to the predictions of the thermodynamic models GFEMN [Ansari and Pandis, 1999] and AIM (http://mae.ucdavis.edu/wexler/aim) [Clegg et al., 1998] in order to estimate the contribution of organic material to water absorption. Because $\mathrm{PM}_{2.5}$ water content data are not available for months after August 2001, a direct comparison with $\mathrm{PM}_{2.5}$ chemical data was only possible for the summer months and the modeling was limited to July 2001 only.

[22] The GFEMN and AIM models calculated the amount of aerosol bound water based on the measured relative humidity and the $\mathrm{PM}_{2.5}$ aerosol concentrations of sulfate, nitrate and ammonium. The contributions of chloride and sodium to water absorption were neglected, because the concentrations of these compounds were negligible in comparison to the other components. Both models assumed that organic compounds do not contribute to water absorption. Thus any difference between the observations and the model predictions may be an indication of the influence of organics on the absorption of water.

[23] The models assumed that the aerosol is dry during the low RH measurement of the DAASS. The neutral aerosol, composed mostly of ammonium sulfate is expected to crystallize at the $\mathrm{RH}$ of the dried measurements, which did not exceed $25 \%$ RH. Only when the aerosol is acidic, i.e., contains ammonium bisulfate, the assumption may not be justified. However, even on those occasions the amount of water in the aerosol at the RH of dried measurements is expected to be small. For this reason, the amount of water in the aerosol during the dried measurements was assumed to

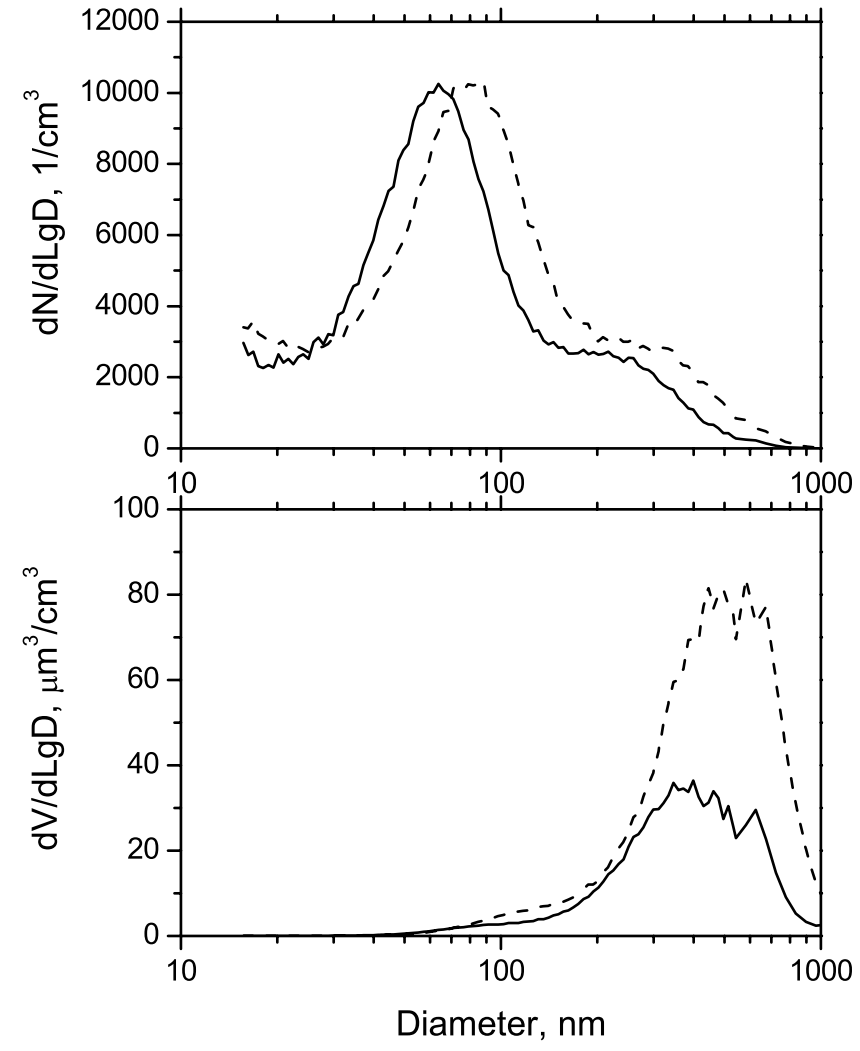

Figure 1. An example of the effect of relative humidity on the ambient aerosol size distribution measured on 1 July 20010130 EST, 73\% RH. The solid lines represent the dried distributions, and the dashed lines are for the wet distributions.

be negligible. In addition, there is no reliable data available for the activities of aerosol components in water solution below $30 \% \mathrm{RH}$ to accurately model the water content at such a low RH.

[24] A Latin hypercube sampling (LHS) routine [McKay, 1988] was used to estimate the uncertainty in the model output due to the uncertainties in the input variables $(\mathrm{RH}$, ammonia, nitrate and sulfate). Owing to the computational intensity of the LHS routine the uncertainty of the model output was assessed only with the GFEMN. Normal distributions were chosen for the input variables, with coefficient of variation of 0.15 for sulfate, nitrate and ammonia, and $5 \%$ for $\mathrm{RH}$. To avoid generating artificial correlations among the different input variables and to allow the statistics (e.g., mean and variance) of the output distribution to converge, a sampling size of 200 was selected. Thus for each input point the model simulated 200 possible scenarios according to the Latin hypercube sampling. These 200 points were then used to calculate the mean and the standard deviation, the latter being a measure of the model uncertainty due to the uncertainty in the input variables.

\section{Results and Discussion}

\subsection{Aerosol Water Content}

[25] The effect of relative humidity on the aerosol size distribution is shown in Figure 1. The number concentration 


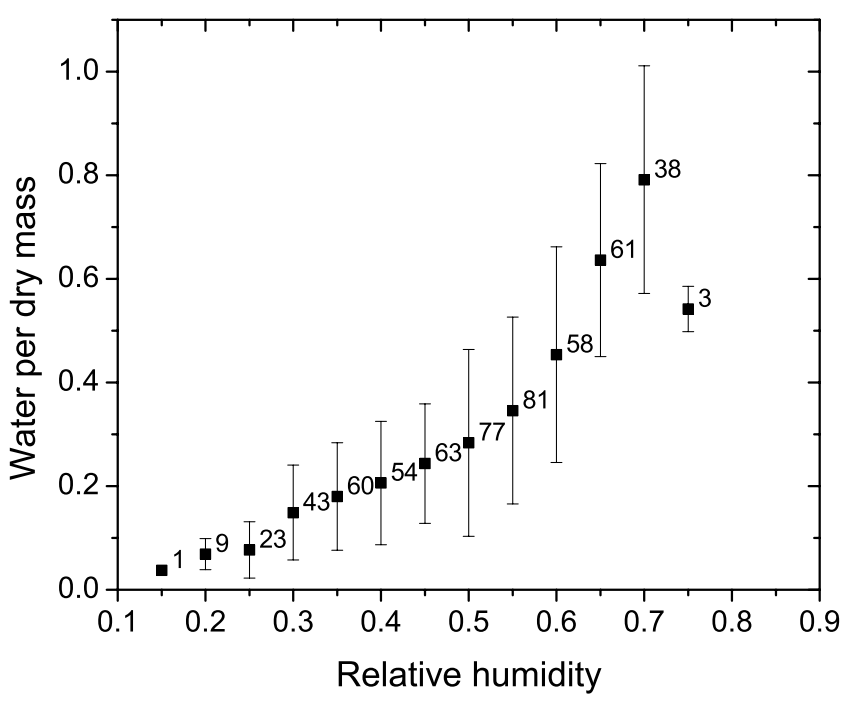

Figure 2. Mass of $\mathrm{PM}_{2.5}$ aerosol bound water per $\mathrm{PM}_{2.5}$ dry aerosol mass as a function of relative humidity during July 2001. The error bars indicate the standard deviation, and the labels show the number of observations per each point.

is preserved as well as the shape of the distribution. However, the wet distribution is shifted to larger sizes relatively to the dry distribution due to the hygroscopic growth of particles. The volume size distributions, shown on the lower panel of Figure 1, indicate that the volume concentration of the wet aerosol is larger than that of the dried aerosol, the difference being the amount of absorbed water, i.e., the aerosol water content.

[26] The aerosol water content shows a strong dependence on the ambient relative humidity. Figure 2 shows average ratios of $\mathrm{PM}_{2.5}$ aerosol bound water to the dry mass of $\mathrm{PM}_{2.5}$ aerosol measured with the TEOM during July 2001. At 70\% RH approximately half of the ambient aerosol mass was composed of water. The point at $75 \% \mathrm{RH}$ does not follow the overall trend because it represents only 3 observations during which the aerosol contained a relatively small fraction of water-soluble material (sulfate comprised $20 \%$ of the aerosol mass as compared to the average of $40 \%$ ). Figure 2 also shows that in July 2001 aerosol contained water even at a relative humidity as low as $30 \%$, at which point water constituted, on average, about $15 \%$ of the wet aerosol mass. As will be shown below, the presence of water at low relative humidities is characteristic of summer aerosol in the Pittsburgh area.

[27] The volume-based growth factors, $\mathrm{GF}_{\mathrm{v}}$, (the aerosol volume at ambient conditions divided by the volume at low $\mathrm{RH}$ ) as a function of ambient RH during different months of the study are shown in Figure 3. A growth factor larger than one indicates that aerosol contains water. The aerosol was found to always contain water when the relative humidity is above $60 \%$. However, the aerosol water content below $60 \%$ RH exhibits a seasonal behavior. The volume growth factors for summer months were practically always above one indicating that the aerosol contained water. Thus the summertime aerosol in the Pittsburgh area may be assumed to be always wet. During winter months, however, the volume ratios at $\mathrm{RH}$ below about $60 \%$ did not differ from unity within experimental error most of the time $(90 \%$ of observations), indicating absence of aerosol bound water. The spring months show a transitional behavior: the fraction of observations when the GF is higher than 1 below $55 \% \mathrm{RH}$ progressively increases from March to May (Figure 4).

\subsection{Aerosol Crystallization and Aerosol Acidity}

[28] The seasonal behavior of the presence of aerosolbound water below $60 \% \mathrm{RH}$ follows that of the aerosol acidity in the Pittsburgh area. The average ammonium to sulfate ratio (moles ammonium to moles sulfate) in summer is 1.4 which corresponds to an approximately equal mixture of ammonium sulfate, $\left(\mathrm{NH}_{4}\right)_{2} \mathrm{SO}_{4}$, and ammonium bisulfate, $\left(\mathrm{NH}_{4}\right) \mathrm{HSO}_{4}$ [Rees et al., 2004]. The ammonium bisulfate crystallizes at 0-22\% RH [Tang and Munkelwitz, 1994]. Such low relative humidities did not occur during the study to induce crystallization. However, the ambient RH often exceeds its deliquescence point, 40\% [Tang and Munkelwitz, 1994]. Even if the ambient RH at the ground level is lower than $40 \%$, the deliquescence point of ammonium bisulfate may be reached at the upper layers of the boundary layer. Thus the particles during their lifetime (a few days) have multiple chances to become hydrated. In contrast to summer, the ammonium to sulfate mole equivalent ratio in winter is higher than 2 indicating that all of sulfate is neutralized by ammonium. The ratio is higher than 2 because some of ammonium is bound to nitrate forming ammonium nitrate. Sulfate is thus in the form of ammonium sulfate that crystallizes at about $40 \%$ $\mathrm{RH}$, which is frequently encountered during the daytime in the mixed boundary layer. Spring months exhibit a transitional behavior as the aerosol becomes progressively more acidic.

[29] The monthly average aerosol acidity is not representative for all observation points: the aerosol was not always acidic and there were periods when it was neutral. However, it appears that most periods of low RH (below 40\%) are associated with acidic conditions. For illustration purposes we consider one week in July 2001. Figure 5 shows the ambient $\mathrm{RH}$, the $\mathrm{GF}_{\mathrm{v}}$ and the periods during which the aerosol was acidic (i.e., had anion to cation mol-equivalent ratio larger than one). During the periods when the $\mathrm{RH}$ was close or below $40 \%$ (in the afternoon hours of most days), the aerosol was acidic and thus was able to retain some water $\left(\mathrm{GF}_{\mathrm{v}}>1\right)$. The only period when the aerosol appears to crystallize $\left(\mathrm{GF}_{\mathrm{v}} \sim 1\right)$ was on 13 July, when the RH decreased from $90 \%$ to around $40 \%$ and the aerosol was neutral.

\subsection{Error Analysis}

[30] The consistent presence of water $\left(\mathrm{GF}_{\mathrm{v}}>1\right)$ during the summer could be a result of a loss of semivolatile aerosol components during the drying process. Even though the temperature is kept close to the ambient, a lower $\mathrm{RH}$ may cause evaporation of a volatile species, such as ammonium nitrate. The dried volume concentration will be then biased low relatively to the wet measurements, and the $\mathrm{GF}_{\mathrm{v}}$ will be thus above 1. This problem, however, should be small during the summer months, as the ammonium nitrate content was only $3 \%$ of the total $\mathrm{PM}_{2.5}$ mass on average. The loss of semivolatile organic material, on the other hand is hard to quantify, because the volatile fraction 

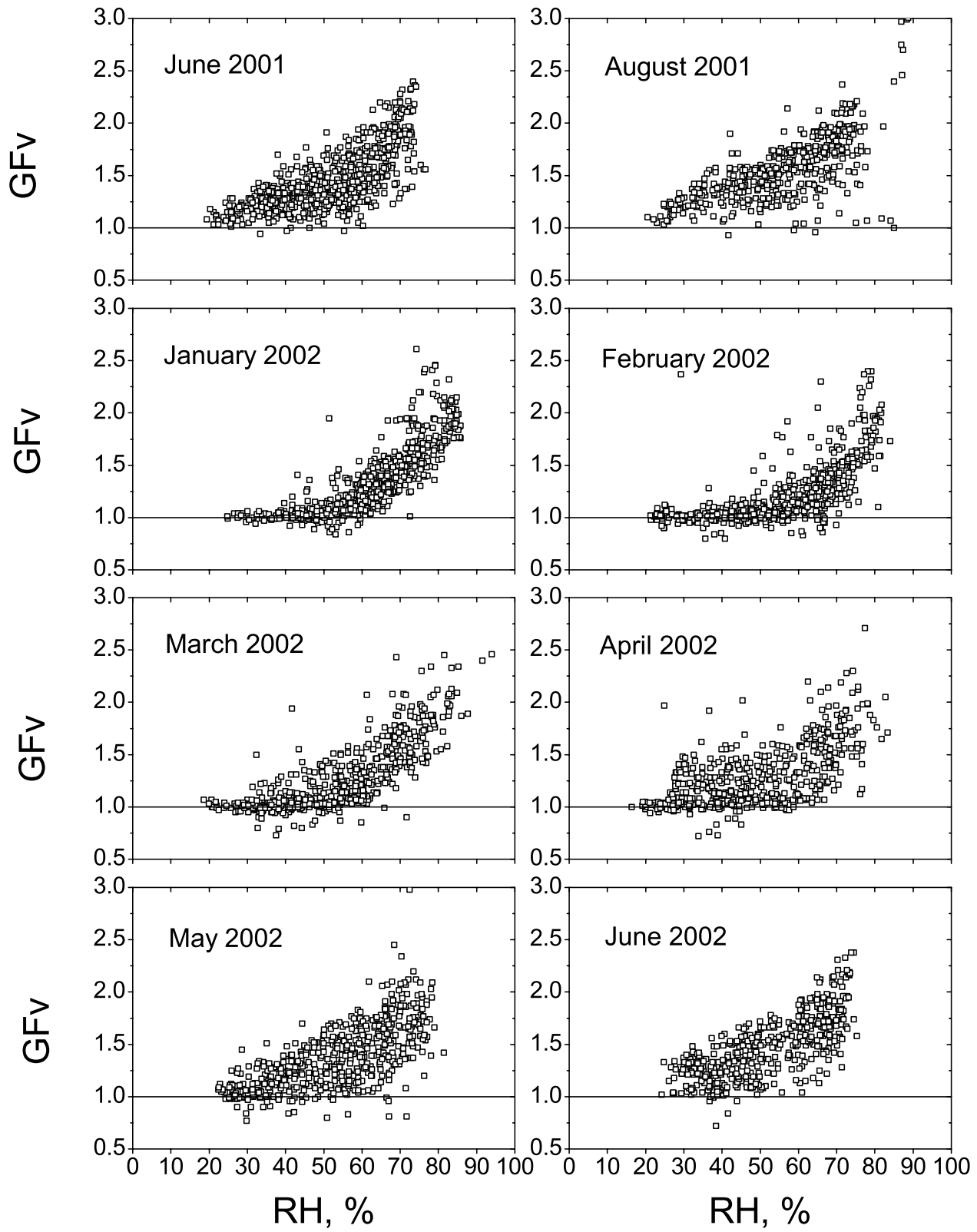

Figure 3. Measured volume growth factors during different months of the study. Each point corresponds to a one-hour average of the growth factors.

of organic aerosol and organic aerosol and its volatility as a function of RH are not known. On the other hand, measurements during the winter indicate that the dried and wet volumes are identical within the experimental error, if the ambient RH is below $60 \%$. If the drying process had a large effect on the growth measurements, the winter measurements would have been biased too. Further, the "dry" DAASS data agree very well with the TEOM $\mathrm{PM}_{2.5}$ mass data when the measured volume is translated to mass using a density based on the aerosol chemical composition [Stanier et al., 2004]. If there was a substantial loss of semivolatile material, the DAASS would have been biased low relatively to the TEOM, which was not the case. Consequently, the effect of evaporation of semivolatile material during the drying process is expected to be negligible in our summer measurements.
[31] In winter months during periods when the temperature was below $9^{\circ} \mathrm{C}$ the aerosol passed through a heated enclosure before it was re-equilibrated to the ambient $\mathrm{RH}$ at which the measurements were done (see experimental section). If the aerosol crystallizes during the passage through the heated section (where the relative humidity is accordingly low) it would remain dry even after re-equilibration, unless the ambient RH is above the deliquescence point of the aerosol. This possibility was investigated by examining the growth factors during periods when the ambient RH was below $60 \%$ (assuming that this is the deliquescence point) while the RH in the heated section was above $40 \%$ (i.e., ammonium sulfate and nitrate should not crystallize). It was found that even at these conditions the aerosol was dry, i.e., ambient aerosol was dry if the ambient $\mathrm{RH}$ is below $60 \%$. Likewise, on a few occasions when the ambient $\mathrm{RH}$ was around $50 \%$ 


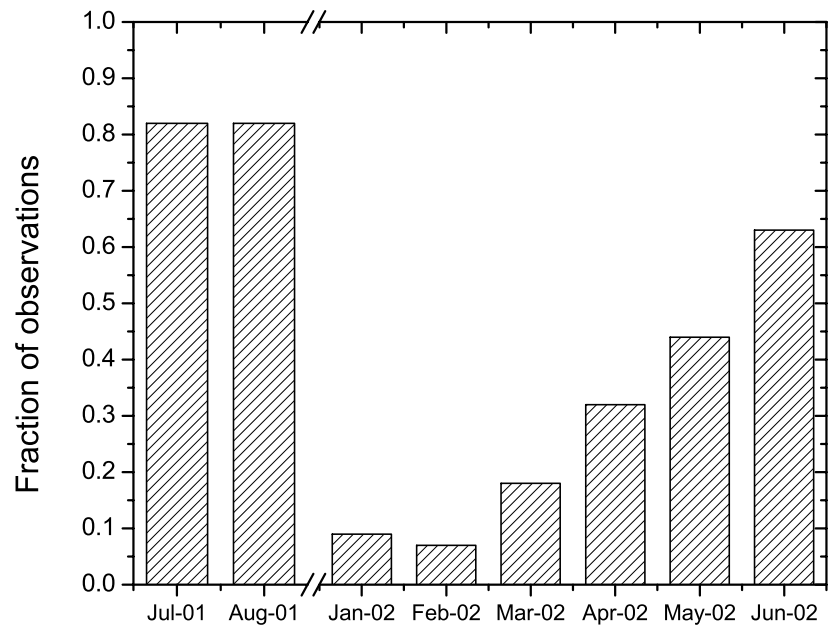

Figure 4. Fraction of observations below 55\% RH when the GF was larger than 1.15; i.e., aerosol had a substantial amount of water.

and the heated section $\mathrm{RH}$ was below $40 \%$, the growth factor was around 1.1 indicating again that the aerosol was not crystallizing.

[32] Another possible problem associated with the heated section during cold periods is evaporation of ammonium nitrate aerosol. Even though the system is re-equilibrated back to the original temperature, some of the nitric acid vapor may be lost to the walls leading to a loss of ammonium nitrate. The effect of the evaporative loss of ammonium nitrate on the growth factor measurements was assessed using the average aerosol chemical composition during January 2002. Even if all ammonium nitrate was lost in the heated section, the measured volume growth factor would be underestimated by about $10 \%$ at $\mathrm{RH}$ between $60 \%$ and $90 \% \mathrm{RH}$. This number represents the worst-case scenario and the actual underestimation should be even lower.

\subsection{Comparison With the Thermodynamic Models}

[33] The observations with the DAASS during July 2001 were compared to simulations using the thermodynamic

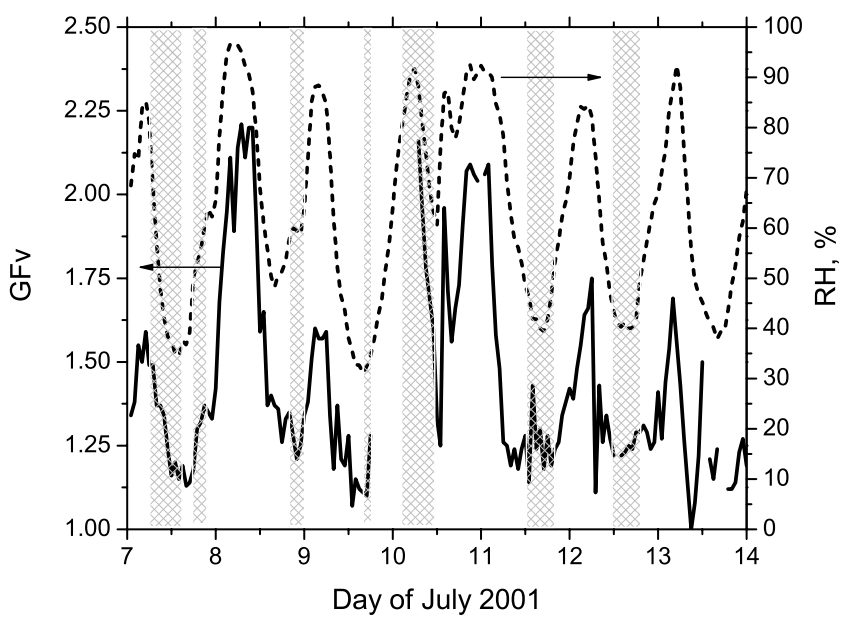

Figure 5. Detailed examination of the effect of aerosol acidity on water retention during summer months. (Solid line, GFv; dotted line, RH; shaded areas, periods when aerosol was acidic).

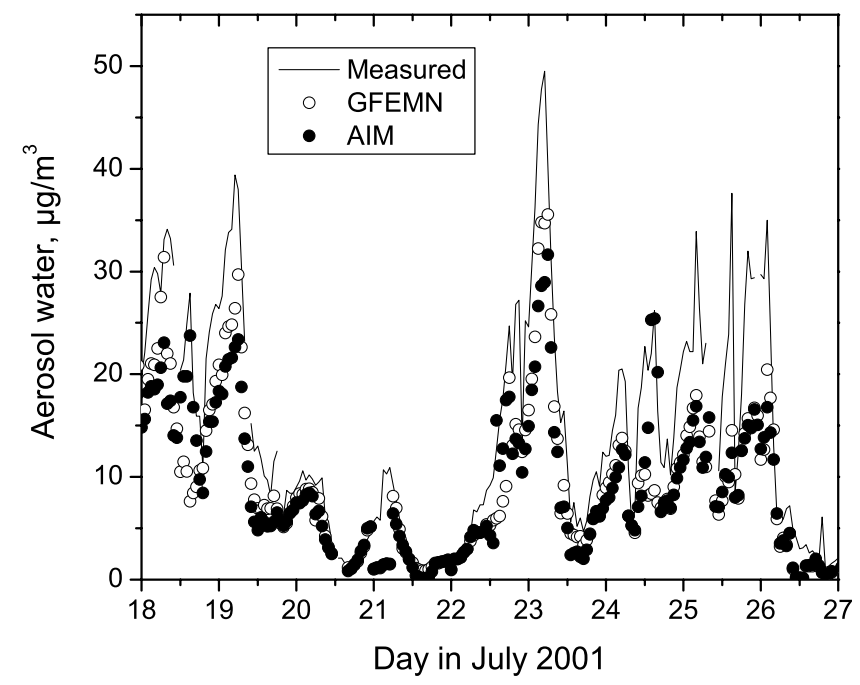

Figure 6. An example of time series of the observed and modeled aerosol water content during a period in July 2001.

models GFEMN [Ansari and Pandis, 1999] and AIM [Clegg et al., 1998] to estimate the contribution of organic aerosol to water absorption. Because the models assume that the organic aerosol does not participate in water absorption, the difference between the observations and the model predictions can be used as a measure of the absorption by organic compounds. The comparison with the models was limited to July 2001 only, because measurements of $\mathrm{PM}_{2.5}$ water concentration were not available after August 2001.

[34] Figure 6 shows an example of time series of the observed and modeled aerosol water content. The models follow the observations, though they often underestimate the water content, which is clear from Figures 7 and 8.

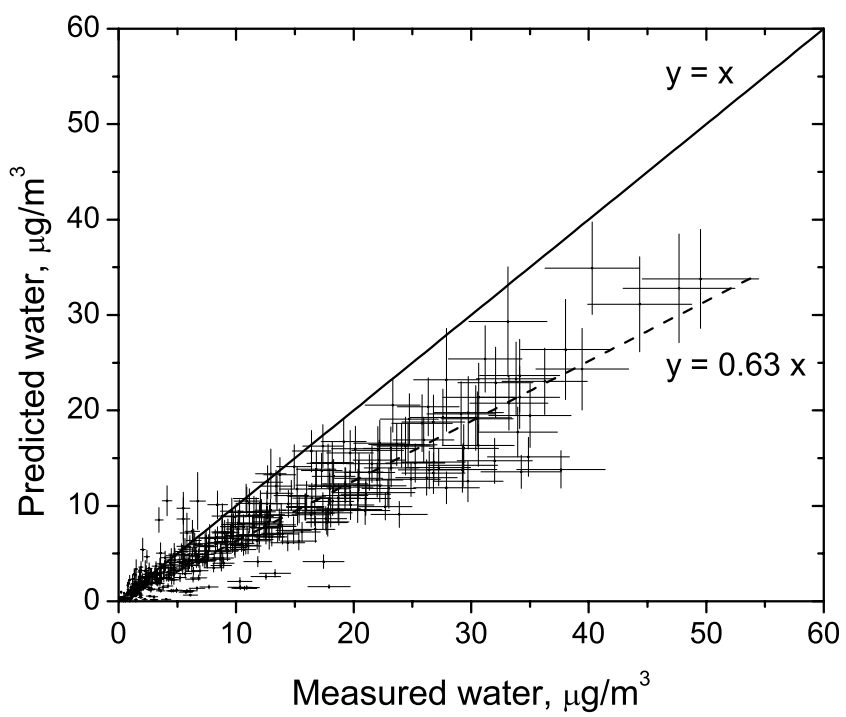

Figure 7. Comparison of the observed and predicted hourly average water concentrations for July 2001. The GFEMN model neglects the organic component contribution to the aerosol water content. The Y-error bars correspond to the standard deviation from the 200 Latin hypercube simulations. The X-error bars represent the measurement uncertainty of the DAASS. A linear regression curve is also shown. 


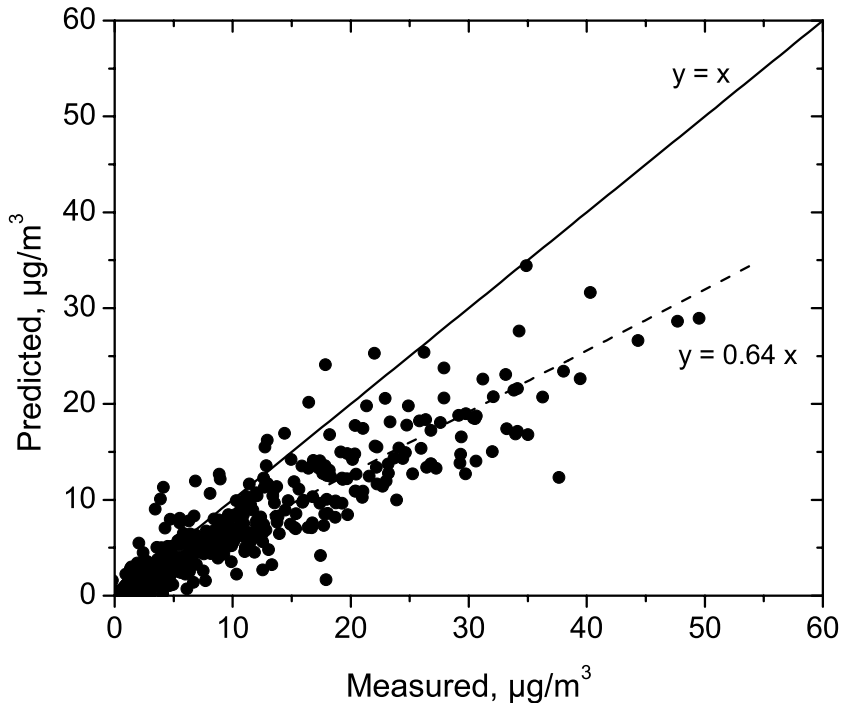

Figure 8. Comparison of the observed hourly average water concentrations and predicted by the AIM model for July 2001.

Figure 7 shows a point-by-point comparison of the aerosol water content during July 2001 observed with the DAASS and predicted with the GFEMN. The model underestimates the water content on average by $37 \%$. Even though this underestimation is not substantially larger than the measurement and modeling uncertainties, it is statistically significant given the number of observations (554). The AIM runs yielded qualitatively similar results to the GFEMN simulations (Figure 8), underestimating the water content, on average, by $36 \%$ (Figure 8 ).

[35] Cabada et al. [2004] modeled light scattering by ambient aerosol during the PAQS using a model developed by [Pilinis, 1989] that calculates the light scattering based on the inorganic concentration data and thermodynamic modeling of water absorption by the aerosol. Similarly to our observations, the model under-predicted the measured scattering coefficient during July 2001. When the water content measured with the DAASS was used to calculate the light scattering, the predictions agreed with the observations. This agreement with independent measurements using an integrating nephelometer suggests that the water content measured with the DAASS is accurate. It also shows that modeling of aerosol water content based only on inorganic aerosol concentrations under-estimates water concentration.

\subsection{Model Under-Prediction and Organic Aerosol}

[36] Because the GFEMN and AIM assumed no contribution to water absorption by organic material in the aerosol, the observed underestimation may be an indication of absorption of water by the organics. However, we have found no clear correlation between the organic mass and the under-prediction by the models (Figure 9). For this comparison the excess water was scaled with $\mathrm{RH} /(1-\mathrm{RH})$ to exclude the large effect of relative humidity on water absorption [Hanel, 1979]. This factor follows from the Rault's law, which states that the equilibrium RH of a solution droplet is equal to the molar fraction of water in the solution. From this follows that the ratio of the absorbed water to the dissolved mass is proportional to the factor $\mathrm{RH} /$ (1-RH).

[37] Dick et al. [2000] in their study conducted in the Great Smoky Mountain National Park have found a good correlation between the excess water (measured minus predicted) and organic carbon concentration, if the inorganic fraction of the aerosol was assumed to be crystalline and all the water absorption was due to the organic aerosol when the $\mathrm{RH}$ was below $80 \%$. The water absorbed per unit organic mass was within the range expected for dicarboxylic acids. However, no such relationship was found in our study, if the inorganic aerosol is assumed to be crystalline below $80 \% \mathrm{RH}$. Also, if only organic matter is assumed to contribute to water absorption below $80 \% \mathrm{RH}$, it would have to have unrealistically high water absorption per unit organic mass, on average 4 times higher than that of pure ammonium sulfate. Thus the assumption that inorganic salts are dissolved below $80 \% \mathrm{RH}$ is more realistic for our study.

[38] A possible explanation for the lack of correlation between the excess water and organic concentration is that the contribution of organics to water absorption is not constant and may change with time. For example, photochemical activity during the day may increase the fraction of water-soluble organics. This possibility was investigated by calculating the diurnal profile of the ratio of the excess water (under-prediction by the model) to the organic carbon (OC) mass (Figure 10). The excess water scaled with RH/

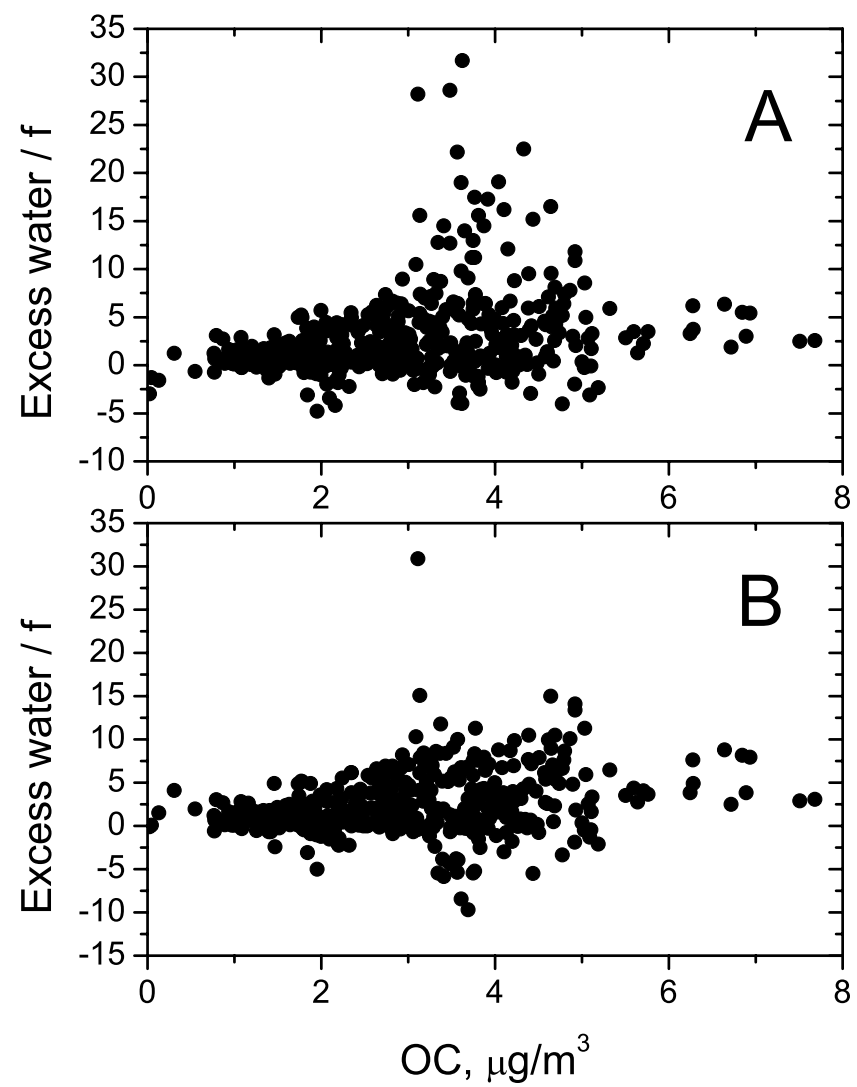

Figure 9. Water deficit versus the concentration of organic carbon. To remove the effect of relative humidity, the excess water was scaled with $f=\mathrm{RH} /(1-\mathrm{RH})$ : (a) using GFEMN predictions and (b) using AIM predictions. 


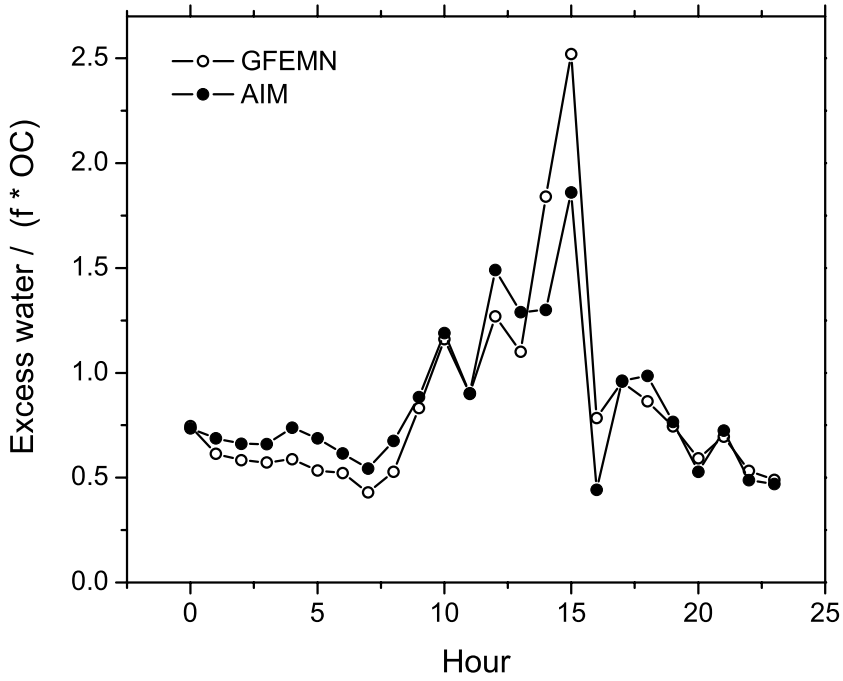

Figure 10. Diurnal profile of the excess water per OC mass. The excess water was scaled with $f=\mathrm{RH} /(1-\mathrm{RH})$ to account for the effect of relative humidity.

(1-RH) per unit carbon mass shows, on average, higher values during the afternoon hours. These observations suggest that during afternoon hours organic compounds contribute more to water absorption and that absorption by the organics can explain the "excess" water. However, it should be noted, that during the afternoon the absorption of water per unit organic mass is quite high, being close to that of pure dicarboxylic acids. Even though this cannot be ruled out, because no data is available for the concentration of these acids during our measurements, it is quite improbable that all of the organic mass was composed of these compounds.

[39] The following observations offer a different explanation for the discrepancy between the models and the observations. It was found that the excess water scaled with $\mathrm{RH} /(1-\mathrm{RH})$ correlates with $\mathrm{PM}_{2.5}$ sulfate concentration stronger than with most of the other parameter measured during the study (Table 1). Concentrations of $\mathrm{PM}_{2.5}$ water, mass and sulfate strongly correlate with each other and thus show similar correlation coefficients with the scaled excess water. Given the fact that sulfate concentration in our study was measured with 4 different instruments/methods and that the concentrations measured at the central cite agreed within the experimental error with those measured at the other sites in and around the city, this correlation is not due to a bias in sulfate measurements. A more probable explanation is that the absorption of water by sulfate is enhanced by other substances, organics in particular. Cruz and Pandis [2000] have shown in a laboratory study that organic compounds can enhance water absorption by ammonium sulfate by a factor of 2-3 relatively to the absorption by the pure salt. If we assume that the organics do not contribute to water absorption, but enhance that of inorganic salts, the enhancement factor in our study is on average 1.5. It should be noted, that Cruz and Pandis [2000] observed the enhancement of absorption at aerosol organic content above 50\% and did not observe a noticeable enhancement when the aerosol organic content was close that of our study (on average $20 \%$ ). It is possible though that organic compounds in ambient aerosol, which were not tested in the study by Cruz and Pandis [2000], enhance water absorption even at lower organic content. However, whether this is the case remains to be resolved.

[40] Modeling of water absorption by atmospheric organic aerosol is extremely difficult due to the complexity of the organic chemical composition confounded by the lack of thermodynamic data and models applicable to organic compounds of various structures and their mixtures. Several attempts have been made to use semiempirical models to predict water absorption by organics using information on the functional organic groups [Ansari and Pandis, 2000; Clegg et al., 2001; Griffin et al., 2003; Ming and Russell, 2002; Pividal and Sandler, 1990]. Unfortunately, no information on functional groups of organic compounds in ambient aerosol is available in our study. Therefore we could not test this approach. Thermodynamic interactions between organic components and inorganic salts in atmospheric aerosol are even less understood. Our data indicate that the observed discrepancy between the models and the observations is unlikely to be explained solely by the contribution of organics, thus suggesting that organic material was changing the activity coefficients of inorganic salts. Analysis of such interactions is beyond the scope of this work. However, our results indicate the apparent importance of such interactions in ambient aerosol.

\section{Conclusions}

[41] The hygroscopic behavior of ambient aerosol in Pittsburgh exhibits a seasonal behavior. During summer months the aerosol always contained water, even when the relative humidity was as low as $30 \%$ at which point water contributed on average about $15 \%$ the aerosol mass. In contrast to summer, winter aerosol was generally dry below $60 \% \mathrm{RH}$. The spring months showed a transitional behavior. The seasonal behavior of aerosol water content follows that of the aerosol acidity. In summer ammonium bisulfate present in the particles retains water even at $\mathrm{RH}$ below $40 \%$, preventing the aerosol from drying. In contrast, the winter aerosol is neutral and has a chance to crystallize at ambient conditions. Aerosol in winter became wet only when the relative humidity reached the deliquescence point of ammonium nitrate.

Table 1. Correlation Coefficients Between the Excess Water Scaled With RH/(1-RH) and Other Variables Measured During July 2001

\begin{tabular}{lc}
\hline Component & Correlation Coefficient \\
\hline $\mathrm{PM}$ water & 0.70 \\
$\mathrm{PM}_{2.5}$ mass & 0.67 \\
Sulfate & 0.64 \\
Ammonium & 0.38 \\
$\mathrm{RH}$ & 0.29 \\
$\mathrm{OC}$ & 0.28 \\
Total nitrate & 0.24 \\
Temperature & 0.22 \\
$\mathrm{O}_{3}$ & 0.04 \\
$\mathrm{PM}_{2.5}$ nitrate & 0.04 \\
$\mathrm{NO}_{\mathrm{x}}$ & 0.02 \\
$\mathrm{SO}$ & -0.03 \\
$\mathrm{NO}$ & -0.05 \\
$\mathrm{CO}$ & -0.10 \\
\hline
\end{tabular}


[42] The observations during July 2001 were compared with the predictions of the thermodynamic models GFEMN and AIM assuming no contribution of the organic aerosol to water absorption. The models under-predicted water concentrations by about $35 \%$. This indicates the magnitude of the possible contribution of organic material to water absorption in July 2001. However, no clear correlation between organic mass and the excess water was observed, which is probably due to a variable nature of the organic aerosol. On average, the contribution of the organics to water absorption appeared to be higher during the afternoon hours. However, this work supports the hypothesis that water absorption by sulfate is influenced by the organics, in addition to the absorption of water by organics themselves.

[43] Acknowledgments. The particulate matter measurements were conducted as part of the Pittsburgh Air Quality Study, which was supported by U.S. Environmental Protection Agency (EPA) under contract R82806101 and by the U.S. Department of Energy National Energy Technology Laboratory under contract DE-FC26-01NT41017. This research was supported by NSF ATM-336296.

\section{References}

Ansari, A. S., and S. N. Pandis (1999), Prediction of multicomponent inorganic atmospheric aerosol behavior, Atmos. Environ., 33(5), 745757.

Ansari, A. S., and S. N. Pandis (2000), Water absorption by secondary organic aerosol and its effect an inorganic aerosol behavior, Environ Sci. Technol., 34(1), 71-77.

Cabada, J. C., A. Khlystov, A. E. Wittig, C. Pilinis, and S. N. Pandis (2004), Light scattering by fine particles during the Pittsburgh Air Quality Study: Measurements and modeling, J. Geophys. Res., 109, D16S03, doi:10.1029/2003JD004155.

Choi, M. Y., and C. K. Chan (2002), The effects of organic species on the hygroscopic behaviors of inorganic aerosols, Environ. Sci. Technol. 36(11), 2422-2428.

Clegg, S. L., P. Brimblecombe, and A. S. Wexler (1998), Thermodynamic model of the system $\mathrm{H}+-\mathrm{NH} 4+-\mathrm{SO} 42--\mathrm{NO} 3--\mathrm{H} 2 \mathrm{O}$ at tropospheric temperatures, J. Phys. Chem. A, 102(12), 2137-2154.

Clegg, S. L., J. H. Seinfeld, and P. Brimblecombe (2001), Thermodynamic modelling of aqueous aerosols containing electrolytes and dissolved organic compounds, J. Aerosol Sci., 32(6), 713-738.

Cocker, D. R., N. E. Whitlock, R. C. Flagan, and J. H. Seinfeld (2001), Hygroscopic properties of Pasadena, California aerosol, Aerosol Sci. Technol., 35(2), 637-647.

Cruz, C. N., and S. N. Pandis (2000), Deliquescence and hygroscopic growth of mixed inorganic-organic atmospheric aerosol, Environ. Sci. Technol., 34(20), 4313-4319.

Dick, W. D., P. Saxena, and P. H. McMurry (2000), Estimation of water uptake by organic compounds in submicron aerosols measured during the Southeastern Aerosol and Visibility Study, J. Geophys. Res., 105(D1), $1471-1479$

Griffin, R. J., K. Nguyen, D. Dabdub, and J. H. Seinfeld (2003), A coupled hydrophobic-hydrophilic model for predicting secondary organic aerosol formation, J. Atmos. Chem., 44(2), 171-190.

Han, J., H. Hung, and S. T. Martin (2002), Size effect of hematite and corundum inclusions on the efflorescence relative humidities of aqueous ammonium nitrate particles, J. Geophys. Res., 107(D10), 4086, doi:10.1029/2001JD001054.

Hanel, G. (1979), The properties of atmospheric aerosol particles as functions of the relative humidity at thermodynamic equilibrium with the surrounding moist air, Adv. Geophys., 19, 73-188.

Khlystov, A., G. P. Wyers, and J. Slanina (1995), The steam-jet aerosol collector, Atmos. Environ., 29(17), 2229-2234.
Khlystov, A., C. Stanier, and S. N. Pandis (2004), An algorithm for combining electrical mobility and aerodynamic size distributions data when measuring ambient aerosol, Aerosol Sci. Technol., 38, suppl. 1, 229-238.

Martin, S. T., J. H. Han, and H. M. Hung (2001), The size effect of hematite and corundum inclusions on the efflorescence relative humidities of aqueous ammonium sulfate particles, Geophys. Res. Lett., 28(13), 2601-2604

McKay, M. D. (1988), Sensitivity and uncertainty analysis using a statistical sample of input values, in Uncertainty Analysis, edited by Y. Ronen, pp. 145-186, CRC Press, Boca Raton, Fla.

Ming, Y., and L. M. Russell (2002), Thermodynamic equilibrium of organic-electrolyte mixtures in aerosol particles, AIChE J., 48(6), 1331-1348. Nessler, R., N. Bukowiecki, S. Henning, E. Weingartner, B. Calpini, and U. Baltensperger (2003), Simultaneous dry and ambient measurements of aerosol size distributions at the Jungfraujoch, Tellus, Ser. B, 55(3), 808819.

Pilinis, C. (1989), Numerical simulation of visibility degradation due to particulate matter: Model development and evaluation, J. Geophys. Res., 94(D7), 9937-9946.

Pividal, K. A., and S. I. Sandler (1990), Neighbor effects on the group contribution method - Infinite dilution activity-coefficients of binary-systems containing primary amines and alcohols, J. Chem. Eng. Data, 35(1), 53-60.

Rader, D. J., and P. H. Mcmurry (1986), Application of the tandem differential mobility analyzer to studies of droplet growth or evaporation, J. Aerosol Sci., 17(5), 771-787.

Ramaswamy, V. (2001), Radiative forcing of climate change, in Climate Change 2001. Scientific Basis: Contribution of the Working Group 1 to the Third Assessment Report of the IPCC, edited by J. T. Houghton et al., Cambridge Univ. Press, New York.

Rees, S. L., A. L. Robinson, A. Khlystov, C. O. Stanier, and S. N. Pandis (2004), Mass balance closure and the Federal Reference Method for PM2.5 in Pittsburgh, Pennsylvania, Atmos. Environ., 38(20), 33053318

Saxena, P., L. M. Hildemann, P. H. Mcmurry, and J. H. Seinfeld (1995), Organics alter hygroscopic behavior of atmospheric particles, J. Geophys. Res., 100(D9), 18,755-18,770.

Stanier, C. O., A. Khlystov, W. R. Chan, M. Mandiro, and S. N. Pandis (2004), A method for the in-situ measurement of aerosol water content of ambient aerosols: The Dry-Ambient Aerosol Size Spectrometer (DAASS), Aerosol Sci. Technol., 38, suppl. 1, 215-228.

Subramanian, R., A. Y. Khlystov, J. C. Cabada, and A. L. Robinson (2004), Positive and negative artifacts in particulate organic carbon measurements with denuded and undenuded sampler configurations, Aerosol. Sci. Technol., 38, suppl. 1, 27-48.

Swietlicki, E., et al. (1999), A closure study of sub-micrometer aerosol particle hygroscopic behaviour, Atmos. Res., 50(3-4), 205-240.

Takahama, S., A. E. Wittig, D. V. Vayenas, C. I. Davidson, and S. N. Pandis (2004), Modeling the diurnal variation of nitrate during the Pittsburgh Air Quality Study, J. Geophys. Res., 109, D16S06, doi:10.1029/ 2003JD004149.

Tang, I. N. (1997), Thermodynamic and optical properties of mixed-salt aerosols of atmospheric importance, J. Geophys. Res., 102(D2), 18831893.

Tang, I. N., and H. R. Munkelwitz (1994), Water activities, densities, and refractive-indexes of aqueous sulfates and sodium-nitrate droplets of atmospheric importance, J. Geophys. Res., 99(D9), 18,801-18,808.

Tang, W., T. Raymond, B. Wittig, C. Davidson, S. Pandis, L. Robinson, and K. Crist (2004), Spatial variations of PM2.5 during the Pittsburgh Air Quality Study, Aerosol Sci. Technol., 38, suppl. 2, 80-90.

Wittig, B., N. Anderson, A. Y. Khlystov, S. N. Pandis, C. Davidson, and A. L. Robinson (2004), Pittsburgh Air Quality Study overview and preliminary scientific findings, Atmos. Environ., 38(20), 3107-3125.

A. Khlystov, Department of Civil and Environmental Engineering, Duke University, Durham, NC 27708, USA.

S. N. Pandis, C. O. Stanier, and S. Takahama, Department of Chemical Engineering, Carnegie Mellon University, Pittsburgh, PA 15213, USA. (spyros@andrew.cmu.edu) 


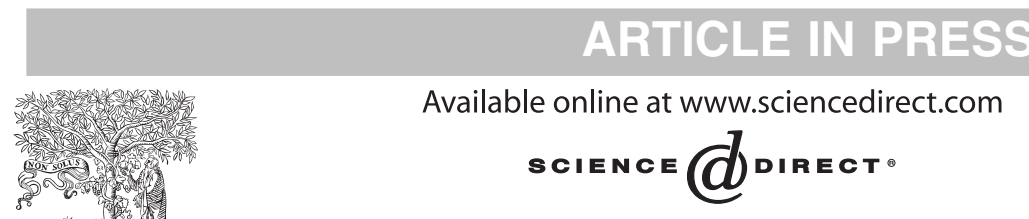

Aerosol Science II (II) II-II
Journal of

Aerosol Science

www.elsevier.com/locate/jaerosci

\title{
In situ concentration of semi-volatile aerosol using water-condensation technology
}

\author{
Andrey Khlystov ${ }^{\mathrm{a}, *}$, Qi Zhang ${ }^{\mathrm{b}}$, Jose L. Jimenez ${ }^{\mathrm{b}}$, Charlie Stanier ${ }^{\mathrm{c}}$, Spyros N. Pandis ${ }^{\mathrm{c}}$, \\ Manjula R. Canagaratna ${ }^{\mathrm{d}}$, Philip Fine ${ }^{\mathrm{e}}$, Chandan Misra ${ }^{\mathrm{e}}$, Constantinos Sioutas ${ }^{\mathrm{e}}$ \\ ${ }^{a}$ Department of Civil and Environmental Engineering, Duke University, Box 90287, Durham, NC 27708, USA \\ ${ }^{\mathrm{b}}$ Department of Chemistry and CIRES, University of Colorado, Boulder, CO 80309, USA \\ ${ }^{\mathrm{c}}$ Department of Chemical Engineering, Carnegie Mellon University, Pittsburgh, PA 15213, USA \\ ${ }^{\mathrm{d}}$ Center for Aerosol and Cloud Chemistry, Aerodyne Research Inc., Billerica, MA 01821, USA \\ ${ }^{\mathrm{e}}$ Department of Civil and Environmental Engineering, University of Southern California, Los Angeles, CA 90089, USA
}

Received 12 July 2004; received in revised form 6 November 2004; accepted 8 November 2004

\begin{abstract}
The effect of concentrating semi-volatile aerosols using a water-condensation technology was investigated using the Versatile Aerosol Concentration Enrichment System (VACES) and the Aerodyne Aerosol Mass Spectrometer (AMS) during measurements of ambient aerosol in Pittsburgh, PA. It was found that the shape of the sulfate mass-weighed size distribution was approximately preserved during passage through the concentrator for all the experiments performed, with a mass enhancement factor of about 10-20 depending on the experiment. The size distributions of organics, ammonium and nitrate were preserved on a relatively clean day (sulfate concentration around $7 \mu \mathrm{g} / \mathrm{m}^{3}$ ), while during more polluted conditions the concentration of these compounds, especially nitrate, was increased at small sizes after passage through the concentrator. The amount of the extra material, however, is rather small in these experiments: between $2.4 \%$ and $7.5 \%$ of the final concentrated PM mass is due to "artifact" condensation. An analysis of thermodynamic processes in the concentrator indicates that the extra particle material detected can be explained by redistribution of gas-phase material to the aerosol phase in the concentrator. The analysis shows that the condensation of extra material is expected to be larger for water-soluble semi-volatile material, such as nitrate, which agrees with the observations. The analysis also shows that artifact formation of nitrate will be more pronounced in ammonia-limited conditions and virtually undetectable in ammonia-rich conditions.
\end{abstract}

(C) 2004 Elsevier Ltd. All rights reserved.

Keywords: Particle concentrator; AMS; Semi-volatile aerosol; Nitrate; Organic aerosol

\footnotetext{
* Corresponding author. Tel.: +19196605209; fax: +1919660 5219.

E-mail address: andrey@duke.edu (A. Khlystov).
} 


\section{Introduction}

Several epidemiological studies have proposed that increased concentrations of ambient particulate matter (PM) are responsible for adverse human health effects (Peters, Dockery, Heinrich, \& Wichmann, 1997; Pope, Dockery, \& Schwartz, 1995; Thurston, Ito, Hayes, Bates, \& Lippmann, 1994). However, the exact mechanism by which inhaled particles affects human health remains a subject of much research and debate. In order to facilitate studies of the adverse effects of PM on health, particle concentrators have been developed (Kim et al., 2001a; Kim, Jaques, Chang, Froines, \& Sioutas, 2001b; Sioutas, Kim, \& Chang, 1999). These devices allow exposure of human or animal subjects to controlled elevated concentrations of ambient PM and, thus, are extremely useful research tools. In addition to their application in the health research, particle concentrators can also be used to reach lower detection limits with instruments measuring aerosol concentration and/or composition, as well as to reduce the effect of gaseous artifacts on aerosol measurements (Eatough, Pang, \& Eatough, 1999).

The heart of a particle concentrator is a virtual impactor (Willeke \& Baron, 1993). Particles larger than a certain cut-off size, which depends on the impactor design, are concentrated in the minor flow of the impactor, with the concentration factor being approximately the ratio of the total sampling flow rate of the impactor to the flow rate of its minor flow. Concentration factors of up to 40 can typically be achieved. Cut-off sizes of less than about $100 \mathrm{~nm}$, however, require a very high pressure drop across the impactor and are therefore not practical. In addition, high pressure drops may cause evaporative losses of semi-volatile aerosol during the concentration process, since the partial pressure of the species in the gas phase has been significantly decreased. For these reasons most concentrators enhance concentration of fine aerosol, while concentration of ultra-fine particles (smaller than $100 \mathrm{~nm}$ ) remains unchanged. Since ultra-fine particles are also proposed as one of the causative agents in the adverse health effects (Peters et al., 1997), the Versatile Aerosol Concentration Enrichment System (VACES) (Kim et al., 2001a,b) has been developed which uses water-condensation technology to concentrate ultra-fine particles.

The operation principle of the VACES is similar to that of other particle concentrators, but differs in that particles are grown by water condensation prior to passage through the virtual impactor. The instrument creates a super-saturation of water vapor by passing the air first through a warm saturator and then through a cold condenser. The super-saturation causes water condensation onto the particles, which as a result grow in size and can be concentrated without the need of a high pressure drop. The VACES was demonstrated to be capable of enriching the concentration of particles in the range of $0.01-10 \mu \mathrm{m}$ by a factor of up to 40 (Kim et al., 2001a,b). Its small size and modular design makes it well suited for studies using mobile exposure platforms. In addition, the VACES can be readily adapted to accommodate higher output flow rates, which are desirable in conducting human exposure studies. The portability and the high concentration enhancement factors of these particle concentrators make them also very attractive for measurements of an aerosol component which is below or close to the detection limit of the available measurement techniques for the available time. Application of the VACES to increase the aerosol concentration in front of the instruments during sampling may decrease the detection limit of the instrument by up to a factor of 40 .

Water condensation and the changes in temperature of the air during the passage through the concentrator raise concerns of possible changes in gas/aerosol partitioning, i.e. sampling artifacts. However, a study by Zhao et al. (2004) found no detectable sampling artifacts for the VACES concentrator. Here we describe our observations during field measurements in which the VACES concentrator was coupled to an Aerodyne Aerosol Mass Spectrometer (AMS) (Jayne et al., 2000; Jimenez et al., 2003). The 
measurements were conducted during the Pittsburgh Air Quality Study at the central monitoring site in Pittsburgh, PA, during September 2002. The main aim of this study was to determine the applicability of the VACES-AMS combination to measurements of aerosol chemical composition during nucleation events. Because of the different nature of the problem, namely the very low aerosol concentrations during these events, attention has been given to small changes in aerosol composition (of the order of 5\% of the concentrated mass) that were not relevant to the previous characterization studies of the VACES (Geller et al., 2002; Kim et al., 2001b; Misra, Fine, Singh, \& Sioutas, 2004).

\section{Methods}

\subsection{VACES}

The VACES concentrator is described in detail elsewhere (Kim et al., 2001a,b). The concentrator used in our studies consisted of a single sampling line operating at an intake flow rate of 112 1/min (LPM), which was checked before and after experiments using a gas flow meter. In the VACES the air stream is saturated with water vapor in a humidifier, which is a 101 aluminum vessel half-filled with water and maintained at $38^{\circ} \mathrm{C}$. The air stream is directed at and passes above the water surface. The residence time in the humidifier is about $3 \mathrm{~s}$. Doubly de-mineralized water $(18.1 \mathrm{M} \Omega / \mathrm{cm})$ was used in the humidifier. By passing through the humidifier the air is saturated with water vapor and warmed up to about $30{ }^{\circ} \mathrm{C}$ ( $\mathrm{Kim}$ et al., 2001a). After leaving the humidifier the air enters a condenser, a stainless steel pipe that is surrounded by a mixture of water and rock-salt, which was regularly stirred. The temperature of the cooling mixture in the condenser was $-8 \pm 0.5^{\circ} \mathrm{C}$ and was checked using a digital thermometer. The actual temperature of the air stream in the condenser is $20-21^{\circ} \mathrm{C}$ (Kim et al., 2001a). Due to the sharp drop in temperature (about $10^{\circ} \mathrm{C}$ ) the air in the condenser becomes strongly supersaturated. The supersaturation causes water vapor to condense onto particles as small as $20 \mathrm{~nm}$ in size, which rapidly grow to $2.5-3 \mu \mathrm{m}$ water droplets. These droplets are subsequently concentrated by a virtual impactor, exiting via its minor air flow. After leaving the virtual impactor, the droplets were dried with a silica-gel diffusion dryer, thus bringing the concentrated aerosol particles to their original size. The ideal concentration factor is equal to the ratio of the inlet flow to the minor flow (5 LPM in this study) of the virtual impactor, i.e. in this study it was 22. The minor flow was regularly checked with a Gillibrator (Sensidyne, Inc. Clearwater, Florida) and constantly monitored by observing the pressure drop across the virtual impactor.

\subsection{Aerodyne AMS}

The Aerodyne AMS measures size-resolved mass distributions and total mass loadings of non-refractory chemical species in/on submicron particles. This instrument and the associated quantification procedures have been described in detail in other publications (Alfarra et al., 2004; Allan et al., 2004; Jayne et al., 2000; Jimenez et al., 2003), and only a brief summary will be given here. The results from the deployment of this instrument in Pittsburgh are described in Zhang, Canagaratna, Jayne, Worsnop, and Jimenez (2004a) and Zhang et al. (2004b). The AMS uses an aerodynamic lens to focus the particles into a narrow beam, a roughened cartridge heater to vaporize them under high vacuum, and an electron impact ionizer coupled to a quadrupole mass spectrometer to analyze the vaporized molecules. Particle size is measured via particle time-of-flight. The AMS is operated in two modes: (1) a continuous mass spectrum 
(MS) mode, which produces mass concentrations of non-refractory species without size information; and (2) a particle time-of-flight (P-TOF) size distribution measurement mode for selected $\mathrm{m} / \mathrm{z}$ settings of the quadrupole (Jimenez et al., 2003). The AMS alternated between the P-TOF and MS modes approximately every $25 \mathrm{~s}$ during this study. The method detection limits of $\mathrm{NO}_{3}^{-}, \mathrm{SO}_{4}^{2-}, \mathrm{NH}_{4}^{+}$, and organics are estimated to be $0.01,0.05,0.11$, and $0.15 \mu \mathrm{g} \mathrm{m}^{-3}$, for a $10 \mathrm{~min}$ averaging time, respectively, based on sampling particle free ambient air (filtered by a HEPA filter) (Zhang et al., 2004a). The standard errors of the other reported parameters, such as concentration factors and artifacts, were calculated through error propagation. Detailed information on AMS operation and data analysis is described in a separate paper (Zhang et al., 2004a).

\subsection{Experimental set-up}

The VACES-AMS measurements were carried out on 10, 18, and 19 September 2002 at the central monitoring site of the Pittsburgh Air Quality Study (Wittig et al., 2004). The site was located in an urban park, approximately $6 \mathrm{~km}$ from the downtown Pittsburgh. Roughly $1 \mathrm{~km}$ of parkland exists between the site and the residential areas in the predominant upwind direction (south and west). The site was several hundred meters from any major sources.

The performance of the concentrator was assessed by performing several back-to-back tests during which the AMS was alternately used with and without the concentrator at its inlet. On September 10 and 18, 2002, three back-to-back runs were made and on September 19, 2002 two runs were made. Each run was about 20-40 min, half with the concentrator and half without it. The integrated concentrations of sulfate, nitrate, ammonium and organics, as well as their size distributions during the part of the runs with the concentrator were compared with those during the part of the runs without the concentrator.

When the AMS was sampling behind the concentrator, its sampling inlet was connected to the minor flow of the virtual impactor via a silica-gel diffusion drier. The sampling flow of the AMS was the nominal $0.0851 \mathrm{~min}^{-1}$, the rest of the minor follow $\left(\sim 4.91 \mathrm{~min}^{-1}\right)$ was drawn by a separate vacuum pump. The concentration factor of the VACES was monitored regularly by attaching a TSI CPC 3022 particle counter to the minor flow of the concentrator and comparing the counts with the counts without the concentrator.

In parallel to the measurements with the AMS, the ambient aerosol size distributions in the size range from $10 \mathrm{~nm}$ to $2.5 \mu \mathrm{m}$ in diameter were continuously monitored with two TSI SMPS systems (TSI 3936N25 and TSI 3936L10, TSI Inc.) and an APS system (TSI 3320, TSI Inc.).

\section{Results and discussion}

\subsection{Effect of concentrator on semi-volatile aerosol material}

The concentrations of PM sulfate, nitrate, ammonium, and organics measured during the experiments, as well as the corresponding concentration factors are given in Table 1. During the first 2 days the concentration factors for sulfate and ammonium were about 2 times lower than the concentration factor calculated from the ratio of the inlet and minor flows of the virtual impactor. The measured concentration factors were verified with the CPC measurements that gave the same concentration factor as the AMS sulfate. The flows were also checked regularly and were correct. Thus, the reason for this discrepancy is 
Table 1

Average concentrations and standard deviations in $\mu \mathrm{g} / \mathrm{m}^{3}$ of the measured aerosol components and the corresponding concentration factors

\begin{tabular}{|c|c|c|c|c|c|c|c|c|c|c|c|c|}
\hline \multirow[t]{2}{*}{ Date } & \multicolumn{3}{|l|}{ Sulfate } & \multicolumn{3}{|c|}{ Ammonium } & \multicolumn{3}{|l|}{ Nitrate } & \multicolumn{3}{|l|}{ Organics } \\
\hline & $\mathrm{OFF}^{\mathrm{a}}$ & $\mathrm{ON}^{\mathrm{b}}$ & $\mathrm{F}^{\mathrm{c}}$ & OFF & $\mathrm{ON}$ & $\mathrm{F}$ & OFF & ON & $\mathrm{F}$ & OFF & $\mathrm{ON}$ & F \\
\hline $10 / 9 / 02$ & $19.3 \pm 0.9$ & $201 \pm 3.1$ & $10.4 \pm 0.5$ & $4.8 \pm 0.5$ & $61.3 \pm 1.7$ & $12.7 \pm 1.3$ & $0.4 \pm 0.1$ & $10.0 \pm 0.3$ & $24.1 \pm 3.3$ & $5.8 \pm 0.2$ & $60.5 \pm 0.7$ & $10.4 \pm 0.4$ \\
\hline $18 / 9 / 02$ & $21.3 \pm 1.0$ & $278 \pm 3.6$ & $13.1 \pm 0.6$ & $7.3 \pm 0.6$ & $95.3 \pm 2.1$ & $13.0 \pm 1.1$ & $0.6 \pm 0.1$ & $21.3 \pm 0.4$ & $33.9 \pm 3.8$ & $7.6 \pm 0.2$ & $122 \pm 1.0$ & $16.1 \pm 0.5$ \\
\hline $19 / 9 / 02$ & $6.6 \pm 0.6$ & $143 \pm 2.6$ & $21.8 \pm 1.9$ & $2.4 \pm 0.3$ & $50.7 \pm 1.5$ & $21.5 \pm 3.1$ & $0.2 \pm 0.0$ & $5.2 \pm 0.2$ & $26.4 \pm 5.3$ & $2.9 \pm 0.1$ & $68.9 \pm 0.7$ & $24.0 \pm 1.3$ \\
\hline
\end{tabular}

${ }^{\mathrm{a}} \mathrm{OFF}$ : when the concentrator was off.

${ }^{\mathrm{b}} \mathrm{ON}$ : when the concentrator was on.

${ }^{\mathrm{c}} \mathrm{F}$ : the concentration factor.

unknown. The agreement between the AMS and the CPC also indicates that there was no change in the particle collection efficiency of the AMS (Alfarra et al., 2004; Allan et al., 2003) between the ambient and concentrated aerosol experiments, which would be expected given the relatively low ambient relative humidities (25-58\%) and the similar RH expected after the concentration and the diffusion dryer.

The concentrator factors for sulfate during each of the experiments were used as the reference for other measured components. This was done for the following reasons: (1) sulfate is a stable aerosol component with least likely gaseous artifacts, due to the very low vapor pressure of sulfuric acid, and to the very short time the air spends in the concentrator $(0.2 \mathrm{~s})$ for $\mathrm{SO}_{2}$-to-sulfate conversion via aqueous chemistry to be significant (maximum formation was estimated to be of the order of $1 \mathrm{ng} / \mathrm{m}^{3}$ ); (2) The CPC and the AMS sulfate measurements showed virtually the same enhancement factor. The CPC counts are mostly determined by particles smaller than $100 \mathrm{~nm}$ and the AMS measurements (of aerosol species mass) are mostly determined by particles larger than $100 \mathrm{~nm}$. The fact that the enhancement factors measured with these two different instruments agreed is an indication that the deviation from the theoretical enhancement efficiency (based on the VACES flows) is not due to changes in the AMS collection efficiency. The AMS can have lower than unity particle collection efficiency mainly due to particle bounce at the vaporizer (Allan et al., 2004; T. Onasch, Aerodyne Research, Personal Communication, 2004), which can be a function of particle composition and water content. Given that concentrated particles were equilibrated back to ambient conditions after the concentrator and before AMS analysis and that the observed condensation of additional material is very small, we believe that the AMS collection efficiency stays essentially the same during each experiment.

On 18/9/02 and 19/9/02 the concentration factors for nitrate, organics, and ammonium were about two times higher than those of sulfate, indicating that some extra particulate material had been formed in the concentrator. The size distributions of organics and especially nitrate on these 2 days were distorted after the concentrator (Fig. 1). Note that the original mode of nitrate mass size distribution changes approximately by the factor close to that of sulfate, while the increase in mass is due to the appearance of the second mode at smaller sizes. This change in the size distributions is another indication that an extra amount of nitrate, ammonia and organics was formed in the concentrator.

The amount of the extra material (artifact) was calculated using the following formula:

$$
\Delta C=\frac{C-C_{\mathrm{a}} X}{X}
$$


(A)
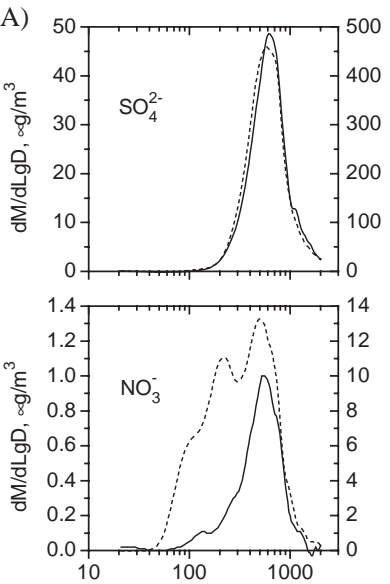

Vacuum aerodynamic diameter, $\mathrm{nm}$
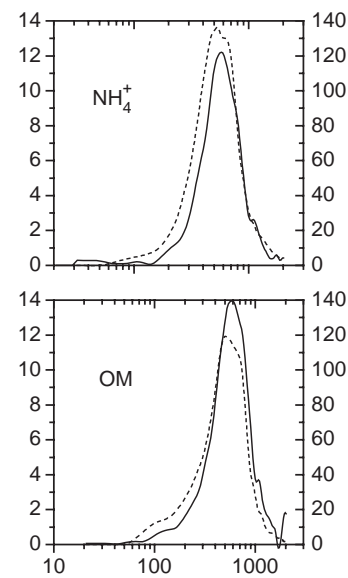

Vacuum aerodynamic diameter, $\mathrm{nm}$
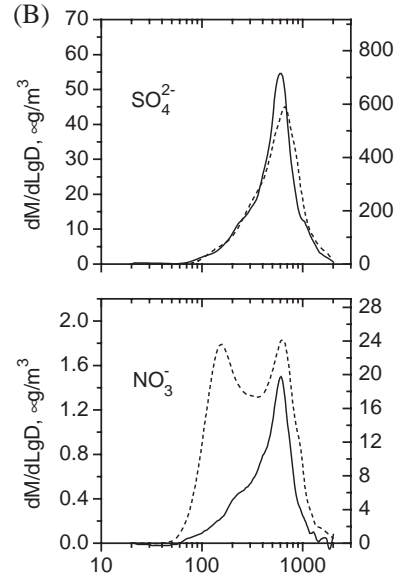

Vacuum aerodynamic diameter, $\mathrm{nm}$
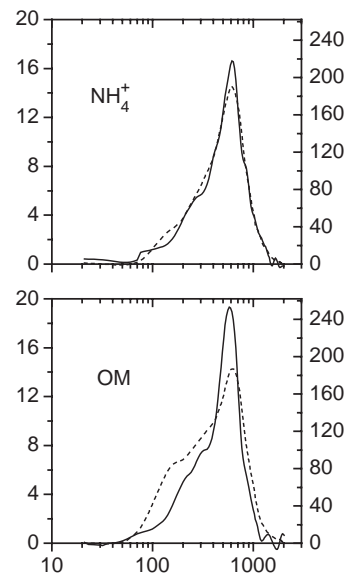

Vacuum aerodynamic diameter, $\mathrm{nm}$
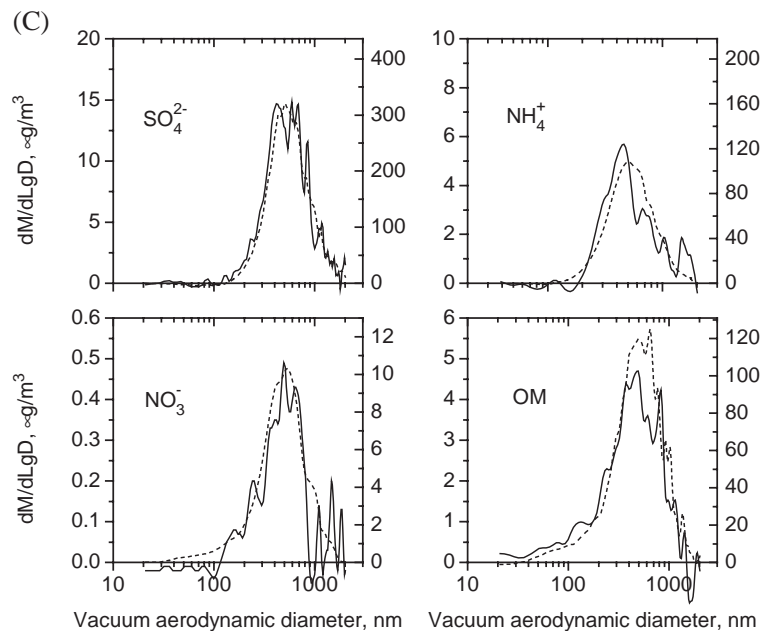

Fig. 1. Size distributions of sulfate, ammonium, nitrate, and organics measured without and with the VACES concentrator (solid and broken lines, respectively). Measurements with the concentrator are plotted using the right-hand axes, which were scaled by the corresponding concentration factor of sulfate. Note the difference in scales for sulfate and nitrate. (A) September 10, 2002; (B) September 18, 2002; (C) September 19, 2002.

in which $\Delta C$ is the concentration of the substance that has formed in the concentrator; $C$ and $C_{\mathrm{a}}$ are the concentrations measured with and without the concentrator, respectively; $X$ is the actual (or true) concentration factor taken to be that of sulfate, which is the least likely subjected to the condensation artifacts.

The calculated artifacts are given in Table 2. The table also contains relative magnitude of the artifacts: relatively to the original concentration of the species as well as relatively to the total $\left(\mathrm{PM}_{2.5}\right)$ aerosol mass. It is clear that even though the relative magnitude of the artifact may be large, its absolute value is small. For example, for the 3 days of the study the average nitrate artifact was found to be $131 \%, 159 \%$, and $21 \%$ of the original nitrate concentration. However, the corresponding average absolute artifact concentrations 
Table 2

Average concentrations of the extra ammonium, nitrate and organics formed in the concentrator (see Eq. (1)), their fractions of the original and concentrated concentration of each of the species $\left(\mathrm{d} C / C_{\mathrm{a}}, \mathrm{d} C / C_{\mathrm{c}}\right.$, respectively), and their fractions of the total aerosol mass $(\mathrm{d} C / M)$. Standard deviations of each value are also given

\begin{tabular}{|c|c|c|c|c|c|c|c|c|c|c|c|c|}
\hline \multirow[t]{2}{*}{ Date } & \multicolumn{3}{|l|}{ Ammonium } & \multicolumn{3}{|c|}{ Nitrate } & \multicolumn{3}{|r|}{ Organics } & \multirow[b]{2}{*}{$\begin{array}{l}\mathrm{d} C / C_{\mathrm{a}} \\
(\%)\end{array}$} & \multirow[b]{2}{*}{$\begin{array}{l}\mathrm{d} C / C_{\mathrm{c}} \\
(\%)\end{array}$} & \multirow[b]{2}{*}{$\begin{array}{l}\mathrm{d} C / M \\
(\%)\end{array}$} \\
\hline & $\begin{array}{l}\mathrm{d} C \\
\left(\mu \mathrm{g} / \mathrm{m}^{3}\right)\end{array}$ & $\begin{array}{l}\mathrm{d} C / C_{\mathrm{a}} \\
(\%)\end{array}$ & $\begin{array}{l}\mathrm{d} C / C_{\mathrm{c}} \\
(\%)\end{array}$ & $\begin{array}{l}\mathrm{d} C / M \\
(\%)\end{array}$ & $\begin{array}{l}\mathrm{d} C \\
\left(\mu \mathrm{g} / \mathrm{m}^{3}\right)\end{array}$ & $\begin{array}{l}\mathrm{d} C / C_{\mathrm{a}} \\
(\%)\end{array}$ & $\begin{array}{l}\mathrm{d} C / C_{\mathrm{c}} \\
(\%)\end{array}$ & $\begin{array}{l}\mathrm{d} C / M \\
(\%)\end{array}$ & $\begin{array}{l}\mathrm{d} C \\
\left(\mu \mathrm{g} / \mathrm{m}^{3}\right)\end{array}$ & & & \\
\hline $10 / 9 / 02$ & $1.05 \pm 0.6$ & $21.7 \pm 12.3$ & $1.7 \pm 0.6$ & $3.5 \pm 1.9$ & $0.55 \pm 0.1$ & $131 \pm 26$ & $5.5 \pm 1.0$ & $1.8 \pm 0.3$ & $-0.01 \pm 0.4$ & $-0.1 \pm 6.4$ & $0.0 \pm 0.7$ & $0.0 \pm 1$ \\
\hline $18 / 9 / 02$ & $-0.01 \pm 0.7$ & $-0.2 \pm 1.4$ & $0.0 \pm 0.7$ & $0.0 \pm 0.2$ & $1.0 \pm 0.1$ & $159 \pm 25$ & $4.7 \pm 0.5$ & $2.7 \pm 0.3$ & $1.75 \pm 0.5$ & $23.1 \pm 6.8$ & $1.4 \pm 0.4$ & $4.8 \pm 1.4$ \\
\hline $19 / 9 / 02$ & $-0.04 \pm 0.4$ & $-1.6 \pm 16$ & $-0.1 \pm 0.8$ & $-0.3 \pm 0.5$ & $0.04 \pm 0.0$ & $20.9 \pm 23$ & $0.8 \pm 0.0$ & $0.3 \pm 0.4$ & $0.29 \pm 0.3$ & $10.0 \pm 10.8$ & $0.4 \pm 0.4$ & $2.4 \pm 2.6$ \\
\hline
\end{tabular}

are $0.55,1.0$, and $0.04 \mu \mathrm{g} / \mathrm{m}^{3}$. Because of the low absolute values of the artifact, its contribution to the total aerosol mass is very small (less than $3 \%$ for nitrate).

\subsection{Partitioning of semi-volatile components during concentration}

The most probable reason for formation of the extra nitrate in the concentrator is a redistribution of nitric acid from the gas to the aerosol phase in the concentrator. Even though the aerosol is re-equilibrated to the original conditions (relative humidity and temperature) after the concentrator, some of the semivolatile material that was in the gas phase upstream of the concentrator may be left in the aerosol phase after the concentrator, as is explained in the following analysis. It should be kept in mind that this analysis assumes an "ideal" situation, i.e. the aerosol and the gas phase are in equilibrium at any time during the passage through the concentrator (i.e. there are no kinetic limitations for reaching equilibrium) and there are no losses of gaseous species or aerosol particles within the concentrator.

Let us assume that in the ambient air there is a semi-volatile substance that is in thermodynamic equilibrium with its solid (aerosol) phase at ambient temperature $\left(T_{\mathrm{a}}\right)$ and relative humidity $\left(\mathrm{RH}_{\mathrm{a}}\right)$. Let the concentration of the substance in the aerosol phase be $C_{\mathrm{a}}$ and its saturation concentration (vapor pressure) as a function of temperature and relative humidity is $C_{\text {sat }}(T, \mathrm{RH})$. Further, let the sample air in the condenser be at temperature $T_{\mathrm{c}}$ and relative humidity $\mathrm{RH}_{\mathrm{c}}$.

When the air is cooled in the condenser the saturation vapor pressure of the substance is decreased and some of the gas condenses on the particles. The amount of condensed material $\left(C_{\text {con }}\right)$ per unit volume of air is

$$
C_{\text {con }}=C_{\text {sat }}\left(T_{\mathrm{a}}, \mathrm{RH}_{\mathrm{a}}\right)-C_{\mathrm{sat}}\left(T_{\mathrm{c}}, \mathrm{RH}_{\mathrm{c}}\right)
$$

After the condenser the sample air passes through the virtual impactor, which is at conditions close to those of the condenser. The aerosol and some of the gas exit the impactor with the minor flow. Let the aerosol concentration in the minor flow be increased relatively to the inlet flow by a concentration factor $X$. In the minor flow, the concentration of species in the gas phase is $C_{\mathrm{sat}}\left(T_{\mathrm{c}}, \mathrm{RH}_{\mathrm{c}}\right)$ and the concentration in the aerosol is

$$
C_{\text {minor }}=\left(C_{\mathrm{a}}+C_{\mathrm{con}}\right) X \text {. }
$$


After the minor flow is re-equilibrated to the ambient temperature and dried to $\mathrm{RH}_{\mathrm{d}}$, some of the substance in the aerosol phase is evaporated. The amount of evaporated substance is

$$
C_{\text {evap }}=C_{\text {sat }}\left(T_{\mathrm{a}}, \mathrm{RH}_{\mathrm{d}}\right)-C_{\text {sat }}\left(T_{\mathrm{c}}, \mathrm{RH}_{\mathrm{c}}\right) .
$$

The concentration in the aerosol phase after re-equilibration is the concentration of the aerosol in the minor flow before re-equilibration minus the amount of evaporated material during the equilibration. Using Eqs. (2) and (3) into Eq. (4), the aerosol concentration after re-equilibration, i.e. the actual output from the concentrator is

$$
C_{\text {final }}=C_{\mathrm{a}} X+\Delta C_{\mathrm{a}}
$$

in which $\Delta C_{\mathrm{a}}$ is the amount of extra substance that was formed in the aerosol phase by passage through the concentrator:

$$
\Delta C_{\mathrm{a}}=\left(C_{\mathrm{sat}}\left(T_{\mathrm{a}}, \mathrm{RH}_{\mathrm{a}}\right)-C_{\mathrm{sat}}\left(T_{\mathrm{c}}, \mathrm{RH}_{\mathrm{c}}\right)\right) X-C_{\text {sat }}\left(T_{\mathrm{a}}, \mathrm{RH}_{\mathrm{d}}\right)+C_{\mathrm{sat}}\left(T_{\mathrm{c}}, \mathrm{RH}_{\mathrm{c}}\right),
$$

which, in turn, can be approximated by neglecting the difference in $C_{\text {sat }}\left(T_{\mathrm{a}}, \mathrm{RH}_{\mathrm{a}}\right)$ and $C_{\text {sat }}\left(T_{\mathrm{a}}, \mathrm{RH}_{\mathrm{d}}\right)$ :

$$
\Delta C_{\mathrm{a}}=\left(C_{\mathrm{sat}}\left(T_{\mathrm{a}}, \mathrm{RH}_{\mathrm{a}}\right)-C_{\mathrm{sat}}\left(T_{\mathrm{c}}, \mathrm{RH}_{\mathrm{c}}\right)\right)(X-1) .
$$

The amount of extra aerosol species that is formed in the concentrator is, thus, proportional to the difference in the saturation concentration (vapor pressure) of the species at the conditions of the ambient air and inside the condenser.

The apparent concentration factor (the ratio of the aerosol concentration after the concentrator to the aerosol concentration in the ambient air) is then:

$$
X_{\mathrm{a}}=X+(X-1) \frac{\Delta C_{\mathrm{sat}}}{C_{\mathrm{a}}}
$$

in which $\Delta C_{\text {sat }}$ is the difference in the saturation concentration (vapor pressure) of the species at the conditions of the ambient air and inside the condenser.

A better measure of the formation of extra material is the relative error in the concentration factor:

$$
E_{\mathrm{x}}=\frac{X_{\mathrm{a}}-X}{X}=\left(1-\frac{1}{X}\right) \frac{\Delta C_{\mathrm{sat}}}{C_{\mathrm{a}}} .
$$

In our analysis so far we have neglected the effect of the humidifier. The humidifier has no effect on the output aerosol concentration because the process is reversible (the system is closed during transit from the humidifier to the condenser, while after the separation in the virtual impactor it is not).

From Eq. (9) it follows, that the relative error in the concentration factor is higher at higher concentration factors and at lower aerosol ambient concentrations. It is also proportional to $\Delta C_{\text {sat }}$. According to the Clausius-Clapeyron equation, substances that have a larger enthalpy of vaporization will have a larger $\Delta C_{\text {sat }}$ and thus exhibit larger errors. It should be noted that an aerosol species can be formed even if its gaseous concentration is lower than the saturation pressure at ambient conditions, but is higher than the saturation pressure at the conditions in the condenser.

$\Delta C_{\text {sat }}$, and, thus, the relative error in the concentration factor (Eq. (9)), also depends on the difference in temperatures between the ambient air and the air inside the condenser. The VACES concentrator was designed in such a way that the air in the condenser is at $20^{\circ} \mathrm{C}$. This prevents much of a temperature 
difference between the condenser and the sample air, especially if it is equilibrated to room temperature, which is often the case, especially in the human and animal exposure studies. Thus, most of the potential artifact formation for species, which vapor pressure does not depend on relative humidity, is avoided. If the sample air temperature differs from $20^{\circ} \mathrm{C}$, artifact formation according to Eq. (8) may be expected. In that case it is recommended that the temperature setting of VACES be adjusted such that the temperature of the air in the condenser is equal to the ambient temperature.

$\Delta C_{\text {sat }}$, also depends on the relative humidity, especially for water-soluble species. For example, ammonium nitrate has a negligible vapor pressure at $100 \% \mathrm{RH}$, the conditions of the air in the condenser. In this case all of the gas phase will be condensed onto the particles in the condenser (assuming no losses) and the formation of extra material would be proportional to the amount of the substance in the gas phase at ambient conditions. Thus, the effect of the concentrator on water-soluble species is expected to be stronger than that for water-insoluble substances. This agrees with our observations that show a larger change in nitrate mass in comparison to that of organics, which are often not or less hygroscopic and which vapor pressure is not expected to depend strongly on RH. It should be noted that organics partition to the aerosol before their saturation vapor pressure is reached by adsorption and absorption, depending on the amount and properties of the pre-existing aerosol organic phase. For a more rigorous treatment of organic artifacts, the analysis here would need to be modified using the formulation of Pankow (1994).

The observations of the increased nitrate mass in the concentrator were used to calculate the amount of the gas that has condensed onto the particles in the concentrator using the following formula, which follows from Eq. (8):

$$
\Delta C_{\mathrm{NO}_{3}}=\frac{C_{\text {final }}-C_{\mathrm{a}} X}{X-1}
$$

in which $\Delta C_{\mathrm{NO}_{3}}$ is the concentration of nitrate that has condensed onto particles in the condenser; $C_{\text {final }}$ and $C_{\mathrm{a}}$ are the concentrations of nitrate measured with and without the concentrator, respectively; $X$ is the actual (or true) concentration factor taken to be that of sulfate, which is the species least likely subjected to condensation artifacts. It should be noted that the amount of the condensed gas is larger than the observed artifact mass (Eq. (1)), because a part of the condensed gas has re-evaporated after the aerosol was equilibrated after exiting the condenser.

During the experiments on September 10, 18 and 19 on average $0.6,1.1$ and less than $0.1 \mu \mathrm{g} / \mathrm{m}^{3}$ of nitrate, respectively, has condensed to the aerosol in the concentrator (Table 1). The difference between the first 2 days and the last one was mostly in the air pollution conditions, with the former two being more polluted than the latter. The $\mathrm{PM}_{2.5}$ sulfate concentration on 10 and 18 September was 19 and $21 \mu \mathrm{g} / \mathrm{m}^{3}$, respectively. On 19 September the concentration decreased to $6.6 \mu \mathrm{g} / \mathrm{m}^{3}$. Therefore, the virtual absence of extra nitrate formation on 19 September is probably due to limited availability of nitric acid.

It should be also noted that the condensed amounts on 10 and 18 September are relatively low in comparison to the maximum possible under the conditions of the experiments. Unfortunately, no measurements of nitric acid or ammonia are available for this period to perform a direct comparison. However, under similar conditions nitric acid concentrations of $5-10 \mu \mathrm{g} / \mathrm{m}^{3}$ were measured in Pittsburgh a year prior to this study. If there are no losses of the gas-phase components in the VACES, practically all of the available nitric acid should have been condensed to the particles. In fact, only a small fraction (about $10 \%$ ) of the estimated nitric acid concentration has condensed on the particles. This is most probably due to the losses of gas-phase nitric acid which is stripped by water in the saturator of the VACES as well as other wall losses. 


\subsection{Size dependence of the artifact}

Fig. 1 shows size distributions of the measured chemical species during the three experiments. The size distributions during the VACES runs were scaled by the concentration factors of sulfate for each of the runs. The size distributions of sulfate are preserved after the passage through the concentrator, while that of nitrate, and to a lesser degree those of ammonium and organics, are not, except for the run of 19 September. The striking feature on 10 and 18 September is the increase of nitrate concentration at small sizes, in the size range of 100-200 nm. Similarly, on these days the enhancement factor for the integrated sulfate concentration is smaller than the factor for nitrate (Table 1). The change of size distribution of nitrate, together with its concentration factor that is higher than that of sulfate, suggests formation of nitrate in VACES on 10 and 18 September. The changes in the size distributions of nitrate on September 10 and 18 are far larger than the uncertainties of the AMS measurement.

As Fig. 1 shows, the extra nitrate (as well as some organics) was mostly formed at small sizes (smaller than about $300 \mathrm{~nm}$ ), while the shape of the distribution was mostly preserved at and around the mode of the mass distribution (500-700 nm). The probable reason for such behavior is the shape of the size distribution of the aerosol surface area. Condensation of the gas-phase species will proceed mostly to the size range of maximum condensational sink, as described in Pirjola et al. (1999). The average size distributions of condensational sink of ambient aerosol during the experiments, as measured with the SMPS, are shown in Fig. 2. The maximum of the condensational sink distribution is located at sizes smaller than the mode of the volume (mass distribution), which explains why the extra nitrate was forming mostly at small sizes. However, the exact position of the extra nitrate on the size distribution will be controlled by the kinetics of nitrate condensation, which occurs simultaneously to water condensation. Because nitrate condenses during the droplet growth, which changes the aerosol surface distribution, the final result may only be determined by numerical calculations of condensational processes. Such calculations, however, are beyond the scope of this work.

\subsection{Modeling of artifact sensitivity to availability of ammonia and nitric acid}

We have used the GFEMN thermodynamic model (Ansari \& Pandis, 1999) to assess the effect of ammonia and nitric acid availability on the error in the concentration factor for nitrate aerosol. The aerosol was assumed to contain either 10 or $20 \mu \mathrm{g} / \mathrm{m}^{3}$ of sulfate, while concentrations of total available ammonia and nitric acid were varied. For these simulations the ambient temperature was set to $20{ }^{\circ} \mathrm{C}$ and relative humidity to $50 \%$. Based on our observations (see above) we assumed that $90 \%$ of the gas-phase ammonia and nitric acid were lost in the humidifier and the remaining $10 \%$ of the gases condensed onto the particles prior to their entrance into the virtual impactor. The concentration of the aerosol was then increased by the concentration factor, which was taken to be 20 . After that the model "equilibrated" the concentrated aerosol to the conditions found after the VACES $\left(20^{\circ} \mathrm{C}\right.$ and $\left.35 \% \mathrm{RH}\right)$.

The ratio of the predicted concentration factor for nitrate to the theoretical concentration factor (set to be 20) as a function of the total available ammonia and nitric acid is shown in Fig. 3. The modeling results indicate that the error is higher in ammonia-limited conditions, while in ammonia-rich environment the modeled factor approaches the theoretical one. It should be noted that the high increase in the concentration factor at low ammonia concentrations is due to the low initial aerosol nitrate concentrations at those conditions. The absolute artifact in the aerosol nitrate after the concentrator as determined by Eq. (1) is shown in Fig. 4. 


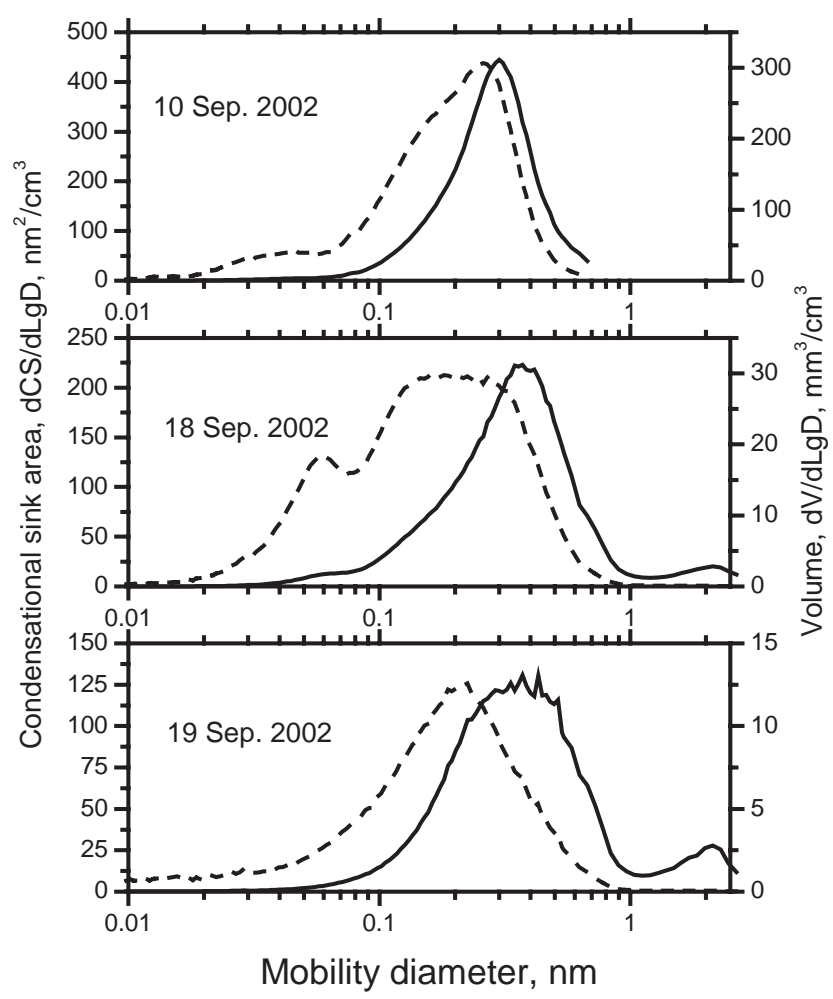

Fig. 2. Volume (solid lines) and condensational sink (broken lines) size distributions measured with the SMPS.

Our observations support the trend predicted by the model. In our experiments the artifact was observed on 10 and 18 September, when the measured $\mathrm{NH}_{4}^{+}$mass concentrations were approximately $10-30 \%$ in deficit to fully neutralize the measured $\mathrm{NO}_{3}^{-}$and $\mathrm{SO}_{4}^{2-}$, indicating ammonia-limited conditions. On September 19, when no discernable artifact was observed, the aerosol was neutral, indicating that the conditions were not ammonia-limited. The sensitivity of the artifact to availability of ammonia is probably also the reason why no artifact was observed in the previous tests which were carried out either in California in ammonia-rich conditions (Geller et al., 2002; Kim et al., 2001b; Misra et al., 2004) or in Pittsburgh in March (Zhao et al., 2004) when the aerosol was neutral.

\subsection{Recommendations}

Given the potential of artifact formation in the concentrators using water-condensation technology it is recommended to use a denuder to remove interfering species from the air stream prior to its entrance into the humidifier. This, however, may lead to particle losses in the ultra-fine size range. Even though no artifact formation was observed on the cleanest day of the study (19 September), care should be taken when using VACES for nucleation and new particle growth studies, since even a small artifact can be large compared to the very small mass of these particles. For the exposure studies we would recommend to monitor concentration of species before and after the concentrator such that concentration factors for each 


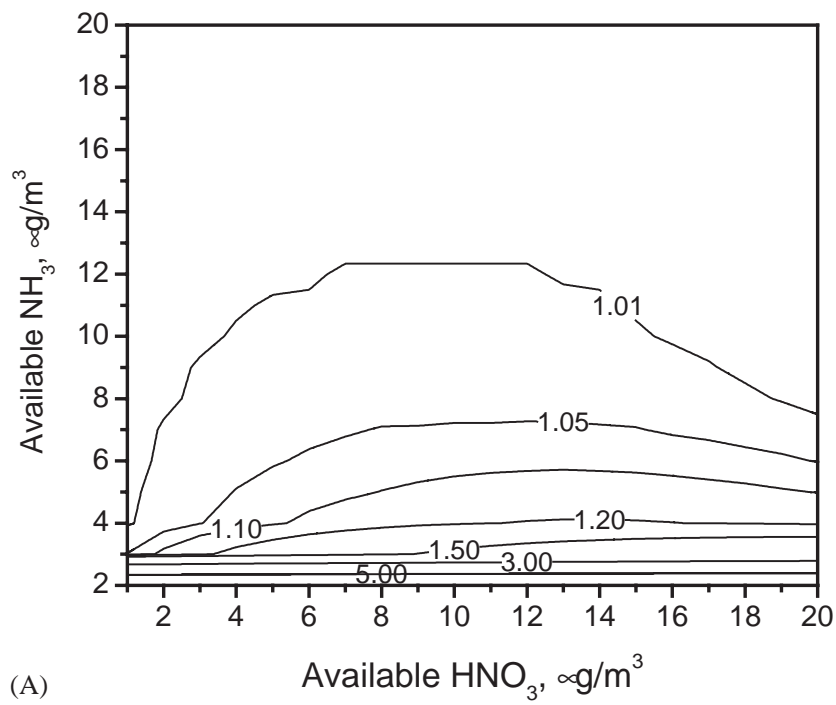

(A)

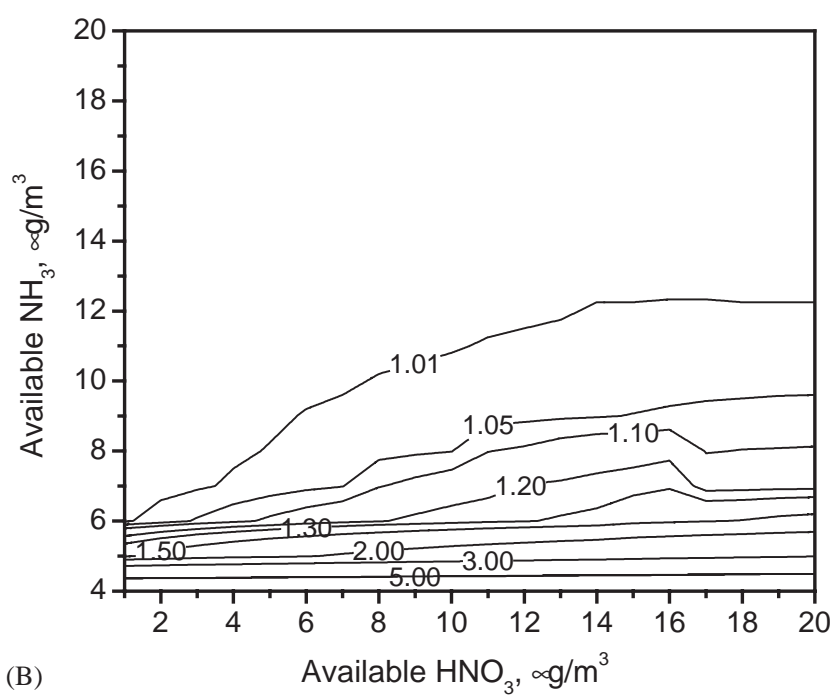

Fig. 3. Modeled increase in the concentration factor for aerosol nitrate relatively to the theoretical concentration factor at different amounts of available ammonia and nitric acid. (A) sulfate concentration is $10 \mu \mathrm{g} / \mathrm{m}^{3}$; (B) sulfate concentration is $20 \mu \mathrm{g} / \mathrm{m}^{3}$.

compound are known. These individual concentration factors can be then used to assess health effects (or lack thereof) of each compound. We would also like to reiterate, that the observed artifact formation is small in comparison to the total mass concentration of the concentrated aerosol.

\section{Summary and conclusions}

The effect of water condensation on semi-volatile aerosol species during their passage through a particle concentrator was assessed using the VACES concentrator coupled to the Aerodyne Aerosol Mass 

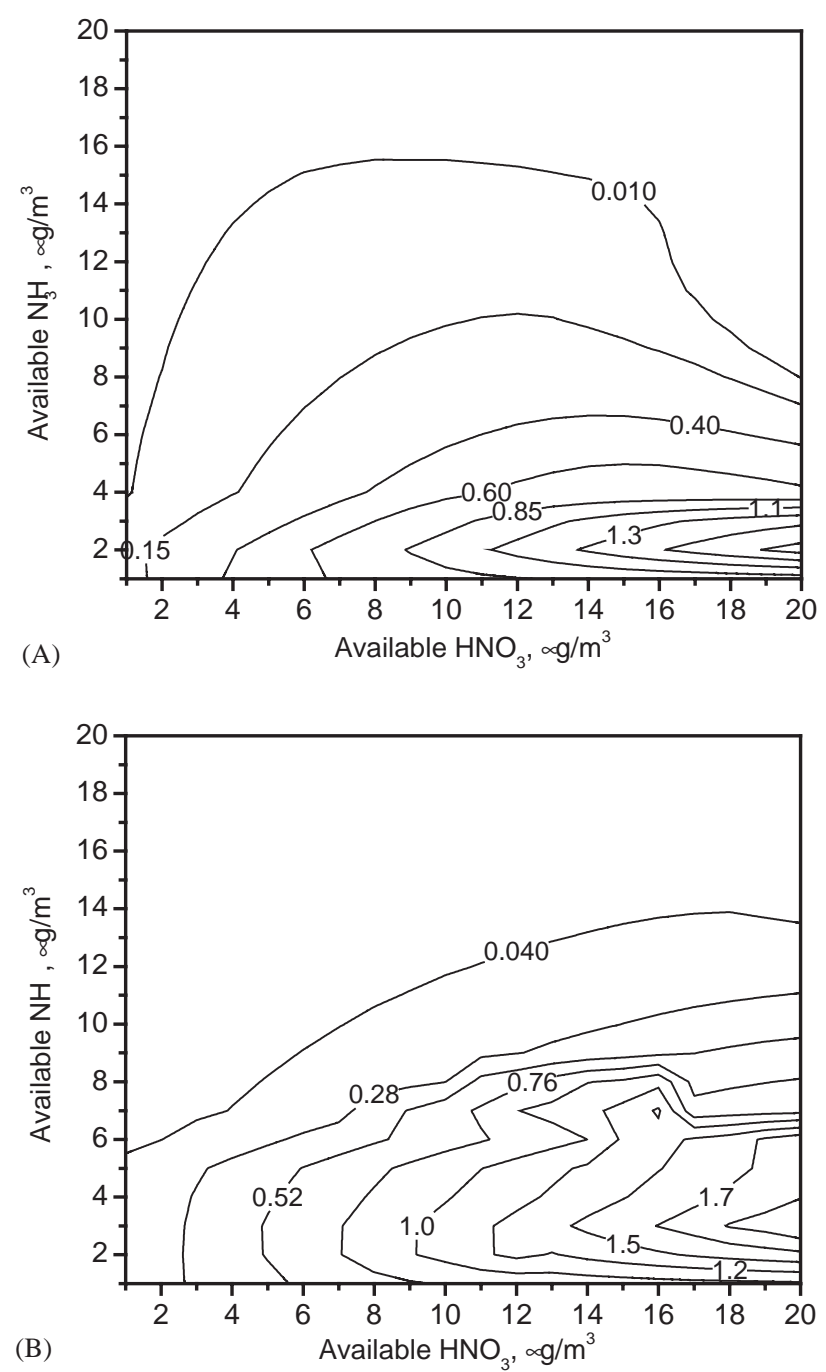

Fig. 4. Modeled increase in the concentration aerosol nitrate $\left(\mu \mathrm{g} / \mathrm{m}^{3}\right)$ due to the artifact after the concentrator at different amounts of available ammonia and nitric acid. (A) sulfate concentration is $10 \mu \mathrm{g} / \mathrm{m}^{3}$; (B) sulfate concentration is $20 \mu \mathrm{g} / \mathrm{m}^{3}$.

Spectrometer during measurements of ambient aerosol, with the following conclusions:

- The size distribution of sulfate is preserved after passage through the concentrator at all conditions encountered during the study. The concentration factors determined for sulfate mass do not always equal the theoretical concentration factors, but are very close to those simultaneously determined for total particle number using a CPC.

- The size distributions of ammonium, organics and nitrate are preserved at relatively clean conditions (sulfate concentrations around $7 \mu \mathrm{g} / \mathrm{m}^{3}$ ), while under more polluted conditions (sulfate concentrations around $20 \mu \mathrm{g} / \mathrm{m}^{3}$ ) they exhibit an increase at small sizes (50-300 $\mathrm{nm}$ in vacuum aerodynamic diameter). Such an increase is especially pronounced for nitrate. 
- The increase of nitrate and organic concentrations at small sizes after the passage through the concentrator corresponds to the sizes of maximum aerosol surface area and fastest gas-to-particle transfer.

- The absolute increase in concentration of nitrate observed in this study is rather small, of the order of $1 \mu \mathrm{g} / \mathrm{m}^{3}$ or less (i.e., $0.3-2.7 \%$ of the total aerosol mass concentration) and can be neglected for many practical purposes.

- An analysis of thermodynamic processes in the concentrator indicates that the formation of extra material in the concentrator is likely due to redistribution of the gas-phase material to the aerosol phase. The modeling indicates that nitrate artifact will be most pronounced in ammonia-limited conditions, while in ammonia-rich environment it becomes negligible.

- Even though the extent of the artifact is limited, to avoid its formation completely it is recommended to operate VACES with a denuder and/or with its condenser temperature set equal to the ambient temperature. If no denuder is used, we recommend that concentration of individual compounds be measured both before and after concentrator, such that concentration factors for individual species are known.

\section{Acknowledgements}

This research was conducted as part of the Pittsburgh Air Quality Study, which was supported by US Environmental Protection Agency under Contract R82806101 and the US Department of Energy National Energy Technology Laboratory under Contract DE-FC26-01NT41017. Q. Zhang and J.L. Jimenez's participation in this study was sponsored by the University of Colorado (J.L. Jimenez's startup funds). This work was additionally supported by the Southern California Particle Center and Supersite (SCPCS), funded by the US EPA (STAR award \#R82735201) and the California Air Resources Board (ARB; Grant \# 98-316). This manuscript has not been subjected to the EPA and ARB's peer and policy review, and therefore does not necessarily reflect the views of the Agencies. No official endorsement should be inferred.

\section{References}

Alfarra, M. R., Coe, H., Allan, J. D., Bower, K. N., Boudries, H., Canagaratna, M. R., Jimenez, J. L., Jayne, J. T., Garforth, A., Li, S. M., \& Worsnop, D. R. (2004). Characterization of urban and regional organic aerosols in the lower Fraser Valley using two aerodyne aerosol mass spectrometers. Atmospheric Environment, 38, 5745-5758.

Allan, J. D., Bower, K. N., Coe, H., Boudries, H., Jayne, J. T., Canagaratna, M. R., Millet, D. B., Goldstein, A. H., Quinn, P. K., Weber, R. J., \& Worsnop, D. R. (2004). Submicron aerosol composition at Trinidad Head, CA during ITCT 2K2, its relationship with gas phase volatile organic carbon and assessment of instrument performance. Journal of Geophysical Research-Atmospheres, 109(D23) D23S24, doi: 10.1029/2003JD004208.

Allan, J. D., Jimenez, J. L., Williams, P. I., Alfarra, M. R., Bower, K. N., Jayne, J. T., Coe, H., \& Worsnop, D. R. (2003). Quantitative sampling using an aerodyne aerosol mass spectrometer: 1. Techniques of data interpretation and error analysis (vol 108, art no 4090, 2003). Journal of Geophysical Research-Atmospheres, 108(D9).

Ansari, A. S., \& Pandis, S. N. (1999). Prediction of multicomponent inorganic atmospheric aerosol behavior. Atmospheric Environment, 33(5), 745-757.

Eatough, D. J., Pang, Y. B., \& Eatough, N. L. (1999). Determination of PM2.5 sulfate and nitrate with a PC-BOSS designed for routine sampling for semi-volatile particulate matter. Journal of the Air \& Waste Management Association, 49, 69-75.

Geller, M. D., Kim, S., Misra, C., Sioutas, C., Olson, B. A., \& Marple, V. A. (2002). A methodology for measuring size-dependent chemical composition of ultrafine particles. Aerosol Science and Technology, 36(6), 748-762. 
Jayne, J. T., Leard, D. C., Zhang, X. F., Davidovits, P., Smith, K. A., Kolb, C. E., \& Worsnop, D. R. (2000). Development of an aerosol mass spectrometer for size and composition analysis of submicron particles. Aerosol Science and Technology, 33(1-2), 49-70.

Jimenez, J. L., Jayne, J. T., Shi, Q., Kolb, C. E., Worsnop, D. R., Yourshaw, I., Seinfeld, J. H., Flagan, R. C., Zhang, X. F., Smith, K. A., Morris, J. W., \& Davidovits, P. (2003). Ambient aerosol sampling using the aerodyne aerosol mass spectrometer. Journal of Geophysical Research-Atmospheres 108(D7).

Kim, S., Jaques, P. A., Chang, M. C., Barone, T., Xiong, C., Friedlander, S. K., \& Sioutas, C. (2001a). Versatile aerosol concentration enrichment system (VACES) for simultaneous in vivo and in vitro evaluation of toxic effects of ultrafine, fine and coarse ambient particles_-Part II: field evaluation. Journal of Aerosol Science, 32(11), 1299-1314.

Kim, S., Jaques, P. A., Chang, M. C., Froines, J. R., \& Sioutas, C. (2001b). Versatile aerosol concentration enrichment system (VACES) for simultaneous in vivo and in vitro evaluation of toxic effects of ultrafine, fine and coarse ambient particles-Part I: development and laboratory characterization. Journal of Aerosol Science, 32(11), 1281-1297.

Misra, C., Fine, P. M., Singh, M., \& Sioutas, C. (2004). Development and evaluation of a compact facility for exposing humans to concentrated ambient ultrafine particles. Aerosol Science and Technology, 38(1), 27-35.

Pankow, J. F. (1994). An absorption-model of gas-particle partitioning of organic-compounds in the atmosphere. Atmospheric Environment, 28(2), 185-188.

Peters, A., Dockery, D. W., Heinrich, J., \& Wichmann, H. E. (1997). Short-term effects of particulate air pollution on respiratory morbidity in asthmatic children. European Respiratory Journal, 10(4), 872-879.

Pirjola, L., Kulmala, M., Wilck, M., Bischoff, A., Stratmann, F., \& Otto, E. (1999). Formation of sulfuric acid aerosols and cloud condensation nuclei: an expression for significant nucleation and model comparison. Journal of Aerosol Science, $30(8), 1079$ -1094 .

Pope, C. A., Dockery, D. W., \& Schwartz, J. (1995). Review of epidemiological evidence of health-effects of particulate airpollution. Inhalation Toxicology, 7(1), 1-18.

Sioutas, C., Kim, S., \& Chang, M. (1999). Development and evaluation of a prototype ultrafine particle concentrator. Journal of Aerosol Science, 30(8), 1001-1017.

Thurston, G. D., Ito, K., Hayes, C. G., Bates, D. V., \& Lippmann, M. (1994). Respiratory hospital admissions and summertime haze air-pollution in Toronto, Ontario-consideration of the role of acid aerosols. Environmental Research, 65(2), $271-290$.

Willeke, K., \& Baron, P. A. (1993). Aerosol measurement: principles, techniques, and applications. New York: Van Nostrand Reinhold.

Wittig, A. E., Anderson, N., Khlystov, A. Y., Pandis, S. N., Davidson, C., \& Robinson, A. L. (2004). Pittsburgh Air Quality Study overview and preliminary scientific findings. Atmospheric Environment, 38, 3107-3125.

Zhang, Q., Canagaratna, M. C., Jayne, J. T., Worsnop, D. R., \& Jimenez, J. L. (2004a). Size-Resolved chemical composition and temporal variation of submicron particles in Pittsburgh-implications on aerosol sources and processes. Journal of Geophysical Research-Atmospheres, in press.

Zhang, Q., Stanier, C. O., Canagaratna, M. C., Jayne, J. T., Worsnop, D. R., Pandis, S. N., \& Jimenez, J. L. (2004b). Insights into the chemistry of nucleation bursts and new particle growth events in Pittsburgh based on aerosol mass spectrometry. Environmental Science and Technology, 38(18), 4797-4809.

Zhao, Y., Bein, K. J., Wexler, A. S., Misra, C., Fine, P. M., \& Sioutas, C. (2004). Using a particle concentrator to increase the hit rates of single particle mass spectrometers. Journal of Geophysical Research-Atmospheres, in press. 


\title{
An Algorithm for Combining Electrical Mobility and Aerodynamic Size Distributions Data when Measuring Ambient Aerosol
}

\author{
A. Khlystov, C. Stanier, and S. N. Pandis \\ Carnegie Mellon University, Department of Chemical Engineering, Pittsburgh, Pennsylvania
}

\begin{abstract}
Ambient aerosol particles vary in size from a few nanometers to several micrometers. No instrument is currently available to cover such a wide size range, and so a combination of several instruments is usually used. One such combination is that of electrical mobility classifiers and an aerodynamic sizer. Because of the differences in measurement principles between the instruments, difficulties arise in the combination of the measurements into a single size distribution. Here we report a simple algorithm that was developed to combine aerosol size distributions measured with commercially available scanning mobility particle sizers (SMPS; TSI Inc.) and an aerodynamic particle sizer (APS; TSI Inc.). This algorithm was tested during July 2001 in the Pittsburgh Air Quality Study. The aerosol during the study had both urban and regional origin and is characteristic of urban atmosphere in the Northeastern U.S. The integrated volume concentrations from the SMPS-APS showed a good correlation with $\mathbf{P M}_{2.5}$ mass concentration measurements using a TEOM. The relation of the aerosol mass to its volume is an "effective" density, a ratio of the bulk aerosol density to the shape factor. As a result of the comparison with the TEOM the ambient aerosol in the Pittsburgh area was found to have an effective density of $1.5 \pm 0.3 \mathrm{~g} \mathrm{~cm}^{-3}$. Given that the aerosol during the study was found to always contain water, the particles are expected to be spherical and thus the shape factor may be assumed to be 1 . This assumption has been supported by a comparison with the MOUDI, using the aerosol density of $1.5 \mathrm{~g} / \mathrm{cm}^{3}$. It should be noted that the estimated aerosol density and the shape factor are applicable to this study only and may be different in other locations.
\end{abstract}

Received 23 October 2002; accepted 25 April 2003.

This research was conducted as part of the Pittsburgh Air Quality Study, which was supported by US Environmental Protection Agency under contract R82806101 and the US Department of Energy National Energy Technology Laboratory under contract DE-FC26-01NT41017. This article has not been subject to the EPA's required peer and policy review, and therefore does not necessarily reflect the views of the Agency. No official endorsement should be inferred.

Address correspondence to S. N. Pandis, Carnegie Mellon University, Department of Chemical Engineering, Pittsburgh, PA 15213. E-mail: spyros@andrew.cmu.edu

\section{INTRODUCTION}

Ambient aerosol plays an important role in the atmosphere, influencing visibility, affecting global climate, and participating in atmospheric chemistry (Seinfeld and Pandis 1998). Recently, increased concentrations of ambient aerosol smaller than $2.5 \mu \mathrm{m}$ $\left(\mathrm{PM}_{2.5}\right)$ have been associated with increased morbidity and mortality rates (Schwartz and Dockery 1992; Dockery et al. 1993). Size distribution of atmospheric particles spans a wide size range from a few nanometers to several micrometers. In order to study aerosol dynamics in such a broad size range a combination of several separate instruments is necessary. A typical combination has been a scanning mobility particle sizer (SMPS; TSI Inc., St. Paul, MN) with an aerodynamic particle sizer (APS; TSI Inc.). This combination was employed in several recent studies to measure ambient aerosol (Shen et al. 2002; Hand and Kreidenweis 2002; Shi et al. 2001). The integrated volume concentration measured with the SMPS-APS can be used to estimate the mass concentration using an assumed bulk aerosol density (Shen et al. 2002). If this approach proves accurate, the SMPS-APS can be used as high time resolution substitution for the filter-based mass measurements.

The SMPS and the APS have different measurement principles. The SMPS classifies particles according to their mobility in an electric field (Wang and Flagan 1989). The electrical mobility size depends on the particle cross section, and for a spherical particle this is the same as the physical size. Unlike the SMPS, the APS measures aerodynamic size; particles are accelerated in a nozzle and their time-of-flight is related to their aerodynamic size (Armendariz and Leith 2002). The aerodynamic size is proportional to the physical size and the square root of particle density (Hinds 1999). Thus, for a sphere the aerodynamic size is equal to the physical size only for unit density particles.

Because of the different measurement principles (i.e., electrical mobility versus aerodynamic sizing), difficulties arise in attempts to create a single size spectrum from the data measured with the SMPS and APS. Some researchers have approached this problem by selecting certain kinds of data from each instrument; 
for example, the SMPS is used up to a certain size, and beyond that size the APS is used (Shen et al. 2002). Hand and Kreidenweis (2002) have developed an algorithm that combines electrical mobility, optical particle counter (OPC), and APS data into a single spectrum. The OPC data are first matched with the SMPS spectrum by finding an optimal refractive index. Then the APS data are matched to the modified OPC spectrum by finding an effective density that would provide the best fit with the OPC. The reason for matching the APS to the OPC and not directly to the SMPS was that the size overlap between the APS and the SMPS is rather small.

In this study we attempted to fit the APS size distribution to the SMPS distribution without using an optical particle counter as a middle ground. A simple algorithm was developed to combine the aerodynamic and electrical mobility spectra into a single distribution. The algorithm provides a ratio of the particle density to the shape factor of particles in the $540-800 \mathrm{~nm}$ size range, where the SMPS and the APS measurement ranges overlap. The ratio of aerosol density to its shape factor is usually called the "effective" density. However, the algorithm does not provide means of separating the density and the shape factor. It should also be noted that the particle shape factor and density may differ through the aerosol spectrum, as the composition and the physical properties of ambient particles usually vary with size. Instead of concentrating on the narrow overlap size range, we have determined the average (bulk) aerosol "effective" density during a month of ambient aerosol measurements within the Pittsburgh Air Quality Study (PAQS).

The measurements were carried out at the central site of the PAQS, in an urban park approximately $5 \mathrm{~km}$ from the downtown Pittsburgh. The aerosol originated from both urban and long-range sources. Ammonium salts of sulfate were the dominant aerosol components, comprising approximately $50 \%$ of the $\mathrm{PM}_{2.5}$ aerosol mass. Carbonaceous material was the second largest component, contributing approximately $25 \%$ to the mass of fine particles (Wittig et al. 2003). The results obtained with the SMPS-APS system were compared to the simultaneous measurements of the aerosol $\mathrm{PM}_{2.5}$ mass concentration using a tapered element oscillating microbalance (TEOM; R\&P Co., Albany, NY, USA) and size-fractionated mass measurements using MOUDI (MSP Co., Minneapolis, MN, USA) cascade impactor. The comparison of the $\mathrm{PM}_{2.5}$ particle volume concentration from the merged SMPS-APS distribution with the TEOM $\mathrm{PM}_{2.5}$ mass concentration has provided us with an estimate of a bulk "effective" particle density.

\section{EXPERIMENTAL}

\section{General Description of the SMPS-APS System}

The SMPS-APS system used in the PAQS was a part of the Dry-Ambient Aerosol Size Spectrometer (DAASS) described in Stanier et al. (2003). The system consists of an ultrafine SMPS system for 3-80 nm (TSI 3936N25), an SMPS for 13-680 nm (TSI 3936L10), and an APS (TSI APS 3320) covering 0.5-
$10 \mu \mathrm{m}$. In this study we used data up to $2.5 \mu \mathrm{m}$ in aerodynamic diameter. The DAASS system is equipped with a number of computer-controlled solenoid valves that direct the sample and sheath flows of the instruments either directly to the instruments or through nafion driers (Perma Pure Inc.). Singlechannel nafion dryers in stainless steel casing were used in the sample lines to minimize particle losses. The sheath lines were equipped with multichannel dryers, because of the higher capacity needed to dry larger flows and because particle loss is not an issue in particle-free flows. To avoid losses of semivolatile aerosol components the whole system was maintained at a temperature that was within $2^{\circ} \mathrm{C}$ of the ambient temperature. When the aerosol is sampled through the dryers, the DAASS provides measurements of dried ambient aerosol (at the relative humidity of $10-35 \%)$. When the sample bypasses the dryers, the system measures the aerosol at ambient relative humidity. Four dried and four "ambient" (or "wet") size distributions are measured each hour.

No impactor was used in front of the SMPS systems. It was found that the cutoff characteristics of the TSI impactor change as the sampling progresses because of accumulation of material on the impactor plate. Even with daily cleaning, it was observed that the impactor was cutting into progressively smaller-thannominal sizes. The purpose of the impactor in SMPS systems is to facilitate the data inversion. The impactor removes particles larger than the measurement range of the SMPS, such that there is no contribution of multiple-charge particles to the last channels of the instrument. If no impactor is used, the concentration in the last channels will be overestimated due to the contribution of multiple-charge particles from particles larger than the upper instrument size limit.

The effect of multiple charging was minimal in the present study. During this study the ambient aerosol number concentration was rapidly declining with size for the particles larger than $100 \mathrm{~nm}$, with the number size distribution at those sizes closely following the power law function of -3 to -6 power. In other words, when moving a factor of 2 in size the concentration drops by $2^{-3}$ to $2^{-6}$ (there are 8 to 32 times less particles). If an SMPS channel measures $700 \mathrm{~nm}$ single-charge particles, it will also measure approximately 2 times larger double-charge particles. Even though the charging for single charge at $700 \mathrm{~nm}$ is approximately equal to that for double-charge particles at $1400 \mathrm{~nm}$, the maximum contribution of double charge particles to the last SMPS channel would be about $10 \%$. A more rigorous analysis of the possible error due to the presence of multiple-charge particles in the last SMPS channels was done using the MICRON software package (Wolfenbarger and Seinfeld 1991). The results confirm that the error due to the presence of multiple-charge particles at sizes larger than the upper size limit of the SMPS is less than $10 \%$ in this study. It should also be noted that the SMPS is not designed to allow a $100 \%$ penetration of particles larger than $1 \mu \mathrm{m}$. Particle losses of the supermicrometer particles will further reduce the error due to particles carrying multiple charges outside the SMPS size range. 
The APS can be operated in two modes: "summed," in which the time-of-flight (TOF) and the optical signals are saved separately, and "correlated," in which the TOF and optical signal are saved together on a per particle basis. Since less memory storage is required in the summed mode, more bits of the TOF information can be stored. Thus, the size resolution of the instrument in that mode is better. In this study the APS was operated in the summed mode, because the correlated mode has been reported to have problems at low aerosol concentrations (Armendariz and Leith 2002).

To facilitate the matching of electrical-mobility size distributions with the aerodynamic size data, the APS was "mobility" calibrated using ammonium sulfate aerosol. Ammonium sulfate was chosen because it is the dominant aerosol component in ambient aerosol in the Pittsburgh area (Rees et al. 2003). The almost monodisperse ammonium sulfate aerosol was produced by selecting a narrow mobility range with a differential mobility analyzer (DMA; 3081, TSI Inc.) from a wider spectrum of artificially generated ammonium sulfate particles. The artificial aerosol was produced by spraying aqueous ammonium sulfate solution with a constant output atomizer (Model 3076, TSI Inc.) and drying it with a silica-gel diffusion drier. After passing the drier the aerosol was fed to the DMA. The sheath and aerosol flows of the DMA were set to be $21 \mathrm{~min}^{-1}$ and $0.21 \mathrm{~min}^{-1}$, respectively. This was done to extend the size range of the DMA to about a $1.2 \mu \mathrm{m}$ mobility diameter. The monodisperse output of the DMA was diluted with $0.81 \mathrm{~min}^{-1}$ of clean filtered air. The resulting flow of $11 \mathrm{~min}^{-1}$ containing the monodisperse aerosol was fed directly to the inner inlet nozzle of the APS. The size of the monodisperse particles was adjusted by changing the voltage of the DMA. Monodisperse ammonium sulfate aerosol with sizes from 0.4 to $1 \mu \mathrm{m}$ was used to calibrate the APS. The DMA output contained smaller amounts of double- and triplecharge particles, that have approximately 2 and 3 times larger sizes than the main peak of single-charge particles. Because the original polydisperse aerosol size distribution was rapidly decreasing with size and because the size resolution of the APS is substantially better than a factor of 2 in size, these double- and triple-charge particle peaks were not interfering with the analysis of calibration results. Since it was impossible to produce particles larger than $1.2 \mu \mathrm{m}$ with the DMA, the calibration curve obtained at smaller sizes was extrapolated to the larger sizes.

Sizing precision of the SMPS was checked using monodisperse PSL aerosol as well as by sizing monodisperse ammonium sulfate in 14 different size ranges from $20 \mathrm{~nm}$ to $900 \mathrm{~nm}$. The monodisperse ammonium sulfate particles were produced using a DMA. Similar to the APS, the double- and triple-charge particles did not interfere with the analysis of the calibration results. Differences in particle sizing were less than $3 \%$ across the entire size range.

Prior to the ambient study the APS was tested in the laboratory using artificial ammonium sulfate aerosol. As will be shown later, the merging algorithm requires the counting efficiency of the SMPS and the APS to be equal in the overlap range. The inte- grated counting efficiency of the SMPS was compared to that of a standalone condensation particle counter (CPC Model 3025, TSI Inc) and was found to be within $10 \%$ of that of the CPC. The counting efficiency of the APS in the summed mode relative to that of the SMPS was assessed by comparing its counts with those of the SMPS in the overlap size range of $0.5-1 \mu \mathrm{m}$ in mobility diameter. The APS was mobility calibrated with monodisperse ammonium sulfate aerosol, as described above, and the calibration information for this laboratory intercomparison was stored in the APS with the particle density and the shape factor set both to 1 . In this way, no postprocessing was required for the APS data: the APS was reporting "mobility-equivalent" size of ammonium sulfate particles.

\section{Merging of the SMPS and the APS Size Distributions}

Data obtained with the SMPS/APS were combined into one single-size distribution in two ways. In the first approach, similar to Shen et al. (2002), the APS data were merged with the SMPS assuming the aerodynamic size is the same as the physical size. In the second, similar to Hand and Kreidenweis (2002), a size correction factor was searched for the APS data to be merged with the SMPS data. Unlike Hand and Kreidenweis (2002), who used optical counter measurements as an intermediate to match the distributions, we converted the APS data to the mobility spectrum by finding a size correction factor that gives the best least-squares fit directly with the SMPS in the overlap size range.

The fitting of the APS to the SMPS was done in the following way. The SMPS and APS systems overlapped from 542-680 nm for unit density particles. The SMPS data were fitted with a power-law (Junge size distribution) function (Willeke and Baron 1992) in this overlap size range. A size correction was then found for the APS size distribution, expressed as $d N / d \log (D)$, to have the least-squares fit with the power-law approximation of the SMPS data. Applying the correction factor preserves the shape of the APS distribution while shifting it along the $d \log (D)$-axis to achieve a good fit with the SMPS size distribution. Given the narrow size range of the overlap region, the size-correction factor is assumed to be constant within the overlapping size range.

The size-correction factor is selected to minimize the difference between the SMPS and shifted APS size distributions, with the objective function $S^{2}$ shown in the following equation:

$$
S^{2}(x)=\frac{1}{n_{2}-n_{1}} \sum_{i=n 1}^{n 2}\left[\log \left(N_{s}\left(D_{i}\right)\right)-\log \left(N_{a}\left(D_{i} x\right)\right)\right]^{2},
$$

where $N_{s}$ is the power-law function representing the smoothed $d N / d \log (D)$ size distribution measured by the SMPS; $N_{a}$ is the APS size distribution; $x$ is the size-correction factor; and $n_{1}$ and $n_{2}$ are the numbers of the first and the last APS channel that fit, after applying the correction, into the 540-800 nm mobility size range. The first two channels of the APS were not used for the fitting procedure because of their unreliable counting. The logarithms of the size distribution values are used to give the same relative weight to all points in the overlap range. 
It should be noted that a $d N / d \log (D)$ distribution should be used for the minimization procedure. If a $d N / d D$ distribution is used, the APS distribution will need to be shifted not only along the abscissa but also vertically:

$$
\left(\frac{d N}{d D_{p}}\right)_{\mathrm{SMPS}}=\left(\frac{d N}{d D_{a}}\right)_{\mathrm{APS}} \frac{d D_{a}}{d D_{p}}=\left(\frac{d N}{d D_{a}}\right)_{\mathrm{APS}} x,
$$

where $D_{a}$ is the aerodynamic size, $D_{p}$ is the physical size, and $x$ is the size-correction factor $\left(D_{a}=x D_{p}\right)$. In contrast, under the assumption that the shift factor does not vary within the overlap size range, the $d N / d \log (D)$ distribution does not need a vertical shift:

$$
\begin{aligned}
\left(\frac{d N}{d \log \left(D_{p}\right)}\right)_{\mathrm{SMPS}} & =\left(\frac{d N}{d \log \left(D_{a}\right)}\right)_{\mathrm{APS}} \frac{d \log \left(D_{a}\right)}{d \log \left(D_{p}\right)} \\
& =\left(\frac{d N}{d \log \left(D_{a}\right)}\right)_{\mathrm{APS}} \frac{d \log \left(D_{p} x\right)}{d \log \left(D_{p}\right)} \\
& =\left(\frac{d N}{d \log \left(D_{a}\right)}\right)_{\mathrm{APS}}
\end{aligned}
$$

The size-correction factor relates the ratio of the aerosol density to its shape factor in the size range used for the fitting and is usually called an "effective" density (Hand and Kreidenweis 2002). This relationship can be seen from the formula relating the mobility size to the aerodynamic size (Hinds 1999):

$$
D_{p}=D_{a} \sqrt{\chi \frac{C_{s}\left(D_{a}\right)}{C_{s}\left(D_{p}\right)} \frac{\rho_{0}}{\rho_{p}}},
$$

where $D_{p}$ and $D_{a}$ are the mobility and the aerodynamic diameters, respectively; $C_{s}$ is the Cunningham slip correction factor; $\rho_{0}$ is the reference density $\left(1 \mathrm{~g} \mathrm{~cm}^{-3}\right) ; \rho_{p}$ is the density of the particle; and $\chi$ is the shape factor. At sizes of the APS-SMPS overlap size range the slip correction can be neglected; for the particle density of $2 \mathrm{~g} \mathrm{~cm}^{-3}$, the shape factor of 1 , and the physical diameter of $500 \mathrm{~nm}$ the error in calculating the aerodynamic diameter is $4 \%$. At lower particle densities and larger shape factors the error will be even smaller. Neglecting the slip correction, Equation (4) becomes

$$
D_{p}=D_{a} \sqrt{\chi \frac{\rho_{0}}{\rho_{p}}}=\frac{D_{a}}{x} .
$$

Thus, the size-correction factor is

$$
x=\sqrt{\frac{\rho_{p}}{\chi \rho_{0}}} .
$$

The size-correction factor depends on both the particle density and the shape factor in the overlap size range. Consequently, it is not possible to determine each of these parameters from the size-correction factor without complementary measurements. However, the particle density in the overlap size range may differ from the average bulk aerosol density, which will introduce errors in determination of the shape factor from the size-correction factor. This problem can be avoided by using mass measurements within the narrow overlap size range. For practical purposes, however, it is more useful to obtain integrated aerosol properties in the $\mathrm{PM}_{2.5}$ size range such that, for example, the aerosol volume measured with the SMPS-APS can be converted to the aerosol mass, substituting filter-based measurements, and the volume size distribution can be converted to the aerodynamic size distribution to substitute cascade impactor measurements. For this reason we concentrated our efforts to find the bulk $\mathrm{PM}_{2.5}$ aerosol effective density from comparisons of the SMPS-APS with a TEOM and a MOUDI cascade impactor.

It should be noted that errors may be introduced into the merged-size distribution if the SMPS and the APS have different counting efficiencies in the overlapping size range. To demonstrate the magnitude of the errors due to miscounting, a short analysis is performed here. As an example we will assume the SMPS to be correct and the APS is counting $\mathrm{Z}$ times lower than the SMPS. If the $d N / d \log (D)$ size distribution follows the power-law function proportional to $D^{-B}$, it can be shown that the merging procedure would shift the APS distribution by $Z^{1 / B}$ times extra, relative to what the correction factor should be if the APS is correct. If the size distribution in physical sizes is $f_{p}(D)=A D^{-B}$, then the size distribution in aerodynamic sizes, $f_{a}(D)$, will be

$$
f_{a}(D)=A D_{p}^{-B}=A\left(x D_{a}\right)^{-B}=x^{-B} f_{p}(D) .
$$

Consequently, the size correction factor is

$$
x=\left(\frac{f_{p}(D)}{f_{a}(D)}\right)^{\frac{1}{B}} .
$$

An error in concentration measurements of aerodynamic distribution of a factor of $Z$ will translate to an error in the correction factor of a factor $Z^{1 / B}$. In other words, a flatter distribution (lower B) will have a larger error in the shift factor. However, if the APS is miscounting with a constant factor throughout its size range and the distribution is power law, there will be no error in the volume concentration measurements after the merging procedure.

\section{Comparison of the SMPS-APS with TEOM and MOUDI Measurements}

The SMPS-APS was compared with the TEOM (Model 1400a, R\&P Co.) and MOUDI cascade impactor (Model 110, MSP Co., Minneapolis, MN, USA) during measurements of ambient aerosol in Pittsburgh, PA during July 2001.

The integrated aerosol volume concentrations in the $\mathrm{PM}_{2.5}$ size range were compared to the simultaneous measurements with the TEOM. Dried (low relative humidity) measurements of the DAASS were used for this comparison, because the relative humidity conditions were close to those of the TEOM (below 
$30 \% \mathrm{RH})$. The TEOM was operated at $30^{\circ} \mathrm{C}$ and was equipped with a Nafion diffusion dryer Sample Equilibration System (SES, R\&P Co.) to minimize evaporative losses of volatile aerosol components. During the study, the TEOM has shown very good agreement with the federal reference method (FRM). The TEOM was on average $1.5 \%$ higher than the FRM, both instruments giving readings within $10 \%$ of each other during the whole study (Rees et al. 2004). Because of the relatively small fraction of ammonium nitrate in July in Pittsburgh, and because the TEOM was operated at $30^{\circ} \mathrm{C}$, the volatilization artifact in the TEOM was relatively small during this study. The accuracy and the artifacts of the FRM observed during the study are discussed in detail in Rees at al. (2004).

Two combined spectra were calculated from the SMPS-APS measurements for comparison with the TEOM: (1) without converting the APS data to mobility diameter, similar to Shen et al. (2002) and (2) with the conversion of APS data to the mobility size using the procedure described in the Experimental section. These two spectra were used to calculate the volume concentrations in $\mathrm{PM}_{2.5}$ range. These volume concentrations were compared to the $\mathrm{PM}_{2.5}$ mass measured with the TEOM.

The MOUDI was operated with $47 \mathrm{~mm}$ ring-supported Teflon membrane filters (7592-104, Whatman) as the stage substrates to reduce bounce during the sampling. The mass size distribution of the aerosol collected with the MOUDI was determined gravimetrically by weighing the MOUDI stages before and after the sample using a precision microbalance (UMX2, Mettler-Toledo, Columbus, OH, USA). The MOUDI stages were weighed in a controlled relative humidity $(35 \pm 2 \% \mathrm{RH})$ and temperature $\left(22 \pm 1{ }^{\circ} \mathrm{C}\right)$ glove box. Prior to the weighing the stages were equilibrated for $24 \mathrm{~h}$ at the conditions of the weighing box. The combined accuracy of determination of the mass was $\pm 5 \mu \mathrm{g}$ per impactor stage.

The $\mathrm{PM}_{2.5}$ mass collected with the MOUDI agreed well with the FRM measurements (Cabada et al. 2004). It was, however, found that volatile material was lost from the MOUDI stages. During the summer more than $70 \%$ of nitrate was lost from the MOUDI stages (Cabada et al. 2003). The nitrate contribution to the $\mathrm{PM}_{2.5}$ mass in summer is small in the Pittsburgh region, so the effect of nitrate loss on the fine mass is negligible. No evident losses of organic carbon from Teflon MOUDI substrates were observed.

The MOUDI was sampling at ambient conditions (temperature and relative humidity). Because of the hygroscopic growth at high relative humidities (especially at night), the aerosol mass size distribution is shifted to larger sizes relative to low relative humidity conditions. For the comparison with MOUDI size distributions, merged dried SMPS-APS distributions need to be converted to aerodynamic size and corrected for the hygroscopic growth. This was done in the following way.

The DAASS measures both dried and wet size distributions. Comparing the integrated volumes from the dried and ambient measurements provides us with the increase in volume due to water accretion. Assuming volume additivity, the aerosol density at ambient conditions is

$$
\rho_{a}=\rho_{w}+\left(\rho_{d}-\rho_{w}\right) \frac{V_{d}}{V_{a}},
$$

where $\rho_{d}$ and $\rho_{a}$ are the dried and ambient aerosol densities, respectively; $\rho_{w}$ is the density of water; and $V_{d}$ and $V_{a}$ are the dried and ambient aerosol integrated volumes, respectively. The aerodynamic size at ambient relative humidity was then found using the following equation (slip correction is ignored here):

$$
D_{a}=D_{p}\left(\frac{V_{a}}{V_{d}}\right)^{1 / 3} \sqrt{\rho_{a}},
$$

where $D_{p}$ is the physical size and $D_{a}$ is the corresponding size at ambient relative humidity.

The dry density of $1.5 \mathrm{~g} \mathrm{~cm}^{-3}$ was used (Tuch et al. 1997; Hand and Kreidenweis 2002). As will be shown in the Results section, this value of the aerosol density is also applicable to this study. Because during this study the ambient aerosol contained water even at low relative humidities (Stanier et al. 2003), the particles are expected to be spherical and thus the shape factor of 1 was used to convert physical to aerodynamic size.

The geometric mean diameters and geometric mean standard deviations of the distributions in the $\mathrm{PM}_{2.5}$ range measured with the MOUDI and the SMPS-APS were compared to assess the size-distribution comparability of the two instruments. To compare the SMPS-APS data with the size-fractionated mass measurements of the MOUDI, the SMPS-APS data have been summed up to match the MOUDI stage size ranges. Then, values from individual stages have been summed up such that the $\mathrm{PM}_{2.5}$ range is divided into 4 fractions: $<0.1 \mu \mathrm{m}, 0.1-0.3 \mu \mathrm{m}$, $0.3-1 \mu \mathrm{m}, 1-2.5 \mu \mathrm{m}$. The grouping corresponds to summing 2 impactor stages. The values found with the MOUDI and the SMPS-APS within these size fractions are compared.

\section{RESULTS AND DISCUSSION}

\section{Laboratory Tests of the APS}

The counting efficiency of the APS in the submicrometer size range is assessed by comparing it to the SMPS in the overlap size range. The APS was mobility calibrated using ammonium sulfate aerosol. The calibration information was stored with the density and the shape factor both being one. In this way the APS was measuring the mobility equivalent size of ammonium sulfate particles and no further data reduction was needed. The APS was found to agree well with the SMPS in the overlap size range of 580-720 nm (mobility equivalent size), with the counting efficiency differences of no more than $10 \%$ (Figure 1). The first APS channel, however, had a lower efficiency of about $70 \%$. Armendariz and Leith (2002) suggested that the counting efficiency was steadily going up from about $30 \%$ at $0.5 \mu \mathrm{m}$ to about $90 \%$ at $1 \mu \mathrm{m}$. However, they did not test the efficiency between these sizes. Our results indicate, however, that the counting efficiency is constant and close to $100 \%$ except for the first APS channel. 


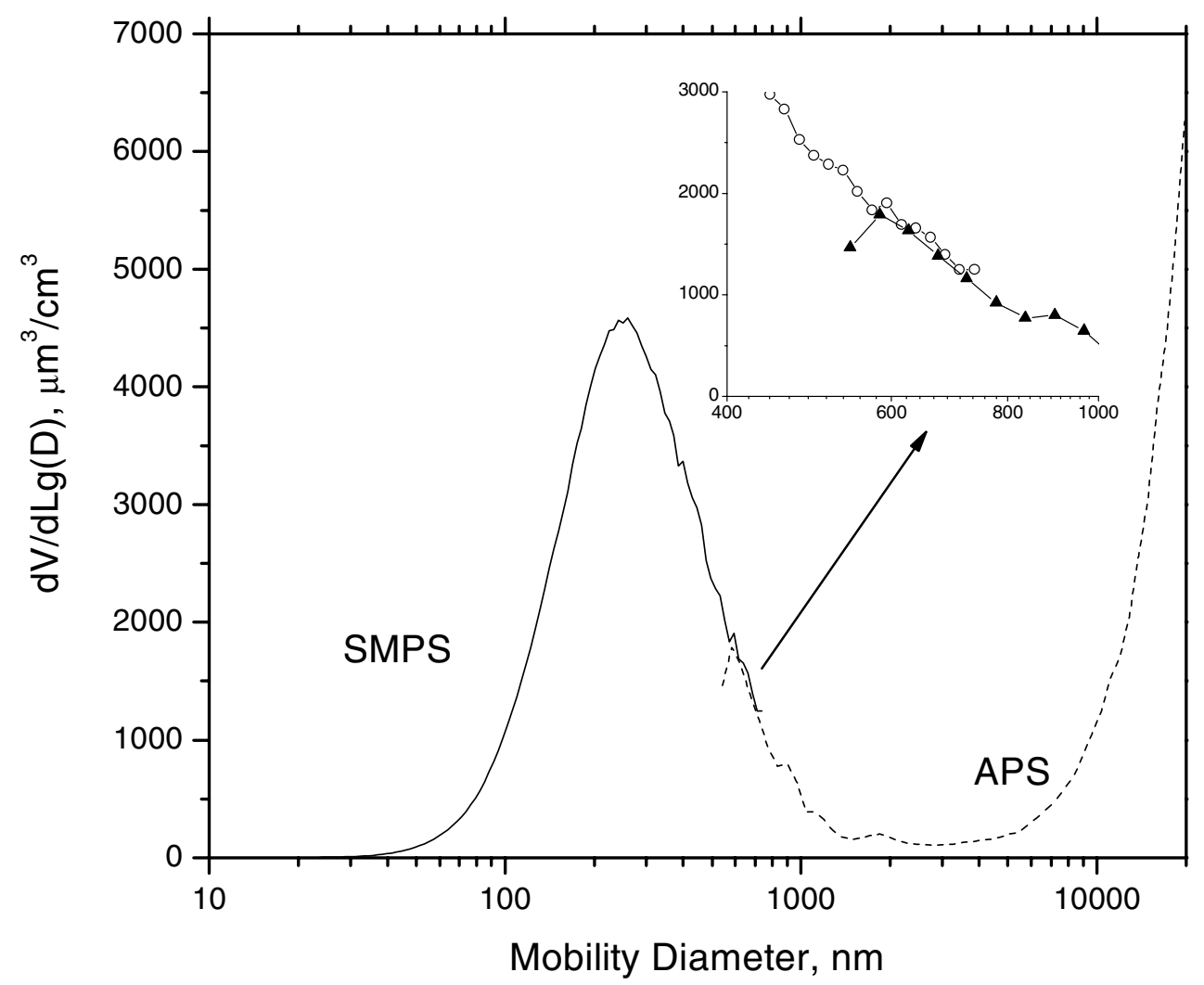

Figure 1. Comparison of the APS and the SMPS in the overlap size range using ammonium sulfate aerosol.

\section{Comparison with TEOM}

Figure 2 shows a comparison of the integrated ambient volume concentrations with the TEOM measurements. The agreement between the SMPS-APS and the TEOM is considerably improved when the APS is fitted to the SMPS distribution. The ratio of the aerosol mass to the aerosol volume is a measure of the aerosol effective density, which is equal to the bulk aerosol density if the shape factor is 1 . If the APS data are used without any corrections, the density is unrealistically low (often below $1 \mathrm{~g} \mathrm{~cm}^{-3}$ ). However, when the APS is fitted to the SMPS, the estimated density is $1.52 \mathrm{~g} \mathrm{~cm}^{-3}$, which is very close to the values reported in the literature, around $1.5 \mathrm{~g} \mathrm{~cm}^{-3}$ (Tuch et al. 1997; Hand and Kreidenweis 2002). The density data appear to be normally distributed around the mean value with the standard deviation of $0.26 \mathrm{~g} \mathrm{~cm}^{-3}$. It is thus concluded that the SMPSAPS size distribution data, in which the APS data are fitted to the SMPS, can be used to estimate the $\mathrm{PM}_{2.5}$ mass concentration with an average standard error of about $20 \%$ by using an average aerosol density of $1.5 \mathrm{~g} \mathrm{~cm}^{-3}$.

The estimated density is in good agreement with the density estimated from the average chemical composition of the aerosol during this study. The aerosol consisted of about 50\% ammonium salts of sulfate, $25 \%$ organic carbon, $3 \%$ elemental carbon, $2 \%$ nitrate, and $2 \%$ crustal material (Wittig et al. 2003). It was also shown that the FRM and the TEOM retained water, which comprised on average about $20 \%$ of the aerosol mass measured with those instruments. Assuming the density of organics to be $1.4 \mathrm{~g} / \mathrm{cm}^{3}$ and the density of crustal material and elemental carbon to be $2 \mathrm{~g} / \mathrm{cm}^{3}$, and taking the density of salts of ammonium to be $1.8 \mathrm{~g} / \mathrm{cm}^{3}$, the average density of the aerosol according to its chemical composition is estimated to be $1.56 \mathrm{~g} / \mathrm{cm}^{3}$, which is close to the density estimated from the SMPS-APS and TEOM comparison. This suggests that the aerosol during this study had a shape factor close to 1 .

\section{Comparison with MOUDI}

Comparisons of the geometric mean diameters (GMD) and the geometric mean standard deviations (GSTD) of the ambient aerosol size distributions measured with the MOUDI and the SMPS-APS converted to the aerodynamic size and corrected for the hygroscopic growth are shown in Figures 3 and 4, respectively. Both the GMD and GSTD show a good correlation between the instruments. The slope of the regression line for GMD is virtually 1 . A shape factor of 1 was used to convert the SMPS-APS distributions to aerodynamic sizes. The equality of the GMDs suggests that the shape factor of 1 is justified for this study. During this study the aerosol was found to be always wet (i.e., contain water), even at relative humidities as low as $30 \%$. Thus, particles are expected to be spherical droplets and have the shape factor of 1 , which explains our observation. 


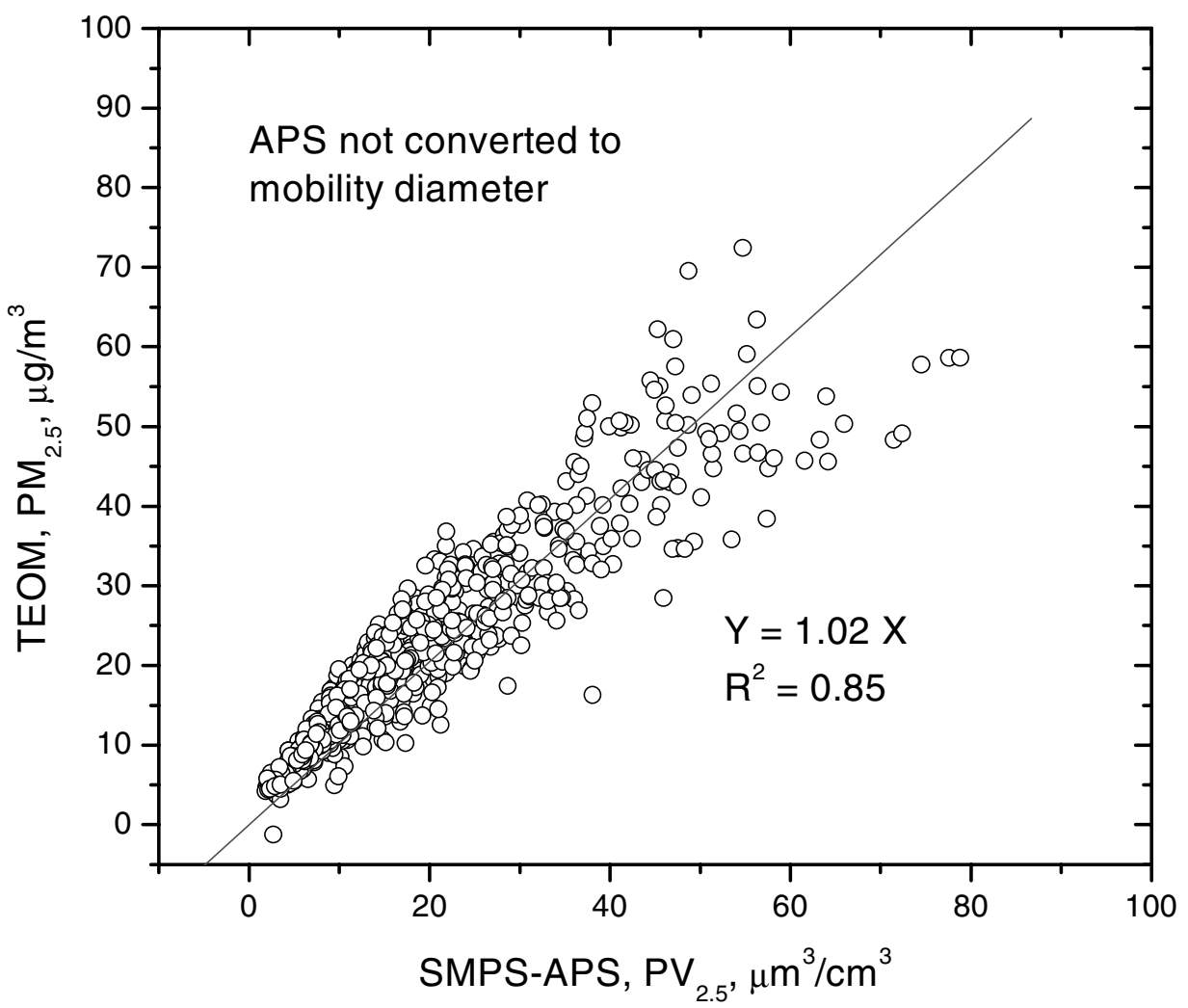

(a)

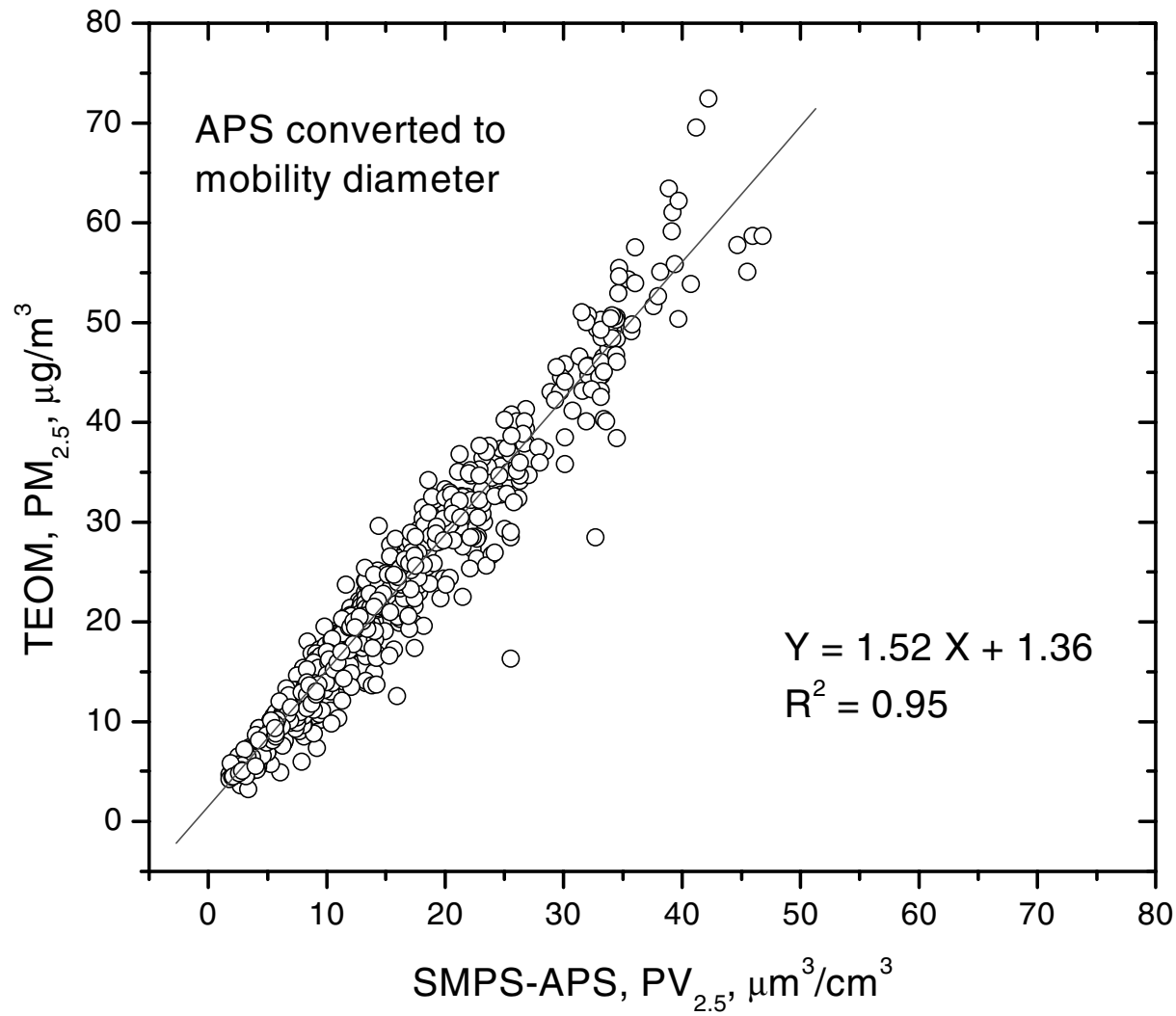

(b)

Figure 2. Comparison of mass concentrations with volume concentration in the $\mathrm{PM}_{2.5}$ range. (a) APS size distributions were not converted to electrical mobility; (b) APS size distributions were converted to electrical mobility using the algorithm. 


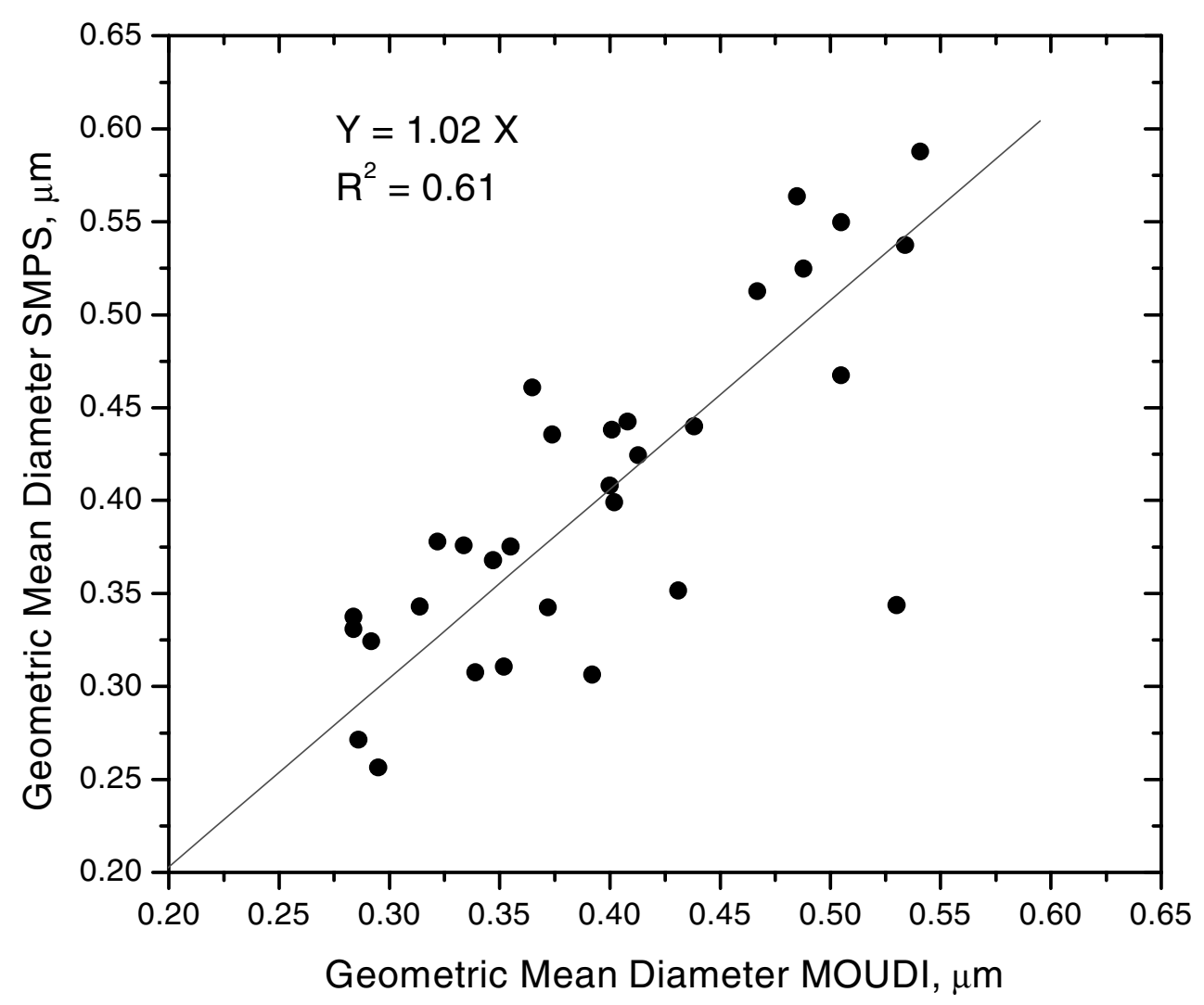

Figure 3. Comparison of geometric mean diameters.

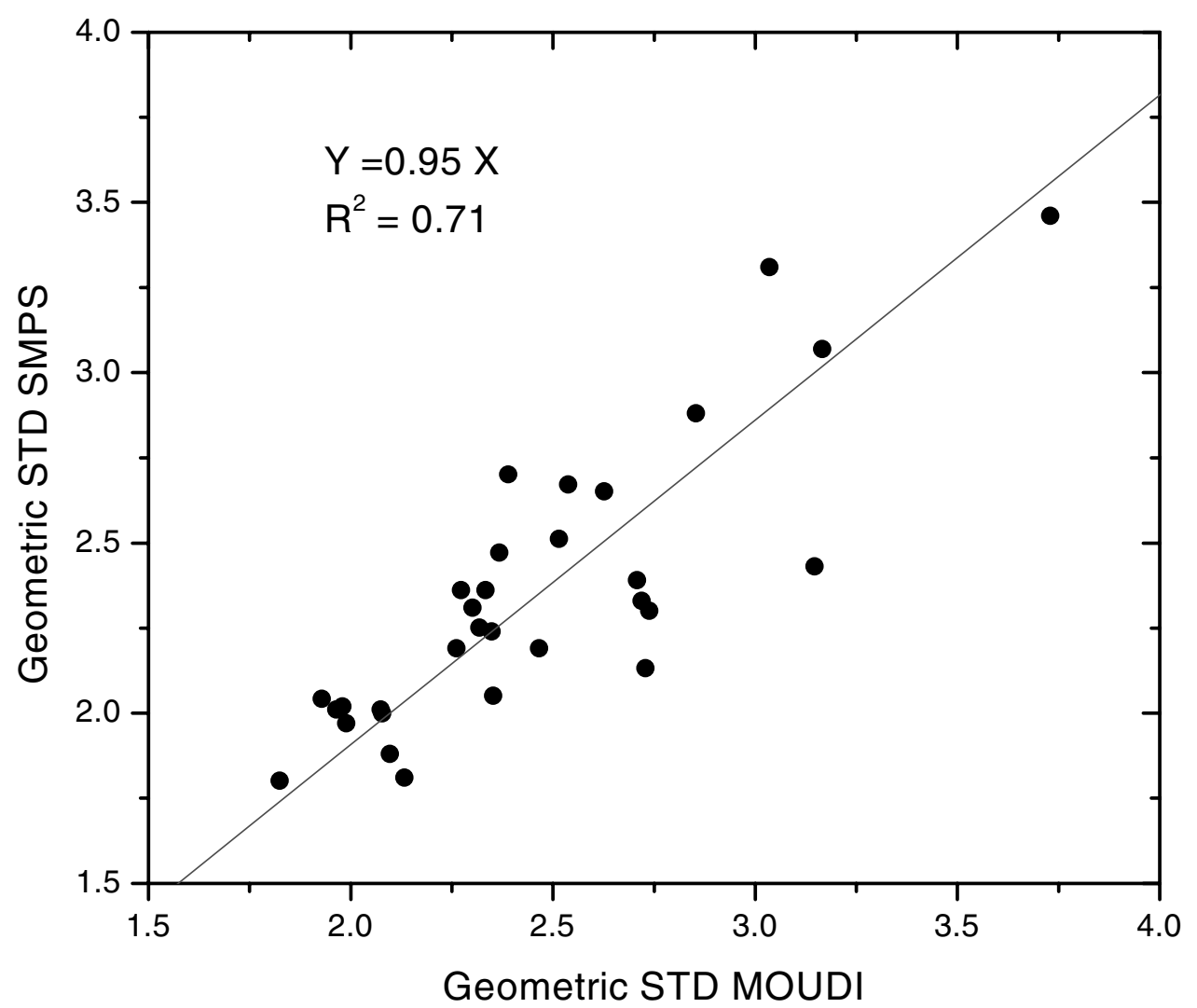

Figure 4. Comparison of geometric standard deviations. 

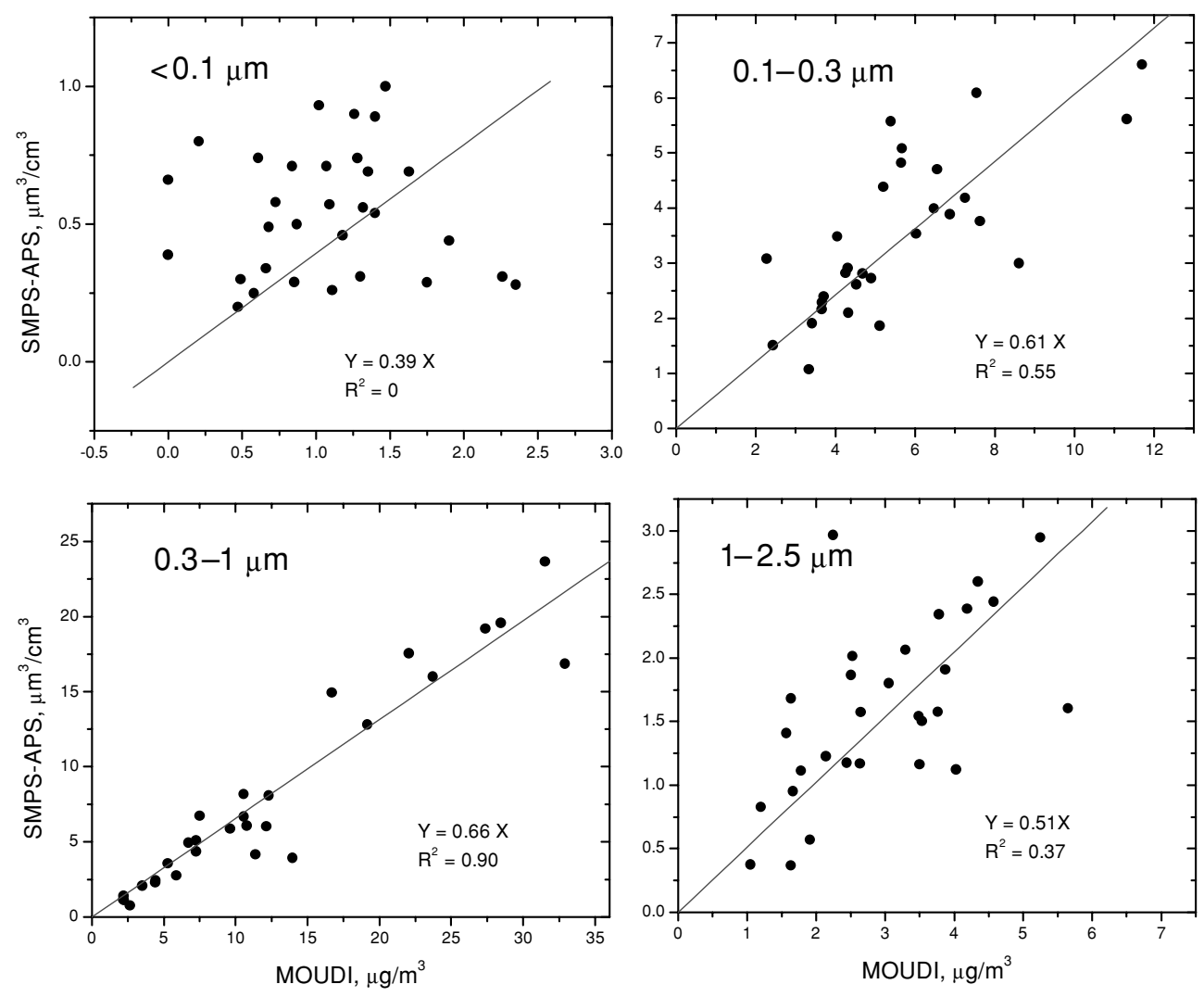

Figure 5. Comparison of size-fractionated mass concentrations from MOUDI and volume concentrations from SMPS-APS.

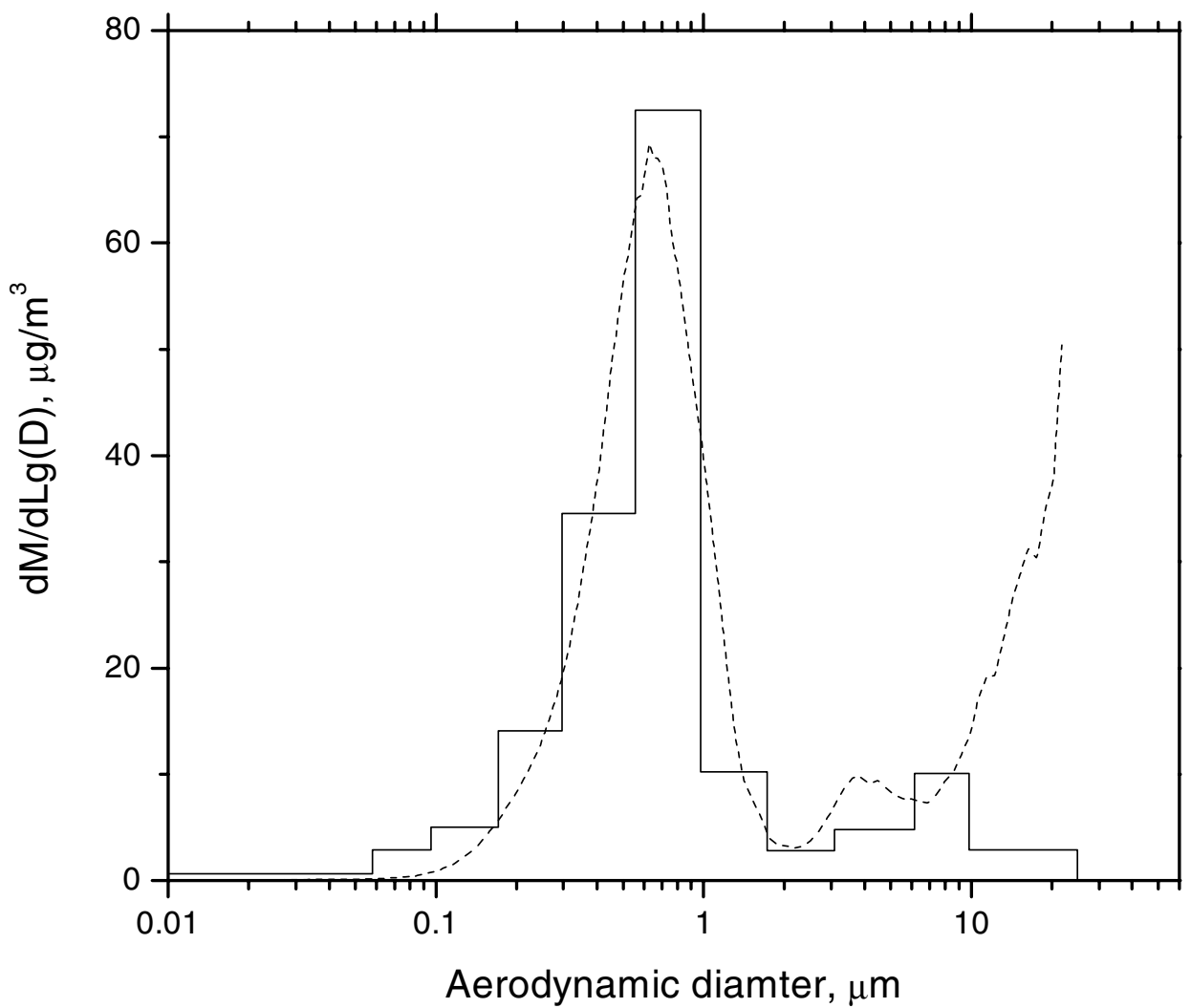

Figure 6. Comparison of size distributions measured with SMPS-APS (dashed line) and MOUDI (solid line). 
Figure 5 shows a size-fractionated comparison of the MOUDI and the SMPS-APS. With the exception of the lowest stages (below $0.1 \mu \mathrm{m}$ ) the MOUDI and the SMPS-APS appear to be well correlated. The $\mathrm{R}^{2}$ values are similar or higher than those found during a similar study by Shen et al. (2002). The slopes for the stages between 0.1 and $1 \mu \mathrm{m}$ are $0.61-0.66$. Such slopes correspond to aerosol densities between 1.52 and $1.64 \mathrm{~g} \mathrm{~cm}^{-3}$, which again are close to ones reported in the literature and to the density found during the comparison with the TEOM. The slope for the $1-2.5 \mu \mathrm{m}$ range indicates a density of about $2 \mathrm{~g} \mathrm{~cm}^{-3}$, which is probably due to a larger contribution of the crustal material to this size range.

The absence of correlation between the SMPS-APS and the MOUDI below $0.1 \mu \mathrm{m}$ is similar to the observations by Shen et al. (2002). Similarly, we found that the MOUDI measures on average more than the SMPS-APS in that size range. A possible explanation for this observation is the particle bounce from the upper stages of the MOUDI (Pak et al. 1992). The mass loading on the last stages of the MOUDI is usually small, and even a small contribution due to the bounce would introduce a large relative error on those stages, while losses from the larger stages will probably remain insignificant.

The comparison of representative size distributions measured with the SMPS-APS and the MOUDI is shown in Figure 6. The SMPS-APS distribution was converted to the mass size distribution in aerodynamic space using the density of $1.5 \mathrm{~g} \mathrm{~cm}^{-3}$ and correcting for the hygroscopic growth as explained in the Experimental section. Qualitatively similar distributions are measured with the two instruments. The sharp increase in the volume concentration after $7 \mu \mathrm{m}$ measured with the SMPS-APS is most probably due to the well-documented artifact counts in the APS in the large size range (Stein et al. 2002). In this study, however, these artifact counts were not observed to contribute significantly to the $\mathrm{PM}_{2.5}$ range.

\section{CONCLUSIONS}

A simple algorithm was developed to combine electrical mobility and aerodynamic size distribution data into a single size distribution. This algorithm was tested during a month of ambient aerosol measurements by comparing size distributions measured using an SMPS-APS combination with simultaneous measurements using a MOUDI cascade impactor and $\mathrm{PM}_{2.5}$ mass concentration measurements using a TEOM.

Size distributions obtained by using the algorithm have better correlations with $\mathrm{PM}_{2.5}$ measurements than do size distributions in which aerodynamic sizes were not converted to electrical mobility diameters.

A comparison of the TEOM PM 2.5 mass concentrations with the volume concentrations from the SMPS-APS data indicates that the ambient aerosol during the study had an effective density of $1.52 \pm 0.26 \mathrm{~g} \mathrm{~cm}^{-3}$. This density is close to $1.56 \mathrm{~g} \mathrm{~cm}^{-3}$, the density estimated from the average aerosol chemical composition during this study. This suggests that the aerosol shape factor during this study is close to 1 .
The SMPS-APS size distributions obtained using the algorithm agree with the mass distributions measured with MOUDI cascade impactors within $0.1-2.5 \mu \mathrm{m}$ size range, if a density of $1.5 \mathrm{~g} \mathrm{~cm}^{-3}$ and a shape factor of 1 are used, and the hygroscopic growth of aerosol is taken into account. However, there was no agreement below $0.1 \mu \mathrm{m}$, probably due to contamination of the low impactor stages because of the bounce of large particles, or a different shape factor based on the composition in this size range, which may affect the particle mobility of ultrafine particles.

\section{REFERENCES}

Armendariz, A. J., and Leith, D. (2002). Concentration Measurement and Counting Efficiency for the Aerodynamic Particle Sizer 3320, J. Aerosol Sci. 33:133-148.

Cabada, J. C., Rees, S., Takahama, S., Khlystov, A., Pandis, S. N., Davidson, C., and Robinson, A. L. (2004). Mass Size Distributions and Size Resolved Chemical Composition of Fine Particulate Matter at the Pittsburgh Supersite, Atmos. Environ. in print.

Dockery, D. W., Pope, C. A., Xu, X., Splengler, J. D., Ware, J. H., Ferris, B. G., and Speizer, F. E. (1993). Mortality Risks of Air Pollution: A Prospective Cohort Study, N. Engl. J. Med. 329:1753-1759.

Hinds, W. C. (1999). Aerosol Technology: Properties, Behavior and Measurements of Airborne Particles, 2nd ed. John Wiley \& Sons, New York.

Hand, J. L., and Kreidenweis, S. M. (2002). A New Method for Retrieving Particle Refractive Index and Effective Density from Aerosol Size Distribution Data, Aerosol Sci. Technol. 36:1012-1026.

Pak, S. S., Liu, B. Y. H., and Rubuw, K. L. (1992). Effect of Coating Thickness on Particle Bounce in Inertial Impactor, Aerosol Sci. Technol. 16:141150.

Rees, S. L., Robinson, A. L., Khlystov, A., Stanier, C. O., and Pandis, S. N. (2004). Performance of the U.S. Federal Reference Method for $\mathrm{PM}_{2.5}$ in Pittsburgh, PA, Atmos. Environ. in print.

Seinfeld, J. H., and Pandis, S. N. (1998). Atmospheric Chemistry and Physics: From Air Pollution to Climate Change, John Wiley \& Sons, Inc., New York. Schwartz, J., and Dockery, D. W. (1992). Increased Mortality in Philadelphia Associated with Daily Air Pollution Concentration, Am.J. Epidimeol. 135:1219.

Shen, S., Jaques, P. A., Zhu, Y., Geller, M. D., and Siuotas, C. (2002). Evaluation of the SMPS-APS System as a Continuous Monitor for Measuring $\mathrm{PM}_{2.5}$, $\mathrm{PM}_{10}$ and Coarse $\mathrm{PM}_{2.5-10}$, Atmos. Environ. 36:3939-3950.

Shi, J. P., Harrison, R. M., and Evans, D. (2001). Comparison of Ambient Particle Surface Area Measurement by Epiphaniometer and SMPS/APS, Atmos. Environ. 35:6193-6200.

Stanier, C. O., Khlystov, A., Chan, W. R., Mandiro, M., and Pandis, S. N. (2004). A Method for the in-situ Measurement of Aerosol Water Content of Ambient Aerosols: The Dry-Ambient Aerosol Size Spectrometer (DAASS), Aerosol Sci. Technol. 38:215-228.

Stein, S. W., Gabrio, B. J., Oberreit, D., Hairston, P., Myrdal, P. B., and Beck, T. J. (2002). An Evaluation of Mass-Weighted Size Distribution Measured with the Model 3320 Aerodynamic Particle Sizer, Aerosol Sci. Technol. 36:845854 .

Tuch, Th., Brand, P., Wichmann, H. E., and Heyder, J. (1997). Variation of Particle Number and Mass Concentration in Various Size Ranges of Ambient Aerosols in Eastern Germany, Atmos. Environ. 31:4193-4197.

Wang, S. C., and Flagan, R. C. (1989). Scanning Electrical Monbility Spectrometer, J. Aerosol Sci. 20:1485-1489.

Willeke, K., and Baron, P. A. (1992). Aerosol Measurement: Principles, Techniques, and Applications. John Wiley \& Sons, New York.

Wittig, B., Anderson, N., Khlystov, A. Y., Pandis, S. N., Davidson C., and Robinson A. L. (2004). Pittsburgh Air Quality Study Overview and Preliminary Scientific Findings, Atmos. Environ. in print.

Wolfenbarger, J. K., and Seinfeld, J. H. (1991). Regularized Solutions to the Aerosol Data Inversion Problem, SIAM J. Sci. Stat. Comput. 12:342-361. 


\title{
Detection of Negative Ions from Individual Ultrafine Particles
}

\author{
David B. Kane, ${ }^{\dagger}$ J injin Wang, ${ }^{\ddagger}$ Keith Frost, and Murray V. J ohnston* \\ Department of Chemistry and Biochemistry, University of Delaware, Newark, Delaware 19716
}

Aerosol mass spectrometers can be used to classify individual airborne particles on the basis of chemical composition. While positive ion mass spectra are normally used to characterize ultrafine particles (defined here as particles smaller than $200 \mathrm{~nm}$ in diameter), negative ion mass spectra can provide complementary information. To effectively utilize the negative ion mass spectra of ultrafine particles, it is important to understand biases in the formation and detection of negative ions. It is found that the intensity of negative ions is generally less than that of positive ions, due to the creation of electrons in the ablation process that must react to form negative ions. The ablation efficiency, defined as the probability that an ablated particle produces a detectable ion signal, exhibits both size and composition dependencies. The ablation efficiency for detection of negative ions follows the same trends as the ablation efficiency for the detection of positive ions: sodium chloride and ammonium nitrate have higher ablation efficiencies than oleic acid, and the ablation efficiency decreases with the particle diameter. The ablation efficiency of negative ions is less than or equal to the ablation efficiency of positive ions, and the relative difference increases as the particle diameter decreases. Pure ammonium sulfate particles exhibit an ablation efficiency too low to be measured in the present experiments. However, trace amounts of sulfate in mixedcomposition particles can be readily detected in the negative ion mass spectra.

Aerosol mass spectrometers are becoming an important tool for the chemical characterization of ambient particulate matter..$^{1,2}$ With these instruments, aerosol particles are sampled directly from ambient conditions into the source region of a mass spectrometer. The most common version of an aerosol mass spectrometer couples laser ablation with time-of-flight mass spectrometry for ionization and mass analysis. ${ }^{3}$ This combination gives the advantages of rapid sampling and particle-by-particle analysis.

Recently, the lower size limit of laser ablation aerosol mass spectrometers was extended for composition analysis of ultrafine

\footnotetext{
${ }^{\dagger}$ Current address: Philip M orris USA Research Center, Richmond, VA 23261

₹ Current address: Department of Chemistry M S-60, Rice University, P.O. Box 1892, Houston, TX 77251.

(1) Johnston, M. V. J. M ass Spectrom. 2000, 35, 585-595.

(2) Suess, D. T.; Prather, K. A. Chem. Rev. 1999, 99, 3007-3035.

(3) Johnston, M. V.; Wexler, A. S. Anal. Chem. 1995, 67, 721A-726A.
}

particles, defined here as particles smaller than $200 \mathrm{~nm}$ in diameter. ${ }^{4-10}$ In this range, size and composition biases of the hit rate, defined as the rate at which single-particle mass spectra are collected, cause the probability of detection to be different for different size and composition particles. ${ }^{10}$ These detection biases must be understood if aerosol mass spectrometers are to be used to infer aerosol compositions.

Field studies of particles smaller than $200 \mathrm{~nm}$ have been limited to the use of positive ion mass spectra because free electrons dominate the negative ion mass spectra. ${ }^{6}$ In principle, positive and negative ion mass spectra can provide complementary information on the composition of particles. ${ }^{11}$ Some species, such as sulfate, are only detected in negative ion mass spectra, while in other instances, the negative ion mass spectra can be used to confirm the presence of a particular species.

Previously we reported on the biases in the detection of positive ions from ultrafine particles. ${ }^{10}$ These biases are found to result from two parameters, both of which exhibit size and composition biases. The first is the sampling rate (SR) of particles into the mass spectrometer. This is the rate at which particles of a given size and composition are sampled from the aerosol into the path of the ablation laser beam when the laser fires. The second is the ablation efficiency $\left(E_{a}\right)$. This is the fraction of particles irradiated by the laser beam that ablate in a manner that produces a detectable ion signal. The relationship between the observed hit rate (HR, particles per unit time that are detected), sampling rate, and ablation efficiency is

$$
H R=n_{\text {aerosol }}(S R) E_{a}
$$

where $\mathrm{n}_{\text {aerosol }}$ is the particle number density of the aerosol being sampled. While the sampling rate is independent of the polarity of the detected ions, the ablation efficiency is expected to be dependent on the ion polarity.

(4) Reents, W. D.; Downey, S. W.; Emerson, A. B.; M ujsce, A. M .; M uller, A. M.; Siconolfi, D. J.; Sinclair, J. D.; Swanson, A. G. Plasma Sources Sci. Technol. 1994, 3, 369-372.

(5) Reents, W. D.; Ge, Z. Aerosol Sci. Technol. 2000, 33 (1-2), 122-134.

(6) Carson, P. G.; J ohnston, M. V.; Wexler, A. S. Rapid Commun. Mass Spectrom. 1997, 11, 993-996

(7) Ge, Z.; Wexler, A. S.; J ohnston, M. V. Environ. Sci. Technol. 1998, 32, 32183223.

(8) Kane, D. B.; Oktem, B.; J ohnston, M. Aerosol Sci. Technol. 2001, 35, 990 997.

(9) Kane, D. B.; Oktem, B.; Johnston, M. Aerosol Sci. Technol. 2001, 34, 520 527.

(10) Kane, D. B.; J ohnston, M. V. Environ. Sci. Technol. 2000, 34, 4887-4893.

(11) Galli, M.; Guazzotti, S. A.; Prather, K. A. Aerosol Sci. Technol. 2001, 43 (4), 381-385.

10.1021/ac011126x CCC: $\$ 22.00$ @ 2002 American Chemical Society Published on Web 03/21/2002 
In this study, positive and negative ion detection are compared for single-component particles having compositions representative of ambient particles. M easurements are restricted to particles greater than $40 \mathrm{~nm}$ in diameter because the hit rate decreases significantly with decreasing particle size. The detection of sulfate ions in mixed-composition particles is discussed, as are the implications of these measurements for the characterization of ambient aerosol.

\section{EXPERIMENTAL DETAILS:}

The design of the aerosol mass spectrometer is similar to that previously described by Kane et al.9,10 In the present investigation, the mass analyzer was operated in the linear mode with a postacceleration detector (bipolar time-of-flight detector, Burle Optoelectronics, Inc., Sturbridge, M A). I dentical acceleration and postacceleration fields were used in both positive and negative ion detection modes. Because previous investigations showed that free electrons produced by laser ablation of ultrafine particles can saturate the detector, obscuring the negative ion signal, ${ }^{6}$ a slight deflection field ( $100 \mathrm{~V}$ ) was applied at the end of the ion source to divert electrons from the detector. The deflection field was used in both positive and negative ion modes to maintain equivalent field gradients. An Ar-F excimer laser (PSX-100, M PB Technologies, Pointe Claire, PQ, Canada) was used for ablation. The laser beam was focused with a 20 -cm-focal length lens to give a fluence of $2.0 \times 10^{4} \mathrm{~J} / \mathrm{m}^{2}$ (193 nm, $8 \mathrm{~ns}, 2.5 \mathrm{~mJ}$ pulse) in the source region of the mass spectrometer.

The aerosol mass spectrometer was used to collect singleparticle mass spectra and measure normalized hit rates for particles representative of ambient compositions in both positive and negative ion modes. 9,10 The normalized hit rate is the rate at which single-particle mass spectra are collected for a given particle number density of the aerosol. It should be noted that the hit rates reported in this work are not comparable to those reported previously, ${ }^{10}$ due to differences in the ionization laser and mass analyzer. The particle beam profile was determined by measuring the hit rate as the laser beam was translated across the ion source region of the mass spectrometer. ${ }^{9,10}$ Since the mass spectrometer used in these experiments was not capable of simultaneous positive and negative ion detection, the average mass spectra of a set of particles were compared rather than those of individual particles. In this manner, changes in the particle mass spectra resulting from shot-to-shot variations were minimized.

M onodisperse test aerosols used in these experiments were generated with a differential mobility analyzer to size select particles produced by atomization of a solution containing the analyte in either ethanol or an ethanol-water mixture. A second differential mobility analyzer was used in-line with the aerosol mass spectrometer to measure the size and concentration of the particles.

\section{RESULTS AND DISCUSSION:}

Particle Mass Spectra. Negative and positive ion mass spectra of oleic acid, ammonium nitrate, and sodium chloride are compared in Figure 1. Each spectrum is the average of 100 singleparticle mass spectra. The negative ion mass spectrum of each particle composition shows distinctive ions representative of that composition. Oleic acid particles give strong ion signals for $\mathrm{H}^{-}$, $\mathrm{C}_{2}{ }^{-}$, and $\mathrm{C}_{2} \mathrm{H}^{-}$. Ammonium nitrate gives strong ion signals for
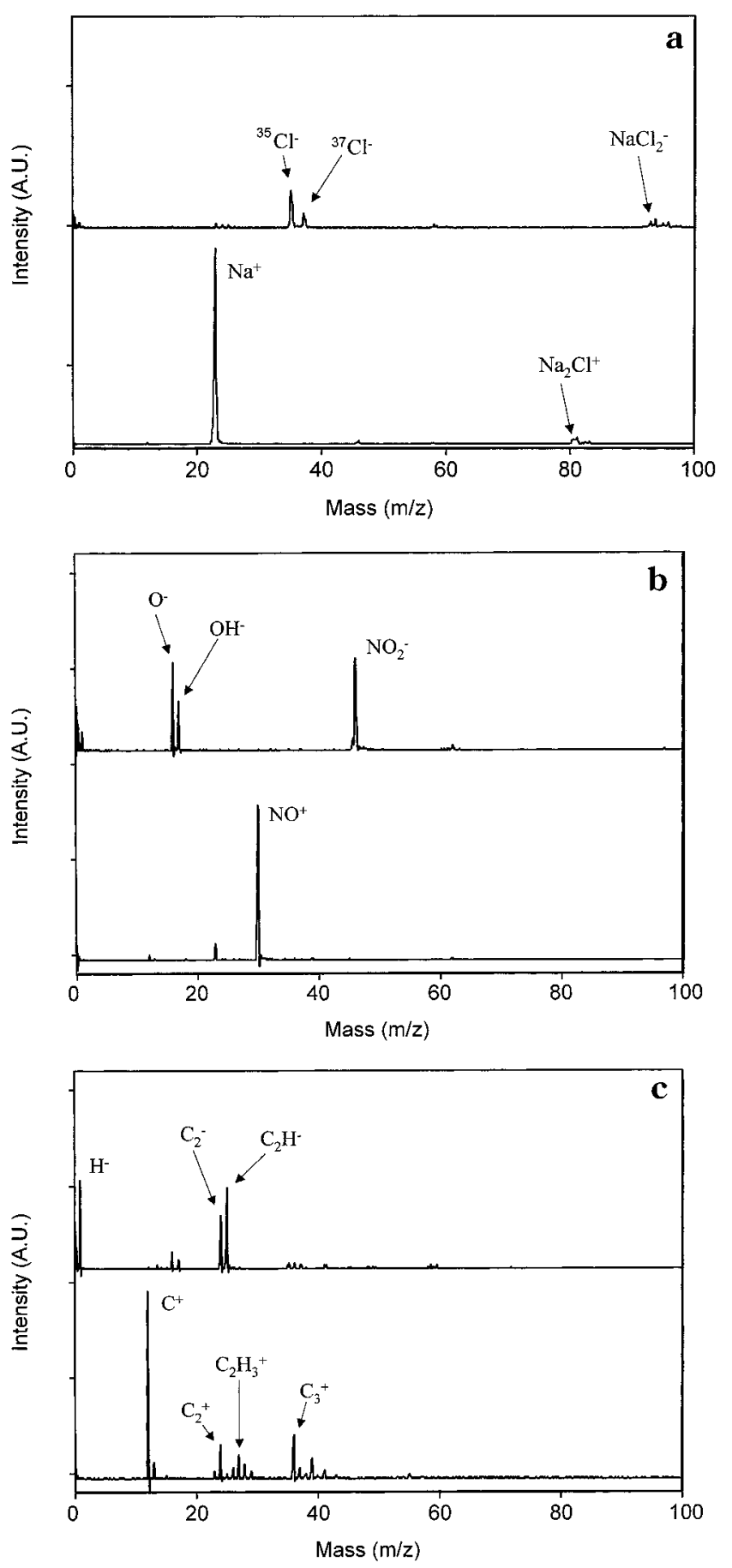

Figure 1. Positive and negative ion mass spectra of (a) sodium chloride, (b) ammonium nitrate, and (c) oleic acid. Each spectrum is the average of 100 single-particle mass spectra from 100-nm mobility diameter particles.

$\mathrm{O}^{-}, \mathrm{OH}^{-}$, and $\mathrm{NO}_{2}^{-}$. Sodium chloride particles are characterized by $\mathrm{Cl}^{-}$and small peaks for $\mathrm{NaCl}_{2}^{-}$. The positive ion mass spectra of these compounds are qualitatively the same as observed previously with a reflectron time-of-flight mass spectrometer. ${ }^{10}$ The negative ion mass spectra of these particles have peaks similar to the negative ion mass spectra of micrometer-diameter particles. ${ }^{3,12,13}$ However, the mass spectra are dominated by smaller

(12) Carson, P. G.; J ohnston, M. V.; Wexler, A. S. Aerosol Sci. Technol. 1997, 26, 291-300. 

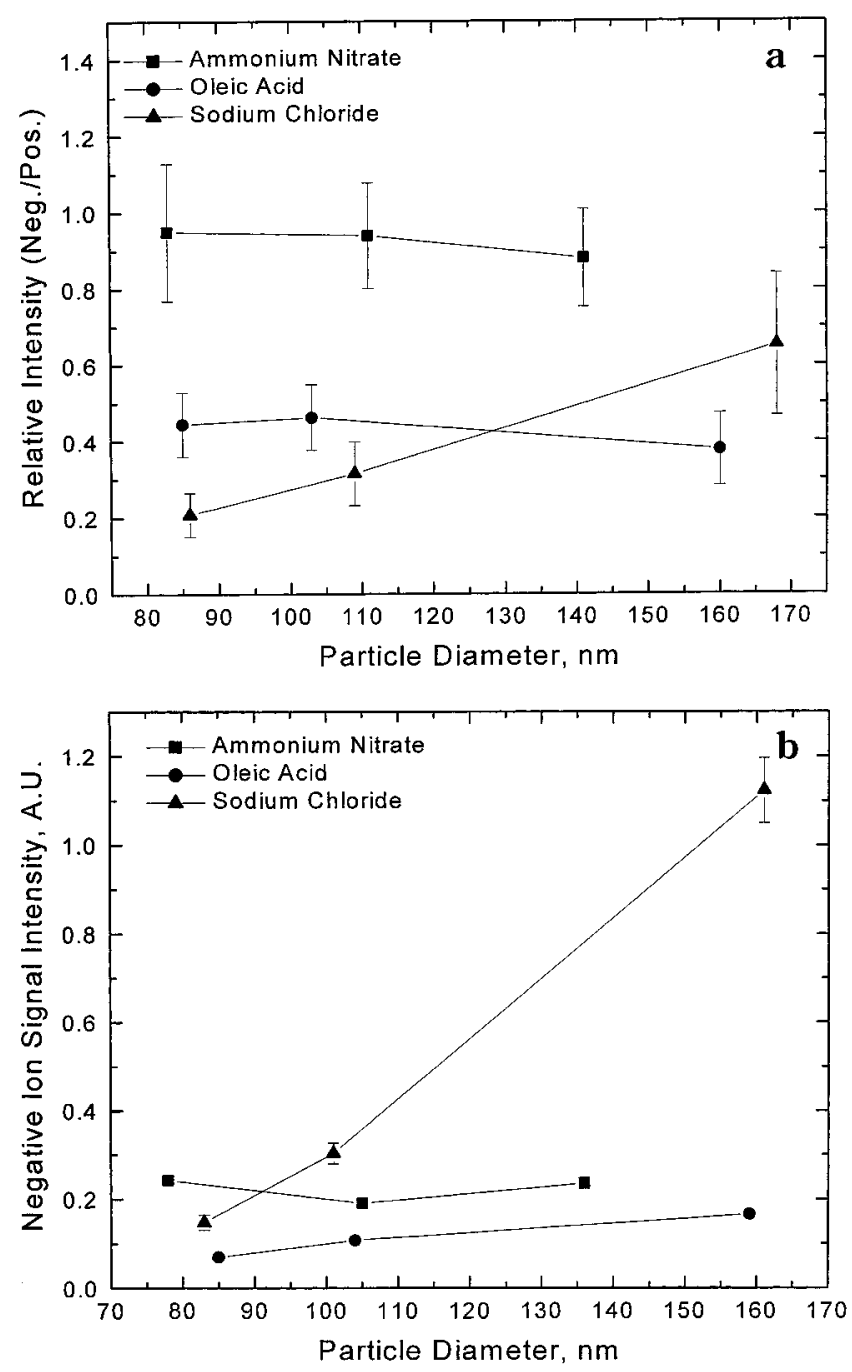

Figure 2. (a) Relative intensities of negative ions to positive ions from ammonium nitrate, sodium chloride, and oleic acid as a function of particle mobility diameter. Error bars are 50\% confidence limits from at least three measurements, 100 particles each. (b) Integrated intensity of the negative ion signal for the compounds above as a function of particle mobility diameter. Error bars are the standard deviation of at least three measurements, 100 particles each.

ions and usually do not contain the larger cluster ions observed from the micrometer-diameter particles. A similar observation was made previously for positive ion mass spectra of ultrafine particles. ${ }^{6}$ The greater fragmentation observed for ultrafine particles is most likely a combination of effects: as the optical penetration depth of the laser beam into the particle approaches the particle size, the increased photon flux per unit volume gives a higher probability of multiphoton absorption and fragmentation; and the decreasing plume size and density as the particle size decreases results in fewer collisions and less efficient cooling as the plume expands.

Figure $2 \mathrm{a}$ is a plot of the integrated negative ion signal relative to the positive ion signal as a function of the particle size. Each point is the average of at least three 100-particle measurements taken over the course of several experiments over several days.

(13) Neubauer, K. R.; J ohnston, M. V.; Wexler, A. S. Int. J. M ass Spectrom. Ion Processes 1997, 163, 29-37.
While the absolute ion signals exhibited a high degree of variability, the relative intensities in Figure 2a were consistent in all of the experiments. For oleic acid and sodium chloride, the negative ion signal from detected particles is significantly less than the positive ion signal. Since overall charge neutrality must be preserved, this illustrates the dominant role that free electrons can play. Ammonium nitrate produces comparable positive and negative ion signal intensities suggesting that electron capture in the vaporized plume is more efficient than with the other compounds. Sodium chloride exhibits a significant decrease in the relative intensity of negative ions to positive ions as the particle diameter decreases, while oleic acid and ammonium nitrate show little size dependence. The reason for these dependencies is apparent upon examination of Figure $2 \mathrm{~b}$, which shows the absolute intensities of negative ions as a function of particle size for the compounds shown in Figure 2a. The dependencies in Figure $2 b$ are similar to those in Figure 2a: the negative ion signal from sodium chloride decreases rapidly with decreasing particle size, while ammonium nitrate and oleic acid show little size dependence. Previously we showed that the positive ion signal from sodium chloride particles decreases only slightly with decreasing particle diameter in this size range. ${ }^{6,9}$

Differences in the positive and negative ion signal intensities and their dependencies on the particle diameter suggest that multiple mechanisms may be responsible for the formation of negative ions from ablated particles. It is expected that laser ablation results in the production of free electrons, and negative ions must be formed by the capture of these electrons by the vaporized molecules or fragments. One would expect that the negative ions formed by the ablation process will be biased toward those species with the greatest electron affinities, provided that the energy released by electron attachment is removed by collisional cooling. However, negative ion formation also depends on the electron capture cross sections of the vaporized species and the residence time of the electrons in the plume. The electron capture cross section is dependent on the electron kinetic energy as well as properties of the molecule or fragment. Since the electron kinetic energy distribution is determined by complex and ill-defined processes associated with photon absorption, ionization, and particle disintegration, a quantitative explanation of these dependencies is not possible. However, the size dependence of the negative ion signal from sodium chloride suggests that the electron capture cross section of $\mathrm{Cl}$ is low enough that the probability of $\mathrm{Cl}^{-}$formation is proportional to the electron residence time in the plume. Larger particles produce more dense plumes resulting in a larger percentage of negative ions. In contrast, ammonium nitrate and oleic acid do not show significant size dependencies. Evidently, the electron capture cross sections of polyatomic species from these particles must be large enough that the production of negative ions is relatively independent of the plume density and hence the particle diameter.

Ablation Efficiency. By measuring the hit rate and determining the diameter of the particle beam from the beam profile, it is possible to determine the ablation efficiency for a given particle composition and size. ${ }^{10} \mathrm{M}$ easurements of the ablation efficiency for negative ion detection show the same trends that have been observed for positive ion detection. ${ }^{10}$ Sodium chloride and ammonium nitrate exhibit higher ablation efficiencies than oleic acid, 


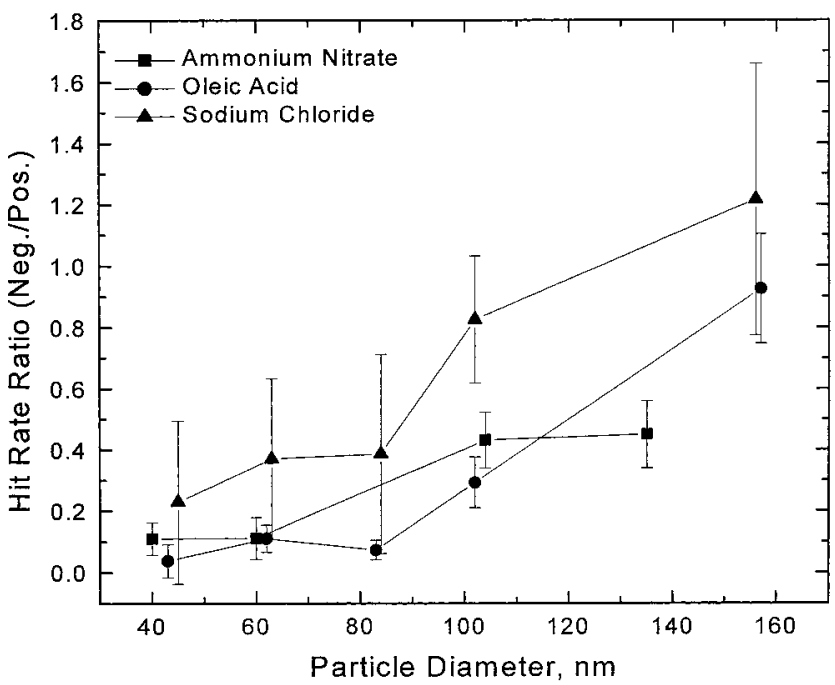

Figure 3. Size (mobility) and composition dependencies of the hit rate for negative ion detection relative to the hit rate for positive ion detection. The relative hit rates are equivalent to the relative ablation efficiencies. Error bars are the standard deviation of at least three measurements, 100 particles each.

and the ablation efficiencies for all three decrease with decreasing particle size. It is not surprising that the trends in the negative ion ablation efficiency are similar to the positive ion ablation efficiency, since the positive and negative ions are created simultaneously.

Figure 3 shows the ablation efficiency for negative ion detection relative to that for detection for positive ion detection. Since the sampling rate of particles into the laser beam is independent of the ion polarity, the relative hit rate is equivalent to the relative ablation efficiency (see eq 1 ). In most cases, the negative ion ablation efficiency is much less than the positive ion ablation efficiency. This behavior indicates that the conversion of free electrons to negative ions varies from particle to particle and does not always yield sufficient ions to exceed the detection threshold. Relative to the positive ion ablation efficiencies, the negative ion ablation efficiencies of all three compounds decrease with decreasing particle diameter. As the particles decrease in diameter, fewer are detected in the negative ion mode, suggesting that free electrons constitute a greater fraction of the negative ion signal. For sodium chloride and oleic acid, the data also suggest that the positive and negative ion ablation efficiencies become equivalent in the limit of a large particle size, although this possibility was not explored for particles above $200 \mathrm{~nm}$ in diameter.

It should be noted that the size and composition dependencies of the relative hit rates in Figure 3 do not match those of the relative signal intensities in Figure $2 \mathrm{a}$. These differences are not surprising since different quantities are measured. The ablation efficiency is a measure of the fraction of all sampled particles that produce an ion signal exceeding the detection threshold level, while the relative signal intensities are measured only for the subset of particles where the ion signal exceeded the threshold.

Detection of Sulfate in Mixed-Composition Particles. Although sulfate is an important component of atmospheric aerosols, the sensitivity of laser ablation mass spectrometry to sulfate is very low in the positive ion mode. Sulfate particles 3 $\mu \mathrm{m}$ in diameter give negative ion signals that correspond to the

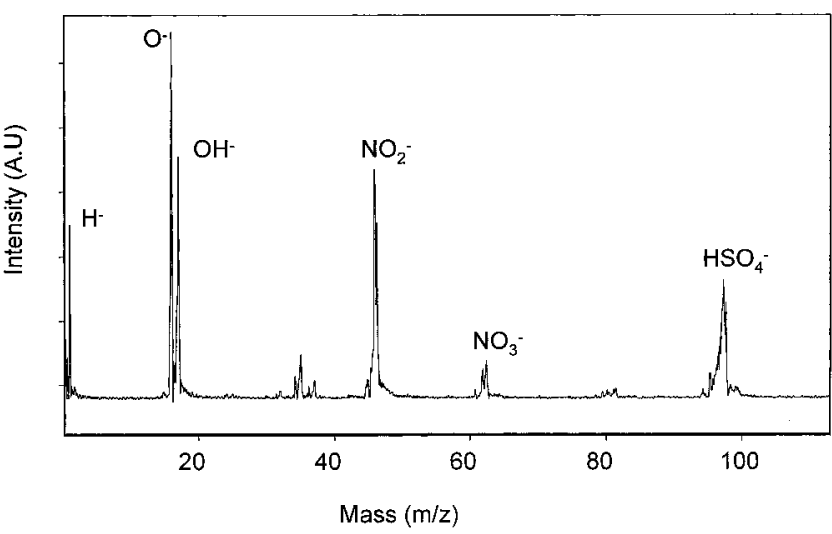

Figure 4. Negative ion mass spectrum of $77-\mathrm{nm}$ mobility diameter particles composed of ammonium nitrate with $1 \%$ ammonium sulfate. The spectrum is an average of 100 single-particle spectra.

sulfate species present in the particle, for example, the $\mathrm{HSO}_{4}{ }^{-}$ ion from ammonium sulfate. ${ }^{14} \mathrm{H}$ owever, we were unable to detect pure ammonium sulfate particles of $<200 \mathrm{~nm}$ in diameter in the negative ion mode, suggesting that these particles have negative ion ablation efficiencies below 0.01 . It is not surprising that the negative ion ablation efficiency is low, since we reported previously that the ablation efficiency of ammonium sulfate in the positive ion mode is less than 0.02 and that the mass spectra of "pure" ammonium sulfate particles are dominated by trace impurities. ${ }^{10,15}$ This suggests that only ammonium sulfate particles containing impurities are detected.

Since particles in the atmosphere are likely to consist of a mixture of components, we investigated the detection of sulfate in mixed-composition particles. The average mass spectrum from $77-n m$ ammonium nitrate particles with $1 \%$ ammonium sulfate is shown in Figure 4. In this spectrum, the $\mathrm{HSO}_{4}{ }^{-}$ion (m/ z 97 ) from sulfate is clearly observed above the noise level. Also present are $\mathrm{O}^{-}(\mathrm{m} / \mathrm{z} \mathrm{16}), \mathrm{OH}^{-}(\mathrm{m} / \mathrm{z} \mathrm{17})$, and $\mathrm{NO}_{2}^{-}(\mathrm{m} / \mathrm{z} 46)$. Although, ammonium nitrate is present at a concentration 100 times greater than ammonium sulfate, the signals from $\mathrm{NO}_{2}^{-}$and $\mathrm{HSO}_{4}^{-}$are similar, suggesting that sulfate competes favorably for charge in the vaporized plume.

While small amounts of sulfate can be detected in mixedcomposition particles, the hit rate decreases as the amount of sulfate in the particle increases. Figure 5 shows the effect of sulfate concentration on the particle hit rate for ammonium nitrateammonium sulfate particles. The hit rate drops significantly as the sulfate mass fraction increases above 20\% Similar behavior was observed for other sizes of ammonium nitrate-ammonium sulfate particles and for oleic acid-ammonium sulfate particles. At high sulfate concentrations, the particles are difficult to detect, since neither positive nor negative ions are efficiently produced by laser ablation. As the ammonium sulfate mass fraction approaches 1, the ablation efficiency becomes dependent on the presence of trace impurities in the particle. It is these impurities that promote ionization and allow sulfate to be detected by electron capture or charge transfer in the vaporized plume.

Under the conditions used in this work, the relationship between particle composition and the relative signal intensities

(14) Neubauer, K. R.; Sum, S. T.; Johnston, M. V. J. Geophys. Res. 1996, 101 (D13), 701-18, 707

(15) Kane, D. B.; Johnston, M. V. Anal. Chem. 2001, 73, 5365-5369. 


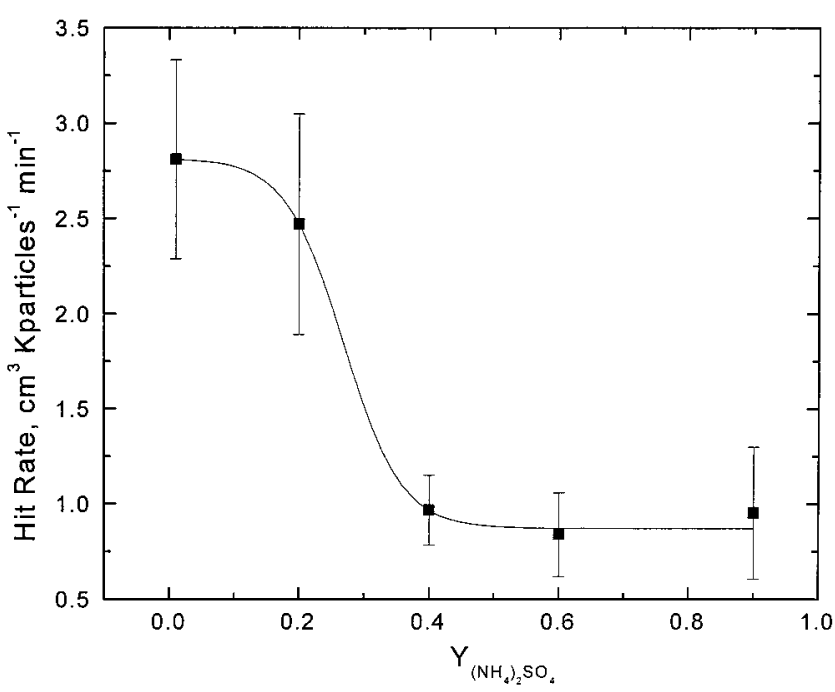

Figure 5. Dependence of the normalized particle hit rate (particles detected per second for an aerosol containing 1000 particles $/ \mathrm{cm}^{3}$ ) on the mass fraction of ammonium sulfate for $150-\mathrm{nm}$ mobility diameter ammonium nitrate-ammonium sulfate particles. Error bars are the standard deviation of at least three measurements, 100 particles each.

of the corresponding ions is complex and cannot be used to quantitatively determine the sulfate concentration from the mass spectra. This complexity reflects the strong coupling of ionization, particle disintegration, and electron capture processes induced by laser ablation.

Implications for Ambient Particle Measurements. The goal of a single-particle experiment is to determine the composition of an ambient aerosol from the distribution of single-particle mass spectra. With this goal in mind, several factors must be taken into consideration when negative ion detection is used for a singleparticle mass spectrometer. First is the need to detect species that do not give strong ion signals in the positive ion mode, such as sulfate. Second is the limitation imposed by lower particle hit rates and lower ion signal intensities. If it is necessary to detect species such as sulfates, which do not give strong ion signals in positive ion mode, then negative ion detection must be used. The poor ablation efficiencies of these particles can potentially be overcome by increasing the sampling rate of particles into the laser beam, either by increasing the laser repetition rate or by decreasing the size of the particle beam. However, if the detection of species in the negative ion mode is not of paramount importance, then positive ion detection is preferred.

Aerosol mass spectrometers capable of dual-polarity ion detection have been reported in the literature. ${ }^{16,17}$ We recently experi-

(16) Hinz, K.-P.; Kaufmann, R.; Spengler, B. Aerosol Sci. Technol. 1996, 24, 233242.

(17) Gard, E.; M ayer, J. E.; M orrical, B. D.; Dienes, T.; Fergenson, D. P.; Prather, K. A. Anal. Chem. 1997, 69, 4083-4091.

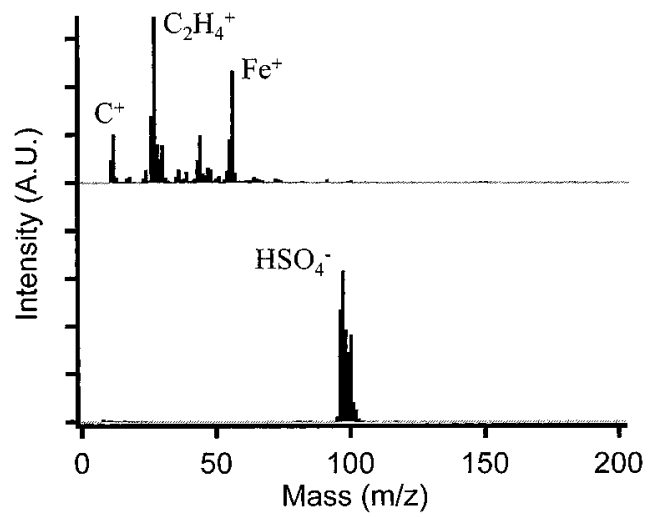

Figure 6. Dual-polarity mass spectra of a single ambient particle containing sulfate. The particle size is nominally $70 \mathrm{~nm}$ in diameter (aerodynamic).

mented with a dual-polarity instrument designed for field measurements of ultrafine particles. This instrument is similar in design to a previous field instrument having single-polarity detection. ${ }^{18}$ Dual-polarity detection combines the advantage of triggering on the positive ion mass spectrum while simultaneously collecting the negative ion spectrum. An example of a dual-polarity ambient particle spectrum is shown in Figure 6 . In the negative ion mode, $\mathrm{HSO}_{4}^{-}$indicates the presence of sulfate, while the positive ion mode indicates the presence of organic components and iron.

While low concentrations of sulfate can be readily detected in particles composed primarily of other materials, particles containing nearly $100 \%$ ammonium sulfate are difficult to detect at all. Recently, we demonstrated that by coating particles with a UVabsorbing compound it is possible to increase the ablation efficiency of "pure" ammonium sulfate particles by a factor of 20.15 When combined with dual-polarity detection, this provides a reliable method for detecting particles with a high concentration of ammonium sulfate.

\section{ACKNOWLEDGMENT}

This research was supported by Grant CHE-0098831 from the National Science Foundation. Ambient particle measurements with the dual-polarity mass spectrometer were supported by the Department of Energy National Energy Technology Laboratory through cooperative agreement no. DE-FC26-0INT 41017 and were performed in collaboration with Anthony Wexler, Derek Lake, M ichael Tolocka, and Yongjing Zhou.

Received for review October 29, 2001. Accepted February 5, 2002.

\section{AC011126X}

(18) M allina, R. V.; Wexler, A. S.; Rhoads, K. P.; Johnston, M. V. Aerosol Sci. Technol. 2000 33, 87-104. 


\title{
Laser-Induced Breakdown Spectroscopy Detection and Classification of Biological Aerosols*
}

\author{
JOHN D. HYBL, GREGG A. LITHGOW, and STEVEN G. BUCKLEY $\dagger$ \\ MIT Lincoln Laboratory, 244 Wood Street, Lexington, Massachusetts 02420-9108 (J.D.H.); and Department of Mechanical and \\ Aerospace Engineering, University of California, San Diego, California 92093 (G.A.L., S.G.B.)
}

\begin{abstract}
Laser-induced breakdown spectroscopy (LIBS) is examined as a potential method for detecting airborne biological agents. A spectrally broadband LIBS system was used for laboratory measurements on some common biological agent simulants. These measurements were compared to those of common, naturally occurring biological aerosol components (pollen and fungal spores) to determine the potential of LIBS for discriminating biological agents from natural background aerosols. A principal components analysis illustrates that linear combinations of the detected atomic lines, which are present in different ratios in each of the samples tested, can be used to discriminate biological agent simulants from other biological matter. A more sensitive, narrowband LIBS instrument was used to demonstrate the detection of single simulant (Bg) particles in the size range 1-5 $\mu \mathrm{m}$. $\mathrm{Ca}, \mathrm{Mg}$, and $\mathrm{Na}$, which are present in varying concentrations between 0.3 and $11 \%$ (by mass) in the $\mathrm{Bg}$ particles, were observed in single particles using LIBS.
\end{abstract}

Index Headings: Laser-induced breakdown spectroscopy; LIBS; Plasma spectroscopy; Biological agent detection; Biological aerosols.

\section{INTRODUCTION}

Biological warfare agents pose a serious threat to both military establishments and civilian population centers. A potential method for disseminating biological agents is via aerosols with particles in the respirable size range of

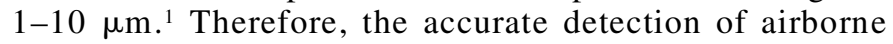
pathogens amidst the ambient background of dirt, biological debris, and combustion products is of considerable importance. In addition to defense applications, fast and accurate detection and classification of biological aerosols could be important in preventing the spread of disease in hospitals and for monitoring outdoor allergy-causing molds and pollens.

In most concepts for complete biowarfare-agent detection systems, a fast, consumables-free sensor continuously samples air and functions as a "trigger" for subsequent, usually wet-chemistry-based, identification of threatening aerosol particles. ${ }^{1}$ Considerable progress has

Received 25 April 2003; accepted 18 June 2003.

* This work was sponsored in part by the Department of the Army under Air Force Contract F19628-00-C-0002. Opinions, interpretations, conclusions, and recommendations are those of the authors and are not necessarily endorsed by the United States Government.

$\dagger$ Author to whom correspondence should be sent. been made in applying various combinations of particle sizing, particle counting, and fluorescence measurements to the trigger component of bioaerosol detection..$^{2-7}$ Fluorescence-based aerosol triggers provide reasonable rejection of inorganic background clutter, but fluorescent combustion products and natural biological aerosols may cause interference. ${ }^{1}$ High false-trigger rates due to background aerosols can drive up system operating costs by increasing the use of consumables such as chemical reagents in the identifier. Technologies that offer discrimination against fluorescent, non-threat aerosols would substantially reduce the deployment costs of bioagent aerosol detection systems and subsequently increase their utility. Additionally, consumables-free technologies with the ability to classify biological aerosols into broad categories such as fungal spores, pollens, bacteria, viruses, and toxins would further reduce operating costs, add another level of confirmation to the identifier, and allow the optical trigger/classifier to operate independent of a full detection system in certain limited-role applications.

Most likely, optimal false-trigger rates and classification capabilities will require the fusion of two or more orthogonal measurement technologies. The addition of elemental analysis to fluorescence/sizing measurement is one example of such a concept. Biological organisms contain a wide variety of readily detectable inorganic elements such as $\mathrm{Ca}, \mathrm{Mg}, \mathrm{Mn}, \mathrm{Fe}, \mathrm{P}, \mathrm{Na}, \mathrm{K}$, and $\mathrm{Si}^{8}{ }^{8}$ In some cases, the relative amounts of these elements vary between different classes of organisms. For example, it is well known that during sporulation bacterial spores concentrate calcium dipicolinate. ${ }^{9}$ Accordingly, Table I shows a laboratory analysis of seven elements in three types of sporulated Bacillus subtilis var. niger (Bg), a common simulant for Bacillus anthracis, and three types of fungal spores. Although the total weight percent of the inorganic species varies within the Bg samples, the relative ratios remain fairly similar. More interesting are the significant differences in the ratios of $\mathrm{Ca}, \mathrm{K}, \mathrm{P}$, and $\mathrm{Na}$ between $\mathrm{Bg}$ and the fungal spores. This suggests that elemental analysis offers substantial potential for broad classification of biological aerosols.

Several techniques exist in atomic spectroscopy for the analysis of laboratory samples. However, many of these techniques are not easily translatable into field instru- 
TABLE I. Elemental analysis of Bg and smut.

\begin{tabular}{|c|c|c|c|c|c|c|}
\hline \multirow[b]{2}{*}{ Element $^{\mathrm{a}}$} & \multicolumn{3}{|c|}{$\mathrm{Bg}$} & \multicolumn{3}{|c|}{ Fungal spores (smuts) } \\
\hline & 1 & 2 & 3 & Oat & Wheat & Corn \\
\hline $\mathrm{Ca}$ & 1.16 & 1.08 & 10.21 & 0.16 & 0.0147 & 0.12 \\
\hline $\mathrm{Mg}$ & 0.30 & 0.37 & 2.80 & 0.20 & 0.0937 & 0.19 \\
\hline $\mathrm{Na}$ & 0.45 & 0.38 & 5.82 & 0.0132 & 0.0110 & 0.0171 \\
\hline $\mathrm{K}$ & 0.49 & 0.49 & 0.68 & 1.60 & 2.24 & 1.63 \\
\hline $\mathrm{Fe}$ & 0.67 & 0.57 & 0.0088 & 0.0253 & 0.0032 & 0.0081 \\
\hline $\mathrm{P}$ & 2.30 & 2.32 & 8.52 & 0.44 & 0.41 & 0.58 \\
\hline $\mathrm{Mn}$ & 0.0081 & 0.0122 & 0.10 & 0.006 & 0.0024 & 0.0037 \\
\hline
\end{tabular}

${ }^{\mathrm{a}}$ Elemental concentrations as percent by weight.

ments. Laser-induced breakdown spectroscopy (LIBS) is one of the most practical means for bringing elemental analysis to the field. ${ }^{10}$ The technique involves tightly focusing a laser pulse to create a microplasma via laser breakdown of a sample or ambient air. Material inside the plasma volume is ionized and decomposed into atomic constituents. The hot plasma $(10000-15000 \mathrm{~K})$ provides a thermal excitation source for the atomized species within the plasma volume. Emission from these electronically excited atoms and ions is spectrally resolved and used to determine the elemental composition of the sample. The lack of sample preparation, experimental simplicity, and portability of modern lasers makes LIBS an attractive and practical technique for deployment in field sensors.

A rough gauge of the ability of LIBS to detect single bioaerosol particles can be made by considering the absolute mass of each element (Table I) present in a representative bioaerosol particle, relative to the LIBS detection limits reported in the literature. A single bacterial spore represents a generally lower size limit for a typical bioaerosol particle. It can be approximated as a cylindrical particle with $2.5-\mu \mathrm{m}$ length, $1-\mu \mathrm{m}$ diameter, and a density of $\sim 1 \mathrm{~g} / \mathrm{cm}^{3}$. This representative particle would have a mass of approximately $2 \mathrm{pg}$. Recently, Hahn and co-workers used a portable LIBS instrument to measure $\mathrm{Na}, \mathrm{Ca}, \mathrm{Mg}$, and $\mathrm{Al}$ from ambient aerosols. They reported absolute mass detection limits of $0.5,1.2$, and $3.3 \mathrm{fg}$ for $\mathrm{Ca}, \mathrm{Mg}$, and $\mathrm{Na}$, respectively. ${ }^{11}$ These detection limits for $\mathrm{Ca}$ and $\mathrm{Mg}$ are similar to previously reported limits determined using a differential mobility analyzer to measure a monodisperse aerosol stream. ${ }^{12}$ Based on these limits, one would expect to be able to detect these elements in a bioaerosol particle at concentrations of $0.1 \%$ by weight. Given the concentrations of these elements in Bg (Table I), it is expected that LIBS should be able to detect them in single spores. In reality, a bioaerosol will have a distribution of particle sizes so that a significant fraction of the bioaerosol particles would actually be larger.

A large body of work on the LIBS technique exists; a general introduction to the technique and a survey of the literature can be found in a number of reviews. ${ }^{13-16}$ Particularly relevant to this study is the literature associated with detection of aerosol particles, which reveals limitations to the LIBS technique. Because the plasma is responsible for both particle ablation/vaporization and excitation of elements in the sample, plasma stability is critical for reproducibility and a favorable signal-to-noise ratio $(\mathrm{S} / \mathrm{N})$ in LIBS. Air plasmas exhibit a saturation effect with increasing laser power; it is observed that an in- creasing percentage of the incident laser energy is absorbed by the plasma until a saturation of approximately $60 \%$ of the incident laser power is reached. Operation in this saturated regime has been shown to increase the reproducibility of the LIBS signal. ${ }^{17}$

The potential of incomplete vaporization of particles also produces uncertainty in LIBS analysis. It is commonly accepted that the energy in a typical breakdown is sufficient to vaporize micrometer-sized particles and atomize molecules in the plasma volume. Estimates of the largest particles that can be vaporized, with ensuing LIBS detection of the particles, have ranged from $\sim 2.5$ $\mu \mathrm{m}$ to $\sim 10 \mu \mathrm{m}$ and are expected to depend on the particle composition and on experimental conditions such as laser power. Such analysis has not been completed for the chemical matrices relevant to bioaerosol detection. In addition, an optimal detection window for peak $\mathrm{S} / \mathrm{N}$ exists for each element based on the transition energy and the transition probability..$^{18,19}$

Once optimal $\mathrm{S} / \mathrm{N}$ is achieved, a robust method is required to distinguish particles from noise generated by the plasma and detection system. Schechter et al. suggest that spectral sorting based on various characteristics could be used to discriminate aerosol particles suspended in gas and reject ill-conditioned spectra, which may have variations due to matrix effects, poor atomization of the particle in the plasma, or laser power fluctuations. ${ }^{20,21}$ Following this concept, Hahn presented an analysis of LIBS-based aerosol detection, elemental analysis, and particle sizing, ${ }^{12}$ which was subsequently confirmed for laboratory-generated aerosol particles and polydisperse particles of known composition using a differential mobility analyzer to size-segregate the particles prior to LIBS. ${ }^{22}$ These strategies have enabled LIBS to be used successfully for measurements of particulate-bound metals in effluents from thermal treatment processes. ${ }^{23,24} \mathrm{Ad}-$ ditional aspects of LIBS for aerosol detection are covered in a recent review by Martin et al. ${ }^{10}$

Here, we report on the investigation of LIBS as a biotrigger technology. Two different LIBS instruments were used to explore the following aspects of the technique fundamental to its utility for bioaerosol detection: (1) its discrimination potential for bioaerosol sensing, and (2) the detection of the discriminatory elements in singlebioagent-simulant particles. To address discrimination potential, a broadband LIBS instrument was used to acquire spectra of dense aerosols over the range 200-825 $\mathrm{nm}$. These spectra were subjected to principal components analysis (PCA) to gauge the overall discrimination ability of the technique. Since the broadband system was 


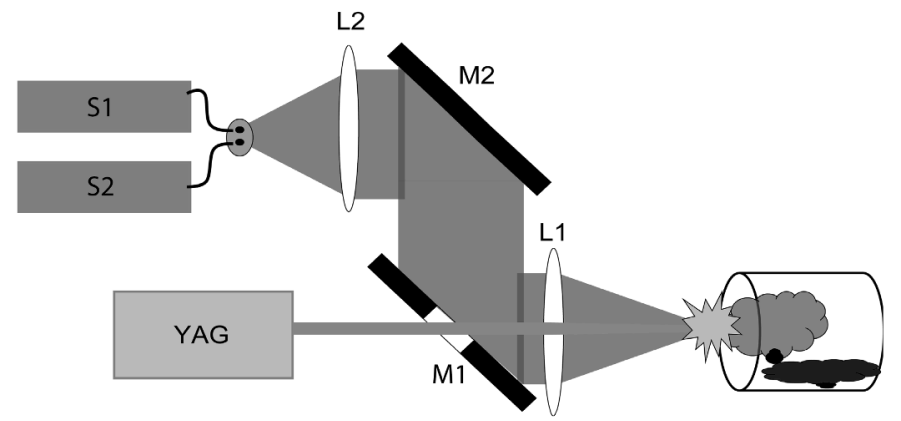

FIG. 1. Experimental LIBS apparatus. A Big Sky Ultra CFR-GRM $\mathrm{Nd}$ : YAG laser ( $\boldsymbol{Y A G}$ ) was used to produce the plasma. A 75-mm-focallength lens $(\boldsymbol{L I})$ was used to both focus the laser pulse and collect the plasma emission in the backward direction. A pierced aluminum mirror (MI) directed the plasma emission towards the collection optics. A 100$\mathrm{mm}$-focal-length lens $(\boldsymbol{L} 2)$ was used to focus the emission into a bifurcated fiber bundle $(\boldsymbol{B F})$ consisting of two $600-\mu \mathrm{m}$-core UV-rated fibers. The fibers then directed the emission into two Ocean Optics HR2000 spectrometers (S1: 613-825 $\mathrm{nm}$ and $\boldsymbol{S 2}: 200-650 \mathrm{~nm}$ ).

not capable of detecting single particles, single-particle detection of $\mathrm{Bg}$ was demonstrated over narrow spectral windows with a more sensitive spectrometer/intensified charge-coupled device (CCD) system.

\section{EXPERIMENTAL}

Broadband, Multi-particle Laser-Induced Breakdown Spectroscopy of Aerosols. Broadband, multi-particle LIBS spectra were recorded using the apparatus shown in Fig. 1. A Big Sky Ultra CFR-GRM Nd:YAG laser was used to generate the plasma. Typical of lasers used for LIBS experiments, it produces 7-ns, 50-mJ pulses at $1064 \mathrm{~nm}$ with a maximum repetition rate of $20 \mathrm{~Hz}$. The laser pulses were focused with a 2-in.-diameter, 75$\mathrm{mm}$-focal-length UV-grade fused-silica lens (L1). CCD images of the resulting plasma (in air) indicate a plasma volume on the order of $10^{-4} \mathrm{~cm}^{3}$ (1-mm-long, 0.5-mmradial-diameter ellipsoid). Optical emission from the plasma was collected with $\mathrm{L} 1$ and focused into a bifurcated fiber bundle $(\mathrm{BF}, \mathrm{NA}=0.22$, diameter $=600 \mu \mathrm{m}$ for each fiber) using a 2-in.-diameter, $100-\mathrm{mm}$-focal-length UV fused-silica lens (L2). Each fiber was connected to the input port of an Ocean Optics HR2000 spectrometer, each with a $5-\mu \mathrm{m}$ slit width. The spectrometers were equipped with electronic triggering so that signal integration could be delayed in increments of $500 \mathrm{~ns}$ from the time the laser fired (total integration time is fixed at 2 $\mathrm{ms})$. For the experiments presented here, this delay was set to $3.5 \mu$ s to prevent unwanted ion lines and broadband plasma emission from decreasing $\mathrm{S} / \mathrm{N}$. The UV/VIS spectrometer (S2) covered $200-650 \mathrm{~nm}$ with $0.35-\mathrm{nm}$ nominal resolution, and the NIR spectrometer (S1) covered 613-825 nm with 0.15-nm nominal resolution. Since an aerosol LIBS sensor will likely encounter a variety of particle sizes, it was assumed that any discrimination algorithm would rely on the ratios of line intensities as opposed to absolute intensities. Therefore, the acquired LIBS spectra were not corrected for chromatic focusing aberrations, wavelength-dependent CCD response, or wavelength-dependent grating efficiency, since these effects should not vary from sample to sample.

An issue in obtaining background-free LIBS spectra of
TABLE II. Samples tested.

\begin{tabular}{ll}
\hline \multicolumn{1}{c}{ Sample class } & \multicolumn{1}{c}{ Sample type } \\
\hline Bacterial spore & 3 types of Bg (A, B, C) \\
Media/protein & Ovalbumin (Ov) \\
& Brain heart infusion (BH) \\
& LB Broth (LB) \\
Fungal/mold spores & Penicillium (Pn) \\
& Corn Smut (Cn) \\
& Oat Smut (Ot) \\
Pollen & Ragweed (Rg) \\
& Red Mulberry (Rm) \\
& Red Oak (Ro) \\
\hline
\end{tabular}

powders is finding a suitable means to bring the sample into the plasma volume for measurement. One approach is to adhere the sample to a surface such as double-sided adhesive tape or a silicon wafer. However, any substrate can contribute atomic lines to the LIBS spectrum that may hide features due to the sample alone. Additionally, because complex materials such as tape include many of the same elements present in the bioagent simulants, they might produce erroneous discriminating features that could vary from sample to sample, or shot to shot, depending on the relative amounts of substrate and sample consumed in each measurement. Therefore, as shown in Fig. 1, a small pile of sample was placed in a microcentrifuge tube for measurement. The tube was positioned so that the air plasma formed at its mouth. The shock wave resulting from each laser shot aerosolized sufficient material that the subsequent laser pulse encountered a dense sample cloud. By continually running the laser at $5 \mathrm{~Hz}$, single-shot spectra were obtained from a continually refreshed, dense aerosol cloud. No signal was observed from empty tubes, indicating that the plasma was not ablating the tube walls. In this way, clean, substratefree spectra were obtained for each sample. Table II contains a list of the samples investigated. Bioagent simulants included three types of Bg and three "protein/media" that simulate viruses, toxins, or growth media. The various types of $\mathrm{Bg}$ differ from each other in growth history and washing methods. Background materials included three types of fungal spores, three types of pollen, and dirt.

Narrowband Laser-Induced Breakdown Spectroscopy of Single Aerosol Particles. Single Bg particles were measured using the more sensitive intensified CCD system, in which a frequency-doubled Big Sky CFR-400 $\mathrm{Nd}$ : YAG laser with a nominal pulse energy of $200 \mathrm{~mJ} /$ pulse fires at an adjustable rate between 1 and $10 \mathrm{~Hz}$ to generate an air plasma inside a sample cell. We note that previous work has shown the similarity of the $1064 \mathrm{~nm}$ and $532 \mathrm{~nm} \mathrm{Nd}$ :YAG wavelength-generated plasmas for elemental analysis. ${ }^{25}$ A 3:1 Galilean telescope expands the $0.6-\mathrm{cm}$ beam from the laser to a diameter of $1.8 \mathrm{~cm}$, and the expanded beam is focused using a $10-\mathrm{cm}$-focallength plano-convex lens. Emission from the plasma is detected at right angles using a 7.5-cm, 1-in.-diameter, plano-convex lens to collimate the light. A $5.2-\mathrm{cm}$-focallength, 3-cm-diameter reflective collection system (Multichannel Instruments CC-52) efficiently couples the plasma light into a UV-compatible, $0.22 \mathrm{NA}$ optical fiber bundle. The optical collection system is characterized by an overall N.A. of $\sim 0.11$, which corresponds to a 0.44 


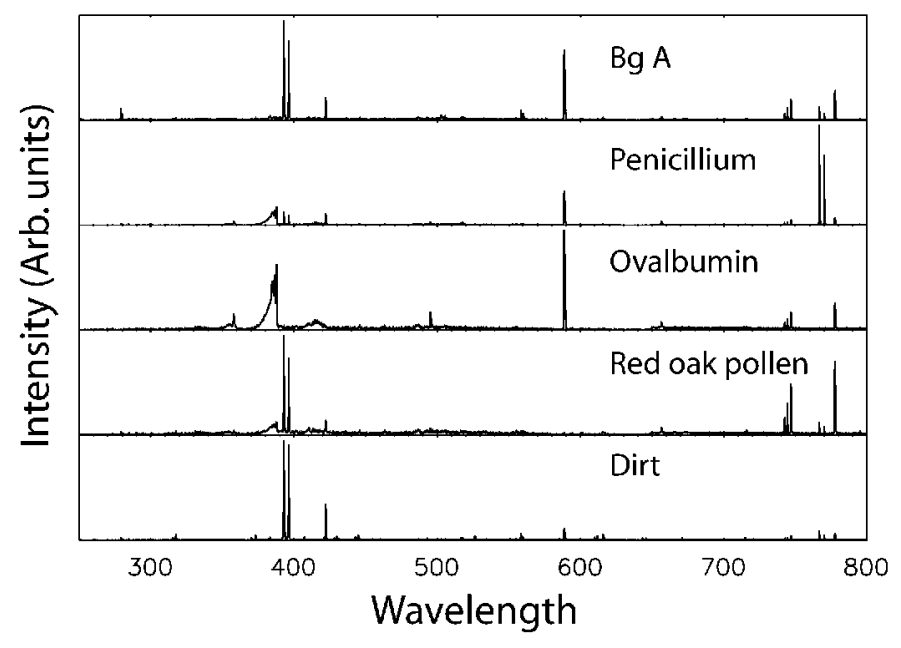

FIG. 2. Representative LIBS spectra of bioagent simulants and background materials. Each spectrum is an average of 50-100 shots. The prominent atomic lines are: $\mathrm{Mg}(\sim 285 \mathrm{~nm}), \mathrm{Ca}(393,396$, and 422.7 $\mathrm{nm}), \mathrm{Na}(\sim 589 \mathrm{~nm})$, and $\mathrm{K}(\sim 766 \mathrm{~nm})$; approximate wavelengths are given since some are unresolved or partially resolved multiplets. Molecular CN appears as a broad peak around $387 \mathrm{~nm}$ in some of the spectra. Atmospheric $\mathrm{N}$ is visible at roughly 741,743 , and $746 \mathrm{~nm}$, and a prominent $\mathrm{O}$ line is apparent at $777 \mathrm{~nm}$.

sr solid angle of collection. The fiber transmits the light to a Roper Scientific PI-Max gated, intensified CCD camera (UV enhanced) mated to a $0.3-\mathrm{m}$ Acton SpectraPro $300 \mathrm{i}$ spectrometer. This setup, collectively referred to here as the Roper system, allows extremely accurate time gating of the collected emission signal, on the order of 5 $\mathrm{ns}$. The effective dispersion of the system using a grating with 1200 grooves per millimeter is approximately 0.05 nm per pixel, allowing spectral window segments of $\sim 50$ $\mathrm{nm}$ to be recorded with each shot.

An aerosol stream was generated from a dry powder. Aerosol particles were suspended in an air stream (5-20 L pm of HEPA-filtered air) using a $60-\mathrm{Hz}$ excitation current driving a loudspeaker. Software processing of each acquired spectrum detects individual aerosol hits by monitoring wavelengths corresponding to atomic emission lines. The operation of the aerosol generator was varied to result in a measured aerosol hit rate in the sample cell between a maximum of one hit per 10 shots and a minimum of approximately one hit per 50 shots. As a result, to first order, the frequency of double particle hits is between 0.01 and 0.0025 .

\section{RESULTS AND DISCUSSION}

Broadband, Multi-particle Measurements. Figure 2 displays averaged LIBS spectra for the various classes of biological simulants and representative background materials. The illustrated spectra are normalized by their total spectral power (integration of the entire spectrum), and in the following discussion ratios of individual elements are determined by integration of the relevant atomic peaks. Figure 2 also shows the LIBS spectrum of a dirt sample in order to give some idea of inorganic aerosols likely to be encountered in the real environment. A few conclusions can be drawn from visual inspection of the individual LIBS spectra. Most important, each class of biological sample has a distinctive feature: $\mathrm{Ca}: \mathrm{K}$ ratio $\gg 1$ in $\mathrm{Bg}, \mathrm{Ca}: \mathrm{K}$ ratio $\ll 1$ for fungal spores (e.g., Penicillium), an overwhelming $\mathrm{Na}$ signal in the ovalbumin spectrum, and a minimal sodium signal (combined with $\mathrm{Ca}: \mathrm{K}>1$ ) in pollen. In most of the biological samples a relatively broad peak is seen around $387 \mathrm{~nm}$, attributed to recombination of $\mathrm{CN}$ in the cooling plasma. The peak is also evident in diesel and spark-ignited engine exhaust, where it has recently been used in conjunction with atomic $\mathrm{O}$ and $\mathrm{N}$ measurements to quantify engine equivalence ratio. ${ }^{26}$ Potentially, this molecular peak could be used to separate predominantly carbon-based particles (mostly biological) from inorganic particles. Phosphorus, with a peak near $255 \mathrm{~nm}$, is not readily visible in the bulk LIBS spectra. This is likely due to the combined effect of delaying detection by $3.5 \mu \mathrm{s}$ (phosphorus emission should peak early because of its large excitation energy) and the low $(<30 \%)$ efficiency of the Ocean Optics grating and CCD detector at this wavelength.

Even when operating significantly above the detection limits, as is the case with bulk samples, shot-to-shot reproducibility of LIBS spectra can be poor because of variations in the plasma volume, temperature, and electron density. Variations of these plasma characteristics affect both absolute and relative intensities of spectral lines. Therefore, it is important to understand the magnitude of these fluctuations relative to differences between samples. Additionally, in order to be of utility for discriminating classes of organisms from one another, the variation in LIBS signatures between samples in the same class must be significantly less than the differences between samples from different classes (i.e., LIBS signatures of two samples of Bg must be more similar to one another than to corn smut). This issue is addressed in Fig. 3, which summarizes the statistics of the major atomic lines in the LIBS spectra of all 12 biological samples. The height of each bar represents the average value of all the singleshot, sum-normalized spectra of a given sample type, and the error bars are drawn at $\pm 1 \sigma$. When the LIBS results in Fig. 3 are compared with the analysis of Bg (different samples of $\mathrm{Bg}$ ) and fungal spores in Table I, there is very good semiquantitative agreement across all four elements. Among these four elements, the discriminating features of each sample class are above the noise level set by the shot-to-shot variations. In single-particle measurements, other noise sources such as shot noise and variable particle ablation become important since many of the elemental concentrations are near the LIBS detection limits. Nonetheless, these initial results are encouraging in that the reproducibility of the LIBS technique is sufficient for discriminating among this limited set of bioagent simulants and interferants. It is interesting to note that, even using only $\mathrm{Mg}, \mathrm{K}, \mathrm{Ca}$, and $\mathrm{Na}$ lines, differences between types of $\mathrm{Bg}$, growth media, and fungal spores are significant, indicating that LIBS measurement of a few elements may be able to go beyond broad classification and differentiate more specifically, e.g., between different types of fungal spores or bacterial spores grown under different conditions.

To visualize and assess the discrimination potential of LIBS (multi-particle, broadband), PCA was applied to the 30 most prominent lines in the data set. This analysis provides a lower-dimensional representation of a data set in a coordinate system that preserves the maximum 

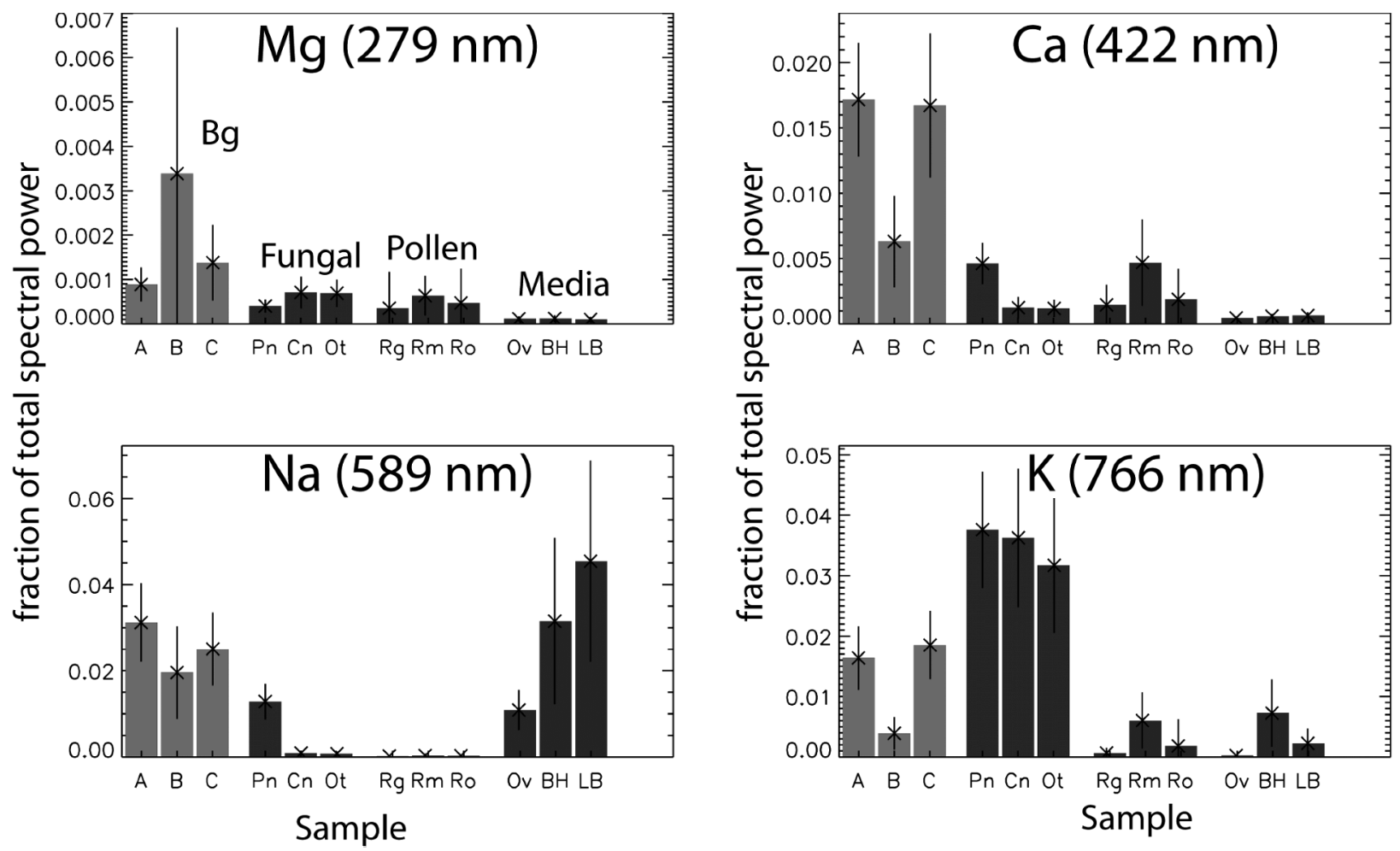

FIG. 3. Bar plots representing major atomic lines in the LIBS spectra of the 12 biological samples. Each bar is drawn to the average value of the atomic line intensity for each type of sample (intensity is normalized by total spectral power). The error bars are drawn at \pm 1 standard deviation and can be used as a guide to shot-to-shot reproducibility. Samples are annotated using the abbreviations in Table II.

amount of the data set's variance (roughly its information content). ${ }^{27}$ For discrimination between biological sample classes, a reduced "training set" was constructed by determining average spectra for each sample class (one for $\mathrm{Bg}$, one for fungal spores, etc.). These four spectra were then used with a basic PCA procedure to generate a threedimensional coordinate system that best represented the differences between each sample class. Each coordinate is composed of a linear combination of atomic line intensities weighted by their contribution to the training set's variance (they are the eigenvectors of the data's covariance matrix). The atomic line intensities from each individual, normalized spectrum were then used to put each single-shot LIBS spectrum into the PCA space as a single point. Figure 4 illustrates this three-dimensional representation of the data, in which all four biological sample classes are fairly well separated. The pollen data set shows significant spread, much of which is due to the lower S/N of the pollen spectra. The lower S/N of the pollen is probably due to the larger particle size of pollen (20-60 $\mu \mathrm{m})$, which makes it difficult to aerosolize and fully ablate. The spread in each sample cluster is the combined effect of shot-to-shot reproducibility and the true variation between the different samples in each class (e.g., oat smut vs. penicillium), which can both be seen in the error bars of Fig. 2.

As suggested by Fig. 3, there are indications that LIBS spectra can be used to differentiate samples within the same class. This type of capability could be useful in determining the source or identity of a particular bioaerosol. A PCA analysis on each sample class, using training spectra constructed from averages of each sample type, such as the various Bg samples, was used to explore this type of high-level discrimination. The results are shown in Fig. 5. It appears that the LIBS spectra do provide significant discrimination between the different types of fungal spores, growth media, and Bg samples. Since these spectra are not single particles, it is not clear whether the different Bg spores are actually different or if their spectral signatures come from impurities such as residual growth media. Spectrally broadband single-particle measurements will be required to determine the origin of these differences in LIBS signatures.

Some cautionary statements should be made about the discriminatory power of LIBS. First, nearly all the "signature" elements of the biological samples can be found in dirt. An example of this is the dirt in Fig. 2, which contains $\mathrm{Mg}, \mathrm{Ca}, \mathrm{K}$, and $\mathrm{Na}$, albeit in different ratios than either Bg or fungal spores. It is not hard to imagine, given the diversity of the natural environment, that other types of inorganic materials would have mineral signatures very similar to the biological materials. The $\mathrm{CN}$ peak does offer some discrimination but varies significantly among the samples of biological origin. Therefore, although LIBS potentially offers better discrimination between biological materials, it might not perform as well as current methods, such as fluorescence, in separating dirt particles from biological aerosol particles. Further, we should emphasize that the use of PCA in this work is merely illustrative of the potential for LIBS-based discrimination, but that more robust algorithms would likely be required in real-world applications.

The presence of these signature elements in the inorganic material also has significant consequences for LIBS 


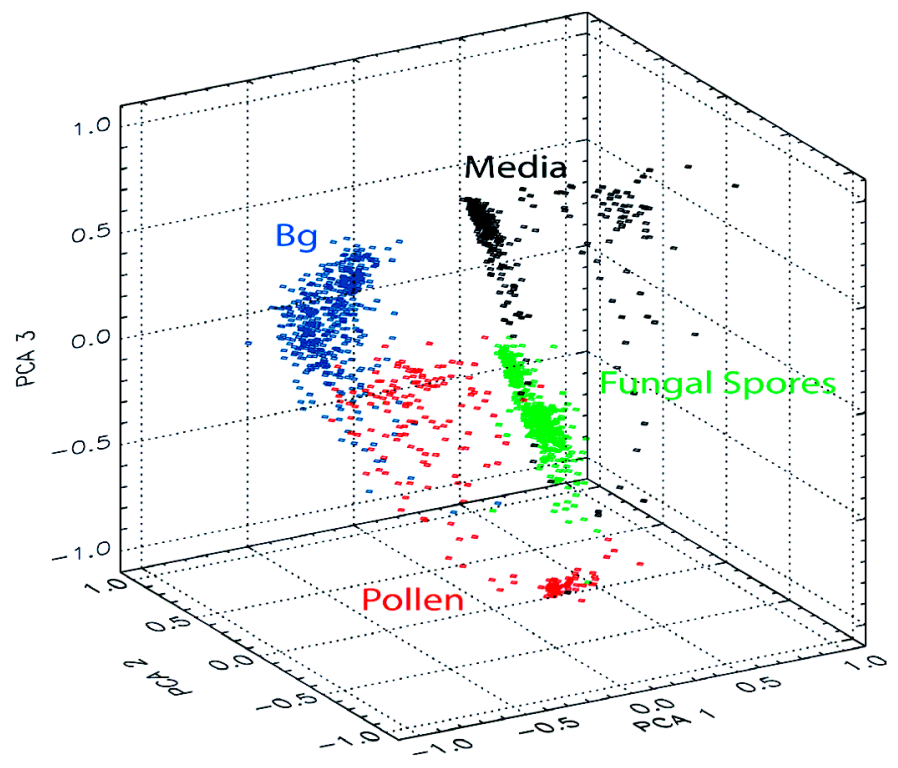

of mixtures. Since the signatures of the simulants rely on the relative intensities of multiple lines, rather than the presence of a single unique line, it is not hard to imagine that LIBS analysis of mixtures could easily cause inaccurate classification. An example, taken from this data set, would be a mixture of ovalbumin (mostly $\mathrm{Na}$ and $\mathrm{CN}$ ) and dirt (mainly $\mathrm{Ca}$ and $\mathrm{K}$ ). In the correct proportions, this would have a nearly identical signature to the LIBS spectrum of Bg. Although this study does not push the limits of chemometrics, the exploitation of matrix effects, or pattern recognition, LIBS of single particles will

FIG. 4. Three-dimensional scatter plot of the biological data set in the principal component coordinates. Each data point represents a single spectrum acquired from a single laser shot. The principal components were computed using three linear combinations of the intensities of 30 lines from the spectra. Each class of bioaerosol contains three different types, e.g., the fungal spore category is composed of penicillium, corn smut, and oat smut.
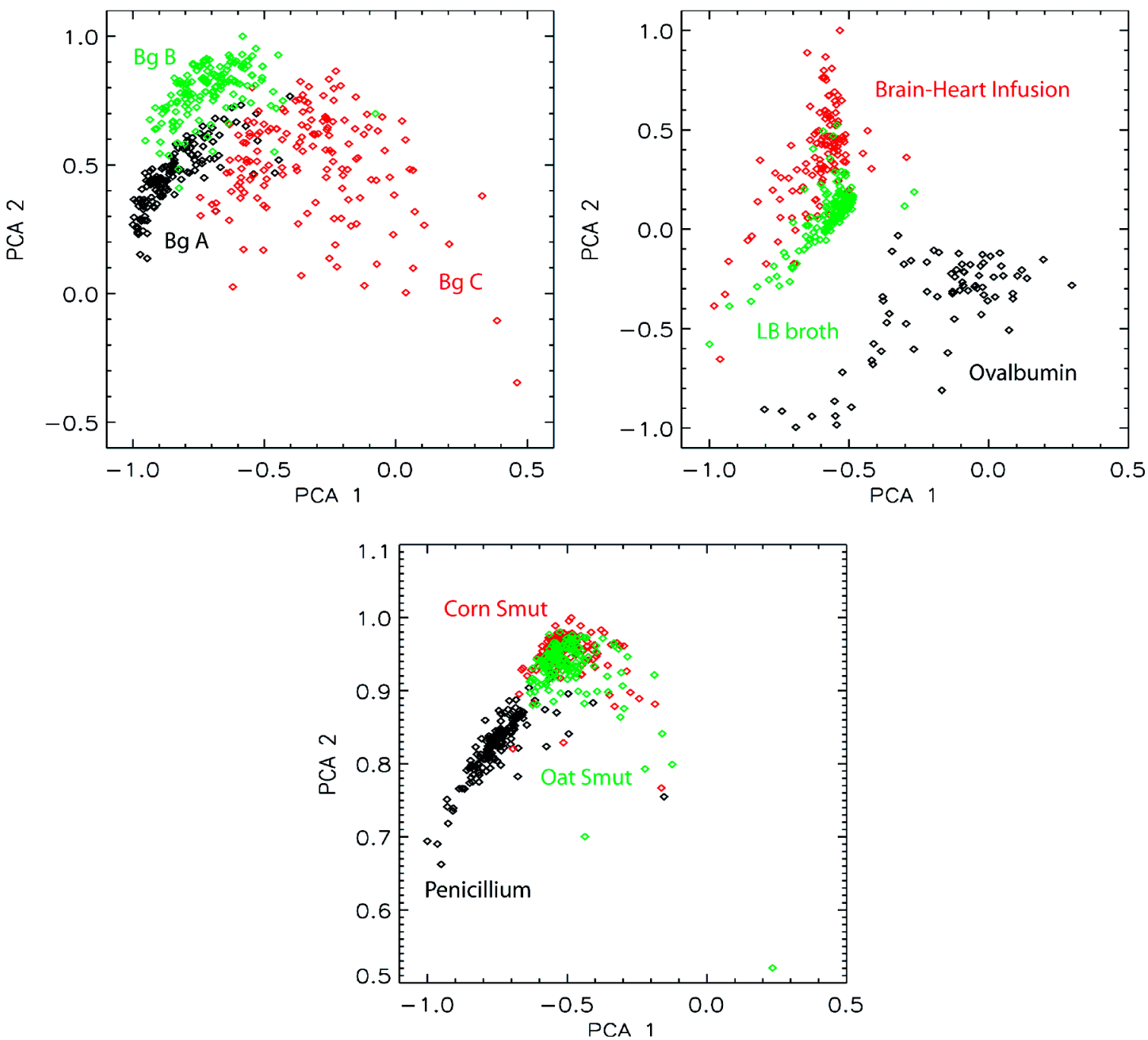

FIG. 5. PCA plots of each bioaerosol class. In this case, the principal components were determined from training sets comprising average spectra of each specific type of bioaerosol. In all three cases, the first two principal components (PCA 1 and PCA 2) account for $>95 \%$ of the training set's variance. 
TABLE III. Detection limits with Roper system at 5- $\mu$ s delay, 60us timing.

\begin{tabular}{cccc}
\hline Element & $\lambda(\mathrm{mm})$ & $\begin{array}{c}\text { Mass detection } \\
\text { limit }(3 \sigma) \\
(\mathrm{fg})\end{array}$ & $\begin{array}{c}\text { Minimum percent } \\
\text { concentration in } \\
\text { representative Bg } \\
\text { particle }(w t \%)\end{array}$ \\
\hline $\mathrm{Ca}$ & 422.67 & 30 & 1.5 \\
$\mathrm{Na}$ & 589.0 & 100 & 5 \\
$\mathrm{Mg}$ & 285.2 & 20 & 1 \\
\hline
\end{tabular}

most likely be very useful for detecting bioagents and discriminating them from the natural background.

Single-Particle, Narrowband Measurements. In order to address the question of whether or not LIBS is capable of detecting the amounts of $\mathrm{Ca}, \mathrm{Na}, \mathrm{Mg}$, etc., present in single biological particles, narrowband singleshot spectra of individual $\mathrm{Bg}$ particles in dilute flows were measured using the Roper system. Although the detection limits mentioned earlier ${ }^{12}$ indicate that the relevant concentrations should be readily detectable, the bioaerosol particles are complex matrices, and it is important to verify that matrix effects do not elevate the detection limits above the expected absolute masses. To obtain these measurements, the aerosol generator was operated in a regime in which the maximum particle hit rate is roughly $1: 10$. Thus, as noted above, the expectation is that the incidence of simultaneous two-particle hits should be roughly $1: 100$, but there was no way to measure coincidence of particles directly in these experiments. Typical mean particle sizes for the $\mathrm{Bg}$ aerosols, determined by aerodynamic particle sizing, are roughly $2.5 \mu \mathrm{m}$.

Quantification of LIBS peaks can be accomplished using several methods, such as by integrating the peak area following baseline subtraction or by ratioing the peak area to the baseline area. The latter quantity is referred to as the peak/base ratio and has the benefit of attenuating fluctuations attributed to variations in the laser power as both the peak strength and the baseline strength are functions of the laser power. For measurements described in this paper, the peak area and the peak/base ratio are closely correlated. The peak/base ratio was used as the measurement variable (e.g., Fig. 7), as it has been shown (e.g., Ref. 18) that relatively robust calibrations, appli-

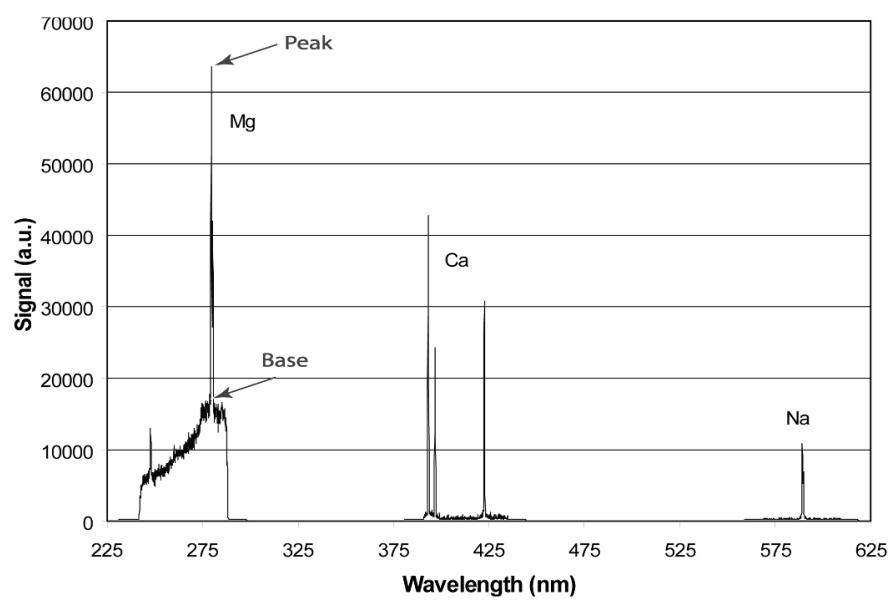

FIG. 6. Representative composite single-shot spectra of Bg, with intensities unadjusted.

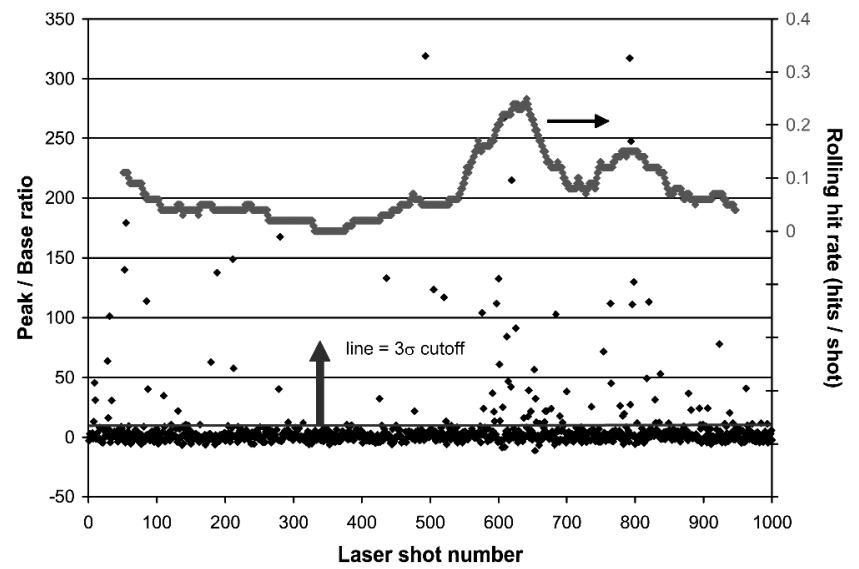

FIG. 7. Peak/base ratio corresponding to $\mathrm{Ca}$ for 1000 shots with $\mathrm{Bg}$ flowing through the sample cell. The upper trace, associated with the right-hand axis, indicates the particle hit rate averaged over $10 \mathrm{~s}$.

cable in the field where conditions may cause fluctuations in laser power and/or transmitted light, are possible using the peak/base ratio. Dedicated software analyzed individual spectra to determine the peak/base ratio for each element, which was then converted to the measured mass of each element using laboratory calibrations.

A timing of a 5 - $\mu$ s delay after the laser pulse and a $60-\mu s$ gate width after the shutter opening allows relatively good detection of all of the measured species. Detection limits for $\mathrm{Ca}, \mathrm{Mg}$, and $\mathrm{Na}$ (obtained with standard aerosols of each element) are shown in Table III. These are not optimized for each element, as were the detection limits mentioned earlier. ${ }^{12}$ The detection limits shown in Table III are related to the minimum percent concentration of the element in the hypothetical single-spore particle described in the Introduction. Based on the elemental analysis of Bg shown in Table I, these detection limits suggest that individual spores are close to the detection limit of our LIBS system as presently configured, but that agglomerated particles should be readily detectable. As agglomerates are expected in realistic situations, these results are equally relevant to the detection of real bioaerosol clouds. In addition, with the present system, detection limits for individual elements could be improved with optimized delay and gate for each element.

With the limited spectral range of the Roper system, it is not possible to measure all of the spectral lines at once, as one may do with an echelle spectrometer or a suitably configured linear array spectrometer. Hence, to visualize the entire spectrum that may result from a single-particle hit, a composite spectrum must be generated from a combination of spectra from each of the spectral windows. Figure 6 shows a composite single-shot, single-particlebased spectrum of $\mathrm{Bg}$. $\mathrm{Ca}, \mathrm{Na}$, and $\mathrm{Mg}$ are visible in the spectra of single $\mathrm{Bg}$ particles. Although the ratios of atomic lines in separate spectral windows are meaningless because they are not obtained from the same particle, the composite spectrum can be used to gauge the relative $\mathrm{S} / \mathrm{N}$ of each element present in the bioaerosol. The background noise, which is primarily from the intensifier and the plasma itself, is somewhat more evident in singleshot spectra than the average spectra, but the signals are still much stronger than the background.

For these experiments, sets of 1000 spectra were taken 


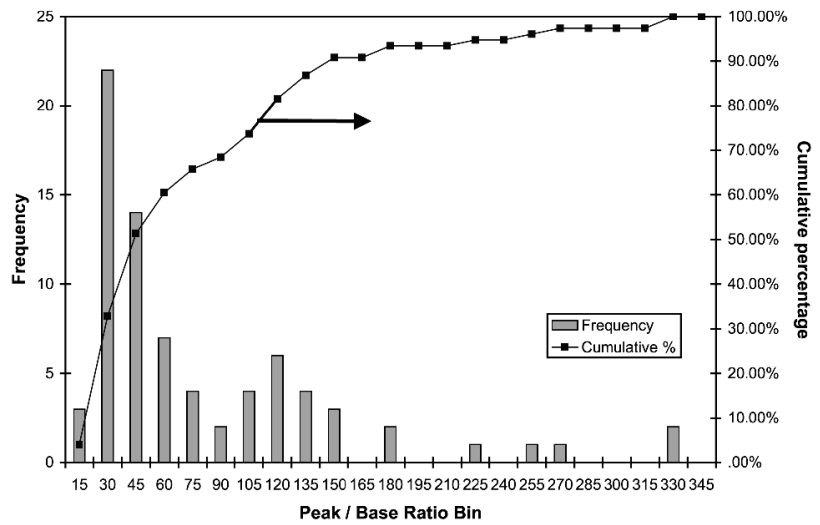

FIG. 8. Histogram of $\mathrm{Ca}$ hits in aerosolized $\mathrm{Bg}$, with peak/base ratio $>3 \sigma$, for 1000 shots. In these spectra $3 \sigma \approx 12.5$.

in each spectral window. Following the data acquisition, software triggers were set to discriminate elemental peaks (as relevant to the spectral window) using an adjustable criterion for peak detection based on the measured rms noise $(\sigma)$ in the spectral region immediately adjacent to the relevant peak. Peaks and noise are both integrated over the spectral line width. For many elements, including all measured here, two lines are available in a single spectral region, allowing software detection on one line and verification/quantification on the other. For purposes of these experiments, a $3 \sigma$ criterion is used; a spectrum is classified as a "hit" if the measured variable at the peak is three or more times the rms value of the measured variable in a region immediately adjacent to the peak. Following software detection of the atomic lines, all spectra classified as hits were hand-checked to verify the hit, and no false hits were detected.

Figures 7 and 8 illustrate the detection of $\mathrm{Ca}$ in aerosolized Bg. Figure 7 shows the measured peak/base ratio for 1000 laser shots. The $\mathrm{Bg}$ concentration fluctuates somewhat during the experiment; the upper trace in Fig. 7 illustrates the particle hit rate, averaged over $10 \mathrm{~s}$, as a function of time. The average hit rate is approximately 1:10, but went as high as 1:5 for a short time near the end of the experiment. The $3 \sigma$ hit criterion for $\mathrm{Ca}$ in the Bg spectrum, based on a spectral region immediately adjacent to the 422.7-nm peak of Ca, corresponds to a peak/ base ratio of 12.5 , which is shown in Fig. 7 as a horizontal line near the bottom of the figure. Points above this line correspond to hits of $\mathrm{Ca}$-containing $\mathrm{Bg}$. Figure 8 illustrates the 91 measured $\mathrm{Ca}$ hits from Fig. 7 in a histogram, and the cumulative percentage (by number) of hits as a function of peak/base ratio. In summary, based on the frequency of hits, the majority can be assigned to single-particle events with a high degree of confidence.

Measured elemental signal strengths for Bg are shown in Table IV, along with the standard deviation of each of the measured peaks (some of this variation is due to the distribution of particle sizes within the aerosol) and the $\mathrm{rms}$ noise in that spectral region. The average $\mathrm{S} / \mathrm{N}$ for typical Bg particles is also shown in Table IV. This experimental data shows that detection of elemental concentrations relevant to single-particle detection of biological particles is possible. Based on the data from Table $\mathrm{IV}$, each of the elements $\mathrm{Ca}, \mathrm{Mg}$, and $\mathrm{Na}$ has a $\mathrm{S} / \mathrm{N}$ well in excess of 3 , sufficient to discriminate individual particles, provided the spectral signatures of the single particles match those obtained in the multi-particle measurements.

In addition to the technique's sensitivity in terms of optical signal strength, a central issue for any technology being considered for bioaerosol detection is its sensitivity in terms of minimum detectable particle concentration. In the case of a bioagent release, detection of agent concentrations as low as 10 particles per liter within a time span of less than 10 min may be required to prevent infection. ${ }^{1}$ In urban environments, the background aerosol concentration in the size range $1-10 \mu \mathrm{m}$ can be up to 10000 particles per liter. ${ }^{28}$ Therefore, any aerosol sensor must sort through a significant amount of air and benign particles in order to interrogate the offensive agent. This is particularly problematic for LIBS, which usually samples $\sim 1 \mathrm{~mm}^{3} /$ laser shot at repetition rates $<50 \mathrm{~Hz}$. Without significant modification or new laser technology, a standalone LIBS sensor would not be capable of achieving the roughly $20-\mathrm{L} / \mathrm{min}$ sampling rate required for detecting realistic concentrations of bioagents. The addition of air concentrators and/or separate "cueing" detection technologies will most likely be required to increase the sensitivity of LIBS so that it can deal with scenarios of this type.

\section{CONCLUSION}

We have demonstrated that LIBS has significant potential as a bioaerosol classifier. The technique is able to resolve differing elemental ratios in biowarfare-agent simulants and in common biological and environmental interferants. Measurements combined with PCA suggest that it may be possible to readily discriminate bioaerosols from interferences. Single-particle analysis of $\mathrm{Bg}$ spores reveals sufficient sensitivity to detect $\mathrm{Ca}, \mathrm{Na}$, and $\mathrm{Mg}$, each with $\mathrm{S} / \mathrm{N}>3$, illustrating that LIBS can be used to detect single bioaerosol particles.

When considering the detection of a minority aerosol species in the outdoor environment, laboratory measurements can only go so far. The capabilities of LIBS must be evaluated in the real outdoor environment. Future studies may consider using sensitive echelle spectrometers coupled with intensified CCD cameras or a polychromator/photomultiplier tube detector configuration to

TABLE IV. Atomic line statistics for single-particle spectra of Bg.

\begin{tabular}{lcccr}
\hline \multicolumn{1}{c}{ Line } & $\begin{array}{c}\text { Number of hits } \\
\left(\begin{array}{c}\times \text { rms }) \\
\text { criterion }\end{array}\right.\end{array}$ & $\begin{array}{c}\text { Peak/base } \\
\text { ratio } \\
\text { (hits) }\end{array}$ & $\begin{array}{c}\text { Peak/base } \\
\text { rms noise }\end{array}$ & $\begin{array}{c}\text { Deviation in } \\
\text { peak/base }\end{array}$ \\
\hline $\mathrm{Mg} 278 \mathrm{~nm}$ & 42 & 36.6 & 3.78 & 17.3 \\
$\mathrm{Ca} 422.67 \mathrm{~nm}$ & 76 & 72.8 & 4.43 & 68.8 \\
$\mathrm{Na} 589.0 \mathrm{~nm}$ & 91 & 137.8 & 15.69 & 98.3 \\
\hline
\end{tabular}


obtain both wide spectral range and the high sensitivity required to detect single particles.

\section{ACKNOWLEDGMENTS}

S. G. Buckley and the University of California, San Diego, laboratory are supported in this work by funding from National Science Foundation Grant \#BES-0093853 and Office of Naval Research Grant \#N000140110698. G. A. Lithgow was additionally supported by the U.S. Department of Energy through subcontract \#1070008-1114402 from the Pittsburgh Air Quality Supersite. J. D. Hybl would like to acknowledge the Joint Service Chemical Biological Defense Program, Joint Service Materiel Group, Joint Science \& Technology Panel for Chemical Biological Defense for supporting this work. J. D. Hybl would also like to acknowledge Tom Jeys, William Herzog, Dan Ripin, and Antonio Sanchez for many useful discussions and ideas.

1. C. A. Primmerman, Linc. Lab. J. 12, 3 (2000).

2. P. P. Hariston, J. Ho, and F. R. Quant, J. Aerosol Sci. 28, 471 (1997).

3. J. Ho, M. Spence, and P. Hairston, Aerobiologia 15, 281 (1999).

4. G. A. Luoma, P. P. Cherrier, and L. A. Retflavi, Field Anal. Chem. Technol. 3, 260 (1999).

5. P. H. Kaye, J. E. Barton, E. Hirst, and J. M. Clark, Appl. Opt. 39, 3738 (2000).

6. J. D. Eversole, W. K. Cary, C. S. Scotto, R. Pierson, M. Spence, and A. K. Campillo, Field Anal. Chem. Technol. 5, 205 (2001).

7. F. L. Reyes, T. H. Jeys, N. R. Newbury, C. A. Primmerman, G. S. Rowe, and A. Sanchez, Field Anal. Chem. Technol. 3, 240 (1999).

8. M. T. Madigan, J. M. Martinko, and J. Parker, Brock Biology of Microorganisms (Prentice Hall, Upper Saddle River, New Jersey, 1997), 8th ed., p. 5.
9. G. Gould and A. Hurst, The Bacterial Spore (Academic Press, London, 1969), p. 247.

10. M. Z. Martin, M. D. Cheng, and R. C. Martin, Aerosol Sci. Technol. 31, 409 (1999).

11. J. E. Carranza, B. T. Fisher, G. D. Yoder, and D. W. Hahn, Spectrochim. Acta, Part B 56, 851 (2001).

12. D. W. Hahn and M. A. Lunden, Aerosol Sci. Technol. 33, 30 (2000).

13. K. Song, Y. I. Lee, and J. Sneddon, Appl. Spectrosc. Rev. 32, 183 (1997).

14. K. Song, Y. I. Lee, and J. Sneddon, Appl. Spectrosc. Rev. 37, 89 (2002).

15. I. Schechter, Rev. Anal. Chem. 16, 173 (1997).

16. L. J. Radziemski, Microchem. J. 50, 218 (1994).

17. J. E. Carranza and D. W. Hahn, Spectrochim. Acta, Part B 57, 779 (2002).

18. B. T. Fisher, H. A. Johnsen, S. G. Buckley, and D. W. Hahn, Appl. Spectrosc. 55, 1312 (2001).

19. U. Panne, C. Haisch, M. Clara, and R. Niessner, Spectrochim. Acta, Part B 53, 1957 (1998).

20. I. Schechter, J. Korean Soc. Anal. Sci. 8, 779 (1995).

21. L. Xu, V. Bulatov, V. Gridin, and I. Schechter, Anal. Chem. 69, 2103 (1997).

22. D. W. Hahn, Appl. Phys. Lett. 72, 2960 (1998).

23. S. G. Buckley, H. A. Johnsen, K. R. Hencken, and D. W. Hahn, Int. J. Integr. Waste Manage. Sci. Technol. 20, 455 (2000).

24. H. Zhang, F.-Y. Yueh, and S. Singh, Appl. Opt. 38, 1459 (1999).

25. S. Yalcin, D. R. Crosley, G. P. Smith, and G. W. Faris, Hazard. Waste Hazard. Mat. 13, 51 (1996).

26. F. Ferioli, P. V. Pusinauskas, and S. G. Buckley, 57, 1183 (2003).

27. D. L. Massart, B. G. Vandeginste, S. N. Deming, Y. Michotte, and L. Kaufman, Chemometrics: A Textbook (Elsevier, Amsterdam, 1988).

28. R. Jaenicke, in Aerosol-Cloud-Climate Interactions, P. V. Hobbs, Ed. (Academic, San Diego, 1993), p. 1. 


\title{
Fine particle emission factors from vehicles in a highway tunnel: Effects of fleet composition and season
}

\author{
Andrew P. Grieshop ${ }^{\mathrm{a}, \mathrm{d}}$, Eric M. Lipsky ${ }^{\mathrm{a}}$, Natalie J. Pekney ${ }^{\mathrm{b}}$, \\ Satoshi Takahama ${ }^{\mathrm{c}}$, Allen L. Robinson ${ }^{\mathrm{a}, \mathrm{d}, *}$ \\ a Department of Mechanical Engineering, Carnegie Mellon University, Pittsburgh, PA, USA \\ ${ }^{\mathrm{b}}$ Department of Civil and Environmental Engineering, Carnegie Mellon University, Pittsburgh, PA, USA \\ ${ }^{\mathrm{c}}$ Department of Chemical Engineering, Carnegie Mellon University, Pittsburgh, PA, USA \\ ${ }^{\mathrm{d}}$ Department of Engineering and Public Policy, Carnegie Mellon University, Pittsburgh, PA, USA
}

Received 30 July 2005; received in revised form 16 March 2006; accepted 24 March 2006

\begin{abstract}
In-use, fuel-based motor vehicle emission factors were determined using measurements made in a highway tunnel in Pittsburgh, Pennsylvania. Concentrations of $\mathrm{PM}_{2.5}$ mass, $\mathrm{CO}, \mathrm{CO}_{2}$, and $\mathrm{NO}_{x}$ were measured continuously. Filter-based measurements included $\mathrm{PM}_{2.5}$ mass, organic and elemental carbon (OC and $\mathrm{EC}$ ), inorganic ions and metals. Fuel-based emission factors for each pollutant were calculated using a fuel-carbon balance. The weekday traffic volume and fleet composition varied in a consistent diurnal pattern with the estimated fraction of fuel consumed by heavy-duty diesel vehicle (HDDV) traffic ranging from $11 \%$ to $36 \%$. The emission rate of most species showed a significant dependence on sample period. $\mathrm{NO}_{x}, \mathrm{PM}_{2.5}, \mathrm{EC}$ and $\mathrm{OC}$ emission factors were significantly larger during the early morning, truckdominated period. Emissions of particulate metals associated with brake wear $(\mathrm{Cu}, \mathrm{Sb}, \mathrm{Ba}$ and potentially $\mathrm{Ga})$ were emitted at higher rates during the rush-hour period, which is characterized by slower, stop-and-go traffic. Emission rates of crustal elements ( $\mathrm{Fe}, \mathrm{Ca}, \mathrm{Mg}, \mathrm{Li}), \mathrm{Zn}$ and $\mathrm{Mn}$ were highest during the early-morning period when there was more heavytruck traffic. A seasonal shift in average OC/EC ratio for the rush-hour period was observed; fall and summer OC/EC ratios are $1.0 \pm 0.6$ and $0.26 \pm 0.06$, respectively. Potential causes for this shift are increased partitioning of semi-volatile organic compounds into the gas phase during the summer months and/or effects of seasonal changes in fuel formulation. Emission factors for HDDV and light-duty vehicles (LDV) classes were estimated using a linear regression of emission factor as a function of fleet composition. The extrapolated emission factors generally agree with previously published measurements, though a substantial range in published values is noted.
\end{abstract}

(C) 2006 Elsevier Ltd. All rights reserved.

Keywords: $\mathrm{PM}_{2.5}$; Organic carbon; Elemental carbon; Trace metals; Emission inventories

\footnotetext{
*Corresponding author. Department of Mechanical Engineering, Carnegie Mellon University, Pittsburgh, PA, USA.

Tel.: + 14122683657 ; fax: + 14122683348 .

E-mail address: alr@andrew.cmu.edu (A.L. Robinson).
}

\section{Introduction}

Motor vehicles are among the most important sources of gas- and particle-phase pollutants in the urban atmosphere. Experiments in roadway tunnels provide information about pollutant emissions from 
a large sample of in-use vehicles. Tunnel experiments have been used to measure vehicle emission rates in a range of geographic locations (see, e.g., Pierson et al., 1996; Kirchstetter et al., 1999; Gertler et al., 2002; Chellam et al., 2005; Lough et al., 2005). Substantial prior work has been done to quantify gas-phase emissions on the East Coast (Pierson et al., 1996), but fine particle emissions have not been as well characterized, and there is more disagreement between measured emission factors and those derived from regulatory models (Kuhns et al., 2004). Geographical variation in fleet age and condition are important issues; for instance, highemitting or 'smoker' light-duty vehicles (LDV) can emit large amounts of particulate matter (Zielinska et al., 2004), and their portion of the fleet can vary significantly with geographical location (Mazzoleni et al., 2004).

The goal of this paper is to measure in-use motor vehicle emissions in a highway tunnel in Pittsburgh, Pennsylvania. Fleet average emission factors were determined for gaseous species and PM mass, organic carbon (OC), elemental carbon (EC) and elemental metals. Measurements are combined with traffic data to investigate the impact of vehicle fleet composition and operating mode on emission rates. Data taken during both fall and summer periods allows the examination of seasonality in emissions.

\section{Experimental methods}

\subsection{The Squirrel Hill Tunnel}

The experiments were conducted in the two-lane westbound bore of the Squirrel Hill Tunnel on Interstate 376 in Pittsburgh, Pennsylvania. The tunnel is $1.3 \mathrm{~km}$ long and has a $2.5 \%$ up-grade in the westerly direction. The tunnel is ventilated mechanically through ducts situated in the tunnel ceiling and by the effects of traffic motion. The sampling location was roughly $50 \mathrm{~m}$ from the tunnel exit; at this position the mechanical ventilation system was under positive pressure and thus pushing fresh air into the tunnel. The sample inlets were inserted through an otherwise sealed ventilation slit in the tunnel ceiling; several slits up- and down-stream of the sample location were also blocked. The majority of the measurements were made during a two-week period in November of 2002. A more limited study was also performed in the summer of 2004 to examine seasonal dependence of organic aerosol emissions.
Traffic count and speed data were gathered via a Remote Traffic Microwave Sensor (RTMS) operated for the Pennsylvania Department of Transportation (PennDOT). Fleet composition was determined from PennDOT video by manually counting heavy-duty (HD) vehicles. HD vehicles include tractor-trucks, large single-unit trucks and buses. City buses did not regularly pass through the tunnel. While this approach separates vehicles by size and not fuel-type, the assumption that most large vehicles are diesel-powered and smaller ones gasoline-powered is reasonable. Less than $5 \%$ of HD vehicles with more than three axles in the US fleet are gasoline-powered (VIUS, 2002). The portion of LDV powered by diesel fuel is similarly small; around $1 \%$ of vehicles in US households were diesel powered (EIA, 1994) and at most $15 \%$ of light-duty trucks are diesel powered (VIUS, 2002). Therefore, we assume that all of the HD vehicles are diesels (HDDV) and LDV are gasoline powered.

The portion of fuel use in the tunnel by HDDV was calculated based on the HD vehicle number counts from the video,

$\%$ fuel $_{\mathrm{HD}}=\frac{f_{\mathrm{HD}} U_{\mathrm{HD}}}{f_{\mathrm{HD}} U_{\mathrm{HD}}+\left(1-f_{\mathrm{HD}}\right) U_{\mathrm{LD}}}$,

where $f_{\mathrm{HD}}$ is the fraction of traffic identified as HD and $U_{\mathrm{HD}}$ and $U_{\mathrm{LD}}$ are the fuel consumption rates for HDDV and cars, respectively. Fuel consumption values for cars and HDDV on an incline were assumed to be $12 \pm 2$ and $47 \pm 91(100 \mathrm{~km})^{-1}$, respectively (Pierson et al., 1996). Fuel consumption rates of the US LDV and HD vehicle fleets have remained essentially unchanged over the past 15 years (BTS, 2001; USDOT, 2004). Therefore, these values represent a best estimate of in-use fuel consumption rates as they were derived from measurements taken under similar conditions (in-use vehicles under load).

\subsection{Air-quality measurements}

The suite of continuous instruments and manual samplers used to measure pollutant species concentrations during the November 2002 study is listed in Table 1. Continuous instruments measured $\mathrm{CO}$, $\mathrm{CO}_{2}, \mathrm{NO}, \mathrm{NO}_{2}$ and $\mathrm{PM}_{2.5}$ mass. The Tapered Element Oscillating Microbalance (TEOM) operated at $30{ }^{\circ} \mathrm{C}$ with a $\mathrm{PM}_{2.5}$ cyclone and Sample Equilibration System that incorporates a Nafion dryer on the inlet. The $\mathrm{CO}_{2}$ monitor was the only 
Table 1

Instrumentation used in tunnel sampling site

\begin{tabular}{llll}
\hline Species measured & Method & Instrument & Time resolution \\
\hline $\mathrm{CO}_{2}$ & Non-dispersive infrared & Li-Cor LI-820 & $1 \mathrm{~min}$ \\
$\mathrm{NO}_{X} \mathrm{NO}$ & Chemiluminensce & API 200A & $1 \mathrm{~min}$ \\
$\mathrm{PM}_{2.5}$ & TEOM with SES & R\&P 1400a & $5 \mathrm{~min}$ \\
$\mathrm{CO}$ & Non-dispersive infrared & API 300A & $1 \mathrm{~min}$ \\
Elemental and organic carbon & Quartz/quartz-behind teflon & Sunset Laboratory TOT & $2 / 4 / 6.5 \mathrm{~h}$ \\
Size resolved mass and OC/EC & MOUDI with Teflon/foil filters & Sunset Laboratory TOT & $2 / 4 / 6.5 \mathrm{~h}$ \\
Inorganic ions & Teflon/nylon/cellulose filters with denuder & Dionex DX-600/120 IC & $2 / 4 / 6.5 \mathrm{~h}$ \\
$\mathrm{PM}_{2.5}$ metals & Teflon/cellulose filters & Agilent 4500 ICP-MS & $6.5 / 8 / 12 \mathrm{~h}$ \\
\hline
\end{tabular}

continuous instrument used during the summer 2004 study.

Integrated filter samples were collected to characterize $\mathrm{PM}_{2.5}$ levels inside the tunnel. Based on the diurnal patterns in tunnel traffic (discussed below), three manual sampling periods (12-6 AM, 7-9 AM and $10 \mathrm{AM}$ to $4: 30 \mathrm{PM}$ ) were defined to characterize variation in emissions with fleet composition and traffic characteristics. All manual sampling was carried out during these time periods.

$\mathrm{PM}_{2.5}$ OC and EC were measured with a twochannel sampler: one channel consisted of a quartz filter (Bare-Q) and the other of a backup quartz filter behind a Teflon (QBT) membrane filter (Subramanian et al., 2004). The quartz filters were analyzed using a modified version of the NIOSH 5040 thermal-optical transmittance (TOT) protocol in a Sunset Laboratory OC/EC Analyzer (Subramanian et al., 2004). OC measured by the backup quartz filter (QBT) is used to correct for positive sampling artifact (Turpin et al., 2000). Unless otherwise noted, all OC data reported in the paper is artifact-corrected by subtracting the $\mathrm{OC}$ on the backup filter from the bare quartz filter (Q-QBT).

Samples to measure $\mathrm{PM}_{2.5}$ and gas-phase inorganic ions were collected using a two-channel sampler with each channel consisting of a filter pack with a PTFE Teflon membrane filter, a nylon filter, and a backup citric-acid-coated cellulose-fiber filter in series; one channel operated with $\mathrm{MgO}$ and citric acid denuders and the second channel operated without the denuders (Takahama et al., 2004). Ion chromatography was performed using the method of Chow and Watson (1998) to yield concentrations of $\mathrm{PM}_{2.5} \mathrm{Na}, \mathrm{K}, \mathrm{Mg}, \mathrm{Ca}, \mathrm{Cl}$, ammonium, nitrate and sulfate and gas-phase ammonia, hydrochloric acid, and nitric acid. Gasphase measurements were determined by taking the difference of semi-volatile inorganic species concentrations between the two sampling lines.

$\mathrm{PM}_{2.5}$ samples for elemental analysis were collected using a two-channel sampler with one channel containing a Teflon filter and the other a cellulose filter. Exposed filters were processed using microwave-assisted digestion in a solution containing $\mathrm{HNO}_{3}, \mathrm{HF}$, and $\mathrm{H}_{2} \mathrm{O}_{2}$. Digested samples were then analyzed by inductively coupled plasma mass spectrometry (ICP-MS). This method was used to quantify the airborne concentrations of $\mathrm{Ag}, \mathrm{Al}, \mathrm{As}$, $\mathrm{Ba}, \mathrm{Be}, \mathrm{Ca}, \mathrm{Cd}, \mathrm{Ce}, \mathrm{Co}, \mathrm{Cr}, \mathrm{Cs}, \mathrm{Cu}, \mathrm{Fe}, \mathrm{Ga}, \mathrm{K}, \mathrm{Li}$, $\mathrm{Mg}, \mathrm{Mn}, \mathrm{Mo}, \mathrm{Ni}, \mathrm{Pb}, \mathrm{Rb}, \mathrm{Se}, \mathrm{Sb}, \mathrm{Sr}, \mathrm{Ti}, \mathrm{Tl}, \mathrm{V}$, and $\mathrm{Zn}$ as described by Pekney and Davidson (2005).

To measure particle size distribution, two 8-stage Micro-Orifice Uniform Deposit Impactor (MOUDI, MSP Corp.) were operated during the study. One MOUDI was operated with aluminum foil substrates and a quartz-fiber after-filter, and the second with Teflon substrates. The Teflon filters were used for gravimetric analysis followed by elemental analysis by ICP-MS using the procedures described above. The aluminum substrates were used for OC/EC analysis as described by Cabada et al. (2004).

\subsubsection{Calculating fuel-based emission factors}

Emission factors are calculated on a fuel basis using a carbon balance of the major carboncontaining exhaust constituents (Miguel et al., 1998; Kirchstetter et al., 1999):

$\mathrm{EF}_{P}=\left[\frac{\Delta[P]}{\Delta\left[\mathrm{CO}_{2}\right]+\Delta[\mathrm{CO}]}\right]\left[\frac{\mathrm{MW}_{p}}{\mathrm{MW}_{C}}\right] w_{\mathrm{c}}$,

where $\mathrm{EF}_{P}$ is the emission factor of pollutant $P$ in grams $(\mathrm{kg} \text { fuel })^{-1}, \Delta[P], \Delta\left[\mathrm{CO}_{2}\right]$ and $\Delta[\mathrm{CO}]$ are the background-corrected pollutant, $\mathrm{CO}_{2}$ and $\mathrm{CO}$ concentrations measured in the tunnel, $\mathrm{MW}_{P}$ and $\mathrm{MW}_{C}$ are the molecular weights of the pollutant 
and carbon, and $w_{\mathrm{c}}$ is the weight fraction of carbon in fuel. VOCs and particulate carbon were assumed to contribute negligibly to the overall carbon mass balance. The carbon weight fractions for gasoline and diesel fuel were assumed to be 0.85 and 0.87 , respectively; a weighted average value for $w_{\mathrm{c}}$ was determined based on fleet fuel use for mixed fleet conditions.

\subsubsection{Background pollutant concentrations}

All measurements in the tunnel were background corrected using measurements made at the Pittsburgh Air Quality Study (PAQS) main site on the Carnegie Mellon University (CMU) campus and other sampling sites operated throughout Allegheny County, none of which were strongly impacted by local sources. Although the PAQS main site was approximately $2 \mathrm{~km}$ to the northwest of the tunnel sampling location, the strong regional character of fine particle concentrations in the Pittsburgh area supports the use of regional background measurements to correct the tunnel data (Tang et al., 2004). In addition, tunnel concentrations were typically much higher than background concentrations, minimizing uncertainty associated with the background correction. For example, the average diurnal profiles of in-tunnel and regional background $\mathrm{PM}_{2.5}$ mass concentrations are shown in Fig. 1. Tunnel concentrations are significantly above background levels throughout the day.

Background $\mathrm{PM}_{2.5}$ mass was measured with a TEOM operated at the CMU site. Background $\mathrm{PM}_{2.5}$ OC and EC data were measured with 24-h quartz and quartz-behind-Teflon filters collected at

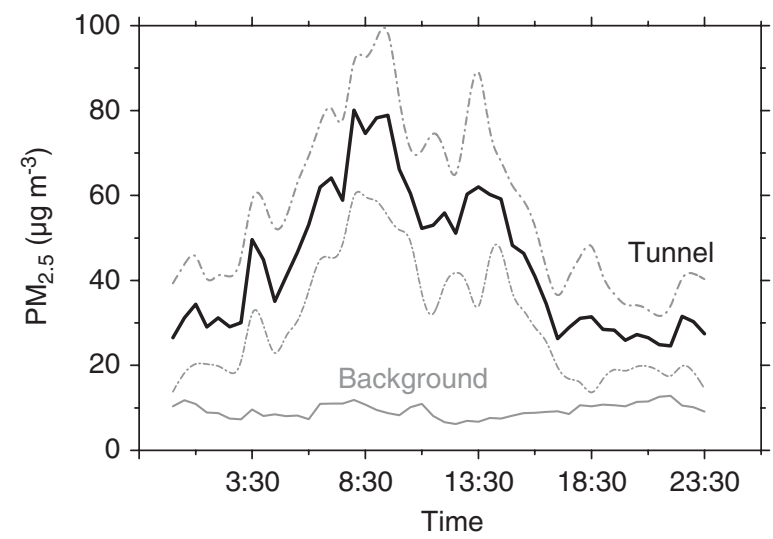

Fig. 1. Average diurnal profile of in-tunnel (heavy solid line) and background (light solid line) $\mathrm{PM}_{2.5}$ mass concentrations. Dashed lines indicate + one standard deviation of tunnel concentration. the CMU site. OC and EC levels during traffic sampling periods were estimated by scaling the $24-\mathrm{h}$ background filter results with the time-resolved TEOM data (Weitkamp et al., 2005).

Background metal levels were estimated using the average ambient concentrations from PAQS daily measurements taken during November and December 2001 with obvious plume-impaction events removed. Elements with tunnel concentrations that were not significantly above detection limits or background levels were removed from the analysis; tunnel concentrations of the species considered were, on average, a factor of 7 higher than in the background. Tunnel data for trace metals that had concentrations less than the mean background concentration plus two times the background standard deviation were discarded.

Background levels of inorganic ion concentrations were estimated based on averages of US EPA's Speciation Trends Network (STN) measurements from two sites in Pittsburgh taken during the study period. Concentrations of inorganic ions in tunnel $\mathrm{PM}_{2.5}$ were not consistently significantly elevated relative to the variable background levels.

Background $\mathrm{NO}, \mathrm{NO}_{2}$, and $\mathrm{CO}$ mixing ratios were taken as the average values of monitors operated at 5 sites in Allegheny County (Weitkamp et al., 2005); $\mathrm{NO}_{x}$ concentrations in the tunnel were at least a factor of 14 above background levels. A composite diurnal profile for background $\mathrm{CO}_{2}$ was constructed based on measurements taken periodically from the ventilation air.

\section{Results and discussion}

\subsection{Fleet composition and traffic characteristics}

The average diurnal traffic flow rate, vehicle speed and fleet-fuel usage are shown in Fig. 2a-c. A consistent, weekday diurnal traffic pattern was evident throughout the study. Average traffic characteristics for the specific periods selected for manual sampling are listed in Table 2. These periods encompass a range of fleet compositions and vehicle operating conditions. The morning rush-hour period (6:30-9:00 AM) was dominated by slow-moving light-duty traffic; on a number basis trucks comprised only $3 \%$ of vehicles, which is equivalent to $11 \%$ of fuel use (Eq. (1)). During the mid-day period from $10 \mathrm{AM}$ to $4: 30 \mathrm{PM}$, the traffic volume decreased slightly and both the average speed and the proportion of HDDV increased. HDDV were 
most prevalent during the early morning hours (between 12 and $6 \mathrm{AM}$ ); during this period traffic flow thinned to a quarter of its daytime level and average speed increased to $57 \mathrm{mph}$. The data indicate that the diurnal variation in fleet composition was largely due to changes in LDV volume; the HDDV traffic volume was relatively constant throughout the day.

Adequate video data for fleet composition identification were available for slightly more than half of the manual sampling periods. In cases where direct counting of vehicles was not possible (for example when it was raining), fleet composition was defined using the ratio of background-corrected $\mathrm{NO}_{x}$ and $\mathrm{CO}_{2}$ mixing ratios measured in the tunnel. A linear regression of $\mathrm{NO}_{x} / \mathrm{CO}_{2}$ data from high traffic periods onto HDDV fuel consumption fraction yielded an excellent fit $\left(R^{2}=0.92\right)$. This regression equation was then used to derive estimated fleet fuel

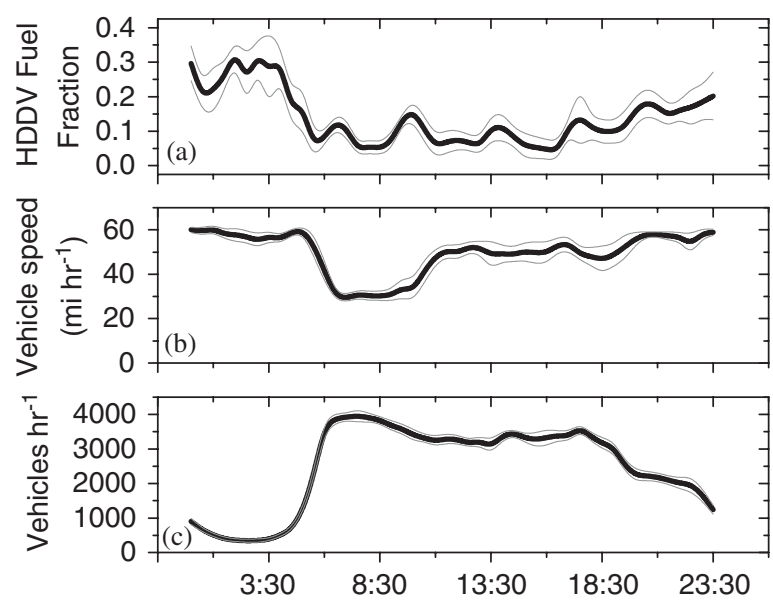

Fig. 2. Average diurnal profile of (a) heavy duty fuel use, (b) vehicle speed and (c) traffic volume in the Squirrel Hill Tunnel during the November 2002 study. In-use fleet fuel use is expressed as a fraction of fuel consumed by diesel vehicles and was estimated from traffic composition data using Eq. (1). Heavy line indicates average profile with light lines indicating \pm one standard deviation. use for those periods when video data were not available.

\subsection{Period average emission characteristics}

Aggregated fuel-based emission factors for each sampling period were calculated by averaging emission factors weighted with the average fuel consumption during the individual sample periods. The sum of the background-corrected $\mathrm{CO}$ and $\mathrm{CO}_{2}$ concentrations was used as the measure of fuel consumption.

Period average fleet fuel use and emission factors for $\mathrm{PM}_{2.5}$, $\mathrm{OC}$ (with and without positive artifact correction), EC and elemental metals are shown in Table 3. Background-corrected fine particle mass in the tunnel was largely carbonaceous in nature, with study average composition of $44 \%$ organic material $(\mathrm{OM}=$ artifact-corrected $\mathrm{OC} \times 1.2$ (Turpin and Lim, 2001)), 42\% EC, 5\% sulfate, 2\% ammonium, $2 \%$ chloride, $1 \%$ other inorganic and $4 \%$ elemental metals. Filter measurements for speciated fine particle concentrations collected in the tunnel exhibited good mass closure with gravimetric mass measurements with the sum of background-corrected $\mathrm{PM}_{2.5}$ components accounting for an average of $97 \pm 18 \%$ of the gravimetrically determined $\mathrm{PM}_{2.5}$ mass. The OC positive artifact correction for each sampling period was $18 \pm 6 \%$ of the total OC mass measured on the bare quartz filter.

The results in Table 3 indicate that the emission factors for many pollutants depend on fleet composition. For example, the fleet-wide fuel-based $\mathrm{PM}_{2.5}$ emission factor is two times higher during the HDDV dominated early-morning hours than during the LDV-dominated rush-hour period. EC and OC emissions show a similar trend. Therefore, the emission factors for HDDV are significantly higher than those for LDV. Variability between measurements made on different days during the same sampling period is relatively small compared to the variations between sampling periods.

Table 2

Summary of sampling period traffic conditions

\begin{tabular}{|c|c|c|c|c|c|}
\hline Period & Time & Speed $\left(\right.$ miles $\left.\mathrm{h}^{-1}\right)$ & $\begin{array}{l}\text { Traffic volume } \\
\left(\text { vehicles } \mathrm{h}^{-1} \text { ) }\right.\end{array}$ & $\begin{array}{l}\% \text { HDDV } \\
\text { (count basis) }\end{array}$ & $\begin{array}{l}\text { \% HDDV } \\
\text { (fuel basis) }\end{array}$ \\
\hline Early morning (high truck) & $12 \mathrm{AM}-6 \mathrm{AM}$ & $56.5 \pm 1.5$ & $860 \pm 70$ & $14.5 \pm 4.0$ & $36 \pm 8$ \\
\hline Rush hour (low speed) & 7 AM-9 AM & $30.4 \pm 3.0$ & $3860 \pm 180$ & $3.4 \pm 0.5$ & $11.0 \pm 1.6$ \\
\hline Mid-day (high speed) & 10 AM-4:30 PM & $49.3 \pm 3.8$ & $3290 \pm 150$ & $6.0 \pm 1.1$ & $19.2 \pm 2.1$ \\
\hline
\end{tabular}


Table 3

Measured tunnel emission factors

\begin{tabular}{|c|c|c|c|c|}
\hline & Units & High-speed (mid-day) & Low-speed (Rush-hour) & High truck (early morning) \\
\hline$\%$ HDDV & $\%$ fuel & $\underline{19 \pm 2}$ & $\underline{11 \pm 2}$ & $\underline{36 \pm 8}$ \\
\hline $\mathrm{PM}_{2.5}$ & $\mathrm{mg}(\mathrm{kg} \text { fuel })^{-1}$ & $\underline{158 \pm 29}$ & $\overline{189 \pm 23}$ & $\underline{437 \pm 76}$ \\
\hline $\mathrm{EC}$ & $\operatorname{mg}(\mathrm{kg} \text { fuel })^{-1}$ & $\overline{110 \pm 44}$ & $74 \pm 27$ & $\overline{153 \pm 27}$ \\
\hline OC (Q-QBT) & $\mathrm{mg}(\mathrm{kg} \text { fuel })^{-1}$ & $64 \pm 28$ & $\overline{61 \pm 7}$ & $\overline{115 \pm 21}$ \\
\hline OC (Bare Q) & $\operatorname{mg}(\mathrm{kg} \text { fuel })^{-1}$ & $82 \pm 29$ & $\overline{74 \pm 5}$ & $\overline{139 \pm 12}$ \\
\hline $\mathrm{NO}_{X}$ & $\mathrm{G}(\mathrm{kg} \text { fuel })^{-1}$ & $\overline{11 \pm 2}$ & $\overline{8 \pm 1}$ & $\overline{18 \pm 4}$ \\
\hline $\mathrm{NH}_{3}$ & $\operatorname{mg}(\mathrm{kg} \text { fuel })^{-1}$ & $\overline{183 \pm 87}$ & $27 \overline{4 \pm 72}$ & $2 \overline{72 \pm 46}$ \\
\hline $\mathrm{Al}$ & $\mu \mathrm{g}(\mathrm{kg} \text { fuel })^{-1}$ & $1 \overline{660 \pm 930}$ & $\overline{850 \pm 690}$ & $\overline{\mathrm{N} / \mathrm{A}}$ \\
\hline As & $\mu \mathrm{g}(\mathrm{kg} \text { fuel })^{-1}$ & $\overline{\mathrm{N} / \mathrm{A}}$ & $\overline{2.3 \pm 1.0}$ & $5 \pm 13$ \\
\hline $\mathrm{Ba}$ & $\mu \mathrm{g}(\mathrm{kg} \text { fuel })^{-1}$ & $360 \pm 50$ & $450 \pm 110$ & $250 \pm 50$ \\
\hline $\mathrm{Ca}$ & $\mu \mathrm{g}(\mathrm{kg} \mathrm{fuel})^{-1}$ & $1 \overline{270 \pm 240}$ & $\overline{840 \pm 490}$ & $2 \overline{200 \pm 1200}$ \\
\hline $\mathrm{Ce}$ & $\mu \mathrm{g}(\mathrm{kg} \text { fuel })^{-1}$ & $\overline{2.9 \pm 0.7}$ & $\overline{2.3 \pm 1.2}$ & $\underline{4.4 \pm 2.1}$ \\
\hline Cs & $\mu \mathrm{g}(\mathrm{kg} \text { fuel })^{-1}$ & $\overline{4.0 \pm 1.0}$ & $\overline{2.2 \pm 1.0}$ & $\overline{4.1 \pm 2.8}$ \\
\hline $\mathrm{Cu}$ & $\mu \mathrm{g}{(\mathrm{kg} \mathrm{fuel})^{-1}}^{-1}$ & $\overline{145 \pm 19}$ & $\overline{209 \pm 54}$ & $\overline{98 \pm 65}$ \\
\hline $\mathrm{Fe}$ & $\mu \mathrm{g}(\mathrm{kg} \text { fuel })^{-1}$ & $5 \overline{400 \pm 800}$ & $4 \overline{900 \pm 1300}$ & $\underline{7000 \pm 2100}$ \\
\hline $\mathrm{Ga}$ & $\mu \mathrm{g}(\mathrm{kg} \text { fuel })^{-1}$ & $\overline{15.1 \pm 1.8}$ & $24.7 \pm 5.3$ & $6.7 \pm 9.9$ \\
\hline $\mathrm{Li}$ & $\mu \mathrm{g}(\mathrm{kg} \text { fuel })^{-1}$ & $\overline{\mathrm{N} / \mathrm{A}}$ & $\overline{4.1 \pm 2.4}$ & $47 \pm 26$ \\
\hline $\mathrm{Mg}$ & $\mu \mathrm{g}(\mathrm{kg} \text { fuel })^{-1}$ & $\underline{163 \pm 20}$ & $\underline{590 \pm 150}$ & $11 \overline{00 \pm 300}$ \\
\hline $\mathrm{Mn}$ & $\mu \mathrm{g}(\mathrm{kg} \text { fuel })^{-1}$ & $\overline{48 \pm 7}$ & $92 \pm 25$ & $400 \pm 90$ \\
\hline Mo & $\mu \mathrm{g}(\mathrm{kg} \text { fuel })^{-1}$ & $4 \overline{2.4 \pm 5.1}$ & $10 \overline{10.5 \pm 2.4}$ & $\overline{3.9 \pm 8.0}$ \\
\hline $\mathrm{Pb}$ & $\mu \mathrm{g}(\mathrm{kg} \text { fuel })^{-1}$ & $\overline{\mathrm{N} / \mathrm{A}}$ & $\overline{19 \pm 6}$ & $45 \pm 54$ \\
\hline $\mathrm{Rb}$ & $\mu \mathrm{g}(\mathrm{kg} \text { fuel })^{-1}$ & $\mathrm{~N} / \mathrm{A}$ & $3.0 \pm 0.6$ & $3.6 \pm 8.0$ \\
\hline $\mathrm{Sb}$ & $\mu \mathrm{g}(\mathrm{kg} \text { fuel })^{-1}$ & $\underline{31 \pm 4}$ & $\underline{57 \pm 12}$ & $\underline{17 \pm 8}$ \\
\hline $\mathrm{Sr}$ & $\mu \mathrm{g}(\mathrm{kg} \text { fuel })^{-1}$ & $1 \overline{11.2 \pm 1.4}$ & $1 \overline{11.1 \pm 2.8}$ & $10.6 \pm 5.3$ \\
\hline $\mathrm{Ti}$ & $\mu \mathrm{g}(\mathrm{kg} \text { fuel })^{-1}$ & $\overline{53.7 \pm 7.0}$ & $\overline{89.6 \pm 23.3}$ & $\overline{54.9 \pm 37.8}$ \\
\hline $\mathrm{Zn}$ & $\mu \mathrm{g}(\mathrm{kg} \text { fuel })^{-1}$ & $70 \pm 9$ & $\overline{290 \pm 70}$ & $\overline{2100 \pm 820}$ \\
\hline
\end{tabular}

Emission factors that are statistically significant relative to inter-sample variation and background concentrations are in underlined, bold text. $\mathrm{N} / \mathrm{A}=$ tunnel concentrations below background.

\subsubsection{Metals emissions}

Fig. 3 and Table 3 indicate that fine particle metal emissions were dominated by $\mathrm{Fe}, \mathrm{Ca}, \mathrm{Al}, \mathrm{Zn}$ and $\mathrm{Mg}$. Concentrations of $\mathrm{Ba}, \mathrm{Cu}, \mathrm{Mn}, \mathrm{Ti}, \mathrm{Ce}, \mathrm{Cs}$, Ga, $\mathrm{Li}, \mathrm{Mo}, \mathrm{Sb}$ and $\mathrm{Sr}$ were also significantly above background levels during all sampling periods, while $\mathrm{Ga}, \mathrm{Li}$ and Mo were not significantly elevated relative to background levels during some sampling periods.

$\mathrm{Fe}, \mathrm{Ca}, \mathrm{Al}$ and $\mathrm{Mg}$ are associated with crustal components of road dust (Lough et al., 2005). Ca, $\mathrm{Ti}, \mathrm{Fe}, \mathrm{Zn}, \mathrm{Ba}, \mathrm{Cu}, \mathrm{Sr}$ and $\mathrm{Sb}$ particles have been associated with brake-wear (Garg et al., 2000; Sternbeck et al., 2002), but there is large variation in reported emission rates due to variations in sampling conditions and brake-pad compounds. $\mathrm{Rb}, \mathrm{Pt}$, and $\mathrm{Pd}$ emissions are associated with degradation of catalytic converters (Lough et al., 2005); $\mathrm{Rb}$ and $\mathrm{Pd}$ were not elevated relative to background, and $\mathrm{Pt}$ was not measured.

Fig. 3 shows that $\mathrm{Ca}, \mathrm{Mg}, \mathrm{Zn}, \mathrm{Mn}, \mathrm{Mo}, \mathrm{Sb}$, and to a lesser extent $\mathrm{Fe}$, emissions all exhibit significant variation with sample period and thus appear influenced by the fleet composition and/or vehicle speed. Emissions of $\mathrm{Ca}, \mathrm{Mg}, \mathrm{Zn}$ and $\mathrm{Mn}$ were significantly higher during the early-morning, HDvehicle-dominated testing period. Gertler et al. (2002) found HD vehicles to emit significantly more $\mathrm{Ca}$ and $\mathrm{Fe}$ than $\mathrm{LD}$ vehicles. However, they also report a lower $\mathrm{Mg}$ emission factor for HD vehicles than LDV - the opposite of what was observed here. $\mathrm{Zn}$ in particular has been has been found to be emitted in larger quantities by HDDV in other studies (Lowenthal et al., 1994; Gillies et al., 2001).

$\mathrm{Ba}, \mathrm{Cu}$ and $\mathrm{Sb}$ are associated with brake wear particles (Garg et al., 2000); emissions of these elements were all significantly higher during the morning rush-hour period. Average speed during this period was $30 \mathrm{mph}$ and is much more variable, indicating more brake usage than other periods. The ratio of $\mathrm{Cu}$ and $\mathrm{Sb}$ measured in the tunnel match the diagnostic criteria of $4.6 \pm 2.3$ proposed by Sternbeck et al. (2002) for brake wear. Ga emissions were also highest during this period. 

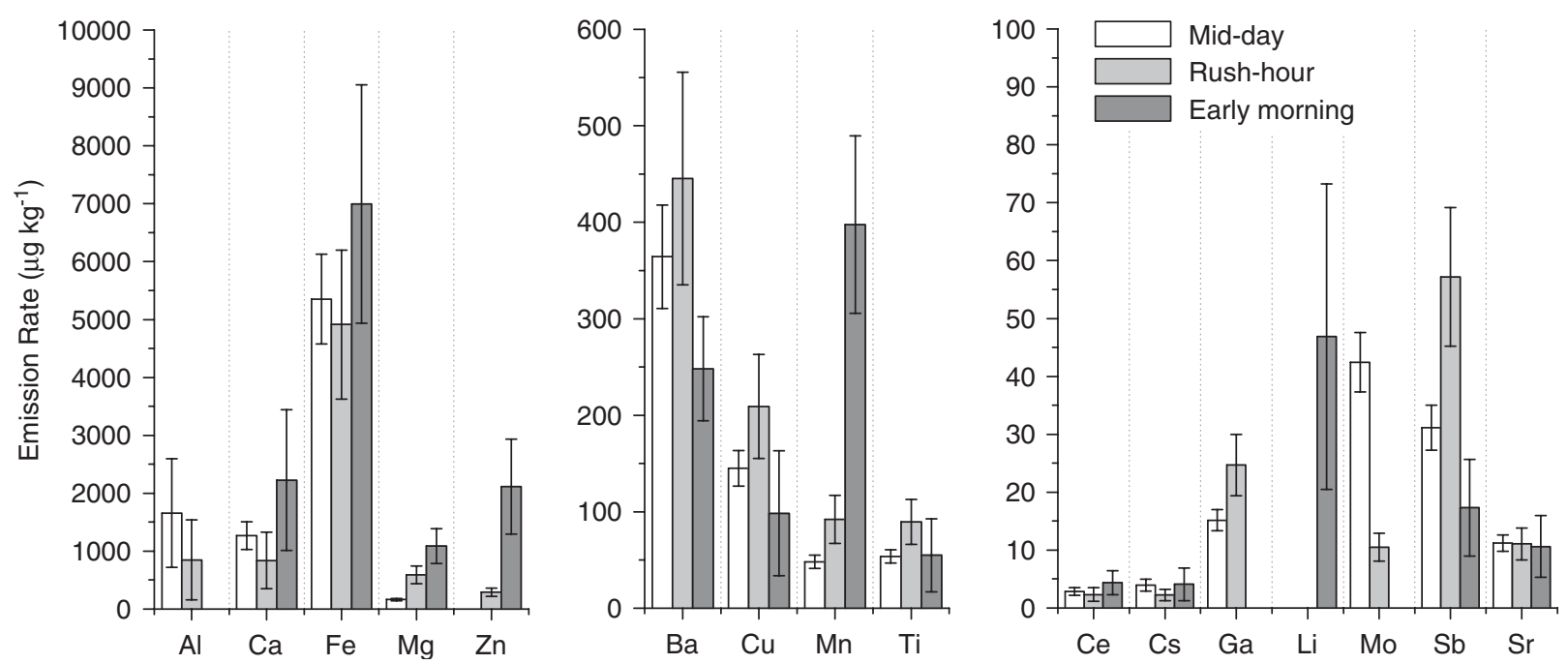

Fig. 3. Period-average emission factors for elemental metals from the three different manual sampling periods. Error bars represent the standard deviation of samples collected on different days.

Table 4

$\mathrm{PM}_{2.5}$, EC and OC emission factors measured during the rush-hour period in November 2002 and July/August, 2004

\begin{tabular}{|c|c|c|c|}
\hline & & Nov. 2002 & July/Aug. 2004 \\
\hline & Temp. $\left({ }^{\circ} \mathrm{C}\right)$ & $9 \pm 3$ & $26 \pm 2$ \\
\hline & $N$ & 3 & $14^{-}$ \\
\hline & $\mathrm{PM}_{2.5}\left(\mathrm{mg}\left(\mathrm{kg}_{\text {fuel }}{ }^{-1}\right)\right.$ & $188 \pm 23$ & $213 \pm 31$ \\
\hline & $\mathrm{EC}\left(\mathrm{mg}-\mathrm{C}\left(\mathrm{kg} f u e l^{-1}\right)\right.$ & $74 \pm 27$ & $111 \pm 19$ \\
\hline \multirow[t]{2}{*}{ Artifact-corrected (Q-QBT) ${ }^{\mathrm{a}}$} & $\mathrm{OC}\left(\mathrm{mg}-\mathrm{C}(\mathrm{kg} \text { fuel })^{-1}\right)$ & $61 \pm 7$ & $29 \pm 4$ \\
\hline & $\mathrm{OC} / \mathrm{EC}$ & $1.0 \pm 0.6$ & $0.29 \pm 0.05$ \\
\hline \multirow[t]{2}{*}{ Not artifact-corrected (BareQ) $)^{\mathrm{b}}$} & OC (mg-C $\left.(\mathrm{kg} \text { fuel })^{-1}\right)$ & $74 \pm 5$ & $60 \pm 5$ \\
\hline & $\mathrm{OC} / \mathrm{EC}$ & $1.2 \pm 0.6$ & $0.48 \pm 0.08$ \\
\hline Artifact Correction & QBT/Q & $18 \pm 6 \%$ & $35 \pm 7 \%$ \\
\hline
\end{tabular}

\footnotetext{
${ }^{\mathrm{a}}$ Artifact-corrected OC is determined by subtracting the OC measured on quartz backup filter (QBT) from the OC measured on the Bare Q filter.

${ }^{\mathrm{b}}$ Bare quartz filter (Bare Q)-OC measurement includes positive artifact gas-phase material adsorbed onto filter.
}

The Mo emission rate is significantly higher during the mid-day sampling period - a period of higher speed, more car-dominated traffic than other periods. Mo is used as a component of automotive catalysts and as an anti-friction additive in lubricating oils.

\subsubsection{Seasonal shift in particle $O C$ emissions}

Comparing the measurements of $\mathrm{PM}_{2.5}$ mass, EC and OC emissions made during the November 2002 and the July 2004 studies provides insight into seasonal changes in emissions. Both studies measured emissions during the early morning rush-hour period (6:30-9:30 AM). Fleet composition and traffic flow during this period were similar in both studies.
Emission factors and OC sample characteristics for the fall and summer studies are compared in Table 4. The overall $\mathrm{PM}_{2.5}$ emission rate is fairly consistent between the summer and fall periods, but there is a significant shift in the composition of the emissions. EC emissions are modestly higher in the summer, but artifact-corrected $\mathrm{OC}$ emissions are reduced by a factor of 2 . During the summer the $\mathrm{OC} / \mathrm{EC}$ ratio of emissions was $0.26 \pm 0.06$ versus $1.0 \pm 0.6$ in the fall.

A potential explanation for this shift in emissions is that changes in ambient temperature influence the gas-particle phase partitioning of the OC emissions. The average temperature during the summer study was $16^{\circ} \mathrm{C}$ higher than during the fall study. The hypothesis is that under cooler fall conditions a 
larger fraction of the semi-volatile emissions exists in the particle phase compared to summer conditions. Recent dilution sampling measurements have shown that sampling conditions can cause large shifts in the partitioning of semi-volatile organics in engine emissions (Lipsky and Robinson, 2006). A roadside study also found significant volume loss of volatile material from freshly emitted particles upon heating (Kuhn et al., 2005).

Careful examination of the quartz filter OC data provides evidence that supports the hypothesis that there are significant seasonal differences in the gasparticle partitioning of the OC emissions. As is seen in Table 4, little seasonal dependence exists in the OC emission factors calculated with non-artifactcorrected bare quartz filters; however, a factor of 2 more OC is collected by the back up quartz filter (QBT) during the summer than during the fall. This is consistent with a much larger fraction of the emitted organic material remaining in the gas phase during the summer sampling period. The sampling flow rates, total sample volumes and filter loadings were essentially the same in both sets of measurements, minimizing potential biases due to the dependence of sampling artifacts on collection time, face velocity, and OC loading on the filter (Subramanian et al., 2004). While artifact corrections are imperfect measures of gas-particle partitioning of organic matter (Turpin et al., 2000), the data strongly suggests that ambient conditions are having a significant impact on the partitioning of freshly emitted particles.

A comparison of the $\mathrm{PM}_{2.5}$ mass measured with the TEOM and MOUDI during the fall study provides additional evidence for the loss of semivolatile mass with increased temperature. A linear regression analysis of data from the two instruments indicates that the TEOM operating at $30^{\circ} \mathrm{C}$ measured $13 \%$ less $\mathrm{PM}_{2.5}$ mass than the MOUDI during the November 2002 period $\left(R^{2}=0.81\right.$, $N=9$ ). The average temperature inside the tunnel was $9{ }^{\circ} \mathrm{C}$ versus $30^{\circ} \mathrm{C}$ in the TEOM. Roughly half the $\mathrm{PM}_{2.5}$ mass in the tunnel is organic material, while only $3 \%$ is ammonium nitrate. Therefore, a significant fraction of the mass discrepancy between the TEOM and the MOUDI data is likely due to loss of semi-volatile organic matter in the TEOM. Assuming all mass lost in the TEOM is volatilized $\mathrm{OM}$ explains the seasonal discrepancy in measured OC emission rates.

Another potential explanation for the seasonal shift in OC/EC emissions is changes in fuel composition. Regulations require gasoline sold in southwestern Pennsylvania to have substantially lower Reid vapor pressure (RVP) during the May-September ozone season (EPA, 2003). Reformulated gasoline (RFG) has been demonstrated to have a large impact on gaseous emissions (Kirchstetter et al., 1996). However, Norbeck et al. (1998) found little difference in the fine particle emissions from LDVs operating on California Phase 2 summer time RFG and pre-reformulation gasoline.

\subsubsection{Gas-phase species emission rates}

$\mathrm{NO}_{x}$ and $\mathrm{NH}_{3}$ emission factors for each sample period are given in Table 3. Concentrations of both of these pollutants inside the tunnel were significantly higher than background levels. Fleet composition has a significant impact on $\mathrm{NO}_{x}$ emission factors; the early-morning (high-truck) period emission factor is twice that during the rush-hour period. The emission factor for gas-phase $\mathrm{NH}_{3}$ was relatively constant across testing periods (Table 3 ). The study average ammonia emission factor is

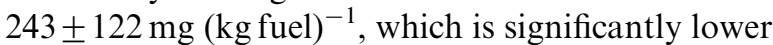
than values reported in a recent tunnel study conducted in California by Kean et al. (2000).

\section{3. $L D V$ and $H D D V$ emission rates}

Table 3 indicates there is a strong dependence of overall emission factors of several species on the fleet composition in the tunnel, with HDDV emitting significantly more on a fuel basis than LDV. Previous studies have performed linear regressions of emission data as a function of fleet composition to estimate HDDV and LDV emission factors (Gertler et al., 2002; Fraser et al., 2003). Results from a similar analysis of the Squirrel Hill tunnel data are summarized in Figs. 4a-d.

Fig. 4a shows $\mathrm{NO}_{x}$ emission factors plotted versus HDDV fuel use; a least-squares linear regression fit to the data is used to estimate HDDV and LDV emission factors. The results show that $\mathrm{NO}_{x}$ emission factor is well correlated with estimated diesel and gasoline fuel consumption $\left(R^{2}=0.74\right)$. The extrapolated value for HDDV agrees well with values from the literature shown in Fig. 4a. The extrapolated value for LDV $\mathrm{NO}_{x}$ emission factor is somewhat lower than many published values but similar to those found by Durbin et al. (1999) in a dynamometer study of model-year 1991-97 gasoline vehicles. 

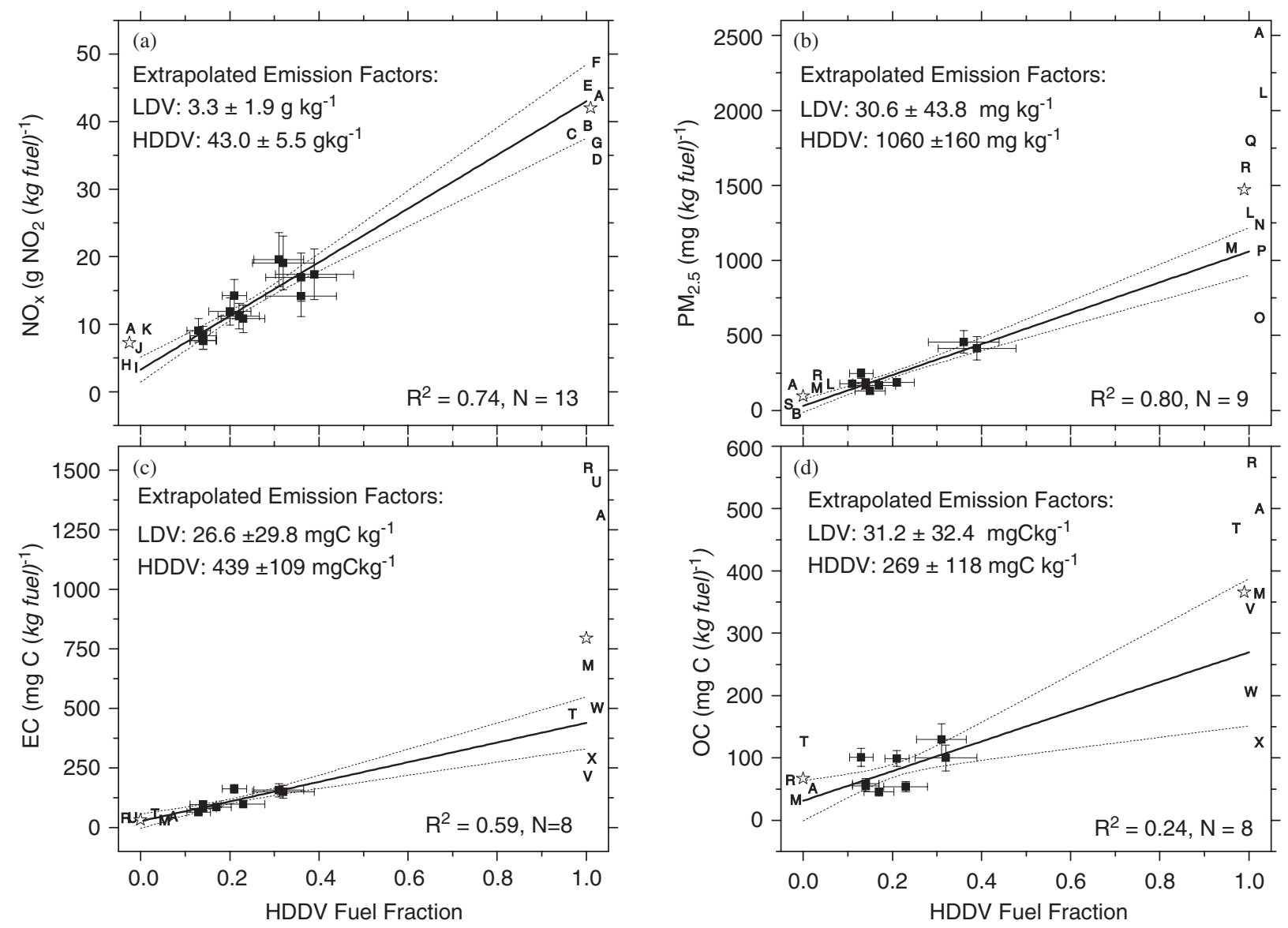

Fig. 4. (a) $\mathrm{NO}_{x}$, (b) $\mathrm{PM}_{2.5}$, (c) EC, and (d) OC emission factors as a function of the fraction of fuel used by HDDV. Best-fit linear regression line is shown along with $69 \%$ confidence intervals for the fit. OC is artifact-corrected OC. Letters indicate emission factors reported in the literature: (A) (Kirchstetter et al., 1999), (B) (Pierson et al., 1996) Tuscarora Tunnel, (C) (Pierson et al., 1996) Ft. McHenry Uphill, (D) (Pierson et al., 1996) Ft. McHenry Downhill, (E) (Jimenez et al., 2000), (F) (Rogak et al., 1998), (G) (Yanowitz et al., 1999), (H) (Cadle et al., 1999), (I) (Durbin et al., 1999), (J) (Kean et al., 2003), (K) (McGaughey et al., 2004), (L) (Mazzoleni et al., 2004), (M) (Allen et al., 2001), (N) (Lowenthal et al., 1994), (O) (Morris et al., 1998), (P) (Fraser et al., 2002), (Q) (Yanowitz et al., 2000), (R) (Hildemann et al., 1991), (S) (Zielinska et al., 2004), (T) (Rogge et al., 1993), (U) (Miguel et al., 1998), (V) (Shah et al., 2004) Creep, (W) (Shah et al., 2004) Transient, (X) (Shah et al., 2004) Cruise. Average of published emission factors for gasoline and diesel vehicles are shown with stars.

The agreement of the emission factors estimated here with those from others' work suggests that vehicle classes are being accurately separated using traffic count and video data.

Similar regressions for $\mathrm{PM}_{2.5}, \mathrm{EC}$ and $\mathrm{OC}$ emission factors versus fleet composition yield estimates for LDV and HDDV emission factors; in all cases the fuel-based emission factors for HDDV are significantly larger than for LDV. Fig. 4b shows that the $\mathrm{PM}_{2.5}$ mass emission factors based on the MOUDI measurements are well correlated with fleet fuel use $\left(R^{2}=0.80\right)$. On a fuel basis, HDDV emit a factor of 25-40 more $\mathrm{PM}_{2.5}$ mass than LDV.
EC emission factors, shown in Fig. 4c, are modestly correlated with fleet fuel usage $\left(R^{2}=0.58\right)$. The extrapolated LDV EC emission factor is highly uncertain but significantly smaller than the extrapolated HDDV EC emission factor. Under the hot-stabilized operating conditions inside the tunnel, LDV EC emissions are a factor of 10-20 lower than HDDV EC emissions.

Fig. 4d shows OC emission factors based on the positive-artifact-corrected (Bare Q-QBT) filter measurements. Again HDDV emissions are higher, but the correlation between $\mathrm{OC}$ emission rate and diesel fuel fraction is poor $\left(R^{2}=0.24\right)$, significantly worse than for other species. This indicates wider 
variability in $\mathrm{OC}$ emissions from vehicles and/or inconsistent artifact corrections. OC emission rates calculated based on Bare-Q data (not-artifactcorrected) are modestly higher but do not provide better correlations with fuel use, suggesting that variation in $\mathrm{OC}$ emission rates is not associated with artifact correction.

Comparison of the estimated vehicle-class $\mathrm{PM}_{2.5}$, $\mathrm{EC}$ and $\mathrm{OC}$ emission factors to values from the literature show that they generally fit within the wide range of published values. Fig. $4 b-d$ plot a selection of published $\mathrm{PM}_{2.5}, \mathrm{EC}$ and $\mathrm{OC}$ emission factors for LDV and HDDV. The LDV $\mathrm{PM}_{2.5}$ emission factor estimated here is a factor of 2 lower than many of the literature values shown in Fig. 4b, while the estimated HDDV $\mathrm{PM}_{2.5}$ emission factor shows better agreement with prior work. The extrapolated LDV EC emission factor shown in Fig. $4 \mathrm{c}$ is consistent with values from the literature, but the extrapolated HDDV EC emission factor is roughly a factor of 3 lower than that found in many other studies. Extrapolated LDV and HDDV OC emission factors are both lower than values reported in many studies, the value for HDDV significantly so. The seasonal variation in measured OC emission factors discussed above provides a potential explanation for large variability in the published values.

\section{Conclusions}

This study was carried out to provide a detailed characterization of motor vehicle emissions in conjunction with the Pittsburgh Air Quality Study. Emission factors were determined from tunnel data collected during three distinct periods: early morning (high proportion of heavy-duty vehicles), midday (high speed, mixed fleet) and rush hour (low speed, low proportion of heavy-duty vehicles). Emissions of $\mathrm{NO}_{x}, \mathrm{PM}_{2.5}, \mathrm{OC}$ and $\mathrm{EC}$ had a strong dependence on sample period, as did many of the metals sampled, indicating a strong influence of fleet composition and operating conditions on emissions. Estimates for emission factors for light-duty gasoline vehicles and heavy-duty diesel vehicles were determined by performing a linear regression on the emissions and fleet composition data.

OC emissions were more variable than other pollutants. Measurements made in the summer also indicate a significant seasonality in $\mathrm{OC}$ emission factors. There is evidence that these changes may be due to changes in partitioning of semi-volatile organic compounds associated with seasonal changes in ambient temperature. If this is the case, the impact of ambient conditions on sampling of organic particulate matter must be considered when measuring and interpreting emissions data. Further work in this area is necessary to elucidate the nature and evolution of organic PM from vehicles and other combustion sources.

\section{Acknowledgments}

The authors acknowledge the contributions of many individuals who provided valuable assistance in this study: Heather Leifeste, R. Subramanian, Rob Pinder, Emily Weitkamp, Charles Stanier, Mark Prack, Jessica Chiu and Beth Wittig. This research was conducted as part of the Pittsburgh Air Quality Study, which was supported by US Environmental Protection Agency under contract R82806101 and the US Department of Energy National Energy Technology Laboratory under contract DE-FC26-01NT41017. This paper has not been subject to EPA's required peer and policy review, and therefore does not necessarily reflect the views of the Agency. No official endorsement should be inferred.

\section{References}

Allen, J.O., Mayo, P.R., et al., 2001. Emissions of size-segregated aerosols from on-road vehicles in the Caldecott Tunnel. Environmental Science \& Technology 35 (21), 4189-4197.

BTS, 2001. Transportation Indicators Report, Bureau of Transportation Statistics.

Cabada, J.C., Rees, S., et al., 2004. Mass size distributions and size resolved chemical composition of fine particulate matter at the Pittsburgh supersite. Atmospheric Environment 38 (20), 3127-3141.

Cadle, S.H., Mulawa, P.A., et al., 1999. Composition of lightduty motor vehicle exhaust particulate matter in the Denver, Colorado area. Environmental Science \& Technology 33 (14), 2328-2339.

Chellam, S., Kulkarni, P., et al., 2005. Emissions of organic compounds and trace metals in fine particulate matter from motor vehicles: a tunnel study in Houston, Texas. Journal of the Air \& Waste Management Association 55 (1), 60-72.

Chow, J.C., Watson, J.G., 1998. Guideline on Speciated Particulate Monitoring, DRI.

Durbin, T.D., Norbeck, J.M., et al., 1999. Particulate emission rates from light-duty vehicles in the South Coast air quality management district. Environmental Science \& Technology 33 (24), 4401-4406.

EIA, 1994. Residential Transportation Energy Consumption Survey. US Energy Information Administration. 
EPA, 2003. Guide on Federal and State Summer RVP Standards for Conventional Gasoline Only. US Environmental Protection Agency.

Fraser, M.P., Buzcu, B., et al., 2003. Separation of fine particulate matter emitted from gasoline and diesel vehicles using chemical mass balancing techniques. Environmental Science \& Technology 37 (17), 3904-3909.

Fraser, M.P., Lakshmanan, K., et al., 2002. Variation in composition of fine particulate emissions from heavy-duty vehicles. Journal of Geophysical Research 107 (D21).

Garg, B.D., Cadle, S.H., et al., 2000. Brake wear particulate matter emissions. Environmental Science \& Technology 34 (21), 4463-4469.

Gertler, A.W., Gillies, J.A., Pierson, W.R., Rogers, C.F., Sagebiel, J.C., Abu-Allaban, M., et al., 2002. Real-world particulate matter and gaseous emissions from motor vehicles in a highway tunnel. Health Effects Institute, 1-92.

Gillies, J.A., Gertler, A.W., et al., 2001. On-road particulate matter $\left(\mathrm{PM}_{2.5}\right.$ and $\left.\mathrm{PM}_{10}\right)$ emissions in the Sepulveda Tunnel, Los Angeles, California. Environmental Science \& Technology 35 (6), 1054-1063.

Hildemann, L.M., Markowski, G.R., et al., 1991. Chemicalcomposition of emissions from urban sources of fine organic aerosol. Environmental Science \& Technology 25 (4), 744-759.

Jimenez, J.L., McRae, G.J., et al., 2000. Remote sensing of NO and $\mathrm{NO}_{2}$ emissions from heavy-duty diesel trucks using tunable diode lasers. Environmental Science \& Technology 34 (12), 2380-2387.

Kean, A.J., Harley, R.A., et al., 2000. On-road measurement of ammonia and other motor vehicle exhaust emissions. Environmental Science \& Technology 34 (17), 3535-3539.

Kean, A.J., Harley, R.A., et al., 2003. Effects of vehicle speed and engine load on motor vehicle emissions. Environmental Science \& Technology 37 (17), 3739-3746.

Kirchstetter, T.W., Singer, B.C., et al., 1996. Impact of oxygenated gasoline use on California light-duty vehicle emissions. Environmental Science \& Technology 30 (2), 661-670.

Kirchstetter, T.W., Harley, R.A., et al., 1999. On-road measurement of fine particle and nitrogen oxide emissions from lightand heavy-duty motor vehicles. Atmospheric Environment 33 (18), 2955-2968.

Kuhn, T., Biswas, S., et al., 2005. Physical and chemical characteristics and volatility of PM in the proximity of a light-duty vehicle freeway. Aerosol Science and Technology 39 (4), 347-357.

Kuhns, H.D., Mazzoleni, C., et al., 2004. Remote sensing of PM, $\mathrm{NO}, \mathrm{CO}$ and $\mathrm{HC}$ emission factors for on-road gasoline and diesel engine vehicles in Las Vegas, NV. Science of the Total Environment 322 (1-3), 123-137.

Lipsky, E.M., Robinson, A.L., 2006. Effects of dilution on fine particle mass and partitioning of semivolatile organics in diesel exhaust and wood smoke. Environmental Science \& Technology 40 (1), 155-162.

Lough, G.C., Schauer, J.J., et al., 2005. Emissions of metals associated with motor vehicle roadways. Environmental Science \& Technology 39 (3), 826-836.

Lowenthal, D.H., Zielinska, B., et al., 1994. Characterization of heavy-duty diesel vehicle emissions. Atmospheric Environment 28 (4), 731-743.
Mazzoleni, C., Kuhns, H.D., et al., 2004. On-road vehicle particulate matter and gaseous emission distributions in Las Vegas, Nevada, compared with other areas. Journal of the Air \& Waste Management Association 54 (6), 711-726.

McGaughey, G.R., Desai, N.R., et al., 2004. Analysis of motor vehicle emissions in a Houston tunnel during the Texas Air Quality Study 2000. Atmospheric Environment 38 (20), 3363-3372.

Miguel, A.H., Kirchstetter, T.W., et al., 1998. On-road emissions of particulate polycyclic aromatic hydrocarbons and black carbon from gasoline and diesel vehicles. Environmental Science \& Technology 32 (4), 450-455.

Morris, J.A., Bishop, G.A., et al., 1998. On-road remote sensing of heavy-duty diesel truck emissions in the Austin-San Marcos Area. University of Denver, Denver, CO.

Norbeck, J.M., Durbin, T.D., et al., 1998. Characterization of particulate emissions from gasoline-fueled vehicles. California Air Resources Board.

Pekney, N.J., Davidson, C.I., 2005. Determination of trace elements in ambient aerosol samples. Analytica Chimica Acta 540 (2), 269-277.

Pierson, W.R., Gertler, A.W., et al., 1996. Real-world automotive emissions - summary of studies in the Fort McHenry and Tuscarora Mountain Tunnels. Atmospheric Environment 30 (12), 2233-2256.

Rogak, S.N., Pott, U., et al., 1998. Gaseous emissions from vehicles in a traffic tunnel in Vancouver, British Columbia. Journal of the Air \& Waste Management Association 48 (7), 604-615.

Rogge, W.F., Hildemann, L.M., et al., 1993. Sources of fine organic aerosol. 2. Noncatalyst and catalyst-equipped automobiles and heavy-duty diesel trucks. Environmental Science \& Technology 27 (4), 636-651.

Shah, S.D., Cocker, D.R., et al., 2004. Emission rates of particulate matter and elemental and organic carbon from in-use diesel engines. Environmental Science \& Technology 38 (9), 2544-2550.

Sternbeck, J., Sjodin, A., et al., 2002. Metal emissions from road traffic and the influence of resuspension-results from two tunnel studies. Atmospheric Environment 36 (30), 4735-4744.

Subramanian, R., Khlystov, A.Y., et al., 2004. Positive and negative artifacts in particulate organic carbon measurements with denuded and undenuded sampler configurations. Aerosol Science and Technology 38, 27-48

Takahama, S., Wittig, A.E., et al., 2004. Modeling the diurnal variation of nitrate during the Pittsburgh Air Quality Study. Journal of Geophysical Research-Atmospheres 109 (D16).

Tang, W., Raymond, T., et al., 2004. Spatial variations of $\mathrm{PM}_{2.5}$ during the Pittsburgh air quality study. Aerosol Science and Technology 38, 80-90.

Turpin, B.J., Lim, H.J., 2001. Species contributions to $\mathrm{PM}_{2.5}$ mass concentrations: Revisiting common assumptions for estimating organic mass. Aerosol Science and Technology 35 (1), 602-610.

Turpin, B.J., Saxena, P., et al., 2000. Measuring and simulating particulate organics in the atmosphere: problems and prospects. Atmospheric Environment 34 (18), 2983-3013.

USDOT, 2004. Summary of Fuel Economy Performance. US Department of Transportation.

VIUS, 2002. Vehicle Inventory and Use Survey. US Census Bureau. 
Weitkamp, E., Lipsky, E., et al., 2005. Fine particle emission profile for a large coke production facility based on highly time resolved fence line measurements. Atmospheric Environment 39 (36), 6719-6733.

Yanowitz, J., Graboski, M.S., et al., 1999. Chassis dynamometer study of emissions from 21 in-use heavy duty diesel vehicles. Environmental Science \& Technology 33 (2), 209-216.
Yanowitz, J., McCormick, R.L., et al., 2000. In-use emissions from heavy-duty diesel vehicles. Environmental Science \& Technology 34 (5), 729-740.

Zielinska, B., Sagebiel, J., et al., 2004. Emission rates and comparative chemical composition from selected in-use diesel and gasoline-fueled vehicles. Journal of the Air \& Waste Management Association 54 (9), 1138-1150. 


\section{Development and Application of a Three- dimensional Aerosol Chemical Transport Model, PMCAMx+}

Timothy M. Gaydos ${ }^{\mathrm{a}}$, Rob Pinder ${ }^{\mathrm{b}}$, Bonyoung Koo ${ }^{\mathrm{a}}$, Kathleen M. Fahey ${ }^{\mathrm{a}}$, Gregory Yarwood ${ }^{\mathrm{c}}$, and Spyros N. Pandis ${ }^{\mathrm{a}, \mathrm{b}, \mathrm{d}}$

${ }^{a}$ Department of Chemical Engineering, Carnegie Mellon University, Pittsburgh, PA 15213, USA, ${ }^{\mathrm{b}}$ Department of Engineering and Public Policy, Carnegie Mellon University, Pittsburgh, PA 15213, USA,

ENVIRON, 101 Rowland Way, Novato, CA 94945, USA

${ }^{\mathrm{d}}$ Department of Chemical Engineering, University of Patras, Rio, 26500 Patras, Greece

\section{Abstract}

Three-dimensional chemical transport models have been previously applied to several particulate matter (PM) episodes in California, but fewer studies have been done in regions such as the eastern United States, where PM mass is dominated by sulfate and organics, in contrast to the high ammonium nitrate concentrations seen in California. Here, a three-dimensional transport model $(\mathrm{PMCAMx}+)$ is used to simulate PM mass in the eastern United States for a July 2001 pollution episode. The performances of the model in this region is evaluated, taking advantage of the highly time and size-resolved PM and gas-phase data collected during the Pittsburgh Air Quality Study (PAQS). PMCAMx+ uses the framework of CAMx, which models the processes of horizontal and vertical advection, horizontal and vertical dispersion, wet and dry deposition, and gas-phase chemistry. Three detailed aerosol modules have been added to PMCAMx+ with the goal of maintaining accuracy while improving efficiency in three areas: inorganic aerosol 
growth, aqueous phase chemistry, and secondary organic aerosol formation and growth. The model predictions are compared to hourly measurements of $\mathrm{PM}_{2.5}$ mass and composition at Pittsburgh, as well as to measurements from the AIRS and IMPROVE networks. The performance of the model is evaluated and the main challenges encountered during PM simulations in this region are discussed.

\section{Introduction}

Atmospheric aerosols have been implicated in the development of adverse effects on human health, visibility reduction, the formation of acid rain and acid fogs, and changes in the energy balance of the planet. Air quality standards have been proposed in the US for the daily and yearly average concentrations of $\mathrm{PM}_{2.5}$ and $\mathrm{PM}_{10}$ (particulate matter less than $2.5 \mu \mathrm{m}$ and 10

$\mu \mathrm{m}$ in diameter, respectively). Three-dimensional chemical transport models that can accurately and efficiently describe the physical and chemical atmospheric transformations of atmospheric PM are crucial for developing emission control strategies to achieve these air quality standards.

Evaluation of model performance requires both time and spatially-resolved measurements of particulate matter (including speciation), as well as the precursor gases. Many field campaigns have been conducted in California, where the first ozone and PM chemical transport models were developed and applied (Russell and Cass, 1986; Pilinis and Seinfeld, 1988; Lurmann et al., 1997; Meng et al., 1998; Sun and Wexler, 1998). However, fewer studies have been conducted in areas such as the eastern United States (Mebust, 2003) where PM mass is dominated by sulfate and organics, in contrast to the high ammonium nitrate concentrations seen in California.

Several chemical transport models have previously been applied with some success to various regions. The CIT (Meng et al., 1998; Harley et al., 1993), GATOR (Jacobson, 1997), 
GATOR-GCMM (Jacobson, 2001), UAM-AERO (Lurmann et al., 1997), SAQM-AERO (Dabdub et al., 1998), and UAM-AIM (Sun and Wexler, 1998) models have all been applied to episodes in southern California. The URM model (Odman and Russell, 1991) has been applied to both southern California (Kumar et al., 1996) and an episode in the southern Appalachian Mountains (Boylan et al., 2002). Finally, the Models-3/CMAQ (Binkowski and Shankar 1995; Mebust et al., 2003) and CMAQ-MADRID (Zhang et al., 2004) models have been applied more extensively, including to the entire contiguous United States.

One of the challenges in modeling chemical and aerosol transport is the tradeoff between accuracy and speed. Simulation of aerosol processes consumes most (95\%) of the computational time in these models. As a result simplifying assumptions, such as assuming equilibrium between the gas and particle phases (i.e., Lurmann et al., 1997), are often used. In this work, three detailed aerosol modules are presented, developed with the goal of maintaining accuracy while achieving good efficiency in three areas: inorganic aerosol growth (Gaydos et al., 2003; Koo et al., 2003a), aqueous-phase chemistry (Fahey and Pandis, 2001), and secondary organic aerosol formation and growth (Koo et al., 2003b).

These modules are implemented in a state-of-the-art chemical transport model, PMCAMx + . In addition to the detailed aerosol modules, PMCAMx + uses the framework of CAMx (ENVIRON, 2002), which simulates horizontal and vertical advection, horizontal and vertical dispersion, wet and dry deposition, and gas-phase chemistry. PMCAMx+ is applied to a July air pollution episode in the eastern United States. The model predictions are evaluated against hourly measurements of PM and gas-phase data collected during the Pittsburgh Air Quality Study (PAQS) (Wittig et al., 2004), as well as daily average PM measurements taken 
throughout the eastern United States by the IMPROVE (IMPROVE, 1995) and AIRS (U.S. EPA, 2002) monitoring networks.

\section{Model Description}

The general equation solved in the model to describe the change in concentration over time for a given species, $\mathrm{c}_{\mathrm{i}}$, is:

$$
\begin{aligned}
& \frac{\partial c_{i}}{\partial t}=\left.\frac{\partial c_{i}}{\partial t}\right|_{\text {Advection }}+\left.\frac{\partial c_{i}}{\partial t}\right|_{\text {Dispersion }}+\left.\frac{\partial c_{i}}{\partial t}\right|_{\begin{array}{l}
\text { Gas-phase } \\
\text { Chemistry }
\end{array}}+\left.\frac{\partial c_{i}}{\partial t}\right|_{\text {Emission }} \\
& -\left.\frac{\partial c_{i}}{\partial t}\right|_{\substack{\text { Wet/Dry } \\
\text { Deposition }}}+\left.\frac{\partial c_{i}}{\partial t}\right|_{\text {Aerosol }}+\left.\frac{\partial c_{i}}{\partial t}\right|_{\substack{\text { Aqueous-phase } \\
\text { Chemistry }}}
\end{aligned}
$$

The change in concentration resulting from advection, dispersion, gas-phase chemistry, emission, wet and dry deposition, aerosol processes (coagulation, condensation, and nucleation), and aqueous phase production, are all represented and will be discussed in more detail below. The aerosol species are modeled using a sectional representation (Gelbard et al., 1980; Jacobson and Turco, 1995), assuming a uniform concentration for the particles in a given size section. In equation (1) $c_{i}=c_{i, k}$ represents the concentration of aerosol species $i$ in section $k$.

As is typical in chemical transport models, an operator-splitting approach is used, with each process simulated separately for each time step. The operator time step changes to ensure numerical stability for horizontal advection, and typically ranges anywhere from five to fifteen minutes. Smaller time steps are used internally in the modules describing individual processes, such as condensation and aqueous phase-chemistry, where smaller time steps are often needed to obtain an accurate solution. The simulation order of the processes is: emissions, horizontal advection, vertical advection, vertical dispersion, horizontal dispersion, wet deposition, gas- 
phase chemistry, aerosol processes (nucleation, coagulation, inorganic aerosol condensation and evaporation), secondary organic aerosol growth, and aqueous phase-chemistry. The approaches used are summarized below:

Horizontal advection. The advection equations are solved using the Piecewise Parabolic Method of Colella and Woodward (1984) as implemented by Odman and Ingram (1996). The order of directions of horizontal advection is alternated to avoid any numerical biases that can develop when this order is constant.

Vertical advection. The equation for vertical transport is

$$
\left.\frac{\partial c_{i}}{\partial t}\right|_{\text {Zadvection }}=\frac{\partial\left(c_{i} w\right)}{\partial z}
$$

where $w$ is the net vertical velocity. To calculate the vertical velocity profile, the atmospheric continuity equation is locally integrated through the depth of the vertical column.

Vertical dispersion. Vertical dispersion is represented by the equation:

$$
\left.\frac{\partial c_{i}}{\partial t}\right|_{\text {Zdiffusion }}=\frac{\partial}{\partial z}\left[\rho K_{v} \frac{\partial\left(c_{i} / \rho\right)}{\partial z}\right]
$$

where $K_{v}$ is the vertical dispersion coefficient and is provided as a meteorological input and $\rho$ is the atmospheric density.

Horizontal dispersion. Horizontal dispersion is simulated using the equation:

$$
\left.\frac{\partial c_{i}}{\partial t}\right|_{X Y \text { diffusion }}=\frac{\partial}{\partial x}\left[\rho K_{X} \frac{\partial\left(c_{i} / \rho\right)}{\partial x}\right]+\frac{\partial}{\partial y}\left[\rho K_{Y} \frac{\partial\left(c_{i} / \rho\right)}{\partial y}\right]
$$

where $K_{X}$ and $K_{Y}$ are the horizontal dispersion coefficients determined using a deformation approach based on the methods of Smagorinsky (1963). $K_{X}$ and $K_{Y}$ are calculated for each face of the grid cell and a maximum value is set to maintain numerical stability. 
Gas-phase chemistry. CAMx supports chemical mechanisms based on the Carbon Bond mechanism version 4 (CB4; Gery et. al., 1989) and the SAPRC99 mechanism (Carter, 2000). The chemical mechanism used in this application is based on the CB4 mechanism (Gery et al., 1989). The mechanism includes 100 reactions for 46 species (34 gas species and 12 radicals). The thirty-four gas-phase species modeled in PMCAMx + are listed in Table 1. The gas-phase chemistry reactions are numerically integrated using the Chemical Mechanism Compiler (CMC) solver (Environ, 2003). The CMC solver is a fast and robust solver. It uses the steady-state approximation for fast reacting species (radicals) while the slower reacting (state) species are separated into two groups. Fast state species (with chemical lifetimes of seconds to a few minutes) are solved using a second order implicit Runge-Kutta method, while slow state species (with longer chemical lifetimes) are solved explicitly. In the base case simulations, the nighttime chemistry for the formation of $\mathrm{HNO}_{3}(\mathrm{~g})$ is neglected, as will be discussed in more detail below. Wet deposition. The change in concentration within or below a cloud due to precipitation is parameterized using a scavenging coefficient, $\Lambda$ :

$$
\frac{\partial c_{i}}{\partial t}=-\Lambda c_{i}
$$

The scavenging coefficient is calculated separately for gases and particles, based on Seinfeld and Pandis (1998). The mass transfer coefficient below the clouds depends on the droplet diameter and falling speed, which are calculated based on the empirical estimates of Scott (1978) and modified to better agree with the data provided by Seinfeld and Pandis (1998). Within a cloud, Henry's Law is used to partition the total gas concentration of a species between the aqueous and gas phases. For the case of aerosols, all aerosol particles are assumed to be within the cloud water. Below the cloud layer, the collection efficiency depends on the particle diameter, $d_{p}$ (Seinfeld and Pandis, 1998). 
Dry deposition. Dry deposition is modeled using the resistance model of Weseley (1989). For gases, the deposition velocity, $v_{d}$, is calculated from three resistances in series: the aerodynamic resistance $r_{a}$, surface resistance $r_{s}$, and canopy resistance $r_{c}\left(v_{d}=1 /\left[r_{a}+r_{s}+r_{c}\right]\right)$. For strong acids such as nitric and hydrochloric acid, the surface resistance is set to zero because of their high rates of uptake onto most surfaces.

For aerosol particles, the resistance approach of Slinn and Slinn (1980) as implemented by Kumar et al. (1996) is used. The deposition velocity is given as:

$$
v_{d}=v_{\text {sed }}+\frac{1}{r_{a}+r_{b}+r_{a} r_{b} v_{\text {sed }}}
$$

where $v_{\text {sed }}$ is the gravitational settling velocity.

Aerosol species. Thirteen aerosol species (listed in Table 2) are included in the model. Several of these species, primary organic aerosol, primary elemental carbon, crustal material and sodium are present only in the aerosol phase. The secondary organic species, chloride, ammonium, nitrate, and sulfate partition between the gas and aerosol phases.

Nucleation. The nucleation rate is calculated using the ternary $\mathrm{NH}_{3}-\mathrm{H}_{2} \mathrm{SO}_{4}-\mathrm{H}_{2} \mathrm{O}$ parameterization of Napari et al. (2002). The parameterization uses the $\mathrm{NH}_{3}$ gas-phase concentration, $\mathrm{H}_{2} \mathrm{SO}_{4}$ gasphase concentration, temperature, and relative humidity as inputs, and provides a nucleation rate as output. Because of the relatively large concentrations of water vapor, water is assumed to be in equilibrium with the aerosol phase. The amount of water in the aerosol phase is calculated using the thermodynamic module ISORROPIA (Nenes et al., 1998).

Coagulation. The coagulation rate of aerosol particles was modeled using the approach of Tambour and Seinfeld (1980). The generalized coagulation coefficient for the collision of two particles is defined as: 


$$
K_{12}=2 \pi\left(D_{p 1}+D_{p 2}\right)\left(D_{1}+D_{2}\right) \beta
$$

where $\beta$ is the Fuchs correction factor (Fuchs, 1964). A high-resolution distribution is used for the coagulation calculations by subdividing each section of the original distribution into three sections.

Inorganic aerosol formation. PMCAMx + allows the user to choose between three methods for solving the inorganic aerosol condensation/evaporation equation, depending on the computational efficiency and level of accuracy desired. The simplest and most efficient approach is the bulk equilibrium approach, with equilibrium being assumed between the bulk aerosol and gas phase for inorganic species. The equilibrium model employed in this work has been described by Capaldo et al. (2000). The amount of each species transferred between gas and aerosol phases is determined by bulk aerosol thermodynamics using ISORROPIA (Nenes et al., 1998), and is distributed over the aerosol size distribution by using a weighting factor for each size section $k, f_{k}$, based on the surface area of each size section (Pandis et al., 1993; Lurmann et al., 1997):

$$
f_{k}=\frac{N_{k} d_{k} /\left(\beta_{k}+1\right)}{\sum_{k} N_{k} d_{k} /\left(\beta_{k}+1\right)}
$$

where $N_{k}$ and $d_{k}$ are the number and diameter, respectively of particles in section $k, \beta_{k}=2 \lambda / a d_{k}, a$ is the accommodation coefficient, and $\lambda$ is the mean free path of the species transferred (Pandis et al., 1993).

The second approach is the hybrid approach, which assumes equilibrium for the smaller particles $(<0.625 \mu \mathrm{m})$ and solves mass transfer explicitly for the larger particles (Capaldo et al., 2000). Finally, a fully dynamic approach can be used, where mass transfer is simulated explicitly for all particles (Pilinis et al., 2000). This is the most accurate method, but requires 
significant computational resources for use in three-dimensional transport models at this time. In the hybrid and fully dynamic approaches, the mass transfer equations are solved using the trajectory-grid method (Gaydos et al., 2003). The equilibrium approach requires about 7.5 CPU hours per simulation day on a $1.8 \mathrm{GHz}$ AMD Opteron CPU.

Secondary organic aerosol (SOA). Four secondary organic aerosol species are modeled. The condensable organic gases are represented by the species CG1 to CG4, while the corresponding secondary organic aerosol species are labeled SOA1 to SOA4. The condensable gases, CG1 and CG2 correspond to the low and high yield products, respectively, from oxidation of toluene and the xylenes. The condensable oxidation products from paraffins, anthropogenic olefins (model species OLE), and cresol are lumped into CG3. The oxidation products from biogenic olefins (OLE2) are assigned to CG4. The VOC precursor, aerosol yield, saturation concentration, and heat of vaporization of each of the SOA condensables is shown in Table 3.

Equilibrium is assumed between the gas and aerosol phase using the Secondary Organic Aerosol Model (SOAM II) of Strader et al. (1999) as implemented by Koo et al. (2003b). The condensable gas products are partitioned between the gas and aerosol phases similar to the inorganics (Eq. 8) with an additional factor to account for the aerosol composition using the pseudo-ideal solution assumption (Koo et al., 2003b). The temperature dependence of saturation concentrations is considered by the Clausius-Clapeyron equation.

Aqueous phase chemistry. The variable sizes resolution model (VSRM) of Fahey and Pandis (2001) is used to model aqueous phase chemistry. The model is based on the chemical mechanism of Pandis and Seinfeld (1989), with the addition of $\mathrm{Ca}^{2+}$ to the list of aqeuous-phase species and $\mathrm{H}_{2} \mathrm{SO}_{4}$ to the gas phase (Fahey and Pandis, 2001). When the liquid water content is larger than $0.05 \mathrm{~g} \mathrm{~m}^{-3}$, aerosol particles larger than the activation diameter of $0.7 \mu \mathrm{m}$ (Strader et 
al., 1998) are assumed to instantly form cloud droplets. The model selects whether to use a bulk or two-section (split at $2.5 \mu \mathrm{m}$ dry diameter) approach for each operator time step for each cell using the decision algorithm of Fahey and Pandis (2001).

\section{Model application}

PMCAMx+ is applied to a seventeen day (July 12-28, 2001) episode in the eastern United States. The simulation begins on a day (July 12) when the measured PM concentrations were low in the northeast US to limit the effect of the initial conditions on the results. For initial and boundary conditions, low or zero background concentrations are used for all species (see Tables 1 and 2). The aerosol module includes ten size sections varying in size from $40 \mathrm{~nm}$ to 10 $\mu \mathrm{m}$, with the equilibrium approach being used to model aerosol condensation and evaporation. The modeling domain covers a $3492 \times 3240 \mathrm{~km}$ region in the eastern United States with 36x36 km grid resolution and fourteen vertical layers totaling $6 \mathrm{~km}$ (Figure 1). Inputs to the model include horizontal wind components, temperature, pressure, water vapor, vertical diffusivity, clouds, and rainfall, all created using the meteorological model MM5 (Grell et al., 1995).

Emission inventory. The emission inventory used is Midwest Regional Planning Organization's Base E inventory (LADCO, 2003). This inventory (including primary carbonaceous material) is based on the U.S. EPA's 1999 National Emissions Inventory (version 2.0) (U.S. EPA, 2001). Spatial and temporal improvements have been made to the EPA inventory. The improvements include the ammonia emissions (Pinder, 2004), the use of MOBILE6 for vehicular sources (U.S. EPA, 2003), BIOME3 for biogenic emissions (Wilkinson and Janssen, 2001), and the updated dust emissions. Three different representative days are available from the inventory: an average weekday, Saturday, and Sunday. There are two types of emissions, elevated point sources and 
gridded area sources, which include mobile sources, area/non-road mobile sources, and biogenic sources.

\subsection{Predicted PM Concentrations}

The average predictions over the entire period of simulation (July 12-28, 2001) for $\mathrm{PM}_{2.5}$ mass, sulfate, nitrate, ammonium, total carbon, and ozone are shown in Figure 2. The highest concentrations for most PM species are seen over the Midwest, while similar ozone concentrations are seen over a significant portion of the modeling domain. Overall, sulfate accounts for $36.5 \%$ of total $\mathrm{PM}_{2.5}$ at ground level averaged over the entire domain, followed by OC (31.3\%), ammonium (13.1\%), EC (4.4\%), and finally nitrate (2.6\%). The remaining $12.3 \%$ is from crustal material and other metal oxides. The model predictions are explored in more detail in the next section where they are compared to the measurements.

\subsection{Model Performance}

The model predictions are first compared to hourly measurements (for both particulate matter and gases) taken during the Pittsburgh Air Quality Study (PAQS) (Wittig et al., 2004). To cover a larger spatial scale, the model results are also compared to daily average measurements from the U.S. EPA's AIRS monitoring network (U.S. EPA, 2002) and the IMPROVE network (IMPROVE, 1995). The fractional bias (FBIAS), fractional error (FERROR), mean bias (BIAS), and mean error (ERROR) are calculated to assess the model performance:

$$
\text { FERROR }=\frac{2}{N} \sum_{i=1}^{N} \frac{\left|P_{i}-O_{i}\right|}{P_{i}+O_{i}}
$$




$$
\begin{aligned}
& \text { FBIAS }=\frac{2}{N} \sum_{i=1}^{N}\left(\frac{P_{i}-O_{i}}{P_{i}+O_{i}}\right) \\
& \text { ERROR }=\frac{1}{N} \sum_{i=1}^{N}\left|P_{i}-O_{i}\right| \\
& \text { BIAS }=\frac{1}{N} \sum_{i=1}^{N}\left(P_{i}-O_{i}\right)
\end{aligned}
$$

where $N$ is the number of measurements, $P_{i}$ is the predicted concentration, and $O_{i}$ is the corresponding observed concentration. The comparisons for the inorganic $\mathrm{PM}_{2.5}$ species and total $\mathrm{PM}_{2.5}$ are shown in Figure 3 and the comparisons for total atmospheric concentrations (gas + particulate matter) are shown in Figure 4.

$P M_{2.5}$ sulfate. Sulfate makes up the largest portion of the $\mathrm{PM}_{2.5}$ mass in Pittsburgh, both for the measurements and predictions. The sulfate predictions generally compare well with the observations although the model underpredicts on the $18^{\text {th }}-19^{\text {th }}$, and $22^{\text {nd }}-24^{\text {th }}$. The model performs poorly for all species during these two periods, with the systematic underpredictions and simultaneous decrease in all species indicating a poor representation of wet deposition in the model. The meteorological model predicts too frequent rainfall and also does not accurately represent the timing of rainfall. For example, significant rainfall is predicted a day earlier (on the $18^{\text {th }}$ ) than it actually occurred in Pittsburgh. The supplied meteorological inputs exhibit a significant overprediction of convective rainfall (LADCO, 2004), that leads to the above discrepancies. With this overprediction of rainfall, the mean bias and error are -1.75 and 3.94 $\mu \mathrm{g} / \mathrm{m}^{3}$, respectively. The measured average sulfate concentration was $10.03 \mu \mathrm{g} / \mathrm{m}^{3}$ compared to the predicted average of $8.29 \mu \mathrm{g} / \mathrm{m}^{3}$. The daily average predicted concentrations exhibit this 
same underprediction throughout most of the domain (Tables 5 and 6), again largely because of the overprediction of convective rainfall. The average daily average sulfate concentration measured at the AIRS monitoring stations was $6.21 \mu \mathrm{g} / \mathrm{m}^{3}$ and the model predictions have a mean bias and mean error of $-1.2 \mu \mathrm{g} / \mathrm{m}^{3}$ and $2.3 \mu \mathrm{g} / \mathrm{m}^{3}$, respectively. The mean bias and error compared to the IMPROVE measurements are similar, -1.62 and $2.68 \mu \mathrm{g} / \mathrm{m}^{3}$, respectively.

$P M_{2.5}$ nitrate. Measurements of both total nitrate (particulate + gas) and $\mathrm{PM}_{2.5}$ nitrate were taken during PAQS. $\mathrm{PM}_{2.5}$ nitrate shows a strong diurnal pattern in Pittsburgh, with the concentrations typically peaking early in the morning when the low temperatures are more favorable to nitrate transfer to the particulate phase. During the day, when the nitric acid tends to stay in the gas phase, the model predictions compare well with the observations except on the days where the poor representation of wet deposition affects the model performance. However, the model significantly underpredicts the daytime peaks of total $\mathrm{HNO}_{3}$ on many days (Figure 4), although the agreement is better during the night. The total nitric acid predictions will be discussed in more detail later as these results are compared to the results when nighttime $\mathrm{HNO}_{3}$ chemistry is included. The measured average $\mathrm{PM}_{2.5}$ nitrate in Pittsburgh is $0.57 \mu \mathrm{g} / \mathrm{m}^{3}$, with a mean bias and mean error of the predictions -0.20 and $0.56 \mu \mathrm{g} / \mathrm{m}^{3}$, respectively. These results are consistent with the results of Takahama et al. (2004), who evaluate the ability of current thermodynamic models to predict the partitioning of nitrate between the gas and aerosol phase and also detail the uncertainty and some of the issues regarding the measurements taken during PAQS. Comparison with the AIRS and IMPROVE data reveal under and overpredictions throughout the modeling domain. Overall, the mean bias and error of the predictions are -0.12 and $0.57 \mu \mathrm{g} / \mathrm{m}^{3}$ compared with the AIRS measurements (with a measured mean of $0.7 \mu \mathrm{g} / \mathrm{m}^{3}$ ), and the mean bias and error 
of the predictions are -0.01 and $0.28 \mu \mathrm{g} / \mathrm{m}^{3}$ compared with the IMPROVE measurements (with a measured mean of $\left.0.29 \mu \mathrm{g} / \mathrm{m}^{3}\right)$.

Ammonium. In Pittsburgh, the total ammonium (particulate + gas) concentration was measured using the steam sampler of Khlystov et al. (1995). The model performs well compared to the observations (Figure 4), again with the exceptions of the $18^{\text {th }}-19^{\text {th }}$ and $22^{\text {nd }}-24^{\text {th }}$. The mean bias is only $-0.31 \mu \mathrm{g} / \mathrm{m}^{3}$, although the mean error is higher at $1.12 \mu \mathrm{g} / \mathrm{m}^{3}$ (Table 3). The $\mathrm{PM}_{2.5}$ ammonium predictions are unbiased compared with the AIRS measurements, with a fractional bias of -0.03 and a mean bias of $-0.10 \mu \mathrm{g} / \mathrm{m}^{3}$. The fractional error of the ammonium measurements is 0.54 and the mean error is $0.83 \mu \mathrm{g} / \mathrm{m}^{3}$ compared to the measured average of $1.80 \mu \mathrm{g} / \mathrm{m}^{3}$. These higher errors are probably related to the poor correlation of the nitrate predictions, since the partitioning of ammonia and $\mathrm{HNO}_{3}(\mathrm{~g})$ are directly related. These encouraging results for ammonia reflect the improvement in the corresponding inventory made by Pinder et al. (2004). Most of the IMPROVE stations had many missing values for $\mathrm{PM}_{2.5}$ ammonium and the model predictions were not compared to the few available measurements.

$P M_{2.5}$ carbon. For carbonaceous PM, the model predictions are compared against OC, EC, and the combined total carbon (TC) measured. A multiplier of 1.2 was applied by LADCO to convert $\mathrm{OC}$ to organic mass $(\mathrm{OM})$ when creating the emission inventories, so this factor is used to convert the modeled primary OM back to $\mathrm{OC}$ when comparing with the measurements. For secondary organic aerosol, where a significant portion of the organic mass comes from oxygen and nitrogen, a multiplier of 1.8 is used. The methods used in measuring OC and EC during PAQS are described in Cabada et al. (2004). In Pittsburgh, the predicted total carbon and OC 
concentrations compare reasonably well with the measurements, while the predicted EC concentrations are a factor of two higher than the measurements on average (Figure 5). The discrepancies are more pronounced at night. Although there are some days where the nighttime peak observed EC concentrations are similar to the predictions, on most nights the observed concentrations are considerably lower. The model performs the worst compared to the AIRS measurements, where the NIOSH TOT protocol (NIOSH, 1999) is used for measuring EC and OC. The EC predictions are nearly a factor of three higher than the measurements on average while $\mathrm{OC}$ and TC are significantly underpredicted. The comparison with the IMPROVE measurements is better, where the TOR protocol (Chow et al., 1993) is used to analyze the filters. Many of the EC predictions agree well with the observations, although there are four IMPROVE monitoring stations where the model significantly overpredicts the amount of EC. The comparison with the OC measurements is unbiased, although there are many points that are significantly over or underpredicted, as with total carbon. Chow et al. (2001) directly compared measurements analyzed using the NIOSH TOT and IMPROVE TOR protocols and found NIOSH EC was typically less than half of IMPROVE EC, consistent with our findings. Since the inventories of most of the major sources of carbonaceous material were constructed using the TOR protocol (Bhave, 2004), the better agreement with the IMPROVE measurements is encouraging. In addition, the total carbon measurements between the two methods compare better if a blank correction, which corrects for collection of gaseous carbonaceous material on the filters, is applied to the NIOSH TOT measurements (the IMPROVE measurements already include this correction). Graham (2004) found that a blank correction of $\sim 1 \mu \mathrm{g} / \mathrm{m}^{3}$ on average is needed to bring the two methods into agreement. With this correction, the model predictions for total carbon will than have a slight positive bias compared to the AIRS measurements, consistent 
with the positive biases observed when the model predictions are compared to the Pittsburgh and IMPROVE measurements.

$P M_{2.5}$ mass. $\mathrm{PM}_{2.5}$ mass is equal to the sum of the above components, with the addition of crustal material and other metal oxides. As with most of the individual species, the model predictions generally agree with the measurements at Pittsburgh (Figure 3) with the exception of the two periods where the poor representation of wet deposition affects the model performance. Even with these periods where the model significantly underpredicts, the overall performance of the model is encouraging, with a fractional bias of -0.16 and a fractional error of 0.31 . The mean bias is $-4.65 \mu \mathrm{g} / \mathrm{m}^{3}$ and the mean error is $7.42 \mu \mathrm{g} / \mathrm{m}^{3}$, while the measured and predicted $\mathrm{PM}_{2.5}$ mass concentrations average $24.2 \mu \mathrm{g} / \mathrm{m}^{3}$ and $19.7 \mu \mathrm{g} / \mathrm{m}^{3}$, respectively, over the 17 day period. Comparing the preditions to the measurements from the AIRS network reveals a similar mean bias and error, $-4.11 \mu \mathrm{g} / \mathrm{m}^{3}$ and $7.39 \mu \mathrm{g} / \mathrm{m}^{3}$, respectively. These errors represent a greater fraction of the measured values, however, as the observed average $\mathrm{PM}_{2.5}$ concentrations are 6 $\mu \mathrm{g} / \mathrm{m}^{3}$ less than in Pittsburgh. The comparison with the IMPROVE observations again is similar, with the same fractional error (0.48) as the comparison with the AIRS measurements, and a similar fractional bias (-0.29 compared to -0.23$)$. Comparing the biases of the individual species with the bias for overall mass indicates that a significant portion of the error is a result of underprediction of the "other" or "unidentified" species. Only $30.7 \%$ of the underprediction is accounted for by the individual species measured in Pittsburgh, $62.3 \%$ for the AIRS network, and $42.9 \%$ for the IMPROVE network. Rees et al. (2004) provided strong evidence that this unexplained mass found in $\mathrm{PM}_{2.5}$ mass measurements in the Eastern US is due to water retention in the filters used for these mass measurements. 
The modeling domain has been broken down into six regions (Figure 6) to help to identify how the model performs in different areas against the AIRS measurements. The performance metrics of the model predictions compared with the AIRS measurements in each region are shown in Table 7. The model underpredicts $\mathrm{PM}_{2.5}$ throughout most of the domain, with a significant negative bias in regions I, III, V, and VI. The exception again is in region II, where the model overpredicts by an average of $25 \%$. Assuming the overprediction of convective rainfall is also present in this region, this tendency to underpredict must be counteracted by some other factor, such as a poor understanding of the ammonia and/or $\mathrm{SO}_{2}$ emissions inventory in this area. The model performs the best in region IV, with the predictions $11 \%$ lower than the measurements on average, although the mean error is still significant at $6.59 \mu \mathrm{g} / \mathrm{m}^{3}$ compared to the measured average of $17.44 \mu \mathrm{g} / \mathrm{m}^{3}$ in this region.

Gases: Ozone, $\mathrm{NO}_{x}, \mathrm{SO}_{2}$. Predictions for four gas species, ozone, $\mathrm{NO}, \mathrm{NO}_{2}$, and $\mathrm{SO}_{2}$ were also compared with hourly measurements from PAQS (Figure 7). The ozone predictions exhibit the strong diurnal pattern seen in the measurements, with the predictions comparing fairly well with the nighttime lows and the daytime concentrations generally underpredicted. For $\mathrm{NO}_{2}$ the diurnal pattern is opposite that of ozone, with a bimodal peak usually seen during the night. The model tends to predict higher concentrations than observed for the first peak, with the second peak is in better agreement. NO peaks around 6:00 or 7:00 EST and remains at a low value the remainder of the time. The peak model predictions agree well with the observations, but the predicted NO drops down to zero on many nights while the observed NO typically remains at or above $2 \mathrm{ppb}$. Unlike the other gases, no typical diurnal pattern is seen for $\mathrm{SO}_{2}$. 
Sensitivity to $\mathrm{HNO}_{3}$ chemistry. Most species do not exhibit significant changes when the $\mathrm{HNO}_{3}$ nighttime reactions are included. The $\mathrm{PM}_{2.5}$ concentrations of sulfate and carbonaceous species do not change significantly compared to the base case, as the increased amount of $\mathrm{HNO}_{3}(\mathrm{~g})$ does not affect the partitioning of these species between the gas and aerosol phase. Ozone is also not affected significantly (increasing by about $1 \mathrm{ppb}$ compared to the study average of $40-50 \mathrm{ppb}$ throughout much of the domain). Both $\mathrm{PM}_{2.5}$ nitrate and ammonium increase, however, as equilibrium between the gas and aerosol phase for these species is directly affected by the increase in $\mathrm{HNO}_{3}(\mathrm{~g})$. Although the total ammonium (gas + particulate) does not change significantly, the partitioning of ammonium between the gas and aerosol phase is affected. The largest changes are seen in the area surrounding Philadelphia and in the Midwest (Region II), where the $\mathrm{PM}_{2.5}$ predictions are about $1.5 \mu \mathrm{g} / \mathrm{m}^{3}$ higher than the base case (a $30-40 \%$ increase). The $\mathrm{PM}_{2.5}$ nitrate predictions increase by an even larger amount, up to $5 \mu \mathrm{g} / \mathrm{m}^{3}$ in some places, while the total nitrate increases by up to $10 \mu \mathrm{g} / \mathrm{m}^{3}$.

The predictions from both cases were compared with hourly total nitrate measurements, which were taken during PAQS using the steam sampler of Khlystov (1995) and corrected by comparing them with 4-6 hourly average measurements collected using filter-based methods (Wittig et al., 2004). The results from the base case are shown in Figure 4, while the results including nighttime $\mathrm{HNO}_{3}$ production (Case II) are shown in Figure 8. The measurements show a strong diurnal pattern, with high concentrations during the day and lower concentrations at night, similar to the bas case predictions. In contrast, the highest predictions for Case II are seen at night, with the predictions an order of magnitude higher than the measurements at midnight on the $16^{\text {th }}, 18^{\text {th }}, 23^{\text {rd }}, 25^{\text {th }}$, and $26^{\text {th }}$ ( $29 \%$ of the days). The mean bias and error calculated for each scenario for both the nighttime (20:00 to 6:00 EST) and daytime (6:00 to 20:00 EST) are shown 
in Table 8. For the base case, the amount of total nitrate is underpredicted at night, with a mean bias of $-0.30 \mu \mathrm{g} / \mathrm{m}^{3}$ and a mean error of $1.64 \mu \mathrm{g} / \mathrm{m}^{3}$. This comparison is much better than for Case II, however, where the predictions are almost a factor of five higher than the measurements (with a mean bias of $6.43 \mu \mathrm{g} / \mathrm{m}^{3}$ and a mean error of $6.83 \mu \mathrm{g} / \mathrm{m}^{3}$ compared to the measured average of $1.63 \mu \mathrm{g} / \mathrm{m}^{3}$. Case II compares better with the measurements during the day, although this is largely because of the high nighttime concentrations carrying over into the daylight hours on some days. Both cases are unable to reproduce the high peak concentrations $\left(20-30 \mu \mathrm{g} / \mathrm{m}^{3}\right)$ measured on several days. This poor agreement does not affect the PM predictions significantly, however, as most of the nitric acid remains in the gas phase at the higher temperatures observed during the day. This comparison indicates that the nighttime nitrate production is significantly overpredicted in the model for reasons that are not clearly understood at the moment.

\section{Conclusions}

A detailed three-dimensional chemical transport model, $\mathrm{PMCAMx}+$, is presented and applied to an episode in the eastern United States. Although the model predictions generally agree with the observations in Pittsburgh, there are two periods where the model underpredicts significantly for all modeled species, indicating a poor representation of wet deposition (overprediction of convective rainfall) in the model. This systematic underprediction also appears in the comparison with the daily average predictions throughout the modeling domain. The exception is in Region II (the Midwest), where the model significantly overpredicts the $\mathrm{PM}_{2.5}$ mass and speciated concentrations.

Simulations both with and without the uncertain nighttime $\mathrm{HNO}_{3}$ chemistry are included, and the results from both cases are compared to hourly total $\mathrm{HNO}_{3}$ concentrations in Pittsburgh. 
The base case results, without this uncertain chemistry, provide a more accurate representation of the nighttime $\mathrm{HNO}_{3}$ levels, while including this chemistry results in predictions that are a factor of five higher than the observations on average, and an order of magnitude higher on $29 \%$ of the modeled days. Neither case accurately predicts the high $\mathrm{HNO}_{3}$ levels observed during the day. This does not affect the $\mathrm{PM}_{2.5}$ nitrate predictions significantly, however, as most of the nitric acid remains in the gas-phase because of the temperatures observed during the day. The simulation of nighttime chemistry is a topic of ongoing research.

Finally, OC and EC predictions are compared to measurements taken using two different analysis methods, the TOR and TOT protocols. The comparisons with the TOR protocol are better than with the TOT protocol. This is not surprising since the emission inventories for most of the major OC and EC sources were constructed using this protocol. Even when compared with the IMPROVE TOR improvements, however, EC is significantly overpredicted and there is a high level of inconsistency in the prediction of OC and TC, indicating that large areas of uncertainty still remain in these emission inventories. Overall, these initial results in the eastern United States are encouraging, but indicate that significant improvements are needed in several uncertain areas.

\section{Acknowledgements}

The authors wish to thank the Lake Michigan Air Directors Consortium (LADCO) for providing the meteorological files and the area and point emission files. This research was conducted as part of the Pittsburgh Air Quality Study, which was supported by the US Environmental Protection Agency (EPA) under contract R830961 and the US Department of Energy National Energy Laboratory under contract DE-FC26-01NT41017. This paper has not 
been subject to EPA's peer and policy review, and therefore does not necessarily reflect the views of the Agency. No official endorsement should be inferred.

\section{References}

Bhave, P.V. 2004. Measurement Needs for Evaluation Model Calculations of Carbonaceous Aerosol. Presented at the EMEP Workshop on Particulate Matter Measurement \& Modeling, April 20-23, New Orleans, LA.

Binkowski, F.S., and Shankar, U., 1995. The Regional particulate Matter Model. 1: Model Description and Preliminary Results, Journal of Geophysical Research 100, 26,19126,209 .

Boylan, J.W., M.T. Odman, J.G. Wilkinson, A.G. Russell, K.G. Doty, W.B. Norris and R.T. McNider, 2002. Development of a comprehensive, multiscale "one-atmosphere" modeling system: application to the Southern Appalachian Mountains, Atmospheric Environment 36, 3721-3734.

Cabada, J.C., and S. N. Pandis. 2004. Estimating the secondary organic aerosol contribution to $\mathrm{PM}_{2.5}$ using the $\mathrm{EC}$ tracer method, Aerosol Science and Technology, in press.

Capaldo, K.P., C. Pilinis, and S.N. Pandis. 2000. A computationally efficient hybrid approach for dynamic gas/aerosol transfer in air quality models. Atmospheric Environment 34, 3617-3627.

Chow, J.C. et al. 1993. The DRI Thermal/Optimal Reflectance Carbon Analysis System: Description, Evaluation and Applications in U.S. Air Quality Studies, Atmospheric Environment 27A, 1185-1201.

Chow, J.C., J.G. Watson, D. Crow, D.H. Lowenthal, and T. Merrifield. 2001. Comparison of IMPROVE and NIOSH Carbon Measurements. Aerosol Science and Technology 34, 2334.

Colella, P., and P.R. Woodward. 1984. The Piecewise Parabolic Method (PPM) for Gasdynamical Simulations. Journal of Computational Physics 54, 174-201. 
Dabdub, D., Dehaan, L., Kumar, N., Lurmann, Fl., and Seingfeld, J.H., 1998. Computationally Efficient Acid Deposition Model for California, Draft Report Contract 92-304, California Air Resources Board, Sacremento, CA.

Environ. 2003. User's guide to the comprehensive air quality model with extensions (CAMx). Version 4.02. Report prepared by ENVIRON International Corporation, Novato, CA.

Fahey, K.M., and S.N. Pandis. 2001. Optimizing model performance: variable size resolution in cloud chemistry modeling. Atmospheric Environment 35, 4471-4478.

Gaydos, T.M., B. Koo, S.N. Pandis, and D.P. Chock. 2003. Development and application of an efficient moving sectional approach for the solution of the atmospheric aerosol condensation/evaporation equations. Atmospheric Environment 37, 3303-3316.

Gelbard, F., 1990. Modeling multicomponent aerosol particle growth by vapor condensation. Aerosol Science and Technology 12, 399-412.

Gery, M.W., G.Z. Whitten, J.P. Killus, and M.C. Dodge. 1989. A photochemical kinetics mechanism for urban and regional scale computer modeling. Journal of Geophysical Research 94, 925-956.

Graham, J. 2004. IMPROVE/STN Comparison \& Implications for Visibility and $\mathrm{PM}_{2.5}$. Presented at the MANE-VU/MARAMA 2004 Science Meeting, January 27-29, Baltimore, MD.

Grell, G.A.., J. Dudhia, and D.R. Stauffer, 1995. A Description of the Fifth-Generation Penn State/NCAR Mesoscale Model (MM5). NCAR/TN-398+STR. http://www.mmm.ucar.edu/mm5/documents/mm5-desc-doc.html.

Harley, R.A., Russell, A.G., McRae, G.J., Cass, G.R., and Seinfeld, J.H., 1993. Photochemical Modeling of the Southern California Air Quality Study, Environmental Science and Technology 27, 378-388.

IMPROVE, 1995. IMPROVE Data Guide. University of California Davis, August, 1995. http://vista.cira.colostate.edu/improve/Publications/OtherDocs/IMPROVEDataGuide/IM PROVEDataGuide.htm.

Jacobson, M.Z., 1997. Development and Application of a New Air Pollution Modeling System. II. Aerosol Module Structure and Design, Atmospheric Environment 31, 131-144. 
Jacobson, M. Z., 2001. GATOR-GCMM: 2. A study of day- and nighttime ozone layers aloft, ozone in national parks, and weather during the SARMAP Field Campaign, Journal of Geophysical Research, 106, 5403-5420.

Jacobson, M.Z., and Turco, R.P., 1995. Simulating condensational growth, evaporation, and coagulation of aerosols using a combined moving and stationary grid. Aerosol Science and Technology. 22, 73-92.

Jaecker-Voirol, A., and P. Mirabel. 1989. Heteromolecular nucleation in the sulfuric acid-water system. Atmospheric Environment 23, 2053-2057.

Koo B., T. M. Gaydos, and S. N. Pandis. 2003a Evaluation of the equilibrium, dynamic, and hybrid aerosol modeling approaches, Aerosol Sci. Technol., 37, 53-64.

Koo, B.Y., A.S. Ansari, and S.N. Pandis. 2003b. Integrated approaches to modeling the organic and inorganic atmospheric aerosol components. Atmospheric Environment 37, 47574768.

Khlystov, A., G.P. Wyers, and J. Slanina. 1995. The Steam-Jet Aerosol Collector. Atmospheric Environment 29, 2229-2234.

Kumar, N., F.W. Lurmann, A.S. Wexler, S. Pandis, and J.H. Seinfeld. 1996. Development and Application of a Three Dimensional Aerosol Model. Presented at the A\&WMA Specialty Conference on Computing in Environmental Resource Management, Research Triangle Park, NC, December 2-4, 1996.

LADCO. 2003. Midwest Regional Planning Organization: Base E modeling inventory. Report prepared by Lake Michigan Air Directors Consortium. http://www.ladco.org/tech/emis/BaseE/baseEreport.pdf.

Lurmann, F.W., A.S. Wexler, S.N. Pandis, S. Musarra, N. Kumar, J.H. Seinfeld. 1997. Modeling urban and regional aerosols - II. Application to California's south coast air basin. Atmospheric Environment 31, 2695-2715.

Mebust, M. R., B. K. Eder, F. S. Binkowski, and S. J. Roselle. 2003. Models-3 Community Multiscale Air Quality (CMAQ) model aerosol component, 2, Model evaluation, Journal of Geophysical Research 108(D6), 4184, doi:10.1029/2001JD001410.

Meng, Z, D. Dabdub, and J. H. Seinfeld. 1998. Size-resolved and chemically resolved model of atmospheric aerosol dynamics, Journal of Geophysical Research 103, 3419-3435. 
Napari, I., M. Noppel, H. Vehkamaki, and M. Kulmala, 2002. Parameterization of ternary nucleation rates for $\mathrm{H}_{2} \mathrm{SO}_{4}-\mathrm{NH}_{3}-\mathrm{H}_{2} \mathrm{O}$ vapors, Journal of Geophysical Research 107(D19), 4381.

Nenes, A., S.N. Pandis, and C. Pilinis. 1998. ISORROPIA: a new thermodynamic model for inorganic multicomponent atmospheric aerosols. Aquatic Geochemistry 4, 123-152.

NIOSH, 1999. Method 5040 Issue 3 (Interim): Elemental Carbon (Diesel Exhaust). In NIOSH Manual of Analytical Methods. National Institute of Occupational Safety and Health, Cincinatti, $\mathrm{OH}$.

Odman, M. T. and Ingram, C. L., 1993. "Multiscale Air Quality Simulation Platform (MAQSIP): Source Code Documentation and Validation." Technical report, 83 pp., ENV-96TR002, MCNC-North Carolina Supercomputing Center, Research Triangle Park, North Carolina, 1996.

Odman M.T. and Russell A.G.,1991. A multiscale finite element pollutant transport scheme for urban and regional modeling. Atmospheric Environment 25A, 2385-2394.

Pandis, S.N., and J.H. Seinfeld. 1989. Sensitivity analysis of a chemical mechanism for aqueous-phase atmospheric chemistry. Journal of Geophysical Research 94, 1105-1126.

Pandis, S.N., A.S. Wexler, and J.H. Seinfeld. 1993. Secondary organic aerosol formation and transport. 2. Predicting the ambient secondary organic aerosol-size distribution. Atmospheric Environment 27, 2403-2416.

Pinder, R.W., Strader, R, Davidson, C.I., and Adams, P.J., 2004. A Temporally and Spatially Resolved Ammonia Emission Inventory for Dairy Cows in the United States, Atmospheric Environment 38, 3747-3756.

Pilinis, C., and Seinfeld, J. H., 1988. Development and evaluation of an Eulerian photochemical gas-aerosol model, Atmospheric Environment 22, 1985-2001.

Pilinis, C., Capaldo, K.P., Nenes, A., and Pandis, S.N., 2000. MADM - A new multicomponent aerosol dynamics model. Aerosol Science and Technology 32, 482-502.

Rees S. L., A. L. Robinson, A. Khlystov, C. O. Stanier, and S. N. Pandis, 2004. Mass balance closure and the $\mathrm{PM}_{2.5}$ Federal Reference Method in Pittsburgh, Pennsylvania, Atmos. Environ., 38, 3305-3318. 
Russell, A. G., and Cass G. R., 1986. Verification of a mathematical model for aerosol nitrate and nitric acid formation and its use for control measure evaluation, Atmospheric Environment 20, 2011-2025.

Russell, L.M., S.N. Pandis, and J.H. Seinfeld. 1994. Aerosol production and growth in the marine boundary layer. Journal of Geophysical Research 99, 20,989-21,003.

Seinfeld, J.H., and S.N. Pandis. 1998. Atmospheric Chemistry and Physics, From Air Pollution to Climate Change. John Wiley and Sons, Inc., NY.

Slinn, S.A., and W.G.N. Slinn. 1980. Predictions for particle deposition on natural-waters. Atmospheric Environment 14, 1013-1016.

Smagorinsky, J. 1963. General Circulation Experiments with the Primitive Equations: I. The Basic Experiment. Monthly Weather Review 91, 99-164.

Strader, R., C.S. Gurciullo, S.N. Pandis, N. Kumar, and F.W. Lurmann. 1998. Development of gas-phase chemistry, secondary organic aerosol, and aqueous-phase chemistry modules for PM modeling. STI Final Report STI-997510-1822-FR.

Strader, R., F. Lurmann, and S.N. Pandis. 1999. Evaluation of secondary organic aerosol formation in winter. Atmospheric Environment 33, 4849-4863.

Sun, Q., and Wexler, A.S., 1998. Modeling Urban and Regional Aerosols near Acid Neutrality Application to the June 24-25 SCAQS Episode, Atmospheric Environment 32, 35333545.

Takahama S., Wittig A.E., Vayenas D.V., Davidson, C.I., Pandis, S.N., 2004. Modeling the diurnal variation of nitrate during the Pittsburgh Air Quality Study, Journal of Geophysical Research - Atmospheres 109 (D16): Art. No. D16S06.

Tambour, Y., and Seinfeld, J.H., 1980. Solution of the discrete coagulation equation, Journal of Colloid Interface Science 74, 260-272.

U.S. EPA. 2001. Procedures Document for National Emission Inventory, Criteria Air Pollutants, 1985-1999. EPA-454/R-01-006. Office of Air Quality Planning and Standards. March. http://www.epa.gov/ttn/chief/trends/procedures/neiproc_99.pdf.

U.S. EPA. 2003. User's Guide to MOBILE6.1 and MOBILE6.2 - Mobile Source Emission Factor Model. EPA420-R-03-010. Air and Radiation. August. http://www.epa.gov/otaq/models/mobile6/420r03010.pdf.

U.S. EPA. 2002. User Guide: Air Quality System. Report prepared by the U.S. EPA. April. 
http://www.epa.gov/ttn/airs/aqs/softw/AQSUserGuide_v1.pdf.

Wesely, M.L. 1989. Parameterization of Surface Resistances to Gaseous Dry Deposition in Regional-Scale Numerical Models. Atmospheric Environment 23, 1293-1304.

Wilkinson, J., and M. Janssen. 2001. BIOME3. Prepared for the National Emissions Inventory Workshop, Denver, CO, May 1-3, 2001. http://www.epa.gov/ttn/chief/conference/ei10/modeling/wilkenson.pdf.

Wittig, B., N. Anderson, A. Y. Khlystov, S. N. Pandis, C. Davidson and A. L. Robinson. 2004. Pittsburgh Air Quality Study overview and preliminary scientific findings, Atmospheric Environment, in press.

Zhang, Y., B. Pun, K. Vijayaraghavan, S.-Y. Wu, C. Seigneur, S. Pandis, M. Jacobson, A. Nenes and J.H. Seinfeld, 2004. Development and Application of the Model of Aerosol Dynamics, Reaction, Ionization and Dissolution (MADRID), J. Geophys. Res., 109, D01202, doi:10.1029/2003JD003501. 
Table 1. Gas phase species in the PMCAMx + CB4 chemical mechanism

\begin{tabular}{|c|c|c|c|}
\hline $\begin{array}{l}\text { PMCAMx+ } \\
\text { Name }\end{array}$ & Description & $\begin{array}{l}\text { Initial } \\
\text { Condition } \\
\text { (ppb) }\end{array}$ & $\begin{array}{l}\text { Boundary } \\
\text { Condition } \\
\text { (ppb) }\end{array}$ \\
\hline NO & Nitric oxide & 0.08 & 0.08 \\
\hline $\mathrm{NO} 2$ & Nitrogen dioxide & 0.17 & 0.17 \\
\hline $\mathrm{O} 3$ & Ozone & 35 & 35 \\
\hline PAN & Peroxyacyl nitrates & 0.0 & 0.0 \\
\hline NXOY & Nitrogen in $\mathrm{NO}_{3}$ and $\mathrm{N}_{2} \mathrm{O}_{5}$ & 0.0 & 0.0 \\
\hline OLE & CB4 olefins (anthropogenic) & 0.0 & 0.0 \\
\hline PAR & CB4 paraffin & 0.0 & 0.0 \\
\hline TOL & CB4 toluene & 0.0 & 0.0 \\
\hline XYL & CB4 xylene & 0.0 & 0.0 \\
\hline FORM & Formaldehyde & 0.0 & 0.0 \\
\hline ALD2 & CB4 higher aldehyde & 0.0 & 0.0 \\
\hline ETH & Ethene & 0.0 & 0.0 \\
\hline CRES & CB4 cresol & 0.0 & 0.0 \\
\hline MGLY & CB4 methylglyoxal & 0.0 & 0.0 \\
\hline OPEN & $\mathrm{CB} 4$ aromatic ring opening product & 0.0 & 0.0 \\
\hline PNA & Peroxynitric acid & 0.0 & 0.0 \\
\hline $\mathrm{CO}$ & Carbon monoxide & 0.0 & 0.0 \\
\hline HONO & Nitrous acid & 0.0 & 0.0 \\
\hline $\mathrm{H} 2 \mathrm{O} 2$ & Hydrogen peroxide & 0.0 & 0.0 \\
\hline HNO3 & Nitric acid & 0.05 & 0.05 \\
\hline ISOP & Isoprene & 0.0 & 0.0 \\
\hline MEOH & Methanol & 0.0 & 0.0 \\
\hline ЕTOH & Ethanol & 0.0 & 0.0 \\
\hline ISPD & Isoprene product & 0.0 & 0.0 \\
\hline NTR & Organic nitrates & 0.0 & 0.0 \\
\hline OLE2 & CB4 olefins (biogenic) & 0.0 & 0.0 \\
\hline CG1 & Condensable gas precursor (forms SOA1) & 0.0 & 0.0 \\
\hline CG2 & Condensable gas precursor (forms SOA2) & 0.0 & 0.0 \\
\hline CG3 & Condensable gas precursor (forms SOA3) & 0.0 & 0.0 \\
\hline CG4 & Condensable gas precursor (forms SOA4) & 0.0 & 0.0 \\
\hline NH3 & Ammonia & 0.1 & 0.1 \\
\hline $\mathrm{HCL}$ & Hydrogen chloride & 0.0 & 0.0 \\
\hline $\mathrm{SO} 2$ & Sulfur dioxide & 0.3 & 0.3 \\
\hline SULF & Sulfuric acid & 0.0 & 0.0 \\
\hline
\end{tabular}


Table 2. Aerosol species in PMCAMx+, along with their initial and boundary conditions.

\begin{tabular}{|c|c|c|c|}
\hline $\begin{array}{l}\text { PMCAMx+ } \\
\text { Name }\end{array}$ & Description & $\begin{array}{l}\text { Initial } \\
\text { Conditions } \\
\left(\mu \mathrm{g} \mathrm{m}^{-3}\right)\end{array}$ & $\begin{array}{l}\text { Boundary } \\
\text { Conditions } \\
\left(\mu \mathrm{g} \mathrm{m}^{-3}\right) \\
\end{array}$ \\
\hline SOA1 & $\begin{array}{l}\text { Secondary organic } \\
\text { aerosol } 1\end{array}$ & 0.0 & 0.0 \\
\hline SOA2 & $\begin{array}{l}\text { Secondary organic } \\
\text { aerosol } 2\end{array}$ & 0.0 & 0.0 \\
\hline SOA3 & $\begin{array}{l}\text { Secondary organic } \\
\text { aerosol } 3\end{array}$ & 0.0 & 0.0 \\
\hline SOA4 & $\begin{array}{l}\text { Secondary organic } \\
\text { aerosol } 4\end{array}$ & 0.0 & 0.0 \\
\hline $\mathrm{POC}^{\mathrm{a}}$ & $\begin{array}{l}\text { Primary organic } \\
\text { aerosol }\end{array}$ & 2.0 & 1.0 \\
\hline PEC & $\begin{array}{l}\text { Primary elemental } \\
\text { carbon }\end{array}$ & 0.0 & 0.0 \\
\hline CRST & Crustal material & 0.0 & 0.0 \\
\hline $\mathrm{PH} 2 \mathrm{O}$ & Water & 0.0 & 0.0 \\
\hline PCL & Chloride ion & 0.0 & 0.0 \\
\hline NA & Sodium ion & 0.0 & 0.0 \\
\hline PNH4 & Ammonium ion & 0.0 & 0.0 \\
\hline PNO3 & Nitrate ion & 0.0 & 0.0 \\
\hline PSO4 & Sulfate ion & $1.77 \mathrm{E}-04^{\mathrm{b}}$ & 0.0 \\
\hline
\end{tabular}

${ }^{a}$ Primary organic particulate matter and not just organic carbon

${ }^{\mathrm{b}}$ A small non-zero value is used to avoid numerical problems at start-up. 
Table 3. VOC precursor, aerosol yield, saturation concentration, and heat of vaporization for modeled SOA species.

\begin{tabular}{llllll}
\hline $\begin{array}{l}\text { VOC } \\
\text { precursor }\end{array}$ & $\begin{array}{l}\text { SOA } \\
\text { product } \\
\text { name }\end{array}$ & $\begin{array}{l}\text { Molecular } \\
\text { weight of } \\
\text { product } \\
\left(\mathrm{g} \text { mole } \mathrm{e}^{-1}\right)\end{array}$ & $\begin{array}{l}\text { Aerosol } \\
\text { Yield } \\
(\mathrm{ppm} / \mathrm{ppm})\end{array}$ & $\begin{array}{l}\text { Saturation } \\
\text { concentration } \\
\left(\mu \mathrm{g} / \mathrm{m}^{3} \text { at }\right. \\
281.5 \mathrm{~K})\end{array}$ & $\begin{array}{l}\text { Heat of } \\
\text { vaporization } \\
\left(\mathrm{kJ} \mathrm{mole}^{-1}\right)\end{array}$ \\
\hline PAR & CG3 & 150 & 0.0088 & 0.007 & 0 \\
OLE & CG3 & 150 & 0.0024 & 0.007 & 0 \\
TOL & CG1 & 150 & 0.07 & 0.023 & 156 \\
TOL & CG2 & 150 & 0.137 & 0.674 & 156 \\
XYL & CG1 & 150 & 0.044 & 0.023 & 156 \\
XYL & CG2 & 150 & 0.192 & 0.674 & 156 \\
CRES & CG3 & 150 & 0.036 & 0.007 & 0 \\
OLE2 & CG4 & 180 & 0.136 & 0.008 & 0
\end{tabular}


Table 4. Comparison of inorganic and total $\mathrm{PM}_{2.5}$ mass predictions at Pittsburgh with hourly measurements taken during the Pittsburgh Air Quality Study.

\begin{tabular}{lcccccc}
\hline Species & $\begin{array}{c}\text { Predicted } \\
\text { Average }^{\mathrm{a}} \\
\left(\mu \mathrm{g} / \mathrm{m}^{3}\right)\end{array}$ & $\begin{array}{c}\text { Measured } \\
\text { Average }^{\mathrm{a}} \\
\left(\mu \mathrm{g} / \mathrm{m}^{3}\right)\end{array}$ & FBIAS & FERROR & $\begin{array}{c}\mathrm{BIAS}^{\mathrm{a}} \\
\left(\mu \mathrm{g} / \mathrm{m}^{3}\right)\end{array}$ & $\begin{array}{c}\text { ERROR }^{\mathrm{a}} \\
\left(\mu \mathrm{g} / \mathrm{m}^{3}\right)\end{array}$ \\
\hline $\mathrm{PM}_{2.5}$ mass & 19.59 & 24.24 & -0.16 & 0.31 & -4.65 & 7.42 \\
$\mathrm{PM}_{2.5} \mathrm{SO}_{4}$ & 8.29 & 10.03 & -0.09 & 0.47 & -1.75 & 3.94 \\
$\mathrm{PM}_{2.5} \mathrm{NO}_{3}$ & 0.37 & 0.57 & -0.77 & 1.30 & -0.20 & 0.56 \\
Total NH$_{3}$ & 2.91 & 3.22 & -0.06 & 0.38 & -0.31 & 1.12 \\
Total HNO & 2.68 & 4.85 & -0.20 & 0.88 & -2.05 & 3.16 \\
$\mathrm{PM}_{2.5} \mathrm{TC}_{3}$ & 4.34 & 3.81 & 0.17 & 0.38 & 0.52 & 1.41 \\
$\mathrm{PM}_{2.5} \mathrm{OC}$ & 2.81 & 3.05 & -0.01 & 0.40 & -0.24 & 1.10 \\
$\mathrm{PM}_{2.5} \mathrm{EC}$ & 1.53 & 0.77 & 0.67 & 0.71 & 0.76 & 0.81 \\
$\mathrm{Oz}_{2} \mathrm{NO}$ & 33.35 & 43.9 & -0.30 & 0.45 & -10.55 & 14.62 \\
$\mathrm{NO}_{2}$ & 19.50 & 14.43 & 0.30 & 0.41 & 5.07 & 7.45 \\
$\mathrm{NO}$ & 3.21 & 4.53 & -0.79 & 1.04 & -1.31 & 3.27 \\
$\mathrm{SO}_{2}$ & 10.8 & 9.46 & 0.19 & 0.50 & 1.38 & 5.15
\end{tabular}

${ }^{\mathrm{a}}$ The concentrations of the PM species and total ammonium and $\mathrm{HNO}_{3}$ are in $\mu \mathrm{g} / \mathrm{m}^{3}$, while the concentrations of the gases are in $\mathrm{ppb}$. 
Table 5. Comparison of $\mathrm{PMCAMx}+$ predictions with daily average measurements taken at monitoring stations in the U.S. EPA's AIRS network.

\begin{tabular}{lcccccc}
\hline Species & $\begin{array}{c}\text { Predicted } \\
\text { Average } \\
\left(\mu \mathrm{g} / \mathrm{m}^{3}\right)\end{array}$ & $\begin{array}{c}\text { Measured } \\
\text { Average } \\
\left(\mu \mathrm{g} / \mathrm{m}^{3}\right)\end{array}$ & FBIAS & FERROR & $\begin{array}{c}\mathrm{BIAS} \\
\left(\mu \mathrm{g} / \mathrm{m}^{3}\right)\end{array}$ & $\begin{array}{c}\text { ERROR } \\
\left(\mu \mathrm{g} / \mathrm{m}^{3}\right)\end{array}$ \\
\hline $\mathrm{PM}_{2.5}$ mass & 14.13 & 18.24 & -0.29 & 0.48 & -4.11 & 7.38 \\
$\mathrm{PM}_{2.5} \mathrm{SO}_{4}$ & 4.79 & 6.21 & -0.32 & 0.55 & -1.42 & 2.70 \\
$\mathrm{PM}_{2.5} \mathrm{NH}_{4}$ & 1.70 & 1.80 & -0.03 & 0.54 & -0.10 & 0.83 \\
$\mathrm{PM}_{2.5} \mathrm{NO}_{3}$ & 0.58 & 0.70 & -0.76 & 1.06 & -0.12 & 0.57 \\
$\mathrm{PM}_{2.5} \mathrm{TC}$ & 3.85 & 4.77 & -0.24 & 0.47 & -0.92 & 1.98 \\
$\mathrm{PM}_{2.5} \mathrm{OC}$ & 2.49 & 4.29 & -0.52 & 0.59 & -1.80 & 2.01 \\
$\mathrm{PM}_{2.5} \mathrm{EC}$ & 1.36 & 0.48 & 0.82 & 0.90 & 0.88 & 0.91
\end{tabular}


Table 6. Comparison of $\mathrm{PMCAMx}+$ predictions with daily average measurements taken at monitoring stations in the IMPROVE network.

\begin{tabular}{lcccccc}
\hline Species & $\begin{array}{c}\text { Predicted } \\
\text { Average } \\
\left(\mu \mathrm{g} / \mathrm{m}^{3}\right)\end{array}$ & $\begin{array}{c}\text { Measured } \\
\text { Average } \\
\left(\mu \mathrm{g} / \mathrm{m}^{3}\right)\end{array}$ & FBIAS & FERROR & $\begin{array}{c}\mathrm{BIAS} \\
\left(\mu \mathrm{g} / \mathrm{m}^{3}\right)\end{array}$ & $\begin{array}{c}\text { ERROR } \\
\left(\mu \mathrm{g} / \mathrm{m}^{3}\right)\end{array}$ \\
\hline $\mathrm{PM}_{2.5}$ mass & 10.06 & 13.54 & -0.23 & 0.48 & -3.54 & 5.88 \\
$\mathrm{PM}_{2.5} \mathrm{SO}_{4}$ & 4.10 & 5.72 & -0.30 & 0.57 & -1.62 & 2.68 \\
$\mathrm{PM}_{2.5} \mathrm{NO}_{3}$ & 0.26 & 0.29 & -0.54 & 0.93 & -0.01 & 0.28 \\
$\mathrm{PM}_{2.5} \mathrm{TC}$ & 2.24 & 2.13 & 0.09 & 0.43 & 0.11 & 0.90 \\
$\mathrm{PM}_{2.5} \mathrm{OC}$ & 1.72 & 1.78 & 0.04 & 0.43 & -0.06 & 0.71 \\
$\mathrm{PM}_{2.5} \mathrm{EC}$ & 0.52 & 0.35 & 0.20 & 0.56 & 0.17 & 0.28
\end{tabular}


Table 7. Comparison of $\mathrm{PMCAMx}+$ daily average $\mathrm{PM}_{2.5}$ mass predictions with measurements taken at monitoring stations in the U.S. EPA's AIRS network in six different regions.

\begin{tabular}{lllllll}
\hline Region & $\begin{array}{l}\text { Predicted } \\
\text { Average } \\
\left(\mu \mathrm{g} / \mathrm{m}^{3}\right)\end{array}$ & $\begin{array}{l}\text { Measured } \\
\text { Average } \\
\left(\mu \mathrm{g} / \mathrm{m}^{3}\right)\end{array}$ & FBIAS & FERROR & $\begin{array}{l}\text { BIAS } \\
\left(\mu \mathrm{g} / \mathrm{m}^{3}\right)\end{array}$ & $\begin{array}{l}\text { ERROR } \\
\left(\mu \mathrm{g} / \mathrm{m}^{3}\right)\end{array}$ \\
\hline I & 8.23 & 14.4 & -0.54 & 0.57 & -6.17 & 6.47 \\
II & 21.12 & 16.91 & 0.19 & 0.40 & 4.21 & 8.07 \\
III & 17.35 & 25.27 & -0.29 & 0.35 & -7.92 & 8.74 \\
IV & 15.49 & 17.44 & -0.12 & 0.41 & -1.96 & 6.59 \\
V & 8.46 & 14.28 & -0.58 & 0.65 & -5.82 & 6.67 \\
VI & 14.88 & 20.76 & -0.35 & 0.50 & -5.87 & 7.91
\end{tabular}


Table 8. Comparison of PMCAMx + total nitrate predictions during the day (7:00 - 20:00 EST) and night (20:00 - 7:00 EST) with measurements taken during the Pittsburgh Air Quality Study.

\begin{tabular}{lcccccc}
\hline Case & $\begin{array}{c}\text { Predicted } \\
\text { Average } \\
\left(\mu \mathrm{g} / \mathrm{m}^{3}\right)\end{array}$ & $\begin{array}{c}\text { Measured } \\
\text { Average } \\
\left(\mu \mathrm{g} / \mathrm{m}^{3}\right)\end{array}$ & FBIAS & FERROR & $\begin{array}{c}\text { BIAS } \\
\left(\mu \mathrm{g} / \mathrm{m}^{3}\right)\end{array}$ & $\begin{array}{c}\text { ERROR } \\
\left(\mu \mathrm{g} / \mathrm{m}^{3}\right)\end{array}$ \\
\hline $\begin{array}{l}\text { Base case } \\
\text { (day) }\end{array}$ & 3.70 & 6.84 & -0.24 & -0.77 & -3.14 & 4.10 \\
Case II (day) & 5.34 & 6.84 & 0.08 & 0.75 & -1.50 & 4.31 \\
$\begin{array}{l}\text { Base case } \\
\text { (night) }\end{array}$ & 1.34 & 1.63 & -0.15 & 1.06 & -0.30 & 1.64 \\
Case II (night) & 8.06 & 1.63 & 1.27 & 1.32 & 6.43 & 6.83
\end{tabular}




\section{Figure Captions}

Figure 1. The PMCAMx + modeling domain for the eastern United States. The locations of the monitoring stations for the IMPROVE network are shown.

Figure 2. Average predicted ground-level concentrations over the entire simulation period (July 12-28, 2001) for $\mathrm{PM}_{2.5}$ mass, sulfate, nitrate, ammonium, total carbon $\left(\mu \mathrm{g} / \mathrm{m}^{3}\right)$, and ozone gas (ppb).

Figure 3. Comparison of model predictions with hourly measurements taken during the Pittsburgh Air Quality Study (PAQS) in July 2001.

Figure 4. Comparison of model predictions with hourly measurements of total (gas and aerosol) ammonium and nitrate taken during the Pittsburgh Air Quality Study (PAQS) in July 2001.

Figure 5. Comparison of model predictions with hourly measurements taken during the Pittsburgh Air Quality Study (PAQS) in July 2001.

Figure 6. The modeling domain broken down into six subregions (I-VI), with the AIRS monitoring stations in each region shown.

Figure 7. Comparison of model predictions with hourly measurements taken during the Pittsburgh Air Quality Study (PAQS) in July 2001.

Figure 8. Comparison of predicted total (particulate + gaseous) $\mathrm{HNO}_{3}$ concentrations when $\mathrm{HNO}_{3}(\mathrm{~g})$ nighttime production is turned on with hourly measurements taken during the Pittsburgh Air Quality Study (PAQS). 


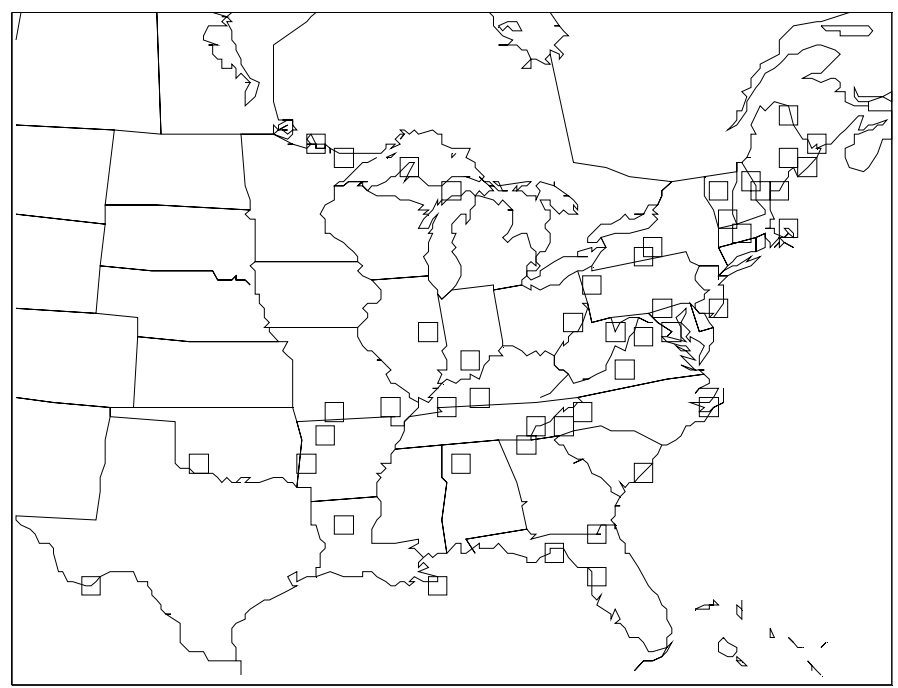

Figure 1. The PMCAMx+ modeling domain for the eastern United States. The locations of the monitoring stations for the IMPROVE network are shown. 
$\mathrm{PM}_{2.5}$ mass
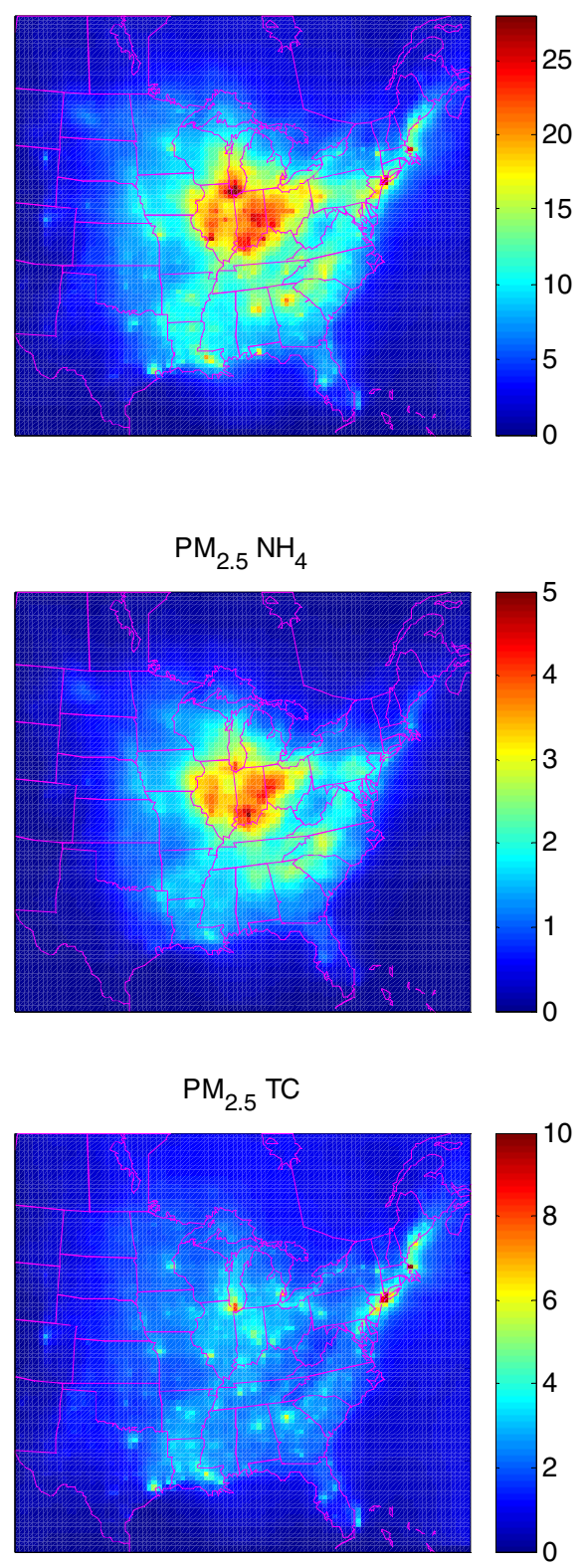
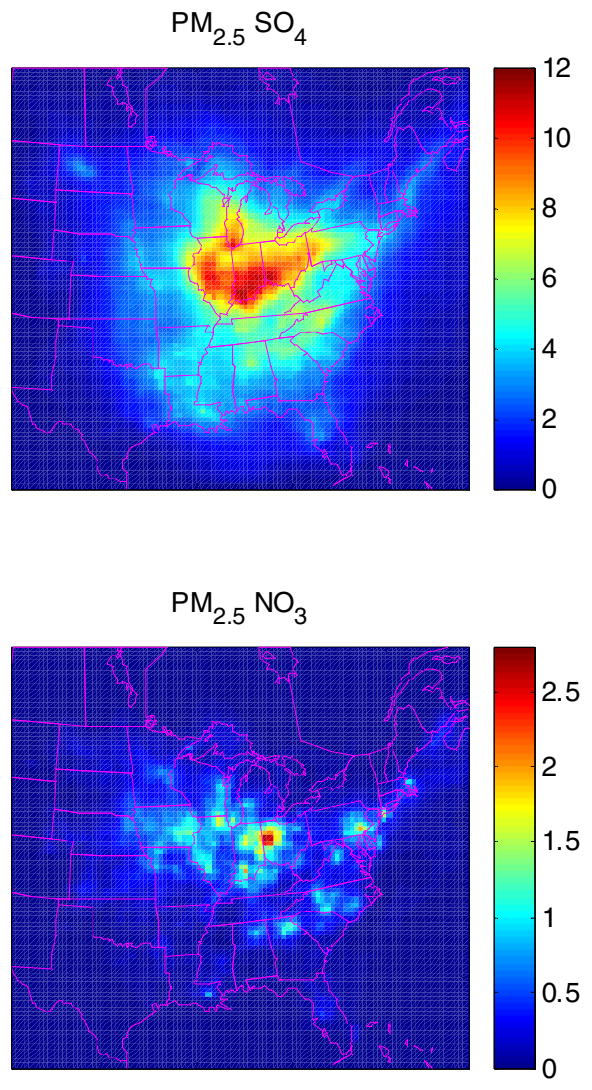

$\mathrm{O}_{3}$

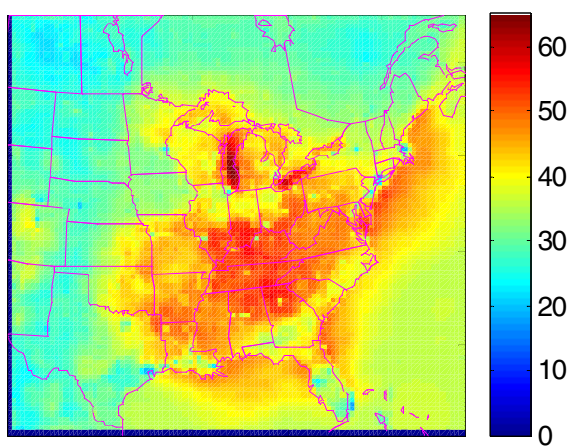

Figure 2. Average predicted ground-level concentrations over the entire simulation period (July $12-28,2001$ ) for $\mathrm{PM}_{2.5}$ mass, sulfate, nitrate, ammonium, total carbonaceous $\mathrm{PM}$ (all in $\mu \mathrm{g} / \mathrm{m}^{3}$ ), and ozone gas (ppb). 

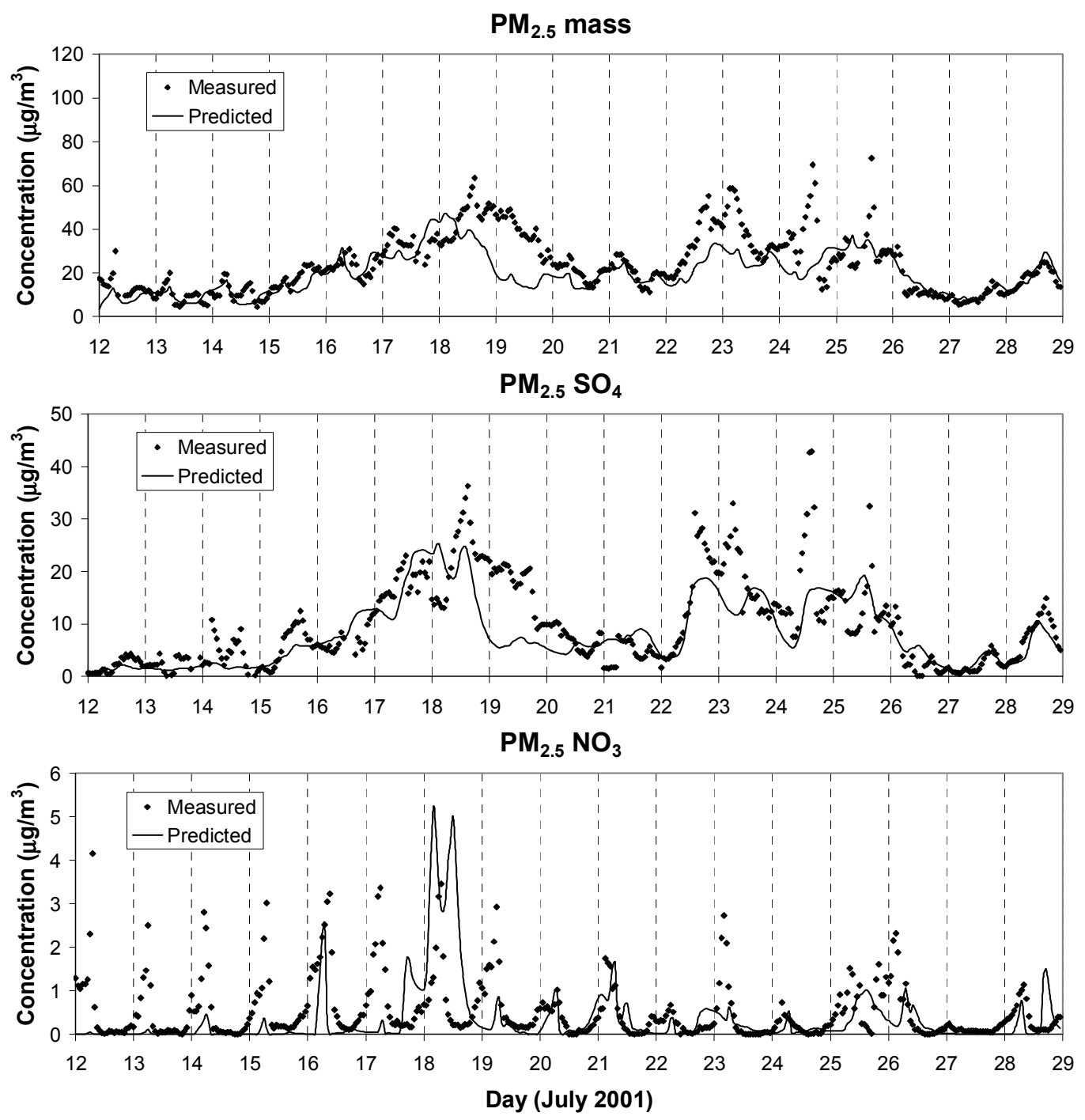

Figure 3. Comparison of model predictions with hourly measurements taken during the Pittsburgh Air Quality Study (PAQS) in July 2001. 
Total $\mathrm{NH}_{4}$

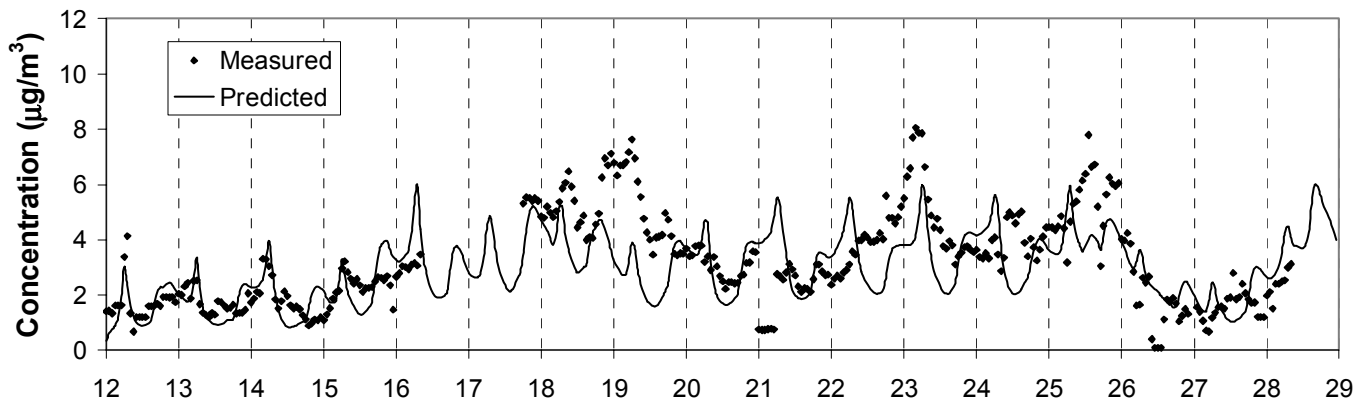

Total $\mathrm{NO}_{3}$

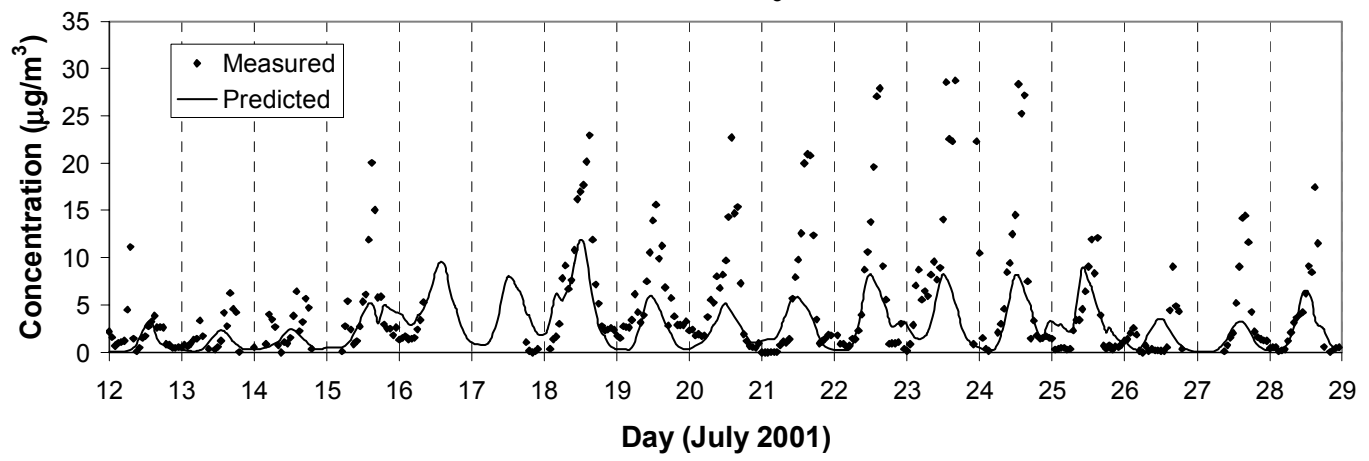

Figure 4. Comparison of model predictions with hourly measurements of total (gas and aerosol) ammonium and nitrate taken during the Pittsburgh Air Quality Study (PAQS) in July 2001. 

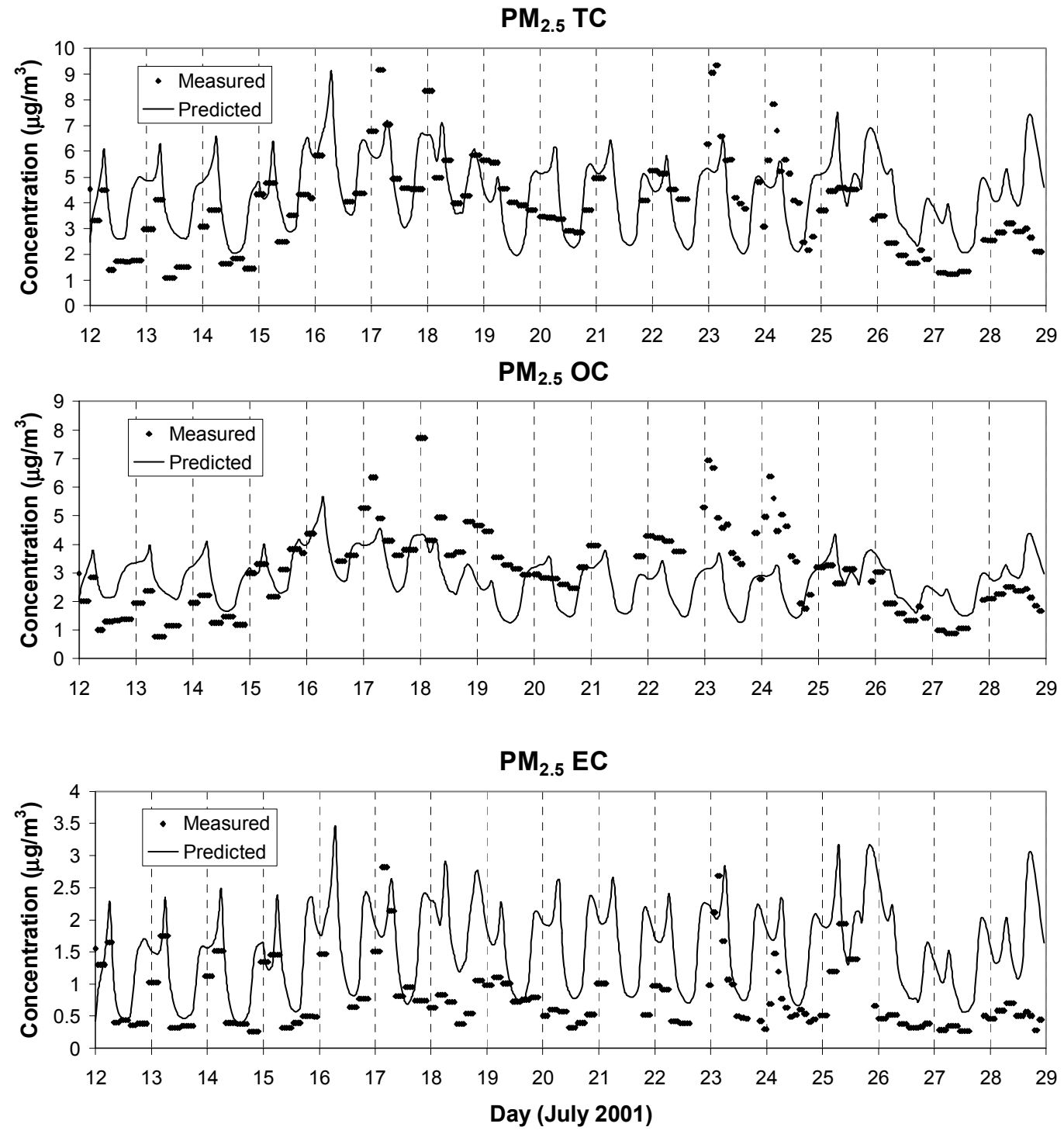

Figure 5. Comparison of model predictions with hourly measurements of organic $\mathrm{PM}_{2.5}$ taken during the Pittsburgh Air Quality Study (PAQS) in July 2001. 


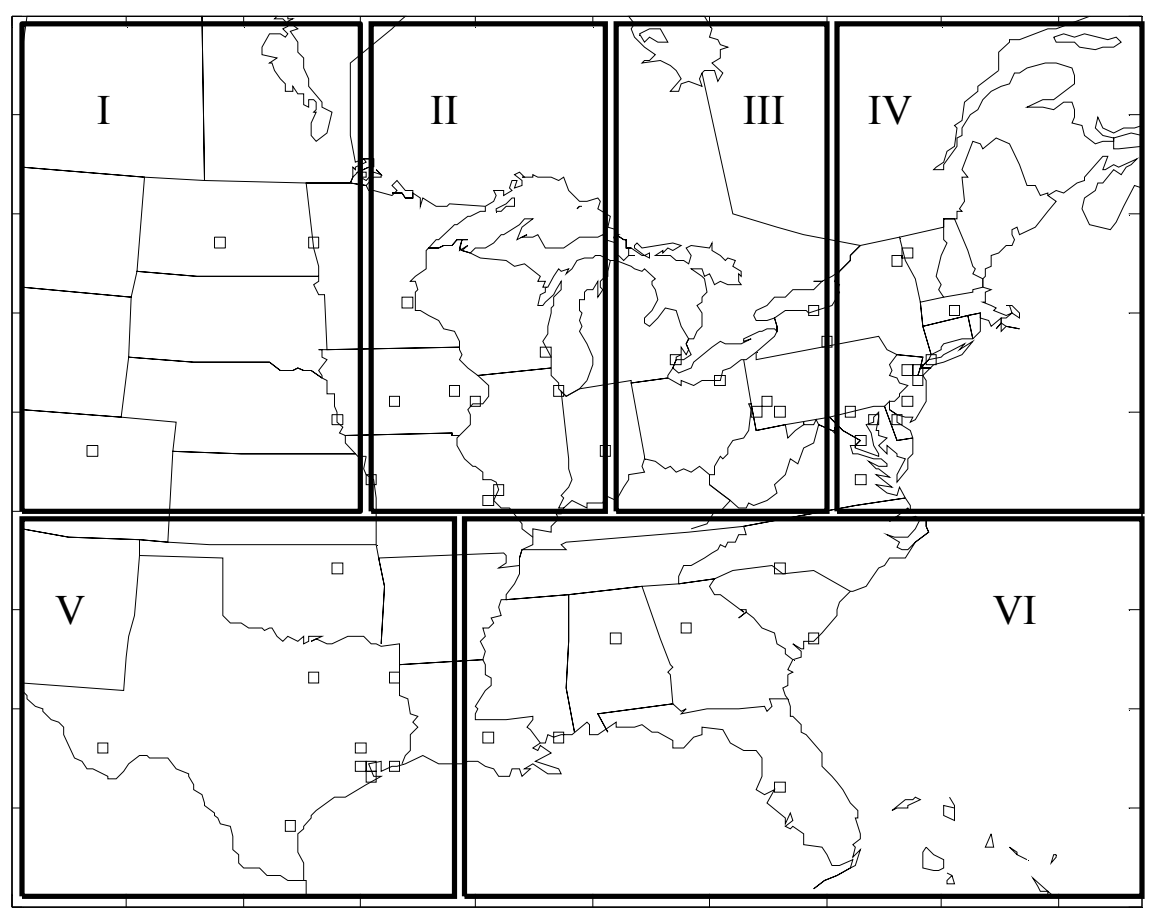

Figure 6. The modeling domain broken down into six subregions (I-VI), with the AIRS monitoring stations in each region shown. 

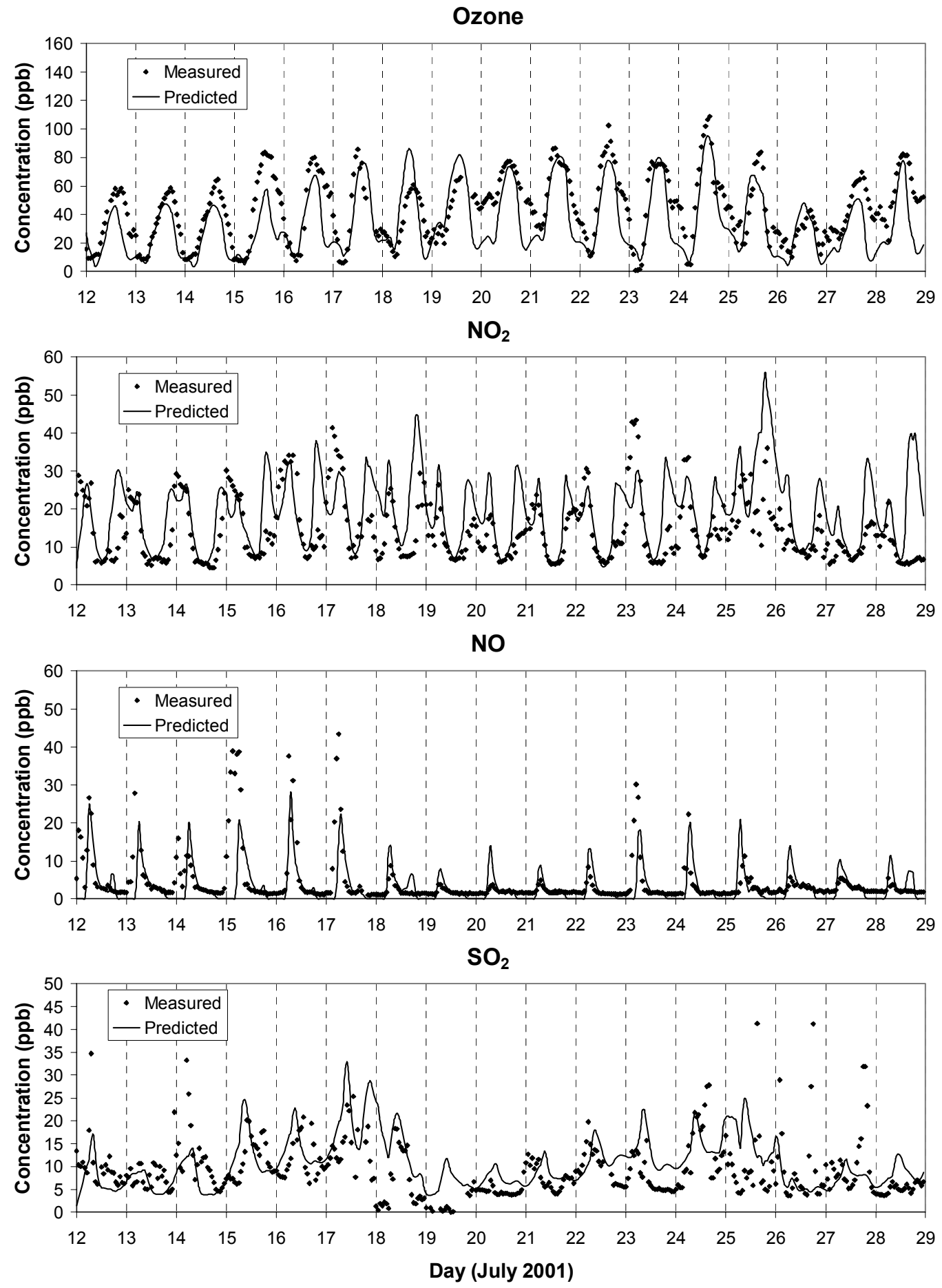

Figure 7. Comparison of model predictions with hourly measurements taken during the Pittsburgh Air Quality Study (PAQS) in July 2001. 


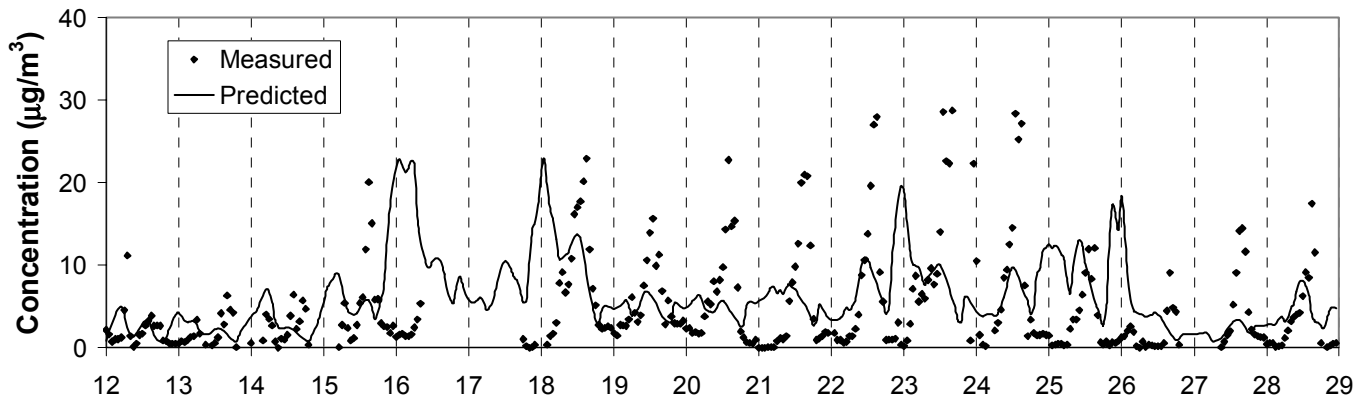

Figure 8. Comparison of predicted total (particulate + gaseous) $\mathrm{HNO}_{3}$ concentrations when $\mathrm{HNO}_{3}(\mathrm{~g})$ nighttime production is turned on with hourly measurements taken during the Pittsburgh Air Quality Study (PAQS). 


\title{
Modeling of in situ ultrafine atmospheric particle formation in the eastern United States
}

\author{
Timothy M. Gaydos and Charles O. Stanier \\ Department of Chemical Engineering, Carnegie Mellon University, Pittsburgh, Pennsylvania, USA
}

Spyros N. Pandis

Departments of Chemical Engineering and Engineering and Public Policy, Carnegie Mellon University, Pittsburgh, Pennsylvania, USA

Received 24 February 2004; revised 26 April 2004; accepted 17 May 2004; published 27 January 2005.

[1] The creation of new atmospheric particles from in situ nucleation influences climate through cloud-aerosol interactions and may negatively impact human health. Although recent observations show that nucleation is widespread in the eastern United States, the corresponding pathways remain uncertain. Combining extensive field measurements in Pittsburgh, Pennsylvania, with an aerosol dynamics and chemistry model assuming ternary $\mathrm{NH}_{3}-\mathrm{H}_{2} \mathrm{SO}_{4}-\mathrm{H}_{2} \mathrm{O}$ nuclei formation, we show excellent model-measurement agreement and predictive capability. The ternary $\mathrm{NH}_{3}-\mathrm{H}_{2} \mathrm{SO}_{4}-\mathrm{H}_{2} \mathrm{O}$ nucleation model is successful in predicting the presence or lack of nucleation on 19 out of 19 days with complete data sets in July 2001 and on 25 out of 29 days in January 2002. Reductions of ammonia emissions are predicted to decrease the frequency of nucleation events during both summer and winter, with a more dramatic effect during the summer. The response to changes in emissions of sulfur dioxide during the summer is counterintuitive. Reductions of sulfur dioxide and the resulting sulfate by up to $40 \%$ actually increase the frequency of the summer nucleation events. Modeling predicts the opposite effect in winter, with reductions of sulfur dioxide leading to fewer nucleation events.

Citation: Gaydos, T. M., C. O. Stanier, and S. N. Pandis (2005), Modeling of in situ ultrafine atmospheric particle formation in the eastern United States, J. Geophys. Res., 110, D07S12, doi:10.1029/2004JD004683.

\section{Introduction}

[2] The two major processes introducing new particles into the atmosphere are in situ nucleation and direct emission from sources such as combustion, sea spray, dust, etc. A variety of field measurements show that in situ nucleation occurs in many places around the globe [Weber et al., 1999; Kulmala et al., 2001a; O'Dowd et al., 2002], including urban areas [Woo et al., 2001] where it was previously thought particularly unlikely due to high concentrations of aerosol surface area, which suppress nucleation. These events alter the number and chemical composition of ultrafine particles and cloud condensation nuclei, with implications for human health [Oberdorster et al., 1992; Ibald-Mulli et al., 2002; Li et al., 1999; Nemmar et al., 2002; Peters et al., 1997; Oberdorster et al., 2002] and climate [Adams and Seinfeld, 2003]. For example, recent studies comparing the health effects of differently sized particles show that ultrafine $(<100 \mathrm{~nm})$ atmospheric particles can have negative health effects, possibly due to their high surface area. Climate can be affected as these particles grow to larger than $100 \mathrm{~nm}$ in size.

Copyright 2005 by the American Geophysical Union. 0148-0227/05/2004JD004683\$09.00
[3] Several mechanisms have been proposed for the production of new atmospheric particles including sulfuric acid-water binary nucleation [Nilsson and Kulmala, 1998], sulfuric acid-ammonia-water ternary nucleation [Coffman and Hegg, 1995; Weber et al., 1998; Kulmala et al., 2002], nucleation of organic vapors [Hoffman et al., 1997], ioninduced nucleation [Laakso et al., 2002], and halogen-oxide nucleation [Hoffman et al., 2001]. Another important issue is what species are involved in the growth of these nuclei. Field measurements [Eisele and McMurray, 1997; Weber et al., 1999; Janson et al., 2001] and model simulations [Kerminen et al., 2001; Kulmala et al., 2001b; Pirjola and Kulmala, 2001; Anttila and Kerminen, 2003] have indicated that the condensation of sulfuric acid alone is often not sufficient to grow these nuclei to detectable sizes, since these particles have a very short lifetime before being lost through coagulation with larger existing particles. To aid in the growth of these particles, the condensation of organic species [Kerminen et al., 2000], heterogeneous reactions [Zhang and Wexler, 2002], and ion-enhanced condensation have been suggested.

[4] During the Pittsburgh Air Quality Study (PAQS) [Wittig et al., 2004], a recent field campaign focused on elucidating aerosol sources, chemistry, and processes, ultrafine particle concentrations were monitored for 15 months. During this period, over 130 days with regional in situ new particle formation were observed. An example is shown in 


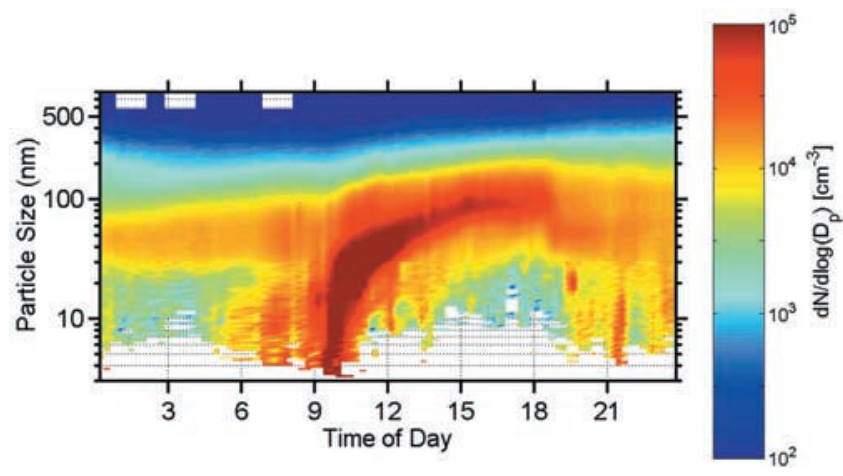

Figure 1. Evolution of the particle size distribution on 11 August 2001, a day with new particle formation and growth. Particle number concentration ( $z$ axis) is plotted against time of day ( $x$ axis) and particle diameter ( $y$ axis). A traffic signal can be seen between 0700 and 0800 EST, followed by new particle formation occurring at just after $0900 \mathrm{EST}$, and finally growth to around $100 \mathrm{~nm}$ in size.

Figure 1, with the smooth progression from small particle sizes at the beginning of events to larger sizes strongly suggesting a regional process.

[5] In this paper, data taken during July 2001 and January 2002 during PAQS is used to evaluate a model for the simulation of these regional nucleation events. Ternary $\mathrm{NH}_{3}-\mathrm{H}_{2} \mathrm{SO}_{4}-\mathrm{H}_{2} \mathrm{O}$ has been cited as an explanatory mechanism for new particle formation in several field studies, and the mechanism is used here as the core of an aerosol dynamics model to quantitatively test model-measurement agreement for new particle formation timing, strength, and evolution of size distributions. Sensitivity analysis is performed on uncertain parameters and to determine how future changes in emissions might affect nucleation, and the differences in nucleation between the summer and winter months are also explored.

\section{Model Description}

[6] A photochemical box model based on the framework of Capaldo et al. [1999] is used to model regional nucleation events occurring in July 2001 and January 2002. Atmospheric processes included in the model are gas-phase chemistry, condensation, aerosol coagulation, and nucleation. The model is a fixed sectional model with 221 size sections: 186 size sections between $3 \mathrm{~nm}$ and $10 \mu \mathrm{m}$ corresponding to the measured size distribution of particles and 35 additional size sections evenly distributed in logarithmic space by diameter for particles below the detection limit of the field instruments. The concentration of $\mathrm{SO}_{2}$ was measured directly and is an input to the model and the input concentrations of $\mathrm{NH}_{3}$ and $\mathrm{OH}$ were calculated from measurement data and are discussed in section 3. The governing equations for the model, giving the change over time of the concentration of gaseous sulfuric acid, $\mathrm{C}_{\mathrm{H}_{2} \mathrm{SO}_{4}}$, and the number concentration of particles in section $i, N_{i}$, are:

$$
\begin{aligned}
\frac{\partial \mathrm{C}_{\mathrm{H}_{2} \mathrm{SO}_{4}}}{\partial t}= & R_{\text {gas }}\left(\mathrm{SO}_{2}, \mathrm{OH}, \mathrm{T}, \mathrm{P}\right)+n^{*} R_{\text {nuc }}\left(\mathrm{C}_{\mathrm{H}_{2} \mathrm{SO}_{4}}, \mathrm{NH}_{3}, \mathrm{~T}, \mathrm{RH}\right) \\
& +R_{\text {cond }}^{\mathrm{H}_{2} \mathrm{SO}_{4}}\left(\mathrm{C}_{\mathrm{H}_{2} \mathrm{SO}_{4}}, \mathrm{RH}\right)-R_{\text {dep }}\left(\mathrm{C}_{\mathrm{H}_{2} \mathrm{SO}_{4}}\right)
\end{aligned}
$$

$$
\begin{aligned}
\frac{\partial N_{i}}{\partial t}= & R_{\text {nuc }}\left(C_{\mathrm{H}_{2} \mathrm{SO}_{4}}, \mathrm{NH}_{3}, T, R H\right)+R_{\text {coag }}\left(N_{j}, R H\right) \\
& +R_{\text {cond }}^{N_{i}}\left(C_{\mathrm{H}_{2} \mathrm{SO}_{4}}, R H\right)-R_{\text {dep }}\left(N_{i}\right),
\end{aligned}
$$

where $R_{\text {gas }}$ is the rate of change due to gas-phase chemistry, $R_{n u c}$ is the rate of nucleation, $n^{*}$ is the number of sulfuric acid molecules in the critical nucleus, $R_{\text {coag }}$ is the coagulation rate, $R_{\text {cond }}$ is the condensation rate, and $R_{d e p}$ is the rate of dry deposition, with dependence on the relative humidity $\mathrm{RH}$, temperature $T$, pressure $\mathrm{P}$, dry particle size distribution, $N_{j}$, and gas-phase concentrations shown. The rate terms are discussed in detail below.

\subsection{Gas-Phase Chemistry}

[7] Sulfuric acid is produced from the reaction of $\mathrm{OH}$ and $\mathrm{SO}_{2}$ :

$$
R_{\text {gas }}=k_{\mathrm{SO}_{2}}\left[\mathrm{SO}_{2}\right][\mathrm{OH}]
$$

where $k_{\mathrm{SO}_{2}}$ is the reaction constant for the reaction of $\mathrm{SO}_{2}$ with OH from DeMore et al. [1994].

\subsection{Nucleation}

[8] The rate of nucleation, $R_{\text {nuc }}$, is calculated using the ternary $\mathrm{NH}_{3}-\mathrm{H}_{2} \mathrm{SO}_{4}-\mathrm{H}_{2} \mathrm{O}$ parameterization of Napari et al. [2002]. The parameterization uses the $\mathrm{NH}_{3}$ gas-phase concentration, $\mathrm{H}_{2} \mathrm{SO}_{4}$ gas-phase concentration, temperature, and relative humidity as inputs, and provides a nucleation rate as output. The upper limit of the nucleation rate for which the parameterization is valid is $10^{6}$ particles $\mathrm{cm}^{-3} \mathrm{~s}^{-1}$, so rates higher than this are capped at this value. Napari et al. [2002] also give an approximation for the radius of the initial nuclei as a function of the nucleation rate and temperature. The initial nuclei diameter was calculated to be $0.8 \mathrm{~nm}$ for the winter and $1.0 \mathrm{~nm}$ during the summer, assuming average temperatures of $275 \mathrm{~K}$ and $298 \mathrm{~K}$, respectively. The nuclei diameter was assumed to be constant for each period. The number of sulfuric acid molecules in the critical nucleus, $n^{*}$, is also calculated using the approximation given in the work of Napari et al. [2002]. There were roughly two sulfuric acid molecules in the critical cluster during the winter and four during the summer.

[9] Figure 2a shows the nucleation rates given by the ternary $\mathrm{NH}_{3}-\mathrm{H}_{2} \mathrm{SO}_{4}-\mathrm{H}_{2} \mathrm{O}$ parameterization for typical July daytime conditions: $298 \mathrm{~K}, 60 \% \mathrm{RH}$, and $\mathrm{H}_{2} \mathrm{SO}_{4}$ from $10^{7}$ to $3 \times 10^{8}$ molecules $/ \mathrm{cm}^{3}$ (0.4 to $\left.4.0 \mathrm{ppt}\right)$. Ammonia is allowed to vary over the entire range of the parameterization, 0.1 to $100 \mathrm{ppt}$. The nucleation rates are not high enough for nucleation to occur unless some gas-phase ammonia is present, although ammonia concentrations above $10 \mathrm{ppt}$ and sulfuric concentrations above $2.4 \mathrm{ppt}$ result in nucleation rates of at least 10 particles $\mathrm{cm}^{-3} \mathrm{~s}^{-1}$, so even this small amount of gas-phase ammonia can be enough for nucleation to occur on summer days in Pittsburgh.

[10] In contrast to July, where the presence of gas-phase ammonia gives the best indication of when nucleation will occur, both the $\mathrm{H}_{2} \mathrm{SO}_{4}$ concentrations and gas-phase ammonia can influence whether or not nucleation will occur during the winter. Figure $2 \mathrm{~b}$ shows the nucleation rates for typical January conditions: $275 \mathrm{~K}, 0.7 \mathrm{RH}, 10^{5}-$ 

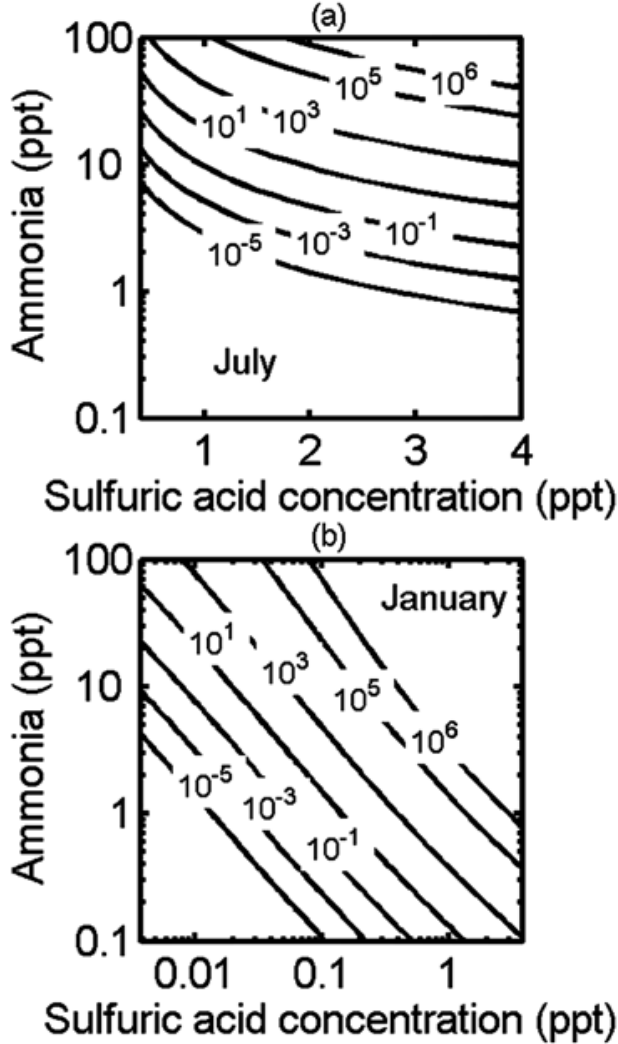

Figure 2. Nucleation rates (in particles $\mathrm{cm}^{-3} \mathrm{~s}^{-1}$ ) for typical conditions in (a) July and (b) January. The conditions for July are: temperature $298 \mathrm{~K}$, relative humidity 0.6, sulfuric acid 0.4-4 ppt, and gas-phase ammonia 0.1-100 ppt. The conditions for January are: temperature $275 \mathrm{~K}$, relative humidity 0.7 , sulfuric acid $0.00375-3.75 \mathrm{ppt}$, and gas-phase ammonia $0.1-100 \mathrm{ppt}$.

$10^{8}$ molecules $/ \mathrm{cm}^{3} \mathrm{H}_{2} \mathrm{SO}_{4}$ (0.00375 to $3.75 \mathrm{ppt}$ ), and $0.1-$

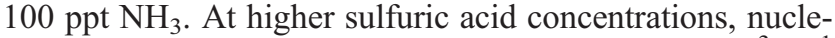
ation rates can still reach about 1000 particles $\mathrm{cm}^{-3} \mathrm{~s}^{-1}$ even with very low gas-phase ammonia concentrations. At lower sulfuric concentrations, nucleation rates can be less than $100 \mathrm{~cm}^{-3} \mathrm{~s}^{-1}$ even with high gas-phase ammonia concentrations. Consequently, the amount of $\mathrm{H}_{2} \mathrm{SO}_{4}$, which varies more than in the summer months, plays a more important role during the winter.

\subsection{Condensation}

[11] The condensation rate, $R_{c n d}$, is described using the modified form of the Fuchs-Sutugin equation [Fuchs and Sutugin, 1971; Hegg, 1990; Kreidenweis et al., 1991]. The condensation rate $J$ to a particle of diameter $D_{p}$ is given by:

$$
J=2 \pi D D_{p} F(K n) A\left(p-p_{0}\right),
$$

where $D$ is the diffusivity of sulfuric acid in air (set to $\left.0.1 \mathrm{~cm}^{2} \mathrm{~s}^{-1}\right), K n$ is the Knudsen number, and $F(K n)$ is a coefficient correcting for free molecular effects:

$$
F(K n)=\frac{1+K n}{1+1.71 K n+1.33 K n^{2}} .
$$

$A$ is a coefficient correcting for the interfacial mass transport limitations described by the accommodation coefficient $a_{e}$ :

$$
A=\left[1+1.33 K n F(K n)\left(\frac{1}{a_{e}}-1\right)\right]^{-1} .
$$

Here $p$ is the bulk partial pressure of sulfuric acid and $p_{0}$ is its partial pressure at the particle surface. The value of the accommodation coefficient depends on the composition of the particle, with the presence of organic species in the aerosol likely to result in a lower accommodation coefficient. Jefferson et al. [1997] report values of 0.31 and 0.19 for the accommodation coefficient onto a $\mathrm{NaCl}$ aerosol coated with stearic acid with high and low coverage, respectively, compared to values $0.73 \pm 0.21$ for ammonium sulfate particles and $0.79 \pm 0.23$ for $\mathrm{NaCl}$. Since the preexisting aerosol mass is likely to be a mixture of inorganic and organic species, the accommodation coefficient is set at 0.2 in this work. Sensitivity analysis is performed to see how changes in this parameter affect the model results.

[12] $\mathrm{H}_{2} \mathrm{SO}_{4}$ is assumed to be the major condensing species. Although it is likely that other species, such as nitrate, ammonium, and organic compounds, also are involved in the growth of the nuclei, their respective roles remain unclear. Measurements taken during PAQS in September 2002 indicate that sulfuric acid is the primary initial species involved in the growth of the nuclei, followed by ammonium and then organics, whose presence lags behind sulfuric acid by .5 hours to up to 2.5 hours in the smallest measured particles (AMS measurements of particles 22$40 \mathrm{~nm}$, estimated physical diameter) [Zhang et al., 2004]. Although contributions of other species cannot be ruled out, condensation of sulfuric acid alone produces growth that is similar to the observations, as will be discussed in more detail below.

[13] The vapor pressure of sulfuric acid at the surface of the aerosol can be estimated from the data of Bolsaitis and Elliott [1990]. For example, at a temperature of $293 \mathrm{~K}$ and a relative humidity of $90 \%$, the value of $p_{0}$ is approximately $10^{-5} \mathrm{ppt}$. Since the calculated values of $p_{0}$ are much smaller than ambient sulfuric acid concentrations, $p_{0}$ is assumed to be zero.

[14] For the $i$ th aerosol size section from $x_{i}=\log _{10}\left(D_{i}\right)$ to $x_{i+1}=\log _{10}\left(D_{i+1}\right)$, the sulfuric acid condensation rate is given by

$$
J_{i}=K_{m t}^{i} N_{i} C_{H_{2} S_{4}},
$$

where the sectional mass transfer coefficients $K_{m t}^{i}$ are calculated by

$$
K_{m t}^{i}=\frac{2 \pi D}{x_{i+1}-x_{i}} \int_{x}^{x_{i+1}} 10^{x} F(x) A(x) d x .
$$

The total change in the gas-phase concentration of sulfuric acid due to condensation is equal to the sum of $J_{i}$ over all size sections $i$.

[15] The diameter of the aerosol particle is adjusted to be in equilibrium with the ambient RH before the calculation. 
The dry aerosol diameter, $D_{p, d r y}$, is increased due to the addition of water vapor according to the following parameterization for ammonium bisulfate based on data presented by Seinfeld and Pandis [1998]:

$$
\frac{D_{p, w e t}}{D_{p, d r y}}=17.8(R H-50 \%)^{3.5}+1.28 .
$$

The parameterization is valid for $\mathrm{RH}$ between $50 \%$ and $98 \%$. When $\mathrm{RH}$ is below $50 \%$, the particles are assumed to have negligible amounts of water. This parameterization neglects the effect of curvature, which can be significant for particles less than $10 \mathrm{~nm}$ in size.

[16] Finally, the change in the number concentration in section $i, N_{i}$, is calculated from the relation:

$$
R_{\text {cond }}^{N_{i}}=F_{i-1} N_{i} C_{H_{2} \mathrm{SO}_{4}}-F_{i} N_{i} C_{H_{2} \mathrm{SO}_{4}},
$$

where $F_{i}$ is the flux from section $i$ into section $\mathrm{i}+1$ :

$$
F_{i}=\frac{6 M_{\mathrm{H}_{2} \mathrm{SO}_{4}} K_{m t}^{i} D_{p, w e t}}{R T \rho \pi\left[\left(D_{p, d r y}^{i+1}\right)^{3}-\left(D_{p, d r y}^{i}\right)^{3}\right]} .
$$

$M_{\mathrm{H}_{2} \mathrm{SO}_{4}}$ is the molecular weight of sulfuric acid, $\rho$ is the density of the particles, and $R$ is the ideal gas constant.

\subsection{Coagulation}

[17] The coagulation rate of aerosol particles, $R_{\text {coag, }}$, is modeled according to Seinfeld and Pandis [1998], using linear interpolation to preserve both mass and particle number concentrations:

$$
R_{\text {coag }}=\frac{1}{2} \sum_{j=1}^{k} f_{k} K_{j, k-j} N_{j} N_{k-j}-N_{k} \sum_{j=1}^{\infty} K_{k, j} N_{j}, \quad k \geq 2
$$

where $K_{k, j}$ is the coagulation coefficient of particles in section $k$ and $j$, and $f_{k}$ is a correction factor to preserve mass, giving the fraction of the newly formed particle that will go into section $k$. The generalized coagulation coefficient for the collision of two particles is defined as:

$$
K_{12}=2 \pi\left(D_{p 1}+D_{p 2}\right)\left(D_{1}+D_{2}\right) \beta
$$

where $\beta$ is the Fuchs correction factor [Fuchs, 1964]. Linear interpolation is then used to determine the value of $f_{k}$. For example, if $V_{p}$ is in between sections $k$ and $k+1$, with volumes $V_{k}$ and $V_{k+1}$ respectively, then $f_{k}$ is defined as:

$$
\begin{gathered}
f_{k}=\frac{V_{k+1}-V_{p}}{V_{k+1}-V_{k}}, \\
f_{k+1}=\frac{V_{p}-V_{k}}{V_{k+1}-V_{k}} .
\end{gathered}
$$

\subsection{Dry Deposition}

[18] Dry deposition is modeled using a species and aerosol size-dependent deposition velocity, $v_{d e p}$, such that

$$
R_{d e p}=\frac{v_{d e p} c_{i}}{H},
$$

where $c_{i}$ is the concentration of gas species or aerosol size section $i$, and $H$ is the mixing height. Aerosol dry deposition rates range from 0.1 to $0.015 \mathrm{~cm} \mathrm{~s}^{-1}$ dependent on particle size according to Hummelshoj et al. [1992] while the deposition velocity of $\mathrm{H}_{2} \mathrm{SO}_{4}$ is assumed to be $1.0 \mathrm{~cm} \mathrm{~s}^{-1}$ [Brook et al., 1999].

\section{Simulation Inputs}

[19] Meteorological data, gas-phase concentrations, and aerosol number concentrations were available as inputs for these time periods from ground-level observations during the Pittsburgh Air Quality Study (PAQS) [Wittig et al., 2004]. Temperature, relative humidity, and UV radiation are updated from 15-min averages during July, and 10-min averages during January. $\mathrm{SO}_{2}$ concentrations are averaged over 10-min intervals for both months. Initial aerosol number distributions are available from dry size distributions measured every $15 \mathrm{~min}$ [Stanier et al., 2004a]. Each simulation starts sometime between 0000 and 0900 (depending on meteorology), and ends at midnight.

\subsection{Gas-Phase Ammonia Concentrations}

[20] Gas-phase $\mathrm{NH}_{3}$ was not measured directly during this period and was estimated from related quantities using two independent methods. First, total $\mathrm{NH}_{3}\left(\mathrm{PM}_{2.5}\right.$ ammonium $+\mathrm{NH}_{3}(\mathrm{~g})$, sampled hourly during July and every 2 hours during January using a steam sampler [Khylstov et al., 1995] and quantified by ion chromatography) was compared to the particulate sulfate and nitrate. Hourly particulate sulfate and nitrate concentrations are composites of measurements made using several methods, including filterbased and semicontinuous methods [Wittig et al., 2004]. If enough $\mathrm{NH}_{3}$ was present to fully neutralize the particulate sulfate and nitrate, then the excess $\mathrm{NH}_{3}$ was assumed to be in the gas phase. Second, total $\mathrm{NH}_{3}$, total nitrate and sulfate (all from steam sampler/ion chromatography) were assumed to be in thermodynamic equilibrium, with gas and particulate phase concentrations determined by the model GFEMN [Ansari and Pandis, 1999]. The two methods showed excellent agreement for the time periods studied.

\subsection{OH Concentration}

[21] To estimate the $\mathrm{OH}$ concentration, a maximum value is assumed for both July and January, and then this value is scaled linearly based on the 15-min average measured UV radiation. The summer maximum was chosen as $5 \times$ $10^{6}$ molecules $/ \mathrm{cm}^{3}$. Observations in New York City during July 2001 had an average maximum $\mathrm{OH}$ concentration of $7 \times 10^{6}$ molecules $/ \mathrm{cm}^{3}$ [Ren et al., 2003a, 2003b] and other measurements during the summer months have given maximum $\mathrm{OH}$ concentrations in a similar range [Tan et al., 2001; Creasey et al., 2002; Mauldin et al., 2001; George et al., 1999]. Fewer studies have been done in the winter, although Heard et al. [2001] have compared measurements 


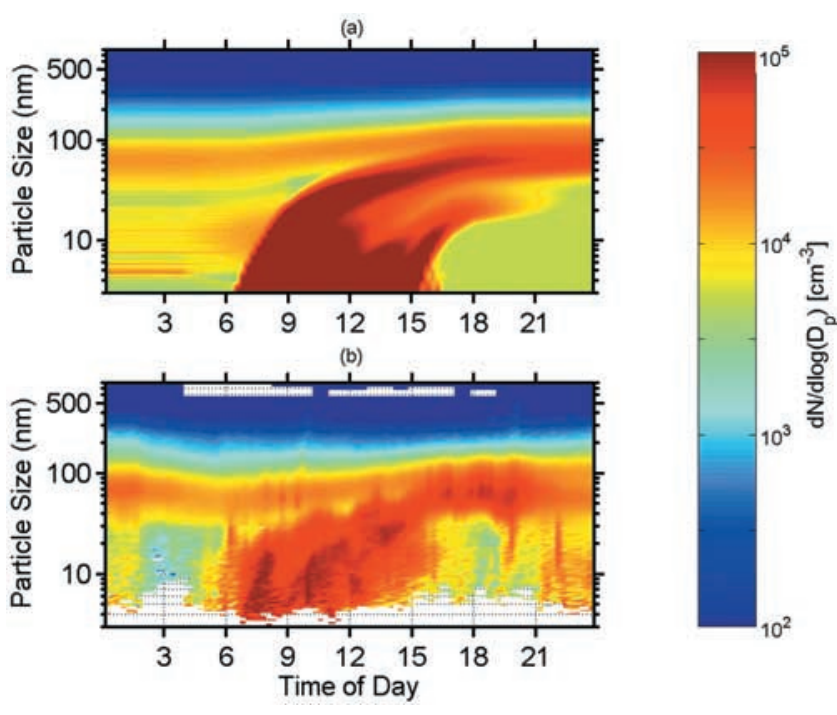

Figure 3. Comparison of modeled (a) and measured (b) size distributions as a function of time for one day with nucleation activity (27 July 2001). Particle number ( $z$ axis) is plotted against time of day ( $x$ axis) and particle size ( $y$ axis). The increased number concentrations observed between 0600 and 0700 EST and after 2100 EST are due to local emissions of ultrafine particles. The observed onset of nucleation at $\sim 0700$ EST is captured in the model, and the qualitative features of particle growth are also captured by the model.

taken in Birmingham, England during June 1999 and January 2000. They reported that winter $\mathrm{OH}$ noontime maximum concentrations in Birmingham ranged from $0.6-5 \times 10^{6}$ molecules $/ \mathrm{cm}^{3}$. Although the ozone photolysis was around 20 times lower compared to July, these winter $\mathrm{OH}$ concentrations were on average only $2-3$ times lower than in the summer, where the noontime maxima ranged from $3-8 \times 10^{6}$ molecules $/ \mathrm{cm}^{3}$. In this paper, a base case value of $1 \times 10^{6}$ molecules $/ \mathrm{cm}^{3}$ is used for the January simulations, a factor of 5 different from July. Sensitivity analysis is performed on both the summer and winter maxima to assess how these assumptions affect the results.

\subsection{Local Emissions of Ultrafine Particles}

[22] From the measurements, it is clear that there are a number of local sources of ultrafine particles which affect the observed number concentrations. The most prominent local source is automobile traffic, which is strongest during morning rush hour between 0600 and 0900 EST. On the basis of the observations, these local emissions appear to have little effect on the regional nucleation events, which usually begin after the morning traffic has returned to normal levels. Even when there are relatively high numbers of ultrafine particles present when nucleation occurs, the number of particles produced by nucleation is much greater than from local sources, and the smooth growth of these nucleated particles is the dominant behavior observed. The fresh particles from these local sources have a relatively low surface area, and they do not appear to have much effect on the nucleation either through depletion of gaseous sulfuric acid concentrations or through coagulation with the nucle- ated particles. Because of this, these local sources of ultrafine particles can be ignored as a first approximation.

\section{Observations}

[23] Overall, significant new particle formation was observed on seventeen days during July 2001. Twelve days of July 2001 were not modeled due to missing measurement data ( 8 days), local primary source influences (3 days), and meteorology that severely violated the box model assumptions (1 day). Of the 19 days modeled, nucleation occurred on 13 of them, while there was no evidence of new particle formation on the other 6 . Most of the nucleation events in July started at about 0900 EST, just a few hours after sunrise. Nucleation also tends to occur on days with below average $\mathrm{PM}_{2.5}$ concentrations and clear skies [Stanier et al., 2004b]. Many of these events are regional in nature, taking place over several hours with smooth growth, indicating that nucleation is taking place over a large geographic area. The events are observed to sometimes coincide with mixing of the stable nighttime ground-level atmosphere. This is similar to observations of nucleation coincident with atmospheric mixing seen in Germany [Birmili et al., 2003] and Finland [Nilsson et al., 2001] and suggests that vertical mixing can be important to nucleation. However, a significant fraction of nucleation events observed in Pittsburgh did not coincide with atmospheric mixing, and occurred either earlier or later than the rise in boundary layer height [Stanier et al., 2004b]. The degree to which vertical transport is important to nucleation in the eastern United States is currently unknown and vertical transport is not included in the box model developed here.

[24] In January, 19 days had no observed nucleation. The first 2 days of the month cannot be modeled because of missing data, leaving 17 days with no nucleation and 12 days where nucleation was observed in our data set. Nucleation typically began around noon, presumably once sufficient sulfuric acid was produced for nucleation and growth to occur. The nucleation events tended to be weaker and shorter-lived than during the summer months, and particles did not grow as much. This is consistent with lower levels of sulfuric acid (predicted by the model) and lower secondary organic aerosol during the winter [Cabada et al., 2002].

\section{Results}

\subsection{Base Case}

[25] An example simulation is shown for 27 July 2001, when new particle formation occurred over a 9-hour span (Figure 3), beginning shortly after 0600 and continuing to nearly 1500 EST. The model prediction compares well with the observed behavior, with the timing of the event and the size and shape of the growth curve similar to the observations. The model does predict higher number concentrations, however, producing a peak hourly average of $93,000 \mathrm{~cm}^{-3}$ for particles greater than $10 \mathrm{~nm}$ in diameter, compared to a maximum of $47,000 \mathrm{~cm}^{-3}$ from the measurements, and the model-predicted nucleation is also stronger between noon and 1500 EST.

[26] As shown in Table 1, the model successfully predicted the presence or lack of new particle formation on all 
Table 1. Predicted and Observed New Particle Formation During July 2001

\begin{tabular}{|c|c|c|c|c|c|c|c|c|c|}
\hline \multirow{2}{*}{$\begin{array}{c}\text { Day of } \\
\text { July } \\
2001 \\
\end{array}$} & \multirow{2}{*}{$\begin{array}{l}\text { Observed } \\
\text { Strength of } \\
\text { Nucleation }^{\mathrm{a}}\end{array}$} & \multicolumn{2}{|c|}{ Time of Nucleation, EST } & \multicolumn{2}{|c|}{$\begin{array}{c}\text { Final Mode Diameter of } \\
\text { Nucleation Mode, } \mathrm{nm} \\
\end{array}$} & \multicolumn{2}{|c|}{$\begin{array}{c}\text { Daily Average Number } \\
\text { Concentration }>10 \mathrm{~nm} \text {, } \\
10^{3} \mathrm{~cm}^{-3}\end{array}$} & \multicolumn{2}{|c|}{$\begin{array}{c}\text { Peak Hourly Number } \\
\text { Concentration }>10 \mathrm{~nm}, \\
10^{3} \mathrm{~cm}^{-3} \\
\end{array}$} \\
\hline & & Observed & Predicted & Observed & Predicted & Observed & Predicted & Observed & Predicted \\
\hline 1 & moderate & 0900 & 0800 & 20 & 60 & 10 & 53 & 19 & 158 \\
\hline 2 & weak & 0800 & 0800 & 80 & 60 & 21 & 50 & 32 & 150 \\
\hline 3 & weak & 0900 & 0900 & 10 & $65^{b}$ & 17 & $50^{\mathrm{b}}$ & 22 & $184^{\mathrm{b}}$ \\
\hline 4 & none & none & none & - & - & 9 & 6 & 12 & 8 \\
\hline 6 & weak & 0900 & 0900 & 60 & 65 & 24 & 54 & 42 & 159 \\
\hline 7 & none & none & none & - & - & 12 & 13 & 26 & 20 \\
\hline 8 & none & none & none & - & - & 11 & 10 & 18 & 11 \\
\hline 9 & strong & 1000 & 0900 & 90 & 100 & 24 & 73 & 54 & 259 \\
\hline 11 & moderate & 0900 & 0900 & 40 & 50 & 24 & 35 & 47 & 105 \\
\hline 12 & moderate & 0900 & 0900 & 70 & 60 & 21 & 59 & 37 & 164 \\
\hline 13 & weak & 0900 & 0900 & 50 & 40 & 19 & 37 & 27 & 86 \\
\hline 14 & strong & 0800 & 0900 & 60 & 60 & 19 & 46 & 36 & 127 \\
\hline 15 & moderate & 0800 & 0900 & 50 & 60 & 25 & 48 & 44 & 145 \\
\hline 18 & none & none & none & - & - & 16 & 10 & 24 & 18 \\
\hline 19 & none & none & none & - & - & 11 & 7 & 17 & 17 \\
\hline 23 & none & none & none & - & - & 8 & 9 & 16 & 17 \\
\hline 24 & moderate & 0800 & 0800 & 70 & 80 & 19 & 47 & 44 & 170 \\
\hline 26 & moderate & 1600 & 1700 & 40 & 20 & 17 & 31 & 35 & 108 \\
\hline 27 & strong & 0600 & 0600 & 70 & 70 & 28 & 45 & 47 & 140 \\
\hline
\end{tabular}

${ }^{a}$ Events were classified as no new particle formation, weak, moderate, or strong according to the observed rate of change of particle number between 3

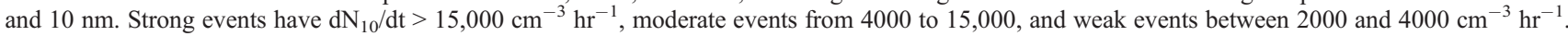
No new particle formation refers to a $\mathrm{dN}_{10} / \mathrm{dt}$ that was not distinguishable from the natural variability in nuclei mode particle concentrations due to local primary sources. Usually the noise threshold was about $2000 \mathrm{~cm}^{-3} \mathrm{hr}^{-1}$.

${ }^{b}$ Multiple air masses moved through the region on this day, probably violating the box model assumptions. This day was modeled to see if the observed 0900 burst in number concentration was replicated by the model, rather than to compare the observed and modeled growth.

19 modeled days in July. The timing of the events also compared well with the observations, with the model predicting the start of new particle formation within 1 hour for all 13 events. The size and shape of the growth curve were consistent with measurements, with the predicted average final size of the nucleated particles within $10 \mathrm{~nm}$ of the actual size in 9 out of the 13 nucleation days. Three of the days with poor agreement are days where observed growth is significantly less than the majority of days. Two of these days (the 1st and 3rd) are characterized by fronts moving through the region, which could effect the performance of the model, and on the other day nucleation is delayed until late afternoon because of cloud cover (26th).

[27] A strong correlation between the presence of free $\mathrm{NH}_{3}$ in the gas phase and new particle formation contributed to the success of the model in July. During the 13 days where particle production was observed, gas-phase $\mathrm{NH}_{3}$ concentrations exceeded $100 \mathrm{ppt}$ while nucleation was occurring. Although Pittsburgh has a high average sulfate concentration, on the 13 days in question there was sufficient $\mathrm{NH}_{3}$ to neutralize the aerosol sulfate. In contrast, during the 6 days without new particle formation, the aerosol sulfate levels were higher, leaving less $\mathrm{NH}_{3}$ in the gas-phase (typically a few ppt). Other parameters, such as $\mathrm{SO}_{2}$, temperature, relative humidity, and UV radiation, all varied widely over the nineteen days. The preexisting surface area tended to be less on the days with particle formation than those without, although there was some overlap.

[28] The ternary $\mathrm{NH}_{3}-\mathrm{H}_{2} \mathrm{SO}_{4}-\mathrm{H}_{2} \mathrm{O}$ parameterization is also successful in predicting the presence of nucleation in January (Table 2). Peak and average number concentrations are not shown for the month of January because the local sources of ultrafine particles occasionally produce similar number of particles as the nucleation events, which produce fewer particles than in July. The difference between particles produced by these local sources and the nucleation events is clear, however, and there appears to be no interaction between the particles produced by these different phenomena. The model is successful in predicting 11 of the 12 observed events, with the exception being the 14th. This day has a sudden drop in surface area shortly after the observed nucleation begins, which is difficult to capture in the model. The model also suffers from three "false positive" cases in January, predicting nucleation events on 3 days where it was not observed in the field. Two of these predicted events are very weak and do not exhibit growth above $10 \mathrm{~nm}$ (the 24th and 29th), while the particles from the third additional event grow only to $15 \mathrm{~nm}$ in size.

[29] A comparison of the model prediction and observations for 28 January 2002 is shown in Figure 4. The predicted onset of nucleation compares well with the observations, beginning shortly after noon. The predicted nucleation event lasts about 1.5 hours longer, and produces more particles than observed. This overprediction of number concentrations is typical in January, as it was in July. In addition, the overall timing of the events does not agree as well as in July, with one event predicted to occur 3 hours earlier than the observed event (on the 9th) and two other events occurring at least 2 hours before nucleation is observed. Most of the predicted nucleation events show growth that is similar or less than the observed growth, although two events show considerably more growth than observed (the 18th and 22nd).

[30] The good agreement with the growth of the particles is somewhat surprising, with sulfuric acid as the only condensing species included in the model. However, due the cubic relation between diameter and volume seemingly 
Table 2. Predicted and Observed New Particle Formation During January 2002

\begin{tabular}{|c|c|c|c|c|c|}
\hline \multirow[b]{2}{*}{ Day of January 2002} & \multirow{2}{*}{$\begin{array}{c}\text { Observed Strength } \\
\text { of Nucleation }\end{array}$} & \multicolumn{2}{|c|}{ Time of Nucleation, EST } & \multicolumn{2}{|c|}{$\begin{array}{l}\text { Final Mode Diameter of } \\
\text { Nucleation Mode, nm }\end{array}$} \\
\hline & & Observed & Predicted $^{\mathrm{a}}$ & Observed & Predicted $^{\mathrm{a}}$ \\
\hline 3 & none & - & - & - & - \\
\hline 4 & none & - & - & - & - \\
\hline 5 & weak & 1200 & 1100 & 20 & 20 \\
\hline 6 & none & - & - & - & - \\
\hline 7 & none & - & - & - & - \\
\hline 8 & none & - & - & - & - \\
\hline 9 & weak & 1200 & 1200 & 30 & 20 \\
\hline 10 & none & - & - & - & - \\
\hline 11 & none & - & - & - & - \\
\hline 12 & weak & 1300 & 1000 & 30 & 20 \\
\hline 13 & moderate & 1200 & 1330 & 25 & $15-20$ \\
\hline 14 & weak & 1100 & - & 20 & - \\
\hline 15 & none & - & - & - & - \\
\hline 16 & none & - & - & - & - \\
\hline 17 & none & - & - & - & - \\
\hline 18 & weak & 1300 & 1330 & 8 & $10-15$ \\
\hline 19 & none & - & 1100 & - & $10-15$ \\
\hline 20 & none & - & - & - & - \\
\hline 21 & none & - & - & - & - \\
\hline 22 & moderate & 1200 & 0930 & 25 & 40 \\
\hline 23 & none & - & - & - & - \\
\hline 24 & none & - & 1330 & - & 10 \\
\hline 25 & strong & 1130 & 1000 & 30 & 25 \\
\hline 26 & strong & 0915 & 1000 & 25 & 25 \\
\hline 27 & strong & 1100 & 1030 & 30 & 20 \\
\hline 28 & moderate & 1200 & 1230 & 25 & 20 \\
\hline 29 & none & - & 1300 & - & 8 \\
\hline 30 & none & - & - & - & - \\
\hline 31 & weak & 1200 & 1000 & $10-15$ & 15 \\
\hline
\end{tabular}

${ }^{\mathrm{a}}$ To classify as an event, the predicted nucleation had to last for at least an hour and produce particles that grew to at least 8 nm in size (the smallest size for an observed event). Events that do not meet these criteria would probably not be observed.

large differences in mass will not greatly affect the diameter. For example, AMS measurements indicate that the final mode of the particles (around $100 \mathrm{~nm}$ ) for three measured events in September 2002 are approximately 25\%, 25\%, and $40 \%$ organic by mass [Zhang et al., 2004]. Leaving out this organic mass would result in a reduction of only $9 \%$ to $16 \%$ in the final diameter of the particles. Thus it is possible that organic and other inorganic species are also involved in the growth, although sulfuric acid alone appears to be sufficient to grow the particles to significant sizes in Pittsburgh during the periods studied.

\subsection{Sensitivity to Changes in the Maximum $\mathrm{OH}$}

[31] One of the major uncertainties in the model is $\mathrm{OH}$ concentration. The July simulations were repeated with maximum $\mathrm{OH}$ concentrations of $10^{7}$ molecules $/ \mathrm{cm}^{3}(200 \%$ of the base case value) and $2.5 \times 10^{6}$ molecules $/ \mathrm{cm}^{3}(50 \%$ of the base case value). No change was seen in the number of nucleation events for either of these additional cases, with the model still predicting nucleation to occur on the same 13 days (68\% of the modeled days). The growth of the particles changed significantly, however. A maximum $\mathrm{OH}$ concentration of $10^{7}$ molecules $/ \mathrm{cm}^{3}$ resulted in significantly more growth than in the base case simulations. For 12 of the 13 events, the nucleated particles grew to at least $80 \mathrm{~nm}$, with the final mode of nine of these events at least $100 \mathrm{~nm}$ in size. With a maximum $\mathrm{OH}$ concentration of $2.5 \times 10^{6}$ molecules $/ \mathrm{cm}^{3}$, the final mode size of the nucleated particles is $20 \%$ less than for the base case on average.
[32] In January, both the number of nucleation events and the growth of the nucleated particles are sensitive to changes in the maximum $\mathrm{OH}$ concentration (Table 3). Again, the simulations are performed with $\mathrm{OH}$ levels $200 \%$ of the base

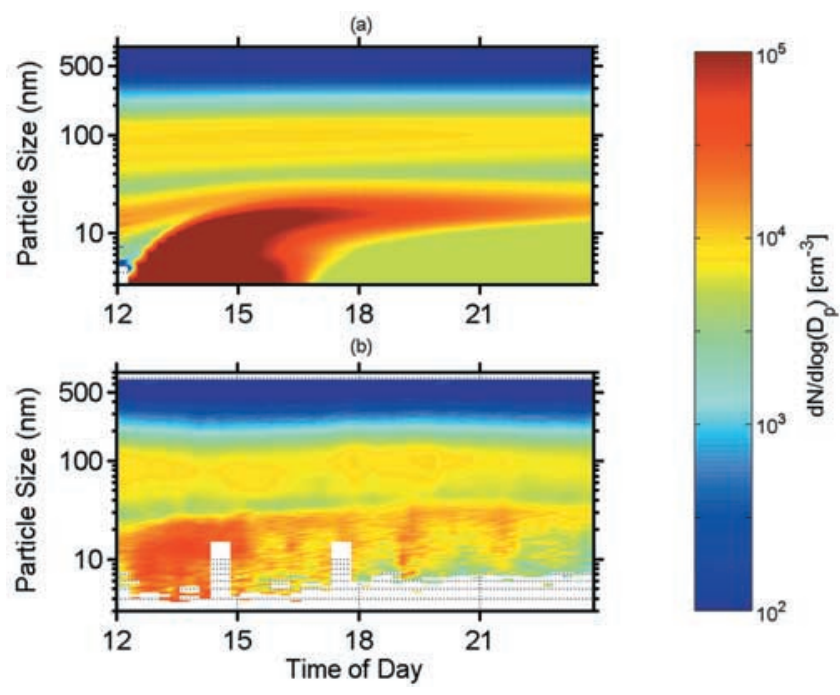

Figure 4. Comparison of modeled (a) and measured (b) size distributions as a function of time for a day in January with nucleation activity (28 July 2001). Particle number ( $z$ axis) is plotted against time of day ( $x$ axis) and particle size ( $y$ axis). 
Table 3. OH Sensitivity Analysis for January 2002

\begin{tabular}{|c|c|c|c|c|c|c|}
\hline \multirow{2}{*}{$\begin{array}{c}\text { Day of } \\
\text { January } 2002 \\
\end{array}$} & \multicolumn{2}{|c|}{$\mathrm{OH}, 0.5 \times 10^{6}$ molecules $/ \mathrm{cm}^{3}$} & \multicolumn{2}{|c|}{$\begin{array}{c}\mathrm{OH}, 1 \times 10^{6} \text { molecules } / \mathrm{cm}^{3} \\
(\text { Base Case) }\end{array}$} & \multicolumn{2}{|c|}{$\mathrm{OH}, 2 \times 10^{6}$ molecules $/ \mathrm{cm}^{3}$} \\
\hline & Time, EST & Final Mode & Time, EST & Final Mode & Time, EST & Final Mode \\
\hline 4 & - & - & - & - & 1100 & 25 \\
\hline 5 & - & - & 1100 & 20 & 0930 & 45 \\
\hline 6 & - & - & - & - & - & - \\
\hline 7 & - & - & - & - & - & - \\
\hline 8 & - & - & - & - & 1200 & 15 \\
\hline 9 & 1200 & 10 & 1200 & 20 & 1100 & 30 \\
\hline 10 & - & - & - & - & - & - \\
\hline 11 & - & - & - & - & - & - \\
\hline 12 & 1000 & 10 & 1000 & 20 & 0900 & 35 \\
\hline 13 & 1300 & 10 & 1330 & $15-20$ & 1330 & 25 \\
\hline 14 & - & - & - & - & 1100 & 9 \\
\hline 15 & - & - & - & - & 1300 & 10 \\
\hline 16 & - & - & - & - & 1100 & 15 \\
\hline 18 & - & - & 1330 & $10-15$ & 1300 & 25 \\
\hline 19 & - & - & 1100 & $10-15$ & 1030 & 25 \\
\hline 20 & - & - & - & - & 1030 & 15 \\
\hline 21 & - & - & - & - & 1000 & 15 \\
\hline 22 & 1000 & 20 & 1000 & 40 & 0900 & 55 \\
\hline 23 & - & - & - & - & - & - \\
\hline 24 & - & - & 1330 & 10 & 1300 & 20 \\
\hline 25 & 1100 & 10 & 1000 & 25 & 0930 & 45 \\
\hline 26 & 1200 & 15 & 1000 & 25 & 0930 & 45 \\
\hline 27 & 1130 & 10 & 1030 & 20 & 1030 & 40 \\
\hline 28 & 1230 & 9 & 1230 & 20 & 1200 & 30 \\
\hline 29 & - & - & 1300 & 8 & 0900 & 20 \\
\hline 30 & - & - & - & - & 1000 & 10 \\
\hline 31 & - & - & 1030 & 15 & 1000 & 30 \\
\hline
\end{tabular}

case value (a maximum of $2 \times 10^{6}$ moleclues $/ \mathrm{cm}^{3}$ ) and $50 \%$ of the base case value $\left(5 \times 10^{5}\right.$ molecules $\left./ \mathrm{cm}^{3}\right)$. The number of events predicted ranges from 8 with $\mathrm{OH}$ levels $50 \%$ of the base case (28\% of the modeled days) to 21 with $\mathrm{OH}$ levels $200 \%$ of the base case ( $72 \%$ of the days). The growth of the nucleated particles is significantly greater with a maximum $\mathrm{OH}$ concentration of $2 \times 10^{6}$ moleclues $/ \mathrm{cm}^{3}$, with 16 events having particles grow to at least $20 \mathrm{~nm}$ in size, compared to 8 for the base case. With a maximum $\mathrm{OH}$ concentration of $5 \times 10^{5}$ molecules $/ \mathrm{cm}^{3}$, particles from only 2 of the 8 events grow above $10 \mathrm{~nm}$ in size, and only 1 event exhibits growth to $20 \mathrm{~nm}$. The difficulties of the model in the simulation of a few winter days could be due to this sensitivity to the uncertain $\mathrm{OH}$ concentration. Because of the sensitivity of nucleation in January to the maximum $\mathrm{OH}$ concentrations, additional field measurements of $\mathrm{OH}$ would help to improve our understanding of nucleation during the winter months.

\subsection{Sensitivity to Accommodation Coefficient}

[33] Another source of uncertainty is the value of the accommodation coefficient. In addition to the base case value of 0.2 , simulations were run with the accommodation coefficient set to values of 0.5 and 1.0. Higher values of the accommodation coefficient will tend to increase the uptake of sulfuric acid onto the preexisting aerosol mass, lowering the gaseous sulfuric acid concentrations (and, consequently, the nucleation rates). The higher accommodation coefficient also has the potential to increase the growth of the nucleated particles, despite the lower sulfuric acid concentrations.

[34] In July, the average predicted sulfuric acid concentrations are reduced by a factor of 2 for an accommodation coefficient of 0.5 , and 3 for an accommodation coefficient of 1.0. The corresponding reductions in the nucleation rate are $22 \%$, and $31 \%$, respectively. However, with the presence of ammonia as the major factor in determining when nucleation occurs in July, the number of days which exhibit nucleation does not change. The final mode of the nucleated particles increases by about 5 nanometers for 2 of the 13 events with an accommodation coefficient of 0.5 , and for 5 of the 13 events with an accommodation coefficient of 1.0 .

[35] In January, changes in the accommodation coefficient had mixed effects, although the number of events was not affected. The average sulfuric acid concentrations between 1000 and 1400 are reduced by a similar amount, a factor of 2 for an accommodation of coefficient of 0.5 , and a factor of 3 using a value of 1.0. The nucleation rates are reduced by $8 \%$ and $15 \%$, respectively. The reduction does not affect the number of events. With an accommodation coefficient of 0.5 , four events are either shorter, weaker, or exhibit less growth than for the base case, while three events produced particles that grew larger than in the base case. Using an accommodation coefficient of 1.0 in the model, five events exhibit more growth than the base case, while four events are either weaker, shorter, or exhibit less growth. The final mode of the nucleated particles was within 5 to $10 \mathrm{~nm}$ of the base case in all cases.

\subsection{Binary Nucleation}

[36] Several binary $\left(\mathrm{H}_{2} \mathrm{SO}_{4}-\mathrm{H}_{2} \mathrm{O}\right)$ parameterizations were also used in the model for comparison. The parameterization of Vehkamäki et al. [2002] predicted no nucleation events in either the summer or winter. Comparisons with 
(a)

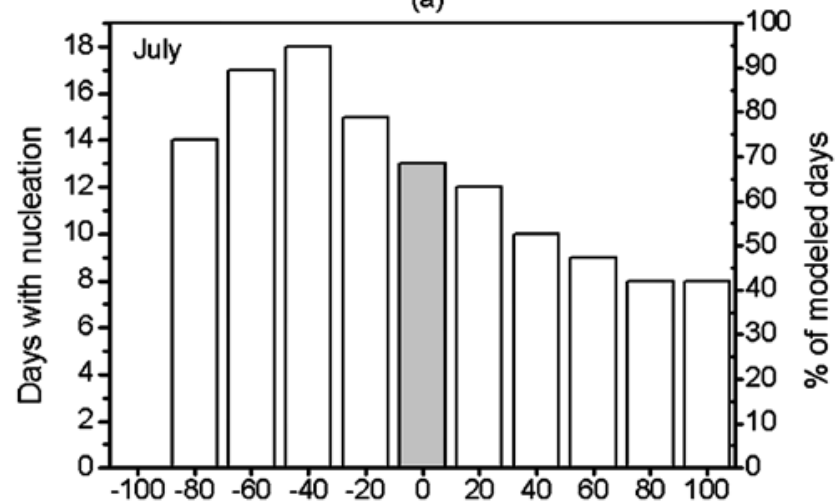

(b)

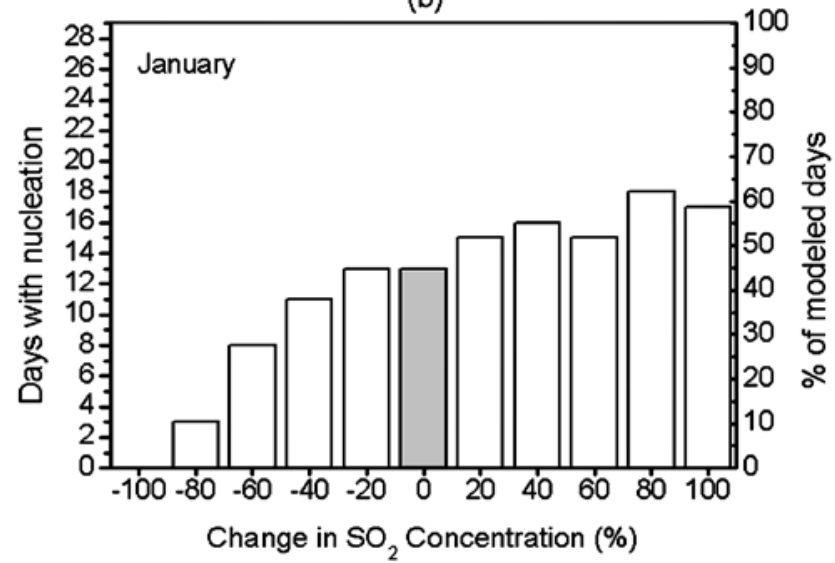

Figure 5. Sensitivity to changes in $\mathrm{SO}_{2}$ concentrations for (a) July and (b) January. The shaded bars represent the base case scenario.

nucleation thresholds given by Wexler et al. [1994] and Pirjola et al. [1999] also revealed that estimated sulfuric acid concentrations were below the threshold needed for $\mathrm{H}_{2} \mathrm{SO}_{4}-\mathrm{H}_{2} \mathrm{O}$ nucleation to occur in both months at the relative humidity and temperature corresponding to the ground level, as well as the top of the mixed layer [Stanier et al., 2004b]. Finally, the parameterization of JaeckerVoirol and Mirabel [1989] was applied, both with and without a nucleation tuner. Without the tuner, no nucleation events were predicted. Although there is a lot of uncertainty as to what value to use for the tuner, Raes et al. [1992] have suggested a value of between $10^{4}$ to $10^{7}$ to bring the results into better agreement with measurements. Even with a value of $10^{7}$, however, only four nucleation events were predicted in January, and no events were predicted in July.

[37] The poor performance of the binary models is consistent with results by other researchers who have found that sulfuric acid concentrations are generally not high enough to induce binary nucleation in the lower troposphere [Korhonen et al., 2003; Kulmala et al., 2002; Weber et al., 1996, 1999]. In fact, the predicted sulfuric acid concentrations are similar on days with and without nucleation for both the summer and winter simulations. In July, the average sulfuric acid concentration on days with nucleation is $6.5 \times 10^{7}$ molecules $/ \mathrm{cm}^{3}$ between 0900 and 1500 EST, about $10 \%$ less than the corresponding value of $7.2 \times 10^{7}$ molecules $/ \mathrm{cm}^{3}$ on days without nucleation.
The daily maximum concentrations predicted for July are $2.8 \times 10^{8}$ and $2.9 \times 10^{8}$ molecules $/ \mathrm{cm}^{3}$ on days with and without nucleation, respectively, while the concentrations are generally at least $10^{7}$ molecules $/ \mathrm{cm}^{3}$. In January, the average sulfuric acid concentration on days with nucleation $\left(1.8 \times 10^{7}\right.$ molecules $\left./ \mathrm{cm}^{3}\right)$ is about $30 \%$ higher than on days without nucleation $(1.3 \times$ $10^{7}$ molecules $/ \mathrm{cm}^{3}$ ). The maximum sulfuric acid concentrations are again similar, $1.3 \times 10^{8}$ and $1.4 \times$ $10^{8}$ molecules $/ \mathrm{cm}^{3}$, respectively, while the predicted concentrations are generally above $10^{5}$ molecules $/ \mathrm{cm}^{3}$.

\section{Effect of Changes in $\mathrm{SO}_{2}$ and $\mathrm{NH}_{3}$ Emissions}

[38] Sensitivity analysis was also performed to determine how changes in emissions of $\mathrm{SO}_{2}$ and $\mathrm{NH}_{3}$ would influence nucleation during summer and winter conditions. Scenarios were modeled changing the emissions of one gas while leaving the other at the base case level, as well as with both ammonia and $\mathrm{SO}_{2}$ being simultaneously controlled. The following changes were made to the model inputs with changes in $\mathrm{SO}_{2}$ emissions: (a) a proportional change was made in the gas-phase $\mathrm{SO}_{2}$ and aerosol sulfate concentrations; (b) the gas-phase ammonia concentrations were recalculated using the adjusted aerosol sulfate concentrations and conserving the total ammonia; (c) the initial surface area was reduced proportional to the change in total aerosol mass. Changes in aerosol mass are calculated accounting for the change in sulfate concentrations, the mass fraction of sulfate in the aerosol phase, and adjusting for replacement of sulfate by nitrate and ammonium when sufficient $\mathrm{NH}_{3}$ and $\mathrm{HNO}_{3}$ are available [Seinfeld and Pandis, 1998]. The adjustment to the surface area implicitly assumes that there is no change in the shape of the aerosol distribution. In reality, there would be a shift in the distribution with changes in total mass. However, sensitivity analysis on the preexisting surface area showed that the results were insensitive to changes of up to $20 \%$ (the number of events remains the same while the growth of nucleated particles is affected only slightly), so the error introduced by this simplifying assumption can be ignored. Changes in $\mathrm{NH}_{3}$ emissions are handled in a similar manner: (a) the total ammonia is changed proportionally to the change in $\mathrm{NH}_{3}$ emissions; (b) the gas-phase ammonia concentrations are recalculated with the new values for total ammonia; (c) the initial surface area is adjusted proportionately to the change in aerosol mass resulting from the increase of total ammonia.

[39] Figure 5 shows the effects of changes in $\mathrm{SO}_{2}$ emissions for both the January and July simulations. For the July simulations, increasing the $\mathrm{SO}_{2}$ emissions decreases the number of nucleation events, as the additional aerosol sulfate lowers the amount of gas-phase ammonia and reduces the nucleation rates. The fresh particles that are created grow to larger sizes, however, as there is more sulfuric acid being produced to contribute to the growth of the particles. Lowering $\mathrm{SO}_{2}$ emissions initially increases the number of nucleation events, as gas-phase ammonia concentrations increase and raise the nucleation rates. A maximum of 18 events out of the 19 modeled days occurs with a $40 \%$ reduction in $\mathrm{SO}_{2}$. Additional reductions in $\mathrm{SO}_{2}$ decrease the number of nucleation events and substantially 
(a)

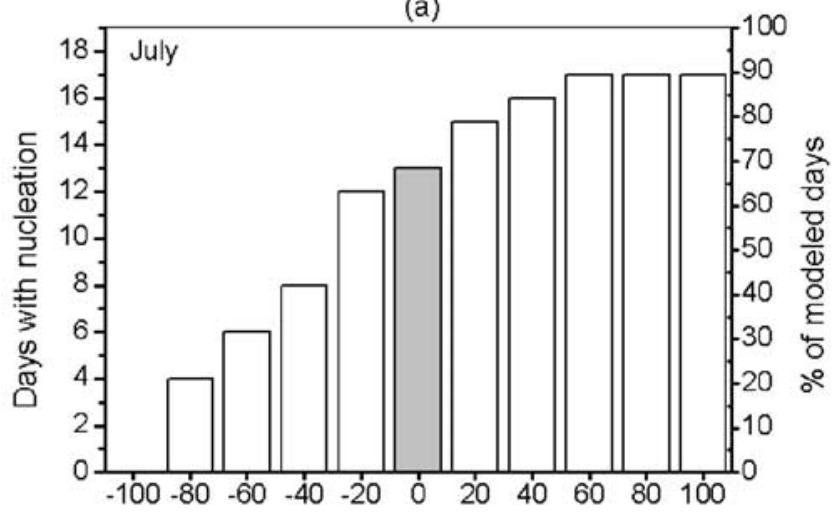

(b)

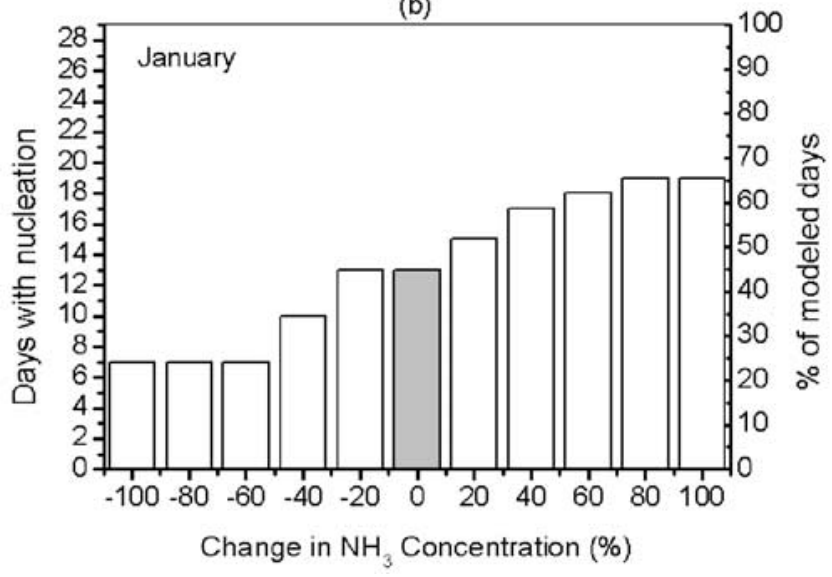

Figure 6. Sensitivity to changes in ammonia emissions for (a) July and (b) January. The shaded bars represent the base case scenario.

reduce the amount of growth seen. Even with an $80 \%$ reduction in $\mathrm{SO}_{2}$ emissions, however, fourteen nucleation events occur ( $74 \%$ of the modeled days), although eight of these events are very weak with the nucleated particles not growing above $20 \mathrm{~nm}$ in size.

[40] In January, changes in $\mathrm{SO}_{2}$ emissions have the opposite effect, with increases in $\mathrm{SO}_{2}$ emissions tending to cause additional events, and vice versa. This is indicative of the fact that sulfuric acid, in conjunction with ammonia, plays an important role in determining the nucleation rate in January, as opposed to in the summer when ammonia controls the system. The model predicts a maximum of 18 events $(62 \%$ of the modeled days) with an $80 \%$ increase in $\mathrm{SO}_{2}$ emissions, 5 more than for the base case. The nucleation events also exhibit increased growth as the $\mathrm{SO}_{2}$ emissions are increased. With a $40 \%$ reduction in $\mathrm{SO}_{2}$, nucleation is still seen on $38 \%$ of the days, although the particles from only 2 of the 11 events grow to $20 \mathrm{~nm}$ in size. An $80 \%$ reduction in $\mathrm{SO}_{2}$ leaves only 3 events ( $10 \%$ of the modeled days), with the mode of the particles only reaching $10 \mathrm{~nm}$ in size for all three events.

[41] In July, a strong positive correlation is seen between changes in total ammonia and the number of nucleation events (Figure 6). In July, the model predicts 17 events when the total ammonia is increased by more than $60 \%$ ( $89 \%$ of the modeled days). A $40 \%$ reduction in ammonia eliminates 5 nucleation events seen in the base case (leaving
$42 \%$ of the days showing nucleation), while a $100 \%$ reduction eliminates all the events. The length of the nucleation events is also affected as ammonia decreases, with several of the events becoming significantly shorter.

[42] Although a similar trend is seen in January, ammonia does not have as great an effect as in July. Even with a $100 \%$ reduction in total ammonia, seven events still occur ( $24 \%$ of the modeled days), with the particles from 5 of these events growing to at least $20 \mathrm{~nm}$ in size. For these cases, the lower limit of ammonia for the parameterization was used, $0.1 \mathrm{ppt}$, so these cases do not reduce to the binary $\mathrm{H}_{2} \mathrm{SO}_{4}-\mathrm{H}_{2} \mathrm{O}$ case. A doubling of ammonia results in nineteen predicted nucleation events (66\% of the days), although 8 of these events are weak and do not exhibit growth above $20 \mathrm{~nm}$ in size.

[43] Although $\mathrm{SO}_{2}$ emissions have been reduced substantially since the 1980 s by the Clean Air Act, further reductions are planned [e.g., U.S. Congress, 2003] to help reduce fine particulate matter mass concentrations in the eastern United States, which have been linked to adverse health effects [Samet et al., 2000; U.S. Environmental Protection Agency, 1996]. With these further reductions in $\mathrm{SO}_{2}$ already planned, additional scenarios were simulated for July in which $\mathrm{NH}_{3}$ was controlled in addition to $\mathrm{SO}_{2}$. As shown previously, a $40 \%$ reduction in sulfate results in five additional events during this month. When coupled with a $10 \%$ reduction in $\mathrm{NH}_{3}$, however, three of these additional events are eliminated. A reduction of $\mathrm{NH}_{3}$ by $30 \%$ eliminates the other two additional events, leaving just the original thirteen.

\section{Conclusions}

[44] A strong correlation between the presence of gasphase ammonia in July and observed nucleation indicates that ternary $\mathrm{NH}_{3}-\mathrm{H}_{2} \mathrm{SO}_{4}-\mathrm{H}_{2} \mathrm{O}$ nucleation is the likely mechanism for nucleation in Pittsburgh. The ternary $\mathrm{NH}_{3}-\mathrm{H}_{2} \mathrm{SO}_{4}-$ $\mathrm{H}_{2} \mathrm{O}$ nucleation parameterization of Napari et al. [2002] is shown to accurately predict the presence or lack of nucleation during both the summer and winter: 19 out of 19 days in July, and 25 out of 29 days in January. The success of the model using $\mathrm{H}_{2} \mathrm{SO}_{4}$ as the sole condensing species and without simulating the effect of organics suggests that the role of organics in the formation and growth of these nucleated particles is probably secondary.

[45] The predicted size and shape of the growth curve, as well as the timing of the onset of nucleation, is in good agreement with observations, particularly in July. The predicted timing of the events is within 1 hour of the observations for all 13 events in July, and 6 of the 12 events in January. Nine of the thirteen predicted events show very similar growth compared to the observations in July, while the model generally predicts less growth than observed in January. The model tends to predict higher number concentrations of particles on days with nucleation for both the summer and winter months. In July, for example, the predicted daily average and peak hourly number concentrations of particles larger than $10 \mathrm{~nm}$ are 2.5 and 4.3 times higher, respectively, than the observed number concentrations on these days. Although this discrepancy in number concentrations might be partially explained by the model not accounting for coagulational 
scavenging of particles smaller than the critical cluster as well as dilution as the mixing layer rises during the day, this issue needs to be further explored.

[46] Sensitivity analysis on the $\mathrm{OH}$ concentrations reveals that the number of nucleation in July events is insensitive to the $\mathrm{OH}$ concentration, while the number of events in January ranges from 8 (28\% of the modeled days) with $\mathrm{OH}$ concentrations $50 \%$ of the base case value to $21(72 \%$ of the days) with $\mathrm{OH}$ concentrations twice the base case value. The growth of the nucleated particles due to sulfuric acid condensation changes significantly for both months as a function of the assumed maximum $\mathrm{OH}$. Field measurements of $\mathrm{OH}$ concentrations during the winter months would greatly enhance our understanding of nucleation during the winter.

[47] Additional sensitivity analysis shows what effect future changes in ammonia and $\mathrm{SO}_{2}$ emissions would have on the number and strength of nucleation events. In both July and January, the number of events shows a strong correlation with ammonia emissions. In July, reducing the ammonia emissions by $100 \%$ eliminates all the nucleation events, while doubling ammonia emissions results in 17 events ( $89 \%$ of the modeled days). In January, the relationship is not quite as strong, with the percentage of days exhibiting nucleation rising from $24 \%$ with no ammonia to $66 \%$ with double ammonia. The effect of changes in $\mathrm{SO}_{2}$ emissions is different depending on the season. In January, there is again a positive correlation with the number of events. An $80 \%$ reduction in $\mathrm{SO}_{2}$ emissions leaves only 3 events, while doubling the $\mathrm{SO}_{2}$ results in 19 events (66\% of the days). In July, the simulated effect of $\mathrm{SO}_{2}$ emissions is nonlinear. For the July simulations, $\mathrm{SO}_{2}$ reductions can either increase or decrease the frequency of nucleation. Reductions up to about $40 \%$ will increase the number of days with nucleation, while larger reductions will ultimately decrease the frequency. Doubling the $\mathrm{SO}_{2}$ in summer decreases the nucleation frequency substantially. Like the changes in $\mathrm{OH}$ concentrations, the amount of $\mathrm{SO}_{2}$ also affects the growth of the nucleated particles significantly in both months, as the amount of sulfuric acid available for condensation is affected.

[48] Acknowledgments. The authors wish to thank Qi Zhang at the University of Colorado, Boulder, for her input regarding the role of organics in condensational growth. This research was conducted as part of the Pittsburgh Air Quality Study, which was supported by the U.S. Environmental Protection Agency under contract R82806101 and the U.S. Department of Energy National Energy Technology Laboratory under contract DE-FC26-01NT41017. This paper has not been subject to EPA's peer and policy review and therefore does not necessarily reflect the views of the agency. No official endorsement should be inferred.

\section{References}

Adams, P. J., and J. H. Seinfeld (2003), Disproportionate impact of particulate emissions on global cloud condensation nuclei concentrations, Geophys. Res. Lett., 30, 1239, doi:10.1029/2002GL016303.

Ansari, A. S., and S. N. Pandis (1999), Prediction of multicomponent inorganic atmospheric aerosol behavior, Atmos. Environ., 33, 745-757.

Anttila, T., and V.-M. Kerminen (2003), Condensational growth of atmospheric nuclei by organic vapours, J. Aerosol Sci., 34, 41-61.

Birmili, W., H. Berresheim, C. Plass-Dülmer, T. Elste, S. Gilge, A. Wiedensohler, and U. Uhrner (2003), The Hohenpeiseenberg aerosol formation experiment (HAFEX): A long-term study including sizeresolved aerosol, $\mathrm{H}_{2} \mathrm{SO}_{4}, \mathrm{OH}$, and monoterpenes measurements, Atmos. Chem. Phys., 3, 361-376.

Bolsaitis, P., and J. F. Elliott (1990), Thermodynamic activities and equilibrium partial pressures for aqueous sulfuric acid solutions, J. Chem. Eng Data, 35, 69-85.
Brook, J. R., L. Zhang, L. Yifan, and D. Johnson (1999), Description and evaluation of a model of deposition velocities for routine estimates of dry deposition over North America. part II: Review of past measurements and model results, Atmos. Environ., 33, 5053-5070.

Cabada, J. C., S. N. Pandis, and A. L. Robinson (2002), Sources of atmospheric carbonaceous particulate matter in Pittsburgh, Pennsylvania, J. Air Waste Manage. Assoc., 52(6), 732-741.

Capaldo, K. P., P. Kasibhatla, and S. N. Pandis (1999), Is aerosol production within the remote marine boundary layer sufficient to maintain observed concentrations?, J. Geophys. Res., 104, 3483-3500.

Coffman, D. J., and D. A. Hegg (1995), A preliminary study of the effect of ammonia on particle nucleation in the MBL, J. Geophys. Res., 100, $7147-7160$.

Creasey, D. J., D. E. Heard, and J. D. Lee (2002), Eastern Atlantic Spring Experiment 1997 (EASE97): 1. Measurements of $\mathrm{OH}$ and $\mathrm{HO}_{2}$ concentrations at Mace Head, Ireland, J. Geophys. Res., 107(D10), 4091, doi:10.1029/2001JD000892.

DeMore, W. B., et al. (1994), Chemical kinetics and photochemical data for use in stratospheric modeling, Tech. Rep. Eval. 10, Jet Propul. Lab., Pasadena, Calif.

Eisele, F. L., and P. H. McMurray (1997), Recent progress in understanding particle nucleation and growth, Philos. Trans. R. Soc. London, Ser. B, 352(1350), 191-200

Fuchs, N. A. (1964), The Mechanics of Aerosols, Elsevier, New York.

Fuchs, N. A., and A. G. Sutugin (1971), Highly Dispersed Aerosols, Elsevier, New York.

George, L. A., T. M. Hard, and R. J. O'Brien (1999), Measurements of free radicals $\mathrm{OH}$ and $\mathrm{HO}_{2}$ in Los Angeles smog, J. Geophys. Res., 104(D9), $11,643-11,655$.

Heard, D. E., J. D. Lee, M. J. Pilling, and N. Carslaw (2001), Measurements of $\mathrm{OH}$ and $\mathrm{HO}_{2}$ in urban environments and comparison of the predictions of photochemical models, paper presented at XXVI General Assembly, Eur. Geophys. Soc., Nice, France, 25-30 March

Hegg, D. A. (1990), Heterogeneous production of cloud condensation nuclei in the marine atmosphere, Geophys. Res. Lett., 17, 2165-2168.

Hoffman, T., et al. (1997), Formation of organic aerosols from the oxidation of biogenic hydrocarbons, J. Atmos. Sci., 26, 189-222.

Hoffmann, T., C. D. O’Dowd, and J. H. Seinfeld (2001), Iodine oxide homogeneous nucleation: An explanation for coastal new particle production, Geophys. Res. Lett., 28, 1949-1952.

Hummelshoj, P., N. O. Jensen, and S. E. Larsen (1992), Particle dry deposition to a sea surface, in Precipitation Scavenging and Atmosphere Surface Exchange, edited by S. E. Schwartz and W. G. N. Slinn, p. 829-840, Taylor and Francis, Philadelphia, Pa.

Ibald-Mulli, A., H. E. Wichmann, W. Kreyling, and A. Peters (2002), Epidemiological evidence on health effects of ultrafine particles, J. Aerosol Med., 15, 189-201.

Jaecker-Voirol, A., and P. Mirabel (1989), Hetermolecular nucleation in the sulfuric acid-water system, Atmos. Environ., 23, 2053-2057.

Janson, R., K. Rosman, A. Karlsson, and H.-C. Hansson (2001), Biogenic emissions and gaseous precursors to forest aerosols, Tellus, Ser. B, 53, $423-440$

Jefferson, A., F. L. Eisele, P. J. Ziemann, R. J. Weber, J. J. Marti, and P. H. McMurry (1997), Measurements of the $\mathrm{H}_{2} \mathrm{SO}_{4}$ mass accommodation coefficient onto polydisperse aerosol, J. Geophys. Res., 102, 19,02119,028 .

Kerminen, V.-M., A. Virkkula, R. Hillamo, A. S. Wexler, and M. Kulmala (2000), Secondary organics and atmospheric cloud condensation nuclei production, J. Geophys. Res., 105(D7), 9255-9264.

Kerminen, V.-M., L. Pirjola, and M. Kulmala (2001), How significantly does coagulational scavenging limit atmospheric particle production?, J. Geophys. Res., 106(D20), 24,119-24,125.

Khlystov, A., G. P. Wyers, and J. Slanina (1995), The steam-jet aerosol collector, Atmos. Environ., 29, 2229-2234.

Korhonen, H. K. E. J. Lehtinen, L. Pirjola, I. Napari, H. Vehkamäki, M. Noppel, and M. Kulmala (2003), Simulation of atmospheric nucleation mode: A comparison of nucleation models and size distribution representations, J. Geophys. Res., 108(D15), 4471, doi:10.1029/2002JD003305.

Kreidenweis, S. M., F. Yin, S. C. Wang, D. Grosjean, R. C. Flagan, and J. H. Seinfeld (1991), Aerosol formation during photooxidation of organosulfur species, Atmos. Environ. Part A, 25, 2491-2500.

Kulmala, M., et al. (2001a), On the formation, growth, and composition of nuclei mode particles, Tellus, Ser. B, 53(4), 479-490.

Kulmala, M., L. Pirjola, and J. M. Makela (2001b), Stable sulphate clusters as a source of new atmospheric particles, Nature, 404(6773), 66-69.

Kulmala, M., P. Korhonen, I. Napari, A. Karlsson, H. Berresheim, and C. D. O'Dowd (2002), Aerosol formation during PARFORCE: Ternary nucleation of $\mathrm{H}_{2} \mathrm{SO}_{4}, \mathrm{NH}_{3}$, and $\mathrm{H}_{2} \mathrm{O}, J$. Geophys. Res., 107(D19), 8111, doi:10.1029/2001JD000900. 
Laakso, L., J. M. Mäkelä, L. Pirjola, and M. Kulmala (2002), Model studies on ion-induced nucleation in the atmosphere, J. Geophys. Res. 107(D20), 4427, doi:10.1029/2002JD002140.

Li, X. Y., D. Brown, S. Smith, W. MacNee, and K. Donaldson (1999) Short-term inflammatory responses following intratracheal instillation of fine and ultrafine carbon black in rats, Inhal. Toxicol., 11, 709-731.

Mauldin, R. L., III, et al. (2001), Measurements of OH aboard the NASA P-3 during PEM-Tropics B, J. Geophys. Res., 106(D23), 32,657-32,666.

Napari, I., M. Noppel, H. Vehkamäki, and M. Kulmala (2002), Parametrization of ternary nucleation rates for $\mathrm{H}_{2} \mathrm{SO}_{4}-\mathrm{NH}_{3}-\mathrm{H}_{2} \mathrm{O}$ vapors, J. Geophys. Res., 107(D19), 4381, doi:10.1029/2002JD002132.

Nemmar, A., et al. (2002), Passage of inhaled particles into the blood circulation in humans, Circulation, 105(4), 411-414

Nilsson, E. D., and M. Kulmala (1998), The potential for atmospheric mixing processes to enhance the binary nucleation rate, J. Geophys Res., 103(D1), 1381-1389.

Nilsson, E. D., U. Rannik, M. Kulmala, G. Buzorius, and C. D. O'Dowd (2001), Effects of continental boundary layer evolution, convection, turbulence and entrainment, on aerosol formation, Tellus, Ser. B, 53(4), $441-461$

Oberdorster, G., J. Ferin, R. Gelein, S. C. Soderholm, and J. Finkelstein (1992), Role of the alveolar macrophage in lung injury-Studies with ultrafine particles, Environ. Health Perspect., 97, 193-199.

Oberdorster, G., et al. (2002), Extrapulmonary translocation of ultrafine carbon particles following whole-body inhalation exposure of rats, J. Toxicol. Environ. Health A, 65(20), 1531-1543.

O'Dowd, C. D., et al. (2002), A dedicated study of New Particle Formation and Fate in the Coastal Environment (PARFORCE): Overview of objectives and achievements, J. Geophys. Res., 107(D19), 8108, doi:10.1029/ 2001JD000555.

Peters, A., H. E. Wichmann, T. Tuch, J. Heinrich, and J. Heyder (1997), Respiratory effects are associated with the number of ultrafine particles, Am. J. Respir. Critical Care Med., 155(4), 1376-1383.

Pirjola, L., and M. Kulmala (2001), Development of particle size and composition distributions with a novel aerosol dynamics model, Tellus, Ser. B, 53(4), 491-509.

Pirjola, L., et al. (1999), Formation of sulphuric acid aerosols and cloud condensation nuclei: An expression for significant nucleation and model comparison, J. Aerosol Sci., 30, 1079-1094.

Raes, F., A. Saltelli, and R. V. Dingenen (1992), Modelling formation and growth of $\mathrm{H}_{2} \mathrm{SO}_{4}-\mathrm{H}_{2} \mathrm{O}$ aerosols: Uncertainty analysis and experimental evaluation, J. Aerosol Sci., 23, 759-771.

Ren, X. R., et al. (2003a), $\mathrm{HO}_{\mathrm{x}}$ concentrations and $\mathrm{OH}$ reactivity observations in New York City during PMTACS-NY2001, Atmos. Environ., 37 $3627-3637$.

Ren, X. R., et al. (2003b), $\mathrm{OH}$ and $\mathrm{HO}_{2}$ Chemistry in the urban atmosphere of New York City, Atmos. Environ., 37, 3639-3651.

Samet, J. M., F. Dominici, F. C. Curriero, I. Coursac, and S. L. Zeger (2000), Fine particulate air pollution and mortality in 20 U.S. cities, 1987-1994, N. Engl. J. Med., 343, 1742-1749.

Seinfeld, J. H., and S. N. Pandis (1998), Atmospheric Chemistry and Physics From Air Pollution to Climate Change, John Wiley, Hoboken, N. J.
Stanier, C. O., A. Khylstov, and S. N. Pandis (2004a), Ambient aerosol size distributions and number concentrations measured during the Pittsburgh Air Quality Study (PAQS), Atmos. Environ., 38, 32753284.

Stanier, C., A. Khlystov, and S. N. Pandis (2004b), Nucleation events during the Pittsburgh Air Quality Study: Description and relation to key meteorological, gas phase, and aerosol parameters, Aerosol Sci. Technol., 38, suppl. 1, 253-264.

Tan, D., et al. (2001), $\mathrm{HO}_{\mathrm{x}}$ budgets in a deciduous forest: Results from the PROPHET summer 1998 campaign, J. Geophys. Res., 106(D20), 24,407-24,427

U.S. Congress (2003), Clear Skies Act, S. 485, 108th Congress, 27 Feb.

U.S. Environmental Protection Agency (1996), Air quality criteria for particulate matter, Rep. EPA/600/P-95/-1aF-cF.v3, Natl. Cent. for Environ. Assess., Research Triangle Park, N. C.

Vehkamäki, H., M. Kulmala, I. Napari, K. E. J. Lehtinen, C. Timmreck, M. Noppel, and A. Laaksonen (2002), An improved parameterization for sulfuric acid-water nucleation rates for tropospheric and stratospheric conditions, J. Geophys. Res., 107(D22), 4622, doi:10.1029/ 2002JD002184.

Weber, R. J., et al. (1996), Measured atmospheric new particle formation rates: Implications for nucleation mechanisms, Chem. Eng. Commun., $151,53-64$

Weber, R. J., et al. (1998), A study of new particle formation and growth involving biogenic and trace gas species measured during ACE 1, J. Geophys. Res., 103, 16,385-16,396.

Weber, R. J., et al. (1999), New particle formation in the remote troposphere: A comparison of observations at various sites, Geophys. Res. Lett., 26, 307-310.

Wexler, A. S., F. W. Lurmann, and J. H. Seinfeld (1994), Modelling urban and regional aerosols-I. Model development, Atmos. Environ., 28(3), $531-546$

Wittig, B., N. Anderson, A. Y. Khlystov, S. N. Pandis, C. Davidson, and A. L. Robinson (2004), Pittsburgh Air Quality Study overview, Atmos. Environ., 38, 3107-3125.

Woo, K. S., D. R. Chen, D. Y. H. Pui, and P. H. McMurry (2001), Measurement of Atlanta aerosol size distributions: Observations of ultrafine particle events, Aerosol Sci. Technol., 34, 75-87.

Zhang, K. M., and A. S. Wexler (2002), A hypothesis for growth of fresh atmospheric nuclei, J. Geophys. Res., 107(D21), 4577, doi:10.1029/ 2002JD002180

Zhang, Q., C. Stanier, M. Caragaratna, J. T. Jayne, D. Worsnop, S. N. Pandis, and J. L. Jimenez (2004), Insights into the chemistry of nucleation bursts and particle growth events in Pittsburgh based on aerosol mass spectrometry, Environ. Sci. Technol., 38, 4797-4809.

T. M. Gaydos, S. N. Pandis, and C. O. Stanier, Department of Chemical Engineering, Carnegie Mellon University, Pittsburgh, PA 15213, USA. (spyros@andrew.cmu.edu) 


\title{
Natural radionuclides in fine aerosols in the Pittsburgh area
}

\author{
Jeffrey S. Gaffney*, Nancy A. Marley, Mary M Cunningham \\ Environmental Research Division, Argonne National Laboratory, Bldg 203/ER, 9700 South Cass Avenue, Cass Ave, \\ Argonne, IL 60439, USA
}

\begin{abstract}
Natural radionuclides have been proposed for use as tracers in assessing the transport of ozone and aerosols in the troposphere. Beryllium-7 is produced in the upper troposphere and lower stratosphere and after formation rapidly attaches itself to fine aerosol particles. Measurements of ${ }^{7} \mathrm{Be}$ at ground level can therefore be used as a tracer of stratospheric/tropospheric folding events, leading to injection of ozone and upper atmospheric aerosols into the lower atmosphere. Since its concentration varies little on regional scales it can also be used to detect and correct for sampling problems in particulate monitoring networks. Lead-210 and its progeny, ${ }^{210} \mathrm{Bi}$, and ${ }^{210} \mathrm{Po}$ can also be used to determine the apparent tropospheric residence times for fine aerosols by looking at the ${ }^{210} \mathrm{Bi} /{ }^{210} \mathrm{~Pb}$ and ${ }^{210} \mathrm{Po} /{ }^{210} \mathrm{~Pb}$ activity ratios. Reported here are measurements of the natural radionuclides ${ }^{7} \mathrm{Be}$ and ${ }^{210} \mathrm{~Pb}$, taken at two sites near Pittsburgh, $\mathrm{PA}$ in the summer of 2001 and at a site near Centerton, New Jersey in 1999 during the NEOPS field campaign. Beryllium-7 results show no evidence of upper atmospheric input during the sampling period. Apparent residence times as calculated from ${ }^{210} \mathrm{Po} /{ }^{210} \mathrm{~Pb}$ ratios are shorter for Pittsburgh than for Centerton, and shorter for both sites than those obtained previously in other areas, indicating a local aerosol source as well as a higher loading of water-soluble species such as sulfate and nitrate. A comparison of fine and course aerosol lifetimes shows no contribution of excess ${ }^{210} \mathrm{Po}$ from wind-blown soil or from coal-fired power plants.
\end{abstract}

(C) 2004 Elsevier Ltd. All rights reserved.

Keywords: Aerosols; Fine particulates; Aerosol residence times; ${ }^{210} \mathrm{~Pb} ;{ }^{210} \mathrm{Po} ;{ }^{210} \mathrm{Bi}$; Natural radioactivity in aerosols; Lead-210 in aerosols

\section{Introduction}

We are now becoming more aware of the importance of atmospheric aerosols in the chemistry and physics of

\footnotetext{
The submitted manuscript has been created by the University of Chicago as operator of Argonne National Laboratory under Contract No. W-31-109-ENG-38 with the US Department of Energy. The US government retains for itself, and others acting on its behalf, a paid-up, nonexclusive, irrevocable worldwide license in said article to reproduce, prepare derivative works, distribute copies to the public, and perform publicly and display publicly, by or on behalf of the government. This effort was supported by the US Department of Energy, Office of Science, Office of Biological and Environmental Research, Atmospheric Chemistry Program, under contract W-31-109-Eng-38.

*Corresponding author. Tel.: + 1-630-252-5178; fax: + 1-630252-7415.

E-mail address: gaffney@anl.gov (J.S. Gaffney).
}

the atmosphere and as a means of transporting materials over long distances. In the stratosphere, atmospheric aerosols provide important surfaces for heterogeneous chemical processes leading to enhancement of the catalytic destruction of stratospheric ozone by chlorine. Aerosols are important as cloud condensation nuclei and play key roles in the hydrological cycle through their influence on the types and formation rates of clouds. Aerosols, as well as the clouds they produce, can also scatter or absorb incoming radiation from the sun or outgoing radiation from Earth, with important consequences for the thermal balance of the planet as well as for climate and weather.

Aerosols at high concentrations also have health impacts, particularly when they are associated with other air pollutant exposures (Holland et al., 1979). The killer smog episodes in London during the 1940s and 1950s were due to high levels of carbon soot and sulfur dioxide trapped in a shallow boundary layer. In the 
United States, similar events in Donora, Pennsylvania, caused a number of deaths and led thousands of residents to be hospitalized. The result was the passage of legislation aimed at reduction of the levels of these primary pollutants. More recently, studies have indicated a relationship between lower levels of atmospheric particulate exposure and increases in the incidences of cardiopulmonary illnesses (Pope, 2000; Schwartz et al., 2001), cancer (Beeson et al., 1998) and mortality rates in general (Lipfert et al., 2000; Schwartz et al., 1996). The effects are particularly strong in individuals with preexisting conditions. This correlation has motivated a call for stricter air quality standards for particulate matter even though a toxicological mechanism is still unknown. Evidence has suggested that aerosol mass concentration is not the most appropriate measure of potential health effects (Lighty et al., 2000). Other characteristics such as size, surface area, particle number, chemical composition, and morphology have been suggested as important measurements to consider.

Critical to our understanding of the roles that aerosols play in the atmosphere as well as their effects on human health is the determination of their size, chemical composition, and physical properties. It is especially important to determine the ability of aerosols to take up water and grow to a size that can be removed by gravitational settling or washout processes. We now recognize that the secondary formation of aerosols in the atmosphere is also important. The oxidation of sulfur dioxide and nitrogen oxides to form submicron sulfate and nitrate aerosols is well documented. Large organic hydrocarbons, such as the natural monoterpenes and anthropogenic-sourced hydrocarbons, can also react with ozone and hydroxyl radical to form secondary organic aerosols. In many cases, these submicron secondary aerosols formed in the boundary layer can be transported for some distances, as they are removed slowly by gravitational settling.

The dominant radioactive isotopes attached to fine aerosols are ${ }^{7} \mathrm{Be}$ and ${ }^{210} \mathrm{~Pb}$, and the ${ }^{210} \mathrm{~Pb}$ progeny, ${ }^{210} \mathrm{Bi}$ and ${ }^{210} \mathrm{Po}$ (Junge, 1963). The sources, radioactive lifetimes, and decay schemes of these radionuclides are shown in Fig. 1. Cosmogenic particles hitting the atmosphere lead to the production of ${ }^{7} \mathrm{Be}$ through spallation processes with light atomic nuclei (e.g. nitrogen and oxygen). About $66 \%$ of the total ${ }^{7} \mathrm{Be}$ is produced in the stratosphere and $33 \%$ in the upper troposphere (Gäggeler, 1995), with peak production at about $15 \mathrm{~km}$ (Bhandari et al., 1970). Once ${ }^{7} \mathrm{Be}$ is produced in the upper troposphere and lower stratosphere, it attaches itself to the available aerosol surfaces. This leads to a mean diameter of $0.3 \mu \mathrm{m}$ for the size distribution of ${ }^{7} \mathrm{Be}$-containing aerosol particles. Concentrations of ${ }^{7} \mathrm{Be}$ in surface air depend on cosmic ray intensity, stratosphere-troposphere exchange, downward transport in the troposphere, and wet and dry

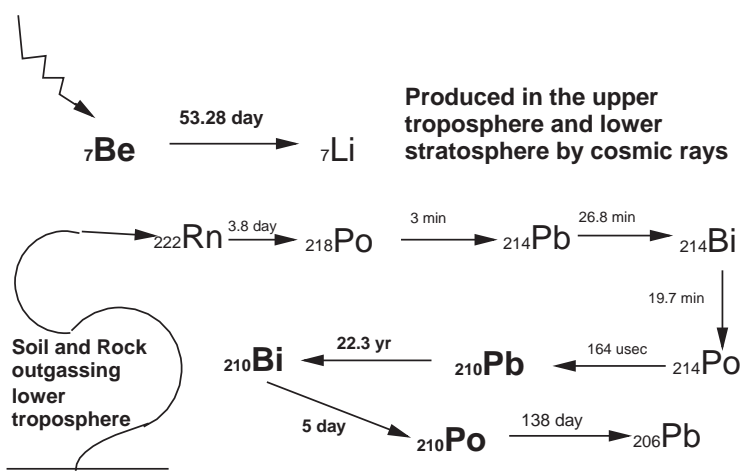

Fig. 1. The sources, radioactive lifetimes, and decay schemes of the natural radioactive tracers ${ }^{7} \mathrm{Be}$ and ${ }^{210} \mathrm{~Pb}$ and its progeny, ${ }^{210} \mathrm{Bi}$ and ${ }^{210} \mathrm{Po}$ (in bold).

deposition of the carrier aerosols. Consequently, ${ }^{7} \mathrm{Be}$ is a sensitive indicator of intrusions of stratospheric air into the troposphere. Thus, the determination of the ${ }^{7} \mathrm{Be}$ content in fine aerosols can give a measure of the transport of this fine aerosol material from the upper troposphere/lower stratosphere to the surface where it is sampled. Past work has also suggested that this might be a useful way to determine stratospheric intrusions into the lower atmosphere in order to estimate whether stratospheric ozone is affecting tropospheric sites during these events (Gaffney et al., 1994, 1995).

Uranium-238 is common throughout the Earth's crust and decays to the inert gas ${ }^{222} \mathrm{Rn}$, which escapes into the atmosphere. The ${ }^{222} \mathrm{Rn}$ decay leads to the formation of ${ }^{218} \mathrm{Po}$, which rapidly becomes attached to fine aerosols, with average attachment times of $40 \mathrm{~s}$ to $3 \mathrm{~min}$ (Whittlestone, 1990), and subsequently decays to form the long-lived ${ }^{210} \mathrm{~Pb}$ (see Fig. 1). The ${ }^{210} \mathrm{~Pb}$ is attached to aerosol particles in the submicron size range and is therefore considered to be a good tracer of secondary aerosols produced by gas-to-particle conversion (Sanak et al., 1981). The size distribution for the ${ }^{210} \mathrm{~Pb}$ attached particles in the boundary layer is somewhat larger than that for ${ }^{7} \mathrm{Be}$, with a median diameter upon initial attachment of $0.4 \mu \mathrm{m}$. Removal of ${ }^{210} \mathrm{~Pb}$ from the atmosphere occurs mainly by wet and dry deposition, with precipitation being the dominant pathway (Gaffney et al., 1994).

In theory, the activity ratio of either ${ }^{210} \mathrm{Po}$ or ${ }^{210} \mathrm{Bi}$ to the parent ${ }^{210} \mathrm{~Pb}$ can be used to estimate the atmospheric residence times of the carrier aerosols (Nevissi, 1991). However, residence times calculated by these methods have led to controversy in the past. In general, the ${ }^{210} \mathrm{Bi} /{ }^{210} \mathrm{~Pb}$ activity ratios obtained on total atmospheric particulate samples have yielded residence times shorter than those calculated from the ${ }^{210} \mathrm{Po} /{ }^{210} \mathrm{~Pb}$ ratios (Gavini et al., 1974; Tsunogai and Fukuda, 1974; Lambert et al., 1979; Tokieda et al., 1996; Kim et al., 2000). Although ${ }^{210} \mathrm{Bi}^{210} \mathrm{~Pb}$ ratios often result in aerosol 
residence times of approximately one week (Fry and Menon, 1962; Poet et al., 1972; Rangarajan et al., 1986), ${ }^{210} \mathrm{Po} /{ }^{210} \mathrm{~Pb}$ ratios yield residence times on the order of a month or more (Burton and Stewart, 1960; Pierson et al., 1966; Anand and Rangarajan, 1990). The larger than expected ${ }^{210} \mathrm{Po} /{ }^{210} \mathrm{~Pb}$ ratios have been attributed to additional sources of ${ }^{210} \mathrm{Po}$ besides the radioactive decay of ${ }^{210} \mathrm{~Pb}$ (Moore et al., 1976).

In contrast to ${ }^{7} \mathrm{Be}$, which has a 53 -day half-life, ${ }^{210} \mathrm{~Pb}$, with a half-life of 22.3 years, is present long enough in soil to be measurable in wind-blown dusts. Contamination of aerosol samples with resuspended surface materials will result in excess ${ }^{210} \mathrm{Po}$ and subsequently, ${ }^{210} \mathrm{Po} /{ }^{210} \mathrm{~Pb}$ activity ratios that are extremely high resulting in very long apparent residence times. Samples taken across a variety of sites with an eight-stage cascade impactor demonstrated that submicron analyses are needed to avoid contamination and the subsequent long apparent lifetimes that occur in the larger size ranges (Marley et al., 2000a,b) from wind-blown dust and soil. In this work (Marley et al., 2000a, b), aerosol samples in the size ranges $>1 \mu \mathrm{m}$ gave apparent residence times of $>300$ days and a wide disagreement between ${ }^{210} \mathrm{Bi} /{ }^{210} \mathrm{~Pb}$ and ${ }^{210} \mathrm{Po} /{ }^{210} \mathrm{~Pb}$ ratios. While sample sizes below $1 \mu \mathrm{m}$ gave apparent residence times of 33-66 days and a general agreement between results obtained from ${ }^{210} \mathrm{Bi} /{ }^{210} \mathrm{~Pb}$ and ${ }^{210} \mathrm{Po} /{ }^{210} \mathrm{~Pb}$ activity ratios.

An unexpected disequilibrium has been recently reported in fine aerosol fractions in the atmosphere inside a mine environment where very high ${ }^{222} \mathrm{Rn}$ levels were observed (Wallner et al., 2002). Large excess of ${ }^{210} \mathrm{Po}$ was seen attached to fine aerosol fractions leading to a significant disequilibrium and was attributed to the attachment of ${ }^{210} \mathrm{Po}$ to aerosols independent of the parent ${ }^{210} \mathrm{~Pb}$. This large disequilibrium requires a separation and/or a new source of ${ }^{210} \mathrm{Po}$ from the parent ${ }^{210} \mathrm{~Pb}$ that might normally be due to high temperature volatilization and separation of the ${ }^{210} \mathrm{Po}$ from the ${ }^{210} \mathrm{~Pb}$ (Lambert et al., 1979) or from chemical fractionation (Gaffney et al., 1992). Although there is no clear explanation for the large disequilibrium observed in this work (Wallner et al., 2002), this unexpected observation might be due to the high concentrations of the gaseous parent reported in the enclosed environment inside the mine. This fractionation effect has not been observed in ambient atmospheric samples. Under conditions of atmospheric levels of ${ }^{222} \mathrm{Rn}$, the daughter ${ }^{218} \mathrm{Po}$ becomes attached to fine aerosols within $40 \mathrm{~s}$ to 3 min after formation (Whittlestone, 1990). Therefore subsequent decay to form the long-lived ${ }^{210} \mathrm{~Pb}$ and its progeny will normally occur after initial attachment has taken place.

In the absence of wind-blown dust contamination or other sources of excess ${ }^{210} \mathrm{Bi}$ and $/$ or ${ }^{210} \mathrm{Po}$, a measure of the activity ratios of ${ }^{210} \mathrm{Bi} /{ }^{210} \mathrm{~Pb}$ and ${ }^{210} \mathrm{Po} /{ }^{210} \mathrm{~Pb}$ have been shown to be useful estimates of the atmospheric residence times of the attached particles (Baskaran and Shaw, 2001; Marley et al., 2000a). Key to the success of this approach is the ability to rapidly separate and determine the activities of ${ }^{210} \mathrm{~Pb},{ }^{210} \mathrm{Bi}$, and ${ }^{210} \mathrm{Po}$ in fine aerosol samples. A quick and easy method for the separation of these species in size-fractionated air samples has been developed based on solid phase extraction of their chloride complexes (Marley et al., 1999).

Reported here are measurements of the natural radionuclides ${ }^{7} \mathrm{Be}$ and ${ }^{210} \mathrm{~Pb}$, taken at two sites near Pittsburgh, PA in the summer of 2001 and at a site near Centerton, New Jersey in 1999 during the North East Oxidant and Particulate Study (NEOPS). Measurements of ${ }^{210} \mathrm{~Pb}$ and ${ }^{210} \mathrm{Po}$ were used to estimate fine aerosol residence times at all sites. Data was taken at the Pittsburgh sites with high-volume, slotted cascade impactors, which enable the determination of 1-day averaged apparent aerosol ages for the first time. The use of ${ }^{210} \mathrm{Po}$ to identify soil aerosol sources is presented and discussed in light of data from recent studies. Beryllium-7 was used for tracing and assessing the effects of upper air parcels on the lower atmosphere in this area. We also discuss how ${ }^{7} \mathrm{Be}$ measurements can be used to address PM-2.5 network sampling problems. Errors in the assessment of aerosol distributions can be large if sampling volumes are not determined accurately. Beryllium-7 values, which should be reasonably consistent on network scales, can help both to identify problems when they occur and to present a basis for correction of data.

\section{Experimental methods}

\subsection{Sample collection}

We define fine aerosols for this study as being $0.1-$ $1 \mu \mathrm{m}$ in aerodynamic diameter. Larger aerosols are considered coarse, and smaller aerosols are considered ultrafine. Initial studies have made use of an eight-stage Lundgren-type impactor with a flow rate of $11 \mathrm{~min}^{-1}$. Samples were collected on Teflon, with the final stage being quartz (Marley et al., 2000a, b). Because of the low flow used by this impactor, a sampling duration of 3-7 days, depending on the aerosol density, was required to obtain sufficient sample for analysis. We have examined the fine and coarse fractions of aerosols collected with this impactor from a number of urban and rural sites for ${ }^{7} \mathrm{Be}$ and for ${ }^{210} \mathrm{~Pb},{ }^{210} \mathrm{Bi}$, and ${ }^{210} \mathrm{Po}$ (Marley et al., $2000 \mathrm{a}, \mathrm{b})$. The results are consistent with the findings of others in that ${ }^{7} \mathrm{Be}$ cannot be detected on aerosols above $1 \mu \mathrm{m}$ in diameter (Papastefanou and Ioannidou, $1996 \mathrm{a}$ b). We have since used this fact to advantage to obtain samples of higher volume and shorter time 
resolution for ${ }^{7} \mathrm{Be}$ determination, by collecting the entire aerosol below a $2.5-\mu \mathrm{m}$ cutoff for direct gamma counting of the ${ }^{7} \mathrm{Be}$. This approach has allowed us to examine the concentrations of ${ }^{7} \mathrm{Be}$ in the Deer Park, Texas area as part of the recent TexAQS 2000 field study (Gaffney and Marley, 2002).

For the determination of ${ }^{210} \mathrm{~Pb},{ }^{210} \mathrm{Bi}$, and ${ }^{210} \mathrm{Po}$, we have found, based on measurements at a number of sites, that a sample collection system, which separates the fine fraction (diameters of $0.1-1.0 \mu \mathrm{m}$ ) from the coarse fraction $(>1.0 \mu \mathrm{m})$, allows the separation of fine combustion-related and secondary aerosols from coarse wind-blown soil and dust. By using a slotted-plate cascade impaction system equipped with a single separation stage, we have been able to collect samples of both fine and coarse aerosol fractions for the determination of ${ }^{210} \mathrm{~Pb},{ }^{210} \mathrm{Bi}$, and ${ }^{210} \mathrm{Po}$.

Samples were collected by using the low flow eightstage Lundgren-type impactor from 24 July to 9 August 1999 (Julian Day 205-222) at Centerton, New Jersey, a rural site, 30 miles south of Philadelphia and 25 miles north of Delaware Bay during the NEOPS. Samples were collected at two sites in the Pittsburgh area using the high flow Sierra impactor from 22 July to 30 July 2001 (Julian Day 202-210). Sierra impactors calibrated to operate at $40 \mathrm{cfm}\left(1.13 \mathrm{~m}^{3} \mathrm{~min}^{-1}\right)$ were set to collect 24-h samples beginning at midnight, Eastern Standard Time. The impactors were equipped with the number 4 plate for a particle size cut-off $\left(D_{50}\right)$ between $0.95 \mu \mathrm{m}$ at a flow rate of $40 \mathrm{cfm}$ and $1.10 \mu \mathrm{m}$ at a flow rate of $30 \mathrm{cfm}$. Aerosol samples were collected on quartz and cellulose filters. Air sample volumes ranged from 1320 to $1600 \mathrm{~m}^{3}$ of air.

The first Pittsburgh site was located approximately $5 \mathrm{~km}$ east of the city center on a building rooftop adjacent to Schenley Park (SP) $\left(40.4395^{\circ} \mathrm{N}\right.$ latitude and $79.9405^{\circ} \mathrm{W}$ longitude) at an altitude of approximately $310 \mathrm{~m}$. At this site the wind was predominantly from the south and the west during the study. The second site was located at the US Department of Energy National Energy Technology Laboratory (NETL) Ambient Air Monitoring Station. This site is approximately $15 \mathrm{~km}$ south of downtown Pittsburgh $\left(40.30655^{\circ} \mathrm{N}\right.$ latitude and $79.9794^{\circ} \mathrm{W}$ longitude) at an elevation of $325 \mathrm{~m}$ above sea level. The wind at this site was predominantly from the south and southwest. A total of nine samples were taken at the NETL site, and six were taken at the SP site.

\subsection{Beryllium 7 measurements}

Beryllium-7 was determined by direct gamma counting of the samples on the filter collection membranes with a high purity, low background, germanium (HPGe) crystal detector coupled to a 4096 channel multichannel analyzer (EG\&G Ortec, 92X Spectrum Master). The $478 \mathrm{keV}$ emission peak was used to determine ${ }^{7} \mathrm{Be}$ attached to fine aerosol particles. Sample counting rates are corrected for counting geometry, detector response factors, and the relative decay rates of the gamma radiation versus total decay processes.

Counting statistical errors were $<5 \%$.

\subsection{Radon daughter measurements}

The method of separation of ${ }^{210} \mathrm{Bi}$ and ${ }^{210} \mathrm{Po}$ from ${ }^{210} \mathrm{~Pb}$ has been described in detail elsewhere (Marley et al., 1999). Briefly, the samples were leached in $50 \%$ nitric acid over gentle heat for $24 \mathrm{~h}$. Filters were removed and washed with distilled water. After the leachate was taken to dryness overnight, approximately $25 \mathrm{ml}$ of $1 \mathrm{M} \mathrm{HCl}$ was added to redissolve the dried samples. Samples obtained on glass fiber membranes were then prefiltered with $0.45-\mu \mathrm{m}$ Millipore filters to remove particulate matter.

Samples were filtered slowly through $0.45-\mathrm{mm}, 0.2-\mu \mathrm{m}$ solid phase extraction disk membranes (3M Empore ${ }^{\mathrm{TM}}$ ) to extract the chloride complexes of ${ }^{210} \mathrm{Bi}$ and ${ }^{210} \mathrm{Po}$ and leave those of the parent ${ }^{210} \mathrm{~Pb}$ in the solution. Samples were rinsed several times with $1 \mathrm{M} \mathrm{HCl}$ to remove traces of lead from the membrane. After drying the membranes and neutralizing the $\mathrm{H}^{+}$, the filter disks were then placed directly in the low background beta counter (Tennelec LB4000 proportional counter) for simultaneous beta and alpha counting of the $1.16 \mathrm{MeV} \beta_{\max }$ of ${ }^{210} \mathrm{Bi}$ and the $5.3 \mathrm{MeV} \alpha$ particle of ${ }^{210} \mathrm{Po}$. The counting efficiencies were 0.4 for beta and 0.18 for alpha. Both ${ }^{210} \mathrm{~Pb}$ and ${ }^{210} \mathrm{Bi}$ were determined by beta counting of the ${ }^{210} \mathrm{Bi}$ daughter, while ${ }^{210} \mathrm{Po}$ was determined by simultaneous alpha counting. After sufficient time is allowed ( $\geqslant 30 \mathrm{~d})$ for regrowth of the ${ }^{210} \mathrm{Bi}$, a second extraction allows for the subsequent determination of ${ }^{210} \mathrm{~Pb}$. Counting statistical errors were $<5 \%$.

\subsection{Determination of atmospheric residence times}

Atmospheric residence times were calculated from ${ }^{210} \mathrm{Po} /{ }^{210} \mathrm{~Pb}$ and ${ }^{210} \mathrm{Bi} /{ }^{210} \mathrm{~Pb}$ ratios by the method described by Nevissi (Nevissi et al., 1974; Nevissi, 1991; Kuroda et al., 1978).

Briefly, the mean residence time of a sample of atmospheric aerosols in days $\left(T_{\mathrm{R}}\right)$ can be calculated from the activity ratio of the attached ${ }^{210} \mathrm{Po} /{ }^{210} \mathrm{~Pb}$ according to the theoretical decay rates as

$$
{ }^{210} \mathrm{Po} /{ }^{210} \mathrm{~Pb}=T_{\mathrm{R}}^{2} /\left(T_{\mathrm{R}}+1 / \lambda_{\mathrm{Bi}}\right)\left(T_{\mathrm{R}}+1 / \lambda_{\mathrm{Po}}\right) .
$$

Similarly, $T_{\mathrm{R}}$ can be calculated from the ${ }^{210} \mathrm{Bi} /{ }^{210} \mathrm{~Pb}$ activity ratio as

${ }^{210} \mathrm{Bi} /{ }^{210} \mathrm{~Pb}=T_{\mathrm{R}} /\left(T_{\mathrm{R}}+1 / \lambda_{\mathrm{Bi}}\right)$,

where $\lambda$ is the radioactive decay constant for each species. 
Note that the observed ages reported are not absolute but are an average representative of the compositions of the aerosols. For example, a mixture of $90 \%$ of a 5-dayold aerosol and $10 \%$ of a 100-day-old aerosol would have an apparent age of 14.5 days. Similarly, a 50:50 mixture of a 5-day-old aerosol and a 100-day-old aerosol would have a 52.5-day apparent residence time.

\section{Results and discussion}

\subsection{Beryllium-7}

Observed ${ }^{7} \mathrm{Be}$ levels in milli-Becquerels per cubic meter of air $\left(\mathrm{mBq} \mathrm{m}^{-3}\right)$ for the Pittsburgh samples are given in Table 1 and shown graphically in Fig. 2. All of the samples collected were fine aerosol fractions except for one coarse sample taken at the same time as the fine sample on 22 July at the NETL site. This course sample was examined and found to contain negligible amounts of ${ }^{7} \mathrm{Be}$ above the counting background observed in sample blanks. This is consistent with earlier findings that the ${ }^{7} \mathrm{Be}$ is exclusively attached to aerosols in the size range of $0.3-0.6 \mu \mathrm{m}$ diameter.

Very good agreement for ${ }^{7} \mathrm{Be}$ samples taken on 22,23 , 26, and 27 July (Julian Dates 202, 203, 206, and 207) can be seen for the two sampling stations. On the 28th (Julian Date 208) the NT site was about a factor of two higher than the SP site, albeit these are still fairly low levels of ${ }^{7} \mathrm{Be}$ in air. The sample on the 29th (Julian Date
209) was just the opposite, with the SP site being higher than the NT site. Generally we have seen that sites that are within a 20-mile distance of each other track fairly well, indicating that ${ }^{7} \mathrm{Be}$ is a fairly good measure of regional air transport. Also, over a short time period $(12 \mathrm{~h})$ in a citywide area, ${ }^{7} \mathrm{Be}$ levels are usually quite constant. For most of this period we observed this same trend. Problems with sample pumping rates were suspected for SP on the 28 July and for NT on 29 July. The reduced sample flows were reflected in the ${ }^{7} \mathrm{Be}$ results when compared with the nearby site. This observation could serve as a quality control measurement for PM-2.5 network sampling problems. The

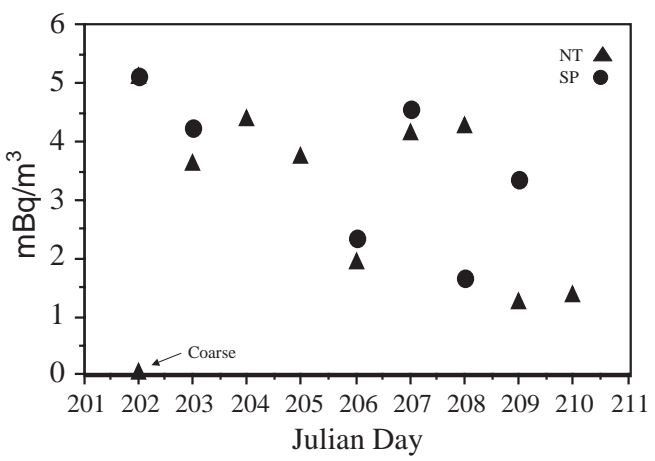

Fig. 2. Observed ${ }^{7}$ Be levels in milli-Becquerels per cubic meter of air $\left(\mathrm{mBq} \mathrm{m}^{-3}\right)$ for samples collected at NETL and SP. All samples were on the fine fractions with the exception of one coarse sample taken at NETL on Julian Day 202.

Table 1

${ }^{7}$ Be measurements on samples collected at the NETL (NT) and SP field sites. All samples were on the fine fractions with the exception of one coarse sample (C) taken at NETL at the beginning of the sampling period. Values in $\mathrm{mBq} \mathrm{m}^{-3}$ are corrected for the time delay in analysis and the radioactive decay of the ${ }^{7} \mathrm{Be}$

\begin{tabular}{lllcrr}
\hline Date & Site & Volume $\left(\mathrm{m}^{3}\right)$ & ${ }^{7} \mathrm{Be}(\mathrm{mBq})$ & ${ }^{7} \mathrm{Be}\left(\mathrm{mBq} \mathrm{m}^{-3}\right)$ & Atoms $^{7} \mathrm{Be}\left(\mathrm{m}^{-3}\right)$ \\
\hline 7.22 .01 & NT & 1471 & 7336 & 5.120 & 34,016 \\
7.22 .01 & NT $(\mathrm{C})$ & 1471 & 108 & 0.077 & 509 \\
7.22 .01 & SP & 1353 & 6587 & 5.125 & 34,052 \\
7.23 .01 & NT & 1432 & 4879 & 3.635 & 24,151 \\
7.23 .01 & SP & 1355 & 5237 & 4.235 & 28,139 \\
7.24 .01 & NT & 1462 & 5895 & 4.416 & 29,339 \\
7.25 .01 & NT & 1410 & 4825 & 3.748 & 24,903 \\
7.26 .01 & NT & 1572 & 2813 & 1.960 & 13,021 \\
7.26 .01 & SP & 1545 & 3296 & 2.367 & 15,728 \\
7.27 .01 & NT & 1605 & 5886 & 4.178 & 27,758 \\
7.27 .01 & SP & 1472 & 5846 & 4.583 & 30,451 \\
7.28 .01 & NT & 1358 & 5032 & 4.275 & 28,401 \\
7.28 .01 & SP & 1385 & 2011 & 1.675 & 11,130 \\
7.29 .01 & NT & 1589 & 1750 & 1.271 & 8444 \\
7.29 .01 & SP & 1609 & 4638 & 3.370 & 22,388 \\
7.30 .01 & NT & 1329 & 1580 & 1.372 & 9114 \\
Blank & & & 29 & & \\
Blank & & & 13 & & \\
\hline
\end{tabular}


reliability of high-volume measurements is often an issue in determining mass loadings of aerosols. Errors can be large if sampling volumes are not determined accurately. Power loss, heavy sample loading leading to reduction in air flow, and a variety of other problems in aerosol networks can result in an inaccurate assessment of aerosol distributions. Beryllium-7 values, which should be reasonably consistent on network scales, can help both to address errors in the volume of air sampled by high-volume filters and could be a means for correcting data when these problems occur.

Concentrations of ${ }^{7} \mathrm{Be}$ in $\mathrm{mBq} \mathrm{m}^{-3}$ in Pittsburgh were observed to range from 0.08 to 5.1 during the sample period. This is very comparable to levels observed in Houston during much of the study in 2000 (Gaffney and Marley, 2002), and lower by a factor of 3-7 than values observed in the Phoenix area during the summer of 2001 (Gaffney and Marley, 2003). The data do not indicate any significant "events" that would be interpreted as upper air mass transport into the Pittsburgh area during the sampling period. Examination of the data and comparison with maximum ozone values for this period also did not reveal significant correlation with ${ }^{7} \mathrm{Be}$ activity, consistent with results seen in the Houston area. Whereas, computer modeling by Lawrence Livermore National Laboratory (IMPACT) has indicated that there may have been some downward transport during the Phoenix study as indicated by higher ${ }^{7} \mathrm{Be}$ levels $(25-$ $30 \mathrm{mBq} \mathrm{m}^{-3}$ ) observed in the beginning of the sampling period. From these data, we derived an upper limit of 20-30 ppb for ozone background from stratospheric input (Gaffney and Marley, 2002).

\subsection{Radon daughters}

The results for ${ }^{210} \mathrm{~Pb}$ collected in Centerton, New Jersey in July-August 1999, with the low flow eightstage Lundgren-type impactor are given in Table 2.
Lead-210 levels range from 0.041 to $0.545 \mathrm{mBq} \mathrm{m}^{-3}$ with the highest values occurring in the size ranges below $2 \mu \mathrm{m}$. These levels were generally less than seen in previous work with samples taken in more arid areas (Marley et al., 2000a). Results for ${ }^{210} \mathrm{~Pb}$ determined on aerosol samples collected at NETL and SP in July 2001, with the high flow, single stage impactor are given in Table 3. Values range from 0.107 to $0.903 \mathrm{mBq} \mathrm{m}^{-3}$ in the fine fraction and $0.031-0.809 \mathrm{mBq} \mathrm{m}^{-3}$ in the coarse fraction. Lead-210 values reported on total atmospheric particulate samples obtained in the Chesapeake Bay have ranged from 0.268 to $1.412 \mathrm{mBq} \mathrm{m}^{-3}$ (Kim et al., 2000). This corresponds to the total ${ }^{210} \mathrm{~Pb}$ levels of 1.46 $1.75 \mathrm{mBq} \mathrm{m}^{-3}$ obtained in this study.

The apparent residence times, as calculated from the ${ }^{210} \mathrm{Po} /{ }^{210} \mathrm{~Pb}$ activity ratios, for the aerosol samples collected at Centerton are given in Table 4. As expected, apparent ages are longer for the larger sample sizes where contamination from resuspended material can be a problem. For the smaller sizes $(<1 \mu \mathrm{m})$, the residence times range from 24 to 44 days with an average of 28 days. These results are compared with the residence times calculated from ${ }^{210} \mathrm{Bi} /{ }^{210} \mathrm{~Pb}$ activity ratios in the size fractions below $2 \mu \mathrm{m}$. These values range from 25 to 47 days with an average difference between ages calculated by the two ratios of 8 days.

The apparent residence times for aerosols collected at NETL and SP are given in Table 5. The fine fractions gave results, which range from 17 to 37 days with an average of 22 days. The coarse fractions ranged from 42 to 14 days and averaged an apparent lifetime of 26 days. The lifetimes estimated for the Pittsburgh aerosols are somewhat shorter than those seen at Centerton. However, both areas yield lifetimes shorter than those previously observed in other areas of the country (Marley et al., 2000a). This is consistent with a local production of aerosols as well as a higher loading of water-soluble species in the northeast. Past results

Table 2

${ }^{210} \mathrm{~Pb}$ measurements on samples collected at the Centerton, NJ field site in July-August, 1999. All samples were collected with an eightstage low flow impactor

\begin{tabular}{|c|c|c|c|c|c|c|}
\hline \multirow[t]{2}{*}{ Diameter $(\mu \mathrm{m})$} & \multicolumn{2}{|l|}{$7 / 24-30$} & \multicolumn{2}{|l|}{$7 / 30-8 / 6$} & \multicolumn{2}{|l|}{$8 / 6-12$} \\
\hline & $\mathrm{mBq}$ & $\mathrm{MBq} \mathrm{m}^{-3}$ & $\mathrm{mBq}$ & $\mathrm{mBq} \mathrm{m}^{-3}$ & $\mathrm{mBq}$ & $\mathrm{mBq} \mathrm{m}^{-3}$ \\
\hline 9.2 & $13(0.7)$ & 0.052 & $11(0.6)$ & 0.041 & $11(0.6)$ & 0.045 \\
\hline 5.8 & $16(0.8)$ & 0.064 & $12(0.6)$ & 0.047 & $18(0.9)$ & 0.077 \\
\hline 4.2 & $10(0.5)$ & 0.042 & $9(0.5)$ & 0.035 & $12(0.6)$ & 0.049 \\
\hline 3.1 & $11(0.6)$ & 0.044 & $74(3.7)$ & 0.286 & $11(0.6)$ & 0.045 \\
\hline 2 & $16(0.8)$ & 0.065 & $36(1.8)$ & 0.139 & $56(2.8)$ & 0.236 \\
\hline 1.05 & $25(1.3)$ & 0.102 & $56(2.8)$ & 0.217 & $66(3.3)$ & 0.278 \\
\hline 0.62 & $51(2.6)$ & 0.210 & $86(4.3)$ & 0.331 & $46(2.3)$ & 0.193 \\
\hline 0.43 & $132(6.6)$ & 0.545 & $86(4.3)$ & 0.331 & $77(3.8)$ & 0.320 \\
\hline 0.1 & $81(4.0)$ & 0.333 & $82(4.1)$ & 0.316 & 87 (4.4) & 0.045 \\
\hline
\end{tabular}

Measurement uncertainties are given in parenthesis. 
Table 3

${ }^{210} \mathrm{~Pb}$ measurements from the NETL (NT) and SP field sites. All samples were on both fine $(<1 \mu \mathrm{m})$ and course $(>1 \mu \mathrm{m})$ fractions

\begin{tabular}{|c|c|c|c|c|c|c|}
\hline \multirow[t]{2}{*}{ Date } & \multirow[t]{2}{*}{ Site } & \multirow[t]{2}{*}{ Volume $\left(\mathrm{m}^{3}\right)$} & \multicolumn{2}{|l|}{ Fine } & \multicolumn{2}{|l|}{ Coarse } \\
\hline & & & ${ }^{210} \mathrm{~Pb}(\mathrm{mBq})$ & $\mathrm{mBq} \mathrm{m}^{-3}$ & ${ }^{210} \mathrm{~Pb}(\mathrm{mBq})$ & $\mathrm{mBqm}^{-3}$ \\
\hline 7.22 .01 & NT & 1471 & $1113(55)$ & 0.757 & $1190(60)$ & 0.809 \\
\hline 7.22 .01 & SP & 1353 & $1190(60)$ & 0.879 & $166(8.3)$ & 0.123 \\
\hline 7.23 .01 & NT & 1432 & $1223(61)$ & 0.854 & $305(15)$ & 0.213 \\
\hline 7.23 .01 & SP & 1355 & $1223(61)$ & 0.903 & $295(15)$ & 0.218 \\
\hline 7.24 .01 & NT & 1462 & $1285(64)$ & 0.879 & $505(25)$ & 0.345 \\
\hline 7.25 .01 & NT & 1410 & 869 (43) & 0.616 & $406(20)$ & 0.288 \\
\hline 7.26 .01 & NT & 1572 & 597 (30) & 0.380 & $86(4.3)$ & 0.055 \\
\hline 7.26 .01 & SP & 1545 & $550(27)$ & 0.356 & $174(8.7)$ & 0.113 \\
\hline 7.27 .01 & NT & 1605 & 281 (14) & 0.175 & $49(2.5)$ & 0.031 \\
\hline 7.27 .01 & SP & 1472 & 254 (13) & 0.173 & $49(2.5)$ & 0.033 \\
\hline 7.28 .01 & NT & 1358 & $792(40)$ & 0.583 & 97 (4.9) & 0.072 \\
\hline 7.28 .01 & SP & 1385 & $361(18)$ & 0.261 & $54(2.7)$ & 0.039 \\
\hline 7.29 .01 & NT & 1589 & $170(8.5)$ & 0.107 & $104(5.2)$ & 0.065 \\
\hline 7.29 .01 & SP & 1609 & 409 (20) & 0.255 & $691(35)$ & 0.429 \\
\hline 7.30 .01 & NT & 1329 & $197(9.9)$ & 0.148 & $48(2.4)$ & 0.036 \\
\hline
\end{tabular}

Measurement uncertainties are given in parenthesis.

Table 4

Apparent atmospheric residence times (ages in days) calculated from the ${ }^{210} \mathrm{Po} /{ }^{210} \mathrm{~Pb}$ and ${ }^{210} \mathrm{Bi} /{ }^{210} \mathrm{~Pb}$ ratios for aerosol samples collected at the Centerton, NJ field site in July-August, 1999. All samples were collected with an eight-stage low flow impactor

\begin{tabular}{|c|c|c|c|c|c|c|}
\hline \multirow[t]{2}{*}{ Diameter $(\mu \mathrm{m})$} & \multicolumn{2}{|l|}{$7 / 24-30$} & \multicolumn{2}{|l|}{$7 / 30-8 / 6$} & \multicolumn{2}{|l|}{$8 / 6-12$} \\
\hline & Age $\left({ }^{210} \mathrm{Po} /{ }^{210} \mathrm{~Pb}\right)$ & Age $\left({ }^{210} \mathrm{Bi} /{ }^{210} \mathrm{~Pb}\right)$ & Age $\left({ }^{210} \mathrm{Po} /{ }^{210} \mathrm{~Pb}\right)$ & Age $\left({ }^{210} \mathrm{Bi}^{/ 210} \mathrm{~Pb}\right)$ & Age $\left({ }^{210} \mathrm{Po} /{ }^{210} \mathrm{~Pb}\right)$ & Age $\left({ }^{210} \mathrm{Bi} /{ }^{210} \mathrm{~Pb}\right)$ \\
\hline 9.2 & 83 & & 112 & & 71 & \\
\hline 5.8 & 37 & & 93 & & 37 & \\
\hline 4.2 & 47 & & 117 & & 45 & \\
\hline 3.1 & 88 & & 19 & & 31 & \\
\hline 2 & 57 & 29 & 24 & 19 & 23 & 25 \\
\hline 1.05 & 44 & 30 & 16 & 13 & 24 & 29 \\
\hline 0.62 & 35 & 47 & 25 & 27 & 26 & 39 \\
\hline 0.43 & 25 & 41 & 27 & 27 & 25 & 34 \\
\hline 0.1 & 37 & 26 & 24 & 32 & 26 & 25 \\
\hline
\end{tabular}

(Gaffney et al., 1994) had indicated apparent lifetimes of approximately 10 days for aerosols being removed by wet deposition, in good agreement with the current understanding of the washout cycles in the atmosphere. Water-soluble aerosols such as the sulfates, in particular, will be removed more readily leading to apparently shorter residence times. The results from Pittsburgh may indicate that sulfate removal processes are occurring leading to the slightly shorter lifetimes than previously observed at other sites.

We have found that ages for aerosol size fractions $<1 \mu \mathrm{m}$ were very consistent from site to site, indicating that the aerosols had apparent atmospheric residence times of 10-40 days. The shorter lifetimes are consistent with typical values of 10 days observed in precipitation. The remaining interstitial aerosol would then be expected to have a longer apparent lifetime. It is likely to be composed of carbonaceous soot and other organic components that require some surface oxidation before they can become wettable and undergo subsequent washout. The concept here is that the wet deposition of soluble aerosols such as sulfate and nitrate would be consistent with these observations for precipitation and that the longer lifetimes observed for the air samples were due to non-hygroscopic fine particulate matter such as carbonaceous aerosols that will not immediately wet deposit.

The results from this work and from past studies indicate that a substantial amount of the fine background aerosol entered the study areas through longrange transport (Marley et al., 2000a,b). The longer lived aerosol species are more likely to be associated 
Table 5

Apparent atmospheric residence times (ages) for aerosol samples collected at the NETL (NT) and SP field sites in July 2001 calculated from the ${ }^{210} \mathrm{Po} /{ }^{210} \mathrm{~Pb}$ ratio. All samples were on both fine $(<1 \mu \mathrm{m})$ and course $(>1 \mu \mathrm{m})$ fractions

\begin{tabular}{llll}
\hline Date & Site & Fine & Coarse \\
\cline { 3 - 4 } & & Age (days) & Age (days) \\
\hline 7.22 .01 & NT & 20 & 14 \\
7.22 .01 & SP & 17 & 23 \\
7.23 .01 & NT & 23 & 24 \\
7.23 .01 & SP & 21 & 30 \\
7.24 .01 & NT & 21 & 21 \\
7.25 .01 & NT & 17 & 19 \\
7.26 .01 & NT & 24 & 25 \\
7.26 .01 & SP & 21 & 25 \\
7.27 .01 & NT & 37 & 33 \\
7.27 .01 & SP & 27 & 28 \\
7.28 .01 & NT & 18 & 25 \\
7.28 .01 & SP & 20 & 30 \\
7.29 .01 & NT & 21 & 27 \\
7.29 .01 & SP & 19 & 18 \\
7.30 .01 & NT & 25 & 42 \\
\hline
\end{tabular}

with carbonaceous aerosols that are not as rapidly wet or dry deposited, as sulfate and nitrate aerosols. This aerosol component probably contributes to the fine submicron aerosol number, with a less significant addition to the PM-2.5 micron mass. The consistency of the data at many sites also indicates that this background concentration of fine aerosols is fairly constant over wide areas. Recent studies on arctic haze have found similar lifetimes of 0-39 days for aerosol samples that were not size fractionated (Baskaran and Shaw, 2001). These data indicate that a significant fraction of fine aerosols can be transported long distances and that the background fine aerosol will still have a significant contribution from long-range transport. This transport of fine aerosols will lead to difficulties in determining local source contributions to the fine particulate mass. This should be considered when determining control strategies for this important health related material.

Samples collected in areas where wind-blown dust is significant, such as Phoenix and Mexico City, have exhibited very high apparent ages in the coarse fractions from soil contamination. Samples collected at Pittsburgh and Centerton do not show this trend. The coarse and fine aerosol fractions at both Pittsburgh and Centerton had similar apparent residence times, indicating that little excess ${ }^{210}$ Po could have come from soils or from coal-fired power plants, which previously had been suggested as a source of excess ${ }^{210}$ Po (Carvalho, 1995; Moore et al., 1976). These results indicate that the ${ }^{210} \mathrm{Po}$ content in the coarse fraction is an excellent indicator of wind-blown dust or soil contamination, because it is not dependent on soil type.

\section{Conclusions}

Natural radioactivity can be a very useful tool in understanding the physical processes affecting aerosols during their evolution in the atmosphere. These studies have shown that useful information can be obtained about the washout and transport of aerosols in the troposphere from the measurement of ${ }^{7} \mathrm{Be}$ and ${ }^{210} \mathrm{~Pb}$ and its progeny. As anticipated, ${ }^{7} \mathrm{Be}$ is a good tracer for fine aerosols. It is also a good indicator for upper air mass intrusions into the lower troposphere, which can be useful for addressing downward transport of ozone and aerosols and impacts on regional and urban air masses. High-volume sampling allows for sampling time periods of $6 \mathrm{~h}$ or shorter for direct gamma counting of ${ }^{7} \mathrm{Be}$ on sample collection media directly. This method is non-destructive and can allow other chemical analyses to be conducted as well, after the gamma counting is completed. Minimal upper air intrusions were evident during the sampling period from the ${ }^{7} \mathrm{Be}$ data collected in the Pittsburgh area. Comparison of ${ }^{7} \mathrm{Be}$ measurements between nearby sampling sites can be used to address PM-2.5 network sampling problems. Errors in the assessment of aerosol distributions can be large if sampling volumes are not determined accurately. Beryllium-7 values, which should be reasonably consistent on the citywide network scales, can help both to identify problems when they occur and to present a basis for correction of data.

In the absence of wind-blown dust contamination or other sources of excess ${ }^{210} \mathrm{Bi}$ and/or ${ }^{210} \mathrm{Po}$, a measure of the activity ratios of ${ }^{210} \mathrm{Bi} /{ }^{210} \mathrm{~Pb}$ and ${ }^{210} \mathrm{Po} /{ }^{210} \mathrm{~Pb}$ can be useful estimates of the apparent atmospheric residence times of the attached particles. High-volume sampling coupled with a single $1 \mu \mathrm{m}$ impactor stage enables rapid aerosol collection for determination of ${ }^{210} \mathrm{~Pb}$ and its progeny. Apparent residence times of aerosols collected in the Pittsburgh area are shorter than those collected at Centerton, New Jersey and both areas are shorter than those observed in other areas, indicating a local source of aerosols as well as a higher loading of water-soluble species such as sulfate or nitrate. Results from fine and course fractions were similar for both Pittsburgh and Centerton sites, indicating the presence of little excess ${ }^{210}$ Po from wind-blown soil or from coal-fired power plants in coarse fractions. Clearly, a significant fraction of fine aerosols is transported for more than 20 days. This means that regional if not global control strategies are needed to maintain adequate air quality and reduce the impacts of elevated levels of fine aerosols on our weather and climate. 


\section{Acknowledgements}

The authors wish to thank Donald V. Martello, and Natalie J. Anderson for their help in collecting the Pittsburgh samples. The authors also want to thank Paul C. Rohar and Karl Waldner at NETL for their assistance with the hi-volume sampling.

This work was supported by the US Department of Energy, Office of Science, Office of Biological and Environmental Research, Atmospheric Chemistry Program and Global Change Education Program, under contract W-31-109-Eng-38. This work was performed at Argonne National Laboratory.

\section{References}

Anand, S.J.S., Rangarajan, C., 1990. Studies on the activity ratios of polonium-210 to lead-210 and their dry-deposition velocities at Bombay in India. Journal of Environmental Reaction 11, 235-250.

Baskaran, M., Shaw, G.E., 2001. Residence time of arctic haze aerosols using the concentrations and activity ratios of ${ }^{210} \mathrm{Po}$, ${ }^{210} \mathrm{~Pb}$ and ${ }^{7} \mathrm{Be}$. Journal of Aerosol Science 32, 443-452.

Beeson, W.L., Abbey, D.E., Knutsen, S.F., 1998. Long-term concentrations of ambient air pollutants and incident lung cancer in California adults: results from the AHSMOG study. Environmental Health Perspectives 106, 813-822.

Bhandari, N., Lad, D., Rama, 1970. Vertical structure of the troposphere as revealed by radioactive tracer studies. Journal of Geophysical Research 75, 2974-2980.

Burton, W.M., Stewart, N.G., 1960. Use of long-lived natural radioactivity as an atmospheric tracer. Nature 186, 584-589.

Carvalho, F.P., 1995. Origins and concentrations of ${ }^{222} \mathrm{Rn}$, ${ }^{210} \mathrm{~Pb},{ }^{210} \mathrm{Bi}$, and ${ }^{210} \mathrm{Po}$ in the surface air at Lisbon, Portugal, at the Atlantic edge of the European continental landmass. Atmospheric Environment 29, 1809-1819.

Fry, L.M., Menon, K.K., 1962. Determination of tropospheric residence times of ${ }^{210} \mathrm{~Pb}$. Science 137, 994-995.

Gaffney, J.S., Marley, N.A., 2002. Measurements of beryllium7 and ozone at Deer Park during the Texas 2000 Air Quality Study. Fourth Conference on Atmospheric Chemistry Symposium: Urban, Regional, and Global-Scale Impacts of Air Pollutants. 82nd American Meteorological Society National Meeting, Orlando, FL, 2002, Proceedings Volume, Paper P1.4, pp. 187-191.

Gaffney, J.S., Marley, N.A., 2003. Natural radioactivity in aerosols and what it can tell us. 83rd American Meteorological Society National Meeting, Fifth Conference on Atmospheric Chemistry, American Meteorological Society National Meeting, Long Beach, CA, Proceedings Volume: Paper 7.2, 4pp.

Gaffney, J.S., Marley, N.A., Orlandini, K.A., 1992. Evidence for thorium isotopic disequilibria in natural waters due to organic complexation: geochemical implications. Environmental Science and Technology 26, 1248-1250.

Gaffney, J.S., Orlandini, K.A., Marley, N.A., Popp, C.J., 1994. Measurement of ${ }^{7} \mathrm{Be}$ and ${ }^{210} \mathrm{~Pb}$ in rain, snow, and hail. Journal of Applied Meteorology 33, 869-873.
Gaffney, J.S., Orlandini, K.A., Marley, N.A., Popp, C.J., 1995. Reply to comments on measurement of ${ }^{7} \mathrm{Be}$ and ${ }^{210} \mathrm{~Pb}$ in rain, snow, and hail. Journal of Applied Meteorology 34, 2106-2109.

Gäggeler, H.W., 1995. Radioactivity in the atmosphere. Radiochimica Acta 70/71, 345-353.

Gavini, M.B., Beck, J.N., Kuroda, P.K., 1974. Mean residence times of the long-lived radon daughters in the atmosphere. Journal of Geophysical Research 79, 4447-4452.

Holland, W.W., Bennett, A.E., Cameron, I.R., Florey, C.V., Leeder, S.R., Schilling, R.S.F., Swan, A.V., Walker, R.E., 1979. Health effects of particulate pollution: reappraising the evidence. American Journal of Epidemiology 110, 525-659.

Junge, C.E., 1963. Air Chemistry and Radioactivity. Academic Press, San Diego, CA, pp. 209-288.

Kim, G., Hussain, N., Church, T.M., 2000. Excess ${ }^{210}$ Po in the coastal atmosphere. Tellus 52B, 74-80.

Kuroda, P.K., Daniel, P.Y., Nevissi, A., Beck, J.N., Meason, J.L., 1978. Atmospheric residence times of strontium-90 and lead-210. Journal of Radioanalytical Chemistry 43, 443-450.

Lambert, G., Buisson, A., Sanak, J., Ardouin, B., 1979. Modification of the atmospheric polonium 210 to lead 210 ratio by volcanic emission. Journal of Geophysical Research 84, 6980-6986.

Lighty, J.S., Veranth, J.M., Sarofim, A.F., 2000. Combustion aerosols: factors governing their size and composition and implications to human health. Journal of the Air and Waste Management Association 50, 1565-1681.

Lipfert, F.W., Morris, S.C., Wyzga, R.E., 2000. Daily mortality in the Philadelphia metropolitan area and size-classified particulate matter. Journal of the Air and Waste Management Association 50, 1501-1513.

Marley, N.A., Gaffney, J.S., Orlandini, K.A., Drayton, P.J., Cunningham, M.M., 1999. An improved method for the separation of ${ }^{210} \mathrm{Bi}$ and ${ }^{210} \mathrm{Po}$ from ${ }^{210} \mathrm{~Pb}$ using solid phase extraction disk membranes: environmental applications. Radiochimica Acta 85, 71-78.

Marley, N.A., Gaffney, J.S., Cunningham, M.M., Orlandini, K.A., Paode, R., Drayton, P.J., 2000a. Measurement of ${ }^{210} \mathrm{~Pb},{ }^{210} \mathrm{Po}$, and ${ }^{210} \mathrm{Bi}$ in size fractionated atmospheric aerosols: an estimate of fine aerosol residence times. Aerosol Science and Technology 32, 569-583.

Marley, N.A., Gaffney, J.S., Drayton, P.J., Cunningham, M.M., Mielcarek, C., Ravelo, R., Wagner, C., 2000 b. Residence times of fine tropospheric aerosols as determined by ${ }^{210} \mathrm{~Pb}$ progeny. Symposium on Atmospheric Chemistry Issues in the 21st Century, 80th National Meeting of the American Meteorological Society (AMS) 2000, Long Beach, CA, January 2000, Proceedings Volume: AMS Preprint 1.1, pp. $1-5$.

Moore, H.E., Martell, E.A., Poet, S.F., 1976. Sources of polonium-210 in atmosphere. Environmental Science and Technology 10, 586-591.

Nevissi, A.E., 1991. Measurement of lead-210, bismuth-210, and polonium-210 in environmental samples. Journal of Radioanalytical and Nuclear Chemistry 148, 121-131.

Nevissi, A.E., Beck, J.N., Kurda, P.K., 1974. Long-lived radon daughters as atmospheric radioactive tracers. Health Physics 27, 181-188. 
Papastefanou, C., Ioannidou, A., 1996a. Beryllium \& aerosols in ambient air. Environment International 22, S125-S130.

Papastefanou, C., Ioannidou, A., 1996b. Influence of air pollutants in the ${ }^{7} \mathrm{Be}$ size distribution of atmospheric aerosols. Aerosol Science and Technology 24, 102-106.

Pierson, D.H., Cambray, R.S., Spicer, G.S., 1966. Lead-210 and polonium-210 in the atmosphere. Tellus 18, 428-433.

Poet, S.E., Moore, H.E., Martell, E.A., 1972. ${ }^{210} \mathrm{~Pb},{ }^{210} \mathrm{Bi}$ and ${ }^{210} \mathrm{Po}$ in the atmosphere: accurate ratio measurement and application to aerosol residence time determination. Journal of Geophysical Research 77, 6515-6527.

Pope, C.A., 2000. Review: epidemiological basis for particulate air pollution health standards. Aerosol Science and Technology 32, 4-14.

Rangarajan, C., Madhavan, R., Gopalakrishnan, S.S., 1986. Spatial and temporal distribution of lead-210 in the surface layers of the atmosphere. Journal of Environmental Radioactivity $3,23-33$.

Schwartz, J., Dockery, D.W., Neas, L.M., 1996. Is daily mortality associated specifically with fine particles? Journal of the Air and Waste Management Association 46, 927-939.
Schwartz, J., Ballester, F., Saez, M., Perez-Hoyos, S., Bellido, J., Cambra, K., Arribas, F., Canada, A., Perez-Boillos, M.J., Sunyer, J., 2001. The concentration-response relationship between air pollution and daily deaths. Environmental Health Perspectives 109, 1001-1006.

Sanak, J., Gaudry, A., Lambert, G., 1981. Size distribution of $\mathrm{Pb}-210$ aerosols over oceans. Geophysical Research Letters 8, 1067-1069.

Tokieda, T., Yamanaka, K., Harada, K., Tsunogai, S., 1996. Seasonal variations of residence time and upper atmospheric contribution of aerosols studied with $\mathrm{Pb}-210$, Bi-210, Po-210 and Be-7. Tellus 48B, 690-702.

Tsunogai, S., Fukuda, K., 1974. ${ }^{210} \mathrm{~Pb},{ }^{210} \mathrm{Bi}$ and ${ }^{210} \mathrm{Po}$ in meteoric precipitation and the residence time of tropospheric aerosol. Geochemical Journal 8, 141-152.

Wallner, G., Berner, A., Irlweck, K., 2002. Aerosols: unexpected disequilibrium phenomena between airborne radio activities of lead-210 and its progenies bismuth-210 and polonium-210. Naturwissenschaften 89, 569-574.

Whittlestone, S., 1990. Radon daughter disequilibrium in the lower marine boundary layer. Journal of the Atmospheric Chemistry $11,27-42$. 


\title{
Light scattering by fine particles during the Pittsburgh Air Quality Study: Measurements and modeling
}

\author{
Juan C. Cabada, Andrey Khlystov, and Ann E. Wittig \\ Department of Chemical Engineering, Carnegie Mellon University, Pittsburgh, Pennsylvania, USA \\ Christodoulos Pilinis \\ Department of Environmental Studies, University of Aegean, Mytilene, Greece
}

Spyros N. Pandis

Departments of Chemical Engineering and Engineering and Public Policy, Carnegie Mellon University, Pittsburgh, Pennsylvania, USA

Received 16 September 2003; revised 3 May 2004; accepted 10 May 2004; published 17 July 2004.

[1] Light scattering by fine particulate matter was measured during the Pittsburgh Air Quality Study (PAQS) as close to ambient conditions as possible. Several approaches are used for the theoretical calculation of the scattering coefficient and the results are compared to the direct measurements. The first approach uses ambient high time and daily resolved $\mathrm{PM}_{2.5}$ composition concentrations to estimate the scattering coefficient assuming that the aerosol is an external mixture. The second approach uses a thermodynamic model and Mie theory to predict the scattering coefficient of aerosols from daily size composition distributions. The third approach introduces high time and daily resolved ambient aerosol water concentrations and concentrations of sulfate, nitrate, organic material, and soil with fixed scattering efficiencies. During the summer the first two approaches underestimate the measured scattering coefficient by around $20 \%$. Agreement within experimental error is obtained between the measured scattering coefficient and the model, incorporating measured water aerosol concentrations. During the winter the first two approaches tend to overpredict the measured scattering by around $15 \%$. This overprediction is weakly correlated to the organic mass. The modeling approaches suggest that sulfate and the associated water contribute $65-73 \%$ to the scattering coefficient during the summer, with organic material contributing $25-30 \%$. During the winter, sulfate accounts for $35-43 \%$, nitrate accounts for $24-32 \%$, and organic material accounts for $30-40 \%$ of the scattering coefficient. INDEX TERMS: 0305 Atmospheric Composition and Structure: Aerosols and particles $(0345,4801) ; 0345$ Atmospheric Composition and Structure: Pollutionurban and regional (0305); 0365 Atmospheric Composition and Structure: Troposphere-composition and chemistry; KEYWORDS: atmospheric aerosols, optical properties, Pittsburgh Air Quality Study

Citation: Cabada, J. C., A. Khlystov, A. E. Wittig, C. Pilinis, and S. N. Pandis (2004), Light scattering by fine particles during the Pittsburgh Air Quality Study: Measurements and modeling, J. Geophys. Res., 109, D16S03, doi:10.1029/2003JD004155.

\section{Introduction}

[2] Visibility degradation is probably the most readily perceived impact of atmospheric pollution. The protection of good visibility conditions for scenic views and the reduction of haze in rural and urban areas is one of the main goals of the Clean Air Act (U.S. Environmental Protection Agency, 1999). Figure 1 shows the effect of fine particle mass concentrations on visibility impairment for the Pittsburgh metropolitan area. High fine particulate concentrations can deteriorate visibility reducing the visual range to a few hundred meters.
[3] Fine particulate matter (below 2.5 microns in aerodynamic size) is efficient in scattering light in the atmosphere and is the main cause of visibility degradation over urban and remote areas. Light scattering from fine particles depends on various factors such as chemical composition, ambient relative humidity, and size distribution of the aerosol [Ouimette and Flagan, 1982; Sloane, 1984; Seinfeld and Pandis, 1998; Watson, 2002]. Sulfate and organic material are the most common components of the fine particulate matter (below $2.5 \mu \mathrm{m}$ ) for the eastern United States [Malm, 2000; U.S. Environmental Protection Agency, 2002; NARSTO, 2003]. The mass of these particles tends to accumulate between 0.1 and $1 \mu \mathrm{m}$. Scattering by particles of sizes comparable to the wavelength of visible light (0.4$0.7 \mu \mathrm{m}$ ) is mostly responsible for visibility reduction in the atmosphere. 

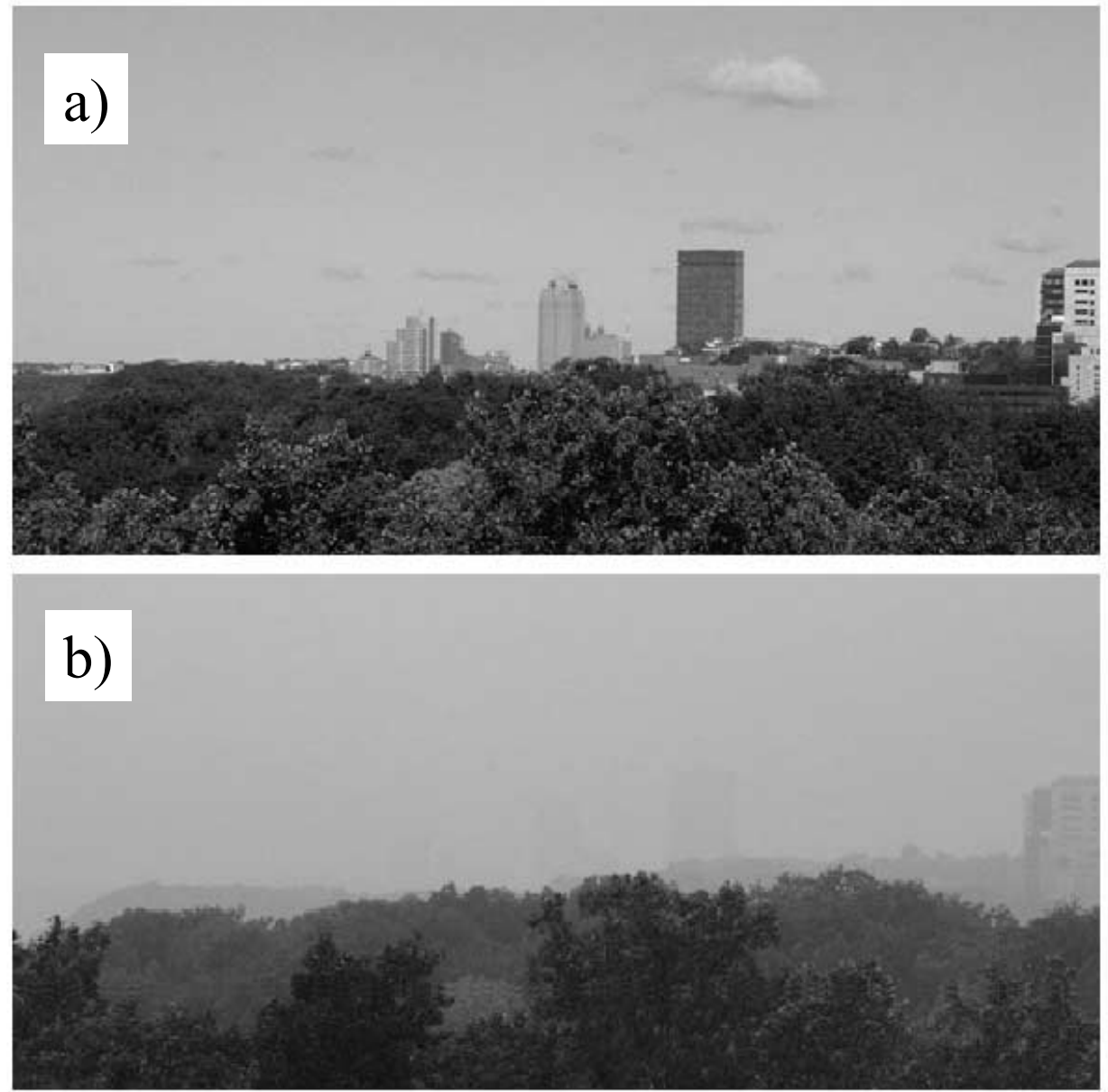

Figure 1. Comparison of visual characteristics during PAQS. View of downtown Pittsburgh from the PAQS main site at Schenley Park. (a) Day with low aerosol concentrations: $\mathrm{PM}_{2.5}=6 \mu \mathrm{g} / \mathrm{m}^{3}, \mathrm{~b}_{\mathrm{sp}}=$ $5 \mathrm{Mm}^{-1}, \mathrm{RH}=60 \%$. (b) Polluted day: $\mathrm{PM}_{2.5}=70 \mu \mathrm{g} / \mathrm{m}^{3}, \mathrm{~b}_{\mathrm{sp}}=255 \mathrm{Mm}^{-1}, \mathrm{RH}=74 \%$. See color version of this figure at back of this issue.

[4] The presence of water in the aerosol phase increases the ability of these particles to scatter light. Hygroscopic growth of inorganic salts as a function of relative humidity has been thoroughly studied by several authors [Tang et al., 1981; Tang, 1996; Cruz and Pandis, 1998, 2000; Hand et al., 2000]. Thermodynamic models that calculate the equilibrium of the inorganic aerosol with the gas phase have been developed, and the estimates of these models are usually in agreement with the laboratory measurements [Pilinis and Seinfeld, 1987; Wexler and Seinfeld, 1990, 1991; Nenes et al., 1998; Ansari and Pandis, 1999].

[5] The role of organic material in the aerosol water uptake is not well understood. In some cases an increase in aerosol water is observed, while in others a decrease in aerosol water content is reported when organics are added to atmospheric inorganic aerosols [Cruz and Pandis, 1998, 2000; Saxena et al., 1995]. Relatively little theoretical work has been done trying to explain the water uptake from organic aerosol, mainly because of the difficulties in estimating the physical properties of organic aerosols, which are composed of hundreds of different compounds [Saxena and Hildemann, 1997; Jang and Kamens, 1998; Ansari and Pandis, 2000; Hemming and Seinfeld, 2001; Koo et al., 2003]. However, Ansari and Pandis [2000] reported that the secondary organic aerosol (SOA) fraction of the aerosol could account for around $20 \%$ of the aerosol water. Koo et al. [2003] reported that SOA increased the water uptake from aerosols by $2-15 \%$, depending on the conditions.

[6] Several authors have used approaches of different complexity to estimate the visibility degradation due to aerosols in the atmosphere [Watson, 2002; Molenar et al., 1994; White, 1986]. The simpler models are based on bulk concentrations of fine particulate matter (PM) [Trijonis et al., 1988; Charlson et al., 1992; Gebhart et al., 1994; Malm et al., 1994, 2000a]. These models assume an external mixture of the aerosol components and average scattering efficiencies for each individual compound to determine the increase in the scattering efficiency as a function of relative humidity. In more complex models, additional characteristics of the aerosol (i.e., mixing state, size distributions of the different compounds, and the physical shape of the particles) are taken into account, and Mie theory is used to calculate the optical properties [Heintzenberg, 1980; Jaggard et al., 1981; Ouimiette and Flagan, 1982; Sloane, 1984; Wu et al., 1996; Hegg et al., 1993; Zhang et al., 1994; Lowenthal et al., 1995; Tang, 1996]. In the most advanced models the aerosol water 
content is calculated by thermodynamic models or estimated from semiempirical relationships [Sloane and Wolff, 1985; Sloane, 1983, 1986; Larson et al., 1988; Pilinis, 1989; Nemesure et al., 1995; Pilinis et al., 1995; Quinn et al., 1995; McMurry et al., 1996; West et al., 1998; Koloutsou-Vakakis et al., 1998; Malm et al., 2000a, 2000b].

[7] Most previous studies found that measured scattering was generally consistent with the aerosol composition measurements [White et al., 1994; Pilinis et al., 1995; Malm et al., 1996], assuming that the inorganic aerosol components were responsible for the PM water uptake [Malm et al., 2003]. Discrepancies between predictions and observations have been attributed to PM composition measurement errors or lack of high temporal resolution composition measurements, lack of information about the aerosol size distribution, lack of understanding of the particle hygroscopic growth, and uncertainties in the scattering efficiencies of the different components.

[8] Despite the advances, there are a number of remaining issues. The ability of the more advanced theoretical tools to simulate visibility reduction has rarely been tested in the eastern United States. The few available studies [Sloane and Wolff, 1985; Lowenthal et al., 1995; Malm et al., 2000a; Kreisberg et al., 2001; Malm and Day, 2000] have relied on daily or semidaily average composition measurements and not on higher-resolution information. The causes of the discrepancies between models and measurements are not well understood because the aerosol water concentration has not been measured in any of the previous field campaigns. Finally, the role of organics in the water uptake by aerosols and therefore their contribution to scattering is yet to be elucidated.

[9] In this work, several modeling approaches of varying complexity are used to predict the scattering coefficient of aerosol particles using composition and size distribution measurements during the Pittsburgh Air Quality Study (PAQS) during the summer of 2001 (July-August) and during the winter of 2002 (January). The results of each approach are compared to the measured scattering coefficient from nephelometry. High time resolution (1 hour) and daily measurements of the principal components of the fine particulate matter are used for the estimations of the scattering coefficient for the simplest approach proposed. Daily concentrations and size distributions are used for a thermodynamic model applying Mie theory to estimate the scattering coefficient. Finally, for the first time we use continuous measurements of the aerosol water concentration to directly calculate its contribution to light scattering. We conclude by addressing the role of aerosol water and organics in the optical properties of the aerosol measured at PAQS.

\section{Experiment}

\subsection{Scattering Coefficient Measurements}

[10] An integrating nephelometer (NGN-3, Optec, Inc., Lowell, Michigan) was used to measure the scattering coefficient of $\mathrm{PM}_{2.5}$ continuously during the summer of 2001 (July-August) and during the winter of 2002 (January) at PAQS. The main sampling site was located on the top of a hill just outside the Carnegie Mellon University campus, around $5 \mathrm{~km}$ from downtown Pittsburgh.

[11] Scatter of light inside the instrument chamber is measured over a $170^{\circ}$ angle, from $5^{\circ}$ to $175^{\circ}$ at a range of wavelengths, peaking near $550 \mathrm{~nm}$ for typical atmospheric aerosols [Molenar, 1997]. A cyclone is used in the sampling inlet for the collection of $\mathrm{PM}_{2.5}$ from ambient air. Raleigh scattering (clean air) calibrations were performed automatically by the instrument approximately every 12 hours. The nephelometer was operated as close to ambient conditions as possible, without using the heater or any treatment of the incoming sample air. However, small differences in the ambient air temperature and the chamber were still observed. Air temperature inside the chamber was on average $3^{\circ} \mathrm{C}$ higher than the ambient air temperature for the length of the project. Differences in the chamber and the ambient temperature affect the relative humidity of the sampled air. The chamber relative humidity was estimated on the basis of the Clausius-Clapeyron equation [Gebhart et al., 1994]:

$$
\mathrm{RH}_{\text {cham }}=\mathrm{RH}_{\mathrm{amb}} \cdot \exp \left(5210.5 \cdot\left(\frac{\mathrm{T}_{\text {amb }}-\mathrm{T}_{\text {cham }}}{\mathrm{T}_{\mathrm{amb}} \cdot \mathrm{T}_{\text {cham }}}\right)\right) \text {, }
$$

where $\mathrm{RH}_{\text {cham }}$ is the relative humidity inside the nephelometer measuring chamber, $\mathrm{RH}_{\mathrm{amb}}$ is the ambient relative humidity, $\mathrm{T}_{\mathrm{amb}}$ is the ambient temperature $\left({ }^{\circ} \mathrm{K}\right)$, and $\mathrm{T}_{\text {cham }}$ is the nephelometer chamber temperature $\left({ }^{\circ} \mathrm{K}\right)$. Relative humidity inside the chamber of the nephelometer ranged from 15 to $92 \%$ during the measurements.

[12] Ambient relative humidity (RH) was measured by the meteorological station installed at the main site of the PAQS with a Campbell Scientific HMP45C probe. The probe contained a Vaisala HUMICAP $^{\circledR} 180$ capacitive relative humidity sensor (expected accuracy at $20^{\circ} \mathrm{C}$ is $\pm 2 \%$ from 0 to $90 \% \mathrm{RH}$ and $\pm 3 \%$ from 90 to $100 \% \mathrm{RH})$.

\subsection{Aerosol Sampling}

[13] Several samplers were operated during the Pittsburgh Supersite project for the determination and speciation of $\mathrm{PM}_{2.5}$ aerosol mass. Continuous and semicontinuous instruments were used for the determination of total $\mathrm{PM}_{2.5}$ mass, organic and elemental carbon, sulfate, nitrate, and ammonium aerosol concentrations from July 2001 to July 2002 [Wittig et al., 2004a]. The uncertainties in the measurements of the major ions are described by Wittig et al. [2004a, $2004 \mathrm{~b}$ ] and the merging of the different data sets for the same parameter to one self-consistent data set in the work of Takahama et al. [2004].

[14] A tamper element oscillating microscale (TEOM, Series 1400a, Rupprecht and Patashnick Co., Inc., Albany, New York) was operated continuously during PAQS, reporting $\mathrm{PM}_{2.5}$ mass concentrations. The TEOM operated at $30^{\circ} \mathrm{C}$ and was equipped with a Nafion diffusion dryer sample equilibration system (SES, Rupprecht and Patashnick Co., Inc.). Atmospheric aerosol was collected on quartz fiber filters using several sampler configurations for the determination of the carbonaceous material concentrations [Cabada et al., 2004a; Subramanian et al., 2004]. High time resolution samples were collected with a denuded in situ semicontinuous sampler (Carbon aerosol analysis field instrument, Sunset Lab, Tigard, Oregon), 
with 2-4 hour time resolution. The denuder was used to remove gas-phase organics and to minimize the positive organic aerosol sampling artifact. Daily samples were collected using an undenuded sampler with the use of backup filters for the correction of positive and negative artifacts [Subramanian et al., 2004]. The ambient organic and elemental carbon concentrations were determined following the National Institute for Occupational Safety and Health protocol with the thermal/optical transmittance method and a flame ionozation detector (FID) [Cabada et al., 2004a]). Concentrations of secondary organic aerosol (SOA) were estimated using the elemental carbon (EC) tracer method [Cabada et al., 2004a]. Aerosol concentrations of sulfate and nitrate were measured on a 10-min basis using Rupprecht and Patashnick (R\&P) instrument models $8400 \mathrm{~N}$ and $8400 \mathrm{~S}$, respectively [Wittig et al., 2004b]. High time resolution total (gas + aerosol) ammonium, nitrate, and chloride concentrations were measured using a steam sampler [Khlystov et al., 1995]. The crustal material concentrations were less than $5 \%$ of the $\mathrm{PM}_{2.5}$ concentration [Rees et al., 2004]. The comparison of the aerosol mass concentration (measured using the federal reference method) and the sum of the component concentrations during PAQS are discussed by Rees et al. [2004]. After accounting for the water retained on the filters when the aerosol was acidic, mass balance was achieved within $10 \%$ for all days, well within experimental error. The aerosol was neutral during the winter but was often acidic during the daytime of the summer days [Rees et al., 2004].

[15] Size distributions of the major inorganic and carbonaceous material were measured using a micro-orifice uniform deposit impactor (MOUDI). Daily samples were collected for 15 days during the summer (July 2001) and for 8 days during the winter intensive (January 2002) [Cabada et al., 2004b]. Samples were collected on aluminum foils for analysis of carbonaceous material and on Teflon filters for analysis of particle mass and inorganic compounds. Carbonaceous concentrations from the MOUDI samples were determined by the thermal/optical method (same as the filter-based samplers), and the inorganic compound concentrations were determined by ion chromatography. During the summer, around $50 \%$ of the organic material was lost from the aluminum foils as compared to a filterbased sampler. The organic aerosol losses were negligible during the winter. The corrected MOUDI values (uniform correction for all stages), based on the artifact corrected filter measurements, were used in the present study. Cabada et al. [2004b] showed that these corrections satisfy the mass balance of all MOUDI stages.

[16] The comparison of the inorganic aerosol measurements is discussed by Wittig et al. [2004a], the comparison of the organic aerosol measurements is discussed by Subramanian et al. [2004] and Cabada et al. [2004a], and the comparison of the MOUDI results with the bulk measurements is discussed by Cabada et al. [2004b]. The results of all of these approaches with different time and size resolutions were merged into one self-consistent data set [Takahama et al., 2004; Cabada et al., 2004b] using the artifact-corrected traditional filter measurements as the basis. When more than one continuous method was used, the average of the corrected values was used for each hour. In the present study the daily average values of the continuous measurements are the same as these of the daily filter measurements, and the sum of the MOUDI concentrations are equal to the artifact corrected bulk measurements.

\subsection{Aerosol Water Content}

[17] A dry-ambient aerosol size spectrometer (DAASS) system [Stanier et al., 2004] operated during PAQS reporting number, surface area, and volume distributions of aerosols. The system consists of two scanning mobility particle sizers (SMPS, TSI 3936N25 and TSI 3936L10) and an aerodynamic particle sizer (APS, TSI APS 3320) that measure the aerosol size distribution between $3 \mathrm{~nm}$ and $10 \mu \mathrm{m}$ in diameter. The inlets of the instruments and their sheath air lines were equipped with computer controlled valves that direct air through Nafion dryers or bypass them. The Nafion dryers reduce the RH to below $30 \%$, at which ambient particles are expected to lose most or all of the water. The instrument cycles between dried and the ambient conditions every $7 \mathrm{~min}$ and is synchronized with the scan times of the aerosol spectrometers. The aerosol water concentrations measurements are described in detail by A. Khlystov et al. (Water content of ambient aerosol during the Pittsburgh Air Quality Study, submitted to Journal of Geophysical Research, 2004, hereinafter referred to as Khlystov et al., submitted manuscript, 2004). The amount of aerosol water can be found from the difference of the integrated dry and wet aerosol volumes. Owing to malfunctions in the APS system, aerosol water concentrations are only calculated from this approach for July and August 2001.

\section{Scattering Coefficient Calculation 3.1. Approach 1a: Use of Bulk $\mathbf{P M}_{2.5}$ Composition (High Time Resolution)}

[18] The scattering coefficient was calculated assuming external mixing of the aerosol components and utilizing bulk $\mathrm{PM}_{2.5}$ concentrations of the different compounds with the ability to scatter light, using the relationship proposed by Malm et al. [2000a]:

$$
\begin{aligned}
\mathrm{Bsp}= & 4.125 \times\left[\mathrm{SO}_{4}^{2-}\right] \times \mathrm{f}(\mathrm{RH})_{\text {salt }}+3.87 \times\left[\mathrm{NO}_{3}^{-}\right] \times \mathrm{f}(\mathrm{RH})_{\text {salt }} \\
& +4 \times[\mathrm{OM}] \times \mathrm{f}(\mathrm{RH})_{\text {org }}+1 \times[\mathrm{SOIL}],
\end{aligned}
$$

where $\left[\mathrm{SO}_{4}^{2-}\right]$ is the sulfate concentration $\left(\mu \mathrm{g} / \mathrm{m}^{3}\right),\left[\mathrm{NO}_{3}^{-}\right]$is the nitrate concentration $\left(\mu \mathrm{g} / \mathrm{m}^{3}\right),[\mathrm{OM}]$ is the organic mass concentration $(1.8 \times[\mathrm{OC}])\left(\mu \mathrm{g} / \mathrm{m}^{3}\right)$ and [SOIL] is the crustal material ambient concentration, estimated here to be $4 \%$ of the TEOM fine mass $\left(\mu \mathrm{g} / \mathrm{m}^{3}\right)$. The dry scatter efficiency of sulfates and nitrates is assumed to be $3 \mathrm{~m}^{2} / \mathrm{g}$, $4 \mathrm{~m}^{2} / \mathrm{g}$ corresponds to the organic material dry scattering efficiency, and $1 \mathrm{~m}^{2} / \mathrm{g}$ corresponds to the crustal material scattering efficiency [Trijonis et al., 1988; Gebhart et al., 1994]. The above parameters were estimated from measurements mainly in the western United States. Sulfate was assumed to be fully neutralized (i.e., ammonium sulfate) for both summer and winter, and the 4.125 factor in equation (2) accounts for both the scatter efficiency and the ammonium corresponding to the sulfate. The increase in scattering efficiency as a function of relative humidity for the inorganic hygroscopic aerosols, $\mathrm{f}(\mathrm{RH})_{\text {salt }}$, was calculated 
Table 1. Average Conditions Used to Calculate the Scattering Efficiency Curves During Summer and Winter

\begin{tabular}{|c|c|c|}
\hline & Summer & Winter \\
\hline Temperature, K & 300 & 280 \\
\hline Total $\mathrm{NH}_{3},{ }^{\mathrm{a}} \mu \mathrm{g} / \mathrm{m}^{3}$ & 2.6 & 1.6 \\
\hline Total $\mathrm{HNO}_{3},{ }^{a} \mu \mathrm{g} / \mathrm{m}^{3}$ & 1.4 & 2.0 \\
\hline Total $\mathrm{HCl},{ }^{\mathrm{a}} \mu \mathrm{g} / \mathrm{m}^{3}$ & 0.4 & 0.3 \\
\hline Size range, $\mu \mathrm{m}$ & Sulfate, $\mu \mathrm{g} / \mathrm{m}^{3}$ & Sulfate, $\mu \mathrm{g} / \mathrm{m}^{3}$ \\
\hline $0.056-0.10$ & 0.1 & 0.0 \\
\hline $0.10-0.18$ & 0.5 & 0.2 \\
\hline $0.18-0.32$ & 1.3 & 0.6 \\
\hline $0.32-0.56$ & 2.2 & 0.6 \\
\hline $0.56-1.00$ & 3.9 & 0.9 \\
\hline $1.00-1.80$ & 0.6 & 0.1 \\
\hline $1.80-2.50$ & 0.1 & 0.0 \\
\hline
\end{tabular}

from Mie theory and the average size distribution of sulfate (Table 1) in PAQS using the model described by Pilinis [1989] (see also the description of approach 2). For the summer the aerosol was assumed to be always liquid based on the PM water concentration measurements of Khlystov et al. (submitted manuscript, 2004). For the winter the particles were assumed to be solid below approximately $60 \% \mathrm{RH}$ and liquid for higher $\mathrm{RH}$ based on the same measurements. The same $\mathrm{f}(\mathrm{RH})_{\text {salt }}$ was used both for sulfate and nitrate since the scattering efficiency curves are very similar for these two compounds [Tang, 1996]. Figure 2 shows the calculated scattering efficiencies for the two seasons. A higher scattering efficiency at high $\mathrm{RH}$ is calculated for the winter. During the winter the geometric mean diameter of the aerosol is smaller than during the summer [Cabada et al., 2004b]. For this approach the contribution of the organic matter to aerosol water is considered to be negligible; therefore $f(\mathrm{RH})_{\text {org }}$ is set to 1 .

[19] Most mass balance studies use a value of 1.4 for the organic carbon (OC) multiplier. Recent work by Turpin and Lim [2001] examines this factor, recommending values ranging from 1.1 for fresh emissions to $1.6-$ 2.1 for an aged aerosol. Comparison of PAQS main site data with satellite sites indicates that the air quality in Pittsburgh is dominated by regional transport [Tang et al., 2004]. We therefore used a multiplication factor of 1.8 , which is representative of an aged, regional aerosol to estimate total organic mass from OC measurements. Our choice of the 1.8 for the conversion of the OC to organic aerosol concentration is supported by the mass closure study of Rees et al. [2004].

\subsection{Approach 1b: Use of Bulk $\mathbf{P M}_{2.5}$ Composition (Daily Resolution)}

[20] For this approach the scattering from particles was also calculated from equation (2), but daily averaged concentrations were used. Since the effect of relative humidity on the scattering from particles is nonlinear, daily averaged values need to account for this to avoid biasing the calculations of the model low. To estimate the effect, the daily average of $f(\mathrm{RH})$ is calculated from hourly resolved data (from approach 1a). From the daily average value of $f(\mathrm{RH})$, the effective value of relative humidity is estimated. On average, this effective relative humidity value is $4 \%$ higher than the arithmetic average of relative humidity for each day. Therefore the $\mathrm{f}(\mathrm{RH})_{\text {salt }}$ is calculated at the daily averaged RH plus $4 \%$ for all days.

\subsection{Approach 2: Use of Mie Theory, Size Composition Distributions, and a Thermodynamic Model}

[21] In this approach the scattering coefficient was calculated using a thermodynamic model and Mie theory. The model is described in detail by Pilinis [1989]. Briefly, the thermodynamic model uses as inputs the measured size distributions of sulfate, sodium, organic material, elemental carbon, crustal material (Tables 2 and 3), and total concentrations (gas + aerosol) of ammonium, nitrate, and chloride together with the ambient temperature and RH. The model finds the appropriate form of the sulfate in the particulate phase (sulfate, bisulfate, or sulfuric acid), partitions the total inorganic semivolatile species (nitrate, ammonium, chloride) between the gas and aerosol phases, calculates their size distribution, and also calculates the aerosol water size distribution. Therefore the thermodynamic model provides the full size composition distribution of the particles including water. After the thermodynamic calculation, Mie theory is used to estimate the scattering coefficient of the aerosol. The relative humidity used is the effective relative humidity used in approach $1 \mathrm{~b}$. The performance of one size-resolved equilibrium calculation per day reduces significantly the computational cost and the potential numerical problems of this method. For the days where no size/composition measurements were available (e.g., August 2001, second half of January 2002), the average distribution for the month was used together with the bulk concentrations measured during that day.

\subsection{Approach 3a: Use of Measured Aerosol Water Concentrations (High Time Resolution)}

[22] Since ambient aerosol water concentrations are available from the DAASS system, the contribution of water to the scattering coefficient can be directly estimated. For this analysis, equation (2) is modified to account for the water aerosol as an ambient concentration as follows:

$$
\begin{aligned}
\mathrm{Bsp}= & 4.125 \times\left[\mathrm{SO}_{4}^{2-}\right]+3.87 \times\left[\mathrm{NO}_{3}^{-}\right]+4 \times[\mathrm{OM}]+1 \\
& \times[\mathrm{SOIL}]+3 \times[\text { WATER }] .
\end{aligned}
$$

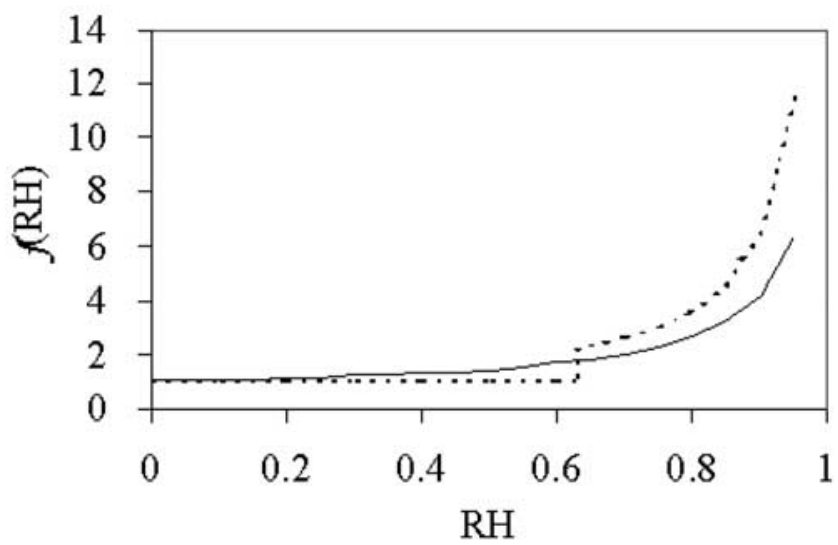

Figure 2. Scattering efficiency curve, $f(\mathrm{RH})$, as a function of relative humidity for the summer (solid line) and winter (dashed line) during PAQS. 
Table 2. Summer Average Size Distributions $\left(\mu \mathrm{g} / \mathrm{m}^{3}\right)$ of Compounds Used and Predicted by the Thermodynamic Model

\begin{tabular}{lccccccc}
\hline Size Range, $\mu \mathrm{m}$ & Sodium & Sulfate & OM & EC & Dust & Nitrate & Ammonium \\
\hline $0.056-0.10$ & 0.00 & 0.1 & 0.4 & 0.0 & 0.00 & 0.00 & 0.03 \\
$0.10-0.18$ & 0.01 & 0.5 & 0.7 & 0.1 & 0.00 & 0.01 & 0.01 \\
$0.18-0.32$ & 0.01 & 1.3 & 1.1 & 0.1 & 0.01 & 0.1 & 0.01 \\
$0.32-0.56$ & 0.01 & 2.2 & 1.7 & 0.1 & 0.01 & 0.02 & 0.0 \\
$0.56-1.00$ & 0.01 & 3.9 & 1.4 & 0.1 & 0.02 & 0.0 \\
$1.00-1.80$ & 0.01 & 0.6 & 0.7 & 0.1 & 0.02 & 0.02 & 0.03 \\
$1.80-2.50$ & 0.01 & 0.1 & 0.4 & 0.0 & 0.05 & 0.03 \\
\hline
\end{tabular}

The terms used in this equation are the same as in equation (2), with the introduction of the water aerosol concentration [WATER] $\left(\mu \mathrm{g} / \mathrm{m}^{3}\right)$. Note that for equation (3) the dependence of the scattering efficiency as a function of relative humidity, $f(\mathrm{RH})$, is not needed. The aerosol water scattering coefficient is assumed to be $3 \mathrm{~m}^{2} / \mathrm{g}$. The choice of the water mass scattering efficiency is based on the similarity of the optical properties and size distribution of water with that of the sulfates and nitrates [Seinfeld and Pandis, 1998]. The use of the same scattering efficiency for the inorganic salts and water is implicitly consistent with the previous studies of aerosol optical properties that calculated $f(\mathrm{RH})$ on the basis of the measured PM hygroscopic growth.

\subsection{Approach 3b: Use of Measured Aerosol Water Concentrations (Daily Resolution)}

[23] For this approach, daily averaged concentrations of sulfate, nitrate, organic mass, and water were used to estimate the scattering coefficient from equation (3).

\section{Results and Discussion}

\subsection{Approach 1a: Use of Bulk $\mathbf{P M}_{2.5}$ Composition (High Time Resolution)}

[24] The time series of measured and estimated scattering coefficient using this approach is shown in Figure 3. For both seasons the model predictions track the actual measurements with the exception of localized episodes mostly during the summer. Figure 4 depicts the comparison between the measured scattering coefficient from the nephelometer and the estimated value from equation (2) for the high time resolution measurements. Good correlation between the measured and the reconstructed scatter is shown during the summer, and a weaker correlation is observed during the winter due to lower values (Table 4). The uncertainty of the predicted values because of the PM composition measurement uncertainty is estimated to be around $15 \%$ and can explain most but not all the discrepancies between predictions and measurements.
[25] During the daytime of the summer months, Pittsburgh aerosol is often acidic, whereas for the other seasons the aerosol is mostly neutralized [Rees et al., 2004]. During periods when the aerosol is acidic, the assumption of neutralized particles may introduce errors. Malm et al. [2000a] showed that errors in the assumed degree of neutralization can explain some of the discrepancies between predictions and observations. However, for our case, these errors are expected to be less than $5 \%$ on a daily average basis because of the small difference in the mass between ammonium sulfate and bisulfate and because aerosol was acidic only during the daytime when the RH was low. This is consistent with the weak correlation $\left(\mathrm{R}^{2}=0.08\right)$ of the model error for scattering to the sulfate concentrations. The error was also not correlated with the nitrate $\left(\mathrm{R}^{2}=0.12\right)$ and the organic aerosol $\left(\mathrm{R}^{2}=0.14\right)$ concentrations. However, a relatively high and statistically significant correlation (at the $99.5 \%$ level) was found between this error and the measured concentration of ambient aerosol water $\left(\mathrm{R}^{2}=0.53\right)$. The link of the error to the aerosol water indicates a potential limitation of the model used to deal with the effect of hygroscopic growth on the scattering from particles.

[26] In relative terms, greater discrepancies between the model and the measurements are observed for the winter (Table 4). There is practically no correlation between the ambient concentrations of the different compounds and the error although there is a downward trend in all of them. The organic mass seems to inversely correlate weakly $\left(\mathrm{R}^{2} \approx 0.2\right)$ with the error. This could be due to an overestimation of the organic mass since the mass conversion factor used $(1.8 \times[\mathrm{OC}])$ might be too high for the winter carbonaceous aerosol. The hydrophobic organics could also have a negative effect on the ability of the inorganics to absorb water.

[27] The assumption of an externally mixed aerosol is not physically realistic but provides a good approximation of the scattering properties of atmospheric aerosol. The error in the scattering coefficient introduced by neglecting the

Table 3. Winter Average Size Distributions $\left(\mu \mathrm{g} / \mathrm{m}^{3}\right)$ of Compounds Used by the Thermodynamic Model

\begin{tabular}{|c|c|c|c|c|c|c|c|}
\hline Size Range, $\mu \mathrm{m}$ & Sodium & Sulfate & $\mathrm{OM}$ & $\mathrm{EC}$ & Dust & Nitrate & Ammonium \\
\hline $0.056-0.10$ & 0.00 & 0.0 & 0.2 & 0.0 & 0.02 & 0.0 & 0.0 \\
\hline $0.10-0.18$ & 0.00 & 0.2 & 0.7 & 0.1 & 0.04 & 0.2 & 0.1 \\
\hline $0.18-0.32$ & 0.01 & 0.6 & 0.8 & 0.1 & 0.05 & 0.4 & 0.25 \\
\hline $0.32-0.56$ & 0.01 & 0.6 & 1.0 & 0.1 & 0.03 & 0.4 & 0.25 \\
\hline $0.56-1.00$ & 0.01 & 0.9 & 0.8 & 0.1 & 0.06 & 0.8 & 0.4 \\
\hline $1.00-1.80$ & 0.02 & 0.1 & 0.3 & 0.0 & 0.08 & 0.1 & 0.05 \\
\hline $1.80-2.50$ & 0.02 & 0.0 & 0.1 & 0.0 & 0.08 & 0.1 & 0.02 \\
\hline
\end{tabular}



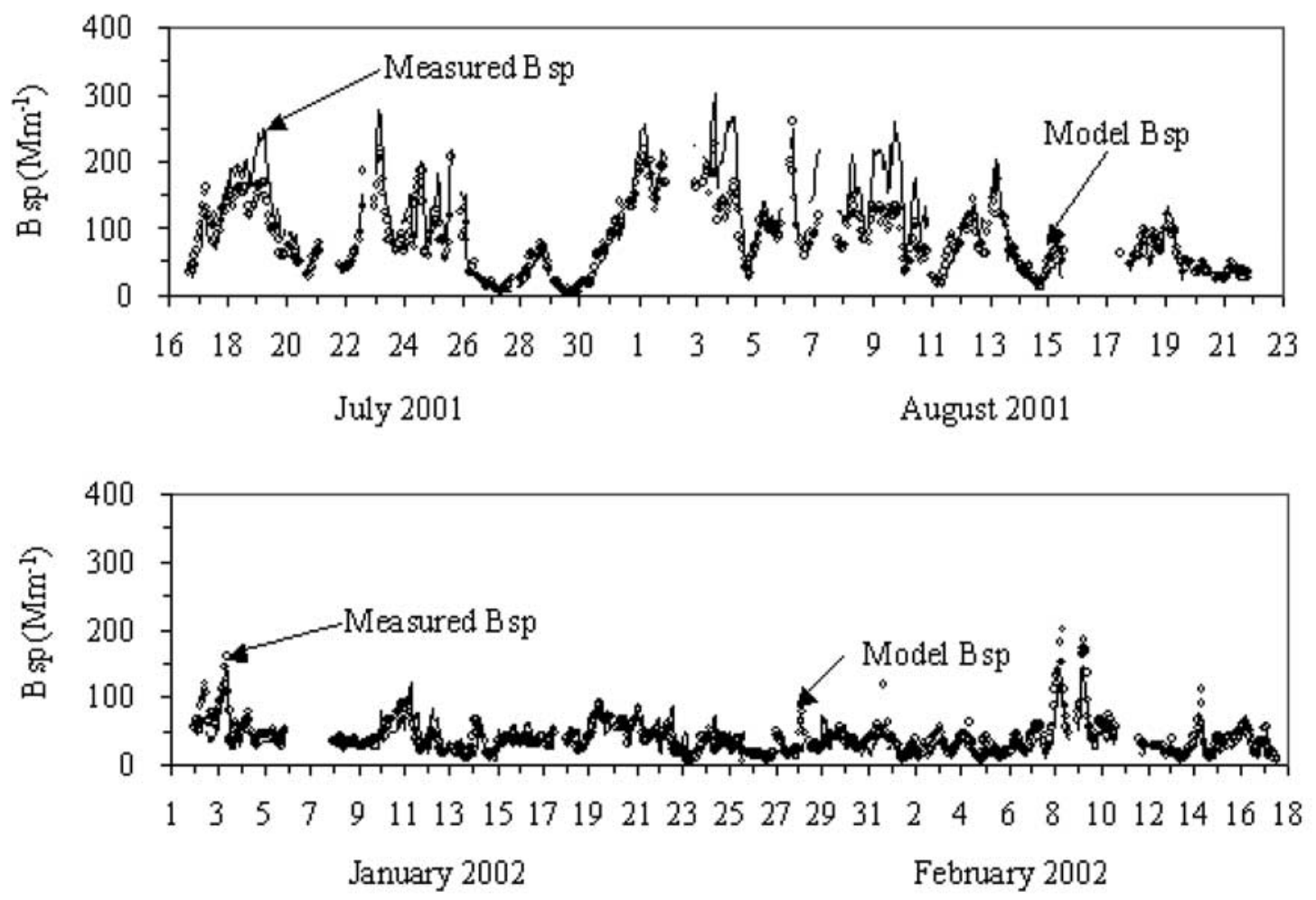

Figure 3. Time series of measured scattering coefficient (line) and estimated scattering coefficient (symbols) from approach 1a. Each point corresponds to 1-hour average.

interactions among the different aerosol species coexisting in the same particle is only a few percent [Seinfeld and Pandis, 1998]. One of the advantages of this approach is the direct apportionment of the scattering to the aerosol components [White, 1986]. On average, for the summer, $70 \%$ of the estimated scattering is due to sulfate, followed by the organic material with $25 \%$ and the remaining fraction is due to nitrates and soil. During the winter, sulfate accounts for $43 \%$ of the scattering, organics account for $31 \%$, and nitrates contribute $24 \%$ on average. Changes in one of the aerosol components may result in changes in the other components (e.g., an increase in nitrate may accompany a decrease in sulfate), so these results should be used with caution [Seinfeld and Pandis, 1998]. The reported contributions are based on the external mixture assumption neglecting these interactions.

\subsection{Approach 1b: Use of Bulk $\mathbf{P M}_{2.5}$ Composition} (Daily Resolution)

[28] Figure 5 shows time series of measured and estimated scattering coefficient applying equation (2) with daily averaged measurements. Model predictions for scattering track the measurements both for summer and winter. The scatterplot of measurements versus predictions for the two seasons analyzed is shown in Figure 6. The effect of averaging the concentrations over 24 -hour periods reduces
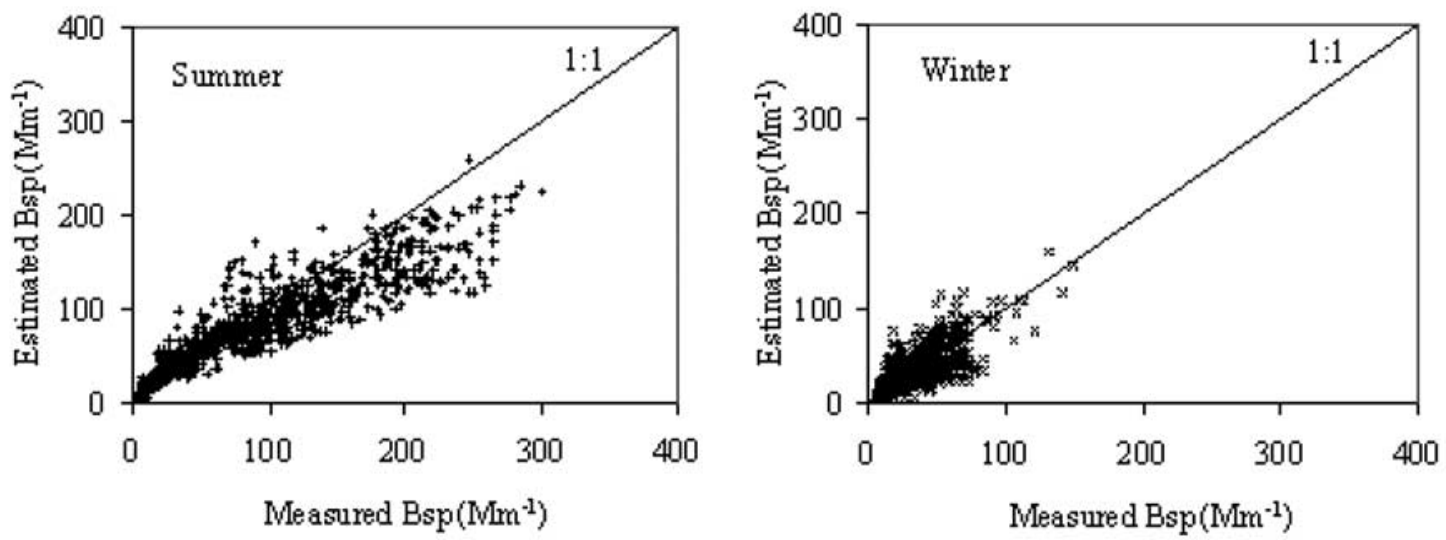

Figure 4. Comparison of measured scattering coefficient versus the calculated value approach 1a for two seasons during PAQS. 
Table 4. Model Performance Statistics for the Different Approaches Used to Estimate Scattering

\begin{tabular}{|c|c|c|c|c|c|}
\hline Summer & $\mathrm{N}$ & Average, $\mathrm{Mm}^{-1}$ & $\mathrm{R}^{2}$ & Error, $\mathrm{Mm}^{-1}$ & Bias \\
\hline Measured & 690 & 99 & & & \\
\hline Approach 1a & 690 & 84 & 0.80 & 23.7 & -15.1 \\
\hline Approach $1 \mathrm{~b}$ & 32 & 89 & 0.87 & 19.1 & -10.4 \\
\hline Approach 2 & 32 & 89 & 0.83 & 19.6 & -10.6 \\
\hline Approach 3a & 690 & 98 & 0.89 & 16.0 & -1.1 \\
\hline Approach 3b & 32 & 104 & 0.95 & 11.6 & 4.9 \\
\hline Winter & $\mathrm{N}$ & Average, $\mathrm{Mm}^{-1}$ & $\mathrm{R}^{2}$ & Error, $\mathrm{Mm}^{-1}$ & Bias \\
\hline Measured & & 39 & & & \\
\hline Approach 1a & 639 & 34 & 0.44 & 12.2 & -1.5 \\
\hline Approach 1b & 28 & 37 & 0.61 & 9.2 & 1.0 \\
\hline Approach 2 & 28 & 40 & 0.47 & 9.0 & 2.3 \\
\hline
\end{tabular}

the random errors of the measurements used as input by the model and therefore reduces model errors in both summer and winter (Table 4). McMurry et al. [1996] reported that significant uncertainty is introduced by this type of models when the scattering efficiencies are assumed constant for long periods of analysis since the characteristics of the aerosol populations are likely to vary within hours. This uncertainty is present in all approaches using daily average values.

\subsection{Approach 2: Use of Mie Theory, Size Composition Distributions, and a Thermodynamic Model}

[29] The measured and calculated scattering coefficients for the summer and winter seasons during PAQS are shown in Figure 7. The model predictions and the measurements are in good agreement with the exception of the period of 4-9 August. Figure 8 shows the scatterplot of measure- ments versus predictions from the model for the two different seasons analyzed. Relatively good correlation is shown between the measurements and the predictions for the summer and the winter (Table 4). During the summer the model tends to under-predict the measured scattering coefficient, although this model shows better agreement between the measurements and the predictions. The errors once more is only weakly correlated to sulfate $\left(\mathrm{R}^{2}=0.17\right)$, nitrate $\left(\mathrm{R}^{2}=0.06\right)$, and organics $\left(\mathrm{R}^{2}=0.10\right)$. The error of this model shows a weak correlation with ambient aerosol water $\left(\mathrm{R}^{2}=0.22\right)$ and $\mathrm{PM}_{2.5}$ mass concentrations $\left(\mathrm{R}^{2}=\right.$ 0.37). The weak correlation of the model and $\mathrm{PM}_{2.5}$ from the TEOM, which also includes some water, suggests that at least part of the error may be related to the hygroscopic properties of the aerosol. Water aerosol concentrations are calculated from the model assuming that only the inorganic material uptakes water at high relative humidity. In the case
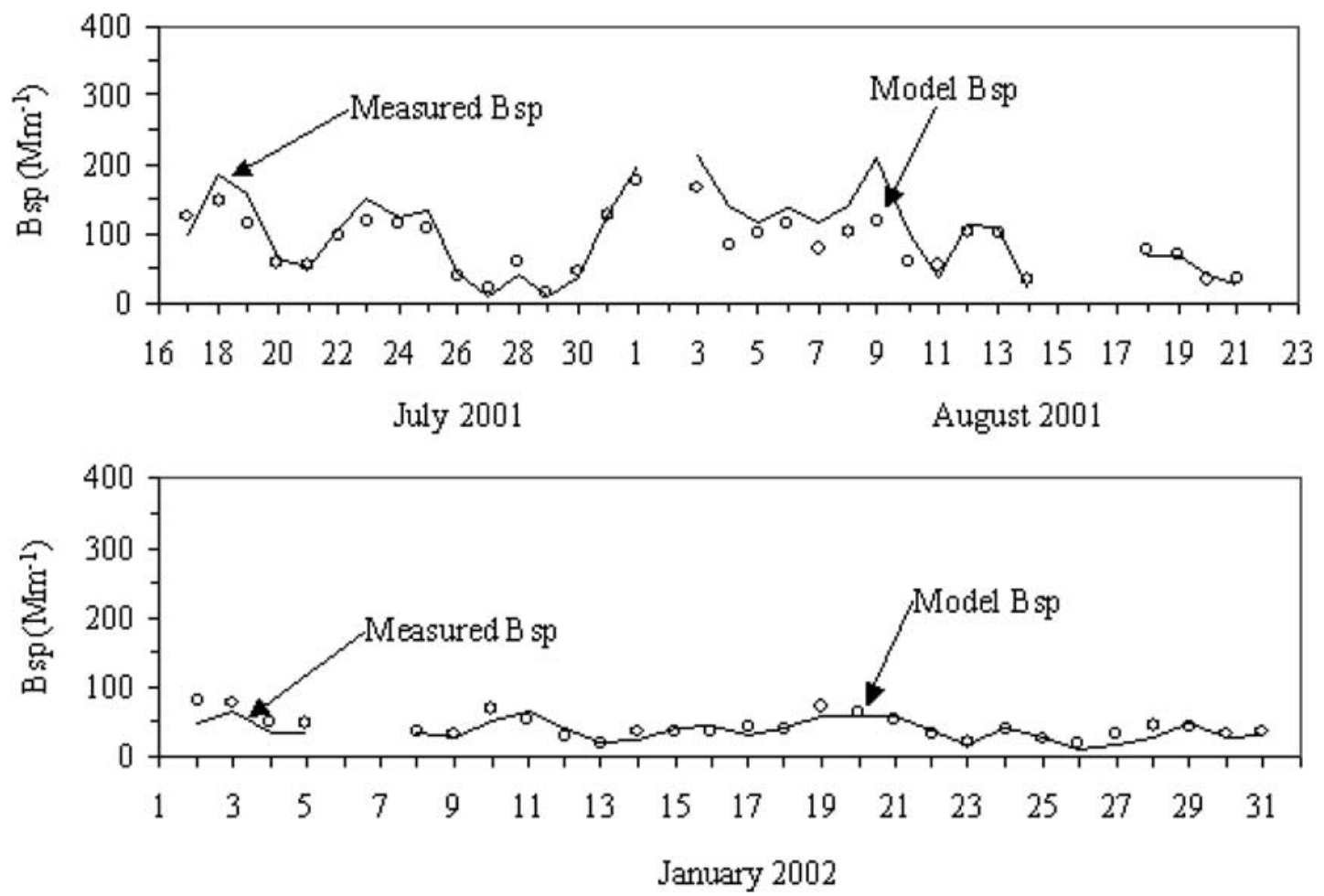

Figure 5. Time series of measured scattering coefficient (line) and estimated scattering coefficient (symbols) from approach 1b. Each point corresponds to 24-hour averages. 

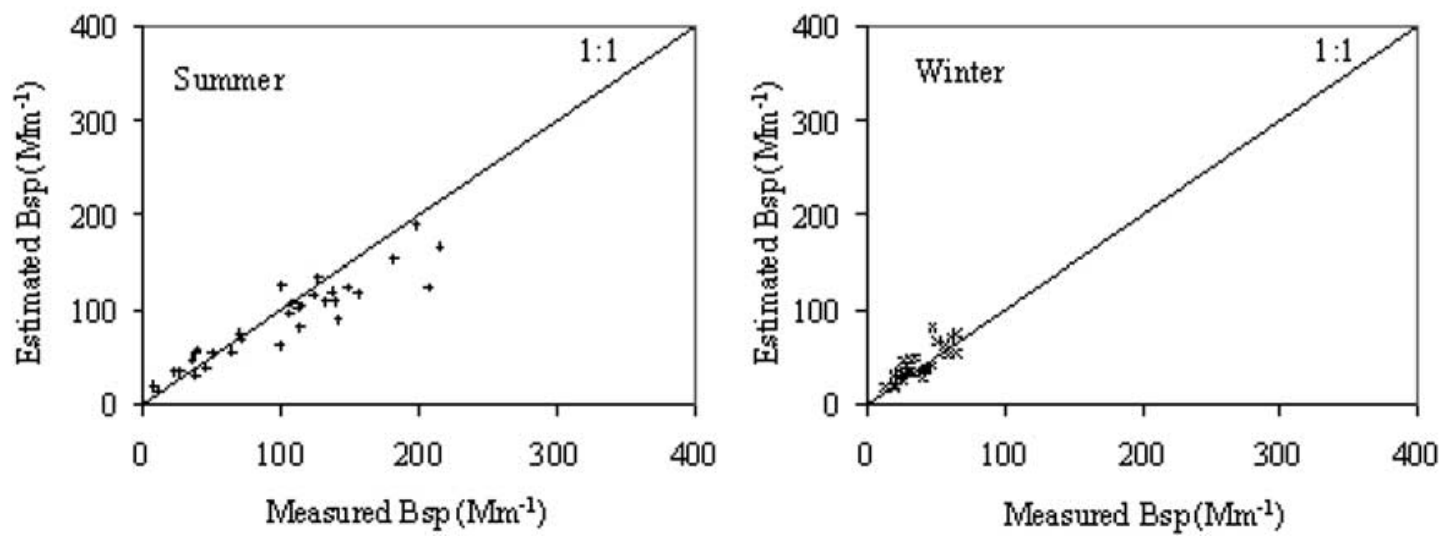

Figure 6. Comparison of measured scattering coefficient versus the calculated value from approach $1 \mathrm{~b}$ for two seasons during PAQS.

that carbonaceous material contributes to the water uptake into the aerosol phase, this could not be predicted from the model, and the actual scattering would be underestimated. Khlystov et al. (submitted manuscript, 2004) also reported that during certain periods in the summer the measured aerosol water was underestimated from theoretical thermodynamic calculations. The effect of using averaged size distributions during the summer does not seem to affect significantly the overall performance of the thermodynamic model. The corresponding scattering efficiencies for the major aerosol components were approximately the same as those used in approach 1.
[30] Winter estimates from this model tend to overpredict the measured scattering coefficient. The model shows a weaker correlation with the measurements as compared to the other approaches used, but the overall error is smaller (Table 4). No correlation was observed between the aerosol components and the error. The organic mass showed a weak anticorrelation $\left(\mathrm{R}^{2} \approx 0.2\right)$, which indicates either an overestimation of the organic mass from the organic carbon measurements or a negative effect of the organics on the water absorption by the inorganics.

[31] Summer contribution of sulfates and nitrates to the scattering coefficient from this model is $67 \%$, followed by
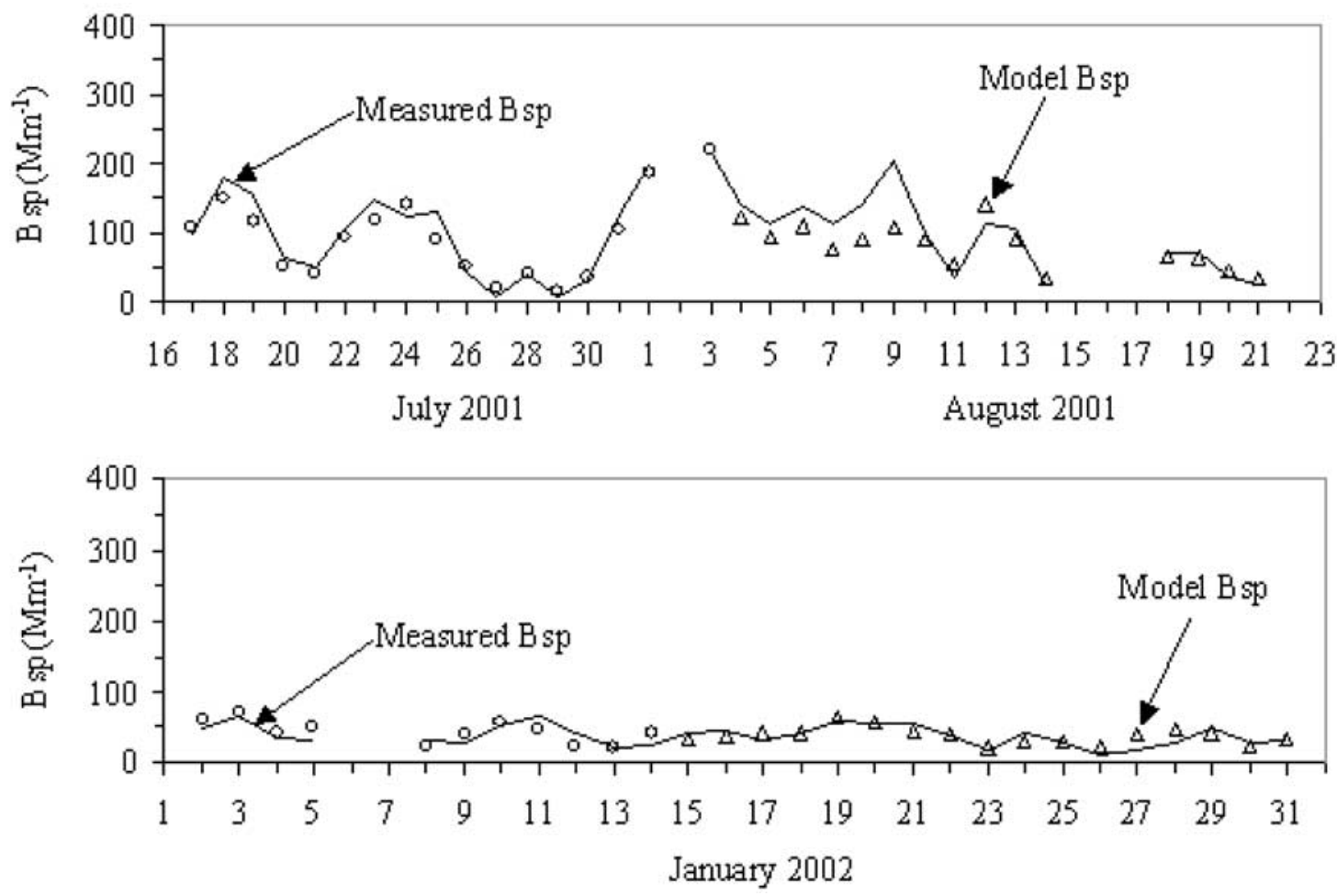

Figure 7. Time series of measured scattering coefficient (line) and estimated scattering coefficient from approach 2. Each point corresponds to 24-hour averages. Open circles point correspond to model calculations using actual size distributions. Open triangles were calculated used the average size distributions. 

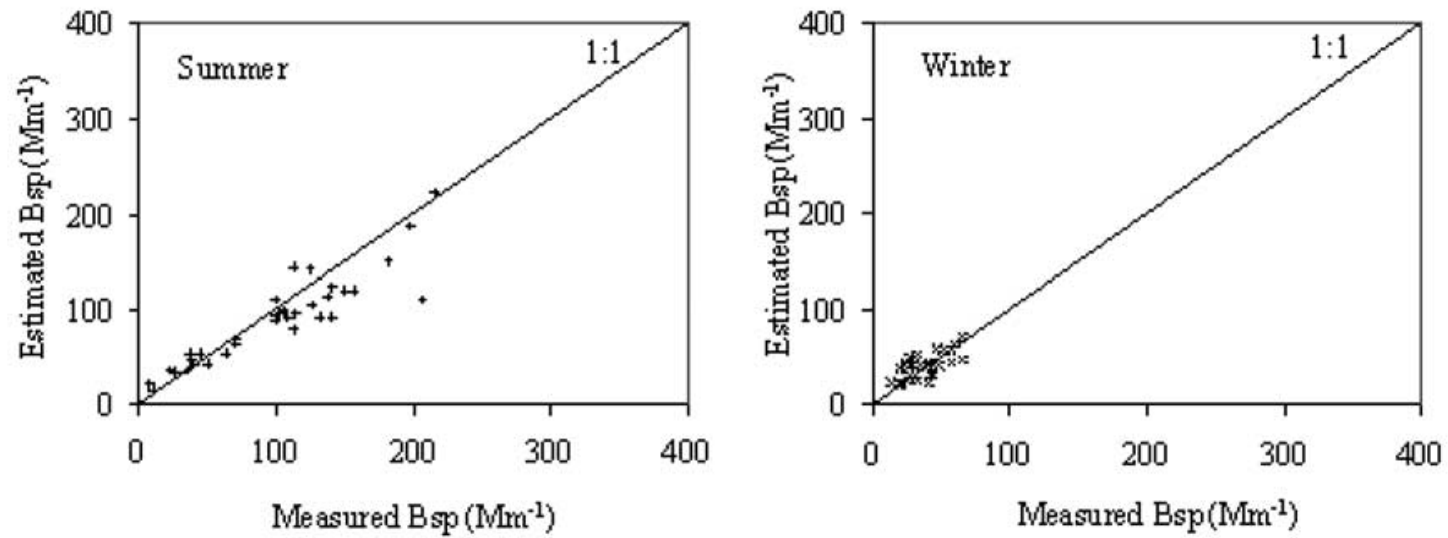

Figure 8. Comparison of measured scattering coefficient versus the calculated value from a thermodynamic model using Mie theory for two different seasons during PAQS. Each point corresponds to a 24-hour sampling period.

the organic material with $30 \%$. Negligible contributions are estimated from the other components. These contributions are estimated comparing the base case with the case where the component is not present. For winter, sulfates and nitrates account for $56 \%$ of the estimated scattering followed by $39 \%$ from the organic material. Other inorganic material and elemental carbon contribute $4 \%$ of the estimated scattering for the winter.

\subsection{Approach 3a: Use of Measured Aerosol Water Concentrations (High Time Resolution)}

[32] Figure 9 shows the time series of measured scattering coefficient and the estimated scattering coefficient for equation (3). The model does a good job in reproducing the observations including the higher peaks. The scatterplot of the measurements and the estimates for the scattering coefficient is shown in Figure 10. Good correlation $\left(\mathrm{R}^{2}=\right.$ 0.89 ) is observed between the model predictions and the measurements (Table 4). This model has the lowest absolute error (less than 10\%) and a low absolute bias (5\%). The consistency of the results is encouraging and indicates that the aerosol water content measurements by the DAASS are quite accurate. The improved agreement also suggests that a large fraction of the error of the previous approaches is indeed due to the estimation of the water uptake of the particles.
[33] A small source of error in this approach is that the water aerosol is measured with the DAASS system at slightly different conditions of relative humidity than the nephelometer. The measurements of aerosol size distributions and scattering coefficient are done at slightly lower than ambient relative humidity because of heating of the sample in both instruments. This difference in RH is a source of discrepancy between the model predictions and the corresponding scattering coefficient measurements. The magnitude of the discrepancy depends on the ambient $\mathrm{RH}$ and is on average around $10 \%$. The error introduced by this $\mathrm{RH}$ difference is similar in magnitude to the model error.

[34] The scattering budget estimated from this model indicates that, for the summer, sulfates account for around $38 \%$ of the estimated scattering coefficient, water aerosol accounts for $35 \%$, and organic material contributes with $24 \%$. Negligible contributions from nitrate and soil are estimated. In this approach the sulfates, organics, and the corresponding water are decoupled.

\subsection{Approach 3b: Use of Measured Aerosol Water Concentrations (Daily Resolution)}

[35] Time series of predictions from the model and daily measurements are shown in Figure 11. The model predicts values closely to the measurements (Figure 12). A high correlation $\left(\mathrm{R}^{2}=0.95\right)$ exists between the model and the

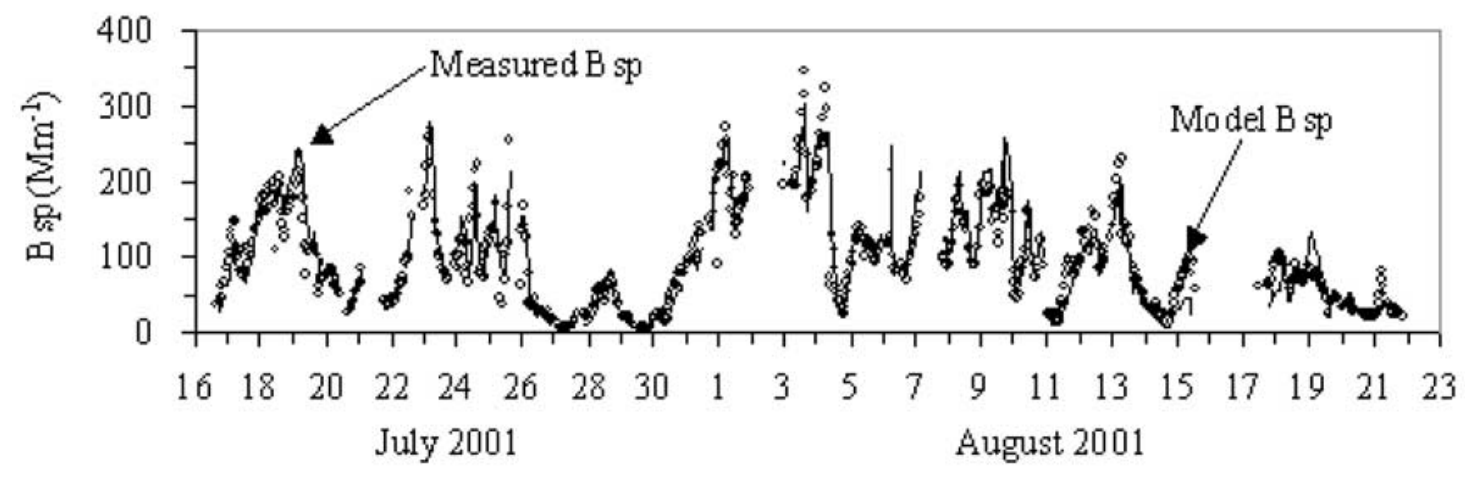

Figure 9. Time series of measured (line) and estimated scattering coefficient (symbols) from approach 3a. Aerosol water concentrations are only available for July and August 2001 during PAQS. Each point corresponds to 1-hour average sampling times. 


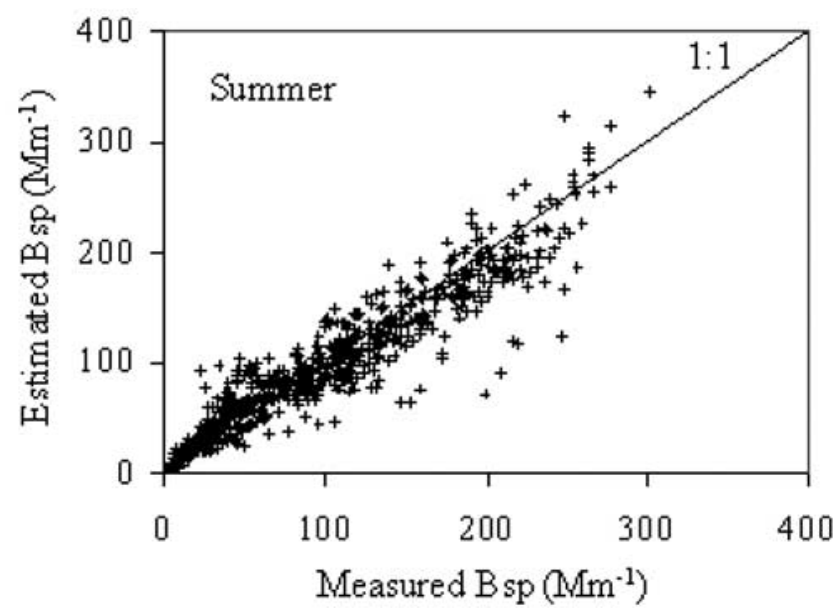

Figure 10. Scatterplot of measured scattering coefficient versus predicted values from approach 3 .

measured scattering (Table 4). The absolute error of the model is less than $10 \%$ for the summer and can be easily explained by the experimental errors. The model slightly overpredicts the scattering coefficient by around 5\% (Table 4). The good agreement and performance of this model indicates that the other approaches do not describe accurately the water uptake by fine particles in Pittsburgh.

\section{Conclusions}

[36] The three modeling approaches used for the calculation of the scattering coefficients (bulk composition and average scattering coefficients, size-resolved composition with Mie theory and a thermodynamic model, and use of the measured water) were able to reproduce the hourly observations with an absolute error less than $25 \%$ and a bias of less than $15 \%$. The approach using the measured aerosol water concentration reduces the error to $15 \%$ and the bias to $1 \%$. This error is similar to the uncertainty of the predictions due to the PM composition measurement uncertainty. These results suggest that while our water measurements are consistent with the observed optical properties, the current models have still some difficulties predicting the aerosol

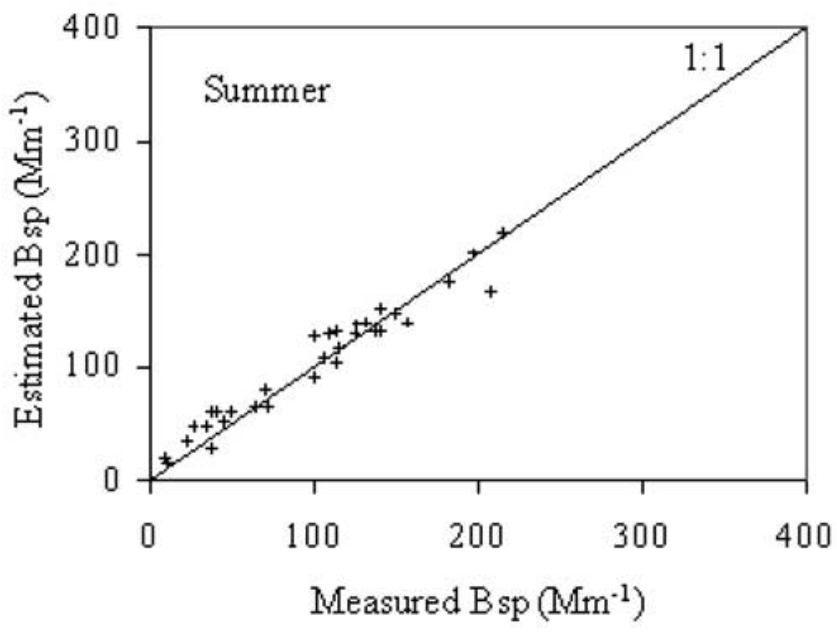

Figure 12. Scatterplot of measured scattering coefficient versus predicted values from approach 3 .

water content. The fact that the thermodynamic model is underestimating the scattering coefficient during the summer is an indication that organics may be contributing to water uptake into the aerosol phase. However, the weak correlation between the error and the organic PM mass suggests that the effect is complex and is probably related not to the total organic matter (OM) but to the hydrophilic fraction of the organic component.

[37] The simplest of the approaches used, bulk $\mathrm{PM}_{2.5}$ composition and average scattering efficiencies, was relatively successful in reproducing the observations even if its parameters were derived from measurements in other parts of the United States [Malm et al., 2000a], and the aerosol acidity during the summer was neglected. This indicates that the model is relatively robust in describing aerosol scattering over not only the western but also the northeastern United States. The use of high time resolution measurements did not result in a significant improvement of the scattering coefficient prediction over using daily resolved concentrations. However, a correction has to be made to the daily relative humidity to account for the nonlinear effect in the scattering coefficient. Also, the relative success (and sometimes failure) of the approach to reproduce hour by

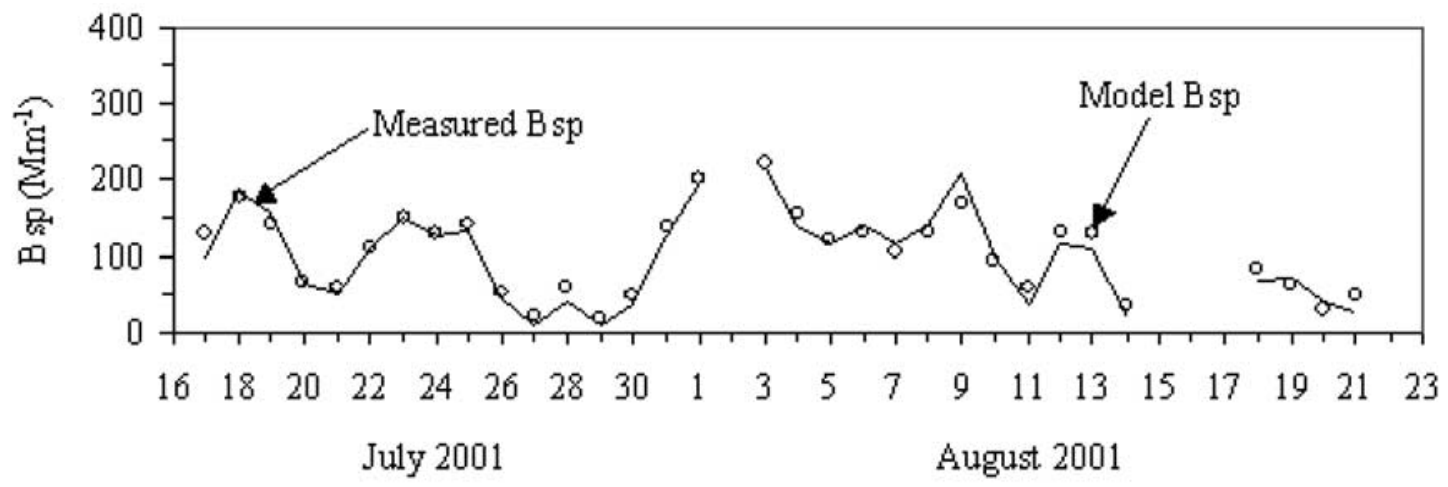

Figure 11. Time series of measured (line) and estimated scattering coefficient (symbols) from approach 3b. Aerosol water concentrations are only available for July and August 2001 during PAQS. Each point corresponds to 1-hour average sampling times. 
hour variations of the scattering coefficient provides an excellent test for our ability to perform accurate semicontinuous measurements of $\mathrm{PM}_{2.5}$ composition and of our understanding of the $\mathrm{PM}_{2.5}$ properties.

[38] A size-resolved thermodynamic model, using daily averaged concentrations, has practically the same performance during the summer as the simpler bulk compositionbased model of approach 1 . The fact that the growth curves, $f(\mathrm{RH})$, in the bulk model were calculated from Mie theory and a thermodynamic model for the average conditions over the two seasons explains part of its success. During the winter the use of size-resolved thermodynamic model marginally improves the estimates of the scattering coefficient.

[39] The summer scattering budget indicates that sulfates and the associated water contribute around $70 \%$ and organic material contributes with around 30\%. Negligible contributions are estimated for nitrate and soils. During the winter, sulfates are responsible for around $40 \%$, organics for $30 \%$, and nitrates for $25 \%$ of the scattering. Negligible contributions to scattering are estimated from the soil ambient concentrations. The budget estimates of the different modeling approaches are within a few percent of each other.

[40] Acknowledgments. This research was conducted as part of the Pittsburgh Air Quality Study that was supported by U.S. Environmental Protection Agency under contract R82806101 and the U.S. Department of Energy National Energy Technology Laboratory under contract DE-FC2601NT41017. This paper has not been subject to EPA's required peer and policy review and therefore does not necessarily reflect the views of the Agency. No official endorsement should be inferred.

\section{References}

Ansari, A. S., and S. N. Pandis (1999), Prediction of multicomponent inorganic atmospheric aerosol behavior, Atmos. Environ., 33, 745-757.

Ansari, A. S., and S. N. Pandis (2000), Water absorption by secondary organic aerosol and its effect on inorganic aerosol behavior, Environ. Sci. Technol., 34, 71-77.

Cabada, J. C., S. N. Pandis, R. Subramanian, A. L. Robinson, A. Polidori, and B. J. Turpin (2004a), Estimating the secondary organic aerosol contribution to $\mathrm{PM}_{2.5}$ using the OC to EC tracer method, Aerosol Sci. Technol., 38, suppl. 1, 140-155.

Cabada, J. C., S. Rees, S. Takahama, A. Khlystov, S. N. Pandis, C. I. Davidson, and A. L. Robinson (2004b), Mass size distributions and size resolved chemical composition of fine particulate matter at the Pittsburgh supersite, Atmos. Environ., 38, 3127-3141.

Charlson, R. J., S. E. Schwartz, J. M. Hales, R. D. Cess, J. A. Coakley, J. E. Hansen, and D. J. Hofmann (1992), Climate forcing by anthropogenic aerosols, Science, 255, 423-430.

Cruz, C., and S. N. Pandis (1998), The effect of organic coatings on the cloud condensation nuclei activation of inorganic atmospheric aerosol, J. Geophys. Res., 103, 13,111-13,123.

Cruz, C., and S. N. Pandis (2000), Deliquescence and hygroscopic growth of mixed inorganic-organic atmospheric aerosol, Environ. Sci. Technol., $34,4313-4319$.

Gebhart, K. A., W. C. Malm, and D. Day (1994), Examination of the effects of sulfate acidity and relative humidity on light scattering at Shenandoah national park, Atmos. Environ., 28, 841-849.

Hand, J. L., R. B. Ames, S. M. Kreidenweis, D. E. Day, and W. C. Malm (2000), Estimates of particle hygroscopicity during the southern aerosol and visibility study, J. Air Waste Manage. Assoc., 50, 677-685.

Hegg, D., T. Larson, and P. F. Yuen (1993), A theoretical study on the effect of relative humidity on light scattering by tropospheric aerosols, J. Geophys. Res., 98, 18,435-18,439.

Heintzenberg, J. (1980), Particle size distribution and optical properties in arctic haze, Tellus, 32, 251-260.

Hemming, B. L., and J. H. Seinfeld (2001), On the hygroscopic behavior of atmospheric organic aerosols, Indust. Eng. Chem. Res., 40, 41624171.

Jaggard, D. L., C. Hill, R. W. Shorthill, D. Stuart, M. Glantz, F. Rosswog, B. Taggart, and S. Hammond (1981), Light scattering from particles of regular and irregular shape, Atmos. Environ., 15, 2511-2519.
Jang, M., and R. M. Kamens (1998), A thermodynamic approach for modeling partitioning of semivolatile organic compounds on atmospheric particulate matter: Humidity effects, Environ. Sci. Technol., 32, 12371243.

Khlystov, A., G. P. Wyers, and J. Slanina (1995), The Steam-Jet Aerosol Collector, Atmos. Environ., 29, 2229-2234.

Koloutsou-Vakakis, S., M. J. Rood, A. Nenes, and C. Pilinis (1998), Modeling of aerosol properties related to radiative forcing, J. Geophys. Res., 103, 17,009-17,032.

Koo, B., A. Ansari, and S. N. Pandis (2003), Integrated approaches to modeling the organic and inorganic atmospheric aerosol components, Atmos. Environ., 37, 4757-4768.

Kreisberg, N. M., M. R. Stolzenburg, S. V. Hering, W. D. Dick, and P. H. McMurry (2001), A new method for measuring the dependence of particle size distributions on relative humidity, with application to the Southeastern Aerosol and Visibility Study, J. Geophys. Res., 106, 14,93514,949 .

Larson, S. M., G. R. Cass, K. J. Hussey, and F. Luce (1988), Verification of image processing based visibility models, Environ. Sci. Technol., 22, $629-637$.

Lowenthal, D. H., C. F. Rogers, P. Saxena, J. Watson, and J. C. Chow (1995), Sensitivity of estimated light extinction coefficients to model assumptions and measurement errors, Atmos. Environ., 29, 751-766.

Malm, W. C. (2000), Spatial and seasonal patterns and temporal variability of haze and its constituents in the United States, Rep. III, Coop. Inst. for Res. in the Atmos., Fort Collins, Colo.

Malm, W. C., and D. E. Day (2000), Optical properties of aerosols at Grand Canyon National Park, Atmos. Environ., 34, 3373-3391.

Malm, W. C., J. F. Sisler, D. Huffman, R. A. Eldred, and T. A. Cahill (1994), Spatial and seasonal trends in particle concentrations and optical extinction in the United States, J. Geophys. Res., 99, 1347-1370.

Malm, W. C., J. V. Molenar, R. A. Eldred, and J. F. Sisler (1996), Examining the relationship among atmospheric aerosols and light scattering and extinction in the Grand Canyon area, J. Geophys. Res., 101, 19,25119,265 .

Malm, W. C., D. E. Day, and S. M. Kreidenweis (2000a), Light scattering characteristics of aerosols as a function of relative humidity: Part I. A comparison of measured scattering and aerosol concentrations using theoretical models, J. Air Waste Manage. Assoc., 50, 686-700.

Malm, W. C., D. E. Day, and S. M. Kreidenweis (2000b), Light scattering characteristics of aerosols as a function of relative humidity: Part II. A comparison of measured scattering and aerosol concentrations using statistical models, J. Air Waste Manage. Assoc., 50, 701-709.

Malm, W. C., D. E. Day, S. M. Kreidenweis, J. L. Collett, and T. Lee (2003), Humidity-dependent optical properties of fine particles during the Big Bend Regional Aerosol and Visibility Observational Study, J. Geophys. Res., 108(D9), 4279, doi:10.1029/2002JD002998.

McMurry, P. H., X. Zhang, and C.-T. Lee (1996), Issues in aerosol measurement for optics assessments, J. Geophys. Res., 101, 19,189-19,197.

Molenar, J. V. (1997), Theoretical and experimental analysis of the Optec NGN nephelometer, Air Resour. Spec. Inc., Fort Collins, Colo.

Molenar, J. V., W. C. Malm, and C. E. Johnson (1994), Visual air quality simulation techniques, Atmos. Environ., 28, 1055-1063.

NARSTO (2003), Particulate matter science for policy makers: A NARSTO assessment, NARSTO Manage. Coord. Off., Eagle Reach, Pasco, Wash. Nemesure, S., R. Wagener, and S. E. Schwartz (1995), Direct shortwave forcing of climate by the anthropogenic sulfate aerosol: Sensitivity to particle size, composition, and relative humidity, J. Geophys. Res., 100, 26,105-26,116.

Nenes, A., S. N. Pandis, and C. Pilinis (1998), ISORROPIA: A new thermodynamic equilibrium model for multiphase multicomponent inorganic aerosols, Aquat. Geochem., 4, 123-152.

Ouimette, J., and R. Flagan (1982), The extinction coefficient of multicomponent aerosols, Atmos. Environ., 16, 2405-2419.

Pilinis, C. (1989), Numerical simulation of visibility degradation due to particulate matter: Model development and evaluation, J. Geophys. Res., 94, 9937-9946.

Pilinis, C., and J. H. Seinfeld (1987), Continued development of a general equilibrium model for inorganic multicomponent atmospheric aerosols, Atmos. Environ., 21, 2453-2466.

Pilinis, C., S. N. Pandis, and J. H. Seinfeld (1995), Sensitivity of direct climate forcing by atmospheric aerosols to aerosol size and composition, J. Geophys. Res., 100, 18,739-18,754.

Quinn, P. K., S. F. Marshall, T. S. Bates, D. S. Covert, and V. N. Kapustin (1995), Comparison of measured and calculated aerosol properties relevant to the direct radiative forcing of tropospheric sulfate aerosol on climate, J. Geophys. Res., 100, 8977-8991.

Rees, S. L., A. L. Robinson, A. Khylstov, C. O. Stanier, and S. N. Pandis (2004), Mass balance closure and the Federal Reference Method for $\mathrm{PM}_{2.5}$ in Pittsburgh, Pennsylvania, Atmos. Environ., 38, 3305-3318. 
Saxena, P., and L. M. Hildemann (1997), Water absorption by organics: Survey of laboratory evidence and evaluation of UNIFAC for estimating water activity, Environ. Sci. Technol., 31, 3318-3324.

Saxena, P., L. Hildemann, P. H. McMurry, and J. H. Seinfeld (1995), Organics alter hygroscopic behavior of atmospheric particles, J. Geophys. Res., 100, 18,755-18,770.

Seinfeld, J. H., and S. N. Pandis (1998), Atmospheric Chemistry and Physics. From Air Pollution to Global Change, John Wiley, Hoboken, N. J.

Sloane, C. S. (1983), Optical properties of aerosols: Comparisons of measurements with model calculations, Atmos. Environ., 17, 409-416.

Sloane, C. S. (1984), Optical properties of aerosols of mixed compositions, Atmos. Environ., 18, 871-878.

Sloane, C. S. (1986), Effect of compositions on aerosol light scattering efficiencies, Atmos. Environ., 20, 1025-1037.

Sloane, C. S., and G. Wolff (1985), Prediction of ambient light scattering using a physical model responsive to relative humidity: Validation with measurements from Detroit, Atmos. Environ., 19, 669-680.

Stanier, C. O., A. Y. Khlystov, W. R. Chan, M. Mandiro, and S. N. Pandis (2004), A method for the in situ measurement of fine aerosol water content of ambient aerosols: The Dry-Ambient Aerosol Size Spectrometer (DAASS), Aerosol Sci. Technol., 38, suppl. 1, 215-228.

Subramanian, R., A. Y. Khlystov, J. C. Cabada, and A. L. Robinson (2004), Positive and negative artifacts in particulate organic carbon measurements with denuded and undenuded sampler configurations, Aerosol Sci. Technol., 38, suppl. 1, 27-48.

Takahama, S., B. Wittig, D. V. Vayenas, C. I. Davidson, and S. N. Pandis (2004), Modeling the diurnal variation of nitrate during the Pittsburgh Air Quality Study, J. Geophys. Res., 109, doi:10.1029/2003JD004149, in press.

Tang, I. N. (1996), Chemical and size effects of hygroscopic aerosols on light scattering coefficients, J. Geophys. Res., 101, 19,245-19,250.

Tang, I. N., W. T. Wong, and H. R. Mulkelwitz (1981), The relative importance of atmospheric sulfates and nitrates in visibility reduction, Atmos. Environ., 15, 2463-2571.

Tang, W., T. Raymond, B. Wittig, C. I. Davidson, S. N. Pandis, A. L. Robinson, and K. Crist (2004), Spatial variations in $\mathrm{PM}_{2.5}$ during the Pittsburgh Air Quality Study, Aerosol Sci. Technol., in press

Trijonis, J., et al., (1988), RESOLVE final project report: Visibility conditions and causes of visibility degradation in the Mojave Desert of California, Rep. NWC TP 6869, Nav. Weapons Cent., China Lake, Calif.

Turpin, B. J., and H. J. Lim (2001), Species contributions to $\mathrm{PM}_{2.5}$ mass concentrations: Revisiting common assumptions for estimating organic mass, Aerosol Sci. Technol., 35, 602-610.
U.S. Environmental Protection Agency (2002), Air quality criteria for particulate matter, Rep. EPA/600/P-99/002aC, Washington, D. C.

Watson, J. G. (2002), Visibility: Science and regulation, J. Air Waste Manage. Assoc., 52, 628-713.

West, J. J., C. Pilinis, A. Nenes, and S. N. Pandis (1998), Marginal direct climate forcing by atmospheric aerosols, Atmos. Environ., 32, $2531-$ 3542 .

Wexler, A. S., and J. H. Seinfeld (1990), The distribution of ammonium salts among a size and composition dispersed aerosol, Atmos. Environ., $24,1231-1246$

Wexler, A. S., and J. H. Seinfeld (1991), Second-generation inorganic aerosol model, Atmos. Environ., 12, 2731-2748.

White, W. H. (1986), On the theoretical and empirical basis for apportioning extinction by aerosols: A critical review, Atmos. Environ., 20, 16591672 .

White, W. H., E. S. Macias, R. C. Ninger, and S. Schoran (1994), Sizeresolved measurements of light scattering by ambient particles in the southwestern USA, Atmos. Environ., 28, 909-921.

Wittig, A. E., S. Takahama, A. Y. Khylstov, S. N. Pandis, S. Hering, B. Kirby, and C. Davidson (2004a), Semi-continuous $\mathrm{PM}_{2.5}$ inorganic composition measurements during the Pittsburgh Air Quality Study, Atmos. Environ., 38, 3201-3213.

Wittig, A. E., N. Anderson, A. Y. Khlystov, S. N. Pandis, C. Davidson, and A. L. Robinson (2004b), Pittsburgh Air Quality Study overview, Atmos. Environ., 38, 3107-3125.

Wu, X. A., C. Seigneur, and R. Bergstrom (1996), Evaluation of a sectional representation of size distributions for calculating aerosol optical properties, J. Geophys. Res., 101, 19,277-19,283.

Zhang, X., B. J. Turpin, P. H. McMurry, S. V. Hering, and M. R. Stolzenburg (1994), Mie theory evaluation of species contributions to 1990 wintertime visibility reduction in the Grand Canyon, J. Air Waste Manage. Assoc., 44, $153-162$

J. C. Cabada, A. Khlystov, and A. E. Wittig, Department of Chemical Engineering, Carnegie Mellon University, Pittsburgh, PA 15213, USA.

S. N. Pandis, Departments of Chemical Engineering and Engineering and Public Policy, Carnegie Mellon University, Pittsburgh, PA 15213, USA. (spyros@andrew.cmu.edu)

C. Pilinis, Department of Environmental Studies, University of Aegean, 17 Karadoni St., Mytilene GR-81100, Greece. 

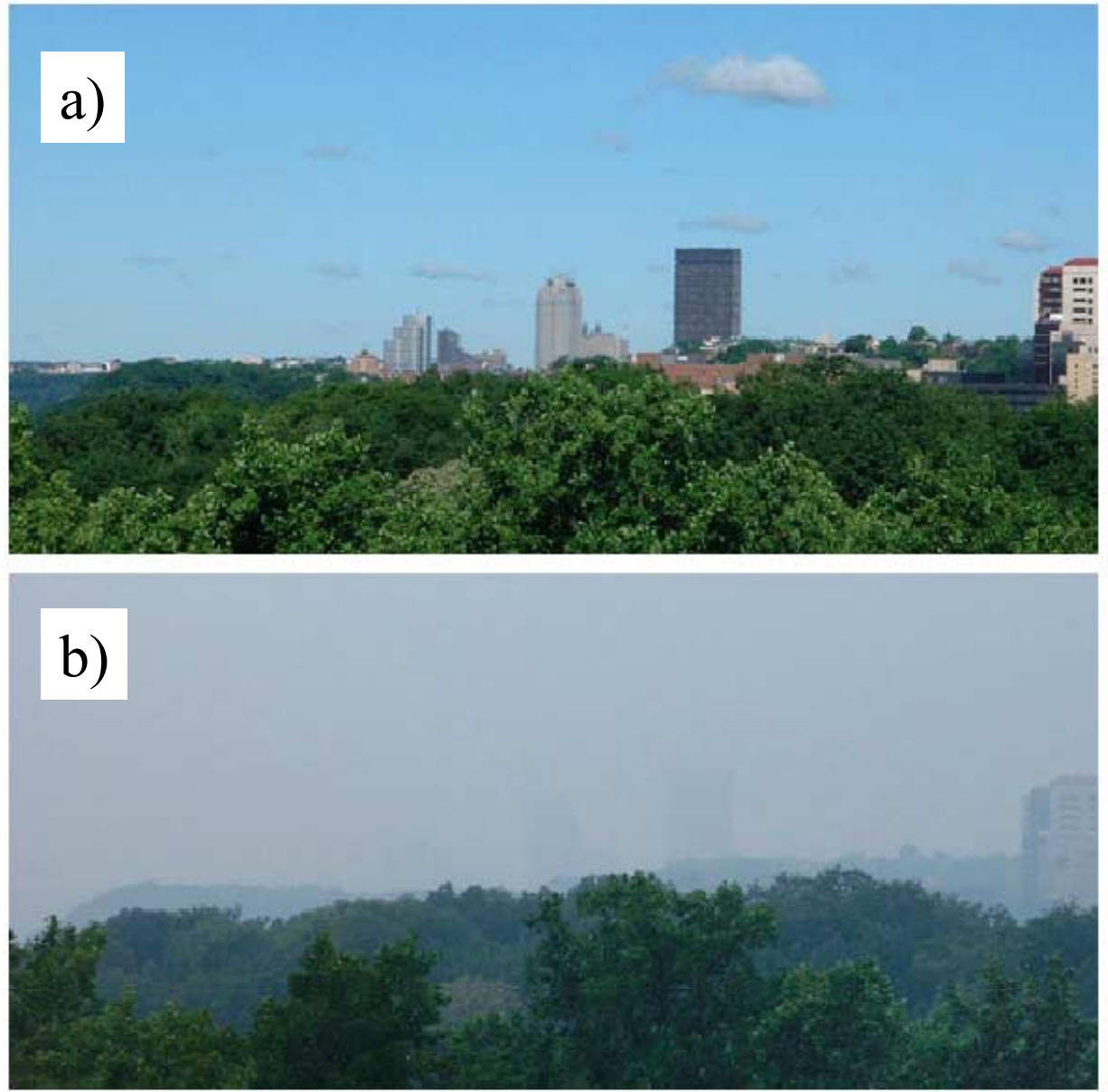

Figure 1. Comparison of visual characteristics during PAQS. View of downtown Pittsburgh from the PAQS main site at Schenley Park. (a) Day with low aerosol concentrations: $\mathrm{PM}_{2.5}=6 \mu \mathrm{g} / \mathrm{m}^{3}, \mathrm{~b}_{\mathrm{sp}}=$ $5 \mathrm{Mm}^{-1}, \mathrm{RH}=60 \%$. (b) Polluted day: $\mathrm{PM}_{2.5}=70 \mu \mathrm{g} / \mathrm{m}^{3}, \mathrm{~b}_{\mathrm{sp}}=255 \mathrm{Mm}^{-1}, \mathrm{RH}=74 \%$. 


\title{
Sources of Atmospheric Carbonaceous Particulate Matter in Pittsburgh, Pennsylvania
}

\author{
Juan C. Cabada and Spyros N. Pandis \\ Department of Chemical Engineering, Carnegie Mellon University, Pittsburgh, Pennsylvania
}

\author{
Allen L. Robinson \\ Department of Mechanical Engineering, Carnegie Mellon University, Pittsburgh, Pennsylvania
}

\begin{abstract}
The organic carbon (OC)/elemental carbon (EC) tracer method is applied to the Pittsburgh, PA, area to estimate the contribution of secondary organic aerosol (SOA) to the monthly average concentration of organic particulate matter (PM) during 1995. An emissions inventory is constructed for the primary emissions of OC and EC in the area of interest. The ratio of primary emissions of OC to those of EC ranges between 2.4 in the winter months and 1.0 in the summer months. A mass balance model and ambient measurements were used to assess the accuracy of the emissions inventory. It is estimated to be accurate to within $50 \%$. The results from this analysis show a strong monthly dependence on SOA contribution to the total organic PM concentration, varying from near zero during winter months to $50 \%$ or more of the total $\mathrm{OC}$ concentration in the summer.
\end{abstract}

\section{INTRODUCTION}

Atmospheric aerosol is a complex mixture of multicomponent particles whose size distribution, composition, and morphology can vary significantly in space and time. Atmospheric aerosol size ranges from a few nanometers to tens of micrometers. Major components include $\mathrm{SO}_{4}{ }_{4}^{2-}$, $\mathrm{NO}_{3}{ }^{-}, \mathrm{NH}_{4}^{+}, \mathrm{Cl}^{-}, \mathrm{Na}^{+}$, trace elements (including toxic and transition metals), organic material, elemental carbon (EC) (or soot), and crustal components. ${ }^{1,2}$ Particulate matter (PM) is emitted directly from anthropogenic sources (combustion, industrial, etc.) and natural sources (dust, sea salt, etc.) and also is formed in the atmosphere during the

\section{IMPLICATIONS}

During the summer in southwestern Pennsylvania, as much as $50 \%$ of the organic aerosol is estimated to be the result of secondary production processes. Therefore, implementation of effective control strategies for organic $\mathrm{PM}_{2.5}$ may require regulation not only of organic $\mathrm{PM}$ but also of SOA. oxidation of gaseous precursors $\left(\mathrm{SO}_{2}, \mathrm{NO}_{x^{\prime}}\right.$ high-molecularweight organic compounds, etc.). In 1997, the U.S. Environmental Protection Agency (EPA) proposed new standards for the mass concentrations of PM less than $2.5 \mu \mathrm{m}$ in diameter $\left(\mathrm{PM}_{2.5}\right)$, because of the hazards that these particles represent to human health. ${ }^{3}$ Fine particles also contribute to visibility impairment in the United States ${ }^{4,5}$ and other parts of the world, ${ }^{6-8}$ influence the energy balance of the planet, and are related to the global change problem. ${ }^{9,10}$

Between 10 and $65 \%$ of $\mathrm{PM}_{2.5}$ in the United States consists of carbonaceous material either directly emitted from sources in particulate form (primary) or produced from gas-to-particle conversion processes (secondary).,11-14 Therefore, control strategies to meet new $\mathrm{PM}_{2.5}$ requirements likely will need to account for carbonaceous PM. Carbonaceous aerosol is usually measured and reported as EC and organic carbon (OC) ${ }^{4,15}$ Elemental carbon resembles impure graphite and only is emitted directly from combustion sources (primary PM). Organic carbon consists of thousands of organic compounds; it either can be emitted directly from sources (primary PM) or can be generated from gaseous precursors in atmospheric oxidation reactions (secondary PM).

Several approaches have been used to estimate the primary and secondary contribution to the total OC concentration, including tracer compounds for primary and secondary OC, computational models describing the formation and transport of secondary organic aerosol (SOA),

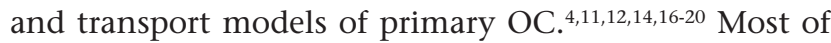
these methods have been applied to areas in the western United States. ${ }^{11,12,14,16,17,19-21}$

Studies conducted in southern and central California have shown that $\mathrm{SOA}$ is an important contributor to the organic $\mathrm{PM}_{2.5}$ concentration. It has been shown that SOA contributes between 15 and $40 \%$ of the total OC on an annual average basis, ${ }^{11,14,18,19}$ with a $60 \%$ contribution during the summer and $20 \%$ contribution during winter. ${ }^{20,22}$ For air pollution episodes, SOA contributions can 
be as high as $75 \%$ on summer days and $50 \%$ on winter days in southern and central California. ${ }^{20,22}$ Most of the SOA produced in this region of the country is related to high photochemical activity episodes (accompanied by high $\mathrm{O}_{3}$ concentrations), generally occurring at midday and in areas downwind of gaseous precursors emitters. ${ }^{11,20,22}$

The results of these studies cannot be generalized to other places in the country because of differences in primary gas and particulate emissions and in meteorological conditions. Relatively little information exists about the extent of SOA formation for other areas of the United States, mainly because there is no analytical technique that permits its direct separation from primary OC. One of the simplest approaches has been the use of EC as a tracer for primary OC in the atmosphere. ${ }^{11,20,22}$

This study applies the OC/EC ratio technique to identify the SOA contribution to the total OC concentration for the Pittsburgh, PA, metropolitan area. The method uses OC and EC measurements obtained in 1995 and emissions inventories of OC and EC constructed for this area. A mass balance is used to evaluate the accuracy of the emissions inventory.

\section{THE OC/EC APPROACH}

The ratio of the ambient concentrations of particulate OC to EC includes information about the extent of secondary OC formation. Ambient OC/EC ratios greater than those that are characteristic of the primary emissions are an indication of secondary aerosol formation. The OC/ EC ratio method takes advantage of the fact that primary OC and EC are mostly emitted by the same sources. Primary ratios of OC to EC can be determined either by using ambient measurements, if a large data set is available, ${ }^{22}$ or by developing an emissions inventory of the principal sources for an area of interest..$^{23}$ The contribution of secondary OC can be estimated by

$$
[\mathrm{OC}]_{\mathrm{S}}=[\mathrm{OC}]-[\mathrm{OC}]^{0}-\left\{[\mathrm{EC}]-[\mathrm{EC}]^{0}\right\} \cdot\left[\frac{\mathrm{OC}}{\mathrm{EC}}\right]_{\mathrm{p}}
$$

where $[\mathrm{OC}]_{\mathrm{S}}$ is the $\mathrm{SOA}$ contribution to the total $\mathrm{OC},[\mathrm{OC}]$ is the measured OC concentration, $[\mathrm{EC}]$ is the measured $\mathrm{EC}$ concentration, $[\mathrm{OC} / \mathrm{EC}]_{\mathrm{p}}$ is the ratio of $\mathrm{OC}$ to $\mathrm{EC}$ for the local primary sources affecting the site of interest, and $[\mathrm{OC}]^{0}$ and $[\mathrm{EC}]^{0}$ are the $\mathrm{OC}$ and $\mathrm{EC}$ concentrations of the air parcels entering the area of interest (background concentrations). All of these parameters are time-dependent because of the temporal variations in anthropogenic emissions and in meteorology. The application of this method requires measurements of $[\mathrm{OC}],[\mathrm{EC}],[\mathrm{OC}]^{0}$, and $[\mathrm{EC}]^{0}$ and the determination of the $[\mathrm{OC} / \mathrm{EC}]_{\mathrm{p}}$ ratio for the area of interest.

A challenge for the implementation of the OC/EC ratio approach analysis is that $\mathrm{OC}$ and EC are operationally defined by the analysis method. The result is that the amount of OC and EC measured using different methodologies, such as the Desert Research Institute (DRI) thermal optical reflectance (TOR) method and the National Institute of Occupational Safety and Health (NIOSH) thermal optical transmittance (TOT) method, do not agree. ${ }^{15,24-26}$ Ideally, all of the samples would be analyzed with the same method. However, this is often impossible because emission factors for the different sources reported in the literature have been measured with one method or the other. To use OC and EC data determined with different techniques, the differences between the techniques must be reconciled before proceeding with the OC/EC ratio analysis.

This study uses emission factors, reported in the literature, that were analyzed using the NIOSH TOT method and ambient data for Pittsburgh analyzed with the DRI TOR method. The comparison of NIOSH and DRI methods by Chow et al. ${ }^{27}$ indicates that for urban aerosol samples, TOR OC and EC values can be corrected to agree with the NIOSH method by reducing EC by $\sim 50 \%$ and adding this difference to OC. For this analysis, we have adopted the Chow et al. ${ }^{27}$ approach to correct the OC and EC values of the ambient data. No corrections were made to the emissions data, which were all measured with the NIOSH technique.

Etyemezian et al. ${ }^{28}$ reported weekly measurements (continuous sampling for 7 days) of EC and OC concentrations in Pittsburgh for 1995. Monthly averages computed from this data are shown in Figure 1. The samples were collected in Oakland, an urban neighborhood 3 miles from downtown Pittsburgh. DRI analyzed the samples for OC and EC content using the TOR method. ${ }^{29}$ Briefly, the TOR method measures the carbon content of the aerosol by exposing the sample collected on a quartz filter to a prescribed temperature profile. The first stage of the analysis volatilizes organic material in an inert environment (He). The second stage of the analysis exposes the filter to a $98 \% \mathrm{He}$ and $2 \% \mathrm{O}_{2}$ environment, and the pyrolized OC

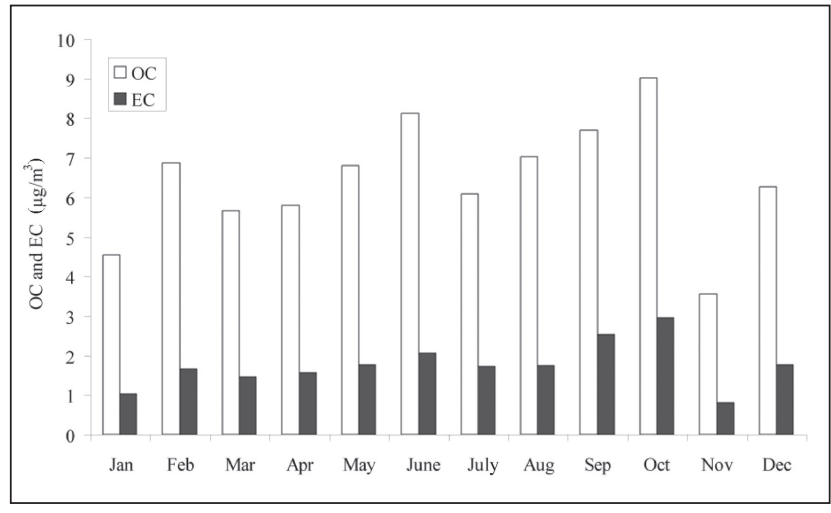

Figure 1. Monthly mean ambient $\mathrm{OC}$ and $\mathrm{EC}$ concentration measured in the Pittsburgh area during 1995. ${ }^{28}$ Data were analyzed using the DRI-TOR method and then corrected to NIOSH equivalent values. 
and the EC are oxidized. The carbon in the off gases from the filter are converted to $\mathrm{CH}_{4}$ through a hydrogen-enriched nickel catalyst and quantified with a flame ionization detector. The TOR method determines OC and EC from the sample by measuring the reflectance of the sample. During the initial stages, the reflectance of the sample decreases because of pyrolization of the carbonaceous material; however, as the sample is oxidized in the second part of the analysis ( $98 \% \mathrm{He}$ and $2 \% \mathrm{O}_{2}$ environment), the reflectance increases. The OC and EC split is defined at the point where the reflectance of the filter reaches its initial value.

Past studies using the OC/EC method to estimate SOA production in California assumed that the impact of background concentrations of carbon was negligible. ${ }^{12,20}$ This assumption is not valid for the eastern United States; data in the literature suggest typical background OC concentrations around $2.5-3.3 \mathrm{mg} \mathrm{C} / \mathrm{m}^{3}$ and $\mathrm{EC}$ concentrations around $0.4-0.6 \mathrm{mg} / \mathrm{m}^{3} .2,13,28$ For the purposes of this study, constant background concentrations of $2.9 \mathrm{mg} \mathrm{C} / \mathrm{m}^{3}$ for OC and $0.5 \mathrm{mg} / \mathrm{m}^{3}$ for EC concentrations were assumed. ${ }^{13}$ The sensitivity of our results to these estimated values is discussed in a subsequent section.

Application of the OC/EC approach also requires the calculation of the $[\mathrm{OC} / \mathrm{EC}]_{\mathrm{p}}$ ratio (see eq 1). This can be obtained by compiling an inventory of carbonaceous PM emissions in the area of interest. In general, inventories are developed by multiplying source activity data by a sourcespecific emission factor of EC and OC. The main OC and EC sources for western Pennsylvania are expected to be wood burning, transportation, and industrial sources. Activity data for these sources were obtained from the Pennsylvania Department of Transportation (PennDot), ${ }^{30}$ the Energy Information Administration (EIA), ${ }^{31}$ EPA, ${ }^{32}$ the U.S. Census Bureau, ${ }^{33}$ and the Allegheny County Health Department (ACHD).$^{34}$ Activity data from the literature are reported at the county level; therefore, for this study, the Pittsburgh metropolitan area is represented by Allegheny County (Figure 2). Emissions factors are obtained from the literature. ${ }^{13,23,35-37}$

\section{EMISSIONS INVENTORY DEVELOPMENT}

The inventory estimates emissions of primary OC and EC in the Pittsburgh area for 1995, with a monthly time resolution. Sources have been classified into mobile and nonmobile according to the characteristics of their activities. Emissions from sources outside the Pittsburgh metropolitan area and regional transport of carbonaceous material are considered to be background OC and EC.

\section{Mobile Sources}

On-Road Vehicles. Emissions from on-road vehicles were determined by multiplying the vehicle miles

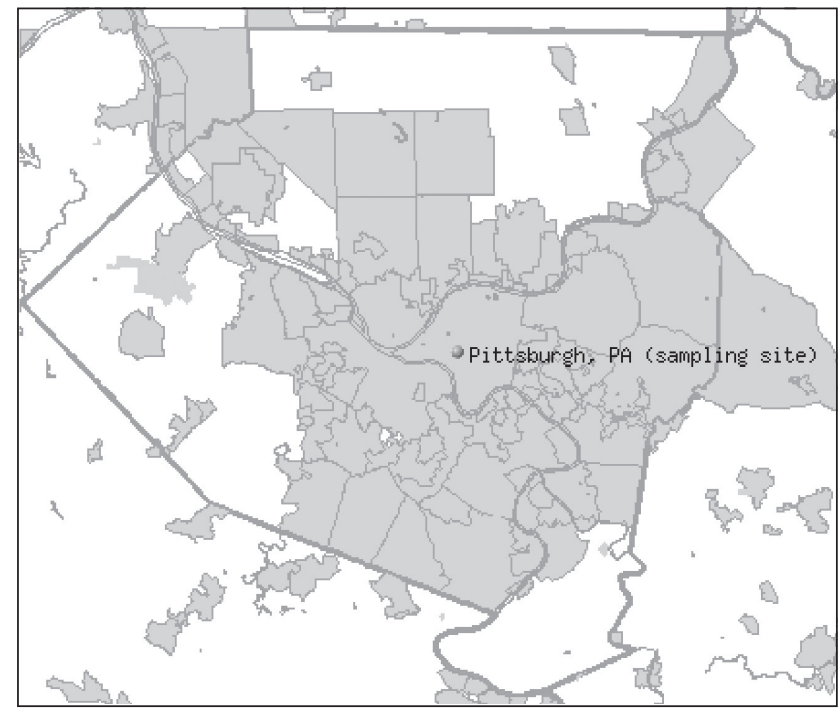

Figure 2. Area map of Allegheny County. The Pittsburgh metropolitan area is indicated by the shadowed area inside county boundaries. The dot represents the geographical location of the sampling site.

traveled (VMT) by each type of vehicle and the measure of total traveled miles by each type of vehicle ${ }^{30}$ (Table 1) by the corresponding emissions factor (Table 2). ${ }^{36}$ The available VMT data are reported on a yearly average basis for each type of vehicle (automobiles and light and heavy diesel trucks). To derive the monthly variation, we assumed that the monthly variation of VMT in southwestern Pennsylvania is the same as the U.S. average reported by the U.S. Department of Transportation (Table 3 ).$^{40}$ For all other sources involving the use of the miles traveled by the vehicles (tire and brake attrition and paved road dust), this same approach, multiplication of reported annual VMT by the Table 3 weighting factors, was adopted to estimate the monthly contributions of primary OC and EC.

Tire and Brake-Lining Attrition. The emissions caused by tire and brake-lining attrition in on-road vehicles were calculated as the product of the VMT for vehicle type times the vehicle-specific emission factor (see Table 2). ${ }^{23}$ For the tire attrition emissions, we assumed four tires for catalytic and non-catalytic automobiles, eight for light-duty trucks, and 18 for heavy-duty trucks. The numbers of tires for each type of vehicle were deduced from

Table 1. Average daily VMT in Allegheny County for $1995^{31}$

\begin{tabular}{lcccc}
\hline \hline Cat. Autos & Non-Cat. Autos $^{\mathrm{a}}$ & $\begin{array}{c}\text { Light-Duty } \\
\text { Diesel Trucks }\end{array}$ & $\begin{array}{c}\text { Heavy-Duty } \\
\text { Diesel Trucks }\end{array}$ \\
\hline VMT & $14,000,000$ & 880,000 & 980,000 & 720,000 \\
\hline \hline
\end{tabular}

${ }^{a}$ Non-catalytic automobiles were considered to be $6 \%$ of the total automobile fleet. ${ }^{38}$ 
Table 2. $\mathrm{OC}$ and $\mathrm{EC}$ emissions factors used in the construction on the inventory. ${ }^{\mathrm{a}}$

\begin{tabular}{|c|c|c|c|c|}
\hline Source & EC & OC & Units & Ref \\
\hline Catalytic automobiles & 4 & 9 & (mg C/mi) & 36 \\
\hline Non-catalytic automobiles & 7.6 & 63 & (mg C/mi) & 36 \\
\hline Diesel trucks & 270 & 210 & (mg C/mi) & 36 \\
\hline Tire attrition & 0.1 & 0.1 & ( $\mu \mathrm{g}$ C/tire-mi) & 22 \\
\hline Brake lining attrition & 0.1 & 0.2 & $\left(\mathrm{~kg} \mathrm{C} / 10^{6} \mathrm{mi}\right)$ & 22 \\
\hline Paved road & 1.1 & 14 & $\left(\%\right.$ of $\left.\mathrm{PM}_{25}\right)$ & 36 \\
\hline Wood burning (softwood) & 680 & 6300 & (mg C/kg wood) & 36 \\
\hline Wood burning (hardwood) & 150 & 2500 & (mg C/kg wood) & 36 \\
\hline Charbroiled meat & - & 23,000 & (mg C/kg meat) & 36 \\
\hline Fried meat & - & 740 & (mg C/kg meat) & 36 \\
\hline Cigarette smoking & 0.01 & 12 & (mg C/cigarette) & 36 \\
\hline Coal & 1.2 & 4.2 & (kg C/t of coal) & 22 \\
\hline Natural gas & 3.1 & 40 & (ng C/kJ) & 36 \\
\hline Distillate fuel & 2.7 & 0.5 & $(\mu \mathrm{g} C / \mathrm{kJ})$ & 22 \\
\hline Residual fuel & 0.4 & 1.4 & (kg C/billion Btu) & 22 \\
\hline Railroad & 50 & 15 & (kg C/billion Btu) & 22 \\
\hline Gasoline & 0.5 & 4.2 & (kg C/billion Btu) & 22 \\
\hline Turbojet (4 engines) ${ }^{b}$ & 1.8 & 0.6 & (kg C/LTO) & 34 \\
\hline Turbojet (3 engines) ${ }^{b}$ & 0.4 & 0.1 & (kg C/LTO) & 34 \\
\hline Turbojet (2 engines) $^{\mathrm{b}}$ & 0.3 & 0.1 & (kg C/LTO) & 34 \\
\hline Turboprop ${ }^{b}$ & 0.2 & 0.1 & (kg C/LTO) & 34 \\
\hline River vessels ${ }^{c}$ & 4.3 & 1.3 & (kg C/ship-day) & 22,34 \\
\hline Structural fires $^{d}$ & 0.5 & 1.0 & (kg C/ton burned) & 22,34 \\
\hline
\end{tabular}

${ }^{a}$ As reported by the authors, OC and EC analyzed by NIOSH protocol for all emission

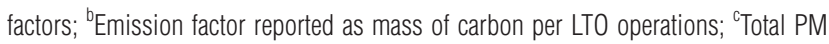
emissions calculated from EPA procedures, ${ }^{39}$ and carbon emissions determined by emission fractions reported by Gray; ${ }^{23 \mathrm{~d}}$ Total PM emissions calculated from EPA procedures, ${ }^{13}$ and carbon emissions determined by emission fractions reported by Gray. ${ }^{23}$

the traffic data reported by PennDOT for the Pittsburgh area. PennDOT reports axle number or directly reports the number of tires for each type of vehicle (automobiles, light-duty diesels, and heavy-duty diesels). The number of tires for each category is a weighted average for each category according to the VMT.
Paved Road. Emissions caused by resuspension of PM from paved roads were calculated based on the following empirical formula: ${ }^{32}$

$$
\mathrm{PM}_{2.5}=\mathrm{VMT}^{*} k\left(\frac{s L}{2}\right)^{0.65}\left(\frac{W}{3}\right)^{1.5}
$$

where $k$ is the $\mathrm{PM}_{2.5}$ particle size multiplier (g/VMT), $s L$ is the road surface silt loading $\left(\mathrm{g} / \mathrm{m}^{2}\right)$, and $W$ is the mean vehicle weight $(\mathrm{Mg})$. The constants for $k, s L$, and $W$ were chosen considering the Pittsburgh road network as "high annual daily traffic roads" in normal conditions, based on EPA recommendations. ${ }^{32}$ The particulate emissions from paved roads calculated by eq 2 were multiplied by the fraction of EC and OC within these particles (see Table 2$)^{36}$ to obtain the carbon emissions from this source.

Railroad. Railroad emissions in Pennsylvania were calculated by multiplying the amount of distillate fuel sold for railroad operations (Table 4) times the fuel-based emission factor for EC and OC (see Table 2). ${ }^{23}$ The emissions for the Pittsburgh area were estimated using the ratio of railway miles inside the Pittsburgh metro area to the total railway miles in the state of Pennsylvania. ${ }^{44}$ The emissions from railroad activity were assumed to have no monthly variation for the year of analysis.

Airplanes. Emissions of EC and OC from airplanes in the Pittsburgh region were estimated by multiplying the number of landing and takeoff (LTO) operations by the appropriate emission factor (see Table 2). ${ }^{35}$ Data for the total LTO operations and the types of planes at Pittsburgh International Airport were obtained from the Federal Aviation Administration (Table 5).$^{45}$ It was assumed that the operations of the airplanes have no monthly variation for the year of analysis.

River Vessels. Emissions of EC and OC from river vessels (towboats and commercial vessels) operating on the rivers

Table 3. Monthly variations for mobile, stationary sources, and residential combustion and structural fires occurrence

\begin{tabular}{|c|c|c|c|c|c|c|c|c|c|c|c|c|}
\hline & Jan & Feb & March & April & May & June & July & Aug & Sept & Oct & Nov & Dec \\
\hline \multicolumn{13}{|l|}{$\%$ Total } \\
\hline \multicolumn{13}{|l|}{ Miles Traveled } \\
\hline a Year ${ }^{\mathrm{a}}$ & 7.0 & 7.7 & 7.8 & 8.1 & 8.7 & 9.0 & 8.8 & 9.0 & 9.2 & 9.1 & 8.1 & 7.6 \\
\hline \multicolumn{13}{|l|}{$\%$ Heating } \\
\hline Fuel Consumed ${ }^{\mathrm{b}}$ & 20.2 & 17.0 & 13.3 & 7.7 & 3.6 & 0.6 & 0.1 & 0.2 & 1.7 & 6.7 & 11.4 & 17.4 \\
\hline \multicolumn{13}{|l|}{$\%$ Structural } \\
\hline Fire Ocurrence ${ }^{c}$ & 12.6 & 9.8 & 9.1 & 8.2 & 7.4 & 7.5 & 7.4 & 6.9 & 6.6 & 7.5 & 8.0 & 9.0 \\
\hline
\end{tabular}

${ }^{\mathrm{a}}$ Ref 40; ${ }^{\mathrm{b}}$ Based on heating-degree days, as reported from the National Weather System; ${ }^{41} \mathrm{C}$ Federal Emergency Management Agency. ${ }^{42}$ 
Table 4. Fuel consumption in the Pittsburgh area for $19955^{43}$

\begin{tabular}{lcc}
\hline \hline Fuel & Quantity & Units \\
\hline Railroad fuel (distillate oil) & 302,000 & Barrels $^{\mathrm{a}}$ \\
Wood (residential) & 170,000 & Cords $^{\mathrm{b}}$ \\
Wood (commercial) & 12,000 & Cords $^{\mathrm{b}}$ \\
Coal (residential) & 2000 & Tons \\
Coal (commercial) & 70,000 & Tons \\
Natural gas (residential) & 1500 & Billion Btu \\
Natural gas (commercial) & 250 & Billion Btu \\
Distillate fuel (residential) & $9.8 \times 10^{11}$ & $\mathrm{~kJ}$ \\
Distillate fuel (commercial) & $4.6 \times 10^{12}$ & $\mathrm{~kJ}$ \\
Residual fuel (residential) & 1000 & Billion Btu \\
Gasoline (commercial) & 60 & Billion Btu \\
\hline \hline
\end{tabular}

${ }^{a}$ Energy content, $5.8 \times 10^{6} \mathrm{Btu} /$ barrel; ${ }^{23 \mathrm{~b}}$ The density of a cord is $79 \mathrm{ft}^{3} / \mathrm{cord}$, and the density of wood $\left(\mathrm{kg} / \mathrm{ft}^{3}\right)$ is 11 for "softwoods" and 16 for "hardwoods."39

around the Pittsburgh area were estimated by multiplying the amount of ships operating each day on the rivers by the appropriate emission factor (see Table 2). ${ }^{23,37}$ Data for the total river vessel operations in the Pittsburgh area were obtained from the U.S. Army Corps of Engineers (USACE) ${ }^{46}$ Monthly variations on these emissions were constructed according to the data reported by USACE.

\section{Non-Mobile Sources}

Industrial. Industrial emissions of EC and OC were calculated based on the PM emissions reported by the Allegheny County Health Department ${ }^{34}$ (Table 6). Emissions from each industry were obtained by multiplying the total PM emissions by the corresponding percentage of EC and OC in the particulate phase (see Table 6). It is assumed that industrial sources have no significant monthly variation in the emissions.

Structural Fires. Emissions of OC and EC from structural fires were calculated based on the occurrences of structural fires in the Pittsburgh area ${ }^{52}$ by multiplying the mass of material burned by the carbon emitted per mass of material burned (see Table 2). ${ }^{23}$ Material burned in structural fires was estimated based on EPA procedures ${ }^{53}$ and

Table 5. Distribution of LTO operations for Pittsburgh International Airport. ${ }^{\text {a }}$

\begin{tabular}{lc}
\hline \hline Aircraft Type & \% of LTO \\
\hline Turbojet (4 engines) & $6 \%$ \\
Turbojet (3 engines) & $16 \%$ \\
Turbojet (2 engines) & $40 \%$ \\
Turboprop & $24 \%$ \\
Others & $13 \%$ \\
\hline
\end{tabular}

a203,000 LT0 operations are reported for Pittsburgh International Airport for $1995 .{ }^{45}$ considering an average residence size of $160 \mathrm{~m}^{2}$. Monthly variations of structural fires in Pittsburgh were assumed to follow U.S. averages (see Table 3), ${ }^{42}$ and OC and EC monthly emissions were calculated multiplying the monthly percentages of fires by the yearly emissions.

Meat Cooking. Meat cooking emissions were calculated based on the total population of the Pittsburgh area and the annual per capita consumption of meat for the United States. ${ }^{54}$ For the commercial and residential cooking practices, it was assumed that $90 \%$ of the meat is fried and $10 \%$ is charbroiled..$^{23}$ The EC and OC emissions were estimated by multiplying the amount of meat cooked times the emission factor for each type of cooking practice (see Table 2). ${ }^{36}$ Meat cooking operations were assumed to remain constant during all months of the year of analysis.

Cigarettes. The active population of smokers in the Pittsburgh area was estimated from data from the Center for Disease Control and Prevention. ${ }^{55}$ In the state of Pennsylvania, $18 \%$ of the population are active smokers. The average smoker in Pittsburgh consumes 19 cigarettes a day. ${ }^{55}$ Emissions from smoking practices were calculated by multiplying the number of cigarettes consumed times the carbon emission factor (see Table 2) ${ }^{36}$ It was assumed that there was no monthly variation in the cigarette emissions.

Heating Fuels. Emissions from fuel combustion (coal, distillate fuel, residual fuel, natural gas, and gasoline) for residential space heating and commercial operations were obtained by multiplying the amount of fuel used in the Pittsburgh area (see Table 4$)^{31}$ times the emission factor for each type of fuel (see Table 2). Pittsburgh area fuel consumption was estimated by scaling Pennsylvania data with the ratio of households and commercial businesses in the Pittsburgh area using each type of heating fuel (ratios obtained from the 1990 census data). ${ }^{54}$ Monthly variations of emissions for non-industrial stationary and area sources of EC and OC (wood, coal, distillate and residual fuels, and natural gas) were estimated using the heatingdegree days approach. ${ }^{43}$ For each degree below $18{ }^{\circ} \mathrm{C}$ on the average ambient temperature, a "heating-degree day" is registered. Data about the heating-degree days of the Pittsburgh area were obtained for $1995 .{ }^{41}$ The monthly variation in emissions from space-heater sources was determined by multiplying the fraction of the total annual heating-degree days by the total emissions for each source. Table 3 shows the monthly variations in the heatingdegree days for Pittsburgh in 1995.

Wood Burning. Emissions of OC and EC from wood burning in residential fireplaces were calculated based on the amount of wood consumed in the Pittsburgh area multiplied by the 
Table 6. Industrial PM emissions and factors used to estimate EC/OC contributions to inventory. ${ }^{34}$

\begin{tabular}{|c|c|c|c|c|c|}
\hline Industrial Source & $\mathrm{PM}_{2.5}(\mathrm{~kg} / \mathrm{day})$ & Classification & $\%$ EC & $\%$ OC & $\operatorname{Ref}^{a}$ \\
\hline Steel works & 4500 & Blast furnaces and steel mills & 0.0 & 10 & 47 \\
\hline Power plants & 2500 & Steam supply & 0.2 & 0.9 & 48 \\
\hline Steel works (coke burning) & 800 & Blast furnaces and steel mills & 74 & 10 & 49 \\
\hline Cement works & 440 & Cement, hydraulic & 0.2 & 5.4 & 49 \\
\hline Glass works & 310 & Glass containers & 0.1 & 0.7 & 49 \\
\hline Construction & 70 & Construction sand and gravel & 1.4 & 0.0 & 48 \\
\hline Copper works & 40 & Copper rolling and drawing & 0.0 & 1.6 & 47 \\
\hline Paving mixtures & 60 & Paving mixtures and blocks & 1.0 & 23 & 50 \\
\hline Plastics works & 30 & Plastics materials and resins & 1.8 & 9.2 & 47 \\
\hline Refuse systems & 20 & Refuse systems & 36 & 5.8 & 51 \\
\hline
\end{tabular}

${ }^{a} \mathrm{OC}$ and EC contents analyzed with NIOSH protocol or equivalent.

Table 7. Carbon emissions inventory for the Pittsburgh area in 1995.

\begin{tabular}{|c|c|c|c|c|}
\hline Source & Primary OC (t/year) & $\%$ & Primary EC (t/year) & $\%$ \\
\hline Residential wood burning & 551 & 32.6 & 37 & 3.6 \\
\hline Steel works & 190 & 11.2 & - & 0.0 \\
\hline Structural fires & 181 & 10.7 & 85 & 8.2 \\
\hline Paved road & 130 & 7.7 & 10 & 1.0 \\
\hline Meat cooking & 106 & 6.2 & - & 0.0 \\
\hline Heavy-duty trucks & 84 & 4.9 & 218 & 21 \\
\hline Light-duty trucks & 76 & 4.5 & 95 & 9.1 \\
\hline Catalytic autos & 46 & 2.7 & 21 & 2.0 \\
\hline Commercial wood burning & 43 & 2.6 & 3 & 0. \\
\hline River vessels & 40 & 2.4 & 130 & 13 \\
\hline Residential natural gas & 37 & 2.2 & 18 & 1.8 \\
\hline Brake attrition & 33 & 2.0 & 7 & 0.7 \\
\hline Tire attrition & 27 & 1.6 & 13 & 1.3 \\
\hline Railroad & 26 & 1.6 & 86 & 8.3 \\
\hline Cigarettes & 20 & 1.2 & 0 & 0.0 \\
\hline Non-catalytic autos & 20 & 1.2 & 2 & 0.2 \\
\hline Aircraft & 20 & 1.2 & 64 & 6.2 \\
\hline Commercial natural gas & 11 & 0.7 & 5 & 0.5 \\
\hline Steel works (coke burning) & 10 & 0.6 & 218 & 21 \\
\hline Cement works & 9 & 0.5 & 0 & 0.0 \\
\hline Residential coal & 8 & 0.5 & 2 & 0.2 \\
\hline Paving mixtures & 5 & 0.3 & 0 & 0.0 \\
\hline Commercial coal & 4 & 0.2 & 1 & 0.1 \\
\hline Power plants & 3 & 0.2 & 1 & 0.1 \\
\hline Commercial distillate fuel & 2 & 0.1 & 12 & 1.2 \\
\hline Commercial residual fuel & 1 & 0.1 & 0 & 0.0 \\
\hline Plastics works & 1 & 0.1 & 0 & 0.0 \\
\hline Others & 2 & 0.1 & 6 & 0.6 \\
\hline Total carbon (t/year) & 1690 & 100 & 1040 & 100 \\
\hline
\end{tabular}

${ }^{a}$ Other sources included in $\mathrm{OC}$ emissions inventory: glass works ( $\left.0.8 \mathrm{t} / \mathrm{year}\right)$, refuse systems ( $0.5 \mathrm{t} / \mathrm{year}$ ), residential distillate fuel (0.4 t/year), commercial gasoline ( $0.3 \mathrm{t} / \mathrm{year})$, and copper works ( $0.2 \mathrm{t} / \mathrm{year}){ }^{\mathrm{b}}{ }^{\mathrm{O}}$ Others sources included in EC emissions inventory: refuse systems ( $3 \mathrm{t} / \mathrm{year}$ ), residential distillate fuel ( $3 \mathrm{t} / \mathrm{year})$, construction ( $0.4 \mathrm{t} / \mathrm{year}$ ), and glass works ( $0.1 \mathrm{t} / \mathrm{year})$. carbon emitted per mass of wood burned (see Table 2). ${ }^{36}$ Wood consumption was estimated based on data for the state of Pennsylvania, ${ }^{54}$ and the ratio of households in Pittsburgh to the total households in the state of Pennsylvania. ${ }^{54}$ For the commercial wood-burning operations, the ratio of commercial businesses in Pittsburgh to the total in the state was used..$^{54}$ Based on sales, $90 \%$ of the wood burned is of the "hardwood" type, and the rest is "softwood." Monthly variations of wood-burning emissions were estimated according to the previously described heating-degree days approach.

\section{OC AND EC EMISSIONS}

Table 7 summarizes the particulate EC and OC primary emissions by various source categories for Pittsburgh. The $\mathrm{PM}_{2.5}$ inventory for the area includes a contribution of $1690 \mathrm{t} /$ year from OC and $1040 \mathrm{t} /$ year from EC. On an annual basis, the major sources of primary OC emissions are wood burning (33\%), fugitive sources (22\%), steel industries (12\%) and mobile sources (13\%). On an annual basis, the major sources of EC are heavy- and light-duty diesel trucks (30\%), coke-burning steelrelated industries (21\%), stationary fuel combustion sources (11\%), and fugitive sources (11\%).

Figures 3 and 4 show the monthly resolved emissions inventory for primary EC and OC in the Pittsburgh area. The OC emissions in winter are 3 times higher 


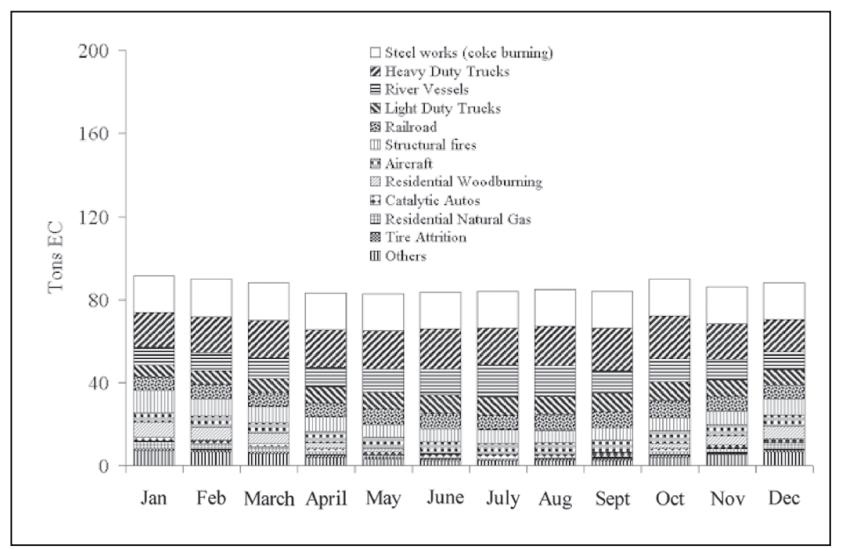

Figure 3. Pittsburgh area EC local primary emissions inventory. Monthly averages for 1995.

than the OC emissions in summer. For EC, the seasonal variation is not as large, but the winter emissions are still $30 \%$ more than the emissions during the summer. The main reason for these seasonal variations is changes in the emissions related to heating purposes (wood, coal, distillate fuel, and residual fuel burning). These sources have a strong seasonal dependence in the area and dominate over the other sources, which are assumed to have a weaker temporal variability (on-road vehicles, airplanes, industrial). Figure 5 shows the $[\mathrm{OC} / \mathrm{EC}]_{\mathrm{p}}$ ratio of emissions during 1995. The OC/EC primary ratio varies between 2.4 in winter and 1.0 in summer.

Uncertainty estimates for the inventories and the $[\mathrm{OC} / \mathrm{EC}]_{\mathrm{p}}$ ratio were developed based on the reported uncertainties in the emissions factors and in the source activity data. Uncertainties are higher during the winter, mostly because of the uncertainty in emissions from wood-burning operations. Several studies have reported large variations in OC and EC emissions from wood-burning operations, ${ }^{36,56,57}$ because these emissions are highly dependent on the nature of the wood consumed and the characteristics of the combustion. Figures 3 and 4 show that coal combustion, the steel industry, and

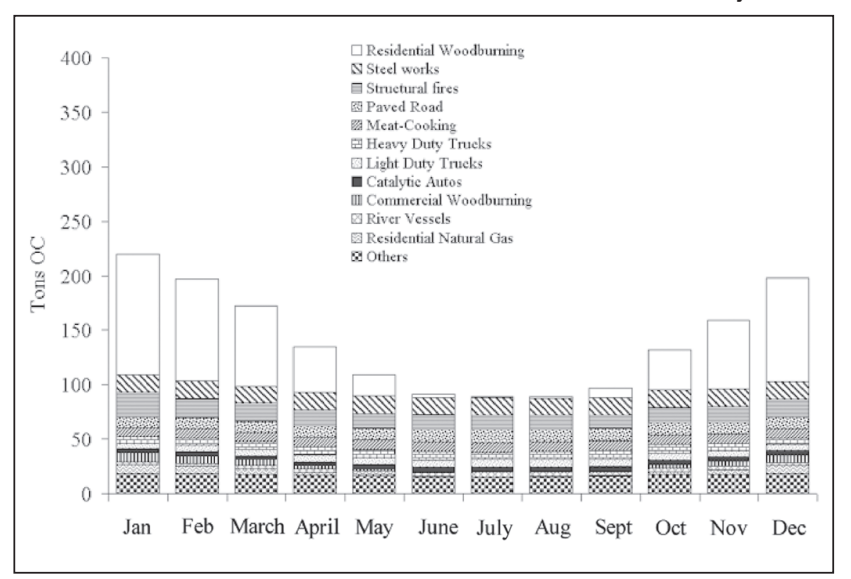

Figure 4. Pittsburgh area OC local primary emissions inventory. Monthly averages for 1995 .

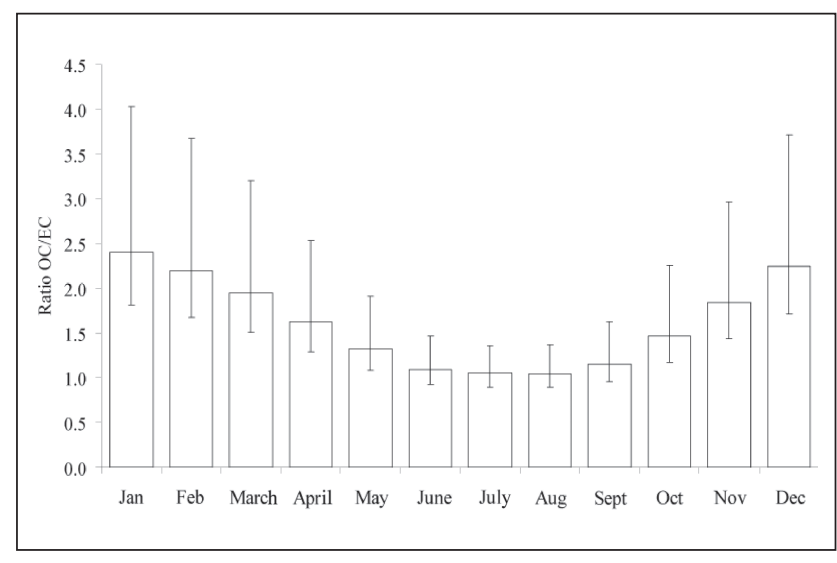

Figure 5. Ratio of $\mathrm{OC}$ to $\mathrm{EC}$ primary emissions in the Pittsburgh region for 1995. Uncertainties are based on the reported uncertainties of emission factors and activity data for the different sources of primary $\mathrm{OC}$ and $\mathrm{EC}$

diesel trucks are important sources of primary organic PM in the Pittsburgh region.

\section{SOA PRODUCTION}

Using the ambient OC and EC measurements for 1995, the $[\mathrm{OC} / \mathrm{EC}]_{\mathrm{p}}$ ratio from the emissions inventory, and the background concentrations of $\mathrm{OC}$ and $\mathrm{EC}$, it is possible to estimate the contribution of SOA to the total OC on a monthly basis applying eq 1 . The estimated SOA concentrations vary from a high of $\sim 3.8 \mu \mathrm{g} \mathrm{C} / \mathrm{m}^{3}$ in June to a low of almost zero during the winter months (Figure 6). The average yearly SOA contribution to the total OC concentration is $\sim 25 \%$, ranging from near zero in the winter to $\sim 50 \%$ in the summer. Monthly variations in the SOA production are consistent with the expected seasonal variations in the photochemical activity and the gaseous precursors concentrations. ${ }^{2,17}$ Winter conditions in western Pennsylvania limit photochemical activity, leading

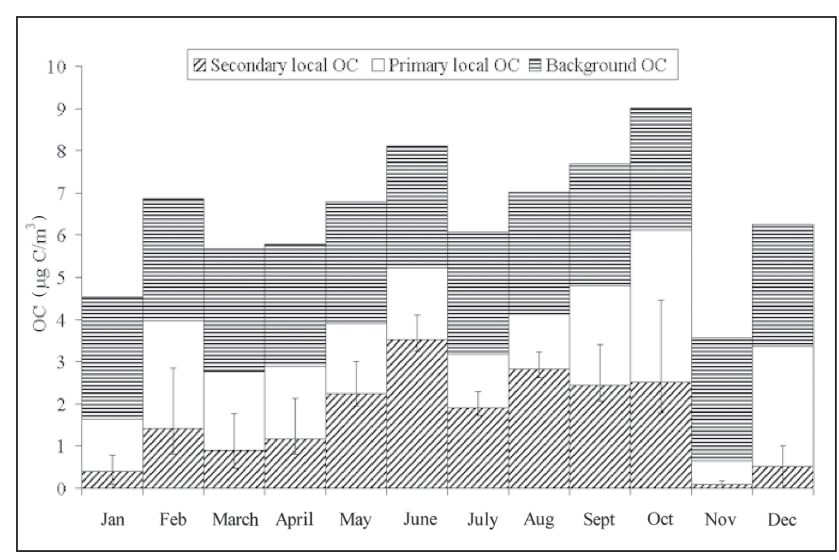

Figure 6. Contribution of $\mathrm{SOA}$, primary emissions, and background to total OC PM 2.5 in Pittsburgh in 1995, based on results from [OC/EC] ratio method, assuming average background concentrations of $2.9 \mu \mathrm{g}$ $\mathrm{C} / \mathrm{m}^{3}$ for $\mathrm{OC}$ and $0.5 \mu \mathrm{g} / \mathrm{m}^{3}$ for $\mathrm{EC}$. Uncertainties are based on the uncertainties of the $[\mathrm{OC} / \mathrm{EC}]_{\mathrm{p}}$. 
to less production of SOA. The current approach cannot explain the origin of the background concentration of OC, which is a significant contributor to the OC in the area. Therefore, the previous estimates represent a lower bound for the SOA contribution to Pittsburgh aerosol.

The OC and EC background concentrations are an important factor in estimating SOA production for areas like Pittsburgh using the OC/EC ratio approach. Figure 7 shows the calculated SOA concentration throughout the year for a range of background concentrations. Increasing background concentrations of OC from 2.5 to $3.3 \mu \mathrm{g}$ $\mathrm{C} / \mathrm{m}^{3}$, and EC from 0.4 to $0.6 \mu \mathrm{g} / \mathrm{m}^{3}$, changes the estimates of the contribution of SOA to the total OC concentration from zero to $2 \mu \mathrm{g} \mathrm{C} / \mathrm{m}^{3}$ in winter, and from 2.0 to $4.1 \mu \mathrm{g} \mathrm{C} / \mathrm{m}^{3}$ in summer. The corresponding contributions of SOA to the total organic aerosol vary from 0 to $20 \%$ in winter and from 20 to $50 \%$ in summer. Because of the uncertainties of background concentrations, SOA contributions to the total OC concentration vary from 10 to $35 \%$ on a yearly basis. Uncertainties in background OC and EC concentrations can lead to variations in the Pittsburgh SOA estimates of more than 50\%. Future measurement campaigns should include sufficient upwind measurements of OC and EC concentrations to determine their seasonal patterns and to provide a better estimate of the Pittsburgh carbonaceous aerosol composition.

\section{EVALUATION OF THE OC AND EC INVENTORIES}

The quality of the emissions inventory is evaluated by comparing predictions of a mass balance model to the measured ambient OC and EC concentrations. The mass balance model predicts the ambient OC and EC concentrations from the emissions inventory and the background concentrations. The model assumes the air above the Pittsburgh area is well mixed and the primary emissions are uniformly distributed throughout the area.

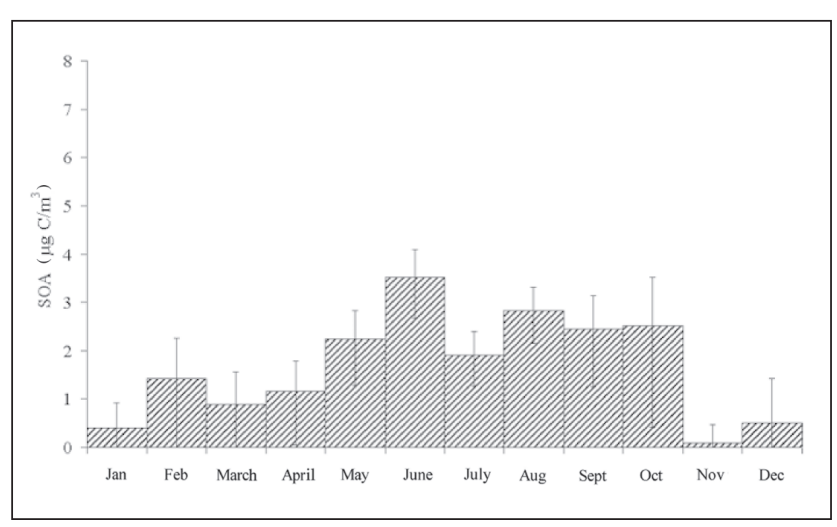

Figure 7. Local SOA concentration estimates under different background concentrations. The error bars represent the range in the estimated SOA concentrations caused by variations of OC from 2.5 to $3.3 \mu \mathrm{g} \mathrm{C} / \mathrm{m}^{3}$, and variations in the EC background concentrations from 0.4 to $0.6 \mu \mathrm{g} / \mathrm{m}^{3}$.
A box model approach, represented by eq 3, was adopted to calculate $\mathrm{OC}$ and EC concentrations in the area.

$$
\frac{d c_{\mathrm{s}}}{d t}=\frac{q_{\mathrm{s}}}{H}-\frac{v_{\mathrm{s}}}{H} c_{\mathrm{s}}+\frac{c_{\mathrm{s}}^{0}-c_{\mathrm{s}}}{\tau_{\mathrm{r}}}+R_{\mathrm{s}}
$$

where $c_{\mathrm{s}}$ is the concentration of the species of interest (EC or OC), $q_{\mathrm{s}}$ is the emission rate per $\mathrm{m}^{2}, H$ is the mixing height, $v_{\mathrm{s}}$ is the removal velocity, $c_{\mathrm{s}}^{0}$ is the background concentration, $\tau_{\mathrm{r}}$ is the residence time inside the system, and $R_{\mathrm{s}}$ is the SOA formation rate. For the evaluation of the emissions inventory, the background concentrations for EC and OC $\left(c_{\mathrm{s}}^{0}\right)$ are considered to be constant for the whole year. The monthly averaged mixing heights are based on the 1991 data for the area, and the average residence time $\left(\tau_{\mathrm{r}}\right)$ of the air parcels is determined according to the monthly averaged wind speeds in Pittsburgh (Table 8 ). The removal velocity is assumed to be constant throughout the year at $v_{\mathrm{s}}=1 \mathrm{~m} / \mathrm{hr}$. The Pittsburgh metro surface area is $1900 \mathrm{~km}^{2}$.

From the emissions inventory monthly data, the meteorological variables, and the background concentrations of carbonaceous material, it is possible to estimate the ambient concentration of "primary" OC and EC. The formation rate of SOA is calculated based on the monthly contribution of SOA to the total OC determined by eq 1 . Depending on the background OC and EC concentrations, the monthly SOA contribution is calculated, and by applying eq 3, a mass balance over the area can be performed.

The mass balance model indicates that the yearly average concentration for a number of scenarios varies from 3.7 to $6.6 \mu \mathrm{g} \mathrm{C} / \mathrm{m}^{3}$ for OC and from 1.1 to $1.6 \mu \mathrm{g} / \mathrm{m}^{3}$ for EC. These ranges were calculated assuming background concentrations of OC ranging from 2.5 to $3.3 \mu \mathrm{g} \mathrm{C} / \mathrm{m}^{3}$ and of EC ranging from 0.4 to $0.6 \mu \mathrm{g} / \mathrm{m}^{3}$. Figures 8 and 9 show the comparison between the monthly model predictions and the measurements for the concentrations of EC and OC for 1995. Reasons for the discrepancies include the simplicity of the model (neglecting the spatial variability of concentrations and sources), assuming constant background concentrations throughout the year, and simplifying the description of aerosol removal by dry and wet deposition. Based on these results, it is estimated that the emissions inventory is probably accurate within 50\% for the whole year for the different scenarios.

\section{CONCLUSIONS}

Accurate estimates of the background OC and EC concentrations are necessary for the estimation of the local OC and EC influences. Our analysis indicates that SOA can represent at least $10-35 \%$ of the total organic PM in the Pittsburgh area on a yearly basis. The primary or secondary origin of the background concentrations cannot be determined by the present analysis. Future measurement 
Table 8. Average mixing height and residence time of air parcels for the Pittsburgh area in $1995{ }^{40}$

\begin{tabular}{|c|c|c|c|c|c|c|c|c|c|c|c|c|}
\hline & Jan & Feb & March & April & May & June & July & Aug & Sept & Oct & Nov & Dec \\
\hline Mixing Height (m) & 750 & 800 & 950 & 1100 & 1000 & 1200 & 1150 & 900 & 1050 & 900 & 700 & 700 \\
\hline \multicolumn{13}{|l|}{ Air Parcel } \\
\hline \multicolumn{13}{|l|}{$\begin{array}{l}\text { Residence Time } \\
\text { in Analysis Area, }\end{array}$} \\
\hline$\tau_{\mathbf{r}}(\mathbf{h r})$ & 9 & 9 & 8 & 9 & 10 & 11 & 12 & 13 & 12 & 11 & 9 & 9 \\
\hline
\end{tabular}

campaigns should include sufficient upwind measurements for their determination.

The calculations of organic aerosol production for the Pittsburgh area indicate that, on average for 1995, SOA contributed at least $10-35 \%$ to the total organic mass of $\mathrm{PM}_{2.5}$. The monthly contributions vary from extremely low levels during the winter to as much as 50\% during the summer.

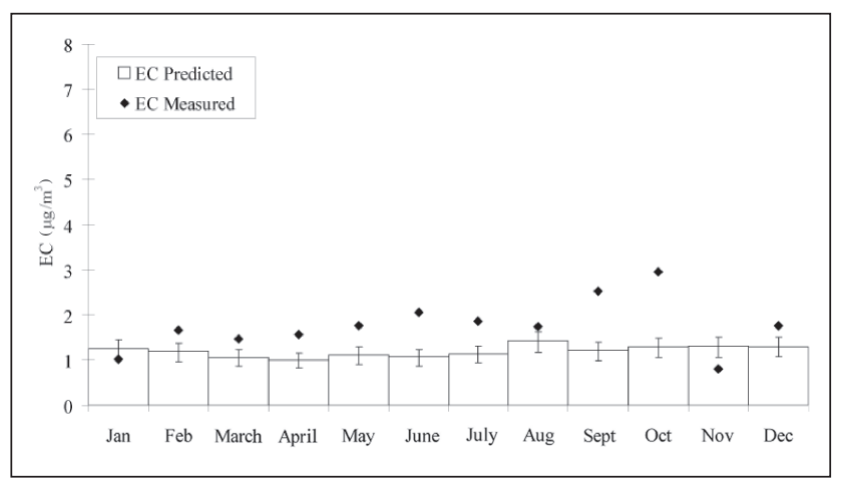

Figure 8. Box model results of predicted $\mathrm{EC}$ concentrations for the Pittsburgh area. The error bars represent the range of estimated EC concentrations caused by variations in the background EC concentrations from 0.4 to $0.6 \mu \mathrm{g} / \mathrm{m}^{3}$ and caused by the uncertainties in the emissions inventory.

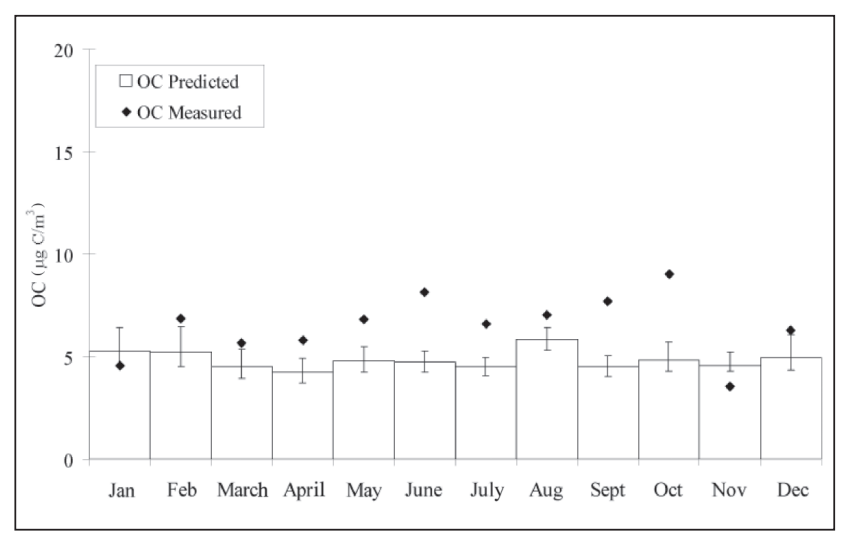

Figure 9. Box model results and measurements of $\mathrm{OC}$ concentrations for the Pittsburgh area. The error bars represent the range of estimated OC concentrations caused by variations in OC background concentrations from 2.5 to $3.3 \mu \mathrm{g} \mathrm{C} / \mathrm{m}^{3}$ and EC background concentrations varying from 0.4 to $0.6 \mu \mathrm{g} / \mathrm{m}^{3}$ and caused by the uncertainties in the emissions inventory.
This finding is consistent with the expected higher photochemical activity during the summer. The winter estimates are highly uncertain because of the uncertainty of wood-burning emissions, which is the dominant primary organic particulate source during these periods. The emissions inventory is consistent with the measured concentration and is probably accurate within $50 \%$.

Local wood and fugitive sources combustion are major sources of primary OC in western Pennsylvania on an annual basis (33 and 22\%, respectively), and wood burning is the dominant source during winter months. The coke production industry and diesel combustion are the dominant sources of the primary EC emissions (21 and 30\%, respectively). The EC emissions show a weaker monthly dependence compared with that shown by OC sources.

\section{ACKNOWLEDGMENTS}

The authors of this paper would like to thank EPA for the Pittsburgh PM Supersite Program grant (EPA cooperative agreement 99-NCERQA-X1), Cliff Davidson and Vicken Etyemezian for providing the database of OC and EC measurements for Pittsburgh, and the ACHD for their help in estimating the PM emissions from industrial sources.

\section{REFERENCES}

1. Daisey, J.M. Ann. N.Y. Acad. Sci. 1980, 338, 50-69.

2. Seinfeld, J.H.; Pandis, S.N. In Atmospheric Chemistry and Physics: From Air Pollution to Global Change; Wiley and Sons: New York, 1998; pp 49-120.

3. Dockery, D.W.; Pope, C.A., III. Annu. Rev. Public Health 1994, 15, 107-132.

4. Wolff, G.T.; Groblicki, P.J.; Cadle, S.H.; Countess, R.J. In Particulate Carbon: Atmospheric Life Cycle; Wolff, G.T.; Klimisch, R.L., Eds; Plenum: New York, 1983; pp 317-324.

5. Malm, W.C.; Gebhart, K.A. Atmos. Environ. 1996, 30, 843-845

6. Lee, C.T.; Tsai, J.J. J. Aerosol Sci. 1998, 29, S651-S652.

7. Carrico, C.M.; Rood, M.J.; Ogren, J.A. J. Geophys. Res. 1998, 103 $16,565-16,574$

8. Bergin, M.H.; Cass, G.R.; Xu, J.; Fang, C.; Zeng, L.M.; Yu, T.; Salmon, L.G.; Kiang, C.S.; Tang, X.Y.; Zhang, Y.H.; Chameides, W.L. J. Geophys. Res. 2001, 106, 17,969-17,980.

9. Rosen, H.; Novakov, T. Atmos. Environ. 1981, 15, 1371-1374.

10. Schwartz, S.E. J. Aerosol Sci. 1996, 27, 359-382.

11. Gray, H.A.; Cass, G.R.; Huntzicker, J.J.; Heyerdahi, E.K.; Rau, J.A Environ. Sci. Technol. 1986, 20, 580-589.

12. Turpin, B.J.; Huntzicker, J.J.; Larson, S.M.; Cass, G.R. Environ. Sci. Technol. 1991, 25, 1788-1793.

13. Air Quality Criteria for PM; EPA/600/P-65/001; U.S. Environmental Protection Agency: Washington, DC, 1996.

14. Schauer, J.J.; Rogge, W.F.; Hildemann, L.M.; Mazurek, M.A.; Cass, G.R Simoneit, B.T. Atmos. Environ. 1996, 30, 3837-3855.

15. Hintzenberger, R.; Jennings, S.G.; Larson, S.M.; Dillner, A.; Cachier, H.; Galambos, Z.; Rouc, A.; Spain, T.G. Atmos. Environ. 1999, 33 2823-2833. 
16. Huntzicker, J.J.; Heyerdahl, E.K.; Rau, J.A.; Griest, W.H.; McDougall, C.S. J. Air Pollut. Control Assoc. 1986, 36, 705-709.

17. Pandis, S.N.; Seinfeld, J.H.; Harley, R.; Cass, G. Atmos. Environ. 1992 26A, 2269-2282.

18. Rogge, W.F.; Mazurek, M.A.; Hildemann, L.M.; Cass, G.R.; Simoneit, B.R.T. Atmos. Environ. 1993, 27, 1309-1330.

19. Hildemann, L.M.; Cass, G.R.; Mazurek, M.A.; Simoneit, B.R.T. Environ. Sci. Technol. 1993, 27, 2045-2055

20. Strader, R.; Lurmann, F.; Pandis, S. Atmos. Environ. 1999, 33, 4849-4863.

21. Schauer, J.J.; Cass, G.R. Environ. Sci. Technol. 2000, 34, 1821-1832.

22. Turpin, B.J.; Huntzicker, J.J. Atmos. Environ. 1995, 33, 3527-3544.

23. Gray, H.A. Ph.D. Thesis, California Institute of Technology, 1986.

24. Birch, M.E. Analyst 1998, 123, 851-857.

25. Countess, R.J. Aerosol Sci. Technol. 1990, 12, 114-121.

26. Turpin, B.J.; Huntzicker, J.J.; Adams, K.M. Atmos. Environ. 1990, 24 A, 1831-1835.

27. Chow, J.C.; Watson, J.G.; Crow, D.; Lowenthal, D.H.; Merrifield, T. Aerosol Sci. Technol. 2001, 34, 23-34.

28. Etyemezian, V.; Davidson, C.; Finger, S.; Chow, J. J. Amer. Inst. for Conserv. 1998, 37, 31-58.

29. Chow, J.C.; Watson, J.G.; Pritchett, L.C.; Pierson, W.R.; Frazier, C.A.; Purcell, R.G. Atmos. Environ. 1993, 27A, 1185-1201.

30. Highway Statistics Report; Pennsylvania State Department of Transportation: Harrisburg, PA, 1998

31. Household Energy Consumption and Expenditures, Supplement: Regional; U.S. Department of Energy, Energy Information Administration: Washington, DC, 1997.

32. Emission Inventory Improvement Program (EIIP) Technical Report Series, Volumes I-X; EPA-454/R-97-004a-g; U.S. Environmental Protection Agency: Washington, DC, 1999.

33. Statistical Abstract of the United States; U.S. Census Bureau, Administrative and Customer Services Division, Statistical Compendia Branch: 1998

34. Air Quality Annual Report; Allegheny County Health Department: Pittsburgh, PA, 1998.

35. Off-Highway Mobile Sources, Part II; U.S. Environmental Protection Agency: Washington, DC, 1984.

36. Hildemann, L.M.; Markowski, G.R.; Cass, G.R. Environ. Sci. Technol. 1991, 24, 744-759.

37. Commercial Marine Emissions Inventory for EPA Category 2 and 3 Compressions Ignition Marine Engines in the United States Continental and Inland Waterways; EPA-420-R-98-020; U.S. Environmental Protection Agency: Pittsburgh, PA, 1998.

38. Dill, J. Older Vehicles and Air Pollution: Insights from the 1995 NPTS; Federal Highway Administration and Bureau of Transportation Statistics: Washington, DC,1995.

39. Introduction to Area Source Emissions Inventory Development; Area Sources Committee, Emissions Inventory Improvement Program, U.S. Environmental Protection Agency: Morrisville, NC, 1996.

40. Hallenbeck, M.; Rice, M.; Smith, B.; Cornell-Martinez, C.; Wilkinson, J. Chaparral Systems; Contract: DTFH61-93-C-00078; Federal Highway Administration: McLean, VA, 1997.

41. National Weather System. Pittsburgh Climate Data; National Oceanographic and Atmospheric Administration: Washington, DC, 2000.

42. U.S. Fire Administration; Federal Emergency Management Association, Publications Center: Emmetsburg, MD, 1996.
43. Energy Information Administration. State Energy Data Report, 1997; Office of Energy Markets and End Use, U.S. Department of Energy: Washington, DC, 1999.

44. Railroads and States Pennsylvania Report; Prepared by the Policy, Legislation and Communications Department of the Association of American Railroads: Washington, DC, 1996.

45. Statistical Handbook, Chapters IV and V, Airport Activity Statistics of Certificated Route Air Carriers; Federal Aviation Administration: Washington, DC, 1996.

46. U.S. Army Corps of Engineers, 1995 Water Resources Support Center Navigation Data Center: Alexandria, VA.

47. Shareef, G.S. Engineering Judgment; EPA Contract 68-02-3884; Radian Corp.: Washington, DC, 1987.

48. Cooper, J.A. PM 10 Source Composition Library for the South Coast Air Basin, Volumes I and II; Prepared for the South Coast Air Quality Management District, El Monte, CA, 1987.

49. Cooper, J.A.; Frazier, C.A.; Houck, J.E. Seattle-Tacoma Aerosol Characterization Study; Draft Final Report to the Puget Sound Air Pollution Control Agency by NEA, Inc.: Washington, DC, 1983.

50. Taback, H.J.; Brienza, A.R.; Macko, J.F.; Brunetz, N. Fine Particle Emissions from Stationary and Miscellaneous Sources in the South Coast Air Basin; No. 5806-783; NTIS No. PB293923; KVB Corp.: Tustin, CA 1979.

51. Watson, J.G. Ph.D. Thesis, Oregon Graduate Center, Portland, OR, 1979.

52. 1999 Fire Bureau Response Statistics; Pittsburgh Fire Bureau: Pittsburgh, PA, 1999.

53. Meteorological Data from National Climatic Data Center, Asheville, NC; U.S. Environmental Protection Agency: Asheville, NC, 1998.

54. USA Counties Database 1998; State and Metropolitan Data Book; U.S. Census Bureau: Washington, DC, 1998.

55. Report: Behaviors; National Center for Chronic Disease Prevention and Health Promotion Centers for Disease Control and Prevention, Office on Smoking and Health: Atlanta, GA, 1997.

56. Rogge, W.; Hildemann, L.; Mazurek, M.; Cass, G. Environ. Sci. Technol. $1998,33,13-22$

57. McDonald, J.; Zielinska, B.; Fujita, E.; Sagebiel, J.; Chow, J.; Watson, J. Environ. Sci. Technol. 2000, 34, 2080-2091.

\section{About the Authors}

Juan C. Cabada is a graduate student in the Department of Chemical Engineering, Carnegie Mellon University, 5000 Forbes Ave., Pittsburgh, PA 15213. Spyros N. Pandis (corresponding author) is a professor in the Department of Chemical Engineering, Carnegie Mellon University. Allen L. Robinson is an assistant professor in the Department of Mechanical Engineering, Carnegie Mellon University. 


\title{
Mass size distributions and size resolved chemical composition of fine particulate matter at the Pittsburgh supersite
}

\author{
Juan C. Cabada ${ }^{\mathrm{a}}$, Sarah Rees ${ }^{\mathrm{b}, \mathrm{d}}$, Satoshi Takahama ${ }^{\mathrm{a}}$, Andrey Khlystov ${ }^{\mathrm{a}}$, \\ Spyros N. Pandis ${ }^{\mathrm{a}, \mathrm{d}, *}$, Cliff I. Davidson ${ }^{\mathrm{b}, \mathrm{d}}$, Allen L. Robinson ${ }^{\mathrm{c}, \mathrm{d}}$ \\ a Department of Chemical Engineering, Carnegie Mellon University, Pittsburgh, PA 15213-7139, USA \\ ${ }^{\mathrm{b}}$ Department of Civil and Environmental Engineering, Carnegie Mellon University, Pittsburgh, PA 15213-7139, USA \\ ${ }^{\mathrm{c}}$ Department of Mechanical Engineering, Carnegie Mellon University, PA 15213-7139, USA \\ ${ }^{\mathrm{d}}$ Department of Engineering and Public Policy, Carnegie Mellon University, Pittsburgh, PA 15213-7139, USA
}

\begin{abstract}
Size-resolved aerosol mass and chemical composition were measured during the Pittsburgh Air Quality Study. Daily samples were collected for 12 months from July 2001 to June 2002. Micro-orifice uniform deposit impactors (MOUDIs) were used to collect aerosol samples of fine particulate matter smaller than $10 \mu \mathrm{m}$. Measurements of $\mathrm{PM}_{0.056}, \mathrm{PM}_{0.10}$, $\mathrm{PM}_{0.18}, \mathrm{PM}_{0.32}, \mathrm{PM}_{0.56}, \mathrm{PM}_{1.0}, \mathrm{PM}_{1.8}$ and $\mathrm{PM}_{2.5}$ with the MOUDI are available for the full study period. Seasonal variations in the concentrations are observed for all size cuts. Higher concentrations are observed during the summer and lower during the winter.

Comparison between the $\mathrm{PM}_{2.5}$ measurements by the MOUDI and other integrated PM samplers reveals good agreement. Good correlation is observed for $\mathrm{PM}_{10}$ between the MOUDI and an integrated sampler but the MOUDI underestimates $\mathrm{PM}_{10}$ by $20 \%$. Bouncing of particles from higher stages of the MOUDI $\left(>\mathrm{PM}_{2.5}\right)$ is not a major problem because of the low concentrations of coarse particles in the area. The main cause of coarse particle losses appears to be losses to the wall of the MOUDI.

Samples were collected on aluminum foils for analysis of carbonaceous material and on Teflon filters for analysis of particle mass and inorganic anions and cations. Daily samples were analyzed during the summer (July 2001) and the winter intensives (January 2002). During the summer around 50\% of the organic material is lost from the aluminum foils as compared to a filter-based sampler. These losses are due to volatilization and bounce-off from the MOUDI stages. High nitrate losses from the MOUDI are also observed during the summer (above $70 \%$ ). Good agreement between the gravimetrically determined mass and the sum of the masses of the individual compounds is obtained, if the lost mass from organics and the aerosol water content are included for the summer. For the winter no significant losses of material are detected and there exists reasonable agreement between the gravimetrical mass and the sum of the concentrations of the individual compounds.

Ultrafine particles (below $100 \mathrm{~nm}$ ) account on average, for $<5 \%$ of the $\mathrm{PM}_{2.5}$ mass, and show different composition for the summer and the winter. During the summer the ultrafine mass is $50 \%$ carbonaceous material (organic material and elemental carbon) and 50\% inorganic (mainly sulfate and ammonium); during the winter these percentages are $70 \%$ and $30 \%$, respectively.
\end{abstract}

(C) 2004 Elsevier Ltd. All rights reserved.

Keywords: MOUDI; $\mathrm{PM}_{x}$; PM size/composition; Condensation mode; Droplet mode; Ultrafine PM; PAQS

\footnotetext{
*Corresponding author. Department of Chemical Engineering, Carnegie Mellon University, Pittsburgh, PA 15213-7139, USA.

E-mail address: spyros@andrew.cmu.edu (S.N. Pandis).
} 


\section{Introduction}

Fine atmospheric aerosol mass concentrations (particulate matter below $2.5 \mu \mathrm{m}, \mathrm{PM}_{2.5}$ ) are of importance because of their impact on human health (Dockery et al., 1993), their ability to scatter light affecting visibility, and their role in global climate change (Seinfeld and Pandis, 1998). Fine particulate matter size distributions can provide information about the chemical and physical processes affecting aerosols as they are transported in the atmosphere (Wall et al., 1988). Aerosol size distributions also are important for the optical properties and the effect of aerosols on the planet energy balance (Seinfeld and Pandis, 1998).

Particles emitted from combustion sources (primary emissions) and produced by nucleation start their atmospheric lifetime in the smaller particle sizes (diameters $<200 \mathrm{~nm}$ ). Condensation of organic compounds after the reaction of atmospheric oxidants and organic gases (secondary organic aerosol, SOA) and secondary production of sulfate affects mainly the accumulation mode between 200 and $500 \mathrm{~nm}$. Longrange transported and cloud-processed aerosol tend to be in the 200-1000 nm diameter range (Whitby, 1978; Wexler and Ondov, 1998; Seinfeld and Pandis, 1998).

Recently, attention has been drawn to the ultrafine (diameter below $100 \mathrm{~nm}$ ) aerosol concentrations. Ultrafine particles account for practically all the aerosol number concentration in the urban atmosphere, though their mass contribution is in general small. These particles can penetrate deep into the interstitial space of the lungs and overwhelm the alveolar macrophages, thereby impeding the natural cleaning mechanisms of the lungs (Oberdorster et al., 1994; Oberdorster, 1995, 2001; Ferin et al., 1992). Furthermore, inhalation of highly acidic particles can cause inflammation and irritation of the lung tissue; if the chemical constituents of the particles are carcinogenic, these particles may induce cancer tumors (Oberdorster and $\mathrm{Yu}, 1990$; Donalson et al., 2002). Fine-particle regulations in the US are focused on controlling the PM mass concentration $\left(\mathrm{PM}_{10}\right.$ and $\left.\mathrm{PM}_{2.5}\right)$ (Environmental Protection Agency, 1996). The effect of reductions of the $\mathrm{PM}_{2.5}$ and $\mathrm{PM}_{10}$ on the concentration of ultrafine particles is not well understood. Ultrafine particles are emitted mainly from mobile sources (automobiles and diesel trucks) and stationary combustion sources. However, they are also formed during the nucleation of sulfuric acid and organic gases (Seinfeld and Pandis, 1998).

Results of studies combining measurements of aerosol number concentrations and chemically speciated size distributions, using micro-orifice uniform deposit impactors (MOUDIs), are limited to southern and central California (Hughes et al., 1998; Chung et al., 2001). Both of these studies were done during the winter. For Southern California (Pasadena) carbonaceous material was the largest fraction of the $\mathrm{PM}_{2.5}$, followed by nitrate. Small contributions of sulfate, ammonium and trace compounds were reported for this winter aerosol. An "unknown" component was determined by the difference between the gravimetrically measured mass and the sum of the masses of the individual compounds on each MOUDI stage. Larger values of "unknown" material are associated with the upper stages of the MOUDI (between 0.32 and $1.0 \mu \mathrm{m}$ ). The fraction of this "unknown" material is reported to be of the order of 10-30\%. Central California (Bakersfield) was characterized by periods of clean and polluted air during the winter. Clean days were characterized by high fractions of carbonaceous material, nitrate and ammonium in the $\mathrm{PM}_{2.5}$. During polluted days particles contained mainly sulfate, nitrate, sodium and chloride. On the clean days, the reported "unknown" mass was negligible, although this discrepancy was significantly larger on polluted days, occasionally over $30 \%$.

The studies of Hughes et al. (1998) and Chung et al. (2001) also provided information on ultrafines particles. Both of these studies reported a lack of correlation between ultrafine number and ultrafine mass concentrations. Carbonaceous compounds were the largest fraction of the ultrafine mass. Significant contributions of sulfate, nitrate and trace compounds were reported in both studies. The unknown fraction of material in the ultrafine particles was reported to be between $0 \%$ and $50 \%$.

In this paper we present data on particle mass concentrations in several size ranges $(0.056-10 \mu \mathrm{m})$ collected in the Pittsburgh Air Quality Study (PAQS) from July 2001 to June 2002. For two intensive periods, during 15 days in the summer of 2001 and 9 days in the winter of 2002, impactor samples were chemically resolved for inorganic components and carbonaceous compounds.

\section{Experimental}

MOUDIs (Marple et al., 1991) (Model 110, MSP Co., Minneapolis, MN) were used to collect daily sizeresolved aerosol samples during PAQS, from July 2001 to June 2002. Aerodynamic size cuts for the impactor are $0.056,0.10,0.18,0.32,0.56,1.0,1.8,2.5,5.6$ and $10 \mu \mathrm{m}$. The MOUDI sampling flow rate is $301 \mathrm{~min}^{-1}$. The PAQS main site was located in Schenley park on the top of a hill adjacent to the Carnegie Mellon University campus, around $6 \mathrm{~km}$ east of downtown Pittsburgh.

Samples for the determination of total mass were collected using $37 \mathrm{~mm}$ ring-supported Teflon filters (7592-104, Whatman, Clifton, NJ). The mass size distribution of the aerosol collected by the MOUDI stages was determined by weighing the filters using a high-precision microbalance (UMX2, Mettler-Toledo, 
Columbus, $\mathrm{OH}$ ). The MOUDI filters were weighed inside a glove box with a controlled relative humidity $(35 \pm 2 \%)$ and temperature $\left(21 \pm 1^{\circ} \mathrm{C}\right)$. Prior to weighing, the filters were equilibrated for $24 \mathrm{~h}$ inside the glove box. During two intensive sampling periods, July of 2001 and January of 2002, two MOUDI samplers were operated in parallel using different filter media to collect aerosol particles. Teflon filters were used for determination of total mass and inorganic compounds, and uncoated aluminum foils (MSP Corp.) were used for the determination of carbonaceous aerosol concentrations. Inorganic compound concentrations were determined by ion chromatography (Dionex DX-600 for anions and DX-120 for cations) and carbonaceous aerosol was analyzed using a thermal/optical transmittance carbon analyzer (Sunset Laboratory Inc., OC-EC Aerosol Carbon Analyzer Model-3) and temperature program based on the NIOSH protocol (Cabada et al., 2003). In the case of aluminum foils it is not possible to determine the organic carbon (OC), elemental carbon (EC) split by the optical transmittance method. Since aluminum foil loads are low, the possible pyrolisis of material is reduced. For this case, the OC concentrations were determined by the amount of carbon evolved during the helium atmosphere in the analysis and the EC was considered to be the carbon evolved during the helium/oxygen atmosphere. Viidanoja et al. (2002) reported that defining organic and elemental carbon without an optical correction for pyrolisis leads to an upper limit in the estimation of EC and an error within $30 \%$ of the real EC concentration. This change in definition has a small effect on the OC concentration $(<10 \%)$.

Aluminum foils were baked at $550^{\circ} \mathrm{C}$ for $24 \mathrm{~h}$ prior to collection. The MOUDI collecting carbonaceous material was operated without rotating the impactor stages, as a multi-orifice impactor (MOI). OC concentrations measured by the carbon analyzer are converted to organic mass $(\mathrm{OM})$ multiplying by a factor of 1.8 to account for the hydrogen, oxygen, sulfur and nitrogen associated with the organic compounds (Turpin and Lim, 2001).

Measurements of $\mathrm{PM}_{0.056}, \mathrm{PM}_{0.01}, \mathrm{PM}_{0.18}, \mathrm{PM}_{0.32}$, $\mathrm{PM}_{0.56}, \mathrm{PM}_{1.0}, \mathrm{PM}_{1.8}$ and $\mathrm{PM}_{2.5}$ with the MOUDI are available for the full study period, while $\mathrm{PM}_{5.6}$ and $\mathrm{PM}_{10}$ measurements were conducted only during 2001 . Independent measurements of $\mathrm{PM}_{10}$ with a dichotomous sampler are available for the full study.

Particle bounce-off in cascade impactors has been documented by several authors, particles as small as $0.2 \mu \mathrm{m}$ can bounce from their respective collection stage and are collected on stages corresponding to smaller sizes (Hinds, 1998; Pak et al., 1992). This problem is more common in the upper stages of the impactors. Experimental data show that once a large particle bounces-off its proper stage it is likely to continue bouncing-off other stages in the impactor and may not be collected at all (Hinds, 1998). The use of fine PM cyclones in front of cascade impactors and the use of greased substrates can reduce this problem (Hinds, 1998). For this study greased Teflon substrates were used for MOUDI stages collecting particles larger than $10 \mu \mathrm{m}$. No greased stages were used for the MOUDI with aluminum foils substrates to avoid contamination of organic material.

A dry-ambient aerosol size spectrometer (DAASS) system (Stanier et al., 2003) operated during PAQS reporting number, surface area and volume distributions of aerosols. The system consists of two scanning mobility particle sizers (SMPS, TSI 3936N25 and TSI 3936L10) and an aerodynamic particle sizer (APS, TSI APS 3320) that measure the aerosol size distribution between $3 \mathrm{~nm}$ and $10 \mu \mathrm{m}$ in diameter. The inlets of the instruments and their sheath air lines were equipped with computer-controlled valves that direct air through Nafion dryers or bypass them. The Nafion dryers reduce the $\mathrm{RH}$ to below $30 \%$, at which ambient particles are expected to lose most or all of the water and thus be virtually dry. The instrument cycles between dried and the ambient conditions every $7 \mathrm{~min}$ and is synchronized with the scan times of the aerosol spectrometers.

In addition to the size-resolved measurements using the MOUDI and the DAASS system, integrated $\mathrm{PM}_{10}$ and $\mathrm{PM}_{2.5}$ samplers were operated at PAQS from July 2001 to July 2002. A dichotomous sampler (Series 241, Thermo Andersen) measured daily average $\mathrm{PM}_{10}$ and $\mathrm{PM}_{2.5}$ mass on Teflon filters (7592-104, Whatman), with operational flow of $16.71 \mathrm{~min}^{-1}$. An FRM sampler (Partisol-2000, Rupprecht \& Patashnick Co., Inc.) measured daily average $\mathrm{PM}_{2.5}$ mass on Teflon substrates (7592-104, Whatman), operating at $16.71 \mathrm{~min}^{-1}$. Determination of mass from these filters followed the same procedure as for the MOUDI Teflon filters. A tapered element oscillating microbalance (TEOM, Series 1400a, Rupprecht \& Patashnick Co., Inc.) operated continuously for the length of the PAQS project, reporting $\mathrm{PM}_{2.5}$ mass. The TEOM sampled at $30^{\circ} \mathrm{C}$ and was equipped with a Nafion diffusion dryer sample equilibration system (SES, Rupprecht \& Patashnick Co., Inc.).

Several $\mathrm{PM}_{2.5}$ speciation samplers were used during PAQS. Filter-based organic material measurements were collected on quartz fiber filters $(47 \mathrm{~mm}$ Pallflex, QAOT) on a daily basis using a denuded sampler during the summer. During the winter, an undenuded sampler using a front quartz filter and a backup quartz filter was used (Cabada et al., 2004). For the winter, the concentrations measured by the undenuded sampler were corrected for positive artifact by subtracting the measured organic concentration in the backup filter (Subramanian et al., 2004). Measurements of total concentrations of nitrate and ammonium $\left(\mathrm{PM}_{2.5}\right.$ and 
gas), aerosol sulfate, sodium, potassium, magnesium, calcium, chloride, nitrite and oxalate were made using Teflon (Whatman Cat No. 7592-104) and nylon filters (Whatman Cat No. 7410-004). Daily samples were collected during the length of the project except for the summer 2001 intensive period (July 2001), where five samples a day were collected (4-6h samples). Two sampling lines were used for these species, one denuded and one undenuded, to provide data for both gas and particulate phase compounds. Analysis of the inorganic material was performed by ion chromatography (Wittig et al., 2003a).

\section{3. $\mathbf{P M}_{10}$ and $\mathbf{P M}_{2.5}$ measurements}

Fig. 1 shows the intercomparison of $\mathrm{PM}_{2.5}$ and $\mathrm{PM}_{10}$ mass concentrations measured by the MOUDI with corresponding measurements by the dichotomous sampler, FRM and TEOM. The correlation coefficient $\left(R^{2}\right)$ exceeded 0.75 , for the comparisons between the different samplers. However, the original data indicate coarse particle losses from the MOUDI. On average, the MOUDI collects $15-20 \%$ less $\mathrm{PM}_{10}$ mass than the dichotomous sampler. The integrated fine mass collected by the MOUDI (sum of all stages up to $2.5 \mu \mathrm{m}$ ) and the filter-based gravimetrical methods (FRM and dichotomous) are in good agreement. These results are consistent with losses to the walls of some mainly coarse particles in the MOUDI and limited bounce-off from the larger to the smaller stages. Particles during PAQS are expected to be liquid during most of the study because of their ability to retain water at low $\mathrm{RH}$ during the summer (Stanier et al., 2003) and their high organic content during the winter (Wittig et al., 2003b). The comparison between the MOUDI and the TEOM for $\mathrm{PM}_{2.5}$ shows that the MOUDI measures, on average, around $5 \%$ less mass than the TEOM. Statistical analyses show no significant difference between these two measurements.

\section{4. $\mathbf{P M}_{x}$ mass concentrations}

The MOUDI results were used to construct time series of the different PM concentrations measured. Fig. 2 shows the monthly averaged concentrations for the different size cuts, from $\mathrm{PM}_{10}$ to $\mathrm{PM}_{0.056}$. A seasonal dependence is observed for all PM sizes, with higher concentrations observed during the summer and lower during the winter. Lower size cuts mass concentrations show more variability.

Comparisons between mass concentrations measured in the different stages of the MOUDI show that good correlations exist between $\mathrm{PM}_{2.5}$ and $\mathrm{PM}_{x}$, when $\mathrm{PM}_{x}$ is close to $\mathrm{PM}_{2.5}$. The correlation between the different concentrations decreases significantly as $\mathrm{PM}_{x}$ is smaller than $\mathrm{PM}_{0.56}$. On average, around $80 \%$ of the $\mathrm{PM}_{10}$ is
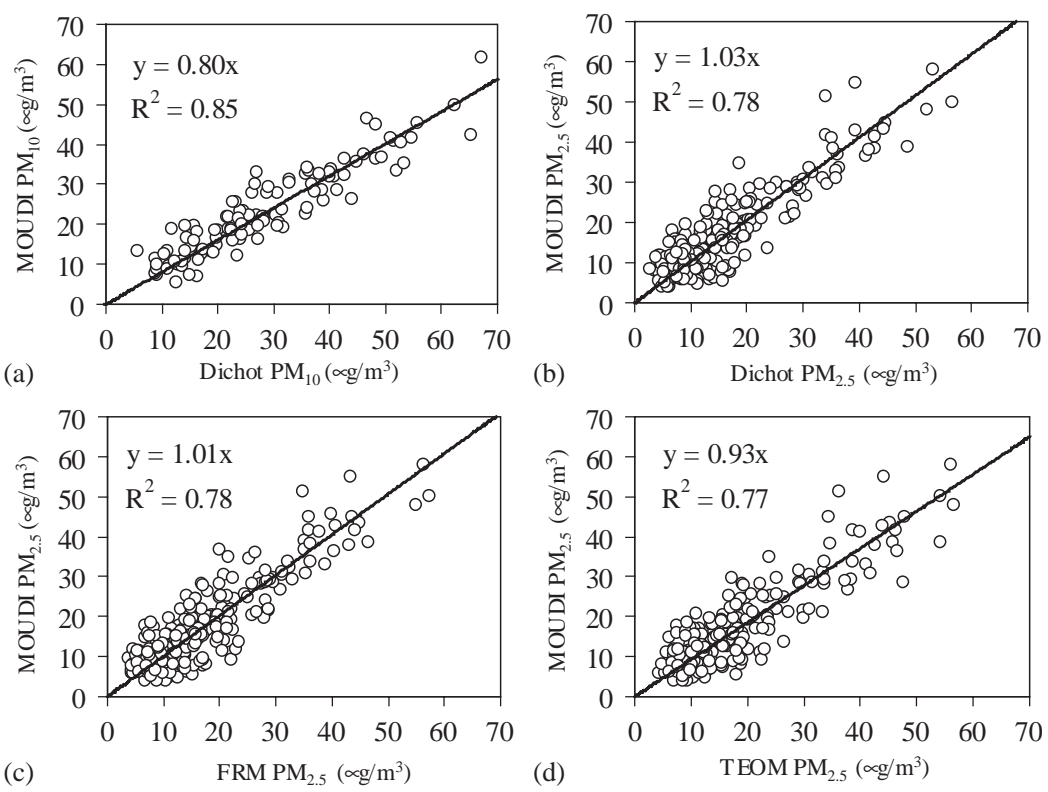

Fig. 1. Sampler inter-comparisons at PAQS, with forced fits through the origin. (a) MOUDI PM 10 vs. Dichot PM 10 for 2001. (b) MOUDI $\mathrm{PM}_{2.5}$ vs. Dichot $\mathrm{PM}_{2.5}$ for 2001 and 2002. (c) MOUDI $\mathrm{PM}_{2.5}$ vs. FRM $\mathrm{PM}_{2.5}$ for 2001 and 2002. (d) MOUDI PM 2.5 vs. TEOM $\mathrm{PM}_{2.5}$ for 2001 and 2002. All measurements are daily averages. 


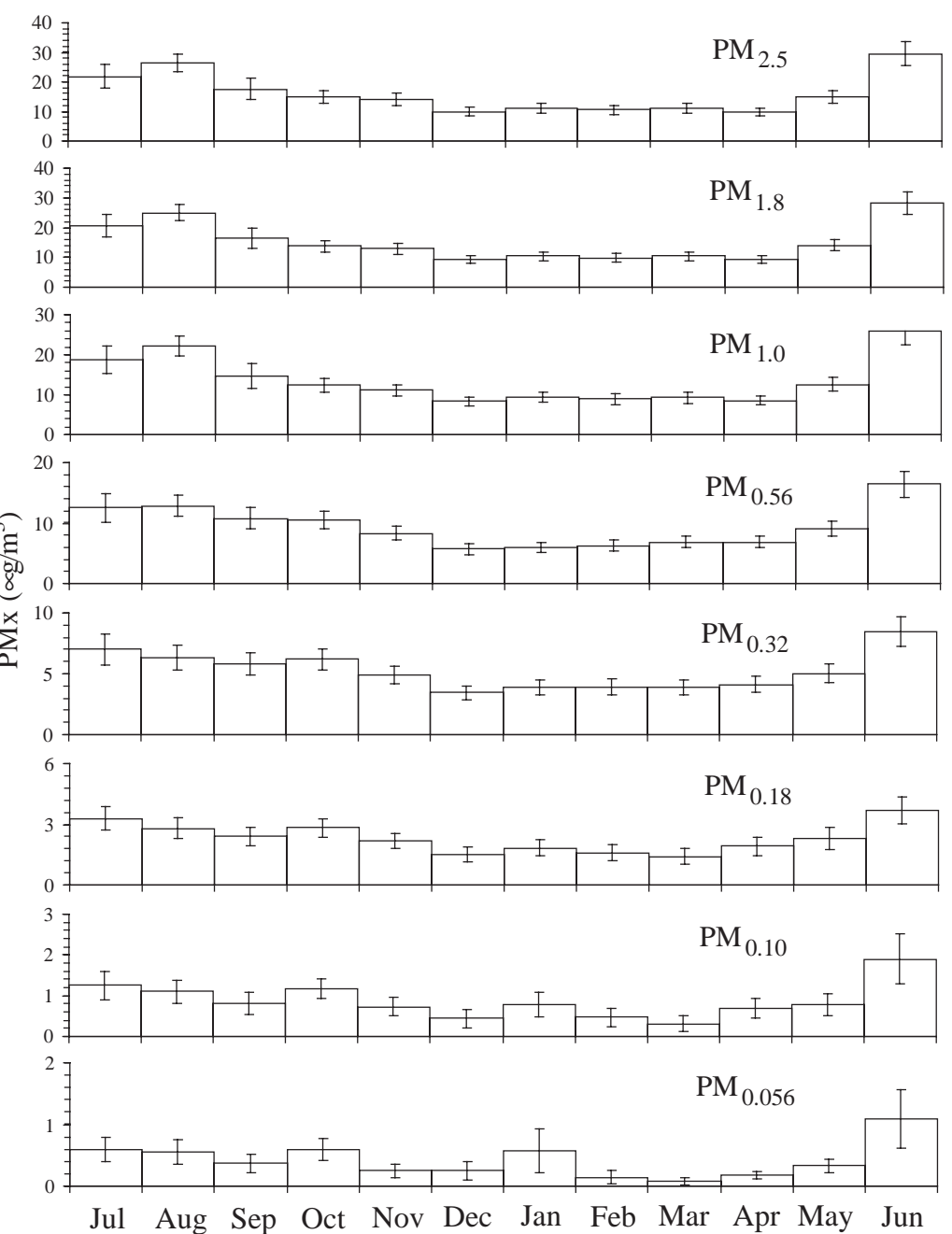

Fig. 2. Monthly averages of $\mathrm{PM}_{x}$ measured with the MOUDI during PAQS. The error bars correspond to the measurement standard errors.

below $2.5 \mu \mathrm{m}$ in Pittsburgh (Fig. 3a). Eighty-five percent of the $\mathrm{PM}_{2.5}$ mass is in aerosol particles smaller than $1.0 \mu \mathrm{m}$ in aerodynamic size (Fig. 3b). Fifty-five percent of the $\mathrm{PM}_{2.5}$ is $\mathrm{PM}_{0.56}$ (Fig. 3c). $\mathrm{PM}_{0.32}$ and $\mathrm{PM}_{0.18}$ constitute around $30 \%$ and $12 \%$ of the total fine aerosol mass, respectively (Fig. $3 \mathrm{~d}$ and e). The ultrafine mass contribution (below $100 \mathrm{~nm}$ ) to $\mathrm{PM}_{2.5}$ is, on average, around 5\% (Fig. 3f).

Ultrafine particles contribute $<5 \%$ of the total fine particle mass and the filter loads are low, making the measurement of the mass of these particles prone to errors during sampling and analysis. Even a few large particles bouncing from higher stages can have a big impact on the mass on the lower stages. Khlystov et al. (2004) showed that good agreement is achieved between the MOUDI and the DAASS system for particles bigger than $100 \mathrm{~nm}$, but not for the ultrafine particles (Fig. 4a).
Improved agreement is obtained when the ratios of ultrafines mass to $\mathrm{PM}_{2.5}$ and ultrafines volume to $V_{2.5}$ are compared (Fig. 4b). Comparing the ratios from the two systems can give an estimate of particles bouncing from higher stages of the MOUDI and that are collected by lower stages, in this case stages corresponding to the ultrafine particulate matter. If bounce-off is a significant problem, the ratio of masses $\left(\mathrm{PM}_{0.10} / \mathrm{PM}_{2.5}\right)$ would exceed the ratio of volumes $\left(V_{0.10} / V_{2.5}\right)$ and the corresponding points would lie in the upper left corner of Fig. 4b, which is not the case. The correlation coefficient between these two measurements is reasonable $\left(R^{2}=0.55\right)$ given the uncertainty of the ultrafine mass concentration (estimated to be around $30 \%$ ).

Variability in the absolute estimates could be due to the measurement of the ultrafine mass in the MOUDI or the fundamental differences in the measurement 

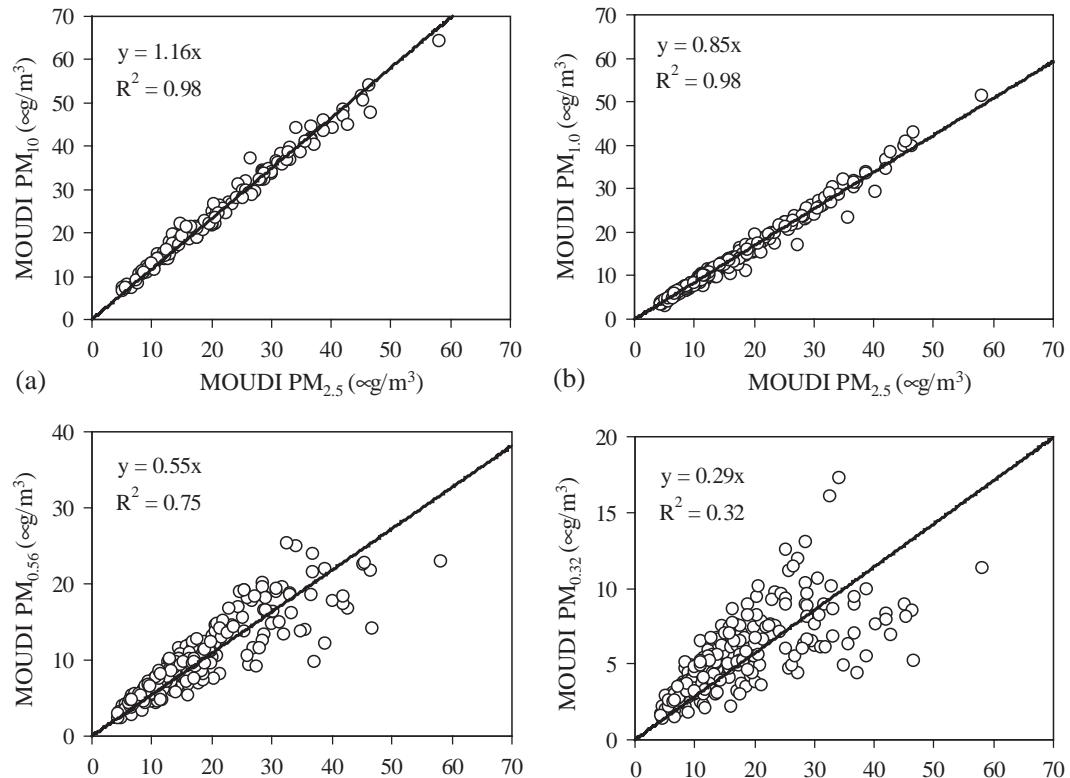

(c)
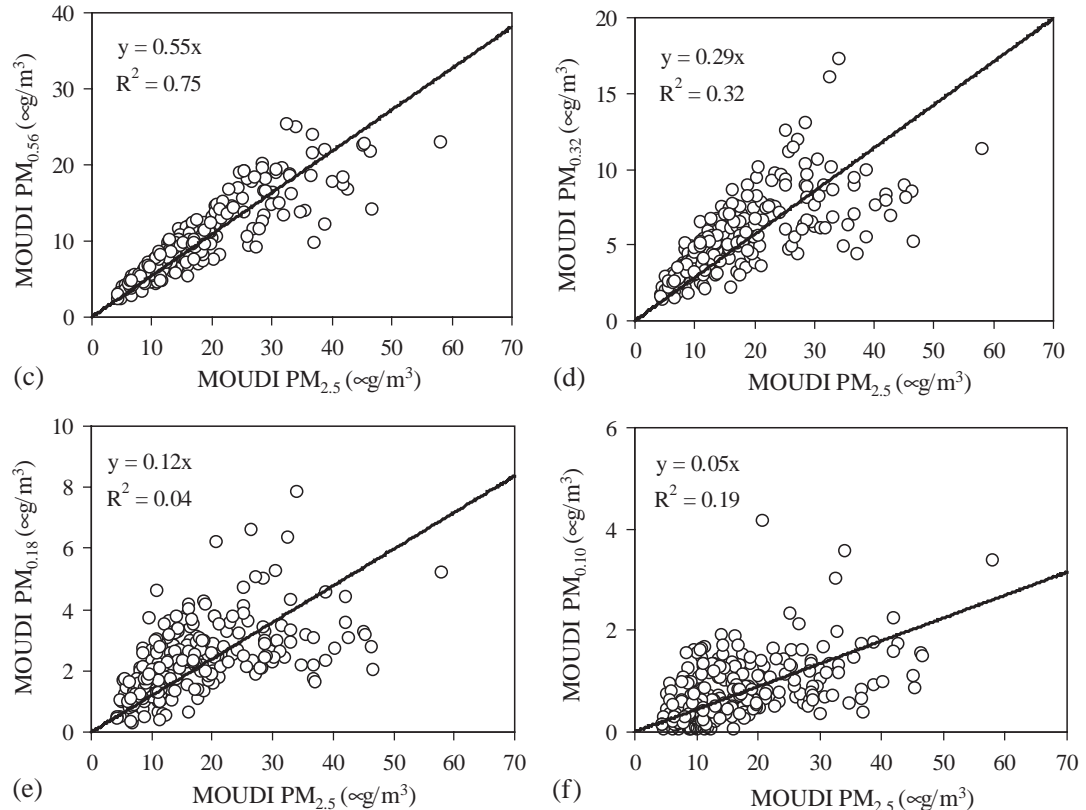

Fig. 3. Correlation of $\mathrm{PM}_{2.5}$ mass collected by the MOUDI and the integrated mass for different size bins of interest. (a) $P M_{10}$ vs. $\mathrm{PM}_{2.5}$ for 2001 , (b) $\mathrm{PM}_{1.0}$ vs. $\mathrm{PM}_{2.5}$, (c) $\mathrm{PM}_{0.56}$ vs. $\mathrm{PM}_{2.5}$, (d) $\mathrm{PM}_{0.32}$ vs. $\mathrm{PM}_{2.5}$, (e) $\mathrm{PM}_{0.18}$ vs. $\mathrm{PM}_{2.5}$, and (f) $\mathrm{PM}_{0.10}$ vs. $\mathrm{PM}_{2.5}$. Comparisons for size cuts below $\mathrm{PM}_{2.5}$ are for 2001 and 2002.
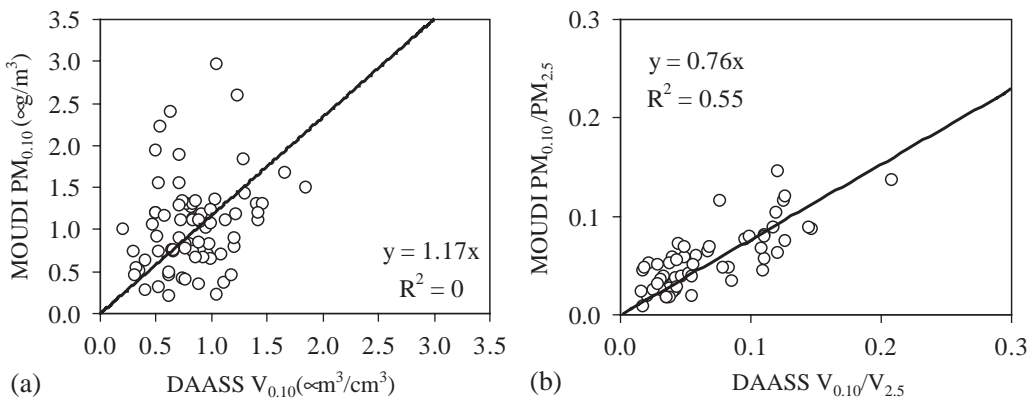

Fig. 4. Comparison of ultrafine particles between the DAASS system and the MOUDI. (a) PM $_{0.10}$ measured with the MOUDI vs. $V_{0.10}$ measured with the DAASS system. (b) Ratio of $\mathrm{PM}_{0.10}$ to $\mathrm{PM}_{2.5}$ from the MOUDI vs. ratio of $V_{0.10}$ to $V_{2.5}$ particle volume from the DAASS system.

methods employed by the MOUDI and the SMPS techniques (i.e. aerosol aerodynamic diameter vs. mobility diameter). Ambient conditions and character- istics of the aerosols (e.g. non-spherical shapes of fresh particles) could also influence these estimates (Shen et al., 2002). 


\section{Size-resolved mass distributions}

Seasonal average mass distributions collected with the MOUDI are shown in Fig. 5. Mass size distributions for each stage of the MOUDI were inverted using MICRON (Wolfenbarger and Seinfeld, 1990). For all seasons, most of the fine aerosol mass is concentrated between 0.10 and $1.0 \mu \mathrm{m}$. Summer size distributions show multiple modes. The droplet mode (around $0.7 \mu \mathrm{m})$ dominates over the size distribution. The condensation mode (around $0.2 \mu \mathrm{m}$ ) is clear only after the data is inverted to create a smooth size distribution. Fall size distributions show tri-modal distribution but in this case the condensation mode is slightly larger than the droplet mode. Winter size distributions show four modes distribution with both, the condensation and the droplet modes contributing equally to the distribution. Spring size distributions are similar to the summer, with a dominating droplet mode and lower condensation mode.

Data collected during SCAQS in southern California, using both MOUDIs and SMPS systems, showed two modes in the fine mass distributions. One mode was observed around $0.20 \mu \mathrm{m}$ (condensation mode) and the second and larger mode was at around $0.70 \mu \mathrm{m}$ (droplet mode) (John et al., 1990; Eldering et al., 1994). Size distributions measured at Fresno, CA, for two different seasons (winter and spring) with an SMPS system showed a bi-modal distribution (Lawless et al., 2001). During the winter, size distributions showed a dominant mode around $0.20 \mu \mathrm{m}$ and a smaller mode around $1.0 \mu \mathrm{m}$. The same two modes existed in the spring, but the $1.0 \mu \mathrm{m}$ mode was dominant.

\section{PM composition measurements}

Comparisons of the major components of $\mathrm{PM}_{2.5}$ in Pittsburgh between the MOUDIs and filter-based samplers are shown in Fig. 6. The organic material (OM, OC multiplied by 1.8) measurements show reasonable correlation between the samplers for both seasons $\left(R^{2}=0.55\right.$ for summer and $R^{2}=0.74$ for winter $)$. However, around $50 \%$ of the OM, is lost from the MOUDI aluminum foils during the summer (Fig. 6a). EC measurements from the MOUDI (not shown) and the filter-based sampler show agreement within experimental uncertainty. Good agreement of the organic material collected by the MOUDI and the filter-based measurements is observed during the winter intensive (Fig. 6b). EC is slightly overestimated during the winter using the MOUDI, but still within experimental error.

There are a couple of possible reasons for the loss of OC during the summer. The first is evaporation of semivolatile material, especially SOA. Volatilization of semivolatile material from impactor samplers has been reported to be as large as $50 \%$ depending on the pressure drop of the sampler, the prevalent sampling conditions, and the ratio of gas to particle concentrations in the sampled air (Zhang and McMurry, 1991). In addition, McMurry and Zhang (1989) found significant SOA volatilization from MOUDI impactors using aluminum foils as collecting media. Anderson et al. (2002) estimated that around $20 \%$ of the total $\mathrm{PM}_{2.5}$ consisted of semi-volatile organic compounds during the summer of 2000 in the Pittsburgh area. Furthermore, Cabada et al. (2003) report that between 20 and 50\% of the organic PM in the Pittsburgh area during the
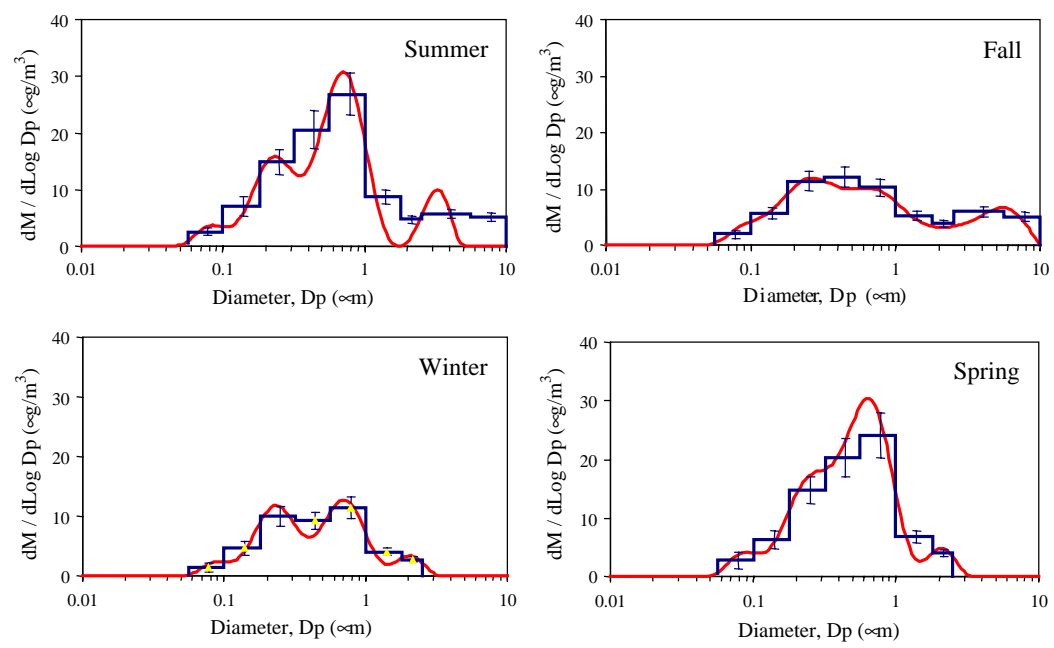

Fig. 5. Average mass size distributions from the MOUDI sampler for different seasons during PAQS. PM 10 size distributions were only collected during the summer and the fall 2001. Smoothed lines correspond to the MICRON inverted size distributions for each season. The error bars represent the sampling and analytical standard errors. 

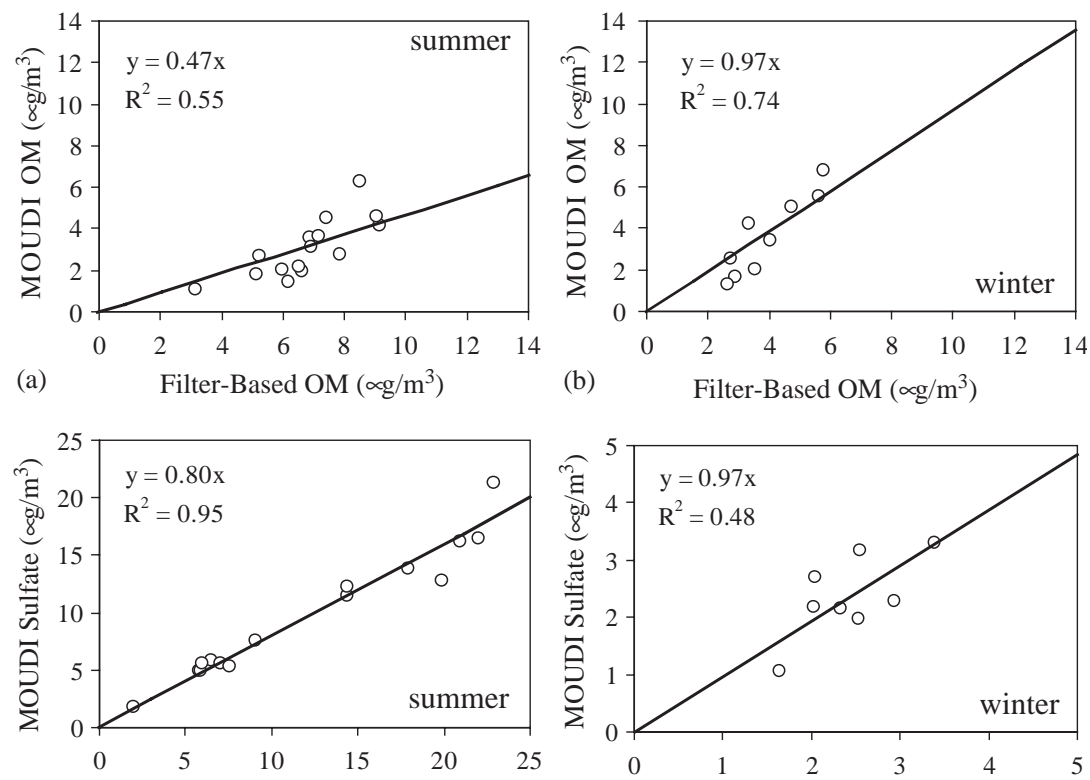

(c) Filter-Based Sulfate $\left(\mu \mathrm{g} / \mathrm{m}^{3}\right)$
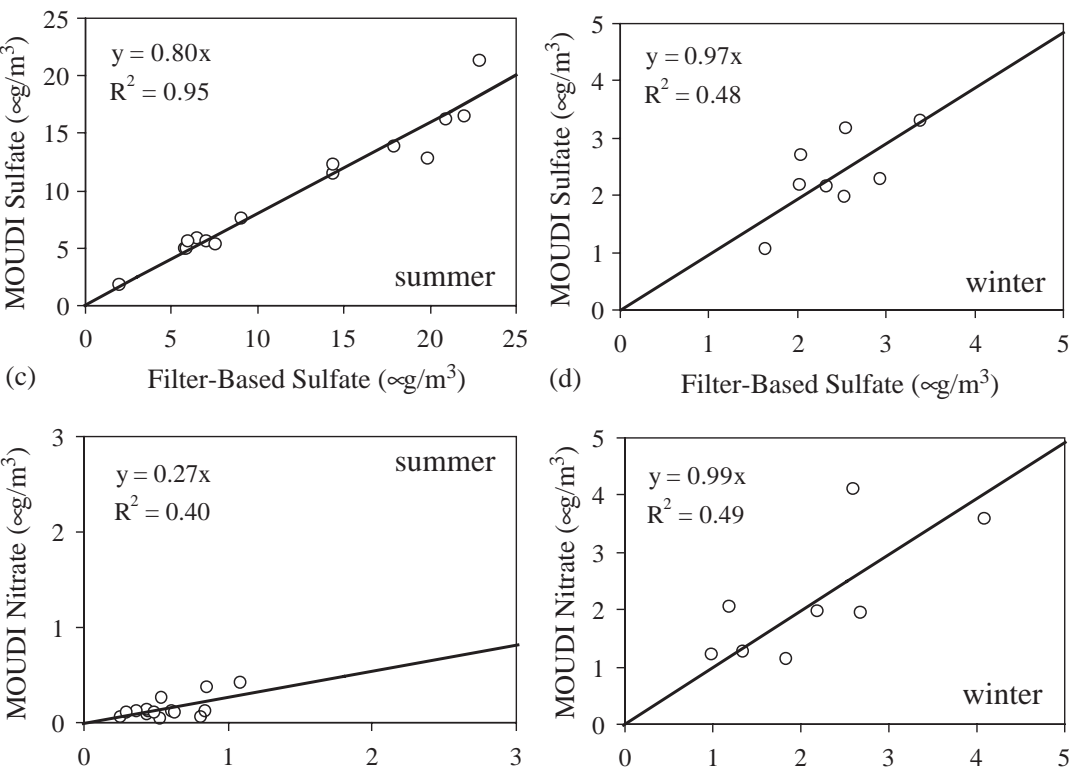

(d)

Filter-Based Sulfate $\left(\mu \mathrm{g} / \mathrm{m}^{3}\right)$

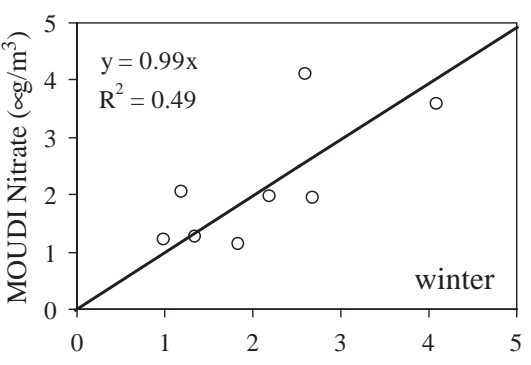

(e) Filter-Based Nitrate $\left(\mu \mathrm{g} / \mathrm{m}^{3}\right)$

(f)

Filter-Based Nitrate $\left(\mu \mathrm{g} / \mathrm{m}^{3}\right)$

Fig. 6. Comparison between measurements of individual $\mathrm{PM}_{2.5}$ compounds by the MOUDI and the integrated samplers for the two intensive periods. (a) Organic material $(\mathrm{OM}, 1.8 \times \mathrm{OC})$ measured with the MOUDI foils vs. OM measured using a denuder-based sampler during the summer intensive. (b) OM measured with the MOUDI foils vs. OM measured using an undenuded sampler during the winter intensive. The winter filter-based measurements were corrected for the positive artifact by the use of a backup quartz filter. (c) Sulfate measured with the MOUDI vs. sulfate measured with a PM speciation sampler during the summer intensive. (d) Sulfate measured with the MOUDI vs. sulfate measured on Teflon filters during the winter intensive. (e) Nitrate measured with the MOUDI vs. nitrate measured with a speciation sampler during the summer intensive. (f) Nitrate measured with the MOUDI vs. nitrate measured on Teflon filters during the summer intensive.

summer is SOA material. The contribution of SOA to the organic PM during the winter is $<10 \%$ (Cabada et al., 2002a). If all of the SOA is assumed to be semivolatile material, these estimates agree reasonably well with the mass losses from the MOUDI sampler, using aluminum foils, for both seasons.

The second explanation is related to the collection efficiency of particles on aluminum foils substrates. The use of non-greased substrates reduces the collection efficiency; especially for larger particles which are more likely to experience bounce-off. From analysis of the geometrical mean diameters (GMD) of the size distributions collected by the MOUDI using Teflon substrates, summer intensive GMDs are larger than those for the winter (summer GMD $=0.18 \mu \mathrm{m}$; winter $\mathrm{GMD}=$ $0.09 \mu \mathrm{m})$. Thus, particle loss by bounce-off may be a factor in losses of organic material from the MOUDI during the summer.

Offenberg and Baker (1999) compared PM $_{10}$ mass data from a Berner impactor using aluminum foils as substrates with data from a dichotomous sampler. The Berner impactor integrated mass accounted for only $45 \%$ of the PM mass collected by the dichotomous sampler at a Chicago urban site. They also report no significant difference between summer and winter samples, though only three data points are reported for the winter. McMurry et al. (1996) reported that MOUDIs with aluminum foil substrates in Southern 
California collected only about $50 \%$ of the organic aerosol mass compared to quartz fiber filters. In contrast, Chung et al. (2001) found good agreement for similar sampling in Central California in winter. Overall, the results of all three studies are consistent with the findings of PAQS reported here.

Fig. 6c and d show the comparison of sulfate collected by the MOUDI and the filter-based sampler for the two intensive periods. These results indicate around $20 \%$ loss of sulfate from the MOUDI during the summer. No significant difference between the MOUDI and the filterbased sampler is detected during the winter. The correlation between the samplers is high $\left(R^{2}>0.95\right)$ during the summer. Lower correlation is observed at lower sulfate levels during the winter. Comparisons in other studies between MOUDI and integrated filter samplers have shown $<10 \%$ differences between the sulfate collected by cascade impactors and sulfate collected by filter-based samplers (Sloane et al., 1991; Wall et al., 1988; Chung et al., 2001).

Fig. 6e and $\mathrm{f}$ show the comparison between the nitrate collected by the MOUDI and the filter-based sampler. Nitrate is an important constituent of $\mathrm{PM}_{2.5}$ during the winter. During the summer, however, aerosol nitrate concentrations are low due to the higher ambient temperatures and the relatively high vapor pressure of ammonium nitrate. More than $70 \%$ of the nitrate mass is lost from the MOUDI during the summer. Good agreement between the MOUDI and the filter-based sampler is observed during the winter. Several studies have reported small discrepancies in the measurement of nitrate with cascade impactors and filter-based samplers in California. Zhang and McMurry (1992) reported that a cascade impactor measures from $10 \%$ to $20 \%$ less nitrate than a denuded filter-based sampler. Wall et al. (1988) also reported an under-sampling of nitrate in a cascade impactor for a study done in Claremont, CA. Sloane et al. (1991) reported a difference of around 15\% between the mass collected by a MOUDI and a filterbased sampler, although they report that this difference is not statistically significant. California is characterized by nitrate concentrations that are one or two orders of magnitude higher than Pittsburgh. This could explain the significant losses as a fraction of the total in PAQS, during the summer, that still represent $0.5 \mu \mathrm{g} \mathrm{m}^{-3}$ of nitrate or less.

\section{Size-resolved mass composition}

\subsection{Summer}

The average size distributions of the major components of $\mathrm{PM}_{2.5}$ during the summer are shown in Fig. 7. The inorganic material, predominantly sulfate, has a practically bi-modal distribution, with a higher peak around $0.7 \mu \mathrm{m}$ (droplet mode) and a smaller peak around $0.2 \mu \mathrm{m}$ (condensation mode). The two modes are overlapping and the condensation mode appears as a "shoulder" in the raw measurements. Several authors (Hering and Friedlander, 1982; Wall et al., 1988; John et al., 1990; McMurry and Wilson, 1983) have reported the existence of these two modes in the size distribution of the inorganic aerosol from ambient samples. The sulfate condensation mode is associated with the gas phase oxidation of $\mathrm{SO}_{2}$ and the droplet mode is the product of heterogeneous reactions mainly in clouds and the accumulation of material from the lower mode. Measurements $50 \mathrm{~km}$ upwind (west of the central site) in Florence, PA, indicate that more than $90 \%$ of $\mathrm{PM}_{2.5}$ sulfate concentration measured at the PAQS site during July was the result of long-range transport into the area. Sulfate is an important constituent of $\mathrm{PM}_{2.5}$ in Pittsburgh during the summer (around $40 \%$ of total $\mathrm{PM}_{2.5}$ ). The effect of long-range transport of emissions and the high relative humidity in the northeast US during the summer are reflected by the sulfate concentration in the droplet mode. Ammonium size distributions are similar to those of sulfate, showing a smaller condensation mode, indicating formation of aerosol from the product of gas phase reactions, and a dominant droplet mode, indicating formation of aerosol by heterogeneous reactions (Wall et al., 1988; Walter et al., 1990). The GMD of the inorganic material for the summer is $0.36 \mu \mathrm{m}$.

The OM and EC have wide size distributions, with a peak between around $0.45 \mu \mathrm{m}$, for both compounds. This mode is the effect of long-range transport of carbonaceous material as particles grow. The condensation of secondary components (organic and sulfate) onto primary emitted particles is another mechanism of particle growth into the droplet mode (Vankatamaran and Friedlander, 1994; Pandis et al., 1993). Cabada et al. (2004) estimated that $20-50 \%$ of the total OC concentration is of secondary in origin for the Pittsburgh area, providing some material for the growth of the particles. Sulfate mass distributions indicate the influence of cloud processing for the Pittsburgh aerosol. Cloud processed organic aerosol or a higher fraction of water-soluble organics could also contribute to the increase the particle diameter. The GMD for OM during the summer is around $0.30 \mu \mathrm{m}$.

Fig. 8a shows the gravimetrically determined mass and the sum of the masses of each chemical compound identified. The sum of the identified compounds accounts for $70 \%$ of the MOUDI mass. This discrepancy is mainly the result of the ability of the Teflon filters used for the determination of mass, to retain more OM than the aluminum foils. Other potential explanations for the discrepancy are that the Teflon filters promote less bounce-off of carbonaceous particles and possibly absorb organic gases. Another possible reason 

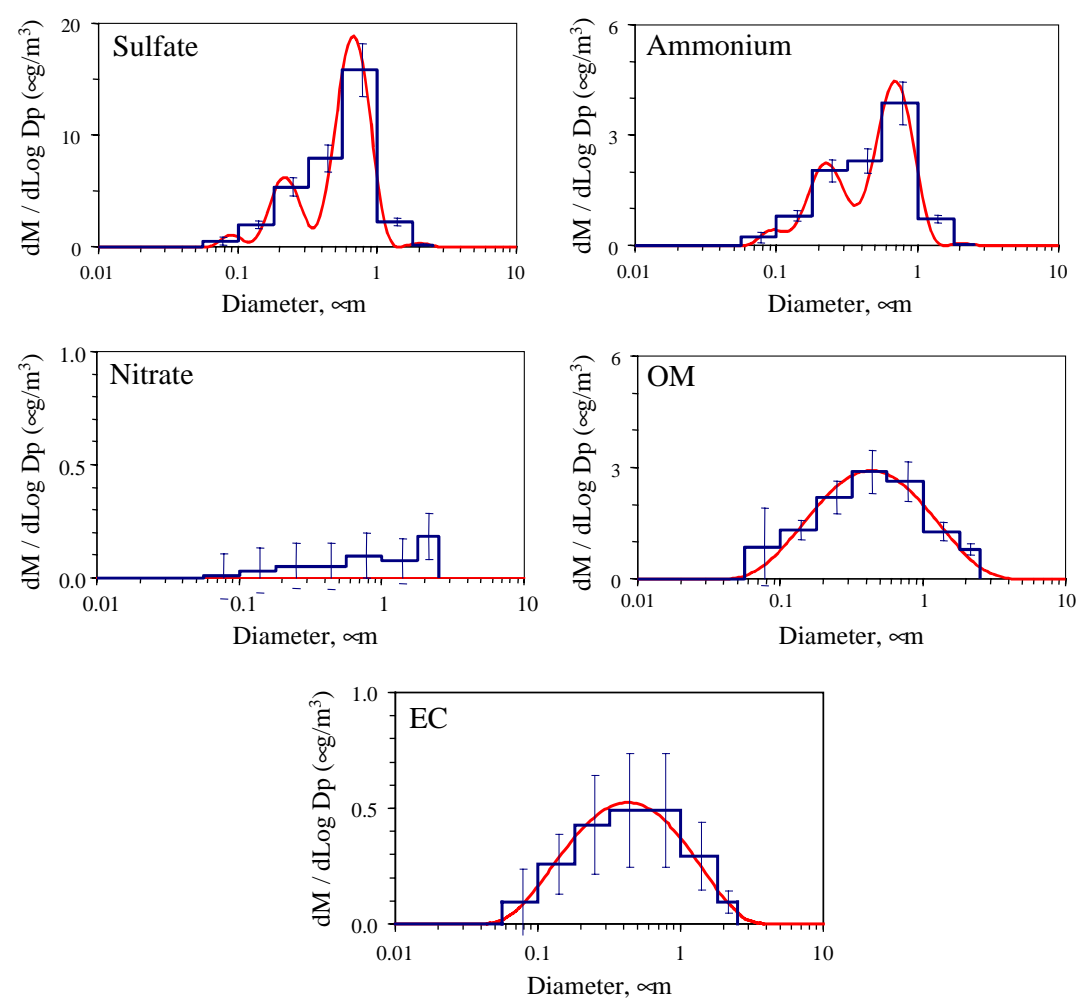

Fig. 7. Summer intensive size resolved chemical composition during PAQS, for the major components of the PM 2.5 . Also shown the MICRON-inverted size distributions (smooth lines). The error bars represent the sampling and analytical standard errors for each compound.
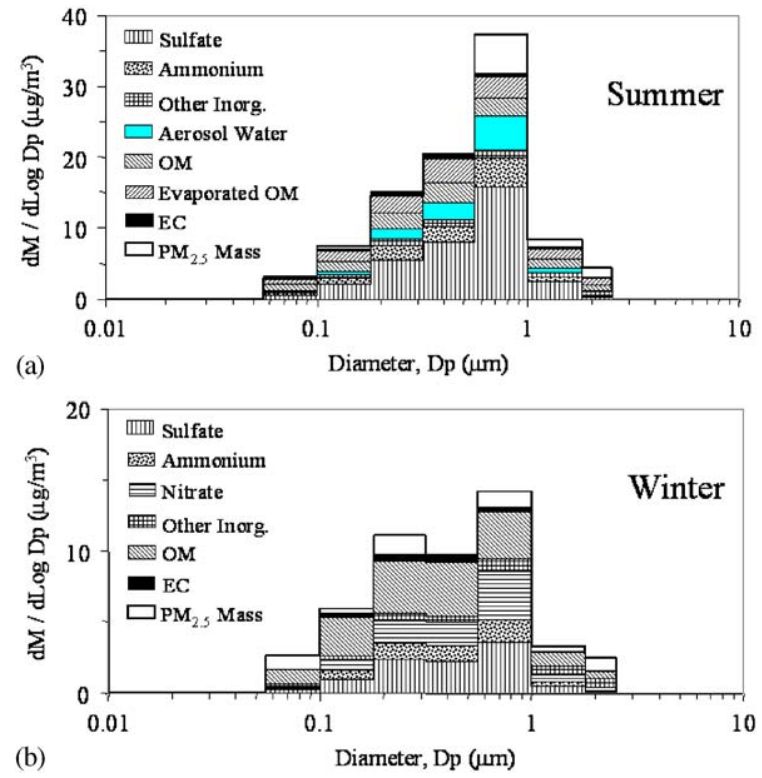

Fig. 8. Chemically resolved average distribution during the two intensive periods at PAQS (July 2001 and January 2002). The $\mathrm{PM}_{2.5}$ mass corresponds to the gravimetrically determined mass from the MOUDI stages. for the discrepancy is aerosol water. Water associated with the inorganic salts and possibly the organic material is not measured by the chemical analyses of the various compounds. Rees et al. (2004) report that Pittsburgh summer aerosol is "wet" even at low relative humidity, indicating that the mass collected by the MOUDI and measured gravimetrically contains aerosol water. Most of the "unknown" mass, namely the difference between the total gravimetric mass and the sum of the chemical species, is between 0.1 and $1.0 \mu \mathrm{m}$, where most of the mass of the inorganic salts is located. Trajectory analyses of air masses passing thru an urban area and measured at different downwind sites show that the amount of "unknown" material increases as the air mass ages in the atmosphere (Hughes et al., 1999, 2000). This is because fresh primary emissions and secondary production of aerosols modify the composition during transport. Increases in concentration of hydrophilic aerosol (via primary emissions as well as secondary production) can increase the amount of aerosol water content, leading to more "unknown" mass. For this work, if the MOUDI losses of organic material are taken into consideration (assuming they are size independent), the sum of masses of the individual compounds accounts for $85 \%$ of the gravimetrically 
determined MOUDI mass. Viidanoja et al. (2002) reported that gaseous or semi-volatile material collected on quartz filters using a cascade impactor only modify the total mass of carbon collected but had no significant impact on the shape of the original size distribution measured. Aerosol water content has been estimated to be from $10 \%$ to $15 \%$ of the $\mathrm{PM}_{2.5}$ during the summer for PAQS (Rees et al., 2004). The estimated aerosol water mass was distributed into the different MOUDI stages according to the size distribution presented by the inorganic aerosol. These two corrections are sufficient for the closure of the summer mass balance for all stages within experimental error (Fig. 8a). The closure of mass balance suggests that the shape of the OM size distribution (Fig. 7) is correct despite the significant losses during the sampling.

\subsection{Winter}

Average winter size distributions are shown in Fig. 9. Sulfate shows practically a bi-modal distribution as the summer, but in this case the droplet mode peak is not as high. Production of sulfate from gas-phase reactions (condensation mode, around $200 \mathrm{~nm}$ ) is evident during the winter, but in lower intensity than the summer. The lower mass accumulated in the droplet mode indicates that even if heterogeneous sulfate formation is the dominant pathway during the winter it is either slower or the removal of these particles occurs faster. During the winter, nitrate accounts for around $20 \%$ of the $\mathrm{PM}_{2.5}$ mass. The nitrate droplet mode suggests cloud processing and growth of the condensation mode of ammonium nitrate. Ammonium size distributions follow the same pattern as shown by sulfate and nitrate, indicating reactions of nitric acid and existing ammonia in the particulate phase and sulfur dioxide accumulate mass in the droplet mode. The GMD of the inorganic material for the winter is $0.34 \mu \mathrm{m}$ practically the same as the GMD during the summer. Unlike the summer where most of the ammonia reacts to form ammonium sulfate, a significant fraction of the ammonia in winter forms ammonium nitrate.

Carbonaceous material size distributions, $\mathrm{OM}$ and $\mathrm{EC}$, are slightly different from the summer. In winter the single mode peaks around $0.32 \mu \mathrm{m}$, indicating a greater influence of primary particles in the size distribution (Seinfeld and Pandis, 1998). The wide shape of the size distribution indicates that growth of material and longrange transport are still important factors. During the winter, the GMD for the total carbonaceous $\mathrm{PM}_{2.5}$ size
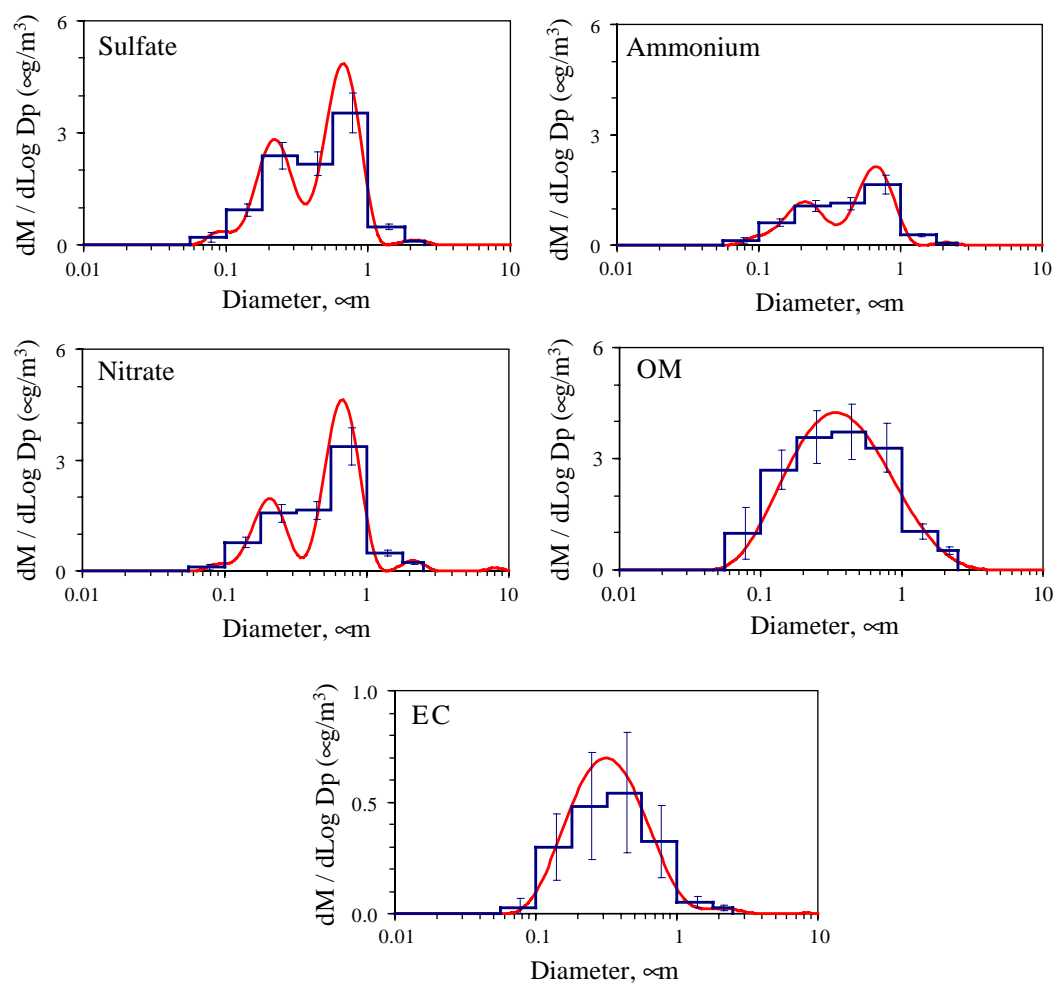

Fig. 9. Winter intensive (January 2002) size-resolved chemical composition during PAQS, for the major components of the PM 2.5 . Also shown the MICRON-inverted size distributions (smooth lines). The error bars represent the sampling and analytical standard errors for each compound. 
distributions is $0.25 \mu \mathrm{m}$, reflecting the increased winter influence of primary emissions and the slower growth to larger particle sizes.

Fig. $8 \mathrm{~b}$ shows the gravimetrically determined mass and the sum of the masses of each chemical compound identified for the winter. The sum of the identified compounds is in agreement with the measured $\mathrm{PM}_{2.5}$, within experimental errors, without any corrections.

\section{Ultrafine particles mass composition}

The chemical composition of ultrafine particles $\left(0.056<D_{\mathrm{p}}<0.10 \mu \mathrm{m}\right.$, collected by the last MOUDI stage) is shown in Fig. 10. Most of the ultrafine mass is collected by the last stage of the MOUDI. For the two intensive periods, ultrafine mass concentrations are comparable, around $0.6 \mu \mathrm{g} \mathrm{m}^{-3}$. The fraction of ultrafine mass to the total $\mathrm{PM}_{2.5}$ ranges from $0 \%$ to $11 \%$ for the summer and from $0 \%$ to $18 \%$ for the winter. Number concentrations of ultrafine particles are $23 \times 10^{3} \mathrm{~cm}^{-3}$ for the summer and $19 \times 10^{3} \mathrm{~cm}^{-3}$ for the winter (Stanier et al., 2004).

During the summer, on average $70 \%$ of the measured ultrafine mass can be explained by the sum of the concentrations from the identified components. Depending on the errors of the measurements the identified percentage ranges from $40 \%$ to $100 \%$ of the summer ultrafine mass. Carbonaceous aerosol (OM and EC) is the largest identified component of the ultrafine mass (around 50\%). Sulfate and ammonium contribute 28\%
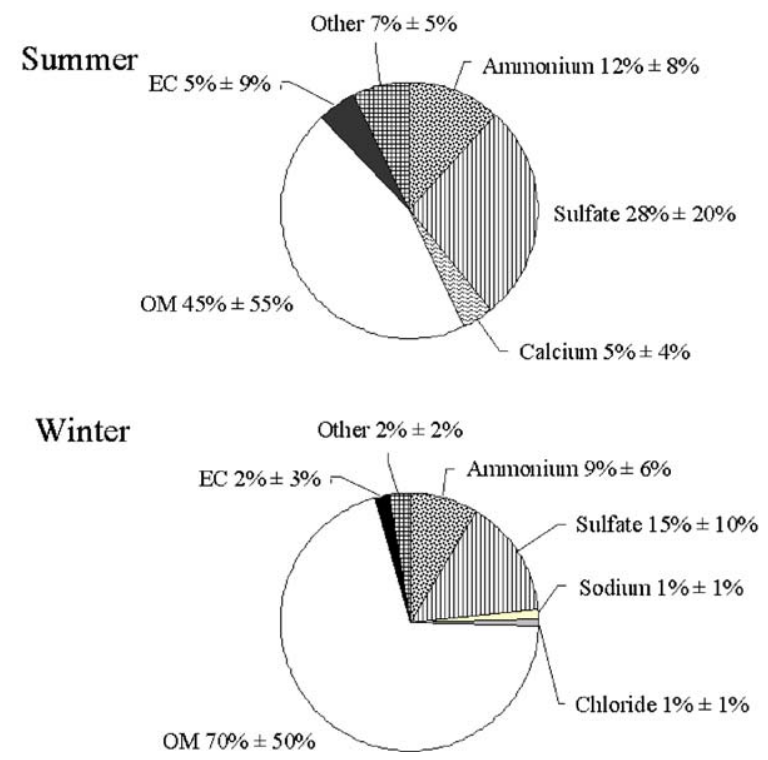

Fig. 10. Identified ultrafine mass composition $\left(56 \mathrm{~nm}<D_{\mathrm{p}}<\right.$ $100 \mathrm{~nm}$ ) for the summer and the winter intensive at PAQS. and $12 \%$, respectively. These three compounds account for around $90 \%$ of the identified ultrafine mass during the summer. The "other" fraction is inorganic material (e.g. sodium, oxalate, potassium, magnesium and chloride) with individual mass contribution $<2 \%$ of the ultrafines.

For the winter, on average $65 \%$ of the mass is accounted for. The identified chemical species explain from $40 \%$ to $100 \%$ of the ultrafine mass during the winter. The chemical composition of the ultrafine particles is different during the winter. Carbonaceous material accounts for more than $70 \%$ of the mass. Ammonium sulfate accounts for around $25 \%$ of the ultrafine mass. The high contribution of carbonaceous material during the winter is consistent with the increase in carbon emissions from combustion of material for heating purposes (Cabada et al., 2002b). A large fraction of crustal material in the ultrafine mass has been identified in studies in Central California, during the winter (Chung et al., 2001). For PAQS, the contribution of crustal compounds to the ultrafine mass is negligible, with $<1 \%$ for both summer and winter.

Southern and Central California studies indicate similar mass composition of ultrafine particles during the winter. Hughes et al. (1998) reported that most of the ultrafine particle composition in Pasadena, California, was carbonaceous material (OM and EC) during the winter $(>40 \%)$, followed by around $5-25 \%$ of trace metals. Negligible contributions of sulfate and nitrate were reported. The unidentified fraction of material in the ultrafine mass is reported to be from $23 \%$ to $43 \%$. Chung et al. (2001) reported that the ultrafine aerosol composition for Bakersfield, California, during the winter is mostly composed of inorganic material, crustal compounds, and trace metals. However, one of the major components identified is OC, contributing about $20 \%$ of the ultrafine mass. Calcium concentrations in the ultrafine mass were reported to be around $20 \%$. Considerable amounts of nitrate, sodium and sulfate were also found $(>5 \%)$. The "unknown" material in the ultrafine mass was reported to be between $0 \%$ and $50 \%$.

During September 2002, an aerosol mass spectrometer (AMS, Aerodyne Inc.) measured size distributions of sulfate, nitrate and $\mathrm{OM}$ between $30 \mathrm{~nm}$ and $2 \mu \mathrm{m}$ at PAQS main site. On average, the ultrafine mass was mostly composed of organic material (50-70\%). Sulfate was detected in the ultrafine section for particle diameters larger than $70 \mathrm{~nm}$. The sulfate contribution to the ultrafine mass varied from $20 \%$ to $50 \%$. Negligible quantities of nitrate are reported for the ultrafine mass. Results by the Aerodyne AMS are consistent with the MOUDI ultrafine mass composition for the summer. Both approaches show that $\mathrm{OM}$ is a major component of the ultrafine mass in the area followed by sulfates. 


\section{Conclusions}

Size-resolved mass distributions have been measured using a MOUDI impactor for 12 months, during the PAQS. Measurements of $\mathrm{PM}_{0.056}, \mathrm{PM}_{0.10}, \mathrm{PM}_{0.18}$, $\mathrm{PM}_{0.32}, \mathrm{PM}_{0.56}, \mathrm{PM}_{1.0}, \mathrm{PM}_{1.8}$ and $\mathrm{PM}_{2.5}$ are available for the length of the study. A seasonal dependence of all the mass fractions is observed, with higher concentrations during the summer and lower concentrations for the winter and spring. High correlation exists between $\mathrm{PM}_{2.5}$ and $\mathrm{PM}_{1.0}$.

The MOUDI $\mathrm{PM}_{10}$ concentration was around $20 \%$ less than the gravimetrically determined $\mathrm{PM}_{10}$ mass. The discrepancy is probably due to losses of coarse particles to the walls of the impactor or bounce-off from the collecting stages. Good agreement between the $\mathrm{PM}_{2.5}$ collected by the MOUDI and other integrated mass samplers is obtained for the length of the study.

Mass distributions for all seasons are dominated by the accumulation mode, with the condensation mode around $0.2 \mu \mathrm{m}$, and the droplet mode around $0.7 \mu \mathrm{m}$. Size distributions of the major inorganic compounds and carbonaceous material were measured for two intensive periods (July 2001 and January 2002). The summer is characterized by a higher peak in the droplet mode, indicating a greater influence of heterogeneous production of inorganic material (sulfate, ammonium) and significant cloud processing. OM is also abundant in the droplet mode. During the winter and fall, the condensation mode becomes more important indicating more influence from primary emissions of carbonaceous material and slower photochemical production and growth.

More than $50 \%$ of the OM is lost from the MOUDI aluminum foils as compared to a filter-based sampler during the summer. Good agreement is observed during the winter. OM losses during the summer are mainly due to volatilization of material due to higher ambient temperatures and higher concentrations of secondary organic species (i.e. SOA). Significant volatilization of nitrate from the MOUDI is also observed in the summer. Reasonable agreement between the gravimetrically determined mass and the sum of the masses of the individual compounds is achieved if the losses of $\mathrm{OM}$ and nitrate are considered and if the water content of the aerosol is taken into account.

Reasonable correlation is observed between the MOUDI and the DAASS when the ratios of $\mathrm{PM}_{0.1}$ to $\mathrm{PM}_{2.5}$ and $V_{0.1}$ to $V_{2.5}$ are compared between each other. Possible bouncing of particles in the MOUDI is introducing an error $<30 \%$ for the ultrafine mass and composition. Winter and summer intensives ultrafine concentrations are somewhat different, with higher contribution of carbonaceous material during the winter. Number concentrations are slightly higher during the winter. During the summer, carbonaceous material contributes around $50 \%$ and sulfate and ammonium contribute $28 \%$ and $12 \%$ of the ultrafine mass, respectively. Small amounts of other inorganic material (e.g. sodium, oxalate, potassium, magnesium and chloride) account for around $10 \%$ of the ultrafine mass. During the winter, carbonaceous material accounts for more than $70 \%$ of the ultrafine mass, indicating a greater influence of primary emissions from combustion sources to the Pittsburgh ultrafine aerosol. Sulfate and ammonium are the most important inorganic compounds in the ultrafine regime accounting for around $25 \%$ of the mass. Negligible amounts of crustal material are detected in the ultrafine particles in either summer or winter.

\section{Acknowledgements}

This research was conducted as part of the Pittsburgh Air Quality Study that was supported by US Environmental Protection Agency under contract R82806101 and the US Department of Energy National Energy Technology Laboratory under contract DE-FC2601NT41017. This paper has not been subject to EPA's required peer and policy review, and therefore does not necessarily reflect the views of the Agency. No official endorsement should be inferred.

\section{References}

Anderson, R.R., Martello, D.V., Rohar, P.C., Straizar, B.R., Tamilia, J.P., Waldner, K., White, C.M., Modey, W.K., Mangelson, N.F., Eatough, D.J., 2002. Sources and composition of $\mathrm{PM}_{2.5}$ at the National Energy Technology Laboratory in Pittsburgh during July and August 2000. Energy and Fuels 16, 261-269.

Cabada, J.C., Pandis, S.N., Robinson, A.L., Subramanian, R., Polidori, A., Turpin, B.J. 2002a. The contribution of secondary organic aerosol to $\mathrm{PM}_{2.5}$ concentrations in Pittsburgh. Presented at the 21st Annual AAAR Conference, Charlotte, NC.

Cabada, J.C., Pandis, S.N., Robinson, A.L., 2002b. Sources of atmospheric carbonaceous particulate matter in Pittsburgh, Pennsylvania. Journal of the Air and Waste Management Association 52, 732-741.

Cabada, J.C., Pandis, S.N., Robinson, A.L., Subramanian, R., Polidori, A., Turpin, B.J., 2004. Estimating the secondary organic aerosol contribution to $\mathrm{PM}_{2.5}$ using the $\mathrm{OC}$ to $\mathrm{EC}$ ratio method. Aerosol Science and Technology, submitted for publication.

Chung, A., Herner, J.D., Kleeman, M.J., 2001. Detection of ultrafine particles at Bakersfield, California. Environmental Science and Technology 35, 2184-2190.

Dockery, D.W., Pope, C.A., Xu, X., Splenger, J.D., Ware, J.H., Ferris, B.G., Speitzer, F.E., 1993. Mortality risks of air pollution: a prospective cohort study. New England Journal of Medicine 329, 1753-1759. 
Donalson, K., Brown, D., Clouter, A., Duffin, R., MacNee, W., Renwick, L., Tran, L., Stone, V., 2002. The pulmonary toxicology of ultrafine particles. Journal of Aerosol Medicine 15, 213-220.

Eldering, A., Cass, G.R., Moon, K.C., 1994. An air monitoring network using continuous particles size distribution monitors: connecting pollutant properties to visibility via Mie scattering calculations. Atmospheric Environment 28, 2733-2749.

Environmental Protection Agency, 1996. Air quality criteria for particulate matter. EPA/600/P-65/001.

Ferin, J., Oberdorster, G., Penney, D.P., 1992. Pulmonary retention of ultrafine and fine particles in rats. American Journal of Respiratory Cell and Molecular Biology 6, 535,542 .

Hering, S.V., Friedlander, S.K., 1982. Origins of aerosol sulfur size distributions in the Los Angeles basin. Atmospheric Environment 16, 2647-2656.

Hinds, W.C., 1998. Aerosol Technology: Properties, Behavior and Measurement of Airborne Particles, 2nd Edition. Wiley, New York.

Hughes, L.S., Cass, G.R., Gone, J., Ames, M., Olmez, I., 1998. Physical and chemical characterization of atmospheric ultrafine particles in the Los Angeles area. Environmental Science and Technology 32, 1153-1157.

Hughes, L.S., Allen, J.O., Kleeman, M.J., Johnson, R.J., Cass, G.R., Gross, D.S., Gard, E.E., Galli, M.E., Morrical, B.D., Fergenson, D.P., Diennes, T., Noble, C.A., Liu, D.Y., Silva, P., Prather, K.A., 1999. Size and composition distribution of atmospheric particles in southern California. Environmental Science and Technology 33, 3506-3515.

Hughes, L.S., Allen, J.O., Bhave, P., Kleeman, M.J., Cass, G.R., Lui, D.-Y., Fergenson, D.P., Morrical, B.D., Prather, K.A., 2000. Evolution of atmospheric particles along trajectories crossing the Los Angeles basin. Environmental Science and Technology 34, 3058-3068.

John, W., Wall, S.M., Ondo, J.L., Winklmayr, W., 1990. Modes in the size distributions of atmospheric inorganic aerosol. Atmospheric Environment 24A, 2349-2359.

Khlystov, A., Stanier, C.O., Pandis, S.N., 2004. An algorithm for combining electrical mobility and aerodynamic size distributions data when measuring ambient aerosol. Aerosol Science and Technology, submitted for publication.

Lawless, P.A., Rodes, C.E., Evans, G., Sheldon, L., Creason, J., 2001. Aerosol concentrations during the 1999 Fresno exposure studies as functions of size, season and meteorology. Aerosol Science and Technology 34, 66-74.

Marple, V.A., Rubow, K.L., Behm, S.M., 1991. A microorifice uniform deposit impactor (MOUDI): description, calibration and use. Aerosol Science and Technology 14, 434-446.

McMurry, P.H., Wilson, J.C., 1983. Droplet phase (heterogeneous) and gas phase (homogeneous) contribution to secondary ambient aerosol formation as functions of relative humidity. Journal of Geophysical Research 88, 5101-5108.

McMurry, P.H., Zhang, X.Q., 1989. Size distributions of ambient organic and elemental carbon. Aerosol Science and Technology 10, 430-437.

McMurry, P.H., Zhang, X., Chung-Te, L., 1996. Issues in aerosol measurement for optics assessments. Journal of Geophysical Research 101, 19189-19197.
Oberdorster, G., 1995. Lung particle overload, implications for occupational exposures to particles. Regulatory Toxicology and Pharmacology 21, 123-135.

Oberdorster, G., 2001. Pulmonary effects of inhaled ultrafine particles. International Archives of Occupational and Environmental Health 74, 1-8.

Oberdorster, G., Yu, C.P., 1990. The carcinogenic potential of inhaled diesel exhaust, a particle effect. Journal of Aerosol Science 21, 397-401.

Oberdorster, G., Ferin, J., Lehnert, B.E., 1994. Correlation between particle size, in-vivo particle persistence, and lung injury. Environmental Health Perspectives 102, 173-179.

Offenberg, J.H., Baker, J.E., 1999. Aerosol size distributions of polycyclic aromatic hydrocarbons in urban and over-water atmospheres. Atmospheric Environment 33, 3324-3331.

Pak, S.S., Liu, B.Y.H., Rubow, KL., 1992. Effect of coating thickness on particle bounce in intertial impactors. Aerosol Science and Technology 16, 141-150.

Pandis, S.N., Wexler, A.S., Seinfeld, J.H., 1993. Secondary organic aerosol formation and transport-II. Predicting the ambient secondary organic aerosol size distribution. Atmospheric Environment 27A, 2403-2416.

Rees, S.L., Robinson, A.L., Khylstov, A.Y., Stanier, C.O., Subramanian, R., Cabada, J.C., Wittig, B., Takahama, S., Davidson, C., Pandis, S.N., 2004. Mass Balance Closure and the Federal Reference Method for $\mathrm{PM}_{2.5}$ in Pittsburgh, PA. Atmospheric Environment, this issue, doi:10.1016/ j.atmosenv.2004.03.016.

Seinfeld, J.H., Pandis, S.N., 1998. Atmospheric Chemistry and Physics: From Air Pollution to Global Change. Wiley, New York.

Shen, S., Jaques, P.A., Zhu, Y., Geller, M.D., Sioutas, C., 2002. Evaluation of the SMPS-APS system as a continuous monitor for measuring $\mathrm{PM}_{2.5}, \mathrm{PM}_{10}$ and coarse (PM2.5-10) concentrations. Atmospheric Environment 36, 3939-3950.

Sloane, C.S., Watson, J., Chow, J., Pritchett, L., Richards, L.W., 1991. Size-segregated fine particulate measurements by chemical species and their impact on visibility impairment in Denver. Atmospheric Environment 25A, 1013-1024.

Stanier, C.O., Khlystov, A.Y., Chan, W.R., Mandiro, M., Pandis, S.N., 2004. A method for the in-situ measurement of aerosol water content of ambient aerosols: The dry-ambient aerosol size spectrometer (DAASS). Aerosol Science and Technology, submitted for publication.

Subramanian, R., Khlystov, A., Cabada, J.C., Robinson, A.L., 2004. Measurement of Positive and Negative Artifacts with Denuded and Undenuded Sampler Configurations. Aerosol Science and Technology, submitted for publication.

Turpin, B.J., Lim, H.J., 2001. Species contributions to $\mathrm{PM}_{2.5}$ mass concentrations: revisiting common assumptions for estimating organic mass. Aerosol Science and Technology $35,602-610$.

Vankatamaran, C., Friedlander, S.K., 1994. Size distributions of polycyclic aromatic hydrocarbons and elemental carbon. 2. Ambient measurements and effects of atmospheric processes. Environmental Science and Technology 28, 563-572.

Viidanoja, J., Kerminen, V.-M., Hillamo, R., 2002. Measuring the size distribution of atmospheric organic and black carbon using impactor sampling coupled with thermal carbon analysis: method development and uncertainties. Aerosol Science and Technology 36, 607-616. 
Wall, S.M., John, W., Ondo, J.L., 1988. Measurement of aerosol size distributions for nitrate and major ionic species. Atmospheric Environment 22, 1649-1656.

Wexler, A.S., Ondov, J.M., 1998. Where do particulate toxins reside? An improved paradigm for the structure and dynamics of the urban mid-Atlantic aerosol. Environmental Science and Technology 32, 2547-2555.

Whitby, K.T., 1978. The physical characteristics of sulfur aerosols. Atmospheric Environment 12, 135-159.

Wittig, B., Pandis, S.N., Hering, S., Kirby, B., Khylstov, A.Y., Takahama, S., Davidson, C., 2004a. Semi-continous PM2.5 inorganic composition measurements during the Pittsburgh Air Quality Study. Atmospheric Environment, this issue, doi:10.1016/j.atmosenv.2004.03.002.
Wittig, B., Anderson, N., Khlystov, A.Y., Pandis, S.N., Davidson, C., Robinson, A.L., 2004b. Pittsburgh Air Quality Study overview. Atmospheric Environment, this issue, doi:10.1016/j.atmosenv.2004.03.003.

Wolfenbarger, J.K., Seinfeld, J.H., 1990. Inversion of aerosol size distribution data. Journal of Aerosol Science 21, 227-247.

Zhang, X.Q., McMurry, P.H., 1991. Theoretical analysis of evaporative losses of adsorbed and absorbed species during atmospheric aerosol sampling. Environmental Science and Technology 25, 456-459.

Zhang, X.Q., McMurry, P.H., 1992. Evaporative losses of fine particulate nitrates during sampling. Atmospheric Environment 26A, 3305-3312. 


\title{
Estimating the Secondary Organic Aerosol Contribution to $\mathrm{PM}_{2.5}$ Using the EC Tracer Method
}

\author{
Juan C. Cabada, ${ }^{1}$ Spyros N. Pandis, ${ }^{1}$ Ramachandran Subramanian, ${ }^{2}$ \\ Allen L. Robinson, ${ }^{2}$ Andrea Polidori, ${ }^{3}$ and Barbara Turpin ${ }^{3}$ \\ ${ }^{1}$ Department of Chemical Engineering, Carnegie Mellon University, Pittsburgh, Pennsylvania \\ ${ }^{2}$ Department of Mechanical Engineering, Carnegie Mellon University, Pittsburgh, Pennsylvania \\ ${ }^{3}$ Department of Environmental Sciences, Rutgers University, New Brunswick, New Jersey
}

The EC tracer method is applied to a series of measurements by different carbonaceous aerosol samplers in the Pittsburgh Air Quality Study (PAQS) in order to estimate the concentration of secondary organic aerosol. High-resolution measurements (2-6 h) and daily averaged concentrations were collected during the summer 2001 intensive (1 July to 4 August 2001) and are used for the analysis. The various samplers used during PAQS show differences in the measured concentrations of $\mathrm{OC}$ and $\mathrm{EC}$ due to the different sampling artifacts and sampling periods.

A systematic approach for the separation of periods where SOA contributes significantly to the ambient $\mathrm{OC}$ levels from the periods where organic and elemental carbon concentrations are dominated by primary emissions is proposed. Ozone is used as an indicator of photochemical activity to identify periods of probable secondary organic aerosol production in the area. Gaseous tracers of combustion sources $\left(\mathrm{CO}, \mathrm{NO}\right.$, and $\mathrm{NO}_{\mathrm{x}}$ ) are used to identify periods where most of the $\mathrm{OC}$ is primary. Periods dominated by primary emissions are used to establish the relationship between primary $\mathrm{OC}$ and EC, a tracer for primary combustion-generated carbon for the different sets of measurements for July 2001. Around 35\% of the organic carbon concentration in Western Pennsylvania during July of 2001 is estimated to be secondary in origin.

\section{INTRODUCTION}

Carbonaceous aerosol is an important constituent of the $\mathrm{PM}_{2.5}$ (particulate matter with aerodynamic diameters less than $2.5 \mathrm{mi}$ -

Received 22 October 2003; accepted 21 May 2003.

This research was conducted as part of the Pittsburgh Air Quality Study that was supported by US Environmental Protection Agency under contract R82806101 and the US Department of Energy National Energy Technology Laboratory under contract DE-FC26-01NT41017. This article has not been subject to EPA's required peer and policy review and therefore does not necessarily reflect the views of the Agency. No official endorsement should be inferred.

Address correspondence to Spyros N. Pandis, Department of Chemical Engineering, Carnegie Mellon University, 5000 Forbes Avenue, Pittsburgh, PA 15213. E-mail: spyros@andrew.cmu.edu crons) mass in most of the U.S. Between $10-65 \%$ of the fine particulate mass has been identified as carbonaceous material for various regions of the country (Gray et al. 1986; Turpin et al. 1991; Seinfeld and Pandis 1998; Tolocka et al. 2001; Lim and Turpin 2002). Aerosol carbon is commonly classified as organic carbon (OC) and elemental carbon (EC). OC can be directly emitted to the atmosphere in the particulate form (primary) or can be produced by gas-to-particle conversion processes (secondary). EC is emitted from combustion sources. Since primary OC and EC are mostly emitted from the same sources, EC can be used as a tracer for primary combustion-generated OC (Gray et al. 1986; Turpin and Huntzicker 1995; Strader et al. 1999). The formation of secondary organic aerosol (SOA) increases the ambient concentration of OC and the ambient OC/EC ratio. OC-to-EC ratios exceeding the expected primary emission ratio are an indication of SOA formation. For Southern and Central California, 30-80\% of the total OC has been identified as secondary in summer (Gray et al. 1986; Pandis et al. 1992; Hildemann et al. 1993; Turpin and Huntzicker 1995; Schauer et al. 1996).

The relationship between primary OC and EC depends also on the sampling and analyses techniques used to determine the ambient OC and EC concentrations. Sample collection (i.e., the use or not of denuders, filter face velocities, etc.) and different analysis techniques (i.e., thermal optical transmittance versus thermal optical reflectance) affect the reported concentrations for OC and EC (Countess 1990; Birch 1998; Chow et al. 2001; Schmid et al. 2001). Sampling carbonaceous particulate matter from the atmosphere is challenging because of interferences from gaseous material that is adsorbed on the filters and evaporation of the collected organic material during sampling (Turpin and Huntzicker 1994; Fitz 1990; Hering et al. 1990). Different sampling arrangements (e.g., using backup quartz filters, placing denuders upstream of the filter to remove organic gases, etc.) have been proposed in order to reduce, measure, and correct for the positive and negative artifacts that 
affect the measured carbonaceous concentrations (Turpin et al. 2000).

Primary ratios of OC to EC vary from source to source and show temporal and diurnal patterns (Gray 1986; Cabada et al. 2002a), but since EC is only emitted by combustion sources, gaseous tracers of combustion $\left(\mathrm{CO}, \mathrm{NO}, \mathrm{NO}_{\mathrm{x}}\right)$ can be used to determine periods dominated by primary aerosol emissions. Ozone is an indicator of photochemical activity, and it also can be used as a tracer for periods where secondary organic aerosol production is expected. In this case, increases in the OC-to-EC ratio correlated to ozone episodes are indicative of SOA production.

In this work a relationship between primary OC and EC is established for each of the different types of measurements and artifact estimation approaches. An algorithm is proposed for the determination of the primary OC-to-EC ratio and secondary organic aerosol concentrations are estimated. SOA results based on high-resolution and daily-averaged samples are compared. The effect of sampling frequency on the estimates of the primary ratios is also discussed.

\section{EXPERIMENTAL METHODS AND EQUIPMENT}

The Pittsburgh Air Quality Study (PAQS) main site was located in Schenley park on the top of a hill just outside of Carnegie Mellon University campus, around three miles to the east of downtown Pittsburgh. The Pittsburgh supersite operated three different samplers for collecting carbonaceous aerosol (one undenuded and two denuded samplers). The undenuded sampler and a denuded in situ analyzer collected samples every 2-6 h, and the denuder-based sampler collected daily samples, during the 2001 summer intensive (1 July to 4 August, 2001).

Quartz fiber filters (47 mm Tissuquartz 2500 QAO-UP), Teflon filters (2 $\mu \mathrm{m}$ pore, Whatman 7592-104), and carbonimpregnated filters (Schleicher and Schuell, GF-3649) were used to sample carbonaceous material in three different samplers. Quartz fiber filters were baked at $550^{\circ} \mathrm{C}$ for more than $12 \mathrm{~h}$ and stored in previously cleaned glass jars until sampling and analysis. Carbon-impregnated filters were baked at $370^{\circ} \mathrm{C}$ for more than $3 \mathrm{~h}$ in a nitrogen atmosphere.

\section{Undenuded Sampler}

$\mathrm{PM}_{2.5}$ carbonaceous aerosol samples were collected on quartz fiber filters using filter packs in a nondenuded line. This sampler consisted of two parallel lines, the first line holding a quartz fiber filter followed by a backup quartz filter and the second line having a Teflon filter followed by a backup quartz fiber filter (Figure 1). The two backup quartz fiber filters are used to estimate the positive and negative artifact (Turpin et al. 2000). Five samples a day, with intervals between sampling times of 4 $6 \mathrm{~h}$, were collected during the summer intensive. Samples were collected during 0-6, 6-10, 10-14, 14-18, and 18-24 h (all in EST). The filter configuration allows two different estimates of the adsorption artifacts on the front quartz fiber filter $\left(\mathrm{Q}_{\mathrm{F}}\right)$. The first correction is done by subtracting the $\mathrm{OC}$ collected in the backup quartz fiber filter behind the front quartz $\left(\mathrm{Q}_{\mathrm{B}, \mathrm{F}}\right)$ from the OC collected by the $\mathrm{Q}_{\mathrm{F}}$. This approach assumes that the $\mathrm{Q}_{\mathrm{F}}$ collects $100 \%$ of the carbonaceous particulate matter (no evaporation) and that both the $\mathrm{Q}_{\mathrm{F}}$ and the $\mathrm{Q}_{\mathrm{B}, \mathrm{F}}$ adsorb organic gases and reach equilibrium with them during the sampling period. The second correction approach subtracts the OC collected in the $\mathrm{Q}_{\mathrm{B}, \mathrm{F}}$ behind the Teflon filter $\left(\mathrm{Q}_{\mathrm{B}, \mathrm{T}}\right)$ from the $\mathrm{OC}$ in the $\mathrm{Q}_{\mathrm{F}}$. This approach assumes that the Teflon filter collects all of the particles from the sampled flow with $100 \%$ efficiency and that the $\mathrm{Q}_{\mathrm{B}, \mathrm{F}}$ from this line adsorbs the same quantity of gases as the $\mathrm{Q}_{\mathrm{F}}$. The EC concentration reported by the $\mathrm{Q}_{\mathrm{F}}$ is used for all datasets from the undenuded sampler. More details on the undenuded sampler and its operation are described in Subramanian (2004).
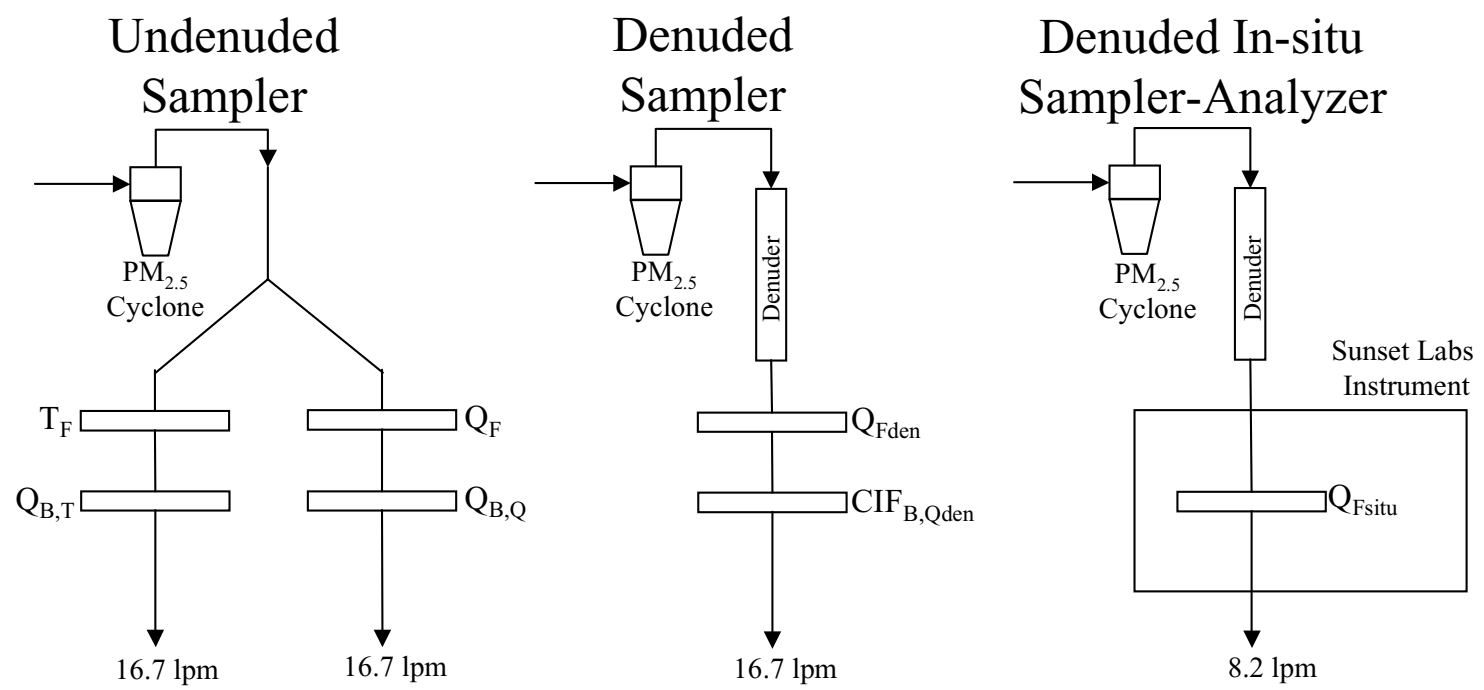

Figure 1. Schematic of the Pittsburgh Air Quality Study carbonaceous aerosol samplers. Subscript "F" denotes the front filter in the samplers. Backup filters are indicated as a subscript "B" followed by the type of filter they are after (T, Teflon; Q, quartz). 


\section{Denuded Sampler}

Filter packs holding a quartz filter in front of a carbonimpregnated filter (CIF) were used to collect carbonaceous material from a denuded sampling line (Figure 1). A carbon annular denuder (Novacarb monolith synthetic carbon, Mast Carbon Ltd., Guilford, UK) was used to remove organic gases and minimize the positive artifact in the quartz filter. The CIF organic carbon concentration was intended to correct for evaporation of semivolatile material from the quartz filter (negative artifact). Sampling frequency for this unit was $24 \mathrm{~h}$, from midnight to midnight (EST). More details on the undenuded sampler and its operation are described in Subramanian (2004).

\section{Semicontinuous Denuded in situ Analyzer}

An in situ semicontinuous carbon analyzer (Sunset Labs, Carbon Aerosol Analysis Field Instrument), similar in design to that described by Turpin et al. (1990), was used to collect and analyze carbonaceous aerosol with sampling periods of 2-4 h (100 to $220 \mathrm{~min}$ sampling time plus $20 \mathrm{~min}$ for analysis). Instrument performance and PAQS protocols are described in detail by Lim et al. (2002). A parallel plate diffusion denuder (CIF; Schleicher Schuell, Keene, NH, USA) was placed upstream of a quartz filter, which was mounted inside the analyzer (Figure 1). Cycles of sampling and analysis were alternated in order to determine the ambient concentrations of OC and EC (Lim et al. 2002).

Quartz filters from the filter-pack-based samplers were analyzed using a Thermal/Optical transmittance carbon analyzer (Sunset Laboratory Inc., OC-EC Aerosol Carbon Analyzer Model-3) using the temperature steps of the NIOSH protocol (Birch and Cary 1996; NIOSH 1999) for the determination of OC and EC. Table 1 shows the experimental parameters for the analysis of the quartz and carbon-impregnated filters during

Table 1

Temperature programs used by the Thermal/Optical Transmittance methods for the analysis of carbonaceous material during PAQS

\begin{tabular}{lccc}
\hline & \multicolumn{3}{c}{ PAQS analysis method } \\
\cline { 2 - 4 } Carrier & $\begin{array}{c}\text { Filter } \\
\text { pack-based } \\
\text { gamplers }\end{array}$ & $\begin{array}{c}\text { In situ } \\
\text { carbon } \\
\text { analyzer }\end{array}$ & $\begin{array}{c}\text { Carbon impregnated } \\
\text { filters }\end{array}$ \\
\hline $\mathrm{He}$ & $340^{\circ} \mathrm{C}, 120 \mathrm{~s}$ & $340^{\circ} \mathrm{C}, 60 \mathrm{~s}$ & $20^{\circ} \mathrm{C} / \mathrm{min}$ up to $330^{\circ} \mathrm{C}$ \\
$\mathrm{He}$ & $500^{\circ} \mathrm{C}, 120 \mathrm{~s}$ & $500^{\circ} \mathrm{C}, 60 \mathrm{~s}$ & $330^{\circ} \mathrm{C}, 300 \mathrm{~s}$ \\
$\mathrm{He}$ & $615^{\circ} \mathrm{C}, 120 \mathrm{~s}$ & $615^{\circ} \mathrm{C}, 60 \mathrm{~s}$ & \\
$\mathrm{He}$ & $870^{\circ} \mathrm{C}, 180 \mathrm{~s}$ & $870^{\circ} \mathrm{C}, 90 \mathrm{~s}$ & \\
$\mathrm{He} / \mathrm{O}_{2}$ & $575^{\circ} \mathrm{C}, 45 \mathrm{~s}$ & $575^{\circ} \mathrm{C}, 45 \mathrm{~s}$ & \\
$\mathrm{He} / \mathrm{O}_{2}$ & $650^{\circ} \mathrm{C}, 45 \mathrm{~s}$ & $650^{\circ} \mathrm{C}, 45 \mathrm{~s}$ & \\
$\mathrm{He} / \mathrm{O}_{2}$ & $725^{\circ} \mathrm{C}, 45 \mathrm{~s}$ & $725^{\circ} \mathrm{C}, 45 \mathrm{~s}$ & \\
$\mathrm{He} / \mathrm{O}_{2}$ & $800^{\circ} \mathrm{C}, 45 \mathrm{~s}$ & $800^{\circ} \mathrm{C}, 45 \mathrm{~s}$ & \\
$\mathrm{He} / \mathrm{O}_{2}$ & $910^{\circ} \mathrm{C}, 100 \mathrm{~s}$ & $910^{\circ} \mathrm{C}, 100 \mathrm{~s}$ & \\
\hline
\end{tabular}

PAQS. The time length of the different temperatures steps in the method was modified to get a better split between OC and EC (Yu et al. 2002). Carbon-impregnated filters were analyzed using a temperature ramp up to $340^{\circ} \mathrm{C}$ during 25 min under a helium atmosphere. All concentrations of OC and EC were corrected for field blanks. Concentrations reported from the in situ carbon analyzer were corrected for dynamic blanks generated by sampling with a Teflon filter upstream of the denuder.

\section{CARBONACEOUS AEROSOL MEASUREMENTS}

Differences exist among the measured concentrations of organic and elemental carbon collected by the different samplers. For example, adsorption of organic gases on the $\mathrm{Q}_{\mathrm{F}}$ of the undenuded lines (positive artifact) is evident as the OC measured by this line is higher than that of the denuded samplers. The magnitudes of the positive and negative artifacts depend not only on the sampling method and the atmospheric composition but also on the length of the sampling period. For the high-time resolved samples the most adequate correction for the concentration measured with the $\mathrm{Q}_{\mathrm{F}}$ in the undenuded sampler is the subtraction of the OC concentration measured in the backup quartz filter behind the Teflon filter. A detailed discussion of the artifacts using these datasets is presented by Subramanian et al. (2004).

Figure 2 shows time-resolved concentrations for the different samplers during a six-day period. Overall samplers indicate similar patterns of OC and EC concentrations, but the $\mathrm{OC}$ and EC concentrations from the undenuded line are almost always higher than those of the other two samplers.

The summer intensive averaged concentrations of OC and EC for all types of samplers and all artifact correction approaches at the Pittsburgh supersite is shown in Table 2. Subtracting the measured OC concentration on the $\mathrm{Q}_{\mathrm{B}, \mathrm{F}}$ and $\mathrm{Q}_{\mathrm{B}, \mathrm{T}}$ from the $\mathrm{Q}_{\mathrm{F}}$ reduces the OC concentrations by $20 \%$ on average for the summer intensive. Subtracting the $\mathrm{OC}$ concentration of the $\mathrm{Q}_{\mathrm{B}, \mathrm{F}}$ behind the Teflon filter in the parallel line of the undenuded sampler results in an average correction of around $50 \%$ on average for the summer intensive. The reported OC concentrations of the two

Table 2

Monthly averaged OC and EC concentrations for the summer intensive 2001 at PAQS

\begin{tabular}{lcc}
\hline \multicolumn{1}{c}{ Measurement type } & $\mathrm{OC}\left(\mu \mathrm{g} \mathrm{C} / \mathrm{m}^{3}\right)$ & $\mathrm{EC}\left(\mu \mathrm{g} \mathrm{C} / \mathrm{m}^{3}\right)$ \\
\hline Undenuded $\left(\mathrm{Q}_{\mathrm{F}}\right)$ & 4.1 & 0.8 \\
Undenuded $\left(\mathrm{Q}_{\mathrm{F}}-\mathrm{Q}_{\mathrm{B}, \mathrm{Q}}\right)^{1}$ & 3.6 & 0.8 \\
Undenuded $\left(\mathrm{Q}_{\mathrm{F}}-\mathrm{Q}_{\mathrm{B}, \mathrm{T}}\right)^{1}$ & 2.2 & 0.8 \\
Denuded in situ analyzer & 2.8 & 0.8 \\
$\quad\left(\mathrm{Q}_{\mathrm{F}, \text { situ }}\right)$ & & \\
Denuded sampler & 3.1 & 0.5 \\
\hline
\end{tabular}

${ }^{1}$ The undenuded sampler OC concentrations are corrected by subtracting the OC collected in the backup quartz filter behind the front quartz or by subtracting the $\mathrm{OC}$ collected in the backup quartz filter behind the Teflon filter from the parallel sampling line. 

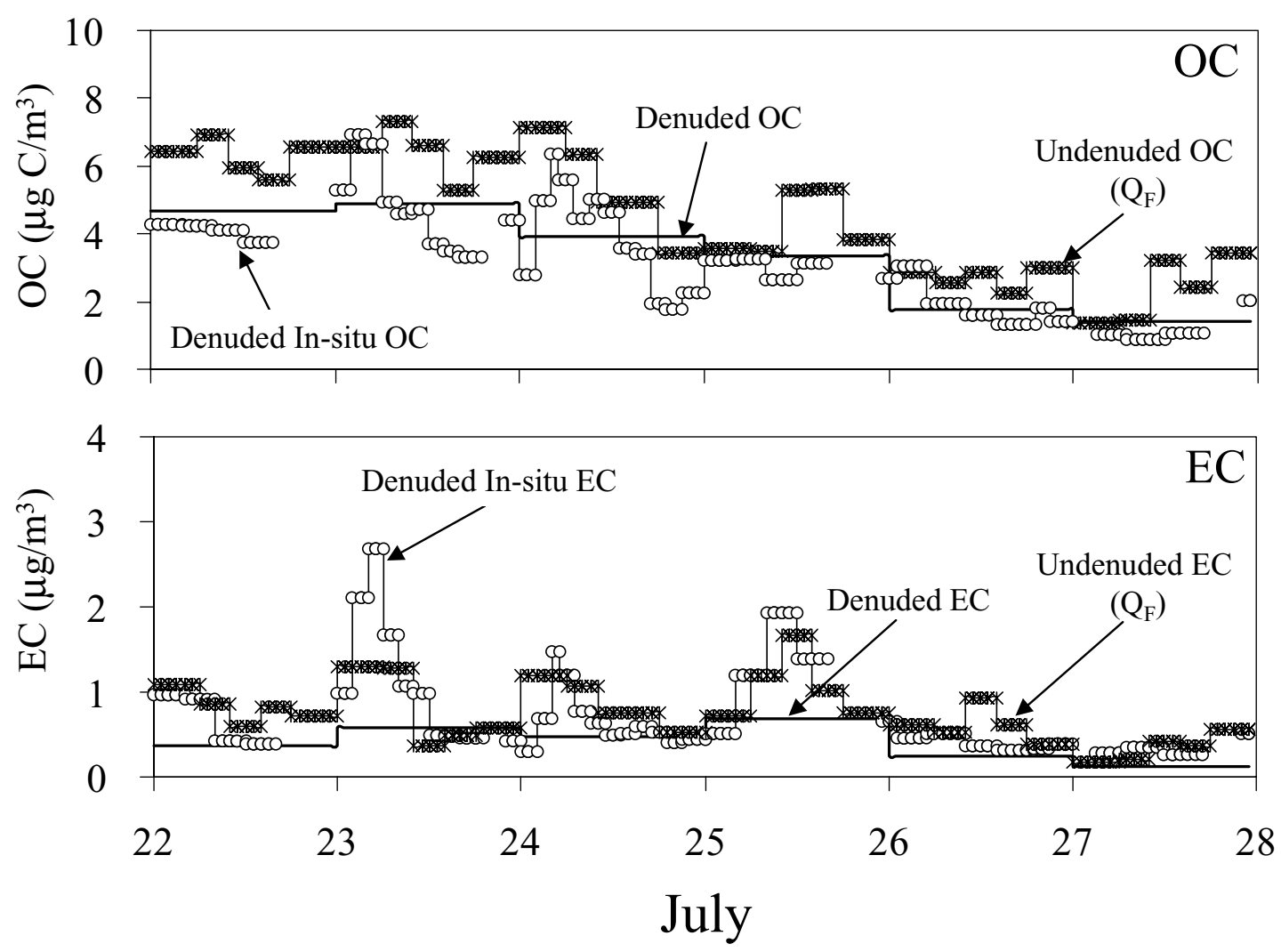

Figure 2. Time-resolved concentrations for the different organic samplers at the Pittsburgh supersite project. The filter-based undenuded samples and the denuded in situ analyzer samples were collected in high resolution time periods ( $2-6 \mathrm{~h})$. The filter-based denuded samples were collected in $24 \mathrm{~h}$ periods.

denuded samplers agree within $10 \%$ and give particulate OC concentrations between the $\mathrm{Q}_{\mathrm{F}, \mathrm{B}}$ and $\mathrm{Q}_{\mathrm{B}, \mathrm{T}}$ corrected undenuded sampler concentrations. However, the reported average EC concentrations differ by $50 \%$. EC concentrations reported by the undenuded and denuded in situ analyzer agree within $10 \%$. A detailed discussion of the potential reasons for these differences is provided by Subramanian et al. (2004).

In this work we examine the effect of the difference in sampler configuration and sampling periods of OC and EC measurements on the SOA estimates by applying the EC tracer method.

\section{THE EC TRACER METHOD}

The ratio of the ambient concentrations of particulate $\mathrm{OC}$ to EC includes information about the extent of secondary OC formation. Ambient OC/EC ratios greater than those characteristic of the primary emissions for a specific area are an indication of secondary aerosol formation. The EC tracer method takes advantage of the fact that primary OC and EC are mostly emitted by the same combustion sources. Primary ratios of OC to EC can be determined from a subset of ambient measurements if a large data set is available and conditions to produce SOA are unlikely (Turpin and Huntzicker 1995; Strader et al. 1999), or by developing an emissions inventory of the principal sources for an area of interest (Gray 1986; Cabada et al. 2002a).
Assuming that OC primary can be defined by,

$$
[\mathrm{OC}]_{\mathrm{p}}=\left[\frac{\mathrm{OC}}{\mathrm{EC}}\right]_{\mathrm{p}} \cdot[\mathrm{EC}]+b,
$$

the contribution of secondary OC can be estimated as

$$
[\mathrm{OC}]_{\mathrm{S}}=[\mathrm{OC}]-[\mathrm{OC}]_{\mathrm{p}}
$$

where $[\mathrm{OC}]_{\mathrm{p}}$ is the primary organic aerosol concentration, $[\mathrm{OC} / \mathrm{EC}]_{\mathrm{p}}$ is the ratio of $\mathrm{OC}$ to $\mathrm{EC}$ for the primary sources affecting the site of interest, and $b$ is the noncombustion contribution to the primary OC and sampling artifacts (Turpin and Huntzicker 1995; Strader et al. 1999). Sources that emit mainly OC and would contribute to this intercept $b$ include meatcooking operations, biogenic sources (i.e., plant detritus, resuspension of other biogenic material), etc. [EC] is the measured EC concentration, $[\mathrm{OC}]_{S}$ is the secondary organic aerosol contribution to the total $\mathrm{OC}$, and $[\mathrm{OC}]$ is the measured $\mathrm{OC}$ concentration. All of these parameters are time dependent because of the temporal variations in anthropogenic emissions and meteorology. The application of this method requires measurements of [OC], [EC], and the determination of the $[\mathrm{OC} / \mathrm{EC}]_{\mathrm{p}}$ ratio, as well as the noncombustion primary $\mathrm{OC}$ contribution $(b)$ for the area and period of interest (Turpin and Huntzicker 1995). 
Calculation of the Primary OC-to-EC Ratio and Intercept

Diurnal variations of the ambient OC-to-EC ratio were observed for all the high-resolution measurements taken during the summer intensive of 2001 at PAQS. Photochemical activity, meteorology, and primary emissions all contribute to these variations. Ozone concentration can be used as an indicator of photochemical activity. Carbon monoxide (CO) and nitrogen oxides ( $\mathrm{NO}$ and $\mathrm{NO}_{\mathrm{x}}$ ) can be used as tracers of combustion-related primary emissions. The primary ratio and intercept are determined from a dataset by identifying the periods where the ambient concentrations are dominated by primary emissions.

The first step in the determination of the primary OC/EC ratio is the subtraction from the original dataset of the points where rain and the corresponding storms cause significant changes to the OC/EC ratio (Figure 3a). These changes have a variety of causes (removal of aged particles and increased importance of the locally produced ones, preferential removal of secondary OC, etc.). These periods are excluded from the analysis to avoid unnecessary complications.

The second step consists of identifying the $\mathrm{OC}$ and EC concentrations where there is high probability of SOA production. The OC-to-EC ratio usually showed a strong correlation with ozone, but a lag time between the ozone peak and the actual OC-to-EC ratio was observed. In an effort to account for those events, the "history" of the ozone peak was taken into account, and the peak ozone concentration for the period preceding the sample was compared to the OC-to-EC ratio to evaluate its influence. For example, Figure $3 \mathrm{~b}$ shows the measured OC-to-EC ratio and $\mathrm{O}_{3}$ concentrations during a 4-day period in July. Periods of significant photochemical activity are evident during the afternoon of each day. The corresponding afternoon OC and EC measurements are "deleted" from the dataset because they probably include some SOA contribution. This does not mean that the other periods have only primary OC. Secondary aerosol can be produced elsewhere, maybe even during the previous day, and transported into the site.

Figures $3 \mathrm{c}$ and $\mathrm{d}$ show periods where combustion-related sources were dominating over the area. The last step in this methodology consists of identifying these periods. As the $\mathrm{NO}_{\mathrm{x}}$, $\mathrm{NO}$, and $\mathrm{CO}$ concentrations peaked during the night and early morning of 3 July and 1 August, the OC-to-EC ratios decreased, indicating the influence of primary sources over the area of analysis. The corresponding samples during these periods are kept in the dataset and are used to estimate the primary OC-to-EC ratio for the period of analysis.
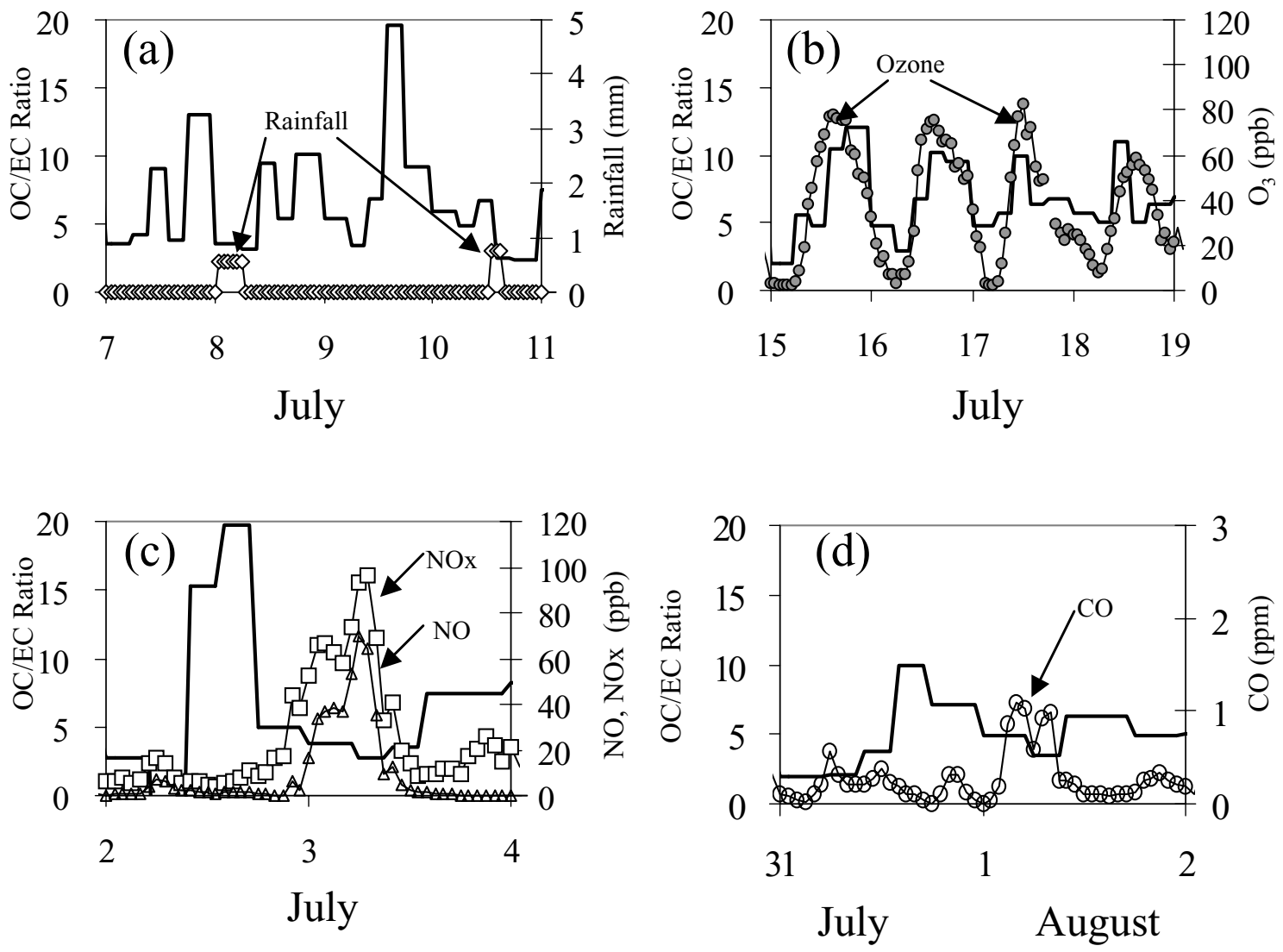

Figure 3. Time series of $\mathrm{OC} / \mathrm{EC}$ ratio (undenuded $\mathrm{Q}_{\mathrm{F}}$ ) and gaseous tracers of photochemical activity and primary emissions for different periods in July 2001 during the summer intensive at the Pittsburgh Supersite. (a) OC/EC ratio affected by rain. (b) Ozone and OC/EC ratio. (c) Nitrogen oxides and OC/EC ratio. (d) OC/EC ratio and carbon monoxide. 

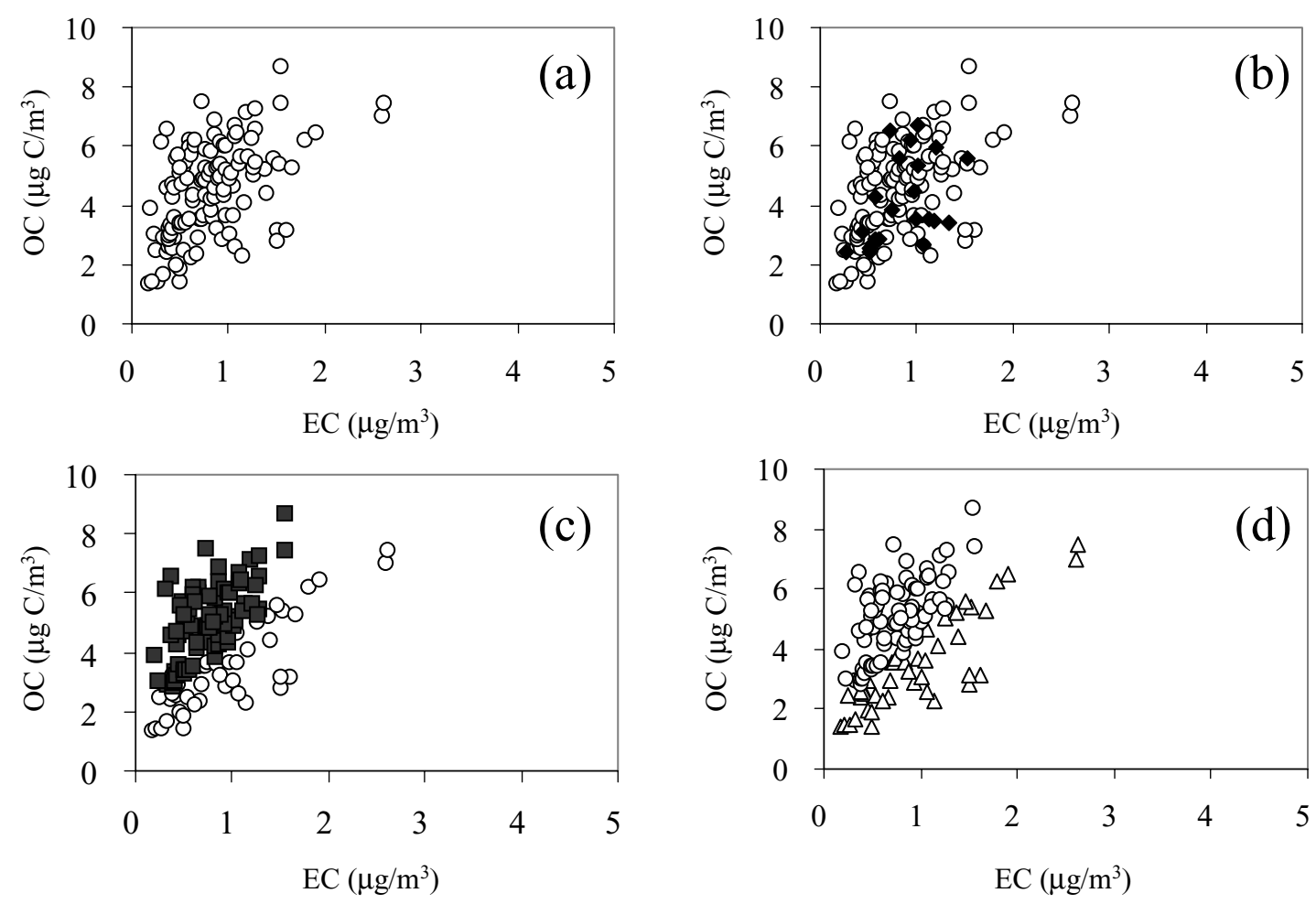

Figure 4. Scatter plot of OC versus $\mathrm{EC}$ for all samples collected during the period (summer intensive 2001). Concentrations shown are from the undenuded sampler, front quartz data. Hollow circles represent concentrations that have not been classified according to the criteria used. (a) OC versus EC for all samples collected during the period of analysis. (b) Concentrations eliminated from the dataset because they are affected by rain (solid rhombus). (c) Concentrations strongly influenced by photochemical activity are deleted from the dataset (solid squares). (d) Final set of concentrations influenced by primary emissions (hollow triangles). OC/EC primary ratio and intercept, $b$, are estimated by a linear fit of these data.

Figure 4 shows the sequence of how the OC versus EC plot is evolving during the different steps of the analysis. Once all the points that are dominated by primary OC are determined (those influenced by combustion sources as described by the algorithm), a linear regression by least squares minimization is fitted to the "primary" concentrations. The slope of the fit represents the OC-to-EC primary ratio, and the intercept represents the "noncombustion organic carbon" contribution to the primary OC concentration (see Equations (1) and (2)).

Table 3 shows the classification of concentrations between primary and secondary influenced for a two-day period during the summer intensive using the undenuded $\mathrm{Q}_{\mathrm{F}}$ data. Most of the OC during the first morning (6:00-10:00) appears to be primary, as the area was heavily influenced by primary emissions showing higher values of $\mathrm{NO}_{\mathrm{x}}$ and CO. OC-to-EC ratios increased rapidly during the day as the ozone concentration increased, suggesting that the formation of SOA was probably taking place. A period where SOA material is probably transported into the area can be observed during the late hours of 1 August and the first hours of 2 August. These periods are characterized by relatively high ozone and $\mathrm{NO}_{\mathrm{x}}$, leading to the classification of this period as SOA dominated. Periods when most of the OC is primary are characterized by average concentrations of $25 \mathrm{ppb}$ of $\mathrm{NO}_{\mathrm{x}}, 8 \mathrm{ppb}$ of NO, $0.3 \mathrm{ppm}$ of $\mathrm{CO}$, and $30 \mathrm{ppb}$ of ozone. The remaining periods where there may be significant amounts of SOA present have average concentrations of $17 \mathrm{ppb}$ of $\mathrm{NO}_{\mathrm{x}}, 2 \mathrm{ppb}$ of $\mathrm{NO}$, $0.2 \mathrm{ppm}$ of $\mathrm{CO}$, and $50 \mathrm{ppb}$ of ozone.

For the analysis of the daily samples (or daily-averaged concentrations) the above algorithm needs to be modified. Daily averages of the $\mathrm{O}_{3}, \mathrm{CO}$, and $\mathrm{NO}_{\mathrm{x}}$ concentrations are used to determine the periods when the OC concentrations are influenced by primary sources. Primary-dominated concentrations for the daily-averaged concentrations show an average concentration of $17 \mathrm{ppb}$ of $\mathrm{NO}_{\mathrm{x}}, 4 \mathrm{ppb}$ of $\mathrm{NO}, 0.2 \mathrm{ppm}$ of $\mathrm{CO}$, and $30 \mathrm{ppb}$ of ozone. Secondary-dominated concentrations show and average concentration of $20 \mathrm{ppb}$ of $\mathrm{NO}_{\mathrm{x}}, 4 \mathrm{ppb}$ of $\mathrm{NO}, 0.2 \mathrm{ppm}$ of $\mathrm{CO}$, and $45 \mathrm{ppb}$ of ozone.

\section{Estimated $[O C / E C]_{p}$ and $\mathrm{b}$}

Figure 5 summarizes the classification of points between primary and secondary influenced for all high-resolution datasets. The estimated primary ratio of $\mathrm{OC}$ to $\mathrm{EC}\left([\mathrm{OC} / \mathrm{EC}]_{\mathrm{p}}\right)$ and the intercept, $b$, vary depending on the dataset analyzed and the averaging period used (Table 4). A consistent set of concentrations of OC and EC should be used in order to estimate the 
Table 3

Selection criteria for OC and EC concentrations (undenuded $\mathrm{Q}_{\mathrm{F}}$ dataset) influenced by primary emissions or SOA formation

\begin{tabular}{|c|c|c|c|c|c|c|c|c|c|c|}
\hline Date & $\begin{array}{c}\mathrm{EC} \\
\mu \mathrm{g} / \mathrm{m}^{3}\end{array}$ & $\begin{array}{c}\mathrm{OC} \\
\mu \mathrm{g} \mathrm{C} / \mathrm{m}^{3}\end{array}$ & $\begin{array}{c}\text { OC/EC } \\
\text { Ratio }\end{array}$ & $\begin{array}{c}\mathrm{O}_{3} \text { avg. } \\
(\mathrm{ppb})\end{array}$ & $\begin{array}{c}\mathrm{O}_{3} \text { peak } \\
(\mathrm{ppb})\end{array}$ & $\begin{array}{c}\mathrm{O}_{3} \text { peak } \\
(i-1)^{*}(\mathrm{ppb})\end{array}$ & $\begin{array}{c}\mathrm{CO} \\
(\mathrm{ppm})\end{array}$ & $\begin{array}{l}\text { NO } \\
(\mathrm{ppb})\end{array}$ & $\begin{array}{l}\mathrm{NO}_{x} \\
(\mathrm{ppb})\end{array}$ & $\begin{array}{c}\text { Source } \\
\text { influence }\end{array}$ \\
\hline \multicolumn{11}{|l|}{ 8/1/01 } \\
\hline $10: 00-14: 00$ & 1.1 & 6.7 & 6.3 & 75 & 97 & 29 & 0.2 & 3 & 20 & Secondary \\
\hline $14: 00-18: 00$ & 0.8 & 5.0 & 6.3 & 92 & 98 & 97 & 0.1 & 1 & 13 & Secondary \\
\hline 0:00-6:00 & 1.1 & 5.6 & 5.0 & 13 & 29 & 80 & 0.3 & 5 & 38 & Secondary \\
\hline $6: 00-10: 00$ & 1.1 & 4.6 & 4.4 & 21 & 35 & 29 & 0.2 & 4 & 25 & Primary \\
\hline $10: 00-14: 00$ & 0.9 & 6.4 & 7.5 & 86 & 107 & 35 & 0.1 & 1 & 12 & Secondary \\
\hline 14:00-18:00 & 0.6 & 4.9 & 8.4 & 104 & 105 & 107 & 0.1 & 0.2 & 8 & Secondary \\
\hline $18: 00-24: 00$ & 0.9 & 4.6 & 5.3 & 69 & 90 & 105 & 0.2 & 0.1 & 15 & Secondary \\
\hline
\end{tabular}

${ }^{*} i-1$, corresponds to the average concentration of ozone during the previous sampling interval of carbonaceous material.
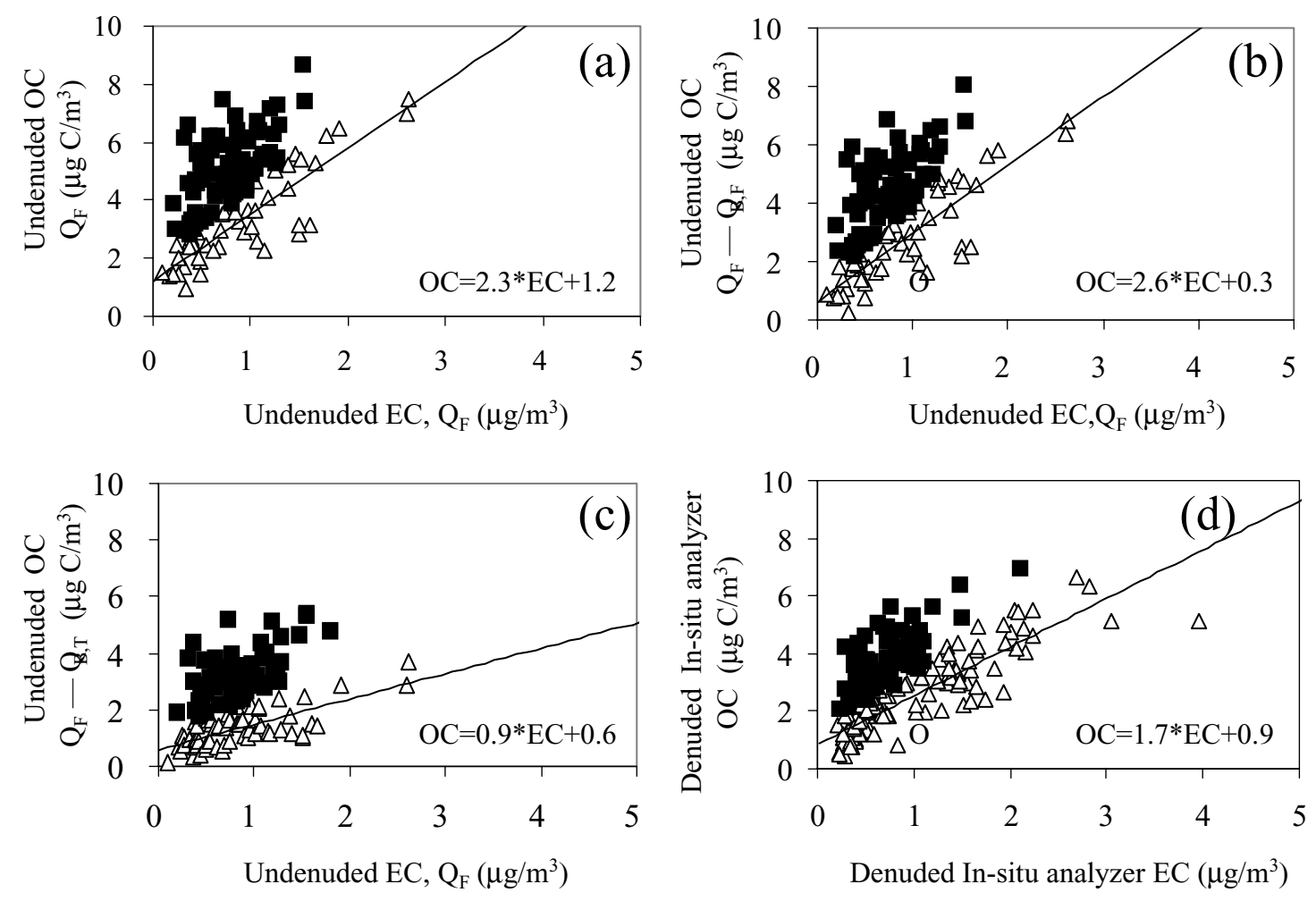

Figure 5. Estimated carbonaceous concentrations influenced by primary emissions and SOA production for high time resolution measurements (2-6 h samples) during the summer intensive at PAQS. Solid squares correspond to SOA-influenced concentrations and hollow triangles to primary-dominated concentrations. (a) Front quartz $\mathrm{OC}\left(\mathrm{Q}_{\mathrm{F}}\right)$ and EC concentrations. (b) Correcting the OC measurement with the backup quartz filter behind the front quartz $\left(Q_{F}-Q_{B, F}\right)$. (c) Correcting the $O C$ concentrations with the backup quartz filter behind the Teflon filter in the parallel undenuded line $\left(\mathrm{Q}_{\mathrm{F}}-\mathrm{Q}_{\mathrm{B}, \mathrm{T}}\right)$. (d) Denuded in situ analyzer. 
Table 4

Estimated parameters for the linear fit of the "primary" OC and EC concentrations: High-resolution data (2-6 h) and daily averages and low-resolution measurements (24 h) from the July 2001 summer intensive at Pittsburgh Air Quality Study

\begin{tabular}{|c|c|c|c|}
\hline Measurement type & {$[\mathrm{OC} / \mathrm{EC}] \mathrm{p}$} & $\begin{array}{c}\text { Noncombustion } \\
\text { primary OC, } b\left(\mu \mathrm{g} / \mathrm{m}^{3}\right)\end{array}$ & $\begin{array}{c}\text { Correlation } \\
\text { coefficient }\left(\mathrm{R}^{2}\right)\end{array}$ \\
\hline Undenuded ( $Q_{F} 4-6$ h samples $)$ & $2.3 \pm 0.4$ & $1.2 \pm 0.4$ & 0.60 \\
\hline Undenuded $\left(\mathrm{Q}_{\mathrm{F}}-\mathrm{Q}_{\mathrm{B}, \mathrm{Q}}\right.$ 4-6 h samples $)$ & $2.6 \pm 0.4$ & $0.3 \pm 0.4$ & 0.76 \\
\hline Denuded in situ analyzer (2-4 h samples) & $1.7 \pm 0.2$ & $0.9 \pm 0.2$ & 0.78 \\
\hline Undenuded $\left(\mathrm{Q}_{\mathrm{F}}-\mathrm{Q}_{\mathrm{B}, \mathrm{T}}\right.$ 4-6 h samples $)$ & $0.9 \pm 0.2$ & $0.6 \pm 0.2$ & 0.53 \\
\hline Denuded sampler (24 h samples) & $3.1 \pm 0.8$ & $1.0 \pm 0.4$ & 0.80 \\
\hline Undenuded ( $\mathrm{Q}_{\mathrm{F}} 24 \mathrm{~h}$ averages $)$ & $2.7 \pm 0.5$ & $1.3 \pm 0.4$ & 0.90 \\
\hline Undenuded $\left(\mathrm{Q}_{\mathrm{F}}-\mathrm{Q}_{\mathrm{B}, \mathrm{Q}} 24 \mathrm{~h}\right.$ averages $)$ & $2.3 \pm 0.5$ & $1.2 \pm 0.3$ & 0.88 \\
\hline Undenuded $\left(\mathrm{Q}_{\mathrm{F}}-\mathrm{Q}_{\mathrm{B}, \mathrm{T}} 24 \mathrm{~h}\right.$ averages $)$ & $1.6 \pm 0.5$ & $0.5 \pm 0.4$ & 0.62 \\
\hline Denuded in situ analyzer ( $24 \mathrm{~h}$ averages) & $1.9 \pm 0.5$ & $0.7 \pm 0.4$ & 0.80 \\
\hline
\end{tabular}

SOA concentration using the approach proposed in the previous sections. Estimates of the primary ratio vary from 0.9 to 3.1 , and the intercept, $b$, varies from 0.3 to $1.2 \mu \mathrm{g} \mathrm{C} / \mathrm{m}^{3}$. Variations in the estimated parameters for the different sets of measurements are due to the different characteristics of the samplers (Subramanian et al. 2003). In general primary ratios and intercepts from the fitting of the daily-averaged concentrations influenced by primary emissions are higher than those calculated from the high time resolution measurements (Figure 6). This can be explained by the fact that high-resolution measurements have the ability to more accurately identify and separate periods of secondary organic aerosol formation from those dominated by primary emissions. Higher correlations coefficients are achieved for the daily samples because the datasets show less variability among the points considered to be influenced by primary emissions (Table 4). The effect of the sampling artifacts can also be seen in the estimated primary ratios for the different datasets. Those datasets with corrections of the artifact (using the backup quartz filters in the undenuded sampler) or having a denuded line have lower primary ratios. The exception to this rule is the denuded sampler.

Higher contributions from the noncombustion primary OC are calculated for the undenuded front quartz datasets. This is due to the addition of the adsorbed organic gases to the actual primary noncombustion OC, and the calculated values $\left(1.2-1.4 \mu \mathrm{g} \mathrm{C} / \mathrm{m}^{3}\right)$ are probably overestimates of the real noncombustion primary OC. Lower intercepts are calculated for the undenuded datasets where corrections are done for the front quartz artifact, and these values are probably closer to the true concentrations (Table 4).

Since the primary ratio and intercept are expected to vary temporally, primary ratios and intercepts were estimated for the different high time resolution samplers, segregating the data for the different times of the day sampled. This analysis showed no significant diurnal variations for the estimated ratios and intercepts. This lack of temporal dependence is associated with the characteristics of the organic $\mathrm{PM}_{2.5}$ in the Northeast U.S. The Northeast U.S. is characterized by long-range transport of PM and shorter residence times over the large urban areas. The dominance of regional sources reduces the effects of local emissions and their temporal patterns. Studies done in Southern and Central California, where most of these types of analyses have been performed, are able to identify different patterns of emissions for different times of the day since there is little background transport into the area (Gray 1986; Turpin and Huntzicker 1995).

\section{SOA CONCENTRATIONS}

Once the primary ratio and the noncombustion primary OC contribution are calculated for each of the different datasets of carbonaceous measurements, the primary and secondary components of the Pittsburgh organic aerosol can be determined by applying Equations (1) and (2).

\section{SOA Based on High Time Resolution Measurements}

The calculated SOA concentrations for the different highresolution measurements are in a qualitative agreement (Figure 7), predicting the same periods of SOA production for the summer intensive. For example, from 15 July to 25 July a high pressure system dominated the area, allowing high production of SOA. The effect of two other high-pressure systems can be seen from 8 July to 11 July and in the beginning of August. Production of SOA can be observed during the mid-afternoon of each of those days, and significant transport of secondary material into the area occasionally occurs during the nighttime. The first days of July were characterized by a strong contribution of SOA during the daylight hours.

Ozone and solar radiation (UV) are triggers of secondary organic aerosol production. Estimated SOA concentrations show the same behavior as the ozone and UV radiation during the day (Figure 8). This qualitative agreement provides some additional confidence on the estimated concentrations. The first hours of 2 August show a period of pollution transport to the area. SOA concentration increases and a peak in the ozone concentration is observed during the middle of the night. The effect of ambient 

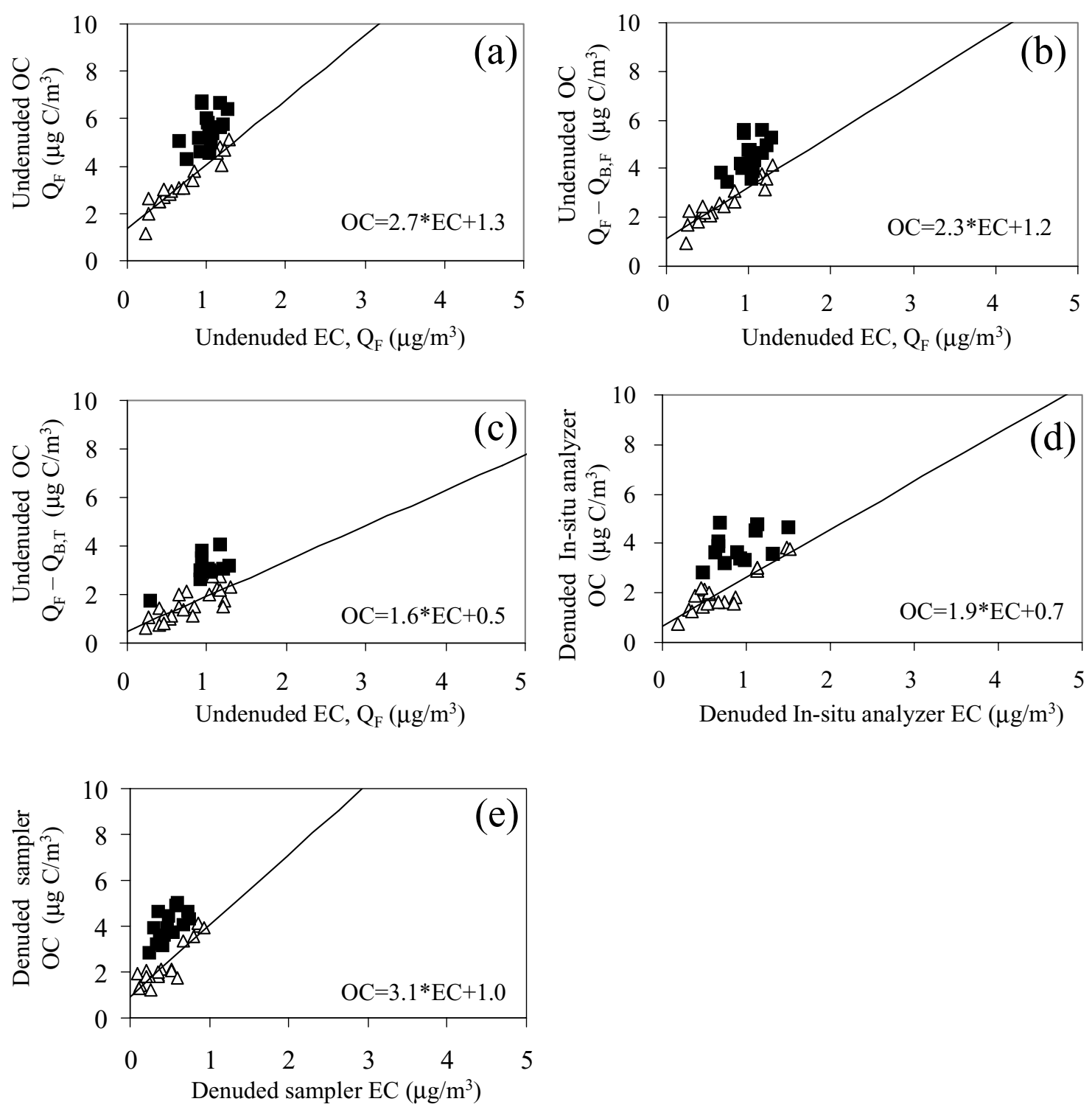

Figure 6. Estimated carbonaceous concentrations influenced by primary emissions and SOA production for daily averaged and $24 \mathrm{~h}$ measurements during the summer intensive at PAQS. Solid squares correspond to SOA-influenced concentrations and hollow triangles to primary-influenced concentrations. (a) Front quartz $\mathrm{OC}\left(\mathrm{Q}_{\mathrm{F}}\right)$ and EC concentrations. (b) Correcting the OC measurement with the backup quartz filter behind the front quartz $\left(\mathrm{Q}_{\mathrm{F}}-\mathrm{Q}_{\mathrm{B}, \mathrm{F}}\right)$. (c) Correcting the OC concentrations with the backup quartz filter behind the Teflon filter in the parallel undenuded line $\left(\mathrm{Q}_{\mathrm{F}}-\mathrm{Q}_{\mathrm{B}, \mathrm{T}}\right)$. (d) Denuded in situ analyzer. (e) Denuded sampler.

temperature can also be observed-as the temperature increases more SOA is produced in the area (Figure 8c). Temperature increases are associated with high-pressure systems over the Pittsburgh area during the summer. In general these periods provide the ideal conditions for the production of SOA, like stagnant air masses and high photochemical activity. Little correlation exists between relative humidity and SOA production (Figure 8d). For the summer intensive 2001, in Pittsburgh low relative humidity periods are associated with high temperatures at the middle of the day, and high relative humidity periods correspond to fronts entering the area and lowering the SOA production.

Figure 9 shows the average daily concentrations of the estimated SOA and primary OC for all high-resolution datasets analyzed. In order to make comparisons between the datasets, average concentrations for each are normalized to the daily average primary OC and SOA for the period. The diurnal variation of primary $\mathrm{OC}$ is relatively small because during the summer intensive 2001 the majority of the OC is transported to the region from elsewhere (Cabada et al. 2002b). SOA patterns 

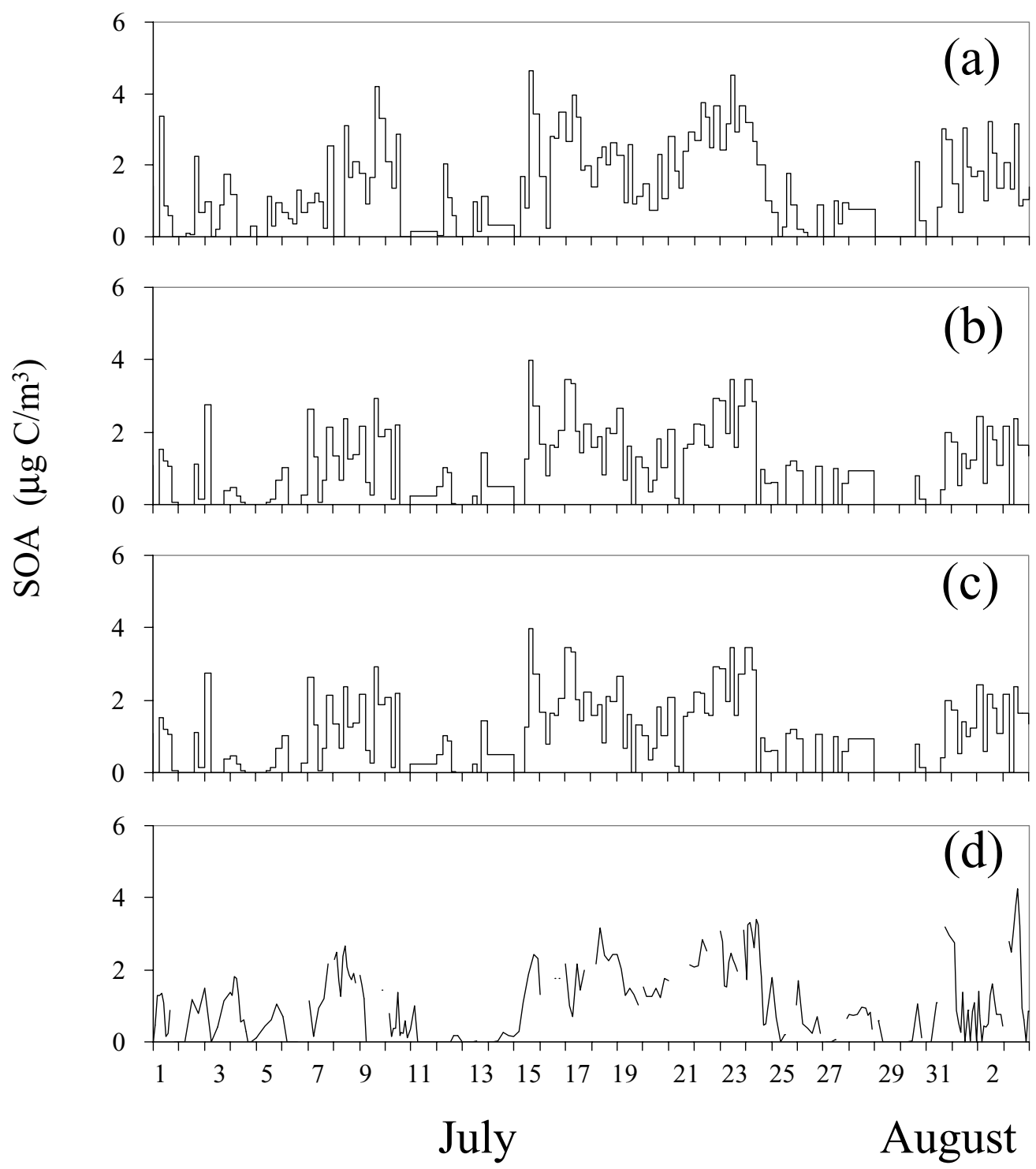

Figure 7. Estimated SOA concentrations based on the higher resolution measurements of OC and EC. (a) Undenuded sampler,

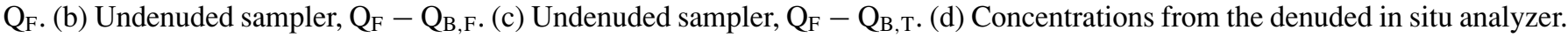

(Figure 9) show some differences among the datasets used. Overall, all samplers show a minimum concentration of SOA in the early morning hours (6:00-9:00 EST) and SOA increase as the photochemical activity increases during the day. The SOA production peak varies depending on the dataset analyzed, but it follows the ozone average daily peak occurring around 15:00 EST (Figure 9). All datasets suggest that a significant amount of SOA is due to long-range transport during the late afternoon and night hours. Minimum production of SOA coincides with periods where the ozone concentration is at its minimum (4:00 8:00 EST).

The daily averaged SOA concentrations for all high-resolution datasets show high episodes of SOA formation in the middle of
July and at the beginning on August (Figure 10). Practically all days during the summer intensive show some contribution of SOA material to the total OC concentrations.

Table 5 summarizes the monthly averaged SOA contribution for all the high-resolution measurements. Estimates of SOA vary from $30 \%$ for the denuded in situ analyzer to a high of $45 \%$ for the undenuded sampler doing the correction to the front quartz artifact with the backup filter behind the Teflon filter. Error bars are estimated from the uncertainties in the linear fit (95\% confidence level) from the point influenced by primary emissions determined for each dataset. Taking into account these uncertainties all methods agree in the average contribution of SOA around $35 \%$. 

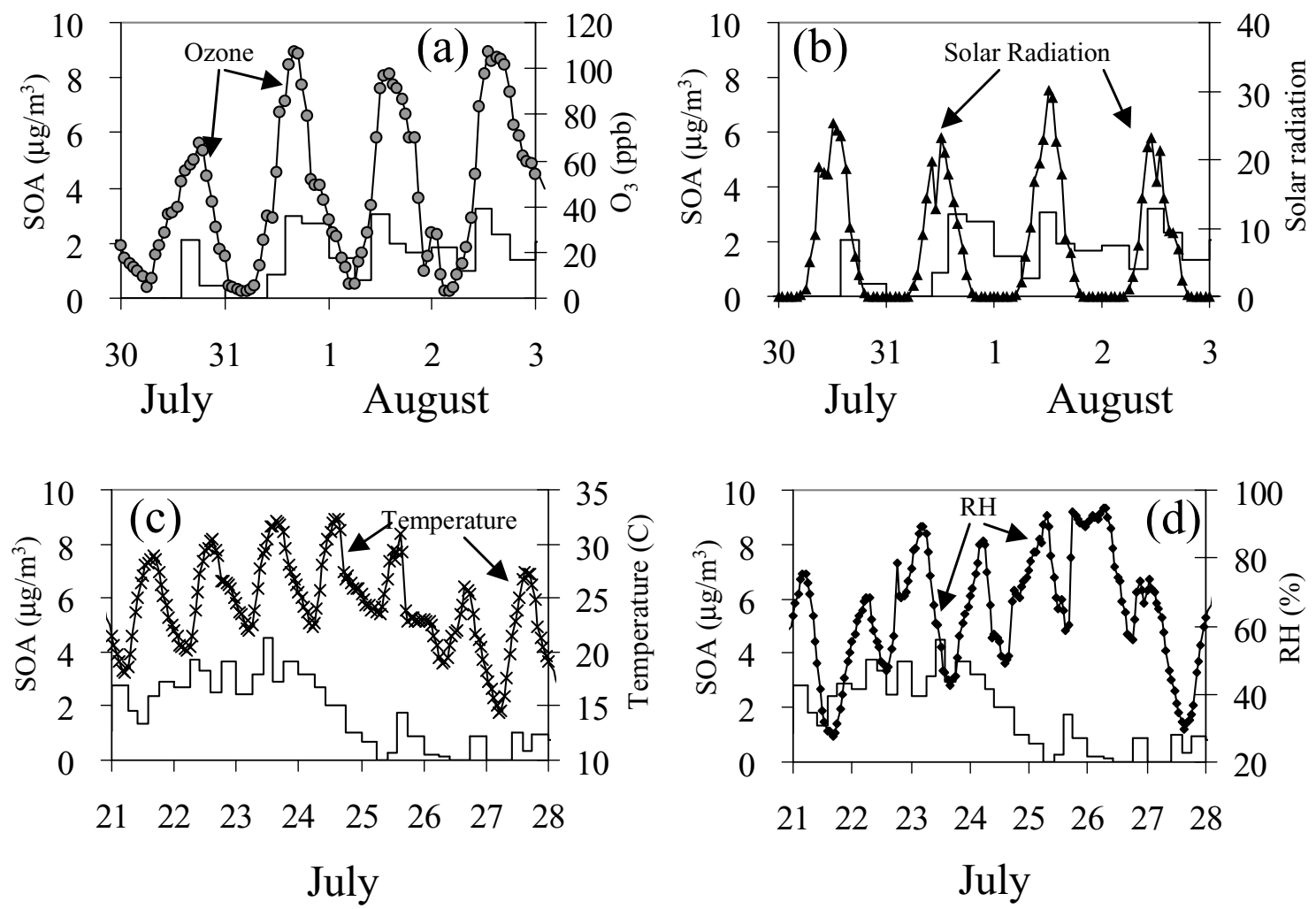

Figure 8. Hourly SOA production patterns for various periods during the summer intensive 2001 at PAQS. SOA concentrations calculated from the undenuded front quartz dataset $\left(\mathrm{Q}_{\mathrm{F}}\right)$. (a) Ozone concentration and SOA production. (b) Solar radiation (UV) and SOA production. (c) Ambient temperature influences over the SOA production in Pittsburgh. (d) Relative humidity has a minor role on the production of SOA.

\section{SOA Based on Daily Results, Summer Intensive 2001}

Figure 11 shows the estimated organic carbon composition (primary and secondary) for all different carbonaceous concentrations datasets using daily concentrations averages to estimate the primary ratio and the primary OC intercept. The SOA concentration variation during the period is qualitatively similar to that estimated using the high-resolution measurements (Figure 10). Periods of high SOA production are evident in the middle of July and the beginning of August. Unlike the estimates of SOA from the high-resolution measurements, primary

\section{Table 5}

Summer intensive average SOA fraction of the Pittsburgh organic aerosol for all high-resolution datasets (2-6 h sampling times)

\begin{tabular}{lc}
\hline \multicolumn{1}{c}{ Measurement type } & SOA (\%) \\
\hline Undenuded, $\mathrm{Q}_{\mathrm{F}}(4-6 \mathrm{~h})$ & $32 \pm 14$ \\
Undenuded, $\mathrm{Q}_{\mathrm{F}}-\mathrm{Q}_{\mathrm{B}, \mathrm{Q}}(4-6 \mathrm{~h})$ & $47 \pm 17$ \\
Undenuded, $\mathrm{Q}_{\mathrm{F}}-\mathrm{Q}_{\mathrm{B}, \mathrm{T}}(4-6 \mathrm{~h})$ & $36 \pm 14$ \\
Denuded in situ analyzer, $\mathrm{Q}_{\mathrm{F}, \text { situ }}(2-6 \mathrm{~h})$ & $29 \pm 10$ \\
\hline
\end{tabular}

ratios and intercepts calculated with the daily averaged concentrations show a number of days when no SOA is produced. SOA is probably present in all days but the estimated primary ratio and intercept is probably too high for this dataset, so these results can be viewed as a lower bound for the SOA concentrations. On average from all the methods, $22 \%$ of the total OC concentrations are estimated to be SOA (Table 6). All estimates agree within 5\% from each other. The higher estimate is given from the denuded

Table 6

Summer intensive average SOA fraction of the Pittsburgh organic aerosol for all datasets $(24 \mathrm{~h}$ averaged concentrations)

\begin{tabular}{lc}
\hline \multicolumn{1}{c}{ Measurement type } & SOA (\%) \\
\hline Undenuded, $\mathrm{Q}_{\mathrm{F}}(24 \mathrm{~h})$ & $19 \pm 14$ \\
Undenuded, $\mathrm{Q}_{\mathrm{F}}-\mathrm{Q}_{\mathrm{B}, \mathrm{Q}}(24 \mathrm{~h})$ & $20 \pm 18$ \\
Undenuded, $\mathrm{Q}_{\mathrm{F}}-\mathrm{Q}_{\mathrm{B}, \mathrm{T}}(24 \mathrm{~h})$ & $23 \pm 15$ \\
Denuded In-situ analyzer, $\mathrm{Q}_{\mathrm{F}, \mathrm{situ}}(24 \mathrm{~h})$ & $24 \pm 19$ \\
Denuder sampler $(24 \mathrm{~h})$ & $25 \pm 18$ \\
\hline
\end{tabular}



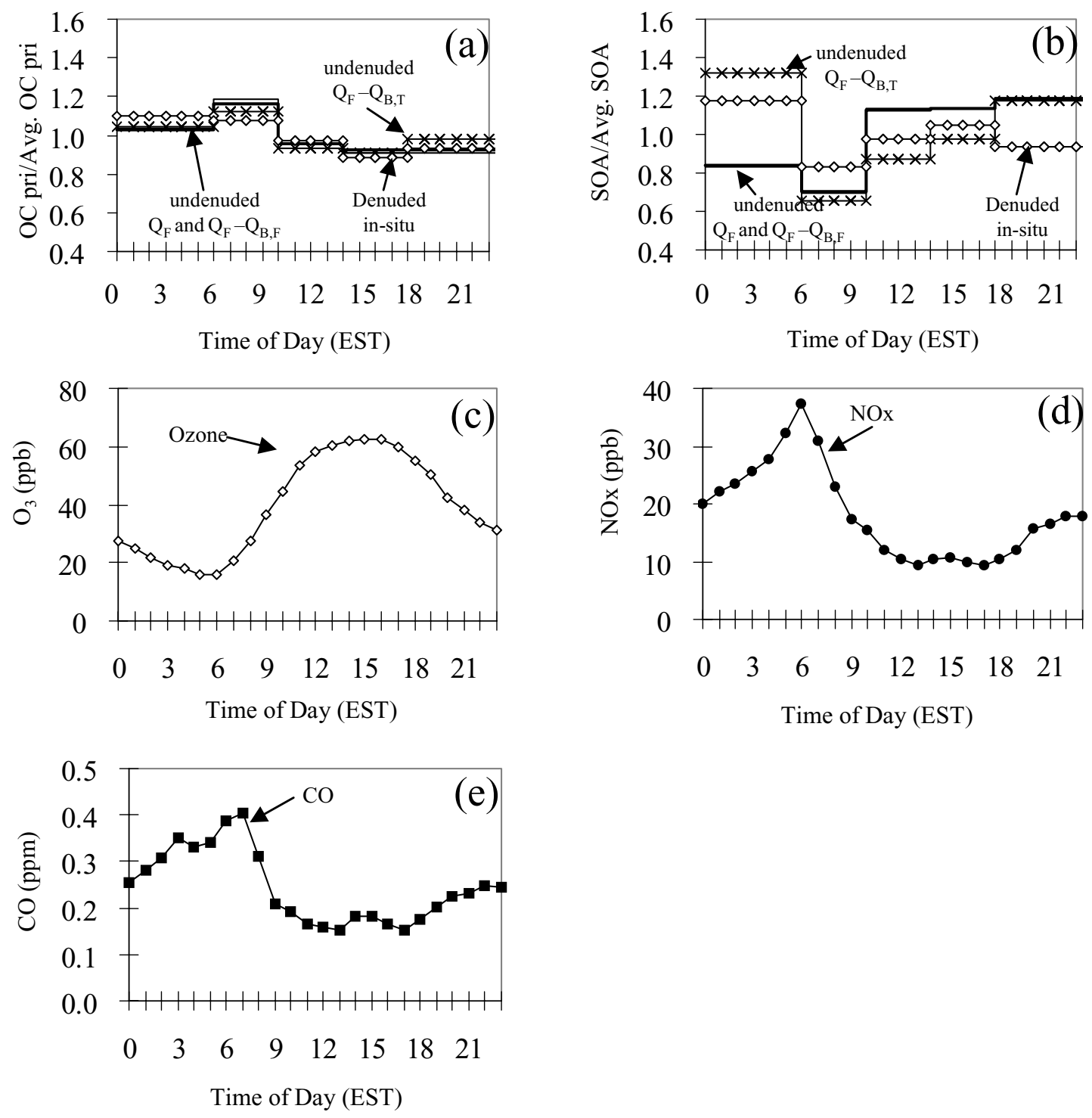

Figure 9. Average daily pattern of SOA and primary OC concentrations (normalized) during the summer intensive at PAQS, plotted along with daily averaged concentrations of various atmospheric variables. (a) Daily pattern primary OC estimated from high-resolution samplers. (b) Daily pattern SOA estimated from high-resolution samplers (c) Ozone averaged daily concentrations. (d) $\mathrm{NO}_{\mathrm{x}}$ averaged daily concentrations. (e) $\mathrm{CO}$ averaged daily concentrations.

sampler dataset, where SOA contributes $25 \%$. The lower estimate is $18 \%$ from the undenuded front quartz dataset. Error bars are calculated from the uncertainties in the linear fitting of the point identified as influenced by primary emissions for each dataset.

Figure 12 shows a scatter plot of the daily fraction of the different samplers for both high-resolution and daily-averaged samplers. For both cases all samplers agree within $20 \%$ on the estimated SOA fraction for each day. Higher differences are shown at the lower fractions. The reason for this could be that the lower fractions correspond to lower concentrations of OC, magnifying small variations over the SOA estimations.

\section{CONCLUSIONS AND DISCUSSION}

Application of the EC tracer method analysis to the different types of high-resolution measurements suggests an average of $35 \%$ SOA contribution to the monthly average OC concentration during the summer intensive of 2001. Overall estimates range from a low of $20 \%$ to a high of $50 \%$. A preliminary study trying to identify the sources of carbonaceous aerosol for western Pennsylvania estimated a SOA contribution of $30-50 \%$ to the total OC concentration during the summer of 1995 (Cabada et al. 2002b). These previous results are in good agreement with the estimates that are obtained applying this new method, giving some confidence about the results. 

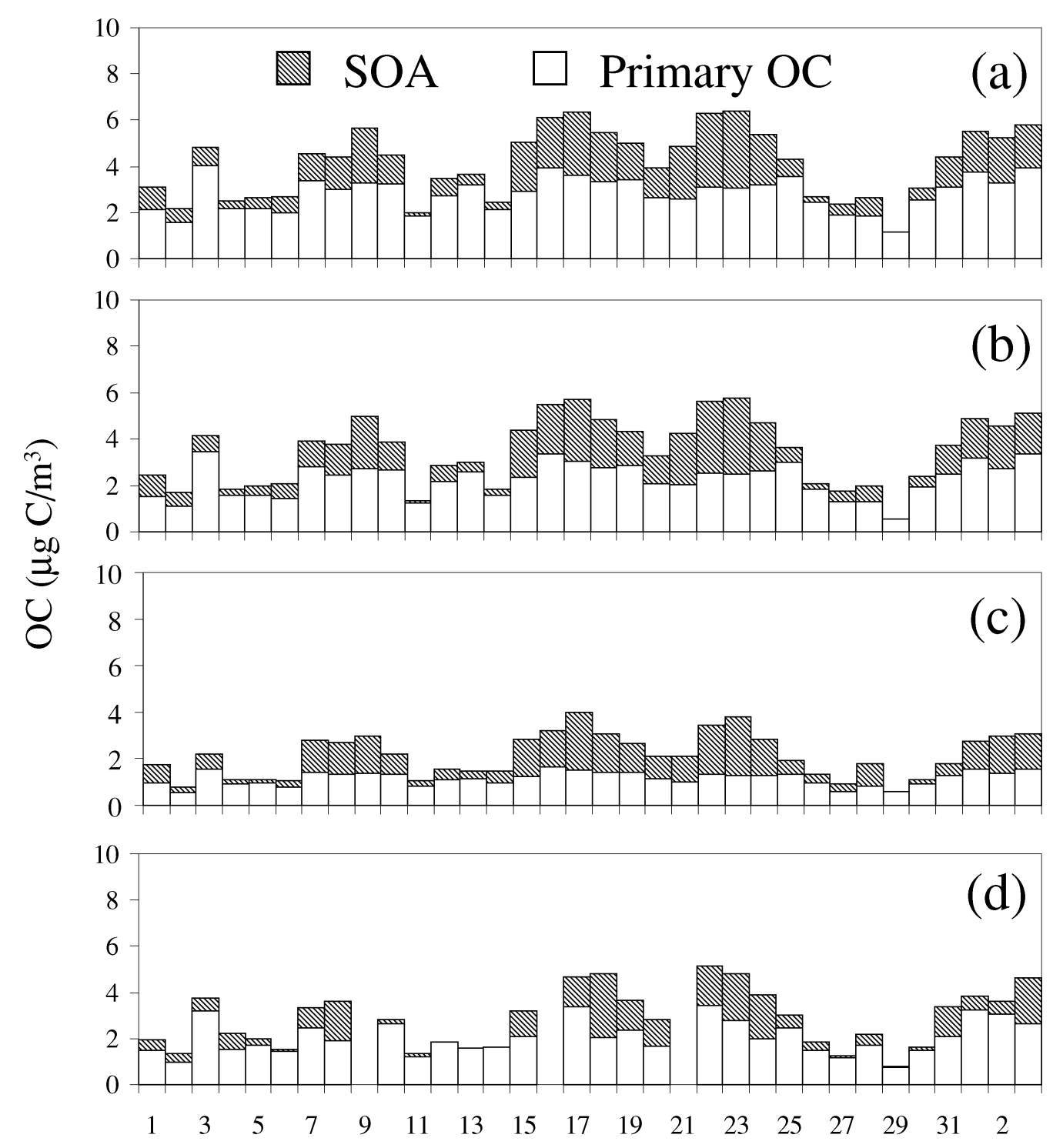

July

August

Figure 10. Daily-averaged SOA and primary OC concentrations during the summer intensive, estimated from the high-resolution measurements. (a) Undenuded sampler, $Q_{F}$. (b) Undenuded sampler, $Q_{F}-Q_{B, F}$. (c) Undenuded sampler, $Q_{F}-Q_{B, T}$. (d) Concentrations from the denuded in situ analyzer.

Higher time resolution measurements result in the highest estimation of SOA. Events that trigger SOA production have a strong diurnal dependence (i.e., ozone and sunlight daily cycles), so high-resolution measurements are more likely to identify periods of primary or secondary production dominance. The use of daily-averaged measurements probably tends to underpredict the SOA concentration, especially for relatively small datasets, because it may be impossible to find days without any SOA present. On average, the SOA concentrations are around 5-10\% higher if high-resolution measurements are used compared to the daily-averaged concentrations.

The EC tracer method is a simple approach for the determination of contribution of SOA to the total OC concentration measured in a sampling site. It relies on simultaneous measurements of gaseous pollutants that could be indicators of primary emissions or secondary aerosol production. The major weakness of the method is its reliance on the assumption of a constant primary OC/EC and constant $b$ during the analysis period 

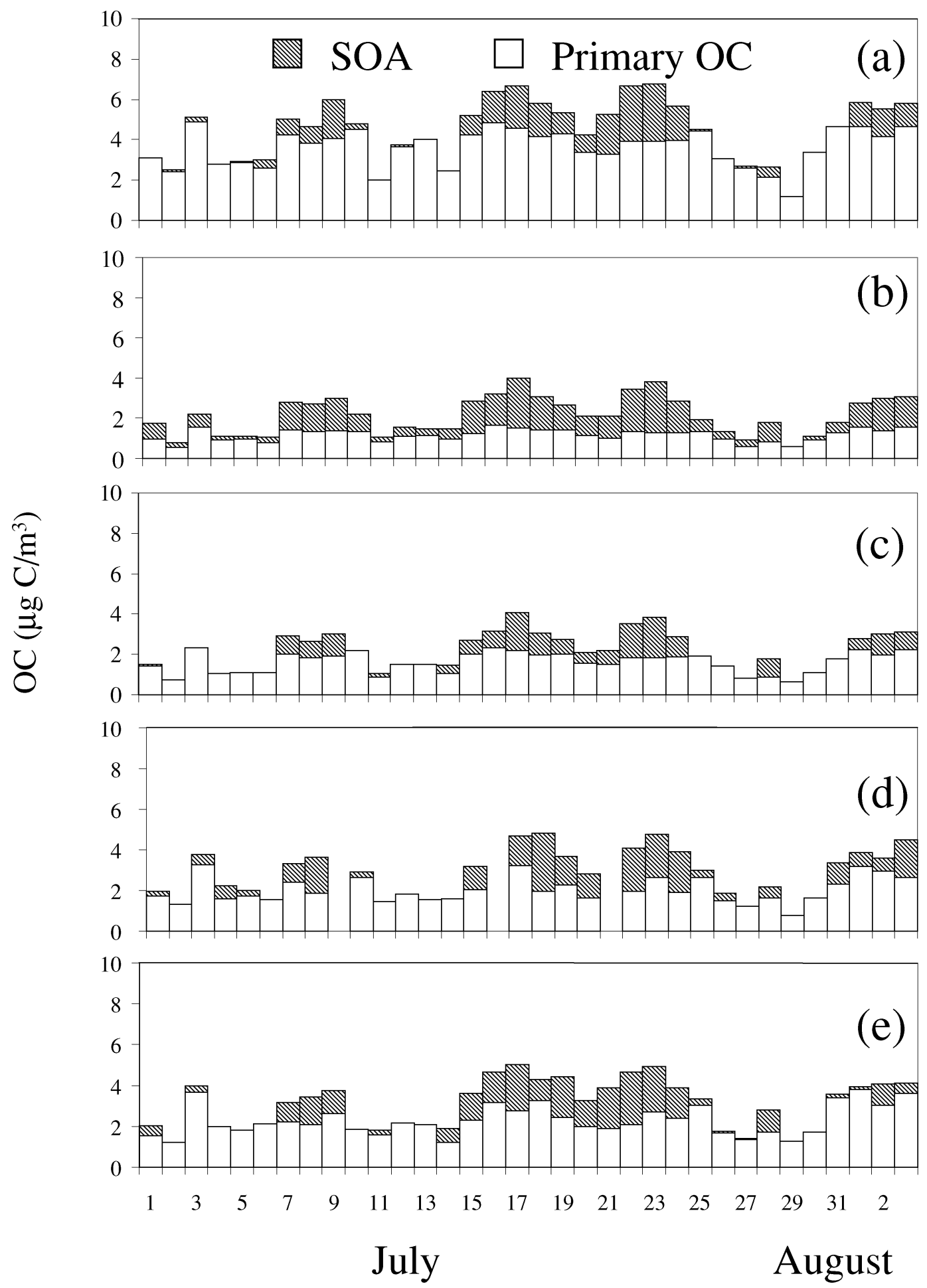

Figure 11. Daily-averaged SOA and primary OC concentrations during the summer intensive, estimated from the daily-averaged concentrations (24 h averages). (a) Undenuded sampler, $Q_{F}$. (b) Undenuded sampler, $Q_{F}-Q_{B, F}$. (c) Undenuded sampler, $Q_{F}-$ $\mathrm{Q}_{\mathrm{B}, \mathrm{T}}$. (d) Concentrations from the denuded in situ analyzer. (e) Concentrations from the denuder sampler. 


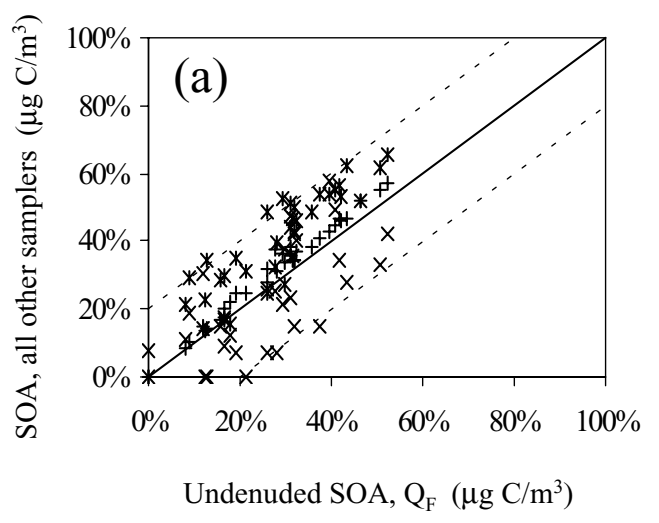

$+\%$ SOA, Undenuded Sampler $\left(\mathrm{Q}_{\mathrm{F}}-\mathrm{Q}_{\mathrm{B}, \mathrm{F}}\right)$
* \% SOA, Undenuded Sampler $\left(\mathrm{Q}_{\mathrm{F}}-\mathrm{Q}_{\mathrm{B}, \mathrm{T}}\right)$

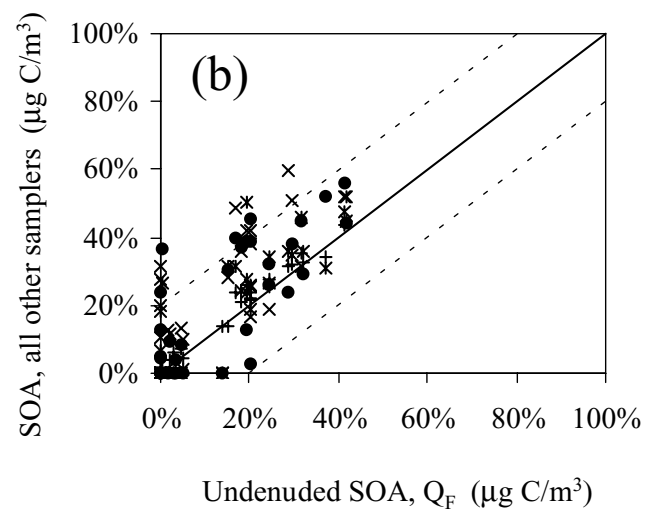

X $\%$ SOA, Denuded in-situ analyzer

- \% SOA, Denuded sampler

Figure 12. Correlation of daily percentage SOA contribution to the total OC for the different samplers for the summer intensive at PAQS for all datasets. Solid line represents the 1:1 fit and dashed lines are $20 \%$ error lines. (a) \% SOA contributions from high-resolution measurements. (b) \% SOA contributions from daily-averaged measurements.

(the whole month, or the few hours of the measurement period). Variations of sources strengths, meteorology, etc. are expected to change the $(\mathrm{OC} / \mathrm{EC})_{\mathrm{p}}$ even for the same 4-h period from day to day. This variability introduces significant uncertainties (see Tables 5 and 6 ).

\section{REFERENCES}

Birch, M. E., and Cary, R. A. (1996). Elemental Carbon-Based Method for Monitoring Occupational Exposures to Particulate Diesel Exhaust, Aerosol Sci. Technol. 25:221-241.

Birch, E. M. (1998). Analysis of Carbonaceous Aerosols: Interlaboratory Comparison, Analyst. 123:851-857.

Cabada, J. C., Pandis, S. N., Davidson, C. I., Robinson, A. L., Subramanian, R., Tang, W., and Raymond, T., (2002b). The Contribution of Long-Range Transport and Secondary Organic Aerosol to PM2.5 in Pittsburgh. In DOE-NETL $\mathrm{PM}_{2.5}$ and Electric Power Generation: Recent Findings and Implications. NETL Publications, Pittsburgh, PA.

Cabada, J. C., Pandis, S. N., and Robinson, A. L. (2002a). Sources of Atmospheric Carbonaceous Particulate Matter in Pittsburgh, Pennsylvania, J. Air Waste Manag. Assoc. 52:732-741.

Chow, J. C., Watson, J. G., Crow, D., Lowenthal, D. H., and Merrifield, T. (2001). Comparison of IMPROVE and NIOSH Carbon Measurements, Aerosol Sci. Technol. 34:23-34.

Countess, R. J. (1990). Interlaboratory Analyses of Carbonaceous Aerosol Samples, Aerosol Sci. Technol. 12:114-121.

Fitz, D. R. (1990). Reduction of the Positive Artifact in Quartz Filters, Aerosol Sci. Technol. 12:142-148.

Gray, H. A. (1986). Ph.D. Thesis, California Institute of Technology, Pasadena, CA.

Gray, H. A., Cass, G. R., Huntzicker, J. J., Heyerdahi, E. K., and Rau, J. A. (1986). Characteristics of Atmospheric Organic and Elemental Carbon Particle Concentrations in Los Angeles, Environ. Sci. Technol. 20:580-589.

Hering, S. V., Appel, B. R., Cheng, W., Salaymeh, F., Cadle, S. H., Mulawa, P. A., Cahill, T. A., Eldred, R. A., Surovik, M., Fitz, D., Howes, J. E., Knapp, K. T., Stockburger, L., Turpin, B. J., Huntzicker, J. J., Zhang, X. Q., and McMurry, P. H. (1990). Comparison of Sampling Methods for Carbonaceous Aerosol in Ambient Air, Aerosol Sci. Technol. 12:200-213.
Hildemann, L. M., Cass, G. R., Mazurek, M. A., and Simoneit, B. R. T. (1993). Mathematical Modeling of Urban Organic Aerosol-Properties Measured by High Resolution Chromatography, Environ. Sci. Technol. 27:2045-2055.

Lim, H. J., and Turpin, B. J. (2002). Origins of Primary and Secondary Organic Aerosol in Atlanta: Results of Time-Resolved Measurements During the Atlanta Supersite Experiment, Environ. Sci. Technol. 36:4489-4496.

NIOSH. (1999). Method 5040 Issue 3 (Interim): Elemental Carbon (diesel exhaust). In NIOSH Manual of Analytical Methods. 4th edition, Paula Fey O'Connor, ed., National Institute of Occupational Safety and Health, Cincinnati, $\mathrm{OH}$.

Pandis, S. N., Seinfeld, J. H., Harley, R., and Cass, G. (1992). Secondary Organic Aerosol Formation and Transport, Atmos. Environ. 26A:2269-2282.

Schauer, J. J., Rogge, W. F., Hildemann, L. M., Mazurek, M. A., Cass, G. R., and Simoneit, B. T. (1996). Source Apportionment of Airborne Particulate Matter Using Organic Compounds as Tracers, Atmos. Environ. 30:3837-3855.

Schmid, H., Laskus, L., Abraham, J. H., Baltensperger, U., Lavanchy, V., Bizjak, M., Burba, P., Cachier, H., Crow, D., Chow, J., Gnauk, T., Even, A., ten Brink, H. M., Giesen, K. P., Hitzenberger, R., Hueglin, C., Maenhaut, W., Pio, C., Carvalho, A., Putaud, J. P., and Toom-Sauntry D. (2001). Results of the "Carbon Conference" International Aerosol Carbon Round Robin Test Stage I, Atmos. Environ. 35:2111-2121.

Seinfeld, J. H., and Pandis, S. N. (1998) Atmospheric Chemistry and Physics: From Air Pollution to Global Change, John Wiley and Sons Inc., New York. Strader, R., Lurmann, F., and Pandis, S. (1999). Evaluation of Secondary Organic Aerosol Formation in Winter, Atmos. Environ. 33:4849-4863.

Subramanian, R., Khlystov, A., Cabada, J. C., and Robinson, A. L. (2004). Measurement of Positive and Negative Artifacts with Denuded and Undenuded Sampler Configurations, Aerosol Sci Technol. 38:27-48.

Tolocka, M. P., Solomon, P. A., Mitchel, W., Norris, G. A., Gemmill, D. B., Weiner, R. W., Vanderpool, R. W., Homolya, J. B., and Rice, J. (2001). East Versus West in the US: Chemical Characteristics of PM2.5 During the Winter of 1999, Aerosol Sci. Technol. 34:88-96.

Turpin, B. J., and Huntzicker, J. J. (1994). Investigation of Organic Aerosol Sampling Artifacts in the Los Angeles Basin, Atmos. Environ. 28:30613071.

Turpin, B. J., and Huntzicker, J. J. (1995). Identification of Secondary Organic Aerosol Episodes and Quantitation of Primary and Secondary Organic Aerosol Concentrations During SCAQS, Atmos. Environ. 29:35273544 . 
Turpin, B. J., Cary, R. A., and Huntzicker, J. J. (1990). An In Situ, Time Resolved Analyzer for Aerosol Organic and Elemental Carbon, Aerosol Sci. Technol. 12:161-171.

Turpin, B. J., Huntzicker, J. J., Larson, S. M., and Cass, G. R. (1991). Los Angeles Summer Midday Particulate Carbon: Primary and Secondary Aerosol, Environ. Sci. Technol. 25:1788-1793.
Turpin, B. J., Saxena P., and Andrews, E. (2000). Measuring and Simulating Particle Organics in the Atmosphere: Problems and Prospects. Atmos. Environ. 34:2983-3013.

Yu, J. Z., Xu, J., and Yang, H. (2002). Charring Characteristics of Atmospheric Organic Particulate Matter in Thermal Analysis, Environ. Sci.Technol. 36:754-761. 


\title{
Identification of sources of atmospheric PM at the Pittsburgh Supersite-Part II: Quantitative comparisons of single particle, particle number, and particle mass measurements
}

\author{
Keith J. Bein ${ }^{\mathrm{a}, *}$, Yongjing Zhao ${ }^{\mathrm{b}}$, Natalie J. Pekney ${ }^{\mathrm{c}}$, Cliff I. Davidson ${ }^{\mathrm{d}}$, \\ Murray V. Johnston ${ }^{\mathrm{e}}$, Anthony S. Wexler ${ }^{\mathrm{f}}$ \\ ${ }^{a}$ Department of Land, Air and Water Resources, University of California, Davis, CA, USA \\ ${ }^{\mathrm{b}}$ Department of Mechanical and Aeronautical Engineering, University of California, Davis, CA, USA \\ ${ }^{\mathrm{c}}$ Department of Civil and Environmental Engineering, Carnegie Mellon University, Pittsburgh, PA \\ ${ }^{\mathrm{d}}$ Departments of Civil and Environmental Engineering and Public Policy, Carnegie Mellon University, Pittsburgh, PA \\ ${ }^{\mathrm{e}}$ Department of Chemistry and Biochemistry, University of Delaware, Newark, DE \\ ${ }^{\mathrm{f}}$ Departments of Mechanical and Aeronautical Engineering, Civil and Environmental Engineering and Land, Air and Water Resources, \\ University of California, Davis, CA, USA
}

Received 30 July 2005; received in revised form 17 December 2005; accepted 5 January 2006

\begin{abstract}
A single particle mass spectrometer, RSMS-3, and a MOUDI were deployed during the Pittsburgh Air Quality Study (PAQS), July 2001-September 2002, to obtain size resolved measurements of elemental composition for particulate matter (PM) within the Pittsburgh area. Elemental mass distributions from analysis of the MOUDI stages were directly compared to those constructed using the single particle data, in conjunction with coincident SMPS measurements, for specific days within the PAQS.

Results from one episode on 27 October 2001 showed that approximately $80 \%$ of the metal containing particles detected on this day belonged to the $\mathrm{Na} / \mathrm{Si} / \mathrm{K} / \mathrm{Ca} / \mathrm{Fe} / \mathrm{Ga} / \mathrm{Pb}$ particle class. The density and shape factor of these particles were estimated to be $3.9 \pm 0.8 \mathrm{~g} / \mathrm{cc}$ and $1.5 \pm 0.2$, respectively, and the relative sensitivity factors for individual metals showed little variation with respect to particle diameter over the size range of $70-800 \mathrm{~nm}$.

Compared to the 27 October 2001 episode, there was a larger degree of variability in the metal containing particles detected during another episode on 14 March 2002. The $\mathrm{Ca}$ and $\mathrm{Pb}$ mass distributions from this day represent an ensemble of externally mixed particles. Estimates of particle density were provided for the dominant particle types, including $\mathrm{EC} / \mathrm{OC} / \mathrm{Ca}, \mathrm{Al} / \mathrm{Si} / \mathrm{Ca} / \mathrm{Fe}$, $\mathrm{EC} / \mathrm{OC} / \mathrm{Pb}$ and $\mathrm{Na} / \mathrm{K} / \mathrm{Zn} / \mathrm{Pb}$, and estimates of particle shape factor were provided for the $\mathrm{EC} / \mathrm{OC} / \mathrm{Ca}$ and $\mathrm{Na} / \mathrm{K} / \mathrm{Zn} / \mathrm{Pb}$ classes. Comparison with the 27 October $2001 \mathrm{Ca}$ and $\mathrm{Pb}$ mass distributions revealed that the RSMS data reconstructed the MOUDI mass much better from the $\mathrm{Ca} / \mathrm{Pb}$ containing particles detected on 14 March 2002 than those observed on 27 October 2001, suggesting that the single particle instrument sensitivity to both $\mathrm{Ca}$ and $\mathrm{Pb}$ depends on the particle matrix.
\end{abstract}

(C) 2006 Elsevier Ltd. All rights reserved.

Keywords: Single particle mass spectrometry; Elemental mass distribution; Composition-resolved number distribution; Particle density; Particle shape factor; Pittsburgh Supersite experiment

*Corresponding author. Tel.: + 15305702562 ; fax: + 15307544962.

E-mail address: kjbein@ucdavis.edu (K.J. Bein). 


\section{Nomenclature}

\section{Particle number and mass distributions}

$D_{i, \mathrm{RSMS}}$ vacuum aerodynamic diameter focused by orifice $i(\mathrm{~nm})$

$\sigma_{g} \quad$ geometric standard deviation of the transmission function for the RSMS inlet (independent of particle size)

$\dot{\forall}_{i} \quad$ volume flow rate through orifice $i\left(\mathrm{~m}^{3} / \mathrm{s}\right)$

$N_{i} \quad$ number of particles measured at orifice $i$

$\Delta t_{i} \quad$ length of time spent sampling from orifice $i$ (s) (see footnote 1)

$\left(\mathrm{d} N / \mathrm{d} \log D_{\mathrm{p}}\right)_{i, \mathrm{RSMS}}$ value of the number concentration distribution function measured at orifice $i$ by RSMS-3 (particles/ $\mathrm{cm}^{3}$ ) (see footnote 1)

$\left(\mathrm{d} N / \mathrm{d} \log D_{\mathrm{p}}\right)_{i \text { SMPS }}$ value of the number concentration distribution function measured by the SMPS for the mobility diameter most closely matching the vacuum aerodynamic diameter focused by orifice $i$ (particles $/ \mathrm{cm}^{3}$ ) (see footnote 1)

$\mathrm{DE}_{i}$ detection efficiency for orifice $i$ (see footnote 1)

$N_{i, g} \quad$ number of group $g$ particles measured at orifice $i$ (see footnote 1)

$\left(\mathrm{d} N / \mathrm{d} \log D_{\mathrm{p}}\right)_{i, q}$ value of the number concentration distribution function for group $g$ particles measured at orifice $i$ (particles/ $\mathrm{cm}^{3}$ ) (see footnote 1)

$\left(\mathrm{d} M / \mathrm{d} \log D_{\mathrm{p}}\right)_{i, q}$ value of the mass concentration distribution function for group $g$ particles measured at orifice $i\left(\mu \mathrm{g} / \mathrm{m}^{3}\right)$ (see footnote 1,2)

\section{Single particle mass fractions}

$\zeta_{n} \quad$ a particular molecule found within an individual particle

$M_{n} \quad$ molar mass of $\zeta_{n}(\mathrm{~g} / \mathrm{mol})$

$\gamma_{n} \quad$ sum of the integrated ion current over all peaks in the single particle mass spectrum due to $\zeta_{n}$

$\sigma_{k} \quad$ a particular element found within $\zeta_{n}$

$\alpha_{k} \quad$ stoichiometric coefficient of $\sigma_{k}$ in $\zeta_{n}$

$M_{k} \quad$ molar mass of $\sigma_{k}(\mathrm{~g} / \mathrm{mol})$

$\phi_{n, k} \quad$ fraction of single particle mass due to $\sigma_{k}$ as a result of $\zeta_{n}$ alone $\phi_{k} \quad$ fraction of single particle mass due to all $\sigma_{k}$-containing molecules

$\left(\mathrm{d} M / \mathrm{d} \log D_{\mathrm{p}}\right)_{i, k}$ value of the mass concentration distribution function for $\sigma_{k}$ due to all $\sigma_{k^{-}}$ containing particles measured at orifice $i$ $\left(\mu \mathrm{g} / \mathrm{m}^{3}\right)$ (see footnote 1,2$)$

\section{Particle diameter distributions}

$D_{\mathrm{m}} \quad$ electrical mobility diameter (nm)

$D_{\mathrm{a}} \quad$ aerodynamic diameter $(\mathrm{nm})$

$D_{\text {va }} \quad$ vacuum aerodynamic diameter $(\mathrm{nm})$

$D_{\text {ve }} \quad$ volume equivalent diameter $(\mathrm{nm})$

$D_{p} \quad$ true (geometric) diameter (nm)

$\rho \quad$ particle density in $\mathrm{g} / \mathrm{cc}$ (includes internal voids)

$\chi \quad$ dynamic shape factor

$C_{\mathrm{c}}(D)$ Cunningham slip correction factor evaluated using the particle diameter within the parentheses

\section{Relative sensitivity factors}

$\Delta m_{k, s} \quad$ mass of element $k$ measured on stage $s$ of the MOUDI (g)

$\Delta m_{j, s} \quad$ mass of element $j$ measured on stage $s$ of the MOUDI $(\mathrm{g})$

$D_{a 1, s} \quad$ lower diameter cut point of stage $s(\mathrm{~nm})$

$D_{a 2, s} \quad$ upper diameter cut point of stage $s(\mathrm{~nm})$

$\bar{D}_{a, s} \quad$ mean diameter of stage $s(\mathrm{~nm})$

$\left(\mathrm{d} M / \mathrm{d} \log D_{\mathrm{p}}\right)_{\mathrm{RSMS}, k}$ mass concentration distribution function for element $k$ measured by RSMS-3 $\left(\mu \mathrm{g} / \mathrm{m}^{3}\right)$ (see footnote 2 )

$\left(\mathrm{d} M / \mathrm{d} \log D_{\mathrm{p}}\right)_{\mathrm{RSMS}, j}$ mass concentration distribution function for element $j$ measured by RSMS-3 $\left(\mu \mathrm{g} / \mathrm{m}^{3}\right)$ (see footnote 2,3 )

$\mathrm{RSF}_{k, j}\left(\bar{D}_{a, s}\right)$ sensitivity of RSMS-3 to element $k$ ions, relative to element $j$ ions, for particles with diameter $\bar{D}_{a, s}$

\section{Particle volume fraction}

$X$ a particular metal found within an individual particle

$\Delta m_{X}$ mass of $X$ measured on the MOUDI stage corresponding to the peak in the dominant mode within the MOUDI distributions $(\mathrm{g})$

$M_{X} \quad$ molar mass of $X(\mathrm{~g} / \mathrm{mol})$

$\bar{\gamma}_{X^{+}} \quad$ integrated ion current due to $X^{+}$in each spectrum as averaged over all $X$-contain- 
ing particles detected at the orifice corresponding to the peak in the dominant mode within the RSMS distributions

$X_{n} O_{m}$ a particular metal oxide found within an individual particle ( $n$ metal atoms coordinated to $m$ oxygen atoms)

$M_{X_{n} O_{m}}$ molecular weight of $X_{n} O_{m}(\mathrm{~g} / \mathrm{mol})$
$\Delta m_{X_{n} O_{m}}$ mass of $X_{n} O_{m}$ measured on the MOUDI stage corresponding to the peak in the dominant mode within the MOUDI distributions $(\mathrm{g})$

$\rho_{X_{n} O_{m}} \quad$ bulk density of $X_{n} O_{m}\left(\mathrm{~g} / \mathrm{cm}^{3}\right)$

$\delta_{X_{n} O_{m}}$ Fraction of the volume of an individual particle that is occupied by $X_{n} O_{m}$

\section{Introduction}

In an ongoing effort to broaden our understanding of the effects of particulate air pollution, it is becoming increasingly important to provide more accurate and complete descriptions of ensembles of airborne particles. As a result, it is common in large field studies, like the EPA Supersite experiments, to include a wide variety of instruments that measure complementary attributes of the same particle population. The attributes of greatest interest are particle size, composition, number and mass, where it is common to report the latter two in terms of the distribution of these moments with respect to particle size. Numerous techniques are now available for obtaining these measurements and each has its own advantages and limitations.

Counting and sizing particles can be done very accurately over a broad range of particle diameters using the combination of a nano differential mobility analyzer (NDMA), long differential mobility analyzer (LDMA), condensation particle counter (CPC) and aerosol particle sizer (APS). Although these instruments provide highly time resolved measurements of particle number distribution, no information about particle composition is obtained. Conventional methods for determining particle composition are centered around collecting particulate matter (PM) on filters for subsequent chemical analysis using various techniques, including gas chromatography-mass spectrometry (GCMS) for organic tracers, Inductively coupled plasma-mass spectrometry (ICP-MS) for trace metals, elemental carbon/organic carbon (EC/OC) ovens for carbon, and ion chromatography (IC) for major ions. All of these chemical analysis techniques are considered quantitative and make it possible to determine the ambient mass concentration of a particular chemical species present in PM during sampling. Size distributed composition can be obtained using impactors like the micro-orifice uniform deposit impactor (MOUDI). There are two major limitations with these measurements: (1) a significant amount of time is required to collect sufficient mass for chemical analysis such that the temporal resolution of these measurements is low, typically $24 \mathrm{~h}$, and (2) it is not possible to determine which chemical species were originally present together in individual particles.

Single particle mass spectrometry represents a recent advancement in aerosol sampling technology and has considerable potential to bridge the gap between size-resolved number-based data and chemically speciated mass-based data by providing highly time resolved measurements of single particle size and composition. Instruments built upon this technique, like the third generation rapid singleultrafine-particle mass spectrometer (RSMS-3), have been deployed in a growing number of field studies and their success in these efforts has been well documented (Bein et al., 2005; Lake et al., 2003; Phares et al., 2003; Rhoads et al., 2003; Liu et al., 2003). Currently, a major limitation of these instruments is that they only measure a small sample of the ambient population such that the data must be scaled to obtain quantitative information. Since single particle instruments are number based, concurrent SMPS measurements are appropriate for scaling the data and the combination yields quantitative estimates of composition-resolved particle number distribution (Lake et al., 2003; Tolocka et al., 2004). The accuracy of these estimates depends primarily on the extent to which the sampled population correctly represents the true population. As a result, choosing events that minimize potential sampling biases is essential to these calculations. Furthermore, a direct comparison with chemically speciated mass-based data requires the ability to quantitatively determine the distribution of different chemical species within 
individual particles from their single particle mass spectra. Since little is known about the relative sensitivity of the single particle instrument to different species in a given particle, it is not clear how accurately the distribution of ion signal within the mass spectrum represents the original distribution of the corresponding species within the particle. Once again, choosing events that highlight the strengths of the single particle instrument is essential to this effort.

Due to these uncertainties, little effort has been made to quantify single particle data, especially using ambient data, and each dataset is typically analyzed and interpreted independently (Liu et al., 2000; Fergenson et al., 2001; Bhave et al., 2002; Wenzel et al., 2003). In the current work, a framework is developed for quantitatively comparing RSMS data to chemically speciated mass-based data. The framework is then applied to elemental mass distributions constructed from the RSMS output and ICP-MS bulk chemistry data. We show that the combination of the single particle, particle number and bulk chemistry results provide a more complete characterization of PM than can be obtained from any method alone. This analysis is performed for select days of ambient measurements collected during the Pittsburgh Air Quality Study (PAQS).

\section{Experimental}

The PAQS took place from July 2001 to September 2002 in Schenley Park, adjacent to Carnegie Mellon University. A general overview of this study, including the various instruments deployed, can be found in Wittig et al. (2004). RSMS3 , a laser ablation time-of-flight single particle mass spectrometer, was used to obtain high-temporalresolution measurements of single particle size (in terms of the vacuum aerodynamic diameter $D_{\text {va }}$ ) and composition, and was operated semi-continuously for 306 of the possible 372 operation days during this study. Details of the instrument and its operation, information about data processing and analysis, as well as general conclusions about the types of particles observed and their sources can be found in Bein et al. (2005). Select days were chosen and the single particle data from these days were combined with coincident measurements of particle number distribution, obtained using SMPSs (NDMA and LDMA), to construct elemental mass distributions for several metals commonly observed throughout the PAQS. For these same days, sizesegregated PM collected by a collocated MOUDI was analyzed for the same metals using ICP-MS. Details of the MOUDI/ICP-MS analysis can be found in Part I of this work (Pekney et al., 2005).

During the PAQS, RSMS-3 sampled on a semicontinual basis with sampling intervals starting every $3 \mathrm{~h}$ for the first 4 months (September-December, 2001) and every $2 \mathrm{~h}$ for the remainder of the study (January-September, 2002). Each sampling interval consisted of cycling through nine flowlimiting orifices, corresponding to nine different particle sizes transmitted into the instrument ranging from about $30 \mathrm{~nm}$ to $1.1 \mu \mathrm{m}$. The instrument was operated at each orifice until either $10 \mathrm{~min}$ expired or 30 particles were sampled, whichever came first. For each particle sampled, a positive and negative ion mass spectrum, as well as particle size and time of detection, were obtained.

\subsection{Data analysis}

For a given sampling interval, the value of the number concentration distribution function measured at each orifice may be written as

$\left(\frac{\mathrm{d} N}{\mathrm{~d} \log D_{\mathrm{p}}}\right)_{i, \text { RSMS }}=\frac{N_{i} / \Delta t_{i}}{\dot{\forall}_{i} \log \left(\sigma_{g}^{2}\right)}$,

where the subscript $i$ denotes the orifice number, ${ }^{1} N_{i}$ is the number of particles measured during time interval $\Delta t_{i}, \dot{\forall}_{i}$ is the volume flow rate through orifice $i, \sigma_{g}$ is the geometric standard deviation of the transmission function for the instrument inlet, which is considered independent of particle size for the diameter range measured (Phares et al., 2002), and $\log \left(\sigma_{g}^{2}\right)$ is the $\log$ width $\left(\Delta \log D_{\mathrm{p}}\right)$ of the transmission function between $15.9 \%$ and $84.1 \%$ of the size distribution.

Using collocated and coincident SMPS measurements, the detection efficiency of each orifice, $\mathrm{DE}_{i}$, may be defined as

$\mathrm{DE}_{i}=\frac{\left(\mathrm{d} N / \mathrm{d} \log D_{\mathrm{p}}\right)_{i, \mathrm{RSMS}}}{\left(\mathrm{d} N / \mathrm{d} \log D_{\mathrm{p}}\right)_{i, \mathrm{SMPS}}}$,

where $\left(\mathrm{d} N / \mathrm{d} \log D_{\mathrm{p}}\right)_{i, \mathrm{RSMS}}$ is calculated using Eq. (1) and $\left(\mathrm{d} N / \mathrm{d} \log D_{\mathrm{p}}\right)_{i, \mathrm{SMPS}}$ is the value of the number concentration distribution function measured by the SMPS for the electrical mobility diameter, $D_{\mathrm{m}}$, most closely matching the vacuum aerodynamic diameter focused by orifice $(i)$,

\footnotetext{
${ }^{1}$ Determined for each RSMS sampling interval.
} 


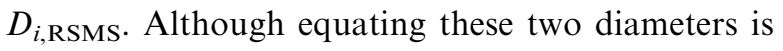
likely to be incorrect in many cases, as long as the features of the SMPS distribution can be matched with corresponding features in the RSMS distribution, the detection efficiencies resulting from Eq. (2) can be adjusted to account for this.

Sampling biases can significantly alter the accuracy of the detection efficiencies. Several studies have investigated these biases using both laboratory generated aerosol and ambient data (Allen et al., 2000; Kane and Johnston, 2000; Kane et al., 2001; Bhave et al., 2002; Wenzel et al., 2003). Although useful in providing selection criteria, the results of these studies depend strongly on the particles being considered and cannot be generalized to accommodate all situations. For ambient data, the specific events being considered must be directly investigated to determine the validity of using Eq. (2) and the accuracy of the resulting values. As a result, a thorough discussion of this will only be pursued within the context of the data being presented and will be addressed in a following section.

Single particle mass spectra are typically used to group particles based upon similarities in composition. Detection efficiencies are then applied to these groups of particles to obtain quantitative estimates of composition-resolved number distribution. For a group of particles, $N_{g}$, within the sampled population, the value of the number distribution function within that group for a given sampling interval and orifice may be written as

$\left(\frac{\mathrm{d} N}{\mathrm{~d} \log D_{\mathrm{p}}}\right)_{i, g}=\frac{N_{i, g} / \Delta t_{i}}{\dot{\forall}_{i} \log \left(\sigma_{g}^{2}\right) \mathrm{DE}_{i}}$,

where $N_{i, g}$ is the number of particles from group $g$ measured at orifice $i$ during time interval $\Delta t_{i}{ }^{2}$ and $\mathrm{DE}_{i}$ is calculated using Eq. (2).

Assuming a particle density $\rho$, Eq. (3) can be transformed into the mass distribution within the group, for the same sampling interval and orifice, as follows:

$$
\left(\frac{\mathrm{d} M}{\mathrm{~d} \log D_{\mathrm{p}}}\right)_{i, g}=\rho \frac{\pi}{6}\left(D_{i, \mathrm{RSMS}}\right)^{3}\left(\frac{\mathrm{d} N}{\mathrm{~d} \log D_{\mathrm{p}}}\right)_{i, g} .
$$

For consistency, $D_{\text {RSMS }}$ has been used in this equation to represent the calculation of particle volume. However, since ambient particles tend to be non-spherical with densities greater than $1.0 \mathrm{~g} / \mathrm{cc}$, and since particle volume varies as the cube of the

\footnotetext{
${ }^{2}$ Detection efficiency of orifice $i$ has been taken into consideration.
}

diameter, this is a potentially significant source of error. Estimates of the appropriate volume corrections for the data being presented will be given in the results section. Detailed compositional data from each single particle mass spectrum, in conjunction with Eq. (4), can be used to calculate elemental mass distributions.

In the current study, only the positive ion mass spectra were used for this effort since a large majority of the compositional information is in these spectra. Secondary components are avoided by focusing on small metal-containing particles $(\sim 50-300 \mathrm{~nm}$ in diameter) which can be attributed to local sources. For these particles, the transport time from source to receptor is short and the likelihood of atmospheric processing is greatly reduced. In addition, data obtained from a collocated, continuous $\mathrm{PM}_{2.5}$ sulfate monitor and TEOM were used to select events with low sulfate mass. For example, during the events being considered, the average fraction of $\mathrm{PM}_{2.5}$ which is sulfate is only $\sim 14-16 \%$.

It is important to note that the original molecular composition information is destroyed during the laser ablation process and atomic and fragment ions are predominantly seen in the mass spectra. The specific molecular structures assumed for the ions observed in the mass spectra of the particles considered in this study will be detailed in a following section.

First it is necessary to outline the mathematical framework for calculating elemental mass fractions from single particle mass spectra. Consider an individual particle composed of $N$ different types of molecules where each molecule, $\zeta_{n}$, contains $k$ elements, $\sigma_{k}$, with stoichiometric coefficients $\alpha_{k}$ and molecular weights $M_{k}$ such that the molecular weight of $\zeta_{n}$ may be written as

$M_{n}=\sum_{k=1}^{K} \alpha_{k} M_{k}$.

Now consider the mass spectrum of this particle within which a binned ion current for each $\mathrm{m} / \mathrm{z}$ value has been obtained by integrating $\pm 0.5 \mathrm{Da}$ about each integer $m / z$ value. Let $\gamma_{n}$ be the sum of the integrated ion current over all peaks in the spectrum that correspond to molecule $\zeta_{n}$. Note that atomic ions, fragment ions, cluster ions or any combination of the three can be produced from the ablation of $\zeta_{n}$. In the case of atomic and fragment ions, the integrated ion current represents the 
number of moles of $\zeta_{n}$ originally within the particle on a one to one basis. However, for cluster ions, the integrated ion current must be multiplied by the number of $\zeta_{n}$ molecules each cluster represents before it is included in $\gamma_{n}$.

For a particular element $\sigma_{k}$ found within molecule $\zeta_{n}$, the fraction of the particle mass that is due to $\sigma_{k}$ as a result of $\zeta_{n}$ alone, $\phi_{n, k}$, may be written as

$\phi_{n, k}=\frac{\alpha_{k} M_{k} \gamma_{n}}{\sum_{n=1}^{N} \gamma_{n} M_{n}}$.

If $\sigma_{k}$ is found in more than one type of molecule within the particle, then $\phi_{n, k}$ must be calculated for each type of molecule and then summed over all $\sigma_{k}$ containing molecules to obtain $\phi_{k}$, which is the fraction of the total particle mass due to $\sigma_{k}$. Furthermore, if $\sigma_{k}$ has isotopes, then the $\zeta_{n}$ that results from each isotope should be considered a different type of molecule with its own $\gamma_{n}, M_{n}$ and $\phi_{n, k}$, and should be included as such in the sum that determines $\phi_{k}$. The constant of proportionality between the number of moles of a particular ion and the integrated ion current in the mass spectrum is assumed to be the same for all ions and particle sizes, as well as independent of the presence of other species in the particle. Based on results from previous studies (Mansoori et al., 1994; Ge et al., 1998; Gross et al., 2000; Woods et al., 2001; Bhave et al., 2002), which show that the instrument sensitivities associated with single particle mass spectrometers vary significantly for different chemical species within a particle due to differences in ionization efficiencies, this is considered a potentially significant source of error in the calculations that follow. During the course of the current work, we were able to estimate the size-dependent relative sensitivity factors for several different elements in a given particle matrix. In addition, evidence that the sensitivity of the instrument to a particular element depends on the other species in the particle, or the particle matrix, was also observed. Both of these topics will be addressed in more detail in the results section.

The next step in this process is to isolate all single particle spectra within the data set that contain the element of interest, $\sigma_{k}$. Eq. (4) may be applied to this group of spectra, assuming a particle density of $1.0 \mathrm{~g} / \mathrm{cc}$, to determine the value of the mass distribution function within that group for a given sampling interval and orifice. This value is used only as a convenient starting point.
On a particle by particle basis, Eq. (6) may be used to calculate $\phi_{k}$ for each spectrum within the group. Summing $\phi_{k}$ over all orifice $i$ particles in the group, $N_{i, g}$, the fraction of the total mass measured at orifice $i$ during the given sampling interval due to element $\sigma_{k}, \phi_{i, k}$, is obtained. The value of the elemental mass distribution for the same sampling interval and orifice may now be written as

$\left(\frac{\mathrm{d} M}{\mathrm{~d} \log D_{\mathrm{p}}}\right)_{i, k}=\phi_{i, k}\left(\frac{\mathrm{d} M}{\mathrm{~d} \log D_{\mathrm{p}}}\right)_{i, g}$.

However, since the goal of this work is a direct comparison between ambient RSMS and MOUDI/ ICP-MS data and the sampling interval of the MOUDI is $24 \mathrm{~h}$, the method described above was slightly modified to account for this. The products $\left(\phi_{i, k} N_{i, g} / \mathrm{DE}_{i}\right)$ and $\left(\dot{\forall}_{i} \Delta t_{i}\right)$ were individually summed over all sampling intervals within a given $24 \mathrm{~h}$ period (8-12 intervals depending on the sampling protocol) and the ratio of the resulting values was multiplied by $\left(\rho(\pi / 6)\left(\left(D_{\mathrm{RSMS}, i}\right)^{3} / \log \left(\sigma_{g}^{2}\right)\right)\right.$ to obtain the $24 \mathrm{~h}$ value of $\left(\mathrm{d} M / \mathrm{d} \log D_{\mathrm{p}}\right)_{i, k}$. This was done for each orifice and then the points were fitted with the following lognormal distribution function:

$M\left(D_{\mathrm{p}}\right)=M_{0}+\sum_{j=1}^{n} M_{j} \exp \left[-\left(\frac{\ln \left(D_{\mathrm{p}} /\left(\bar{D}_{\mathrm{p} g}\right)_{j}\right)}{\sqrt{2} \ln \left(\left(\sigma_{g}\right)_{j}\right)}\right)^{2}\right]$,

where $n$ is the number of modes in the distribution, the subscript $j$ denotes the mode number, $M_{j}$ is the amplitude of mode $j,\left(\bar{D}_{\mathrm{p} g}\right)_{j}$ is the geometric mean diameter and $\left(\sigma_{g}\right)_{j}$ is the geometric standard deviation. The Levenberg-Marquardt algorithm was used to identify coefficient values. The resulting curve fits are included in Figs. 1-4 and 10-11, as well as values for $\left(\bar{D}_{\mathrm{p}}\right)_{j},\left(\sigma_{g}\right)_{j}$ and $24 \mathrm{~h}$ elemental $\mathrm{PM}_{1.2}$, as determined by integrating under the curves.

\section{Results and discussion}

Graphical comparison of the RSMS and MOUDI mass distributions are presented only for those situations in which both RSMS and MOUDI detection criteria are met; particles containing the element must have a significant number of hits on that day and the element mass measured on the MOUDI filters must be above detection on at least one of the stages. In addition, the airborne concentrations as determined by the MOUDI must 
be within $35 \%$ of the airborne concentrations measured by a collocated high volume sampler (hivol). Details of sample collection, preparation, and analysis for the hi-vol samples are given by Pekney and Davidson (2005). For Figs. 1-4 and 10-11, the MOUDI filter stages with measured mass below detection limits (BDL) are shown as hatched bars that give an upper limit to the element mass concentration. Mass distributions for calcium, iron, gallium and lead from the 27 October 2001 sampling date (Figs. 1-4), and calcium and lead mass distributions from the 14 March 2002 sampling date (Figs. 10-11) have been included for comparison.

\subsection{October 2001 data}

Approximately $80 \%$ of the metal containing particles detected on 27 October 2001 were composed of $\mathrm{Na}, \mathrm{Si}, \mathrm{K}, \mathrm{Ca}, \mathrm{Fe}, \mathrm{Ga}$, and $\mathrm{Pb}$. This single particle composition represents a subclass of the $\mathrm{Si} / \mathrm{K} / \mathrm{Fe} / \mathrm{Ga}$ class, which was detected frequently throughout the duration of the PAQS (Bein et al., 2005). In Part I of this work evidence is presented connecting this class of particles to coal combustion sources in Pittsburgh and surrounding area (Pekney et al., 2005). As a result, it was assumed that these metals were originally in the form of metal oxides within the particle for the purposes of calculating elemental mass fractions $\left(\chi_{i, k}\right)$. The oxidation states used and corresponding metal oxides are as follows: $\mathrm{Na}(\mathrm{I}): \mathrm{Na}_{2} \mathrm{O} ; \mathrm{Si}(\mathrm{IV}): \mathrm{SiO}_{2} ; \mathrm{K}(\mathrm{I}): \mathrm{K}_{2} \mathrm{O} ; \mathrm{Ca}(\mathrm{II}): \mathrm{CaO}$; Fe(III): $\mathrm{Fe}_{2} \mathrm{O}_{3} ; \mathrm{Ga}(\mathrm{III}): \mathrm{Ga}_{2} \mathrm{O}_{3} ; \mathrm{Pb}(\mathrm{IV}): \mathrm{PbO}_{2}$ (Wigley and Williamson, 1998; Font et al., 2005).

Of the remaining metal containing particles detected on this day, $\sim 95 \%$ of them belong to the $\mathrm{Na} / \mathrm{K} / \mathrm{Zn} / \mathrm{Pb}$ class, which was also detected consistently throughout the Pittsburgh Supersite experiment. For these types of particles, both chlorine and oxygen are frequently observed in the mass spectra such that the possibility the metals in these particles existed as chloride salts and/or oxides must be addressed. In the current study, $\mathrm{NaCl} / \mathrm{KCl} /$ $\mathrm{ZnCl}_{2} / \mathrm{PbO}_{2}$ was chosen as the molecular makeup because it minimizes the error between the values of $\phi_{i, k}$ obtained using this combination versus any other combination of chlorides and oxides. Assuming that $\gamma_{\mathrm{Na}} \approx \gamma_{\mathrm{K}} \approx \gamma_{\mathrm{Zn}} \approx \gamma_{\mathrm{Pb}}$, which is approximately true in most observed particles, it can be shown that this error is less than $7 \%$.

The clean separation of a large group of metals between two distinct particle classes $(\mathrm{Na} / \mathrm{Si} / \mathrm{K} / \mathrm{Ca} /$

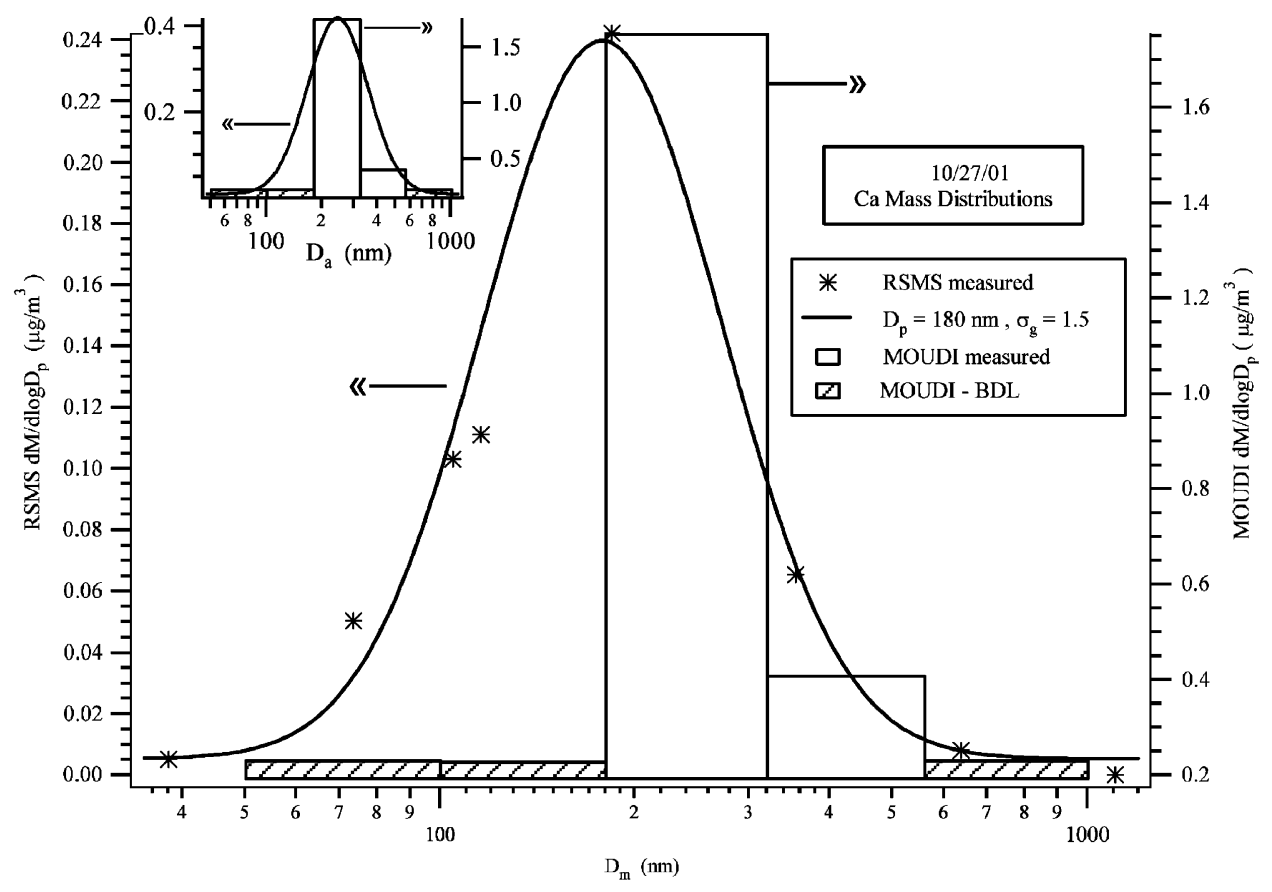

Fig. 1. Airborne concentration on 27 October 2001 was $0.046 \mu \mathrm{g} / \mathrm{m}^{3} \mathrm{PM}_{3.2} \mathrm{Ca}$ as measured by the MOUDI and $0.050 \mu \mathrm{g} / \mathrm{m}^{3} \mathrm{PM}{ }_{2.5} \mathrm{Ca}$ as measured by the hi vol. The mass distribution scale for the RSMS data is on the left $y$-axis and the MOUDI mass distribution scale is on the right $y$-axis. 


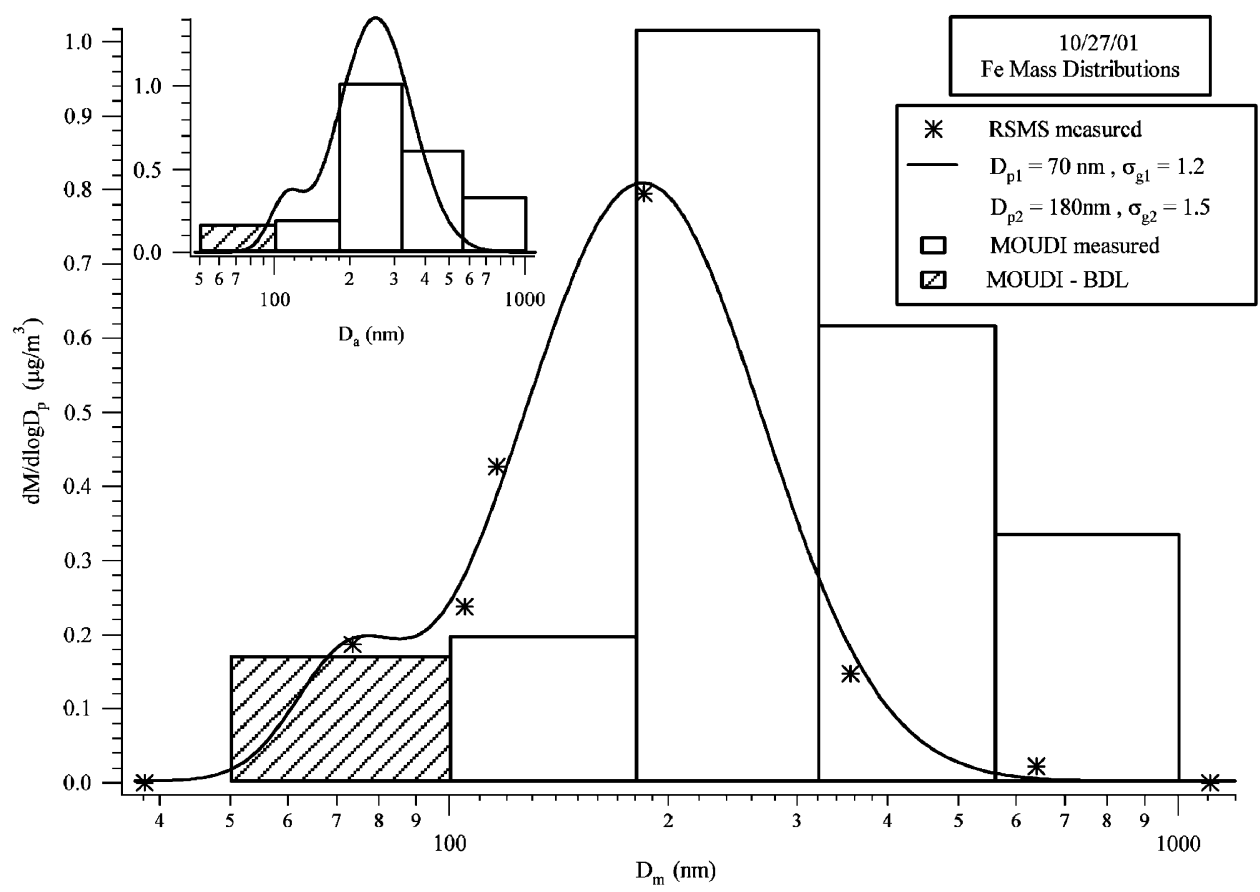

Fig. 2. Airborne concentration on 27 October 2001 was $0.056 \mu \mathrm{g} / \mathrm{m}^{3} \mathrm{PM}_{3.2} \mathrm{Fe}$ as measured by the MOUDI and $0.088 \mu \mathrm{g} / \mathrm{m}^{3} \mathrm{PM}{ }_{2.5} \mathrm{Fe}$ as measured by the hi vol.

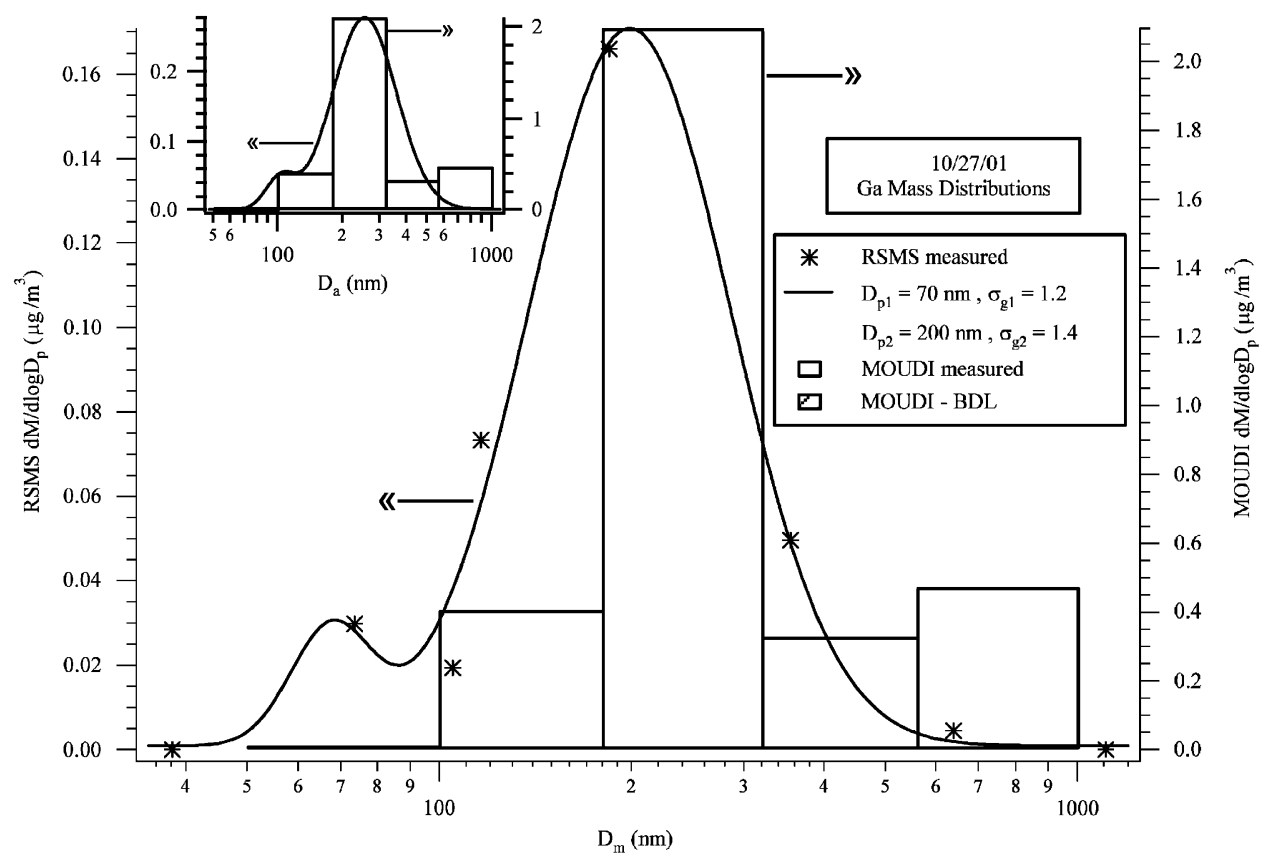

Fig. 3. Airborne concentration on 27 October 2001 was $0.0029 \mu \mathrm{g} / \mathrm{m}^{3} \mathrm{PM}_{3.2} \mathrm{Ga}$ as measured by the MOUDI and $0.0026 \mu \mathrm{g} / \mathrm{m}^{3} \mathrm{PM}{ }_{2.5} \mathrm{Ga}$ as measured by the hi vol. The mass distribution scale for the RSMS data is on the left $y$-axis and the MOUDI mass distribution scale is on the right $y$-axis. 


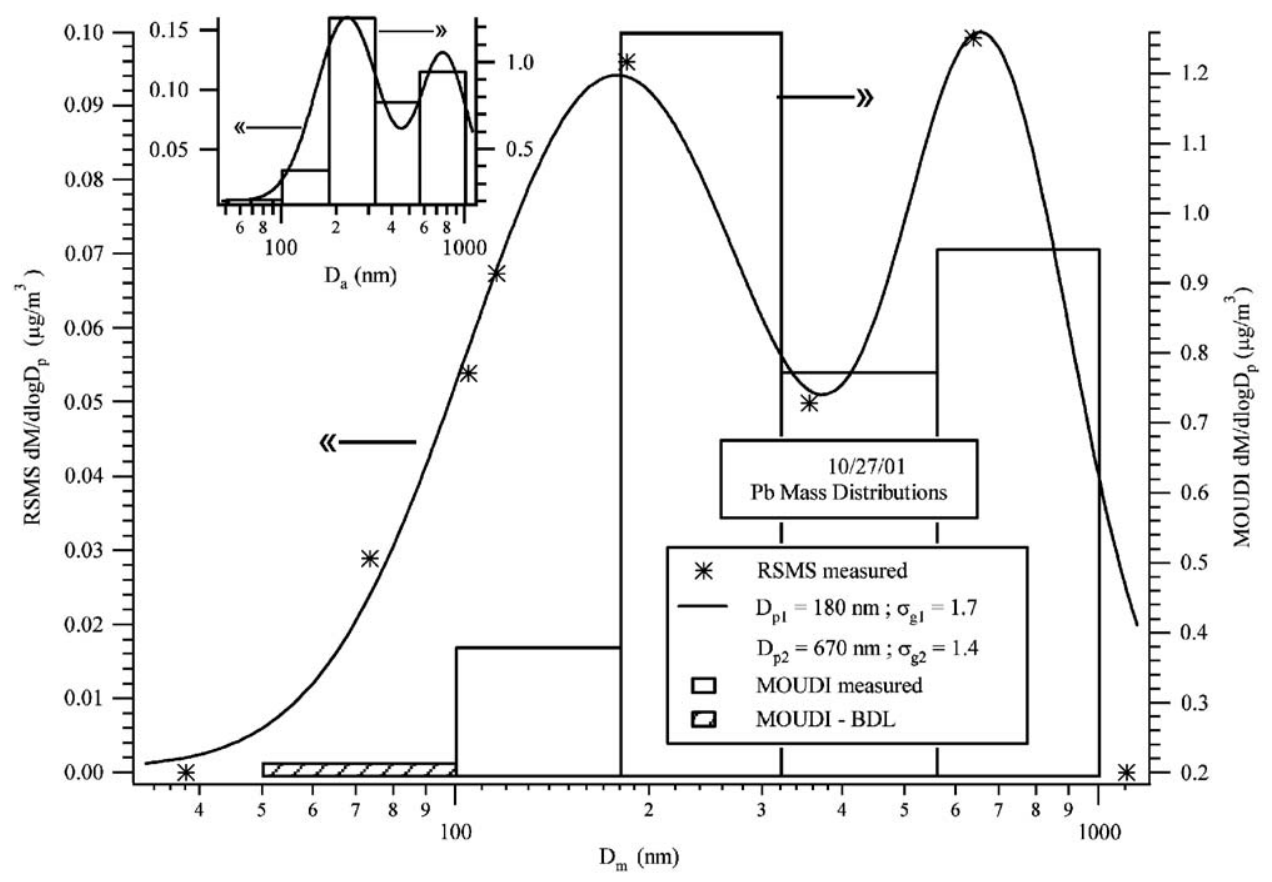

Fig. 4. Airborne concentration on 27 October 2001 was $0.0056 \mu \mathrm{g} / \mathrm{m}^{3} \mathrm{PM}_{3.2} \mathrm{~Pb}$ as measured by the MOUDI and $0.0044 \mu \mathrm{g} / \mathrm{m}^{3} \mathrm{PM}{ }_{2.5} \mathrm{~Pb}$ as measured by the hi vol. The mass distribution scale for the RSMS data is on the left $y$-axis and the MOUDI mass distribution scale is on the right $y$-axis.

$\mathrm{Fe} / \mathrm{Ga} / \mathrm{Pb}$ and $\mathrm{Na} / \mathrm{K} / \mathrm{Zn} / \mathrm{Pb}$ ) that account for $99 \%$ of the metal containing particles detected on a particular day is a rare situation, but allows for a direct investigation of the sensitivity of the single particle instrument to those metals in the given matrix. This can be seen explicitly in Figs. 1-4 by noticing the similarity in the shape of the mass distributions from metal to metal in both the RSMS and MOUDI data. All of the distributions have a dominant fine mode centered at $\sim 200 \mathrm{~nm}$ which can be attributed solely to the $\mathrm{Na} / \mathrm{Si} / \mathrm{K} / \mathrm{Ca} / \mathrm{Fe} / \mathrm{Ga} / \mathrm{Pb}$ particle class. The coarse mode in the RSMS lead distribution is a result of the $\mathrm{Na} / \mathrm{K} / \mathrm{Zn} / \mathrm{Pb}$ class only and allows for lead to be included in this analysis because the distinction between the two particle classes is clear within the distribution. The MOUDI lead distribution is in excellent agreement showing the same two modes and thus the same separation between the two particle classes.

\subsubsection{Comparison of RSMS, SMPS and MOUDI} size data: differences in size distribution peaks

Let us now reconcile the size distributions of RSMS, SMPS, and MOUDI data. On 27 October 2001 , the observation of $\mathrm{Na} / \mathrm{Si} / \mathrm{K} / \mathrm{Ca} / \mathrm{Fe} / \mathrm{Ga} / \mathrm{Pb}$ particles was an isolated event, occurring during three consecutive sampling intervals only. Fig. 5a shows the RSMS number distribution, as determined using Eq. (1), the SMPS number distribution and the number distribution of $\mathrm{Na} / \mathrm{Si} / \mathrm{K} / \mathrm{Ca} / \mathrm{Fe} / \mathrm{Ga} /$ $\mathrm{Pb}$ particles, as determined using Eq. (3), for the first of the three sampling intervals. Results from the other two sampling intervals are almost identical to those shown. There are several things to note in this figure.

First, there is excellent agreement between the features of the SMPS and RSMS distributions, i.e. both are bimodal with a dominant ultrafine mode and smaller fine mode, but the distributions are shifted. The shift is a direct result of plotting the distributions with respect to a common particle diameter $\left(D_{\text {va }}\right)$. To understand this, consider an ensemble of ambient particles composed of $n$ independent particle types, each with its own density, shape factor, and size distribution. The vacuum aerodynamic and electrical mobility diameters of each particle type are related to each other through the particle density $\rho$ and shape factor $\chi$ as follows:

$\frac{D_{\mathrm{va}}}{D_{\mathrm{m}}}=\frac{\rho}{\chi^{2} C_{\mathrm{c}}\left(D_{\mathrm{m}}\right)} C_{\mathrm{c}}\left[\frac{D_{\mathrm{m}}}{\chi} \frac{C_{\mathrm{c}}\left(D_{\mathrm{m}} / \chi\right)}{C_{\mathrm{c}}\left(D_{\mathrm{m}}\right)}\right]$, 


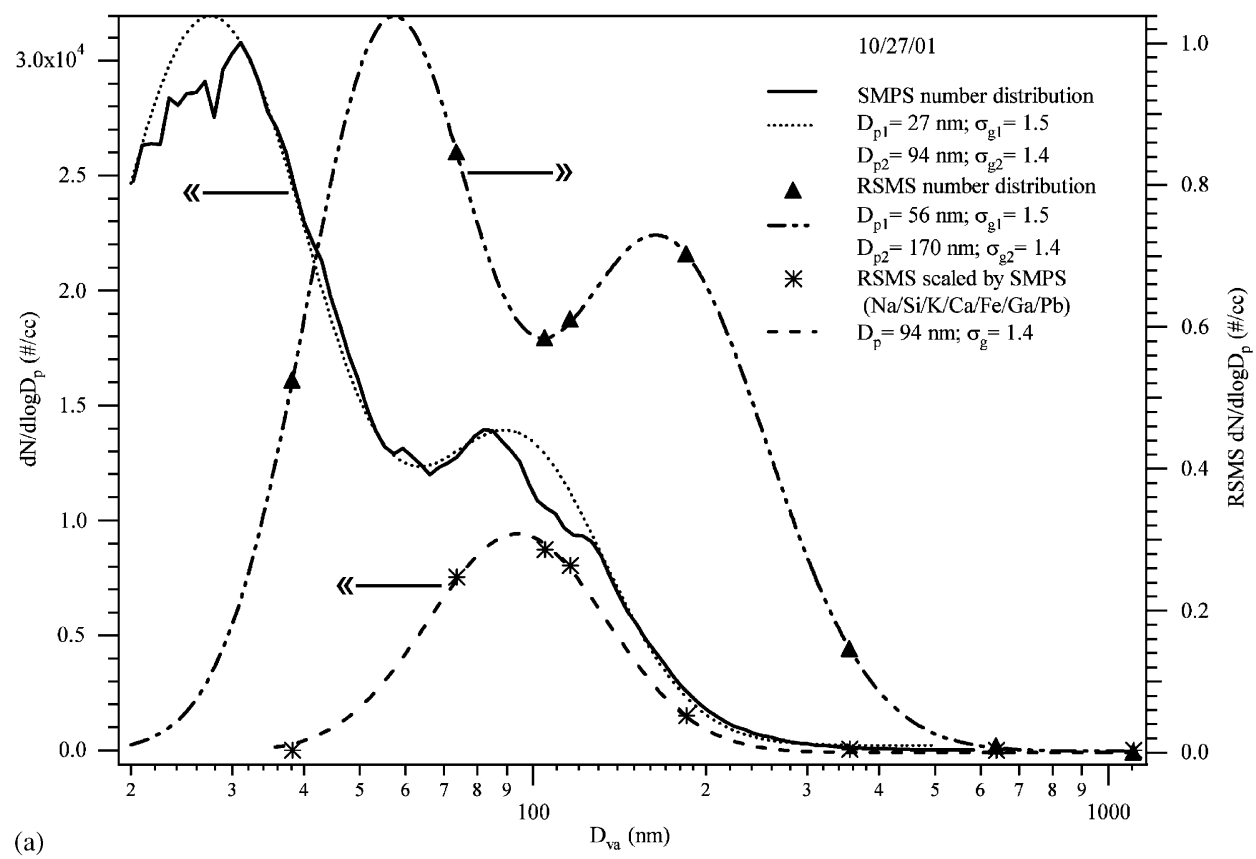

(a)

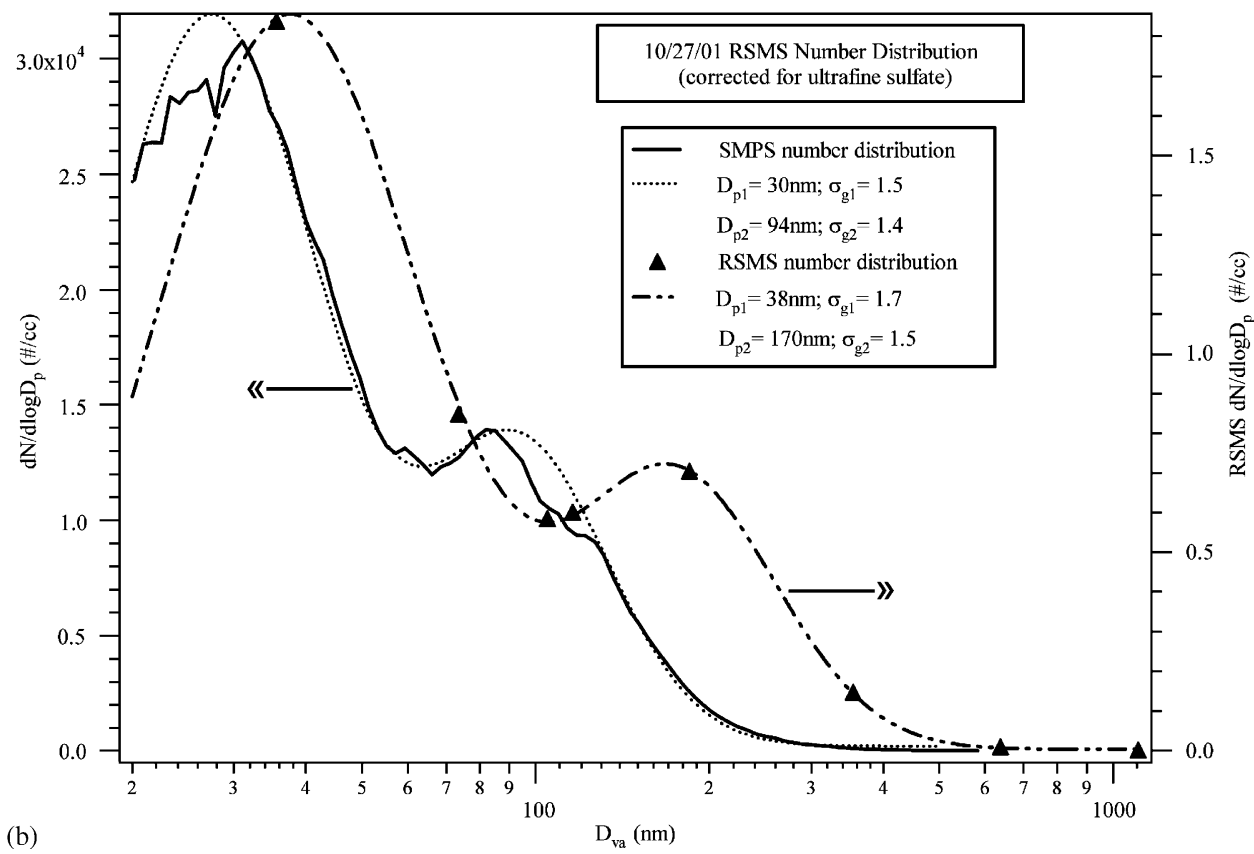

Fig. 5. (a) The RSMS and SMPS number distributions and the scaled number distribution of $\mathrm{Na} / \mathrm{Si} / \mathrm{K} / \mathrm{Ca} / \mathrm{Fe} / \mathrm{Ga} / \mathrm{Pb}$ particles for the first of three sampling intervals during which these particles were detected. (b) The RSMS number distribution for the same sampling interval after adjusting the hit rate of the orifice corresponding to the lower size limit of the instrument for an undetected particle type. The SMPS and $\mathrm{Na} / \mathrm{Si} / \mathrm{K} / \mathrm{Ca} / \mathrm{Fe} / \mathrm{Ga} / \mathrm{Pb}$ distributions are plotted with respect to the left $y$-axis and the RSMS distribution is plotted with respect to the right $y$-axis.

where $C_{\mathrm{c}}(D)$ is the Cunningham slip correction factor calculated using the diameter, or expression, within the parentheses and the dependence of $\chi$ on the flow regime, or Knudsen number, has been neglected (Jimenez et al., 2003; DeCarlo et al., 2004). Since the total size distribution is a superposition of the independent, type-specific distributions, the general agreement between the features of 
the RSMS and SMPS distributions (\# of modes and mean diameter and standard deviation of each mode) depends strongly on the observed particle types. For the 27 October 2001 data, a large majority of the particles detected during the sampling intervals being considered were $\mathrm{Na} / \mathrm{Si} / \mathrm{K} /$ $\mathrm{Ca} / \mathrm{Fe} / \mathrm{Ga} / \mathrm{Pb}$ particles. For example, at the orifices within the $50-400 \mathrm{~nm}$ size range, they represent as much as $70-90 \%$ of the total number of particles detected. As a result, calculating the detection efficiencies for these orifices, as in Eq. (2), and then applying the detection efficiencies to this group of particles, as in Eq. (3), essentially returns the corresponding section of the SMPS distribution. This can be seen explicitly in Fig. 5a by looking at the scaled distribution of $\mathrm{Na} / \mathrm{Si} / \mathrm{K} / \mathrm{Ca} / \mathrm{Fe} / \mathrm{Ga} / \mathrm{Pb}$ particles and noting that the scaling has simply collapsed the RSMS fine mode onto the SMPS fine mode such that the shift between these modes can be explained by the physical properties of the particles, as described in Eq. (9).

Secondly, the apparent agreement between the ultrafine modes in this figure is misleading. The hit rate falls off by an order of magnitude between the lower size limit of RSMS ( $\sim 40 \mathrm{~nm})$ and that of the adjacent orifice. This suggests that the particles represented by the ultrafine mode in the SMPS distribution are not being detected by the single particle instrument. Similar size-dependent hit rates were observed by Wenzel et al. (2003). In that study, the decrease in hit rate was correlated with a missing particle type composed primarily of ammonium sulfate that was undetected by the single particle instrument. This represents a composition-based sampling bias. Similar biases have been observed in laboratory studies investigating particles of comparable compositional simplicity; i.e. relatively pure single component and very simple multi-component laboratory generated aerosol (Kane and Johnston, 2000; Kane et al., 2001). Calculating the detection efficiency for this orifice using Eq. (2) would represent a significant source of error if the missing particle type is not accounted for. In the current work, this issue is avoided as the number of $\mathrm{Na} / \mathrm{Si}$ / $\mathrm{K} / \mathrm{Ca} / \mathrm{Fe} / \mathrm{Ga} / \mathrm{Pb}$ particles goes to zero at the smallest sizes and the corresponding detection efficiencies are not necessary. However, a first-order approximate solution to this problem is to use the hit rate of the adjacent orifice to adjust the RSMS data at the smallest particle size. The adjusted distribution is shown in Fig. 5b. Note the improved agreement between the features of the RSMS and
SMPS distributions. The shift between the ultrafine modes can be explained in a similar fashion as the fine modes and Eq. (9) describes the physical properties of the missing particle type. If the missing particle type is assumed to represent relative pure ammonium sulfate particles, similar to Wenzel et al. (2003), then using a particle density of $\sim 1.8 \mathrm{~g} / \mathrm{cc}$ in Eq. (9) yields a particle shape factor of $\sim 1.1$. Using these values to convert to mass, the integrated ultrafine mode ammonium sulfate mass concentration is $1.2 \mu \mathrm{g} / \mathrm{m}^{3}$ which is in close agreement with the continuous $\mathrm{PM}_{2.5}$ sulfate monitor reading of $\sim 1.1 \mu \mathrm{g} / \mathrm{m}^{3}\left(\sim 1.5 \mu \mathrm{g} / \mathrm{m}^{3} \mathrm{PM}_{2.5}\right.$ ammonium sulfate).

Finally, it is clear in Fig. 5 that equating the electrical mobility and vacuum aerodynamic diameters when calculating detection efficiencies, as described for Eq. (2), can result in significant errors since the RSMS and SMPS distributions are based on different physical size measures. In the case that the features of the distributions can be matched, for example the fine modes in Fig. 5a, the error is clear and the detection efficiencies can be adjusted. If the features cannot be matched, then the error is not clear and nothing can be said about the accuracy of the resulting detection efficiencies. In this specific example, however, the adjustment is unnecessary because for the size range within which the $\mathrm{Na} / \mathrm{Si}$ / $\mathrm{K} / \mathrm{Ca} / \mathrm{Fe} / \mathrm{Ga} / \mathrm{Pb}$ particles exist $(\sim 60-200 \mathrm{~nm})$, their number distribution essentially is the SMPS distribution. Adjusting the detection efficiencies has an insignificant impact on the amplitude or width of the distribution and simply shifts the scaled distribution from the SMPS fine mode back to the RSMS fine mode.

With this in mind, the shift between the peaks in the RSMS and MOUDI distributions can be understood by considering the relationship between the aerodynamic diameter $\left(D_{\mathrm{a}}\right)$, as measured by the MOUDI, and the electrical mobility diameter. This relationship may be written as

$$
\begin{aligned}
\frac{D_{\mathrm{a}}}{D_{\mathrm{m}}}= & \sqrt{\frac{\rho}{\chi^{3} C_{\mathrm{c}}\left(D_{\mathrm{a}}\right)\left[C_{\mathrm{c}}\left(D_{\mathrm{m}}\right)\right]^{2}}} \\
& \times\left[C_{\mathrm{c}}\left[\sqrt{\frac{\chi}{\rho} \frac{C_{\mathrm{c}}\left(D_{\mathrm{a}}\right)}{C_{\mathrm{c}}\left(\sqrt{\chi / \rho} D_{\mathrm{a}}\right)}} D_{\mathrm{a}}\right]\right]^{3 / 2} .
\end{aligned}
$$

Since the dominant fine mode in Figs. 1-4 is due solely to one class of particles, Eq. (10) can be used to convert between electrical mobility and aerodynamic diameter if the density and shape factor of the particles are known. Although the application of 
Eq. (9) to the fine modes in Fig. 5a yields information about the combined effect of particle density and shape factor, as discussed above, it cannot be used to directly estimate either variable without knowledge of the other. In the following section, an alternative method for determining particle density, based upon particle composition considerations, is presented.

\subsubsection{Particle characteristics}

Considering that the dominant fine mode in each of the MOUDI distributions (Figs. 1-4) represents only one class of particles and that each particle within that class contains all four metals, the ratio of the mass collected on the corresponding MOUDI stage for one metal, $\Delta m_{X}$, to that of another represents the relative amounts of these metals in each particle as averaged over all particles within the class that were collected on that stage. As a result, the fraction of the particle volume that is occupied by the metal oxide of each element, $\delta_{X_{n} O_{m}}$, can be calculated. This calculation requires values of $\Delta m_{X}$ in conjunction with the single particle mass spectra collected from the orifice that corresponds to the same dominant fine mode, and literature

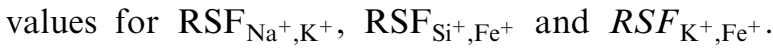
For particles in the $\mathrm{Na} / \mathrm{Si} / \mathrm{K} / \mathrm{Ca} / \mathrm{Fe} / \mathrm{Ga} / \mathrm{Pb}$ class, $\delta_{X_{n} O_{m}}$ may be written as

$\delta_{X_{n} O_{m}}=\frac{(\Delta m / \rho)_{X_{n} O_{m}}}{\sum_{X=\mathrm{Na}}^{X=\mathrm{Pb}}(\Delta m / \rho)_{X_{n} O_{m}}}$,

where the sum in the denominator is over all metals in the particle. In the case of metals for which MOUDI data are available $(\mathrm{Ca}, \mathrm{Fe}, \mathrm{Ga}$ and $\mathrm{Pb})$, the ratio $(\Delta m / \rho)_{X_{n} O_{m}}$ may be determined using

$\left(\frac{\Delta m}{\rho}\right)_{X_{n} O_{m}}=\frac{\Delta m_{X} M_{X_{n} O_{m}}}{n \rho_{X_{n} O_{m}} M_{X}}$,

where $M_{X_{n} O_{m}}$ is the molecular weight of metal oxide $X_{n} O_{m}, n$ is the number of atoms of metal $X$ in each molecule of $X_{n} O_{m}, \rho_{X_{n} O_{m}}$ is the bulk density of $X_{n} O_{m}$ and $M_{X}$ is the molecular weight of $X$. The single particle data, reported values for the relative sensitivity factors listed above, and the value of $\Delta m_{\mathrm{Fe}}$ can be used to calculate $(\Delta m / \rho)_{X_{n} O_{m}}$ for sodium, silicon and potassium, since no MOUDI data are available. For example, the value of this ratio for $\mathrm{K}_{2} \mathrm{O}$ can be calculated as

$\left(\frac{\Delta m}{\rho}\right)_{\mathrm{K}_{2} \mathrm{O}}=\frac{\Delta m_{\mathrm{Fe}} M_{\mathrm{K}_{2} \mathrm{O}}}{2 \rho_{\mathrm{K}_{2} \mathrm{O}} \mathrm{RSF}_{\mathrm{K}^{+}, \mathrm{Fe}^{+}} M_{\mathrm{Fe}}} \frac{\bar{\gamma}_{\mathrm{K}^{+}}}{\bar{\gamma}_{\mathrm{Fe}^{+}}}$, where $\bar{\gamma}_{X^{+}}$is the value of the total integrated ion current that is due to $X^{+}$in each single particle averaged over all $\mathrm{Na} / \mathrm{Si} / \mathrm{K} / \mathrm{Ca} / \mathrm{Fe} / \mathrm{Ga} / \mathrm{Pb}$ spectra collected at the orifice that corresponds to the dominant fine mode. Similar equations can be obtained for $\mathrm{Na}_{2} \mathrm{O}$ and $\mathrm{SiO}_{2}$. Yokozawa et al. (1987) used laser microprobe mass spectrometry (LMMS) on samples of coal fly ash collected from a heavy-oil fired testing furnace to obtain $\mathrm{RSF}_{\mathrm{Si}^{+}, \mathrm{Fe}^{+}}=0.2$ and $\mathrm{RSF}_{\mathrm{K}^{+}, \mathrm{Fe}^{+}}=1.7$. Gross et al. (2000) reported a value of 5.1 for $\mathrm{RSF}_{\mathrm{Na}^{+}, \mathrm{K}^{+}}$. Combining all of these data, $\delta_{X_{n} O_{m}}$ was calculated for each metal. The resulting values, plotted in Fig. 6, may be used to estimate the density of these particles according to

$\rho=\sum_{X=\mathrm{Na}}^{X=\mathrm{Pb}} \delta_{X_{n} O_{m}} \rho_{X_{n} O_{m}}$.

Using Eq. (14), the particle density was determined to be $3.9 \pm 0.8 \mathrm{~g} / \mathrm{cc}$, where the error estimate was obtained through the propagation of the standard deviation in both the MOUDI/ICP-MS data and $\bar{\gamma}_{X^{+}}$values. Using this density in Eq. (9), the particle shape factor is $1.5 \pm 0.2$.

Given the particle density and shape factor listed above, there are several corrections that must be made to the RSMS distributions shown in Figs. 1-4. First, these values must be used in Eq. (10) to convert from electrical mobility to aerodynamic diameter so that the RSMS and MOUDI distributions are with respect to the same particle diameter. Second, as discussed previously, assuming a particle density of $1.0 \mathrm{~g} / \mathrm{cc}$ in Eq. (4) is a serious source of error in the value of $\mathrm{d} M / \mathrm{d} \log D_{\mathrm{p}}$ and the distributions must be adjusted using the true particle density to account for this. Finally, since the number distributions originally used in Eq. (4) to obtain the mass distributions are with respect to the electrical mobility diameter, these diameters were used to calculate particle volume. This assumes that the particles are spherical which is incorrect and the volume equivalent diameter is the appropriate diameter to use in these calculations. The correction factor $(\beta)$ which must be applied to the RSMS distributions to account for both the density and volume corrections may be written as

$\beta\left(D_{\mathrm{m}}\right)=\left[\frac{\rho}{\chi} \frac{C_{\mathrm{c}}\left(D_{\mathrm{ve}}\right)}{C_{\mathrm{c}}\left(D_{\mathrm{m}}\right)}\right]^{3}$.

Using the particle density and shape factor listed above, and the electrical mobility diameters shown 


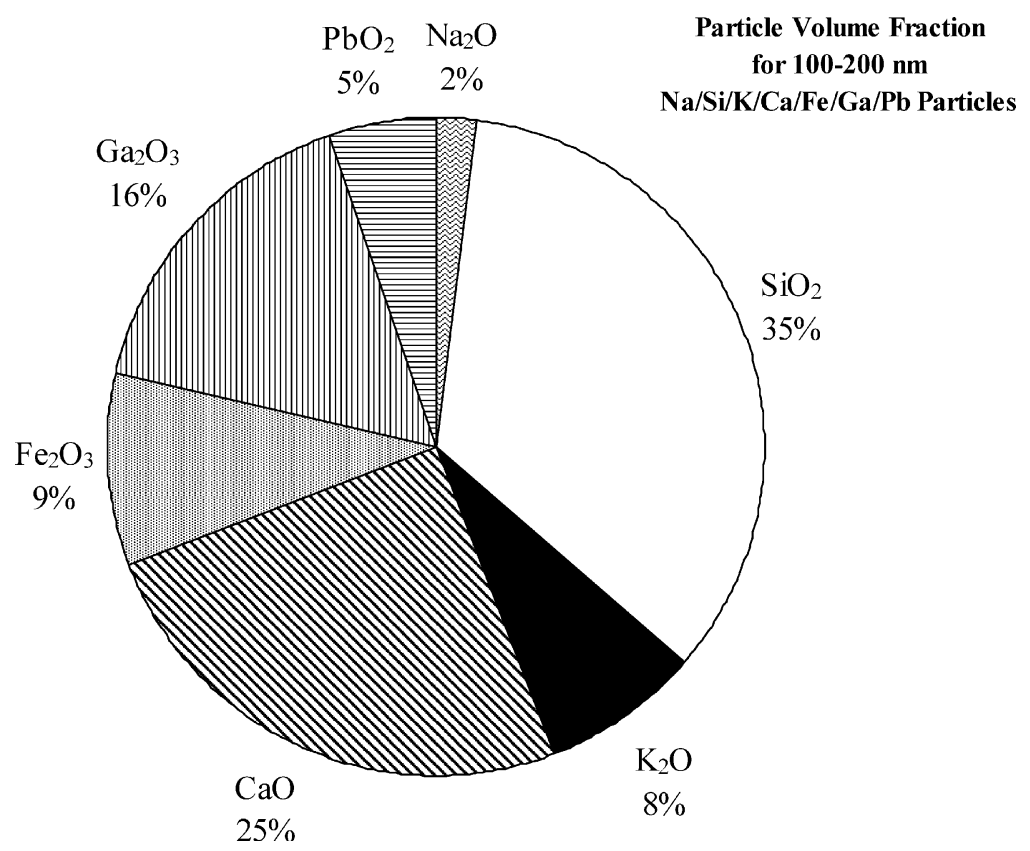

Fig. 6. The particle volume fraction of $100-200 \mathrm{~nm} \mathrm{Na} / \mathrm{Si} / \mathrm{K} / \mathrm{Ca} / \mathrm{Fe} / \mathrm{Ga} / \mathrm{Pb}$ particles measured on 27 October 2001; shows the fraction of the total particle volume occupied by each of the metal oxides believed to be present in the particle.

in the figures, Eq. (15) may be solved numerically to obtain $\beta\left(D_{\mathrm{m}}\right)$. All of these corrections have been applied to the RSMS distributions shown in Figs. 1-4 and the corrected distributions are shown in the inset of each figure.

For the coarse mode $\mathrm{Pb}$ distribution (Fig. 4), all of the particles within this mode belong to the $\mathrm{Na}$ / $\mathrm{K} / \mathrm{Zn} / \mathrm{Pb}$ class. As will be shown in a later section, the density of these particles is $\sim 3.0 \mathrm{~g} / \mathrm{cc}$. Using the shift between the RSMS and MOUDI distributions for this mode in Eq. (10), in conjunction with the density listed above, the particle shape factor of these particles is $\sim 1.4$. These values were used in the analyses presented above to correct this mode.

There are several things to note about the corrected distributions. First, the excellent agreement between the position of the fine mode peaks in the RSMS and MOUDI distributions is evidence of the accuracy of the particle density and shape factor estimates, obtained from independent analyses, used to convert from electrical mobility to aerodynamic diameter. Second, although the density and volume corrections tend to offset each other, there is a general increase in agreement between the peak values of $\mathrm{d} M / \mathrm{d} \log D_{\mathrm{p}}$ in the RSMS and MOUDI distributions. For example, the agreement between the peak value of the fine mode in the RSMS and MOUDI distributions increases by a factor of $\sim 2$ for $\mathrm{Ca}, \mathrm{Ga}$, and $\mathrm{Pb}$. In the RSMS Fe distribution, the peak value switches from a $20 \%$ underestimation of the MOUDI peak to a $30 \%$ overestimation, but the total integrated $\mathrm{PM}_{1.2}$ is in excellent agreement; $0.57 \mu \mathrm{g} / \mathrm{m}^{3}$ for the RSMS distribution and $0.5 \pm 0.1 \mu \mathrm{g} / \mathrm{m}^{3}$ for the MOUDI distribution.

Finally, note that the discrepancy between the peak value of $\mathrm{d} M / \mathrm{d} \log D_{\mathrm{p}}$ for the dominant fine mode in the RSMS distributions versus the MOUDI distributions ranges from $30 \%$ for iron to a factor of 4 for $\mathrm{Ca}$ and an order of magnitude for gallium and lead. The fact that the $\mathrm{Fe}$ distributions are in such good agreement, coupled to the fact that the MOUDI values of $\mathrm{d} M / \mathrm{d} \log D_{\mathrm{p}}$ differ by less than a factor of 2 from element to element, is strong evidence that the variation in the discrepancy between the distributions for iron versus the other three metals $(\mathrm{Ca}, \mathrm{Ga}$ and $\mathrm{Pb})$ is directly a result of the sensitivity of the single particle instrument to each. If it is assumed that the remainder of the error between the RSMS and MOUDI distributions can be accounted for in the relative sensitivity factors, then these factors can be calculated. An outline of these calculations is presented in the following section.

Similar arguments can be made for the coarse mode in the $\mathrm{Pb}$ distribution, which represents $\mathrm{Na} / \mathrm{K} / \mathrm{Zn} / \mathrm{Pb}$ particles. The error between the RSMS and MOUDI data for the coarse mode is 


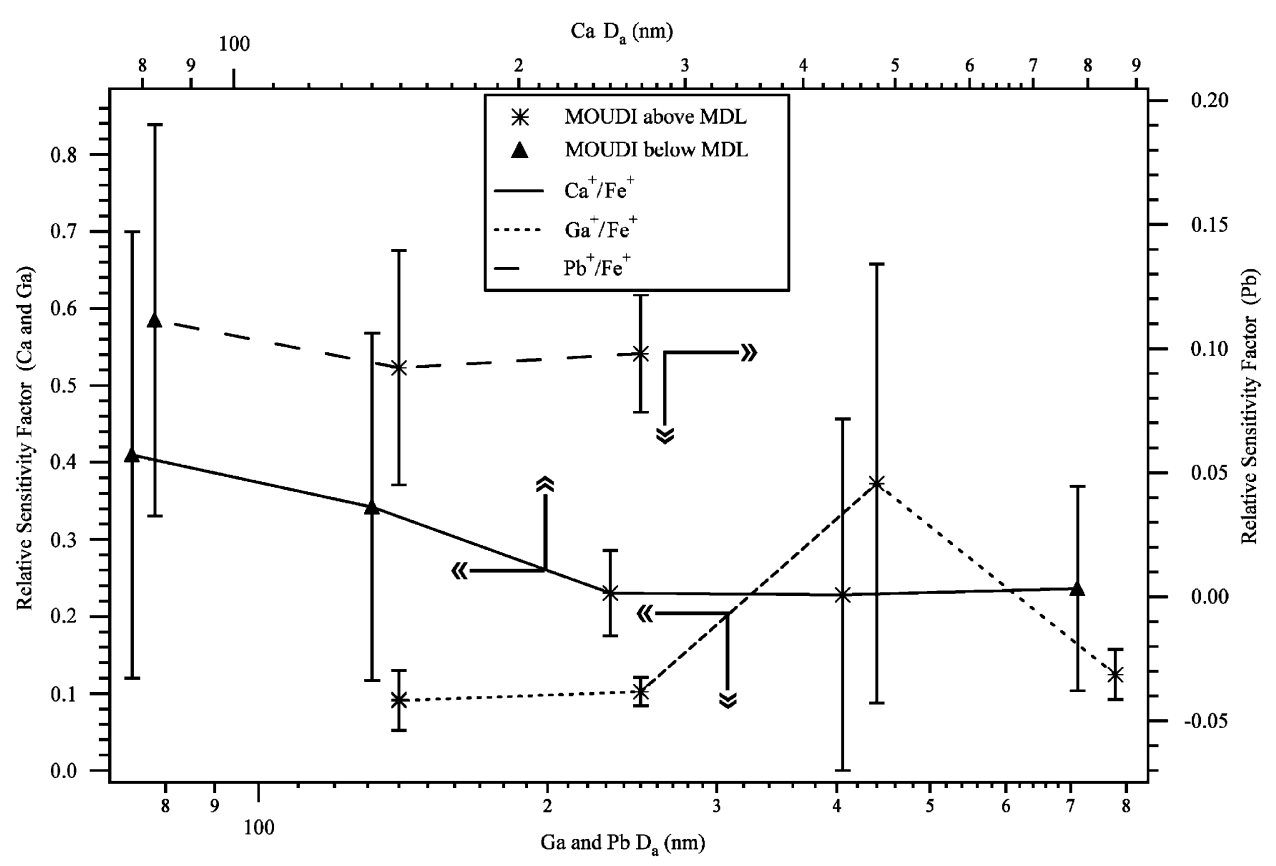

Fig. 7. The relative sensitivity factors (RSF) for $\mathrm{Ca}, \mathrm{Ga}$ and $\mathrm{Pb}$, normalized by $\mathrm{Fe}$, as a function of particle diameter. The RSF values for $\mathrm{Ca}$ and $\mathrm{Ga}$ are plotted on the left $y$-axis and the values for $\mathrm{Pb}$ are plotted on the right $y$-axis. $\mathrm{Ga}$ and $\mathrm{Pb}$ are with respect to the bottom $x$ axis and $\mathrm{Ca}$ is with respect to the top $x$-axis. The triangles represent RSF values calculated using MOUDI data below the method detection limit (MDL) and the asterisks represent RSF values calculated using MOUDI data above the MDL.

comparable to that of the fine mode. This suggests that the $\mathrm{Pb}$ mass fractions are being underestimated for these particles and the single particle instrument is more sensitive to one, or more, of the other species in these particles. A direct calculation of this requires MOUDI data for the other species, which is not available.

\subsubsection{Sensitivity of RSMS-3 to particle composition}

Letting $\Delta m_{k, s}$ represent the mass of element $k$ collected on stage $s$ of the MOUDI, where $D_{a 1, s}$ and $D_{a 2, s}$ are the lower and upper diameter cut points of stage $s$, respectively, and $\bar{D}_{a, s}$ is the mean diameter, then the sensitivity of the single particle instrument to element $k$ ions, relative to element $j$ ions, for particles with diameter $\bar{D}_{a, s}, \operatorname{RSF}_{k, j}\left(\bar{D}_{a, s}\right),{ }^{3}$ may be written as

$$
\begin{aligned}
\operatorname{RSF}_{k, j}\left(\bar{D}_{a, s}\right)= & \frac{\int_{D_{a 1, s}}^{D_{a 2, s}}\left(\mathrm{~d} M / \mathrm{d} \log D_{\mathrm{p}}\right)_{\mathrm{RSMS}, k} \mathrm{~d} \log D_{\mathrm{p}}}{\int_{D_{a, 1, s}}^{D_{a 2, s}}\left(\mathrm{~d} M / \mathrm{d} \log D_{\mathrm{p}}\right)_{\mathrm{RSMS}, j} \mathrm{~d} \log D_{\mathrm{p}}} \\
& \times\left(\frac{\Delta m_{j, s}}{\Delta m_{k, s}}\right) .
\end{aligned}
$$

\footnotetext{
${ }^{3} 24 \mathrm{~h}$ value
}

Using $\mathrm{Fe}^{+}$as the normalizing ion in Eq. (16), the RSF values for calcium, gallium and lead have been calculated and are plotted versus aerodynamic diameter in Fig. 7. The error bars represent the propagation of errors from the standard deviation in the MOUDI/ICP-MS measurements. Notice that the RSF value for a particular element within this particle matrix is approximately constant with respect to particle diameter within the range of $\sim 70-800 \mathrm{~nm}$.

\subsection{March 2002 data}

In contrast to the situation presented above for 27 October 2001, there was a large degree of variability in the metal containing particles detected on 14 March 2002. This is more representative of what was typically observed throughout the PAQS. Figs. 10 and 11 show the RSMS and MOUDI $24 \mathrm{~h}$ elemental mass distributions for calcium and lead, respectively, on 14 March 2002. Calcium and lead were chosen because they are the only two metals detected on this day that were also detected on 27 October 2001 and satisfied the detection criteria described previously.

Since $\mathrm{Ca}$ and $\mathrm{Pb}$ were observed in multiple types of particles, each with their own density, the 
features of the elemental mass distributions will ultimately depend on the distribution of densities. As a result, rather than assuming a constant density for all particles, like the 27 October 2001 analysis, each particle type was considered independently when using Eq. (4) in what follows.

According to the single particle data, $\sim 80 \%$ of the Ca-containing particles detected on this day belong to one of two classes; $\mathrm{EC} / \mathrm{OC} / \mathrm{Ca}(\sim 60 \%)$ or $\mathrm{Al} / \mathrm{Si} / \mathrm{Ca} / \mathrm{Fe}(\sim 20 \%)$. Similarly, $\sim 85 \%$ of the $\mathrm{Pb}-$ containing particles belong to either the $\mathrm{EC} / \mathrm{OC} / \mathrm{Pb}$ class $(\sim 45 \%)$ or $\mathrm{Na} / \mathrm{K} / \mathrm{Zn} / \mathrm{Pb}$ class $(\sim 40 \%)$. However, the remaining $\mathrm{Ca}$ and $\mathrm{Pb}$-containing particles detected on this day were of highly variable composition, containing mixtures of different metals, and a constant density of $3 \mathrm{~g} / \mathrm{cc}$ was assumed for these particles. This is simply the number-weighted average density of the major species observed in these particles. Although the true densities will likely be slightly different, this is not considered a significant source of error since these particles only represent a small fraction of the total number of $\mathrm{Ca}$ and $\mathrm{Pb}$ containing particles detected $(\sim 20 \%$ and $15 \%$, respectively).

For EC/OC/Ca particles, the single particle mass fraction for $\mathrm{Ca}$, as averaged over all $\mathrm{EC} / \mathrm{OC} / \mathrm{Ca}$ particles, is $\sim 5 \%$. Assuming that the remaining $95 \%$ of the single particle mass is EC/OC, with an average density of $1.5 \mathrm{~g} / \mathrm{cc}$ (Zhang et al., 2005), the particle volume fraction for $\mathrm{CaO}(\rho=3.34 \mathrm{~g} / \mathrm{cc})$ is 0.023 and the volume-weighted particle density is $1.5 \mathrm{~g} / \mathrm{cc}$. Of the remaining Ca-containing particles detected on this day, $\sim 50 \%$ of them belong to the $\mathrm{Al} / \mathrm{Si} / \mathrm{Ca} / \mathrm{Fe}$ class. Numerous other elements were detected in these particles, including $\mathrm{Na}, \mathrm{K}, \mathrm{Ti}, \mathrm{V}$, $\mathrm{Mn}, \mathrm{Sr}, \mathrm{Rh}$ and $\mathrm{Ba}$, but were observed with a high degree of variability and only in trace amounts. The average mass fraction of $\mathrm{Ca}$ within these particles is $\sim 14 \%$ and a large majority of the remaining mass is $\mathrm{Al}$ and $\mathrm{Si}$. These particles are considered to be associated with coal combustion where the $\mathrm{Al}, \mathrm{Si}$ and $\mathrm{Ca}$ were originally present as minerals within the coal, predominantly quartz $(\rho=2.65 \mathrm{~g} / \mathrm{cc})$ and kaolinite $(\rho=2.65 \mathrm{~g} / \mathrm{cc})$ for $\mathrm{Al}$ and $\mathrm{Si}$ and calcite $\left(\mathrm{CaCO}_{3}\right)$ for $\mathrm{Ca}$ (Ward, 2002), which were directly transferred to PM during combustion. The calcite is decarboxylated to $\mathrm{CaO}$ and the quartz and kaolinite form a melt which traps nonvolatile trace elements, including those listed above, as it solidifies into aluminosilicate glass (Clarke, 1993; Reifenstein et al., 1999). Using these data, and ignoring the trace elements, the particle volume fraction of $\mathrm{CaO}$ is $\sim 0.11$ and the volume-weighted density is $2.7 \mathrm{~g} / \mathrm{cc}$ for this class of particles.

The density of $\mathrm{EC} / \mathrm{OC} / \mathrm{Pb}$ particles can be estimated in a similar fashion as the $\mathrm{EC} / \mathrm{OC} / \mathrm{Ca}$ particles. Averaged over all $\mathrm{EC} / \mathrm{OC} / \mathrm{Pb}$ particles, the mass fraction of lead is $\sim 0.2$ and, assuming the remaining mass is $\mathrm{EC} / \mathrm{OC}(\rho=1.5 \mathrm{~g} / \mathrm{cc})$, the volume-weighted density is $\sim 1.8 \mathrm{~g} / \mathrm{cc}$. More than $70 \%$ of the remaining $\mathrm{Pb}$-containing particles belong to the $\mathrm{Na} / \mathrm{K} / \mathrm{Zn} / \mathrm{Pb}$ class. Assuming the molecular makeup described previously and $\gamma_{\mathrm{Na}} \approx \gamma_{\mathrm{K}} \approx \gamma_{\mathrm{Zn}}$, the average mass fraction of lead $(\sim 0.3)$ may be used to obtain a volume-weighted density of $\sim 3.0 \mathrm{~g} / \mathrm{cc}$.

For the purposes of calculating the lead and calcium single particle mass fractions, it was assumed that all of the metals detected on this day were originally in the form of metal oxides with the highest oxidation state that is stable under atmospheric conditions. The $\mathrm{Na} / \mathrm{K} / \mathrm{Zn} / \mathrm{Pb}$ class is an exception and was treated in the same manner as was discussed in the 27 October 2001 analysis. In addition, for the combustion-oriented $\mathrm{EC} / \mathrm{OC} / \mathrm{Ca}$ and $\mathrm{EC} / \mathrm{OC} / \mathrm{Pb}$ classes, in which metal and carbon are found together in individual particles, it was assumed that the sum of signal intensities, weighted by the appropriate molecular weights, over all carbon based ions within the spectrum correctly represents the mass of $\mathrm{EC} / \mathrm{OC}$ originally in the particle.

\subsubsection{Comparison of RSMS, SMPS and MOUDI} size data: differences in size distribution peaks

Similar to the 27 October 2001 analysis, when using Eq. (2) to calculate detection efficiencies for the 14 March 2002 data, it is important to consider any potential errors associated with mismatch in the RSMS and SMPS distributions. Fig. 8 shows the RSMS number distribution, as determined from Eq. (1), and the SMPS number distribution for the sampling interval during which a majority of the fine mode, Ca-containing particles were detected $(\sim 55 \%)$. In contrast to the situation for the 27 October 2001 data (Fig. 5a), note the excellent agreement between the position of the RSMS and SMPS peaks. The implication is that $D_{\mathrm{va}} \approx D_{\mathrm{m}}$ for the particles within this mode such that equating these diameters when calculating detection efficiencies is appropriate. The inset of Fig. 8 shows the number distribution of $\mathrm{Ca}$-containing particles for the case of scaled versus un-scaled data. Note that 


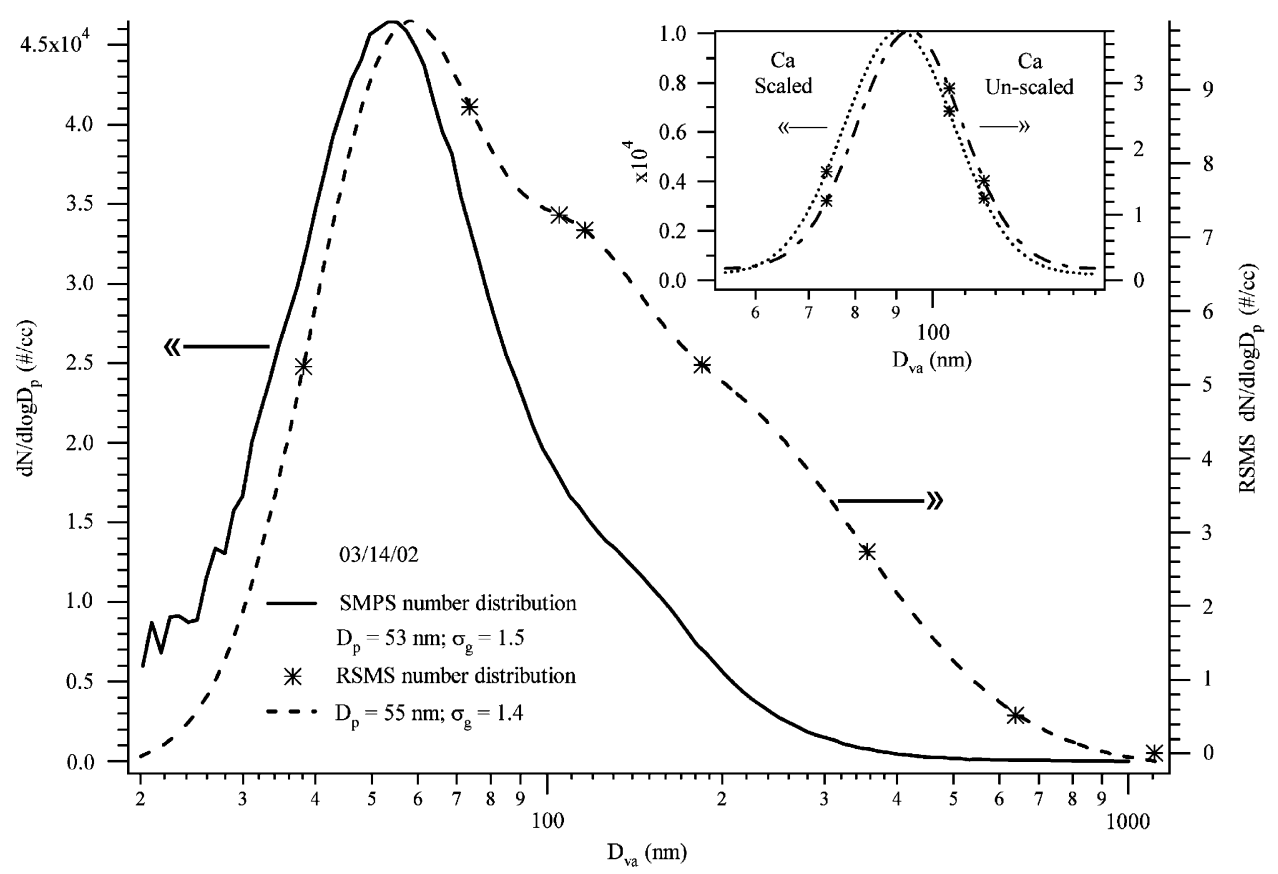

Fig. 8. The RSMS and SMPS number distributions for the sampling interval during which a majority of the fine mode Ca-containing particles were detected $(\sim 55 \%)$. The inset shows the number distribution of Ca-containing particles for the case of scaled versus un-scaled data. The SMPS and scaled distributions are with respect to the left $y$-axis and the RSMS and un-scaled distributions are with respect to the right $y$-axis.

the scaling has no effect on the shape of the distribution or the position of the peak.

According to the single particle data, $\sim 80 \%$ of the particles detected during this interval were $\mathrm{EC} /$ OC particles; the $\mathrm{EC} / \mathrm{OC} / \mathrm{Ca}$ particles are a small subset of this population. As a result, the distributions shown in Fig. 8 can be attributed almost entirely to the EC/OC class and the fact that $D_{\text {va }} \approx$ $D_{\mathrm{m}}$ describes the relationship between the density and shape factor of these particles during this particular event. Using a density of $1.5 \mathrm{~g} / \mathrm{cc}$ for $\mathrm{EC} /$ OC particles, Eq. (9) can be used to obtain a particle shape factor of $\sim 1.2$.

The RSMS and SMPS number distributions for the sampling interval during which a majority of the remaining $\mathrm{Ca}$ and $\mathrm{Pb}$-containing particles were detected is shown in Fig. 9a. Similar to the 27 October 2001 data, there is excellent agreement between the features of the two distributions, i.e. both are tri-modal and peak in the ultrafine range, but they are shifted. If the SMPS and RSMS number distributions can be fit with lognormal distributions, according to Eq. (8), and the modes of the distributions can be matched so that, conceivably, the distributions could be aligned before calculating detection efficiencies, then the correction factor $(\psi)$ can be written as

$$
\begin{aligned}
& \psi\left(D_{\mathrm{va}}\right) \\
& =\frac{\sum_{j} N_{\mathrm{SMPS}, j} \exp \left[-\left(\left(\ln \left(D_{\mathrm{va}} / \bar{D}_{m, j}\right) / \sqrt{2} \ln \left(\sigma_{m, j}\right)\right)\right)^{2}\right]}{\sum_{j} N_{\mathrm{SMPS}_{j} j} \exp \left[-\left(\ln \left(D_{\mathrm{va}} / \bar{D}_{\mathrm{va}, j}\right) / \sqrt{2} \ln \left(\sigma_{m, j}\right)\right)^{2}\right]},
\end{aligned}
$$

where the subscript $j$ denotes the mode number, $N_{\mathrm{SMPS}, j}$ is the amplitude of mode $j$ in the SMPS distribution, $\bar{D}_{m, j}$ and $\sigma_{m, j}$ are the geometric mean diameter and standard deviation of the SMPS mode, $\bar{D}_{\mathrm{va}, j}$ is the geometric mean diameter of the corresponding mode in the RSMS distribution, and the summations are over all modes within the distribution. Using the lognormal fits shown in Fig. 9a, these correction factors have been calculated and are plotted in Fig. $9 \mathrm{~b}$ in terms of the relative error $(\varepsilon)$, which is defined simply as $\varepsilon \equiv 1-\psi$. Also shown in this figure is the number distribution of $\mathrm{Ca}$ and $\mathrm{Pb}$-containing particles for the un-scaled data, the data scaled using the detection efficiencies from the mismatched distributions, and the data scaled using 


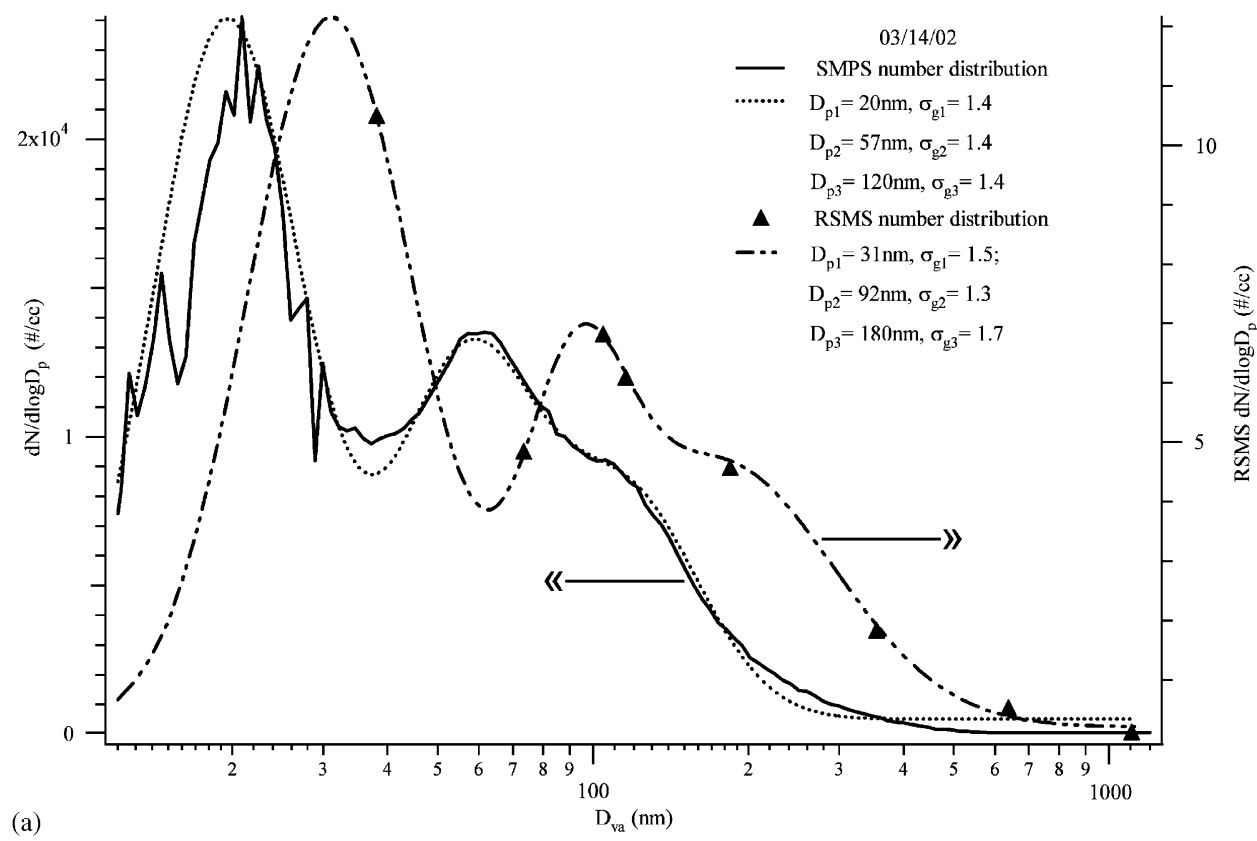

(a)

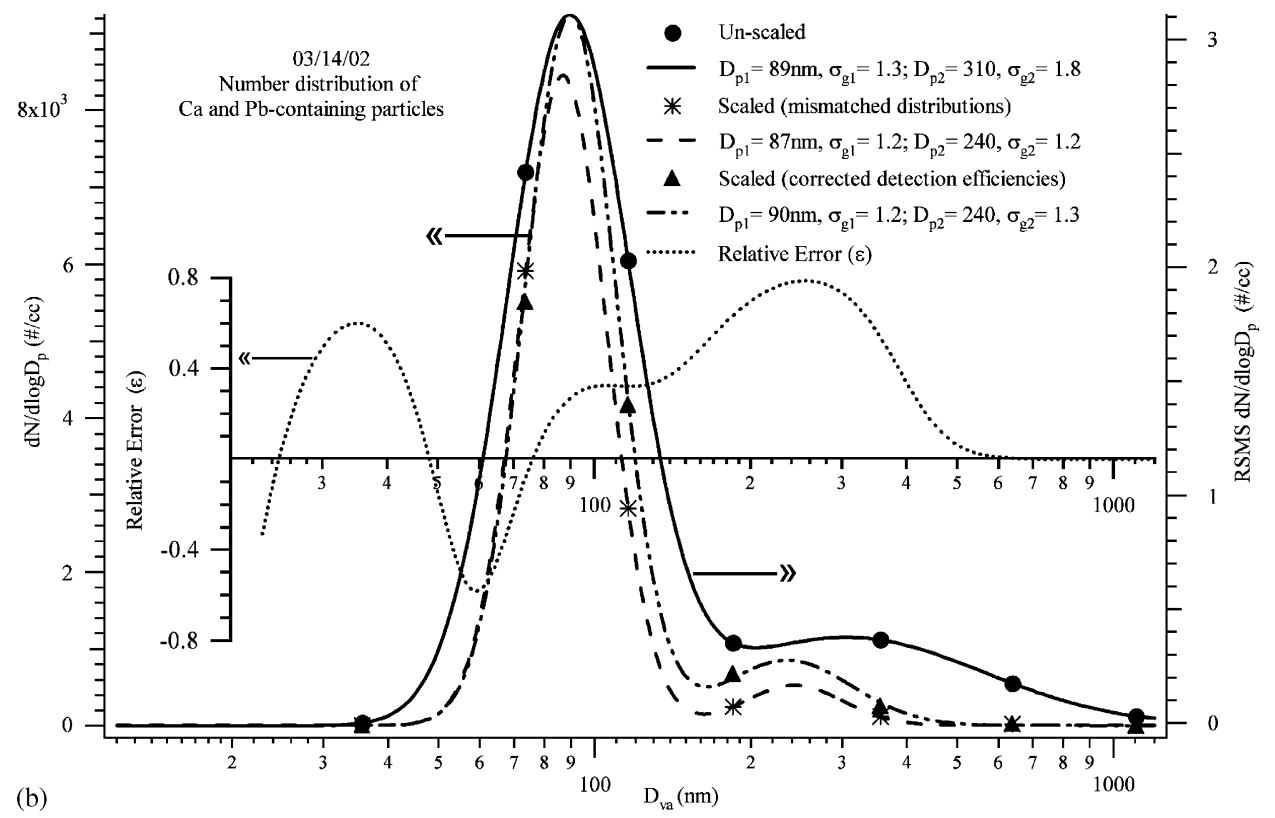

Fig. 9. (a) The RSMS and SMPS number distributions for the sampling interval during which a majority of the remaining $\mathrm{Ca}$ and $\mathrm{Pb}$ containing particles were detected. (b) The number distribution of $\mathrm{Ca}$ and $\mathrm{Pb}$-containing particles for un-scaled data, data scaled using the detection efficiencies from the mismatched distributions, and data scaled using the corrected detection efficiencies. The relative error in the value of the detection efficiency, for the cases of matched versus mismatched RSMS and SMPS number distributions, is plotted on the second pair of axes. The SMPS and scaled distributions are with respect to the left $y$-axis and the RSMS and un-scaled distributions are with respect to the right $y$-axis.

the corrected detection efficiencies. Note that using the corrected detection efficiencies scales the value of $\mathrm{d} N / \mathrm{d} \log D_{\mathrm{p}}$ by significant factors ranging from $\sim 0.9$ to 3 .
Since the distributions shown in Figs. 10 and 11 represent several different types of particles, a direct investigation of the relative position of the RSMS and MOUDI peaks is significantly more complicated 
than the 27 October 2001 data so it is difficult to resolve the affects of a single particle type. The fine mode $\mathrm{Ca}$ distribution is an exception considering that $\sim 80 \%$ of the particles in this mode belong to the $\mathrm{EC} / \mathrm{OC} / \mathrm{Ca}$ class. In this case, the agreement between the position of the RSMS and MOUDI peaks is due to the physical properties of these particles and can be understood by considering the relationship between the aerodynamic and vacuum aerodynamic diameters, which can be written as

$\frac{D_{\mathrm{a}}}{D_{\text {va }}}=\sqrt{\frac{\chi}{\rho} \frac{C_{\mathrm{c}}\left(\chi D_{\mathrm{va}} / \rho\right)}{C_{\mathrm{c}}\left(D_{\mathrm{a}}\right)}}$.

Using the density determined previously for $\mathrm{EC} /$ $\mathrm{OC} / \mathrm{Ca}$ particles, and the positions of the fine mode peaks in Fig. 10, the particle shape factor is $\sim 1.3$.

It was mentioned previously that since $\mathrm{Ca}$ and $\mathrm{Pb}$ were observed in multiple types of particles, each with their own density, the features of the elemental mass distributions will ultimately depend on the distribution of densities such that each particle type must be considered independently when using Eq. (4). A further implication of this is that using the vacuum aerodynamic diameter in this equation to calculate the particle volume is incorrect and the volume equivalent diameter is the appropriate diameter to use. If a constant density of $1.0 \mathrm{~g} / \mathrm{cc}$ is used in Eq. (4) to determine the mass distribution, then the correction factor which must be applied to the resulting distribution to account for the combined effect of scaling by the true particle density and correcting for overestimating particle volume may be written as

$\beta_{i}=\sum_{g} \frac{N_{i, g}}{N_{i}} \frac{\chi_{g}^{3}}{\rho_{g}^{2}}$

where the subscript $i$ denotes the orifice number, $N_{i}$ is the total number of particles detected at orifice $i$, $N_{i, g}$ is the number of group $g$ particles, $\chi_{g}$ is the shape factor of group $g$ particles and $\rho_{g}$ is their density. The distributions shown in Figs. 10 and 11 already include these corrections. The distribution of particle types, and corresponding densities, described previously were used in those calculations. In terms of the particle shape factor, direct estimates are available for the $\mathrm{EC} / \mathrm{OC} / \mathrm{Ca}$ and $\mathrm{Na} / \mathrm{K} / \mathrm{Zn} / \mathrm{Pb}$ classes. The value obtained for $\mathrm{EC} / \mathrm{OC} / \mathrm{Ca}$ particles was also used for the $\mathrm{EC} / \mathrm{OC} / \mathrm{Pb}$ class, the value obtained for the $\mathrm{Na} / \mathrm{Si} / \mathrm{K} / \mathrm{Ca} / \mathrm{Fe} / \mathrm{Ga} / \mathrm{Pb}$ particles (27 October 2001 analysis) was applied to the $\mathrm{Al} / \mathrm{Si} /$ $\mathrm{Ca} / \mathrm{Fe}$ particles, since both are related to coal combustion, and the value obtained for the $\mathrm{Na} / \mathrm{K} /$ $\mathrm{Zn} / \mathrm{Pb}$ particles was used to represent the remaining mixed-metal particles.

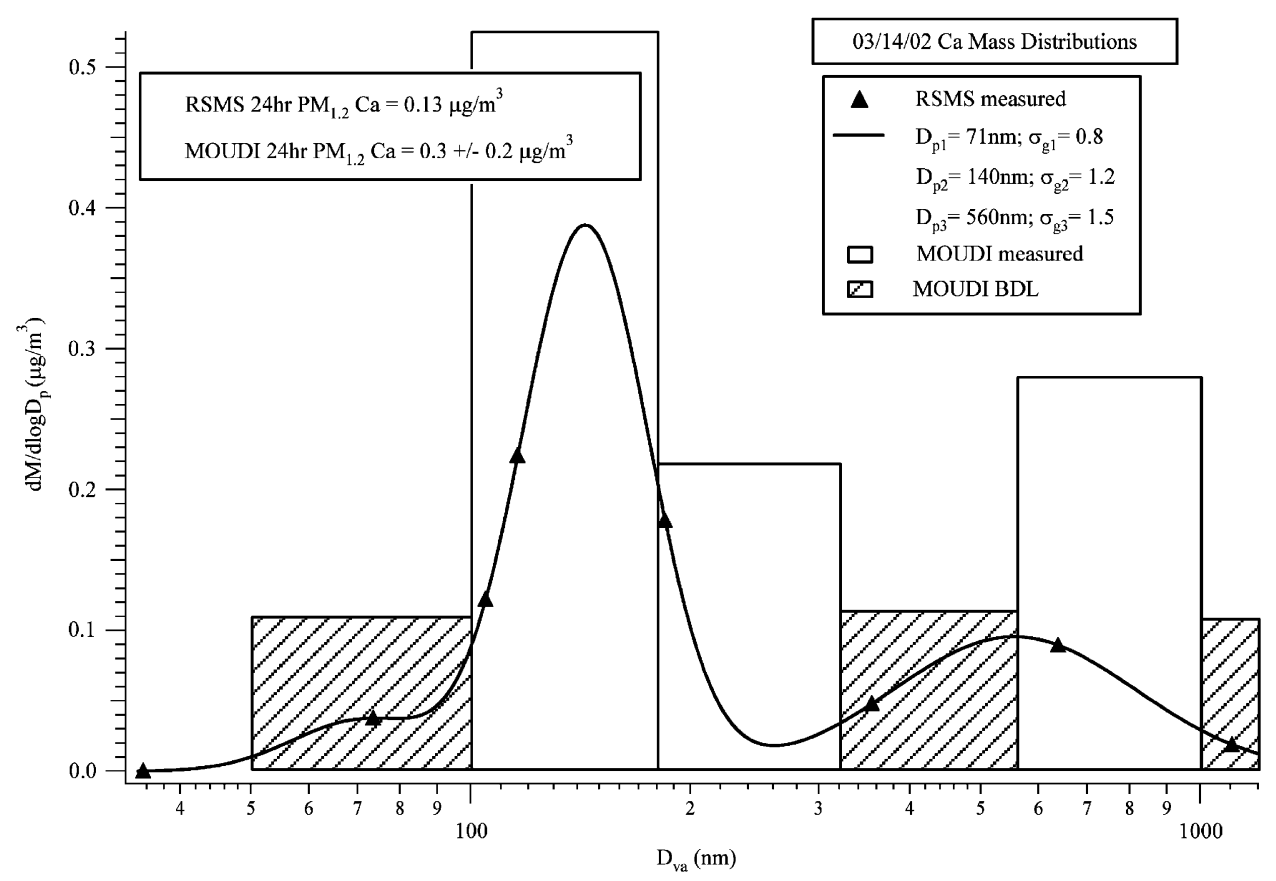

Fig. 10. Airborne concentration on 14 March 2002 was $0.10 \mu \mathrm{g} / \mathrm{m}^{3} \mathrm{PM}_{3.2} \mathrm{Ca}$ as measured by the MOUDI and $0.11 \mu \mathrm{g} / \mathrm{m}^{3} \mathrm{PM}{ }_{2.5} \mathrm{Ca}$ as measured by the hi vol. 


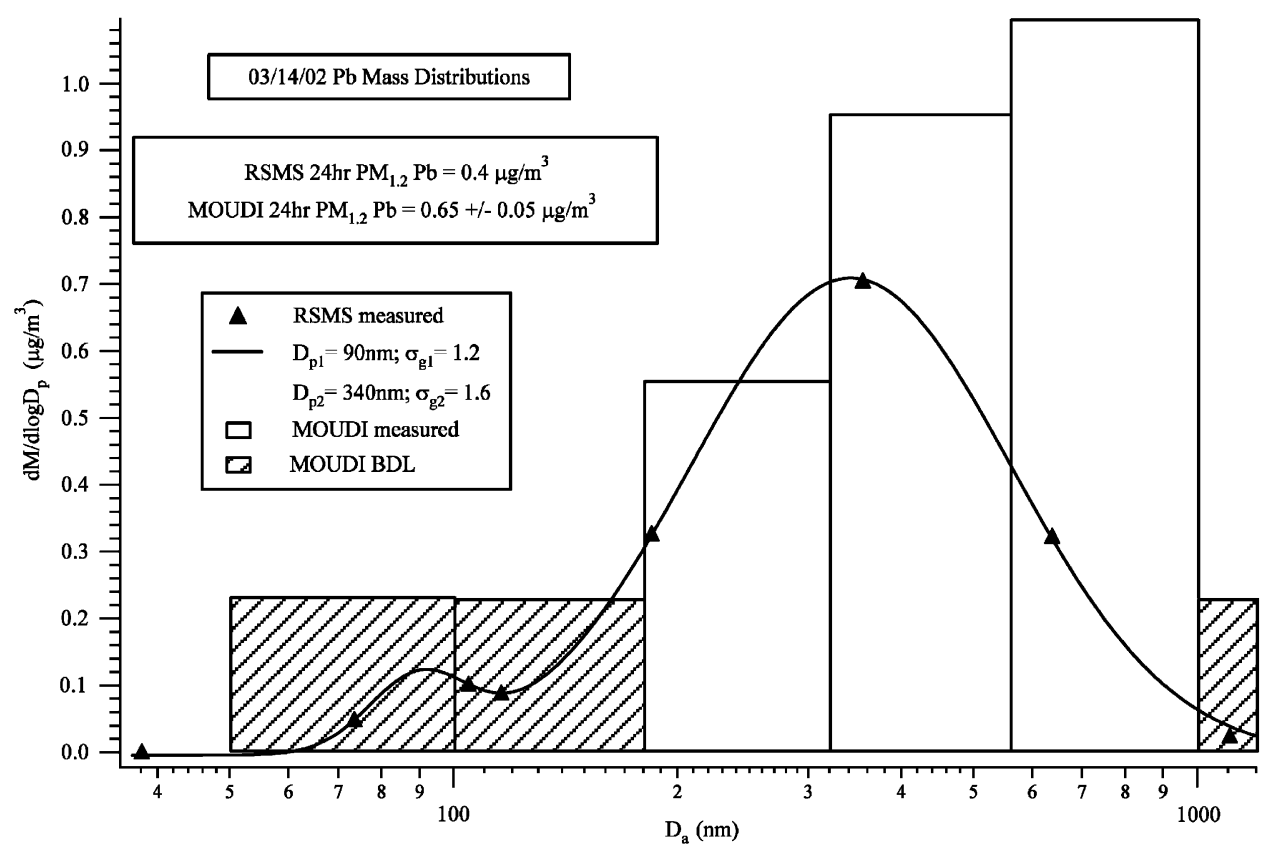

Fig. 11. Airborne concentration on 14 March 2002 was $0.0050 \mu \mathrm{g} / \mathrm{m}^{3} \mathrm{PM}_{3.2} \mathrm{~Pb}$ as measured by the $\mathrm{MOUDI}$ and $0.0065 \mu \mathrm{g} / \mathrm{m}^{3} \mathrm{PM} 2.5 \mathrm{~Pb}$ as measured by the hi vol.

\subsubsection{Comparisons of mass distributions on different dates}

When comparing the $\mathrm{Ca}$ and $\mathrm{Pb}$ distributions from 27 October 2001 to those obtained for 14 March 2002, it is evident that, in general, the ability of the RSMS data to reconstruct the MOUDI mass is much better for the particles detected on 14 March 2002. For example, considering the fine mode in the $\mathrm{Ca}$ distributions, the agreement between the RSMS and MOUDI mass is a factor of 4 better for the 14 March 2002 data than the 27 October 2001 data. Assuming that, similar to the 27 October 2001 analysis, the error in the 14 March 2002 data represents the relative sensitivity of the single particle instrument to $\mathrm{Ca}$, the increase in agreement for this day suggests that $\mathrm{Ca}$ is detected more efficiently in $\mathrm{EC} / \mathrm{OC} / \mathrm{Ca}$ particles than $\mathrm{Na} / \mathrm{Si}$ / $\mathrm{K} / \mathrm{Ca} / \mathrm{Fe} / \mathrm{Ga} / \mathrm{Pb}$ particles. The implication is that the sensitivity of the single particle instrument to a particular species depends on the other species in the particle; i.e. RSFs depend on particle matrix. Similar arguments can be made for the $\mathrm{Pb}$ distributions. For the MOUDI stage corresponding to the largest particle sizes $(\sim 560-1000 \mathrm{~nm})$, the particles detected at the corresponding RSMS orifices were almost exclusively $\mathrm{Na} / \mathrm{K} / \mathrm{Zn} / \mathrm{Pb}$ particles in both the 27 October 2001 and 14 March 2002 distributions. It was suggested earlier in the context of the 27
October 2001 analysis that the $\mathrm{Pb}$ mass fractions were underestimated and that the single particle instrument is more sensitive to one, or more, of the other species in the particle. The fact that the relative error between the MOUDI and RSMS mass for the 14 March 2002 data is almost identical to that of the 27 October 2001 data ( $<10 \%$ difference) is further evidence of this conclusion. Progressing from larger to smaller particles, the distribution of particle type changes, $\mathrm{EC} / \mathrm{OC} / \mathrm{Pb}$ particles begin to dominate the distribution, especially in the fine range $(\sim 150-500 \mathrm{~nm})$, and the agreement between the RSMS and MOUDI mass increases considerably. Comparing the relative error between the RSMS and MOUDI fine mode in the 27 October 2001 distribution to the error in the corresponding section of the 14 March 2002 distribution, it is evident that the agreement between the 14 March 2002 distributions is an order of magnitude better. This corroborates the results for the $\mathrm{EC} / \mathrm{OC} / \mathrm{Ca}$ particles; the sensitivity of the single particle instrument to both $\mathrm{Ca}$ and $\mathrm{Pb}$ depends on the other species in the particle, or particle matrix.

\section{Conclusions}

Elemental mass distributions were constructed using the RSMS single particle data, in conjunction 
with concurrent SMPS measurements, and compared to mass distributions obtained from MOUDI/ICP-MS analysis for two separate days during the PAQS. Calcium, iron, gallium and lead mass distributions from 27 October 2001 and calcium and lead mass distributions from 14 March 2002 were included for comparison.

Approximately $80 \%$ of the metal-containing particles detected on 27 October 2001 belonged to the $\mathrm{Na} / \mathrm{Si} / \mathrm{K} / \mathrm{Ca} / \mathrm{Fe} / \mathrm{Ga} / \mathrm{Pb}$ particle class and for the sampling intervals during which they were detected, they represent $\sim 70-90 \%$ of the total number of particles detected. As a result, it was possible to use a combination of the RSMS, SMPS and MOUDI data to investigate the physical properties of these particles. The density and shape factors of the $\mathrm{Na}$ / $\mathrm{Si} / \mathrm{K} / \mathrm{Ca} / \mathrm{Fe} / \mathrm{Ga} / \mathrm{Pb}$ particles were estimated to be $3.9 \pm 0.8 \mathrm{~g} / \mathrm{cc}$ and $1.5 \pm 0.2$, respectively. In addition, a direct comparison between the magnitudes of the RSMS and MOUDI distributions allowed investigation of the sensitivity of the single particle instrument for different metals in the $\mathrm{Na} / \mathrm{Si} / \mathrm{K} / \mathrm{Ca} /$ $\mathrm{Fe} / \mathrm{Ga} / \mathrm{Pb}$ particle matrix. Relative sensitivity factors (RSF) were calculated for $\mathrm{Ca}, \mathrm{Ga}$, and $\mathrm{Pb}$, normalized by $\mathrm{Fe}$, and showed little variation with respect to particle diameter over the range of $70-800 \mathrm{~nm}$.

In contrast to the particles observed on 27 October 2001, there was a large degree of variability in the metal containing particles detected on 14 March 2002. As a result, the calcium and lead mass distributions from this day represent an ensemble of externally mixed calcium and lead containing particles. Estimates of particle density, based upon compositional considerations, were provided for the dominant particle types, including $\mathrm{EC} / \mathrm{OC} / \mathrm{Ca}, \mathrm{Al} /$ $\mathrm{Si} / \mathrm{Ca} / \mathrm{Fe}, \mathrm{EC} / \mathrm{OC} / \mathrm{Pb}$ and $\mathrm{Na} / \mathrm{K} / \mathrm{Zn} / \mathrm{Pb}$. Furthermore, direct estimates of particle shape factor were given for the $\mathrm{EC} / \mathrm{OC} / \mathrm{Ca}$ and $\mathrm{Na} / \mathrm{K} / \mathrm{Zn} / \mathrm{Pb}$ classes. Comparison with the 27 October 2001 calcium and lead mass distributions showed that the ability of the RSMS data to reconstruct the MOUDI mass is much better for the $\mathrm{Ca} / \mathrm{Pb}$-containing particles observed on 14 March 2002 than for those detected on 27 October 2001, suggesting that the sensitivity of the single particle instrument to both calcium and lead depends on the particle matrix. In addition, inspection of the ultrafine mass measured by RSMS, as compared to the method detection limits of the MOUDI/ICP-MS analysis, revealed that it is difficult to detect trace amounts of metal in ultrafine particles using MOUDI/
ICP-MS, highlighting another benefit of single particle analysis.

\section{Acknowledgements}

This research was conducted as a part of the Pittsburgh Air Quality Study which was supported by the US Environmental Protection Agency under contract R82806101 and the US Department of Energy National Energy Technology Laboratory under contract DE-FC26-01NT41017. This paper has not been subjected to EPA's peer and policy review, and therefore does not necessarily reflect the views of the Agency. No official endorsement should be inferred.

\section{Appendix A. Supplementary Material}

Supplementary data associated with this article can be found in the online version at doi:10.1016/ j.atmosenv.2006.01.064.

\section{References}

Allen, J.O., Fergenson, D.P., Gard, E.E., Hughes, L.X., Morrical, B.D., Kleeman, M.J., Gross, D.S., Galli, M.E., Prather, K.A., Cass, G.R., 2000. Particle detection efficiencies of aerosol time of flight mass spectrometers under ambient sampling conditions. Environmental Science and Technology 34 (1), 211-217.

Bein, K.J., Zhao, Y., Wexler, A.S., Johnston, M.V., 2005. Speciation of size-resolved individual ultrafine particles in Pittsburgh, Pennsylvania. Journal of Geophysical Research 110, D07S05.

Bhave, P.V., Allen, J.O., Morrical, B.D., Fergenson, D.P., Cass, G.R., Prather, K.A., 2002. A field-based approach for determining ATOFMS instrument sensitivities to ammonium and nitrate. Environmental Science and Technology 36, 4868-4879.

Clarke, L.B., 1993. The fate of trace elements during coal combustion and gasification: an overview. Fuel 72, 731-736.

DeCarlo, P.F., Slowik, J.G., Worsnop, D.R., Davidovits, P., Jimenez, J.L., 2004. Particle morphology and density characterization by combined mobility and aerodynamic diameter measurements. Part 1: theory. Aerosol Science and Technology $38,1185-1205$.

Fergenson, D.P., Song, X.-H., Ramadan, Z., Allen, J.O., Hughes, L.S., Cass, G.R., Hopke, P.K., Prather, K.A., 2001. Quantification of ATOFMS data by multivariate methods. Annals of Chemistry 73, 3535-3541.

Font, O., Querol, X., Huggins, F.E., Chimenos, J.M., Fernández, A.I., Burgos, S., García Peña, F., 2005. Speciation of major and selected trace elements in IGCC fly ash. Fuel 32, 1364-1371. 
Ge, Z., Wexler, A.S., Johnston, M.V., 1998. Laser desorption/ ionization of single ultrafine multi-component aerosols. Environmental Science and Technology 32, 3218-3223.

Gross, D.S., Galli, M.E., Silva, P.J., Prather, K.A., 2000. Relative sensitivity factors for alkali metal and ammonium cations in single-particle aerosol time-of-flight mass spectra. Annals of Chemistry 72, 416-422.

Jimenez, J.L., Bahreini, R., Crocker, D.R., Zhuang, H., Varutbangkul, V., Flagan, R.C., Seinfeld, J.H., O’Dowd, C.D., Hoffman, T., 2003. New particle formation from photooxidation of diiodomethane (CH2I2. Journal of Geophysical Research-Atmosphere 108 (D10), 4318.

Kane, D.B., Johnston, M.V., 2000. Size and composition biases on the detection of individual ultrafine particles by aerosol mass spectrometry. Environmental Science and Technology 34, 4887-4893.

Kane, D.B., Oktem, B., Johnston, M.V., 2001. Nanoparticle detection by aerosol mass Spectrometry. Aerosol Science and Technology 34, 520-527.

Lake, D.L., Tolocka, M.P., Wexler, A.S., Johnston, M.V., 2003. Mass spectrometry of individual particles between 50 and $750 \mathrm{~nm}$ in diameter at the Baltimore Supersite. Environmental Science and Technology 37, 3268-3274.

Liu, D.-Y., Prather, K.A., Hering, S.V., 2000. Variations in the size and chemical composition of nitrate-containing particles in Riverside, CA. Aerosol Science and Technology 33, 71-86.

Liu, D.-Y., Wenzel, R.J., Prather, K.A., 2003. Aerosol time-offlight mass spectrometry during the Atlanta Supersite Experiment: 1. Measurements. Journal of Geophysical Research 108 (D7), 8426.

Mansoori, B.A., Johnston, M.V., Wexler, A.S., 1994. Quantitation of ionic species in single micro-droplets by on-line laser desorption/ionization. Annals of Chemistry 66, 3681-3687.

Pekney, N.J., Davidson, C.I., 2005. Determination of trace elements in ambient aerosol samples. Analytica Chimica Acta 540 (2), 269-277.

Pekney, N.J., Bein, K.J., Zhao, Y., Wexler, A.S., Johnston, M.V., Davidson, C.I., 2005. Identification of sources of atmospheric PM at the Pittsburgh Supersite. Part I: single particle analysis and filter-based Positive Matrix Factorization. Atmospheric Environment, in press, doi:10.1016/j.atmosenv.2005.12.072.

Phares, D.J., Rhoads, K.P., Wexler, A.S., 2002. Performance of a single-ultrafine-particle mass spectrometer. Aerosol Science and Technology 36, 583-592.
Phares, D.J., Rhoads, K.P., Wexler, A.S., Johnston, M.V., 2003. Size resolved ultrafine particle composition analysis, 2, Houston. Journal of Geophysical Research 108 (D7) art. no. 8420 .

Reifenstein, A.P., Kahraman, H., Coin, C.D.A., Calos, N.J., Millar, G., Uwins, P., 1999. Behavior of selected minerals in an improved ash fusion test: quartz, potassium feldspar, sodium feldspar, kaolinite, illite, calcite, dolomite, siderite, pyrite and apatite. Fuel 78, 1449-1461.

Rhoads, K.P., Phares, D.J., Wexler, A.S., Johnston, M.V., 2003. Size-resolved ultrafine particle composition analysis, 1, Atlanta. Journal of Geophysical Research 108 (D7) art. no. 8418.

Tolocka, M.P., Lake, D.A., Johnston, M.V., Wexler, A.S., 2004. Number concentrations of fine and ultrafine particles containing metals. Atmospheric Environment 38, 3263-3273.

Ward, C.R., 2002. Analysis and significance of mineral matter in coal seams. International Journal of Coal Geology 50, 135-168.

Wenzel, R.J., Liu, D.-Y., Edgerton, E.S., Prather, K.A., 2003. Aerosol time-of-flight mass spectrometry during the Atlanta Supersite Experiment: 2. Scaling procedures. Journal of Geophysical Research 108 (D7), 8427.

Wigley, F., Williamson, J., 1998. Modeling fly ash generation for pulverized coal combustion. Progress in Energy and Combustion Science 24, 337-343.

Wittig, A.E., Anderson, N., Khlystov, A.Y., Pandis, S.N., Davidson, C.I., Robinson, A.L., 2004. Pittsburgh air quality study overview. Atmospheric Environment 38, $3107-3125$.

Woods III, E., Smith, G.D., Dessiaterik, Y., Baer, T., Miller, R.E., 2001. Quantitative detection of aromatic compounds in single aerosol particle mass spectrometry. Annals of Chemistry 73, 2317-2322.

Yokozawa, H., Kikuchi, T., Furuya, K., Ando, S., Hoshino, K., 1987. Characterization of coal fly-ash particles by laser microprobe mass spectrometry. Analytica Chimica Acta 195, 73-80.

Zhang, Q., Canagaratna, M.R., Jayne, J.T., Worsnop, D.R., Jimenez, J.-L., 2005. Time- and size-resolved chemical composition of submicron particles in Pittsburgh: implications for aerosol sources and processes. Journal of Geophysical Research 110, D07S09. 


\title{
Speciation of size-resolved individual ultrafine particles in Pittsburgh, Pennsylvania
}

\author{
Keith J. Bein, ${ }^{1}$ Yongjing Zhao, ${ }^{2}$ Anthony S. Wexler, ${ }^{3}$ and Murray V. Johnston ${ }^{4}$ \\ Received 28 February 2004; revised 8 June 2004; accepted 30 July 2004; published 4 February 2005.
}

[1] Approximately 236,000 single particle mass spectra were collected throughout the duration of the Pittsburgh Supersite experiment using the third-generation rapid single particle mass spectrometer (RSMS-3). The instrument was operated semicontinuously for 306 days, sampling particles with aerodynamic diameters in the range of $30-1100 \mathrm{~nm}$ and collecting both positive and negative ion spectra, particle size, and time of detection for each particle measured. The entire data set has been fully processed and analyzed. Spectra have been clustered into 20 distinct particle classes on the basis of the distribution of their positive ion mass peaks. Negative ion spectra were classified independently within each positive ion class. Frequency of occurrence versus particle size, month of the year, and wind direction has also been calculated for the full data set, as well as within each class. Results indicate a rich array of multicomponent ultrafine particles composed primarily of carbon and ammonium nitrate. Approximately 54\% of all the particles measured fell into the carbonaceous ammonium nitrate (CAN) class. These particles were observed in all size bins and from most wind directions for the entirety of this study. Ubiquitous sources throughout the area, including vehicular emissions and secondary organic aerosol formation, are considered to be responsible for a larger fraction of these particles. In terms of particle number, metal containing aerosol dominated the remainder of the particle classes identified. These particles were rich in $\mathrm{K}^{+}, \mathrm{Na}^{+}, \mathrm{Fe}^{+}$, and $\mathrm{Pb}^{+}$and to a lesser extent, $\mathrm{Ga}^{+}$and $\mathrm{Zn}^{+}$. They tended to be smaller in size and were highly correlated with specific wind directions, facilitating the isolation of specific sources.

Citation: Bein, K. J., Y. Zhao, A. S. Wexler, and M. V. Johnston (2005), Speciation of size-resolved individual ultrafine particles in Pittsburgh, Pennsylvania, J. Geophys. Res., 110, D07S05, doi:10.1029/2004JD004708.

\section{Introduction}

[2] Numerous advances have been made in understanding the effects of anthropogenic particle emissions on the atmosphere including the mechanism and feedback of aerosol radiative forcing, the correlation between fine atmospheric particles and adverse health effects, visibility reduction due to particle loading and ecological degradation due to particle deposition. These advances have facilitated and necessitated the continual development of aerosol instruments capable of measuring particle concentrations, size distribution and chemical composition with increasing accuracy for smaller and smaller particle sizes.

[3] Conventional instruments for these measurements were designed with the idea of collecting particles onto a

\footnotetext{
${ }^{1}$ Department of Land, Air and Water Resources, University of California, Davis, California, USA.

${ }^{2}$ Department of Mechanical and Aeronautical Engineering, University of California, Davis, California, USA.

${ }^{3}$ Department of Mechanical and Aeronautical Engineering, Department of Civil and Environmental Engineering, and Department of Land, Air and Water Resources, University of California, Davis, California, USA.

${ }^{4}$ Department of Chemistry and Biochemistry, University of Delaware, Newark, Delaware, USA.

Copyright 2005 by the American Geophysical Union. 0148-0227/05/2004JD004708\$09.00
}

substrate, which can be analyzed later for mass and chemical composition using traditional techniques. Although these instruments are useful, they are limited by the amount of detail they can capture. Essential information, such as the original chemical composition of individual particles, the distribution of compositions among different particle sizes and time resolution of particle detection, is obscured or lost. Recent technological developments have allowed for improvements in the way that atmospheric aerosol is sampled. A new technique has been established that is capable of counting, sizing, and analyzing particles one by one in real time. The general design of an instrument implementing such a technique encompasses size-selective particle focusing, particle detection, laser ablation, mass spectrometry, and signal digitization [Wexler and Johnston, 2001]. Several groups around the world have built instruments based upon this method of single particle sampling [Thomson and Murphy, 1994; Prather et al., 1994; Hinz et al., 1994; Murphy and Thomson, 1995; Suess and Prather, 1999; Jayne et al., 2000]. One of the original prototypes [Johnston and Wexler, 1995; Carson et al., 1995] is referred to as a rapid single particle mass spectrometer (RSMS). Instruments like RSMS are clearly superior in their capacity to obtain the original composition of individual particles at the time of detection as well as counting and sizing particles of similar or different composition. 
[4] One inherent drawback of these instruments is the use of light scattering to detect particles. Particles with diameters less than the wavelength of light cannot be easily detected. This puts a practical lower detection limit of approximately 200 nanometers on the instrument [Wexler and Johnston, 2001]. Unfortunately, some of the most fundamental physics and chemistry behind particle formation and evolution can only be captured in the analysis of ultrafine particles, typically defined as having aerodynamic diameters less than $100 \mathrm{~nm}$. In addition, both toxicological and epidemiological studies show an increased risk of respiratory health effects associated with exposure to ultrafine particles, as compared to accumulation mode or coarse mode particulate matter (PM), making their characterization even more important [Peters et al., 1997; Pekkanen et al., 1997; Donaldson et al., 1998; Li et al., 2003]. The instrumental modifications necessary to overcome this obstacle have only recently been implemented though they have been known for several years. The essence of eliminating the need to detect a particle lies in the ability to fire the ablation laser with a constant energy output at a high frequency and to only collect spectra when a particle is present [Reents et al., 1995]. This technique was first deployed in the development of the second-generation rapid single particle mass spectrometer, RSMS-2 [Carson et al., 1997]. Further improvements to RSMS-2 were made by the inclusion of a size-selective focusing inlet, originally introduced by Mallina et al. [2000]. The design of the instrument and results from laboratory experiments have been described in detail elsewhere [Ge et al., 1998; Mallina et al., 2000; Johnston, 2000; Phares et al., 2002; Lake et al., 2003]. In addition, RSMS-2 has been successfully deployed in two major field studies, the 1999 Atlanta Supersite experiment [Rhoads et al., 2003] and the 2000 Houston Supersite experiment [Phares et al., 2003]. During the Atlanta study four different types of single particle instruments, including RSMS-2, were deployed simultaneously [Lee et al., 2002; Liu et al., 2003; Jimenez et al., 2003; Rhoads et al., 2003; Wenzel et al., 2003; Lee et al., 2003]. For an inter comparison of the measurements obtained from these techniques, see Middlebrook et al. [2003].

[5] RSMS-3 is the latest version in the RSMS family. Improvements in RSMS-3 over earlier versions include: (1) the addition of a negative ion mass spectrometer to run in conjunction with the positive one such that both positive and negative ion spectra are obtained for each particle, (2) two $\mathrm{A} / \mathrm{D}$ converters on each spectrometer to increase dynamic range, and (3) software improvements that enable automation and remote control of the instrument. RSMS-3 was specifically constructed for deployment in a yearlong air quality field study located adjacent to Carnegie Mellon University in Pittsburgh, Pennsylvania as a part of the U.S. Environmental Protection Agency (EPA) Supersites program. The purpose of RSMS-3 in this study was to provide semicontinuous, time and size resolved measurements of single particle composition for ambient air pollution in the Pittsburgh area. These measurements are extremely valuable not only in assessing Pittsburgh air quality but also for numerous other applications. Many issues of interest in this field, ranging from health affects to global climate, are believed to be strongly linked to the composition as well as the size of the particles involved.
This paper, however, will focus strictly on statistical analysis of the data and the application of these statistics to initiating possible source-receptor relationships.

\section{Experiment}

\subsection{Instrument}

[6] As mentioned in the introduction, the details of RSMS have been described in previous work such that only the major points will be revisited here. RSMS-3 is depicted schematically in Figure 1. The measurement technique involves size-selective particle sampling, particle beam generation, laser desorption/ionization of individual particles and mass spectrometry. During this experiment, ambient air was drawn from outside the trailer at a height of 6.4 meters above ground through a $10 \mathrm{~cm}$ diameter stainless steel duct. Aerosol was sampled from the center of the duct through $1 / 4$ inches OD copper tubing and dried with a Nafion dryer (Perma Pure, Inc., Toms River, New Jersey) prior to entering the inlet. Using a computer controlled 10-position rotary valve (Valco Instruments Co., Houston, Texas), the particle flow is then directed through one of nine differently sized flow-limiting orifices contained in an orifice bank (O'Keefe Controls Co., Trumbull, Connecticut). The size of the orifice determines the pressure just upstream of the critical focusing orifice. This inlet pressure, in conjunction with the geometry of the critical orifice, determines the particle size focused and transmitted into the instrument. Aerodynamic diameter of the focused particles can be approximated by

$$
D_{p}=\left[(1.66 \lambda)^{2}+\left(\frac{18 \mu d_{n} S t k_{f}}{\rho_{p} U_{\text {sonic }}}\right)\right]^{\frac{1}{2}}-166 \lambda
$$

where $\lambda$ is the mean free path evaluated at the critical orifice conditions [Wexler and Johnston, 2001]. The maximum particle diameter that can be focused in this manner is given by the quantity within the inner brackets, where $\mu$ is gas viscosity, $d_{n}$ is the diameter of the critical orifice, $\rho_{p}$ is particle density, $\mathrm{Stk}_{\mathrm{f}}$ is the stokes number that is focused $(\sim 1.1)$ and $U_{\text {sonic }}$ is the speed of the gas through the critical orifice, which is sonic since the flow is choked. The particle beam then travels through several differentially pumped skimming stages in which the carrier gas is removed using both mechanical pumps (BOC Edwards, Wilmington, Massachusetts; Varian Inc., Lexington, Massachusetts) and split turbo pumps (Pfeiffer Vacuum, Nashua, New Hampshire). Particles then enter the source region where they are vaporized and ionized by a colinear, counter propagating and free-fired $193 \mathrm{~nm}$ excimer laser (GAM Laser, Orlando, Florida). Ions are initially accelerated by a dual gradient in the source region and then travel at constant velocity down their respective time of flight tubes until they impinge upon the microchannel plate detectors (Burle Electro-optics Inc., Sturbridge, Massachusetts). Current from each detector is recorded and digitized by two separate $500 \mathrm{MHz}$ digitizer channels (Acqiris, Monroe, New York). Overlapping high and low sensitivity channels increases signal resolution and enhances dynamic range by optimizing the offset between signal saturation and limit of detection. Triggered by each laser pulse $(50 \mathrm{~Hz})$, the digitizer collects 5000 sample points 


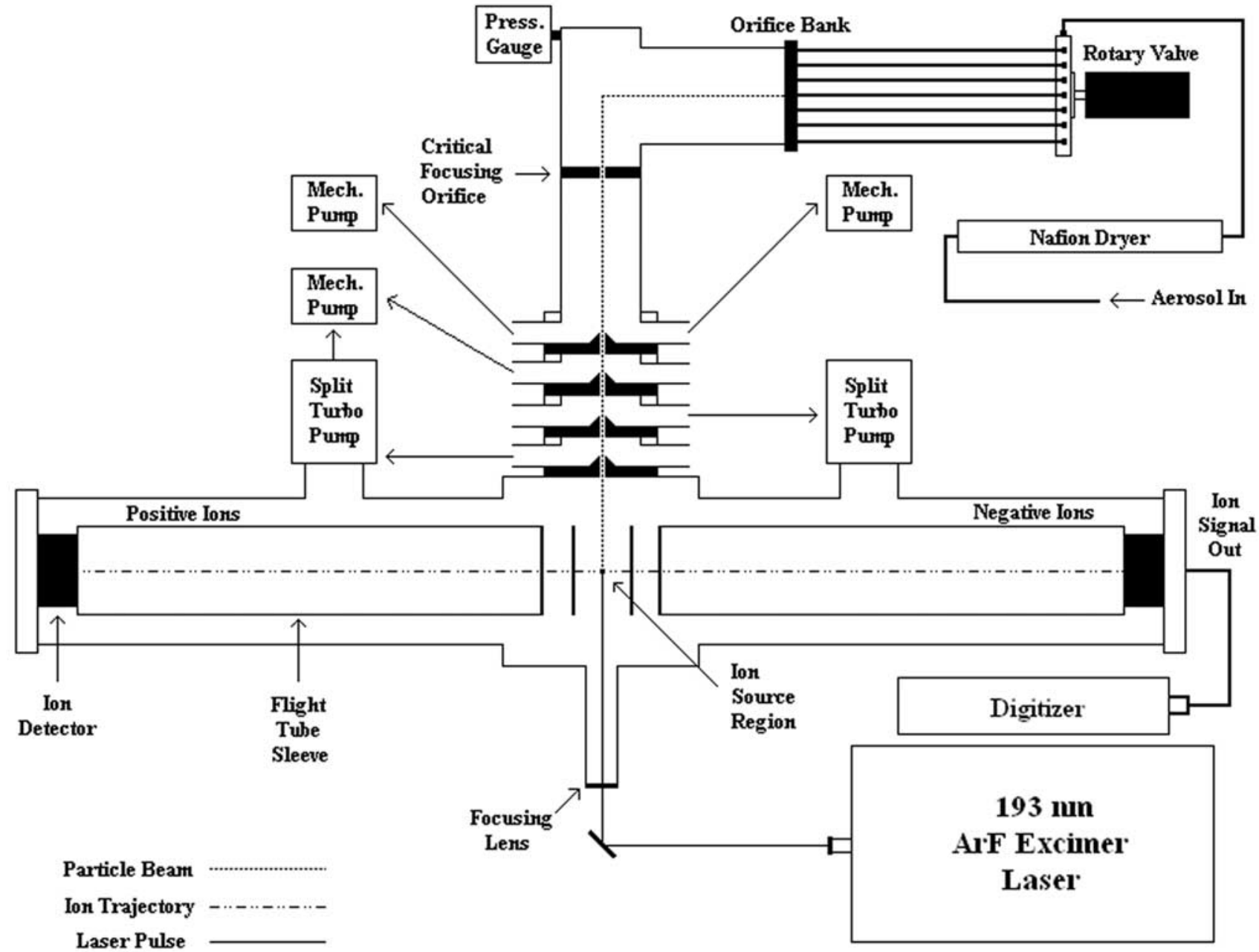

Figure 1. A schematic of the RSMS-3 instrument.

with a sampling rate of $2 \mathrm{~ns}$ covering a mass-to-charge ratio range of $\sim 1-300 \mathrm{Da}$ for each detector. As part of the data acquisition software, written in LabView ${ }^{\circledR}$ (National Instruments Inc., Austin, Texas), signal intensity from each mass spectrum is checked against an experimentally determined threshold to ensure that only spectra from true particle hits are recorded. Peaks from either positive or negative ions can trigger the spectrum to be saved. Threshold values are different for different windows within the spectrum and certain sections were left out to avoid triggering on background peaks. Since the background peaks are associated with gas phase contamination originating from inside the instrument, their relative peak heights are independent of the particle size being sampled, and thus the same threshold values were used for all nine orifices. For each particle hit, positive and negative ion intensity, time of detection and particle size are recorded.

\subsection{Sampling Protocol}

[7] RSMS-3 was fully automated for the entirety of the Pittsburgh Supersite experiment, controlled solely by the data acquisition software, except during times of maintenance or special studies. The measurement protocol was based on sampling intervals starting every three hours for the first four months (September-December 2001) and every two hours for the remainder of the study (JanuarySeptember 2002). Each sampling interval consisted of cycling through the nine flow-limiting orifices, corresponding to nine different particle sizes transmitted into the instrument ranging from about $30 \mathrm{~nm}$ to 1.1 micrometers. The instrument was operated at each orifice until either $10 \mathrm{~min}$ expired or 30 particles were sampled, whichever came first. On average, $\sim 1$ hour was required to step through the entire orifice bank. This protocol was adopted to allow for instrument down time between sampling intervals to optimize the balance between instrument sustainability and robustness of the data set. Over the course of a day, the average number of single particle mass spectra acquired was $\sim 1100$. Of these $1100, \sim 15 \%$ had detectable signal from both positive and negative ions while the remainder had signal from positive ions only.

\subsection{Data Processing and Analysis}

[8] Remote control software, pcAnywhere ${ }^{\text {TM }}$ (Symantec Inc., Cupertino, California), was used to monitor the performance of the instrument and to transfer data from the instrument's hard drive to University of California-Davis computers for storage, postprocessing and analysis. Each single particle mass spectrum collected has been processed from its original state as follows: (1) It has been time to 
mass calibrated according to the relation $m / z=(a t+b)^{1 / 2}$ where $m / z$ is mass to charge ratio, $a$ and $b$ are experimentally determined constants and $t$ is the time interval during which the ion current was detected. (2) A binned ion current for each $\mathrm{m} / \mathrm{z}$ value was obtained by integrating $\pm 0.5 \mathrm{Da}$ about each integer $\mathrm{m} / \mathrm{z}$ value. (3) The spectrum has been normalized across all $\mathrm{m} / \mathrm{z}$ values according to a Euclidian norm. Calibration is the single most important step in this procedure. It is vital to the interpretation of the spectral peaks. Since the calibration constants are moderately dependent on the degree to which the particle and laser beams are coincident, they have a tendency to vary slightly from spectrum to spectrum. As a result, each spectrum has been inspected and calibrated individually to ensure the quality of the data processing and the integrity of the analysis.

[9] Owing to recent progress in digital capture, processing and storage technology, RSMS-3 presents a unique challenge to data analysis in its ability to amass enormous sets of data in relatively short time durations. For instance, over 200,000 dual-polarity single particle mass spectra were collected during this campaign alone. Confounding the issue is that each spectrum itself can have as many as 500 independent components, or dimensions, each with its own inherent uncertainty. Perhaps the single most challenging task in working with such data sets is creating an unbiased and self-consistent mechanism for reducing the amount of data without compromising resolution or robustness. An obvious starting point is to construct an algorithm that organizes individual data points (spectra) into unique clusters (particle classes) based upon some metric of similarity between them. The process should include a set of conditions that control the evolution of the classification while simultaneously allowing for convergence to a unique solution.

[10] Adaptive Resonance Theory version 2a (ART-2a) [Carpenter et al., 1991], originally introduced to the aerosol community by Hopke and Song [1997], is an example of such a classification algorithm currently in use by a number of groups. Spectra obtained from laboratory generated aerosol of known composition were used to validate its application to single particle data [Phares et al., 2001]. It uses the vector dot product as its metric of similarity and is controlled by two parameters, one of which sets the condition for similarity and the other which determines the rate at which the algorithm learns. A new algorithm, based on ART-2a, has been developed specifically for the analysis of this data set encompassing several improvements. First, the process by which clusters are seeded has been reconstructed to allow for the identification of "natural seeds" in a data set, rather than picking random seeds. "Natural seeds" are defined as those spectra having the largest number of most similar partners. This makes the seeding process definitive, rather than random, and ensures that the outcome of the classification is no longer dependent on the choice of spectra used to seed it, since the same spectra will always be chosen. Secondly, the dynamics of the algorithm have been de-coupled from parameterization to allow for unbiased learning from iteration to iteration. Specifically, the learning rate parameter has been eliminated in favor of equal-weighted averaging; allowing the variance in the mass peaks of each cluster to be calculated. This ensures that the clustering is based on true statistics rather than an arbitrary parameter. Finally, a variance-weighted similarity metric, based on the geometric distance rather than the dot product, has been adopted. Both the dot product and the geometric distance are inherently a measure of the angle of separation between two vectors (spectra), but the geometric distance is proportional to the sine of the angle, rather than the cosine, and thus is more sensitive at small angles; similar vectors. Using either metric alone, however, assumes that each dimension is equally important when comparing spectra and therefore each dimension should be equally weighted. In attempts to relax this notion, a more realistic approach has been taken that weights each dimension by its own inherent variance. This allows clusters to adopt an ellipsoidal shape, rather than being strictly spherical. The algorithm has been thoroughly tested and validated on a well characterized subset of data collected during this experiment. Results indicate convergence from different sets of initial conditions, stability from iteration to iteration and reproducibility of results.

[11] In addition to data clustering, numerous other analysis techniques have been applied which sort, organize, correlate and count spectra. These have been used on the entire data set to determine the fraction of total particle hits by month, size and particle class. They were also applied independently to each particle class to establish the distribution of particle hits within the class by month, time of day, size and wind direction. Correlating single particle spectra with wind data is possible due to the time signature associated with each spectrum. It allows for the identification of the direction(s) from which each particle class is most frequently observed. This, in conjunction with knowledge of local industry, can be used to associate specific classes with nearby sources and has proven to be a very powerful technique for studying source attribution.

\subsection{Site}

[12] During this field study, RSMS-3 was housed at the Schenley Park site located adjacent to Carnegie Mellon University in Pittsburgh, Pennsylvania. Pittsburgh can be characterized as a largely industrialized and urbanized city surrounded by both suburbs and rural areas. As such, it was an excellent location to capture the true depth and complexity of urban aerosol. The list of emission sources within Pittsburgh is exhausting, let alone the possibility of regional transport from outside the city. Figure 2 depicts a map of some of the major PM2.5 point sources within $24 \mathrm{~km}$ (15 miles) of the site. Notice that the site is surrounded by coal fired power plants. To the northeast are Allegheny Power and the Duquesne Light Co., to the south are Elrama and Mitchell and to the northwest, not drawn on the map, are Orion Power Midwest LLC (Phillips Station, $28.3 \mathrm{~km}, 301^{\circ}$ ) and Bruce Mansfield (1255 metric tons/year PM2.5, $45.5 \mathrm{~km}, 298^{\circ}$ ). There are seven additional major coal fired power plants within $80 \mathrm{~km}$ (50 miles) of the site. All of these power plants are considered large sources of $\mathrm{NO}_{\mathrm{x}}$ and $\mathrm{SO}_{2}$, as well as moderate sources of trace metals. Steel mills and blast furnaces comprise the next largest industrial point source of PM in the area. USX Corporation (Edgar Thompson Works), USX Corporation (Irvin Plant), USS Clairton Works, Universal and Stainless Alloy Products and Shenango Neville Island Coke Works all use steel mills and blast furnaces. Other sources on the map include a 


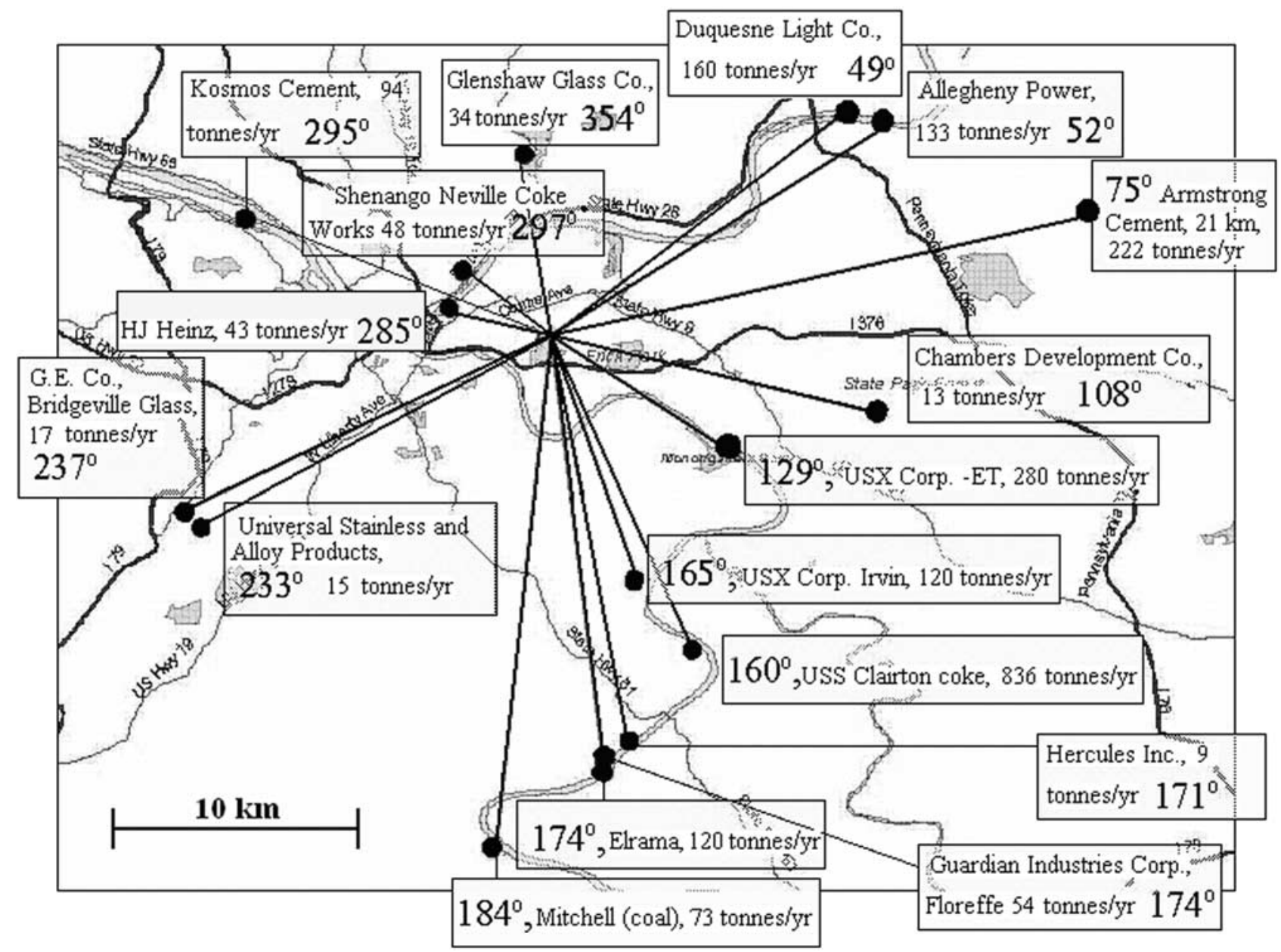

Figure 2. Major PM sources within $24 \mathrm{~km}$ of the site. Bearing from site is measured clockwise from true north. Listed emission rates are for PM2.5.

landfill owned by Chambers Development Co., two cement processing facilities (possibly large sources of coarse mode calcium) and two glass plants. Not included on the map, but still of interest, are a hospital incinerator located adjacent to Shenango Coke Works (General Suburban Hospital, $11.9 \mathrm{~km}$, $310^{\circ}$ ), Allegheny Ludlum Steel (410 metric tons/year PM2.5, $26.5 \mathrm{~km}, 42^{\circ}$ ) and Zinc Corporation of America (363 metric tons/year PM2.5, $41.8 \mathrm{~km}, 307^{\circ}$ ), a company dealing primarily in zinc and other nonferrous metals. Besides industry, a significant fraction of PM measured at the site can be attributed to numerous ubiquitous sources such as commuting, cooking and wood burning.

\section{Results}

[13] RSMS-3 began taking measurements on 20 September 2001 and was successfully sampling for 306 of the possible 372 operation days. During this period, 236,286 single particle mass spectra were acquired, $185,244(78.4 \%)$ of which had positive ion signal only and $51,042(21.6 \%)$ that had both positive and negative ion signal. There were very few instances of spectra with only negative ion signal. Figure 3 a shows the fraction of total particle hits obtained from each of the nine orifices for particles with positive ion spectra only (solid white) and those with positive and negative ion spectra (diagonal stripes). The sum of the two is the total fraction. The nominal aerodynamic diameter of particles focused by each orifice is labeled in nanometers on the $\mathrm{x}$ axis. Please note that this is not a true size distribution, particles are simply being counted at each orifice. There are many confounding factors involved with extracting true size distributions from this data. The difficulty lies in the fact that the hit rate efficiency of the instrument is a function of both particle size and composition, as has been discussed in detail by Kane and Johnston [2000]. It is also important to note that the existence of a complimentary negative ion spectrum is most commonly an indicator of atmospheric aging. This is simply due to the fact that the major negative ions detected are those of secondary particle components, specifically nitrates and sulfates. As a result, negative ion signal is seen predominantly in the spectra of particles from the larger size bins because there is simply more analyte in larger particles, making detection more likely. Note that small particles may have also undergone significant atmospheric transformations but this cannot be detected in the negative ion spectrum due to the combination of insufficient analyte and lower instrument sensitivities for these components in general [Kane et al., 2002]. The fraction of total particle hits by month 

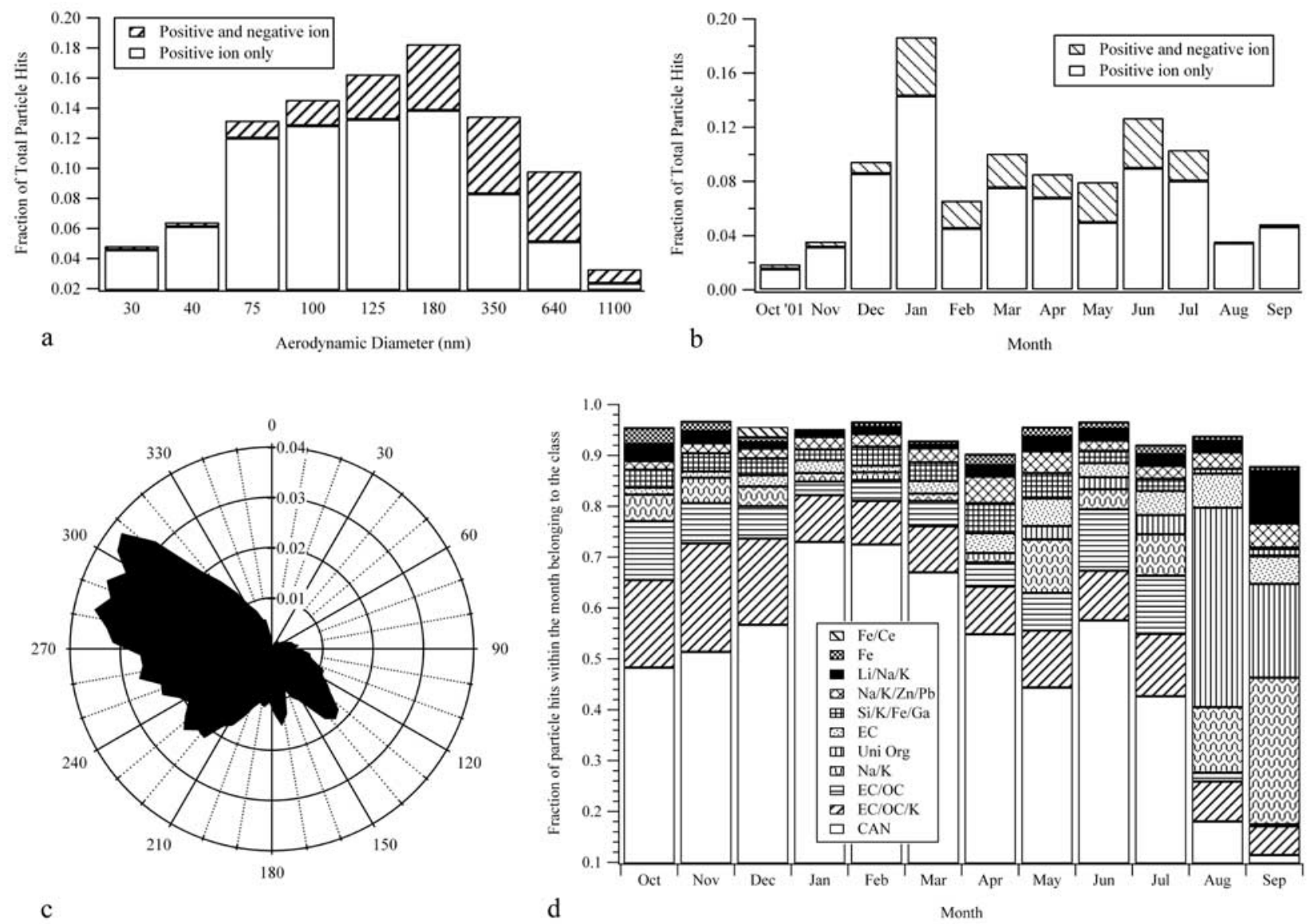

Figure 3. Summary statistics for all particles sampled during the Pittsburgh Supersite experiment. (a) Fraction of total particle hits versus particle aerodynamic diameter for spectra containing both positive and negative ion signal and those containing positive ion signal only. (b) Fraction of total particle hits by month for spectra containing both positive and negative ion signal and those containing positive ion signal only. Reported values have been normalized by the number of days within each month the instrument was operating. (c) Frequency of observation versus wind direction for all wind observations greater than $2 \mathrm{~m} / \mathrm{s}$. Wind direction is measured clockwise from true north. (d) Monthly distribution of the ten largest particle classes, along with the iron/cerium class. The number of spectra, belonging to a given class within each month, has been normalized by the total number of spectra collected during that same month.

of the year is shown in Figure 3b, normalized by the number of days within each month that the instrument was actually sampling. Once again, both the fraction of particles with positive ion spectra only (solid white) and the fraction of those with positive and negative ion spectra (diagonal stripes) have been plotted. Results indicate that the largest fraction of particles sampled, as well as the largest fraction containing negative ion spectra, was observed in the winter during the month of January. However, there are elevated levels in the summer months of June and July as well. Figure 3c displays frequency of occurrence versus wind direction for all wind observations greater than two meters per second, depicting major wind directions in the Pittsburgh area. Notice that the wind comes predominantly from the west to northwest and is almost never observed originating from anywhere inside the first quadrant. This is an important issue to consider when interpreting wind signatures associated with specific particle classes. Particles are observed in the first quadrant, but little statistical significance is given to these observations in the context of identifying source-receptor relationships.

[14] Data classification was performed in a series of steps designed to enhance the quality of the classification and make certain its validity. First, the entire data set was split into three groups based upon periods of similar operating conditions and instrument performance. The clustering algorithm was then applied independently to each of the three groups using positive ion spectra only. In total, over 500 clusters were isolated. Each cluster was individually inspected, peak assignments were made for all known peaks and the normalized peak heights recorded. This information was used in conjunction with a sorting algorithm to organize the clusters into approximately 100 subclasses. Sorting was performed under the condition that all clusters within each subclass contain the same six largest peaks in identical peak height progressions. Subclasses were then categorized into 20 distinct particle classes by grouping together those 
containing the same four dominant peaks, regardless of the distribution of peak heights. Results are summarized in Table 1. The first column contains a general description of each particle class, the second displays the percent of the total number of spectra which belong to that class, the third is the percent of spectra within the class observed to have negative ion signal, the fourth shows the percent of spectra within the class having detectable amounts of $\mathrm{NO}^{+}$, the fifth lists the subclasses within each class and the sixth column lists the subclass distribution within the class. Figure $3 \mathrm{~d}$ depicts the monthly distribution of the ten largest particle classes listed in Table 1, along with the iron/cerium class. The number of spectra, belonging to a given class, within each month has been normalized by the total number of spectra collected during that same month. Notice that all of these classes, with the exception of unidentified organics and iron/cerium, were observed continuously throughout the duration of this study, and that a majority of the particles observed were carbonaceous in nature, both overall and within each month. A more detailed discussion of the temporal distribution of each class will be given below. Negative ion spectra were classified independently within each positive ion class during the final step of classification. Figure 4 depicts the distribution of all negative ion spectra among the identified negative ion classes. As stated above, sulfate and nitrate, secondary components, dominate the entire distribution. Note that every single negative ion class has some form of sulfate in it. The four largest classes contain nitrate/sulfate, sulfate only, chlorine/nitrate/sulfate and elemental carbon/organic carbon/sulfate.

[15] Figures 5-16 summarize the results of the 10 major particle classes $(>1 \%$ of the total number of particles, see Table 1) and two minor classes $(\mathrm{Ce} / \mathrm{Fe}$ and $\mathrm{Cr} / \mathrm{Mo} / \mathrm{W})$. Each figure contains five graphs, $a-e$, that illustrate different ensemble characteristics of the particles found in that class. The first graph (a) is a spectral representation of the class showing normalized peak area versus mass to charge ratio $(\mathrm{m} / \mathrm{z})$, the average of all spectra belonging to the dominant subclass. The bars at the top of each mass peak represent the standard deviation in the average. The second plot (b) depicts both the fraction of particle hits having positive ion signal only (solid white) and the fraction having positive and negative ion signal (diagonal stripes) versus aerodynamic diameter. Third is a wind rose (c) illustrating frequency of observation of the class versus wind direction for all wind speeds greater than $2 \mathrm{~m} / \mathrm{s}$. Real time data acquisition allows each single particle spectrum to be associated with a specific wind speed and direction. As a result, particles can be counted for a given wind direction interval. For the analysis described in this paper, $5^{\circ}$ intervals were used. Values obtained for each interval have been normalized by the total number of wind observations, depicted in Figure 3c, for that same interval. This has been done in attempts to normalize out the major wind directions, isolating regions where particles were observed more frequently than the wind, relative to the other directions. However, as mentioned above, the wind is almost never observed originating from anywhere inside the first quadrant and thus particle observations within this quadrant will tend to be exaggerated, relative to the other three quadrants, during the normalization process. In this sense, peaks occurring in the first quadrant are considered statistically insignificant and will be disregarded.
Next is a graph (d) showing the fraction of particle hits by month for spectra containing positive ion signal only and those containing both positive and negative ion signal. The fractions reported have been obtained by normalizing the number of particle hits, belonging to the class, that were observed during each month by the total number of particles observed during that same month. The final plot (e) is a pie chart displaying the distribution of particles, for which negative ions were observed, among the identified negative ion classes.

\subsection{Carbonaceous Ammonium Nitrate (CAN)}

[16] CAN is by far the largest and perhaps most complex class of particles identified in Pittsburgh. For a large majority of these particles, it is not possible to determine whether the carbon is elemental or organic solely from the peaks in their mass spectra. The laser energy required to vaporize and ionize particles is too large to retain any significant information about the original structure of most organic molecules. As a result, unless stated otherwise, the carbon in these particles can be elemental, organic or a combination of the two. The spectral representation shown in Figure 5 is that of an ammonium nitrate dominant subclass containing smaller carbon peaks (44\% of class). Closely related is the carbon dominant subclass containing smaller ammonium nitrate peaks $(28 \%)$. It is important to note that making a distinction between these two subclasses is a bit arbitrary. In reality, the ratio of signal intensities, $\mathrm{NO}^{+} / \mathrm{C}_{1}^{+}$, is continuously distributed over a range of $\sim 0.1-$ 1.5 [Zhao et al., 2005]. Therefore it is more appropriate to think of only one subclass that displays a wide range of relative peak heights. The other three subclasses, comprising the remaining $28 \%$ of the class, have the same four dominant peaks $\left(\mathrm{C}_{1}^{+}, \mathrm{C}_{2}^{+}, \mathrm{C}_{3}^{+}\right.$and $\left.\mathrm{NO}^{+}\right)$as the previous two, but contain additional peaks, facilitating their isolation as different particle types. Two of them contain carbon that is distinguishably organic and appears to be highly oxygenated (oxy-OCAN), as evidenced by the presence of signature peaks for: $\mathrm{CO}^{+}, \mathrm{C}_{2} \mathrm{H}_{3} \mathrm{O}^{+}, \mathrm{C}_{4} \mathrm{H}_{7} \mathrm{O}^{+}$, etc. The remaining one, as well as one of the oxy-OCAN subclasses, contains distinct positive ion sulfate peaks $\left(\mathrm{SO}^{+}, \mathrm{HSO}_{2}^{+}, \mathrm{HSO}_{3}^{+}\right.$and $\mathrm{NH}_{4} \cdot \mathrm{HSO}_{3}^{+}$) in addition to carbon and ammonium nitrate (CANS, oxy-OCANS), possibly indicating a greater amount of sulfate in these particles. The purpose to making a distinction between these subclasses is to accentuate the fact that this class does not represent a single particle type, but rather a distribution of many carbonaceous aerosols containing various combinations of numerous organic species, elemental carbon, nitrate and sulfate.

[17] Looking at the fraction of total particle hits by size indicates that these particles have undergone a considerable amount of atmospheric aging and thus tend to be skewed toward the larger size bins. This is also evident by the overwhelming amount of ammonium nitrate and sulfate found in both the positive and negative ion spectra. The organic carbon itself is most likely from combustion or secondary organic aerosol. From the wind rose there appear to be numerous sources, as was expected given the complexity of the class and the nature of the particle. The monthly distribution shows the particle fraction slowly climbing during the late fall, peaking during the winter months of January and February, and then falling off as 
Table 1. Positive Ion Classification Statistics ${ }^{\mathrm{a}}$

\begin{tabular}{|c|c|c|c|c|c|}
\hline Positive Ion Class $^{\mathrm{b}}$ & $\begin{array}{l}\text { Percent } \\
\text { of Total }^{\mathrm{c}}\end{array}$ & $\begin{array}{l}\text { Percent } \\
\text { With } \\
\text { Negative } \\
\text { Ion } \\
\text { Signal }^{\mathrm{d}} \\
\end{array}$ & $\begin{array}{c}\text { Percent } \\
\text { With } \\
\text { Ammonium } \\
\text { Nitrate }^{\mathrm{e}} \\
\end{array}$ & Subclasses $^{\mathrm{f}}$ & Percent of Class $^{\mathrm{g}}$ \\
\hline $\begin{array}{l}\text { Carbonaceous ammonium } \\
\text { nitrate (CAN) }\end{array}$ & 54.43 & 22.06 & 100 & $\begin{array}{c}\text { ammonium nitrate, carbon, } \\
\text { oxygenated }+ \text { sulfate, } \\
\text { oxygenated organics, sulfate }\end{array}$ & $\begin{array}{c}44.2,28.3,12.9 \\
11.7,2.9\end{array}$ \\
\hline $\mathrm{EC} / \mathrm{OC} / \mathrm{K}$ & 10.87 & 20.59 & 99.6 & EC/OC_K, EC/OC_K, $\mathrm{Pb}$ & $93.9,6.1$ \\
\hline $\mathrm{EC} / \mathrm{OC}$ & 6.59 & 20.39 & 3.5 & 6 subclasses $\left(\mathrm{C}_{1}, \overline{\mathrm{C}}_{2}, \mathrm{C}_{3}\right)$ & 100 \\
\hline $\mathrm{Na}, \mathrm{K}$ & 5.76 & 14.87 & 72.5 & 5 subclasses $(\mathrm{Fe}, \mathrm{Pb})$ & 100 \\
\hline Unidentified organics & 3.34 & 19.89 & 64.6 & 4 subclasses & 100 \\
\hline Elemental carbon & 3.24 & 20.88 & 63.3 & 3 subclasses & 100 \\
\hline $\mathrm{Si}, \mathrm{K}, \mathrm{Fe}, \mathrm{Ga}$ & 3.12 & 30.52 & 66.7 & $\begin{array}{c}10 \text { subclasses } \\
(\mathrm{Al}, \mathrm{Na}, \mathrm{Li}, \mathrm{Pb}, \mathrm{P}, \mathrm{Cr})\end{array}$ & 100 \\
\hline $\mathrm{Na}, \mathrm{K}, \mathrm{Zn}, \mathrm{Pb}$ & 2.86 & 18.6 & 86.2 & $\begin{array}{l}8 \text { subclasses } \\
(\mathrm{Fe}, \mathrm{Cr}, \mathrm{Li}, \mathrm{Sn})\end{array}$ & 100 \\
\hline $\mathrm{Li}, \mathrm{Na}, \mathrm{K}$ & 2.28 & 11.56 & 71 & 3 subclasses $(\mathrm{Fe}, \mathrm{Pb})$ & 100 \\
\hline $\mathrm{Fe}$ & 1.16 & 24.31 & 16.6 & 3 subclasses & 100 \\
\hline $\mathrm{Na}, \mathrm{K}, \mathrm{Sn}, \mathrm{Pb}$ & 0.8 & 22.24 & 67.1 & 3 subclasses $(\mathrm{Li}, \mathrm{Cr})$ & 100 \\
\hline Amines & 0.68 & 15.03 & 100 & $\begin{array}{l}\mathrm{C}_{3} \mathrm{H}_{8} \mathrm{~N}, \mathrm{C}_{5} \mathrm{H}_{12} \mathrm{~N} \\
\text { hydroxy aliphatic }\end{array}$ & $58.9,37.4,3.8$ \\
\hline $\mathrm{Al}, \mathrm{Si}, \mathrm{Ca}$ & 0.55 & 51.08 & 19.6 & 6 subclasses $(\mathrm{Na}, \mathrm{K}, \mathrm{V}, \mathrm{Pb})$ & 100 \\
\hline Mixed metals & 0.5 & 17.69 & 41.5 & 27 subclasses & 100 \\
\hline Nickel & 0.45 & 0.09 & 0 & 4 subclasses $(\mathrm{Fe}, \mathrm{Ca}, \mathrm{Co})$ & 100 \\
\hline $\mathrm{Ca} / \mathrm{CaO}$ & 0.37 & 24.12 & 66.7 & 4 subclasses $(\mathrm{S}, \mathrm{P}, \mathrm{Na})$ & 100 \\
\hline $\mathrm{Fe}, \mathrm{Ce}$ & 0.29 & 25 & 80.8 & 3 subclasses & 100 \\
\hline Unidentified & 0.26 & 37.14 & N/A & $\mathrm{N} / \mathrm{A}^{\mathrm{h}}$ & N/A \\
\hline Cr, Mo, W & 0.13 & 8.91 & 10.9 & 2 subclasses $(\mathrm{Na}, \mathrm{K})$ & 100 \\
\hline Sulfate only (positive) & 0.02 & 3.7 & 0 & no subclasses & 100 \\
\hline
\end{tabular}

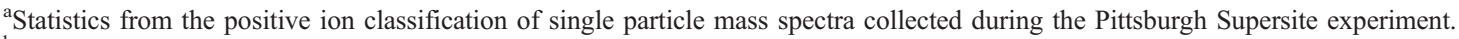

${ }^{\mathrm{b}}$ Generalized description of identified classes.

${ }^{c}$ Percent of the total number of particles sampled that belong to each class.

${ }^{\mathrm{d}}$ Percent of particles within the class observed to have detectable negative ion signal.

${ }^{e}$ Percent of particles within the class containing detectable amounts of $\mathrm{NO}^{+}$(associated with ammonium nitrate).

${ }^{\mathrm{f}}$ General description of subclasses identified within each class.

gercent of particles within the class that belong to each subclass.

${ }^{\mathrm{h}} \mathrm{N} / \mathrm{A}$, not applicable.

spring and summer approach. This trend is most likely associated with a reduction in the vapor pressure of ammonium nitrate accompanying lower temperatures. The reduction in vapor pressure will force the ammonium nitrate into the particle phase and thus increase the frequency with which this class of particles is observed. However, notice that there are elevated levels in the month of June, falling outside the trend. Obviously nitrate and sulfate dominate the negative ion speciation within this class. A bit more surprising is the significant amount of chlorine found in these particles, whose source is unknown.

\subsection{Elemental Carbon/Organic Carbon/Potassium (EC/OC/K)}

[18] In urban and residential settings, biomass particles are typically associated with burning wood for energy or recreation. Rural areas can also be a large source of biomass particles, typically through heating, recreation and waste disposal. The signature spectrum, shown in Figure 6 , includes a potassium ion in conjunction with multiple carbon peaks $\left(\mathrm{C}_{1}^{+}-\mathrm{C}_{4}^{+}\right)$. Similar to $\mathrm{CAN}$, the $\mathrm{K}^{+} / \mathrm{C}_{1}^{+}$ ratio exhibits a rather continuous distribution. Also similar to CAN, it is not possible to discern whether the carbon is elemental or organic from these mass peaks. A majority of the particles measured had aerodynamic diameters in the range of $100-200 \mathrm{~nm}$, consistent with a combustion source. The wind rose suggests numerous sources and the monthly distribution indicates that these particles were observed largely during the late fall and early winter, although there are slightly elevated levels in July as well. The negative ion spectra contain predominantly nitrate and sulfate, again with a significant amount of chlorine. Chlorine in this class is most likely

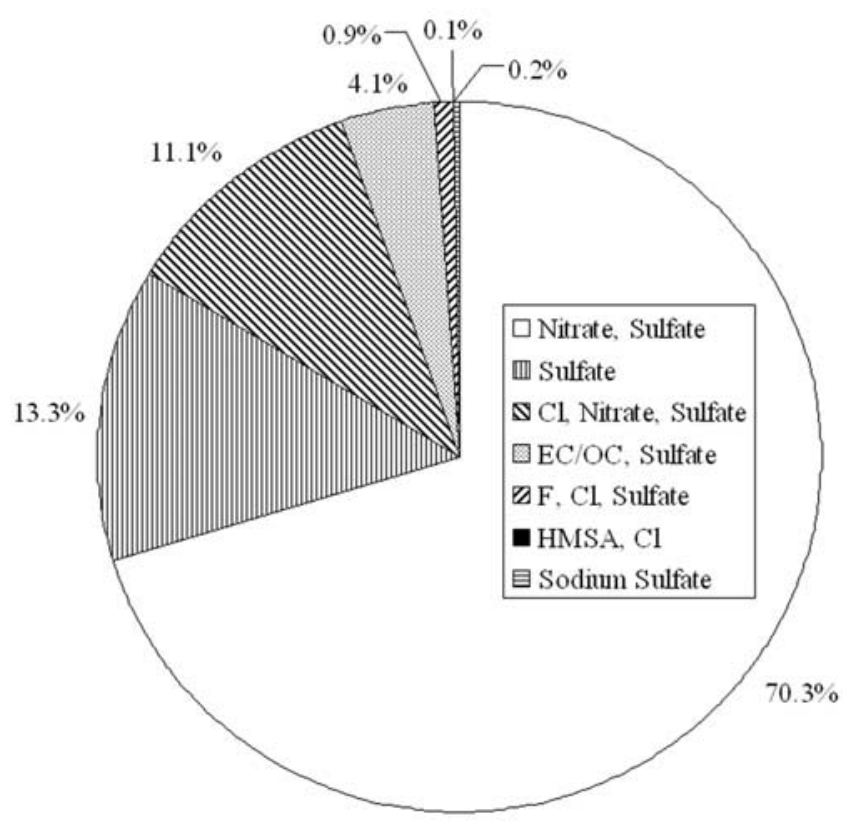

Figure 4. Overall classification of negative ion mass spectra collected during the Pittsburgh Supersite experiment. 

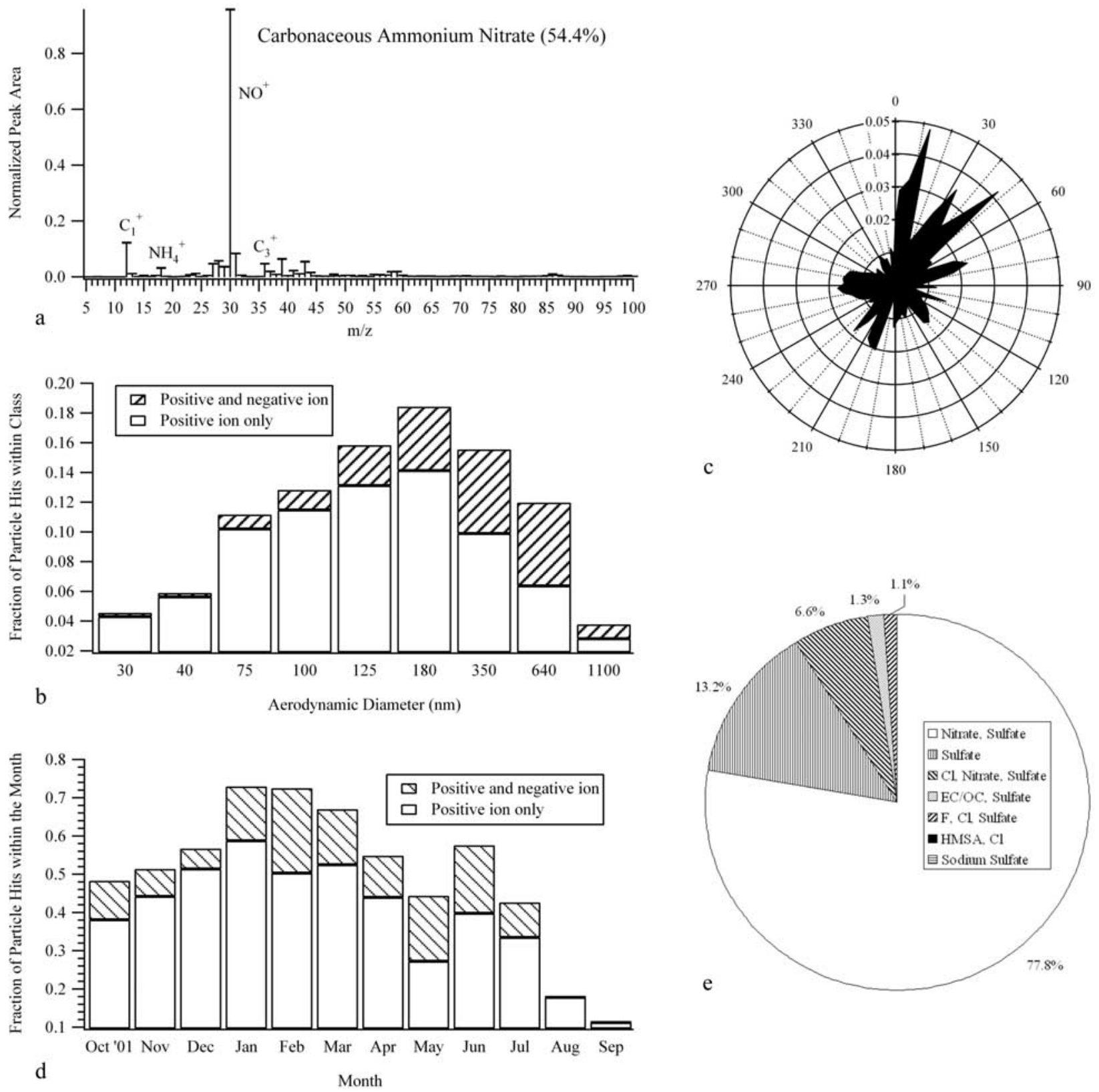

Figure 5. Summary statistics from the analysis of the carbonaceous ammonium nitrate class, containing $54.4 \%$ of the total number of particles sampled. See text for more details. All distributions plotted in this figure refer solely to the particles within this class. (a) Positive ion spectral representation of the carbonaceous ammonium nitrate class depicting average peak area versus mass to charge ratio. The bars at the top of each mass peak represent the standard deviation in the average. (b) Fraction of particle hits versus particle aerodynamic diameter for spectra containing both positive and negative ion signal and those containing positive ion signal only. (c) Frequency of observation versus wind direction for wind speeds greater than $2 \mathrm{~m} / \mathrm{s}$. Values obtained for each wind direction interval have been normalized by the total number of wind observations for that same interval. Wind direction is measured clockwise from true north. (d) Fraction of particle hits by month for spectra containing both positive and negative ion signal and those containing positive ion signal only. Reported fractions have been obtained by normalizing the number of spectra, belonging to the class, that were observed during each month by the total number of particles observed during that same month. (e) Distribution of particles, for which negative ions were observed, among the identified negative ion classes. 

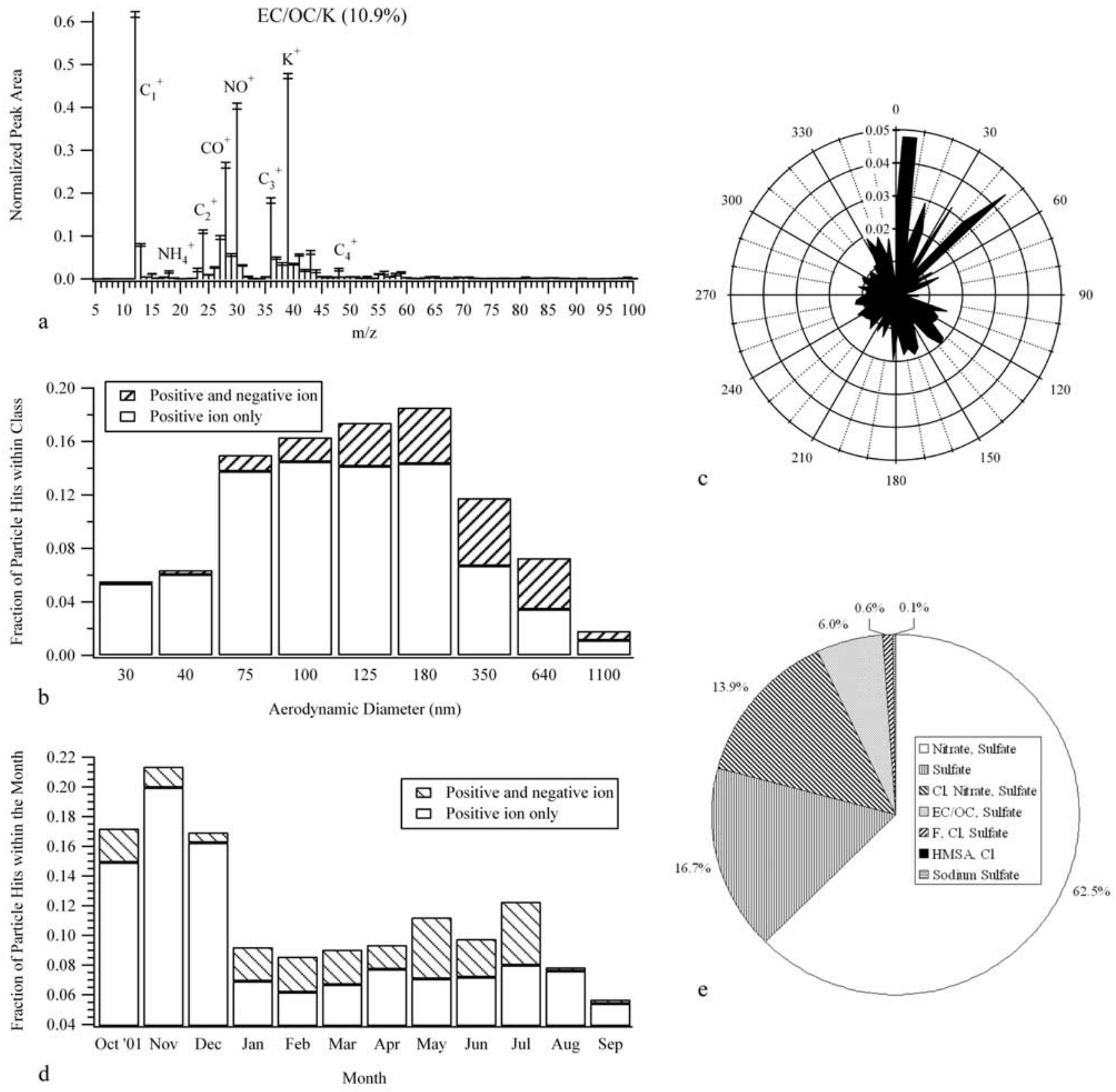

Figure 6. Summary statistics from the analysis of the EC/OC/K class, containing $10.9 \%$ of the total number of particles sampled. See caption of Figure 5 and text for a detailed description of Figures $6 a-6 e$.

associated with wood burning, as chloride ions have been observed in several studies on wood smoke emissions [Kleeman et al., 1999; Schauer et al., 2001]. Depending on the type of wood that is burned, chlorine comprises as much as $0.13-1.7 \%$, by mass, of the fine particulate emissions [Schauer et al., 2001].

\subsection{Elemental Carbon/Organic Carbon (EC/OC)}

[19] Typical spectra in this class contain only the first three carbon cluster ions $\left(\mathrm{C}_{1}^{+}-\mathrm{C}_{3}^{+}\right)$, see Figure 7 . Once again, it is not immediately clear from this series of peaks whether the carbon is elemental, organic, or a combination. However, the sizes of the particles suggest that they are combustion particles since the majority of those detected were in the $75-125 \mathrm{~nm}$ range. The wind rose suggests that this class of particles has been routinely detected from all directions, which is consistent with multiple sources; such as vehicles and cooking. One of the major differences between this class and CAN is the absence of detectable amounts of ammonium nitrate in the positive ion spectra. Notice that the observed monthly distribution for this class is largely anticorrelated with that of CAN, further evidence for the seasonal trend in ammonium nitrate discussed earlier. Carbon particles without ammonium nitrate are observed less frequently in the winter months, when the temperatures are colder, and more frequently in warmer months, with the exception of August and September. Nitrate is still observed in 

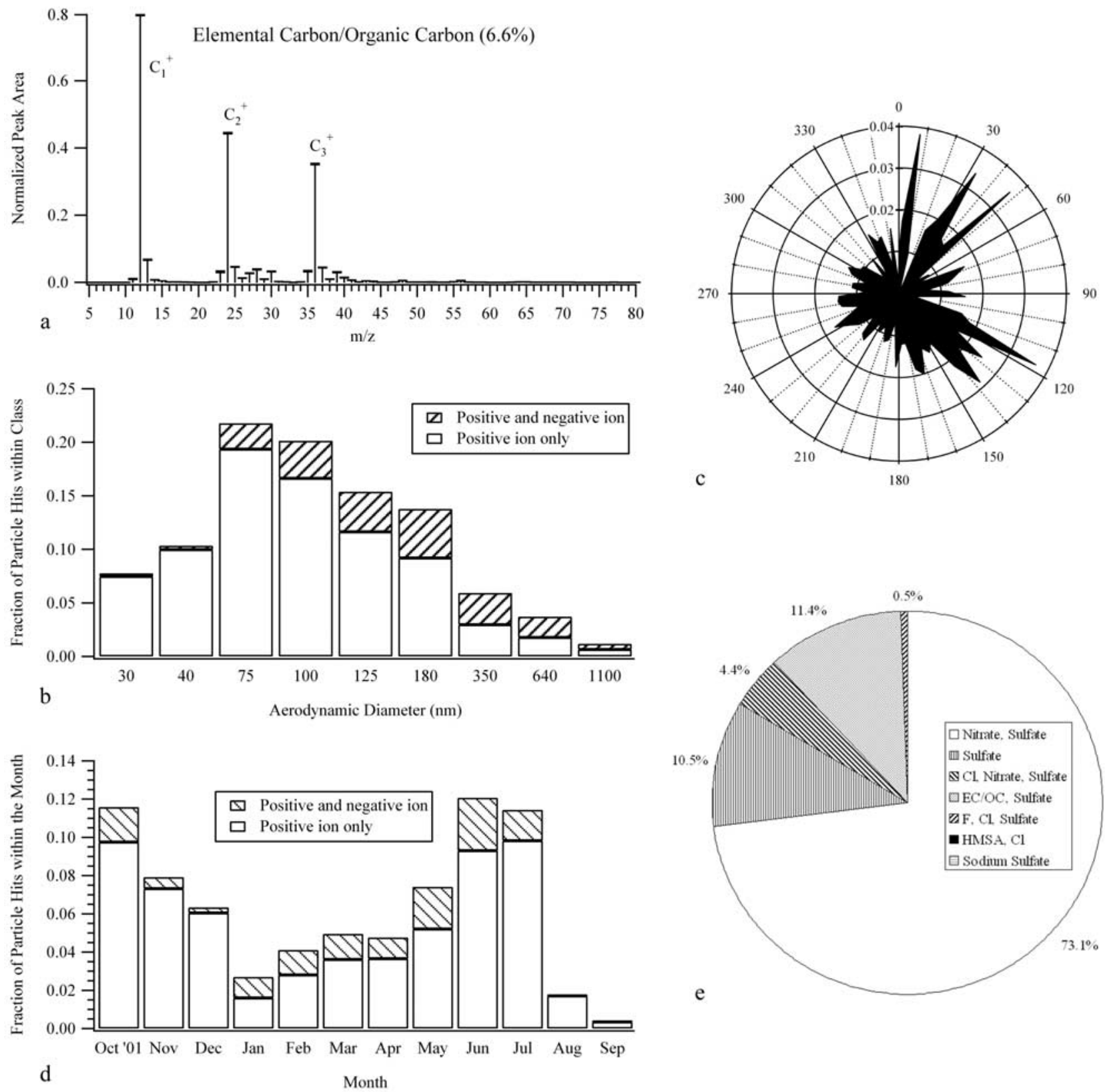

Figure 7. Summary statistics from the analysis of the elemental carbon/organic carbon class, containing $6.6 \%$ of the total number of particles sampled. See caption of Figure 5 and text for a detailed description of Figures $7 \mathrm{a}-7 \mathrm{e}$.

the negative ion spectra, along with sulfate and EC/OC, but note that, similar to CAN, only $\sim 20 \%$ of the particles in the class have detectable negative ion signal.

\subsection{Sodium/Potassium ( $\mathrm{Na} / \mathrm{K})$}

[20] Easily distinguished by the combination of $\mathrm{Na}^{+}$, $\mathrm{K}^{+}, \mathrm{Na}_{2}^{+}$and $\mathrm{NaK}^{+}$ions, as depicted in Figure 8, this is the largest class of non-carbon-based particles. Sodium and potassium are very common earth alkali metals emitted by a variety of sources. Potassium was discussed briefly in the context of biomass burning, but has also been identified in both sea salt and road salt particles, similar to sodium. However, given that the majority of the particles detected were in the size range of 75-200 nm, this class is inconsistent with what one would expect to see for sea salt or road salt particles, suggesting an alternate source. This is supported by the fact that the nearest ocean is almost $500 \mathrm{~km}$ away and the majority of $\mathrm{Na} / \mathrm{K}$ particles were observed in the summer months, when the roads are not being salted. On the other hand, besides the nitrate/sulfate class, the negative ion speciation is dominated by chlorine (33\%), implying that these particles are, in fact, composed primarily of $\mathrm{NaCl}$ and $\mathrm{KCl}$ salts. From the wind rose, it is clear that these particles originate largely from somewhere inside the 

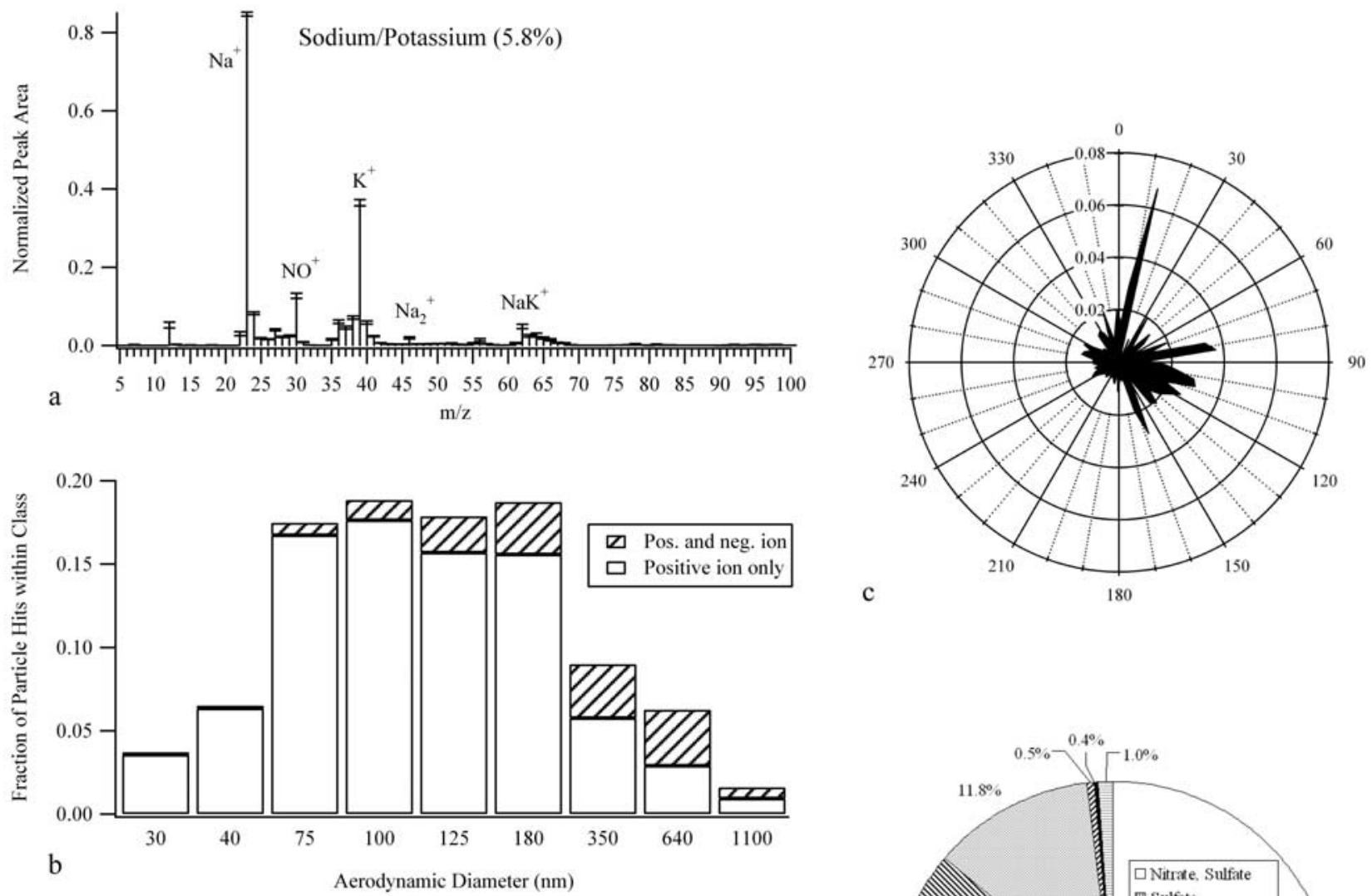

$\mathrm{c}$

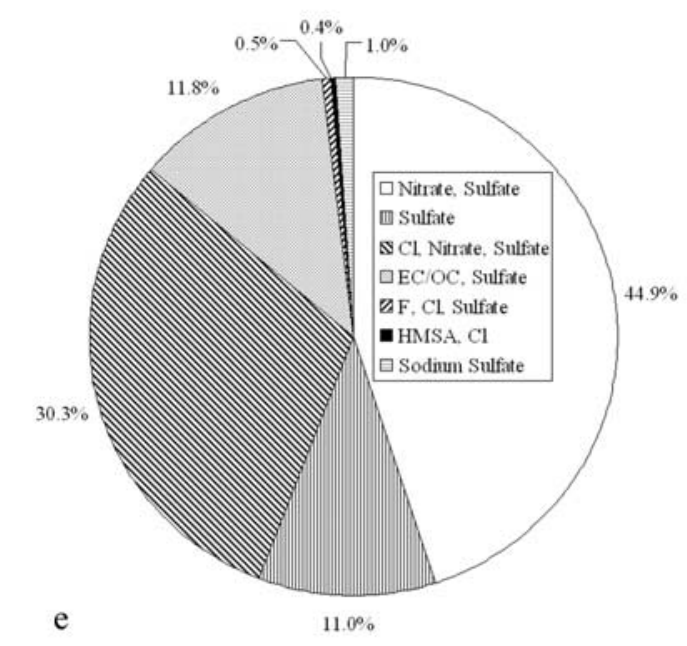

d

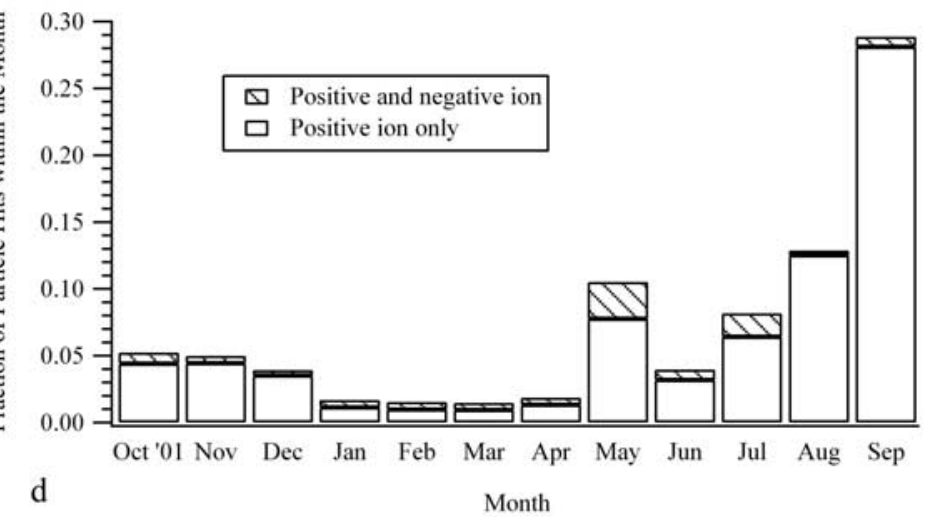

$\mathrm{e}$

Figure 8. Summary statistics from the analysis of the sodium/potassium class, containing $5.8 \%$ of the total number of particles sampled. See caption of Figure 5 and text for a detailed description of Figures $8 \mathrm{a}-8 \mathrm{e}$.

second quadrant. There are numerous sources in this general area so it is hard to attribute the class to any one in particular.

\subsection{Unidentified Organics}

[21] This is a very interesting class of particles. As the name of the class states, the chemical composition of these particles is yet to be completely identified. For this reason, no peak assignments have been made in Figure 9; only the $\mathrm{m} / \mathrm{z}$ values of the dominant and reoccurring peaks have been listed. Notice the structure that begins to develop at $\mathrm{m} / \mathrm{z} 81$. Successive peaks further downfield are separated by 14 Da $(81,95,109,123,135,149$, etc.).
This kind of structure suggests large organic carbon chains perhaps with high degrees of functionality. From the plot of particle fraction versus size, these particles tend to be larger in size, peaking around $200-300 \mathrm{~nm}$, with significant fractions of secondary components in the largest size bins. Looking at the wind rose, there is a very well-defined wind signature for this class of particles. Notice that the direction coincides exactly with Chambers Development Co. at $108^{\circ}$. This is a major landfill and a moderate source of volatile organic compounds (16 metric tons/year). In fact, these particles were seen almost exclusively in the summer months when the VOC emissions from the landfill were probably at a 


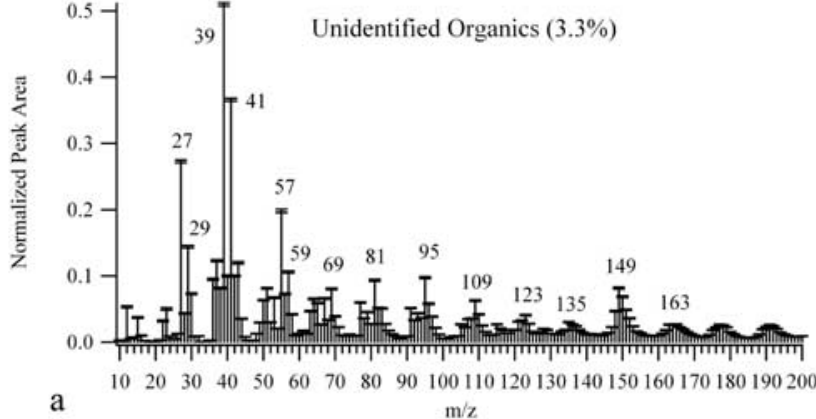

$\mathrm{m} / \mathrm{z}$
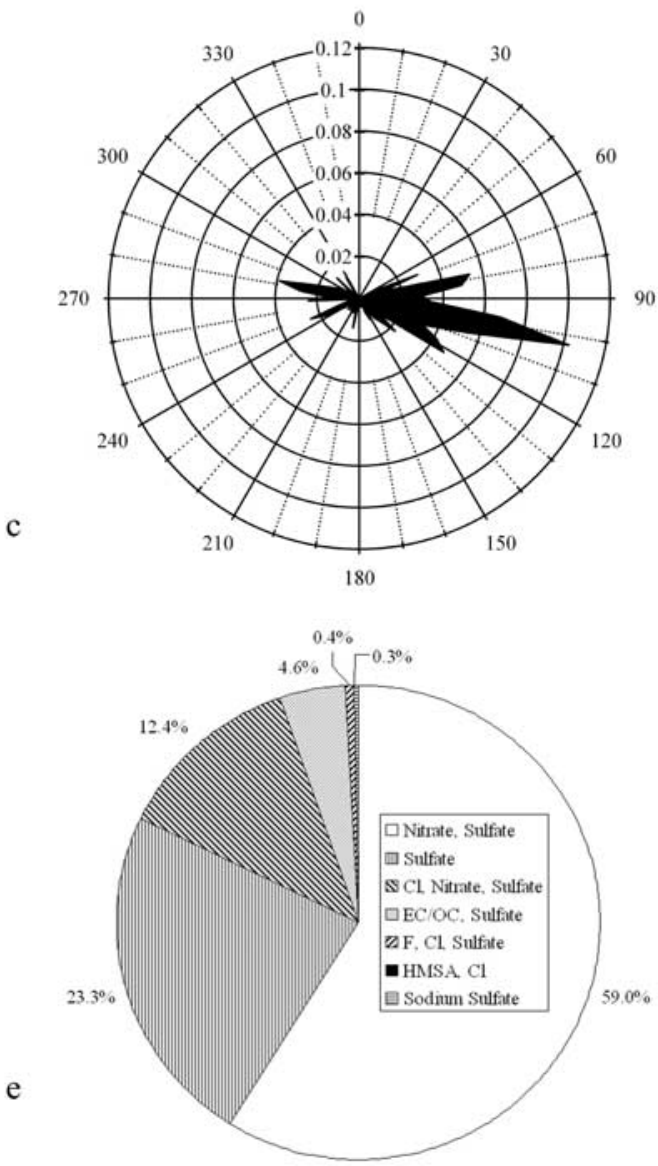
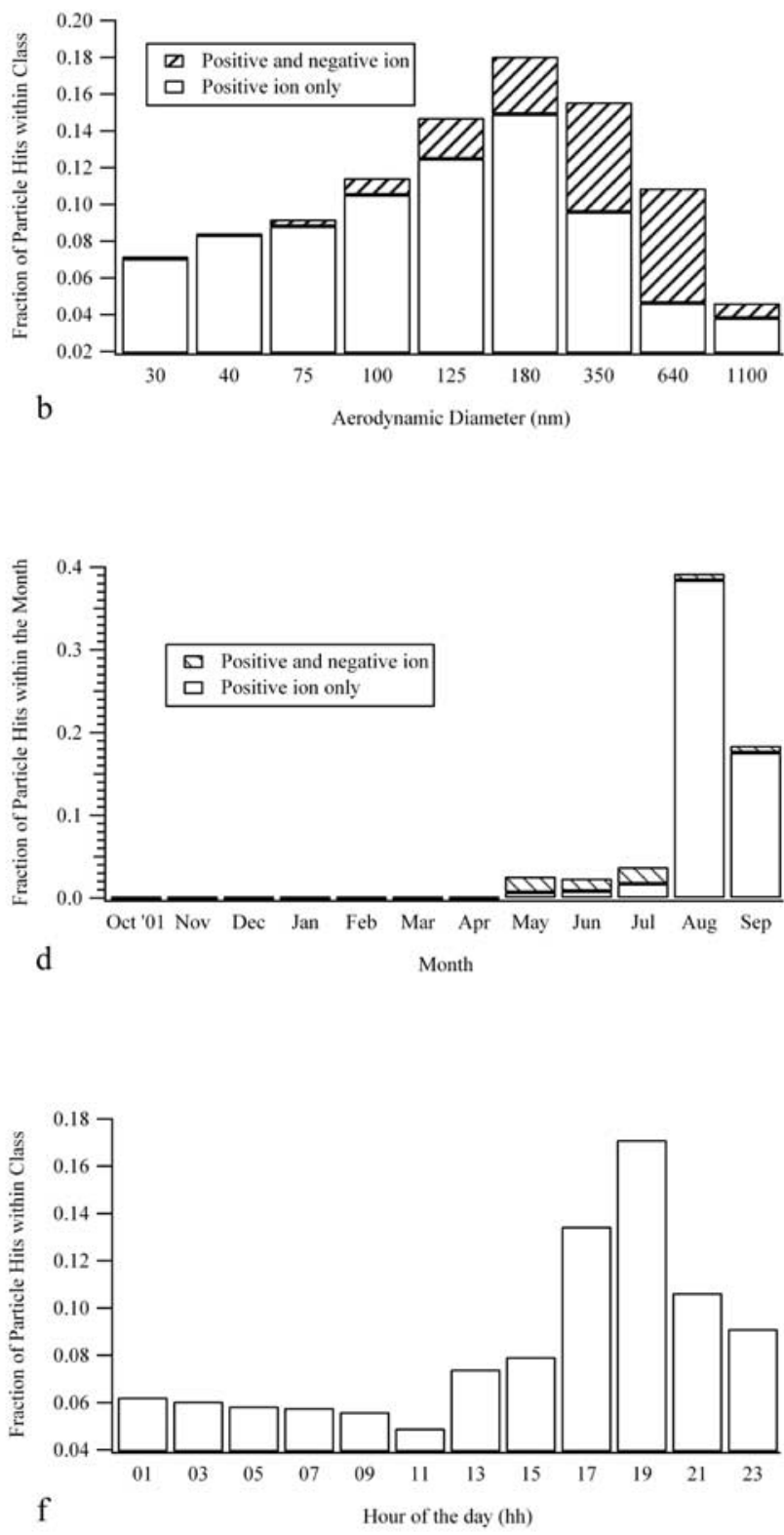

Figure 9. Summary statistics from the analysis of the unidentified organics class, containing $3.3 \%$ of the total number of particles sampled. See caption of Figure 5 and text for a detailed description of Figures $9 \mathrm{a}-9 \mathrm{e}$. (f) Fraction of particle hits by hour of the day.

maximum. Figure 9 includes an additional plot (f) showing the fraction of total particle hits by hour of the day. Notice that this particle type is observed almost entirely during the nighttime hours, after the sun has gone down and the atmosphere begins to cool. Combining this evidence we posit that the sun drives the volatilization of VOCs from the landfill during the day, but the atmosphere cools at night and these vapors condense on preexisting particles. Another possibility, however, must also be considered. Photochemical oxidation may suppress the detection of these particles during the day, but when the sun goes down and the chemistry shuts off, the particles reappear. Both mechanisms most likely play a role in the observed diurnal variation.

\subsection{Elemental Carbon (EC)}

[22] This is the classic elemental carbon particle, also called black carbon. It is easily identified by the long series of carbon cluster ions, typically from $\mathrm{C}_{1}^{+}-\mathrm{C}_{11}^{+}$, seen in Figure 10. Approximately $63 \%$ of these particles also contain detectable amounts of ammonium nitrate, as evidenced by the presence of $\mathrm{NO}^{+}$peaks. Carbon has already been discussed extensively, so this class will only be touched on briefly. Similar to the EC/OC class, the size 

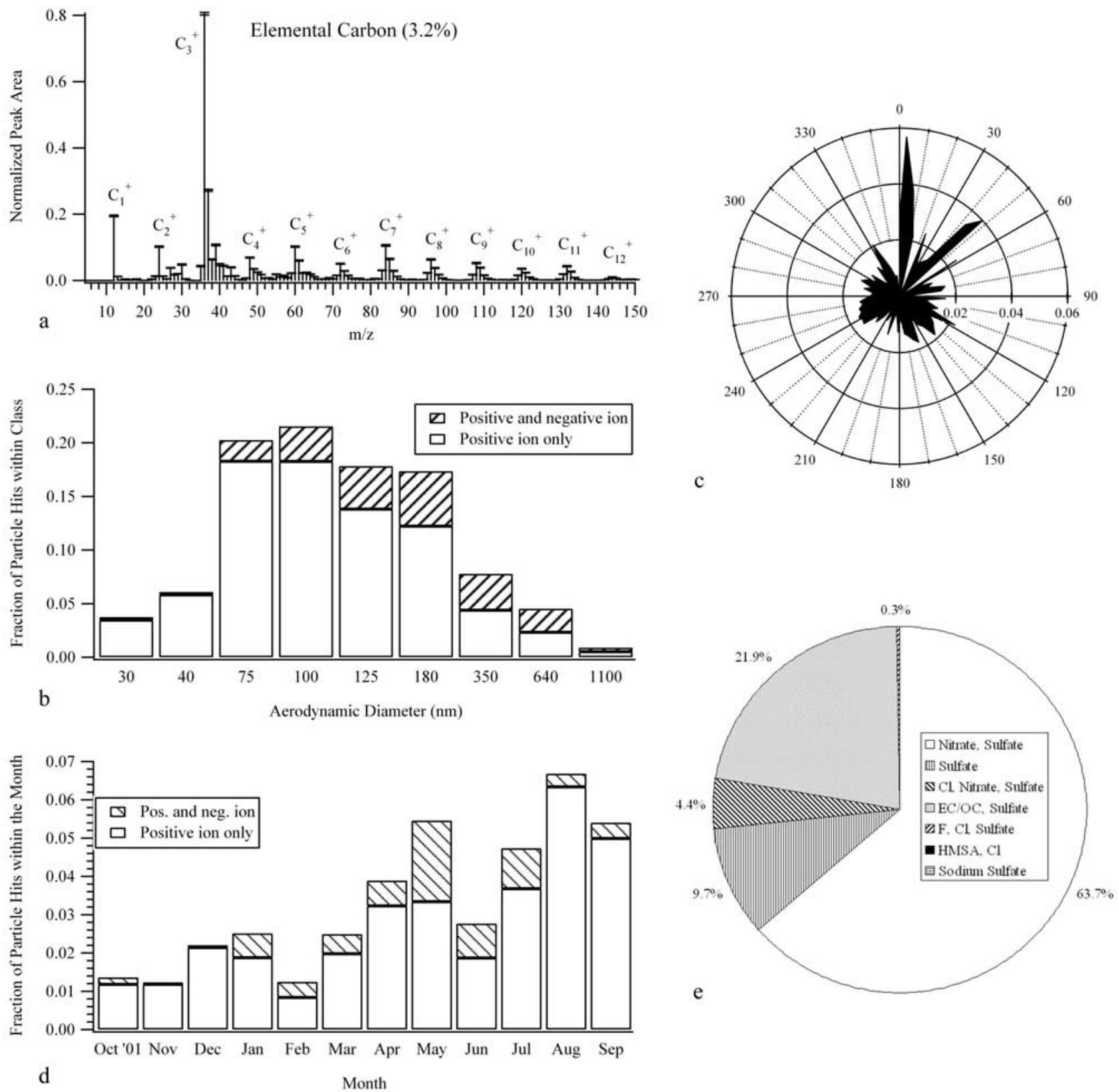

Figure 10. Summary statistics from the analysis of the elemental carbon class, containing $3.2 \%$ of the total number of particles sampled. See caption of Figure 5 and text for a detailed description of Figures $10 \mathrm{a}-10 \mathrm{e}$.

of these particles tend to be skewed toward the smaller size bins $(75-125 \mathrm{~nm})$, consistent with a combustion source. The wind rose shows multiple sources for this particle, also consistent with combustion. They were observed continuously throughout the campaign and the negative ion classification reveals a significantly larger fraction of $\mathrm{EC} / \mathrm{OC}$, in addition to nitrate and sulfate.

\subsection{Silicon/Potassium/Iron/Gallium}

[23] This is the single largest class of metal particles identified in the data set. Sodium and potassium have already been discussed, but only in the context of biomass burning and salts. The spectral representation, shown in Figure 11, contains the $\mathrm{Si}^{+}, \mathrm{K}^{+}, \mathrm{SiO}^{+}, \mathrm{Fe}^{+}$and $\mathrm{Ga}^{+}$peaks. Some of the subclasses within this class contain trace amounts of additional metal, such as aluminum, sodium, lithium and lead, but the dominant ions remain the four listed above. Notice that the majority of the particles fall in the size range of 100-300 nm, implicating combustion or a high temperature furnace as the source. Also notice that there is significant negative ion fraction in each size bin, indicating extensive atmospheric processing and suggesting a more distant source. A very pronounced wind signature for this class is 

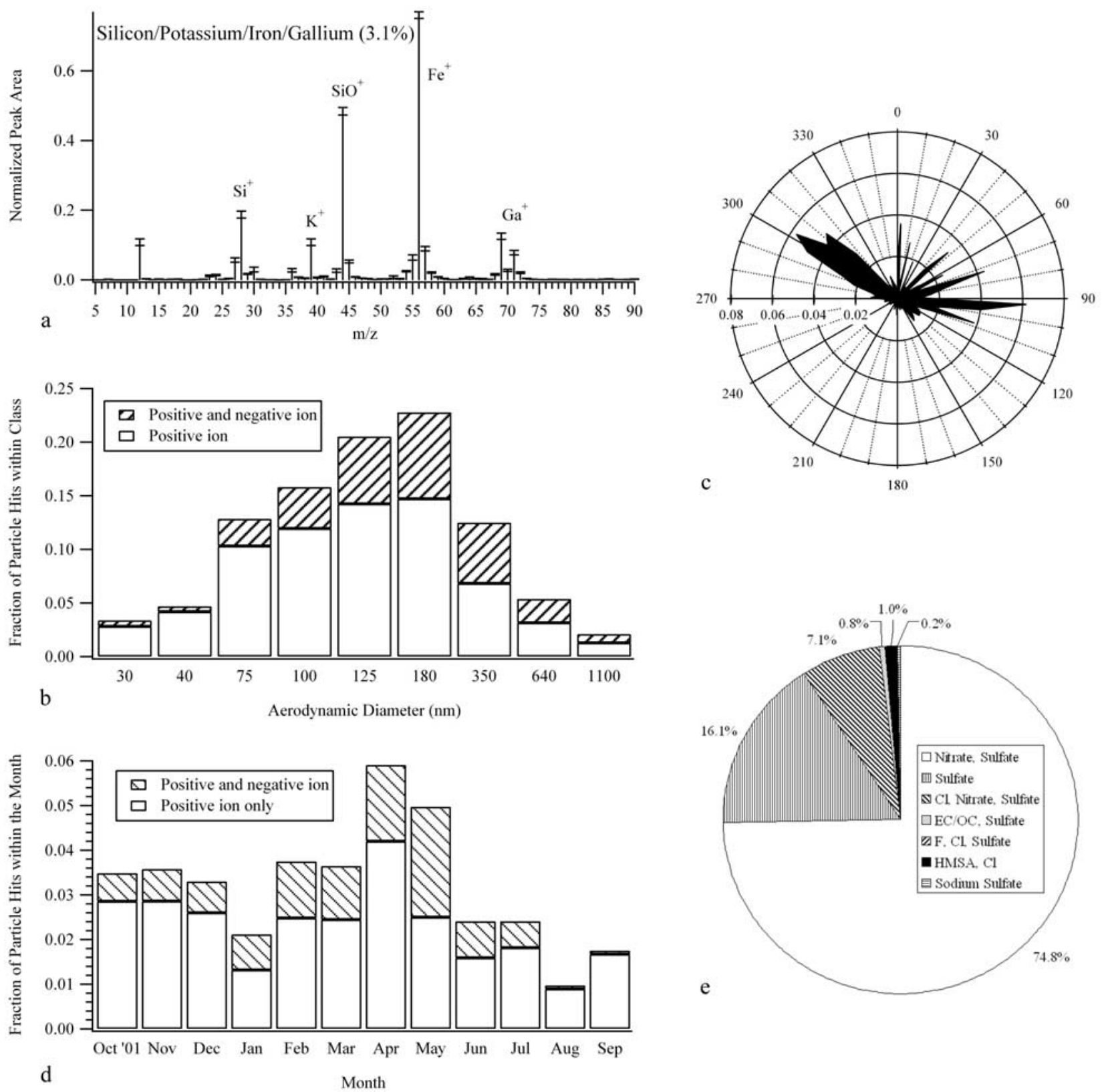

Figure 11. Summary statistics from the analysis of the silicon/potassium/iron/gallium class, containing $3.1 \%$ of the total number of particles sampled. See caption of Figure 5 and text for a detailed description of Figures $11 \mathrm{a}-11 \mathrm{e}$.

centered at $\sim 305^{\circ}$. Numerous possible sources exist in this direction, but not all are equally probable. Shenango Coke plant $\left(297^{\circ}, 13 \mathrm{~km}\right)$ and the hospital incinerator $\left(303^{\circ}, 11.9 \mathrm{~km}\right)$ seem too close to the site to be consistent with the amount of aging, while the Zinc Corporation of America (307, 42.2 miles) deals only in nonferrous metals. Gallium, the least commonly seen of the metals in this class, is actually a trace metal found in coal. As there are very few sources of gallium in the atmosphere, it is most likely that these particles originated from coal combustion. It is also common to see other crustal metals like aluminum, silicon, potassium and iron in coal. Supporting this hypothesis, is the existence of two large coal fired power plants precisely in the direction of interest: Orion Power (Phillips Station, 301 ${ }^{\circ}, 28.3 \mathrm{~km}$ ) and Bruce Mansfield $\left(298^{\circ}, 45.5 \mathrm{~km}, 1255\right.$ metric tons/year PM2.5).

\subsection{Sodium/Potassium/Zinc/Lead}

[24] Besides being composed of different metals, this class of particles closely resembles the previous one. A majority of the particles are in the size range of 100-300 nm, all size bins contain some fraction of negative ion spectra and there is a well resolved wind signature at $\sim 300^{\circ}$ 

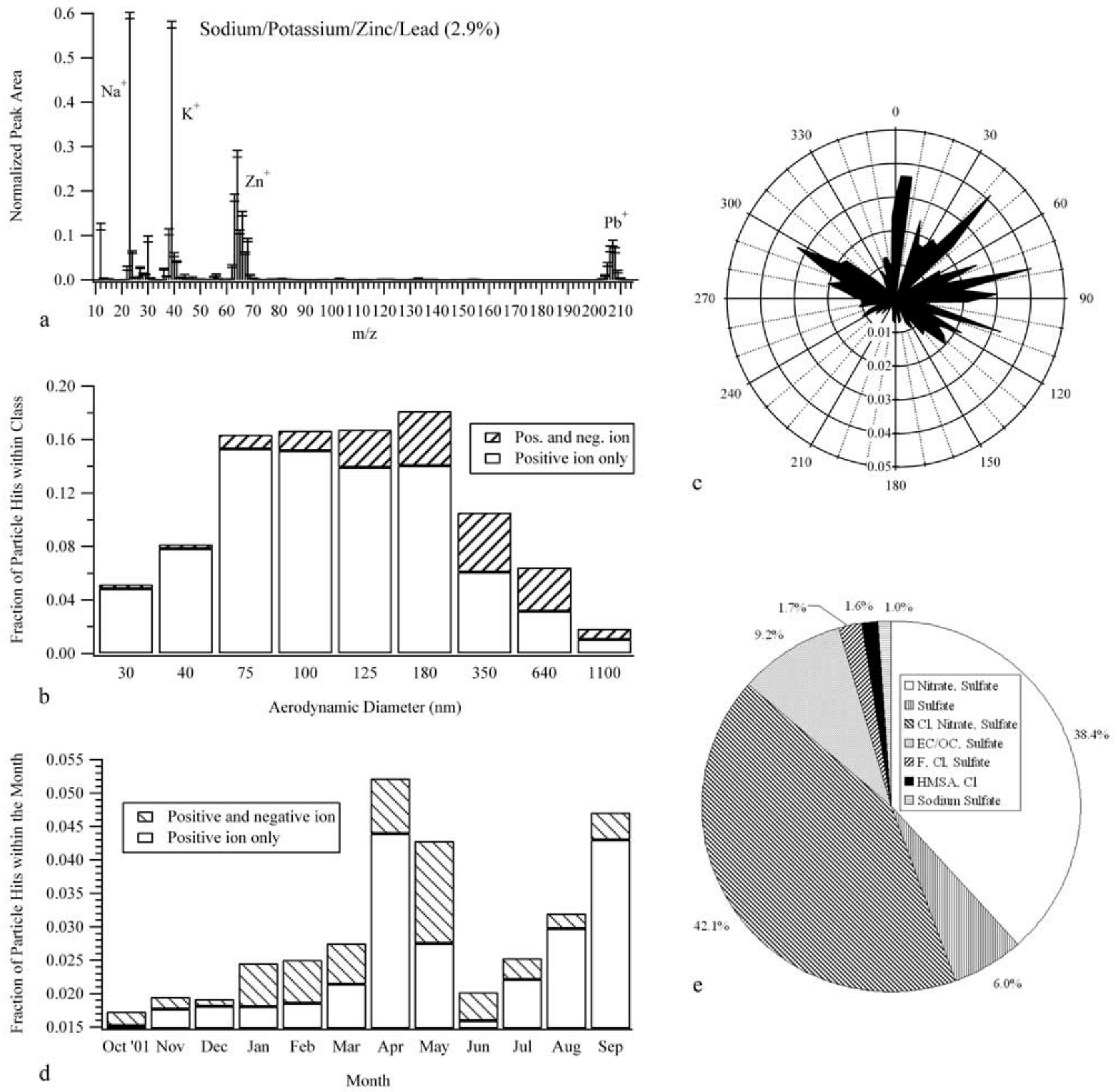

Figure 12. Summary statistics from the analysis of the sodium/potassium/zinc/lead class, containing $2.9 \%$ of the total number of particles sampled. See caption of Figure 5 and text for a detailed description of Figures $12 \mathrm{a}-12 \mathrm{e}$.

(Figure 12). Given this information, and the composition of the class, the most likely source of this particle is Zinc Corporation of America. It is interesting to note, however, that almost half of the negative ions contain chlorine, suggesting that a significant amount of the metal in these particles is bound as a salt.

\subsection{Lithium/Sodium/Potassium}

[25] Except for the addition of lithium, these particles are almost identical to those of the $\mathrm{Na} / \mathrm{K}$ class discussed above, see Figure 13. They were observed consistently throughout the study and a majority of them are in the size range of 75-200 $\mathrm{nm}$. Once again, this is more indicative of a combustion process or high temperature furnace rather than a sea salt particle or crustal material. However, the wind signatures are fewer and more resolved. Of particular interest is the well developed peak at $\sim 120^{\circ}$; the peak near $80^{\circ}$ is not statistically significant. Notice that this coincides exactly with the USX Corporation (Edgar Thompson Works, $128^{\circ}, 8.5 \mathrm{~km}, 280$ metric tons/year PM2.5), a steel manufacturer housing blast furnaces and steel mills. Almost half of the negative ion spectra contain chlorine, suggesting predominantly $\mathrm{NaCl}$ and $\mathrm{KCl}$ particles. Sodium and potassium chloride are both 

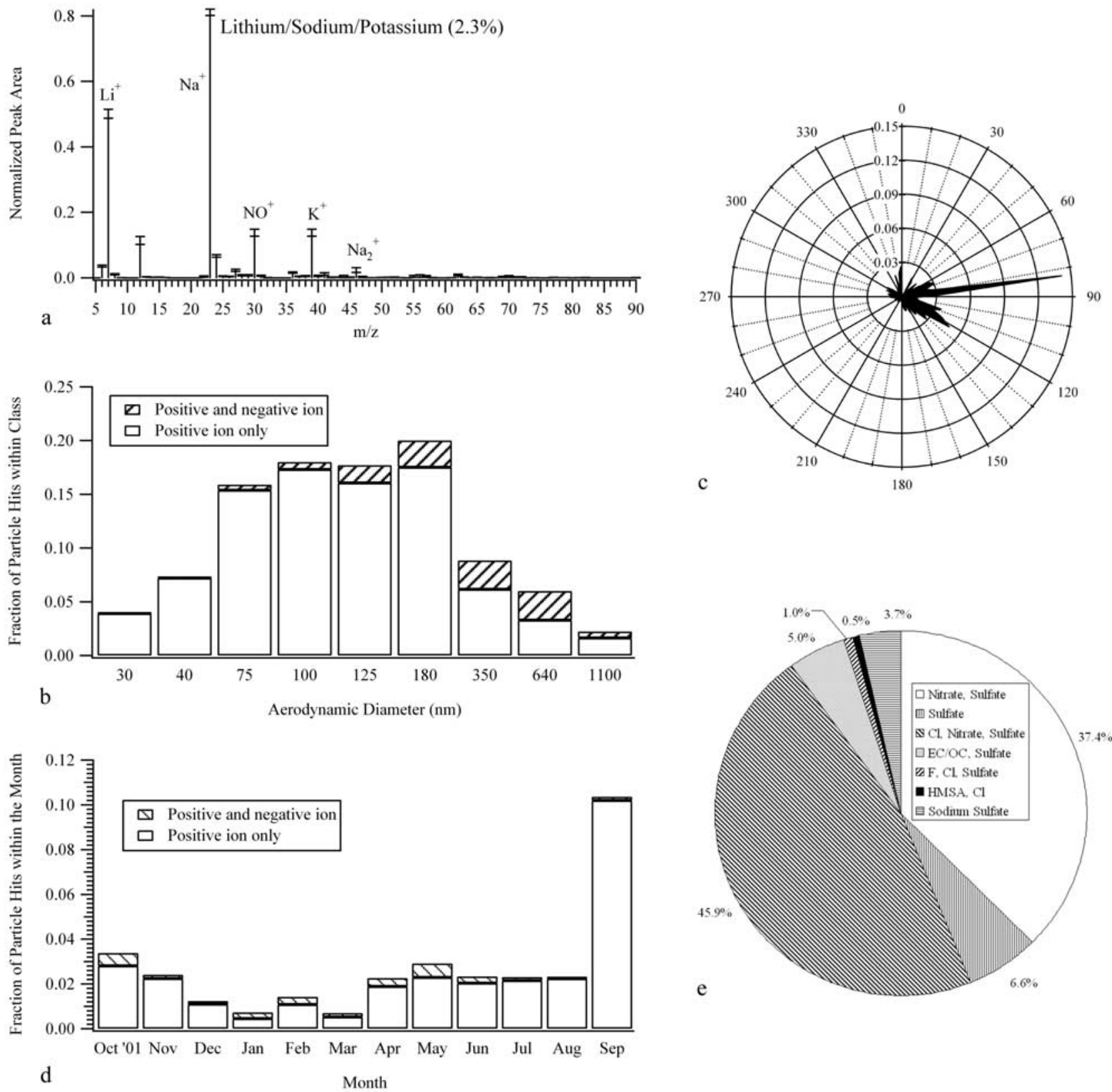

Figure 13. Summary statistics from the analysis of the lithium/sodium/potassium class, containing $2.3 \%$ of the total number of particles sampled. See caption of Figure 5 and text for a detailed description of Figures $13 \mathrm{a}-13 \mathrm{e}$.

used as hot molten alkali salts in salt pots for heat treating steel, suggesting that these particles originate from within the framework of steel processing. Since $48 \%$ of the total PM2.5 (6368 metric tons/year) emitted within 80 kilometers of the site is directly a result of the steel industry (8 separate companies), this could help explain the overwhelming amount of ultrafine sodium and potassium observed in Pittsburgh aerosol.

\subsection{Iron}

[26] Iron is one of the most commonly observed transition metals in urban particles and such is also the case in Pittsburgh. Consisting primarily of the iron isotope peaks $(54,56$ and 57), the spectral representation of this class is shown in Figure 14. Notice from the size plot that the particle fraction distribution tends to be skewed toward the larger size bins, peaking around 200-300 nm, possibly the tail end of a distribution of mechanical abrasion particles, but more likely combustion or high temperature furnace related. Looking at the wind rose, there is a predominant wind signature centered at $\sim 125^{\circ}$, again pointing almost directly at Edgar Thompson Works (USX Corporation). This makes sense given that, besides steel, this company also manufactures iron products. Blast furnaces, used in the casting houses of this facility, are the most probable source of these particles. From 

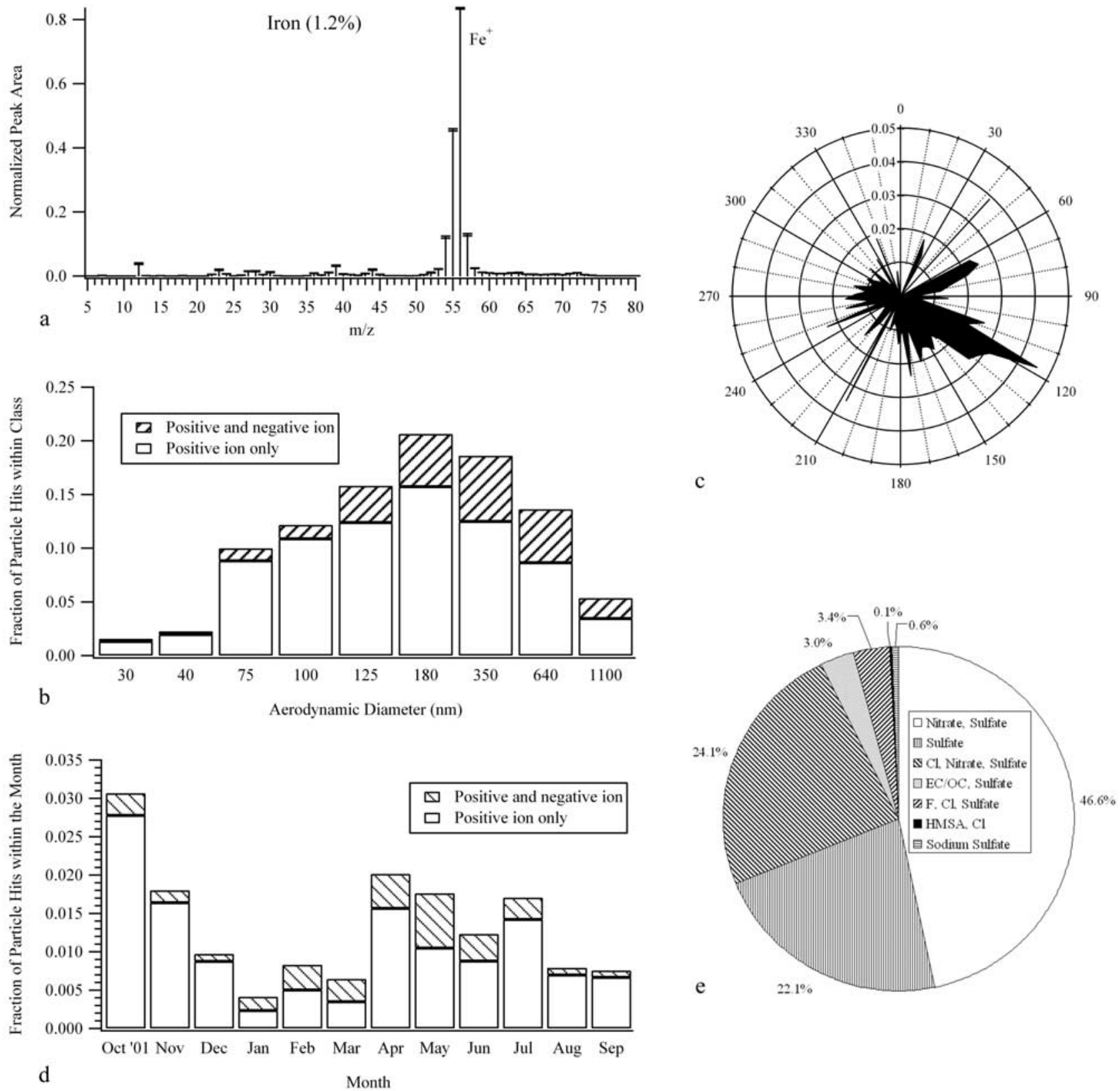

Figure 14. Summary statistics from the analysis of the iron class, containing $1.2 \%$ of the total number of particles sampled. See caption of Figure 5 and text for a detailed description of Figures $14 \mathrm{a}-14 \mathrm{e}$.

the negative ion speciation, it is apparent that, to some degree, all seven negative ion classes are present. However, notice the increased fractions of both fluorine and chlorine in these particles. One possible explanation is the existence of hydrofluoric and hydrochloric acid on the interior wall of the emissions stack. If this is the case, then as iron is emitted it would sequester these acids, eventually transferring the fluorine and chlorine into the particle phase as salts.

\subsection{Iron/Cerium}

[27] As part of one of the most unanticipated classes observed during this campaign, these particles contain the rare earth metal cerium. From Figure 15, it can be seen that a majority of them occupy the largest size bins, $300-1100 \mathrm{~nm}$, unlike any other class. Also unique to this class is the fact that the particles were only observed for a relatively short period of time. Notice from the monthly distribution that they begin to appear in December and then practically disappear by the end of January. As a result, there were not enough data points with wind speeds greater than $2 \mathrm{~m} / \mathrm{s}$ to obtain a good wind signature, making source attribution difficult. However, the two most common uses of cerium are:(1) as part of catalytic crackers used in oil refineries to break down large hydrocarbons in crude oil to smaller, higher grade, hydro- 

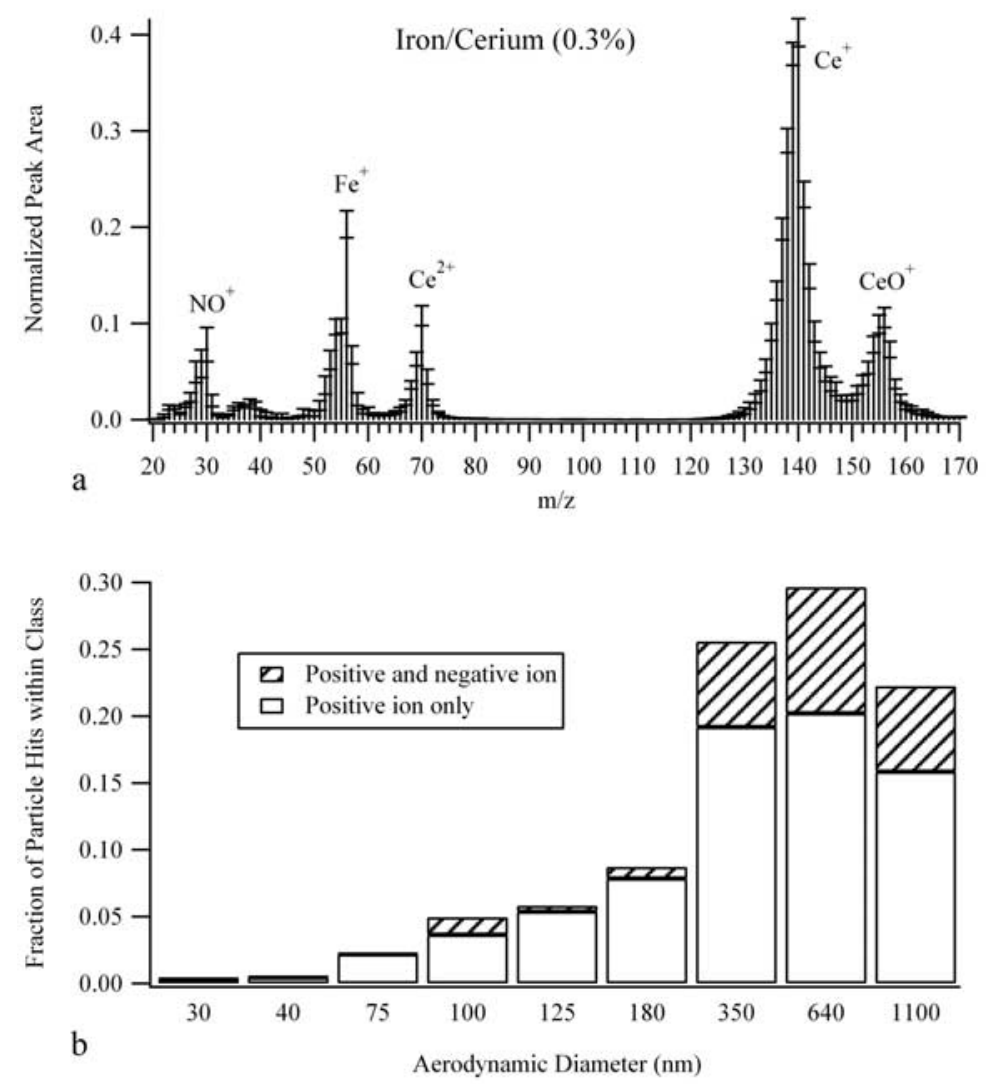

c
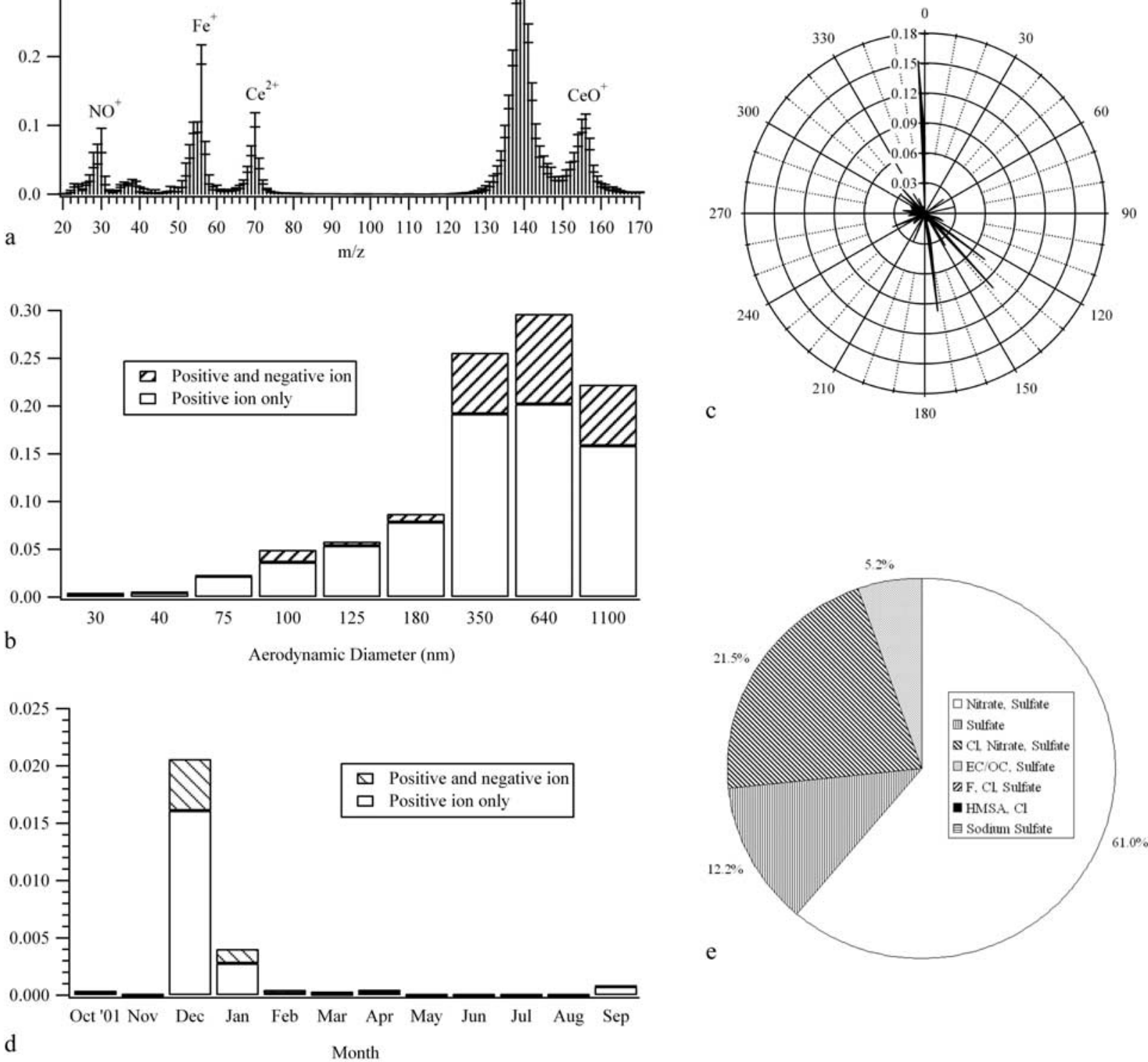

Figure 15. Summary statistics from the analysis of the iron/cerium class, containing $0.3 \%$ of the total number of particles sampled. See caption of Figure 5 and text for a detailed description of Figures $15 \mathrm{a}-15 \mathrm{e}$.

carbons and (2) as an additive to many oxidation and heat resistant alloys. Rare earth metals like cerium, lanthanum and yttrium are added to alloys (microalloying) specifically for oxidation and scaling resistance. Cerium is also found naturally in the Earth's crust, as a trace component, and has been observed in certain types of mineral dust [Utsunomiya et al., 2004].

\subsection{Chromium/Molybdenum/Tungsten}

[28] This is the last class of particles that will be addressed in this paper. A spectral representation, showing the $\mathrm{Cr}^{+}, \mathrm{Mo}^{+}, \mathrm{MoO}^{+}$and $\mathrm{W}^{+}$peaks, can be seen in Figure 16 .
Similar to many of the other metal-based classes, the majority of the particle fraction resides in the smaller size bins, peaking at $\sim 75 \mathrm{~nm}$. In addition, there is only a very small fraction of negative ions across all size bins. Together, this indicates that the particles originated from a high temperature furnace located relatively close to the site. Incorporating data from the wind rose, it is clear that they are most frequently observed from the southwest, at $\sim 235^{\circ}$. This coincides almost exactly with Universal Stainless and Alloy Products $\left(237^{\circ}, 16.9 \mathrm{~km}, 15\right.$ tons/year PM2.5). Not surprisingly, this company is primarily involved with melting and refining alloys, including stainless steel, using 

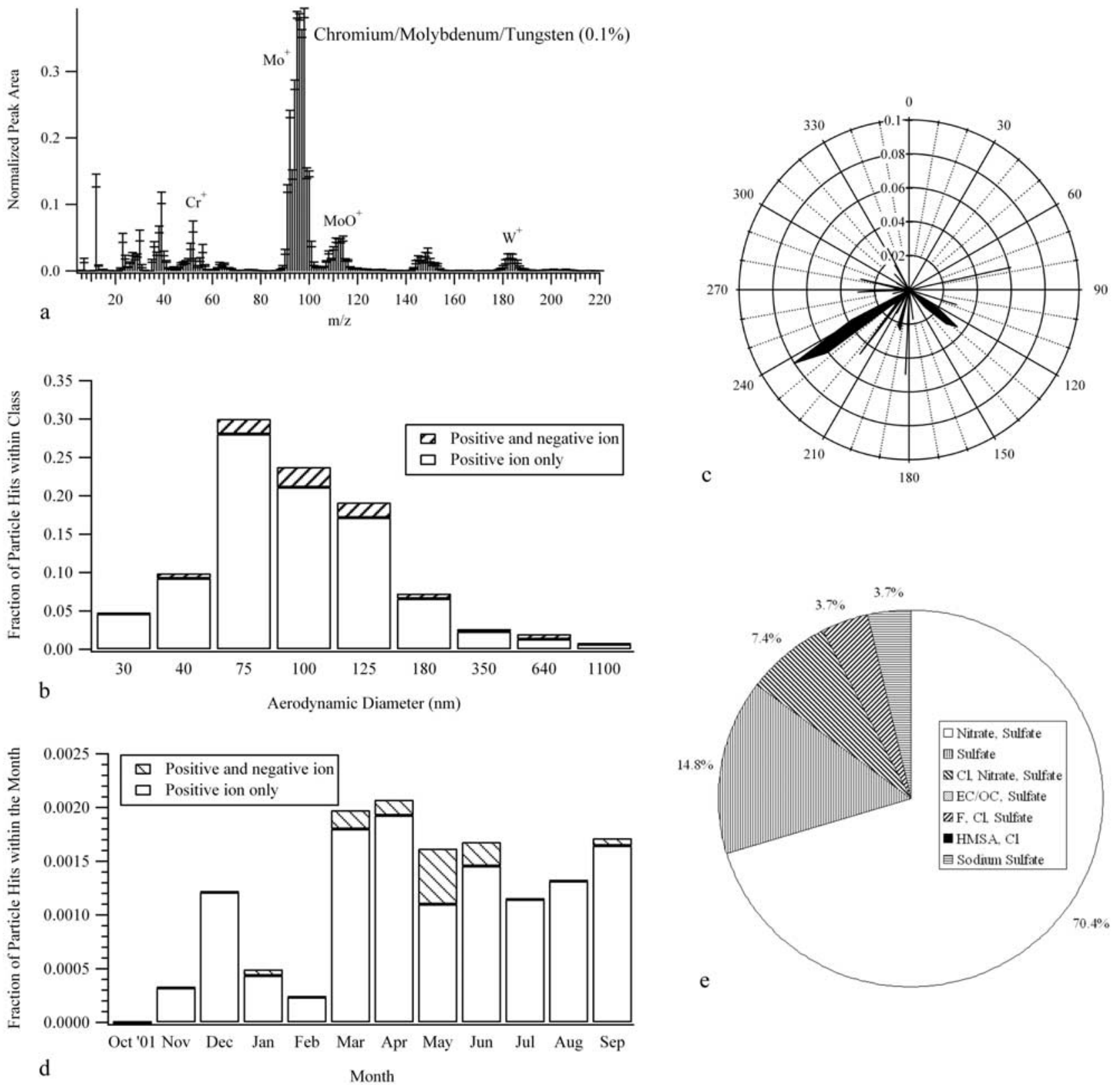

Figure 16. Summary statistics from the analysis of the chromium/molybdenum/tungsten class, containing $0.1 \%$ of the total number of particles sampled. See caption of Figure 5 and text for a detailed description of Figures 16a-16e.

equipment like electric arc furnaces. Note that chromium and molybdenum are two major constituents (along with nickel, iron and silicon) of almost all alloys. Chromium is added for oxidative resistance and strength, while molybdenum is added for strength and weldability. Tungsten, on the other hand, is a major constituent of superalloys, added for additional strength. It is also interesting to note that the other dominant wind signature is aligned with the USX Corporation (Edgar Thompson Works), also stainless steel manufacturers. Chromium is of special interest in this class since, depending on its oxidation state ( $\mathrm{Cr}[\mathrm{III}]$ versus $\mathrm{Cr}[\mathrm{VI}])$, it can be either a nutrient or a carcinogen. Unfor- tunately, RSMS-3 is not able distinguish between the two species [Neubauer et al., 1995].

\section{Conclusions}

[29] RSMS-3, operating semicontinuously for 306 days and sampling ambient aerosol within the size range of $\sim 30-1100 \mathrm{~nm}$, collected approximately 236,000 single particle mass spectra as part of the EPA funded Supersites experiment in Pittsburgh, Pennsylvania. Although negative ion mass spectra were collected for every particle measured, very few $(\sim 22 \%)$ had detectable amounts of negative ion 
signal. This can be attributed to the fact that the sensitivity of the instrument to negative ions increases with increasing particle size. Since atmospheric number concentrations are typically much smaller for larger particles, the hit rate of the instrument is significantly lower at this size range. Consequently, fewer particles are sampled and the fraction of spectra containing negative ion signal is small. For this reason, the negative ion mass spectrometer is able to characterize larger aerosol, but becomes less informative as the size of the particles decrease, especially in the ultrafine regime.

[30] All particles measured during this campaign have been clustered into particle classes based on the spectral distribution of their positive ion mass peaks. Twenty classes, and over 100 subclasses, were identified during this analysis. Negative ion spectra were then classified independently within each positive ion class. Characteristic size distributions, monthly distributions and wind signatures were also obtained within each class. These data were used not only to assess the dominant sizes and composition of urban aerosol in Pittsburgh, but also to construct possible source-receptor relationships between local industry and classes of observed particles.

[31] Particulate pollution in Pittsburgh was found to be predominantly carbonaceous in nature with $\sim 79 \%$ of the particles measured containing some form of carbon. These particles were observed in all size bins and from almost every direction for the duration of this study. Numerous ubiquitous sources scattered throughout the area, such as vehicular traffic, biomass burning and secondary organic aerosol formation, are most likely responsible for a large fraction of these carbon particles. In addition, there was a significant amount of ammonium nitrate observed in these particles.

[32] Besides carbon and secondary components, metals were recognized as the next largest constituent of Pittsburgh aerosol. Although a rich array of multicomponent metal particles was identified, the most commonly observed ions were $\mathrm{K}^{+}, \mathrm{Na}^{+}, \mathrm{Fe}^{+}, \mathrm{Pb}^{+}$, and to a lesser extent, $\mathrm{Ga}^{+}$and $\mathrm{Zn}^{+}$. These particles were typically smaller in size, ranging from about $75-300 \mathrm{~nm}$, and tended to be associated with specific wind directions. The analysis of the wind roses for individual classes has facilitated the isolation of specific local industries to which the observed metal-based classes may be attributed. Results from this analysis alone indicate that high temperature furnaces are the single largest source of ultrafine metal particles in the Pittsburgh area.

[33] Acknowledgments. Although the research described in this paper has been funded by the U. S. Environmental Protection Agency through grants to the Pittsburgh and Baltimore Supersites, it has not been subjected to the agency's required peer and policy review and therefore does not necessarily reflect the views of the agency. No official endorsement should be inferred. The authors acknowledge the assistance of Allen Robinson in compiling emissions inventory for industry-based air pollution sources in the Pittsburgh area, from which emission rates and source locations reported in this paper have been obtained. The authors also acknowledge Jon Ondov and his group for supplying the map of Pittsburgh used in the construction of Figure 2.

\section{References}

Carpenter, G. A., S. Grossberg, and D. B. Rosen (1991), ART2-A-An adaptive resonance algorithm for rapid category learning and recognition, Neural Networks, 4, 493-504.
Carson, P. G., K. R. Neubauer, M. V. Johnston, and A. S. Wexler (1995), On-line chemical analysis of single aerosol particles by rapid singleparticle mass spectrometry, J. Aerosol Sci., 26, 535-545.

Carson, P. G., M. V. Johnston, and A. S. Wexler (1997), Laser desorption ionization of ultrafine aerosol particles, Rapp. Commun. Mass Spectrom., $11,993-996$.

Donaldson, K., X. Y. Li, and W. MacNee (1998), Ultrafine (nanometer) particle mediated lung injury, J. Aerosol Sci., 29, 553-560.

Ge, Z., A. S. Wexler, and M. V. Johnston (1998), Laser desorption/ ionization of single ultrafine multi-component aerosols, Environ. Sci. Technol., 32, 3218-3223.

Hinz, K.-P., R. Kaufmann, and B. Spengler (1994), Laser-induced mass analysis of single particles in the airborne state, Anal. Chem., 66, 2071-2076.

Hopke, P. K., and X. Song (1997), Classification of single particles by neural networks based on the computer-controlled scanning electron microscopy data, Anal. Chim. Acta, 348, 375-388.

Jayne, J. T., et al. (2000), Aerosol mass spectrometer for size and composition analysis of submicron particles, Aerosol Sci. Technol., 33, 49-70.

Jimenez, J. L., et al. (2003), Ambient aerosol sampling with an aerosol mass spectrometer, J. Geophys. Res., 108(D7), 8425, doi:10.1029/ 2001JD001213.

Johnston, M. V. (2000), Sampling and analysis of individual particles by aerosol mass spectrometry, J. Mass Spectrom, 35, 585-595.

Johnston, M. V., and A. S. Wexler (1995), MS of individual aerosol particles, Anal. Chem., 67, 721A-726A.

Kane, D. B., and M. V. Johnston (2000), Size and composition biases on the detection of individual ultrafine particles by aerosol mass spectrometry, Environ. Sci. Technol., 34, 4887-4893.

Kane, D. B., J. J. Wang, K. Frost, and M. V. Johnston (2002), Detection of negative ions from individual ultrafine particles, Anal. Chem., 74, $2092-$ 2096.

Kleeman, M. J., J. J. Schauer, and G. R. Cass (1999), Size and composition distribution of fine particulate matter emitted from wood burning, meat charbroiling and cigarettes, Environ. Sci. Technol., 33, 3516-3523.

Lake, D. A., M. P. Tolocka, M. V. Johnston, and A. S. Wexler (2003), Mass spectrometry of individual particles between 50 and $750 \mathrm{~nm}$ in diameter at the Baltimore Supersite, Environ. Sci. Technol., 37, 3268-3274.

Lee, S.-H., D. M. Murphy, D. S. Thomson, and A. M. Middlebrook (2002), Chemical components of single particles measured with Particle Analysis by Laser Mass Spectrometry (PALMS) during the Atlanta Supersite Project: Focus on organic/sulfate, lead, soot, and mineral particles, J. Geophys. Res., 107(D1), 4003, doi:10.1029/2000JD000011.

Lee, S.-H., D. M. Murphy, D. S. Thomson, and A. M. Middlebrook (2003), Nitrate and oxidized organic ions in single particle mass spectra during the 1999 Atlanta Supersite Project, J. Geophys. Res., 108(D7), 8417, doi:10.1029/2001JD001455.

Li, N., C. Sioutas, J. R. Froines, A. Cho, C. Misra, and A. Nel (2003), Ultrafine particulate pollutants induce oxidative stress and mitochondrial damage, Environ. Health Perspect., 111(4), 455-460.

Liu, D.-Y., R. J. Wenzel, and K. A. Prather (2003), Aerosol time-of-flight mass spectrometry during the Atlanta Supersite Experiment: 1. Measurements, J. Geophys. Res., 108(D7), 8426, doi:10.1029/2001JD001562.

Mallina, R. V., A. S. Wexler, K. P. Rhoads, and M. V. Johnston (2000), High speed particle beam generation: A dynamic focusing mechanism for selecting ultrafine particles, Aerosol Sci. Technol., 33, 87-104.

Middlebrook, A. M., et al. (2003), A comparison of particle mass spectrometers during the 1999 Atlanta Supersite Project, J. Geophys. Res., 108(D7), 8424, doi:10.1029/2001JD000660.

Murphy, D. M., and D. S. Thomson (1995), Laser ionization mass spectroscopy of single aerosol particles, Aerosol Sci. Technol., 22, 249-273.

Neubauer, K. R., M. V. Johnston, and A. S. Wexler (1995), Chromium speciation in aerosols by rapid single-particle mass spectrometry, Int. J. Mass Spectrom. Ion Process., 151, 77-87.

Pekkanen, J., K. L. Timonen, J. Ruuskanen, A. Reponen, and A. Mirme (1997), Effects of ultrafine and fine particles in urban air on peak expiratory flow among children with asthmatic symptoms, Environ. Respir., 74(1), 24-33.

Peters, A., H. E. Wichmann, T. Tuch, J. Heinrich, and J. Heyder (1997), Respiratory effects are associated with the number of ultrafine particles, Am. J. Respir. Crit. Care Med., 155, 1376-1383.

Phares, D. J., K. P. Rhoads, A. S. Wexler, D. B. Kane, and M. V. Johnston (2001), Application of the ART-2a algorithm to laser ablation aerosol mass spectrometry of particle standards, Anal. Chem., 73, 2338-2344.

Phares, D. J., K. P. Rhoads, and A. S. Wexler (2002), Performance of a single-ultrafine-particle mass spectrometer, Aerosol Sci. Technol., 36, $583-592$.

Phares, D. J., K. P. Rhoads, M. V. Johnston, and A. S. Wexler (2003), Sizeresolved ultrafine particle composition analysis: 2. Houston, J. Geophys. Res., 108(D7), 8420, doi:10.1029/2001JD001212. 
Prather, K. A., T. Nordmeyer, and K. Salt (1994), Real-time characterization of individual aerosol particles using time-of-flight mass spectrometry, Anal. Chem., 66, 1403-1407.

Reents, W. D., S. W. Downey, A. B. Emerson, A. M. Mujsce, A. J. Muller, D. J. Siconolfi, J. D. Sinclair, and A. G. Swanson (1995), Single particle characterization by time-of-flight mass spectrometry, Aerosol Sci. Technol., 23, 263-270.

Rhoads, K. P., D. J. Phares, A. S. Wexler, and M. V. Johnston (2003), Sizeresolved ultrafine particle composition analysis: 1. Atlanta, J. Geophys. Res., 108(D7), 8418, doi:10.1029/2001JD001211.

Schauer, J. J., M. J. Kleeman, G. R. Cass, and B. R. T. Simoneit (2001), Measurement of emissions from air pollution sources. 3. $\mathrm{C}_{1}-\mathrm{C}_{29}$ organic compounds from fireplace combustion of wood, Environ. Sci. Technol., $35,1716-1728$.

Suess, D. T., and K. A. Prather (1999), Mass spectrometry of aerosols, Chem. Rev., 99, 3007-3035.

Thomson, D. S., and D. M. Murphy (1994), Analyzing single aerosol particles in real time, Chemtech, 24, 30-35.

Utsunomiya, S., K. A. Jensen, G. J. Keeler, and R. C. Ewing (2004), Direct identification of trace metals in fine and ultrafine particles in the Detroit urban atmosphere, Environ. Sci. Technol., 38(8), 2289-2297.
Wenzel, R. J., D. Liu, E. S. Edgerton, and K. A. Prather (2003), Aerosol time-of-flight mass spectrometry during the Atlanta Supersite Experiment: 2. Scaling procedures, J. Geophys. Res., 108(D7), 8427, doi:10.1029/2001JD001563.

Wexler, A. S., and M. V. Johnston (2001), Real time single particle analysis, in Aerosol Measurement, edited by P. A. Baron and K. Willeke, pp. 365385, Van Nostrand Reinhold, New York.

Zhao, Y., K. J. Bein, A. S. Wexler, C. Misra, P. M. Fine, and C. Sioutas (2005), Field evaluation of the Versatile Aerosol Concentration Enrichment System (VACES) coupled to the third-generation rapid single particle mass spectrometer (RSMS-3), J. Geophys. Res, doi:10.1029/ 2004JD004644, in press.

K. J. Bein, Department of Land, Air and Water Resources, University of California, 1 Shields Avenue, Davis, CA 95616, USA. (kjbein@ucdavis. edu)

M. V. Johnston, Department of Chemistry and Biochemistry, University of Delaware, Newark, DE 19716, USA.

A. S. Wexler and Y. Zhao, Department of Mechanical and Aeronautical Engineering, University of California, Davis, CA 95616, USA. 


\title{
Mining airborne particulate size distribution data by positive matrix factorization
}

\author{
Liming Zhou, ${ }^{1}$ Eugene Kim, and Philip K. Hopke \\ Center for Air Resources Engineering and Science and Department of Chemical Engineering, Clarkson University, Potsdam, \\ New York, USA \\ Charles Stanier ${ }^{2}$ and Spyros N. Pandis \\ Department of Chemical Engineering, Carnegie Mellon University, Pittsburgh, Pennsylvania, USA
}

Received 28 February 2004; revised 13 July 2004; accepted 2 August 2004; published 17 March 2005.

[1] Airborne particulate size distribution data acquired in Pittsburgh from July 2001 to June 2002 were analyzed as a bilinear receptor model solved by positive matrix factorization (PMF). The data were obtained from two scanning mobility particle spectrometers and an aerodynamic particle sampler with a temporal resolution of $15 \mathrm{~min}$. Each sample contained 165 size bins from 0.003 to $2.5 \mu \mathrm{m}$. Particle growth periods in nucleation events were identified, and the data in these intervals were excluded from this study so that the size distribution profiles associated with the factors could be regarded as sufficiently constant to satisfy the assumptions of the receptor model. The values for each set of five consecutive size bins were averaged to produce 33 new size intervals. Analyses were made on monthly data sets to ensure that the changes in the size distributions from the source to the receptor site could be regarded as constant. The factors from PMF could be assigned to particle sources by examination of the number size distributions associated with the factors, the time frequency properties of the contribution of each source (Fourier analysis of source contribution values), and the correlations of the contribution values with simultaneous gas phase measurements $\left(\mathrm{O}_{3}\right.$, $\mathrm{NO}, \mathrm{NO}_{2}, \mathrm{SO}_{2}, \mathrm{CO}$ ) and particle composition data (sulfate, nitrate, organic carbon/ elemental carbon). Seasonal trends and weekday/weekend effects were investigated. Conditional probability function analyses were performed for each source to ascertain the likely directions in which the sources were located. Five factors were separated. Two factors, local traffic and nucleation, are clear sources, but each of the other factors appears to be a mixture of several sources that cannot be further separated.

Citation: Zhou, L., E. Kim, P. K. Hopke, C. Stanier, and S. N. Pandis (2005), Mining airborne particulate size distribution data by positive matrix factorization, J. Geophys. Res., 110, D07S19, doi:10.1029/2004JD004707.

\section{Introduction}

[2] There have been many studies that find relationships between elevated morbidity and mortality and higher particulate matter (PM) concentrations [Dockery and Pope, 1994; Pope et al., 1995; Brunekreef et al., 1995; van Bree and Cassee, 2000]. In order to develop an effective control strategy for airborne particles, the relationship between the sources and receptor concentrations needs to be understood.

[3] Positive matrix factorization (PMF) is a powerful tool for solving receptor models with aerosol composition data and has been used successfully in identifying the sources of the airborne particles in many studies [Xie et al., 1999; Lee

\footnotetext{
${ }^{1}$ Now at Providence Engineering and Environmental Group LLC, Baton Rouge, Louisiana, USA.

${ }^{2}$ Now at Department of Chemical and Biochemical Engineering, University of Iowa, Iowa City, Iowa, USA.

Copyright 2005 by the American Geophysical Union. 0148-0227/05/2004JD004707\$09.00
}

et al., 1999; Ramadan et al., 2000; Chueinta et al., 2000; Polissar et al., 2001; Song et al., 2001]. Recently, the aerosol size distribution data have been analyzed by principal component analysis [Ruuskanen et al., 2001; Wahlin et al., 2001] and PMF [Kim et al., 2004].

[4] It is obvious that particles in different size ranges have unique characteristics and one always needs to consider classifying the size range before detailed analyses. A threemodal structure was widely used to describe continental particle number size distribution, including a nucleation mode at 10-20 nm, an Aitken mode at $40-80 \mathrm{~nm}$, and an accumulation mode at 100-300 nm [Whitby, 1978; Raes et al., 1997; Hussein et al., 2004]. Lognormal distribution functions representing the three modes were used to parameterize size distribution, and the modes fit by the lognormal distribution functions were thought to be associated with particle sources and processes during the transport [Birmili et al., 2001]. Using constant modal distribution functions was also proved possible if particle growth processes from nucleation mode to Aitken or even accumulation mode were 
not considered [Mäkelä et al., 2000]. It is worth mentioning that for size distributions with a stable structure, using variant modal distributions instead of constant ones tends to be an overfitting, which means that the small random variations of the modes and even measurement errors may be fit and, consequently, the features of the modes may be smeared.

[5] The Pittsburgh supersite was located in a strong anthropogenic source region, and the size distribution measured there cannot be fit well by the aforementioned threemodal distribution [Stanier et al., 2004c]. Thus some other methods need to be applied for classifying the size ranges.

[6] Previously, size distribution data from July 2001 measured by the Pittsburgh Air Quality Study (PAQS) have been analyzed to identify the particle sources by PMF [Zhou et al., 2004]. Five sources were identified: secondary aerosol (from distant sources), local stationary combustion, remote traffic (probably from the interstate highway 1 mile away), local traffic (from the street and minor roads close to the receptor site), and nucleation, with decreasing sizes. The PMF method has successfully divided the particles into the five classes on the basis of both their size ranges and temporal behavior without prior assumptions such as lognormal distribution and the number of modes. The PMF analysis requires stationary or quasistationary size distributions measured at the receptor site. In a short period such as one month, conditions that might affect particle size changes such as photochemical activity or temperatures may be taken as relatively constant, and the change of the particle size distribution can be thought to be sufficiently constant to permit the PMF analyses.

[7] Our recent study has indicated linear relationships between size distribution data and chemical composition data simultaneously measured at the Pittsburgh supersite [Zhou et al., 2005], and this is a direct proof of the stationarity of the size distribution.

[8] In this study, a larger data set from PAQS, containing 1 year of size distribution data from July 2001 to June 2002, has been analyzed for source identification. Data mining is a process to discover patterns and relationships in data using various tools. The tools used in this study, including PMF, will be introduced in the next sections. Each factor found by PMF can be thought of as a pattern that represents the variations of a source or a group of sources. The possible sources associated with each factor (or pattern) will be investigated.

[9] Over a full year the atmospheric processes influencing the size distributions vary significantly, and our previous efforts in directly analyzing the full year data set proved to be inappropriate [Zhou et al., 2003]. Therefore this analysis will be performed on a month-by-month basis.

[10] In our previous work [Zhou et al., 2004], the days with extensive nucleation events, especially those events followed by particle growth, were excluded, and this leads to an incomplete description of the summer situation at the Pittsburgh area. In this study, a special method has been designed to remove only the data representing particle growth events, and thus a more thorough and complete investigation will be made.

\section{Description of the Data}

[11] The receptor site was located in Schenley Park, Pittsburgh (latitude $40.4395^{\circ}$, longitude $-79.9405^{\circ}$ ). An

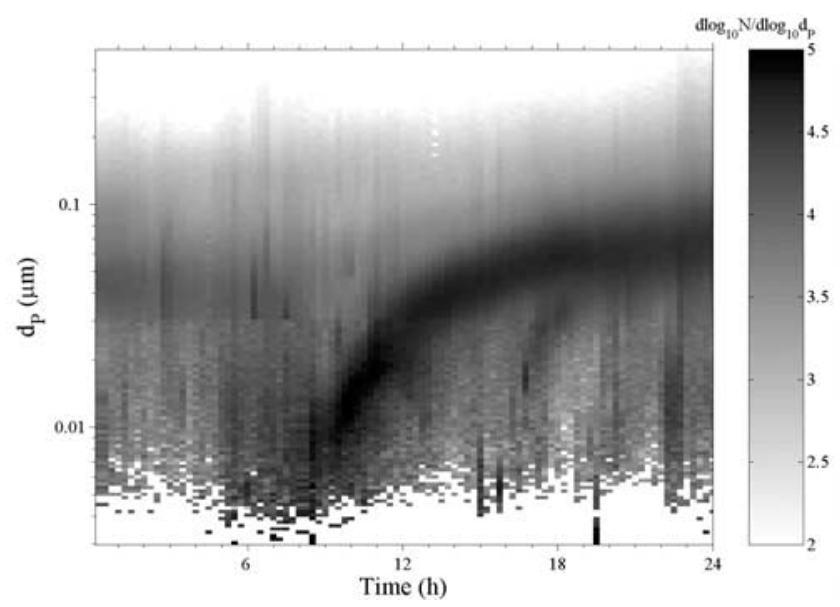

Figure 1. Typical particle growth on 2 July 2001.

overall summary and preliminary results for PAQS were given elsewhere [Wittig et al., 2003]. The size distribution data were obtained from July 2001 to June 2002. Samples were collected and measured continuously every $15 \mathrm{~min}$. The data were from two scanning mobility particle spectrometers (SMPS) and an aerodynamic particle sampler (APS). Above $583 \mathrm{~nm}$, the data used in this study represent the electrical mobility diameter inferred from aerodynamic mobility and estimated density [Khlystov et al., 2004]. The ratio of APS diameter and SMPS diameter is around 1.3. The samples were collected at $25 \%$ relative humidity, and "dry" particle distributions were obtained [Stanier et al., 2004a].

[12] The gas phase concentrations $\left(\mathrm{O}_{3}, \mathrm{NO}, \mathrm{NO}_{x}, \mathrm{SO}_{2}\right.$, and $\mathrm{CO}$, with a $10 \mathrm{~min}$ resolution), particle mass and composition $\left(\mathrm{PM}_{2.5}\right.$, sulfate, and nitrate, with 10 min time resolution; organic carbon (OC) and elemental carbon (EC), with 2 to 4 hour resolution), and meteorological conditions (wind direction, wind speed, etc., with 10 min resolution) were measured at the same time and location as the size distribution data. A detailed description of the instrument and sampling methods can be found elsewhere [Wittig et al., 2003; Stanier et al., 2004b]. Each size distribution sample contained 165 geometrically equal sized intervals covering the particle size range of $0.003-2.5 \mu \mathrm{m}$.

\section{Exclusion of Data Representing Particle Growth}

[13] Nucleation events observed at the Pittsburgh site were classified as regional and short-lived by Stanier et al. [2004b]. In a regional nucleation event, it is often observed that the newly formed particles continue growing. Particle growth events are confined to limited time intervals and size ranges from over $10 \mathrm{~nm}$ up to accumulation mode size. A typical growth event after the nucleation is shown in Figure 1, where the number concentrations on 2 July 2001 are shown. These particle growth events were also observed at many other places, and the experimental and theoretical studies on this phenomenon were recently reviewed by Kulmala et al. [2004]. Discussions of the mechanisms of these events are beyond the scope of this study. 


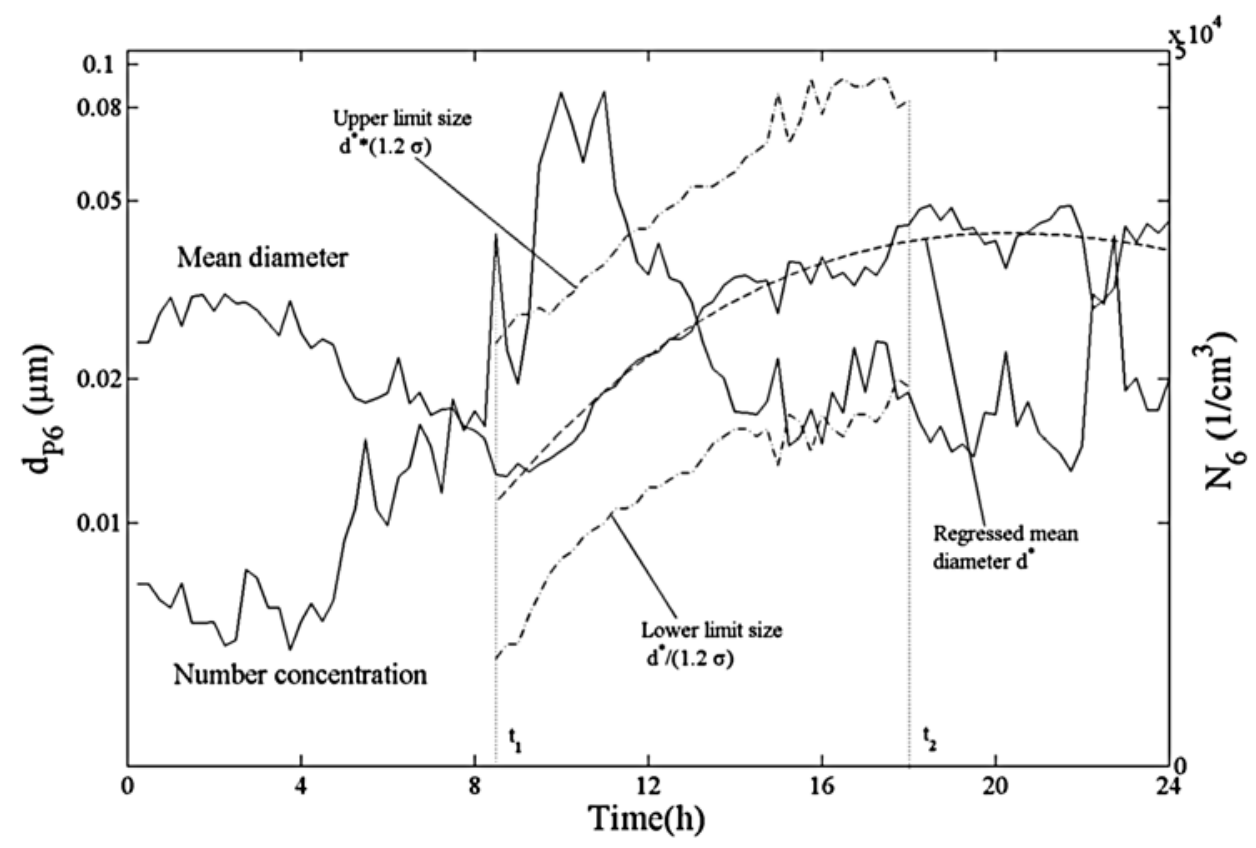

Figure 2. Number concentration and mean size variations on 2 July 2001.

[14] Receptor models require that the property being apportioned be stationary or, in this case, that there must be constant size distribution profiles associated with all factors. The temporal variations of the size distribution caused by particle growth events will deform the model solutions and make the sources unidentifiable. Particle growth during a regional nucleation event was identified, and the data representing the growth were removed by the following method. The number-concentration-weighted mean diameter is defined by the following equation:

$$
\log d_{a v}=\sum_{d_{p}} N\left(d_{p}\right) \log d_{p} / \sum_{d_{p}} N\left(d_{p}\right)
$$

In addition to the variation of number concentration $\mathrm{d} N / \mathrm{d} t$, the number-concentration-weighted diameter can also be used to investigate the nucleation event. If there is a nucleation event, the number concentration increases sharply, and the mean diameter should simultaneously drop sharply since the newly formed particles have the smallest sizes and the largest number concentrations.

[15] Since many particle growth events occurred in the size range above $6 \mathrm{~nm}$, the total number concentration and mean diameter above $6 \mathrm{~nm}, N_{6}$ and $d_{p 6, a v}$, were used. Here, $N_{6}$ is the total number concentration over $6 \mathrm{~nm}$; $d_{p 6, a v}$ is computed by equation (1) for all particles larger than $6 \mathrm{~nm}$. The beginning of particle growth is defined quantitatively as the point at which no significant decrease of mean diameter occurs when the number concentration has a sharp increase and the mean diameter rises gradually afterward. In Figure $2, t_{1}$ is found to be the start of particle growth. The mean diameter $d_{p 6, a v}$ does not change with time when particle growth becomes stable. A polynomial regression of $\log d_{p 6, a v}$ against time was made to estimate the time after which the variation in mean diameter is sufficiently small, as shown in Figure 2. When the first derivative of the regressed value is below a criterion value, such as at $t_{2}$ in Figure 2, the growth can be considered to have terminated.

[16] By assuming the number concentration distribution versus $d_{p 6, a v}$ to be lognormal (during the particle growth after the nucleation, the number concentration distribution over $6 \mathrm{~nm}$ is usually unimodal), we define the data, within $\pm 1.2 \sigma$ from the regressed $d_{p 6, a v}$ and between $t_{1}$ and $t_{2}$, representing particle growth, where $\sigma$ is the standard deviation of the lognormal distribution for each time interval. Figure 3 shows the result of this definition for 2 July 2001. The data thus defined were treated as missing values, which means replacing these values by the mean values and

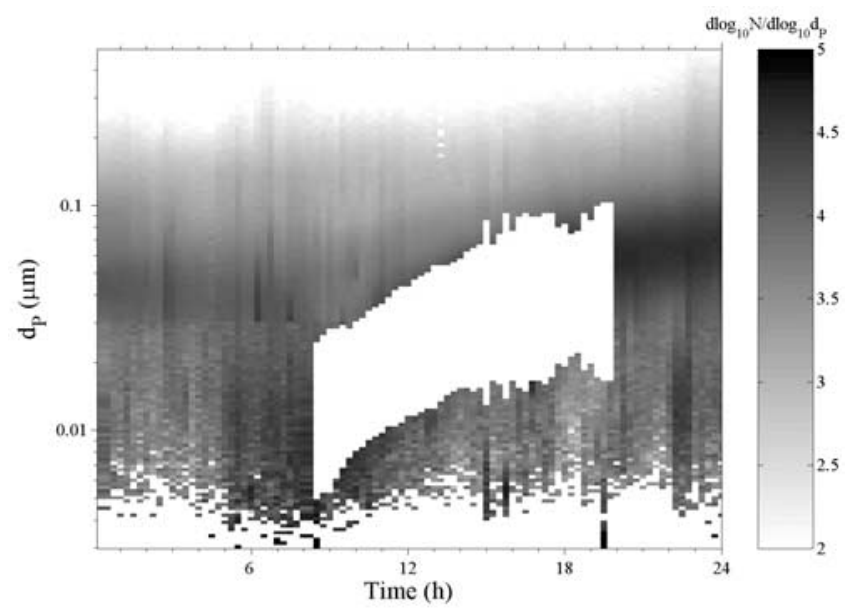

Figure 3. Definition of particle growth zone for 2 July 2001. 
Table 1. Days With a Particle Growth Zone Defined (Data by This Definition Excluded in PMF Analysis)

\begin{tabular}{ll}
\hline \multicolumn{1}{c}{ Month } & \multicolumn{1}{c}{ Days } \\
\hline July 2001 & $2,11,14,15,24$, and 30 July \\
Aug. 2001 & $7,11,14$, and 18 Aug. \\
Sept. 2001 & $1,5,11,14,16$, and 26 Sept. \\
Oct. 2001 & $7,10,11,15,18,20,24,25,29$, and 30 Oct. \\
Nov. 2001 & $3,4,8,9,10$, and 22 Nov. \\
Dec. 2001 & None \\
Jan. 2002 & 25 and 26 Jan. \\
Feb. 2002 & 13,14, and 25 Feb. \\
March 2002 & $6,7,9,15,23,24$, and 29 March \\
April 2002 & $2,10,11,12,16,17,18$, and 19 April \\
May 2002 & $4,5,10,11,15,16,17,23,24,26,27,28,29,30$, \\
& and 31 May \\
June 2002 & $2,3,4,5,8,9,21,22,23,24$, and 29 June \\
\hline
\end{tabular}

assigning them high uncertainties (low weights), such as 10 times their concentrations. Thus these data will not influence the PMF result and will not be reflected in the factors obtained by PMF. The data before and after this operation were inspected visually, and sometimes the parameters were adjusted manually to properly define the particle growth zone. If a regional nucleation event is followed with no particle growth, the corresponding data were not processed by this method. The days processed by this method are listed in Table 1. From this table, it can be seen that this kind of particle growth phenomenon rarely happened in winter.

\section{PMF and Other Tools for Mining the Size Distribution Data}

\subsection{PMF}

[17] A detailed introduction to PMF can be found elsewhere [Paatero, 1997]. In this study, PMF2 (two-way PMF) is used to solve the following two-way receptor model:

$$
X=G F+E
$$

or, in the elemental form,

$$
x_{i j}=\sum_{k=1}^{p} g_{i k} f_{k j}+e_{i j},
$$

where $X$ is the matrix of observed data and the element $x_{i j}$ is the number concentration value of the $i$ th sample at the $j$ th size bin. $G$ and $F$ are the source contributions and size distribution profiles, respectively, of the sources that are unknown and to be estimated from the analysis. To be specific, $g_{i k}$ is the concentration of particles from the $k$ th source associated with the $i$ th sample and $f_{k j}$ is the size distribution associated with $k$ th source. $E$ is a matrix of residuals.

\begin{tabular}{|c|c|c|c|c|c|c|c|c|}
\hline \multirow[b]{2}{*}{$\begin{array}{l}\text { Size, } \\
\mu \mathrm{m}\end{array}$} & \multicolumn{7}{|c|}{ Number Concentration, number $\mathrm{cm}^{-3}$} & \multirow{2}{*}{$\begin{array}{c}\text { Mean Volume } \\
\text { Concentration, } \\
\mu \mathrm{m}^{3} \mathrm{~cm}^{-3}\end{array}$} \\
\hline & $\begin{array}{l}\text { Mean, } \\
\times 10^{3} \\
\end{array}$ & $\begin{array}{l}\mathrm{SD}^{\mathrm{a}} \\
\times 10^{3} \\
\end{array}$ & Min & $\begin{array}{l}\text { Max, } \\
\times 10^{3} \\
\end{array}$ & $\begin{array}{c}\text { Median, } \\
\times 10^{3} \\
\end{array}$ & $\begin{array}{c}\text { Lower } 25 \%, \\
\times 10^{3} \\
\end{array}$ & $\begin{array}{c}\text { Upper } 25 \%, \\
\times 10^{3} \\
\end{array}$ & \\
\hline 0.0032 & 21.5 & 53 & 0 & 1550 & 7.8 & 0 & 23 & 0.00038 \\
\hline 0.0039 & 11.2 & 21 & 0 & 496 & 5.7 & 0 & 13 & 0.00034 \\
\hline 0.0046 & 8.8 & 12.7 & 0 & 241 & 5.4 & 2.4 & 10 & 0.00045 \\
\hline 0.0055 & 8.4 & 10.1 & 0 & 306 & 5.9 & 3.2 & 9.7 & 0.00074 \\
\hline 0.0066 & 8.8 & 9.7 & 0 & 667 & 6.9 & 3.9 & 10 & 0.0013 \\
\hline 0.0079 & 9.9 & 14 & 0 & 2110 & 8.3 & 4.9 & 12 & 0.0026 \\
\hline 0.0095 & 11.2 & 9.1 & 0 & 406 & 10.1 & 5.8 & 13 & 0.0050 \\
\hline 0.0113 & 12.6 & 9.6 & 0 & 304 & 11.5 & 6.7 & 15 & 0.0096 \\
\hline 0.0136 & 13.7 & 10.4 & 0 & 405 & 12.6 & 7.2 & 16 & 0.018 \\
\hline 0.0163 & 14.3 & 12.5 & 0 & 688 & 12.0 & 6.9 & 17 & 0.030 \\
\hline 0.0195 & 14.6 & 12.7 & 0 & 481 & 12.3 & 7.2 & 17 & 0.056 \\
\hline 0.0233 & 15.0 & 13.3 & 0 & 371 & 12.3 & 7 & 17 & 0.10 \\
\hline 0.0279 & 15.5 & 15.1 & 0 & 1170 & 12.7 & 7.5 & 18 & 0.18 \\
\hline 0.0334 & 16.1 & 13.9 & 0.6 & 444 & 13.4 & 7.8 & 20 & 0.31 \\
\hline 0.0400 & 16.2 & 13.2 & 0.44 & 224 & 13.7 & 7.7 & 19 & 0.54 \\
\hline 0.048 & 15.6 & 12.0 & 0 & 150 & 13.6 & 7.6 & 19 & 0.90 \\
\hline 0.057 & 14.6 & 10.8 & 0 & 131 & 12.6 & 7.2 & 17 & 1.4 \\
\hline 0.069 & 13.0 & 9.6 & 0 & 124 & 11.2 & 6.7 & 15 & 2.2 \\
\hline 0.082 & 11.2 & 8.1 & 0 & 118 & 9.8 & 6.0 & 13 & 3.2 \\
\hline 0.098 & 9.5 & 6.7 & 0 & 96.9 & 8.5 & 5.1 & 11 & 4.7 \\
\hline 0.118 & 7.8 & 5.2 & 0 & 51.8 & 6.9 & 4.1 & 9.3 & 6.6 \\
\hline 0.141 & 6.03 & 3.87 & 0 & 38.0 & 5.3 & 3.3 & 7.3 & 8.8 \\
\hline 0.169 & 4.43 & 2.74 & 0 & 23.5 & 3.8 & 2.5 & 5.7 & 11 \\
\hline 0.20 & 3.11 & 1.90 & 0 & 19.2 & 2.6 & 1.7 & 4.1 & 13 \\
\hline 0.24 & 2.09 & 1.36 & 0.32 & 19.0 & 1.7 & 1.1 & 2.7 & 15 \\
\hline 0.29 & 1.31 & 0.99 & 0 & 11.0 & 0.98 & 0.62 & 1.7 & 16.5 \\
\hline 0.35 & 0.74 & 0.68 & 0 & 5.9 & 0.51 & 0.29 & 0.96 & 16 \\
\hline 0.41 & 0.37 & 0.41 & 0 & 4.1 & 0.22 & 0.12 & 0.45 & 14 \\
\hline 0.50 & 0.16 & 0.21 & 0.43 & 2.6 & 0.089 & 0.045 & 0.19 & 11 \\
\hline 0.63 & 0.087 & 0.092 & 1.81 & 0.84 & 0.056 & 0.027 & 0.109 & 11 \\
\hline 0.90 & 0.01 & 0.010 & 0.23 & 0.21 & 0.0075 & 0.0037 & 0.12 & 3.8 \\
\hline 1.29 & 0.0021 & 0.0018 & 0.1 & 0.054 & 0.00190281 & 0.0012 & 0 & 2.3 \\
\hline 1.84 & 0.0011 & 0.0009 & 0 & 0.036 & 0.0009 & 0.0007 & 0.0013 & 3.6 \\
\hline
\end{tabular}

Table 2. Description of the Whole Year Data (Data Representing Particle Growth Excluded)

${ }^{\mathrm{a}} \mathrm{SD}$, standard deviation. 

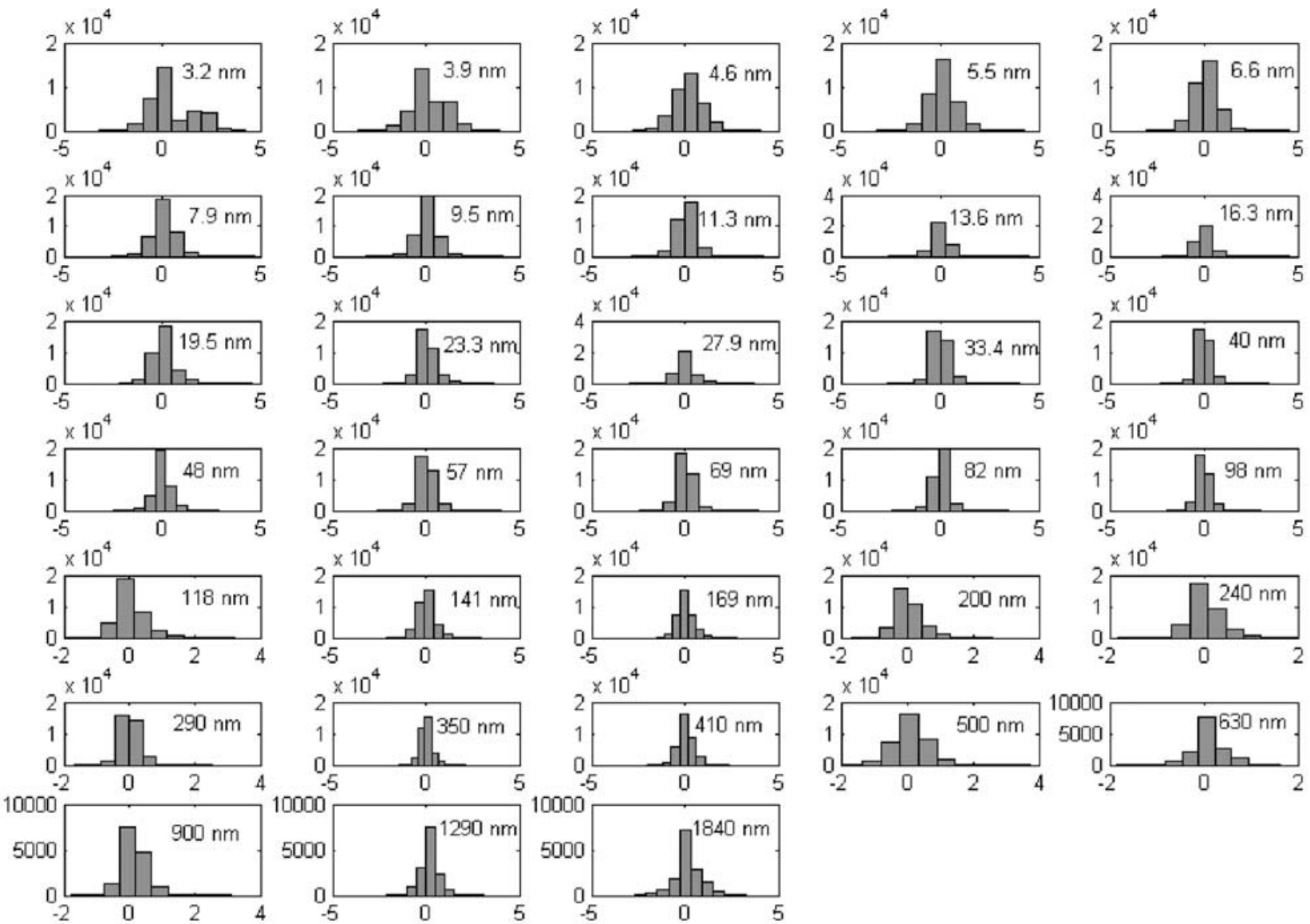

Figure 4. Histograms of the scaled regression residuals. The horizontal axes indicate the scaled residual, and the vertical axes indicate the frequencies.

[18] The residual sum of squares $(Q)$ is minimized by finding the optimal $F$ and $G$ values as indicated in equations (2) and (3):

$$
Q=\left\|\frac{(X-G F)}{s}\right\|_{F, G}^{2}=\sum_{i} \sum_{j}\left(\frac{e_{i j}}{s_{i j}}\right)^{2} .
$$

[19] Rotations are obtained by setting an FPEAK value [Paatero et al., 2002]. When the FPEAK value is positive, the following additional term is included in the object function $Q$ :

$$
Q^{P}=\beta^{2}\left(\sum_{k=1}^{p} \sum_{j=1}^{n} f_{k j}\right)^{2}
$$

where $\beta^{2}$ corresponds to the FPEAK value. The term defined above attempts to pull the sum of all the elements of $F$ toward zero and makes the program do elementary transformations for $F$ and $G$ by subtracting the $F$ vectors from each other and adding corresponding $G$ vectors to obtain a more physically realistic solution. Recently, a method for solving rotational ambiguities by detecting edges in $G$ space have been developed, and the best solution is obtained without clearly defined edges in $G$ space [Paatero et al., 2005]. In this study, the FPEAK value is set to 0.2 for each of the months to eliminate $G$ space edges, and five similar factors were found for each month.

[20] The $F$ values were normalized to make the sum of each size interval equal to 1 for each factor. The $G$ values were then rescaled correspondingly. The uncertainties were estimated with the method described by Zhou et al. [2004]. To smooth the size distribution data and minimize the error caused by the discontinuity between instruments, every five consecutive size bins were combined into one, and 33 new size intervals were produced from the original 165 size bins. The analysis of volume size distribution data does not provide much more information since it generates similar source contributions as number size distribution analyses do [Zhou et al., 2004], and hence it will not be performed in this study. During the period between October 2001 and April 2002 the APS was not working, and only 29 new size intervals were produced for these months. This also caused incomplete information on volume size distribution. Table 2 summarizes the data set after the aforementioned treatment.

\subsection{Correlation Analyses}

[21] Correlations of some compositions and gases with source contributions were investigated. Using only gas data as an input to PMF will not bring much more information, and both data sets need to be averaged to $30 \mathrm{~min}$. The $15 \mathrm{~min}$ resolution has made possible a more thorough time series 

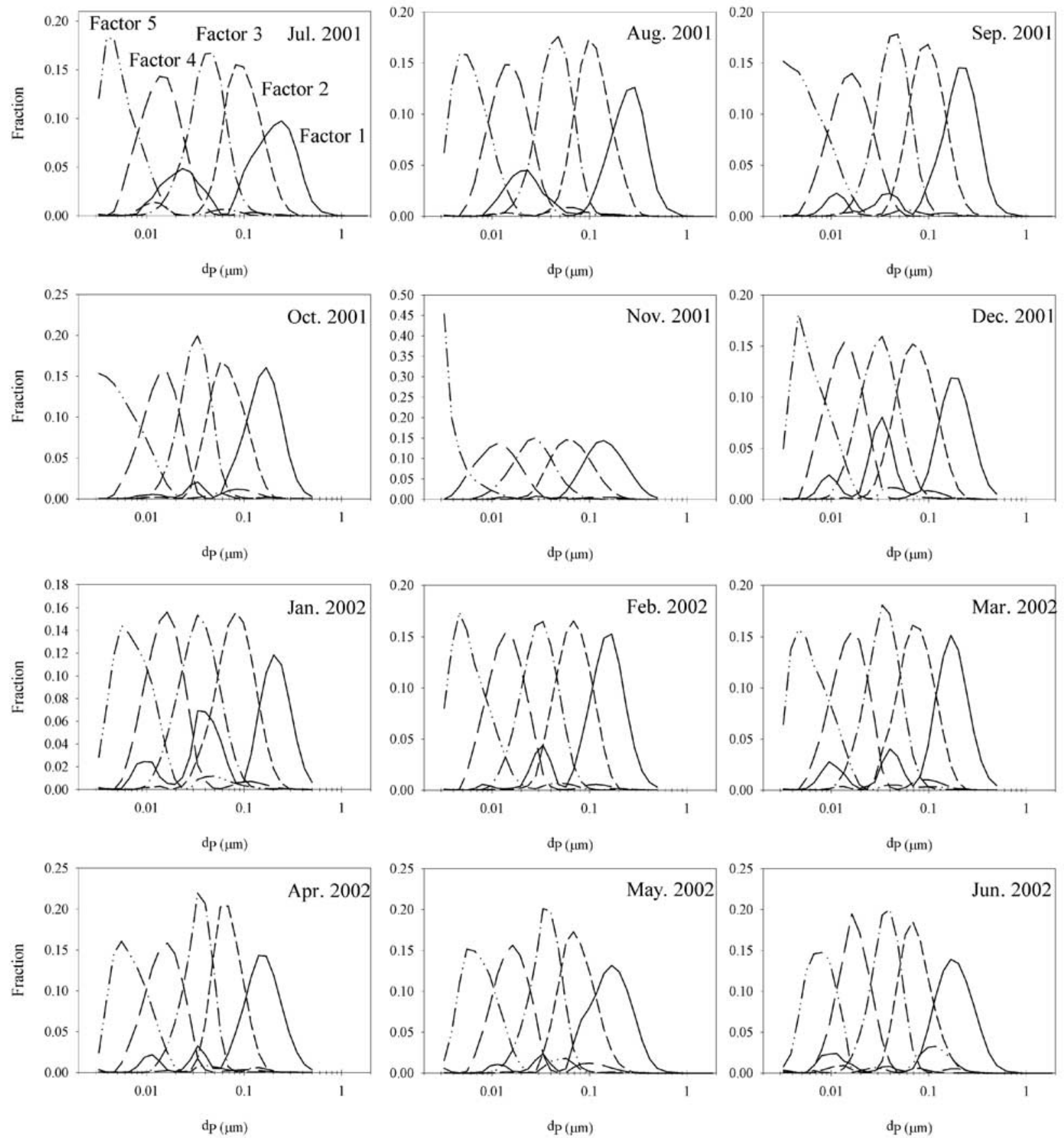

Figure 5. Size distribution profiles for each month.

analysis than $30 \mathrm{~min}$ resolution, such as in the time of day effect.

\subsection{Conditional Probability Function (CPF)}

[22] A conditional probability function [Ashbaugh et al., 1985; Kim et al., 2004] was calculated using the source contributions obtained by PMF2 and wind direction values by the following equation:

$$
C P F=\frac{m_{\Delta \theta}}{n_{\Delta \theta}}
$$

where $m_{\Delta \theta}$ is the number of occurrences in the direction sector that exceeds the threshold, upper 25 th percentile of the fractional contribution from each source, and $n_{\Delta \theta}$ is the total number of wind occurrences in the same direction sector. Fractional contributions are used to avoid the influence of atmospheric dilution on CPF results. The angular width of direction sector is set as $10^{\circ}$, and thus there are 36 directional sectors. Those samples corresponding to a wind speed below $1.0 \mathrm{~m} \mathrm{~s}^{-1}$ are excluded from this study, and two thirds of the total samples were excluded. The sources are thought to be located in the direction sectors 

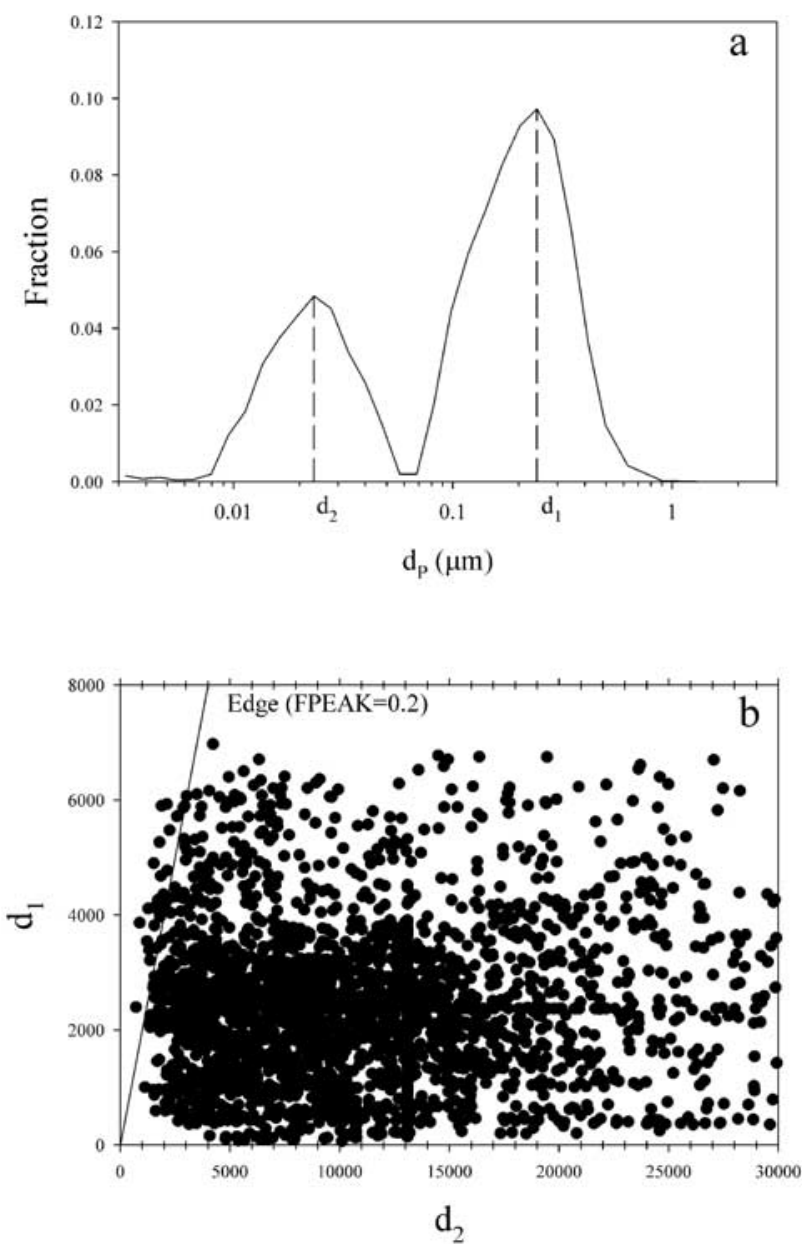

Figure 6. Discussion of the two modes of factor 1 in July 2001. (a) Size distribution of factor 1. (b) Scatterplot of the number concentration of the central size intervals of the two modes.

with high CPF values. When $n_{\Delta \theta}$ is below 10 , the $\mathrm{CPF}$ value is set to zero.

\subsection{Fourier Analysis}

[23] The source contributions from each month are linked in a temporal sequence to form annual source contribution series, and Fourier analyses have been performed for these annual series. In the signal-processing field, it is well known that Fourier analysis has no temporal specificity. For example, if the Fourier analysis says that the series has a daily pattern ( 24 hour period), it cannot determine that the daily pattern exists at any specific time in the series. Therefore many time-frequency analysis techniques have been developed and used in combination with Fourier analyses. To address the problem in this study, the Fourier analysis was performed for the source contributions of each month. The correlations of the source contributions with the gas and particle composition data were calculated for each month.

\section{Results and Discussion}

[24] Table 2 describes the average number and volume size distribution of the full year of data. The volume distribution mode is located between 0.1 and $1 \mu \mathrm{m}$. The smallest particles have the largest mean number concentration, but their median concentrations are not the highest. This situation may be caused by the frequent nucleation events that produce large numbers of new particles. Table 2 also indicates that the minimum number concentrations in all the size intervals are nearly zero, implying very low contributions from certain sources. The 12 months of data were summarized and described in detail elsewhere [Stanier et al., 2004c]. Figure 4 indicates the histograms of the scaled regression residuals for all size intervals of all 12 months. Most of the residuals are within -2 and +2 , and the distributions are symmetric and close to normal distributions. If the size distributions measured at the receptor are not stationary or quasistationary, then these good regressions cannot be obtained. The five factors are arranged in order of decreasing size from factor 1 to factor 5. These five factors are similar to the sources identified in our previous study [Zhou et al., 2004]. Detailed discussion is provided as follows.

[25] Factor 1 has a number mode between 0.15 and $0.25 \mu \mathrm{m}$ and a submode at $0.02 \mu \mathrm{m}$, as shown in Figure 5 . Detecting edges in $F$ space is a method to help resolve rotation problems [Henry, 2003]. For July 2001 the number concentrations of the two size intervals $d_{1}(240 \mathrm{~nm})$ and $d_{2}(23 \mathrm{~nm})$, the center size intervals of the two modes, were plotted in Figure 6. The edge corresponding to $\mathrm{FPEAK}=0.2$ was also plotted. When pulling down the submode by increasing the FPEAK value, the edge will rotate toward the $d_{1}$ axis, but it is clear that the edge cannot overlap with the $d_{1}$ axis, suggesting that the submode cannot be eliminated completely. Nevertheless, for the solutions with different rotations, the major mode and source contributions did not experience large changes, and our analysis and conclusions were not severely influenced.

[26] In Figure 7, the Fourier analysis shows a weak daily pattern for factor 1 . Table 3 indicates that the daily pattern is clear in February and April of 2002 but not that clear in other months. Fourier analyses have also been applied for sulfate and nitrate data for each month. These analyses found that sulfate does not have daily patterns except in the summer months (it had weak daily patterns in July and August of 2001 and June of 2002) and nitrate usually has a strong diurnal pattern during the whole year except in November and December of 2001 and January and March of 2002. On the basis of these facts, one possible explanation for the periodicity of factor 1 is as follows: When the sulfate concentration is low and nitrate has a strong simultaneously diurnal pattern, such as the situation of February and April of 2002, the temporal behavior of factor 1 can be influenced by nitrate and shows observable daily patterns. Another possible reason is that some local emissions have been included in factor 1 . However, the reason cannot be completely clarified within the current data sets.

[27] Factor 1 has a strong correlation with $\mathrm{PM}_{2.5}$ mass over the entire period as indicated in Table 4 and is related to the major components of the $\mathrm{PM}_{2.5}$ mass at the Pittsburgh supersite. In winter the correlation of nitrate with factor 1 increases, suggesting that more nitrate is included in factor 1.

[28] As indicated in Table 4, factor 1 usually has high correlations with sulfate. Secondary sulfate was formed by the oxidation of $\mathrm{SO}_{2}$ through photochemical reactions. 


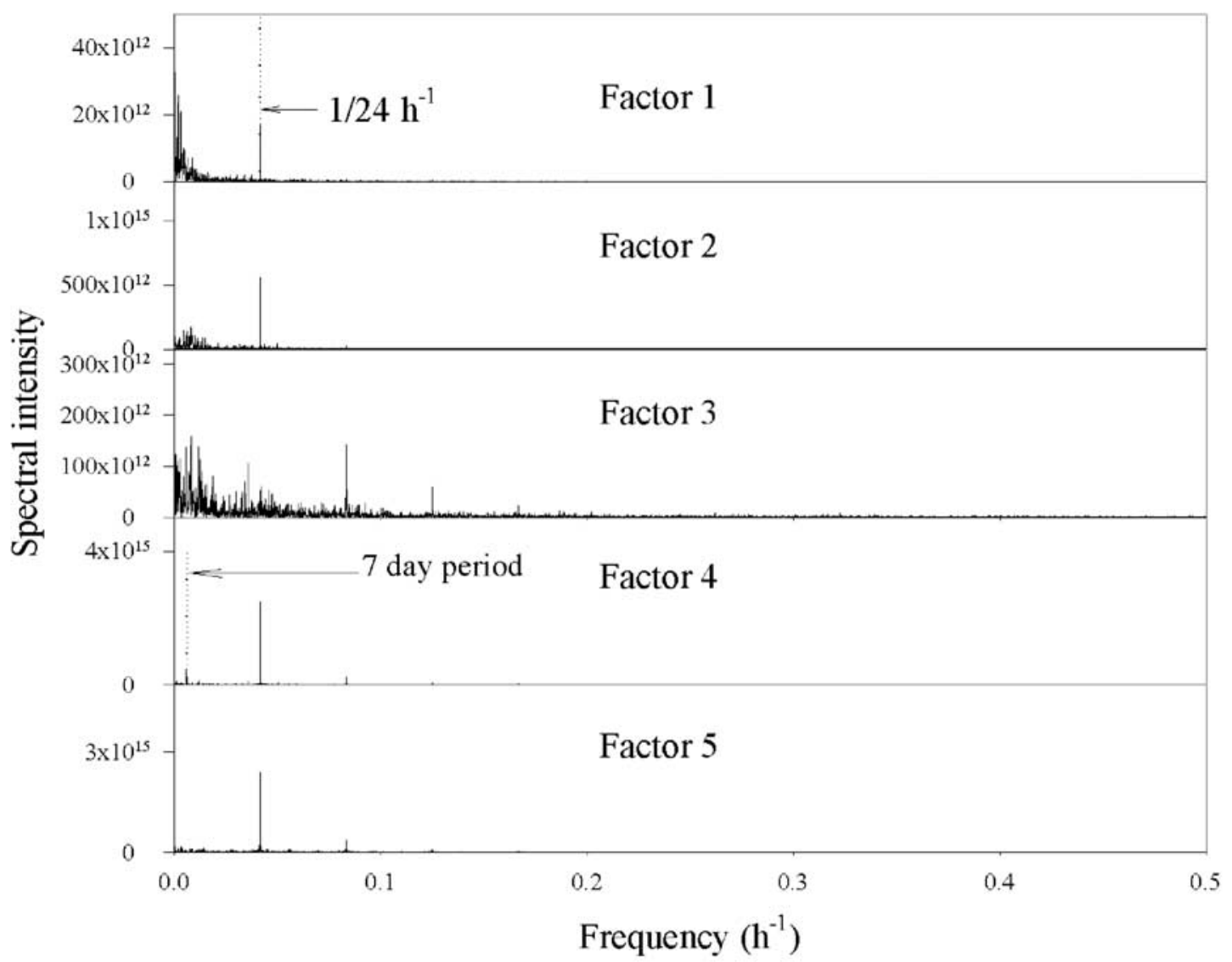

Figure 7. Fourier transformation for the annual contribution of each factor. (The powers are all nearly zero for all factors from $0.2 \mathrm{~h}^{-1}$ to $2 \mathrm{~h}^{-1}$, the Nyquist frequency.)

The main $\mathrm{SO}_{2}$ sources are coal-fired power plants hundreds of kilometers away. In winter the correlation of factor 1 with nitrate increases noticeably. The lower temperatures support ammonium nitrate in the solid phase, and the fraction of nitrate in $\mathrm{PM}_{2.5}$ mass increases. The increase of the correlation coefficient with nitrate may explain the higher correlation of factor 1 with $\mathrm{PM}_{2.5}$ than with sulfate during winter, and this is also consistent with the fact that there was much less sulfate in winter.

[29] Factor 1 has a higher correlation with OC than with EC in the summer and fall of 2001, but the correlations are similar for both species in the first half of 2002. The higher correlation with OC than with EC in the summer may be caused by secondary organic matter condensing onto preexisting particles during the transport as well as more production of secondary $\mathrm{OC}$ in the summer. When primary $\mathrm{OC}$ dominates, the correlations with $\mathrm{OC}$ and with $\mathrm{EC}$ are similar since they are from the same sources and have similar temporal variations. In Figure 8 the CPF analysis shows that factor 1 is from the south. Table 5 summarizes the characteristics of factor 1 and other factors.

[30] Factor 1 typically includes sulfate, nitrate (in winter), and secondary organics as well as primary organics that have aged in the atmosphere and have grown from their original size. The particles of the submodes cannot be from distant places; otherwise, they would be depleted during the transport. The small modes of factor 1 are more likely to be from fresh emissions of some combustion sources south of the site. Since the dominating direction of factor 1 is south, the particles of the small mode have the same variations as other large particles in factor 1, which may explain the correlation with CO. Factor 1 includes secondary, aged primary aerosol particles and also fresh primary particles from local combustion sources.

[31] The number mode of factor 2 is at $0.08 \sim 0.1 \mu \mathrm{m}$ in July, August, and September of 2001. In 2002 the number mode is at $0.06 \sim 0.07 \mu \mathrm{m}$. In Figure 9 a daily pattern, caused by the reduction of mixing height at night, is clearly observed. The nocturnal increase of the source contribution, owing to the reduction of the mixing height, may be the

Table 3. Results of Monthly Fourier Analysis for Daily Patterns ${ }^{\mathrm{a}}$

\begin{tabular}{lllccc}
\hline & Factor 1 & Factor 2 & Factor 3 & Factor 4 & Factor 5 \\
\hline July 2001 & no & weak & no & strong & strong \\
Aug. 2001 & no & no & no & strong & strong \\
Sept. 2001 & very weak & very weak & no & strong & strong \\
Oct. 2001 & very weak & weak & no & strong & strong \\
Nov. 2001 & no & very weak & no & strong & strong \\
Dec. 2001 & no & no & no & strong & strong \\
Jan. 2002 & no & no & no & strong & strong \\
Feb. 2002 & weak & very weak & no & strong & strong \\
March 2002 & no & no & no & strong & strong \\
April 2002 & weak & weak & no & strong & strong \\
May 2002 & no & weak & no & strong & strong \\
June 2002 & no & weak & no & strong & - \\
\hline
\end{tabular}

${ }^{a}$ The strength of the daily patterns was decided by comparing the spectral intensity at $1 / 24 \mathrm{~h}^{-1}$ with other frequencies. 
Table 4. Correlations $r$ of the Source Contributions With Gas and Particle Composition Data ${ }^{\mathrm{a}}$

\begin{tabular}{|c|c|c|c|c|c|c|c|c|c|c|}
\hline & $\mathrm{O}_{3}$ & $\mathrm{NO}$ & $\mathrm{NO}_{x}$ & $\mathrm{SO}_{2}$ & $\mathrm{CO}$ & $\mathrm{PM}_{2.5}$ & Sulfate & Nitrate & $\mathrm{OC}$ & $\mathrm{EC}$ \\
\hline \multicolumn{11}{|c|}{ Factor 1} \\
\hline July 2001 & 0.18 & 0.03 & 0.18 & 0.16 & 0.19 & 0.87 & 0.85 & 0.23 & 0.63 & 0.28 \\
\hline Aug. 2001 & -0.02 & 0.20 & 0.29 & 0.07 & 0.42 & 0.75 & 0.54 & 0.29 & 0.56 & 0.42 \\
\hline Sept. 2001 & 0.10 & 0.26 & 0.34 & 0.21 & 0.38 & 0.80 & 0.78 & 0.46 & $-{ }^{b}$ & $-{ }^{b}$ \\
\hline Oct. 2001 & 0.03 & 0.32 & 0.41 & 0.27 & 0.51 & 0.73 & 0.75 & 0.61 & 0.67 & 0.56 \\
\hline Nov. 2001 & -0.05 & 0.42 & 0.48 & 0.33 & 0.57 & 0.90 & 0.68 & 0.40 & 0.89 & 0.67 \\
\hline Dec. 2001 & -0.47 & 0.46 & 0.45 & 0.14 & 0.61 & 0.81 & 0.47 & 0.54 & 0.74 & 0.76 \\
\hline Jan. 2002 & -0.42 & 0.51 & 0.56 & 0.17 & 0.47 & 0.70 & 0.48 & 0.67 & 0.56 & 0.55 \\
\hline Feb. 2002 & -0.53 & 0.70 & 0.75 & 0.34 & 0.76 & 0.91 & 0.61 & 0.54 & 0.82 & 0.76 \\
\hline March 2002 & -0.50 & 0.43 & 0.57 & 0.30 & 0.59 & 0.88 & 0.62 & 0.57 & 0.65 & 0.69 \\
\hline April 2002 & -0.33 & 0.44 & 0.52 & 0.18 & 0.52 & 0.73 & 0.58 & 0.45 & 0.51 & 0.43 \\
\hline May 02 & -0.12 & 0.39 & 0.45 & 0.22 & 0.50 & 0.85 & 0.74 & 0.31 & 0.80 & 0.66 \\
\hline June 02 & 0.18 & 0.13 & 0.35 & 0.17 & 0.49 & 0.82 & 0.63 & 0.31 & 0.58 & 0.56 \\
\hline \multicolumn{11}{|c|}{ Factor 2} \\
\hline July 2001 & -0.18 & 0.47 & 0.57 & 0.48 & 0.54 & 0.29 & 0.15 & 0.54 & 0.46 & 0.56 \\
\hline Aug. 2001 & -0.15 & 0.34 & 0.48 & 0.32 & 0.35 & 0.25 & 0.23 & 0.31 & 0.31 & 0.39 \\
\hline Sept. 2001 & -0.24 & 0.60 & 0.73 & 0.32 & 0.77 & 0.61 & 0.52 & 0.58 & $-{ }^{b}$ & $-{ }^{b}$ \\
\hline Oct. 2001 & -0.46 & 0.66 & 0.78 & 0.46 & 0.56 & 0.46 & 0.23 & 0.61 & 0.43 & 0.58 \\
\hline Nov. 2001 & -0.51 & 0.74 & 0.79 & 0.42 & 0.79 & 0.48 & 0.23 & 0.34 & 0.41 & 0.75 \\
\hline Dec. 2001 & -0.46 & 0.53 & 0.53 & 0.36 & 0.67 & 0.81 & 0.47 & 0.40 & 0.80 & 0.81 \\
\hline Jan. 2002 & -0.37 & 0.59 & 0.71 & 0.44 & 0.59 & 0.72 & 0.61 & 0.45 & 0.71 & 0.71 \\
\hline Feb. 2002 & -0.61 & 0.71 & 0.78 & 0.58 & 0.70 & 0.79 & 0.56 & 0.38 & 0.66 & 0.71 \\
\hline March 2002 & -0.50 & 0.53 & 0.68 & 0.59 & 0.61 & 0.70 & 0.57 & 0.43 & 0.53 & 0.61 \\
\hline April 2002 & -0.41 & 0.50 & 0.59 & 0.48 & 0.54 & 0.49 & 0.46 & 0.34 & 0.36 & 0.34 \\
\hline May 2002 & -0.29 & 0.60 & 0.68 & 0.47 & 0.64 & 0.65 & 0.41 & 0.38 & 0.64 & 0.71 \\
\hline June 2002 & -0.21 & 0.36 & 0.58 & 0.27 & 0.61 & 0.45 & 0.19 & 0.52 & 0.50 & 0.58 \\
\hline \multicolumn{11}{|c|}{ Factor 3} \\
\hline July 2001 & -0.24 & 0.37 & 0.40 & 0.23 & 0.28 & -0.14 & -0.23 & 0.23 & -0.04 & 0.26 \\
\hline Aug. 2001 & -0.27 & 0.32 & 0.44 & 0.18 & 0.31 & -0.01 & -0.11 & 0.25 & 0.14 & 0.35 \\
\hline Sept. 2001 & -0.31 & 0.36 & 0.51 & 0.29 & 0.43 & 0.13 & 0.07 & 0.30 & $-{ }^{b}$ & $-{ }^{b}$ \\
\hline Oct. 2001 & -0.24 & 0.39 & 0.45 & 0.24 & 0.22 & 0.13 & 0.02 & 0.28 & 0.08 & 0.27 \\
\hline Nov. 2001 & -0.32 & 0.33 & 0.36 & 0.15 & 0.21 & 0.04 & -0.15 & 0.17 & -0.07 & 0.21 \\
\hline Dec. 2001 & -0.25 & 0.20 & 0.18 & 0.34 & 0.24 & 0.34 & 0.13 & 0.31 & 0.27 & 0.30 \\
\hline Jan. 2002 & -0.20 & 0.38 & 0.42 & 0.40 & 0.30 & 0.28 & 0.41 & 0.35 & 0.20 & 0.33 \\
\hline Feb. 2002 & -0.23 & 0.16 & 0.20 & 0.45 & 0.08 & 0.11 & 0.19 & 0.10 & -0.10 & 0.02 \\
\hline March 2002 & -0.32 & 0.35 & 0.42 & 0.52 & 0.28 & 0.31 & 0.36 & 0.22 & 0.14 & 0.25 \\
\hline April 2002 & -0.28 & 0.30 & 0.37 & 0.59 & 0.28 & 0.22 & 0.19 & 0.17 & 0.02 & 0.13 \\
\hline May 2002 & -0.21 & 0.33 & 0.41 & 0.49 & 0.27 & 0.15 & 0.01 & 0.11 & 0.06 & 0.21 \\
\hline June 2002 & -0.17 & 0.22 & 0.30 & 0.21 & 0.20 & 0.02 & -0.05 & 0.20 & 0.04 & 0.17 \\
\hline \multicolumn{11}{|c|}{ Factor 4} \\
\hline July 2001 & -0.10 & 0.18 & 0.19 & 0.06 & 0.01 & -0.03 & -0.06 & 0.09 & -0.11 & 0.10 \\
\hline Aug. 2001 & -0.13 & 0.23 & 0.21 & 0.07 & -0.05 & -0.20 & -0.23 & 0.01 & -0.17 & 0.03 \\
\hline Sept. 2001 & -0.09 & 0.17 & 0.11 & 0.12 & -0.10 & -0.18 & -0.20 & -0.03 & $-{ }^{b}$ & $-{ }^{b}$ \\
\hline Oct. 2001 & 0.01 & 0.11 & 0.07 & 0.18 & -0.03 & -0.11 & -0.03 & 0.02 & -0.07 & 0.04 \\
\hline Nov. 2001 & 0.20 & -0.17 & -0.18 & 0.06 & -0.23 & -0.11 & -0.17 & -0.10 & -0.16 & -0.16 \\
\hline Dec. 2001 & 0.05 & -0.04 & -0.04 & 0.06 & -0.09 & -0.10 & -0.20 & 0.06 & -0.15 & -0.02 \\
\hline Jan. 2002 & 0.05 & 0.16 & 0.14 & 0.16 & 0.07 & -0.10 & 0.02 & 0.16 & -0.15 & 0.03 \\
\hline Feb. 2002 & 0.11 & -0.07 & -0.08 & 0.13 & -0.19 & -0.23 & -0.09 & -0.10 & -0.27 & -0.16 \\
\hline March 2002 & -0.10 & 0.14 & 0.15 & 0.21 & 0.06 & 0.10 & 0.12 & 0.14 & 0.01 & 0.10 \\
\hline April 2002 & -0.03 & 0.08 & 0.07 & 0.34 & -0.08 & -0.05 & -0.07 & -0.03 & -0.19 & -0.02 \\
\hline May 2002 & 0.02 & 0.03 & 0.05 & 0.24 & -0.08 & -0.08 & -0.11 & -0.03 & -0.17 & -0.07 \\
\hline June 2002 & -0.04 & 0.21 & 0.13 & 0.21 & 0.04 & -0.05 & -0.08 & 0.01 & -0.10 & 0.01 \\
\hline \multicolumn{11}{|c|}{ Factor 5} \\
\hline July 2001 & 0.08 & -0.13 & -0.16 & -0.02 & -0.15 & -0.15 & -0.11 & -0.19 & -0.25 & -0.18 \\
\hline Aug. 2001 & 0.12 & -0.05 & -0.12 & 0.03 & -0.17 & -0.22 & -0.18 & -0.19 & -0.24 & -0.17 \\
\hline Sept. 2001 & 0.09 & -0.11 & -0.20 & 0.03 & -0.19 & -0.24 & -0.20 & -0.24 & $-{ }^{b}$ & $-{ }^{b}$ \\
\hline Oct. 2001 & 0.17 & -0.17 & -0.25 & 0.03 & -0.25 & -0.27 & -0.14 & -0.27 & -0.19 & -0.12 \\
\hline Nov. 2001 & 0.17 & -0.12 & -0.15 & 0.13 & -0.14 & -0.11 & -0.14 & -0.07 & -0.14 & -0.17 \\
\hline Dec. 2001 & 0.27 & -0.06 & -0.08 & -0.07 & -0.10 & -0.19 & -0.21 & -0.13 & -0.15 & -0.08 \\
\hline Jan. 2002 & 0.20 & -0.08 & -0.15 & -0.10 & -0.16 & -0.28 & -0.25 & -0.21 & -0.26 & -0.17 \\
\hline Feb. 2002 & 0.35 & -0.16 & -0.21 & -0.03 & -0.20 & -0.27 & -0.20 & -0.22 & -0.22 & -0.20 \\
\hline March 2002 & 0.21 & -0.08 & -0.14 & -0.08 & -0.11 & -0.11 & -0.10 & -0.09 & -0.02 & -0.03 \\
\hline April 2002 & 0.13 & -0.01 & -0.05 & 0.16 & -0.09 & -0.03 & 0.00 & -0.15 & -0.08 & 0.02 \\
\hline May 2002 & 0.18 & -0.11 & -0.14 & 0.11 & -0.14 & -0.08 & -0.11 & -0.16 & -0.14 & -0.13 \\
\hline June 2002 & $-{ }^{\mathrm{c}}$ & $-{ }^{\mathrm{c}}$ & $-{ }^{\mathrm{c}}$ & $-{ }^{\mathrm{c}}$ & $-{ }^{\mathrm{c}}$ & $-{ }^{\mathrm{c}}$ & $-{ }^{\mathrm{c}}$ & $-{ }^{\mathrm{c}}$ & $-{ }^{\mathrm{c}}$ & $-^{\mathrm{c}}$ \\
\hline
\end{tabular}

${ }^{\mathrm{a}}$ The source contributions and the species with $10 \mathrm{~min}$ resolution were averaged to $30 \mathrm{~min}$; for OC and EC the source contributions were averaged to the corresponding OC/EC sampling period.

${ }^{\mathrm{b}} \mathrm{OC}$ or $\mathrm{EC}$ data are missing.

${ }^{\mathrm{c}}$ Half of the data between $3 \mathrm{~nm}$ and $15 \mathrm{~nm}$ were missing during this month because the SMPS was not functioning properly. 

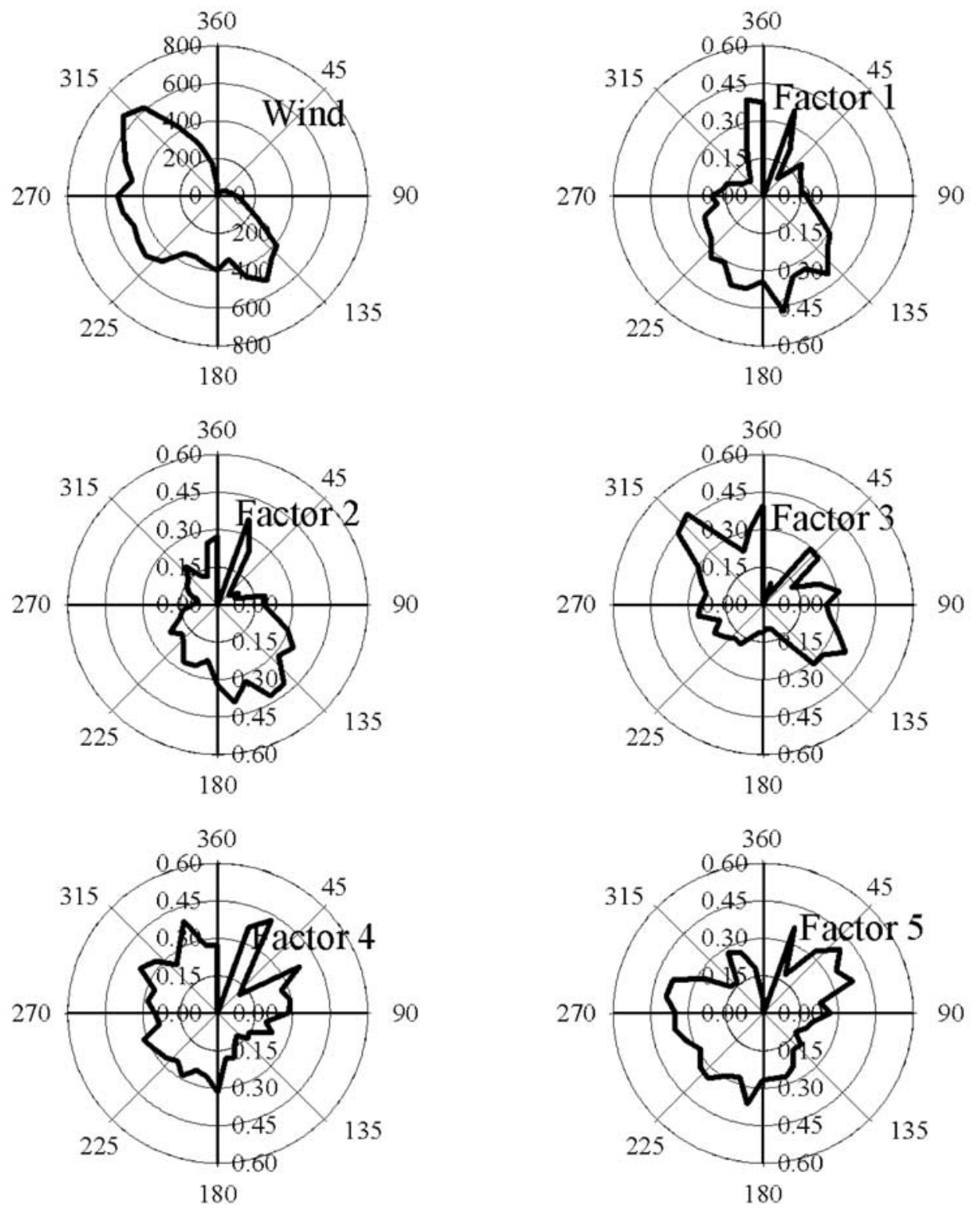

Figure 8. Wind profile and CPF of the whole year contribution of each factor.

cause of the negative correlation with ozone. Table 3 shows that the diurnal pattern of factor 2 does not appear in all months, indicating that this daily pattern is weak and is easily disturbed.

[32] The correlations between factor 2 and these gases, $\mathrm{NO}, \mathrm{NO}_{x}, \mathrm{CO}$, and $\mathrm{SO}_{2}$, suggest that the particles are from combustion sources and arrived at the receptor site accompanied by these gases. The strong correlation with $\mathrm{SO}_{2}$ suggests coal burning, including coal power plants and steel mills. The correlation with $\mathrm{CO}$ can be explained by the emission from steel mills as well as a boiler $1 \mathrm{~km}$ away to the northwest of the site.

[33] Factor 2 has a weak correlation with $\mathrm{OC}$ in summer, and in winter the correlation becomes stronger. Like factor 1, the winter correlations with OC and with EC are similar, indicating primary $\mathrm{OC}$ sources. Factor 2 probably contains both emissions from wood burning and other local com- bustion sources that are too similar in size to separate. The average contribution of factor 2 in the winter months (December 2001, January 2002, February 2002, and March 2002) is higher than that in the summer months (July 2001, August 2001, and September 2001) by 34\%. In comparison, the average number contribution of factor 3 increases from summer to winter by only $17 \%$. The higher increase of the factor 2 contribution may suggest an additional source from wood burning. As shown in Figure 9, factor 2 has no significant differences between weekdays and weekends. The CPF analysis shows it is also from the south to southeast.

[34] Factor 2 is thus assigned as stationary combustion, including emissions from local combustion sources. Probably, it may also include wood burning in winter. The emission from the boiler may also be included, except in summer when it seldom ran. The higher correlation of factor 
2 with $\mathrm{SO}_{2}$ and lower correlation with sulfate suggests that the sources are located close to the receptor site, $<100 \mathrm{~km}$ [Zhou et al., 2004]. This is also consistent with results for a coal-fired power plant that was found to increase $\mathrm{SO}_{2}$ close to the source but that produced little sulfate because of the short distance and time for oxidation [Green et al., 2003].

[35] The number mode of factor 3 is at $0.04 \mu \mathrm{m}$ in summer and $0.03 \mu \mathrm{m}$ in the fall and winter. This factor has been associated with Pittsburgh traffic in a previous paper [Zhou et al., 2004], but its behavior is a little puzzling here. Factor 3 is weakly correlated with $\mathrm{NO}, \mathrm{NO}_{x}$, and $\mathrm{CO}$ during all months. Figure 7 and Table 3 both indicate that it has no daily patterns. Table 6 also indicates that factor 3 has no correlation with the traffic flow on Interstate 376 . One reason may be that the distance of transport weakened the daily pattern and the correlations, and meteorological conditions have more influence than the emissions. Another possible reason is the presence of some particles from local point sources in factor 3. Figure 7 indicates frequency peaks at $1 / 12 \mathrm{~h}^{-1}, 1 / 8 \mathrm{~h}^{-1}$, and $1 / 6 \mathrm{~h}^{-1}$ for factor 3 . These are the harmonic frequencies of $1 / 24 \mathrm{~h}^{-1}$, caused by a nonsinusoidal periodicity of 24 hours. Although these frequencies may suggest a hidden daily pattern, they only appeared in January and February of 2002, and we cannot conclude that factor 3 has daily patterns.

[36] Taking the periodicity of a series as "signal" and the nonperiodicity as "noise," then factor 3 has weak signals as just discussed, and noise is then sufficiently large to prevent a detectable 24 hour periodicity by Fourier analyses. The effect of the noise is random, and it affects any time of day to the same extent. Since there are a large number of samples for each time of weekday and weekend, we can expect a horizontal line with small fluctuations for the daily average in Figure 9. Thereafter, the concentration peak of factor 3 at morning rush hours as well as the weekday/ weekend difference in Figure 9 suggests the influence of traffic.

[37] Although the number size distribution changes significantly within $\sim 100 \mathrm{~m}$ of highways [Zhu et al., 2002a, $2002 \mathrm{~b}, 2004]$, the mode of the size distribution becomes stable at farther distances, and factor 3 is consistent with the unimodal size distributions found several kilometers downwind of the highway in Los Angeles [Kim et al., 2002].

[38] Factor 3 is probably a collection of point sources and remote Pittsburgh traffic. These are particles produced in the city but not close to the measurement station, several kilometers away. The current techniques cannot separate these two source categories, and the source characteristics are not identified well.

[39] Factor 4 has its number mode at $15 \mathrm{~nm}$. Figure 9 clearly shows the concentration peak during the morning rush hour for weekdays and shows no such peak for weekends. Sometimes particles in $10-20 \mathrm{~nm}$ were formed by nucleation followed with no detectable particle growth, and these data were not excluded by the method described in the previous section. These nucleation events keep the average concentration value of factor 4 from decreasing rapidly after 1000 local time (LT) to the afternoon. In Figure 7, Fourier analyses found two frequency peaks corresponding to a 7 day period and a 24 hour period. Factor 4 has no correlations with any gas and particle composition data, and this can be attributed to the small traffic flow around the site. 
Weekday
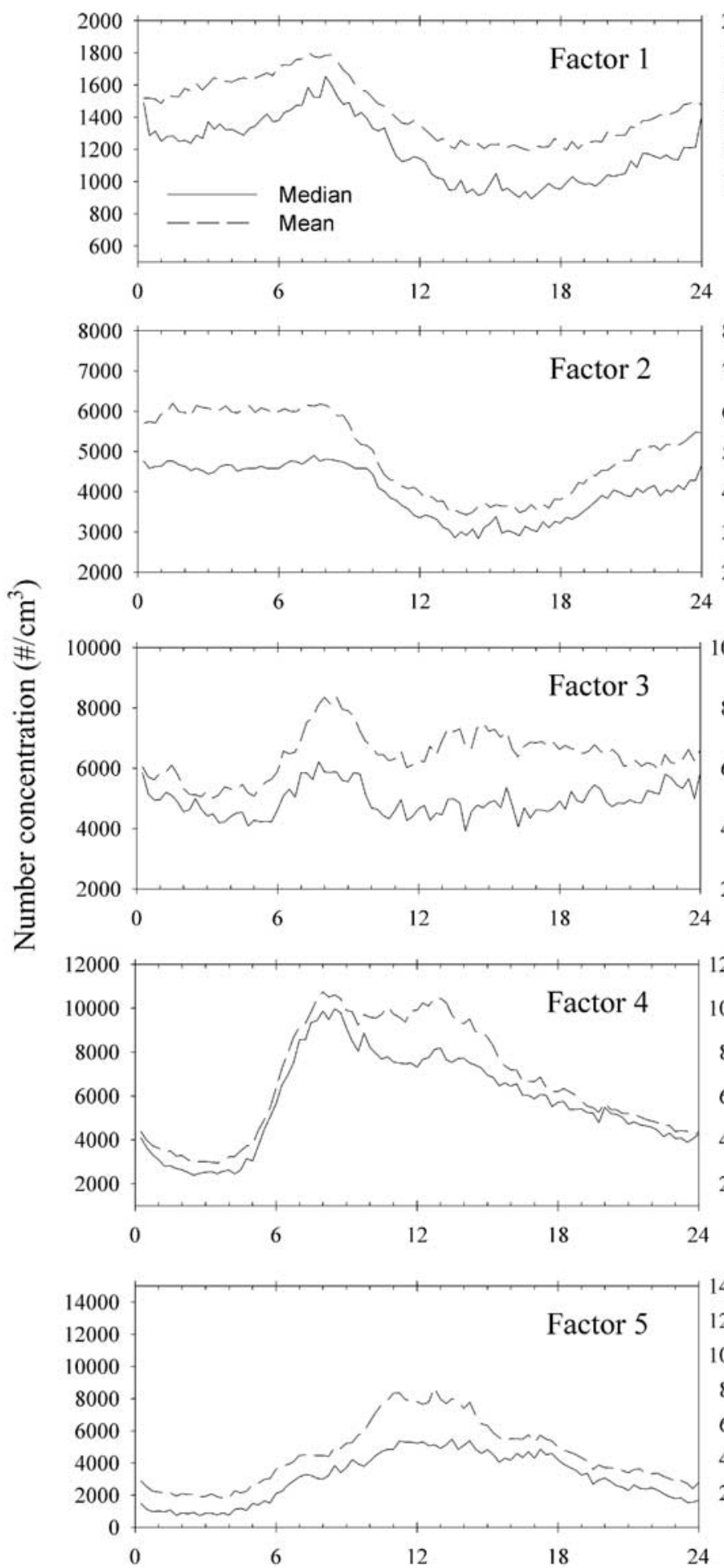

Time (h)

Weekend
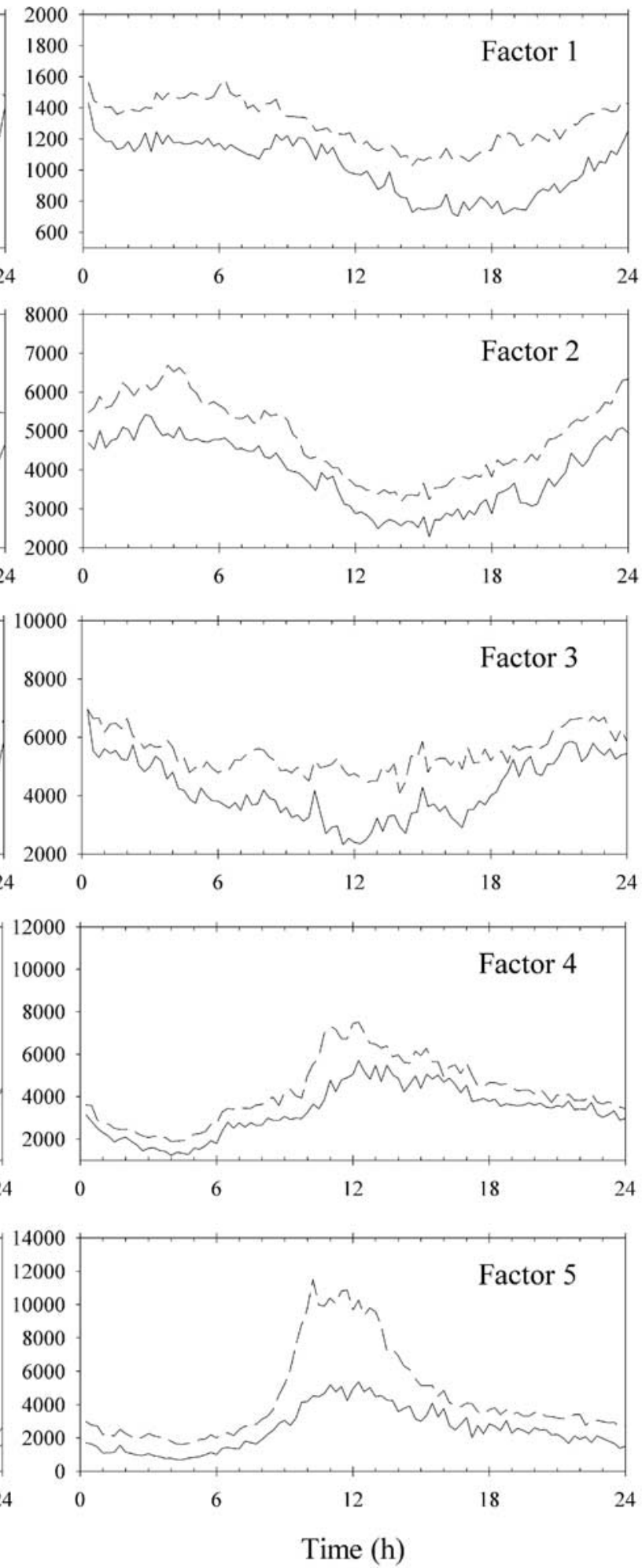

Figure 9. Daily average contribution from each factor for weekdays and weekends. The strength of the daily pattern can be determined by the spectral intensity at $1 / 24 \mathrm{~h}^{-1}$ in Figure 6 . The daily pattern strength is strong for factors 4 and 5 , weak for factors 1 and 2 , and nearly nothing for factor 3 . 
Table 6. Correlations of Gases and Source Contributions With Traffic Flow Volume on Schenley Drive, Forbes Avenue, and Interstate $376^{\mathrm{a}}$

\begin{tabular}{lccccc}
\hline & $\mathrm{NO}$ & $\mathrm{NO}_{x}$ & $\mathrm{CO}$ & Factor 3 & Factor 4 \\
\hline Schenley Drive $^{\mathrm{b}}$ & 0.056 & -0.0024 & -0.045 & 0.066 & 0.30 \\
Forbes Avenue $^{\mathrm{b}}$ & $\mathrm{M} 0.021$ & -0.081 & -0.145 & -0.0069 & 0.27 \\
Interstate 376 $^{\mathrm{c}}$ & -0.032 & -0.125 & -0.154 & 0.046 & 0.39 \\
\hline
\end{tabular}

${ }^{\mathrm{a}}$ Distance to the site: Schenley Drive, $\sim 200 \mathrm{~m}$; Forbes Avenue, $\sim 600 \mathrm{~m}$; and Interstate $376, \sim 1600 \mathrm{~m}$.

${ }^{\mathrm{b}}$ For 1-31 January and 1-30 April 2002.

${ }^{\mathrm{c}}$ For 11-31 January and 1-30 April 2002 .

The traffic flow rate at three places (Schenley Drive, Forbes Avenue, and Interstate 376) near the site were not correlated with $\mathrm{NO}, \mathrm{NO}_{x}$, or $\mathrm{CO}$ as indicated in Table 6 , indicating that the temporal variations of these gases do not show traffic patterns. The contribution of factor 4 has a positive correlation with the traffic flows. These facts support the conclusion that factor 4 is from local traffic while most $\mathrm{NO}, \mathrm{NO}_{x}$, or $\mathrm{CO}$ is not from local traffic.

[40] In 2002, from mid-February to mid-April, the roads near the station were closed in the early weekend mornings (0600-1200 LT) for motorless "buggy" practice. Figure 10 shows the contribution series of factor 4 and also the moving average of this series. The moving average time was chosen as 6 hours so that rapid variations were filtered. Usually, there are low emissions from local traffic on weekends, corresponding to low source contributions of factor 4 . When the roads were closed for buggy practice, factor 4 shows the lowest contributions, as indicated in Figure 10. Factor 4 is thus assigned to be from local traffic. It may be from Forbes Avenue and other minor roads close to the measurement station within a distance of $1 \mathrm{~km}$.

[41] Factor 5 represents particles smaller $10 \mathrm{~nm}$ from nucleation. These small particles are not involved in the particle growth event that we have discussed. A detailed study of nucleation events during PAQS was presented by Stanier et al. [2004b]. On average, the number mode is larger than $3 \mathrm{~nm}$, and this may be caused by the nucleation happening upwind or happening at higher elevations with subsequent downward mixing of the particles. The similarity of data between the upwind (Florence) nucleation and Pittsburgh indicates that the nucleation is occurring upwind of Pittsburgh [Stanier et al., 2004b]. Figure 7 shows a clear daily pattern, and Figure 9 indicates that local nucleation events are more active during the daytime, especially around noon. The similar pattern of mean and median values indicates that nucleation happened frequently in Pittsburgh, and this cannot be explained by occasional

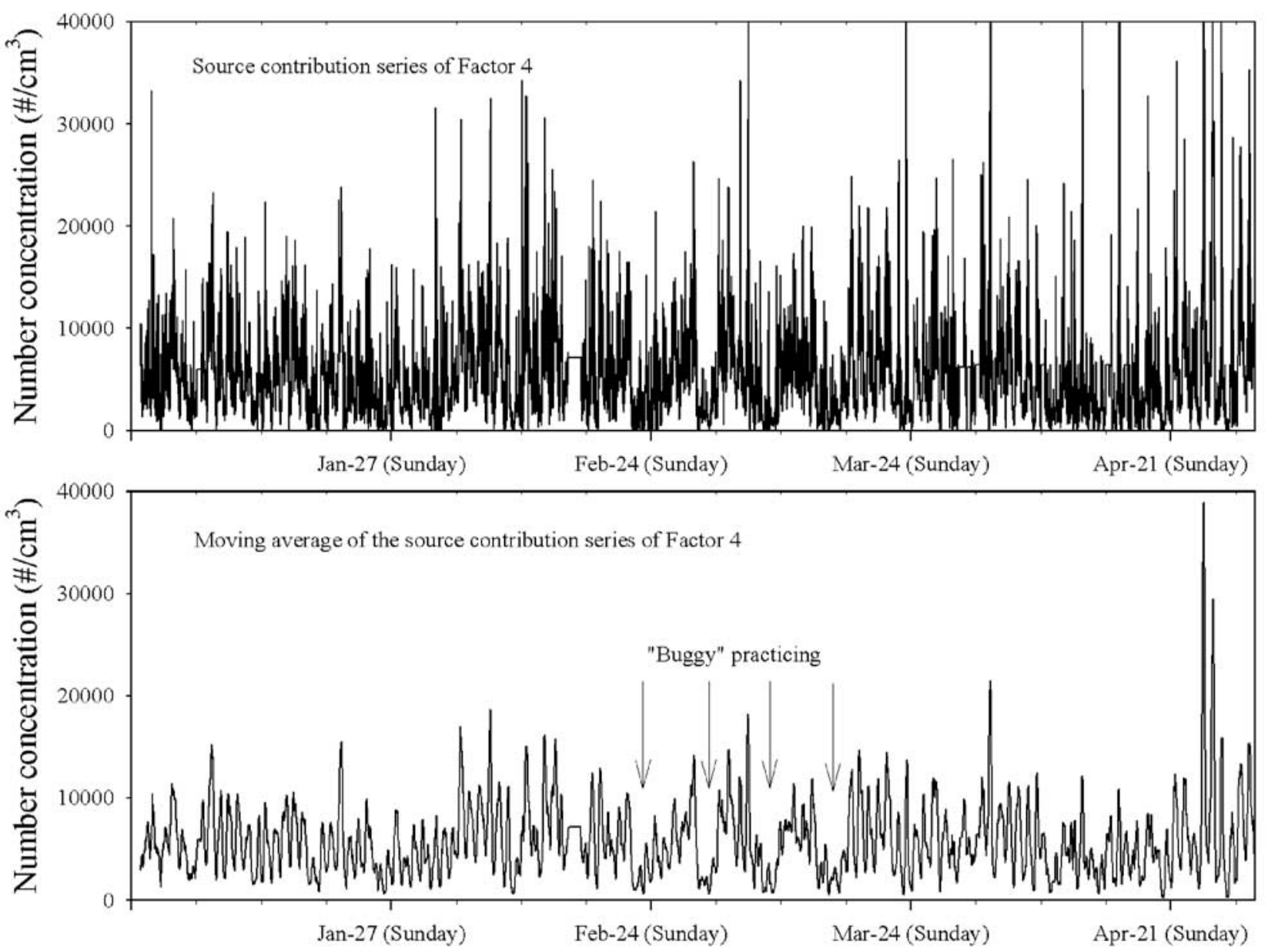

Figure 10. Source contribution series of factor 4 from 1 January to 30 April 2002. 


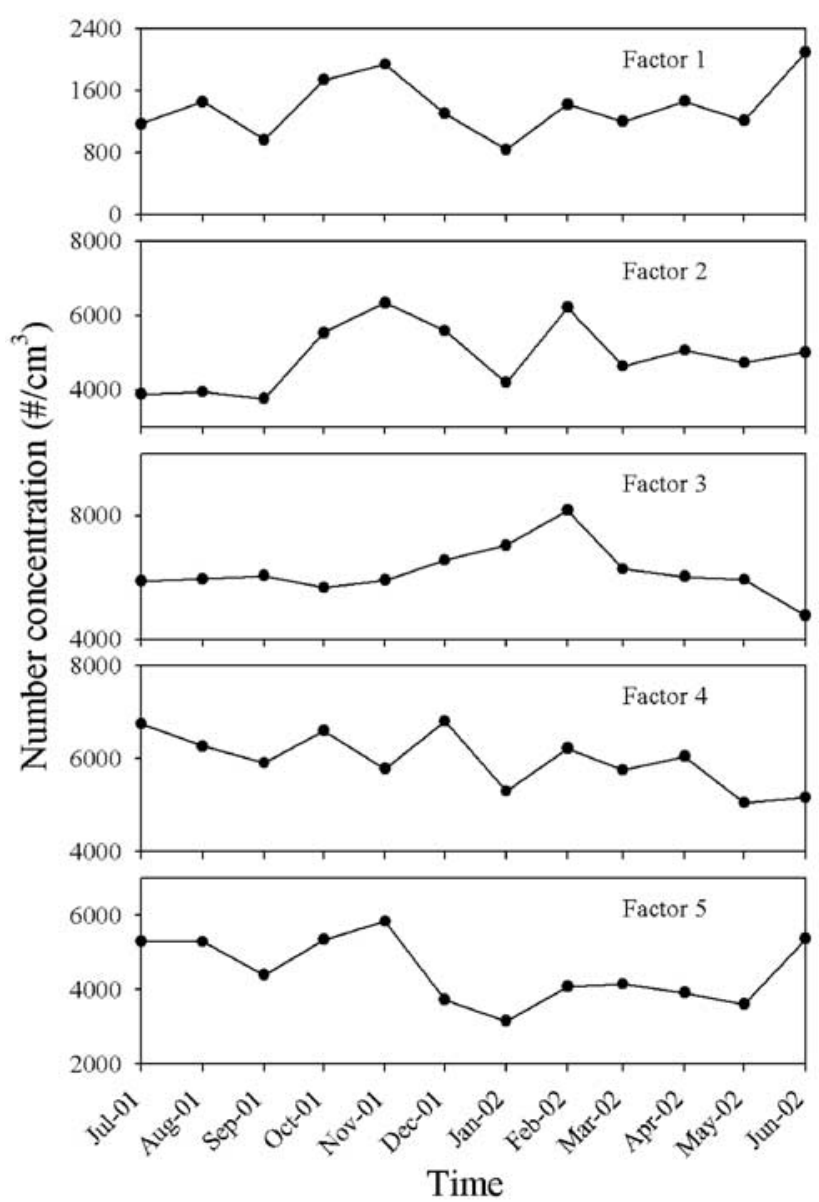

Figure 11. Monthly variations of average number contribution from each factor.

occurrences of nucleation with a large number of new particles produced.

[42] Figure 11 illustrates the variation of the monthly mean number contribution from each factor. The fluctuations are within a factor of 2 . Factors 1 and 2 have similar seasonal trends, high in the fall and low in the winter. They all reach their highest concentrations in November 2001, and the $\mathrm{SO}_{2}$ concentration is also the highest in that month. These high concentrations in November 2001 may be attributed to the dominant wind direction from the south, where more coal power plants are located. Figure 12 indicates the monthly volume contribution variations. The volume contribution is calculated from the number contribution and size distribution of each factor. For factor 1 the volume contribution only includes particles smaller than $0.5 \mu \mathrm{m}$ for all months since the lack of APS data in some months prevents us from investigating the volume contribution over this size. The monthly variation of factor 1 is similar to $\mathrm{PM}_{2.5}$ mass concentration. In the summer of 2001, particles from all sources seem to be larger than in other seasons.

[43] Table 7 summarizes the average contribution from each factor through the full year. The number contributions from factors 2, 3, 4, and 5 are approximately the same, and factor 1 contributes far fewer particles. The volume contribution is dominated by factors 1 and 2 , and factor 5 contributes little to the total volume concentration. The volume contribution of factor 1 is underestimated because of the missing APS data.

\section{Conclusion}

[44] Positive matrix factorization and other data-mining techniques have been applied for extracting source information with the full year size distribution data from the Pittsburgh Air Quality Study. The data representing particle growth after nucleation events were excluded. The analysis was performed for each month, and the same five factors were found for all months.

[45] The five factors found in this analysis represent five different size patterns. Each pattern is caused by a source or source group. This analysis has succeeded in separating and identifying local traffic and nucleation. The effect of this method is limited in separating sources in factors 1,2 , and 3 . Factor 1 includes local sources besides secondary and aged primary aerosol; factor 2 includes power plants, but it cannot be separated further from other stationary combustion sources; factor 3 is probably Pittsburgh traffic, but the evidence is insufficient.

[46] For the purpose of source apportionment, the approach by itself has significant limitations compared with traditional receptor modeling based on chemical composi-

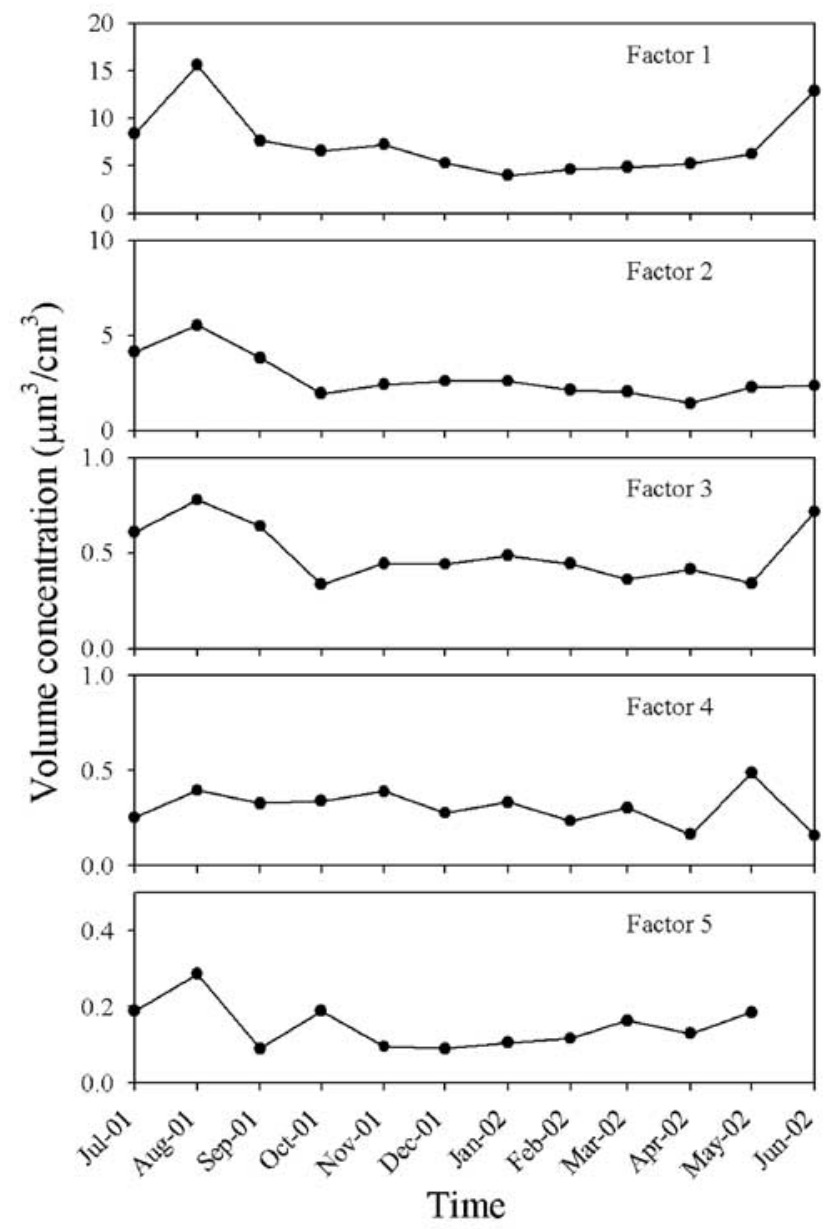

Figure 12. Monthly variations of average volume contribution from each factor. 
Table 7. Average Contribution of All Factors

\begin{tabular}{lccccc}
\hline & Factor 1 & Factor 2 & Factor 3 & Factor 4 & Factor 5 \\
\hline $\begin{array}{c}\text { Number concentration, } \\
\text { number cm }\end{array}$ & 1402 & 4907 & 6182 & 5972 & 4514 \\
$\begin{array}{c}\text { Volume concentration, } \\
\mu \mathrm{m}^{3} \mathrm{~cm}^{-3}\end{array}$ & 7.40 & 2.78 & 0.500 & 0.306 & $0.149^{\mathrm{a}}$ \\
\hline
\end{tabular}

June 2002 is not included.

tion data. Comparison of the results of this approach with other chemical-composition-based analysis in the future will provide more information, especially in identifying the sources included in factors 1,2 , and 3 . However, there is useful information that can be obtained from such analyses. Although the initial cost of the equipment is substantial, the operational costs are low, and they provide far more information than can be obtained from particle counts alone.

[47] Acknowledgments. This research was conducted as part of the Pittsburgh Air Quality Study, which was supported by the U.S. Environmental Protection Agency under contract R82806101 and the U.S. Department of Energy National Energy Technology Laboratory under contract DE-FC26-01NT41017. This paper has not been subject to EPA's required peer and policy review and therefore does not necessarily reflect the views of the Agency. No official endorsement should be inferred.

\section{References}

Ashbaugh, L. L., W. C. Malm, and W. Z. Sadeh (1985), A residence time probability analysis of sulfur concentrations at Grand Canyon National Park, Atmos. Environ., 19, 1263-1270.

Birmili, W., A. Wiedensohler, J. Heintzenberg, and K. Lehmann (2001), Atmospheric particle number size distribution in central Europe: Statistical relations to air masses and meteorology, J. Geophys. Res., 106, $32,005-32,018$

Brunekreef, B., D. W. Dockery, and M. Krzyzanowski (1995), Epidemiologic studies of short-term effects of low levels of major ambient air pollution components, Environ. Health Perspect., 103, 3-13.

Chueinta, W., P. K. Hopke, and P. Paatero (2000), Investigation of sources of atmospheric aerosol at urban and suburban residential area in Thailand by positive matrix factorization, Atmos. Environ., 34, 3319-3329.

Dockery, D. W., and C. A. Pope III (1994), Acute respiratory effects of particulate air pollution, Annu. Rev. Public Health, 15, 107-132.

Green, M., H. Kuhns, M. Pitchford, R. Dietz, L. Ashbaugh, and T. Watson (2003), Application of the tracer-aerosol gradient interpretive technique to sulfur attribution for the Big Bend Regional Aerosol and Visibility Observational Study, J. Air Waste Manage. Assoc., 53, 586-595.

Henry, R. C. (2003), Multivariate receptor modeling by N-dimensional edge detection, Chemom. Intell. Lab. Syst., 65, 179-189.

Hussein, T., A. Puustinen, P. P. Aalto, J. M. Makela, K. Hameri, and M. Kulmala (2004), Urban aerosol number size distributions, Atmos. Chem. Phys., 4, 391-411.

Khlystov, A., C. Stanier, and S. N. Pandis (2004), An algorithm for combining electrical mobility and aerodynamic size distributions when measuring ambient aerosol, Aerosol Sci. Technol., 38, 229-238.

Kim, E., P. K. Hopke, T. V. Larson, and D. S. Covert (2004), Analysis of ambient particle size distributions using UNMIX and positive matrix factorization, Environ. Sci. Technol., 38, 202-209.

Kim, S., S. Shen, C. Sioutas, Y. Zhu, and W. C. Hinds (2002), Size distribution and diurnal and seasonal trends of ultrafine particles in source and receptor sites of the Los Angeles Basin, J. Air Waste Manage. Assoc., $52,297-307$.

Kulmala, M., H. Vehkamaki, T. Petajda, M. Dal Maso, A. Lauri, V. M. Kerminen, W. Birmili, and P. H. McMurry (2004), Formation and growth rates of ultrafine atmospheric particles: A review of observations, J. Aerosol. Sci., 35(2), 143-176.

Lee, E., C. K. Chun, and P. Paatero (1999), Application of positive matrix factorization in source apportionment of particulate pollutants, Atmos. Environ., 33, 3201-3212.

Mäkelä, J. M., E. K. Koponen, P. Aalto, and M. Kulmala (2000), One-year data of submicron size modes of tropospheric background aerosol in southern Finland, J. Aerosol. Sci., 31(5), 595-611.

Paatero, P. (1997), Least squares formulation of robust, non-negative factor analysis, Chemom. Intell. Lab. Syst., 37, 23-55.

Paatero, P., P. K. Hopke, X. H. Song, and Z. Ramadan (2002), Understanding and controlling rotations in factor analytic models, Chemom. Intell. Lab. Syst., 60, 253-264.
Paatero, P., P. K. Hopke, B. A. Begum, and S. K. Biswas (2005), A graphical diagnostic method for assessing the rotation in factor analytical models of atmospheric pollution, Atmos. Environ., 39, 193-201.

Polissar, A. V., P. K. Hopke, and R. L. Poirot (2001), Atmospheric aerosol over Vermont: Chemical composition and sources, Environ. Sci. Technol., $35,4604-4621$.

Pope, C. A., III, D. W. Dockery, and J. Schwartz (1995), Review of epidemiological evidence of health effects of particulate air pollution, Inhalation Toxicol., 7, 1-18.

Raes, F., R. Van Dingenen, E. Cuegas, P. F. J. Van Velthoven, and J. M. Prospero (1997), Observations of aerosols in the free troposphere and marine boundary layer of the subtropical northeast Atlantic: Discussion of processes determining their size distribution, J. Geophys. Res., 102, 21,315-21,318.

Ramadan, Z., X. H. Song, and P. K. Hopke (2000), Identification of sources of Phoenix aerosol by positive matrix factorization, J. Air Waste Manage. Assoc., 50, 1308-1320.

Ruuskanen, J., et al. (2001), Concentrations of ultrafine, fine and $\mathrm{PM}_{2.5}$ particles in three European cities, Atmos. Environ., 35, 3729-3738.

Song, X. H., A. V. Polissar, and P. K. Hopke (2001), Source of fine particle composition in the northeastern U.S., Atmos. Environ., 35, 5277-5286.

Stanier, C., A. Khlystov, W. R. Chan, M. Mandiro, and S. N. Pandis (2004a), A method for the in-situ measurement of fine aerosol water content of ambient aerosol: The Dry-Ambient Aerosol Spectrometer (DAASS), Aerosol Sci. Technol., 38, Suppl. 1, 215-228.

Stanier, C., A. Khlystov, and S. N. Pandis (2004b), Nucleation events during the Pittsburgh Air Quality Study: Description and relation to key meteorological, gas phase, and aerosol parameters, Aerosol Sci. Technol., 38, Suppl. 1, 253-264.

Stanier, C., A. Khlystov, and S. N. Pandis (2004c), Ambient aerosol size distributions and number concentrations measured during the Pittsburgh Air Quality Study (PAQS), Atmos. Environ., 38, 3275-3284.

van Bree, L., and F. R. Cassee (2000), A critical review of potentially causative PM properties and mechanisms associated with health effects, RIVM Rapp. 650010015, Natl. Inst. of Public Health and the Environ., Bilthoven, Netherlands.

Wahlin, P., F. Palmgren, R. V. Dingenen, and F. Raes (2001), Experimental studies of ultrafine particles in streets and the relationship to traffic, Atmos. Environ., 35, Suppl. 1, S63-S69.

Whitby, K. T. (1978), The physical characteristics of sulfur aerosols, Atmos. Environ., 12, 135-159.

Wittig, B., N. Anderson, A. Y. Khlystov, S. N. Pandis, C. Davidson, and A. L. Robinson (2003), Pittsburgh Air Quality Study overview and preliminary scientific findings, Atmos. Environ., 38, 3107-3125.

Xie, Y. L., P. K. Hopke, P. Paatero, L. A. Barrie, and S. M. Li (1999), Identification of source nature and seasonal variations of Arctic aerosol by positive matrix factorization, J. Atmos. Sci., 56, 249-260.

Zhou, L., E. Kim, P. K. Hopke, C. Stanier, and S. N. Pandis (2003), Source apportionment using particle size distribution data from the Pittsburgh Air Quality Study (PAQS), paper presented at AAAR PM Conference, Am. Assoc. for Aerosol Res., Pittsburgh, April.

Zhou, L., E. Kim, P. K. Hopke, C. Stanier, and S. N. Pandis (2004), Advanced factor analysis on Pittsburgh particle size distribution data, Aerosol Sci. Technol., 38, Suppl. 1, 118-132.

Zhou, L., P. K. Hopke, C. O. Stanier, S. N. Pandis, J. M. Ondov, and J. P. Pancras (2005), Investigation of the relationship between chemical composition and size distribution of airborne particles by partial least squares (PLS) and positive matrix factorization (PMF), J. Geophys. Res., D07S18, doi:10.1029/2004JD005050.

Zhu, Y., W. C. Hinds, S. Kim, S. Shen, and C. Sioutas (2002a), Study of ultrafine particles near a major highway with heavy-duty diesel traffic, Atmos. Environ., 36, 4323-4335.

Zhu, Y. F., W. C. Hinds, S. Kim, and C. Sioutas (2002b), Concentration and size distribution of ultrafine particles near a major highway, J. Air Waste Manage. Assoc., 52, 1032-1042.

Zhu, Y., W. C. Hinds, S. Shen, and C. Sioutas (2004), Seasonal trends of concentration and size distribution of ultrafine particles near major highways in Los Angeles, Aerosol Sci. Technol., 38, Suppl. 1, 5-13.

P. K. Hopke and E. Kim, Center for Air Resources Engineering and Science, Clarkson University, P. O. Box 5708, Potsdam, NY 13699-5708, USA. (hopkepk@clarkson.edu)

S. N. Pandis, Department of Chemical Engineering, Carnegie Mellon University, 5000 Forbes Avenue, Pittsburgh, PA 15213, USA.

C. Stanier, Department of Chemical and Biochemical Engineering, University of Iowa, 4122 Seamans Center, Iowa City, IA 52242, USA. (cstanier@engineering.uiowa.edu)

L. Zhou, Providence Engineering and Environmental Group LLC, 6160 Perkins Road, Suite 100, Baton Rouge, LA 70808, USA. (limingzhou@) providencebr.com) 